\title{
Towards a Bayesian Approach in Criminology: A Case Study of Risk Assessment in Youth Justice
}

\author{
Hodges, Helen R.
}

How to cite:

Hodges, Helen R. (2019) Towards a Bayesian Approach in Criminology: A Case Study of Risk Assessment in Youth Justice. Doctoral thesis, Swansea University.

http://cronfa.swan.ac.uk/Record/cronfa48027

Use policy:

This item is brought to you by Swansea University. Any person downloading material is agreeing to abide by the terms of the repository licence: copies of full text items may be used or reproduced in any format or medium, without prior permission for personal research or study, educational or non-commercial purposes only. The copyright for any work remains with the original author unless otherwise specified. The full-text must not be sold in any format or medium without the formal permission of the copyright holder. Permission for multiple reproductions should be obtained from the original author.

Authors are personally responsible for adhering to copyright and publisher restrictions when uploading content to the repository.

Please link to the metadata record in the Swansea University repository, Cronfa (link given in the citation reference above.)

http://www.swansea.ac.uk/library/researchsupport/ris-support/ 


\section{Towards A Bayesian Approach in \\ Criminology: A Case Study of Risk}

Assessment in Youth Justice

\section{Main Body}

(99,079 words with accompanying Technical Annex)

\section{Helen Hodges}

Submitted to Swansea University in fulfilment of the requirements for the Degree of Doctor of Philosophy

Swansea University

2018 
This research makes a significantand original contribution to emerging debates within criminologyand the social sciences more broadly, concerning the academic merit of using Bayesian statistics to analyse complex social problems, such as crime, with a view to promoting progressive and evidence-based policy reform agendas. It uses the risk assessment process in youth justice as a case study to demonstrate the utility of adding Bayesian approaches in the standard analytical tool box used to investigate the aetiology of offending behaviours, particularlywhen dealing with relatively small data-sets.

The findings presented reinforce that it is possible using a Bayesian approach to 'do more with less' in terms of the number of cases analysed, and model the impact on the likelihood of further offending of individual characteristics, offending history, different types of offending and contact with the youth justice system. In considering the implications of its findings, the thesis considers how adopting a post-positivist stance - as called for by critics of the risk assessment process used within youth justice in England and Wales - enables new insights to be offered concerning the complex relationship between the framework of risk and protective factors and offending behaviours.

It is concluded that they are distinct advantages associated with the adoption of novel statistical techniques within criminology, especially at a time where there is an increased emphasis on making greater use of administrative data to develop robust evidence-based policy. 


\section{Contents}

Abstract. ..$i$

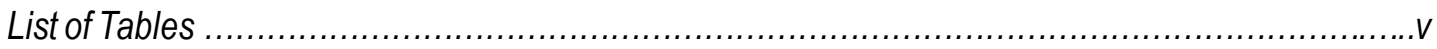

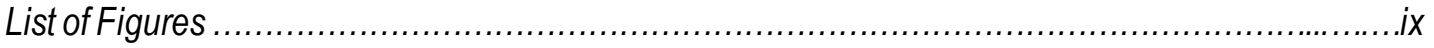

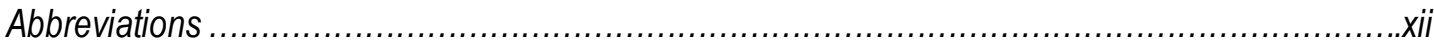

1 THE RATIONALE FOR USING BAYESIAN APPROACHES IN CRIMINOLOGY ......................................

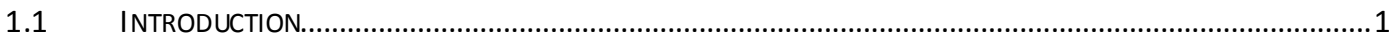

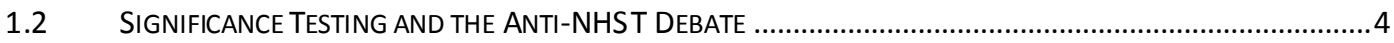

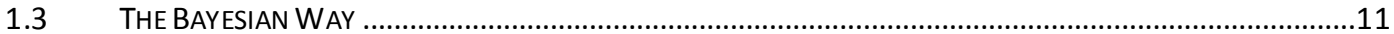

1.4 From the NeW Penology to the AdVENT Of DigitAl CRIMINology ................................................18

1.5 DOING MORE WITH LESS: THE INCREASED USE OF ADMINISTRATIVE DATA .............................................21

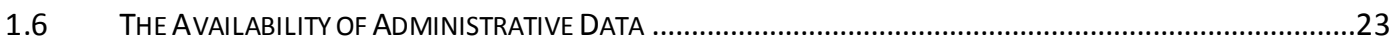

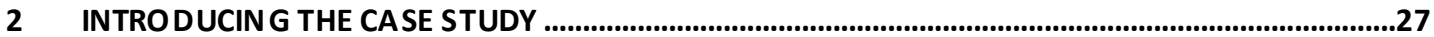

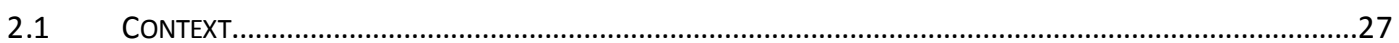

2.2 PROBABILISTIC DECISION MAKING IN THE CRIMINAL JUSTICE SYSTEM .....................................................33

2.3 RISK FACTOR RESEARCH: THE EVIDENCE UNDERPINNING ASSET .....................................................38

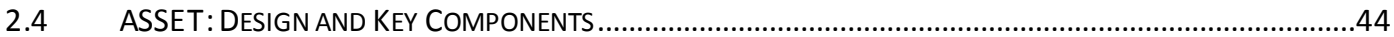

2.5 THE ACTUARIAL FalLACY AND PREDICTIVE ACCURACY...............................................................55

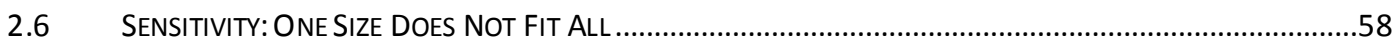

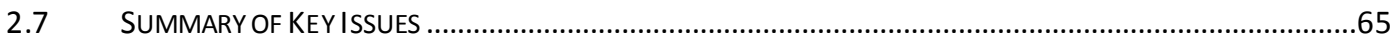

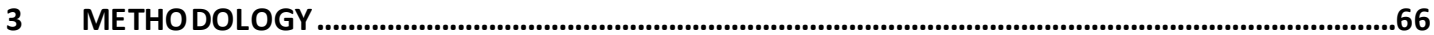

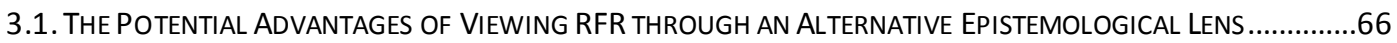

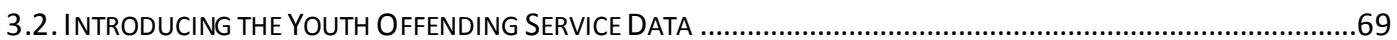

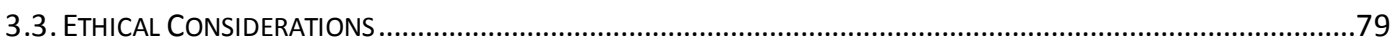

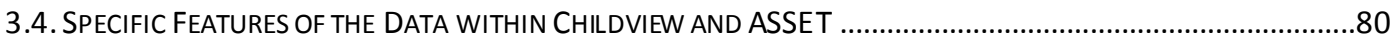

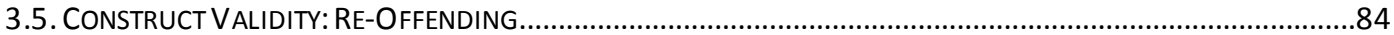

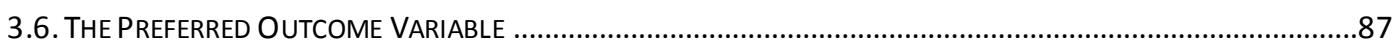

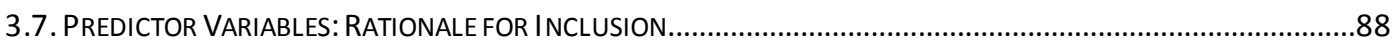

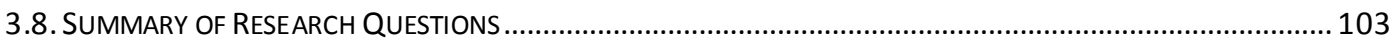




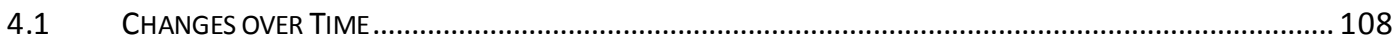

4.2 THE RELATIONSHIP BETWEEN FURTHER OFFENDING AND DOMAIN SCORES.......................................... 115

4.3 THE ROLE OF THE 12 DOMAINS IN PREDICTING FURTHER OFFENDING OVER TIME. HOW DOTHESE FINDINGS EXTEND THE EXISTING EVIDENCE BASE?

4.4 HOW WELL DOES THE 'BASIC’ MODEL REFLECT THE REALITIES OF YOUNG PEOPLE’S LIVES? ....................... 136

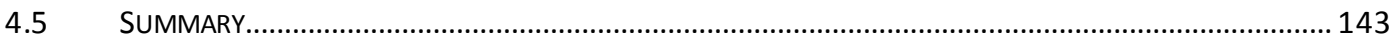

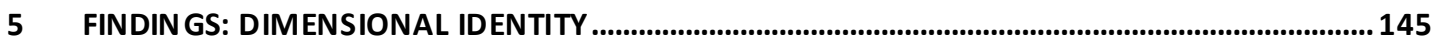

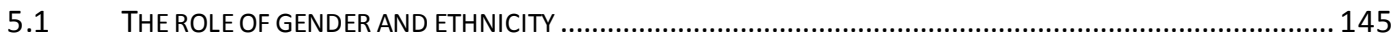

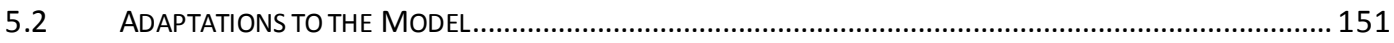

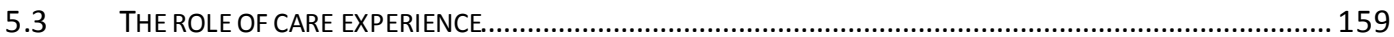

5.4 THE ROLE OF GENDER AND ETHNICITY IN THE CONTEXT OF CARE EXPERIENCE........................................ 165

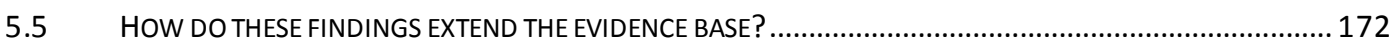

5.6 HOW DOES THE MODEL INVOLVING GENDER, ETHNICITY AND CARE EXPERIENCE REFLECT THE REALITIES OF

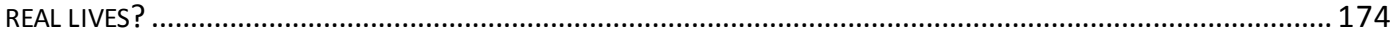

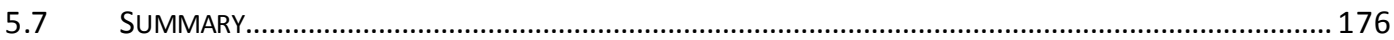

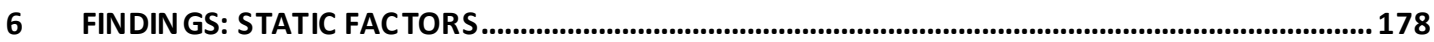

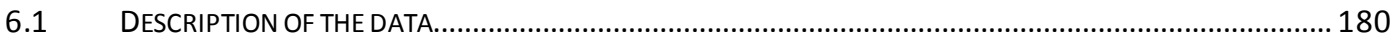

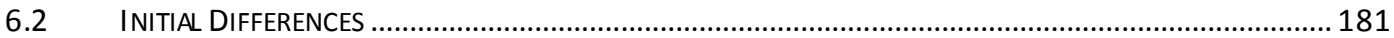

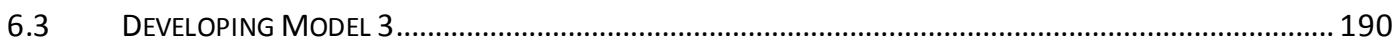

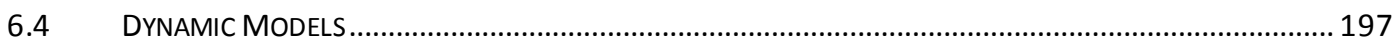

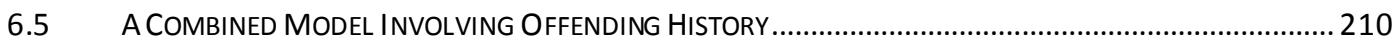

6.6 HOW DO THE MODELS INVOLVING STATIC FACTORS REFLECT THE REALITIES OF REAL LIVES? ..................... 217

6.7 INCREASING THE SENSITIVITY OF THE MODEL BY EXTENDING THE PREDICTORS .....................................225

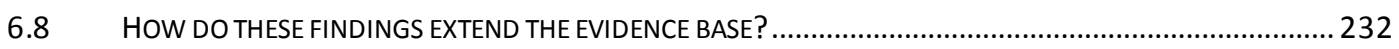

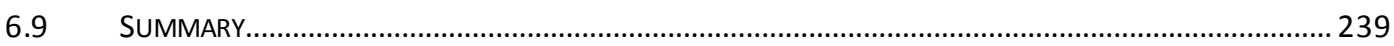

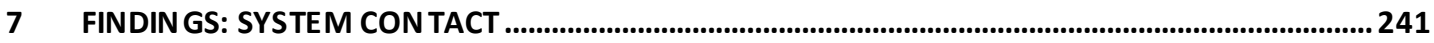

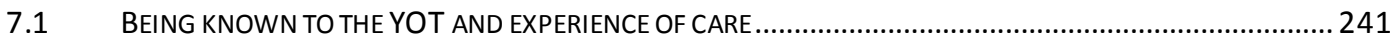

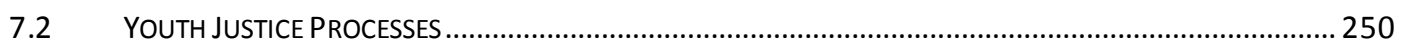

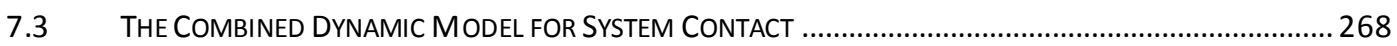

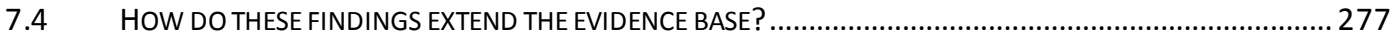

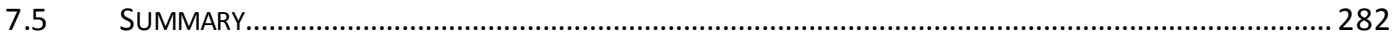




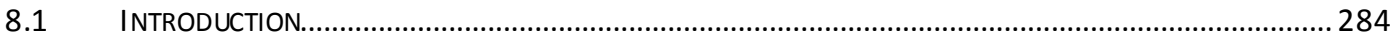

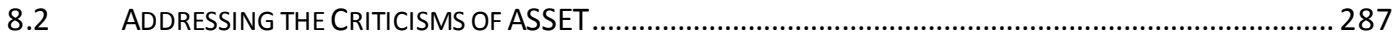

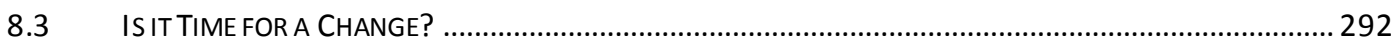

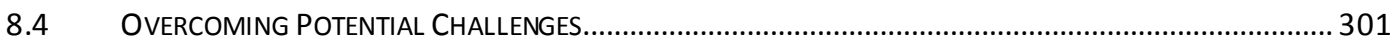

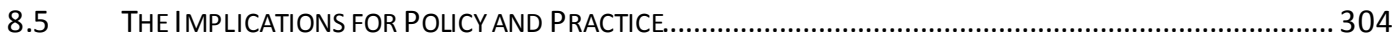

9 APPENDICES

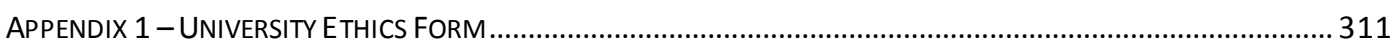

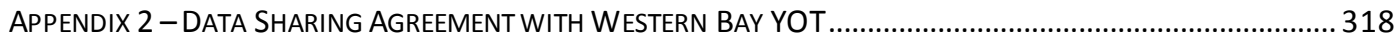

10 REFERENCES 


\section{List of Tables}

Table 1.1: Summary of the Research Questions, by Chapter and Theme ……........................................

Table 2.1: The Subjective Ratings Used within ASSET ………………………………......................46

Table 2.2: Static Risk Factors and the Scores Assigned to these in ASSET (Max=16) Under the Scaled

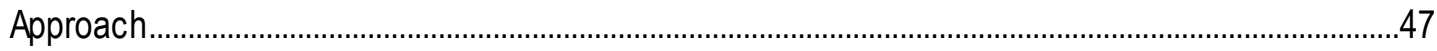

Table 2.3: Determining Intervention Level ...........................................................................................51

Table 2.4: Statutory contacts for assessed intervention levels ...............................................................52

Table 2.5: Plotting Percentage Correctly Predicted: ASSET (Original Version) .....................................57

Table 2.6: The Predictive Accuracy of ASSET and ASSET under the Scaled Approach........................58

Table 2.7: The Proportion of 10-17 year old Offenders who Became Chronic and Serious Re-Offenders,

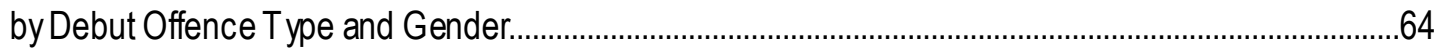

Table 3.1: Fields in the Local YOT Reoffending Spreadsheets 2012/13 and 2013/14 _..........................72

Table 3.2: Outcomes and Outcome Tiers for Young People Pre- and Post-LASPO ...............................75

Table 3.3: Outcome Tier by ASSET Band for all Unique Individuals, Swansea YOT, 2012/13and 2013/14

Table 3.4: Outcome Tier andASSET Bands for those with ASSET Core Profiles, Swansea YOT, 2012/13

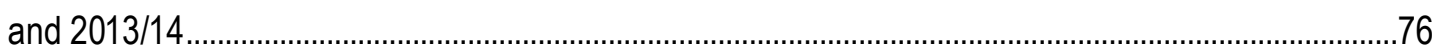

Table 3.5: Ethnicity and Gender Profile of Clients...............................................................................83

Table 3.6: Demographic Profile, Swansea YOT, 2012/13 and 2013/14_.............................................89

Table 3.7: Proven Re-Offending, Local and National Rates, by Demographic Characteristics, 2012/13 and $2013 / 14$

Table 3.8: Demographic Profiles of those with ASSET Core Profiles, Swansea YOT, 2012/13 and $2013 / 14$ 90

Table 3.9:Rates of Further Offending Across the Two Years, by Gender and Ethnicity.........................92

Table 3.10: Rates of Further Offending Across the Two Years, by Care Status.....................................94

Table 3.11:Rates of Further Offending Across the Two Years, by Offending History ..............................95

Table 3.12:PrimaryOffence Categoryof Those with ASSET Core Profiles, with Re-Offending and Further Offending Rates.

Table 3.13:GravityScore of the Primary Offence for Those with ASSET Core Profiles, with Re-Offending and Further Offending Rates ........................................................................................................98

Table 3.14:Primary Offence Category by YJB Gravity Score.................................................................98

Table 3.15: Outcome T ier of the Disposal Received for the Primary Offence, by YJB Gravity Score 101

Table 3.16: Outcome Tier of the Disposal Received for the PrimaryOffence for Those with ASSET Core Profiles, with Re-Offending and Further Offending Rates............................................................... 101

Table 4.1: Subjective Ratings Used in ASSET for the 12 Domains ........................................................ 106

Table 4.2:Changes in Dynamic ASSET Domain Scores between Initial and Second Assessments .. 109 
Table 4.3: Changes in ASSET Domain Scores between Initial and Final Assessments 110

Table 4.4: Total ASSET Score Change by Direction, Between Successive T ime Points. 112

Table 4.5: Total ASSET Score Change by Direction, Between Time 2 and Time 3, by Whether or Not Further Offences Where Committed between ASSETs. (All with Complete ASSET Core Profiles Regardless of Disposal Received). 113

Table 4.6: ASSET Score Change by Direction, Between T ime 3 and T ime 4, by Whether or Not Further Offences Where Committed between ASSETs. (All with Complete ASSET Core Profiles Regardless of Disposal Received) 114

Table 4.7: Random Intercept Model for Further Offending 120

Table 4.8: Random Intercept Model for Further Offending with Single Predictor 120

Table 4.9: Random Intercept Model for Further Offending including ASSET Domains ......................... 123

Table 4.10: Random Intercept and Varying Slope Models for Further Offending. 124

Table 4.11: The Basic Model: Random Intercept and Varying Slope Models for Further Offending including ASSET Domains. 125

Table 4.12: The Basic Dynamic Model Involving the 12 Domains. 126

Table 4.13: The Central Eight, Their Indicators and Associated Needs. 134

Table 4.14: Summary of Key Information for the Three Case Histories. 136

Table 5.1: Summary of Level 2 Predictors for the Nine Females in the Dataset. 146

Table 5.2: Summary of Level 2 Predictors for the Six Non-White Young People in the Dataset......... 146

Table 5.3: Random Intercepts and Varying Slope Models for Further Offending including ASSET Domains and Demographic Characteristics. 150

Table 5.4: The Dynamic Model Involving Gender 153

Table 5.5: The Dynamic Model Involving Ethnicity 154

Table 5.6: FTE Status, by Gender and Ethnicity 157

Table 5.7: FTE Status, by Gender and Ethnicity 157

Table 5.8: Type of Primary Offence, by Gender and Ethnicity 158

Table 5.9: YJB Gravity Score of the Primary Offence, by Gender and Ethnicity .. 158

Table 5.10:The Re-Offending Cohort, By Gender, Ethnicity and Experience of Care 159

Table 5.11: Random Intercepts and Varying Slope Models for Further Offending including ASSET Domains and Experience of Care. 161

Table 5.12: The Dynamic Model Involving Care Experience... 162

Table 5.13: Model 2: The Basic Model plus Demographics and Experience of Care. 166

Table 5.14:The Dynamic Model involving Demographic Characteristics and Experience of Care ..... 168

Table 6.1: Scoring for the Static Risk Factors under the Scaled Approach. 178

Table 6.2: The Reoffending Cohortby FTE Status, Grouped Age at First Offence, Grouped Age at First Conviction and Grouped YJB Offence Category.. 180

Table 6.3: The Reoffending Cohort by FTE Status, Age at First Offence and Age at First Conviction180 
Table 6.4: Random Intercepts and Varying Slope Models for Further Offending including ASSET Domains and the Two Age Related Static Factors

Table 6.5: Random Intercepts and Varying Slope Models for Further Offending including ASSET

Domains and FTE Status. 185

Table 6.6: The Re-Offending Cohort, by YJB Offence Category of Their Primary Offence 186

Table 6.7: The Basic Model plus (a) YJB Offence Category and (b) Grouped YJB Offence Category189

Table 6.8: Model 3: The Basic Model plus Static Factors. 190

Table 6.9: Characteristics of those Convicted of Their First Offence Before Age 14 192

Table 6.10: The Reoffending Cohort by FTE Status, Grouped Age at First Offence and Grouped YJB Offence Category 192

Table 6.11: Model 3a: The Basic Model plus FTE Status, Grouped Age at First Offence and Grouped YJB Offence Category... 193

Table 6.12: The Basic Model plus YJB Gravity Score 194

Table 6.13:The Reoffending CohortbyFTEStatus, Grouped Age at First Offence, Grouped Age at First Conviction and YJB Gravity Score 195

Table 6.14: The Reoffending Cohort by FTE Status, Grouped Age at First Offence and YJB Gravity Score. 195

Table 6.15: Model 3b:The Basic Model plus FTE Status, Grouped Age at First Offence and YJB Gravity Score. 196

Table 6.16: The Dynamic Model Involving FTE Status 197

Table 6.17: The Dynamic Model Involving Grouped Age at First Offence. 199

Table 6.18: The Dynamic Model Involving Grouped YJB Offence Category........................................ 202

Table 6.19: The Dynamic Model Involving YJB Gravity Scores.......................................................... 207

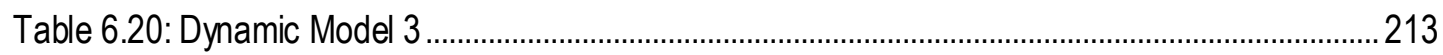

Table 6.21: The Dynamic Model Involving Age at First Offence........................................................ 225

Table 6.22: The Re-Offending Cohort, by Grouped YJB Category and Seriousness of Primary Offence 230

Table 6.23:The Basic Model plus Grouped YJB Offence Category and Gravity Score. 231

Table 6.24: The Reoffending Cohort by FTE Status and Grouped Age at First Offence, with Further Offending Rates... 233

Table 7.1: Dynamic Model 4 .242

Table 7.2: Further Offending by Experience of Care and Grouped Age at First Offence. 245

Table 7.3: Initial Average Domain Scores by FTE Status and Whether or NotFurther Offending Occurred

Table 7.4: Random Intercepts and Varying Slope Models for Further Offending including ASSET

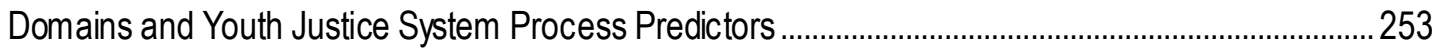

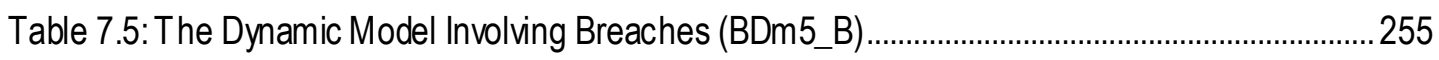




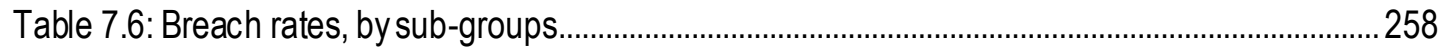

Table 7.7: The Dynamic Model Involving Court Appearances (BDm5_A) .......................................... 259

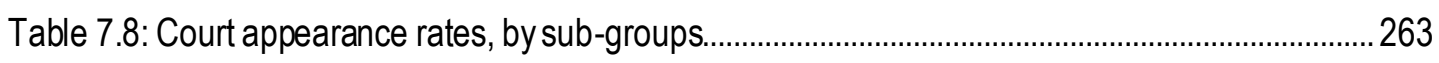

Table 7.9: The Dynamic Model Involving Periods in Custody or On Remand (BDm5_C).................... 265

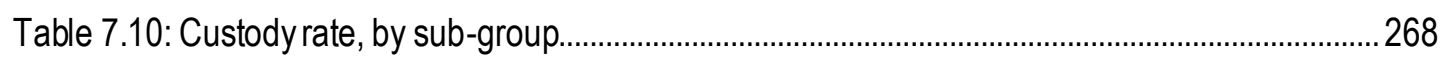

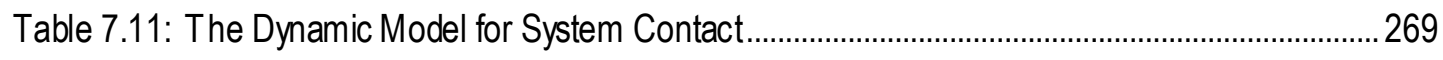

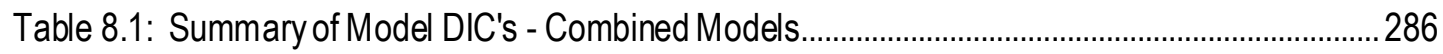

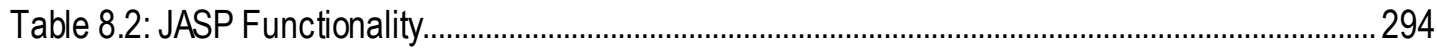




\section{List of Figures}

Figure 1.1: Interpretation of Hypothesis Testing under (a) the Frequentist Framework and (b) the Bayesian Framework 15

Figure 2.1: Components of the ASSET Core Profile.

Figure 2.2: APIS Framework - The continuous cycle of (re)assessment, (re)formulation of sentence planning, and supervision approaches. .50

Figure 3.1: Proven Re-Offending: 2013/14 Cohort ...........................................................................85

Figure 3.2: Re-Offending: A Worked Example Based on Offender A from the 2013/14 Cohort .............85

Figure 3.3: Case 1: A 13-year old Male Appearing in Both Cohorts .......................................................86

Figure 3.4: Case 2: A 17-year old Male Appearing in Both Cohorts .......................................................86

Figure 3.5:National Proven Reoffending Data, by Index Offence, Years Ending March 2013 and 2014

Figure 3.6: National Proven Reoffending Data, by Index Disposal, Years Ending March 2013 and 2014 100

Figure 4.1: Distribution of Domain Scores..................................................................................... 107

Figure 4.2: Distribution of ASSET Core Profiles per Individual ............................................................116

Figure 4.3: Average Domains Scores, by Individual ..........................................................................118

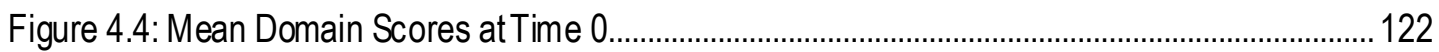

Figure 4.5: Mean Domain Scores by Time.................................................................................. 122

Figure 4.6: Changes in the Probability of Further Offending Over Time ............................................ 127

Figure 4.7: Summary of the Underlying Data for the Basic Dynamic Model (All Individuals) ............... 128

Figure 4.8: Case Study "Fred": Domain Scores at Time 2 and Time 3............................................... 137

Figure 4.9: Estimated Probability of Further Offending Over Time: "Fred" .......................................... 138

Figure 4.10: Case Study "Connor": Domain Scores at Time 2 and Time 3 …...................................... 139

Figure 4.11: Estimated Probability of Further Offending Over Time: "Connor" .................................... 141

Figure 4.12: Case Study "David": Domain Scores at T ime 3 and T ime 4 ........................................... 142

Figure 4.13: Estimated Probability of Further Offending Over Time: "David" ....................................... 143

Figure 5.1: Domain Score Profile, by (a) Gender and (b) Ethnicity, at Time 0 .................................... 147

Figure 5.2: Summary of Average Domain Scores, by Gender (Males).............................................. 156

Figure 5.3: Summary of Average Domain Scores, by Gender (Females)............................................. 156

Figure 5.4: Summary of Average Domain Scores, byEthnicity (White) ............................................... 156

Figure 5.5: Summary of Average Domain Scores, byEthnicity (Non-Whites) ...................................... 156

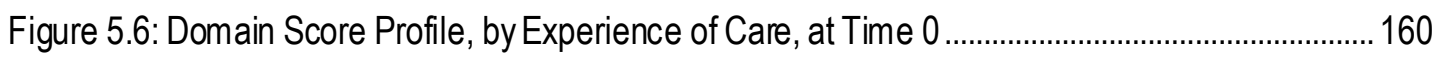

Figure 5.7: Changes in the Probability of Further Offending Over Time, by Care Experience .............. 163

Figure 5.8: Summary of Average Domain Scores, by Care Experience ................................................. 164 
Figure 5.9: Estimated Changes in the Probability of Further Offending for those with No Experience of Care, by Gender and Ethnicity...... 170

Figure 5.10: Estimated Changes in the Probability of Further Offending for those with Experience of Care, by Gender and Ethnicity. 170

Figure 5.11: Comparisons of the Estimated Probability of Further Offending Over T ime: "Fred"........ 174

Figure 5.12: Comparisons of the Estimated Probability of Further Offending Over T ime: "Connor" ... 175

Figure 5.13: Comparisons of the Estimated Probability of Further Offending Over T ime: "David" ...... 175

Figure 6.1: Domain Score Profile, by (a) Grouped Age at First Offence and (b) Grouped Age at First Conviction, at Time 0 .. 181

Figure 6.2: Domain Score Profiles, byFTE Status, at Time 0. 184

Figure 6.3: Domain Score Profiles, by Grouped YJB Offence Category at T ime 0 187

Figure 6.4: Changes in the Probability of Further offending Over Time, byFTE Status. 198

Figure 6.5: Changes in the Probability of Further Offending OverT ime, by Grouped Age at First Offence 200

Figure 6.6: Summary of Average Domain Scores, by Grouped Age at First Offence - Younger Group 201

Figure 6.7: Summary of Average Domain Scores, by Grouped Age at First Offence - Older Group 201 Figure 6.8: Changes in the Probability of Further Offending Over Time - Serious Acquisitive Crimes205 Figure 6.9: Changes in the Probability of Further Offending Over Time - Violence Against the Person 205

Figure 6.10: Changes in the Probability of Further Offending Over T ime - Other Offences 205

Figure 6.11: Summary of Average Domain Scores, by Primary Offence - Serious Acquisitive Crimes 206

Figure 6.12: Summary of Average Domain Scores, by Primary Offence - Violence Against the Person 206

Figure 6.13: Summary of Average Domain Scores, by Primary Offence - Other Offences .................. 206

Figure 6.14: Rate of Further Offending, by YJB Gravity Score....................................................... 208

Figure 6.15: Change in the Probability of Further Offending Over Time, by YJB Gravity Score .......... 209

Figure 6.16: Change in the Probability of Further Offending Over Time, Dynamic Model 3 ................. 215

Figure 6.17: Comparisons of the Estimated Probability of Further Offending Over Time - Individual Dynamic Models: "Fred" 217

Figure 6.18: Changes in the Probability of Further Offending Over T ime: "Fred". 218

Figure 6.19: Comparisons of the Estimated Probability of Further Offending Over Time - Individual Dynamic Models: "Connor" 219

Figure 6.20: Changes in the Probability of Further Offending Over T ime: "Connor". 221

Figure 6.21: Comparisons of the Estimated Probability of Further Offending Over Time - Individual Dynamic Models: "David". .223 
Figure 6.22: Changes in the Probability of Further Offending Over T ime: "David".

Figure 6.23: Changes in the Probability of Further offending Over Time, by Age at First Offence...... 227

Figure 6.24: Summary of Average Domain Scores, by Age at First Offence (T rend in Cohort Size).. 228

Figure 6.25: Summary of Average Domain Scores, by Age at First Offence (T rend in Mean Scores)229

Figure 7.1: Changes in the Probability of Further Offending, Dynamic Model 4 244

Figure 7.2: Number and Percentage of the Cohort Who Breached, by Time. 254

Figure 7.3: Changes in the Probability of Further Offending in Response to a Breach at Different Time Points 257

Figure 7.4: Number and Percentage of the Cohort Who had Court Appearances, by Time. 259

Figure 7.5: Changes in the Probability of Further Offending in Response to a CourtAppearance at Time 0 and Different Time Points. 262

Figure 7.6: Number and Percentage of the Cohort Who had Periods in Custody / On Remand, by Time 264

Figure 7.7: Changes in the Probability of Further Offending in Response to a Period in Custody at DifferentTimes. 267

Figure 7.8: Comparisons of the Estimated Probability of Further Offending Over Time - Individual Dynamic 'Event' Models: "Fred". 271

Figure 7.9: Comparisons of the Estimated Probability of Further Offending Over Time - System Contact Dynamic Models: "Fred" 272

Figure 7.10: Comparisons of the Estimated Probability of Further Offending Over Time - Individual Dynamic 'Event' Models: "Connor". 273

Figure 7.11: Comparisons of the Estimated Probability of Further Offending OverT Time - System Contact Dynamic Models: "Connor" 274

Figure 7.12: Comparisons of the Estimated Probability of Further Offending Over Time - Individual Dynamic 'Event' Models: "David" 275

Figure 7.13: Comparisons of the Estimated Probability of Further Offending Over T ime - System Contact Dynamic Models: "David". 276 


\section{Abbreviations}

\begin{tabular}{|l|l|}
\hline APA & American Psychological Association \\
\hline ASA & American Statistical Association \\
\hline BF $x$ x & $\begin{array}{l}\text { Bayes Factor, } \\
\text { Note: } B F_{01} \text { expresses the likelihood of } H_{0} \text { relative to } H_{1} \text { given the data whereas } B F_{10} \\
\text { expresses the probability of the data given } H_{0}, \text { relative to } H_{1} .\end{array}$ \\
\hline DTO & Detention and Training Order \\
\hline ESRC & Economic and Social Research Council \\
\hline ETE & Education, Training and Employment \\
\hline FTE & First-Time Entrant(into the Youth Justice System) \\
\hline Ho & Null Hypothesis \\
\hline$H_{1}$ & Alternative Hypothesis \\
\hline MCMC & Markov Chain Monte Carlo \\
\hline NHST & Null Hypothesis Significance Testing \\
\hline NHSTP & Null Hypothesis Significance Testing Procedures \\
\hline PPV & Positive Predictive Value \\
\hline RCT & Randomised Control Trial \\
\hline RFR & Risk Factor Research \\
\hline RNR & Risk, Needs and Responsiveness \\
\hline SAC & Serious Acquisitive Crimes eg Burglary, Robbery, Vehicle Theft/ Unauthorised Taking \\
\hline VAP & Violence Against the Person \\
\hline YJB & Youth Justice Board \\
\hline YOS & Youth Offending Service \\
\hline YOT & Youth Offending Team \\
\hline YRD & Youth Restorative Disposal \\
\hline YRO & Youth Rehabilitation Order \\
\hline
\end{tabular}

Additionally Section 5 of the accompanying Technical Annex provides an overview of terminology associated with hierarchical modelling and the associated diagnostic tests. This includes worked examples of the output, highlighting key features forthose who are not familiar with undertaking analysis undera Bayesian framework. 


\section{The Rationale for Using Bayesian Approaches in Criminology}

\subsection{Introduction}

In 2015, the journal Basic and Applied Social Psychology 'banned' null hypothesis significance testing including all vestiges of the procedure ( $p$-values, t-values, F-values, statements about "significant" differences or lack thereof, and so on), suggesting that 'the journal would no longer publish papers containing $p$-values because the statistics were too often used to support lower-quality research' (T rafimowand Marks, cited in Woolston, 2015). Confidence intervals were also banned from the journal. Instead the editors indicated that they require strong descriptive statistics, including effect sizes. The presentation of frequencyor distribution data is encouraged, where feasible. Such was the debate that this prompted that the American Statistical Association published a statementon $p$-values and statistical significance in March 2016. Their statementadvocates that as an alternative, other approaches might be entertained, including confidence intervals and Bayesian methods, acknowledging that whilst these come with their own conceptual challenges, 'they may more directly address the size of an effect (and its associated uncertainty) or whether the hypothesis is correct' (Wasserstein and Lazar, 2016: 11).

The decision by the editors of the Basic and Applied Social Psychology (BASP) and subsequent statementfrom the American Statistical Association(ASA) raise the question as to whether it is time that criminology should similarly be looking beyond null hypothesis significance testing (NHST), especially given that Trafimow and Marks indicate that the motivation for their journal's ban is that it will liberate authors from 'the stultified structure of NHSTP thinking thereby eliminating an important obstacle to creative thinking' (2015:2). This research therefore considers the extent to which creative thinking and innovation in criminologycould be enhanced through the adoption of Bayesian methods.

In doing this, the arguments around why some believe that NHST and all its vestiges should be banned are summarised. Rather than replicate the various arguments that have been posited since the techniques came into common usage and adopt an anti-Frequentist stance, the view here is that in wishing to progress knowledge, the discipline should take advantage of all the tools in the toolbox. Hence, the emphasis within this researchis upon how the discipline maybenefitfrom the use of Bayesian methods. Chapter One therefore focuses upon introducing Bayesian approaches and outlines why criminological policy and practice would benefit from these in the context of the increasing concems about the validity of NHST.

\section{Research Aim and Objectives}

Convention would have it that from the research objectives, specific research questions and hypothesis are then formulated which in turn are used to inform decisions about data and methods (de Vaus, 2001; 
White, 2009). However, as Punch observes 'there are exceptions to this order of events, and it is not mandatory' (2006:27). In this instance the overarching research aim is to explore the utility for using Bayesian approaches within criminology through presentation of a case study focusing on risk assessment in the youth justice system in England and Wales. Hence, this first chapter sets out the rationale for the adoption of new approaches within criminology, whilst Chapter Twoprovides context as to why the case study was chosen and how the development of risk assessment tools could benefit from a shift in the criminological gaze to reflectemerging technologies and techniques.

To achieve the research aim, it has been necessary to adopt a methods-led approach with data being purposefully selected to demonstrate the utility of conducting analysis under a Bayesian framework (details of which are described in Chapter Three). Methods-led approaches have been criticised for going against the logic of interconnectedness between method and research questions (Grix, 2002) with concern being raised about the construction and maintenance of 'mono-methods' which limit professional development (Gorard, 2002). However, since the emphasis here is on demonstrating how the application of novel statistical approaches can further knowledge, the approach adopted represents analysis-led rather than methods-led research which is more in keeping with Marx (1997) when he suggests that possible research questions may arise from the way in which new methods and theories mightbe applied to new settings. With this in mind, the following research objectives are identified:

- What can be learnt from approaches alreadyused in other disciplines which could be applied to criminologyand more specificallyyouth justice?

- How can the relationship between risk factors and (re)offending be explored using Bayesian approaches?

- What methodological challenges would need to be overcome?

The first of these objectives is addressed primarily through discussion within Chapter Two whilst the practical issues pertaining to the second objective are considered within Chapter Three. In keeping with the analysis-led approach, the research questions explored in the analysis chapters (Chapters Four to Seven) are located alongside the discussion of the dataset in Chapter Three, were developed to address common criticisms of the risk assessment tool used until recentlyin the youth justice system in England and Wales.

Notably ASSET and actuarial tools more generally have been criticised for being blunt tools which lack the sensitivity to be able to reflect the realities of real lives. Therefore, research questions have been designed to determine how gender, ethnicity and experience of care impacton the likelihood of further offending. These link into policy concerns around the over-representation of BAME and looked after children in the criminal justice system. 
Young people who offend can live particularly chaotic lives. Therefore, there is also a desire to understand how responsive the risk assessment tool is to changes over time and whether these are affected by the 'event's such as breaching, returning to courtand spending time in custody/ on remand. These issues which have not previously been considered as part of the evaluations of ASSET carried out on behalf of the Youth Justice Board (YJB) / Ministry of Justice. As it was anticipated that those with a prior history of offending would respond differently to those who are first-time entrants, research questions were also designed to explore the role played by the young person's criminal history on the likelihood of further offending behaviours. Table 1.1 summarises the research questions by theme.

Table 1.1: Summary of the Research Questions, by Chapter and Theme

\begin{tabular}{|c|c|c|}
\hline \multicolumn{2}{|c|}{ Chapter and Theme } & \multirow{2}{*}{$\begin{array}{l}\text { Research Questions } \\
\text { What is the relationship between further offending, the } 12 \text { domains and time? }\end{array}$} \\
\hline 4 & Risk Assessment Domains & \\
\hline \multirow{2}{*}{5} & \multirow{2}{*}{ Dimensional Identity } & What is the impact of gender and ethnicity on the likelihood of further offending? \\
\hline & & $\begin{array}{l}\text { What is the impact of having experience of care on the likelihood of further offending } \\
\text { over time? }\end{array}$ \\
\hline \multirow{2}{*}{6} & \multirow{2}{*}{ Static Factors } & $\begin{array}{l}\text { What is the impact of the 'static' factors within ASSET in predicting further offending } \\
\text { over time? }\end{array}$ \\
\hline & & Is it possible to extend the sensitivity of ASSET by extending any of the predictors? \\
\hline \multirow{2}{*}{7} & \multirow{2}{*}{ System Contact } & $\begin{array}{l}\text { How is the likelihood of further offending affected by having experience of care and } \\
\text { a previous offending history? }\end{array}$ \\
\hline & & $\begin{array}{l}\text { What is the impact of coming into contact with facets of the youth justice system on } \\
\text { the likelihood of further offending? }\end{array}$ \\
\hline
\end{tabular}

Cutting across the four analysis chapters, a further research question is posed which considers how well ASSET scores reflect the realities of the young person's change in circumstances during their time under the supervision of the YOT. This final question provides a means of assessing the predictive accuracy of the various models constructed in response to the other questions posed and links back to concems about the predictive accuracyof ASSET and actuarial tools more generally.

The analysis presented in Chapters Four to Seven utilises a range of common statistical techniques performed under the Bayesian framework in order to demonstrate how the relationship between risk factors and (re)offending can be explored. Chapter Four builds upon the work of Baker et al. (2003, 2005) and Wilson and Hinks (2011) by considering changes both in the total ASSET score and in the individual risk domain scores over time. A hierarchical model is constructed which mimics the features of ASSET with the ratings or 'scores' from 12 domains of risk being added together to allow the young person's perceived level of risk and hence the intensity of their contactwith the YOT to be determined. 
In subsequent chapters, this basic 'Dynamic' model is enhanced using time invariant predictors constructed to reflectaspects of dimensional identity, the four static factors and time varying 'events' which represent when the individual comes into contact with facets of the youth justice system. These include dichotomous, categorical and continuous predictors. Sadly, there was insufficient data to enable models to be simulated which would have enabled the impact of gender, ethnicity and age at first conviction to be explored over time. Whilst the it was also necessary to make compromises due to the size of the dataset, working with a comparativelysmall datasetalso offered the opportunity to investigate the underlying data to understand what mightbe contributing to unexpected findings. As such the it has been possible to establish the utility of applying Bayesian approaches, by demonstrating their potential. Although the analysis presented within the case study identifies some of the methodolo gical challenges which would need to be overcome, many of these are associated with the size of the dataset. However, as highlighted in Chapter Eight, there are wider pedagogical and philosophical issues which the discipline will need to address if Bayesian approaches are to be used more widely. This research therefore represents an importantkey step in facilitating the paradigm shiftrequired to enable this to happen.

The remainder of this chapter outlines the extent to which criminology as a discipline has engaged in the NHST debate, introduces Bayesian approaches and argues why now is an appropriate time to be considering their application.

\subsection{Significance Testing and the Anti-NHST Debate}

NHST represents an amalgamation of Fisher's significance testing and Neyman-Pearson's theory of hypothesis testing - something which has been frequently commented upon as part of anti-NHST arguments since they reflect different philosophical perspectives. Kruschke (2010a;2010b;2011;2012) is just one of many who have been highly critical of NHST, emphasizing its inability to tell us what we want to know i.e. the probability of the hypothesis being true given the data. This, along with criticism that many researchers misinterpret the findings (see for example Nickerson, 2000 for a detailed discussion) have been key themes running throughout the anti-NHST literature.

The two most common misconceptions which cause confusion are '(a) that the size of the $p$-value indicates the strength of the relationship and (b) that statistical significance implies theoretical or practical significance' (Gliner etal., 2002:84). To overcome these issues, the advice has been that there should be routine reporting of effect sizes in the form of confidence intervals (see for example Cohen, 1994; Gorard, 2014b) and improvements to the way in which statistics is taught to researchers (Gliner et al. 2002; Kalinowski etal., 2008). In the case of the former, this is now standard practice in mostjournals in keeping with the guidelines issued by the American Psychological Association (American Psychological Association, 2010), although this in itself is problematic since confidence intervals can 
also be readilymisunderstood (Hoekstra etal., 2014) and do nothave the properties atare often claimed on their behalf:

'Confidence interval theory was developed to solve a very constrained problem: how can one constructa procedure that produces intervals containing the true parameter a fixed proportion of the time? Claims that confidence intervals yield an index of precision, that the values within them are plausible, and that the confidence coefficient can be read as a measure of certainty that the interval contains the true value, are all fallacies and unjustified by confidence interval theory.'

(Morey et al., 2015: 118)

The process of NHST has been scathinglyreferred to as being 'the null ritual' (Gigerenzer, 2004) arguing that the process is taught without statistical thinking and crucially without reference to concepts such as statistical power or effect size - a finding supported by Gliner et al. (2002) based on a review of how twelve key educational text books deal with the problems and common misconceptions. Cohen has similarly likened NHST to a ritual, but also alluded to a mechanised approach when he bemoaned the fact that 'after 4 decades of severe criticism, the ritual of null hypothesis significancetesting - mechanical dichotomous decisions around a sacred .05 criterion - still persist' (1994: 997). Certainly, itwould appear that where NHST is blindly followed without an understanding of the formal logic, in particular Modus Tollens associated with statistics, 'researchers are all too readily lulled into a false sense of science' (Lambdin, 2012:71).

The problems of adopting this mechanised approach are further exacerbated by the ease with which analysis can now be undertaken in software such as SPSS, with students often being provided with instructions of how to carry out various statistical tests without having a good grasp of the underlying assumptions. For example, much of the data in the social sciences is not normallydistributed, nor is it always drawn randomlyfrom repeated samples, yet these characteristics underpint-tests, chi-squared and many of the other tests which fall under the NHST umbrella. All too often students are told that if their sample is sufficientlylarge then they can assume normality, but how manyactually check?

McShane and Gal (2016) suggest that whilstmanyresearchers may be aware that statistical significance at the 0.05 level is a mere convention, what started as a rule of thumb has evolved into an ironclad principle that affects the interpretation of evidence. Thus, rote learning and recipe-like teaching/practice can be seen as treating 0.05 as a "magic number" upon which to make a dichotomous decision rather than evaluating evidence as a continuum. Where this happens, there is the potential for researchers to erroneouslydraw unwarranted conclusions because their evidence fails to attain statistical significance, rather than considering alternative explanations. Similarly, there is the potential for spurious find ings based on poor-quality data or lacking a plausible mechanism, to be published simply because they attained statistical significance. 


\section{Positivist Criminology and NHST}

In his popular introductory text book, Newburn (2007) characterises criminology as being a strange beast, with origins in applied medico-legal science, psychiatry, a scientifically-orientated psychologyand more recentlysociology. As a discipline therefore, itis apt at borrowing from its neighbours. However, it could also be argued that it has picked up some questionable habits, some of which stem from the efforts of some criminologists to mimic scholars from the natural and physical sciences, who have sought to make the more discipline more scientific (DiCristina, 1997) and attempts to objectify the study of a social phenomenon.

To appreciate the oft contradictory and eclectic nature of criminology in the first half of the twentieth century, one only needs to look at the career of the criminological thinker Sutherland. He promoted a predominately sociological framework for criminology, pursuing a scientific, objective study of criminological phenomena through attempts to explain individual-level and macro-level differences in crime rates. Regarded as being hugely beneficial for the field of criminology, the imposition of sociological positivism (and hence a shift away from biogenic and psychiatric explanations of crime) brought abouta tendencyto focus on issues of cause and effect, empirical data, replication and public statement of research methods. Notably, Sutherland privileged empirical evidence over revealed truth whilst at the same time 'recognising and advancing a powerful initiative against the inherent biases of the "scientific" criminology of his time in terms of how crime was defined and what type of crime was studied' (Friedrichs, 2016: 4). However, as Laub and Sampson (1991) observe - drawing upon Gottredson and Hirschi, Sutherland held doggedly to the interests of his own discipline, rather than being open to the interests of scientific explanation coming from other sources. In particular he promoted the influence of sociological variables such as peer group, cultureand community. As a result, promotion of his theory of differential association cameat the expense of a number of his contemporaries including the multiple-factor theory of crime advocated by the Gluecks - the latter now considered to be hugely influential in risk factor research.

Much maligned by Sutherland, and certainly not without their critics, the Gluecks' methodological approach with its emphasis on longitudinal and follow-up prediction studies, including where possible control groups for comparison studies; and the importance of triangulation through use of multiple sources in addition to official records exemplifies, at least on paper, qualities today associated with high quality quantitative research. Viewed through a contemporarylens, the flaws in each of their respective bodies of work are apparent, but what is significant is their pursuit of "scientific" approaches and the systematic collection of data to support their endeavours. In this respect, their legacyis unquestionable with elements of their respective methodological approaches now firmly embedded into contemporary criminological practice. 
As the discipline has matured, it has become a more diverse and fragmented enterprise, with less of a sociologicaldominance (Friedrichs, 2016). Both classical and positivist traditions have contributed to its currentform, shaping the development of theoretical approaches and the adoption of scientific principles. Whilst positivist assumptions have been much criticised, Bottoms asserts that:

'Whatever the defects of old-style positivism (and it has many), it has bequeathed to contemporary criminologists a fine tradition of careful observation of the natural and social worlds; of the scientist's duty to report his/her research data dispassionately, even if he/she finds them personally unwelcome; and of the careful search for causes and explanations'

(Bottoms, 2008: 88)

Criminology's battle for recognition as a distinctscientific discipline coincided with the rise of statistical approaches championed bythe likes of Fisher, Neyman and Pearson (see Salsburg, 2001), hence these form the bed rock of statistics within quantitative criminology. Indeed, NHST has been identified as being 'the engine that drives theory testing in criminology' (Barnes et al., 2017:26). However, as criminology has moved further away from psychology and more towards sociology, so the relationship between theory and research has evolved, and crucially the nature of questions has changed.

The growing demand for more reliable evidence of cause and effect, along with a means to evaluate the effectiveness of interventions, has spurred the drive for experimental criminologywhich has encouraged the increased use of meta-analysis and randomised control trials in criminology. Hailed almost as a panacea by some, experimental criminologyhas been promoted as being able to:

'...help make a world in which governments can refuse to waste moneyon ineffective criminal sanctions despite populist pressures; a world in which citizens can demand that governmentmust test policies with well-controlled experiments before spending vast sums in the name of crime prevention.'

(Sherman, 2009:7)

Notably randomised control trials (RCTs) have for some time now been held up as being the 'gold standard' in criminology, being assigned a score of 5 on the Maryland Scientific Methods Scale (Farrington et al., 2002) and have been utilised by the Society of Evidence Based Policing and the College of Policing to contribute to the body of evidence on a range of policing methods - evidence from many of these experiments along with those from quasi-experiments is disseminated through the Global Policing Databaseand the What Works for Crime Reduction website. As Sampson (2010:490) observes, 'claims for RCT superiority are not surprising given that experiments have long been cloaked in the mantle of science, especially the laboratory paradigm of randomisation (or investigator control over allocation to treatment)'. Criminologists have similarlyturned to medicine and experimental methods to evaluate interventionsusing meta-analyses.

Such borrowing from our neighbours is seen as a way to increase the validityand robustness of research, a vital step if we are to see more evidence-based policy. However, in seeking to apply ideas from 
medicine to criminology, the dominance of positivist approaches has meant that empiricism and Frequentistapproaches have been privileged as the route to the acquisition of criminological knowledge. The primarylimitation of the positivistlegacyis the belief that it is both possible and correct to understand notions such as offending behaviour by measuring variables and combining them in linear statistical analysis. But is it appropriate for use within a discipline where there is so much inherent complexity, unpredictability, context-dependence and multidimensionality?

\section{The Anti-NHST Argument in Criminology}

Whilst psychology has been particularlyvocal in the anti-NHST argument, it has also been considered within criminology with Maltz's 1994 paper remaining perhaps one of the best known. He argues that criminologists should no longer make do with techniques which implythat there is a norm of behaviour to which we compare other's behaviour, and advocates for greater use of qualitative data including narrative accounts - a direction which has been taken successfullyby some researchers. Bushway et al's 2006 paper is one of the few that has sought to quantify the extent of the problems arising from misapplication. Theyargue for promotion of more thoughtful application, 'to putNHST in its place: as a tool to facilitate the inferential process, notas the end game for quantitative research' (Bushwayet al., 2006: 14). As part of this, the authors advise that size matters and hence greater attention should be placed on reporting effect sizes and statistical power.

A cursory glance at many criminological journals suggests that we are now seeing increased reporting of effect sizes, confidence intervals and meta-analysis, in part because of the American Psychological Association's (APA) guidelines around appropriate statistical practice. Whilst the guidelines provide the opportunity for many disciplines 'to become cumulative sciences of estimation that deal with quantifying uncertainty rather than simply making dichotomous accept-reject decisions on the basis of individual studies' (Fidler, 2010), the utility of NHST remains a concern especiallywhen such research is used to inform policyand practice. Not only do we need to know the 'effect' size or strength of any pattern or finding that is being reported, butwe also need to have an appreciation of the costs, benefits and possible dangers of using that finding in practice (Gorard, 2014a). The debate has prompted a call for more transparent methodsand the need to eradicate 'black box' statistics. Only then can we get a sense of how trustworthy the findings are.

For research findings to be trustworthy, use of appropriate research designs and the meaningful operationalisation of variables have a fundamental role to play. Here is it perhaps more obvious where the problems lie. In medicine, variables are easy to define and can be measured with a high degree of precision, yet this is considerablymore difficult when considering human behaviour. Thus, efforts to unpick for example, the causes of offending behaviour and identify appropriate treatments or interventions are inevitably limited by the inherent complexity and subjective understandings of the key concepts being measured. In the case of offending, all too often it is necessary to utilise proxy measures 
such as self-reported offending or police recorded crime, or to focus on restricted populations such as those in custody which introduces biases. This use of crude measurements means that there is insufficient sensitivity in initial conditions which then amplifies error in the conclusions drawn. In order to adhere to the assumptions of various statistical tests, it is often necessary to collapse groups, something which reduces the potential to draw out more sensitive measures of, in this case, offending by different sub-groups. Hence what looks like 'science' in criminology, with its sampling rules, methodological coherence, use of statistical tests and certainty of conclusions, is, in reality, a social construct of dubious, real-world validity (Salsburg, 2001).

The situation in criminologyis further complicated bythe reductionist simplicity of methods enshrined in positivist traditions which have privileged nomothetic methods. In the quest to identify stable, predictable, deterministic, replicable relationships with offending, risk factor research (RFR) - prominent in explaining youth offending - has sought to apply linear modelling techniques such as linear regression which suppose that incremental increases in predictor variables produce linear and proportional increases in effects / outcomes suchas offending (Case and Haines, 2014). However, this is predilection compounds problems associated with the way in which key concepts have been operationalised and measurements made. Whilst various theories have been put forward to explain offending behaviour, the fact that such behaviours can be context specific means that there is a lack of research findings which explain why initiatives have worked in some geographical areas or with certain groups, but not others.

\section{Is there a replication crisis in Criminology?}

Replication is an essential component of the scientific process since itestablishes stabilityand rules out the possibility that results are merely due to coincidence. As such replication enables claims to be substantiated and generalised to larger or different populations than used in the original study. Yet Farrington (2000) notes that pure replication in criminological research is rare and few replications studies are published in criminology - a situation which McNeeleyand Warner (2015) have hypothesised may be due to the comparative youth of the discipline. This, theysuggest, has resulted in a bias towards criminological research which examines new topics sincethese are perceived to be more interesting and therefore more worthy. Their review found that replication studies constituted just over 2 percent of articles published between 2006 and 2010 in leading criminological journals, compared to replication rates of between 3 and 10 percentin prestigious journals relating to other disciplines.

Lösel (2017) goes further suggesting there are many social factors in research that form obstacles againsta culture of replication with the academic world reinforcing mass publication in criminologyand other disciplines. This 'publish or perish' ethos means that researchers seem to avoid replications as they wish to be seen to demonstrate their own creativity. Large collaborative projects tend to be promoted by research foundations. However, these can make replication more difficult especially as time and resource issues can hinder replications of complex field experiments that require years of 
follow-up. In primarystudies, it is argued thatin studies with manyvariables, selective data analysis and reporting, and fishing for significance is a danger particularly in areas where there may be financial incentives. There is also a reluctance for scholars to share their own data with others despite policies to encourage open data access and greater transparency.

Whilst it is acknowledged thatnotall criminological studies should necessarilybe expected to replicate, where general theories are being tested or evaluations of interventions undertaken then replication is important, especiallywhere findings maythen be applied to policy or practice, 'Replication of these types of research would allow practitioners to have more confidence in the original findings, lending credibility to the research process and making the integration of research results into policies and practice more attractive' (McNeeleyand Warner, 2015:582). However, where replication is notfeasible, 'confirmatory meta-analyses can play an important role on the path towards more differentiated and replicated knowledge' (Lösel, 2017: 1). In this respect, there is the potential to learn from other disciplines e.g. clinical pharmacy, engineering and climate research where evaluations are undertaken of programme packages rather than focusing on specific components in isolation since factors often have a minor effect in isolation, but in combination, they may show a strong impact. Methodologicallyit is recognised that whilst this is more challenging, adopting such an approach facilitates understandings of the effects of combinations that may potentiate effectiveness or lead to negative side effects. Thus, from a realistic perspective, in promoting replication studies in criminology Lösel acknowledges that 'applied research in criminologyoften has an explanatory character' (2017:14) and hence flexible strategies are required which are not limited by uniform and rigid guidelines.

Although Lösel believes that there is no need for using the term "crisis" within criminology, Barnes et al. (2017) have questioned how powerful the evidence is in criminology, suggesting that the disciplinemay be at risk of a replication crisis. They assert that the crisis in confidence which has struck scientific disciplines like psychology and neuroscience is intimately tied to the low levels of statistical power in many studies in these areas. Their analysis of effect sizes from 80 meta-analyses, covering more than 6,000 individual primary criminology studies published between 1995 and 2015 concluded that more than half of all studies were underpowered to detect the effect sizes that they observed. However, at least $25 \%$ of all criminologyresearch wasfound to be very well powered. As a result, they conclude that 'studies in criminologyhave relatively high levels of statistical power and a relatively high PPV [Positive Predictive Value] compared to other areas of behavioural and social science' (Barnes et al., 2017: 24). However, they caution that this is relative to other behavioural science disciplines like psychology, neuroscience and behavioural genetics which are known to have alarmingly low statistical power and average PPV. Hence their estimated PPV of 0.4 to 0.8 (which suggests that a randomlyselected study from a criminologyjournal would report that the null hypothesis was correctly rejected between $40 \%$ and $80 \%$ of the time) appears to be very favourable when compared with sayDuncan and Keller's estimated 
PPV of approximately 0.05 for candidate gene literature within behavioural genetics (2011, cited in Barnes et al., 2017).

Looking more widelyacross the social sciences, the replication crisis in psychologyhas prom pted much debate about the reliability and robustness of published "scientific" experiments prompting Nature to survey 1,500 scientists as to their views on whether there is a replication crisis within their discipline and what factors could boost reproducibility(Baker, 2016). Of the 11 improvements suggested, nearly $90 \%$ ticked 'More robust experimental design', 'better statistics' and 'better mentoring'. Whilst the Nature article does not address what is meantby 'better statistics', the ASA statement suggests that Bayesian methods mayaddress the problems associated with size effects and whether the hypothesis being tested is true. The following section therefore introduces Bayesian approaches as an alternative to NHST.

\subsection{The Bayesian Way}

The standard Frequentist interpretation of probability which many will have been taught at school describes long-run properties of repeated stochastic i.e. random events and associated standard statistical methods. Bayesian approaches offer alternative probabilistic methods which are essentially a 'subjective' interpretation of probability since they allow uncertainty or 'degree of belief about any unknown but potentially observable quality to be expressed, whether or not it is one of a number of repeated experiments. In terms of the philosophical differences,

'Bayesians view observed data as permanently fixed, but unknown parameters are considered random quantities given distributions based on the current level of knowledge. Conversely, Frequentists view data as stochastic, coming from a neverending stream created by exacting the same generating process, butparameters are quantities fixed by nature and never changing.'

(Gill and Witko, 2013: 459)

The Bayesian starting point is therefore a beliefor assumption - which could be informed by the results of previous research. In contrast Frequentist inference describes the behaviour of test statistics and confidence intervals under hypothetical repeated sampling from an underlying population using a battery of tests which fall under the umbrella of NHST.

\section{Introducing Bayesian Approaches}

At its most basic, Bayesian is a branch of probability that uses a subjective rather than objective or 'Frequentist' interpretation of uncertainty i.e. its starting point is a belief or an assumption rather than being based on relative frequencies, and ithas the advantage that it can be updated as new information becomes available. It can also be applied to situations where an event cannotnecessarilybe repeated under identical conditions (as in the classical or Frequentistapproach) and where the alternatives to the event cannot be reduced to a finite list of equally likely outcomes (as in the objective approach). Its 
subjective interpretation means it is considered to be more intuitive and hence more applicable in the real world.

Although it was independently discovered more than 250 years ago by Thomas Bayes (c. 1701-1761) and Pierre-Simon Laplace (1749-1827), it is this subjectivity - at a time when there was a growing conviction that modern science required objectivity and precision - which led to significant controversy and the theorem languishing in near obscurity until the dawn of the computer age. The increased computational power that becameavailable in the 1980s and 1990s has led to the resurgence in the use of Bayes' rule (the theorem upon which Bayesian approaches are based). Notably the development of Markov Chain Monte Carlo or 'MCMC' methods in the 1990s made iteasier to compute the mathematics required to model some of the more complex relationships that exist in real life data by enabling sequences of random samples to be obtained from probability distributions for which directsampling is difficult. Together these developments have contributed to the proliferation of applications across a range of disciplines including medicine, psychology, physics, ecology, geology, artificial intelligence, economics and finance.

A key reason as to why Bayesian approaches have emerged as a powerful tool with a wide range of applications is that they provide a rigorous method for interpreting evidence in the context of previous experience or knowledge. As a result, one application which many will have benefited from without realising is Google's use of Bayesian techniques to classify spam and pornography and to find related words, phrases and documents within its search engine (McGrayne, 2011). Other examples deemed newsworthy are associated with cryptography - the breaking of the Enigma code is attributed to Alan T uring's use of Bayesian techniques (Good, 1979; Tarran, 2014; see also Simpson, 2010 in relation to the Japanese Naval 25 (JN 25) encryption); the huntfor missing ships and aircraft including the Malaysia Airlines Flight MH370 and Air France Flight 447 in 2011 (Flam, 2014a; Zahriyeh, 2014); establishing if the remains discovered in a Leicester car park really are those of Richard III (King et al., 2014b) and in the presentation of forensic data in legal cases (Blair and Rossmo, 2010; Fenton and Neil, 2013; Stone, 2013).

\section{Bayes' Theorem}

Bayesian statistical analysis relies on Bayes' Theorem (Equation 1.1) - an elegantformula whichenables us to update prior beliefs about parameters and hypotheses in light of data, to yield posterior beliefs. Applying Bayes' rule transforms probabilities that look useful (but are often not), into probabilities that are useful. It does this by combining prior experience (in the form of a prior probability) with observed data (in the form of a likelihood) to enable us to interpret the data (in the form of a posterior probability). This process is known as Bayesian Inference. In the context of an experimentwe are concerned with what is the probability that the proposed hypothesis is correct $(H)$ given the data $(D)$. As can be seen from Equation (1.1), this would be written as $\operatorname{Pr}(H \mid D)$. However, what is observed during an experiment 
is the data given the hypothesis being tested i.e. $\operatorname{Pr}(D \mid H)$ which is not what is required. In terms of interpreting the behaviour of test statistics using Frequentist techniques, this relies on using $p$-values and confidence intervals whilstBayesian inference permits directinferences to be made - see Equation (1.2). Hence the Bayesian probabilistic interpretation of statistical parameters is considered not only to be more intuitive, but since prior assumptions are stated, it is also more transparent.

\section{Equation (1.1): Bayes' Theorem}

$$
\operatorname{Pr}(A \mid B)=\frac{\operatorname{Pr}(B \mid A) \cdot \operatorname{Pr}(A)}{\operatorname{Pr}(B)}
$$

where

- $\quad A$ and $B$ are events and $\operatorname{Pr}(B) \neq 0$.

- $\operatorname{Pr}(A)$ and $\operatorname{Pr}(B)$ are the probabilities of observing $A$ and $B$ without regard to each other.

- $\operatorname{Pr}(A \mid B)$, a conditional probability, is the probability of observing event $A$ given that $B$ is true.

- $\operatorname{Pr}(B \mid A)$ is the probability of observing event $B$ given that $A$ is true.

This can be re-written in the context of observed data $(D)$ and a hypothesis $(H)$, where the interpretation is that the hypothesis is true.

$$
\operatorname{Pr}(H \mid D)=\frac{\operatorname{Pr}(D \mid H) \cdot \operatorname{Pr}(H)}{\operatorname{Pr}(D)}
$$

where

$\operatorname{Pr}(H)$ is the prior probability

- $\operatorname{Pr}(D)$ is the marginal probability

- $\operatorname{Pr}(H \mid D)$ is the probability that the proposed hypothesis is true given some data that were actuallyobserved. This is the posterior probability

- $\operatorname{Pr}(D \mid H)$ is the probability of observing the data given that the hypothesis is true. This is the likelihood.

Bayes Theorem can also be written as:

$$
\text { Posterior Probability }=\frac{\text { Likelihood } x \text { Prior Probability }}{\text { Evidence }}
$$

From this, the posterior probabilityis proportional to the prior probability time the likelihood. 


\section{Equation (1.2)-Bayes' Theorem for Two Hypotheses}

Consider two hypotheses $\mathrm{H}_{0}$ and $\mathrm{H}_{1}$ which are 'mutuallyexhaustive and exclusive' i.e. one and only one is true. Before having access to any evidence, the respective prior probability of each hypothesis is $\operatorname{Pr}\left(H_{0}\right)$ and $\operatorname{Pr}\left(H_{1}\right)$. Suppose that we have observed some data $D$, such as the results of a test and we know from past experience that the probability of observing $y$ under each hypothesis is $\operatorname{Pr}\left(D \mid H_{0}\right)$ and $\operatorname{Pr}\left(D \mid H_{1}\right)$ respectively. These are the likelihoods.

By adapting Equation (1.1), we have the identity:

$$
\operatorname{Pr}\left(H_{0} \mid D\right)=\frac{\operatorname{Pr}\left(D \mid H_{0}\right) \cdot \operatorname{Pr}(D)}{\operatorname{Pr}\left(H_{0}\right)}
$$

where $\operatorname{Pr}(\mathrm{y})=\operatorname{Pr}\left(\mathrm{D} \mid \mathrm{H}_{0}\right) \cdot \operatorname{Pr}\left(\mathrm{H}_{0}\right)+\operatorname{Pr}\left(\mathrm{D} \mid \mathrm{H}_{1}\right) \cdot \operatorname{Pr}\left(\mathrm{H}_{1}\right)$ is the overall probability of $\mathrm{D}$ occurring Now $\mathrm{H}_{1}=$ 'not $\mathrm{H}_{0}$ ' and so $\operatorname{Pr}\left(\mathrm{H}_{0}\right)=1-\operatorname{Pr}\left(\mathrm{H}_{1}\right)$ and $\operatorname{Pr}\left(\mathrm{H}_{0} \mid \mathrm{D}\right)=1-\operatorname{Pr}\left(\mathrm{H}_{1} \mid \mathrm{D}\right)$. In terms of odds rather than probabilities, Bayes' Theorem can be re-expressed as:

$$
\frac{\operatorname{Pr}\left(H_{1} \mid D\right)}{\operatorname{Pr}\left(H_{0} \mid D\right)}=\frac{\operatorname{Pr}\left(D \mid H_{1}\right)}{\operatorname{Pr}\left(D \mid H_{0}\right)} \times \frac{\operatorname{Pr}\left(H_{1}\right)}{\operatorname{Pr}\left(H_{0}\right)}
$$

Now $\operatorname{Pr}\left(H_{1}\right) / \operatorname{Pr}\left(H_{0}\right)$ is the 'prior odds', $\operatorname{Pr}\left(H_{1} \mid D\right) / \operatorname{Pr}\left(H_{0} \mid D\right)$ is the 'posterior odds', and $\operatorname{Pr}\left(D \mid H_{1}\right) /$ $\operatorname{Pr}\left(D \mid H_{0}\right)$ is the ratio of the likelihoods. Hence

$$
\text { Posterior odds }=\text { likelihood ratio } \mathrm{x} \text { priorodds }
$$

In the context of hypothesis testing, this can also be thought of as:

Posterior confidence $=$ Bayes Factor $x$ Prior confidence in $\mathrm{H}_{1}$ rather than $\mathrm{H}_{0}$

Under the Bayesian framework, the Bayes Factor provides a measure of the strength of the evidence for one theory verses another (e.g. $\mathrm{H}_{1}$ verses $\mathrm{H}_{0}$ ). This value is read directlyhence a Bayes Factor of 5 suggests that the data are 5 times more likely under $\mathrm{H}_{1}$ than $\mathrm{H}_{0}$. Thus, if the Bayes Factor is about 1 , the experiment was not sensitive. A value greater than 1 suggests that the data supports $\mathrm{H}_{1}$ over the null whilst values less than 1 support $\mathrm{H}_{0}$ over the theory being tested. As highlighted by Dienes and Mclatchie (2017), Bayes Factors have a distinctadvantage over $p$-values in that they are able to provide a measure of strength of evidence, indicating the extent to which one's belief ought to change. Whereas $\mathrm{p}$-values are interpreted as a dichotomous decision to retain or reject $\mathrm{H}_{0}$ (Figure 1.1a), following the advice set out by Jeffreys that Bayes Factors of more than 3 are worth taking note of, it is possible to distinguish between evidence for the null hypothesis and insensitive data. Hence where the Bayes Factor is less than $1 / 3$, this provides notable supportfor the null. Values between $1 / 3$ and 3 suggest that 
there is no evidence to speak of. Those greater than 3 , provide noticeable support for the theory $\left(\mathrm{H}_{1}\right)$ (Figure 1.1b).

Figure 1.1: Interpretation of Hypothesis Testing under (a) the Frequentist Framework and (b) the Bayesian Framework

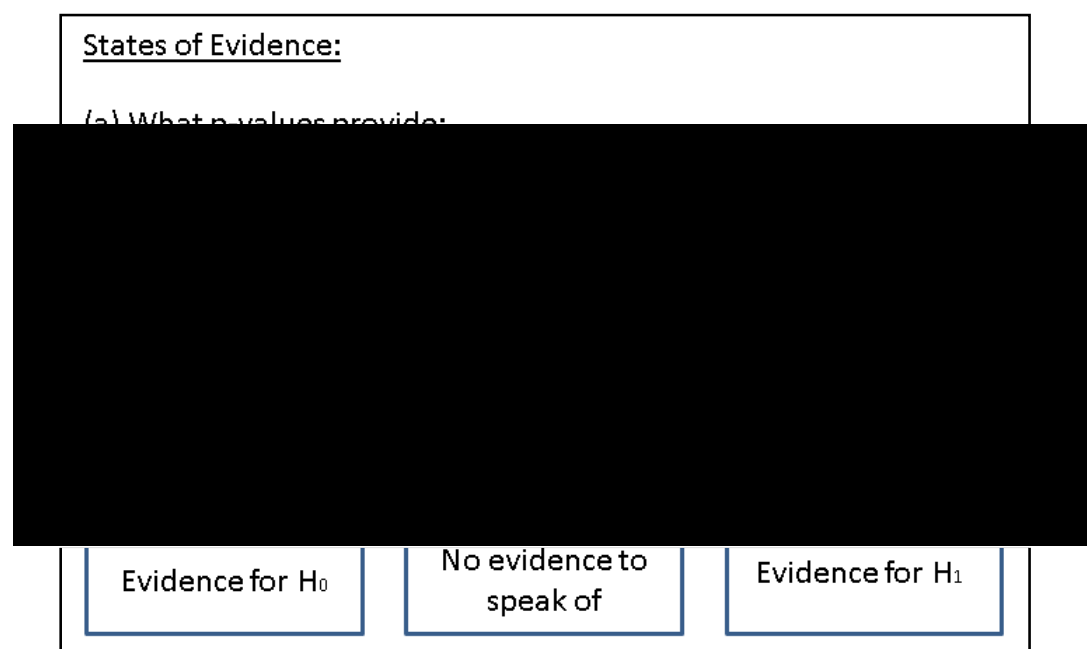

Adapted from Dienes and Mclatchie (2017: 2)

Bayes' Theorem itself is utterly uncontroversial and adheres to the axioms of probability. It is important to reiterate this as many anti-Bayesian arguments have hinged upon creating controversy around the 'whimsical' choice of priors in the absence of genuine prior experience, branding them unscientific (Western, 1999).

Historically critics e.g. Efron (1986) expressed concern that there was also the potential for two researchers analysing the same data to have different prior beliefs and hence arrive at sets of different outcomes or posterior beliefs. However, this is now recognised as a key strength of Bayesian approaches since it enables subjectivity and context to be acknowledged. The result is that 'the prior distribution is explicitlyspecified and justified for a sceptical scientific audience' (Kruschke etal., 2012: 726). This transparency, which is sometimes lacking in Frequentistapproaches, can also be extended by either conducting the analysis with more than one prior to demonstrate the invariant nature of the posterior distribution, or by using a noncommittal broad prior. A further advantage in relation to priors is that the probabilities for events are conditioned on the context, which includes the observer and all the observer's background knowledge and assumptions (Spiegelhalter etal., 2004). Through the choice of prior(s), this subjectivity can be treated with respect, in an open and transparentmanner. This view is particularlyrelevant in the context of evaluations of criminological interventions where the viewpoints of sponsors, investigators, reviewers, politicians, policy makers and 'consumers' (e.g. institutional actors within the criminal justice system or those who engage specificallywith victims / offenders) need to be taken into account(Carr, 2010). Consequently, a range of different priors may be utilised in the design, but potentially not reported as part of the results. 


\section{Bayesian Approaches as an Alternative to NHST: Transferability from OtherDisciplines}

One of the key methodological issues in the social sciences is that the way in which data is collected means that it does not always fit the criteria of being randomly selected cases and/or repeatable experiments - key features of traditional 'Frequentist' approaches. Yet these are the assumptions upon which NHST is based. In seeking to apply techniques - calculating standard errors, confidence intervals and performing significance tests (both explicitlyand disguised within more complex statistical modelling) we risk 'errors, wasted opportunities, vanishing breakthroughs, and unwarranted conclusions' (Gorard, 2014b:5). Doubtover the appropriateness of applying such tests to the types of data that we frequently come across within the social sciences generally has been the topic of much academic debate particularlyin disciplines aligned to criminologysuch as the cognitive sciences (see for example Lambdin (2012), Gigerenzer and Marewski (2015)). With this in mind, the widespread adoption of alternative Bayesian approaches which are not dependent upon sample size, random sampling or repeated experiments have the potential to negate many of the abuses which have become unfortunatelybecome so pervasive in social science research.

Successes in other disciplines, such as medicine, psychologyand ecologysuggest that it will be possible to transplant, with some minor adaptions, Bayesian approaches into criminology. Aready Bayesian approaches have been used to explore different aspects of human behaviour and cognition including the assessment of risk for violent recidivism through multivariate Bayesian classification (Mokros etal., 2010); a Bayesian learning theory of deterrence among serious juvenile offenders (Anwar and Loughran, 2011) and the use of 'Bayesian T ruth Serum' to consider the accuracy of survey responses in perceptual deterrence studies (Loughran etal., 2014). Although these come from psychology, itis easy to see how approaches could be replicated. Within criminology Bayesian quantile regression has been undertaken to analyse potential risk factors for the incidence of violent crime (Wang and Zhang, 2012) whilst Bayesian spatial techniques have been used bygeographers and publichealth specialists to investigate journeyto crime patterns for serial offenders (Levine and Lee, 2009; Levine and Block, 2011). Bayesian hierarchical models have been employed to look atarrest rates (Cohen etal., 1998) whilstBlattenberger et al. (2010) have compared three different types of Bayesian modelling techniques to explore the criminological, sociological and economic factors which predict parolees' return to prison. This latter example coming from academics based in economics.

Within public health literature examples can be found which also have a criminological slant including work estimating the prevalence of injecting drug users at a regional level (King et al., 2009; King et al., 2014a) utilising the small area techniques highlighted by Fienberg (2011) whilst geographers have contributed to understanding about the spatial relationship betweenalc oholoutlets and violence (see for example, Zhu et al., 2006; Cunradi et al., 2012; Mair et al., 2013; Erickson et al., 2015; Fitterer and Nelson, 2015) and have begun to explore the potential for using Bayesian spatio-temporal modelling to 
look at localised crime trends (Law etal., 2014). These bodies of work utilise techniques which 'borrow' strength from other, typically neighbouring areas to refine the quality of the estimate.

Despite these examples, criminologyis yet to embrace the advantages offered by employing Bayesian techniques. One of the few instances where a criminologisthas applied Bayesian approaches to crime and criminal justice is the work byBerk etal. (1992a; 1992b) around policingdomestic abuse in Colorado Spring. Now twenty-five years old, these papers rely on methods of evidence synthesis to consider the deterrent effects of arrest, a feature also utilised by Sullivan and Mieczkowski (2008) in their consideration of the utility of applying Bayesian approaches to synthesize the evidence from intervention studies.

\section{Taking Stock and Looking Forward}

The assertions from Barnes etal. (2017) comealmost twentyyears after the criminologistBruce Westem advocated to sociologists that 'Bayesian statistics offers practical methods for statistical inference that are rooted in the basic rules of probability theory. By using probability to describe uncertainty about parameters, the Bayesian approach converges with standard sociological understanding' (Westem, 1999: 31). Much of this stems from the fact that despite mathematical advances, for a long time within the social sciences, Bayesian statistics was seen as a minority topic with Bayesian approaches often being dismissed despite a growing disquiet about the 'logic' of NHST and use of p-values, because of controversy over the use of subjectivism (see for example Stone, 2013:119-128). This position is slowly changing and there are now several popular text books available which are aimed specificallyat social scientists (see for example, Jackman, 2009; Kruschke, 2015; Kaplan, 2014).

Whilst criminologyis notalone in its reliance on NHST, there are signs that other disciplines have begun to warm to the idea of using Bayesian approaches. In this respect, we cannotafford to be left behind. This is particularly true if we wish to (1) produce quantitatively informed research to inform policy and practice, and (2) make optimum use of the growing amount of administrative data that is collected at differentstages of the criminal justice system. The discipline has previously demonstrated that it is adept at borrowing from its neighbours, with criminologists having shown their willingness to utilise techniques from other disciplines such as data visualization techniques including geospatial applications to consider crime trends (Chainey and Thompson, 2008; Chainey and Radcliffe, 2005) and data linkage to explore re-offending (Ministry of Justice, 2014a). As such they have demonstrated that they can be responsive to the challenge facing all social scientists to 'Import, Introspect and Innovate in order to better answer the questions of interest in the field' (Bushway and Weisburd, 2006: 1). However, in neglecting to explore the potential that Bayesian approaches may bring to criminology, then I believe we risk undermining the progress made thus far as a discipline in gaining credibility and legitimacywhen itcomes to informing policydecisions. It is therefore vital that criminologists continue to be both innovative and introspective in their analytical approaches, importing newand novel ideas whereapplicable from others. 


\subsection{From the New Penology to the Advent of Digital Criminology}

The resurgence in the use of Bayes' rule in the 1980s and 1990s coincided not onlywith the dawn of the computer age, but also with the advent of actuarial justice - Feeley and Simon (1992; 1994) identified this emergence of new discourses, the formation of new objectives for the system and the deployment of new techniques as features of a 'new penology'. This shift marked a radicallydifferent orientation in the way in which crime was governed (known about and acted on), with 'the language of the new penology... anchored in the discourse of systems analysis and operations research. Itconceives crime as systemic phenomenon and crime policyas a problem of actuarial risk management' (Simon and Feeley, 2003: 78). Since this time there have been significantadvances in statistical applicationand the discipline has further evolved to meet the challenges and opportunities facing criminologyin the age of Big Data. Notably, there have been advances in terms of computational power which has both increased the accessibility of the mathematics required to model some of the more complex relationships that exist in real life data and technological advances which have led to the digitalization of administrative data on a near universal scale.

The drive for efficiency and increasingly managerialist approaches adopted by the New Labour Government (1997-2010) under the auspices of risk (T urnbull and Spence, 2011; Brownlee, 1998) has resulted in the exponential growth of 'routine' data being collected. Within criminology, this includes data which enables an individual's progress through the criminal justice system to be monitored; provides details of both workflows and the workforce available to do this, along with the number of crimes recorded and convictions secured. The adoption of case management systems and the use of standardised tools for monitoring and risk assessmentmeans that there is now a phenomenal amount of information about the criminal justice system available through government websites, from high level statistical trends to detailed (but typically anonymised) datasets. However, it is the data held by individual agencies that perhaps have the greatest potential to inform our understandings of the aetiology of crime and societys responses to offending behaviour since it is possible to link this individual level data to other sources including social survey data, victimisation and offending surveys, and administrative data around health, education, family life, employment and benefits. However, the utility of this data for contemporary criminologyis only now starting to be appreciated, with the creation of the Administrative Data Research Network in 2013 making it easier for approved researchers who want to use government data for their social or economic research to access anonymised versions of datasets.

Across the social sciences, ithas been suggested that there has been a digital turn, not just because of the increased digitalization of administrative data, but also the routine use of digital devices. Key developments such as the Internet and mobile technologies have created opportunities and challenges for criminologists, not least because of the emergence of new crime types which has in turn required legislative change and the employment of new, often technologicallybased responses. However, digital 
devices also enable researchers to collect, store and transmitnumerical, textual, aural and visual signals. Thus, the digital turn has provided both the resources and sources of information for criminologists to analyse using emergent computational techniques from the organisational sciences and research rooted in artificial intelligence and expertsystems. Such is the fundamental shift that this has promoted in the way in which research is now being undertaken in the social sciences and humanities has led commentators such as Kitchin (2014) to suggest that Big Data has created new epistemological approaches for making sense of the world and paradigm shifts.

Echoing developments in other social sciences and humanities, Smith et al. suggests that digital criminology'concerns itselfconceptually, methodologicallyand empiricallywith the task of understanding how digital devices/data are mediating experiences, impressions and processes of crime/crime control in familiar and strange ways' (2017:263). Whilst Chan and BennettMoses (2016) predict that the advent of 'Big Data' and machine learning algorithms will transform how criminologists work and think, I believe that in the context of seeking to predict future offending behaviour, we cannot overlook the complexity of human behaviour and social relations. As a result, whilst it may be appropriate in some areas of criminology to 'mine' data looking for new insights, caution needs to be applied before abandoning theory and prior understandings of recidivist behaviour in favour of a seemingly 'neutral' algorithms to aid criminal justice decision making. This is not to say that it may not be necessary to revise our views as a result of new evidence, but in a field where there is already concern about the creation of artefactual risk factors and an over-reliance upon correlations rather than causality (O'Mahony, 2009) enabling data to speak for itself, free from theory is too great a risk.

Much has been made of the potential for machine learning algorithms to generate Minority Report style models which would enable law enforcement to predictand punish crimes before they happen (see for example Mayer-Schibverger and Cukier, 2013). However, since these are still dependent upon the quality of the underlying data, if this has inherent biases then the resulting model will also have these biases (Issac and Dixon, 2017). For example, it has been demonstrated that the Correctional Offender ManagementProfiling for Alternative Sanctions (Compas) used by the a US court for risk assessment, is biased against black prisoners (Buranyi, 2017) whilst PredPol, a program used by predominatelyUS police departments, butalso Kent Police (O'Donoghue, 2016) to predict crime hotspots has been shown to get stuck in a feedback loop of over-policing in communities which are predominately black and/or brown (Lum and Isaac, 2016; Robinson and Koepke, 2016). This has implications for civil rights since equating locations with criminalityam plifies problematic policing patterns (Shapiro, 2017:458) Although there are instances where artificial intelligence has "successfully" predicted the outcomes of trials (see for example Johnson, 2016 in relation to verdicts at the European Court of Human Rights), there are calls for police agencies, software firms and the public to become more aware of the limitations of using machine learning techniques that rely on historical crime data since they risk fuelling a cycle of distorted enforcement(Robinson and Koepke, 2016). 
O'Neil (2017) advocates, that data integrity checks are required so that we do not become overlyreliant upon blindly applying algorithms which maintain the status quo. The distinct advantage of utilising Bayesian approaches with their ability to update models as new information becomes available, is that unlike machine learning, there is scope to incorporate both theory - through to the use of priors - and data from other sources (Berry, 2005). In the context of predicting offending behaviour this includes utilising narrative information held within an individual offender's case historyand practitioner judgement (Deandrea et al., 2014), and accepting that there will be uncertainty within any model. Doing this enables the 'big picture' to be seen and helps counter concerns that relying upon administrative data to inform public policyprovides only a perspective of crime viewed through the lens of the criminal justice system itself (McVie, 2016).

The stance taken by this research is that the advent of Big Data and digital criminology represents an opportunity for criminologists since itprovides it offers the empirical flexibility to probe properly theorised lines of inquiry. Echoing the views of Langton and Bannister (2017), it is suggested that what Big Data does is enable criminologists to 'slice, dice and splice' datasets in multiple ways in order to advance the understanding of causal mechanisms.

Alongside the emergence of new data has come new techniques delivered through progress in computing and data science. This was apparent in the 1980s and 1990s when the increased computational power revolutionised the way in which researchers be it in the environment, economics, health, education or social sciences began to look at their data. Then it was necessary to respond to the curse of high-dimensionality' (McGrayne, 2011) with computers generating a multivariate revolution and spawning a plague of unknowns - the need to analyse more than more than one unknown ata time, and to calculate the relationship between multiple variables and ascertain their impact on each other. This proved to be a challenge for both Frequentist and Bayesian statisticians, but presented an opportunity to learn from those in business schools and theoretical economics who had been utilising Bayes' rule to aid decision making under extreme uncertaintyand in the absence of sample data. The digital turn has similarly brought with it new techniques. Machine learning is possiblya step too far at this stage due to inherentbiases in criminal justice data which currently limitits potential for predicting future offending behaviour, but this does not mean that as a discipline we should not seek to explore the possibilities that the advent of digital criminologyaffords us. It is therefore important that we continue to 'adapt the criminological gaze and imagination' so as not to 'impinge of the quality of the contribution [that] criminology can make to crime and justice processes' (Smith et al., 2017: 264). As part of the epistemological shift that Big Data and digital criminologyhas broughtabout, it is the contention of this research thatnow is an opportune time to expand the boundaries of contemporary criminological theory and research by exploring the utility of adopting Bayesian approaches within the discipline. 


\subsection{Doing More with Less: The Increased Use of Administrative Data}

With increasing demands on an alreadystretched public purse, the focus has become one of doing more with less. As highlighted in the previous section, attention has turned to how to optimise the use of what has become a rich portfolio of data resources with the ESRC identifying as part of the SecondaryData Analysis Initiative that these are ripe for delivering high-quality and innovative research, generating knowledge exchange and policyand practitioner impact (Economic and Social Research Council, 2016). Whilst the volumes associated with 'Big Data' may address the issue of sample size, this emphasis on administrative data and re-using survey data is once again taking the discipline in a new direction and requires the researcher to adapt and learn new skills. It is therefore timely to once again be introspect and innovative, to look to import ideas and techniques from aligned disciplines such as public administration. If we are to do this then we need to bear in mind the observation from Gill and Witko (2013: 457) that public administration research 'generally uses data incompatible with standard Frequentist statistical thinking because theyare usuallypopulation measures that can never be repeated as if in a standard experimental setting'.

\section{Overcoming Methodological Challenges}

The nature of the data utilised within criminologyincludes collections arising from the use of large-scale survey methods to capture snapshots of criminal activity and the victim experience of crime; the results of experiments and evaluations, and increasinglythe systematic collation of data around criminal justice processes/ outcomes. As budgets for research have been scaled back and the challenge of obtaining ethical approval for surveys with vulnerable people has intensified, fewer large-scale surveys are being conducted. The flip side of this is that there is increasing emphasis on using administrative data which is systematicallycollected for monitoring purposes to explore many of key policyissues.

Criminology's growing statistical evidence base is particularly amenable to the application of Bayesian approaches since administrative datasets often suffer from issues relating to collinearity - they typically contain large numbers of variables that can be causallyrelated, with manyalso being non-stochastici.e. the data is generated as a population rather than from a repeatable known probability process. Whilst there may be missing cases or missing values, under the Bayesian framework, these cases are considered to be a cause of bias rather than being a consequence of random sampling variation, an issue which can be addressed through judgement but not through significance testing. These characteristics create conceptual problems for traditional statistical inference:

'First, the data are not generated by probability sampling or random assignment Second ... an apparent population is the result of a data generation mechanism that produces only a single batch of data. In effect the machinery is turned off after a single batch is produced; the data generation mechanism cannot be expected to produce another dataset.'

(Berk et al., 1995: 422) 
In the quest for more reliable evidence of cause and effect along with a means to evaluate the effectiveness of interventions, experimental criminologists have promoted the use of randomisation as the 'best method for drawing causal inferences between treatment programmes and their outcomes' (Weisburd et al., 2007: 3). In exalting the virtues of techniques such as RTCs, it is inferred that nonrandomised studies have less internal validity and that it is much more difficult to control for both measures and unmeasured factors or influences. However, it is worth noting that since Bayesian probability models are derived from subjective judgement, and hence do not require any underlying physical justification for a randomisation mechanism, the latter requirement is irrelevant. Thus, conducting experiments under the Bayesian framework negates issues around the power of the experimentand there is less emphasis upon achieving minimum sample sizes to detect effects. In this respect, Bayesian approaches are considered to be more efficient especially since sample size has become something of an obsession as researchers seek to demonstrate that their findings are empiricallysound:

'a claim is commonlymade that in some ways researchin the social sciences is harder than in the natural sciences because the cases are more variable and less inherently predictable ... In order to make believable claims, social science research would therefore need a larger number of cases than used in other area of investigation.'

(Gorard, 2014a: 50)

Under the Bayesian framework with concerns about randomisation are negated, there is also more scope to undertake sub-group analysis it is possible to do this withoutbeing constrained by minimum sample sizes. This is particularlyadvantageous when looking at rare events since small datasets can be more effectively handled due to the incorporation of prior information in the estimation. That is not say that Bayesian approaches are perfect. Outside of criminologythe difficulties of determining rates for different subgroups using both Frequentist and Bayesian approaches represents a challenge for statisticians wishing to demonstrate the effectiveness of interventions:

'Modern medical science is poorly equipped for identifying characteristics of patients who benefitfrom particular therapies, in large part because of the rigor of frequentist methods. In particular, the frequentist approach is not very good at discriminating subsets of patients who benefit, but many Bayesian methods are "too good" in the sense that they are overfitting. Perhaps a spirit of ecumenism in which Bayesians and frequentists learn from each other will be necessary to begin to crack this knotting but criticallyimportant problem.'

(Berry, 2006:429) 


\subsection{The Availability of Administrative Data}

With the advent of the increased digitalisation, there has been growing recognition of the research potential of administrative data and its ability to inform policy and practice around a range of social, environmental, health and security issues. In 2012, the Cabinet Office published The Open Data White Paper which suggested that '.. data is the $21^{\text {st }}$ century's new raw material' (2012:5). The richness of the largely untapped sources was further acknowledged by the Administrative Data Taskforce who subsequently published a series of recommendations to address the challenges which needed to be overcome if the UK is to become a world leader in research using de-identifiedadministrative data. They found that:

'... access to and use of such data for research purposes in the UK has been difficult, due mainly to the concerns that data holders have had about the possibility that information that identifies individuals could enter the public doma in or because of legal restrictions they face on the uses to which such data can be put.'

(Administrative Data Taskforce, 2012:iii)

A key driving force in moving this agenda forward has been that the data is relatively inexpensive to exploit, compared to the costs of establishing specially commissioned surveys additionally in using routinely collected data, it is perceived that it is more efficient since research findings can be generated quicker. This argumentechoes the views held around secondarydata analysis - that this is a 'relatively quickmethod of research as someone else has already been through the more time consuming job of collecting the data' (Rowlingson, 2004: 139). However, it is not without its limitations as:

'.. the available data do not always perfectly fit the secondary analysts' research question for a number of reasons. Perhaps the population is slightly different. Or perhaps the sample is not large enough to enable certain types of subgroup analysis. Or perhaps some key questions were notasked, or at least were not asked in exactly the way the secondaryanalyst would have liked.'

(Rowlingson, 2004:140)

In the context of British research, it is further acknowledged that we lag behind other jurisdictions (such as the Nordic countries) in our abilities to make optimum use of administrative data due to the lack of unique identifiers with which to routinely link data and the fact that we generally do not seek people's permission to do this (McVie, 2016). This limits our ability to make joined up public policy decision making.

As will be discussed in greater depth in Chapter Three, the administrative data being utilised for this research is drawn from the case management system at a local Youth Offending Team. As such the process of linking multiple sources of information has already been undertaken by members of the administrative staff and practitioners at the Youth Offending Team (YOT) who are familiar with the individuals referred to the service. Hence ithas been possible to create a de-identified linked database containing the young person's risk assessment scores, key socio-demographic characteristics, offending 
and courtrecords. Access to this data has been secured though the Western Bay YOT Managerwho is also the Data Controller. A number of conditions were imposed to protect the individuals whom the data relates to and ensure adherence to both the principles of the Data Protection Act 1998 and also management of information advice set out in Advice on Information Management in Youth Offending Teams (Youth Justice Board, 2011). This includes making provision for the safe storage of any data considered to be personal or sensitive. In order to adhere with these requirements, data has been extracted from Childview - the case management system by the YOT's Information Officer. Working versions of the dataset have been prepared within the secure environment of the YOT office with all identifying information being stripped out and replaced bya unique research ID. The lookup for this has been retained on the YOT's server.

\section{The Application of Bayesian Approaches to Administrative Data}

Gill and Meier (2000) have previously argued that public administration researchers should embrace Bayesian approaches, an argument which has taken on renewed importance in recent years as the volumes of data collected for administrative purposes continue to grow exponentially. Their augments apply equally to criminology where examples can also be found of data representing 'apparent populations' (Berk et al., 1995) - collections which usuallydescribe an entire set of objects of interest, providing details of fluid events. Such one-time events are often situational in time and circumstance and hence why they can never be replicated. That is, we cannot go back and re-survey and 'assume that no attitudes, experiences or administrative events have changed. Thus our datasets represent a fixed, unique look at the phenomenon of interes' (Gill and Witko, 2013). In this instance, since all young people who come into conflict with the law are referred to their local YOT, the administrative data available for this research consists of a rich series of datasets of the 'apparent population' rather than being a sample.

As part of their rationale as to why Bayesian approaches should be adopted in public administration research, Wagner and Gill (2005) highlightan issue which commonlyoccurs within administrative data which reinforces the need to consider adopting alternative approaches:

'Public administration research often suffers from issues relating to collinearity, since scholars regularly obtain datasets with a large number of variables that can be causallyrelated...T his cancreate difficulties in a linear model as collinear explanatory variables carry little independent information, and the least squares estimator does not then provide a means to distinguish one co-efficientfrom another.'

(Wagner and Gill, 2005:3) 
Whereas, in medical epidemiology researchers are very aware that confounding variables, biases and weak measures can lead to the discovery of unreal risk factors, O'Mahony(2009) argues that there is a tendency within RFR to ignore the question of effect sizes as long as statistical significance has been established, and to avoid testing the causal potency of apparent risk factors. This, he maintains had promoted the production of artefactual risk factors.

A key benefit of utilising administrative data to advance understandings of causal mechanisms is that typically within case management systems, when new information is added, it is 'date stamped'. This enables temporal precedence to be established. 


\section{Introducing the Case Study}

\subsection{Context}

In selecting risk assessment in the youth justice system in England and Wales as a case study, it is recognised that the quality of the evidence base that underpins this process has been widely criticised and hence this research also provides an opportunity to extend knowledge and understanding around the relationship between youth offending and the framework of risk and protective factors. This chapter therefore sets out the strengths and limitations of risk assessment processes. This is done with a particular emphasis upon conceptual and methodological criticisms since this is where there is greatest scope to advancement through the use of alternative probabilistic methods.

\section{Responding to Calls for a Post-Positivist Approach}

Drawing upon complexity theory, Case and Haines present an argument in which they posit that 'the crude and imprecise measurement of risk in youth justice processes has fed into insensitive analyses and produced invalid conclusions that risk factors exerta linear, proportionate and deterministic influence on offending behaviour by young people' (2014: 132). Since the validity of research outcomes and conclusions are inherently linked to the tools of measurement and analytical approach utilised in research, they assert that 'using an imprecise and insensitive measurement tool and plugging measurements uncritically into statistical analyses results in alchemy. crude, invalid and artefactual results and conclusions thatare distanced from individual and social realities' (2014:133). Goldson and Muncie whilstmaking their case for youth justice with integrity, similarlyargue that 'the social world and the processes of youth justice formation are far more complex than oversimplified evidence-based and what works discourses often imply'. They make the charge that 'the positivist assumption that quasiscientific laws and rational prediction are not only possible and desirable, but also essential, for modernising youth governance is flawed' (2006: 98).

These arguments advance the limitations summarised in Chapter One around the use of NHST, calling for post-positivist statistical analyses. Whilst Case and Haines (2014) do notprovide any details of what this mightlook like, they advocate as promising approaches such techniques as Bayesian analysis and data visualisation.

Achievement of the research aim i.e. to demonstrate the utility of Bayesian approaches to risk assessment processes within youth justice, is not only in keeping with the ASA statement, but also with the suggestions made by Barnes et al. (2017) for criminologygenerallyand those made by critics such as Case and Haines (2014) with respect to risk assessment within youth justice in England and Wales. Thus, the choice of case study demonstrates the motivation to additionally further knowledge and 
understanding through exploration of the relationship between reoffending and the paradigm of riskand protective factors:

'A different or broader methodological profile in a scientific field often leads to new and varied insights. Moreover, because any methodology or tool associated with probability and statistics is created rather than found, different and broader approaches can be formulated at any time if the will to do so exists'.

(McKee and Miller, 2015:473-474)

\section{Transferability}

In the context of the case study chosen, it is the successes in medicine that are the most encouraging with Bayesian statistics having now permeated all the major areas of medical statistics including clinical trials; epidemiology; meta-analysis and evidence synthesis; spatial modelling; longitudinal modelling; survival modelling; modular genetics and decision making in respect to new technologies. The quasimedical nature of the risk factor prevention framework which underpins the risk assessment tool bodes well conceptuallyfor the application such statistics to youth justice and criminologymore generally.

Particularly in the context of analysing multiple risk factors, use of Bayesian inference in medicine has been used to demonstrate the strength of links between exposure and disease - a key diagnostic feature. As the medical profession have demonstrated, having the correct diagnosis means that an appropriate treatment plan can be developed which is tailored to the individual and their circumstances. In principle, this is what happens in youth justice as well. The problem is however, that in youth justice, 'the science is ... not always as scientific as we would like and in fact substantial problems can exist with the method used to identify risk facts in that quantitative variables are, in fact, constructs of social phenomenon' (France, 2008: 4). In presenting subjective processes as objective and scientific, there is an oversimplification of the potentially complex and dynamic aspects of children's lives, experiences, perceptions and thoughts into readily quantifiable and targetable risk 'factors'. It is these issues along with those associated with the evidence base which are summarised in this Chapter.

\section{The Rise of Risk Orientated Thinking}

The development of a standard risk assessment tool for use with young people who have offended did not occur in isolation. Rather it reflects the growing momentum with which policy and practice has become increasingly focused on risk (Turnbull and Spence, 2011). Indeed Kemshall et al. (1997) suggested that 'risk assessment, risk management, the monitoring of risk and risk-taking itself were rapidly becoming the dominant raison d'etre' for personal social services including probation. Specifically, they observed that:

'Notions of risk are increasinglybecoming embedded in organizational rationales and procedures for both the services and relationships with users and clients. Similarly, 
estimations about risk have become key in identifying priorities and making judgements about the quality of performance and what should the central focus of professional activities. Notions of risk have taken on a strategic significance for rationing services and holding professionals and others accountable in a changing political and economic context where potential need and demand is increasing but where there are insufficientresources.'

(Kemshall etal., 1997:214)

Twenty years later risk orientated thinking is now embedded in almost all aspects of mainstream criminology (Walklate and Mythen, 2011). For example:

- In the prison system the risk of re-offending has to be determined before decisions can be made about releasing offenders, particularly for those who commit serious violent and sex offences who may be subject to an indeterminate sentence (Robinson, 2002; Buchanan and Grounds, 2011).

- In the criminal justice system, the police need to weigh up the chances of suspects absconding / failing to attend court; potential further offending or hindering the investigation in some way e.g. interfering with witnesses or evidence, whilst on bail (Dhami, 2005; Ofili, 2014).

- Associated with fear of crime, there are often individual concerns about the likelihood of becoming a victim of crime which can impact on perceptions of personal safety and security. These are often out of kilter with reality (Gray et al., 2011).

Although some of these decisions are madeessentiallyupon the basis of individual judgementcalls, the criminal justice system has becoming increasinglyreliant upon standardised actuarial risk assessment tools, not just when considering sentencing and release, butalso to make decisions around assignment to treatment. Having moved on from the prediction tables and first-generation risk assessment tools which were predominately unstructured professional judgements of the probability of offending behaviour, many of the second and third-generation risk assessment tools which have emerged largely since the 1990s, are grounded in the statistical association between risk and repeat offending (Schwalbe, 2007). Whereas second-generation tools were limited to prediction and classification, thirdgeneration tools are characterised by their predictive role in informing intervention planning in addition to their classification role. Reflecting their respective roles as well as emerging knowledge about predictor variables and 'What Works', the focus of their content differs - the development of secondgeneration tools emphasized the classification of risk of recidivism irrespective of their content. Hence, they tended to be dominated by static risk factors like offence history. Whilst the dual focus of thirdgeneration tools has meant that they usually consist of an array of dynamic risk factors which it may be possible to change as a result of intervention.

Third-generation tools are also required to gather information about criminogenic needs and responsiveness. The so-called fourth generation-tools take this approach one stage further, actively gathering information to facilitate planning, case management, supervision and service delivery 
(Vaswani and Merone, 2014). A major goal of the fourth-generation instruments is 'to strengthen adherence with the principles of effective treatment and to facilitate clinical supervision devoted to enhance public protection from recidivism crime' (Andrews et al., 2006: 8). Thus '[c]urrent risk assessment practices are promoted on the basis of being able to provide an objective, impartial and rational decision-making process, reduce reoffending and increase public protection' (Lewis, 2014:122). However, as this chapter will highlight, they have their limitations. It is both the design features and limitations of these tools, specifically the standardised tool used until recently within the youth justice system in England and Wales, which make this an ideal case study to demonstrate the utility of applying Bayesian approaches.

\section{The Political Motivation for Commissioning a Standardised Risk Assessment Tool}

Risk now appears across a range of social domains, such as health, welfare, crime, national security and the environment. With this, the terms risk and youth have become synonymous - a view that has become progressively more pervasive in the media and within policy responses. Young people have increasinglybeen perceived as being either 'atrisk' or as 'posing a risk' (Armstrong, 2004; 2006). They are seen 'both as a treasured resource and as endangered and dangerous - at risk from others, to themselves, and to the fabric of communities' (Sharland, 2005:36-37).

The problem of youth was played out in the media throughout the 1990s and 2000s. Panics about joyriding, alcopops, Ecstasy, girl gangs and persistent offenders predominated in the 1990s, to be joined by 'hoodies', 'boy racers', 'mini-moto riders', 'happy slappers', 'video-gamers', 'under-age binge drinkers', and 'feral yobs' the following decade (Muncie, 2009). In fuelling public concern, the media helped to perpetuate the notion of children as evil, a view that then became enshrined in legislation.

As Sharland (2005) observes, politicians and policy makers have become concerned with how best to preventing young people from taking or being exposed to risk, from becoming sociallyexcluded, deviant, unhealthy or unproductive. Care leavers, teenage parents, young homeless, addicts or those with mental health problems have been identified as being discrete populations of children at risk who have tended to require a more welfare orientated approach. Whilstpolicyhas sought to control those seen as being troublesome rather than troubled, at risk of offending or simply being offensive. There has however, within the risk rhetoric, been a blurring of these distinctions, with Goldson (2000; 2002) suggesting that concerns for the former have been subsumed by the 'need' to control.

The Crime and Disorder Act, 1998 restructured the delivery of youth justice in England and Wales, redefining its key purpose as preventing re-offending. Central to this were two concerns: the risk that involvementin the criminal justice system poses to young people's future and the accepted wisdom that young people grow out of crime (Phoenix, 2009b). At a policylevel, notions of young people's needs began to be interpreted almostexclusivelyas 'criminogenic need' or 'risk of re-offending' promoting 'risk', 
its assessmentand management to begin to dominate the 'new youth justice' (Gray, 2005; Armstrong, 2004). Represented as being 'the most radical shake-up of youth justice in 30 years' by then Home Secretary, Jack Straw (quoted in Pitts, 2005: 8), the Act introduced:

'... fully-funded multi-agency groupings dedicated to work with young people who offend; the youth offending teams (YOTs); and the creation of a centralised, governmental body, the Youth Justice Board for England and Wales, charged with the realisation of an accountable, youth justice system and with the power and reach to deal with the problem of justice by geography, address the inequitable distribution of resources, and to effect a thoroughgoing reform of custodial institutions for children and young people'.

(Bateman and Pitts, 2005: xvi)

The various other measures introduced by the Act provided for earlier and more intensive intervention in the lives of children and young people (Brown, 2005). In a climate where itwas considered desirable to identify at an early age those considered to be at risk of future anti-social and offending behaviour, the policy emphasis was very much on the management and control of troublesome young people (Armstrong, 2006). To do this, it becamenecessaryto identify those factors that make someone 'at risk' and for the development of a consistent approach for the youth justice system in England and Wales towards risk assessment.

\section{Requirements for the Standardised Tool}

Keen to promote consistency of practice within the new multi-disciplinary YOT settings, and to encourage practitioners to target interventions at the factors identified as being most closely associated with offending by young people, the Youth Justice Board set out a specification (in December 1998) for the development of a standard assessment profile to be used by the newly created YOTs. According to Baker et al. (2003), the key requirements for the tool were that it should:

- identify the key factors contributing to offending by young people

- provide a prediction of reconviction

- help to identify young people who may present a risk of serious harm to others

- identify situations in which a young offender is vulnerable to being harmed

- identify issues where more in-depth assessmentis required.

There was also an expectation that the profile would be a 'live' document that would inform plans for working with young people (in both community and custodial settings). As such, it would be used to measure change over time when reapplied during, or at the end of, interventions. In addition to assisting in the collection of aggregate data, the Youth Justice Board (YJB) stated that its mostimportantfunction would be 'to help YOTs to assess the needs of young people and the degree of risk they pose and then to match intervention programmes to their assessed need' (Youth Justice Board, 2000 cited in Baker et al., 2003). 
The tender to design and produce the new assessment profile for the YJB was won by The Centre for Criminological Research (University of Oxford). Supported by an advisory panel consisting of representatives from YOTs, the secure estate, the Department of Health, the Departmentfor Education and Skills, the Drugs Prevention Advisory Service, the magistracyand the police, the Centre designed ASSET. A tool which they felt incorporated and reflected a wide range of perspectives on the risks and needs of young people who offend.

ASSET, has in various forms, been the standardised risk assessment tool used across the youth justice system since YOT s came into being in 2000. However, as will be described in later sections, ithas been subject to much criticism from practitioners and academics. It is this criticism, especially that focusing upon its reliance upon RFR and the methodological limitations of the evidence base which make it a particularlyattractive case study for demonstrating the utility of Bayesian approaches.

In making this choice, it is acknowledged that ASSET has now largely been replaced by ASSET Plus. This fourth-generation tool was designed in response to the growing criticism and to address concems around ASSET's usefulness and validity in the context of broader developments across services for children and young people, and the justice system itself. Atthough work to develop ASSETPlus began in 2010 , its rollout across the YOTs and the secure estate has been beset by problems. It first went operational in October 2015, with the first Welsh YOTs getting ASSETPlus the following month. However, the deploymentdid not occur in Western Bay YOT - the YOT from which the data has been drawn from for this research, until April 2016. Given the emphasis upon predicting re-offending - a measure which can take up to 18 months to calculate, the timing of this research means that it was necessary to take this into account when selecting which data to utilise (see Chapter Three for details of the data specification). The roll out of ASSETPlus across the various English and Welsh YOTs was completed at the end of August 2017 (Youth Justice Board, 2017a) having taken almost2 years.

In order to understand the various criticisms made aboutASSET, it is important to recognise that many of these are not unique to youth justice. Whilst predictions of future offending are now an integral part of the criminaljustice decision making process with ideas being incorporated from other disciplines, there are a number of key methodological limitations associated with the use of actuarial tools which undermine the assumption that being able to predict future criminality will reduce crime: firstly, the predictive accuracy of risk assessment tools; secondly, their ability to predictindividual rather than group behaviour; and thirdly, the problem of predicting different types of offending. These limitations are considered within the remainder of this chapter firstly in the context of tools developed predominately for use with adults, before focusing more specificallyupon those developed for young people and the role of risk factor research as the evidence base underpinning tools such as ASSET. 


\subsection{Probabilistic Decision Making in the Criminal Justice System}

\section{Predictive Approaches}

Farrington and Tarling (1985) identifythe following applications of predictive methods in criminology:

- The prediction of rates e.g. crime and imprisonmentrates

- Parole and the evaluation of penal treatments

- Selective Incapacitation

- Dangerousness

- Delinquency

Whilst this list was drawn up more than thirty years ago, it provides a convenient starting point for considering issues faced bythe contem porarycriminal justice system in England and Wales. In outlining the development of actuarial tools in different settings, the intention is to provide contextfor discussions later within this chapter around how the use of Bayesian approaches have the potential to address some of the limitations of the risk assessment tools developed in relation to youth offending.

While the development of mathematical models to predictcrime and imprisonment rates in the event of significant population, policy or legislative changes, fall largely outside the scope of the 'new penology' (Feeley and Simon, 1992; 1994), the other examples given reflect the shift in the direction of criminal justice. Earlier discourses of clinical diagnosis and retributive judgementbegan to be replaced with 'an actuarial language of probabilistic calculations and statistical distributions applied to populations' (1992: 452). Notably the emphasis switches from reforming the individual offender to consider aggregated groups such as "high-rate offenders" and "career criminals". These groups, along with other categories were defined by actuarial classifications. As part of this evolution, it is argued that the criminal justice system has become more concerned with managerial process with its goal no longer being to eliminate crime, butthe identification and management of unruly groups through systemic coordination. This has been achieved through the deployment of new techniques such as statistical applications for assessing risk and predicting dangerousness.

\section{Parole and the Evaluation of Penal Treatments}

Farrington and Tarling (1985) believe that the greatestimpacton policyand practice as a result of using predictive methods has been with regard to parole prediction. Whilst they provide details of a number of pieces of research which have influenced practice in both the USA and UK, it is the Burgess-type prediction devices which formed 'the core of actuarial parole prediction' (Kemshall, 1998:45) until fairly recently. Under these, individuals are given a score based on binary responses to a series of predictor variables depending on whether the parole violation rate for the individual was greater than or less than the average for persons in that category, 
Burgess' methodology, developed in the early part of the twentieth centuryand replicated in subsequent studies, takes the form of a composite predictor, presented as an experience table which divides the sample into different risk groups with different probabilities of offending. Underpinning this is the assumption that a composite variable will predict the criterion more accurately than a single predictor. An English prediction score was constructed during the 1970s using sixteen variables covering offender's previous criminal history (type of offense committed; number of previous convictions and prison sentences; interval at risk since the last conviction; age atfirst convic tion) plus the offender's age, marital status, living arrangements and employmenthistory.

Work on the development of an actuarial reconviction predictor started in the 1990 s, with the statistical analysis of the criminal records of 13,711 offenders being used to create the first national reconviction predictor for use by the Probation Service in England and Wales. Widely used, this second-generation tool was similarly based on static factors (such as sex, age and previous criminal history) but since these factors could not be changed, it failed to address issues which would enable correctional agencies to assess need or to plan or evaluate supervision. Various initiatives were sought to fill this gap, with work on OASys (a fourth-generation risk assessment system, linking assessment and case management (Lewis, 2014)) beginning in 2001. By 2006, this had become the standard system used by Probation throughoutEngland and Wales, aiming to 'produce an assessment of dangerousness or risk of harm in addition to a risk score for reconviction' (Raynor, 2016:31).

Evaluations of differentpenal treatments in the UK contexthave typically been linked to the 'What Works' agenda. Measuring the difference in outcomes for those in treatment and controls, these have rarely considered the mechanism which has broughtabout the change in behaviours, attracting criticism from realist criminologists such as Pawson and Tilly(2000). As such when applied in different geographies and with different types of offenders, initiatives have often had limited success. Where evaluations are being undertake of different penal treatments, propensityscores are often used to match control and test groups as an alternative to random assignment.

\section{Selective Incapacitation}

Interest in methods relating to the assignment of a lengthy sentence or other freedom-restricting penalties to repeat offenders grew in response to concerns that rehabilitation as a penal aim was not being achieved by existing treatments. The research was primarily focused on estimating the number of crimes prevented by mandatorysentence of incarceration for certain categories of detected offenders and depended on having detailed knowledge about criminal careers. Examples include Greenwood's proposed method of predicting which offenders committed offences athigh rates whilst they were in the community. 
In considering 'incapacitation effects' i.e. those crimes prevented while offenders are incarcerated, Greenwood's proposals were based a US survey of incarcerated males, with seven binary variables being selected to make up an additive predictive scale:

1. 'Incarcerated more than half of the two-year period preceding the most recent arrest

2. A prior conviction for the crime type that is being predicted

3. Juvenile conviction up to age 16

4. Commitment to a state or federal juvenile facility

5. Heroin or barbiturate use in the two-year period preceding the currentarrest

6. Heroin or barbiturate use as a juvenile

7. Employed less than half of the two-year period preceding the currentarrest'

(Greenwood, 1982: xv-xvi)

By identifying high-rate offenders, selective incapacitation policies were intended to target the most prolific offenders and design sentencing policies to incarcerate them during their most crime-prone years. During the 1990s, the reach was extended with the passing of 'three strikes' rules firstly in a number of US states before being adopted in the UK.

Reflecting the nature of the types of offences which fall under the definition of being 'grave crimes', there are two areas where risk assessment tools have been developed in the context of dangerous offenders - those assessing the risk of violence and those for sexual offenders. These will often rely more upon clinical prediction, utilizing 'the knowledge base, experience and expertise of professionals to make sense of someone who has been violent, and may include the use of actuarial or otherwise structured assessment protocols to inform the overall decision making' (Milner and Myers, 2007: 29). In the UK contexts, the International Classification of Disease (ICD-10) rather than the Diagnostic and Statistical Manual of Mental Disorders - Fifth Edition (DSM-5) tends to be used to provide the formal diagnosis required within psychologicalassessments of offenders presented to criminal courts prior to sentencing.

Douglas et al. (2016) suggest that there are currently more than 200 structured tools available for assessing the risk of violence in forensic psychiatry and criminal justice settings. Actuarial instruments (as opposed to structured clinical judgement tools) include the Violence Risk Assessment Guide(VRAG) (Quinsey et al., 2006) for serious violence prediction and the Static-99 (Phenix et al., 2016) which is used with sexual offenders. Widely accepted as the definitive resource in assessing psychopathic personality disorders, the Hare Psychopathy Check List - Revised (PCL-R) is also commonly used in research on criminal offenders andforensic psychiatric patients (Hare, 2003). Notablyin the UK context, such risk assessment tools form part of a wider clinical assessment process with risk assessment tools being 'used to roughly classify individuals at the group level, and not to safely determine criminal prognosis in an individual case' (Fazel et al., 2012). 
Criticism of risk assessment within psychiatric literature has focused on the possibilitythat, in deploying risk assessment tools to realise justice or public protection, mental health professionals mayfail to fulfil their professional obligations to their patients (Douglas et al., 2016). There are also considerable concerns around the predictive accuracy of actuarial tools, particularlywhere linked to post-sentencing detention and parole decisions. Where the probability of dangerousness is incorrectly identified, this could lead to low risk offenders being placed on lengthy treatment programmes or subject to prolonged detention in custody. Conversely high-risk offenders may be released prematurely, and there is a high probability of further offending. The implications of false positives and false negatives can therefore be significant. Hence an important element of the use of actuarial tools is that the rationale behind the decision is defensible, adding a layer of transparencywhich was not always afforded when using nonstructured processes.

In the context of sexual offending where offending is relatively rare (i.e. a low base rate), the possibility of false positives is greater (Tully et al., 2013) and there is wide variability associated complex and multifactorial nature of this type of crime (Borum, 1996; Neller and Petris, 2013). Evidence suggests that within the sex offender population, there are differences based on the nature of the offence committed e.g. contact and non-contact, child sexual abusers and rapists, and between highly deviant and low deviant men. However, Craig et al. (2003) highlight that little is known about the differences between late- and early-onset offenders and between those who successfully complete treatment and those who drop out or complete treatment with little or no evidence of cognitive shift. The difficulties in predicting such behaviour is further complicated bythe fact that many sex offenders engage in criminal behaviour that is not limited to deviant sexual activity. Criticisms of existing tools include question marks over their appropriateness in assessing sexual offenders with intellectual disabilities, from minorityethnic groups (Tullyet al., 2013) and female offenders (Abulafia et al., 2015).

Increasingly routine risk assessment protocols are required to address specific factors relating to dual diagnosis. Many individuals who enter and move through the criminal justice system are portrayed as being high risk as a result of having substance misuse issues and/or mental disorders. For the criminal justice system those individuals who experience serious substance misuse and mental disorders representa significantchallenge, often existing at the intersection with the health system (Rose, 2016). Not only does the multi-faceted nature of their risk necessitate a response from across multiple policy, legislative and organisational arenas, but the complexity of their lives and the rarity of the grave crimes committed by dangerous offenders poses a particular challenge for practitioners.

\section{Delinquency}

In 1950, the Gleucks developed a Social Prediction Table for Delinquency based on comparing 500 institutionalized boys and 500 unconvicted boys, matched on age, IQ, national origin and residence in underprivileged areas, against five factors. In determining a score for each boy, the percentage of those 
in his category who were 'delinquent' were summed to give a total which was subsequently used to discriminate between 'delinquents' and 'non-delinquents'. The Gleucks advocated that their prediction device should be used to identify potential 'delinquents' at the time of school entrance (age 6). However, inherent flaws in their work led to criminological prediction in general and in particular in predicting delinquencybeing discredited. Where work was undertaken to predictyouth offending after the 1950s, it was limited to questionnaire-based approaches using multivariate methods within psychology (Farrington and Tarling, 1985).

There has since been a resurgence of interest in predicting youth offending associated with developments in adult contexts which has broadlyfollowed the same generational pattern as outlined by Andrews et al. (2006). As such, the promise of contemporary risk assessment is dependent on their ability to accurately and reliably predict the classification of young offenders so that decisions can be made with levels and intensity of supervision as well as the nature of the treatment required. The principals of risk, need and responsiveness which underpin the tools state that:

'....in order to constitute effective practice, the intensity of the intervention needs to be matched to the young person's level of risk, the intervention should address the specific needs that are contributing to the risk level and the intervention needs to be responsive to the young person's circumstances, cultural requirements, learning style and developmental stage.'

(Vaswani and Merone, 2014:2)

Without an accurate assessment which adheres to these principals there is a danger of unintended and unwanted consequences such as increasing the offending of low risk offenders. These concerns echo those made with regard to adult offenders but take on greater meaning for young people due to the net widening tendencies of the various pieces of legislation introduced by New Labour. Through the interconnectiveness of their social exclusion agenda, risk factors emerged not just for crime but also social problems with marginalised and excluded communities being particularlytargeted. Attempts to manage intractable social ills led to the blurring crimeand social policies, and sanctions being introduced for antisocial behaviour (Kemshall, 2008a). As the strategy for tackling youth crime has evolved under subsequent Governments, there is also a risk that in times of austerity, that inappropriate decisions are made aboutindividual risk and need which place pressure on limited services or resources.

Current risk assessment tools consist of a mixture of instruments which have been adapted from the adultarena and those developed specificallyfor use with under 18 s. For example, the PCL-R has been adapted into the Hare Psychopathy Checklist: Youth Version (PCL:Y) for the assessment of psychopathic traits in male and female offenders aged 12 to 18. In contrast, ASSET was designed as a bespoke tool, drawing upon risk factor research. This has been the dominant paradigm for understanding and addressing youth offending for much of the last 20 years and is also a source of criticism. Notably whilst the pursuit of the risk factor prevention paradigm has proven to be attractive to politicians and others because it provides a framework for conceptualising the risk factors approach to 
researching the origins of youth offending and devising preventative strategies (and hence provides the basis for tools such as ASSET), it is quite distinct to the risk focused epistemological approach which is commonly found in medicine (Case and Haines, 2009).

\subsection{Risk Factor Research: The Evidence Underpinning ASSET Risk Factors for Youth Offending}

Risk factor research utilises the concept of risk factors (Hale et al., 2005: 392-393), with Farrington and West's work with the Cambridge Institute of Criminology dominating the field. Their research, a longitudinal study of 411 working class boys from the age of eight in 1961 has been particularlyinfluential in a UK context in shaping contem porary risk assessment tools. According to Farrington (1996), the major risk factors associated with youth crime are:

- Prenatal and perinatal: Early child-bearing increases the risks of such undesirable outcomes for children as low school attainment, antisocial behaviour, substance use and early sexual activity. An increased risk of offending among children of teenage mothers is associated with low income, poorhousing, absent fathers and poor child-rearing methods.

- Personality: Impulsiveness, hyperactivity, restlessness and limited ability to concentrate are associated with low attainment in school and a poor ability to foresee the consequences of offending.

- Intelligence and attainment: Low intelligence and poor performance in school, although important statistical predictors of offending, are difficult to disentangle from each other. One plausible explanation of the link between low intelligence and crime is its association with a poor ability to manipulate abstractconcepts and to appreciate the feelings of victims.

- Parental supenvision and discipline: Harsh or erratic parental discipline and cold or rejecting parental attitudes have been linked to offending behaviours and are associated with children's lack of internal inhibitions against offending. Physical abuse by parents has been associated with an increased risk of the children themselves becoming violent offenders in later life.

- Parental conflict and separation: Living in a home affected by separation or divorce is more strongly related to offending behaviours than when the disruption has beencaused bythe death of one parent. However, it may not be a 'broken home' that creates an increased risk of offending so much as the parental conflict that led to the separation.

- Socio-economic status: Social and economic deprivation are important predictors of antisocial behaviour and crime, but low family income and poor housing are better measurements than the prestige of parents' occupations.

- 'Delinquent' friends: 'Delinquents' tend to have 'delinquent' friends. Butit is not certain whether membership of a 'delinquent' peer group leads to offending or whether 'delinquents' simply 
gravitate towards each other's company (or both). Breaking up with 'delinquent' friends often coincides with desisting from crime.

- School Influences: The prevalence of offending by pupils varies widely between secondary schools. But it is not clearhow far schools themselves have an effect on offending behaviours (for example, by paying insufficientattention to bullying or providing too much punishmentand too little praise), or whether it is simply that troublesome children tend to go to high 'delinquency'-rate schools.

- Community influences: The risks of becoming criminallyinvolved are higher for young people raised in disorganised inner-city areas, characterised by physical deterioration, overcrowded households, publicly-subsidised renting and high residential mobility. It is not clear, however, whether this is due to a direct influence on children, or whether environmental stress causes familyadversities which in turn cause youth crime.

The realityhowever, is that as Brown highlights 'manythousands of factors may place young people 'at risk' of offending, including, at differentages, 'biological, individual, family, peer, school, neighbourhood, and situational factors' (2005: 100). However, this leads to problems as many children who are technically 'at risk' lead 'successful lives', thus there is also a need to understand factors which in many various combinations actin a protective way, mitigating risk.

\section{The Methodological Limitations of RFR}

Case and Haines (2009) provide a narrative of the origins and development of RFR, covering the role of key longitudinal and cross-sectional surveys have had in shaping our currentunderstanding of the riskreoffending relationship. In charting the chronological journeyfrom the pioneering work of the Glueck's in the 1930s through to the current day, they consider the contributions made by different theoretical perspectives highlighting RFR's developmental origins; the role of ecological, pathways and integra ted approaches of recent years, and the unfulfilled promise of the constructivist strand. Highlighting the significant underpinning of risk factor prevention paradigm (RFPP) provided by findings from the prospective longitudinal Cambridge Studyin Delinquent Development, they lament that:

'Even the more complex contemporary explanatory models have struggled to break the development shackles put in place by the Gleucks and the Cambridge Study. It is difficult to pinpoint an example of RFR that has not taken the developmental influence of psychosocial risk factors as its theoretical starting point and utilised factorisation and statistical analysis of risk as its methodological basis.'

(Case and Haines, 2009: 100-101) 
Dividing their critique of influential studies into longitudinal and cross-sectional designs, means that Case and Haines are able to provide an appraisal of their respective strengths and weaknesses as well as commentmore genericallyon issues that affect these types of studies. O'Mahony (2009: 110) extends the critique, highlighting 'how prominent proponents of the RFPP, like Farrington often appear to pay on perfunctory attention to the paradigm's inherentflaws and continue to oversell the approach or at least allow policymakers to do so'. Notably he points to a 'bizarre, oxymoronic admission' madeby Farrington about how 'typically, prospective prediction (based on the RFPP) is poor, but retrospective prediction is good' to question why it is that ASSET - a tool which by definition is designed to predict the future, is underpinned bythe findings from a prospective study.

Debates about prospective and respective studies aside, the key pertinent design feature is temporal sensitivity since being able to establish temporal precedence enables causality to be explored - whereas longitudinal designs track an individual over time, cross sectional designs are essentially snapshots although a time dimension can be added by having a repeated cross-sectional design and collecting data from different (but comparable) individuals. By default, cross sectional designs put the theory variables and their associations in static form whereas longitudinal designs enable change over to time to be measured and are able to consider dynamic measures. Potentially two importantcharacteristics of change can be captured by the longitudinal designs: (1) within-person change across time, or trajectories, and (2) inter-individual changes that can either be predicted or used for prediction (Ployhart and Vandenberg, 2010).

Through the objectification of the social sciences, positivists have strived to establish a causal connection between aspects of the social world in a bid to explain human behaviour. The positivist school of criminology introduced the problem of causality into criminological thinking applying methods from the natural sciences. The resulthas been a focus on searching for the causes of criminal behaviour which has assumed that this behaviour is predictable and dete rmined. Whilst some aspects of the theory have fallen out of favour, the legacy of others remains within RFR. Notably RFR has tended to rely on demonstrating causality through statistical relationships and as yet there has been little emphasis placed on explaining how risk is related to offending, or what processes link the two:

'Finding out who mightbe prone to offending is onlya first tentative step in the process of answering much more crucial questions abouthow and why people actuallycome to offend. This easily made elision between correlate and cause characterizes both the RFPP and the risk-focused research literatures and generally serves to obscure rather than clarify the complex issue of causation' 
If we return to the rationale behind third-and fourth-generation risk assessment tools, it becomes clearer as to why conceptualisation, operationalization and time sensitivity are so important. With multiple functions, there becomes a need to have a greater understanding of why it is some young people come into conflict with the law and subsequently commit further offences; why certain interventions work for some and not for others, and why others may be more responsive to treatment. Without this 'risk is hidden beneath a plethora of correlations that in themselves tell us little about the socio-historical nature, meaning and significance of crime and its discourses in these time in which we are now living (Armstrong, 2004:113)

\section{The Aetiological Focus}

The basic principle of RFR is to identify those risk factors associated with offending and implement measures or interventions designed to counteract them. However, the developmental focus of RFR has meant that typically researchers have sought early childhood psychosocial factors that are statistically related with the onset of teenage offending. In doing this, RFR has tended to utilise broad (factorised) measures of risk factors, relating them statistically to broad categories of offending (i.e. a single offence of any type as counted as 'offending' and any three offences is taken to be 'serious offending'). As a result, studies of the risk factor-offending relationship for young people have been overly superficial, generalised and insensitive. It has also been suggested that there is a 'psycho-social bias' (France and Homel, 2006) which has resulted in 'an artificial restriction in the range of factors that have been explored' (Case and Haines, 2009:22).

Pitts (2001) has been highlycritical of the 'narrowing of the aetiological focus' relating to youth offending in the political discourse promoted during the 1990s by New Labour which he suggests has resulted in a 'strangelyskewed criminological perspective'. This has manifested itself in the promotion of risk factors derived from the Cambridge Study, which have then been reduced in multiplicityfrom the original list to favour of an emphasis on parenting, schooling and peers. By the time of the No More Excuses White Paper in 1997, the key risk factors being promoted were being male, poor parental discipline, criminal parents and poor school performance - the central practice of the newly formed YOT s being to identify and respond to these identified risk factors.

Critiques relating to the robustness of the evidence base have been further compounded by New Labour's assertions about the 'What Works' and their commitment to 'modernization' and evidencebased policyformulation. Despite the promise that policy decisions should be based on sound decisions, it is notable that in Misspent Youth ' 98 it is observed that 'few programmes for preventing offending by young people in England and Wales have been thoroughlyevaluated' (para 81, AuditCommission, 1998: 59). This, they attribute to the information to undertake the evaluation being absent or incomplete, in part due to the lack of co-ordination between agencies and partly as a result of the low priority given to evaluating public spending by some of the agencies involved. There are hints of the managerialist 
approach to come in youth justice when it is asserted that 'without sound evaluation, it is impossible to judge whether investing in such options saves moneyoverall - so all schemes should be properlycosted and monitored' (para 82, Audit Commission, 1998:59).

Moving forward, the YJB has professed a commitment to knowledge / evidence-based approaches to policy formulation and practice development. However, a number of critics including Case (2007), O'Mahony(2009) and Goldson(2010) have challenged the rhetoric, suggesting that the evidence (where is exists) has only been selectively used, often for political gain. Paylor suggests that 'the new focus on 'risk' is simply a device aimed at winning elections. Electoral anxiety is the motor force of the youth justice, as opposed to a considered and compassionate response to children in trouble' (2010:31). The emphasis on what is considered to be 'modifiable' has meant that the wider socio-economic problems are disregarded, privileging the 'family' under the rhetoric of care and support rather than social hams such as poverty. Thus, by focusing on 'modifiable' risk factors, interventions are most likely to impact on those living in the most challenging material circumstances. (Jamieson, 2005; Bateman, 2011).

A key issue in trying to understand 'What Works' in terms of interventions is that having identified so called risk and protective factors, there remains little understanding about how these impact on an individual basis on offending behaviour. Despite attempts to simplifythe relationship through the RFPP, characterising it as a 'linear risk paradigm' the realityis somewhat more complex (see Case and Haines, 2014). When attempts are made to assess how programmes underpinned by this paradigm work to reduce offending, the absence of a theory of change (Pawson and Tilly, 2000) results in ineffectual evaluation. Drawing on the work of Bateman and Pitts, Case asserts that:

\begin{abstract}
'According to Bateman and Pitts (2005: 253), the RFPP 'relies on an account of the origins of offending based on a combination of correlation and speculations'. The blind faith of politicians and academics in this burgeoning body of technical evidence is fraught with danger. If the available evidence cannot tell us howrisk/protective factors work, how these factors may precipitate youth offending or how programmes underpinned by them can reduce offending, subsequent research conclusions and 'evidence-based' policies and practices are builton sand.'
\end{abstract}

(Case, 2007:98)

Paraphrasing Pawson and Tilly (2000), Case arguments for the realistic evaluation of interventions, stressing that the RFPP as it stands does not inform the youth justice system since we do not know 'what kinds of risk factors have what kind of impact upon what kinds of people under what kinds of circumstances and why?' Case (2007: 98).

\title{
An Epidemiological Approach to Risk
}

In the context of medicine and public health, the RFPP model is used to identify 'risk factors' for physical illnesses and 'protective factors' which can mediate against these illnesses. Knowledge of the risk and protective factors are then used to formulate preventative interventions which are targeted at those 
considered to be 'atrisk' or 'high risk' of developing the illness. The epide miological nature of the RFPP model has made itattractive to policymakers, practitioners andresearchers interested in youth offending with the use of RFR within youth justice policyand practice growing exponentiallyin recent years. As a result, all young people who come into contact with the youth justice system in England and Wales are assessed in terms of their risk level. Considered to be the jewel in the actuarialistcrown, RFPP within youth offending is:

'A pragmatic crime prevision tool that uses risk assessment and survey to identify factors in the key domains of a young person's life (family, school, community and psycho-emotional) that statistically increase the likelihood of (official or self-reported) offending ('risk' factors) or decrease its likelihood ('protective' factors). Identified risk and protective factors are then used to inform 'evidence-led' interventions thataim to reduce risk and prevent offending.'

(Case, 2007:92)

However, manycommentators have been critical of the lack of statistical rigour within the evidence base - notably that there has been a reliance on the analysis of associations or correlations rather than establishing causality (Goldson and Muncie, 2006; Goldson, 2010; Case and Haines, 2010). Additionally, its positivist origins have sought to reduce what is a highly complex area into a tool which is oversimplified, generalised and superficial.

O'Mahony provides a detailed epidemiological critique of RFPP which highlights that risk-factor researchers 'are often guilty of forgetting that the measures in criminological epidemiological research are inherently weak and far less reliable than those used in medical epidemiology, which is the model they emulate' (2009a: 103-104). This is further compounded by their tendency to fail to report effect sizes where statistical significance has been established and to avoid testing for 'the causal potencyof apparent risk factors' (ibid). What are often produced therefore are artefactual risk factors - artefacts rather than facts, based on flawed theory and flawed (proxy) data. The failure of the field to ask:

'Is the association valid?; if valid, doesitrepresenta causal effect?; if there is a causal inference, whatelements in the experience or circumstance provides the risk and by what mechanism does it operate?; and does the risk operate in all people in all circumstances or is it contingent on either particular individual characteristics or a particular social context?'

(Rutter, 2005 cited in O'Mahony, 2009: 105)

has resulted in a long list of 'risk' factors for both anti-social and offending behaviour which O'Mahony argues betray the field's lack of ability to synthesize or produce a coherent explanation for the developmentand maintenance of such behaviour.

Broadly speaking factors are considered to be either static i.e. circumstances or conditions that cannot be changed, such as age at first offence, or dynamic i.e. factors which have the potential to change. Dynamic factors are those which can potentially be changed such as friends or school performance. By assessing both static and dynamic factors, the intention is to assess notonlylevel of risk, butalso identity 
potential ways in which risk can be reduced. In this way, the young person's needs can be addressed, and appropriate decisions made aboutinterventions.

\subsection{ASSET: Design and Key Components}

\section{The Theoretical Basis}

Whilst Farrington and West's Cambridge Study of Delinquent Developmenthas dominated research into understanding and addressing youth crime, as Baker explains, when it came to designing ASSET, this was not the only source that was utilised:

'This drew particularly on 'life course' or developmental perspectives (Sampson and Laub 1993, Loeber and le Blanc 1990) and the 'criminal career' paradigm (Blum stein et al 1998, Graham and Bowling 1995). Research into criminal careers has identified factors relating to the onset, persistence and desistance of offending and has shown that the factors contributing to one aspect of offending, such as onset, may differ from those which relate to persistence or desistance. The classification of risk factors used by Rutter, Giller and Hagell (1998) provided another useful framework. This distinguishes between 'individual characteristics' (such as hyperactivity or impulsivity), 'psychosocial features' (for example, poor parenting or school exclusion) and 'population-wide influences' (including the availability of drugs or weapons) that may contribute to offending behaviour. The aim in designing ASSET was to ensure that all of the key empiricallybased offending related risk factors were included.

Whilst ASSET necessarily focuses on identifying factors contributing to offending behaviour, it also recognises the broad range of needs and problems experienced by this group of young people. Consequently, some items which mightnotcontribute to the prediction of reconviction were included because of their value to practitioners in engaging and working with a young person. ASSET also acknowledges the insights of interactional theory which highlights the 'interactive and reciprocal causal influences that develop over time' (Thornberry 1997 p199). Problems in one part of a young person's life (e.g. education) maycontribute to difficulties in another area (e.g. family relationships) which in turn affects other aspects of his/her behaviour and attitudes.'

(Baker et al., 2003: 10-11)

The literature utilised reflects the implicitaetiology of the Crime and Disorder Act, 1998 in its emphasis on the development of offending and anti-social behaviour; risk factors at different ages and the effects of life events on the course of development. It also drew on the knowledge gained from the risk assessment processes which had been introduced into adult settings - the Offender Group Reconviction Scale (OGRS) had been used within probation from 1996 onwards, and elsewhere.

The inclusion of dynamic risk factors, or criminogenic needs plus the theoretical basis underpinning ASSET distinguishes it from earlier first-generation and second-generation tools that consisted mainly of clinical / professional judgements and risk instruments consisting mostly of static items (Andrews et al., 2006). Consisting of 13 inter-related sections dealing with factors such as 'family and personal 
relationships', lifestyle' and 'thinking behaviour' (Figure 2.1), ASSET was rolled out across the newly formed YOT sin 2000.

Figure 2.1: Components of the ASSETCore Profile

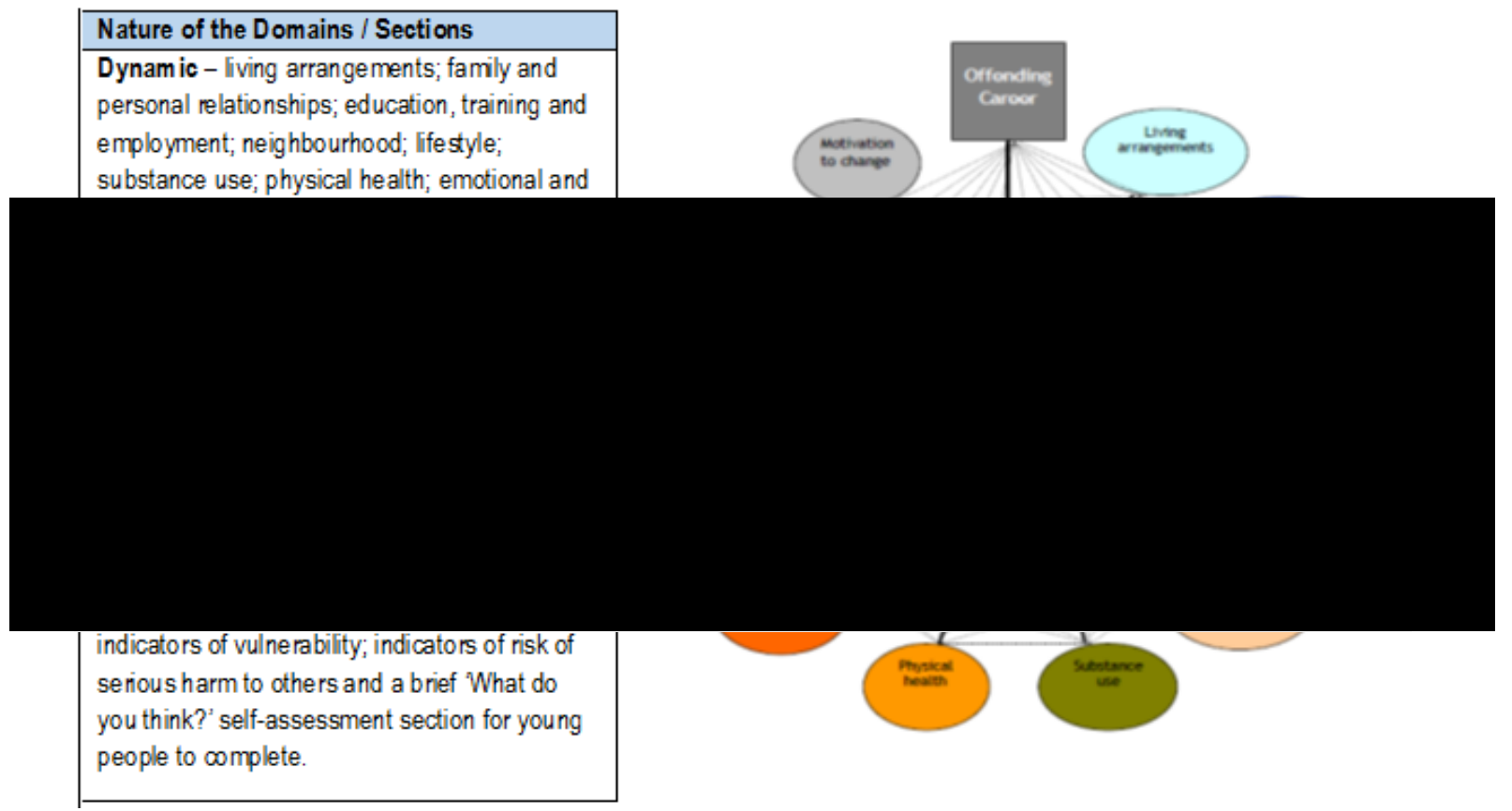

Adapted from Baker et al. (2003: 99), Youth Justice Board (2010b: 17-20); Case and Haines (2015: 102)

Within each section or domain are a series of questions requiring yes, no or don'tknow responses along with a narrative 'evidence box' where further details of the problem or issues identified can be recorded. Practitioners are then asked to rate (using a 0-4 scale) the extent to which each of these sections is related to the likelihood of further offending by the young person. Examples are given within the guidance (Youth Justice Board, 2008a) as to how the ratings mightbe applied specificallyin relation to each domain. Summaries are also provided in the TechnicalAnnex. Generic descriptions can be found in Table 2.1. It is these rating which will form the basis of the hierarchical modelling described in Chapters Four to Seven. 
Table 2.1: The Subjective Ratings Used within ASSET

\begin{tabular}{|c|l|}
\hline Rating & Description \\
\hline 0 & Not associated at all \\
\hline 1 & Slight, occassional or only a limited indirect association \\
\hline 2 & $\begin{array}{l}\text { Moderate but definate association - could be direct or indirect link. May be related to some offending, but } \\
\text { not all. Tends to become offending related when combined with other factors }\end{array}$ \\
\hline 3 & Quite strongly related - normally a direct link, relevant to most types / occassions of his/her offending \\
\hline 4 & $\begin{array}{l}\text { Very strongly related - wil be clearly and directly related to any offending by the young person. Will be a } \\
\text { dominant factor in any cluster of offending-related problems. }\end{array}$ \\
\hline
\end{tabular}

Adapted from Youth Justice Board (2008b: 4)

As highlighted in Figure 2.1, there are also a number of sections which do not require a numerical rating. These include sections on positive factors (intended to capture information aboutaspects of the young person's life which could be considered to be protective factors with respect to re-offending, to be strengthen as part of any intervention), vulnerability (any possibility of the young person being harmed) and an 'indicators' of serious harm. This latter section serves as a screen to identify cases which require a more detailed assessment of the likelihood of a young person causing serious harm to others. For the minority of cases in which some initial indicators of a risk of serious harm to others have been identified, the Risk of Serious Harm (ROSH) form can be utilised to provide the more in-depth assessment. Scores for a series of static factors were subsequently added to improve the predictive accuracy of the ASSET under the Scaled Approach, increasing the maximum potential ASSET score from 48 to 64 . Details of the scores assigned can be found in Table 2.2 .

To support the assessment process, a self-assessmentform 'What do YOU think?' was also designed to provide the opportunity for young people to directly record their views regarding their circumstances as well as explanations for their offending behaviour. Whilst this does not contribute to the ASSET Score, it can provide practitioners which additional information to consider when making an assessment and determining the appropriateness of different types of interventions. The form is also intended to facilitate discussion. Practitioners are required to complete the core assessmentdocument with every young person before any intervention is made. Theyalso need to review and update the assessmentat the end of any intervention. 
Table 2.2: Static Risk Factors and the Scores Assigned to these in ASSET (Max = 16) Under the Scaled Approach

\begin{tabular}{|c|c|c|}
\hline Static Factors & Scoring & Notes \\
\hline Offence type & $\begin{array}{l}\text { Motoring offences/ vehicle theff unauthorised } \\
\text { taking }=4 \\
\text { Burglary (domestic and non-domestic) }=3 \\
\text { Other offence }=0\end{array}$ & $\begin{array}{l}\text { When determining the young person's score for this } \\
\text { static factor category, YOTs will need to ensure that this } \\
\text { is adhered to. The offence type refers to the current } \\
\text { offence. } \\
\text { Future offending episodes will not continue to count any } \\
\text { previous burglary or motoring offences unless the new } \\
\text { primary index offence is one of offences. If their current } \\
\text { primary index offence is not one of these offences then } \\
\text { they will score } 0 \text {. }\end{array}$ \\
\hline $\begin{array}{l}\text { Age at first Reprimand/ } \\
\text { Caution/Warning }\end{array}$ & $\begin{array}{l}10 \text { to } 12=4 \\
13 \text { to } 17=2 \\
\text { No previous Reprimand/ Caution/Warning = } 0\end{array}$ & $\begin{array}{l}\text { If the young person does not have previous } \\
\text { reprimands/ cautions or a final warning they will score } \\
0 \text { in this category. }\end{array}$ \\
\hline Age at first conviction & $\begin{array}{l}10 \text { to } 13=4 \\
14 \text { to } 17=3 \\
\text { No previous convictions }=0\end{array}$ & $\begin{array}{l}\text { If the current conviction is their first conviction the young } \\
\text { person will score a } 0 \text { for this category as the } \\
\text { assessment is in relation to their current offence. }\end{array}$ \\
\hline $\begin{array}{l}\text { Number of previous } \\
\text { convictions }\end{array}$ & $\begin{array}{l}4 \text { or more }=4 \\
1 \text { to } 3=3 \\
\text { No previous convictions }=0\end{array}$ & $\begin{array}{l}\text { YOTs should not count the current conviction to this } \\
\text { score as the assessment is in relation to the current } \\
\text { offence. All previous convictions will count even if there } \\
\text { has been a significant gap in offending. }\end{array}$ \\
\hline
\end{tabular}

Adapted from Youth Justice Board (2010b: 18)

\section{Is ASSET reallyan Asset?}

Taken atface value, ASSET was seen as improving the quality of practice in assessmentand planning. As previously highlighted, prior to its introduction the process of assessing risks and planning interventions was perceived to lack rigour and consistency. The introduction of ASSET heralded in a new era of actuarial justice, bringing a standardised approach to decision making within the youth justice system. However, before ASSET was even commissioned, commentators such as Haines and Drakeford (1998: 217) were cautioning 'the dangers of attempting unthinkingly to transfer actuanial methods into the field of human behaviour'. Drawing on Kemshall $(1995,1996)$, theyhighlighta number of caveats which they assert needed to be borne in mind when drawn into the risk assessment arena. Sadly, as time progressed, many of these concerns were to be realised:

- 'The danger of regarding risk assessment as a neutral, value-free technical operation. In fact it is an enterprise determined bythe political and economic context within which it takes place. 'Risk' is not a shared or unproblematic concept which everyone mightbe expected to take a common view

- The danger represented by reliance on data which has the appearance of reliabilityand 'science', butwhich turns out on closer inspectionto be far less rigorous

- The difficulty with which such methods encounter in encompassing qualitative as well as quantitative information

- The danger which arises from 'the potential for the concept of risk to be used as a mechanism of social regulation, justifying the extension of community surveillance and dis-proportionately affecting some groups of the population 
- 'Play it safe' regulations which elicit cautious practice and which do not, in any case, guarantee risk-free practice

- 'Hindsight bias' which shapes practice on the basis of enquiries into disasters, rather than learning from successes

- The over-use of negative indicators by management'

(Haines and Drakeford, 1998: 217-218)

Although the YJB has previously been very positive aboutASSET, commenting for example in 2002 that 'More than any other aspect of the reformed system, this tool, properly used is capable of preventing further offending' (cited in Baker, 2004:72), as will be seen the tool has been subject to much criticism. However, for 15 years ASSET remained the standard risk assessment tool used across the youth justice system in England and Wales albeitin a modified form under the Scaled Approach.

\section{The Movement towards the Scaled Approach}

The Audit Commission's 2004 review of the youth justice reforms recommended that the YJB should make changes to the National Standards introduced in 2000 to reflecta risk-based approach and should make greater use of the assessment process to inform interventions. In doing this it used the term 'scaled approach' - the name which was later to be given to the new model:

'YOTs should make better use of ASSET to determine the amount as well as the nature of interventions with individuals using a scaled approach'

(Audit Commission, 2004 cited in Monk, 2009)

Consultation on proposals for the new model, revisions to the National Standards and case management guidance beganin November 2007, with morethan 130 responses being received reflecting the growing disquietabout the suitability of ASSET in its original format. Amongst the key issues raised was 'how to make ASSET assessments as reliable and consistent as possible, given that they are the basis for the Scaled Approach' (Youth Justice Board, 2008c:5).

At the time of the consultation, ASSET had been in operation for more than seven years with two evaluations of the validity and reliability of ASSET having been conducted (Baker et al., 2003; Baker et al., 2005). The first of these had included a 'thorough test of ASSET's predictive validity ... to establish its credibility and relevance to YOT practice' (2003:7). Analysis was presented which suggests that the ASSET rating score predicted reconviction with $67 \%$ accuracy - a rate comparable to that found for equivalent tools being used at the time with adult offenders, and considered to be particularly encouraging given the 'greater difficulties in predicting the future behaviour of young people who are often at an earlystage in their criminal careers simplyas a result of their age' (2003:7). It further asserted that this level of predictive accuracy was maintained with respect to specific socio-demographic subcohorts e.g. females, ethnic minorities and younger age groups. The various recommendations made by the report including ways in which predictive reliabilitycould be increased through the inclusion of static scores around offending history. This resulted in a revision to the original ASSET model. 
The 're-launch' of ASSET in summer 2003 included revised versions to the 'What do YOU think?' selfassessment form, Final Warning ASSET, standardised Intervention Plan and explanatorynotes. The Bail ASSET and ROSH forms were also revised in consultation with YOT staff. In the case of the latter, it was designed so that the risk classifications used would be the same as in OASys, the assessment tool used by prison and probation services with adult offenders (Baker et al., 2005).

\section{All Change}

The roll out of the Scaled Approach was timed to reflect the major youth justice provisions of the Criminal Justice and Immigration Act2008 (CJIA2008) including the new Youth Rehabilitation Order(YRO) which had been introduced to replace the then myriad of communitysentencing options. The YRO required a more tailored and targeted approach to the proposals made in court reports, enabling sentencers to tailor sentences on the basis of individual risk and need, drawing on a menu of interventions to tackle offending behaviour. A key part of this was that communitysentences could be returned to on multiple occasions. According to David Monk (2009), Head of Practice at the YJB, the development of the Scaled Approach was informed bya number of factors including a review of evidence to develop the revised Key Elements of Effective Practice. This highlighted that interventions were more effective when:

- the level and intensity of intervention is matched to an assessment of the likelihood of reoffending

- it is focused on the risk factors associated with offending

He also highlights the role played by the growing interest in a risk-led approach with a risk-based pilot being run by the YJB in four YOTs between December 2007 and June 2007. This concluded that the approach was backed byfrontline workers:

'There is very clear evidence that the practitioners in the pilot YOT s considered that adopting the risk-based approach had resulted in better outcomes for young people and these outcomes were measured in terms of better targeting and tailoring of interventions and more appropriate levels of contact,'

(YJB, 2010 cited in Puffet, 2010a)

However, the authors of the report - Matrix Evidence, were critical of the fact that they had not been tasked by the YJB with gauging the success of the system in terms of reconviction rates or value for money. This they felt constrained them 'from making objective assessments of the different practices adopted by pilot YOT s and identifying which were the most effective' (Puffet, 2010a). Rather they were able to make a number of technical recommendations as to how the Scaled Approach should be rolled out nationally, which included the importance of accurate and consistent assessment with rigorous quality checks and that the YJB should implement a method of risk assessment based on the 'highest of any' system that is populated by scores for risk of re-offending and risk of serious harm have been adopted (see Youth Justice Board, 2010a: 113-116). Further recommendations were made which 
focused on the provision of guidance. Judging bythe statistics which featured in the YJB's press release a prior to the launch, significant strides had been made to ensure that practitioners were trained and prepared for the changes, and that the IT systems were ready. Indeed ' $87 \%$ believed that they had had the right support from the YJB to get the Scaled Approach successfullyup and running in line with the YRO' (Youth Justice Board, 2009a). This is a marked contrast to the situation at the time of ASSET's launch in 2000.

With a tiered framework of interventions in the adultsector, it was also acknowledged that there was an opportunity to gather learning from these along with the implementation of Onset - ASSET's sister referral and assessmentframework, in 2007. This 'pre-crime screening tool' (Baker etal., 2005: 8) was intended to identify those 8-13 year olds who would benefit from early intervention, applying a tailored approach to the targeting of prevention services at those at highest risk of anti-social or offending behaviour.

The changes introduced under the Scaled Approach therefore did not bring about a change in the assessmenttool, rather they changed the process of assessmentand the way in ASSET scores would be used to determine the planning of interventions and nature / intensity of supervision. In implementing the Scaled Approach, there was the opportunity to harmonise practice:

'The basis of this 'new' model is the existing framework of 'Assessment, Planning, Interventions and Supervision (APIS). APIS forms the basis for youth justice practice and all elements of this have minimal National Standards for practitioners to adhere to in order to ensure fair, equitable and consistent practice across the system.'

(Sutherland, 2009:44)

Sutherland highlights that the thinking behind the introduction of an explicit risk-led approach was that there would be an intentional differentiation according to the assessed risks and needs of young people in terms of the interventions planned. By improving the practice of ASSET completion, writing of the presentencing reports and intervention planning, it was intended that YOT s would be able to more effectively target their resources. Thus, the Scaled Approach made much more explicit the links between assessments, plans, interventions and reassessments (as per Figure 2.2) and was designed to inform the ongoing case management of children and young people subject to YOT interventions.

Figure 2.2: APIS Framework - The continuous cycle of (re)assessment, (re)formulation of sentence planning, and supervision approaches 


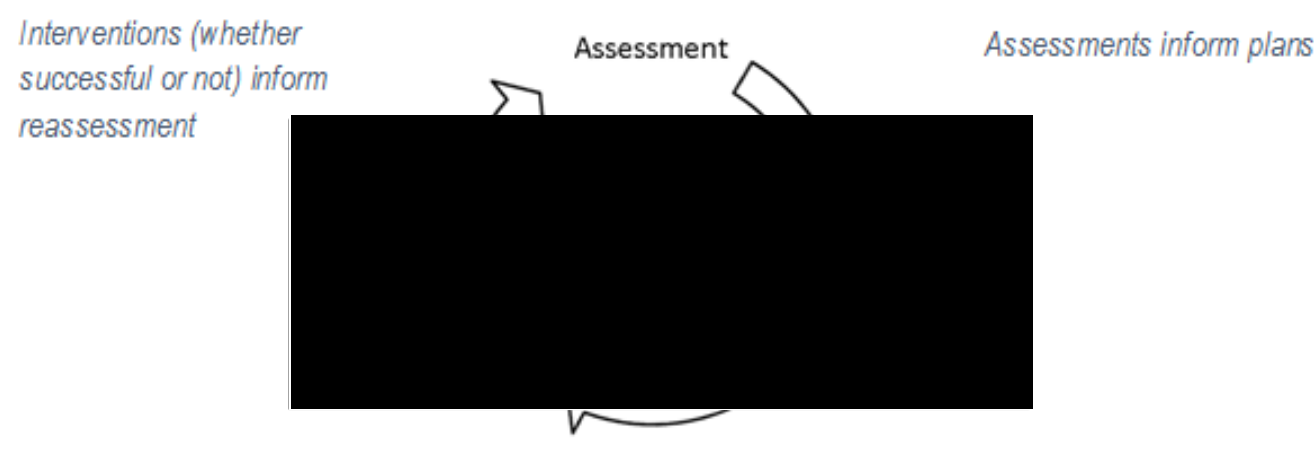

Plans focus interventions

Adapted from Sutherland (2009: 44-45)

\section{How the Scaled Approach works}

In aiming to ensure that interventions are tailored to the individual and based on an assessment of risk and needs, and that the intended outcomes are to reduce the likelihood of re-offending, practitioners were required to determine the appropriate level of YOT intervention based primarilyon two factors:

- Likelihood of reoffending - To assess the likelihood of the young person reoffending, practitioners are required combine the scores from the 12 main sections of ASSET - Core Profile (which relate to dynamic factors affecting offending behaviour, using the ratings given in Table 2.1) and score four 'static' factors (Table 2.2), to arrive at a total score between 0 and 64.

- Risk of serious harm - A full ROSH form is completed if there is a 'Yes' response to any of the questions in the 'Indicators of serious harm to others' section of the ASSET - Core Profile.

Under the Scaled Approach practitioners were required to use the framework set out in Table 2.3 to determine the most suitable level of intervention for managing the young person. This then formed the basis of the proposal to the courtor information for the panel. It is notable that it was felt necessary to revise the thresholds for the intervention levels as it was identified that too many young people were falling into the 'low' category. These changes were reflected in the second version of the postconsultation documentreleased In February2009 (Youth Justice Board, 2009b).

There was scope for practitioners to review the intervention level in the context of all other available information and consider whether there are any factors that indicate the intervention level may need to be amended e.g. where a young person had committed a particularlyserious offence butwas assessed by the YOT as low likelihood of reoffending or low risk of serious harm. Should the responsible officer decide to increase or decrease the initial intervention level then the decision needed to be defensible, discussed and agreed with a manager, with the reasons clearly recorded. The link between the intervention level and the intensity of supervision is summarised in Table 2.4.

Table 2.3: Determining Intervention Level 


\begin{tabular}{|c|c|c|}
\hline Child / Young Person's Profile & $\begin{array}{c}\text { Intervention } \\
\text { Level }\end{array}$ & Revisions Made \\
\hline $\begin{array}{l}\text { Low likelihood of reoffending (as indicated by ASSET score } \\
\text { [dynamic and static factors] between } 0 \text { and } 14 \text { inclusive) } \\
\text { AND } \\
\text { Low risk of serious harm (as indicated by no risk of serious harm } \\
\text { assessment being required, or low risk of serious harm } \\
\text { assessment) }\end{array}$ & STANDARD & $\begin{array}{l}\text { Originally proposed as being 'Low' with } \\
\text { ASSET scores less than } 25\end{array}$ \\
\hline $\begin{array}{l}\text { Medium likelihood of reoffending (as indicated by ASSET score } \\
\text { [dynamic and static factors] between } 15 \text { and } 32 \text { inclusive) } \\
\text { OR } \\
\text { Medium risk of serious harm (as indicated by risk of serious harm } \\
\text { assessment) }\end{array}$ & ENHANCED & $\begin{array}{l}\text { Originally proposed as being 'Medium' } \\
\text { with ASSET scores between } 25 \text { and } 41\end{array}$ \\
\hline $\begin{array}{l}\text { High likelihood of reoffending (as indicated by ASSET score } \\
\text { [dynamic and static factors] between } 33 \text { and } 64 \text { inclusive) } \\
\text { OR } \\
\text { High or very high risk of serious harm (as indicated by risk of } \\
\text { serious harm assessment) }\end{array}$ & INTENSIVE & $\begin{array}{l}\text { Originally proposed as being 'High' with } \\
\text { ASSET scores of } 42 \text { or over }\end{array}$ \\
\hline
\end{tabular}

Adapted from Youth Justice Board (2008d) and Youth Justice Board (2009b)

Table 2.4: Statutory contacts for assessed intervention levels

\begin{tabular}{|l|c|c|}
\hline Intervention Level & $\begin{array}{c}\text { Minimum number of contacts per month } \\
\text { for first three months of order }\end{array}$ & $\begin{array}{c}\text { Minimum number of contacts per month } \\
\text { for remainder of order }\end{array}$ \\
\hline Standard & 2 & 1 \\
\hline Enhanced & 4 & 2 \\
\hline Intensive & 12 & 4 \\
\hline
\end{tabular}

Adapted from Youth Justice Board (2010b: 12)

Scaled Approach intervention levels were not affected by any identified issues around the vulnerability or welfare of the young person. However, as YOT practitioners have ongoing responsibilities for addressing safeguarding and welfare issues as part of wider social / children's services partnerships, then appropriate action could be taken to ensure that these considerations were reflected in the overall intervention plan and vulnerability plan where one was needed. If the child or young person was assessed as being particularlyat risk of harm from themselves or others then the appropriate action could also be identified, with the potential to utilise multi-agencyinput. The final judgment was then used to inform the proposal made to the courtor report to the youth offender panel.

Thus, the Scaled Approach provided a framework for assessment, proposals to courts and youth offender panels, interventions and review. As a model for interventions delivered by the YOT, it reflected the statutory aim of the youth justice system to prevent offending, including reoffending, by children and young people and was designed to help YOTs become more effective in delivering this requirement in their local communities. Prior to its launch, Monk (2009) had promoted the following anticipated benefits:

- More efficientand effective allocation of YOT resources

- Fewer young people in custody

- Strengthened case managementacross the youth justice system 
- Improved practice in assessment quality, pre-sentence reports (PSRs) and intervention planning

- Tailored interventions based on the young person's risks and needs

The combination of these it was believed would reduce reoffending; re duce the risk of serious harm and would lead to increased public confidence in the youth justice system.

\section{Is the Scaled Approach a Failed Approach?}

Despite claims thatall available evidence was utilised to inform the development of the Scaled Approach, the YJB has continued to face criticism about the approach not least for having the potential to lead to different levels of intervention for young people committing the same crime:

'if two young people commit the same offence buthave different risk score, the higher risk one will be required to meet their YOT more often than the low-risk one, and more often than the current system'

(Bateman quoted in Pemberton, 2009)

Just four months after its launch, questions were being raised about how sentence recommendations from the Criminal Justice and Immigration Act 2008 were being calculated and as part of a wider review of the future needs of the youth justice system, the YJB announced a review of the predictive validity of ASSET. The timing of the review was criticised with Nacro's Senior Policy Development Officer, Tim Bateman (quoted in Puffet, 2010b) asserting that 'If the YJB was considering a fundamental review of assessment policy it would have made sense to incorporate it into any plans they had to change the nature of intervention'. However, Monk defended the decision, saying that the review would be 'a longterm projectand itwould have been unrealistic to carryout prior to the introduction of the new sentencing system' (ibid).

The Review reported in December 2011 (Wilson and Hinks, 2011) by which time sponsorship of the YJB had moved to the Ministry of Justice. This found that ASSET was still a good predictor of proven reoffending among young people and that the findings were broadly in line with Baker et al's 2003 evaluation (based on a variant utilising scores from the 12 dynamic domains). It tested a number of models including a simulation of the Scaled Approach, different combinations of dynamic and static scores, and the Offender Group Reconviction Scale (OGRS 3) being used with adult offenders within OASys - use of the latter within youth justice had previously been advocated by Howard et al. (2009) and was considered to be an efficientand quick pre-screening tool sincedata could be extracted directly from the Police National Computer (PNC).

Wilson and Hink's key findings and recommendations were: 
- ASSET 'dynamic plus OGRS 3' was found to be the best predictor of proven re-offending of those tested. Hence the predictive ability of ASSET could be improved byreplacingthe ASSET static component of the Scaled Approach with OGRS3.

- Using OGRS 3 as a predictor of risk of re-offending was as good as using ASSET (both preand Scaled Approach), butit should notbe used for Final Warnings as there was no information aboutcriminal history to calculate a score.

- Of the 12 ASSET dynamic factors, 'lifestyle', 'substance use' and 'motivation to change' were highly statisticallysignificant predictors of proven one-year re-offending. 'Living arrangements', 'family and personal relationships', and 'education, training and employment' were also statistically significant. The remaining six factors, although of less importance to predicting proven re-offending, are likely to still be relevant for understanding the needs experienced by young people. Hence in order to inform intervention planning, the information captured via the 12 domains is also required. This enables areas of need to be identified and addressed.

- Not all young people (72\%) had an ASSET completed within 30 days prior to, or after, the index disposal - for most disposals, an ASSET assessment should be conducted 10-15 days prior to the order being made as the information gathered forms part of the PSR. This suggested that the timeliness and completeness of ASSETs requires further improvement to ensure that assessments are completed in line with National Standards.

As with the risk assessment tools used in adult settings, questions have also been raised about the predictive accuracy of the tools developed for predicting youth offending. However, issues are also raised about the integrity of the evidence base with Case and Haines arguing that:

'... the risk assessment processes promoted by the Youth Justice Board and particularly the risk assessment instruments that underpin these processes, have been founded on the mis-appliance of research findings and have exacerbated the methodological over-simplification, indefinite and imputation that undermine RFR.'

(Case and Haines, 2009:256)

Through a detailed critique of the major studies contributing to the RFR paradigm, they highlight how factorisation and reductionism have over-simplified the context and operation of risk whilst the aggregation of findings has limited the potential to understand some of the complex relationships that exist within youth offending. In particular they question the value of homogenising 'offending' into lifetime, active, general and serious, and promotion of prevention as a dichotomy of risk. In reviewing the respective research designs and data collection methods utilised within these studies, Case and Haines are critical of their ability to determine causalityand of a psychosocial bias which has tended to overlook socio-structural factors such as gender, class, poverty and societal access routes to opportunities. 


\subsection{The Actuarial Fallacy and Predictive Accuracy}

There are significant methodological limitations with actuarial approaches resulting from the 'actuarial fallacy' - this relates to the fact that actuarial methods can only provide an estimate to the probability that an offender with a particular set of characteristics will be reconvic ted within a defined period: it does not provide the means of making an accurate prediction for a specific individual. It is this feature which has also proven to be controversial in medical epidemiology despite its more precise and rigorous measurements (O'Mahony, 2009). In translating criminological risk-focused epidemiological research into a format that can be more readily understood by practitioners, much of the 'daunting detail and conceptual and statistical complexity' (2009:100) has been over-simplified. Many of the key concepts are undefined with this ambiguity being identified as a methodological paradox by Case and Haines (2009). They highlight that although many of the conclusions of RFR are presented as if there is homogeneity, there remains uncertaintyand disagreementin the field. With poorly-defined and partially understood concepts, it calls into question just how scientific the conclusions reallyare.

\section{Methodological Issues Affecting Predictive Accuracy}

The accuracy and utility of actuarial risk assessment tools has been subject to much debate amongst academics and practitioners. Gottfredson and Moriarty (2006), in a follow up to earlier work, highlight that many of the issues they had identified twenty years previously remained a problem. In providing their critique, they point to a number of issues which theyassert are fundamental to the development and implementation of risk assessment tools whichare often ignored including those that affectaccuracy and the nature of the criterion variables chosen. In terms of statistical methods being used in criminological prediction studies, the approaches listed reflect advances made in applied mathematics and the increased computational power available for analysis e.g. 'cross-classification tables; multiple regression; multiple discriminant function analysis; multidimensional contingency table analysis; logit, probit and tobit analysis; a variety of clustering approaches; and neural networks' (Gottfredson and Moriarty, 2006: 183).

Despite these advances, Gottredson and Moriarty express concern thatall too often those developing prediction tools have failed to consider base rates. In the context of prediction tools, the consequence of having a base rate further from 0.5 is that it increases the likelihood of inaccurate prediction. This has the potential to introduce larger errors at the extremes i.e. for more frequent or infrequent events and hence could limit the usefulness of a tool for predicting for example, more serious offences which are committed less frequently by young people. A notable exception to this which comes from Harris and Rice - part of the team responsible for developing the VRAG tool and for promoting the use of receiveroperating characteristic (ROC) statistics as an index of predictive accuracy (Rice and Harris, 1995). Theyhave examined base rates using Bayes Theorem to consider informative priors to actuarial violent risk assessment, concluding that 'what makes a base rate an informative prior partly depends on how 
much it differs from the selection rate, especially, in forensic clinical practice, how low it is' (Harris and Rice, 2013:119). Theydo however, caution that 'more empirical work is required before Bayes' rule can be automaticallyapplied to actuarial assessmentnorms' (Harris and Rice, 2013:120).

In identifying the sample for constructing prediction tools, it is advocated that the sample must be representative of the population on which the device is intended to be used (this is not the same as the being representative of the population as a whole). This is done to ensure that appropriate base rates are used and also as a measure to minimise shrinkage of power. Cross-validation is typicallyundertaken therefore using construction and validation samples. Whilst this is now more common practice, this has not always been the case. Similarly, there are examples of experiments intended to identify predictor variables which were designed withoutcontrol groups (see Case and Haines, 2009 for a critique of the research designs utilised in key surveys which underpin risk assessmentin a UK context).

Perhaps most significantly, risk assessment devices are being used on populations for which they were not designed. This can be because the original research was not representative of the wider youth offender cohortor it because the predictive variables mayprove to be more (orless) predictive for some defined populations when the composition of the population is different. Gottfredson and Moriarty(2006: 190) give the examples of 'race' noting that 'it may be predictive of criminal convictions in some large urban populations and not at all predictive in suburban or rural populations', and age. In the case of the latter they note that 'some items that are predictive during some age ranges may not be if other age ranges are considered.' Theyconclude with the following:

'Despite good and consistentadvice to the contrary, policy makers continue to seek a panacea through 'risk assessment', typically by seeking to adopt a pre-packaged 'one-size-fits-all' risk assessment tool. And despite good and consistentadvice to the contrary, basic methodological requirements of risk assessment development are frequently ignored.'

(Gottfredson and Moriarty, 2006: 195)

\section{The Predictive Accuracy of ASSET}

As previously indicated, Baker et al. (2003) had reported that the original version of ASSET had a predictive validity of $67 \%$. The measure used to determine this was the 'percentage correctlypredicted' which is essentially a crude sum of the proportion of non-reconvicted low scorers (32.7\%) and reconvicted high scorers (34.3\%) and was used to enable comparisons to be made with equivalent models at that time in Probation (for example Raynor et al., 2000). The way in which the percentage correctly predicted is presented within the report serves to highlight the fact that $16.3 \%$ of low scorers went on to be reconvicted within 12 months whilst $16.7 \%$ of higher scorers had no proven reoffending during the following year (Table 2.5). As Smith (2006) highlights this is equivalent to $33 \%$ of young people for whom the outcomes of ASSET profiling have been neither valid nor reliable. When you 
consider that tossing a coin has a predictive accuracy of 50\% does not give must confidence in using ASSET to justify the intensiveness of an intervention as became the case under the Scaled Approach.

Table 2.5: Plotting Percentage Correctly Predicted: ASSET (Original Version)

\begin{tabular}{|c|c|c|c|}
\hline & \multicolumn{2}{|c|}{ Outcome } \\
\hline & & Non-Recidivists & Recidivists \\
\hline \multirow{2}{*}{ Prediction } & Low Risk & $\begin{array}{c}\text { True Negative } \\
(32.7 \%)\end{array}$ & $\begin{array}{c}\text { False Negative } \\
(16.3 \%)\end{array}$ \\
\hline & High Risk & $\begin{array}{c}\text { False Positive } \\
(16.7 \%)\end{array}$ & $\begin{array}{c}\text { True Positive } \\
(34.3 \%)\end{array}$ \\
\hline
\end{tabular}

Notes: Based on Baker et al (2003) which reported on the original version of ASSET, considering re-offending after 12 months

More recently there has been a shift in the reporting effect sizes and hence predictive validity, with increasing use of area under the curve (AUC) - a form of receiver-operating characteristic (ROC) analysis to measure the predictive ability of both adultand juvenile risk assessmentinstruments (Baglivo and Jackowski, 2013). This approach has been shown to be robust to variation in base rates, selection ratios, and truncated distributions - common problems in risk assessment research (Rice and Harris, 1995; Gottfredson and Moriarty, 2006; Schwalbe, 2008). Although comparatively new to criminology, the AUC is the preferred method of predictive or diagnostic accuracy in forensic psychology and psychiatry (Rice and Harris, 2005), and has been used as a measure of effect size when reporting violent and sexual recidivism:

'The AUC statistic illustrates the probability that a score (eg on a risk assessment instrument) of a randomly selected case from one population (eg a youth who recidivates) will be higher than a randomly selected score from a second population (eg a youth who does not recidivate). An additional benefit is that the AUC is less affected by fluctuating or low base rates (eg historically low recidivism rates for females)'

(Baglivio and Jackowski, 2013:27)

Baker et al. (2005) suggest that for the original version of ASSET, the AUC for the construction sample was 0.719 at the 12 -month stage and 0.731 at the 24 -month stage. The AUC measure of predictive validity has since been utilised to assess ASSET as part of the YJB's review of assessment and intervention planning (Wilson and Hinks, 2011). They suggest that the AUC for ASSET pre-Scaled Approach i.e. based on a score out of 48 , was 0.68 whilst for the simulated model intended to represent ASSET under the Scaled Approach was 0.70 (Table 2.6). Their reportfound that the simulated model was significantlydifferent from the earlier version at a significance level of $p<0.001$.

Although the benchmarks for interpreting AUC values are arbitrary, generally the higher the AUC, the higher the predictive validity. Wilson and Hinks (2011) for example suggest that a model is generally considered 'moderate' if the AUC value is $0.64-0.70$ and 'good' if 0.71 or above. This is broadly consistentwith the benchmarks putforward by van der Put et al. (2014) i.e. that an AUC value greater than 0.70 is considered to be 'acceptable', whereas an AUC value of greater than 0.75 is considered to 
be high. To put the findings for ASSET into context, Schwalbe (2007) reports that the weighted average AUC for the third-generation juvenile risk assessment instruments included in his meta-analysis was 0.646 ( $95 \%$ Confidence Interval: 0.588 to 0.704 ) but ranged from 0.57 to 0.78 . This average was calculated using twenty-one third generation tools including ASSET .

Table 2.6: The Predictive Accuracy of ASSET and ASSET under the Scaled Approach

\begin{tabular}{|l|c|c|}
\hline Description of Model & $\begin{array}{c}\text { ASSET 'Dynamic (48)' } \\
\text { This model represents the pre-Scaled } \\
\text { Approach practice of undertaking risk } \\
\text { assessments and remanted the practice for } \\
\text { Final Warning cases. It utilised a score out of } \\
48 \text { for the ASSET dynamic factors }\end{array}$ & $\begin{array}{c}\text { Simulated ASSET 'Static plus Dynamic (64)' } \\
\text { This model was simulated to represent how } \\
\text { assessments were undertaken for } \\
\text { 'sentanced' cases under the Scaled } \\
\text { Approach. It utlised a score out of } 64 \\
\text { derived from both the statsic and dynamic } \\
\text { ASSET factors }\end{array}$ \\
\hline AUC & 0.68 & 0.7 \\
\hline Standard Error (SE) & 0.012 & 0.011 \\
\hline $\begin{array}{l}95 \% \text { Confidence Intervals } \\
\text { around the AUC }\end{array}$ & $0.66-0.70$ & $0.68-0.72$ \\
\hline Interpretation of AUC & Moderate / Acceptable & Acceptable \\
\hline
\end{tabular}

Adapted from Wilson and Hinks (2011: 26)

Notes: Validation sample $(n=2,172)$, sentenced cases only.

Data was taken from the Juvenile Cohort Study. Since this took place before the introduction of the Scaled Approach, it was necessary to simulate scores for the static factors. Hence results referring to the 'simulated' ASSET can only be regarded as indicative. The Standard Error provides an estimate of the uncertainty about a calculated value (here the AUC). The smaller the Standard Error, the more confidence that the reported value is the 'true' value. AUCs of 0.5 are the practical minimum as these could have been obtained by randomly, while AUCs of 1 represent the hypothetical situation where in this instance, all proven re-offenders have higher scores than non-proven re-offenders.

Whilst Wilson and Hinks highlight the limitations of their methodological approach, they are at pains to stress the representativeness of the data they used and the importance of providing timely results to inform the YJB assessment review. In doing this they emphasize that the study 'did notintend to exhaust all the many possible options in designing a risk assessment tool and developing the 'best' possible predictor of youth re-offending' (Wilson and Hinks, 2011:1). The number of cases used is however, significantlyhigher than those used in earlier reviews by Baker et al, thus negating some of the previous challenges made around the robustness of the analysis.

From a generic perspective, reliabilityrelates to the need for significantresult to be more than a one-off finding and therefore inherentlyrepeatable. However, this can be dependentupon the stability with which measurements may be made. As Gottfredson and Moriarty observe, 'statistical validity is constrained by the reliability with which criterion and predictor measurements are made' and they argue that 'no risk assessment device can be better than the data from which it is constructed' (2006:183).

\subsection{Sensitivity: One Size Does Not Fit All}

As a result of factorisation - which also has its origins in the positivist philosophy of science, itis argued that RFR has becomea 'blunt tool' with which to 'carve out risk factors and 'at risk' populations' (Case and Haines, 2009: 19). In seeking to reduce information from a raft of different sources (e.g. surveys, behavioural rating scales, psychometric tests and official records along with qualitative risk data from 
interviews and observations), into subjective scores corresponding to broad 'risk' categories, risk assessment tools are insensitive not just to individual differences but also fundamental differences in terms of the nature and type of offending. Whilst the aggregation of variables and assumptions of homogeneityhelp to make risk assessment tools 'easy to understand and communicate, and ... readily accepted by policymakers, practitioners and the general public' (Farrington, 2000:7), this has come at the cost of sensitivity. This particularlyhas implications due to the multiple functions of fourth-generation tools where it could be argued that in order to respond to individual risk, need and responsiveness, then increased sensitivity is required. Onlythen can scant resources be appropriatelytargeted.

\section{Dimensional Identity}

If we are to consider how to address the insensitivity of many risk assessmentinstruments with respect to demographic characteristics it is necessary to consider the concept of dimensional identity (Schwalbe et al., 2006). The conceptwas developed in the person-centred research paradigm within developmental psychology and is said to exist when a measure such as recidivism in a risk assessmentinstrument is the same for all subpopulations within a sample. If empirical research demonstrates that the predictive validity of the risk assessment tool is greater for some groups than others, then it fails to possess dimensional identity. It is only when the dimensional identity exists that the generalizability of the risk assessment tool can be asserted.

Certainly, if disparities based on race/ethnicityand gender are to be reduced within the criminal justice system, then the predictive validity of risk assessmentinstruments should not differ across demographic groups. Although written in the context of the juvenile justice system in the United States and Canada, Schwalbe etal highlight that:

'Risk instruments are designed to reduce, racial, ethnic and gender disparities and biases by increasing the consistency of assessment through a structured process... Coupled with needs assessment, sentencing guidelines, and other reports, risk assessment is an importantelement of a larger strategy to reduce racial and gender disparities in the treatment of offenders by the juvenile justice system'

(Schwalbe etal., 2006: 306)

Given the way in which ASSET is used within youth offending under the Scaled Approach, there is a need for the predictive validity of the instrument to hold across diverse groups both in terms of assessing the likelihood of further offending and the tiered risk classifications. Thus, a medium-risk classification should convey a similar meaning with respect to the probability of reoffending for both males and females, for different racial/ethnic groups, and for different ages of offenders.

However, as yet there are few examples of where AUC has been utilised to measure the predictive validity of juvenile riskassessment tools by demographic subgroup. Exceptions include Schwalbe (2006; 2007 ;2008) who has undertaken meta-analyses of juvenile risk assessmentinstruments from the United States; Meyers and Schmidt (2008) who has focused specifically on a Canadian clinician-led violence 
risk assessment tool and Emeka and Sorensen (2009) using data from the USA. All have examined differences by gender with Meyers and Schmidtalso looking at race/ethnicity. Whilst the findings from the first two sets of studieswere found to be inconclusive - an issue which is attributed to small sample sizes, Emeka and Sorensen's analysis of data from the Texas Ju venile Probation Commission led to the construction of a risk assessment scale which showed marked gender differences for the respective AUCs despite coming from a pooled sample. It is findings such as these which reinforce the need to periodically review the predictive validity of risk assessment instruments and for innovations such as gender-specific instruments to be explored:

'Gender differences, when they appear, can open the window in the presence of gender biases in juvenile justice decision-making. By routinely testing for gender differences, risk assessment validation studies can expose gender biases that can become the focal point for ongoing research and policyinterventions. In this way, risk assessmentinstruments, and the research that supports them, can serve to increase, rather than undermine, gender equalityin the juvenile justice system.'

(Schwalbe, 2008:1379)

More recentwork by a Dutch team using police data has also asked if there are any sex differences in risk factors for re-offending and in risk profiles (van derPut et al., 2014). Their work categorises the girls into four groups: a low risk group (containing $65 \%$ of the girls) and three high risk groups (girls with 'delinquent' parents, victims of abuse, and repeat offenders), and shows that each has a specific set of risk factors and hence implies the need for specific interventions. These findings sit within the context of the development of new risk assessmenttools for the prediction of first-time offending (Assink et al., 2016) and general recidivism (van der Put, 2014).

The predictive validity of juvenile tools on the basis of race / ethnicityhave been considered by Baglivio and Jackowski (2013) and by Rembertetal. (2014). In both cases, the work has been undertaken using US samples. In the case of the former, it was not possible to determine whether the differences found between genderand race/ethnicitywere due to shortcomings of the instrumentor to external factors in the criminal justice system itself, noting that different law enforcement practices all affect estimates of predictive risk assessmentinstruments. Rembertetal. (2014) excluded Whites from their sample on the basis that inclusion biases the predictability of minority group members. Their research found that the Los Angeles County Needs Assessment Instrumentwas a better predictor of re-arrestfor Hispanics then African American juveniles. The findings suggest that:

'...these offender differential prediction validations warrant more complex statistical techniques. In particular, they support the use of multilevel modelling over the traditional logistical regression, due to the former's ability to decipher the impact of exogenous community-level variables (eg neighbourhood disadvantage, vacant housing, public assistance, crime rates, and law enforcement surveillance) on the respective outcome measures.'

(Rembertet al., 2014: 161-162) 


\section{How Good is ASSET at Correctly Predicting Re-Offending for Sub-Populations?}

In the absence of any published studies which either state the AUC or provide sufficientinformation for the AUC statistic to be calculated in order to measure the predictive validity of ASSET on the basis of subgroups, it is necessary to fall back onto an alternative measure of predictive validity described in Section 2.6. Baker et al present tables which provide the 'percent correctlypredicted' for females, young offenders (10-15 year olds) and ethnic minority offenders with respect to proven reoffending within 1 year (Baker et al., 2003) and 24 months (Baker et al., 2005). This crude rate based on the proportion of those with a 'high' score being reconvicted plus the proportion of those with a 'low' score not being reconvicted, it suggests that there is little difference between the respective sub-groups. For example, overall recidivism was correctly predicted for $67.1 \%$ of the cases from June/July 2000 . Amongstfemales, $66.0 \%$ were correctly predicted compared to $67.3 \%$ for males despite there being significant differences in their respective recidivism base rates $-39.5 \%$ for females compared to $53.1 \%$ for males.

The subsequentevaluation undertaken by Wilson and Hinks (2011) using data from the Juvenile Cohort Study to examine how well ASSET under the Scaled Approach predicted reoffending over one year, found that ASSET accuratelyassigned higher scores to those who went on to reoffend. A finding that was repeated when the analysis for females, BAME groups and those aged 10-15:

- Females: the mean ASSET score for those who reoffended was 24.3 (out of a maximum of 64 i.e. static plus dynamic scores) compared to 16.9 for those who did not reoffend $(\mathrm{t}(921)=-12.6$, $p<0.001$; Effect size eta squared $=0.15$ )

- BAME: mean score of 22.4 amongst those who reoffended compared to 15.4 for those who had not reoffended $(t(747)=-10.6, p<0.001 ;$ Effect size eta squared $=0.13)$

- Young People aged 10-15 years: 23.1 compared to 16.8 amongst those who had not reoffended $(t(3,2182)=-16.5, p<0.001 ;$ Effect size eta squared $=0.11)$

This evaluation also used logistic regression to examine how well each of the 12 dynamic factors predicted reoffending over 1 year. However, the results of this analysis are not broken down by subgroup. As the headline findings have a specific bearing upon the results of the modelling described in Chapters Five and Six, these will be + .

As previously highlighted a key issue which has previously limited the potential for further exploration of gender-specific assessment tools has been small sample sizes - in the case of Baker et al's follow up work there were 399 females compared to 1,834 males whilst Wilson and Hink's work, $18 \%$ of the sentenced sample were females (917 out of 5,107). Echoing the views of van der Put et al. (2014) and Emeka and Sorensen (2009), itmaywell be that because girls make up onlya small percentage of youth offenders, the risk factors that are important to female offending have not been identified and 
incorporated into risk assessment tools. Instead female risk factors have been embedded within male risk factors within generic tools.

This is also the position with regards to ethnicity with the BAME cohortmaking up just $8.4 \%$ of the cases analysed by Baker et al. (2005) - equivalent to 189 of the 2,233 cases. Although the cohort for the Wilson and Hinks (2011) analysis was larger: Black/Black British young people made up 5.7\% (290) whilst $4.4 \%$ were Mixed and $4.4 \%$ Asian/Asian British, the comparatively small number of cases has limited the analysis which could be undertaken using traditional statistical approaches, especiallywhen looking to further segment to the cohort say to focus on non-White females or those who committed different offences.

\section{Different Types of Offending}

In terms of predicting different types of offences, as previously highlighted, tools have been developed specifically for the risk assessment of serious violent and sexual offenders for use in clinical settings. However, it is acknowledged that low base rates, and particularlyin the context of sexual offending, the diverse nature of the offending can limit the utility of these tools - as Kemshall (2008b:10) observes, 'if something doesn't happen very often it is difficult to predict if and when it might happen in the future, although the consequences of it happening could be very high.' Thus, it is particularly difficult to accurately predict 'grave crime' such as murder, with practitioners being tasked to make difficult decisions about risk of harm. Predicting such offending amongst young people is even harder due to the rarity of such offending amongst under $18 \mathrm{~s}$. As a result, where such tools exist for young people, these are typically modified versions of adult tools and tend to focus on the dangerousness of the offender.

Tools for predicting more general youth offending, typically do not differentiate between violent, acquisitive and other types of offending. Although under the Scaled Approach, it should be noted that those young people whose primary index offence was burglary or motoring offences were assigned scores of 3 and 4 respectively within the Offence Type section of the Static Factors (Table 2.2). The higherperceived risk associated with having committed these types of offences was a reflection of the fact that when the research was undertaken, those young people who had committed one or more of these offences were more likely to be reconvicted in the subsequent 12 months than those young people whose index offence was another offence type. When Wilson and Hinks (2011) subsequentlysought to compare the predictive validity of different models using logistic regression techniques, they found that relative to those who have committed other offences, the odds ratio for proven re-offending where the young person had committed motoring offences (including vehicle theft and unauthorised taking) was 0.87 whilst that for burglary (domestic and non-domestic) was 1.20 . However, neitherwere found to be significantpredictors of reconvictions within the model designed to representASSET under the Scaled Approach i.e. including both static and dynamic factors. 
Type of reoffending is considered within Wilson and Hink's evaluation of ASSET, since they look at accuracy in predicting the severity of proven re-offending both by considering (1) the most serious reoffence and (2) the most punitive criminal justice disposal within the one-year follow up period. Generally, is was found that young people with more serious re-offences had on average, higher ASSET scores than those committing non-serious offences. They also found that young people receiving custodial sentences for a re-offence typically had higher ASSET scores than those receiving less punitive disposals. Based on the young person's mostserious re-offence:

- Those whose re-offence was categorised as being a serious violence and sexual offence i.e. offences resulting in death, grievous bodilyharm and serious sexual offences had a mean score of 25.9 (out of a maximum of 64)

- Those committing serious acquisitive crime i.e. robbery, burglary, theft of or from a motor vehicle, had a mean score of 25.8

It is reported that ASSET was unable to differentiate between the types of serious re-offence. However, it was possible to differentiate between those who went on to commitserious re-offences, and those who went on to commit'non-serious' re-offences. This latter group had a mean score of 22.1.

In terms of the seriousness of the disposal received for the re-offence, it was found that those receiving custodial sentences had statistically significantly higher mean ASSET scores than those receiving communityand other penalties. However, ASSET was unable to differentiate between those receiving communitypenalties and those received lower level disposals. Wilson and Hinks suggest that this first finding maybe due to the escalator policywherebythose with a more prolific criminal history (and hence who score higher on the ASSET static factors) have a higher chance of receiving a custodial sentence. Notablythe static factors age atfirst Reprimand/Caution/Warning and age at firstconviction were found to be highly significantpredictors of reconviction in the model designed to represent ASSET under the Scaled Approach.

The number of previous convictions was also shown to be a significant predictor where the young person had 1-3 prior convictions relative to those who had none. However, it was necessary to remove the predictor for 4 or more previous convictions from the model due to a high correlation with age at first conviction. Differences in mean scores between these groups were reported:

- Number of Proven Offences: the mean ASSET score for those who 1-3 re-offences was 212 (out of a maximum of 64 i.e. static plus dynamic scores) compared to 26.2 for those who had more than 3 offences during one year $(t(2560)=12.9, p<0.001$; Effect size eta squared $=.06)$

Theoreticallythere is a basis for being able to understand the different trajectories for different types of offender and offence. For example, Owen and Cooper (2013) found from their analysis of the Police National Computer for all first-time entrants into the criminal justice system in 2001, that the type of debut 
offence committed was significant predictor of both chronic offending status and committing a further serious offence (i.e. robbery, serious violence or a sexual offence). Whilst their analysis excludes lowlevel offences which have resulted in no further action or a restorative sanction which is notrecorded on PNC, it suggests that re-offending rates are higher for those who were first sanctioned at a young age with those aged 10-17 at their first offence being 4 times more likely than those aged 18-25 years and 11 times more likely than those aged over 25 years when committing their debut offence to become chronic offenders i.e. to have committed 15 or more re-offences during the 9 year follow up period from 2001. Those aged 10 to 17 at their debut offence were 2.5 times more likely to commit a serious reoffence compared with $18-25$ year olds ( $23 \%$ and $9 \%$ respectively); and 7 times more likely than older adults (3\%). Table 2.7 summarises the respective proportions who went onto be chronic and serious reoffenders, by debutoffence.

Table 2.7: The Proportion of 10-17 year old Offenders who Became Chronic and Serious Re-Offenders, by Debut Offence Type and Gender

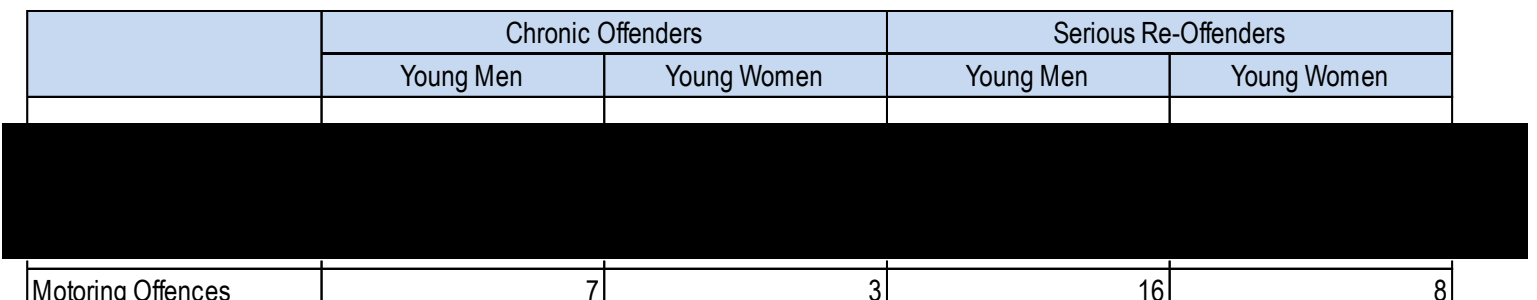

\begin{tabular}{|l|r|r|r|r|}
\hline Motoring Offences & 7 & 3 & 16 & 8 \\
\hline
\end{tabular}

Source: Owen and Cooper (2013: Tables B6 and B7)

Taking into consideration gender and age at debut offence, Owen and Cooper (2013) found that those who committed robberyas their debut offence were 1.7 times more likely to become a chronic offender compared with all other offence types. Those who committed vehicle theft or burglary were 1.6 times and 1.5 times, respectively more likely than offenders who committed other debut offence types to become chronic offenders.

Overall $65 \%$ of those aged $10-17$ at their first offence re-offended within the 9 years compared to $47 \%$ of $18-24$ year olds and $26 \%$ of those aged $25+$. Just over four-fifths ( $81 \%$ ) of $10-17$ year olds whose debut offence was robbery went on to commitfurther offences in the 9 year follow up period along with nearly three-quarters $(74 \%)$ of those who had committed a vehicle theft and $73 \%$ of those who had committed a burglary. Seven out of $10(71 \%)$ of those whose debut offence was a weapons offence had gone on to commitone or more further offences in the follow up period.

Owen and Cooper (2013) also found gender differences in terms of the proportions going on to commit serious re-offences. For example, $44 \%$ of young males whose debut offence had been robberywent on to commita serious re-offence as did around a third of those who had committed a burglary or vehicle theft. This compares to just over a quarter of females who had been 10-17 years when first sanctioned for a robbery debut offence. Similar patterns can be observed for other debut offence types. 
Evaluations of ASSET have tended to focus on one-year and two-year proven reoffending measures and have not followed young people who offend over longer periods. However, a number of typologies have been complied such as that from the Edinburgh Study which differentiate between early onset chronic, early onset desisters, later onset decliners and those with no convictions (McAra and McVie, 2010) which also point to different durations of criminal career. Ultimatelyitwould be desirable to identify how effective risk assessment tools such as ASSET are in identifying where differences lie in terms of the perceived risks of reoffending associated with each of these groups.

\subsection{Summary of Key lssues}

The criminal justice system has become increasinglyreliant upon standardised actuarial risk assessment tools which have become increasingly more reliable in terms of predicting further offending with each successive generation. Predictive risk assessment tools have been developed to facilitate decisions around parole and the evaluation of treatments. In the context of determining the extent to which an offender poses a risk to themselves or others, tools have also been designed specificallyfor use within forensic settings to assess dangerousness, particularly in the case of selective incapacitation, serious violent and sexual offending. In some cases, the tools have been modified specifically for use with juvenile offenders. However, within England and Wales, ASSET and its successor ASSETPlus have been developed specificallyas a tool to assess the likelihood of youth re-offending.

Whilst this Chapter has highlighted a number of fundamental issues in relation to ASSET, it should be noted that a number of these have been addressed through the development of ASSETPlus. Notably the literature published by the YJB suggests that clarification has been provided around the definition of risk being used and there have been attempts to reflectemerging research, policyand practice to reduce the psychosocial bias. However, many of the issues raised by critics relate to the continued use of Frequentistapproaches to develop actuarial risk assessment tools and the use of RFR as an evidence base. With risk assessment tools now such an integral part of the criminal justice decision making process, being promoted as being able to provide an objective, impartial and rational process which reduces reoffending and facilitates increased public protection, common sense suggests that actuarial tools will continue to evolve. This creates an opportunity to continue to develop the evidence base that underpins them so that it remains fit for purpose.

As Harcourt(writing in the context of profiling and policing) argues:

'The general public and most academics generally support the use of prediction in policing. To most, it is a matter of plain common sense. Why would we not use our best social science research and the most advanced statistical methods to improve the efficiency of police investigations, sentencing decisions, parole practices, treatment efforts, and general correctional procedures? Why not display our wealth of new knowledge to fight more effectively? It would be crazy not to take advantage of what we now know about the propensity to commitcrime.'

(Harcourt, 2007: 21) 
The reducing numbers within the formal youth justice system mean that increasingly those referred to the YOT represent more complexcases whilstreviews such as those by Lord Laming around the overrepresentation of those with care experience in the youth justice (Prison Reform Trust, 2016) and David Lammy MP around BAME experiences in the criminal justice system (Lammy, 2017) highlight inequalities. In order to support these young people a greater understanding of the interaction between risks, needs and vulnerabilities is required. Exploring emerging and innovative statistical approaches to examine this relationship therefore enables this to be done, particularlysince Bayesian approaches are better suited to working with smaller datasets.

In terms of new tools that have been developed elsewhere and for more specific forms of recidivism, these have sought to utilise innovative techniques such CHAID (van der Put, 2014; Assink et al., 2016) and logistical regression. Whilst these have enabled more sensitive analysis to be undertaken using these approaches are not without their limitations not least the need for large sample sizes. In other disciplines, the quest for more sophisticated tools continues with Liu et al. (2011) for example having compared the accuracy of logistic regression, classification and regression trees (CART) and neutral networks in the context of predicting violentre-offending on a sample of UK male prisoners. Also, within the context of forensic risk assessment, Harris and Rice (2013) have begun to explore the potential of utilising base rates for violent offending as informative priors within a Bayesian framework whilst Blattenberger et al. (2010) has compared differentBayesian models to predict return to prison.

The following chapters present the methodology employed for exploring the potential for utilising Bayesian approaches along with administrative data from the youth offending service to advance our understandings along with findings from a hierarchical model conducted under a Bayesian framework. In keeping with the analysis led approachoutlined in Chapter One, Chapter Three considers the structure of ASSET and the potential that this affords as well as identifying what can be achieved using the dataset available for this research. This is structured so as to provide the rationale for each of the research questions which underpin the analysis undertaken in Chapters Four to Seven.

\section{Methodology}

\subsection{The Potential Advantages of Viewing RFR through an Alternative Epistemological Lens \\ The Potential to Increase the Sensitivity}

The adoption of Bayesian approaches in youth justice, particularly through their application to administrative datasets represents an opportunity to address some of the key criticisms of RFR. By starting afresh with a suitably large administrative dataset and adopting an alternative epistemological lens, there is the potential for new criterion to be identified which will enable analysis to be undertaken to explore:

Page $\mid 66$ 
- Whether there are differences on the basis of demographic characteristics and experience of being in care.

- Different features of a 'criminal' career. For example, being a first-time entrant (FTE), age at first offence and conviction, and hence the duration of their time within the youth justice system. Associated with this is consideration of the impact of coming into contact with different facets of the youth justice system namely court appearances, the nature of the disposal received, spending time in custody / on remand and breaching.

- More sensitive measures of reoffending based on offence type and the seriousness of that offence. This will be explored through the use of the YJB Offence Categories and Gravity Scores used within the Reoffending Spreadsheet.

Undertaking this work will enable the lack of sensitivity with regard to the risk factor-reoffending relationship to be addressed. Whilst Bayesian approaches are not constrained in the same way by minimum sample sizes as Frequentistapproaches, the permutations of predictor variables and criterion which can be explored is limited by the information captured within the datasetand the time available in order to carry out the analysis. As a result, whilst it is possible to do more with less in terms of sample size, the absence of data and insufficient cases to form subgroups can still limit the analysis. However, it should be noted that when looking atrare events Bayesian approaches are privileged over Frequentist ones since small datasets can be more effectively handled due to the incorporation of prior information in the estimation.

\section{Subgroup Analysis}

Given the interest in developing more sensitive measures of reoffending and examining the impact of different features of a criminal career, the adoption of an approach which is appropriate for research involving a small number of observations and cases with non-stochastic data is desirable. Bayesian methods are advocated since they allow for estimates and predictions when there is insufficient data to fit the desired model using Frequentistmethods. Notablysmall data sets that produce fragile statistical methods based on Frequentistapproaches can be more effectively handled by the Bayesian approach because of the incorporation of prior information in the estimation.

Whilst Bayesian approaches are not constrained in the same way by minimum samples sizes as Frequentist approaches, the extent to which such analysis can be undertaken in relation to say demographic characteristics, particular interventions or sociographic / structural variables is still dependentupon there being sufficientcases within the dataset to form the subgroups. The permutations of predictor variables and criterion which can be explored are therefore limited by the information captured within the dataset and the time available in order to carry out the analysis. 
In opting to utilise Bayesian approaches as is done in subsequentchapters, the intention is to highlight the need to consider using all the tools in the toolbox in order to attempt to unpick what is a highly complex and often dynamic issue.

\section{Sequential Learning and Dynamic Risk}

Due to the monitoring requirements of the YOT it is possible to build up a picture over time of individuals and those sharing key characteristics. Through examination of their case histories and ASSET records, it is possible to establish temporal precedence. Key advantages of adopting a Bayesian approach include the fact that models can be updated as new information becomes available; that Bayesian methods support sequential learning and hence can utilise historical information and the results of other research when setting the prior (Berry, 2005). The approach also lends itself to the triangulation of data from other sources through data synthesis (McMahon et al., 2006). Notably the Bayesian approach is ideal for assessing and conveying uncertainty (Berry, 2006) giving it a distinct advantage over Frequentistapproaches when itcomes utilising information collected via subjective rating scales.

This latter feature is particularly pertinent in the case of the ASSET scores. Although RFR's claims to be culture-free (O'Mahony, 2009), it is anticipated that there may be variations in the interpretation of risk and/or adherence to guidance set out in the National Standards, and the introduction of subjectivity due to practitioner's individual values. As a result, inter-rater agreementis something that could impact on the generalizability of research findings. Agreementanalysis has been an active research area where Bayesian approaches have been employed. Calle-Alonso and Pérez Sánchez (2014) for example suggests a Monte Carlo-basedBayesian approach for measuring agreementin a qualitative scale rather than Cohen's Kappa.

Berry highlights 'Bayesian methods supportsequential learning, allow for finding predictive distributions of future results and enable borrowing strength across studies' (2005: 296). Specifically, he notes that 'the Bayesian paradigm allows for using historical information and results of other trials, whether they involve the same drug, similar drugs or possiblythe same drug but with different patient populations.' It is these qualities of Bayesian analysis make it ideal for exploring the complex relationship between risk and youth offending without completely dismissing existing research. Given RFR's heavy reliance on the findings from a single data source which have since been replicated in a multitude of studies, it would be pragmatic to revisit prior assumptions about risk factors and their relationship with youth offending. This can similarly be achieved using Bayesian analysis with highlighting that some statisticians and scientists are optimistic that Bayesian methods can improve the reliability of research by allowing scientists to crosscheck work done with the more traditional or "classical" approach. 


\section{Complexity}

In the context of advancing the evidence base in youth justice, a further feature which offers potential for extending knowledge is a mechanism for triangulating data from a number of different sources. Whilst Bayesian inference with its use of prior probabilities that can be drawn from previous research offers a formal process for synthesizing data from multiple sources, Bayesian evidence synthesis allows for the inclusion of other pertinent information that would otherwise be excluded as well as the potential to extend models to accommodate more complex, but frequently occurring, scenarios. Unlike in a metaanalysis, multiple treatment comparisons can be made, something which is much more in keeping with the suite of interventions which can make up a young person's action plan. Although the analysis presented in Chapters Four to Seven does not include interventions, the sequential manner in which different variables are added to the models serves to illustrate the potential for this to be done in the future.

\subsection{Introducing the Youth Offending Service Data \\ Strengths and Limitations}

Within youth justice in England and Wales, the Youth Offending Service maintains records on each young person that it comes into contactwith including their ASSET scores over time, journal records of supervision meetings and other contacts, and details of progress made in relation to the both their order and any specified requirements. Additional partnership data may also be held both informing their presentencing report and providing a more detailed picture of their individual circumstances. Whilst the assessment and ongoing monitoring requirements have been oft criticised for being 'managerialist' (Brownlee, 1998; Pitts, 2001; Baker, 2005), the data captured represents a comprehensive picture of key aspects of the young lives of those who have come into conflictwith the law.

In the case of the youth offending data utilised for this research, this is very rich since it incorporates information collected from a number of different sources including the young person, their parent/guardian, the police/courts, case workers along with other statutory and non-statutory agencies that may work or have contact with the individual during their order. However, this information has been collectedfor the purpose of monitoring the clientrather than for researc $h$ purposes. As a result, the data is structured for ease of looking at individual's records rather than extracting large volumes of records.

When the research was originallyconceived, the aspiration was to also look at what evidence existed within the ASSET which could be utilised to consider the prevalence of speech language and communication needs or mental ill health - issues which have emerged as areas for both societal and policyconcern since 2000 and have been incorporated into the ASSETPlus framework. Unfortunately, during the initial familiarisation training received, it became apparent thatalthough individuals mayhave referrals for support / treatment, the outcome of these referrals is not recorded within their record - the 
information being considered to be health data. This is also the case for referrals for substance misuse. This was particularlydisappointing given the Wales specific Youth Justice Key Performance Indicators around mental health, emotional health and wellbeing, and substance misuse which relate to the number of children identified as requiring assessment within 10 days of the screening date (Welsh Assembly Governmentand Youth Justice Board, 2009) and the identification of these as priorities in Children and Young People First (Welsh Governmentand Youth Justice Board, 2014).

In addition to this, it is important to note that whilst individual case workers may have opted to refer to their client's progress within case notes, there is no systematic mechanism via a designated field. To access this information would have required a manual trawl through each individual's record which was not feasible within the time available There is also no guarantee that the information would be included - in the case of speech, language and communication needs (SLCN), for example, these could potentially have been identified by the young person's school or another agency, and unless disclosed by the individual or their parent/guardian, the case worker maynot be aware of their diffic ulties including where there was a formal diagnosis.

\section{Selection Criteria}

\section{a) Geography}

In recent years there have been a number of local changes at Swansea YOT including the merger of Swansea, Neath-Port T albot and Bridgend to create The Western Bay Youth Justice \& EarlyIntervention Service. At the point where the data collection process commenced, the three local authorities each had their own standalone case management system. Although these have since been merged, the timing for this happening was uncertain with the initial attempt to integrate the systems before the roll out of ASSETPlus failing. Hence for pragmatic reasons, the decision was made to focus on those young offenders residentin the City and County of Swansea. This area had the highestcaseload of the three original local authorities and a member of the academic staff within the department was able to facilitate discussions with the data holder to secure permission to utilise the data.

To enable those referred to Swansea YOT to be identified should the merger of the three local authority datasets take place during the data collection period, a unique identifier was created for each individual within Childview. This identifier or 'ResearchID' replaced the Service Reference and was used to anonymise in all working versions of the data. Thus, any individual level information that left the YOT premises also had name and address data stripped out to protect the identify of those that the YOT had worked with. As an additional precaution, the lookup between the Service Reference and ResearchID has been retained on the YOT's server should it be necessaryto check details of anyindividual's record. In 2012, Swansea YOT changed its case management system from YOIS to Childview. The decision was made not to carry over case records relating to those:

Page | 70 
- Where the client was aged 23 years or over or the client's last offence dated before 2007

- Pre-courtcases prior to 2009 or where the clientwas aged over 18

- Blankcases

As a result, the data set in Childview does not represent a full historical record of all clients. Concem was also expressed by the data holder that the older records held in YOIS would not reflectcontemporary recording practices. The decision was therefore made to utilise young people's records from their point of entry into the re-offending cohortonwards rather than including those pre-dating the 2012/13 financial year.

\section{b) The Reoffending Cohort}

As part of the YJB's Reducing Reoffending Programme, each local YOT was provided with a prepopulated PNC Reoffending Data Tool or 'Reoffending Spreadsheet'. At the point of starting this research, spreadsheets were available for $2012 / 13$ and 2013/14. Table 3.1 summarises the fields that appear within these spreadsheets with a single row per young person: 
Table 3.1: Fields in the Local YOT Reoffending Spreadsheets 2012/13 and 2013/14

\begin{tabular}{|c|c|}
\hline Field & Notes \\
\hline PNC ID & (Blank) \\
\hline \multicolumn{2}{|l|}{ YOT } \\
\hline Service Reference & Replaced by ResearchID \\
\hline \multicolumn{2}{|l|}{ Age } \\
\hline \multicolumn{2}{|l|}{ Gender } \\
\hline \multicolumn{2}{|l|}{ Ethnicity } \\
\hline Locality (blank) & (Blank) \\
\hline LAC at Time of Disposal & (Blank) \\
\hline No of Previous Disposals & (Blank) \\
\hline No of Previous Custodial Outcomes & (Blank) \\
\hline \multicolumn{2}{|l|}{ Original Offence } \\
\hline \multicolumn{2}{|l|}{ Outcome } \\
\hline Outcome Type & \multirow[t]{2}{*}{ See Table 3.2 for further information } \\
\hline Outcome Tier & \\
\hline \multicolumn{2}{|l|}{ Date of Outcome (or custody release date) } \\
\hline \multicolumn{2}{|l|}{ Original Gravity Score } \\
\hline \multicolumn{2}{|l|}{ ASSET Score } \\
\hline \multicolumn{2}{|l|}{ ASSET Band } \\
\hline \multicolumn{2}{|l|}{ Intervention Level (amend if requried) } \\
\hline Reoffended (Y/N) & See Section 3.4 for futher information \\
\hline \multicolumn{2}{|l|}{ Reoffending Gravity Score } \\
\hline \multicolumn{2}{|l|}{ Seriousness of Further Offending } \\
\hline \multicolumn{2}{|l|}{ Most Serious Further Offence } \\
\hline \multicolumn{2}{|l|}{ Number of Further Offences } \\
\hline \multicolumn{2}{|l|}{ Date of 1st Further Offence } \\
\hline Time Entering Cohort to 1st Further Offence & In Months and Days \\
\hline Time Entering Cohort to 1stFurther Offence & In Decimalised Months \\
\hline \multicolumn{2}{|l|}{ Reoffence Same Category? } \\
\hline Custodial Establishment (Custody Cases Only) & (Blank) \\
\hline
\end{tabular}

The spreadsheets incorporate local YOT level performance data from the official Ministry of Justice PNC reoffending summarylevel performance data and have been designed so that YOT s can identify areas for improvement and targeting resources; reconciling and identifying gaps between the local and PNC data (in particular for 17-year olds and pre-courts); and comparing performance both over time and with different geographies (Youth Justice Board, 2017b)

\section{c) Identifying those with ASSET Core Profiles}

Swansea YOT operates a diversionary 'Bureau' model and as such their case management system includes details of both statutory and non-statutory ('Bureau') clients. The model is 'designed to divert young people out of the formal processes of the Youth Justice System', with Bureau clients being 'young 
people who have commita low-level offence and who have not previously received a Reprimand, Final Warning or Youth Conditional Caution' (Haines etal., 2013:169). There is nota designated field within the case management system to differentiate between the two. Bureau clients are generally risk assessed using the shorter version of the ASSET tool which was designed for those receiving Final Warnings. Whilst this is captures ratings across for each of the domain scores, there is no necessity to provide accompanying narrative. Given the wish to be able to drill down into the behaviours and circumstances thathave promoted a change in the individual domain risk scores, only those individuals who have ASSET Core Profiles have been included in the model. Cross-referencing the ResearchID's of those with ASSET Core Profiles with the re-offending spreadsheetsuggested an initial profile of the records which could be included in the model.

\section{2/13 Spreadsheet}

This initiallyconsisted of 148 records of which 146 could be matched to ResearchIDs.

Upon investigation, it was possible to identify that

- One of these relates to an individual with no assessments on the system. He was charged with a motoring offence and given a fine.

- There were 17 individuals who could initiallynot be matched to ResearchIDs. 16 of these individuals had service references (the YOT's unique ID) which had previously been identified as being anomalies. The remaining anomaly related to a test case which was subsequentlybe removed.

After removing duplicates (the majority of which had arisen from the service ref anomaly) the resulting spreadsheetconsisted of 134 unique individuals.

These records were then matched against the results of a query designed to pull back details of the ASSET Core Profiles. This identified 63 individuals who were part of the 2012/13 cohort who had been subject to the full risk assessment process. Of these 28 did not reoffend, 35 did reoffend. 


\section{3/14 Spreadsheet}

This initiallyconsisted of 265 records from across Western Bay YOT of which 132 could be matched

to ResearchIDs and hence can be assumed to be Swansea Young Offenders.

Upon investigation, it was possible to identify that

- One of these was a test case which was subsequentlyremoved

- There were 8 individuals who had service references which had previously been identified as being anomalies.

After removing duplicates (the majority of which had arisen from the service ref anomaly) the resulting spreadsheet consisted of 131 individuals.

These records were then matched against the results of a query designed to pull back details of ASSET Core Profiles. This identified 61 individuals who were part of the 2013/14 cohort who had been subject to the full risk assessment process. Of these 45 did not reoffend, 16 did reoffend.

For the purposes of the model, the following criterion were then applied to identify those records which fell within the period of interest:

- 2012/13 reoffending spreadsheet only: those ASSETs dated from the outcome date of their primary offence in 2012/13 to 31 ${ }^{\text {st }}$ March 2014 have been included.

- 2013/14 reoffending spreadsheet only: those ASSETs dated from the outcome date of their primaryoffence in 2013/14 to $31^{\text {st }}$ March 2015 have been included.

- If the young person appeared on both reoffending spreadsheets, then the period of interest runs from the outcome of their first offence in 2012/13 until 31 st March 2015.

\section{d) Harm onising the Outcomes}

During the period of interest, the possible outcomes or 'disposals' for young people changed as a result of the Legal Aid, Sentencing and Punishment of Offenders Act (LASPO), 2012. With effectfrom $8^{\text {th }}$ April 2013, Reprimands and Final Warnings were replaced by Youth Cautions and Youth Conditional Cautions. However, these are not directly comparable. Penalty Notices for Disorder or 'PNDs', more commonly known as 'on the spot fines' could previously be given to 16 and 17 year olds having committed low level offences. As a result of LASPO, PNDs were no longer available for under $18 \mathrm{~s}$ in 2013/14. Table 3.2 summarises the disposals by the outcome tiers used in the two reoffending spreadsheets. 
Table 3.2: Outcomes and Outcome Tiers for Young People Pre- and Post-LASPO

\begin{tabular}{|c|c|c|}
\hline 2012/13 Outcome & Outcome Tier & 2013/14 Outcome \\
\hline Detention and Training Order & Custody & Detention and Training Order \\
\hline Youth Rehabilitation Order & Community & Youth Rehabilitation Order \\
\hline Referral Order & First Tier & Bind Over \\
\cline { 3 - 3 } & & Compensation Order \\
\hline Final Warning & Pre-Court & Youth Caution \\
\hline Conditional Discharge & & No Intervention \\
\cline { 1 - 1 } Reprimand & &
\end{tabular}

Comparing Tables 3.3 and 3.4 , confirms that many of those without ASSET Core Profiles originallyhad no intervention or were subject to pre-court disposals. There are however, a small number who have gone on to commitfurther offences with their ASSET Core Profiles relating to these offences rather than the primary offence. Generally speaking there is a positive association between the assessed risk of reoffending (as measured by ASSET) and the outcome tier, with those receiving custodial sentences more likely to pose a greater risk. Within two cohorts there are a small number who have no ASSET recorded.

Table 3.3: Outcome Tier by ASSET Band for all Unique Individuals, Swansea YOT, 2012/13 and 2013/14

\begin{tabular}{|c|c|c|c|c|c|c|c|}
\hline & \multicolumn{5}{|c|}{ ASSET Band } & \multirow[b]{2}{*}{ Total } \\
\hline & & $\begin{array}{c}\text { No } \\
\text { Intervention }\end{array}$ & $\begin{array}{c}\text { No } \\
\text { ASSET }\end{array}$ & 1 to 14 & 15 to 25 & 26 to 48 & \\
\hline \multirow{5}{*}{$2012 / 13$} & No Intervention & 49 & - & - & - & - & 49 \\
\hline & Pre-Court & - & 3 & 29 & 9 & 2 & 43 \\
\hline & First Tier & - & 2 & 13 & 9 & 3 & 27 \\
\hline & Community & - & 1 & 2 & 3 & 4 & 10 \\
\hline & Custody & - & - & - & 1 & 4 & 5 \\
\hline \multicolumn{2}{|l|}{ Total } & 49 & 6 & 44 & 22 & 13 & 134 \\
\hline \multirow{5}{*}{$2013 / 14$} & No Intervention & 81 & - & - & - & - & 81 \\
\hline & Pre-Court & - & 1 & - & - & - & 1 \\
\hline & First Tier & - & 1 & 6 & 13 & 12 & 32 \\
\hline & Community & - & 3 & - & 5 & 6 & 14 \\
\hline & Custody & - & - & - & 1 & 2 & 3 \\
\hline \multicolumn{2}{|l|}{ Total } & 81 & 5 & 6 & 19 & 20 & 131 \\
\hline
\end{tabular}

Source: Local figures for 2012/13 and 2013/14 taken from locally held versions of the YJB's Re-offending Spreadsheets and may differ from published figures. In 2013/14 data for Swansea was published as part of the figures for Western Bay YOT with individuals being identified as being from Swansea on the basis of their YOT Identifier. 
Table 3.4: Outcome Tier and ASSETBands for those with ASSET Core Profiles, Swansea YOT, 2012/13 and 2013/14

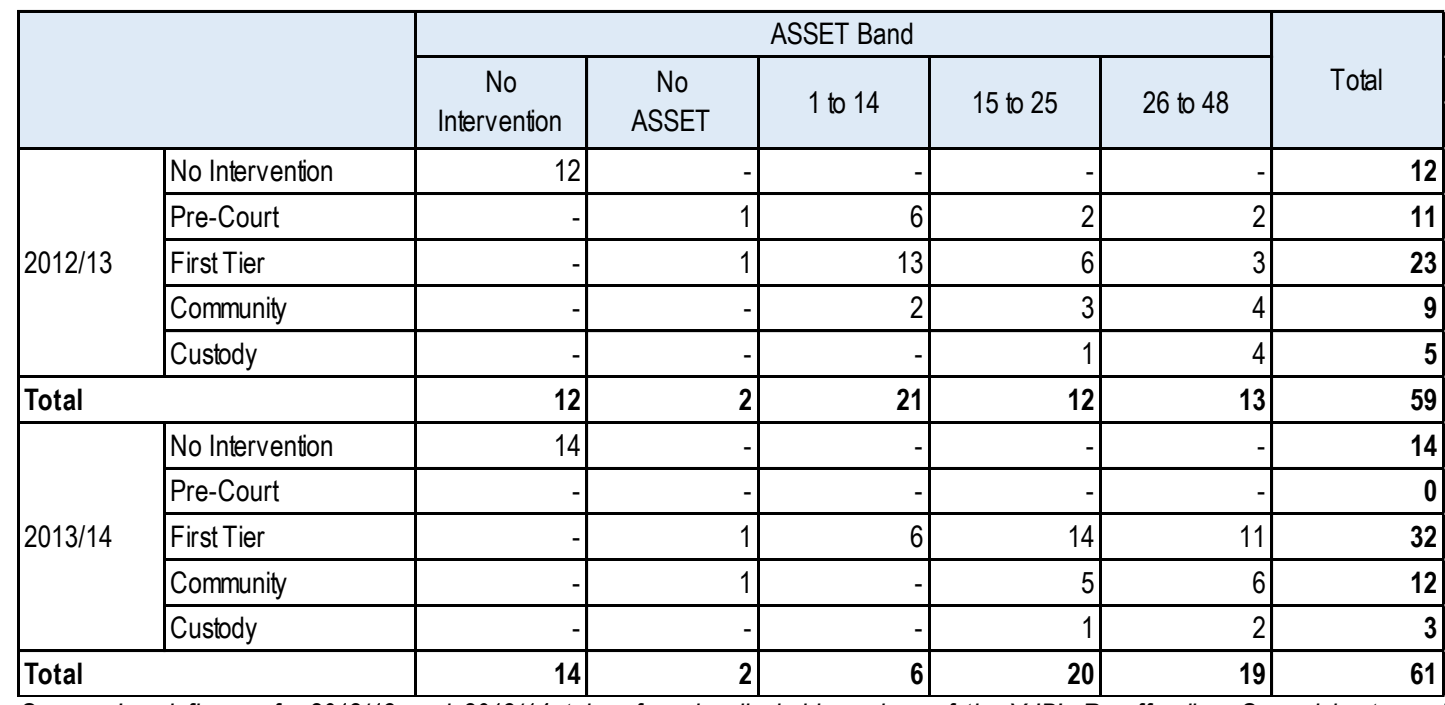

Source: Local figures for 2012/13 and 2013/14 taken from locally held versions of the YJB's Re-offending Spreadsheets and may differ from published figures. In 2013/14 data for Swansea was published as part of the figures for Western Bay YOT with individuals being identified as being from Swansea on the basis of their YOT Identifier. The bands correspond to the pre-Scaled Approach risk levels of Low, Medium and High, and are a sum of the ratings given for the 12 dynamic domains.

\section{e) Matching to ASSET Core Profiles}

The following querywas created to extract data from Childview relating to the ASSET Core Profiles:

\section{Query 1 - ASSET Scores by Domain}

Date specified on the basis of the assessment start date i.e. from $1{ }^{\text {st }}$ April 2012 to $31^{\text {st }}$ March 2015. The queried fields from Childview were:

- YOT Identifier (replaced by ResearchID)

- Birthdate

- Age

- Gender

- Ethnicity

- Start Date

- Stage

- Instance

- Care Order

- Eligible Child

- Relevant Child

\section{For Calculating Static Scores}

- Age at First Reprimand or Caution

- Age at First Conviction

- Number of Previous Convictions

- Number of Custodials

- Primary Offence
ForCalculating Dynamic Scores

- Living Arrangements

- Familyand Personal Circumstances

- Education, Training and Employment

- Neighbourhood

- Lifestyle

- Substance Use

- Physical Health

- Emotional and Mental Health

- Perception of Self and Others

- Thinking Behaviours

- Attitudes to Offending

- Motivation to Change

ASSET Score and Level of Intervention

- ASSET Static Score

- ASSET Dynamic Score

- Indicated Level of Intervention

- Adjusted Intervention Level

- Interim Level 
Unfortunately, it was not possible to pull back all the fields when the query was run. It was therefore necessary to manually populate these fields by looking at the individual records on Childview. The problem largely affected the fields required to calculate the static score i.e. original offence including date, age at first Reprimand/Caution/ Warning, age at first conviction and number of previous convictions. However, stage was also not pulled back - this field denoting the point in the referral process that the assessmentrelated to i.e. Start / Review / End.

Where the fields required to calculate the static score were populated within Childview, inspection revealed that there were a lot of inconsistencies. For example, one practitioner might have identified that the young person was aged 15 at the time of their first conviction, the next identified them as being 13. Whilst these inconsistencies did not prevent Childview from calculating a static score and hence a total ASSET score, where practitioners had failed to specify which offence was the Scaled Approach Offence (typically the one for which they had received the main outcome, or the one with the highest gravity score), then the static score was shown as being incomplete on the system. With an incomplete static score, the total ASSET score generated is misleading.

Having identified this issue with the static score generated within Childview, it was decided to use the information in the young person's offending and court records to populate the relevant fields.

\section{f) Creatingan EnhancedVersion of the Dataset}

The data represented as Queries 2 and 3 below was downloaded from each identified individual's record having previously been entered into Childview by the YOT. The information was then manuallyadded to create an enhanced version of the dataset. From the offence records it was possible to determine when further offences have been committed and when young people had been breached. The court records provided the dates for when the young person had attended court, regardless of the outcome of the proceedings.

\footnotetext{
Queries 2 and 3 - Individual's Offence and Court Records

No date criteria specified

Offences

- YOT Identifier (replaced by ResearchID)

- Offence Date

- Age (Years and Months)

- Offence

- Plea

- Outcome

- Seriousness (Gravity Score)

Court Proceedings

- YOT Identifier (replaced by ResearchID)

- Proceeding Date

- Age (Years and Months)

- Court Action

- Main Offence

- Main Outcome

- Term
} 
Notablyfrom the proceeding dates, itwas possible to identifywhich offence hadled to the young person's inclusion within the reoffending cohortfor that particularyear since the outcome date is used rather than the date that the offence was committed to mark the start of the one year follow up period. The exception to this is those who received a custodial sentence in which case the follow up period starts at the point when the young person has been released. Typically, young people sentenced to a detention and training order or 'DTO' serve half of their term with the remainder of the sentence being served under the supervision of their local YOT in the community.

Where a young person has been returned to the courtfollowing a breach, the outcome for the primary offence was over-written in the offence record. Within the court records, only the main offence is provided, hence it was not always clear when offences had been dealt with, with typically the main offence being the one with the highest seriousness score.

By cross referencing the start dates of the ASSET Core Profiles with the offending and courtrecords it was possible to identify if in the period prior to date of the assessment, the young person had:

- Breached - shown as an offence on the individual's offence record. In keeping with the definition used to identify proven reoffending (Section 3.5), where the young person has breached, this is not treated as a further offence

- Committed an offence - based on the date committed, regardless of the outcome subsequentlyreceived. Al young people are reflected as having committed an offence at Time 0 , marking their entry into the cohort.

- Attended court - proceeding dates are reflected on the courtrecord. Given the lag in cases coming before the court and the potential for cases being withdrawn and adjourned, no distinction is made between the nature of the proceedings. This includes where the young person has been placed on remand and when sentenced. More serious cases typically take less time to court and hence the order of court appearances does not necessarilycorrespond to the order in which offences were committed.

- Spent time in custody / on remand - this information is reflected in the court record as an outcome of the proceedings.

Particularlyin the case of offending and attending court, there maybe multiple occurrences in the period leading up to the ASSET being updated, therefore the flags created reflect simply that such an 'event' has occurred. 


\subsection{Ethical Considerations}

The main areas in which ethical issues can arise relate to whether there is harm or risk to participants; if there is a lack of informed consent; whether deception is involved, if there has been any invasion of privacy and respecting confidentiality (Punch, 2006). In the context of using administrative data, as has been done here, the data are not collected for research, but by the Youth Offending Service during the course of their normal business with data subjects being compelled to provide information about their circumstances and offending behaviour. As such there is a lack of implicit research consent which places a greater emphasis upon safeguarding the data and reporting with integrity.

Unlike administrative data available through the Administrative Data Research Network (ADRN), the data utilised within this research has been drawn directly from a live client database. Therefore, data classified as both personal and sensitive under the Data Protection Act 1998 features within the database. Further to this, data subjects, by virtue of their age are considered to be vulnerable with many having complex lives. For this reason, procedures were put in place to de-identify records onsite with pseudo identifiers being created for research purposes - the ResearchID. The 'lookup' of matched IDs has been retained on the YOT server - a secure environment-along with copies of original queries and datasets created during the process of de-personalising the data. In this way, the integrity of the personal data has been retained.

As part of the assurances made to the YOT during the process of negotiating access to the data, it was necessary to agree on steps to minimise the risks of statistical disclosure. For this reason, as will be seen in Chapters Four to Seven, data has been aggregated with pseudonyms being used when discussing individual cases. In some cases, it has been necessary to 'blur' the details to prevent individuals, their victims or theirfamilies being identified. Where it has been necessaryto do this, it has been flagged in the text.

Although there has been no directcontact with the data subjects, in order to seek clarification, especially in the context of understanding the offending histories of some of the young people, it has been necessary to speak to individual YOT workers about individual cases. This has been done on the understanding thatall information disclosed is confidential. Due to the sensitive subjectmatter and the vulnerability of the young people whose records are held within the YOT client database, it was necessary to secure Disclosure and Barring Service clearance; to sign a Third Party Connection Agreement with the City \& County of Swansea which includes a confidentiality agreement and Memorandum of Understanding about the use of their IT systems. Copies of the latter can be found in Appendix 2 of this volume along with a copy of the completed University's ethics form (Appendix 1). 


\subsection{Specific Features of the Data within Childview and ASSET}

As would be expected from anyadministrative dataset, Childview contains individual level data stored in a number of different formats including subjective rating scales from the ASSET Core Profile, free text, dates and postcodes. The 'date stamping' of activityprovides a means of establishing temporal ordering for example, highlighting where the timing of offences and courtappearances, and changes in their risk scores over time.

\section{Repeated Measures}

Since repeated ASSET Core Profiles are completed for young people over the course of their time with the YOT, the series of assessments for each individual can be thought of as being longitudinal data. As such the data lends itself to analysis by way of a hierarchical or multilevel model. When using hierarchical models to analyse longitudinal data, Level 1 is generally associated with a single measurementin time and Level 2 refers to an individual subject. In this way the advantages associated with the flexibility and power of such models can be maximised. For example, Finch etal. advo cate that modelling longitudinal data in a multilevel framework allows 'the simultaneous modeling of both intraindividual change (how an individual changes over time) and inter-individual change (difference in temporal change across individuals)' (2014:99-100).

Individuals under the supervision of the YOT will typicallyhave a 'Start' and 'Finish' ASSET. Depending upon the duration of their order, they may have further 'Review' ASSETs since the National Standards recommend that assessments are reviewed every three months or where there has been a significant change in the young person's circumstances. As such the data is unbalanced with individuals having differing numbers of assessments. Traditional techniques, such as repeated measures ANOVAs can only analyse balanced datasets whereas multilevel modelling can utilise available data from 'incomplete' observations. Additionally, repeated measures ANOVAs rely upon the assumption of sphericity(i.e. of equal variances of outcome variable differences). This assumption is unreasonable given that variability may change considerably over time. Thus, multilevel models, which do not require this assumption, offer greater flexibility by allowing information to be included in the model specification about the anticipated effects of time on error variation.

Multilevel models also allow for more complex data structures to be explored and can be considered to be a 'powerful and flexible extension to conventional regression frameworks ... extending the linear model and the generalised linear model by incorporating levels directlyinto the model statement, thus accounting for aggregation presentin the data' (Gill and Womack, 2013: 3). Through use of a nested data structure, it is therefore possible to avoid the unaccounted for heterogeneityand correlation which are common in conventional, flatmodelling. This has made hierarchical linear models the main type of application in biological and medical sciences (Snijders and Bosker, 2012: 247). However, as Gill and 
Womack (2013) observe, although hierarchical structures are common in social science data, they are commonlyignored by social science researchers.

Notably it is easy to incorporate both time-varying 'Level 1' predictors and time invariant 'Level 2' or individual level characteristics. In this way, temporal changes associated with both the domain scores and individual characteristics can be explored as per first research objective. This advances the work undertaken by Wilson and Hinks (2011: 10) who utilised 'Only one (Core/Final Warning) ASSET assessmentper offender' when selecting cases to include in their evaluation of the predictive accuracy of the tool.

To demonstrate the utility of the hierarchical modelling approach - the name often given to multilevel models run under a Bayesian framework, the equivalent models have also been run under a Frequentist framework. The R packages identified to undertake the analysis - MCMCglmm (Hadfield, 2010) and Ime4 (Bates et al., 2015), are very similar in terms of the way in which the model is specified, with both packages have been written specifically to enable generalised linear models to be fitted. The former uses Markov chain Monte Carlo techniques to fit models under a Bayesian framework. The latter is recommended by Li et al. (2011) as the most efficient package for logistic random effects regression models for binary or ordinal outcomes undera Frequentistapproach, in terms of usability, flexibility and speed.

Finch etal. (2014) provides an introduction to the glmer function in Ime4 and to MCMCgImm. However, vignettes are available for both packages. This research therefore also draws upon techniques outlined in MCMCgImm Course Notes (Hadfield, 2016), and tutorials provided by Wilson et al. (2010) and de Villemereuil (2012).

\section{Data Structure}

Within Childview, much of the data being utilised has been captured using structured forms. However, these are supported by reports from practitioners, the police / courts and other agencies which are held within free-text and journal fields. Whilst there is a desire to incorporate expert opinion as a distinct advantage of Bayesian approaches is that it is possible to incorporate both qualitative and quantitative data, the volume of information and way in which itis held, limits the extent to which this can be utilised. However, as will be seen in Chapter Five, being able to draw upon this detailed information, aids in the interpretation of findings.

As outlined in Section 3.2, Childview was designed to enable practitioners to monitor individual clients rather than for research purposes. Hence whilst data may be captured and is visible within the various 'pages' of Childview, it is not necessarilypossible to query the underlying data to do bulk extracts. As a result, it is possible for example to bulk download dynamic ASSET score (with accompanying identifiers) whilst the offence and courtrecords can only be downloaded for individual clients. Other fields e.g. the 
static scores appear to be 'locked down'. The situation was further complicated during the data collection period by the transition to an updated version of Childview which was intended as a transition database prior to the roll out of ASSETPlus. The local YOT has since migrated to Childview 3. Subtle changes in field names which occurred during this process meant that a number of the built-in queries ceased to work further reducing access to raw data from the original assessment tool. It is however, possible to view individual historic records for those young people who have committed further offending and have since been assessed using the new tool.

\section{Subjective Rating Scales}

Concerns around the subjective nature of the rating scales within ASSET, are lessened as a result of clients typically having a dedicated key worker for the duration of their order who is responsible for reviewing and updating their risk assessments. However, within the dataset are a proportion of individuals who have been subject to multiple orders and it has not always been possible to have this level of continuity. As part of the negotiations to secure access to the data, it was agreed that individual practitioners would notbe scrutinised. Hence, this potential area for variation has not been explored. In this respect it is prudent to highlight that the guidance which accompanies the ASSET tools is very comprehensive and therefore it is unlikely that within a local YOT there would be much variation ratings assigned by in a given situation.

As was done by Wilson and Hinks (2011), it has been necessary to assume that the ASSETs were completed correctlyby practitioners. Particularlyin the case of the dynamic scores it is not possible to undertake any quality assurance checks or to see if ratings from previous assessments had simplybeen copied. Inconsistencies were however identified in the static scores when the information from the ASSET was compared to the offence and court information held within Childview. As a result, this information has been used rather than that entered by the practitioner at the time of the assessment.

Strictly speaking the domain scores are ordinal data. However, in keeping with the advice given by Gelman (2010), the ratings have been treated as being discrete (from 0 to 4 ).

\section{Missing and Incomplete Data}

The issue of missing and incomplete data is not a modern phenomenon, nor is it unique to Bayesian approaches. Indeed, George B Vold, when writing about the efficiency of prediction in criminology in 1949 observed:

'The most discouraging thing about the whole field of prediction is criminologyis the continued unreliability and general worthlessness of much of the so-called 'information' in original records. Opinions, hearsay, and haphazardly recorded judgements still constitute the bulk of any parole file.'

(Cited in Farrington and Tarling, 1985:15) 
Whilst the view is that the quality of the data captured within the YOT is much higher than this, it has been necessary to follow the approach taken by both Baker et al $(2003,2005)$ and Wilson and Hinks (2011) to exclude ASSET records which were less than $80 \%$ complete. The difficulty encountered, is that for ease, Childview (the case management system within the YOT) permitted ASSET scores to be pre-populated using data from the previous assessment. The idea being that where there was new evidence (and hence a change in score) then this section could be over-written, leaving the remainder unchanged.

A manual check of the number, completeness and dates of the ASSET Core Profiles resulted in a small number of individuals being excluded - largely because their ASSET Core Profiles were blanks, were duplicates (based on start date and domain scores) or pre-dated the individual's entry to the cohort. Across the two years, it was necessaryto exclude a further 12 individuals who only had a single ASSET Core Profile within the relevant period thus limiting the amount that they could contribute to the model. This reduced the size of the reoffending cohortwith ASSET Core Profiles to 88. Between then they had 544 ASSET Profiles.

Issues around missing data within published sources are also a problem within RFR, limiting particularly analysis of trends amongstBME young offenders. Notably in 2013/14, a system errorled to 7\% of young people included in the annual workload statistics were shown as having 'unknown' ethnicity (Youth Justice Board and Ministry of Justice, 2015b). However, perhaps more significantlyit appears that the YOT has adopted a practice of recording clients as being of 'Any other White background' rather than 'White British' - potentially to reflect their national identify as being Welsh. Unfortunately, it is not possible to differentiate between these young people and those who say are of Eastern European heritage. This, plus the low numbers recorded as being from Asian, Black, Mixed or Other backgrounds $(6 / 88,7 \%)$ limits the amount of analysis that can be undertaken around ethnicity, particularly if crossreferenced by gender. (Table 3.5)

Table 3.5: Ethnicity and Gender Profile of Clients

\begin{tabular}{|c|l|r|r|r|}
\hline Ethnicity Recorded & Male & Female & Total \\
\hline \multirow{4}{*}{ White } & White Birtish & 16 & 3 & 19 \\
\cline { 2 - 5 } & White Irish & 1 & & 1 \\
\cline { 2 - 5 } & Any Other White Background & 66 & 7 & 73 \\
\hline \multirow{2}{*}{ Black } & Black Caribbean & 1 & 1 \\
\hline \multirow{2}{*}{ Asian } & Pakistani & 1 & 1 \\
\cline { 2 - 5 } & Any Other Asian Background & 2 & 2 \\
\hline \multirow{3}{*}{ Mixed } & White and Asian & 1 & 1 \\
\cline { 2 - 5 } & White and Black Caribbean & 1 & 1 \\
\cline { 2 - 5 } & Any Other Mixed / Multiple Background & 1 & & 1 \\
\hline Grand Total & & 90 & 10 & 100 \\
\hline
\end{tabular}

Notes: Young people included in the YJB's 2012/13 and /or 2013/14 Reoffending Spreadsheet for whom there are ASSET Core Profiles. 
Snijders and Bosker (2012) note that it can be assumed thatabsent data are missing at random and the fact that there are missing does not in itself provide relevant information about the studied phenomena. However, it should be noted that MCMCgImm and Ime4 do nothandle missing data well and as a result it has been necessary to exclude a small number of records due to one or more domain score being missing.

\subsection{Construct Validity: Re-Offending}

Historically, there has been concerned raised around the measurement of crime as a social phenomenon, with many of the studies which have contributed to the RFR evidence base relying upon self-reported measures of offending. This has led to a body of literature which has sought to compare self-reported and 'official' measures. For example, West and Farrington (1977) have done this with the cohort from the Cambridge Study of Delinquent Development and more recently the self-reported 'delinquency' reported by those involved in the Edinburgh Study of Youth Transitions and Crime have been compared against the 'rich and detailed records that exist in Scotland about young people who have been in contactwith the social work or children's hearing system' (Smith and McVie, 2003: 179). Comparisons have also been madeelsewhere e.g. Sullivan and McGloin (2014) whichadditionallylooks at the impactacross gender and race/ethnicity.

In the context of this piece of work, ASSET is promoted as being an assessment tool for determining the risk of re-offending. However, the reality is that it is concerned with re-conviction. As such the concems around using 'official data' are the most pertinent, with the potential for offending behaviour to occur which is does not come to the attention of the police / courts and hence may represent an underrepresentation of an individual's criminality. Following the Ministry of Justice Consultation on Improvements to Ministry of Justice Statistics, a proven offence is defined as any offence committed in a one year follow-up period that resulted in a court conviction, Caution, Reprimand or Final Warning in the one year follow-up or a further six month waiting period (to allow time for cases to progress through the courts) (Ministry of Justice, 2017a: 5). Although there has since been a move to a three-month cohort for re-offending (Ministry of Justice, 2017b), the data available to this research was based on a 12-month cohort. This is illustrated in Figure 3.1 for the 2013/14 performance year. 
Figure 3.1: Proven Re-Offending: 2013/14 Cohort

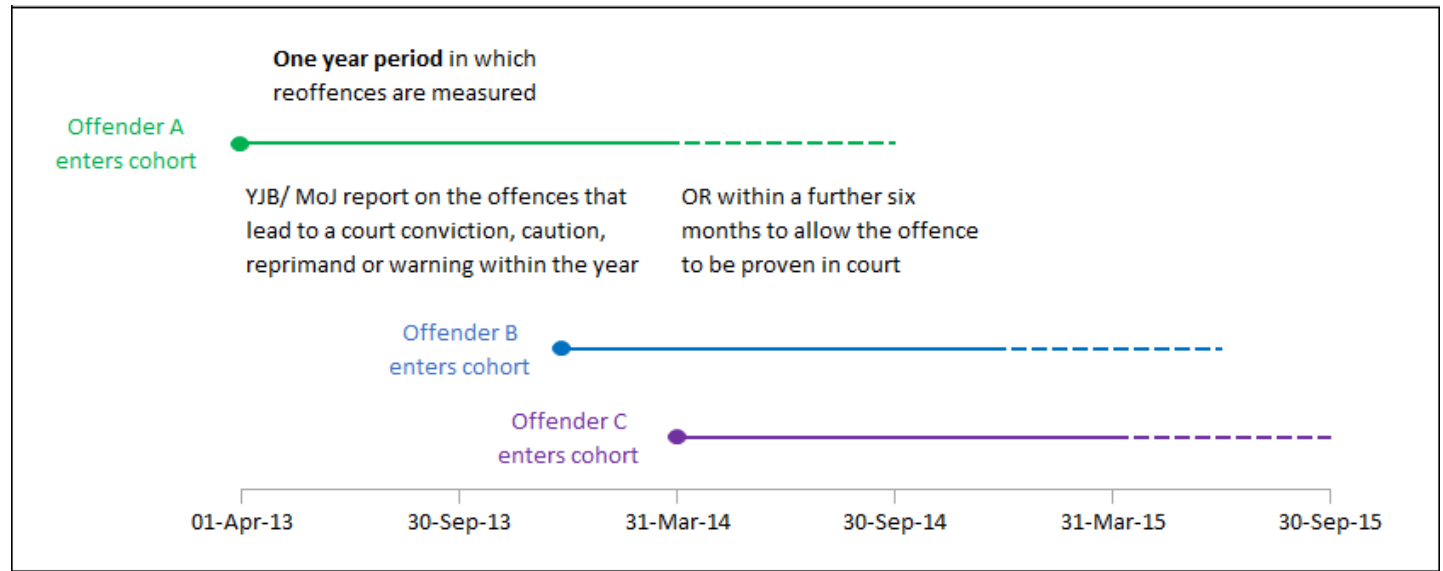

Adapted from Ministry of Justice, 2017

An offender enters the 2013/14 cohort if they are released from custody, received a non-custodial conviction at courtor received an offence in the period April 2013 to March 2014. Potentiallyan offender can enter the cohort on $31^{\text {st }}$ March 2014 and would still fall into scope. The need to wait for the $12-$ month follow up period plus up to six months for any further offending to be proven results in a lag in establishing whether or not the offender has re-offended. This is illustrated in Figure 3.2.

Due to the complexity of this administrative measure, the young person's reoffending status has been taken from the 'Reoffending Spreadsheet' since this was com piled utilising PNC records.

Figure 3.2: Re-Offending: A Worked Example Based on Offender A from the 2013/14 Cohort

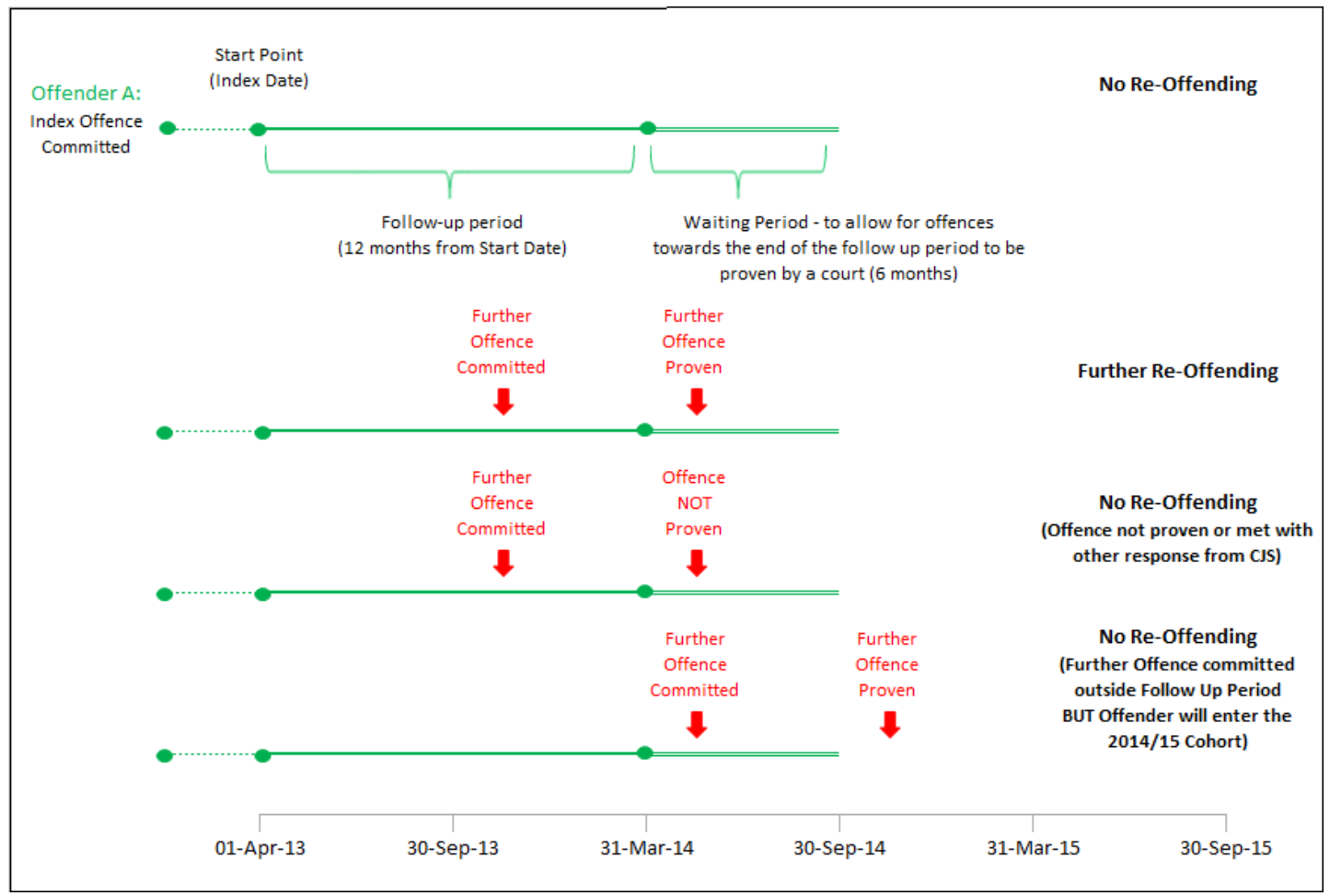


Analysis of the individual cases highlight that by virtue of their proven re-offending, there are 24 young people who feature in both the 2012/13 and 2013/14 cohorts. However, it is important to highlight that there are two young people who are shown as having not reoffended in both years. Figures 3.3 and 3.4 highlighthow using the official Ministry of Justice measure of proven re-offending maynot be as reliable as it initially seems. Whilst the utility of both self-reported and official measures of offending have been widely discussed elsewhere (see for example Farrington and Tarling (1985); Smith and McVie (2003)), the second case in particular illustrates that reliance upon the timing of the sentencing hearing to fulfil the criteria of further offending being proven and receiving a substantive outcome exposes a limitation of this measure. The first case demonstrates the importance of not just relying upon the domain risk scores - in this instance, the move would be reflected in the likelihood of reoffending scores relating to the young person's living arrangements and the family and personal relationships domains - but also having access to the supporting information which helped the practitioner come to their decision.

Figure 3.3: Case 1: A 13-year old Male Appearing in Both Cohorts

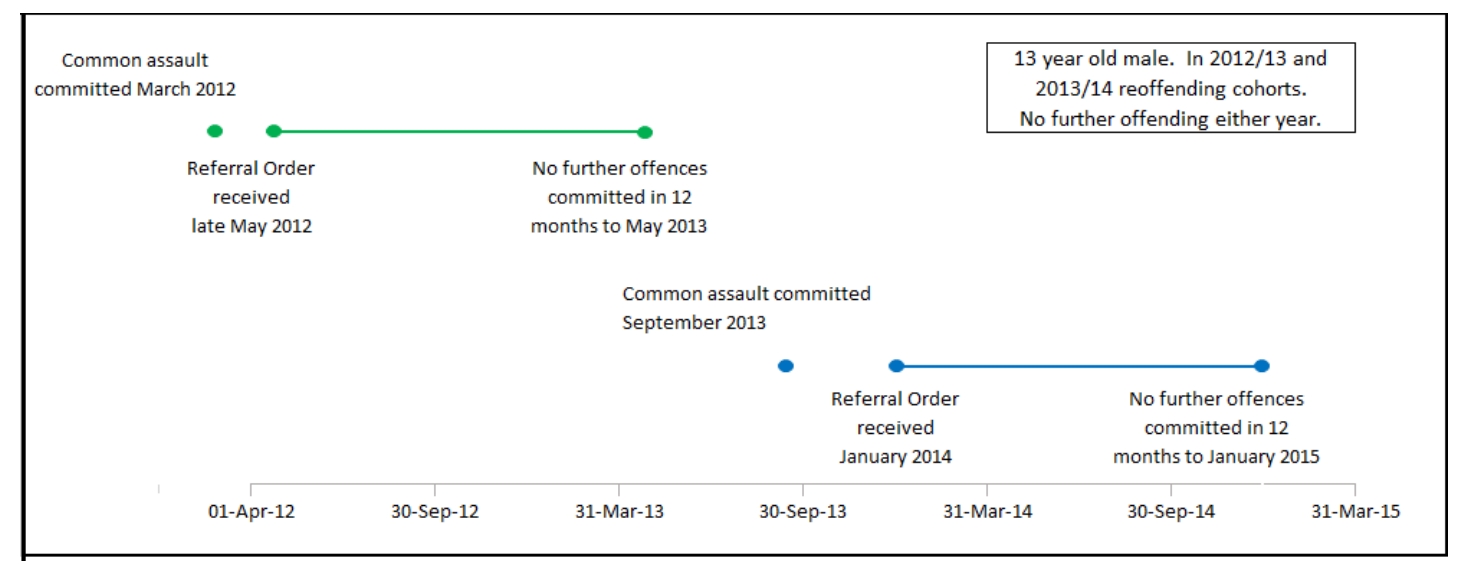

This individual has since gone on to commit further offences for which he has received substantive outcomes. However, there was a period of primary desistance which is understood to have coincided with a move to live with his father - his father being able to exert a stronger influence over his son's behaviour. The nextperiod of offending began in February2016.

Figure 3.4: Case 2: A 17-year old Male Appearing in Both Cohorts

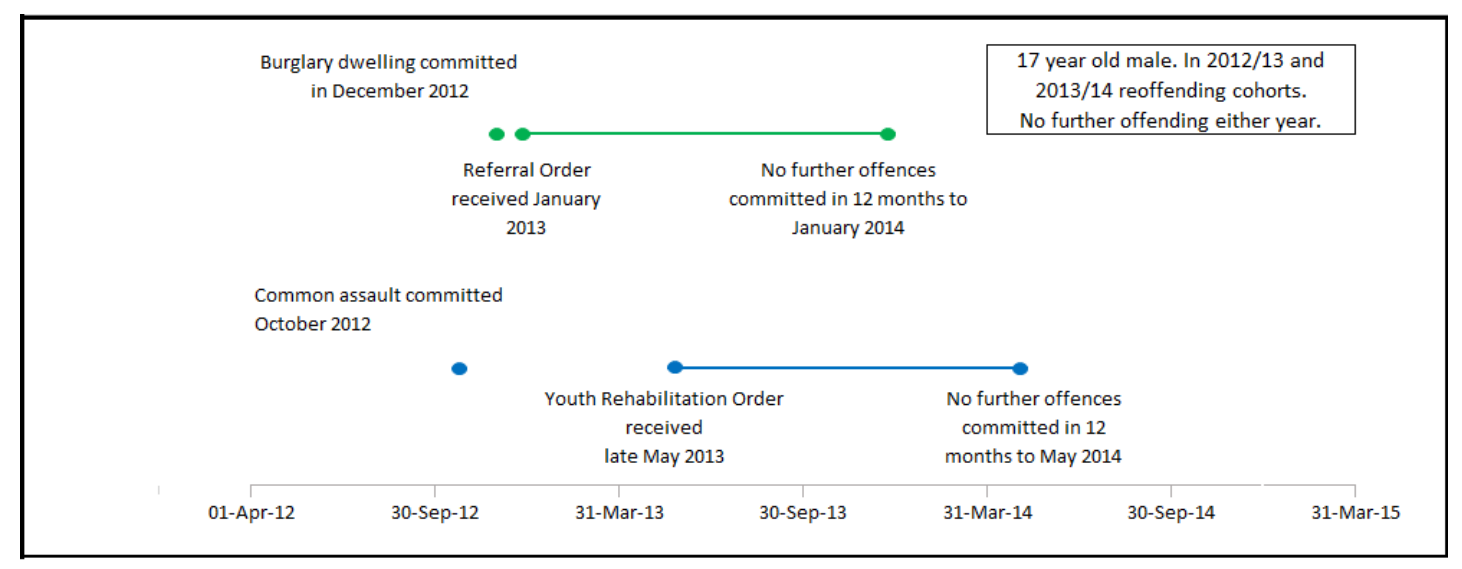


In this second case, the earlier offence took longer to go to court than the more serious burglarydwelling. As a result, when sentenced for the common assault, he was also re-sentenced for the burglary. The referral order for the burglary placed him in the 2012/13 cohort whilst the youth rehabilitation order received for the common assault placed in him in the 2013/14 cohort. At the time of the second sentencing hearing, he was two weeks shy of his eighteenth birthday. No further offences have been committed since late 2012 hence he is reflected as having not reoffended in either year.

It should also be noted that the manual determination of proven reoffending is dependent upon the following criteria being taken into consideration:

'Offences are counted as proven re-offences if they meetall of the following criteria:

- Theyare recordable. Not all offences are on the PNC and more recordable offences are entered than non-recordable offences. Analysis companing offences proven at court with offences recorded on the PNC suggests the most common offences thatare not recorded relates to motor vehicles, e.g. using a motor vehicle whilst uninsured against third party risks, speeding offences, keeping a vehicle on the highway without a driving licence or television licence evasion.

- Theywere committed in England or Wales.

- They are offences that were prosecuted by the police. PNC data are collected and inputby the police and offences prosecuted by the police are likely to be recorded more comprehensively on the PNC than offences that are prosecuted by other organisations.

- Offences are only counted if they are proven through caution, reprimands or final warnings (for juveniles) and court convictions. Offences that are not proven, or which meet with other responses from the Criminal Justice System, are not counted.

- The offence is not a breach offence, i.e. breach of a court order, since we are only interested in new offences.'

(Ministry of Justice, 2017a:7-8)

Without access to PNC, it has been necessary to rely upon the information captured within Childview around the young person's offending and courtappearances.

\subsection{The Preferred Outcome Variable}

As highlighted in the previous section, the 'official' measure of proven reoffending is somewhatartificial and is not withoutits limitations. In the context of the hierarchical modelling exercise, using re-offending which is measured at the individual level (Level 2) means that you no longer have a hierarchical structure i.e. time points nested within individuals. The preferred outcome variable is therefore further offending.

This measure has been added to the dataset to denote whether in the period prior to the assessment, the young person has committed one or more offences. As a result, at T ime 0 (the initial assessment), every young person is reflected as having committed one or more offences. Details of further offences 
have then been added as a flag using information from the young person's offending records recorded within Childview. Since there is often a lag in cases going to court, the measure is not based on proven further offending. As indicated in Section 3.2, flags for breaches, courtappearances and time spend in custody / on remand were added in a similarmanner.

The rationale for including this measure is that regardless of the ultimate outcome, the arrest/ charge is a reflection of the young person's offending behaviours and it is anticipated that this will be reflected in their risk score. The preferred outcome variable is therefore a binary measure. In the context of MCMCgImm, this means that the 'family' that the simulated model belongs to is ordinal whilst in Ime4, the distribution is reflected as being binomial.

\section{Research Question:}

What does the modelling tell us about the relationship between further offending, the 12 domains and time?

\subsection{Predictor Variables: Rationale for Inclusion}

In wishing to achieve the research aims and objectives set out in Chapter Two, a Bayesian hierarchical modelling approach has been adopted which utilises administrative data from the young people's ASSET Core Profiles. The cohorthas been identified using Swansea (and later Western Bay) YOT's prepopulated 2012/13 and 2013/14 reoffending spreadsheets, matched to ASSET Core Profiles.

In 2012/13, Swansea YOT had 134 clients in their Re-Offending Cohortafter test cases and issues with erroneous YOT Identifiers were removed. As a result, the local figures used within this research differ from those published by the YJB and Ministry of Justice. The following year, 131 of the 273 of those on the newly formed Western Bay YOT's spreadsheet were Swansea clients. For consistency, analysis has been limited in the second year to just those known to Swansea. Once matched, their dynamic scores and additional individual level data held within Childview, the resulting dataset has been utilised to explore the role of:

- Individual, demographic characteristics

- Being looked after by the local authority

- The nature of the primaryor index offence

In light of the inconsistencies around the way in which the static domains have been completed, proxy measures have also been developed to represent the young person's offending history and the impact of organisational measures reflecting specific facets of the youth justice system. This section therefore provides the rationale for the various predictor variables which will be used within the modelling. This is Page $\mid 88$ 
done by presenting analysis of both local data and national trends for proven reoffending to provide an indication of where differences mightexist within the wider cohort.

\section{Demographic Characteristics}

Across the two re-offending spreadsheets, less than one in five of the young offenders is female - a figure which is slightlylower than the national proportion receiving substantive outcomes in 2012/13 and 2013/14 i.e. 19.3\% and 18.6\% respectively (Youth Justice Board and Ministry of Justice, 2015a:Tables 3.3 and 3.5) (Table 3.6).

Table 3.6: Demographic Profile, Swansea YOT, 2012/13 and 2013/14

\begin{tabular}{|c|c|c|c|c|c|}
\hline & \multicolumn{2}{|c|}{$2012 / 13$} & \multicolumn{2}{|c|}{$2013 / 14$} \\
\hline & & No & $\%$ & No & $\%$ \\
\hline \multirow{2}{*}{ Gender } & Male & 109 & 81.3 & 108 & 82.4 \\
\hline & Female & 25 & 18.7 & 23 & 17.6 \\
\hline \multirow{2}{*}{ Ethnicity } & White & 123 & 91.8 & 124 & 94.7 \\
\hline & Non-White & 11 & 8.2 & 7 & 5.3 \\
\hline Age & 10 years & 0 & 0 & 0 & 0 \\
\hline \multirow{7}{*}{$\begin{array}{l}\text { (At the time of the } \\
\text { primary offence) }\end{array}$} & 11 years & 0 & 0 & 0 & 0 \\
\hline & 12 years & 4 & 3 & 0 & 0 \\
\hline & 13 years & 6 & 4.5 & 3 & 2.3 \\
\hline & 14 years & 16 & 11.9 & 17 & 13 \\
\hline & 15 years & 32 & 23.9 & 31 & 23.7 \\
\hline & 16 years & 30 & 22.4 & 33 & 25.2 \\
\hline & 17 years & 46 & 34.3 & 47 & 35.9 \\
\hline \multicolumn{2}{|l|}{ All Persons } & 134 & 100 & 131 & 100 \\
\hline
\end{tabular}

Source: Swansea YOT's Internal Re-offending Spreadsheet 2012/13 and Western Bay YOT's Internal Reoffending Spreadsheet 2013/14. 2013/14 Swansea figures identified on the basis of the individual's YOT Identifier.

In part reflecting the ethnic profile of Swansea at the time of the 2011 Census when $92.9 \%$ of Swansea's 10-17 year olds were identified as being White compared to the England and Wales average of $81.7 \%$ (Office of National Statistics, 2013), the numbers identifying as being non-White are low. In 2012/13, the breakdown was 4 Asians, 2 Mixed and 4 Unknown. Thefollowing year there were 2 Asians, 1 Black, 2 Mixed and 2 Unknown.

Nationally, 23.2\% of those receiving substantive outcomes in 2012/13 were aged 10-14 years, falling slightly to $22.1 \%$ the following year (Youth Justice Board and Ministry of Justice, 2015a: Table 3.5). Locally, the proportion was lower $-19.4 \%$ in $2012 / 13$ and $15.3 \%$ the following year, in part a reflection of the local diversionary practices which utilise the Youth Restorative Disposal (YRD) - a non-statutory disposal for young people committing a low-level offence (Haines etal., 2013). Introduced in 2008, this disposal is aimed at those who have not previously received a Reprimand, Final Warning or Youth Conditional Caution and is therefore more commonly given to younger offenders. As a non-statutory disposal YRDs are notconsidered to be a substantive outcome and hencethose receiving it do not reach the threshold to appear on the YJB's reoffending spreadsheet. 
At a headline level, 31.3\% (42/134) of those in the 2012/13 cohortre-offended whilst the following year the proportion fell significantly to $20.6 \%$ (27/131) - the fall, in part linked to the local implementation of the Legal Aid, Sentencing and Punishment of Offenders Act, 2012 which provided for greater flexibility in the sentencing of young offenders. With increased use of diversionary activity, the local trend bucked that experienced nationallywhere the proven reoffending rate increased from $36.1 \%$ in the year ending March 2013 to 38.0\% the following year (Youth Justice Board and Ministry of Justice, 2015a: Table 9.1).

Table 3.7: Proven Re-Offending, Local and National Rates, by Demographic Characteristics, 2012/13 and 2013/14

\begin{tabular}{|c|c|c|c|c|c|}
\hline & \multicolumn{2}{|c|}{$2012 / 13$} & \multicolumn{2}{|c|}{ 2013/14 } \\
\hline & & $\begin{array}{c}\text { Swansea } \\
\text { YOT }\end{array}$ & $\begin{array}{l}\text { England } \\
\text { and Wales }\end{array}$ & $\begin{array}{c}\text { Swansea } \\
\text { YOT }\end{array}$ & $\begin{array}{l}\text { England } \\
\text { and Wales }\end{array}$ \\
\hline \multirow{2}{*}{ Gender } & Male & $\begin{array}{c}34.9 \% \\
(38 / 109)\end{array}$ & $38.6 \%$ & $\begin{array}{l}23.6 \% \\
(25 / 108)\end{array}$ & $40.4 \%$ \\
\hline & Female & $\begin{array}{l}16.0 \% \\
(4 / 25)\end{array}$ & $26.2 \%$ & $\begin{array}{l}8.0 \% \\
(2 / 23)\end{array}$ & $28.4 \%$ \\
\hline \multirow[b]{2}{*}{ Ethnicity } & White & $\begin{array}{c}30.9 \% \\
(38 / 123)\end{array}$ & $36.0 \%$ & $\begin{array}{l}20.2 \% \\
(25 / 124)\end{array}$ & $38.4 \%$ \\
\hline & Non-White & $\begin{array}{r}36.4 \% \\
(4 / 11)\end{array}$ & $\begin{array}{l}\text { Asian - } 31.8 \% \\
\text { Black - } 43.9 \% \\
\text { Other - } 35.2 \%\end{array}$ & $\begin{array}{c}28.6 \% \\
(2 / 7)\end{array}$ & $\begin{array}{l}\text { Asian }-32.6 \% \\
\text { Black }-44.9 \% \\
\text { Other }-35.9 \%\end{array}$ \\
\hline \multirow{2}{*}{ Age } & $10-14$ years & $\begin{array}{l}19.2 \% \\
(5 / 26)\end{array}$ & $35.2 \%$ & $\begin{array}{l}10.0 \% \\
(2 / 20)\end{array}$ & $38.9 \%$ \\
\hline & $15-17+$ years & $\begin{array}{l}34.3 \% \\
(37 / 108)\end{array}$ & $36.4 \%$ & $\begin{array}{l}22.5 \% \\
(25 / 111)\end{array}$ & $37.8 \%$ \\
\hline \multicolumn{2}{|l|}{ Overall } & $\begin{array}{c}31.3 \% \\
(42 / 134)\end{array}$ & $36.1 \%$ & $\begin{array}{c}20.6 \% \\
(27 / 131)\end{array}$ & $38.0 \%$ \\
\hline
\end{tabular}

Notes: National figures taken from Youth Justice Board and Ministry of Justice (2015a: Tables 9.1, 9.2, 9.3 and 9.4) reflecting the years to end of March 2013 and March 2014 respectively. Local figures for 2012/13 and 2013/14 taken from locally held versions of the YJB's Re-offending Spreadsheets and may differ from published figures. In 2013/14 data for Swansea was published as part of the figures for Western Bay YOT with individuals being identified as being from Swansea on the basis of their YOT Identifier.

As can be seen, at both a local and national level, proven re-offending rates vary on the basis of gender, ethnicity and age (Table 3.7). However, the small numbers who have either identified as being NonWhite or nothad their ethnicityrecorded in the local cohorts highlights how the rates may be susceptible to high variability due to the size of the cohorts, with proven reoffending rates being determined for aggregated groups even at a national level. This finding supports the rationale for including sociodemographic characteristics within the hierarchical model. Athough, the low numbers suggest that may be necessary to set some of the variables up as being dichotomous e.g. White - Non-White.

As previously highlighted, by virtue of their offending behaviours, it is possible for some of those in the $2012 / 13$ to also feature on the reoffending spreadsheet for the following year. In total there were 24 individuals who appeared on both spreadsheets. The demographic profile of 88 members of the combined cohortwith ASSET Core Profiles is summarised in Table 3.8. 


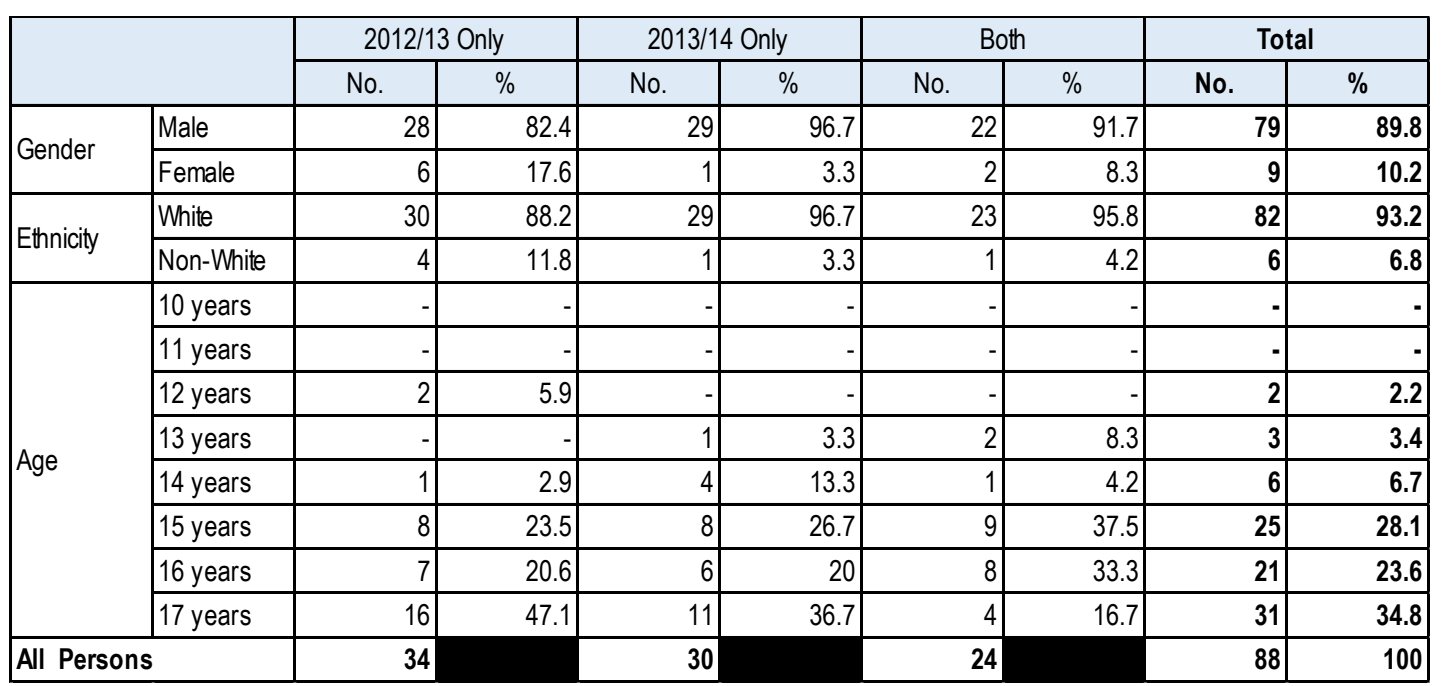

Notes: Age is at time committing the primary offence which led to entry to the cohort. Individuals have been identified as having multiple ASSET Core Profiles having met the criteria to be included on the YOT's Reoffending Spreadsheets 2012/13 and 2013/14.

Of the 82 males in the cohort, 6 are identified as having a non-White background. However, all the females identified as being White. The youngestmale appearing on the reoffending spreadsheetwas 12 at the time of entering the cohortwhilst the youngest female was 14 . Overall, $85 \%(77 / 88)$ of the young people were aged 15 to 17 at the time of their primary offence. This included all 6 of those identifying as being from a non-White background.

Table 3.9 summarises the rates of further offending by gender and ethnicity for those in the reoffending cohort. Since this offending is notnecessarily proven and may reflectmultiple offences committed during the period between ASSET assessments, directcomparisons cannotbe made with the published data. 
Table 3.9:Rates of Further Offending Across the Two Years, by Gender and Ethnicity

\begin{tabular}{|c|c|c|c|c|c|c|c|}
\hline & \multicolumn{2}{|c|}{ Comparator Groups } & No. & $\begin{array}{l}\text { Further } \\
\text { Offences }\end{array}$ & \begin{tabular}{c|c|}
$\%$ \\
Committing \\
Further Offences
\end{tabular} & $\begin{array}{l}\text { Bayes Factor (BF 10) } \\
\text { (H1: Group } 1 \text { f Group 2) }\end{array}$ & $\begin{array}{l}\text { Bayes Factor (BF 10) } \\
\text { (H1: Group } 1>\text { Group 2) }\end{array}$ \\
\hline \multirow[t]{2}{*}{ Gender } & 1 & Male & 79 & 39 & $49.4 \%$ & \multirow{2}{*}{0.591} & \multirow{2}{*}{0.238} \\
\hline & 2 & Female & 9 & 3 & $33.3 \%$ & & \\
\hline \multirow[t]{2}{*}{ Ethnicity } & 1 & White & 82 & 41 & $50.0 \%$ & \multirow{2}{*}{1.442} & \multirow{2}{*}{0.211} \\
\hline & & Non-White & 6 & 1 & $16.7 \%$ & & \\
\hline \multicolumn{3}{|l|}{ Total } & 88 & 42 & $47.7 \%$ & & \\
\hline
\end{tabular}

Notes: Bayes Factors have been calculated using the test for Bayesian Contingency Tables within JASP version 0.8.1.1. This can be thought of as being the equivalent of a $2 \times 2$ chi-squared test. The two sets of Bayes Factors represent the results of (1)

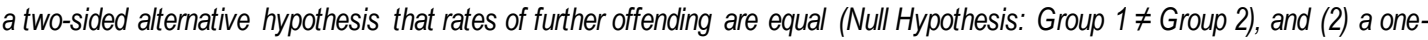
sided alternative hypothesis that the rates for Group 1 are larger than Group 2. Bayes Factors quantify the evidence for the alternative hypothesis relative to the null hypothesis and are interpreted using the categories suggested by Jeffreys (1961).

The rate for females (33.3\%) is notably lower than that for males (49.4\%) which is consistent with the national reoffending rates. There is moderate evidence to support the null-hypothesis that the rate for males is greater than that for female $\left(\mathrm{BF}_{10}=0.238\right)$. Since the Bayes Factor is less than $1 / 3$, this is can be interpreted as a significant result. National figures around the proven reoffending rate by ethnicity suggest that relative to those from a White ethnic background, the reoffending rates for those from an Asian or Other background are typically lower whilst those from a Black background have a higher reoffending rate (Youth Justice Board and Ministry of Justice, 2015a: Table 9.4) therefore a two-side hypothesis test was conducted to establish whether or not the apparent difference was statistically significant. This suggested that within this dataset, there is insufficient evidence for the alternative hypothesis that the reoffending rates are equal for both groups $\left(\mathrm{BF}_{10}=1.442\right)$. For there to be substantial evidence against the null hypothesis, the Bayes Factor would need to be greater than 3 . However, there is moderate evidence to suggest that the rate for the non-White group is significantlyless than that for the White group $\left(\mathrm{BF}_{10}=0.211\right)$ in the one-sided test.

\section{Research Question:}

What does the modelling tell us about the impact of gender and ethnicity on the likelihood of further offending over time? 


\section{Organisational Measure: Care Status}

The young person's care history is recorded within the ASSET Core Profile, with fields populated by the practitioner to reflect whether the young person is, or ever has been:

- Accommodated byvoluntary agreement with parents under section 20 of the Children Act 1989

- An 'eligible' or 'relevant' child

In the case of the former, if a young person who is accommodated under 20 goes into custody, he or she is no longer looked after by the local authority (although the authority may retain responsibility for providing a leaving care service). In such cases, itis necessaryfor the practitioner to update the ASSET after sentencing to reflect the change in status.

Eligible children are those young people still in care aged 16 and 17 who have been looked after for (a total of at least 13 weeks from the age of 14 . Relevant children are young people aged 16 or 17 who have already left care, and who were looked after for (a total of) at least 13 weeks from the age of 14 , and have been looked after at some time while 16 or 17 . The inclusion of these questions was intended to clarify whether a young person is entitled to the local authority's leaving care services under the provisions of the Children (Leaving Care) Act2000. Where applicable, entitlement continues if he or she is remanded or sentenced to custody.

Given the concerns about the over-representation of looked after children in the youth and adultcriminal justice systems, there is a desire to incorporate a measure within the final model. However, an initial examination of the data suggests 43 ASSET records (relating to 6 individuals) reflect that the young person was currently under a care order at the time of their assessment. There are an additional 4 records ( 1 individual) suggesting that the young person had previously been a looked after child. With just 7 young people having experience of being looked after based on this information, this limits the amount of analysis that can potentially be undertaken and the credibility of any findings. A further flag has therefore been created which reflects if (based on ASSET records) the child meets one or more of the following criteria:

- Previously or currentlysubject to a Care Order

- An Eligible child

- A Relevant child

In total, 25 of the 88 members of the cohortmet these criteria, including 2 (out of the 7) females and 23 males. Just one young person with a non-White background had experience of being in care.

Of the cohort with experience of care, 18 have committed further offences since coming under the supervision of the YOT, equivalent to $72.0 \%$ compared to $38.1 \%$ (24/63) for those who have not metany of the criteria. There is very strong evidence in favour of the alternative hypothesis that the rate for those 
with experience of care having a higher likelihood of committing further offences $\left(\mathrm{BF}_{10}\right.$ for the one-sided test $=33.97)($ Table 3.10).

Table 3.10: Rates of Further Offending Across the Two Years, by Care Status

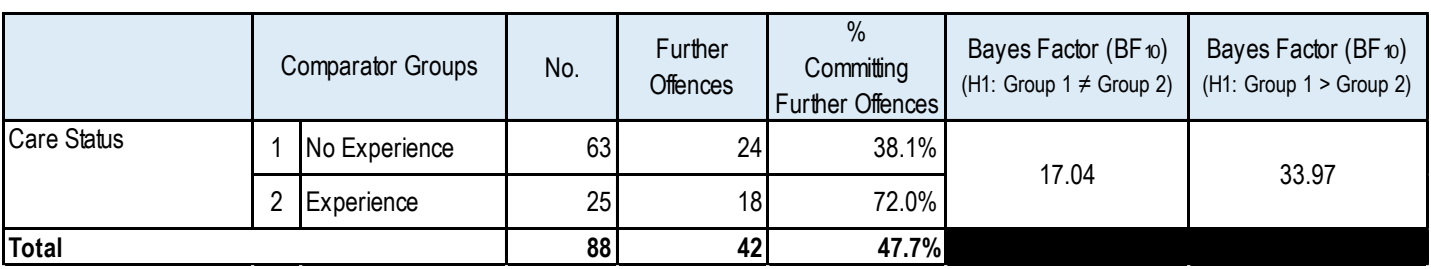

Notes: Bayes Factors have been calculated using the test for Bayesian Contingency Tables within JASP version 0.8.1.1.

\section{Research Question:}

What does the modelling tell us about the impact of having experience of care on the likelihood of further offending over time?

\section{Offending History}

Analysis of the offending history of the 88 individuals suggest that 33 (37.5\%)were first time entrants at the time of entering the cohort i.e. the outcome received for their initial primary offences was their Reprimand, Final Warning, Caution or conviction based on data held on the Police National Computer (PNC). However, 11 of these had previously been in contact with the YOT having receive informal action or a Youth Restorative Disposal (YRD). Whilst for 19, the primary offence had been their first time of offending (or more correctly, being caught), there were also 3 who had previously been in trouble with the police. One of these was the young person whose offending was summarised in Figure 3.4. Another was dealt with informally for their primary offence (and receiving a Youth Caution) having committed an earlier, more serious offence which took 3 months to get to court. There, he received a referral order. The third had his case withdrawn when it got to court.

Published national figures suggest that as the number of previous offences increases, the proportion of offenders who reoffend increases, with the proven re-offending rate for those with no previous proven offences being $36.1 \%$ in 2013 compared to $49.1 \%$ for those who have. The equivalent figures in the year ending March 2014 were $38.0 \%$ for first time entrants (FTEs) and $51.3 \%$ for those already known to the youth justice system. As the number of previous offences increases as does the proven reoffending rate with three-quarters of those who have committed 11 or more previous offences having proven reoffending (Youth Justice Board and Ministry of Justice, 2015a:Table 9.6).

The local figures also suggest a difference in the proven re-offending rate, with $33.3 \%(11 / 33)$ of those identified as being an FTE at their time of entry the cohortwent on to commitfurther offences compared with $57.4 \%(31 / 54)$ of those who had previously offended - the status of one individual is not known. 
There is moderate evidence in favour of the null hypothesis that the rate of offending is higher amongst those who are not FTEs $\left(\mathrm{BF}_{10}\right.$ for the one-sided test $\left.=5.548\right)($ T able 3.11).

Table 3.11:Rates of Further Offending Across the Two Years, by Offending History

\begin{tabular}{|c|c|c|c|c|c|c|c|}
\hline & \multicolumn{2}{|c|}{ Comparator Groups } & No. & $\begin{array}{l}\text { Further } \\
\text { Offences }\end{array}$ & $\begin{array}{c}\% \\
\text { Commiting }\end{array}$ & $\begin{array}{l}\text { Bayes Factor (BF 10) } \\
\text { (H1: Group } 1 \text { ₹ Group 2) }\end{array}$ & $\begin{array}{l}\text { Bayes Factor (BF 10) } \\
\text { (H1: Group } 1>\text { Group 2) }\end{array}$ \\
\hline \multirow[t]{2}{*}{ FTE } & 1 & FTE & 33 & 11 & $33.3 \%$ & \multirow{2}{*}{2.808} & \multirow{2}{*}{5.548} \\
\hline & 2 & Previous Offending & 54 & 31 & $57.4 \%$ & & \\
\hline \multirow[t]{2}{*}{ Age at First Offence } & 1 & 10 to 12 & 22 & 13 & $59.1 \%$ & \multirow{2}{*}{0.623} & \multirow{2}{*}{0.136} \\
\hline & 2 & 13 to 17 & 66 & 29 & $43.9 \%$ & & \\
\hline \multirow[t]{2}{*}{ Age at First Conviction } & 1 & 10 to 13 & 11 & 7 & $63.6 \%$ & \multirow{2}{*}{0.691} & \multirow{2}{*}{0.192} \\
\hline & 2 & 14 to 17 & 77 & 35 & $45.5 \%$ & & \\
\hline \multicolumn{3}{|l|}{ Total } & 87 & 42 & $48.3 \%$ & & \\
\hline
\end{tabular}

Notes: Bayes Factors have been calculated using the test for Bayesian Contingency Tables within JASP version 0.8.1.1.

Under the Scaled Approach, those who received their first Reprimand, Caution or Final Warning before the age of 13 were considered to be at a higher risk of re-offending than older children. The further offending rates have therefore been compared for the two age groups based on the proxy measure of age at first offence. This suggests that there is only anecdotal evidence to support the null hypothesis of there being a difference between the two groups $\left(\mathrm{BF}_{10}=0.623\right)$. A similar trend was observed when the cohort was split on the basis of age at their first conviction - in this instance, those aged 10 to 13 were considered to be a higher risk then those aged 14 plus $\left(\mathrm{BF}_{10}=0.691\right)$.

Despite these findings, the decision has been to retain these measures of the individual's offending history within the model. In terms of the gender profile, only one female received their first Reprimand/ Caution / Final Warning whilst in the younger age group, along with 21 (out of the 79) males. All the females were in the 14-17 year group when they received their first conviction, whilst 11 males were aged 10-13.

\section{The Nature of the Primary Offence}

The YJB's reoffending spreadsheets provide details of the main or primary offence. As such information is only provided about the offence which attracts the most severe sentencing outcome, or if there are two offences in the same case then the offence with the statutory maximum sentence is deemed to be the 'primary offence'. Other offences which are dealtwith by that courtcase or cautioning occasion are ignored. This approach is consistent with that used in the publication of youth justice statistics (Youth Justice Board and Ministry of Justice, 2015c). However, it presents challenges not only during the preparation of the data for the modelling exercise butalso when considering an approach to considering more sensitive measures of re-offending. Published national figures for the years being considered by this research suggest that the proven reoffending rate varies by index or prim aryoffence suggesting that it may be a source of variation at an individual level (Figure 3.5). Inclusion of information about the 
nature of the primary offence also supports the aim of developing a more sensitive measure of reoffending.

Table 3.12 provides a breakdown of the primary offences committed by those individuals in the reoffending cohort with ASSET Core Profiles along with the rate of reoffending and further offending for each category. The most commonly occurring primary offences are categorised as being violence against the person; theft and handling stolen goods; public order offences; drugs; criminal damage and motoring offences. The comparatively low numbers whose offending falls under some of the less commonlyoccurring principleoffence categories maylimit the analysis that can be undertaken. Notably across the two cohorts, there are no young people recorded as having their primary offence being death or injury by dangerous driving or arson.

Table 3.12:Primary Offence Category of Those with ASSET Core Profiles, with Re-Offending and Further Offending Rates

\begin{tabular}{|c|c|c|c|c|c|}
\hline \multirow{2}{*}{ Primary Offence Category } & \multirow{2}{*}{ Total } & \multicolumn{2}{|c|}{ Re-Offended? } & \multicolumn{2}{|c|}{ Further Offending? } \\
\hline & & Number & $\%$ & Number & $\%$ \\
\hline Criminal Damage & 12 & 6 & $50.0 \%$ & 7 & $58.3 \%$ \\
\hline Domestic Burglary & 5 & 3 & $60.0 \%$ & 3 & $60.0 \%$ \\
\hline Drugs & 8 & 5 & $62.5 \%$ & 4 & $50.0 \%$ \\
\hline Motoring Offences & 4 & 1 & $25.0 \%$ & 3 & $75.0 \%$ \\
\hline Non Domestic Burglary & 2 & 1 & $50.0 \%$ & 1 & $50.0 \%$ \\
\hline Other & 1 & & $0.0 \%$ & & $0.0 \%$ \\
\hline Public Order & 11 & 2 & $18.2 \%$ & 3 & $27.3 \%$ \\
\hline Racially Aggravated & 1 & & $0.0 \%$ & & $0.0 \%$ \\
\hline Robbery & 5 & 3 & $60.0 \%$ & 3 & $60.0 \%$ \\
\hline Sexual Offences & 1 & & $0.0 \%$ & & $0.0 \%$ \\
\hline Theft And Handling Stolen Goods & 10 & 3 & $30.0 \%$ & 4 & $40.0 \%$ \\
\hline Vehicle Theft / Unauthorised Taking & 6 & 5 & $83.3 \%$ & 5 & $83.3 \%$ \\
\hline Violence Against The Person & 22 & 8 & $36.4 \%$ & 9 & $40.9 \%$ \\
\hline Grand Total & 88 & 37 & $42.0 \%$ & 42 & $47.7 \%$ \\
\hline
\end{tabular}

Notes: Offence category of the primary offence upon entering the cohort. Individuals have been identified as having multiple ASSET Core Profiles having met the criteria to be included on the YOT's Reoffending Spreadsheets 2012/13 and 2013/14. 
Figure 3.5:National Proven Reoffending Data, by Index Offence, Years Ending March 2013 and 2014

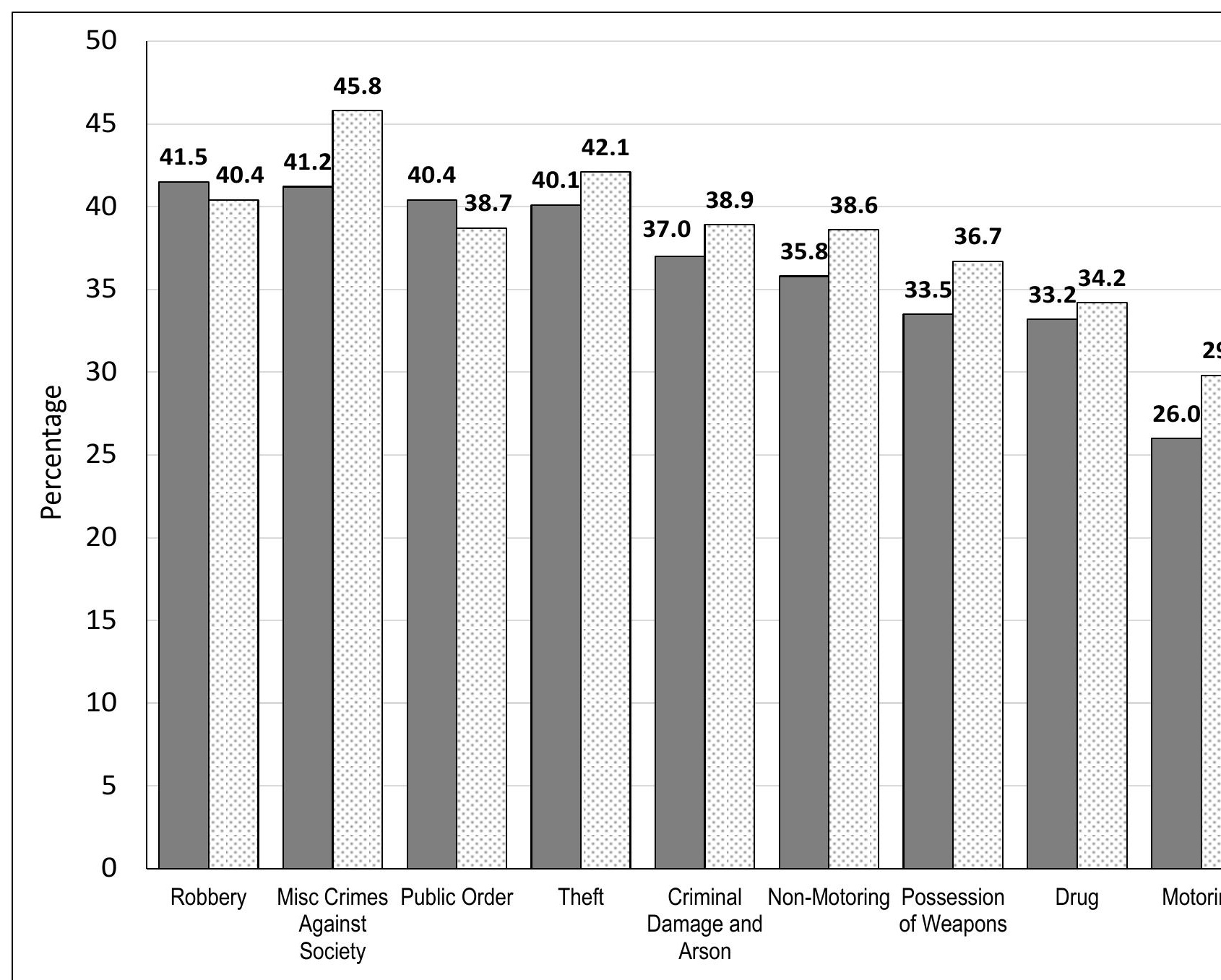

Source: Youth Justice Board and Ministry of Justice (2015a: Table 9.5). The offence categories used here are based on the ONS crime classificatio in the reoffending spreadsheets. 
The criteria set out which defines the Ministry of Justice's measure of reoffending (see Section 3.4) precludes any of the cohorthaving a breach as a primary offence since breaches typically result in the young person being re-sentenced rather than them receiving an additional substantive outcome. Where this has occurred, information about the original offence is given on the reoffending spreadsheet.

An alternative to using the offence category is to use the gravity scores of the primary offence (Table 3.13). Designed with eight levels, there are examples of offencesscoring 2-6 within reoffending cohort Reducing the number of groups to six as opposed to the populated offence categories, will incur less of penalty in any modelling and offers a means of making inferences about those offence categories for which there are no examples within the dataset.

Table 3.13:Gravity Score of the Primary Offence for Those with ASSET Core Profiles, with Re-Offending and Further Offending Rates

\begin{tabular}{|l|r|r|r|r|r|}
\hline \multirow{2}{*}{ Gravity Score of the Primary Offence } & \multirow{2}{*}{ Total } & \multicolumn{2}{|c|}{ Re-Offended? } & \multicolumn{2}{c|}{ Further Offending? } \\
\cline { 3 - 6 } & & Number & \multicolumn{1}{c|}{$\%$} & Number & \multicolumn{1}{c|}{$\%$} \\
\hline 2 & 31 & 13 & $41.9 \%$ & 16 & $51.6 \%$ \\
\hline 3 & 31 & 12 & $38.7 \%$ & 13 & $41.9 \%$ \\
\hline 4 & 9 & 4 & $44.4 \%$ & 5 & $55.6 \%$ \\
\hline 5 & 4 & 2 & $50.0 \%$ & 2 & $50.0 \%$ \\
\hline 6 & 13 & 6 & $46.2 \%$ & 6 & $46.2 \%$ \\
\hline Grand Total & $\mathbf{8 8}$ & $\mathbf{3 7}$ & $\mathbf{4 2 . 0} \%$ & $\mathbf{4 2}$ & $\mathbf{4 7 . 7} \%$ \\
\hline
\end{tabular}

Notes: Gravity score of primary offence upon entering the cohort. Individuals have been identified as having multiple ASSET Core Profiles having met the criteria to be included on the YOT's Reoffending Spreadsheets 2012/13 and 2013/14.

Table 3.14:Primary Offence Category by YJB Gravity Score

\begin{tabular}{|c|c|c|c|c|c|c|}
\hline \multirow{2}{*}{ Primary Offence Category } & \multicolumn{5}{|c|}{ YJB Gravity Score } & \multirow{2}{*}{ Total } \\
\hline & 2 & 3 & 4 & 5 & 6 & \\
\hline Criminal Damage & 11 & 1 & & & & 12 \\
\hline Domestic Burglary & & & & & 5 & 5 \\
\hline Drugs & 8 & & & & & 8 \\
\hline Motoring Offences & 3 & 1 & & & & 4 \\
\hline Non Domestic Burglary & & & 2 & & & 2 \\
\hline Other & & 1 & & & & 1 \\
\hline Public Order & 9 & 1 & & 1 & & 11 \\
\hline Racially Aggravated & & 1 & & & & 1 \\
\hline Robbery & & & & & 5 & 5 \\
\hline Sexual Offences & & & 1 & & & 1 \\
\hline Theft And Handling Stolen Goods & & 10 & & & & 10 \\
\hline Vehicle Theft / Unauthorised Taking & & 1 & 2 & 3 & & 6 \\
\hline Violence Against The Person & & 15 & 4 & & 3 & 22 \\
\hline Grand Total & 31 & 31 & 9 & 4 & 13 & 88 \\
\hline
\end{tabular}

Notes: Offence category and gravity score of the primary offence upon entering the cohort. Individuals have been identified as having multiple ASSET Core Profiles having met the criteria to be included on the YOT's Reoffending Spreadsheets 2012/13 and $2013 / 14$.

A further advantage is that using gravity scores offers a means of differentiating between the seriousness of the offence within an offence category (Table 3.14). For example, violence against the person offences accountfor almostone in four primaryoffence categories recorded (22/88). Of these,

Page $\mid 98$ 
- The majorityhave been identified as having a gravity score of $3(68.2 \%)$. Violence against the person offences attracting a gravity score of 3 include: possession of an offensive weapon; threatening, abusive or insulting words / behaviour; assaulting a police officer and common assault.

- $18.2 \%$ had a gravity score of 4 - equivalent to assaultoccasioning bodilyharm ( $\mathrm{ABH})$

- No members of the cohort had been involved in firearms offences which have a gravity score of 5

- $13.6 \%$ had a gravity score of 6 - equivalent to grievous bodily harm (GBH)

- None of the cohortwere recorded as being involved in more serious offending such as murder (8); manslaughter (8); abduction/ kidnap (7); GBH or wounding with intent (7).

(It should be noted that the gravity scores utilised within this thesis are based on those published within the ASSET Guidance issued by the YJB (Youth Justice Board, 2008a: Appendix B). These have had various incarnations and differ from those used by the Police. A full list of offences by category and gravity score can be found in the Section 2 of the Technical Annex.)

\section{Research Question:}

What does the modelling tell us about the impact of the 'static' factors within ASSET in predicting further offending over time?

Inclusion of the outcome tier in the model is supported by the published reoffending figures by index disposal. Notably, at a national level, those receiving custodial sentences have a higher reoffending rate than those receiving pre-courtdisposals such as cautions (Figure 3.6). 
Figure 3.6: National Proven Reoffending Data, by Index Disposal, Years Ending March 2013 and 2014

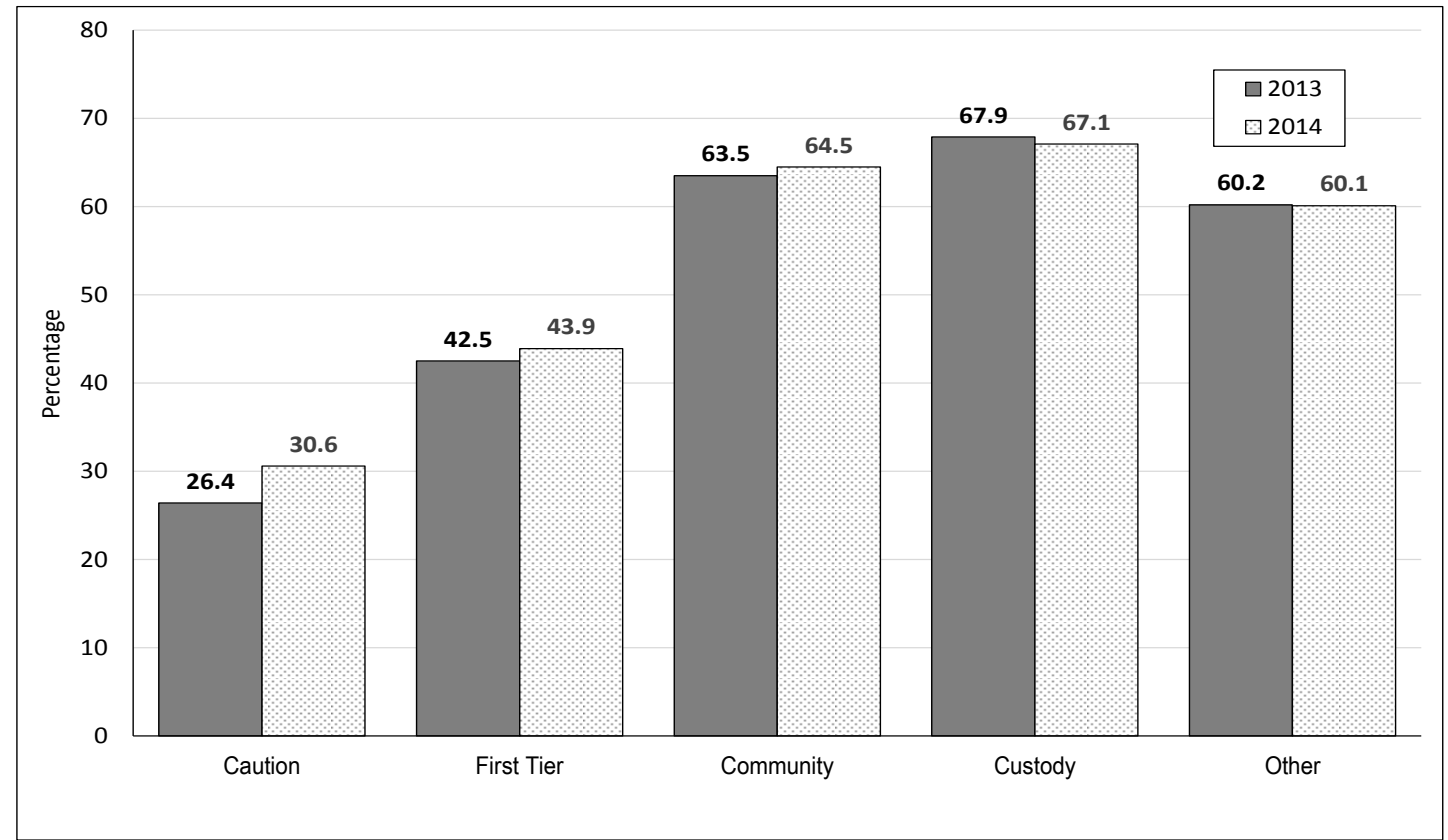

Source: Youth Justice Board and Ministry of Justice (2015a: Table 9.7).

However, in cross referencing the offences and outcomes in the reoffending spreadsheets with those recorded in the individual's offending and court records as held within Childview inconsistencies were found. It is likely that this is as a result of where young people have been returned to court to have their original sentence reviewed:

Individual (1) - committed a drugs offence (seriousness $=2$ ) whilst on conditional bail and tag during the hearing relating to burglary dwelling offences. He was sentenced to an 8-month Detention and Training Order. The reoffending spreadsheet reflects his primary offence as being a drugs offence.

Individual (2) - Originally sentenced in 2011 to a 1 -year conditional discharge for a drugs offence (seriousness = 2). However, he continued to offend and was referred back to the courts where he received a Youth Rehabilitation Order. Further restrictions were later added to this as he committed further offences before being sentenced to a 10-month Detention and Training Order. He is included on the reoffending spreadsheetas having received a custodial sentence for the 2011 drugs offence.

Without access to the original records in PNC, it has not been possible to establish the extent to which this has occurred across the dataset.

As previously highlighted, the timing of the research coincided with the changes in youth disposals coming aboutas a result of LASPO 2012. These changes were implemented between December 2012 and April 2013. To enable comparisons to be made across the period of interestitis therefore necessary to use outcome tiers as per Table 3.2. However, as can be seen from Table 3.15, as sentencing is dependentnotjust upon the seriousness of the offence, there is no directcorrelation between the gravity score and the outcome tier of the disposal received for the primary offence. 
Table 3.15: Outcome Tier of the Disposal Received for the Primary Offence, by YJB Gravity Score

\begin{tabular}{|l|r|r|r|r|r|r|}
\hline \multirow{2}{*}{ Outcome Tier of the Primary Offence } & \multicolumn{5}{|c|}{ YJB Gravity Score } & \multirow{2}{*}{ Total } \\
\cline { 2 - 7 } & 2 & 3 & 4 & 5 & 6 & \\
\hline No intervention & 5 & 5 & & & 1 & 11 \\
\hline Pre-Court & 4 & 3 & 4 & 1 & & 12 \\
\hline First-Tier & 16 & 17 & 5 & 2 & 8 & 48 \\
\hline Community & 4 & 6 & & & 2 & 12 \\
\hline Custody & 2 & & & 1 & 2 & 5 \\
\hline Grand Total & $\mathbf{3 1}$ & $\mathbf{3 1}$ & $\mathbf{9}$ & $\mathbf{4}$ & $\mathbf{1 3}$ & $\mathbf{8 8}$ \\
\hline
\end{tabular}

Notes: Outcome tier of disposal received upon entering the cohort. Individuals have been identified as having multiple ASSET Core Profiles having met the criteria to be included on the YOT's Reoffending Spreadsheets 2012/13 and 2013/14. For details of the disposals falling under each Outcome Tier see Table 3.2.

Initial analysis of the local re-offending rates by outcome tier suggests that they increase amongst those who have had court disposals, with the rate being highest amongst those sentenced to custodial sentences. The re-offending rates for those who were subject to no intervention or who received a precourt disposal are notablya lot higher. It is important to note that since this research focuses upon those within the formal youth justice system. As such the ASSET Core Profiles for these individuals relate not to the primary offence, but to the assessmentundertaken after their first further offence.

Given the comparatively small number of cases, particularly receiving community and custodial sentences, along with the concerns about the reliability of the outcomes recorded, the decision has been made not to investigate the role of outcome tier at this time. The information has however been retained within the dataset to assist in interpreting findings.

For completeness, the re-offending and further offending rates for each outcome tier of those cases included in the modelling exercise have been included (Table 3.16).

Table 3.16: Outcome Tier of the Disposal Received for the Primary Offence for Those with ASSET Core Profiles, with ReOffending and Further Offending Rates

\begin{tabular}{|l|r|r|r|r|r|}
\hline \multirow{2}{*}{ Outcome Tier of the Primary Offence } & \multirow{2}{*}{ Total } & \multicolumn{2}{c|}{ Re-Offended? } & \multicolumn{2}{c|}{ Further Offending? } \\
\cline { 3 - 6 } & & Number & \multicolumn{1}{c|}{$\%$} & Number & \multicolumn{1}{c|}{$\%$} \\
\hline No intervention & 11 & 8 & $72.7 \%$ & 6 & $54.5 \%$ \\
\hline Pre-Court & 12 & 8 & $66.7 \%$ & 8 & $66.7 \%$ \\
\hline First-Tier & 48 & 10 & $20.8 \%$ & 16 & $33.3 \%$ \\
\hline Community & 12 & 7 & $58.3 \%$ & 9 & $75.0 \%$ \\
\hline Custodial & 5 & 4 & $80.0 \%$ & 3 & $60.0 \%$ \\
\hline Grand Total & $\mathbf{8 8}$ & $\mathbf{3 7}$ & $\mathbf{4 2 . 0} \%$ & $\mathbf{4 2}$ & $\mathbf{4 7 . 7 \%}$ \\
\hline
\end{tabular}

Notes: Outcome tier of disposal received upon entering the cohort. Individuals have been identified as having multiple ASSET Core Profiles having met the criteria to be included on the YOT's Reoffending Spreadsheets 2012/13 and 2013/14. 


\section{Organisational Measures: Facets of the Youth Justice System}

Cross-referencing court and offending records from Childview with the ASSET Core Profiles enabled 'flags' to be added to reflect whether or not in the period before the date of the assessment, the young person had:

- Breached (and this is recorded as an offence)

- Attended court

- Spent time in custody, including anytime on remand

Breachescan be interpreted as a measure of non-compliance and can often resultin the young person being returned to the court in order to have their order reviewed. Serious breaches can result in the young person becoming subject to a period in custody. Court appearances do notnecessarilyresult in the young person being sentenced. The case may well be adjourned or even withdrawn. Depending upon the circumstances that have led to the young person appearing in court, they may be subject to further restrictions (including upon their liberty) with bail conditions potentiallybeing applied including the young person placed on an Intensive Supervision and SupportProgramme(ISSP), tagged or remanded. It was originally hypothesised that some young people would be motivated to stay out of any further trouble in the run up to their appearance in courtand hence there would be decrease in thei risk scores. However, there may be others who were anticipating for example a custodial sentence and the 'threat' of this loss of liberty may be sufficient for them to engage in further risky and offending behaviours believing that if they are going down then they might as well go down for everything. Increased risk scores were anticipated for this group.

It is recognised that some young people who have come into contact with the law live very chaotic and complex lives. Therefore, a period in custodycan offer stability and an opportunity to engage in training, receive substance misuse treatment and participate in activities which address their thinking behaviours I attitudes towards offending. It is therefore anticipated that risk scores would go down, certainlyin these domains. However, concerns about resettlement, the loss of liberty and having to face up to the consequences of their actions may have a detrimental effecton for example mental wellbeing.

Along with the domain scores, these predictors have the potential to change at the time of each assessment. Hence, they are included in the model as time-varying, 'Level 1' predictors. 


\section{Research Question:}

What does the modelling tell us about the relationship between further offending and coming into contact with facets of the youth justice system?

\subsection{Summary of Research Questions}

Within this chapter, a number of research questions have been posed. These have been grouped within subsequentchapters:

\begin{tabular}{|c|c|c|}
\hline \multicolumn{2}{|c|}{ Chapter and Theme } & \multirow{2}{*}{$\begin{array}{l}\text { Research Questions } \\
\text { What is the relationship between further offending, the } 12 \text { domains and time? }\end{array}$} \\
\hline 4 & Risk Assessment Domains & \\
\hline \multirow{2}{*}{5} & \multirow{2}{*}{ Dimensional Identity } & What is the impact of gender and ethnicity on the likelihood of further offending? \\
\hline & & $\begin{array}{l}\text { What is the impact of having experience of care on the likelihood of further offending } \\
\text { over time? }\end{array}$ \\
\hline \multirow{2}{*}{6} & \multirow{2}{*}{ Static Factors } & $\begin{array}{l}\text { What is the impact of the 'static' factors within ASSET in predicting further offending } \\
\text { over time? }\end{array}$ \\
\hline & & Is it possible to extend the sensitivity of ASSET by extending any of the predictors? \\
\hline \multirow{2}{*}{7} & \multirow{2}{*}{ System Contact } & $\begin{array}{l}\text { How is the likelihood of further offending affected by having experience of care and } \\
\text { a previous offending history? }\end{array}$ \\
\hline & & $\begin{array}{l}\text { What is the impact of coming into contact with facets of the youth justice system on } \\
\text { the likelihood of further offending? }\end{array}$ \\
\hline
\end{tabular}

Chapter Four presents the development of the hierarchical model to reflect the ASSET Core Profile framework with its repeated measures. This enables the first research question to be explored. The basic dynamic model is then adapted to explore the impact of dimensional identifyin Chapter Five and the impact of 'static' factors within ASSET in Chapter Six. Chapter Seven concentrates on whether having system contactincreases the likelihood of further offending.

The nature of the measures used as proxies for the static factors in Chapter Six provide the greatest scope for considering whether itis possible to extend the sensitivity of ASSET through extending any of the predictors. Hence this research question is also explored within this chapter.

The second considered within Chapter Seven links back to the research questions posed in Chapter Five and Six which utilise the predictors around care and FTE status.

Cutting across the four chapters, a further question is posed which considers How well ASSET scores reflect the realities of the young person's changein circumstances during their time under the supervision of the YOT. As highlighted in Chapter One, this final question provides a means of assessing the predictive accuracy of the various models constructed in response to the other questions posed. 


\section{Findings: Risk Assessment Domains}

As highlighted in Chapter Two, the premise behind the APIS framework is the continuous cycle of (re)assessment, (re)formulation of sentence planning, and supervision approaches. As such the likelihood of reoffending determined by the ASSET Core Profile informs the action plan devised for the young person and determines the nature and level of interventions. The outcome of these interventions in reducing the likelihood of reoffending then inform the reassessment process (Figure 2.2). Having a continuous cycle means that a series of risk assessment scores are available for each young person which can then be analysed to explore the relationship between the twelve dynamic risk factors measures in ASSET and young offending behaviours. This has been done within this Chapter by focusing on the following research questions:

1. What is the relationship between further offending, the 12 domain scores and time?

8. How well do ASSET scores reflect the realities of the young person's change in circumstances during their time under the supervision of the YOT?

The first of these is addressed initially through consideration of the change in total ASSET scores and those relating to individual domains between assessments, building upon the approach taken by Baker et al (2005). Their analysis is then extended in section 4.2 through development of a hierarchical model representing the repeated assessment process undertaken using the ASSET core profile. Theextent to which this model reflects the realities of a young person's change in circumstances during their time with the YOT is considered through presentation of three examples who for the purposes of this research will be referred to as Fred, David and Connor. Their scores will also be used in subsequent chapters to consider how models which reflect dimensional identity, the nature of criminal careers and system contact fit their changing circumstances.

As highlighted in Chapter T wo, as part of the ASSET Core Profile, practitioners are required to provide a subjective rating of the young person's likelihood of reoffending based on a series of questions which are grouped under 12 domains:

- Living arrangements

- Familyand personal relationships

- Education, training and employment(ETE)

- Neighbourhood

- Lifestyle

- Substance use
- Physical health

- Emotional and mental health

- Perception of self and others

- Thinking and behaviour

- Attitudes to behaviour

- Motivation to change

These represent the dynamic component of the overall ASSET score, with a maximum potential score of $48(4 \times 12)$. It is apparent from reviewing the linked reoffending spreadsheet and the ASSET Core Profile records that the score used for the ASSET banding / levels utilises only the dynamic score. 
Guidance is available for practitioners (Youth Justice Board, 2008a) which includes further explanation of the evidence collecting questions and provides exam ples of ratings 1 or 2 and 3 or 4 for each domain. Table 4.1 provides a generic summary of the subjective ratings used. The distribution of domain scores can be found in Figure 4.1 .

Table 4.1: Subjective Ratings Used in ASSET for the 12 Domains

\begin{tabular}{|c|l|}
\hline Rating & Description \\
\hline 0 & Not associated at all \\
\hline 1 & Slight, occasional or only a limited indirect association \\
\hline 2 & $\begin{array}{l}\text { Moderate but definite association - could be direct or indirect link. May be related to some offending, but not } \\
\text { all. Tends to become offending related when combined with other fadors. }\end{array}$ \\
\hline 3 & Quite strongly associated - normally a direct link, relevant to most types / occasions of his/her offending. \\
\hline 4 & $\begin{array}{l}\text { Very strongly associated - will be dearly and directly related to any offending by the young person. Will be a } \\
\text { dominant fador in any duster of offending-related problems. }\end{array}$ \\
\hline
\end{tabular}

Adapted from Youth Justice Board (2008b: 4)

A visual inspection the distributions of each of the domain scores (Figure 4.1) suggests that it is appropriate to assume that each set of measurements is independent and that each potential rating has an equal probability of being assigned. In this instance, the zero is meaningful therefore it has not been necessary to centre or standardise the domain scores. 
Figure 4.1: Distribution of Domain Scores
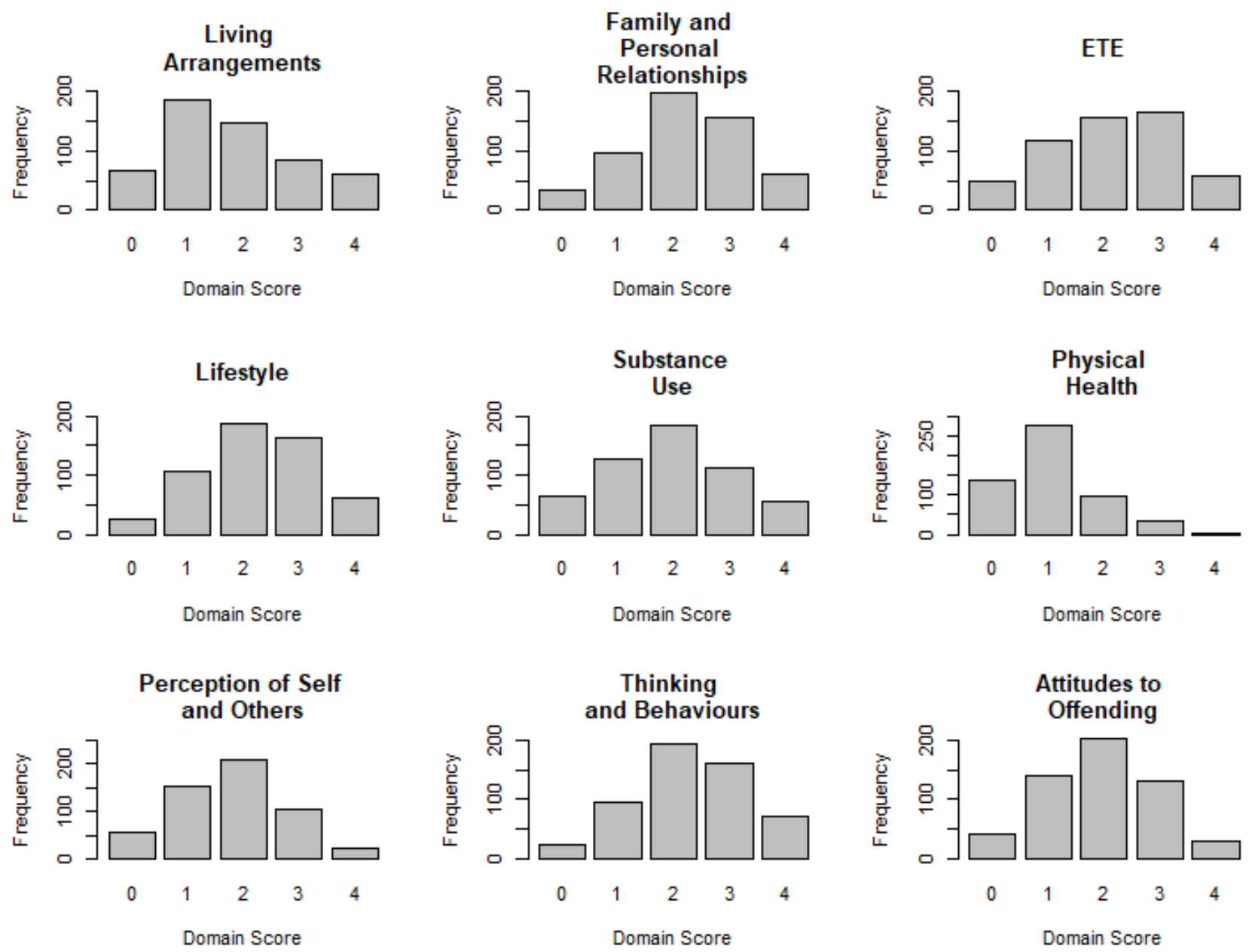

Notes: $n=545$ (All complete ASSETS), 87 Individuals 


\subsection{Changes over Time}

\section{Change in Domain Scores}

Whilst previous reviews of ASSET have focused upon the validity and reliability in terms of predicting reconviction (Baker etal., 2003) and the link between ASSET and Intervention Plans (Baker etal., 2005), the second review also considered the effectiveness of the revised version of ASSET in measuring riskrelated change. This component of the review explored the mean ASSET score change for community cases ( $n=607$ ): measured once, between the first and second assessment. Their findings suggest that although some reductions were cancelled outby increases, seven of the 12 domains showed significant reductions. The most important of these were Thinking and Behaviour (mean score change $=0.20$, $p<0.001)$, Lifestyle $(-0.15, p<0.001)$, ETE $(-0.14, p<0.001)$ and Attitudes to Offending $(-0.12, p<0.001)$. The changes in mean domain scores for Physical Health, Emotional and Mental Health, and Motivation to Change were not found to be significant whilst those for Perception of Self and Others, and Neighbourhood on average did not change (Baker et al., 2005: T able 3.8).

Change was also considered for custodial cases: measured at two points: on release, and after a period of post-release supervision. For this group ( $n=57)$, significant improvements were found between the first and third assessment in relation to 8 domain scores. As with community disposals, Thinking and Behaviour $(-0.63, p<0.01)$, Lifestyle $(-0.60, p<0.001)$ and Attitude to Offending $(-0.54, p<0.001)$ were found to be more significant domains. The domains representing Motivation to Change, Perception of Self and Others, and Neighbourhood were found to not be significant whilst on average there was a marginal (butnot significant) increase in the mean score for Physical Health (Baker et al., 2005: T able 3.12).

Repeating this exercise for the 87 individuals whose risk assessment scores form the basis of the case study suggests that whilst the mean domain scores at Time 0 are typically higher than those at Time 1 (with the exception of those for Emotional and Mental Health), there is no evidence to support this. Indeed, the Bayes Factors $\left(\mathrm{BF}_{01}\right)$ of 3 or more provide moderate evidence for the null hypothesis relative to the alternative hypothesis that the mean score at Time 0 is greater than the mean score at T ime 1 whilst those which are less than 3 provide anecdotal evidence in factor of $\mathrm{H}_{0}(\mathrm{~T}$ able 4.2). 
Table 4.2:Changes in Dynamic ASSET Domain Scores between Initial and Second Assessments (All with ASSET Core Profiles Regardless of Disposal Received)

\begin{tabular}{|c|c|c|c|c|c|}
\hline \multirow{2}{*}{ Domain } & \multicolumn{2}{|c|}{ Mean Domain Score } & \multirow{2}{*}{$\begin{array}{c}\text { Mean Score } \\
\text { Change }\end{array}$} & \multirow{2}{*}{$\mathrm{BF} 01$} & \multirow{2}{*}{ Error \% } \\
\hline & Time 0 & Time 1 & & & \\
\hline Living Arrangements & 1.72 & 1.61 & -0.11 & 3.399 & $\sim 1.798 \mathrm{e}-6$ \\
\hline Family and Personal Relationships & 2.16 & 2.03 & -0.13 & 2.991 & $\sim 9.989 \mathrm{e}-6$ \\
\hline ETE & 2.00 & 1.91 & -0.09 & 3.999 & $\sim 3.151 \mathrm{e}-6$ \\
\hline Neighbourhood & 1.51 & 1.32 & -0.19 & 1.838 & $\sim 3.833 \mathrm{e}-5$ \\
\hline Lifestyle & 2.14 & 1.97 & -0.17 & 1.983 & $\sim 3.795 \mathrm{e}-5$ \\
\hline Substance Use & 1.66 & 1.56 & -0.10 & 3.773 & $\sim 1.067 \mathrm{e}-6$ \\
\hline Physical Health & 1.05 & 1.02 & -0.02 & 5.383 & $\sim 9.405 \mathrm{e}-6$ \\
\hline Emotional and Mental Health & 1.29 & 1.30 & 0.01 & 6.370 & $\sim 5.635 \mathrm{e}-6$ \\
\hline Perception of Self and Others & 1.71 & 1.61 & -0.10 & 3.223 & $\sim 0.002$ \\
\hline Thinking and Behaviour & 2.22 & 2.09 & -0.13 & 2.799 & $\sim 1.556 \mathrm{e}-5$ \\
\hline Attitude to Offending & 1.72 & 1.69 & -0.03 & 5.131 & $\sim 9.582 \mathrm{e}-6$ \\
\hline Motivation to Change & 1.66 & 1.55 & -0.11 & 3.394 & $\sim 0.037$ \\
\hline
\end{tabular}

Notes: Of the 87 individuals, more than half (47) had received a first-tier disposal whilst 23 had received a pre-court disposal for the primary offence which lead to their inclusion in the reoffending cohort. Bayes Factors have been calculated for onesided Bayesian Independent Sample T-Tests using JASP.

Having only 12 who received communitydisposals and 5 with custodial sentences, itis difficult to draw direct comparisons with the original research and it is also necessary to apply caution in interpreting these results due to the low number of cases involved overall. However, it is notable that the weakest evidence for no difference in mean score change is in relation to the Neighbourhood; Lifestyle; Family and Personal Relationships; and Thinking and Behaviour domains.

The general moderating trend that would be expected as a result of working with the YOT is more apparent when differences are considered between the mean domain scores at Time 0 and the individual's final ASSET (Table 4.3). 
Table 4.3: Changes in ASSET Domain Scores between Initial and Final Assessments (All with ASSET Core Profiles Regardless of Disposal Received)

\begin{tabular}{|c|c|c|c|c|c|}
\hline \multirow{2}{*}{ Domain } & \multicolumn{2}{|c|}{ Mean Domain Score } & \multirow{2}{*}{$\begin{array}{c}\text { Mean Score } \\
\text { Change }\end{array}$} & \multirow{2}{*}{ BF01 } & \multirow{2}{*}{ Error \% } \\
\hline & Time 0 & Final & & & \\
\hline Living Arrangements & 1.72 & 1.44 & -0.28 & 1.007 & $\sim 1.100 \mathrm{e}-6$ \\
\hline Family and Personal Relabionships & 2.16 & 1.84 & -0.32 & 1.970 & $\sim 9.652 \mathrm{e}-5$ \\
\hline ETE & 2.00 & 1.77 & -0.23 & 0.535 & $\sim 3.842 \mathrm{e}-5$ \\
\hline Neighbourhood & 1.52 & 1.16 & -0.36 & 3.737 & $\sim 1.191 \mathrm{e}-4$ \\
\hline Lifestyle & 2.14 & 1.77 & -0.37 & 3.560 & $\sim 1.187 \mathrm{e}-4$ \\
\hline Substance Use & 1.66 & 1.52 & -0.15 & 0.350 & $\sim 1.383 \mathrm{e}-5$ \\
\hline Physical Heath & 1.05 & 0.95 & -0.09 & 0.298 & $\sim 0.034$ \\
\hline Emotional and Mental Health & 1.29 & 1.30 & 0.01 & 0.159 & $\sim 5.995 \mathrm{e}-6$ \\
\hline Perception of Self and Others & 1.71 & 1.43 & -0.28 & 1.976 & $\sim 9.682 \mathrm{e}-5$ \\
\hline Thinking and Behaviour & 2.22 & 1.82 & -0.41 & 6.107 & $\sim 1.458 \mathrm{e}-4$ \\
\hline Aftitude to Offending & 1.72 & 1.55 & -0.17 & 0.524 & $\sim 3.840 \mathrm{e}-5$ \\
\hline Motivation to Change & 1.66 & 1.39 & -0.27 & 1.058 & $\sim 1.790 \mathrm{e}-5$ \\
\hline
\end{tabular}

Notes: The number of ASSETs completed per individual varies. For more details see Figure 4.2. Bayes Factors have been calculated for one-sided Bayesian Independent Sample T-Tests using JASP.

One-sided independentt-tests provide moderate evidence of a reduction in the mean domain scores for the Thinking and Behaviour, Neighbourhood and Lifestyle domains. However, itis important to note that not all young people experienced a net reduction in these domains during their time with the YOT, some saw increases, whilstothers had the same rating at the beginning and end of their time with the YOT although this is not to say that it did not vary. Certainly, when change in total ASSET Scores are considered, as in the next section, one in ten (10.6\%) saw an increase of 7 or more between their initial and final assessments with those having the biggest increases subsequentlybeing subject to custodial sentences reflecting the increased threat that they pose as a result of their escalating risky behaviour and/or offending.

Although it is important to stress the difference in the outcome variable being measured: Baker et al were concerned with the administrative measure of proven re-offending whereas this research has focused upon whether or not the young person has gone on to commit further offences, the findings were felt to be indicative of what mightbe expected in a time-varying 'dynamic' model. Indeed, as will be seen in section 4.2, the Lifestyle and Thinking and Behaviour domains were found to be significant predictors of further offending in Dynamic Model 1 (BDm1).

\section{Change in Total ASSET Scores}

Replicating the analysis that Baker et al. (2005) present in relation to the change in direction of total dynamic ASSET scores between assessments at T imes 0 through to $\mathrm{T}$ ime 10 , it is possible to see why some of the apparentlycontradictory upward trajectories which are presented later in this chapter have occurred (Table 4.4), particularly when the cohortis segmented by whether or not the young person has committed a further offence in the intervening period.

Page $\mid 110$ 
Of the 84 young people with a complete set of domain scores at Time 0 and Time 1, nineteen had committed further offences before being re-assessed (at T ime 1), equivalent to $22.6 \%$. Whilst those who had not committed any offences saw an average reduction of 1.77 in their total ASSET score, the reduction was less for those who had offended (mean $=-0.21$ ).

14.1\% (10 out of 71 ) of those assessed at both Time 1 and Time 2 had committed a further offence in the intervening period. On average their total ASSET scores increased by 2.20 whilst those who had not offended had a mean reduction of 0.69 . 
Table 4.4: Total ASSET Score Change by Direction, Between Successive Time Points (All with ASSET Core Profiles Regardless of Disposal Received)

\begin{tabular}{|c|c|c|c|c|c|c|c|c|}
\hline \multirow{2}{*}{$\begin{array}{l}\text { Difference in } \\
\text { Total ASSET Score }\end{array}$} & \multicolumn{2}{|c|}{ Time 0 to 1} & \multicolumn{2}{|c|}{ Time 1 to 2} & \multicolumn{2}{|c|}{ Time 2 to 3} & \multicolumn{2}{|c|}{ Time 3 to 4} \\
\hline & No & $\%$ & No & $\%$ & No & $\%$ & No & $\%$ \\
\hline Reduction of 7 or more & 11 & $13.1 \%$ & 6 & $8.5 \%$ & 7 & $12.1 \%$ & 8 & $17.0 \%$ \\
\hline Reduction of 4 to 6 & 9 & $10.7 \%$ & 5 & $7.0 \%$ & 4 & $6.9 \%$ & 3 & $6.4 \%$ \\
\hline Reduction of 1 to 3 & 13 & $15.5 \%$ & 8 & $11.3 \%$ & 9 & $15.5 \%$ & 14 & $29.8 \%$ \\
\hline No Change & 33 & $39.3 \%$ & 28 & $39.4 \%$ & 15 & $25.9 \%$ & 10 & $21.3 \%$ \\
\hline Increase of 1 to 3 & 11 & $13.1 \%$ & 18 & $25.4 \%$ & 8 & $13.8 \%$ & 3 & $6.4 \%$ \\
\hline Increase of 4 to 6 & 5 & $6.0 \%$ & 2 & $2.8 \%$ & 6 & $10.3 \%$ & 3 & $6.4 \%$ \\
\hline Increase of 7 or more & 2 & $2.4 \%$ & 4 & $5.6 \%$ & 9 & $15.5 \%$ & 6 & $12.8 \%$ \\
\hline Total & 84 & $100.0 \%$ & 71 & $100.0 \%$ & 58 & $100.0 \%$ & 47 & $100.0 \%$ \\
\hline Mean Score Change & -1.42 & & -0.28 & & 0.62 & & -1.02 & \\
\hline
\end{tabular}

\begin{tabular}{|c|c|c|c|c|c|c|c|c|}
\hline \multirow{2}{*}{$\begin{array}{l}\text { Difference in } \\
\text { Total ASSET Score }\end{array}$} & \multicolumn{2}{|c|}{ Time 5 to 6} & \multicolumn{2}{|c|}{ Time 6 to 7} & \multicolumn{2}{|c|}{ Time 7 to 8} & \multicolumn{2}{|c|}{ Time 8 to 9} \\
\hline & No & $\%$ & No & $\%$ & No & $\%$ & No & $\%$ \\
\hline Reduction of 7 or more & 3 & $9.7 \%$ & 4 & $14.8 \%$ & 2 & $8.3 \%$ & 2 & $10.5 \%$ \\
\hline Reduction of 4 to 6 & 2 & $6.5 \%$ & 3 & $11.1 \%$ & 0 & $0.0 \%$ & 1 & $5.3 \%$ \\
\hline Reduction of 1 to 3 & 7 & $22.6 \%$ & 4 & $14.8 \%$ & 2 & $8.3 \%$ & 2 & $10.5 \%$ \\
\hline No Change & 8 & $25.8 \%$ & 10 & $37.0 \%$ & 12 & $50.0 \%$ & 7 & $36.8 \%$ \\
\hline Increase of 1 to 3 & 6 & $19.4 \%$ & 3 & $11.1 \%$ & 1 & $4.2 \%$ & 6 & $31.6 \%$ \\
\hline Increase of 4 to 6 & 3 & $9.7 \%$ & 2 & $7.4 \%$ & 2 & $8.3 \%$ & 0 & $0.0 \%$ \\
\hline Increase of 7 or more & 2 & $6.5 \%$ & 1 & $3.7 \%$ & 3 & $12.5 \%$ & 1 & $5.3 \%$ \\
\hline Total & 31 & $100.0 \%$ & 27 & $100.0 \%$ & 24 & $100.0 \%$ & 19 & $100.0 \%$ \\
\hline Mean Score Change & -0.10 & & -1.19 & & 0.67 & & -0.42 & \\
\hline
\end{tabular}

Notes: Mean change scores have only been calculated where there has been a complete set of domain scores. Hence the total number of cases does not matc 
The situation between T imes 2 and 3 is notable because there is very little difference between the mean ASSET scores of the two groups (Table 4.5). Although overall just over one in four (15 out of 58 ) did not experience a change in their total ASSET scores and $34.5 \%$ experienceda reduction in their scores, the change in the mean ASSET Score is influenced bya small proportion who had significantchanges. For example, the maximum reduction experienced was -15 , whilst there were three individuals whose score increased by more than 10 overall who had not committed any further offences. The circumstances of two of these young people form the basis of the case studies which are used in section 4.4 and subsequent chapters to consider how well the models reflect the realities of the young person's change in circumstances during their time under the supervision of the YOT.

Table 4.5: Total ASSET Score Change by Direction, Between Time 2 and Time 3, by Whether or Not Further Offences Where Committed between ASSETs. (All with Complete ASSET Core Profiles Regardless of Disposal Received)

\begin{tabular}{|c|c|c|c|c|c|c|}
\hline \multirow[t]{2}{*}{$\begin{array}{l}\text { Difference in } \\
\text { Total ASSET Score }\end{array}$} & \multicolumn{4}{|c|}{$\begin{array}{l}\text { Further Offending between } \\
\text { Time } 2 \text { and Time } 3\end{array}$} & \multicolumn{2}{|c|}{ Overall } \\
\hline & No & $\%$ & Yes & $\%$ & Number & $\%$ \\
\hline Reduction of 7 or more & 4 & $9.1 \%$ & 3 & $21.4 \%$ & 7 & $12.1 \%$ \\
\hline Reduction of 4 to 6 & 4 & $9.1 \%$ & 0 & $0.0 \%$ & 4 & $6.9 \%$ \\
\hline Reduction of 1 to 3 & 7 & $15.9 \%$ & 2 & $14.3 \%$ & 9 & $15.5 \%$ \\
\hline No Change & 13 & $29.5 \%$ & 2 & $14.3 \%$ & 15 & $25.9 \%$ \\
\hline Increase of 1 to 3 & 6 & $13.6 \%$ & 2 & $14.3 \%$ & 8 & $13.8 \%$ \\
\hline Increase of 4 to 6 & 2 & $4.5 \%$ & 4 & $28.6 \%$ & 6 & $10.3 \%$ \\
\hline Increase of 7 or more & 8 & $18.2 \%$ & 1 & $7.1 \%$ & 9 & $15.5 \%$ \\
\hline Total & 44 & $100.0 \%$ & 14 & $100.0 \%$ & 58 & $100.0 \%$ \\
\hline Mean Score Change & 0.61 & & 0.64 & & 0.62 & \\
\hline
\end{tabular}

This negligible difference in the change in the total ASSET scores highlights that on average there is little to differentiate between those who had offended during the intervening period and those who had not. The nature of the modelling is such that differences in the average young person are reflected.

Between T imes 3 and 4, 23.4\% (11 out of 47) committed a further offence. Those whohad not committed an offence prior to Time 4 experienced a mean decrease in their total ASSET score of 2.69 whilst those who had offended, typically saw their score increase by 4.45 - a differential of 7.15. However, it is important to note that more than half (53.2\%) of those assessed at Times 3 and 4 saw a reduction in their total dynamic ASSET score. One of those who saw the greatestreduction in their score, forms the basis of the third case study discussed in section 4.4 and subsequent chapters. 
Table 4.6: ASSET Score Change by Direction, Between Time 3 and Time 4, by Whether or Not Further Offences Where Committed between ASSETs. (All with Complete ASSET Core Profiles Regardless of Disposal Received)

\begin{tabular}{|c|c|c|c|c|c|c|}
\hline \multirow[t]{2}{*}{$\begin{array}{l}\text { Difference in } \\
\text { Total ASSET Score }\end{array}$} & \multicolumn{4}{|c|}{$\begin{array}{c}\text { Further Offending between } \\
\text { Time } 3 \text { and Time } 4\end{array}$} & \multicolumn{2}{|c|}{ Overall } \\
\hline & No & $\%$ & Yes & $\%$ & Number & $\%$ \\
\hline Reduction of 7 or more & 8 & $22.2 \%$ & 0 & $0.0 \%$ & 8 & $17.0 \%$ \\
\hline Reduction of 4 to 6 & 3 & $8.3 \%$ & 0 & $0.0 \%$ & 3 & $6.4 \%$ \\
\hline Reduction of 1 to 3 & 12 & $33.3 \%$ & 2 & $18.2 \%$ & 14 & $29.8 \%$ \\
\hline No Change & 8 & $22.2 \%$ & 2 & $18.2 \%$ & 10 & $21.3 \%$ \\
\hline Increase of 1 to 3 & 2 & $5.6 \%$ & 1 & $9.1 \%$ & 3 & $6.4 \%$ \\
\hline Increase of 4 to 6 & 1 & $2.8 \%$ & 2 & $18.2 \%$ & 3 & $6.4 \%$ \\
\hline Increase of 7 or more & 2 & $5.6 \%$ & 4 & $36.4 \%$ & 6 & $12.8 \%$ \\
\hline Total & 36 & $100.0 \%$ & 11 & $100.0 \%$ & 47 & $100.0 \%$ \\
\hline Mean Score Change & -2.69 & & 4.45 & & -1.02 & \\
\hline
\end{tabular}

The difference between the mean scores of those who have committed a further offence between Times 4 and 5 is not as great, but the nine who committed a further offence before T ime 5 experienced a mean increase of 1.56 whilst the twenty-five who did not offend typically saw their total ASSET score reduce by an average of 0.36 . After this time, the numbers whose ASSET scores contribute to the analysis falls to around a third or lower with the number who committed a further offence between measurement points falling to just three between Times 7 and 8 . With such low numbers it is inappropriate to comment on the trends other than to highlight that the mean change in overall scores is negligible after Time7 ( $T$ able 4.4).

If the data is segmented with respect to gender, ethnicityand age, then the differential between the mean changes in ASSET scores it is expected that at each time point to also vary. This was something which was explored by Baker et al. (2005), with the team finding that amongst those receiving community disposals there was no significantamount of change. Theydid however, find that those with higher inital ASSET scores recorded a larger score reduction between their initial and second assessment (equivalent to Times 0 and 1 ) than those with lower initial scores. This they suggested may have been as a result of either regression to the mean or a 'ceiling effect' caused by the fact that high scorescannot rise any further. Rather that replicate this approach with each of the individual characteristics, the remainder of this chapter describes the development of the hierarchical model which represents the way in which the ASSET Core Profile has been used in practice as a series of repeated assessments of an individual's likelihood of reoffending. This basic model will then be enhanced in subsequent chapters to consider the potential impact of dimensional identityand experience of care (Chapter Five), different facets of the criminal career (Chapter Six) and system contact(Chapter Seven). 


\subsection{The Relationship between Further Offending and Domain Scores}

Broadlyspeaking the independent variables being considered can be grouped into time varying and nontime varying. For example, the individual domain scores have the potential to be different at the time of measurement, whilst individual characteristics such as gender and ethnicity are 'fixed'. This section focuses specificallyon the time-dependent domain score predictors which have been considered for the model representing the ASSET Core Profile.

\section{From the Null Model to the 'Basic'Model}

Finch etal. (2014:88) suggest that 'to applymultilevel models to longitudinal data problems, time-varying predictors will appear at Level 1 because they are associated with specific measurements, whereas time-invariant predictors will appear atLevel 2 or higher because theyare associated with the individual (or higher data level) across all measurementconditions. Thus, the structure of the ASSET Core Profile data, with repeated measures being taken for each individual, can be thought of initially as a two-level model. The model will then be extended to consider individuals nested within higher level clusters based on their primary offence.

A random interceptmodel in which an individual's 'response' depends linearlyon time, can be expressed as:

$$
\mathrm{y}_{\mathrm{ti}}=\beta_{0}+\beta_{1} \mathrm{~m}_{\mathrm{ti}}+\mu_{0 \mathrm{t}}+\mathrm{e}_{\mathrm{ti}}
$$

Where $\mathrm{y}_{\mathrm{ti}}$ is the dependent variable i.e. the likelihood of re-offending at occasion $t(t=1, \ldots M)$ for individual $\mathrm{i}(\mathrm{i}=1, \ldots \mathrm{n}) . \mathrm{m}_{\mathrm{ti}}$ is the occasion at which measurement $\mathrm{t}$ was taken on individual $\mathrm{i}$. As such the subscript $t$ refers to the Level 1 units and $i$ to the individuals at Level 2.

The terms in Equation (4.1) are defined as follows:

- $\beta_{0}$ is the overall intercept(averaged across individuals), interpreted as the expected value of $y$ at $\mathrm{m}_{\mathrm{ti}}=0$.

- $\beta_{1}$ is the slope of the regression of $y$ on time, commonly referred to as the growth rate. In a random intercepts model, the growth rate of is assumed to be the same for all individuals.

- $\mu_{0 \mathrm{t}} \sim \mathrm{N}\left(0, \sigma_{\mathrm{u}_{0}}^{2}\right)$ is an individual-specific random effect, capturing the effects on $\mathrm{y}$ of unmeasured individual characterises with values that are fixed over time. The intercept for individual $\mathrm{i}$ is $\beta_{0 \mathrm{i}}=\beta_{0}+\mu_{0 \mathrm{i}}$, so $\mu_{0 \mathrm{i}}$ represents the difference between an individual's value on $y$ (at any occasion) from the overall mean $\beta_{0}$. The variance of $\mu_{0 \mathrm{i}}\left(\sigma_{\mathrm{u}_{0}}^{2}\right)$ is the betweenindividual variance in y after accounting for the linear effect of time.

- $\mathrm{e}_{\mathrm{ti}} \sim \mathrm{N}\left(0, \sigma_{\mathrm{e}}^{2}\right)$ is an occasion-specific (time varying) residual, capturing the effects on y of unmeasured time-varying characteristics. The variance of $\mathrm{e}_{\mathrm{ti}}\left(\sigma_{\mathrm{e}}^{2}\right)$ is the within-individual variance in $\mathrm{y}$. 
The time variable (measurement occasion) has been coded $\mathrm{m}_{\mathrm{ti}}=0,1, \ldots M$. As the first occasion is coded 0 , time is said to be centred at the first occasion. Therefore, the model intercepts for all models can be interpreted as the expected likelihood of reoffending at $\mathrm{m}_{\mathrm{ti}}=0$ i.e. at the time of their initial assessment.

The number of measurements per individual, $\mathrm{m}_{\mathrm{ti}}$ can be anything, including one although for this model, those with only one record have been excluded. This number itself cannotbe informative of the process being studied. However, larger numbers $t_{i}$ give more information about intra-individual differences, and with larger average $\mathrm{m}_{\mathrm{ti}}$ there is greater potential to fit models with a more complicated, and more precise random part.

The number of ASSET Core Profiles will depend upon the duration of a young person's order, the complexity of their personal circumstances and offending behaviours. In this instance $61 \%$ of the individuals had five or less records with the maximum being 19 (Figure 4.2). Since measurement occasions are notfixed, the data is considered to be unbalanced. This can be seen in Figure 4.3 where the trajectory of each individual's average domain scores is represented by a line. Moving from left to right, the chart becomes less congested as the number of young people in the cohort decreases over time.

Figure 4.2: Distribution of ASSET Core Profiles per Individual

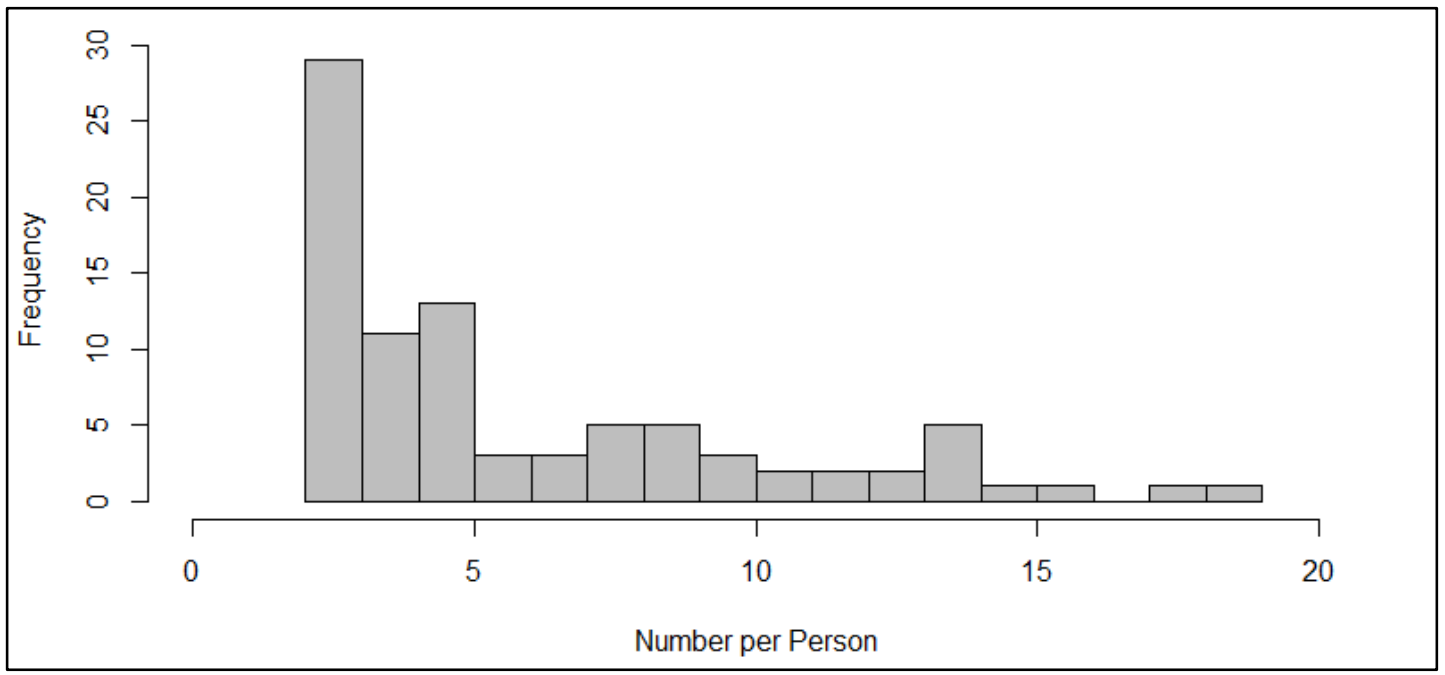

Notes: Individuals have been identified as having multiple ASSET Core Profiles having met the criteria to be included on the YOT's Reoffending Spreadsheets 2012/13 and 2013/14.

To represent the ASSET Core Profile with its 12 domains, the random intercept model (4.1) can be extended. For ease, the domainsare denoted as $\mathrm{x}_{1}$ through to $\mathrm{x}_{12}$ respectively:

$$
\begin{aligned}
\mathrm{y}_{\mathrm{ti}}= & \beta_{0}+\beta_{1} \mathrm{x}_{1 \mathrm{ti}}+\beta_{2} \mathrm{x}_{2 \mathrm{ti}}+\beta_{3} \mathrm{x}_{3 \mathrm{ti}}+\beta_{4} \mathrm{x}_{4 \mathrm{ti}}+\beta_{5} \mathrm{x}_{5 \mathrm{ti}}+\beta_{6} \mathrm{x}_{6 \mathrm{ti}}+ \\
& \beta_{7} \mathrm{x}_{7 \mathrm{ti}}+\beta_{8} \mathrm{x}_{8 \mathrm{ti}}+\beta_{9} \mathrm{x}_{9 \mathrm{ti}}+\beta_{10} \mathrm{x}_{10 \mathrm{ti}}+\beta_{11} \mathrm{x}_{11 \mathrm{ti}}+\beta_{12} \mathrm{x}_{12 \mathrm{ti}} \\
& +\beta_{13} \mathrm{~m}_{\mathrm{ti}}+\mu_{0 \mathrm{t}}+\mathrm{e}_{\mathrm{ti}}
\end{aligned}
$$


Since the dependent variable is dichotomous, with further offending being assigned the value of 1 and no further offending as 0 , the multivariate logistical regression model (4.2) takes the form:

$$
\operatorname{Pr}\left(\mathrm{y}_{\mathrm{ti}}=1\right)=\operatorname{logit}^{-1}\left(\boldsymbol{X}_{\mathrm{t}} \beta\right)
$$

where $\boldsymbol{X}_{t i}$ is a matrix of predictors. Hence, the hierarchical logistic regression model which allows both the interceptand time-trend to vary by individual which involves all 12 domains plus time can be written as:

$$
\begin{gathered}
\operatorname{Pr}\left(\mathrm{y}_{\mathrm{ti}}=1\right)=\operatorname{logit-1}\left(\beta_{0}+\beta_{1} \mathrm{x}_{1 \mathrm{ti}}+\beta_{2} \mathrm{x}_{2 \mathrm{ti}}+\beta_{3} \mathrm{x}_{3 \mathrm{ti}}+\ldots .+\beta_{12} \mathrm{x}_{12 \mathrm{ti}}+\beta_{13} \mathrm{~m}_{\mathrm{ti}}\right. \\
\left.+\mu_{0 \mathrm{i}}+\mu_{1 \mathrm{i}} \mathrm{m}_{\mathrm{ti}}+\mathrm{e}_{\mathrm{ti}}\right)
\end{gathered}
$$


Figure 4.3: Average Domains Scores, by Individual

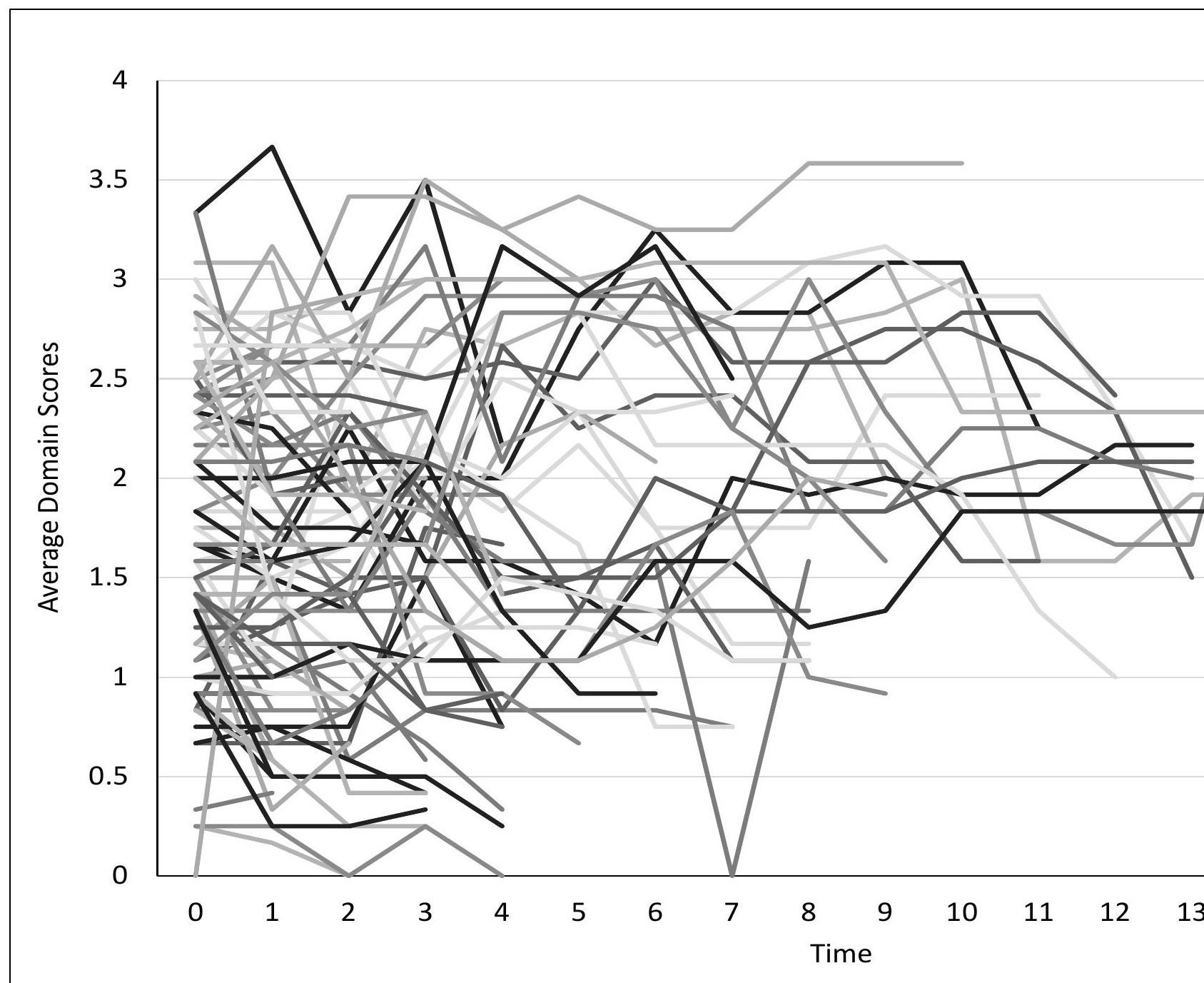

Notes: Average domain scores range from 0 to 4 reflecting the range of the possible ratings that can be assigned for each of the 12 domains. 
Under this model, positive values of the slope indicate that the larger the value of the predictor, the greater the log odds of further offending. Through a transformation of the slope (by taking the natural $\log$ of the coefficient i.e. $\mathrm{e}^{\beta_{n}}$ ), it is possible to obtain the odds of further offending as a function of the predictor(s). To aid interpretation of subsequentmodels, the standardised estimates of the coefficients with their $95 \%$ credible interval are presented alongside the unstandardised estimates.

It is this model (4.4) which is considered to be the 'basic' model which will be expanded upon in subsequentsections to explore the impactof:

- Dimensional identity (non-time varying, binary Level 2 predictors representing gender and ethnicity) and experience of care (a non-time varying, binary Level 2 predictor) (Chapter Five).

- Measures which represent aspects of the young person's criminal career (non-time varying, binary and continuous Level 2 predictors) and the nature of their primary offence (non-time varying, categorical and continuous Level 2 predictors) (Chapter Six)

- Organisational measures associated system contact. These include experience of care and with the young person's journey through the youth justice system such as courtappearances, potential time in custody and non-compliance (time varying, binary, Level 1 predictors) (Chapter Seven)

the preparatory steps undertaken to get to the basic model are outlined below. Throughout, the goodness of fit is evaluated using the Deviance Information Criterion (Spiegelhalter et al., 2002). The Deviance Information Criterion (DIC) is a Bayesian information criterion that quantifies the information in the fitted model by measuring how well the model reduced uncertainty of future predictions. Adding more parameters often improves the fit of the model, but a penalty can be occurred as complexity increases. The DIC simultaneouslyaccounts for model complexity(number of parameters) and model fit, by penalizing based on the number of (effective) parameters. It is calculated based on the sum of the effective number of parameters and the posterior mean of the deviance, with deviance defined as 2 times the log of likelihood function.

Although widely used in papers on applied Bayesian statistics, use of the DIC is not withoutits limitations (Spiegelhalter et al., 2014) including criticisms around its lack of consistency; not being based on a proper predictive criterion and having a weak theoretical justification. As a consequence, text books such as those by Kruschke (2013) and Gelman and Hill (2007) advocate the use of posterior predictive checks to evaluate absolute model fit. The Technical Annex therefore includes both the trace plots and posterior density plots for each of the dynamic models. As a further heuristic guide, models have also been run in the Frequentist paradigm using the Ime4 package. However, it should be noted that warning messages around failure to converge are generated even with the Basic Model. 
The sequential development of the models is described in subsequent sections so that evaluations can be made around the utility of including each predictor in the model. Under the Frequentist paradigm, ANOVAs have been used to compare the goodness of fit of each model relative to the version without the 'new' predictor. The outputfrom these can be found in the Technical Annex alongside the R code and output from each stage of the modelling process.

\section{Preparatory Models}

\section{a) The Emptyor 'Null'Model}

According to Robson and Pevalin (2016) the first step in multilevel modelling is to run a null or empty model $(\mathrm{Bm} 0)$ with no covariates. A two-level model withoutindependent predictors, the null model, can be expressed as:

$$
\operatorname{Pr}\left(\mathrm{y}_{\mathrm{ti}}=1\right)=\operatorname{logit}^{-1}\left(\beta_{0}+\mu_{\mathrm{i}}+\mathrm{e}_{\mathrm{ti}}\right)
$$

Comparisons between this and the hierarchical logistic regression models which sequentiallybuild up to the model representing the ASSET Core Assessment process with its 12 time-varying domains can be seen in Tables 4.7 to 4.12.

Table 4.7: Random Intercept Model for Further Offending

\begin{tabular}{|c|c|c|c|c|c|c|c|}
\hline \multirow[b]{3}{*}{ Fixed Effect: } & \multicolumn{7}{|c|}{ Null Model } \\
\hline & \multicolumn{3}{|c|}{ Unstandardised } & \multicolumn{3}{|c|}{ Standardised } & \multirow{2}{*}{ Significant? } \\
\hline & Post.Mean & Lower $\mathrm{Cl}$ & Upper Cl & Post.Mean & Lower Cl & Upper $\mathrm{Cl}$ & \\
\hline Individual (Intercept) & 0.130 & 1.65E-04 & 0.049 & 1.139 & 1.000 & 1.051 & Yes \\
\hline Random Effect: & Post.Mean & Lower $\mathrm{Cl}$ & Upper Cl & Post.Mean & Lower $\mathrm{Cl}$ & Upper $\mathrm{Cl}$ & Significant? \\
\hline Individual (Intercept) & -0.624 & -0.789 & -0.474 & 0.536 & 0.454 & 0.622 & Yes \\
\hline
\end{tabular}

\begin{tabular}{|l|r|}
\hline DIC & 661.92 \\
\hline
\end{tabular}

Source: Model Bm0, Technical Annex: p25-26

The model (BmT0 - summarised in Table 4.8), which allows each individual to deviate from the overall mean response by a person-specific constant that applies equallyover time can be written as:

$$
\operatorname{Pr}\left(\mathrm{y}_{\mathrm{ti}}=1\right)=\operatorname{logit}^{-1}\left(\beta_{0}+\beta_{1} \mathrm{~m}_{\mathrm{ti}}+\mu_{0 \mathrm{i}}+\mathrm{e}_{\mathrm{ti}}\right)
$$

Where $\mu_{0 \mathrm{i}}$ represents the influence of individual i on his/her repeated observations. Comparing Tables 4.7 and 4.8 , it is possible to see the impact of having likelihood of modelling Individual's assessments varying with time - under this model, the random individual-specific effect, $\mu_{0 \mathrm{i}}$ is estimated to be $\exp (0.039)=1.040$, with a $95 \%$ credible interval of 1.000 to 1.159 . Notably, adding a single predictor (time) to the random intercepts model, adds to its complexity, increasing the DIC from 661.9 to 671.1 . 


\begin{tabular}{|l|r|r|r|r|r|r|r|}
\hline \multirow{2}{*}{} & \multicolumn{5}{c|}{ Null Model, Random Intercept } \\
\cline { 2 - 8 } & \multicolumn{3}{|c|}{ Unstandardised } & \multicolumn{3}{c|}{ Standardised } \\
\hline Fixed Effect: & Post.Mean & Lower Cl & Upper Cl & Post.Mean & Lower Cl & Upper Cl & Significant? \\
\hline Individual (Intercept) & -0.044 & -0.279 & 0.191 & 0.957 & 0.756 & 1.211 & 0.812 \\
\hline Time & -0.159 & -0.208 & -0.108 & 0.853 & 0.898 & Yes \\
\hline Random Effect: & Post.Mean & Lower Cl & Upper Cl & Post.Mean & Lower Cl & Upper Cl & Significant? \\
\hline Individual (Intercept) & 0.039 & $2.06 \mathrm{E}-04$ & 0.147 & 1.040 & 1.00 & 1.159 & Yes \\
\hline
\end{tabular}

\begin{tabular}{|l|l|}
\hline DIC & 671.91 \\
\hline
\end{tabular}

Source: Model BmT0, Technical Annex: p28-29

From these results, time appears to be negatively related to the likelihood of re-offending, suggesing that the likelihood decreases over time. This is what would be expected given the premise that as a result of working with the YOT, the young person's risk of further offending will reduce. Notably, this relationship is statisticallysignificant with the range of estimates or 'c redible interval' for the standardised coefficient being 1.000, 1.159. The odds of further offending as a function of time can be obtained through transformation $\left(\mathrm{e}^{-0.159}=0.853\right)$. Thus, for every additional assessment the young person is subject to, the estimated odds of them committing further offences are multiplied by 0.853 . Since the unstandardised estimate of the credible interval does not include zero, this finding can be considered to be statisticallysignificant.

\section{b) Adding Time Varying Predictors to Represent the ASSET Domains}

Athough there is the potential for the individuals to have a rating of 0 to 4 for each domain, as highlighted in Figure 4.1, the majority receive rating of 1 or 2 . Figure 4.4 summarises the mean scores for each domain at the time of the Individual's initial assessment (Time 0 ). In a small number of cases - where the primary index had been dealt with out of court, the young person's first ASSET Core Profile relates to their referral following their further offence. 
Figure 4.4: Mean Domain Scores at Time 0

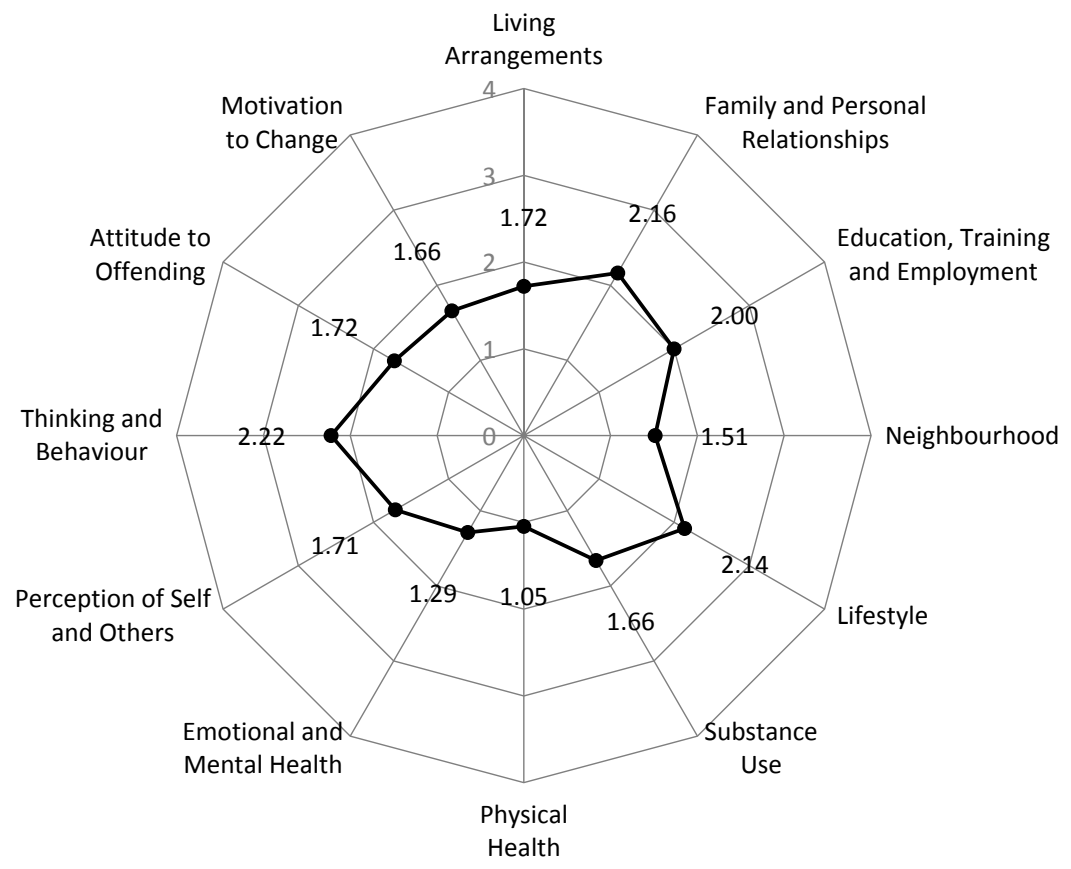

Ratings can vary depending upon how the Individual is progressing during their time with the YOT and in response to changes in their personal circumstances. Figure 4.5 summarises the mean scores for the first five measurementpoints, illustrating the time-varying nature of each these predictors.

Figure 4.5: Mean Domain Scores by Time

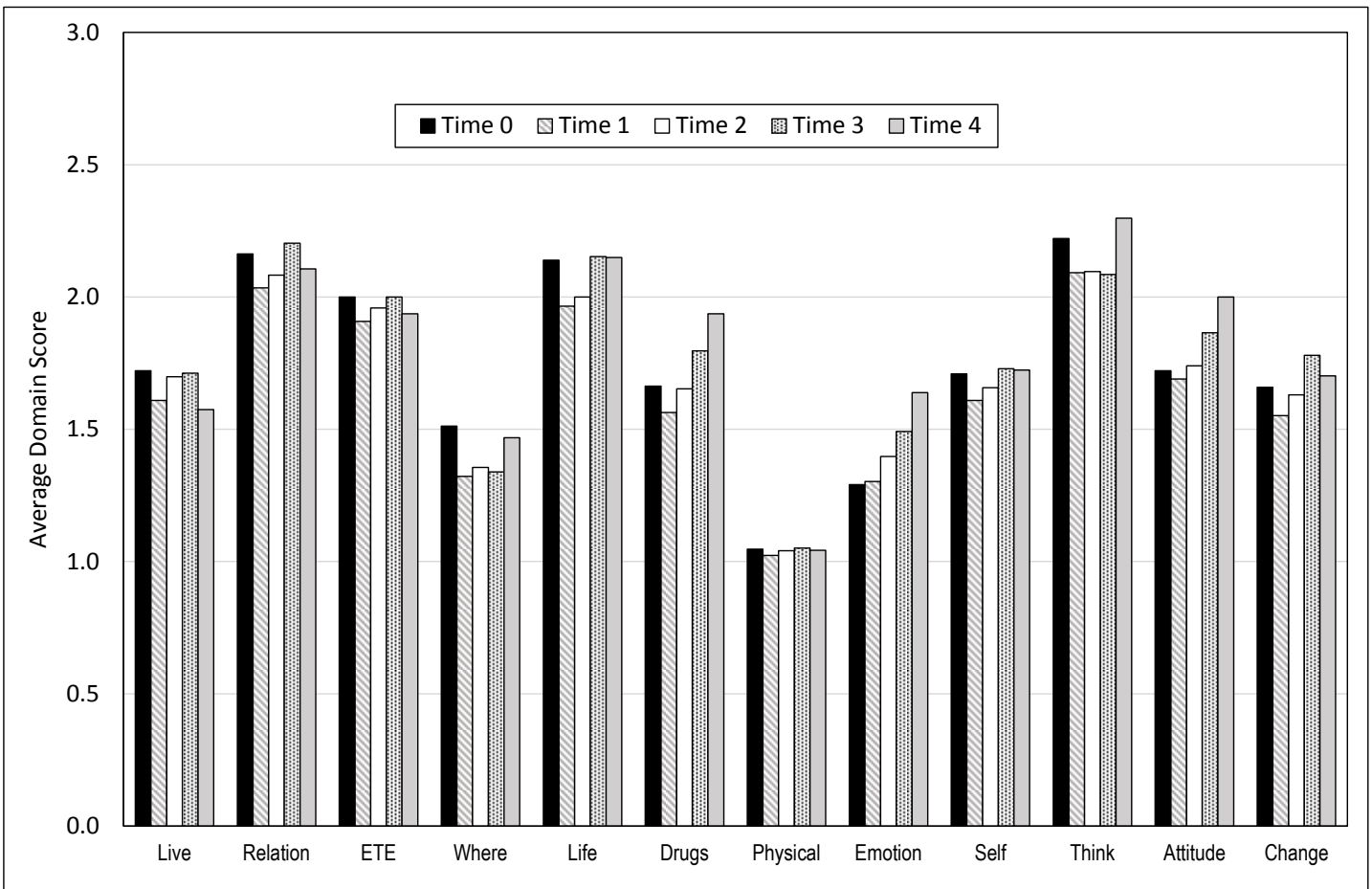

Notes: Time $0=87$ individuals; Time 1= 87 individuals; Time 2= 73 individuals; Time 3= 59 individuals; Time 4= 47 individuals. The number of individuals having 5 or more assessments (Time $>4$ ), is summarised in Figure 4.2. 
Notablythe mean scores for the 'substance misuse' (Drugs) and 'emotional and mental health' (Emotion) domains increase across the firstfive measurementoccasions. Whilst this may in part be a reflection of the more complex lives of those remaining under the supervision of the YOT after Time 3 , Wilson and Hinks (2011) report that practitioners found the' emotional and mental health' domain to be the most difficult to explore with young people, followed by 'family and personal relationships' (Relation) and 'perception of self and others' (Self). This, they suggest, may be a consequence of 'the limited skills of workers in this area' (2011: 55). As a result, it may take time for the practitioner to learn sufficient information about the young person to make an assessment of their risk level.

In the case of ratings for the substance misuse domain, this is perhaps more prone to a disclosure effect (Raynor et al., 2000) whereby the individual may be unwilling to disclose information which could be perceived as potentially getting them into further trouble. Notably, Wilson and Hinks highlight that there may be difficulties in exploring issues around substance use in the presence of a parent/guardian in the interview. Hence disclosure about the true nature and extent of a young person's substance use may only occur after the relationship has had a chance to develop with the practitioner leading to the upward trend in mean scores after Time 0.

Table 4.9: Random Intercept Model for Further Offending including ASSET Domains

\begin{tabular}{|c|c|c|c|c|c|c|c|}
\hline \multirow[b]{3}{*}{ Fixed Effect: } & \multicolumn{7}{|c|}{12 Domains + Time, Random Intercepts } \\
\hline & \multicolumn{3}{|c|}{ Unstandardised } & \multicolumn{3}{|c|}{ Standardised } & \multirow{2}{*}{ Significant? } \\
\hline & Post.Mean & Lower $\mathrm{Cl}$ & Upper Cl & Post.Mean & Lower $\mathrm{Cl}$ & Upper Cl & \\
\hline Individual (Intercept) & -0.894 & -1.378 & -0.425 & 0.409 & 0.252 & 0.654 & Yes \\
\hline Living Arrangements & 0.009 & -0.204 & 0.233 & 1.009 & 0.816 & 1.262 & \\
\hline Family and Personal Relationships & 0.176 & -0.062 & 0.422 & 1.193 & 0.940 & 1.526 & \\
\hline Education, Training and Employment & 0.056 & -0.151 & 0.261 & 1.058 & 0.860 & 1.299 & \\
\hline Neighbourhood & 0.056 & -0.127 & 0.238 & 1.058 & 0.880 & 1.269 & \\
\hline Lifestyle & 0.075 & -0.204 & 0.369 & 1.078 & 0.816 & 1.446 & \\
\hline Substance Use & 0.119 & -0.073 & 0.324 & 1.127 & 0.930 & 1.383 & \\
\hline Physical Health & -0.128 & -0.360 & 0.104 & 0.880 & 0.697 & 1.109 & \\
\hline Emotional and Mental Health & -0.044 & -0.252 & 0.164 & 0.957 & 0.777 & 1.178 & \\
\hline Perceptions of Self and Others & -0.026 & -0.301 & 0.231 & 0.974 & 0.740 & 1.260 & \\
\hline Thinking and Behaviour & -0.006 & -0.274 & 0.277 & 0.994 & 0.760 & 1.319 & \\
\hline Attitude to Offending & -0.050 & -0.345 & 0.260 & 0.952 & 0.708 & 1.297 & \\
\hline Motivation to Change & 0.163 & -0.112 & 0.454 & 1.177 & 0.894 & 1.575 & \\
\hline Time & -0.188 & -0.244 & -0.133 & 0.828 & 0.784 & 0.875 & Yes \\
\hline Random Effect: & Post.Mean & Lower Cl & Upper Cl & Post.Mean & Lower $\mathrm{Cl}$ & Upper Cl & Significant? \\
\hline Individual (Intercept) & 0.040 & $1.80 \mathrm{E}-04$ & 0.158 & 1.041 & 1.000 & 1.171 & Yes \\
\hline
\end{tabular}

\begin{tabular}{|l|l|}
\hline DIC & 608.51 \\
\hline
\end{tabular}

Source: Model BmT1, Technical Annex: p31-37

The random intercepts model including the 12 domains is summarised in Table 4.9. This reflects the mean score for each domain across all measurementoccasions. Compared to the null model and the null + time model (Tables 4.7 and Table 4.8), the DIC is lower despite the increased complexity of the model suggesting that the addition of these predictors helps to reduce the amount of variance. 


\section{c) Varying the Slope to Allow Variation by Individual over Time (Adding Random Coefficients)}

Notably the number of ASSET Core profiles varies by Individual (Figure 4.1) suggesting that time also needs to be included as a random coefficient. The model for further offending where both the intercept and time-trend vary by individual is written as:

$$
\operatorname{Pr}\left(y_{t i}=1\right)=\operatorname{logit}^{-1}\left(\beta_{0}+\beta_{1} m_{t i}+\mu_{0 i}+\mu_{1 i} m_{t i}+e_{t i}\right)
$$

In this model, the fixed effects are represented as being $\beta_{0}$ and $\mu_{0 \mathrm{i}}$, whilst the random effects are written as $\beta_{1} \mathrm{~m}_{\mathrm{ti}}$ and $\mu_{1 \mathrm{i}}$. The term $\mu_{1 \mathrm{i}} \mathrm{m}_{\mathrm{ti}}$ represents the random coefficient, giving the difference between each Individual's coefficientfrom the overall population coefficient $\beta_{1} \mathrm{~m}_{\mathrm{ti}}$ where time is a predictor. The impactis to reduce the DIC to 487.64 .

Table 4.10: Random Intercept and Varying Slope Models for Further Offending

\begin{tabular}{|c|c|c|c|c|c|c|c|}
\hline & & Null Mo & del, Randon & n Intercepts & and Varying & Slope & \\
\hline & & standardis & & & tandardisec & & \\
\hline Fixed Effect: & Post.Mean & Lower Cl & Upper Cl & Post.Mean & Lower $\mathrm{Cl}$ & Upper $\mathrm{Cl}$ & Signiticant? \\
\hline Individual (Intercept) & 0.134 & 2.31E-04 & 0.417 & 1.144 & 1.000 & 1.518 & Yes \\
\hline Time & 1.122 & 0.3061 & 2.329 & 3.071 & 1.358 & 10.268 & Yes \\
\hline Random Effect: & Post.Mean & Lower $\mathrm{Cl}$ & Upper $\mathrm{Cl}$ & PostMean & Lower $\mathrm{Cl}$ & Upper $\mathrm{Cl}$ & Significant? \\
\hline Individual (Intercept) & -0.123 & -1.182 & 0.954 & 0.885 & 0.307 & 2.597 & \\
\hline Time & -0.139 & -0.275 & -0.017 & 0.870 & 0.759 & 0.983 & Yes \\
\hline
\end{tabular}

\begin{tabular}{|l|r|}
\hline DIC & 487.64 \\
\hline
\end{tabular}

Source: Model BmTV0, Technical Annex: p39-40

The estimate for the intercept -0.134 , is fixed with a $95 \%$ credible interval for the unstandardised coefficient of 0.000231 to 0.417 . Since this interval does not contain 0 , this is considered to be significant.

The slope for time indicates that the logit of the probability of further offending increases on average by 1.022 for every additional assessment. Exponentiating this value suggests that the odds of further offending increase bya multiplicative factor of 3.071 -that is, an increase of $307 \%$-for every additional assessment. However, the credible interval suggests that this could range from $35.8 \%$ to $1026.8 \%$ highlighting the high amount of potential variability in this estimate.

\section{d) The Basic Model}

The Basic model (Bm1, summarised in Table 4.11) represents the repeated measurements from the ASSET Core Profile. Despite the increased complexity of the model, the DIC falls from 487.6 to 476.2. This also compares favourably to the DIC for the equivalentmodel without time as a random coefficient (Model BmT1, DIC = 608.5). Time is both significant as a fixed and random effect i.e. 0 is not in the interval. Notably, the posterior mean estimate for time as a fixed effect and its $95 \%$ credible interval are negative suggesting that in addition to the random effect of both time and individual, the probability of further offending decreases as time increases. 
Table 4.11: The Basic Model: Random Intercept and Varying Slope Models for Further Offending including ASSET Domains

\begin{tabular}{|l|r|r|r|r|r|r|r|}
\hline & \multicolumn{6}{|c|}{ 12 Domains + Time, Random Intercepts and and Varying Slope } \\
\cline { 2 - 7 } & \multicolumn{3}{|c|}{ Unstandardised } & \multicolumn{3}{|c|}{ Standardised } \\
\hline \multirow{2}{*}{ Fixed Effect: } & PostMean & Lower Cl & Upper Cl & PostMean & Lower Cl & Upper Cl & \\
\hline Individual (Intercept) & -1.168 & -2.379 & 0.129 & 0.311 & 0.093 & 1.138 & \\
\hline Living Arrangements & 0.033 & -0.216 & 0.293 & 1.033 & 0.806 & 1.340 & \\
\hline Family and Personal Relationships & 0.275 & -0.026 & 0.556 & 1.316 & 0.974 & 1.744 & \\
\hline Education, Training and Employment & 0.094 & -0.152 & 0.342 & 1.099 & 0.859 & 1.408 & \\
\hline Neighbourhood & 0.044 & -0.166 & 0.262 & 1.045 & 0.847 & 1.300 & \\
\hline Lifestyle & 0.024 & -0.316 & 0.371 & 1.024 & 0.729 & 1.450 & \\
\hline Substance Use & 0.158 & -0.087 & 0.388 & 1.172 & 0.917 & 1.473 & \\
\hline Physical Health & -0.114 & -0.394 & 0.165 & 0.892 & 0.674 & 1.180 & \\
\hline Emotional and Mental Health & -0.003 & -0.249 & 0.242 & 0.997 & 0.780 & 1.273 & \\
\hline Perceptions of Self and Others & -0.138 & -0.443 & 0.182 & 0.871 & 0.642 & 1.200 & \\
\hline Thinking and Behaviour & -0.160 & -0.508 & 0.157 & 0.853 & 0.602 & 1.170 & \\
\hline Attitude to Offending & 0.043 & -0.298 & 0.389 & 1.044 & 0.742 & 1.475 & \\
\hline Motivation to Change & 0.231 & -0.095 & 0.582 & 1.260 & 0.909 & 1.790 & \\
\hline Time & -0.153 & -0.283 & -0.018 & 0.858 & 0.753 & 0.982 & Yes \\
\hline Random Effect: & PostMean & Lower Cl & Upper Cl & Post.Mean & Lower Cl & Upper Cl & Significant? \\
\hline Individual (Intercept) & 0.101 & $1.99 \mathrm{E}-04$ & 0.366 & 1.106 & 1.000 & 1.442 & Yes \\
\hline Time & 1.267 & 0.338 & 2.605 & 3.550 & 1.403 & 13.531 & Yes \\
\hline
\end{tabular}

\begin{tabular}{|l|r|}
\hline DIC & 476.20 \\
\hline
\end{tabular}

Source: Model Bm1, Technical Annex: p42-47

Comparing this model with those summarised in Tables 4.7 and 4.8, demonstrates the utility of utilising a hierarchical modelling approach to analyse the data generated by the risk assessment process.

It is apparent from Table 4.11 that time is a significant fixed effect when included in the Basic Model alongside the 12 domains, with the negative coefficient suggesting that if the domain scores remain constant, the probability of further offending is expected to decrease. However, the significantrandom effects suggest that this trend will vary both by individual and time. In the dynamic equivalent of this Basic Model (Table 4.12), time is not a significantmain fixed effect, buttwo of the domain predictors are when there is an interaction between these and time.

It should be noted that the coefficients presented in subsequentmodels have been generated through use of a simulated model. As a result, there can be some variability in the estimates for the fixed effects. 


\section{The Basic Dynamic Model}

Under this model, there is the potential for each individual domain predictor to vary by time, reflecting the dynamic nature of the risk assessmentframework. Full descriptions of each domain can be found in The ASSET Core Profile Guidance (Youth Justice Board, 2008a). Examples of ratings of 1, 2, 3 and 4 are given within this document to assist practitioners. Summaries can also be found in the Technical Annex.

Table 4.12: The Basic Dynamic Model Involving the 12 Domains

\begin{tabular}{|c|c|c|c|c|c|c|c|}
\hline \multirow[b]{3}{*}{ Fixed Effect: } & \multicolumn{7}{|c|}{ Dynamic Model 1} \\
\hline & \multicolumn{3}{|c|}{ Unstandardised } & \multicolumn{3}{|c|}{ Standardised } & \multirow{2}{*}{ Significant? } \\
\hline & Post.Mean & Lower $\mathrm{Cl}$ & Upper $\mathrm{Cl}$ & PostMean & Lower $\mathrm{Cl}$ & Upper $\mathrm{Cl}$ & \\
\hline (Intercept) & -1.099 & -1.768 & -0.483 & 0.333 & 0.171 & 0.617 & \\
\hline Time & -0.008 & -0.109 & 0.088 & 0.992 & 0.897 & 1.092 & \\
\hline Living Arrangements (Live) & 0.274 & -0.473 & 1.000 & 1.316 & 0.623 & 2.719 & \\
\hline Family and Personal Relationships (Relation) & 0.319 & -0.697 & 1.331 & 1.376 & 0.498 & 3.783 & \\
\hline Education, Training and Employment(ETE) & -0.517 & -1.306 & 0.274 & 0.596 & 0.271 & 1.315 & \\
\hline Neighbourhood (Where) & -0.254 & -0.985 & 0.471 & 0.775 & 0.373 & 1.601 & \\
\hline Lifestyle (Life) & 1.454 & 0.237 & 2.692 & 4.280 & 1.267 & 14.768 & Yes \\
\hline Substance Use (Drugs) & -0.320 & -1.041 & 0.364 & 0.726 & 0.353 & 1.439 & \\
\hline Physical Health (Physical) & -0.586 & -1.956 & 0.864 & 0.557 & 0.141 & 2.374 & \\
\hline Emotional and Mental Health (Emotion) & -0.381 & -1.313 & 0.516 & 0.683 & 0.269 & 1.676 & \\
\hline Perceptions of Self and Others (Self) & 1.000 & -0.123 & 2.080 & 2.717 & 0.884 & 8.008 & \\
\hline Thinking and Behaviour (Think) & -1.109 & -2.252 & -0.096 & 0.330 & 0.105 & 0.908 & Yes \\
\hline Attitude to Offending (Attitude) & 0.014 & -1.142 & 1.116 & 1.014 & 0.319 & 3.053 & \\
\hline Motivation to Change (Change) & 1.024 & -0.156 & 2.238 & 2.785 & 0.855 & 9.372 & \\
\hline Time: Live & -0.041 & -0.165 & 0.097 & 0.959 & 0.848 & 1.102 & \\
\hline Time: Relation & -0.063 & -0.270 & 0.151 & 0.939 & 0.763 & 1.163 & \\
\hline Time: ETE & 0.080 & -0.077 & 0.219 & 1.083 & 0.926 & 1.245 & \\
\hline Time: Where & 0.071 & -0.050 & 0.193 & 1.074 & 0.951 & 1.213 & \\
\hline Time: Life & -0.204 & -0.417 & 0.008 & 0.816 & 0.659 & 1.008 & \\
\hline Time: Drugs & 0.089 & -0.054 & 0.236 & 1.093 & 0.947 & 1.267 & \\
\hline Time: Physical & 0.033 & -0.174 & 0.235 & 1.033 & 0.840 & 1.265 & \\
\hline Time: Emotion & 0.104 & -0.082 & 0.301 & 1.110 & 0.921 & 1.352 & \\
\hline Time: Self & -0.265 & -0.488 & -0.040 & 0.767 & 0.614 & 0.961 & Yes \\
\hline Time: Think & 0.288 & 0.051 & 0.533 & 1.334 & 1.052 & 1.704 & Yes \\
\hline Time: Attitude & 0.068 & -0.178 & 0.309 & 1.071 & 0.837 & 1.362 & \\
\hline Time: Change & -0.161 & -0.412 & 0.080 & 0.851 & 0.662 & 1.083 & \\
\hline Random Effect: & Post.Mean & Lower $\mathrm{Cl}$ & Upper Cl & PostMean & Lower $\mathrm{Cl}$ & Upper $\mathrm{Cl}$ & Significant? \\
\hline Individual (Intercept) & 0.180 & $9.49 \mathrm{E}-10$ & 0.697 & 1.197 & 1.000 & 2.007 & Yes \\
\hline Time & 0.126 & $5.32 \mathrm{E}-10$ & 0.514 & 1.134 & 1.000 & 1.672 & Yes \\
\hline DIC & & & 256.77 & & & & \\
\hline
\end{tabular}

Source: Model BDm1, Technical Annex: p50-57

Under this model, the estimates for the fixed effect of the lifestyle and thinking and behaviour domains are flagged as being significantalong with those for the interactions between timean d perception of self, and time and thinking behaviours i.e. 0 is in the interval. These results suggest that the ratings for these two domains are statisticallysignificantlyrelated to further offending. The significantinteractions suggest that how a young person' ratings for these domainschange over time is also related to their likelihood of further offending. 
The anticipation within the risk assessmentframework is that domain scores will decrease over time as the young person works with the YOT. In Figure 4.6, the domain scores have been fixed at their initial values so that the estimated change in the probability of further offending from time 0 to time 10 can be seen. In doing this it is recognised that this is somewhat artificial, especially since individual young people can also have different scores in each of the 12 domains, reflecting their personal circumstances at a given time. The unbalanced nature of the data means that at later time points, there is less information for the model to draw upon and hence the domain scores of highly prolific and hence higher risk young people may have greater influence upon the model.

Figure 4.6: Changes in the Probability of Further Offending Over Time

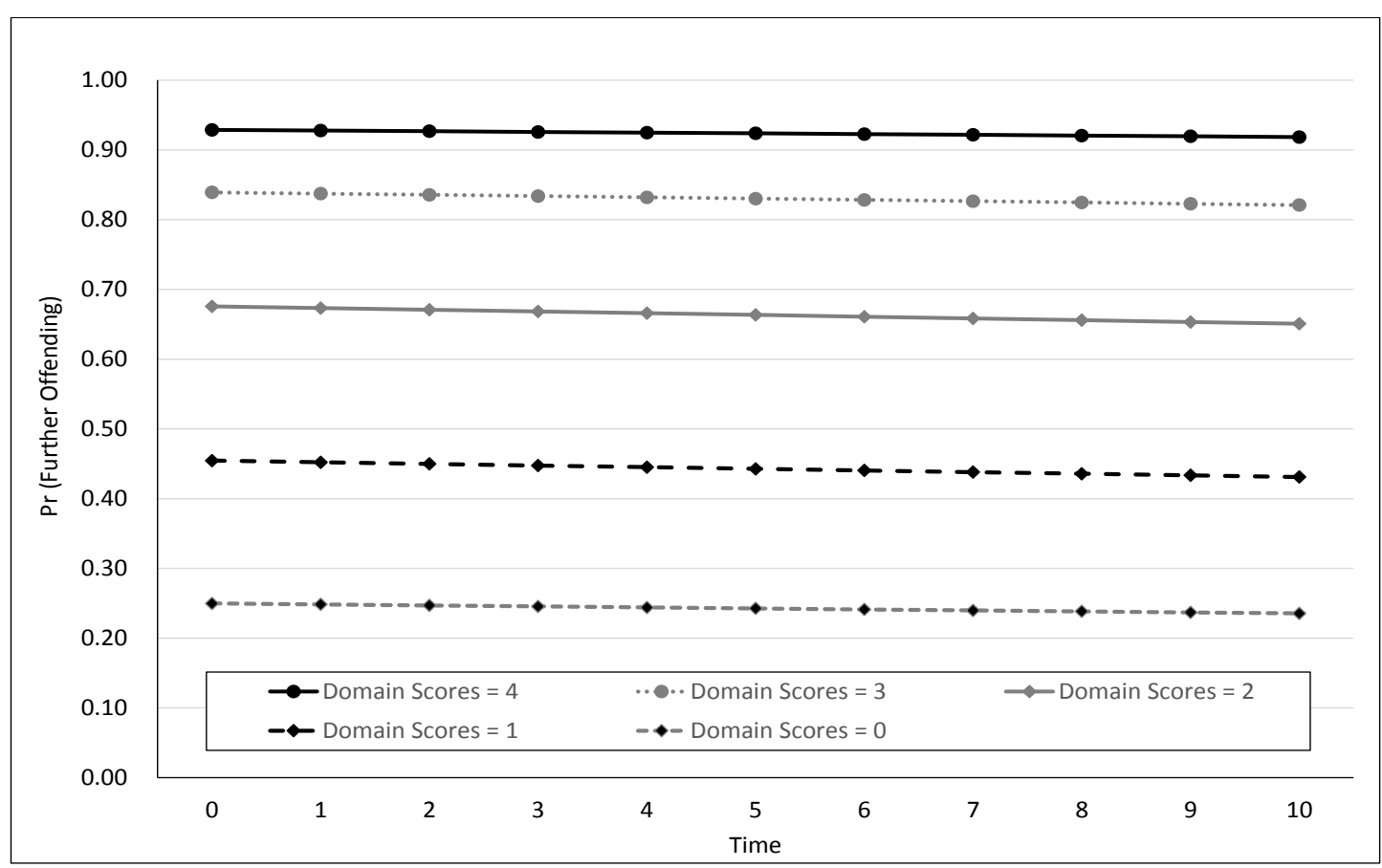

Notes: The domain scores have respectively been shown as being fixed at 0, 1, 2, 3 and 4 respectively to demonstrate the estimated change in the probability of further offending from time 0 to time 10. Estimates derived from Model BDm1.

Figure 4.7 summaries the number of young people with ASSET s at each time pointalong with the range of domain scores. To simplify the visual representation of the data from Figure 4.3, rather than presenting the Individual trajectories, these have been averaged. Hence the average mean scoreacross the 12 domains has been presented along with the minimum and maximum. At time 18, these are all the same since they are based on the data from just one individual. 


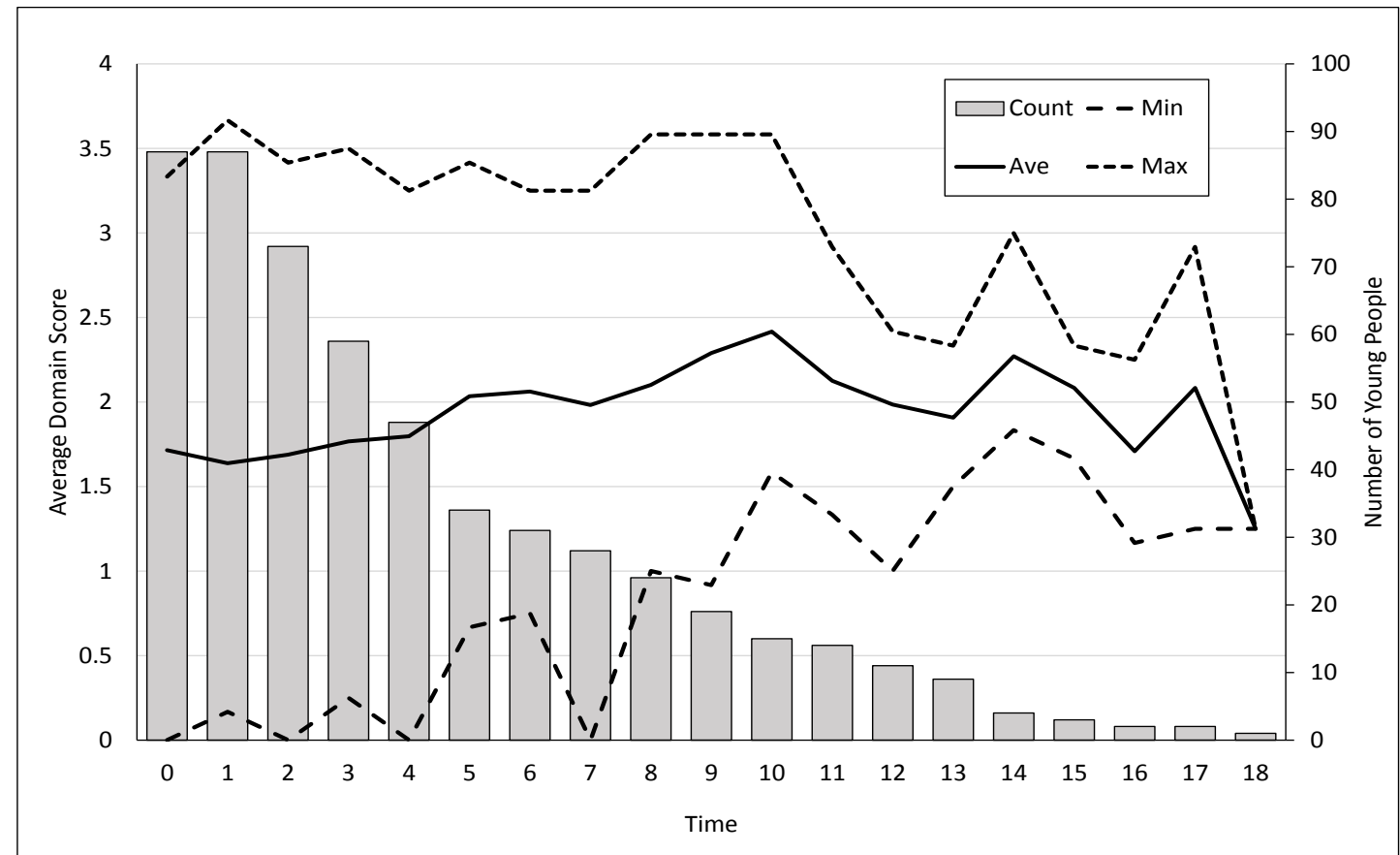

Notes: Average domain scores range from 0 to 4 reflecting the range of the possible ratings that can be assigned for each of the 12 domains.

\subsection{The role of the 12 domains in predicting further offending over time. How do these findings extend the existing evidence base?}

The review of ASSET conducted by Wilson and Hinks (2011) considered the predictive validity of ASSET on proven re-offending over one year using a number of different measures:

- The accuracy of the total score (out of 64) in predicting the proportion of young people assessed using the Core ASSET Profile who re-offended within one year as well as the frequency and severity of re-offences and disposals. The results of this analysis were summarised in Section 2.6.

- A series of binary logistical regression models run to determine which of the 12 dynamic measures was the mostpredictive of re-offending.

In comparing different combinations of static and dynamic factors and the OGRS 3 , this second exercise was undertaken using data relating to both Core Profile (sentenced) and Final Warning cases, with one assessment per person $(n=7,621)$ rather than has been done here, using the repeated measures. Wilson and Hinks also used the administrative measure of proven re-offending rather than whether or not the young person had committing a further offence. As such it is not possible to draw direct comparisons using the 87 cases from Western Bay YOT. However, their results provide useful context when considering the findings presented in section 4.2 . 
Wilson and Hinks (2011) found that Lifestyle, Substance Use and Motivation to Change were highly significant predictors of proven one-year reoffending. Ratings in the Living Arrangements, Family and Personal Relationships and ETE domains were also statisticallysignificant whilst those in the remaining six domains were felt to be relevant. From Dynamic Model 1 (BDm1), it is apparent that the Lifestyle domain is also a significant predictor of further offending along with Thinking and Behaviour. Notably, the interactions between Thinking and Behaviour: Time, and between Perceptions of Self and Others: Time were also found to be significant when no further predictors are included in the model.

\section{Lifestyle}

As can be seen from the description provided in the Technical Annex, the Lifestyle domain focuses upon the young person's friends and associates, what they do in their spare time and money issues. The positive coefficientfor the Lifestyle domain as a main effect(BDm1, $1.454[0.237,2.692])$ suggests that assuming all other predictors remainconstant, an increase of 1 in the rating, increases the probabili ty of further offending by a factor of $4.3(\exp (1.454))$ with the credible interval suggesting that this could be between 1.3 and 14.8. The interaction between the domain and Time is notsignificant.

The fact that ratings for the Lifestyle domain were found to be significant predictors in both previous evaluations of ASSET and within this research fits with the literature. Indeed, as Warr (2012) highlights, few criminologists today would dispute the social nature of youth offending behaviours, with a number of major theories of 'delinquent' peer influencehaving been developed. In particular, ithas been argued that criminal behaviour is learned from others in much the same way as all other forms of human behaviour is learnt. Whilst Sutherland's theory of differential association is commonlysubscribed to in relation to peer influence, the causal mechanism and indeed the direction of this causation has been questioned. Drawing upon the sociological principle of homophily (people make friends with people who are similar to themselves), an argumentcan be made that people do not become 'delinquent' because they acquire 'delinquent' friends. Theyacquire 'delinquent' friends after they themselves have become 'delinquent'.

However, manycriminologists maintain that the relation between 'delinquent' behaviour and 'delinquen' peers over time is likelyto be bidirectional or sequential. Thornberry's interactional theory of delinquency combines aspects of both the socialisation and selection models - steming from the theory of differential association and social control theories respectively, asserting that 'associating with delinquent peers leads to increases in delinquencyvia the reinforcing environmentbythe peer network. In turn, engaging in delinquencyleads to increases in associations with delinquent peers' (Thornberryet al., 1994:74).

Jang (1999) has tested the developmental perspective of the interactional theory of delinquency which hypothesizes that the role of 'delinquent' beliefs varies developmentally, focusing on the age-varying effects of family, school, and 'delinquent' peers on offending behaviours for the crime-prone adolescent 
years i.e. between ages 11 and 20 . Under this hypothesis Thornberryet al argued that although peers already have a significantimpacton the adolescent's behaviour, during the early stages of adolescence (ages 11-13) parents still have a strong influence on their children. The impact of 'delinquent' peers on the adolescent's behaviour continues to grow as the locus of interaction and social influence shifts from the family to peernetworks during middle adolescence (ages 15-16). In the final stages of adolescence, association with 'delinquent' peers still has a strong, primarilydirect, influence on 'delinquent' behaviour, thought its effects are expected to be mediated by other factors e.g. 'delinquent' values and need to compete with activities such as employment, college, education and romantic relationships. Jang's analysis of five waves of the Rochester Youth Development Study using multilevel modelling supports the age-variance hypothesis, although he found that the impact of 'delinquent' peers on offending behaviours tended to peak earlier than hypothesised. A curvilinear pattern was observed which is understood to be the combination of developmental challenges and 'delinquent' activity, with developmental challenges reflecting the young person's struggle to adjust to their interim status (i.e. between child and adult status). Under this developmental perspective, problem behaviour including offending behaviour is seen as being a result of inadequate coping with the challenges of transition especiallywhere they fail to find proper social supportfrom their immediate surroundings.

Other features of the domain reflect participation in a broader range of reckless activities including those which place the young person and/or others at risk of physical injury (e.g. playing on railway lines, building sites or major roads, and racing cars around residential areas); activities done to impress others or to get a 'buzz'; and involving other in their offending. As the data collection process did notdrill down to the responses to the 'Yes' / 'No' questions completed bythe practitioners as part of the assessment process, it is not possible to ascertain the extent to which these are features of the young people's lives as Baker et al. (2003) did in their first published review of ASSET. However, it is notable that at T ime 0 , $73.9 \%$ ( 65 out of 87 ) where judged to be at significant risk of reoffending as a result of their Lifestyle with practitioners assigning ratings of 2 or more. This was one of the highest proportions with only the proportion deemed at significant risk because of their Thinking and Behaviours being higher (76.1\%). The other domain with similar proportions considered to be at significantrisk at Time 0 was Familyand Personal Relationships (73.9\%).

\section{Thinking and Behaviours}

The role of others in a young person's offending is complex, ranging from coercion, threats and manipulation through to committing offences to impress and gain in popularity. As such itis also reflected in the Thinking and Behaviour domain where the practitioner is asked to identify if the young person's actions are characterised byamongst other things, giving in easily to pressure from others and attempts to manipulate/control others. 
The negative coefficientfor the Thinking and Behaviour domain as a main effect(BDm1, -1.109 [-2.252, $-0.096]$ ) suggests that if the rating were to increase by 1 with no other changes, the probability of further offending would decrease by a factor of $0.33(\exp (-1.109))$, although this could potentially be between 0.10 and 0.91 . In addition to the main effect being significant, the interaction between this domain and Time is also significant with a positive coefficient $(0.288$ [0.051, 0.533]). Since the main effect for Time is not significant, it is difficult to draw firm conclusions about the net effect of these within the additive model. However, broadly speaking after Time 4, if all other domain scores remain the same then an increase in the rating for the Thinking and Behaviour domain increases the probability of further offending. The credible intervals for each of the coefficients represent the amount of uncertaintyaround these main effects and interactions involving them.

Notably Wilson and Hinks did not identify this domain as being a statistically significant predictor of reoffending although they note that along with Neighbourhood; Physical Health; Emotional and Mental Health; Perception of Self and Others; and Attitude to Offending, the Thinking and Behaviours domain was 'still likely to be relevant for understanding the needs in the experienced by young people' (2011: 29). In their interviews with practitioners, this domain was 'considered difficult to assess in the initial stages of the contact' (Wilson and Hinks, 2011:55). This view is perhaps understandable given that the domain focuses on problematic patterns of thinking and types of behaviour in a range of different contexts e.g. at home, at school, with friends, in the neighbourhood, with staff and in relation to their offending. As such it is necessary to compile evidence from a number of different sources and to form a relationship with the young person. However, both practitioners and young people who have been assessed using ASSET have previously suggested that the Thinking and Behaviours and Lifestyle domains are clearly related with reoffending along with ETE and a lack of training / qualifications respectively, and in the case of young people, the neighbourhood (Youth Justice Board, 2005a) - a finding which is potentially linked to the perceived potential to be modified by practical and communitybased prevention programmes.

\section{Perception of Self and Others}

This domain concentrates upon the young person's understanding of how they - and others - fit into the world around them. As such itconsiders whether the individual has an inappropriate level of self-esteem; a general mistrust of others; difficulties with self-identity and if they see themselves as an offender. In terms of their relationships with others, they may display discriminatory attitudes or have a lack of understanding for other people.

\section{Individual Factors: Personality Traits, Attitudes, Beliefs and Offending}

Using the groupings of risk and protective factors adopted by the YJB (Youth Justice Board, 2005b), it is apparent that a number of the traits which appear within the Thinking and Behaviour, and Perc eption of Self and Others domains can be considered to be individual or personal factors - the primary 
exception being aggression which is commonlyconsidered to be a school factor due to its links to bullying behaviours although it can also be linked to family factors. These tend to focus upon personality traits, attitudes and beliefs:

- Hyperactivity and impulsivity

- Low intelligence and cognitive impairment

- Alienation and lack of social commitment

- Attitudes that condone offending and drug misuse

- Friends involved in crime and drug use

The association between various personalitytraits, attitudes and beliefs and offending during childhood and adolescence is well documented, featuring in the Gluecks' work undertaken in the 1920s and 1930s. Case and Haines for example highlight that in 500 Criminal Careers (1930), the Gleucks identify'dull or borderline intelligence, psychotic or psychopathic, neuropathic traits - extreme suggestibility, emotional instability, impulsiveness' (2009: 55) as personality / intelligence factors that were present in early childhood and earlyadolescence and related to later onset of recorded offending. In their follow up work One Thousand Juvenile Delinquents (1934), the Gleucks identified 'sub-normal intelligence, marked emotional and personality handicaps' (Case and Haines, 2009: 57) as personality / intelligence factors which increased the risk of the young men coming before the courts as an official offender. Other factors were also identified within these studies under the domains of family, school, lifestyle and employment. In their later study, Unraveling Juvenile Delinquency (1950), the Gluecks identified strong associations between a range of biological, psychological and social risk factors measured in childhood and subsequentyouth offending, notably:

- 'Bodytype - stocky, muscularmesomorphs

- Temperament- restless, impulsive, extroverted, aggressive, destructive

- Attitude - hostile, defiant, resentful, suspicious, stubborn, assertive, adventurous, not submissive to authority

- Psychological - tending to think in concrete (rather than abstract) terms

- Family - lack of parental discipline, poor supervision and low family cohesiveness.'

(Case and Haines, 2009:61-62)

Whilst the work of the Gleucks has been criticised for being overly simplistic, many of the subsequent longitudinal and cross-sectional risk factor research studies have investigated the association between personality traits, attitudes and beliefs, and subsequent offending behaviours. This includes the Cambridge Study in DelinquentDevelopmentand the Edinburgh Study of Youth T ransitions and Crime which have been particularly influential in shaping policy and practice in England and Wales, and Scotland respectively.

Page $\mid 132$ 
Personality traits, attitudes and beliefs also form an integral part of the risk-needs-responsivitymodel of correctional assessmentand rehabilitation (Andrews et al., 1990) which has been widelyadopted within both the youth and wider justice system. The model (Table 4.13) comprises of the Central Eight split between the "Big Four" and the "ModestFour" - the former including a history of criminal behaviour, antisocial behaviour pattern, anti-social attitudes, values, beliefs and cognitive-emotional states, and antisocial associates. In the context of wishing to affect change, the risk-need-responsivity(RNR) model is particularlyrelevant with a number of international meta-analyses showing that if properly implemented, reductions in reoffending can be detected.

Although not withoutits detractors, the RNR model acknowledges the psychological nature of some risk factors which has led to the recognition that these are dynamic and modifiable. Hence the likelihood of further offending behaviour could be reduced by reducing the risk level associated with these factors. In matching services to the assessed risk level of the individual, focus can placed upon those attributes that are predictive of offending and ensure that they are targeted in the individual's rehabilitation plan. In order to maximise the individual's ability to benefit from a rehabilitative intervention, evaluations need to be tailored to the young person's learning style, motivation, abilities and strengths with consideration also being made to their age, gender and ethnicity (Adler et al., 2016). As a result of the theoretical intercorrelations between these factors, some of the most effective intervention approaches are characterised by using a combination of skills training and cognitive behavioural intervention approaches, employing a multi-modal design with a broad range in interventions that address a number of offending related risks (Wilson, 2013). 
Table 4.13: The Central Eight, Their Indicators and Associated Needs

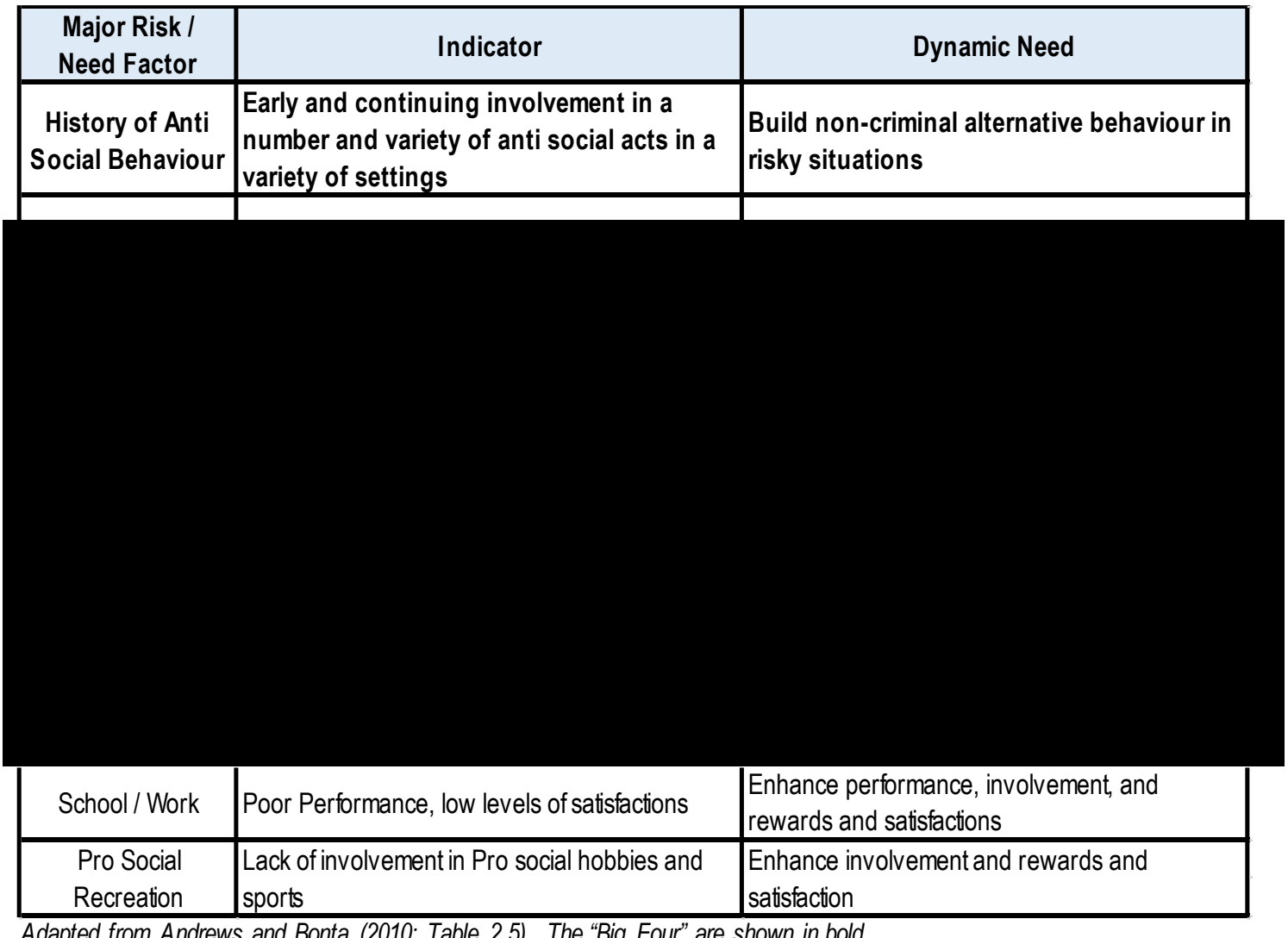

Adapted from Andrews and Bonta (2010: Table 2.5). The "Big Four" are shown in bold.

Assuming that the principles of RNR are being adhered to within the YOT setting when planning interventions, then it follows that activities that counter the "Big Four" are going to be the most significant in promoting desistence. As such it is encouraging that research undertaken by the Ministry of Justice (Wilson, 2013) suggests that YOT s are better at addressing factors such as Lifestyle, Perception of Self and Others, Thinking and Behaviour, Attitudes to Offending and Motivation to Change, than others. However, it was acknowledged that there remained room for improvementin terms of aligning offending related risks and needs, aims in the intervention plan and subsequentprovision. Wilson also identified that it was more challenging for the YOTs to directly address certain needs such as neighbourhood, living arrangements and family and personal relationships. Consequently, these were less likely to be targeted in intervention plans.

Given the transition to ASSETPlus which has happened since this inception of this research, there has been increasing emphasis upon promoting desistence. In particular McNeill (2009) highlights that desistence relates to age and maturing, to social ties or bonds, and to changing personal identities. However, for changes processes (like desistence) to occur, both practitioners and offenders need the motivation to change, capacity to be and to act differently and opportunities to do so, with all three being required for the change to occur. For some young people, the capacity to change is affected by noncriminogenic factors suchas self-esteem, anxiety, victimisation issues and learning disabilities. Notably 
in the context of England and Wales, there has been a growing awareness of the high prevalence of neurodevelopmental disorders amongst those who offend (Hughes et al., 2012) which can hinder both treatment and the ability to actively engage in the youth justice process. Such individuals are more likely to struggle to engage and comply with requirements placed upon them. Speech, language and communication needs similarlymaylimit the young person's comprehension of diversionary/ restorative justice processes and criminal proceedings (The Communication Trust, 2014; Youth Justice Board and Royal College of Speech and Language Therapists, 2015) meaning that they are more likelyto enter the formal youth justice system. With increasing numbers now being diverted from the formaljustice system, this begs the question as to whether those remaining represent more complex cases with more entrenched offending behaviours.

The YJB considered whether or not the cohort is becoming more complex as part of its three-year Reducing Reoffending Programme. In exploring this issue as part of their attempts to increase the sector's knowledge of reoffending and the drivers behind this, the YJB undertook analysis of ASSET data from over a six-year period, looking atchanges in total ASSET scores and individual domain scores over time. Whilst this analysis was based on assessments rather than individual young people, it found that:

'For the year ending March 2016, the dynamic factors with the highest average ASSET scores are Lifestyle and Thinking Behaviour (indicating the strongest association with likelihood of further offending), with average scores of 1.89 and 2.23 respectively. The factors with the lowest values are Physical Health and Neighbourhood with scores of 0.31 and 1.01 respectively.

While the scores or some factors dipped in the firstyears examined, theyall increased over the whole period, indicating an average rise in the level of assessed risk/need that young people present. The factors showing the greatest percentage change in score over time are Perception of Self and Others (identity and self-esteem) and Emotional and Mental Health: between the year ending March2010 and March 2016, these increased by $24 \%$ and $33 \%$ respectively.'

(Youth Justice Board, 2016b: 7-8)

The reportconcluded thaton average case complexity has been increasing over time with those in the higher risk score band falling at a lesser rate than those in the lower score bands, with proportionally now more assessments being in the higher band than in 2009/10. Notably the domains highlighted as having the highest average scores and greatest percentage change have also been highlighted in the findings from this research as having a significant role to playin a young person's likelihood of committing further offence. The exception to this is Emotional and Mental Health. 


\subsection{How well does the 'basic' model reflect the realities of young people's lives?}

Andrews and Bonta (2007) suggest that third generation riskinstruments are sensitive to changes in an offender's circumstances and hence should be able to provide practitioners with information as to what needs should be targeted in their interventions. Theyalso assert that there is evidence that changes in the scores on some of these risk-need instruments are associated with changes in recidivism. Hence it would be expected that changes in risk scores would signal changes in the likelihood of the individual committing a new offence. In this way, such tools provide a means of monitoring the effectiveness, or ineffectiveness of programmes and supervision strategies, and of tailoring the nature of interventions to target accordingly. This section therefore considers the ability of the 'basic dynamic' model to reflect such changes. This has enabled the work of Wilson and Hinks (2011) who undertook a review of ASSET on behalf of the Ministry of Justice to be advanced.

In looking to explain the differences in the trajectories of the various probabilities of further of offending, three case studies are presented which illustrate the complexity of some of the young people's lives and how changes in their circ umstances have impacted uponthe ratings they have received at different time points. This work builds upon that of Baker et al. (2005) who in their review of ASSET had looked at early changes in the total dynamic score. Rather than fixing the domain scores as done in Figure 4.6, these reflect the realities of the young people's lives. To protectindividual identities, pseudonyms have been used and some key identifying details have been omitted.

Table 4.14: Summary of Key Information for the Three Case Histories

\begin{tabular}{|l|c|c|c|}
\hline & Fred & Connor & David \\
\hline Gender & Male & Male & Male \\
\hline Ethnicity & White & White & White \\
\hline Experience of Care? & None & Yes & None \\
\hline FTE Status Upon Entry to Cohort & FTE & Prior Offending History & Prior Offending History \\
\hline Age atFirst Offence & 14 & 14 & 10 \\
\hline Age at First Conviction & 15 & 14 & 16 \\
\hline Primary Offence & 2 & 6 & 2 \\
\hline Seriousness of Primary Offence & Other (Criminal Damage) & SAC (Burglary Dwelling) & Other (Public Order) \\
\hline Breaches & $2,1,2,3,4$ & 0 & 1,2 \\
\hline Court Appearances & 4 & 0,1 & $0,1,2,4$ \\
\hline Periods in Custody / On Remand & None & 1,2 & None \\
\hline Further Offending & 1 & None \\
\hline
\end{tabular}

Notes: The information for breaches, court appearances, periods in custody / on remand and further offending relate to the measurement point after the event occurred. 


\section{Case Study "Fred"}

As a 14-year old, Fred had been given a youth caution for criminal damage. However, with no proven offences during 2012/13 he does not appear on the reoffending spreadsheetfor that year.

The following June he received a further youth caution after committing a theft offence. However, just over a month later Fred took a vehicle without consent and was given a 4-month Referral Order. A couple of months short of his 16th birthday (and whilst still on the Referral Order), Fred committed a burglary. Thisled to him being placed under Intensive Supervision and Surveillance Programme (ISSP) Bail and being tagged. As such he would have been mandated to engage in a structured programme of relevant activities including five core elements around offending behaviour; interpersonal skills; ETE; familysupport and restorative justice. In combining community-based surveillance with comprehensive and sustained focus on tackling the factors contributing to the young person's offending behaviour, the ISSP is the most rigorous, non-custodial intervention available to young people. In total there were seven courtappearances over the following months before he was sentenced to a 10 -month Detention and Training Order.

Figure 4.8: Case Study "Fred": Domain Scores at Time 2 and Time 3

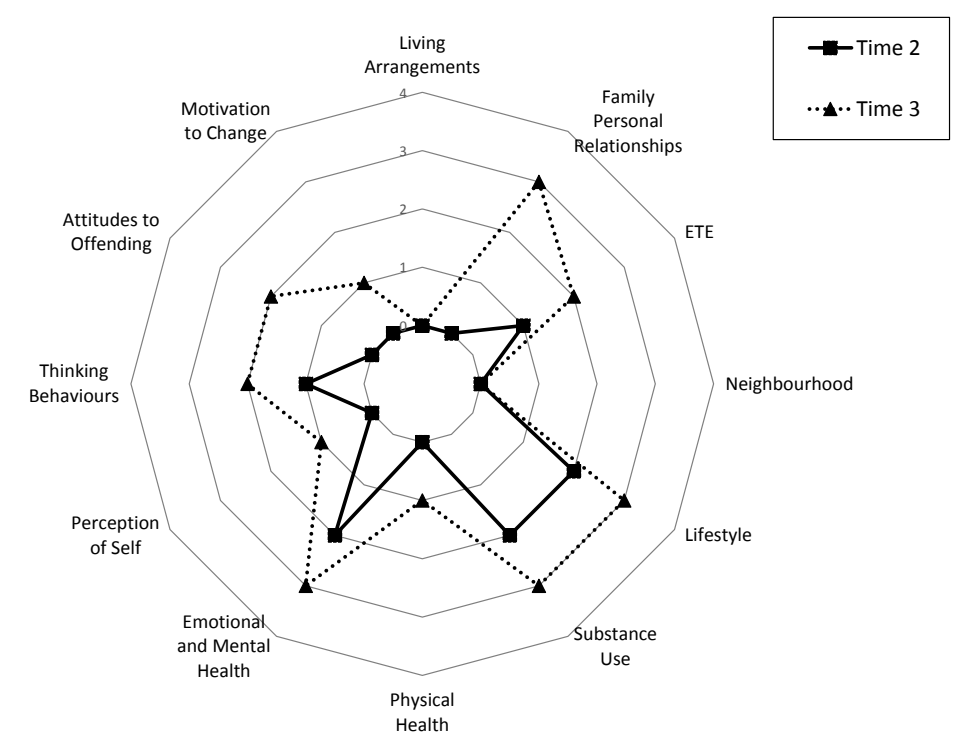

The interval between Time 2 and 3 relate to a 3-month period when Fred was on ISSP Bail and Tag, and he attended courtfour times - his sentencing took place a week after Time 3. During this time Fred's total dynamic ASSET score increases from 8 to 21 i.e. from low-medium to medium-high risk, butas can be seen from Figure 4.8, whilst there were no perceived differences in the level of risk associated with his living arrangements or the neighbourhood, his rating for the family and personal relationships domain increased from 0 to 3 . The practitioner also judged thathis lifestyle; substance use; and emotional and mental health were also quite strongly related to the likelihood of reoffending. 
It is notable that Fred's rating for Attitudes to Offending increases from 0 to 2 suggesting that in the run up to his sentencing hearing he is displaying a lack of understanding about the impact of his offending; a reluctance to accept responsibility and potentially a denial of the seriousness of his behaviour (see description below). When coupled with the increase in the rating for the Family and Personal Relationships domain, this is indicative of a child whose parent(s) are not fully engaged in a supportive manner, perhaps as a response to the young person's increasinglyproblematic behaviour - evidenced by the increases in substance misuse and lifestyle ratings. In this case, accommodation records suggest that Fred was living at home with his mother and her partner, and he returns to this address after his time in custody. Since his living arrangements and the neighbourhood in which he lives are at no time considered to be associated with a risk of reoffending, it must be assumed that this is suitable, stable accommodation.

Figure 4.9 summarises the estimated probability of further offending based on the ASSET scores for Fred using the basic dynamic model based on just the 12 domains (BDm1) and the three models based on the domains, time and the various static factors discussed in the previous section. The in crease in the domain scores between Times 2 and 3 summarised in Figure 4.8, is reflected in the upwards trend in the estimated probability of further offending based on coefficients from the basic dynamic model. Events and total ASSET scores are recorded alongthe x-axis. Although Fred did notcommitanyfurther offences, his increased scores led to him being in custody.

Figure 4.9: Estimated Probability of Further Offending Over Time: "Fred"

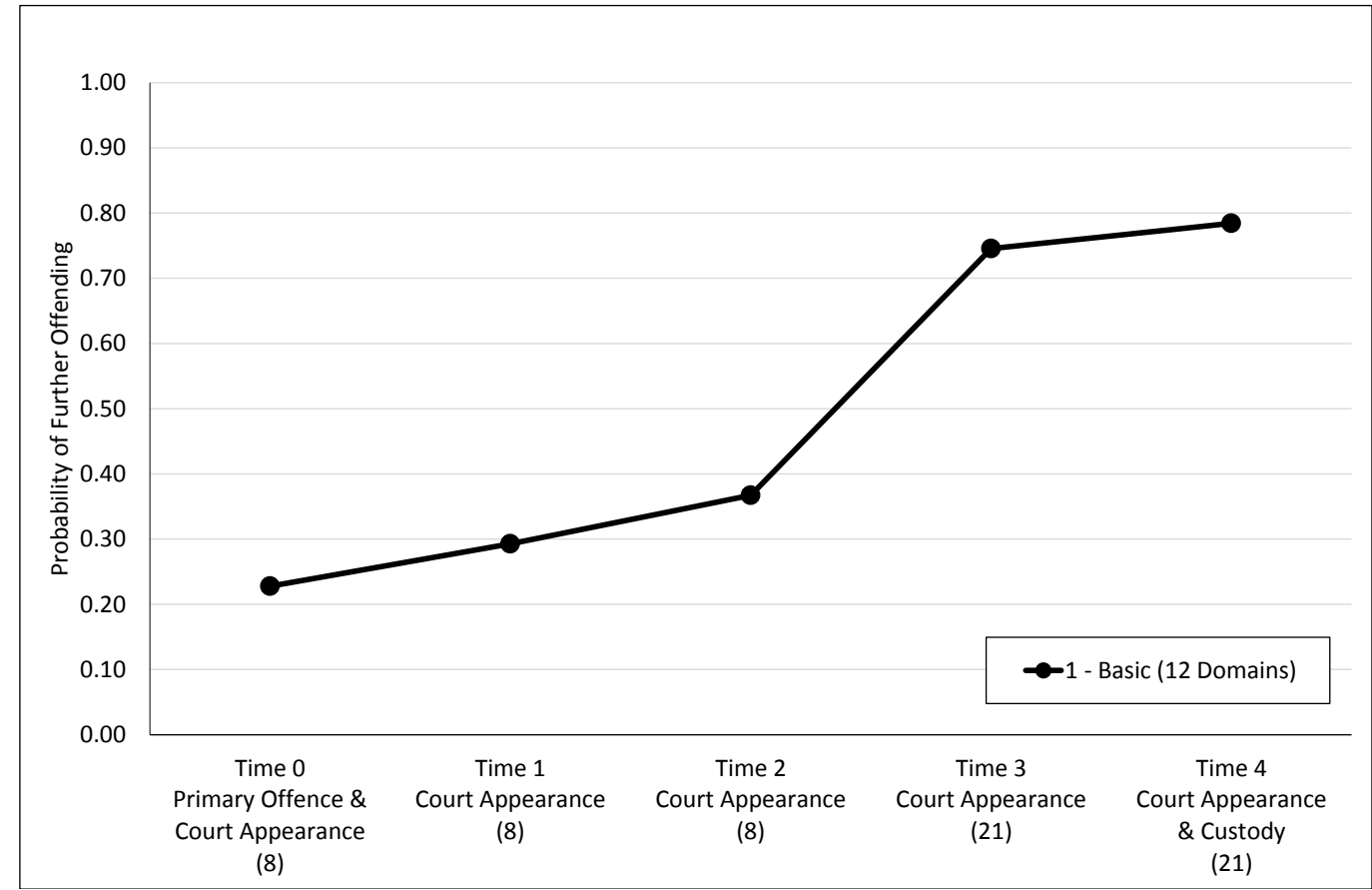




\section{Case Study "Connor"}

In contrast to Fred, "Connor" had already been identified as a prolific offender prior to joining the reoffending cohortin 2012/13. Having received a Final Warning for a non-domestic burglaryin 2009 at the age of 14 , Connor had been charged having committed further non-domestic burglaries on nine separate occasions including the offence which led to him being sentenced to his second Detention and T raining Order (DTO). He had already been subject to a Referral Order which had been extended and Youth Rehabilitation Order as well as a couple of Conditional Discharges with offences including burglary non-dwelling, criminal damage and theft from the person.

His first DTO had been at the age of 16 following convictions for burglary non-dwelling; possession of Class $\mathrm{C}$ drugs and theft of a motor vehicle. Upon release, Connor had breached the terms of his licence and had been returned to custody to serve the remainder of his sentence. Two days after the end of his DTO, he was in court again - this time for an attempted burglary committed prior to his detention. He received conditional discharges for both this and at a subsequent hearing for vehicle interference. $A$ month later he was fined for possession of cannabis. It was the attempted burglary com mitted in June 2012 which lead to Connor's inclusion on the reoffending spreadsheet. Having received a conditional discharge, he did not receive an intervention from the YOT.

During December 2012, Connor committed a theft - it was the theft that led to him being assessed using the Core ASSET Profile. Whilst on conditional bail for this he committed a common assault; two further non-domestic burglaries and been charged for being carried in an aggravated taken without owner's consent. As a result, he was remanded in custody in mid-January 2013 and was subsequently sentenced to an 8-month DTO. Connor's first three Core ASSET Profiles were conducted in January 2013 with Time 3 being post-release.

Figure 4.10: Case Study "Connor": Domain Scores at Time 2 and Time 3

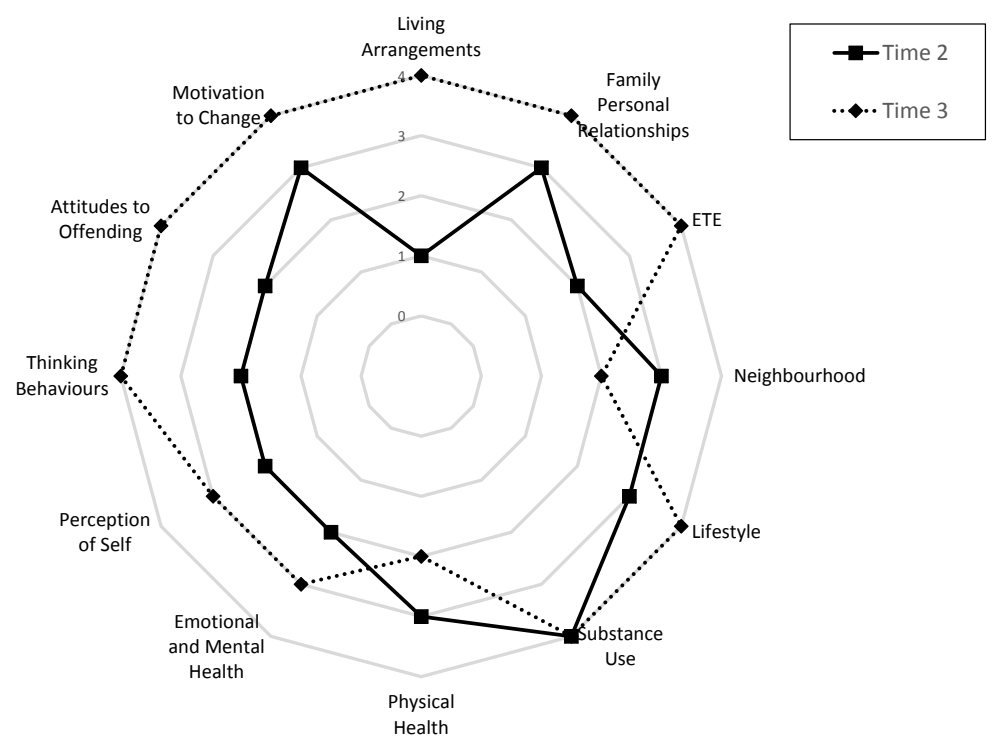


As can be seen from Figure 4.10, Connor was judged to have a high risk of reoffending post-release, with a total dynamic ASSET score of 42 out of a maximum of 48. Notably the ratings for Neighbourhood and Physical Health reduced as a result of him being released from custody.

Connor's background is significantlymore complex than Fred's, having been looked after for periods including spending around 3-months living in a residential care home whilst he was known to the YOT. He has also experienced the loss / bereavement of a close family member.

Connor has a diagnosis of ADHD and it has been identified within the case notes that he has significant issues with anger management. He is known to be violent although based on his offending and court records, with the exception of a charge of common assault when aged 17 which was subsequently dismissed, the incidences which attracted police attention occurred when he was aged 14. This included threatening, abusive or insulting words or behaviour (case dismissed) and two incidences of causing harassment, alarm or distress by threatening words or behaviour which occurred within a 3-week period - the latter resulting in him receiving a Youth Rehabilitation Order.

At differenttimes, Connor has lived with differentmembers of his family, with his accommodation records suggesting thathe had been living with an Aunt prior to being taken into custody. Although upon release he appears to have moved into a bedsit under an independenttenancy. Notably from the time that he became known to the YOT in April 2009, it would appear that he has moved between six addresses described as being "at home", "family (immediate)" and "relatives" as well as being temporarilyhou sed in a B\&B and foster care prior to starting his custodial sentences atHMP Parc. In addition, therefore to a lack of stability in his living arrangements, it is likely that the combination of his ADHD, offending behaviours and drug use will have put a strain on relationships with family members hence the high ratings for these domainsaswell.

Figure 4.11 summarises the estimated trajectory for the probability of further offending using Connor's ASSET scores. Compared to Fred (who had an initial total ASSET score of 8 making him low-medium risk), Connor's was rated as being a high risk. Thus, his initial probability of further offending at Time 0 based on the basic dynamic model is notably higher and continues to be higher for the duration of his time under the supervision of the YOT.

Connor committed the common assault, non-domestic burglaries and vehicle interference at Time 1. Such was the perceived risk that he posed, he was remanded in custody. However, the basic dynamic model does not reflect this increase in the estimated probability of further offending at Time 1 despite the increase in his total ASSET score from 30 to 38. 


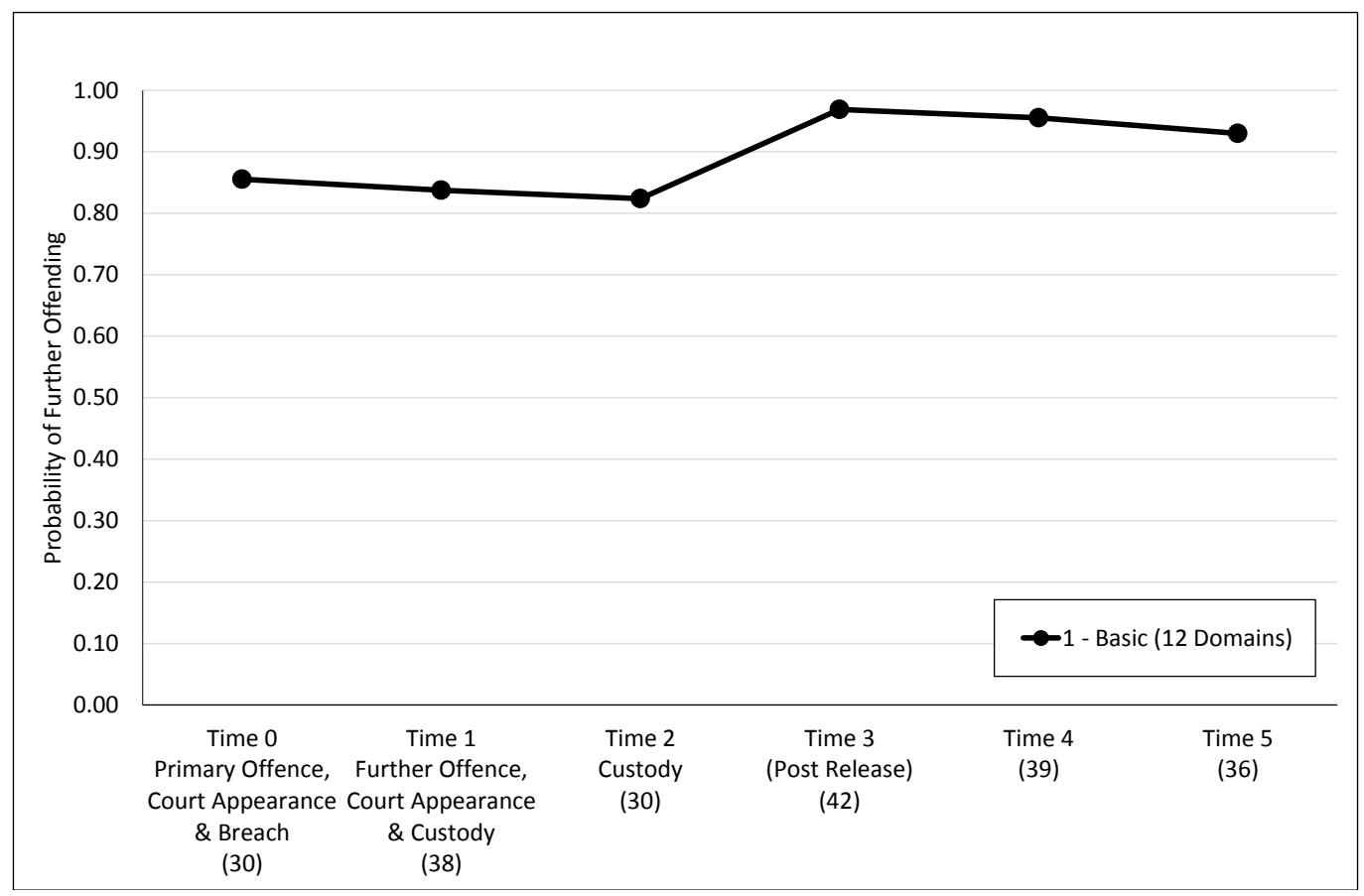

Notes: Although the ASSET scores reflected along the $x$-axis are out of a maximum of 48 with Connor having a total of 30 at Time 0, under the Scaled Approach he would have attracted additional scores due to the fact that his primary offence (for the purposes of this exercise where the information has been taken from the reoffending spreadsheet) was anon-domestic burglary and as a result of his prior convictions.

Having fallen between Time 1 and 2, the model estimates that the probability of further offending increased following his release from custody - the changes in individual domain scores being shown in Figure 4.10. At Time 5 his estimated probability of further offending is higher than when he was initially assessed.

Sadly, without access to PNC it is not possible to ascertain whether Connor committed any further offences after this time. Having turned 18 whilst in custody (Time2), he continued to be supervised by the YOT until the end of his order and would have been too old to have been included in the reoffending spreadsheetfor 2013/14 had he committed any further offences after this time.

\section{Case Study "David"}

This final case study focuses on "David" who in contrast to Fred and Connor who were identified having experienced increases in their total dynamic ASSET score, saw a significant reduction in his score. David committed his first offence at age 10 and received his first conviction at age 16. However, after receiving a Reprimand for a common assault in 2006, there were no further offences until May 2012 when he committed two public order offences (Causing Harassment Alarm Distress by Threatening Words or Behaviour). Although his offending and court records suggest that he received a Referral Order (a First-Tier outcome) for these offences, he does not appear on the 2012/13 reoffending spreadsheetfor Swansea YOT. Rather he appears within the 2013/14 cohortas a 17 -year old having 
received a 12-month Youth Rehabilitation Order (YRO) for stealing a motor vehicle and being caught driving this withoutlicense or insurance.

David has been looked after for periods and it is identified that there are critical familyand relationship issues. This includes a familyhistory of both drug and alcohol use, and his case notes sugge st that he been the victim of domestic violence, neglect, physical and emotional abuse. He is subject to a statement of education special needs and has both poor communication skills and literacy difficulties. There is also recorded that David is lacking some life skills and has mental health problems. He has some issue with anger managementand significantissues with self-esteem. There are alsosome issues with identify / self-image and a critical lack of motivation around his offending behaviour.

David's accommodation records are particularly revealing since they suggest that despite having experienced significant disadvantage, he has managed to maintain a relationship with his mother although this has broken down at different points. After his initial assessment, there are three assessments made within a month, corresponding to when David appeared in courtand was due to be sentenced for breaching his YRO. Time 3 relates to an assessmentcompleted a week after the terms of his YRO were reviewed whilst Time 4 was completed 8 months later.

In the intervening period David's total score reduced by 16 overall with notable decreases in his ratings for the ETE; lifestyle; physical health; emotional and mental health and motivation to change domains (Figure 4.12). In part this reflects the greater stability in his life, having lived with his mother for approximately two and a half months at the time of the assessment. Prior to this he had periods where he was staying in B\&B accommodation, had been homeless and spent time ata night shelter which had contributed to a deterioration in his physical health.

Figure 4.12: Case Study "David": Domain Scores at Time 3 and Time 4

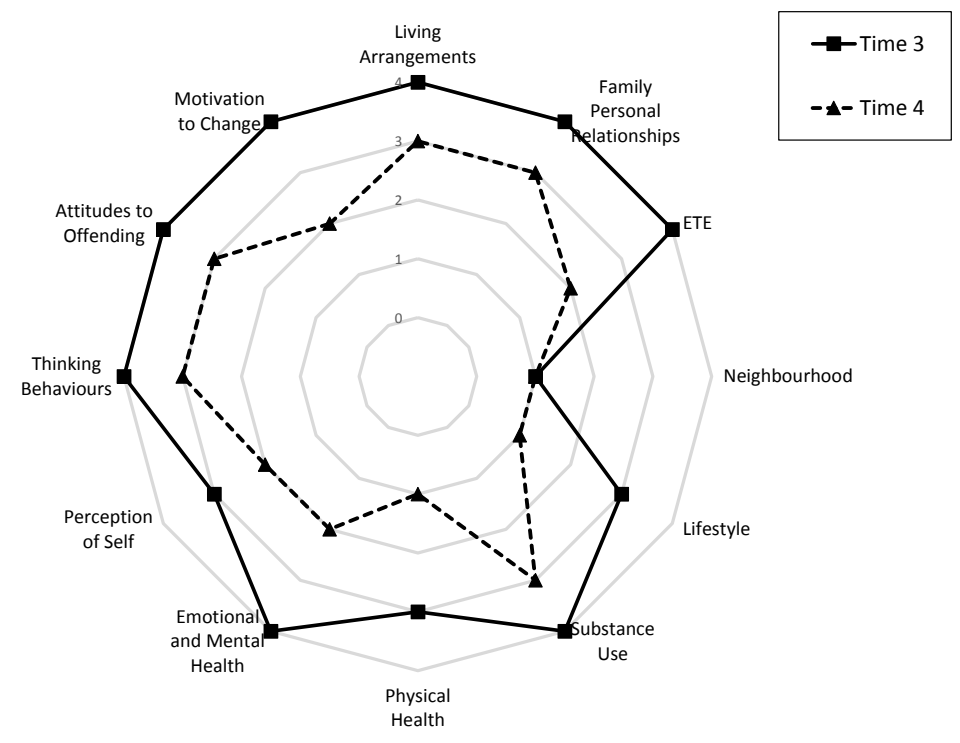


Figure 4.13 summarises the estimated trajectories for the probability of further offending using David's ASSET scores. Like Connor, David was also initiallyrated as being a high risk and he also committed a further offence before his assessmentat Time 1.

Figure 4.13: Estimated Probability of Further Offending Over Time: "David"

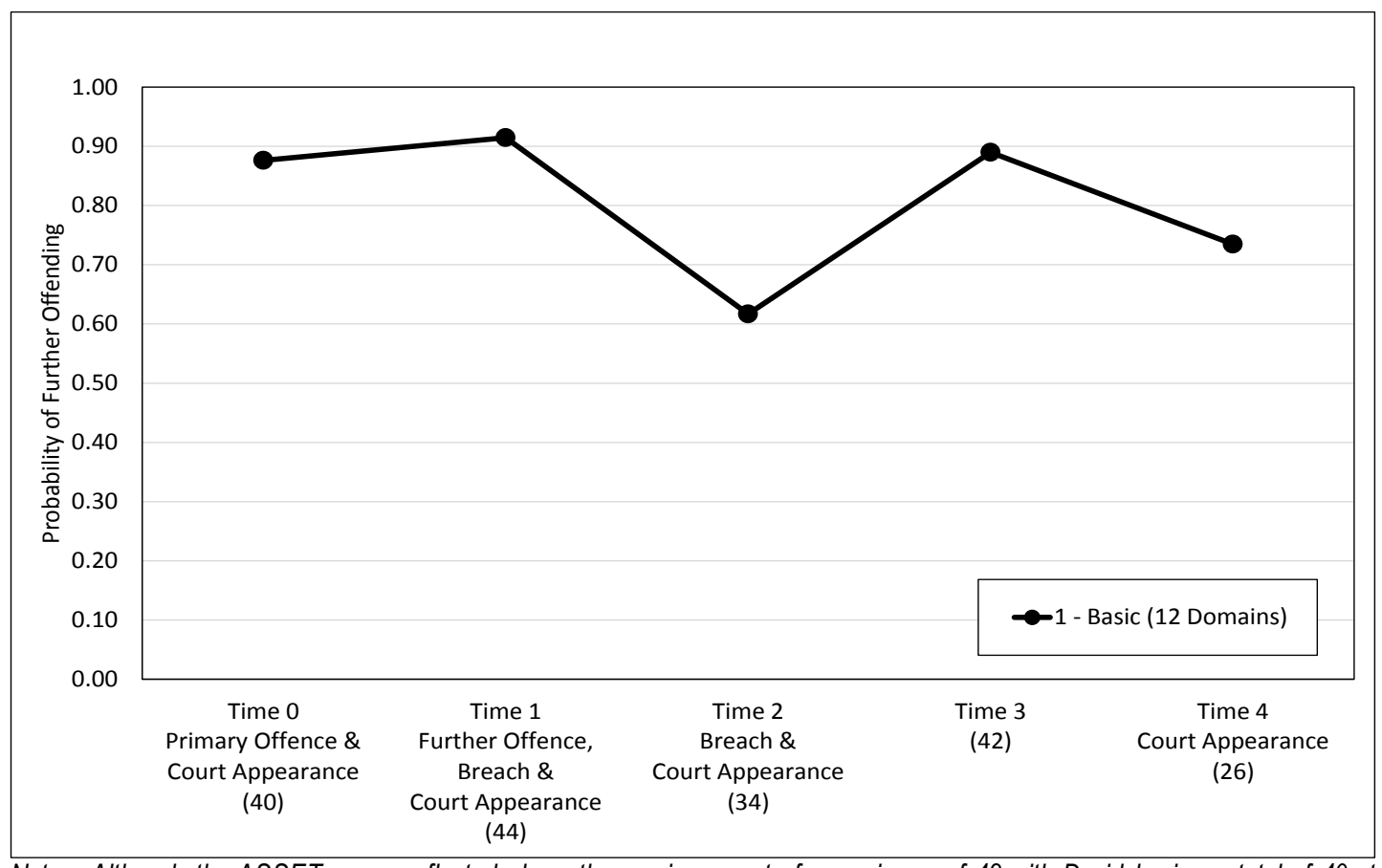

Notes: Although the ASSET scores reflected along the $x$-axis are out of a maximum of 48 with David having a total of 40 at Time 0, under the Scaled Approach he would have attracted additional scores since he was aged 10 at the time of his first Reprimand and as a result of his prior convictions.

The basis dynamic model suggests that there was an increase in the estimated probability of further offending which coincided with when the further offence was committed (at Time 1). There is then a fall (at Time 2) in his total ASSET scores followed by an increase, then a further fall. The two peaks in the trajectory correspond to where David had his highest ASSET scores and hence was considered by practitioners to have the highest likelihood of further offending. However, it is notable that the second peak (at Time 3) corresponds not to when he committed a further offence, but shortly after the terms of his YRO were reviewed.

\subsection{Summary}

The analysis presented in this chapter addresses the first research question What is the relationship between further offending, the 12 domain scores and time? By creating an additive multilevel model which represents the way in which scores from the risk assessment process are used over time, it has been possible to observe:

- That the assumption thata young person's probability of further offending will decrease over time as a result of working with the YOT is supported by the Basic Model (Bm1) where time is a significantfixed main effect with a negative coefficient. 
- The progress that a young person makes through the YOT varies by individual and time, as evidenced by the significant random effects in $\mathrm{Bm} 1$.

- By allowing the individual domain scores to vary by time (the Basic Dynamic Model, BDm1), it is possible to identify which domains are the most significant as the young person progresses through their order. These findings support those previously identified in evaluations of ASSET (Baker et al, 2005, Wilson and Hinks, 2011) and more recentwork by the YJB in relation to its Reducing Reoffending Programme (Youth Justice Board, 2016b), and anticipated, link back to the principals of RNR.

Alongside each of the preparatorymodels, the frequentist equivalent model has been run for comparison. As can be seen from the Technical Annex, running the frequentist version of the Basic Model (M1) resulted in warning signs about convergence (p48). Adding additional predictors and hence adding to the complexity of the basic model results in similar warnings. Whilst various online help pages li ke Stackflow suggest that these should not be taken too seriously, the fact that these are appearing prior to the dynamic model being constructed highlights the limitations of using frequentist approaches and the benefit of generating the models under a Bayesian framework.

Through consideration of the respective probabilities of further offending for Fred, Connor and David, it is possible to see that there some of the trends apparent in the trajectories which coincide with key events. For example, Fred's increasing ASSET scores let to him being detained, a trend that is apparent in Figure 4.9. Post-release, Connor's total ASSET score increased - a trend that was also reflected in the trajectory of his probability of further offending (Figure 4.11). David committed a further offence between Time 0 and Time 1. His ASSET score increased during this period and this is also reflected in the increased probability of further offending estimated by the $\mathrm{BDm} 1$ at this time.

The extent to which ASSET scores reflect the realities of the young person's change in circumstances during their time under the supervision of the YOT (the second research question posed within this chapter) cannotbe fully explored without considering individual differences; the impact of organi sational factors such as having experience of care and as highlighted above understanding more about the impact of coming into contact with the different facets of the youth justice system. Hence, the data pertaining to Fred, Connor and David presented in this chapter will form a baseline against which subsequentmodels can be compared.

The findings in Chapters Five to Seven build upon this Basic Dynamic model by adding predictors to represent dimensional identityi.e. gender and ethnicity; the nature of criminal careers and differentforms of system contact. These predictors have different characteristics and have been selected to demonstrate both the utility of the approach and its abilityto handle different types of predictors, butalso due to their theoretical role in understanding youth offending behaviours. 


\section{Findings: Dimensional Identity}

Section 2.7 highlights concerns as to the insensitivity of manyrisk assessmentinstruments with respect to demographic characteristics. Building upon differences in the published proven reoffending and further offending rates determined for those within the reoffending cohort (summarised in Chapter Three), this Chapter focused upon the following research questions:

2. What is the impact of gender and ethnicity on the likelihood of further offending over time?

3. What is the impact of having experience of care on the likelihood of further offending over time?

8. How well do ASSET scores reflect the realities of the young person's change in circumstances during their time under the supervision of the YOT?

The predictors explored within this chapter are therefore:

- Gender - a dichotomous predictor (Male/Female)

- Ethnicity - a dichotomous predictor(White / Non-White)

- Care Experience-a dichotomous predictor reflecting whether or not the young person has had experience of care

Further individual level predictors relating to the age at first offence, age at first conviction, FTE status and the nature of the offence are considered in Chapter Six. Time-varying predictors reflecting events such as breaching, court appearances and spending time in custody / on remand are considered in Chapter Seven.

\subsection{The role of gender and ethnicity}

\section{Description of the Data}

It was apparent from the early analysis presented in Chapter Three to describe the reoffending cohort that there are low numbers of females and non-White young people within the dataset. Whilst this was to be expected, it is necessary to consider what can be achieved with such small sub-groups. Tables 5.1 and 5.2 summarise the Level 2 predictors for the six non-White young people and the nine females in the reoffending cohortrespectively. 
Table 5.1: Summary of Level 2 Predictors for the Nine Females in the Dataset

\begin{tabular}{|c|c|c|c|c|c|c|}
\hline ID & Gender & Ethnicity & $\begin{array}{c}\text { Care } \\
\text { Experience? }\end{array}$ & FTE? & AgeFirst & AgeCon \\
\hline 1 & Female & White & Yes & No & 12 & 15 \\
\hline 2 & Female & White & No & No & 13 & 14 \\
\hline 3 & Female & White & Yes & No & 13 & 15 \\
\hline 4 & Female & White & No & No & 14 & 14 \\
\hline 5 & Female & White & No & No & 14 & 15 \\
\hline 6 & Female & White & No & Yes & 14 & 16 \\
\hline 7 & Female & White & No & No & 15 & 17 \\
\hline 8 & Female & White & No & No & 15 & 17 \\
\hline 9 & Female & White & No & NotKnown & 17 & 17 \\
\hline
\end{tabular}

Note: Since the FTE Status of the gth $^{\text {themale }}$ is not known, it has been necessary to exclude her ASSETs from the modelling.

None of the females in the reoffending dataset are non-White, with two having experience of care. Just one was an FTE at the point of entry into the cohortalthough the absence of courtand offending records within Childview for the ninth female means that it is not possible to establish whether or not they had a prior offending history. Their ages at the time of their first offence range from 12 to 17 . However, the youngest age of conviction was 14 . The low numbers of females with experience of care and who are FTEs respectively means there is insufficient data to enable reliable estimates to be simulated for the interactions between Gender: Ethnicity, Gender: FTE and Gender: FTE: Care Experience, particularly as further predictors are added to the model.

Table 5.2: Summary of Level 2 Predictors for the Six Non-White Young People in the Dataset

\begin{tabular}{|c|c|c|c|c|c|c|}
\hline ID & Ethnicity & Gender & $\begin{array}{c}\text { Care } \\
\text { Experience? }\end{array}$ & FTE? & AgeFirst & AgeCon \\
\hline 1 & Non-White & Male & Yes & No & 10 & 10 \\
\hline 2 & Non-White & Male & No & No & 12 & 13 \\
\hline 3 & Non-White & Male & No & No & 13 & 14 \\
\hline 4 & Non-White & Male & No & Yes & 15 & 16 \\
\hline 5 & Non-White & Male & No & No & 15 & 17 \\
\hline 6 & Non-White & Male & No & Yes & 16 & 16 \\
\hline
\end{tabular}

As can be seen from Table 5.2, all the non-White young people are male; only one has experience of care whilst there are two who were FTEs at the time of entering the cohort. Their ages at the time of first offence and first conviction vary. The absence of females amongst this sub-group means that estimates cannot be simulated for the Gender: Ethnicity interaction term. The low numbers of nonWhites with experience of care and who are FTEs respectively also means there is very limited data which can be used to enable estimates to be simulated for the interactions between Ethnicity: Care Experience, and Ethnicity: FTE as further predictors are added to the model.

From Table3.9, we know that the rate of further offending across the two years appears to be higher for males than females, and for Whites compared to non-Whites - given the size of the dataset, there is only moderate evidence to support this finding. However, it is born out in the published national data (for example Youth Justice Board and Ministry of Justice, 2015a). To understand why these differences 
occur, a number of factors are considered and hence in order to identify where there may be differences reflected within the model, the first step is to consider whether there are differences in their respective domain score profiles at Time 0 (Figure 5.1).

Figure 5.1: Domain Score Profile, by (a) Gender and (b) Ethnicity, at Time 0
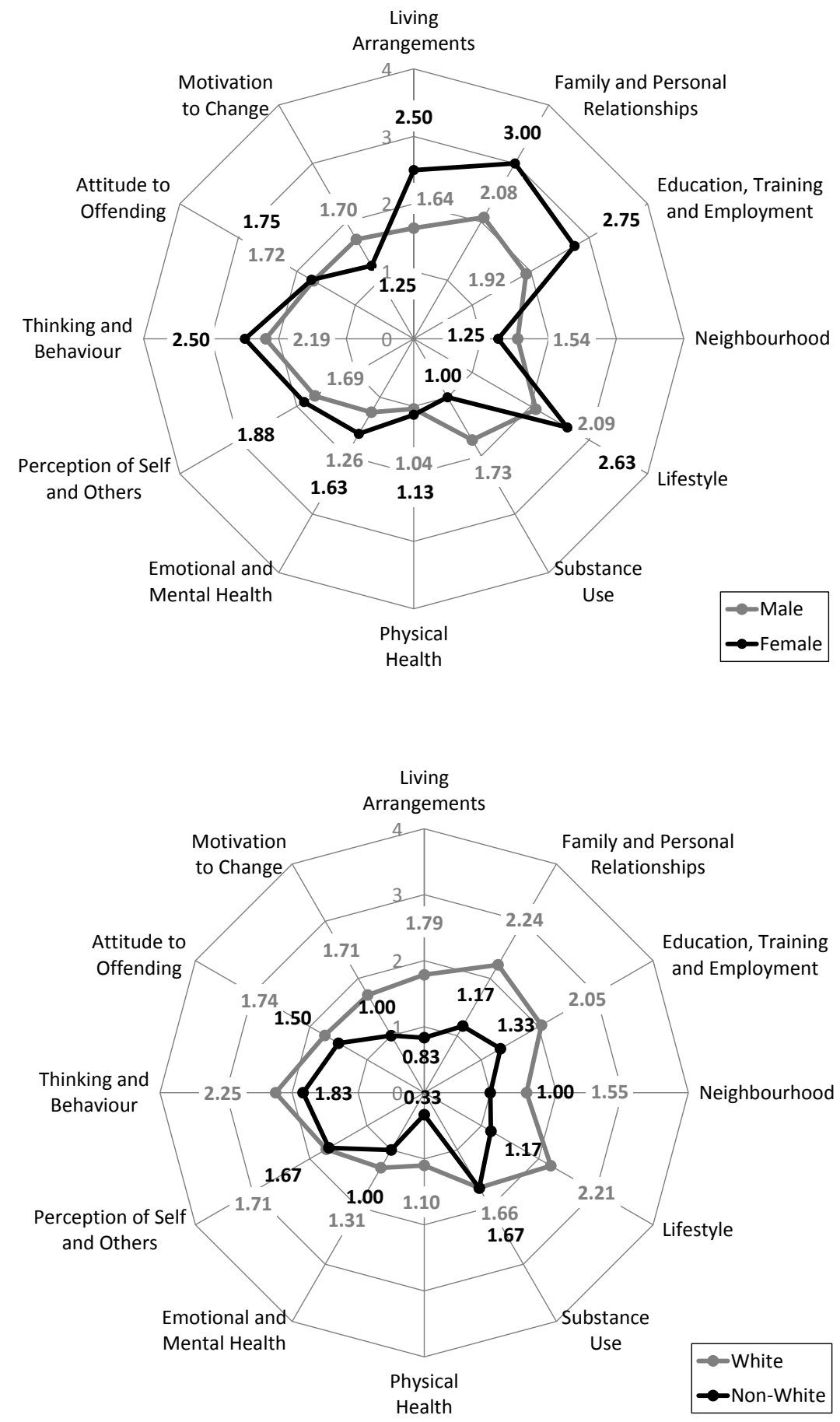

Notes: Of the 87 individuals, 8 are female and 6 identify as being non-White. Time 0 represents the initial assessment undertaken.

One-sided Bayesian independentt-tests (Wagenmakers et al., 2017; Rouder et al., 2009) suggest that females $(\mathrm{N}=8)$ typically have higher ratings than males $(\mathrm{N}=79)$ at the time of their initial assessment for: 
- Living arrangements $\left(\mathrm{BF}_{10}=3.268\right.$ in favour of $\mathrm{H}_{1}$ : Males $<$ Females, $\%$ error $\left.=3.921 \mathrm{e}^{-5}\right)$

- Familyand personal relationships $\left(\mathrm{BF}_{10}=3.616, \%\right.$ error $\left.=3.989 \mathrm{e}^{-5}\right)$

However, there is only anecdotal evidence to suggest that the following mean initial domain scores for males are higher than for females:

- Neighbourhood $\left(\mathrm{BF}_{10}=0.508\right.$ in favour of $\mathrm{H}_{1}$ : Males $>$ Females, $\%$ error $\left.=1.056 \mathrm{e}^{-4}\right)$

- Substance Use $\left(\mathrm{BF}_{10}=1.433, \%\right.$ error $\left.=2.626 \mathrm{e}^{-4}\right)$

- Motivation to Change $\left(\mathrm{BF}_{10}=1.610, \%\right.$ error $\left.=3.060 \mathrm{e}^{-4}\right)$

Figure $5.1(\mathrm{~b})$ suggests that the average initial ratings for those identifying as non-White $(\mathrm{N}=6)$ are typically higher than those for their White peers $(\mathrm{N}=81)$ therefore the t-test considers the alternative hypothesis that the population mean for non-Whites is greater than that for Whites. The t-test suggests that there are differences in terms of ratings for:

- Living arrangements $\left(\mathrm{BF}_{10}=3.259\right.$ in favour of $\mathrm{H}_{1}, \%$ error $\left.=3.613 \mathrm{e}^{-5}\right)$

- Familyand personal relationships $\left(\mathrm{BF}_{10}=5.313, \%\right.$ error $\left.=2.123 \mathrm{e}^{-4}\right)$

- Lifestyle $\left(\mathrm{BF}_{10}=7.278, \%\right.$ error $\left.=2.719 \mathrm{e}^{-4}\right)$

Although the average initial domain scores for Substance Misuse $\left(\mathrm{H}_{1}\right.$ : Non-Whites $\neq$ Whites, $\mathrm{BF}_{10}=$ 0.383) and Perception of Selfand Others $\left(\mathrm{BF}_{10}=0.385\right)$ appear to be very similarfor both groups, there is only anecdotal evidence to support this. It is also important to note that since the non-White group consists of only 6 young people, the credible intervals for their respective mean domain scores are wide suggesting less certainty.

\section{Adding Genderand Ethnicity to the Basic Model}

Prior to running a dynamic model where the domain scores are allowed to vary over time, dummy variables for gender (referenced by males) and ethnicity (referenced by White) have been added to the Basic Model (summarised in Table 4.11). Running this less complex model which requires fewer iterations than the Basic Dynamic Model allows us to test if our assumptions appear to hold true i.e. that when all other factors are equal, males will have a higher further offending rate than females, and Whites have a higher further offending rates than their non-White peers.

Adding dummy variables for gender (referenced by males) and ethnicity (referenced by White) to the Basic Model, does not result in a marked reduction in the DIC for the respective models - the DIC for the Basic Model which did not involve any additional predictors was 476.20 whereas these are 476.52 and 475.19 for the versions involving gender (Model 1.1) and ethnicity(Model 1.2). There also does not appear to be any issues in relation to convergence when these models are run (Table 5.3). 
To determine if all things being equal in terms of the other predictors in the specified model, there is a difference between the two groups, all the domain scores have been 'set' at 2. This has been chosen since it roughly corresponds to the mean scores overall. By summing the coefficients for the fixed effects, it is possible to determine the estimated probability of further offending at a given time e.g. Time 0 . Doing this for each sub-group enables the odds of further offending to be compared. The Basic Models involving gender and ethnicity respectively suggest that:

- The odds of further offending amongstfemales are estimated to be $\exp (0.150)=1.16$ times the odds for male further offending (Model 1.1). [Cl =0.45, 3.03]

- The odds of further offending amongst those from White backgrounds is estimated to be $1 / \exp (-$ $0.735)=2.09$ times higher than the odds for their non-White peers (Model 1.2) $[\mathrm{Cl}=0.76,5.73]$

The wide credible intervals for these is a consequence of the small numbers of females (8) and nonWhite (6) young people in the sample whilst the absence of any non-White females, means that it is not possible to explore the potential for any interaction between the two predictors. However, the additive model (Model 1.3) involving both predictors suggests that there is no notable penalty for adding both gender and ethnicity to the Basic Model.

The odds above reflect the impact of the respective demographic characteristics when all other predictors are equal. However, as highlighted in Figure 5.1, there appear to be different risk profiles at Time 0 for each of the demographic groups suggestingfor example that there are marked differences in terms of average ratings for a number of domains which maybe influenced byfactors not reflected within the models. 
Table 5.3: Random Intercepts and Varying Slope Models for Further Offending including ASSET Domains and Demographic Characteristics Unstandardised Coefficients

\begin{tabular}{|c|c|c|c|c|c|c|c|c|}
\hline \multirow{4}{*}{\begin{tabular}{|l} 
\\
Fixed Effect:
\end{tabular}} & \multicolumn{4}{|c|}{ Model 1.1} & \multicolumn{4}{|c|}{ Model 1.2} \\
\hline & \multicolumn{4}{|c|}{ Basic Model + Gender } & \multicolumn{4}{|c|}{ Basic Model + Ethnicity } \\
\hline & \multicolumn{3}{|c|}{ Unstandardised } & \multirow{2}{*}{ Significant? } & \multicolumn{3}{|c|}{ Unstandardised } & \multirow{2}{*}{ Significan } \\
\hline & Post.Mean & Lower $\mathrm{Cl}$ & Upper Cl & & Post.Mean & Lower $\mathrm{Cl}$ & Upper Cl & \\
\hline Intercept & -1.165 & -2.385 & 0.089 & & -1.071 & -2.295 & 0.209 & \\
\hline Gender (Male $=$ Ref). & 0.150 & -0.798 & 1.107 & & & & & \\
\hline Ethnicity (White = Ref) & & & & & -0.735 & -1.745 & 0.273 & \\
\hline Living Arrangements & 0.033 & -0.023 & 0.293 & & 0.029 & -0.231 & 0.285 & \\
\hline Family and Personal Relationships & 0.271 & -0.017 & 0.566 & & 0.252 & -0.039 & 0.544 & \\
\hline Education, Training and Employment & 0.087 & -0.167 & 0.329 & & 0.081 & -0.164 & 0.329 & \\
\hline Neighbourhood & 0.046 & -0.178 & 0.263 & & 0.059 & -0.162 & 0.278 & \\
\hline Lifestyle & 0.021 & -0.330 & 0.363 & & -0.022 & -0.372 & 0.322 & \\
\hline Substance Use & 0.163 & -0.085 & 0.404 & & 0.175 & -0.058 & 0.417 & \\
\hline Physical Health & -0.117 & -0.396 & 0.172 & & -0.146 & -0.435 & 0.139 & \\
\hline Emotional and Mental Health & -0.001 & -0.248 & 0.241 & & $-3.58 \mathrm{E}-03$ & -0.247 & 0.239 & \\
\hline Perceptions of Self and Others & -0.144 & -0.475 & 0.166 & & -0.114 & -0.439 & 0.199 & \\
\hline Thinking and Behaviour & -0.164 & -0.494 & 0.177 & & -0.129 & -0.470 & 0.201 & \\
\hline Attitude to Offending & 0.055 & -0.289 & 0.412 & & 0.056 & -0.297 & 0.400 & \\
\hline Motivation to Change & 0.241 & -0.109 & 0.582 & & 0.227 & -0.108 & 0.568 & \\
\hline Time & -0.156 & -0.290 & -0.019 & Yes & -0.156 & -0.290 & -0.025 & Yes \\
\hline Random Effect: & PostMean & Lower $\mathrm{Cl}$ & Upper Cl & Significant? & Post.Mean & Lower Cl & Upper Cl & Significan \\
\hline Individual (Intercept) & 0.117 & $1.46 \mathrm{E}-04$ & 0.407 & Yes & 0.107 & $1.51 \mathrm{E}-04$ & 0.377 & Yes \\
\hline Time & 1.287 & 0.339 & 2.641 & Yes & 1.285 & 0.346 & 2.614 & Yes \\
\hline
\end{tabular}

DIC 


\subsection{Adaptations to the Model}

The 'basic' hierarchical generalised linear model summarised in T able 4.12 has variables at two levels:

- Level 1 units are the measurementoccasions (the ASSET Core Profiles) indexed by $t$

- Level 2 units are the individuals measured by $i$

Non-time varying characteristics such as gender and ethnicity represent additional Level 2 predicators to be added. The model proposed here therefore equates to:

Level 1: $\operatorname{Pr}\left(\mathrm{y}_{\mathrm{ti}}=1\right)=\operatorname{logit}^{-1}\left(\beta_{0}+\beta_{1} \mathrm{x}_{1 \mathrm{ti}}+\beta_{2} \mathrm{x}_{2 \mathrm{ti}}+\beta_{3} \mathrm{x}_{3 \mathrm{ti}}+\ldots .+\beta_{12} \mathrm{x}_{12 \mathrm{ti}}+\beta_{13} \mathrm{~m}_{\mathrm{ti}}+\mathrm{e}_{\mathrm{ti}}\right)$ Level 2: $\operatorname{Pr}\left(\beta_{\mathrm{hi}}\right)=\operatorname{logit}^{-1}\left(\gamma_{\mathrm{h} 0}+\gamma_{\mathrm{h} 1} \mathrm{z}_{1 \mathrm{i}}+\gamma_{\mathrm{h} 2} \mathrm{z}_{2 \mathrm{i}}+\gamma_{\mathrm{h} 3} \mathrm{z}_{3 \mathrm{i}}+\mu_{\mathrm{hi}}\right)$

Since this is an additive model - reflecting the way in which the ratings given for each of the 12 domains are added together to give a total ASSET score - the basic dynamic model (BDm1) is specified as a series of interactions between the individual domains and time. To add an additional Level 2 predictor, this is incorporated into each of the interactions as shown in BDmX_L2:

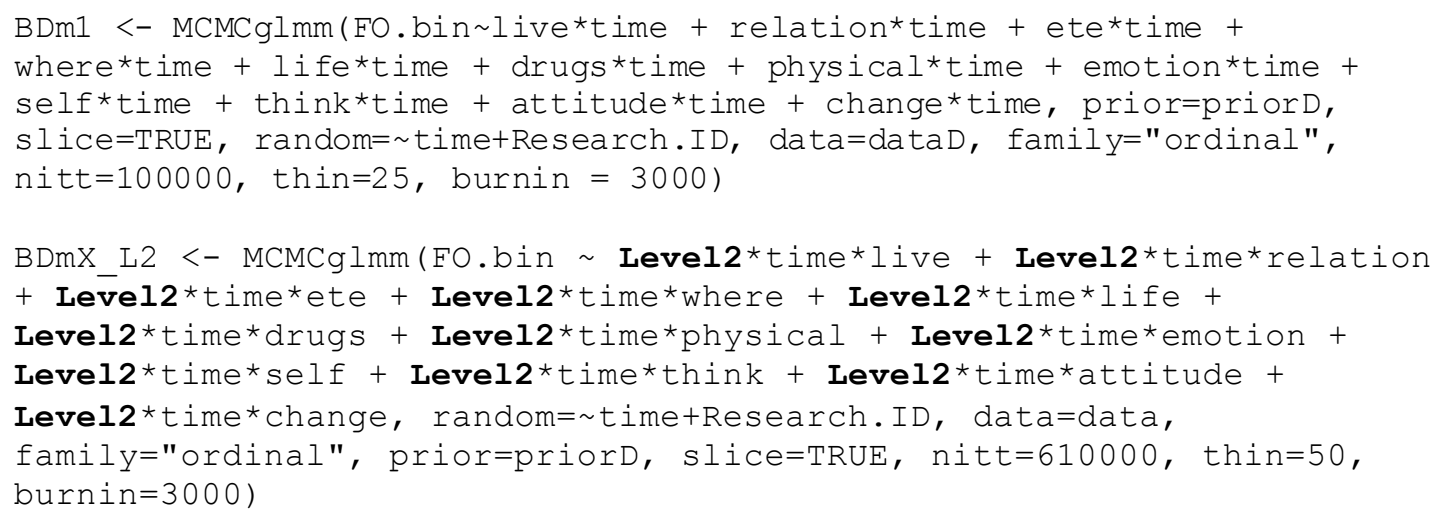

By specifying the interactions in this way, when the resulting model is simulated, then all the terms are included individuallyin the model along with all the 2-way permutations and the 3-way interaction: 


\begin{tabular}{|c|c|c|}
\hline Description & $\begin{array}{l}\text { Basic Dynamic Model } \\
\text { (BDm1) }\end{array}$ & $\begin{array}{l}\text { Dynamic model involving a Level } 2 \text { predictor } \\
\text { (BDmX_L2) }\end{array}$ \\
\hline Main Effects & $\begin{array}{l}12 \times \text { domains } \\
\text { Time }\end{array}$ & $\begin{array}{l}12 \times \text { domains } \\
\text { Time } \\
\text { Level } 2 \text { predictor }\end{array}$ \\
\hline 2-way Interactions & Time $x$ each of the 12 domains & $\begin{array}{l}\text { Time } x \text { each of the } 12 \text { domains } \\
\text { Level } 2 \text { predictor } x \text { Time } \\
\text { Level } 2 \text { predictor } x \text { each of the } 12 \text { domains }\end{array}$ \\
\hline 3-way Interactions & & Level $2 x$ Time $x$ each of the 12 domains \\
\hline
\end{tabular}

Hence, the complexity of the model is significantlyincreased through inclusion of additional parameters. Examples of the outputfrom these modelscan be found in the Technical Annex.

\section{The Dynamic Models Involving Gender and Ethnicity}

Extending the Basic Dynamic model as described in section 5.1, provides a sense of how the individual domains behave over time and if there are differences based on gender and the ethnicity of the young person. The resulting models are summarised in T ables 5.4 and 5.5 respectively. As there are no nonWhite females, no attempt has been made to stimulate a model involving an interaction between these two demographic predictors, thus extending Model 1.3. 
Table 5.4: The Dynamic Model Involving Gender

\begin{tabular}{|c|c|c|c|c|c|c|c|}
\hline \multirow{3}{*}{\begin{tabular}{|l} 
\\
Fixed Effect: \\
\end{tabular}} & \multicolumn{7}{|c|}{$\begin{array}{l}\text { The Dynamic Model including Gender } \\
\text { (BDm2_d1) }\end{array}$} \\
\hline & \multicolumn{3}{|c|}{ Unstandardised } & \multicolumn{3}{|c|}{ Standardised } & \multirow{2}{*}{ Significant? } \\
\hline & Post.Mean & Lower $\mathrm{Cl}$ & Upper $\mathrm{Cl}$ & Post.Mean & Lower $\mathrm{Cl}$ & Upper $\mathrm{Cl}$ & \\
\hline Intercept & -1.117 & -2.745 & 0.538 & 0.327 & 0.064 & 1.713 & \\
\hline Gender (Male = Ref). & -4286.000 & -7928.000 & -153.000 & 0.000 & 0.000 & 0.000 & Yes \\
\hline Time & -0.220 & -0.491 & 0.048 & 0.803 & 0.612 & 1.049 & \\
\hline Living Arrangements (Live) & -0.041 & -0.515 & 0.418 & 0.960 & 0.598 & 1.519 & \\
\hline Family and Personal Relationships (Relation) & 0.300 & -0.159 & 0.822 & 1.350 & 0.853 & 2.276 & \\
\hline Education, Training and Employment(ETE) & -0.327 & -0.740 & 0.044 & 0.721 & 0.477 & 1.045 & \\
\hline Neighbourhood (Where) & 0.048 & -0.349 & 0.434 & 1.049 & 0.705 & 1.543 & \\
\hline Lifestyle (Life) & 0.039 & -0.570 & 0.651 & 1.039 & 0.566 & 1.918 & \\
\hline Substance Use (Drugs) & 0.338 & -0.074 & 0.762 & 1.402 & 0.928 & 2.142 & \\
\hline Physical Health (Physical) & -0.733 & -1.273 & -0.236 & 0.481 & 0.280 & 0.790 & Yes \\
\hline Emotional and Mental Health (Emotion) & -0.126 & -0.514 & 0.284 & 0.882 & 0.598 & 1.329 & \\
\hline Perceptions of Self and Others (Self) & 0.048 & -0.556 & 0.655 & 1.049 & 0.574 & 1.925 & \\
\hline Thinking and Behaviour (Think) & -0.080 & -0.651 & 0.467 & 0.923 & 0.522 & 1.596 & \\
\hline Attitude to Offending (Attitude) & 0.038 & -0.533 & 0.619 & 1.039 & 0.587 & 1.856 & \\
\hline Motivation to Change (Change) & 0.718 & 0.100 & 1.371 & 2.050 & 1.105 & 3.939 & Yes \\
\hline Gender: Time & 1987.000 & -102.000 & 3763.000 & \#NUM! & 0.000 & \#NUM! & \\
\hline Gender: Live & -428.100 & -2063.000 & 542.000 & 0.000 & 0.000 & $2.44 E+235$ & \\
\hline Gender: Relation & 574.300 & -1086.000 & 2229.000 & $2.60 \mathrm{E}+249$ & 0.000 & \#NUM! & \\
\hline Gender: ETE & 828.600 & -1319.000 & 3225.000 & \#NUM! & 0.000 & \#NUM! & \\
\hline Gender: Where & 1921.000 & -69.530 & 3169.000 & \#NUM! & 0.000 & \#NUM! & \\
\hline Gender: Life & -3269.000 & -7131.000 & 276.600 & 0.000 & 0.000 & $1.34 E+120$ & \\
\hline Gender: Drugs & 736.900 & -1511.000 & 2668.000 & \#NUM! & 0.000 & \#NUM! & \\
\hline Gender: Physical & 1387.000 & -128.000 & 3209.000 & \#NUM! & 0.000 & \#NUM! & \\
\hline Gender: Emotion & 1584.000 & -497.200 & 3372.000 & \#NUM! & 0.000 & \#NUM! & \\
\hline Gender: Self & -2575.000 & -4725.000 & 181.400 & 0.000 & 0.000 & $6.04 \mathrm{E}+78$ & \\
\hline Gender: Think & 1307.000 & -747.900 & 3240.000 & \#NUM! & 0.000 & \#NUM! & \\
\hline Gender: Attitude & 1605.000 & -47.730 & 3607.000 & \#NUM! & 0.000 & \#NUM! & \\
\hline Gender: Change & 2017.000 & -355.400 & 4101.000 & \#NUM! & 0.000 & \#NUM! & \\
\hline Time: Live & 0.021 & -0.077 & 0.112 & 1.021 & 0.926 & 1.118 & \\
\hline Time: Relation & -0.014 & -0.127 & 0.099 & 0.986 & 0.881 & 1.104 & \\
\hline Time: ETE & 0.104 & 0.019 & 0.198 & 1.110 & 1.019 & 1.218 & Yes \\
\hline Time: Where & 0.009 & -0.070 & 0.081 & 1.009 & 0.932 & 1.084 & \\
\hline Time: Life & 0.002 & -0.122 & 0.120 & 1.002 & 0.885 & 1.127 & \\
\hline Time: Drugs & -0.040 & -0.130 & 0.039 & 0.960 & 0.878 & 1.039 & \\
\hline Time: Physical & 0.151 & 0.026 & 0.264 & 1.163 & 1.026 & 1.303 & Yes \\
\hline Time: Emotion & 0.038 & -0.046 & 0.118 & 1.038 & 0.955 & 1.126 & \\
\hline Time: Self & -0.082 & -0.198 & 0.046 & 0.921 & 0.821 & 1.047 & \\
\hline Time: Think & -0.011 & -0.126 & 0.115 & 0.989 & 0.881 & 1.122 & \\
\hline Time: Attitude & 0.007 & -0.115 & 0.126 & 1.007 & 0.891 & 1.134 & \\
\hline Time: Change & -0.092 & -0.214 & 0.039 & 0.912 & 0.808 & 1.039 & \\
\hline
\end{tabular}

/continued 


\begin{tabular}{|c|c|c|c|c|c|c|c|}
\hline \multirow[b]{3}{*}{ Fixed Effect: } & \multicolumn{7}{|c|}{$\begin{array}{l}\text { The Dynamic Model including Gender } \\
\text { (BDm2_d1) }\end{array}$} \\
\hline & \multicolumn{3}{|c|}{ Unstandardised } & \multicolumn{3}{|c|}{ Standardised } & \multirow{2}{*}{ Significant? } \\
\hline & PostMean & Lower $\mathrm{Cl}$ & Upper $\mathrm{Cl}$ & PostMean & Lower $\mathrm{Cl}$ & Upper Cl & \\
\hline Gender: Time: Live & -183.100 & -1051.000 & 206.800 & 0.000 & 0.000 & $6.49 \mathrm{E}+89$ & \\
\hline Gender: Time: Relation & -410.400 & -1144.000 & 320.000 & 0.000 & 0.000 & $9.42 E+138$ & \\
\hline Gender: Time: ETE & 42.020 & -953.900 & 1082.000 & $1.77 \mathrm{E}+18$ & 0.000 & \#NUM! & \\
\hline Gender: Time: Where & -580.200 & -1372.000 & 150.400 & 0.000 & 0.000 & $2.08 \mathrm{E}+65$ & \\
\hline Gender: Time: Life & 979.400 & -424.500 & 2278.000 & \#NUM! & 0.000 & \#NUM! & \\
\hline Gender: Time: Drugs & 49.800 & -373.700 & 410.900 & $4.24 \mathrm{E}+21$ & 0.000 & $2.83 E+178$ & \\
\hline Gender: Time: Physical & -1193.000 & -2069.000 & -42.530 & 0.000 & 0.000 & 0.000 & Yes \\
\hline Gender: Time: Emotion & -768.100 & -1842.000 & 658.200 & 0.000 & 0.000 & $7.12 E+285$ & \\
\hline Gender: Time: Self & 1528.000 & -244.000 & 3165.000 & \#NUM! & 0.000 & \#NUM! & \\
\hline Gender: Time: Think & -161.500 & -469.800 & 188.400 & 0.000 & 0.000 & $6.62 E+81$ & \\
\hline Gender: Time: Attitude & -1308.000 & -1979.000 & -333.100 & 0.000 & 0.000 & 0.000 & Yes \\
\hline Gender: Time: Change & -1139.000 & -2395.000 & 608.900 & 0.000 & 0.000 & $2.77 \mathrm{E}+264$ & \\
\hline Random Effect: & Post.Mean & Lower $\mathrm{Cl}$ & Upper $\mathrm{Cl}$ & Post.Mean & Lower $\mathrm{Cl}$ & Upper $\mathrm{Cl}$ & Significant? \\
\hline Individual (Intercept) & 0.216 & $1.41 \mathrm{E}-07$ & 0.611 & 1.241 & 1.000 & 1.842 & Yes \\
\hline Time & 1.962 & 0.467 & 4.205 & 7.114 & 1.595 & 67.021 & Yes \\
\hline DIC & & & 451.57 & & & & \\
\hline
\end{tabular}

Source: Model BDm2_d1, Technical Annex: p70-82

The output from this model points to a number of issues. Notably there are a number of coefficients which when standardised are very large or cannotbe calculated - reflected by\#NUM! in Table5.4. The trace plots for Genderas a main effect and a number of the interactions involving Genderand/or Time do not converge. A similar pattern can be seen with respect to the dynamic model involving ethnicity (Table 5.5).

Table 5.5: The Dynamic Model Involving Ethnicity

\begin{tabular}{|c|c|c|c|c|c|c|c|}
\hline \multirow[b]{3}{*}{ Fixed Effect: } & \multicolumn{7}{|c|}{$\begin{array}{l}\text { The Dynamic Model including Ethnicity } \\
\text { (BDm2_d2) }\end{array}$} \\
\hline & \multicolumn{3}{|c|}{ Unstandardised } & \multicolumn{3}{|c|}{ Standardised } & \multirow{2}{*}{ Significant? } \\
\hline & PostMean & Lower $\mathrm{Cl}$ & Upper $\mathrm{Cl}$ & Post.Mean & Lower $\mathrm{Cl}$ & Upper $\mathrm{Cl}$ & \\
\hline Intercept & -1.161 & -2.883 & 0.480 & 0.313 & 0.056 & 1.617 & \\
\hline Ethnicity (White $=$ Ref) & -709.400 & -2192.000 & 319.400 & 0.000 & 0.000 & $5.17 \mathrm{E}+138$ & \\
\hline Time & -0.178 & -0.483 & 0.070 & 0.837 & 0.617 & 1.072 & \\
\hline Living Arrangements (Live) & -0.045 & -0.488 & 0.418 & 0.956 & 0.614 & 1.518 & \\
\hline Family and Personal Relationships (Relation) & 0.183 & -0.310 & 0.703 & 1.201 & 0.733 & 2.019 & \\
\hline Education, Training and Employment(ETE) & -0.277 & -0.656 & 0.129 & 0.758 & 0.519 & 1.138 & \\
\hline Neighbourhood (Where) & 0.006 & -0.400 & 0.381 & 1.006 & 0.670 & 1.464 & \\
\hline Lifestyle (Life) & 0.059 & -0.546 & 0.662 & 1.061 & 0.579 & 1.939 & \\
\hline Substance Use (Drugs) & 0.373 & -0.042 & 0.769 & 1.452 & 0.959 & 2.157 & \\
\hline Physical Health (Physical) & -0.583 & -1.066 & -0.094 & 0.558 & 0.344 & 0.911 & Yes \\
\hline Emotional and Mental Health (Emotion) & -0.197 & -0.593 & 0.222 & 0.821 & 0.553 & 1.249 & \\
\hline Perceptions of Self and Others (Self) & 0.366 & -0.226 & 0.980 & 1.442 & 0.798 & 2.665 & \\
\hline Thinking and Behaviour (Think) & 0.129 & -0.425 & 0.751 & 1.138 & 0.654 & 2.118 & \\
\hline Attitude to Offending (Attitude) & 0.048 & -0.515 & 0.613 & 1.049 & 0.598 & 1.845 & \\
\hline Motivation to Change (Change) & 0.264 & -0.322 & 0.818 & 1.302 & 0.725 & 2.266 & \\
\hline
\end{tabular}




\begin{tabular}{|c|c|c|c|c|c|c|c|}
\hline \multirow[b]{3}{*}{ Fixed Effect: } & \multicolumn{7}{|c|}{$\begin{array}{l}\text { The Dynamic Model including Ethnicity } \\
\text { (BDm2_d2) }\end{array}$} \\
\hline & \multicolumn{3}{|c|}{ Unstandardised } & \multicolumn{3}{|c|}{ Standardised } & \multirow{2}{*}{ Significant? } \\
\hline & Post.Mean & Lower $\mathrm{Cl}$ & Upper Cl & Post.Mean & Lower $\mathrm{Cl}$ & Upper Cl & \\
\hline Ethnicity: Time & 92.580 & -245.100 & 494.200 & $1.61 \mathrm{E}+40$ & 0.000 & $4.25 E+214$ & \\
\hline Ethnicity: Live & -2992.000 & -6403.000 & 176.800 & 0.000 & 0.000 & $6.07 \mathrm{E}+76$ & \\
\hline Ethnicity: Relation & 302.700 & -1924.000 & 2533.000 & $2.89 E+131$ & 0.000 & \#NUM! & \\
\hline Ethnicity: ETE & 1134.000 & 305.900 & 1902.000 & \#NUM! & $7.09 E+132$ & \#NUM! & Yes \\
\hline Ethnicity: Where & 3182.000 & 1073.000 & 5074.000 & \#NUM! & \#NUM! & \#NUM! & Yes \\
\hline Ethnicity: Life & 1826.000 & -3642.000 & 7020.000 & \#NUM! & 0.000 & \#NUM! & \\
\hline Ethnicity: Drugs & -4159.000 & -7652.000 & -536.600 & 0.000 & 0.000 & 0.000 & \\
\hline Ethnicity: Physical & 2834.000 & -523.000 & 6935.000 & \#NUM! & 0.000 & \#NUM! & \\
\hline Ethnicity: Emotion & 211.400 & -339.200 & 688.000 & $6.45 \mathrm{E}+91$ & 0.000 & $6.23 E+298$ & \\
\hline Ethnicity: Self & 2670.000 & 736.500 & 4807.000 & \#NUM! & \#NUM! & \#NUM! & Yes \\
\hline Ethnicity: Think & -5015.000 & -8640.000 & -1847.000 & 0.000 & 0.000 & 0.000 & \\
\hline Ethnicity: Attitude & 5100.000 & 1193.000 & 8550.000 & \#NUM! & \#NUM! & \#NUM! & Yes \\
\hline Ethnicity: Change & -432.700 & -2133.000 & 1198.000 & 0.000 & 0.000 & \#NUM! & \\
\hline Time: Live & 0.016 & -0.083 & 0.110 & 1.016 & 0.920 & 1.116 & \\
\hline Time: Relation & 0.004 & -0.108 & 0.120 & 1.004 & 0.897 & 1.128 & \\
\hline Time: ETE & 0.106 & 0.019 & 0.198 & 1.112 & 1.019 & 1.219 & Yes \\
\hline Time: Where & 0.013 & -0.063 & 0.086 & 1.013 & 0.939 & 1.090 & \\
\hline Time: Life & -0.020 & -0.154 & 0.098 & 0.980 & 0.858 & 1.103 & \\
\hline Time: Drugs & -0.053 & -0.148 & 0.027 & 0.948 & 0.862 & 1.028 & \\
\hline Time: Physical & 0.144 & 0.025 & 0.263 & 1.155 & 1.026 & 1.301 & Yes \\
\hline Time: Emotion & 0.051 & -0.037 & 0.134 & 1.052 & 0.963 & 1.143 & \\
\hline Time: Self & -0.116 & -0.235 & 0.006 & 0.890 & 0.791 & 1.006 & \\
\hline Time: Think & -0.043 & -0.173 & 0.088 & 0.958 & 0.841 & 1.092 & \\
\hline Time: Attitude & -0.014 & -0.141 & 0.109 & 0.986 & 0.868 & 1.115 & \\
\hline Time: Change & -0.016 & -0.145 & 0.104 & 0.984 & 0.865 & 1.109 & \\
\hline Ethnicity: Time: Live & 253.200 & -255.000 & 811.200 & $9.19 \mathrm{E}+109$ & 0.000 & \#NUM! & \\
\hline Ethnicity: Time: Relation & -750.600 & -2087.000 & 411.100 & 0.000 & 0.000 & $3.46 \mathrm{E}+178$ & \\
\hline Ethnicity: Time: ETE & 105.300 & -212.600 & 423.600 & $5.39 \mathrm{E}+45$ & 0.000 & $9.27 \mathrm{E}+183$ & \\
\hline Ethnicity: Time: Where & 286.000 & -120.500 & 919.300 & $1.62 \mathrm{E}+124$ & 0.000 & \#NUM! & \\
\hline Ethnicity: Time: Life & 333.200 & -1106.000 & 1927.000 & $5.09 \mathrm{E}+144$ & 0.000 & \#NUM! & \\
\hline Ethnicity: Time: Drugs & -289.100 & -924.400 & 432.000 & 0.000 & 0.000 & $4.12 \mathrm{E}+187$ & \\
\hline Ethnicity: Time: Physical & -673.800 & -1751.000 & 254.500 & 0.000 & 0.000 & $3.37 E+110$ & \\
\hline Ethnicity: Time: Emotion & 676.200 & 171.900 & 1145.000 & $4.68 \mathrm{E}+293$ & $4.52 \mathrm{E}+74$ & \#NUM! & Yes \\
\hline Ethnicity: Time: Self & -942.100 & -1468.000 & -251.900 & 0.000 & 0.000 & 0.000 & Yes \\
\hline Ethnicity: Time: Think & 353.500 & -15.060 & 867.600 & $3.34 \mathrm{E}+153$ & 0.000 & \#NUM! & \\
\hline Ethnicity: Time: Attitude & 679.400 & -138.400 & 1745.000 & $1.15 \mathrm{E}+295$ & 0.000 & \#NUM! & \\
\hline Ethnicity: Time: Change & -883.300 & -1613.000 & -212.700 & 0.000 & 0.000 & 0.000 & Yes \\
\hline Random Effect: & Post.Mean & Lower $\mathrm{Cl}$ & Upper Cl & Post.Mean & Lower $\mathrm{Cl}$ & Upper Cl & Significant? \\
\hline Individual (Intercept) & 0.211 & $1.04 \mathrm{E}-08$ & 0.593 & 1.235 & 1.000 & 1.810 & Yes \\
\hline Time & 2.158 & 0.494 & 4.716 & 8.654 & 1.639 & 111.720 & Yes \\
\hline DIC & & & 453.19 & & & & \\
\hline
\end{tabular}

Source: Model BDm2_d2, Technical Annex: p83-95

Reproducing Figure 4.7 with the data split by gender(Figures 5.6 and 5.7) highlights how from Time 10 there is only information about males upon which to base the model, with there being less than 2 girls from $T$ ime 5 . As result, trends from this pointon are dominated bya single female case. A similar trend can be seen in the case of ethnicity (Figures 5.8 and 5.9). Here there is just one non-White case whose domain scores are informing the model from Times 8 to 11 . This contributes to the amount of uncertainty reflected in the wide credible intervals for some of the coefficients. 
Figure 5.2: Summary of Average Domain Scores, by Gender (Males)

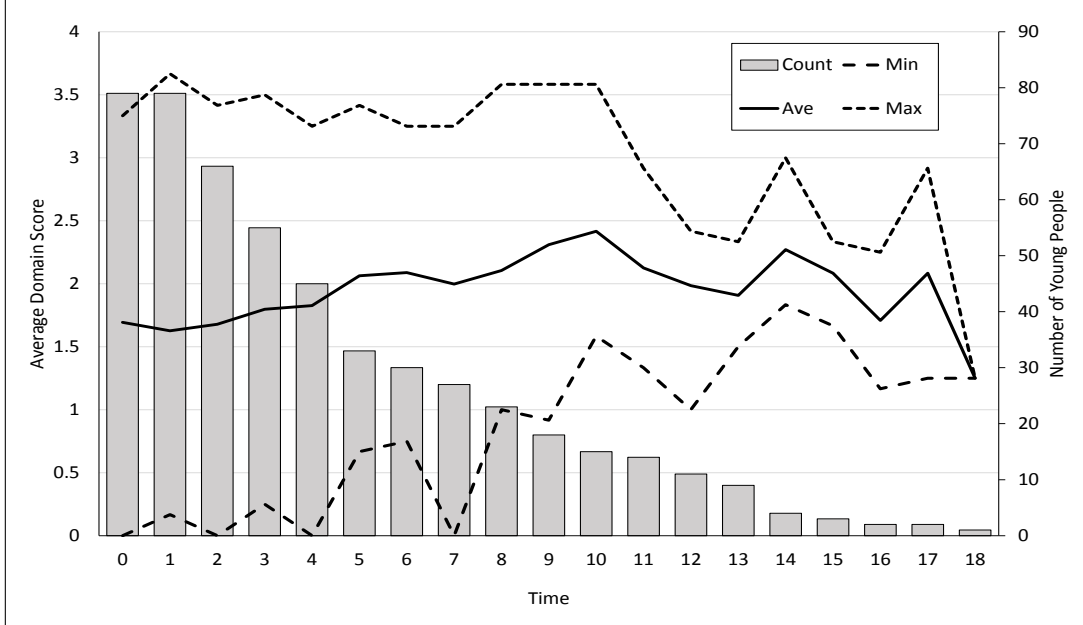

Figure 5.4: Summary of Average Domain Scores, by Ethnicity (White)

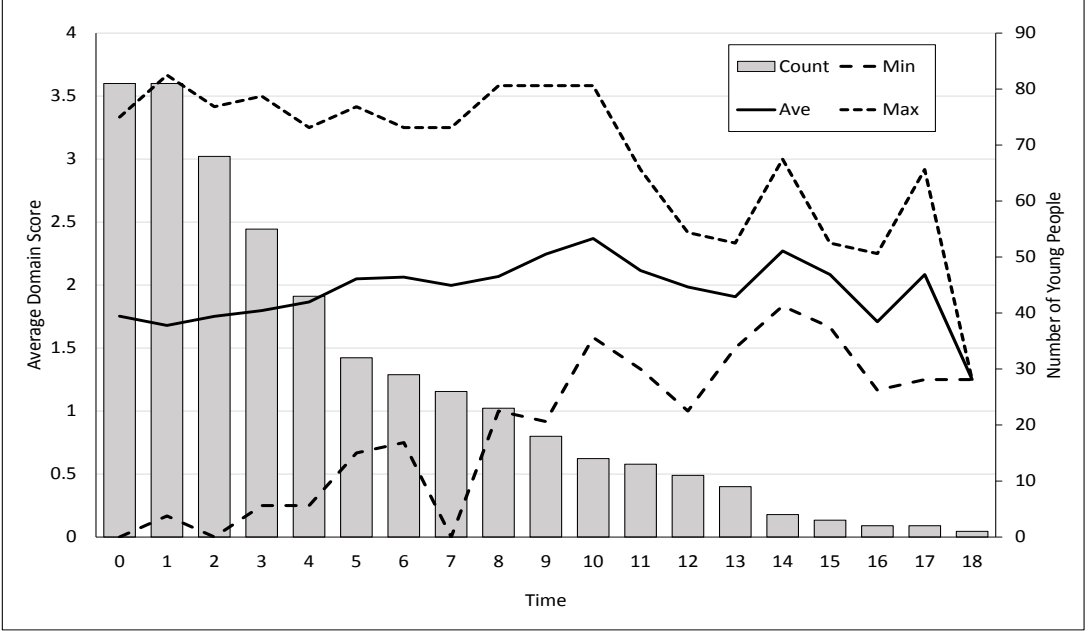

Figure 5.3: Summary of Average Domain Scores, by Genc

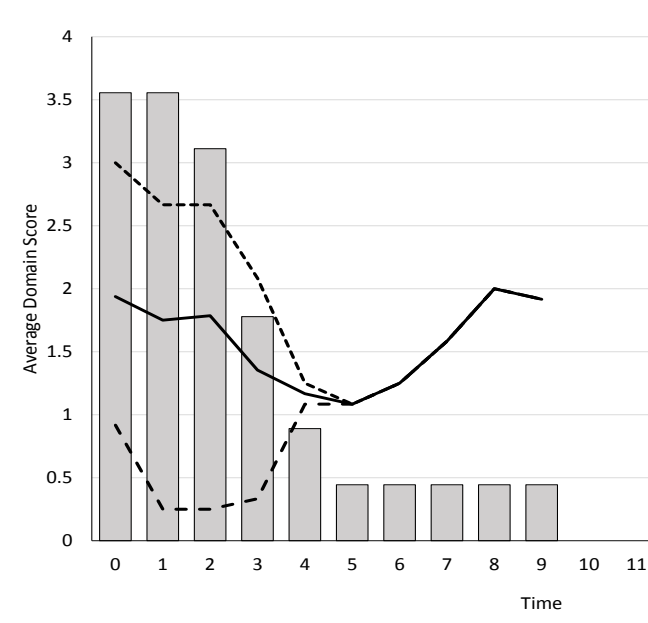

Figure 5.5: Summary of Average Domain Scores, by Ethn

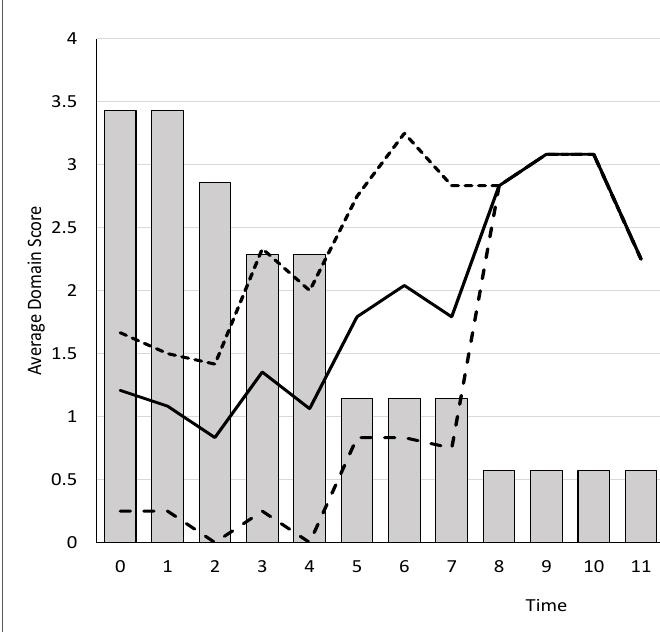




\section{Other potential explanations for the differences}

\section{Experience ofCare}

Table 3.10 suggests that there is very strong evidence to suggest that rates of further offending are higher amongst those who have experience of care than for those who have never being looked after $\left(\mathrm{BF}_{10}\right.$ for the one-sided test $\left.=33.97\right)$. Table 5.6 summarises the respective proportions who have experience of care, by gender and ethnicity.

Table 5.6: FTE Status, by Gender and Ethnicity

\begin{tabular}{|c|c|c|c|c|c|c|c|}
\hline & \multicolumn{2}{|c|}{ Comparator Groups } & No. & Care & $\%$ Care & $\begin{array}{c}\text { Bayes Factor (BF 10) } \\
\text { (H1: Group } 1 \text { F Group 2) }\end{array}$ & $\begin{array}{l}\text { Bayes Factor (BF 10) } \\
\text { (H1: Group 1 > Group 2) }\end{array}$ \\
\hline \multirow[t]{2}{*}{ Gender } & 1 & Male & 79 & 23 & $29.1 \%$ & \multirow{2}{*}{0.383} & \multirow{2}{*}{0.308} \\
\hline & 2 & Female & 9 & 2 & $22.2 \%$ & & \\
\hline \multirow[t]{2}{*}{ Ethnicity } & 1 & White & 82 & 24 & $29.3 \%$ & \multirow{2}{*}{0.472} & \multirow{2}{*}{0.326} \\
\hline & 2 & Non-White & 6 & 1 & $16.7 \%$ & & \\
\hline \multicolumn{3}{|l|}{ Total } & 88 & 25 & $28.4 \%$ & & \\
\hline
\end{tabular}

Notes: Bayes Factors have been calculated using the test for Bayesian Contingency Tables within JASP version 0.8.1.1. The two sets of Bayes Factors represent the results of (1) a two-sided alternative hypothesis that the respective proportions of with experience of care are equal (Alternative Hypothesis: Group $1 \neq$ Group 2), and (2) a one-sided alternative hypothesis that the rates for Group 1 are larger than Group 2. Bayes Factors quantify the evidence for the alternative hypothesis relative to the null hypothesis and are interpreted using the categories suggested by Jeffreys (1961).

There is anecdotal evidence to suggest from the two-sided test and moderate evidence from the onesided test to suggest that the respective proportions with experience of care are the same when the reoffending cohortis segmented by gender and ethnicityrespectively.

\section{FTEStatus}

Table 3.11 suggests that there is moderate evidenceto suggest that the rate of further offending is higher amongst those with a history of previous offending behaviour than those who are FTEs. Table 5.7 summarises the proportion of FTEs by gender and ethnicity respectively. Due to small numbers, the Bayes Factors for the two-sided tests are inconclusive.

Table 5.7: FTE Status, by Gender and Ethnicity

\begin{tabular}{|c|c|c|c|c|c|c|c|}
\hline & & omparator Groups & No. & FTE & $\%$ FTE & $\begin{array}{c}\text { Bayes Factor (BF 10) } \\
\text { (H1: Group } 1 \text { F Group 2) }\end{array}$ & $\begin{array}{l}\text { Bayes Factor (BF 10) } \\
\text { (H1: Group } 1>\text { Group 2) }\end{array}$ \\
\hline \multirow[t]{2}{*}{ Gender } & 1 & Male & 79 & 32 & $40.5 \%$ & \multirow{2}{*}{1.222} & \multirow{2}{*}{0.192} \\
\hline & 2 & Female & 8 & 1 & $12.5 \%$ & & \\
\hline \multirow[t]{2}{*}{ Ethnicity } & 1 & White & 81 & 31 & $38.3 \%$ & \multirow{2}{*}{0.464} & \multirow{2}{*}{0.420} \\
\hline & 2 & Non-White & 6 & 2 & $33.3 \%$ & & \\
\hline \multicolumn{3}{|l|}{ Total } & 87 & 33 & $37.9 \%$ & & \\
\hline
\end{tabular}

Notes: Bayes Factors have been calculated using the test for Bayesian Contingency Tables within JASP version 0.8.1.1. The two sets of Bayes Factors represent the results of (1) a two-sided alternative hypothesis that the respective proportions of firsttime entrants are equal (Alternative Hypothesis: Group $1 \neq$ Group 2), and (2) a one-sided alternative hypothesis that the rates for Group 1 are larger than Group 2. Bayes Factors quantify the evidence for the alternative hypothesis relative to the null hypothesis and are interpreted using the categories suggested by Jeffreys (1961). 
The one-sided test with respect to gender, the Bayes Factor of 0.192 suggests that the data are 5.2 times $(1 / 0.192)$ more likely under the $\mathrm{H}_{1}$. Hence there is moderate evidence to support the finding that males in the reoffending cohort are more likely to be FTEs than females. There is only anecdotal evidence of this with respect to ethnicity.

\section{Nature of the Primary Offence}

As can be seen from Table 5.8, the 18 young people whose index offence had been either a robbery, burglaryor motoring offence i.e. a serious acquisitive crime, were all white males. Thenon-White males were equally split between those who had committed violence against the person offences and those whose primary offence fell into the 'other' category. Three-quarters of the females within the reoffending cohorthad committed VAP offences.

Table 5.8: Type of Primary Offence, by Gender and Ethnicity

\begin{tabular}{|c|c|c|c|c|c|}
\hline \multirow{2}{*}{\multicolumn{2}{|c|}{ Sub-Group }} & \multicolumn{3}{|c|}{ Type of Primary Offence } & \multirow{2}{*}{$\begin{array}{c}\text { Grand } \\
\text { Total }\end{array}$} \\
\hline & & Other & SAC & VAP & \\
\hline \multirow{2}{*}{ Gender } & Female & 2 & & 6 & 8 \\
\hline & Male & 46 & 18 & 15 & 79 \\
\hline \multirow{2}{*}{ Ethnicity } & Non-White & 3 & & 3 & 6 \\
\hline & White & 45 & 18 & 18 & 81 \\
\hline Grand Total & & 48 & 18 & 21 & 87 \\
\hline
\end{tabular}

The low number of females and non-White young people makes it difficult to draw reliable conclusions about whether the differences observed in Figure 5.1 can be attributed to the nature of the primary offence or the seriousness of the offence (Table 5.9).

Table 5.9: YJB Gravity Score of the Primary Offence, by Gender and Ethnicity

\begin{tabular}{|c|c|c|c|c|c|c|c|}
\hline & \multirow{2}{*}{ Sub-Group } & \multicolumn{5}{|c|}{ YJB Gravity Score } & \multirow{2}{*}{ Total } \\
\hline & & 2 & 3 & 4 & 5 & 6 & \\
\hline \multirow{2}{*}{ Gender } & Male & 31 & 24 & 7 & 4 & 13 & 79 \\
\hline & Female & & 6 & 2 & & & 8 \\
\hline \multirow{2}{*}{ Ethnicity } & White & 29 & 28 & 9 & 4 & 11 & 81 \\
\hline & Non-White & 2 & 2 & & & 2 & 6 \\
\hline \multicolumn{2}{|l|}{ Grand Total } & 31 & 30 & 9 & 4 & 13 & 87 \\
\hline
\end{tabular}

On the basis of this analysis, the low number of cases for females and non-Whites make it difficult to establish the role that gender and ethnicity have when combined with other predictors when looking to create a dynamic model of the probability of further offending. However, it is possible to explore the role of having experience of care, being an FTE and the nature of the primary offence, with the latter being explored in Chapter Six. 


\subsection{The role of care experience}

\section{Description of the Data}

Table 5.10 provides a breakdown of those who have never been looked after and those with experience of care, by gender and ethnicity. Whilst there are no non-White females in the reoffending cohort, two of the nine females have experience ofcare. Overall, there are 23 males with experience of care. One of these is non-White. As established in section 5.2, with such low numbers, it is difficult to reliably estimate the role of having experience of care bygender and ethnicity. However, it is useful to get a sense of the profile of the cohortby ethnic group as recorded in Childview.

Table 5.10: The Re-Offending Cohort, By Gender, Ethnicity and Experience of Care

\begin{tabular}{|c|c|c|c|c|c|}
\hline \multirow{2}{*}{ Gender } & & \multirow{2}{*}{ Ethnicity } & \multicolumn{2}{|c|}{ Care Experience } & \multirow{2}{*}{ Total } \\
\hline & & & No & Yes & \\
\hline \multirow{8}{*}{ Male } & \multirow{3}{*}{ White } & White - British & 7 & 7 & 14 \\
\hline & & White - Irish & 1 & & 1 \\
\hline & & Any Other White Background & 43 & 15 & 58 \\
\hline & \multirow{5}{*}{ Non-White } & Black Caribbean & & 1 & 1 \\
\hline & & Pakistani & 1 & & 1 \\
\hline & & Any Other Asian Background & 2 & & 2 \\
\hline & & White and Asian & 1 & & 1 \\
\hline & & White and Black Caribbean & 1 & & 1 \\
\hline \multicolumn{3}{|l|}{ Male Total } & 56 & 23 & 79 \\
\hline \multirow{2}{*}{ Female } & \multirow{2}{*}{ White } & Any Other White Background & 6 & & 6 \\
\hline & & White - British & 1 & 2 & 3 \\
\hline \multicolumn{3}{|c|}{ Female Total } & 7 & 2 & 9 \\
\hline \multicolumn{3}{|c|}{ Grand Total } & 63 & 25 & 88 \\
\hline
\end{tabular}

Notes: The FTE status of one female is unknown. It is therefore necessary to remove this case from subsequent analysis.

Of the 25 young people with experience of care:

- 7 were FTEs at the point of entering the reoffending cohort(28.0\%). This compares to $41.3 \%$ of their peers without this experience.

- The average age of first offence was 13 - slightly younger than for those who have never been looked after (mean $=14$ years). However, amongstboth sub-groups, the youngest were aged 10. Amongst those who have never been looked after, roughly one in five (14/63) were aged 16 or 17 at the time of their first offence whereas the proportion amongstlooked after children in the reoffending cohortwasjust $4 \%(1 / 25)$.

- Similar pattern is observed in terms of age of first conviction, with the average ages being 14 for those with experience of care compared to 15 for those who have never been looked after. 
Lord Lamming's review of looked after children in the criminal justice system (Prison Reform Trust, 2016) presents evidence to suggest that whilst mostchildren in care do not get in trouble with the law, looked after children in England are six times more likely than children in the general population to be convicted of a crime or receive an out of court disposal. The literature review which accompanies the Lamming Review (Staines, 2016), highlights ways in which the needs and vulnerabilities of children who have been looked after may be contributing to their over-representation in the criminal justice system. Some of these are apparentin the initial mean domain scores for those with and without experience of care at Time 0 (Figure 5.6).

Figure 5.6: Domain Score Profile, by Experience of Care, at Time 0

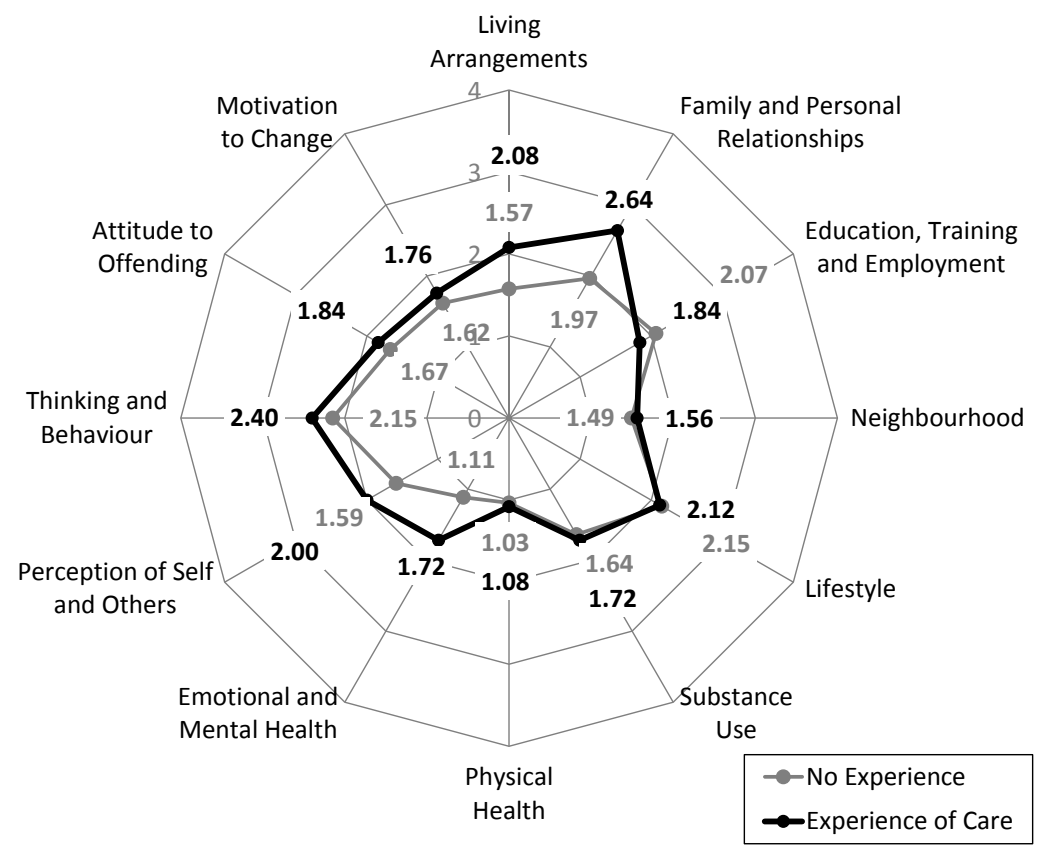

Notes: Of the 87 individuals, 25 have experience of care. Time 0 represents the initial assessment.

Those with experience of care $(\mathrm{N}=25)$ typically have higher initial ratings for:

- Familyand personal relationships $\left(\mathrm{BF}_{10}=9.235 \mathrm{e}^{-4}\right.$ in favour of $\mathrm{H}_{1}$ : No Experience $>$ Experience of Care, $\%$ error $=3.494 \mathrm{e}^{-5}$ )

- Emotion and Mental Health $\left(\mathrm{BF}_{10}=4.332, \%\right.$ error $\left.=6.155 \mathrm{e}^{-4}\right)$

There is moderate evidence to support the trend apparent in Figure 4.9 that there is no difference between the mean ratings for the two groups $\left(\mathrm{H}_{1}\right.$ : No Experience $\neq$ Experience of Care) with respect to:

- $\operatorname{ETE}\left(, \mathrm{BF}_{10}=0.383\right)$

- Neighbourhood $\left(\mathrm{BF}_{10}=0.250\right)$

- Lifestyle $\left(\mathrm{BF}_{10}=0.245\right)$

- Substance Use $\left(\mathrm{BF}_{10}=0.252\right)$

- Physical Health $\left(\mathrm{BF}_{10}=0.252\right)$ 
- Motivation to Change $\left(\mathrm{BF}_{10}=0.250\right)$

Additionally, there is anecdotal evidence in relation to Attitude to Offending $\left(\mathrm{BF}_{10}=0.250\right)$ and Thinking and Behaviour $\left(\mathrm{BF}_{10}=0.433\right)$.

\section{Adding Experience of Care to the Basic Model}

Adding a dummyvariable for care experience (referenced by having experience) to the Ba sic Model, reduces the DIC from 476.20 to 473.48 (Tables 4.12 and 5.11). Inclusion of the additional predictor suggests that at Time 0 :

- The odds of further offending amongst those with experience of care are estimated to be $\exp (0.50)=1.649$ times the odds for their peers without experience of care (Model 1.4). $\quad[\mathrm{Cl}$ $=1.016,3.03]$

The credible interval suggests that having experience of care is a significant predictor of further offending, equivalent to a $64.9 \%$ increase in the odds relative to their peers. Further to this, it highlights that the odds could be as much as three times higher.

Table 5.11: Random Intercepts and Varying Slope Models for Further Offending including ASSET Domains and Experience of Care

\begin{tabular}{|c|c|c|c|c|c|c|c|}
\hline \multirow[b]{3}{*}{ Fixed Effect: } & \multicolumn{7}{|c|}{ Model 1.4: Basic Model + Care } \\
\hline & \multicolumn{3}{|c|}{ Unstandardised } & \multicolumn{3}{|c|}{ Standardised } & \multirow{2}{*}{ Significant? } \\
\hline & PostMean & Lower $\mathrm{Cl}$ & Upper Cl & Post.Mean & Lower $\mathrm{Cl}$ & Upper $\mathrm{Cl}$ & \\
\hline Intercept & -1.232 & -2.472 & 0.006 & 0.292 & 0.084 & 1.006 & \\
\hline Care Experience (None = Ref) & 0.500 & 0.016 & 0.967 & 1.649 & 1.016 & 2.629 & Yes \\
\hline Living Arrangements & 0.018 & -0.222 & 0.282 & 1.018 & 0.801 & 1.326 & \\
\hline Family and Personal Relationships & 0.217 & -0.071 & 0.516 & 1.242 & 0.931 & 1.675 & \\
\hline Education, Training and Employment & 0.139 & -0.106 & 0.392 & 1.150 & 0.899 & 1.480 & \\
\hline Neighbourhood & 0.010 & -0.227 & 0.208 & 1.010 & 0.797 & 1.231 & \\
\hline Lifestyle & 0.099 & -0.248 & 0.460 & 1.104 & 0.781 & 1.584 & \\
\hline Substance Use & 0.147 & -0.092 & 0.385 & 1.159 & 0.912 & 1.469 & \\
\hline Physical Health & -0.088 & -0.376 & 0.179 & 0.916 & 0.686 & 1.196 & \\
\hline Emotional and Mental Health & -0.042 & -0.292 & 0.194 & 0.958 & 0.747 & 1.215 & \\
\hline Perceptions of Self and Others & -0.142 & -0.448 & 0.164 & 0.867 & 0.639 & 1.179 & \\
\hline Thinking and Behaviour & -0.169 & -0.528 & 0.146 & 0.845 & 0.590 & 1.158 & \\
\hline Attitude to Offending & -0.012 & -0.360 & 0.328 & 0.988 & 0.698 & 1.388 & \\
\hline Motivation to Change & 0.247 & -0.102 & 0.583 & 1.281 & 0.903 & 1.791 & \\
\hline Time & -0.160 & -0.304 & -0.035 & 0.852 & 0.738 & 0.965 & Yes \\
\hline Random Effect: & Post.Mean & Lower Cl & Upper Cl & Post.Mean & Lower $\mathrm{Cl}$ & Upper Cl & Significant? \\
\hline Individual (Intercept) & 0.092 & 1.85E-04 & 0.348 & 1.096 & 1.000 & 1.416 & Yes \\
\hline Time & 1.291 & 0.349 & 2.643 & 3.636 & 1.417 & 14.055 & Yes \\
\hline
\end{tabular}

\begin{tabular}{|l|l|}
\hline DIC & 473.48 \\
\hline
\end{tabular}

Source: Model Bm1_ch, Technical Annex: p96-97 
Extending the Basic Dynamic model to involve experience of care (referenced by having never been looked after) results in a model which has no issues with convergence as can be seen from the trace plots in the Technical Annex.

Table 5.12: The Dynamic Model Involving Care Experience

\begin{tabular}{|c|c|c|c|c|c|c|c|}
\hline \multirow[b]{3}{*}{ Fixed Effect: } & \multicolumn{7}{|c|}{$\begin{array}{l}\text { Dymanic Mode including Experience of Care } \\
\text { (BDm2_ch) }\end{array}$} \\
\hline & \multicolumn{3}{|c|}{ Unstandardised } & \multicolumn{3}{|c|}{ Standardised } & \multirow{2}{*}{ Significant? } \\
\hline & PostMean & Lower $\mathrm{Cl}$ & Upper $\mathrm{Cl}$ & PostMean & Lower $\mathrm{Cl}$ & Upper $\mathrm{Cl}$ & \\
\hline (Intercept) & -1.501 & -3.507 & 0.439 & 0.223 & 0.030 & 1.551 & \\
\hline Experience of Care (None $=$ Ref) & 1.462 & -1.419 & 4.309 & 4.313 & 0.242 & 74.393 & \\
\hline Time & -0.335 & -0.753 & 0.066 & 0.715 & 0.471 & 1.068 & \\
\hline Living Arrangements (Live) & -0.091 & -0.729 & 0.552 & 0.913 & 0.482 & 1.737 & \\
\hline Family and Personal Relationships (Relation) & 0.149 & -0.547 & 0.883 & 1.161 & 0.579 & 2.419 & \\
\hline Education, Training and Employment (ETE) & -0.157 & -0.668 & 0.324 & 0.855 & 0.512 & 1.382 & \\
\hline Neighbourhood (Where) & 0.060 & -0.501 & 0.608 & 1.062 & 0.606 & 1.838 & \\
\hline Lifestyle (Life) & 0.611 & -0.239 & 1.484 & 1.843 & 0.788 & 4.412 & \\
\hline Substance Use (Drugs) & 0.125 & -0.435 & 0.672 & 1.133 & 0.647 & 1.959 & \\
\hline Physical Health (Physical) & -0.082 & -0.804 & 0.633 & 0.922 & 0.448 & 1.883 & \\
\hline Emotional and Mental Health (Emotion) & -0.405 & -0.999 & 0.152 & 0.667 & 0.368 & 1.164 & \\
\hline Perceptions of Self and Others (Self) & -0.129 & -0.920 & 0.680 & 0.879 & 0.399 & 1.974 & \\
\hline Thinking and Behaviour (Think) & 0.095 & -0.676 & 0.858 & 1.100 & 0.509 & 2.358 & \\
\hline Attitude to Offending (Attitude) & 0.070 & -0.750 & 0.871 & 1.073 & 0.472 & 2.389 & \\
\hline Motivation to Change (Change) & 0.208 & -0.519 & 0.960 & 1.231 & 0.595 & 2.611 & \\
\hline Care: Time & -0.075 & -0.708 & 0.564 & 0.928 & 0.493 & 1.759 & \\
\hline Care: Live & 0.453 & -0.564 & 1.525 & 1.572 & 0.569 & 4.594 & \\
\hline Care: Relation & -0.046 & -1.249 & 1.222 & 0.955 & 0.287 & 3.393 & \\
\hline Care: ETE & 0.259 & -0.753 & 1.274 & 1.296 & 0.471 & 3.574 & \\
\hline Care: Where & -0.311 & -1.308 & 0.673 & 0.732 & 0.270 & 1.960 & \\
\hline Care: Life & -0.633 & -2.109 & 0.826 & 0.531 & 0.121 & 2.283 & \\
\hline Care: Drugs & 0.326 & -0.614 & 1.267 & 1.385 & 0.541 & 3.551 & \\
\hline Care: Physical & -0.890 & -2.115 & 0.365 & 0.411 & 0.121 & 1.441 & \\
\hline Care: Emotion & 0.392 & -0.649 & 1.400 & 1.480 & 0.523 & 4.056 & \\
\hline Care: Self & 0.872 & -0.559 & 2.304 & 2.391 & 0.572 & 10.012 & \\
\hline Care: Think & -0.841 & -2.314 & 0.618 & 0.431 & 0.099 & 1.855 & \\
\hline Care: Attitude & -0.118 & -1.420 & 1.272 & 0.889 & 0.242 & 3.569 & \\
\hline Care: Change & 0.126 & -1.393 & 1.521 & 1.134 & 0.248 & 4.576 & \\
\hline Time: Live & -0.035 & -0.202 & 0.121 & 0.965 & 0.817 & 1.129 & \\
\hline Time: Relation & 0.005 & -0.175 & 0.183 & 1.005 & 0.840 & 1.201 & \\
\hline Time: ETE & 0.057 & -0.066 & 0.183 & 1.059 & 0.936 & 1.201 & \\
\hline Time: Where & -0.054 & -0.191 & 0.077 & 0.948 & 0.826 & 1.080 & \\
\hline Time: Life & -0.005 & -0.187 & 0.192 & 0.995 & 0.829 & 1.212 & \\
\hline Time: Drugs & 0.036 & -0.100 & 0.172 & 1.037 & 0.905 & 1.187 & \\
\hline Time: Physical & -0.042 & -0.269 & 0.179 & 0.958 & 0.764 & 1.196 & \\
\hline Time: Emotion & 0.053 & -0.089 & 0.198 & 1.054 & 0.915 & 1.219 & \\
\hline Time: Self & 0.072 & -0.105 & 0.263 & 1.075 & 0.901 & 1.301 & \\
\hline Time: Think & -0.012 & -0.207 & 0.180 & 0.988 & 0.813 & 1.197 & \\
\hline Time: Attitude & -0.095 & -0.296 & 0.104 & 0.910 & 0.743 & 1.110 & \\
\hline Time: Change & 0.027 & -0.173 & 0.236 & 1.027 & 0.841 & 1.267 & \\
\hline
\end{tabular}




\begin{tabular}{|c|c|c|c|c|c|c|c|}
\hline \multirow[b]{3}{*}{ Fixed Effect: } & \multicolumn{7}{|c|}{$\begin{array}{l}\text { Dymanic Mode including Experience of Care } \\
\text { (BDm2_ch) }\end{array}$} \\
\hline & \multicolumn{3}{|c|}{ Unstandardised } & \multicolumn{3}{|c|}{ Standardised } & \multirow{2}{*}{ Significant? } \\
\hline & Post.Mean & Lower Cl & Upper Cl & Post.Mean & Lower Cl & Upper Cl & \\
\hline Care: Time: Live & 0.021 & -0.199 & 0.249 & 1.021 & 0.820 & 1.282 & \\
\hline Care: Time: Relation & $-2.07 \mathrm{E}-04$ & -0.283 & 0.278 & 1.000 & 0.753 & 1.321 & \\
\hline Care: Time: ETE & 0.004 & -0.199 & 0.216 & 1.004 & 0.820 & 1.241 & \\
\hline Care: Time: Where & 0.178 & -0.015 & 0.375 & 1.195 & 0.985 & 1.455 & \\
\hline Care: Time: Life & -0.083 & -0.374 & 0.200 & 0.921 & 0.688 & 1.222 & \\
\hline Care: Time: Drugs & -0.072 & -0.291 & 0.143 & 0.930 & 0.747 & 1.154 & \\
\hline Care: Time: Physical & 0.242 & -0.063 & 0.532 & 1.274 & 0.939 & 1.703 & \\
\hline Care: Time: Emotion & 0.040 & -0.195 & 0.279 & 1.041 & 0.823 & 1.322 & \\
\hline Care: Time: Self & -0.341 & -0.644 & -0.045 & 0.711 & 0.525 & 0.956 & Yes \\
\hline Care: Time: Think & 0.085 & -0.209 & 0.388 & 1.088 & 0.812 & 1.474 & \\
\hline Care: Time: Attitude & 0.130 & -0.174 & 0.443 & 1.139 & 0.840 & 1.558 & \\
\hline Care: Time: Change & -0.058 & -0.354 & 0.248 & 0.944 & 0.702 & 1.281 & \\
\hline Random Effect: & Post.Mean & Lower $\mathrm{Cl}$ & Upper Cl & Post.Mean & Lower $\mathrm{Cl}$ & Upper Cl & Significant? \\
\hline Individual (Intercept) & 0.520 & $9.93 \mathrm{E}-08$ & 1.322 & 1.683 & 1.000 & 3.751 & Yes \\
\hline Time & 2.188 & 0.435 & 4.774 & 8.917 & 1.544 & 118.392 & Yes \\
\hline
\end{tabular}

Source: Model BDm2_ch, Technical Annex: p100-112

The model has been used to consider the trajectory of the probability of further offending over time for those with experience of care relative to that for their peers who have never been looked after. In Figure 5.7, the domain scores have been fixed at their initial values so that changes can be seen in the estimated probability of further offending from time 0 to time 10 . In the case of those who have never been looked after (Figure 5.7(a), n=63), there is a distinctive downwards curve which tends towards zero. The estimated probability of further offending at $T$ ime 0 is higher for those with higher initial domain scores which is in keeping with the subjective ratings - those with 3's and 4's are considered to have a higher likelihood of reoffending and hence are subject to more intensive supervision.

Figure 5.7: Changes in the Probability of Further Offending Over Time, by Care Experience
(a) Never Looked After
(b) With Experience of Care 


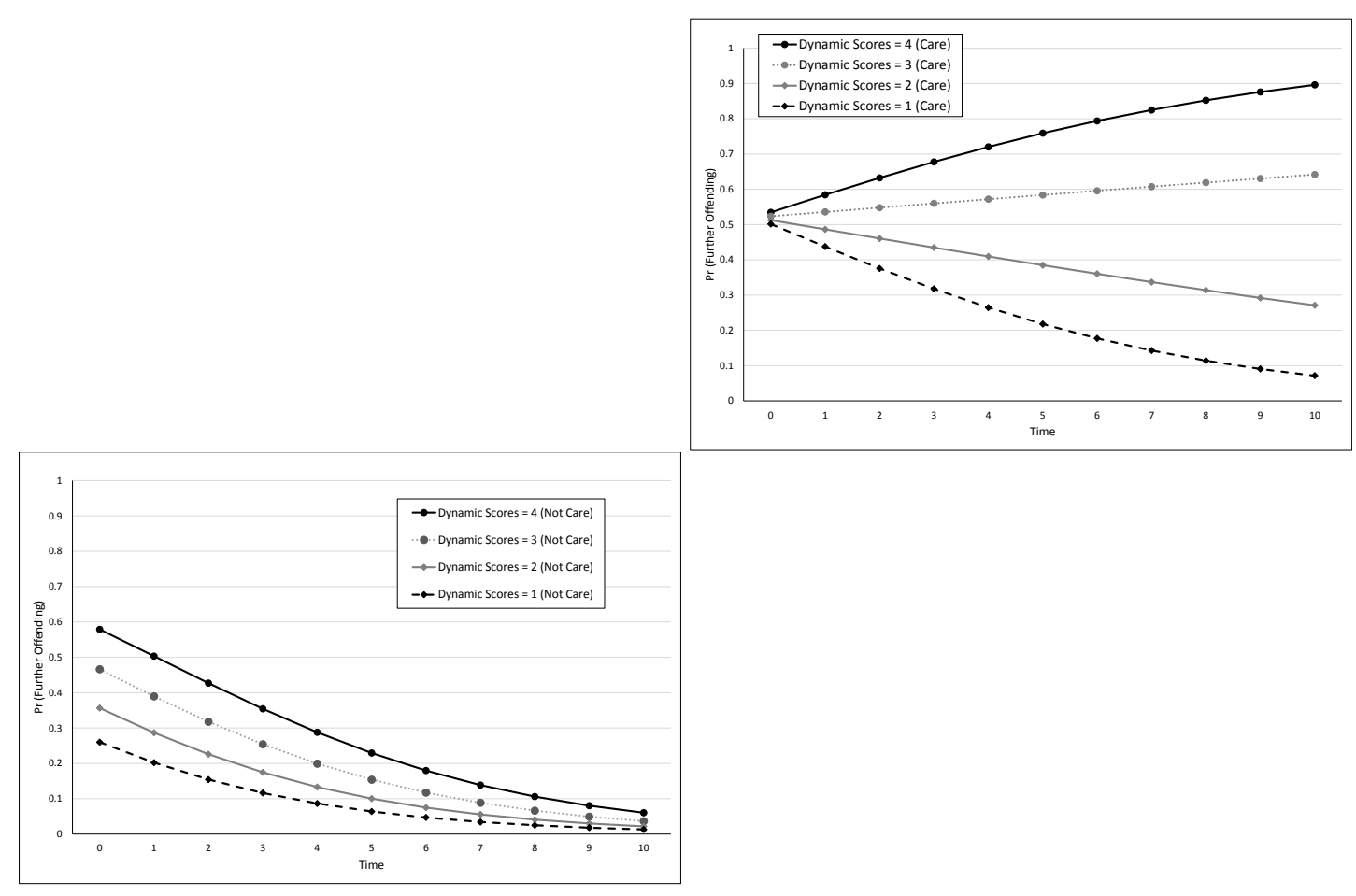

Notes: The domain scores have respectively been shown as being fixed at 1,2, 3 and 4 respectively to demonstrate the estimated change in the probability of further offending from time 0 to time 10. Estimates derived from Model BDm2_ch.

The trends apparent in Figure 5.7(b) are less clear and it is important that these are based on the repeated measurements of the 25 young people who have experience of care. As such there is the potential for the trend, particularlyfor the higher domain scores for these to have been undulyinfluenced by a very small number of cases, especiallyat later time points.

Figure 5.8: Summary of Average Domain Scores, by Care Experience

(b) Never Looked After

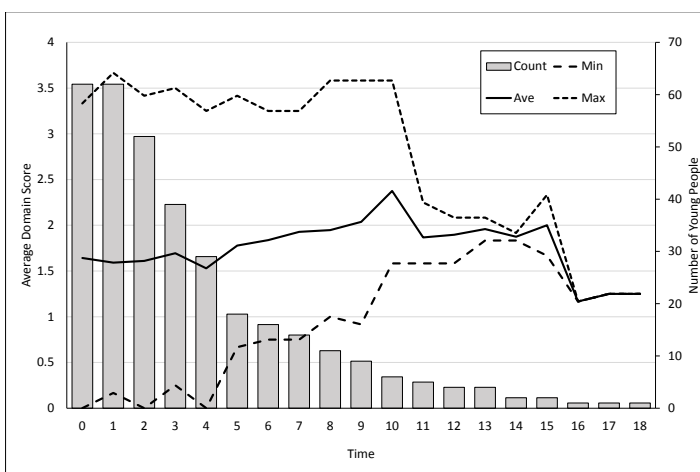

(b) With Experience of Care

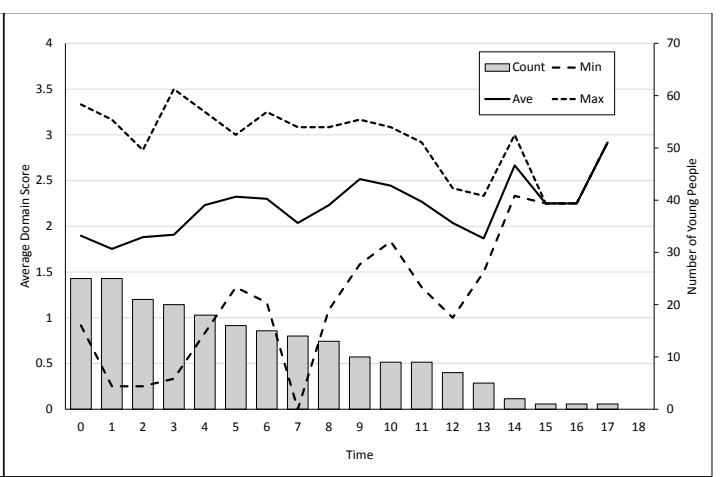

As can be seen from Figure 5.8, the mean domain score for those who have never been looked after has a net downward trend whereas for those with experience of care, there is a net upward trend. Notably after Time 14, there are only 2 or fewer cases relating to children with experience of care and to those who have never been looked after respectively. The domain scores from these cases determine the tail end of these trends. 


\subsection{The role of gender and ethnicity in the context of care experience}

Although it is apparent from section 5.2 that there is insufficient data to support a dynamic model involving gender, ethnicityand experience of care, itis possible to add these three predictors to the Basic Model (described in Table 4.11). This provides an indication of how the odds of further offending are affected when these are allowed to interact. Unfortunately, due to the absence of any non-White females, it is not possible to simulate an estimate of the coefficientfor the Gender: Ethnicity interaction - the model is rank deficient.

The basic model involving demographics and experience of care with interaction terms (Model 2) is summarised in Table 5.13. The addition of the interaction terms impacts on the amount of uncertainty which can be explained by the model with the DIC being lower for Model 2 than for Models 1.1, 1.2 and 1.3 respectively (summarised in Table 5.3) -471.53 compared to around 476 . This is despite the additional complexity. 
Table 5.13: Model 2: The Basic Model plus Demographics and Experience of Care

\begin{tabular}{|c|c|c|c|c|c|c|c|}
\hline \multirow[b]{3}{*}{ Fixed Effect: } & \multicolumn{7}{|c|}{ Model 2: Basic Model + Demographics + Care } \\
\hline & \multicolumn{3}{|c|}{ Unstandardised } & \multicolumn{3}{|c|}{ Standardised } & \multirow{2}{*}{ Significant? } \\
\hline & Post.Mean & Lower $\mathrm{Cl}$ & Upper Cl & Post.Mean & Lower $\mathrm{Cl}$ & Upper Cl & \\
\hline Intercept & -1.096 & -2.413 & 0.230 & 0.334 & 0.090 & 1.259 & \\
\hline Gender (Male = Ref). & 0.482 & -0.577 & 1.557 & 1.620 & 0.562 & 4.746 & \\
\hline Ethnicity (White = Ref) & -1.349 & -2.883 & 0.206 & 0.260 & 0.056 & 1.228 & \\
\hline Care Experience (None = Ref) & 0.526 & 0.009 & 1.044 & 1.692 & 1.009 & 2.840 & Yes \\
\hline Living Arrangements & -0.005 & -0.268 & 0.263 & 0.995 & 0.765 & 1.301 & \\
\hline Family and Personal Relationships & 0.214 & -0.091 & 0.514 & 1.238 & 0.913 & 1.672 & \\
\hline Education, Training and Employment & 0.103 & -0.153 & 0.369 & 1.109 & 0.858 & 1.446 & \\
\hline Neighbourhood & 0.036 & -0.186 & 0.268 & 1.036 & 0.830 & 1.308 & \\
\hline Lifestyle & 0.033 & -0.333 & 0.394 & 1.034 & 0.717 & 1.483 & \\
\hline Substance Use & 0.189 & -0.051 & 0.445 & 1.209 & 0.951 & 1.560 & \\
\hline Physical Health & -0.123 & -0.411 & 0.182 & 0.884 & 0.663 & 1.200 & \\
\hline Emotional and Mental Health & -0.059 & -0.303 & 0.193 & 0.943 & 0.739 & 1.213 & \\
\hline Perceptions of Self and Others & -0.158 & -0.480 & 0.174 & 0.854 & 0.619 & 1.190 & \\
\hline Thinking and Behaviour & -0.147 & -0.492 & 0.188 & 0.864 & 0.612 & 1.206 & \\
\hline Attitude to Offending & 0.030 & -0.341 & 0.390 & 1.031 & 0.711 & 1.477 & \\
\hline Motivation to Change & 0.265 & -0.086 & 0.614 & 1.303 & 0.918 & 1.849 & \\
\hline Time & -0.168 & -0.314 & -0.029 & 0.845 & 0.731 & 0.972 & Yes \\
\hline Gender:Care Experience & -1.715 & -4.508 & 1.065 & 0.180 & 0.011 & 2.901 & \\
\hline Care Experience: Ethnicity & 1.196 & -0.890 & 3.308 & 3.308 & 0.411 & 27.318 & \\
\hline Random Effect: & Post.Mean & Lower $\mathrm{Cl}$ & Upper Cl & PostMean & Lower $\mathrm{Cl}$ & Upper Cl & Significant? \\
\hline Individual (Intercept) & 0.120 & $1.87 \mathrm{E}-04$ & 0.430 & 1.127 & 1.000 & 1.537 & Yes \\
\hline Time & 1.462 & 0.401 & 3.037 & 4.315 & 1.493 & 20.843 & Yes \\
\hline
\end{tabular}

DIC 471.53

Source: Model Bm1_d1.ch_d2.ch, renamed as Model 2, Technical Annex: p113-118

Both care experience and time are significant within Model2, with the positive unstandardised coefficient suggesting a 'penalty' for having experience of care. The negative unstandardised coefficient for time suggests a moderating effectas time progresses, which is consistent with the premise that working with the YOT will reduce a young person's likelihood of further offending behaviours.

Using this model, it is possible to determine estimates for the probability of further offending at a given time point for different permutations of gender, ethnicity and care experience. These suggest that:

Compared to a male with no experience of care, at Time 0 , the odds of further offending amongst

- males with experience of care are estimated to be 1.77 times higher

- females withoutexperience of care, the odds are 1.65 times higher

However, for females who have never been looked after, the odds of further offending are 3.17 times higher than for those females who have experience of care. There is also a further notable gender 
difference amongst those who have experience of care, with the odds of further offending being 3.41 times higher amongstmales than females.

Compared to a young person who is non-White with no experience of care, at T ime 0 , the odds of further offending amongst

- White young people with experience of care are estimated to be 1.14 times higher

- White young people without experience of care, the odds are 1.39 times higher

- Non-Whites with experience of care, the odds are 5.35 times higher

For White young people, the odds of further offending increase by a factor of 1.58 for those with care experience. Having experience of care also increases the odds for non-Whites, with those young people having experience of care having odds of further offending which are 3.86 times higher than for those who have never been a looked after child.

Model 2 can therefore be thought of as being:

$\operatorname{Pr}$ (Further Offending)

$$
\begin{aligned}
& =\text { Logit }^{-1}\left(\text { Intercept }+\beta_{\text {Gender }} x_{1}+\beta_{\text {Ethnicity }} x_{2}+\beta_{\text {Care }} \mathrm{x}_{3}\right. \\
& \left.+\beta_{\text {Gender }} \mathrm{x}_{1} \beta_{\text {Care }} \mathrm{x}_{3}+\beta_{\text {Ethnicity }} \mathrm{x}_{2} \beta_{\text {Care }} \mathrm{x}_{3}+[\text { BASIC MODEL }]\right)
\end{aligned}
$$

Where

Gender $\left(\mathrm{x}_{1}\right)$ is coded as 0 for males and 1 for females

Ethnicity $\left(\mathrm{x}_{2}\right)$ is coded as 0 for Whites and 1 for non-Whites

Care $\left(\mathrm{x}_{3}\right)$ is coded as 0 for no experience, 1 for experience of care

Which becomes:

$\operatorname{Pr}$ (Further Offending)

$$
\begin{aligned}
& =\operatorname{Logit}^{-1}\left(-1.096+0.482 \mathrm{x}_{1}-1.349 \mathrm{x}_{2}+0.526 \mathrm{x}_{3}-1.715 \mathrm{x}_{1} \mathrm{x}_{3}\right. \\
& \left.+1.196 \mathrm{x}_{2} \mathrm{x}_{3}+[\text { BASIC MODEL }]\right)
\end{aligned}
$$

Hence for a White female with experience of care:

$\operatorname{Pr}$ (Further Offending)

$$
\begin{aligned}
& =\operatorname{Logit}^{-1}(-1.096+0.482(1)-1.349(0)+0.526(1)-1.715(1)(1) \\
& +1.196(0)(1)+[\text { BASIC MODEL }]) \\
& \quad=\operatorname{Logit}^{-1}(-1.096+0.482(1)+0.526(1)-1.715(1) \\
& \quad+[\text { BASIC MODEL }])
\end{aligned}
$$


From a methodological point of view, although ithas not been possible to investigate the extent to which individual domains differ with respect to both gender and ethnicity over time, particularlyin the context of care experience, it is possible to stimulate a model which represents a compromise. Table 5.14 builds on the dynamic model involving care (BDm2_ch, Table 5.12) by additionally including gender and ethnicityas main affects along with interactions between care experience and gender, care experienceethnicity, gender-time, and ethnicity-time.

Table 5.14: The Dynamic Model involving Demographic Characteristics and Experience of Care

\begin{tabular}{|c|c|c|c|c|c|c|c|}
\hline \multirow{3}{*}{$\begin{array}{l} \\
\text { Fixed Effect: }\end{array}$} & \multicolumn{7}{|c|}{$\begin{array}{l}\text { The Dynamic Model including Demographics + Care } \\
\text { (BDm 2) }\end{array}$} \\
\hline & \multicolumn{3}{|c|}{ Unstandardised } & \multicolumn{3}{|c|}{ Standardised } & \multirow{2}{*}{ Significant? } \\
\hline & PostMean & Lower $\mathrm{Cl}$ & Upper $\mathrm{Cl}$ & PostMean & Lower $\mathrm{Cl}$ & Upper $\mathrm{Cl}$ & \\
\hline Intercept & -1.187 & -3.288 & 1.019 & 0.305 & 0.037 & 2.770 & \\
\hline Gender (Male = Ref). & 2.425 & 0.153 & 4.799 & 11.302 & 1.165 & 121.389 & Yes \\
\hline Care Experience (None $=$ Ref) & 1.631 & -1.623 & 4.751 & 5.109 & 0.197 & 115.700 & \\
\hline Ethnicity (White $=$ Ref) & -2.263 & -4.420 & -0.043 & 0.104 & 0.012 & 0.958 & Yes \\
\hline Time & -0.359 & -0.801 & 0.067 & 0.698 & 0.449 & 1.069 & \\
\hline Living Arrangements (Live) & -0.298 & -1.011 & 0.417 & 0.742 & 0.364 & 1.517 & \\
\hline Family and Personal Relationships (Relation) & 0.159 & -0.603 & 0.965 & 1.172 & 0.547 & 2.623 & \\
\hline Education, Training and Employment (ETE) & -0.349 & -0.912 & 0.199 & 0.706 & 0.402 & 1.220 & \\
\hline Neighbourhood (Where) & 0.105 & -0.518 & 0.687 & 1.110 & 0.596 & 1.987 & \\
\hline Lifestyle (Life) & 0.200 & -0.731 & 1.209 & 1.222 & 0.482 & 3.350 & \\
\hline Substance Use (Drugs) & 0.462 & -0.195 & 1.070 & 1.587 & 0.823 & 2.915 & \\
\hline Physical Health (Physical) & -0.133 & -0.880 & 0.663 & 0.876 & 0.415 & 1.941 & \\
\hline Emotional and Mental Health (Emotion) & -0.514 & -1.133 & 0.109 & 0.598 & 0.322 & 1.115 & \\
\hline Perceptions of Self and Others (Self) & -0.140 & -0.987 & 0.788 & 0.869 & 0.373 & 2.198 & \\
\hline Thinking and Behaviour (Think) & 0.196 & -0.591 & 1.043 & 1.216 & 0.554 & 2.838 & \\
\hline Attitude to Offending (Attitude) & 0.240 & -0.658 & 1.119 & 1.271 & 0.518 & 3.062 & \\
\hline Motivation to Change (Change) & 0.436 & -0.398 & 1.244 & 1.547 & 0.672 & 3.469 & \\
\hline Care Experience: Gender & -3.429 & -7.727 & 0.575 & 0.032 & 0.000 & 1.778 & \\
\hline Care Experience: Ethnicity & 0.216 & -3.551 & 3.906 & 1.241 & 0.029 & 49.700 & \\
\hline Care Experience: Time & -0.115 & -0.760 & 0.537 & 0.892 & 0.468 & 1.710 & \\
\hline Care Experience: Live & 0.674 & -0.544 & 1.783 & 1.962 & 0.580 & 5.948 & \\
\hline Care Experience: Relation & -0.133 & -1.495 & 1.192 & 0.875 & 0.224 & 3.294 & \\
\hline Care Experience: ETE & 0.388 & -0.650 & 1.531 & 1.474 & 0.522 & 4.623 & \\
\hline Care Experience: Where & -0.354 & -1.392 & 0.719 & 0.702 & 0.249 & 2.052 & \\
\hline Care Experience: Life & -0.183 & -1.699 & 1.520 & 0.833 & 0.183 & 4.572 & \\
\hline Care Experience: Drugs & -0.036 & -1.096 & 0.966 & 0.964 & 0.334 & 2.627 & \\
\hline Care Experience: Physical & -1.068 & -2.521 & 0.193 & 0.344 & 0.080 & 1.213 & \\
\hline Care Experience: Emotion & 0.522 & -0.613 & 1.546 & 1.686 & 0.542 & 4.693 & \\
\hline Care Experience: Self & 0.942 & -0.544 & 2.572 & 2.564 & 0.580 & 13.092 & \\
\hline Care Experience: Think & -1.011 & -2.562 & 0.561 & 0.364 & 0.077 & 1.752 & \\
\hline Care Experience: Attitude & -0.197 & -1.613 & 1.262 & 0.822 & 0.199 & 3.532 & \\
\hline Care Experience: Change & -0.051 & -1.584 & 1.581 & 0.950 & 0.205 & 4.860 & \\
\hline
\end{tabular}




\begin{tabular}{|c|c|c|c|c|c|c|c|}
\hline \multirow[b]{3}{*}{ Fixed Effect: } & \multicolumn{7}{|c|}{$\begin{array}{l}\text { The Dynamic Model including Demographics + Care } \\
\text { (BDm 2) }\end{array}$} \\
\hline & \multicolumn{3}{|c|}{ Unstandardised } & \multicolumn{3}{|c|}{ Standardised } & \multirow{2}{*}{ Significant? } \\
\hline & Post.Mean & Lower Cl & Upper Cl & Post.Mean & Lower $\mathrm{Cl}$ & Upper Cl & \\
\hline Time: Gender & -0.528 & -1.149 & 0.092 & 0.590 & 0.317 & 1.097 & \\
\hline Time: Ethnicity & 0.210 & -0.279 & 0.664 & 1.233 & 0.756 & 1.943 & \\
\hline Time: Live & 0.004 & -0.168 & 0.181 & 1.004 & 0.845 & 1.198 & \\
\hline Time: Relation & -0.011 & -0.207 & 0.179 & 0.990 & 0.813 & 1.196 & \\
\hline Time: ETE & 0.071 & -0.059 & 0.205 & 1.074 & 0.943 & 1.227 & \\
\hline Time: Where & -0.068 & -0.217 & 0.074 & 0.934 & 0.805 & 1.076 & \\
\hline Time: Life & 0.050 & -0.146 & 0.259 & 1.051 & 0.864 & 1.295 & \\
\hline Time: Drugs & 0.000 & -0.144 & 0.140 & 1.000 & 0.866 & 1.151 & \\
\hline Time: Physical & -0.030 & -0.268 & 0.203 & 0.970 & 0.765 & 1.225 & \\
\hline Time: Emotion & 0.082 & -0.069 & 0.241 & 1.085 & 0.933 & 1.272 & \\
\hline Time: Self & 0.094 & -0.103 & 0.315 & 1.099 & 0.902 & 1.370 & \\
\hline Time: Think & -0.030 & -0.235 & 0.175 & 0.970 & 0.790 & 1.192 & \\
\hline Time: Attitude & -0.114 & -0.325 & 0.093 & 0.892 & 0.722 & 1.098 & \\
\hline Time: Change & -0.028 & -0.252 & 0.185 & 0.972 & 0.777 & 1.203 & \\
\hline Care Experience: Time: Live & -0.020 & -0.266 & 0.208 & 0.980 & 0.767 & 1.231 & \\
\hline Care Experience: Time: Relation & 0.025 & -0.267 & 0.331 & 1.026 & 0.766 & 1.392 & \\
\hline Care Experience: Time: ETE & 0.002 & -0.224 & 0.226 & 1.002 & 0.800 & 1.254 & \\
\hline Care Experience: Time: Where & 0.202 & -0.001 & 0.417 & 1.223 & 0.999 & 1.517 & \\
\hline Care Experience: Time: Life & -0.145 & -0.470 & 0.149 & 0.865 & 0.625 & 1.160 & \\
\hline Care Experience: Time: Drugs & -0.033 & -0.262 & 0.209 & 0.968 & 0.770 & 1.232 & \\
\hline Care Experience: Time: Physical & 0.252 & -0.047 & 0.574 & 1.286 & 0.954 & 1.776 & \\
\hline Care Experience: Time: Emotion & 0.018 & -0.219 & 0.273 & 1.018 & 0.803 & 1.314 & \\
\hline Care Experience: Time: Self & -0.381 & -0.712 & -0.054 & 0.683 & 0.491 & 0.947 & Yes \\
\hline Care Experience: Time: Think & 0.121 & -0.203 & 0.430 & 1.129 & 0.816 & 1.537 & \\
\hline Care Experience: Time: Attitude & 0.124 & -0.221 & 0.445 & 1.132 & 0.802 & 1.560 & \\
\hline Care Experience: Time: Change & -0.009 & -0.332 & 0.304 & 0.991 & 0.717 & 1.355 & \\
\hline Random Effect: & Post.Mean & Lower $\mathrm{Cl}$ & Upper $\mathrm{Cl}$ & Post.Mean & Lower $\mathrm{Cl}$ & Upper Cl & Significant? \\
\hline Individual (Intercept) & 0.72 & $1.03 \mathrm{E}-05$ & 1.67 & 2.049 & 1.000 & 5.317 & Yes \\
\hline Time & 2.733 & 0.611 & 6.169 & 15.379 & 1.842 & 477.708 & Yes \\
\hline DIC & & & 466.38 & & & & \\
\hline
\end{tabular}

Source: Model BDm2, Technical Annex p121-134.

The model suggests that the main effects for gender and ethnicity are significant, with the estimate for gender being positive. So that the impact of this can be visualised, the probability of further offending has been calculated using the model with domain scores fixed at 2 so as to broadly represent the 'average' young person at the time of their initial assessment. As can be seen from Figure 5.9, the respective probabilities of further offending for females (males being the reference group for the Gender predictor), are highergenerallyhigher until Time 4.

The estimate for ethnicityis negative, suggesting that those who are non-White have on average a lower probability of further offending than Whites (Figures 5.9 and 5.10). This finding is potentiallylinked not just to the small number of cases but also the net effect of having both Black and Asian young people within this group since national proven reoffending figures suggest that Black boys and girls are more likely to reoffend than Whites peers whilst Asian boys and girls are less likely to reoffend (Ministry of Justice, 2017c). This is also apparent in Figures 5.9 and 5.10. Estimates for non-Whites with and without experience of care have generated by the model due to the inclusion of the interaction term Ethnicity: Care experience. 
Figure 5.9: Estimated Changes in the Probability of Further Offending for those with No Experience of Care, by Gender and Ethnicity

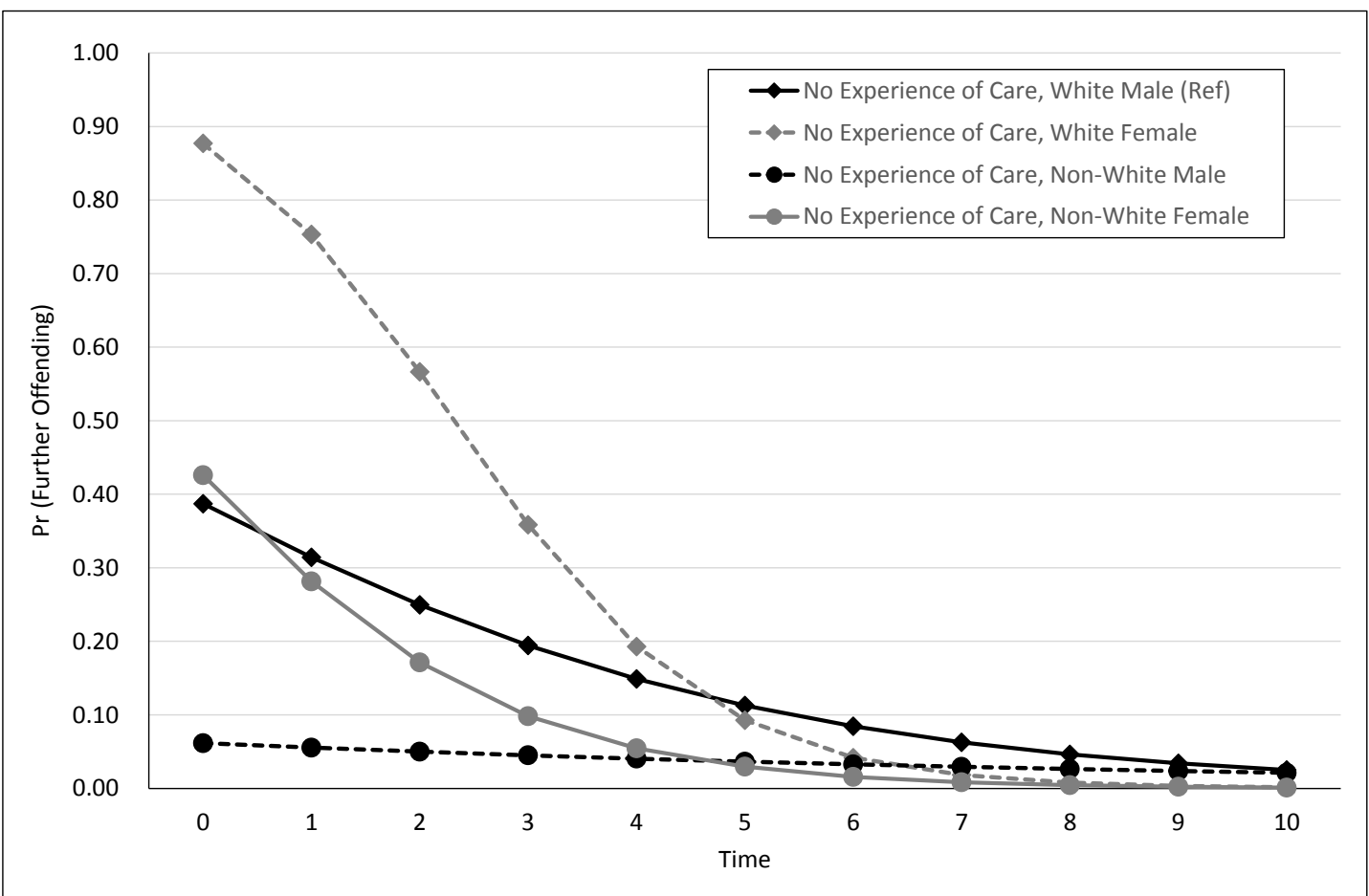

Notes: The domain scores have been fixed at 2 to demonstrate the estimated change in the probability of further offending for different sub-groups, from time 0 to time 10. Estimates derived from Model BDm2.

Figure 5.10: Estimated Changes in the Probability of Further Offending for those with Experience of Care, by Gender and Ethnicity

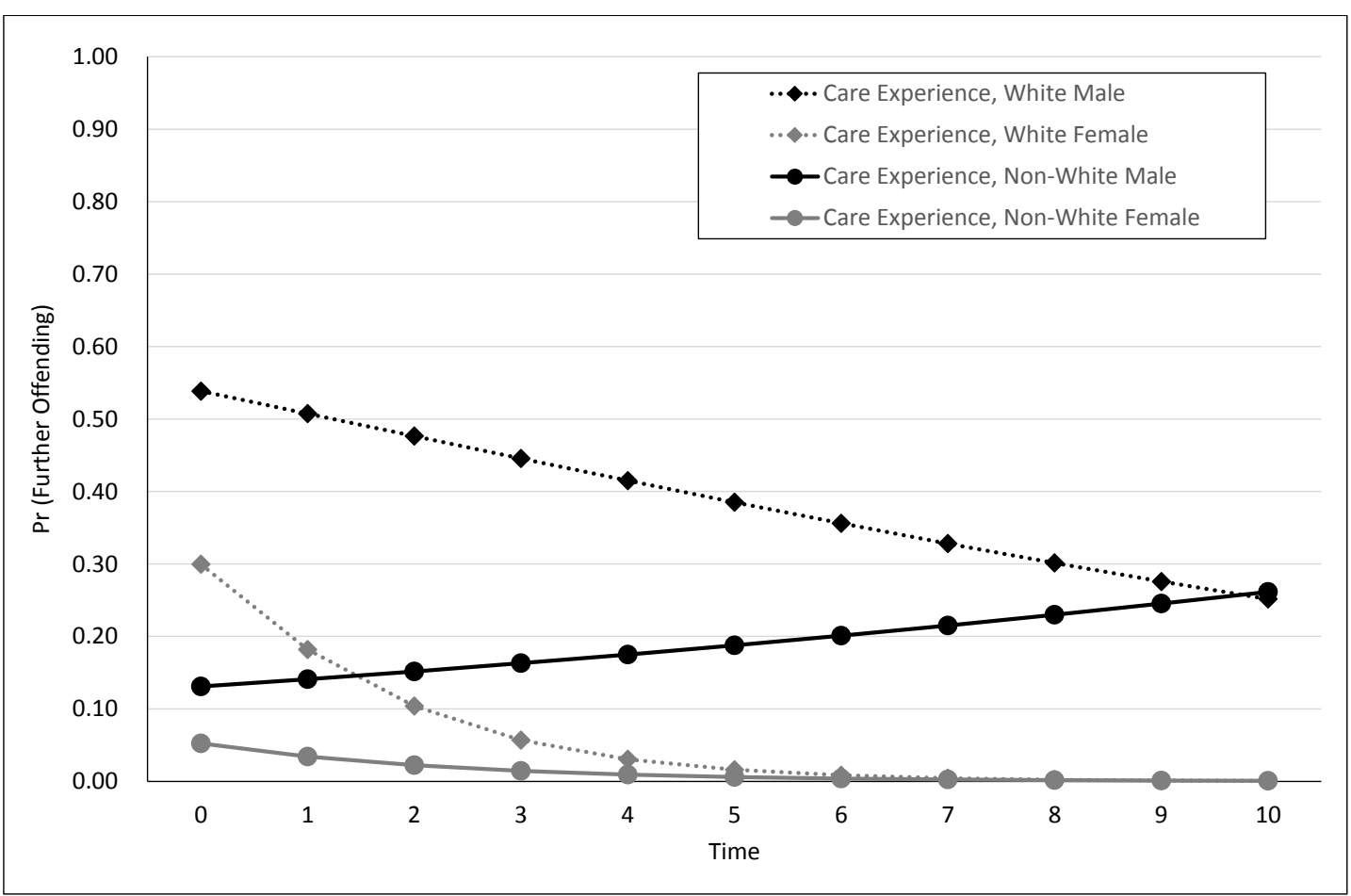

Notes: The domain scores have been fixed at 2 to demonstrate the estimated change in the probability of further offending for different sub-groups, from time 0 to time 10. Estimates derived from Model BDm2. 
Care as a main effect is not significant. However, this is because the interactions between the 12 domains, ethnicity, gender and time help to explain some, but not all of the differences between those with experience of care and those who have never been looked after.

The low numbers offemales and non-Whites in the reoffending cohort, means that there remains a high amount of uncertainty within the model - reflected by the DIC of 466.38 and the wide credible intervals for some of the main and interactionfixed effects. The estimated probability of further offending for these groups therefore also contain a high degree of uncertaintyand especiallyat later time points, may prove to be unreliable.

The estimated trajectory of the reference group of White males with no experience of care (representing $58.0 \%$ of the cohort, $51 / 87$ ), is shown on both Figures 5.9 and 5.10 . Relative to this, the probabilityof further offending amongst White males ( $25 \%$ of the cohort) at each measurement occasion is estimated to be lower. Differences also existfor those with different risk scores. For example, at Time 0 , the odds of a White male who has never been looked after committing further offences are estimated to be:

- 3 times higher than the odds of further offending for White males who have experience of care when their domain scores are fixed at 1.

- 1.9 timeshigher when domain scores are fixed at 2, as in Figure 5.9.

- $11 \%$ higher when domain scores are fixed at 3

The trend for the non-white male with experience of care is contrary to what would be expected. From Table 5.3, it is possible to see that this trend is based on data for just one individual who has exhibited more serious, sustained offending behaviours.

The trend for White females with experience of care also needs to be treated with caution as it is based upon data relating to just two young people. As both of these had comparatively low domain scores, particularly after Time 0 , this casts doubt upon the reliability for estimated probabilities of further offending for higher domain scores. Looking across the females more generally, there were only three girls who committed further offences with these having higher average domain scores at the earlier measurement points than those who did not engage in any further offending. This was also the case for the non-White cohort.

Notably, it is possible to use the model to generate an estimated trajectory of the probability of offending for non-White females with experience of care despite there being no young people who share these characteristics within the dataset. Whilstlooked after girls representa very small proportion of the whole within the criminal justice system (Prison Reform Trust, 2016), it is inconceivable that nationally these would all be White. 


\subsection{How do these findings extend the evidence base?}

Whilst there is a desire to extend what is known in terms of the young people with experience of care and particularlysub-groups within this cohort, there is insufficient data to reliablyexplore this with respect to gender and ethnicity. From BDm2, the positive coefficientfor Genderas a main effect suggests that the non-reference group i.e. females have 'penalty' which if all other factors / scores in the model were equal would result in females having a higher initial probability of further offending. Since the coefficient for Gender: Time is not significant, it is not possible to be certain of the differences over time.

The negative coefficient for Ethnicity as a main effect suggests that the on average it is the reference group i.e. Whites who if all other factors / scores in the model were equal would have a 'penalty' which would result in non-Whites having a lower initial probability of further offending. However, the extent to which this is moderated over time is difficult to ascertain due to the coefficientfor the interaction between Ethnicity: Time not being significant.

Both the dynamic model involving care (BDm2_ch) and the enhanced version which additionally incorporates gender and ethnicity (BDm2) resulted in significantcoefficients for the interaction between Care Experience: Time: Self. This domain is one which Wilson and Hinks (2011) identified that practitioners had difficultyexploring with young people. Notably the coefficientfor Self is not significant as a main effectsuggesting that there are other factors which have not been included in the model which could account for this uncertainty. Potentially this could include gender and ethnicity, but there could also be additional explanatoryfactors which have not been included in these models.

As highlighted in section 4.3, the Perception of Self and Others domain concentrates upon the young person's understanding of how they - and others - fit into the world around them, including their levels of self-esteem; mistrust of others; difficulties with self-identify and if they see themselves as an offender - for a more detailed description see Section 1 of the Technical Annex. Baker et al. (2005) found differences in the ratings on the basis of gender and ethnicity which due to the small number of female and non-White cases within the dataset are not possible to explore in the context of this research. In particular their findings around self-identify and the general mistrust of others amongst BAME young people involved in the youth justice system are consistent with those found by David Lammy MP (Lammy, 2017) in his recentreview of the over-representation of these groups. In the context of gender differences, Smith and McAra (2004) identified through their analysis of self-reported data that low selfesteem was more closelylinked to delinquencyin girls than boys. In terms of the factors that increased serious delinquency more in girls than in boys, these additionally included having a weak belief in conventional moral standards i.e. considering it acceptable to lie / steal / fight. Risk taking was found to be very strongly associated with delinquency for both sexes whilst impulsivity was found to be quite strongly associated. 
Since the responses to the individual questions within the domain did not form part of the datasetcreated, it is notpossible to ascertain where there are differences in the responses for differentsub-groups of the cohort. It is however, possible to use BDm2 to consider the net impact of the probability of further offending should a young person with experience of care experience an increase in their rating for the Perception of Self and Others domain (whilst ratings in the other domains are not altered). Whilst the odds of further offending increase, the inclusion of time in the interaction acts as a moderating factor meaning that relative to a young person with experience of care who has not received the elevated rating, the 'gap' reduces:

- At Time 0 , the odds of a typical young person with experience of care with an elevated rating for the Perception of Self and Others domain (i.e. with domain scores fixed at2, reflecting the average rating for all young people in the dataset, and this domain increased to 3 ) is estimated to be 2.1 times more likely to commit further offences than their peer whose domain scores remained fixed at 2. By Time 2, the former is 1.2 times more likely to commitfurther offences than the young person with experience of care without the elevated rating.

- Amongst those without experience of care, at Time 0 , the young person with the elevated rating is 1.1 times more likely to commitfurther offences.

- Relative to the typical young person with no experience of care, their peer who has been looked after with the elevated rating is estimated to be 4.5 times more like to commit further offences at T ime $0,2.8$ times more likely at T ime 5 and if still under the supervision of the YOT at Time 10, the odds of further offending are estimated to be 1.3 times higher.

More generally, the amount of uncertainty around the predictor for care experience and interactions involving this term can potentially be explained by the range of different reasons which can lead to a young person becoming looked after, the various legal status' that these young people can hold and the time which the child has been looked after. For example, a young person placed in care having previously been identified as being at significant risk of harm may be struggling to overcome these adverse childhood experiences whilsta child in a long-term stable placementmay have formed strong relationships which have had a positive impact upon their perceptions of themselves and others. Similarly, a young person recently removed from the family home as a result of their offending behaviour may have a general distrust of others, especiallythose in authority.

Given the current policy emphasis, the role which experience of care plays in the likelihood of further offending, it will be revisited in Chapter Seven as part of the discussion of the impact of system contact 


\subsection{How does the model involving gender, ethnicity and care experience reflect}

the realities of real lives?

The following section returns to the examples of Fred, David and Connor, to consider how the estimates of their respective probabilities of further offending based on $\mathrm{BDm} 2$ compare with those generated by the Basic Dynamic Model (BDm1, summarised in Table 4.12).

\section{Case Study "Fred"}

"Fred" is a white male who has never been looked after. The trajectory of the probability of further offending based on BDm2 (in grey in Figure 5.11) suggests a slightly higher initial probability of further offending at Time 0 relative to that based on the Basic Dynamic Model (BDm1, in black). However, whilst the BDm1 shows an upwards trend which becomes steeper between Times 2 and 3 when his risk score increases to 21 - this was when he was on ISSP Bail and Tag, before flattening again towards Time 4, the model involving demographics and care has an initial decline before becoming steeper between Times 2 and 3 . There is then a downward trend towards Time 4.

Figure 5.11: Comparisons of the Estimated Probability of Further Offending Over Time: "Fred"

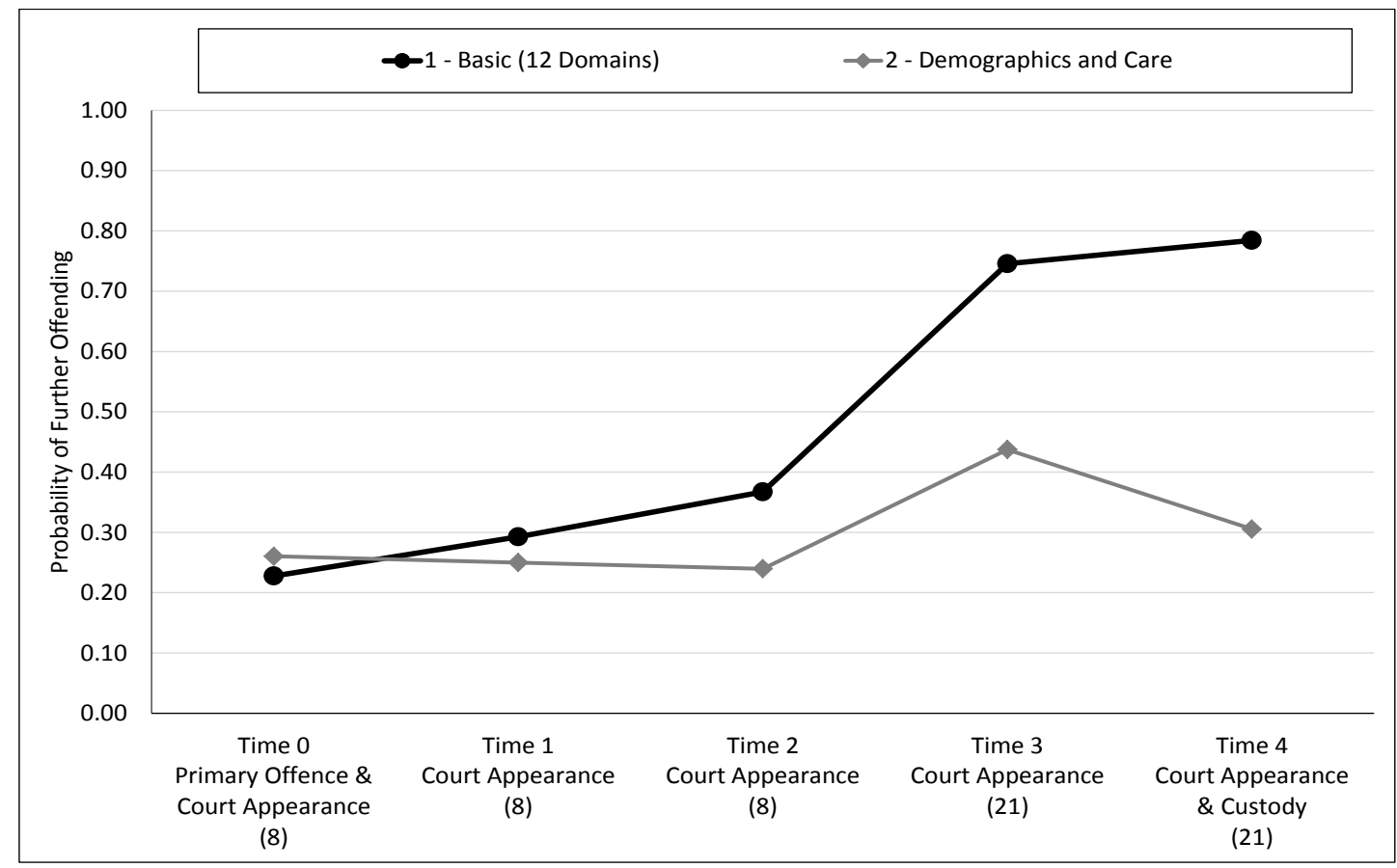

\section{Case Study "Connor"}

"Connor" is also a white male. However, he does have experience of being looked after. He had been identified as a prolific offender prior to entering the 2012/13 reoffending cohortand this is reflected in $\mathrm{h}$ is high-risk scores and the corresponding high probabilities of further offending in Figure 5.12. The effect of using BDm2 to generate the probability of Connor committing further offences is that it now reflects the increase in his total domain scores between Times 0 and 1 when he committed a further offence, 
the subsequent dip whilsthe was in custodyand then the increase post-release (at Time3). BDm1 does not reflect this initial increase between Times 0 and 1 , suggesting that actuallyhis probability of further offending decreases during this time.

Figure 5.12: Comparisons of the Estimated Probability of Further Offending Over Time: "Connor"

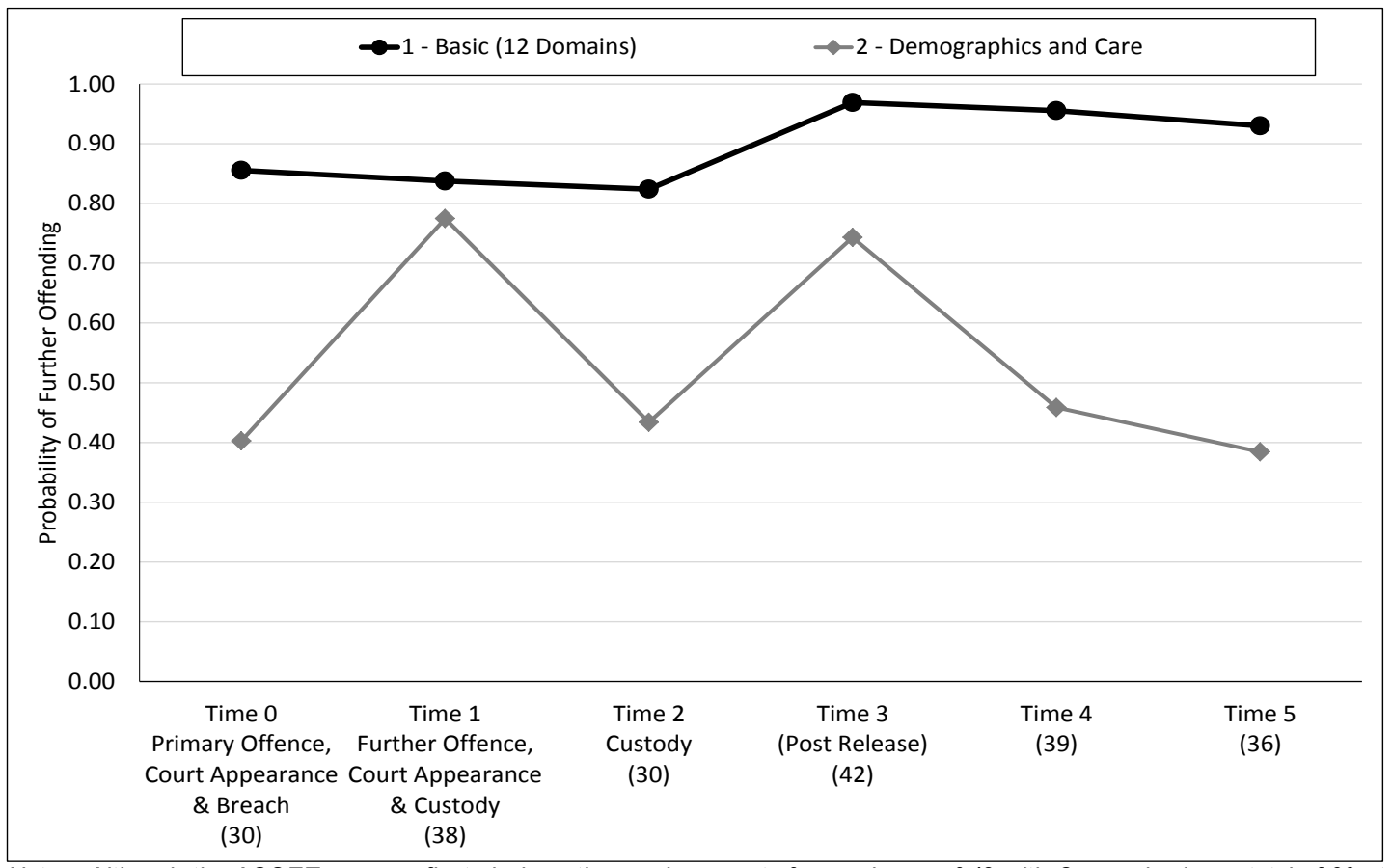

Notes: Although the ASSET scores reflected along the $x$-axis are out of a maximum of 48 with Connor having a total of 30 at Time 0, under the Scaled Approach he would have attracted additional scores due to the fact that his primary offence (for the purposes of this exercise where the information has been taken from the reoffending spreadsheet) was anon-domestic burglary and as a result of his prior convictions.

\section{Case Study "David"}

"David" is a white male who has never been looked after. The respective trajectories of the probability of David committing further offences whilst similar in shape, suggest quite a difference between the two models - at each measurement point, the probability of further offending is lower when based on BDm2.

Between Time 0 and Time 1, the Basic Dynamic Model suggests an increase in David's probability of further offending which corresponds to the increase in his ASSET score from 40 to 44 . However, the trend suggests by estimates from $\mathrm{BDm} 2$ is downwards. This is despite the fact that David committed a further offence during this period. He also breached. Therefore, this is not what would be expected. 


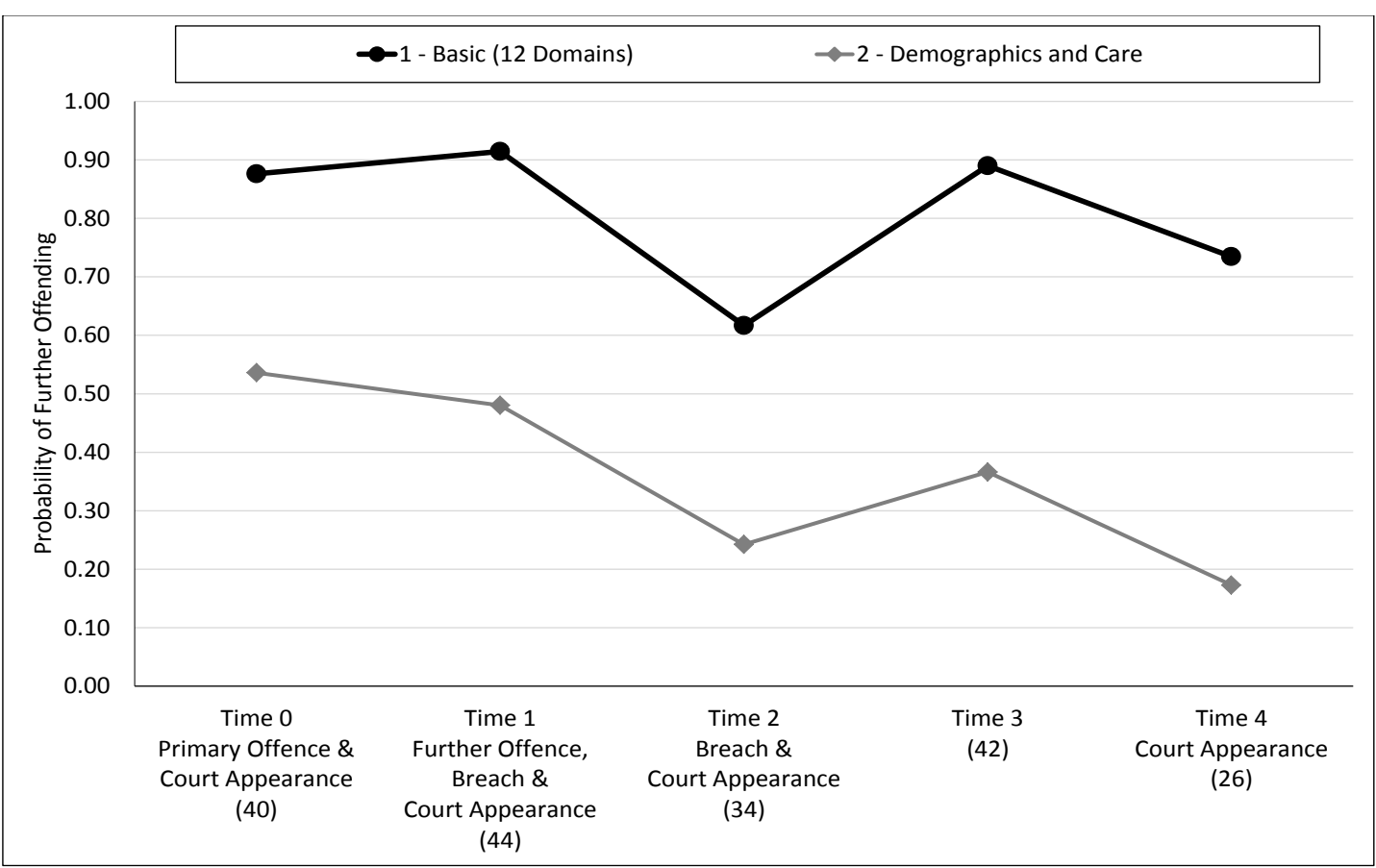

Notes: Although the ASSET scores reflected along the $x$-axis are out of a maximum of 48 with David having a total of 40 at Time 0, under the Scaled Approach he would have attracted additional scores due to the fact that he was aged 10 at the time of his first Reprimand and as a result of his prior convictions.

Whilst it is not possible to measure the predictive accuracy of $\mathrm{BDm} 2$ relative to $\mathrm{BDm} 1$ which was constructed to represent the ASSET Core Profile, it would appear that including care and demographic characteristics has the potential to reduce the estimates of the probability of further offending for white males (reflecting the two reference groups for the Gender and Ethnicity predictors). However, it is notable that whilst the probability of further offending fell for David in the period when he committed a further offence whereas it increased for Connor suggests that further predictors may need to be incorporated into subsequentmodels to increase its sensitivity to changes over time.

\subsection{Summary}

The analysis presented in this chapter sought to address three research questions, with the final question being addressed as part of the previous section:

2. What is the impact of gender and ethnicity on the likelihood of further offending?

3. What is the impact of having experience of care on the likelihood of further offending?

8. How well do ASSET scores reflect the realities of the young person's change in circumstances during their time under the supervision of the YOT?

Sadly, there was insufficient data to fully explore the impact of gender and ethnicityon the likelihood of further offending over time. However, from their respective initial mean domain scores, there does appear to be significant differences in the profiles of males and females, and Whites compared to NonWhites within the reoffending cohort. The extent to which this hold for those in the formal youth justice 
system in England and Wales more generally cannot be established, although it is in keeping with the findings of Schwalbe (2008), van der Putet al. (2014) and others who have explored the need for genderspecific instruments, and with work carried out using American samples which has considered whether there are differences on the basis of race/ethnicity.

Previous evaluations of ASSET have not considered potential differences on the basis of care status. However, the evidence presented as part of Lord Lamming's Review (Prison Reform Trust, 2016) highlights the over-representation of care experiences children within the youth justice system, pointing at the disadvantages that these children may face relative to their peers who have never been looked after. The models presented within this chapter represent a compromise as is not possible to fully differentiate between different sub-groups of the looked after children cohort e.g. on the basis of gender and/or ethnicity or their legal status. Despite this, when the estimated probabilities of further offending at different measurement points have been determined, it becomes apparent from the re sulting charts that there are distinct differences in the estimated initial probability and the subsequent trajectory of change over time. Where it possible to access data from multiple YOT sand hence increase the size of the dataset, this is something I would be keen to explore further, especiallygiven the current policyfocus. From a methodological point of view, what this chapter has demonstrated is the way in which the basic dynamic model can be extended to take into account additional predictors. Doing this increases the complexity of the model as highlighted in section 5.1 and is reflected in the increase in the DIC relative to the Basic Dynamic Model.

Subsequent chapters consider differenttypes of predictors for example, in Chapter Six the predictor for the YJB Offence Category is categorical whilst that for the YJB Gravity Score is continuous. A key learning pointfrom the analysis undertaken with respect to dimensional identityhas been that although cohort has only been split into two groups - through the use of dichotomous predictors, the size of the datasetcan still place limitations upon what can be explored. This is despite hierarchical modelling being promoted as being a more efficient approach. The small size of the non-reference groups has meant that at later measurement points, the model is informed bythe data relating to just one individual, leading is potentially misleading trajectories of the estimated probability of further offending for some groups / fixed domain scores. 


\section{Findings: Static Factors}

As described in Chapter Two, the ASSET Core Profile consists of two elements: the 12 domains or 'dynamic' factors, and four 'static' factors. The scoring for the static factors is summarised in Table 6.1, with a maximum potential score of 16 being assigned by practitioners to reflect the perceived additional risk posed by those with more established criminal careers, who were also committing more

Table 6.1: Scoring for the Static Risk Factors under the Scaled Approach

\begin{tabular}{|c|c|c|}
\hline \multirow{2}{*}{ Static Factor } & \multicolumn{2}{|l|}{ Scoring } \\
\hline & Criteria & Score \\
\hline \multirow{3}{*}{ Age at first reprimand, caution or warning } & 10 to 12 & 4 \\
\hline & 13 to 17 & 2 \\
\hline & No previous reprimand, caution or warning & 0 \\
\hline \multirow{3}{*}{ Age at first conviction } & 10 to 13 & 4 \\
\hline & 14 to 17 & 3 \\
\hline & No previous convictions & 0 \\
\hline \multirow{3}{*}{ Number of previous convictions } & 4 or more & 4 \\
\hline & 1 to 3 & 3 \\
\hline & No previous convictions & 0 \\
\hline \multirow{3}{*}{ Offence Type } & Motoring offences / vehicle theft / unauthorised taking & 4 \\
\hline & Burglary (domestic and non-domestic) & 3 \\
\hline & Other offence & 0 \\
\hline
\end{tabular}

Adapted from Youth Justice Board (2010b: 17)

This chapter considers the role that these static factors play with respect to the 12 dynamic risk factors, in predicting the likelihood of further offending. The following research questions are therefore considered:

4. What is the impact of the 'static' factors within ASSET in predicting further offending over time?

5. Is it possible to extend the sensitivity of ASSET by extending any of the predictors?

8. How well do ASSET scores reflect the realities of the young person's change in circumstances during their time under the supervision of the YOT? 
The following non-time varying, Level 2 measures have therefore been added to the dataset to serve as a proxy for the static risk factors in ASSET:

- G_ageFirst-grouped aged at first offence. A dichotomous variable where the thresholds for the two groups reflect the scoring outlined in Table 6.1 i.e. young (10-12 years) and older (1317 years)

- G_ageCon - grouped aged atfirst conviction. A dichotomous variable where the thresholds for the two groups reflect the scoring in ASSET i.e. young (10-13 years) and older (14-17 years)

- FTE - As it was not possible to check the number of previous convictions recorded on PNC, this dichotomous variable relies upon data held within Childview to determine whether the young person was a first-time entrantat the time of entering the cohort(Y/N)

- I_Cat2 - grouping of the offence categories used by the YJB in relation to the primaryoffence. Structured as a categorical predictor, this predictor has been constructed to differentiate between serious acquisitive crimes (SAC), violence against the person offences (VAP) and other offences.

In looking to establish if the sensitivity of ASSET can be extended, the following predictors are also considered:

- AgeFirst - age at first offence. Since the age of criminal responsibility is 10 , this has been centred (by subtracting 10) to give a meaningful zero.

- AgeCon - age at first conviction. As with AgeFirst, this has also been centred (by subtracting 10) to give a meaningful zero.

- I_Seriousness2 - based upon the YJB Gravity Score of the primary offence, this enables the seriousness of the offence to be considered. Since the focus is on those in the formal youth justice system, this has been centred at 2 (reflecting the lowest gravity score of those in the reoffending cohort) to give a meaningful zero. 


\subsection{Description of the data}

During the data collection process, itwas identified that the static factors were notconsistentlycompleted and therefore it has been necessaryto rely upon the information held within Childview in relation to the individual's offending and courtappearances.

Table 6.2: The Reoffending Cohort by FTE Status, Grouped Age at First Offence, Grouped Age at First Conviction and Grouped YJB Offence Category

\begin{tabular}{|c|c|c|c|c|c|c|}
\hline \multirow{2}{*}{ FTE Status } & \multirow{2}{*}{$\begin{array}{l}\text { Grouped Age at } \\
\text { First Offence }\end{array}$} & \multirow{2}{*}{$\begin{array}{l}\text { Grouped Age at } \\
\text { First Conviction }\end{array}$} & \multicolumn{3}{|c|}{ Grouped YJB Offence Category } & \multirow{2}{*}{ Total } \\
\hline & & & Other & SAC & VAP & \\
\hline \multirow{4}{*}{ FTE } & \multirow{2}{*}{10 to 12 years } & 10 to 13 years & 3 & & 2 & 5 \\
\hline & & 14 to 17 years & 10 & 3 & 1 & 14 \\
\hline & \multirow{2}{*}{13 to 17 years } & 10 to 13 years & 1 & 1 & & 2 \\
\hline & & 14 to 17 years & 16 & 7 & 10 & 33 \\
\hline \multicolumn{3}{|l|}{ FTE Total } & 30 & 11 & 13 & 54 \\
\hline \multirow{4}{*}{ NotFTE } & \multirow{2}{*}{10 to 12 years } & 10 to 13 years & 1 & & 1 & 2 \\
\hline & & 14 to 17 years & & & 1 & 1 \\
\hline & \multirow{2}{*}{13 to 17 years } & 10 to 13 years & & & 2 & 2 \\
\hline & & 14 to 17 years & 17 & 7 & 4 & 28 \\
\hline \multicolumn{3}{|c|}{ Previous Offending History Total } & 18 & 7 & 8 & 33 \\
\hline \multicolumn{3}{|l|}{ Grand Total } & 48 & 18 & 21 & 87 \\
\hline
\end{tabular}

Notes: The individual whose FTE status is not known has been excluded from this summary.

Since some young people received a conviction on their first offence (rather than a Reprimand, Caution or Final Warning), then their age of first offence (AgeFirst) can be equal to their age at the time of their first conviction (AgeCon). This is more apparent when the reoffending cohort is segmented by age rather than grouped age at first offence and first conviction respectively.

Table 6.3: The Reoffending Cohort by FTE Status, Age at First Offence and Age at First Conviction

\begin{tabular}{|c|c|c|c|c|c|c|c|c|c|c|}
\hline \multirow{2}{*}{$\begin{array}{c}\text { FTE } \\
\text { Status }\end{array}$} & \multirow{2}{*}{ Age at First Offence } & \multicolumn{8}{|c|}{ Age at First Conviction } & \multirow{2}{*}{ Total } \\
\hline & & 10 & 11 & 12 & 13 & 14 & 15 & 16 & 17 & \\
\hline \multirow{8}{*}{ FTE } & 10 & & & & & & & & & \\
\hline & 11 & & & & & & & & & \\
\hline & 12 & & & 1 & 1 & & 1 & & & 3 \\
\hline & 13 & & & & 2 & & & & & 2 \\
\hline & 14 & & & & & 1 & 2 & 1 & 1 & 5 \\
\hline & 15 & & & & & & 7 & 4 & & 11 \\
\hline & 16 & & & & & & & 5 & 2 & 7 \\
\hline & 17 & & & & & & 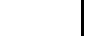 & & 5 & 5 \\
\hline \multicolumn{2}{|l|}{ FTE Total } & & & 1 & 3 & 1 & 10 & 10 & 8 & 33 \\
\hline \multirow{8}{*}{$\begin{array}{l}\text { Not } \\
\text { FTE }\end{array}$} & 10 & 1 & & 1 & & 2 & & 2 & & 6 \\
\hline & 11 & & & 1 & & 2 & 2 & & & 5 \\
\hline & 12 & & & & 2 & 2 & 4 & & & 8 \\
\hline & 13 & & & & 2 & 6 & 2 & & 1 & 11 \\
\hline & 14 & & & & & 5 & 4 & & 3 & 12 \\
\hline & 15 & & & & & & 3 & 2 & 5 & 10 \\
\hline & 16 & & & & & & & 1 & 1] & 2 \\
\hline & 17 & & & & & & & & & \\
\hline \multicolumn{2}{|c|}{ Previous Offending History Total } & 1 & & 2 & 4 & 17 & 15 & 5 & 10 & 54 \\
\hline \multicolumn{2}{|c|}{ Grand Total } & 1 & & 3 & 7 & 18 & 25 & 15 & 18 & 87 \\
\hline
\end{tabular}

Notes: The individual whose FTE status is not known has been excluded from this summary

Page $\mid 180$ 
Looking at the age that the FTEs received their first conviction, there appears to be conflicting information. In the main this is due to the way in which the predictor was set up, with the status upon entry to the reoffending cohortbeing used. Thus, young people who appeared on both the 2012/13 and 2013/14 reoffending spreadsheets were recorded on the basis of their status in 2012/13 - their inclusion the second year reflects the fact that they had gone on to commitfurther offences and hence in 2013/14. This status was carried forward into the second year rather than updating it to reflect that when the entered that cohort they had a previous offending history.

In the case of the FTEs who committed their first offence aged 12 and 14 but were not convicted until age 15 and 17 respectively, their initial offence was dealt with informally. The former appears in Table 6.2 as the FTE aged 10 to 12 years at time of first offence but aged 14 to 17 years at time of first conviction. A small number of FTE also had their birthday in the time that it took for the case to go to courtaccounting for the difference in ages.

\subsection{Initial Differences}

\section{Age at First Offence and Conviction}

Under the Scaled Approach, the way in which the scores for the static factors relating to age were assigned is summarised in Table 6.1. The respective thresholds have been used to segment the reoffending cohort to explore differences in the domain scores at Time 0 .

Figure 6.1: Domain Score Profile, by (a) Grouped Age at First Offence and (b) Grouped Age at First Conviction, at Time 0

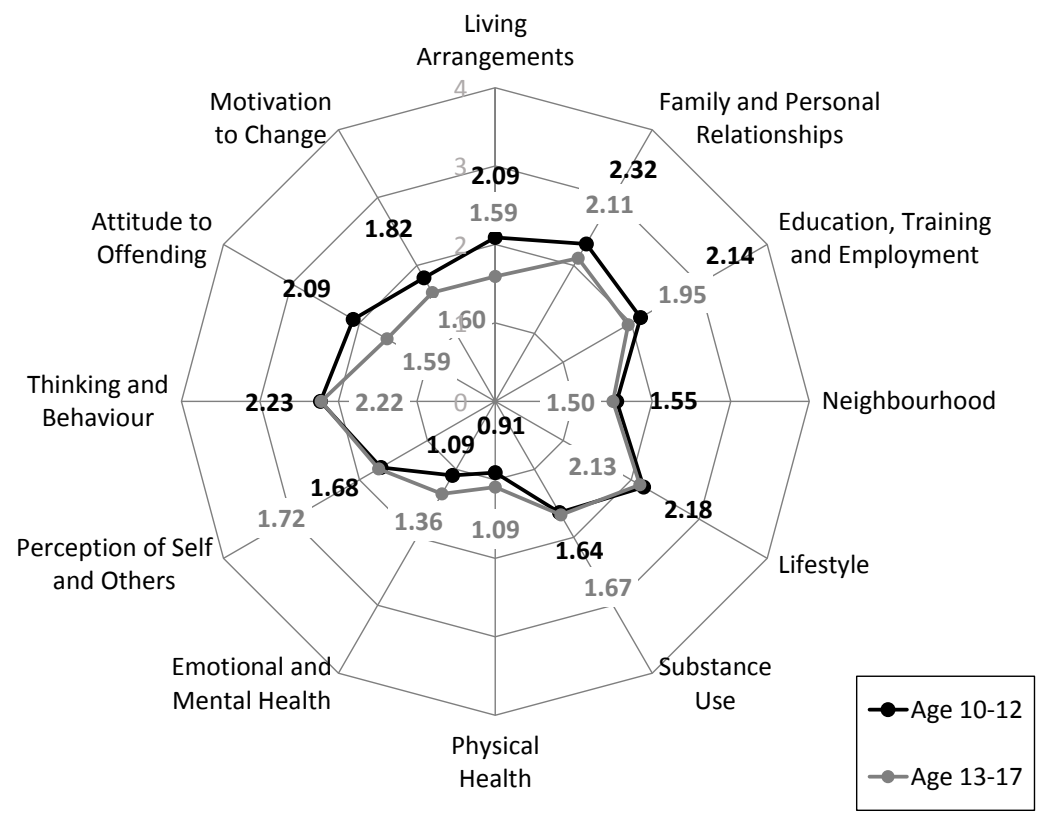




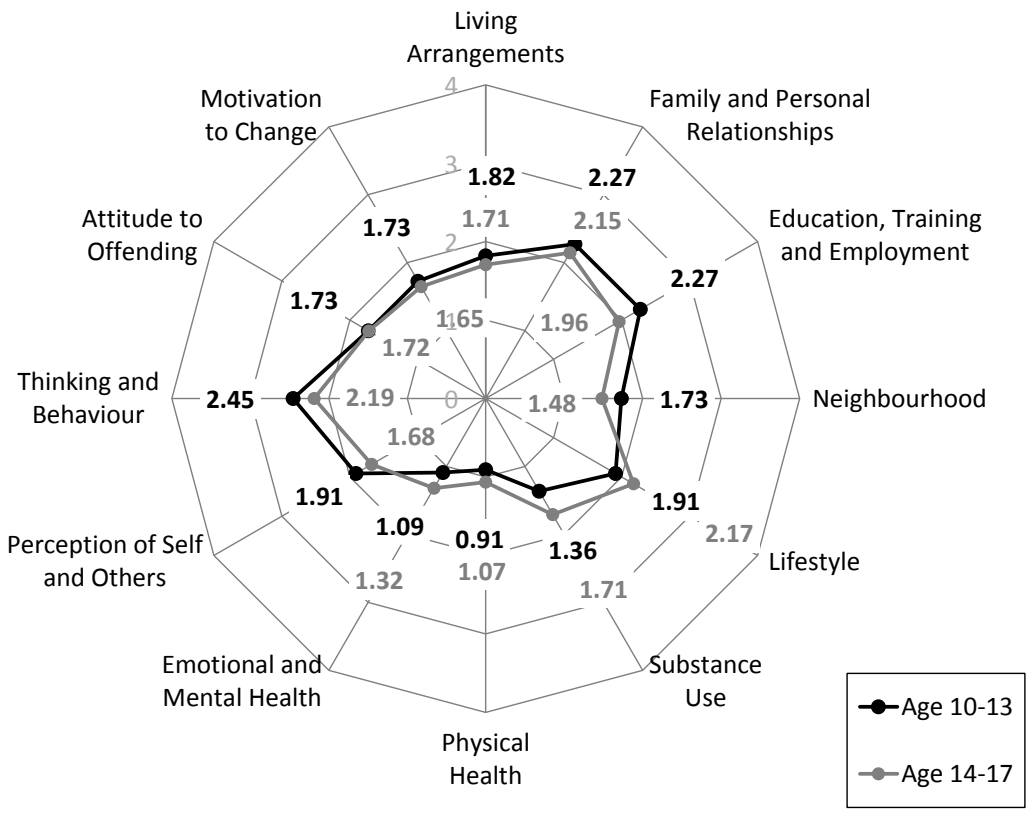

Notes: Of the 87 individuals, 22 were aged 10-12 at the time of their first Offence. 11 were aged 10-13 at the time of their first conviction.

In the context of the grouped age at first offence, one-sided t-tests suggest that there no evidence to support the apparent differences in the mean domain scores at $\mathrm{T}$ ime 0 between the younger and older sub-cohorts whichcanbe seen in Figure 6.1(a). The exception to this is the Attitude to Offending domain where there is moderate evidence to suggest that on average, those aged 10-12 have higher ratings $\left(\mathrm{BF}_{10}=4.046, \%\right.$ error $\left.=3.405 \mathrm{e}^{4}\right)$. There is also anecdotal evidence that this is also the case for the living arrangements domain $\left(\mathrm{BF}_{10}=1.842, \%\right.$ error $\left.=1.028 \mathrm{e}^{-4}\right)$. The equivalent two-sided tests in relation to the age at first conviction suggest that there is anecdotal evidence in favour of $\mathrm{H}_{0}$ that there is no difference between the mean scores for each of the domains for each of the age groups. This is broadly consistent with the trends in Figure 6.1(b). However, this may in part be due to the small number of cases in the young age group - there are only 11 cases as opposed to 76 who received their first conviction after the age of 14 . 
Table 6.4: Random Intercepts and Varying Slope Models for Further Offending including ASSET Domains and the Two Age Related Static Factors

\begin{tabular}{|c|c|c|c|c|c|c|c|}
\hline \multirow[b]{3}{*}{ Fixed Effect: } & \multicolumn{4}{|c|}{$\begin{array}{c}\text { Model 1.5: Basic Model + } \\
\text { Grouped Age at First Offence }\end{array}$} & \multirow{2}{*}{\multicolumn{3}{|c|}{$\begin{array}{l}\text { Model 1.6: Basic Mo } \\
\text { Grouped Age at First C } \\
\text { Unstandardised }\end{array}$}} \\
\hline & \multicolumn{3}{|c|}{ Unstandardised } & \multirow{2}{*}{ Significant? } & & & \\
\hline & Post.Mean & Lower $\mathrm{Cl}$ & Upper $\mathrm{Cl}$ & & \begin{tabular}{|l|} 
Post.Mean \\
\end{tabular} & Lower $\mathrm{Cl}$ & Upper \\
\hline Intercept & -1.090 & -2.518 & 0.249 & & -0.888 & -2.461 & $0 . !$ \\
\hline Grouped Age at First Offence (Ref = 13-17 years) & -0.147 & -0.620 & 0.363 & & & & \\
\hline Grouped Age at First Conviction (Ref $=14-17$ years) & & & & & -0.323 & -0.946 & 0. \\
\hline Living Arrangements & 0.023 & -0.254 & 0.291 & & 0.034 & -0.235 & 0. \\
\hline Family and Personal Relationships & 0.278 & -0.030 & 0.574 & & 0.276 & -0.046 & 0. \\
\hline Education, Training and Employment & 0.074 & -0.172 & 0.332 & & 0.088 & -0.151 & 0. \\
\hline Neighbourhood & 0.054 & -0.169 & 0.278 & & 0.036 & -0.192 & 0. \\
\hline Lifestyle & 0.042 & -0.311 & 0.386 & & 0.041 & -0.323 & 0. \\
\hline Substance Use & 0.173 & -0.064 & 0.434 & & 0.174 & -0.062 & 0. \\
\hline Physical Health & -0.123 & -0.414 & 0.172 & & -0.111 & -0.422 & 0. \\
\hline Emotional and Mental Health & 0.016 & -0.230 & 0.265 & & 0.000 & -0.257 & 0. \\
\hline Perceptions of Self and Others & -0.134 & -0.480 & 0.186 & & -0.157 & -0.496 & 0. \\
\hline Thinking and Behaviour & -0.165 & -0.527 & 0.148 & & -0.177 & -0.506 & 0 . \\
\hline Attitude to Offending & 0.024 & -0.354 & 0.385 & & 0.039 & -0.331 & 0. \\
\hline Motivation to Change & 0.241 & -0.105 & 0.574 & & 0.242 & -0.111 & 0. \\
\hline Time & -0.163 & -0.296 & -0.014 & Yes & -0.160 & -0.312 & -0 . \\
\hline Random Effect: & Post.Mean & Lower Cl & Upper Cl & Significant? & Post.Mean & Lower Cl & Upper \\
\hline Individual (Intercept) & 0.208 & $2.02 \mathrm{E}-07$ & 0.566 & Yes & 0.190 & 1.79E-08 & 0. \\
\hline Time & 1.514 & 0.389 & 3.152 & Yes & 1.520 & 0.397 & 3. \\
\hline DIC & & 473 & & & & & \\
\hline
\end{tabular}

Source: Models Bm1G_cc2 (Grouped Age at First Offence) and Bm1G_cc3 (Grouped Age at First Conviction). Technical Annex: p135-136 and p139-140. 
Inclusion of these predictors alongside the Basic Model does not reduce the DIC relative to the Basic Model despite the inclusion of an additional predictor (Models 1.5 and 1.6). Assuming all other things were equal, at Time 0 :

- The odds of further offending amongst those aged 10-12 years at the time of their first offence are estimated to be $\exp (-0.147)=0.86$ times the odds of who are older when they commit their first offence i.e. aged $13-17$ years. $[\mathrm{Cl}=0.54,1.44]$

- The odds of further offending amongst those aged 10-13 at the time of their first conviction are estimated to be $\exp (-0.323)=0.72$ times the odds of those who are older i.e. aged $14-17$ years when this occurs. $[\mathrm{Cl}=0.39,1.38]$

In each instance, the credible interval straddles one, suggesting that potentially there may be no difference between the odds of further offending amongst the two respective groups. This is not what would be expected given the theoretical underpinnings of the scoring of the static factors.

\section{FTE Status}

Whether or not the young person was a first-time entrant (FTE) at the time of entering the cohort has been determined from a combination of their offending and court records in Childview. Figure 6.2 segments the cohort on the basis of their FTE status, highlighting where there are differences in the mean domain scores at Time 0 .

Figure 6.2: Domain Score Profiles, by FTE Status, at Time 0

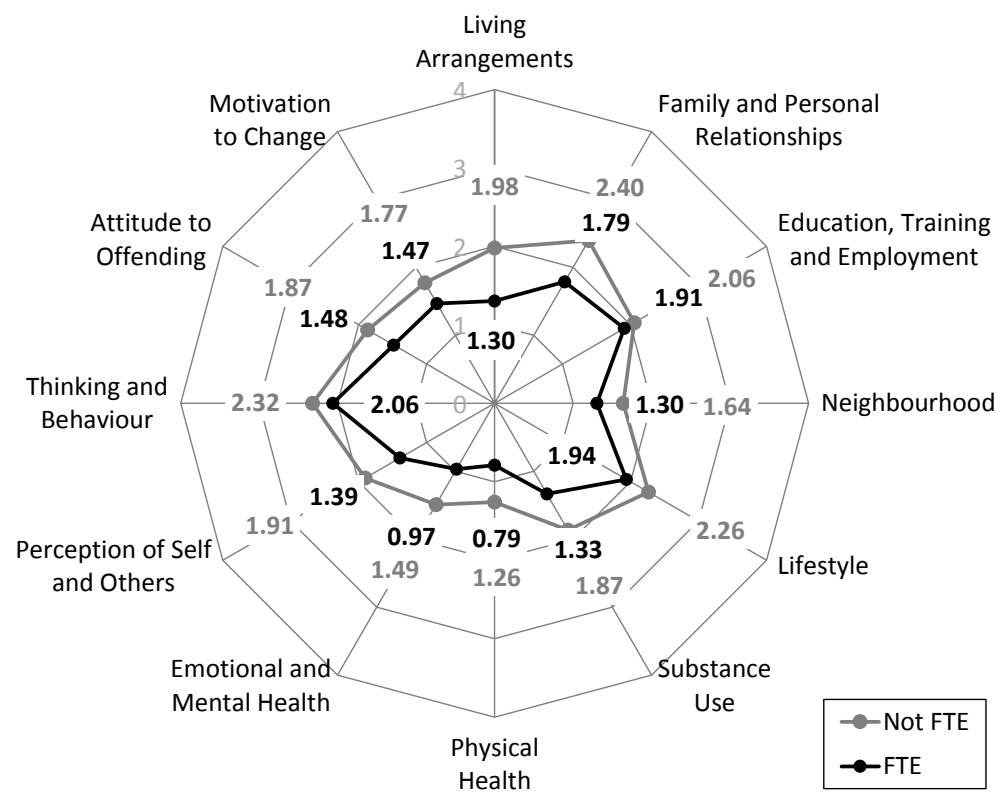

Notes: Of the original 88 individuals, 33 are FTEs, there is also one individual whose status is unknown and hence has been excluded from these figures. 
FTEs generallyappear to have lower initial ratings for each of the 12 domains compared to their peers with history of previous offending. T-tests suggest that there is moderate evidence to support this difference in relation to:

- Living Arrangements $\left(\mathrm{BF}_{10}=12.930\right.$ in favour of $\mathrm{H}_{1:} \mathrm{FTE}<$ Prior, $\%$ error $\left.=9.308 \mathrm{e}^{-5}\right)$

- Familyand personal relationships $\left(\mathrm{BF}_{10}=7.135, \%\right.$ error $\left.=1.775 \mathrm{e}^{-4}\right)$

- Perception of Self and Others $\left(\mathrm{BF}_{10}=6.884, \%\right.$ error $\left.=0.001\right)$

There is moderate evidence to suggest that there is no difference in the average initial domain scores for the two groups with respect to $E T E\left(B F_{10}=0.263\right.$ in favour of $\mathrm{H}_{0}: \mathrm{FTE}=$ Prior, $\%$ error $\left.=0.024\right)$.

A dummyvariable for FTE Status (referenced by FTE) has been added to the Basic Model (Model 1.7, Table 6.5). The inclusion did not result in a marked reduction in the DIC.

Table 6.5: Random Intercepts and Varying Slope Models for Further Offending including ASSET Domains and FTE Status

\begin{tabular}{|l|r|r|r|r|}
\hline \multirow{2}{*}{} & \multicolumn{3}{|c|}{ Model 1.7: Basic Model + } \\
\multicolumn{1}{|c|}{ FTE Status } \\
\cline { 2 - 5 } & \multicolumn{3}{|c|}{ Unstandardised } & \multirow{2}{*}{ Significant? } \\
\hline Fixed Effect: & Post.Mean & Lower Cl & Upper Cl & \\
\hline Intercept & -1.212 & -2.494 & 0.058 & \\
\hline First Time Entant (No = Ref). & 0.083 & -0.377 & 0.541 & \\
\hline Living Arrangements & 0.036 & -0.221 & 0.297 & \\
\hline Family and Personal Relationships & 0.278 & -0.016 & 0.570 & \\
\hline Education, Training and Employment & 0.089 & -0.159 & 0.333 & \\
\hline Neighbourhood & 0.038 & -0.184 & 0.252 & \\
\hline Lifestyle & 0.031 & -0.308 & 0.380 & \\
\hline Substance Use & 0.159 & -0.081 & 0.401 & \\
\hline Physical Health & -0.107 & -0.395 & 0.177 & \\
\hline Emotional and Mental Health & -0.004 & -0.249 & 0.279 & \\
\hline Perceptions of Self and Others & -0.132 & -0.447 & 0.177 & \\
\hline Thinking and Behaviour & -0.155 & -0.485 & 0.174 & \\
\hline Attitude to Offending & 0.038 & -0.316 & 0.379 & \\
\hline Motivation to Change & 0.232 & -0.102 & 0.577 & \\
\hline Time & -0.155 & -0.294 & -0.022 & Yes \\
\hline Random Effect: & Post.Mean & Lower Cl & Upper Cl & Significant? \\
\hline Individual (Intercept) & 0.113 & $1.66 \mathrm{E}-04$ & 0.403 & Yes \\
\hline Time & & 0.357 & 0.267 & Yes \\
\hline
\end{tabular}

Source: Models Bm1_cc1 (FTE Status). Technical Annex: p139-140

From the model it is estimated that at Time 0 , with all other things being equal, the odds of further offending amongst FTEs are $\exp (0.083)=1.09$ times the odds for those with previous offending 
committing further offence. However, the credible interval of 0.69 to 1.72 suggest that it is plausible that the odds for further offending could be higher amongstnon-FTEs which is what would be expected.

\section{Offence Category}

One of the original objectives for this piece of research was to explore the role of risk and protective factors for young people who have committed different types of offences. However, given the sample size, the reliability of using this as a measure needs to considered. Notably, as there is a desire to generate a model which considers offence category alongside other predictors, it has therefore been necessary to group the 13 YJB offence categories in order to reduce the amount of uncertainty around the simulated estimates. This is broadly in keeping with the static factor around offence type under the Scaled Approach (see Table 6.1). However, robbery offences have been grouped alongside domestic and non-domestic burglaries and theft of motor vehicles to form a 'Serious Acquisitive Crime' category. A breakdown of the cohortby offence categoryis provided in Table 6.6.

Table 6.6: The Re-Offending Cohort, by YJBOffence Category of Their Primary Offence

\begin{tabular}{|l|l|r|}
\hline Type of Offence & YJB Offence Category & No. \\
\hline \multirow{5}{*}{ Other } & Criminal Damage & 12 \\
\cline { 2 - 3 } & Drugs & 8 \\
\cline { 2 - 3 } & Motoring Offences & 4 \\
\cline { 2 - 3 } & Other & 1 \\
\cline { 2 - 3 } & Public Order & 11 \\
\cline { 2 - 3 } & Racially Aggravated & 1 \\
\cline { 2 - 3 } & Sexual Offences & 1 \\
\cline { 2 - 3 } & Theft And Handling Stolen Goods & 10 \\
\cline { 2 - 3 } Crimes (SAC) & Other Total & $\mathbf{4 8}$ \\
\hline \multirow{5}{*}{$\begin{array}{l}\text { Serious Acquisitive } \\
\text { Domestic Burglary }\end{array}$} \\
\cline { 2 - 3 } & Non Domestic Burglary & 5 \\
\cline { 2 - 3 } & Robbery & $\mathbf{1 8}$ \\
\cline { 2 - 3 } & Vehicle Theft Unauthorised Taking & $\mathbf{2 1}$ \\
\cline { 2 - 3 } & Serious Acquisitive Crime Total & $\mathbf{8 7}$ \\
\hline
\end{tabular}

Notes: The individual whose FTE status is not known has been excluded from this summary

Grouping the YJB Offence Categories in this way means that $25.0 \%$ of the cohort entered having committed a violence against the person offence; one in five $(20.5 \%)$ had committed a serious acquisitive crime whilst the remainder had committed offences which fell under 'Other'. Figure 6.3 compares the average initial domain scoresfor each sub-group. 
Figure 6.3: Domain Score Profiles, by Grouped YJB Offence Category at Time 0

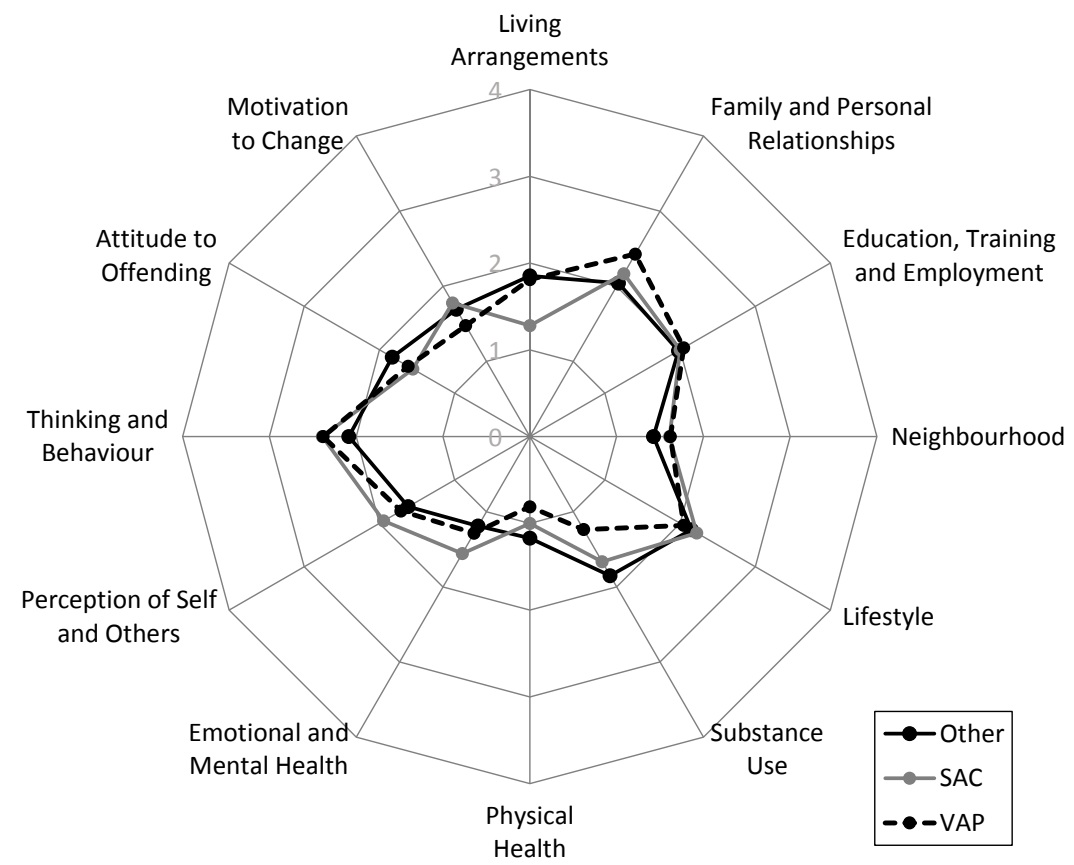

Notes: of the 87 individuals, 48 had committed an 'Other' offence; 18 had committed a serious acquisitive crime (SAC) whilst the remaining 21 had committed a violence against the person offence (VAP). Time 0 represents the initial assessment undertaken.

One-sided Bayesian independentt-tests suggests that those who have committed 'Other' offences have higher ratings than those who have committed VAP offences at the time of their initial assessmentfor:

- Substance misuse $\left(B F_{10}=2.444\right.$ in favour of $\mathrm{H}_{1}, \%$ error $\left.=\sim 0.008\right)$

- Physical health $\left(\mathrm{BF}_{10}=1.226, \%\right.$ error $\left.=\sim 0.001\right)$

There are also differences in the ratings for the living arrangements domain with these being higher for those committing other and violence against the person offences than for those who had committed a SAC offence:

- 'Other' offenders have higher ratings for the living arrangements domain than those committing $\mathrm{SAC}$ offences $\left(\mathrm{BF}_{10}=1.688\right.$ in favour of $\mathrm{H}_{1}, \%$ error $\left.=\sim 9.055 \mathrm{e}^{-4}\right)$

- SAC offenders have lower initial ratings than those committing VAP offences $\left(B_{10}=3.286\right.$ in favour of $\mathrm{H}_{1}, \%$ error $=\sim 5.624 \mathrm{e}^{-4}$ )

- There is moderate evidence to suggest that the ratings for Other offenders are not greater than those for VAP offenders $\left(\mathrm{BF}_{10}=0.285\right.$ in favour of $\mathrm{H}_{0}, \%$ error $\left.=\sim 0.003\right)$

There is no other evidence to support differences in the average domain scores between offenders committing the different types of offences at Time 0 . 
Table 6.7 compares the impact of incorporating both the Grouped and Ungrouped YJB Offence Category into the Basic Model. In Model 1.8 (Bm1_01), the reference category is criminal damage whereas in Model 1.9 (Bm1G_01), the categories are grouped with the reference category being 'Other Offence'. From Table 6.6, it is apparent that there are a number of YJB Offence Categories where there is only one case contributing information to the data e.g. the estimates for raciallyaggravated, sexual and other offences. There are also only two young people who entered the reoffending cohorthaving committed non-domestic burglaries. For this reason, it is felt that a potentially more accurate picture can be obtained by aggregating the YJB Offence Category as in Model 1.9.

Notably where the ungrouped predictor is used (Model 1.8), the substance misuse domain is significant, with the estimate suggesting that as the rating for this domain increases, it increases the probabilityof further offending. The certaintysurrounding the estimate for this domain is lost when the YJB Offence Category is grouped.

Model 1.9 suggest that all other things being equal, the odds of further offending amongst those who committed a serious acquisitive crime are $\exp (0.162)=1.8$ times the odds of those who committed an 'Other Offence' $[\mathrm{Cl}=0.70,2.17]$. Those who committed a violence against the person offence have odds which are $\exp (0.234)=1.3$ times higher $[\mathrm{Cl}=0.70,2.25]$. 
Table 6.7: The Basic Model plus (a) YJB Offence Category and (b) Grouped YJB Offence Category

\begin{tabular}{|c|c|c|c|c|c|c|c|c|c|}
\hline \multirow[b]{3}{*}{ Fixed Effect: } & \multicolumn{7}{|c|}{ Model 1.8: Basic Model + YJB Offence Category } & & \\
\hline & \multicolumn{3}{|c|}{ Unstandardised } & \multicolumn{3}{|c|}{ Standardised } & \multirow{2}{*}{ Significant? } & & \\
\hline & Post.Mean & Lower $\mathrm{Cl}$ & Upper $\mathrm{Cl}$ & Post.Mean & Lower $\mathrm{Cl}$ & Upper $\mathrm{Cl}$ & & Fixed Effect: & Post.Mean \\
\hline (Intercept) & -0.783 & -2.313 & 0.704 & 0.457 & 0.099 & 2.023 & & (Intercept) & -1.297 \\
\hline \multicolumn{8}{|c|}{ YJB Offence Category (Ref = Criminal Damage $)$} & \multirow{8}{*}{\multicolumn{2}{|c|}{$\begin{array}{l}\text { Grouped YJB Offence Category } \\
\text { (Ref }=\text { Other })\end{array}$}} \\
\hline Drugs & -0.982 & -2.063 & 0.123 & 0.374 & 0.127 & 1.131 & & & \\
\hline Motoring Offences & -0.307 & -1.690 & 0.991 & 0.736 & 0.185 & 2.693 & & & \\
\hline Other & -0.744 & -4.289 & 2.689 & 0.475 & 0.014 & 14.720 & & & \\
\hline Public Order & -0.870 & -1.909 & 0.272 & 0.419 & 0.148 & 1.313 & & & \\
\hline Racially Aggravated & -1.457 & -5.018 & 2.069 & 0.233 & 0.007 & 7.919 & & & \\
\hline Sexual Offences & -1.981 & -4.987 & 0.947 & 0.138 & 0.007 & 2.577 & & & \\
\hline Theft And Handling Stolen Goods & -0.407 & -1.466 & 0.623 & 0.666 & 0.231 & 1.865 & & & \\
\hline Domestic Burglary & -0.095 & -1.183 & 1.088 & 0.909 & 0.306 & 2.967 & & \multirow{4}{*}{ Serious Acquisitive Crime } & \multirow{4}{*}{0.162} \\
\hline Non Domestic Burglary & 0.044 & -1.638 & 1.747 & 1.045 & 0.194 & 5.739 & & & \\
\hline Robbery & -0.960 & -2.158 & 0.238 & 0.383 & 0.116 & 1.269 & & & \\
\hline Vehicle Theft / Unauthorised Taking & -0.216 & -1.144 & 0.742 & 0.806 & 0.319 & 2.100 & & & \\
\hline Violence Against The Person & -0.221 & -1.111 & 0.568 & 0.802 & 0.329 & 1.765 & & Violence Against The Person & 0.234 \\
\hline Living Arrangements & 0.097 & -0.176 & 0.402 & 1.102 & 0.839 & 1.495 & & Living Arrangements & 0.052 \\
\hline Family and Personal Relationships & 0.298 & -0.020 & 0.620 & 1.347 & 0.980 & 1.859 & & Family and Personal Relationships & 0.270 \\
\hline Education, Training and Employment & 0.046 & -0.217 & 0.316 & 1.047 & 0.805 & 1.372 & & Education, Training and Employment & 0.081 \\
\hline Neighbourhood & 0.050 & -0.201 & 0.295 & 1.052 & 0.818 & 1.344 & & Neighbourhood & 0.050 \\
\hline Lifestyle & -0.007 & -0.386 & 0.376 & 0.993 & 0.680 & 1.457 & & Lifestyle & 0.036 \\
\hline Substance Use & 0.310 & 0.040 & 0.594 & 1.363 & 1.040 & 1.811 & Yes & Substance Use & 0.183 \\
\hline Physical Health & -0.181 & -0.500 & 0.152 & 0.834 & 0.606 & 1.164 & & Physical Health & -0.116 \\
\hline Emotional and Mental Health & -0.020 & -0.269 & 0.267 & 0.980 & 0.764 & 1.306 & & Emotional and Mental Health & 0.015 \\
\hline Perceptions of Self and Others & -0.114 & -0.459 & 0.226 & 0.892 & 0.632 & 1.254 & & Perceptions of Self and Others & -0.158 \\
\hline Thinking and Behaviour & -0.215 & -0.571 & 0.177 & 0.806 & 0.565 & 1.193 & & Thinking and Behaviour & -0.168 \\
\hline Attitude to Offending & 0.038 & -0.352 & 0.413 & 1.039 & 0.703 & 1.512 & & Attitude to Offending & 0.039 \\
\hline Motivation to Change & 0.220 & -0.158 & 0.570 & 1.246 & 0.854 & 1.769 & & Motivation to Change & 0.234 \\
\hline Time & -0.183 & -0.343 & -0.042 & 0.833 & 0.710 & 0.959 & \begin{tabular}{|c|} 
Yes \\
\end{tabular} & \begin{tabular}{|l|} 
Time \\
\end{tabular} & -0.165 \\
\hline Random Effect: & Post.Mean & Lower $\mathrm{Cl}$ & Upper $\mathrm{Cl}$ & Post.Mean & Lower $\mathrm{Cl}$ & Upper $\mathrm{Cl}$ & Significant? & Random Effect: & PostMean \\
\hline Individual (Intercept) & 0.275 & 2.94E-07 & 0.761 & 1.316 & 1.000 & 2.140 & Yes & Individual (Intercept) & 0.220 \\
\hline Time & 1.660 & 0.439 & 3.541 & 5.259 & 1.551 & 34.501 & Yes & Time & 1.540 \\
\hline DIC & & & 474.54 & & & & & DIC & \\
\hline
\end{tabular}

Source: Models Bm1_01 and Bm1G_01, Technical Annex: p147-152 


\subsection{Developing Model 3}

Since the four predictors are proxies for the static factors in ASSET, there is a theoretical rationale for includingall four in the model for predicting the likelihood of further offending based on offending history.

Table 6.8: Model 3: The Basic Model plus Static Factors

\begin{tabular}{|c|c|c|c|c|c|c|c|}
\hline \multirow{3}{*}{$\begin{array}{l}\text { Fixed Effect: } \\
\end{array}$} & \multicolumn{7}{|c|}{ Model 3: Basic Model + Static Factors } \\
\hline & \multicolumn{3}{|c|}{ Unstandardised } & \multicolumn{3}{|c|}{ Standardised } & \multirow{2}{*}{ Significant? } \\
\hline & Post.Mean & Lower $\mathrm{Cl}$ & Upper Cl & PostMean & Lower $\mathrm{Cl}$ & Upper $\mathrm{Cl}$ & \\
\hline Intercept & -1.493 & -3.529 & 0.300 & 0.225 & 0.029 & 1.350 & \\
\hline First Time Entant $\left(\mathrm{No}_{\mathrm{O}}=\right.$ Ref $)$. & 0.059 & -3.748 & 4.159 & 1.060 & 0.024 & 64.035 & \\
\hline Grouped Age at First Offence (Ref $=13-17$ years) & 1.613 & -0.405 & 3.776 & 5.019 & 0.667 & 43.639 & \\
\hline Grouped Age at First Conviction (Ref $=14-17$ years) & 0.405 & -0.940 & 1.850 & 1.499 & 0.391 & 6.357 & \\
\hline \multicolumn{8}{|l|}{ Grouped YJB Offence Category (Ref = Other) } \\
\hline Serious Aquisitive Crime (SAC) & 0.084 & -4.312 & 4.245 & 1.087 & 0.013 & 69.761 & \\
\hline Violence Against the Person (VAP) & 0.701 & -1.250 & 2.501 & 2.017 & 0.287 & 12.190 & \\
\hline Living Arrangements & 0.047 & -0.245 & 0.337 & 1.048 & 0.782 & 1.401 & \\
\hline Family and Personal Relationships & 0.312 & -0.038 & 0.637 & 1.366 & 0.963 & 1.891 & \\
\hline Education, Training and Employment & 0.058 & -0.223 & 0.324 & 1.060 & 0.800 & 1.382 & \\
\hline Neighbourhood & 0.035 & -0.221 & 0.277 & 1.035 & 0.802 & 1.320 & \\
\hline Lifestyle & -0.050 & -0.421 & 0.351 & 0.951 & 0.656 & 1.421 & \\
\hline Substance Use & 0.266 & -0.017 & 0.540 & 1.305 & 0.983 & 1.716 & \\
\hline Physical Health & -0.141 & -0.486 & 0.203 & 0.869 & 0.615 & 1.225 & \\
\hline Emotional and Mental Health & 0.061 & -0.212 & 0.332 & 1.063 & 0.809 & 1.394 & \\
\hline Perceptions of Self and Others & -0.201 & -0.581 & 0.171 & 0.818 & 0.559 & 1.187 & \\
\hline Thinking and Behaviour & -0.154 & -0.529 & 0.202 & 0.857 & 0.589 & 1.224 & \\
\hline Attitude to Offending & 0.015 & -0.366 & 0.426 & 1.015 & 0.693 & 1.531 & \\
\hline Motivation to Change & 0.248 & -0.134 & 0.605 & 1.282 & 0.874 & 1.831 & \\
\hline Time & -0.198 & -0.347 & -0.038 & 0.821 & 0.707 & 0.963 & Yes \\
\hline FTE: G_AgeFirst & -1.411 & -7.863 & 5.147 & 0.244 & 0.000 & 171.846 & \\
\hline FTE: G_AgeCon & -0.310 & -7.286 & 5.715 & 0.733 & 0.001 & 303.311 & \\
\hline FTE: SAC & 1.131 & -0.413 & 2.754 & 3.100 & 0.662 & 15.713 & \\
\hline FTE: VAP & -0.902 & -6.732 & 4.788 & 0.406 & 0.001 & 120.039 & \\
\hline G_AgeFirst G_AgeCon & -1.705 & -4.131 & 0.447 & 0.182 & 0.016 & 1.564 & \\
\hline G_AgeFirst SAC & -1.483 & -3.074 & 0.071 & 0.227 & 0.046 & 1.073 & \\
\hline G_AgeFirst VAP & 0.394 & -3.461 & 4.479 & 1.483 & 0.031 & 88.159 & \\
\hline G_AgeCon: SAC & 0.819 & -3.527 & 5.025 & 2.268 & 0.029 & 152.201 & \\
\hline G_AgeCon: VAP & -0.905 & -5.341 & 3.252 & 0.404 & 0.005 & 25.842 & \\
\hline FTE: G_AgeFirst: G_AgeCon & 1.518 & -4.181 & 7.408 & 4.563 & 0.015 & 1649.488 & \\
\hline FTE: G_AgeFirst: VAP & 0.849 & -5.209 & 6.817 & 2.337 & 0.005 & 913.141 & \\
\hline Random Effect: & \begin{tabular}{|l} 
Post.Mean \\
\end{tabular} & Lower $\mathrm{Cl}$ & Upper Cl & PostMean & Lower $\mathrm{Cl}$ & Upper $\mathrm{Cl}$ & Significant? \\
\hline Individual (Intercept) & 0.330 & $1.86 \mathrm{E}-08$ & 0.902 & 1.390 & 1.00 & 2.464 & Yes \\
\hline Time & 1.767 & 0.388 & 3.699 & 5.853 & 1.475 & 40.407 & Yes \\
\hline DIC & & & 473.50 & & & & \\
\hline
\end{tabular}

Source: Model Bm1G_cc12301 (Model 3), Technical Annex: p155-156. 
Although the model was specified to include all the potential 2-, 3- and 4-way combinations of the four predictors, it is notable that estimates have not been simulated for the following interactions:

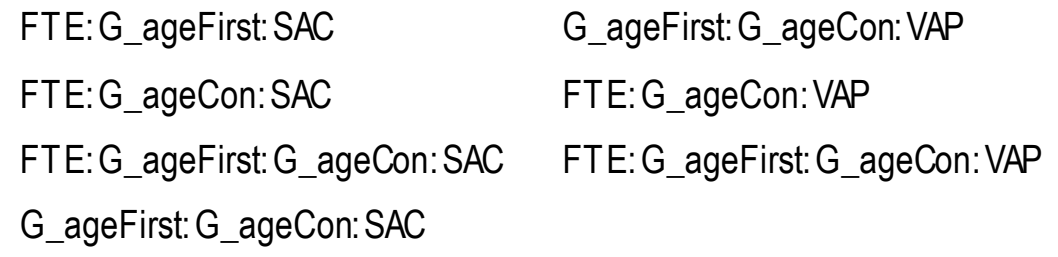

When compared to the underlying data (Table 6.2), only one of those who had committed a serious acquisitive crime had been aged 10-13 at the time of their first conviction. This young person had been an FTE at the time of joining the reoffending cohort. Whilst there were three young people who had committed serious acquisitive offences who had been aged 10-12 at the time of their first offence, these all had a history of prior offending at the time of entering the cohort. In terms of those who had committed violence against the person offences, there were none who had been FTEs aged 13-17 at the time of their first offence and aged 10-13 at the time of their first conviction. Hence the model being rank deficient with respect to interactions involving both $G$ _ageCon and VAP and another predictor.

Table 6.8 additionally highlights the impact of the low numbers for different permutations of the predictors, with wide credible intervals for the estimates for the 2-and 3-way interactions which could be simulated. With insufficient data to supporta model of this complexity, two options present themselves. The firstis to remove the predictors for grouped age atfirst conviction since there are only 11 in the nonreference category. The second is to explore the whether it is appropriate to replace the YJB Offence Category with the predictor for YJB Gravity Score. In the case of the latter, this is still in keeping with the notion that those that have committed more serious offences pose a greater risk. 


\section{Removing Grouped Age of Conviction}

Table 6.9 summarises the characteristics of the 11 individuals who were convicted of their first offence before the age of 14 i.e. those in the non-reference group of $G$ _ageCon. As can be seen four of these were first time entrants at the time of entering the reoffending cohort. Of these, two were aged 13 at the time of their first offence which means that due to the different thresholds for the two age-related predictors employed within ASSET, they were in the older age group for G_ageFirst.

Table 6.9: Characteristics of those Convicted of Their First Offence Before Age 14

\begin{tabular}{|c|c|c|c|c|c|c|}
\hline ID & FTE? & AgeFirst & AgeCon & YJB Offence Category & $\begin{array}{c}\text { Grouped YJB } \\
\text { Offence Category }\end{array}$ & $\begin{array}{c}\text { YJB Gravity } \\
\text { Score }\end{array}$ \\
\hline 1 & No & 10 & 10 & Drugs & Other & 2 \\
\hline 2 & No & 11 & 12 & Drugs & Other & 2 \\
\hline 3 & Yes & 12 & 12 & Theft \& Handling Stolen Goods & Other & 3 \\
\hline 4 & No & 10 & 12 & Violence Against the Person & VAP & 3 \\
\hline 5 & No & 12 & 13 & Public Order & Other & 2 \\
\hline 6 & No & 12 & 13 & Violence Against the Person & VAP & 3 \\
\hline 7 & Yes & 12 & 13 & Violence Against the Person & VAP & 3 \\
\hline 8 & Yes & 13 & 13 & Violence Against the Person & VAP & 3 \\
\hline 9 & Yes & 13 & 13 & Violence Against the Person & VAP & 4 \\
\hline 10 & No & 13 & 13 & Criminal Damage & Other & 3 \\
\hline 11 & No & 13 & 13 & Non Domestic Burglary & SAC & 4 \\
\hline
\end{tabular}

Just one of the younger group had committed a serious ac quisitive crime. However, there was another boy who had committed a violence against the person offence with a gravity score of 4 . In total, there were five who committed violence against the person offences. The remainder committed a variety of Other Offences. Table 6.10 summarises the underlying data with respect to the FTE Status, Grouped Age at First Offence and Grouped YJB Offence category. Notably there are no examples of where a young person with a previous offending history, having committed their first offence aged 10 to 13 years, had committed a serious acquisitive crime. The resulting model (Table 6.11) is therefore unable to simulate an estimate for the interaction between FTE: G_ageFirst: SAC. There is also a lot of uncertainty around some of the estimates where they are based on a low number of cases.

Table 6.10: The Reoffending Cohort by FTE Status, Grouped Age at First Offence and Grouped YJB Offence Category

\begin{tabular}{|c|c|c|c|c|c|}
\hline \multirow{2}{*}{$\begin{array}{l}\text { FTE } \\
\text { Status }\end{array}$} & \multirow{2}{*}{$\begin{array}{c}\text { Grouped Age at } \\
\text { First Offence }\end{array}$} & \multicolumn{3}{|c|}{ Grouped YJB Offence Category } & \multirow{2}{*}{ Total } \\
\hline & & Other & SAC & VAP & \\
\hline \multirow{2}{*}{ FTE } & 10 to 13 years & 13 & 3 & 3 & 19 \\
\hline & 14 to 17 years & 17 & 8 & 10 & 35 \\
\hline \multicolumn{2}{|l|}{ FTE Total } & 30 & 11 & 13 & 54 \\
\hline \multirow{2}{*}{ Not FTE } & 10 to 13 years & 1 & & 2 & 3 \\
\hline & 14 to 17 years & 17 & 7 & 6 & 30 \\
\hline \multicolumn{2}{|l|}{ Not FTE Total } & 18 & 7 & 8 & 33 \\
\hline \multicolumn{2}{|l|}{ Grand Total } & 48 & 18 & 21 & 87 \\
\hline
\end{tabular}

Notes: The individual whose FTE status is not known has been excluded from this summary.

Table 6.11 summarises a version of Model 3 , with Grouped Age of First Conviction excluded. 
Table 6.11: Model 3a: The Basic Model plus FTE Status, Grouped Age at First Offence and Grouped YJB Offence Category

\begin{tabular}{|c|c|c|c|c|c|c|c|}
\hline \multirow[b]{3}{*}{ Fixed Effect: } & \multicolumn{7}{|c|}{$\begin{array}{l}\text { Model 3a: Basic Model + FTE Status, Grouped Age at First Offence } \\
\text { and Grouped YJB Offence Category }\end{array}$} \\
\hline & \multicolumn{3}{|c|}{ Unstandardised } & \multicolumn{3}{|c|}{ Standardised } & \multirow{2}{*}{ Significant } \\
\hline & Post.Mean & Lower $\mathrm{Cl}$ & Upper $\mathrm{Cl}$ & Post.Mean & Lower $\mathrm{Cl}$ & Upper $\mathrm{Cl}$ & \\
\hline Intercept & -1.230 & -2.729 & 0.214 & 0.292 & 0.065 & 1.239 & \\
\hline First Time Entant (No = Ref). & -0.224 & -4.074 & 3.490 & 0.800 & 0.017 & 32.787 & \\
\hline Grouped Age at First Offence (Ref $=13-17$ years) & 0.159 & -0.672 & 0.975 & 1.173 & 0.511 & 2.651 & \\
\hline \multicolumn{8}{|l|}{ Grouped YJB Offence Category (Ref = Other) } \\
\hline Serious Aquisitive Crime (SAC) & 0.970 & -0.062 & 2.018 & 2.638 & 0.940 & 7.520 & \\
\hline Violence Against the Person (VAP) & 0.383 & -0.951 & 1.780 & 1.467 & 0.386 & 5.932 & \\
\hline Living Arrangements & 0.050 & -0.218 & 0.337 & 1.051 & 0.804 & 1.401 & \\
\hline Family and Personal Relationships & 0.300 & -0.041 & 0.618 & 1.350 & 0.960 & 1.855 & \\
\hline Education, Training and Employment & 0.036 & -0.232 & 0.296 & 1.037 & 0.793 & 1.344 & \\
\hline Neighbourhood & 0.042 & -0.206 & 0.283 & 1.043 & 0.814 & 1.327 & \\
\hline Lifestyle & -0.008 & -0.383 & 0.372 & 0.992 & 0.682 & 1.451 & \\
\hline Substance Use & 0.226 & -0.036 & 0.510 & 1.254 & 0.965 & 1.665 & \\
\hline Physical Health & -0.097 & -0.430 & 0.217 & 0.907 & 0.650 & 1.243 & \\
\hline Emotional and Mental Health & 0.070 & -0.182 & 0.342 & 1.072 & 0.833 & 1.408 & \\
\hline Perceptions of Self and Others & -0.198 & -0.563 & 0.128 & 0.821 & 0.569 & 1.137 & \\
\hline Thinking and Behaviour & -0.157 & -0.509 & 0.194 & 0.854 & 0.601 & 1.214 & \\
\hline Attitude to Offending & 0.002 & -0.401 & 0.364 & 1.002 & 0.669 & 1.439 & \\
\hline Motivation to Change & 0.251 & -0.103 & 0.593 & 1.286 & 0.902 & 1.809 & \\
\hline Time & -0.186 & -0.343 & -0.045 & 0.830 & 0.709 & 0.956 & Yes \\
\hline FTE: Grouped AgeFirst & -0.044 & -4.176 & 3.610 & 0.957 & 0.015 & 36.972 & \\
\hline FTE: SAC & 1.210 & -0.272 & 2.622 & 3.355 & 0.762 & 13.761 & \\
\hline FTE: VAP & -0.948 & -5.671 & 3.752 & 0.388 & 0.003 & 42.595 & \\
\hline Grouped Age First SAC & -1.668 & -3.165 & -0.264 & 0.189 & 0.042 & 0.768 & Yes \\
\hline Grouped Age First VAP & -0.369 & -1.987 & 1.360 & 0.692 & 0.137 & 3.897 & \\
\hline FTE: Grouped AgeFirst: VAP & 1.454 & -3.566 & 6.248 & 4.282 & 0.028 & 517.235 & \\
\hline Random Effect: & Post.Mean & Lower $\mathrm{Cl}$ & Upper $\mathrm{Cl}$ & Post.Mean & Lower $\mathrm{Cl}$ & Upper $\mathrm{Cl}$ & Significant? \\
\hline Individual (Intercept) & 0.267 & $3.30 \mathrm{E}-08$ & 0.725 & 1.306 & 1.000 & 2.065 & Yes \\
\hline Time & 1.658 & 0.421 & 3.306 & 5.249 & 1.523 & 27.276 & Yes \\
\hline DIC & & & 473.13 & & & & \\
\hline
\end{tabular}

Source: Model Bm1G_cc1201 (Model 3a), Technical Annex: p160-161. 
The YJB Gravity Score can be used to reflect the seriousness of the young person's primary offence. Table 3.14 summarised the profile of members of the re-offending cohort, showing that none of the reoffending cohort had committed an offence with a gravity score of 1 , or with a gravity score of 7 or 8 . Including this predictor, centred on 2 as an initial value, alongside the Basic Model results in a model with a DIC of 473.7 (T able 6.12).

Table 6.12: The Basic Model plus YJB Gravity Score

\begin{tabular}{|c|c|c|c|c|c|c|c|}
\hline \multirow[b]{3}{*}{ Fixed Effect: } & \multicolumn{7}{|c|}{ Model 1.10: Basic Model + YJB Gravity Score } \\
\hline & \multicolumn{3}{|c|}{ Unstandardised } & \multicolumn{3}{|c|}{ Standardised } & \multirow{2}{*}{ Significant? } \\
\hline & Post.Mean & Lower $\mathrm{Cl}$ & Upper $\mathrm{Cl}$ & Post.Mean & Lower $\mathrm{Cl}$ & Upper Cl & \\
\hline (Intercept) & -1.195 & -2.565 & 0.303 & 0.303 & 0.077 & 1.354 & \\
\hline YJB Gravity Score $(\operatorname{Ref}=2)$ & 0.001 & -0.161 & 0.163 & 1.001 & 0.852 & 1.177 & \\
\hline Living Arrangements & 0.042 & -0.213 & 0.319 & 1.043 & 0.808 & 1.375 & \\
\hline Family and Personal Relationships & 0.277 & -0.007 & 0.596 & 1.319 & 0.993 & 1.815 & \\
\hline Education, Training and Employment & 0.074 & -0.175 & 0.332 & 1.077 & 0.840 & 1.393 & \\
\hline Neighbourhood & 0.047 & -0.196 & 0.257 & 1.048 & 0.822 & 1.293 & \\
\hline Lifestyle & 0.040 & -0.317 & 0.404 & 1.041 & 0.728 & 1.498 & \\
\hline Substance Use & 0.172 & -0.081 & 0.408 & 1.188 & 0.922 & 1.504 & \\
\hline Physical Health & -0.132 & -0.432 & 0.163 & 0.876 & 0.649 & 1.178 & \\
\hline Emotional and Mental Health & 0.004 & -0.248 & 0.251 & 1.004 & 0.780 & 1.285 & \\
\hline Perceptions of Self and Others & -0.137 & -0.446 & 0.195 & 0.872 & 0.640 & 1.216 & \\
\hline Thinking and Behaviour & -0.157 & -0.508 & 0.158 & 0.854 & 0.601 & 1.171 & \\
\hline Attitude to Offending & 0.034 & -0.328 & 0.386 & 1.035 & 0.721 & 1.471 & \\
\hline Motivation to Change & 0.231 & -0.113 & 0.586 & 1.260 & 0.893 & 1.796 & \\
\hline Time & -0.161 & -0.307 & -0.023 & 0.851 & 0.736 & 0.977 & Yes \\
\hline Random Effect: & Post.Mean & Lower $\mathrm{Cl}$ & Upper $\mathrm{Cl}$ & Post.Mean & Lower $\mathrm{Cl}$ & Upper $\mathrm{Cl}$ & Significant? \\
\hline Individual (Intercept) & 0.211 & $2.84 \mathrm{E}-11$ & 0.573 & 1.235 & 1.000 & 1.773 & Yes \\
\hline Time & 1.503 & 0.418 & 3.106 & 4.495 & 1.520 & 22.332 & Yes \\
\hline
\end{tabular}

\begin{tabular}{|l|l|}
\hline DIC & 473.69 \\
\hline
\end{tabular}

Source: Model Bm1_02a, Technical Annex: p164-165.

Model 1.10 provides an estimate for the fixed effect of the seriousness of the primaryoffence. Assuming all other factors are equal, at $\mathrm{T}$ ime 0 , the odds of further offending increases by a multiplicative factor of $\exp (0.001)=1.001$ for each additional level of seriousness over a gravity score of $2[\mathrm{Cl}=0.85,1.18]$. As can be seen from Table 6.13, if the predictor for grouped age at first conviction is used to segment the cohortin addition to the other three predictors, there are a number of empty cells which would lead to the resulting model being rank deficient. Removing G_ageCon (Table 6.14) reduces the number of empty cells. 
Table 6.13: The Reoffending Cohort by FTE Status, Grouped Age at First Offence, Grouped Age at First Conviction and YJB Gravity Score

\begin{tabular}{|c|c|c|c|c|c|c|c|c|}
\hline \multirow{2}{*}{$\begin{array}{l}\text { FTE } \\
\text { Status }\end{array}$} & \multirow{2}{*}{$\begin{array}{l}\text { Grouped Age at } \\
\text { First Offence }\end{array}$} & \multirow{2}{*}{$\begin{array}{l}\text { Grouped Age at } \\
\text { First Conviction }\end{array}$} & \multicolumn{5}{|c|}{ YJB Gravity Score } & \multirow{2}{*}{ Total } \\
\hline & & & 2 & 3 & 4 & 5 & 6 & \\
\hline \multirow{4}{*}{ FTE } & \multirow{2}{*}{10 to 12 years } & 10 to 13 years & 3 & 2 & & & & 5 \\
\hline & & 14 to 17 years & 6 & 5 & 1 & 1 & 1 & 14 \\
\hline & \multirow{2}{*}{13 to 17 years } & 10 to 13 years & & 1 & 1 & & & 2 \\
\hline & & 14 to 17 years & 11 & 11 & 3 & 2 & 6 & 33 \\
\hline \multicolumn{3}{|l|}{ FTE Total } & 20 & 19 & 5 & 3 & 7 & 54 \\
\hline \multirow{4}{*}{ NotFTE } & \multirow{2}{*}{10 to 12 years } & 10 to 13 years & & 2 & & & & 2 \\
\hline & & 14 to 17 years & & 1 & & & & 1 \\
\hline & \multirow{2}{*}{13 to 17 years } & 10 to 13 years & & 1 & 1 & & & 2 \\
\hline & & 14 to 17 years & 11 & 7 & 3 & 1 & 6 & 28 \\
\hline \multicolumn{3}{|l|}{ NotFTE Total } & 11 & 11 & 4 & 1 & 6 & 33 \\
\hline \multicolumn{3}{|l|}{ Grand Total } & 31 & 30 & 9 & 4 & 13 & 87 \\
\hline
\end{tabular}

Notes: The individual whose FTE status is not known has been excluded from this summary.

Table 6.14: The Reoffending Cohort by FTE Status, Grouped Age at First Offence and YJB Gravity Score

\begin{tabular}{|c|c|c|c|c|c|c|c|}
\hline \multirow{2}{*}{$\begin{array}{l}\text { FTE } \\
\text { Status }\end{array}$} & \multirow{2}{*}{$\begin{array}{l}\text { Grouped Age at } \\
\text { First Offence }\end{array}$} & \multicolumn{5}{|c|}{ YJB Gravity Score } & \multirow{2}{*}{ Total } \\
\hline & & 2 & 3 & 4 & 5 & 6 & \\
\hline \multirow{2}{*}{ FTE } & 10 to 12 years & 9 & 7 & 1 & 1 & 1 & 19 \\
\hline & 13 to 17 years & 11 & 12 & 4 & 2 & 6 & 35 \\
\hline \multicolumn{2}{|l|}{ FTE Total } & 20 & 19 & 5 & 3 & 7 & 54 \\
\hline \multirow{2}{*}{ NotFTE } & 10 to 12 years & & 3 & & & & 3 \\
\hline & 13 to 17 years & 11 & 8 & 4 & 1 & 6 & 30 \\
\hline \multicolumn{2}{|l|}{ NotFTE Total } & 11 & 11 & 4 & 1 & 6 & 33 \\
\hline \multicolumn{2}{|l|}{ Grand Total } & 31 & 30 & 9 & 4 & 13 & 87 \\
\hline
\end{tabular}

Notes: The individual whose FTE status is not known has been excluded from this summary.

The three individuals with a prior offending history at the time of entering the re-offending cohort, who had been aged 10 to 12 years when they committed their first offence had all committed offences with a gravity score of 3 . As can be seen from Table 6.10, one of these had been an 'Other' offence whereas two had committed violence against the person offences. In the resulting model (Table 6.15), an estimate for the interaction between grouped age at first offence and gravity score has not been simulated reflecting the lack of cases. However, a 3-way interaction involving all three predictors has been estimated reflecting the fact that it has been possible to determine some information about the likelihood of further offending amongst the younger age group and how this is affected bythe seriousness of their primary offence. 
Table 6.15: Model 3b: The Basic Model plus FTE Status, Grouped Age at First Offence and YJB Gravity Score

\begin{tabular}{|c|c|c|c|c|c|c|c|}
\hline \multirow{3}{*}{ Fixed Effect: } & \multicolumn{7}{|c|}{$\begin{array}{l}\text { Model 3b: Basic Model + FTE Status, Grouped Age at First Offence } \\
\text { and YJB Gravity Score }\end{array}$} \\
\hline & \multicolumn{3}{|c|}{ Unstandardised } & \multicolumn{3}{|c|}{ Standardised } & \multirow{2}{*}{ Significant? } \\
\hline & Post.Mean & Lower $\mathrm{Cl}$ & Upper Cl & PostMean & Lower $\mathrm{Cl}$ & Upper Cl & \\
\hline Intercept & -1.381 & -2.831 & 0.141 & 0.251 & 0.059 & 1.152 & \\
\hline First Time Entant (No = Ref). & -0.998 & -3.230 & 1.031 & 0.369 & 0.040 & 2.804 & \\
\hline Grouped Age at First Offence (Ref $=13-17$ years) & 0.326 & -0.453 & 1.110 & 1.385 & 0.636 & 3.034 & \\
\hline YJB Gravity Score ( 0 = Gravity Score of 2 ) & 0.334 & 0.017 & 0.647 & 1.396 & 1.017 & 1.910 & Yes \\
\hline Living Arrangements & 0.037 & -0.262 & 0.288 & 1.037 & 0.769 & 1.334 & \\
\hline Family and Personal Relationships & 0.274 & -0.035 & 0.580 & 1.315 & 0.965 & 1.787 & \\
\hline Education, Training and Employment & 0.072 & -0.179 & 0.327 & 1.074 & 0.836 & 1.387 & \\
\hline Neighbourhood & 0.078 & -0.140 & 0.322 & 1.082 & 0.869 & 1.380 & \\
\hline Lifestyle & 0.028 & -0.322 & 0.403 & 1.029 & 0.725 & 1.497 & \\
\hline Substance Use & 0.164 & -0.097 & 0.431 & 1.178 & 0.907 & 1.539 & \\
\hline Physical Health & -0.126 & -0.432 & 0.187 & 0.882 & 0.650 & 1.205 & \\
\hline Emotional and Mental Health & 0.038 & -0.212 & 0.289 & 1.038 & 0.809 & 1.335 & \\
\hline Perceptions of Self and Others & -0.122 & -0.461 & 0.217 & 0.885 & 0.631 & 1.243 & \\
\hline Thinking and Behaviour & -0.142 & -0.497 & 0.197 & 0.868 & 0.609 & 1.217 & \\
\hline Attitude to Offending & -0.003 & -0.364 & 0.358 & 0.997 & 0.695 & 1.430 & \\
\hline Motivation to Change & 0.234 & -0.127 & 0.572 & 1.264 & 0.880 & 1.772 & \\
\hline Time & -0.176 & -0.322 & -0.025 & 0.838 & 0.725 & 0.975 & Yes \\
\hline FTE: Grouped AgeFirst & 1.029 & -1.084 & 3.218 & 2.799 & 0.338 & 24.989 & \\
\hline FTE: Seriousness & 0.152 & -0.249 & 0.563 & 1.164 & 0.779 & 1.756 & \\
\hline FTE: Grouped AgeFirst Seriousness & -0.529 & -0.950 & -0.115 & 0.589 & 0.387 & 0.892 & Yes \\
\hline Random Effect: & Post.Mean & Lower $\mathrm{Cl}$ & Upper Cl & Post.Mean & Lower $\mathrm{Cl}$ & Upper $\mathrm{Cl}$ & Significant? \\
\hline Individual (Intercept) & 0.191 & $2.84 \mathrm{E}-11$ & 0.577 & 1.210 & 1.000 & 1.781 & Yes \\
\hline Time & 1.579 & 0.386 & 3.404 & 4.850 & 1.471 & 30.084 & Yes \\
\hline DIC & & & 471.65 & & & & \\
\hline
\end{tabular}

Source: Model Bm1G_cc1202a (Model 3b), Technical Annex: p168-169.

Of the two options for developing a combined model which reflects elements of the four static factors incorporated into ASSET, it is this third model which has the lower DIC (471.7 compared to 473.1 for model 3a). However, the difference is negligible, and both models have limitations as a result of the underlying data.

From the work previously undertaken with respect to gender and ethnicity, it has been concluded that there is insufficient data to pursue a dynamic model involving grouped age atfirst conviction. However, the following section considers the remaining 'static' predictors. 


\subsection{Dynamic Models}

As highlighted in Figures 6.1,6.2 and 6.3, there are differences in the domain score profiles on the basis of the age at which theyyoung person entered the youth justice system, whether or not the young person was an FTE at the time of entry to the reoffending cohortand the nature of their primaryoffence. In this section, these differences are explored to consider how they alter over time.

\section{FTE Status}

The dynamic model involving FTE status is summarised in Table 6.16. Those with a prior history of offending make up the reference group.

Table 6.16: The Dynamic Model Involving FTE Status

\begin{tabular}{|c|c|c|c|c|c|c|c|}
\hline \multirow[b]{3}{*}{ Fixed Effect: } & \multicolumn{7}{|c|}{$\begin{array}{l}\text { Dynamic Basic Model including FTE Status } \\
\text { (BDm3_cc1) }\end{array}$} \\
\hline & \multicolumn{3}{|c|}{ Unstandardised } & \multicolumn{3}{|c|}{ Standardised } & \multirow{2}{*}{ Significant } \\
\hline & PostMean & Lower $\mathrm{Cl}$ & Upper Cl & Post.Mean & Lower $\mathrm{Cl}$ & Upper Cl & \\
\hline (Intercept) & -1.542 & -3.853 & 0.732 & 0.214 & 0.021 & 2.080 & \\
\hline First Time Entant (No = Ref) (FTE) & 0.995 & -1.668 & 3.660 & 2.705 & 0.189 & 38.879 & \\
\hline Time & -0.164 & -0.523 & 0.204 & 0.849 & 0.593 & 1.227 & \\
\hline Living Arrangements (Live) & -0.047 & -0.612 & 0.574 & 0.954 & 0.542 & 1.775 & \\
\hline Family and Personal Relationships (Relation) & 0.640 & -0.018 & 1.344 & 1.896 & 0.982 & 3.833 & \\
\hline Education, Training and Employment(ETE) & -0.301 & -0.828 & 0.165 & 0.740 & 0.437 & 1.179 & \\
\hline Neighbourhood (Where) & 0.198 & -0.318 & 0.737 & 1.218 & 0.728 & 2.091 & \\
\hline Lifestyle (Life) & 0.362 & -0.511 & 1.333 & 1.437 & 0.600 & 3.791 & \\
\hline Substance Use (Drugs) & 0.207 & -0.277 & 0.767 & 1.230 & 0.758 & 2.154 & \\
\hline Physical Health (Physical) & -0.411 & -1.063 & 0.239 & 0.663 & 0.345 & 1.271 & \\
\hline Emotional and Mental Health (Emotion) & 0.006 & -0.480 & 0.551 & 1.006 & 0.619 & 1.734 & \\
\hline Perceptions of Self and Others (Self) & -0.522 & -1.364 & 0.316 & 0.594 & 0.256 & 1.372 & \\
\hline Thinking and Behaviour (Think) & -0.003 & -0.774 & 0.814 & 0.997 & 0.461 & 2.258 & \\
\hline Attitude to Offending (Attitude) & 0.449 & -0.335 & 1.324 & 1.566 & 0.715 & 3.758 & \\
\hline Motivation to Change (Change) & -0.087 & -0.907 & 0.714 & 0.917 & 0.404 & 2.041 & \\
\hline FTE: Time & -0.766 & -1.532 & -0.046 & 0.465 & 0.216 & 0.955 & Yes \\
\hline FTE: Live & 1.099 & -0.321 & 2.548 & 3.001 & 0.725 & 12.775 & \\
\hline FTE: Relation & -1.572 & -3.080 & -0.208 & 0.208 & 0.046 & 0.812 & Yes \\
\hline FTE: ETE & 0.266 & -0.948 & 1.585 & 1.305 & 0.388 & 4.880 & \\
\hline FTE: Where & -1.479 & -2.678 & -0.294 & 0.228 & 0.069 & 0.745 & Yes \\
\hline FTE: Life & 0.992 & -0.652 & 2.701 & 2.696 & 0.521 & 14.889 & \\
\hline FTE: Drugs & -0.697 & $\begin{array}{l}-1.973 \\
\end{array}$ & 0.538 & 0.498 & 0.139 & 1.713 & \\
\hline FTE: Physical & -0.406 & -1.766 & 1.016 & 0.666 & 0.171 & 2.762 & \\
\hline FTE: Emotion & -0.526 & -1.691 & 0.548 & 0.591 & 0.184 & 1.730 & \\
\hline FTE: Self & 2.392 & 0.904 & 3.930 & 10.937 & 2.468 & 50.930 & Yes \\
\hline FTE: Think & -0.484 & -1.928 & 0.990 & 0.617 & 0.145 & 2.690 & \\
\hline FTE: Attitude & -1.249 & -2.686 & 0.231 & 0.287 & 0.068 & 1.259 & \\
\hline FTE: Change & 1.193 & -0.399 & 2.734 & 3.298 & 0.671 & 15.392 & \\
\hline Time: Live & 0.019 & -0.105 & 0.151 & 1.019 & 0.901 & 1.163 & \\
\hline Time: Relation & -0.071 & -0.221 & 0.081 & 0.931 & 0.802 & 1.084 & \\
\hline Time: ETE & 0.053 & -0.058 & 0.158 & 1.055 & 0.944 & 1.172 & \\
\hline Time: Where & -0.005 & -0.112 & 0.097 & 0.995 & 0.894 & 1.102 & \\
\hline Time: Life & -0.007 & -0.180 & 0.182 & 0.993 & 0.836 & 1.200 & \\
\hline Time: Drugs & -0.027 & -0.138 & 0.090 & 0.974 & 0.871 & 1.094 & \\
\hline Time: Physical & 0.115 & -0.053 & 0.275 & 1.122 & 0.949 & 1.316 & \\
\hline Time: Emotion & 0.008 & -0.106 & 0.113 & 1.008 & 0.900 & 1.119 & \\
\hline
\end{tabular}




\begin{tabular}{|c|c|c|c|c|c|c|c|}
\hline \multirow[b]{3}{*}{ Fixed Effect: } & \multicolumn{7}{|c|}{$\begin{array}{l}\text { Dynamic Basic Model including FTE Status } \\
\text { (BDm3_cc1) }\end{array}$} \\
\hline & \multicolumn{3}{|c|}{ Unstandardised } & \multicolumn{3}{|c|}{ Standardised } & \multirow{2}{*}{ Significant? } \\
\hline & Post.Mean & Lower $\mathrm{Cl}$ & Upper $\mathrm{Cl}$ & Post.Mean & Lower $\mathrm{Cl}$ & Upper $\mathrm{Cl}$ & \\
\hline Time: Self & 0.084 & -0.082 & 0.239 & 1.088 & 0.921 & 1.271 & \\
\hline Time: Think & -0.062 & -0.245 & 0.113 & 0.940 & 0.783 & 1.120 & \\
\hline Time: Attitude & -0.101 & -0.285 & 0.065 & 0.904 & 0.752 & 1.068 & \\
\hline Time: Change & 0.047 & -0.113 & 0.215 & 1.048 & 0.893 & 1.240 & \\
\hline FTE: Time: Live & -0.243 & -0.594 & 0.105 & 0.784 & 0.552 & 1.111 & \\
\hline FTE: Time: Relation & 0.381 & 0.024 & 0.751 & 1.463 & 1.024 & 2.119 & Yes \\
\hline FTE: Time: ETE & 0.254 & -0.123 & 0.611 & 1.289 & 0.884 & 1.843 & \\
\hline FTE: Time: Where & 0.251 & 0.029 & 0.496 & 1.285 & 1.029 & 1.642 & Yes \\
\hline FTE: Time: Life & -0.441 & -0.862 & 0.000 & 0.643 & 0.422 & 1.000 & \\
\hline FTE: Time: Drugs & 0.260 & -0.061 & 0.608 & 1.297 & 0.941 & 1.836 & \\
\hline FTE: Time: Physical & -0.048 & -0.431 & 0.347 & 0.953 & 0.650 & 1.415 & \\
\hline FTE: Time: Emotion & 0.157 & -0.093 & 0.426 & 1.170 & 0.911 & 1.531 & \\
\hline FTE: Time: Self & -0.614 & -0.971 & -0.267 & 0.541 & 0.379 & 0.766 & Yes \\
\hline FTE: Time: Think & 0.184 & -0.143 & 0.532 & 1.203 & 0.866 & 1.702 & \\
\hline FTE: Time: Attitude & 0.296 & -0.077 & 0.714 & 1.345 & 0.926 & 2.043 & \\
\hline FTE: Time: Change & -0.227 & -0.606 & 0.156 & 0.797 & 0.546 & 1.169 & \\
\hline Random Effect: & Post.Mean & Lower $\mathrm{Cl}$ & Upper Cl & PostMean & Lower $\mathrm{Cl}$ & Upper Cl & Significant? \\
\hline Individual (Intercept) & 0.71 & $3.54 \mathrm{E}-10$ & 1.74 & 2.039 & 1.000 & 5.703 & Yes \\
\hline Time & 3.136 & 0.523 & 6.932 & 23.012 & 1.686 & 1024.541 & Yes \\
\hline DIC & & & 458.28 & & & & \\
\hline
\end{tabular}

Source: Model BDm3_cc1, Technical Annex: p172-184.

The model has been used to consider the trajectory of the probability of further offending over time for FTEs and those with prior offending history (Figure 6.4). Whilst there remains considerable uncertainty within the model, notably the credible interval for the main effect of being an FTE, it is possible to see that amongst those with previous offending history, the initial probability of further offending is higher amongst those with higher domain scores. For both groups, the probability of further offending amongst those with very high domain scores increases over time. It is likely that this is associated with noncompliance leading to the young person being breached and having further court appearances. The impact of such contacts with youth justice processes is considered in Chapter Seven.

Figure 6.4: Changes in the Probability of Further offending Over Time, by FTE Status

(a) A First Time Entrant

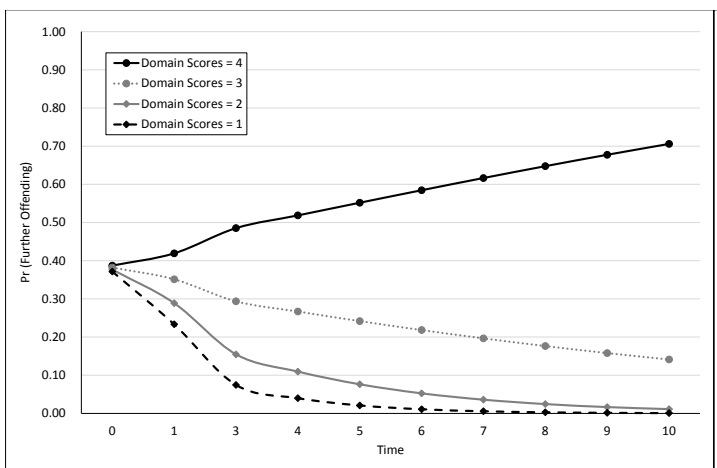

(b) A Young Person with Previous Offending Behaviour

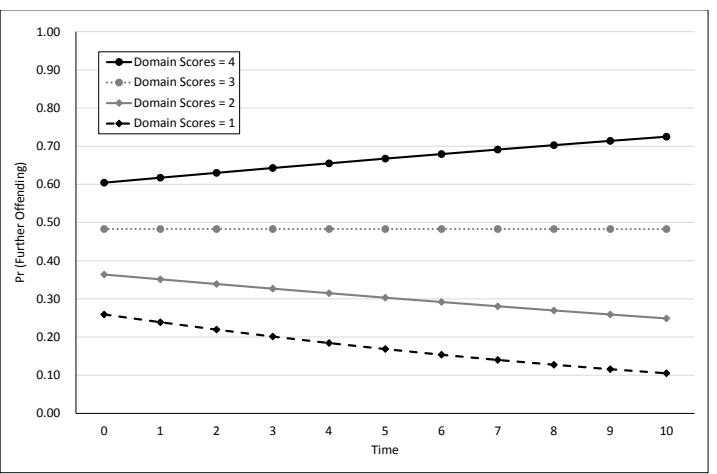

Notes: The domain scores have respectively been shown as being fixed at 1, 2, 3 and 4 respectively to demonstrate the estimated change in the probability of further offending from time 0 to time 10. Estimates derived from Model BDm3_cc1. 
The dynamic model involving the grouped predictor for age at first offence is summarised in Table6.17. This predictor uses the thresholds suggested by the scores assigned for the static factors to segment the cohortwith those aged 10 to 12 being the reference group.

Table 6.17: The Dynamic Model Involving Grouped Age at First Offence

\begin{tabular}{|c|c|c|c|c|c|c|c|}
\hline \multirow[b]{3}{*}{ Fixed Effect: } & \multicolumn{7}{|c|}{$\begin{array}{l}\text { Dynamic Basic Model including Grouped Age at First Offence } \\
\qquad \text { (BDm3G_cc2) }\end{array}$} \\
\hline & \multicolumn{3}{|c|}{ Unstandardised } & \multicolumn{3}{|c|}{ Standardised } & \multirow{2}{*}{ Significant? } \\
\hline & Post.Mean & Lower $\mathrm{Cl}$ & Upper Cl & Post.Mean & Lower $\mathrm{Cl}$ & Upper Cl & \\
\hline (Intercept) & -3.285 & -6.952 & -0.407 & 0.037 & 0.001 & 0.666 & Yes \\
\hline Grouped Age First Offence (Age 10-12 = Ref) & 2.340 & -0.928 & 5.488 & 10.383 & 0.395 & 241.818 & \\
\hline Time & 0.277 & -0.421 & 0.963 & 1.320 & 0.656 & 2.620 & \\
\hline Living Arrangements (Live) & -0.367 & -1.530 & 0.762 & 0.693 & 0.217 & 2.143 & \\
\hline Family and Personal Relationships (Relation) & 1.479 & 0.071 & 2.905 & 4.387 & 1.074 & 18.271 & Yes \\
\hline Education, Training and Employment (ETE) & -0.419 & -1.484 & 0.794 & 0.658 & 0.227 & 2.212 & \\
\hline Neighbourhood (Where) & 0.165 & -0.804 & 1.091 & 1.179 & 0.448 & 2.976 & \\
\hline Lifestyle (Life) & 1.856 & 0.105 & 3.643 & 6.396 & 1.111 & 38.198 & Yes \\
\hline Substance Use (Drugs) & -0.055 & -1.147 & 0.993 & 0.947 & 0.317 & 2.698 & \\
\hline Physical Health (Physical) & -0.947 & -2.066 & 0.283 & 0.388 & 0.127 & 1.327 & \\
\hline Emotional and Mental Health (Emotion) & -0.559 & -1.547 & 0.446 & 0.572 & 0.213 & 1.563 & \\
\hline Perceptions of Self and Others (Self) & -3.417 & -5.411 & -1.480 & 0.033 & 0.004 & 0.228 & Yes \\
\hline Thinking and Behaviour (Think) & 0.622 & -1.169 & 2.247 & 1.862 & 0.311 & 9.458 & \\
\hline Attitude to Offending (Attitude) & 1.706 & -0.163 & 3.783 & 5.505 & 0.849 & 43.951 & \\
\hline Motivation to Change (Change) & 0.286 & -1.359 & 1.917 & 1.331 & 0.257 & 6.802 & \\
\hline Grouped Age First Time & -0.559 & -1.295 & 0.208 & 0.572 & 0.274 & 1.231 & \\
\hline Grouped Age First Live & 0.484 & -0.793 & 1.885 & 1.623 & 0.453 & 6.584 & \\
\hline Grouped Age First Relation & -1.584 & -3.238 & -0.048 & 0.205 & 0.039 & 0.953 & Yes \\
\hline Grouped Age First ETE & 0.135 & -1.095 & 1.307 & 1.144 & 0.334 & 3.697 & \\
\hline Grouped Age First Where & 0.135 & -1.095 & 1.307 & 1.144 & 0.334 & 3.697 & \\
\hline Grouped Age First Life & -1.624 & -3.612 & 0.239 & 0.197 & 0.027 & 1.270 & \\
\hline Grouped Age First Drugs & 0.342 & -0.810 & 1.539 & 1.407 & 0.445 & 4.660 & \\
\hline Grouped Age First Physical & 0.344 & -0.976 & 1.694 & 1.410 & 0.377 & 5.442 & \\
\hline Grouped Age First Emotion & 0.031 & -1.057 & 1.244 & 1.031 & 0.348 & 3.469 & \\
\hline Grouped Age First Self & 4.480 & 2.237 & 6.635 & 88.267 & 9.369 & 761.562 & Yes \\
\hline Grouped Age First Think & -0.594 & -2.532 & 1.216 & 0.552 & 0.079 & 3.374 & \\
\hline Grouped Age First Attitude & -1.357 & -3.479 & 0.703 & 0.258 & 0.031 & 2.020 & \\
\hline Grouped Age First Change & -0.448 & -2.260 & 1.314 & 0.639 & 0.104 & 3.721 & \\
\hline Time: Live & -0.146 & -0.410 & 0.145 & 0.864 & 0.664 & 1.156 & \\
\hline Time: Relation & -0.351 & -0.718 & -0.001 & 0.704 & 0.488 & 0.999 & Yes \\
\hline Time: ETE & 0.158 & -0.086 & 0.412 & 1.171 & 0.918 & 1.509 & \\
\hline Time: Where & -0.118 & -0.315 & 0.096 & 0.888 & 0.730 & 1.101 & \\
\hline Time: Life & -0.419 & -0.817 & -0.016 & 0.658 & 0.442 & 0.984 & Yes \\
\hline Time: Drugs & 0.229 & -0.011 & 0.459 & 1.257 & 0.989 & 1.583 & \\
\hline Time: Physical & 0.231 & -0.068 & 0.541 & 1.260 & 0.934 & 1.718 & \\
\hline Time: Emotion & 0.305 & 0.060 & 0.528 & 1.356 & 1.061 & 1.695 & Yes \\
\hline Time: Self & 0.804 & 0.383 & 1.248 & 2.234 & 1.467 & 3.483 & Yes \\
\hline Time: Think & -0.186 & -0.506 & 0.158 & 0.830 & 0.603 & 1.171 & \\
\hline Time: Attitude & -0.548 & -0.911 & -0.141 & 0.578 & 0.402 & 0.869 & Yes \\
\hline Time: Change & 0.151 & -0.166 & 0.526 & 1.162 & 0.847 & 1.692 & \\
\hline
\end{tabular}




\begin{tabular}{|c|c|c|c|c|c|c|c|}
\hline \multirow[b]{3}{*}{ Fixed Effect: } & \multicolumn{7}{|c|}{$\begin{array}{l}\text { Dynamic Basic Model including Grouped Age at First Offence } \\
\text { (BDm3G_cc2) }\end{array}$} \\
\hline & \multicolumn{3}{|c|}{ Unstandardised } & \multicolumn{3}{|c|}{ Standardised } & \multirow{2}{*}{ Significant? } \\
\hline & Post.Mean & Lower $\mathrm{Cl}$ & Upper $\mathrm{Cl}$ & Post.Mean & Lower $\mathrm{Cl}$ & Upper $\mathrm{Cl}$ & \\
\hline Grouped Age First Time: Live & 0.191 & -0.141 & 0.471 & 1.211 & 0.869 & 1.602 & \\
\hline Grouped Age First Time: Relation & 0.413 & 0.018 & 0.794 & 1.512 & 1.019 & 2.212 & Yes \\
\hline Grouped Age First Time: ETE & -0.049 & -0.323 & 0.233 & 0.952 & 0.724 & 1.262 & \\
\hline Grouped Age First Time: Where & 0.142 & -0.111 & 0.359 & 1.152 & 0.895 & 1.432 & \\
\hline Grouped Age First Time: Life & 0.331 & -0.148 & 0.740 & 1.392 & 0.862 & 2.096 & \\
\hline Grouped Age First Time: Drugs & -0.259 & -0.525 & 0.015 & 0.772 & 0.592 & 1.016 & \\
\hline Grouped Age First Time: Physical & -0.128 & -0.464 & 0.230 & 0.880 & 0.629 & 1.258 & \\
\hline Grouped Age First Time: Emotion & -0.171 & -0.467 & 0.118 & 0.842 & 0.627 & 1.125 & \\
\hline Grouped Age First Time: Self & -1.064 & -1.556 & -0.594 & 0.345 & 0.211 & 0.552 & Yes \\
\hline Grouped Age First Time: Think & 0.189 & -0.209 & 0.533 & 1.209 & 0.811 & 1.704 & \\
\hline Grouped Age First Time: Attitude & 0.425 & 0.010 & 0.870 & 1.529 & 1.010 & 2.386 & Yes \\
\hline Grouped Age First Time: Change & -0.094 & -0.490 & 0.277 & 0.911 & 0.613 & 1.319 & \\
\hline Random Effect: & PostMean & Lower $\mathrm{Cl}$ & Upper $\mathrm{Cl}$ & PostMean & Lower $\mathrm{Cl}$ & Upper $\mathrm{Cl}$ & Significant? \\
\hline Individual (Intercept) & 0.71 & $6.79 \mathrm{E}-06$ & 1.70 & 2.041 & 1.000 & 5.496 & Yes \\
\hline Time & 2.814 & 0.630 & 6.402 & 16.676 & 1.878 & 603.050 & Yes \\
\hline DIC & & & 448.73 & & & & \\
\hline
\end{tabular}

Source: Model BDm3G_cc2, Technical Annex: p185-197.

The model has been used to consider the trajectory of the probability of further offending over time for the younger and older groups based on their age at the time of their first offence (Figure 6.5). Notably, the trajectory for those aged 10-12 increases over time whereas that for those aged 13-17 decreases.

Figure 6.5: Changes in the Probability of Further Offending Over Time, by Grouped Age at First Offence

(a) Age 10-12 years

(b) Age 13-17 years
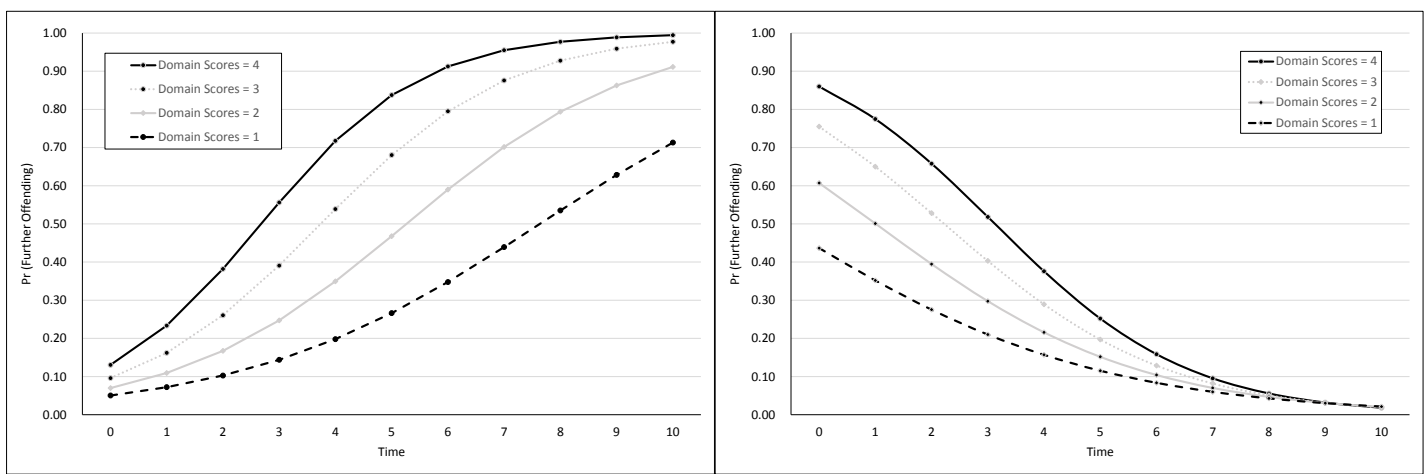

Notes: The domain scores have respectively been shown as being fixed at 1, 2, 3 and 4 respectively to demonstrate the estimated change in the probability of further offending from time 0 to time 10. Estimates derived from Model BDm3G_cc2.

This trend can be explained by looking at the mean domain scores for those aged $10-12$ at the time of their first offence and how these change over time. As can be seen from Figure 6.6, the average domain score increases between T ime 0 and T ime 15 whereas the equivalentfor the older group (Figure 6.7) is less pronounced and there is actuallya net downward trend after Time 10. 
Figure 6.6: Summary of Average Domain Scores, by Grouped Age at First Offence - Younger Group

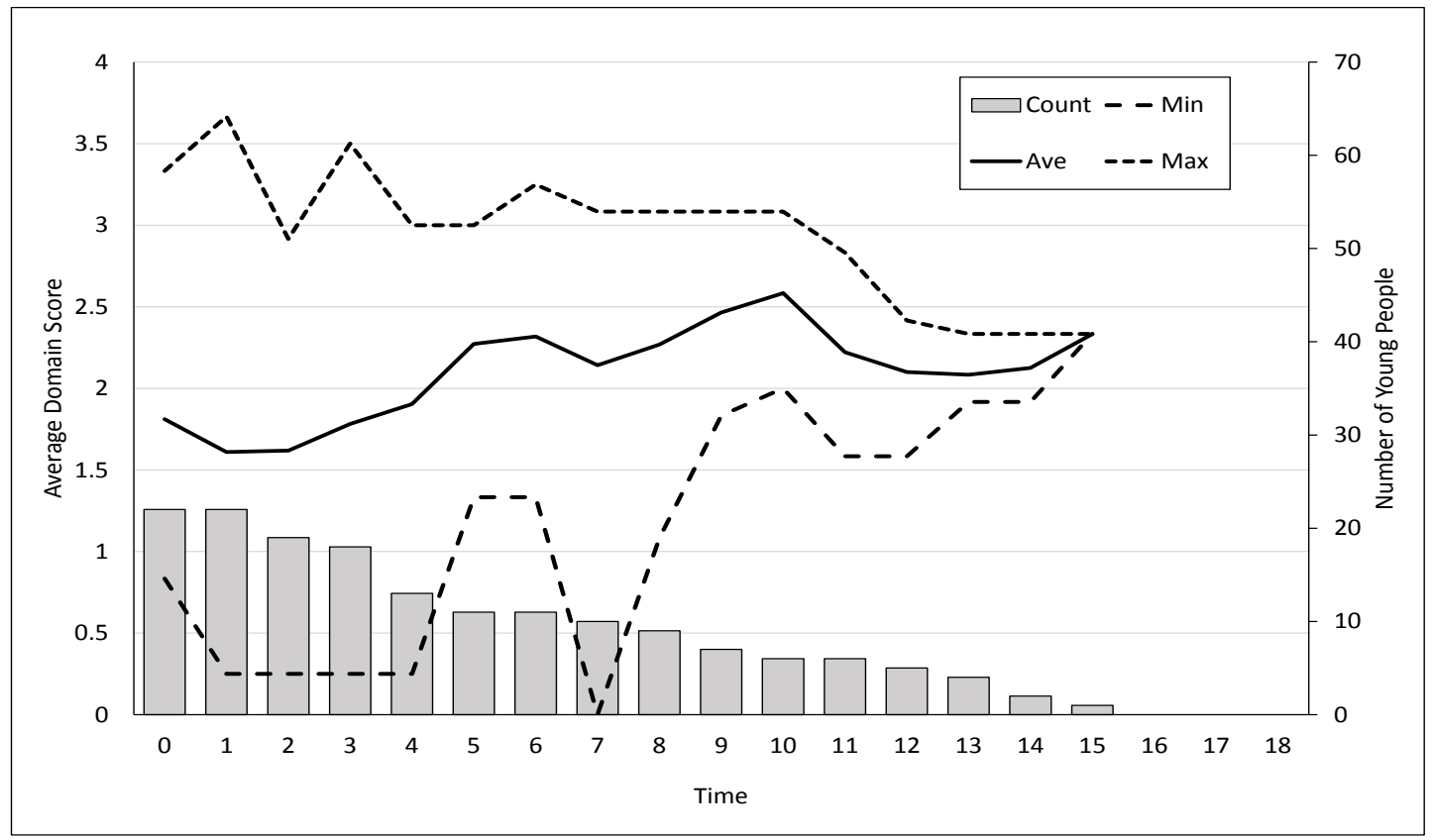

Figure 6.7: Summary of Average Domain Scores, by Grouped Age at First Offence - Older Group

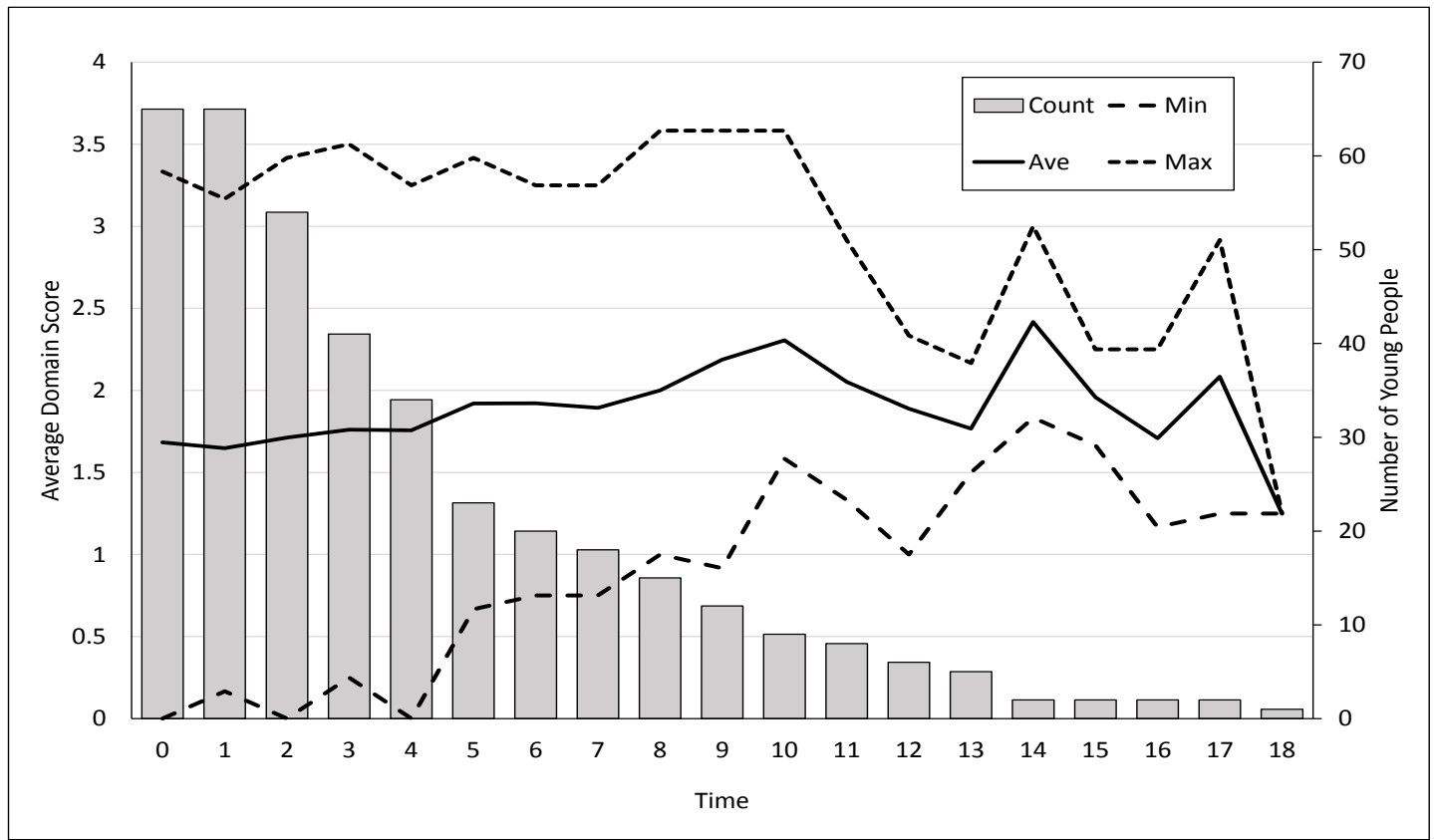

From Time 14, both groups are informed by two or fewer cases. Within the younger group there was one individual who was assessed 16 times. In the older group, there was one individual who was assessed 19 times during the period of interest. This limits the reliability of models involving this predictor at later measurement points. 
The dynamic model involving the categorical predictor which reflects whether the young person had entered the cohort having committed a violent offence, a serious acquisitive crime (SAC) or an 'Other' offence is summarised in Table 6.18. In this model, 'Other' offences are the reference category.

Table 6.18: The Dynamic Model Involving Grouped YJB Offence Category

\begin{tabular}{|c|c|c|c|c|c|c|c|}
\hline \multirow[b]{3}{*}{ Fixed Effect: } & \multicolumn{7}{|c|}{$\begin{array}{l}\text { Dynamic Basic Model including Grouped YJB Offence Category } \\
\qquad \text { (BDm3G_01) }\end{array}$} \\
\hline & \multicolumn{3}{|c|}{ Unstandardised } & \multicolumn{3}{|c|}{ Standardised } & \multirow{2}{*}{ Significant? } \\
\hline & Post.Mean & Lower $\mathrm{Cl}$ & Upper $\mathrm{Cl}$ & Post.Mean & Lower $\mathrm{Cl}$ & Upper $\mathrm{Cl}$ & \\
\hline (Intercept) & -1.743 & -5.109 & 1.287 & 0.175 & 0.006 & 3.621 & \\
\hline \multicolumn{8}{|l|}{ Grouped YJB Offence Category (Ref = Other) } \\
\hline Serious Acquisitive Crime (SAC) & 1.924 & -2.331 & 6.124 & 6.847 & 0.097 & 456.878 & \\
\hline Violence Against the Person (VAP) & -4.070 & -10.078 & 1.331 & 0.017 & 0.000 & 3.785 & \\
\hline Time & -0.233 & -0.830 & 0.284 & 0.792 & 0.436 & 1.329 & \\
\hline Living Arrangements (Live) & -0.165 & -1.120 & 0.777 & 0.848 & 0.326 & 2.174 & \\
\hline Family and Personal Relationships (Relation) & 0.058 & -1.040 & 1.106 & 1.059 & 0.354 & 3.023 & \\
\hline Education, Training and Employment(ETE) & 0.595 & -0.182 & 1.355 & 1.813 & 0.834 & 3.877 & \\
\hline Neighbourhood (Where) & 0.069 & -0.743 & 0.837 & 1.071 & 0.476 & 2.310 & \\
\hline Lifestyle (Life) & -0.325 & -1.832 & 1.123 & 0.723 & 0.160 & 3.075 & \\
\hline Substance Use (Drugs) & 0.170 & -0.691 & 1.064 & 1.185 & 0.501 & 2.899 & \\
\hline Physical Health (Physical) & -0.148 & -1.086 & 0.760 & 0.862 & 0.337 & 2.138 & \\
\hline Emotional and Mental Health (Emotion) & -0.158 & -0.908 & 0.652 & 0.854 & 0.403 & 1.919 & \\
\hline Perceptions of Self and Others (Self) & 0.381 & -0.912 & 1.582 & 1.463 & 0.402 & 4.866 & \\
\hline Thinking and Behaviour (Think) & -0.046 & -1.145 & 0.970 & 0.955 & 0.318 & 2.637 & \\
\hline Attitude to Offending (Attitude) & -0.149 & -1.454 & 1.053 & 0.861 & 0.234 & 2.865 & \\
\hline Motivation to Change (Change) & 0.719 & -0.531 & 1.899 & 2.053 & 0.588 & 6.678 & \\
\hline SAC: Time & -0.746 & -1.623 & 0.182 & 0.474 & 0.197 & 1.199 & \\
\hline SAC: Live & 0.522 & -1.104 & 2.222 & 1.686 & 0.331 & 9.229 & \\
\hline SAC: Relation & 0.777 & -1.044 & 2.587 & 2.176 & 0.352 & 13.285 & \\
\hline SAC: ETE & -0.518 & -2.148 & 1.168 & 0.596 & 0.117 & 3.215 & \\
\hline SAC: Where & 0.897 & -0.564 & 2.341 & 2.452 & 0.569 & 10.391 & \\
\hline SAC: Life & 0.620 & -1.804 & 2.928 & 1.859 & 0.165 & 18.687 & \\
\hline SAC: Drugs & 0.381 & -1.102 & 1.853 & 1.464 & 0.332 & 6.378 & \\
\hline SAC: Physical & -1.795 & -3.842 & 0.215 & 0.166 & 0.021 & 1.240 & \\
\hline SAC: Emotion & 0.045 & -1.556 & 1.694 & 1.046 & 0.211 & 5.440 & \\
\hline SAC: Self & -1.012 & -3.082 & 1.149 & 0.363 & 0.046 & 3.155 & \\
\hline SAC: Think & -0.253 & -2.462 & 1.974 & 0.776 & 0.085 & 7.200 & \\
\hline SAC: Attitude & 0.256 & -1.893 & 2.418 & 1.292 & 0.151 & 11.223 & \\
\hline SAC: Change & -1.331 & -4.312 & 1.750 & 0.264 & 0.013 & 5.754 & \\
\hline VAP: Time & 0.829 & -0.267 & 2.085 & 2.291 & 0.766 & 8.047 & \\
\hline VAP: Live & 1.307 & -1.290 & 3.652 & 3.694 & 0.275 & 38.552 & \\
\hline VAP: Relation & -0.996 & -3.459 & 1.719 & 0.369 & 0.031 & 5.581 & \\
\hline VAP: ETE & -3.461 & -5.758 & -1.407 & 0.031 & 0.003 & 0.245 & Yes \\
\hline VAP: Where & -1.226 & -3.084 & 0.684 & 0.293 & 0.046 & 1.981 & \\
\hline VAP: Life & 3.032 & 0.437 & 5.904 & 20.728 & 1.548 & 366.635 & Yes \\
\hline VAP: Drugs & 0.398 & -1.540 & 2.112 & 1.489 & 0.214 & 8.264 & \\
\hline VAP: Physical & -0.865 & -3.450 & 1.667 & 0.421 & 0.032 & 5.296 & \\
\hline VAP: Emotion & -0.458 & -2.160 & 1.204 & 0.633 & 0.115 & 3.335 & \\
\hline VAP: Self & 0.188 & -2.047 & 2.613 & 1.207 & 0.129 & 13.639 & \\
\hline VAP: Think & 2.361 & -0.712 & 5.592 & 10.599 & 0.491 & 268.378 & \\
\hline VAP: Attitude & 0.385 & -2.933 & 3.469 & 1.469 & 0.053 & 32.119 & \\
\hline VAP: Change & 0.769 & -1.767 & 3.112 & 2.157 & 0.171 & 22.460 & \\
\hline
\end{tabular}




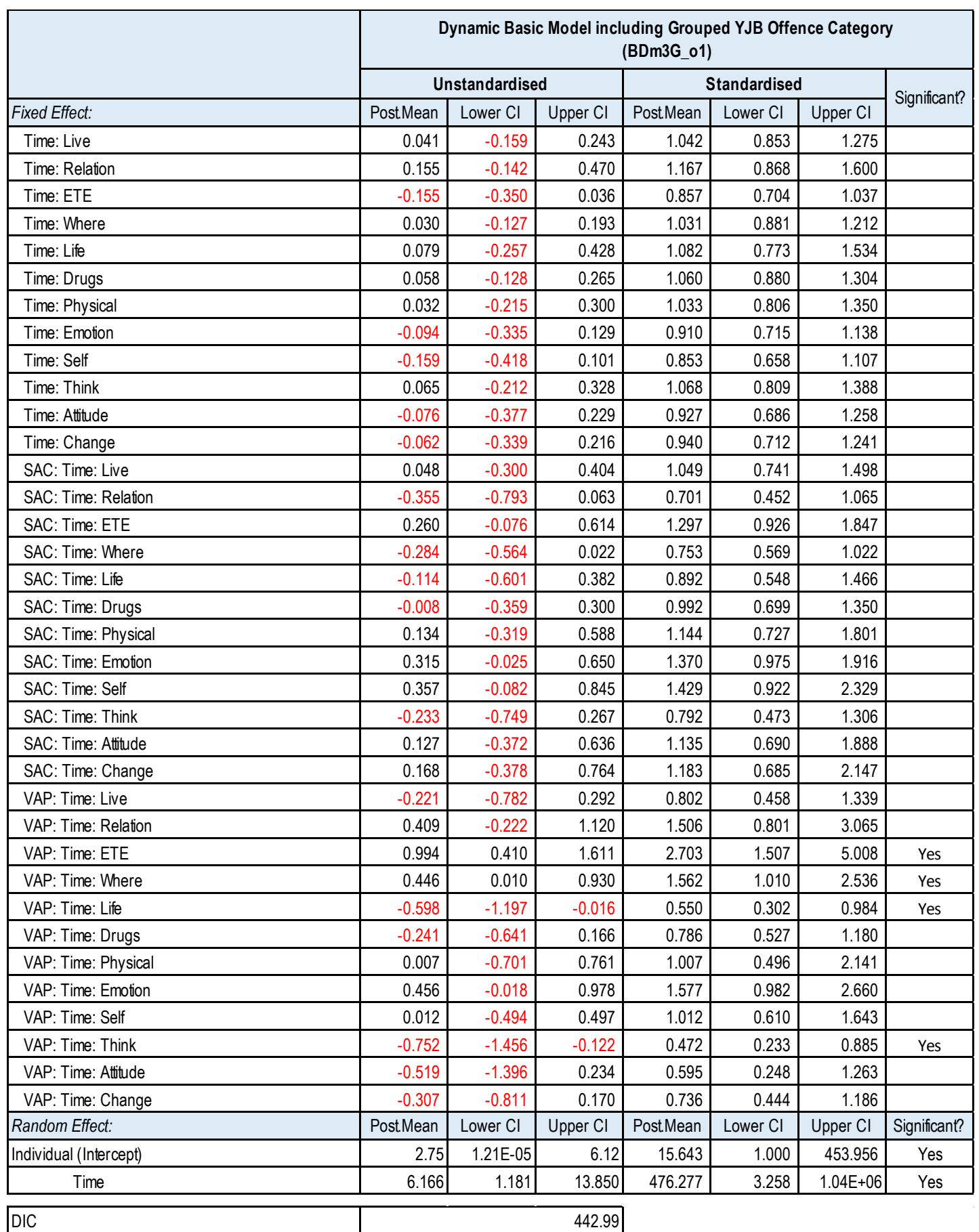

Source: Model BDm3_01, Technical Annex: p198-214.

Compared to where the Grouped YJB Offence Categories have been added to the Basic Model (Table 6.7), allowing the domain scores and the interactions upon these for the different offence types, to vary by time improves the DIC from 473.8 to 443.0 . This suggests that despite the additional parameters, the dynamic modelaccounts for more of the uncertaintyaround the odds of further offending for the average young person. However, despite the trace plots suggesting that there has been convergence, the credible intervals for some of the main and interaction fixed effects are very wide including the estimates for SAC as a main effect and the interactions between VAP and the lifestyle and thinking behaviours 
domain scores. Figures 6.8 to 6.10 summarises the trajectories of the probability of further offending for each type of offence where the domain scores are fixed. The trend for other offences is notably different to that for serious acquisitive crimes and violence against the person offences.

At Time 1, for those with ratings of 2 across the domain scores, the probability of further offending is lowest amongst those who have committed violence against the person offences. The odds of further offending are estimated to be 1.2 times higher if they had committed a serious acquisitive crime, and 2.7 timeshigher if they had committed an 'Other' offence.

If similar comparisons are made at Time 6 (when the intersection between the trajectories occurs for violence against the person offences), the probability of a young person with domain scores of 2 committing further offences is lowest amongst those who have committed a serious acquisitive crime. Relative to this, the odds of further offending, are estimated to be 1.5 timeshigher if the young person's primaryoffence was either an 'Other' or a violence against the person offence.

Figures 6.11 to 6.13 summarise the average domain scores by primary offence. These do not offer an explanation as to why there is an intersection of the trajectories of the probabilities of further offending for the serious acquisitive crimes or the violence against the person offences. 
Figure 6.8: Changes in the Probability of Further Offending Over Time - Serious Acquisitive Crimes

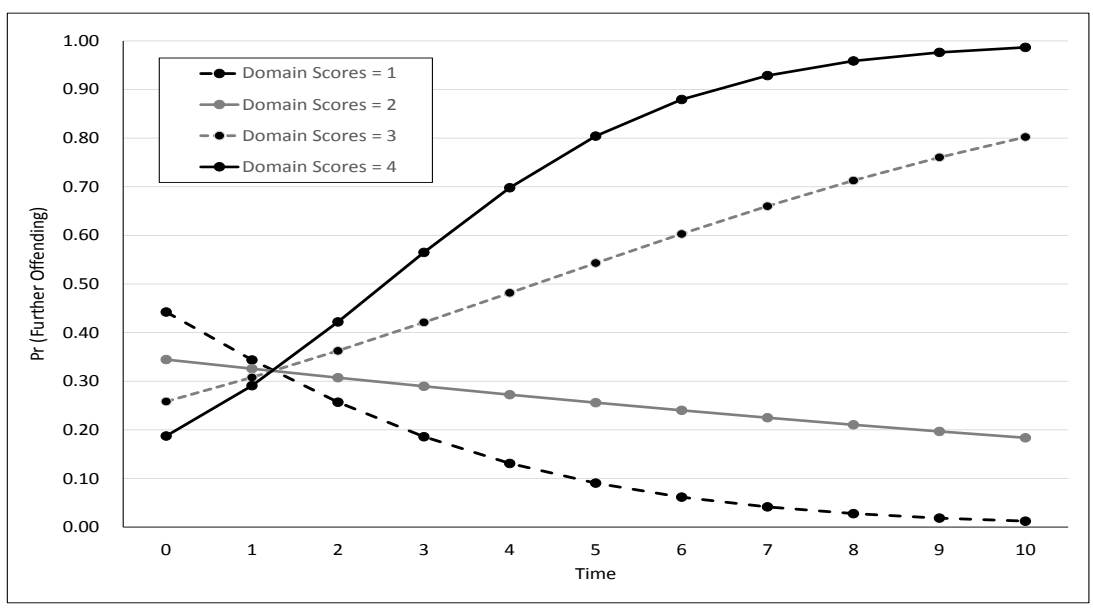

Figure 6.10: Changes in the Probability of Further Offending Over Time - Other Offences

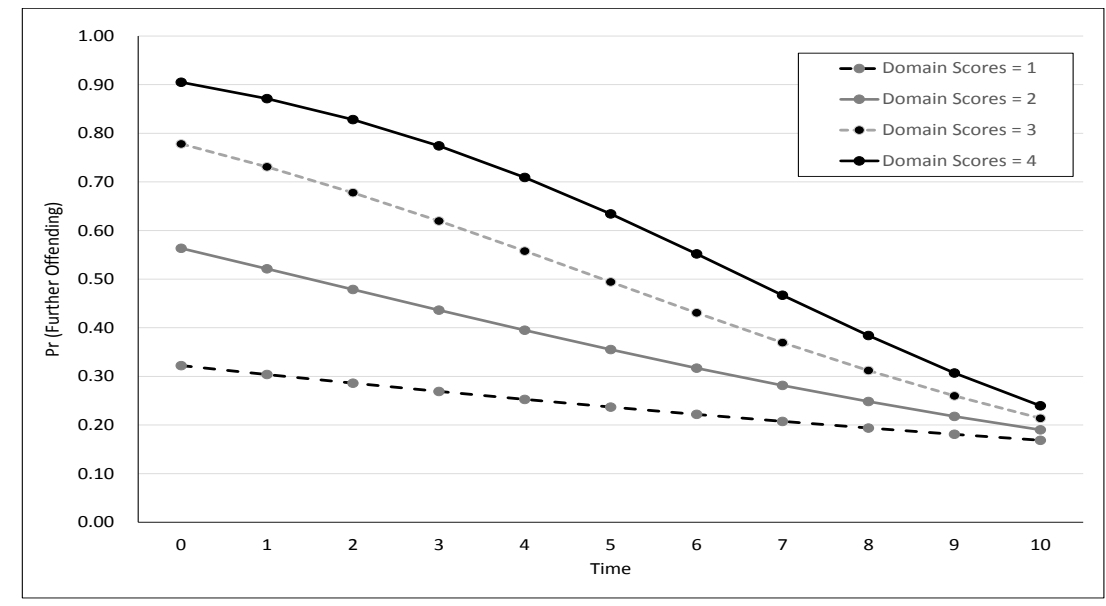

Figure 6.9: Changes in the Probability of Further Offending Person

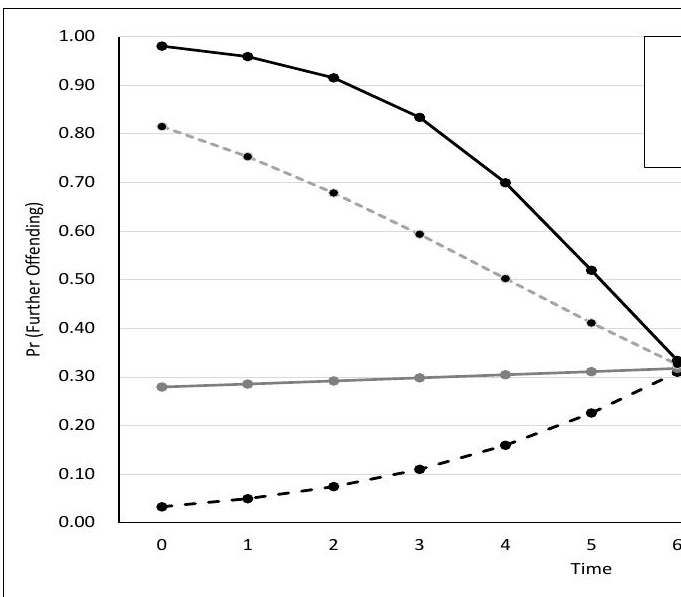

Notes: The domain scores have respectively been shown as the estimated change in the probability of further offending derived from Model BDm3G_01. 
Figure 6.11: Summary of Average Domain Scores, by Primary Offence - Serious Acquisitive Crimes

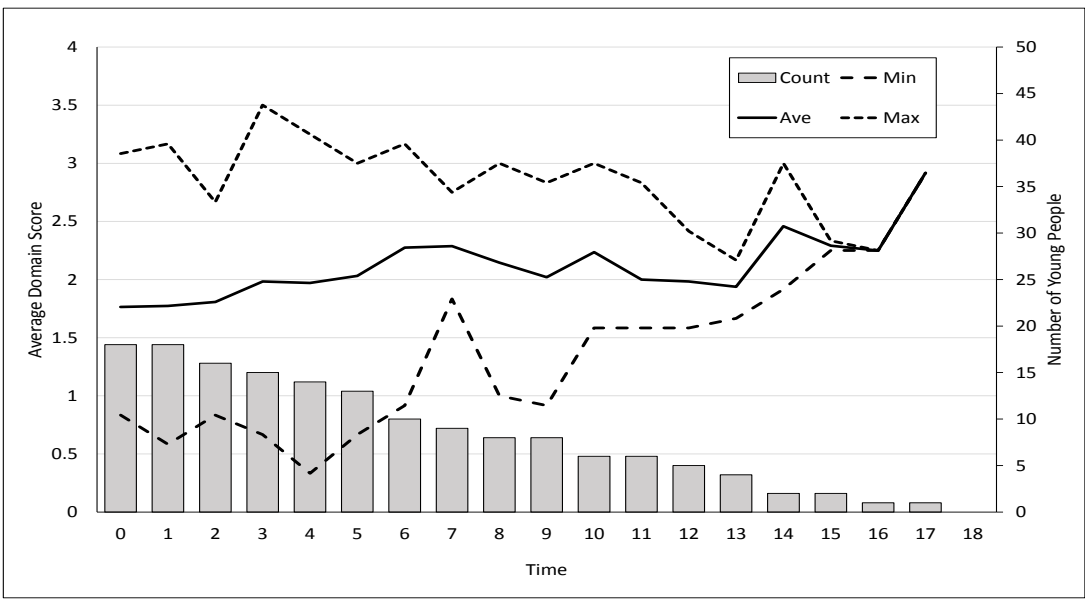

Figure 6.13: Summary of Average Domain Scores, by Primary Offence - Other Offences

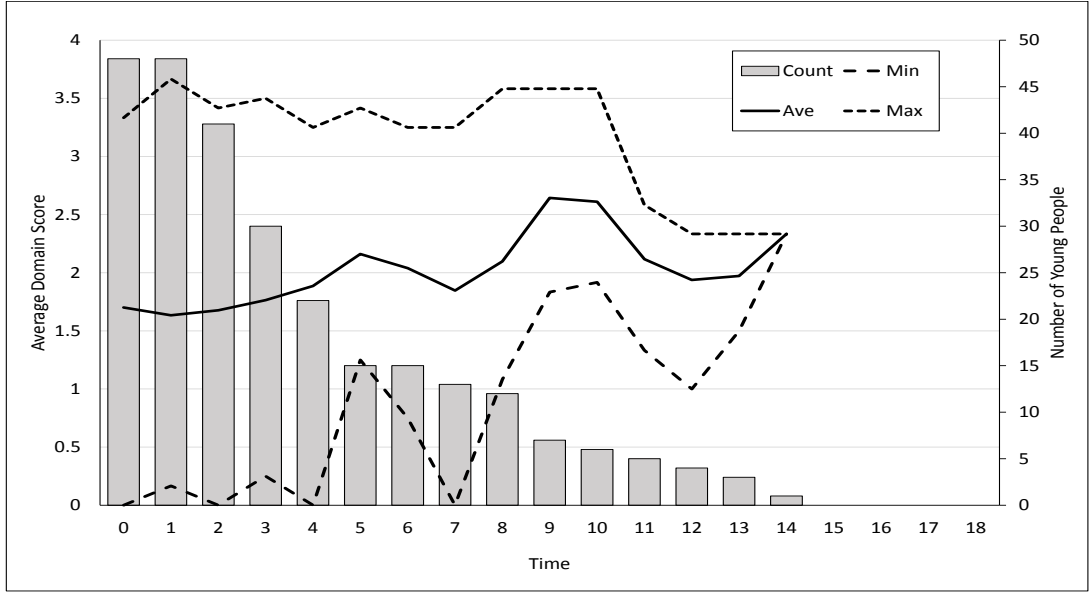

Figure 6.12: Summary of Average Domain Scores, by Prin Person

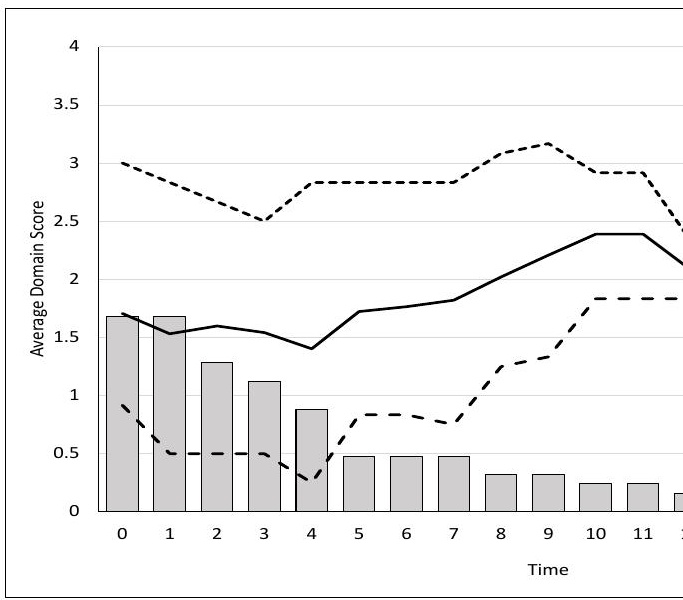


Table 6.19: The Dynamic Model Involving YJB Gravity Scores

\begin{tabular}{|c|c|c|c|c|c|c|c|}
\hline \multirow{3}{*}{\begin{tabular}{|l} 
\\
Fixed Effect: \\
\end{tabular}} & \multicolumn{7}{|c|}{$\begin{array}{l}\text { Dynamic Basic Model including YJB Gravity Score } \\
\text { (BDm3_02a) }\end{array}$} \\
\hline & \multicolumn{3}{|c|}{ Unstandardised } & \multicolumn{3}{|c|}{ Standardised } & \multirow{2}{*}{ Significant? } \\
\hline & Post.Mean & Lower $\mathrm{Cl}$ & Upper $\mathrm{Cl}$ & Post.Mean & Lower $\mathrm{Cl}$ & Upper $\mathrm{Cl}$ & \\
\hline (Intercept) & -1.306 & -3.401 & 0.772 & 0.271 & 0.033 & 2.164 & \\
\hline YJB Gravity Score (Seriousness) (0 = Gravity Score of 2 ) & -0.143 & -1.059 & 0.810 & 0.867 & 0.347 & 2.247 & \\
\hline Time & -0.145 & -0.572 & 0.257 & 0.865 & 0.564 & 1.293 & \\
\hline Living Arrangements (Live) & -0.459 & -1.156 & 0.190 & 0.632 & 0.315 & 1.210 & \\
\hline Family and Personal Relationships (Relation) & 0.207 & -0.552 & 0.960 & 1.230 & 0.576 & 2.613 & \\
\hline Education, Training and Employment (ETE) & -0.064 & -0.664 & 0.487 & 0.938 & 0.515 & 1.628 & \\
\hline Neighbourhood (Where) & -0.091 & -0.669 & 0.533 & 0.913 & 0.512 & 1.705 & \\
\hline Lifestyle (Life) & 0.244 & -0.633 & 1.211 & 1.276 & 0.531 & 3.357 & \\
\hline Substance Use (Drugs) & 0.180 & -0.423 & 0.787 & 1.197 & 0.655 & 2.197 & \\
\hline Physical Health (Physical) & -0.588 & -1.282 & 0.120 & 0.556 & 0.278 & 1.128 & \\
\hline Emotional and Mental Health (Emotion) & -0.032 & -0.630 & 0.526 & 0.969 & 0.532 & 1.693 & \\
\hline Perceptions of Self and Others (Self) & 0.567 & -0.288 & 1.476 & 1.764 & 0.750 & 4.376 & \\
\hline Thinking and Behaviour (Think) & -0.248 & -0.984 & 0.556 & 0.780 & 0.374 & 1.743 & \\
\hline Attitude to Offending (Attitude) & -0.025 & -0.895 & 0.835 & 0.976 & 0.409 & 2.305 & \\
\hline Motivation to Change (Change) & 0.850 & -0.077 & 1.783 & 2.339 & 0.926 & 5.949 & \\
\hline Seriousness: Time & -0.008 & -0.245 & 0.232 & 0.992 & 0.783 & 1.262 & \\
\hline Seriousness: Live & 0.285 & -0.094 & 0.690 & 1.329 & 0.910 & 1.994 & \\
\hline Seriousness: Relation & 0.015 & -0.403 & 0.475 & 1.015 & 0.668 & 1.608 & \\
\hline Seriousness: ETE & -0.110 & -0.495 & 0.246 & 0.896 & 0.610 & 1.279 & \\
\hline Seriousness: Where & 0.145 & -0.193 & 0.504 & 1.156 & 0.825 & 1.656 & \\
\hline Seriousness: Life & 0.140 & -0.406 & 0.729 & 1.150 & 0.667 & 2.072 & \\
\hline Seriousness: Drugs & 0.064 & -0.265 & 0.409 & 1.066 & 0.767 & 1.505 & \\
\hline Seriousness: Physical & -0.060 & -0.521 & 0.393 & 0.942 & 0.594 & 1.481 & \\
\hline Seriousness: Emotion & -0.073 & -0.434 & 0.328 & 0.930 & 0.648 & 1.388 & \\
\hline Seriousness: Self & -0.140 & -0.589 & 0.340 & 0.869 & 0.555 & 1.405 & \\
\hline Seriousness: Think & 0.112 & -0.490 & 0.672 & 1.118 & 0.612 & 1.959 & \\
\hline Seriousness: Attitude & -0.019 & -0.554 & 0.489 & 0.982 & 0.575 & 1.631 & \\
\hline Seriousness: Change & -0.339 & -1.016 & 0.333 & 0.713 & 0.362 & 1.395 & \\
\hline Time: Live & 0.079 & -0.066 & 0.239 & 1.083 & 0.936 & 1.269 & \\
\hline Time: Relation & 0.122 & -0.093 & 0.332 & 1.130 & 0.911 & 1.394 & \\
\hline Time: ETE & 0.022 & -0.133 & 0.182 & 1.022 & 0.876 & 1.200 & \\
\hline Time: Where & 0.094 & -0.027 & 0.221 & 1.099 & 0.973 & 1.247 & \\
\hline Time: Life & -0.092 & -0.312 & 0.124 & 0.912 & 0.732 & 1.132 & \\
\hline Time: Drugs & -0.026 & -0.168 & 0.112 & 0.975 & 0.846 & 1.118 & \\
\hline Time: Physical & 0.200 & -0.002 & 0.399 & 1.221 & 0.998 & 1.491 & \\
\hline Time: Emotion & -0.110 & -0.276 & 0.048 & 0.896 & 0.759 & 1.049 & \\
\hline Time: Self & -0.174 & -0.361 & 0.014 & 0.840 & 0.697 & 1.014 & \\
\hline Time: Think & 0.146 & -0.036 & 0.329 & 1.157 & 0.964 & 1.389 & \\
\hline Time: Attitude & -0.031 & -0.233 & 0.172 & 0.970 & 0.792 & 1.188 & \\
\hline Time: Change & -0.199 & -0.408 & 0.004 & 0.819 & 0.665 & 1.004 & \\
\hline Seriousness: Time: Live & -0.014 & -0.107 & 0.081 & 0.986 & 0.898 & 1.085 & \\
\hline Seriousness: Time: Relation & -0.104 & -0.233 & 0.018 & 0.902 & 0.792 & 1.018 & \\
\hline Seriousness: Time: ETE & 0.061 & -0.045 & 0.163 & 1.062 & 0.956 & 1.177 & \\
\hline Seriousness: Time: Where & -0.087 & -0.167 & -0.006 & 0.917 & 0.846 & 0.994 & Yes \\
\hline Seriousness: Time: Life & 0.028 & -0.110 & 0.161 & 1.029 & 0.896 & 1.174 & \\
\hline Seriousness: Time: Drugs & -0.008 & -0.092 & 0.067 & 0.992 & 0.912 & 1.069 & \\
\hline
\end{tabular}




\begin{tabular}{|l|r|r|r|r|r|r|r|}
\hline \multirow{2}{*}{} & \multicolumn{5}{|c|}{ Dynamic Basic Model including YJB Gravity Score } \\
(BDm3_02a)
\end{tabular}

\begin{tabular}{|l|l|}
\hline DIC & 472.66 \\
\hline
\end{tabular}

Source: Model BDm3_o2a, Technical Annex: p215-227.

The model has been used to estimate the role that the seriousness of the primary offence has on the probability of further offending over time (Figure 6.14). As would be expected, those with higher domain scores have higher initial probabilities of further offending. However, it is those who have committed offences with lower gravity scores who are more likely to commit further offences - a trend that holds over time. This maywell be linked to the type of offences that have a lower gravity score. For example, theft and handling stolen goods - a categorywhich includes shoplifting; criminal damage and possession of drugs have low gravity scores. However, as can be seen from Figure 6.14, there is not a clear relationship between the proportion of further offending and YJB Gravity Score. This is also apparentin the national published figures (Figure 3.5).

Figure 6.14: Rate of Further Offending, by YJB Gravity Score

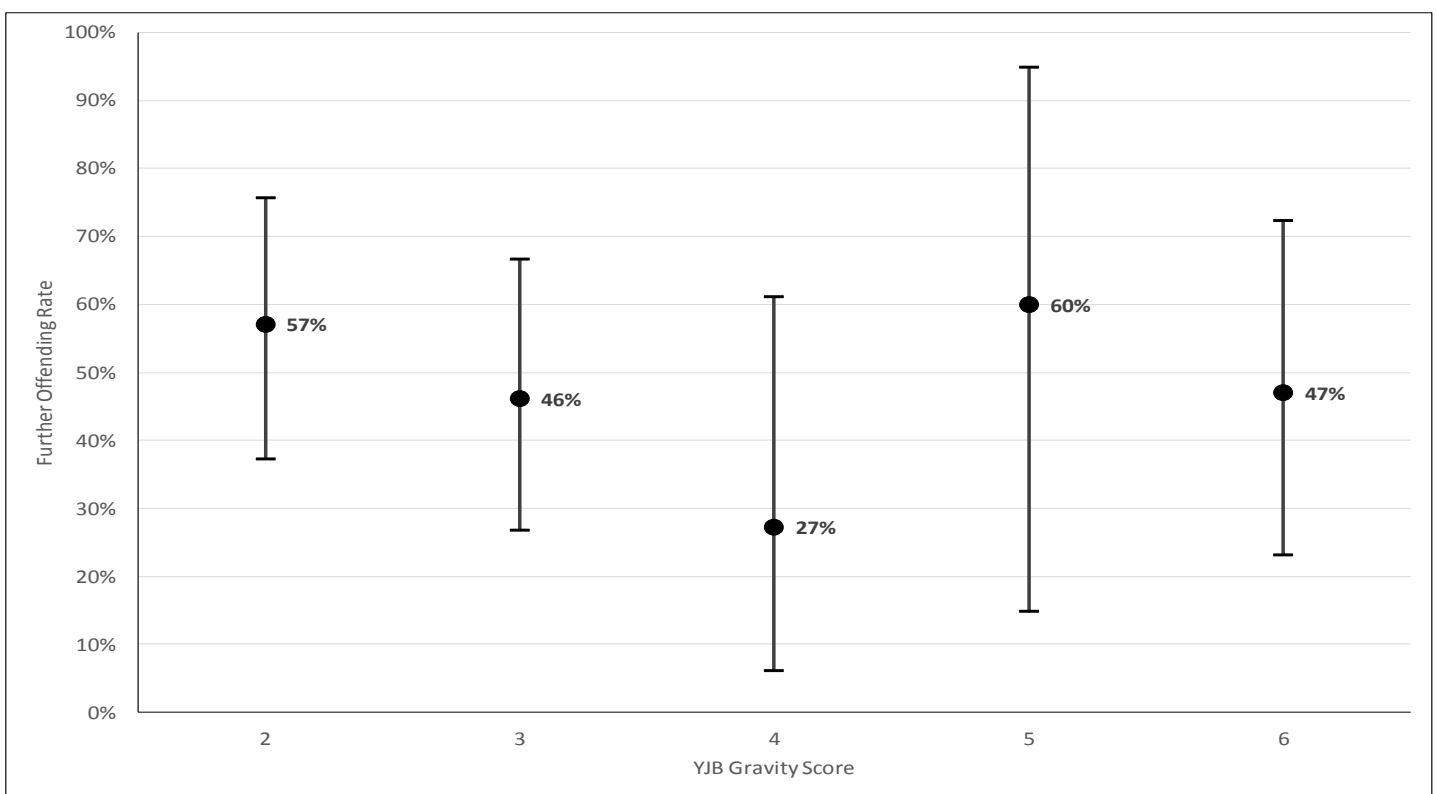

Note: $95 \%$ confidence intervals have been included to take into account the profile of the cohort by YJB Gravity Score. For example, there were 31 individuals whose primary offence had a gravity score of 2 and 30 with a gravity score of 3 . This compares to 9 with a gravity score of 4; just 4 with a gravity score of 5 and 13 with a gravity score of 6 . 
Figure 6.15: Change in the Probability of Further Offending Over Time, by YJB Gravity Score
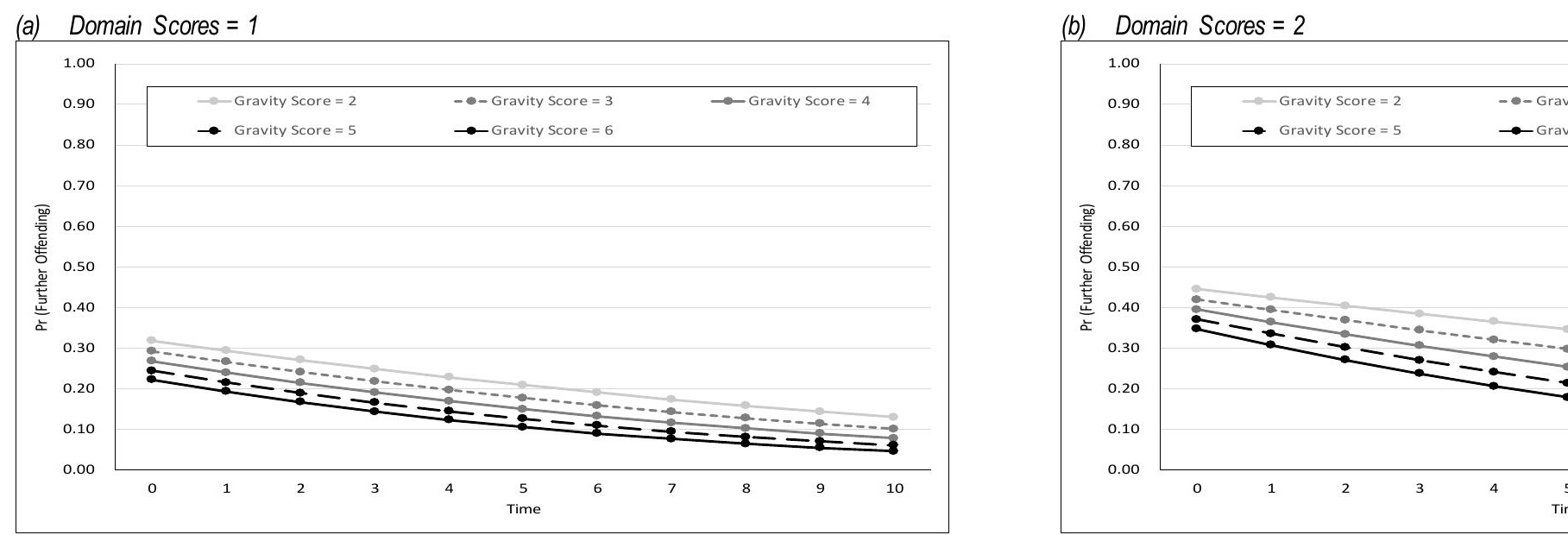

(c) Domain Scores $=3$

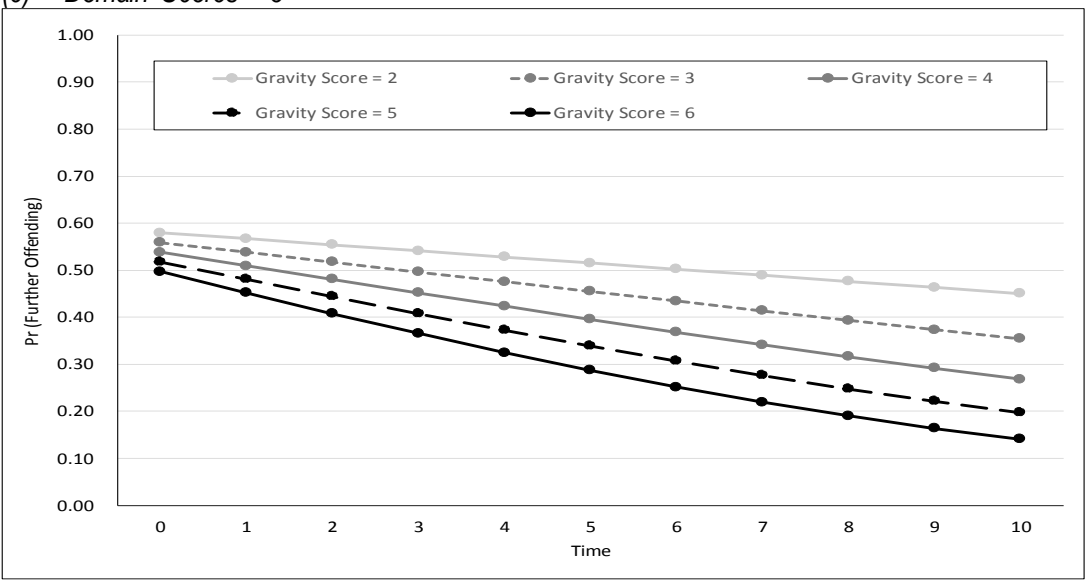

(d) Domain Scores $=4$

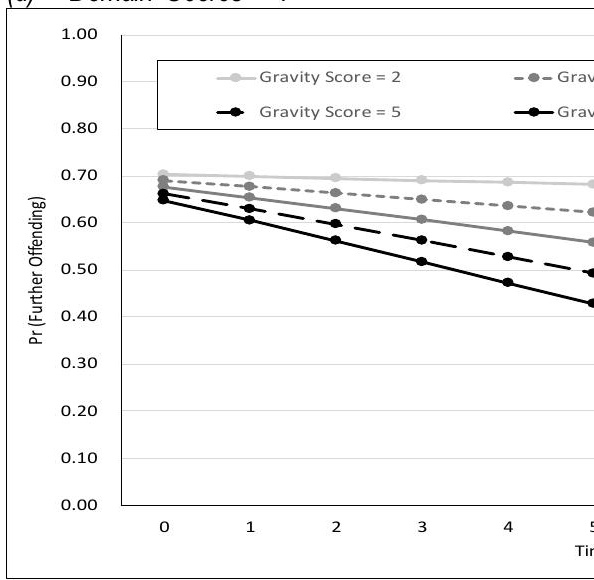




\subsection{A Combined Model Involving Offending History}

Ideally, if there were sufficient data to supportit, the combined model would involve measures reflecting all four of the static factors. However, it has been necessary to compromise. The following dynamic model therefore utilises:

- Grouped Age at First Offence - a dichotomous variable

- FTE Status - a dichotomous variable

- Seriousness of the Offence(I_Seriousness2) - a continuous variable

\section{Version 1}

From Model3b (summarised in Table6.15), ithas been established that is possible to simulate estimates for the interactions between FTE: G_ageFirst, FTE: I_Seriousness2, and G_ageFirst:I_Seriousness2. This knowledge has been used to inform the specification of a combined model:

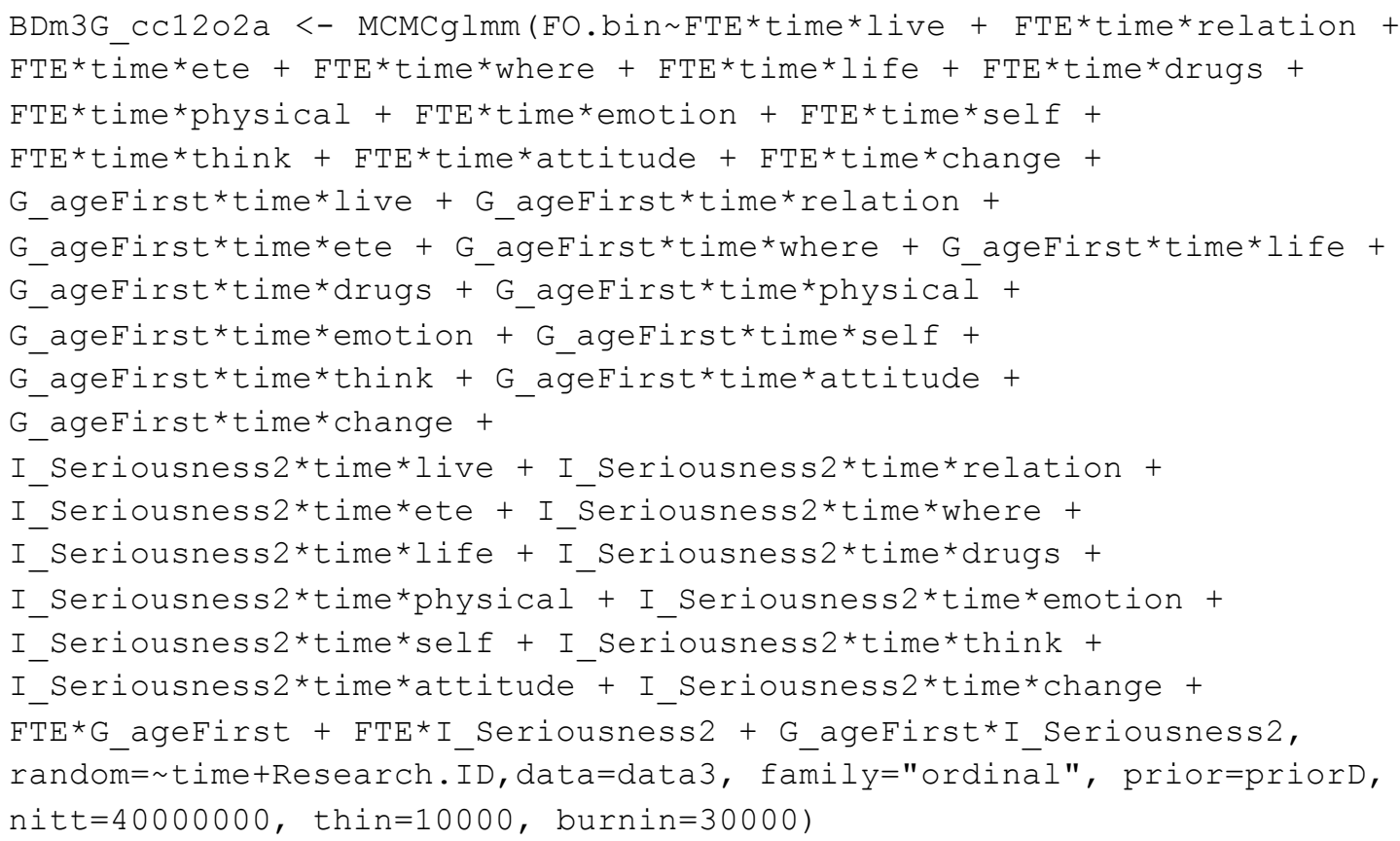

As can be seen from the resulting output (Technical Annex, p228-250), the Raftery-Lewis diagnosic suggests that the required number of iterations should be at least 48 million (rather than 56 million). However, in order to deal with the autocorrelation within the model, the thinning needs to be increased to 50,000 . In order to achieve this level of thinning and achieve the minimum effective sample size, the number of iterations would need to be increased to 188 million. This is not feasible to run as it will take over a week.

The plots do not point to any significant issues, nor does the overall effective sample size of 3,997. However, closer inspection of the model summaryhighlights that the effective sample size for some of Page $\mid 210$ 
the estimates is notably lower. For example, the effective sample size for 'self' as a main effect is 599.7 whereas that for I_Seriousness2 is 531.8. Additionally, the effective sample size for some of the estimates for the interactions e.g. FTE: Time and I_Seriousness: Relation are even lower at 459.5 and 424.1 respectively. This reiterates the need to consider the autocorrelation not just of the random effects, but also the fixed effects in the model.

Whilst the DIC of 294.7 indicated the potential of this model, the wide credible intervals around many of the individual estimates suggest that it would not be possible to use this to determine the probability of further offending.

\section{Version 2}

In accepting that there is insufficient data to simulate models based on a series of all three measures, these have been considered in pairs. For example, the first of these involves a series of interaction between FTE, time and the 12 domains; G_ageFirst, time and the 12 domains; and an interaction between FTE and G_ageFirst:

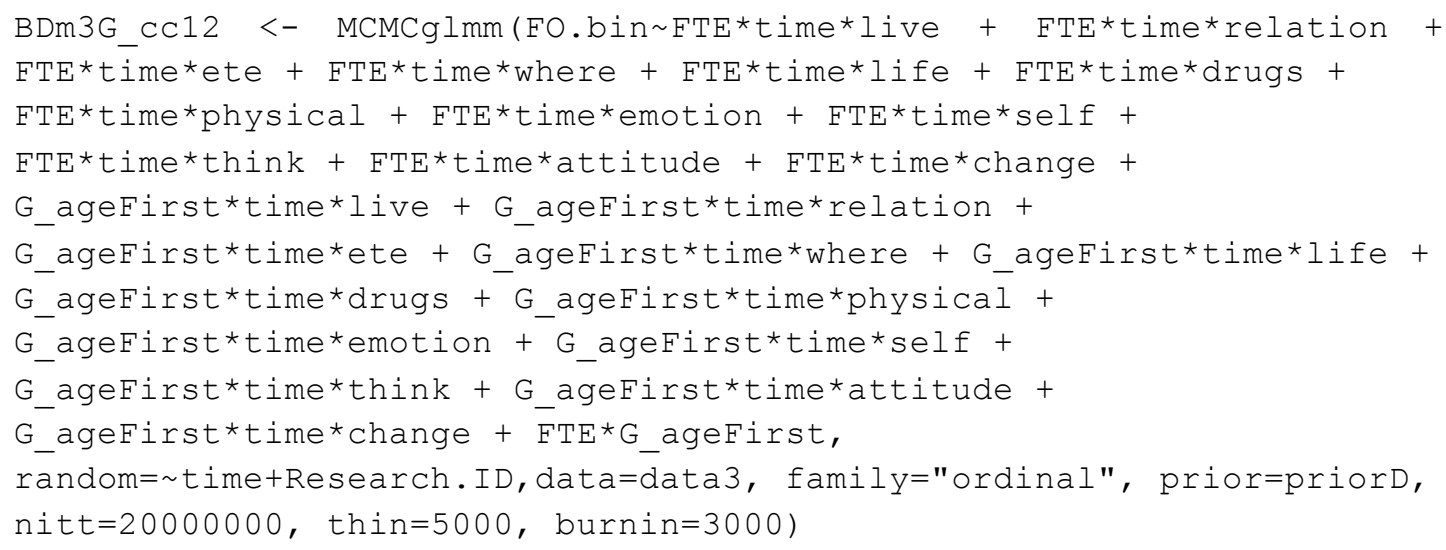

Equivalent models have also been run involving:

- FTE Status and YJB Gravity Score (BDm3_cc102a)

- Grouped Age at First Offence and YJB Gravity Score (BDm3G_cc202a)

The output from these models canbe found in the Technical Annex on pages 251-302. Of the 3 models, it is BDm3G_cc12 i.e. the model specified above which has the lowest DIC.

\begin{tabular}{|l|l|r|}
\hline Model & Static Factors in Model & \multicolumn{1}{c|}{ DIC } \\
\hline BDm3G_cc12 & FTE and Grouped Age at First Offence & 391.2 \\
\hline BDm3_cc102a & FTE and YJB Gravity Score & 432.8 \\
\hline BDm3G_cc2o2a & Grouped Age at First Offence and YJB Gravity Score & 434.8 \\
\hline
\end{tabular}


An examination of the output and plots from the dynamic model involving FTE and I_Seriousness2 (BDm3G_cc102a) suggests that there are no notable issues in terms of convergence or the effective sample sizes, that is the estimates for the number of independent samples (taking into account autocorrelations) generated by the MCMC run. Although, the effective sample size of 3,537 for the estimate for the interaction between FTE, Time and Drugs falls below 3,746, this is the only effective sample size to do this.

It was necessary to run the dynamic model involving G_ageFirstand I_Seriousness2 (BDm3G_cc202a) for slightly longer than for BDm3G_cc102a in order to meet the criteria for the various convergence diagnostics. Although an effective sample size of 4,997 was achieved by simulating a model with 5 million iterations, with a thinning of 1,000 and burn-in of 1000, the resulting output indicates that the effective sample size for the interaction between Neighbourhood (Where) and Time is the only one to fall below 3,746 .

\section{Version 3}

Whilst acknowledging that this represents a compromise since there is insufficient data to support a more complex version, this model builds upon BDm3G_cc12 to include 2-wayinteractions between:

- I_Seriousness2 and Time

- G_ageFirstandI_Seriousness2

- FTE and I_Seriousness2

Whilst this model does not include any interactions between the 12 domains and I_Seriousness2, the inclusion of these interactions does enable some of the potential uncertaintyaround the seriousness of the primary offence to be explored. As a result, three out of the four proxies for the static factors are included.

To address the autocorrelation, it is necessary to set the thinning to 2,000 and increase the burn-in to 5,000 . The resulting model requires at least $7,497,000$ iterations $(3,746 \times 2,000$ plus 5,000$)$. To ensure that the sample size is sufficient, 8 million iterations were run. The outputfrom the various convergence diagnostics can be found in the Technical Annex, pages 303-321. The model is summarised in Table 6.20 and for convenience, is renamed as $\mathrm{BDm} 3$. 
Table 6.20: Dynamic Model 3

\begin{tabular}{|c|c|c|c|c|c|c|c|}
\hline \multirow[b]{3}{*}{ Fixed Effect: } & \multicolumn{7}{|c|}{$\begin{array}{l}\text { Dynamic Basic Model including FTE Status and Grouped Age at First Offence } \\
\text { with 2-way Interactions involving YJB Gravity Score (BDm3G_cc12_o2) }\end{array}$} \\
\hline & \multicolumn{3}{|c|}{ Unstandardised } & \multicolumn{3}{|c|}{\begin{tabular}{|c|} 
Standardised \\
\end{tabular}} & \multirow{2}{*}{ Significant? } \\
\hline & Post.Mean & Lower $\mathrm{Cl}$ & Upper $\mathrm{Cl}$ & Post.Mean & Lower $\mathrm{Cl}$ & Upper Cl & \\
\hline (Intercept) & -5.151 & -11.164 & 0.570 & 0.006 & 0.000 & 1.769 & \\
\hline YJB Gravity Score (zero = 2) (Seriousness) & 0.085 & -1.265 & 1.478 & 1.089 & 0.282 & 4.384 & \\
\hline First Time Entant (No = Ref) (FTE) & -4.720 & -14.578 & 4.547 & 0.009 & 0.000 & 94.371 & \\
\hline Grouped Age at First Offence (10-12 = Ref) & 5.144 & -1.234 & 11.585 & 171.444 & 0.291 & $1.07 \mathrm{E}+05$ & \\
\hline Time & 0.201 & -0.725 & 1.139 & 1.223 & 0.484 & 3.125 & \\
\hline Living Arrangements (Live) & -0.674 & -2.386 & 0.979 & 0.510 & 0.092 & 2.663 & \\
\hline Family and Personal Relationships (Relation) & 2.671 & 0.384 & 4.840 & 14.454 & 1.468 & 126.427 & Yes \\
\hline Education, Training and Employment (ETE) & -1.092 & -2.650 & 0.443 & 0.335 & 0.071 & 1.557 & \\
\hline Neighbourhood (Where) & 0.480 & -0.870 & 1.758 & 1.616 & 0.419 & 5.802 & \\
\hline Lifestyle (Life) & 2.513 & 0.199 & 4.968 & 12.348 & 1.221 & 143.695 & Yes \\
\hline Substance Use (Drugs) & -0.447 & -2.009 & 1.270 & 0.640 & 0.134 & 3.561 & \\
\hline Physical Health (Physical) & -1.160 & -2.987 & 0.611 & 0.314 & 0.050 & 1.842 & \\
\hline Emotional and Mental Health (Emotion) & -0.472 & -1.889 & 1.008 & 0.624 & 0.151 & 2.741 & \\
\hline Perceptions of Self and Others (Self) & -6.736 & -10.171 & -3.456 & 0.001 & 0.000 & 0.032 & Yes \\
\hline Thinking and Behaviour (Think) & 1.840 & -0.618 & 4.406 & 6.296 & 0.539 & 81.908 & \\
\hline Attitude to Offending (Attitude) & 2.819 & -0.321 & 6.040 & 16.757 & 0.725 & 419.973 & \\
\hline Motivation to Change (Change) & 1.065 & -1.535 & 3.995 & 2.901 & 0.215 & 54.335 & \\
\hline Seriousness: FTE & 0.509 & -0.709 & 1.936 & 1.664 & 0.492 & 6.931 & \\
\hline Seriousness: Grouped Age at First Offence & -0.673 & -2.241 & 0.841 & 0.510 & 0.106 & 2.320 & \\
\hline Seriousness: Time & 0.042 & -0.080 & 0.161 & 1.042 & 0.923 & 1.175 & \\
\hline FTE: Grouped Age at First Offence & 4.642 & -4.263 & 13.741 & 103.803 & 0.014 & $9.29 \mathrm{E}+05$ & \\
\hline FTE: Time & -1.046 & -2.577 & 0.474 & 0.351 & 0.076 & 1.606 & \\
\hline FTE: Live & 1.080 & -1.841 & 4.083 & 2.944 & 0.159 & 59.300 & \\
\hline FTE: Relation & -0.025 & -2.811 & 2.747 & 0.976 & 0.060 & 15.602 & \\
\hline FTE: ETE & 1.228 & -1.147 & 3.682 & 3.416 & 0.318 & 39.706 & \\
\hline FTE: Where & -4.412 & -7.223 & -1.939 & 0.012 & 0.001 & 0.144 & Yes \\
\hline FTE: Life & 2.334 & -1.050 & 5.869 & 10.316 & 0.350 & 353.918 & \\
\hline FTE: Drugs & -0.964 & -3.505 & 1.834 & 0.382 & 0.030 & 6.261 & \\
\hline FTE: Physical & 1.310 & -1.556 & 4.124 & 3.707 & 0.211 & 61.798 & \\
\hline FTE: Emotion & -0.687 & -2.927 & 1.532 & 0.503 & 0.054 & 4.628 & \\
\hline FTE: Self & 2.151 & -0.632 & 4.936 & 8.597 & 0.532 & 139.230 & \\
\hline FTE: Think & -2.604 & -5.332 & 0.132 & 0.074 & 0.005 & 1.141 & \\
\hline FTE: Attitude & -3.706 & -6.715 & -1.076 & 0.025 & 0.001 & 0.341 & Yes \\
\hline FTE: Change & 3.256 & 0.291 & 6.521 & 25.958 & 1.338 & 679.214 & Yes \\
\hline Grouped Age at First Offence: Time & -0.587 & -1.873 & 0.828 & 0.556 & 0.154 & 2.289 & \\
\hline Grouped Age at First Offence: Live & 1.274 & -1.152 & 3.454 & 3.575 & 0.316 & 31.641 & \\
\hline Grouped Age at First Offence: Relation & -4.090 & -7.298 & -1.260 & 0.017 & 0.001 & 0.284 & Yes \\
\hline Grouped Age at First Offence: ETE & 0.332 & -1.547 & 2.373 & 1.394 & 0.213 & 10.735 & \\
\hline Grouped Age at First Offence: Where & 1.478 & -0.591 & 3.579 & 4.383 & 0.554 & 35.836 & \\
\hline Grouped Age at First Offence: Life & -2.416 & -5.930 & 0.777 & 0.089 & 0.003 & 2.174 & \\
\hline Grouped Age at First Offence: Drugs & 0.358 & -1.860 & 2.531 & 1.430 & 0.156 & 12.566 & \\
\hline Grouped Age at First Offence: Physical & -1.260 & -3.939 & 1.431 & 0.284 & 0.019 & 4.182 & \\
\hline Grouped Age at First Offence: Emotion & 0.170 & -1.771 & 2.323 & 1.186 & 0.170 & 10.202 & \\
\hline Grouped Age at First Offence: Self & 8.071 & 4.001 & 12.025 & $3.20 E+03$ & 54.646 & $1.67 \mathrm{E}+05$ & Yes \\
\hline Grouped Age at First Offence: Think & -0.318 & -3.186 & 2.640 & 0.727 & 0.041 & 14.016 & \\
\hline Grouped Age at First Offence: Attitude & -0.362 & -4.096 & 3.347 & 0.696 & 0.017 & 28.413 & \\
\hline Grouped Age at First Offence: Change & -3.297 & -6.840 & 0.087 & 0.037 & 0.001 & 1.091 & \\
\hline
\end{tabular}

/ continued 


\begin{tabular}{|c|c|c|c|c|c|c|c|}
\hline \multirow[b]{3}{*}{ Fixed Effect: } & \multicolumn{7}{|c|}{$\begin{array}{l}\text { Dynamic Basic Model including FTE Status and Grouped Age at First Offence } \\
\text { with 2-way Interactions involving YJB Gravity Score (BDm3G_cc12_02) }\end{array}$} \\
\hline & \multicolumn{3}{|c|}{ Unstandardised } & \multicolumn{3}{|c|}{ Standardised } & \multirow{2}{*}{ Significant? } \\
\hline & Post.Mean & Lower $\mathrm{Cl}$ & Upper $\mathrm{Cl}$ & Post.Mean & Lower $\mathrm{Cl}$ & Upper $\mathrm{Cl}$ & \\
\hline Time: Live & -0.143 & -0.517 & 0.258 & 0.867 & 0.596 & 1.294 & \\
\hline Time: Relation & -0.519 & -1.013 & -0.013 & 0.595 & 0.363 & 0.987 & Yes \\
\hline Time: ETE & 0.208 & -0.114 & 0.528 & 1.231 & 0.893 & 1.696 & \\
\hline Time: Where & -0.193 & -0.461 & 0.085 & 0.824 & 0.631 & 1.089 & \\
\hline Time: Life & -0.528 & -1.055 & 0.039 & 0.590 & 0.348 & 1.040 & \\
\hline Time: Drugs & 0.340 & 0.020 & 0.701 & 1.405 & 1.021 & 2.015 & Yes \\
\hline Time: Physical & 0.327 & -0.118 & 0.756 & 1.387 & 0.888 & 2.130 & \\
\hline Time: Emotion & 0.373 & 0.076 & 0.676 & 1.452 & 1.078 & 1.965 & Yes \\
\hline Time: Self & 1.472 & 0.820 & 2.191 & 4.357 & 2.271 & 8.945 & Yes \\
\hline Time: Think & -0.366 & -0.842 & 0.068 & 0.693 & 0.431 & 1.071 & \\
\hline Time: Attitude & -0.859 & -1.421 & -0.282 & 0.423 & 0.241 & 0.754 & Yes \\
\hline Time: Change & 0.099 & -0.413 & 0.605 & 1.105 & 0.661 & 1.830 & \\
\hline FTE: Time: Live & -0.593 & -1.354 & 0.139 & 0.552 & 0.258 & 1.149 & \\
\hline FTE: Time: Relation & -0.083 & -0.805 & 0.602 & 0.920 & 0.447 & 1.826 & \\
\hline FTE: Time: ETE & 0.434 & -0.227 & 1.116 & 1.544 & 0.797 & 3.052 & \\
\hline FTE: Time: Where & 0.606 & 0.102 & 1.065 & 1.833 & 1.108 & 2.900 & Yes \\
\hline FTE: Time: Life & -0.526 & -1.400 & 0.338 & 0.591 & 0.246 & 1.402 & \\
\hline FTE: Time: Drugs & 0.695 & 0.066 & 1.285 & 2.004 & 1.068 & 3.615 & Yes \\
\hline FTE: Time: Physical & -0.920 & -1.851 & -0.108 & 0.398 & 0.157 & 0.897 & Yes \\
\hline FTE: Time: Emotion & 0.498 & -0.122 & 1.135 & 1.646 & 0.885 & 3.111 & \\
\hline FTE: Time: Self & -0.807 & \begin{tabular}{|l|}
-1.497 \\
\end{tabular} & -0.160 & 0.446 & 0.224 & 0.852 & Yes \\
\hline FTE: Time: Think & 0.854 & 0.141 & 1.529 & 2.348 & 1.152 & 4.615 & Yes \\
\hline FTE: Time: Attitude & 1.087 & 0.331 & 1.922 & 2.965 & 1.393 & 6.833 & Yes \\
\hline FTE: Time: Change & -0.987 & -1.799 & -0.236 & 0.373 & 0.165 & 0.789 & Yes \\
\hline Grouped Age at First Offence: Time: Live & 0.325 & -0.283 & 0.940 & 1.384 & 0.754 & 2.561 & \\
\hline Grouped Age at First Offence: Time: Relation & 1.049 & 0.391 & 1.773 & 2.855 & 1.479 & 5.890 & Yes \\
\hline Grouped Age at First Offence: Time: ETE & -0.308 & -0.807 & 0.198 & 0.735 & 0.446 & 1.219 & \\
\hline Grouped Age at First Offence: Time: Where & -0.004 & -0.432 & 0.433 & 0.996 & 0.649 & 1.541 & \\
\hline Grouped Age at First Offence: Time: Life & 0.358 & -0.464 & 1.198 & 1.430 & 0.629 & 3.313 & \\
\hline Grouped Age at First Offence: Time: Drugs & -0.622 & -1.204 & -0.151 & 0.537 & 0.300 & 0.860 & Yes \\
\hline Grouped Age at First Offence: Time: Physical & 0.554 & -0.233 & 1.365 & 1.741 & 0.793 & 3.917 & \\
\hline Grouped Age at First Offence: Time: Emotion & -0.593 & -1.183 & -0.023 & 0.553 & 0.306 & 0.977 & Yes \\
\hline Grouped Age at First Offence: Time: Self & -1.562 & -2.374 & -0.739 & 0.210 & 0.093 & 0.478 & Yes \\
\hline Grouped Age at First Offence: Time: Think & -0.163 & -0.820 & 0.494 & 0.850 & 0.440 & 1.639 & \\
\hline Grouped Age at First Offence: Time: Attitude & 0.106 & -0.695 & 0.908 & 1.112 & 0.499 & 2.479 & \\
\hline Grouped Age at First Offence: Time: Change & 0.654 & -0.129 & 1.402 & 1.923 & 0.879 & 4.064 & \\
\hline Random Effect: & Post.Mean & Lower $\mathrm{Cl}$ & Upper $\mathrm{Cl}$ & Post.Mean & Lower $\mathrm{Cl}$ & Upper $\mathrm{Cl}$ & Significant? \\
\hline Individual (Intercept) & 7.37 & 1.133 & 15.09 & $1.59 \mathrm{E}+03$ & 3.105 & $3.58 \mathrm{E}+06$ & Yes \\
\hline Time & 13.280 & 1.636 & 31.540 & $5.85 \mathrm{E}+05$ & 5.135 & $4.98 \mathrm{E}+13$ & Yes \\
\hline
\end{tabular}

Source: Model BDm3G_cc12_02, renamed as BDm3, Technical Annex: p303-321

With a DIC of 387.9, this model appears to represent an improvement upon Model BDm3G_cc12. However, inclusion of I_Seriousness and the 2-way interactions involving Time, FTE and G_ageFirst does not appear to address the amount of uncertainty around the estimates for FTE and G_ageFirst both as main effects and the interaction between the two. The credible interval around the coefficient for the perception of selfand others (Self) is also particularlywide as is the interval around the coefficient for the interaction G_ageFirst: Self. When the estimated probabilities of further offending are calculated, the implications of this become more apparent. 
Figure 6.16: Change in the Probability of Further Offending Over Time, Dynamic Model 3
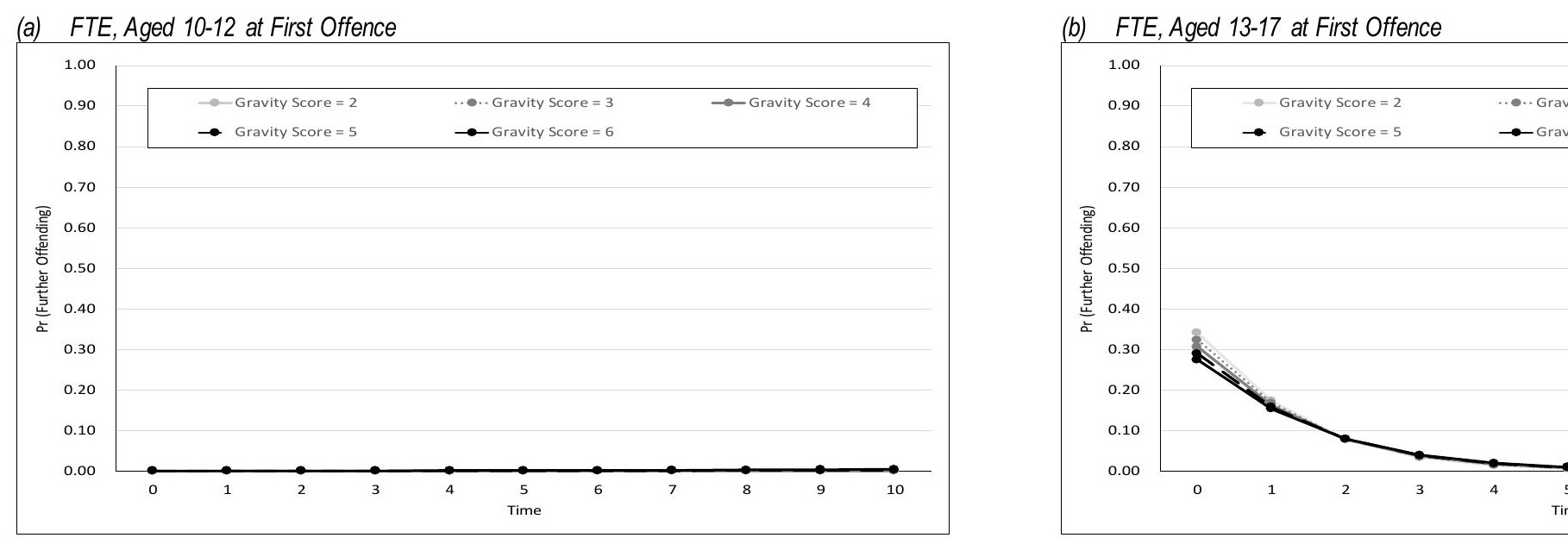

(c) Prior Offending Behaviour, Aged 10-12 at First Offence

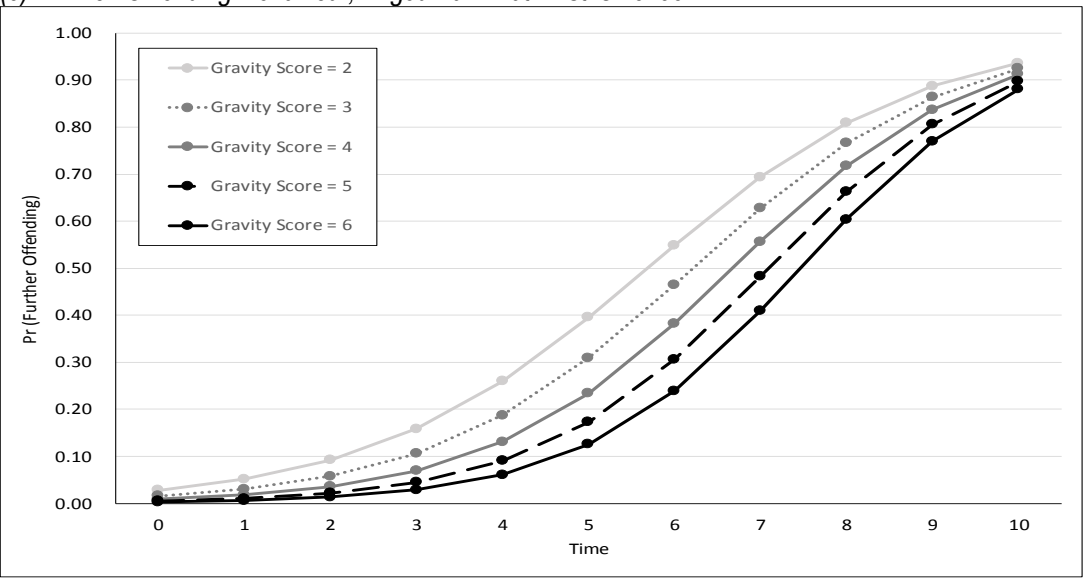

(d) Prior Offending Behaviour, Aged 13-17 at Firs

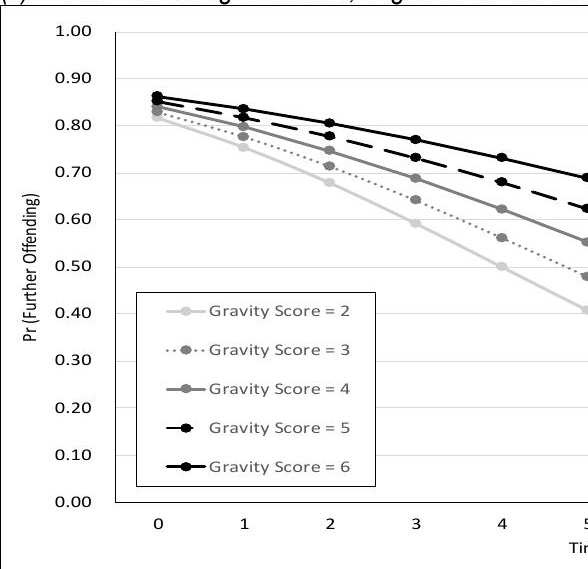


The very low probabilities of further offending amongstFTEs who were aged 10-12 at the time of their first offence (Figure 6.15(a)) are a reflection of the underlying data - there were just 3 cases within the dataset who shared these characteristics. None of these committed further offences hence the inital probability of further offending being zero, regardless of the seriousness of the primary offence.

Amongst FTEs who were aged 13-17 at the time of their first offence, the initial probability of further offending is higher amongst those who committed primaryoffences with a lower gravity score - a trend apparentin the dynamic modelinvolving YJB Gravity Score, (BDm3_02a, summarised in Table 6.19 and Figure 6.14). After Time 2, there is little difference in the downward trajectory of the estimates of probability for each YJB Gravity Score, approaching a probability of zero by Time 7. Amongst the 30 cases which shared these characteristics, the further offending rate was $36.7 \%(11 / 30)$.

Two-thirds $(68 \%, 13 / 19)$ of those who had committed their first offence when aged $10-12$ but had a history of prior offending at the time of entering the reoffending cohortwas involved in further offend ing behaviour. Figure 6.15 (c) summarises the estimates of the probability of further offending over time for this sub-group. Notably the initial probabilities of further offending are very low (as for FTEs aged 1012, Figure 6.15(a)). However, the longer the individual is in contact with the youth justice system, the higher the estimated probability of further of further offending. Once more, the estimated probabilities of further offending are higher for lower gravity scores.

Just over half $(51 \%, 18 / 35)$ of those with a prior offending history, who had been aged $13-17$ at the time of their first offence committed further offences. What stands out in the summary of the estimated probabilities of further offending for this group is that at each measurement point, is that they are higher for the higher gravity scores. As time increases, the probabilities of further offending fall and hence this is quite different to the trend seen in Figure 6.15(c) for the younger group. The trend in Figure 6.15(d) is more in keeping with what would be expected if the premise that as a result of working with the YOT, the risk of further offending is mediated. 


\subsection{How do the models involving static factors reflect the realities of real lives?}

The following section returns once more to the examples of Fred, David and Connor, to consider how the estimates of their respective probabilities of further offending based on (1) the individual dynamic models summarised in section 6.4, (2) the permutations considered under Version 2 and (3) the combined model involving the three static factors. In each instance these are compared to the estimates generated by the Basic Dynamic Model (BDm1, summarised in Table 4.12).

\section{Case Study "Fred"}

"Fred" is a white male who was an FTE at the time of entering the reoffending cohort. He committed his first offence aged 14, receiving his first conviction at age 15. The offence which led to his inclusion in the reoffending cohort was a criminal damage offence (Gravity Score $=2$ ). This type of offence falls within the 'Other' offence category. Using this information along with the domain scores from each ASSET, the following estimates of probability of further offending have been calculated from the individual dynamic models:

Figure 6.17: Comparisons of the Estimated Probability of Further Offending Over Time - Individual Dynamic Models: "Fred"

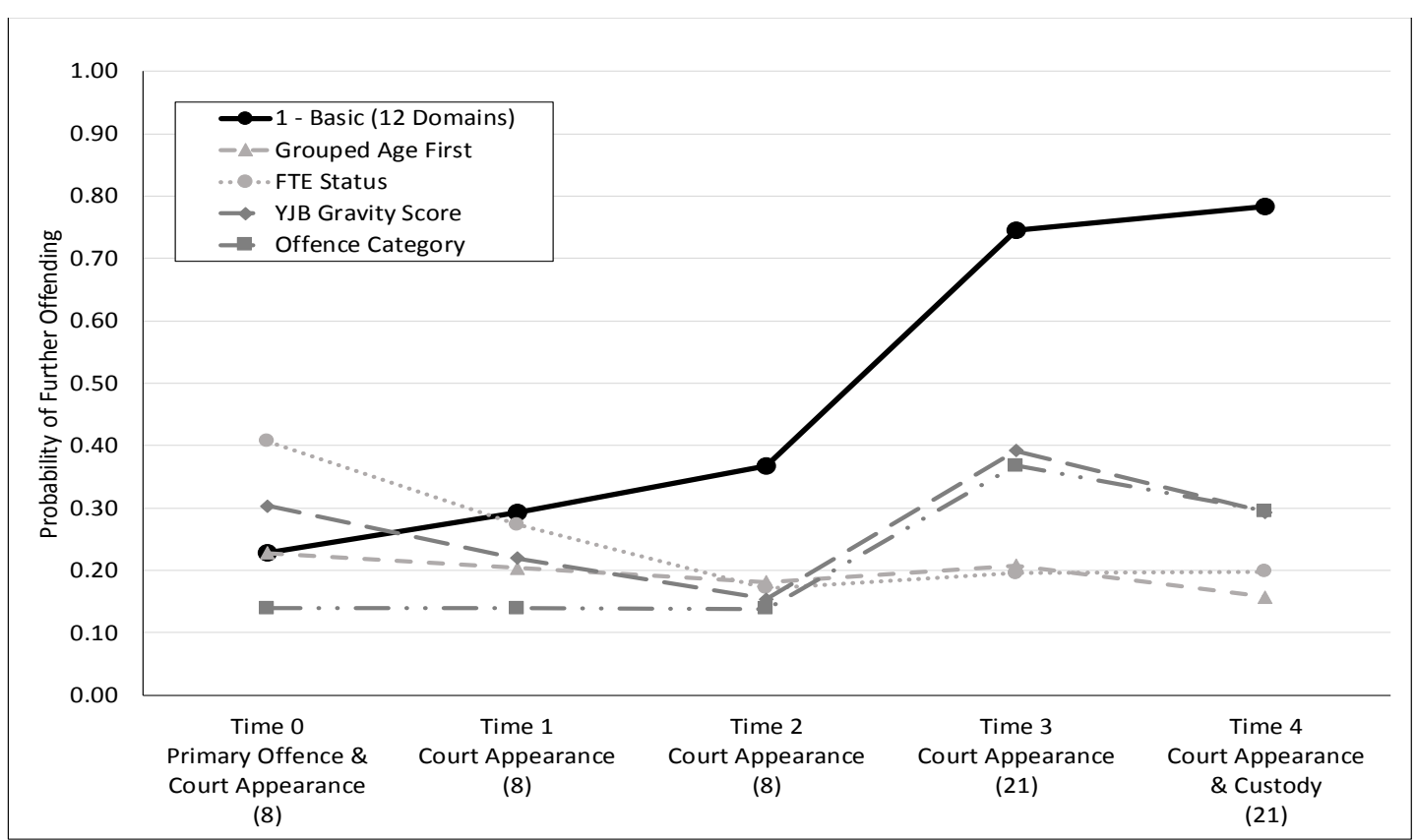

Source: BDm3_cc1 (FTE Status), BDm3G_cc2 (Grouped Age at First Offence), BDm3G_01 (Grouped YJB Offence Category) and BDm3_02a (YJB Gravity Score) along with BDm1.

The four models resultin quite different estimates for Fred's initial probability of furth er offending, one of which is the same as that resulting from the Basic Dynamic model based on the 12 domains. Those for FTE status and the YJB Gravity Score are higher. However, by Time 1, all are lower than the estimate from the Basic Dynamic Model. Theyfell again by Time 2. 
Figure 6.18: Changes in the Probability of Further Offending Over Time: "Fred"

\section{(a) Version 2: FTE and Grouped Age at First Offence}

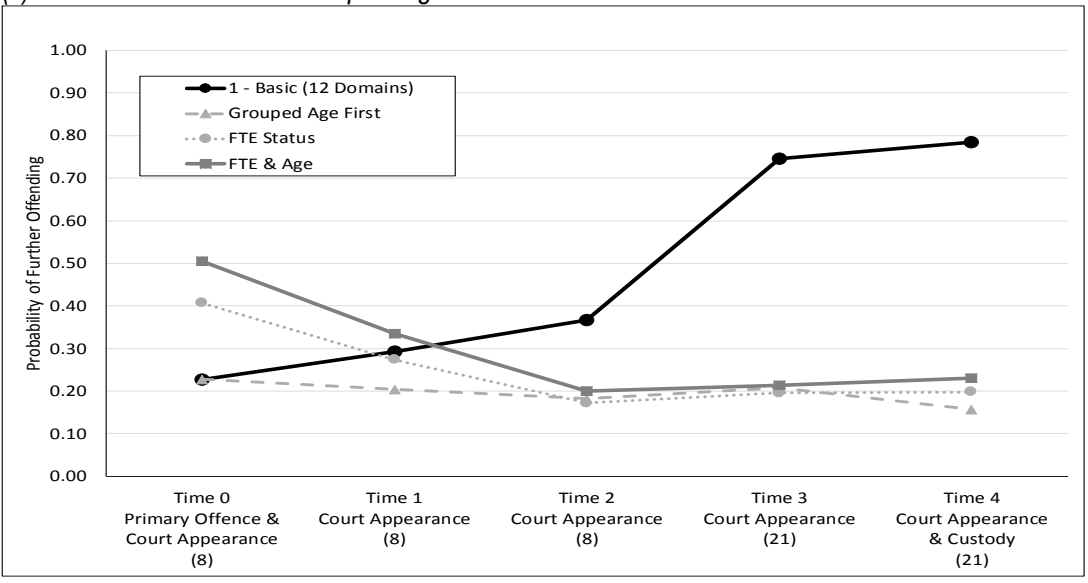

(c) Version 2: Grouped Age at First Offence and YJB Gravity Score

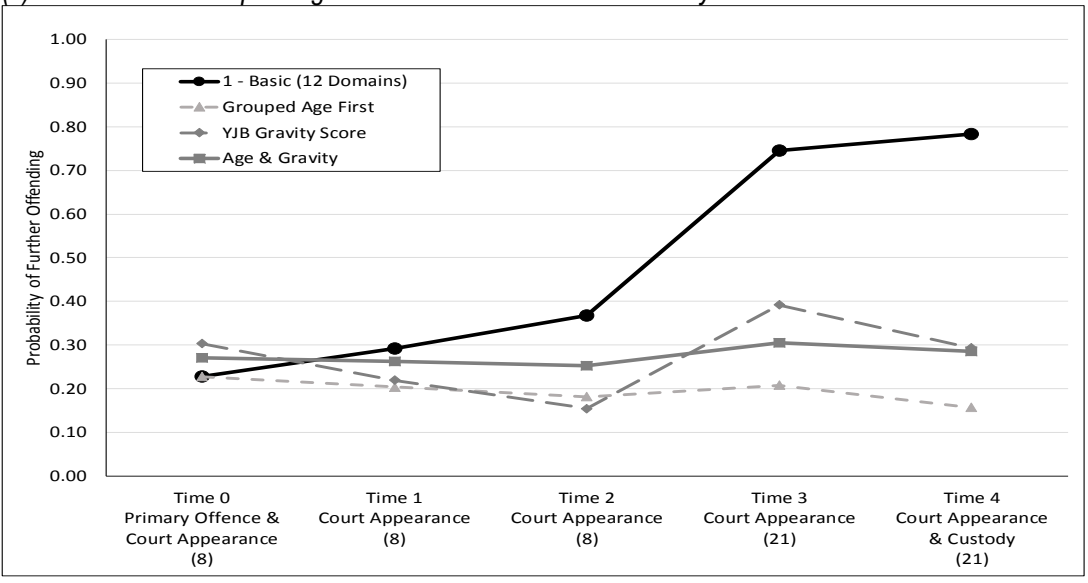

(b) Version 2: FTE and YGB Gravity Score

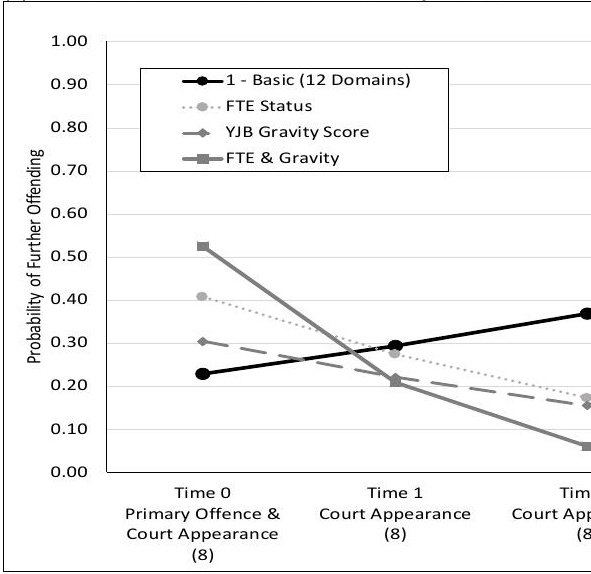

(d) Combined Model: Based Upon Enhancing Ver

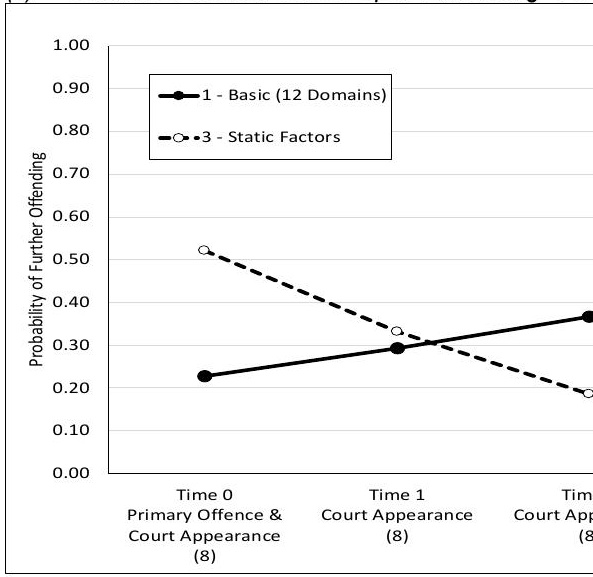


Between Time 2 and Time 3, Fred was on ISSP Bail and Tag, and he attended court four times. His ASSET score increased from 8 to 21 . This increase is reflected in the estimates based on the models involving the YJB Gravity Score and Offence Category, whereas the models involving Grouped Age at First Offence and FTE status do not. Fred was sentenced to custody a week after this assessment at Time 3. Hence during the intervening period, there was little opportunity for the individual domain scores to alter. However, whereas the Basic Dynamic Model suggests an increase in Fred's probability of further offending, the dynamic models involving YJB Gravity Score, Offence Categoryand Grouped Age at First Offence show a downward trend between Time 3 and Time 4.

Of the three models specified under Version 2, it is notable that those involving FTE status in combination with another static factor (summarised in Figures 6.16(a) and (b)) result in higher initial probabilities of further offending then the dynamic models based on the static factors individually.

The model involving both Grouped Age at First Offence and YJB Gravity Score (Figure 6.15(c)) reflects the increase in ASSET scores between Time 2 and 3 and corresponding increase in the probability of further offending that would be expected as a result of this, although not to the extent suggested by the Basic Dynamic Model. The other models do not show this.

Given that the combined model (Figure 6.15(d)) is an enhanced version of that summarised in Figure 6.15(a), the trajectories for the estimated probabilities of further offending over time based upon Fred's domain scores are very similar.

\section{Case Study "Connor"}

"Connor" was also aged 14 at the time of his first offence. However, unlike Fred, he had been identified as a prolific offender prior to entering the 2012/13 reoffending. Connor's primary offence was a burglary (Gravity Score $=6$ ), and therefore is categorised as a serious acquisitive crime. Using this information along with Connor's domainscores at each measurement point, it is possible to dete rmine the estimated probabilities of further offending over time based on each model involving static factors. Figure 6.19 summarises the probabilities of further offending based on the individual dynamic static factors.

The initial probabilities arising from each individual dynamic model are lower than those suggested by the Basic Dynamic Model, with those simulated by the models involving FTE Status and YJB Gravity Score being the lowest. As time progresses, the estimated probabilities of further offending suggested by these two models is perhaps the mosterratic. 


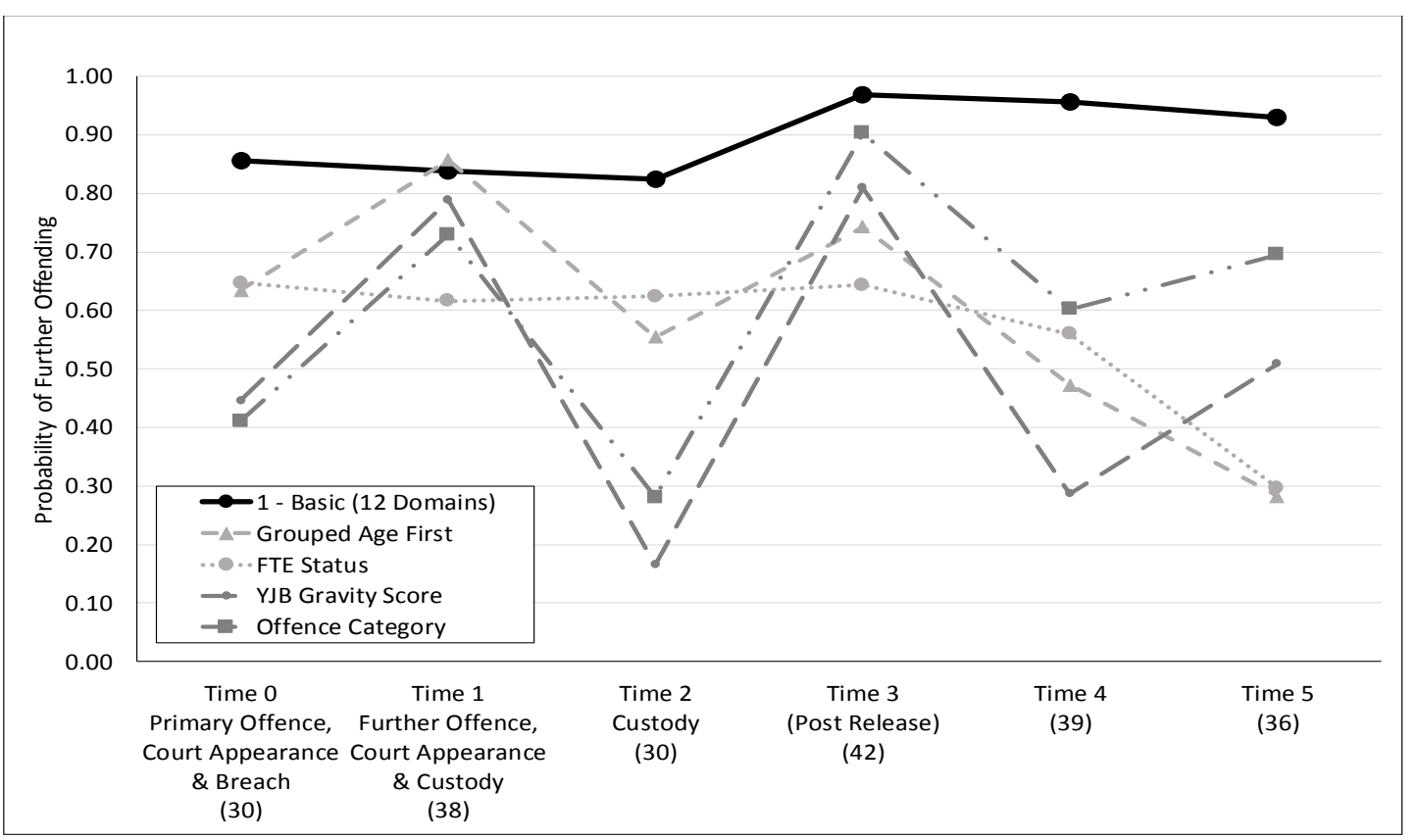

Notes: Although the ASSET scores reflected along the $x$-axis are out of a maximum of 48 with Connor having a total of 30 at Time 0, under the Scaled Approach he would have attracted additional scores due to the fact that his primary offence (for the purposes of this exercise where the information has been taken from the reoffending spreadsheet) was a non-domestic burglary and as a result of his prior convictions.

Connor committed a further offence prior to his assessment at Time 1. The estimated probabilities simulated by the individual dynamic models involving Grouped Age at First Offence, YJB Gravity Score and Offence Categoryincrease - something which is notapparent with the Basic Dynamic model despite the increase in Connor's total ASSET scores (from 30 to 38 ).

The assessment undertaken at Time 2 coincided with when Connor was in custody. Once more the estimated probabilities of further offending based on the models involving Grouped Age atFirst Offence, YJB Gravity Score and Offence Category fell. Connor's total ASSET score at Time 2 was the same as that for Time 0 . However, the estimated probabilities of further offending were lower, potentially an impact of the moderating effect of time.

Post release (T ime 3) all the models, including the Basic Dynamic Model and that involving FTE Status suggestan increase in the risk of further offending. This is as hypothesised and will be explored further in Chapter Seven.

Of the individual models, it is the dynamic model involving FTE Status which potentially most closely follows the shape of that for the Basic Dynamic Model although the drop off between Times 3 and 5 is steeper and at each measurement point, the estimated probabilities of further offending are notably lower. 
Figure 6.20: Changes in the Probability of Further Offending Over Time: "Connor"

\section{(a) Version 2: FTE and Grouped Age at First Offence}

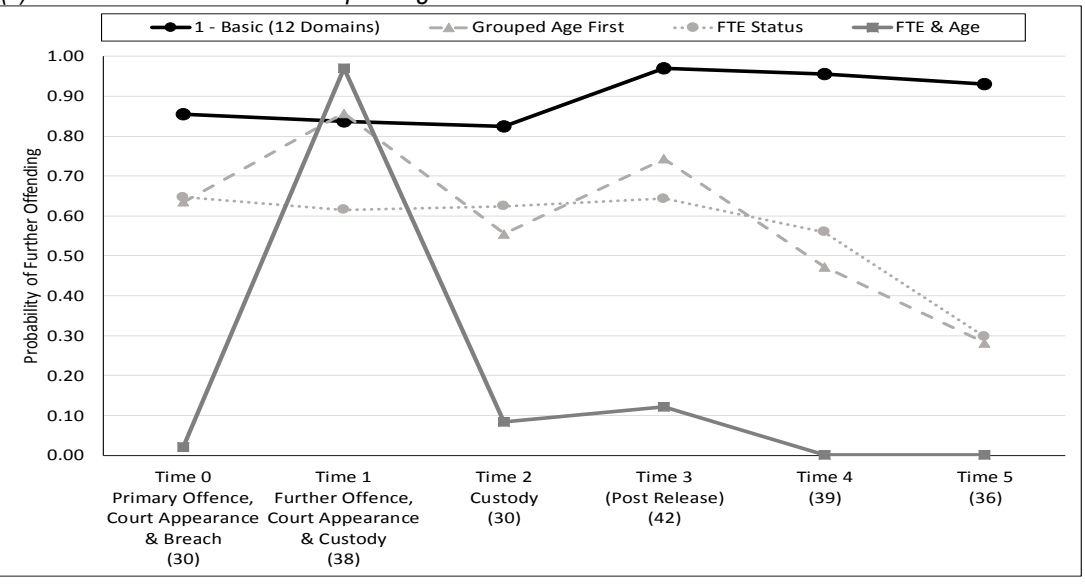

(c) Version 2: Grouped Age at First Offence and YJB Gravity Score

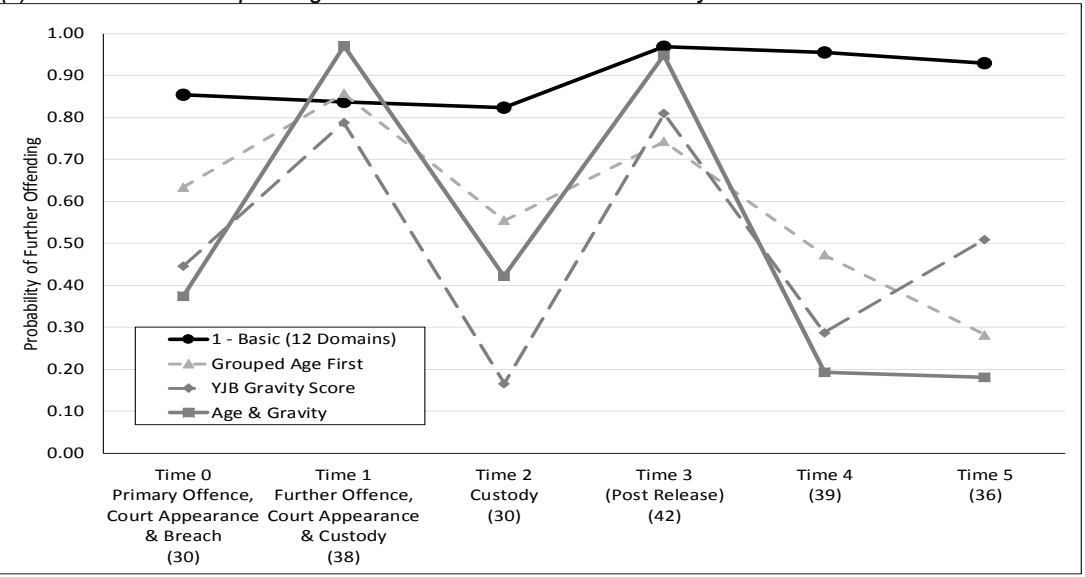

\section{(b) Version 2: FTE and YGB Gravity Score}

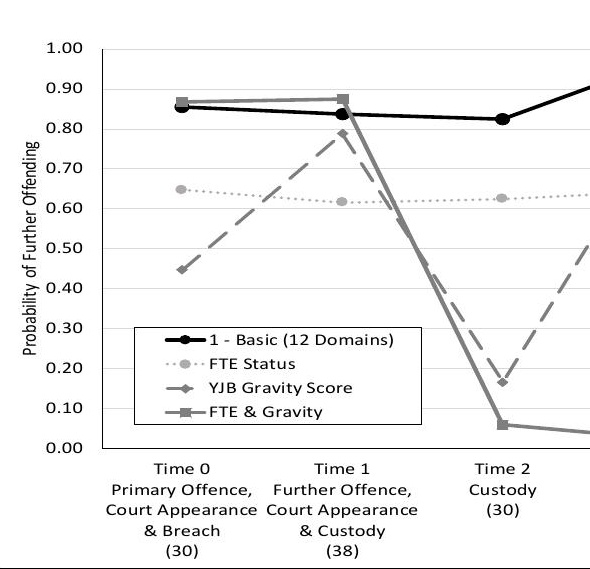

(d) Combined Model: Based Upon Enhancing Ver

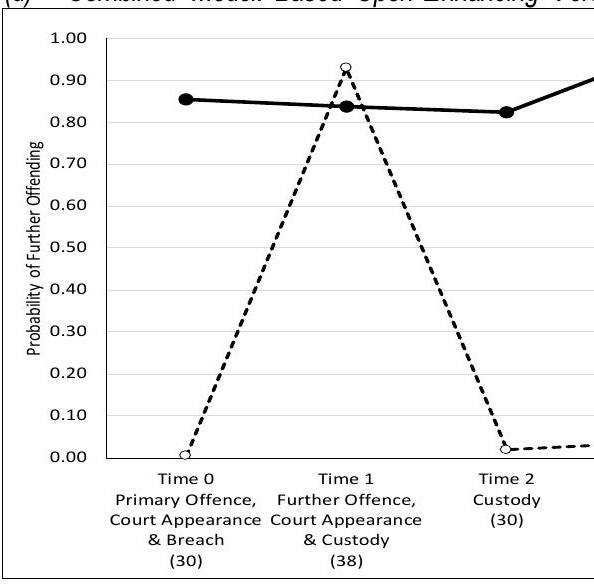


When FTE Status is included alongside another static factor as in Figures 6.20(a) and (b), the resulting set of estimated probabilities are the furthest away from the shape of the Basic Dynamic Model and the individual dynamic models. Significantly the initial estimated probability for Connor simulated by the model involving both FTE Status and Grouped Age of First Offence (Figure 6.20(a)) is very low which does not fit with the level of perceived level of risk as determined by his key worker, with his noncompliance (the breach) or identified status as a prolific offender. Whilst the initial probability of further offending is higher in the other models generated in Version 2, these also does notappear to fit Connor's profile particularlywell.

All three of the Version 2 models (Figures 6.20(a), (b)and (c)) suggest that Connor's probabilityof further offending at the time when he committed a further offence (Time 1) was approaching 1.0. This is also the case with the combined model (Figure 6.20(d)). Three of the four models involving two or more static factors suggest that whilst Connor was in custody (T ime 2), his likelihood of further offending is close to zero despite his ASSET score being 30. This also seems unlikely raising further questions about the how well the various models reflect the realities of real lives.

Particularlyin the case of the combined model (Figure 6.20(d)), Connor's very low estimated probability of further offending post-release - when he was considered to have a high risk of further offending i.e. an ASSET score greater than 25 - additionally does not appear to be realistic. A similar trend is also apparentin the three models involving FTE Status (Figures6.20(a), (b) and (d). However, it should be noted that despite the high score, Connor did not commit any further offences during the remainder of his time under the supervision of the YOT.

\section{Case Study "David"}

"David" has a history of prior offending behaviour, having committed his first offence at age 10 . He became part of the reoffending cohorthaving committed a public order offence -an 'Other' offence with a Gravity Score of 2. The respective trajectories of the probability of David committing further offences based on the models involving the individual static factors have a similar shape with 'peaks' at Time 1 and Time 3 when David's ASSET scores were highest (Figure 6.21).

Of the three case studies, David has the highest initial ASSET score. Whilst the Basic Dynamic model did not suggesta significant difference in the initial probability of further offending between Connor ( 30 , Figure 6.19) and David (40), an inspection of the individual dynamic models suggest that these all reflect David's higher ASSET score. Both David and Connor's sets of initial probabilities are also higher than those for Fred who had an opening ASSET score of 8 (Figure 6.17). 
Figure 6.21: Comparisons of the Estimated Probability of Further Offending Over Time - Individual Dynamic Models: "David"

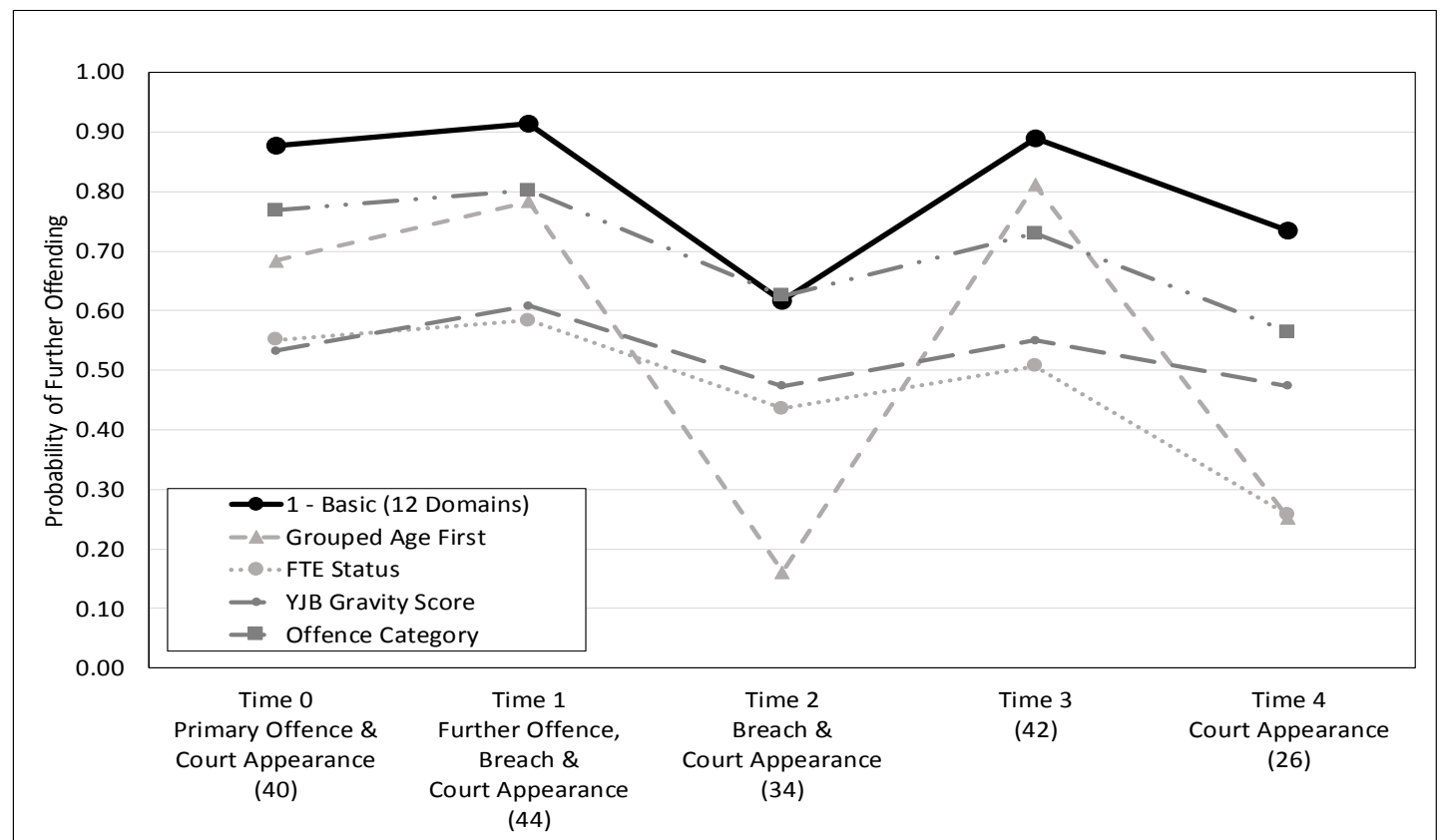

Notes: Although the ASSET scores reflected along the $x$-axis are out of a maximum of 48 with David having a total of 40 at Time 0, under the Scaled Approach he would have attracted additional scores due to the fact that he was aged 10 at the time of his first Reprimand and as a result of his prior convictions.

The models involving Grouped Age at First Offence and a further static factor suggest that David's probability of further offending was higher than that suggested by the Basic Dynamic model at Time 1. This corresponds to an increase in his ASSET score from 40 to 44 , and when he committed a further offence, breached and consequentlyreturned to court. Mirroring the trend apparentin the Basic Dynamic model, David's probability of further offending fell between Time 1 and 2 . However, this decline is more pronounced in the models involving Grouped Age at First Offence (Figures 6.22(a) and (C)).

Notably the model involving FTE and YJB Gravity Score (Figure 6.22(b)) is the only one to not reflectan increase as a result of David's ASSET score then increasing to 42 at T ime3. The model involving both Grouped Age at First Offence and FTE Status suggests a probability of further offending which is approaching 1. However, whilst David's circumstances changed (as discussed in section 4.4), he did not commita further offence, nor did he breach. During the following 8 months, David's ASSET score reduced to 26 with the probability of further offending also falling during this period. The court appearance at T ime 4 for a breach of his YRO. However, this was withdrawn.

Given that the combined model has been constructed by enhancing the dynamic model involving Grouped Age at First Offence and FTE Status, it is not surprising that the estimated probabilities of offending over time are identical. Having centred the YJB Gravity Score at2, this means thatfor David's public order offence (GravityScore =2), there is no additional 'penalty' for the seriousness of the offence. 
Figure 6.22: Changes in the Probability of Further Offending Over Time: "David"

\section{(a) Version 2: FTE and Grouped Age at First Offence}

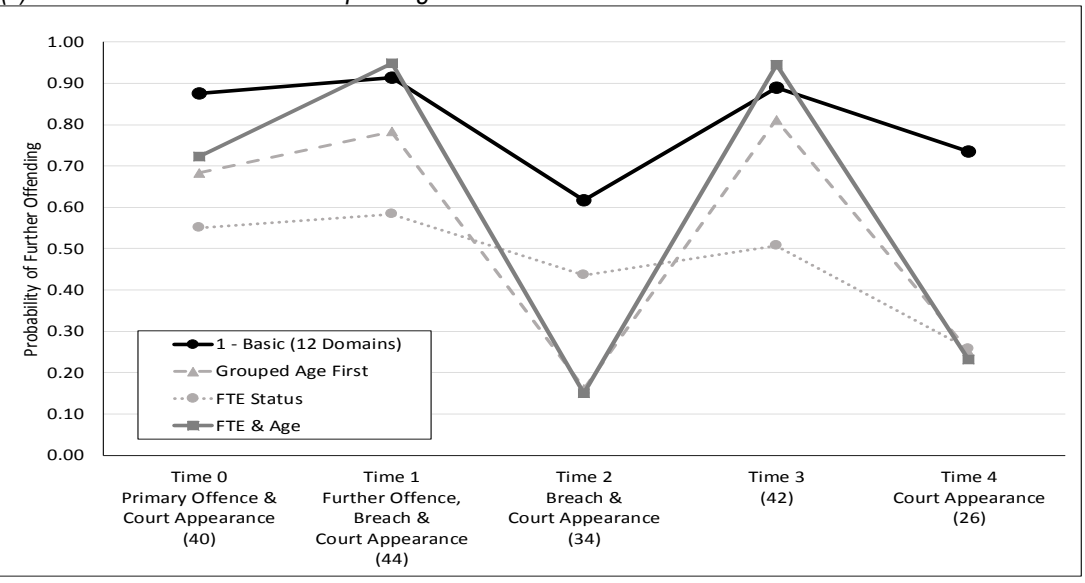

(c) Version 2: Grouped Age at First Offence and YJB Gravity Score

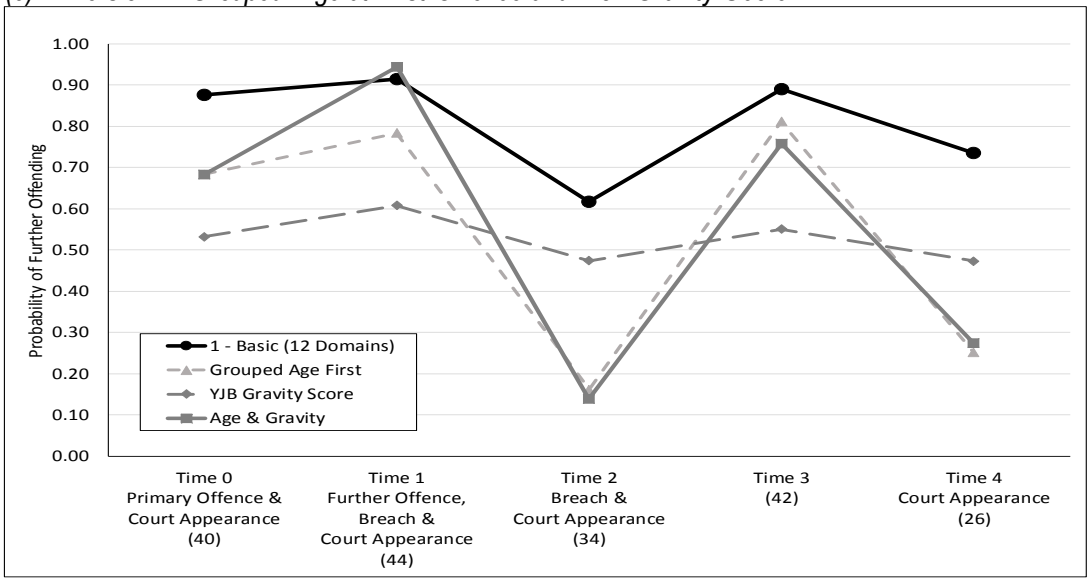

(b) Version 2: FTE and YGB Gravity Score

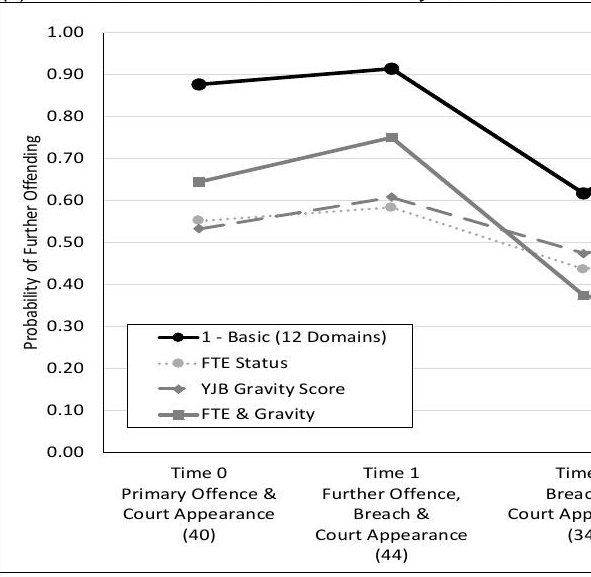

(d) Combined Model: Based Upon Enhancing Ver

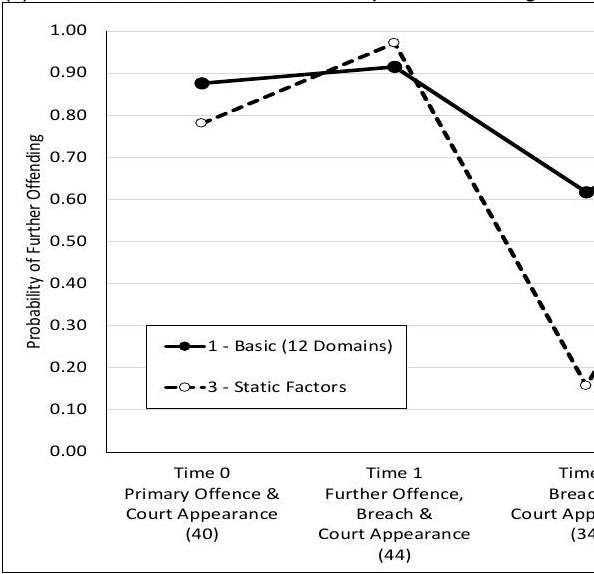


As with $\mathrm{BDm} 2$, it is not possible to measure the predictive accuracy of the combined dynamic model involving static factors. However, the observations made when utilising the ASSET scores of these three individuals does highlight some of its limitations. Notably, these arise from the amount of uncertainty surrounding the coefficients forFTE and Grouped Age at First Offence as main effects and the interaction between these two predictors.

\subsection{Increasing the Sensitivity of the Model by Extending the Predictors}

Whilst acknowledging that the combined model (BDm3) represents a compromise as there is not sufficient data to support a 'full' model involving proxies for all four of the static factors, this section considers the potential for increasing the sensitivity of the model by extending the predictors. This is done in the following ways:

- Treating the age-related predictors as continuous rather than dichotomous

- Revisiting the way in which the nature and severity of the offence is taken into account

\section{Treating AgeFirst as a Continuous Predictor}

Until now, age at first offence has been treated as being a dichotomous predictor, referenced by the younger age group. However, age can also be treated as being continuous, ranging from 10 - the age of criminal responsibility, and 17 . Table 6.21 summarises the dynamic model involving the continuous predictor centred atage 10.

Table 6.21: The Dynamic Model Involving Age at First Offence

\begin{tabular}{|c|c|c|c|c|c|c|c|}
\hline \multirow[b]{3}{*}{ Fixed Effect: } & \multicolumn{7}{|c|}{$\begin{array}{l}\text { Dynamic Basic Model including Age at First Offence } \\
\text { (Bm3_cc2) }\end{array}$} \\
\hline & \multicolumn{3}{|c|}{ Unstandardised } & \multicolumn{3}{|c|}{ Standardised } & \multirow{2}{*}{ Significant? } \\
\hline & Post.Mean & Lower $\mathrm{Cl}$ & Upper $\mathrm{Cl}$ & Post.Mean & Lower $\mathrm{Cl}$ & Upper $\mathrm{Cl}$ & \\
\hline (Intercept) & -2.530 & -5.688 & 0.408 & 0.080 & 0.003 & 1.504 & \\
\hline Age at First Offence ( $0=10$ years) & 0.297 & -0.368 & 0.939 & 1.346 & 0.692 & 2.557 & \\
\hline Time & 0.221 & -0.397 & 0.807 & 1.247 & 0.672 & 2.242 & \\
\hline Living Arrangements (Live) & -0.109 & -1.406 & 1.077 & 0.896 & 0.245 & 2.936 & \\
\hline Family and Personal Relationships (Relation) & 1.607 & 0.207 & 3.022 & 4.985 & 1.229 & 20.528 & Yes \\
\hline Education, Training and Employment (ETE) & -0.409 & -1.571 & 0.718 & 0.664 & 0.208 & 2.051 & \\
\hline Neighbourhood (Where) & 0.252 & -0.766 & 1.219 & 1.286 & 0.465 & 3.384 & \\
\hline Lifestyle (Life) & 0.536 & -1.115 & 2.142 & 1.710 & 0.328 & 8.516 & \\
\hline Substance Use (Drugs) & 0.606 & -0.579 & 1.692 & 1.834 & 0.561 & 5.432 & \\
\hline Physical Health (Physical) & -0.474 & -1.602 & 0.703 & 0.623 & 0.201 & 2.019 & \\
\hline Emotional and Mental Health (Emotion) & -0.361 & -1.405 & 0.673 & 0.697 & 0.245 & 1.960 & \\
\hline Perceptions of Self and Others (Self) & -2.082 & -3.784 & -0.323 & 0.125 & 0.023 & 0.724 & Yes \\
\hline Thinking and Behaviour (Think) & 0.351 & -1.242 & 1.997 & 1.421 & 0.289 & 7.370 & \\
\hline Attitude to Offending (Attitude) & 0.445 & -1.325 & 2.281 & 1.561 & 0.266 & 9.784 & \\
\hline Motivation to Change (Change) & 0.170 & -1.430 & 1.822 & 1.185 & 0.239 & 6.182 & \\
\hline Age at First Offence: Time & -0.140 & -0.312 & 0.025 & 0.869 & 0.732 & 1.025 & \\
\hline Age at First Offence: Live & 0.027 & -0.289 & 0.366 & 1.027 & 0.749 & 1.442 & \\
\hline Age at First Offence: Relation & -0.364 & -0.717 & -0.012 & 0.695 & 0.488 & 0.988 & Yes \\
\hline Age at First Offence: ETE & 0.021 & -0.262 & 0.315 & 1.021 & 0.769 & 1.371 & \\
\hline Age at First Offence: Where & -0.046 & -0.320 & 0.208 & 0.955 & 0.726 & 1.231 & \\
\hline Age at First Offence: Life & -0.052 & -0.458 & 0.370 & 0.949 & 0.632 & 1.447 & \\
\hline
\end{tabular}




\begin{tabular}{|c|c|c|c|c|c|c|c|}
\hline \multirow[b]{3}{*}{ Fixed Effect: } & \multicolumn{7}{|c|}{$\begin{array}{l}\text { Dynamic Basic Model including Age at First Offence } \\
\qquad\left(B m 3 \_c c 2\right)\end{array}$} \\
\hline & \multicolumn{3}{|c|}{ Unstandardised } & \multicolumn{3}{|c|}{\begin{tabular}{|c|} 
Standardised \\
\end{tabular}} & \multirow{2}{*}{ Significant? } \\
\hline & Post.Mean & Lower $\mathrm{Cl}$ & Upper $\mathrm{Cl}$ & Post.Mean & Lower $\mathrm{Cl}$ & Upper $\mathrm{Cl}$ & \\
\hline Age at First Offence: Drugs & -0.086 & -0.376 & 0.196 & 0.918 & 0.687 & 1.216 & \\
\hline Age at First Offence: Physical & -0.047 & -0.364 & 0.259 & 0.954 & 0.695 & 1.295 & \\
\hline Age at First Offence: Emotion & 0.006 & -0.265 & 0.292 & 1.006 & 0.767 & 1.339 & \\
\hline Age at First Offence: Self & 0.666 & 0.238 & 1.116 & 1.947 & 1.268 & 3.053 & Yes \\
\hline Age at First Offence: Think & -0.123 & -0.543 & 0.267 & 0.884 & 0.581 & 1.306 & \\
\hline Age at First Offence: Attitude & -0.020 & -0.471 & 0.421 & 0.981 & 0.624 & 1.524 & \\
\hline Age at First Offence: Change & 0.014 & -0.448 & 0.451 & 1.014 & 0.639 & 1.570 & \\
\hline Time: Live & -0.075 & -0.351 & 0.197 & 0.928 & 0.704 & 1.217 & \\
\hline Time: Relation & -0.361 & -0.703 & -0.020 & 0.697 & 0.495 & 0.980 & Yes \\
\hline Time: ETE & 0.008 & -0.254 & 0.270 & 1.008 & 0.776 & 1.310 & \\
\hline Time: Where & -0.043 & -0.246 & 0.168 & 0.958 & 0.782 & 1.183 & \\
\hline Time: Life & 0.014 & -0.353 & 0.402 & 1.014 & 0.702 & 1.494 & \\
\hline Time: Drugs & -0.115 & -0.381 & 0.117 & 0.892 & 0.683 & 1.124 & \\
\hline Time: Physical & 0.233 & -0.064 & 0.518 & 1.262 & 0.938 & 1.678 & \\
\hline Time: Emotion & 0.115 & -0.125 & 0.362 & 1.122 & 0.882 & 1.436 & \\
\hline Time: Self & 0.515 & 0.153 & 0.903 & 1.673 & 1.165 & 2.467 & Yes \\
\hline Time: Think & -0.157 & -0.482 & 0.170 & 0.855 & 0.618 & 1.185 & \\
\hline Time: Attitude & -0.096 & -0.451 & 0.252 & 0.908 & 0.637 & 1.286 & \\
\hline Time: Change & 0.071 & -0.278 & 0.424 & 1.074 & 0.758 & 1.528 & \\
\hline Age at First Offence: Time: Live & 0.031 & -0.048 & 0.104 & 1.031 & 0.953 & 1.110 & \\
\hline Age at First Offence: Time: Relation & 0.093 & -0.002 & 0.181 & 1.098 & 0.998 & 1.199 & \\
\hline Age at First Offence: Time: ETE & 0.032 & -0.044 & 0.113 & 1.033 & 0.957 & 1.120 & \\
\hline Age at First Offence: Time: Where & 0.019 & -0.038 & 0.076 & 1.019 & 0.962 & 1.079 & \\
\hline Age at First Offence: Time: Life & -0.024 & -0.130 & 0.086 & 0.977 & 0.878 & 1.090 & \\
\hline Age at First Offence: Time: Drugs & 0.030 & -0.045 & 0.105 & 1.031 & 0.956 & 1.111 & \\
\hline Age at First Offence: Time: Physical & -0.019 & -0.102 & 0.065 & 0.981 & 0.903 & 1.067 & \\
\hline Age at First Offence: Time: Emotion & 0.002 & -0.073 & 0.076 & 1.002 & 0.930 & 1.079 & \\
\hline Age at First Offence: Time: Self & -0.183 & -0.293 & -0.083 & 0.833 & 0.746 & 0.920 & Yes \\
\hline Age at First Offence: Time: Think & 0.040 & -0.046 & 0.127 & 1.041 & 0.955 & 1.136 & \\
\hline Age at First Offence: Time: Attitude & -0.008 & -0.109 & 0.092 & 0.992 & 0.896 & 1.096 & \\
\hline \begin{tabular}{|l|} 
Age at First Offence: Time: Change \\
\end{tabular} & -0.016 & -0.116 & 0.090 & 0.984 & 0.890 & 1.094 & \\
\hline Random Effect: & Post.Mean & Lower $\mathrm{Cl}$ & Upper $\mathrm{Cl}$ & Post.Mean & Lower $\mathrm{Cl}$ & Upper $\mathrm{Cl}$ & Significant? \\
\hline Individual (Intercept) & $\begin{array}{r}0.66 \\
\end{array}$ & $6.91 \mathrm{E}-07$ & 1.53 & 1.938 & 1.000 & 4.609 & Yes \\
\hline Time & 2.768 & 0.576 & 5.972 & 15.927 & 1.778 & 392.289 & Yes \\
\hline
\end{tabular}

\begin{tabular}{|l|r|}
\hline DIC & 465.27 \\
\hline
\end{tabular}

Source: Model BDm3_cc2, Technical Annex: p322-334.

As with previous dynamic models, the trajectory of the probability of further offending over time has been determined for each year from age 10 to 17 . Figure 6.23 summarises these trajectories where the domain scores are fixed at 1, 2, 3 and 4 respectively. These charts suggest that that at Time 0 , younger children have a lower probability of further offending than those who are older supporting the trend apparentin Figure 6.5. 
Figure 6.23: Changes in the Probability of Further offending Over Time, by Age at First Offence
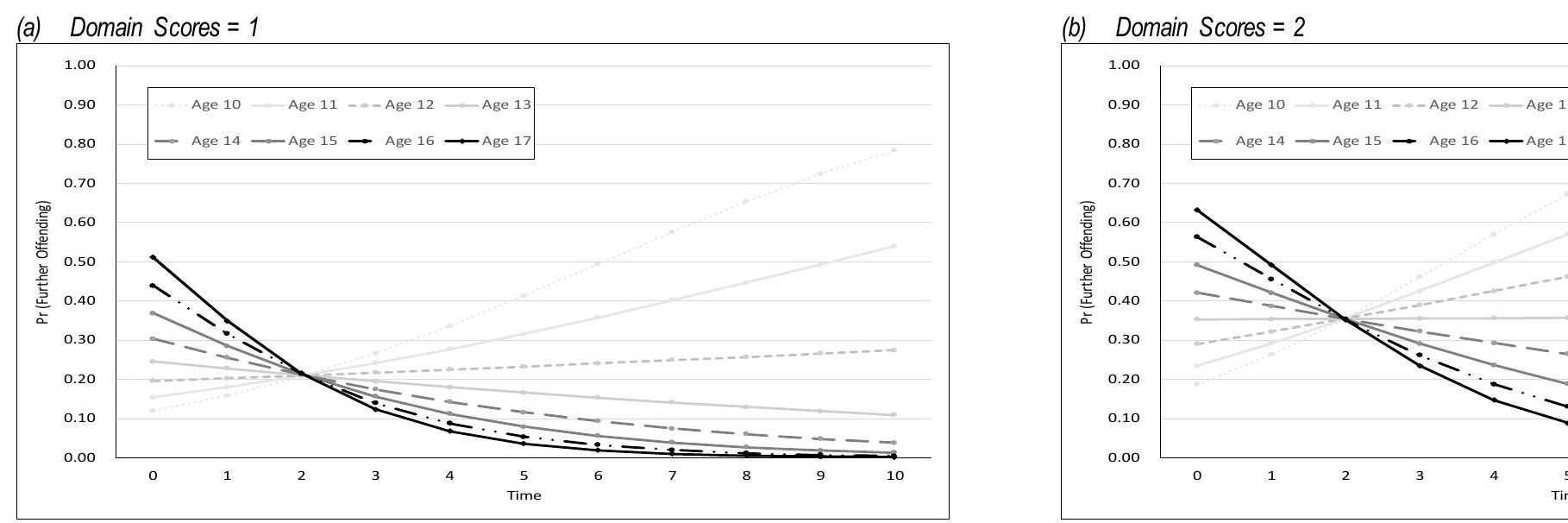

(c) Domain Scores $=3$
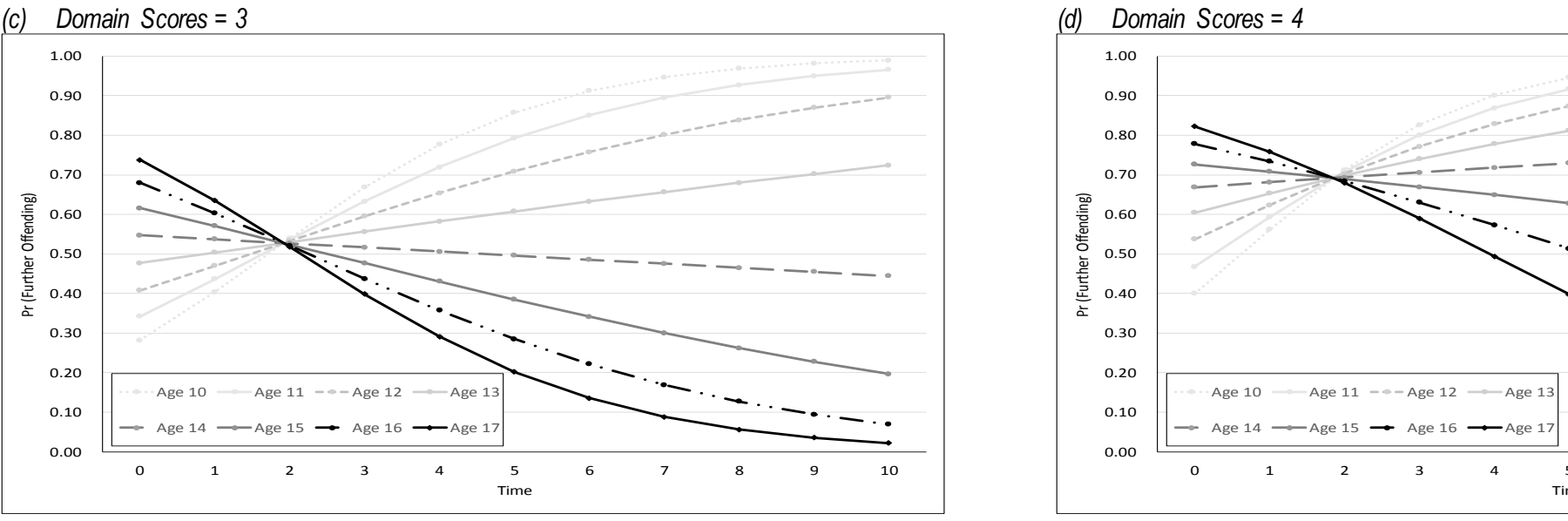

Notes: The domain scores have respectively been shown as being fixed at 1, 2, 3 and 4 respectively to demonstrate the estimated change in the probability of fu derived from Model BDm3_cc2. 
For those under 12 who have lower domain scores, the probability of offending appears to increase, the longer they spend under the supervision of the YOT. This also appears to be the case for those aged 13 or 14 at the time of their first Offence who had higher domain scores. Particularlyafter Time 2, the number in remaining in the cohortwhose ASSET s have been used to determine the fixed effects in the model fall. As a result, later estimates of the probability of further offending are less reliable.

The probability of further offending for those aged 14 or over suggest a gradual downward trend, more like those seen in Figure 6.14 (the dynamic model involving YJB Gravity Scores). Despite those who are older at the time of their first offence having higher initial probabilities of further offending at Time 0 , 17 -year olds have a greater rate of decline than those aged 14 . This trend can be observed where the domain scores are fixed at 1, 2 and 3 . When the domain scores are fixed at 4 , those aged 14 appear to have an upwards trajectory. It is likely that this apparent trend has resulted from the small number of cases that these estimates are based upon (Figure 6.24).

Figure 6.24: Summary of Average Domain Scores, by Age at First Offence (Trend in Cohort Size)

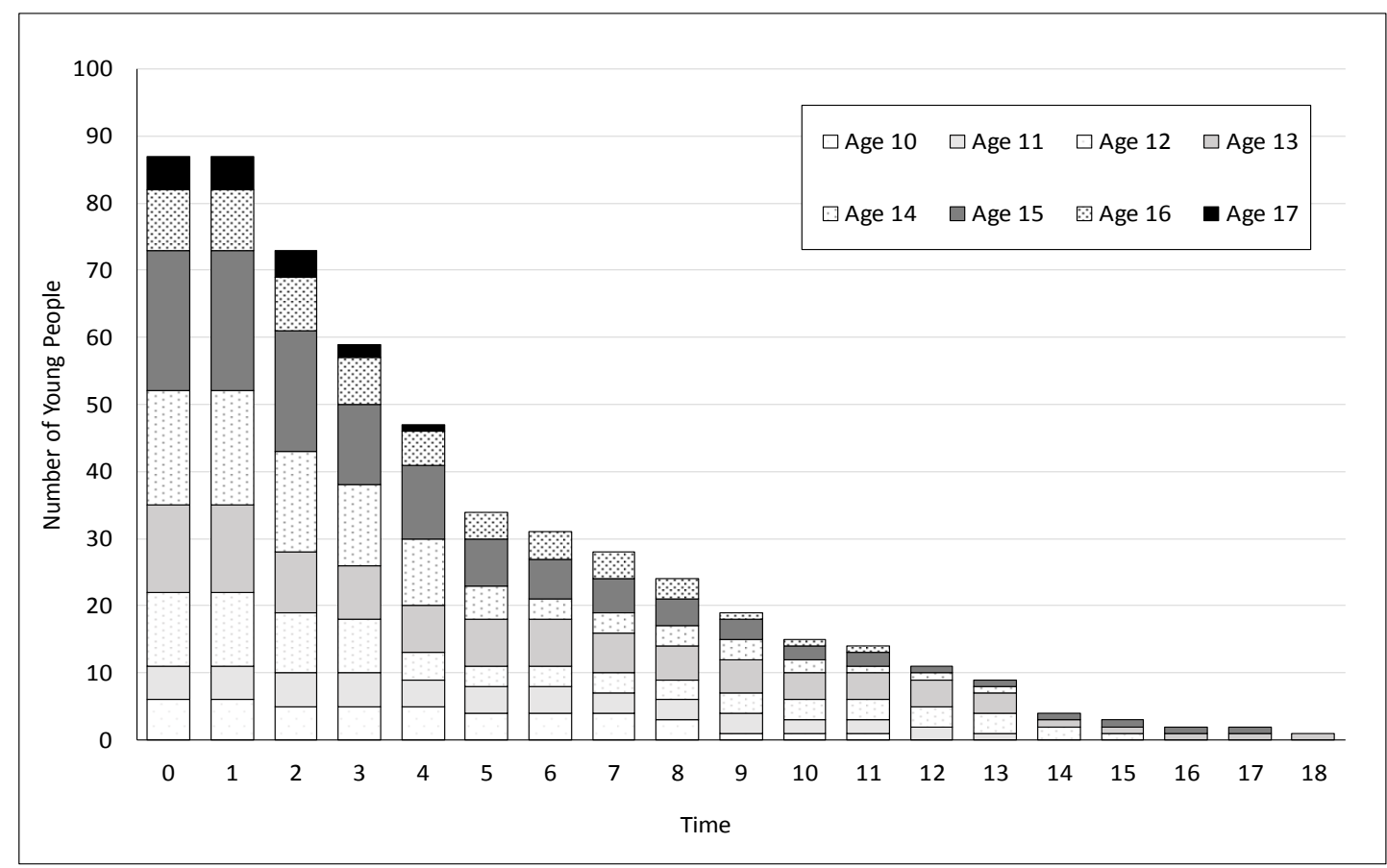

Notably there are only 5 young people whose first offence was at age 17 . By Time 3 , the number in the sub-group had fallen to 2 with one person having their final ASSET for Time 4. As a result, the amount that 17 -year olds can contribute to the model is quite limited. Of the 9 young people aged 16 at the time of their first offence, there was just one person who was risk assessed after Time 8. Hence the mean domain score for 16 -year olds at Times 9 to 11 is based on just one person (Figure 6.25). 


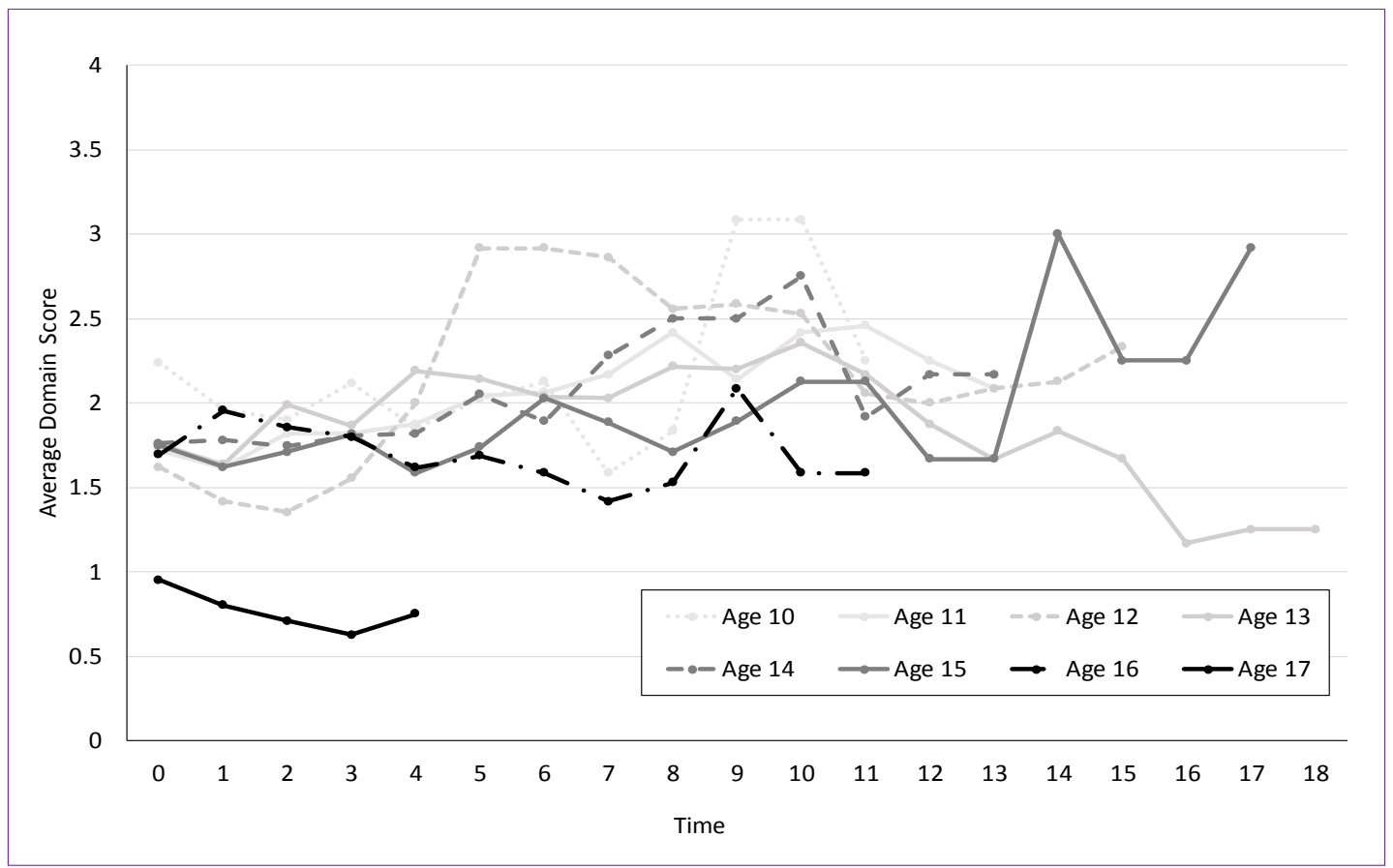

At the otherend of the age range, there were 6 young people who were aged 10 at the time of their first offence with one individual having 12 ASSETs ( $T=11$ ). The last 3 observations for 10 -year olds are based on just this individual. There were 5 young people whose first offence was at age 11 . Al but the last average domain score (at Time 11) is based on more than one ASSET.

Of the two dynamic models involving age at first offence, the one involving the grouped predictor has the lower DIC suggesting that it accounts for more uncertainty ( 448.73 compared to 465.27 for the continuous predictor). However, what is not clear is the extent to which the model with the continuous predictor is paying a penalty for having increased complexity. This is something that would onlybecome apparentif there was more data with which to run the model.

\section{Treating Age Con as a Continuous Predictor}

Theoretically it would also have been possible to treat the age at first conviction as a continuous predictor. However, as identified in Section 6.3, there are insufficientcases in the reoffending cohortto supporta dynamic version of the model involving this predictor.

Further to this, T able 6.3 highlights that there would also be issues arising from multicollinearitybetween the two continuous age-related predictors should theybe combined in the same model. Multicollinearity arises when there is a very high correlation between two or more predictor variables. Although overall the Pearson's correlation between the two age-related continuous predictors is $0.651(\mathrm{Cl}=0.502,0.753$, $\left.\mathrm{BF}_{10}=1.222 \mathrm{e}^{9}\right)$, then the data is split on the basis of FTE Status, it suggests that the $R_{\text {Prior }}=0.554(\mathrm{Cl}=$ $\left.0.325,0.760, \mathrm{BF}_{10}=1660\right)$ whilst $\mathrm{R}_{\mathrm{FTE}}=0.823\left(\mathrm{Cl}=0.642,0.904, \mathrm{BF}_{10}=3.066 \mathrm{e}^{6}\right)$. The consequence of multicollinearityis that 'the posterior distribution will say that a very large range of parameter variables 
are plausible, from tiny associations to massive ones, even if all the variables are in reality strongly associated with the outcome variable' (McElreath, 2016:141-142).

Although overall the level of correlation is not that high, within this dataset, $63.6 \%(21 / 33)$ of the FTEs had been convicted of their first offence hence their age at first offence and age at first conviction was the same. Amongst those with a prior offending history the proportion was $14.8 \%(8 / 54)$, meaning that across the cohort the proportion was $33.3 \%$ with a further $28.7 \%$ having a 1 -year difference (although this could be due to them having their birthday in the time between committing the offence and attending court).

The Raftery Lewis diagnostic suggests that to achieve convergence in a combined dynamic model involving the static factors where both age at first offence and age at first conviction are continuous, more than 150 million iterations would be required with a substantial burn-in. To address the autocorrelation within the model, it would be necessary to set the lag to at least 50,000. This is not feasible to run.

\section{Nature and Seriousness of the Primary Offence}

Table 6.22 summarises the profile of the cohort on the basis of the nature of their primary offence illustrating why it has been necessary to group the offence categories. As highlighted in Section 3.6, using a predictor based on the seriousness of the offence means that it is possible to differentiate between for example violence against the person offences which can vary in severity as well as potentially enabling inferences to be made about offences which fall under YJB Categories for which there are no cases within the dataset.

Table 6.22: The Re-Offending Cohort, by Grouped YJB Category and Seriousness of Primary Offence

\begin{tabular}{|l|r|r|r|r|r|r|}
\hline \multirow{2}{*}{ Grouped YJB Offence Category } & \multicolumn{9}{|c|}{ YJB Gravity Score } & \multirow{2}{*}{ Total } \\
\cline { 2 - 7 } & 2 & 3 & 4 & 5 & 6 & \\
\hline Other & 31 & 15 & 1 & 1 & & 48 \\
\hline SAC & & 1 & 4 & 3 & 10 & 18 \\
\hline VAP & & 14 & 4 & & 3 & 21 \\
\hline Grand Total & $\mathbf{3 1}$ & $\mathbf{3 0}$ & $\mathbf{9}$ & $\mathbf{4}$ & $\mathbf{1 3}$ & $\mathbf{8 7}$ \\
\hline
\end{tabular}

Notes: The individual whose FTE status is not known has been excluded from this summary. 
Table 6.23: The Basic Model plus Grouped YJB Offence Category and Gravity Score

\begin{tabular}{|c|c|c|c|c|c|c|c|}
\hline \multirow{3}{*}{\begin{tabular}{|l} 
\\
Fixed Effect: \\
\end{tabular}} & \multicolumn{7}{|c|}{$\begin{array}{l}\text { Model 1.11: Basic Model + Grouped YJB Offence Category } \\
\text { and YJB Gravity Score }\end{array}$} \\
\hline & \multicolumn{3}{|c|}{ Unstandardised } & \multicolumn{3}{|c|}{ Standardised } & \multirow{2}{*}{ Significant? } \\
\hline & Post.Mean & Lower $\mathrm{Cl}$ & Upper $\mathrm{Cl}$ & Post.Mean & Lower $\mathrm{Cl}$ & Upper $\mathrm{Cl}$ & \\
\hline & -1.229 & -3.390 & 0.781 & 0.293 & 0.034 & 2.183 & \\
\hline \multicolumn{8}{|c|}{ Grouped YJB Offence Category (Ref = Other $)$} \\
\hline Serious Acquisitive Crime & 0.865 & -1.911 & 3.481 & 2.376 & 0.148 & 32.493 & \\
\hline Violence Against The Person & 1.313 & -1.087 & 4.067 & 3.718 & 0.337 & 58.396 & \\
\hline YJB Gravity Score (Serious) & -0.015 & -0.663 & 0.587 & 0.985 & 0.516 & 1.799 & \\
\hline Living Arrangements & 0.047 & -0.241 & 0.315 & 1.048 & 0.786 & 1.370 & \\
\hline Family and Personal Relationships & 0.253 & -0.055 & 0.575 & 1.288 & 0.947 & 1.778 & \\
\hline Education, Training and Employment & 0.065 & -0.194 & 0.340 & 1.067 & 0.824 & 1.404 & \\
\hline Neighbourhood & 0.064 & -0.190 & 0.288 & 1.066 & 0.827 & 1.333 & \\
\hline Lifestyle & 0.049 & -0.321 & 0.411 & 1.050 & 0.725 & 1.509 & \\
\hline Substance Use & 0.207 & -0.039 & 0.486 & 1.230 & 0.962 & 1.626 & \\
\hline Physical Health & -0.115 & -0.422 & 0.192 & 0.891 & 0.656 & 1.212 & \\
\hline Emotional and Mental Health & 0.022 & -0.229 & 0.282 & 1.022 & 0.795 & 1.326 & \\
\hline Perceptions of Self and Others & -0.142 & -0.496 & 0.178 & 0.868 & 0.609 & 1.195 & \\
\hline Thinking and Behaviour & -0.166 & -0.521 & 0.174 & 0.847 & 0.594 & 1.190 & \\
\hline Attitude to Offending & 0.029 & -0.343 & 0.391 & 1.029 & 0.710 & 1.479 & \\
\hline Motivation to Change & 0.213 & -0.139 & 0.567 & 1.238 & 0.870 & 1.763 & \\
\hline Time & -0.177 & -0.324 & -0.025 & 0.838 & 0.723 & 0.975 & Yes \\
\hline Serious: SAC & -0.130 & -0.910 & 0.623 & 0.878 & 0.402 & 1.864 & \\
\hline Serious: VAP & -0.316 & -1.155 & 0.562 & 0.729 & 0.315 & 1.754 & \\
\hline Random Effect: & Post.Mean & Lower $\mathrm{Cl}$ & Upper $\mathrm{Cl}$ & Post.Mean & Lower $\mathrm{Cl}$ & Upper $\mathrm{Cl}$ & Significant? \\
\hline Individual (Intercept) & 0.287 & $1.22 \mathrm{E}-07$ & 0.720 & 1.332 & 1.000 & 2.055 & Yes \\
\hline Time & 1.607 & 0.376 & 3.364 & 4.988 & 1.456 & 28.905 & Yes \\
\hline
\end{tabular}

DIC

Source: Model Bm1G_012a, Technical Annex: p335-336.

There is insufficient data to run a dynamic model involving both the type and seriousness of the primary offence. However, where these two predictors have been included alongside the basic model (Model 1.11), it suggests that all other things being equal, at Time 0 :

- Relative to a young person with domain scores of 2 who has committed an 'Other' offence with a gravity score of 3 , the odds of further offending are estimated to be 1.6 times higher if they had committed an equivalent serious acquisitive crime. The odds are 1.4 times higher if they commitan equivalent violence against the person offence.

- Comparing equivalent offences with a gravityscore of 4 , the odds of further offending are lowest if that offence is an 'Other' offence. Relative to this, the odds of further offending are estimated to be 1.4 times higher if the young person has committed a serious acquisitive crime but only 1.05 times higher if they committed a violent offence.

It is also possible to estimate the impact of committing a more serious offence within each offence type. Based once again upon equivalentyoung people with domain scores of 2 , at T ime 0 : 
- The odds of further offending are on average 1.02 times higher amongst those who committed an 'Other' offence with gravity score of 3 , than for those who committed an equivalent offence with a gravity score of 4 .

- Violence against the person: the odds of further offending appear to increase as the gravity score decreases. Relative to a VAP offence with a gravity score of 4 , the odds of further offending are estimated to be 1.4 times higheramongst those who committed an offence with a gravity score of 3 .

- Serious acquisitive crime: the odds of further offending appear to increase as the gravity score increases. Relative to a SAC offence with a gravity score of 3 , the odds of further offending are estimated to be 1.16 times higher amongst those who committed an offence with a gravityscore of 4 .

Had there been sufficient data to support the model, it is anticipated that the trajectories of the probability of further offending as time progresses would be quite different. Published data suggests that amongst those who reoffend, the time to reoffence can differ significantly (Youth Justice Board and Ministry of Justice, 2016). As with reoffending rates, there is evidence to suggest that this varies by index offence, number of previous convictions and age (Owen and Cooper, 2013).

\subsection{How do these findings extend the evidence base?}

\section{Static Factors}

It is notable that in the individual dynamic and combined models involving FTE and Grouped Age atFirst Offence, there is a lot of uncertaintyaround the estimates for the static factors both as main effects and when in an interaction. In the combined model the estimated unstandardised coefficients are:

- $\mathrm{FTE},-4.720$ [-14.578, 4.547]. In BDm3_cc1, the estimate is 0.995 [-1.668, 3.660]

- G_ageFirst, $5.144[-1.234,11.585]$. In BDm3G_cc2, the estimate is $2.340[-0.928,5.488]$

- FTE:G_ageFirst, $4.642[-4.263,13.741]$

This uncertaintyis associated with the comparativelylow number of cases, and particularlythe fact that none of those who were FTEs aged 10-12 committed anyfurther offences (Table 6.24). With just three individuals within this sub-group, there is little information, especiallyatlater measurement points, which can be used to inform the estimates of the probability of further offending. 
Table 6.24: The Reoffending Cohort by FTE Status and Grouped Age at First Offence, with Further Offending Rates

\begin{tabular}{|c|l|c|c|c|}
\hline \multicolumn{2}{|c|}{} & \multicolumn{2}{|c|}{ Grouped Age at First Offence } & \multirow{2}{*}{ Total } \\
\cline { 3 - 4 } \multicolumn{2}{|c|}{} & $10-12$ years & $13-17$ years & \\
\hline \multirow{2}{*}{$\begin{array}{c}\text { FTE } \\
\text { Status }\end{array}$} & Prior Offending & $68 \%(13 / 19)$ & $51 \%(18 / 35)$ & $57 \%(31 / 54)$ \\
\cline { 2 - 4 } & FTE & $0 \%(0 / 3)$ & $37 \%(11 / 30)$ & $33 \%(11 / 33)$ \\
\hline \multicolumn{2}{|l|}{ Total } & $59 \%(13 / 22)$ & $45 \%(29 / 65)$ & $48 \%(42 / 87)$ \\
\hline
\end{tabular}

The amount of uncertainty around the estimated coefficients suggests that the other predictors in the models i.e. time, the 12 domains and, in the case of the combinedmodel, the YJB Gravity Score, do not adequatelyexplain the amount of variation. Potential explanations include the role of gender, ethnicity and experience of care. However, there may be other factors which are notmeasured as part of the risk assessment process. As seen in Chapter Five, there was insufficient data within the reoffending cohort to explore these more fully.

The variable representing FTE status was constructed as a proxy measure for the number of previous convictions. Previous research by Wilson and Hinks (2011) identified that relative to having no previous convictions, having 1-3 convictions decreased the odds of proven reconviction by a factor of 0.44 . However, it was not possible to calculate the odds ratio for those who had 4+ convictions due to a high correlation with age at first conviction. Given the practical difficulties in establishing the number of prior convictions from the offence and court records within Childview, the pragmatic decision was made to use a binary indicator to reflect the young person's status at the time of entering the reoffending cohort. For those appearing on both the 2012/13 and 2013/14 reoffending spreadsheets, their status was taken at the time of the primaryoffence in 2012/13. This leads to a small number of seeminglycontradictory trends at an individual level.

The trajectory of the predicted probability of further offending for FTEs makes very little distinction at Time 0 on the basis of the domain scores (Figure 6.4(a)) - reflecting the difficulties in predicting further offending for those who have little previous contact with the youth justice system. In contrast practitioners will know more about those with a prior offending history. Having this knowledge enables the YOT to make a more informed judgement about the likelihood of re-offending basedon the individual circumstances of the young person. For both groups the trajectory suggested for those who pose the greatest risk (reflected by fixing the domain scores at 4) is upwards which is contrary to what would be expected. However, the reality is that there are no young people who at any time were judged to have a total ASSET score of 48 although there are four who at different times had scores greater than or equal to 40 , with the maximum total score being 44 .

A notable feature of the underlying data is that due to the emphasis on those who are in the formal youth justice system and hence have been assessed using the Core ASSET Profile, many of those aged 10 12 at the time of their first offence already have a history of prior offending. Indeed, of the twenty-two young people within this younger group, just three were FTEs. Whilst two had had no previous contact 
with the YOT. Theydid however, commita further offence within just a couple of weeks of the first and as a result were sentenced for both offences as the same time. Thus, differences in their age at the time of their first offence and conviction reflectonly the time that the case took to get to court. However, the third young person had previously had informal action taken against him for an offence committed when aged 12 but was not convicted of an offence until he was aged 15. This meant that he was an FTE at the time of entering the cohorthaving not previously committed any proven offences. This scenario exposes a weakness in the construction of the predictor.

Under the Scaled Approach, those receiving their first reprimand, caution or warning aged 10-12 had 4 added to their total ASSET score. In translating this to also reflect the situation post-LASPO, I opted to use a wider definition whereby the age at first offence was used, drawing information from the offence and courtrecords held within Childview. This meant that a small number who were known to the YOT as a result of having informal action taken against them or receiving a Youth Restorative Disposal typically meaning that they had been subject to Swansea's Bureau proceedings, have similar inconsistencies in their age at first offence and FTE status. This had unintended implications in the analysis given the comparativelylow numbers in the dataset especiallyat later measurement points.

From the basic model involving YJB Gravity Score (Bm1_02a) it is unclear how the likelihood of further offending is affected by an increase in the seriousness of the primaryoffence, with the credible interval for the standardised coefficient straddling one. However, in the equivalent basic model which additionally involves FTE Status, Grouped Age at First Offence and interactions with seriousness, the role of YJB Gravity Score becomes more apparent. The significant positive coefficient (Bm1G_cc1202a, 0.334 $[0.017,0.647])$ suggests that increasing the seriousness of the offence, will increase the likelihood of further offending. Whilst it was not possible to simulate an estimate for the interaction for G_ageFirst Seriousness, the model suggests that at Time 0 , with all domain scores setat 2 , the impact of increasing the YJB Gravity Score from 2 to 3 increases the probability of further offending:

- For those with a history of prior offending (the reference category for FTE Status), aged 10-12 at the time of their first offence from 0.502 to 0.585 i.e. the odds are increased by $16.5 \%$

- For those with prior offending, aged 13-17 at the time of their first off offence the probability increased from 0.421 to 0.504 i.e. the odds increased by $19.6 \%$

- ForFTEs aged 10-12, having committed an offence with a YJB Gravity Score of 3 rather than 2 , the likelihood of further offending increased from 0.271 to 0.499 i.e. the odds increased by $84.2 \%$

- For FTEs aged 13-17, the impact of increasing the YJB Gravity Score was that their odds of further offending increased by $43.5 \%$ (from a probability of 0.212 at Time 0 to 0.304 if they had committed a primaryoffence with a gravity score of 3 ). 
Whilst the figures presented to not represent the trend over time and are somewhatartificial in that they assume that of the domain scores are fixed at 2, they appear to support the rationale for diversionary activities which in the case of Swansea tend to be used with those who have committed low level offences, particularlywhere there is less evidence of a pattern of offending behaviour. By keeping these young people out of the formal youth justice system, it avoids the negative aspects of system contact, especially the stigma associated with labelling theory. Sadly, when this model was transformed into a dynamic model (BDm3, summarised in Table 6.20), there was insufficientdata to support the additional complexity. As a result, it was not possible to explore this more fully.

The dynamic model involving Grouped YJB Offence Category (BDm3G_01) was set up with Other offences as the reference category. It is notable that there is a lot of uncertaintyaround the estimate for SAC offences (1.924 [-2.331, 6.124]) and VAP offences (-4.070 [-10.078, 1.331]), with neither being significant. Where interactions occur, particularlybetween VAP and the individual domains, there is also a fair amount of uncertainty. For example, the positive significantinte raction between VAP: Life (3.032 $[0.437,5.904])$ and that between VAP: Think $(2.361[-0.712,5.592])$. There is more certaintywhen VAP and the individual domains interact with time, with those involving ETE and Where being positive whilst those involving Life and Think are negative. There is less certaintyaround the estimates for interactions involving SAC.

It is important to remember that the VAP offences, whilst largely less serious in nature e.g. Common Assault and Actual Bodily Harm (ABH), do include a small number of serious violent offences such as Grievous Bodily Harm (GBH) which attract a much higher Gravity Score. In contrast, the majority of Other offences are less serious in nature with Gravity Scores of $2(65 \%, 31 / 48)$ and $3(31 \%, 15 / 48)$. Without knowing the circumstances of the individual offences or events leading up to the assault, it is difficult in the case of the VAP offences to know if these acts of violence were one-offs or part of a prolonged pattern of aggressive behaviours. The im plication being that those demonstrating aggressive behaviours being likely to be referred to interventions which are intended to help them to address their anger management issues, and potentially have recommendations within their order which limit who they can associate with. As a result, you would expect to see an improvement in their behaviour over time hence the negative estimate for the interaction between VAP: Time: Life. These types of interventions will also include work to help the young person understand the impact of their behaviour on others, and as a result, this would also be reflected in their scores within the Thinking and Behaviours domain.

Where aggressive and disruptive behaviour has involved in school settings, potentiallydirected at other pupils or staff, this can result in the young person being excluded. In keeping with the RNR approach placing the young person in alternative provision or arranging a place on a training course, typically has a moderating effecton their risk offurther offending behaviour. Within the dynamic model (BDm3G_01), this is reflected by the significant positive interaction between VAP: Time: ETE and the significant 
negative interaction between VAP:ETE. This is also consistent with the views of practitioners and those who have been assessed thatETE and a lack of training / qualifications are related to reoffending (Youth Justice Board, 2005a) which were raised in section 4.3. However, it also highlights that responses to youth crime need to be tailored according to the nature and seriousness of the offence committed. In this respect it is disappointing that there was insufficient data to develop a model which included both YJB Offence Category and YJB Gravity Score so that this could be explored further.

\section{Domain Scores}

In the context of this element of the research, it is notable the Lifestyle domain is significant in the dynamic model involving the grouped predictor for age at first offence (BDm3G_cc2, 1.856 [0.105, 3.643]) and the combined dynamic model for offending history where the model also includes the grouped predictor for age atfirst offence (BDm3, 2.636 [0.219,5.213]). This finding does nothold where the predictor for age at first offence is continuous (BDm3_cc2, $0.536[-1.115,2.142]$. The positive coefficients suggest that as the rating for Lifestyle increases by 1 , then the probability of further offending increases. However, this is mediated by the interaction between Time: Lifestyle (BDm3G_cc2, -0.419 $[-0.817,-0.016])$ with the negative coefficient suggesting that as time progresses this impactdecreases.

One of the features of this domain is that it reflects participation in a broader range of reckless activities including those which place the young person and/or others at risk of physical injury (e.g. playing on railway lines, building sites or major roads, and racing cars around residential areas); activities done to impress others or to get a 'buzz'; and involving other in their offending. As the data collection process did not drill down to the responses to the 'Yes' / 'No' questions completed bythe practitioners as part of the assessment process, it is not possible to ascertain the extent to which these are features of the young people's lives as Baker et al. (2003) did in their first published review of ASSET. However, it is notable that at Time $0,73.9 \%$ (65 out of 87 ) where judged to be at significant risk of reoffending as a result of their Lifestyle with practitioners assigning ratings of 2 or more. This was one of the highest proportions with only the proportion deemed at significant risk as a result of their Thinking and Behaviours being higher $(76.1 \%)$. The other domain with similar proportions considered to be at significantrisk at Time 0 was Familyand Personal Relationships (73.9\%).

Given that two of the four examples of high ratings given by the YJB for the Lifestyle domain (Youth Justice Board, 2008a: 10-11) relate to co-offending - a well-documented criminological phenomenon, it is notable that there is also evidence to suggest that the prevalence of co-offending also varies by age. Carrington (2015) for example, has explored the structure of age homophily in co-offending groups through consideration of the mean age differences. His results indicate a strong age homophilyin the population which decreases with increasing age of co-offenders. However, the trend is structured within four aggregated age groups, the first three of which are bounded by the age of criminal responsibility and criminal majorityin Canada i.e. between 12 and 18 years. Pertinent to this research is the finding 
that youth (12-17 year olds) were found to have the strongestand mostnarrowly defined age homophily, tending to co-offend with others who were within a year or so of their own age. Those aged 3 to 11 years were also found to exhibitstrong age homophily, but their co-offending was less narrowly age-exclusive and more dispersed among members of their own aggregated age group, regardless of their specific age.

Kivivuori (2007) asserts that there is a distinct, but related type of offending: crime by proxy where the offender commits the crime for someone rather than with someone. In exploring whether or not shoplifting is a proxy crime and addressing fears in Finland that adolescents who werecriminallyculpable were coercing those younger than them to commit crimes as their proxies, Kivivuori draws upon historical accounts including some which are akin to Dickensian descriptions in Oliver Twist of Fagin like characters who entice youngsters into crime to suggest that there is a historical precedent for this type of offending. Although he found that these concerns had been exaggerated, Kivivuori found that $7.2 \%$ of respondents had shoplifted for someone else and that it appeared to be related to peer-group activities. Notably males who had acted as proxies were more often paid or threatened to steal whereas females shoplifted as proxies to increase their popularity.

Carrington (2015) also investigated the so called "Fagin" hypothesis that offenders below the age of criminal responsibility are particularly attractive as co-offenders for older offenders - a phenomenon which is specificallyidentified within the examples of higher ratings for Lifestyle. This was disconfimed with children below 12 being found to be unlikely to co-offend with 12-17 year olds, and very unlikelyto co-offend with adult offenders once the population age distribution was controlled for. Despite this finding, there is an established narrative, according to Lammy (2017), associated with gang activity whereby vulnerable young people are coerced into committing criminal acts by powerful adults. In particular drawing upon findings from police records and the National Crime Agency (2017), Lammy makes reference to many children and young adults from BAME backgrounds being drawn into the criminal justice system having been recruited by gang leaders to sell drugs or to carry weapons. Within this gang culture girls and young women who 'become involved are targeted because they are vulnerable, potentiallyclass A drug users; and they can often find themselves controlled through threats and intimidation' (2017:20).

The substance use domain focuses upon the types of substances used, when used and age at first use. It was found to be a highlystatisticallysignificant predictor of proven one-year reoffending in the logistical regression model based upon the 12 'dynamic' domains and the significant at the 0.05 level in the simulated model representing ASSET under the Scaled Approach (Wilson and Hinks, 2011). In the case of the latter, Lifestyle and Motivation to Change were also found to be significant.

Whilst BDm 3 is a compromise since there was insufficient data to incorporate all four static factors, it is notable that the estimates for substance misuse (Drugs) are significantwhen in interactions with Time. 
This includes the 3-way interactions G_ageFirst: Drugs: Time and FTE: Drugs: Time, with the former being a significantnegative coefficientwhereas the latter is a significantpositive coefficient. Given the respective reference categories for these two predictors, it suggests that those with a history of prior offending with substance misuse issues will see a greater 'penalty' over time than FTEs as will those aged 10-12 at the time of their first offence who have alcohol or drug problems. More generally, the significant positive coefficient for the interaction between Time:Drugs suggests that if the young person's substance misuse issues are not addressed, they will continue to be at a higher risk of further offending behaviour. For this reason, it is recognised in Welsh policythat:

\begin{abstract}
'Having access to the rightservices at the right time, designed to minimise the impact of substance misuse, is essential. The relationship between crime, anti-social behaviour and impacton personal health is well understood and documented. Service access to those agencies set up and funded to provide help for young people who misuse drugs and alcohol should be based on an assessment of need. Referral pathways should be simple and understood by a range of professionals likely to encounter young people involved with the criminal justice system. This extends beyond YOTs and social workers. It should include schools based nurses and counsellors, youth and sports developmentworkers, and teachers with pastoral care duties.'
\end{abstract}

(Welsh Governmentand Youth Justice Board, 2014:10)

This priority is also reflected in the devolved outcome indicators around access for those in the youth justice system to assessment and treatment for substance misuse need: In 2013/14, 90.4\% of assessments were made within 5 working days of referral, which was a 3 percentage point increase compared to the previous year, whilst the proportion of young people with identified needs that started interventions within 10 working days was $96.6 \%$. This was a 3.7 percentage point increase on the $92.9 \%$ in 2012/13 (Youth Justice Board and Ministry of Justice, 2015c).

The estimated coefficients for the Relationships with Familyand Friends domain and Perception of Self and Others domain are significant main effects in BDm3 and the dynamic model involving Grouped Age at First offence. In the case of the latter, the large positive significant coefficient for the interaction

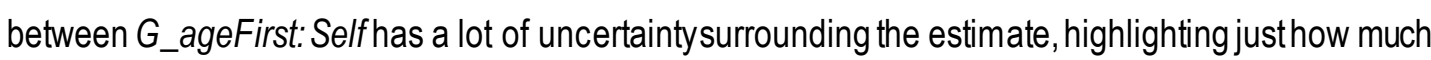
those aged 13-17 at the time of their first offence may be struggling with issues associated with selfesteem and self-identity. This includes potentially having adopted a criminal identify and displaying discriminatoryattitudes towards others.

With so few cases in the dataset, it is not possible to explore whether there is a clearer pattern for those who have a history of prior offending or FTEs respectively. Similarly, it has notbeen possible to explore this in the context of the nature and / or seriousness of the primary offence. Had it been possible to do this, it would have provided additional information to inform the evidence base about criminal careers and the need for a tailored approach to interventions. However, it is apparent from Table 6.20, that 
having a history of prior offending explains a significant amount of uncertaintyover time around variations in the Neighbourhood; Substance Misuse; Physical Health; Thinking and Behaviour; Attitude to Offending and Motivation to Change domains.

\subsection{Summary}

The analysis presented in this chapter sought to address three research questions, with the final question being addressed within section 6.6 :

4. What is the impact of the 'static' factors within ASSET in predicting further offending over time?

5. Is it possible to extend the sensitivity of ASSET by extending any of the predictors?

8. How well do ASSET scores reflect the realities of the young person's change in circumstances during their time under the supervision of the YOT?

As in Chapter Five, the low number of cases within the reoffending cohortlimits the construction of the combined model. Had there been sufficientcases, it would have been desirable to include all fo ur static factors rather than make a compromise. There wouldalso have been the potential to more fully explore how the sensitivity of the model could have been increased for example, by:

- treating the age-related predictors as a continuous rather than grouping them using the thresholds used in the Scaled Approach

- combining YJB Gravity Score and the Grouped YJB Offence Category;

- including gender, ethnicityand experience of care

It should be noted however, as highlighted in section 6.7 , including continuous versions of age at first conviction alongside age at first offence introduces problems with collinearity. One alternative to get around this would be to consider age at the time of the primary offence and a measure that takes into account the time known to the YOT.

From a methodological point of view, whilst the potential for addressing the two research questions was limited bythe low number of cases, in exploring the static factors, it has been possible to explore the use of different types of predictors. For example, the Grouped Age at First Offence was set up as a dummy variable whereas in treating age at first offence as a continuous variable meant that it became a multiplicative variable. The variable was also centred at 10 - the age of criminal responsibility. The DIC for the latter model was higher than that involving the dichotomous predictor (465 compared to 448), reflecting the increased complexity of the model. Comparing the resulting trajectories of the probability of further offending for the model involving the continuous predictor (Figure 6.23), it is possible to see features of both Figure 6.5(a) and (b). For those who were younger when then committed their first 
offence, the probability of further offending increases the longer the individual is in the formal youth justice system whereas that for the older group starts higher and then decreases.

The YJB Gravity Score is similarlya continuous predictor. In this case, the variable is centred at 2 reflecting the lowest score of primary offences committed by members of the reoffending cohort. Notably, none of the cohortcommitted veryserious offences. However, it is possible to use the individual dynamic model and the combined model to estimate the probability of further offending for those who had committed offences attracting a gravity score of 7 or 8 .

The predictor for Grouped YJB Offence Categoryis a categorical variable - in this case with three levels or 'factors'. The reference categorywas selected on the basis of the average initial scores, with those for SAC and VAP offences being higher than those for Other offences. If access could be secured to the national dataset, it would be worth exploring whether it would be possible to ungroup the categories. Based on published data, it is anticipated that there would be a very low number of individuals who had committed either serious sexual or serious violent offences including murder. The distinctadvantage of using Bayesian approaches is that low incidence crime types can explored. 


\section{Findings: System Contact}

This chapter concentrates on whether having system contact increases the likelihood of further offending. As such the following research questions are considered:

6. How is the likelihood of further offending affected by having experience of care and a previous offending history?

7. What is the impact of coming into contact with facets of the youth justice system on the likelihood of further offending?

8. How well do ASSET scores reflect the realities of the young person's change in circumstances during their time under the supervision of the YOT?

The first of these questions links back to the research questions posed in Chapter Five and Six which considered the predictors around care and FTE status. The additional predictors explored within this chapter are therefore:

- Breach - whether the young person has breached in the period before the ASSET

- Appear - whether the young person has had a court appearance (regardless of outcome) before the ASSET

- Custody - whether the young person has spent time in custody either on remand or as part of a custodial sentence before the ASSET

These time-varying Level 1 measures represent key features of the youth justice process and will be explored in Section 7.2.

\subsection{Being known to the YOT and experience ofcare}

Analysis in Chapter Six highlights that there are only three young people who were young FT Esi .e. who committed their first offence aged 10-12. As none of these went on to commit any further offences, it limits the extent to which FTE Status can be used as a predictor alongside grouped age at first offence - the implications of this can be seen in Figure 6.16(a).

Of the remaining predictors, grouped age at first offence providers the greatestopportunity for exploring the impact of being known to the YOT. Using their age at the time of their primary offence from the reoffending spreadsheet, it is possible to determine the difference between this and their age at the time of their first offence as recorded in their offence record in Childview. Within the reoffending cohort, the average difference was 1.9 years. However, amongst those in the younger group i.e. those at 10-12 at the time of their first offence, the average difference was 3.8 years ( $\max =7$ years), whereas amongst the oldergroup, the average difference was 1.2 years ( $\max =4$ years). From this it can be inferred that those in the younger group have typically been in contact with the YOT for longer. 
Whilst, ideally the impact of system contact on the probability of further offending would be modelled using FTE Status and Care Experience, as a compromise, G_ageFirst has been used to demonstrate the potential of using Bayesian approaches. The resulting dynamic model, renamed as Dynamic Model 4 (BDm4), is summarised in Table 7.1.

Table 7.1: Dynamic Model 4

\begin{tabular}{|c|c|c|c|c|c|c|c|}
\hline \multirow{3}{*}{ Fixed Effect: } & \multicolumn{7}{|c|}{$\begin{array}{l}\text { Dynamic Basic Model including Grouped Age at First Offence } \\
\text { and Care Experience (BDm4G_cc2_ch) }\end{array}$} \\
\hline & \multicolumn{3}{|c|}{ Unstandardised } & \multicolumn{3}{|c|}{ Standardised } & \multirow{2}{*}{ Significant? } \\
\hline & Post.Mean & Lower $\mathrm{Cl}$ & Upper $\mathrm{Cl}$ & Post.Mean & Lower $\mathrm{Cl}$ & Upper Cl & \\
\hline (Intercept) & -3.872 & -8.998 & 0.955 & 0.021 & 0.000 & 2.599 & \\
\hline Grouped Age at First Offence (10-12 = Ref $)$ & 2.135 & -2.230 & 6.925 & 8.455 & 0.108 & $1.02 E+03$ & \\
\hline Care Experience (No = Ref) & -0.228 & -5.272 & 5.155 & 0.796 & 0.005 & $1.73 \mathrm{E}+02$ & \\
\hline Time & 0.057 & -0.986 & 1.101 & 1.059 & 0.373 & 3.008 & \\
\hline Living Arrangements (Live) & -0.889 & -2.551 & 0.638 & 0.411 & 0.078 & 1.892 & \\
\hline Family and Personal Relationships (Relation) & 1.942 & -0.205 & 3.994 & 6.969 & 0.815 & 54.287 & \\
\hline Education, Training and Employment (ETE) & -1.103 & -2.870 & 0.388 & 0.332 & 0.057 & 1.474 & \\
\hline Neighbourhood (Where) & 0.085 & -1.248 & 1.379 & 1.089 & 0.287 & 3.970 & \\
\hline Lifestyle (Life) & 3.334 & 1.002 & 5.708 & 28.041 & 2.723 & $3.01 \mathrm{E}+02$ & Yes \\
\hline Substance Use (Drugs) & -0.852 & -2.458 & 0.820 & 0.426 & 0.086 & 2.271 & \\
\hline Physical Health (Physical) & -0.654 & -2.717 & 1.082 & 0.520 & 0.066 & 2.951 & \\
\hline Emotional and Mental Health (Emotion) & -1.227 & -2.674 & 0.252 & 0.293 & 0.069 & 1.287 & \\
\hline Perceptions of Self and Others (Self) & -5.760 & -8.833 & -2.635 & 0.003 & 0.000 & 0.072 & Yes \\
\hline Thinking and Behaviour (Think) & 1.798 & -0.472 & 4.183 & 6.037 & 0.624 & 65.578 & \\
\hline Attitude to Offending (Attitude) & 3.034 & 0.211 & 5.908 & 20.772 & 1.235 & $3.68 \mathrm{E}+02$ & Yes \\
\hline Motivation to Change (Change) & 0.467 & -1.929 & 2.912 & 1.596 & 0.145 & 18.387 & \\
\hline Grouped Age atFirst Offence: Care Experience & 2.550 & -0.514 & 5.636 & 12.809 & 0.598 & $2.80 \mathrm{E}+02$ & \\
\hline Grouped Age at First Offence: Time & -0.264 & -1.195 & 0.726 & 0.768 & 0.303 & 2.066 & \\
\hline Grouped Age at First Offence: Live & 0.632 & -1.052 & 2.535 & 1.880 & 0.349 & 12.617 & \\
\hline Grouped Age at First Offence: Relation & -1.886 & -4.096 & 0.330 & 0.152 & 0.017 & 1.391 & \\
\hline Grouped Age at First Offence: ETE & 0.948 & -0.688 & 2.782 & 2.580 & 0.502 & 16.148 & \\
\hline Grouped Age at First Offence: Where & -0.084 & -1.549 & 1.334 & 0.919 & 0.213 & 3.797 & \\
\hline Grouped Age at First Offence: Life & -2.567 & -5.160 & 0.006 & 0.077 & 0.006 & 1.006 & \\
\hline Grouped Age at First Offence: Drugs & 1.200 & -0.501 & 2.914 & 3.321 & 0.606 & 18.436 & \\
\hline Grouped Age at First Offence: Physical & 0.655 & -1.418 & 2.664 & 1.925 & 0.242 & 14.351 & \\
\hline Grouped Age at First Offence: Emotion & -0.205 & -1.844 & 1.463 & 0.815 & 0.158 & 4.319 & \\
\hline Grouped Age at First Offence: Self & 6.721 & 3.484 & 10.099 & 829.405 & 32.596 & $2.43 E+04$ & Yes \\
\hline Grouped Age at First Offence: Think & -1.034 & -3.533 & 1.418 & 0.356 & 0.029 & 4.129 & \\
\hline Grouped Age at First Offence: Attitude & -2.351 & -5.355 & 0.683 & 0.095 & 0.005 & 1.981 & \\
\hline Grouped Age at First Offence: Change & -1.600 & -4.253 & 1.068 & 0.202 & 0.014 & 2.910 & \\
\hline Care Experience: Time & -0.074 & -1.013 & 0.759 & 0.928 & 0.363 & 2.135 & \\
\hline Care Experience: Live & 1.024 & -0.689 & 2.681 & 2.786 & 0.502 & 14.597 & \\
\hline Care Experience: Relation & -0.406 & -2.258 & 1.661 & 0.666 & 0.105 & 5.265 & \\
\hline Care Experience: ETE & 0.053 & -1.458 & 1.547 & 1.054 & 0.233 & 4.696 & \\
\hline Care Experience: Where & 0.435 & -0.985 & 1.843 & 1.545 & 0.373 & 6.314 & \\
\hline Care Experience: Life & -1.019 & -3.178 & 1.298 & 0.361 & 0.042 & 3.660 & \\
\hline Care Experience: Drugs & 0.140 & -1.247 & 1.584 & 1.151 & 0.287 & 4.873 & \\
\hline Care Experience: Physical & -1.779 & -3.694 & 0.284 & 0.169 & 0.025 & 1.329 & \\
\hline Care Experience: Emotion & 1.582 & -0.022 & 3.174 & 4.865 & 0.978 & 23.915 & \\
\hline Care Experience: Self & 1.493 & -0.856 & 3.922 & 4.450 & 0.425 & 50.495 & \\
\hline Care Experience: Think & -1.937 & -4.050 & 0.118 & 0.144 & 0.017 & 1.125 & \\
\hline Care Experience: Attitude & -0.278 & -2.230 & 1.611 & 0.758 & 0.108 & 5.007 & \\
\hline Care Experience: Change & 0.460 & -1.800 & 2.657 & 1.585 & 0.165 & 14.255 & \\
\hline
\end{tabular}




\begin{tabular}{|c|c|c|c|c|c|c|c|}
\hline \multirow[b]{3}{*}{ Fixed Effect: } & \multicolumn{7}{|c|}{$\begin{array}{l}\text { Dynamic Basic Model including Grouped Age at First Offence } \\
\text { and Care Experience (BDm4G_cc2_ch) }\end{array}$} \\
\hline & \multicolumn{3}{|c|}{ Unstandardised } & \multicolumn{3}{|c|}{ Standardised } & \multirow{2}{*}{ Significant? } \\
\hline & Post.Mean & Lower $\mathrm{Cl}$ & Upper Cl & Post.Mean & Lower Cl & Upper Cl & \\
\hline Time: Live & -0.240 & -0.624 & 0.127 & 0.787 & 0.536 & 1.136 & \\
\hline Time: Relation & -0.411 & -0.863 & 0.057 & 0.663 & 0.422 & 1.059 & \\
\hline Time: ETE & 0.185 & -0.149 & 0.512 & 1.203 & 0.862 & 1.668 & \\
\hline Time: Where & -0.230 & -0.505 & 0.078 & 0.794 & 0.604 & 1.081 & \\
\hline Time: Life & -0.622 & -1.172 & -0.101 & 0.537 & 0.310 & 0.904 & Yes \\
\hline Time: Drugs & 0.480 & 0.117 & 0.838 & 1.616 & 1.124 & 2.311 & Yes \\
\hline Time: Physical & 0.315 & -0.228 & 0.821 & 1.370 & 0.796 & 2.273 & \\
\hline Time: Emotion & 0.397 & 0.070 & 0.720 & 1.487 & 1.073 & 2.055 & Yes \\
\hline Time: Self & 1.497 & 0.917 & 2.227 & 4.468 & 2.501 & 9.270 & Yes \\
\hline Time: Think & -0.437 & -0.925 & 0.012 & 0.646 & 0.396 & 1.012 & \\
\hline Time: Attitude & -0.953 & -1.519 & -0.418 & 0.386 & 0.219 & 0.658 & Yes \\
\hline Time: Change & 0.289 & -0.281 & 0.807 & 1.334 & 0.755 & 2.242 & \\
\hline Grouped Age at First Offence: Time: Live & 0.325 & -0.115 & 0.784 & 1.383 & 0.891 & 2.190 & \\
\hline Grouped Age at First Offence: Time: Relation & 0.375 & -0.139 & 0.893 & 1.455 & 0.870 & 2.442 & \\
\hline Grouped Age at First Offence: Time: ETE & -0.217 & -0.612 & 0.162 & 0.805 & 0.542 & 1.176 & \\
\hline Grouped Age at First Offence: Time: Where & 0.137 & -0.163 & 0.441 & 1.146 & 0.849 & 1.555 & \\
\hline Grouped Age at First Offence: Time: Life & 0.491 & -0.091 & 1.056 & 1.633 & 0.913 & 2.875 & \\
\hline Grouped Age at First Offence: Time: Drugs & -0.391 & -0.773 & -0.025 & 0.677 & 0.462 & 0.976 & Yes \\
\hline Grouped Age at First Offence: Time: Physical & -0.435 & -0.979 & 0.040 & 0.647 & 0.376 & 1.041 & \\
\hline Grouped Age at First Offence: Time: Emotion & -0.160 & -0.559 & 0.217 & 0.852 & 0.572 & 1.243 & \\
\hline Grouped Age atFirst Offence: Time: Self & -1.643 & -2.358 & -0.985 & 0.193 & 0.095 & 0.374 & Yes \\
\hline Grouped Age at First Offence: Time: Think & 0.327 & -0.181 & 0.830 & 1.387 & 0.834 & 2.293 & \\
\hline Grouped Age at First Offence: Time: Attitude & 0.571 & -0.067 & 1.216 & 1.769 & 0.935 & 3.375 & \\
\hline Grouped Age at First Offence: Time: Change & 0.165 & -0.408 & 0.740 & 1.179 & 0.665 & 2.096 & \\
\hline Care Experience: Time: Live & 0.019 & -0.373 & 0.442 & 1.020 & 0.688 & 1.556 & \\
\hline Care Experience: Time: Relation & 0.009 & -0.451 & 0.425 & 1.009 & 0.637 & 1.529 & \\
\hline Care Experience: Time: ETE & 0.163 & -0.144 & 0.498 & 1.177 & 0.866 & 1.645 & \\
\hline Care Experience: Time: Where & 0.199 & -0.110 & 0.469 & 1.220 & 0.896 & 1.598 & \\
\hline Care Experience: Time: Life & 0.022 & -0.430 & 0.435 & 1.022 & 0.650 & 1.544 & \\
\hline Care Experience: Time: Drugs & -0.170 & -0.534 & 0.156 & 0.844 & 0.586 & 1.169 & \\
\hline Care Experience: Time: Physical & 0.166 & -0.301 & 0.617 & 1.180 & 0.740 & 1.854 & \\
\hline Care Experience: Time: Emotion & -0.155 & -0.526 & 0.192 & 0.857 & 0.591 & 1.212 & \\
\hline Care Experience: Time: Self & -0.546 & -1.019 & -0.069 & 0.579 & 0.361 & 0.933 & Yes \\
\hline Care Experience: Time: Think & 0.452 & 0.002 & 0.899 & 1.571 & 1.002 & 2.457 & Yes \\
\hline Care Experience: Time: Attitude & 0.293 & -0.196 & 0.801 & 1.341 & 0.822 & 2.229 & \\
\hline Care Experience: Time: Change & -0.390 & -0.861 & 0.091 & 0.677 & 0.423 & 1.095 & \\
\hline Random Effect: & Post.Mean & Lower $\mathrm{Cl}$ & Upper Cl & Post.Mean & Lower $\mathrm{Cl}$ & Upper $\mathrm{Cl}$ & Significant? \\
\hline Individual (Intercept) & 3.206 & 0.305 & 7.460 & 24.68 & 1.357 & $1.74 \mathrm{E}+03$ & Yes \\
\hline Time & 5.241 & 0.779 & 12.170 & 188.86 & 2.178 & $1.93 \mathrm{E}+05$ & Yes \\
\hline DIC & & & 425.90 & & & & \\
\hline
\end{tabular}

Source: Model BDm4G_cc2_ch, renamed as BDm4, Technical Annex: p339-355.

Despite the additional complexity, including both grouped age at first offence and care experience in a dynamic model, reduces the DIC to 425.9. In comparison the dynamic model involving grouped age at first offence (BDm3G_cc2) has a DIC of 448.7 whilst that involving care experience has a DIC of 471.4 . Using the model to estimate the probability of further offending over time results in the following sets of trajectories (Figure 7.1). 
Figure 7.1: Changes in the Probability of Further Offending, Dynamic Model 4

(a) Age 10-12 at First Offence, Never Looked After

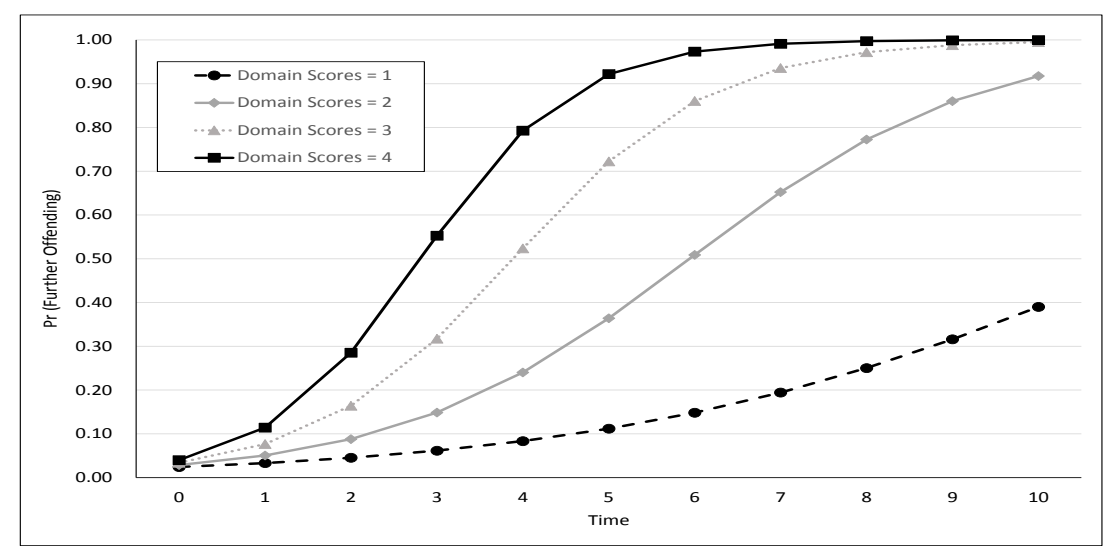

(c) Age 13-17 at First Offence, Never Looked After

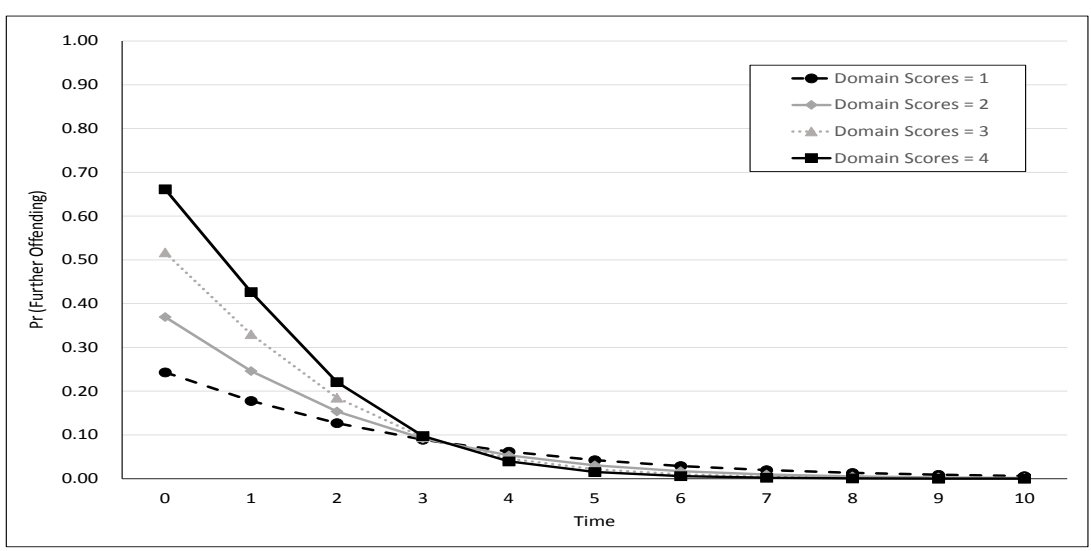

(b) Age 10-12 at First Offence, Experience of Care

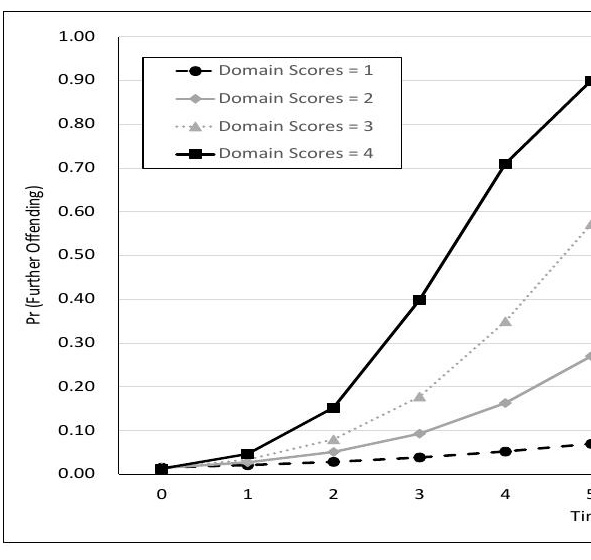

(d) Age 13-17 at First Offence, Experience of Car

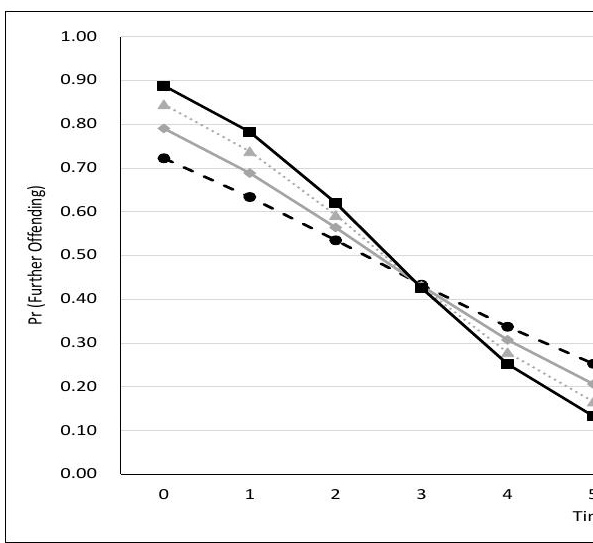

Notes: The domain scores have respectively been shown as being fixed at 1, 2, 3 and 4 respectively to demonstrate the estimated change in the probability of fu derived from Model BDm4. 
Generallyspeaking, the initial estimated probability of further offending for those aged 10-12 at the time of their first offence are low. However, as can be seen from Figures 7.1(a) and (b), their estimated probability of further offending increases at later measurement points. This is broadly consistent with the trend observed as a result of estimates derived from the dynamic model involving grouped age of first offence (BDm3G_cc2, Figure 6.5). When the respective trajectories are compared for those in the younger group who have experience of care and those who have never been looked after, it is apparent that with the exception of when the domain scores were fixed at 1 , the estimated probability of further offending is higher at each time point for those who have never been looked after. This is contrary to what would be expected but may well reflect the low numbers - there are only 8 young people who committed their first offence aged 10-12 who have experience of care.

In contrast, Figures 7.1(c) and (d) have a downward trajectory with the probability of further offending decreasing at later measurement points. The trend suggests that for those aged 13-17 at the time of their first offence, their estimated probability of further offending decreases the longer theyare in contact with the YOT. However, it is notable that the initial probabilities of further offending are higher for those who have experience of care. This is apparent when the domain scores are fixed at 1, 2, 3 and 4 respectively. This difference is also apparentat all later measurement points, with the estimates for the probability of further offending taking longer to tend towards zero.

Notably when the further offending rates are compared for the four different groups, there is no evidence to suggest that amongst those who first offended aged 10-12, there is onlyanecdotal evidence to suggest that the rates are different on the basis of having experience of care relative to those who have never been looked after $\left(\mathrm{BF}_{10}=0.882\right.$ for the two-sided test). However, there is strong evidence to support the rate within the older group being higher amongst those with experience of care $\left(\mathrm{BF}_{10}=16.48\right)$. Table 7.2 summarises the respective rates.

Table 7.2: Further Offending by Experience of Care and Grouped Age at First Offence

\begin{tabular}{|c|c|c|c|c|}
\hline & \multicolumn{2}{|c|}{ Grouped Age at First Offence } & \multirow{2}{*}{ Total } \\
\hline & & $10-12$ years & $13-17$ years & \\
\hline \multirow{2}{*}{$\begin{array}{l}\text { Care } \\
\text { Status }\end{array}$} & $\begin{array}{c}\text { Never } \\
\text { Looked After }\end{array}$ & $\begin{array}{l}50 \% \\
(7 / 14)\end{array}$ & $\begin{array}{l}34.7 \% \\
(17 / 49)\end{array}$ & $\begin{array}{l}38.1 \% \\
(24 / 63)\end{array}$ \\
\hline & $\begin{array}{c}\text { Experience } \\
\text { of Care }\end{array}$ & $\begin{array}{l}75 \% \\
(6 / 8)\end{array}$ & $\begin{array}{l}70.6 \% \\
(12 / 17)\end{array}$ & $\begin{array}{c}72.0 \% \\
(18 / 25)\end{array}$ \\
\hline \multicolumn{2}{|l|}{ Total } & $\begin{array}{l}59.1 \% \\
(13 / 22)\end{array}$ & $\begin{array}{l}43.9 \% \\
(29 / 66)\end{array}$ & $\begin{array}{l}47.7 \% \\
(42 / 88)\end{array}$ \\
\hline
\end{tabular}

From Table 7.1, it is clear that the perception of self and others domain has a key role to play. It has previously been discussed a number of times, but in this particular context, the lack of uncertaintyaround the estimates is indicative of the issues around self-identity which maybe faced by those who have had prolonged contact with the youth justice system, having committed their first offence at a young age 
and/or being a looked after child. Notably the guidance relating to this domain makes specific reference to the following common factors that may contribute to a poor or confused sense of self-identity:

- 'A lack of knowledge of personal and family history (eg a young person subject to a care order who has little knowledge abouthis/herbirth family);

- Experience of discrimination;

- A feeling of cultural/social isolation;

- A very unstable or highly dysfunctional familybackground.'

(Youth Justice Board, 2008a: 16)

There may also be issues associated with having a mistrust of others and perceptions of having a criminal identity which could also be factors associated with having prolonged exposure to 'T heSystem' and the impact of labelling. Having potentiallyestablished a criminal identity, the young person may well feel that further offending behaviour is inevitable hence the significance of the attitudes to offending domain both as a main effect and in the interaction with time. With this there is an increased likelihood of 'falling in with the wrong crowd' and participation in reckless activities including substance misuse. This pattern can similarlybe seen within the model with the lifestyle domain being significantas a main effect and in the interaction with time. In the case of substance use, if this is seen as being highly associated with their likelihood of reoffending, attracting higher ratings, then this will need to be addressed. The negative significantestimate for $G$ _ageFirst: Time: Drugs suggests that time has less of a mediating effectif the young person was aged 10-12 at the time of their first offence.

\section{Having Experience of Being Looked After}

Looked after children and Care Leavers have long been over-represented in the criminal justice system, and as such there is a growing body of research whichinvestigates the relationshipbetween involvement in the care system and negative outcomes for children. Notably Staines (2016) produced a systematic review and narrative synthesis of the international literature to accompany Lord Laming's review of looked after children in the criminal justice system. Given the comprehensive nature of this review, the intention is not to reproduce the findings here.

As highlighted in Section 4.3, there were significant difference in the initial mean ratings for the Family and Personal Relationships and Emotional and Mental Wellbeing domains. This is consistent with the findings summarised by Staines which suggests that looked after children have significantly worse emotional, psychological and behavioural health, and to experience difficulties in interpersonal relationships. However, whilst research suggests that looked after children are at greater risk of having poor educational attainment and having fewer employment/ training opportunities; of living in more deprived areas; having poorer physical health including being involved in substance misuse, in this study, there was moderate evidence to suggest the average initial scores for looked after children in the 
reoffending cohort are not that dissimilar to those for their peers in these domains. It is perhaps the absence of these differences that have contributed to the lack of significantcoefficients in the Dynamic Model involving Care (BDm2_ch). The only significant coefficient in the model was for the Care Experience: Time: Self interaction (BDm2_ch, -0.341 [-0.644,-0.045]).

Blades et al. (2011) identified a number of inconsistencies as a result of speaking with young people with relevant experiences which are pertinent to this research. For example, some children with a history of offending said they had offended prior to going into care, citing peer pressure as the most common reason, although other reasons given included difficulties controlling anger, a lack of money, being bored and living in a high crime area. However, there were also children who felt that being in care was the primaryreason for their offending behaviour, or at leastbeing in the care system increased the likelihood of offending. A small number believed that being in care had no real affect, or even reduced their chances of offending.

To attempt to unpick this further, it would be necessaryto take into account the individual experiences and pathways before and after entering care. It is known for example that there are links between adverse family experiences and proven offending, with maltreatment and going into care as a teenager potentially having a stronger association with youth offending than maltreatment or care only being experienced in earlychildhood (Fortyand Sturrock, 2017). The type and instability of the placement can also have a bearing with those in residential care homes not only being criminalised at excessively high rates (Howard League for Penal Reform, 2017) but may also not be receiving an equivalent level of parental-type supportwhen they come before the court (Sentencing Council, 2017).

\section{Having Experience of the Youth Justice System}

Analysis by the Ministry of Justice (Sutherland etal., 2017) suggest that the characteristics of FTEs have changed over time in a way that was consistent with increasing numbers of young people who commit first time low-level offences being diverted from the formal youth justice system. Compared to those entering the system in 2003/04, FTEs in 2012/13 (which coincidently is within the period of interest for this research) were, on average:

- More likely to be older (aged 15 to 17 years)

- Less likely to be female

- Less likely to be White

- More likely to have committed a more serious offence

In investigating possible explanations for these changes, it was observed that 2009 onwards, 'a new 'class' of FTEs emerged who were older, black, violent and female (although the majority in this group were still male) and/or who had committed a violent offence' (Sutherland et al., 2017: 16). This group 
were more likely to have received court outcomes. As the profile of FTEs changed, the probability of proven reoffending within a year increased.

Since the young people's whose risk assessment score were utilised for this research were all in the formal youth justice system, comparisons cannotbe made with the typology described by Sutherland et al. However, there are notable differences in the profiles of FTEs and those with a prior history of offending: Of the 33 FTEs within the cohort, all but one was male. $69.7 \%$ (23/33) were aged 15-17, $30.3 \%(10 / 33)$ were aged 12-14, with none of the FTEs being aged 10 or 11 . Two identified as being non-White. Their average total ASSET score was 18.0 (out of 48) at Time 0.

In contrast, $87.0 \%$ (47/54) of the young people with a prior history of offending at the time of entering the cohort were male. The majority $(77.8 \%, 42 / 54)$ had been aged $10-14$ at the time of their first offence with 11 of these being aged under 12. Four young people were identified as being non-White. There are no significant differences in terms of the nature of the primary offence or its severity as determined by the YJB Offence Categories and Gravity Scores respectively. However, the disposals received for the primary offence do have a different profile, with none of the FTEs having received community or custodial sentences. Their average total ASSET score at T ime 0 was 22.8. Since this score is across the 12 dynamic domains, it does not take into account their prior offending history or the nature of their primaryoffence i.e. the static factors.

As highlighted in Chapter Six, FTEs typically had lower initial ratings for Family and Personal Relationships, Emotional and Mental Health, and Perception of Self and Others. The estimated coefficients for these domains were also are main effects in the Dynamic Model 3 (BDm3) whilst in the dynamic model involving FTE Status (BDm3_cc1), these were significant when in interactions with FTE. Interactions terms involving Neighbourhood were also significantin the FTE dynamic model.

These domains link to findings from the Cambridge Study which suggest that protective factors, particularlythose from high risk backgrounds include:
'...having a resilient temperament; a warm and affectionate relationship with at least one parent; parents who provide effective supervision, pro-social beliefs and consistent supervision; and parents who maintain a strong interest in their children's education.'

(Farrington, 2002:427)

Conversely, family variables such as a parental rejection, erratic and harsh discipline, marital confict and weak emotional attachment to parents have been consistently identified as being significant predictors of anti-social behaviours such as drug use and offending behaviour (Haines and Case, 2005) whilst resilience is key to how a young person coming into contact with different facets of the youth justice system copes. In particular those whose offending behaviour has contributed to a deterioration of the relationship that they have with their family / carers are likely to struggle without appropriate 
support. For those young people passed between members of the extended family / placements, this can be particularly distressing adding to feelings of shame, rejection and self-worth which in turn can affect their emotional wellbeing and mental health.

When the initial domain scores for FTEs and those with a prior offending history are segmented according to whether or not they went on to commitfurther offences (Table 7.3), this highlights distinct differences between the two groups which were not as evident in the modelling as would perhaps have been expected.

Table 7.3: Initial Average Domain Scores by FTE Status and Whether or Not Further Offending Occurred

\begin{tabular}{|c|c|c|c|c|c|c|}
\hline \multirow[b]{2}{*}{ Domain } & \multicolumn{3}{|c|}{ FTE } & \multicolumn{3}{|c|}{ Prior Offending } \\
\hline & $\begin{array}{l}\text { No Further } \\
\text { Offending } \\
(\mathrm{N}=22)\end{array}$ & $\begin{array}{l}\text { Further } \\
\text { Offending } \\
(\mathrm{N}=11)\end{array}$ & $\begin{array}{c}\text { Bayes Factor } 10 \\
\text { (H1: Group } 0<\text { Group 1) }\end{array}$ & $\begin{array}{l}\text { No Further } \\
\text { Offending } \\
(\mathrm{N}=22)\end{array}$ & $\begin{array}{c}\text { Further } \\
\text { Offending } \\
(\mathrm{N}=31)\end{array}$ & $\begin{array}{c}\text { Bayes Factor } 10 \\
\text { (H1: Group } 0<\text { Group 1) }\end{array}$ \\
\hline Living Arrangements & 1.182 & 1.545 & 0.879 & 1.591 & 2.258 & 3.124 \\
\hline Family and Personal Relationships & 1.727 & 1.909 & 0.478 & 2.045 & 2.645 & 3.378 \\
\hline ETE & 1.682 & 2.364 & 2.467 & 1.773 & 2.258 & 1.014 \\
\hline Neighbourhood & 1.045 & 1.818 & 3.369 & 1.455 & 1.774 & 0.727 \\
\hline Lifestyle & 1.727 & 2.364 & 1.801 & 1.864 & 2.548 & 8.794 \\
\hline Substance Use & 1.091 & 1.818 & 2.119 & 1.455 & 2.161 & 4.067 \\
\hline Physical Health & 0.591 & 1.182 & 2.095 & 1.000 & 1.355 & 0.946 \\
\hline Emotional and Mental Health & 0.909 & 1.091 & 0.506 & 1.273 & 1.645 & 0.768 \\
\hline Percpetion of Self and Others & 1.091 & 2.000 & 9.148 & 1.682 & 2.065 & 1.507 \\
\hline Thinking Behaviour & 1.773 & 2.636 & 5.830 & 2.045 & 2.516 & 1.982 \\
\hline Attitudes to Offending & 1.227 & 2.000 & 4.519 & 1.682 & 2.000 & 0.917 \\
\hline Motivation to Change & 1.143 & 2.091 & 10.243 & 1.545 & 1.935 & 1.005 \\
\hline
\end{tabular}

Notes: One-sided Bayesian independent t-tests have been conducted using JASP version 0.8.5 (JASP Team, 2017b). Bayes Factors quantify the evidence for the alternative hypothesis relative to the null hypothesis and are interpreted using the categories suggested by Jeffreys (1961).

Whilst those who committed further offences typically had higher initial domain scores than their peers who did not, amongst FTEs there are significant differences in the average scores for Motivation to Change $\left(B F_{10}=10.2\right)$, Perception of Self and Others $\left(B F_{10}=9.1\right)$, Thinking Behaviours $\left(B F_{10}=5.8\right)$ and Attitudes to Offending $\left(\mathrm{BF}_{10}=4.5\right)$. In the case of Motivation to Change, there is strong evidence to support this difference amongst FTEs whilst for the other domains, there is only moderate evidence. Notably it is this domain which considers whether the young person displays an appropriate understanding of the problematic aspects of his/her own behaviour; an understanding of the consequences for him/herself of further offending; has identified clear reasons or incentives for him/her to avoid further offending or shows real evidence of wanting to stop offending. The other domain for which there is comparativelystrong evidence relates to the perception of self and others. As discussed in Sections 4.2 and 5.5, this domain is concerned with the young person's understanding of how they and others - fit into the world around them and is very much concerned with the formation of an offender identity.

Amongst those with a prior history of offending, there are significant differences in the initial domain scores for Lifestyle $\left(\mathrm{BF}_{10}=8.8\right)$ and Substance Use $\left(\mathrm{BF}_{10}=4.1\right)$ when the group is segmented on the 
basis of whether they went on to commit further offences. Whilst both domains had proved to be significant in published evaluations of ASSET, the basic dynamic model (BDm1, Table 4.11) did not suggestany significantcoefficients either for Substance Use as a main effector in an interaction. Of the various models presented in Chapter Six, it is only the Combined Dynamic Model for Offending History (BDm3) which include significant coefficients for this domain. Due to the compromises itwas necessary to make in the combined model - see Section 6.5 , it is difficult to interpret the relationship between FTE status, substance misuse and grouped age at first offence. However, as Haines and Case (2005) highlight in relation to their research involving Swansea YOT, there are significant overlaps in the risk and protective factors related to offending behaviours and drug misuse, with the wider literature prompting the inclusion of the availability of drugs in the neighbourhood and parental substance misuse within the ASSET framework.

A possible explanation as to why substance misuse is not significantin the Dynamic Model involving in Demographics and Care (BDm2) is that whilst the characteristics of the neighbourhoods in whichyoung people live play a role in influencing the offending and drug using behaviour, these have been found to be relatively weakin comparison to individual characteristics suchas personalityand gender (McVie and Norris, 2006). In this instance the low number of females potentially contributed to this effect not becoming observed in the models involving gender.

Recognising the impact that substance misuse can have on the physical health, attitudes, problematic behaviours and poor decision making, substance misuse workers are embedded within YOTs with treatment representing an importantelement of the rehabilitation process - hence its identification within the Central Eight (Table 4.13). Sadly, those with more entrenched offending behaviours typicallyalso have more entrenched substance misuse problems and their chaotic lifestyles can lead to noncompliance and hence being sucked further into the youth justice system.

\subsection{Youth Justice Processes}

Concern has grown in the last twenty years over the stigmatising, labelling and criminogenic effects that formal system processing has on young people (Haines et al., 2013). Notably, research suggests that processing youth people through the justice system increases the likelihood that they will offend again and re-enter the system (McAra and McVie, 2007; 2015; Petrosino et al., 2010) hence both local and jurisdictional initiatives to increase diversionary activity. Whilst Swansea was operating a diversionary model during the period of interest for this research which sought 'to mediate national policyprescriptions and to develop a local response to the excessive criminalisation of young people' (Haines et al., 2013: 171), the young people whose Core ASSET S were utilised for this research were processed through the formal youth justice system. Consequently, rates of system contactare higher than for the wider youth offending cohort. 
Much of the quantitative work on system contact focuses upon individual's contact with the police, policing practices such as stop and search, and the impact of arrests. However, there is also research which considers whether or not contact with the youth justice system increases the likelihood of later offending. This work builds upon a long pedigree of criminological theory including theories of anomie, labelling and symbolic interactionism, with criminology having recently rediscovered social identify, and particularly the idea that criminal justice institutions can create and shape the objective and subjective identities of those that they police, sentence or incarcerate (Bradford etal., 2014). Notably Bradford et al suggest that fair process, legitimacy and compliance are linked by social identify with those who perceive that they have been treated fairly by police and other agencies, having their respect for that organisation enhanced, strengthening legitimacy as a result. In contrast, unfair treatment signals to people that they do not belong, undermining both identification and the legitimacy of the criminal justice system. Hence the nature of the encounters thatyoung people have with the system have the potential not just to shape individual's perceptions of themselves, but also their sense of self-worth.

McAra and McVie suggest that there is a group of 'usual suspects' who the police may be unfairly targeting. Whilst it is the volume and seriousness of offending which is what first brings a young person to the attention of the police, having been 'identified as a troublemaker, 'this status appears to suck young people into a spiral of amplified contact, regardless of whether they continue to be involved in serious levels of offending (according to their self-reports)' (2005: 9). Hence the adversarial nature of their contacts with the police, can result in certain categories of young people becoming permanent suspects rather than suspects for a particular offence. This, argue McAra and McVie, has the effect of recycling these young people whilst other serious offenders escape the tutelage of the formal system altogether. Importantly, selection effects at each stage of the youth justice process mean that the deeper the usual suspects penetrate the youth justice system, the more this is associated with inhibited desistence from offending (McAra and McVie, 2007).

Conventional thinking suggests that unfair (and thus labelling) system contact promotes 'delinquent" identities which in turn makes peoplemore likely to actlike one. Since people's social identities canalso be shaped by encounters with other authority figures and are subject to alteration throughout people's lives, this suggests that they are potentiallyamenable to positive change when treated in a sensitive and principled manner. Hence the relationship between the young person and their practitioner is integral if entrenched negative perceptions of self-worth are to be countered. However, McAra and McVie (2007) suggest that once ascribed, young people find ithard to shrug off labels, creating a self-fulfilling prophecy which maybe damaging to the young person in the long term. Notably they observe that:

... youngsters are powerless to alter the majority of the factors that propel them further and further into the system at age 15 (including fam ily structure, social deprivation, gender, and being known to the police and Reporter in earlier years). The only real certaintyfor such children is that the master status of troubled/troublesome youngster results in amplified levels of intervention. 
The findings from the Edinburgh Study in particular highlight the negative consequences of system contact, providing the theoretical context for exploring the relationship between notjustFTE status, but also contact with different facets of the youth justice system. Since these can occur at any time during the young person's time under the supervision with the YOT, the predictors are time variant.

Adding dummy variables for breaches (referenced by breached) and custody (referenced by having spenttime in custody or on remand) to the Basic Model, does notresultin a marked reduction in the DIC for the respective models. However, as can be seen from Table 7.4, the inclusion of the dummyvariable reflecting courtappearances (referenced byappearance, regardless of outcome) reduced the DIC from 476.2 to 429.6 thus reducing the amount of uncertaintyin the model.

The individual inclusion of the additional criminal justice related predictors suggests:

- The odds of further offending following a breach is estimated to be $\exp (0.190)=1.21$ times the odds for those who have not breached (Model 1.12). $\quad[\mathrm{Cl}=0.67,2.16]$

- The odds of further offending following a courtappearance is estimated to be $\exp (1.659)=525$ times the odds for those who have not had to attend court (Model 1.13) $[\mathrm{Cl}=3.34,8.26]$

- The odds of further offending following time in custody decreases, with the odds for those who have not spend time in custody between ASSETS, being estimated to be $1 / \exp (-0.528)=1.70$ times the odds for those who have spenttime in custody (Model 1.14) $[\mathrm{Cl}=0.88,3.33]$

The credible interval for court appearance is a significant predictor of further offending, equivalent to a $525 \%$ increase in the odds relative to those who have not needed to attend courtin the period between assessments. 
Table 7.4: Random Intercepts and Varying Slope Models for Further Offending including ASSET Domains and Youth Justice System Process Predic Unstandardised Coefficients

\begin{tabular}{|c|c|c|c|c|c|c|c|c|}
\hline \multirow[b]{3}{*}{ Fixed Effect: } & \multicolumn{4}{|c|}{$\begin{array}{c}\text { Model 1.12: Basic Model + } \\
\text { Breach }\end{array}$} & \multicolumn{4}{|c|}{$\begin{array}{c}\text { Model 1.13: Basic Model + } \\
\text { Court Appearance (Y/N) }\end{array}$} \\
\hline & \multicolumn{3}{|c|}{ Unstandardised } & \multirow{2}{*}{ Significant? } & \multicolumn{3}{|c|}{ Unstandardised } & \multirow{2}{*}{ Significan } \\
\hline & PostMean & Lower $\mathrm{Cl}$ & Upper Cl & & Post.Mean & Lower $\mathrm{Cl}$ & Upper Cl & \\
\hline Intercept & -1.143 & -2.387 & 0.071 & & -1.833 & -2.925 & -0.720 & Yes \\
\hline Breach $($ Compliance $=$ Ref) & 0.190 & -0.397 & 0.772 & & & & & \\
\hline Court Appearance (None = Ref) & & & & & 1.659 & 1.206 & 2.112 & Yes \\
\hline \multicolumn{9}{|l|}{ Period in Custody (None=Ref) } \\
\hline Living Arrangements & 0.031 & -0.230 & 0.289 & & 0.022 & -0.244 & 0.289 & \\
\hline Family and Personal Relationships & 0.267 & -0.034 & 0.547 & & 0.271 & -0.016 & 0.573 & \\
\hline Education, Training and Employment & 0.094 & -0.158 & 0.336 & & 0.045 & -0.204 & 0.289 & \\
\hline Neighbourhood & 0.046 & -0.173 & 0.264 & & 0.050 & -0.173 & 0.268 & \\
\hline Lifestyle & 0.007 & -0.342 & 0.354 & & -0.123 & -0.471 & 0.233 & \\
\hline Substance Use & 0.158 & -0.079 & 0.396 & & 0.103 & -0.135 & 0.346 & \\
\hline Physical Health & -0.112 & -0.392 & 0.168 & & 0.010 & -0.275 & 0.303 & \\
\hline Emotional and Mental Health & 0.006 & -0.241 & 0.245 & & 0.014 & -0.230 & 0.264 & \\
\hline Perceptions of Self and Others & -0.144 & -0.460 & 0.166 & & -0.216 & -0.554 & 0.101 & \\
\hline Thinking and Behaviour & -0.156 & -0.489 & 0.168 & & -0.156 & -0.488 & 0.188 & \\
\hline Attitude to Offending & 0.041 & -0.303 & 0.387 & & 0.032 & -0.323 & 0.386 & \\
\hline Motivation to Change & 0.236 & -0.108 & 0.575 & & 0.282 & -0.078 & 0.626 & \\
\hline Time & -0.154 & -0.291 & -0.024 & Yes & -0.125 & -0.244 & -0.010 & Yes \\
\hline Random Effect: & PostMean & Lower $\mathrm{Cl}$ & Upper Cl & Significant? & Post.Mean & Lower Cl & Upper Cl & Significan \\
\hline Individual (Intercept) & 0.10 & $1.63 \mathrm{E}-04$ & 0.37 & Yes & 0.03 & $1.47 \mathrm{E}-04$ & 0.15 & Yes \\
\hline Time & 1.26 & 0.34 & 2.61 & Yes & 0.82 & 0.16 & 1.72 & Yes \\
\hline
\end{tabular}

DIC

476.73

429.55

Source: Models Bm1_cj1 (Breach), Bm1_cj2 (Court Appearance) and Bm1_cj3 (Custody), Technical Annex: p356-365. 
Table 7.4 summarises the various permutations considered in relation to enhancing the Basic Model through inclusion of the three time-varying predictors, and the resulting impacton the DIC. In terms of the impact of the individual predictors at a given point in time, the dummyvariable representing whether or not the young person has had a court appearance (regardless of the outcome), had the greatest impact. In contrast, the inclusion of predictors to represent breaches and time in custody/ on remand does not result in a decrease in the DIC relative to that for the Basic Model. However, there is a theoretical rationale for including these ultimately within a dynamic model involving predictors reflecting dimensional identifyand static factors which is responsive to having contact with facets of the youth justice system.

\section{The Dynamic Models for Individual Youth Justice Processes or 'Events'}

\section{a) Breaches}

Across the dataset, $42 \%$ of the cohort have breached with the average number of breaches amongst this group being 1.8. The most common time to breach was before their initial assessment although there is a 'spike' at T ime 5 when almosta quarter of those remaining in the cohort breached (Figure 7.2). Taking into account the reductions in the size of the cohort at successive measurement points, it is apparent that the proportion who breached progressivelydecreases to Time, 4 . After this time there is not no clear trend.

Figure 7.2: Number and Percentage of the Cohort Who Breached, by Time

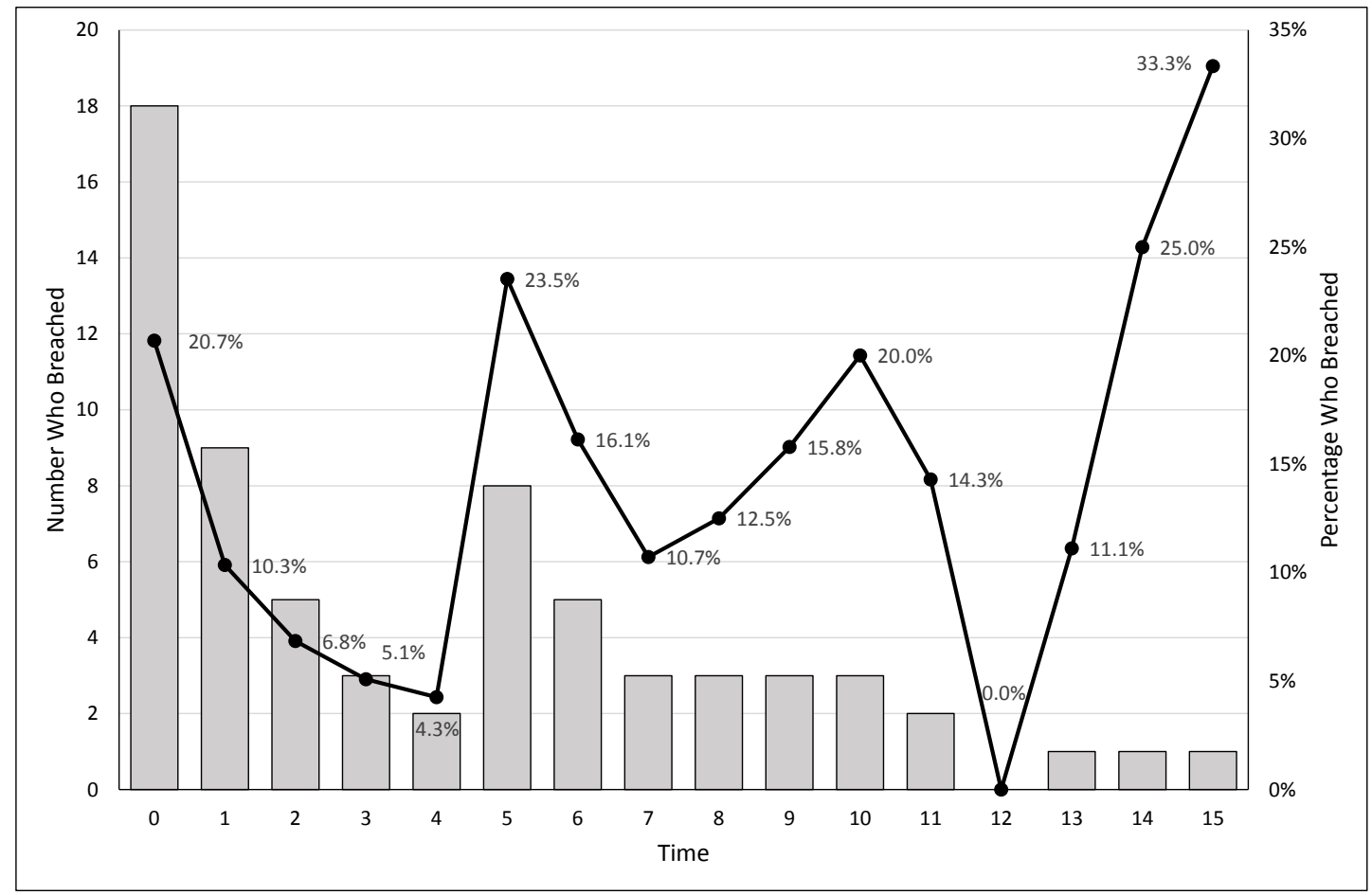


Table 7.5: The Dynamic Model Involving Breaches (BDm5_B)

\begin{tabular}{|c|c|c|c|c|c|c|c|}
\hline \multirow[b]{3}{*}{ Fixed Effect: } & \multicolumn{7}{|c|}{ Dymanic Model 5: Breaches } \\
\hline & \multicolumn{3}{|c|}{ Unstandardised } & \multicolumn{3}{|c|}{ Standardised } & \multirow{2}{*}{ Significant } \\
\hline & Post.Mean & Lower $\mathrm{Cl}$ & Upper $\mathrm{Cl}$ & Post.Mean & Lower $\mathrm{Cl}$ & Upper $\mathrm{Cl}$ & \\
\hline (Intercept) & -1.093 & -2.931 & 0.834 & 0.335 & 0.053 & 2.304 & \\
\hline Breach (None $=$ Ref) & 14.801 & 5.234 & 24.736 & $2.68 \mathrm{E}+06$ & 187.600 & $5.53 \mathrm{E}+10$ & Yes \\
\hline Time & -0.298 & -0.613 & 0.016 & 0.742 & 0.542 & 1.017 & \\
\hline Living Arrangements (Live) & 0.058 & -0.453 & 0.594 & 1.060 & 0.636 & 1.811 & \\
\hline Family and Personal Relationships (Relation) & 0.125 & -0.454 & 0.691 & 1.133 & 0.635 & 1.995 & \\
\hline Education, Training and Employment(ETE) & -0.244 & -0.686 & 0.175 & 0.783 & 0.503 & 1.191 & \\
\hline Neighbourhood (Where) & 0.082 & -0.361 & 0.621 & 1.085 & 0.697 & 1.861 & \\
\hline Lifestyle (Life) & 0.257 & -0.447 & 0.955 & 1.293 & 0.639 & 2.599 & \\
\hline Substance Use (Drugs) & 0.319 & -0.116 & 0.806 & 1.376 & 0.891 & 2.239 & \\
\hline Physical Health (Physical) & -0.730 & -1.312 & -0.166 & 0.482 & 0.269 & 0.847 & Yes \\
\hline Emotional and Mental Health (Emotion) & -0.353 & -0.844 & 0.141 & 0.703 & 0.430 & 1.152 & \\
\hline Perceptions of Self and Others (Self) & 0.270 & -0.371 & 0.932 & 1.310 & 0.690 & 2.539 & \\
\hline Thinking and Behaviour (Think) & 0.071 & -0.528 & 0.750 & 1.074 & 0.590 & 2.118 & \\
\hline Attitude to Offending (Attitude) & 0.140 & -0.477 & 0.826 & 1.150 & 0.620 & 2.284 & \\
\hline Motivation to Change (Change) & 0.387 & -0.299 & 1.020 & 1.473 & 0.742 & 2.773 & \\
\hline Breach: Time & -6.005 & -9.873 & -2.506 & 0.002 & 0.000 & 0.082 & Yes \\
\hline Breach: Live & -10.356 & -15.968 & -4.401 & 0.000 & 0.000 & 0.012 & Yes \\
\hline Breach: Relation & 15.299 & 7.367 & 23.776 & $4.41 \mathrm{E}+06$ & 1582.266 & $2.12 \mathrm{E}+10$ & Yes \\
\hline Breach: ETE & -10.431 & -16.322 & -5.105 & 0.000 & 0.000 & 0.006 & Yes \\
\hline Breach: Where & -0.794 & -2.869 & 1.165 & 0.452 & 0.057 & 3.205 & \\
\hline Breach: Life & 4.784 & -0.908 & 10.398 & 119.611 & 0.403 & $3.28 \mathrm{E}+04$ & \\
\hline Breach: Drugs & -3.051 & -6.599 & 0.049 & 0.047 & 0.001 & 1.050 & \\
\hline Breach: Physical & 4.932 & 1.218 & 9.213 & 138.666 & 3.381 & $1.00 E+04$ & Yes \\
\hline Breach: Emotion & 7.798 & 3.609 & 12.234 & 2436.834 & 36.934 & $2.06 \mathrm{E}+05$ & Yes \\
\hline Breach: Self & 6.533 & 1.589 & 11.397 & 687.764 & 4.898 & $8.90 \mathrm{E}+04$ & Yes \\
\hline Breach: Think & -19.325 & -29.823 & -7.734 & 0.000 & 0.000 & 0.000 & Yes \\
\hline Breach: Attitude & -4.251 & -9.508 & 0.888 & 0.014 & 0.000 & 2.431 & \\
\hline Breach: Change & 10.737 & 4.120 & 17.669 & $4.60 E+04$ & 61.561 & $4.71 \mathrm{E}+07$ & Yes \\
\hline Time: Live & -0.004 & -0.113 & 0.112 & 0.996 & 0.893 & 1.119 & \\
\hline Time: Relation & 0.028 & -0.112 & 0.164 & 1.028 & 0.894 & 1.179 & \\
\hline Time: ETE & 0.089 & -0.019 & 0.198 & 1.093 & 0.982 & 1.219 & \\
\hline Time: Where & 0.015 & -0.076 & 0.112 & 1.015 & 0.927 & 1.118 & \\
\hline Time: Life & -0.057 & -0.205 & 0.094 & 0.945 & 0.815 & 1.098 & \\
\hline Time: Drugs & -0.021 & -0.120 & 0.087 & 0.980 & 0.887 & 1.091 & \\
\hline Time: Physical & 0.152 & 0.020 & 0.291 & 1.164 & 1.020 & 1.338 & Yes \\
\hline Time: Emotion & 0.119 & 0.003 & 0.233 & 1.126 & 1.003 & 1.263 & Yes \\
\hline Time: Self & -0.096 & -0.229 & 0.037 & 0.908 & 0.795 & 1.038 & \\
\hline Time: Think & -0.046 & -0.191 & 0.090 & 0.955 & 0.827 & 1.094 & \\
\hline Time: Attitude & -0.052 & -0.212 & 0.109 & 0.949 & 0.809 & 1.115 & \\
\hline Time: Change & -0.035 & -0.192 & 0.105 & 0.966 & 0.825 & 1.110 & \\
\hline
\end{tabular}

/ continued 


\begin{tabular}{|c|c|c|c|c|c|c|c|}
\hline \multirow[b]{3}{*}{ Fixed Effect: } & \multicolumn{7}{|c|}{ Dymanic Model 5: Breaches } \\
\hline & \multicolumn{3}{|c|}{ Unstandardised } & \multicolumn{3}{|c|}{ Standardised } & \multirow{2}{*}{ Significant? } \\
\hline & Post.Mean & Lower $\mathrm{Cl}$ & Upper $\mathrm{Cl}$ & Post.Mean & Lower $\mathrm{Cl}$ & Upper $\mathrm{Cl}$ & \\
\hline Breach: Time: Live & 1.101 & 0.092 & 2.151 & 3.009 & 1.096 & 8.592 & Yes \\
\hline Breach: Time: Relation & -0.704 & -2.249 & 0.774 & 0.494 & 0.106 & 2.168 & \\
\hline Breach: Time: ETE & 2.291 & 0.897 & 3.973 & 9.887 & 2.451 & 53.166 & Yes \\
\hline Breach: Time: Where & -1.073 & -1.858 & -0.243 & 0.342 & 0.156 & 0.784 & Yes \\
\hline Breach: Time: Life & 1.490 & -0.087 & 3.071 & 4.439 & 0.917 & 21.569 & \\
\hline Breach: Time: Drugs & 1.378 & 0.642 & 2.218 & 3.965 & 1.900 & 9.185 & Yes \\
\hline Breach: Time: Physical & -1.865 & -3.357 & -0.635 & 0.155 & 0.035 & 0.530 & Yes \\
\hline Breach: Time: Emotion & -2.807 & -4.447 & -1.452 & 0.060 & 0.012 & 0.234 & Yes \\
\hline Breach: Time: Self & -1.365 & -2.244 & -0.504 & 0.255 & 0.106 & 0.604 & Yes \\
\hline Breach: Time: Think & 2.389 & 0.681 & 4.157 & 10.907 & 1.975 & 63.890 & Yes \\
\hline Breach: Time: Attitude & 1.433 & 0.347 & 2.469 & 4.189 & 1.414 & 11.814 & Yes \\
\hline Breach: Time: Change & -3.626 & -5.677 & -1.558 & 0.027 & 0.003 & 0.211 & Yes \\
\hline Random Effect: & \begin{tabular}{|l|} 
Post.Mean \\
\end{tabular} & Lower $\mathrm{Cl}$ & Upper Cl & PostMean & Lower $\mathrm{Cl}$ & Upper $\mathrm{Cl}$ & Significant? \\
\hline Individual (Intercept) & 0.583 & $2.89 \mathrm{E}-05$ & 1.315 & 1.791 & 1.000 & 3.725 & Yes \\
\hline \begin{tabular}{|c|} 
Time \\
\end{tabular} & 2.273 & 0.452 & 4.976 & 9.708 & 1.571 & 144.894 & Yes \\
\hline DIC & & & 449.91 & & & & \\
\hline
\end{tabular}

Source: Model BDm5_B, Technical Annex: p368-380.

The model can be used to estimate the impact of breaching atanygiven time on the probability of further offending (Figure 7.2). However, as can be seen from Table 7.5, there is a considerable amount of uncertaintyrelating to some of the estimates - evident in the wide credible intervals for estimates of the 2-way interactions between breaching and the individual domain scores, and by issues with the convergence formany of the 3-way interactions (see Technical Annex: p368-380). Whilstat Time0 and Time 1, the impact of breaching is for the probability of further of offending to be close to 1 , the shape of the trend at Time 2 (where the domain scores have been fixed at 4 - Figure 7.3(c)) is a reflection of this uncertaintyas there is insufficient data to accuratelyestimate the probability of further offending at later measurement points amongst those with higher ratings. The fall in predicted probability reflected following a breach at Time 3 (Figures 7.3(d)) is contrary to what would be expected and is likely a result of the low number of cases upon which the model is based upon. This suggests that the model's ability to predict the probability of further offending as time progresses is potentiallypoor.

As with previous charts showing how the probability of further offending changes over time, fixing the domain scores provides a somewhat artificial impression of perceived levels of risk as typically the circumstances which lead to the breach will also have been reflected in increases in individual do main scores. Hence there would not be a return to the previous trajectory. 
Figure 7.3: Changes in the Probability of Further Offending in Response to a Breach at Different Time Points

(a) Time $=0$

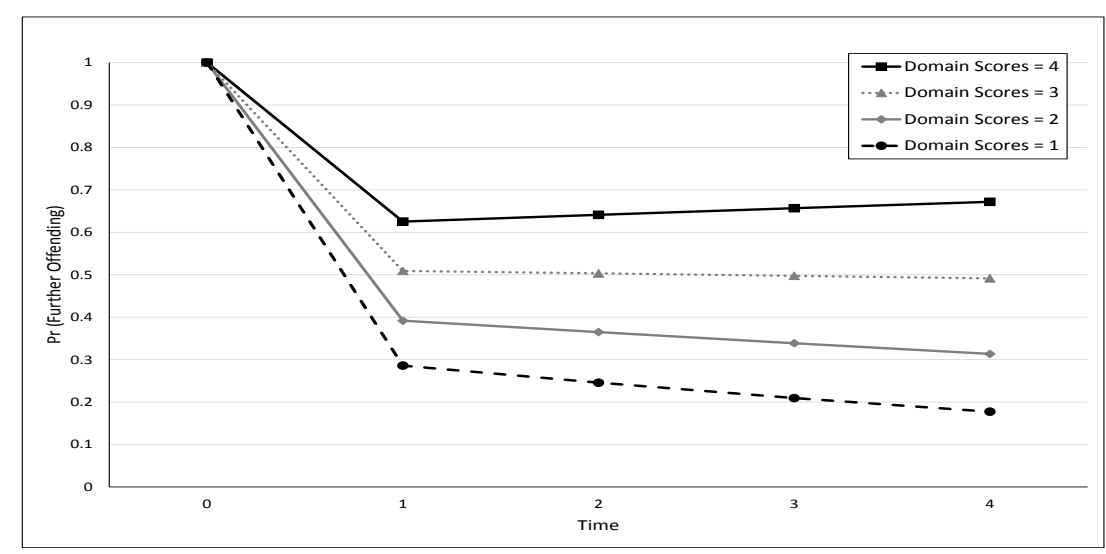

(c) Time $=2$

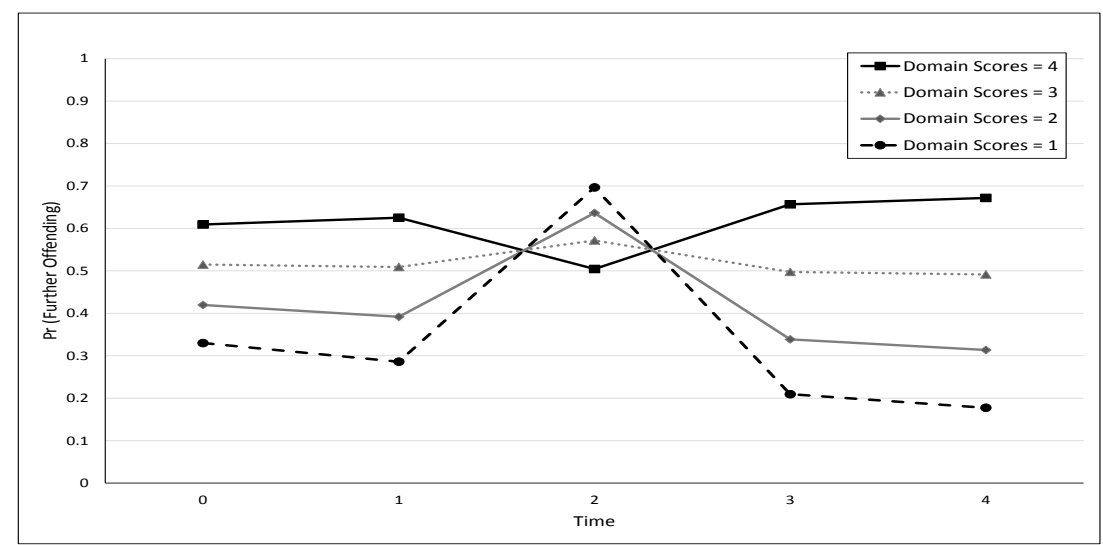

(b) Time $=1$

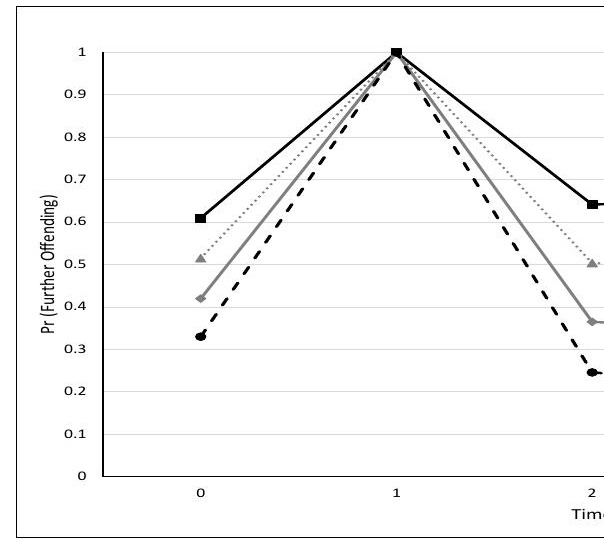

(d) Time $=3$

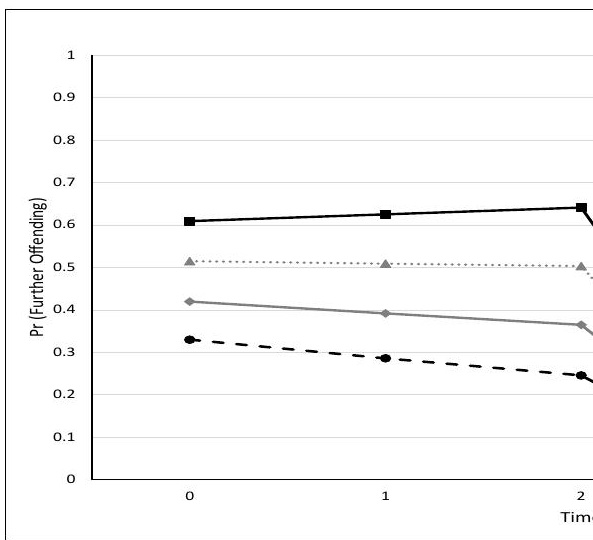

Notes: The domain scores have respectively been shown as being fixed at 1, 2, 3 and 4 respectively to demonstrate the estimated change in the probability of fu derived from Model BDm5_B. 
It would also be anticipated that there would differences on the basis of individual characteristics and their criminal history. Table 7.6 summarises the breach rate for different sub-groups. Whilst the approach here mirrors that used in Chapter Three with respect to further offending i.e. that a flag has been created indicating whether or not the young person breached at any time whilst under the supervision of the YOT, regardless of when or how manytimes theywere breached, it gives an indication of where there are differences.

Table 7.6: Breach rates, by sub-groups

\begin{tabular}{|c|c|c|c|c|c|c|c|}
\hline & \multicolumn{2}{|r|}{ Comparator Groups } & No. & Breached & $\begin{array}{c}\% \\
\text { Breaching }\end{array}$ & $\begin{array}{c}\text { Bayes Factor (BF 10) } \\
\text { (H1: Group } 1 \text { ₹ Group 2) }\end{array}$ & $\begin{array}{l}\text { Bayes Factor (BF 10) } \\
\text { (H1: Group } 1>\text { Group 2) }\end{array}$ \\
\hline \multirow[t]{2}{*}{ Gender } & 1 & Male & 79 & 35 & $44.3 \%$ & \multirow{2}{*}{0.824} & \multirow{2}{*}{0.196} \\
\hline & 2 & Female & 9 & 2 & $22.2 \%$ & & \\
\hline \multirow[t]{2}{*}{ Ethnicity } & 1 & White & 82 & 36 & $43.9 \%$ & \multirow{2}{*}{0.955} & \multirow{2}{*}{0.229} \\
\hline & 2 & Non-White & 6 & 1 & $16.7 \%$ & & \\
\hline \multirow[t]{2}{*}{ Care Status } & 1 & No Experience & 63 & 21 & $33.3 \%$ & \multirow{2}{*}{8.235} & \multirow{2}{*}{16.38} \\
\hline & 2 & Experience & 25 & 16 & $64.0 \%$ & & \\
\hline \multirow[t]{2}{*}{ Age at First Offence } & 1 & 10 to 12 & 22 & 15 & $68.2 \%$ & \multirow{2}{*}{16.15} & \multirow{2}{*}{0.094} \\
\hline & 2 & 13 to 17 & 66 & 22 & $33.3 \%$ & & \\
\hline \multirow[t]{2}{*}{ Age at First Conviction } & 1 & 10 to 13 & 11 & 5 & $45.5 \%$ & \multirow{2}{*}{0.388} & \multirow{2}{*}{0.290} \\
\hline & 2 & 14 to 17 & 77 & 32 & $41.6 \%$ & & \\
\hline \multirow[t]{2}{*}{$\mathrm{FTE}^{*}$} & 1 & FTE & 33 & 10 & $30.3 \%$ & \multirow{2}{*}{1.315} & \multirow{2}{*}{0.095} \\
\hline & 2 & Previous Offending & 54 & 27 & $50.0 \%$ & & \\
\hline \multicolumn{3}{|l|}{ Total } & 88 & 37 & $42.0 \%$ & & \\
\hline
\end{tabular}

Notes: The individual whose FTE status is not known has been excluded from this summary. Bayes Factors have been calculated using the test for Bayesian Contingency Tables within JASP version 0.8.1.1 and are interpreted using the categories suggested by Jeffreys (1961).

With $a \mathrm{BF}_{10}$ of 16.38 for the one-sided test, there is strong evidence that those with experience of care have a higher breach rate than their peers in the formal youth justice system without this experience. Similarly, the two-sided suggest with respect to grouped age at first offence points provides strong evidence to suggest that rates for the two groups are different. When the appropriate one-sided test is run, it confirms that there is very strong evidence in favour of the breach rate being higher for those who committed their first offence aged 10-12 $\left(\mathrm{BF}_{10}=32.21\right.$ that Group $1<$ Group 2$)$. 


\section{b) CourtAppearances}

All those young people whose ASSET Core Profiles have been considered as part of this research received court disposals and were within the formal youth justice system. As a result, court appearances after the time of the initial assessment are considered. Almost two-thirds of the cohort $(65 \%)$ have additional court appearances, with the average being 3.6 appearances. This includes occasions when the case has been adjourned, they have been bailed, remanded or sentenced, or the case dismissed/withdrawn. The number of young people attending court decreases over time along with the size of the cohortsubject to ASSET assessments decreases. Figure 7.4 includes a trend line reflects the proportion of the cohortat each measurementoccasion who had attended court.

Figure 7.4: Number and Percentage of the Cohort Who had Court Appearances, by Time

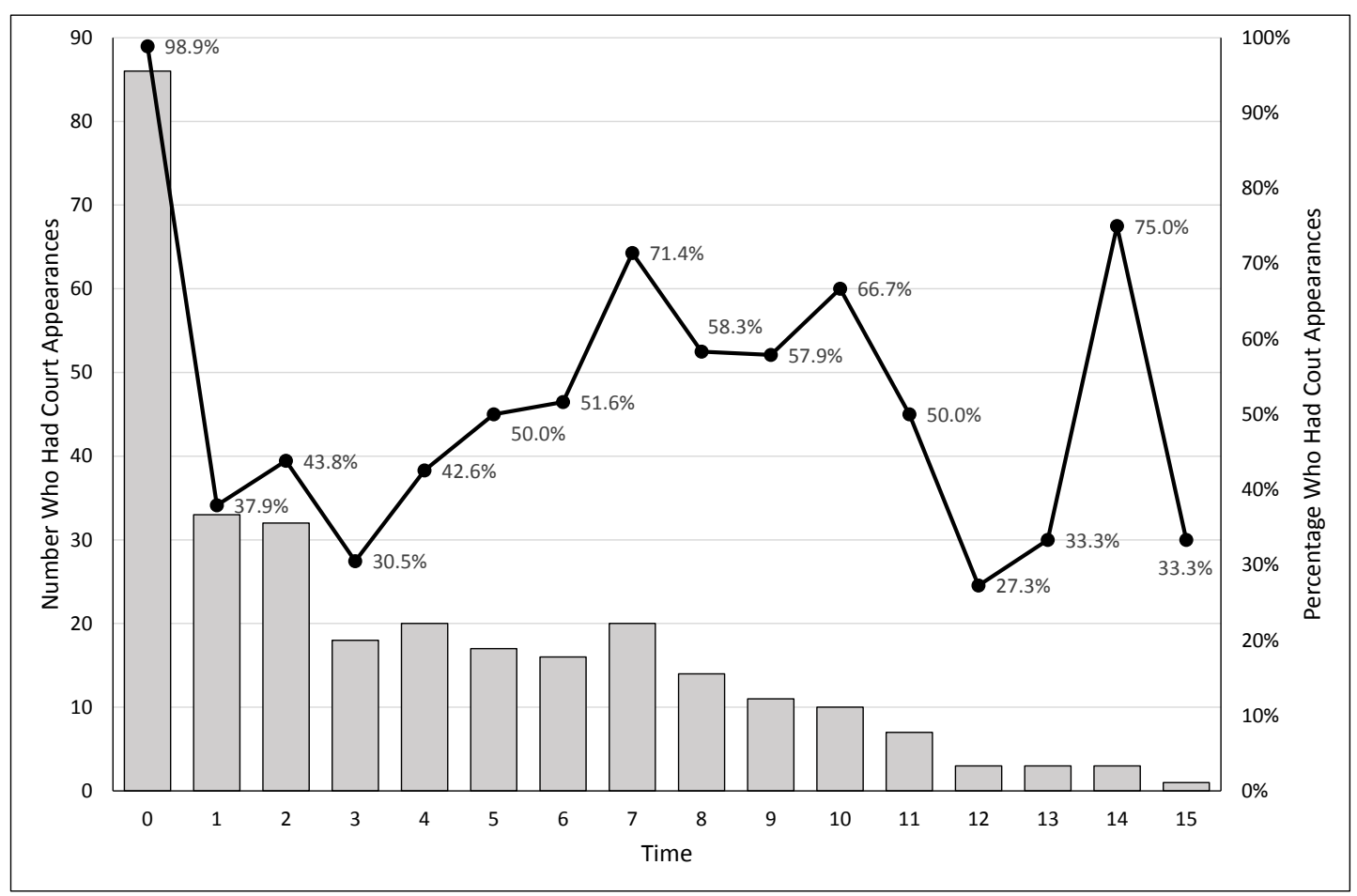

As with breaches, a dynamic model -summarised in Table 7.7, has been simulated to enable the impact of courtappearances on the probability of further offending to be estimated (Figure 7.5). However, with the higher numbers who have had court appearances, there is less uncertainty within the model. The only significantestimate is that for the fixed effect of the interaction between the courtappearance and time. Since the estimate of the fixed effect is negative, this suggests that at later measurement occasions, the effect of courtappearances have less of an impacton the probability of further offending although over time, the domain scores also change as a result of appearing in court. Athough these changes help to accountfor some of the uncertainty around the impact of attending court, there remains some unaccounted-for uncertaintyhence the wide credible interval for this main effect. 


\begin{tabular}{|c|c|c|c|c|c|c|c|}
\hline \multirow[b]{3}{*}{ Fixed Effect: } & \multicolumn{7}{|c|}{ Dymanic Model 5: Court Appearances } \\
\hline & \multicolumn{3}{|c|}{ Unstandardised } & \multicolumn{3}{|c|}{ Standardised } & \multirow{2}{*}{ Significant? } \\
\hline & Post.Mean & Lower $\mathrm{Cl}$ & Upper Cl & Post.Mean & Lower $\mathrm{Cl}$ & Upper Cl & \\
\hline (Intercept) & -5.053 & -7.815 & -2.257 & 0.006 & 0.000 & 0.105 & \\
\hline Court Appearance $($ None $=$ Ref) & 5.710 & 2.913 & 8.884 & 301.795 & 18.416 & 7218.360 & Yes \\
\hline Time & 0.410 & -0.133 & 0.977 & 1.506 & 0.875 & 2.657 & \\
\hline Living Arrangements (Live) & -0.066 & -1.035 & 1.023 & 0.936 & 0.355 & 2.782 & \\
\hline Family and Personal Relationships (Relation) & 1.349 & 0.127 & 2.514 & 3.854 & 1.135 & 12.352 & \\
\hline Education, Training and Employment(ETE) & -0.510 & -1.380 & 0.420 & 0.601 & 0.252 & 1.521 & \\
\hline Neighbourhood (Where) & 0.719 & -0.228 & 1.657 & 2.053 & 0.796 & 5.243 & \\
\hline Lifestyle (Life) & -0.106 & -1.453 & 1.173 & 0.899 & 0.234 & 3.230 & \\
\hline Substance Use (Drugs) & 0.896 & -0.060 & 1.808 & 2.451 & 0.942 & 6.096 & \\
\hline Physical Health (Physical) & -1.017 & -2.169 & 0.008 & 0.362 & 0.114 & 1.008 & \\
\hline Emotional and Mental Health (Emotion) & 0.133 & -0.803 & 0.989 & 1.142 & 0.448 & 2.689 & \\
\hline Perceptions of Self and Others (Self) & 0.157 & -1.156 & 1.484 & 1.170 & 0.315 & 4.409 & \\
\hline Thinking and Behaviour (Think) & 0.091 & -1.153 & 1.382 & 1.095 & 0.316 & 3.984 & \\
\hline Attitude to Offending (Attitude) & 0.122 & -1.078 & 1.424 & 1.129 & 0.340 & 4.154 & \\
\hline Motivation to Change (Change) & -0.216 & -1.721 & 1.221 & 0.806 & 0.179 & 3.390 & \\
\hline Appear: Live & 0.053 & -1.075 & 1.263 & 1.054 & 0.341 & 3.535 & \\
\hline Appear: Relation & -1.045 & -2.364 & 0.303 & 0.352 & 0.094 & 1.353 & \\
\hline Appear: ETE & 0.330 & -0.662 & 1.337 & 1.391 & 0.516 & 3.808 & \\
\hline Appear: Where & -0.795 & -1.854 & 0.227 & 0.452 & 0.157 & 1.254 & \\
\hline Appear: Life & 0.107 & -1.362 & 1.656 & 1.113 & 0.256 & 5.238 & \\
\hline Appear: Drugs & -0.789 & -1.797 & 0.247 & 0.454 & 0.166 & 1.280 & \\
\hline Appear: Physical & 0.802 & -0.438 & 2.019 & 2.229 & 0.645 & 7.532 & \\
\hline Appear: Emotion & -0.185 & -1.265 & 0.800 & 0.831 & 0.282 & 2.227 & \\
\hline Appear: Self & -0.244 & -1.696 & 1.254 & 0.783 & 0.183 & 3.503 & \\
\hline Appear: Think & -0.379 & -1.788 & 1.045 & 0.685 & 0.167 & 2.842 & \\
\hline Appear: Attitude & -0.127 & -1.495 & 1.352 & 0.881 & 0.224 & 3.866 & \\
\hline Appear: Change & 0.666 & -1.006 & 2.152 & 1.947 & 0.366 & 8.601 & \\
\hline Appear: Time & -0.805 & -1.432 & -0.166 & 0.447 & 0.239 & 0.847 & Yes \\
\hline Time: Live & -0.120 & -0.366 & 0.126 & 0.887 & 0.694 & 1.134 & \\
\hline Time: Relation & -0.257 & -0.556 & 0.032 & 0.773 & 0.574 & 1.033 & \\
\hline Time: ETE & 0.098 & -0.115 & 0.304 & 1.103 & 0.892 & 1.355 & \\
\hline Time: Where & -0.013 & -0.181 & 0.170 & 0.987 & 0.834 & 1.185 & \\
\hline Time: Life & 0.029 & -0.245 & 0.349 & 1.029 & 0.783 & 1.417 & \\
\hline Time: Drugs & -0.116 & -0.302 & 0.080 & 0.890 & 0.739 & 1.083 & \\
\hline Time: Physical & 0.196 & -0.066 & 0.448 & 1.217 & 0.936 & 1.564 & \\
\hline Time: Emotion & 0.053 & -0.171 & 0.273 & 1.054 & 0.843 & 1.314 & \\
\hline Time: Self & -0.058 & -0.318 & 0.216 & 0.944 & 0.727 & 1.241 & \\
\hline Time: Think & 0.012 & -0.283 & 0.309 & 1.013 & 0.753 & 1.362 & \\
\hline Time: Attitude & -0.111 & -0.440 & 0.212 & 0.895 & 0.644 & 1.236 & \\
\hline Time: Change & 0.071 & -0.244 & 0.378 & 1.073 & 0.784 & 1.460 & \\
\hline
\end{tabular}

/continued 


\begin{tabular}{|c|c|c|c|c|c|c|c|}
\hline \multirow[b]{3}{*}{ Fixed Effect: } & \multicolumn{7}{|c|}{ Dymanic Model 5: Court Appearances } \\
\hline & \multicolumn{3}{|c|}{ Unstandardised } & \multicolumn{3}{|c|}{ Standardised } & \multirow{2}{*}{ Significant? } \\
\hline & PostMean & Lower $\mathrm{Cl}$ & Upper $\mathrm{Cl}$ & Post.Mean & Lower $\mathrm{Cl}$ & Upper $\mathrm{Cl}$ & \\
\hline Appear: Time: Live & 0.188 & -0.077 & 0.470 & 1.207 & 0.926 & 1.600 & \\
\hline Appear: Time: Relation & 0.226 & -0.083 & 0.563 & 1.254 & 0.920 & 1.757 & \\
\hline Appear: Time: ETE & -0.043 & -0.275 & 0.202 & 0.958 & 0.760 & 1.223 & \\
\hline Appear: Time: Where & 0.004 & -0.200 & 0.187 & 1.004 & 0.819 & 1.205 & \\
\hline Appear: Time: Life & -0.046 & -0.380 & 0.287 & 0.955 & 0.684 & 1.332 & \\
\hline Appear: Time: Drugs & 0.107 & -0.093 & 0.340 & 1.113 & 0.912 & 1.405 & \\
\hline Appear: Time: Physical & -0.095 & -0.389 & 0.198 & 0.910 & 0.678 & 1.219 & \\
\hline Appear: Time: Emotion & -0.035 & -0.280 & 0.212 & 0.966 & 0.756 & 1.236 & \\
\hline Appear: Time: Self & 0.010 & -0.278 & 0.314 & 1.010 & 0.757 & 1.369 & \\
\hline Appear: Time: Think & -0.007 & -0.336 & 0.329 & 0.993 & 0.715 & 1.389 & \\
\hline Appear: Time: Attitude & 0.143 & -0.203 & 0.505 & 1.154 & 0.816 & 1.658 & \\
\hline Appear: Time: Change & -0.094 & -0.422 & 0.257 & 0.910 & 0.656 & 1.293 & \\
\hline Random Effect: & PostMean & Lower $\mathrm{Cl}$ & Upper Cl & Post.Mean & Lower Cl & Upper Cl & Significant? \\
\hline Individual (Intercept) & 0.16 & $3.30 \mathrm{E}-08$ & 0.59 & 1.178 & 1.000 & 1.800 & Yes \\
\hline Time & 1.219 & 0.221 & 2.817 & 3.384 & 1.247 & 16.727 & Yes \\
\hline
\end{tabular}

Source: Model BDm5_A, Technical Annex: p381-393.

The trajectory of the fall in the probability of further offending for those with lower average domain scores in Figures 7.5(c) and (d) is perhaps not what would be expected since the probabilityappears to be close to 0 from time 1 onwards. For those with ratings of 3 across each of the Domains being around $19 \%$ at Time 1 , around $6 \%$ at Time 2 , and $2 \%$ at $\mathrm{T} \mathrm{ime} 3$. As with the model involving breaches, the small number of cases at the later time points make predictors of the probability of further offending less reliable as time progresses.

In the case of court appearances, it is likely that domain scores will be revised depending upon the outcome of the court appearance with some young people being subject to bail or remand restrictions, or depending upon their sentence, having further restrictions imposed upon them as part of their order. This could include receiving a custodial sentence. Later court appearances may be as a result of breaches or having committed further offences including where an offence committed earlier than that which led to the referral has taken longer to get to court. Notably of the 57 young people who appeared in court after time 0,17 also had periods in custody / on remand although not necessarily in the same period. 
Figure 7.5: Changes in the Probability of Further Offending in Response to a Court Appearance at Time 0 and Different Time Points

(a) Time $=1$

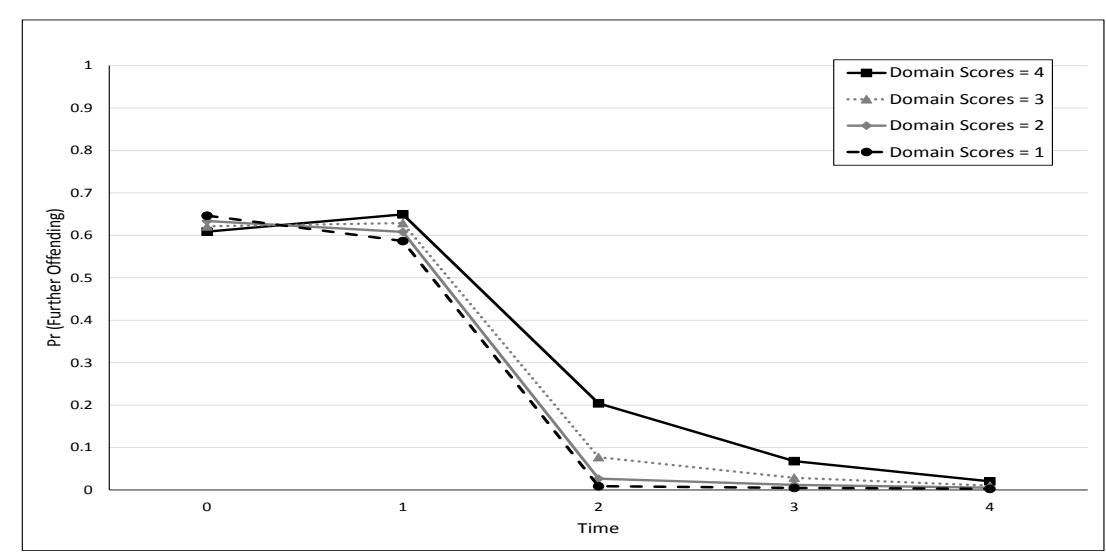

(c) Time $=3$

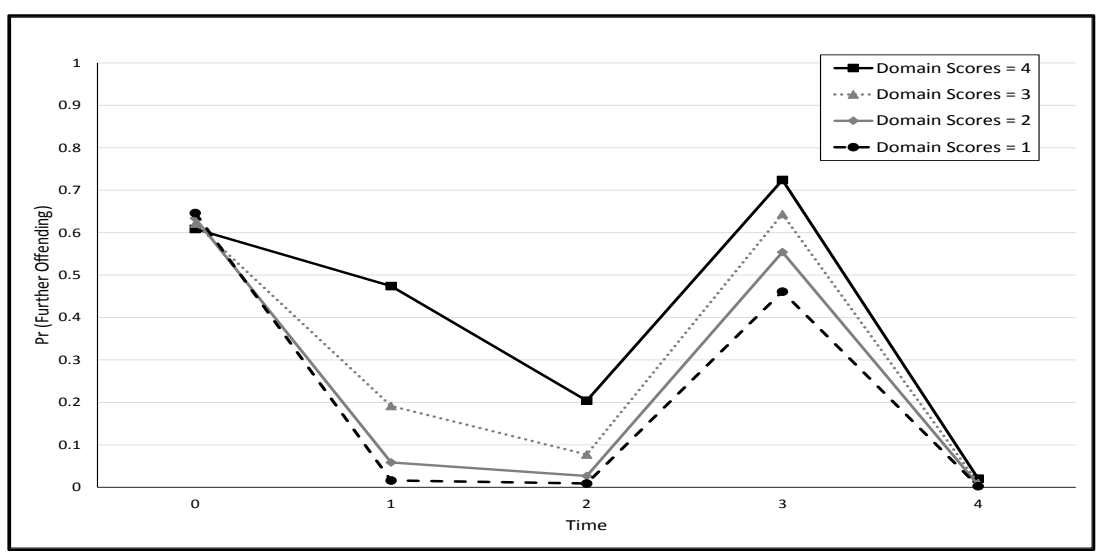

(b) Time $=2$

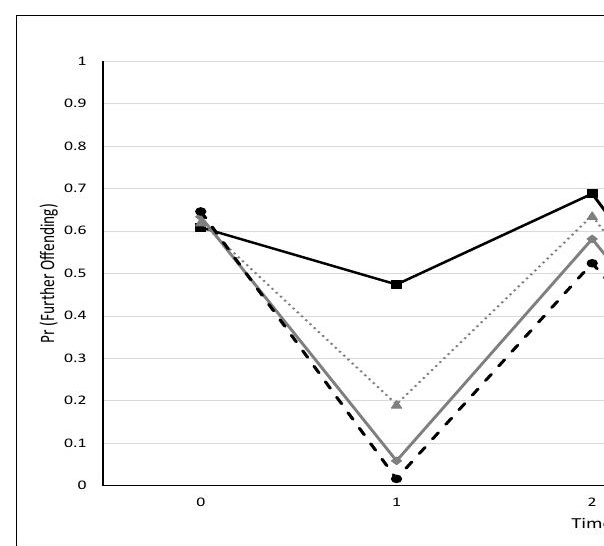

(d) Time $=4$

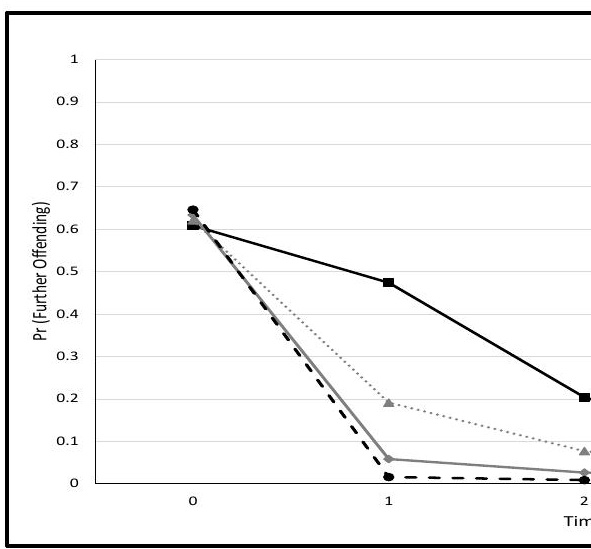

Notes: The domain scores have respectively been shown as being fixed at 1, 2, 3 and 4 respectively to demonstrate the estimated change in the probability of fu derived from Model BDm5_A. 
The respective rates have been calculated based on whether or not a young person during their time under the supervision of the YOT the 'event' has occurred one or more times. As a result, were for example a young person has returned to courtmultiple times, this is only counted once. As can be seen from Table 7.8, the likelihood of a young person returning to courtafter their initial appearance at Time 0 does notappear to differ for different sub-groups, with no evidence to suggest differences between the rates for each sub-group.

Table 7.8: Court appearance rates, by sub-groups

\begin{tabular}{|c|c|c|c|c|c|c|c|}
\hline & & omparator Groups & No. & $\begin{array}{l}\text { Returned } \\
\text { to Court }\end{array}$ & $\begin{array}{c}\% \\
\text { Returned } \\
\text { to Court }\end{array}$ & $\begin{array}{l}\text { Bayes Factor (BF 10) } \\
\text { (H1: Group } 1 \text { F Group 2) }\end{array}$ & $\begin{array}{l}\text { Bayes Factor (BF 10) } \\
\text { (H1: Group } 1>\text { Group 2) }\end{array}$ \\
\hline \multirow[t]{2}{*}{ Gender } & 1 & Male & 79 & 53 & $67.1 \%$ & \multirow{2}{*}{0.408} & \multirow{2}{*}{0.169} \\
\hline & 2 & Female & 9 & 4 & $44.4 \%$ & & \\
\hline \multirow[t]{2}{*}{ Ethnicity } & 1 & White & 82 & 55 & $67.1 \%$ & \multirow{2}{*}{0.517} & \multirow{2}{*}{0.169} \\
\hline & 2 & Non-White & 6 & 2 & $33.3 \%$ & & \\
\hline \multirow[t]{2}{*}{ Care Status } & 1 & No Experience & 63 & 38 & $60.3 \%$ & \multirow{2}{*}{0.617} & \multirow{2}{*}{1.255} \\
\hline & 2 & Experience & 25 & 19 & $76.0 \%$ & & \\
\hline \multirow[t]{2}{*}{ Age at First Offence } & 1 & 10 to 12 & 22 & 14 & $63.6 \%$ & \multirow{2}{*}{0.239} & \multirow{2}{*}{0.330} \\
\hline & 2 & 13 to 17 & 66 & 43 & $65.2 \%$ & & \\
\hline \multirow[t]{2}{*}{ Age at First Conviction } & 1 & 10 to 13 & 11 & 7 & $63.6 \%$ & \multirow{2}{*}{0.184} & \multirow{2}{*}{0.412} \\
\hline & 2 & 14 to 17 & 77 & 50 & $64.9 \%$ & & \\
\hline \multirow[t]{2}{*}{ FTE * $^{*}$} & 1 & FTE & 33 & 18 & $54.5 \%$ & \multirow{2}{*}{1.069} & \multirow{2}{*}{0.091} \\
\hline & 2 & Previous Offending & 54 & 39 & $72.2 \%$ & & \\
\hline \multicolumn{3}{|l|}{ Total } & 88 & 57 & $64.8 \%$ & & \\
\hline
\end{tabular}

Notes: The individual whose FTE status is not known has been excluded from this summary. Bayes Factors have been calculated using the test for Bayesian Contingency Tables within JASP version 0.8.1.1 and are interpreted using the categories suggested by Jeffreys (1961).

Within the reoffending cohort, nearly all of those who breached at some point returned to court $(94.6 \%$, 35 out 37 ). As would be expected, there is also a strong relationship between returning to court and spending time either on remand or in custody. However, there are also a small number who received a custodial sentence following their primaryoffence. 


\section{c) Periods in Custody}

Almost a quarter of those in the cohort $(23 \%)$ have spent time in custody or on remand. This includes 3 young people who were in custody at the time of their initial assessment after joining the cohort. On average, those young people were in custody during the period of interest were in custody prior to 3.35 measurement occasions. Of the 20 young people who had time in custody, 6 had multiple periods in custody. Figure 7.6 summarises the size of the cohort subject to ASSET assessments at each measurementoccasion with a trend line reflecting the proportion of the cohort that this represents.

Figure 7.6: Number and Percentage of the Cohort Who had Periods in Custody / On Remand, by Time

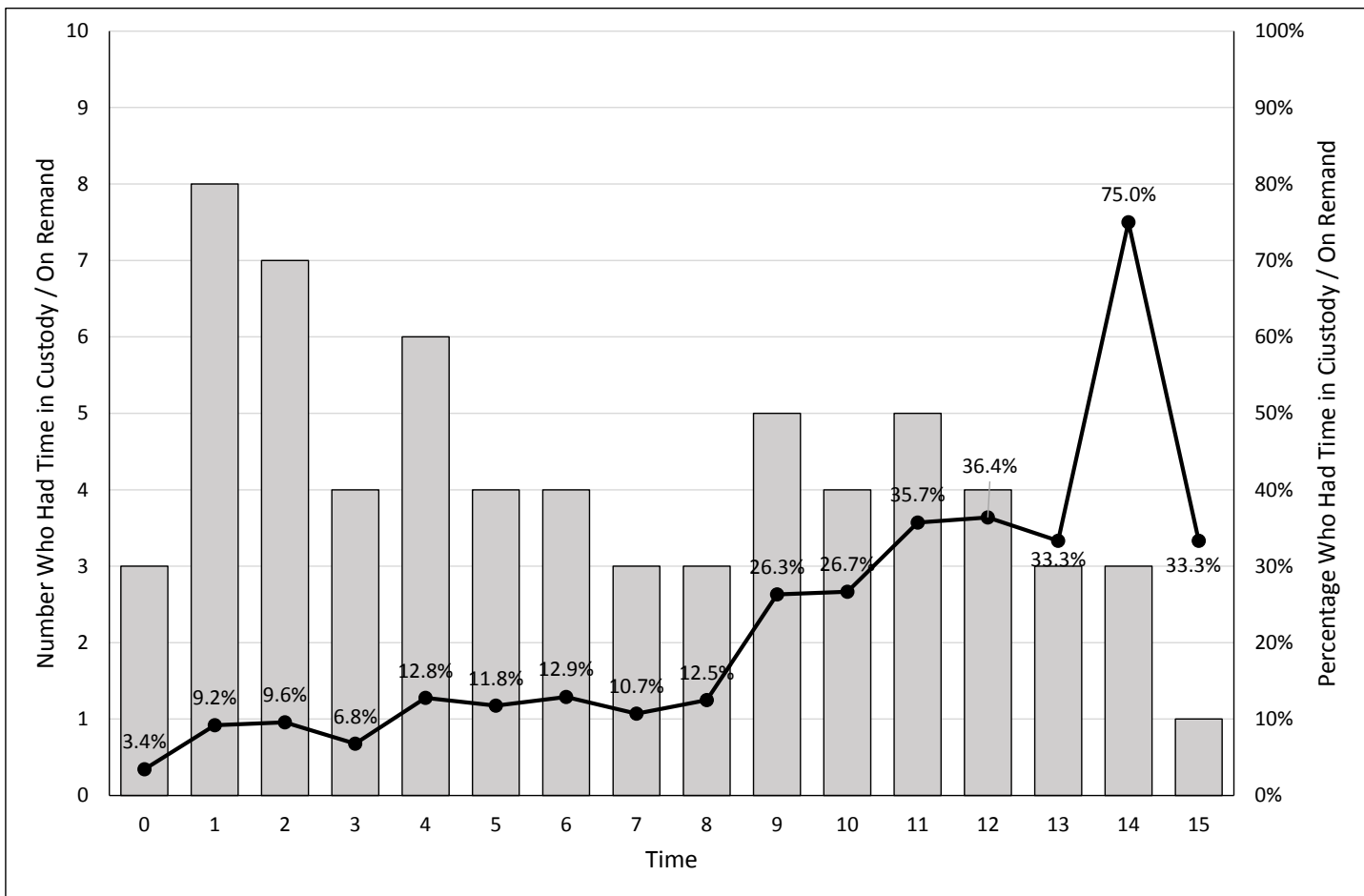

This model (Table 7.9) suffers less from issues around convergence (see Technical Annex, p394-406) than that for breaches, despite the lower number of cases involved. As would be expected the fixed effect between custody and time is significant - as with court appearances, suggesting that at later measurementoccasions, spending time in custody/ on remand has less of an impacton the probability of further offending. This may be because the period of custody has followed a breach rather than a further offence, or because the detention and training order was for a longer period. 
Table 7.9: The Dynamic Model Involving Periods in Custody or On Remand (BDm5_C)

\begin{tabular}{|c|c|c|c|c|c|c|c|}
\hline \multirow[b]{3}{*}{ Fixed Effect: } & \multicolumn{7}{|c|}{ Dymanic Model 5: Custody } \\
\hline & \multicolumn{3}{|c|}{ Unstandardised } & \multicolumn{3}{|c|}{ Standardised } & \multirow{2}{*}{ Significant? } \\
\hline & Post.Mean & Lower $\mathrm{Cl}$ & Upper Cl & Post.Mean & Lower $\mathrm{Cl}$ & Upper Cl & \\
\hline (Intercept) & -1.380 & -3.256 & 0.545 & 0.252 & 0.039 & 1.725 & \\
\hline Custody (None = Ref) & 8.295 & -10.670 & 26.221 & 4005.485 & 0.000 & $2.44 \mathrm{E}+11$ & \\
\hline Time & -0.142 & -0.451 & 0.149 & 0.868 & 0.637 & 1.160 & \\
\hline Living Arrangements (Live) & -0.059 & -0.548 & 0.414 & 0.942 & 0.578 & 1.513 & \\
\hline Family and Personal Relationships (Relation) & 0.393 & -0.123 & 0.940 & 1.482 & 0.884 & 2.560 & \\
\hline Education, Training and Employment (ETE) & -0.304 & -0.689 & 0.100 & 0.738 & 0.502 & 1.105 & \\
\hline Neighbourhood (Where) & 0.190 & -0.232 & 0.576 & 1.209 & 0.793 & 1.778 & \\
\hline Lifestyle (Life) & 0.251 & -0.389 & 0.922 & 1.286 & 0.678 & 2.513 & \\
\hline Substance Use (Drugs) & 0.367 & -0.047 & 0.803 & 1.443 & 0.955 & 2.233 & \\
\hline Physical Health (Physical) & -0.588 & -1.136 & -0.081 & 0.555 & 0.321 & 0.922 & Yes \\
\hline Emotional and Mental Health (Emotion) & -0.157 & -0.573 & 0.269 & 0.854 & 0.564 & 1.309 & \\
\hline Perceptions of Self and Others (Self) & 0.186 & -0.418 & 0.800 & 1.204 & 0.658 & 2.225 & \\
\hline Thinking and Behaviour (Think) & -0.139 & -0.743 & 0.457 & 0.871 & 0.476 & 1.579 & \\
\hline Attitude to Offending (Attitude) & -0.081 & -0.703 & 0.502 & 0.922 & 0.495 & 1.652 & \\
\hline Motivation to Change (Change) & 0.436 & -0.174 & 1.000 & 1.547 & 0.840 & 2.717 & \\
\hline Custody: Time & -4.947 & -8.971 & -1.293 & 0.007 & 0.000 & 0.274 & Yes \\
\hline Custody: Live & 1.741 & -2.684 & 6.318 & 5.705 & 0.068 & 554.284 & \\
\hline Custody: Relation & 0.300 & -10.651 & 11.071 & 1.349 & 0.000 & $6.43 E+04$ & \\
\hline Custody: ETE & -0.710 & -7.560 & 5.366 & 0.492 & 0.001 & 214.025 & \\
\hline Custody: Where & -0.907 & -6.603 & 4.644 & 0.404 & 0.001 & 104.010 & \\
\hline Custody: Life & -7.234 & -13.231 & -0.957 & 0.001 & 0.000 & 0.384 & Yes \\
\hline Custody: Drugs & -1.734 & -7.219 & 3.397 & 0.177 & 0.001 & 29.884 & \\
\hline Custody: Physical & -1.560 & -6.104 & 2.726 & 0.210 & 0.002 & 15.275 & \\
\hline Custody: Emotion & 2.792 & -8.357 & 14.399 & 16.313 & 0.000 & $1.79 E+06$ & \\
\hline Custody: Self & 0.659 & -8.023 & 9.339 & 1.933 & 0.000 & $1.14 \mathrm{E}+04$ & \\
\hline Custody: Think & 1.833 & -9.165 & 13.883 & 6.253 & 0.000 & $1.07 E+06$ & \\
\hline Custody: Attitude & 7.786 & -4.113 & 20.235 & 2405.834 & 0.016 & $6.14 \mathrm{E}+08$ & \\
\hline Custody: Change & -8.794 & -24.027 & 6.465 & 0.000 & 0.000 & 642.177 & \\
\hline Time: Live & 0.026 & -0.079 & 0.135 & 1.027 & 0.924 & 1.145 & \\
\hline Time: Relation & -0.043 & -0.164 & 0.089 & 0.957 & 0.849 & 1.094 & \\
\hline Time: ETE & 0.102 & 0.012 & 0.202 & 1.108 & 1.012 & 1.223 & Yes \\
\hline Time: Where & -0.029 & -0.114 & 0.055 & 0.971 & 0.892 & 1.057 & \\
\hline Time: Life & -0.031 & -0.174 & 0.104 & 0.969 & 0.840 & 1.110 & \\
\hline Time: Drugs & -0.055 & -0.146 & 0.035 & 0.947 & 0.864 & 1.036 & \\
\hline Time: Physical & 0.124 & -0.014 & 0.257 & 1.132 & 0.986 & 1.293 & \\
\hline Time: Emotion & 0.057 & -0.038 & 0.149 & 1.058 & 0.962 & 1.160 & \\
\hline Time: Self & -0.083 & -0.207 & 0.040 & 0.920 & 0.813 & 1.040 & \\
\hline Time: Think & 0.001 & -0.139 & 0.131 & 1.001 & 0.870 & 1.140 & \\
\hline Time: Attitude & 0.019 & -0.113 & 0.152 & 1.019 & 0.893 & 1.164 & \\
\hline Time: Change & -0.052 & -0.190 & 0.077 & 0.949 & 0.827 & 1.080 & \\
\hline
\end{tabular}

/continued 


\begin{tabular}{|c|c|c|c|c|c|c|c|}
\hline \multirow[b]{3}{*}{ Fixed Effect: } & \multicolumn{7}{|c|}{ Dymanic Model 5: Custody } \\
\hline & \multicolumn{3}{|c|}{ Unstandardised } & \multicolumn{3}{|c|}{ Standardised } & \multirow{2}{*}{ Significant? } \\
\hline & Post.Mean & Lower $\mathrm{Cl}$ & Upper $\mathrm{Cl}$ & \begin{tabular}{|l|} 
Post.Mean \\
\end{tabular} & Lower $\mathrm{Cl}$ & Upper $\mathrm{Cl}$ & \\
\hline Custody: Time: Live & -0.782 & -1.750 & 0.132 & 0.458 & 0.174 & 1.141 & \\
\hline Custody: Time: Relation & -0.360 & -1.713 & 1.061 & 0.698 & 0.180 & 2.889 & \\
\hline Custody: Time: ETE & 0.940 & -0.578 & 2.381 & 2.561 & 0.561 & 10.811 & \\
\hline Custody: Time: Where & 0.903 & 0.094 & 1.710 & 2.466 & 1.099 & 5.529 & Yes \\
\hline Custody: Time: Life & 0.907 & -0.441 & 2.230 & 2.478 & 0.644 & 9.300 & \\
\hline Custody: Time: Drugs & 0.989 & -0.023 & 1.912 & 2.688 & 0.978 & 6.769 & \\
\hline Custody: Time: Physical & 0.812 & -0.051 & 1.712 & 2.253 & 0.950 & 5.543 & \\
\hline Custody: Time: Emotion & -0.286 & -1.736 & 1.166 & 0.751 & 0.176 & 3.210 & \\
\hline Custody: Time: Self & 0.050 & -1.715 & 1.804 & 1.052 & 0.180 & 6.076 & \\
\hline Custody: Time: Think & -0.332 & -2.214 & 1.371 & 0.718 & 0.109 & 3.938 & \\
\hline Custody: Time: Attitude & -1.339 & -3.097 & 0.214 & 0.262 & 0.045 & 1.239 & \\
\hline Custody: Time: Change & 0.868 & -1.458 & 3.101 & $\begin{array}{r}2.382 \\
\end{array}$ & 0.233 & 22.225 & \\
\hline Random Effect: & Post.Mean & Lower $\mathrm{Cl}$ & Upper Cl & \begin{tabular}{|l|} 
Post.Mean \\
\end{tabular} & Lower $\mathrm{Cl}$ & Upper Cl & Significant? \\
\hline Individual (Intercept) & 0.215 & $9.57 \mathrm{E}-08$ & 0.669 & 1.239 & 1.000 & 1.953 & Yes \\
\hline \begin{tabular}{|c|} 
Time \\
\end{tabular} & 2.654 & 0.502 & 6.329 & 14.211 & 1.652 & 560.596 & Yes \\
\hline DIC & & & 477.99 & & & & \\
\hline
\end{tabular}

Source: Model BDm5_C, Technical Annex: p394-406.

The main effectreflecting the physical health domain as a main effect is significantas are those for the interaction effects between Custody: Life; Time: ETE; and Custody: Time: Neighbourhood. A fuller discussion is provided in Section 7.5. However, it is plausible that the structure and routine of the custody environment means that young people have access to appropriate health care services including GPS and dentists, and to treatment which enables them to address risky behaviours such as substance misuse. Their loss of liberty will also see them moved away from associating with friends who influence their behaviour, and from problematic neighbourhoods albeit temporarily. Whilst in the custodial environment there will be a requirement for young people to engage in education and training, and to gain skills to enable them to access employmentupon release.

Estimates of the probability of further offending in response to a period in custodyare given in Figure 7.7. Whilst the probability of further offending following a breach or court appearance increases, following a period in custody the probabilitydecreases (Figures 7.7(a) and (b)). Since the way that the dummy variable has been set up can reflect that the young person is in custody at the time of the assessment, this reflects the lack of opportunity that the young person has to commitfurther offences at that time. However, there are indications of the poor fit of this model at later time points, especially where the ratings are fixed at 2 and 3 at Time 3 (Figure 7.7(c)). At this time point, the estimates are based on just 4 cases. The number of cases increases at Time 4 which may explain the trajectories evident in Figure 7.7(d). Following time in custody, the probability of further offending remains high especiallyfor those with lower ratings. 
Figure 7.7: Changes in the Probability of Further Offending in Response to a Period in Custody at Different Times

(a) Time $=1$

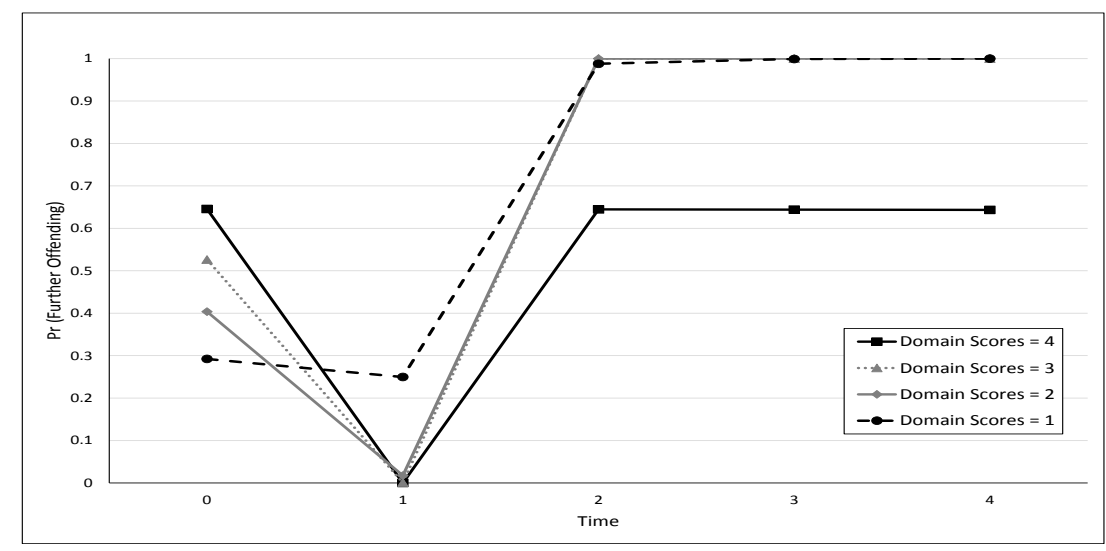

(c) Time $=3$

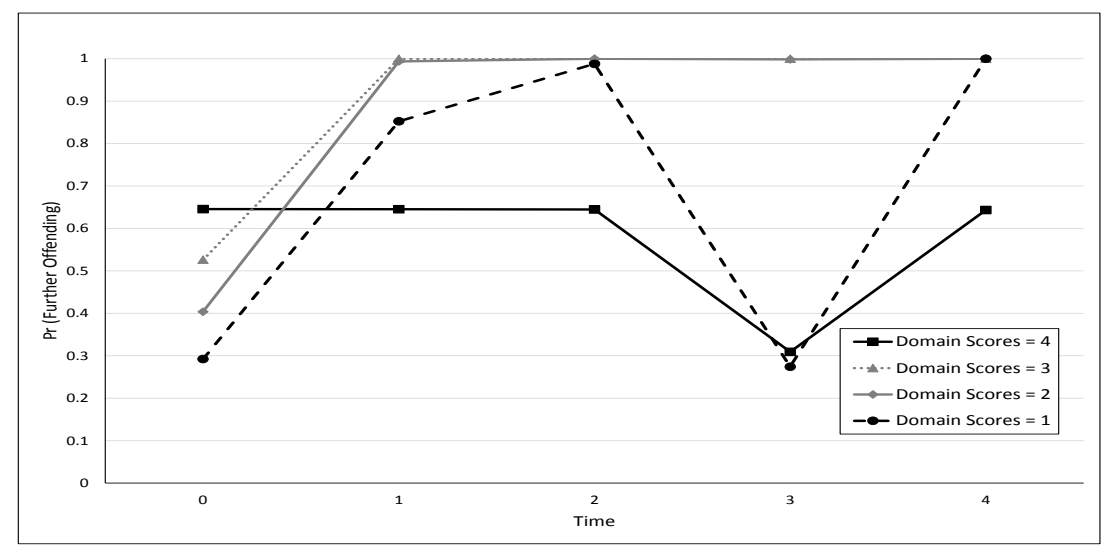

(b) Time $=2$

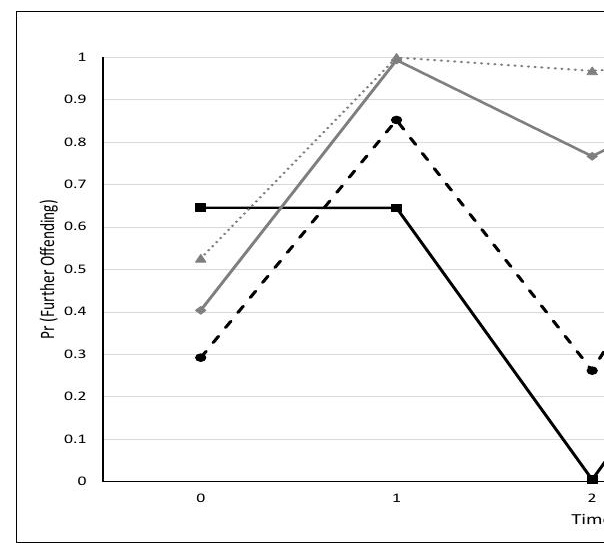

(d) Time $=4$

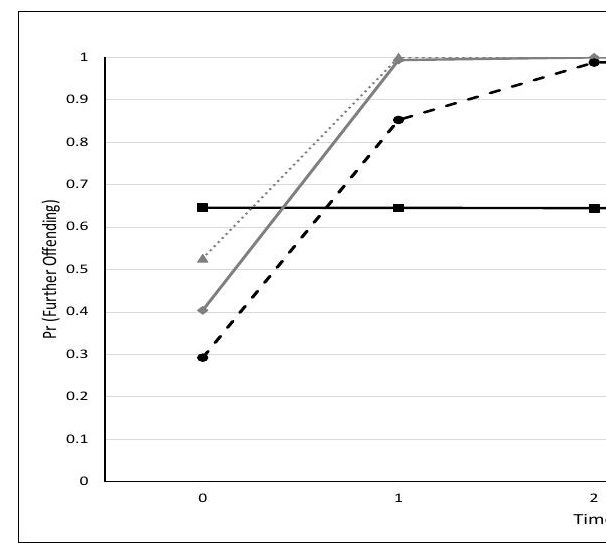

Notes: The domain scores have respectively been shown as being fixed at 1, 2, 3 and 4 respectively to demonstrate the estimated change in the probability of fu derived from Model BDm5_C. 
With only 20 young people having spent time in custody / on remand, when divided bysub-group, the numbers are quite low with all being male. Since the nature of the resulting ordercan be influenced by the young person's previous offending behaviours, there is moderate evidence in the appropriate onesided test that their custody rate is higher than that for $\mathrm{FTE}\left(\mathrm{BF}_{10}=8.626\right)$.

Table 7.10: Custody rate, by sub-group

\begin{tabular}{|c|c|c|c|c|c|c|c|}
\hline & \multicolumn{2}{|r|}{ Comparator Groups } & No. & $\begin{array}{l}\text { Remand / } \\
\text { Custody }\end{array}$ & $\begin{array}{c}\% \\
\text { Remand / } \\
\text { Custody }\end{array}$ & $\begin{array}{l}\text { Bayes Factor (BF 10) } \\
\text { (H1: Group } 1 \text { F Group 2) }\end{array}$ & $\begin{array}{l}\text { Bayes Factor (BF 10) } \\
\text { (H1: Group } 1>\text { Group 2) }\end{array}$ \\
\hline \multirow[t]{2}{*}{ Gender } & 1 & Male & 79 & 20 & $25.3 \%$ & \multirow{2}{*}{0.712} & \multirow{2}{*}{0.154} \\
\hline & 2 & Female & 9 & 0 & $0.0 \%$ & & \\
\hline \multirow[t]{2}{*}{ Ethnicity } & 1 & White & 82 & 18 & $22.0 \%$ & \multirow{2}{*}{0.215} & \multirow{2}{*}{0.859} \\
\hline & 2 & Non-White & 6 & 2 & $33.3 \%$ & & \\
\hline \multirow[t]{2}{*}{ Care Status } & 1 & No Experience & 63 & 8 & $12.7 \%$ & \multirow{2}{*}{93.8} & \multirow{2}{*}{163.2} \\
\hline & 2 & Experience & 25 & 12 & $48.0 \%$ & & \\
\hline \multirow[t]{2}{*}{ Age at First Offence } & 1 & 10 to 12 & 22 & 7 & $31.8 \%$ & \multirow{2}{*}{0.541} & \multirow{2}{*}{0.117} \\
\hline & 2 & 13 to 17 & 66 & 13 & $19.7 \%$ & & \\
\hline \multirow[t]{2}{*}{ Age at First Conviction } & 1 & 10 to 13 & 11 & 2 & $18.2 \%$ & \multirow{2}{*}{0.210} & \multirow{2}{*}{0.368} \\
\hline & 2 & 14 to 17 & 77 & 18 & $23.4 \%$ & & \\
\hline \multirow[t]{2}{*}{$\mathrm{FTE}^{*}$} & 1 & FTE & 33 & 3 & $9.1 \%$ & \multirow{2}{*}{5.690} & \multirow{2}{*}{0.064} \\
\hline & 2 & Previous Offending & 54 & 17 & $31.5 \%$ & & \\
\hline \multicolumn{3}{|l|}{ Total } & 88 & 20 & $22.7 \%$ & & \\
\hline
\end{tabular}

Notes: The individual whose FTE status is not known has been excluded from this summary. Bayes Factors have been calculated using the test for Bayesian Contingency Tables within JASP version 0.8.1.1 and are interpreted using the categorie s suggested by Jeffreys (1961).

The custody rate for those with experience of care is also notably higher than that for their peers who have never been looked after $\left(\mathrm{BF}_{10}=163.2\right)$.

\subsection{The Combined Dynamic Model for System Contact}

The individual dynamic models for coming into contacts with facets of the youth justice system highlight a number of issues with convergence which limit the potential to explore the three predictors in combination i.e. incorporating interactions between Breach: Appear, Appear: Custody, Breach: Custody and Breach: Appear: Custody. From BDm5_B (summarised in Table 7.5), it was also recognised that there was a lot of uncertaintysurrounding estimates of the coefficients relating to interactions involving Breach and the individual domains. There were similar issues in relation to interactions between Custody and the 12 domains (BDm5_C, Table 7.9). As a result, the combined model has been based on expanding upon BDm5_A.

From Table 7.6 it is apparent that there is a strong evidence that the breach rate for those who have experience of care is higher than that for those who have never been looked after whilst Table 7.10 highlights that there is a very strong evidence that the custodyrate is also higher for this group. Although there is insufficient data to investigate interactions between these 'events', experience of care and the 
individual domains, the interactions between CareExp:Breach: Time and CareExp:Appear: Time have been included in the combined dynamic model for system contact. The resulting model is summarised in Table 7.11.

Table 7.11: The Dynamic Model for System Contact

\begin{tabular}{|c|c|c|c|c|c|c|c|}
\hline \multirow{3}{*}{\begin{tabular}{|l} 
\\
Fixed Effect: \\
\end{tabular}} & \multicolumn{7}{|c|}{$\begin{array}{c}\text { Dymanic Model including Experience of Care and Appearing in Court, with 3- } \\
\text { way Interactions Involving Breach and Custody (BDm6) }\end{array}$} \\
\hline & \multicolumn{3}{|c|}{ Unstandardised } & \multicolumn{3}{|c|}{\begin{tabular}{|l|} 
Standardised \\
\end{tabular}} & \multirow{2}{*}{ Significant? } \\
\hline & Post.Mean & Lower $\mathrm{Cl}$ & Upper $\mathrm{Cl}$ & Post.Mean & Lower $\mathrm{Cl}$ & Upper $\mathrm{Cl}$ & \\
\hline (Intercept) & -7.070 & -11.138 & -2.705 & $8.50 \mathrm{E}-04$ & $1.45 \mathrm{E}-05$ & 0.067 & Yes \\
\hline Experience of Care (None $=$ Ref) & 3.260 & -1.268 & 7.553 & 26.055 & 0.281 & $1.91 \mathrm{E}+03$ & \\
\hline Court Appearance (Appear) (None = Ref) & 7.794 & 3.531 & 12.264 & $2.43 \mathrm{E}+03$ & 34.143 & $2.12 E+05$ & Yes \\
\hline Breach (None = Ref) & -0.844 & -2.463 & 0.825 & 0.430 & 0.085 & 2.283 & \\
\hline Custody (None = Ref) & -2.095 & -5.608 & 1.259 & 0.123 & 0.004 & 3.523 & \\
\hline Time & 0.332 & -0.508 & 1.136 & 1.394 & 0.602 & 3.115 & \\
\hline Living Arrangements (Live) & -0.288 & -1.899 & 1.365 & 0.750 & 0.150 & 3.917 & \\
\hline Family and Personal Relationships (Relation) & 1.767 & -0.150 & 3.563 & 5.854 & 0.861 & 35.285 & \\
\hline Education, Training and Employment (ETE) & -0.776 & -2.187 & 0.504 & 0.460 & 0.112 & 1.655 & \\
\hline Neighbourhood (Where) & 0.868 & -0.526 & 2.178 & 2.381 & 0.591 & 8.825 & \\
\hline Lifestyle (Life) & 0.373 & -1.585 & 2.356 & 1.452 & 0.205 & 10.550 & \\
\hline Substance Use (Drugs) & 1.181 & -0.292 & 2.625 & 3.259 & 0.747 & 13.807 & \\
\hline Physical Health (Physical) & -0.891 & -2.530 & 0.890 & 0.410 & 0.080 & 2.434 & \\
\hline Emotional and Mental Health (Emotion) & -0.322 & -1.717 & 0.990 & 0.725 & 0.180 & 2.691 & \\
\hline Perceptions of Self and Others (Self) & -0.019 & -1.885 & 1.904 & 0.981 & 0.152 & 6.711 & \\
\hline Thinking and Behaviour (Think) & 0.123 & -1.597 & 1.876 & 1.130 & 0.202 & 6.525 & \\
\hline Attitude to Offending (Attitude) & 1.127 & -0.759 & 3.071 & 3.087 & 0.468 & 21.564 & \\
\hline Motivation to Change (Change) & -1.060 & -3.278 & 1.201 & 0.347 & 0.038 & 3.322 & \\
\hline Care: Appear & -0.746 & -3.411 & 1.979 & 0.474 & 0.033 & 7.236 & \\
\hline Care: Breach & 2.540 & -0.441 & 5.566 & 12.685 & 0.643 & 261.296 & \\
\hline Care: Custody & 1.192 & -2.725 & 5.286 & 3.294 & 0.066 & 197.611 & \\
\hline Care: Time & -0.256 & -1.153 & 0.611 & 0.774 & 0.316 & 1.843 & \\
\hline Care: Live & 0.591 & -0.839 & 2.049 & 1.806 & 0.432 & 7.759 & \\
\hline Care: Relation & -0.175 & -1.882 & 1.453 & 0.840 & 0.152 & 4.274 & \\
\hline Care: ETE & 0.303 & -1.098 & 1.635 & 1.354 & 0.333 & 5.130 & \\
\hline Care: Where & -0.420 & -1.709 & 1.026 & 0.657 & 0.181 & 2.790 & \\
\hline Care: Life & -0.888 & -2.924 & 1.115 & 0.411 & 0.054 & 3.050 & \\
\hline Care: Drugs & -0.072 & -1.344 & 1.155 & 0.930 & 0.261 & 3.174 & \\
\hline Care: Physical & -1.271 & -3.167 & 0.530 & 0.280 & 0.042 & 1.699 & \\
\hline Care: Emotion & 0.901 & -0.489 & 2.245 & 2.462 & 0.613 & 9.437 & \\
\hline Care: Self & 1.197 & -0.765 & 3.070 & 3.311 & 0.465 & 21.541 & \\
\hline Care: Think & -1.347 & -3.491 & 0.726 & 0.260 & 0.030 & 2.067 & \\
\hline Care: Attitude & -0.924 & -2.768 & 1.040 & 0.397 & 0.063 & 2.831 & \\
\hline Care: Change & 1.169 & -0.860 & 3.318 & 3.218 & 0.423 & 27.609 & \\
\hline Time: Appear & -1.272 & -2.174 & -0.375 & 0.280 & 0.114 & 0.687 & Yes \\
\hline Time: Breach & 0.117 & -0.205 & 0.437 & 1.124 & 0.815 & 1.547 & \\
\hline Time: Custody & -0.011 & -0.520 & 0.493 & 0.989 & 0.594 & 1.637 & \\
\hline Time: Live & -0.282 & -0.651 & 0.121 & 0.754 & 0.522 & 1.129 & \\
\hline Time: Relation & -0.264 & -0.689 & 0.149 & 0.768 & 0.502 & 1.161 & \\
\hline Time: ETE & 0.144 & -0.171 & 0.466 & 1.154 & 0.843 & 1.593 & \\
\hline Time: Where & 0.019 & -0.263 & 0.280 & 1.019 & 0.769 & 1.324 & \\
\hline Time: Life & -0.043 & -0.484 & 0.421 & 0.958 & 0.616 & 1.524 & \\
\hline Time: Drugs & -0.011 & -0.315 & 0.274 & 0.990 & 0.730 & 1.315 & \\
\hline Time: Physical & -0.003 & -0.436 & 0.460 & 0.997 & 0.647 & 1.584 & \\
\hline Time: Emotion & 0.161 & -0.172 & 0.522 & 1.175 & 0.842 & 1.685 & \\
\hline Time: Self & 0.135 & -0.269 & 0.500 & 1.144 & 0.764 & 1.648 & \\
\hline Time: Think & 0.001 & -0.395 & 0.395 & 1.001 & 0.674 & 1.484 & \\
\hline Time: Attitude & -0.512 & -1.041 & -0.021 & 0.599 & 0.353 & 0.979 & Yes \\
\hline Time: Change & 0.395 & -0.102 & 0.924 & 1.485 & 0.903 & 2.519 & \\
\hline
\end{tabular}


/ continued

\begin{tabular}{|c|c|c|c|c|c|c|c|}
\hline \multirow[b]{3}{*}{ Fixed Effect: } & \multicolumn{7}{|c|}{$\begin{array}{c}\text { Dymanic Model including Experience of Care and Appearing in Court, with 3- } \\
\text { way Interactions Involving Breach and Custody (BDm6) }\end{array}$} \\
\hline & \multicolumn{3}{|c|}{\begin{tabular}{|l|} 
Unstandardised \\
\end{tabular}} & \multicolumn{3}{|c|}{ Standardised } & \multirow{2}{*}{ Significant? } \\
\hline & Post.Mean & Lower $\mathrm{Cl}$ & Upper Cl & Post.Mean & Lower $\mathrm{Cl}$ & Upper Cl & \\
\hline Appear: Live & 0.153 & -1.509 & 1.842 & 1.165 & 0.221 & 6.310 & \\
\hline Appear: Relation & -1.536 & -3.394 & 0.398 & 0.215 & 0.034 & 1.489 & \\
\hline Appear: ETE & 0.355 & -1.100 & 1.733 & 1.426 & 0.333 & 5.659 & \\
\hline Appear: Where & -1.000 & -2.417 & 0.413 & 0.368 & 0.089 & 1.511 & \\
\hline Appear: Life & 0.232 & -1.702 & 2.492 & 1.261 & 0.182 & 12.090 & \\
\hline Appear: Drugs & -1.025 & -2.551 & 0.379 & 0.359 & 0.078 & 1.461 & \\
\hline Appear: Physical & 1.166 & -0.532 & 2.960 & 3.210 & 0.588 & 19.305 & \\
\hline Appear: Emotion & -0.207 & -1.582 & 1.265 & 0.813 & 0.206 & 3.543 & \\
\hline Appear: Self & -0.562 & -2.678 & 1.491 & 0.570 & 0.069 & 4.443 & \\
\hline Appear: Think & 0.136 & -1.875 & 2.072 & 1.146 & 0.153 & 7.944 & \\
\hline Appear: Attitude & -1.144 & -3.125 & 0.900 & 0.319 & 0.044 & 2.460 & \\
\hline Appear: Change & 1.419 & -0.890 & 3.806 & 4.134 & 0.411 & 44.950 & \\
\hline Care: Time: Appear & 0.435 & -0.173 & 1.075 & 1.545 & 0.841 & 2.929 & \\
\hline Care: Time: Breach & -0.669 & -1.226 & -0.056 & 0.512 & 0.293 & 0.946 & \\
\hline Care: Time: Custody & 0.093 & -0.556 & 0.701 & 1.098 & 0.574 & 2.015 & \\
\hline Care: Time: Live & 0.080 & -0.212 & 0.391 & 1.083 & 0.809 & 1.478 & \\
\hline Care: Time: Relation & -0.137 & -0.533 & 0.251 & 0.872 & 0.587 & 1.286 & \\
\hline Care: Time: ETE & 0.013 & -0.288 & 0.318 & 1.013 & 0.750 & 1.375 & \\
\hline Care: Time: Where & 0.237 & -0.023 & 0.514 & 1.268 & 0.978 & 1.671 & \\
\hline Care: Time: Life & -0.040 & -0.459 & 0.351 & 0.961 & 0.632 & 1.421 & \\
\hline Care: Time: Drugs & -0.106 & -0.402 & 0.197 & 0.899 & 0.669 & 1.218 & \\
\hline Care: Time: Physical & 0.404 & -0.005 & 0.826 & 1.498 & 0.995 & 2.283 & \\
\hline Care: Time: Emotion & -0.116 & -0.459 & 0.200 & 0.890 & 0.632 & 1.221 & \\
\hline Care: Time: Self & -0.631 & -1.069 & -0.196 & 0.532 & 0.343 & 0.822 & Yes \\
\hline Care: Time: Think & 0.437 & 0.031 & 0.898 & 1.547 & 1.032 & 2.456 & Yes \\
\hline Care: Time: Attitude & 0.380 & -0.065 & 0.817 & 1.462 & 0.937 & 2.264 & \\
\hline Care: Time: Change & -0.409 & -0.841 & 0.017 & 0.664 & 0.431 & 1.017 & \\
\hline Appear: Time: Live & 0.272 & -0.113 & 0.654 & 1.313 & 0.893 & 1.923 & \\
\hline Appear: Time: Relation & 0.324 & -0.104 & 0.765 & 1.383 & 0.901 & 2.149 & \\
\hline Appear: Time: ETE & -0.123 & -0.455 & 0.216 & 0.884 & 0.634 & 1.241 & \\
\hline Appear: Time: Where & -0.116 & -0.399 & 0.173 & 0.891 & 0.671 & 1.188 & \\
\hline Appear: Time: Life & 0.066 & -0.405 & 0.555 & 1.069 & 0.667 & 1.742 & \\
\hline Appear: Time: Drugs & 0.108 & -0.191 & 0.421 & 1.114 & 0.826 & 1.523 & \\
\hline Appear: Time: Physical & -0.176 & -0.615 & 0.252 & 0.839 & 0.541 & 1.286 & \\
\hline Appear: Time: Emotion & -0.048 & -0.411 & 0.315 & 0.953 & 0.663 & 1.371 & \\
\hline Appear: Time: Self & 0.091 & -0.354 & 0.496 & 1.096 & 0.702 & 1.643 & \\
\hline Appear: Time: Think & -0.173 & -0.607 & 0.275 & 0.841 & 0.545 & 1.317 & \\
\hline Appear: Time: Attitude & 0.425 & -0.068 & 0.908 & 1.529 & 0.934 & 2.479 & \\
\hline Appear: Time: Change & -0.230 & -0.742 & 0.240 & 0.795 & 0.476 & 1.271 & \\
\hline Random Effect: & Post.Mean & Lower $\mathrm{Cl}$ & Upper $\mathrm{Cl}$ & Post.Mean & Lower $\mathrm{Cl}$ & Upper $\mathrm{Cl}$ & Significant? \\
\hline Individual (Intercept) & 1.795 & $1.56 \mathrm{E}-07$ & 4.759 & 6.019 & 1.000 & 116.629 & Yes \\
\hline \begin{tabular}{|c|} 
Time \\
\end{tabular} & 1.982 & 0.235 & 4.818 & 7.257 & 1.265 & 123.717 & Yes \\
\hline
\end{tabular}

\begin{tabular}{|l|r|}
\hline DIC & 433.64 \\
\hline
\end{tabular}

Source: Model BDm6_ch_A_BC, renamed as BDm6, Technical Annex: p407-425.

The 'events' are all time variant whereas the predictor representing experience of care, for the purposes of this analysis, is being treated as being time-invariant. To demonstrate how well this model represents the realities of the young person's change in circumstances during their time under the supervision of the YOT (RQ8), estimated probabilities of further offending have been calculated for each of the three case histories. 


\section{Case Study "Fred"}

"Fred" entered the cohort having committed an offence with a gravity score of 2 . As an FTE, he was initially judged to be a low risk of reoffending with a total ASSET score of just 8 . As a member of the formal youth justice system, Time 0 relates to the initial ASSET Core Profile having committed an offence and attending court. In Fred's case, as he was already on a referral order having committed a theft offence (his primary offence), he was placed on ISSP Bail and Tag whilst he awaited sentencing for a burglary dwelling. This occurred between Time 3 and Time 4, with intervening court appearances relating to bail hearings. His final assessment occurs after he has been sentenced to a DTO for 10 months.

Figure 7.8: Comparisons of the Estimated Probability of Further Offending Over Time - Individual Dynamic 'Event' Models: "Fred"

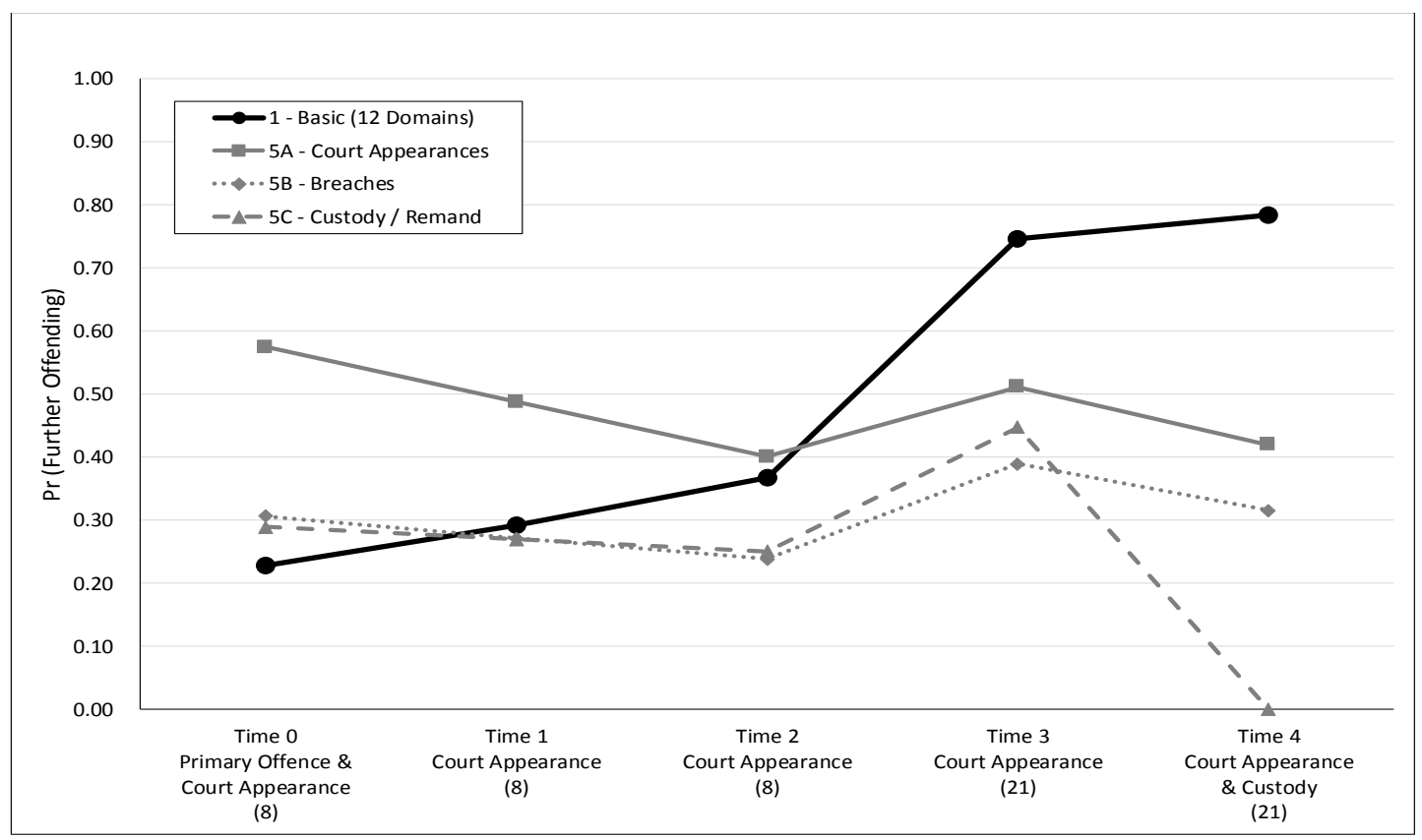

Source: BDm5_B (Breaches), BDm5_A (Court Appearances) and BDm5_C (Custody/Remand) along with BDm1.

If the initial estimated probabilities of further offending are compared in Figure 7.8, it is notable that the estimate derived from BDm5_Ais 0.57 whereas that based the Basic Dynamic Model (BDm1) is 0.23. Those based on BDm5_B and BDm5_C are around 0.30. At subsequent measurement points, the estimated probabilities based the three 'event' dynamic models initially decline, reflecting the positive impact of working with the YOT to reduce the likelihood of further offending behaviours. This is in contrast to the trajectory of the probabilities of further offending between Time 0 and Time 2 based on BDm 1 which is upward despite Fred's ASSET score remaining at 8 during this period.

At Time 3, when Fred was due backin courtfor sentencing, his ASSET score increased to 21. His final assessment was undertaken 10 days later, three days after sentencing. As would be expected, given 
that he is then in the secure estate, his probability of further offending has fallen despite no change in his ASSET score. All three of the event models suggest that there is a decrease in his estimated probability of further offending between Time 3 and Time 4. Although, BDm5_C suggests the most dramatic fall. This is potentially more realistic that the trend suggested by BDm1.

Figure 7.9: Comparisons of the Estimated Probability of Further Offending Over Time - System Contact Dynamic Models: "Fred"

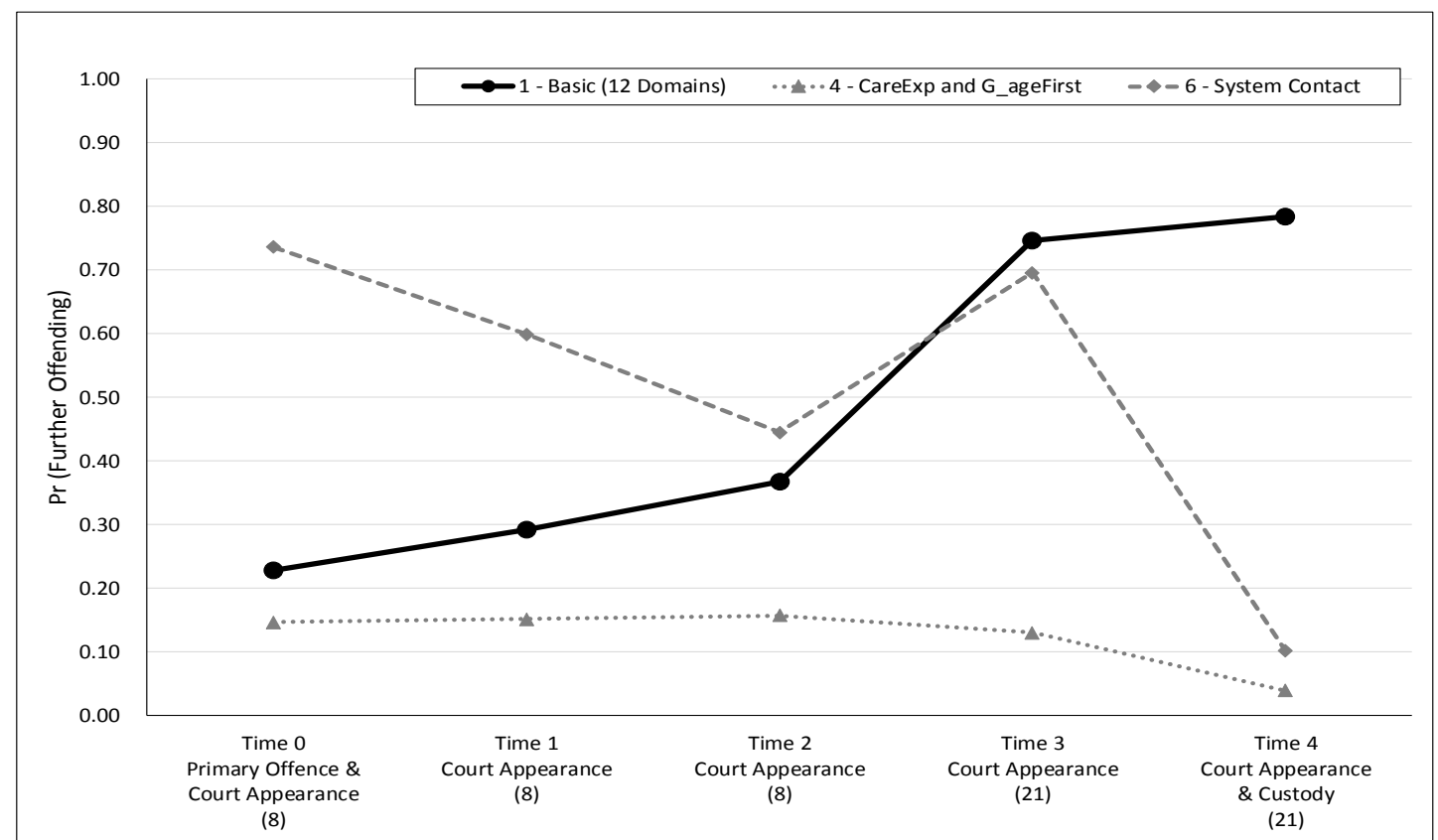

Source: BDm4 (CareExp and G_ageFirst), BDm6 (System Contact) along with BDm1.

BDm6 also takes into account Fred's court appearances and being sentenced to custody - had he breached, this would also have been reflected. However, it the model was specified to reflect the impact of court appearances on the individual domains and how these changed over time, along with the experience of care. Whilst it shares features with BDm5_A, the initial probability of further offending is higher (estimated to be 0.74$)$ as is the estimate for Time 3 ( 0.70 compared to 0.51$)$. However, the response to entering the secure estate between Time 3 and 4 is greater with the probability of Fred committing anyfurther offences estimated to be around 0.10 .

Having never been looked after and being aged 14 at the time of his first offence, Fred falls into the nonreference groups for both elements of $\mathrm{BDm} 4$, with the estimated probabilities of further offending based on this model being notably lower at each measurement point than those based on BDm1. Although his ASSET score increased at Time 3, Fred's estimated probability of further offending fell at that time whereas this was reflected in estimated based on $\mathrm{BDm} 1$ and $\mathrm{BDm} 6$. 


\section{Case Study "Connor"}

The high initial estimated probabilities of further offending based on BDm1 along with the 'event' models for court appearances and custody/ remand reflect "Connor's" prior offending history including having previously been sentenced to an 8-month DTO, and the seriousness of his primaryoffence (an attempted burglary dwelling). However, it is notable that despite his non-compliance at Time 0 , this is not reflected in BDm5_B. As a result, this model suggests that Connor's initial probability of further offending is negligible which is somewhatunrealistic.

Figure 7.10: Comparisons of the Estimated Probability of Further Offending Over Time - Individual Dynamic 'Event' Models: "Connor"

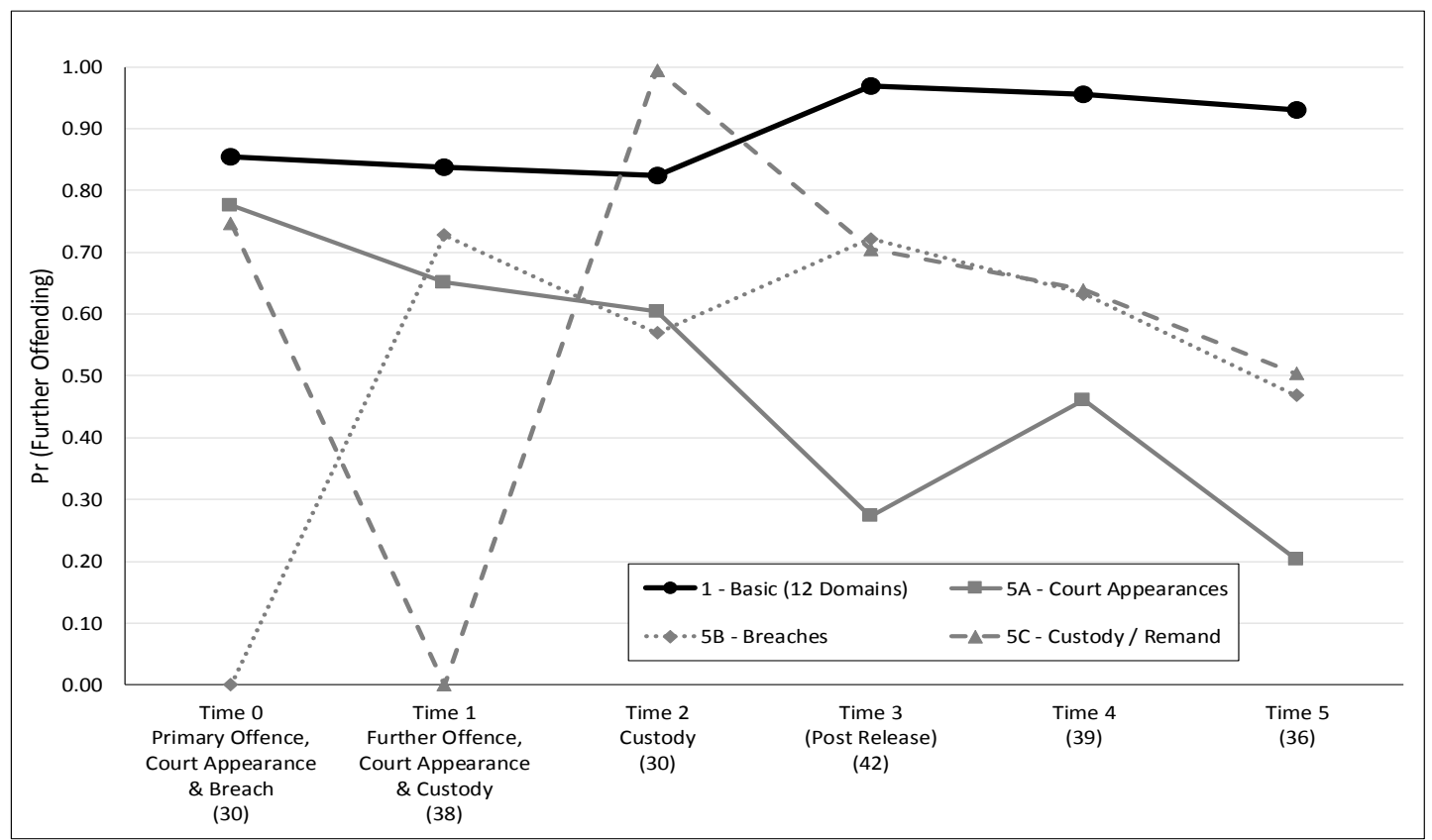

Source: BDm5_B (Breaches), BDm5_A (Court Appearances) and BDm5_C (Custody/Remand) along with BDm1.

Notes: Although the ASSET scores reflected along the $x$-axis are out of a maximum of 48 with Connor having a total of 30 at Time 0, under the Scaled Approach he would have attracted additional scores due to the fact that his primary offence (for the purposes of this exercise where the information has been taken from the reoffending spreadsheet) was anon-domestic burglary and as a result of his prior convictions.

Connor was returned to court between Time 0 and Time 1 having committed further offences (the offending occurred the dayafter his initial assessment). The offences were committed whilsthe was on conditional bail. As a result, he was remanded to custody prior to being sentenced. The assessmentat Time 2 took place 4 days after Time 1 by which time Connor was almost 2 weeks into his second 8 month DTO.

As such the opportunity to engage in any further offending behaviours is limited which is reflected in the estimated probability of further offending based on BDm5_C at Time1. However, the same model suggests that despite a decrease in his ASSET score between Time 1 and Time 2, Connor's likelihood of further offending increased significantly to around 1.0. The estimates based on the other models summarised in Figure 7.10 reflect the anticipated decrease associated with being in the secure estate. 
The assessment at T ime 3 would have been approximately one month after Connor was released so that he could serve the second half of his DTO under the supervision of the YOT. By this time, he had had his $18^{\text {th }}$ birthday. Time 4 reflects a review assessment whilst Time 5 reflects the end of his DTO.

Between Time 3 and Time 5, Connor's ASSET scores decreased. This is reflected in BDm1 and the 'event' models for breaches and custody / on remand, but not that for court appearances. BDm5_A suggest that the probability of further offending increased before falling again at Time 4 despite there being no 'events' and Connor's ASSET score falling from 42 to 38.

Figure 7.11 summarises the change in the estimated probabilities of further offending based on the system contactdynamic models for Connor. As discussed in Chapter Four, Connor has experience of care and was aged 14 at the time of his first offence. He was 17 at the time of his primary offence and therefore have been in the formal youth justice for around 3 years when he entered the 2012/3 reoffending cohort.

Figure 7.11: Comparisons of the Estimated Probability of Further Offending Over Time - System Contact Dynamic Models: "Connor"

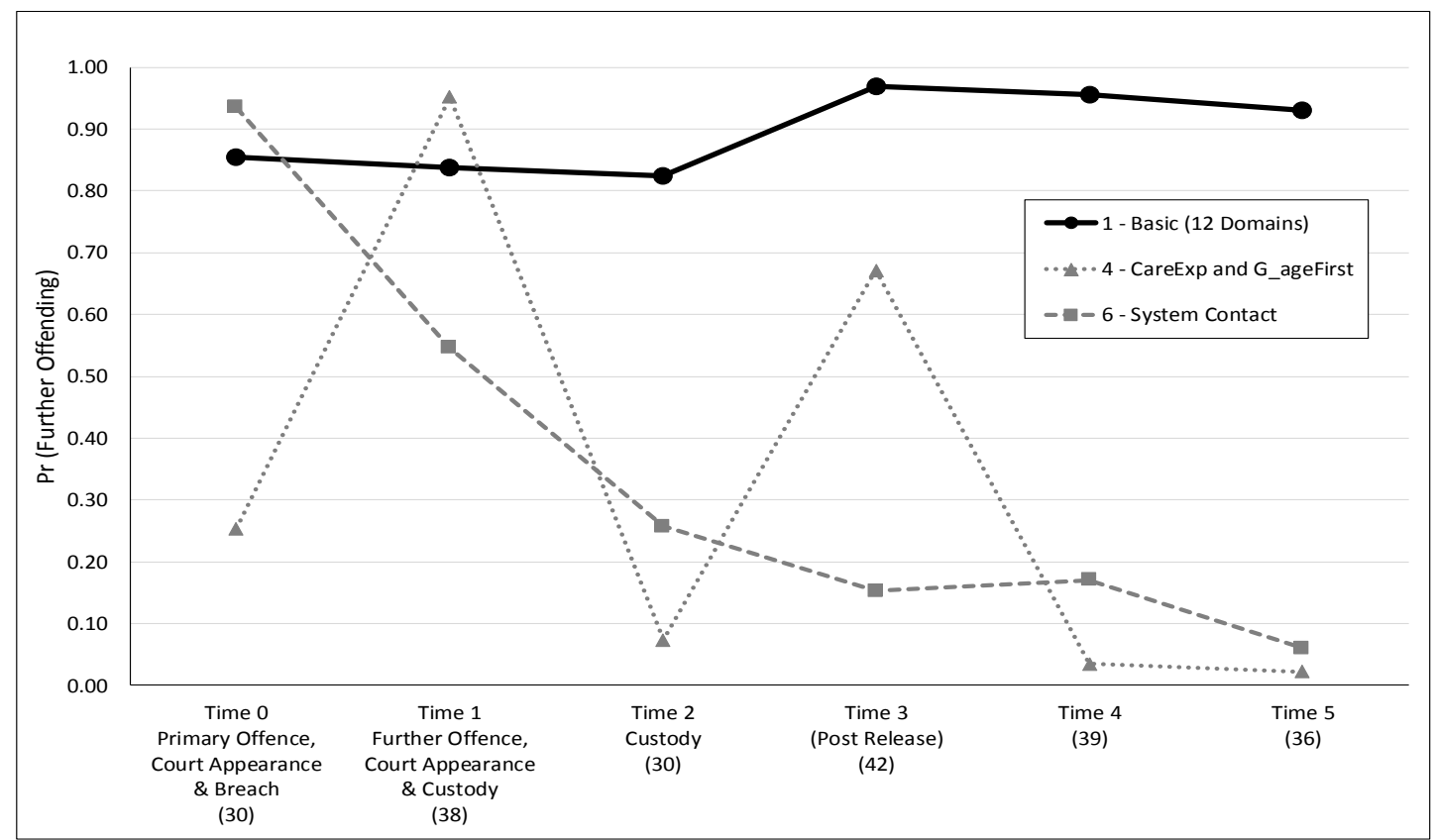

Source: BDm4 (CareExp and G_ageFirst), BDm6 (System Contact) along with BDm1.

Notes: Although the ASSET scores reflected along the $x$-axis are out of a maximum of 48 with Connor having a total of 30 at Time 0, under the Scaled Approach he would have attracted additional scores due to the fact that his primary offence (for the purposes of this exercise where the information has been taken from the reoffending spreadsheet) was a non-domestic burglary and as a result of his prior convictions.

Given Connor's history of offending and non-compliance, the high initial probability of further of offending suggested by $\mathrm{BDm} 4$ seems unrealistic whereas that based on $\mathrm{BDm} 6$ is. This latter model also reflects the increased ASSET score between Time 0 and Time 1 when Connor committed further offences following the decrease when he was in custody (Time 2 ) and the increase that corresponds to his assessment post-release. In contrastBDm6 does not reflect these changes. 


\section{Case Study "David"}

"David", like Connor has a history of prior offending and non-compliance. He committed his first offence aged 10 and therefore had been in the youth justice system for almost 7 years when he committed his primaryoffence. At the time of his initial assessment, David was on unconditional bail having committed a criminal damage offence and stolen a vehicle during the previous month. As a result, he was sentenced just before Time 1 to a 12-month YRO with an intensive supervision and surveillance (ISS) requirement.

The high intensity supervision phase for David's ISS would have entailed a minimum of 25 hours per week of purposeful, timetabledactivity with 2 contacts per week. He would also have a curfew monitored by an electronic tag. Given his chaotic background and communication issues, it is not surprising that he struggled to complywith the requirements.

Figure 7.12: Comparisons of the Estimated Probability of Further Offending Over Time - Individual Dynamic 'Event' Models: "David"

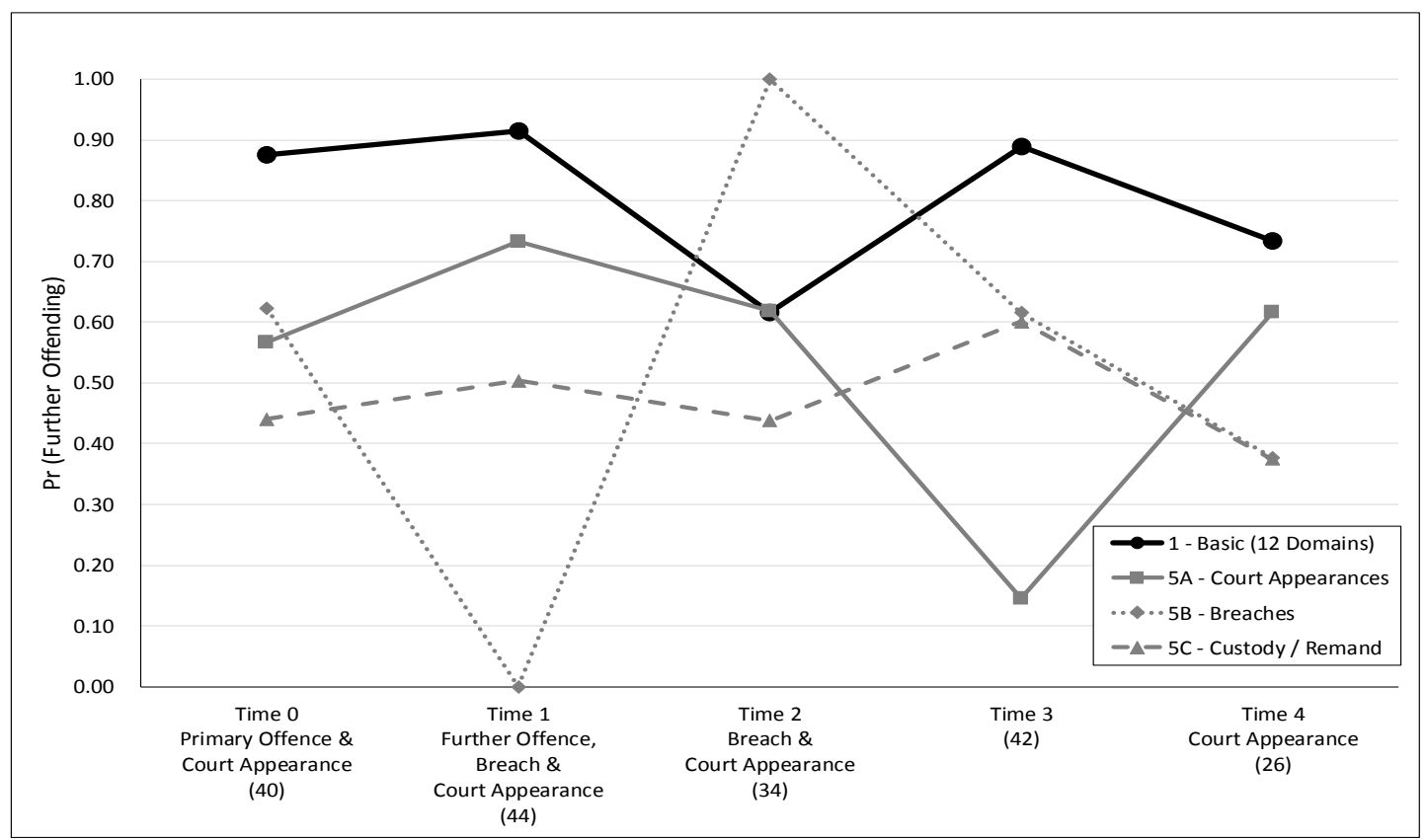

Source: BDm5_B (Breaches), BDm5_A (Court Appearances) and BDm5_C (Custody/Remand) along with BDm1.

Notes: Although the ASSET scores reflected along the $x$-axis are out of a maximum of 48 with David having a total of 40 at Time 0, under the Scaled Approach he would have attracted additional scores due to the fact that he was aged 10 at the time of his first Reprimand and as a result of his prior convictions.

The initial estimated probabilities based on the 'events' models are lower than that based on BDm1, with that estimated using BDm5_B being the highest of the three. This model, however, is potentially the least realistic: at Time 1 when David was considered to have a high risk of reoffending with an ASSET score of 42 , had breached and returned to court after committing a further offence, his estimated probability of further offending was negligible. When his ASSET score declined between Time 1 and Time 2, under BDm5_B, his probability of further offending increased to 1.0. Just over a week later (Time 3), he was sentenced for the breaches of his YRO. 
Since David did not spend any time in custody / on remand, he was in the reference category for BDm5_C. The resulting shape of the trajectory of the estimates for the probability of further offending over time, whilst lower, is not that dissimilar to that for BDm1.

As with Connor, the estimates for David based on BDm5_A, 'dip' at a time when the young person's ASSET scores increased - for David, this occurred at Time 3. This was the only measurement point where there was not an 'event'.

Figure 7.13: Comparisons of the Estimated Probability of Further Offending Over Time - System Contact Dynamic Models: "David"

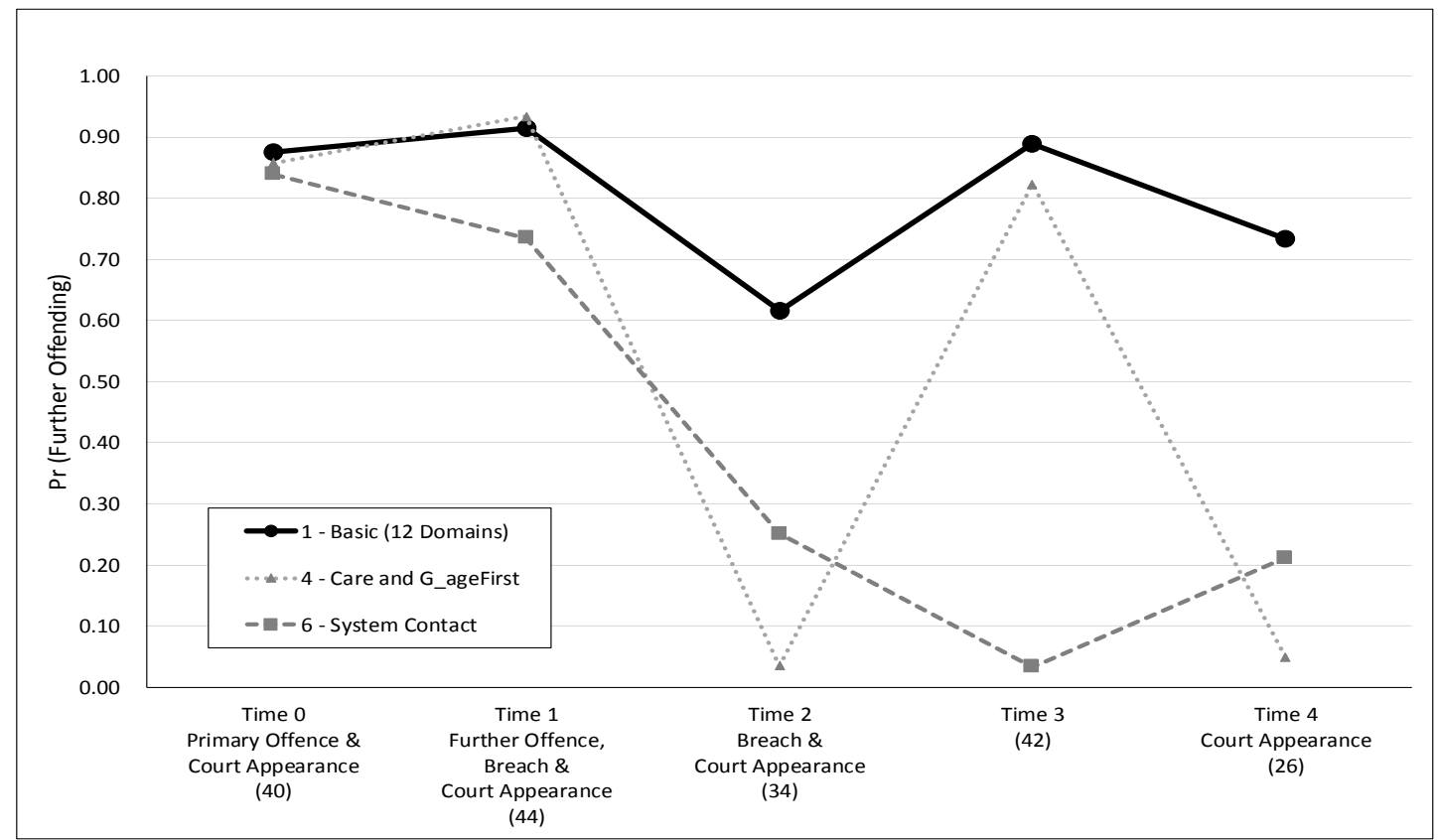

Source: BDm4 (CareExp and G_ageFirst), BDm6 (System Contact) along with BDm1.

Notes: Although the ASSET scores reflected along the x-axis are out of a maximum of 48 with David having a total of 40 at Time 0, under the Scaled Approach he would have attracted additional scores due to the fact that he was aged 10 at the time of his first Reprimand and as a result of his prior convictions.

The initial estimated probabilities of further offending are similar for BDm4 and BDm6. However, whilst BDm6 suggests that over time, David's probability of further offending decreases until T ime 4 , the trend based on BDm4 is somewhatmore erratic. Despite this, it does broadly reflect the changes in David's ASSET scores.

In utilising the ASSET scores of these three young people, some of the limitations of the models presented in this chapter are exposed. Had there been access to a larger dataset, then it is anticipated that there would be more examples of where young people had experienced multiple events. This would have enabled interactions between Breach: Appear: Time, Appear: Custody: Time and Breach: Custody: Time, to have been included within the dynamic models. This would have enabled a more responsive model for system contact to have been developed. 


\subsection{How do these findings extend the evidence base?}

\section{Breaches}

YOTs are expected to bring breach proceedings after three instances of non-compliance within a youth justice order. However, they are also required to 'ensure that every effort is made to support the child or young person or parent/carer(s) in successfullycompleting all orders including those made in the civil courts and effectively manage compliance and enforcement issues in accordance with relevant legislation' (Youth Justice Board, 2013: 30). In ensuring compliance, they are obliged to take account of 'the young person's or parent/carer's individual needs in relation to mental health problems, learning disabilities/difficulties, and speech, language and communication difficulties'(Youth Justice Board, 2013: 30).

If a child or young person is found guilty of breaching an order or commits a further offence during the period of an order, the court has various options available depending upon the nature of the order. These include revoking the order and resentencing, imposing a custodial sentence for the breach, or if the young person is on license, theycan be recalled to custody.

For the purposes of this research no distinction is made between the types of statutory order that the young person had breached. Although the dataset included examples of where the young person had been returned to court following breach and where the breach lead to a custodial sentence / return to custody, the number of cases were small hence it there remained considerable uncertainty within the models involving interactions between Breach:Appearand Breach: Custody.

Since information pertaining to this predictor has been taken from the young person's offending record held within Childview, ithas notbeen feasible to investigate the circumstances which led to the individual being breached. However, Grandi and Adler (2016) in their research into the circumstances and characteristics of young people from a single urban YOT who breached between June and December 2012 found that the most common reason was missed appointments - a finding which concurs with (Hart, 2011a; 2011b), and failure to adhere to their electronically monitored curfew. Other reasons reported included unacceptable behaviour and entering an exclusion zone. Whilst both pieces of research involved small sample sizes and include caveats around the generalisability of findings, it is anticipated that many of the young people whose risk assessments were utilised for this research will have had similar experiences to those involved in qualitative work in this area.

Although the recommendation is that the court should take care to ensure that the requirements imposed 'are not too onerous so as to make breach of the order almost inevitable' (Sentencing Guidelines Council, 2009: s10.27), both Hart (2011a) and Grandi and Adler (2016) suggest that those who found it hardest to comply are those facing the greatest disadvantage, including family factors, cognitive or communication difficulties or social pressures. 
The dynamic model involving breaches (BDm5_B) suggests that the likelihood of further offending increases if the young person has breached although the very wide credible interval highlights the amount of uncertainty around this main effect and in relation to the significant interactions between Breach and the individual domains (Table 7.7). Time helps to explain some of the uncertainty - as evidenced by the various significant 3-way interactions between Breach, Time and the individual domains. However, it would appear that there must be other factors (not included in the model) which help to explain the uncertainty.

Of the 12 domains, it is Physical Health that is a significantmain effect within the dynamic model. It is also significant in interaction terms with Breach and/or Time. As can be seen from the domain description in the Technical Annex, this domain includes problematic issues around the physical immaturity/ delayed development of the young person and where they are considered to be putting their own health at risk through their own behaviour along with having a health condition which affects everyday life functioning, notbeing registered with a GP or other health care services. In particular the domain's links with physical immaturity / delayed development tie in with Hart's finding that some children's abilityand level of maturity were factors related to compliance with some being impulsive and having problem-solving skills meaning that they were easily discouraged (Hart, 2011a).

Hart also found that perhaps the mostimportantfactors in enabling compliancewas having a reason not to breach: causing distress to family or ruining the 'normal life' that they aspired to. Familyrelationships were also important in both a positive and negative sense. The chaos described in the households of some of the children interviewed by Hart led directlyto non-compliance and a number had been kicked out by their parents or passed between warring ex-partners causing distress and conflict. When parents were supportive, this was on a practical level - with parents writing appointments on the calendar, getting them up and making sure that theyhad the means to get there. Practitioners similarlycited family support - or lack of it - as being a vital factor related to compliance along with good accommodation and thinking / communication skills. In particular they highlighted problems experienced by looked after children, especially those in residential care where staff did not always remind them of their appointments or generallysupport their compliance. The large significantpositive coefficientfor the Breach: Familyand Personal Relationships interaction with its wide credible interval is potentially a reflection of this.

\section{Court Appearances}

Cleghorn et al. (2011) found that although those who had attended court were fairly ambivalent, with Court being a necessary stage to 'get through' if they were being convicted of an offence, there were some, particularlythose who were younger that reported feeling anxious and nervous both prior to and whilstin court. Notablythey reported feeling unsupported, with courtproceedings 'going over their head'. Deuchar and Sapouna (2016) similarlyidentified the need for young people to be supported, reassured and aware of their rights so that they could adequately understand court proceedings. In providing 
adequate support during this stage in the criminal justice system, they argue that it ensures that young peoples' prospects for desistence are not inadvertently damaged. However, it also needs to be acknowledged that for those who had had a long wait before their case came to trial, the time spent waiting was considered to be 'wasted' time during which their ability and motivation to engage in education and start planning for their future was severely restricted (Cleghorn etal., 2011). During this time, they may be open to temptation and can become resigned to further offending as a consequence of the constrained opportunities arising from having had contact with the youth justice system. Thus, as Corr (2014) observes criminal justice responses can serve to further marginalize young people's positions as they begin to appreciate potential barriers to 'moving on' with their lives, particularlythrough access to education and em ployment.

Whilst going to courtwas considered a daunting experience bythe young people spoken to by both Corr and Cleghorn etal, they felt that 'it would notnecessarilyfunction as a deterrent to all young people who had offended. Rather, it wasfelt that it would actas a deterrent to some, whilst for others perhaps those committing more serious offences, going to court would have a very limited preventative effect' (2011: 29). Indeed, there may be some for whom going to Courtenhanced their criminal status. The extent to which members of the reoffending cohort felt this cannot be ascertained from the data available. However, the rate of further offending suggests that attending court did not serve as a deterrent - whilst some were required to return to courtfollowing a breach and at least two for offences committed before their primary offence went to court, there were also individuals who committed further offences.

For those with 'previous form', the consequences of having an offender identity, can be deleterious in courtparticularlyif they have become distrustful of authority. Hart (2011b) for example found that some of the factors which influenced sentencing decisions in relation to breaches included:

- 'The young person is sorry and pleads guilty

- If they are totally disengaged with the process, with a real attitude, that's going to be the same outside court

- If the parent comes to court for the breach, l'd feel more confident that the parentwas engaged with the order

- The young person's attitude, motivation and family support

- Are they remorseful?

- A good home background, a sense of self-worth and basic education'

(Hart, 2011b: 22)

Those young people whosedemeanour in courtwas interp reted as cheeky were identified as being more likely to receive a punitive response as were those lacking social or communication skills. However, it is also perceivable that these factors also apply when determining sentences for other offences, with magistrates not knowing what to do with particularly chaotic young people who have 'form' for not complying with the requirements of their order or who are perceived to be lacking the support of family 
members. Inadvertently, since those who have appeared in court previously may have a better understanding of what to expect, those FTEs who are lacking a comprehension of court processes may be disadvantaged if they not appear to be engaging.

The dynamic model involving courtappearance (BDm5_A) suggests that as with breaches, the likelihood of further offending increases if the young person attends court (Table 7.7). This coefficient is similarly moderated bythe Appear: Time interaction - the significantnegative coefficient suggesting that the effect of having to return to court after the initial appearance decreases with time. None of the other main effects or interactions are significant within the model whilst the DIC of 445.9 suggests that even taking into account the additional complexity of the model, there is a significant amount of uncertainty unaccounted for relative to the equivalentmodel involving just the 12 domains (BDm1). Had there been sufficient data to enable the Level 2 'individual' characteristics to be incorporated into the dynamic model, based on the findings, it is anticipated thatFTE status, experience of care, age atfirst offence and other vulnerabilities would have helped to explain some of this uncertainty. Dynamic Model 6, whichincluded experience of care alongside court appearances and interactions relating to breaching and spending time in custody/ on remand illustrates this, with the DIC falling to 433.6 despite the additional complexity of the model.

\section{Custody}

Custody as a main effect was not found to be significant in this dynamic model (BDm5_C). However, the interaction between Custody and Time was significant, with the negative coefficientsuggesting that if the time in custody / on remand occurs later in the young person's time under the supervision of the YOT, then it has less impacton the likelihood of further offending. As with BDm5_B, the Physical Health domain was a significantas a main effect. However, none of the interactions involving Physical Health were found to be significant.

YOT staff believe that young people with learning disabilities, communication difficulties, mental health problems, ADHD, and low levels of literacy are more likely than children without such impairments to receive a custodial sentence (Talbot, 2010). This, it suggested, is the coming together of a number of factors including:

- the lack of routine screening and assessment to identify the particular supportneeds of children who offend - an issue which ASSETPlus has sought to address

- a poor understanding across youth justice services of how impairments and difficulties can affect behaviours, which can be particularlysignificant during court proceedings

- limited availability of appropriate youth justice programmes, activities and support, linked to which, the increased likelihood that children with impairments and difficulties will fail to comply. 
Subsequent research and pressure from organisations such as the Royal College of Speech and Language Therapist, the Prison Reform Trust and Howard League for Penal Reform have contributed to an increased awareness of these issues with the youth justice system within the YJB and individual YOTs playing an integral role in the development of good practice. However, as independent reviews such as those by Lord Lamming and David LammyPM highlight, some of the most vulnerable groups of children continue to be over-represented, particularlyin the formal youth justice system and the secure estate.

For those who have spent time in custody, the neighbourhood to which theyreturn along with their living arrangements has been shown to have a significantbearing upon the likelihood of reoffending. Hence in recentyears there has been a policyemphasis upon resettlementas part of the Transforming Youth Justice agenda. Research by Beyond Youth Custody (Bateman and Hazel, 2015) suggests that young people experience a period of reorientation and transition immediately following release during which they can become overwhelmed, lost and confused. Even though they may be returning to familiar surroundings, inevitably there will be elements of their environment which have changed whilst in custody. Interactions with people, even family and friends, can be problematic causing stress, confusion and anxiety. WhilstBateman and Hazel suggest that there is a dearth of literature on how young people experience resettlementafter prison, they emphasise that this area needs to be explored given findings from research amongstadultex-prisoners suggesting that they are extremely vulnerable to suicide and that the first two weeks following release are associated with higher rates of drug-related mortality.

The transition from custody to communitycan be disorientating and destabilising, with young people not only having to renegotiate relationships and adjust to the new lifestyle, but also because the structural support such as stable accommodation, education, training, employmentand financial stabilityon which to build their reorientation may well not be available at the time of release. This can be particularly challenging since a number of vulnerable groups are over-represented in the custody population e.g. those with poor mental health (Berelowitzand Hibbert, 2011); neurodisabilities including traumatic brain injuries and attention-deficithyperactivity disorder (Young and Goodwin, 2010; Hughes et al., 2012; Hughes etal., 2015); looked after children (Prison Reform T rust, 2016) and having experienced different types of trauma (Liddle et al., 2016). For these young people, their needs and vulnerabilities mean that they may not have developed strategies to cope with transitions and are more likely to have to orientate themselves around a chaotic home environment.

The low number of individuals in the reoffending cohortwith experience of custody or being on remand means that it is difficult to draw firm conclusions about the impact of release from custody on the likelihood of further offending. However, the second case study presented in Section 7.4 'Connor' focuses on the changes in dynamic risk associated with a young male with $A D H D$ upon his release from custody. 


\subsection{Summary}

The analysis presented in this chapter sought to address three research questions:

6. How is the likelihood of further offending affected by having experience of care and a previous offending history?

7. What is the impact of coming into contact with facets of the youth justice system on the likelihood of further offending?

8. How well do ASSET scores reflect the realities of the young person's change in circumstances during their time under the supervision of the YOT?

In doing this, Chapter Seven built upon findings from previous chapters. Whilst the size of the dataset limited the extent to which different forms of system contact could be explored, it was possible to constructa model which considered both experience of care and grouped age at first offence (BDm4). As highlighted in section 7.4, when applied using the ASSET scores for the three case studies rather than artificially fixing the domain scores, some of the limitations of the model were exposed. Notably, although the various diagnostic tests were satisfied and there were no convergence issues, the comparatively small sample size meant that amongst those who had first offended aged $10-12$ years, there were few cases to inform the model, particularlyatlater measurements. As a result, the trajectory of the estimates of further offending were more heavily influenced by the ASSET scores of those considered bypractitioners to have a high risk of reoffending, resulting in an upward trend over time.

This chapter also considered system contact by considering how 'events' such as breaching, returning to courtand spending time in custody/ on remand affected the likelihood of further offending (BDm5_B, BDm5_A and BDm5_C respectively). This is not something which has previously been explored in evaluations of ASSET, largelysince these have confined to looking at a singleassessment per individual. Whilst the 12 domains are time varying, reflecting the scores at each assessment, by extending the Basic Dynamic Model to include both a Level 2 characteristic and an 'event' brings the model closer to representing the realities of a young person's experience under the supervision of the YOT.

Had the datasetbeen larger, it would be desirable to look at combinations of the Level 2 predictors and the different permutations of encounters with different facets of the youth justice system for to get a greater understanding of how these typically impact upon the likelihood of further offending. From a policy and practice perspective, it would be particularly useful to increase our understandings for the following sub-groups:

- a looked after child with a prior offending history relative to their peers who have either no history of offending or have never been looked after. Ideally this should take into account the legal status of the child; whether they were looked after at the time of their initial offence; if they continued to be looked after and how they are being accommodated. 
- for those who enter the youth justice system at a young age and have an established offending career relative to the young FTE and the older FTE. Potentially this require an additional predictor which takes into account their age at the time of the primary offence as well as their age at the time of their first offence and conviction.

- females with different characteristics/experiences relative to their male peers.

- BAME children with different characteristics / experiences relative to their peers, ideally with the potential to enable individual ethnic groups to be considered.

Across Chapters Five to Seven, different types of predictors have been used to extend the Basic Dynamic Model constructed in Chapter Four. This has included dichotomous, categorical and continuous measures which represent data collected by the YOT to assess the risk of individual further offending behaviour and to determine the most appropriate interventions for the child. One of the key tools used in conducting this analysis was the reoffending spreadsheets compiled by the YJB which include individual level data, to measure the performance of the YOT. Courtand offending records held within Childview were also utilised with records being matched using a unique identifier. Theseillustrate the potential for using data linkage approaches to provide additional contextual in formation about the child. This could include education, health and social services records as well as the outcome for referrals for substance misuse treatment, speech-language therapy, mental health supportetc.

As these three chapters have demonstrated, utilising Bayesian approaches offers a flexible means to incorporate different types of data so that new hierarchical models can be developed as and when additional information becomes available. This could either be abouta specific individual e.g. as a result of further assessments being made, or about a particular issue. Whilst having a small sample size did mean that compromises needed to be made, this did have advantages since it was possible to look in more detail at the underlying data in order to understand why unexpected outcomes had occurred.

In addition to using hierarchical models, analysis has also been taken using one- and two-sided $2 \times 2$ chi squared tests to considered whether or not there was a difference in the rates for different groups. A Pearson's correlation was also undertaken to look at the relationship between the age at first offence and age at first conviction (in Section 6.7). These are just some of the commonlyused statistical tests which it is possible to perform in a Bayesian framework, with Bayes Factors being used to interpret findings rather than $p$-values. Together, these offer practical examples of how the relationship between risk factors and (re)offending can be explored using Bayesian approaches, thus addressing the second research objective. A number of challenges were identified which have been touched upon within this analysis. These are considered further in Chapter Eight along with implications for policyand practice of adopting Bayesian approaches more widel ywithin criminology. 


\subsection{Introduction}

The overarching research aim of this research is to explore the utility for using Bayesian approaches within criminology. This has been done by way of a case study focusing upon risk assessment in the youth justice system in England and Wales. The case study was chosen because the evidence base has been widely criticised and hence there is the opportunity to demonstrate how, by applying novel statistical approaches to a comparativelysmall dataset, knowledge and understanding can be extended. Athough a series of research questions were posed, these were designed as part of the analysis led approach to demonstrate specific features of the data and how these could be explored using Bayesian techniques.

The findings presented in Chapters Four to Seven illustrate some of the strengths and limitations of applying these techniques to a comparatively small number of cases drawn from administrative data relating to the youth justice system in England and Wales. Whilst the size of the dataset is somewhat smaller than in other reviews / evaluations of ASSET (Baker et al., 2003; 2005; Wilson and Hinks, 2011), it was possible to build upon these by specificallyfocusing on the dynamic element of risk and to look at the impact of breaching, having to attend court and spending time in custody / on remand. However, the comparatively small size meant that it was not possible to explore some of the permutations of individual characteristics e.g. ethnicityand gender. This is frustrating in the context of the recent reviews:

- The Lammy Review(2017) which emphasised the diversity of the BAME community, including the potential to investigate differences by age, gender and heritage, with looked after children who are Black or from other minority ethnic groups and looked after girls identified as being minoritygroups with particular needs, and

- In Care, Out of Trouble (Prison Reform Trust, 2016) which specificallyidentified looked after children from minoritygroups e.g. those from black or from other minorityethnic backgrounds, and children and young people of Muslim faith; looked after girls; children with disabilities, learning difficulties and speech, language and communication needs; foreign national children including asylum seekers and those who have been trafficked.

Utilising the comparatively small dataset has afforded this research a distinct advantage in that it has been possible to drill down to individual cases to investigate trends and provide contextual information to assist in the interpretation of the findings. Notably understanding the 'weight' of the observed data for different sub-groups at respective measurementpoints assists in understanding issues around why some models have struggled to converge whilst others have generated spurious trends. Significantly the unbalanced nature of the dataset means that at later measurement points, there are fewer cases and these relate to individuals who have committed further offences. As such the influence that these 
cases have in interactions involving time can be distorted by what has happened to a single individual. This is perhaps mostapparent for the gender predictor where the average number of measurements for each female is 4.25 compared to 6.53 for males whilst the maximums are 10 (T ime 9) and 19 (T ime 18) respectively (Figures 5.2 and 5.3). Similarly, when predictors are combined, there are instances where only a small number of individuals shared a particular combination of the characteristics reflected in the model. This meant that compromises needed to be made, for example using groupedage atfirst offence rather than FTE status alongside experience of care when exploring system contactin BDm4.

A key feature of the use of Bayesian approaches is that they enable low base rates to be taken into consideration and henceoffer the potential to increase the sensitivity of risk assessment tools to explore within- and between-individual differences. Whilst there were insufficient cases to establish within the reoffending cohort any issues around dimensional identity, the inclusion of predictors for Gender and Ethnicity alongside the 12 domains does not disconfirm the notion that there may be distinct trends for females and non-White groups. It was similarly not possible to differentiate between the severities of violent offending nor to explore any differences that might exist on the basis of the type of offence. However, the potential for this has been demonstrated should access to a larger number of cases be secured.

Had the datasetbeen larger, it would also have been desirable to investigate potential difference in terms of the type and severity of further offences committed. Not only would this have enabled 'offending' to be de-homogenised (Case and Haines, 2009) to consider different typologies of offender, it would also have enabled theories around the persistence, escalation and degree of specialisation which form important aspects of the criminal careers approach to be considered. It is anticipated that access to PNC would be required to enable young people's offending to be tracked beyond the age of 18 as the information included within the YJB's reoffending spreadsheetwas somewhatlimited.

What it was possible to determine from the data was the impact of system contact whether itbe 'events' representing facets of the youth justice system, having first offended at an early age or being a looked after child. Notably what was apparent in many of the models was the role of individual domains, particularlythose relating to Lifestyle, Thinking and Behaviours; Perception of Selfand Others, and those relating to individual factors. These highlight the strong and enduring relationship between punishment and offender identify which McAra and McVie (2015) argue we have an obligation to investigate if we wish to build a youth justice system which protects the rights of children, promotes the well -being of those young people who have offended and hence come into contact with differentfacets of the system, reduces crime and promotes social justice.

The hierarchical models generated were intended to mimic those which features of the ASSET Core Profile, with the motivation being to identify if a more sensitive tool could be developed - hence the choice of research questions. 
The posterior mean distribution for the random effects of time and individual were found to be significant in each model suggesting that the probability of further offending differs across individuals which is what would be expected given the role of agency. Whilst there remains uncertainty within the models, it is likely that some of this could potentiallybe explained by the role of other variables not included in the model specification. The nature of these could as yet also be unknown.

From the trace plots and histograms for each model, the convergence is satisfactory whilst the autocorrelations of the estimates were small suggesting that adequate thinning and burnin had been allowed for. Given the comparatively small size of the dataset upon which these models have been simulated, it is anticipated that the predictive accuracy could be improved upon through the inclusion of more cases. However, in adding to the complexity of the Basic Dynamic Model (BDm1), the DIC tends to increase and therefore this is not necessarilyindicative of increased predictive accuracy.

The DIC is currently the predictive measure of choice in Bayesian applications, in part because of its incorporation into the popular BUGS package (Spiegelhalter et al., 2002; 2014). It also features within the MCMCgImm package (Hadfield, 2010) used for this research. Based upon the DIC's of the respective models (Table 8.1), Dynamic Model 1 appears to be the 'best' model. However, this is also the simplest model - a limitation of the way in which the DIC is constructed is that it tends to favour simpler models with those involving more parameters being penalised for their complexity (Plummer, 2008). Of the models involving additional predictors, it is Dynamic Model 3 (BDm3) which has the lowest DIC and hence provides the 'optimal' fit (Finch etal., 2014). However, when 'real' domainscores relating to "Fred", "Connor" and "David" were used to estimate their probability of further of offending over time and compared to 'events', this revealed some of the limitations of these models.

Table 8.1: Summary of Model DIC's - Combined Models

\begin{tabular}{|l|l|r|}
\hline Description & Model & DIC \\
\hline 1 - The Basic Dynamic Model (12 Domains) & BDm1 & 256.8 \\
\hline 2 - Demographics and Care & BDm2 & 466.4 \\
\hline 3 - Static Factors & BDm3 & 387.9 \\
\hline 4 - Age at First Offence and Care & BDm4 & 425.9 \\
\hline 5A - Court Appearances & BDm5_A & 445.9 \\
\hline 5B - Breaching & BDm5_B & 449.9 \\
\hline 5C - Custody / on Remand & BDm5_C & 478.0 \\
\hline 6 - System Contact & BDm6 & 433.6 \\
\hline
\end{tabular}

It is anticipated thatwere this work to be repeated using a larger number of cases, then it would enable the role of the 'individual' level predictors to be explored more thoroughly. Notably there would be the potential to model permutations with more certainty, for example to explore the role of age and FTE status, and also to gain a greater understanding of multiple events e.g. where a breach leads to a court appearance and where the outcome of a court appearance is a custodial sentence / remand. A key insight gained from undertaking this research is that whilst the techniques employed enable more to be 
done with less, there is still a need to have cases which share the various characteristics in order to inform the model.

Whilst Chapters Four to Seven demonstrate from a practical perspective how the relationship between risk factors and (re)offending be explored using Bayesian approaches (the second research objective) the next section addresses this from a more theoretical perspective. As such it focuses upon how the approaches described can potentiallyaddress the criticisms of ASSET and hence issues more generally with risk assessment processes used within the criminal justice system.

\subsection{Addressing the Criticisms of ASSET}

As previously outlined, ASSET was chosen as a case study because it has been widely criticised by both practitioners and academics. It has since been replaced by ASSET Plus - a tool designed to address many of the criticisms and to reflect emerging practice. The main criticisms of ASSET with regard to the methodology, contentand its practicalityhave been identified by Stephenson et al. (2011) as being:

- Factorisation

- Marginalisation of young people's perspectives

- Technicised practice

- Developmentalisation and psychosocial bias

- Predictive utility

- Repressive welfarism

Given the breadth of the criticisms, notall of these could be addresses within this research. Hence the focus has been upon those relate to the application of actuarial approaches i.e. factorisation and predictive utility. However, the findings do, in some instances, have implications which have the potental to indirectlyaddress other aspects raised by critics.

\section{Adulterisation and Zombification: the application and use of ASSET}

In response to accusations that ASSET is an adult-led assessment which neglects the views of young people (see for example Case, 2006), Stephenson et al. suggest that this is 'perhapslargelya failure to implementASSET properly rather than a function of the tool itself (2011:51). Whilst this is not a view that I share, I feel that both this and issues around the articulation of children's rights within the assessment process fall outside the scope of this research. Notablythis is an issue which Baker (2014a) asserts has been addressed within the ASSET Plus framework through the inclusion of self-assessments for young people and parents/carers whichcan be builtupon as the intervention progresses and through the engagementand participation section of Foundations for Change. 
Accusations of technicised practice and the 'zombification' of practitioners (Pitts, 2001) stem from ASSET being characterised as a mechanised tick-box system. This, Stephenson et al suggests is associated with the 'abundantevidence of inconsistency of practice and neglect of guidance' (2011:52). However, it has been acknowledged in Breaking the Cycle: Effective Punishment, Rehabilitation and Sentencing of Offenders that there was a need 'to move towards a lighter touch performance monitoring capability which supports a more risk-based inspection programme and increases professional discretion' (Ministry of Justice, 2010: 76). Indeed, the impetus for the review of youth justice assessments was the recognition that mechanisms were needed which 'increase discretion and reduce the amount of time frontline workers spend in front of their computers, so as to free up their time to work with young offenders' (Ministry of Justice, 2010, quoted by Teli, 2011:5). The extent to which this has been achieved through the ASSETPlus framework has yet to be explored. However, the way in which practitioners engage with the risk assessment process similarlyfalls outside of the scope of this research. Athough, it is recognised that it continues to be desirable to have a framework for determining the likelihood of reoffending which can be readilyunderstood and operationalised for practitioners. Indirectly this piece of work has the potential to contribute towards this.

\section{Privileged Policies: Repressive Welfarism, Developmentalisation and Psychosocial Bias}

Of the remaining main criticisms, it could be argued that developmentalisation and psychosocial bias, and repressive welfarism are a reflection of policy. In the case of the former, the bias arises from the selective use of evidence (Case, 2007; O'Mahony, 2009; Goldson, 2010), which Case (2010) argues has privileged three developmental explanations of offending behaviour: Farrington's criminal careers model; Sampson and Laub's theory of age-graded informal social control and Thornberry's interactional theory - these explain 'offending as the predetermined product of exposure to psychosocial risk factors at different developmental stages (particularly childhood), and therefore, privileges risk factors located within the individual' (Case, 2010:91). The emphasis on what is considered to be 'modifiable' has meant that the wider socio-economic problems are disregarded, privileging the 'family' under the rhetoric of care and support rather than social harms such as poverty, class and societal access to opportunities. In this way a psychosocial bias has been reinforced through the artificial restriction of the range of risk factors being explored.

Repressive welfarism is, according to Phoenix (2009a), similarly a reflection of the previous Labour government and the YJB's approach to youth crime. However, this arises from the tension between welfare and justice approaches with some risk factors for reoffending equallybeing constructed as social welfare needs. In acknowledging that perceptions of risk and need have become very blurred, Phoenix (2009a) asserts that in their attempts to highlight service gaps which have created a raft of unmetneeds for young people and operate to push young people into less than law abiding behaviour, prac titioners have inadvertently created conditions whereby'needy' young people are rendered 'punishable'. Thus, 
the recognition that there is a lack of appropriate state responses to social welfare issues is resulting in vulnerable, marginalised and excluded young people being subject to more punitive (and especially penal responses).

At the heart of both of these issues, is what is being assessed in term s of contentand the extent to which the assessment considers the individual risk, needs and vulnerabilities of the young person. Notably, ASSET was particularlycriticised for its emphasis on negative risk factors over positive protective factors; that the approach served to identify and act upon risks associated with the young person without necessarily addressing their needs, and that it stereotyped and demonised young people, thereby facilitating a culture of control (Stephenson et al., 2007). Under the Scaled Approach, there were also concerns that in the interests of eradicating potential threats, there were also issues raised about fairness and equality due to the potential for two young people who committed the same crime being treated differently as a result of their perceived level of risk.

The YJB have sought to reflectemerging evidence in developing ASSETPlus as a successor to ASSET, particularly through the incorporation of issues reflecting heightened societal and policy concerns that have emerged since 2000. Significantly the new framework is underpinned byan evidence base that extends beyond the risk and protective factor paradigm of which many commentators had been so critical, to reflect the 'growing emphasis on the development of theoryand practice models based around factors which increase the likelihood of young people desisting from offending' (Youth Justice Board, 2014). Reflecting its mandate as an evidence-based organisation, the YJB has also been keen to ensure that lessons learnt from other fields e.g. social work, health care and probation with regards to assessment practice have been incorporated along with the perceptions of practitioners and young people. As such ASSETPlus includes the following new areas:

- Speech, language and communication needs screening

- Optional alcohol use screening tools

- Financial circumstances of the young person

- Risk of/identification of radicalisation

- Gang associations

- Gambling and inappropriate uses of technology

- Identification of parental responsibilities of the young person

- Identification of carer responsibilities of the young person

Whilst this research has not explicitly explored newground since ithas focused on variables which reflect the scoring system from the Scaled Approach, the adoption of Bayesian approaches provides a mechanism for continually updating the risk assessment process as more is learnt about the young person and also about the cohort within the formal youth justice system. The flexibility that the approach 
affords also means that the risk assessment process could continue to evolve to reflectemerging crime types and to respond to evidence around 'What Works' with young people who offend.

A frustrating feature of the administrative data used for this research is that the information relating to these 'new' concerns, if captured would have been noted within narrative fields which are more difficult to systematically interrogate - although the Core Profile includes questions about whether the young person has a formal diagnosis of mental illness or has a statement of special educational need, the details would be recorded in the evidence box within that domain. There may also be details in information provided by partner agencies to support the writing of the pre-sentence report. As such it was not possible to explore these issues within this research. Similarly, whilstit mightbe recorded that a young person has been referred by the YOT say to substance misuse treatmentservices or CAHMS, the outcome was not routinely recorded since this data would be considered 'health' data. However, this is not to say that if concerns begin to emerge about a particular issue then, assuming relevant data could be captured, this could be explored through a modification to the model if a Bayesian approach were adopted. In this way the range of factors associated with the likelihood of further offending and/or promoting desistence could be enhanced to offer a more fit for purpose holistic assessmentprocess.

\section{A Blunt Tool? The Application of Actuarial Approaches}

Whereas the previous criticisms relate to the application and use of ASSET by practitioners and the evidence underpinning ASSET, the criticisms around factorisation and predictive utility relate to the application of actuarial approaches. Associated this are issues around how to operationalise measures and outcomes (O'Mahony, 2009). These have a particular bearing on the 'What Works' agenda since one of the key variables in determining outcomes of interventions designed to reduce the likelihood of reoffending should logicallybe the ASSET Score/ Band.

As highlighted in Chapter Two, there have been particular concerns about the predictive accuracy of ASSET with Smith questioning the efficacynotjust of having the authoritative decision making based on unreliable information and subjective judgements, but also whether 'it is acceptable to 'get it wrong' sometimes in order to manage and control risk?' (2006: 102). In particular, he points to the very high reported rates of both false positives and false negatives (summarised in Table 2.5), highlighting that cost-benefitanalysis of the kind undertake by the Audit Commission (2004), often tend to gloss over the 'massive human and financial waste resulting from the failures of pre-emptive and excessive interventions'. At a time when there is little public money, there is perhaps now even less justification for there being routine error within the technologies of assessment and classification applied in youth justice especiallywhen it has been suggested that increased diversionary activity and the declining number of FTEs, means that those in the formal youth justice system although smaller in number, now represents a 'greater concentration of young people with complex needs and risky, entrenched 
behaviours. As a group, they are more likely to be challenging to work with and more likely to reoffend' (Youth Justice Board, 2016a: 13).

Issues around the predictive utility, notjust of ASSET but of actuarial tools generallyarise from attempts to reduce complex and interrelated experiences and circumstances into a series of ratings which indicate the likelihood of reoffending. Critics in particular point to the reductionisttendencies which have led to such tools becoming an oversimplified technical fix to the complex social realities of young people's lives (Case, 2010). They have also expressed concern that ASSET is concerned not with predicting reoffending, but with reconviction. The limitations of using proven reoffending as an outcome measure where highlighted in Chapter Three with two examples of pseudo-reconvictions being found amongst the 88 young people whose data was used for this research. However, italso needs to be acknowledged that 'reconviction is not the same as reoffending and can be influenced by the local practice of the police and other CJS agencies in securing convictions' (Howard and Kershaw, 2000:1). Similarly, convictions can representmore than one offenceand levels of attrition within the justice process can mean that there can be a significant difference between the number of offences committed, recorded, detec ted and ultimately resulting in conviction. Having a risk assessment tool therefore that is based upon an inherentlyflawed outcomemeasure, the risk of which is determined on the basis of statistical correlations and associations, renders many of the so-called risk and protective factors as being artefactual. The decision to use further offending as an outcome rather than reconviction represents an attempt to minimisethe impact of this. However, as will be highlighted in later sections, issues emerged as a result of the construction of the proxy indicators designed to mimic the static factors included within ASSET which may have inadvertently added to the uncertainty, particularlyin relation to the model involving offending history.

Whilst it is important to reiterate that ASSET was not intended to be an exact science, rather the individuals' ASSET score was intended to provide an indication of the likelihood of reoffending and was designed to aid practitioners in consistently and transparently identifying those domains associated with offending in order to construct robust interventions. However, it was felt that the chances of disproportionate intervention were increased under the Scaled Approach, for example a low-risk young person who had committed a serious offence receiving too intensive an intervention and vice versa. Certainly, having an approach where around one-third of profile outcomes were neither valid nor reliable was both damaging and counter-productive to the young people concerned. With their dual-purpose objectives, second- / third-generation tools such as ASSET are subject to significantmethodological issues associated with the actuarial fallacy. As a result, the tools lack the sensitivity to be specific to the individual and their circumstances. With so many uncontrolled and unknown (in the sense of not being measured) variables, ithas been suggested that this makes analysis using traditional statistical methods meaningless. This black box approach, in a youth justice context, means that the transferability of good 
practice is not always possible since the reason why some interventions work can be context specific. Hence why it is so important to shed lighton the mechanisms involved.

This thesis has been concerned with the utility of Bayesian approaches for criminology. In considering this, the framework of youth justice risk and protective factors and associated risk assessment tools has been used as a case study to demonstrate what can be achieved through use of Bayesian approaches using a comparativelysmall administrative dataset. As demonstrated in Chapters Four to Seven it has been possible to enhance knowledge and understanding about the dynamic nature of risk and how this differs depending upon the circumstances and characteristics of the young person. Although it was necessary to make compromises due to the size of the dataset, the findings presented in Chapters Four to Seven provide the building blocks for potentially advancing this work whilst also acknowledging common problems associated with working with administrative data.

Having discussed the implications for youth justice in the previous section, the emphasis in the remainder of this chapter is upon the wider benefits that could be realised if the discipline were to embrace the adoption of Bayesian approaches. In doing this is recognised that as yet the merits of Bayesian approaches have yet to be realised by many social scientists. However, in the context of the ASA's statement advocating that other approaches should be entertained as an alternative to NHST and the advent of the digital turn in criminology, it is my belief that the time is ripe for paradigm shift.

Notably the advent of digital criminologyand 'Big Data' affords significantopportunities for criminology especially given the amount of routine data captured at different points in the criminal justice system. This has been acknowledged through the creation of the ADRN and initiatives such as the ESRC's Secondary Data Analysis Initiative. Yet this also comes at a time when there are concerns about the mathematics and quantitative skills of future generations (Smith, 2017). Thus, in discussing the opportunities, it is also necessaryto recognise that there will be philosophical and pedagogical barriers to overcome before the discipline can truly benefit from adopting novel statistical techniques such as Bayesian. Hence the key theme within the next section is addressing the third research objective: What methodological challenges will need to be overcome?

\subsection{Is it Time for a Change?}

\section{Out with the Old...}

In outlining the rationale for using Bayesian approaches in criminology in Chapter One, a key motivation is the ongoing debate about the appropriateness of using NHST and all its vestiges, and concerns that they are often used to support lower-qualityresearch. Such is the strength of this debate that the ASA published a statement on $p$-values and statistical significance in which they advocated that alternatives such as Bayesian approaches should be entertained whilst acknowledging that these come with their own conceptual problems (Wasserstein and Lazar, 2016). 
The position taken by this research is that the continued use of NHST is an obstacle to creative thinking and innovation within criminology. Associated with this is the movement towards post-positivist approaches in the discipline. Whilst both classical and positivist traditions have played a role in the development of theoretical approaches and the adoption of scientific principles within the discipline, in order for it to continue to evolve, it is necessary for criminologists to rise to the 'challenge to Import, Introspect and Innovate in order to better answer the questions of interest to the field' (Bushway and Weisburd, 2006: 1). The eclectic origins of the discipline mean that criminologists have a history of drawing upon 'sophisticated and cutting-edge approaches from other fields' and have 'given significant attention to the ways such approaches must be adapted to fit criminological problems' (Bushway and Weisburd, 2006:1). The adoption of Bayesian approaches therefore would representa continuation of this tradition, adapting techniques which have been successfully used in other disciplines to advance knowledge and understanding around the aetiology of offending behaviour and informing what works in terms of society responses.

Along with many of the social science disciplines, as criminologyhas continued to mature as a discipline, it has continued to be innovative, taking advantage for example of data visualization techniques including geospatial applications to consider crimetrends (Chaineyand Radcliffe, 2005; Chaineyand Thompson, 2008) whilst the UK's Ministry of Justice has made inroads into data linkage to explore re-offending as part of its Justice Lab initiative (Ministry of Justice, 2014a). However, the extent to which the discipline can claim to be introspective with regard to the appropriate use of different techniques could be called into question since ithas yet to shake off the shackles of NHST. Criminologyis not alone in this, but as other social science disciplines start to warm to the idea of using Bayesian approaches, then I believe that it cannot afford to be left behind. This is particularly true if we wish to (1) produce quantitatively informed research to inform policy and practice, and (2) make optimum use of the growing amount of administrative data that is collected at different stages of the criminal justice system. It is therefore vital that the discipline continue to be both innovative and introspective in our analytical approaches, importing new and novel ideas where applicable from others.

\section{In with the (not so) New}

The analytical techniques utilised in Chapters Four to Seven should be familiar to most quantitative researchers and include t-tests; contingencytables to compare proportions; Pearson's correlations and logistical regression. However, these have been conducted in under a Bayesian framework. As such Bayes Factors rather than $p$-values have been used to interpret the significance of the various tests. Bayes Factors have the advantage that they can be interpreted directly and provide a measure of the strength of the evidence of one theory verses another. In using these rather than $p$-values, the potential source of confusion associated with the interpretation of hypothesis testing conducted under the Frequentist framework can avoided. Whilst not used here, equivalent tests for many of the common 
tools in the analytical toolboxalso exist with further tests also being developed. Notablydevelopment of the open source software JASP (JASP Team, 2017b) 'provides a straightforward means of performing Bayesian analysis using a graphical "point and click" environment that will be familiar to researchers conversant with other graphical statistical packages, such as SPSS' (Quintana and Williams, Preprint 1). As such it opens up a toolbox which had previously been inaccessible for many criminologists and social researchers. Since it offers both Frequentistand Bayesian analysis methods, JASP supports the conversion process for those whose statistical schooling has been grounded in classical approaches. Not only is this useful as a teaching aid, but when both sets of results point in the same direction, this bolsters one's confidence in the conclusions.

A similar approach was adopted with respect to the packages used to perform the hierarchical modelling which made up the majority of the analysis presented in Chapters Four to Seven. The functionality to undertake this is not available in JASP - see T able 8.2 for a list of functionality. However, it was possible to identify packages which could be used in $\mathrm{R}$ which had a similar syntax so that both Frequentistand Bayesian versions of the models could be specified. As can be seen from the Technical Annex, this highlighted the limitations of the Frequentist versions of the models, particularlyonce the complexity was extended beyond the basic dynamic model. Despite the limitations arising from the size of the dataset, it was possible to achieve more with less when conducting the analysis under the Bayesian framework using the MCMCgImm package (Hadfield, 2017).

Table 8.2: JASP Functionality

\begin{tabular}{|lcc|}
\hline Analysis & Frequentist & Bayesian \\
ANOVA & $\checkmark$ & $\checkmark$ \\
ANCOVA & $\checkmark$ & $\checkmark$ \\
Binomial Test & $\checkmark$ & $\checkmark$ \\
Contingency Tables (incl. Chi-Squared Test) & $\checkmark$ & $\checkmark$ \\
Correlation: Pearson, Spearman, Kendall & $\checkmark$ & - \\
Exploratory Factor Analysis (EFA) & $\checkmark$ & $\checkmark$ \\
Linear Regression & $\checkmark$ & - \\
Log-Linear Regression & $\checkmark$ & - \\
Logistic Regression & $\checkmark$ & $\checkmark$ \\
Principal Component Analysis (PCA) & $\checkmark$ & - \\
Repeated Measures ANOVA & $\checkmark$ & $\checkmark$ \\
Reliability Analyses: $\alpha$, Yō, and $\omega$ & $\checkmark$ & $\checkmark$ \\
Structural Equation Modeling (SEM) & $\checkmark$ & \\
Summary Stats & - & \\
T-Tests: Independent, Paired, One-Sample & $\checkmark$ & \\
\hline Note: Correct as at January 2018 (JASP Team, 2017b) & & \\
Big Data, Big Opportunities? & &
\end{tabular}

Efficiency, effectiveness, accountabilityand fairness have been at the heart of successive governments strategies for the criminal justice system in England and Wales (HM Government, 2007; Ministry of Justice, 2014b) with evidence suggesting that the criminal justice system can prevent crime through four 
principal mechanisms - deterrence, legitimacy, incapacitation and rehabilitation. Under the Transforming the Criminal Justice System agenda, there has been a particular emphasis upon digitalising the criminal justice system with reforms being mooted which will facilitate the seamless transfer of information from police to prosecution through to defence and the courts. Whilstmuch of this relates to speeding up the courtprocess, there are also plans to equip police officers with the tools they need to start capturing evidence digitallyat the scene of a crime, taking statements and uploading digital case information. Such steps are intended to reduce costs and delays. However, they will also mean that the amount of information collected by the different facets of the criminal justice system are set to grow exponentially. Whilst not all the data collected will be appropriate for applying Bayesian approaches and there will be a greater emphasis upon reducing duplication, it could lead to greater consistencyin data collection methods across differentag encies. Hence, I believe that the potential that this element of the digital turn affords to researchers interested in the aetiology of crime and the formulation of appropriate responses is significant.

Much has been made of the potential of Big Data and machine learning to the extent that these are seen almost as being a panacea. However, in the context of understanding offending behaviour and identifying appropriate and effective responses to reduce re-offending, then accountability and legitimacy should be at the fore. In particulargiven the concerns about the over-representation of minoritygroups within the criminal justice system e.g. the BAME population and looked after children/ care leavers, if historic information is used to formulate interventions, then there is a risk that the practices which contributed to these biases will be amplified. This has already occurred with some of the machine learning applications intended to predict parole violations and inform predictive policing in the USA notably, as highlighted in Chapter One, PredPol has generated significantmedia coverage on both sides of the Atlantic. Such problems can undermine the public's confidence in the justice system. I therefore believe that until these issues can be resolved, it is actually small sample research which affords the greatest potential for reducing disparities and identifying appropriate responses to some of the most vulnerable in the criminal justice system.

Having access to administrative data, notjustfrom the criminal justice system, butalso potentiallyhealth, education, social services and in relation to em ploymentadditionallywill assist in construct validity since it will negate the need to use proxy measures, particularly with respect to offending. By facilitating criminologists in their endeavours to operationalise key concepts of interest, this will not only enable more robust analysis to be undertaken, but it will also support the investigation of new lines of enquiry. Sullivan and McGloin (2014) caution that:

'The reliance on existing data containing onlya subset of measures on key constructs generally leads to some compromises in specifying models that fully capture the theory of interest, which can in turn color the scope of work and conclusions reached in entire bodies of literature around a theory.' 
Looking forward, they advocate that in developing measures, it is important to start small, pilot and generalise. It is therefore necessary to acknowledge the contribution that data linkage could make to small sample research. The data matching techniques employed particularlylend themselves to small sample research, where there is the opportunity to investigate in more depth individual cases and supplement with the use of triangulated measures. The flexibility of conducting modelling under a Bayesian framework is that as new information comes to light, it can be systematically added to the model with priors being revised accordingly. Notably Bayes Factors can be used to compare the strength of evidence for and against the use of new or alternative constructs developed to measure potential risk and protective factors, with administrative data also assisting in determining temporal precedence.

Used properly, Bayesian reasoning has 'the potential to improve dramatically the efficiency, transparency, and fairness of the criminal justice system and the accuracy of verdicts, by enabling the relevance of evidence to be meaningfully evaluated and communicated' (Fenton and Neil, 2013: 407). Notably in the legal context, Bayesian reasoning can be used help in formulating accurate and informative opinions and it is this experience which I believe will contribute to enhancing the acceptability of Bayesian approaches in the area of risk assessmentand formulating appropriate interventions. Key to this will be demonstrating the value of such novel approaches to facilitate the rigorous assessment of interventions. In this respect there is a lot to be gained from the path being taken by prevention science and public administration researchers in their employment of Bayesian .approaches.

\section{Lessons from Public Administration Research}

The nature of the data utilised within criminologyincludes collections arising from the use of large-scale survey methods to capture snapshots of criminal activity and the victim experience of crime; the results of experiments and evaluations, and increasinglythe systematic collation of data around criminal justice processes / outcomes. Criminology's growing statistical evidence base is particularlyamenable to the application of Bayesian approaches since administrative datasets often suffer from issues relating to collinearity - this was an issue within the dataset used in the case study, with the two continuous variables for age being highlycorrelated forFTEs. A further advantage of Bayesian approaches is that they can be applied to situations where an event can not necessarily be repeated under identical conditions and where the alternatives to the event cannot be reduced to a finite list of equally likely outcomes (as in the objective approach). This is particularly useful in criminology where we typically get a dataset that represents a fixed, unique look at the phenomenon of interest since the data is situational in time and circumstance and hence why theycan never be replicated - as with data collected from risk assessments used to inform decision making at different stages of the criminal justice system. 
In addition to learning from medicine, criminologists can gain a lot from the experiences of public administrators particularlygiven the advent of Big Data and the digital turn in criminology. Whilst public administration researchers have been slow to embrace Bayesian approaches (Gill and Witko, 2013), there are common methodological challenges for those working with quantitative data. In both disciplines, Bayesian approaches offer a workable alternative to those who have reached the limits of what can be done using Frequentist techniques; whose data is not appropriate for their application or where the problems are too complex for classical methods. As evidenced by the case study, Bayesian approaches are particularly applicable where data can be modelled as a time-series and/or using multilevel models.

In 2000, Gill and Meier set out a manifesto for methodological change in public administration research which called for investment in methods having relied heavily upon related disciplines for its methodological tools: tools which when faced with the demands made by public administrators make them less promising. As I believe is the case in criminology, Gill and Meier express concern that the implications of making a mistake can be more significant because of the way in which the evidence generated by public administrators can influence policy:

'If a political scientist makes a major error in his or her study of the 1992 election, it matters little. Clinton still wins. If a public administration scholar commits a major error in analyzing an education program, it can have major implications simply because itcould influence public policy.'

(Gill and Meier, 2000:158)

Gorard goes further in his arguments as to why social scientists need to stop clinging on to the NHST approach to analyse their carefullycollected evidence, emphasising that should they continue to do so they not only risk devaluing their efforts, but also risk damaging people's lives:

'The confused use of significance testing has practical and damaging consequences for people's lives. Ending the use of significancetesting is a pressing ethical issue for research. Anyone knowing the problems, as described in over one hundred years, who continues to teach, use of publish significance tests is acting unethically, and knowingly risking the damage that ensues'.

(Gorard, 2016:1)

Some of the methodological advances that Gill and Meier promote in their manifesto for public administrators alongside the use of Bayesian approaches, have been realised in criminology including the creation of data archives which not only ensure that key data sets are not lost, but also that researchers can gain access to core datasets for training and research purposes. The existence of data archives also facilitates time series analysis - a technique which is well suited to before and after programme evaluation designs, butin 2000 was being under-utilised bypublic administrators. Certainly, in the UK context the work of the UK Data Service has been instrumental in reducing the barriers to accessing keysurveys such as the Crime Survey for England and Wales; the Scottish Crime and Justice 
Survey; the Edinburgh Study; the Cambridge Study in DelinquentDevelopment; and the Offending, Crime and Justice Survey (UK Data Service, 2018).

The ADRN has secured access to data to enable for example, criminal careers and the crime drop in Scotland to be investigated; an examination of the links between homelessness and recidivism; finding work after leaving prison; and assessing the feasibility of using administrative data to examinerisk factors for domestic violence and child sexual exploitation (Administrative Data Research Network, 2017). Whilst these projects are all at different stages, they illustrate ways in which, particularly linked administrative data is being used to understand and tackle some of the contemporary major social challenges. The approaches adopted represent cutting edge methodological developments pertinent to both criminologyand public administration researchers.

As indicated, linking the youth offending data to other sources such as health, education and social services data would enable a more detailed model of the role of risk and protective factors and their relationship with offending behaviour to be developed. Unfortunately, this was not possible within the scope of this thesis and would have added to the already protracted process of securing access to the data. However, having this data would potentiallyadd to understandings for example around substance misuse and treatment; mental health; learning disabilities and other developmental problems; problems in the home environmentetc. A key feature of Bayesian approaches is that models can be updated as and when new information becomes available. As demonstrated through the approach adopted in relation to the case study, 'Bayesian methods support sequential learning, allow for finding predictive distributions of future results and enable borrowing strength across studies' (Berry, 2005: 296). In this way, they lend themselves not only to situations such as dynamic models of risk where there is a need to update the assessmentas more is learnt about the individual, butalso where there is scope to continue to add new variables to reflect changes in circumstances. Berry also highlights that 'the Bayesian paradigm allows for using historical information and results of other trials, whether they involve the same drug, similardrugs or possibly the same drug but with different patient populations.' The equivalent to this in a criminological context could relate using information from the evaluation of an intervention to reduce recidivism, to explore the potential for transferability to other localities or groups of offenders.

\section{Rigorous Designs: Small Sample Research}

Whilst the ability to harness the benefits of Big Data has had a revolutionary impacton the both the types of research questions that can be addressed and the nature of analysis that can be undertaken, as Srinivasan et al. (2015) emphasises in the context of public health, small sample research is also essential if progress is to be made in addressing inequalityand ensure that it is not just majoritygroups which benefitfrom interventions. Concerns around statistical power have already been alluded to with the perception being that rigorous research requires large samples. Therefore, argue Etz and Arroyo (2015) if the importance of small sample research is to be recognised, then there will need to be 'an 
expansion of what constitutes rigor in analysis and design strategies ... Advances with also require making room for the adoption of innovative design and statistical analysis approaches' (2015: 1033). This includes taking steps to ensure that alternatives to RCTs are accepted into the repertoire of available design and assessment tools by those funding and approving research in prevention science. Athough a number of potential solutions are put forward within the special section that they are commentating on Etz and Arroyo assert that:

'It would be a failure of science and the imagination if newly discovered or rediscovered (i.e. Bayesian) strategies are not employed to facilitate rigorous assessment of interventions in small samples. It is imperative that the tools of science do not limitour ability to address pressing public health questions. New app roaches can be used to address contemporary research questions, including providing solutions to the undue burden of disease that can and often does occur in small populations. It must be the pressing nature of the questions, not the limitations of our methods, that determines what science is undertaken.'

(Etz and Arroyo, 2015: 1035)

Relating the observations from prevention science, to criminology and more specifically to risk assessmentand responses to offending behaviour, there is mounting evidence thatcertain minorityand vulnerable groups are over-represented in the criminal justice system, particularly within the secure estate (see for example Hughes et al., 2012; Prison Reform Trust, 2016; Lammy, 2017; Youth Justice Board and Ministry of Justice, 2018). These representboth a challenge to practitioners in terms of the often complex lives that many of these individuals led, but also in research terms with investigators limited by the small numbers involved and the heterogeneity of the subgroups - all too often, due to the small numbers in the general population that the group represents, when these groups are considered in the context of the offender population, they are aggregated. Common examples include components of the BAME population (typically using the headline ethnic groups although this will depend upon the geographical coverage of the analysis) and the grouping together of all 'looked after children' without distinguishing between the types of setting that the child is in or the reason that they have entered care. Within these groups there are further subgroups of particular intereste.g. on the basis of their age and/or gender, their offending history or the nature of their primary offence / disposal. When the data is 'sliced and diced' to consider some these permutations the subpopulations can start to become quite small even in the national data.

From a policyperspective, if we wish to understand why it is that these groups are over-represented in the youth justice system, then undertaking analysis which identifies whether there are differences in terms of risk and protective factors within these subgroups is key. The currentanalytical strategies which assume homogeneity within aggregated groups tend to mask the nuances which existand hence impact upon the generalisability of findings. To this end, within- rather than between- group designs are advocated as a potential option when undertaking small sample research. The advantage here being 
that within-group designs use the sample as its own control, reducing by half the sample size required for accurate sample comparisons. In the context of longitudinal designs (as employed within this research) it is recognised that within-subject designs require more data than between-subjectdesigns. This need for more data emerged as an issue within the hierarchical modelling conducted here when considering gender, with females typicallyhaving fewer measurement points than males.

Other instances where small subpopulations represent a challenge in the context of the youth justice system can be identified on the basis of the type of offences that they have committed with the low base rates for example for serious violent or sexual offences. These high-risk groups present difficulties especially in the context of sub-national research. In terms of risk assessment tools and interventions for designed for use with these groups, the low number of cases typically means that they have been adapted from adult versions. Here a more appropriate option for the small sample research maybe the use of qualitative and mixed methods. Again, there is the potential to undertake this undera Bayesian framework since expert opinion can be utilised to inform the choice of prior.

The approach used within this research explored both within- and between-individual differences using hierarchical models. These involve two sample sizes: the number of independent sampling units, $N$ (i.e. groups) and the number of secondary sampling units. Since the number of level one units vary, the average number of measurement units is denoted as $\check{n}$. As Hoyle and Gottfredson (2015) highlight in the context of longitudinal research designs where the Level 1 units representmeasurement points and Level 2 units representindividuals, for a researcher who wishes to make claims aboutdevelopmentit is essential to have enough over-time information (i.e. a relatively large n̆). Level 1 sample size is also important for reliability estimating group level measures. For this between-group processes rely upon aggregate within-group information for proxy measures of intergroup differences.

This proved to be a limitation notjust in the context of gender, but also ethnicityand when looking at the age of first conviction, particularlyin combination with other Level 2 predictors. This is reflected within models including these predictors both as main and interaction terms by the amount of uncertaintythat surrounds the estimated coefficients. Thus whilstacknowledging thatboth $N$ and $n$ are importantin the context of the type of question that was being tackled using the hierarchical models, the key limitation was found to be the fact that where using default priors within software packages, Bayesian approaches still require cases which reflect the diverse nature of the data being explored, and depending upon the level of hegemony, sufficient examples upon which to determine trends under different permutations of the various predictors being tested. Despite this, it was still possible to advance the work of Wilson and Hinks (2011) using a significantlysmaller sample size. 


\title{
8.4 Overcoming Potential Challenges
}

In 1975, the statistician and leading advocate of Bayesian statistics Dennis Lindley (1923-2013) predicted that the 21 st century would be Bayesian. In his speech at a conference on the Directions for Mathematical Statistics, he asserted, that:

\begin{abstract}
'Statistics had had its greatest successes in the fields of science where the long-run frequencyview of probabilityis appropriate .... But with the widening of the notion of probability to embrace non-repeatable situations the potential scope for statistics is enormously increased. We can now enter into fields that were previously denied to us, without any loss in the traditional ones, where propensity and exchangeability replace long-run frequencies and randomisation'.
\end{abstract}

(Lindley, 1975: 113)

Particularly in the context of risk assessment in the youth justice system, there is growing awareness that this point has been reached with calls being made for post-positivist statistical analyses which reflect the complexities of the real world. The nature of the data available to researchers is changing, in particular the amount of administrative data from across the criminal justice system and beyond is growing exponentially and increasingly reflects apparent populations rather than those which need to be randomlysampled. To consider how Bayesian approaches maybe used to respond to this, it is important to acknowledge the factors that have contributed to the acceptance of such techniques in other disciplines.

One of the principal reasons for presenting risk assessmentin the youth justice system in England and Wales as a case study to demonstrate the utility of using Bayesian approaches in criminology is the quasi-medical nature of the risk and protective model framework that underpins it the risk assessment tool, ASSET. Associated with this is the recognition not only making an assessment of the issue, but also determining an appropriate course of action. Since third-generation tools such as ASSET are characterised bytheir predictive role in informing intervention planning in addition to their classification role, there are inevitable links to the 'What Works' agenda and determining the effectiveness of interventions. These mirror the diagnostic and treatment elements of epidemiological approaches in medicine.

As highlighted in Chapter Two, Bayesian statistics have now permeated all the major areas of medical statistics including clinical trials; epidemiology; meta-analysis and evidence synthesis; spatial modelling; longitudinal modelling; survival modelling; modular genetics and decision making in respect to new technologies. Such is the extent of its acceptance within the medical profession that both the National Institute of Clinical Excellence (NICE) in the UK and the Food and Drug Administration (FDA) in the United States have been open to Bayesian submissions for more than a decade, particularlyin the area of medical devices (O'Hagan and Luce, 2003). 


\section{Having the Right Tools for the Job}

Software such as WinBUGS, STAN and JAGS has helped to make the techniques more accessible to social scientists so that they may tackle real world problems, with the JASP interface being designed to allow statistical practitioners 'to conduct statistical analyses in seconds, and without having to leam programming or risking a programming mistake' (JASP Team, 2017c). Indeed, the JASP Team are keen to stress that they are motivated to:

'... make it easier for statistical practitioners to conductBayesian analyses. We firmly believe that Bayesian statistics deserves to be applied more often and more widely than it is today, and that there is more to statistical inference than the frequentist $p$ value.'

(JASP Team, 2017c)

Alongside the development of software, those charting the adoption of Bayesian approaches have also highlighted the significant role played by the emergence of popular text books (Aldrich, 2002; Fienberg, 2006; Kruschke, 2011; McGrayne, 2011; Andrews and Baguley, 2013). Notably Harold Jeffrey's book Theory of Probability, published in 1939 has played an important part in the revival of the Bayesian view of probabilityindeed in recognition of his pioneering role, JASP stands for Jeffreys's Amazing Statistics Program (JASP Team, 2017a). Whilst the 1950s and 1960s saw the publication of a number of significant mathematical texts which had a Bayesian slant on the decision-theoretic formulation of statistical inference and/or the notion of personal probability (also known as subjective probability), more recently there have been a number of texts aimed specifically at social scientists. These include Bayesian Analysis for the Social Sciences, Jackman (2009); Bayesian Statistics, Lee (2012); Bayesian Data Analysis, Gelman et al. (2013); Bayesian Methods: A Social and Behavioral Sciences Approach, Gill (2014); Bayesian Statistics for the Social Sciences, Kaplan (2014); and Doing Bayesian Data Analysis: A Tutorial with R, JAGS and STAN, Kruschke (2015). These have predominatelybeen written by political scientists and psychologists, and as such incorporate real-world examples which itcould be argued are more accessible than those found in texts aimed at statisticians. Alongside this are more specialist texts such as those by Gelman and Hill (2007) and Banerjee et al. (2015) which focus specifically on niche areas such as regression and multilevel/hierarchical models, and spatio-temporal techniques. Manyof these have accompanying websites and in keeping with the spirit of openness and transparencywhich prevails amongstBayesians, these typically include sample code and data so that others can follow the worked examples in the texts.

Together these developments provide the building blocks for the curious criminologist who is looking for solutions for many of the complex real-world problems that exist within criminologywhich are at or are beyond the limits of Frequentistapproaches. Whilst there are some with psychology who see Bayesian as a means to address the replication crisis that their discipline is experiencing (Andrews, 2016; Dienes, 2016; Morey, 2016), what the Bayesian paradigm offers to youth justice and more widely to criminology 
is an opportunity to improve the reliability of research by allowing scientists to crosscheck work undertaken using more traditional or classical approaches without completelydismissing existing work. Given the assertions from critics such as Haines and Case, that RFR is heavy reliant upon a single data source which has since been replicated in a multitude of studies it would be pragmatic to revisit prior assumptions about risk factors and their relationship with youth offending. These should be re-examined with the benefit of the fresh perspective afforded by Bayesian approaches.

\section{Do Criminologists have the Skills to Rise to the Challenges that This Would Bring?}

When Lindley predicted that the twenty-first century would be Bayesian, he questioned where the next generation of statisticians would come from:

'The future of statistics is bright. We can expand greatly. but where are the recruits to come from? We need to attractable young people into the field: people who have the mathematical experience, and exposure to scientific ideas, to make good statisticians. My hope is that by teaching Bayesian ideas we shall succeed in this. The formal system will make it easier to teach, and will appeal to the mathematical mind. The fact that it works will bring in the interested scientist.'

(Lindley, 1975: 115)

Four decades later, concerns around whether future generations of crime scholars and practitioners have the necessary statistical skills continue. Additionally, the British Society of Criminology has expressed concern as to whether they will possess the thinking and research skills necessary to engage, as public-serving intellectuals, with politics and public policy. Chamberlain (2016) observes that undergraduate criminology students worldwide tend to possess high levels of statistical anxiety and a concurrent tendency to avoid numerical study tasks, including quantitative forms of data analysis. Initiatives such as the $\mathrm{Q}$-Step programme are intended to promote a step-change in quantitative social science training in the UK. However, they will take some time to bear fruit. Whilst criminology is not uniquely placed in terms of this skills deficit, if the generally low levels of quantitative literacy and research skills possessed by criminologystudents are not addressed through high quality, relevant and engaging skills training, then there is a risk that the legacy will be graduates who are at best proficient at blindly following instructions on what to click and then which figure to report after performing their analysis in SPSS. Ideally, I would wish to see a shift away from $p$-values and the routine teaching of Bayesian approaches which are generally considered to be more intuitive and easier to interpret. However, to achieve this we must first generate an interest in Bayesian approaches amongstlecturing staff and ensure that they are sufficiently curious and enthusiastic to be able to pass on the necessary skills to their students. Initially it may be necessary to utilise expertise from other disciplines to do this. 


\subsection{The Implications for Policy and Practice}

\section{Errors, wasted opportunities, vanishing breakthroughs, and unwarranted conclusions?}

One of the key methodological issues in the social sciences is that the way in which data is collected means that it does not always fit the criteria of being randomly selected cases and/or repeatable experiments. Yet these are the assumptions upon which NHST is based. In seeking to apply te chniques - calculating standard errors, confidence intervals and performing significance tests (both explicitlyand disguised within more complex statistical modelling) we risk 'errors, wasted opportunities, vanishing breakthroughs, and unwarranted conclusions' (Gorard, 2014b: 3). With this in mind, the widespread adoption of Bayesian approaches which are not dependent upon sample size, random sampling or repeated experiments have the potential to negate manyof the abuses which have become unfortunately become so pervasive in social science research.

Sample size has become something of an obsession as researchers seek to demonstrate that their findings are empirically sound. As budgets for research have been scaled back and the challenge of obtaining ethical approval for surveys with vulnerable people has intensified, fewer large-scale surveys are being conducted. The flip side of this is that there is increasing emphasis on using administrative data which is systematically collected for monitoring purposes to explore many of key policy issues. Such datasets frequently represent a population and whilst there may be missing cases or missing values, this is a cause of bias rather than being a consequence of random sampling variation, an issue which can be addressed through judgementbutnot through significance testing. Given the disparities that exist and the desire to identify interventions which are effective with all the population and not just the majority group, the role of small sample research is an importantone which cannotbe ignored at a time when the emphasis is on Big Data.

The inappropriateness of applying NHST to population data is one which is particularly relevant to the use of administrative datasets for social science research, necessitating the adoption of new and more novel techniques including borrowing from other disciplines so that we are well positioned to inform policy makers and practitioners alike, providing a robust evidence base upon which decisions can be made. Notablythere are lessons which can be learnt from public administration researchers, who have gra ppled with issues such as collinearity and are becoming increasingly adept at considering data which is structurally nested such as pupils within schools, patients within hospitals and different geographical units. Criminologists can also learn from the experiences of public health and prevention researchers with respect to small sample research, although itis probablymedicine thathas the most to offer to the discipline. However, it is necessaryto caveat this.

In medicine, the RFPP model has an epidemiological nature, utilising knowledge of the 'risk factors' for physical illnesses and 'protective factors' which mediate against these illnesses to formulate preventative interventions which are targeted at those considered to be 'at risk' or 'high risk' of developing the illness. 
Given that Bayesian statistics have now permeated all the major areas of medical statistics, the quasimedical nature of this model bodes well conceptually for the application of such approaches to youth justice and criminologymore generally. Particularlyin the context of analysing multiple riskfactors, use of Bayesian inference in medicine has been used to demonstrate the strength of links between exposure and disease - a key diagnostic feature. As the medical profession have demonstrated, having the correct diagnosis means that an appropriate treatment plan can be developed which is tailored to the individual and their circumstances. In principle, this is what happens in youth justice as well. The problem is however, that in youth justice, 'the science is ... not always as scientific as we would like and in fact substantial problems can exist with the method used to identify risk facts in that quantitative variables are, in fact, constructs of social phenomenon' (France, 2008:4). In presenting subjective processes as objective and scientific, there is an oversimplification of the potentially complex and dynamic aspects of children's lives, experiences, perceptions and thoughts into readily quantifiable and targetable risk 'factors'.

\section{Flexibility, Efficiency and Effectiveness}

A distinct advantage of employing Bayesian approaches is that it is possible to 'feed' the model and update it as new information is gathered. This fits with the idea of evolving assessments which follow the individual as reflected bythe ASSETPlus framework. There is also the potential to explore emerging issues through enhancing the data collection process e.g. by adding additional fields to the minimum dataset, employing data mining or linkage techniques, or having a targeted data collection. Anecdotal feedback about ASSETPlus is critical of how long it takes to complete the new assessment process therefore it would be necessary to be mindful of striking a balance between being motivated to incr ease the sophistication of the assessment process and notincreasing the burden upon the practitioner.

In the context of advancing the evidence base in youth justice, a further feature which offers potential for extending knowledge is a mechanism for triangulating data from a number of different sources. Whilst Bayesian inference with its use of prior probabilities that can be drawn from previous research offers a formal process for synthesizing data from multiple sources, Bayesian evidence synthesis allows for the inclusion of other pertinent information that would otherwise be excluded as well as the potential to extend models to accommodate morecomplex, but frequentlyoccurring, scenarios (Sutton and Abrams, 2001). Unlike in a meta-analysis, multiple treatment comparisons can be made, something which is much more in keeping with the suite of interventions which can be incorporated into a young person's action plan.

The transition from ASSET to ASSETPlus has meant from a policy perspective, that the aetiology of youth offending, and the need to understand the relationship with risk and protective factors, including their contribution to desistence has become increasingly important. The YJB has been at pains to stress that emerging evidence was one of the key drivers for change, acknowledging thatRFPP has been the 
subject of increasing debate in academic literature over the past decade (Teli, 2011;Cabey, 2013) and it is not enough to just note the occurrence of risk and protective factors in a young person's life (Baker, 2014b). However, much of the evidence base relies upon just this.

Given RFR's heavy reliance on the findings from a single data source which have since been replicated in a multitude of studies, it would be pragmatic to revisit prior assumptions about risk factors and their relationship with youth offending. This can similarly be achieved using Bayesian analysis with Flam (2014b) highlighting that some statisticians and scientists are optimistic that Bayesian methods can improve the reliability of research by allowing scientists to crosscheck work undertaken with the more traditional or "classical" approach. In this way it may be possible to refine thinking which has been based on proxy measures where linked administrative data can now provide a more accurate measurement. This is something that could prove to be invaluable given the increasing complexity of those in the formal youth justice system and emerging policy concerns around for example neurodisabilities and mental ill health.

\section{Managing an Increasingly Complex Cohort}

With an increasinglycomplex, albeitsmaller cohort in the youth offending system, the question of how policy and practice should best respond in order to control those exhibiting or at risk of offending behaviours. There are a number of emerging policyconcerns associated with participatoryapproaches and the promotion of children's rights, and increasingly practice, especially in Wales and Scotland is being informed by an appreciation of the impact of adverse childhood experiences. However, fundamentally, there remains the need for justice to be seen to be served in the most efficientand cost effective manner.

With the advent of the new peneology, the earlier discourses of clinical diagnosis and retributive judgement were replaced with 'an actuarial language of probabilistic calculations and statistical distributions applied to populations' (Feeleyand Simon, 1992). The emphasis switched from reforming the individual offender to consider aggregated groups such as "high-rate offenders" and "career criminals" with these groups, along with other categories were defined byactuarial classi fications. From there the criminal justice system has evolved to become one which is more concerned with managenial process with its goal no longer being to eliminate crime, but the identification and management of unruly groups through systemic coordination. To some extent this was achieved through the deployment of techniques in both the youth and adult systems such as statistical applications for assessing risk and predicting dangerousness, and the use of electronic monitoring systems. However, it could be argued that the successes achieved were limited to the simpler cases, and if we are to stand a chance at effectively tackling the offending behaviour of the more persistent and complex cases then we need a more nuanced and flexible approach. 
Moving forwards therefore, there needs to be increased recognition that 'positivism has long ceased to be a viable option (though the message has still not got through to some researchers)' (Robson and McCartan, 2016: 176). This is certainly the case in youth justices where the hegemony of positivist criminology has resulted in a system dependent up on correlations and statistical associations rather than a deeper understanding of the aetiology of youth offending. As Armstrong (and others) have highlighted 'risk is hidden beneath a plethora of correlations that in themselves tell us little about the socio-historical nature, meaning and significance of crime and its discourses in these times in which we are now living' (2004:113). If we are to address this then we need to the right tools for the job - an approach which deals with uncertainty and is flexible enough to enable models to be refined as more information becomes available. In this way knowledge can be advanced beyond the boundaries imposed by Frequentistapproaches and a more responsive means prediction developed.

Within-individual analysis has been largely neglected along with investigations to establish temporal ordering, hence findings are typically based on aggregation, imputation and extrapolation. However, a distinct advantage associated with the adoption of standardised, actuarial approaches to risk assessment as has been done in youth justice is that they necessitate the routine updating of case management systems. As a result, the temporal ordering of events can often be established in administrative datasets - a key requirementifwe wish to establishing causality. In this way the multiple aims of third- and fourth-generation tools such as ASSET and ASSETPlus offer a rich resource for exploring the complex relationship between a range of different factors, further offending behaviour and cruciallydesistence.

The application of Bayesian techniques to administrative data representing a population, albeitata local youth offending team level, means that risk factors can be explored in terms of their absolute rather than relative risk. This helps to address some of the concerns raised by Gottfredson and Moriarty (2006) who suggest that problems with predictive accuracy may arise as a result of the original research failing to be representative of the wider youth offending cohort - this is not the same as being representative of the population as a whole. By additionallyconsidering base rates and hence focusing on absolute risk, it becomes possible to increase the predictive accuracyfor more frequent or infrequentevents such as the more serious offences which are committed less frequentlyby young people.

Utilising Bayesian approaches supplemented byother new and emerging form s of analysis such as data linkage affords the opportunity to re-visit the assumptions of existing RFR and to unpick the complexities of the risk factor-reoffending relationship. The same techniques can also be applied to existing survey data, offering the opportunity to drill down further than has previously been permitted by Frequentist approaches. Since Bayesian approaches incorporate prior information, it is possible to use what is alreadyknown as a starting point. In this way, existing RFR can be used as the foundation for furthering knowledge about this complex and dynamic area. However, we do not need to limitourselves to RFR, 
there are a plethora of scenarios where researchers have perhaps reached the limit of what can be explored using Frequentist approaches or when it is not appropriate to apply these techniques due to the nature of the data. As suggested, a key related area to looking at the evidence base that underpins risk assessment processes is to examine 'What Works' in terms of interventions to reduce further offending. Within this thesis I have highlighted many of the qualities of Bayesian analysis which make it ideal for exploring similarlycomplex issues without dismissing existing research and for this learning to be extended to inform both the aetiology of offending more generallyand the development of appropriate societal responses.

\section{Next Steps}

Much has been made of the notion that NHST stifles creativity. However, its continued use has potential ramifications within public policy. For example, Gorard (2014b) questions whathappens if mistakes are made interpreting the findings of social research? What the costs are to the public purse when policyis developed on the basis of these findings? We therefore need to consider whether we can afford to continue to have a process of risk assessment that has been based on questionable evidence? Those involved in the development of ASSET Plus have been at pains to pointout that in developing the tool, they have sought to incorporate emerging evidence, but the extent to which this has been gathered through the questionable application of methodologies and partial analysis is unknown. This means that our knowledge of the mechanisms and processes which explain the risk factor-youth offending relationship remains incomplete. Without this understanding we risk the perpetuation of a flawed system of youth justice which fails to provide timely and appropriate support to those children and young people who come into conflictwith the law. The costs to the individuals, their families and the communities in which they live is difficult to measure, but what is certain is that the result is a system that is not fit for the twenty-first century.

It is my beliefthat if criminologyis going to be able to address of the more perplexing issues that affect today's society then it needs to continue to import, introspect and innovate. Through the adoption of Bayesian approaches, there is the potential for new and varied insights to be realised which can inform policyand practice. Whilst there are pedagogical challenges to overcome, significant progress has been made in aligned disciples which has meant that as software has been developed and applied Bayesian text books written, Bayesian techniques are now becoming more accessible to social scientists, providing them with the tools to tackle real world problems.

To date criminologists have been slow to embrace Bayesian approaches. However, for the curious criminologist, they offer a much-needed additional tool in the analytical toolbox, permitting robust evidence to be accumulated in a more efficient and transparent way - something which is particularly important at a time of austerity. With the advent of digital criminology, the rise in the 'Big Data' and increased use of data linkage techniques, the raw materials required for enable new measures to be 
operationalised and support the investigation of new lines of enquiry have become more readilyavailable to researchers. Hence, I believe that the time is now ripe to explore the potential that Bayesian approaches offer to criminology. 


\section{Appendices}

Appendix 1 - University Ethics Form

Appendix 2 - Data Sharing Agreementwith Western Bay YOT 


\section{Appendix 1 - University Ethics Form}

Application for Standard Ethical Approval

\section{PLEASE COMPLETE THE FORM USING TYPESCRIPT}

(hand-written applications will not be considered)

\begin{tabular}{|l|l|}
\hline $\begin{array}{l}\text { Principal } \\
\text { Investigator }\end{array}$ & Helen HODGES \\
\hline Date & $6^{\text {th }}$ October 2014 \\
\hline School/Department & Criminal Justice and Criminology \\
\hline E-mail address & \\
\hline $\begin{array}{l}\text { Title of Proposed } \\
\text { Research }\end{array}$ & $\begin{array}{l}\text { A Bayesian Approach to Risk Assessment and Youth Offending } \\
\text { Relationships }\end{array}$ \\
\hline $\begin{array}{l}\text { Type of } \\
\text { Researcher } \\
\text { (please tick) }\end{array}$ & Postgraduate student \\
\hline Name of supervisor & Professor Kevin Haines \\
\hline
\end{tabular}

1. Briefly describe the main aims of the research you wish to undertake, including a statement of the intended benefits of the research. Please use non-technical language wherever possible.

To determine whether a Bayesian approach can be applied to refine the risk assessment process currently being used in the context of youth offending and hence enhance our understanding of relationships which it has previously not been possible to explore using frequentist analysis.

2. Briefly describe the overall design of the project

There are two key strands to this project:

1) Theoretical Advancement - Consideration of whether a Bayesian approach* combined with regression methods would be appropriate for advancing work around risk assessment and youth offending relationship. This will include a systematic review of literature relating to where Bayes Theorem has already been used to consider issues within the criminal justice system generally and also with young people.

2) Application - Identification of known risk and protective factors within administrative data using Bayesian techniques, followed by the exploration of further potential factors. This will be an iterative process and is likely to involve data fusion and triangulation as well as the views of subject experts including practitioners.

The research will primarily utilise existing data held by the Youth Offending Service about their clients. Secondary data analysis of qualitative and quantitative material will be employed such as official statistics provided by the Home Office or Ministry of Justice concerning youth justice and youth offending, and data already collected in pertinent studies locally in Swansea.

The Bavesian Approach

Bayes' theorem is the foundation of Bayesian inference. It shows the relation between two conditional probabilities that are the reverse of each other. This theorem is named after Reverend Thomas Bayes (1702-1761), and is also referred to as Bayes' law or Bayes' rule. Bayes' theorem expresses the conditional probability, or 'posterior probability', of an event $A$ after $B$ is observed in terms of the 'prior probability' of $A$, prior probability of $B$, and the conditional probability of $B$ given $A$, denoted $B \mid A$. Bayes' theorem is valid in all common interpretations of probability. 
Bayesian inference estimates are based on developing hypothesis given the data. As a result prior knowledge or the results of a previous model can be used to inform the current model. As a result, the approach is iterative in nature.

3. Briefly describe the methods of data collection and analysis. Please describe all measures to be employed. If questionnaire or interviews are to be used, please provide the questionnaire / interview questions and schedule - if available.

The iterative nature of the approach means that it is difficult to be specific about the methodology that will be employed at this stage. However, it is recognised that having access to the case managements data held by the Youth Offending Team (YOT) about their clients in the Youth Offending Information System (YOIS) will afford the opportunity to 'test' the Bayesian approach and enable the exploration of the often complex relationships that impact on the likelihood of offending and any subsequent desistence / recidivist behaviours

\section{The Primary Dataset}

The YOIS is a case management system used by the YOT to capture information about their clients. It includes client level information such as:

- Asset* scorings along with those from associated tools where appropriate

- Socio-demographic information

- Details of their offence plus any subsequent offending, including where they have been involved in any antisocial behaviour

- Sentences and specific orders as determined by the court

- A record of all Interventions and Action plans with progress reports

- Case work and records of meetings with the client and other agencies

- The young person's views

- Multi-agency intelligence such as information from schools and social workers

- A record of education and/or training that the client has participated in

It has since been superseded by Childview. As a result, it is an archived version of the dataset that will be utilised.

* Asset is the structured assessment tool used by YOTs in England and Wales on all young offenders who come into contact with the criminal justice system. Its aim is to look at the young person's offence or offences and identify a multitude of factors or circumstances - ranging from lack of educational attainment to mental health problems - which may have contributed to their behaviour. The information gathered from Asset is used to inform court reports so that appropriate intervention programmes can be drawn up. Additionally it highlights any particular needs or difficulties the young person has, so that these can be addressed.

Since its inception in 2000, the intention has been that the information collected from Asset would help to increase knowledge about offending by particular groups of young people and young people in general. Overtime it was envisaged that it would provide detailed information about the needs and problems of different groups of young people, with aggregate data from Asset highlighting the most significant and/ or significant issues associated with offending. Locally the intelligence gathered is used to guide decisions about the partnerships and programmes that will be most relevant to young offenders in that particular area whilst at a national level it is used to informs strategic planning in order to improve the use and allocation of resources.

Plans are in place to replace Asset, with AssetPlus. AssetPlus has been designed to provide a holistic end-to-end 
assessment and intervention plan, allowing one record to follow a young person throughout their time in youth justice system. The earliest delivery date for trance 1 is currently June 2015.

Potential Additional Data Sources

As the research progresses, it is envisaged that additional data sources may be used to triangulate findings. These could potentially include:

- performance and socio-demographic data held by the local authority

- published statistics from the Home Office / Ministry of Justice or relevant Government departments

- archived survey data such as that held by the UK Data Service

- data collected in pertinent studies undertaken in Swansea

It is anticipated that there will be a reliance on published data which has been validated and conforms to the standards set out by the Office of National Statistics.

Analysis

Initially the intention is to use WinBUGS to conduct analysis as this is a specialist piece of software which has been developed specifically for Bayesian analysis. The statistical techniques are likely to evolve as the research progresses and will be determine by the nature of the data itself - it will be a combination of qualitative and quantitative data which by necessity will require different analytical approaches if they are to inform the model.

Given that Bayes' rule is used to combine prior experience (in the form of a prior probability) with observed data (in the form of a likelihood) in order to determine a posterior probability, initially the analysis will be descriptive in nature.

Techniques such as regression analysis may be required to expose the relationship between the response variable and predictor variables whilst appropriate techniques may need to be employed if it is identified that there are significant issues with missing data.

4. Location of the proposed research (i.e., Departmental labs, schools, etc.)

City and County of Swansea

5. Describe the participants: give the age range, gender, inclusion and exclusion criteria, and any particular characteristics pertinent to the research project.

NA - All data subjects will have come from a variety of socio-demographic backgrounds and will feature within the dataset because they have entered into / engaged with the criminal justice system. All clients would have been aged between 10 and 17 at the time of their engagement with the YOT, and living in Swansea.

No exclusion criteria will be applied.

6. How will the participants be selected and recruited?

NA - The data subjects will have been Swansea YOT clients between 2001 and 2012. 


\begin{tabular}{l}
\hline 7. What procedures (e.g., interviews, computer-based learning tasks, etc.) will be carried out \\
on the participants? \\
\hline NA \\
\hline
\end{tabular}

8. What potential risks to the participants do you foresee and how do you propose to ameliorate/deal with potential risks? For instance, provide contact details of counselling services and/or relevant community support organizations etc.

NA - There will be no direct contact with YOT clients. Analysis will be retrospective and it is anticipated that were issues were identified, the YOT would have taken timely and appropriate action to safeguard their client / others, and to respond to their individual needs.

However, it is recognised that other individuals (eg family members, friends, victims) may be named within the client records. In order to protect their identities, analysis will be presented in an aggregate form with reference only being made to their relationship to the client eg 'sibling', 'parent', 'friend', 'victim' - see also sections 17 and 18.

9. What potential risks to the interests of the researchers do you foresee and how will you ameliorate/deal with potential risks?

\section{Personal Safety and Wellbeing}

As there is no direct contact with clients and the research is taking the form of secondary data analysis, it is anticipated that there will be negligible risks to the researcher's personal safety. However, there is the potential given that the client records will include information about their offending behaviours that there may be details which may cause distress to the researcher. Should this scenario arise, then it will be raised with supervision so that appropriate safeguards can be put in place. As the researcher has previously worked as a police analyst, she is accustomed to viewing offender / victim records and maintaining a professional distance.

Data Security

The greatest risks are around data security as the client records will include personal and sensitive personal information about children and young people who may have additional vulnerabilities by virtue of being YOT clients. As a result, all relevant guidance relating to the secure storage and use of the data supplied will be adhered to.

It is envisaged that the file size will limit the potential to save the file on $P: /$ drive (max capacity $=2 G B$ ) therefore a working version of the database will need to be stored on an encrypted external hard drive purchased by the department specifically for this research. As outlined in section 18 , there will be two versions of the database - the first being a depersonalised version which will be used by the researcher on a day to day basis, the second being retained in its original format for reference purposes only. The later will be securely held by the supervisor on University premises. Access to the data will be limited to the researcher and supervision with advice being sought as appropriate in a timely manner from the data controller and named practitioners at the YOT.

To minimise the risks of any security breach, the dataset will be password protected on the encrypted external hard drive. All versions of the data will be appropriately disposed of at the end of the research.

10. How will you brief and debrief participants? (Please attach copy of debrief information to be given to participants)

NA 


\begin{tabular}{|c|c|c|}
\hline $\begin{array}{l}\text { 11. Will informed consent be sought from } \\
\text { participants? }\end{array}$ & $\begin{array}{l}\text { Yes (Please attach a copy of } \\
\text { the consent form) }\end{array}$ & \\
\hline & No & $\mathrm{X}$ \\
\hline If no, please explain below: & & \\
\hline $\begin{array}{l}\text { Data is being made available via Section } 115 \text { of the Crime and } \\
\text { YOTs, as local authority partnerships, to share information } \mathrm{W} \\
\text { sharing the YOIS data for research purposes can be found und } \\
\text { Controller. }\end{array}$ & $\begin{array}{l}\text { Disorder Act } 1998 \text { which provides an enab } \\
\text { nen it is in the public interest. Further } \\
\text { er the Data Protection Act } 1998 \text { as agree }\end{array}$ & $\begin{array}{l}\text { ling power for } \\
\text { exemption for } \\
\text { d by the Data }\end{array}$ \\
\hline
\end{tabular}

$\begin{aligned} & \text { 12. Will participants be informed of the } \begin{array}{l}\text { Yes (Please attach details of } \\ \text { proposed use of the data? } \\ \text { the information to be given to } \\ \text { participants) }\end{array} \\ & \\ & \text { If no please explain below: }\end{aligned}$
See section 11

13. If there are doubts about participants' abilities to give informed consent, what steps have you taken to ensure that they are willing to participate?

NA

14. If participants are under 18 years of age, please describe how you will seek informed consent. If the proposed research is to be conducted in a school, please describe how you will seek general consent from the relevant authorities and attach a copy of any written consent. NA

15. How will consent be recorded?

NA

16. Will participants be informed of the right to withdraw without Yes penalty?

If no, please detail the reasons for this:

NA - see section 11

17. How do you propose to ensure participants' confidentiality and anonymity? Please state who will have access to the data and what measures will be adopted to maintain the confidentiality of research subjects and to comply with data protection requirements.

The data is being supplied with individual identifiers and there is the potential that other individuals may be named in narrative case reports. To protect the identity of any named individuals - clients or otherwise - analytical findings will be presented in a de-personalised form. Where the named individual is not the client, references to that individual will only be made by way of relationship labels eg 'sibling', 'parent', friend'. 
The intention is to make a second copy of the database which will become the working version. As the dataset will include multiple identifiers for each client, these will be utilised to create a unique client identifier which can then be used to create a depersonalised version of the file. This is the version that will be utilised on a day to day basis by the researcher.

The original version will be retained for reference purposes only and will be securely retained by the supervisor Professor Kevin Haines in a locked cabinet on University premises.

Throughout the process the framework set out in the Data Protection Act will be adhered to along with any locally agreed information sharing protocols set out by the YOT, the University of Swansea and the ESRC.

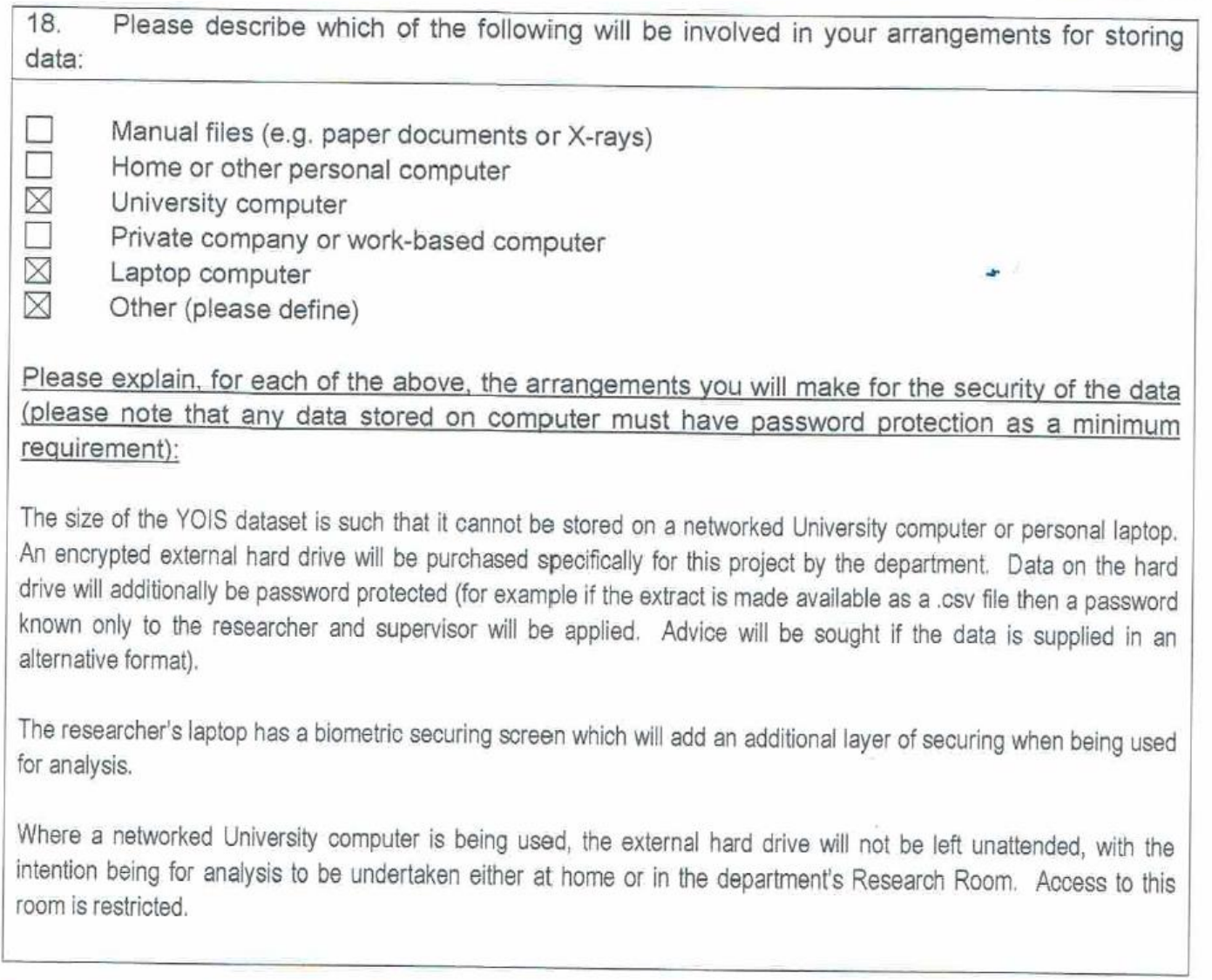

19. Does your research require the written consent of a public or private body, e.g. school, local authority or company? If so, please attach letter of consent

Permission to draw data down has been granted by Eddie Isles, Data Controller for Swansea YOS as part of the ongoing research partnership between the Criminology Department and the YOS.

20. If your proposed research is with 'vulnerable' groups (e.g., children, people with a disability etc.), please attach a copy of your Criminal Records Bureau check (if UK) or equivalent 
non-UK clearance.

NA - There will be no direct contact with YOT clients. However, the researcher has prior experience of working with sensitive personal data having been a police analyst before embarking on this research. As a result she has been subject to management vetting. This can be confirmed by contacting West Midlands Police.

A DSB check is currently being completed which is being administered by the YOS.

\section{DECLARATION:}

I am satisfied that all ethical issues have been identified and that satisfactory procedures are in place to deal with those issues in this research project. I will abide by the procedures described in this form.

Signature of Applicant:

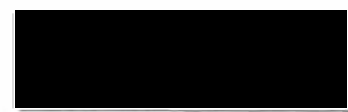

Date:

$6 / 10 / 14$

Supervisor declaration (for student research only)

I have discussed the ethics of the proposed research with the student and am satisfied that all ethical issues have been identified and that satisfactory procedures are in place to deal with those issues in this research project.

Signature of Supervisor:

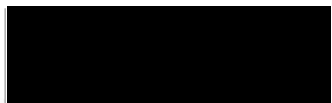

Date:

$6 / 10 / 14$

CHECKLIST OF ATTACHMENTS:

PLEASE REMEMBER TO ATTACH COPIES OF EACH OF THE FOLLOWING (WHERE RELEVANT)

*INCOMPLETE APPLICATIONS WILL NOT BE CONSIDERED*

Copy of Participant Information Sheet

Copy of Consent Form

Copy of Participant debrief

Copy of any questionnaires and/or interview schedules to be employed

Copy of written consent from local authorities or other government bodies

If your proposed research is with 'vulnerable' groups (e.g., children, people with developmental disorder), please attach a copy of your clearance letter from the Criminal Records Bureau (if UK) or equivalent non-UK clearance.

PLEASE ATTACH A COPY OF THE RESEARCH PROPOSAL TO THIS APPLICATION

**RESEARCH MAY ONLY COMMENCE ONCE ETHICAL APPROVAL HAS BEEN OBTAINED** 
Appendix 2 - Data Sharing Agreementwith Western Bay YOT

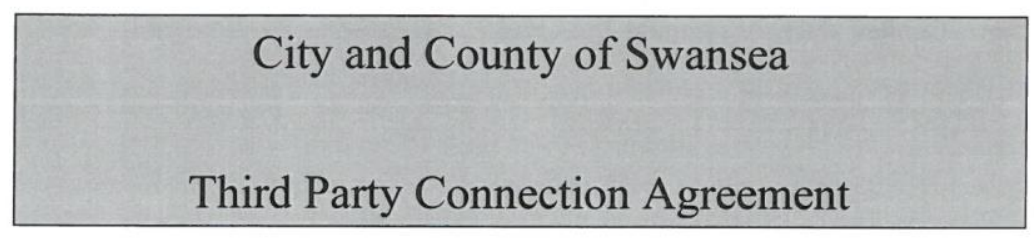

City and County of Swansea

Third Party Connection agreement

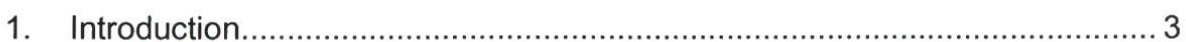

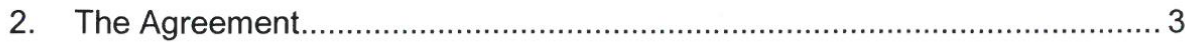

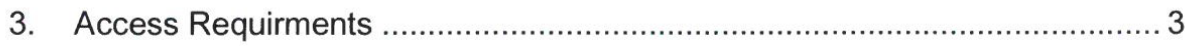

4. Purpose of Connection ........................................................................... 3

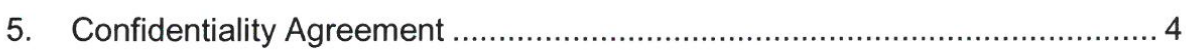


1. Introduction

1.1 This document details the terms and conditions applicable to the connection of third party access to an Authority Information System.

1.2 The agreement will allow an authorised individual to access data held on Authority systems for specific purpose(s) with specific means of access.

1.3 The system which authorised employees of Swansea University (Helen Hodges) will be allowed to access is Childview.

2. The Agreement

2.1 Details of the person who will be required to access the data held on the City and County of Swansea (CCoS) systems must be supplied to the ICT Division.

2.2 The person granted a user account will agree to abide by CCOS ICT policies and by the terms and conditions of this agreement whenever they connect to CCOS systems.

2.3 When an employee, granted a user account, leaves the position or changes post which means that access to the CCOS system is no longer required the line manager must immediately inform the IT Division of the change.

2.4 The data accessed by the non-Authority employee must not be used for any purpose not specified in this agreement and must not be supplied to any person(s) or company who are not party to this agreement.

2.5 The data will remain the property of CCOS.

3. Access Requirements

3.1 Access will be enabled using authentication controlled by CCOS.

3.2 The person given a user account will not supply details of this account i.e. user id or password, etc or allow the device used to enable the authentication to be used by any non-authorised individual.

3.3 The user will be responsible for all access from the account allocated.

4. Purpose of Connection

4.1 The connection to the database will be to allow the authorised user to interrogate data relating to Youth Offending services. 


\section{5. $\quad$ Confidentiality Agreement}

5.1 All data accessed will be treated as confidential and will not be supplied to unauthorised parties or made viewable to unauthorised persons without the approval of CCOS.

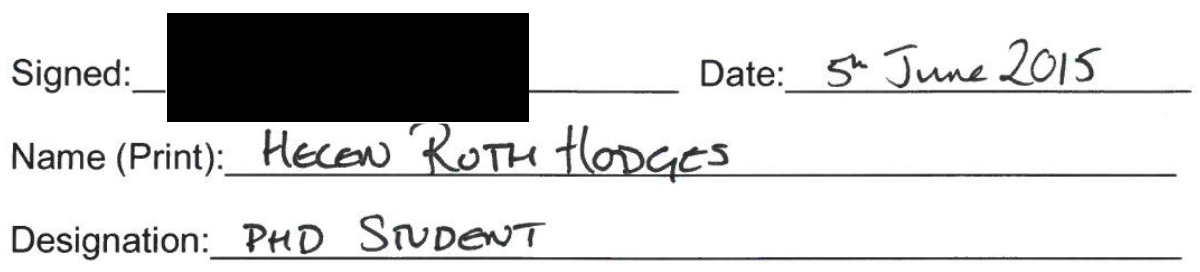

Authorised by:

Job Title: 


\section{Agreement Between Bridgend County Borough Council and SWANSEA UNIVERSITY}

This agreement forms a Memorandum of Understanding between Bridgend County Borough Council (BCBC) and Helen Hodges (Swansea University) with respect to the methods and procedures for employees of Swansea University to access services, machines and networks detailed in Schedule 1 below for BCBC Network access.

All information contained in this agreement and the appended schedules is confidential between BCBC and Swansea University. Unauthorised disclosure except as required under UK or local law will be treated as a breach of the agreement.

For the purposes of this agreement, the responsible person for the $\mathrm{BCBC}$ customer is Caroline Dyer, the responsible person for the BCBC Information and Communications Technology Department (ICT) is Martin Morgans.

Variations on this agreement, including the schedules are permitted only by signed agreement of the responsible persons above (fax and electronic signatures will be deemed acceptable). Following each change, Martin Morgans will deliver new electronic copies of the agreement to the other responsible persons and file the completed agreement with the ICT Service Desk.

This agreement will remain in force until:

1. The primary contract between $B C B C$ and Swansea University expires. For the purposes of this document, the primary contract is the Student Placement.

2. Either party requests termination

3. A breach of the agreement occurs that either party regards as serious enough to warrant termination

Confidentiality and non-disclosure of data acquired during the agreement must be respected even after the agreement terminates.

No part of this agreement restricts either party from undertaking other remedies in the event of a breach of the agreement.

Access to the BCBC network will be via Network Access. No other method of access to the network may be used, and installation of alternate access software/hardware outside this agreement will be treated as a violation of the agreement.

BCBC will supply appropriate tokens for access (e.g. usernames and passwords). Connection software and hardware will be supplied by BCBC as required. Swansea University must use the software provided in a legal manner under the applicable laws and abide by any license agreements applying to it. Any software or hardware supplied remains the property of BCBC and must be returned or disposed as appropriate at the end of the agreement.

Access will only be permitted to services, machines and networks detailed in Schedule 1 below. Any attempt to access other services, machines or networks agreement will be treated as a breach of the agreement.

Access will only be granted to the named individuals detailed in Schedule 2 below. All persons on the list must be employees of Swansea University, and, where appropriate, may be required to be vetted under access procedures as required under UK law or professional practice.

All named individuals on the list will have individual passwords, which are for use by that individual only. It is the responsibility of Swansea University to ensure that the list is kept up to date, and in particular that $\mathrm{BCBC}$ are informed of contract terminations so that individual accounts can be removed.

Swansea University employees must have received suitable security and awareness training within their own organisation for the role they will be performing within $\mathrm{BCBC}$, and to be aware

$\begin{array}{ll}\text { Author: Steve Durbin } & \text { Last } \\ \text { Page: } 1 \text { of } 4 & \\ \text { Filename: C:IUsersI807913.TAWE.035VAppDatalLocallMicrosoftIWindowsITemporary Internet }\end{array}$

FilesIContent.IE5IEUH7X34Y IExternal Local Access Agreement - Helen Hodges.doc 


\section{Agreement Between Bridgend County Borough Council And SWANSEA UNIVERSITY}

of the protection requirements imposed on $\mathrm{BCBC}$ by legislative and compliance requirements, in particular the Government Secure Intranet, the Payment Card Industry Data Security

Standard, the Computer Misuse Act, the Regulation of Investigatory Power Act and the Data Protection Act.

Swansea University employees are required to abide by the ICT code of practice for BCBC employees when using BCBC equipment and accounts. This code of practice is supplied separately by $\mathrm{BCBC}$ and must be read and accepted by every Swansea University employee in schedule 2 below.

Swansea University undertakes to discipline their employees for breach of the BCBC ICT code of practice with appropriate severity. $\mathrm{BCBC}$ reserves the right to withdraw access from any Swansea University employee who has committed or is under investigation for a breach of the code. 
Agreement Between Bridgend County Borough Council and SWANSEA UNIVERSITY

\section{Schedule 1 - Accessible Services, Machines and Networks}

[Details of each service, machine and network to be accessed. Example below]

\begin{tabular}{|l|l|}
\hline Type of Access & Identifiers \\
\hline BCBC Domain Controllers & Domain Controllers \\
\hline & \\
\hline & \\
\hline
\end{tabular}


Agreement Between Bridgend County Borough Council and

SWANSEA UNIVERSITY

\section{Schedule 2 - Named Individuals Permitted to Use} Services

[Name, job title and signature for each user. Passwords will be issused over the telephone and should be at least 8 characters long and not be a word in any language. It is suggested for clarity that phonetic alphabet spellings are given for each one to reduce confusion. All passwords in a schedule must be unique.

Helen Hodges

Signature

BCBC Line Manager

Date 


\section{References}

Abulafia J, Bukshizki M and Cohen D. (2015) Risk Assessments of Female Sex Offenders: Actuarial Tools Versus Clinical Criteria. The 23rd European Congress of PsychiatryVienna, Austria, 2831 March. ScienceDirect, doi:10.1016/S0924-9338(15)31364-X.

Adler JR, Edwards SK, ScallyM, et al. (2016) What Works in Managing Young People Who Offend? A Summary of the International Evidence. Available at: https://www.gov.uk/government/publications/what-works-in-managing-young-people-whooffend. (Accessed 31/1/18).

Administrative Data Research Network. (2017) Featured Research and Case Studies. Available at: https://adrn.ac.uk/research-impact/research/(Accessed 31/1/18).

Administrative Data Taskforce. (2012) The UK Administrative Research Network: Improving Access for Research and Policy. Available at: http://www.esrc.ac.uk/research/our-research/administrativedata-research-network/administrative-data-taskforce-adt. (Accessed 31/1/18).

Aldrich J. (2002) How Likelihood and Identification WentBayesian. International Statistical Review70(1): 79-98.

American Psychological Association. (2010) Publication Manual of the American Psychological Association (6th Edition). Washington: APA.

Andrews DA and Bonta J. (2007) Risk-Need-Responsivity Model for Offender Assessment and Rehabilitation Available at: https://www.publicsafety.gc.ca/cnt/rsrcs/pblctns/rsk-ndrspnsvty/index-en.aspx. (Accessed 31/1/18).

Andrews DA and Bonta J. (2010) The Psychology of Criminal Conduct. Abingdon: Anderson Publishing. Andrews DA, Bonta J and Hoge RD. (1990) Classification for Effective Rehabilitation:Rediscovering Psychology. Criminal Justice and Behavior 17(1): 19-52.

Andrews DA, Bonta J and Wormith JS. (2006) The Recent Past and near Future of Risk and/or Need Assessment. Crime \& Delinquency 52(1):7-27.

Andrews M. (2016) What Role Can Bayesian Methods Play in Resolving the Replication Crisis? ESRC Research Methods Festival, University of Bath, 7th July. Available at: http://www.ncrm.ac.uk/RMF2016/programme/session.php?id=R6. (Accessed 31/1/18).

Andrews M and Baguley T. (2013) Prior Approval: The Growth of Bayesian Methods in Psychology. British Journal of Mathematical and Statistical Psychology 66(1):1-7.

Anwar S and Loughran TA. (2011) Testing a Bayesian Learning Theory of Deterrence among Serious Juvenile Offenders. Criminology 49(3):667-698.

Armstrong D. (2004) A Risky Business? Research, Policy, Governmentalityand Youth Offending. Youth Justice 4(2): 100-116.

Armstrong D. (2006) Becoming Criminal: The Cultural Politics of Risk. International Journal of Inclusive Education 10(2-3): 265-278.

Ashby D. (2006) Bayesian Statistics in Medicine:A25 Year Review. Statistics in Medicine 25(21):35893631.

Assink M, van der Put CE and Stams G. (2016) The Development and Validation of an Actuarial Risk Assessment Tool for the Prediction of First-Time Offending. International Journal of Offender Therapy and Comparative Criminology60(7): 847-864.

Audit Commission. (1998) Misspent Youth '98: The Challenge for Youth Justice. London: Audit Commission.

Audit Commission. (2004) Youth Justice 2004: A Review of the Reformed Youth Justice System. London:Audit Commission.

Baglivio MT and Jackowski K. (2013) Examining the Validity of a Juvenile Offending Risk Assessment Instrumentacross Gender and Race/Ethnicity. Youth Violence and Juvenile Justice 11(1): 2643.

Baker K. (2004) Is ASSET Really an Asset? Assessment of Youth Offenders in Practice. In: Burnett R and Roberts C (eds) What Works in Probation and Youth Justice: Developing Evidence-Based Practice. Cullompton, Devon: Willan Publishing, 70-87. 
Baker K. (2005) Assessment in Youth Justice: Professional Discretion and the Use of ASSET. Youth Justice 5(2): 106-122.

Baker K. (2014) ASSETPLUS Rationale. London: Youth Justice Board.

Baker K, Jones S, Merrington S, et al. (2005) Further Development of ASSET. London: Youth Justice Board.

Baker K, Jones S, Roberts C, et al. (2003) The Evaluation of the Validity and Reliability of the Youth Justice Board's Assessment for Young Offenders. Oxford: Probation Studies Unit, Centre for Criminological Research, University of Oxford.

Baker M. (2016) 1,500 Scientists Lift the Lid on Reproducibility. Nature 533:452-454.

Banerjee S, Carlin BP and Gelfand AE. (2015) Hierarchical Modelling and Analysis for Spatial Data (2nd Edition). Boca Raton, FL: Chapman and Hall/CRC.

Barnes J, TenEyck M and Pratt TC. (2017) How Powerful Is the Evidence in Criminology? On Whether We Should Feara Coming Crisis of Confidence. Open Science Framework.

Bateman T . (2011) Punishing Poverty: The 'Scaled Approach' and Youth Justice Practice. The Howard Journal of Criminal Justice 50(2): 171-183.

Bateman T and Hazel N. (2015) Custody to Community: How Young People Cope with Release. London: Beyond Youth Custody.

Bateman T and Pitts J. (2005) The RHP Companion to Youth Justice. Lyme Regis: Russell House Publishing Ltd.

Bates D, MächlerM, Bolker B, et al. (2015) Fitting Linear Mixed-Effects Models Using Ime4. Journal of Statistical Software 67(1): 48.

BerelowitzS and HibbertP. (2011) 'I Think I Must Have Been Born Bad' Emotional Wellbeing and Mental Health of Children and Young People in the Youth Justice System. London: Office of the Children's Commissioner.

Berk RA, Campbell A, Klap R, et al. (1992a)A Bayesian Analysis of the Colorado Springs Spouse Abuse Experiment. Journal of Criminal Law \& Criminology 83(1): 170-200.

Berk RA, Campbell A, Klap R, et al. (1992b) The Deterrent Effect of Arrest in Incidents of Domestic Violence - a Bayesian-Analysis of 4 Field Experiments. American Sociological Review 57(5): 698-708.

Berk RA, Western B and Weiss RE. (1995) Statistical Inference for Apparent Populations. Sociological Methodology 25:421-458.

Berry D. (2005) Introduction to Bayesian Methods III: Use and Interpretation of Bayesian Tools in Design and Analysis. Clinical Trials 2: 295-300.

Berry D. (2006) Bayesian Statistics. Medical Decision Making 26: 429-430.

Blades R, Hart D, Lea J, et al. (2011) Care-a Stepping Stone to Custody: The Views of Children in Care on the Links between Care, Offending and Custody. Available at: http://www.prisonreformtrust.org.uk/Portals/0/Documents/careasteppingstonetocustody.pdf. (Accessed 31/1/18).

BlairP and Rossmo K. (2010) Evidence in Context: Bayes' Theorm and Investigations. Police Quarterly 13(2): 123-135.

Blattenberger G, Fowles R and KrantzJ. (2010) Bayesian Models to Predict the Return to Prison. Section on Bayesian Statistical Science-JSM: 5216-5229.

Borum R. (1996) Improving the Clinical Practice of Violence Risk Assessment: Technology, Guidelines, and Training. American psychologist 51 (9): 945-956.

Bottoms A. (2008) The Relationship between Theory and Empirical Observations in Criminology. In: King $\mathrm{R}$ and Wincup E (eds) Doing Research on Crime and Justice. (2nd Edition). Oxford: Oxford University Press, 75-116.

Bradford B, MurphyK and Jackson J. (2014) Officers as Mirrors: Policing, Procedural Justice and the (Re)Production of Social Identity. The British Journal of Criminology 54(4):527-550.

Brown S. (2005) Understanding Youth Crime:Listening to Youth? Buckingham: Open Univeristy Press.

Brownlee I. (1998) New Labour - New Penology? Punitive Rhetoric and the Limits of Managerialism in Criminal Justice Policy. Journal of Law and Society 25(3): 313-335.

Buchanan A and Grounds A. (2011) Forensic Psychiatry and Public Protection. The British Journal of Psychiatry 198(6): 420-423. 
Buranyi S. (2017) Rise of the RacistRobots - How Al Is Learning All Our Worst Impulses. The Guardian, 8th August 2017. Available at:

$\mathrm{https://www.theguardian.com/inequality/2017/aug/08/rise-of-the-racist-robots-how-ai-is-}$ learning-all-our-worst-impulses. (Accessed 31/1/18).

Bushway S and Weisburd D. (2006) Acknowledging the Centrality of Quantitative Criminology in Criminology and Criminal Justice. American Society of Criminology, 1-4.

Bushway SD, Sweeten G and Wilson DB. (2006) Size Matters: Standard Errors in the Application of Null Hypothesis Significance Testing in Criminology and Criminal Justice. Journal of Experimental Criminology 2(1): 1-22.

Cabey C. (2013) Assessment and Planning Interventions Framework - ASSETPLUS: Model Document (V1.0). London:Youth Justice Board.

Cabinet Office. (2012) Open Data White Paper: Unleashing the Potential. London:HM Government.

Calle-Alonso F and Pérez SánchezCJ. (2014)A Monte Carlo-Based Bayesian Approach for Measuring Agreementin a Qualitative Scale. Applied Psychological Measurement39(3):189-207.

Carr PJ. (2010) The Problem with Experimental Criminology: A Response to Sherman's 'Evidence and Liberty'. Criminology \& Criminal Justice 10(1): 3-10.

Carrington PJ. (2015) The Structure of Age Homophily in Co-Offending Groups. Journal of Contemporary Criminal Justice 31(3): 337-353.

Case S. (2006) Young People 'at Risk' of What? Challenging Risk-Focused Early Intervention as Crime Prevention. Youth Justice 6(3): 171-179.

Case S. (2007) Questioning the 'Evidence' of Risk That Underpins Evidence-Led Youth Justice Interventions. Youth Justice 7(2): 91-105.

Case S. (2010)Preventing and Reducing Risk. In: TaylorW, Earle R and Hester R (eds) Youth Justice Handbook: Theory, Policy and Practice. Cullompton: Willian Publishing.

Case S and Haines K. (2009) Understanding Youth Offending: Risk Factor Research, Policy and Practice. Cullompton: Willan Publishing.

Case S and Haines K. (2010) Risky Business? The Risk in Risk Factor Research. Criminal Justice Matters 80(1): 20-22.

Case S and Haines K. (2014) Youth Justice: From Linear Risk Paradigm to Complexity. In: Pycroft A and Bartollas C (eds) Applying Complexity Theory. Bristol: PolicyPress, 113-139.

Case S and Haines K. (2015) Risk Managementand Early Intervention: A Critical Analysis. In: Goldson B and Muncie J (eds) Youth Crime and Justice. London: Sage Publications Ltd, 100-118.

ChaineyS and Radcliffe J. (2005) GIS and Crime Mapping. Chichester: John Wiley \& Sons, Inc.

Chainey S and Thompson L. (2008) Crime Mapping Case Studies: Practice and Research. Chichester. John Wiley \& Sons, Inc.

Chamberlain JM. (2016) Ensuring the Criminological Skills of the Next Generation:A Case Study on the Importance of Enhanced Quantitative Method Teaching Provision. Journal of Further and HigherEducation: 1-12.

Chan J and Bennett Moses L. (2016) Is Big Data Challenging Criminology? Theoretical Criminology 20(1): 21-39.

Cleghorn N, Kinsella R and McNaughton NC. (2011) Engaging with the Views of Young People with Experience of the Youth Justice System. London:The Police Foundation, NatCen and the Paul Hamlyn Foundation.

Cohen J. (1994) The Earth Is Round ( $\mathrm{P}<.05)$. American psychologist 49: 997-1003.

Cohen J, Nagin D, Wallstrom G, et al. (1998) Hierarchical Bayesian Analysis of Arrest Rates. Journal of American Statsitical Association 93(444): 1260-1270.

Corr M-L. (2014) Young People's Offending Careers and Criminal Justice Contact: A Case for Social Justice. Youth Justice 14(3): 255-268.

Craig LA, Browne KD and Stringer I. (2003) Risk Scales and Factors Predictive of Sexual Offence Recidivism. Trauma, Violence, \& Abuse 4(1): 45-69.

Cunradi CB, Mair C, Ponicki W, et al. (2012) Alcohol Outlet Density and Intimate Partner ViolenceRelated Emergency Department Visits. Alcoholism-Clinical and Experimental Research 36(5): 847-853.

de Vaus D. (2001) Research Design in Social Research. London: SAGE Publications Ltd. 
de Villemereuil P. (2012) Estimation of a Biological TraitHeritability Using the Animal Model. How to Use the MCMCgImm R Package. Available at:

https://www.researchgate.net/profile/Pierre_De_Villemereuil/publication/257729224_Tutorial_ Estimation_of_a_biological_trait_heritability_using_the_animal_model_How_to_use_the_MC MCgImm_R_package/links/0c960525c32aaa92e3000000/Tutorial-Estimation-of-a-biologicaltrait-heritability-using-the-animal-model-How-to-use-the-MCMCglmm-R-package.pdf. (Accessed 31/1/18).

Deandrea S, Negri E and Ruggeri F. (2014) Integrating Clinicians' Opinion in the Bayesian Meta-Analysis of Observational Studies: The Case of the Risk Factors for Falls in Community-Dwelling Older People. Epidemiology Biostatistics and Public Health 11(1): e8909-8901 - e8909-8914.

DeucharR and Sapouna M. (2016) 'It's Harder to Go to Court Yourself Because You Don't ReallyKnow What to Expect': Reducing the Negative Effects of Court Exposure on Young People - Findings from an Evaluation in Scotland. Youth Justice 16(2):130-146.

Dhami MK. (2005) From Discretion to Disagreement: Explaining Disparities in Judges' Pretrial Decisions. Behavioral Sciences \& the Law 23(3): 367-386.

DiCristina B. (1997) The Quantitative Emphasis in Criminal Justice Education. Journal of Criminal Justice Education 8(2): 181-199.

Dienes Z. (2016) What Role Can Bayesian Methods Play in Resolving the Replication Crisis? ESRC Research Methods Festival, University of Bath, 7th July. Available at: http://www.ncrm.ac.uk/RMF2016/programme/session.php?id=R6. (Accessed 31/1/18).

Dienes Z and Mclatchie N. (2017) Four Reasons to Prefer Bayesian Analyses over Significance Testing. Psychonomic Bulletin \& Review. doi: 10.3758/s13423-017-1266-z

Douglas T, Pugh J, Singh I, et al. (2016) Risk Assessment Tools in Criminal Justice and Forensic Psychiatry. The Need for Better Data. European Psychiatry 42(134-137.

Economic and Social Research Council. (2016) Secondary Data Analysis Initiative. Available at: $\mathrm{http} / / / w w w . e s r c . a c . u k / r e s e a r c h / o u r-r e s e a r c h / s e c o n d a r y-d a t a-a n a l y s i s-i n i t i a t i v e / . \quad$ (Accessed $31 / 1 / 18)$.

Efron B. (1986) Why Isn't Everyone a Bayesian? The American Statistician 40(1): 1-5.

Emeka TQ and Sorensen JR. (2009) Female Juvenile Risk: Is There a Need for Gendered Assessment Instruments? Youth Violence and Juvenile Justice 7(4): 313-330.

Erickson DJ, Carlin BP, Lenk KM, et al. (2015) Do Neighborhood Attributes Moderate the Relationship between Alcohol EstablishmentDensityand Crime? Prevention Science 16(2):254-264.

Etz KE and Arroyo JA. (2015) Small Sample Research: Considerations Beyond Statistical Power. Prevention Science 16(7): 1033-1036.

Farrington DP. (1996) Understanding and Preventing Youth Crime. York: Joseph Rowntree Foundation.

Farrington DP. (2000) Explaining and Preventing Crime: The Globalization of Knowledge - the American Society of Criminology 1999 Presidential Address. Criminology 38(1):1-24.

Farrington DP. (2002) Understanding and Preventing Youth Crime. In: Muncie J, Hughes G and McLaughlin E (eds) Youth Justice: Critical Readings. London: Sage Publications Ltd, 425-430.

Farrington DP, Gottfredson DC, Sherman LW, et al. (2002) The Maryland Scientific Methods Scale. In: Sherman LW, Farrington DP, Welsh BC, et al. (eds) Evidence-Based Crime Prevention. Oxon: Routledge, 13-21.

Farrington DP and Tarling R. (1985) Prediction in Criminology. Albany, USA: State Unversity of New York Press.

Fazel S, Singh JP, Doll H, et al. (2012) Use of Risk Assessment Instruments to Predict Violence and Antisocial Behaviour in 73 Samples Involving 24827 People: Systematic Review and MetaAnalysis. British Medical Journal 345: 12.

FeeleyMM and Simon J. (1992) The New Penology: Notes on the Emerging Strategy of Corrections and Its Implications. Criminology 30(4):449-474.

Feeley MM and Simon J. (1994) Actuarial Justice: The Emerging New Criminal Law. In: Nelken D (ed) The Futures of Criminology. London: Sage Publications Ltd, 173-201.

Fenton Nand Neil M. (2013) Risk Assessment and Decision Analysis with Bayesian Networks. Croydon: Taylor \& Francis Group.

Fidler F. (2010) The American Psychological Association Publication Manual Sixth Edition: Implications for Statistics Education. Eighth International Conference on Teaching Statistics (ICOTS8), 
Ljubljana, Slovenia, 11-16 July. Available at:

http://citeseerx.ist.psu.edu/viewdoc/download?doi=10.1.1.205.600\&rep=rep1\&type=pdf.

(Accessed 31/1/18).

Fienberg SE. (2006) When Did Bayesian Inference Become "Bayesian"? Bayesian Analysis 1(1): 1-40.

Fienberg SE. (2011) Bayesian Models and Methods in Public Policyand Government Settings. Statistical Science 26(2): 212-226.

Finch WH, Bolin JE and Kelley K. (2014) Multilevel Modeling Using R. Boca Raton, FL: CRC Press.

Fitterer JL and Nelson TA. (2015) A Review of the Statistical and Quantitative Methods Used to Study Alcohol-Atributable Crime. PLOS One 10(9): 24.

Flam F. (2014) The Odds, Continually Updated. The New York Times, 29th September. Available at: http://www.nytimes.com/2014/09/30/science/the-odds-continually-updated.html?_r=2. (Accessed 31/1/18).

Forty R and Sturrock R. (2017) Using Family Court Data to Explore Links between Adverse Family Experiences and Proven Youth Offending. London: Ministry of Justice.

France A. (2008) RiskFactor Analysis and the Youth Question. Journal of Youth Studies 11(1):1-15.

France A and Homel R. (2006) Societal Access Routes and Developmental Pathways: Putting Social Structure and Young People's Voice into the Analysis of Pathways into and out of Crime. Australian and New Zealand Journal of Criminology 39(3):295-309.

Friedrichs DO. (2016) Edwin H. Sutherland: An Improbable Criminological Key Thinker-for Critical Criminologists and for Mainstream Criminologists. Critical Criminology: 1-15.

Gelman A. (2010) What Do Practitioners Need to Know AboutRegression? Statistical Modeling, Causal Inference, and Social Science, 5 December. Available at: http://andrewgelman.com/2010/12/05/what_do_practit. (Accessed 31/1/18).

Gelman A, Carlin JB, Stern HS, et al. (2013) Bayesian Data Analysis (3rd Edition). Boca Raton, FL: Chapman and Hall/CRC.

Gelman A and Hill J. (2007) Data Analysis Using Regression and Multilevel/Hierarchical Models. New York: Cambridge University Press.

Gigerenzer G. (2004) Mindless Statistics. The Journal of Socio-Economics 33(5): 587-606.

Gigerenzer $\mathrm{G}$ and Marewski JN. (2015) Surrogate Science: The Idol of a Universal Method for Scientific Inference. Journal of Management 41(2):421-440.

Gill J. (2014) Bayesian Methods: A Social and Behavioral Sciences Approach (3rd Edition). New York: Chapman and Hall/CRC.

Gill J and Meier KJ. (2000) Public Administration Research and Practice: AMethodological Manifesto. Journal of Public Administration Research and Theory: J-PART 10(1): 157-199.

Gill J and Witko C. (2013) Bayesian Analytical Methods: A Methodological Prescription for Public Administration. Journal of Public Administration Research and Theory 23(2): 457-494.

Gill J and WomackA. (2013) The Multilevel Model Framework. London: SAGE Publications Ltd, 3-20.

Gliner J, Leech N and Morgan G. (2002) Problems with Null Hypothesis Significance Testing (NHST): What Do the Textbooks Say? Journal of Experimental Education 71(1): 83-92.

Goldson B. (2000) 'Children in Need' or' Young Offenders'? Hardening Ideology, Organizational Change and New Challenges for Social Work with Children in T rouble. Child \& Family Social Work 5(3): 255-265.

Goldson B. (2002) New Labour, Social Justice and Children: Political Calculation and the DeservingUndeserving Schism. British Journal of Social Work 32(6):683-695.

Goldson B. (2010) The Sleep of (Criminological) Reason: Knowledge - PolicyRupture and New Labour's Youth Justice Legacy. Criminology \& Criminal Justice 10(2):155-178.

Goldson B and Muncie J. (2006) Rethinking Youth Justice: Comparative Analysis, International Human Rights and Research Evidence. Youth Justice 6(2): 91-106.

Good IJ. (1979) Studies in the History of Probabilityand Statistics. XXXVI A. M. Turing's StatisticalWork in World War II. Biometrika 66(2): 393-396.

Gorard S. (2002) How Can We Overcome the Methodological Schism? (or Can There be a 'Compleat' Researcher)? Annual Conference of the British Educational Research Association, University of Exeter, 12-14 September. Available at:

http://www.leeds.ac.uk/educol/documents/00002205. (Accessed 31/1/18). 
Gorard S. (2014a)A Proposal for Judging the Trustworthiness of Research Findings. Radical Statistics 110: 47-59.

Gorard S. (2014b) The Widespread Abuse of Statistics by Researchers: What ls the Problem and What Is the Ethical Way Forward? Psychology of Education Review 38(1): 3-10.

Gorard S. (2016) Damaging Real Lives through Obstinacy: Re-Emphasising Why Significance Testing Is Wrong. Sociological Research Online 21(1):2.

Gottfredson SD and Moriarty LJ. (2006) Statistical Risk Assessment: Old Problems and New Applications. Crime \& Delinquency52(1): 178-200.

Grandi LD and Adler JR. (2016)A Study into Breaches of Youth Justice Orders and the Young People Who Breach Them. Youth Justice 16(3):205-225.

Gray E, Jackson J and Farrall S. (2011) Feelings and Functions in the Fear of Crime: Applying a New Approach to Victimisation Insecurity. The British Journal of Criminology 51(1):75-94.

Gray P. (2005) The Politics of Risk and Young Offenders' Experiences of Social Exclusion and Restorative Justice. British Journal of Criminology 45(6): 938-957.

Greenwood PW. (1982) Selective Incapacitation. Santa Monica, CA: The RAND Corporation.

Grix J. (2002) Introducing Students to the Generic Terminology of Social Research. Politics 22(3): 175186.

Hadfield JD. (2010) MCMC Methods for Multi-Response Generalized Linear Mixed Models: The MCMCgImm R Package. Journal of Statistical Software 33(2): 22.

Hadfield JD. (2017a) MCMCgImm (Version 2.25) [Computer Software]. Available at: https://cran.rproject.org/web/packages/MCMCglmm/index.html. (Accessed 31/1/18).

Hadfield JD. (2017b) MCMCgImm Course Notes Available at: https://cran.rproject.org/web/packages/MCMCglmm/vignettes/CourseNotes.pdf. (Accessed31/1/18).

Haines K and Case S. (2005) Promoting Prevention: Targeting Family-Based Risk and Protective Factors for Drug Use and Youth Offending in Swansea. The British Journal of Social Work 35(2): 169-187.

Haines K, Case S, Davies K, et al. (2013)The Swansea Bureau: A Model of Diversion from the Youth Justice System. International Journal of Law, Crime and Justice 41(2): 167-187.

Haines K and Drakeford M. (1998) Young People and Youth Justice. London: Macmillian Press Ltd.

Hale C, Hayward K, Wahidin A, et al. (2005) Criminology. Oxford: Oxford University Press.

HarcourtBE. (2007) Against Prediction: Profiling, Policing, and Punishing in an Actuarial Age. Chicago: The University of Chicago Press.

Hare RD. (2003) The Hare Psychopathy Checklist-Revised (2ndEdition). Toronto: Multi-Health Systems.

Harris GT and Rice ME. (2013) Bayes and Base Rates: What Is an Informative Prior for Actuarial Violence RiskAssessment? Behavioral Sciences \& the Law31(1): 103-124.

Hart D. (2011a) Into the Breach: The Enforcement of Statutory Orders in the Youth Justice System. London: Prison Reform Trust.

Hart D. (2011b) Public Protection or Public Protection? Breach Action within the Youth Justice System. Safer Communities 10(4): 19-27.

HM Government. (2007) Working Together to Cut Crime and Deliver Justice. London: HMSO.

Hoekstra R, Morey RD, Rouder JN, et al. (2014) Robust Misinterpretation of Confidence Intervals. Psychonomic Bulletin \& Review 21(5): 1157-1164.

Howard League for Penal Reform. (2017) Ending the Criminalisation of Children in Residential Care: Briefing One. London: Howard League for Penal Reform.

Howard P, Francis B, Soothill K, et al. (2009) OGRS 3: The Revised Offender Group Reconviction Scale. London: Ministry of Justice.

Howard P and Kershaw C. (2000) Using Criminal Career Data in Evaluation. British Society of Criminology, Liverpool, July 1999. Available at: http://britsoccrim.org/new/volume3/005.pdf. (Accessed 28/11/17).

Hoyle RH and Gottredson NC. (2015) Sample Size Considerations in Prevention Research Applications of Multilevel Modeling and Structural Equation Modeling. Prevention Science 16(7):987-996.

Hughes N, Williams H, Chitsabesan P, et al. (2012) Nobody Made the Connection: The Prevalence of Neurodisability in Young People Who Offend. Available at:

https://www.childrenscommissioner.gov.uk/publication/nobody-made-the-connection/.

(Accessed 31/1/18). 
Hughes N, Williams WH, Chitsabesan P, et al. (2015) The Prevalence of T raumatic Brain Injuryamong Young Offenders in Custody: A Systematic Review. The Journal of Head Trauma Rehabilitation 30(2): 94-105.

Issac W and Dixon A. (2017) Why Big-Data Analysis of Policy Activity Is Inherently Biased. The Conversation, 10 May. Available at: http://theconversation.com/why-big-data-analysis-ofpolice-activity-is-inherently-biased-72640. (Accessed 31/1/18).

Jackman S. (2009) Bayesian Analysis for the Social Sciences. Chichester:Wiley.

Jamieson J. (2005) New Labour, Youth Justice and the Question of Respect. Youth Justice 5(3): 180.

Jang SJ. (1999) Age-Varying Effects of Family, School, and Peers on Delinquency: A Multilevel Modeling Test of Interactional Theory Exchange. Criminology 37(643-686.

JASP Team. (2017a) FAQ: What Does JASP Stand For? Available at: https://jasp-stats.org/faq/. (Accessed 31/1/18).

JASP Team. (2017b) JASP (Version 0.8.5)[Computer Software]. Available at: https://jasp-stats.org/. (Accessed 31/1/18).

JASP Team. (2017c) OurGoals. Available at: https://jasp-stats.org/aboutl. (Accessed 31/1/18).

Jeffreys H. (1961) Theory of Probability (3rd Edition). Oxford: Oxford University Press.

Johnson C. (2016) Artificial Intelligence 'Judge' Developed by Ucl Computer Scientists The Guardian, 24th October. Available at: https:/www.theguardian.com/technology/2016/oct/24/artificialintelligence-judge-university-college-london-computer-scientists. (Accessed 31/1/18).

Kalinowski P, Fidler F and Cumming G. (2008) Overcoming the Inverse Probability Fallacy: A Comparison of Two Teaching Interventions. Experimental Psychology 4(4): 152-158.

Kaplan D. (2014) Bayesian Statistics for the Social Sciences. New York: The Gulford Press.

Kemshall H. (1995) Risk in Probation Practice: The Hazards and Dangers of Supervision. Probation Journal 42(2): 67-72.

Kemshall H. (1996) Risk Assessment: Fuzzy Thinking or 'Decisions in Action'? Probation Journal 43(1): 2-7.

Kemshall H. (1998) Risk in Probation Practice. Aldershot: Ashgate Publishing Ltd.

Kemshall H. (2008a) Risks, Rights and Justice: Understanding and Responding to Youth Risk. Youth Justice 8(1): 21-37.

Kemshall H. (2008b) Understanding the Community Management of High Risk Offenders. Maidenhead: Open University Press.

Kemshall H, Parton N, Walsh M, et al. (1997) Concepts of Risk in Relation to Organizational Structure and Functioning within the Personal Social Services and Probation. Social Policy and Administration 31(3): 213-232.

King R, Bird SM, Hay G, et al. (2009) Estimating Current Injectorsin Scotland and TheirDrug-Related Death Rate by Sex, Region and Age-Group Via Bayesian Capture-Recapture Methods. Statistical Methods in Medical Research 18(4):341-359.

King R, Bird SM, Overstall AM, et al. (2014a) Estimating Prevalence of Injecting Drug Users and Associated Heroin-Related Death Rates in England by Using Regional Data and Incorporating Prior Information. Journal of the Royal Statistical Society: Series A (Statistics in Society) 177(1): 209-236.

King TE, Fortes GG, Balaresque P, etal. (2014b) Identification of the Remains of King Richard III. Nature Communications 5

Kitchin R. (2014) Big Data, New Epistemologies and Paradigm Shifts. Big Data \& Society 1(1): 1-12.

Kivivuori J. (2007) Crime by Proxy - Coercion and Altruism in Adolescent Shoplifting. British Journal of Criminology 47(5):817-833.

Kruschke JK. (2010a) Bayesian Data Analysis. Wiley Interdisciplinary Reviews: Cognitive Science,658676.

Kruschke JK. (2010b) What to Believe: Bayesian Methods for Data Analysis. Trends in Cognitive Sciences 14(7): 293-300.

Kruschke JK. (2011) Introduction to Special Section on Bayesian Data Analysis. Perspectives on Psychological Science 6(3): 272-273.

Kruschke JK. (2013) Posterior Predictive Checks Can and Should Be Bayesian: Commenton Gelman and Shaliz, 'Philosophyand the Practice of Bayesian Statistics'. British Journal ofMathematical and Statistical Psychology66(1): 45-56. 
Kruschke JK. (2015) Doing Bayesian Data Analysis: A Tutorial with R and Bugs (2nd Edition). Burlington, MA: Elsevier.

Kruschke JK, Aguinis H and Joo H. (2012) The Time Has Come: Bayesian Methods for Data Analysis in the Organizational Sciences. Organizational Research Methods 15(4): 722-752.

Lambdin C. (2012) Significance Tests as Sorcery: Science Is Empirical-Significance Tests Are Not. Theory \& Psychology22(1): 67-90.

LammyD. (2017) The Lammy Review: Final Report. London: Gov.UK.

Langton S and Bannister J. (2017) Space, Place and Crime in an Era of 'Big Data'. The GeographerNewsletter of the Royal Scottish Geographical Society Spring 2017:23.

Laub JH and Sampson RJ. (1991)The Sutherland-Glueck Debate: On the Sociology of Criminological Knowledge. American Journal of Sociology 96(6):1402-1440.

Law J, Quick M and Chan P. (2014) Bayesian Spatio-Temporal Modeling for Analysing Local Pattems of Crime over Time at the Small-Area Level. Journal of Quantitative Criminology 30(1):57-78.

Lee PM. (2012) Bayesian Statistics: An Introduction (4th Edition). Chichester: Wiley.

Levine N and Block R. (2011) Bayesian Journey-to-Crime Estimation: An Improvement in Geographic Profiling Methodology. The Professional Geographer63(2):213-229.

Levine N and Lee P. (2009) Bayesian Journey-to-Crime Modelling of Juvenile and Adult Offenders by Gender in Manchester. Journal of Investigative Psychology and Offender Profiling 6(3): 237252.

Lewis D-M. (2014) The Risk Factor - (Re-) Visiting Adult Offender Risk Assessments within Criminal Justice Practice. Risk Management - Journal of Risk Crisis and Disaster 16(2): 121-136.

Li B, Lingsma HF, Steyerberg EW, et al. (2011) Logistic Random Effects Regression Models: A Comparison of Statistical Packages for Binary and Ordinal Outcomes. BMC Medical Research Methodology 11(1): 77.

Liddle M, Boswell G, Wright S, et al. (2016) Trauma and Young Offenders: A Review of the Research and Practice Literature. London: Beyond Youth Custody.

LindleyDV. (1975) The Future of Statistics: A Bayesian 21st Century. Advances in Applied Probability 7:106-115.

Liu Y, Yang M, Ramsay M, et al. (2011) A Comparison of Logistic Regression, Classification and Regression Tree, and Neural Networks Models in Predicting Violent Re-Offending. Journal of Quantitative Criminology 27(4):547-573.

Lösel F. (2017) Evidence Comes by Replication, butNeeds Differentiation: The Reproducibility Issue in Science and Its Relevance for Criminology. Journal of Experimental Criminology. doi: 10.1007/s11292-017-9297-z

Loughran TA, Paternoster R and Thomas KJ. (2014) Incentivizing Responses to Self-Report Questions in Perceptual Deterrence Studies: An Investigation of the Validity of Deterrence TheoryUsing Bayesian Truth Serum. Journal of Quantitative Criminology 30(4):677-707.

Lum K and Isaac W. (2016) To Predictand Serve? Significance 13(5): 14-19.

Mair C, Gruenewald PJ, Ponicki WR, etal. (2013) Varying Impacts of Alcohol OutletDensities on Violent Assaults: Explaining Differences across Neighborhoods. Journal of Studies on Alcohol and Drugs 74(1): 50-58.

Maltz MD. (1994) Deviating from the Mean: The Declining Significance of Significance. Journal of Research in Crime and Delinquency 31(4): 434-463.

Marx GT . (1997) OfMethods and Manners for Aspiring Sociologists: 37 Moral Imperatives. The American Sociologist 28(1): 102-125.

Mayer-SchibvergerV and CukierK. (2013) Should We Use Big Data to Punish Crimes before They're Committed? Popular Science, 6 March. Available at: http://www.popsci.com/science/article/2013-03/should-we-use-big-data-to-punish-crimesbefore-theyre-committed. (Accessed 31/1/18).

McAra L and McVie S. (2005) The Usual Suspects? Street-Life, Young People and the Police. Criminal Justice 5(1): 5-36.

McAraL and McVie S. (2007) Youth Justice? The Impact of System Contacton Patterns of Desistance from Offending. European Journal of Criminology 4(3):315-345.

McAra L and McVie S. (2010) Youth Crime and Justice: Key Messages from the Edinburgh Study of Youth T ransitions and Crime. Criminology \& Criminal Justice 10(2): 179-209. 
McAra L and McVie S. (2015) The Case for Diversion and Minimum Necessary Intervention. In: Goldson B and Muncie J (eds) Youth Crime and Justice (2nd Edition). London: Sage, 119-136.

McElreath R. (2016) Statistical Rethinking: A Bayesian Course with Examples in R and Stan. Boca Raton, FL: CRC Press.

McGrayne SB. (2011) The Theory That Would NotDie : How Bayes' Rule Cracked the Enigma Code Hunted Down Russian Submarines, and Emerged Triumphant from Two Centuries of Controversy. New Haven and London: Yale University Press.

McKee RA and Miller CC. (2015) Institutionalizing Bayesianism within the Organizational Sciences: A Practical Guide Featuring Comments from Eminent Scholars. Journal of Management 41(2): 471-490.

McMahon P, ZaslavskyA, Weinstein M, etal. (2006) Estimation of Mortality Rates for Disease Simulation Models Using Bayesian Evidence Synthesis. Medical Decision Making 26: 497-511.

McNeeleyS and Warner JJ. (2015) Replication in Criminology: ANecessaryPractice. European Joumal of Criminology 12(5):581-597.

McNeill F. (2009) Towards Effective Practice in Offender Supervision. Glasgow: Scottish Centre for Crime and Justice Research.

McShane BB and Gal D. (2016) Blinding Us to the Obvious? The Effect of Statistical Training on the Evaluation of Evidence. Management Science 62(6): 1707-1718.

McVie S. (2016) It's a Criminal Waste: How Using Administrative Data About Crime Could Better Inform Public Policy. ADRN Blog, 15/11/16. Available at: https://adrn.ac.uk/understand-data/blog/it-sa-criminal-waste/. (Accessed 5/4/17).

McVie S and Norris P. (2006) Neighbourhood Effects on Youth Delinquency and Drug Use Edinburgh: Centre for Law and Society, The University of Edinburgh.

Meyers JR and SchmidtF. (2008) Predictive Validity of the Structured Assessment for Violence Risk in Youth (Savry) with Juvenile Offenders. Criminal Justice and Behavior 35(3): 344-355.

Milner J and Myers S. (2007) Working with Violence: Policies and Practices in Risk Assessment and Management. Basingstoke: Palgrave Macmillan.

Ministry of Justice. (2010) Breaking the Cycle: Effective Punishment, Rehabilitation and Sentencing of Offenders. London: The StationaryOffice.

Ministry of Justice. (2014a) Accessing the Justice Data Lab Service. Available at: https://www.gov.uk/government/publications/justice-data-lab. (Accessed 31/1/18).

Ministry of Justice. (2014b) Transforming the Criminal Justice System Strategy and Action Plan Implementation Update. London:HMSO.

Ministry of Justice. (2017a) Guide to Proven Reoffending Statistics. Available at: https://www.gov.uk/government/uploads/system/uploads/attachment_data/file/585963/guideto-proven-reoffending-statistics-Jan17.pdf. (Accessed 31/1/18).

Ministry of Justice. (2017b) How the Measure of Proven Reoffending Has Changed and the Effect of These Changes. Available at:

https:/www.gov.uk/government/uploads/system/uploads/attachment_data/file/658380/howthe-measure-of-reoffending-has-changed-and-the-effect-of-these-changes.pdf. (Accessed 31/1/18).

Ministry of Justice. (2017c) Proven Reoffending Statistics Quarterly Bulletin, April 2014 to March 2015. Available at: https://www.gov.uk/government/statistics/proven-reoffending-statistics-april2014-to-march-2015. (Accessed 31/1/18).

Mokros A, Stadtland C, OsterheiderM, et al. (2010) Assessment of Risk for Violent Recidivism through Multivariate Bayesian Classification. Psychology, Public Policy and Law 16(4): 418-450.

Monk D. (2009) The Scaled Approach: Improving Positive Outcomes for Young People in the Youth Justice System. Available at: http://embed.policyreview.tv/media/documents/LI281_DAVID_MONK.pdf. (Accessed 31/1/18).

Morey RD. (2016) What Role Can Bayesian Methods Play in Resolving the Replication Crisis? ESRC Research Methods Festival, University of Bath, 7th July. Available at: http://www.ncrm.ac.uk/RMF2016/programme/session.php?id=R6. (Accessed 31/1/18).

Morey RD, Hoekstra R, Rouder JN, et al. (2016) The Fallacy of Placing Confidence in Confidence Intervals. Psychonomic Bulletin \& Review 23(1): 103-123. 
Muncie J. (2009) Youth and Crime. London: Sage Publications Ltd.

National Crime Agency. (2017) County Lines Violence, Exploitation \& Drug Supply 2017. London: National Crime Agency.

NellerDJand Petris G. (2013) Sexually Violent Predators: Toward Reasonable Estimates of Recidivism Base Rates. Behavioral Sciences \& the Law 31(4): 429-443.

Newburn T . (2007) Criminology. Cullompton: Willian Publishing.

Nickerson RS. (2000) Null Hypothesis Significance Testing: A Review of an Old and Continuing Controversy. Psychological Methods 5(2):241-301.

O'Donoghue R. (2016) Is Kent's Predictive Policing Project the Future of Crime Prevention? . KentOnline, 5th April. Available at: http://www.kentonline.co.uk/sheerness/news/what-ifpolice-could-detect-93715/. (Accessed 31/1/18).

O'Hagan A and Luce B. (2003) A Primer on Bayesian Statistics in Health Economics and Outcomes Research. Available at: https://www.sheffield.ac.uk/polopoly_fs/1.80635!/file/primer.pdf (Accessed 31/1/18).

O'Mahony P. (2009) The Risk Factors Prevention Paradigm and the Causes of Youth Crime: A Deceptively Useful Analysis? Youth Justice 9(2): 99-114.

O'Neil C. (2017) The Era of Blind Faith in Big Data Must End. New York: TED Conferences, LLC

Office of National Statistics. (2013) Census 2011 Detailed Characteristics - Ethnic Group by Sex and Age (DC2101EW). Available at: https://www.nomisweb.co.uk/census/2011/dc2101ew. (Accessed 31/1/18).

Ofili OU. (2014) Bail Decision Support System. The International Journal of Engineering and Science 3(8): 45-66.

Owen N and Cooper C. (2013) The Start of a Criminal Career:Does the Type of Debut Offence Predict Future Offending? London: Home Office.

Pawson R and TillyN. (2000) Realistic Evaluation. London: SAGE Publications.

Paylor I. (2010) The Scaled Approach to Youth Justice: A Risky Business. Criminal Justice Matters 81(1): 30-31.

Pemberton C. (2009) Youth Justice: Scaled Approach and YRO Launch. Community Care, 30th November. Available at: http://www.communitycare.co.uk/2009/11/30/youth-justice-scaledapproach-and-yro-launch. (Accessed 31/1/18).

Petrosino A, Turpin-Petrosino C and Guckenburg S. (2010) Formal System Processing of Juveniles: Effects on Delinquency. Campbell Systematic Reviews. Woburn, Mass: The Campbell Collaboration.

Phenix A, Fernandez Y, Harris AJR, et al. (2016) Static-99R Coding Rules, Revised. Available at: http://www.static99.org/. (Accessed 31/1/18).

Phoenix J. (2009a) Beyond Risk Assessment: The Return of Repressive Welfare? In: Barry M and McNeill F (eds) Youth Offending and Youth Justice. London: Jessica KingsleyPublishers, 113131.

Phoenix J. (2009b) Doing Youth Justice: Analysing Risk and Need Assessments in Youth Justice Practice, 2004-2005. [User Guide]. SN: 5831. Essex: UK Data Service.

Pitts J. (2001) Korrectional Karaoke: New Labour and the Zombification of Youth Justice. Youth Justice 1(2): 3-16.

Pitts J. (2005) The Recent History of Youth Justice in England and Wales. In: Bateman T and Pitts J (eds) The RHP Companion to Youth Justice. Lyme Regis: Russeel House Publishing Ltd, 2-11.

Ployhart RE and Vandenberg RJ. (2010) Longitudinal Research: The Theory, Design, and Analysis of Change. Journal of Management 36(1): 94-120.

Plummer M. (2008) Penalized Loss Functions for Bayesian Model Com parison. Biostatistics 9(3): 523539.

Prison Reform Trust. (2016) In Care, out of Trouble London: Prison Reform Trust.

Puffet N. (2010a) Cautious Welcome for Scaled Approach System. Children and Young People Now, 2nd July. Available at: http://www.cypnow.co.uk/cyp/news/1052628/cautious-welcome-scaledapproach (Accessed 31/1/18)

Puffet N. (2010b) YJB Considers Shake-Up. Children and Young People Now, 1st March. Available at: http://www.cypnow.co.uk/cyp/news/1042636/yjb-considers-assessment-shake. (Accessed $31 / 1 / 18)$. 
Punch K, F. (2006) Developing Effective Research Proposals. London: SAGE.

Quinsey V, Harris GT, Rice ME, et al. (2006) Violent Offenders: Appraising and Managing Risk (2nd Edition). Washington DC: American Psychological Association.

Quintana DS and Williams DR. (Preprint) Bayesian Alternatives for Common Null-Hypothesis Significance Tests in Psychiatry: A Non-Technical Guide Using JASP. Open Science Framework.

Raynor P. (2016) Three Narrratives of Risk: Corrections, Critique and Context. In: Trotter C, Mclvor G and McNeill F (eds) Beyond the Risk Paradigm in Criminal Justice. London: Palgrave, 24-45.

Raynor P, Kynch J, Roberts C, et al. (2000) Risk and Need Assessment in Probation Services: An Evaluation. Home Office London.

Rembert DA, Henderson H and Pirtle D. (2014) Differential Racial/Ethnic Predictive Validity. Youth Violence and Juvenile Justice 12(2): 152-166.

Rice ME and Harris GT. (1995) Violent Recidivism:Assessing Predictive Validity. Journal of consulting and clinical psychology 63(5): 737.

Rice ME and Harris GT . (2005) Comparing Effect Sizes in Follow-up Studies: Roc Area, Cohen's D, and R. Law and Human Behavior 29(5): 615-620.

Robinson D and Koepke L. (2016) Stuck in a Pattern: Early Evidence on "Predictive Policing" and Civil Rights Washington DC: Upturn.

Robinson G. (2002) Exploring Risk Management in Probation Practice. Punishment \& Society 4(1): 525.

Robson C and McCartan K. (2016) Real World Research. Wiley.

Robson K and Pevalin D. (2016) Multilevel Modeling in Plain Language. London: Sage.

Rose D. (2016)Drugs, Mental Disorder and Risk. In: TrotterC (ed) Beyond the Risk Paradigm in Criminal Justice. London: Palgrave, 92-107.

Rouder JN, Speckman PL, Sun D, et al. (2009) Bayesian T Tests for Accepting and Rejecting the Null Hypothesis. Psychonomic Bulletin \& Review 16(2): 225-237.

Rowlingson K. (2004) Secondary Data Analysis. In: Becker S and Bryman A (eds) Understanding Research for Social Policy and Practice. Bristol: The PolicyPress, 138-142.

Salsburg D. (2001) The Lady Tasting Tea: How Statistics Revolutionised Science in the Twentieth Century. New York: HoltPaperbacks.

Sampson RJ. (2010) Gold Standard Myths: Observations on the Experimental Turn in Quantitative Criminology. Journal of Quantitative Criminology 26(4): 489-500.

Schwalbe CS. (2007) Risk Assessmentfor Juvenile Justice: AMeta-Analysis. Law and Human Behavior 31(5): 449-462.

Schwalbe CS. (2008) A Meta-Analysis of Juvenile Justice Risk Assessment Instruments: Predictive Validity by Gender. Criminal Justice and Behavior35(11):1367-1381.

Schwalbe CS, Fraser MW, Day SH, et al. (2006) Classifying Juvenile Offenders According to Risk of Recidivism Predictive Validity, Race/Ethnicity, and Gender. Criminal Justice and Behavior 33(3): 305-324.

Sentencing Council. (2017) Sentencing Children and Young People: Definitive Guide. London: Sentencing Council.

Sentencing Guidelines Council. (2009) Overarching Principles - Sentencing Youths: Definitive Guidelines. London: Sentencing Guidelines Council.

Shapiro A. (2017) Reform Predictive Policing. Nature 541:458-460.

Sharland E. (2005) Young People, Risk Takingand Risk Making: Some Thoughts for Social Work. British Journal of Social Work 36(2): 247-265.

Sherman LW. (2009) Evidence and Liberty: The Promise of Experimental Criminology. Criminology \& Criminal Justice 9(1): 5-28.

Simon J and Feeley MM. (2003) The Form and Limits of the New Penology. In: Blomberg T and Cohen $S$ (eds) Punishmentand Social Control. New York: Walter de Gruyter Inc, 75-116.

Simpson E. (2010)Edward Simpson: Bayes at BletchleyPark. Significance 7(2): 76-80.

Smith A. (2017) Report of Professor Sir Adrian Smith's Review of Post-16 Mathematics. Available at: https://www.gov.uk/government/uploads/system/uploads/attachment_data/file/630488/AS_rev iew_report.pdf. (Accessed 31/1/18).

Smith DJ and McAra L. (2004) Genderand Youth Offending. Edinburgh:University of Edinburgh. 
Smith DJ and McVie S. (2003) Theoryand Method in the Edinburgh Study of Youth Transitions and Crime. The British Journal of Criminology 43(1): 169-195.

Smith GJD, Bennett Moses L and Chan J. (2017) The Challenges of Doing Criminologyin the Big Data Era: Towards a Digital and Data-Driven Approach. The British Journal of Criminology 57(2): 259-274.

Smith R. (2006) Actuarialism and Early Intervention in Contemporary Youth Justice. In: Goldson B and Muncie J (eds) Youth Crime and Justice. London: Sage Publications Ltd, 92-109.

Snijders TAand BoskerRJ. (2012) Multilevel Analysis: An Introduction to Basic and Advanced Multilevel Modeling (2nd Edition). London: SAGE.

SpiegelhalterDJ, Abrams KR and Myles JP. (2004) Bayesian Approaches to Clinical Trials and HealthCare Evaluation. Chichester: John Wiley \& Sons.

Spiegelhalter DJ, Best NG, Carlin BP, et al. (2002) Bayesian Measures of Model Complexityand Fit. Journal of the Royal Statistical Society: Series B (Statistical Methodology) 64(4):583-616.

Spiegelhalter DJ, Best NG, Carlin BP, et al. (2014) The Deviance Information Criterion: 12 Years On. Journal of the Royal Statistical Society: Series B (Statistical Methodology) 76(3):485-493.

Srinivasan S, Moser RP, Willis G, etal. (2015) Small Is Essential: Importance of SubpopulationResearch in Cancer Control. American Journal of Public Health 105(Suppl 3): S371-S373.

Staines J. (2016) Risk, Adverse Influence and Criminalisation: Understanding the over-Representation of Looked after Children in the Youth Justice System. London: Prison Reform Trust.

Stephenson M, Henri G and Brown S. (2007) Effective Practice in Youth Justice. Cullompton: Willian Publishing.

Stephenson M, Henri G and Brown S. (2011) Effective Practice in Youth Justice. Abingdon, Oxon: Routledge.

Stone J. (2013) Bayes' Rule: A Tutorial Introduction to Bayesian Analysis. Milton Keynes: Sebtel Press.

Sullivan CJ and McGloin JM. (2014) Looking Back to Move Forward. Journal of Research in Crime and Delinquency 51(4): 445-466.

Sullivan CJ and Mieczkowski T. (2008) Bayesian Analysis and the Accumulation of Evidence in Crime and Justice Intervention Studies. Journal of Experimental Criminology 4(4):381-402.

Sutherland A. (2009) The Scaled Approach in Youth Justice: Fools Rush in.... Youth Justice 9(1): 4460.

Sutherland A, DisleyE, Cattell J, et al. (2017) An Analysis of Trends in First Time Entrants to the Youth Justice System. Available at: https://www.gov.uk/government/publications/analysis-of-trendsin-first-time-entrants-to-the-youth-justice-system. (Accessed 31/1/18).

Sutton AJ and Abrams KR. (2001) Bayesian Methods in Meta-Analysis and Evidence Synthesis. Statistical Methods in Medical Research 10(4):277-303.

TalbotJ. (2010) Seen and Heard: Supporting Vulnerable Children in the Youth Justice System. London: Prison Reform Trust.

Tarran B. (2014) How Turing - and Bayes - Cracked Enigma. Available at: http://www.statslife.org.uk/history-of-stats-science/1909-how-turing-and-bayes-cracked-theenigma-code. (Accessed 31/1/18).

Teli B. (2011) Assessment and Planning Interventions: Review and Redesign Project. London: Youth Justice Board.

The Communication Trust. (2014) Doing Justice to Speech, Language and Communication Needs: Proceedings of a Round Table on Speech Language and Communication Needs in the Youth Justice Sector - November 2014. London: The Communication Trust.

ThornberryTP, Lizotte AJ, Krohn MD, et al. (1994) DelinquentPeers, Beliefs, and DelinquentBehavior. A Longitudinal Test of Interactional Theory. Criminology 32:47-84.

Trafimow D and Marks M. (2015) Editorial. Basic Applied Social Psychology 37: 1-2.

TullyRJ, Chou SN and Browne KD. (2013) A Systematic Review on the Effectiveness of Sex Offender Risk Assessment Tools in Predicting Sexual Recidivism of Adult Male Sex Offenders. Clinical Psychology Review 33(2):287-316.

Turnbull G and Spence J. (2011) What's at Risk? The Proliferation of Risk across Child and Youth Policy in England. Journal of Youth Studies 14(8): 939-959.

UK Data Service. (2018) Data by Theme: Crime. Available at: https://www.ukdataservice.ac.uk/getdata/themes/crime. (Accessed 31/1/18). 
van der Put CE. (2014) Youth Actuarial RiskAssessment Tool (Y-Arat): The Development of an Actuarial Risk Assessment Instrumentfor Predicting General Offense Recidivism on the Basis of Police Records. Assessment21(3): 340-351.

van der Put CE, Deković M, Hoeve M, et al. (2014) Risk Assessment of Girls: Are There Any Sex Differences in Risk Factors for Re-Offending and in Risk Profiles? Crime \& Delinquency 60(7): 1033-1056.

Vaswani N and Merone L. (2014) Are There Risks with Risk Assessment? A Study of the Predictive Accuracy of the Youth Level of Service-Case Management Inventory with Young Offenders in Scotland. British Journal of Social Work44(8): 2163-2181.

Wagenmakers E-J, Love J, Marsman M, et al. (2017) Bayesian Inference for Psychology. Part II: Example Applications with JASP. Psychonomic Bulletin \& Review. doi: 10.3758/s13423-0171323-7

Wagner K and Gill J. (2005) Bayesian Inference in Public Administration Research: Substantive Differences from Somewhat Different Assumptions. Journal of Public Administration 28:5-35.

Walklate S and Mythen G. (2011) Beyond Risk Theory. Experiential Knowledge and 'Knowing Otherwise'. Criminology \& Criminal Justice 11(2): 99-113.

Wang M and Zhang L. (2012) A Bayesian Quantile Regression Analysis of Potential Risk Factors for Violent Crimes in USA. Open Journal of Statistics 2: 526-533.

Warr M. (2012) The Social Side of DelinquentBehavior. In: Feld BC and Bishop DM (eds) The Oxford Handbook of Juvenile Crime and Juvenile Justice. New York: Oxford University Press, 226245.

Wasserstein RL and Lazar NA (2016) The ASA's Statement on P-Values: Context, Process, and Purpose. The American Statistician 70(2): 129-133.

Weisburd D, Mazerolle L and Petrosino A. (2007) The Academy of Experimental Criminology: Advancing Randomized Trials in Crime and Justice. The Criminologist 32(3):1-7.

Welsh Assembly Governmentand Youth Justice Board. (2009) All Wales Youth Offending Strategy: Delivery Plan 2009-11. Available at:

http://dera.ioe.ac.uk/9616/13/fileDownload.asp_file\%3DAIl\%2BWales\%2BYouth\%2BOffendin g\%2BStrategy\%2B-\%2BDelivery\%2BPlan\%2B2009-11_Redacted.pdf. (Accessed 31/1/18).

Welsh Governmentand Youth Justice Board. (2014) Youth Justice Strategy for Wales: Children and Young People First. Available at:

https:/www.gov.uk/government/uploads/system/uploads/attachment_data/file/374572/Youth_ Justice_Strategy_English.PDF.(Accessed 31/1/18).

West DJ and Farrington D. (1977) The Delinquent Way of Life. London: Heinemann.

Western B. (1999) Bayesian Analysis for Sociologists: An Introduction. Sociological Methods \& Research 28(1): 7-34.

White P. (2009) Developing Research Questions: A Guide for Social Scientists. Basingstoke: Palgrave Macmillan.

Wilson AJ, Réale D, Clements MN, et al. (2010) An Ecologist's Guide to the Animal Model. Journal of Animal Ecology 79(1): 13-26.

Wilson E. (2013) Youth Justice Interventions - Findings from the Juvenile Cohort Study (JCS).

Available at:

https://www.gov.uk/government/uploads/system/uploads/attachment_data/file/266405/juvenile -cohort-study.pdf. (Accessed 31/1/18).

Wilson E and Hinks S. (2011) Assessing the Predictive Validity of the ASSET Youth Risk Assessment Tool Using the Juvenile Cohort Study (JCS). London: Ministry of Justice.

Woolston C. (2015) Psychology Journal Bans P-Values. Nature. doi: 0.1038/519009f

Young S and Goodwin E. (2010) Attention-Deficit/Hyperactivity Disorder in Persistent Criminal Offenders: The Need for Specialist T reatmentPrograms. Expert Review of Neurotherapeutics 10(10): $1497-1500$.

Youth Justice Board. (2005a) Risk and Protective Factors. London: Youth Justice Board.

Youth Justice Board. (2005b) Risk and Protective Factors (Summary Report). London: Youth Justice Board.

Youth Justice Board. (2008a) ASSET Core Profile - Guidance. Available at: https://www.gov.uk/government/publications/asset-documents. (Accessed 31/1/18). 
Youth Justice Board. (2008b) ASSET Core Profile - Introduction. Available at: https://www.gov.uk/government/publications/asset-documents. (Accessed 31/1/18).

Youth Justice Board. (2008c) Youth Justice: The Scaled Approach Consultation Summary. Summary of Issues Raised in Consultation and Next Steps.

Youth Justice Board. (2008d) Youth Justice: The Scaled Approach: A Framework for Assessment and Interventions: Post-Consultation.

Youth Justice Board. (2009a) Scaled Approach Making Strides. Available at: http://www.cjp.org.uk/news/archive/scaled-approach-making-strides-15-10-2009/. (Accessed 31/1/18).

Youth Justice Board. (2009b) Youth Justice: The Scaled Approach: A Framework for Assessment and Interventions: Post-Consultation (Version 2). Available at: http://webarchive.nationalarchives.gov.uk/+/http://www.yjb.gov.uk/publications/Resources/Do wnloads/Youth\%20Justice\%20-\%20The\%20Scaled\%20Approach\%202009.pdf. (Accessed 31/1/18).

Youth Justice Board. (2010a) Process Evaluation of the Pilot of a Risk-Based Approach to Interventions. London:Youth Justice Board.

Youth Justice Board. (2010b) Youth Justice: The Scaled Approach - a Framework for Assessment and Interventions. Available at:

http://webarchive.nationalarchives.gov.uk/20110601215311/https://www.yjb.gov.uk/Publicatio ns/Resources/Downloads/Youth\%20Justice\%20the\%20Scaled\%20Approach\%20-

$\% 20 \mathrm{~A} \% 20$ framework\%20for\%20assessment\%20and\%20interventions.pdf. (Accessed $31 / 1 / 18)$.

Youth Justice Board. (2013) National Standards for Youth Justice Services. Available at: https://www.gov.uk/government/uploads/system/uploads/attachment_data/file/296274/nationa I-standards-youth-justice-services.pdf. (Accessed 31/1/18).

Youth Justice Board. (2014) ASSETPLUS Framework Model. Available at: https://www.gov.uk/government/uploads/system/uploads/attachment_data/file/362278/AssetPI us_framework_diagram.pdf. (Accessed 31/1/18).

Youth Justice Board. (2016a) Understanding and Improving Reoffending Performance: A Summary of Learning from the YJBs Reoffending Programme with Implications for Practice. Available at: https://yjresourcehub.uk/yjb-effective-practice/youth-justicekits/item/download/563_c323b3ab846cf1babaf74c698a02ed12.html.

Youth Justice Board. (2016b) Understanding and Improving Reoffending Performance:Annex B WhatDoes ASSET Data Tell Us About Changes in the Youth Justice Cohortover Time? Available at: https://yiresourcehub.uk/yjb-effective-practice/youth-justicekits/item/download/567_5f1df82e058308c67a2ec3cdb35b294e.html. (Accessed 31/1/18).

Youth Justice Board. (2017a) ASSETPLUS Deployment List 30-08-17. Available at: https://www.gov.uk/government/uploads/system/uploads/attachment_data/file/658941/AssetPI us_deployment_list_30-08-2017.xls. (Accessed 21/11/17).

Youth Justice Board. (2017b) Youth Justice Resource Hub:How to Reduce Reoffending by Children and Young People. Available at: https://yiresourcehub.uk/yjb-effective-practice/youth-justicekits/item/469-how-to-reduce-reoffending-by-children-and-young-people.html. (Accessed $31 / 1 / 18)$.

Youth Justice Board and Ministry of Justice. (2015a) Youth Justice Statistics 2013/14 - England and Wales (Supplementary Volumes). Available at: https://www.gov.uk/government/uploads/system/uploads/attachment_data/file/399654/youthjustice-stats-2013-14-supp-volumes.zip. (Accessed 31/1/18).

Youth Justice Board and Ministry of Justice. (2015b) Youth Justice Statistics 2013/14 for England and Wales. Available at: https://www.gov.uk/government/uploads/system/uploads/attachment_data/file/399379/youthjustice-annual-stats-13-14.pdf. (Accessed 31/1/18).

Youth Justice Board and Ministry of Justice. (2015c) Youth Justice Statistics Glossary. Available at: https://www.gov.uk/government/uploads/system/uploads/attachment_data/file/399562/youthjustice-stats-glossary.pdf. (Accessed 31/1/18). 
Youth Justice Board and Ministry of Justice. (2018) Youth Justice Statistics 2016/17 for England and Wales. Available at: https://www.gov.uk/government/uploads/system/uploads/attachment_data/file/676072/youth_j ustice_statistics_2016-17.pdf. (Accessed 31/1/18).

Youth Justice Board and Royal College of Speech and Language Therapists. (2015) Practice Advice: Speech, Language and Communication Needs (SLCN) in the Youth Justice System. Available at: https://www.gov.uk/government/publications/speech-language-andcommunication-needs-in-the-youth-justice-system/practice-advice-speech-language-andcommunication-needs-sicn-in-the-youth-justice-system. (Accessed 31/1/18).

Zahriyeh E. (2014) Maths Equation Could Help Find Missing Malaysian Plan. Available at: http://america.aljazeera.com/articles/2014/3/12/mathematicalequationcouldhelpfindmissingmalaysianplane.html\#. (Accessed 31/1/18).

Zhu L, Gorman DM and Horel S. (2006) Hierarchical Bayesian Spatial Models for Alcohol Availability, Drug "Hot Spots" and Violent Crime. International Journal of Health Geographics 5(54):1-12.

Zwahlen M. (2009) Commentary: Cornfield on Cigarette Smoking and Lung Cancer and How to Assess Causality. International Journal of Epidemiology38(5):1197-1198. 


\section{Towards A Bayesian Approach in Criminology: A Case Study of Risk Assessment in Youth Justice}

\section{Technical Annex}

\section{Helen Hodges}

Submitted to Swansea University in fulfilment of the requirements for the Degree of Doctor of Philosophy

Swansea University

2018 



\section{Contents}

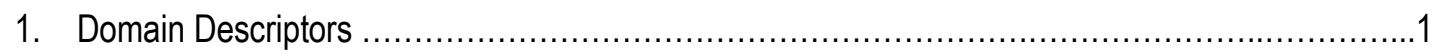

2. Youth Justice Board Gravity Scores, by Offence Category .............................................

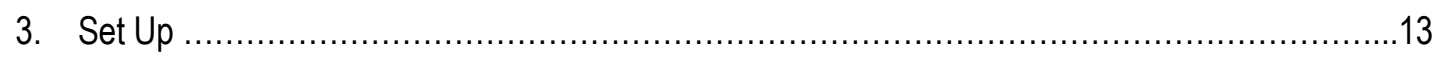

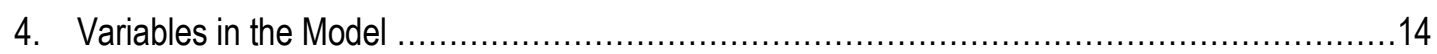

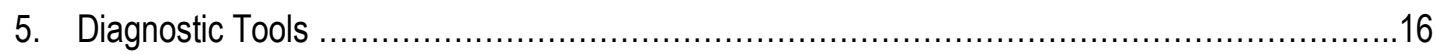

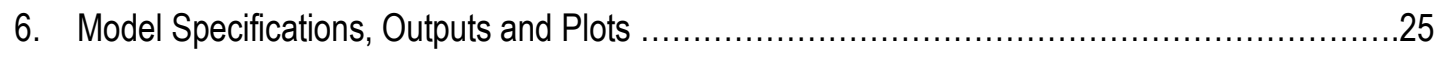

Chapter Four - Preparatory Models to Explore the Risk Assessment Domains......................25

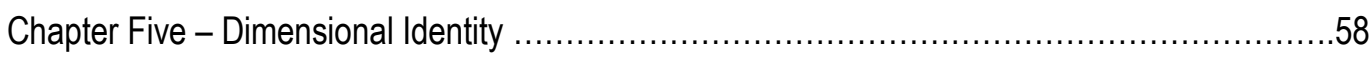

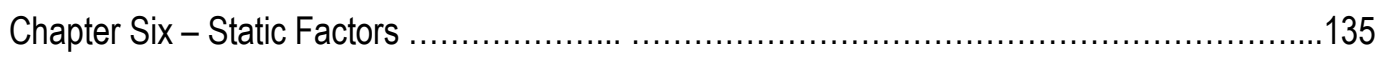

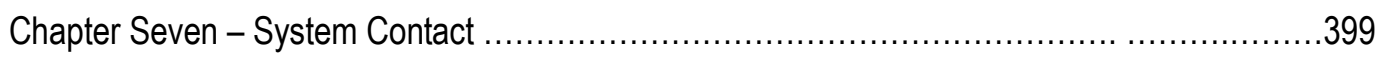

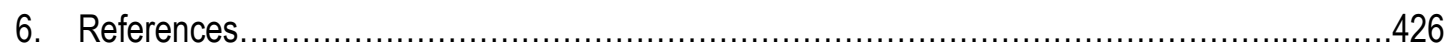





\section{List of Models}

Chapter Four - Preparatory models to explore the risk assessment domains..................................25

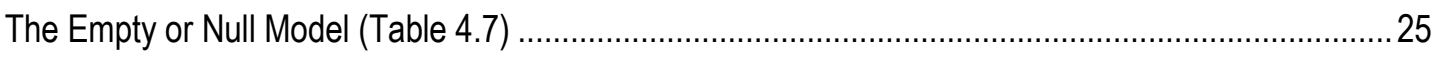

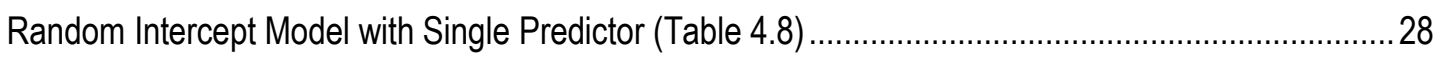

Random Intercept Model with ASSET Domain Predictors (Table 4.9) ................................................ 31

Random Intercept and Varying Slope Model, with Single Predictor (Table 4.10)................................ 39

The Basic Model: Random Intercept and Varying Slope Model, with ASSET Domain Predictors (Table

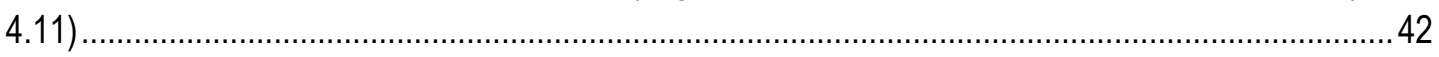

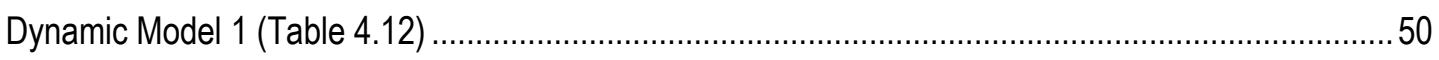

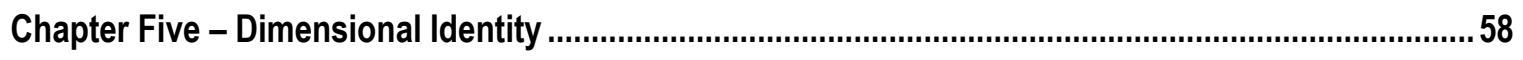

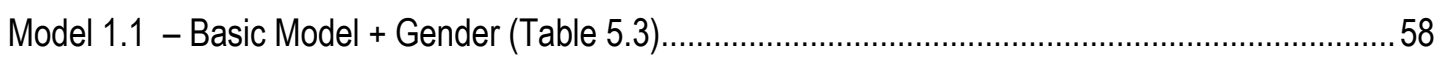

Model 1.2 - Basic Model + Ethnicity (Table 5.3) ...................................................................... 62

Model 1.3 - Basic Model + Demographics (Table 5.3) …...................................................... 66

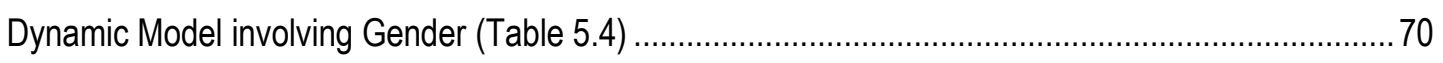

Dynamic Model involving Ethnicity (Table 5.5).................................................................... 83

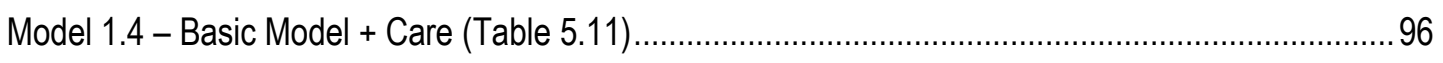

Dynamic Model involving Care (Table 5.12) .................................................................... 100

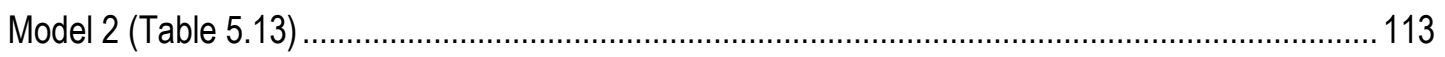

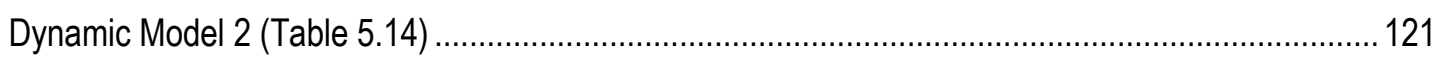

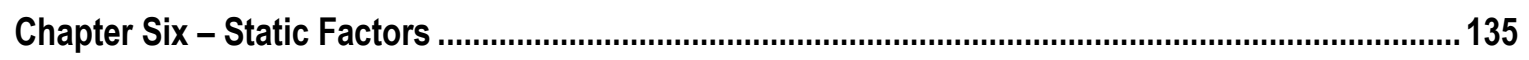

Model 1.5 - Basic Model + Grouped Age at First Offence (Table 6.4) .......................................... 135

Model 1.6 - Basic Model + Grouped Age at First Conviction (Table 6.4) ...................................... 139

Model 1.7 - Basic Model + FTE (Table 6.5) ......................................................................... 143

Model 1.8 - Basic Model + Offence Category (Table 6.7) …..................................................... 147

Model 1.9 - Basic Model + Grouped YJB Offence Category (Table 6.7) ...................................... 151

Model 3 - Basic Model + Static Factors (Table 6.6) ................................................................... 155

Model 3a - Basic Model + FTE Status, Grouped Age at First Offence and Grouped YJB Offence

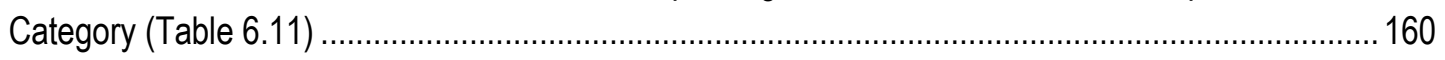

Model 1.10 - Basic Model + YJB Gravity Score (Table 6.12) .................................................... 164

Model 3b - Basic Model + FTE Status, Grouped Age at First Offence and YJB Gravity Score (Table

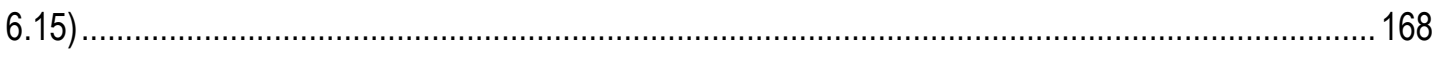

Dynamic Model involving FTE Status (Table 6.16) ….......................................................... 172

Dynamic Model involving Grouped Age at First Offence (Table 6.17) .......................................... 185

Dynamic Model involving Grouped YJB Offence Category (Table 6.18)......................................... 198 



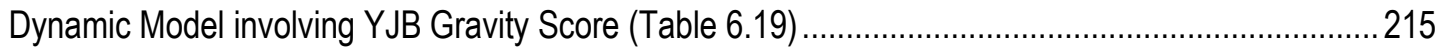

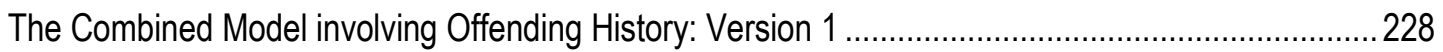

The Combined Model involving Offending History: Version 2a .................................................... 251

The Combined Model involving Offending History: Version 2b .................................................. 269

The Combined Model involving Offending History: Version 2c ................................................... 286

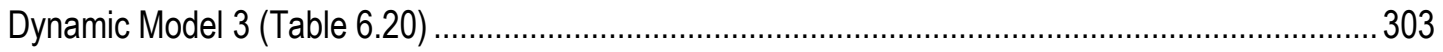

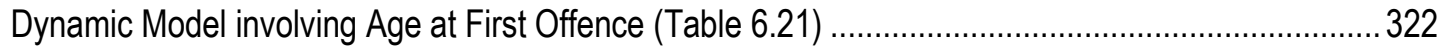

Model 1.11 - Basic Model + Grouped YJB Offence Category and YJB Gravity Score (Table 6.23)...335

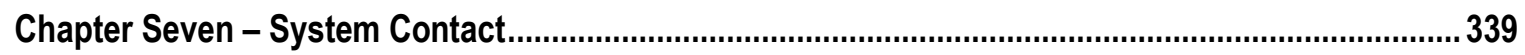

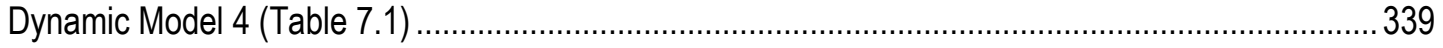

Model 1.12 - Basic Model + Breach (Table 7.4) ...................................................................... 356

Model 1.13 - Basic Model + Court Appearance (Table 7.4) …...................................................... 360

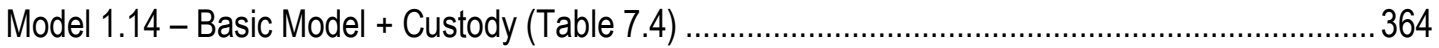

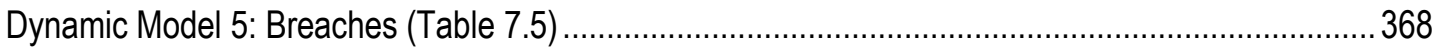

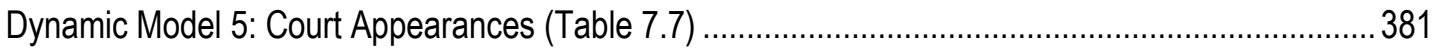

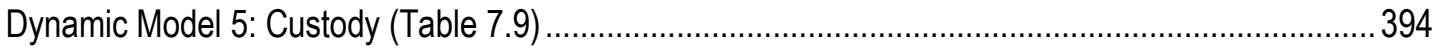

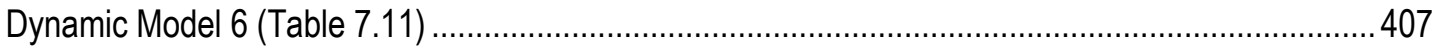





\section{Domain Descriptors}

The following is a summary of the descriptors of the 12 domains taken from the ASSET Core Profile Guidance (Youth Justice Board, 2008a). The document was provided to practitioners to support them in their completion of the assessment. Although the roll out of ASSETPlus was completed in July 2017, it is currently possible to access a full set of the paperwork associated with the ASSET Core Profile from:

\section{https://www.gov.uk/government/publications/asset-documents}

\section{Living Arrangements}

This domain is concerned with the extent to which the young person's living arrangements are associated with the likelihood of further offending. In addition to considering who the young person has mostly been living with over the last six months (or if in custody, the six months prior to this), practitioners are asked to identify if any of the following apply:

- No fixed abode

- Unsuitable, does not meet his/her needs

- Living with known offender/s

- Disorganised/chaotic

- Other problems eg isolation, accommodation provides opportunities for offending, availability of drugs within the house / home.

Examples of high ratings ( 3 or 4 ) include:

- The young person lives with known offenders who are clearly involving or encouraging him/her in offending behaviour.

- The young person is living on the streets and is offending to survive.

- Accommodation is stable, but s/he is living with someone they have previously stolen from or assaulted.

- Living arrangements give the young person access to potential vulnerable victims (e.g. younger siblings).

\section{Family and Personal Relationships}

This section focuses on some of the key relationships in a young person's life and highlights situations where s/he may have lost contact with someone. The phrase 'in contact with' captures a variety of interactions, both positive and negative (eg personal contact, letters, phone calls and so on). Problematic issues considered as part of the assessment include:

- Evidence of family members or carers with whom the young person has been in contact over the last six months being involved in criminal activity, heavy alcohol misuse, drug or solvent misuse

- Significant adults failing to communicate with or show care/interest in the young person

- Experience of abuse

- Witnessing other violence in family context

- Significant bereavement or loss

- Difficulties with care of his/her own children 
Examples of high ratings ( 3 or 4 ) include:

- There is a close family member who is criminally active and is involving him/her in offending.

- Supervision is inconsistent and parents/carers do not know where the young person goes or who s/he is with.

- The young person is offending to obtain attention from carers who show no interest in him/her.

- Combinations of problems present at the same time (e.g. one parent with a mental health problem and one who is criminally active).

\section{Education, Training and Employment (ETE)}

Assessments should draw on a range of evidence including:

- educational records such as test/exam results, educational plans (in particular Statements of Educational Need and Personal Education Plans for young people in the care of the local authority), school/college reports, records of achievement/progress files;

- interviews/discussion with young people, their parents/carers and other professionals such as teachers/tutors;

- practitioner observation of the way in which a young person speaks, listens, reads, writes and approaches concepts related to number, time, directions etc.

The assessment focuses upon:

- Engagement in education, training or employment ie whether of compulsory school age, number of hours of ETE arranged each week; number of hours of ETE currently engaged in / receiving each week and if there is evidence of non-attendance

- Educational attainment including if special education needs (SEN) has been identified

- Other factors eg being bullied or being a bully

Examples of high ratings ( 3 or 4 ) include:

- Most of his/her offending occurs when s/he is not attending school/college/training/ employment.

- The young person offends whilst on school premises and sees school as providing opportunities for offending.

- The young person thinks that getting a job is a waste of time because it won't pay as much as s/he could get from crime.

- The young person regularly uses work opportunities (e.g. access to certain people or resources) for offending.

- The young person lacks training/work, is persistently bored and offends to fill up the time.

\section{Neighbourhood}

Practitioners are required to provide a brief description of the neighbourhood in which the young person spends most of their time and to consider issues such as:

- Obvious signs of drug dealing and/or usage

- Isolated location / lack of accessible transport

- Lack of age-appropriate facilities eg youth clubs, sports facilities

- Racial or ethnic tensions 
- Other problems eg lack of amenities such as shops or post office, opportunities to sell stolen goods, red-light district, tensions between police and the local community

Examples of high ratings ( 3 or 4 ) include:

- All of the young person's offending occurs within the same neighbourhood.

- Many opportunities for offending in the area which seem attractive and profitable to him/her.

\section{Lifestyle}

This domain considers if the young person:

- Has a lack of age-appropriate friendships (if friends/ associates are noticeably younger / older)

- Is associating with predominately pro-criminal peers

- Has a lack of non-criminal friends

- Is participating in reckless activities (not just offending)

- Has inadequate legitimate personal income

Examples of high ratings (3 or 4$)$ include:

- All of the young person's offending occurs with a particular group of friends

- The young person is offending to obtain money for a gambling habit

- The young person is bored, has little to do and sees offending as a necessary way of getting some excitement in life

- The young person is involving younger friends in offending

\section{Substance Use}

This domain focuses on the young person's attitudes and choices about substance use, and in particular occasions when s/he has used substances independently or with friends / associates. It is not limited to drug use as issues about tobacco, alcohol and solves, especially at a young age are also taken into account. Ratings are based upon:

- Historic substance use including experimental and one-off use

- Recent use ie usage that is an on-going aspect of the young person's life (although this does not necessarily relate to frequent use)

- Practices which put him / her at particular risk

- Seeing substance use as a positive and/or essential to life

- Noticeably detrimental effect on education, relationships, daily functioning

Practitioners are advised that some information will not always be disclosed, particularly at first interview and hence ratings may be revised as a result of information coming to light later on as well as if there is a real difference observed in the pattern of substance use.

Examples of high ratings ( 3 or 4 ) include:

- A 'yes' response to the question about offending to obtain money for substances.

- All his/her offending occurs whilst under the influence of substances. 
- The young person's attitudes and willingness to experiment with substances increases the likelihood of him/her being found in possession of illegal drugs.

\section{Physical Health}

Practitioners are advised that a comprehensive assessment of the young person requires some consideration of his/her physical health and development. Health problems may have an adverse impact on many other aspects of his/her life, including educational and school experiences, peer group interactions, self-presentation and self-esteem.

Health needs will clearly vary according to age and gender and this needs to be borne in mind throughout the section. Consideration should also be given to any cultural or religious beliefs of the young person and his/her family which may affect health care. Problematic issues include:

- Health condition which significantly affects everyday life functioning

- Physical immaturity / delayed development

- Problems caused by not being registered with GP

- Lack of access to other appropriate health care services eg dentist

- Health put at risk through his/her own behaviour

The links between physical health and offending behaviour will usually be indirect and consequently there will be a tendency towards lower ratings in this section. For example:

- a condition which leads to disruptive behaviour at school and possible exclusion

- an impairment which makes it more difficult for him/her to find suitable work or training

- the young person's frustration with a health problem contributes to aggressive behaviour

- other negative effects e.g. poor school attendance, low self-esteem

\section{Emotional and Mental Health}

In making their assessment practitioners are reminded that mental and emotional well-being will be influenced by issues such as personal relationships and social environment as well as medical factors. Different cultural groups will vary in their views about what constitutes emotional well-being and this needs to be borne in mind. The following three factors, however, may provide a useful framework for understanding the young person's mental health needs within the context of his/her particular situation.

- Events/circumstances

- Support networks

- Coping abilities

This section may raise some issues which cannot be fully assessed in the context of the Core Profile eg issues about mental illness or suicide attempts. The expectation is therefore that ASSET should act as a 'trigger' to highlight areas where further specialist assessment may be required.

The focus of the assessment is upon if:

- The young person's daily functioning is significantly affected by emotions or thoughts resulting from coming to term with significant past event/s; their current circumstances or concerns about the future

- There has been a formal diagnosis of mental illness 
- Any other contact with, or referrals to, mental health services

- There are indications that any of the following apply to the young person

- S/he is affected by other emotional or psychological difficulties

- S/he has deliberately harmed her/himself

- S/he has previously attempted suicide

Examples of high ratings ( 3 or 4 ) include:

- There is a direct link with symptoms of mental illness (e.g. offending due to hallucinations, delusions, hearing voices).

- The young person is struggling to cope with strong feelings of anger/hatred and is likely to take this out on other people.

- Offending happens at specific times (e.g. when s/he fails to take medication or misses appointments with psychiatrist).

\section{Perception of Self and Others}

This domain is concerned with whether s/he:

- has difficulties with self-identity

- has inappropriate self-esteem

- has a general mistrust of others

- displays discriminatory attitudes towards others

- perceives him/herself as having a criminal identity

Examples of high ratings (3 or 4 ) include:

- Discriminatory attitudes that provide a clear motive for his/her offending

- The young person sees crime as his/her 'career' and thinks that s/he will always be involved in offending

- The young person's self-esteem is dependent on the sense of achievement that $s /$ he gets from offending

\section{Thinking and Behaviour}

This section draws together information about the young person from other sections of the ASSET to identify patterns of thinking and types of behaviours which cause difficulties for him/her. As such the practitioner is asked to identify if the young person's actions are characterised by any of the following:

- Lack of understanding of consequences

- Impulsiveness

- Need for excitement

- Giving in easily to pressure from others

- Inappropriate social and communication skills

And of the young person displays any of the following types of behaviour in different settings:

- Destruction of property

- Aggression towards others

- Sexually inappropriate behaviour 
- Attempts to manipulate / control others

Examples of high ratings ( 3 or 4$)$ include:

- Combination of impulsiveness, poor temper control and aggression means a high risk of violent behaviour

- The young person's need for excitement frequently leads him/her into offending situations

\section{Attitudes to Offending}

Ratings this domain are based upon whether the young person displays any of the following attitudes in relation to the offences which triggered the assessment. However, if there were any significant issues about attitudes to past offences then these could also be included:

- Denial of the seriousness of his/her behaviour

- Reluctance to accept responsibility for involvement in most recent offence(s)

- Lack of understanding about the impact of his/her behaviour on victims

- Lack of remorse

- Lack of understanding about impact of his/her behaviour on family / carers

- A belief that certain types of offences are acceptable

- A belief that certain people /groups are acceptable 'targets' of offending behaviour

- $\mathrm{S} / \mathrm{he}$ thinks that further offending is inevitable

Examples of high ratings ( 3 or 4 ) include:

- Attitude that provides a direct motive for his/her offending.

- The young person's genuine belief that further offending is inevitable.

- Clusters of these attitudes.

\section{Motivation to Change}

This domain considers whether the young person displays any of the following attitudes:

- An appropriate understanding of the problematic aspects of his/her own behaviour

- Understanding of the consequences for him/herself of further offending

- Has identified clear reasons or incentives for him/her to avoid further offending

- Shows real evidence of wanting to stop offending

Examples of high ratings ( 3 or 4 ) include:

- The young person has no understanding of the problematic aspects of his/her behaviour.

- The young person cannot identify any incentives to stop offending.

- There is no evidence from his/her behaviour of a desire to change. 


\section{Youth Justice Board Gravity Scores, by Offence Category}

The following has been taken from Appendix B of the ASSET Guidance (Youth Justice Board, 2008a: 3-9)

Violence against the person

\begin{tabular}{|c|c|}
\hline $\begin{array}{l}\text { Abduction/Kidnapping } \\
\text { - } \quad \text { Abduction of female by force } \\
\text { - } \quad \text { Child abduction } \\
\text { - } \quad \text { False imprisonment } \\
\text { - } \quad \text { Hijacking } \\
\text { - } \quad \text { Kidnapping }\end{array}$ & 7 \\
\hline $\begin{array}{l}\text { Assault police officer } \\
\text { - Assault with intent to resist arrest or assaulting a person assisting a police constable }\end{array}$ & 3 \\
\hline $\begin{array}{l}\text { Common assault } \\
\text { - } \quad \text { Assault and battery } \\
\text { - }\end{array}$ & 3 \\
\hline Grievous Bodily Harm & 6 \\
\hline $\begin{array}{l}\text { Manslaughter } \\
\text { - } \quad \text { Child destruction, infanticide or manslaughter due to diminished responsibility }\end{array}$ & 8 \\
\hline $\begin{array}{l}\text { Murder } \\
\text { - } \quad \text { Attempted murder }\end{array}$ & 8 \\
\hline $\begin{array}{l}\text { Indictable firearms offences } \\
\text { - Possessing a real or imitation firearm at the time of committing or being arrested for an offence } \\
\text { - Ppecified in Schedule } 1 \text { of the Firearms Act } 1968 \\
\text { - } \text { including resisting arrest } \\
\text { - Possession of real or imitation firearms/explosives with intent to cause violence }\end{array}$ & 5 \\
\hline $\begin{array}{l}\text { Other wounding } \\
\text { - } \quad \text { Administering poison with intent to injure or annoy } \\
\text { - } \quad \text { Assault occasioning actual bodily harm }(\mathrm{ABH})\end{array}$ & 4 \\
\hline $\begin{array}{l}\text { Possession of an offensive weapon } \\
\text { - Having an article with a blade or point in a public place }\end{array}$ & 3 \\
\hline Threatening, abusive or insulting words or behaviour & 3 \\
\hline $\begin{array}{l}\text { Threat or conspiracy to murder } \\
\text { - Soliciting to commit murder }\end{array}$ & 5 \\
\hline $\begin{array}{l}\text { Wounding or other act endangering life } \\
\text { - } \quad \text { Attempting to choke, suffocate with intent to commit an indictable offence (garrotting) } \\
\text { - } \quad \text { Burning or maiming by explosion } \\
\text { - } \text { equipment } \\
\text { - Causing explosions or casting corrosive fluids with intent to do grievous bodily harm } \\
\text { - Endangering life or causing harm by administering poison } \\
\text { - } \text { Endangering railway passengers (by placing anything on railway, taking up rails, changing points } \\
\text { - } \quad \text { Pausing danger to road users (throwing stones etc.) } \\
\text { - Using chloroform to commit or assist in committing an indictable offence } \\
\text { - Using firearms or imitation firearms with intent to resist arrest }\end{array}$ & 7 \\
\hline Wounding with intent to cause grievous bodily harm (section 18$)^{*}$ & 7 \\
\hline Other/unspecified violence against the person & 4 \\
\hline
\end{tabular}




\begin{tabular}{|l|c|}
\hline Buggery & 7 \\
\hline Gross indecency with a child & 5 \\
\hline $\begin{array}{l}\text { Incest } \\
\text { - Incest with a female under 13 }\end{array}$ & 7 \\
\hline$\quad$ Inciting a girl under 16 to have incestuous sexual intercourse & \\
\hline Indecent assault & 5 \\
\hline Indecent behaviour/exposure & 4 \\
\hline $\begin{array}{l}\text { Rape } \\
\text { - Assault with intent to commit rape or buggery }\end{array}$ & 8 \\
- Attempted rape & \\
\hline Unlawful sexual intercourse with female under 13 & \\
\hline Unlawful sexual intercourse with female under 16 & 4 \\
\hline Other/unspecified sexual offences & 3 \\
\hline
\end{tabular}

\section{Death or Injury by Dangerous Driving}

\section{Death by dangerous driving}

- Causing death by aggravated vehicle taking

- Causing death by dangerous driving when under the influence of drink or drugs

Injury by dangerous driving

- Causing injury by aggravated vehicle taking

- Causing injury by dangerous driving when under the influence of drink or drugs

\section{Motoring Offences}

\begin{tabular}{|l|c|}
\hline Dangerous driving & $\mathbf{5}$ \\
\hline Driving when under the influence of drink / drugs & 3 \\
\hline Driving whilst disqualified & $\mathbf{5}$ \\
\hline Interfering with a motor vehicle & 3 \\
\hline Refusing to give a breath test & 4 \\
\hline Road Traffic / Additional Offences & $\mathbf{2}$ \\
- Driving without due care and attention & \\
- Driving on a footpath or/and common land & \\
- Driving defective motor vehicle & \\
- Exceeding speed limit & \\
- Failure to wear a seatbelt & \\
- Failure to comply with a road traffic sign & \\
- Failure to give particulars after an accident & \\
- Failure to produce documents & \\
- Failure to report an accident & \\
- Failure to stop when requested by a constable & \\
- Forge vehicle records/licence & \\
- No insurance & \\
- No L plates & \\
- No licence & \\
- No MOT & \\
\hline
\end{tabular}


- Not wearing protective headgear

- Not well maintained indicators/stop/hazard and light reflectors

- Pedal cycle offences

Other / Unspecified motoring offences

\section{Robbery}

Robbery

- Assault with intent to rob

- Conspiracy to rob

\section{Domestic Burglary}

\section{Aggravated burglary of a dwelling}

- Burglary with violence or threat of violence

Burglary in a dwelling

- Conspiracy to commit burglary of a dwelling

Other/unspecified domestic burglary

Non-Domestic Burglary

\begin{tabular}{|l|c|}
\hline $\begin{array}{l}\text { Aggravated burglary of a non-dwelling } \\
-\quad \text { Burglary with violence or threat of violence }\end{array}$ & 7 \\
\hline $\begin{array}{l}\text { Burglary in a non-dwelling } \\
-\quad \text { Conspiracy to commit burglary of a non-dwelling }\end{array}$ & 4 \\
\hline Found on enclosed premises & 3 \\
\hline Other/unspecified non-domestic burglary & 4 \\
\hline
\end{tabular}

Vehicle theft / unauthorised vehicle taking

\begin{tabular}{|l|c|}
\hline $\begin{array}{l}\text { Aggravated vehicle taking } \\
-\quad \text { Injury to person, damage to property or car }\end{array}$ & $\mathbf{5}$ \\
\hline $\begin{array}{l}\text { Being carried } \\
-\quad \text { Being carried (aggravated) }\end{array}$ & 3 \\
\hline $\begin{array}{l}\text { Vehicle taking } \\
-\quad \text { Theft of motor vehicle } \\
-\quad \text { Unauthorised vehicle taking (TWOC/TADA) }\end{array}$ & 4 \\
\hline Other/unspecified vehicle theft/taking & 4 \\
\hline
\end{tabular}

\section{Theft and Handling Stolen Goods}

Handling stolen goods

- Receiving stolen goods

- Undertaking or assisting in the retention, removal, disposal or realisation of stolen goods, or arranging to do so

Theft

- Extracting electricity

- Making off without payment

- Going equipped for stealing

- Intent to steal

Other/unspecified theft and handling 


\begin{tabular}{|l|c|}
\hline Forgery & 3 \\
\hline Fraud & \\
\hline - Acting as a peddler without certificate & 3 \\
- Counterfeiting & \\
- $\quad$ Fraudulent use of documents & \\
- Obtaining pecuniary advantage by deception & \\
- Obtaining property by deception & \\
\hline Public / private service vehicle and rail fare evasion & 1 \\
\hline Other/unspecified fraud and forgery & 2 \\
\hline
\end{tabular}

\begin{tabular}{|l|c|}
\hline $\begin{array}{l}\text { Arson endangering life } \\
-\quad \text { Arson reckless as to whether life is in danger }\end{array}$ & 6 \\
\hline Arson not endangering life & 5 \\
\hline Other/unspecified arson & 5 \\
\hline
\end{tabular}

\section{Criminal Damage}

\section{Criminal damage endangering life}

- Forgery, or use of false prescription

Other criminal damage over $£ 2,000$

- Equipped with intent to commit criminal damage

- Threat to commit criminal damage

Other criminal damage under $£ 2,000$

- Equipped with intent to commit criminal damage

- Threat to commit criminal damage

Other/unspecified criminal damage

\section{Drugs}

\begin{tabular}{|l|c|}
\hline Permitting use of premises for use of Class B or Class C drug & 3 \\
\hline Possession - Class A drug & 3 \\
\hline Possession - Class B drug & 2 \\
\hline Possession - Class C drug & 2 \\
\hline $\begin{array}{l}\text { Supply - Class A drug } \\
\text { - Possessing a class A drug with intent to supply } \\
\text { - Offering to supply a class A drug }\end{array}$ \\
\hline $\begin{array}{l}\text { Supply - Class B drug } \\
\text { - Possessing a class B drug with intent to supply } \\
\text { - Offering to supply a class B drug }\end{array}$ \\
\hline $\begin{array}{l}\text { Supply - Class C drug } \\
\text { - Possessing a class C drug with intent to supply }\end{array}$ \\
\hline Offering to supply a class C drug & 4 \\
\hline Unlawful importation or exportation of a controlled drug & \\
\hline Other/unspecified drug offence & 2 \\
\hline
\end{tabular}




\begin{tabular}{|l|c|}
\hline Affray & $\mathbf{4}$ \\
\hline $\begin{array}{l}\text { Bomb hoax } \\
\text { - Supply false information about the presence of bombs }\end{array}$ & $\mathbf{5}$ \\
\hline $\begin{array}{l}\text { Breach of the peace } \\
\text { Behaviour likely to cause breach of the peace }\end{array}$ & $\mathbf{2}$ \\
\hline Drunk and disorderly & $\mathbf{1}$ \\
\hline $\begin{array}{l}\text { Other Public Order Act offences } \\
\text { - Section 4 Public Order Act 1986 (fear or provocation of violence) } \\
\text { - Section 4a Public Order Act 1986 (intentional harassment, alarm or distress) } \\
\text { - Section 5 Public Order Act 1986 (harassment, alarm or distress) } \\
\text { - Placing people in fear of violence }\end{array}$ & $\mathbf{2}$ \\
\hline Rioting & \\
\hline Violent Disorder & $\mathbf{6}$ \\
\hline Other/unspecified drug offence & $\mathbf{5}$ \\
\hline
\end{tabular}

Other

Other specified offences

- Absconding from lawful custody

- Air weapons offences

- Blackmail

- Cruelty to animals or unlawful killing of animals

- Firearms Act Offences (e.g. no firearm licence)

- Interfering with witness/perverting justice

- Obstruct police or fire service

- Public nuisance (common law offence)

- Resisting arrest

- Sending indecent/offensive articles

- Trespassing on a railway

Other minor offences

- Abusive language

- Begging

- Consuming alcohol under the age of 18 in a public place

- Concealment of birth

- Cycling in pedestrian area

- Failure to make children attend school

- Infuriating an animal (Section1 (1) (a) Protection of Animals Act 1911)

- Inciting a child away from local authority care

- Littering

- Nuisance on educational premises

- Urinating in a public place

- Vagrancy

- Making hoax/abusive or malicious telephone calls

- Non-payment of financial penalty

- Purchasing alcohol under the age of 18

- Wasting police time

Other / unspecified offence 
Criminal damage - racially aggravated

Other wounding - racially aggravated

- Actual bodily harm

- Common assault

- Intentional harassment, alarm or distress

- Putting people in fear of violence

- Threatening, abusive or insulting words or behaviour

Wounding or other act endangering life - racially aggravated

- Wounding with intent to do grievous bodily harm

Other/unspecified racially aggravated offence

\section{Breach of conditional discharge}

This only applies where the breach has resulted in an additional substantive outcome. Where a young person has been re-sentenced, please refer back to the original offence for the seriousness score.

\section{Breach of conditions of discharge}

\section{Breach of bail}

This only applies where the breach has resulted in an additional substantive outcome. Where a young person has been re-sentenced, please refer back to the original offence for the seriousness score.

Breach of conditions of bail

Breach of statutory order

This only applies where the breach has resulted in an additional substantive outcome. Where a young person has been re-sentenced, please refer back to the original offence for the seriousness score. 


\section{Set Up}

Set Up the Workspace and Install Relevant Packages

\#\# Clear global environment from existing objects

rm(list $=1 s())$

\#\# Set working directory

setwd("G:/_My Research - current/_A. Hierarchical Bayesian/Modelling")

\#\# Load packages

\#\# Save package names as a vector of strings

pkgs <- c("arm", "foreign", "scales","rjags", R2WinBUGS", "superdiag", "lme4", "BayesFactor", "MCMCglmm", "sjmisc", "sjPlot")

\#\# Install uninstalled packages

lapply (pkgs [! (pkgs 으을 installed.packages())], install.packages)

\#\# Load all packages to library

lapply (pkgs, library, character.only = TRUE)

Data

\#\# Load data

dataFULL <- read.cSv("Asset_R_Version.csv", header = TRUE, skip = 0) head (dataFULL)

$\operatorname{dim}(\operatorname{dataFULL})$

\# [1] $55261 \quad$ - $552 \times$ x ASSETS with 61 variables, some of which have missing data

\#\# Load data modified version of the dataset as MCMCgImm doesn't handle missing data

dataNM <- read.csv("Asset_R_Version - No Miss.CSV", header = TRUE, skip $=0$ )

head (dataNM)

data.nomiss <- na.omit (datanM)

$\operatorname{dim}($ data.nomiss)

\# [1] 54530 - have lost 7 ASSET records as a result of doing this, but now no missing data

data $<-$ data.nomiss 


\section{Variables in the Model}

\begin{tabular}{|c|c|c|c|}
\hline Type & \multicolumn{2}{|c|}{ Coding } & Notes \\
\hline $\begin{array}{l}\text { Outcome } \\
\text { Variable }\end{array}$ & \multicolumn{2}{|c|}{ FO.bin <- data\$Further_Offending } & $\begin{array}{l}\text { Time-varying, dichotomous measure } \\
\text { reflecting where further offending } \\
\text { has occurred prior to the ASSET. } 1 \\
\text { = Further Offending }\end{array}$ \\
\hline Time & \multicolumn{2}{|l|}{ time $<-$ data\$time } & $\begin{array}{l}\text { Time } 0=\text { initial assessment. As a } \\
\text { result, the intercept can be } \\
\text { interpreted as the predicted outcome } \\
\text { for the baseline. Continuous, max = } \\
19\end{array}$ \\
\hline Research.ID & \multicolumn{2}{|c|}{$\begin{array}{l}\text { Generate a new Individual ID with consecutive } \\
\text { integers: }\end{array}$} & \multirow{2}{*}{$\begin{array}{l}\text { There are } 87 \text { individuals in the } \\
\text { dataset since offending and court } \\
\text { records for } 1 \text { individual were not } \\
\text { available. }\end{array}$} \\
\hline Individual & \multicolumn{2}{|c|}{ 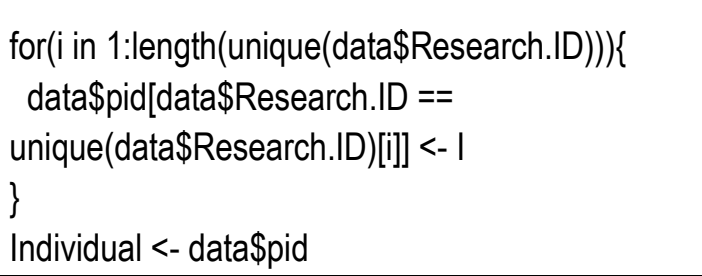 } & \\
\hline \multirow[t]{12}{*}{12 Domains } & live <- data\$live & Living Arrangements & \multirow{12}{*}{$\begin{array}{l}\text { Finch et al. (2014) suggest that to } \\
\text { apply multilevel models to } \\
\text { longitudinal data problems time- } \\
\text { varying predictors will appear at } \\
\text { Level } 1 \text { because they are associated } \\
\text { with specific measurements, } \\
\text { whereas time-invariant predictors will } \\
\text { appear at Level } 2 \text { or higher because } \\
\text { they are associated with the } \\
\text { individual (or higher level) across all } \\
\text { measurement conditions. }\end{array}$} \\
\hline & $\begin{array}{l}\text { relation <- } \\
\text { data\$relation }\end{array}$ & $\begin{array}{l}\text { Family and Personal } \\
\text { Relationships }\end{array}$ & \\
\hline & ete $<-$ data\$ete & $\begin{array}{l}\text { Education, Training and } \\
\text { Employment }\end{array}$ & \\
\hline & where <- data\$where & Neighbourhood & \\
\hline & life <- data\$life & Lifestyle & \\
\hline & drugs <- data\$drugs & Substance Use & \\
\hline & $\begin{array}{l}\text { physical <- } \\
\text { data\$physical }\end{array}$ & Physical Health & \\
\hline & $\begin{array}{l}\text { emotion <- } \\
\text { data\$emotion }\end{array}$ & $\begin{array}{l}\text { Emotional and Mental } \\
\text { Health }\end{array}$ & \\
\hline & self <- data\$self & $\begin{array}{l}\text { Perception of Self and } \\
\text { Others }\end{array}$ & \\
\hline & think <- data\$think & Thinking and Behaviours & \\
\hline & $\begin{array}{l}\text { attitude }<- \\
\text { data\$attitude }\end{array}$ & Attitude to Change & \\
\hline & $\begin{array}{l}\text { change }<- \\
\text { data\$change }\end{array}$ & Motivation to Change & \\
\hline
\end{tabular}




\begin{tabular}{|c|c|c|}
\hline Type & Coding & Notes \\
\hline \multirow[t]{2}{*}{ Demographics } & female <- data\$Gender & Dummy Variable, female $=1$ \\
\hline & $\begin{array}{l}\text { Create dichotomous variables for ethnicity: } \\
\text { ethnic.bin <-(ifelse(data\$Head_Ethnic >0, 1, } \\
\text { 0)) } \\
\text { bme <- ethnic.bin }\end{array}$ & $\begin{array}{l}\text { Headline ethnicity originally set up as a } \\
\text { factor, differentiating between White, } \\
\text { Black, Asian, Mixed and Other. } \\
\text { Aggregated into a dummy variable with } \\
\text { non-Whites = } 1\end{array}$ \\
\hline Care Experience & careExp $<-$ data $\$$ careExp & $\begin{array}{l}\text { Experience of care (subject to a care } \\
\text { order, eligible or relevant child). Dummy } \\
\text { variable, experience of care }=1\end{array}$ \\
\hline \multirow[t]{5}{*}{$\begin{array}{l}\text { Offending } \\
\text { History }\end{array}$} & fte <- data\$FTE & $\begin{array}{l}\text { Dummy variable, FTE at time of primary } \\
\text { offence, } \mathrm{FTE}=1\end{array}$ \\
\hline & $\begin{array}{l}\text { ageFirst }<- \text { data\$AgeFirst } \\
\text { ageFirst } 10<-(\text { data\$AgeFirst)-10 }\end{array}$ & $\begin{array}{l}\text { Age at first reprimand, warning, caution, } \\
\text { youth restorative disposal or informal } \\
\text { action. Centred to reflect the age of } \\
\text { criminal responsibility at } 10 \text { years. } \\
\text { Continuous, max }=7 \text { ( } 17 \text { years) }\end{array}$ \\
\hline & G_ageFirst <- data\$G_ageFirst & $\begin{array}{l}\text { Age at first offence originally set up as a } \\
\text { continuous variable. Aggregated into a } \\
\text { dummy variable based on the thresholds } \\
\text { used in the Scaled Approach, those aged } \\
13-17=1\end{array}$ \\
\hline & $\begin{array}{l}\text { ageCon }<- \text { data\$AgeCon } \\
\text { ageCon10 <- (data\$AgeCon)-10 }\end{array}$ & $\begin{array}{l}\text { Age at first conviction. Centred to reflect } \\
\text { the age of criminal responsibility at } 10 \\
\text { years. Continuous, } \max =7 \text { (17 years) }\end{array}$ \\
\hline & G_ageCon <- data\$G_ageCon & $\begin{array}{l}\text { Age at first conviction originally set up as } \\
\text { a continuous variable. Aggregated into a } \\
\text { dummy variable based on the thresholds } \\
\text { used in the Scaled Approach, those aged } \\
14-17=1\end{array}$ \\
\hline \multirow[t]{2}{*}{ Primary Offence } & $\begin{array}{l}\text { I_Cat <- data\$I_Cat } \\
\text { I_Cat2 <- data\$I_Cat2 }\end{array}$ & $\begin{array}{l}\text { YJB Offence Category. Originally set up } \\
\text { as a factor. However, it was necessary } \\
\text { to group the offences into Other, Serious } \\
\text { Acquisitive Crimes (SAC) and Violence } \\
\text { Against the Person (VAP). }\end{array}$ \\
\hline & $\begin{array}{l}\text { I_Seriousness <- data\$I_Seriousness } \\
\text { I_Seriousness2 <- (data\$I_Seriousness)-2 }\end{array}$ & $\begin{array}{l}\text { YJB Gravity Score. Centred to reflect the } \\
\text { lowest gravity score within the dataset ie } \\
\text { 2. Continuous, } \max =4 \text { (Gravity =6) }\end{array}$ \\
\hline \multirow{3}{*}{$\begin{array}{l}\text { Facets of the } \\
\text { Youth Justice } \\
\text { System }\end{array}$} & breach <- data\$breach & $\begin{array}{l}\text { Dummy variable, Breach before ASSET, } \\
\text { breach }=1\end{array}$ \\
\hline & appear <- data\$appear & $\begin{array}{l}\text { Dummy variable, court appearance } \\
\text { before ASSET, appear }=1\end{array}$ \\
\hline & custody <- data\$custody & $\begin{array}{l}\text { Dummy variable, time in custody before } \\
\text { ASSET, custody = } 1\end{array}$ \\
\hline
\end{tabular}




\section{Diagnostic Tools}

Bayesian

The following descriptions are taken from https://cran.r-project.org/web/packages/coda/coda.pdf

\section{(1) Convergence}

\section{- The Raftery and Lewis's Diagnostic}

raftery.diag is a run length control diagnostic based on a criterion of accuracy of estimation of the quantile q. It is intended for use on a short pilot run of a Markov chain. The number of iterations required to estimate the quantile $q$ to within an accuracy of $+/-r$ with probability $p$ is calculated. Separate calculations are performed for each variable within each chain.

If the number of iterations in data is too small, an error message is printed indicating the minimum length of pilot run. The minimum length is the required sample size for a chain with no correlation between consecutive samples. Positive autocorrelation will increase the required sample size above this minimum value. An estimate I (the 'dependence factor') of the extent to which autocorrelation inflates the required sample size is also provided. Values of I larger than 5 indicate strong autocorrelation which may be due to a poor choice of starting value, high posterior correlations or 'stickiness' of the MCMC algorithm.

The number of 'burn in' iterations to be discarded at the beginning of the chain is also calculated.

\section{- Heidelberger and Welch's Convergence Diagnostic}

heidel . diag is a run length control diagnostic based on a criterion of relative accuracy for the estimate of the mean. The default setting corresponds to a relative accuracy of two significant digits. heidel .diag also implements a convergence diagnostic, and removes up to half the chain in order to ensure that the means are estimated from a chain that has converged.

\section{(2) Autocorrelation of the Fixed and Random Effects}

autocorr calculates the autocorrelation function for the Markov chain mcmc.obj at the lags given by lags. The lag values are taken to be relative to the thinning interval if relative=TRUE. High autocorrelations within chains indicate slow mixing and, usually, slow convergence. It may be useful to thin out a chain with high autocorrelations before calculating summary statistics: a thinned chain may contain most of the information, but take up less space in memory. Re-running the MCMC sampler with a different parameterization can help to reduce autocorrelation.

When using the MCMCgImm package, the autocorrelation of the random and fixed effects are determined using autocorr (Model\$VCV) and autocorr (Model\$Sol) respectively.

\section{(3) Diagnosing the results using plots}

Plotting the samples drawn by MCMCgImm provides an indication as to whether or not they are an accurate representation of the true posterior. 
Two plots are generated:

(1) Trace Plots (on the left)

These show the values that the parameter took during the runtime of the chain. The index of the sample is on the $x$-axis and the value of the parameter in that sample is on the $y$-axis.

(2) Marginal Density Plot (on the right)

Basically, it is the (smoothened) histogram of the values in the trace-plot, i.e. the distribution of the values of the parameter in the chain, ignoring burn-in samples. Ideally, we would like to have something like the following:

Trace of Var1

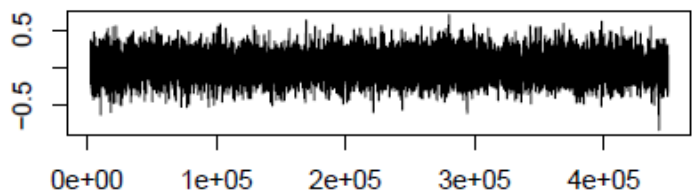

Density of Var1

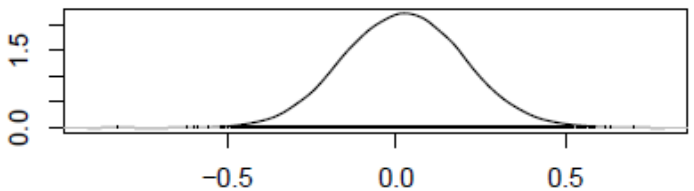

In this trace plot of random data, there is no autocorrelation of consecutive samples and the distribution of samples is stationary. It is very likely that taking more samples would not shift the distribution substantially. Hence, if we see a plot like this, we would be more confident that our posterior is a good approximation of the true posterior.

The following illustrate the impact of altering number of iterations and the thining. This worked example uses generated from this dataset used within the research. However, only selected output has been included so that particular features can be highlighted:

a) Iterations $=45,000$, thinning $=10$, no burn in

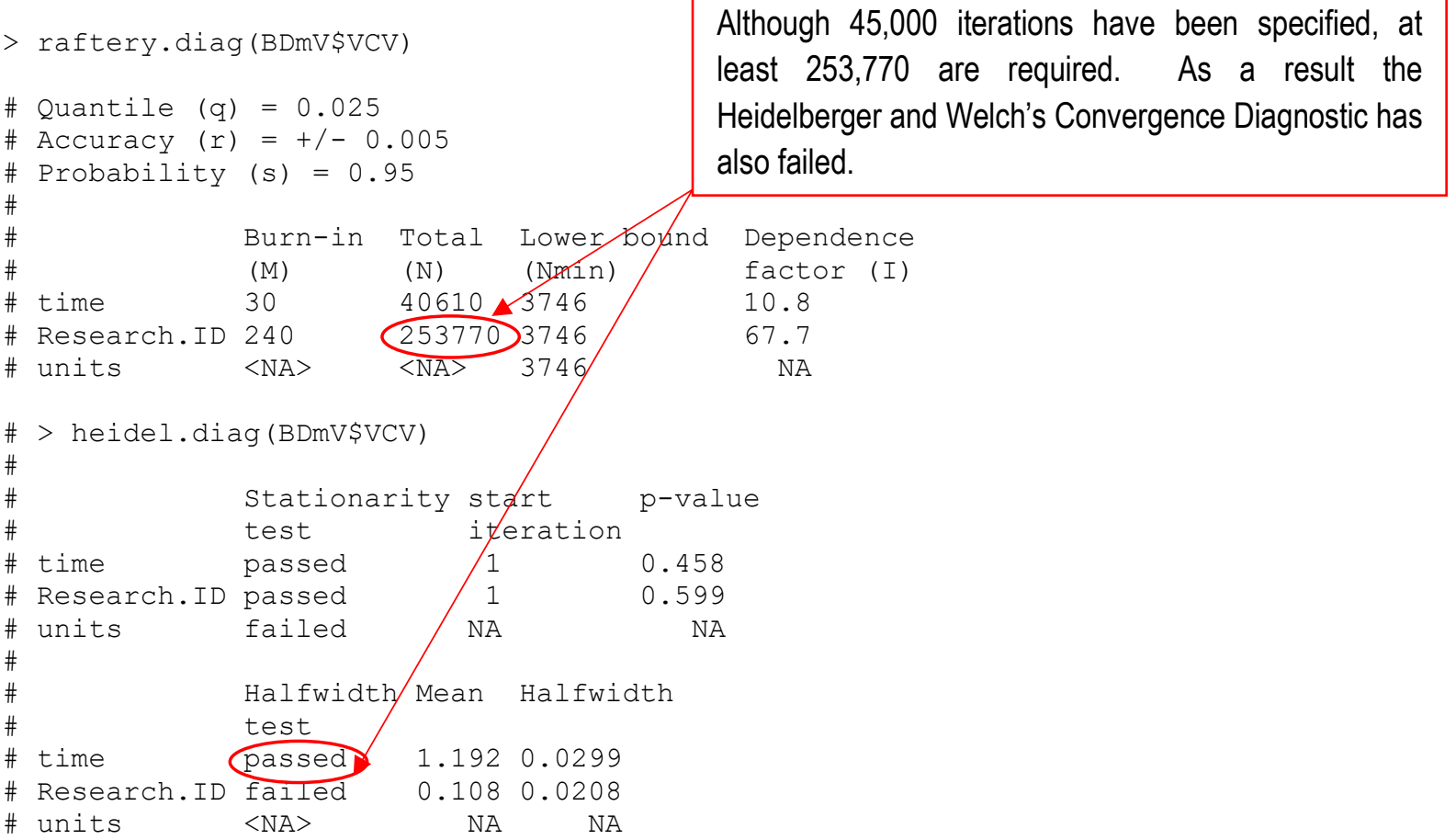




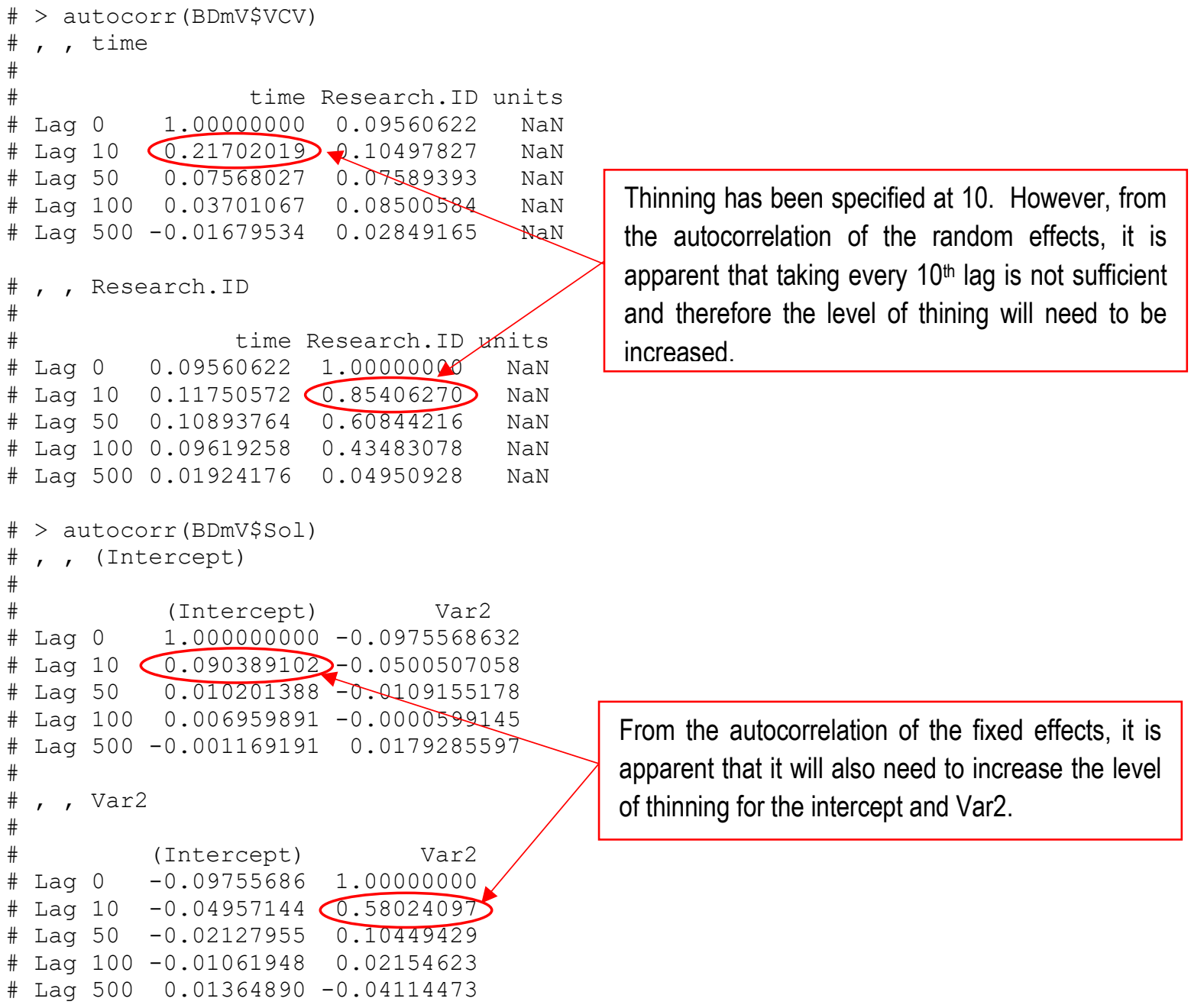

\section{Trace of Var2}

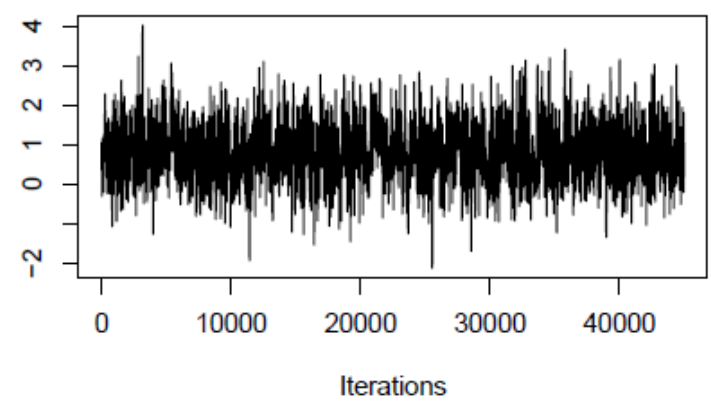

Density of Var2

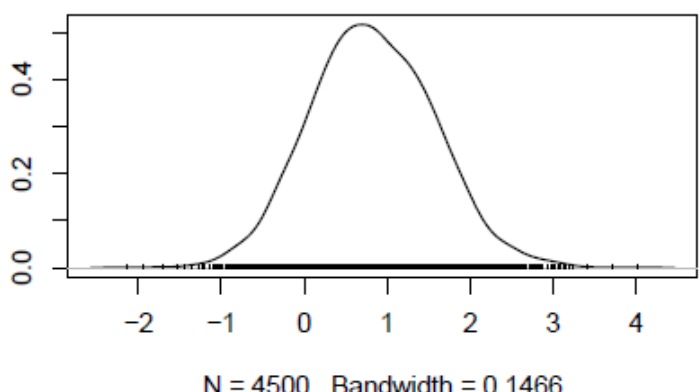


b) Adding a burn-in: Iterations $=45,000$, thinning $=10$, burn-in $=3000$

Trace of Var2

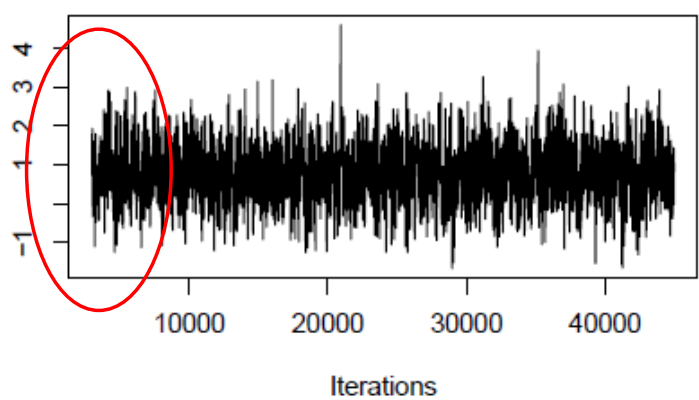

Density of Var2

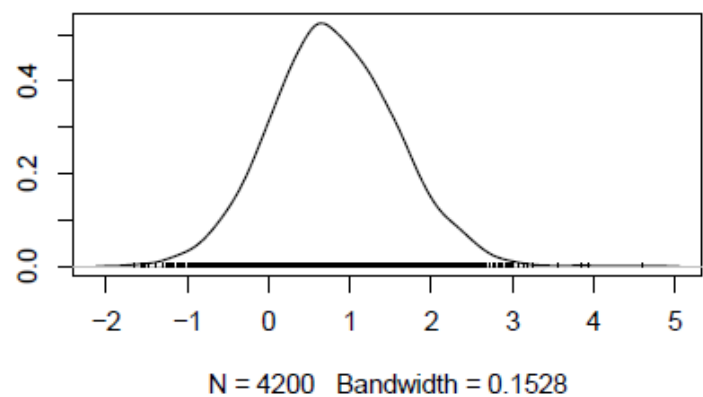

As a result of the burn-in, the initial 3,000 iterations are disgarded. The marginal density plot also ignores the burn-in samples.

c) Increasing the number of iterations: Iterations $=450,000$, thinning $=10$, burn-in $=3000$

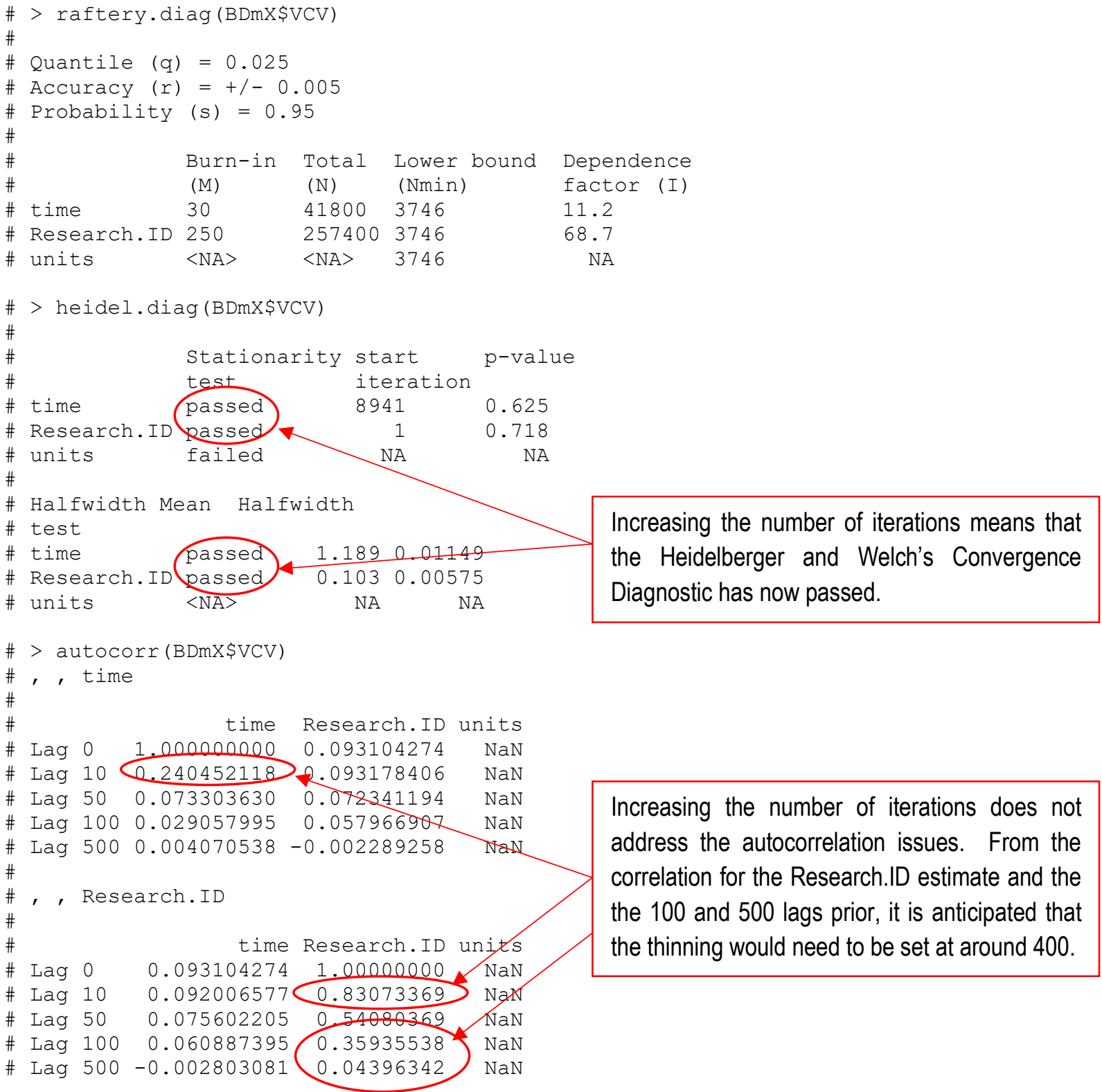




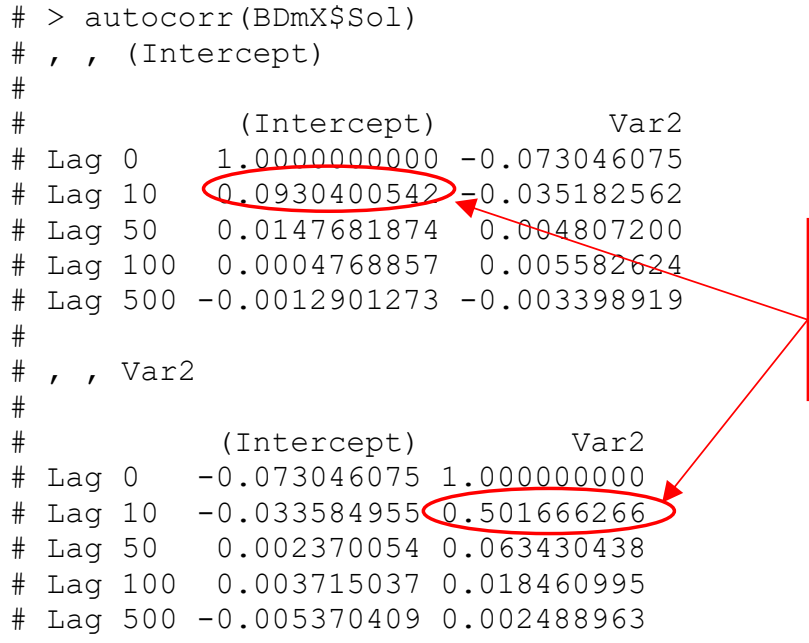

Trace of Var2

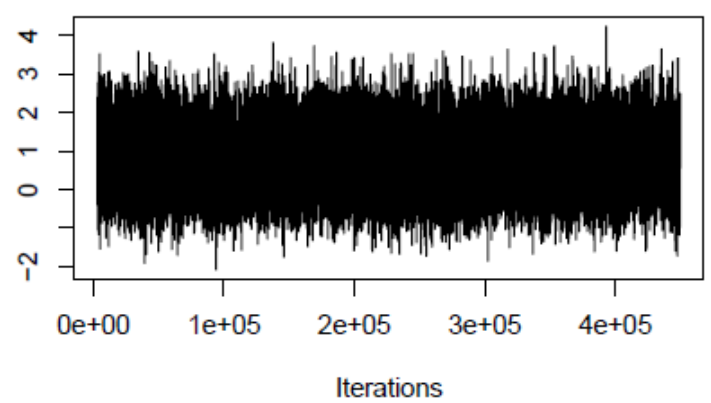

Iterations
From the autocorrelation of the fixed effects, it is apparent that increasing the thinning to 400 would also address the autocorrelation for Var2.

\section{d) Increasing the thinning: Iterations $=450,000$, thinning $=400$, burn-in $=3000$}

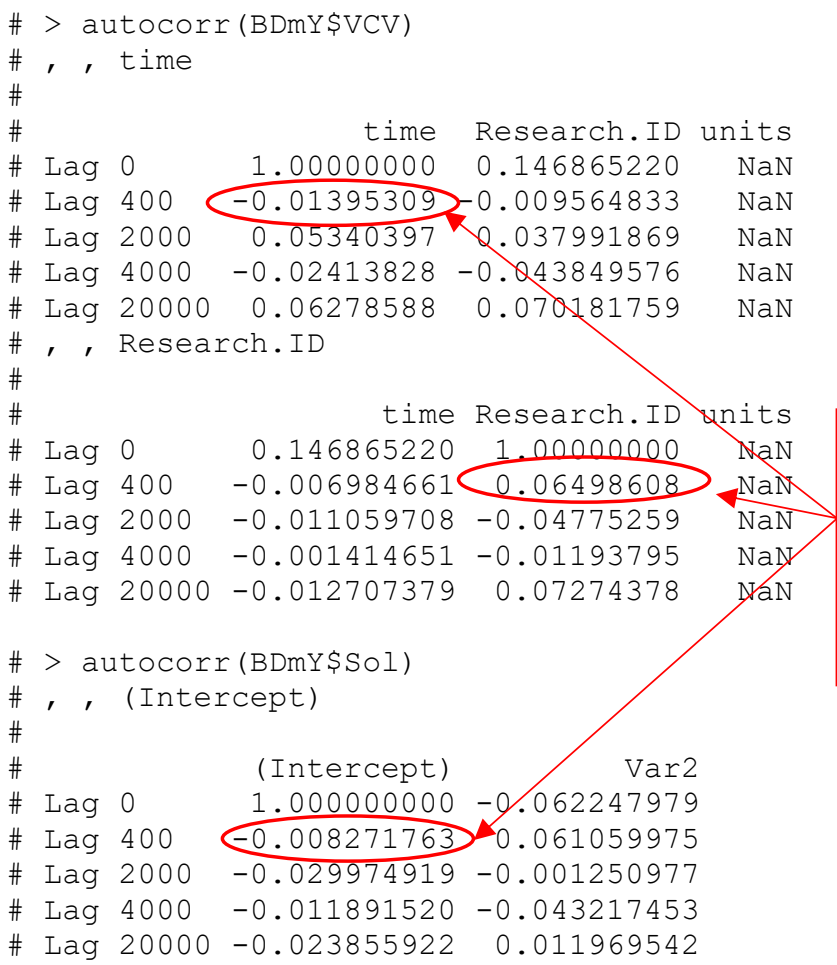

\section{Density of Var2}

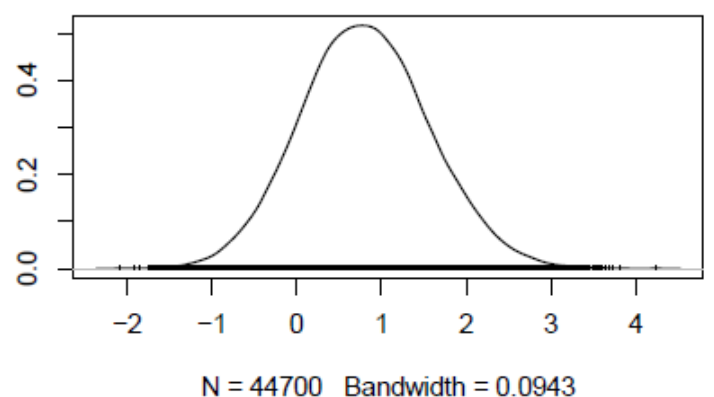

From the autocorrelation of the random and fixed effects (continued on next page), the effect of increasing the thinning is that the autocorrelation at a lag of 400 is sufficiently small for us to have confidence in our thinning the results at 400 . 
$\begin{array}{lll}\#, & , & \text { Var2 } \\ \# & & \\ \# & & \\ \# & \text { Lag } & 0 \\ \# & \text { Lag } 400 \\ \# & \text { Lag } 2000 \\ \# & \text { Lag } 4000\end{array}$

(Intercept)

$-0.06224798 \quad 1.000000000$

$-0.059295860 .001477662$

$-0.03636059-0.019774663$

$0.02452572 \quad 0.011603689$

$0.04105996-0.004374761$

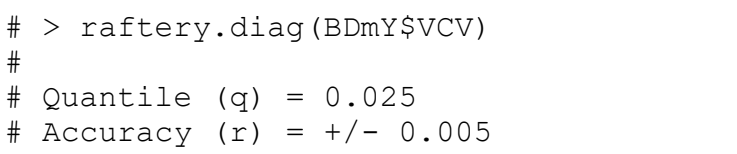

Increasing the thinning unfortunately means that the sample size is now too small. The number of iterations needs to be increased:

$3,746 \times 400=1,498,400$ where 400 is the level of thinning. $1,498,400+3,000=1,501,400$ where 3,000 is the burn-in.

The suggested number of iterations is therefore at least $1,501,400$.

$\#$

\# You need a sample size of at least 3746 with these values of $q$, $r$ and $s$

\# heidel.diag (BDmY\$VCV)

Stationarity start p-value

test iteration

time passed

Research.ID passed

units failed

\#

\# Halfwidth Mean Halfwidth

test

\# time passed 1.19900 .04129

\# Research.ID passed 0.09760 .00906

\# units $\quad\langle$ NA $\quad$ NA

\section{e) Increasing the number of iterations to reflect the level of thinning required: Iterations = $1,600,000$, thinning $=400$, burn-in $=3000$}

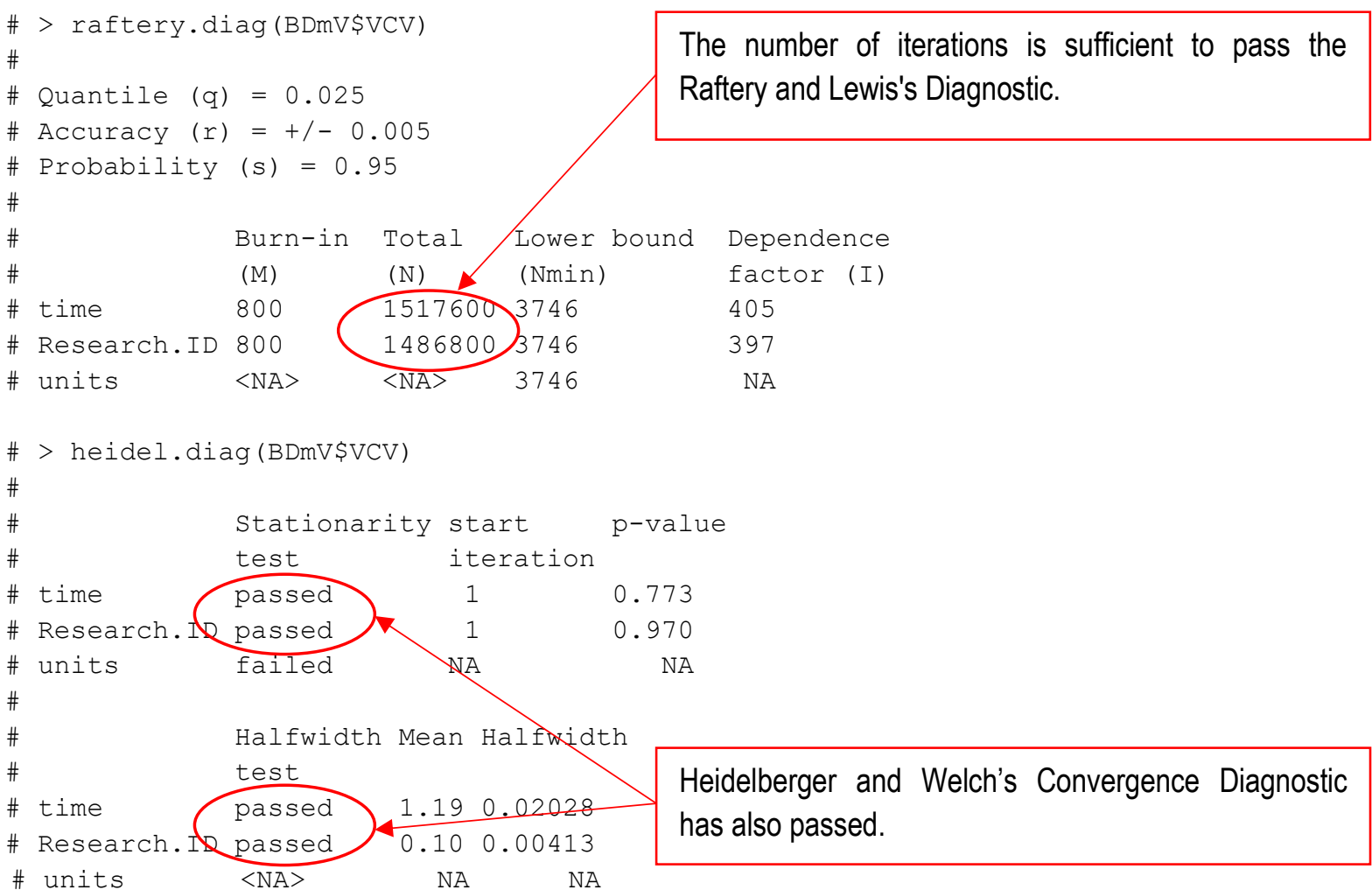




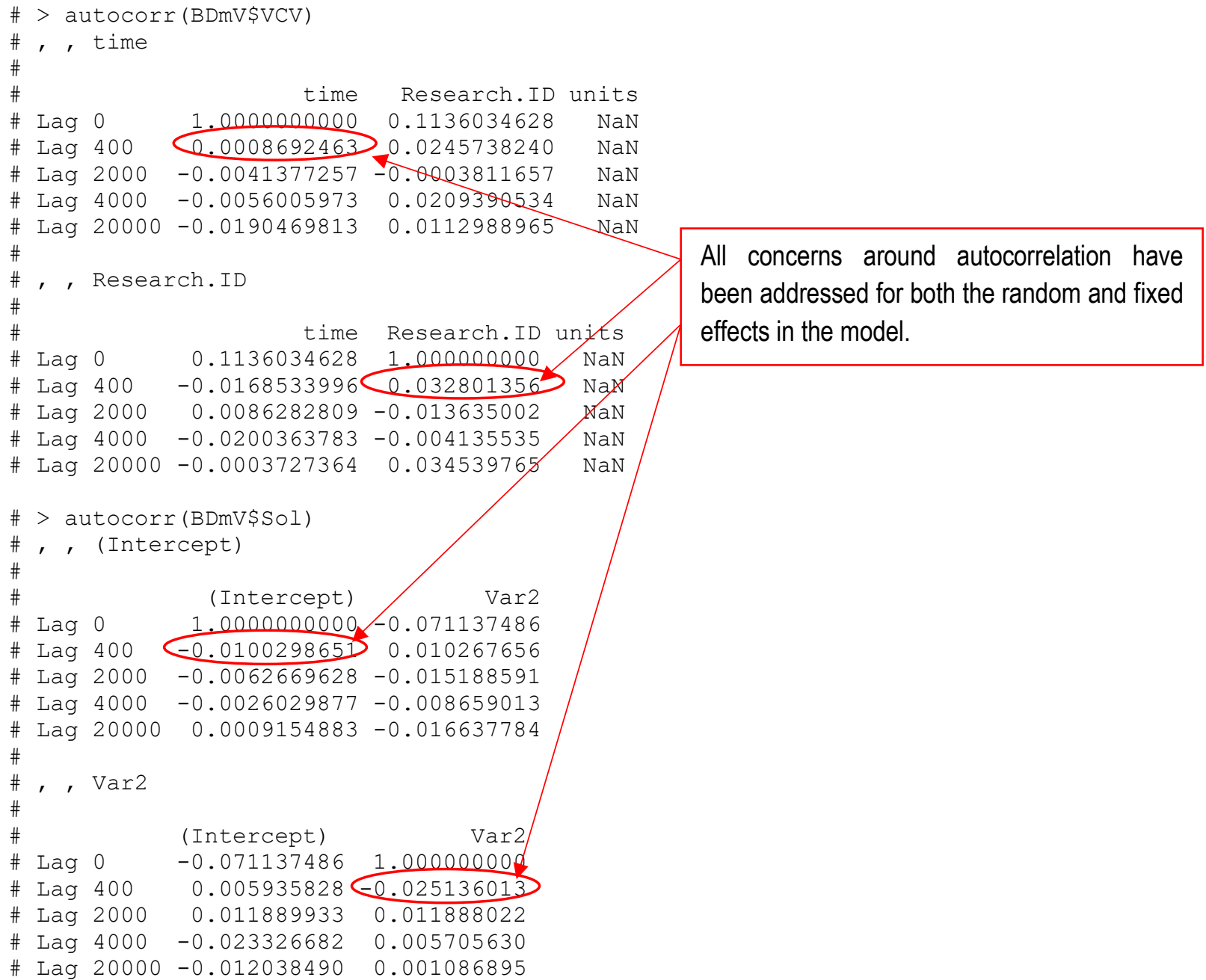

\section{Fixed Effects}

Trace of Intercept

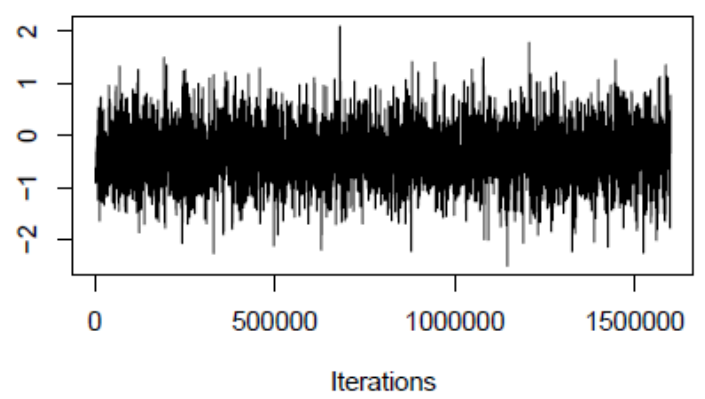

Trace of Var2

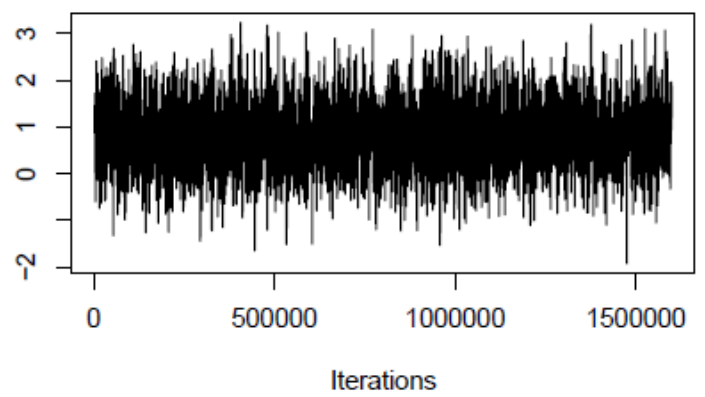

Density of Intercept

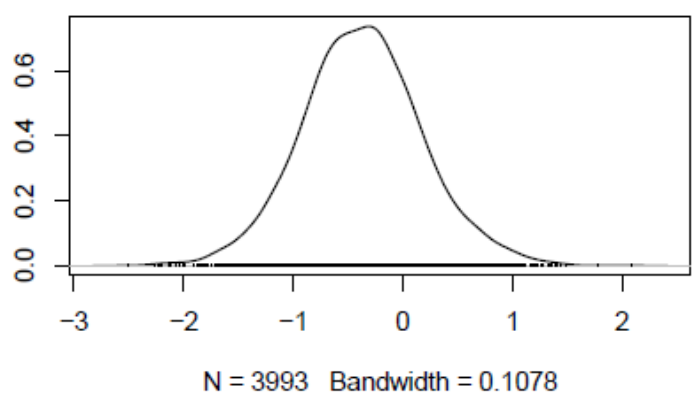

Density of Var2

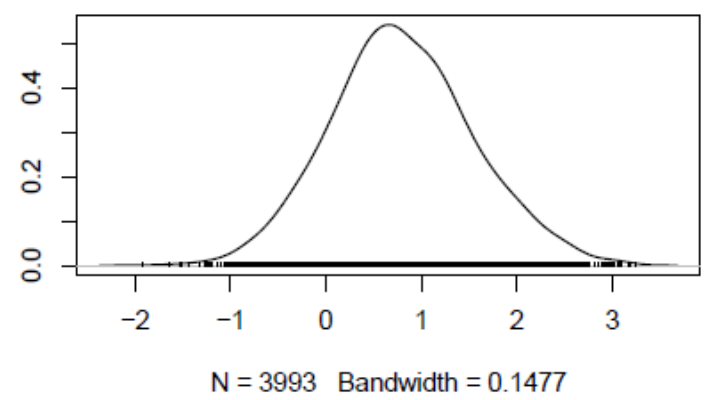


Trace of Time

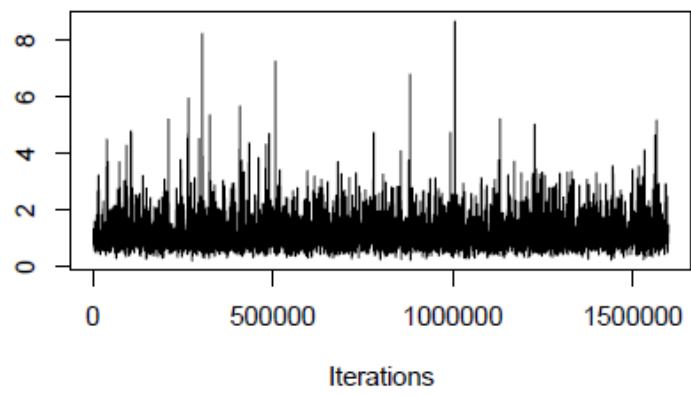

Trace of Research.ID

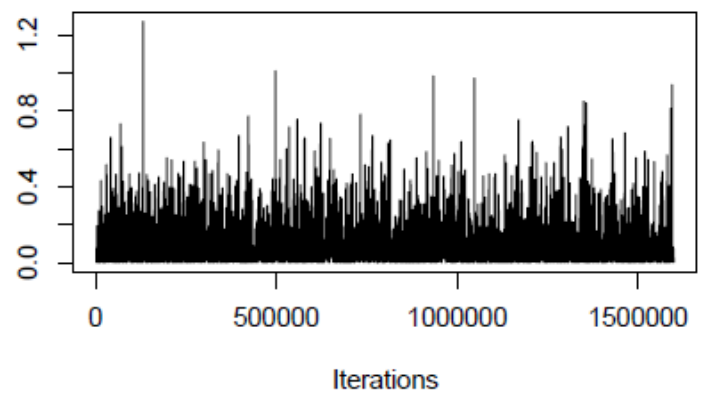

Density of Time

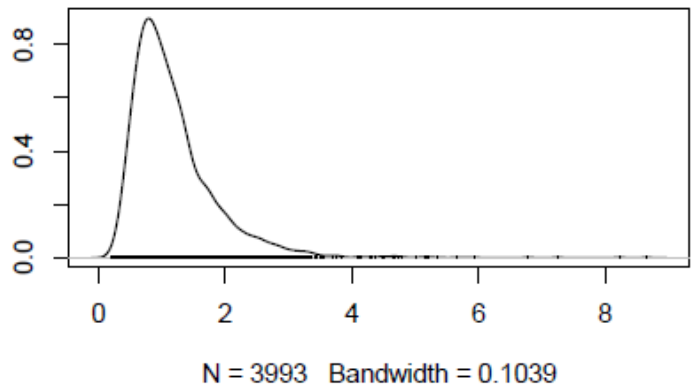

Density of Research.ID

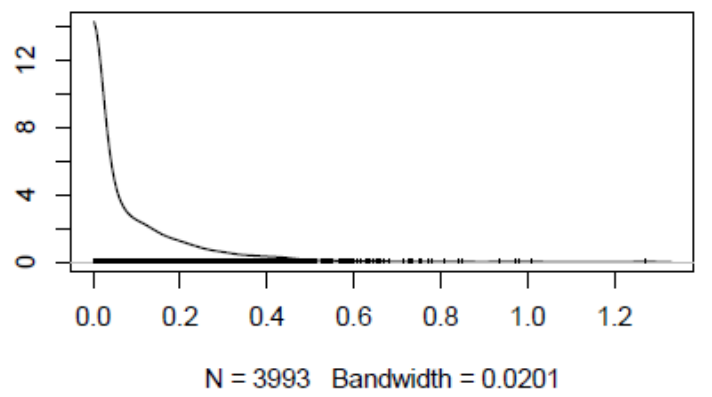

Whilst the worked example, illustrates where it is possible to improve the level of convergence by taking into account any autocorrelation, this is not always feasible to address. The following trace plots and marginal density plots reflect where there continue to be issues with convergence. Typically these reflect where the distrubtion of the samples is not stationary ie the sampling process dwells in one part of the parameter sample and then visits other parts of the parameter space. In the case of Var3, around $1.5 \mathrm{e}^{7}$ iterations, the trace suddenly moves to more negative values not visited before.

The unstable posterior reflected in the marginal density plots is also reflected in the summary output for the model, with the credible interval being particularly wide.

Trace of Var3

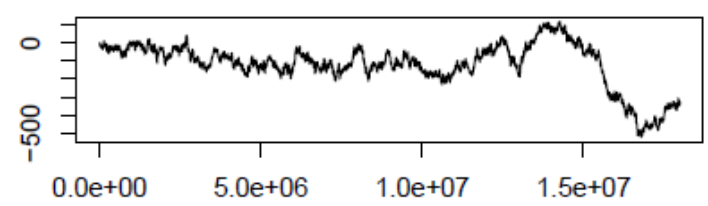

Trace of Var4

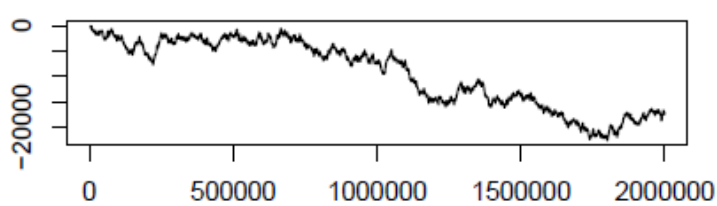

Density of Var3

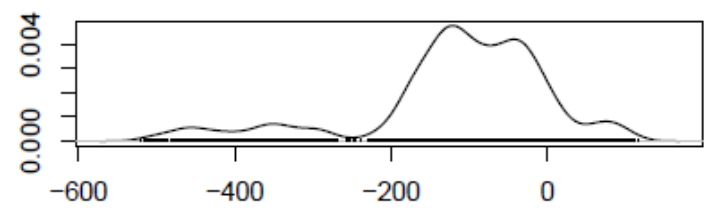

Density of Var4

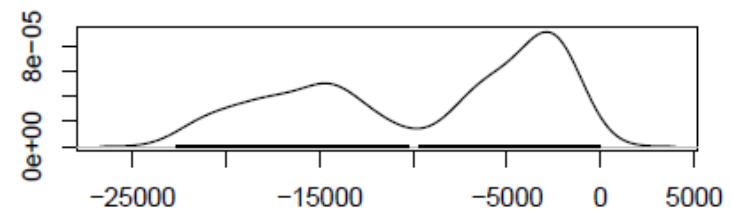


Trace of Var5

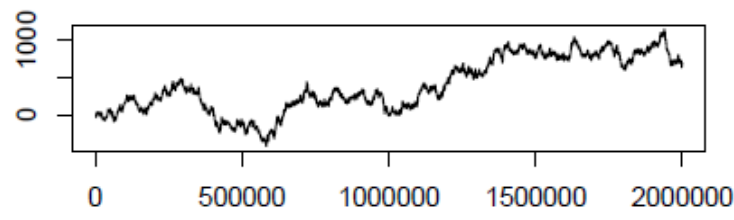

Trace of Var6

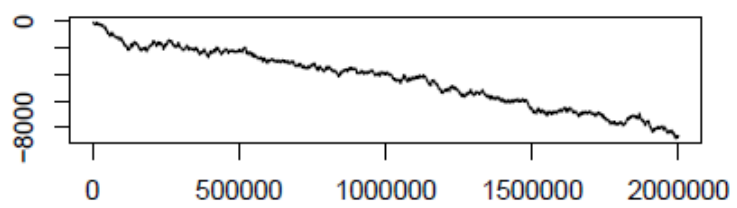

Density of Var5

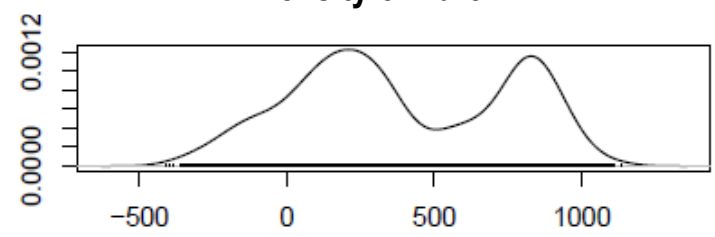

Density of Var6

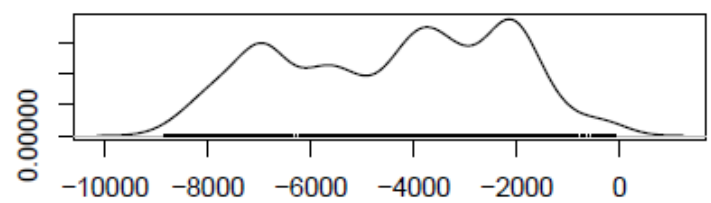

Frequentist

The Interclass Correlation (ICC) - used to inspect the variance components at different levels of the model

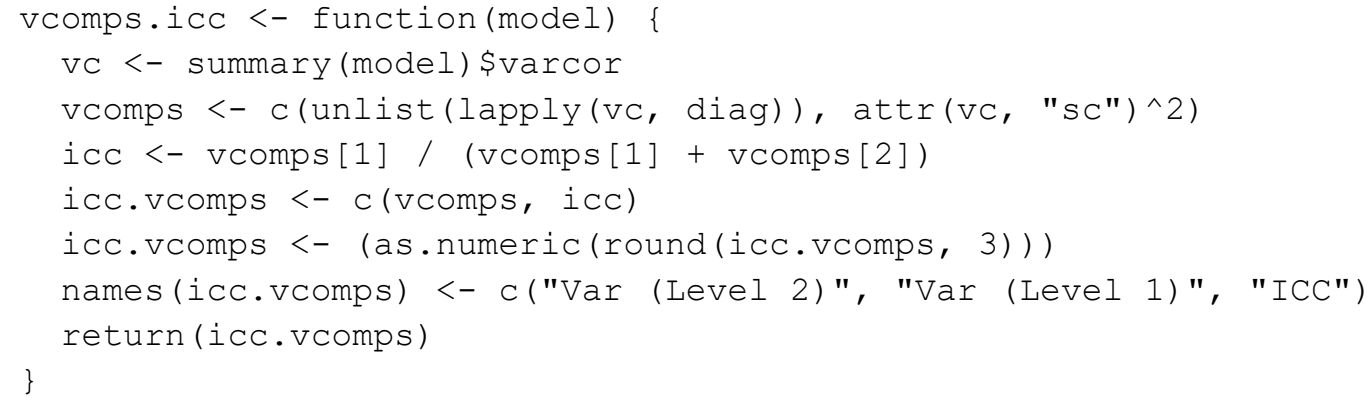

In cases where the measurements are clustered or nested within a higher level unit (eg individuals), it is possible to estimate the correlation among measurements within the nested structure using the ICC. It ranges from 0 (no variation amongst clusters) to 1 (variance among clusters but no within-cluster variance).

The ICC is an important tool in multilevel modelling, because it indicates the degree to which a multilevel structure may impact the outcome variable of interest. Larger ICC values are indicative of a greater amount of clustering (Finch, 2014). The ICC is obtained using vcomps . icc () .

ANOVA (Goodness of Fit) - when the fits of nested models are compared, the difference in the chisquare values for each model deviance can be used to compare the model fit. After each of the models in question has been run, the difference in chi-squares values can be obtained using the anova ( ) function call (Finch, 2014). For example to compare Model 1 and Model 2, this would be specified as anova (m1, $\mathrm{m} 2)$. 


\section{Model Specifications, Outputs and Plots}

Chapter Four - Preparatory models to explore the risk assessment domains The Empty or Null Model (Table 4.7)

Bayesian Model (BmO)

\#\# Set Prior. This is equivalent to an inverse-gamma prior with shape and scale equal to 0.001

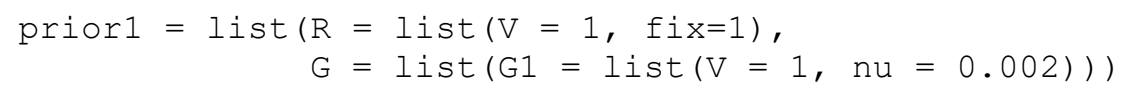

\section{\#\# Define the model}

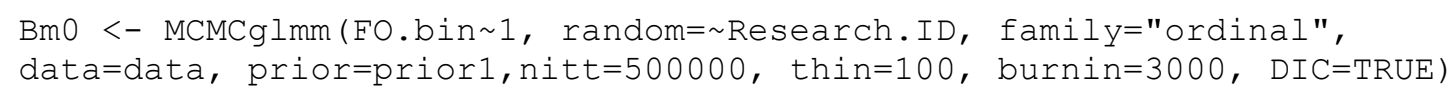

\section{\#\# Checks for suitable convergence}

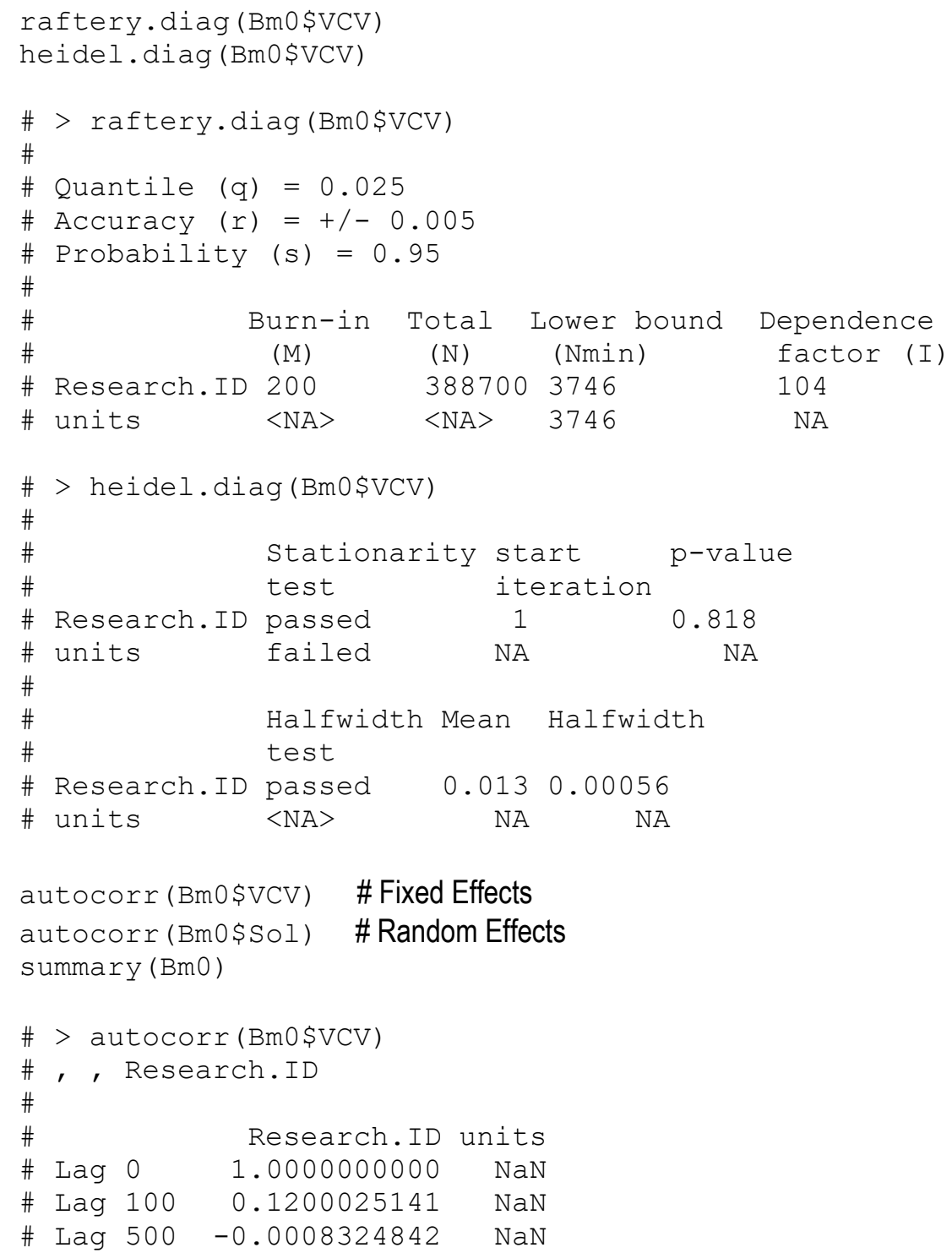




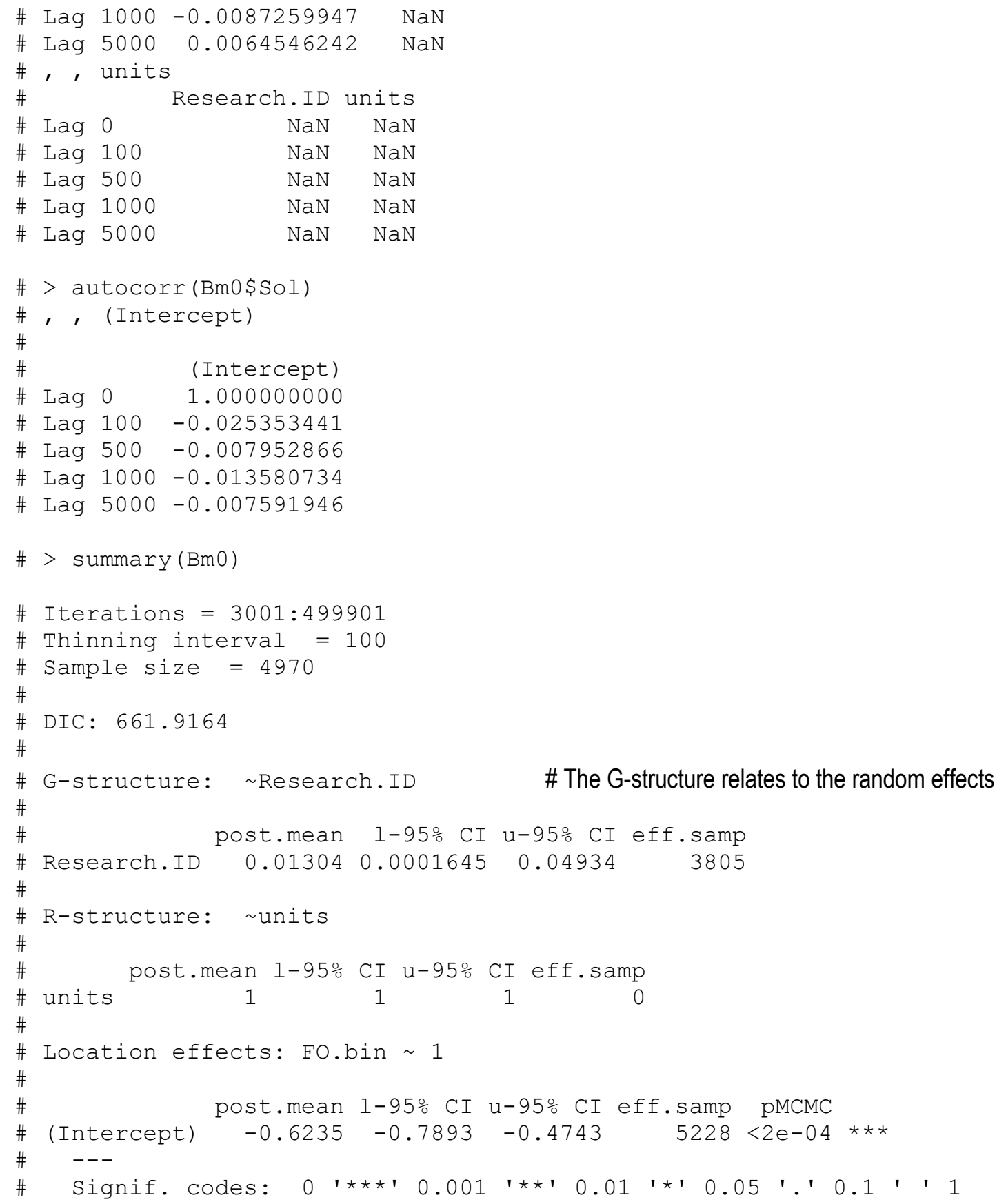




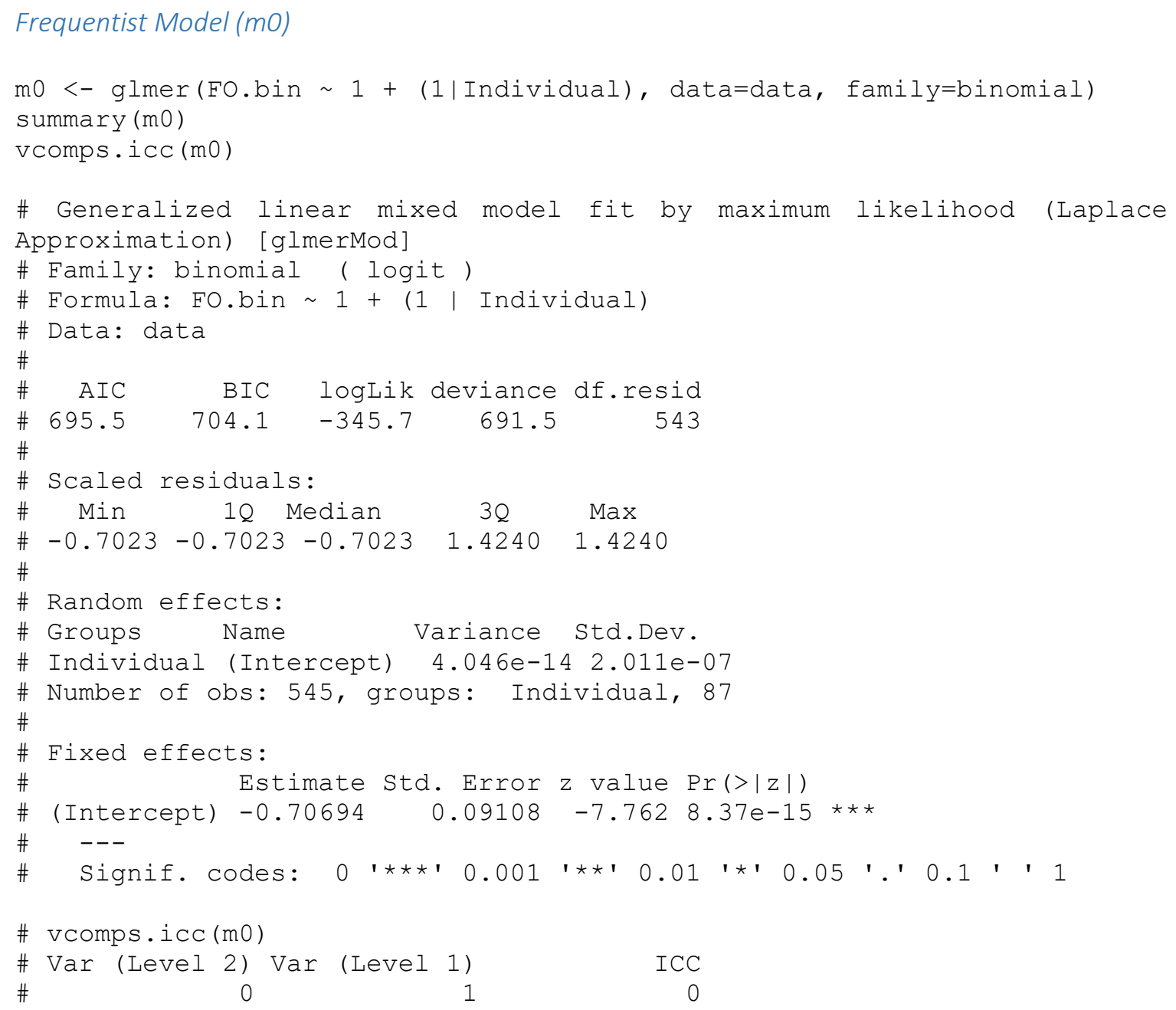


Random Intercept Model with Single Predictor (Table 4.8)

Bayesian (BmTO)

\section{\#\# Define the model}

BmT0 <- MCMCglmm(FO.bin time, random= Research.ID, family="ordinal", data=data.nomiss, prior=priorl, nitt $=400000$, thin=100, burnin=3000, DIC=TRUE)

\section{\# Checks for suitable convergence}

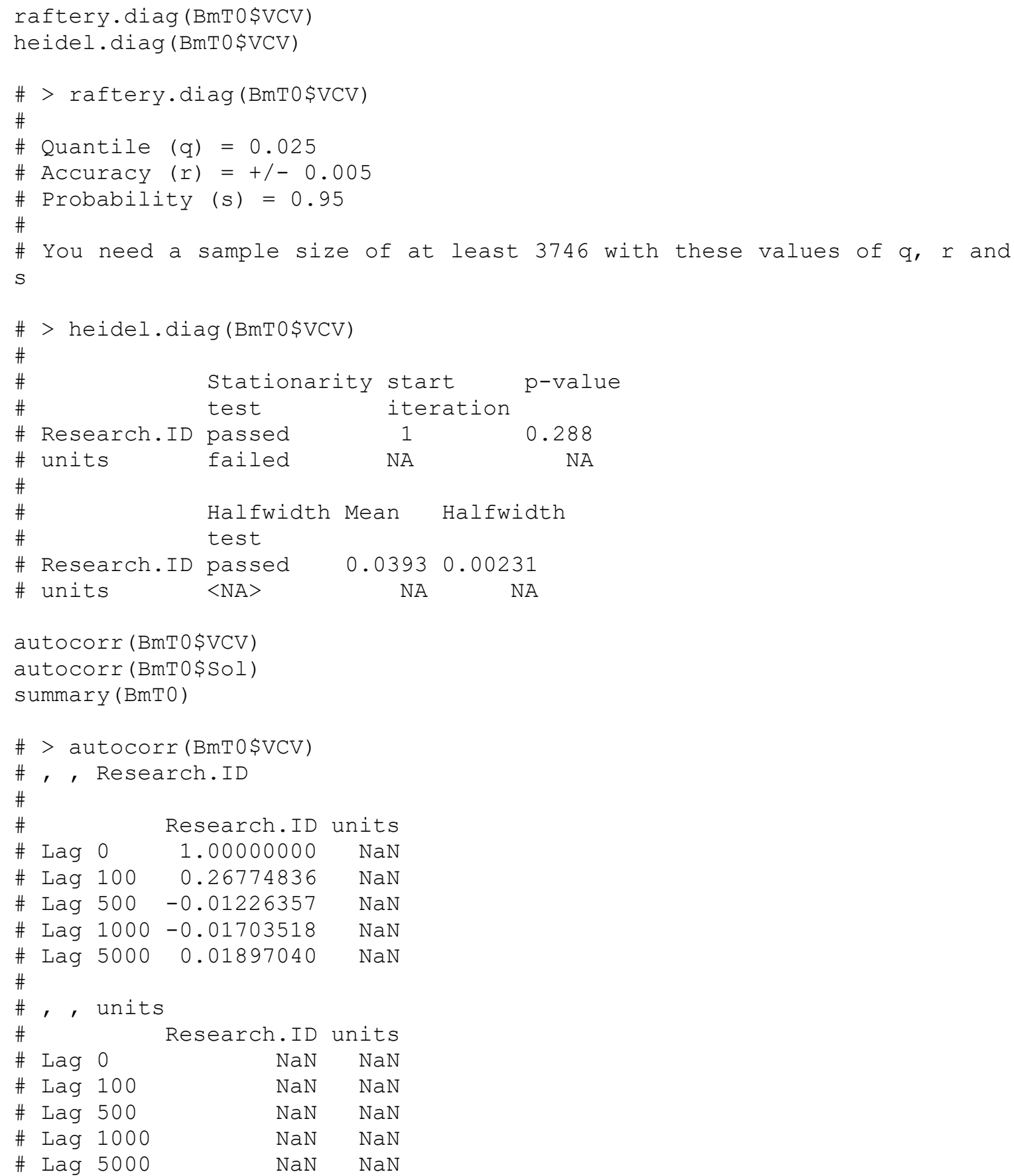




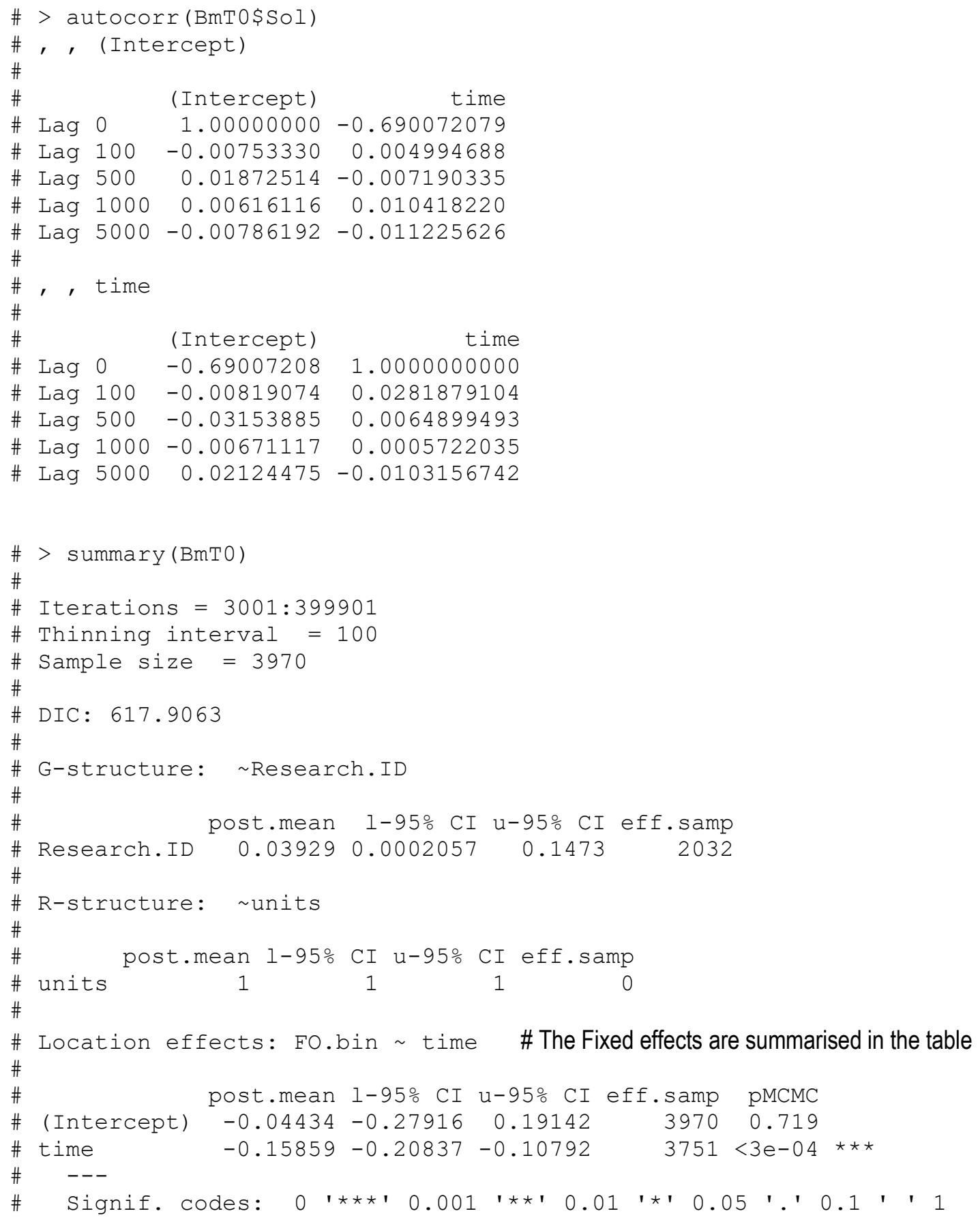




\section{\# To compare two mixed effects models (eg containing one or two random factors), use the 'anova()' function.}

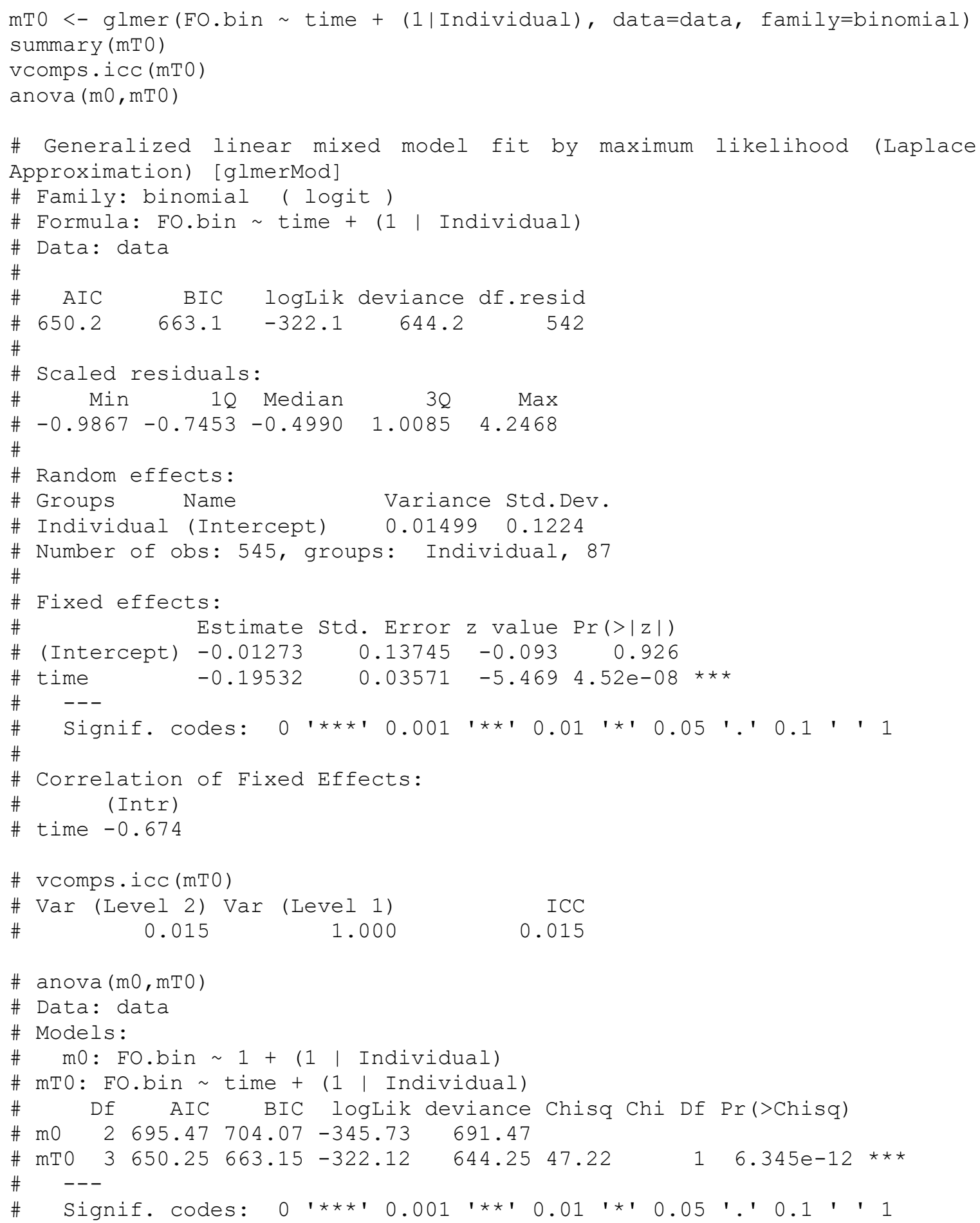


Random Intercept Model with ASSET Domain Predictors (Table 4.9)

Bayesian Model (BmT1)

\section{\#\# Define the model}

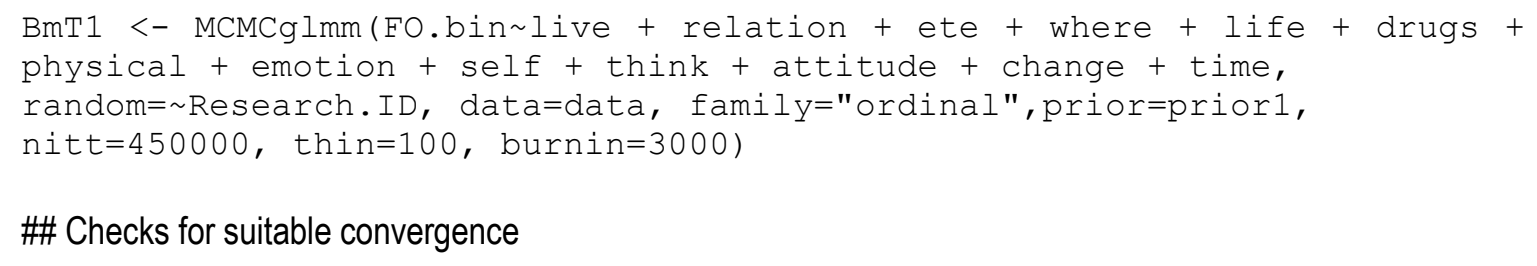

\section{\#\# Checks for suitable convergence}

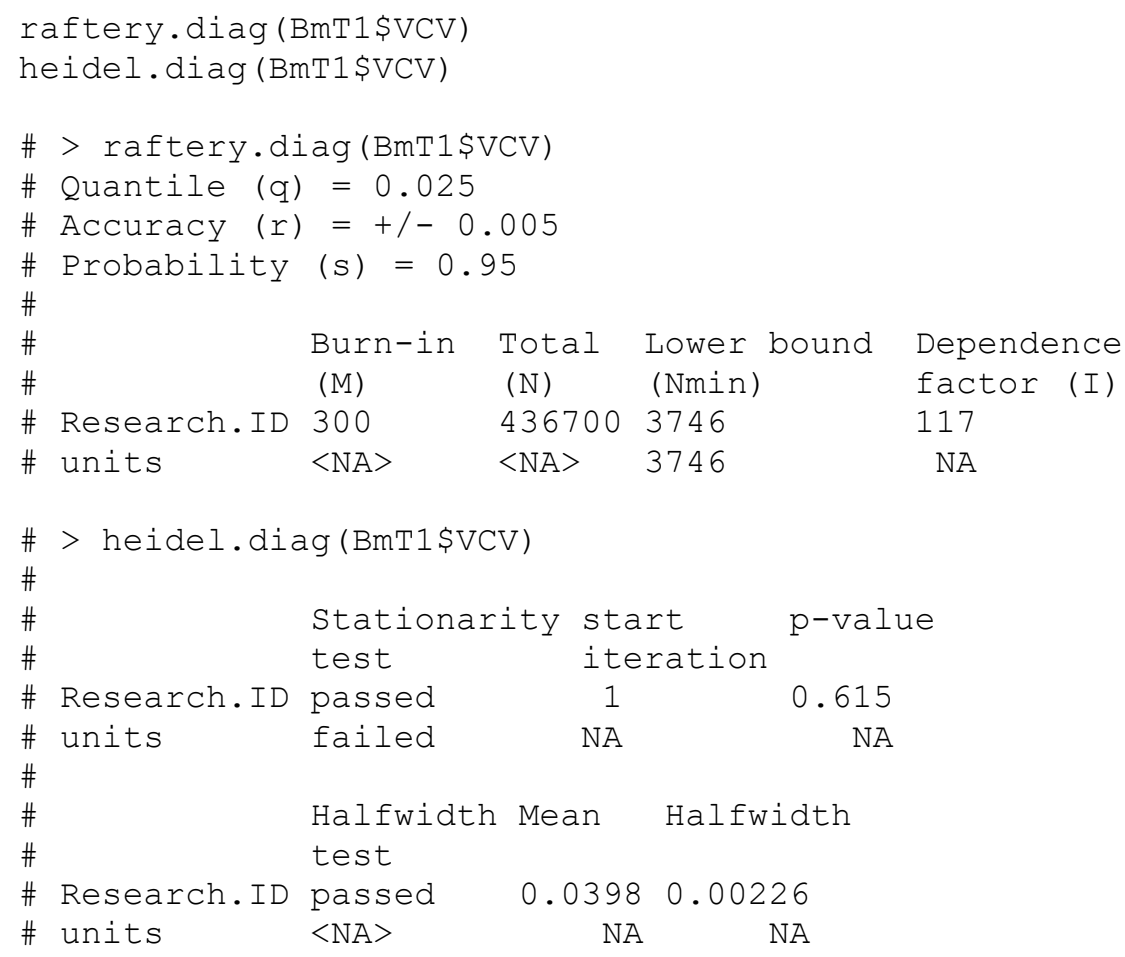




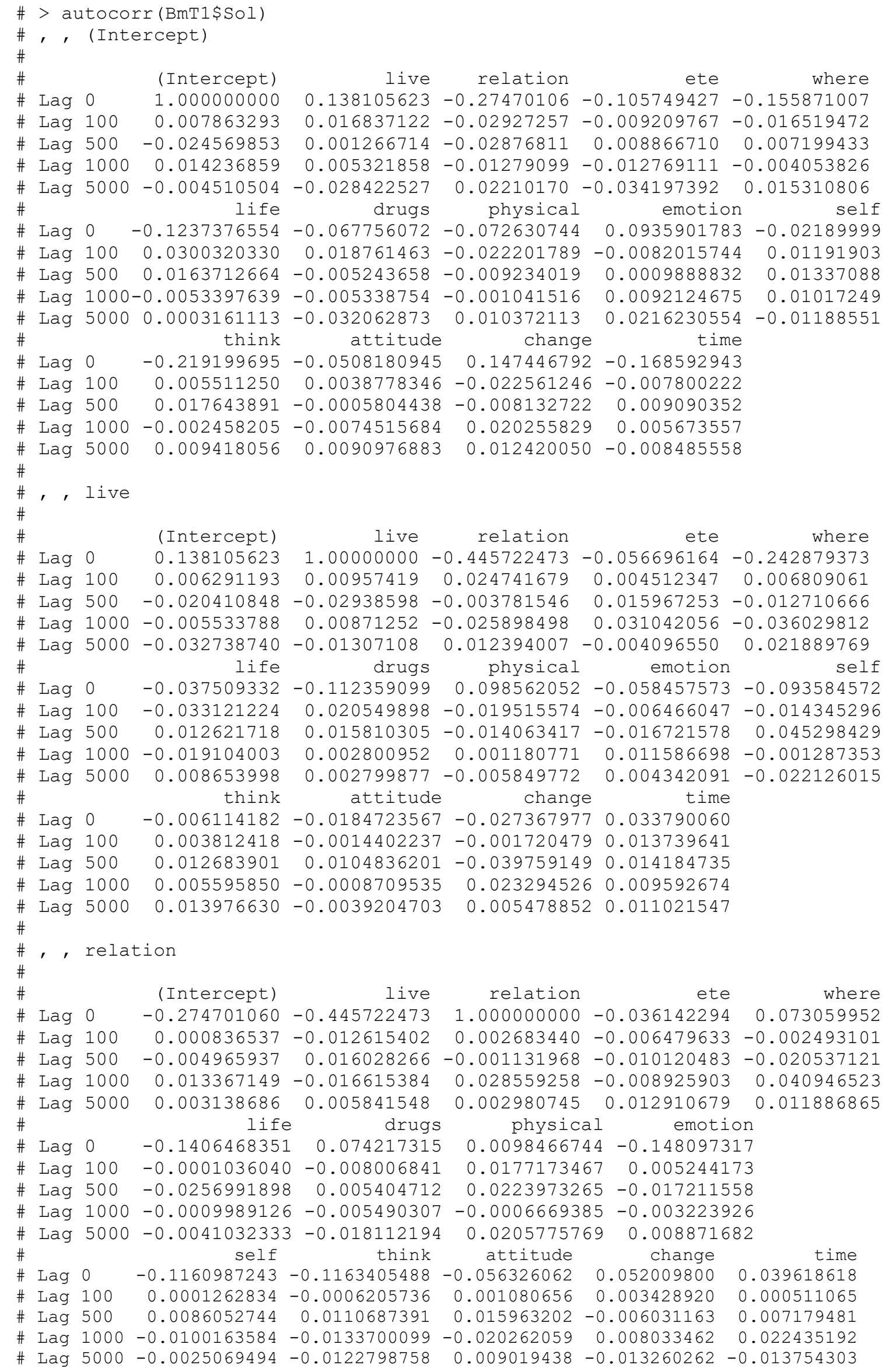




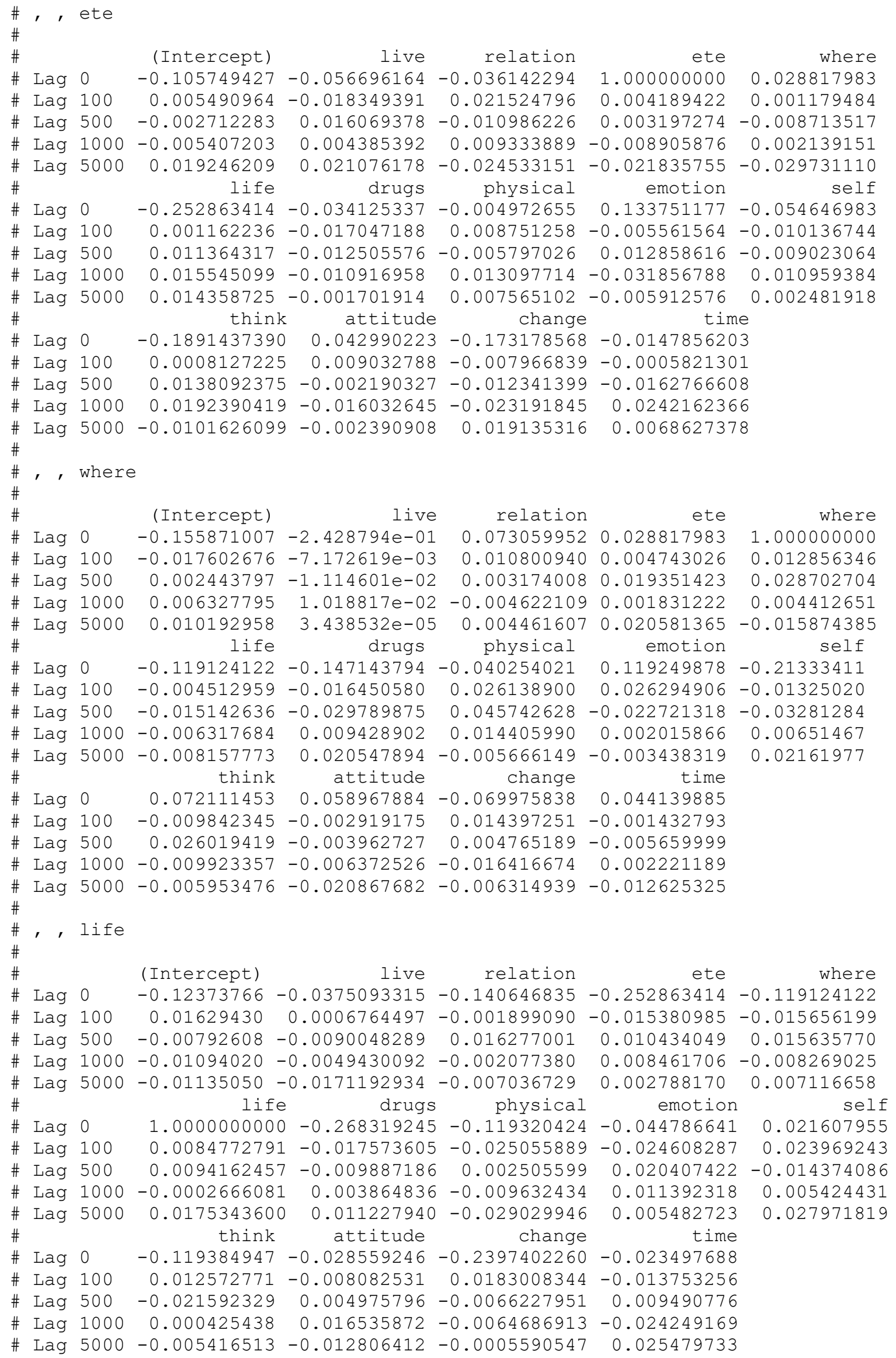




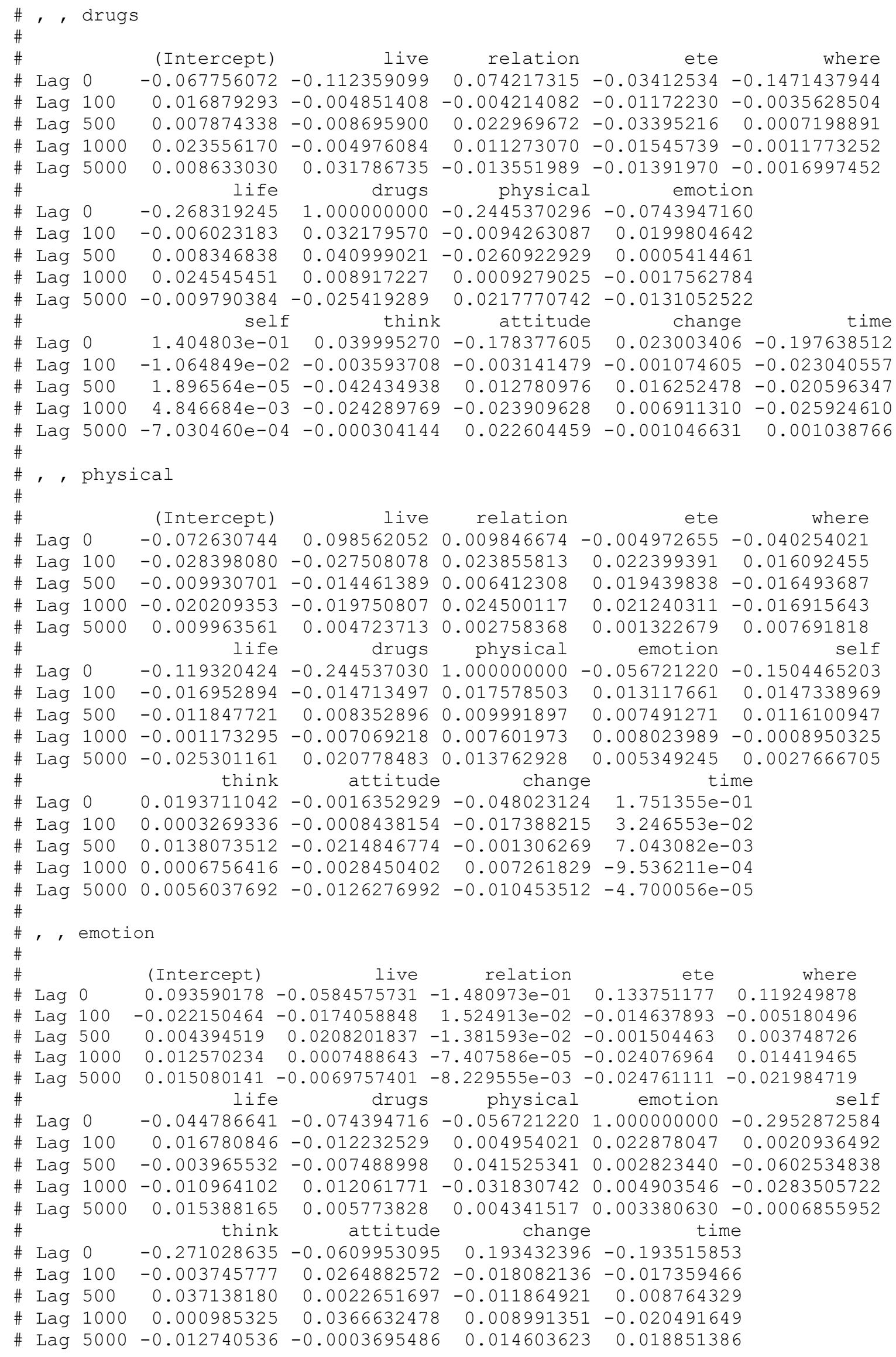




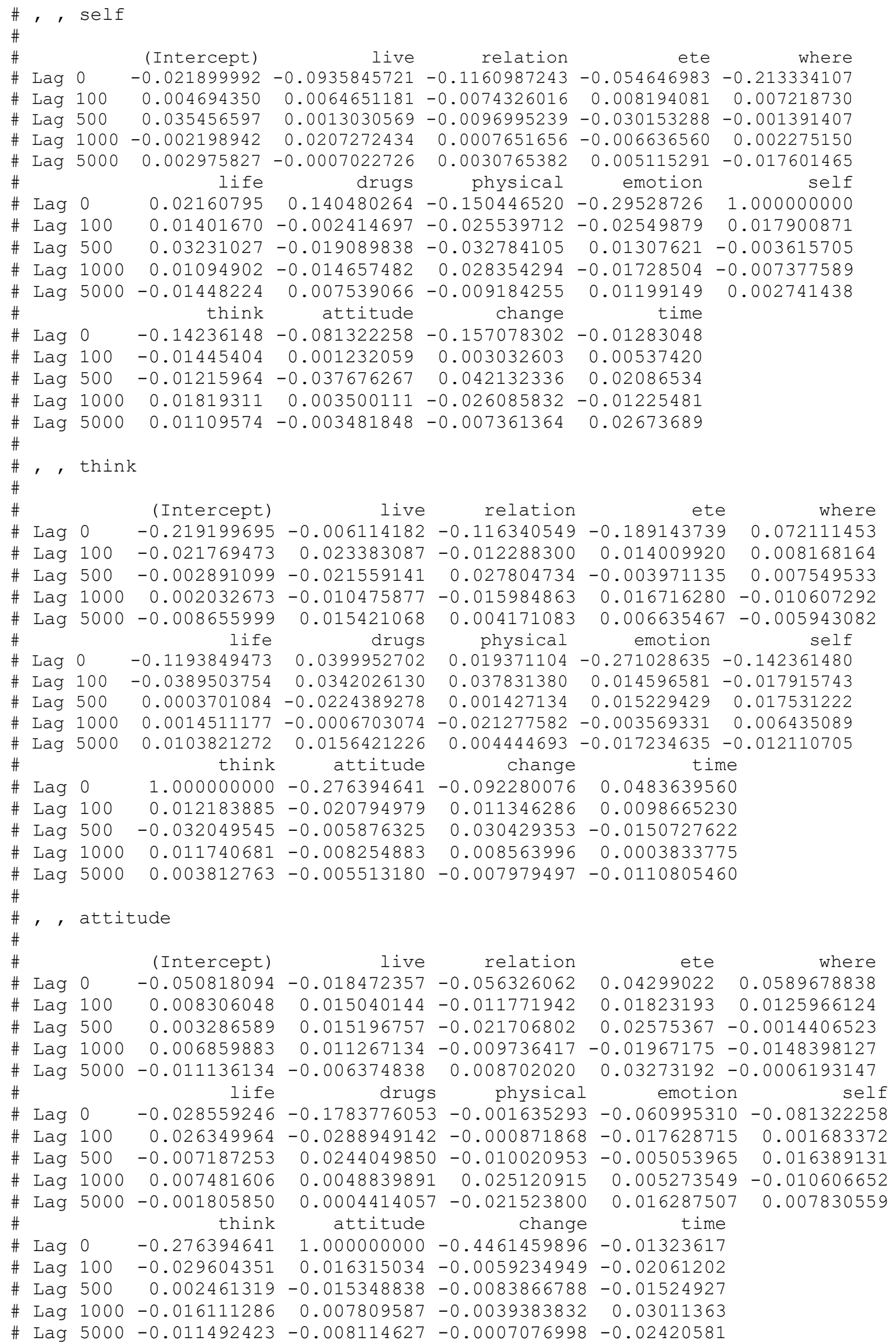




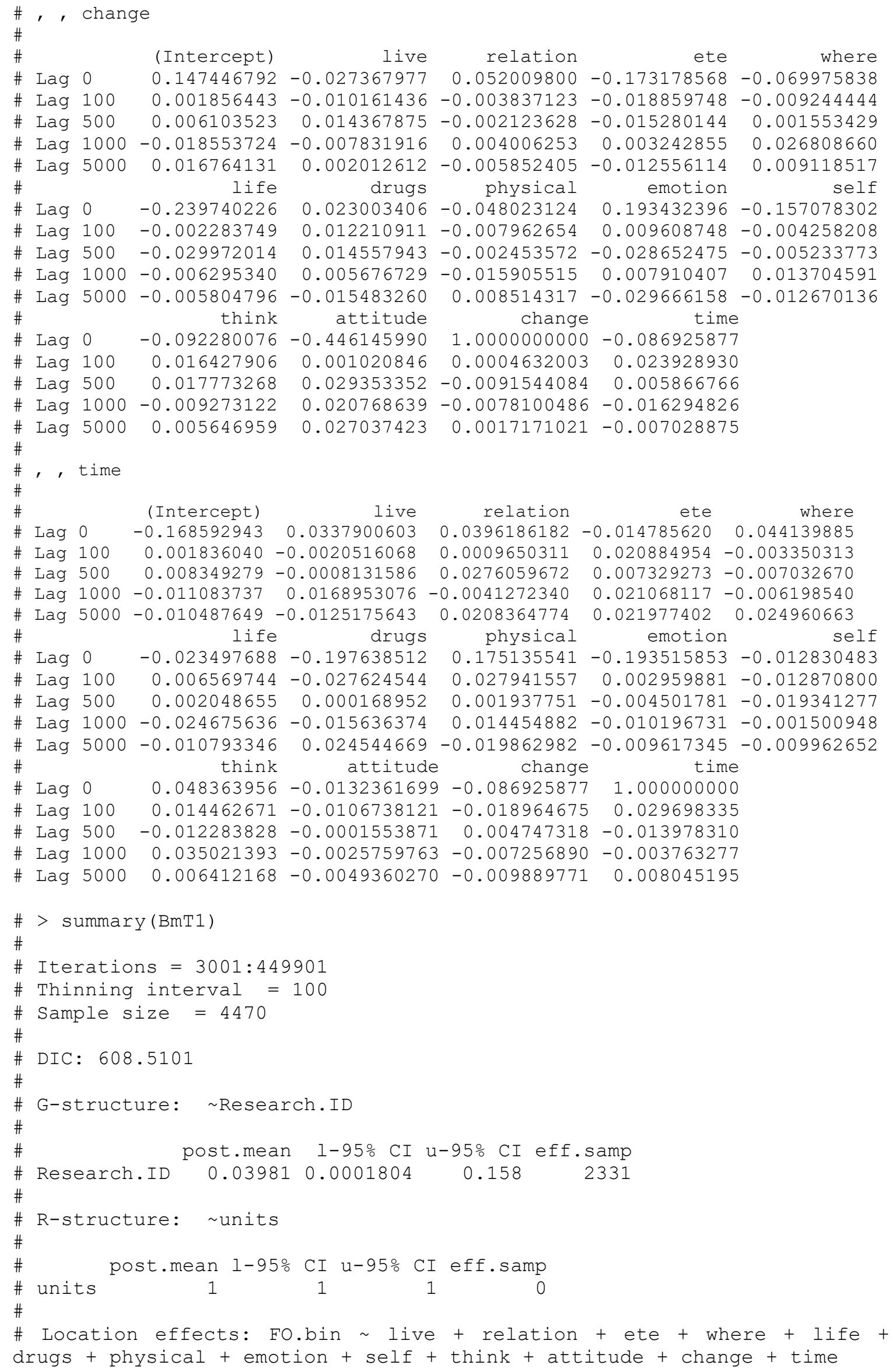




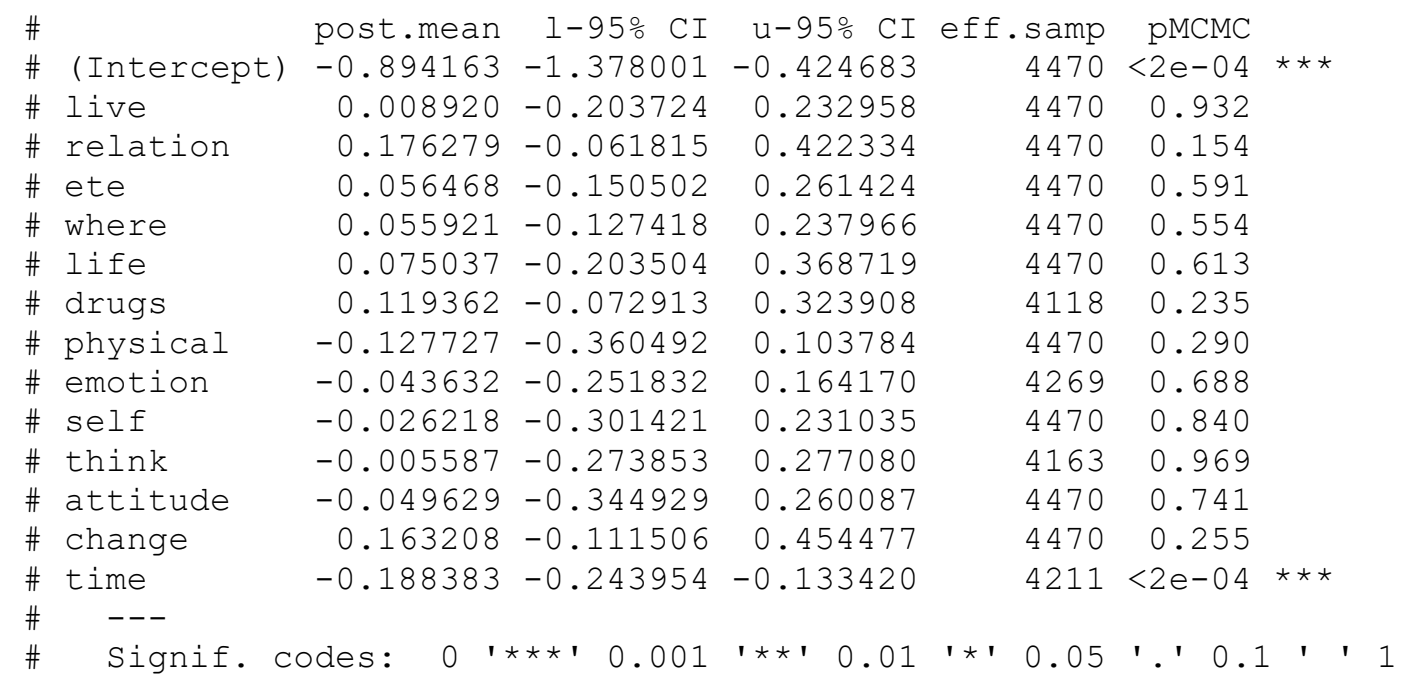




\section{Frequentist Model (riT12)}

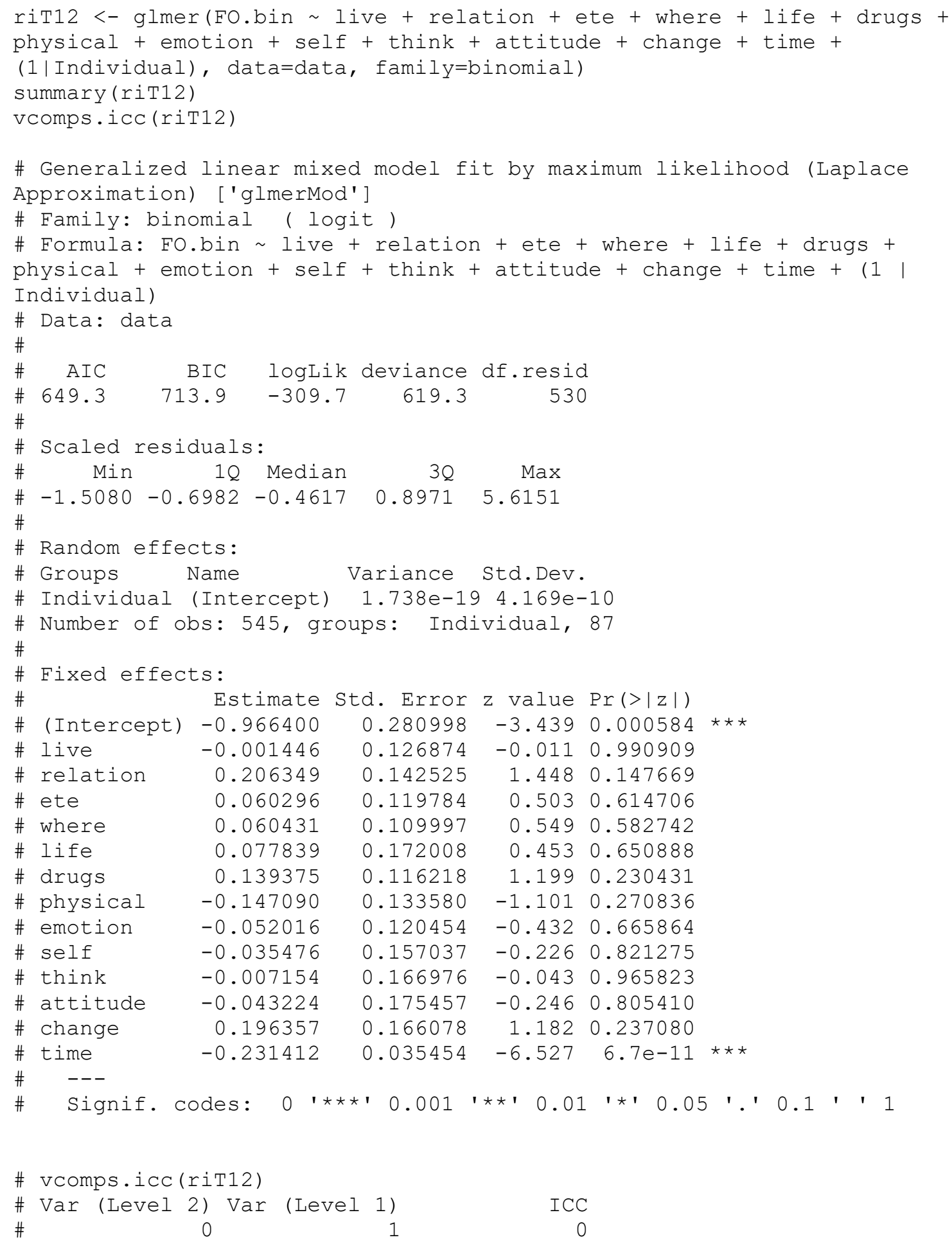


Random Intercept and Varying Slope Model, with Single Predictor (Table 4.10)

Bayesian Model (BmTVO)

\#\# Define the Prior - Adding an additional random effect means that it is necessary to revise the prior. The specified prior is equivalent to an inverse-gamma prior with shape and scale equal to 0.001

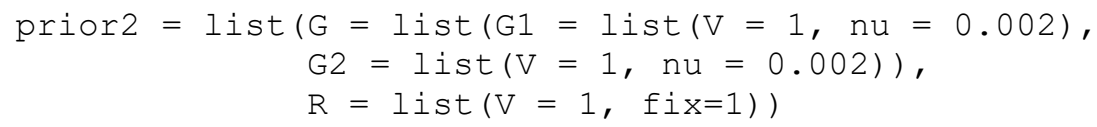

\#\# Define the Model

BmTV0 <- MCMCglmm(FO.bin time, random= time+Research.ID, data=data, family="ordinal", prior=prior2, nitt $=400000$, thin=10, burnin=3000)

\section{\#\# Checks for suitable convergence}

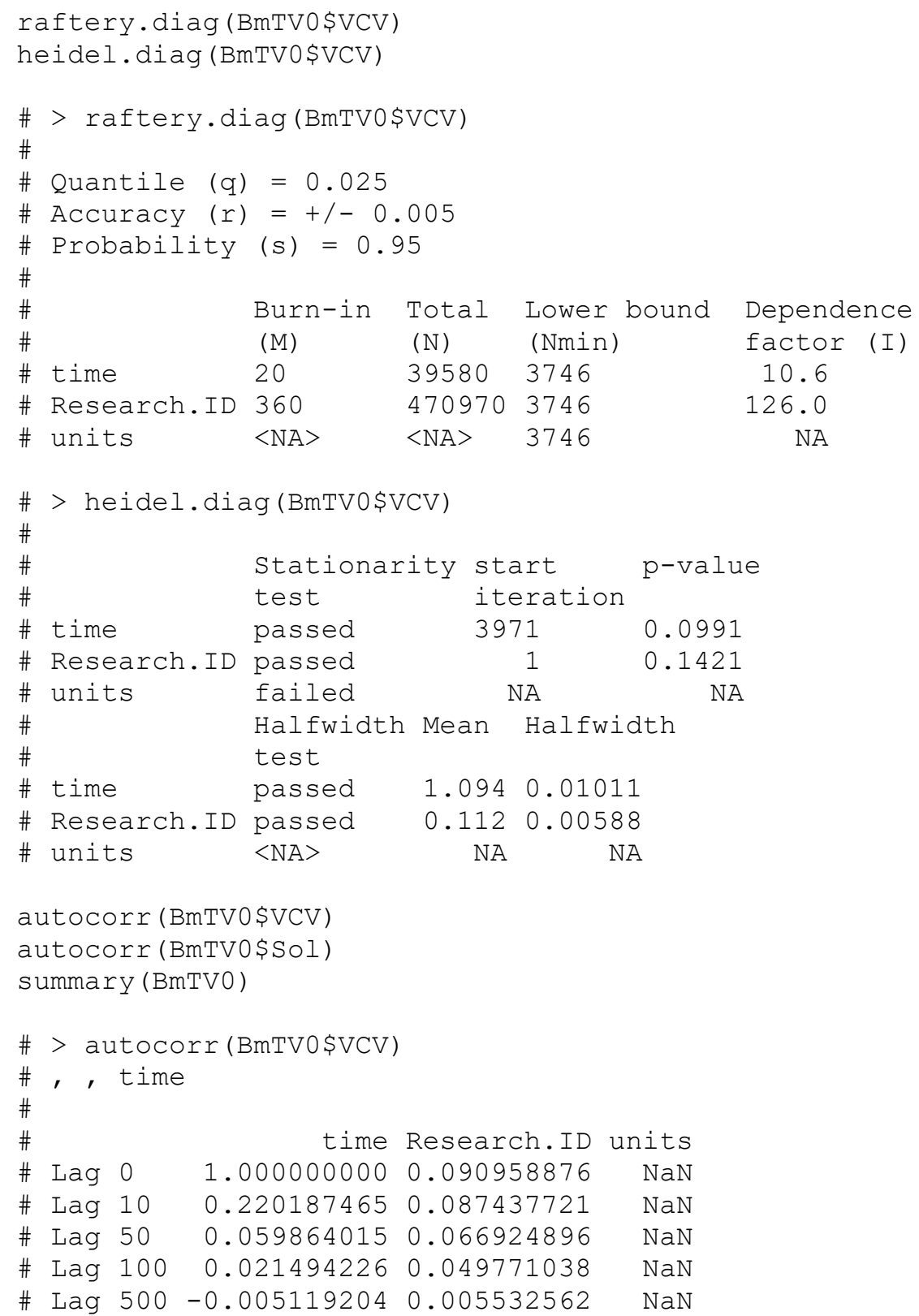




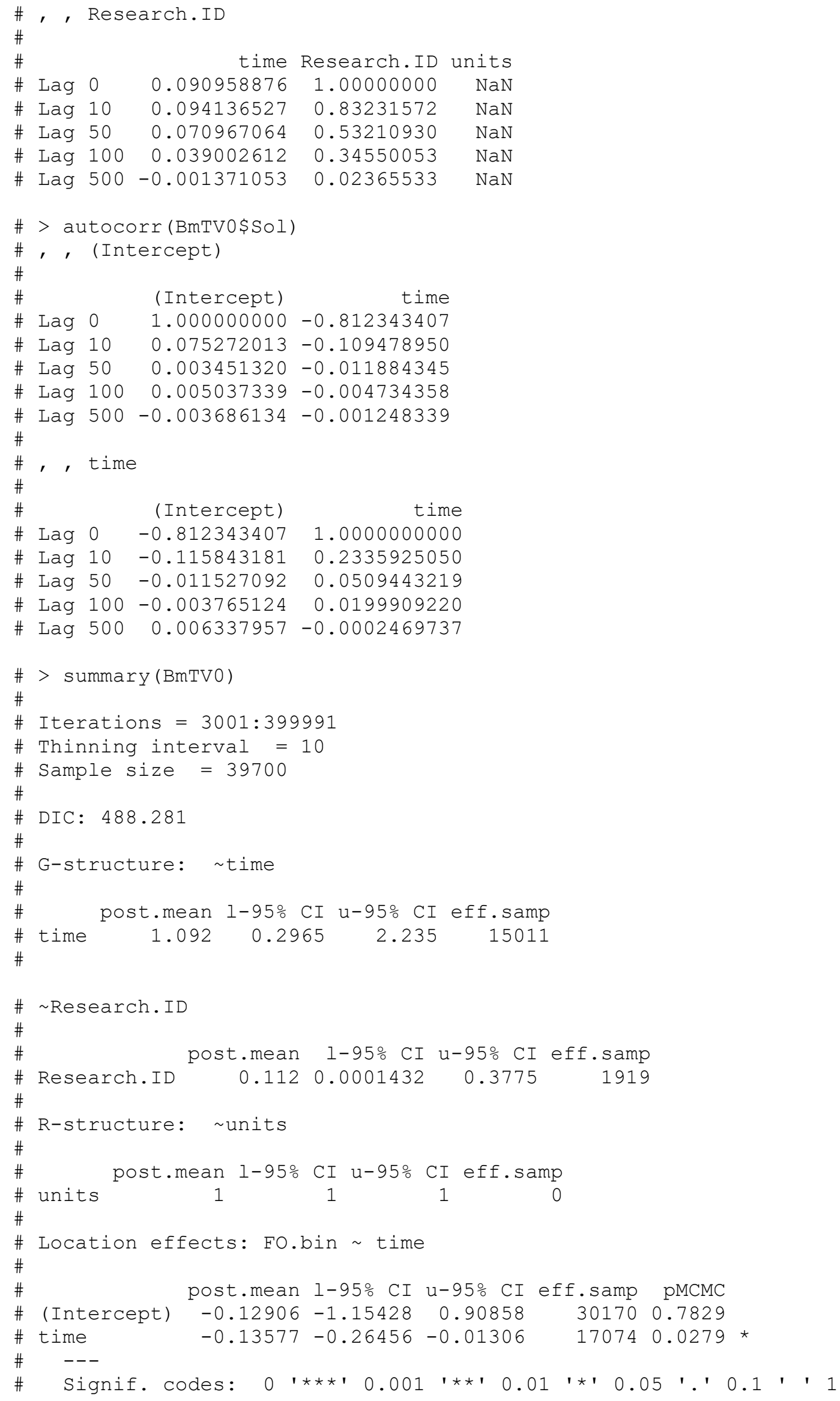




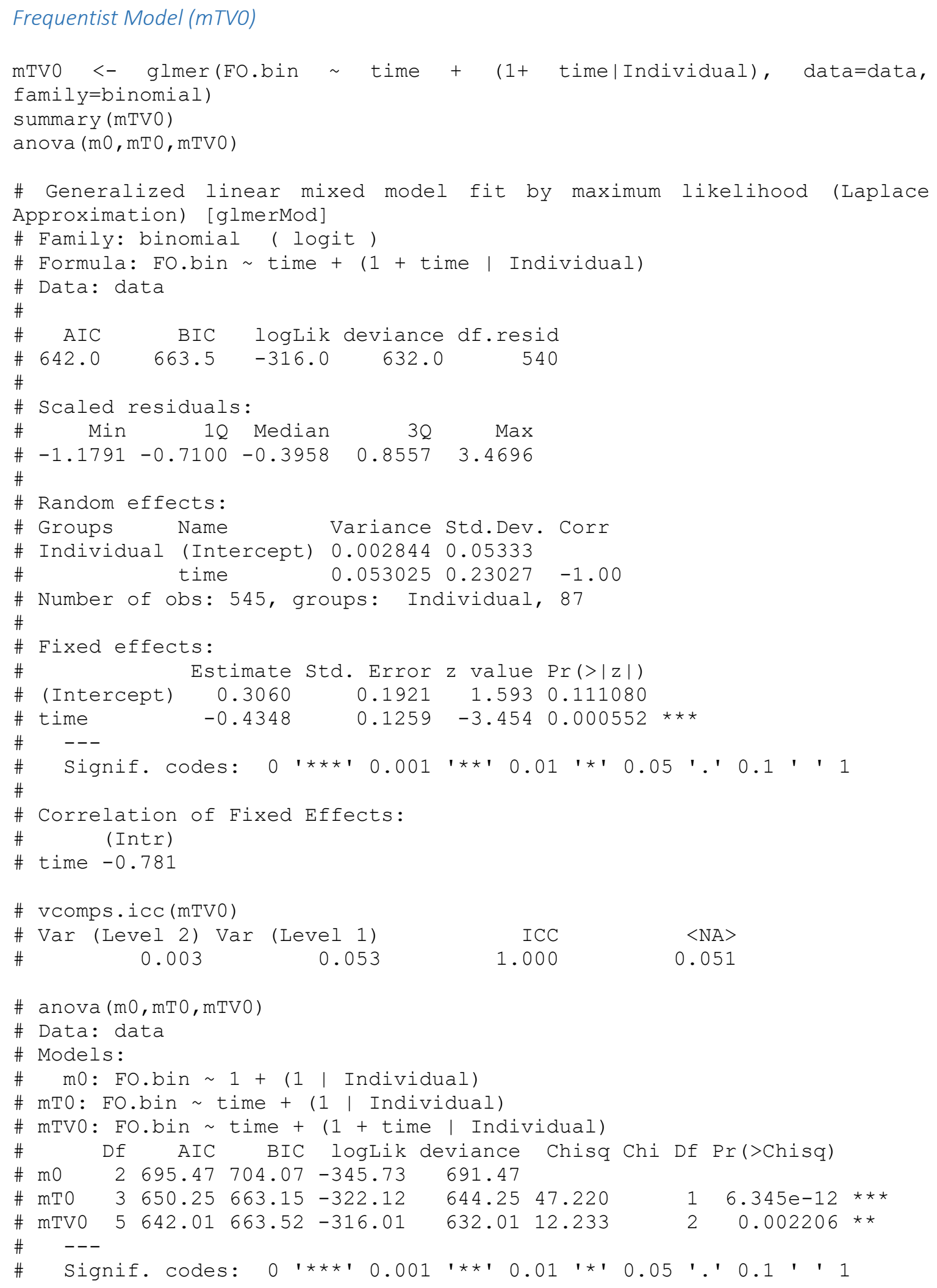


The Basic Model: Random Intercept and Varying Slope Model, with ASSET Domain Predictors (Table 4.11)

Bayesian Model (BmTV1)

\section{\#\# Define the model}

BmTV1 <- MCMCglmm(FO.bin live + relation + ete + where + life + drugs + physical + emotion + self + think + attitude + change + time, random= time+Research.ID, data=data, family="ordinal", prior=prior2, nitt $=450000$, thin=50, burnin=3000)

\section{\# Checks for suitable convergence}

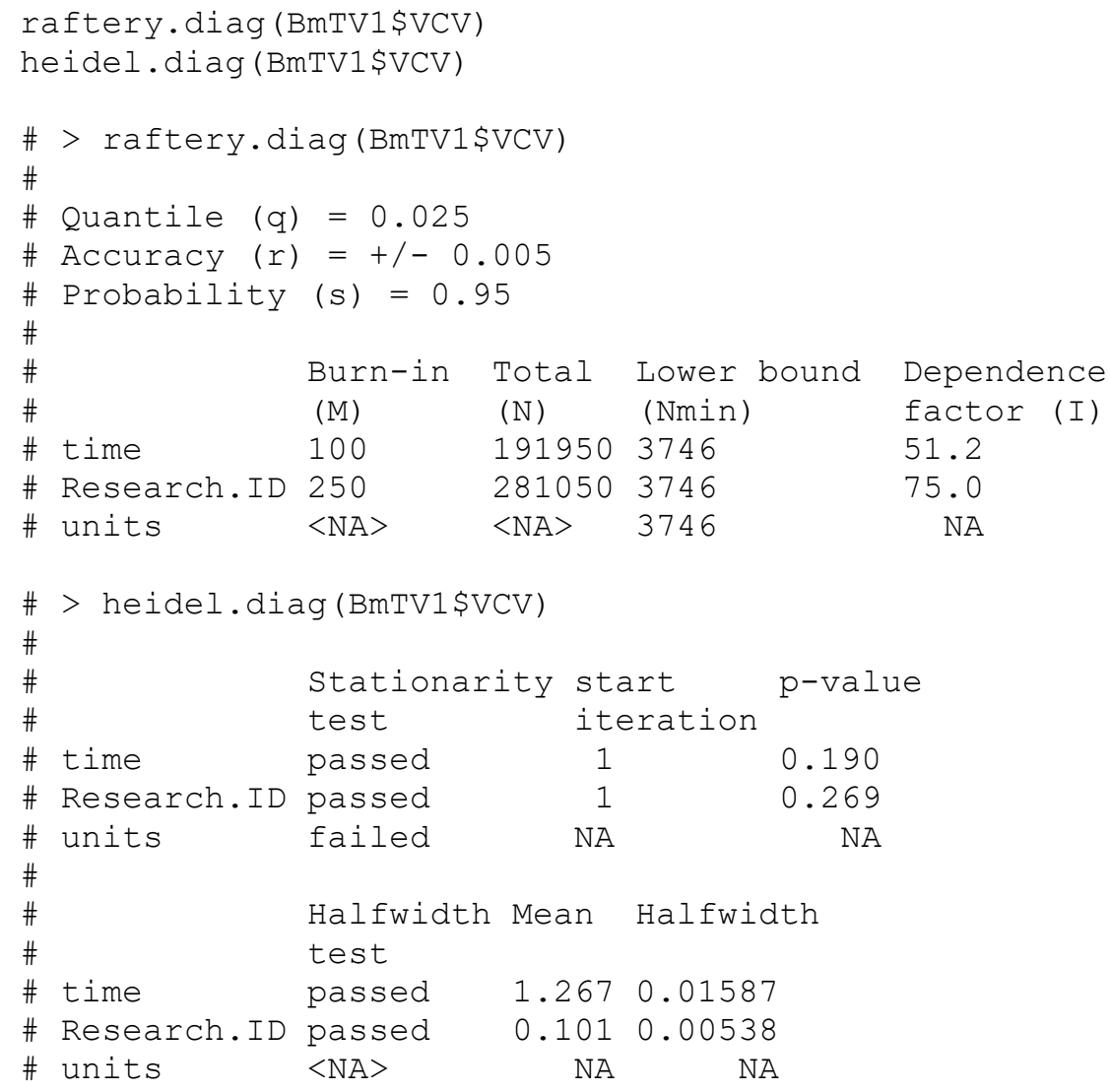




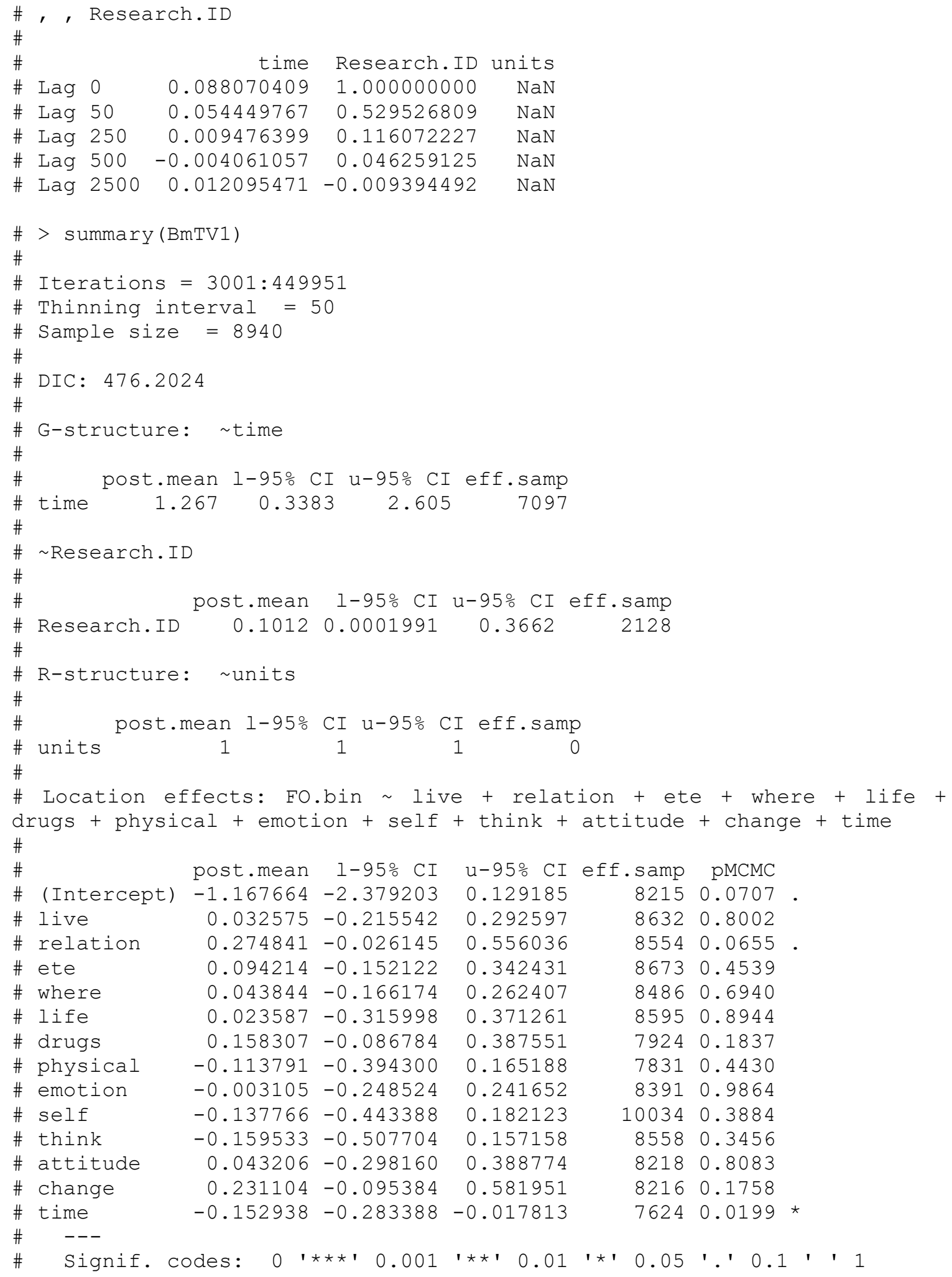


\# To simplify naming conventions, BmTV1 is renamed as Bm1 whilst the Frequentist equivalent riTV12 is renamed $\mathrm{m} 1$. This represents the Basic model.

\section{m1 <- riTV12}

Bm1 <- BmTV1

Fixed Effects

Trace of (Intercept)

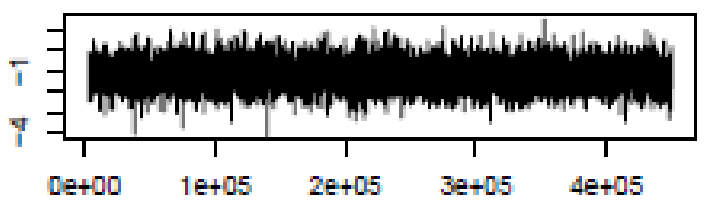

Iterations

Trace of live

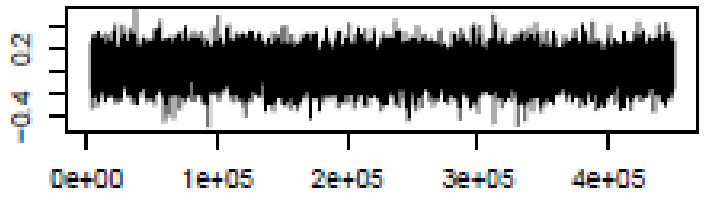

Iterations

Trace of relation

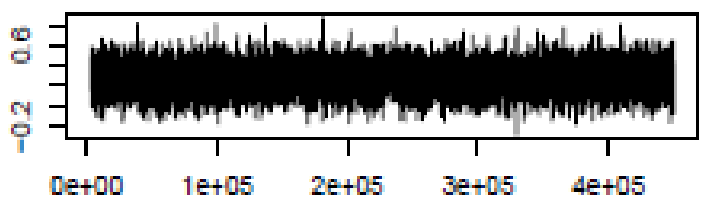

Iterations

Trace of ete

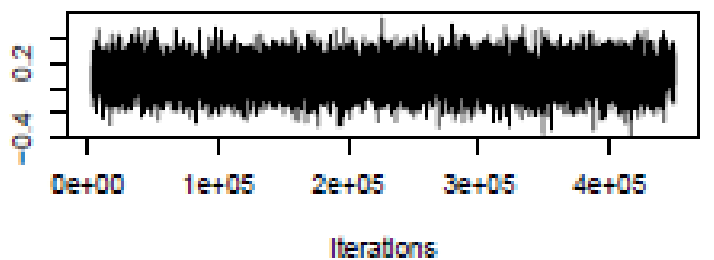

Density of (Intercept)

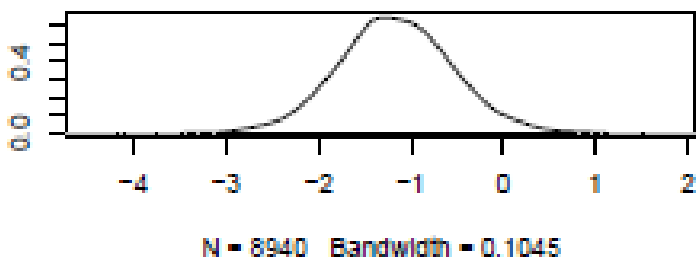

Density of live

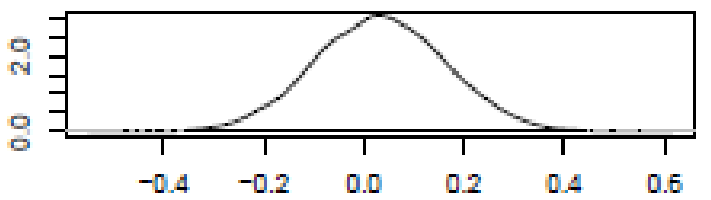

$\mathrm{N}=8940$ Bandwidth $=0.0224$

Density of relation

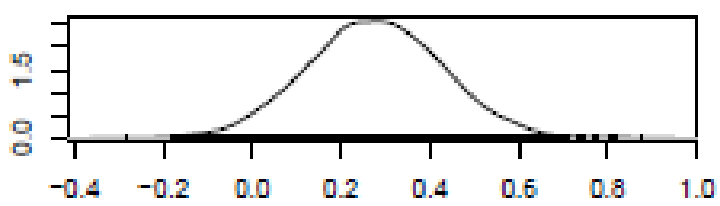

$\mathrm{N}=8940$ Bandwidth -0.02558

Density of ete

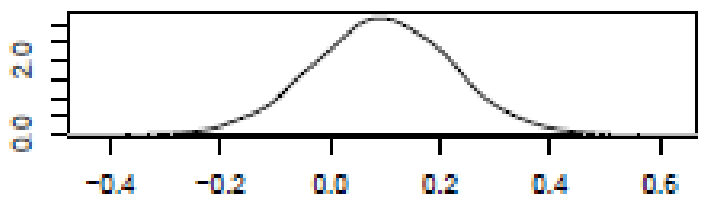

$\mathrm{N}=8940$ Bandwidth $=0.02146$ 
Trace of where

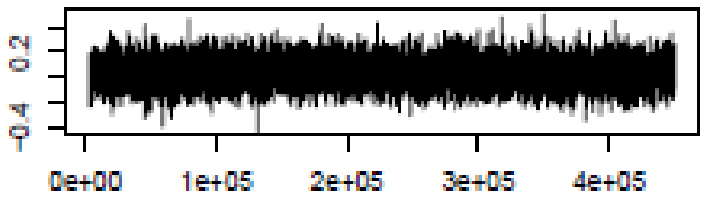

Iterations

Trace of life

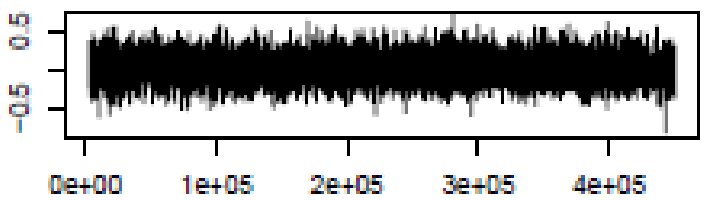

Iterations

Trace of drugs

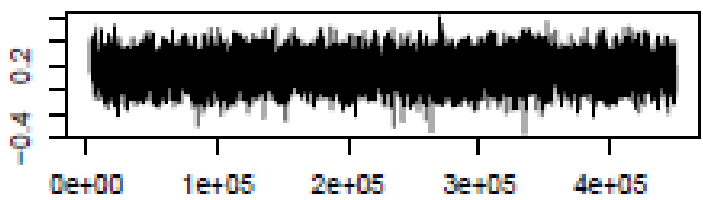

Iterations

Trace of physical

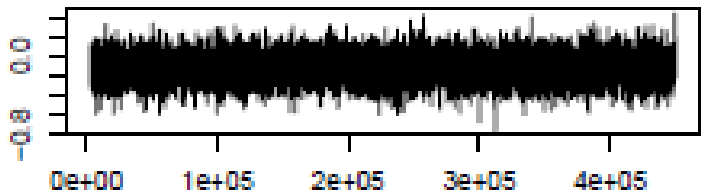

Iterations

Trace of emotion

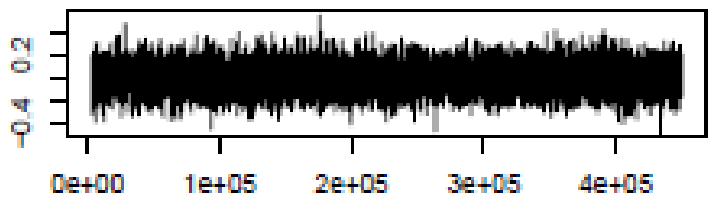

Iterations

Trace of self

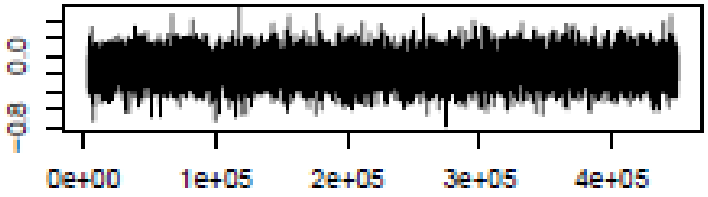

Iterations
Density of where

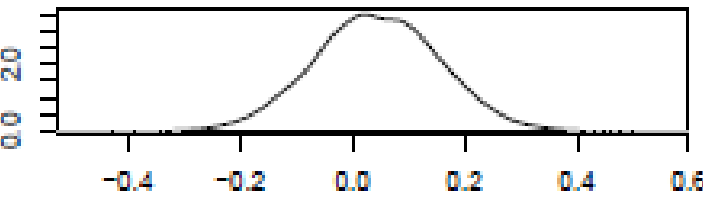

$\mathrm{N}=8940$ Bandwidth $=0.01895$

Density of life

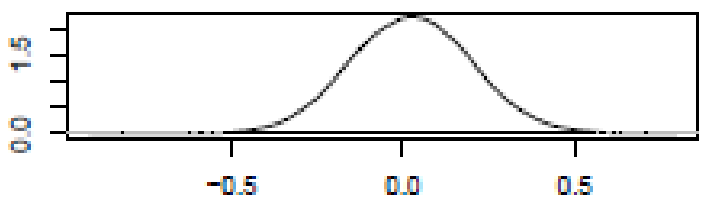

$\mathrm{N}=8940$ Bandwidth $=0.03032$

Density of drugs

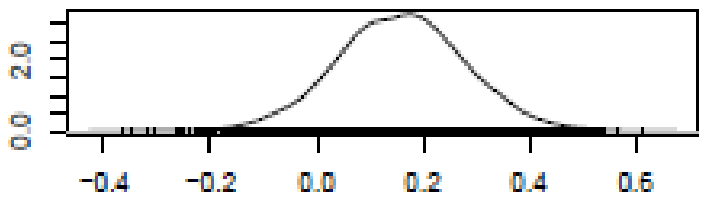

$\mathrm{N}=8940$ Bandwidth $=0.02063$

Density of physical

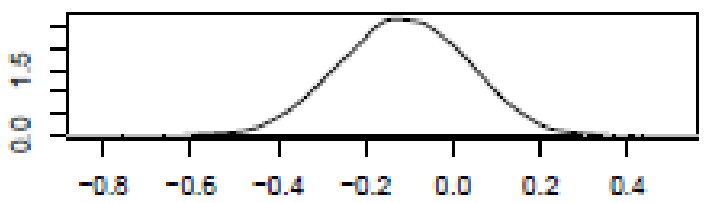

$\mathrm{N}=8940$ Bandwidth $\mathbf{0} 0.02491$

Density of emotion

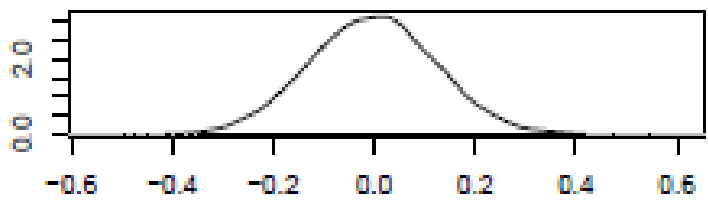

$\mathrm{N}=8940$ Bandwidth $\mathbf{0} 0.02144$

Density of self

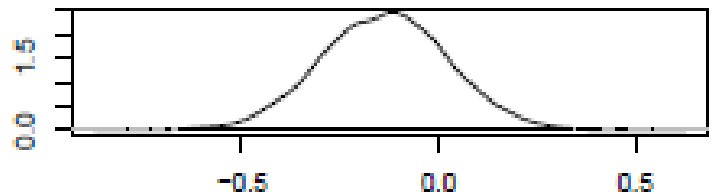

$\mathrm{N}=8940$ Bandwidth $=0.02773$ 
Trace of think

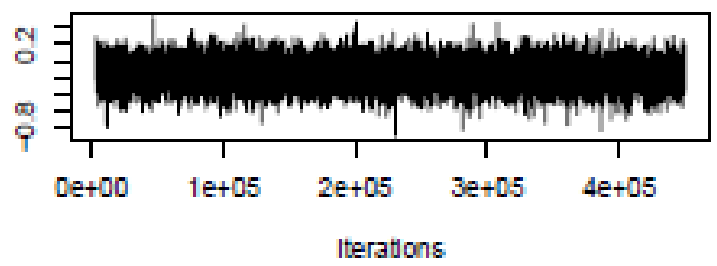

Trace of attitude

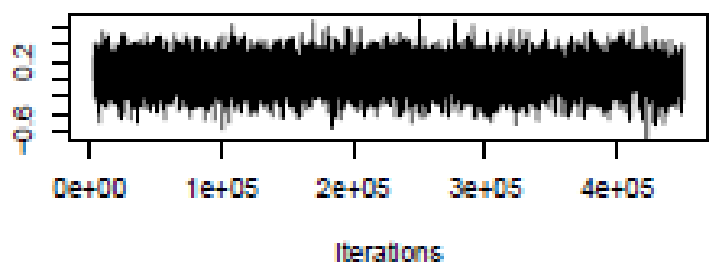

Trace of change

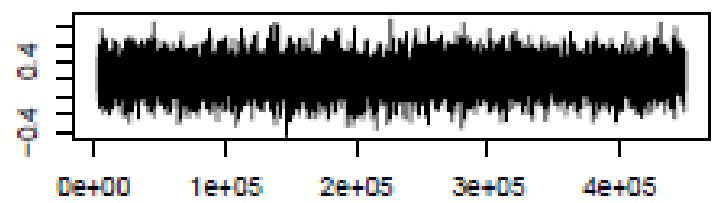

Iterations

Trace of time

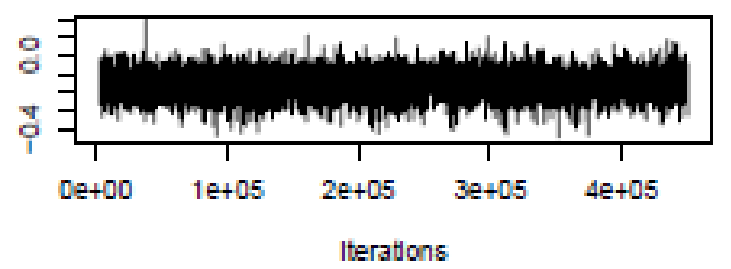

Density of think

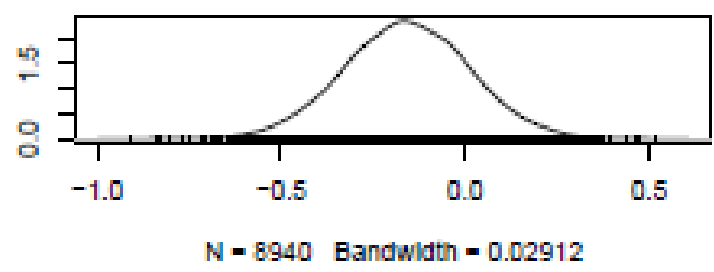

Density of attitude

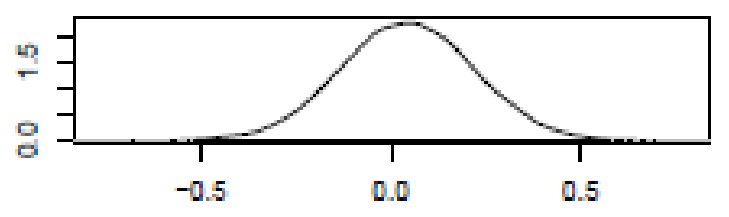

$\mathrm{N}=8940$ Bandwidth -0.03004

Density of change

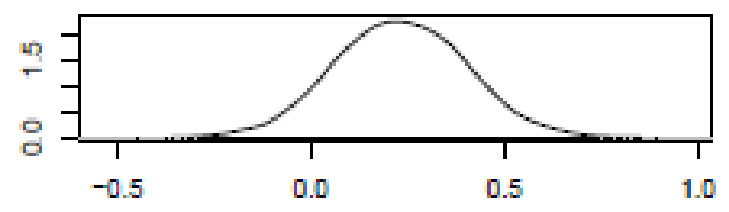

$N=8940$ Bandwidth -0.0297

Density of time

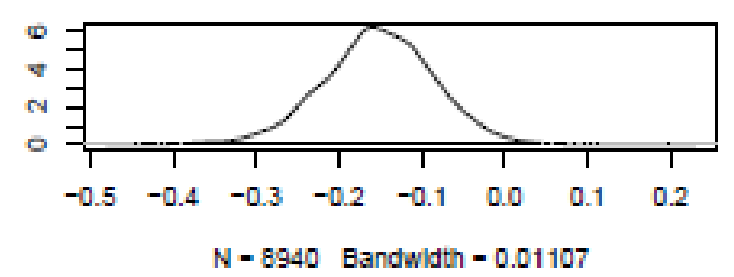


$\underline{\text { Random Effects }}$

Trace of time

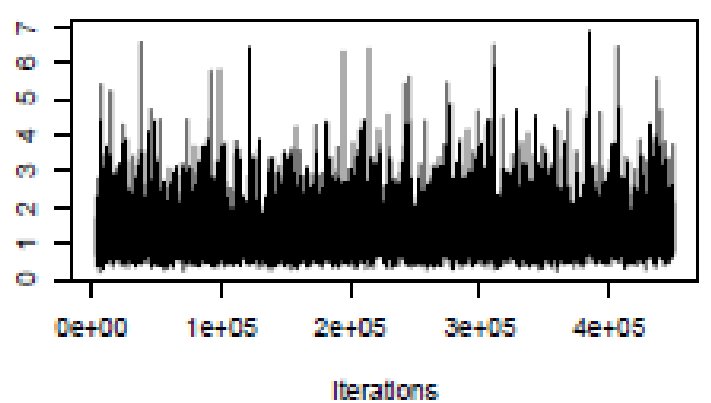

Trace of Research.ID

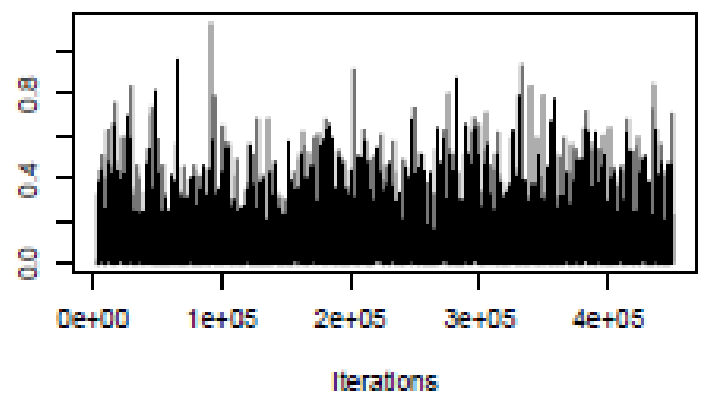

Density of time

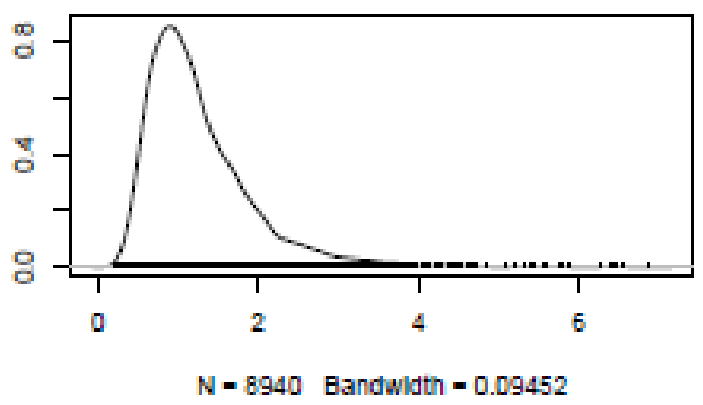

Density of Research.ID

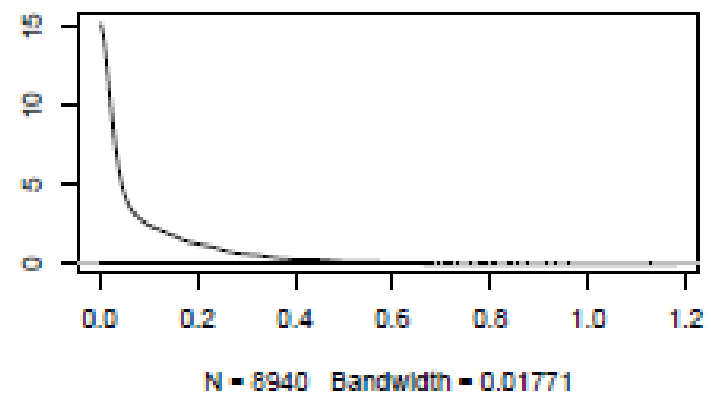




\section{Frequentist Model (riTV12)}

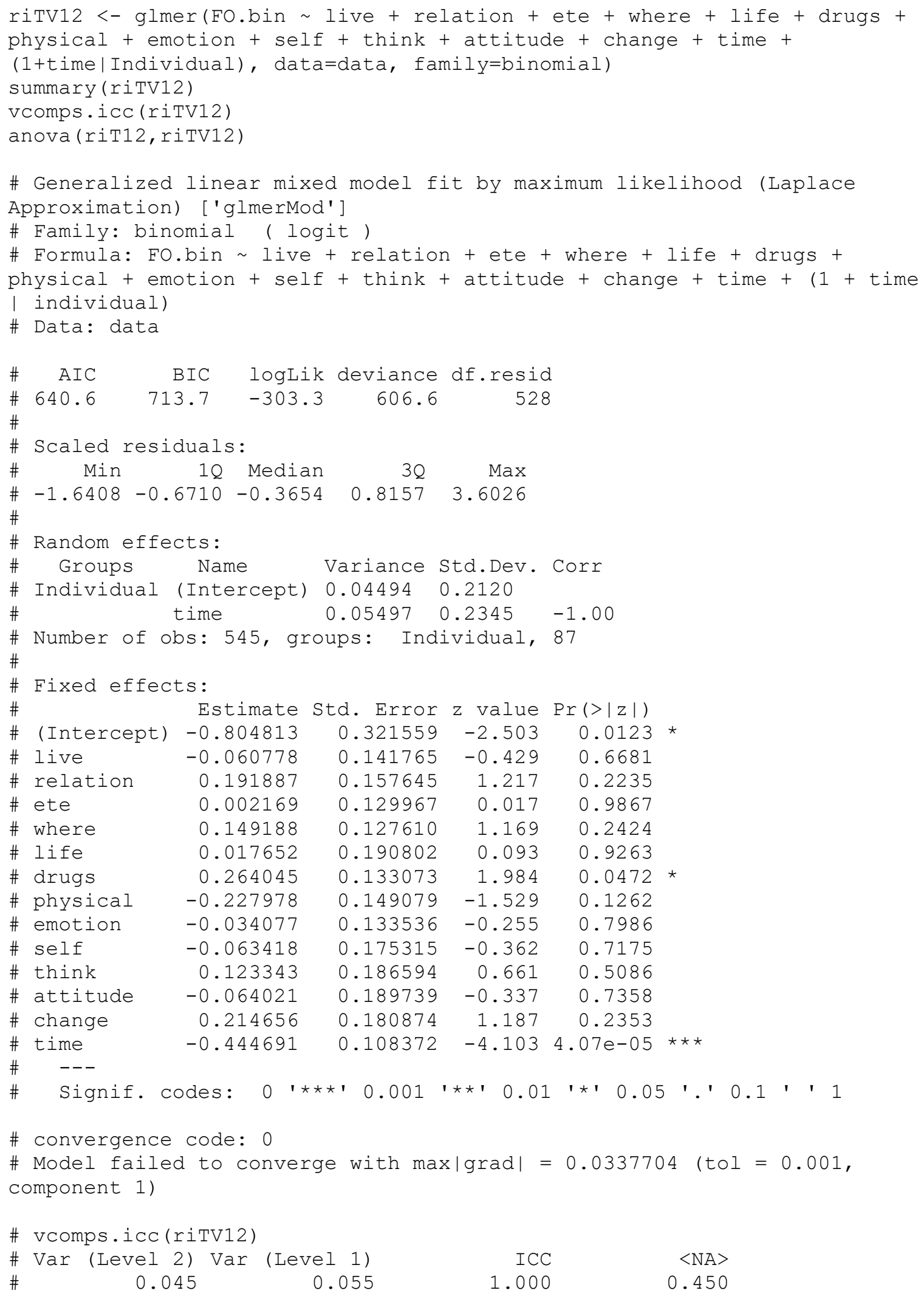




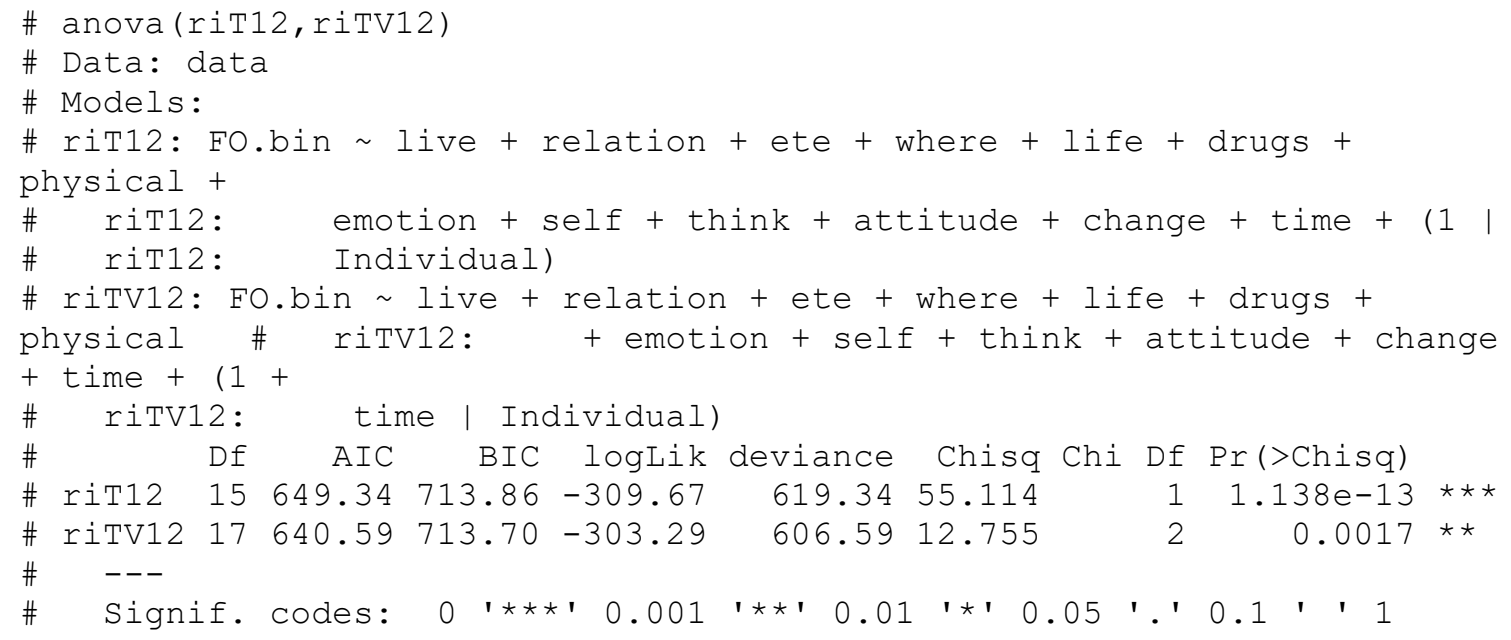


Dynamic Model 1 (Table 4.12)

Bayesian (BDm1)

\section{\# Revise the prior}

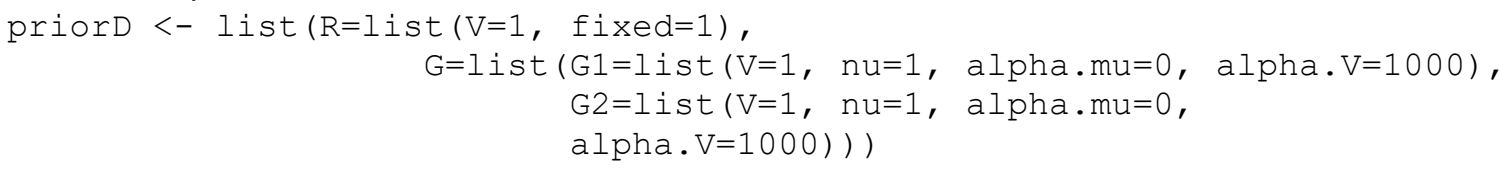

\section{\#\# Define the model}

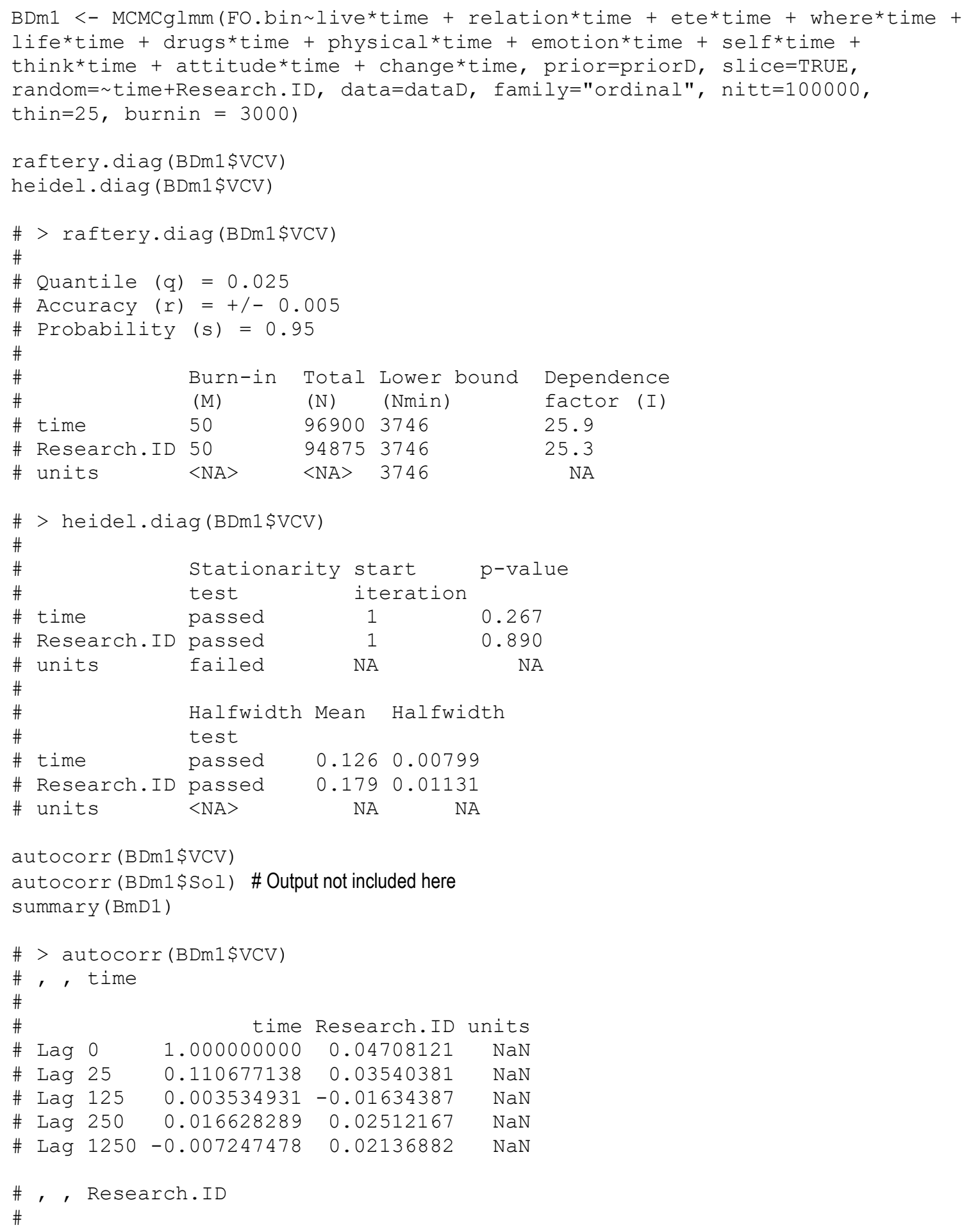




$\begin{array}{lllrrr}\# & & \text { time } & \text { Research.ID } & \text { units } \\ \# \text { Lag } 0 & 0.047081205 & 1.000000000 & \text { NaN } \\ \# \text { Lag 25 } & 0.024996348 & 0.267709123 & \text { NaN } \\ \text { \# Lag 125 } & -0.018980326 & 0.008959189 & \text { NaN } \\ \text { \# Lag 250 } & -0.003648318 & 0.024686331 & \text { NaN } \\ \text { \# Lag 1250 } & 0.010969437 & -0.011849702 & \text { NaN }\end{array}$

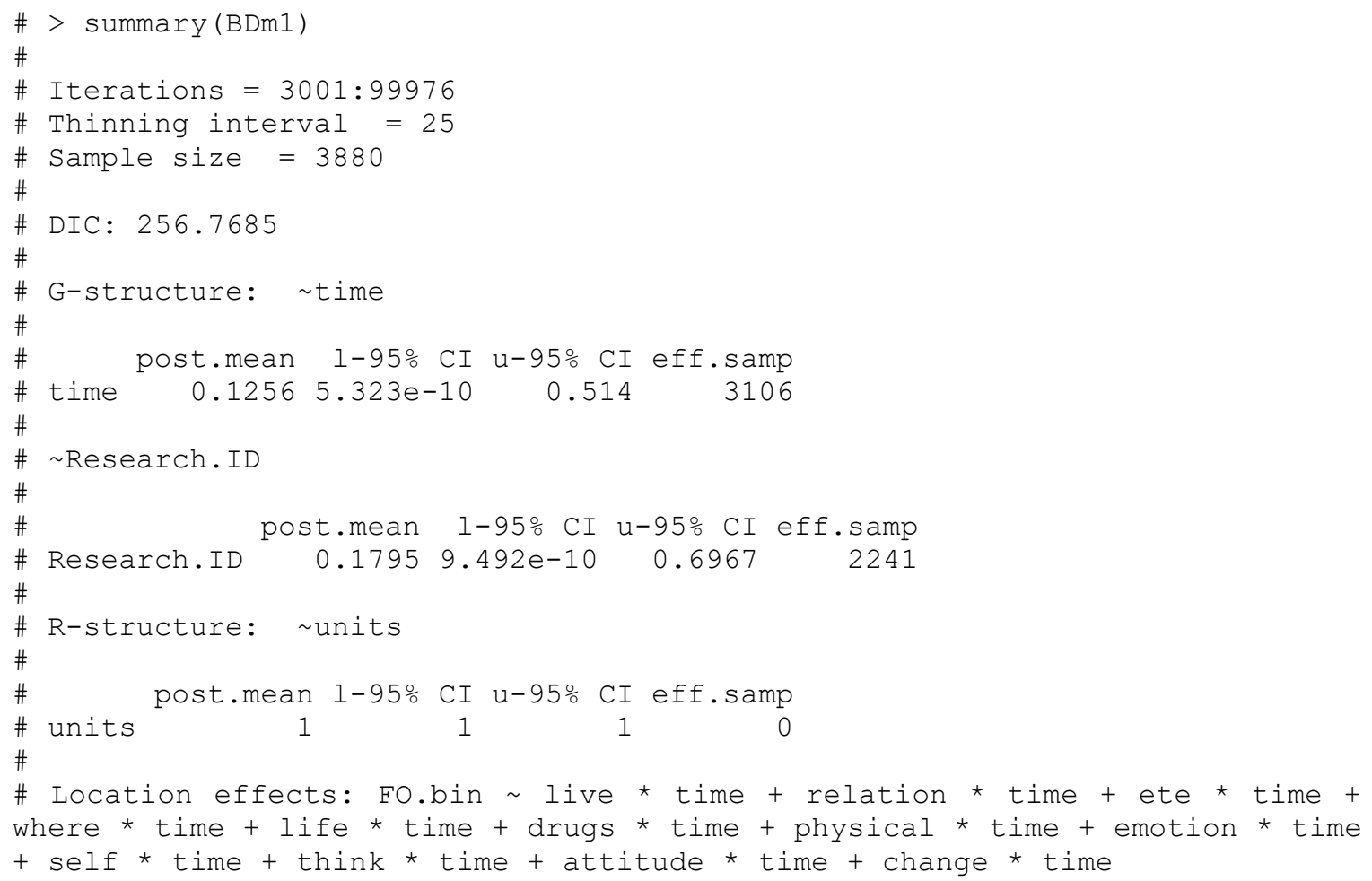


Fixed Effects

Trace of (Intercept)

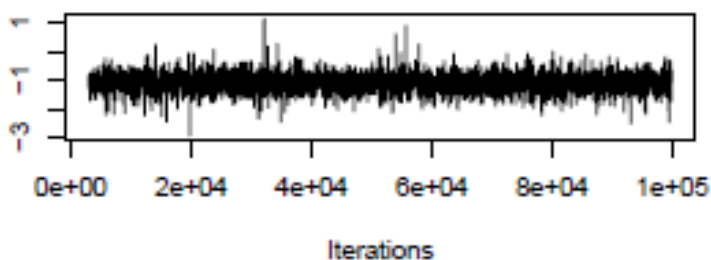

Trace of live

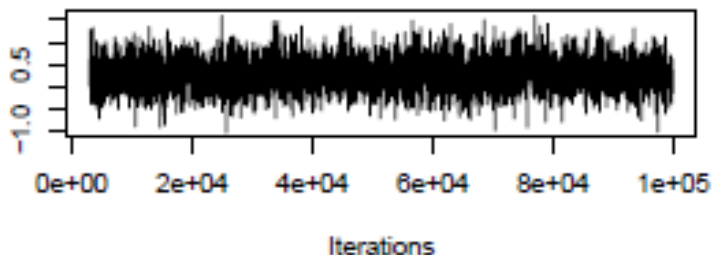

Trace of time

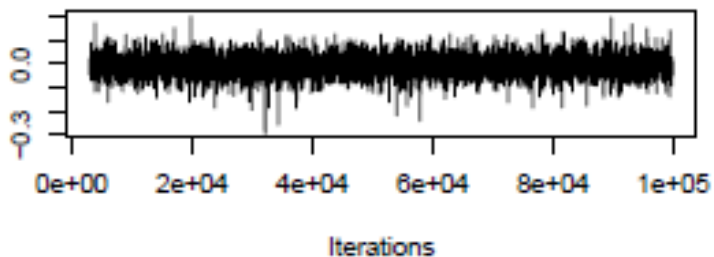

Trace of relation

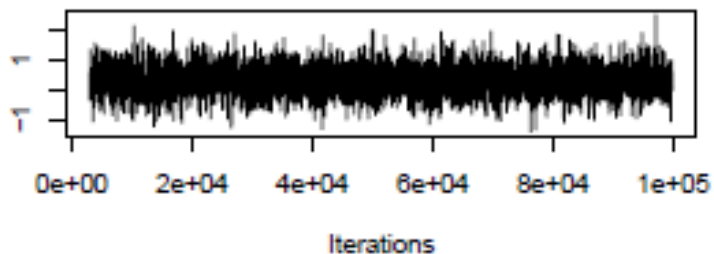

Trace of ete

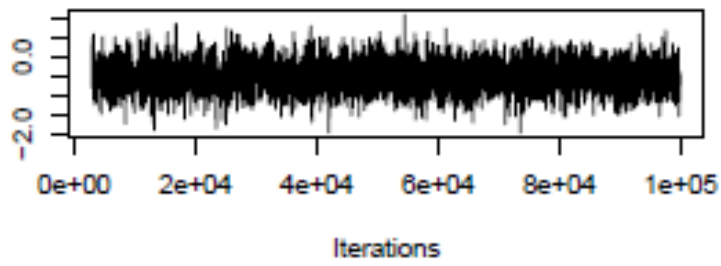

Density of (Intercept)

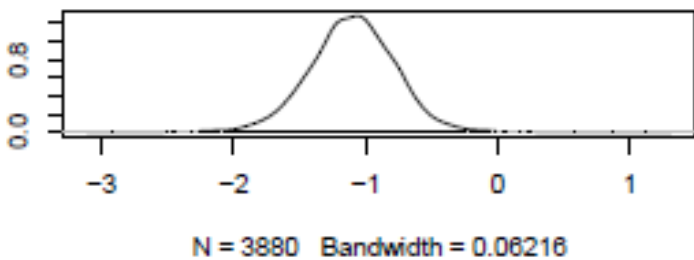

Density of live

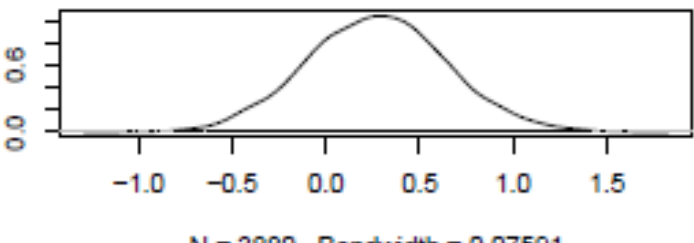

Density of time

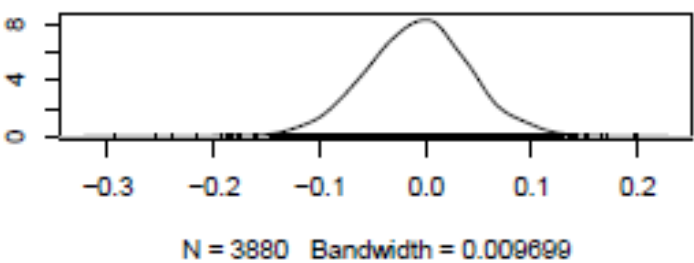

Density of relation

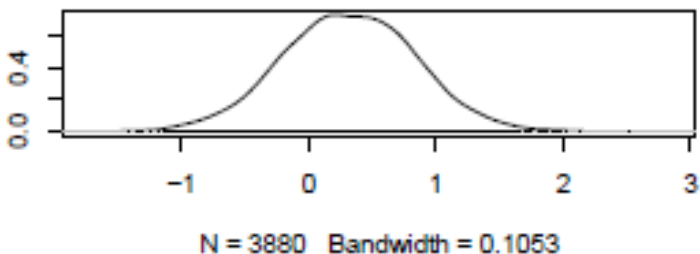

Density of ete

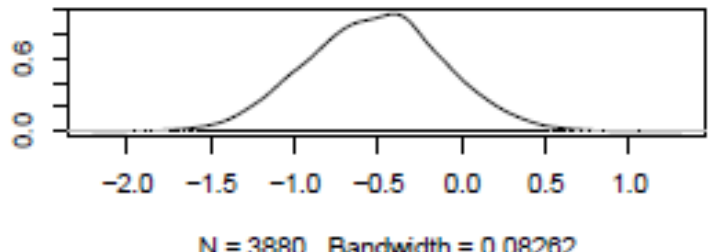


Trace of where

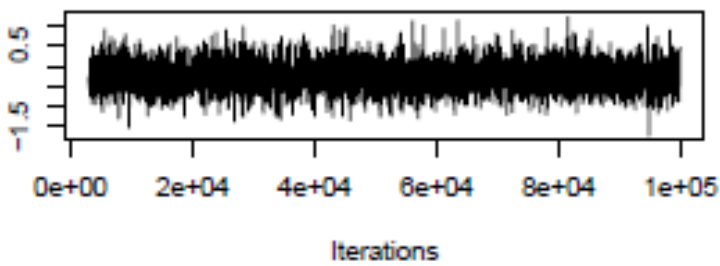

Trace of life

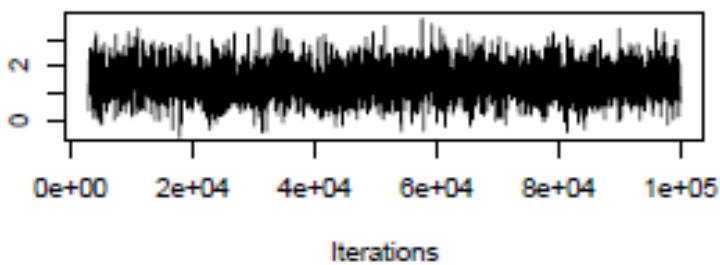

Trace of drugs

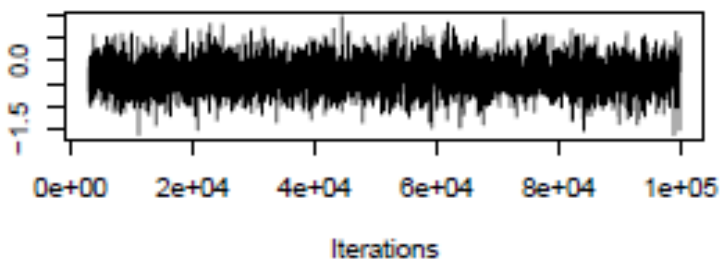

Trace of physical

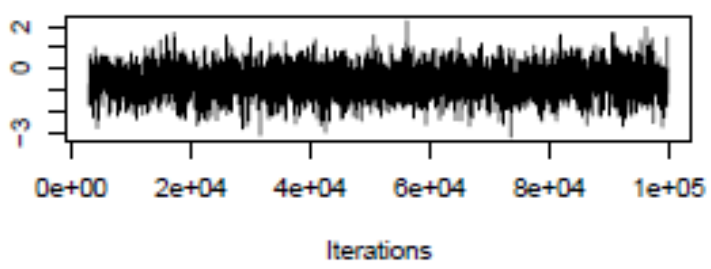

Trace of emotion

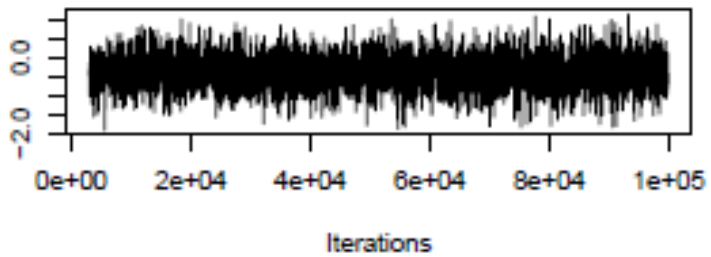

Trace of self

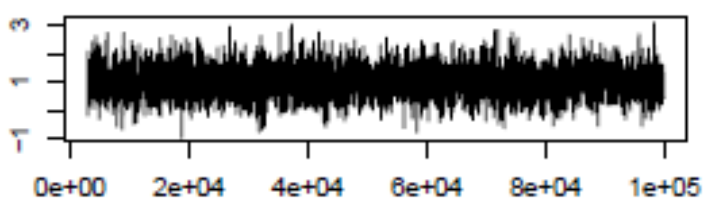

Iterations
Density of where

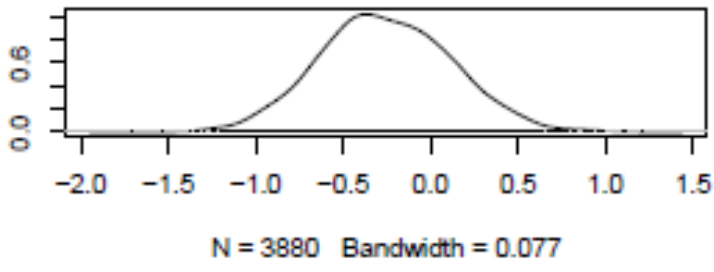

Density of life

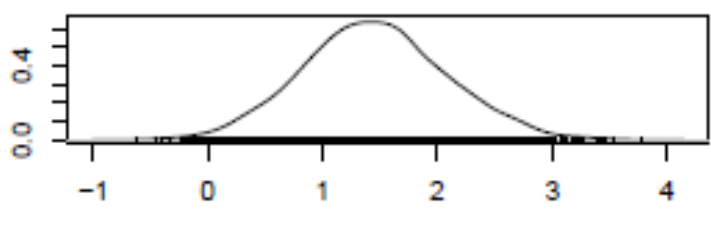

$\mathrm{N}=3880$ Bandwidth $=0.1256$

Density of drugs

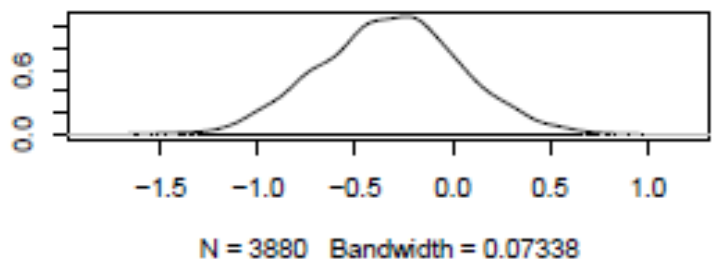

Density of physical

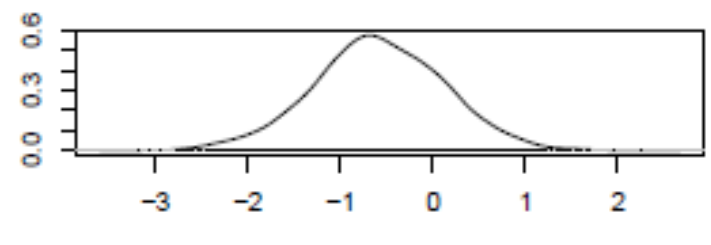

$\mathrm{N}=3880$ Bandwidth $=0.1411$

Density of emotion

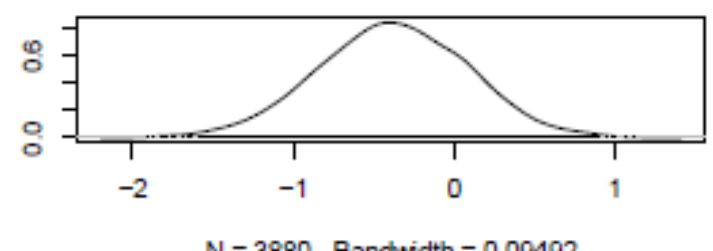

Density of self

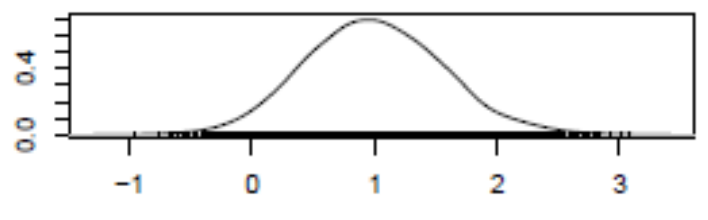

$\mathrm{N}=3880$ Bandwidth $=0.1139$ 
Trace of self

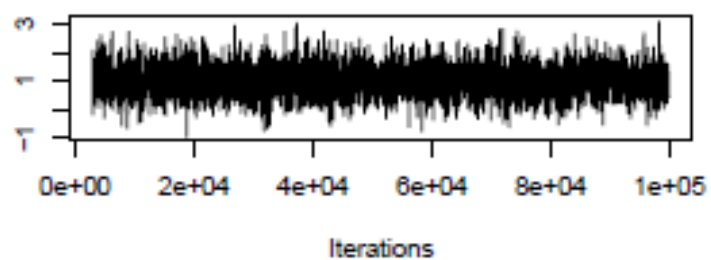

Trace of think

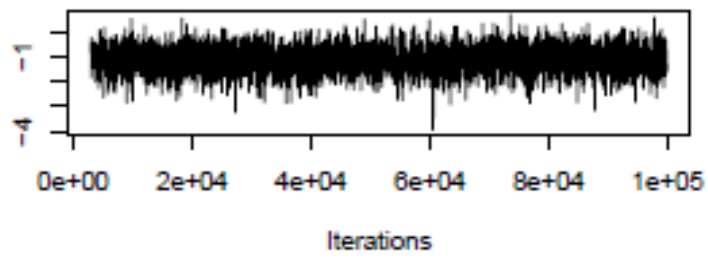

Trace of attitude

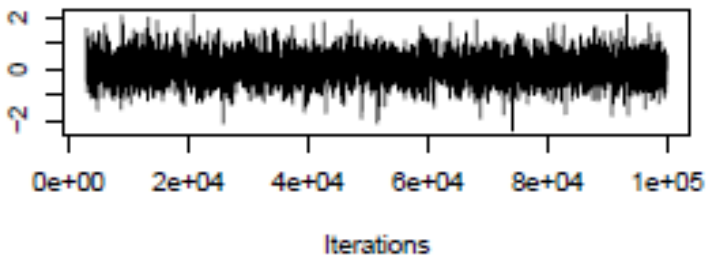

Trace of change

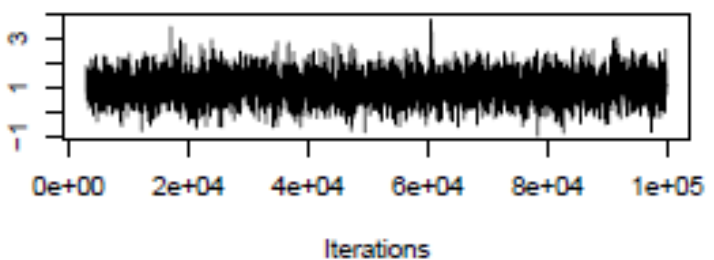

Trace of live:time

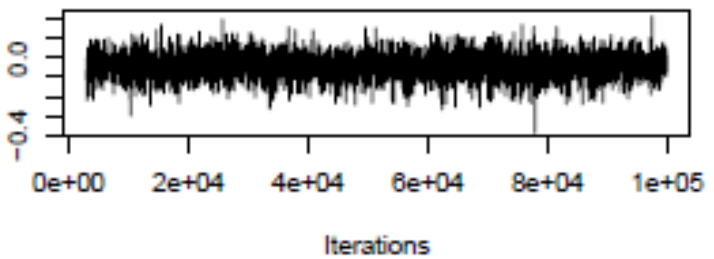

Trace of time:relation

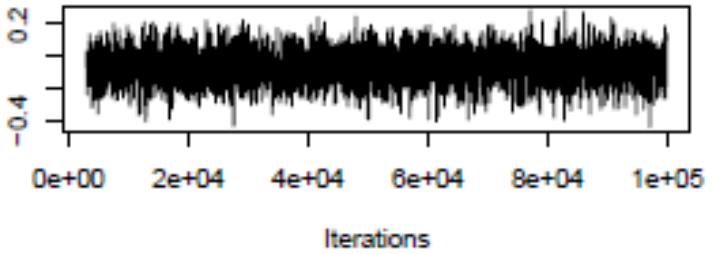

Density of self

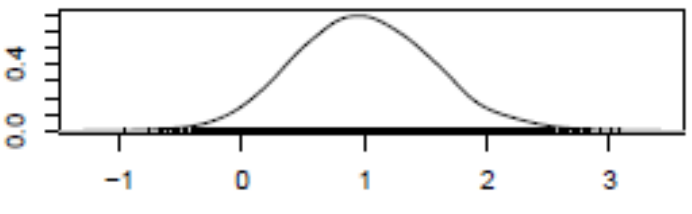

$\mathrm{N}=3880$ Bandwidth $=0.1139$

Density of think

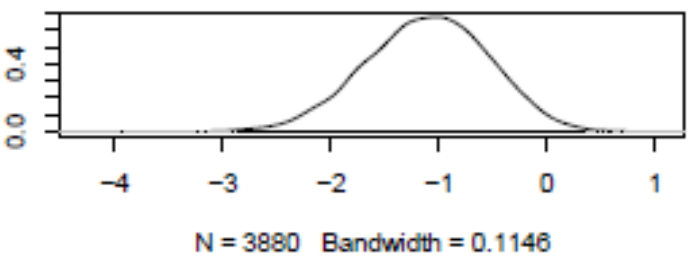

Density of attitude

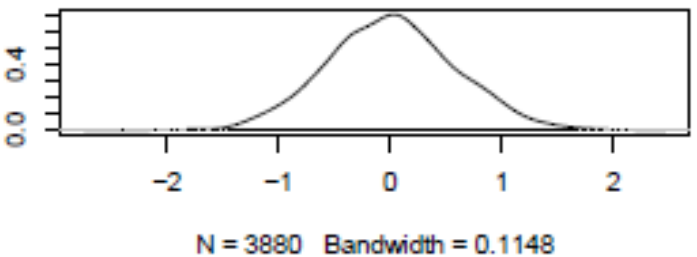

Density of change

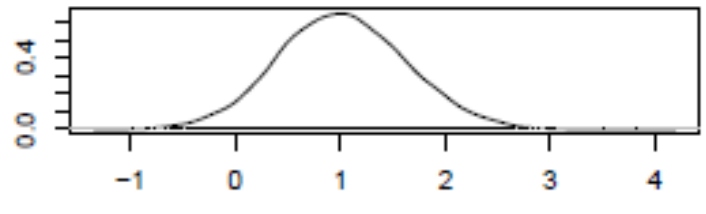

$\mathrm{N}=3880$ Bandwidth $=0.1236$

Density of live:time

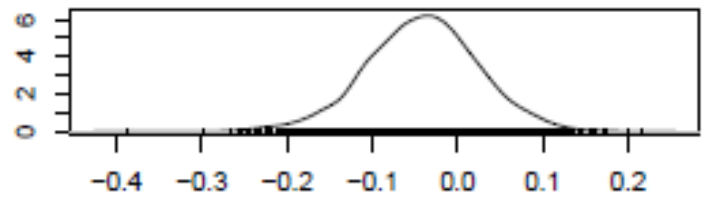

$\mathrm{N}=3880$ Bandwidth $=0.01285$

Density of time:relation

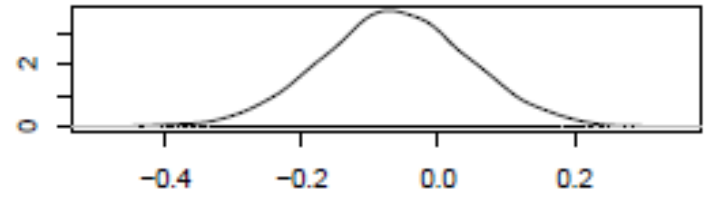

$\mathrm{N}=3880$ Bandwidth $=0.02136$ 
Trace of time:ete

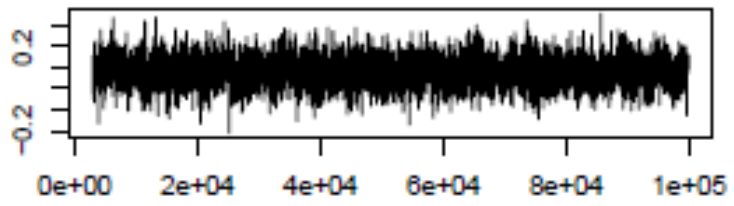

Iterations

Trace of time:where

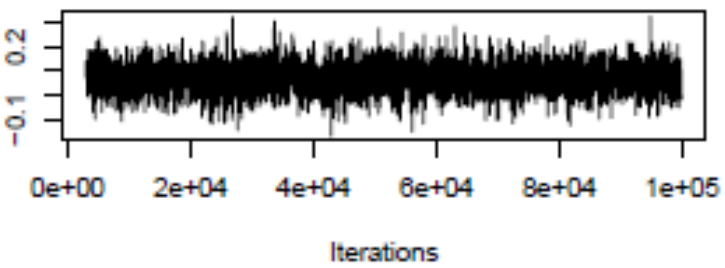

Trace of time:life

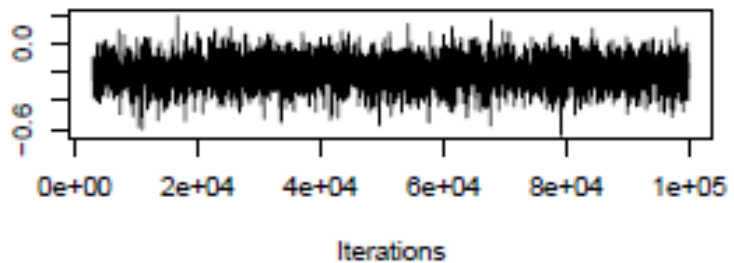

Trace of time:drugs

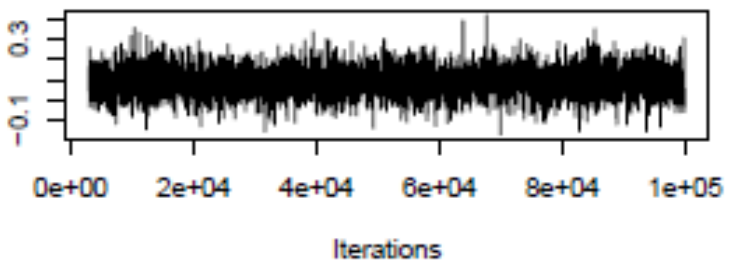

Trace of time:physical

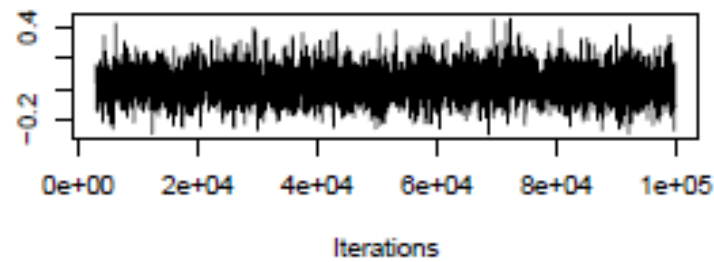

Trace of time:emotion

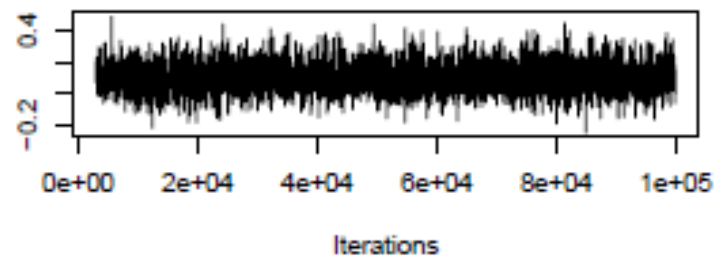

Density of time:ete

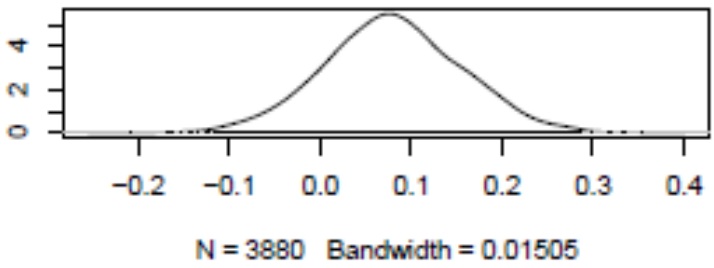

Density of time:where

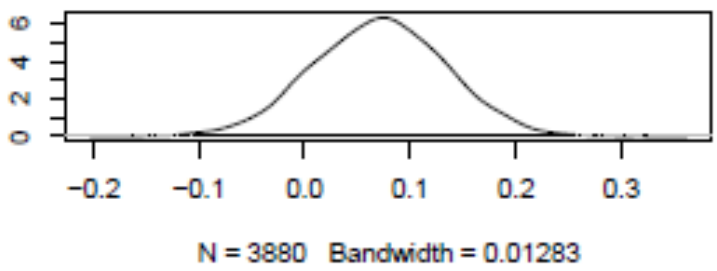

Density of time:life

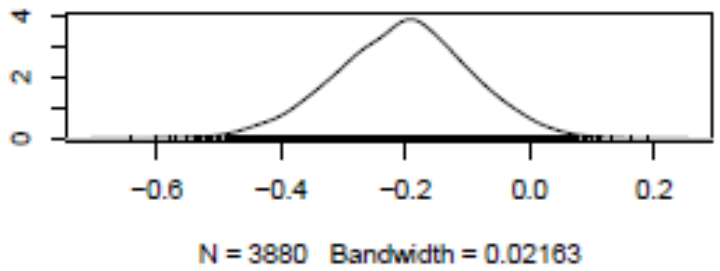

Density of time:drugs

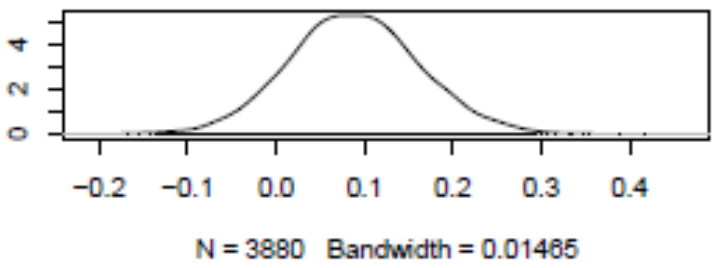

Density of time:physical

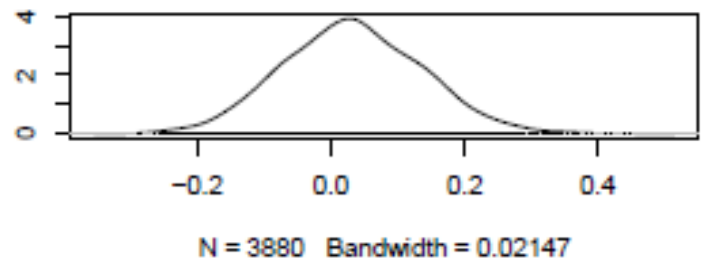

Density of time:emotion

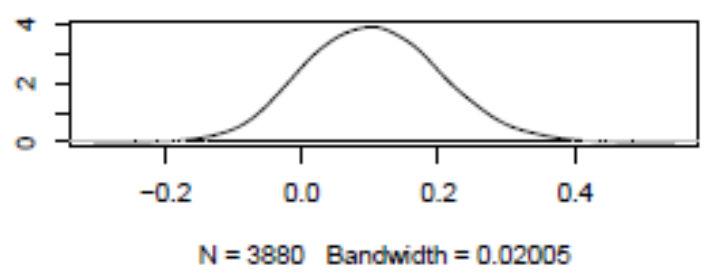


Trace of time:self

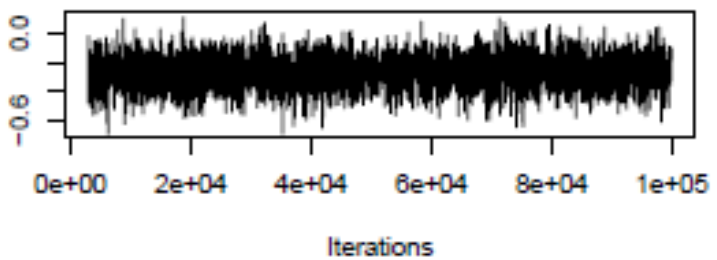

Trace of time:think

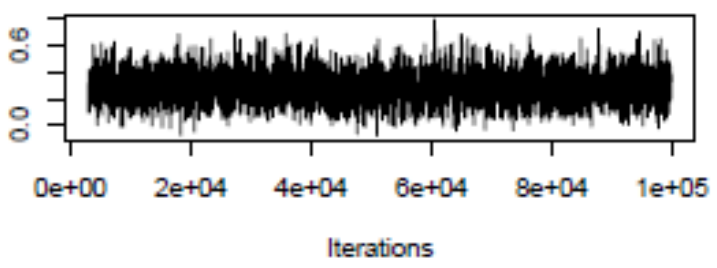

Trace of time:attitude

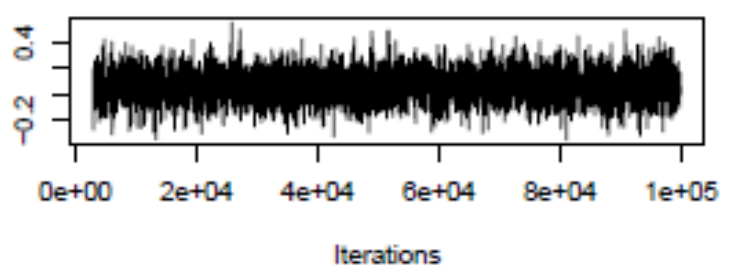

Trace of time:change

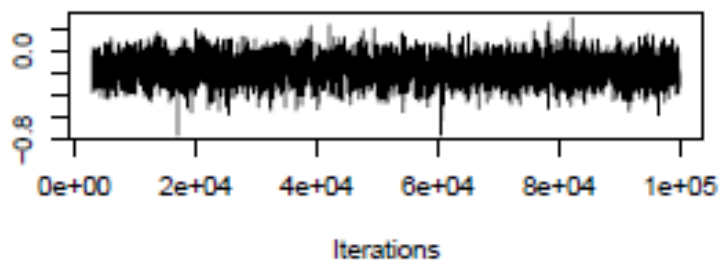

$\underline{\text { Random Effects }}$

Trace of time

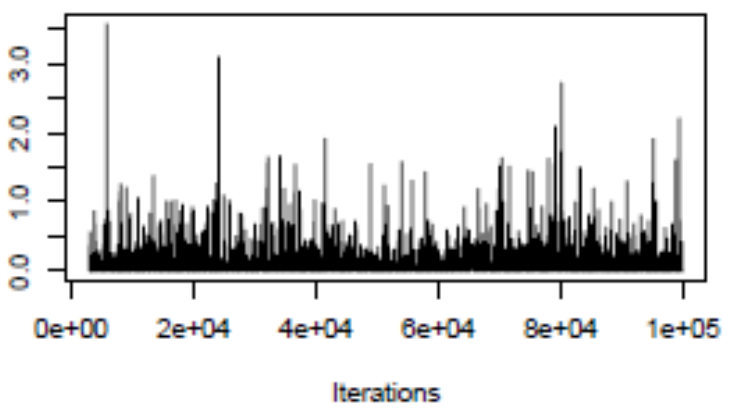

Density of time:self

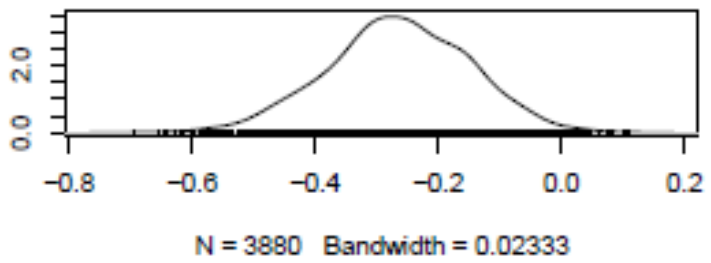

Density of time:think

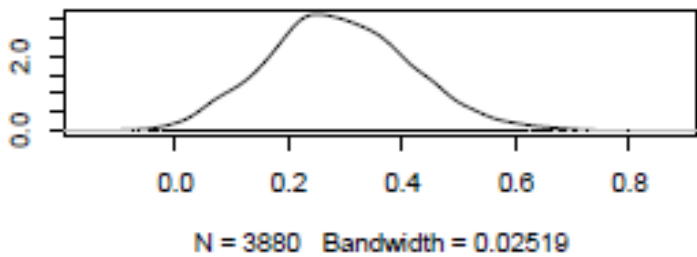

Density of time:attitude

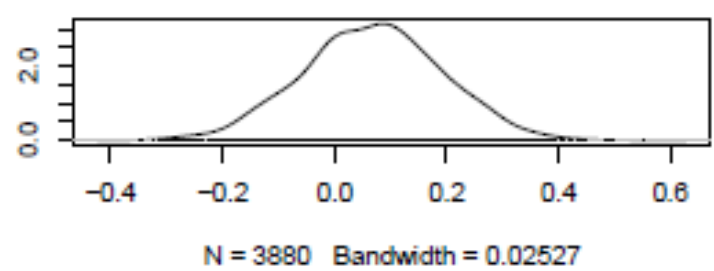

Density of time:change

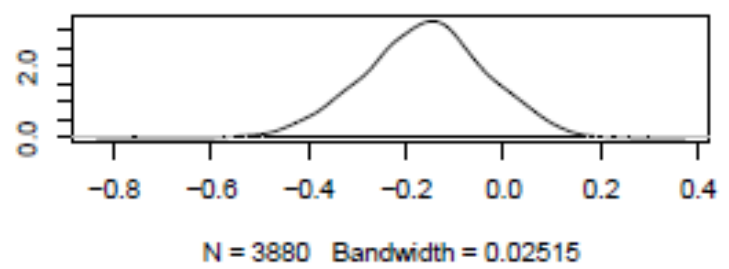

Density of time

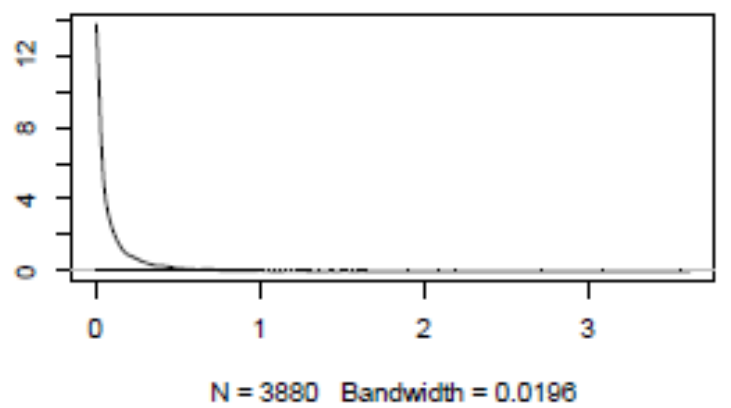


Trace of Research.ID

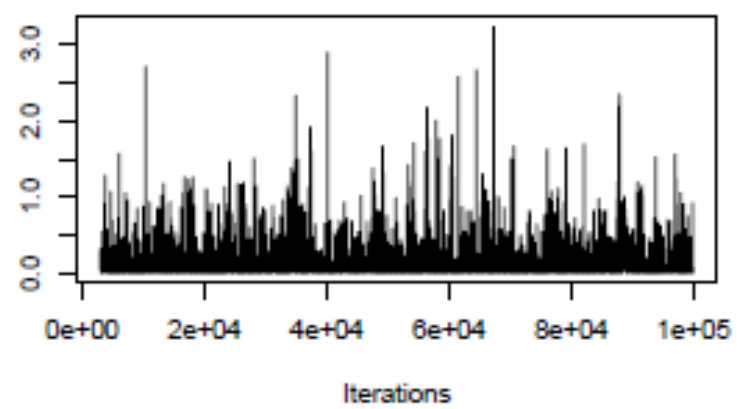

Density of Research.ID

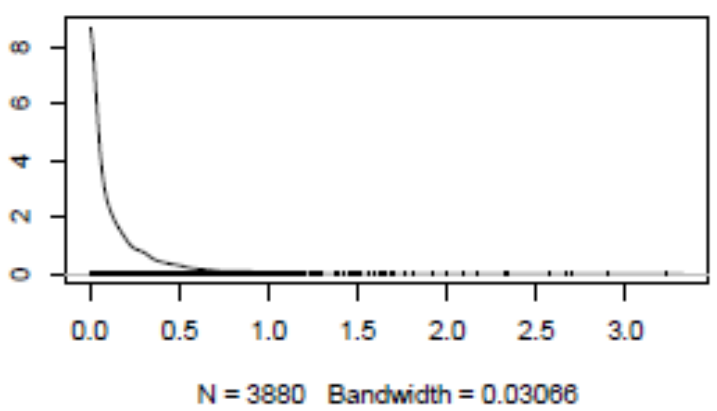


Chapter Five - Dimensional Identity

Model 1.1 - Basic Model + Gender (Table 5.3)

Bayesian Model (Bm1_d1)

\section{\# Define the Model}

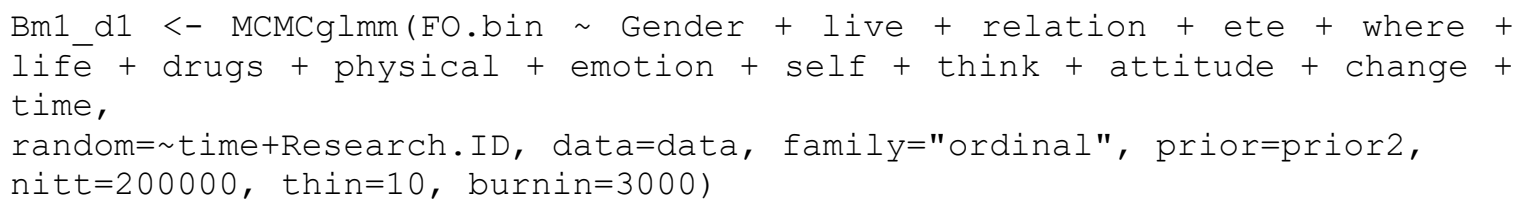

\section{\# Checks for suitable convergence}

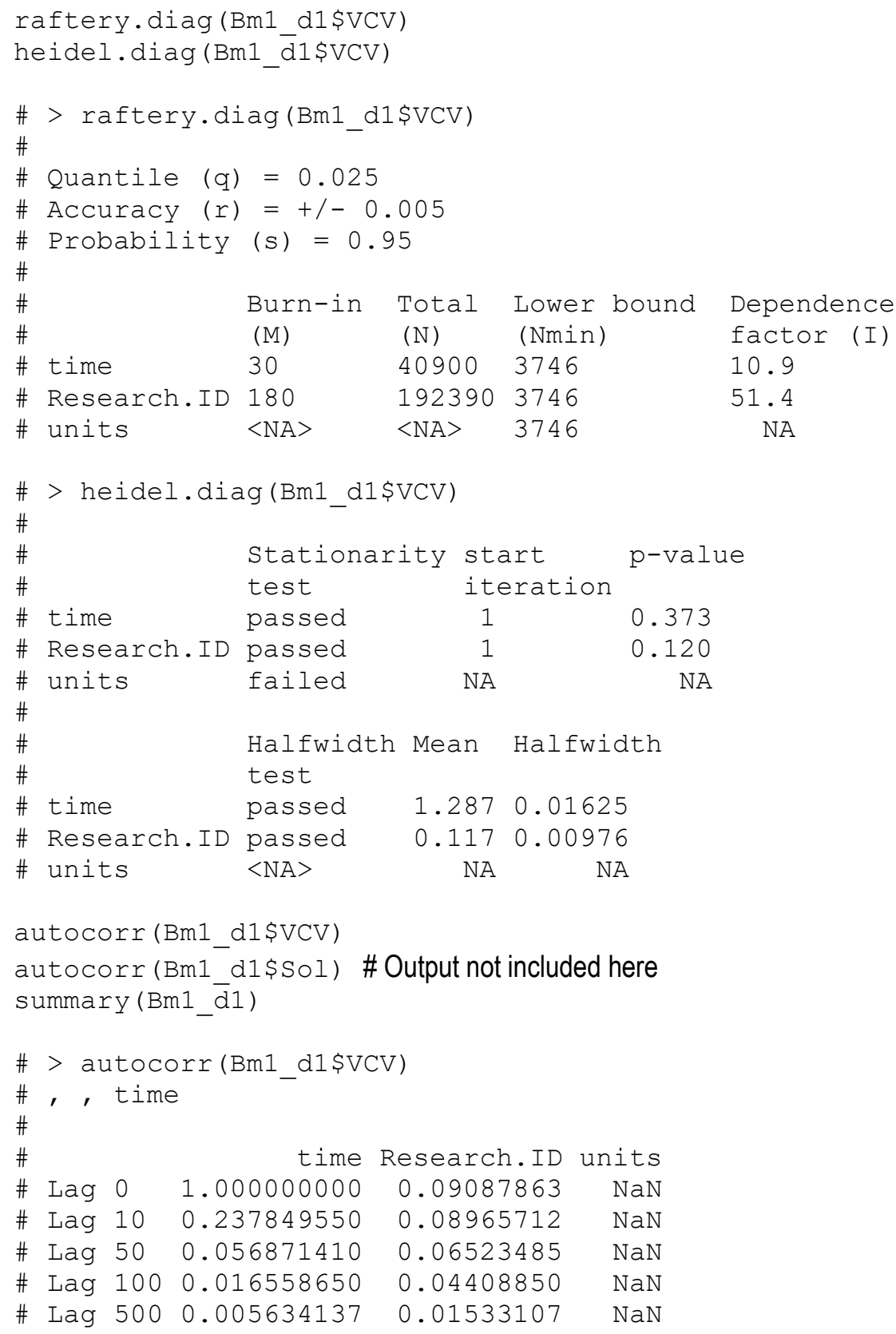




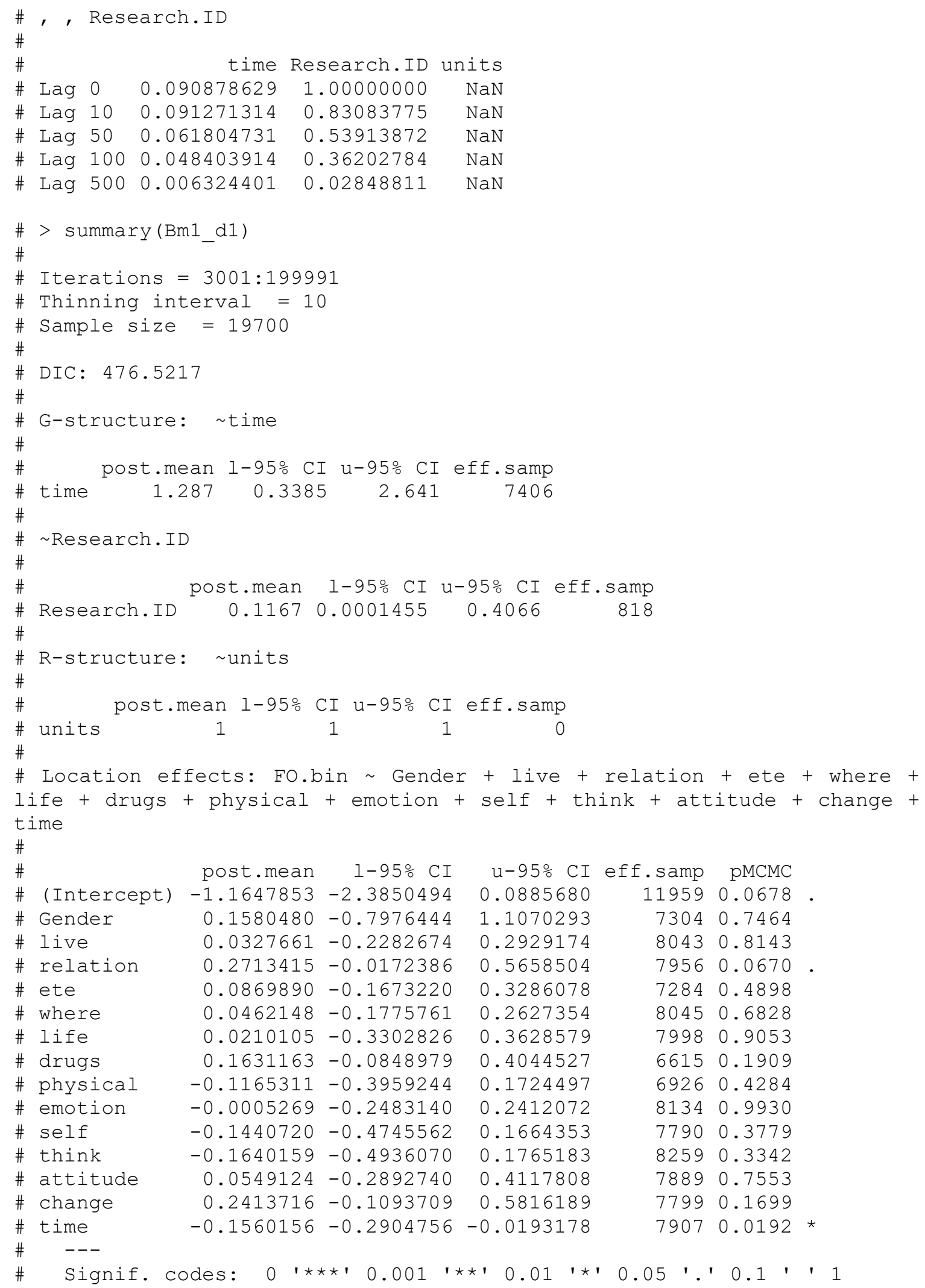




\section{Frequentist Model (m1_d1)}

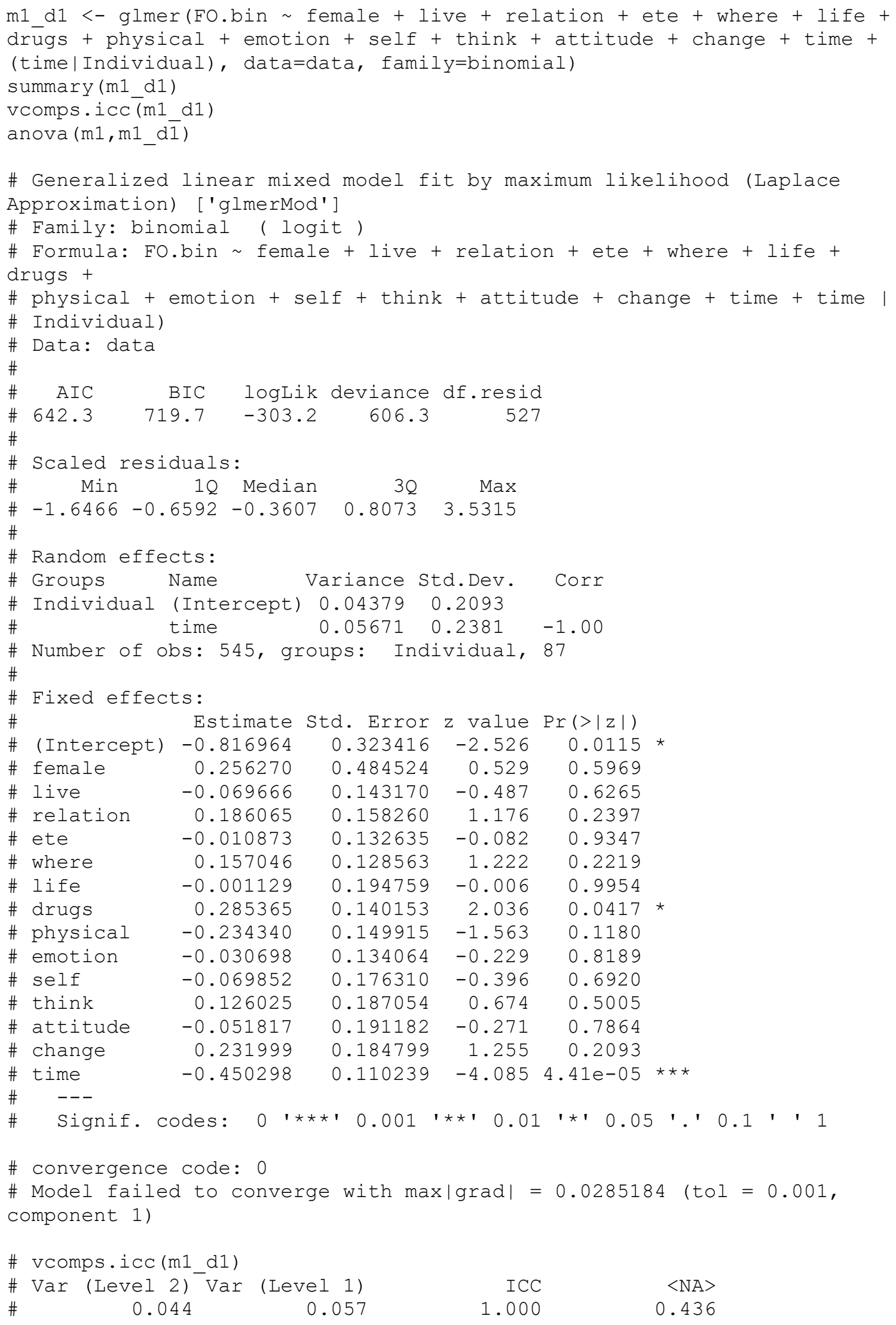




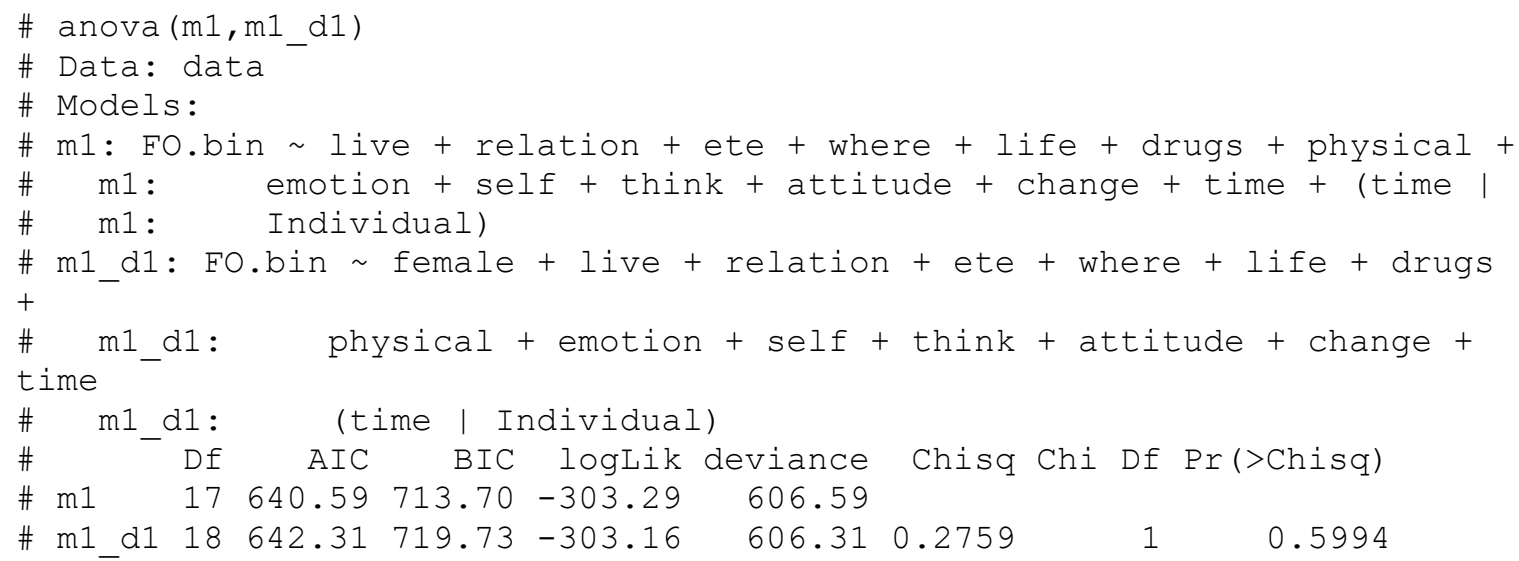


Model 1.2 - Basic Model + Ethnicity (Table 5.3)

Bayesian Model (Bm1_d2)

\section{\#\# Define the model}

Bm1_d2 <- MCMCglmm(FO.bin bme + live + relation + ete + where + life + drugs + physical + emotion + self + think + attitude + change + time, random= time+Research.ID, data=data, family="ordinal", prior=prior2, nitt $=350000$, thin=10, burnin=3000)

\section{\#\# Checks for suitable convergence}

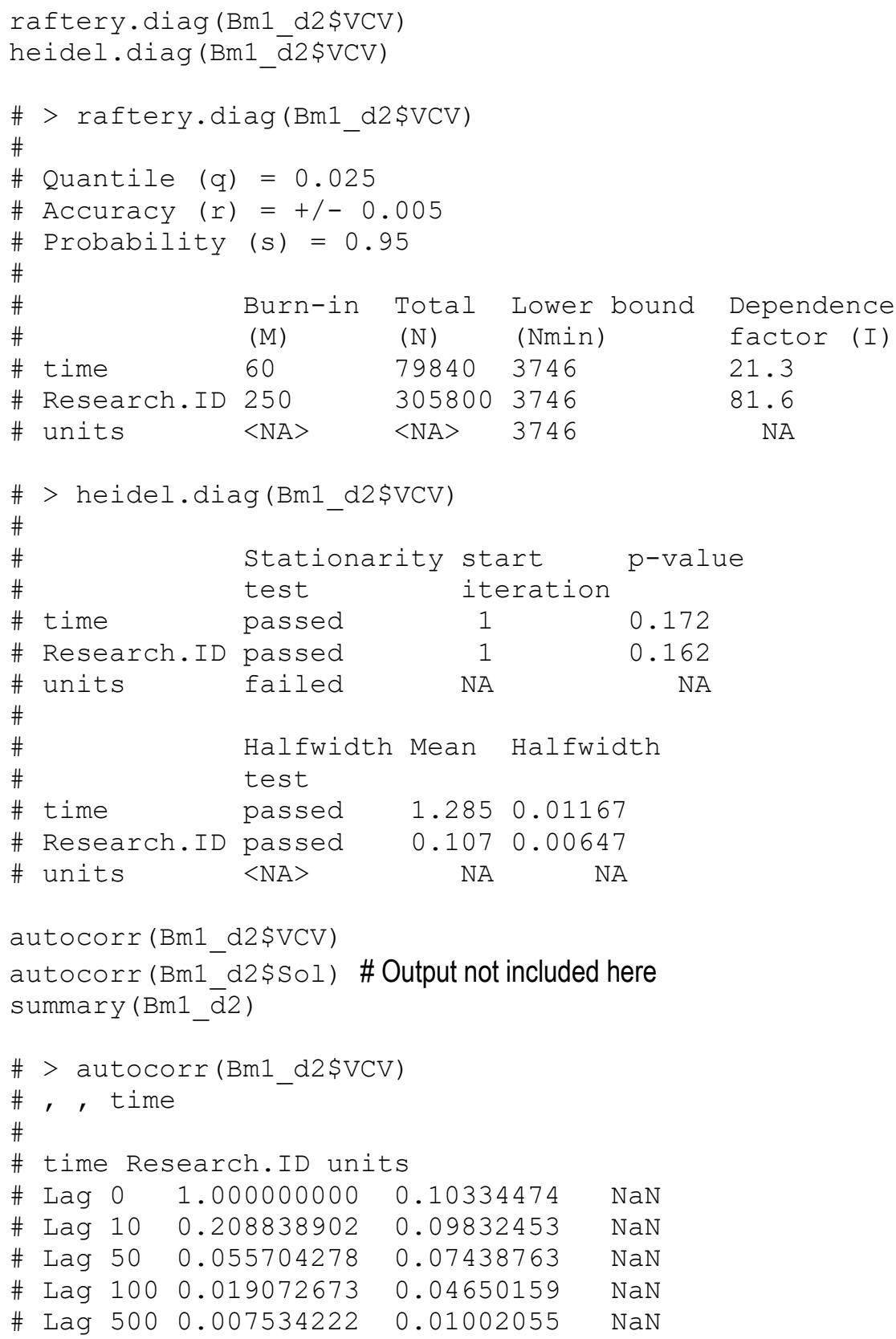




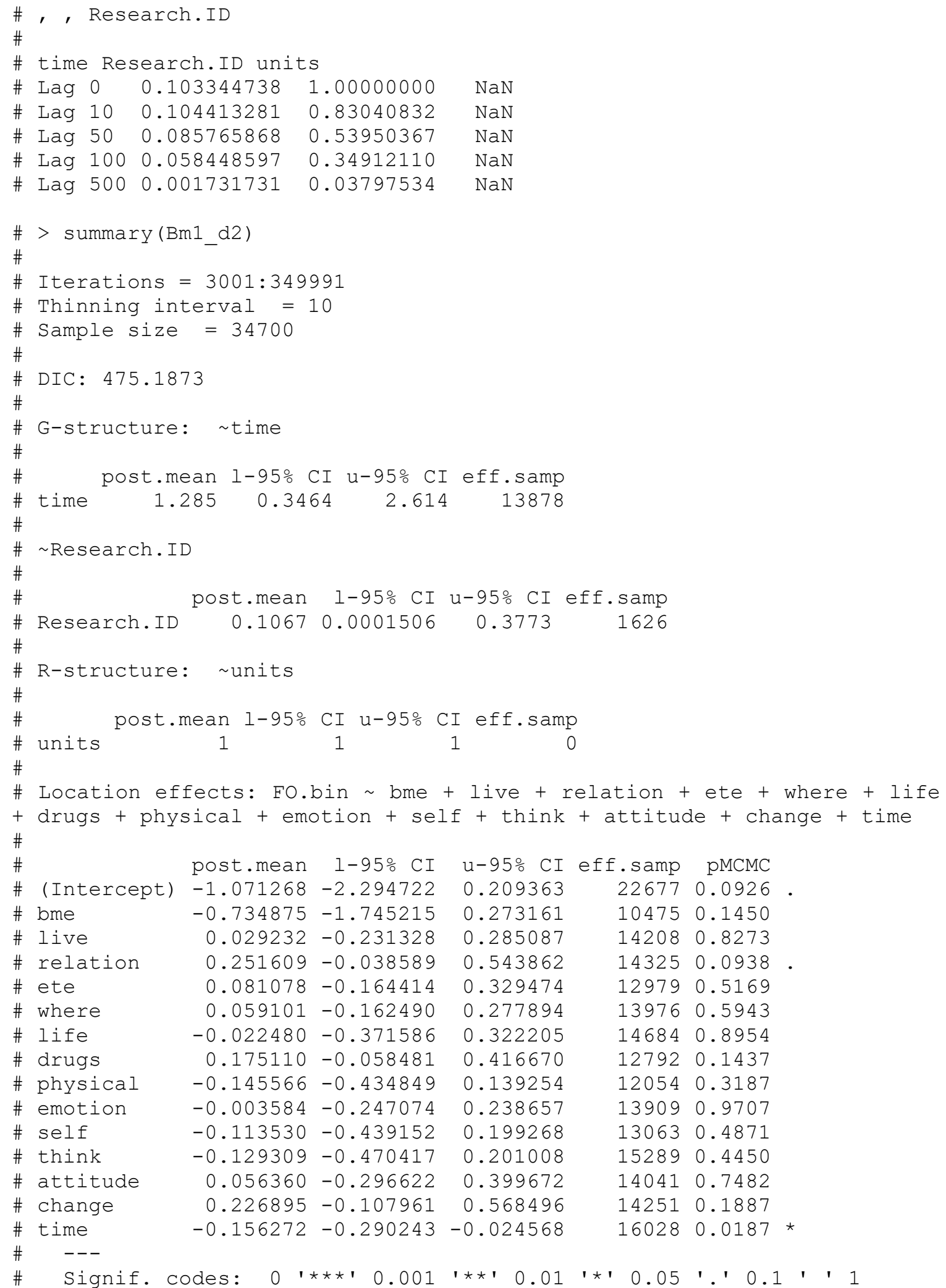




\section{Frequentist Model (m1_d2)}

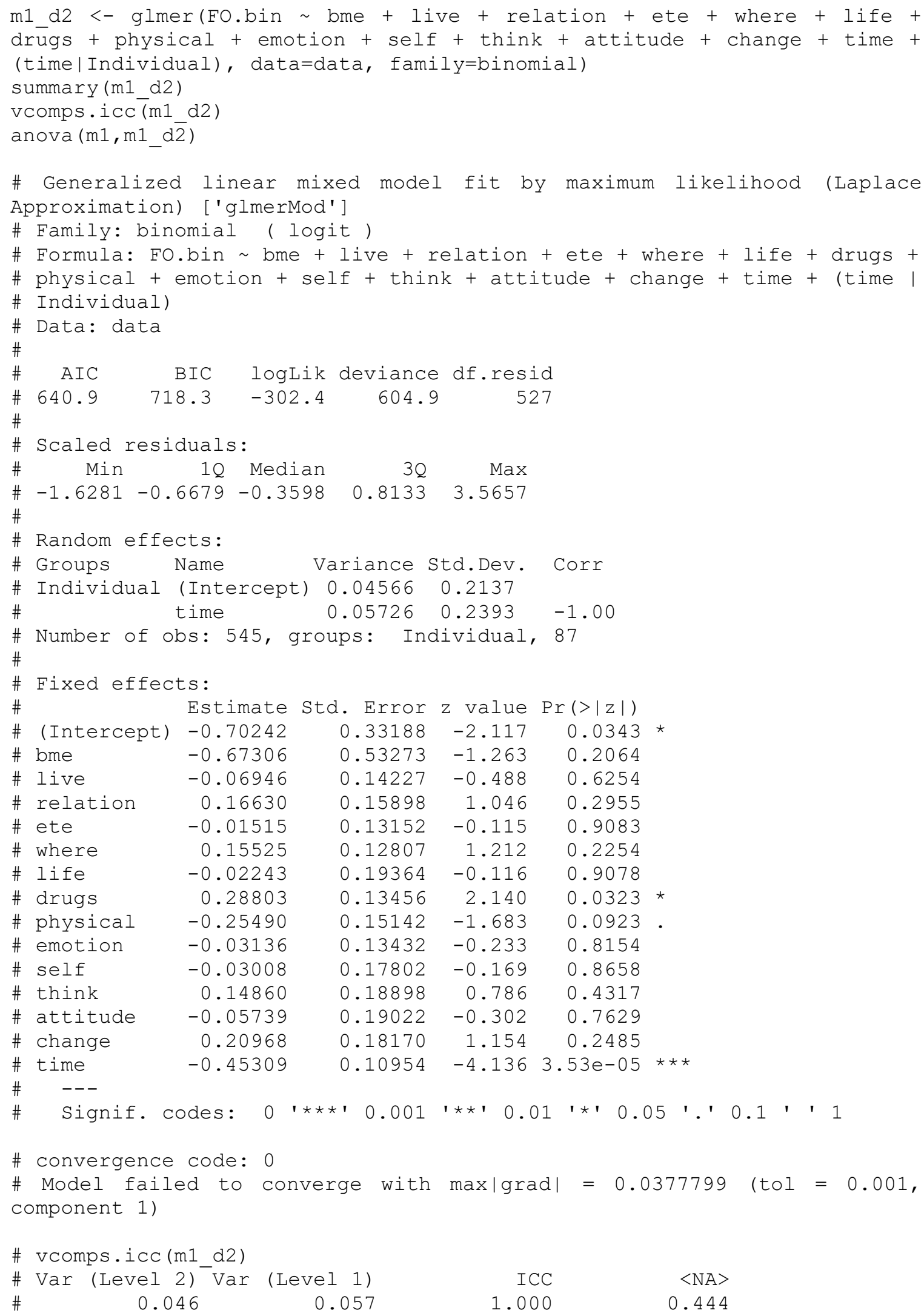




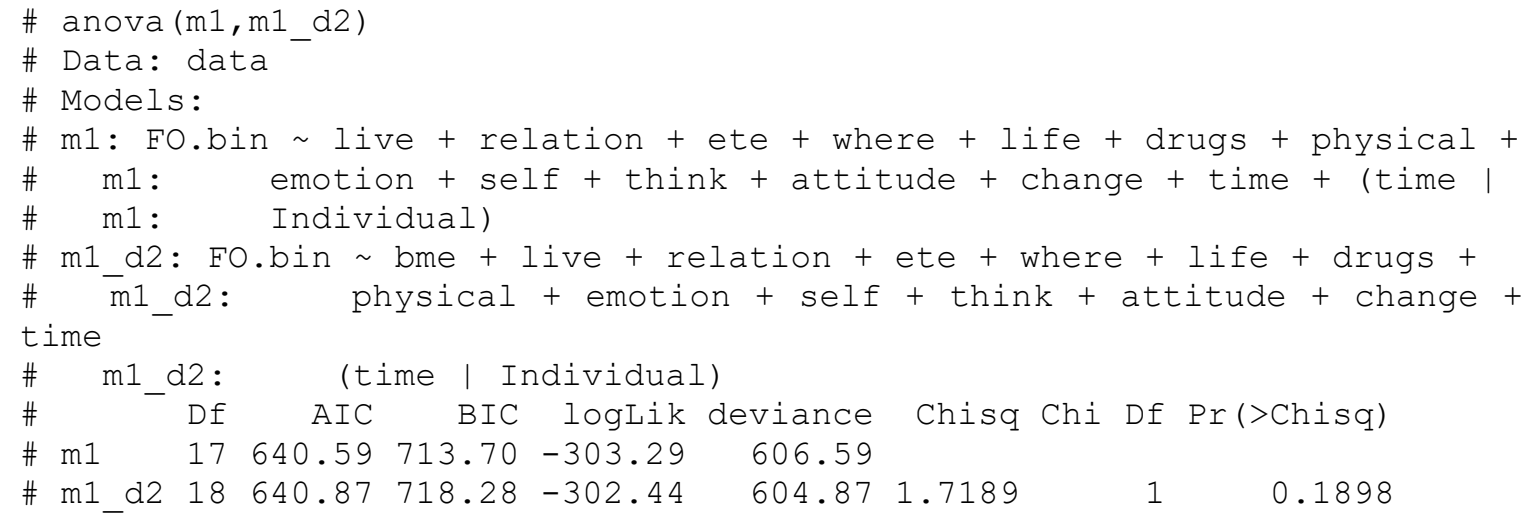


Model 1.3 - Basic Model + Demographics (Table 5.3)

Bayesian Model (Bm_d12)

\section{\# Define the model}

Bm1 d12 <- MCMCglmm(FO.bin Gender + bme + live + relation + ete + where + life + drugs + physical + emotion + self + think + attitude + change + time,

random= time+Research.ID, data=data, family="ordinal", prior=prior2, nitt $=400000$, thin=10, burnin=3000)

\section{\# Checks for suitable convergence}

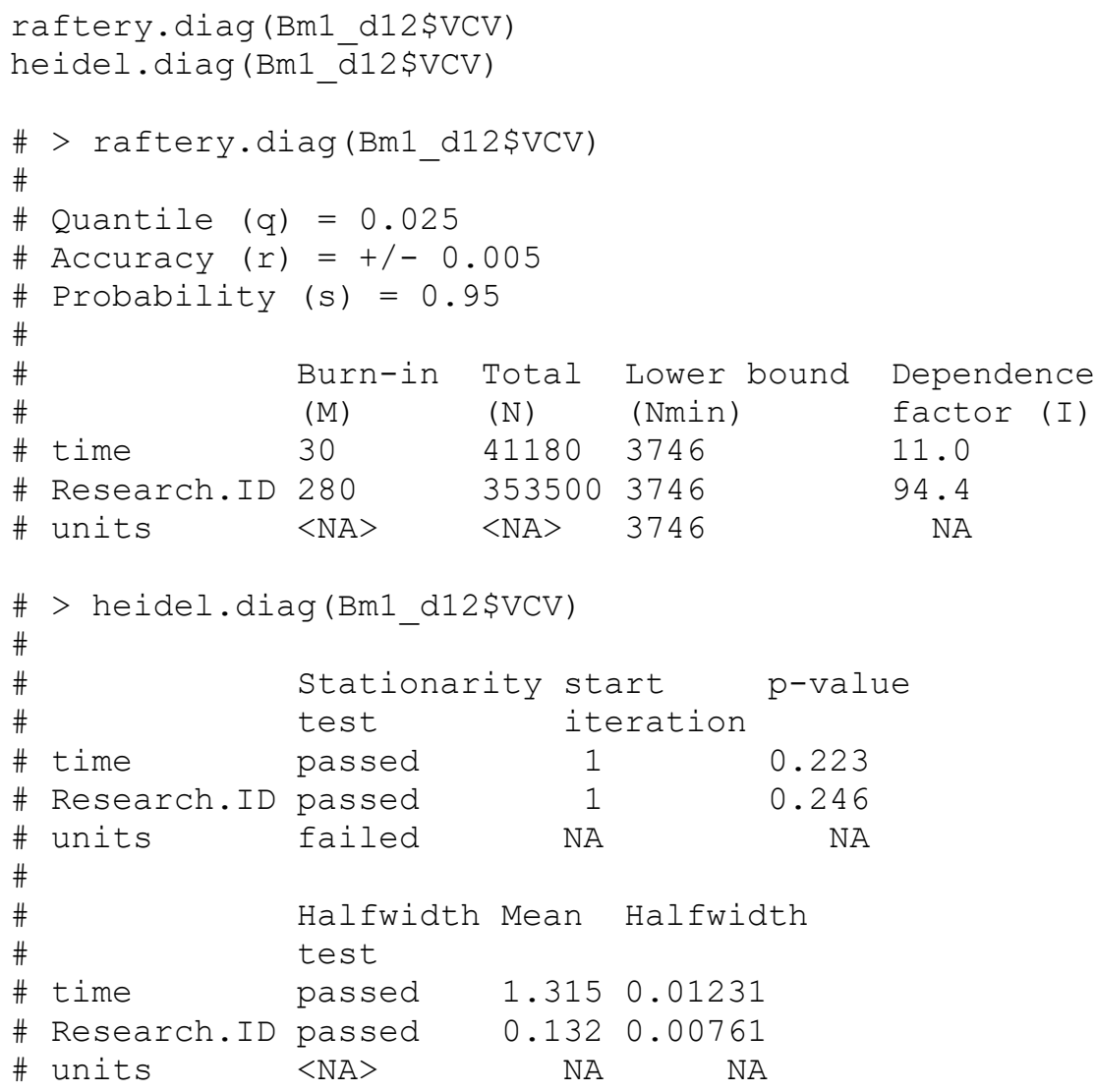

autocorr (Bm1_d12\$VCV)

autocorr (Bm1_d12\$Sol) \# Output not included here

summary (Bm1_ $\bar{d} 12)$

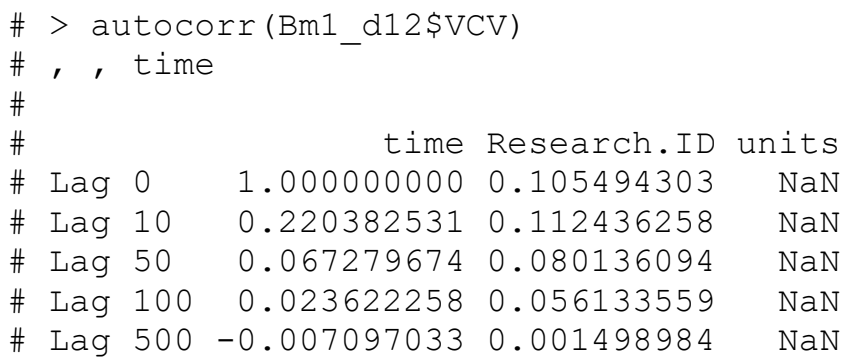




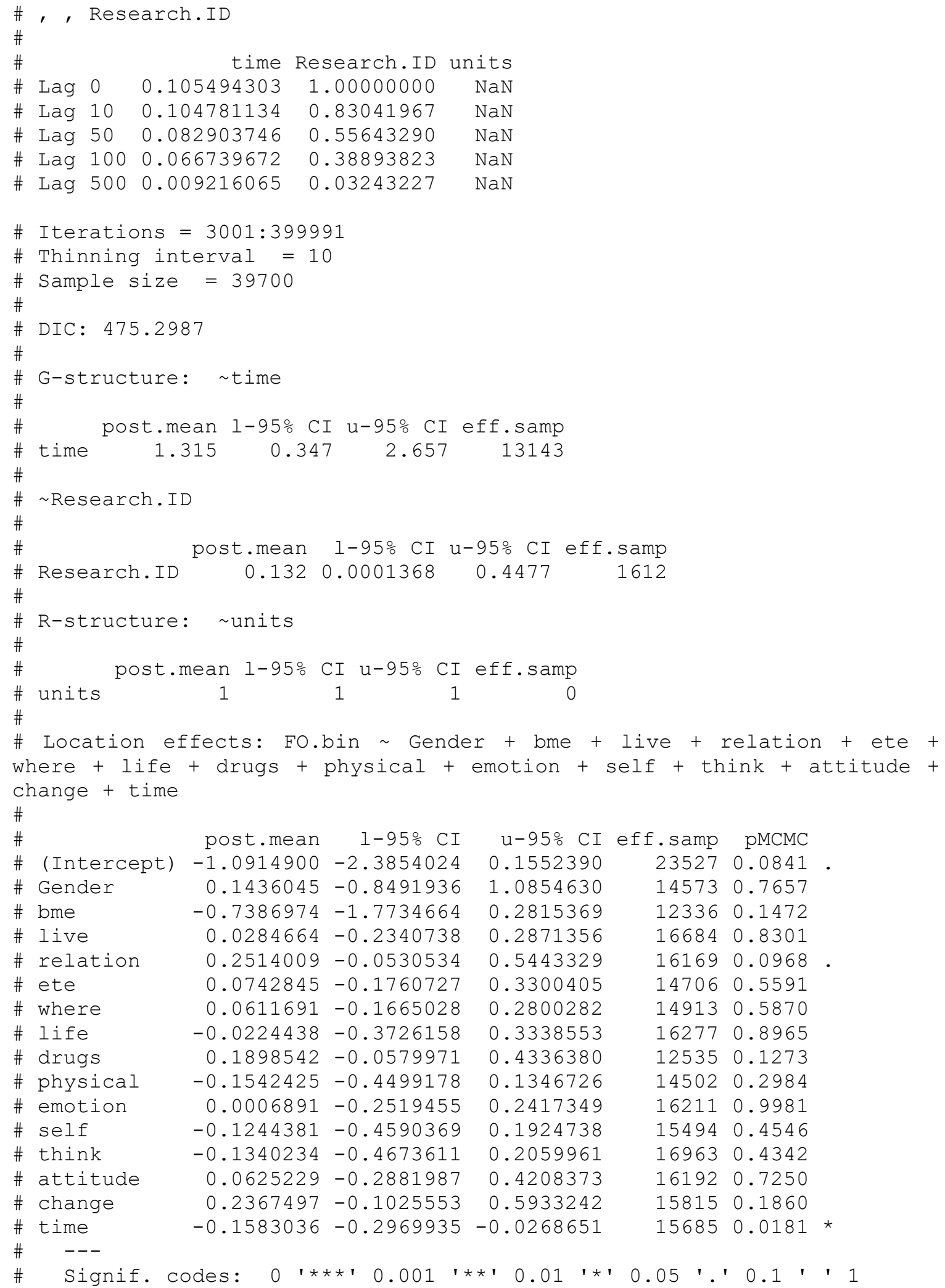




\section{Frequentist Model (m1_d12)}

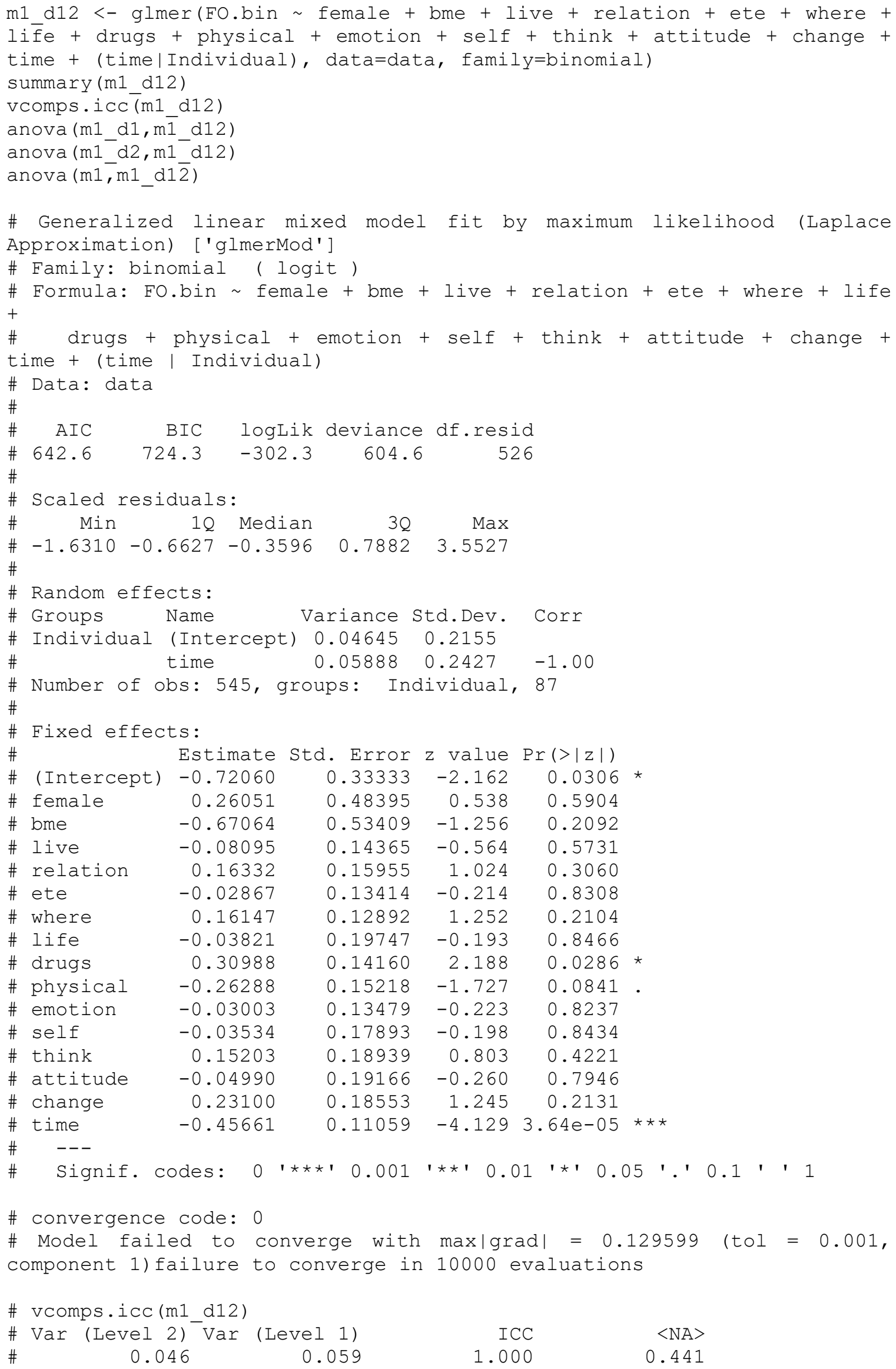




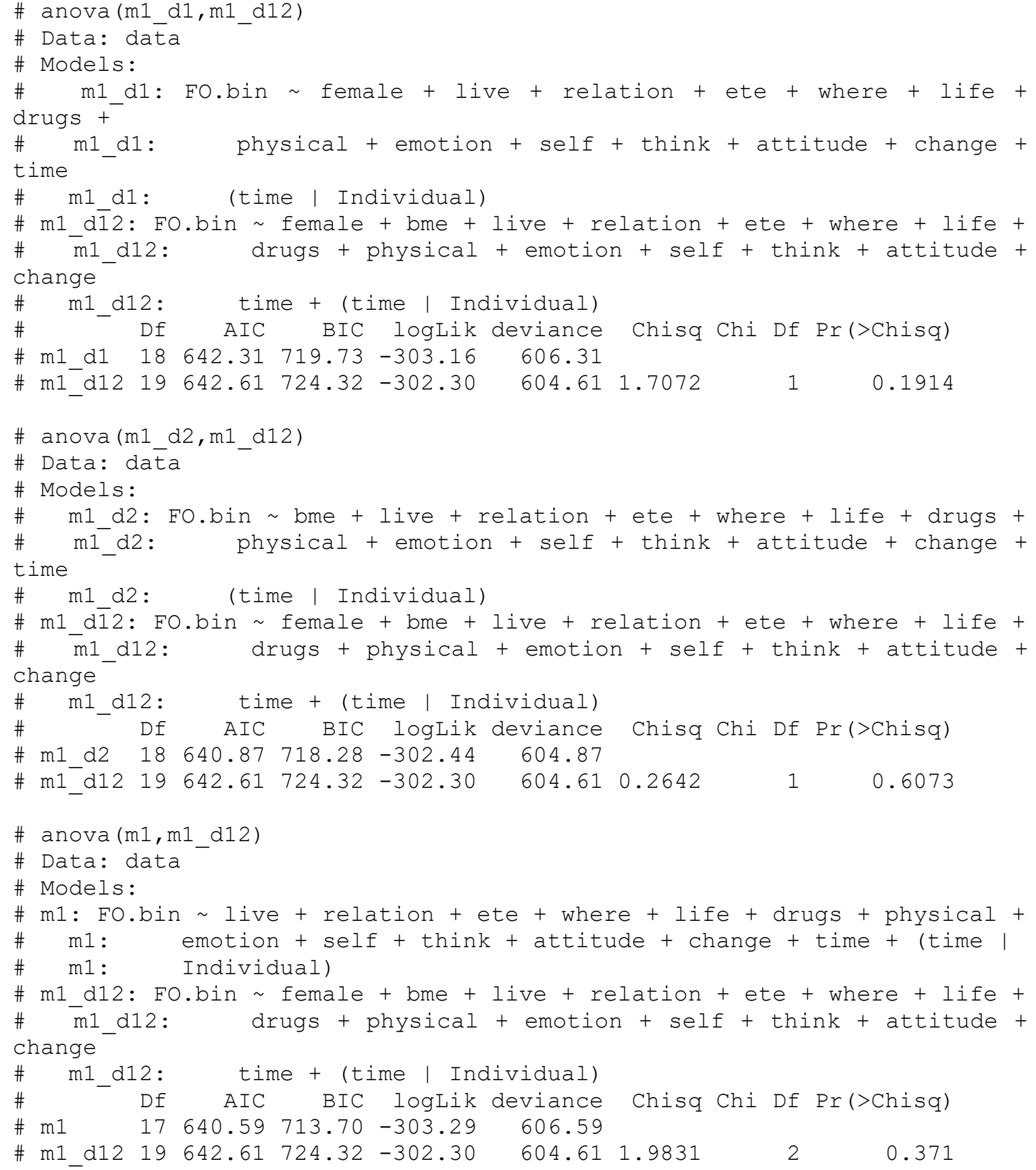


Dynamic Model involving Gender (Table 5.4)

Bayesian Model (BDm2 d1)

\section{\#\# Define the model}

BDm2 d1 <- MCMCglmm(FO.bin Gender*time*live + Gender*time*relation + Gender*time*ete + Gender*time*where + Gender*time*life + Gender*time*drugs + Gender*time*physical + Gender*time*emotion + Gender*time*self + Gender*time*think + Gender*time*attitude + Gender*time* change, random $=$ time+Research.ID, data=data, family="ordinal", prior=priord, slice=TRUE, nitt=2000000, thin=500, burnin=3000)

\section{\# Checks for suitable convergence}

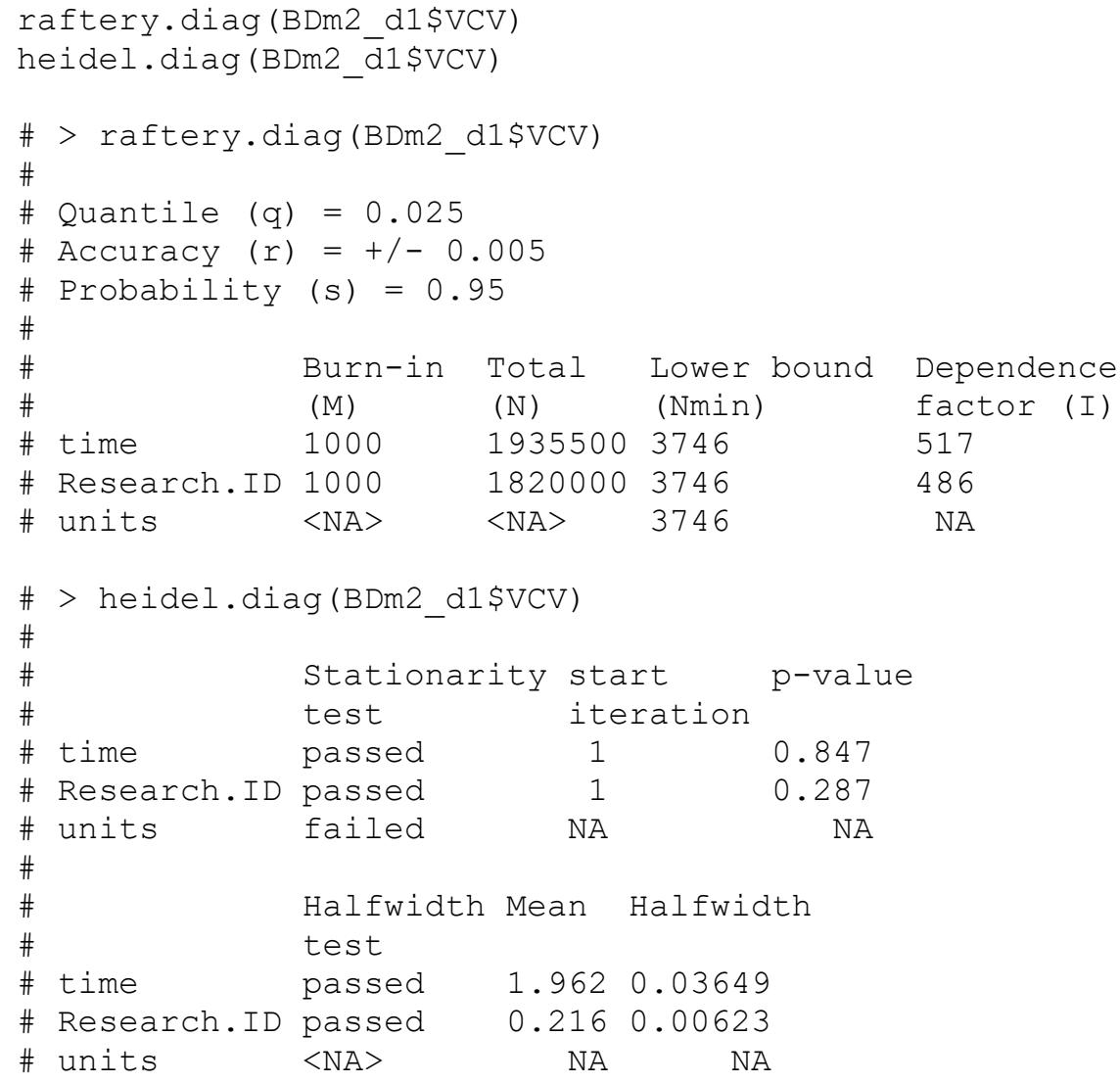




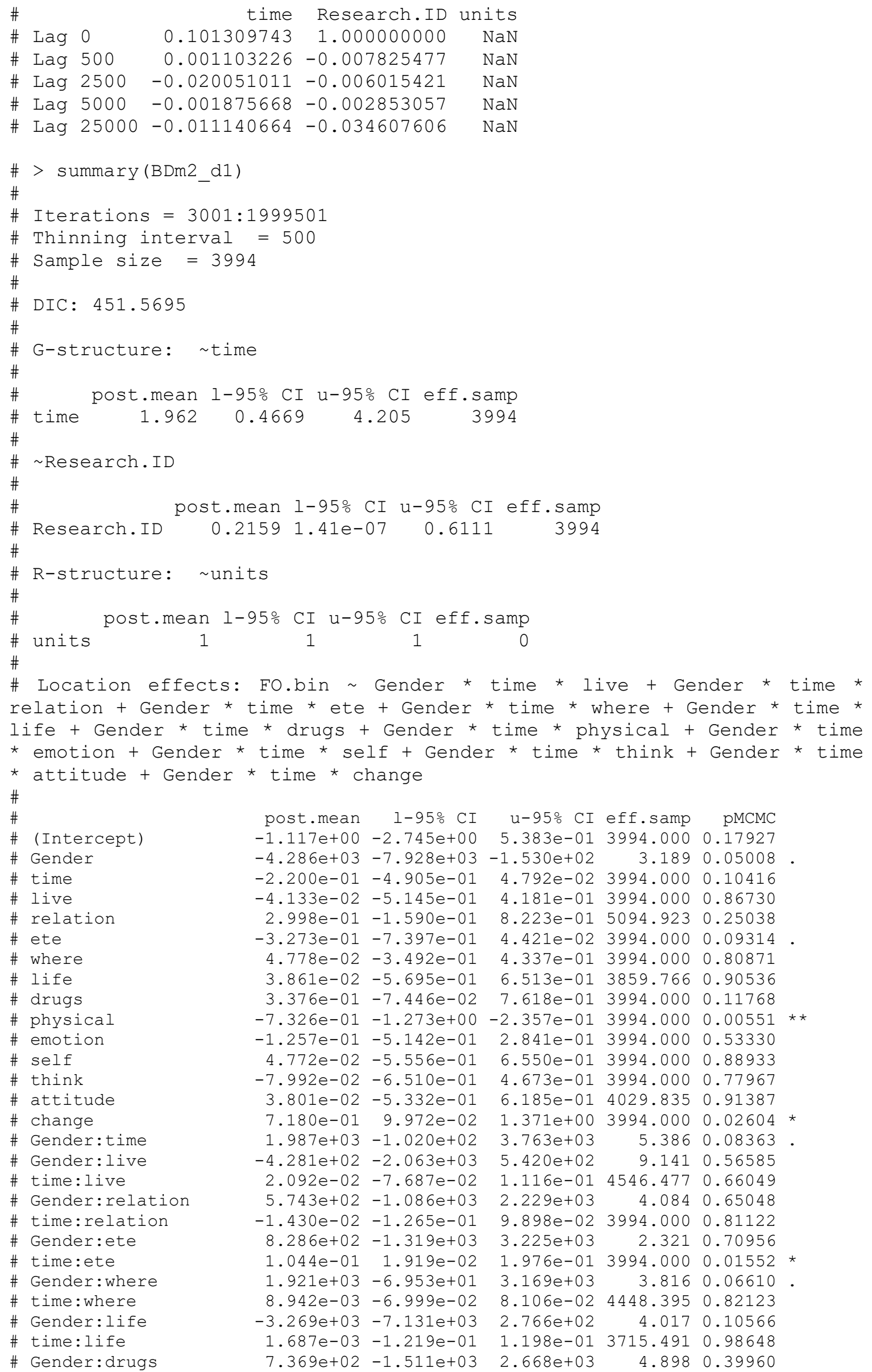




\begin{tabular}{|c|c|c|c|c|c|c|c|}
\hline \# & time:drugs & $-4.043 e-02$ & $-1.296 e-01$ & $3.850 e-02$ & 3994.000 & 0.34352 & \\
\hline \# & Gender:physical & $1.387 e+03$ & $-1.280 e+02$ & $3.209 e+03$ & 8.101 & 0.01753 & * \\
\hline \# & time:physical & $1.511 e-01$ & $2.579 e-02$ & $2.643 e-01$ & 3994.000 & 0.01452 & * \\
\hline \# & Gender:emotion & $1.584 e+03$ & $-4.972 e+02$ & $3.372 e+03$ & 4.550 & 0.27942 & \\
\hline & time: emotion & $3.766 e-02$ & $-4.556 e-02$ & $1.184 e-01$ & 3994.000 & 0.38508 & \\
\hline 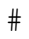 & Gender:self & $-2.575 e+03$ & $-4.725 e+03$ & $1.814 e+02$ & 3.270 & 0.14422 & \\
\hline \# & time:self & $-8.228 e-02$ & $-1.977 e-01$ & $4.638 e-02$ & 4012.090 & 0.17426 & \\
\hline 开 & Gender:think & $1.307 e+03$ & $-7.479 e+02$ & $3.240 e+03$ & 4.347 & 0.3 & \\
\hline & time:think & $-1.076 e-02$ & $-1.262 e-01$ & $1.151 e-01$ & 3994.000 & 0.8 & \\
\hline & Gender:attitude & $1.605 e+03$ & $-4.773 e+01$ & $3.607 e+03$ & 3.055 & 0.0 & * \\
\hline \# & time:attitude & $7.461 e-03$ & $-1.149 e-01$ & $1.255 e-01$ & 4066.529 & 0.9 & \\
\hline$f$ & Gender: change & $2.017 e+03$ & $-3.554 e+02$ & $4.101 e+03$ & 5.061 & 0.1 & \\
\hline 开 & time: change & $-9.188 e-02$ & $-2.136 e-01$ & $3.857 e-02$ & 3994.000 & 0.1 & \\
\hline \# & Gender:time:live & $-1.831 e+02$ & $-1.051 e+03$ & $2.068 e+02$ & 8.403 & 0.4 & \\
\hline \# & Gender:time:relation & $-4.104 e+02$ & $-1.144 e+03$ & $3.200 e+02$ & 7.176 & 0.3 & \\
\hline 牛 & Gender:time:ete & 4. $202 e+01$ & $-9.539 e+02$ & $1.082 e+03$ & 2.804 & 0.97847 & \\
\hline \# & Gender:time: where & $-5.802 e+02$ & $-1.372 e+03$ & $1.504 e+02$ & 6.364 & 0.2 & \\
\hline \# & Gender:time:life & $9.794 e+02$ & $-4.245 e+02$ & $2.278 e+03$ & 3.357 & 0.1 & \\
\hline \# & Gender:time:drugs & $4.980 e+01$ & $-3.737 e+02$ & $4.109 e+02$ & 5.642 & 0.7 & \\
\hline \# & Gender:time:physical & $-1.193 e+03$ & $-2.069 e+03$ & $3 e+01$ & 7.112 & 0.0 & * \\
\hline \# & Gender:time:emotion & $-7.681 e+02$ & $-1.842 e+03$ & $6.582 e+02$ & 2.356 & 0.5 & \\
\hline \# & Gender:time:self & $1.528 e+03$ & $-2.440 e+02$ & $3.165 e+03$ & 2.438 & 0.1 & \\
\hline \# & Gender:time:think & $-1.615 e+02$ & $-4.698 e+02$ & $1.884 e+02$ & 4.057 & $0.47^{7}$ & \\
\hline \# & Gender:time:attitude & $-1.308 e+03$ & $-1.979 e+03$ & $-3.331 e+02$ & 10.187 & $<3 e-04$ & \\
\hline \# & Gender:time:change & $-1.139 e+03$ & $-2.395 e+03$ & $6.089 e+02$ & 3.372 & 0.19529 & \\
\hline & & & & & & & \\
\hline & iff. codes: & & 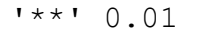 & 5 & .11 & 1 & \\
\hline
\end{tabular}


Trace of the Sampled Output and Density Estimates

Fixed Effects

Trace of (Intercept)

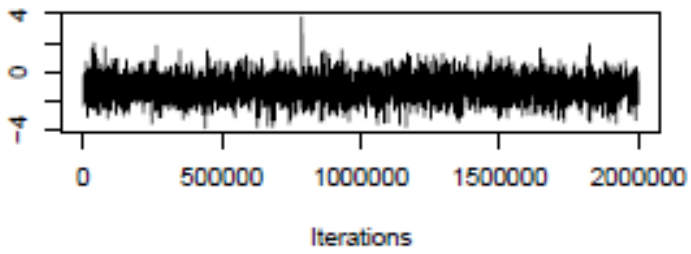

Trace of Gender

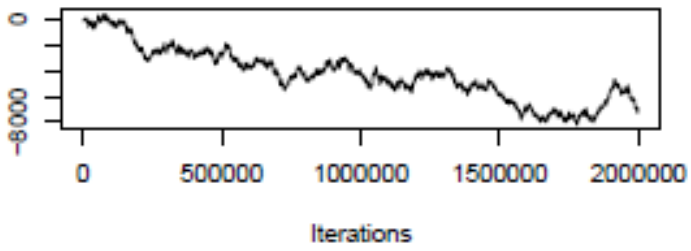

Trace of time

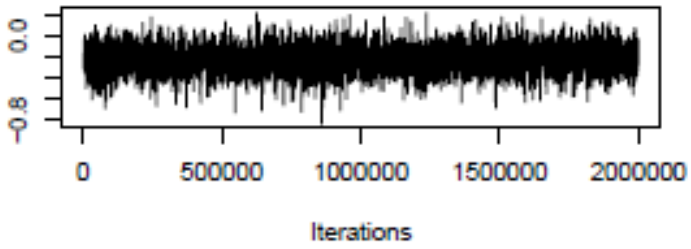

Trace of live

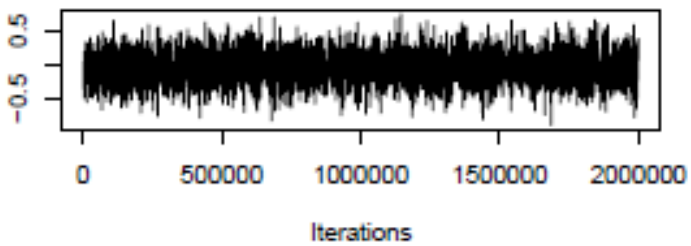

Trace of relation

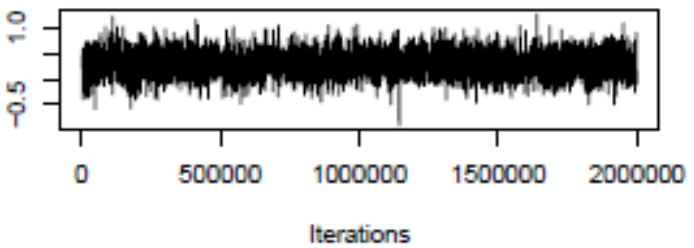

Trace of ete

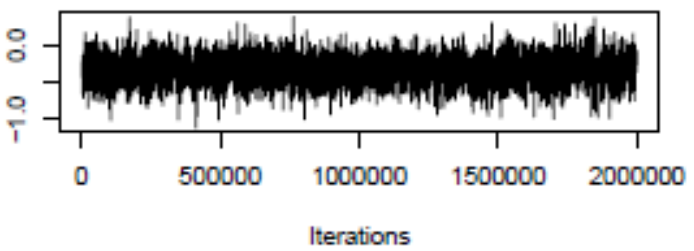

Density of (Intercept)

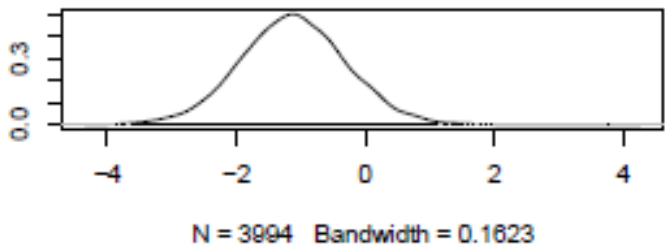

Density of Gender

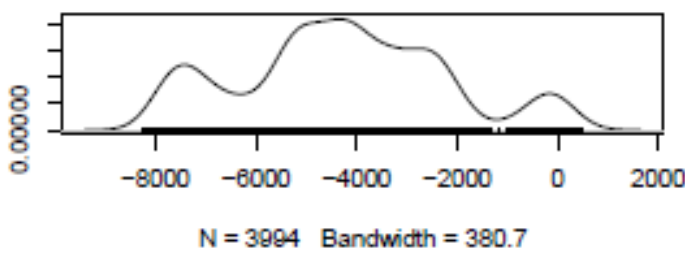

Density of time

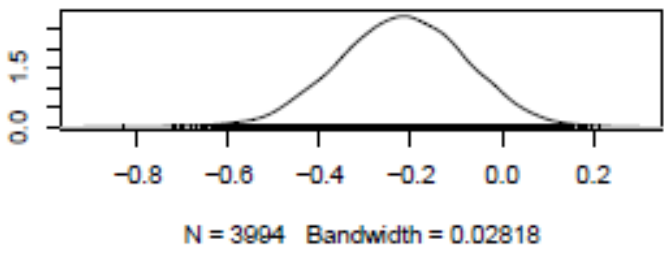

Density of live

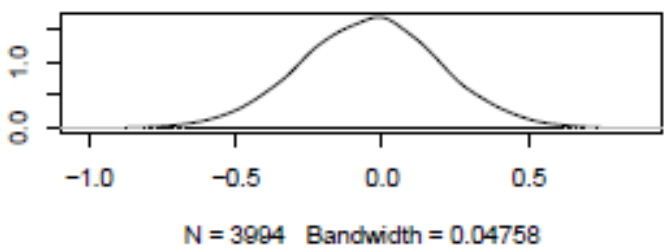

Density of relation

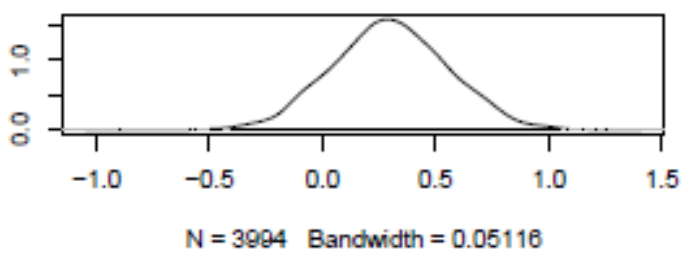

Density of ete

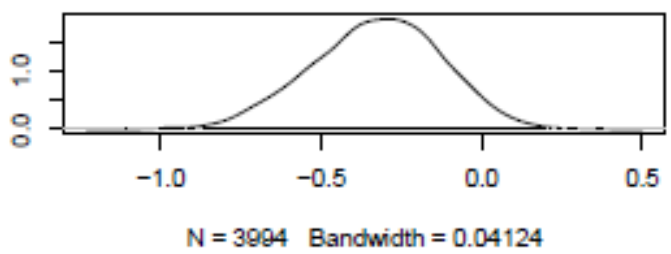


Trace of where

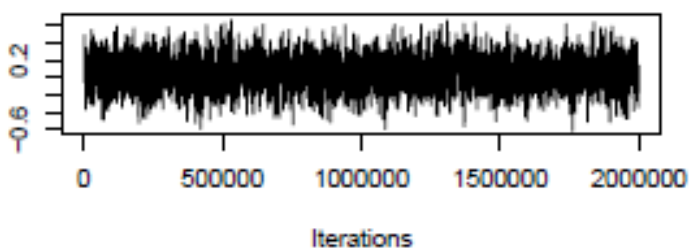

Trace of life

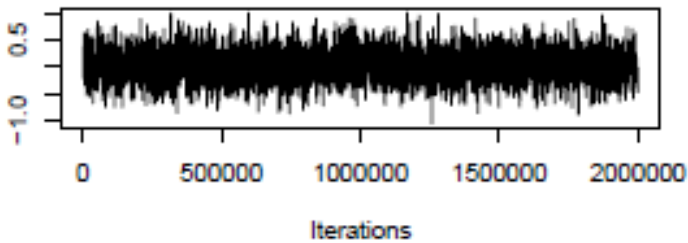

Trace of drugs

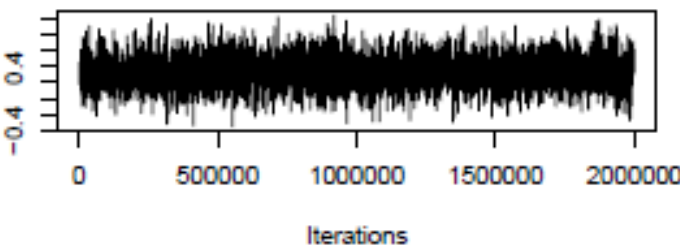

Trace of physical

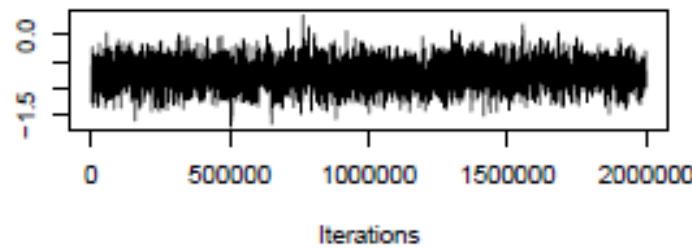

Trace of emotion

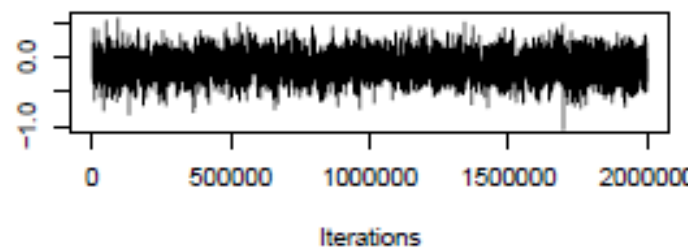

Trace of self

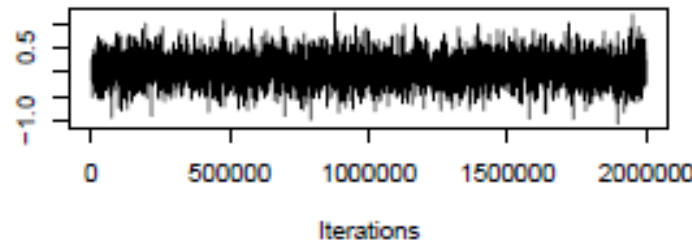

Density of where

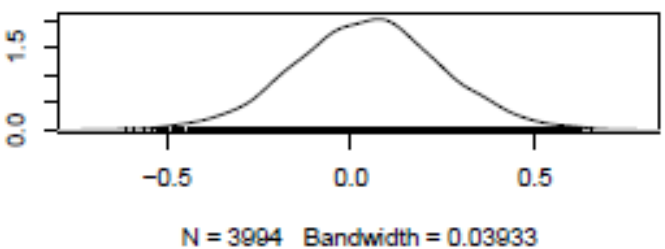

Density of life

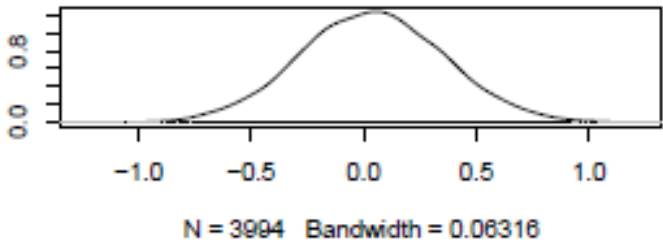

Density of drugs

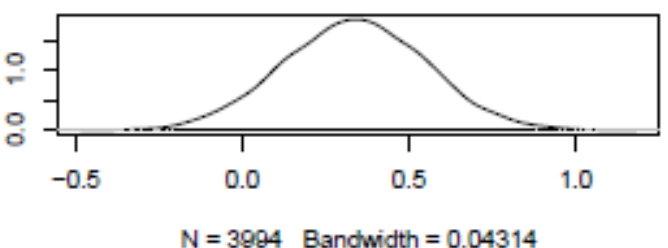

Density of physical

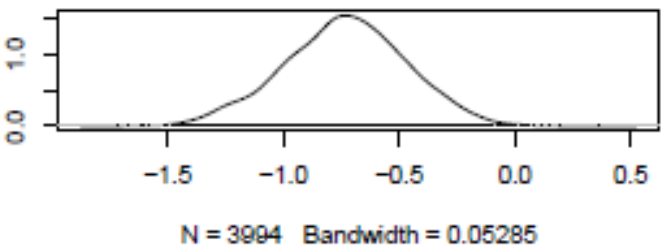

Density of emotion

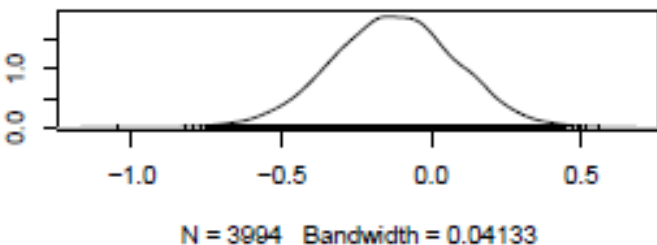

Density of self

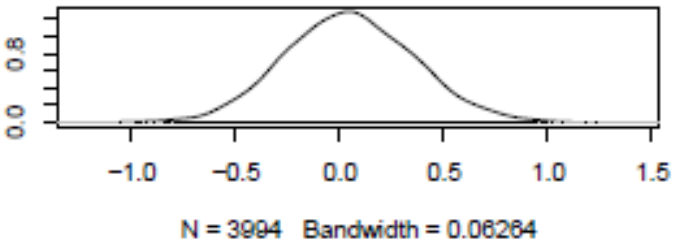


Trace of think

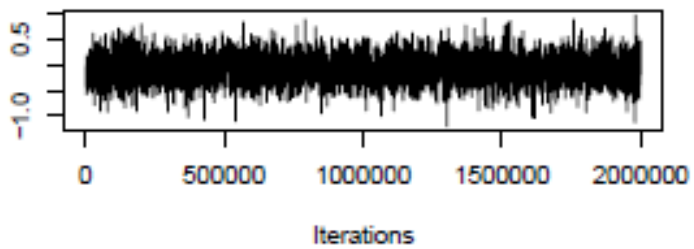

Trace of attitude

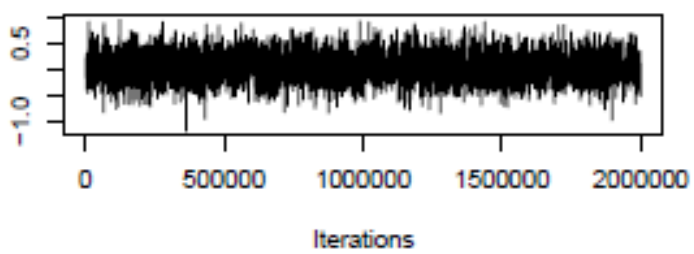

Trace of change

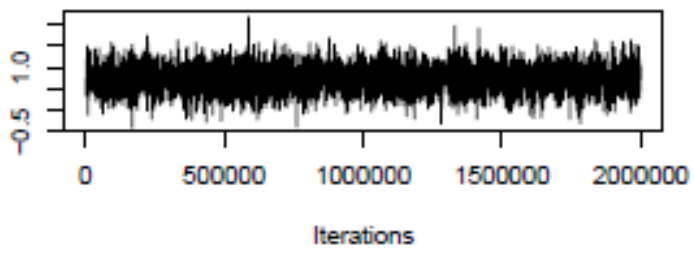

Trace of Gender:time

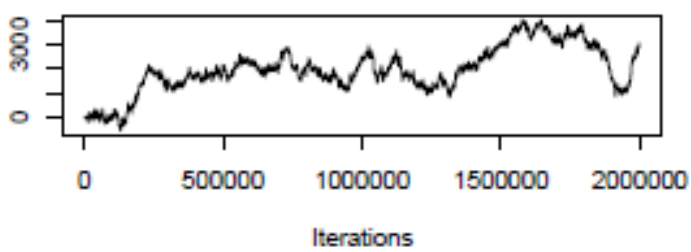

Trace of Gender:live

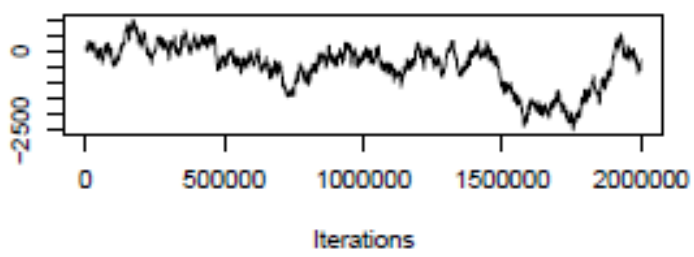

Trace of time:live

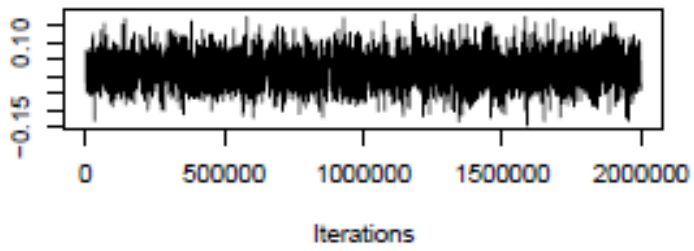

Density of think

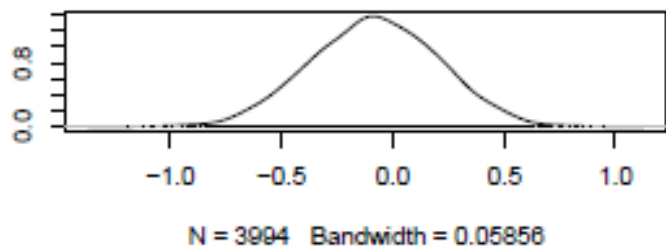

Density of attitude

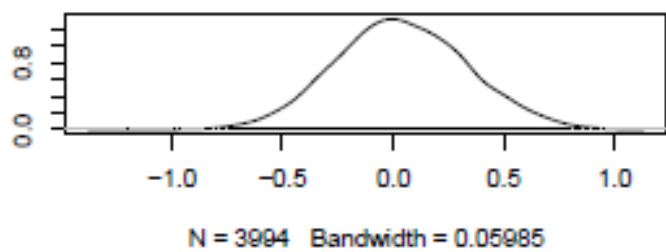

Density of change

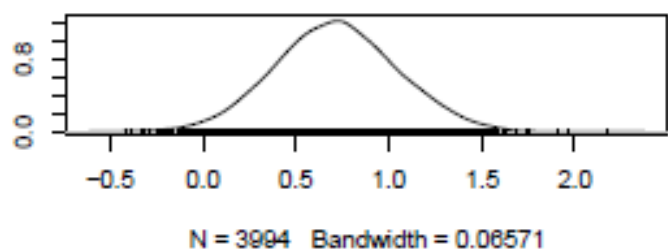

Density of Gender:time

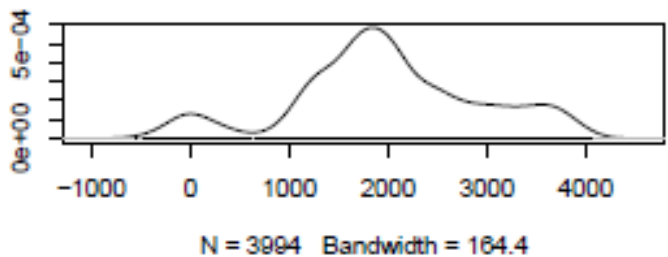

Density of Gender:live

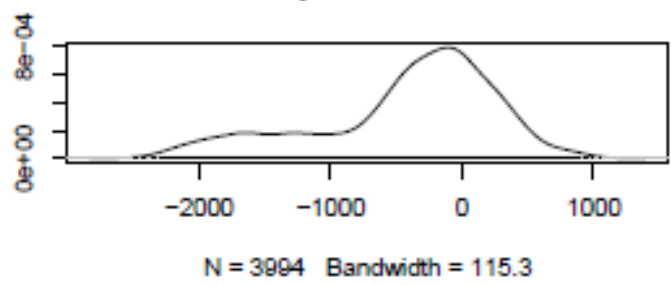

Density of time:live

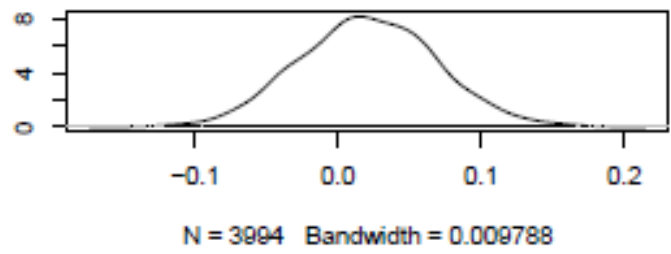


Trace of Gender:relation

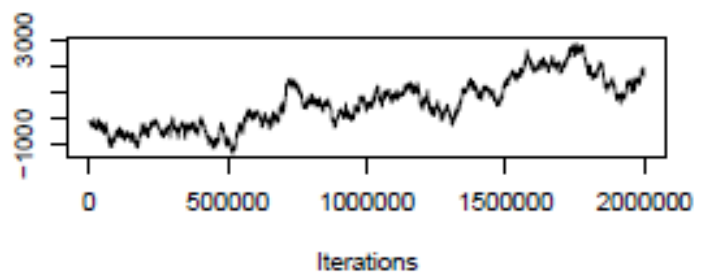

Trace of time:relation

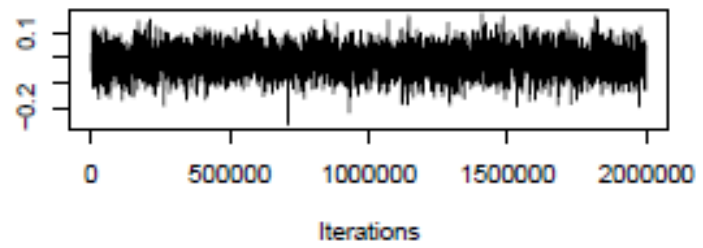

Trace of Gender:ete

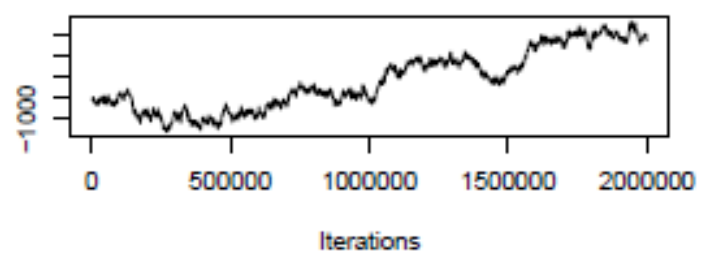

Trace of time:ete

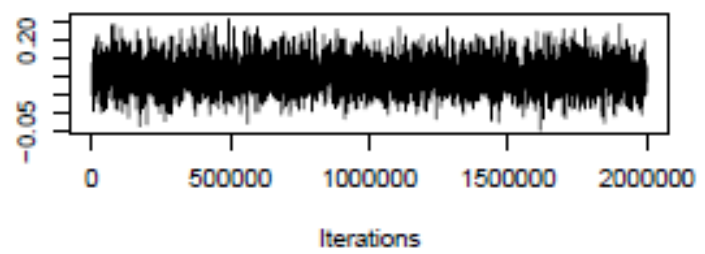

Trace of Gender:where

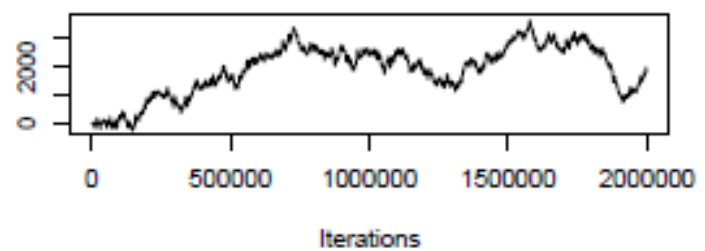

Trace of time:where

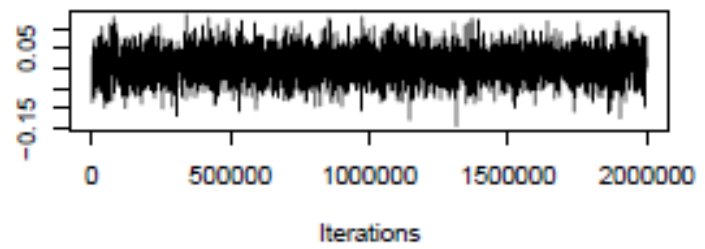

Density of Gender:relation

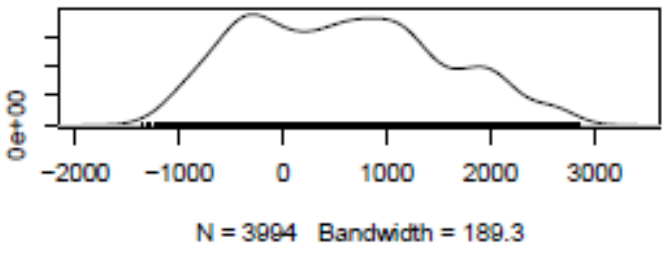

Density of time:relation

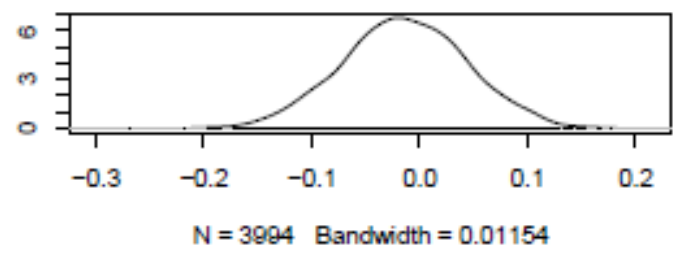

Density of Gender:ete

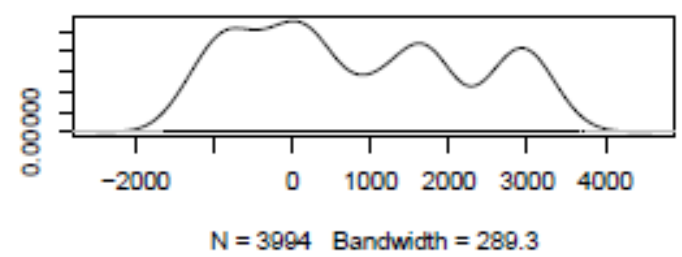

Density of time:ete

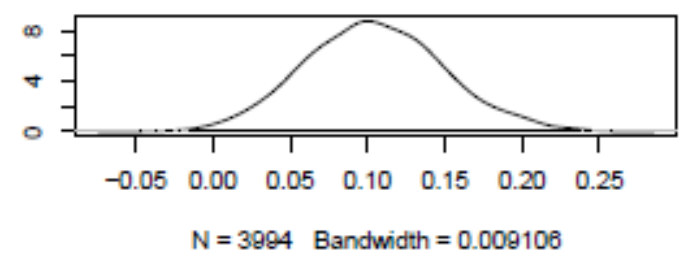

Density of Gender:where

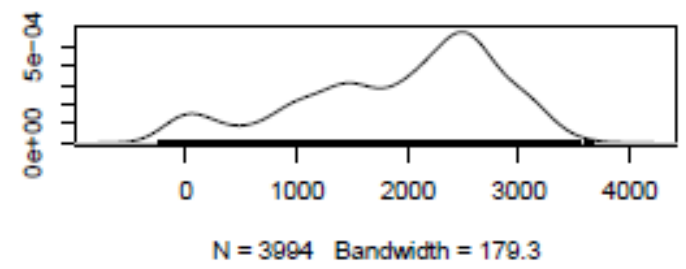

Density of time:where

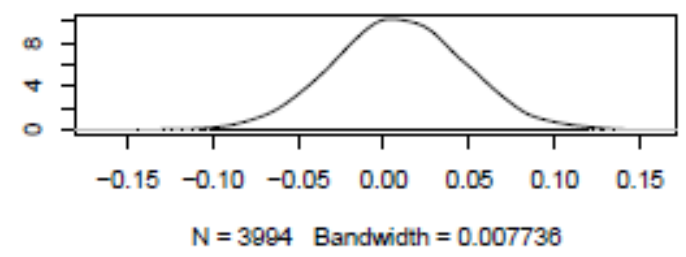


Trace of Gender:life

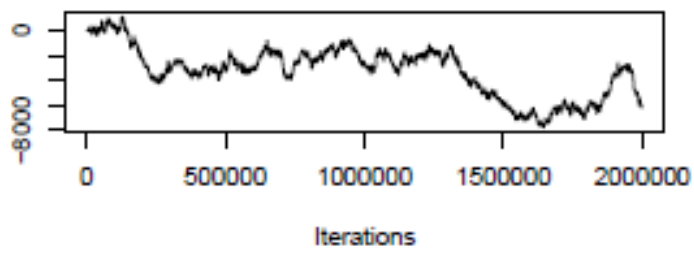

Trace of time:life

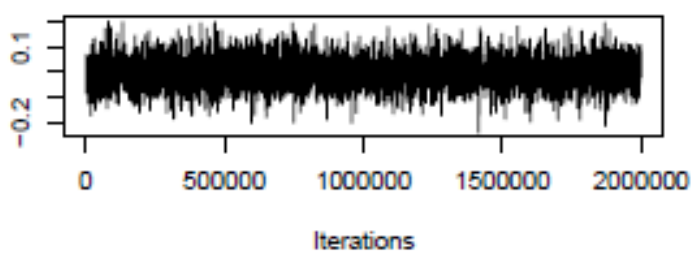

Trace of Gender:drugs

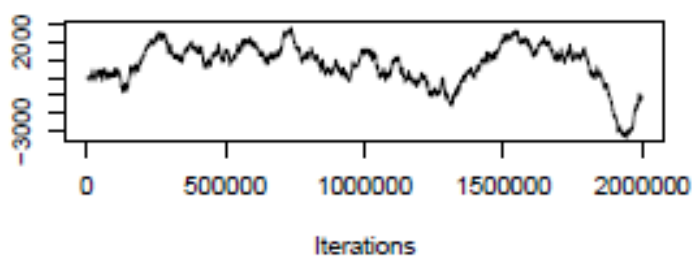

Trace of time:drugs

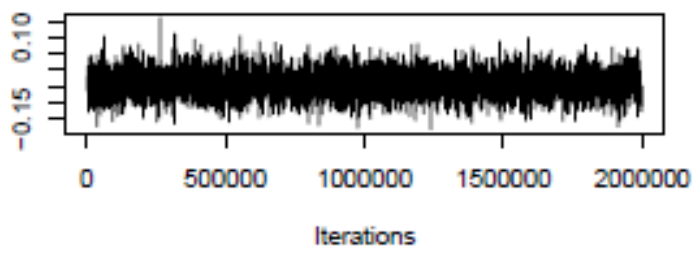

Trace of Gender:physical

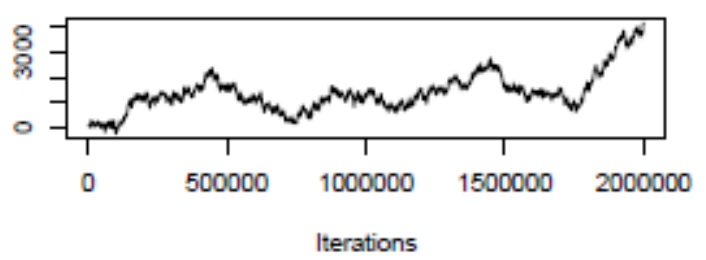

Trace of time:physical

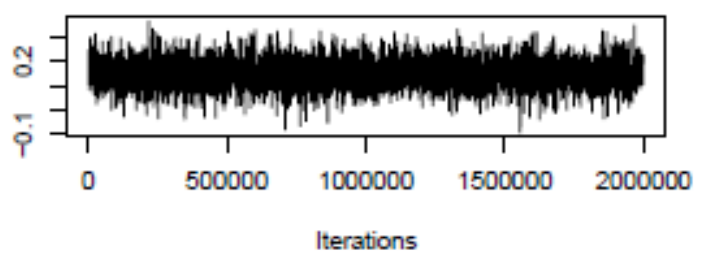

Density of Gender:life

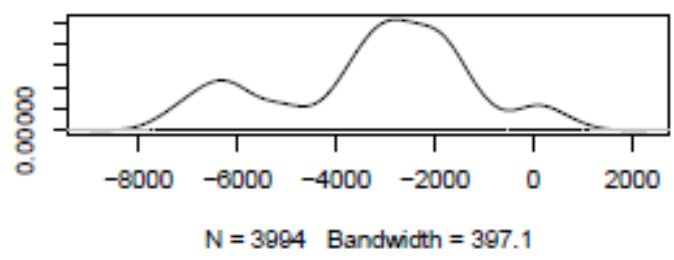

Density of time:life

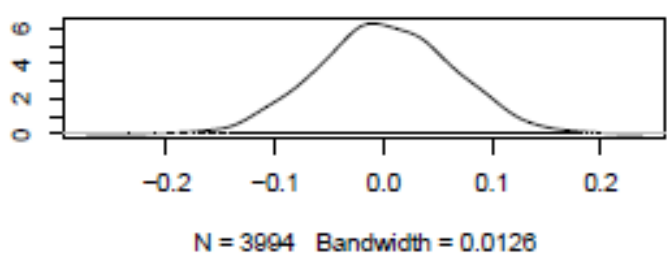

Density of Gender:drugs

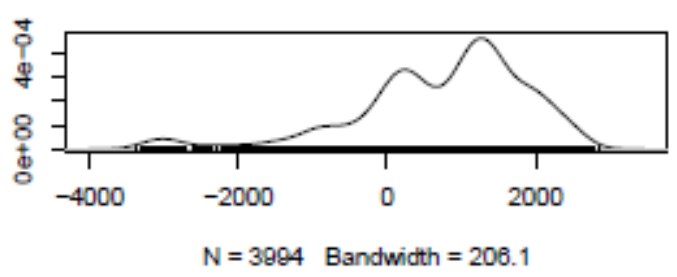

Density of time:drugs

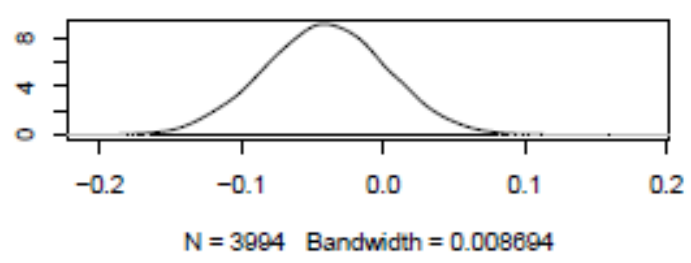

Density of Gender:physical

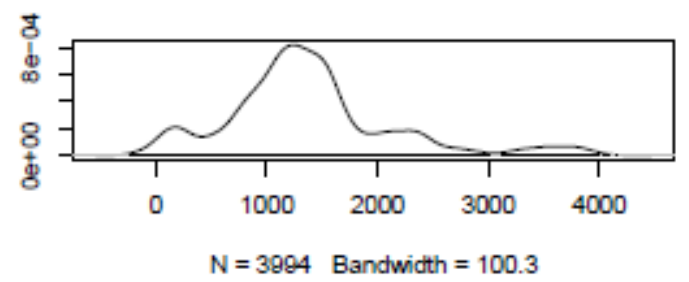

Density of time:physical

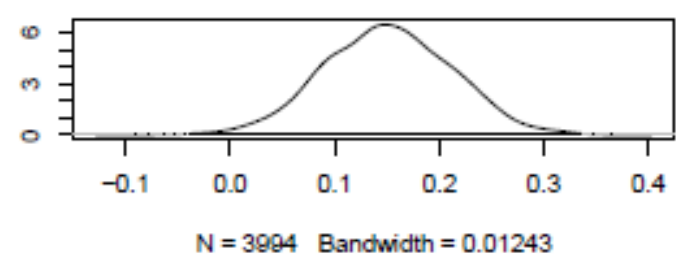


Trace of Gender:emotion

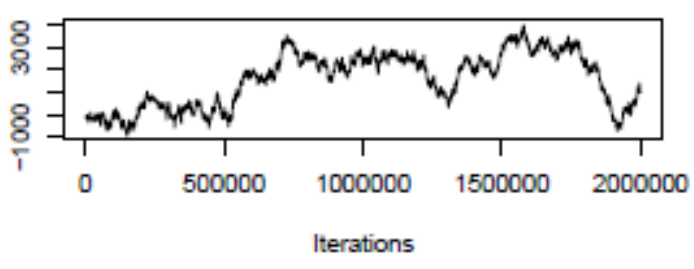

Trace of time:emotion

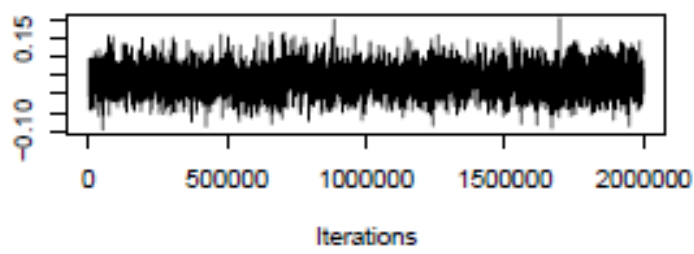

Trace of Gender:self

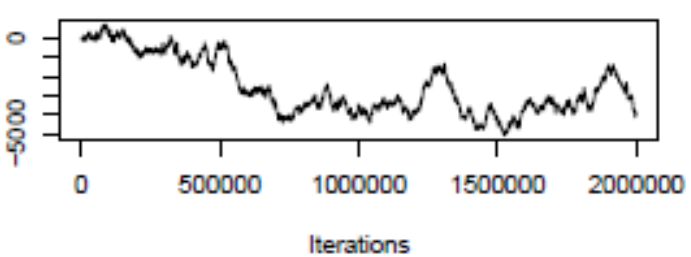

Trace of time:self

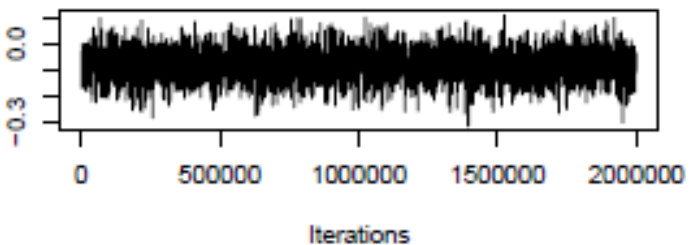

Trace of Gender:think

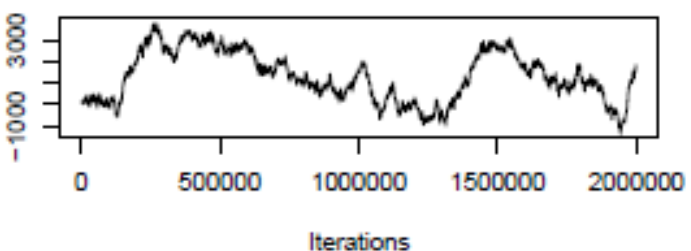

Trace of time:think

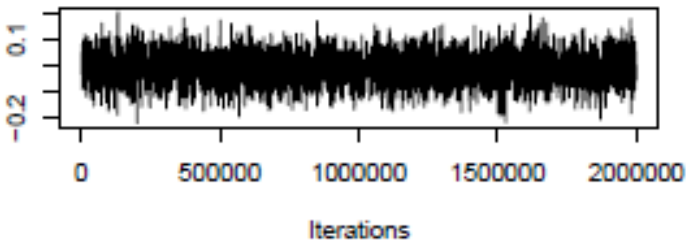

Density of Gender:emotion

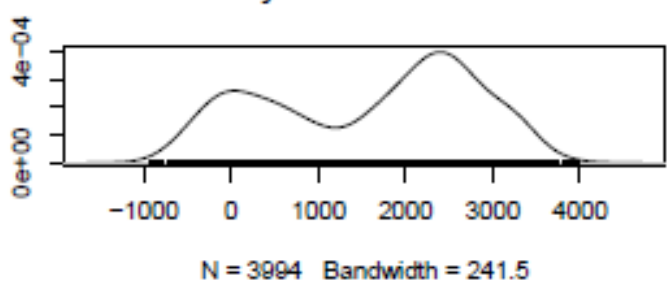

Density of time:emotion

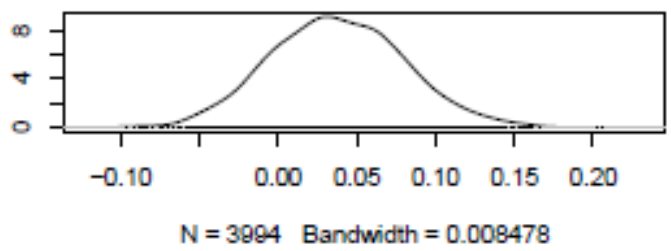

Density of Gender:self

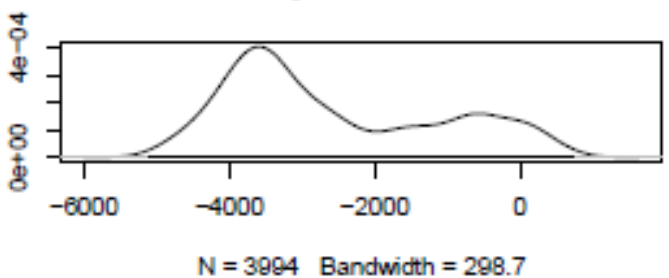

Density of time:self

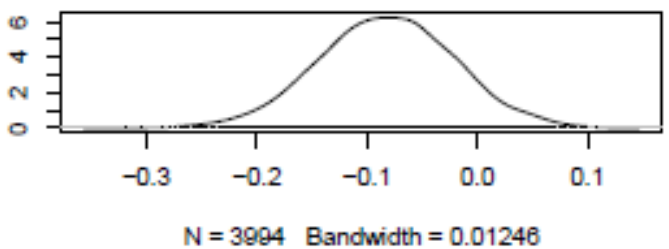

Density of Gender:think

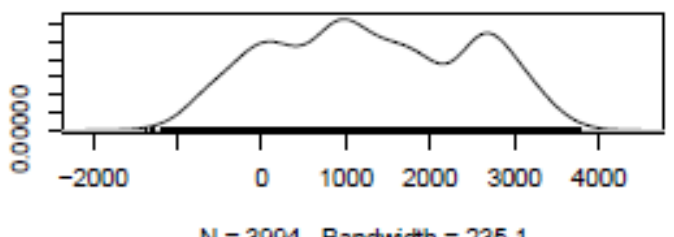

Density of time:think

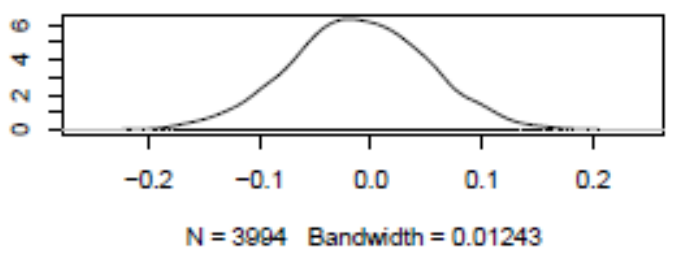


Trace of Gender:attitude

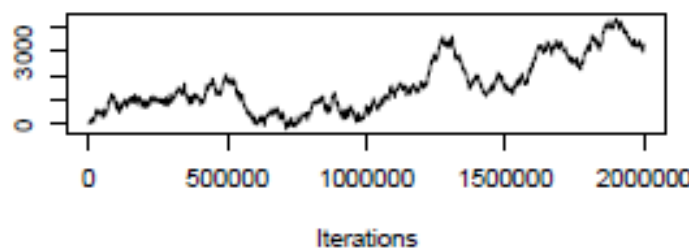

Trace of time:attitude

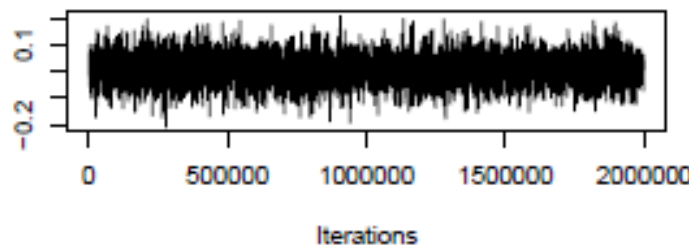

Trace of Gender:change

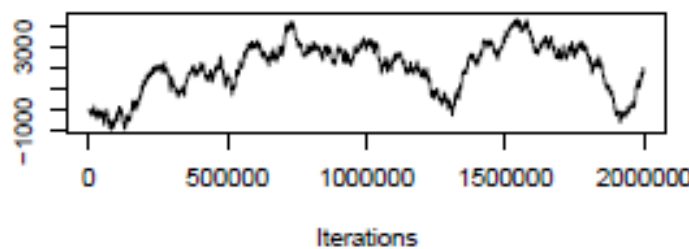

Trace of time:change

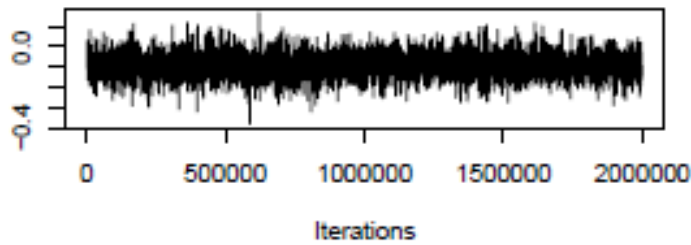

Trace of Gender:time:live

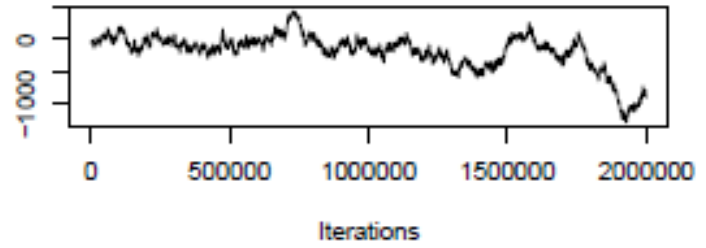

Trace of Gender:time:relation

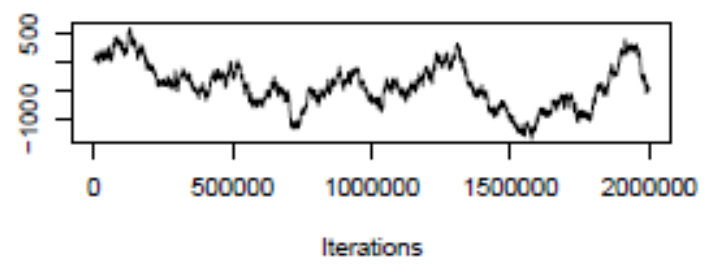

Density of Gender:attitude

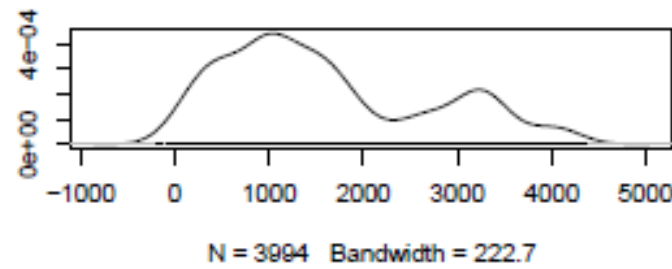

Density of time:attitude

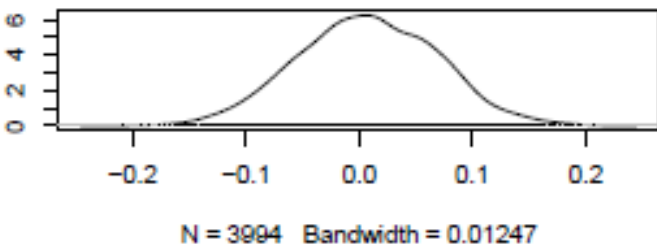

Density of Gender:change

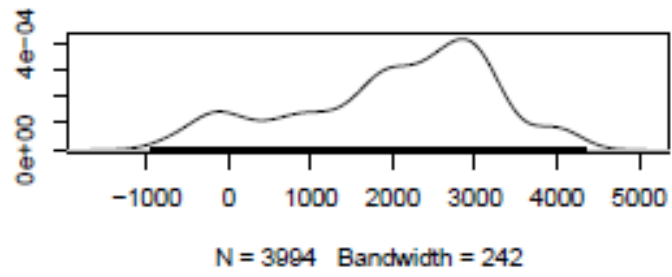

Density of time:change

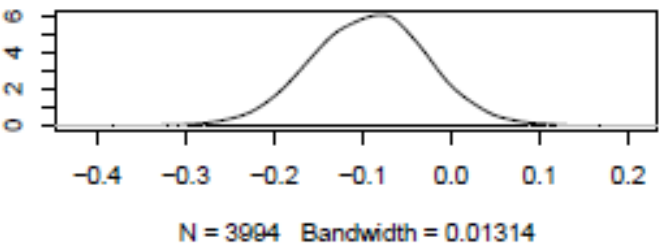

Density of Gender:time:live

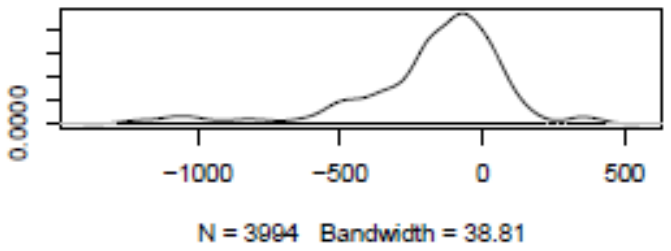

Density of Gender:time:relation

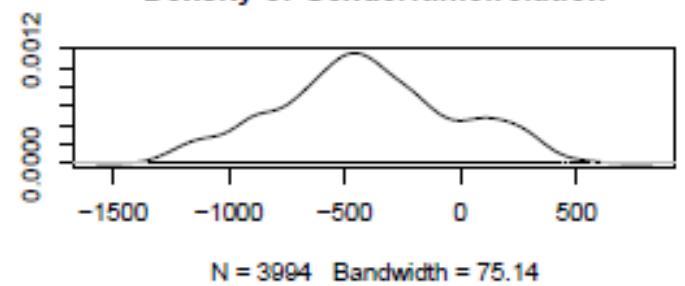


Trace of Gender:time:ete

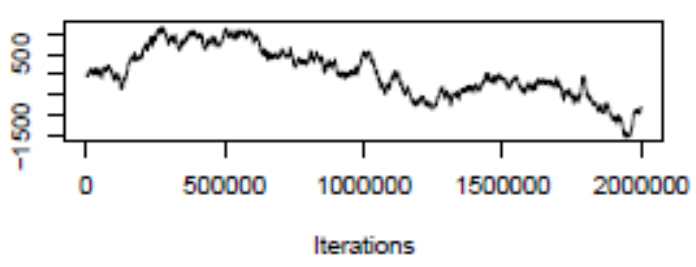

Trace of Gender:time:where

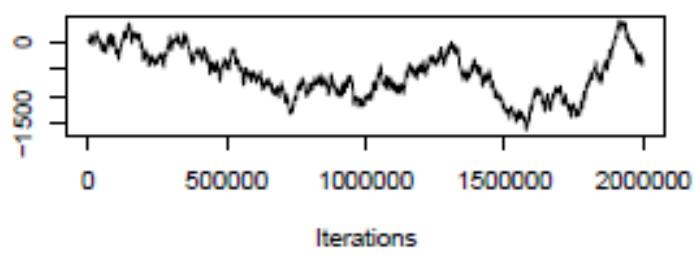

Trace of Gender:time:life

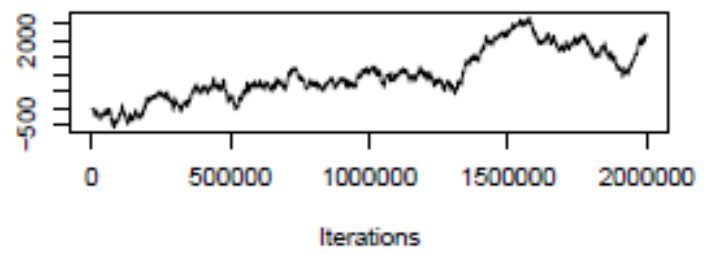

Trace of Gender:time:drugs

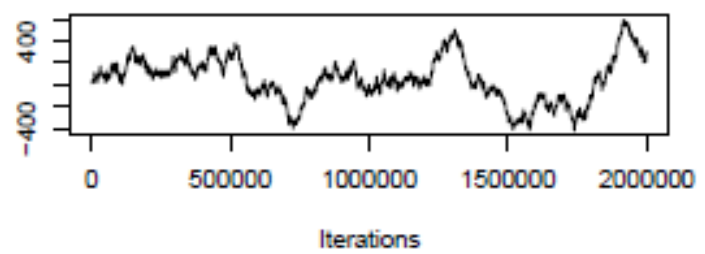

Trace of Gender:time:physical

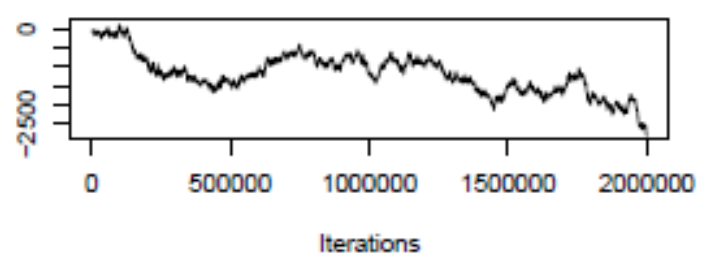

Trace of Gender:time:emotion

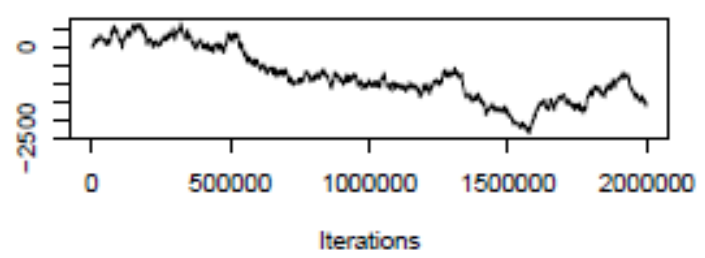

Density of Gender:time:ete

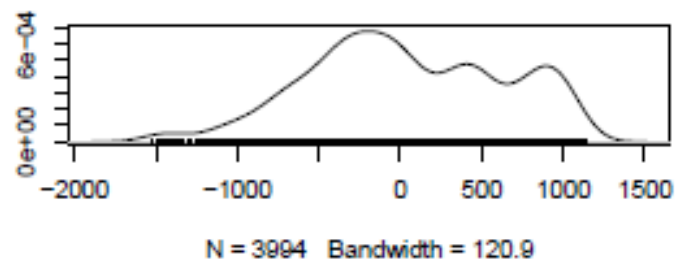

Density of Gender:time:where

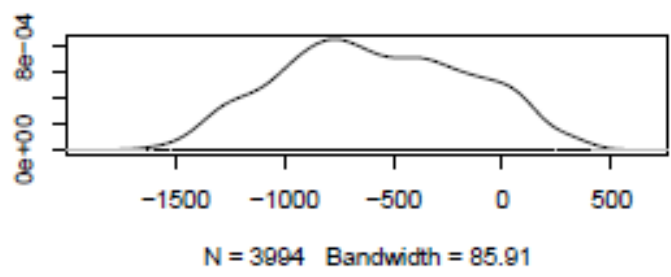

Density of Gender:time:life

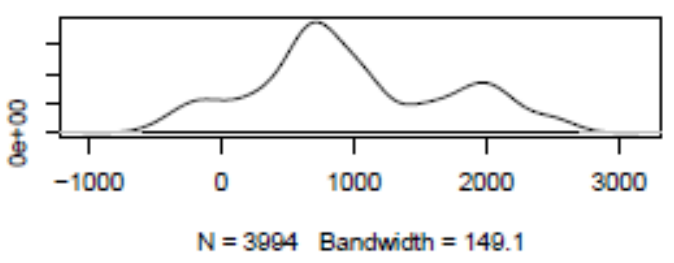

Density of Gender:time:drugs

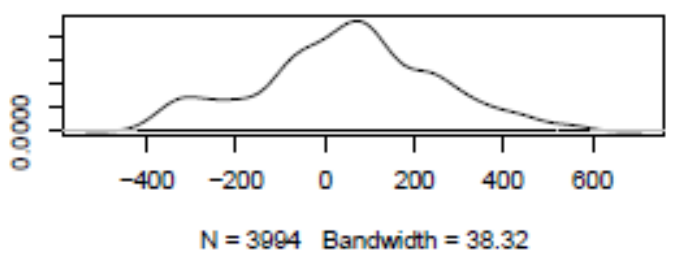

Density of Gender:time:physical

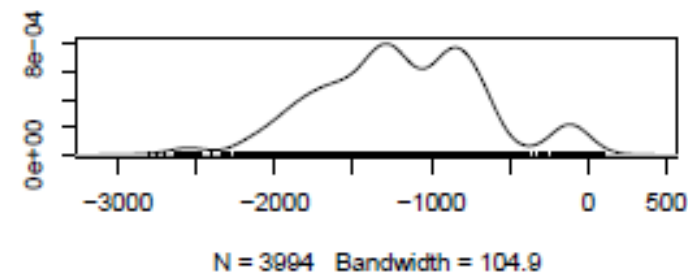

Density of Gender:time:emotion

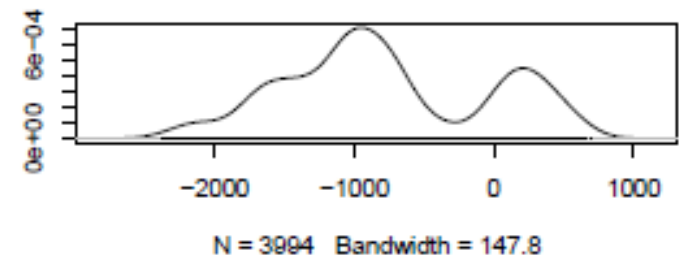


Trace of Gender:time:self

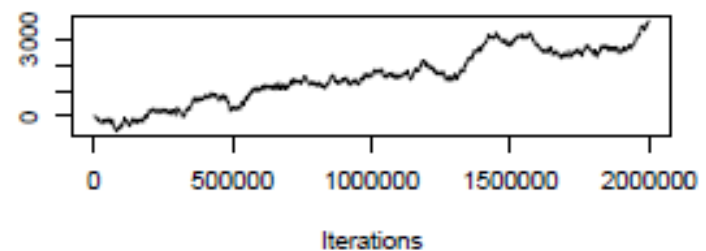

Trace of Gender:time:think

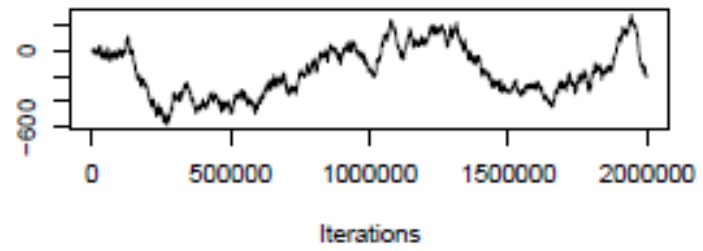

Trace of Gender:time:attitude

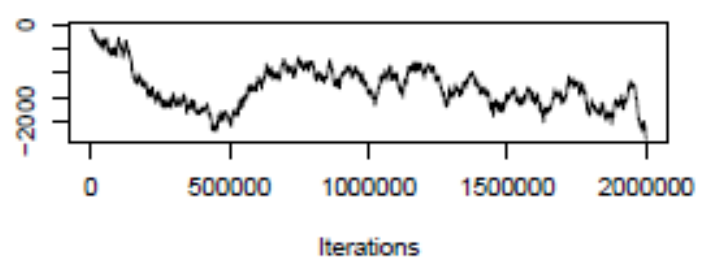

Trace of Gender:time:change

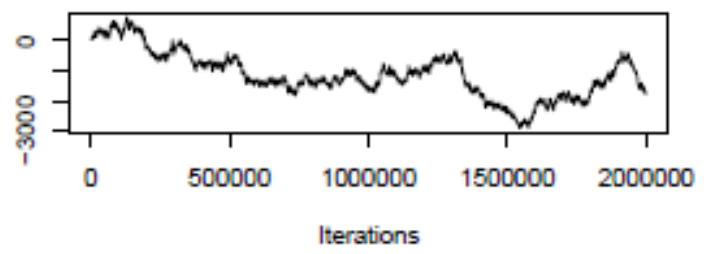

Density of Gender:time:self

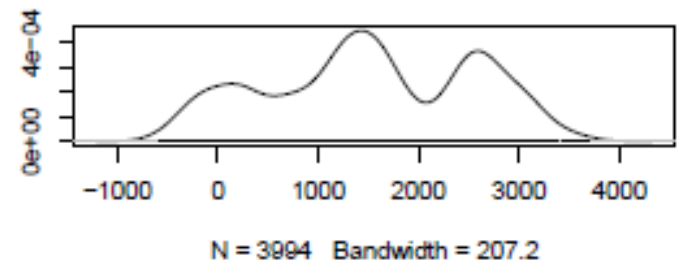

Density of Gender:time:think

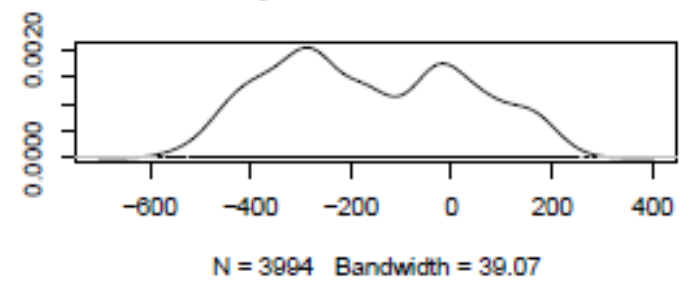

Density of Gender:time:attitude

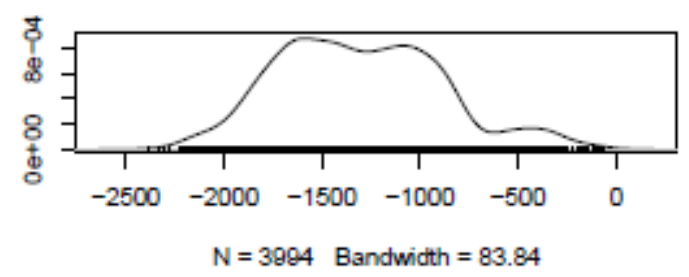

Density of Gender:time:change

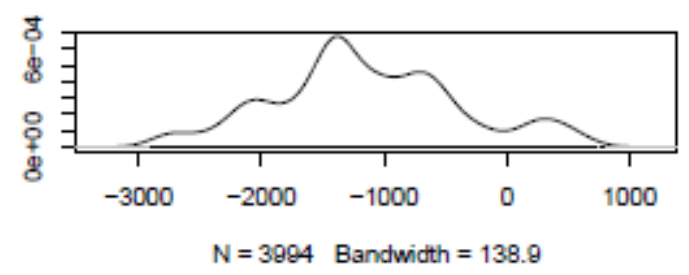


$\underline{\text { Random Effects }}$

Trace of time

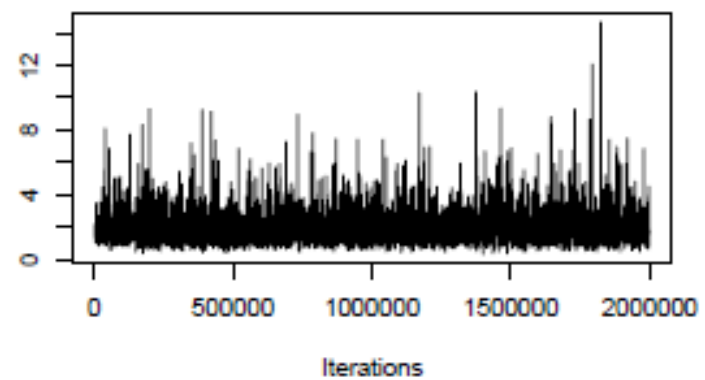

Trace of Research.ID

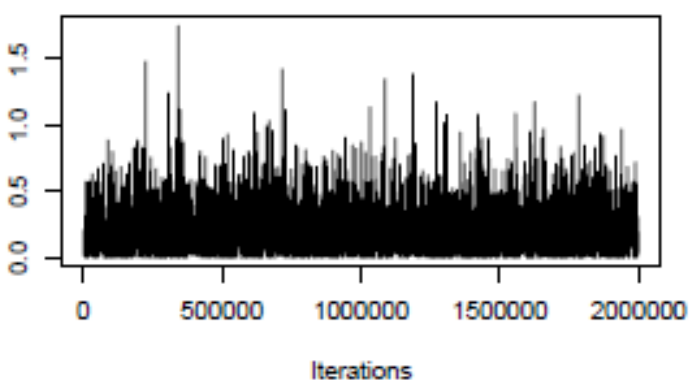

Density of time

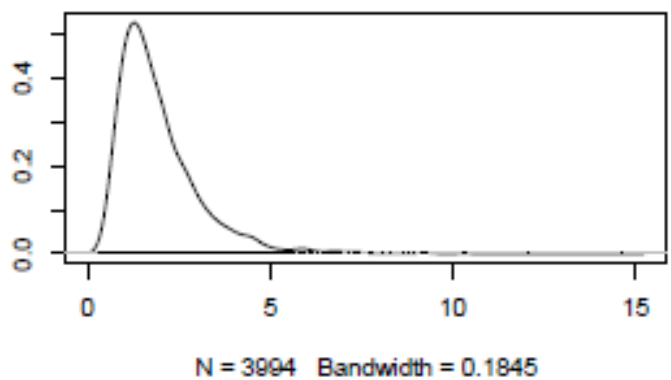

Density of Research.ID

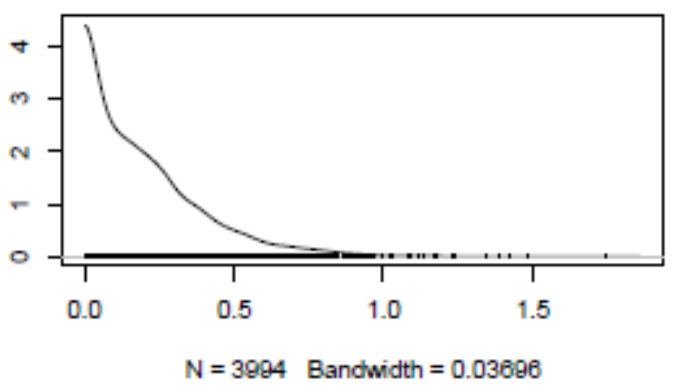


Dynamic Model involving Ethnicity (Table 5.5)

Bayesian Model (BDm2_d2)

\section{\#\# Define the model}

BDm2_d2 <- MCMCglmm(FO.bin bme*time*live + bme*time*relation + bme*time*ete + bme*time*where + bme*time*life + bme*time*drugs + bme*time*physical + bme*time*emotion + bme*time*self + bme*time*think + bme*time*attitude + bme*time* change, random= time+Research.ID, data=data, family="ordinal",prior=priorD, slice=TRUE, nitt $=950000$, thin=250, burnin=3000)

\section{\#\# Checks for suitable convergence}

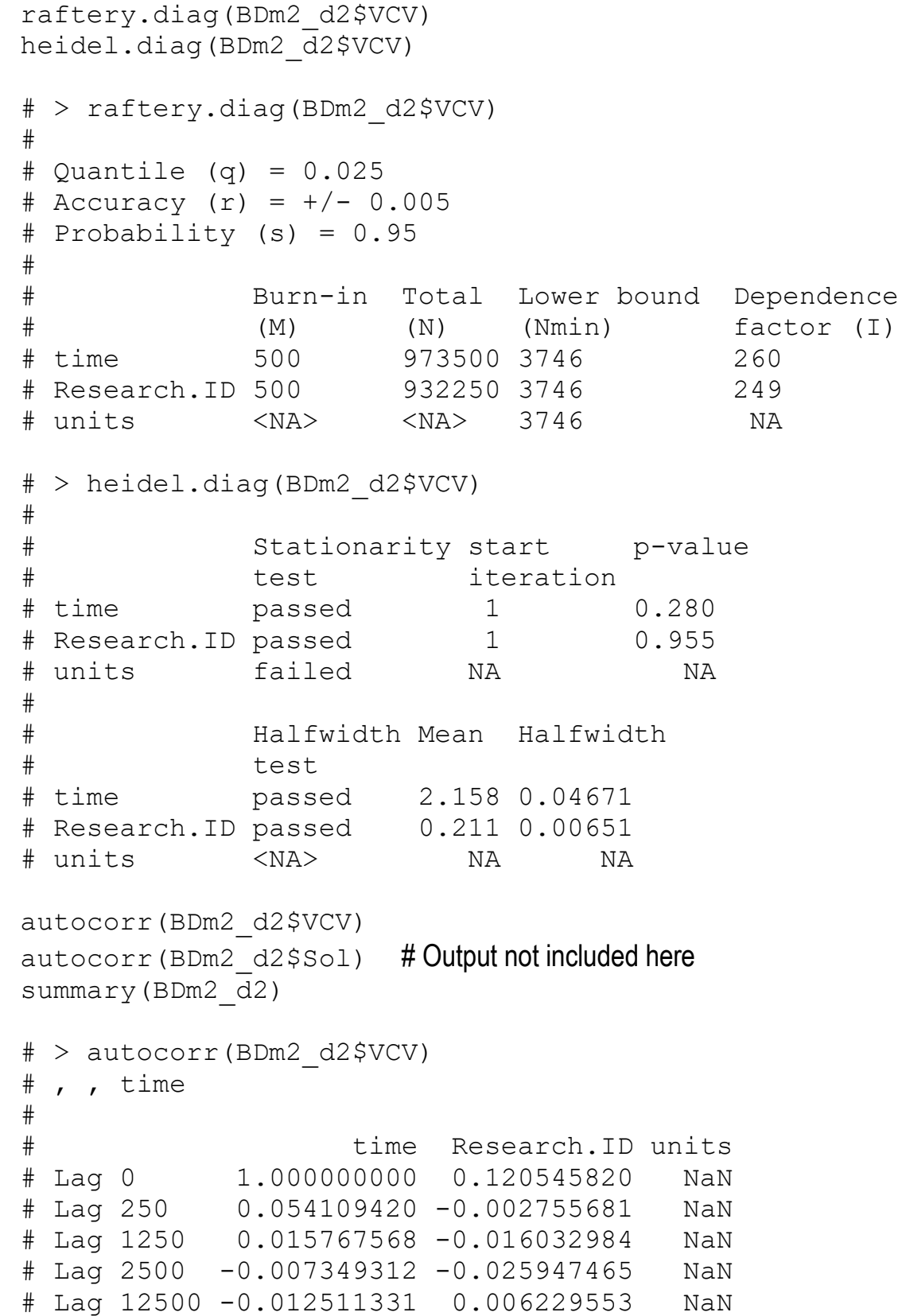




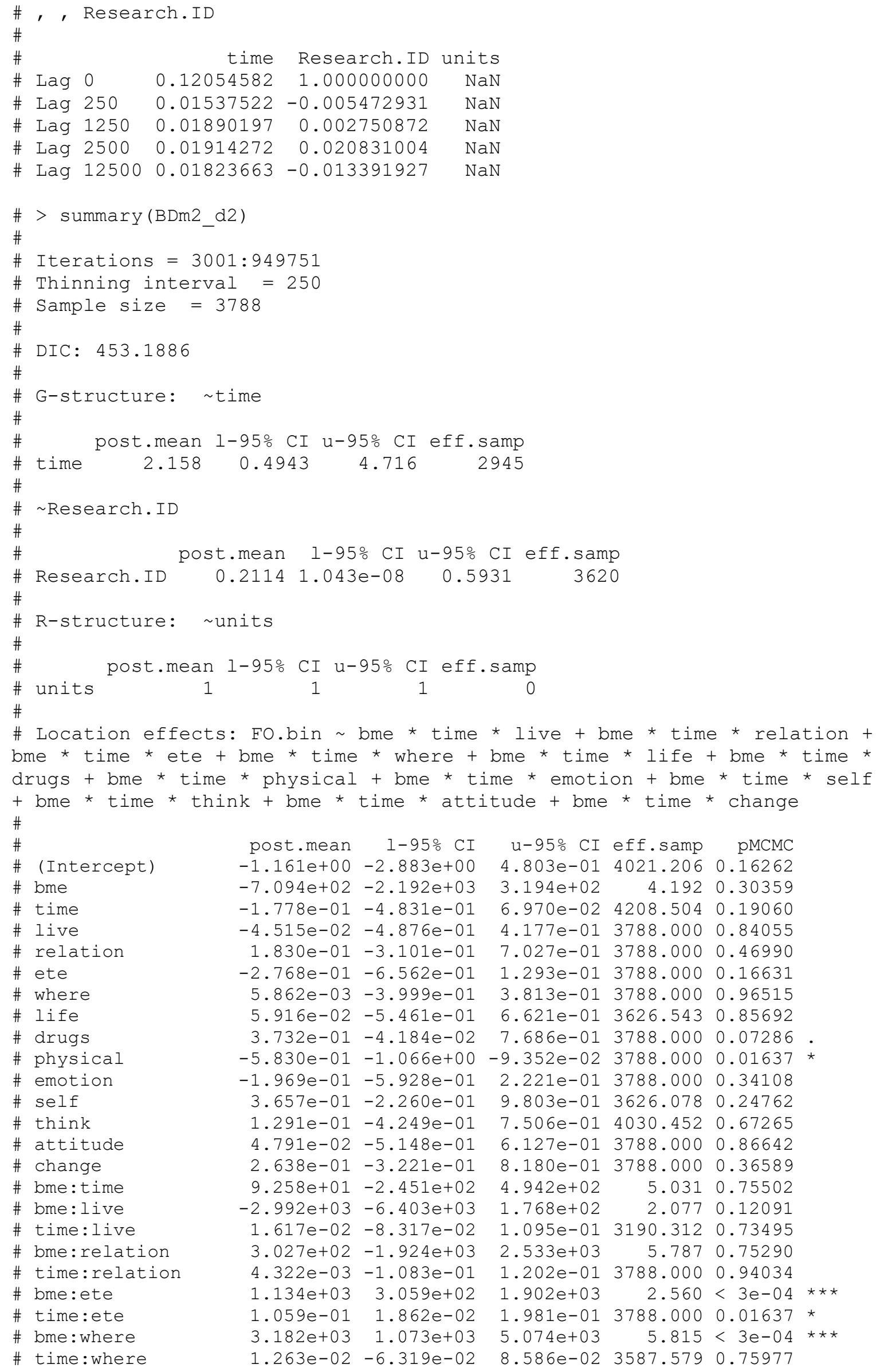




\begin{tabular}{|c|c|c|c|c|c|c|}
\hline \# bme:life & $1.826 e+03$ & $-3.642 e+03$ & $7.020 e+03$ & 3.417 & 0.68532 & \\
\hline \# time:life & $-1.983 e-02$ & $-1.535 e-01$ & $9.794 e-02$ & 3411.550 & 0.76346 & \\
\hline bme: drugs & $-4.159 e+03$ & $-7.652 e+03$ & $-5.366 e+02$ & 4.040 & 0.01584 & * \\
\hline time:drugs & $-5.296 e-02$ & $-1.480 e-01$ & $2.746 e-02$ & 3788.000 & 0.21595 & \\
\hline bme:physical & $2.834 e+03$ & $-5.230 e+02$ & $6.935 e+03$ & 4.854 & 0.17 & \\
\hline time:physical & $1.437 e-01$ & $2.539 e-02$ & $2.628 e-01$ & 3788.000 & 0.01 & * \\
\hline bme: emotion & $2.114 e+02$ & $-3.392 e+02$ & $6.880 e+02$ & 5.331 & 0.5 & \\
\hline ime:emotion & $5.068 e-02$ & $-3.738 e-02$ & $1.338 e-01$ & 3426.153 & 0.2 & \\
\hline me: self & $2.670 e+03$ & $7.365 e+02$ & $4.807 e+03$ & 2.603 & $<3$ & $\star \star \star$ \\
\hline ime:self & $-1.164 e-01$ & $-2.346 e-01$ & $5.888 e-03$ & 3788.000 & 0.0 & . \\
\hline me: think & $-5.015 e+03$ & $-8.640 e+03$ & $-1.847 e+03$ & 4.417 & $<3$ & $\star \star \star$ \\
\hline ime: think & $-4 \cdot 33$ & $-1.732 e-01$ & $8.765 e-02$ & 4052.388 & 0.4 & \\
\hline me:attitude & $5.100 e+03$ & 1.19 & $50 e+03$ & 882 & & $\star \star \star$ \\
\hline time:attitude & $-1 \cdot 41$ & $-1 \cdot 4]$ & $1 e-01$ & 3788.000 & 0.8 & \\
\hline bme: change & $-4 \cdot 32$ & $3 e+03$ & $8 e+03$ & 4.3 & 0.7 & \\
\hline time: change & $9 e-02$ & -1 & $6 e-01$ & 35.0 & 0.7 & \\
\hline bme: time:live & $2 e+02$ & -2.5 & $2 e+02$ & 3.5 & 0.3 & \\
\hline bme: time:relation & -7.50 & $7 e+03$ & $\mathrm{Le}+02$ & 5.1 & 0.4 & \\
\hline bme: time:ete & 1.05 & $6 e+02$ & $5 e+02$ & 9.685 & 0.5 & \\
\hline ne: where & $0 e+02$ & $-1 \cdot 2$ & $3 e+02$ & 3.853 & 0.3 & \\
\hline ne: life & $3.332 e+02$ & $6 e+03$ & $e+03$ & & 0.8 & \\
\hline me:drugs & $-2.891 e+02$ & -9.24 & $0 e+02$ & & 0.6 & \\
\hline bme: time:physical & $8 e+02$ & -1.75 & $5 e+02$ & 4.368 & 0.2 & \\
\hline bme: time:emotion & $6.762 e+02$ & $1.719 e+02$ & $5 e+03$ & 4.573 & 0.0 & * \\
\hline bme: time:self & $-9.421 e+02$ & $-1.468 e+03$ & $9 e+02$ & & $<3 e$ & $\star \star \star$ \\
\hline bme:time:think & $3.535 e+02$ & $-1.506 e+01$ & $6 e+02$ & & $0.0^{-}$ & $\cdot$ \\
\hline bme: time:attitude & $6.794 e+02$ & $-1.384 e+02$ & 1.74 & & 0.2 & \\
\hline $\begin{array}{l}\text { bme: } \text { time: change } \\
---\end{array}$ & $-8.833 e+02$ & $-1.613 e+03$ & $-2.127 e+02$ & 4.370 & 0.00264 & $\star \star$ \\
\hline 土. codes: & $\star 1 \quad 0$. & & 0.05 & $\cdot \quad 0.1$ & 1 & \\
\hline
\end{tabular}


Trace of (Intercept)

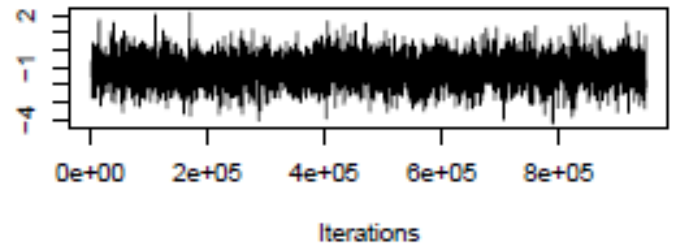

Trace of bme

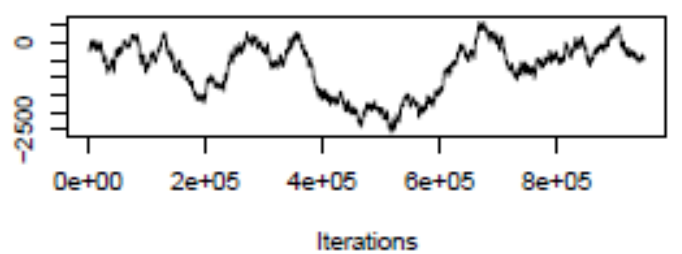

Trace of time

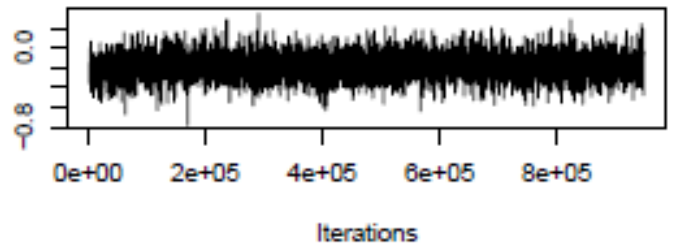

Trace of live

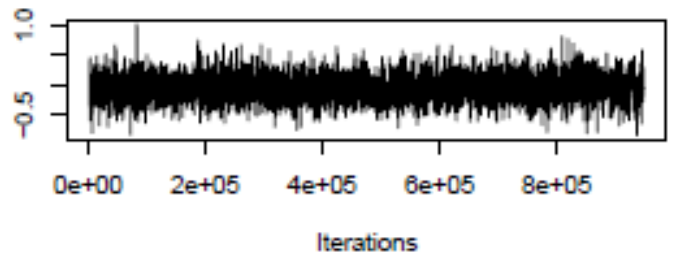

Trace of relation

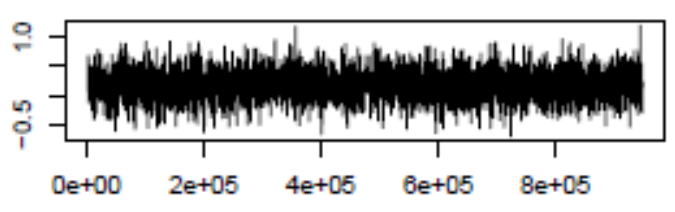

Iterations

Trace of ete

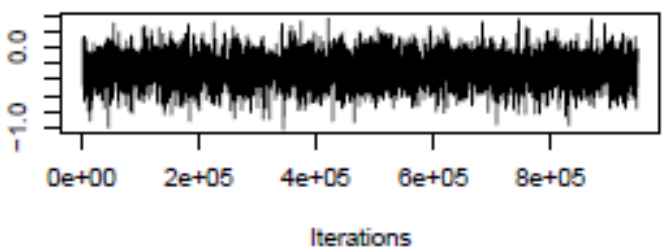

Density of (Intercept)

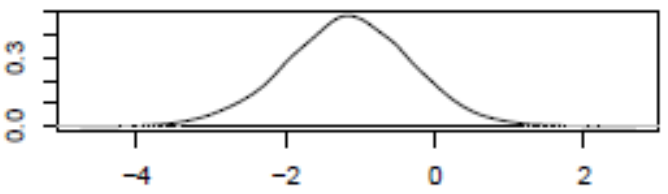

$\mathrm{N}=3788$ Bandwidth $=0.1705$

Density of bme

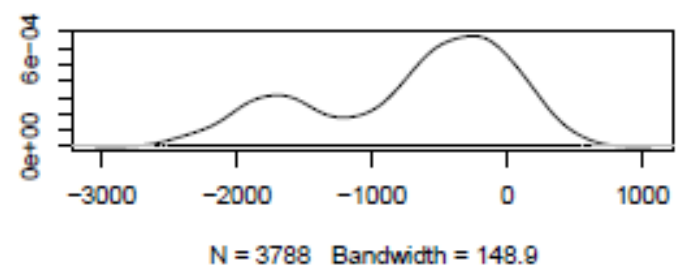

Density of time

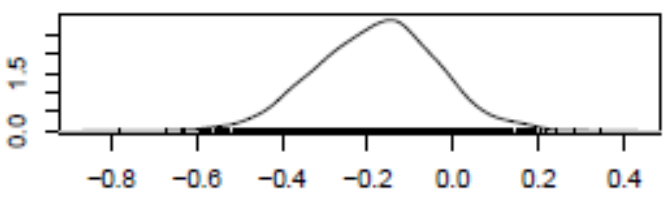

$\mathrm{N}=3788$ Bandwidth $=0.0287$

Density of live

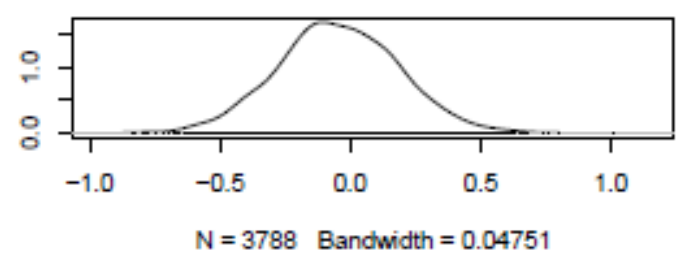

Density of relation

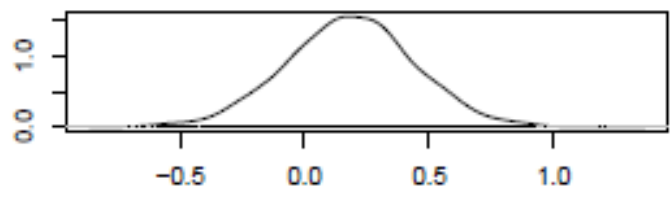

$\mathrm{N}=3788$ Bandwidth $=0.05148$

Density of ete

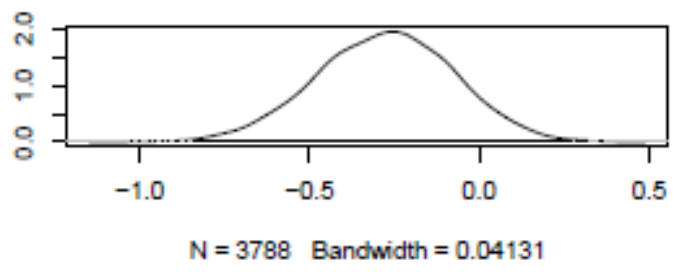


Trace of where

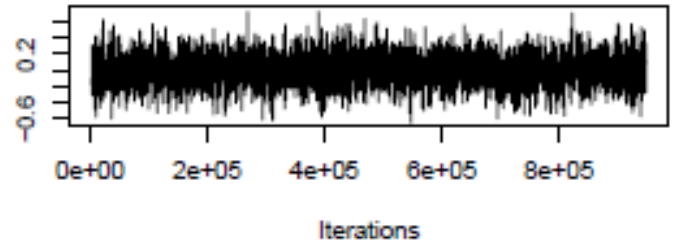

Trace of life

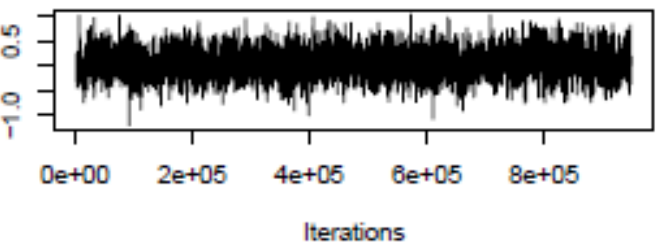

Trace of drugs

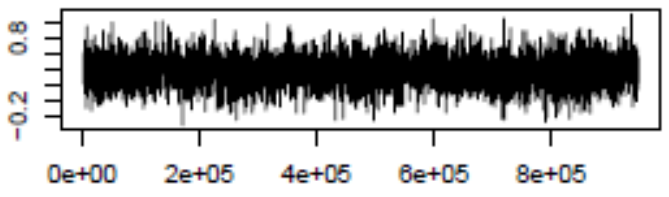

Iterations

Trace of physical

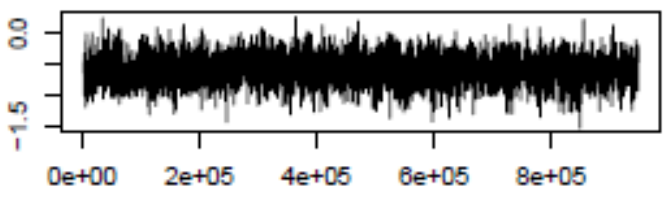

Iterations

Trace of emotion

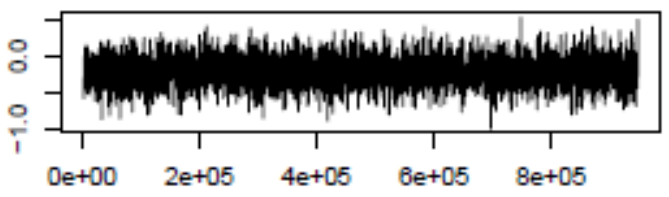

Iterations

Trace of self

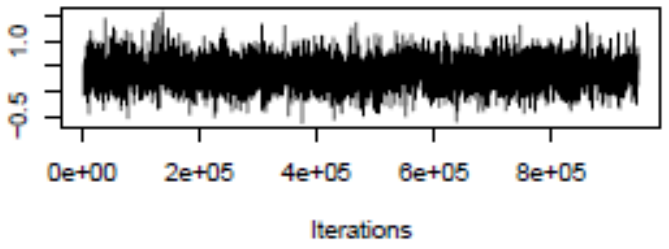

Density of where

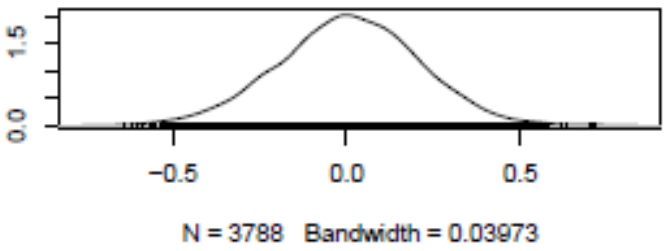

Density of life

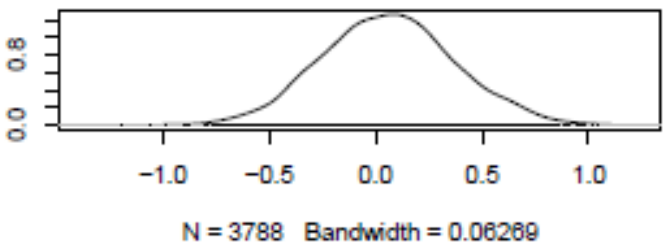

Density of drugs

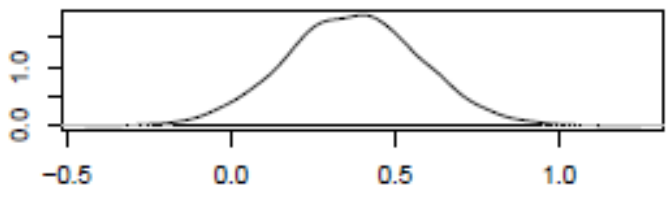

$\mathrm{N}=3788$ Bandwidth $=0.04201$

Density of physical

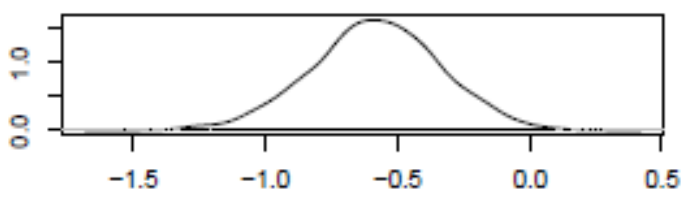

$N=3788$ Bandwidth $=0.04884$

Density of emotion

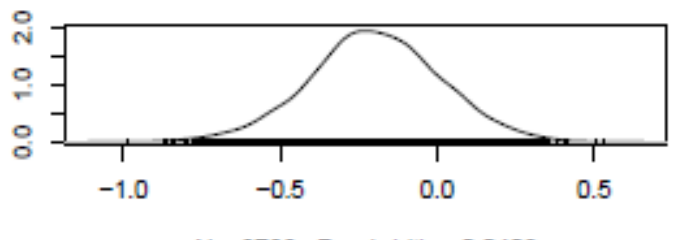

$\mathrm{N}=3788$ Bandwidth $=0.0409$

Density of self

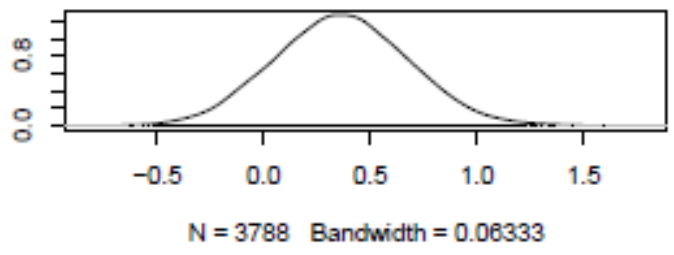


Trace of think

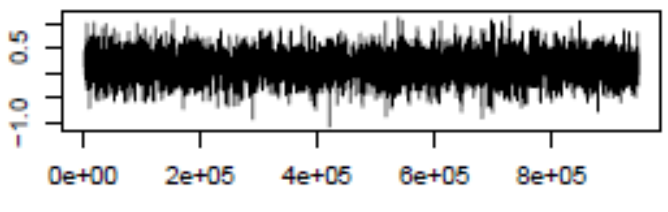

Iterations

Trace of attitude

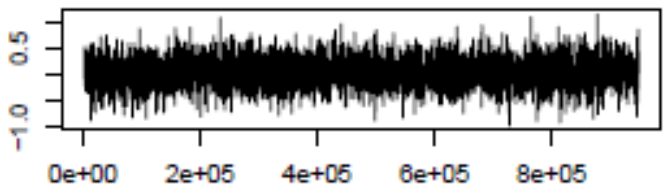

Iterations

Trace of change

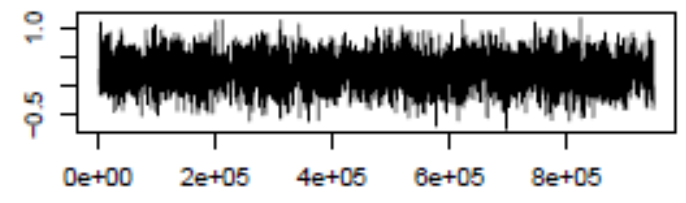

Iterations

Trace of bme:time

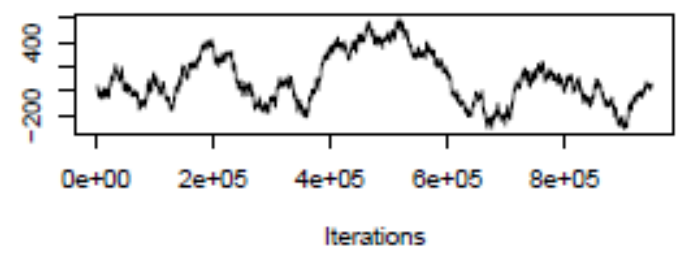

Trace of bme:live

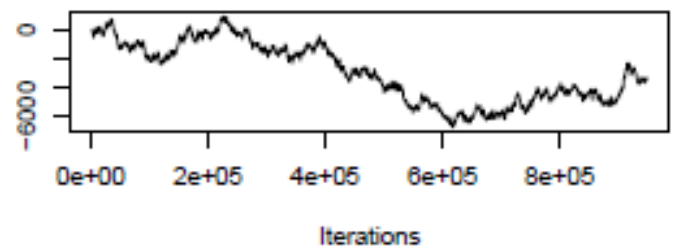

Trace of time:live

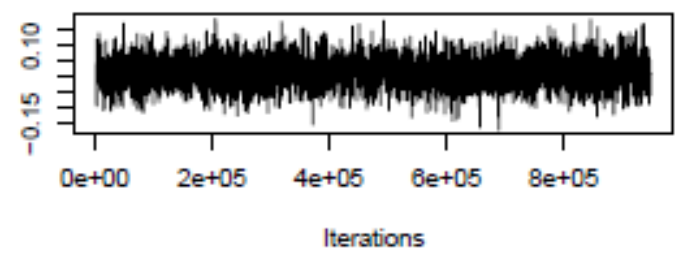

\section{Density of think}

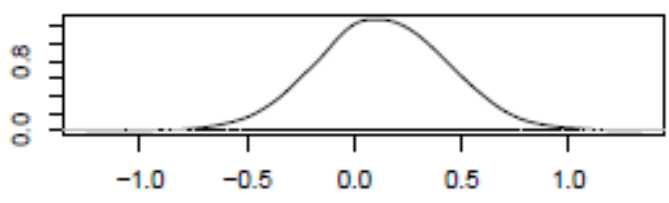

$\mathrm{N}=3788$ Bandwidth $=0.06163$

Density of attitude

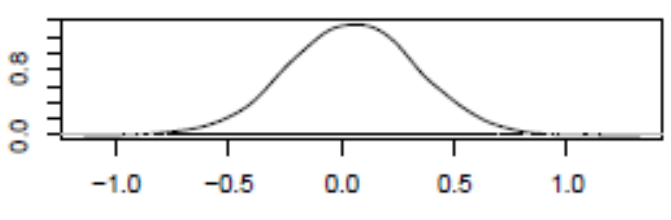

$\mathrm{N}=3788$ Bandwidth $=0.05859$

Density of change

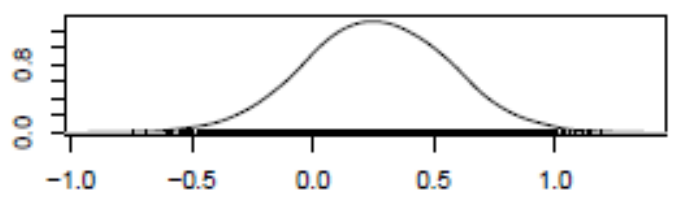

$\mathrm{N}=3788$ Bandwidth $=0.05965$

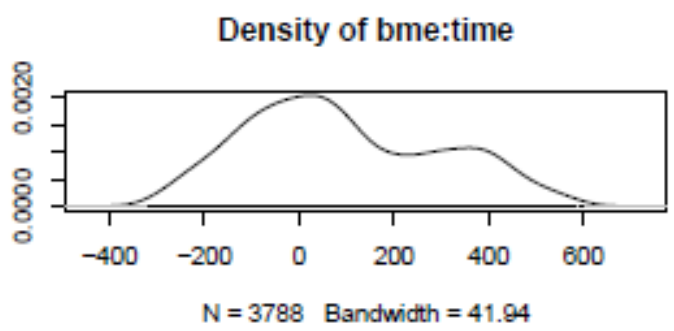

Density of bme:live

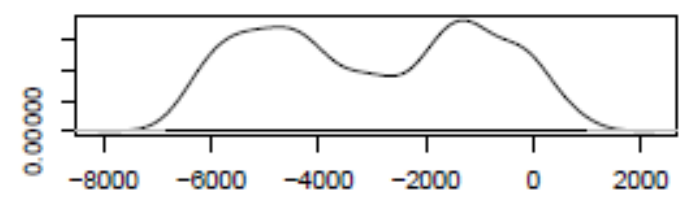

$\mathrm{N}=3788$ Bandwidth $=426.1$

Density of time:live

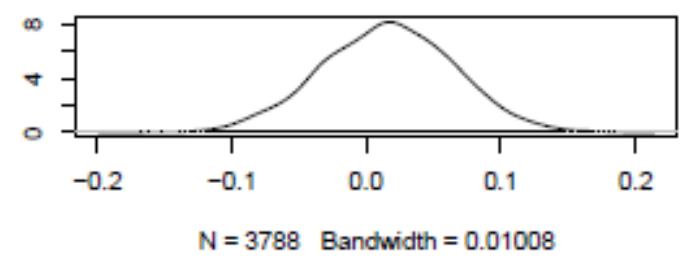


Trace of bme:relation

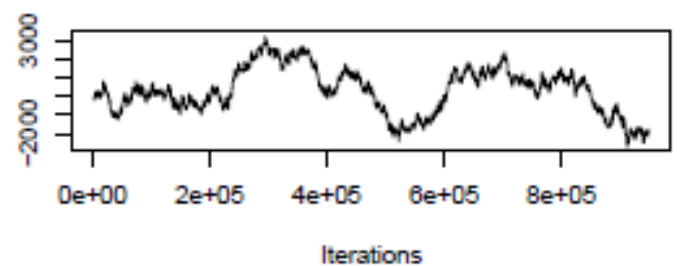

Trace of time:relation

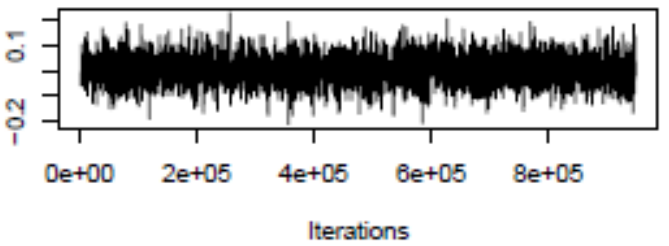

Trace of bme:ete

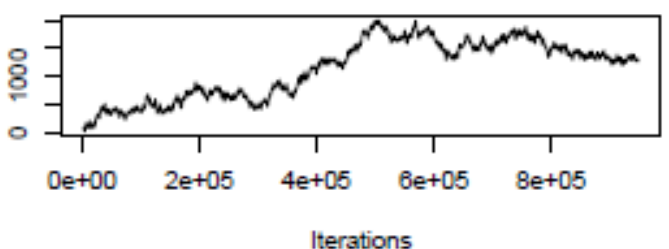

Trace of time:ete

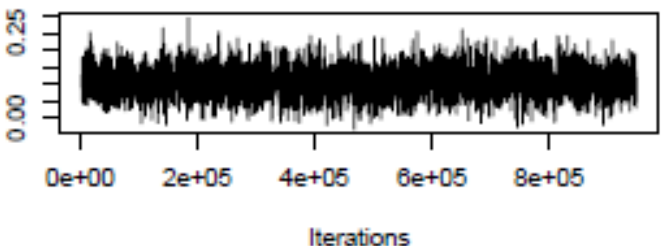

Trace of bme:where

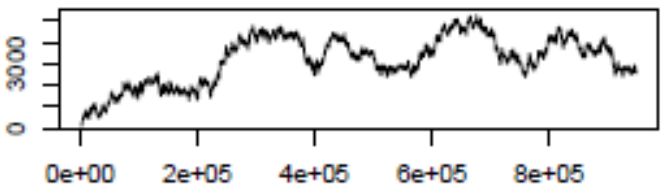

Iterations

Trace of time:where

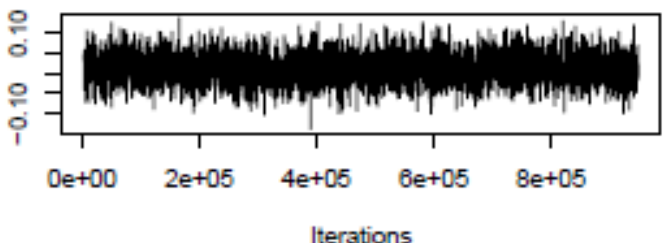

Density of bme:relation

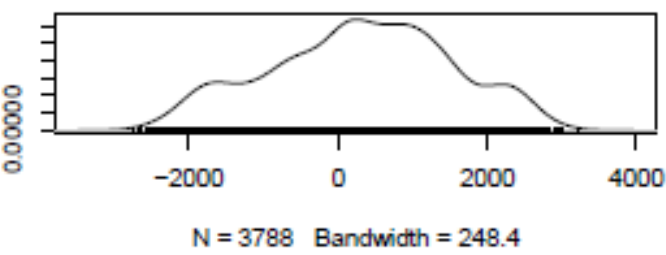

Density of time:relation

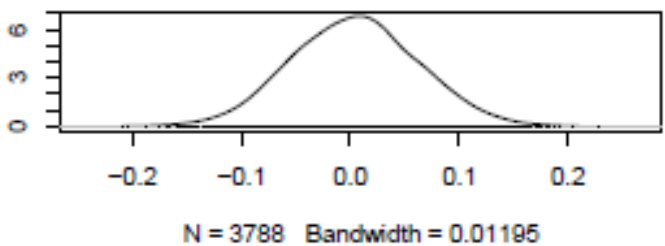

Density of bme:ete

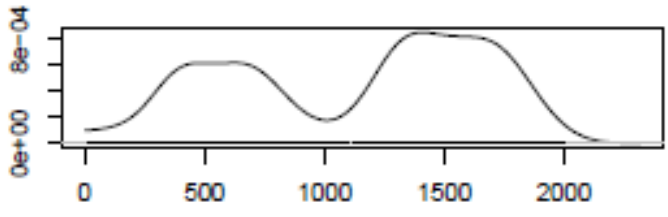

$\mathrm{N}=3788$ Bandwidth $=106.4$

Density of time:ete

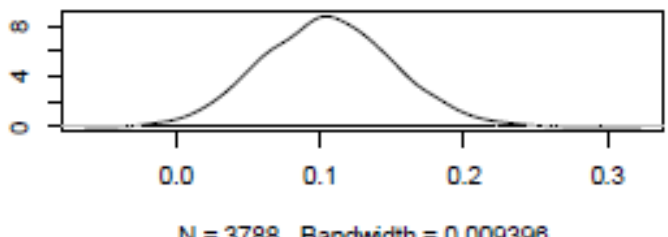

Density of bme:where

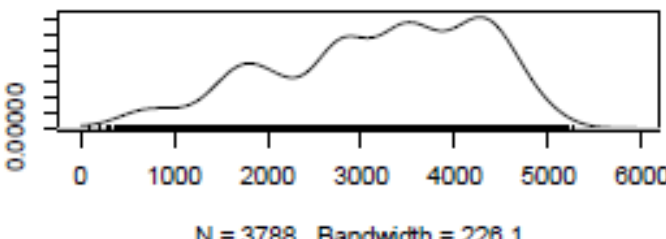

Density of time:where

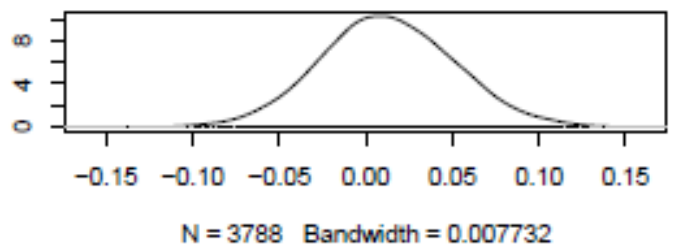


Trace of bme:life

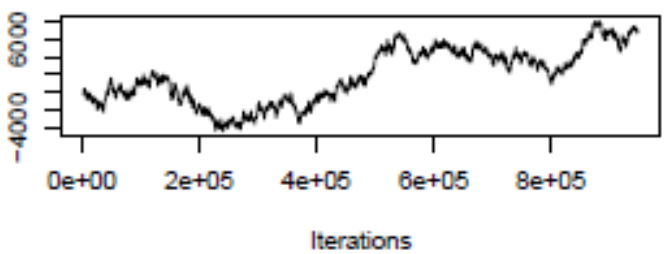

Trace of time:life

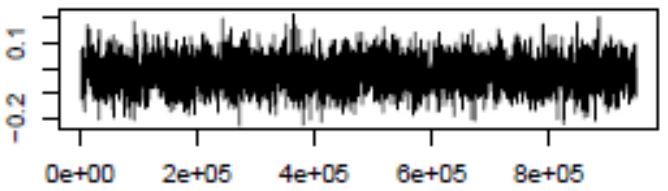

Iterations

Trace of bme:drugs

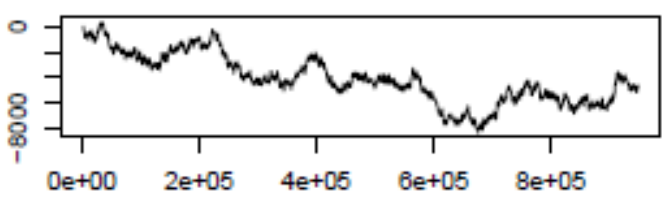

Iterations

Trace of time:drugs

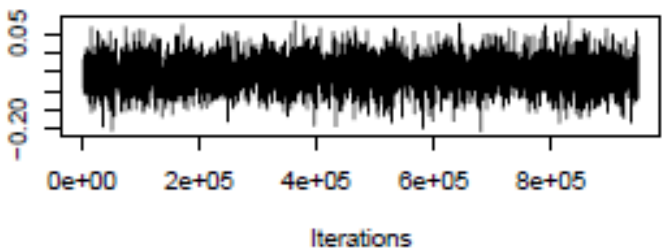

Trace of bme:physical

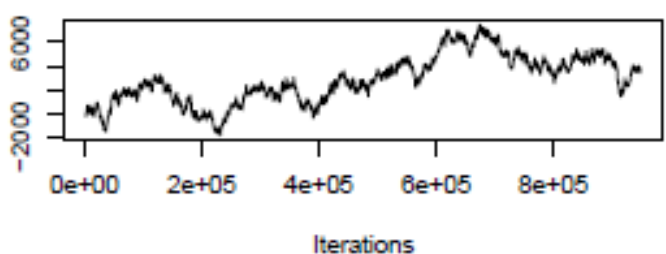

Trace of time:physical

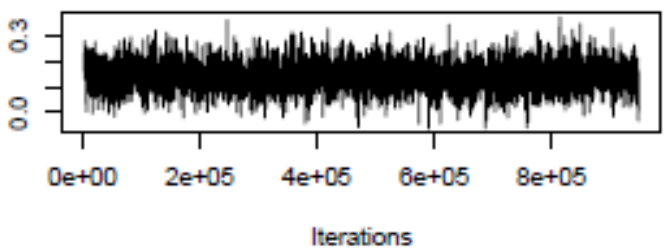

Density of bme:life

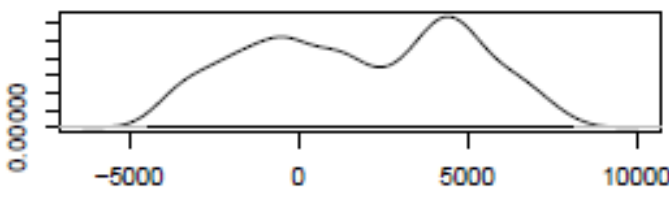

$\mathrm{N}=3788$ Bandwidth $=640.4$

Density of time:life

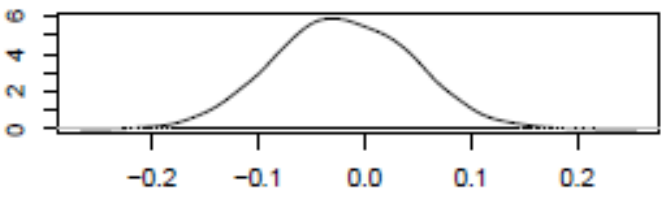

$\mathrm{N}=3788$ Bandwidth $=0.01325$

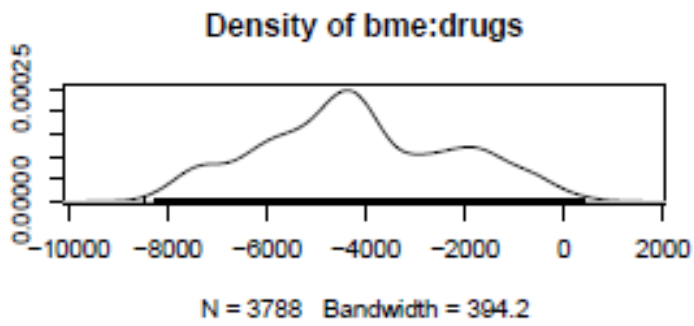

Density of time:drugs
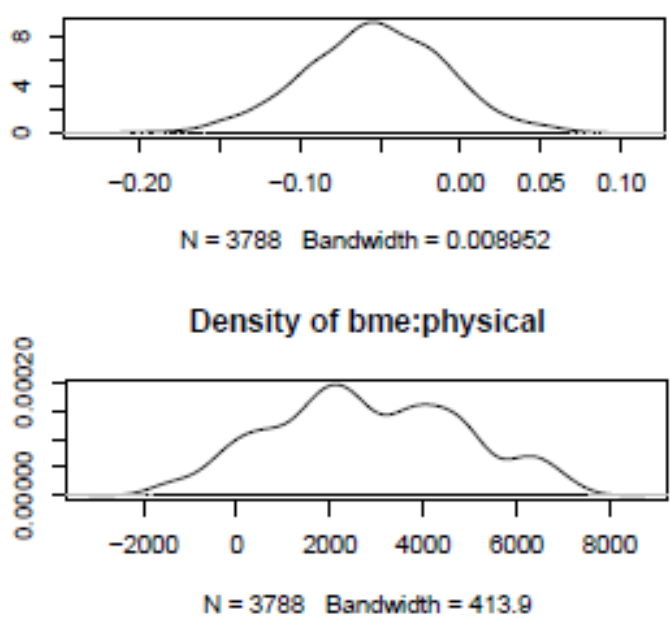

Density of time:physical

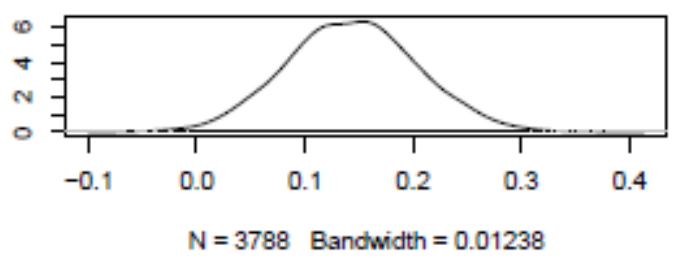


Trace of bme:emotion

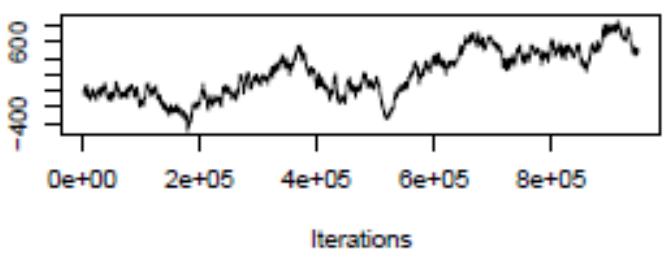

Trace of time:emotion

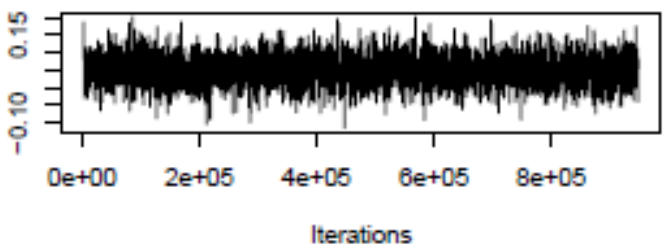

Trace of bme:self

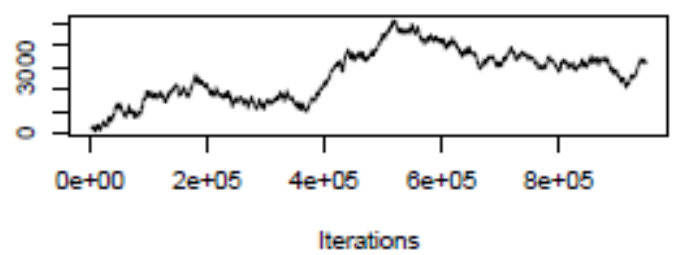

Trace of time:self

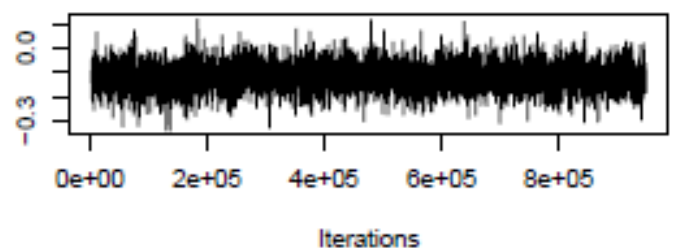

Trace of bme:think

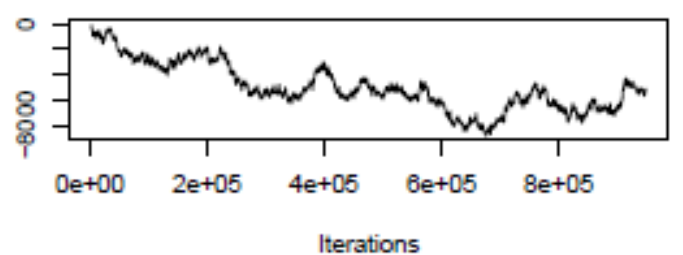

Trace of time:think

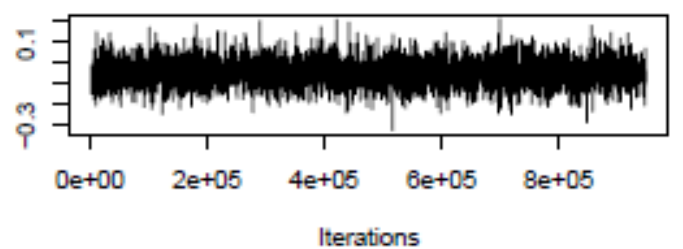

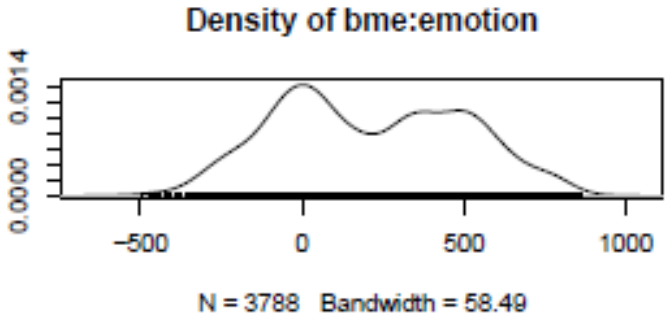

Density of time:emotion

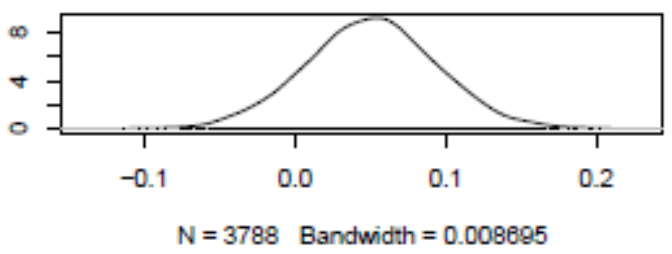

Density of bme:self

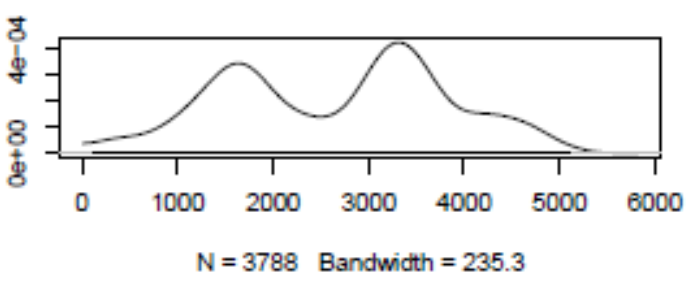

Density of time:self

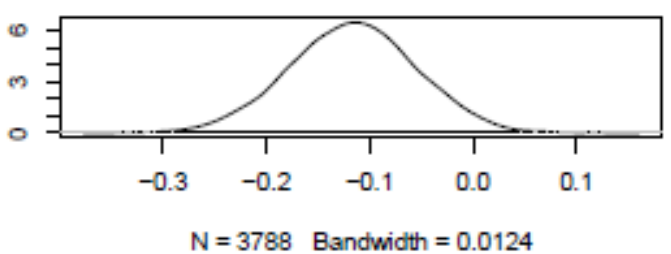

Density of bme:think

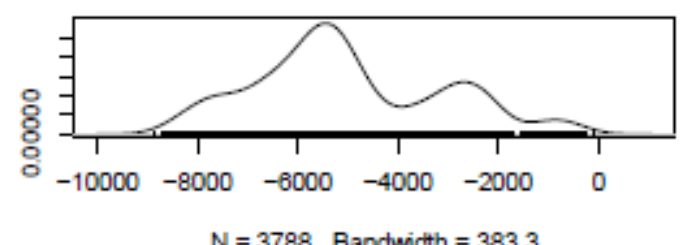

Density of time:think

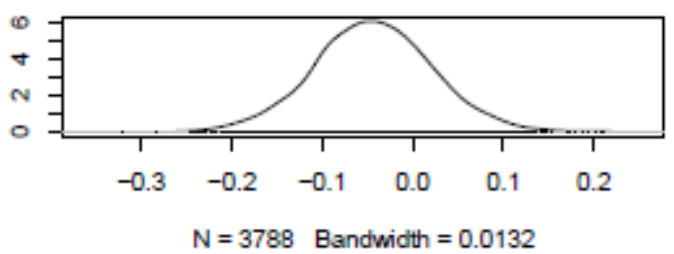


Trace of bme:attitude

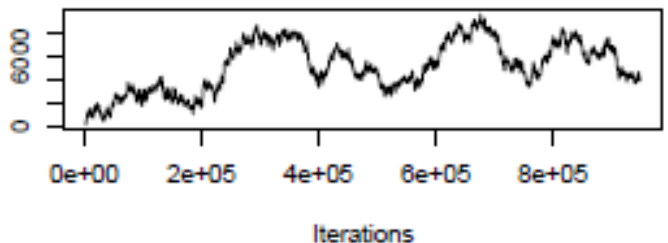

Trace of time:attitude

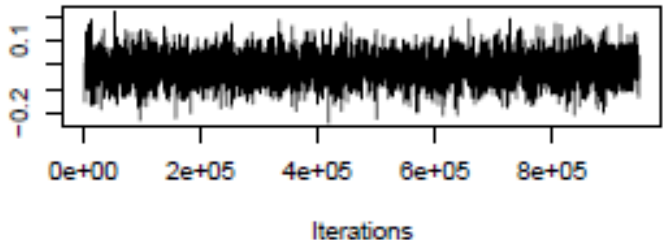

Trace of bme:change

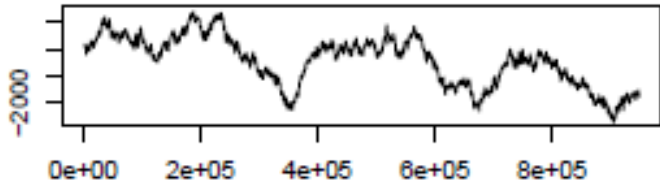

Iterations

Trace of time:change

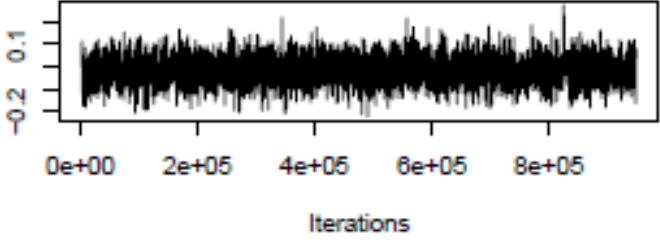

Trace of bme:time:live

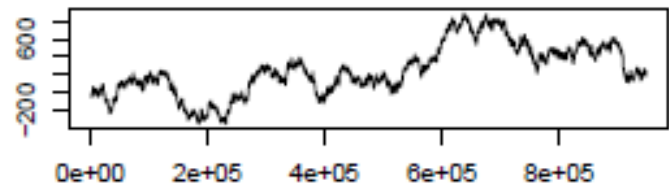

Iterations

Trace of bme:time:relation

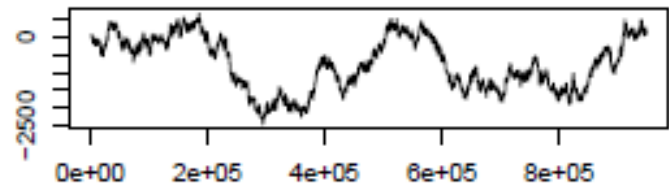

Iterations
Density of bme:attitude

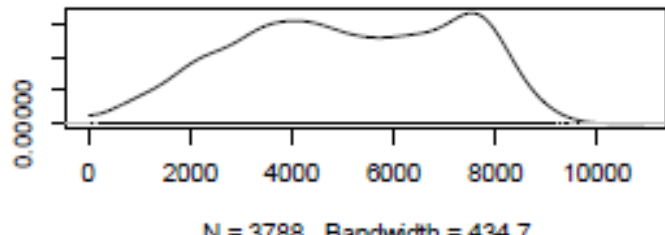

Density of time:attitude

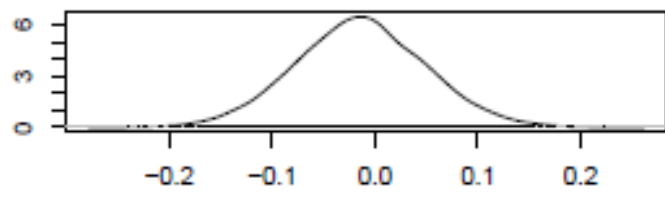

$\mathrm{N}=3788$ Bandwidth $=0.0128$

Density of bme:change

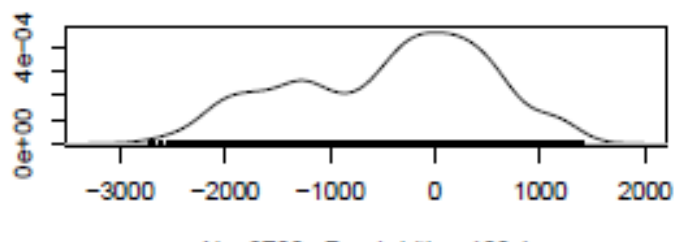

$\mathrm{N}=3788$ Bandwidth $=189.1$

Density of time:change

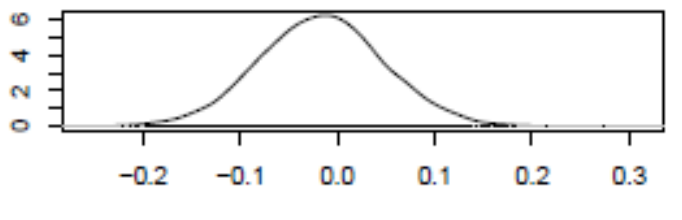

$\mathrm{N}=3788$ Bandwidth $=0.01283$

Density of bme:time:live

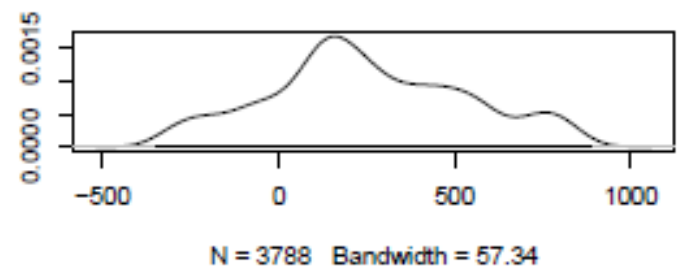

Density of bme:time:relation

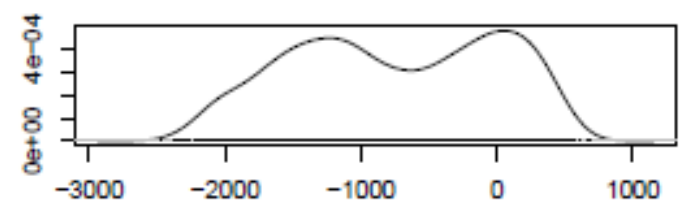

$\mathrm{N}=3788$ Bandwidth $=153.8$ 
Trace of bme:time:ete

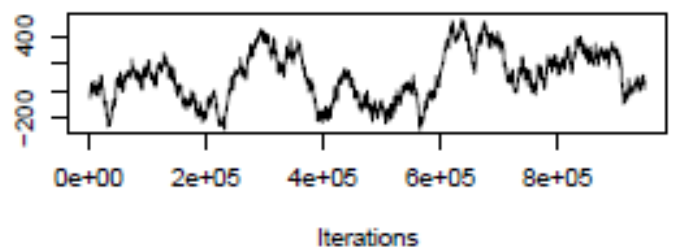

Trace of bme:time:where

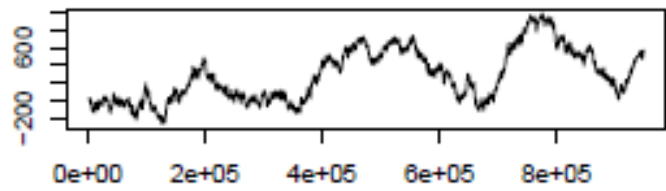

Iterations

Trace of bme:time:life

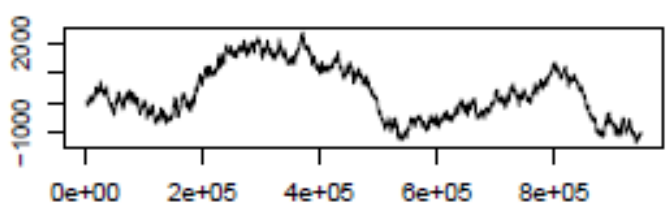

Iterations

Trace of bme:time:drugs

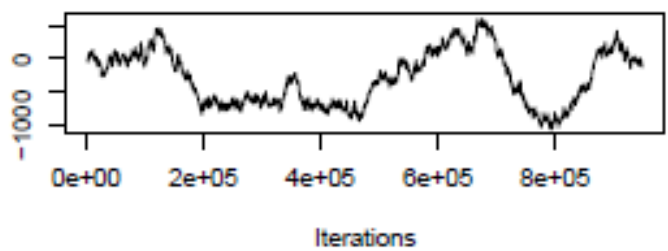

Trace of bme:time:physical

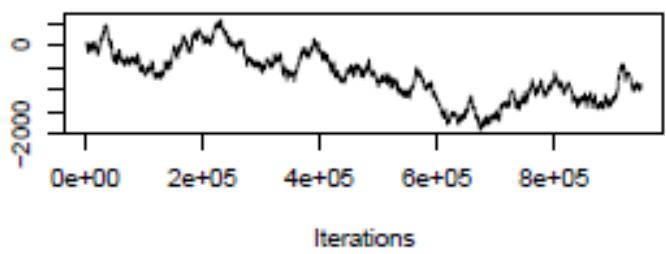

Trace of bme:time:emotion

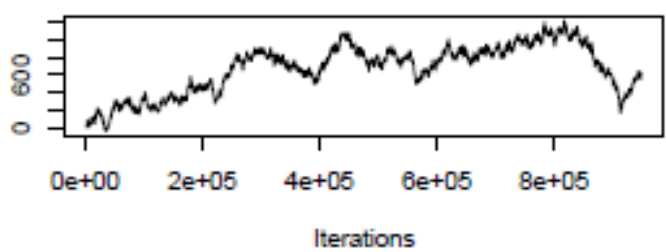

Density of bme:time:ete

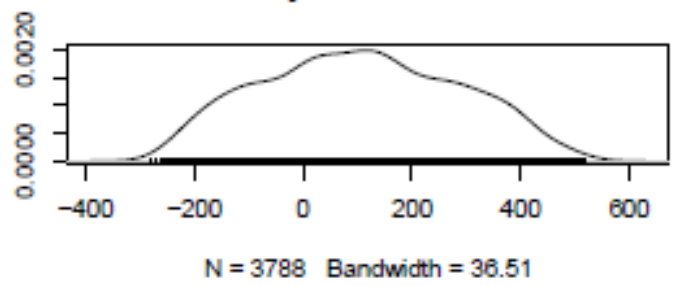

Density of bme:time:where

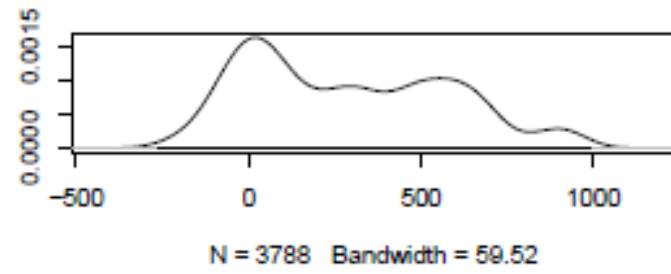

Density of bme:time:life

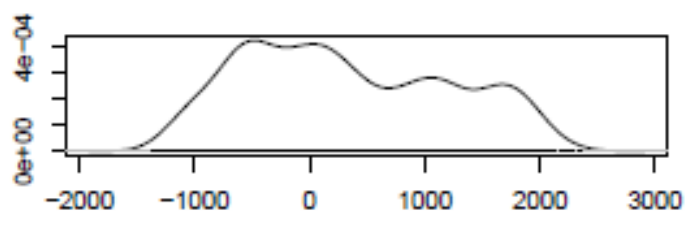

$\mathrm{N}=3788$ Bandwidth $=183$

Density of bme:time:drugs

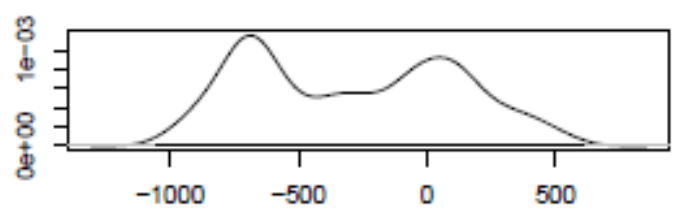

$\mathrm{N}=3788$ Bandwidth $=82.89$

Density of bme:time:physical

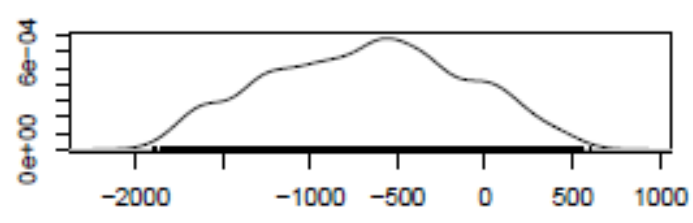

$\mathrm{N}=3788$ Bandwicth $=112.4$

Density of bme:time:emotion

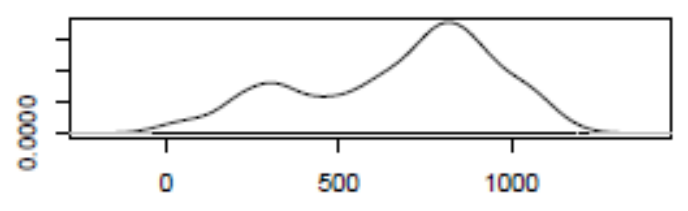

$\mathrm{N}=3788$ Bandwidth $=\mathbf{5 7 . 0 1}$ 
Trace of bme:time:self

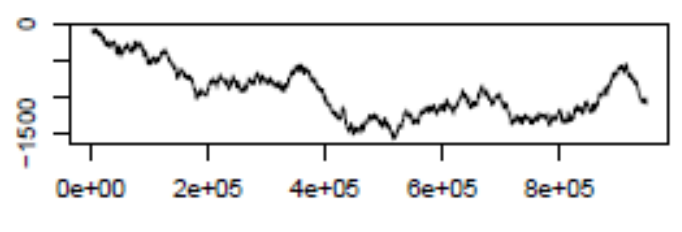

Iterations

Trace of bme:time:think

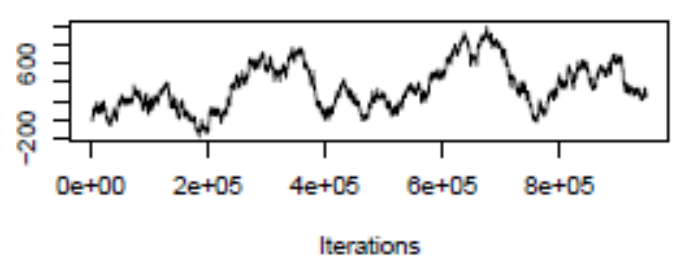

Trace of bme:time:attitude

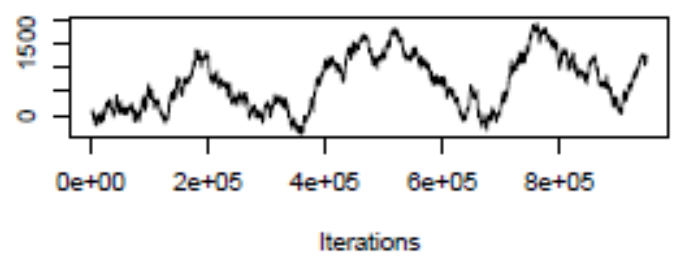

Trace of bme:time:change

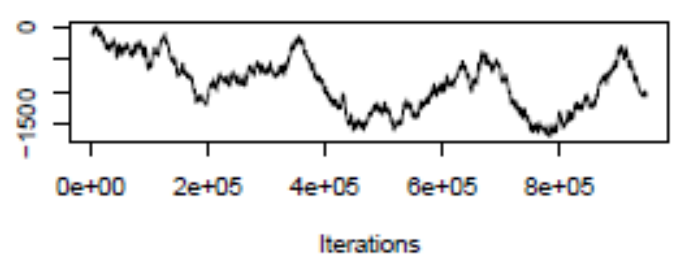

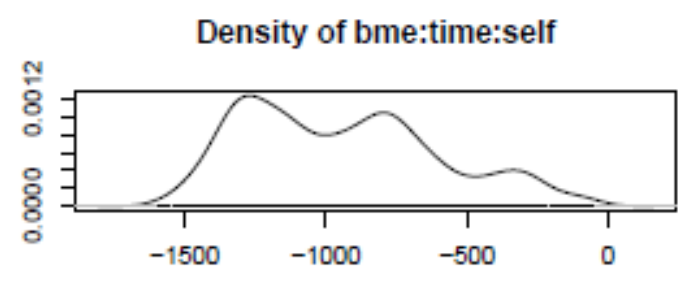

$\mathrm{N}=3788$ Bandwidth $=70.71$

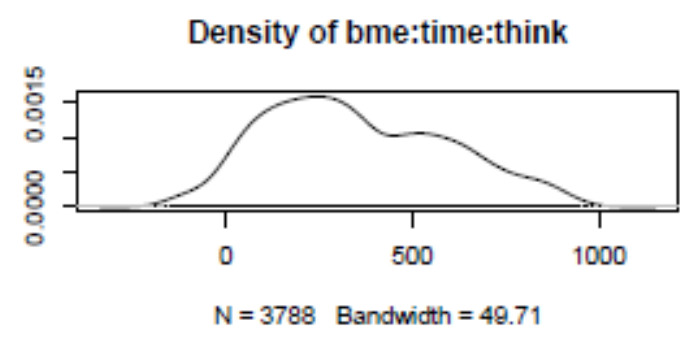

Density of bme:time:attitude

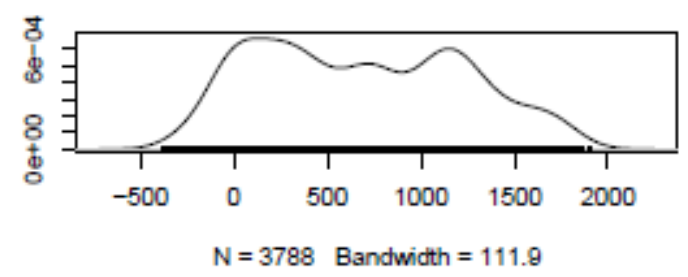

Density of bme:time:change

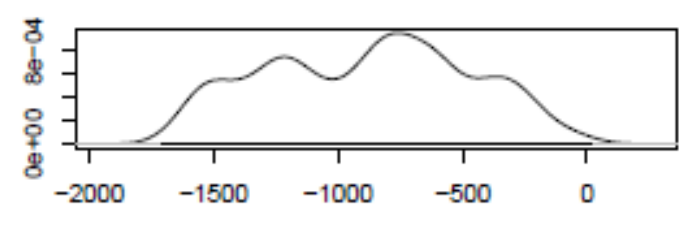

$\mathrm{N}=3788$ Bandwidth $=83.69$ 
$\underline{\text { Random Effects }}$

Trace of time

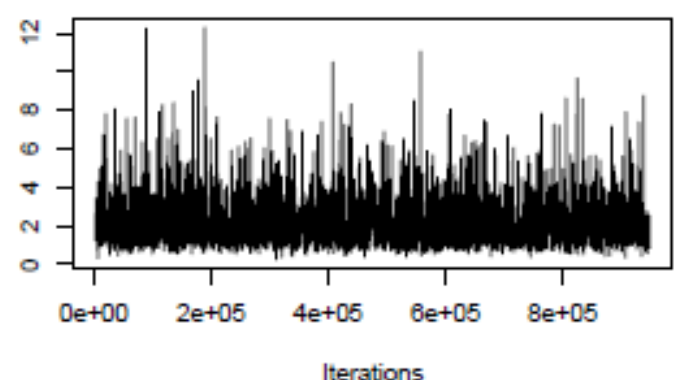

Trace of Research.ID

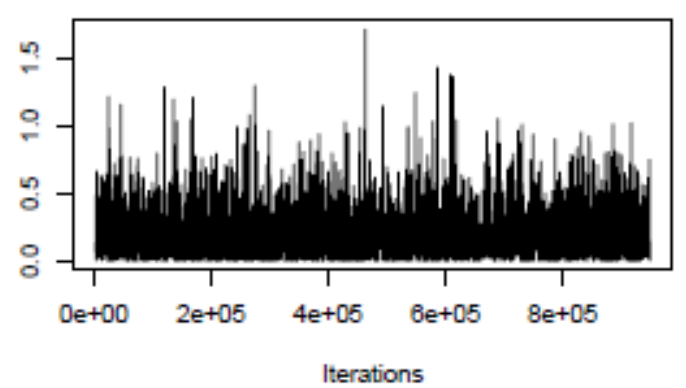

Density of time

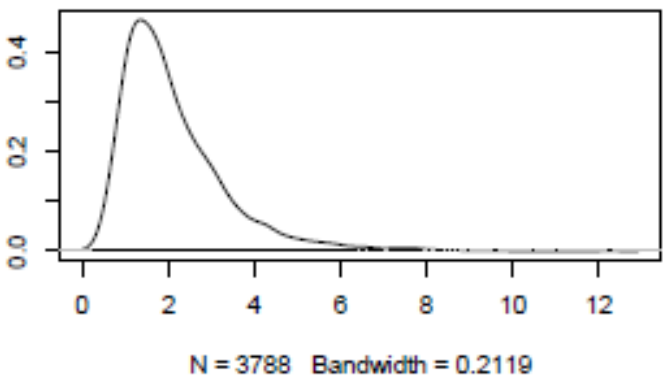

Density of Research.ID

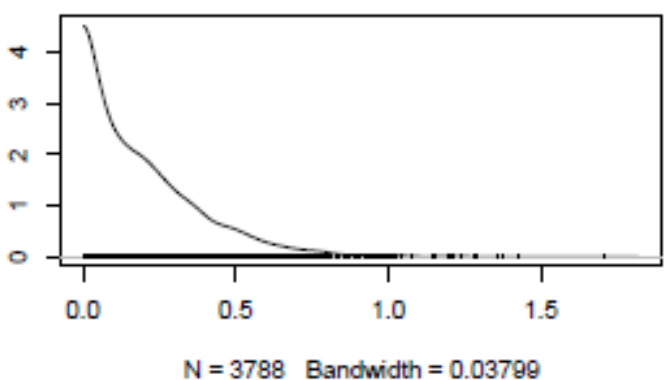


Model 1.4 - Basic Model + Care (Table 5.11)

Bayesian Model (Bm1_ch)

\section{\# Define the model}

Bm1 ch <- MCMCglmm(FO.bin careExp + live + relation + ete + where + life + drugs + physical + emotion + self + think + attitude + change + time, random= time+Research.ID, data=data, family="ordinal", prior=prior2, nitt $=45000$, thin=10, burnin=3000)

\section{\# Checks for suitable convergence}
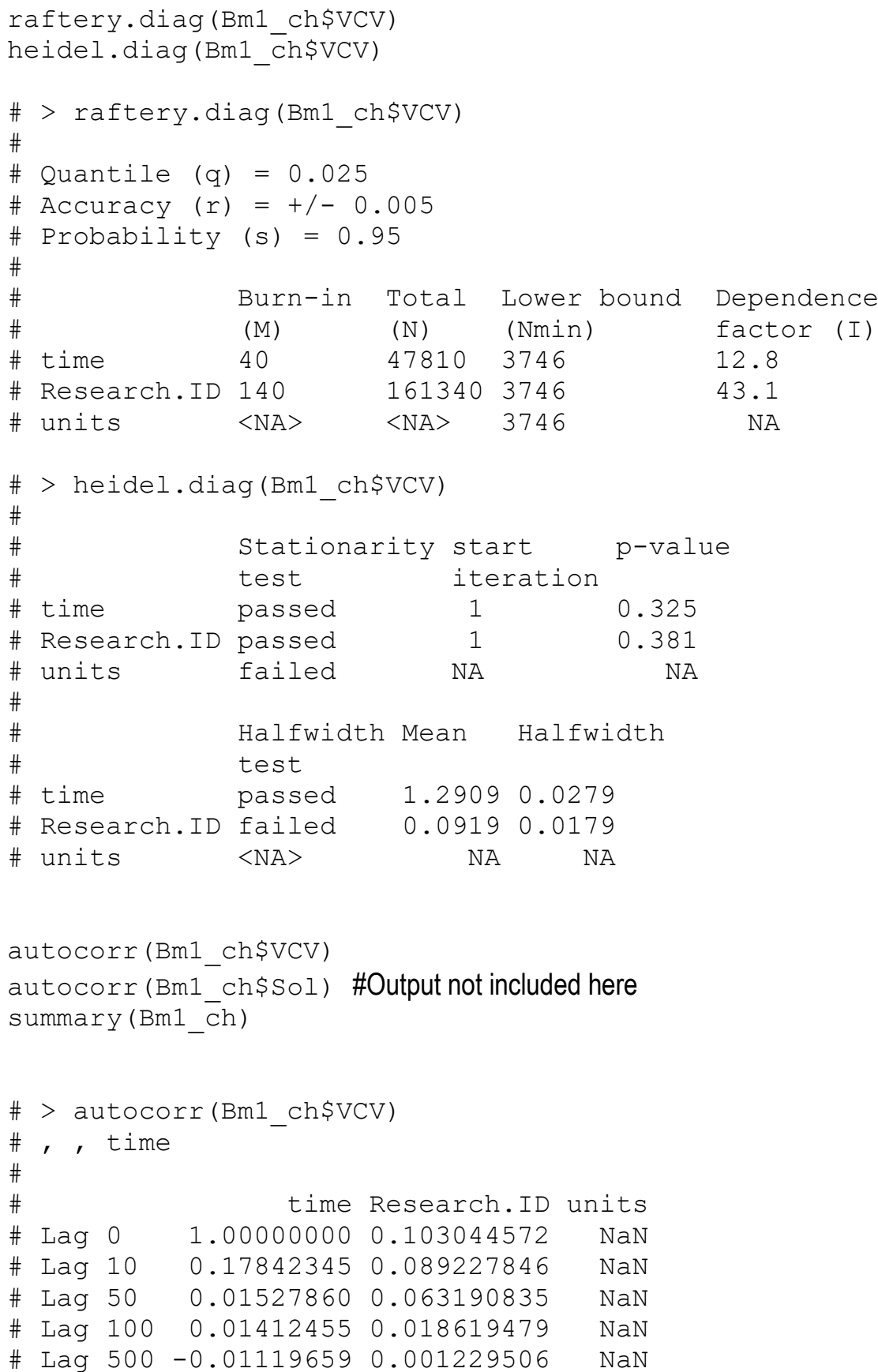


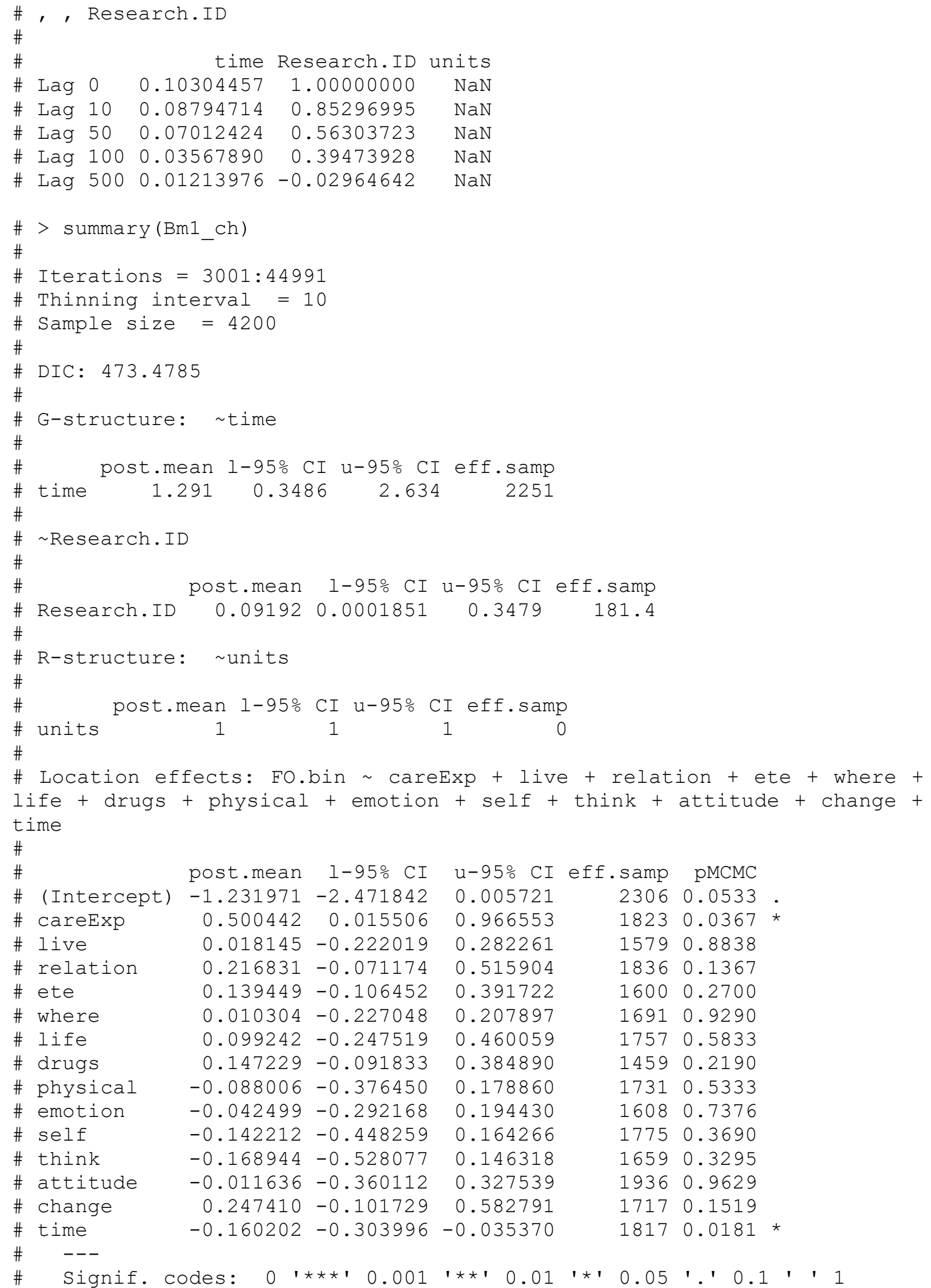




\section{Frequentist Model (m1_ch)}

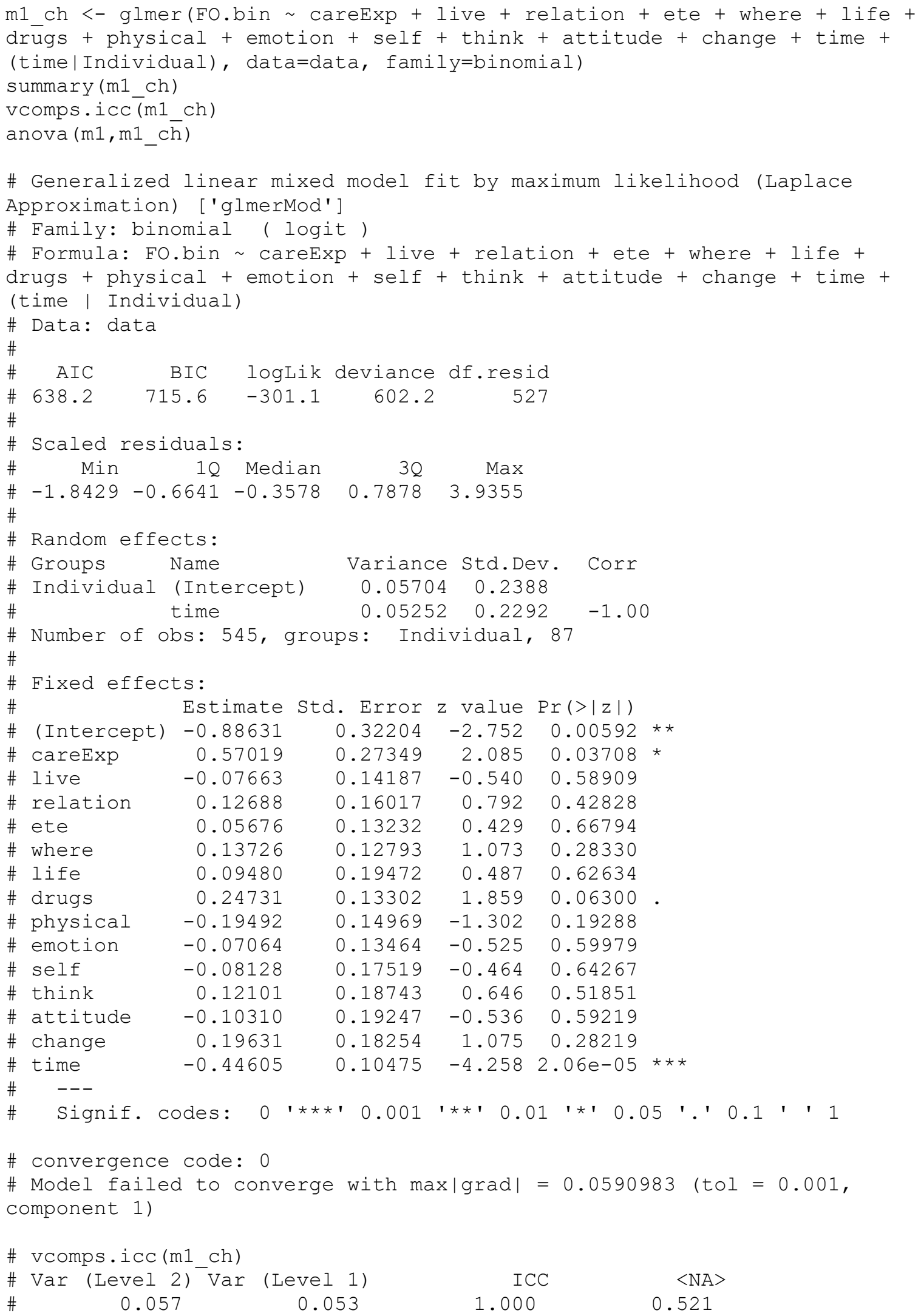




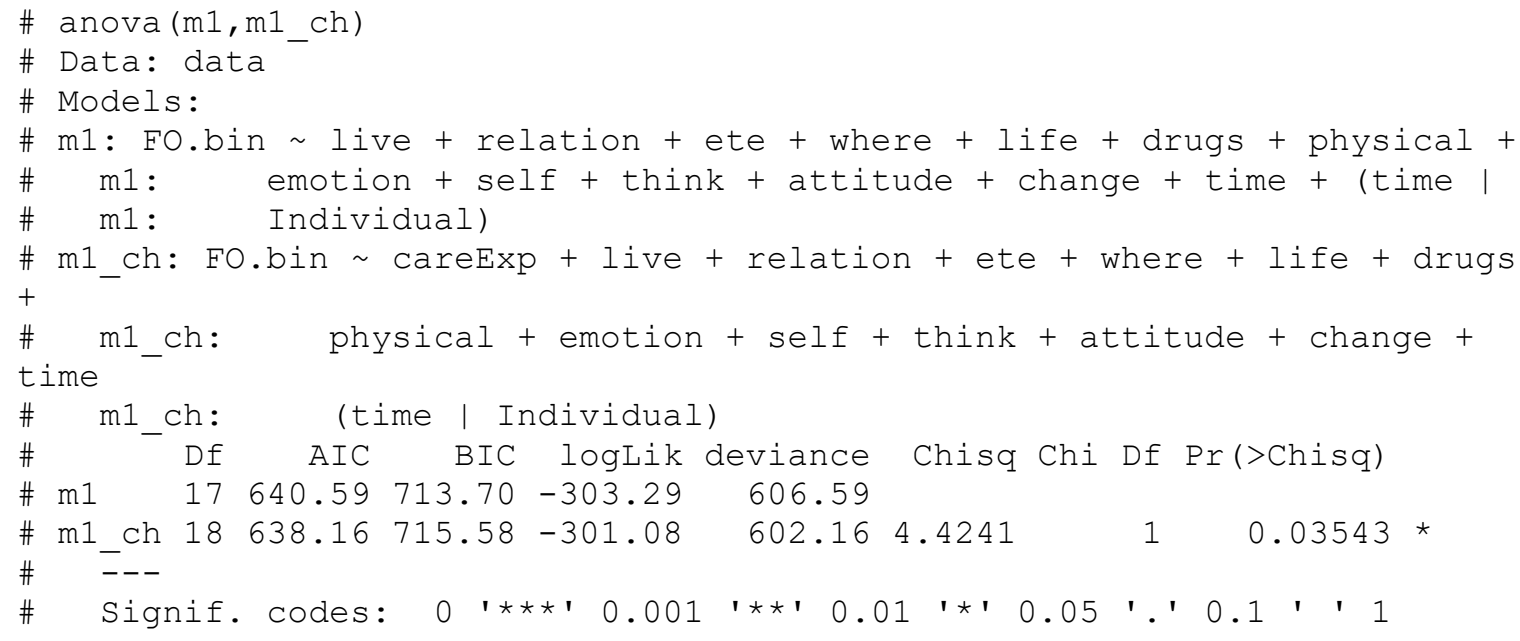


Dynamic Model involving Care (Table 5.12)

Bayesian Model (BDm2_ch)

\section{\#\# Define the model}

BDm2 ch <- MCMCglmm(FO.bin careExp*time*live + careExp*time*relation + careExp*time*ete + careExp*time*where + careExp*time*life + careExp*time*drugs + careExp*time*physical + careExp*time*emotion + careExp*time*self + careExp*time*think + careExp*time*attitude + careExp*time*change, random= time+Research. ID, data=data, family="ordinal", prior=priorD, slice=TRUE, nitt=610000, thin=50, burnin=3000)

\section{\# Checks for suitable convergence}

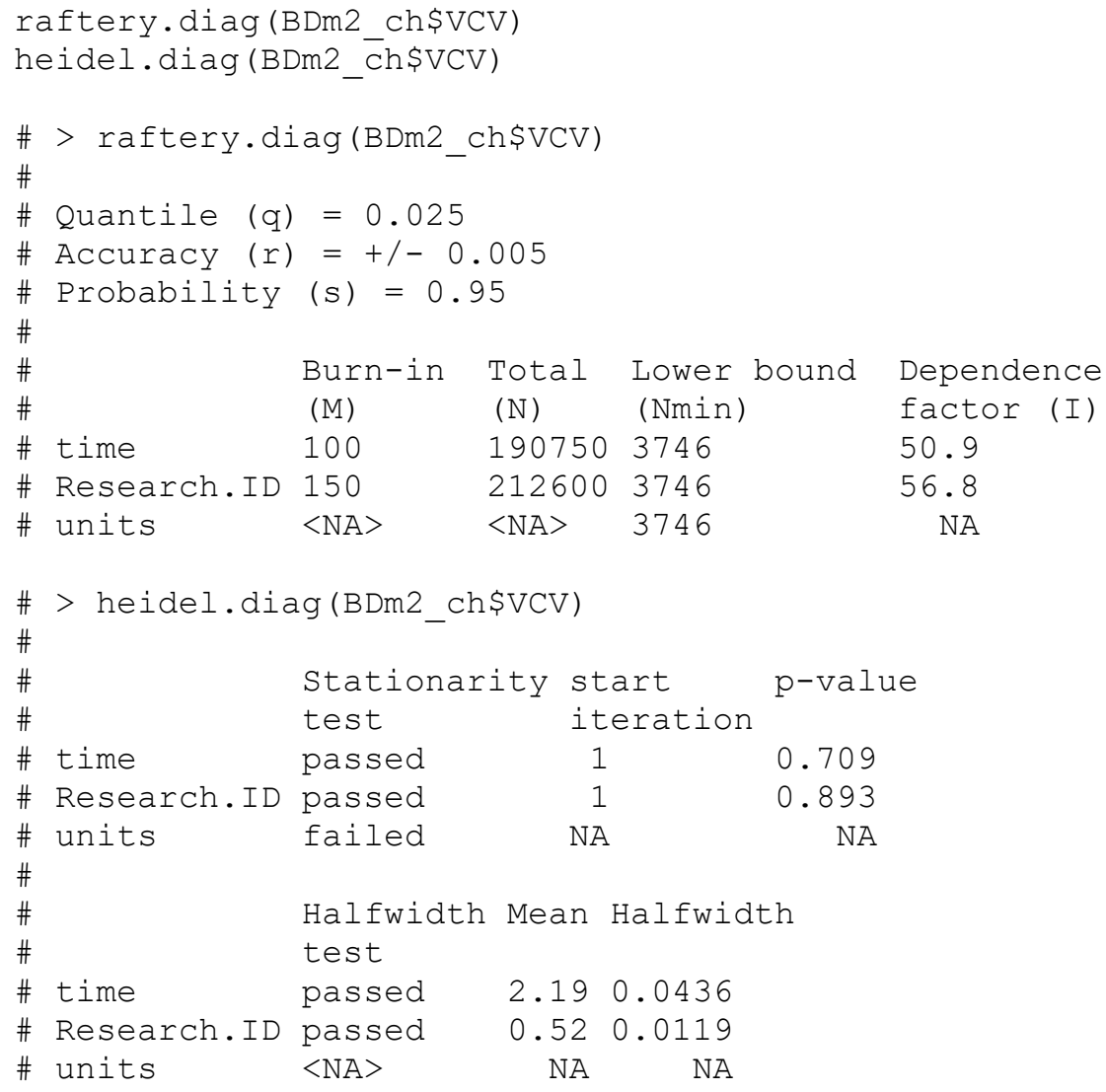




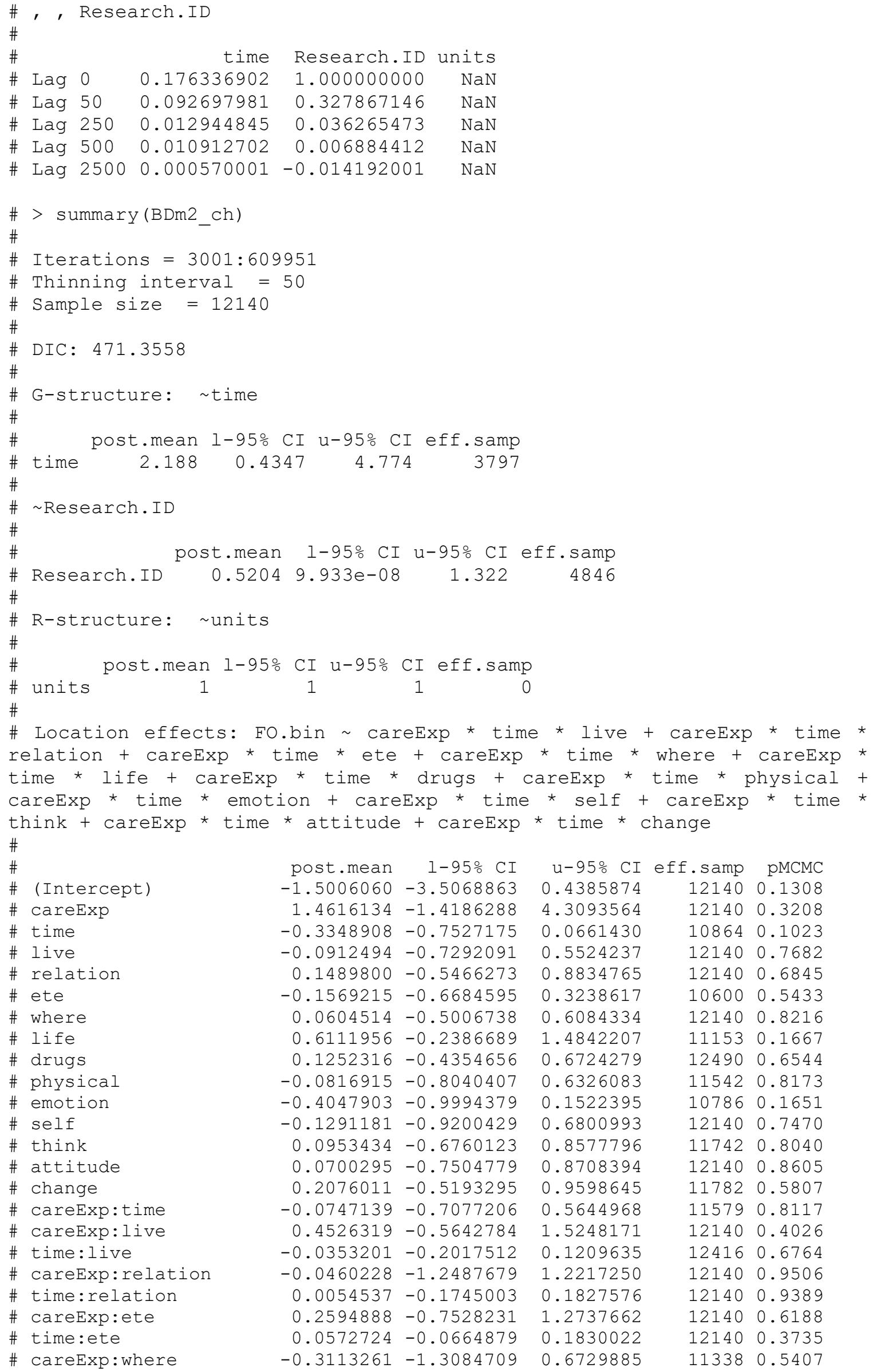

$\begin{array}{rrrrr}\text { post.mean } & 1-95 \% \text { CI } & \text { u-95\% CI } & \text { eff.samp } & \text { pMCMC } \\ -1.5006060 & -3.5068863 & 0.4385874 & 12140 & 0.1308 \\ 1.4616134 & -1.4186288 & 4.3093564 & 12140 & 0.3208 \\ -0.3348908 & -0.7527175 & 0.0661430 & 10864 & 0.1023 \\ -0.0912494 & -0.7292091 & 0.5524237 & 12140 & 0.7682 \\ 0.1489800 & -0.5466273 & 0.8834765 & 12140 & 0.6845 \\ -0.1569215 & -0.6684595 & 0.3238617 & 10600 & 0.5433 \\ 0.0604514 & -0.5006738 & 0.6084334 & 12140 & 0.8216 \\ 0.6111956 & -0.2386689 & 1.4842207 & 11153 & 0.1667 \\ 0.1252316 & -0.4354656 & 0.6724279 & 12490 & 0.6544 \\ -0.0816915 & -0.8040407 & 0.6326083 & 11542 & 0.8173 \\ -0.4047903 & -0.9994379 & 0.1522395 & 10786 & 0.1651 \\ -0.1291181 & -0.9200429 & 0.6800993 & 12140 & 0.7470 \\ 0.0953434 & -0.6760123 & 0.8577796 & 11742 & 0.8040 \\ 0.0700295 & -0.7504779 & 0.8708394 & 12140 & 0.8605 \\ 0.2076011 & -0.5193295 & 0.9598645 & 11782 & 0.5807 \\ -0.0747139 & -0.7077206 & 0.5644968 & 11579 & 0.8117 \\ 0.4526319 & -0.5642784 & 1.5248171 & 12140 & 0.4026 \\ -0.0353201 & -0.2017512 & 0.1209635 & 12416 & 0.6764 \\ -0.0460228 & -1.2487679 & 1.2217250 & 12140 & 0.9506 \\ 0.0054537 & -0.1745003 & 0.1827576 & 12140 & 0.9389 \\ 0.2594888 & -0.7528231 & 1.2737662 & 12140 & 0.6188 \\ 0.0572724 & -0.0664879 & 0.1830022 & 12140 & 0.3735 \\ -0.3113261 & -1.3084709 & 0.6729885 & 11338 & 0.5407\end{array}$




\begin{tabular}{|c|c|c|c|c|c|c|}
\hline & time:where & -0.0538686 & -0.1914446 & 0.0765889 & 11230 & 0.4288 \\
\hline & careExp:life & -0.6325248 & -2.1092239 & 0.8256715 & 12406 & 0.3923 \\
\hline & time:life & -0.0051764 & -0.1871814 & 0.1923293 & 11861 & 0.9540 \\
\hline & careExp:drugs & 0.3260131 & -0.6140927 & 1.2670934 & 12534 & 0.4932 \\
\hline & time:drugs & 0.0363598 & -0.1003575 & 0.1718449 & 11731 & 0.6097 \\
\hline & careExp:physical & -0.8900624 & -2.1145396 & 0.3651294 & 10770 & 0.1590 \\
\hline & time:physical & -0.0423870 & -0.2686424 & 0.1790588 & 11347 & 0.7147 \\
\hline & careExp:emotion & 0.3923488 & -0.6489381 & 1.4002455 & 11614 & 0.4549 \\
\hline & time:emotion & 0.0528995 & -0.0889530 & 0.1980607 & 11667 & 0.4723 \\
\hline & careExp:self & 0.8715772 & -0.5587058 & 2.3038163 & 11113 & 0.2329 \\
\hline & time:self & 0.0719019 & -0.1046936 & 0.2634116 & 12140 & 0.4445 \\
\hline & careExp:think & -0.8408783 & -2.3139680 & 0.6180410 & 11816 & 0.2603 \\
\hline & time:think & -0.0121284 & -0.2074019 & 0.1795310 & 11074 & 0.9056 \\
\hline \# & careExp:attitude & -0.1176196 & -1.4198052 & 1.2722378 & 1550 & 0.8639 \\
\hline & time:attitude & -0.0945612 & -0.2964184 & 0.1043443 & 12140 & 0.3677 \\
\hline & careExp: change & 0.1257902 & -1.3930247 & 1.5208195 & 11563 & 0.8524 \\
\hline & time: change & 0.0266534 & -0.1733894 & 0.2363857 & 12140 & 0.806 \\
\hline & careExp:time:live & 0.0210127 & -0.1987465 & 0.2485695 & 11663 & 0.8619 \\
\hline & careExp:time:relation & -0.0002072 & -0.2833647 & 0.2782756 & 11624 & 0.9911 \\
\hline & careExp:time:ete & 0.0044299 & -0.1989134 & 0.2156877 & 11565 & 0.9634 \\
\hline & careExp:time:where & 0.1777905 & -0.0152111 & 0.3747486 & 12140 & 0.0697 \\
\hline & careExp:time:life & -0.0828341 & -0.3738983 & 0.2001525 & 11541 & 0.575 \\
\hline & careExp:time:drugs & -0.0723851 & -0.2913360 & 0.1429795 & 11502 & 0.511 \\
\hline & careExp:time:physical & 0.2420940 & -0.0631206 & 0.5322557 & 11487 & 0.1119 \\
\hline & careExp:time:emotion & 0.0402425 & -0.1949566 & 0.2788018 & 12140 & 0.7331 \\
\hline & careExp:time:self & -0.3409189 & -0.6439077 & -0.0449812 & 10560 & 0.021 \\
\hline & careExp:time:think & 0.0847188 & -0.2086670 & 0.3877198 & 10400 & 0.581 \\
\hline & careExp:time:attitude & 0.1298185 & -0.1740126 & 0.4431976 & 11599 & 0.418 \\
\hline & careExp:time:change & -0.0580154 & -0.3539014 & 0.2478403 & 11777 & 0.7025 \\
\hline & & & & & & \\
\hline & codes: 0 & & & & & \\
\hline
\end{tabular}


Trace of the Sampled Output and Density Estimates

Fixed Effects

Trace of (Intercept)

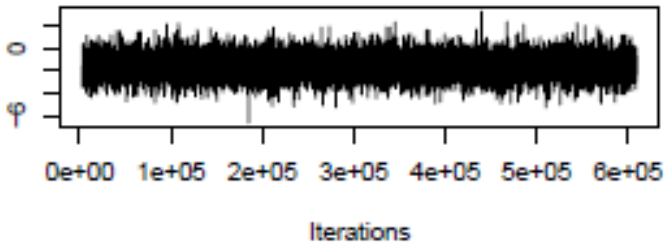

Trace of careExp

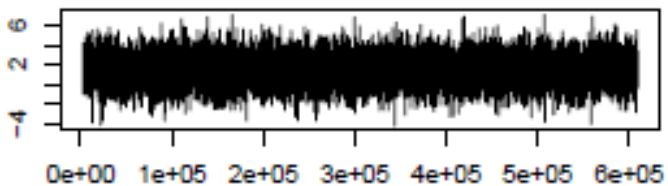

Iterations

Trace of time

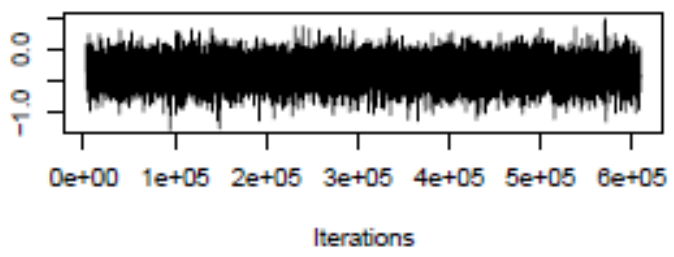

Trace of live

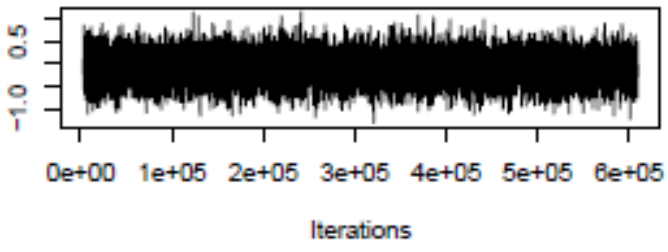

Trace of relation

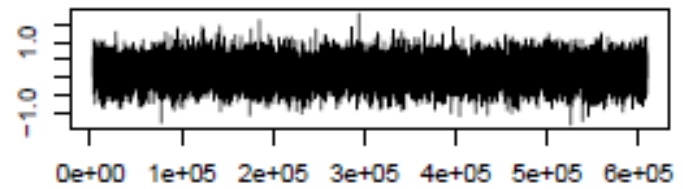

Iterations

Trace of ete

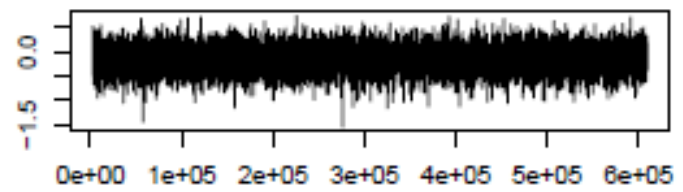

Iterations
Density of (Intercept)

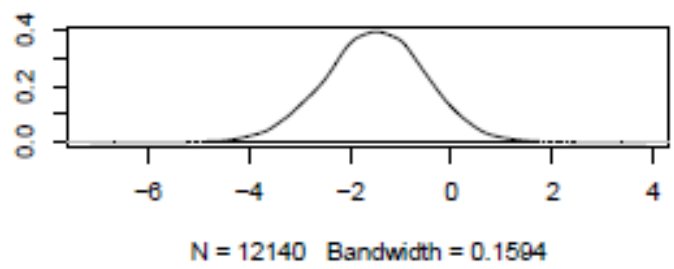

Density of careExp

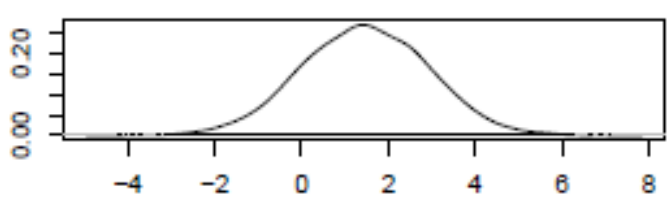

$\mathrm{N}=12140$ Bandwidth $=0.2382$

Density of time

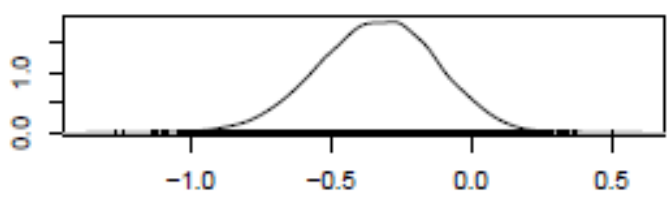

$\mathrm{N}=12140$ Bandwidth $=0.03417$

Density of live

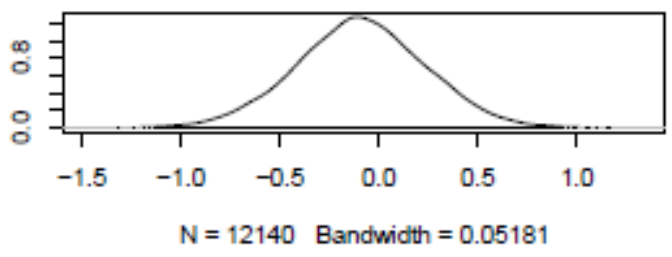

Density of relation

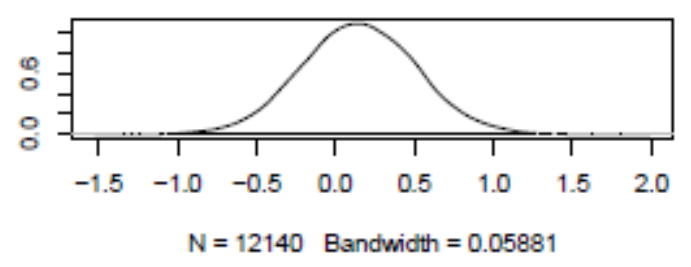

Density of ete

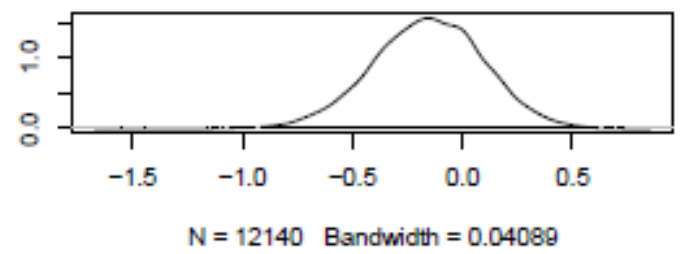


Trace of where

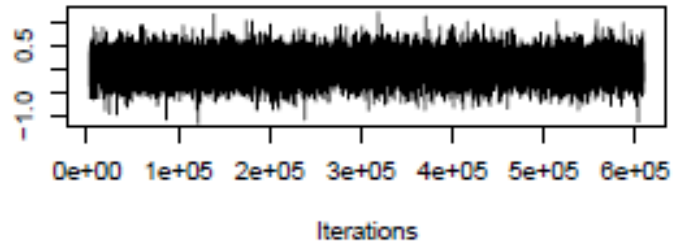

Trace of life

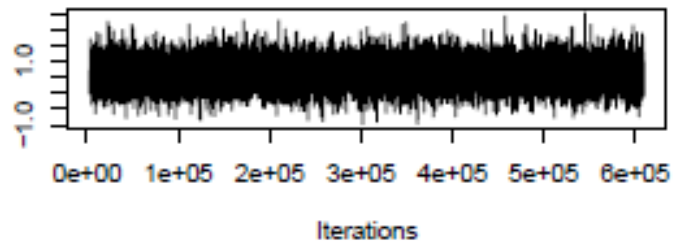

Trace of drugs

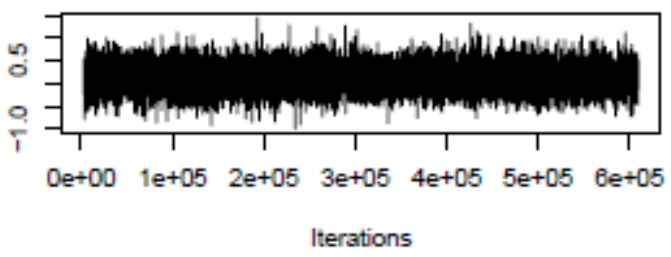

Trace of physical

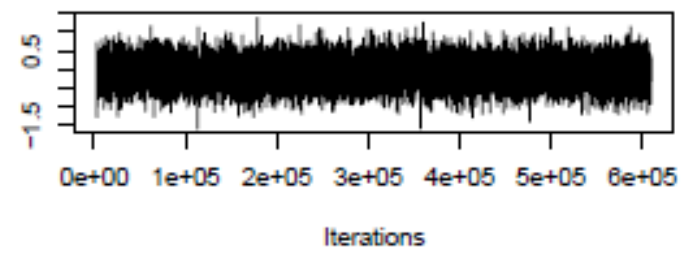

Trace of emotion

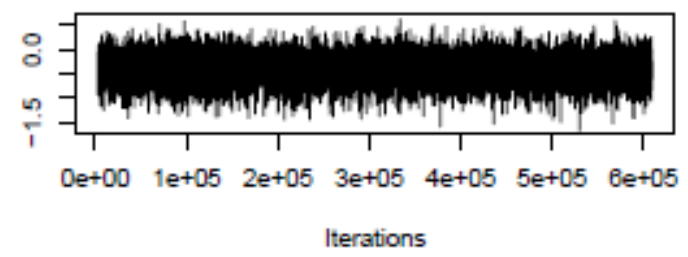

Trace of self

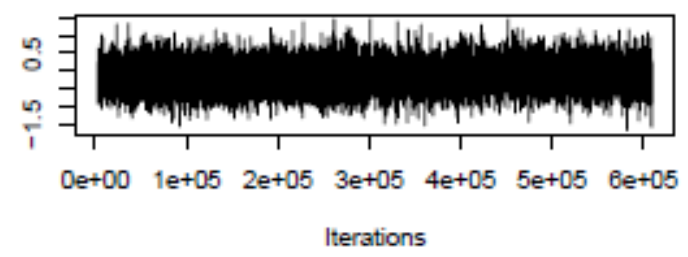

Density of where

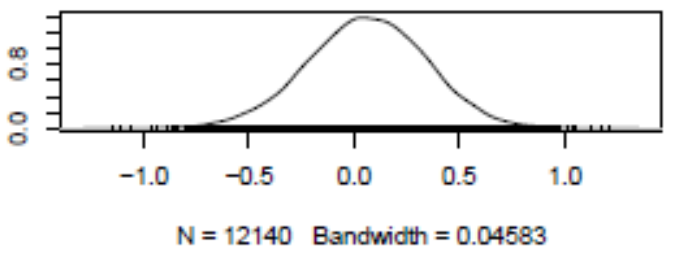

Density of life

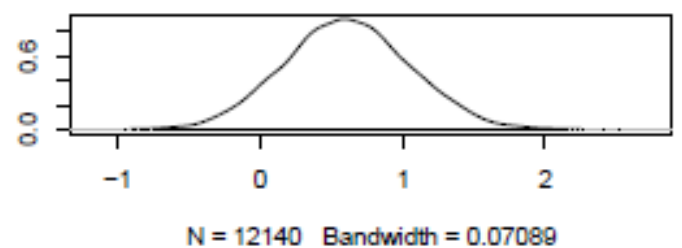

Density of drugs

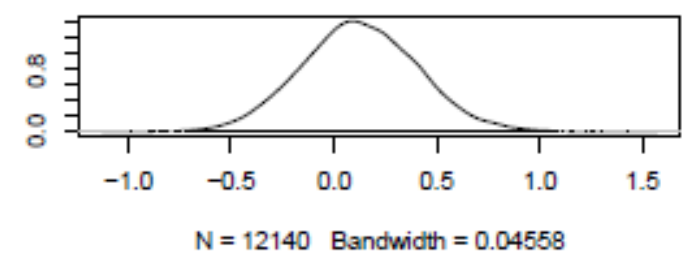

Density of physical

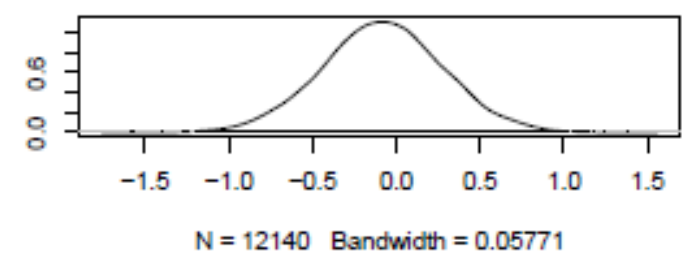

Density of emotion

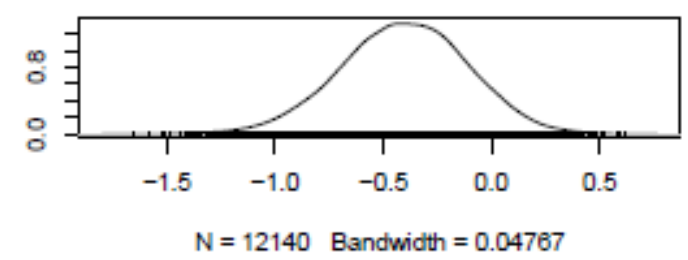

Density of self

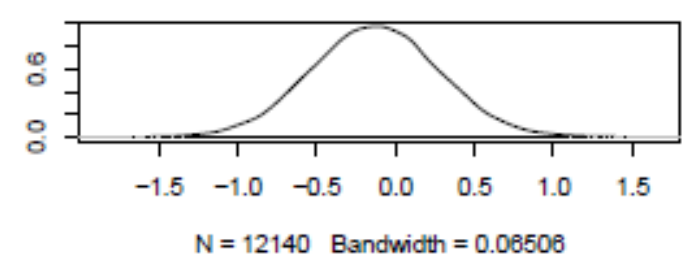


Trace of think

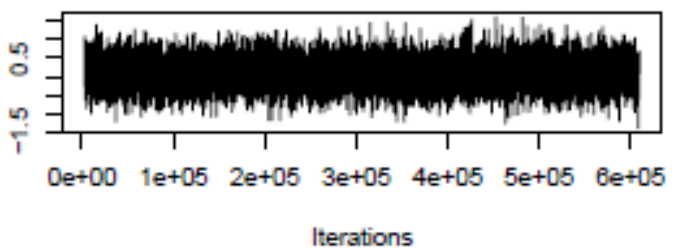

Trace of attitude

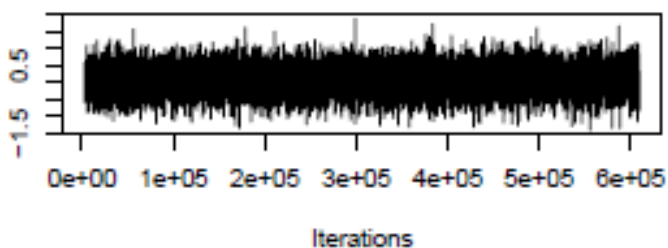

Trace of change

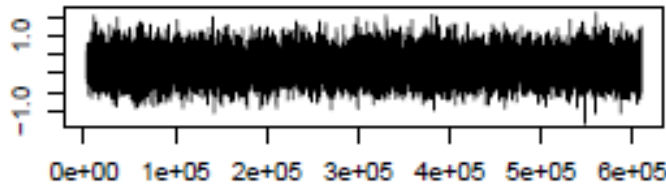

Iterations

Trace of careExp:time

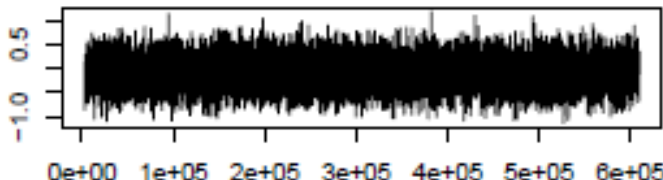

Iterations

Trace of careExp:live

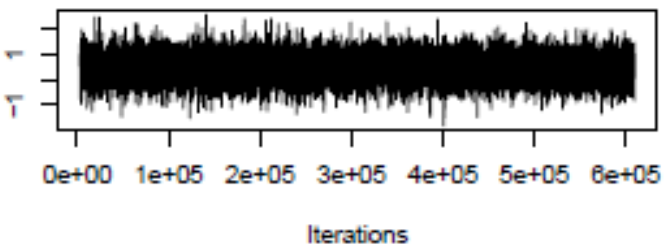

Trace of time:live

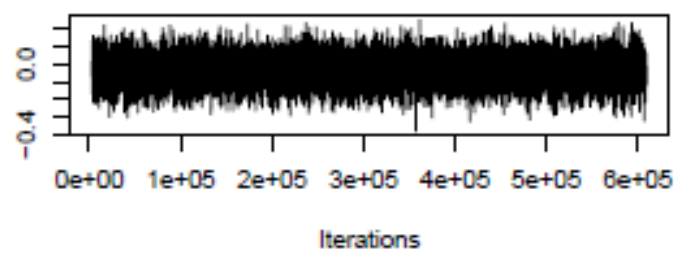

Density of think

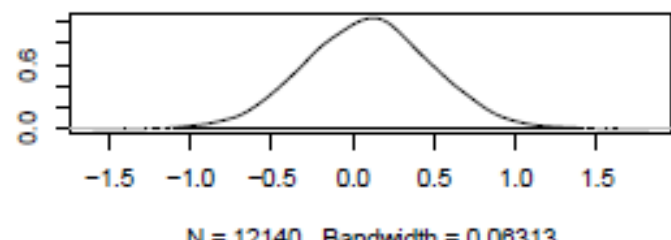

Density of attitude

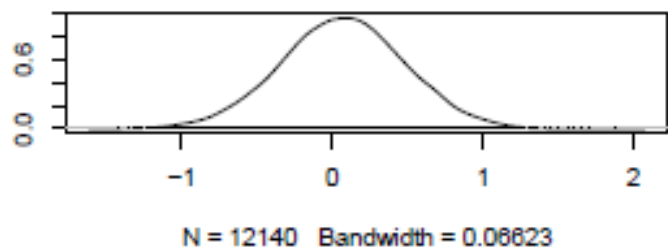

Density of change

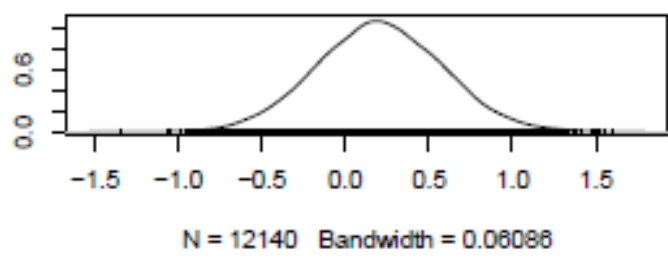

Density of careExp:time

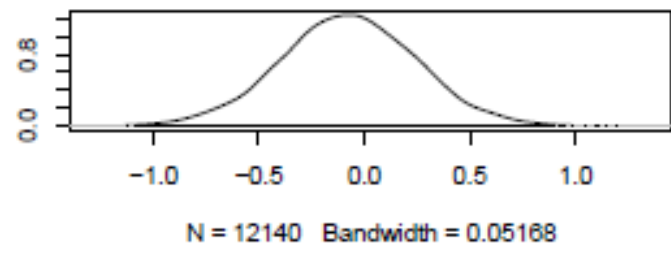

Density of careExp:live

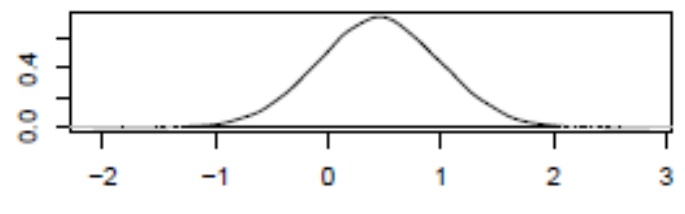

$N=12140$ Bandwidth $=0.08688$

Density of time:live

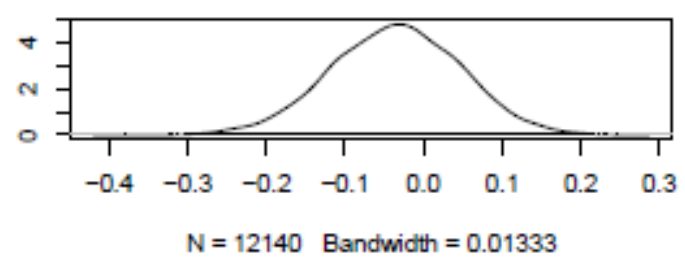


Trace of careExp:relation

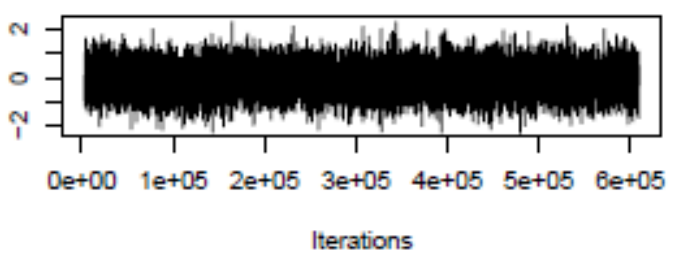

Trace of time:relation

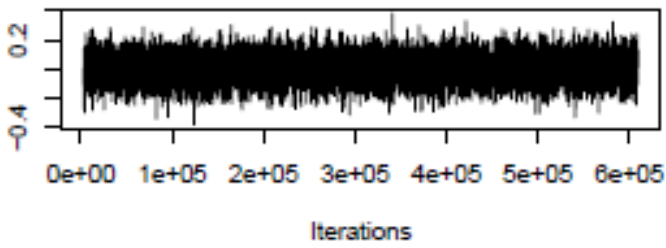

Trace of careExp:ete

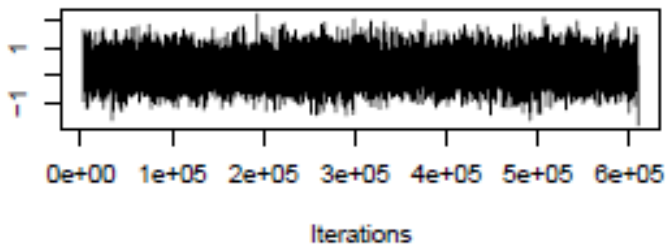

Trace of time:ete

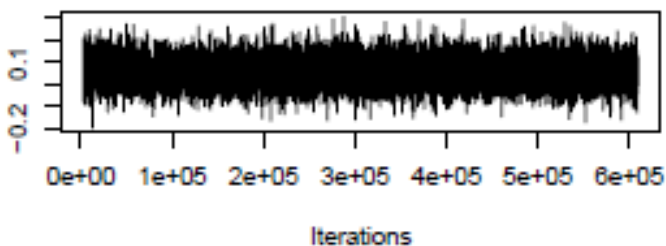

Trace of careExp:where

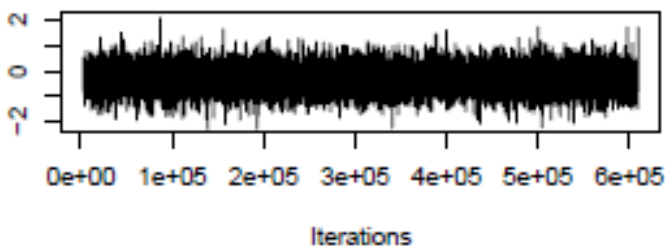

Trace of time:where

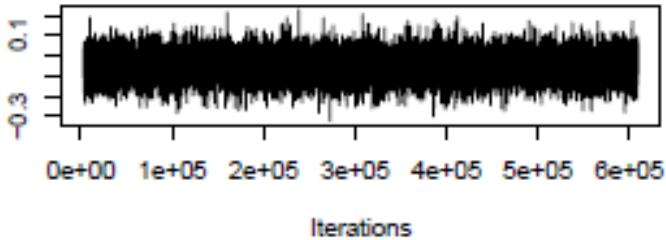

Density of careExp:relation

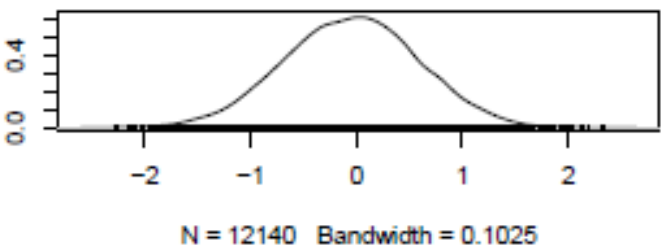

Density of time:relation

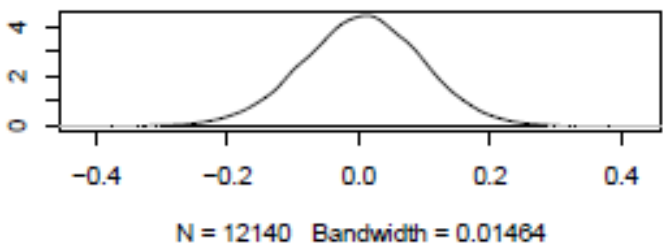

Density of careExp:ete

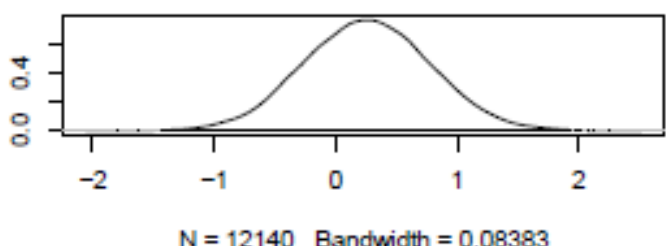

Density of time:ete

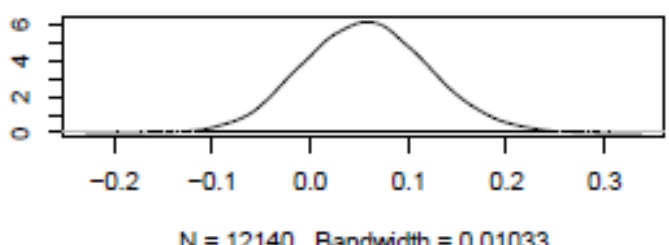

Density of careExp:where

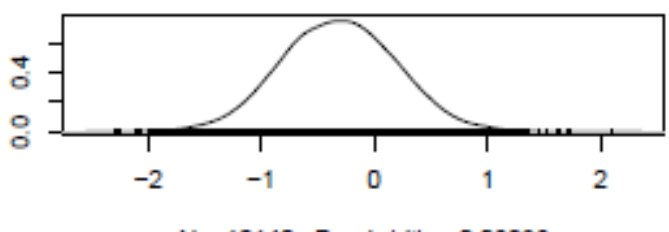

$\mathrm{N}=12140$ Bandwidth $=0.08236$

Density of time:where

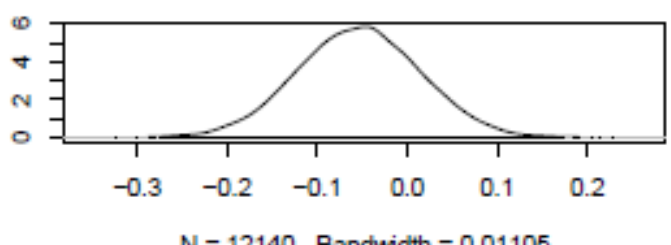

$N=12140$ Bandwidth $=0.01105$ 
Trace of careExp:life

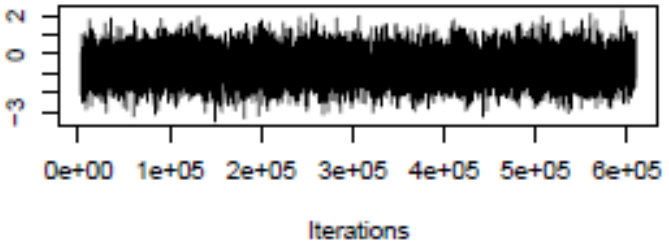

Trace of time:life

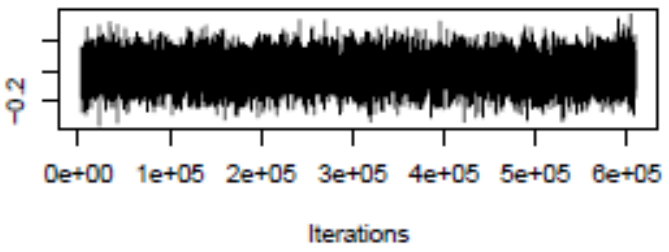

Trace of careExp:drugs

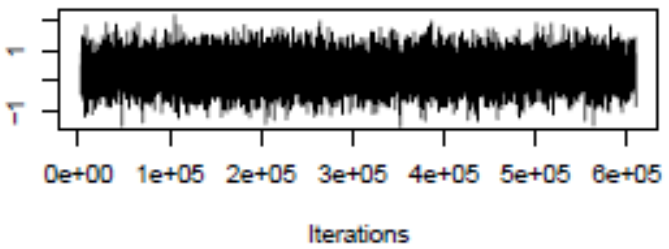

Trace of time:drugs

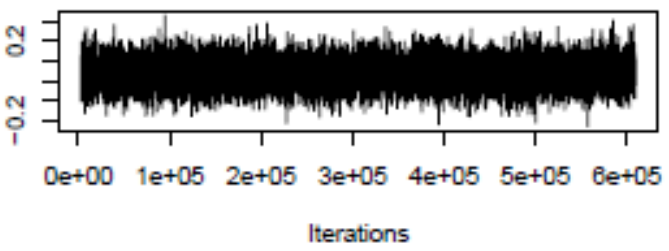

Trace of careExp:physical

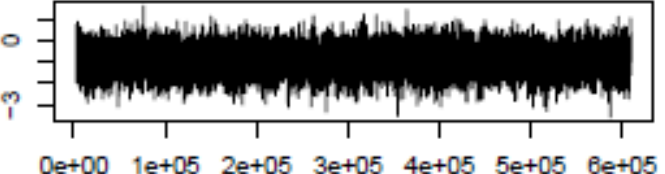

Iterations

Trace of time:physical

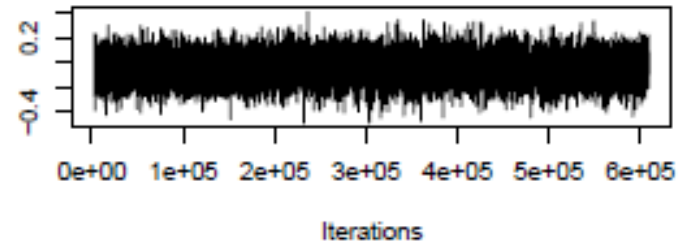

Density of careExp:life

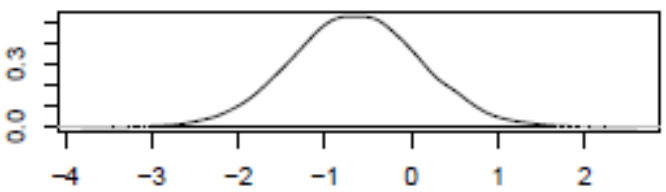

$\mathrm{N}=12140$ Bandwidth $=0.12$

Density of time:life

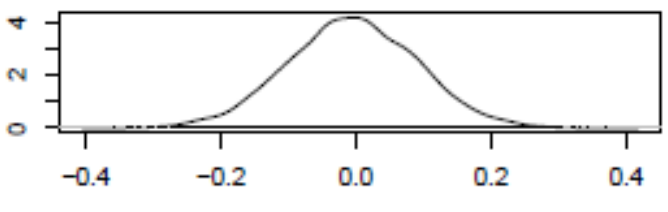

$N=12140$ Bandwidth $=0.01556$

Density of careExp:drugs

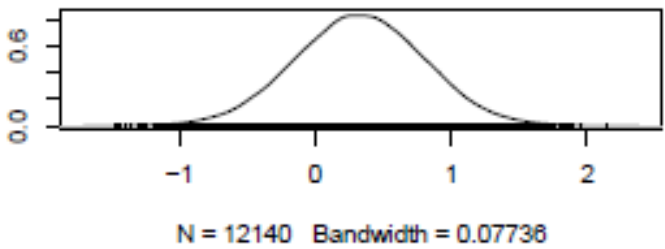

Density of time:drugs

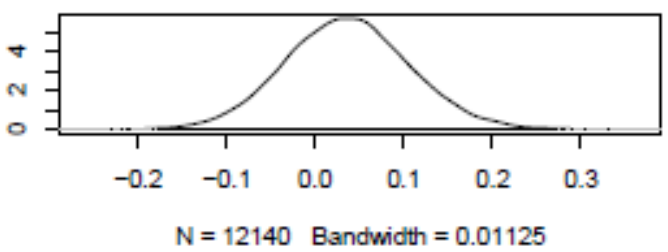

Density of careExp:physical

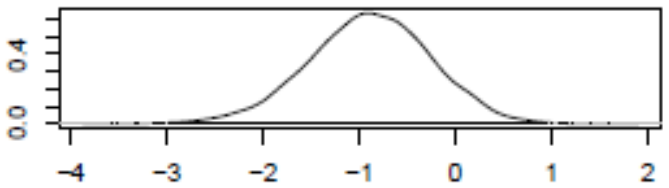

$\mathrm{N}=12140$ Bandwidth $=0.1018$

Density of time:physical

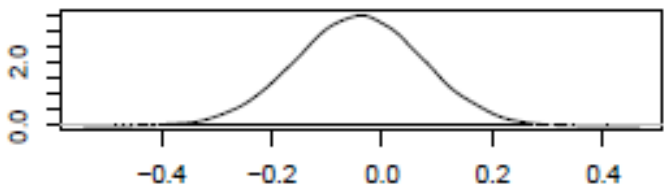

$\mathrm{N}=12140$ Bandwidth $=0.0185$ 
Trace of careExp:emotion

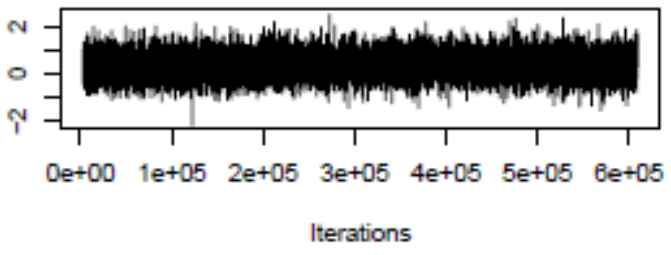

Trace of time:emotion

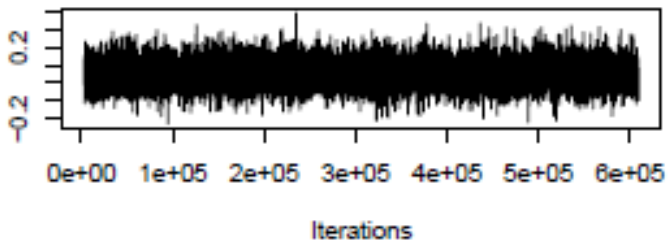

Trace of careExp:self

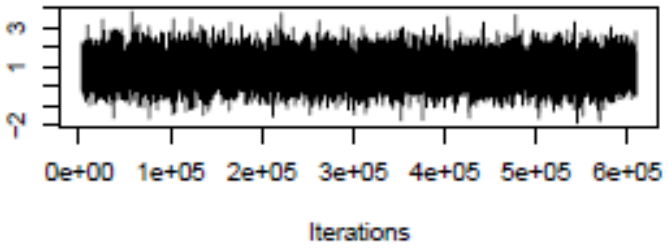

Trace of time:self

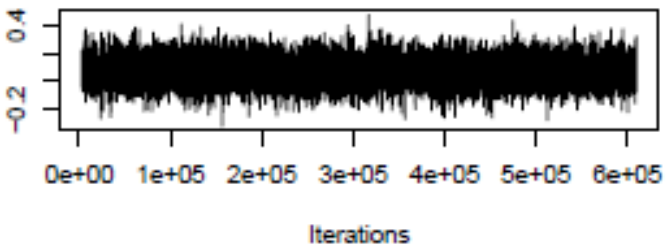

Trace of careExp:think

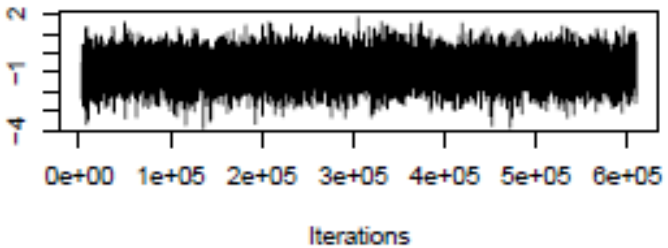

Trace of time:think

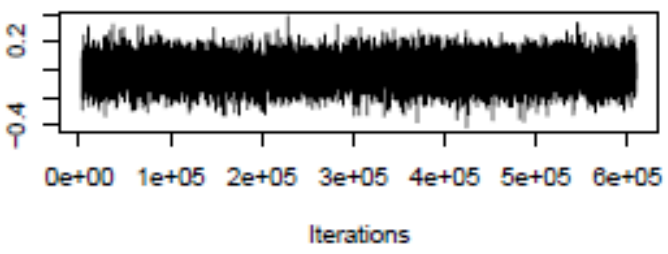

Density of careExp:emotion

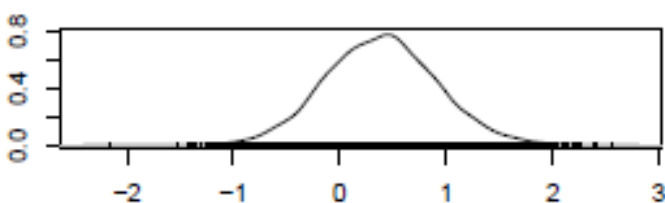

$\mathrm{N}=12140$ Bandwidth $=0.08424$

Density of time:emotion

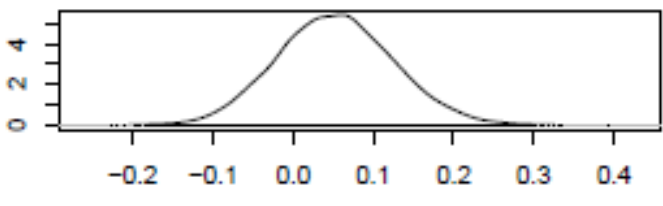

$\mathrm{N}=12140$ Bandwidth $=0.01183$

Density of careExp:self

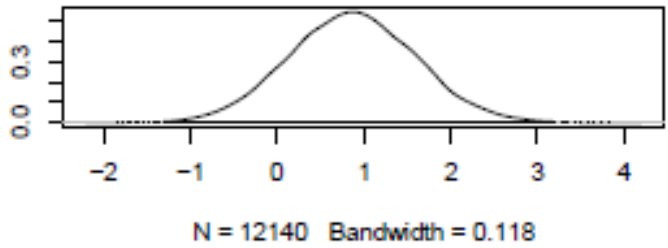

Density of time:self

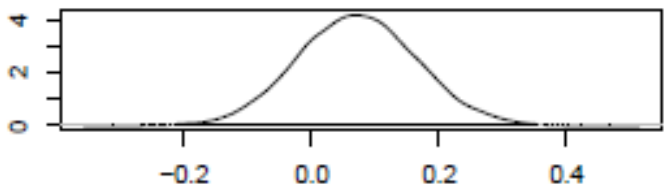

$N=12140$ Bandwidth $=0.01517$

Density of careExp:think

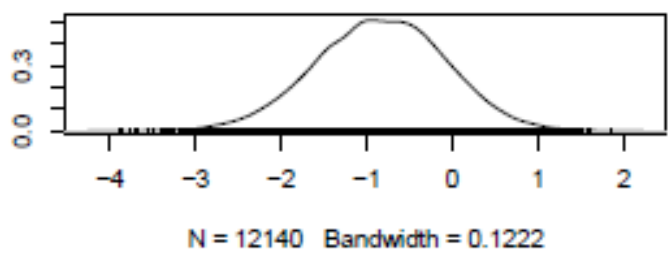

Density of time:think

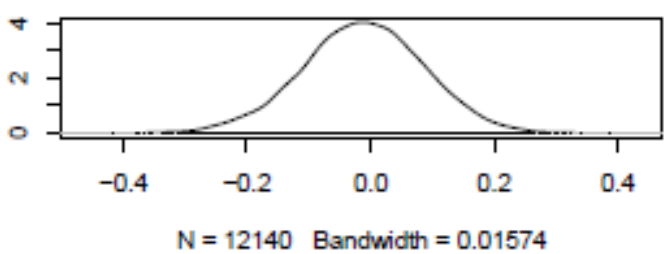


Trace of careExp:attitude

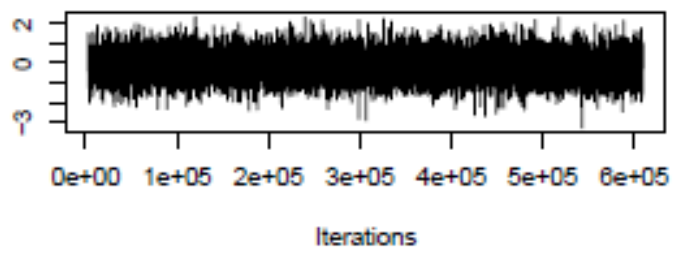

Trace of time:attitude

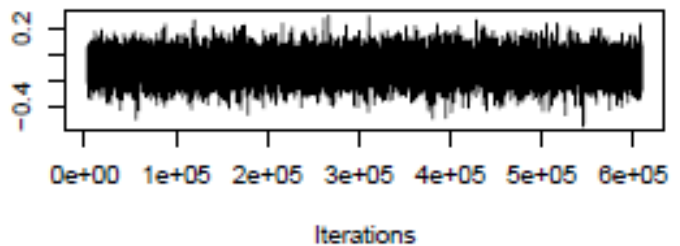

Trace of careExp:change

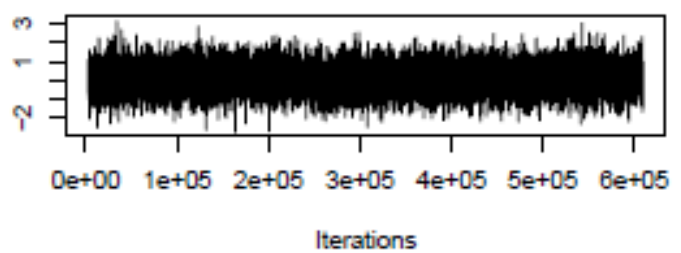

Trace of time:change

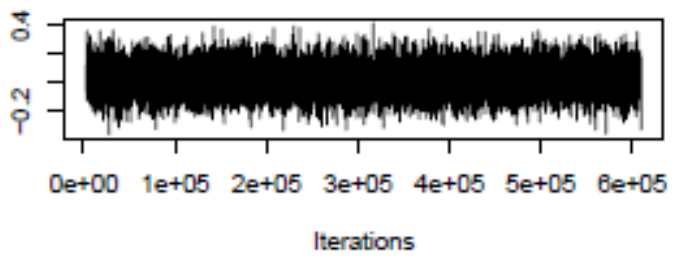

Trace of careExp:time:live

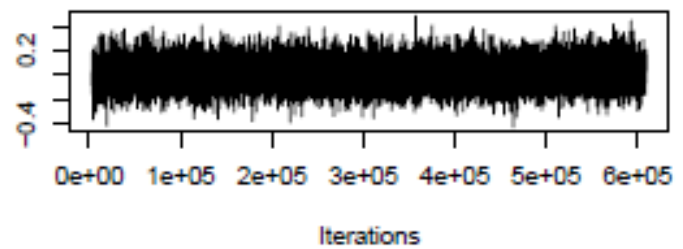

Trace of careExp:time:relation

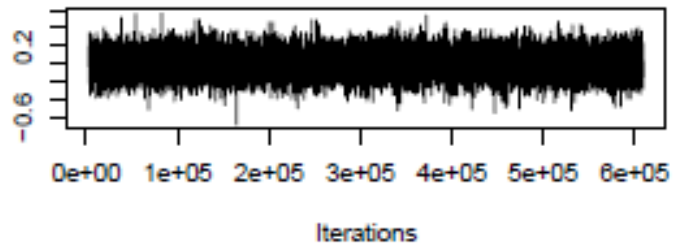

Density of careExp:attitude

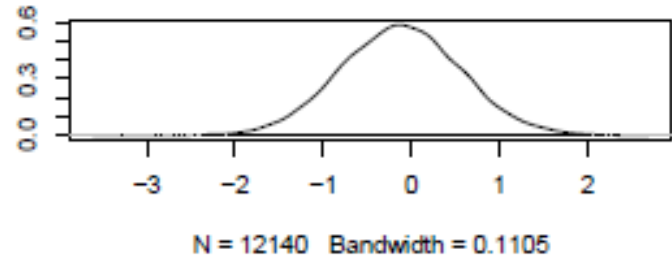

Density of time:attitude

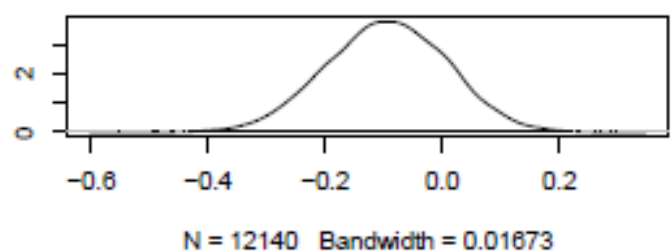

Density of careExp:change

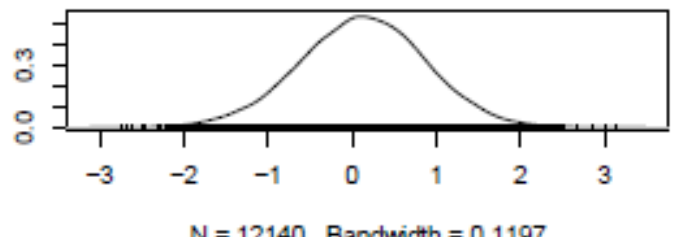

Density of time:change

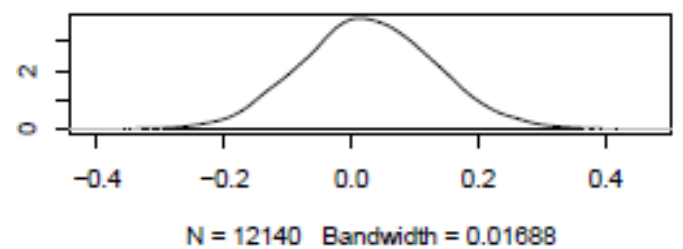

Density of careExp:time:live

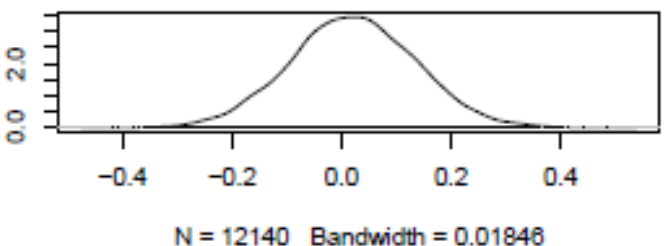

Density of careExp:time:relation

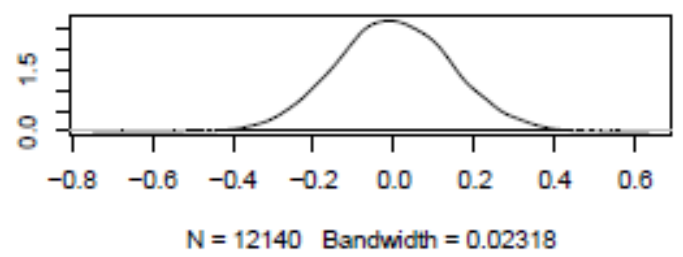


Trace of careExp:time:ete

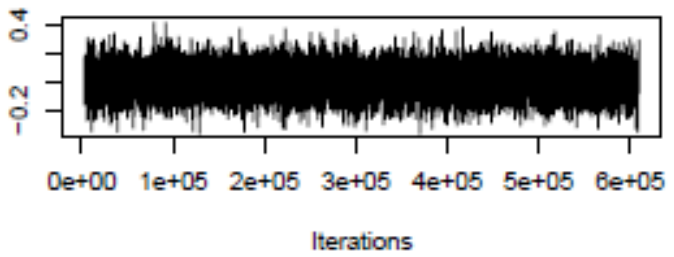

Trace of careExp:time:where

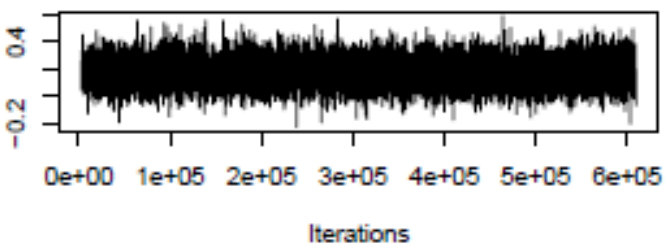

Trace of careExp:time:life

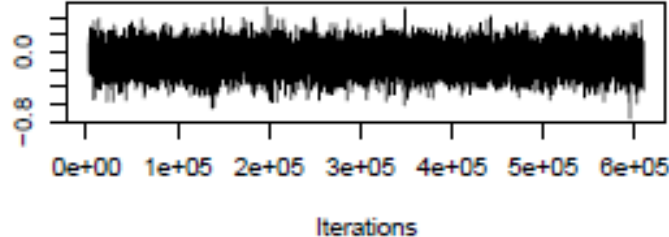

Trace of careExp:time:drugs

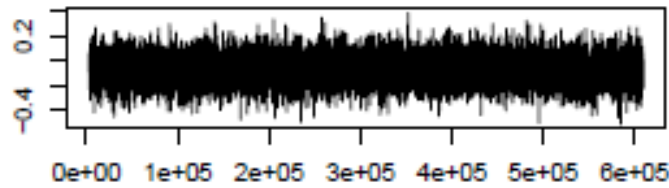

Iterations

Trace of careExp:time:physical

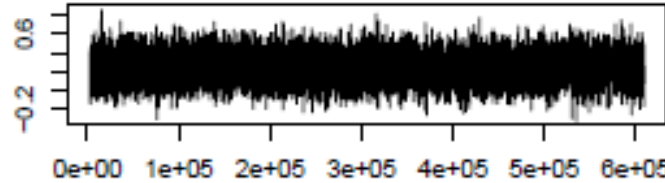

Iterations

Trace of careExp:time:emotion

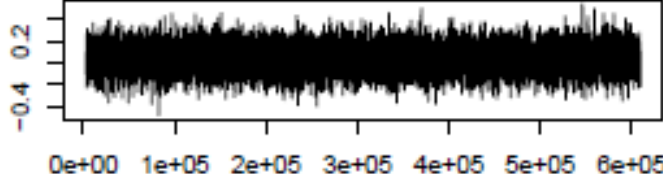

Iterations
Density of careExp:time:ete

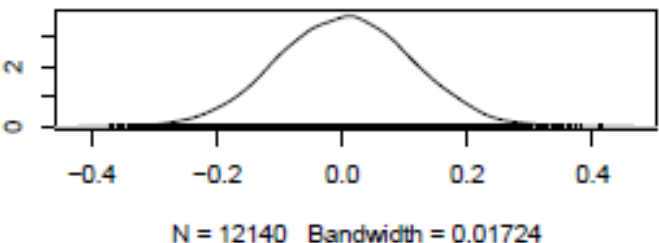

Density of careExp:time:where

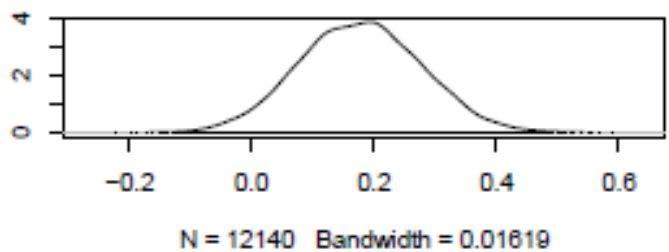

Density of careExp:time:life

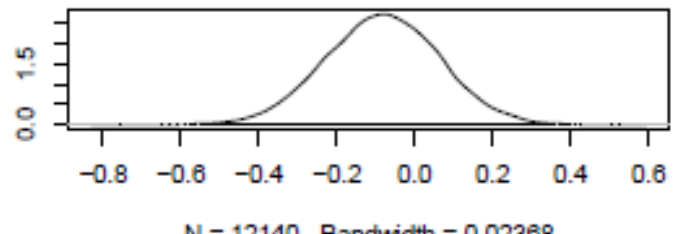

Density of careExp:time:drugs

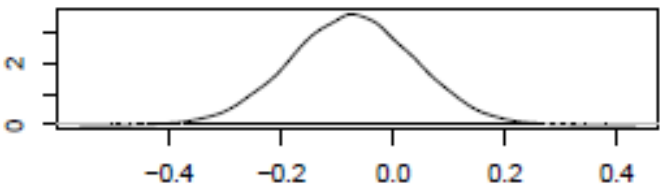

$N=12140$ Bandwidth $=0.01799$

Density of careExp:time:physical

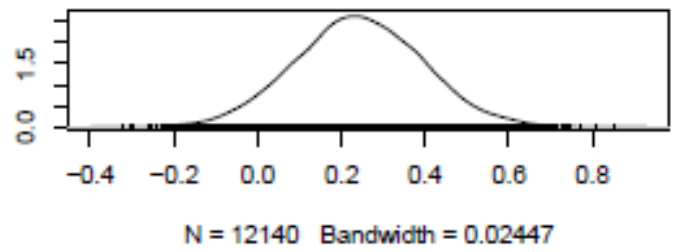

Density of careExp:time:emotion

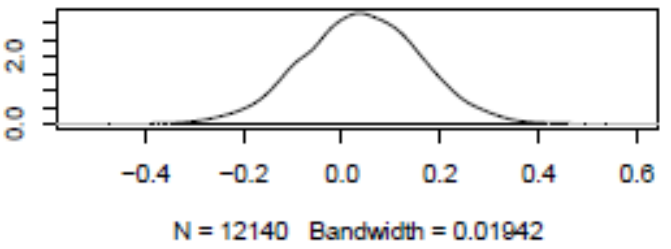


Trace of careExp:time:self

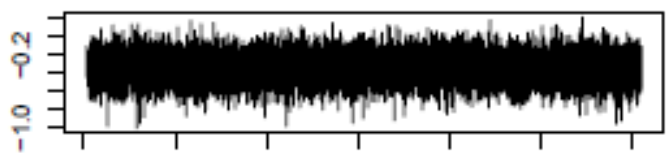

0e+00 1e+05 2e+05 3e+05 4e+05 5e+05 6e+05

Iterations

Trace of careExp:time:think

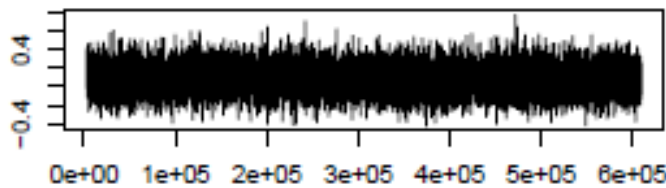

Iterations

Trace of careExp:time:attitude

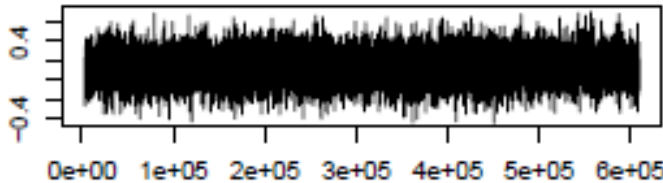

Iterations

Trace of careExp:time:change

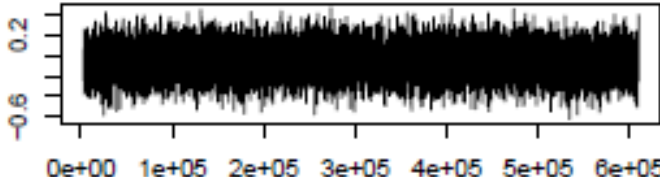

Iterations
Density of careExp:time:self

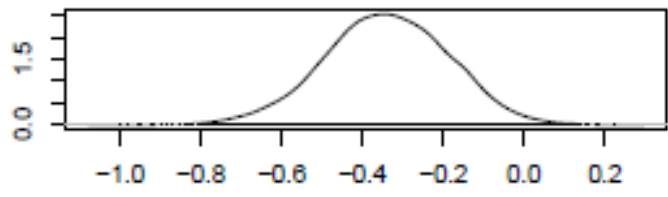

$\mathrm{N}=12140$ Bandwidth $=0.02481$

Density of careExp:time:think

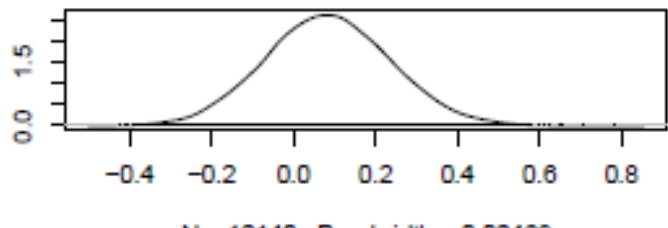

$\mathrm{N}=12140$ Bandwidth $=0.02469$

Density of careExp:time:attitude

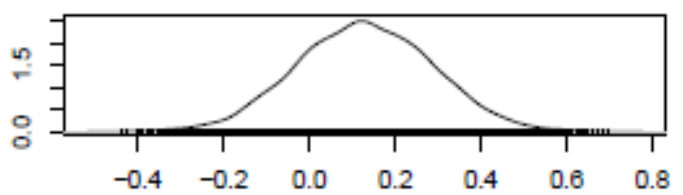

$N=12140$ Bandwidth $=0.02578$

Density of careExp:time:change

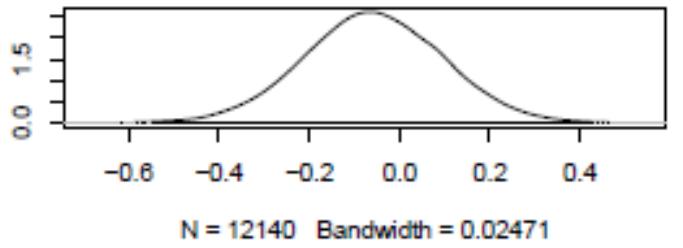


$\underline{\text { Random Effects }}$

Trace of time

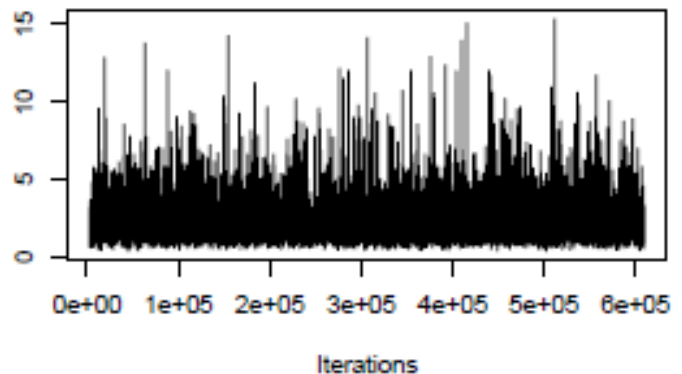

Trace of Research.ID

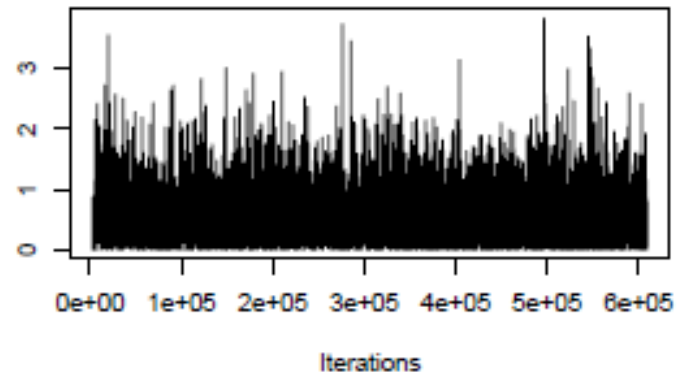

Density of time

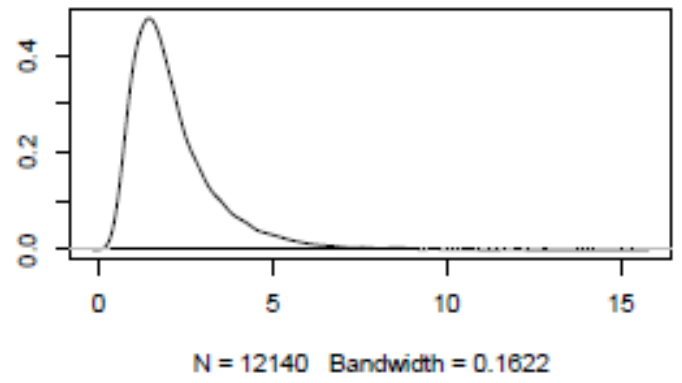

Density of Research.ID

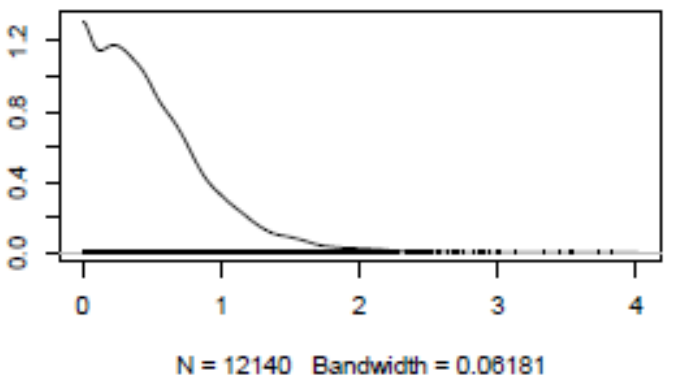


Model 2 (Table 5.13)

Bayesian Model (Bm2)

\section{\#\# Define the model}

Bm1_d1.ch_d2.ch <- MCMCglmm(FO.bin Gender*CareExp + careExp* bme + live + relation + ete + where + life + drugs + physical + emotion + self + think + attitude + change + time,

random= time+Research.ID, data=data, family="ordinal",prior=prior2, nitt $=300000$, thin=10, burnin=3000)

\section{\#\# Checks for suitable convergence}

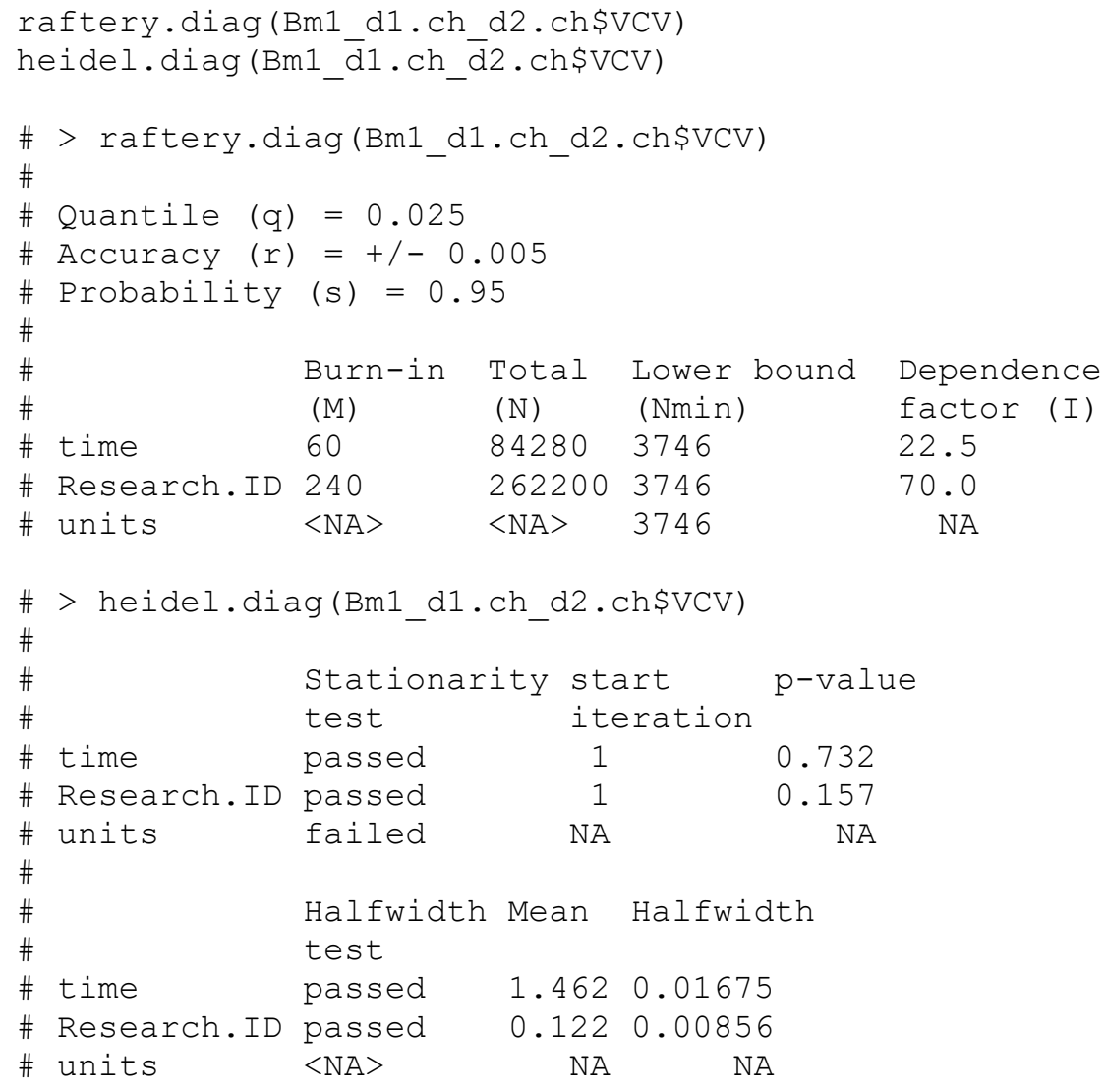




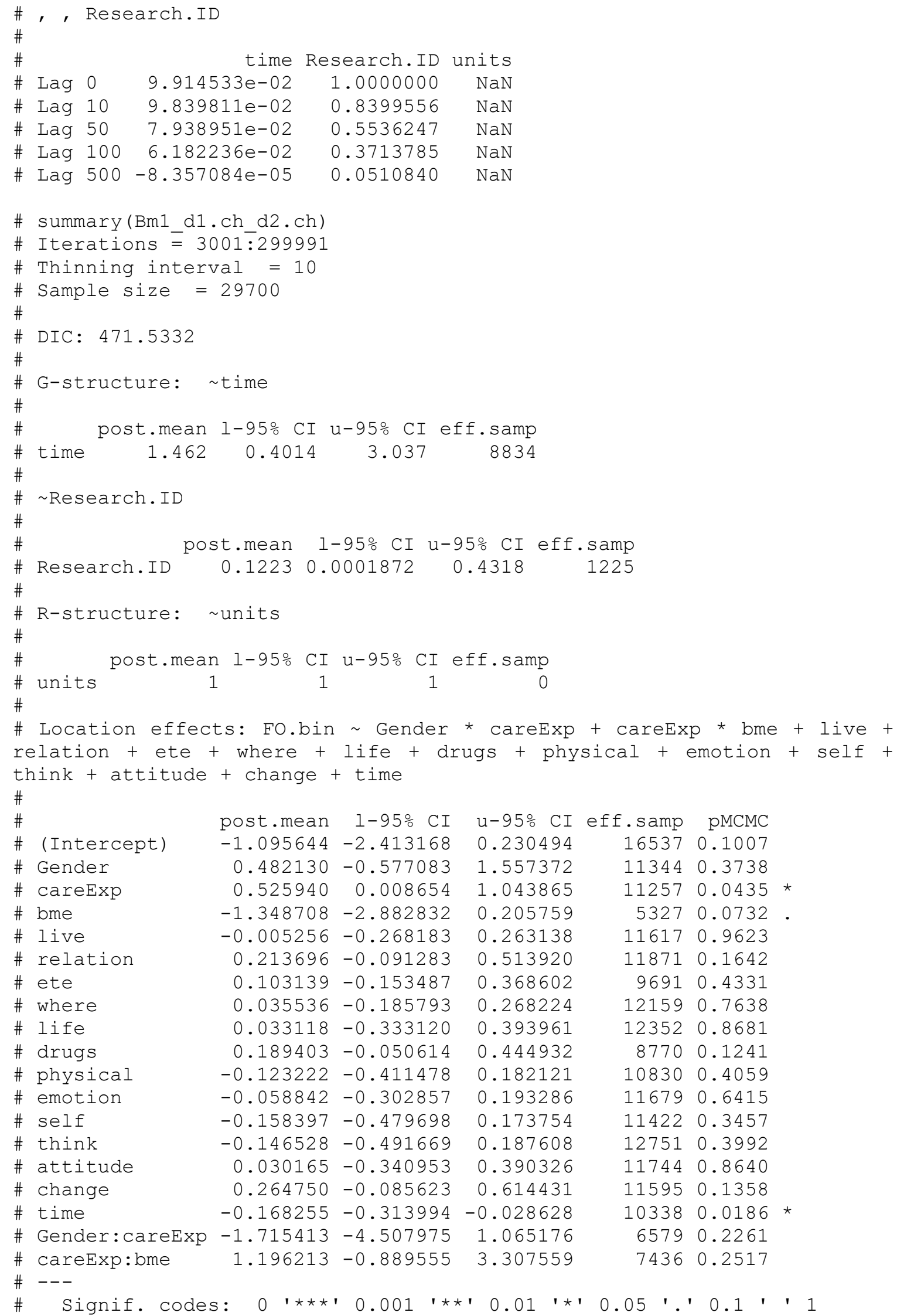


\#\# To simplify naming conventions, Bm1_d1.ch_d2.ch is renamed as Bm2 whilst the Frequentist equivalent m1_d1.ch_d2_ch is renamed as m2.

\section{$\mathrm{Bm} 2<-\mathrm{Bm} 1$ d1. ch d2. ch \\ $\mathrm{m} 2<-\mathrm{m} 1 \_\mathrm{d} \overline{1} \cdot \mathrm{ch} \_\mathrm{d} \overline{2} \cdot \mathrm{ch}$}

Fixed Effects

Trace of (Intercept)

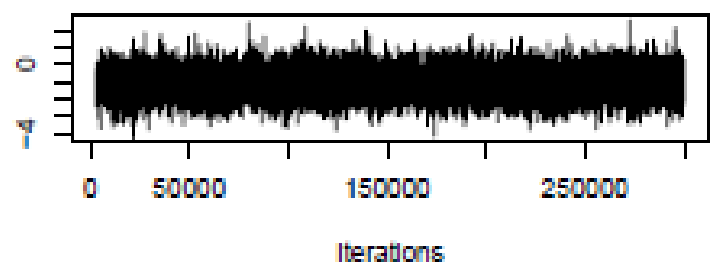

Trace of Gender

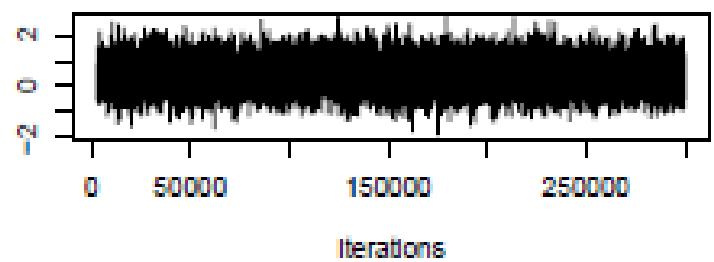

Trace of careExp

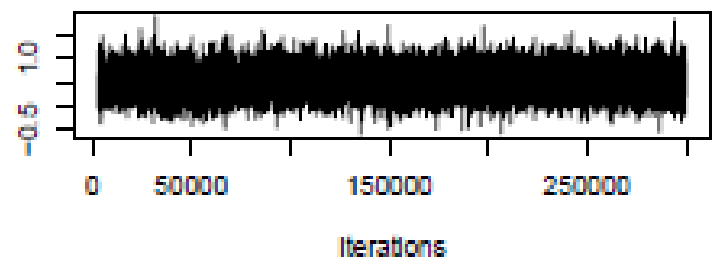

Trace of bme

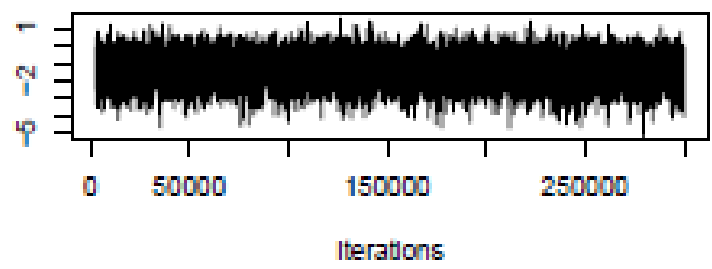

Density of (Intercept)

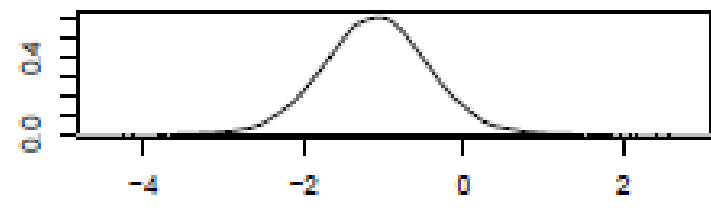

$\mathrm{N}=29700$ Bandwidh -0.08785

Density of Gender

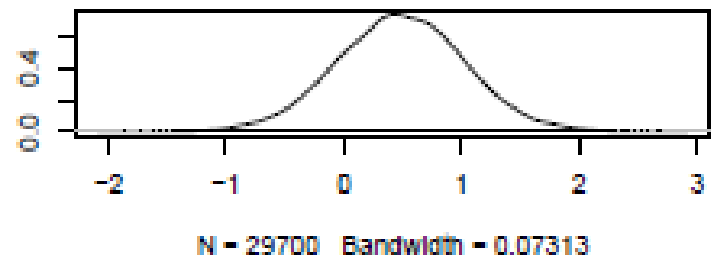

Density of careExp

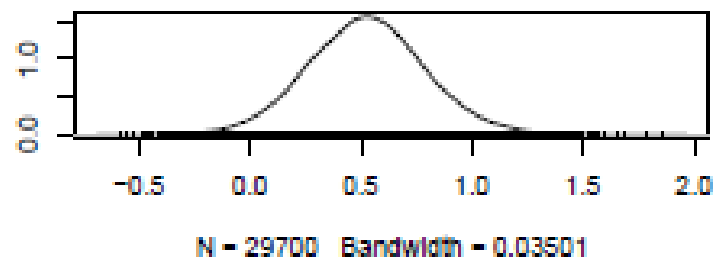

Density of bme

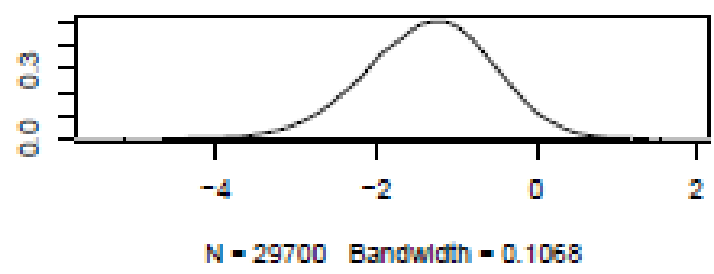


Trace of live

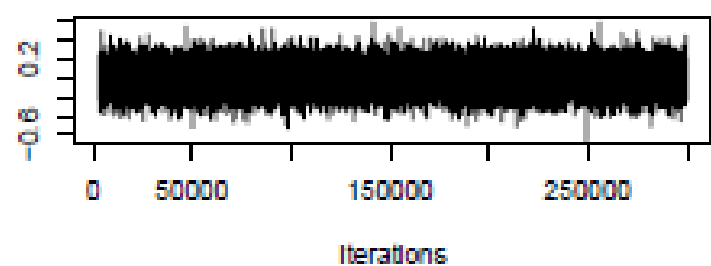

Trace of relation

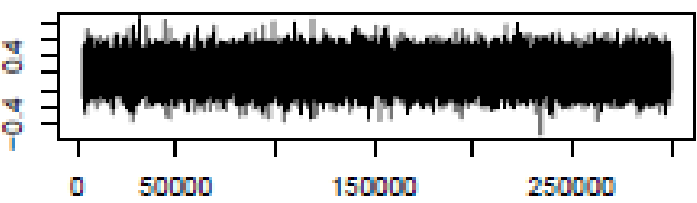

Iterations

Trace of ete

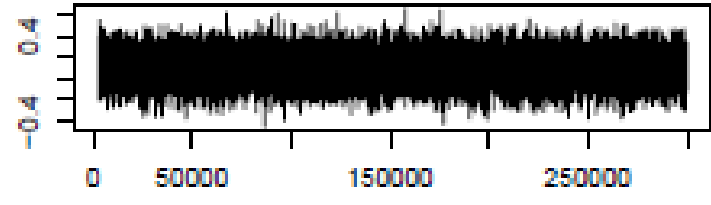

Iterations

Trace of where

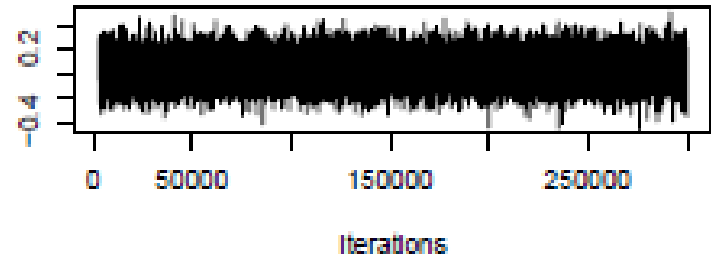

Trace of life

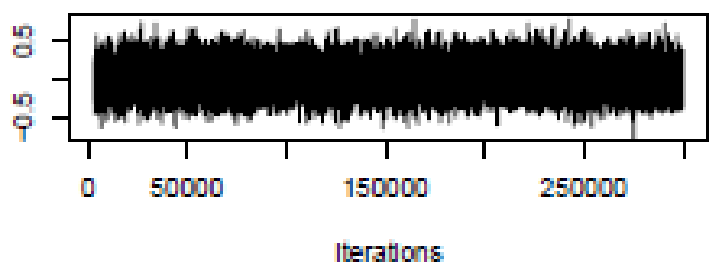

Trace of drugs

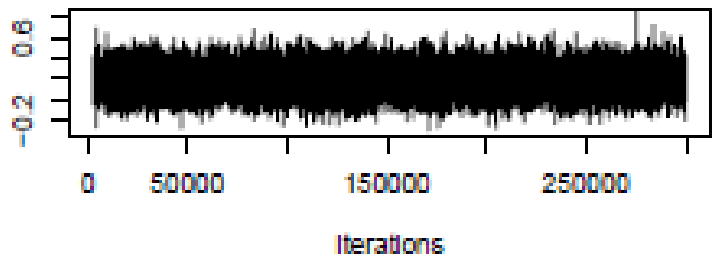

Density of live

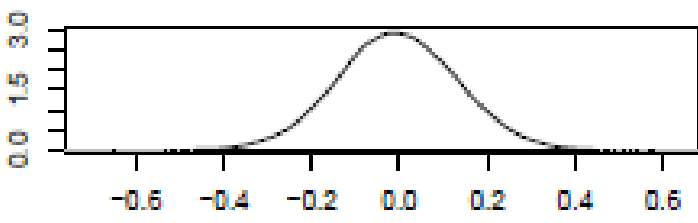

$\mathrm{N}=29700$ Bandwidth -0.01832

Density of relation

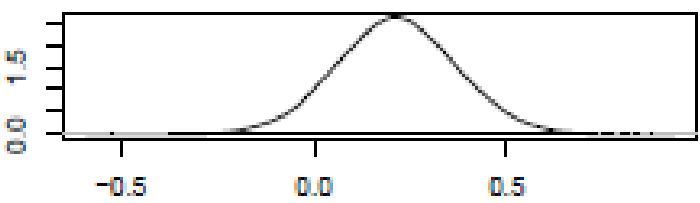

$\mathrm{N}=29700$ Bandwidh $=0.02085$

Density of ete

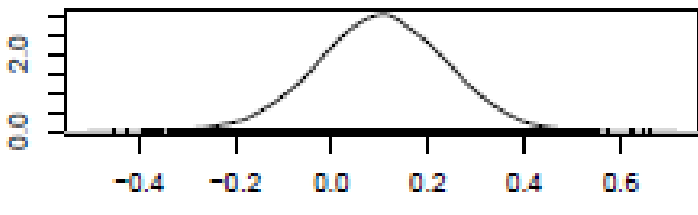

$\mathrm{N}=29700$ Bandwidh -0.01801

Density of where

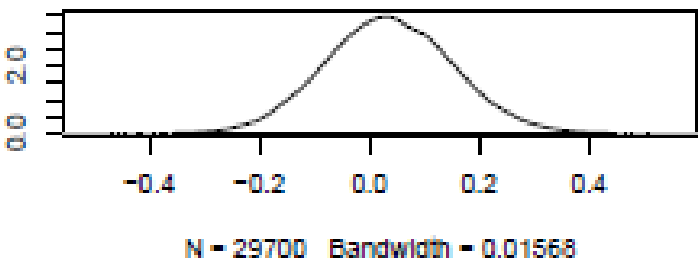

Density of life

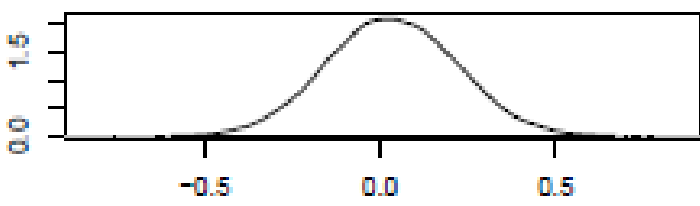

$\mathrm{N}=29700$ Bandwidth $\boldsymbol{0} 0.02512$

Density of drugs

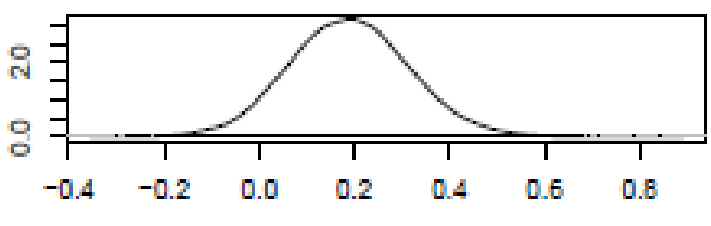

$\mathrm{N}=29700$ Bandwidth $=0.017$ 
Trace of physical

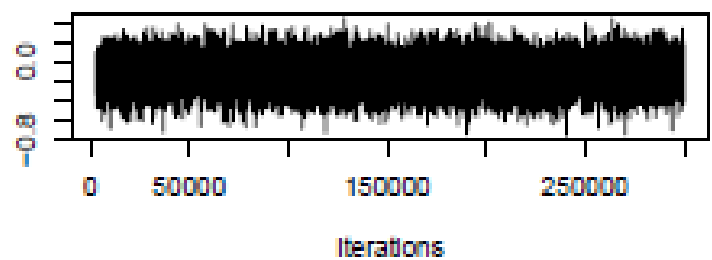

Trace of emotion

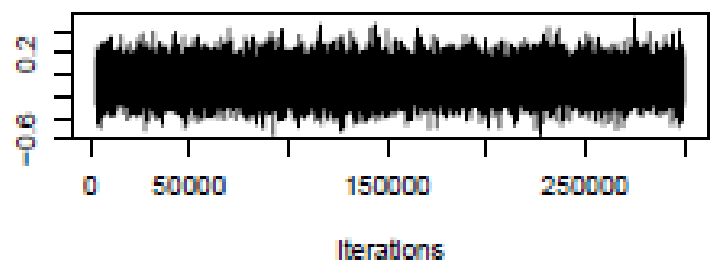

Trace of self

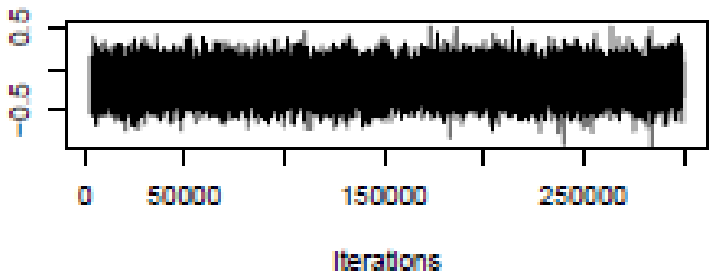

Trace of think

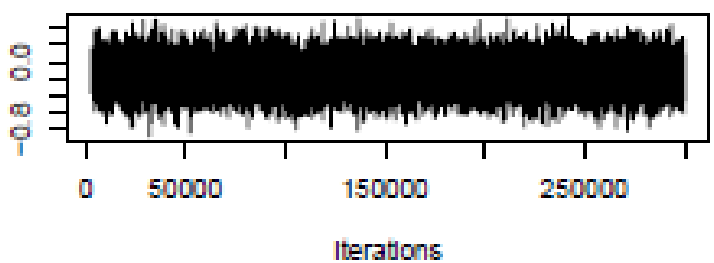

Trace of attitude

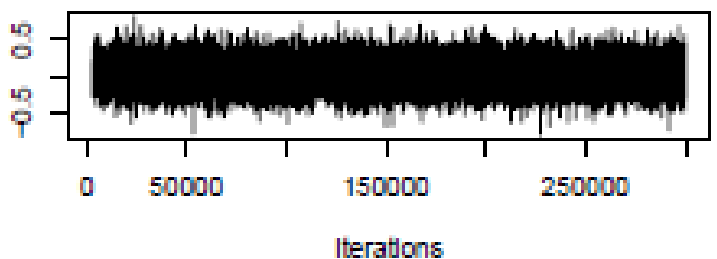

Trace of change

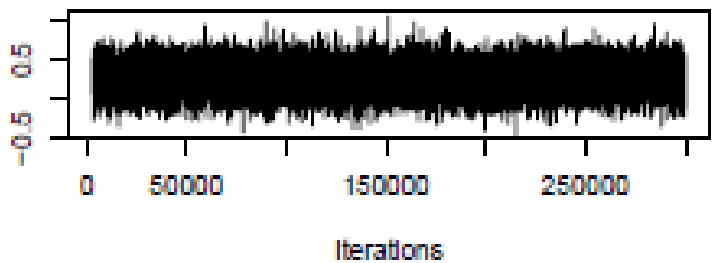

Density of physical

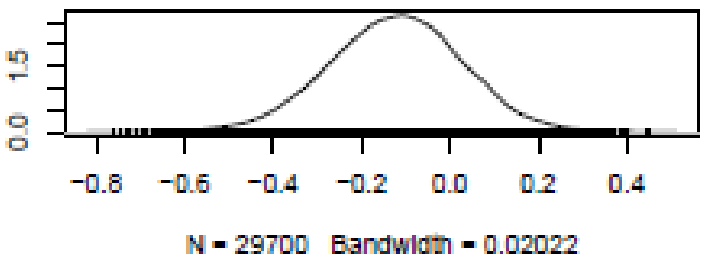

Density of emotion

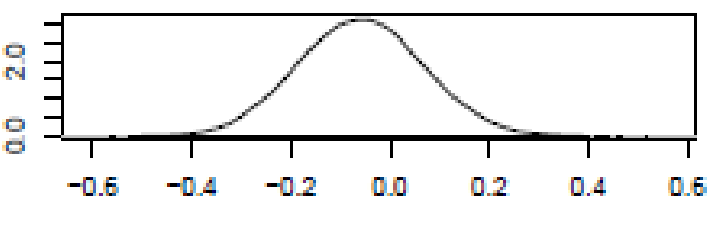

$N=29700$ Bandwidh -0.01726

Density of self

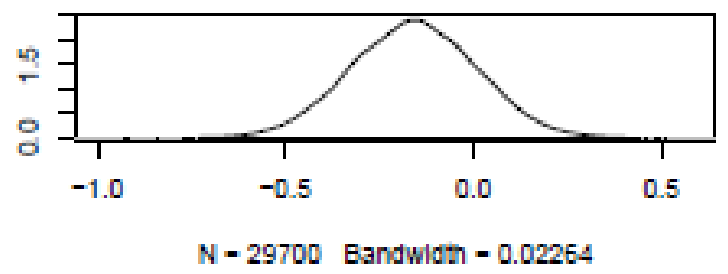

Density of think

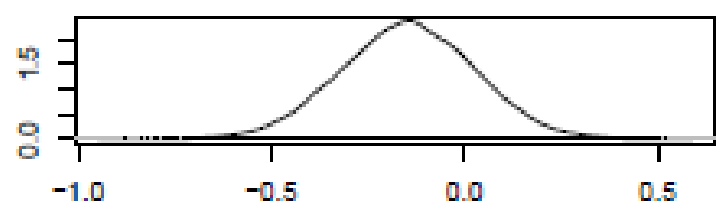

$\mathrm{N}-29700$ Bandwidth -0.02351

Density of attitude

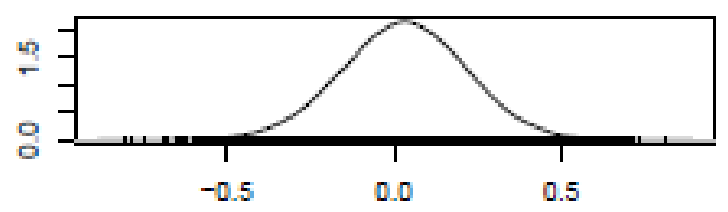

$\mathrm{N}=29700$ Bandwidth $=0.02504$

Density of change

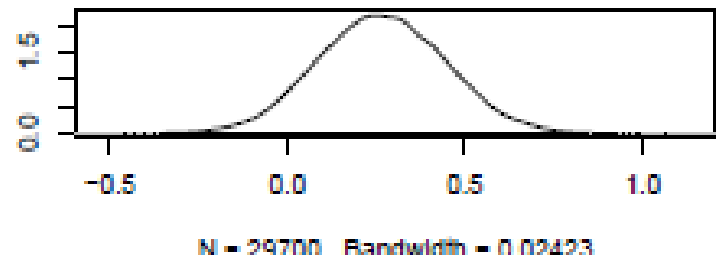

Page | 117 
Trace of time

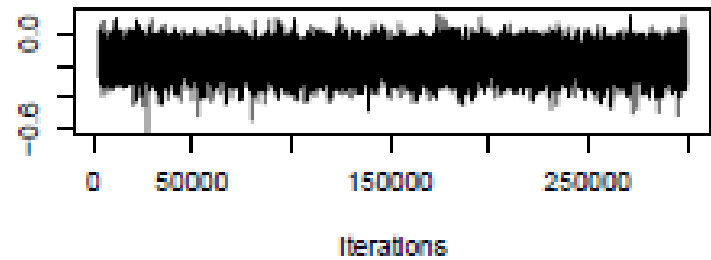

Trace of Gender:careExp

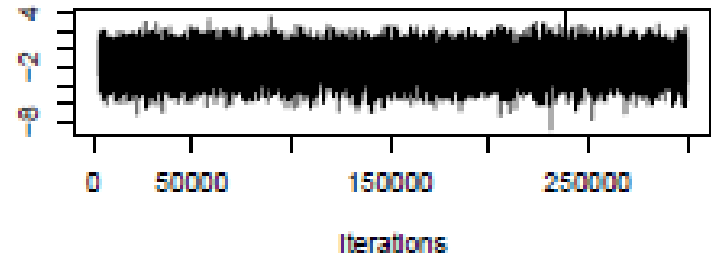

Trace of careExp:bme

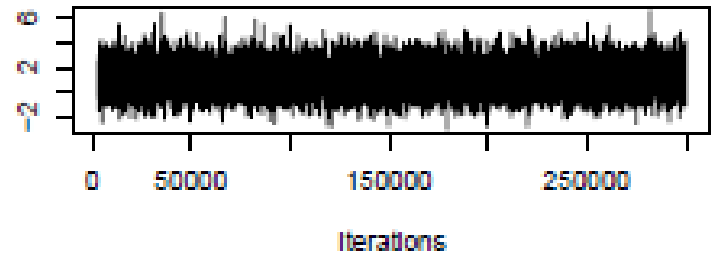

$\underline{\text { Random Effects }}$

\section{Trace of time}

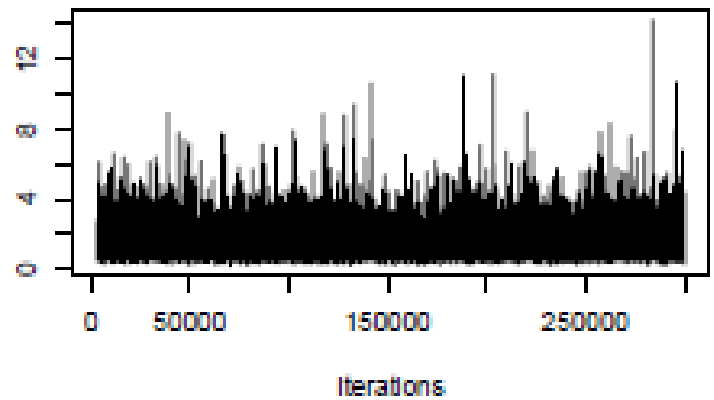

Trace of Research.ID

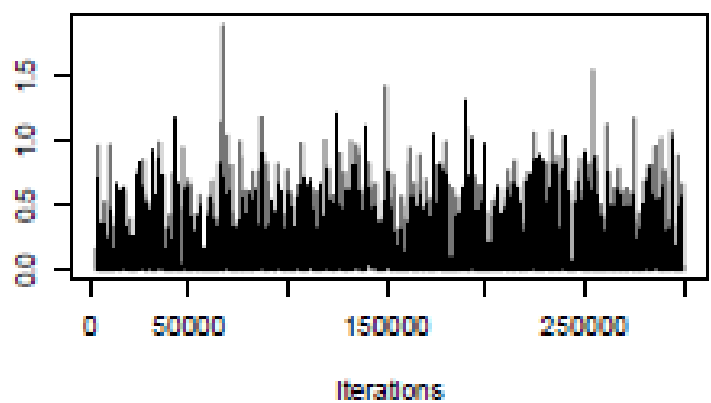

Density of time

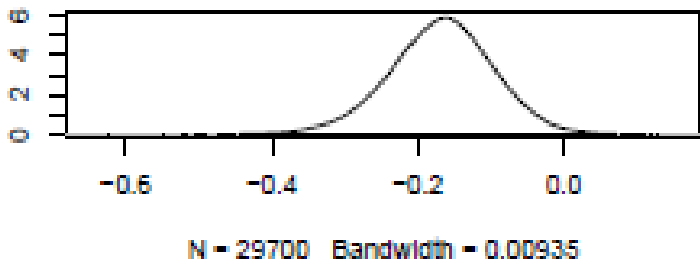

Density of Gender:careExp

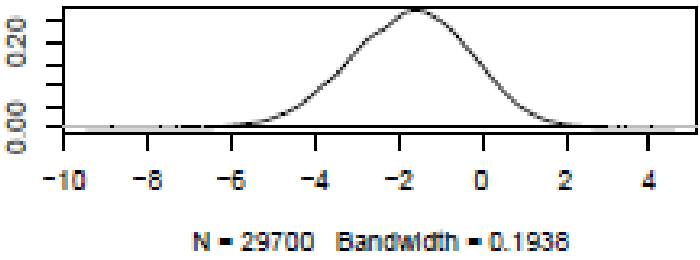

Density of careExp:bme

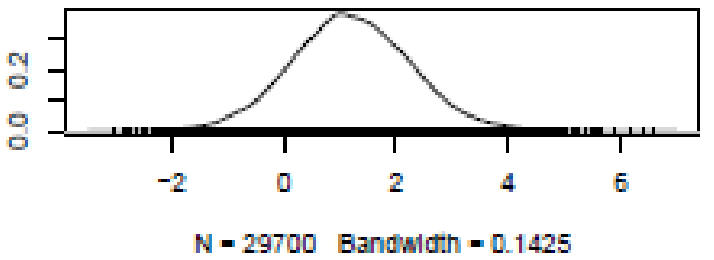

Density of time

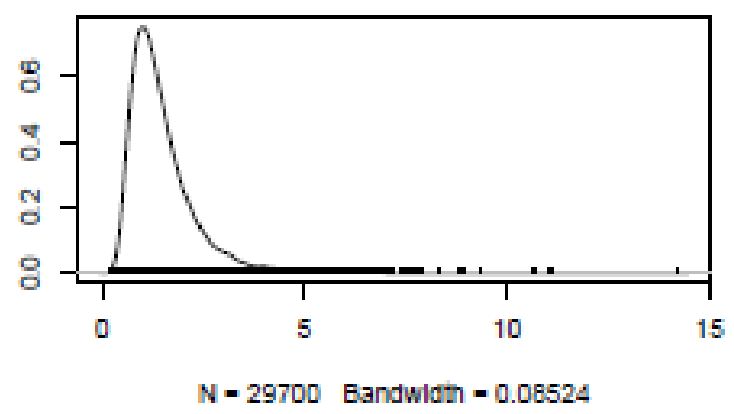

Density of Research.ID

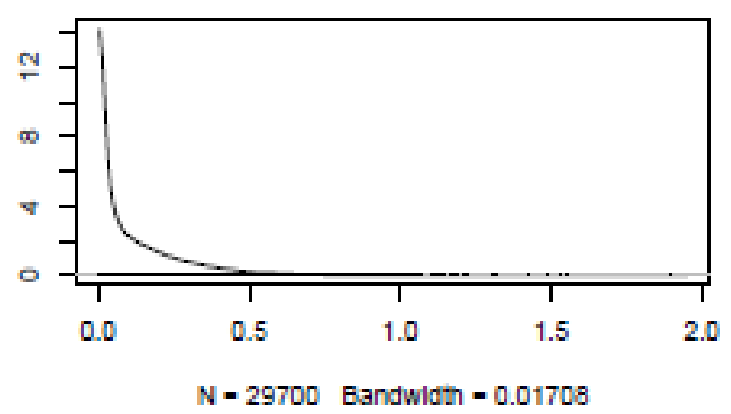


m1_d1.ch_d2.ch <- glmer(FO.bin female*careExp + careExp*bme + live + relation ${ }^{-}$ete + where + life + drugs + physical + emotion + self + think + attitude + change + time + (timelIndividual), data=data, family=binomial)

summary (m1_d1.ch_d2.ch)

vcomps.icc (m1 d1. ch d2.ch)

anova (m1_d12_ch, m1_ $\left.\bar{d} 1 . c h \_d 2 . c h\right)$

anova (m1, $\left.{ }^{\mathrm{m} 1} \mathrm{~d} 1 . \mathrm{ch} \_\overline{\mathrm{d}} 2 . \mathrm{ch}\right)$

\# Generalized linear mixed model fit by maximum likelihood (Laplace Approximation) ['glmerMod']

\# Family: binomial ( logit)

\# Formula: FO.bin female * careExp + careExp * bme + live + relation +

\# ete + where + life + drugs + physical + emotion + self +

\# think + attitude + change + time + (time | Individual)

\# Data: data

\#

$\begin{array}{rrrrr}\# & \text { AIC } & \text { BIC } & \text { logLik deviance df.resid } \\ \text { \# } 643.6 & 738.3 & -299.8 & 599.6 & 523\end{array}$

\#

\# Scaled residuals:

\# $\quad$ Min 10 Median $30 \quad$ Max

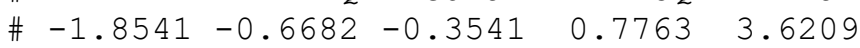

\#

\# Random effects:

\# Groups Name Variance Std.Dev. Corr

\# Individual (Intercept) $0.07066 \quad 0.2658$

\# $\quad$ time $\quad 0.05808 \quad 0.2410 \quad-1.00$

\# Number of obs: 545, groups: Individual, 87

\#

\# Fixed effects:

\#

\# (Intercept)

Estimate Std. Error z value $\operatorname{Pr}(>|z|)$

\# female

\# careExp

\# bme

\# live

\# relation

$-0.764603$

$0.339776-2.250$

0.524890

$0.566987 \quad 0.926$

$0.0244 *$

0.598552

$0.290209 \quad 2.062$

0.3546

\# ete

\# where

$-0.773253$

0.656496

$-1.178$

$0.0392 *$

\# life

\# drugs

\# physical

$-0.114424$

$0.145383-0.787$

0.2389

0.111470

0.162349

0.687

0.4313

0.015565

0.137881

0.113

0.4923

0.163501

0.130230

1.255

0.009882

0.203963

0.048

0.9101

0.303134

0.142475

2.128

0.2093

\# emotion

$-0.212755$

0.153957

$-1.382$

0.9614

\# self

\# think

$-0.079389$

0.137445

$-0.091114$

0.182496

$-0.578$

0.0334

\# attitude

0.145743

0.191514

$-0.499$

0.1670

\# change

$-0.058874$

0.197060

0.761

.5635

\# time

0.224052

0.187956

$-0.299$

0.6176

\# female:careExp

$-0.461982$

0.108437

1.192

0.4467

$-0.894353$

1.159862

$-4.260$

0.7651

\# car

\# Signif. codes:

1.119857

$-0.771$

0.2332

0.560683

0.501

$2.04 \mathrm{e}-05$

$01 * \star \star x+1$

$0.001, * \star \prime$

$0.01 ' * \prime 0.05$ '.' 0.1 ' 1

\# convergence code: 0

\# Model failed to converge with max|grad $=0.260456$ (tol $=0.001$, component 1) 


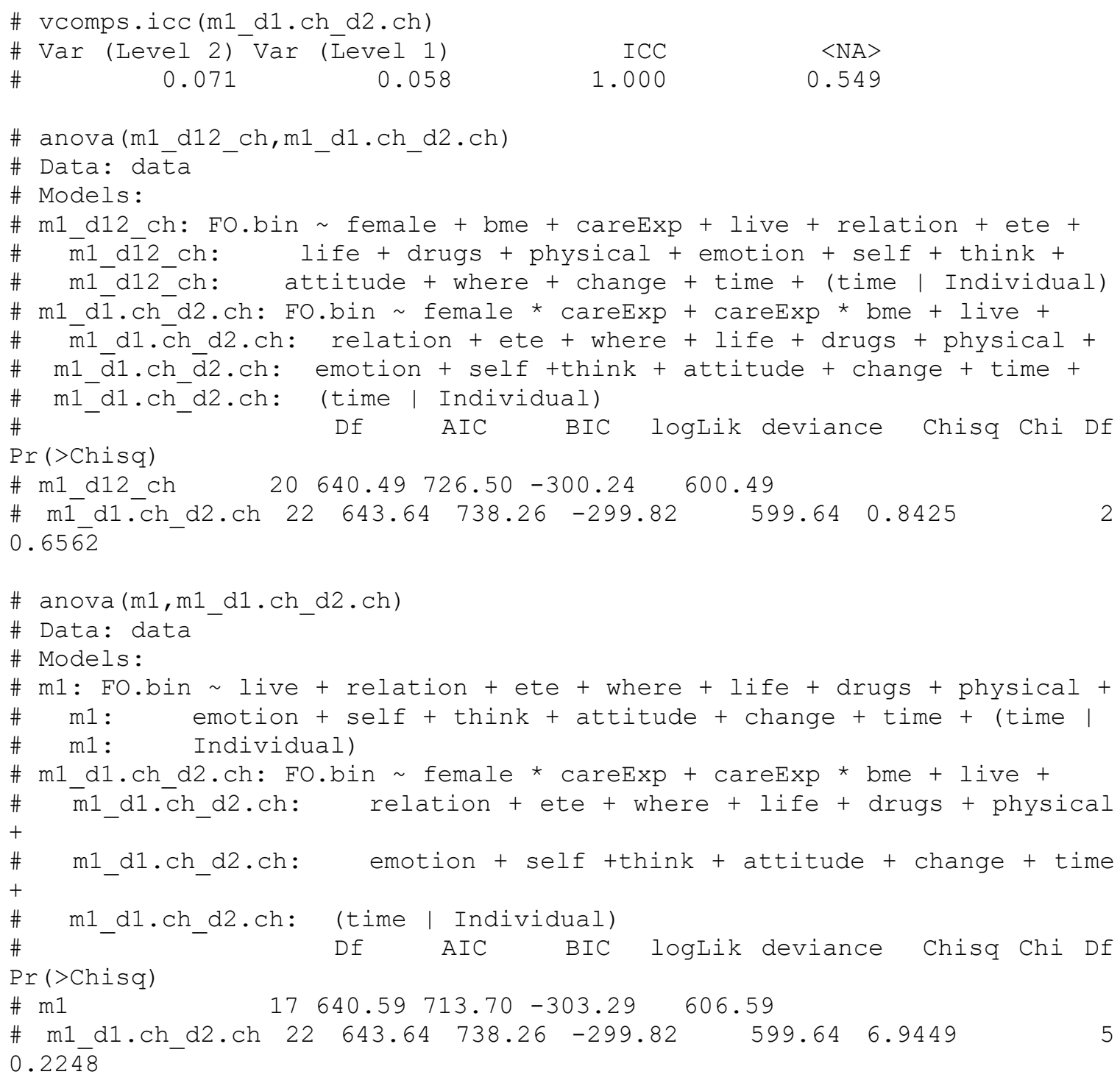




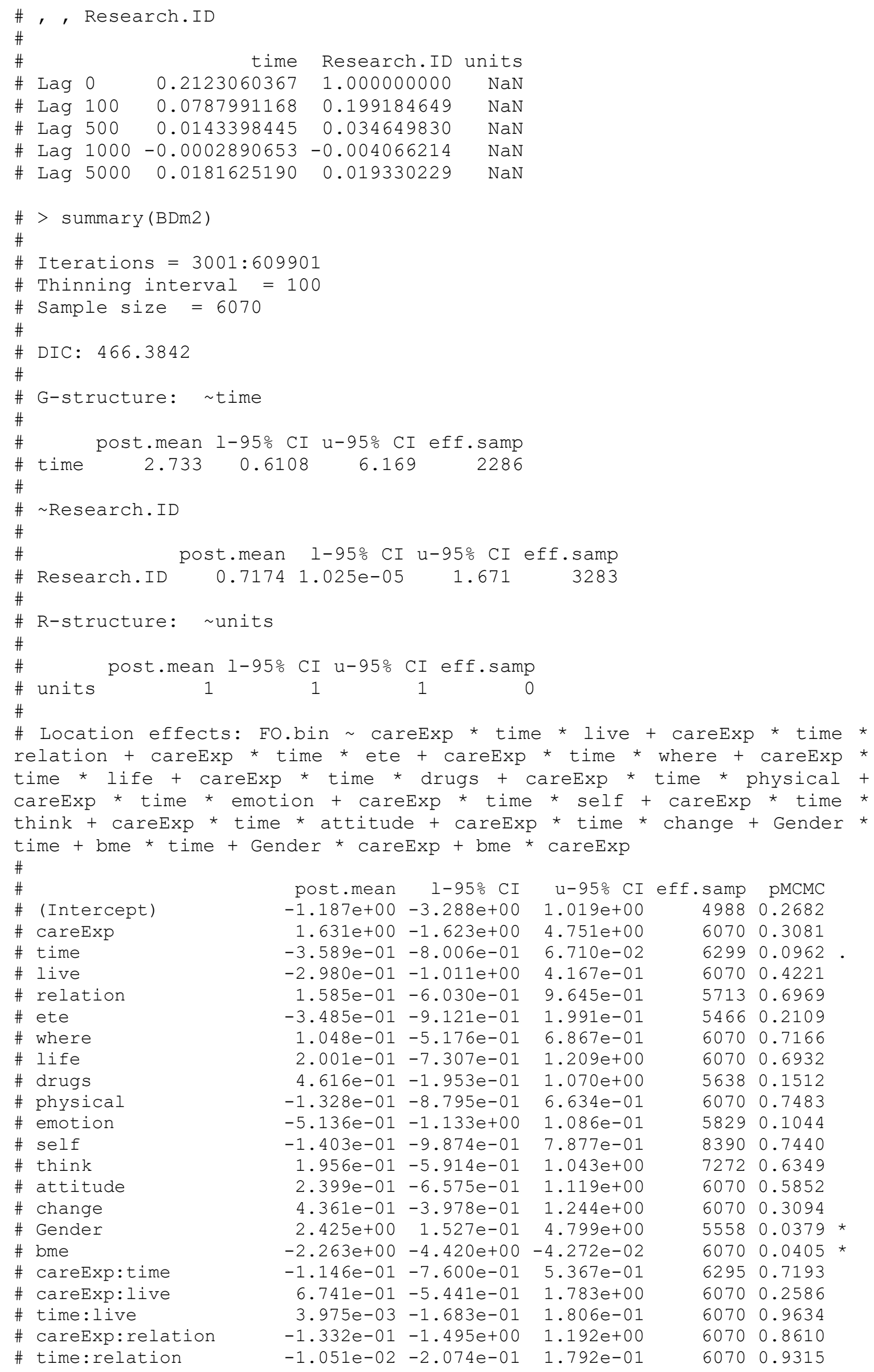




\begin{tabular}{|c|c|c|c|c|c|c|}
\hline \# & careExp:ete & $3.877 e-01$ & $-6.496 e-01$ & $1.531 e+00$ & 6070 & 0.4909 \\
\hline \# & time:ete & $7.096 e-02$ & $-5.909 e-02$ & $2.048 e-01$ & 6070 & 0.2903 \\
\hline \# & careExp: where & $-3.539 e-01$ & $-1.392 e+00$ & $7.186 e-01$ & 6070 & 0.5041 \\
\hline \# & time:where & $-6.834 e-02$ & $-2.171 e-01$ & $7.370 e-02$ & 6070 & 0.3631 \\
\hline \# & careExp:life & $-1.832 e-01$ & $-1.699 e+00$ & $1.520 e+00$ & 6543 & 0.8191 \\
\hline \# & time:life & $4.953 e-02$ & $-1.457 e-01$ & $2.588 e-01$ & 6070 & 0.6333 \\
\hline \# & careExp:drugs & $-3.639 e-02$ & $-1.096 e+00$ & $9.659 e-01$ & 5757 & 0.9562 \\
\hline \# & time:drugs & $3.633 e-05$ & $-1.437 e-01$ & $1.402 e-01$ & 5744 & 0.9875 \\
\hline \# & careExp:physical & $-1.068 e+00$ & $-2.521 e+00$ & $1.933 e-01$ & 5711 & 0.1031 \\
\hline \# & time:physical & $-3.015 e-02$ & $-2.675 e-01$ & $2.032 e-01$ & 5828 & 0.8102 \\
\hline \# & careExp:emotion & $5.222 e-01$ & $-6.132 e-01$ & $1.546 e+00$ & 830 & 0.3417 \\
\hline \# & time:emotion & $8.171 e-02$ & $-6.889 e-02$ & $2.408 e-01$ & 6070 & 0.2988 \\
\hline \# & careExp:self & $9.415 e-01$ & $-5.440 e-01$ & $2.572 e+00$ & 5767 & 0.2244 \\
\hline \# & time:self & $9.441 e-02$ & $-1.029 e-01$ & $3.149 e-01$ & 7403 & 0.3667 \\
\hline \# & careExp:think & $-1.011 e+00$ & $-2.562 e+00$ & $5.609 e-01$ & 6070 & 0.1931 \\
\hline \# & time:think & $-3.026 e-02$ & $-2.352 e-01$ & $1.753 e-01$ & 6070 & 0.7746 \\
\hline \# & careExp:attitude & $-1.966 e-01$ & $-1.613 e+00$ & $1.262 e+00$ & 5794 & 0.7951 \\
\hline \# & time:attitude & $-1.142 e-01$ & $-3.252 e-01$ & $9.315 e-02$ & 6070 & 0.28 \\
\hline \# & careExp: change & $-5.140 e-02$ & $-1.584 e+00$ & $1.581 e+00$ & 070 & 0.9562 \\
\hline \# & time:change & $-2.803 e-02$ & $-2.524 e-01$ & $1.847 e-01$ & 6070 & 0.8020 \\
\hline \# & time:Gender & $-5.284 e-01$ & $-1.149 e+00$ & $9.237 e-02$ & 6070 & 0.0867 \\
\hline \# & time:bme & $2.098 e-01$ & $-2.793 e-01$ & $6.640 e-01$ & 5609 & 0.3855 \\
\hline$\#$ & careExp: Gender & $-3.429 e+00$ & $-7.727 e+00$ & $5.753 e-01$ & 5706 & 0.091 \\
\hline \# & careExp:bme & $2.162 e-01$ & $-3.551 e+00$ & $3.906 e+00$ & 6070 & 0.900 \\
\hline \# & careExp:time:live & $-2.034 e-02$ & $-2.656 e-01$ & $2.079 e-01$ & 6344 & 0.8662 \\
\hline \# & careExp:time:relation & $2.541 e-02$ & $-2.671 e-01$ & $3.308 e-01$ & 6070 & 0.8738 \\
\hline \# & careExp:time:ete & $2.410 e-03$ & $-2.235 e-01$ & $2.260 e-01$ & 6070 & 0.9769 \\
\hline \# & careExp:time: where & $2.015 e-01$ & $-6.136 e-04$ & $4.166 e-01$ & 503 & 0.0583 \\
\hline \# & careExp:time:life & $-1.447 e-01$ & $-4.702 e-01$ & $1.487 e-01$ & 5262 & 0.3674 \\
\hline \# & careExp:time:drugs & $-3.269 e-02$ & $-2.616 e-01$ & $2.086 e-01$ & 5782 & 0.7740 \\
\hline \# & careExp:time:physical & $2.517 e-01$ & $-4.746 e-02$ & $5.744 e-01$ & 6070 & 0.1077 \\
\hline \# & careExp:time:emotion & $1.780 e-02$ & $-2.191 e-01$ & $2.731 e-01$ & 6070 & 0.8896 \\
\hline \# & careExp:time:self & $-3.814 e-01$ & $-7.122 e-01$ & $-5.440 e-02$ & 6070 & 0.0194 \\
\hline \# & careExp:time:think & $1.209 e-01$ & $-2.028 e-01$ & $4.299 e-01$ & 6070 & 0.454 \\
\hline \# & careExp:time:attitude & $1.237 e-01$ & $-2.212 e-01$ & $4.450 e-01$ & 6718 & 0.464 \\
\hline \# & careExp:time:change & $-8.874 e-03$ & $-3.321 e-01$ & $3.035 e-01$ & 6070 & 0.9651 \\
\hline & & & & & & \\
\hline & Signif. codes: & 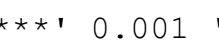 & & 00 & & \\
\hline
\end{tabular}


Trace Plots and Posterior Density Plots

Trace of (Intercept)

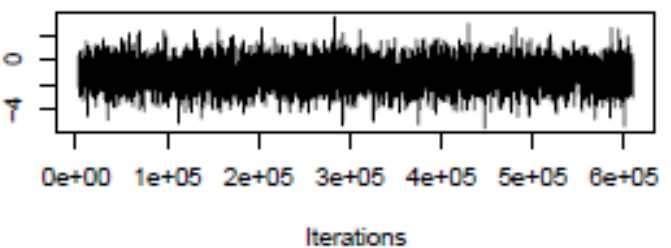

Trace of careExp

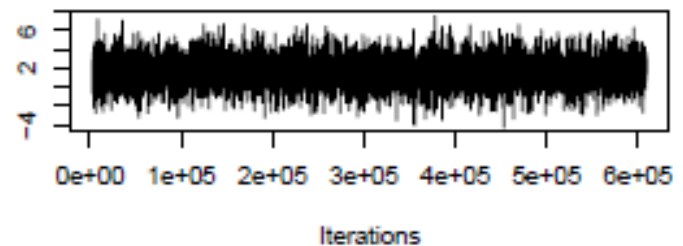

Trace of time

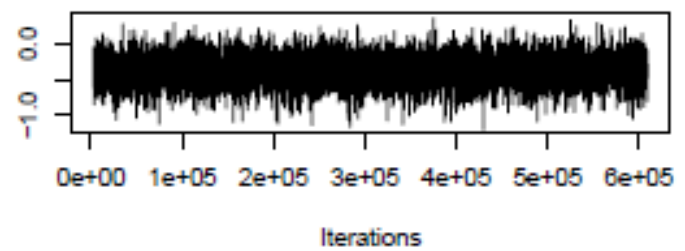

Trace of live

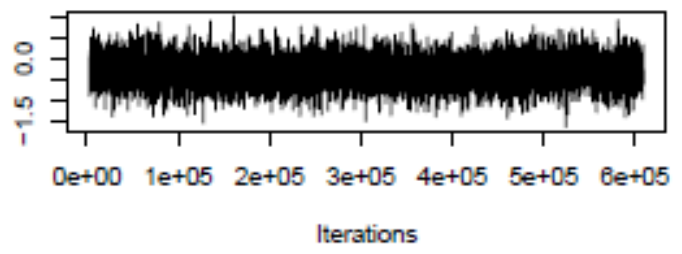

Trace of relation

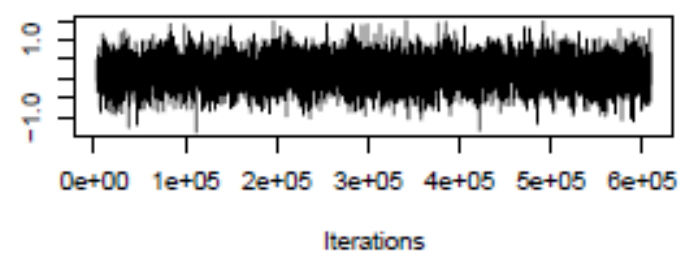

Trace of ete

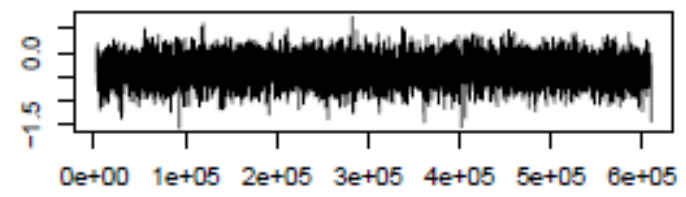

Iterations
Density of (Intercept)

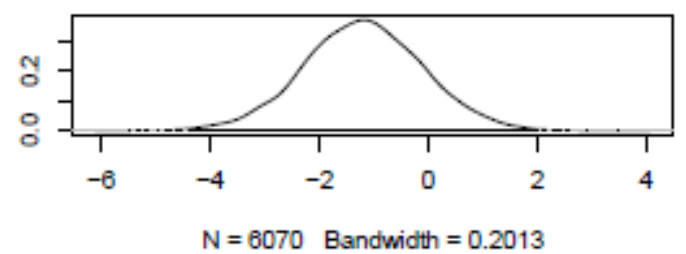

Density of careExp

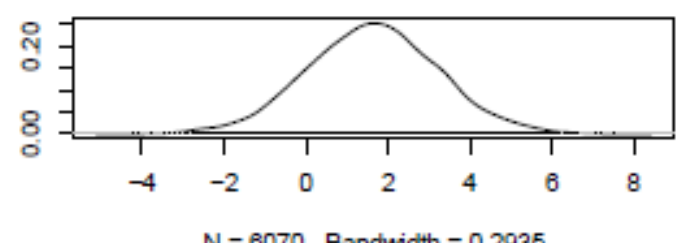

Density of time

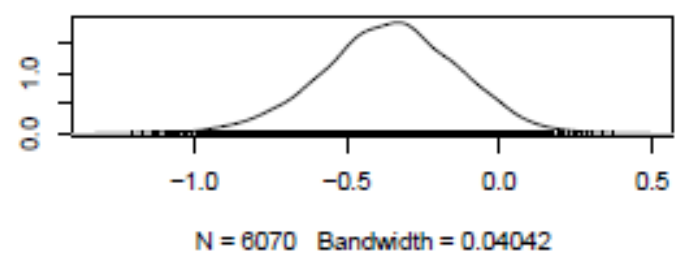

Density of live

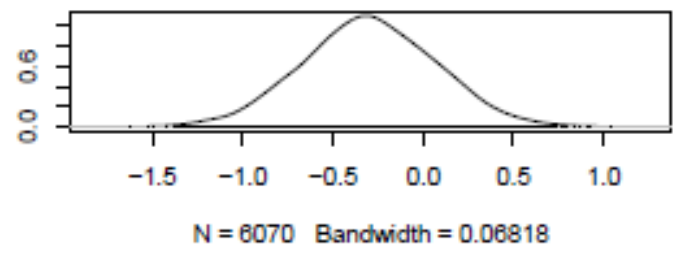

Density of relation

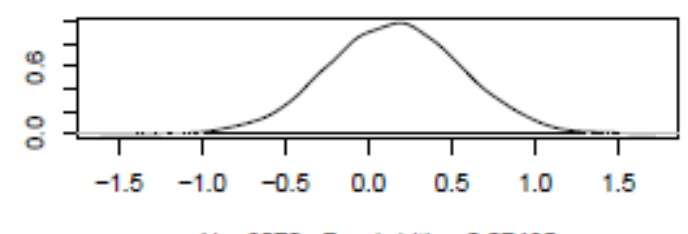

$\mathrm{N}=6070$ Bandwidth $=0.07435$

Density of ete

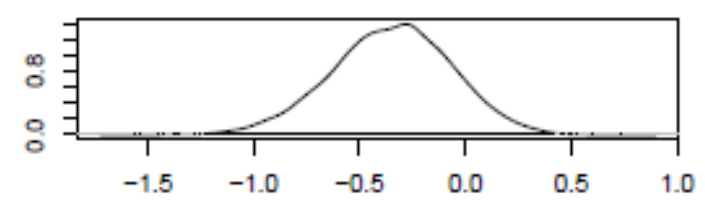

$\mathrm{N}=6070$ Bandwidth $=0.05263$ 
Trace of where

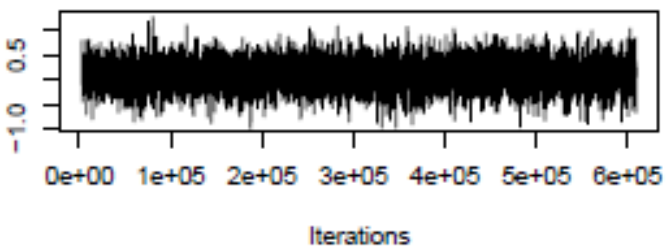

Trace of life

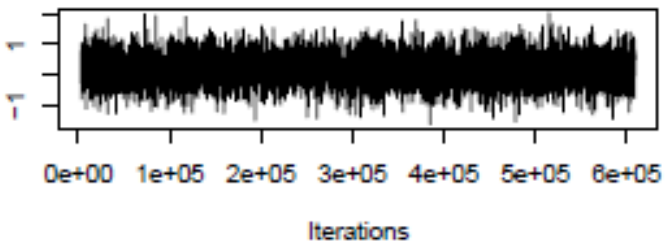

Trace of drugs

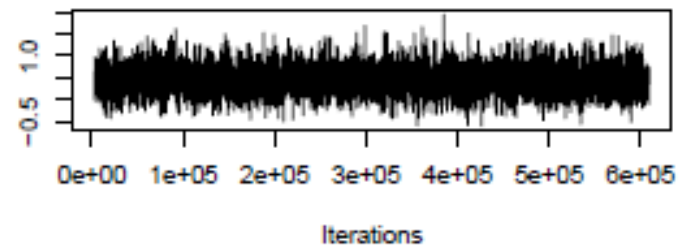

Trace of physical

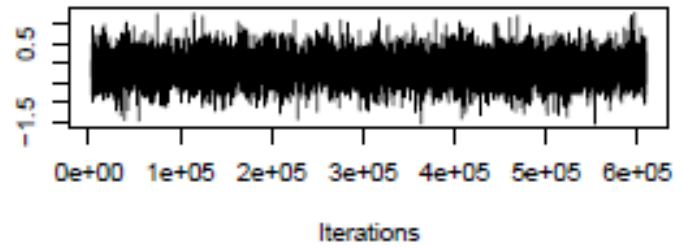

Trace of emotion

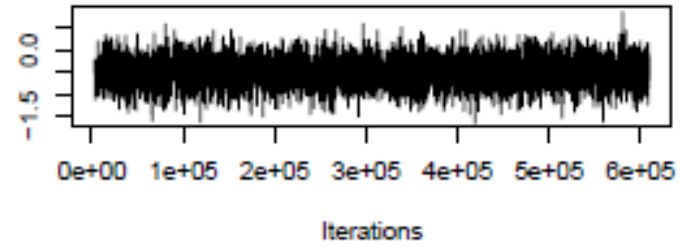

Trace of self

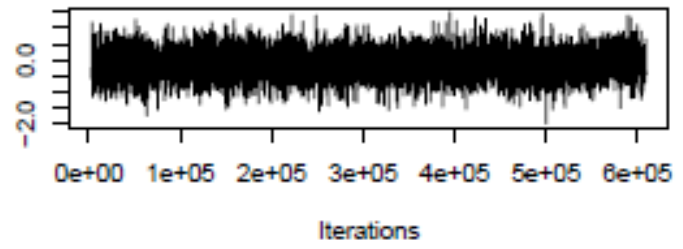

Density of where

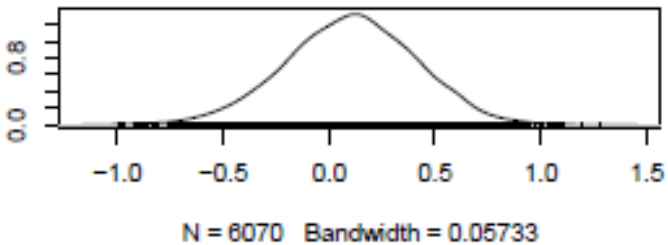

Density of life

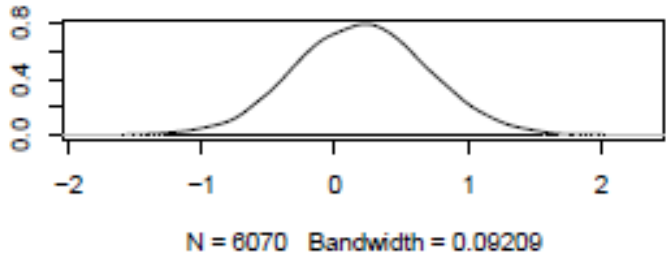

Density of drugs

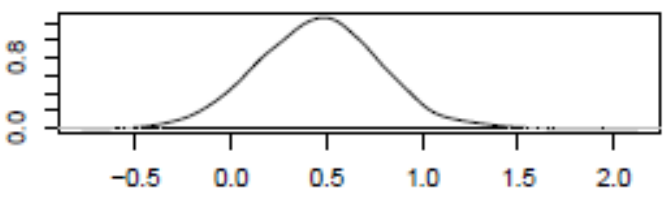

$\mathrm{N}=6070$ Bandwidth $=0.05947$

Density of physical

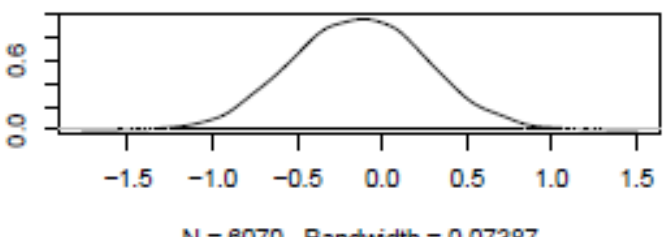

Density of emotion

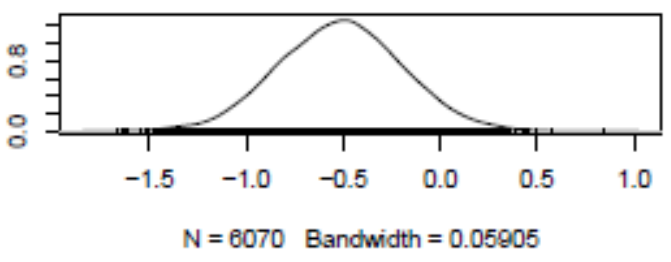

Density of self

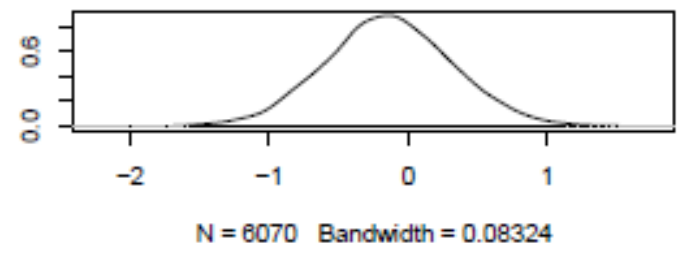


Trace of think

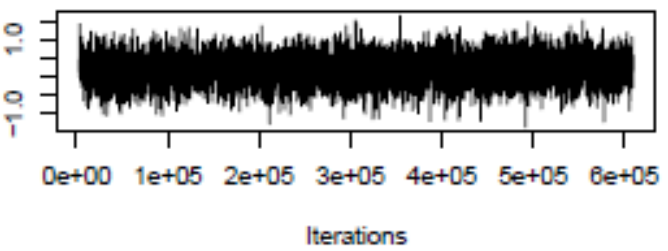

Trace of attitude

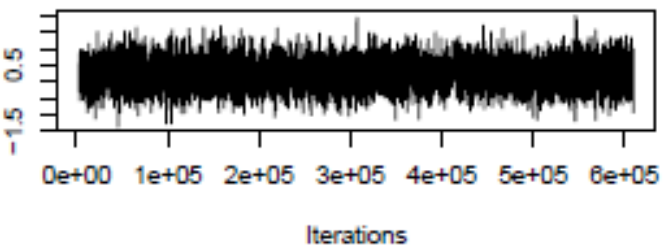

Trace of change

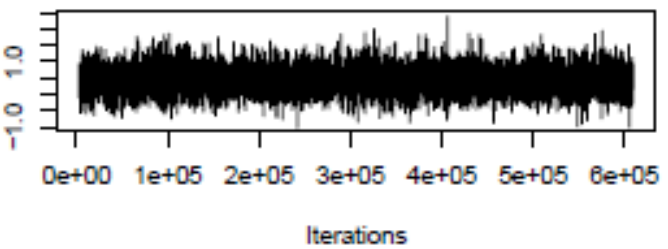

Trace of Gender

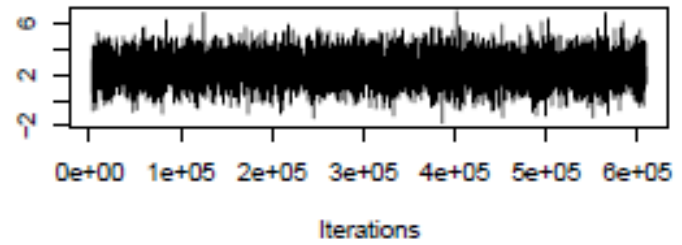

Trace of bme

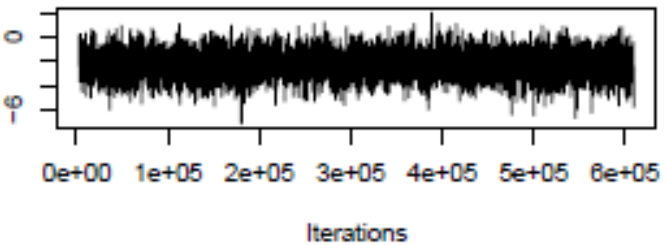

Trace of careExp:time

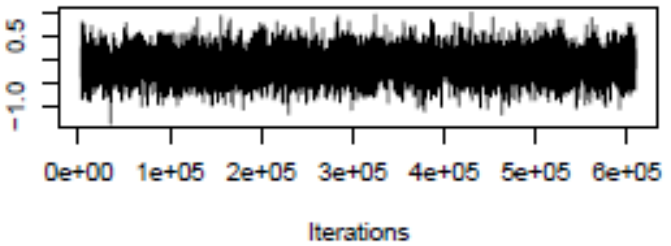

Density of think

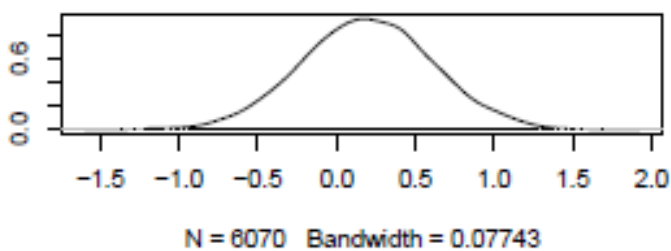

Density of attitude

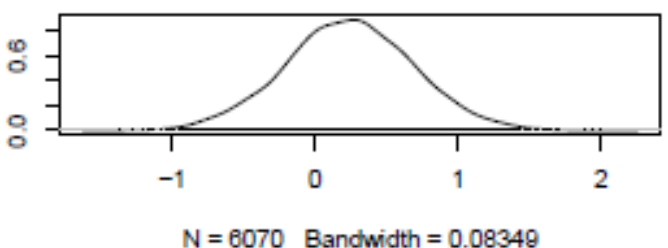

Density of change

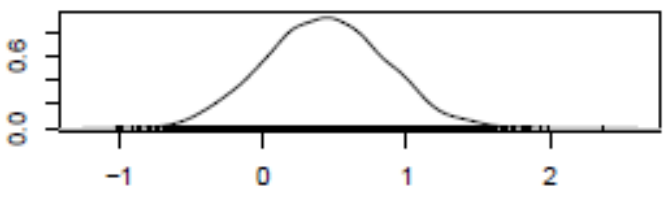

$\mathrm{N}=6070$ Bandwidth $=0.07882$

Density of Gender

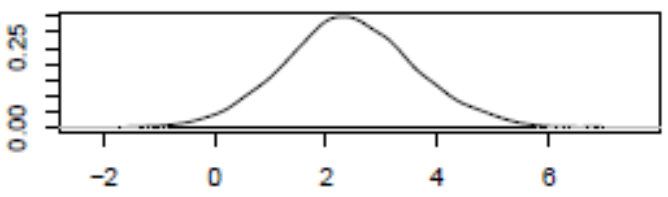

$\mathrm{N}=6070$ Bandwidth $=0.2112$

Density of bme

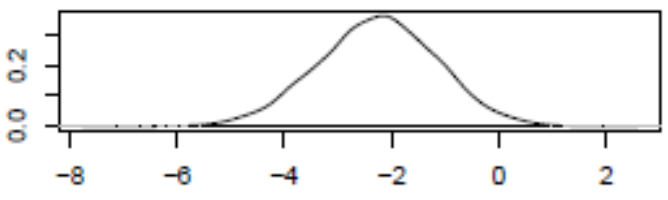

$\mathrm{N}=6070$ Bandwidth $=0.2062$

Density of careExp:time

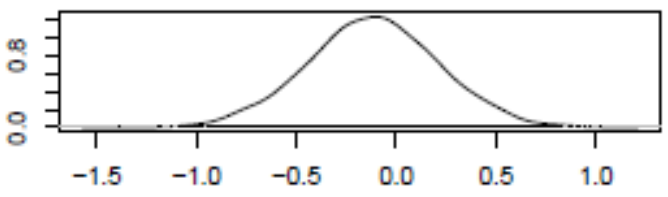

$\mathrm{N}=6070$ Bandwidth $=0.06028$ 
Trace of careExp:live

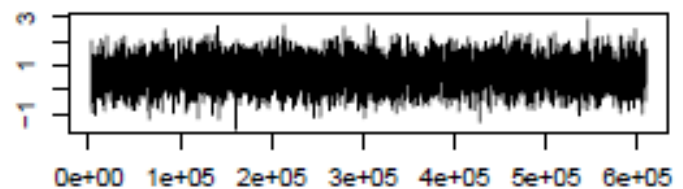

Iterations

Trace of time:live

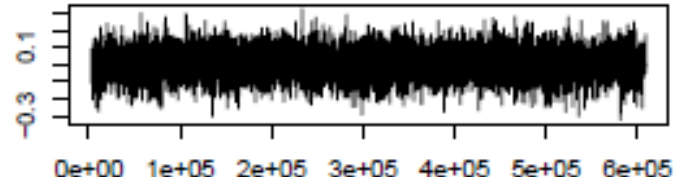

Iterations

Trace of careExp:relation

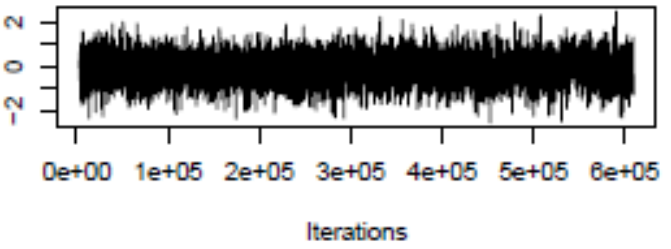

Trace of time:relation

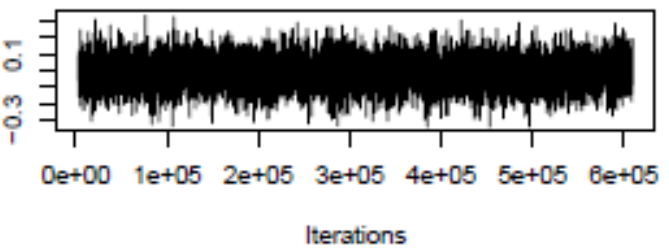

Trace of careExp:ete

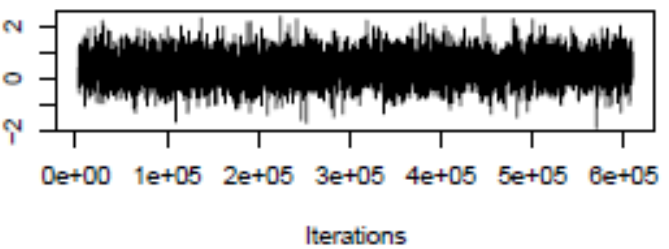

Trace of time:ete

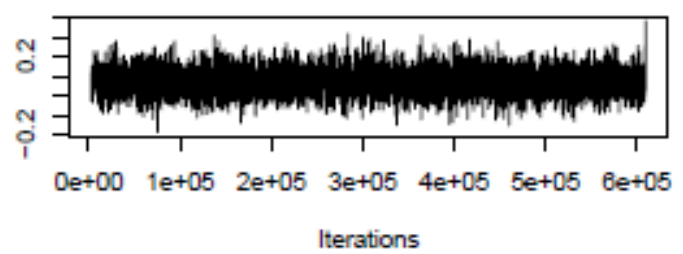

Density of careExp:live

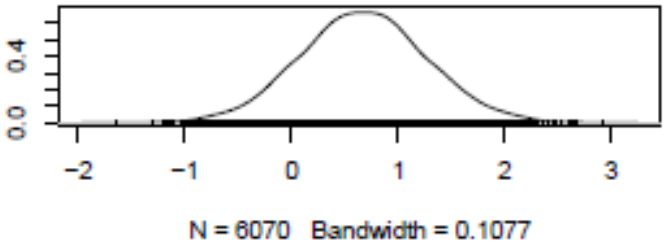

Density of time:live

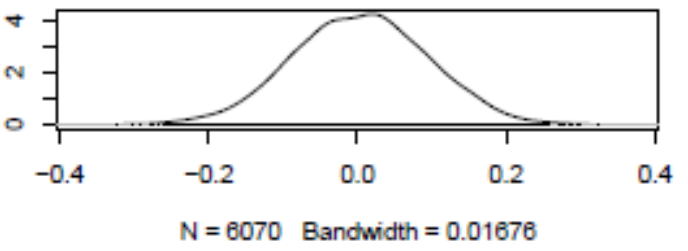

Density of careExp:relation

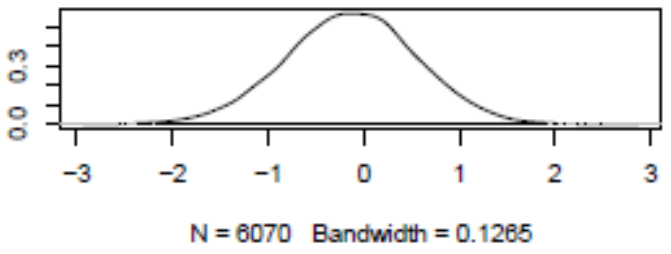

Density of time:relation

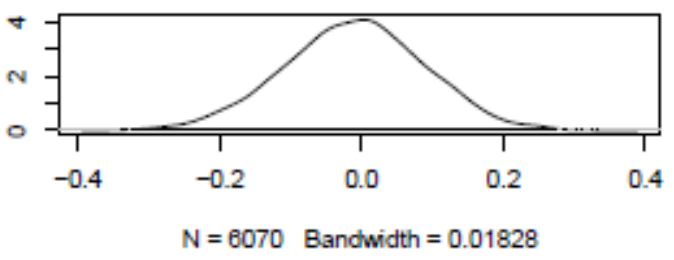

Density of careExp:ete

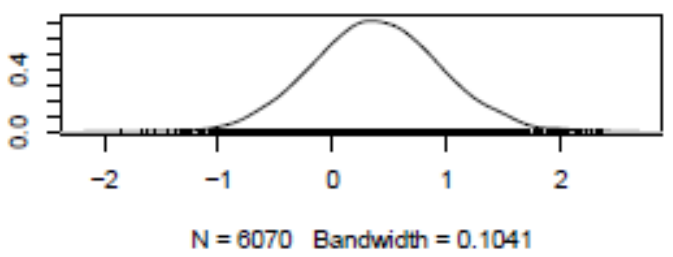

Density of time:ete

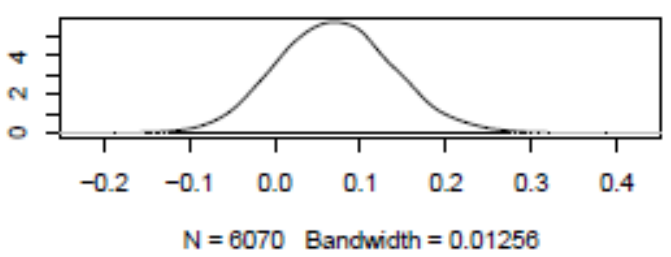


Trace of careExp:where

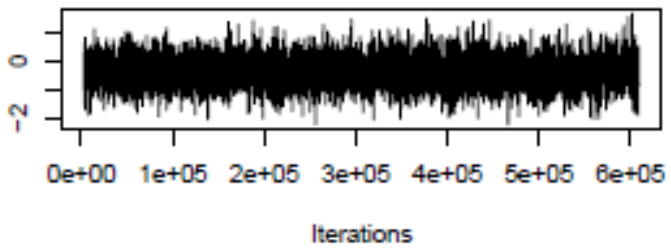

Trace of time:where

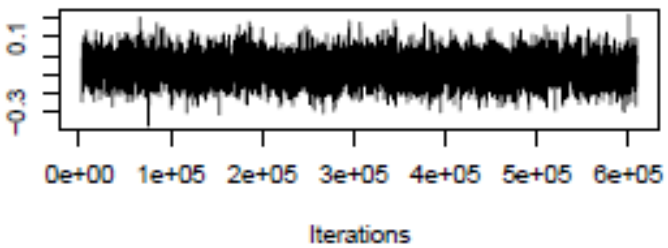

Trace of careExp:life

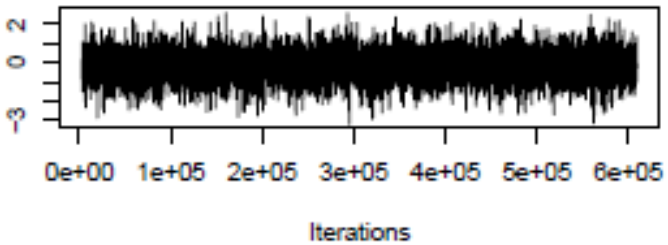

Trace of time:life

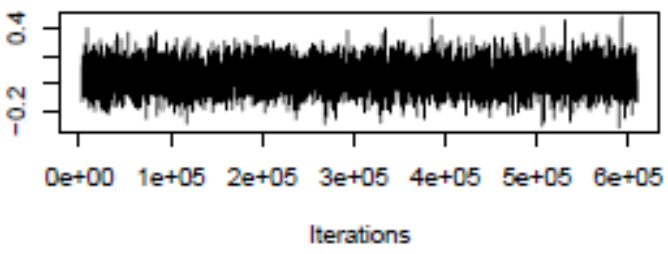

Trace of careExp:drugs

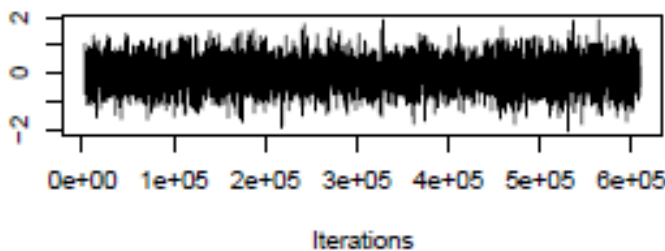

Trace of time:drugs

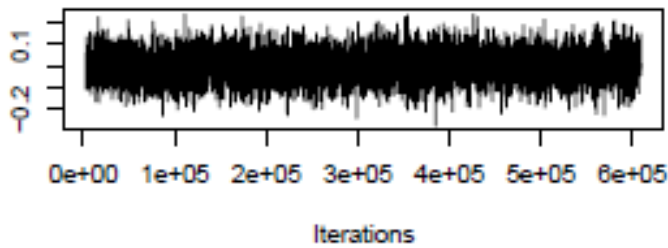

Density of careExp:where

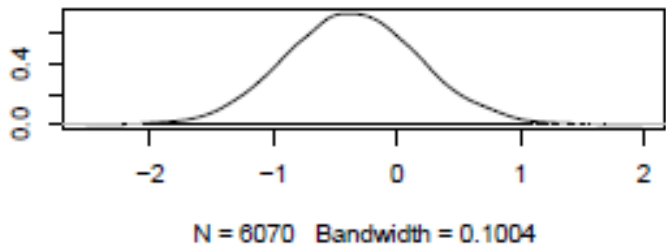

Density of time:where

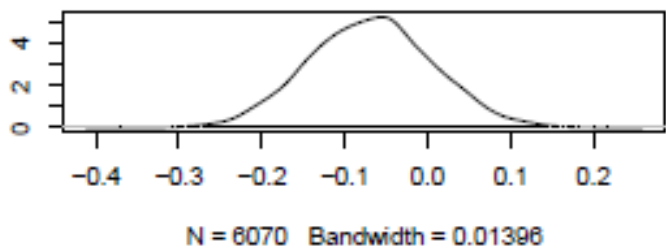

Density of careExp:life

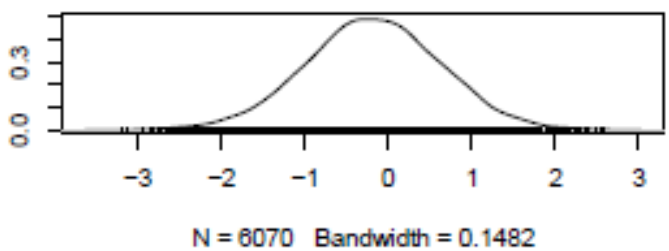

Density of time:life

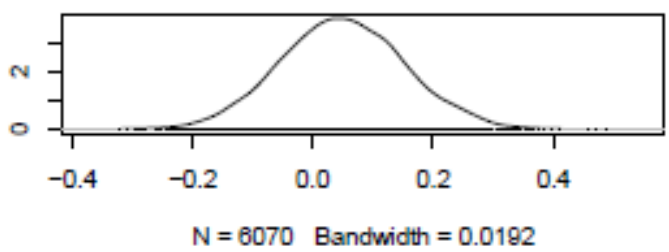

Density of careExp:drugs

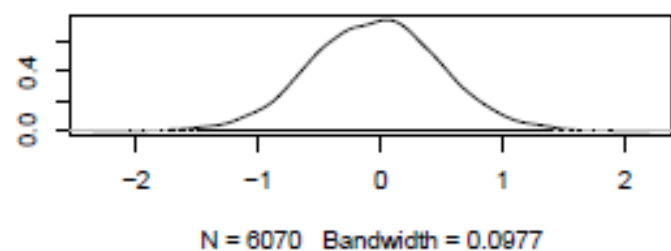

Density of time:drugs

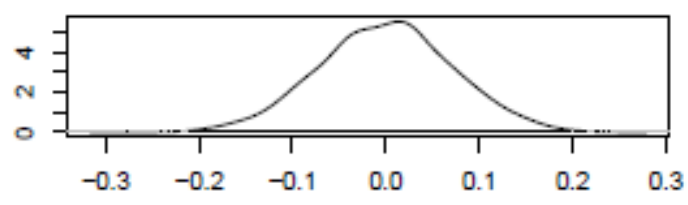

$\mathrm{N}=6070$ Bandwidth $=0.01328$ 
Trace of careExp:physical

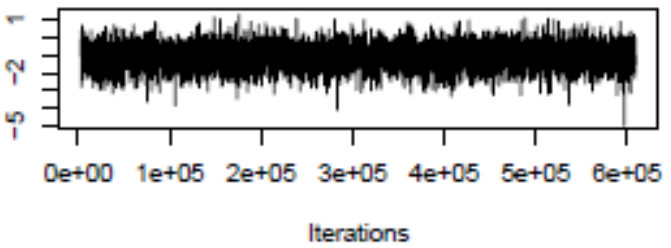

Trace of time:physical

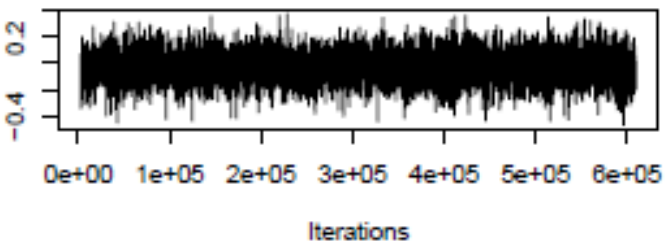

Trace of careExp:emotion

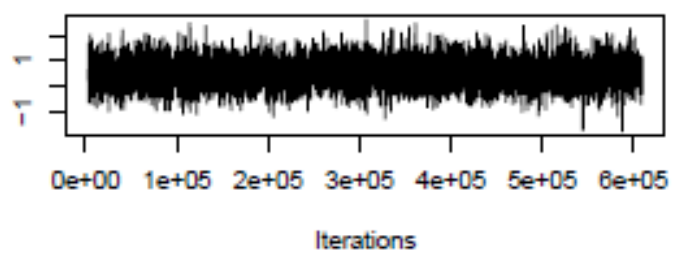

Trace of time:emotion

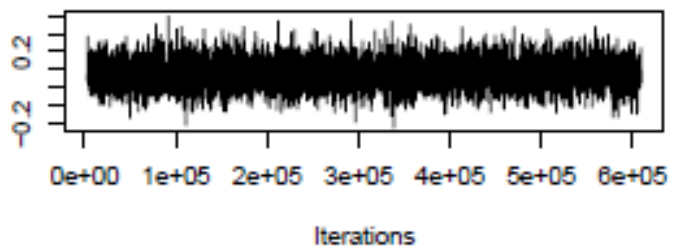

Trace of careExp:self

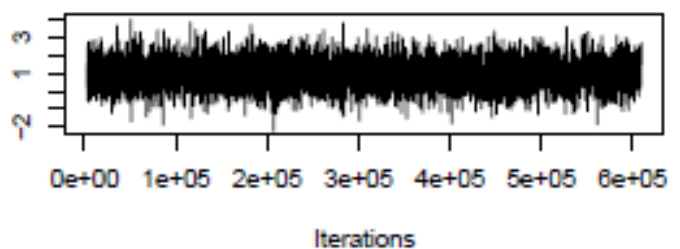

Trace of time:self

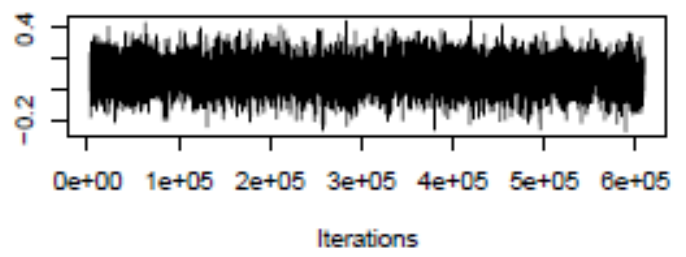

Density of careExp:physical

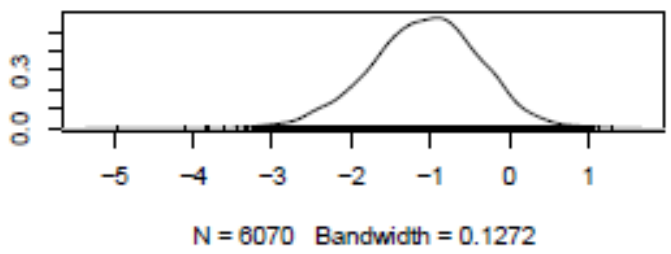

Density of time:physical

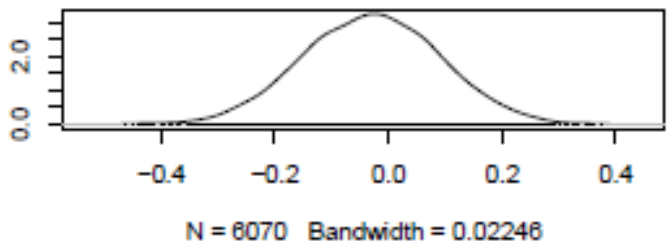

Density of careExp:emotion

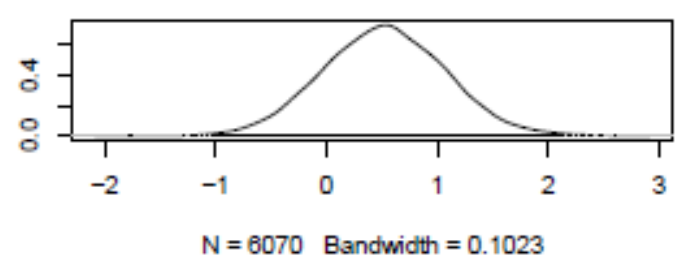

Density of time:emotion

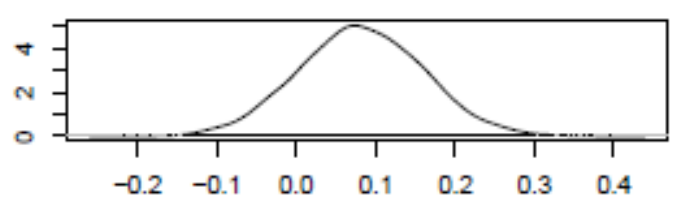

$\mathrm{N}=6070$ Bandwidth $=0.01466$

Density of careExp:self

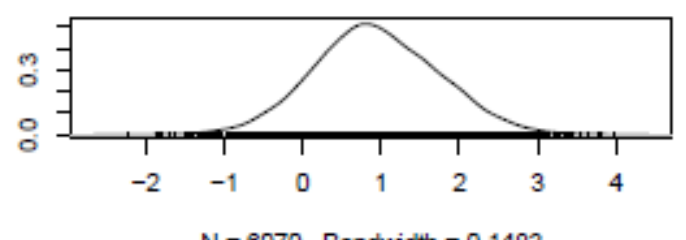

Density of time:self

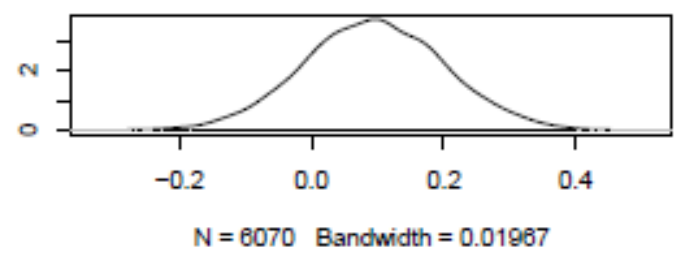


Trace of careExp:think

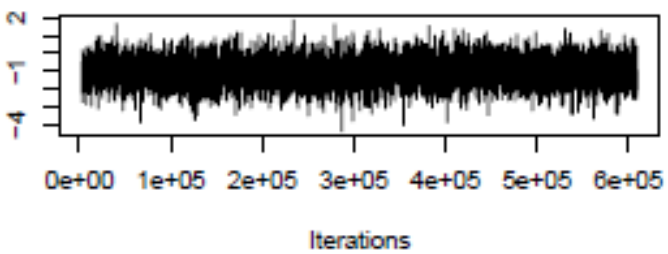

Trace of time:think

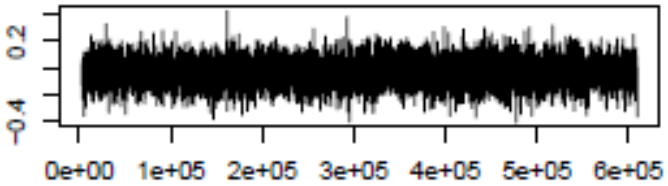

Iterations

Trace of careExp:attitude

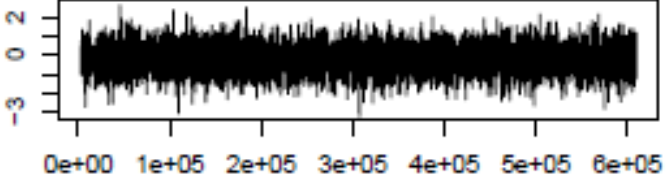

Iterations

Trace of time:attitude

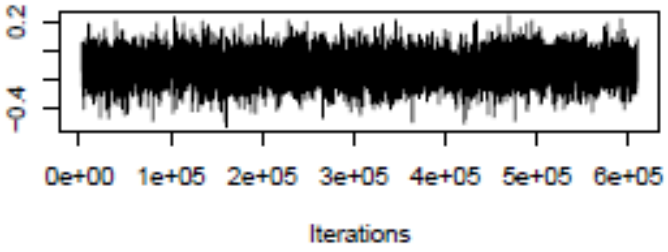

Trace of careExp:change

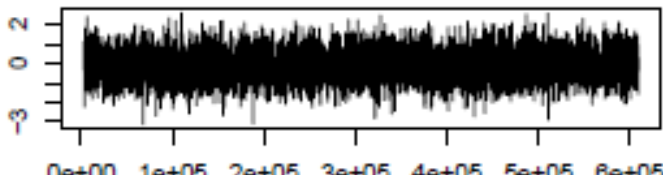

Iterations

Trace of time:change

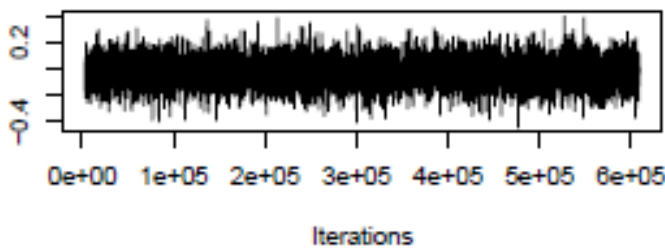

Density of careExp:think

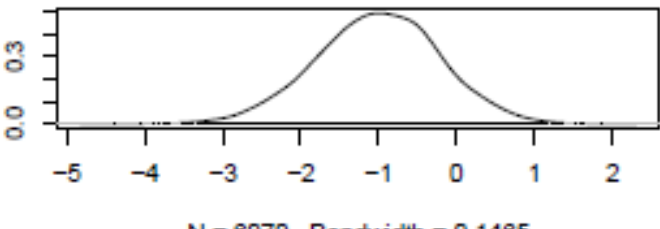

Density of time:think

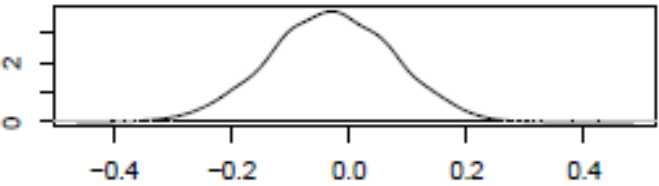

$\mathrm{N}=6070$ Bandwidth $=0.01962$

Density of careExp:attitude

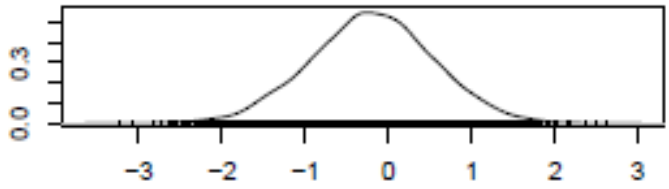

$\mathrm{N}=6070$ Bandwidth $=0.1343$

Density of time:attitude

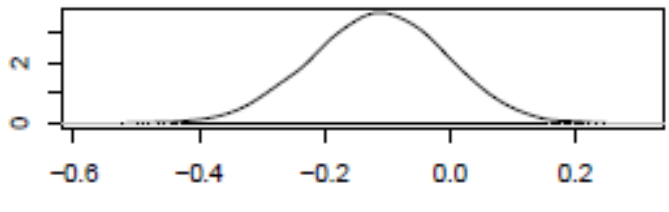

$\mathrm{N}=6070$ Bandwidth $=0.01995$

Density of careExp:change

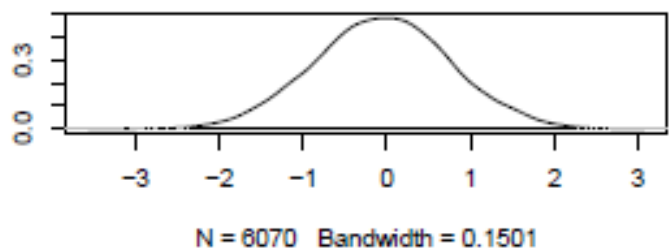

Density of time:change

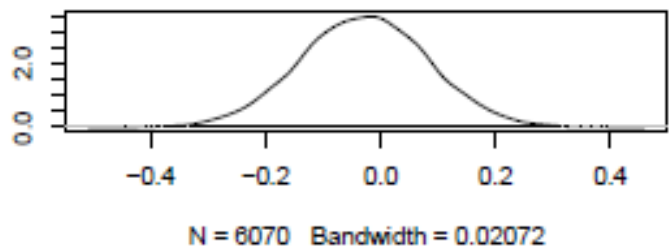


Trace of time:Gender

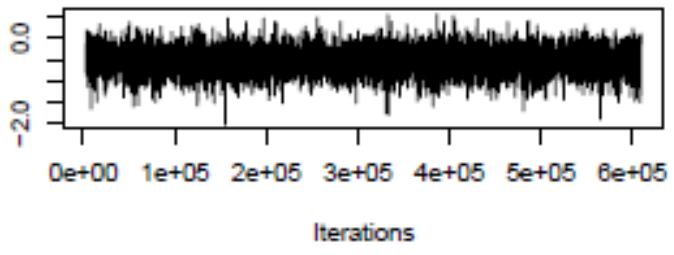

Trace of time:bme

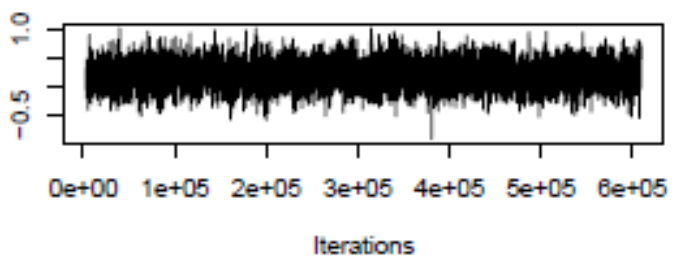

Trace of careExp:Gender

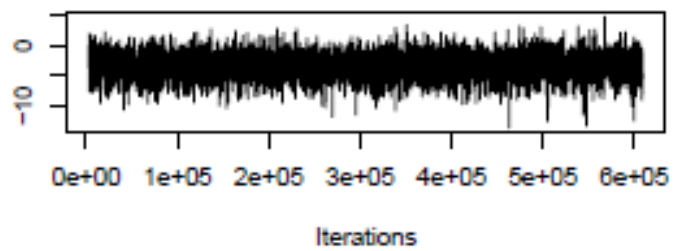

Trace of careExp:bme

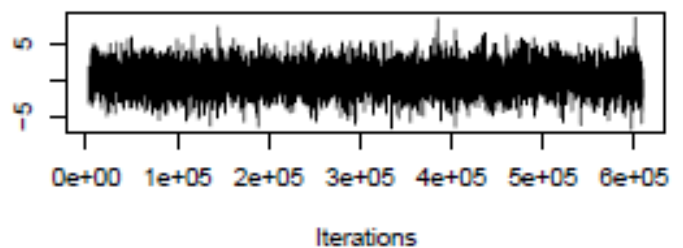

Trace of careExp:time:live

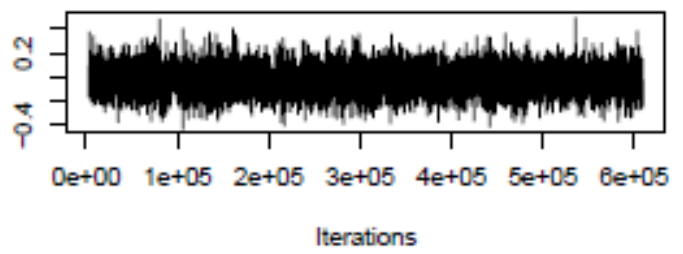

Trace of careExp:time:relation

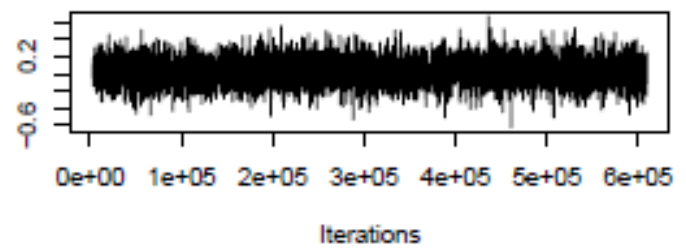

Density of time:Gender

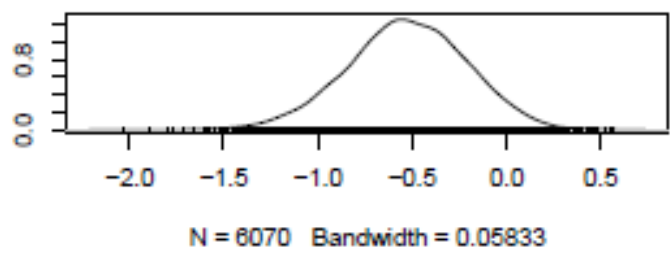

Density of time:bme

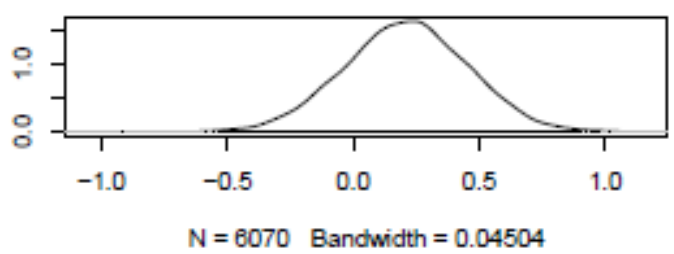

Density of careExp:Gender

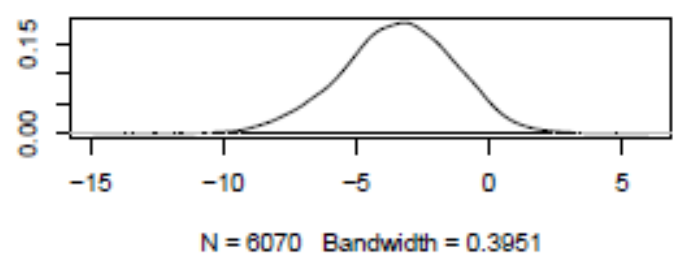

Density of careExp:bme

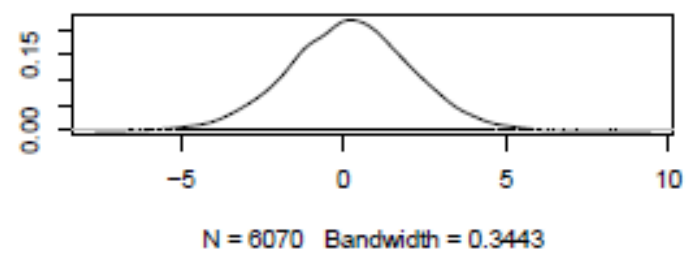

Density of careExp:time:live

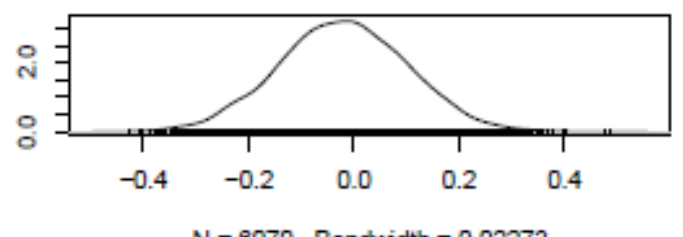

$\mathrm{N}=6070$ Bandwidth $=0.02273$

Density of careExp:time:relation

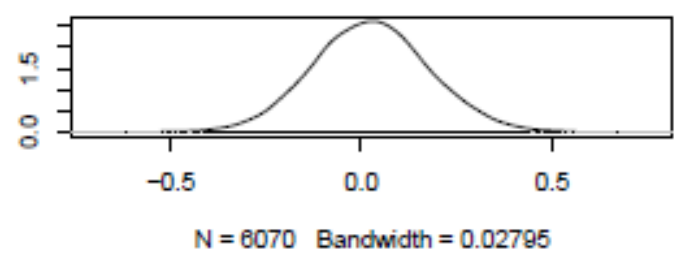


Trace of careExp:time:ete

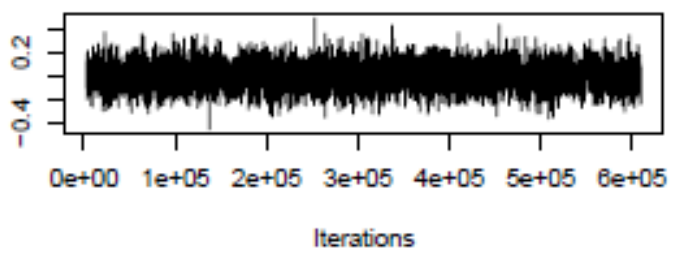

Trace of careExp:time:where

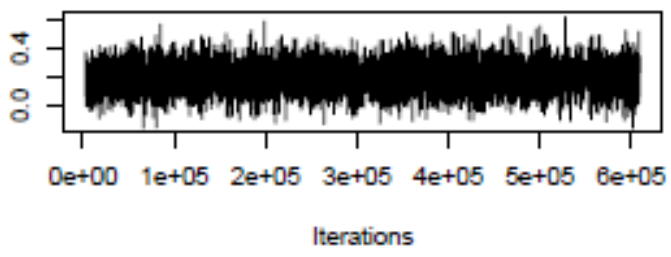

Trace of careExp:time:life

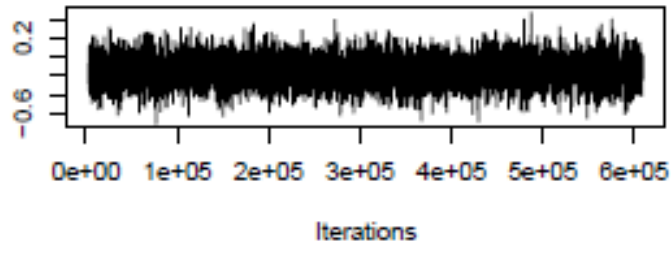

Trace of careExp:time:drugs

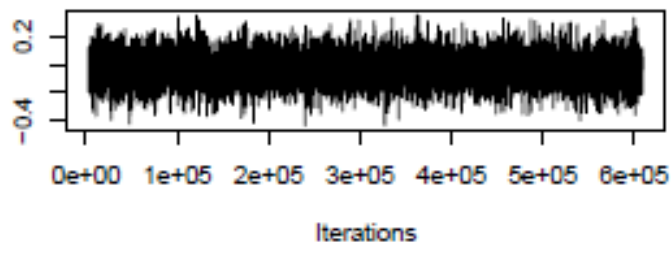

Trace of careExp:time:physical

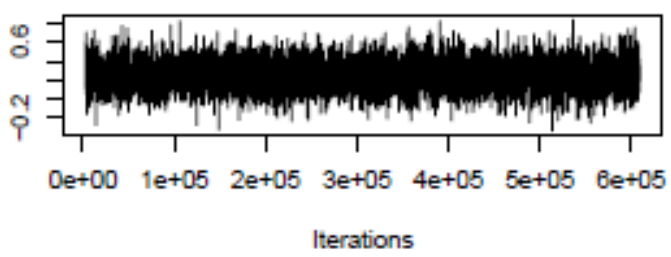

Trace of careExp:time:emotion

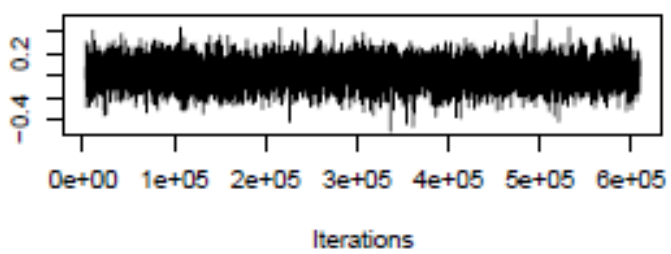

Density of careExp:time:ete

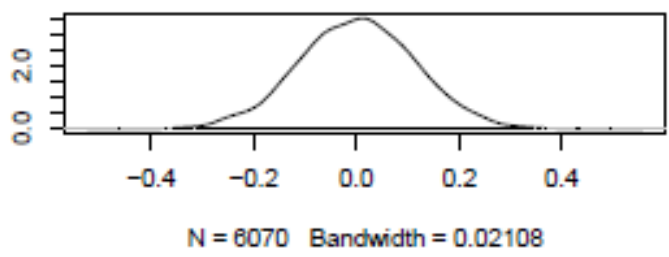

Density of careExp:time:where

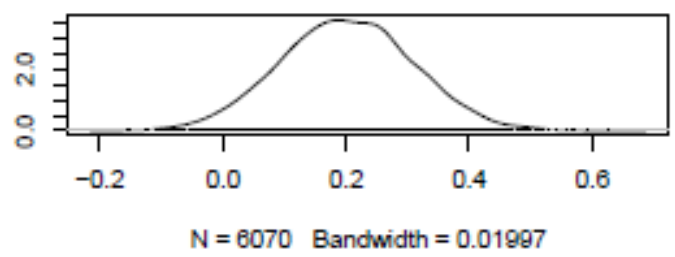

Density of careExp:time:life

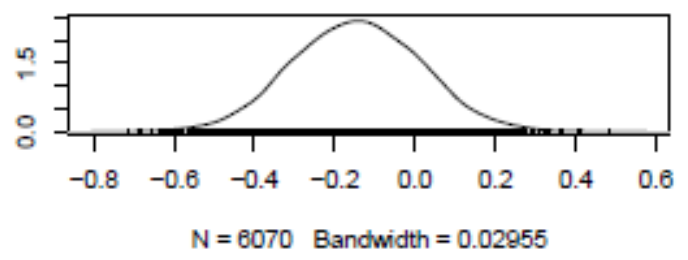

Density of careExp:time:drugs

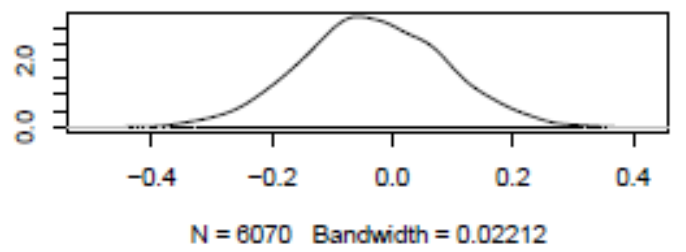

Density of careExp:time:physical

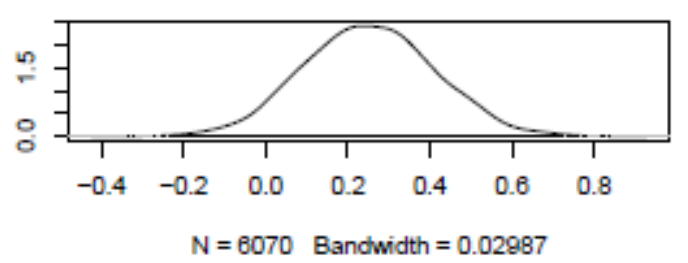

Density of careExp:time:emotion

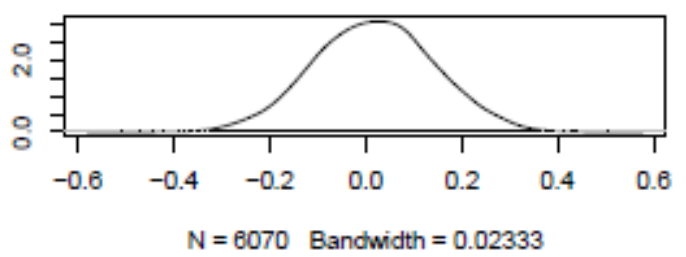


Trace of careExp:time:self

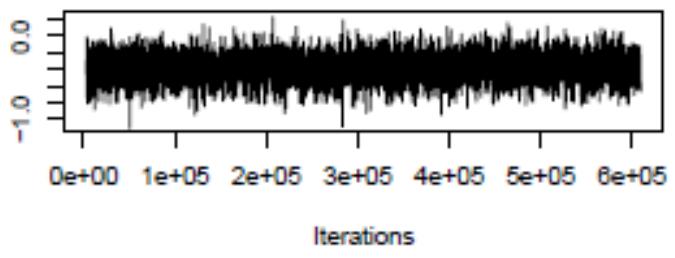

Trace of careExp:time:think

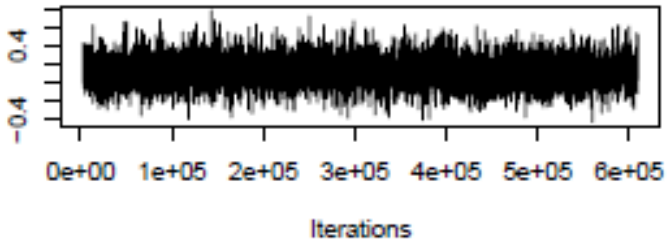

Trace of careExp:time:attitude

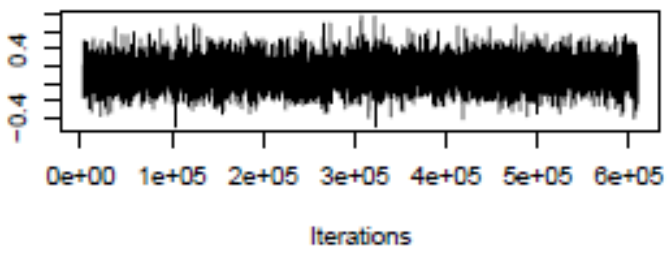

Trace of careExp:time:change

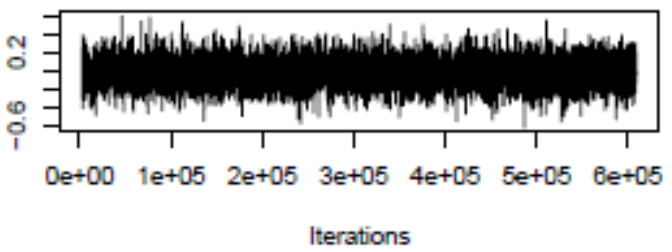

Density of careExp:time:self

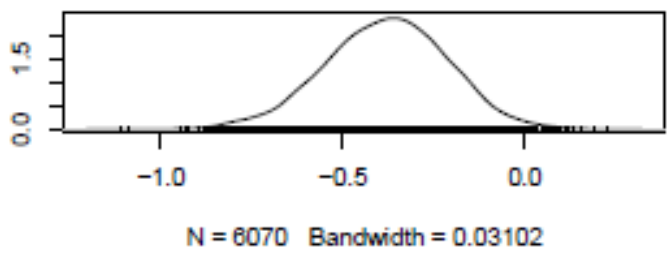

Density of careExp:time:think

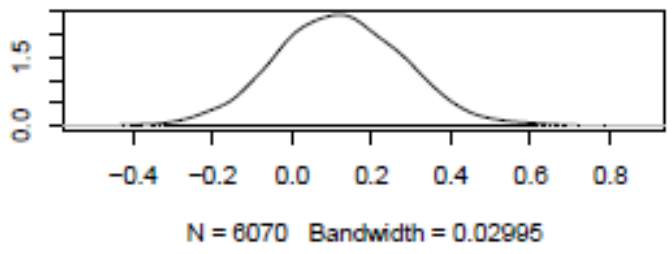

Density of careExp:time:attitude

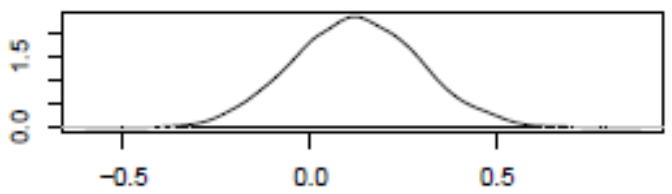

$\mathrm{N}=6070$ Bandwidth $=0.03154$

Density of careExp:time:change

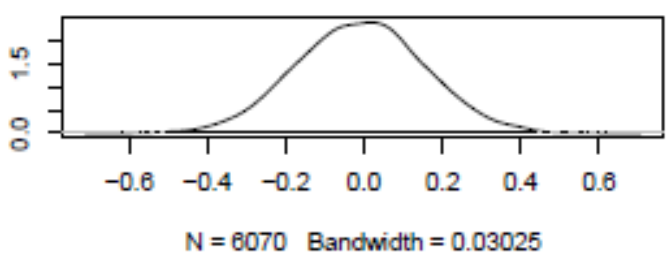


$\underline{\text { Random Effects }}$

Trace of time

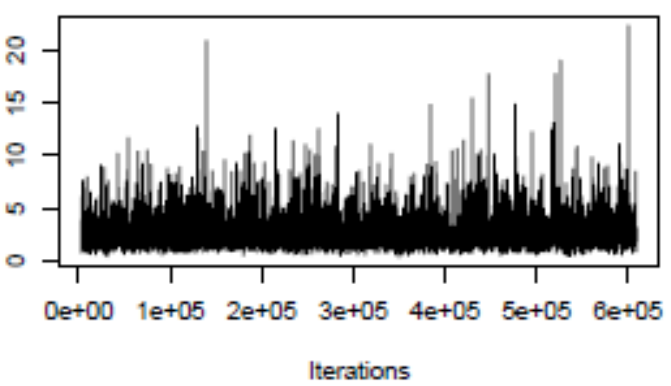

Trace of Research.ID

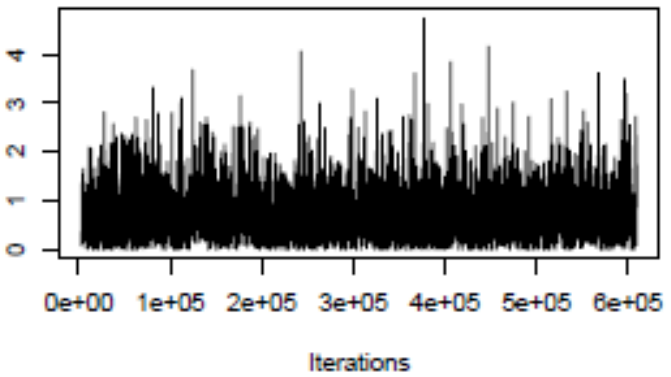

Density of time

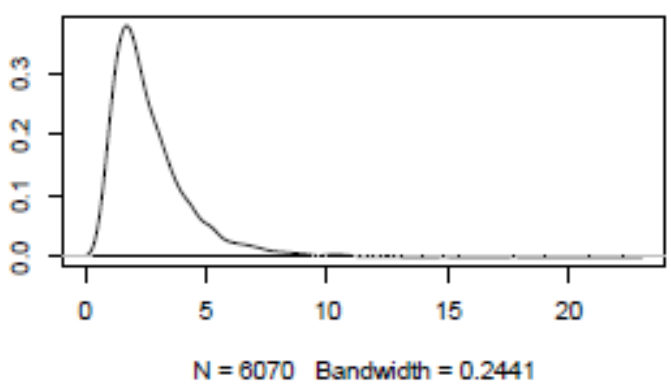

Density of Research.ID

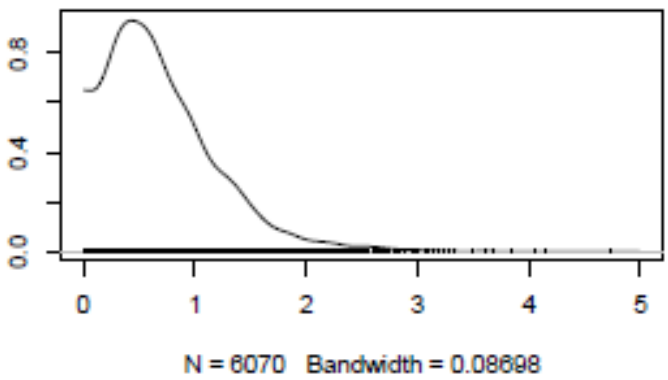




\section{Chapter Six - Static Factors}

Model 1.5 - Basic Model + Grouped Age at First Offence (Table 6.4)

Bayesian Model (Bm1G_cc2)

\section{\#\# Define the model}

Bm1G CC2 <- MCMCglmm(FO.bin G ageFirst + live + relation + ete + where + life + drugs + physical + emotion + self + think + attitude + change + time, random= time+Research.ID, data=data3, family="ordinal", prior=priorD, slice=TRUE, nitt=200000, thin=50, burnin=3000)

\section{\#\# Checks for suitable convergence}

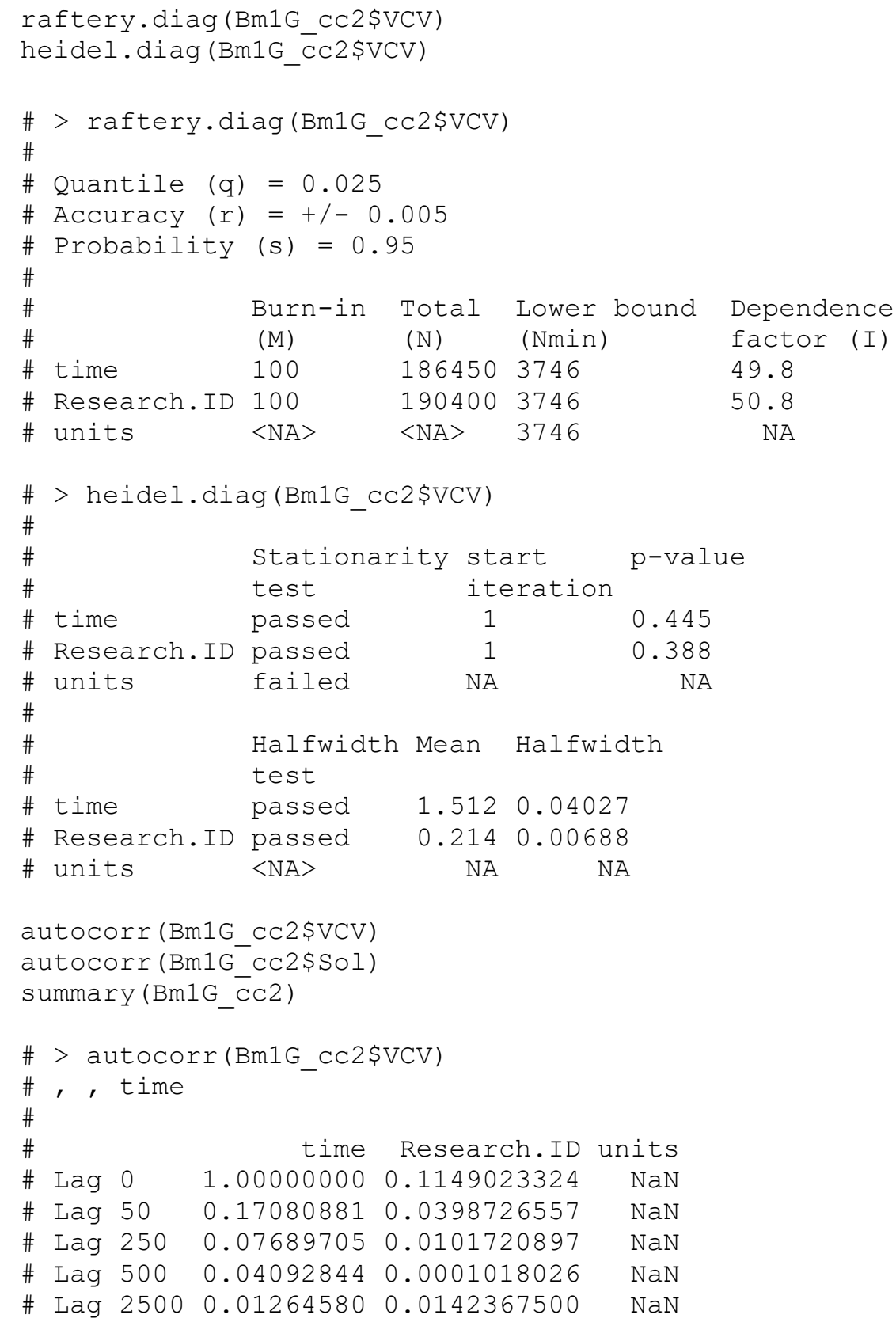




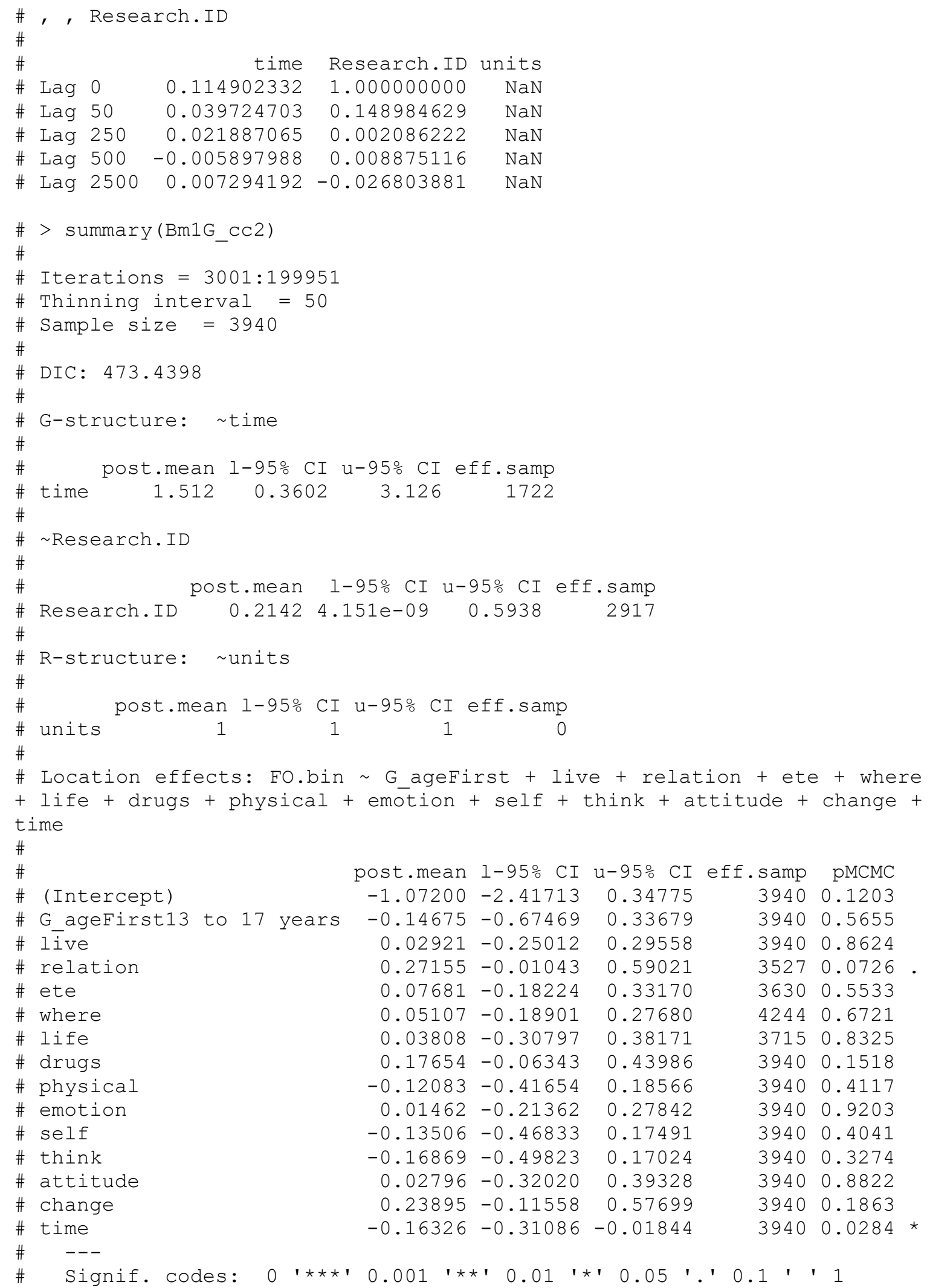


m1G CC2 <- glmer(FO.bin G ageFirst + live + relation + ete + where + life + drugs + physical + emotion + self + think + attitude + change + time + (timelIndividual), data=data, family=binomial)

summary (m1G_cc2)

vcomps.icc ( $\left.\overline{\mathrm{m}} 1 \mathrm{G} \_\mathrm{cc} 2\right)$

anova (m1, m1G_c $\bar{c} 2)$

\section{Warning message:}

In checkConv(attr (opt, "derivs"), opt\$par, ctrl = control\$checkConv, : Model failed to converge with max $\mid$ grad $=0.0360707$ (tol $=0.001$, component 1)

\# > summary (m1G_cc2)

\# Generalized linear mixed model fit by maximum likelihood (Laplace Approximation) ['glmerMod']

\# Family: binomial ( logit)

\# Formula: FO.bin G_ageFirst + live + relation + ete + where + life +

\# drugs + physical + emotion + self + think + attitude + change +

time + (time | Individual)

\# Data: data

$\#$

\# AIC BIC logLik deviance df.resid

\# $642.5 \quad 719.9 \quad-303.3 \quad 606.5 \quad 527$

$\#$

\# Scaled residuals:

\# $\quad$ Min 10 Median $30 \quad$ Max

$\# \begin{array}{lllll}\#-1.6688 & -0.6683 & -0.3657 & 0.8210 & 3.6161\end{array}$

$\#$

\# Random effects:

\# Groups Name Variance Std.Dev. Corr

\# Individual (Intercept) $0.04508 \quad 0.2123$

\# time $0.05330 \quad 0.2309-1.00$

\# Number of obs: 545, groups: Individual, 87

\#

\# Fixed effects:

\# Estimate Std. Error z value Pr $(>|z|)$

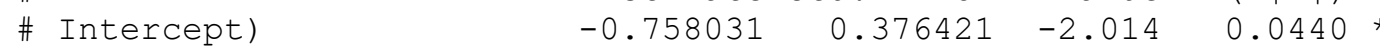

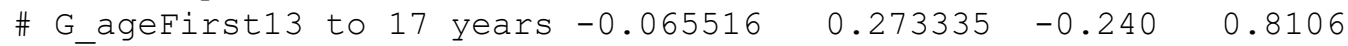

\# live

\# relation

relation

$-0.068365$

0.143999

$-0.475$

0.6350

0.002639

0.157399

1.226

0.2202

\# where

0.150261

0.129792

0.020

0.9838

\# life

0.020749

0.127519

1.178

0.2387

\# drugs

\# physical

0.264647

0.190939

0.109

0.9135

\# emotion

$-0.223508$

0.132960

1.990

0.0465

\# self

\# think

\# attitude

$-0.033675$

$-1.492$

0.1357

\# change

\# time

$-0.060519$

0.133387

$-0.252$

0.8007

0.121178

0.175111

$-0.346$

0.7296

$-0.068246$

.186468

0.650

0.5158

0.215122

0.190813

$-0.358$

0.7206

$-0.441566$

0.180713

1.190

0.2339

\# ---

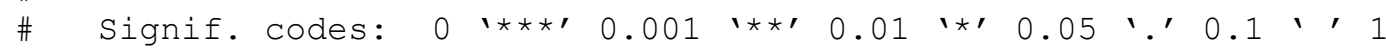

\# convergence code: 0

\# Model failed to converge with max|grad $=0.0360707$ (tol $=0.001$, component 1) 


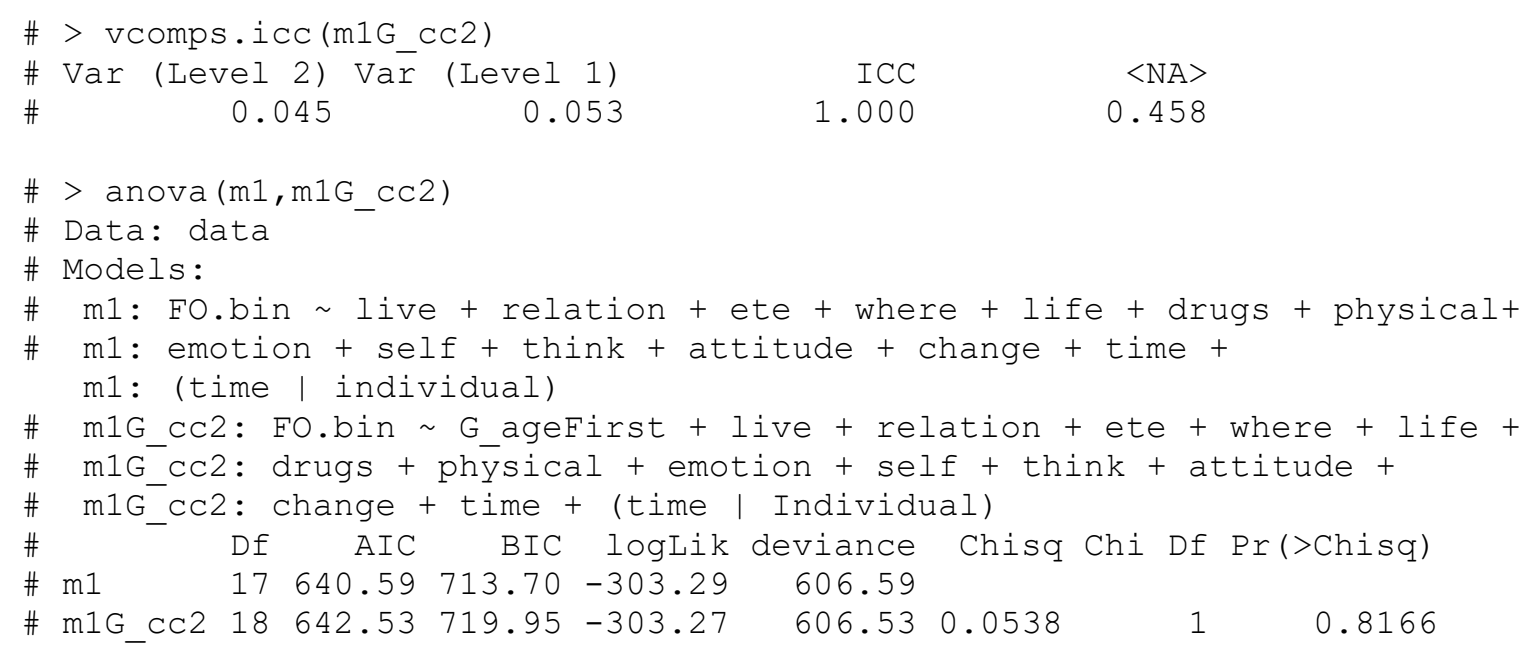


Model 1.6 - Basic Model + Grouped Age at First Conviction (Table 6.4)

Bayesian Model (Bm1G_cc3)

\section{\#\# Define the model}

Bm1G_CC3 <- MCMCglmm(FO.bin G_ageCon + live + relation + ete + where + life + drugs + physical + emotion + self + think + attitude + change + time, random= time+Research. ID, data=data3, family="ordinal",prior=priorD, slice=TRUE, nitt $=200000$, thin=50, burnin=3000)

\section{\#\# Checks for suitable convergence}

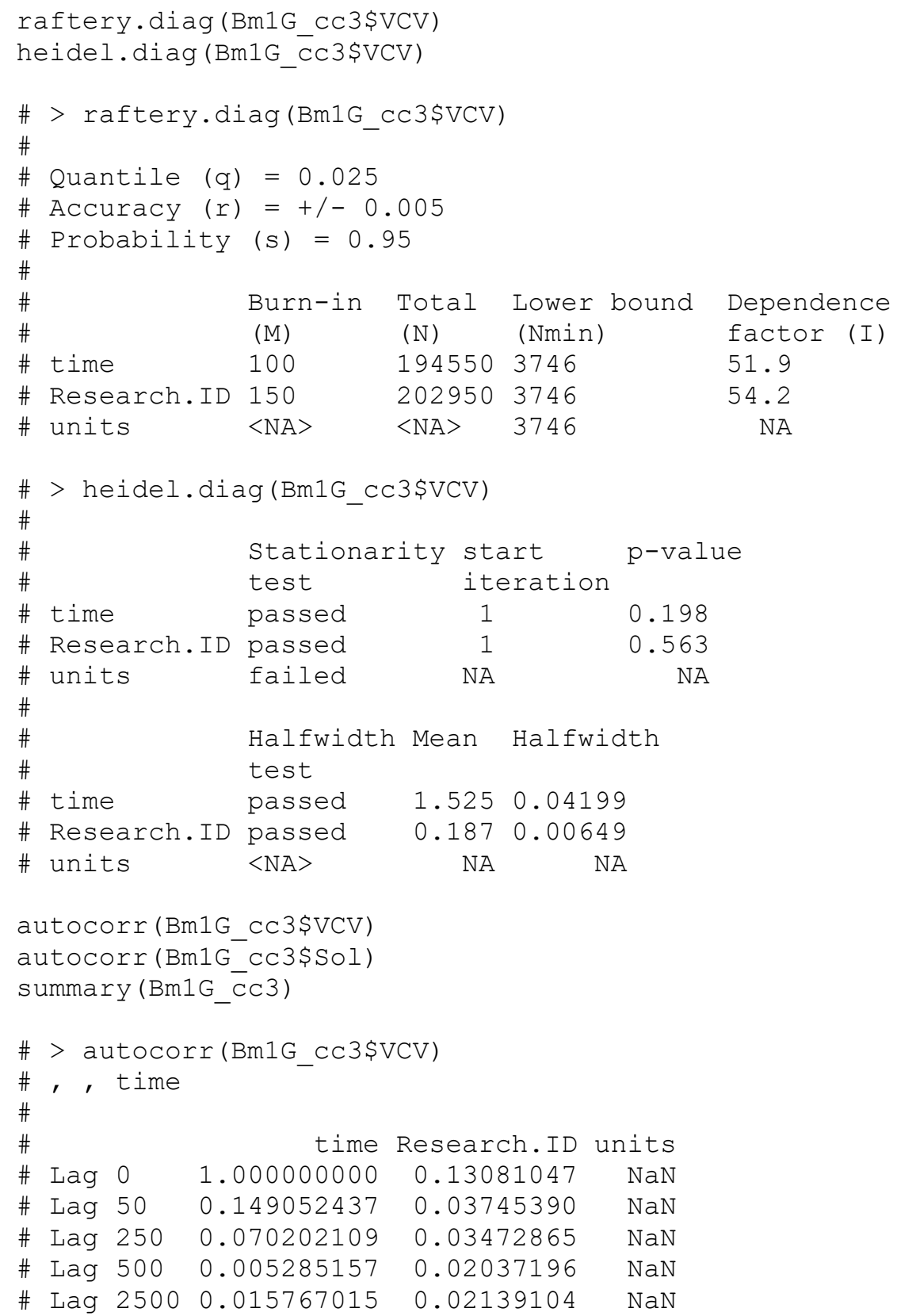




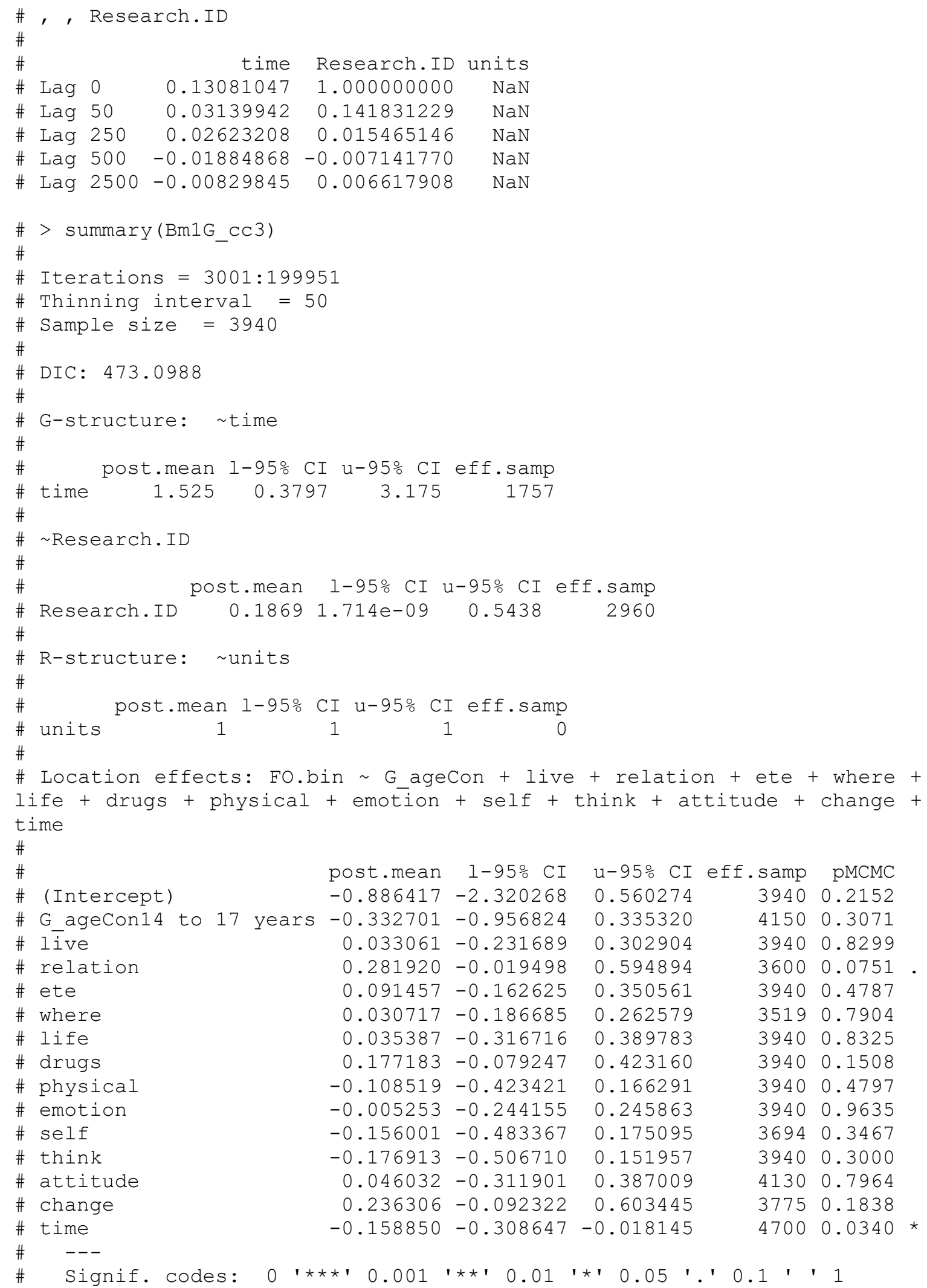


m1G cc3 <- glmer(FO.bin G ageCon + live + relation + ete + where + life + drugs + physical + emotion + self + think + attitude + change + time + (timelIndividual), data=data, family=binomial)

summary (m1G_cc3)

vcomps.icc ( $\left.\overline{\mathrm{m}} 1 \mathrm{G} \_\mathrm{cc} 3\right)$

anova (m1, m1G_cc 3 )

\section{Warning message:}

In checkConv(attr (opt, "derivs"), opt\$par, ctrl = control\$checkConv, : Model failed to converge with max|gradl $=0.118701$ (tol $=0.001$, component 1)

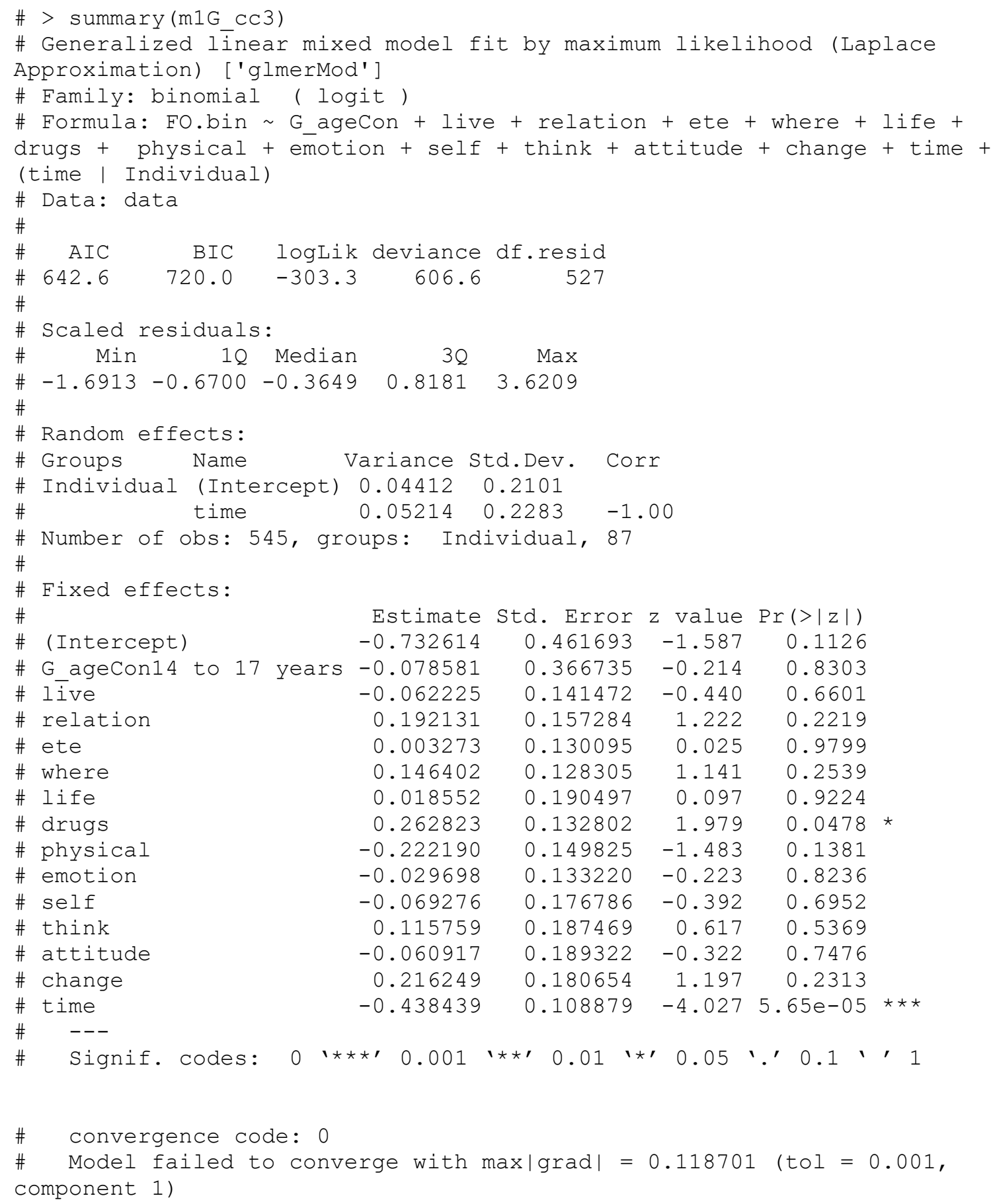




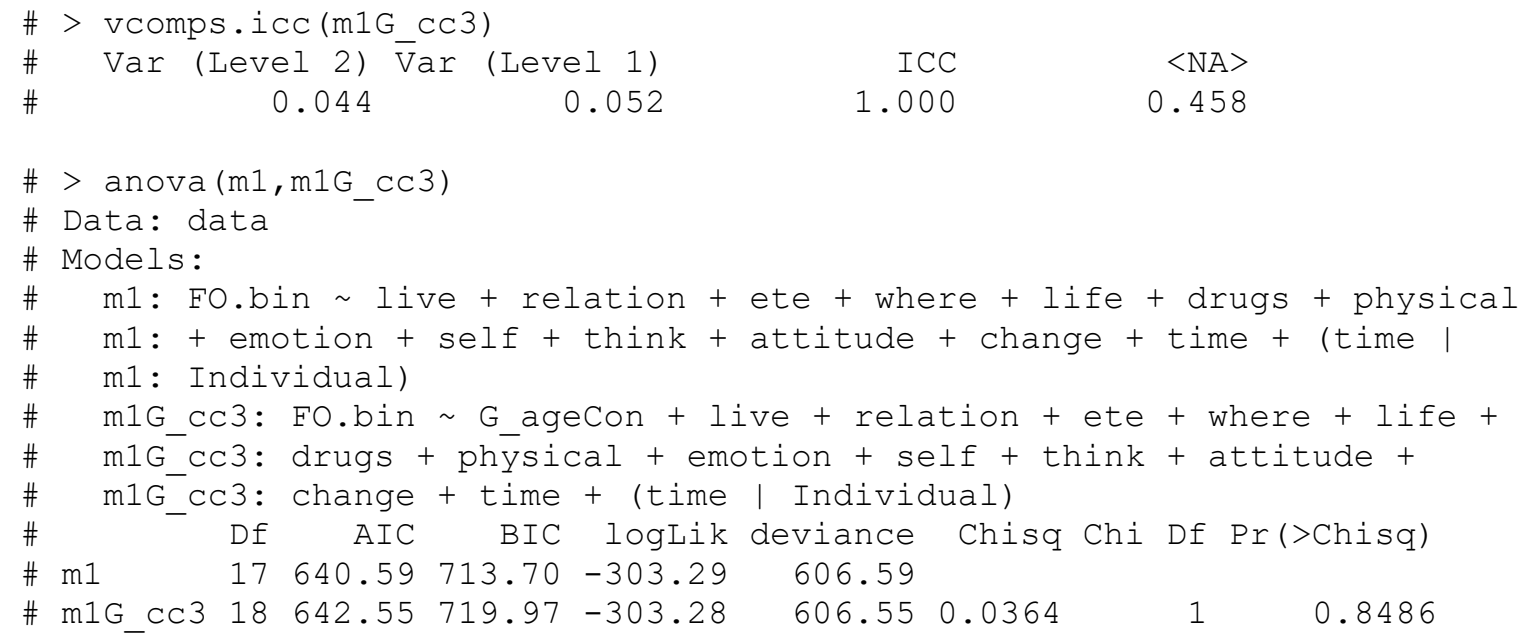


Model 1.7 - Basic Model + FTE (Table 6.5)

Bayesian Model (Bm1_cc1)

\section{\#\# Define the model}

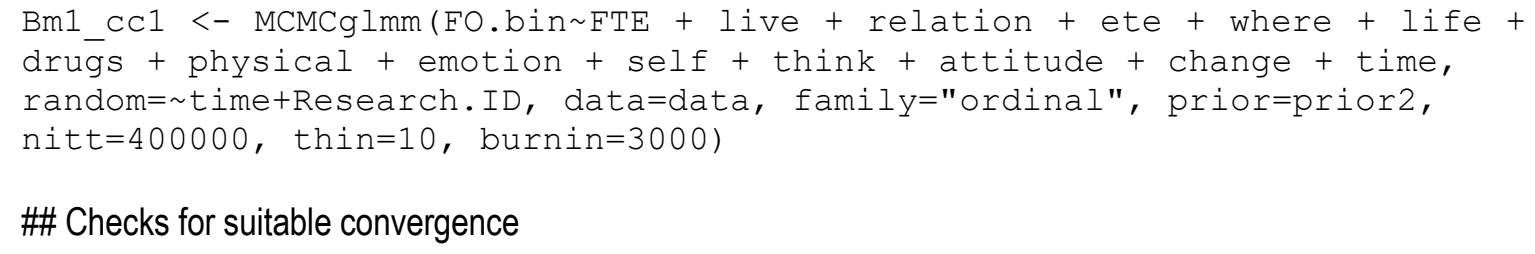

\section{\#\# Checks for suitable convergence}

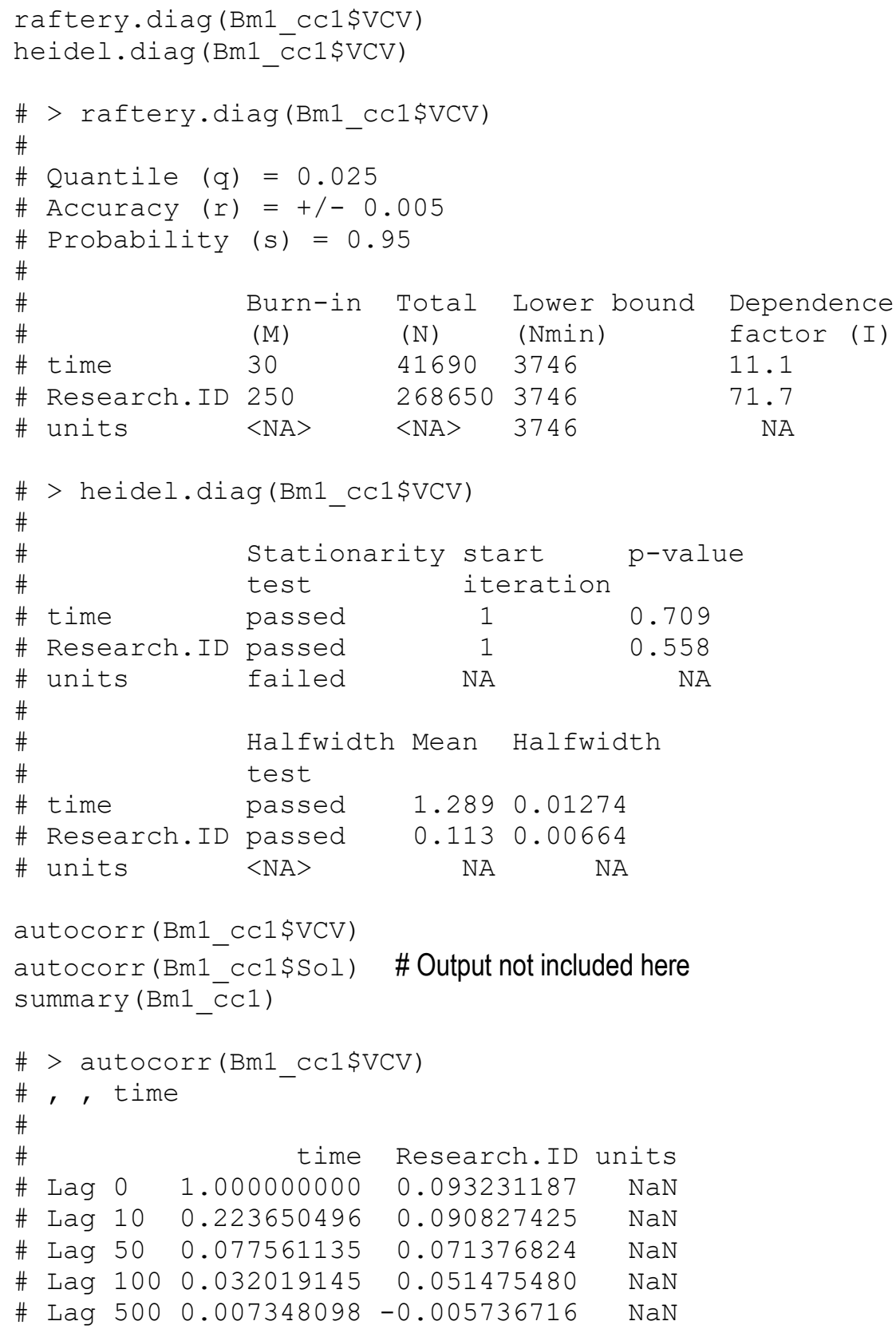




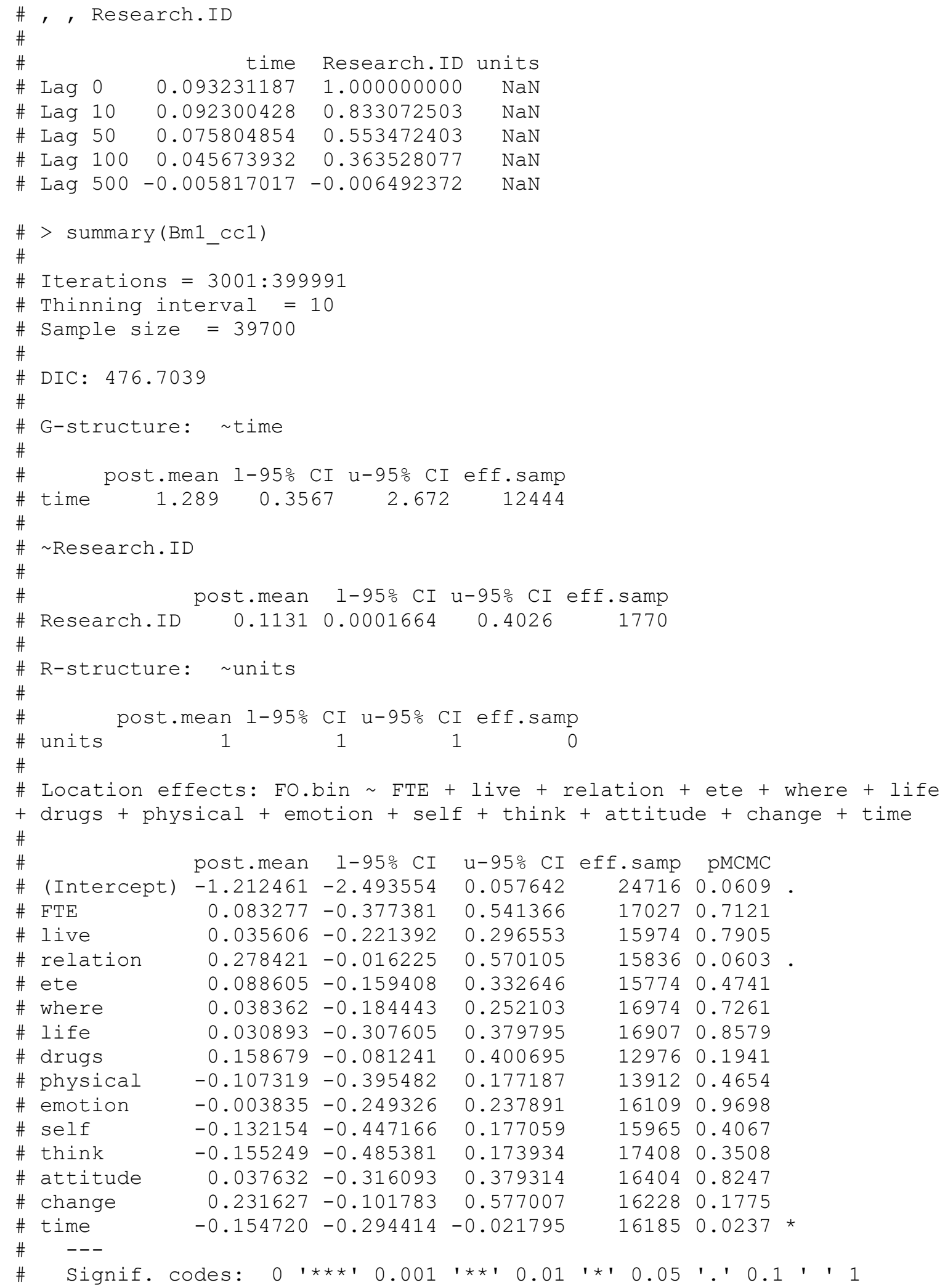




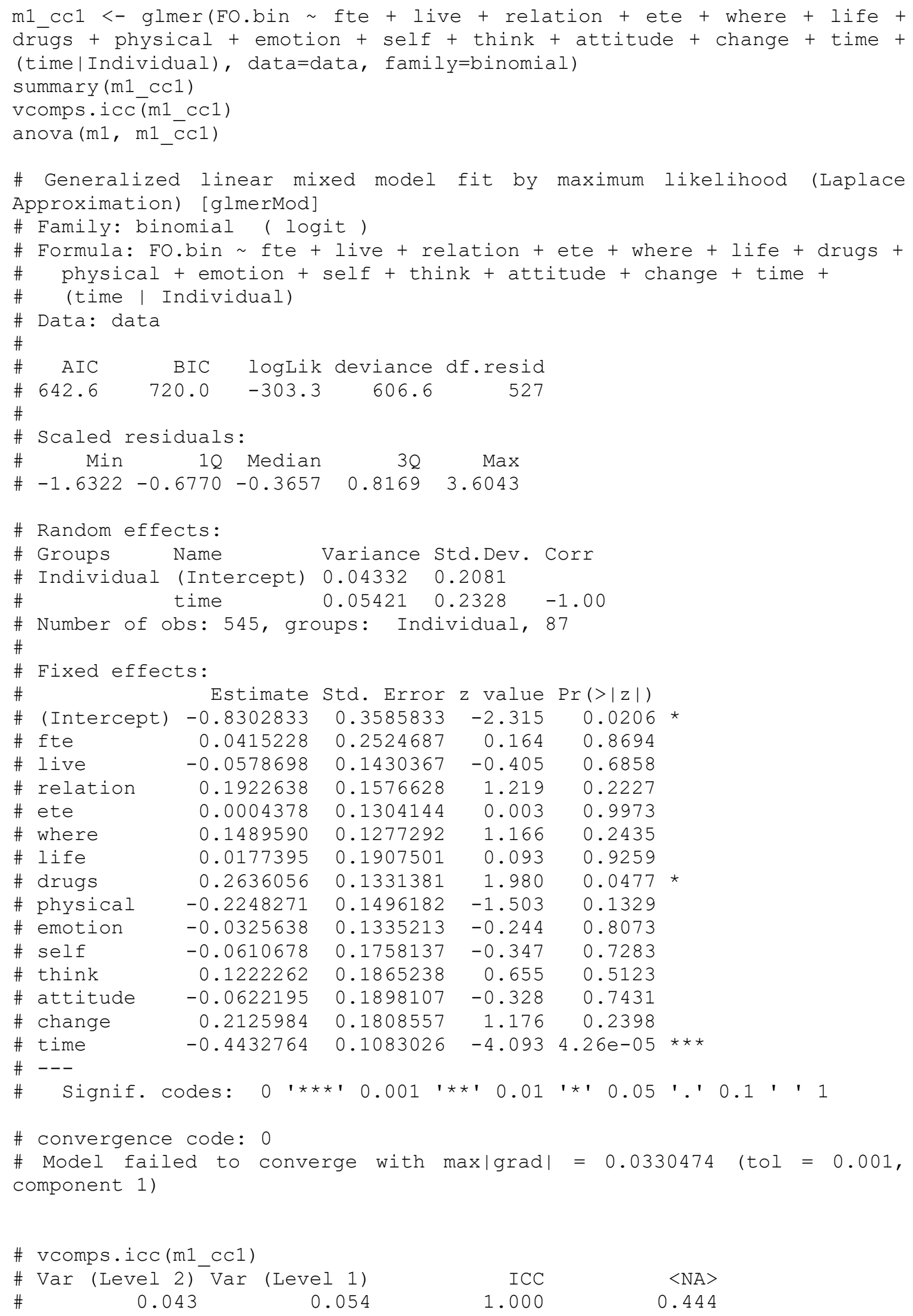




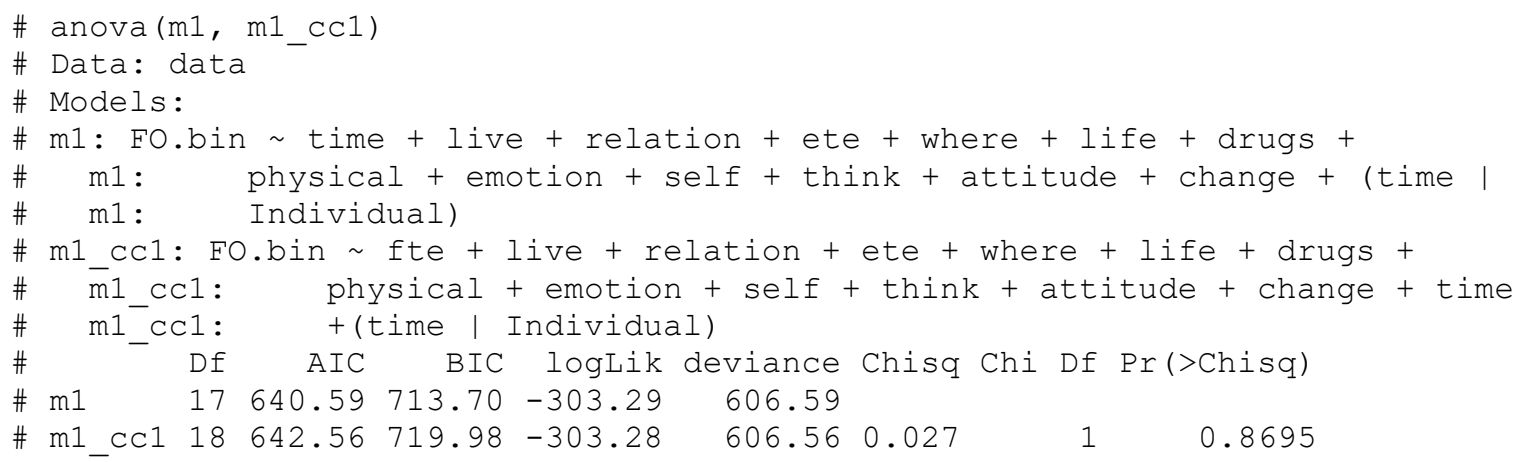




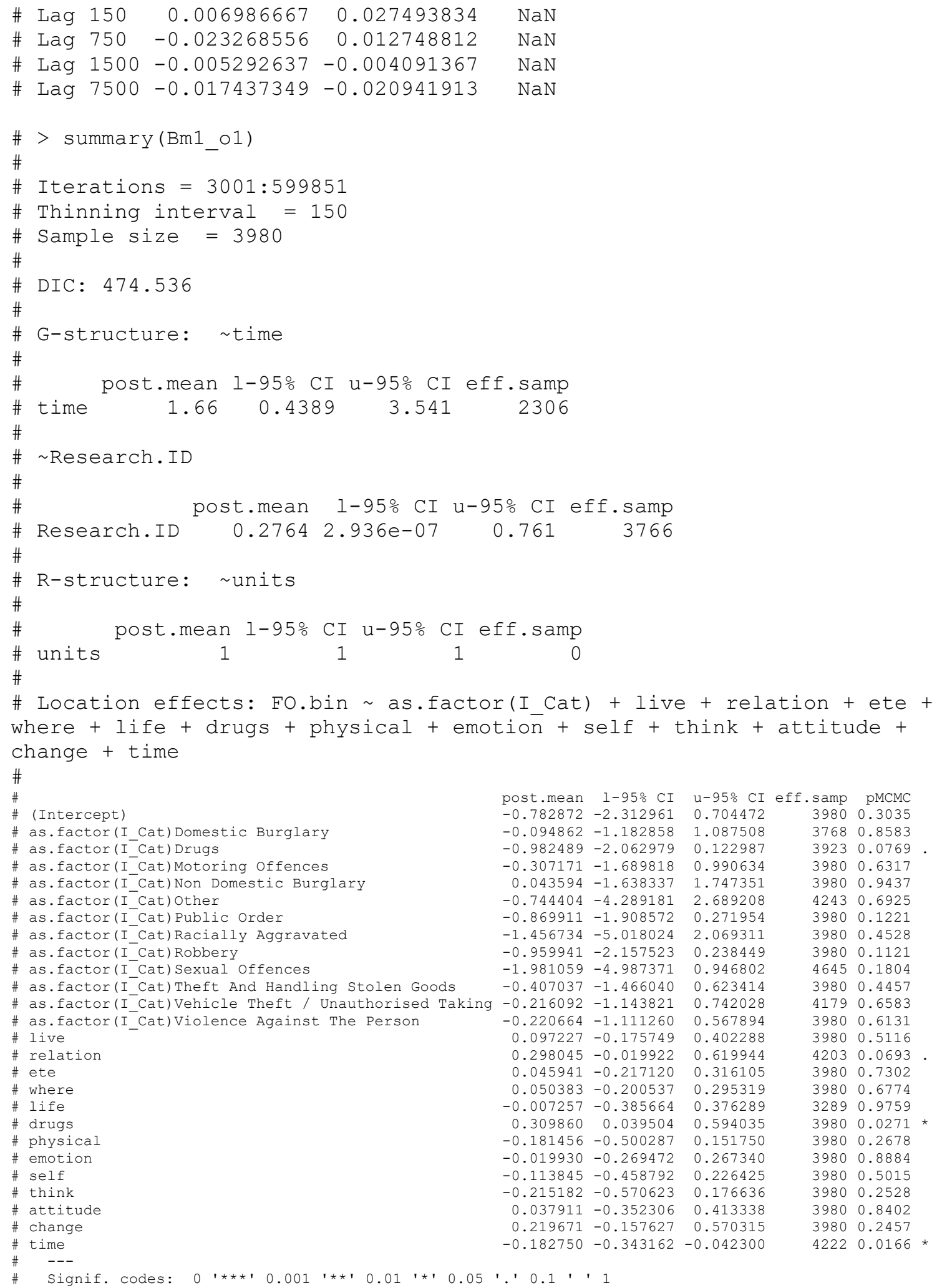


Frequentist Model (m1_o1)

m1 ol <- glmer(FO.bin as.factor(I Cat) + live + relation + ete + where + Iife + drugs + physical + emotion ${ }^{-}$self + think + attitude + change + time + timelIndividual), data=data3, family=binomial)

summary (m1 o1)

vcomps.icc $\left(\mathrm{m} 1 \_\right.$ol)

anova (m1_o1, mí_ola)

Warning message:

In checkConv(attr (opt, "derivs"), opt\$par, ctrl =
control\$checkConv, : Model failed to converge with max|gradl =
0.109368 (tol $=0.001$, component 1)

\# > summary $\left(m 1 \_01\right)$

\# Generalized linear mixed model fit by maximum likelihood (Laplace Approximation) ['glmerMod']

\# Family: binomial ( logit)

\# Formula: FO.bin as.factor(I_Cat) + live + relation + ete + where +

life + drugs + physical + emotion + self + think + attitude + change + time + (time | Individual)

\# Data: data3

\#

\# AIC BIC logLik deviance df.resid

$\begin{array}{llllr}\text { \# } 657.1 & 781.8 & -299.6 & 599.1 & 516\end{array}$

\#

\# Scaled residuals:

\# Min $\quad 10$ Median $3 Q \quad$ Max

$\begin{array}{llllll}\#-2.0267 & -0.6646 & -0.3588 & 0.8112 & 3.7450\end{array}$

\#

\# Random effects:

\# Groups Name Variance Std.Dev. Corr

\# Individual (Intercept) 0.072140 .2686

\# $\quad$ time $\quad 0.04347 \quad 0.2085 \quad-1.00$

\# Number of obs: 545, groups: Individual, 87

\#

\# Fixed effects:

\#

\# (Intercept)

\# as.factor(I Cat) Domestic Burglary

\# as.factor (I-Cat) Drugs

\# as.factor(I_Cat) Motoring offences

\# as.factor(I Cat) Non Domestic Burglary

\# as.factor (I Cat) Other

\# as.factor(I_Cat) Public Order

\# as.factor(I_Cat) Racially Aggravated

\# as.factor (I Cat) Robbery

\# as.factor ( I Cat) Sexual offences

\# as.factor(I_Cat) Theft And Handling Stolen Goods

\# as.factor(I Cat)Vehicle Theft / Unauthorised Taking

\# as.factor(I_Cat)Violence Against The Person

\# live

\# relation

\# ete

\# where

\# life

\# drugs

\# physical

\# emotion

\# self

\# think

\# attitude

\# change

\# time

$\#$ Hime

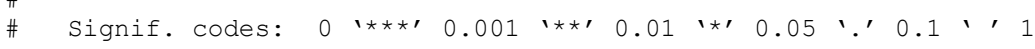

Estimate Std. Error z value $\operatorname{Pr}(>|z|)$

$\begin{array}{rrr}-0.414796 \quad 0.456019 & -0.910 & 0.3630\end{array}$

$\begin{array}{llll}-0.225663 & 0.542671 & -0.416 & 0.6775\end{array}$

$\begin{array}{llll}-0.836899 & 0.538080 & -1.555 & 0.1199\end{array}$

$\begin{array}{llll}-0.189013 & 0.687615 & -0.275 & 0.7834\end{array}$

$\begin{array}{llll}0.456535 & 0.931904 & 0.490 & 0.6242\end{array}$

$\begin{array}{llll}-0.286017 & 1.339457 & -0.214 & 0.8309\end{array}$

$\begin{array}{llll}-0.783258 & 0.491401 & -1.594 & 0.1110\end{array}$

$\begin{array}{llll}-0.396151 & 1.308967 & -0.303 & 0.7622\end{array}$

$\begin{array}{llll}-0.925634 & 0.566008 & -1.635 & 0.1020\end{array}$

$\begin{array}{llll}-0.827565 & 1.253581 & -0.660 & 0.5092\end{array}$

$\begin{array}{llll}-0.397216 & 0.496988 & -0.799 & 0.4241\end{array}$

$\begin{array}{llll}-0.101326 & 0.501862 & -0.202 & 0.8400\end{array}$

$\begin{array}{llll}-0.255274 & 0.425643 & -0.600 & 0.5487\end{array}$

$\begin{array}{llll}0.024074 & 0.149557 & 0.161 & 0.8721\end{array}$

$\begin{array}{llll}0.163467 & 0.161519 & 1.012 & 0.3115\end{array}$

$\begin{array}{llll}-0.008100 & 0.133417 & -0.061 & 0.9516\end{array}$

$\begin{array}{llll}0.130701 & 0.132094 & 0.989 & 0.3224\end{array}$

$\begin{array}{llll}0.002379 & 0.196768 & 0.012 & 0.9904\end{array}$

$\begin{array}{llll}0.328044 & 0.141160 & 2.324 & 0.0201\end{array}$

$\begin{array}{llll}-0.240586 & 0.163264 & -1.474 & 0.1406\end{array}$

$\begin{array}{llll}-0.060676 & 0.136754 & -0.444 & 0.6573\end{array}$

$\begin{array}{llll}-0.071118 & 0.181263 & -0.392 & 0.6948\end{array}$

$\begin{array}{llll}0.067143 & 0.202506 & 0.332 & 0.7402\end{array}$

$\begin{array}{llll}-0.039646 & 0.203323 & -0.195 & 0.8454\end{array}$

$\begin{array}{llll}0.201639 & 0.185684 & 1.086 & 0.2775\end{array}$

$\begin{array}{lllll}-0.416487 & 0.098703 & -4.220 & 2.45 e-05 * * *\end{array}$ 


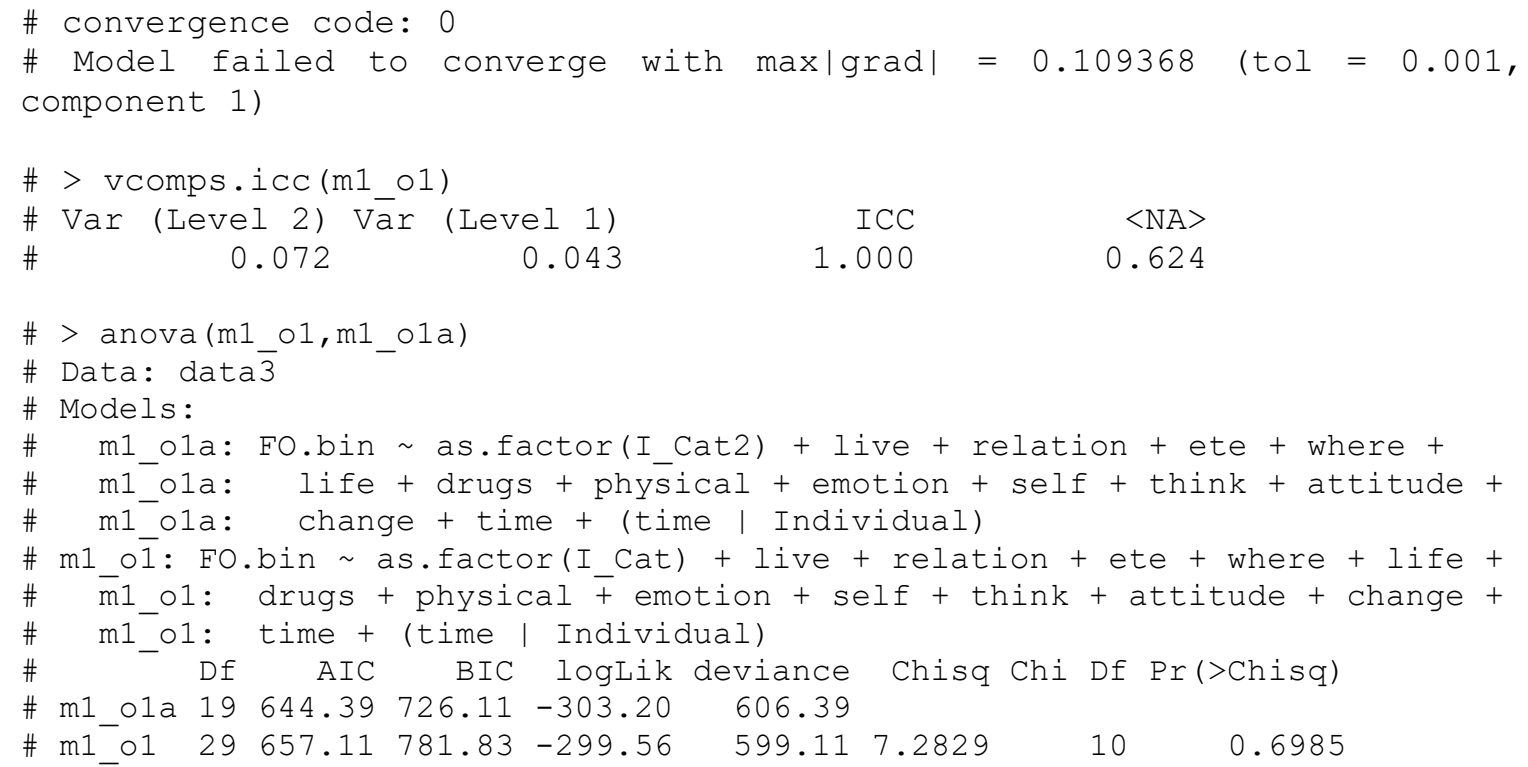


Model 1.9 - Basic Model + Grouped YJB Offence Category (Table 6.7)

Create a new predictor which groups the YJB Offence Categories

- $\quad \mathrm{VAP}=$ Violence against the person

- $S A C=$ burglary (domestic and non-domestic), robbery and vehicle theft $/$ TWOC

- $\quad$ Other = Everything else

Since factors default to being in alphabetical order, 'Other' will be the reference category

Bayesian Model (Bm1G_o1)

\#\# Define the model

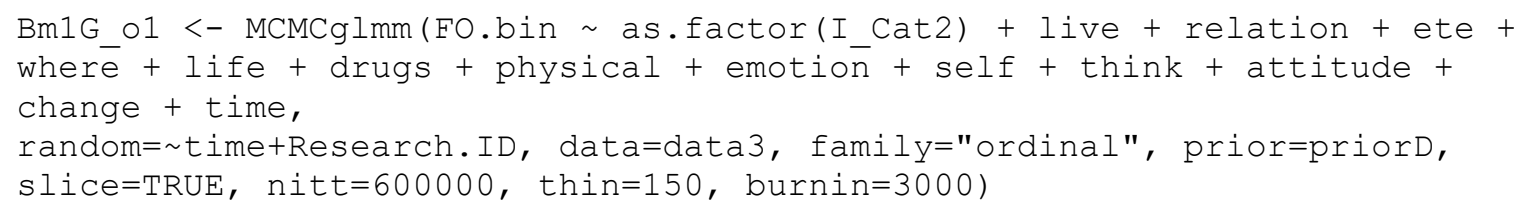

\#\# Checks for suitable convergence

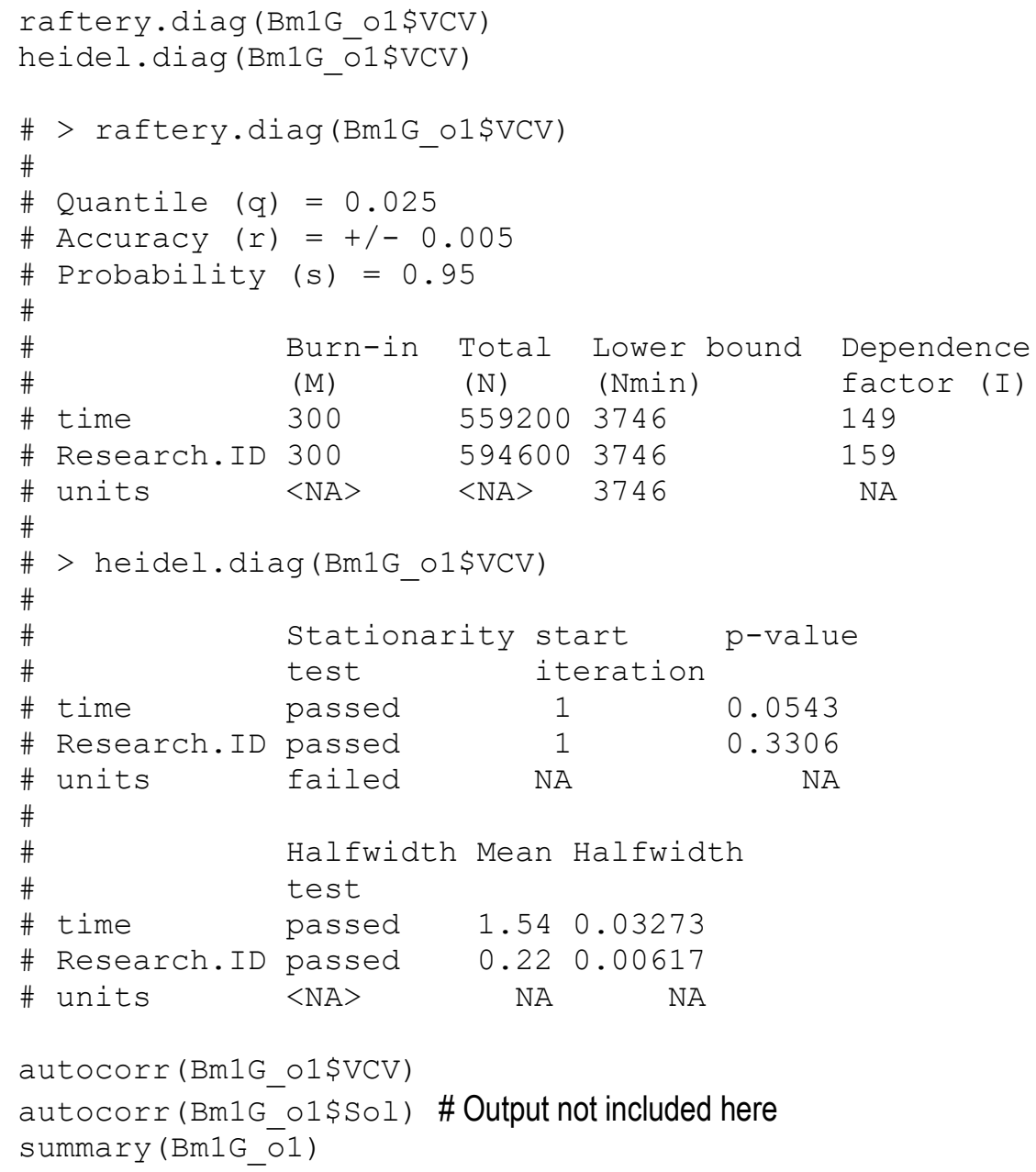




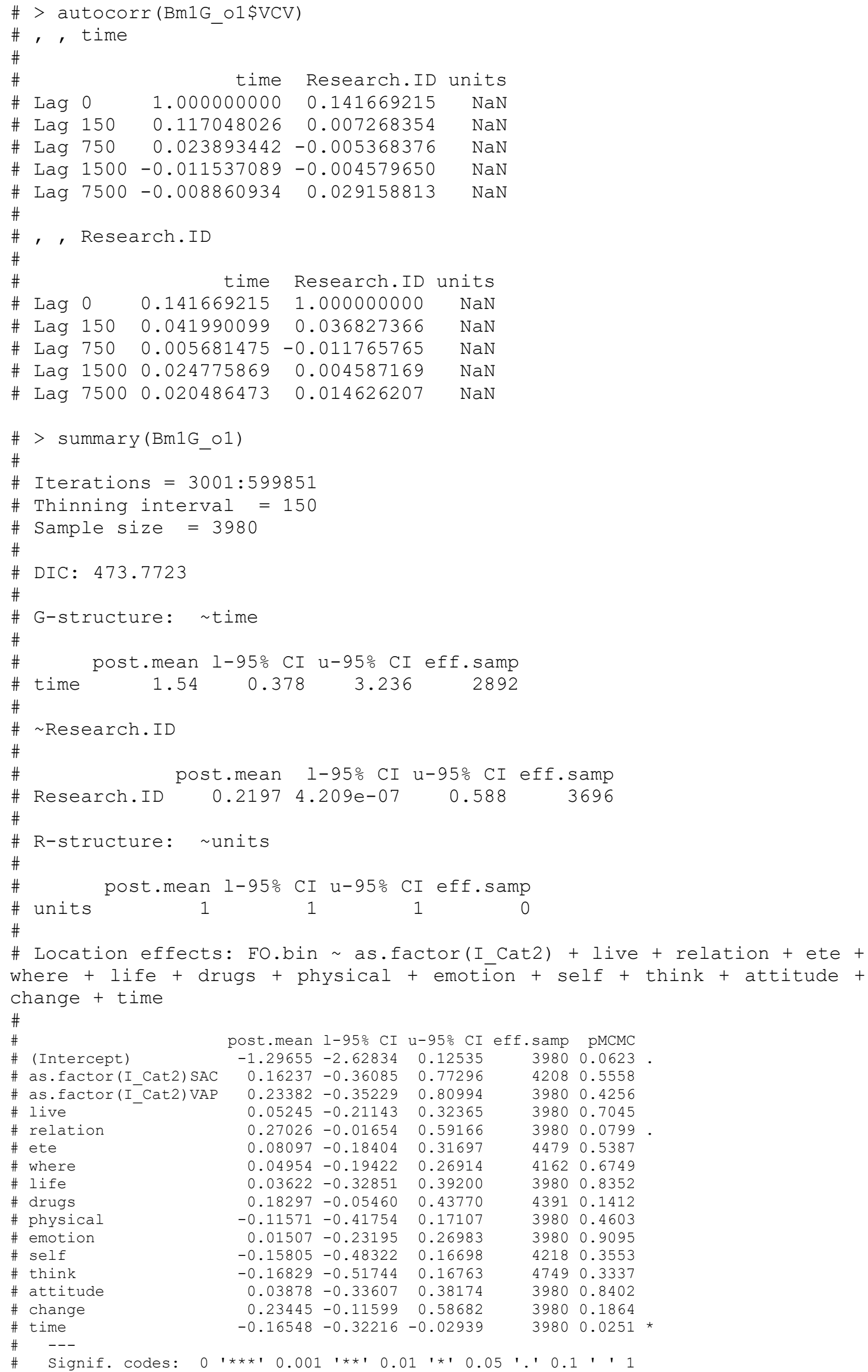




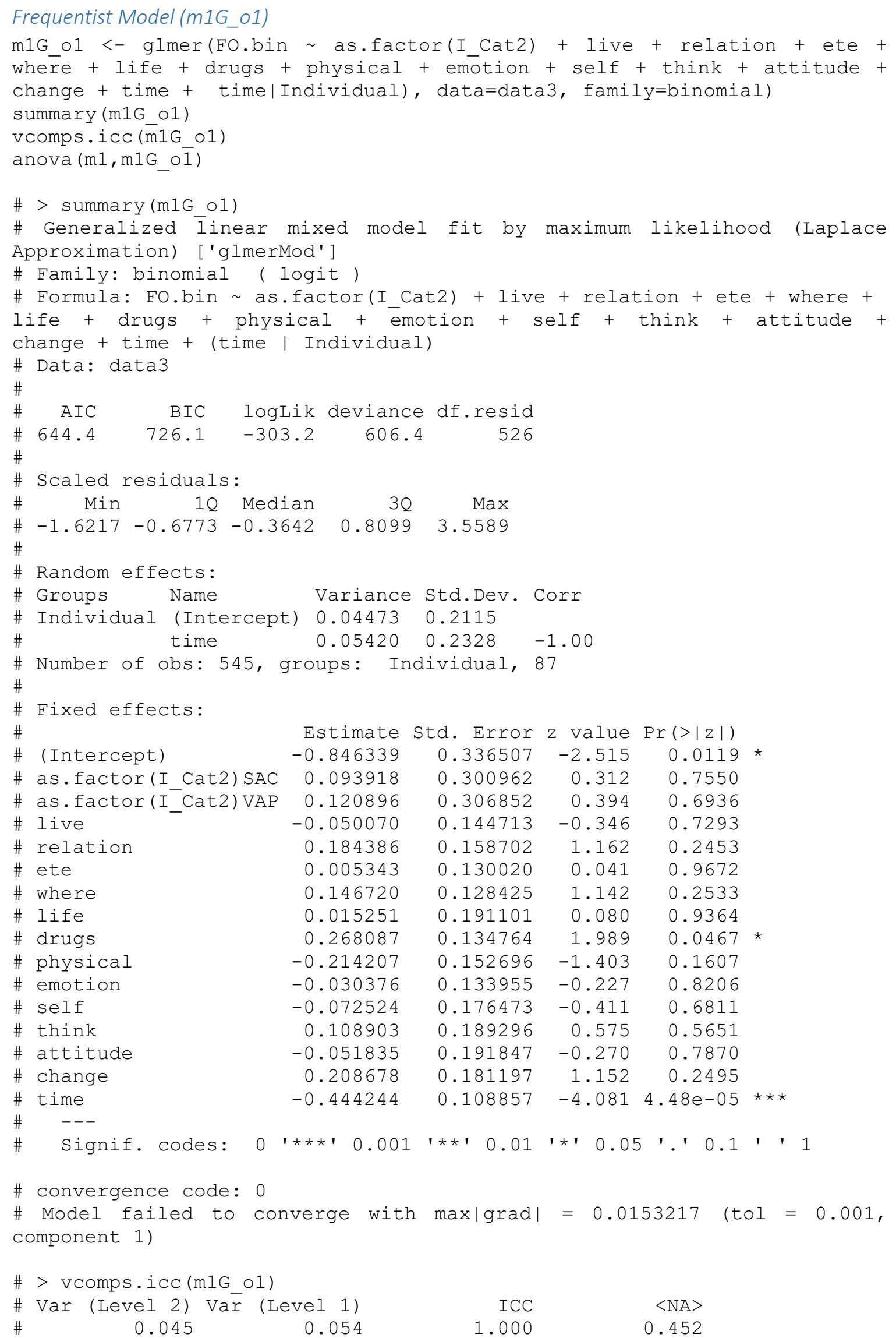




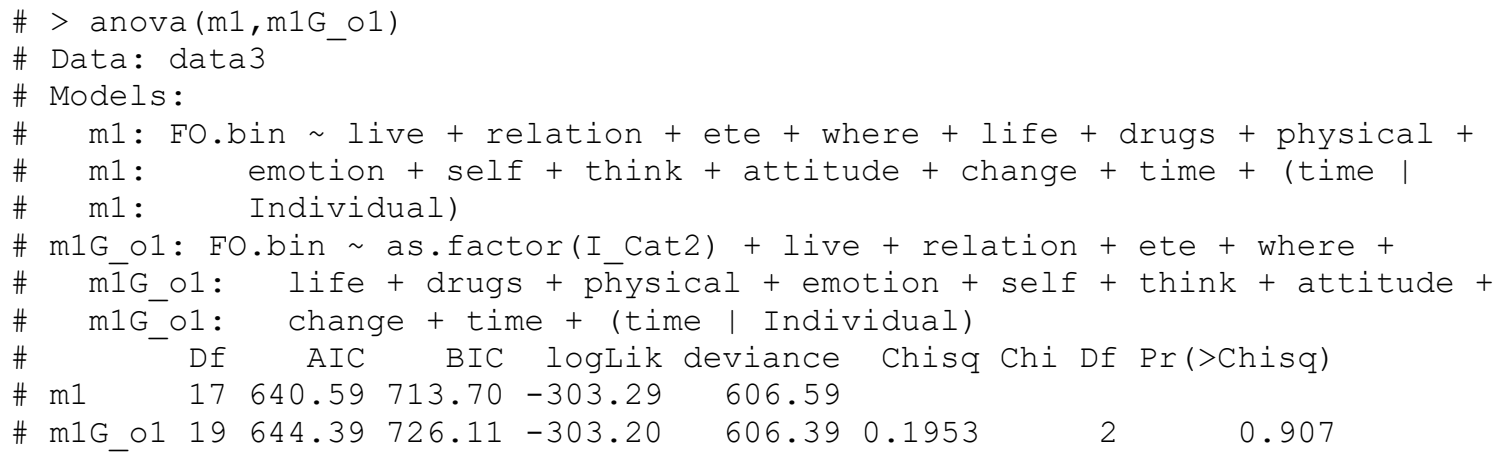


Model 3 - Basic Model + Static Factors (Table 6.8)

Bayesian Model (Bm1G_cc12301)

\section{\#\# Define the model}

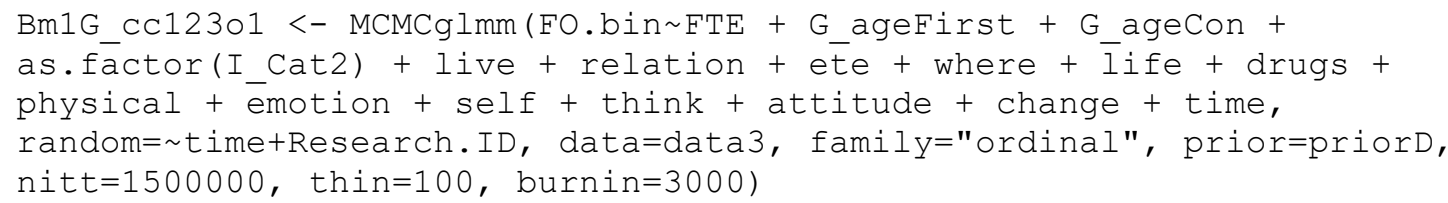

\section{\#\# Checks for suitable convergence}

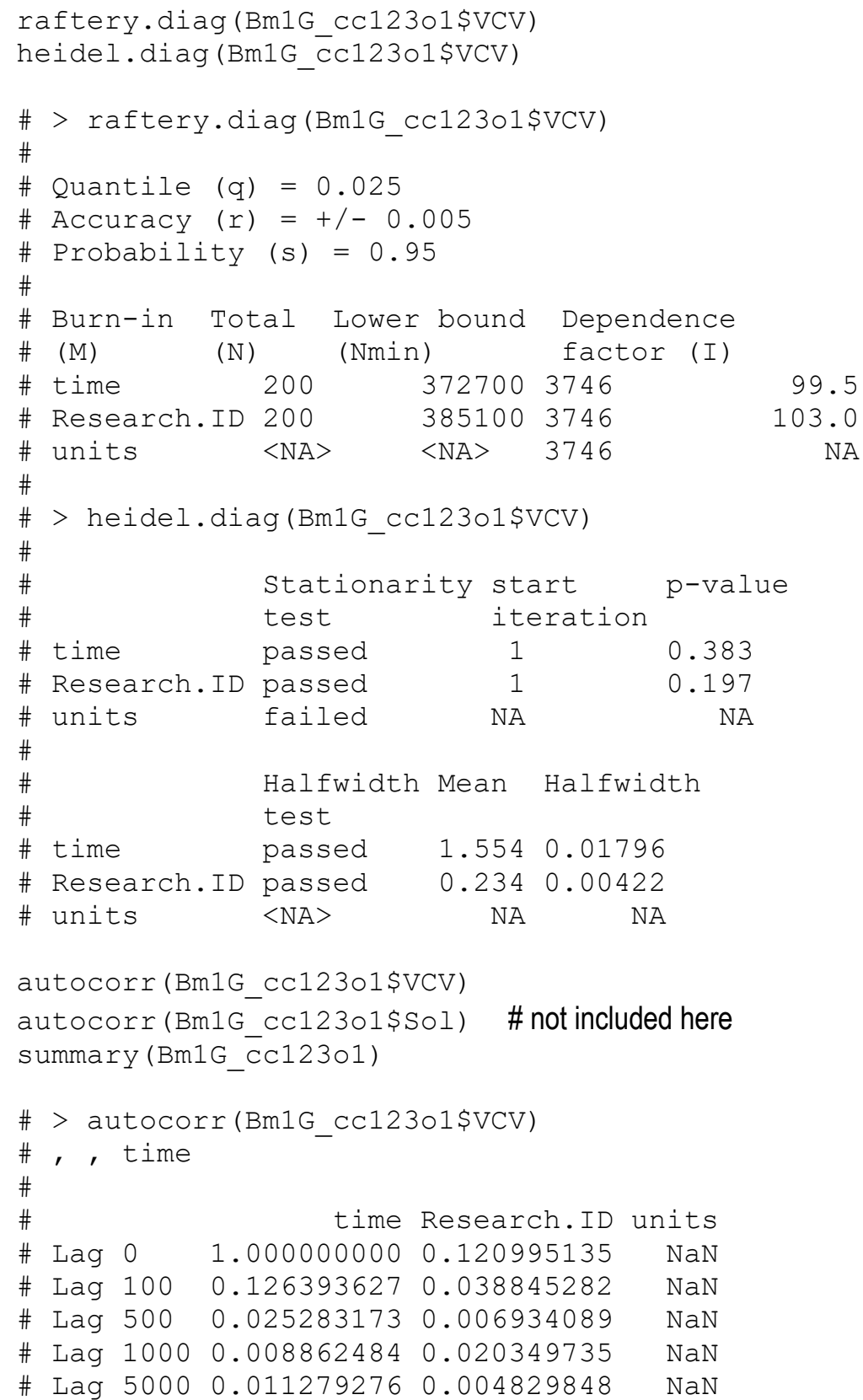




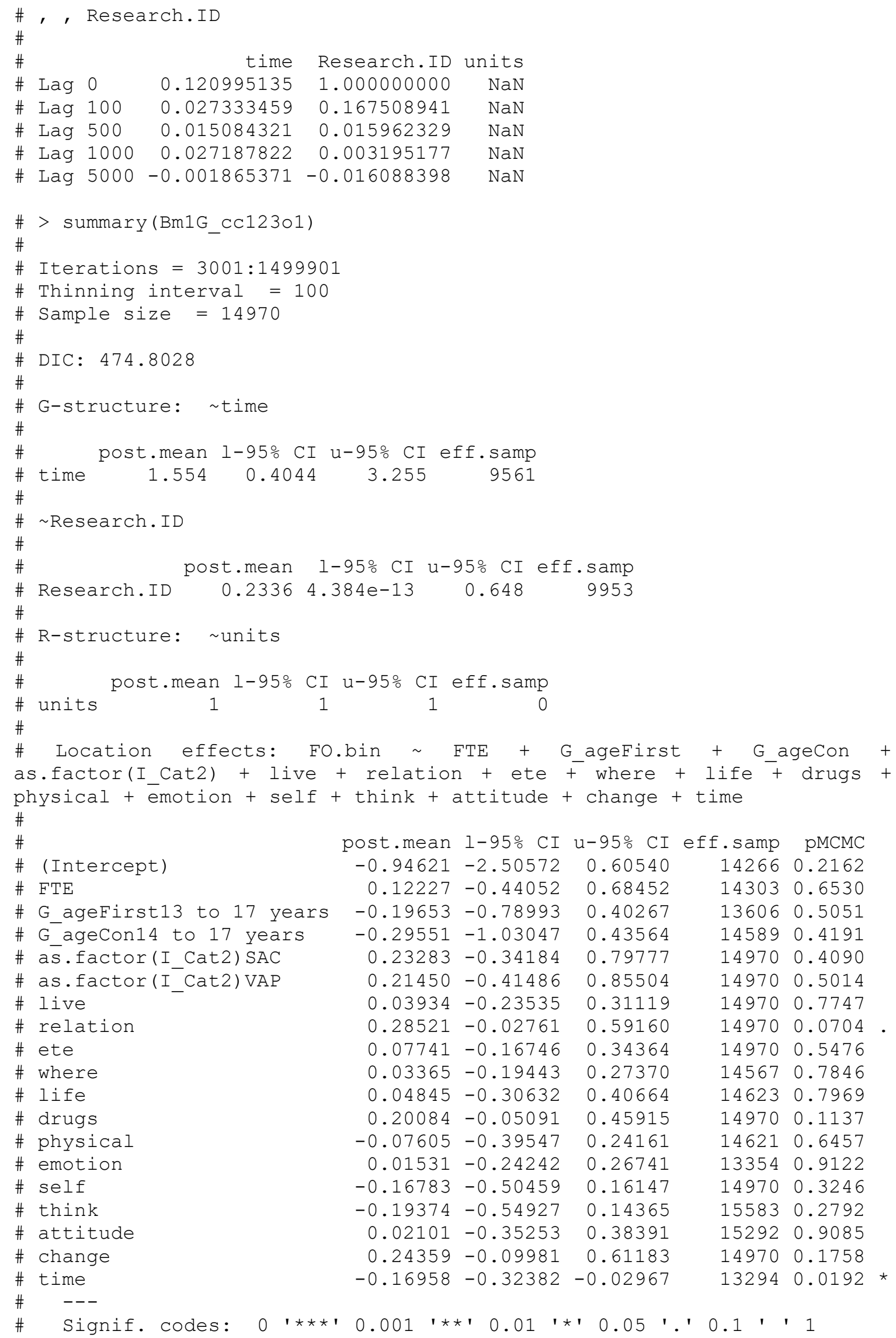

\section{Page | 156}




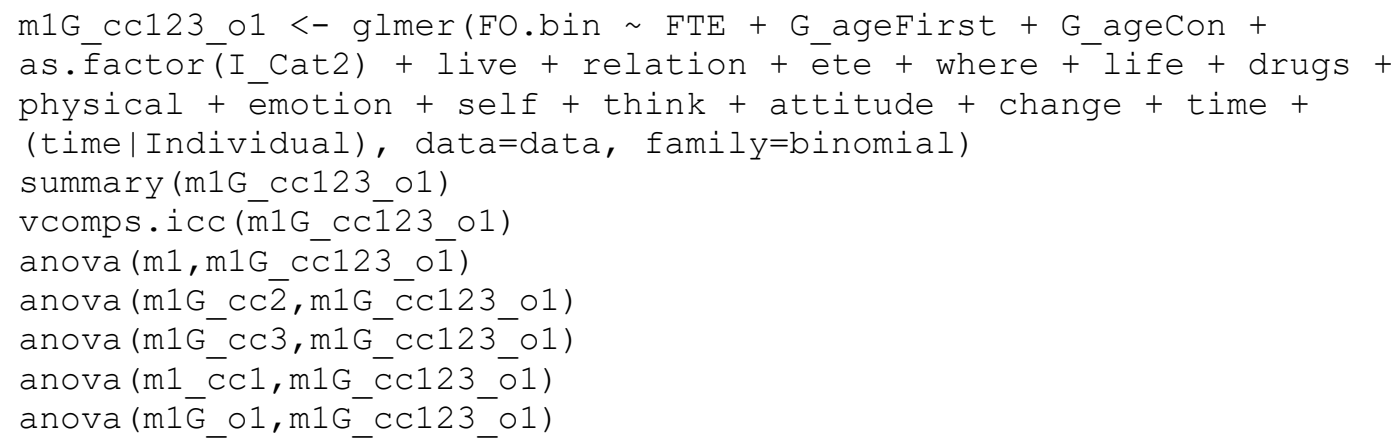

Warning message:

In checkConv(attr (opt, "derivs"), opt\$par, ctrl = control\$checkConv, : Model failed to converge with $\max \mid$ grad $\mid=0.120329$ (tol $=0.001$, component 1)

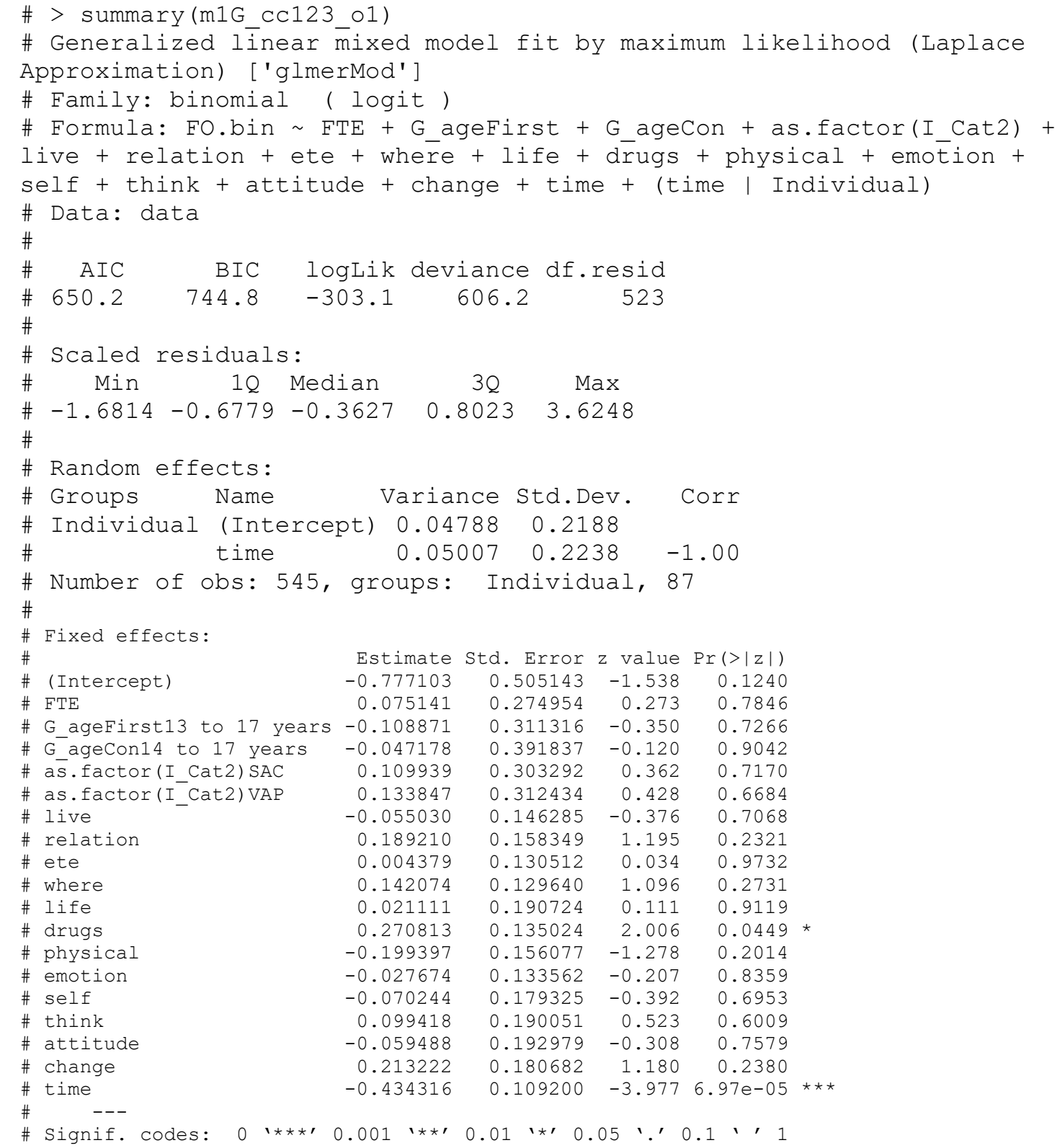




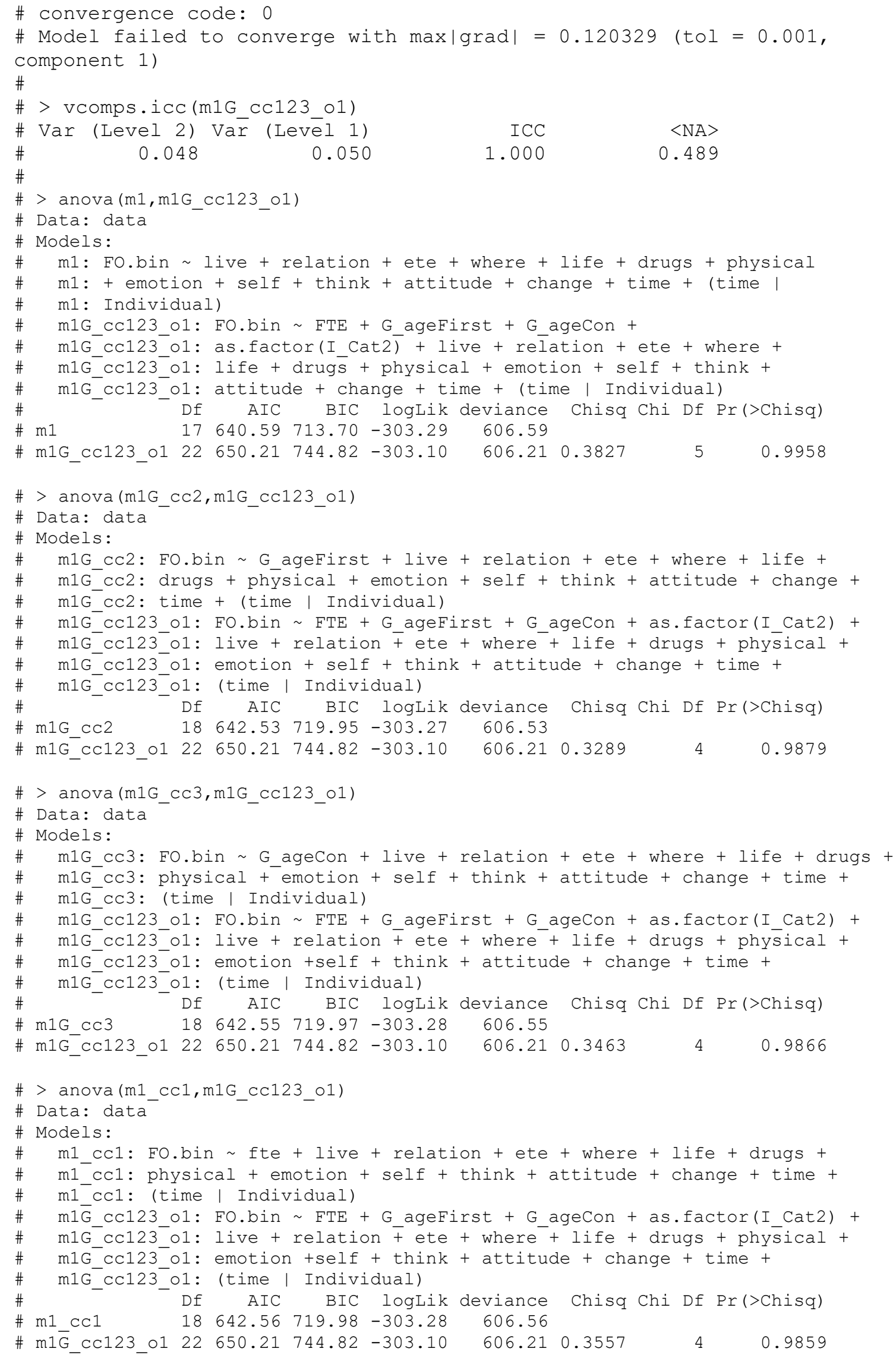




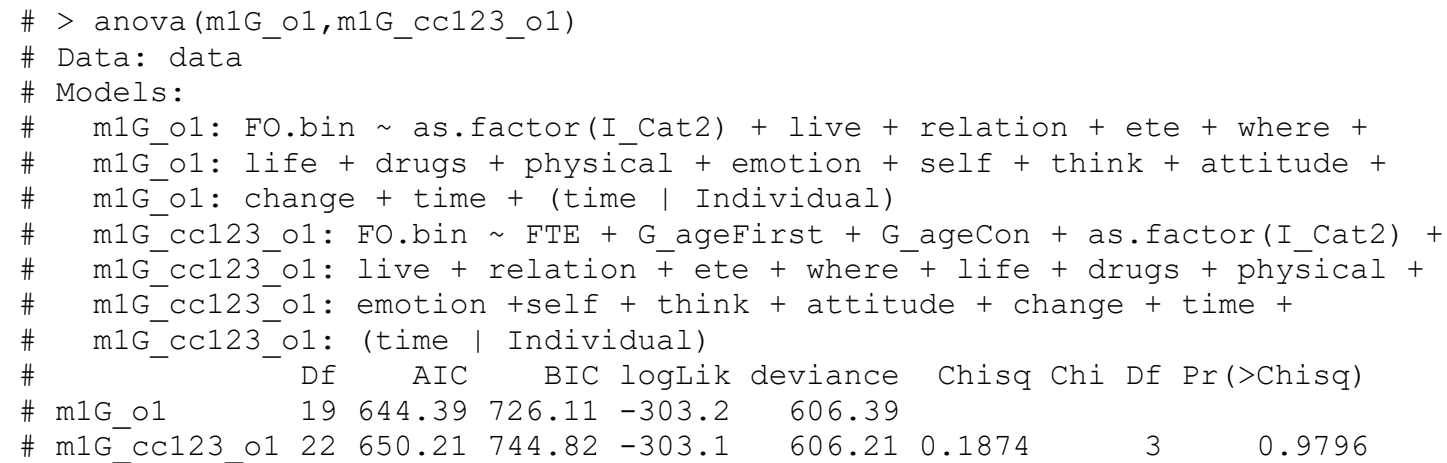


Model 3a - Basic Model + FTE Status, Grouped Age at First Offence and Grouped YJB Offence Category (Table 6.11)

Bayesian Model (Bm1G_cc12_o1)

\section{\#\# Define the model}

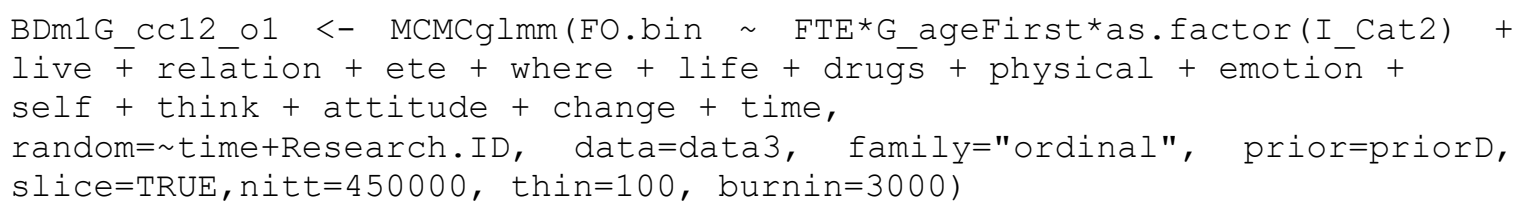

\# Checks for suitable convergence

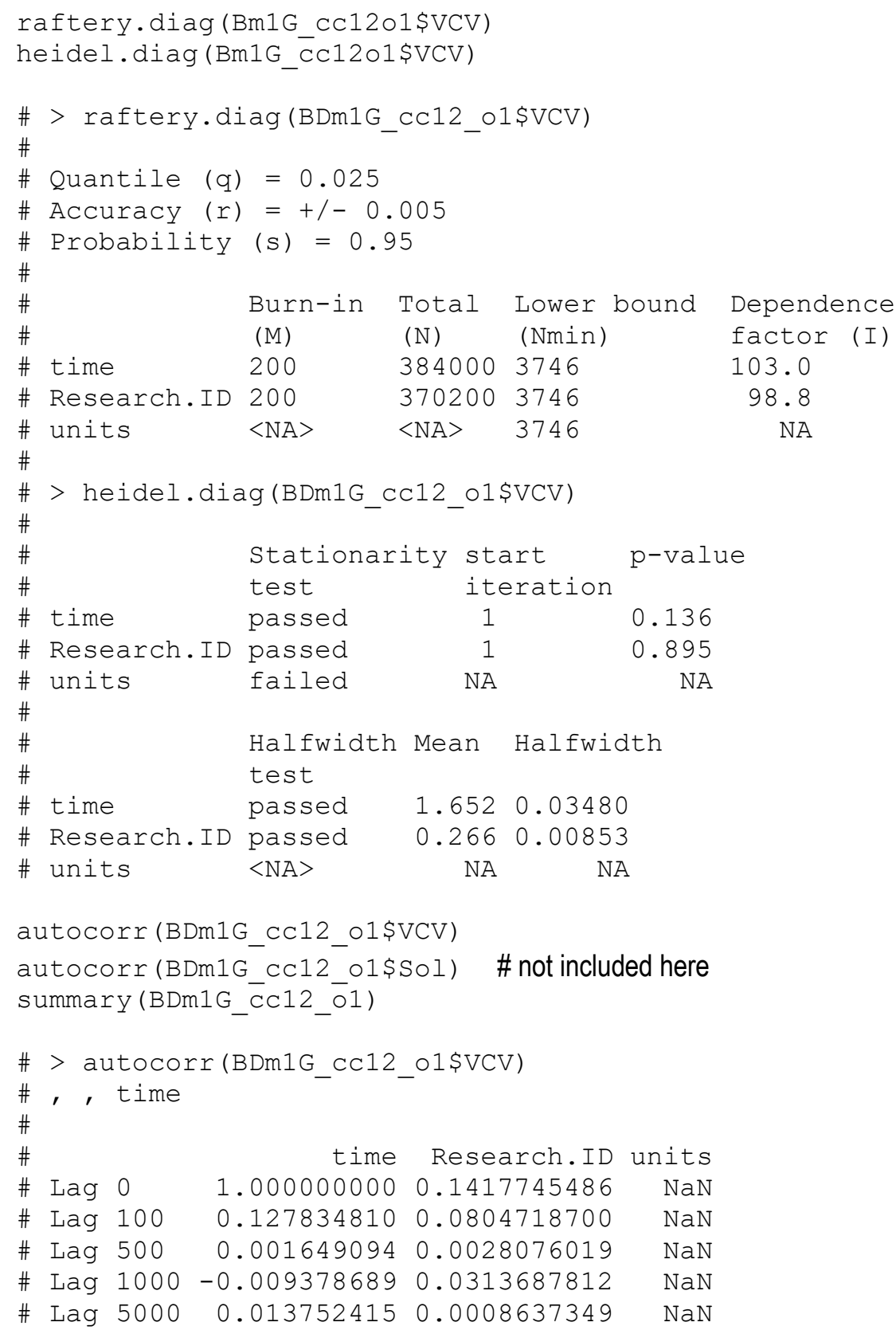




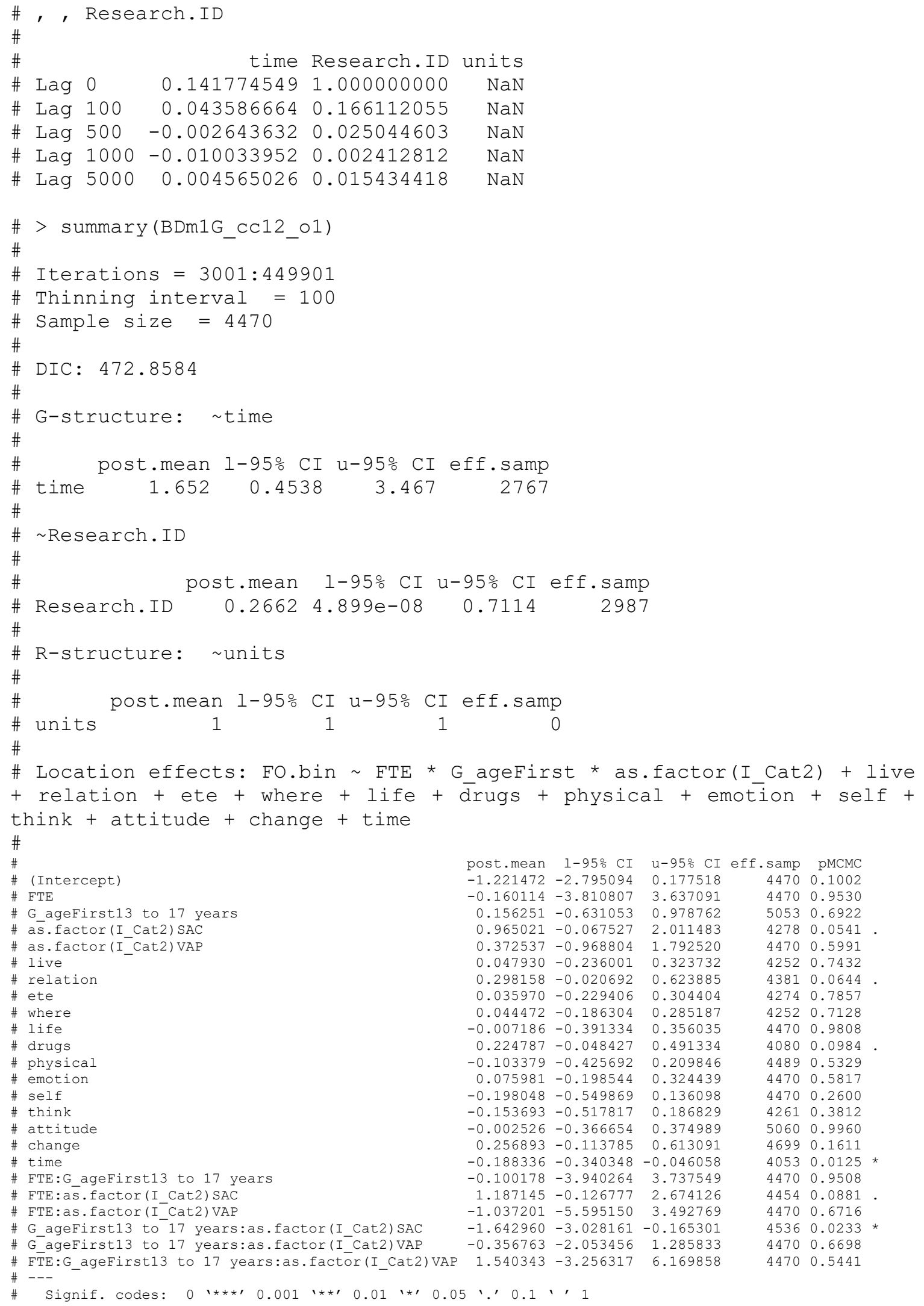


m1G cc12 o1 <- glmer(FO.bin FTE + G ageFirst + as.factor (I Cat2) + live + rélation + ete + where + life + drugs + physical + emotion + self + think + attitude + change + time + (timelIndividual), data=data, family=binomial) summary (m1G_cc12 o1) vcomps.icc ( $\left.\overline{\mathrm{m}} 1 \mathrm{G} \_\mathrm{c} \overline{\mathrm{C}} 12 \_\mathrm{ol}\right)$ anova (m1, m1G_c $\left.\bar{c} 12 \_o \overline{1}\right)$

\section{Warning message:}

In checkConv (attr (opt, "derivs"), opt\$par, ctrl = control\$checkConv, : Model failed to converge with max|grad| $=0.0334511$ (tol $=0.001$, component 1)

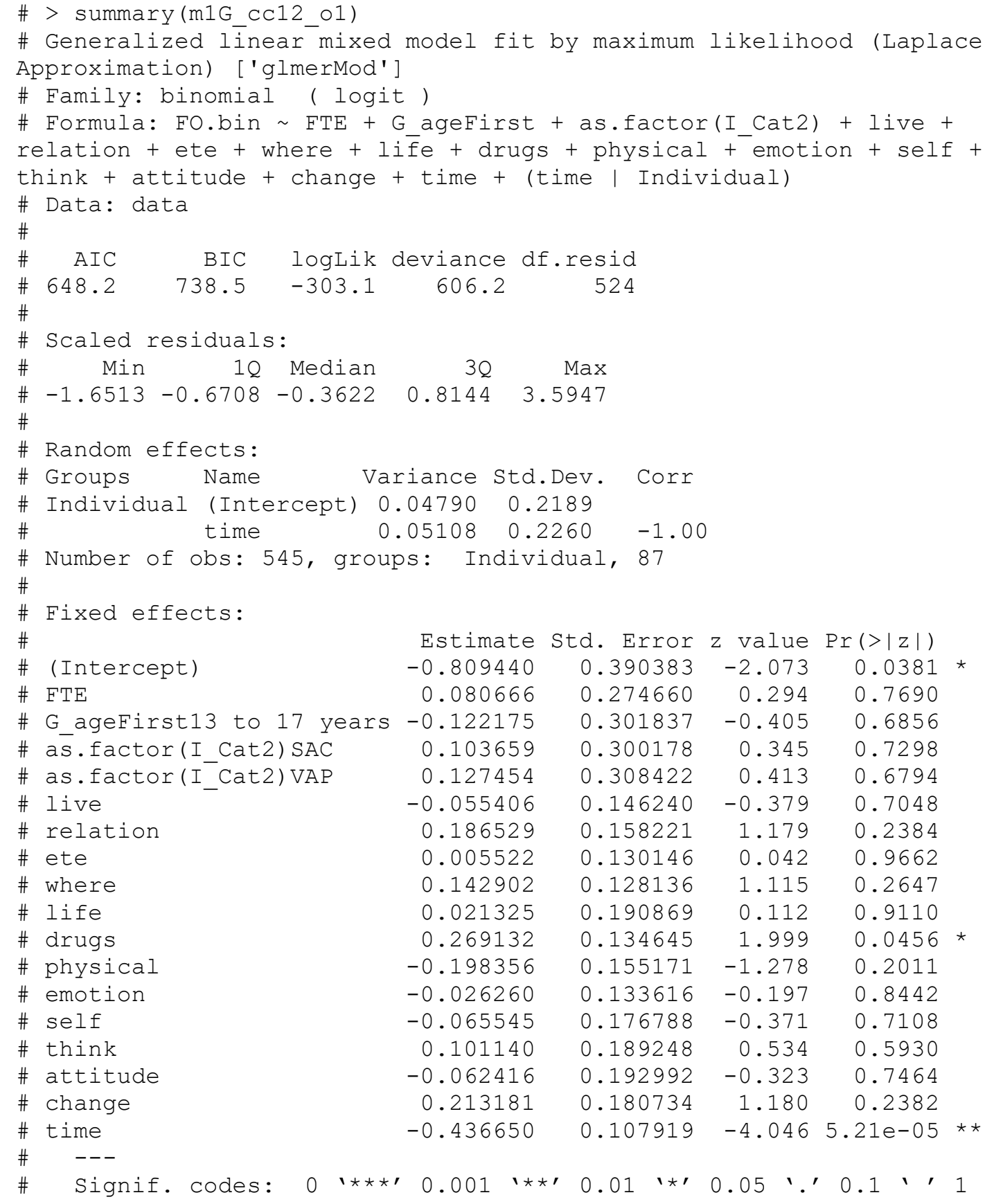




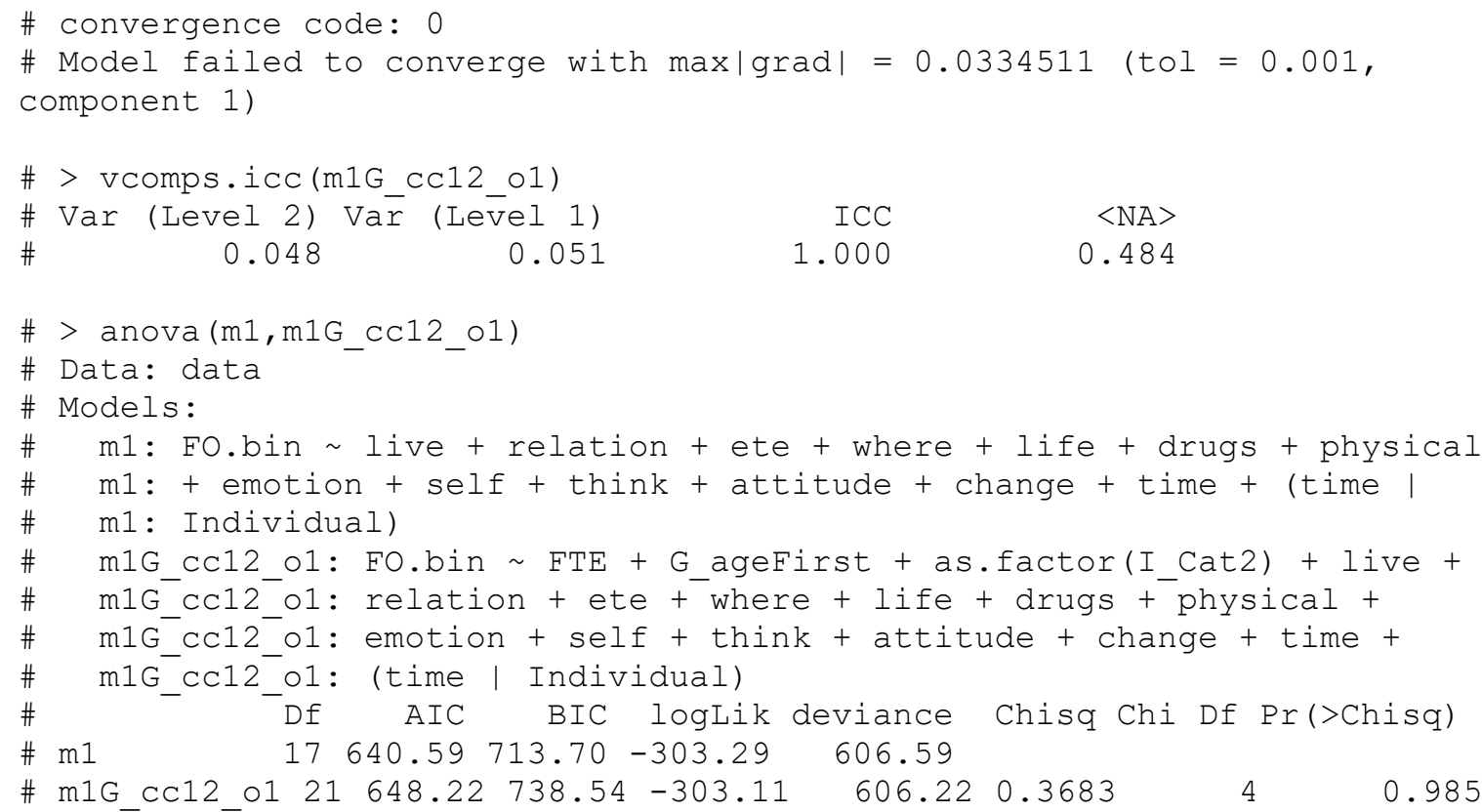


Model 1.10 - Basic Model + YJB Gravity Score (Table 6.12)

Bayesian Model (Bm1_o2a)

\section{\#\# Define the model}

Bm1_o2a <- MCMCglmm(FO.bin I_Seriousness2 + live + relation + ete + where + life + drugs + physical + emotion + self + think + attitude + change + time, random= time+Research.ID, data=data3, family="ordinal", prior=priorD, slice=TRUE, nitt $=600000$, thin=150, burnin=3000)

\section{\# Checks for suitable convergence}

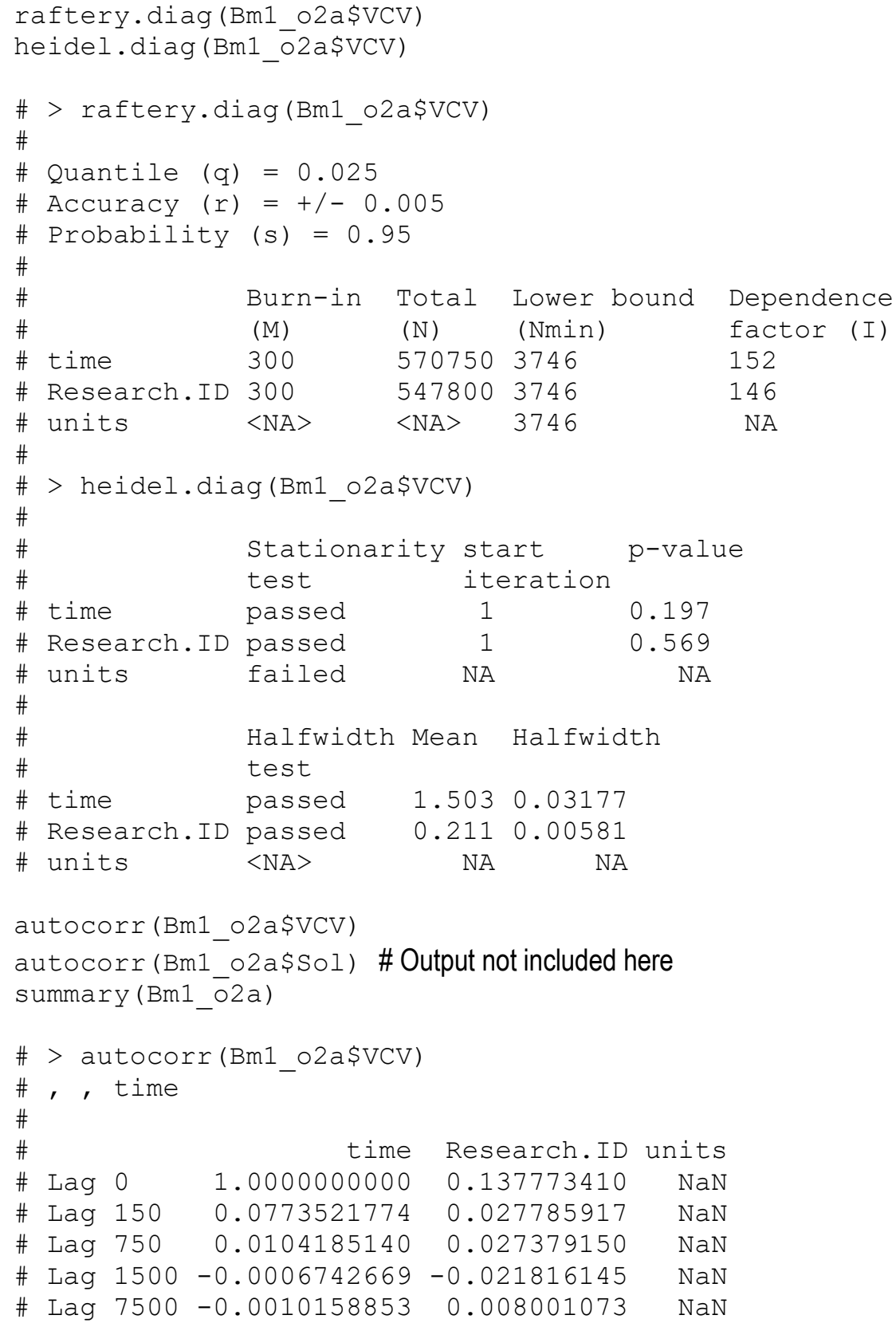




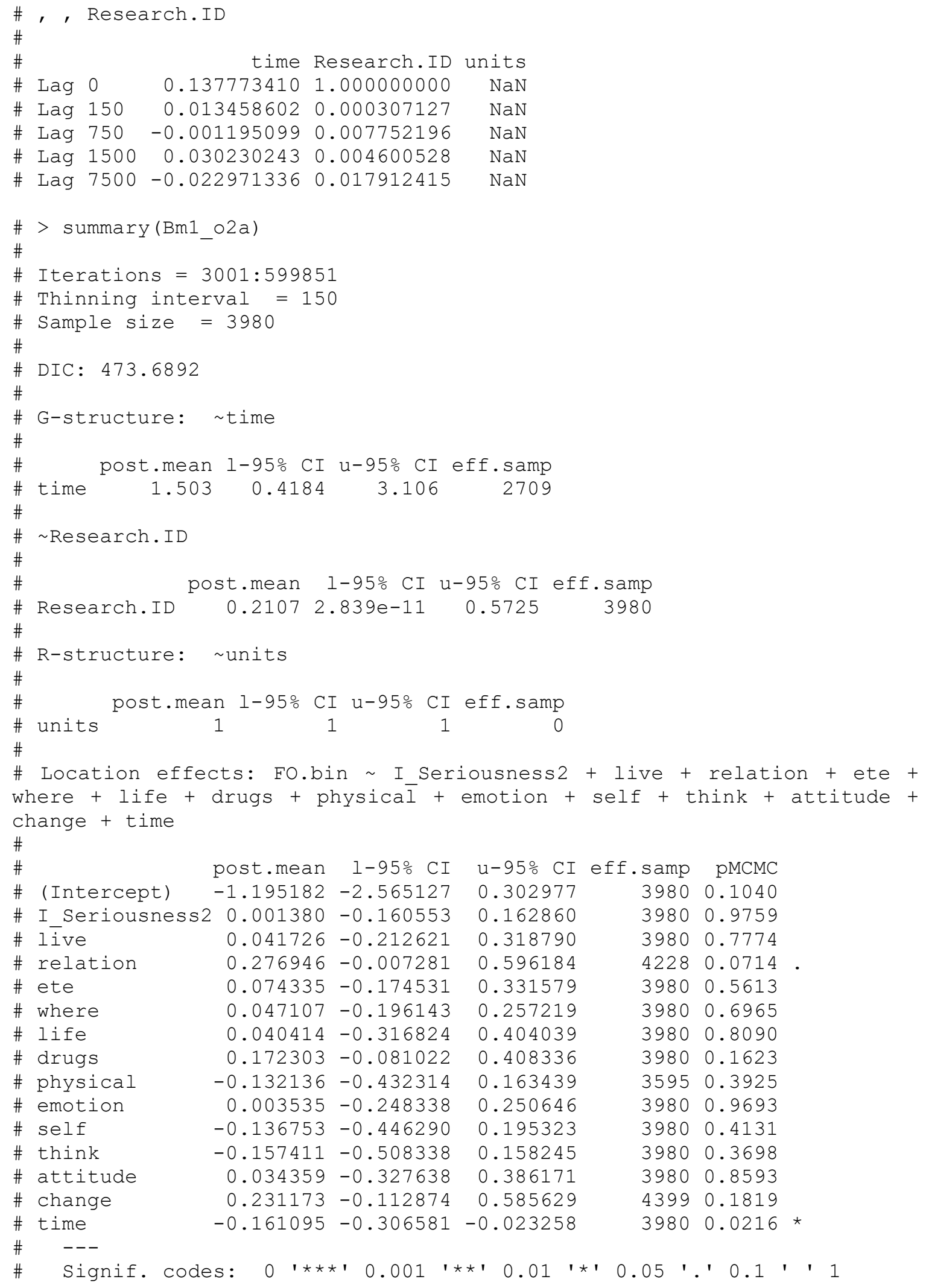




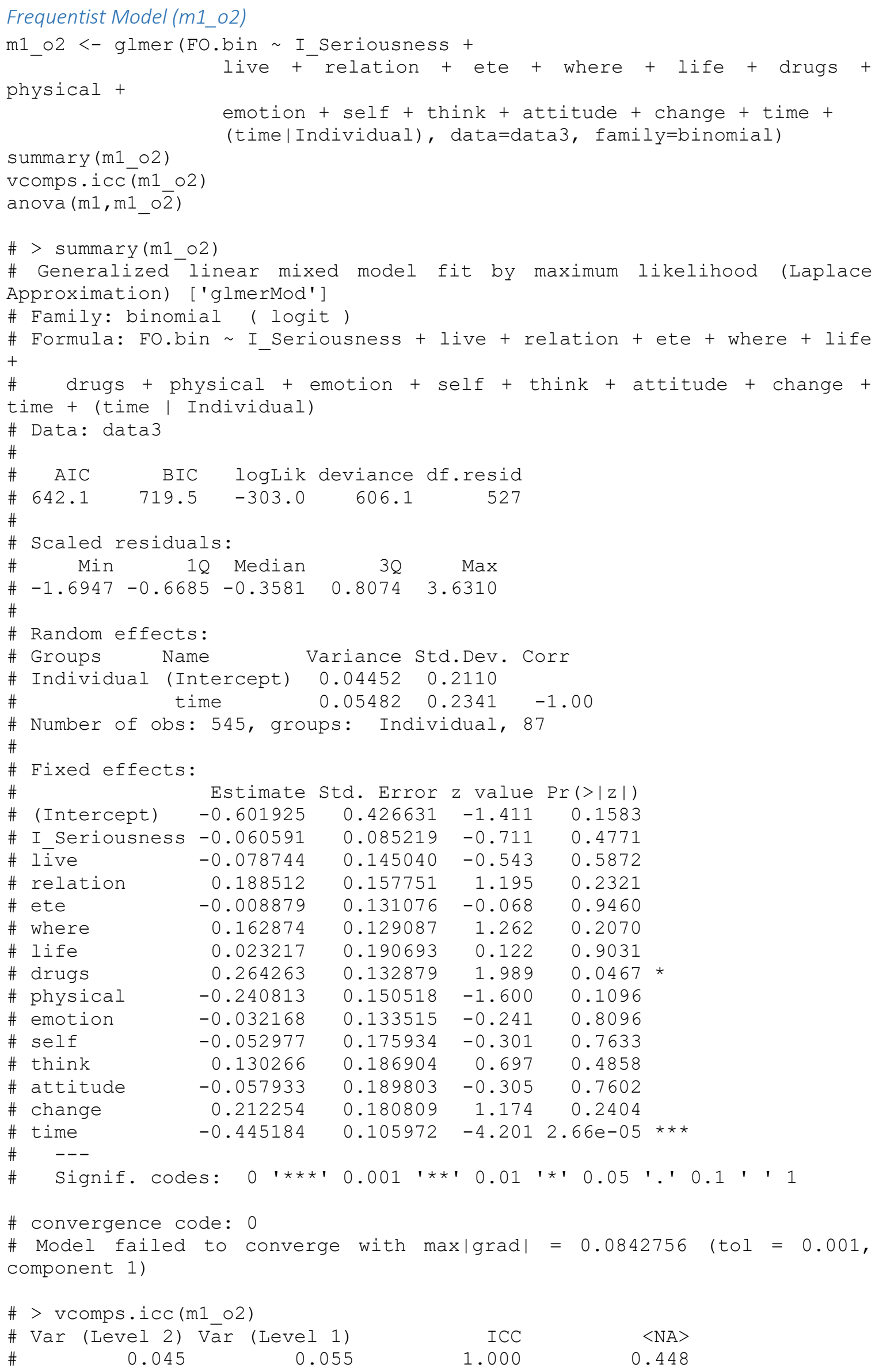




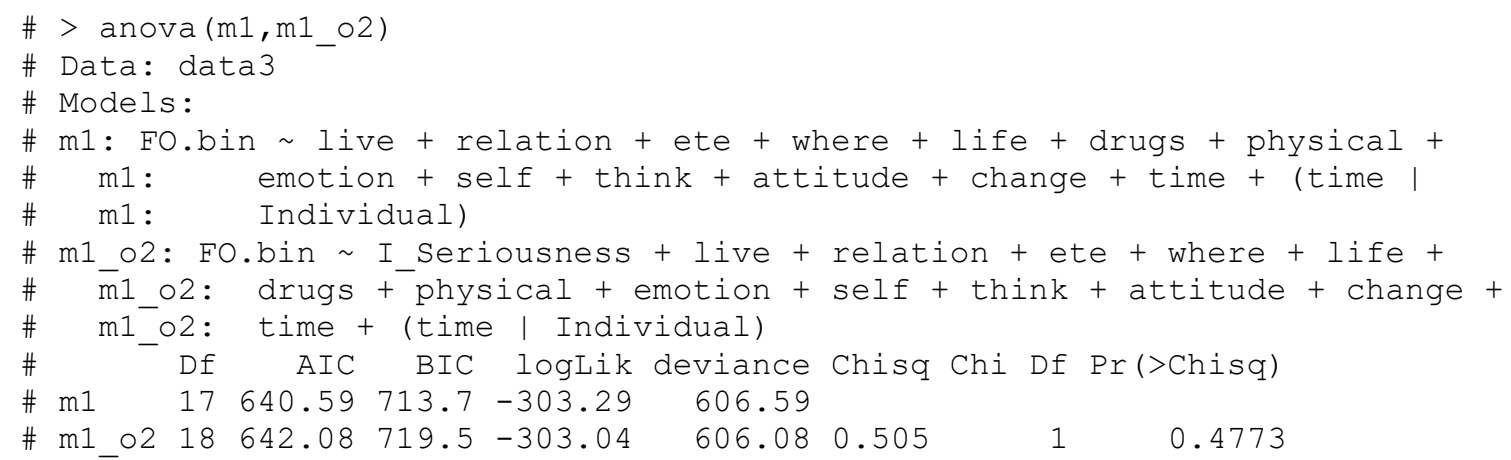


Model 3b - Basic Model + FTE Status, Grouped Age at First Offence and YJB Gravity Score (Table 6.15)

Bayesian Model (Bm1G_cc12o2a)

\section{\# Define the model}

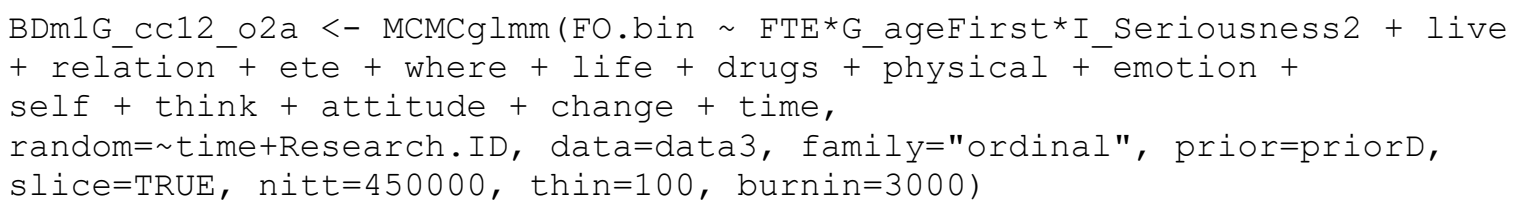

\# Checks for suitable convergence

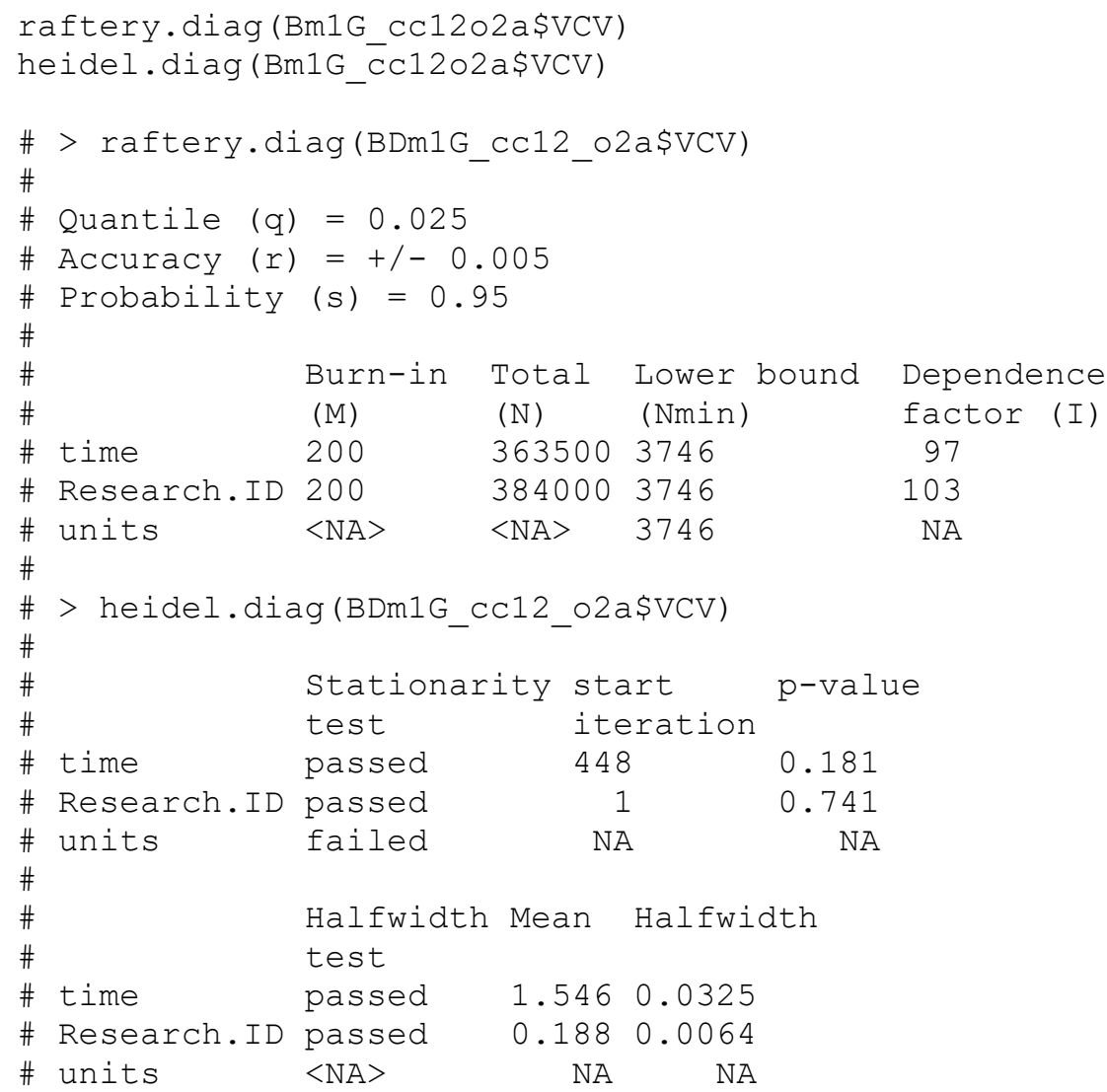




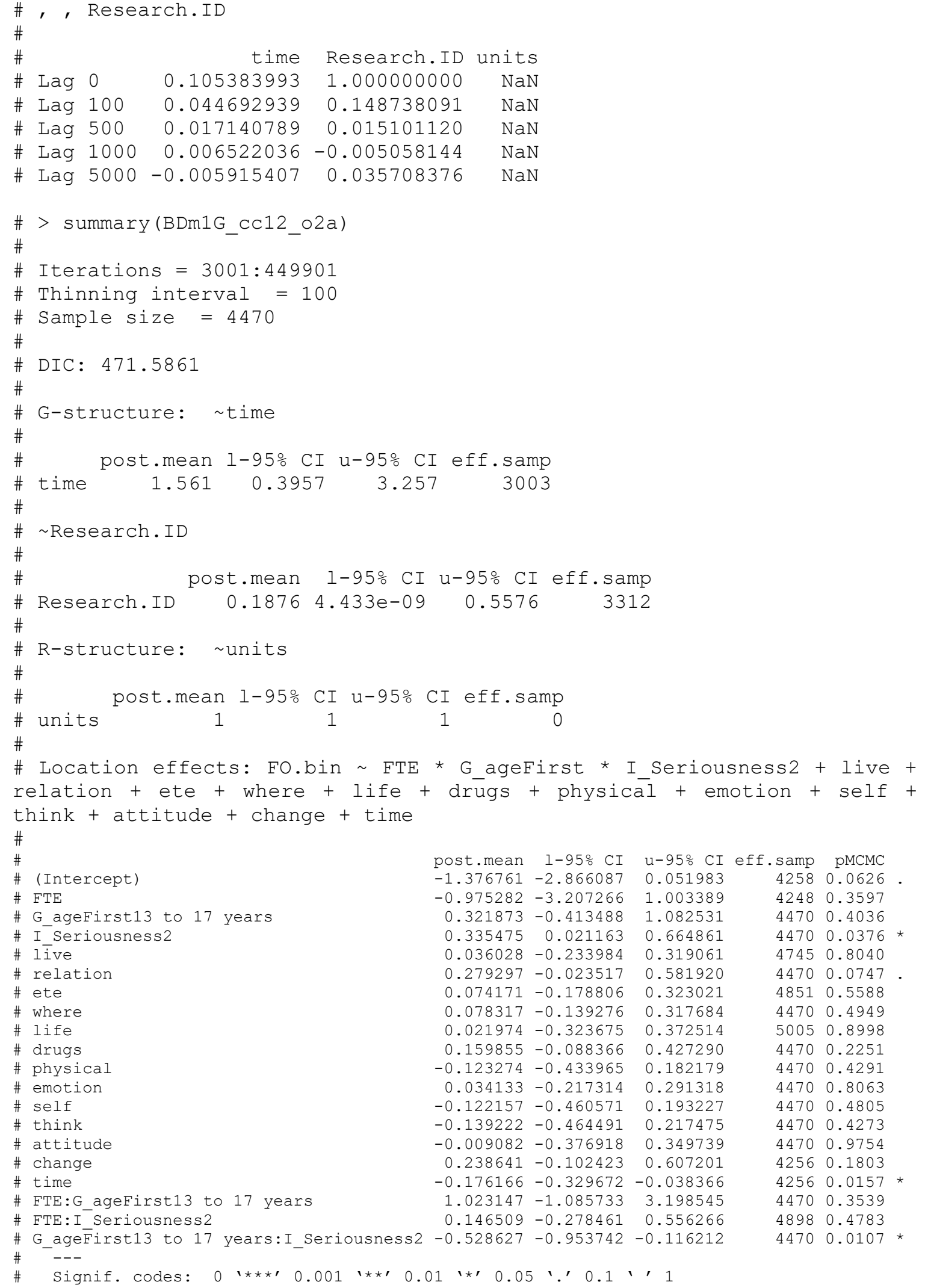




\section{Frequentist Model (m1G_cc12_o2a)}

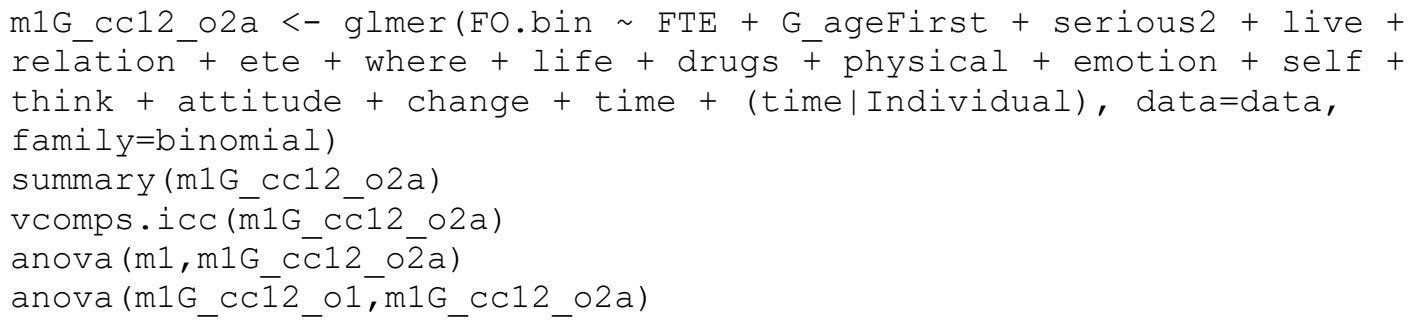

\section{Warning message:}

In checkConv(attr(opt, "derivs"), opt\$par, ctrl = control\$checkConv, : Model failed to converge with max|grad $\mid=0.0181997$ (tol $=0.001$, component 1)

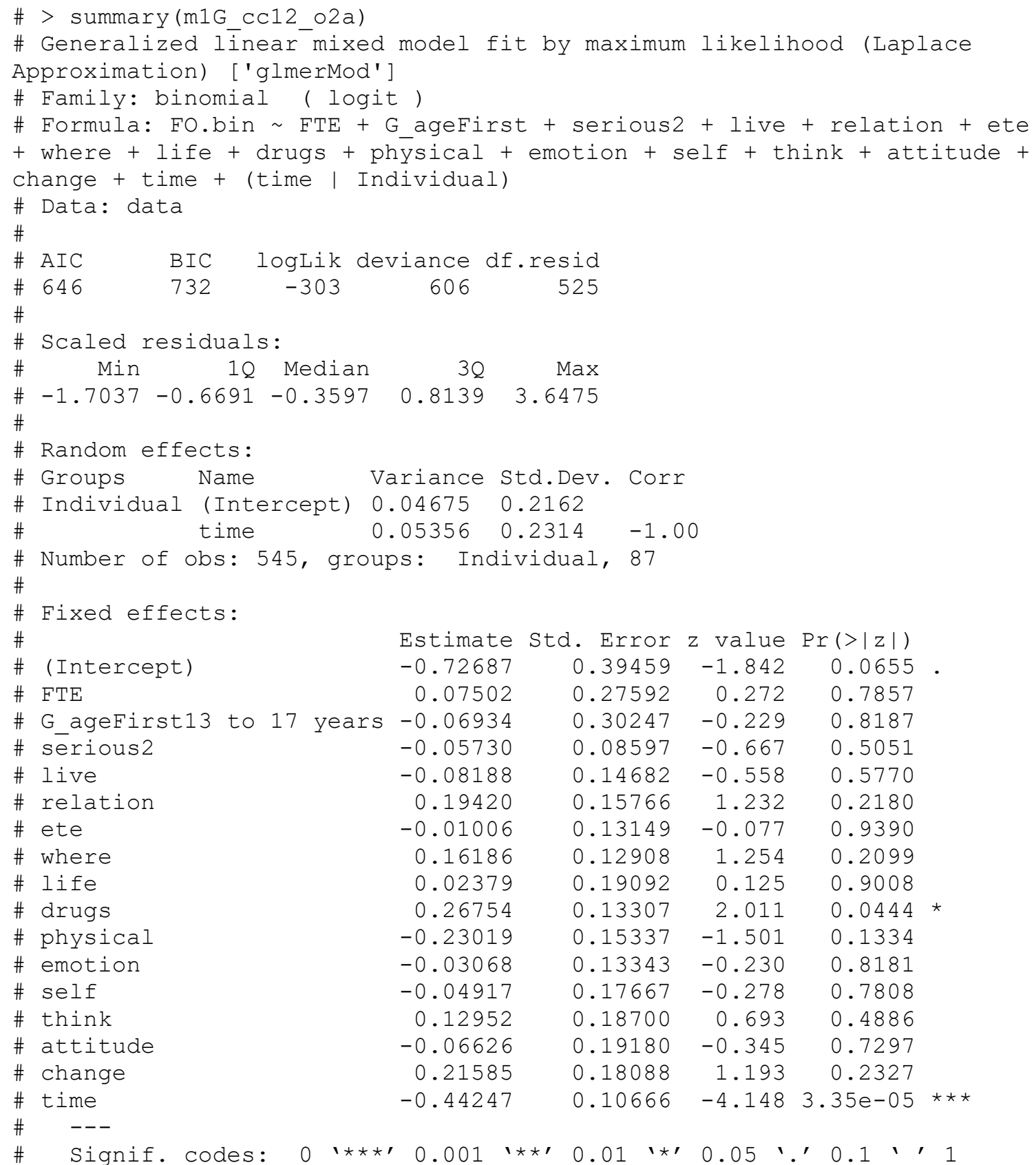




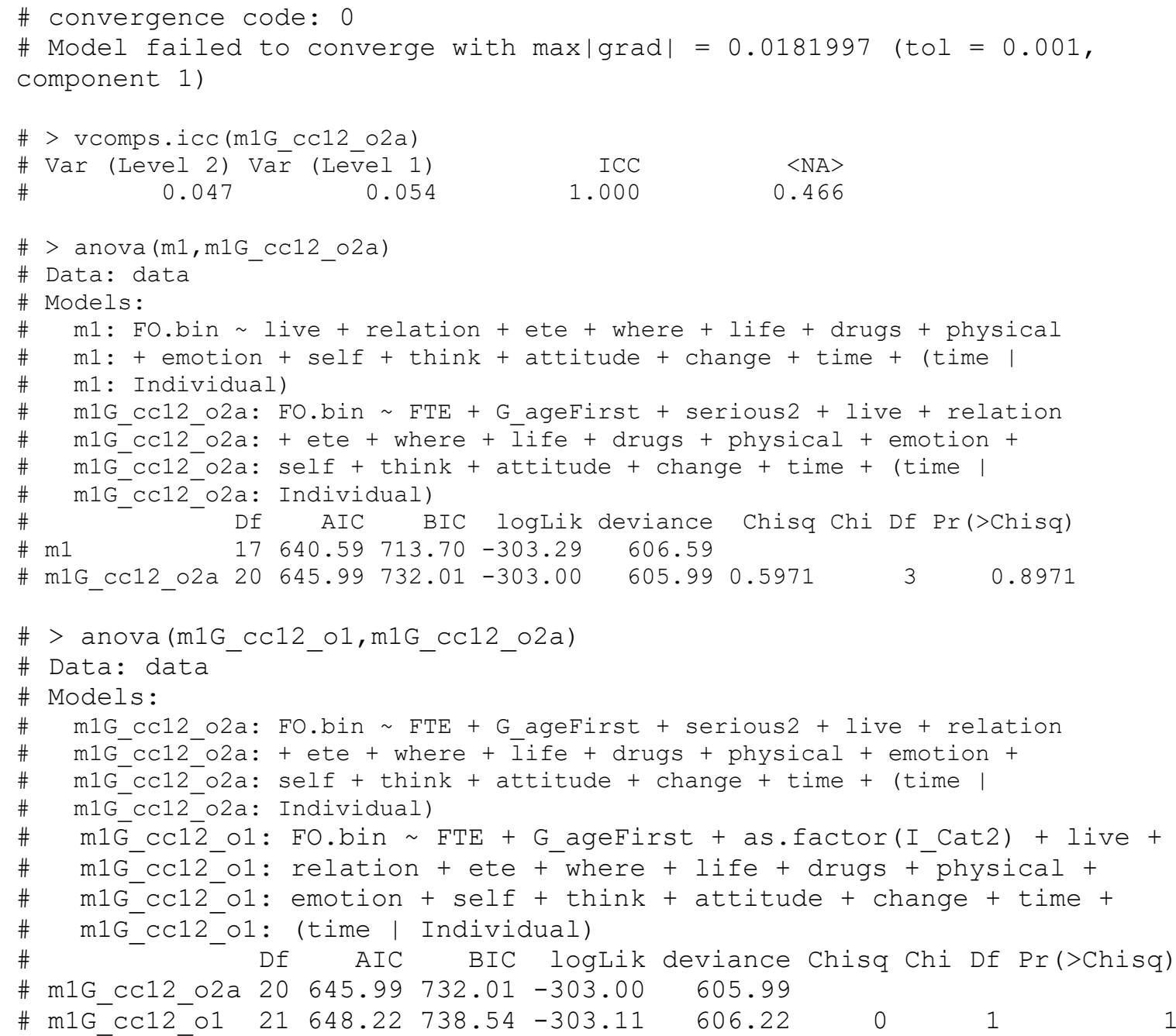


Dynamic Model involving FTE Status (Table 6.16)

Bayesian Model (BDm3_cc1)

\section{\#\# Define the model}

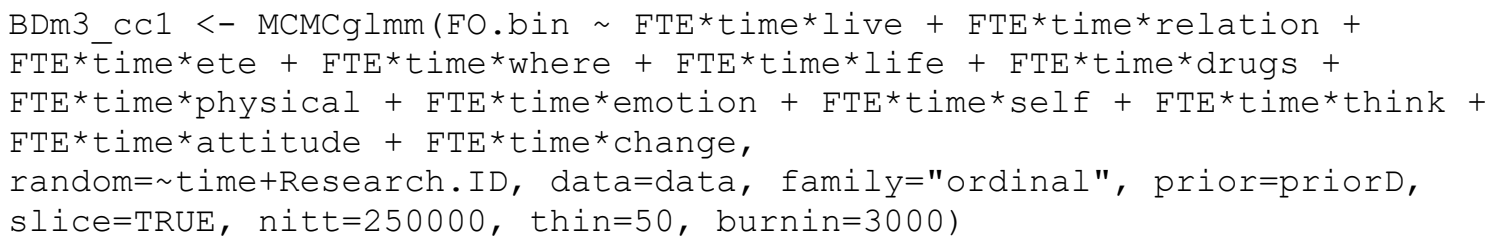

\section{\# Checks for suitable convergence}

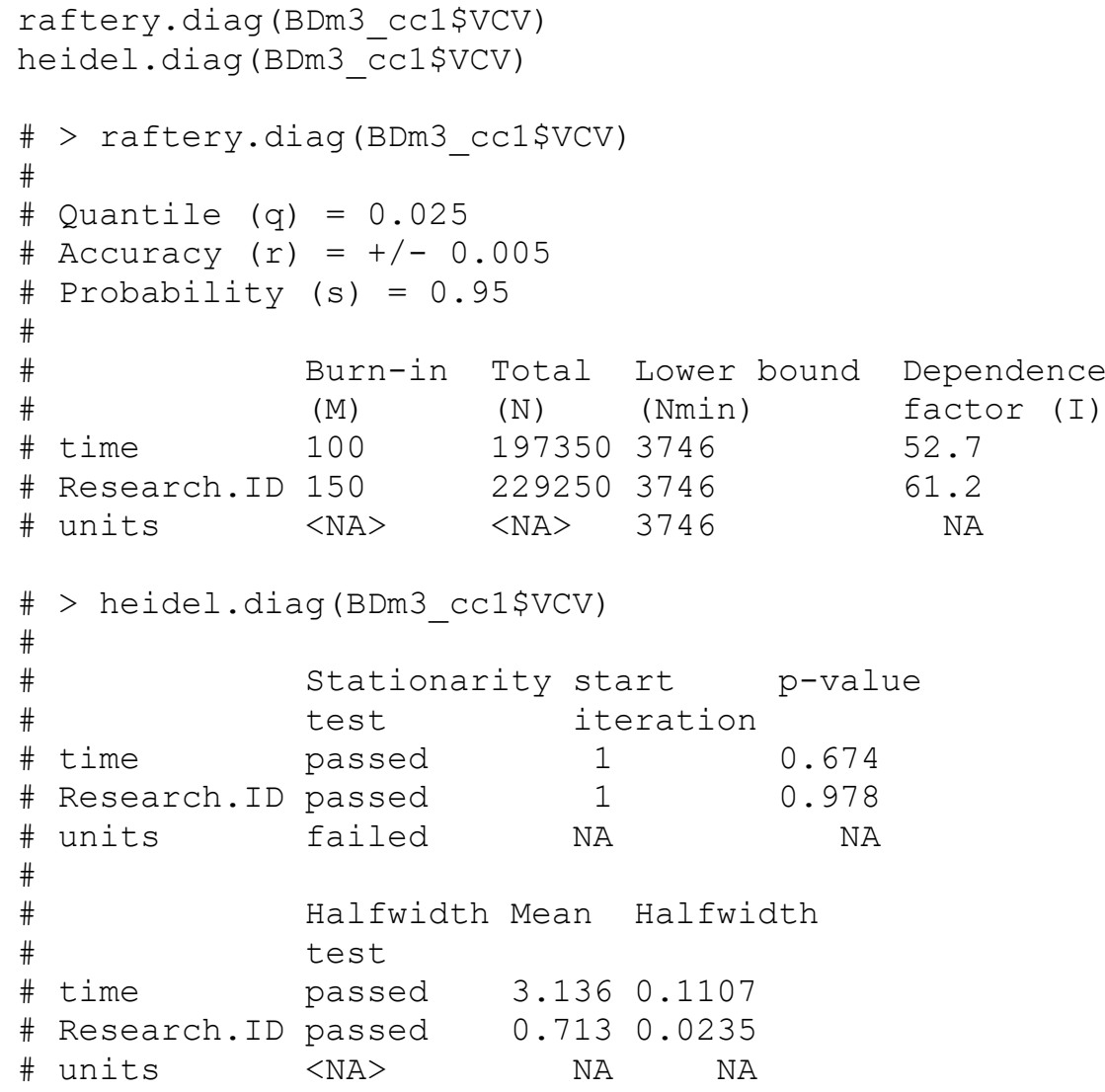




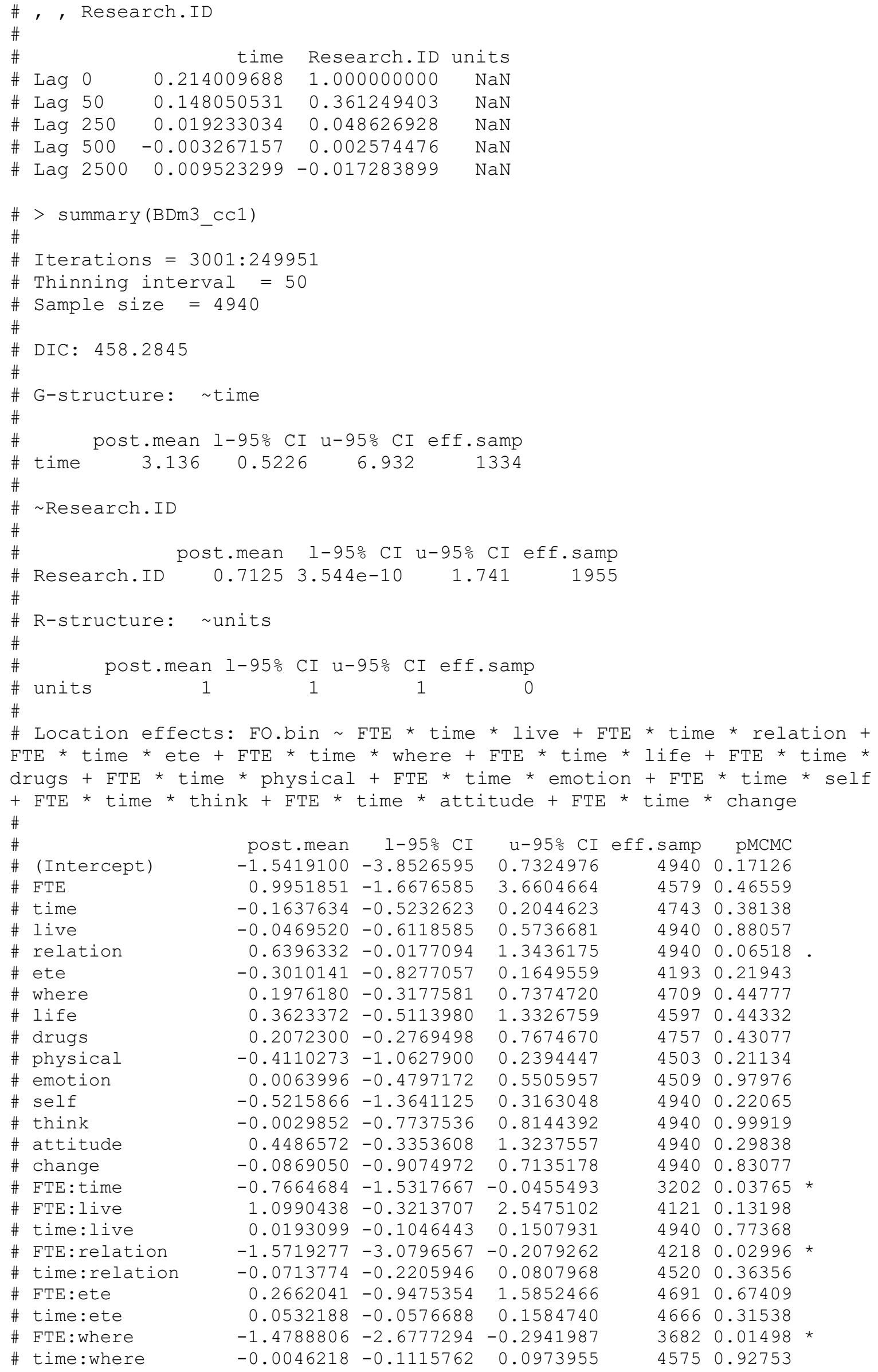




\begin{tabular}{|c|c|c|c|c|c|c|c|}
\hline \# & FTE:life & 0.9916816 & -0.6515429 & 2.7006459 & 4237 & 0.24615 & \\
\hline \# & time:life & -0.0068196 & -0.1795075 & 0.1819226 & 4659 & 0.95061 & \\
\hline \# & FTE:drugs & -0.6965924 & -1.9733197 & 0.5383916 & 4430 & 0.28623 & \\
\hline$\#$ & time: drugs & -0.0266508 & -0.1375709 & 0.0896788 & 4940 & 0.62753 & \\
\hline$\#$ & FTE:physical & -0.4060459 & -1.7663980 & 1.0158650 & 4112 & 0.58097 & \\
\hline \# & time:physical & 0.1148929 & -0.0526034 & 0.2748870 & 4624 & 0.16194 & \\
\hline$\#$ & FTE:emotion & -0.5261318 & -1.6914322 & 0.5482584 & 4355 & 0.35992 & \\
\hline \# & time:emotion & 0.0083038 & -0.1055163 & 0.1125855 & 517 & 0.88502 & \\
\hline \# & FTE:self & 2.3921137 & 0.9035888 & 3.9304515 & 943 & 0.0 & $\star \star$ \\
\hline \# & time:self & 0.0843226 & -0.0823312 & 0.2394500 & 4640 & 0.30486 & \\
\hline \# & FTE:think & -0.4835213 & -1.9276197 & 0.98950 & 4738 & 0.50729 & \\
\hline \# & time:think & -0.0623785 & -0.2446857 & 0.11295 & 4672 & 0.48907 & \\
\hline \# & FTE:attitude & -1.2487718 & -2.6864267 & 0.23 & 74 & 0.0 & . \\
\hline \# & time: attitude & -0.1005846 & -0.2852418 & 0.06542 & 40 & 0.2 & \\
\hline \# & FTE: change & 1.1932741 & -0.3988753 & 2.73385 & & 0.1 & \\
\hline \# & time: change & 0.0469474 & -0.1133549 & 0.2148000 & & 0.5 & \\
\hline$\#$ & FTE: time:live & -0.2429895 & -0.5944883 & 0.1048186 & 04 & 0.17 & \\
\hline \# & FTE: time:relation & 0.3806582 & 0.0238632 & 0.7509608 & 55 & 0.0 & * \\
\hline \# & FTE:time:ete & 0.2541658 & -0.1228007 & 0.6112065 & & 0.1 & \\
\hline \# & FTE:time: where & 0.2509095 & 0.0286526 & 0.4960120 & & 0.0 & 夫 \\
\hline \# & FTE:time:life & -0.4413192 & -0.8623134 & 0.0001852 & & 0.03 & * \\
\hline \# & FTE:time:drugs & 0.2602919 & -0.0610515 & 0.6078128 & & 0.1 & \\
\hline \# & FTE:time:physical & -0.0480137 & -0.4309057 & 0.3473896 & & 0.8 & \\
\hline \# & FTE:time:emotion & 0.1573710 & -0.0932918 & 0.4260784 & & 0.2 & \\
\hline \# & FTE:time:self & -0.6139503 & -0.9711916 & -0.2666042 & & $<2 e-04$ & $\star \star \star$ \\
\hline \# & FTE:time:think & 0.1844859 & -0.1434362 & 0.5318857 & & 0.27611 & \\
\hline \# & FTE: time:attitude & 0.2963801 & -0.0768483 & 0.7144638 & & 0.14089 & \\
\hline \# & FTE:time:change & -0.2266278 & -0.6057061 & 0.1558307 & 4541 & 0.25668 & \\
\hline & & & & & & & \\
\hline & f. codes: & 0 & & $1 \quad 0.05$ & '. ' & ' 1 & \\
\hline
\end{tabular}


Trace Plots and Posterior Density Plots

Fixed Effects

Trace of (Intercept)

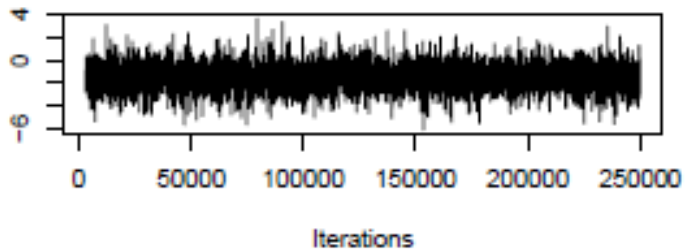

Trace of FTE

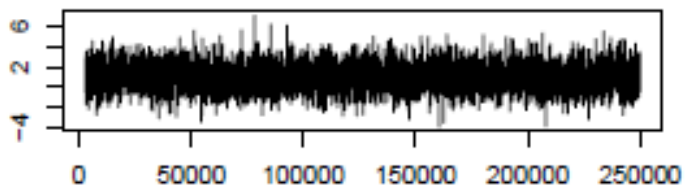

Iterations

Trace of time

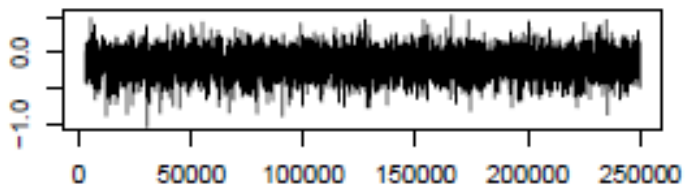

Iterations

Trace of live

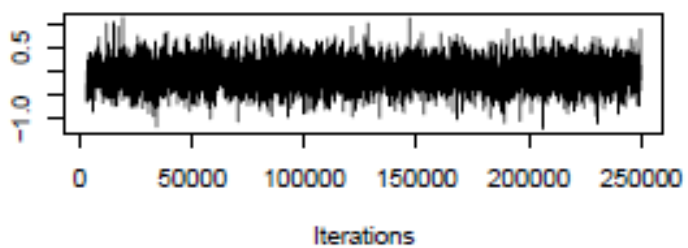

Trace of relation

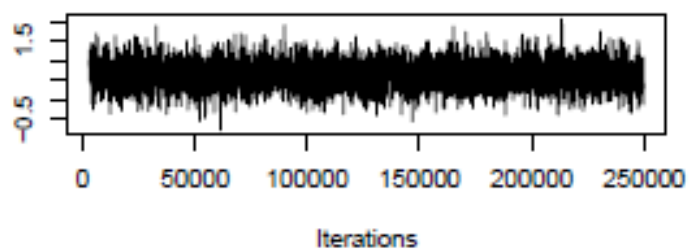

Trace of ete

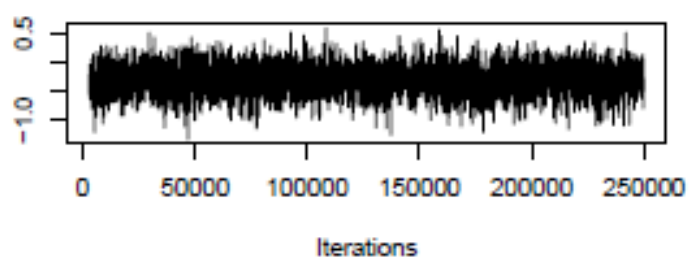

Density of (Intercept)

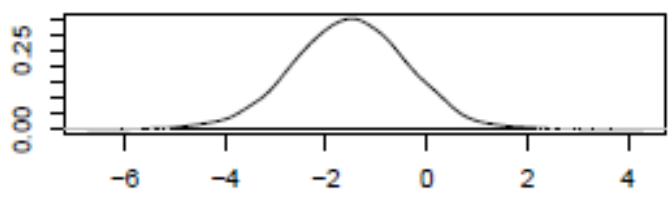

$\mathrm{N}=4940$ Bandwidth $=0.2181$

Density of FTE

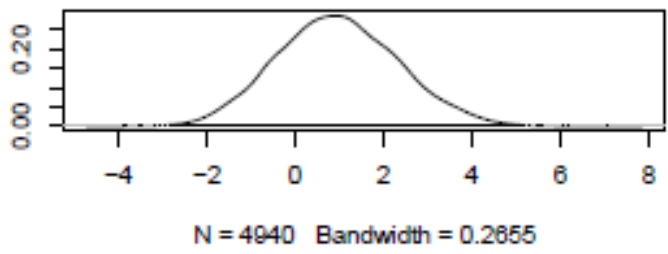

Density of time

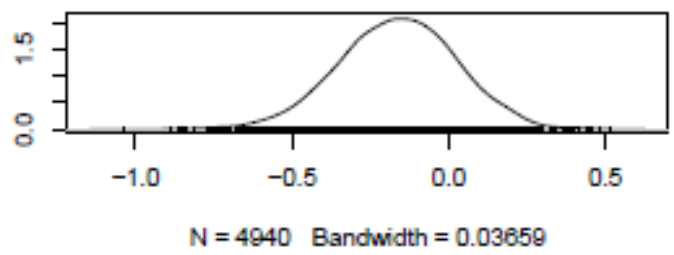

Density of live

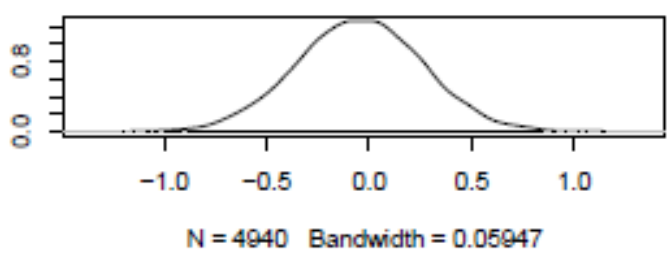

Density of relation

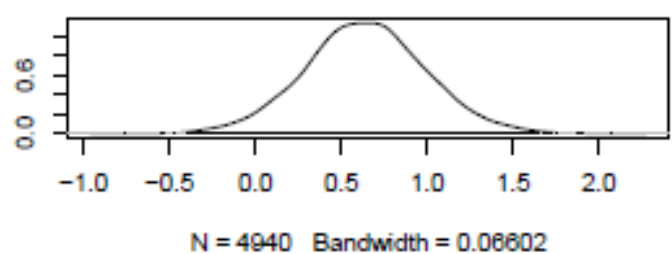

Density of ete

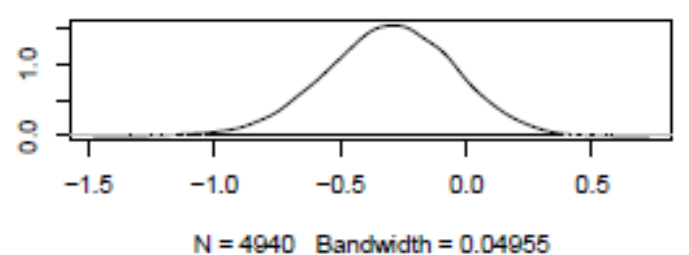


Trace of where

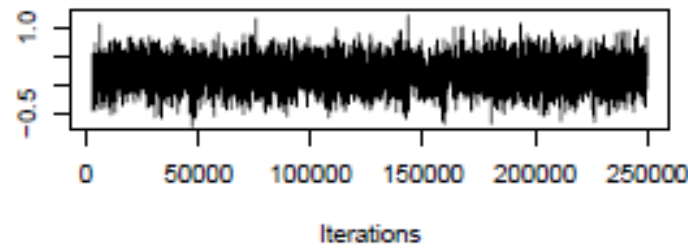

Trace of life

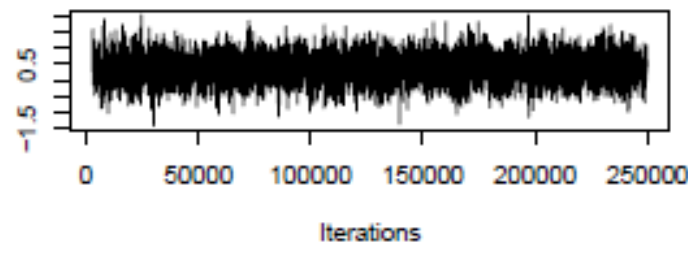

Trace of drugs

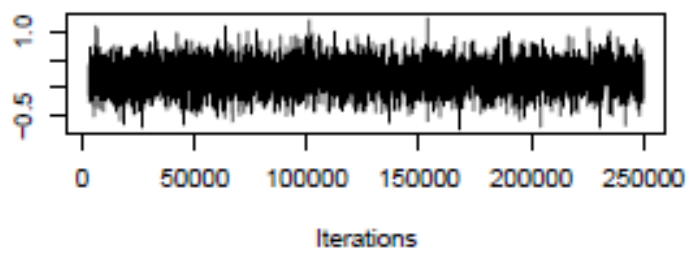

Trace of physical

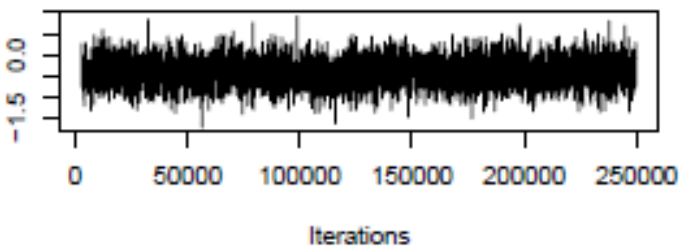

Trace of emotion

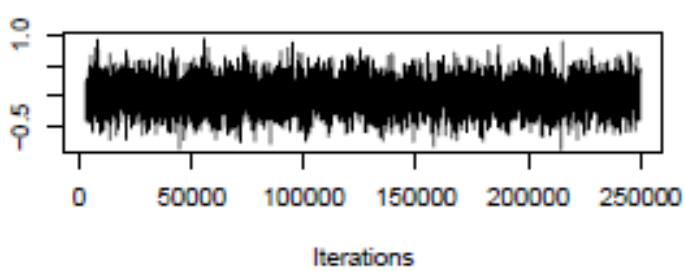

Trace of self

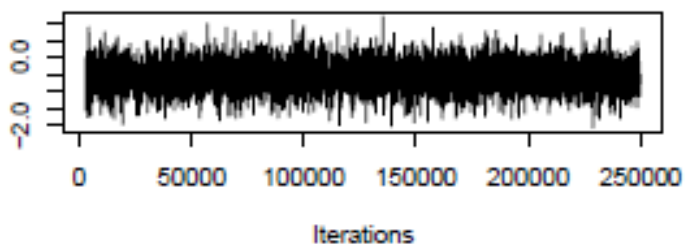

Density of where

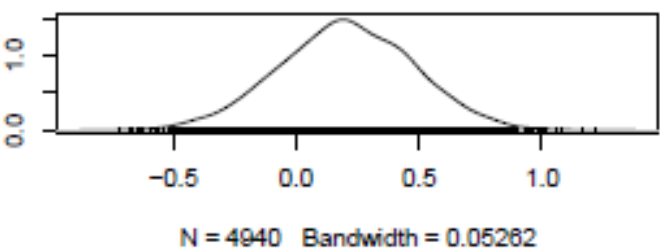

Density of life

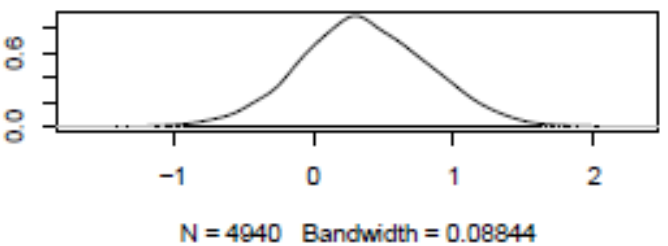

Density of drugs

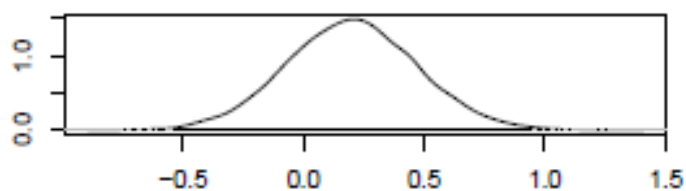

$\mathrm{N}=4940$ Bandwidth $=0.0518$

Density of physical

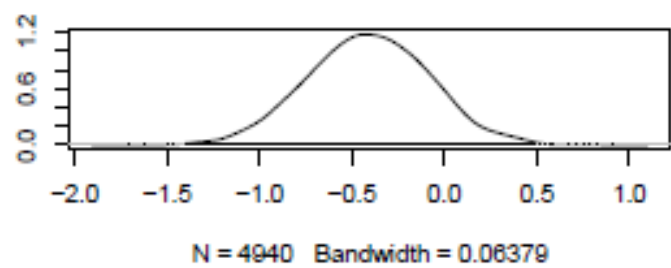

Density of emotion

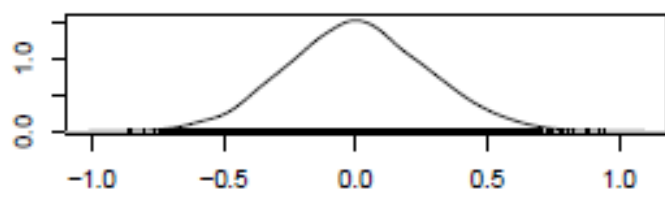

$\mathrm{N}=4940$ Bandwidth $=0.05064$

Density of self

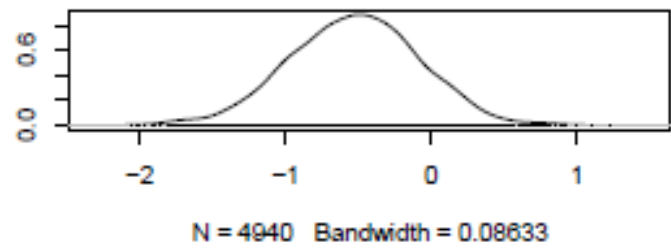


Trace of think

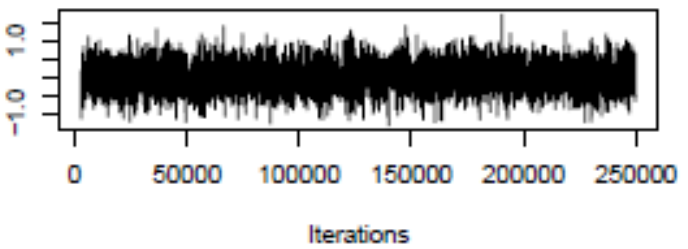

Trace of attitude

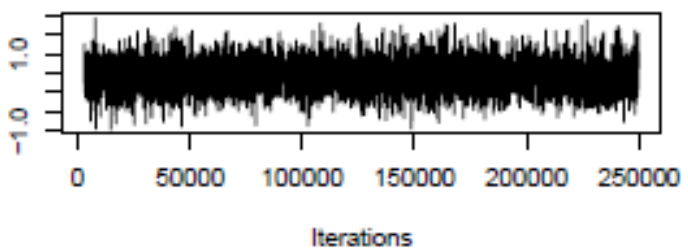

Trace of change

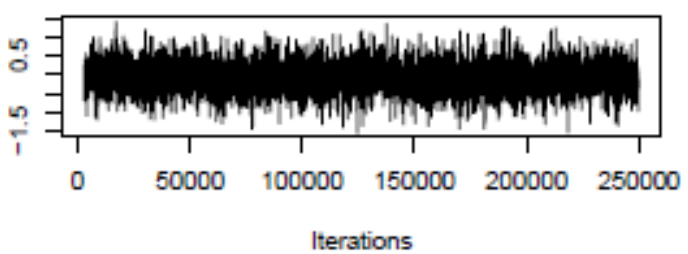

Trace of FTE:time

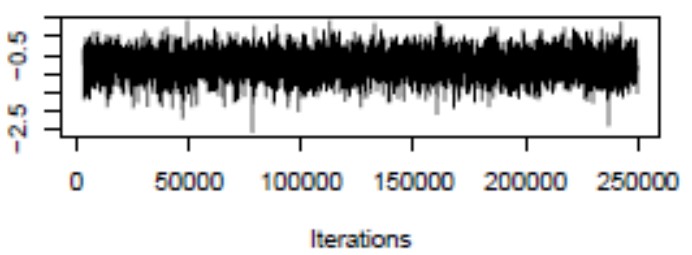

Trace of FTE:live

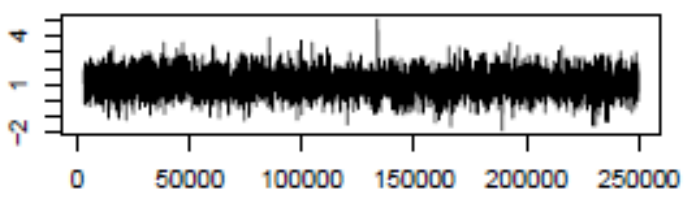

Iterations

Trace of time:live

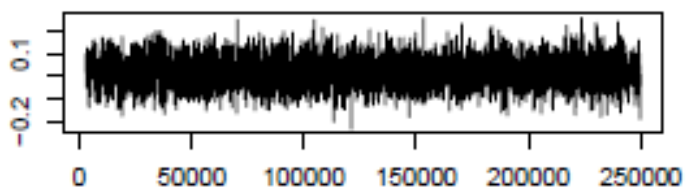

Iterations
Density of think

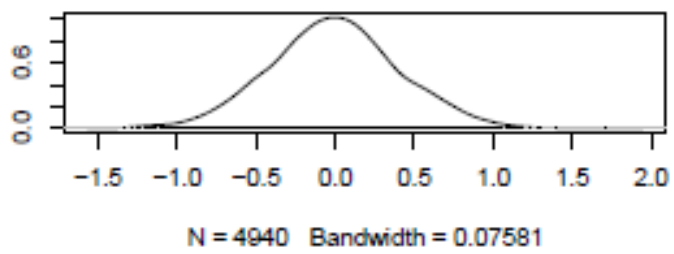

Density of attitude

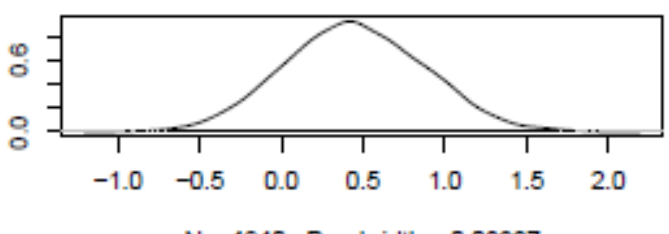

$\mathrm{N}=4940$ Bandwidth $=0.08337$

Density of change

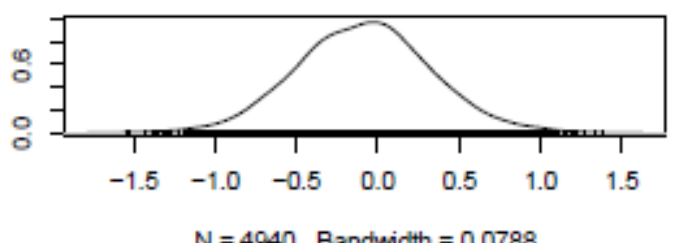

Density of FTE:time

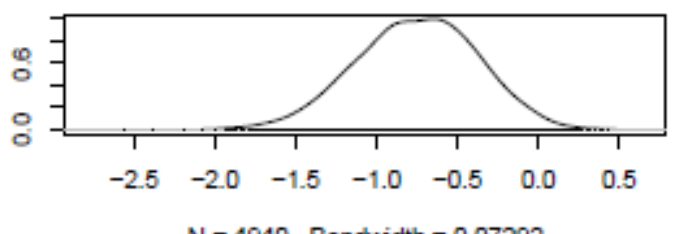

$\mathrm{N}=4940$ Bandwidth $=0.07292$

Density of FTE:live

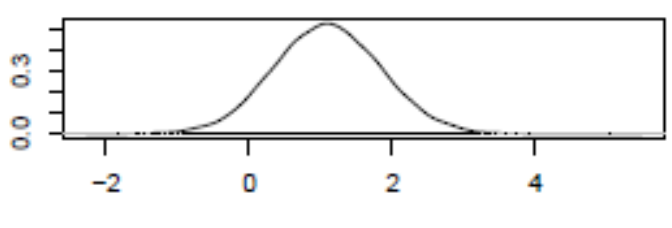

$\mathrm{N}=4940$ Bandwidth $=0.1453$

Density of time:live

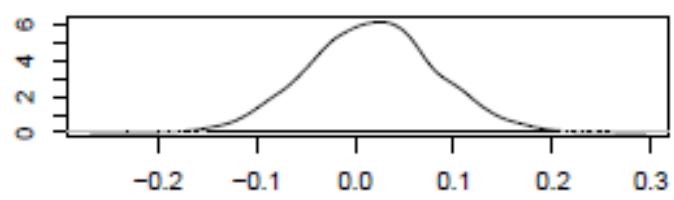

$\mathrm{N}=4940$ Bandwidth $=0.01228$ 
Trace of FTE:relation

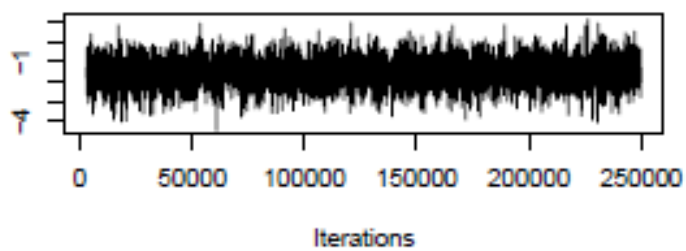

Trace of time:relation
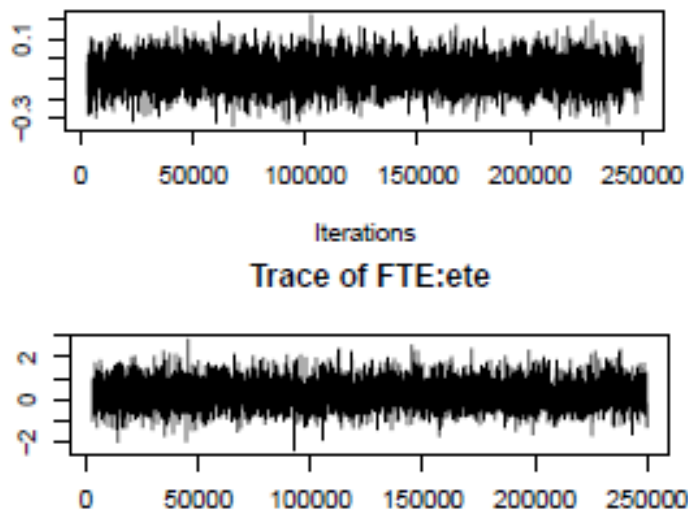

Iterations

Trace of time:ete

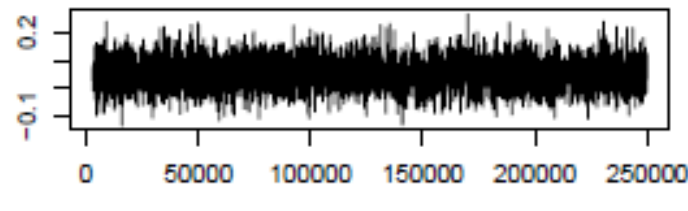

Iterations

Trace of FTE:where

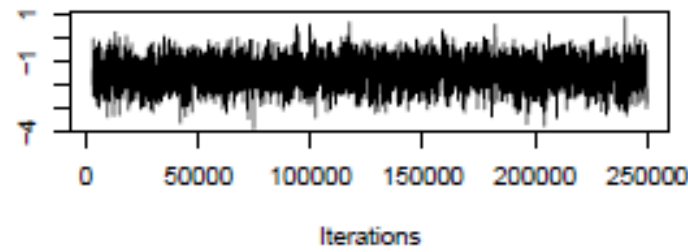

Trace of time:where

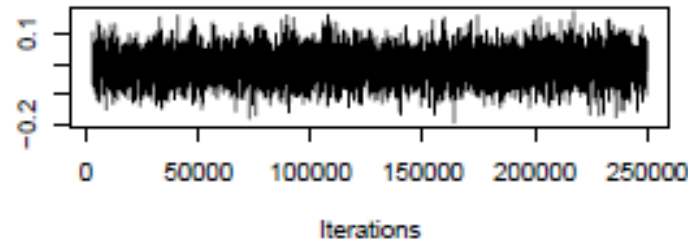

Density of FTE:relation

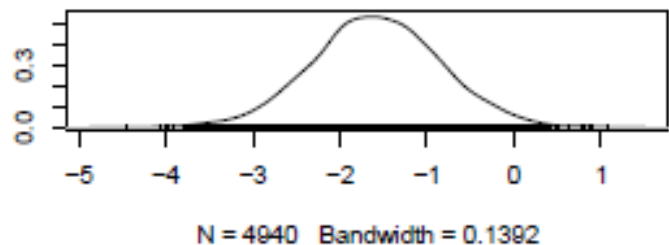

Density of time:relation

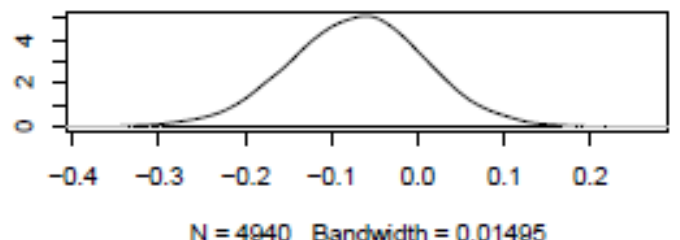

Density of FTE:ete

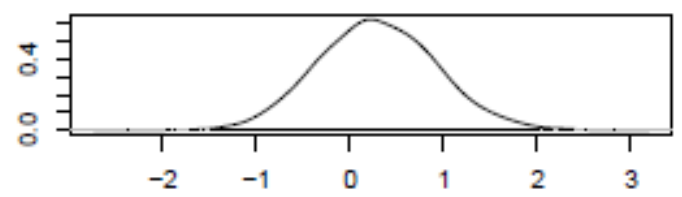

$\mathrm{N}=4940$ Bandwidth $=0.1228$

Density of time:ete

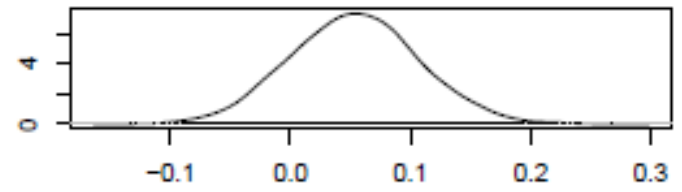

$\mathrm{N}=4940$ Bandwidth $=0.01045$

Density of FTE:where

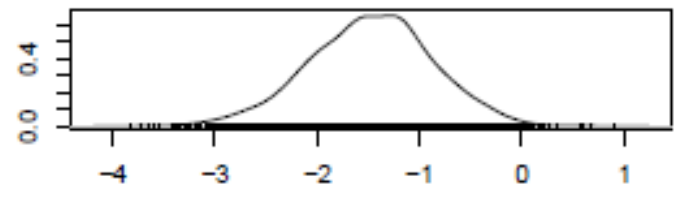

$\mathrm{N}=4940$ Bandwidth $=0.1157$

Density of time:where

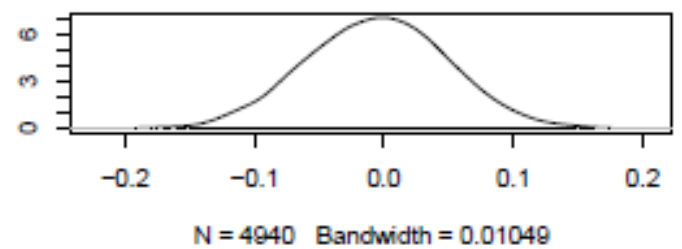


Trace of FTE:life

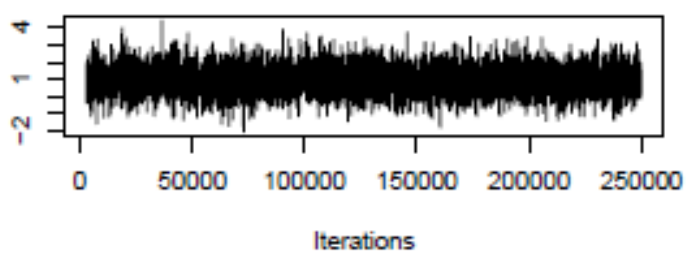

Trace of time:life

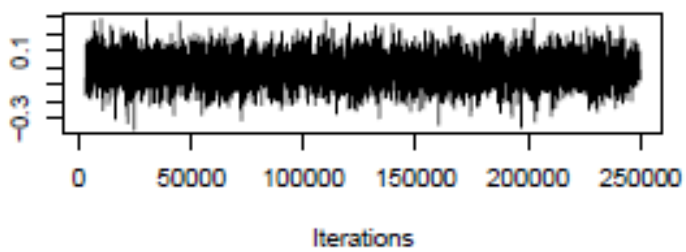

Trace of FTE:drugs

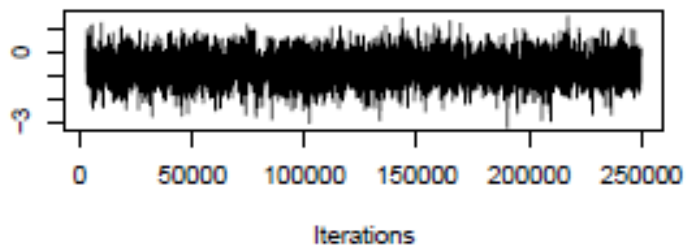

Trace of time:drugs

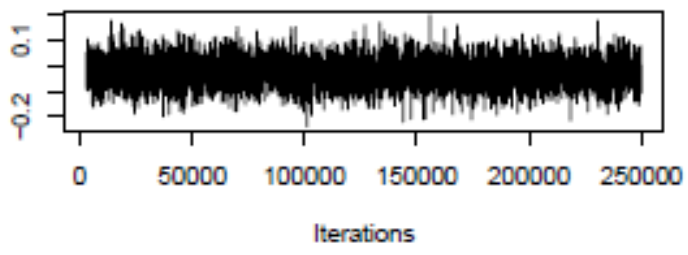

Trace of FTE:physical

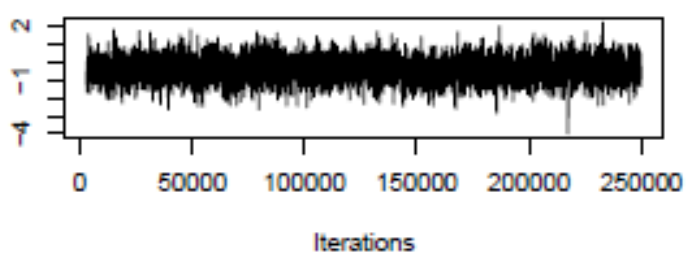

Trace of time:physical

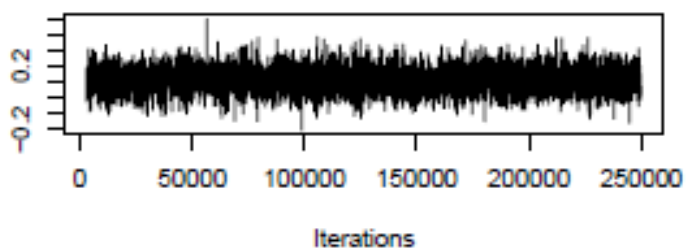

Density of FTE:life

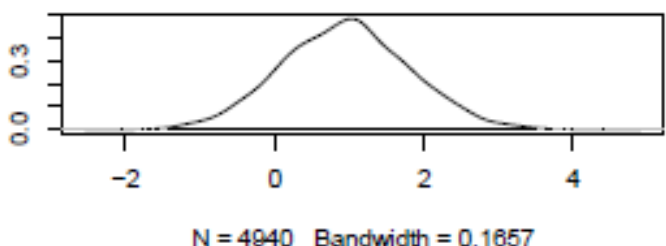

Density of time:life

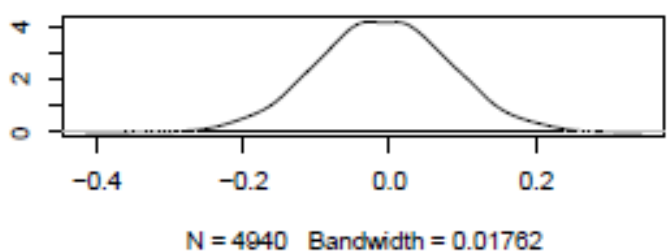

Density of FTE:drugs

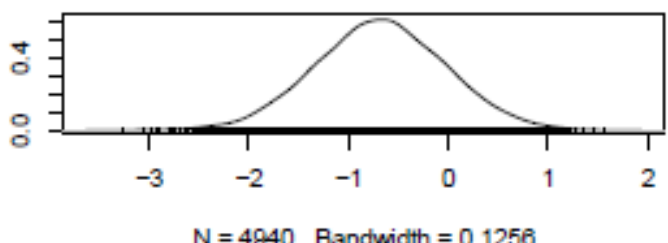

Density of time:drugs

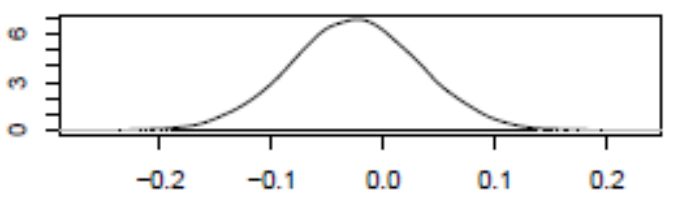

$\mathrm{N}=4940$ Bandwidth $=0.01104$

Density of FTE:physical

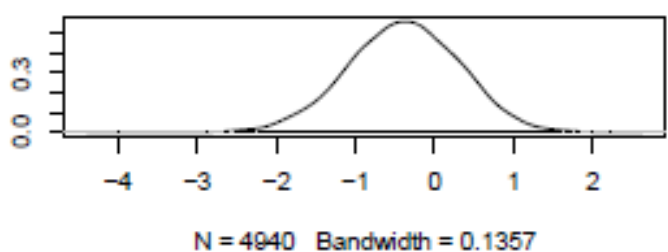

Density of time:physical

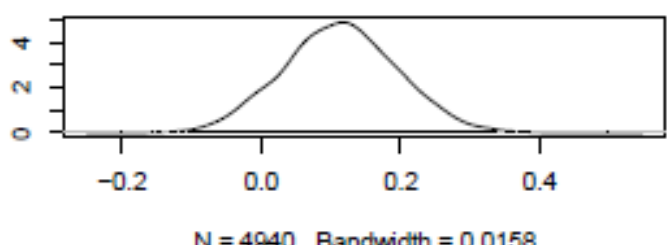

$\mathrm{N}=4940$ Bandwidth $=0.0158$ 
Trace of FTE:emotion

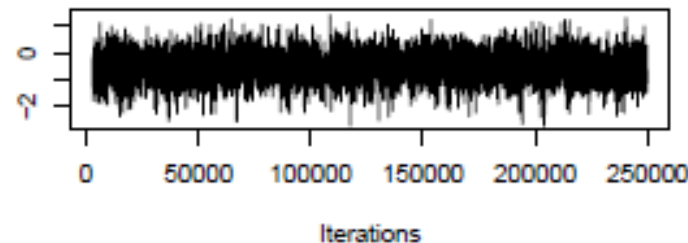

Trace of time:emotion

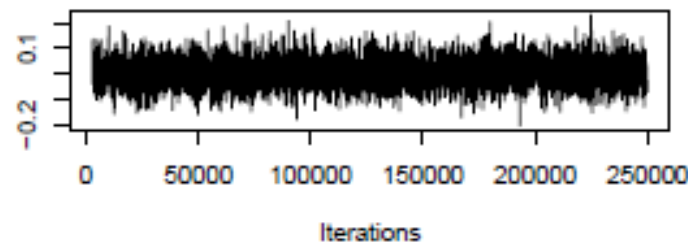

Trace of FTE:self

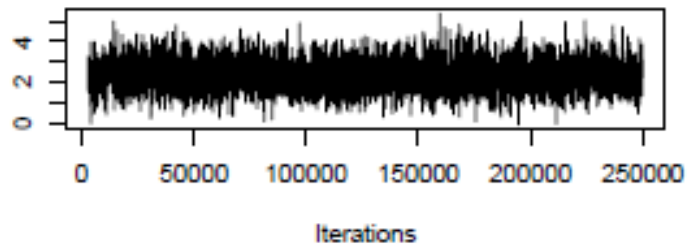

Trace of time:self

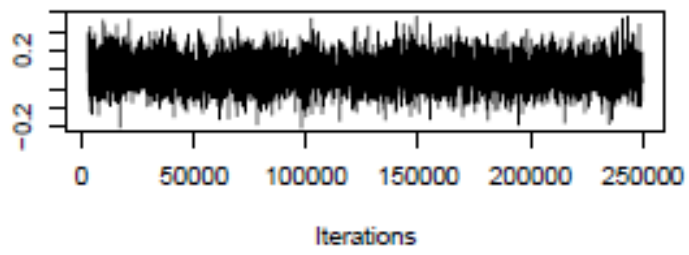

Trace of FTE:think

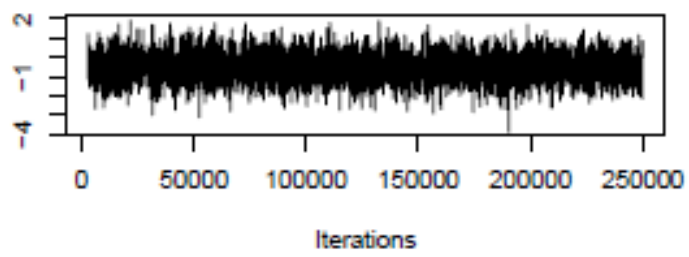

Trace of time:think

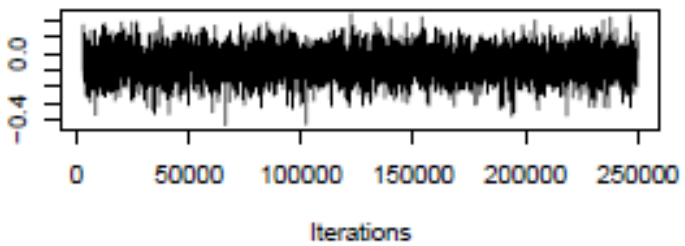

Density of FTE:emotion

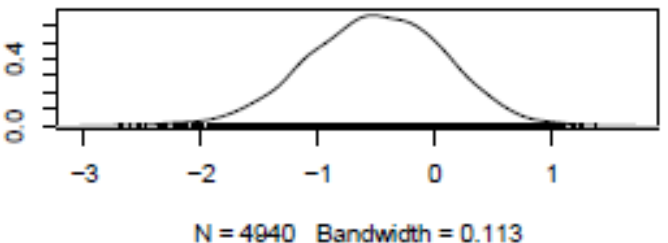

Density of time:emotion

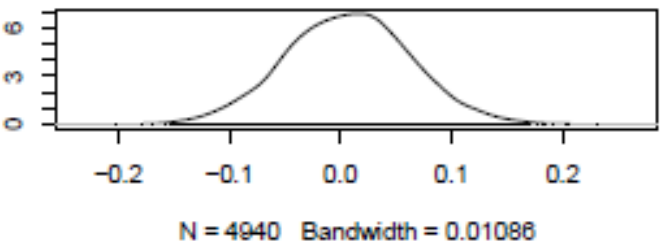

Density of FTE:self

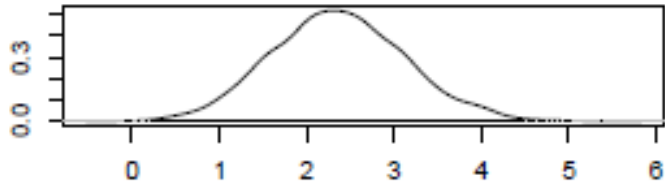

$N=4940$ Bandwidth $=0.1477$

Density of time:self

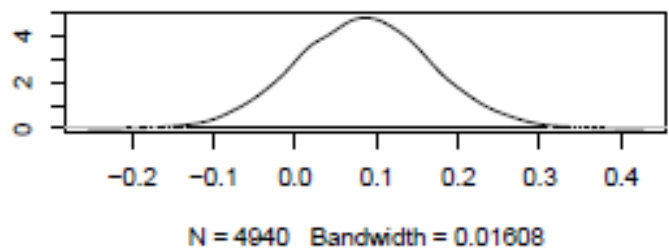

Density of FTE:think

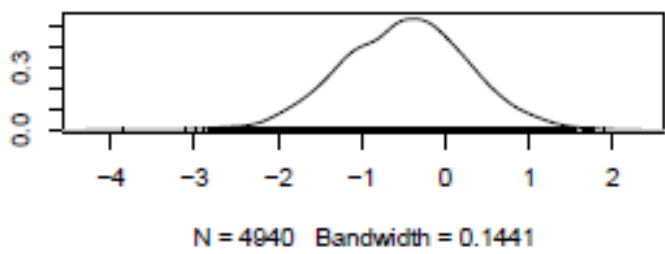

Density of time:think

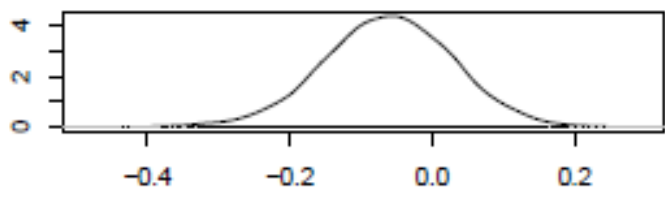

$\mathrm{N}=4940$ Bandwidth $=0.01748$ 
Trace of FTE:attitude

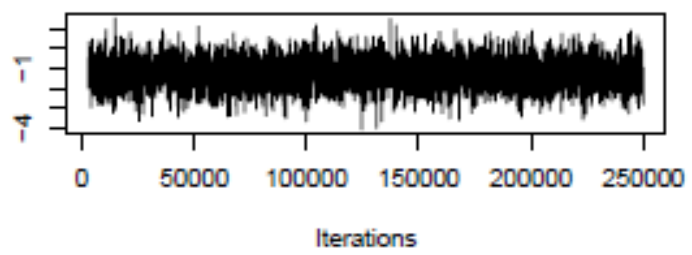

Trace of time:attitude

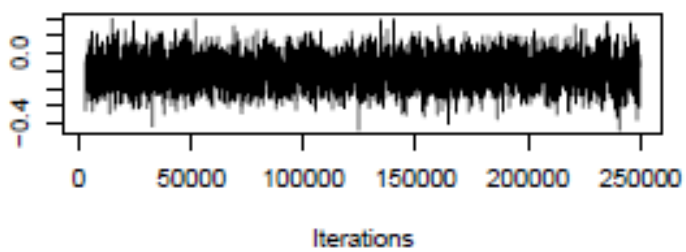

Trace of FTE:change

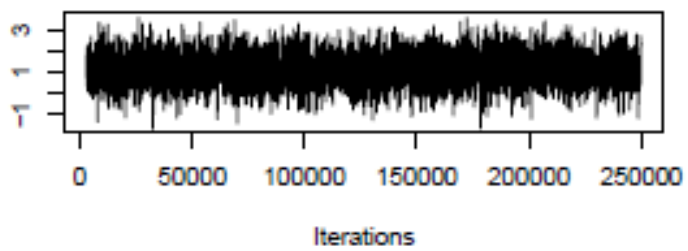

Trace of time:change

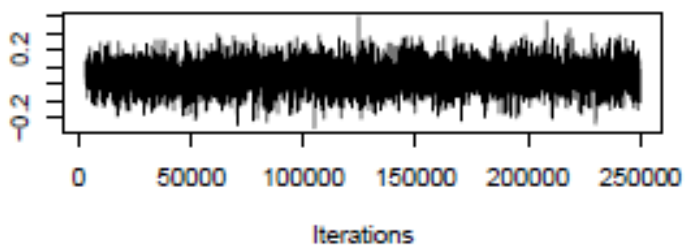

Trace of FTE:time:live

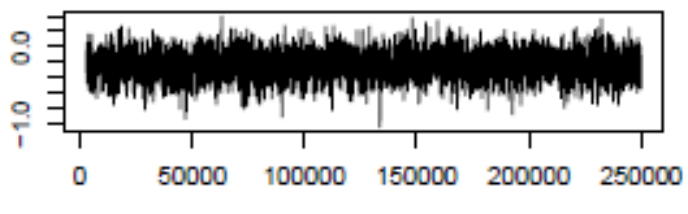

Iterations

Trace of FTE:time:relation

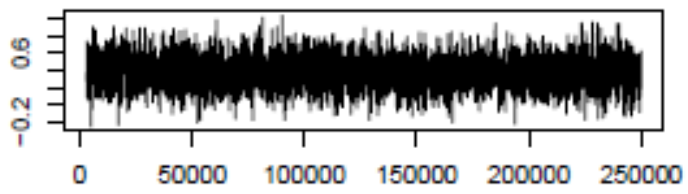

Iterations
Density of FTE:attitude

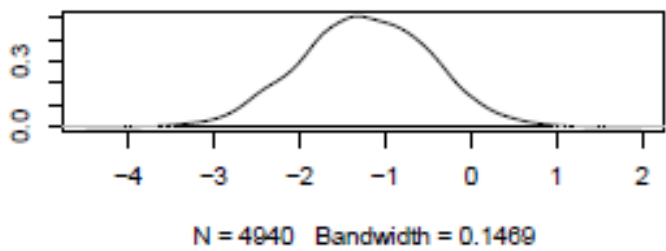

Density of time:attitude

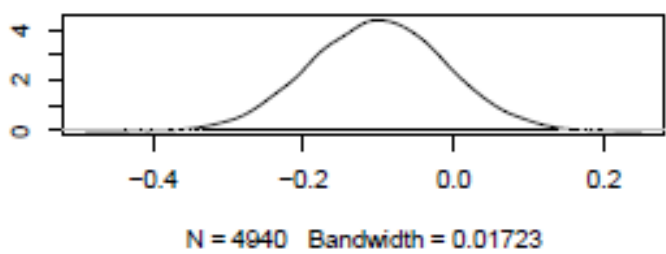

Density of FTE:change

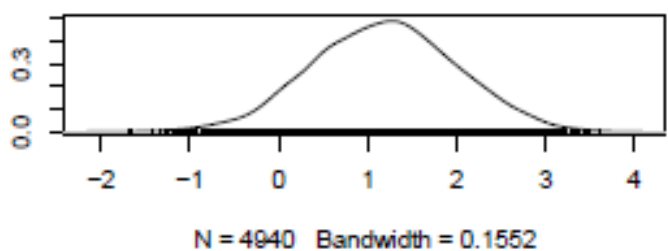

Density of time:change

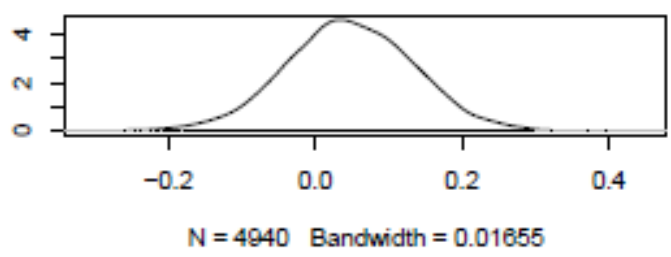

Density of FTE:time:live

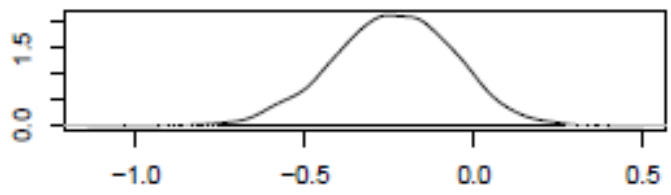

$\mathrm{N}=4940$ Bandwidth $=0.03495$

Density of FTE:time:relation

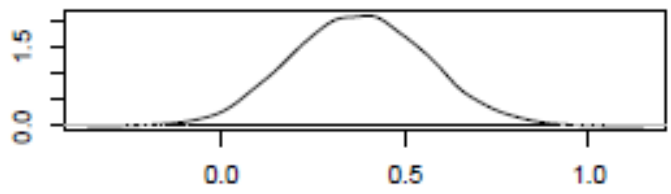

$\mathrm{N}=4940$ Bandwidth $=0.03597$ 
Trace of FTE:time:ete

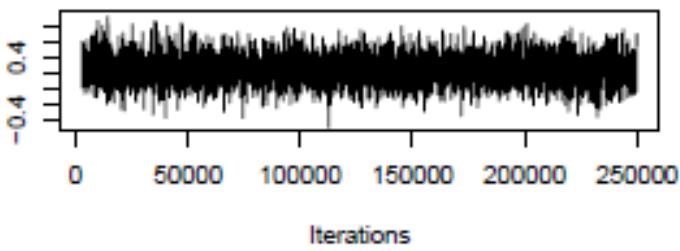

Trace of FTE:time:where

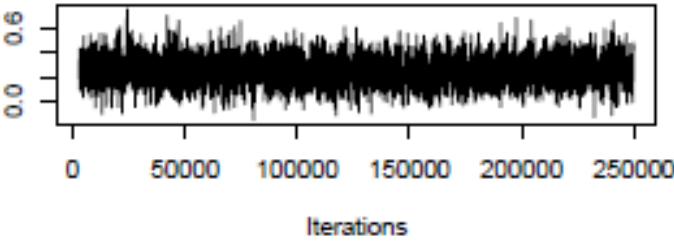

Trace of FTE:time:life

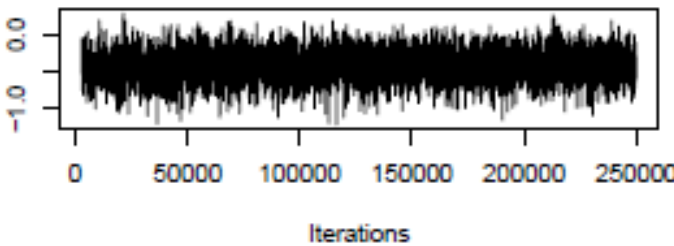

Trace of FTE:time:drugs

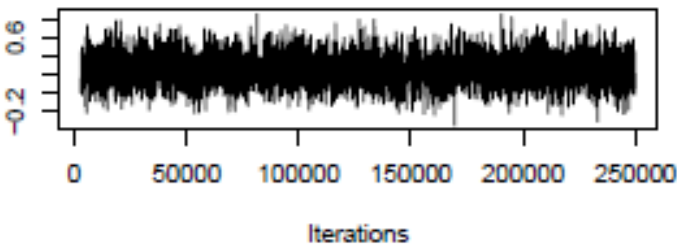

Trace of FTE:time:physical

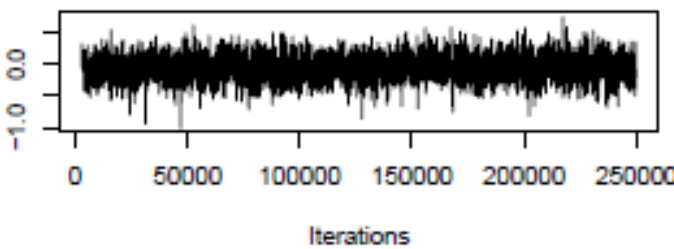

Trace of FTE:time:emotion

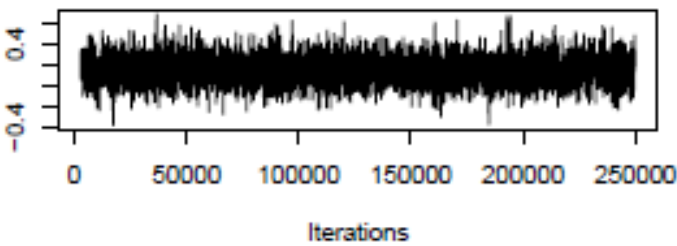

Density of FTE:time:ete

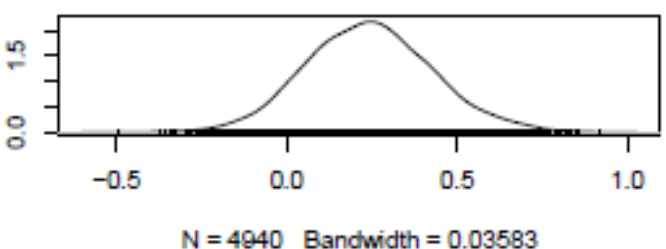

Density of FTE:time:where

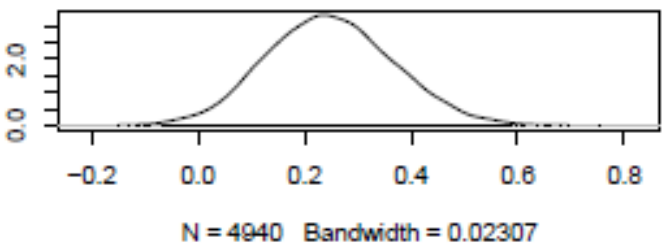

Density of FTE:time:life

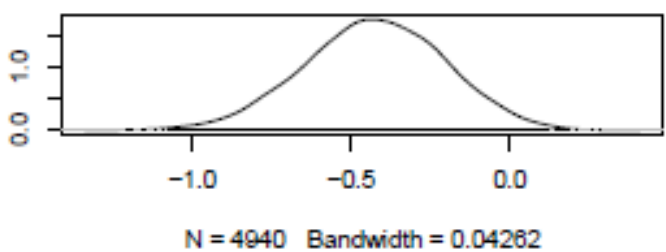

Density of FTE:time:drugs

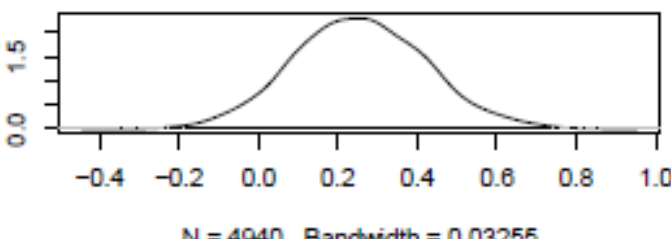

Density of FTE:time:physical

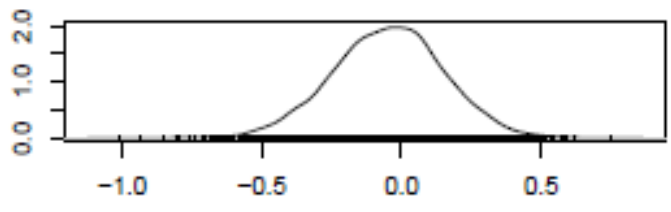

$\mathrm{N}=4940$ Bandwidth $=0.03771$

Density of FTE:time:emotion

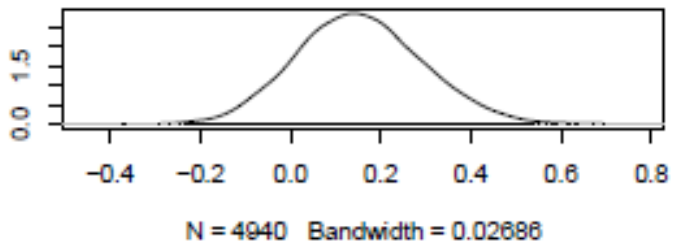


Trace of FTE:time:self

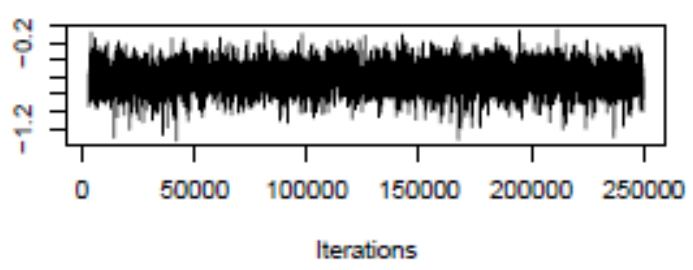

Trace of FTE:time:think

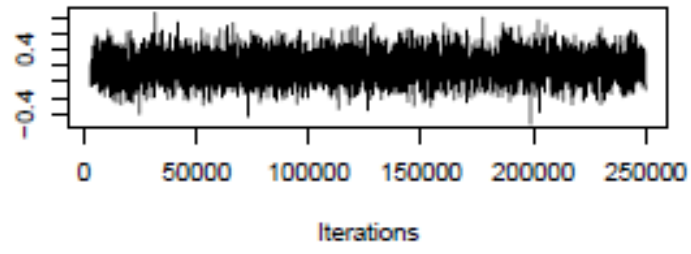

Trace of FTE:time:attitude

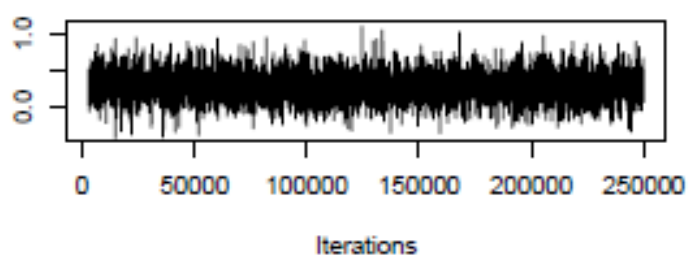

Trace of FTE:time:change

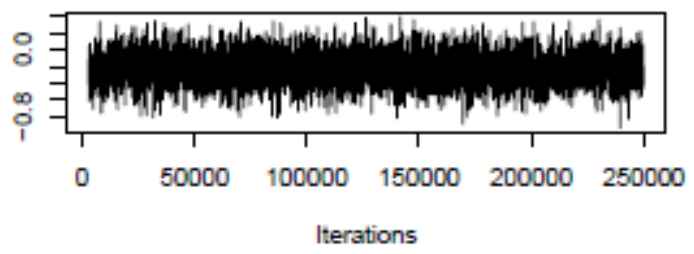

Density of FTE:time:self

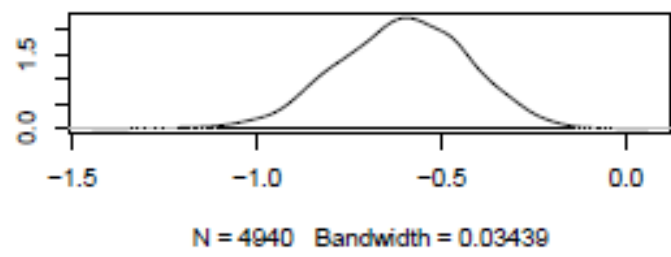

Density of FTE:time:think

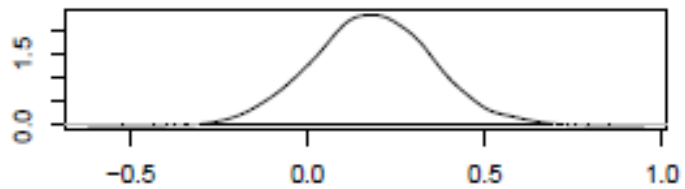

$\mathrm{N}=4940$ Bandwidth $=0.0324$

Density of FTE:time:attitude

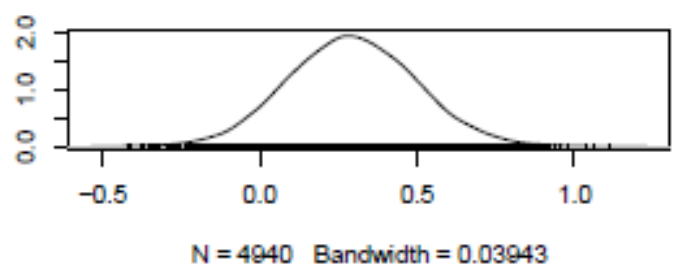

Density of FTE:time:change

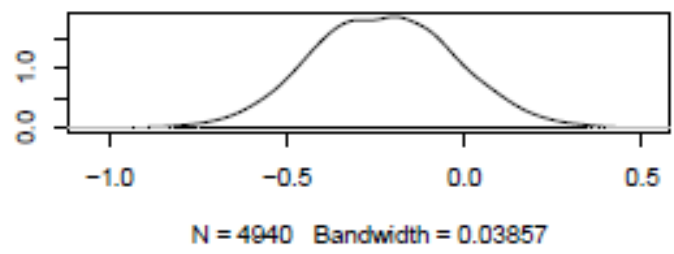


$\underline{\text { Random Effects }}$

Trace of time

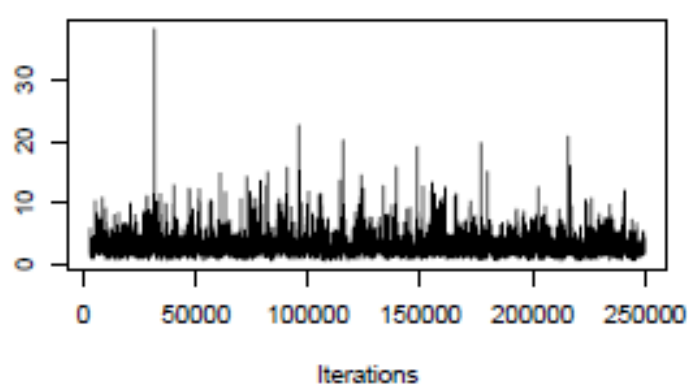

Trace of Research.ID

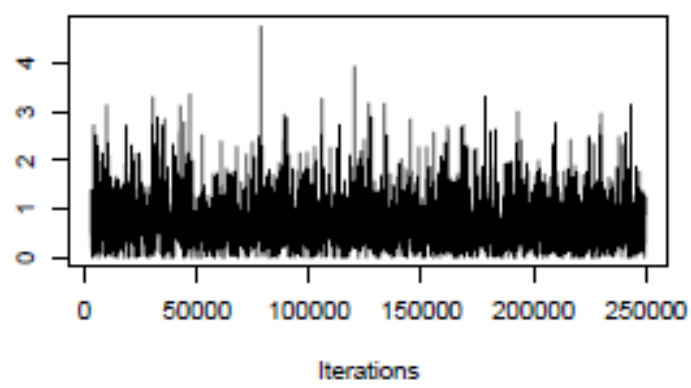

Density of time

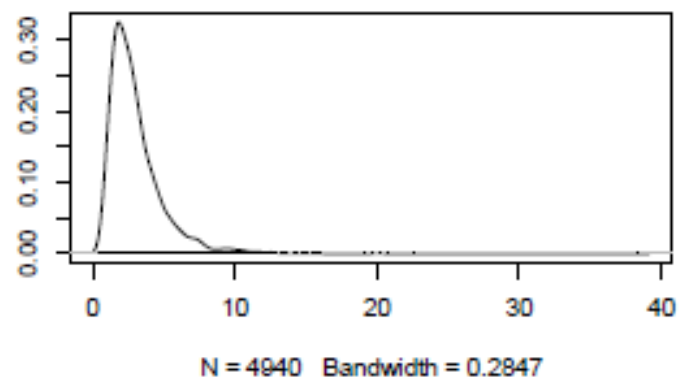

Density of Research.ID

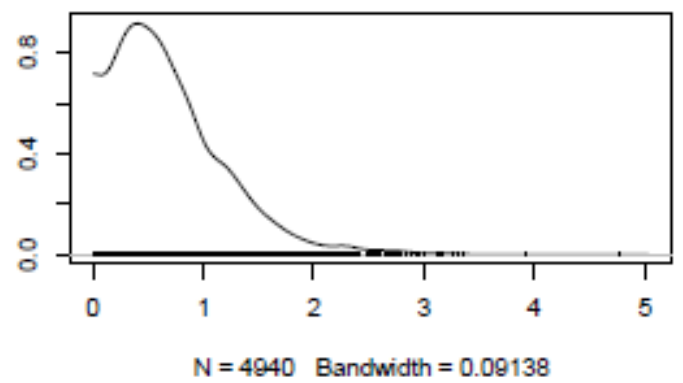


Dynamic Model involving Grouped Age at First Offence (Table 6.17)

Bayesian Model (BDm3G_cc2)

\section{\#\# Define the model}

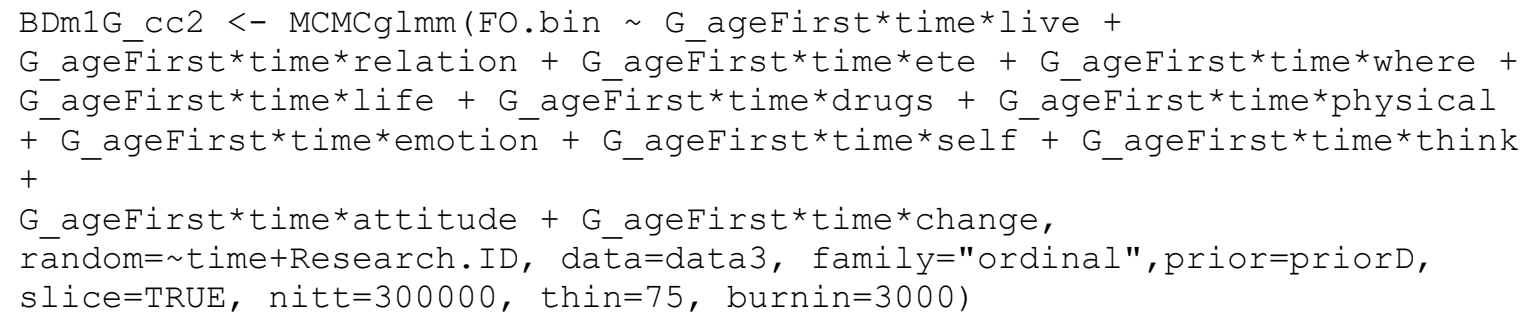

\section{\#\# Checks for suitable convergence}

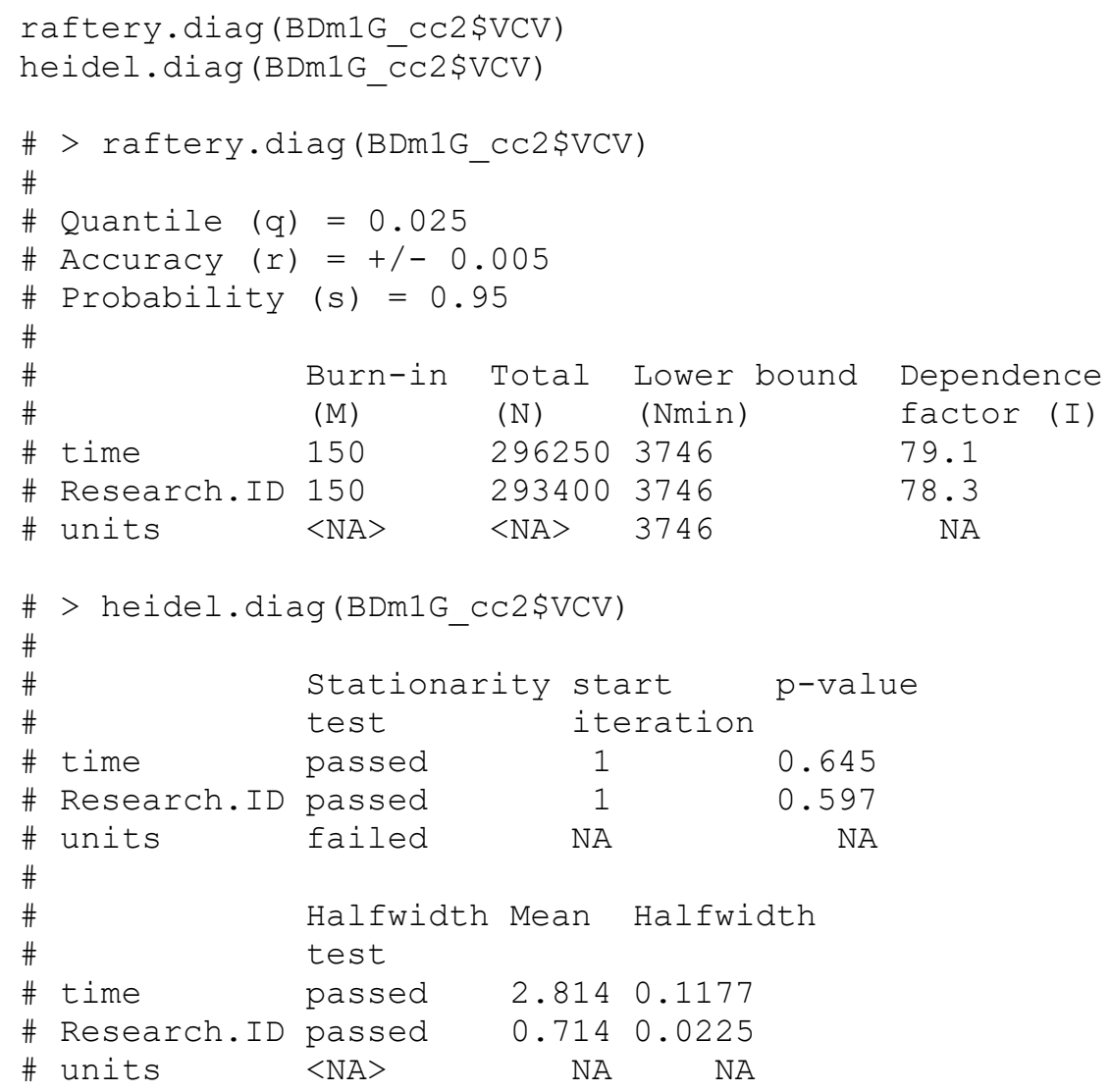



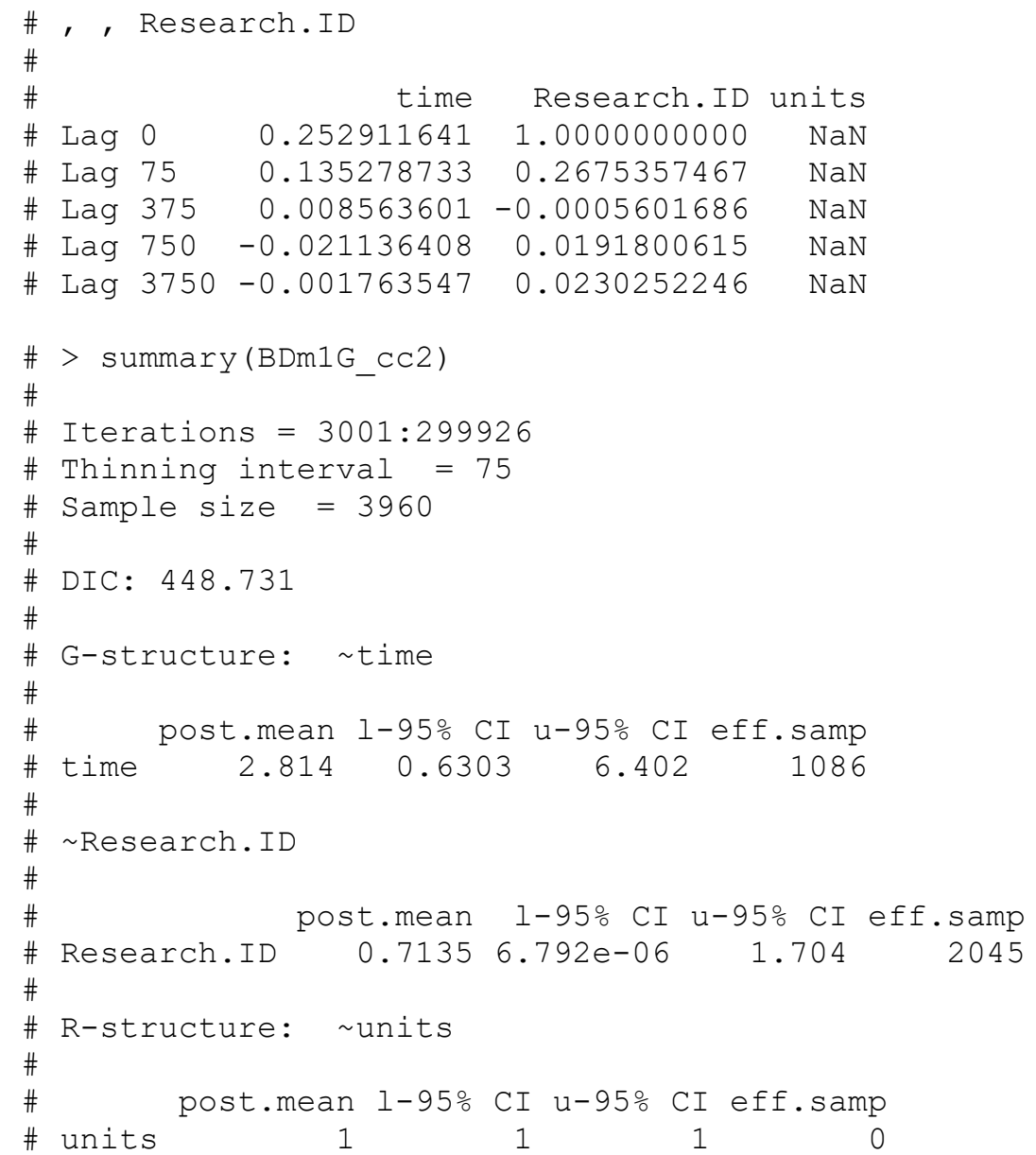


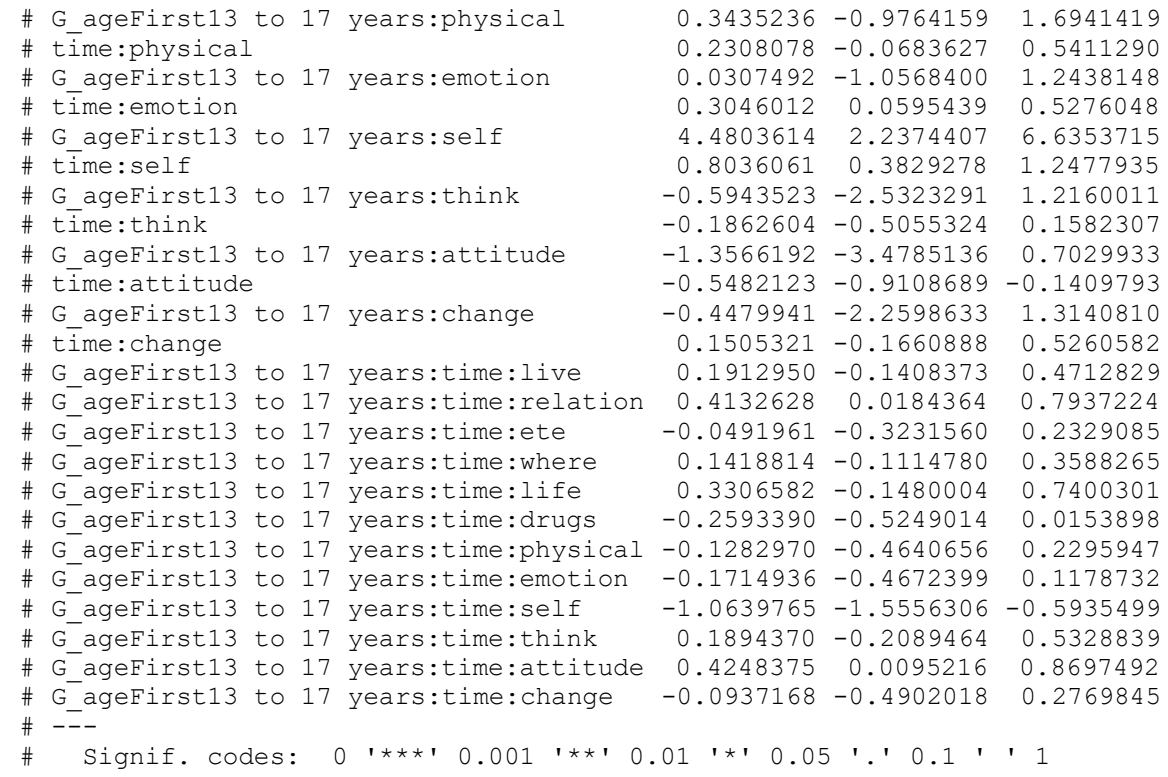

37530.62828

$\begin{array}{lll}3545 & 0.13333\end{array}$

39600.94091

35310.00606 *

$3028<3 e-04 * * *$

$3147<3 e-04 * \star *$

36750.52323

$\begin{array}{lll}3652 & 0.26313\end{array}$

39600.21061

35950.00253 **

$3960 \quad 0.63535$

$\begin{array}{lll}3960 & 0.39697\end{array}$

36940.21717

36240.02576 *

39600.73333

$3960 \quad 0.22879$

35120.14747

35890.06212

$\begin{array}{lll}3818 & 0.45960\end{array}$

$3410 \quad 0.23384$

$3077<3 e-04 * * *$

$\begin{array}{lll}3488 & 0.32273\end{array}$

37170.04848 *

$3960 \quad 0.63232$ 
Trace of (Intercept)

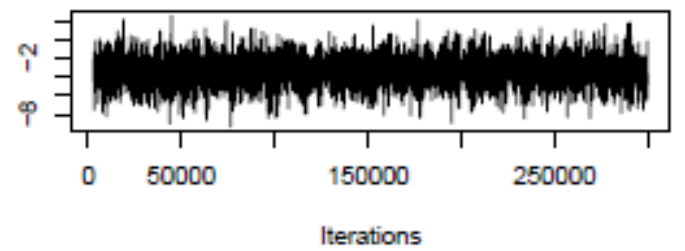

Trace of G_ageFirst13 to 17 years

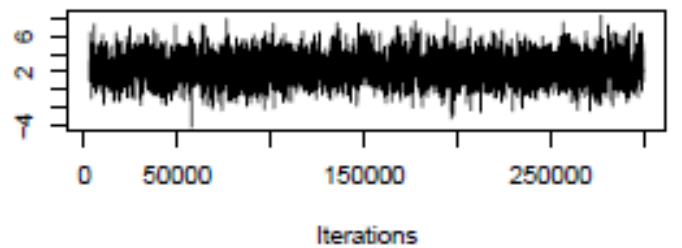

Trace of time

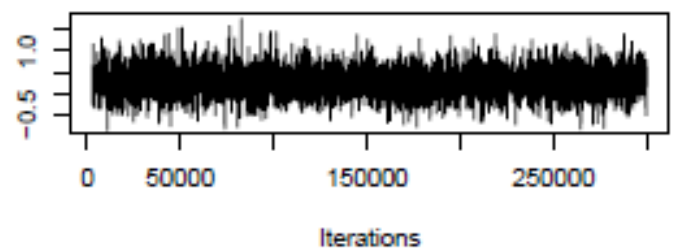

Trace of live

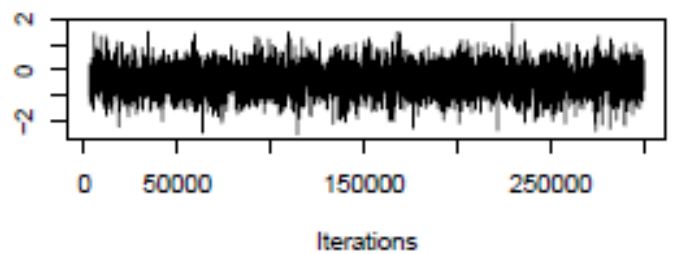

Trace of relation

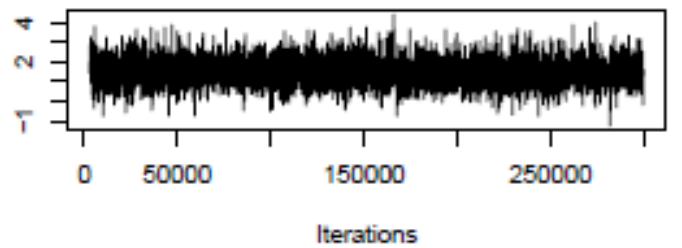

Trace of ete

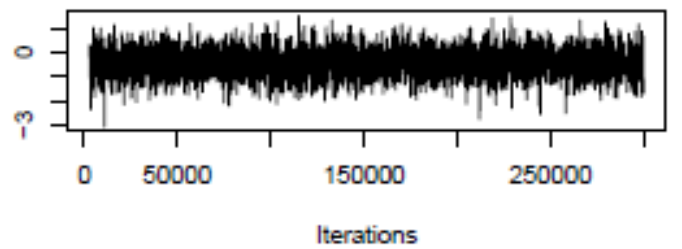

Density of (Intercept)

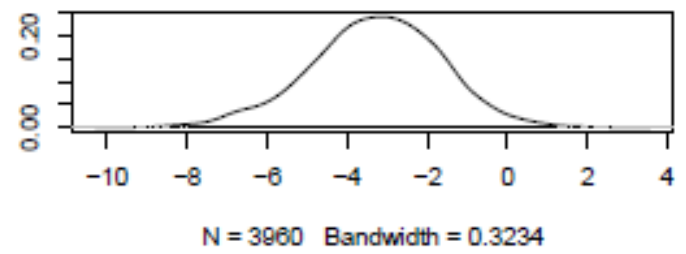

Density of G_ageFirst13 to 17 years

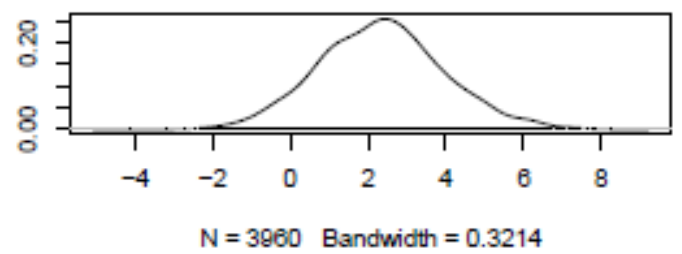

Density of time

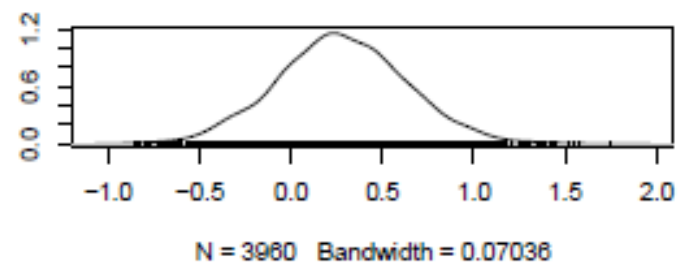

Density of live

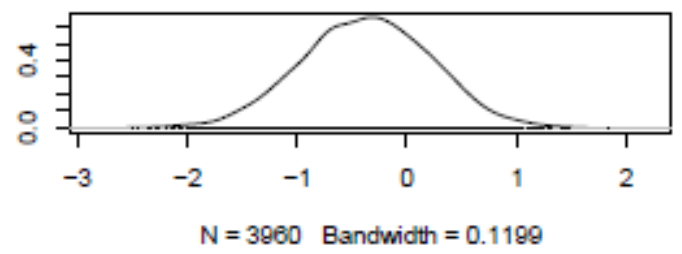

Density of relation

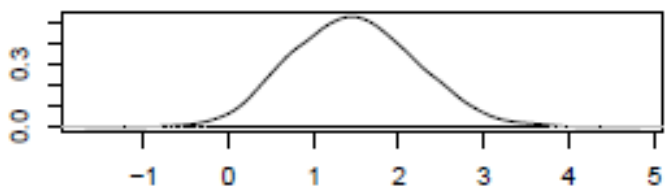

$\mathrm{N}=3960$ Bandwidth $=0.1497$

Density of ete

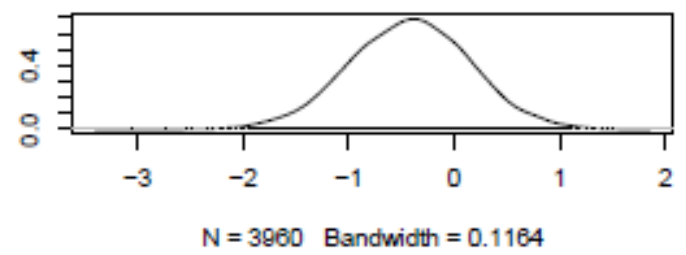


Trace of where

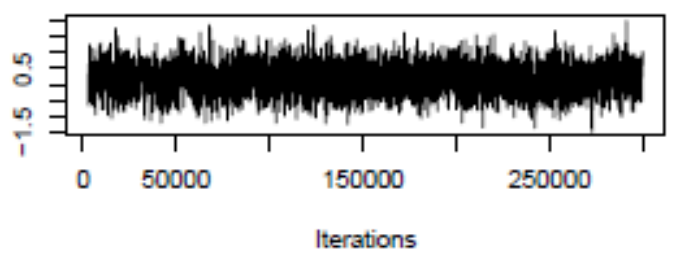

Trace of life

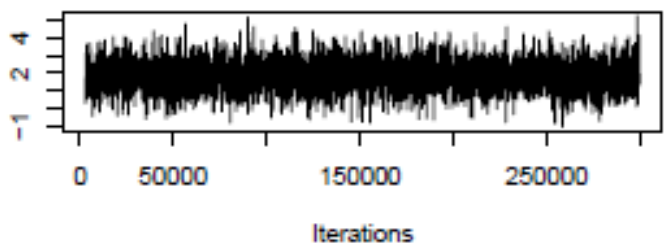

Trace of drugs

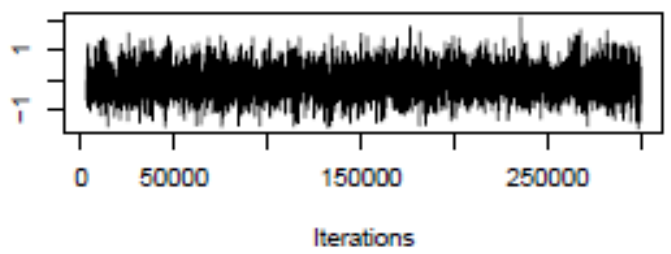

Trace of physical

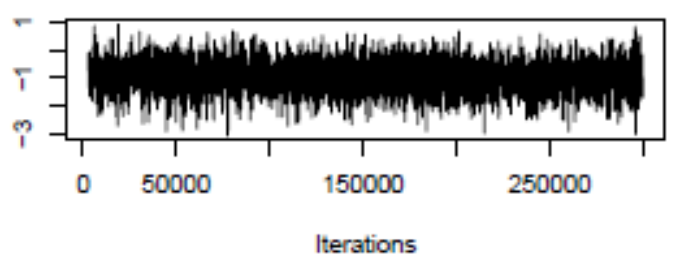

Trace of emotion

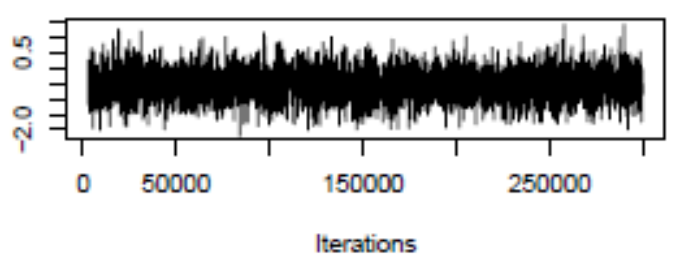

Trace of self

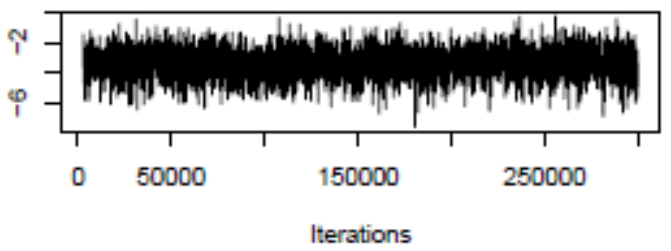

Density of where

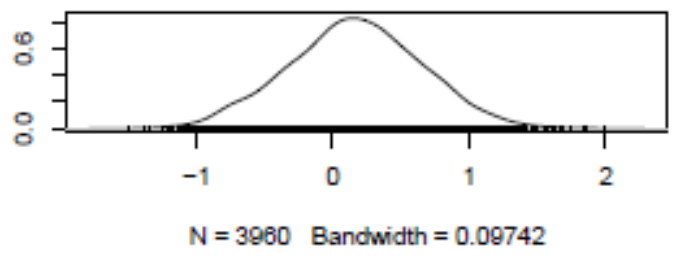

Density of life

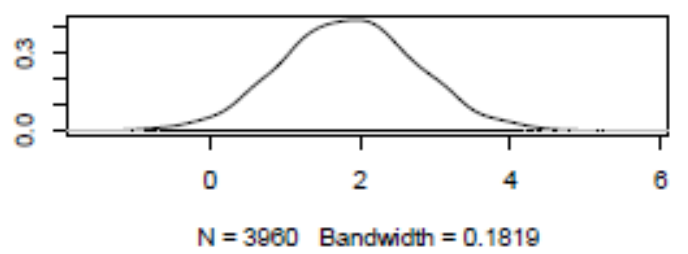

Density of drugs

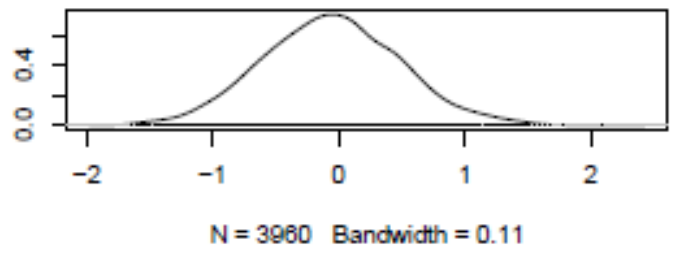

Density of physical

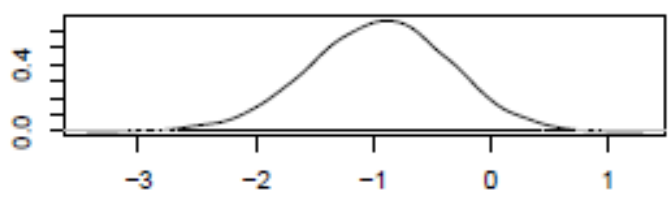

$\mathrm{N}=3960$ Bandwidth $=0.1199$

Density of emotion

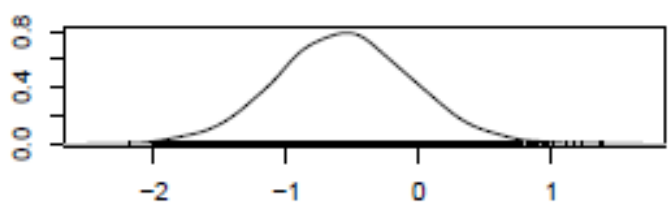

$\mathrm{N}=3960$ Bandwidth $=0.1022$

Density of self

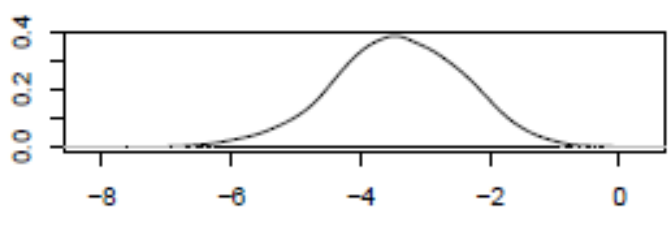

$\mathrm{N}=3960$ Bandwidth $=0.2037$ 
Trace of think

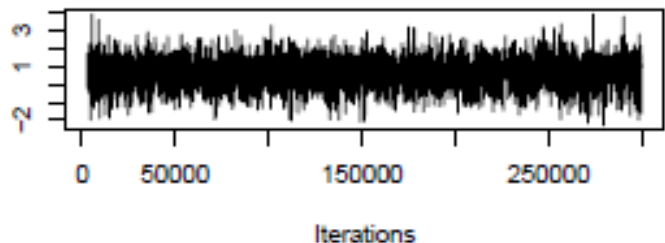

Trace of attitude

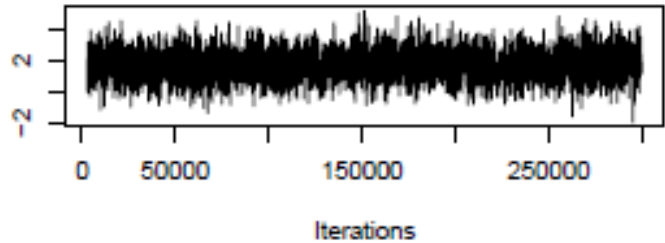

Trace of change

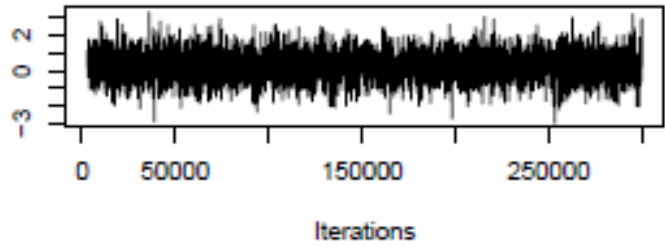

Trace of G_ageFirst13 to 17 years:time

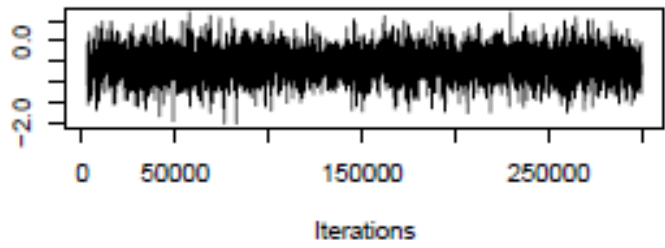

Trace of G_ageFirst13 to 17 years:live

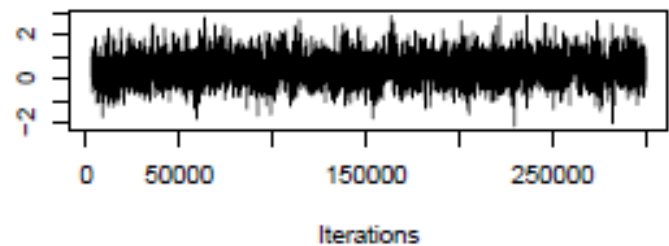

Trace of time:live

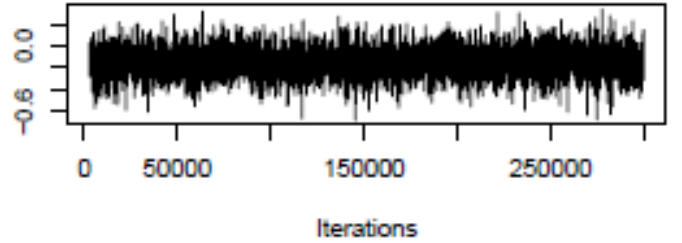

Density of think

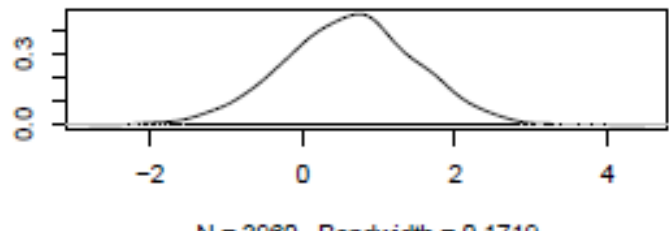

Density of attitude

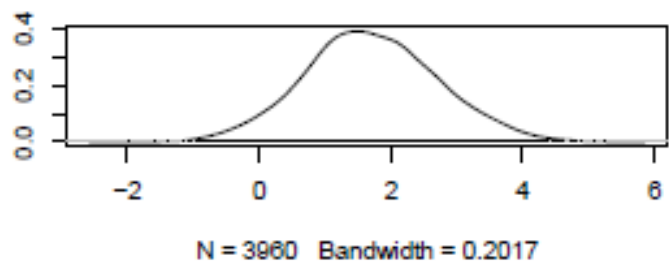

Density of change

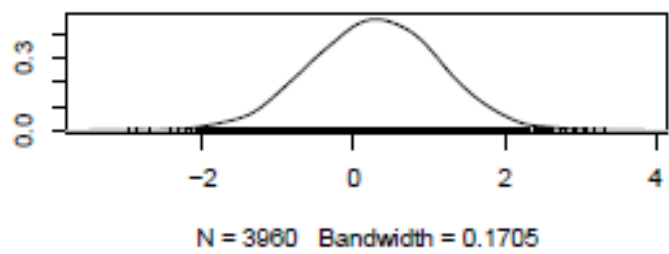

Density of G_ageFirst13 to 17 years:time

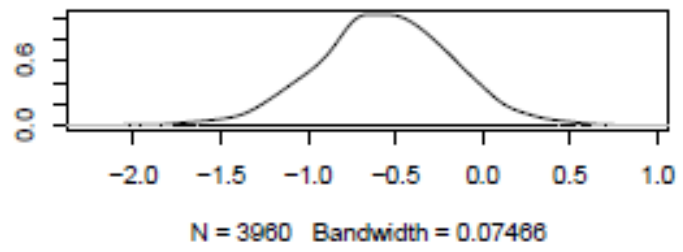

Density of G_ageFirst13 to 17 years:live

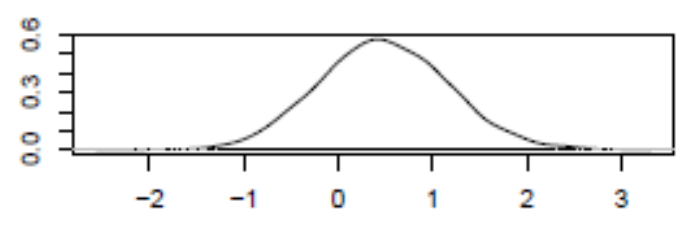

$\mathrm{N}=3960$ Bandwidth $=0.139$

Density of time:live

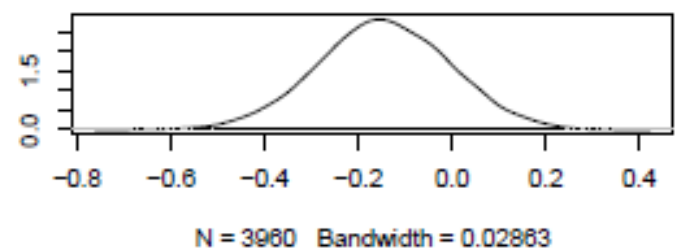


Trace of G_ageFirst13 to 17 years:relation

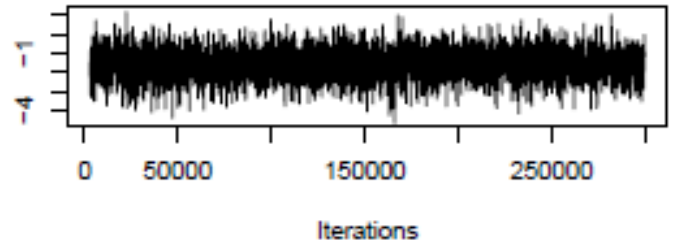

Trace of time:relation

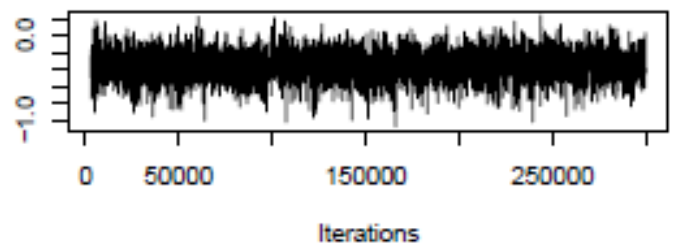

Trace of G_ageFirst13 to 17 years:ete

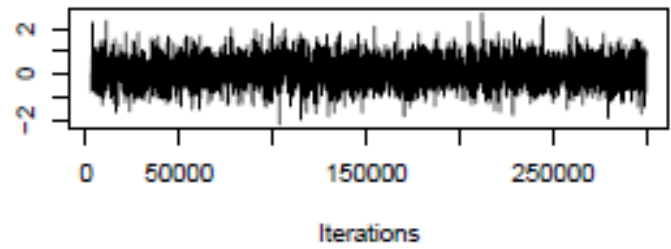

Trace of time:ete

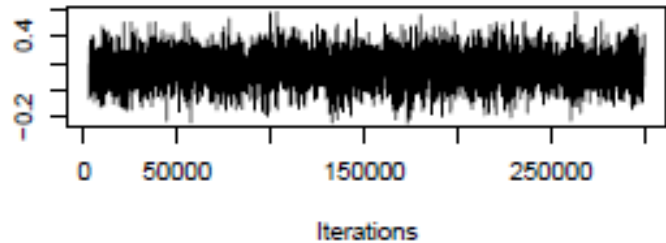

Trace of G_ageFirst13 to 17 years:where

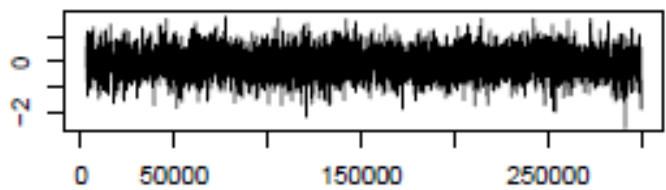

Iterations

Trace of time:where

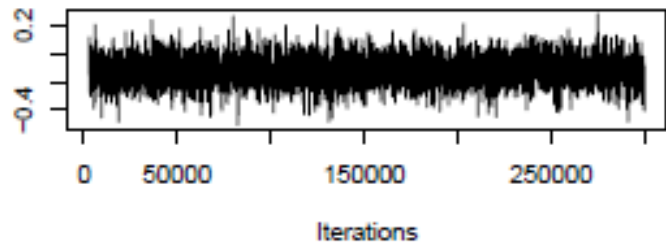

Density of G_ageFirst13 to 17 years:relation

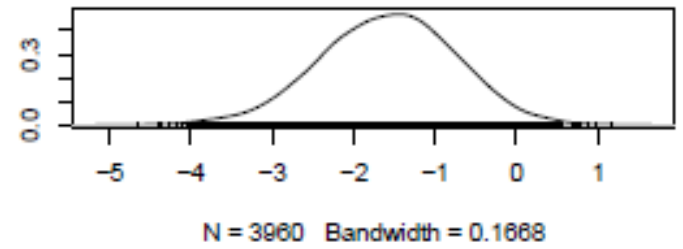

Density of time:relation

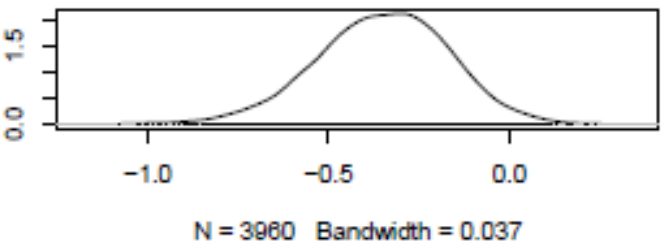

Density of G_ageFirst13 to 17 years:ete

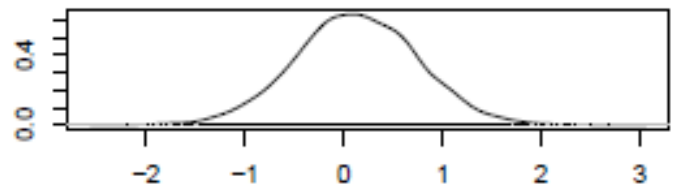

$\mathrm{N}=3960$ Bandwidth $=0.1236$

Density of time:ete

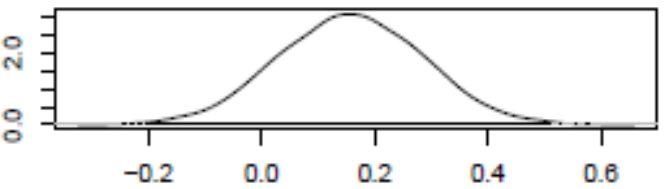

$\mathrm{N}=3960$ Bandwidth $=0.0258$

Density of G_ageFirst13 to 17 years:where

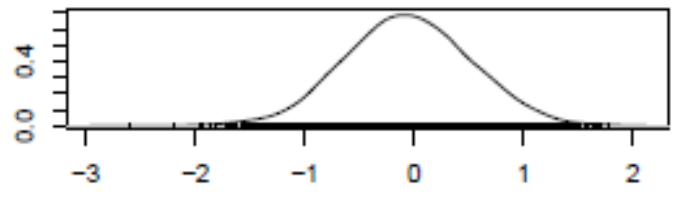

$\mathrm{N}=3960$ Bandwidth $=0.1156$

Density of time:where

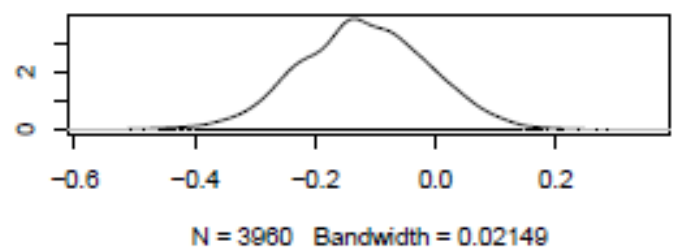


Trace of G_ageFirst13 to 17 years:life

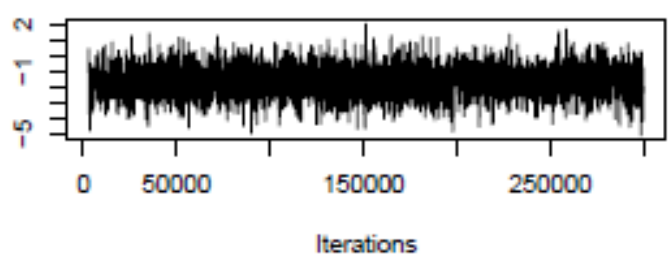

Trace of time:life

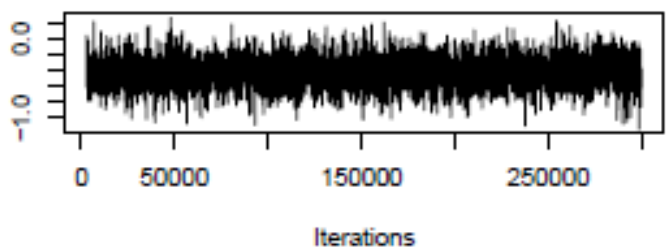

Trace of G_ageFirst13 to 17 years:drugs

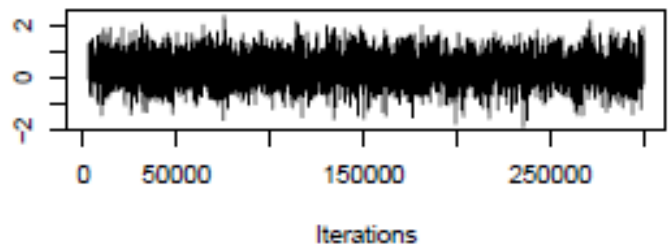

Trace of time:drugs

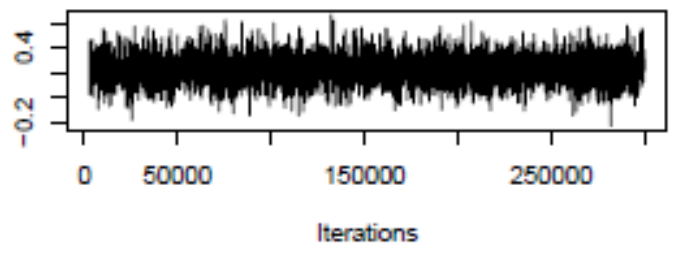

Trace of G_ageFirst13 to 17 years:physical

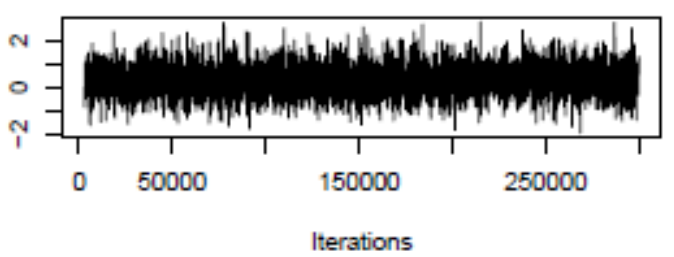

Trace of time:physical

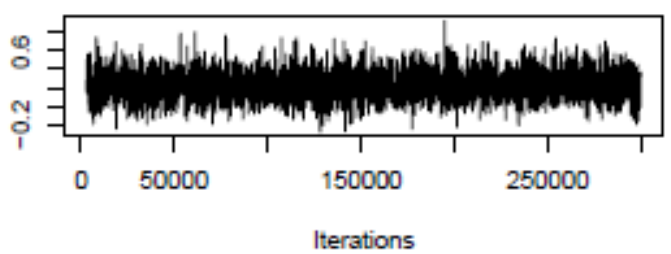

Density of G_ageFirst13 to 17 years:life

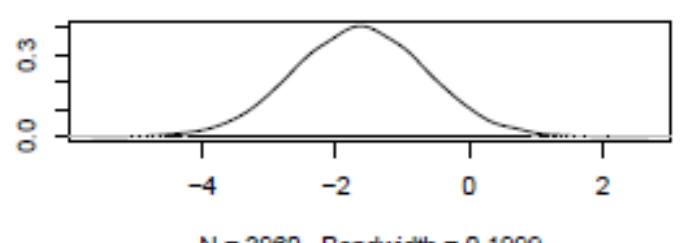

Density of time:life

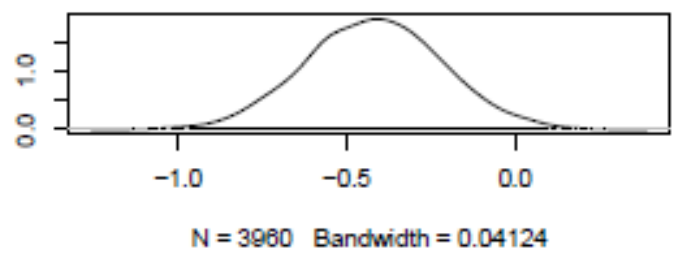

Density of G_ageFirst13 to 17 years:drugs

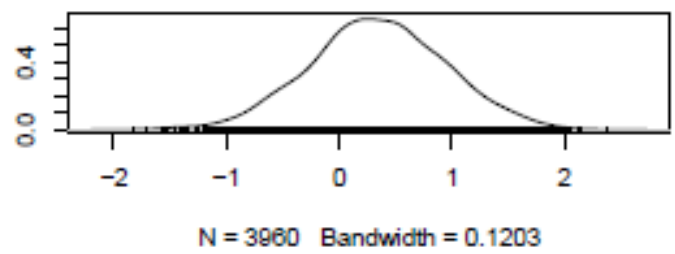

Density of time:drugs

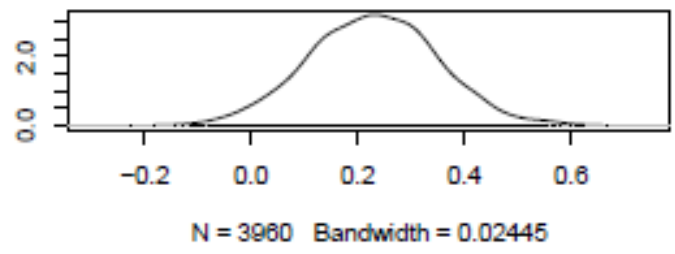

Density of G_ageFirst13 to 17 years:physical

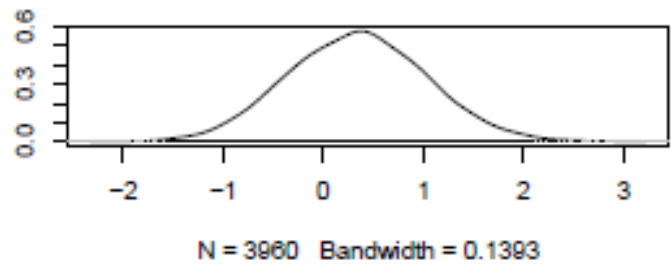

Density of time:physical

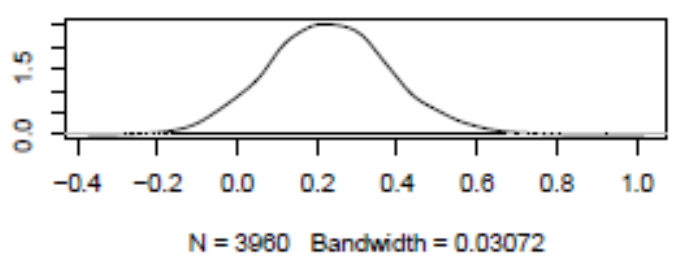


Trace of G_ageFirst13 to 17 years:emotion

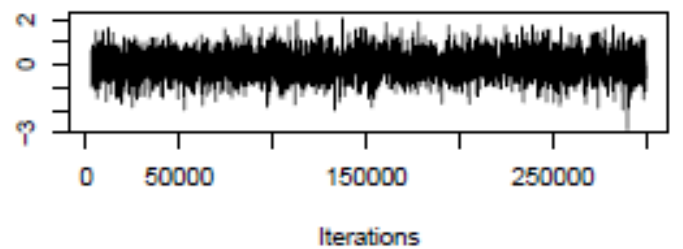

Trace of time:emotion

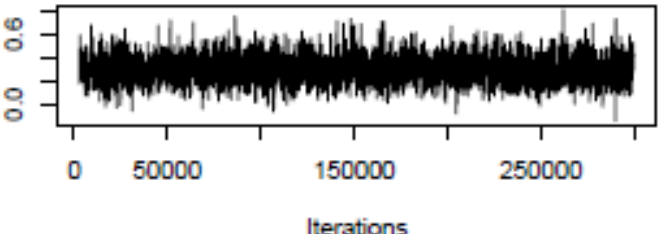

Trace of G_ageFirst13 to 17 years:self

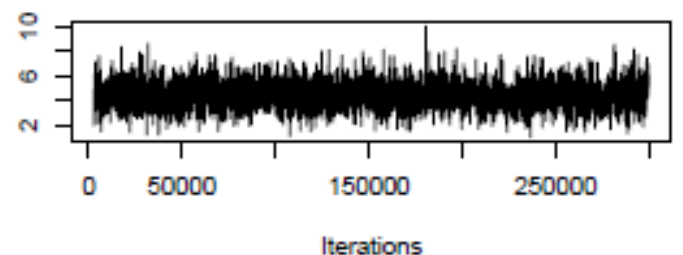

Trace of time:self

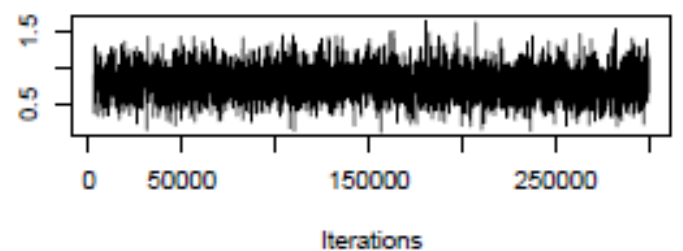

Trace of G_ageFirst13 to 17 years:think

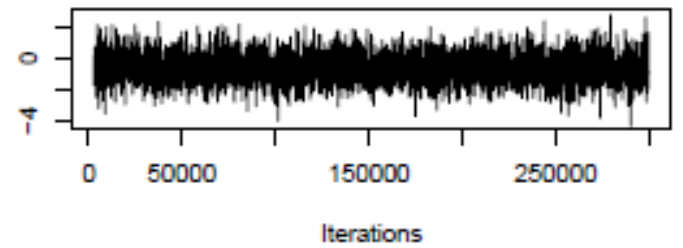

Trace of time:think

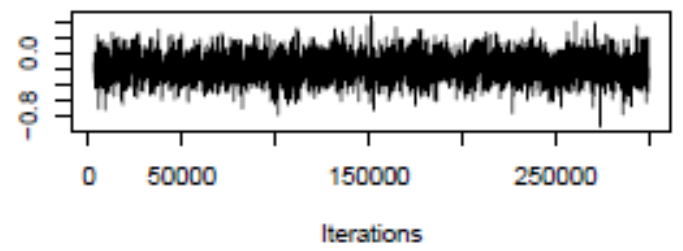

Density of G_ageFirst13 to 17 years:emotion

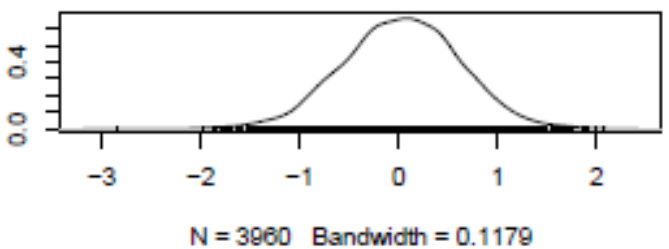

Density of time:emotion

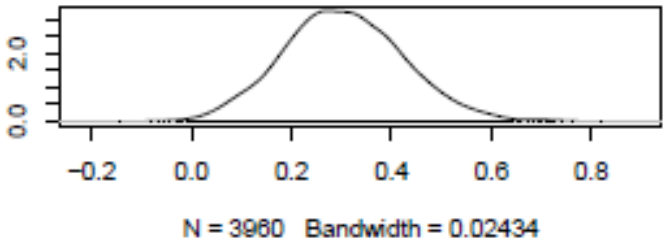

Density of G_ageFirst13 to 17 years:self

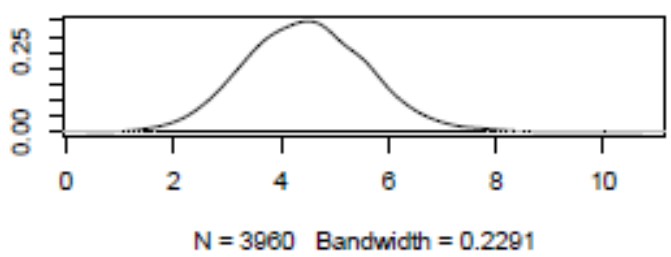

Density of time:self

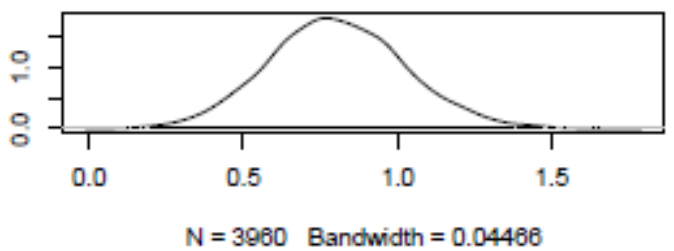

Density of G_ageFirst13 to 17 years:think

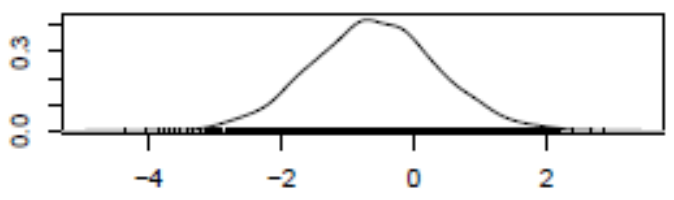

$\mathrm{N}=3960$ Bandwidth $=0.1908$

Density of time:think

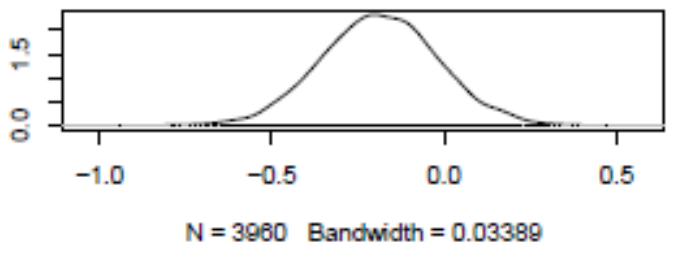


Trace of G_ageFirst13 to 17 years:attitude

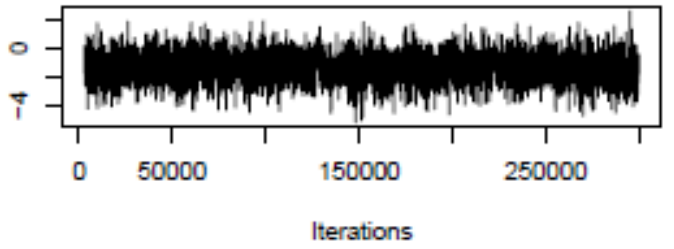

Trace of time:attitude

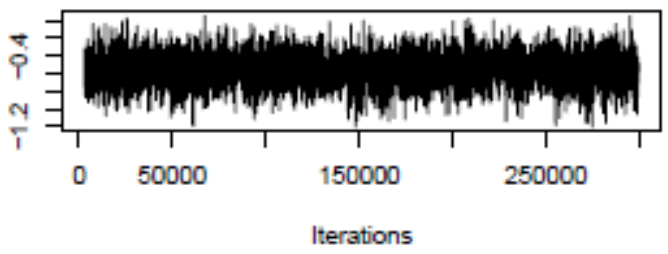

Trace of G_ageFirst13 to 17 years:change

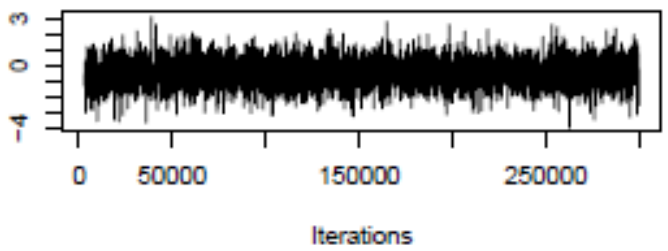

Trace of time:change

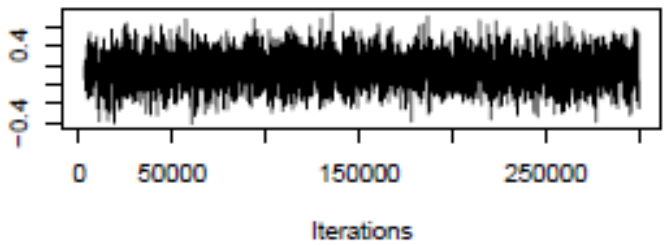

Trace of G_ageFirst13 to 17 years:time:live

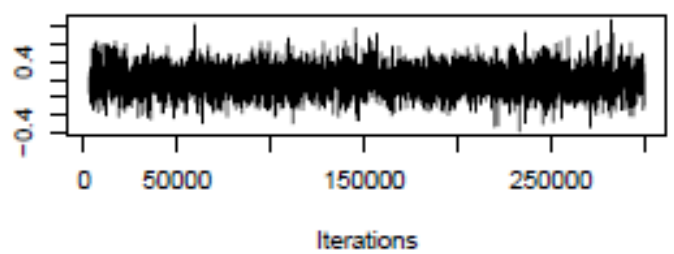

Trace of G_ageFirst13 to 17 years:time:relation

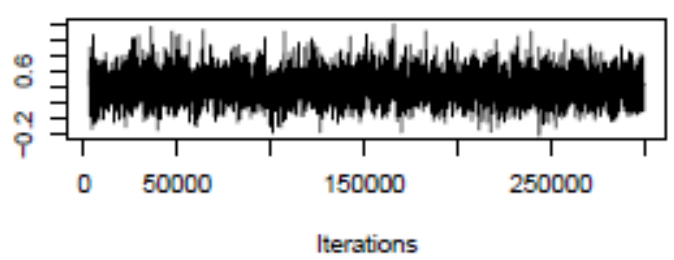

Density of G_ageFirst13 to 17 years:attitude

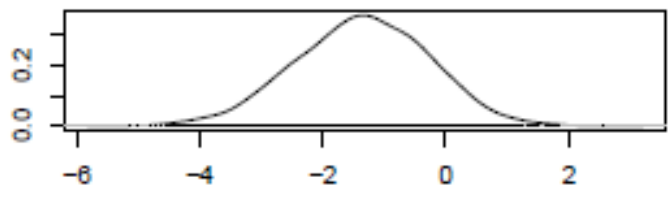

$\mathrm{N}=3960$ Bandwidth $=0.2193$

Density of time:attitude

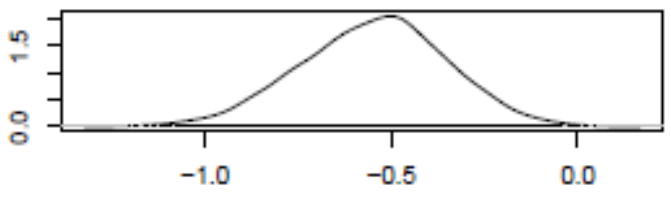

$\mathrm{N}=3960$ Bandwidth $=0.0397$

Density of G_ageFirst13 to 17 years:change

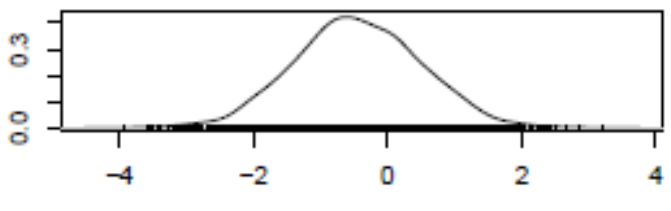

$\mathrm{N}=3960$ Bandwidth $=0.1895$

Density of time:change

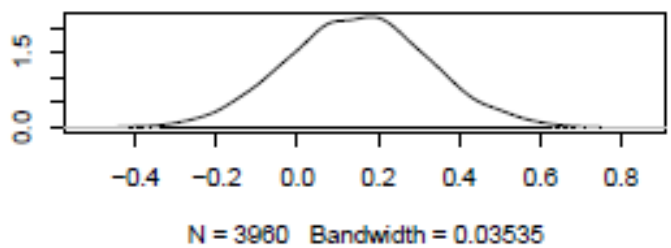

Density of G_ageFirst13 to 17 years:time:live

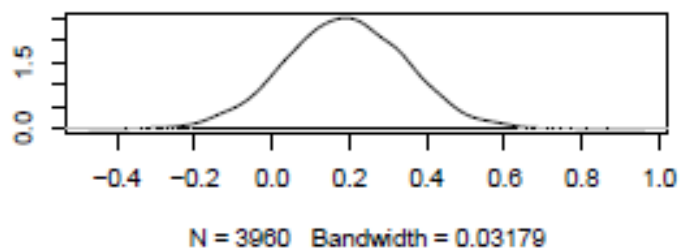

Density of G_ageFirst13 to 17 years:time:relation

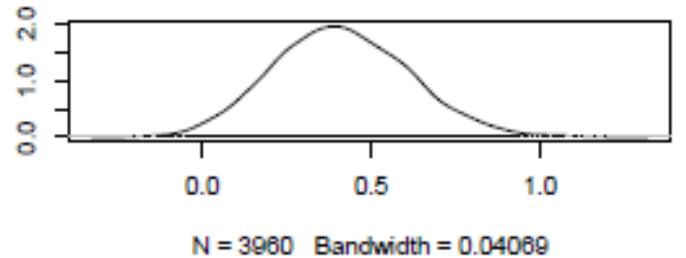


Trace of G_ageFirst13 to 17 years:time:ete

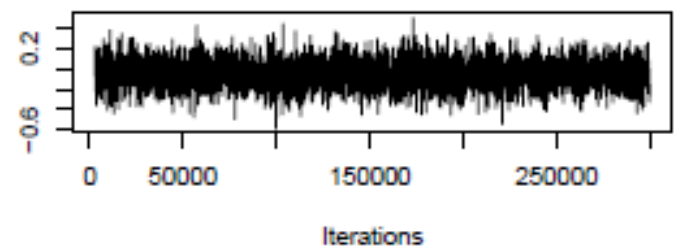

Trace of G_ageFirst13 to 17 years:time:where

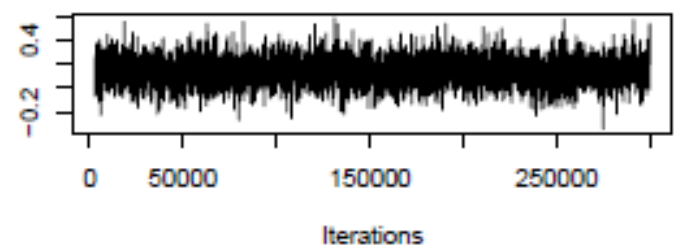

Trace of G_ageFirst13 to 17 years:time:life

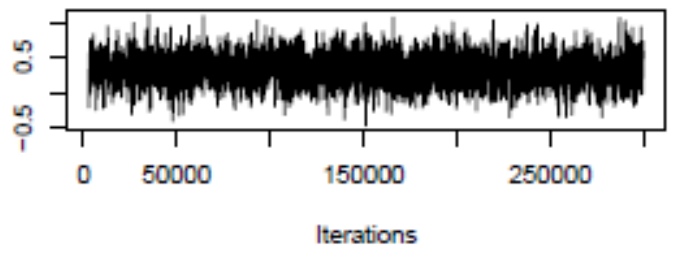

Trace of G_ageFirst13 to 17 years:time:drugs

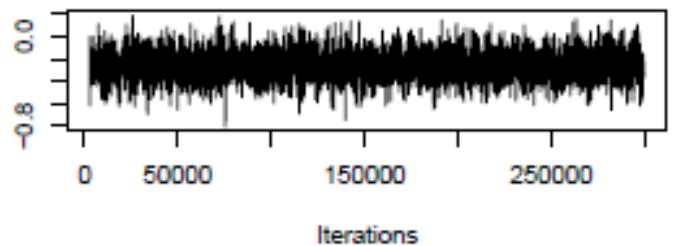

Trace of G_ageFirst13 to 17 years:time:physical

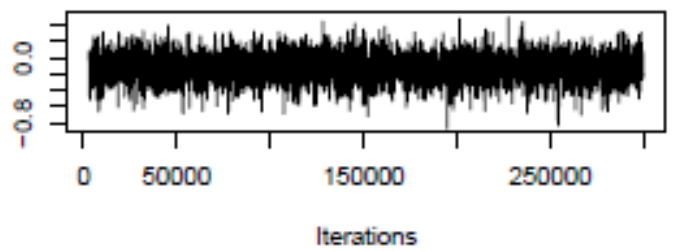

Trace of G_ageFirst13 to 17 years:time:emotion

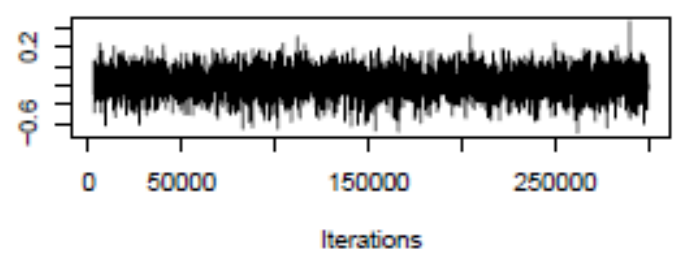

Density of G_ageFirst13 to 17 years:time:ete

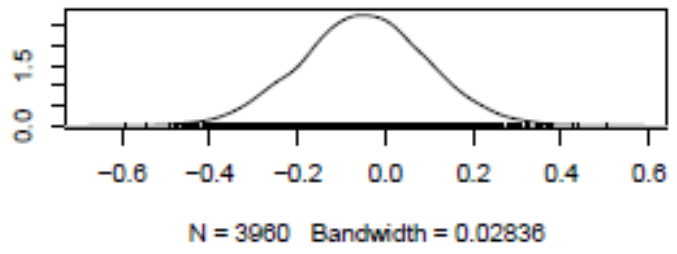

Density of G_ageFirst13 to 17 years:time:where

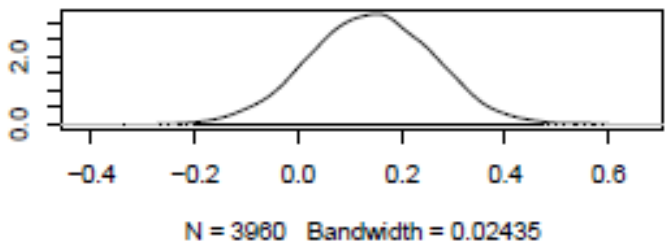

Density of G_ageFirst13 to 17 years:time:life

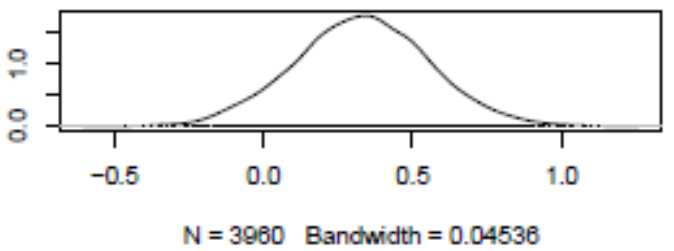

Density of G_ageFirst13 to 17 years:time:drugs

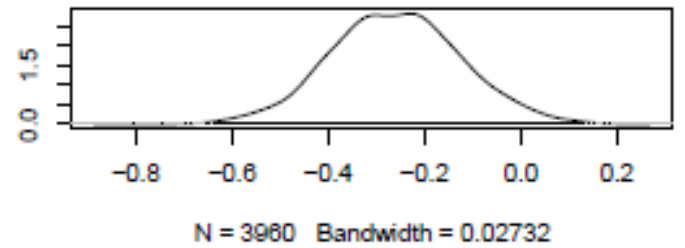

Density of G_ageFirst13 to 17 years:time:physica

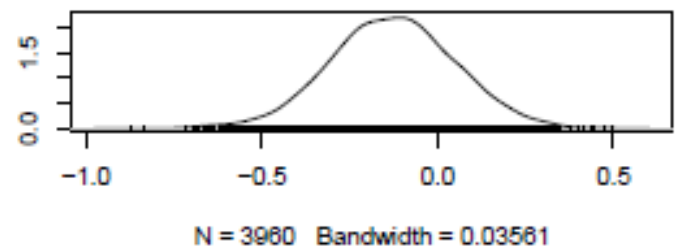

Density of G_ageFirst13 to 17 years:time:emotior

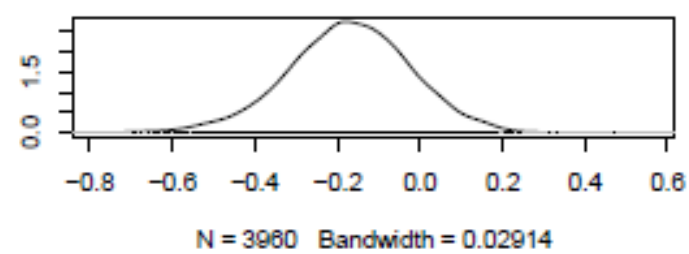


Trace of G_ageFirst13 to 17 years:time:self

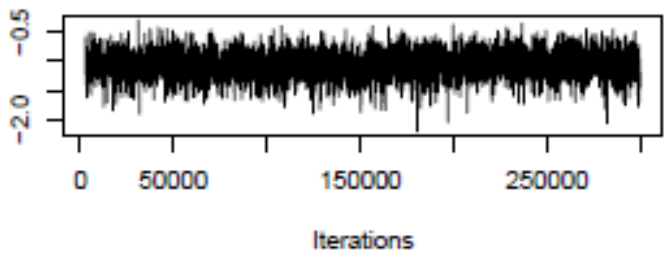

Trace of G_ageFirst13 to 17 years:time:think

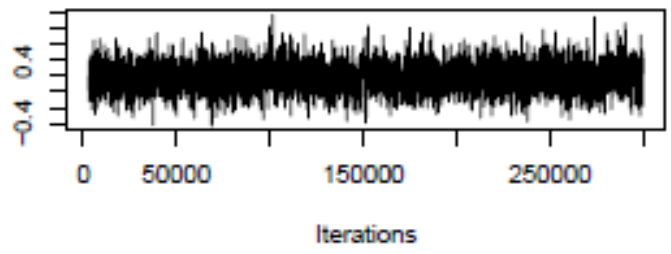

Trace of G_ageFirst13 to 17 years:time:attitude

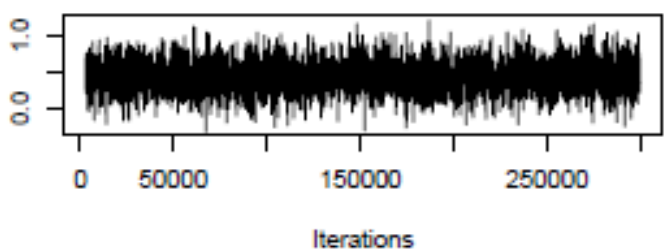

Trace of G_ageFirst13 to 17 years:time:change

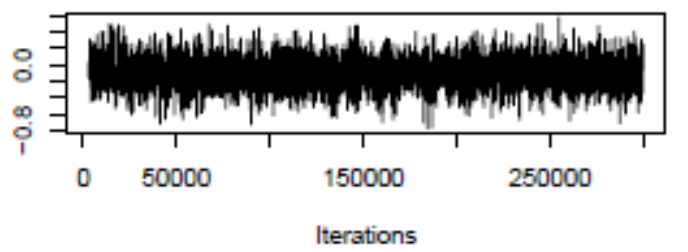

Density of G_ageFirst13 to 17 years:time:self

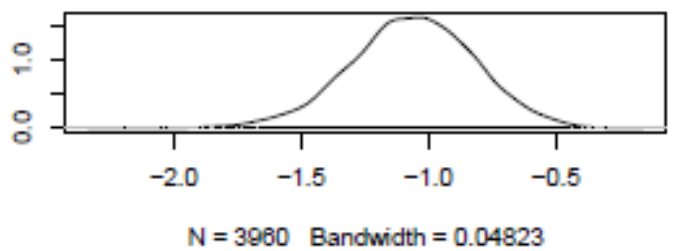

Density of G_ageFirst13 to 17 years:time:think

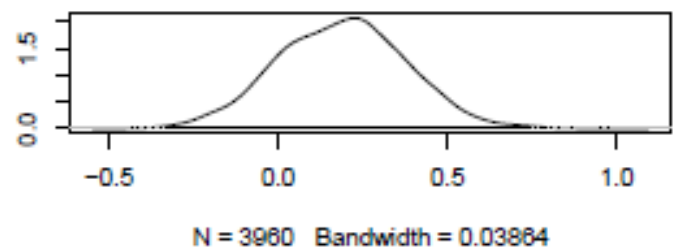

Density of G_ageFirst13 to 17 years:time:attitude

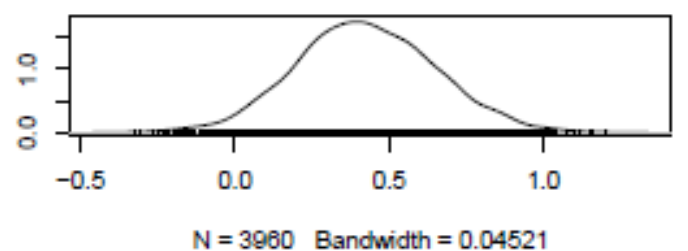

Density of G_ageFirst13 to 17 years:time:chang€

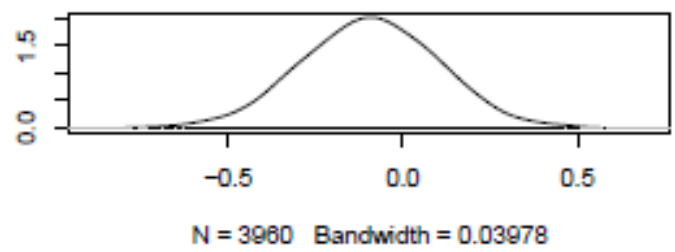


$\underline{\text { Random Effects }}$

Trace of time

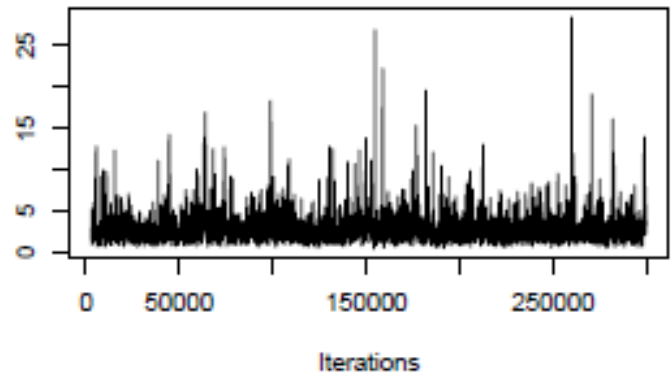

Trace of Research.ID

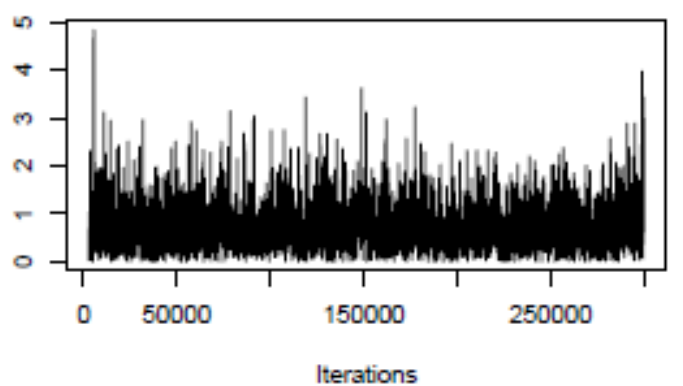

Density of time

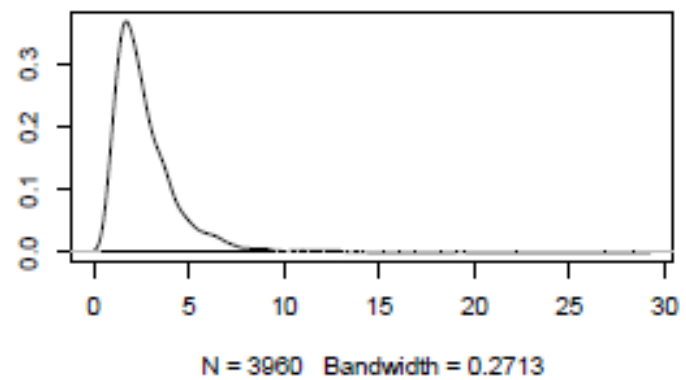

Density of Research.ID

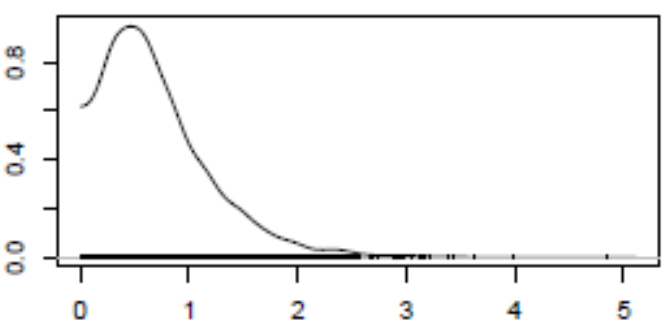

$\mathrm{N}=3960$ Bandwidth $=0.09278$ 
Dynamic Model involving Grouped YJB Offence Category (Table 6.18)

Bayesian Model (BDm3G_o1)

\section{\#\# Define the model}

BDm3G_o1 <- MCMCglmm(FO.bin as.factor(I_Cat2)*time*live + as.factor (I_Cat2)*time*relation + as.factor(I_Cat2)*time*ete + as.factor (I Cat2)*time*where + as.factor (I Cať2)*time*life + as.factor ( I- Cat2)*time*drugs + as.factor (I-Cat2)*time*physical + as.factor (I_Cat2)*time*emotion + as.factor(I_Cat2)*time*self + as.factor (I_Cat2)*time*think + as.factor (I_Cāt2)*time*attitude + as.factor ( I Cat2) *time* change, random= timétResearch.ID, data=data3, family="ordinal", prior=priorD, slice=TRUE, nitt $=800000$, thin=150, burnin=3000)

\section{\# Checks for suitable convergence}

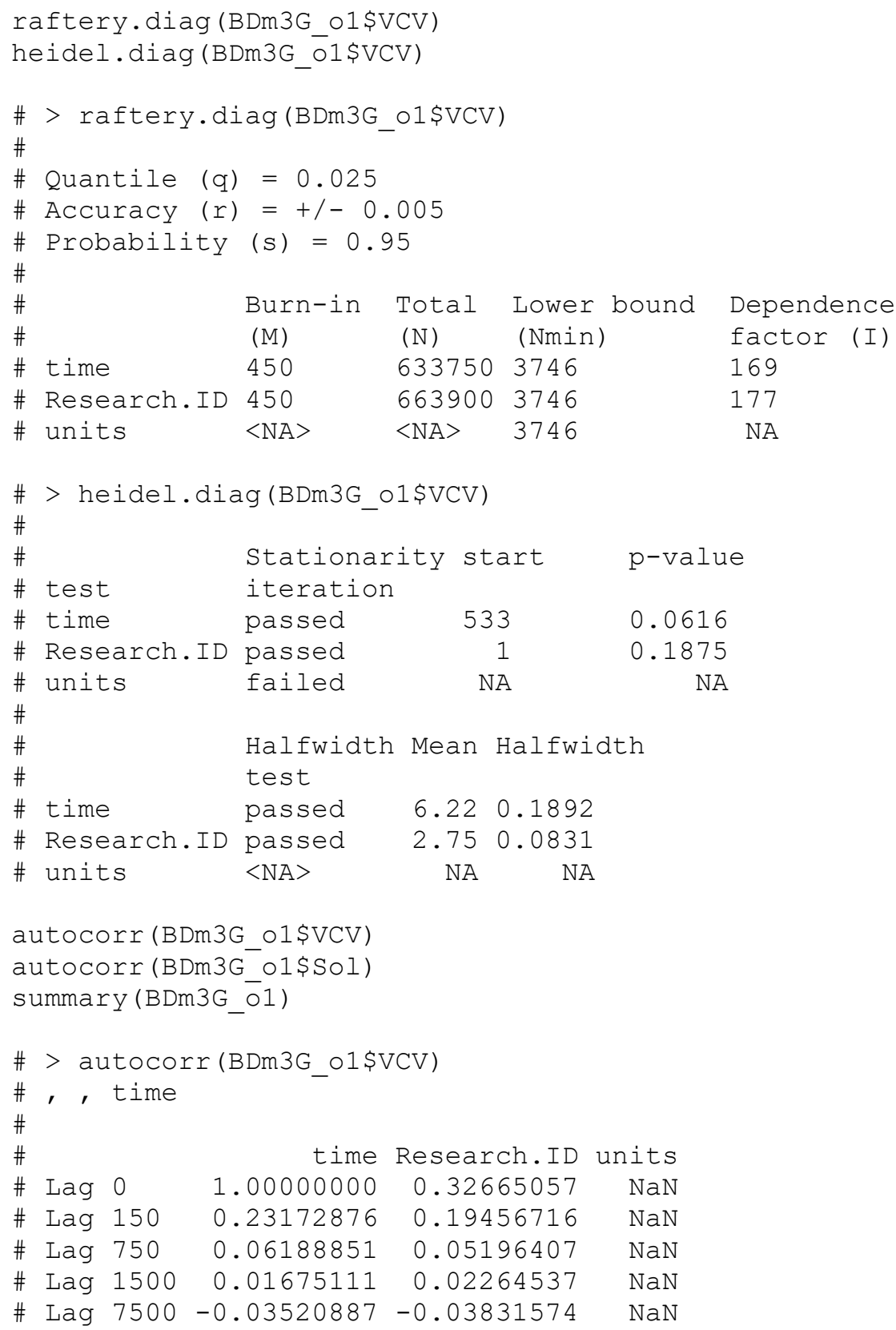




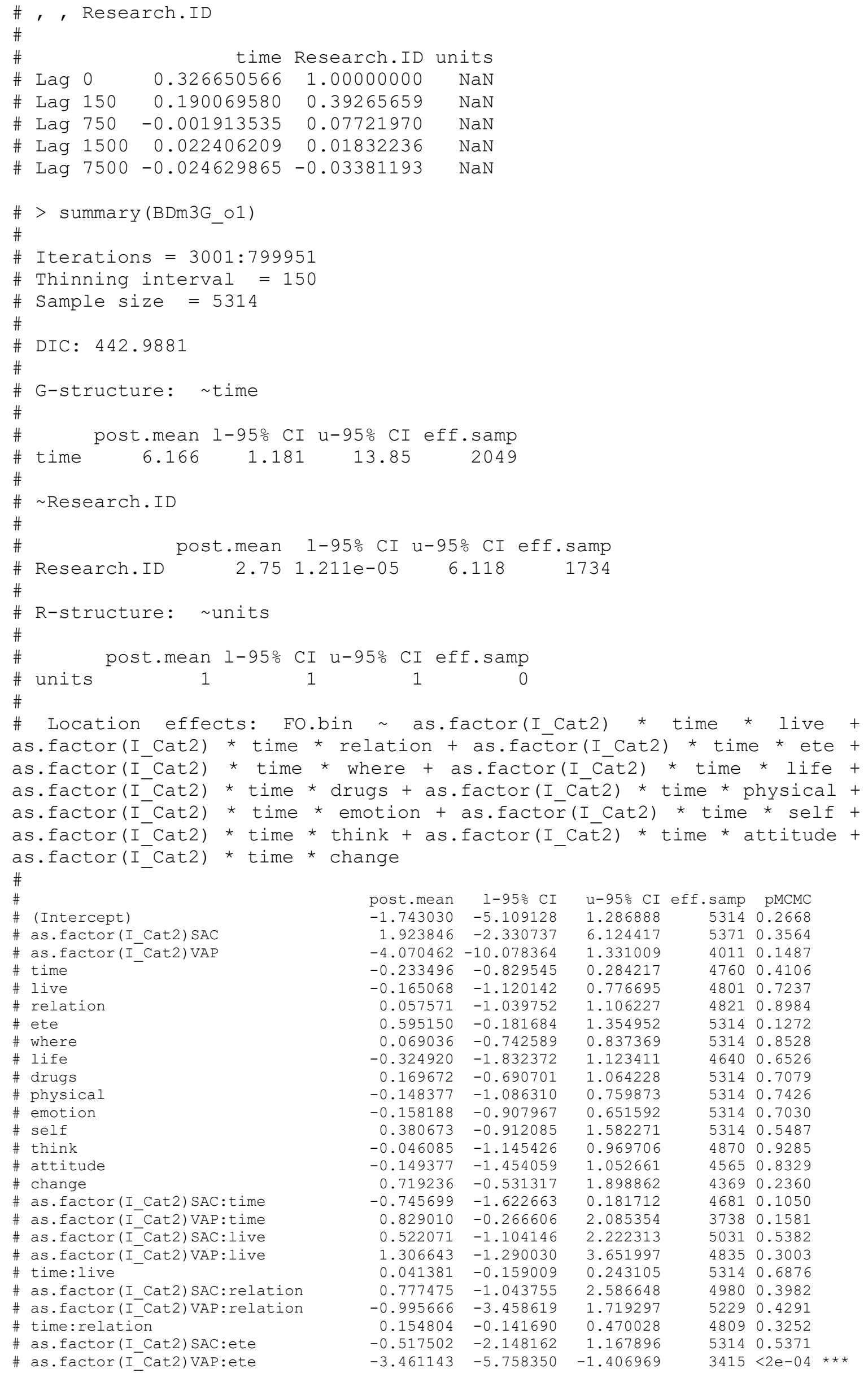

post.mean 1-95\% CI $-1.743030-5.109128$ $1.923846-2.330737$ $-4.070462-10.078364$ $-0.233496-0.829545$ $-0.165068-1.120142$ $0.057571-1.039752$

$0.595150-0.181684$

$0.069036-0.742589$

$-0.324920-1.832372$

$0.169672-0.690701$

$-0.148377-1.086310$

$-0.158188-0.907967$

$0.380673-0.912085$

$-0.046085-1.145426$

$-0.149377-1.454059$

$0.719236-0.531317$

$-0.745699-1.622663$

$0.829010-0.266606$

$0.522071-1.104146$

$1.306643-1.290030$

$0.041381-0.159009$

$0.777475-1.043755$

$-0.995666-3.458619$

$0.154804-0.141690$

$-0.517502-2.148162$

$-3.461143$

u-95\% CI eff.samp pMCMC

$\begin{array}{lll}1.286888 & 5314 & 0.2668\end{array}$

$\begin{array}{lll}6.124417 & 5371 & 0.3564\end{array}$

$1.331009 \quad 40110.1487$

$0.284217 \quad 4760 \quad 0.4106$

$\begin{array}{llll}0.776695 & 4801 & 0.7237\end{array}$

$\begin{array}{llll}1.106227 & 4821 & 0.8984\end{array}$

$\begin{array}{lll}1.354952 & 5314 & 0.1272\end{array}$

$0.837369 \quad 53140.8528$

$\begin{array}{llll}1.123411 & 4640 & 0.6526\end{array}$

$\begin{array}{lll}1.064228 & 5314 & 0.7079\end{array}$

$\begin{array}{llll}0.759873 & 5314 & 0.7426\end{array}$

$0.651592 \quad 5314 \quad 0.7030$

$\begin{array}{lll}1.582271 & 5314 & 0.5487\end{array}$

$0.969706 \quad 4870 \quad 0.9285$

$1.052661 \quad 45650.8329$

$\begin{array}{lll}1.898862 & 4369 & 0.2360\end{array}$

$0.181712 \quad 46810.1050$

$2.085354 \quad 3738 \quad 0.1581$

$2.222313 \quad 50310.5382$

$\begin{array}{lll}3.651997 & 48350.3003\end{array}$

$0.243105 \quad 5314 \quad 0.6876$

$\begin{array}{llll}2.586648 & 4980 & 0.3982\end{array}$

$\begin{array}{lll}1.719297 & 5229 & 0.4291\end{array}$

$0.470028 \quad 48090.3252$

$\begin{array}{lll}1.167896 & 5314 & 0.5371\end{array}$

$-1.406969 \quad 3415<2 e-04 * \star *$ 
\# time:ete

\# as.factor (I_Cat2) SAC: where

\# as.factor ( I Cat2) VAP: where

\# time:where

\# as.factor (I Cat2) SAC:Iife

\# as.factor(I_Cat2) VAP:Iife

\# time:life

\# as.factor (I Cat2) SAC:drugs

\# as.factor(I_Cat2) VAP:drugs

\# time:drugs

\# as.factor(I Cat2) SAC:physical

\# as.factor(I_Cat2) VAP:physical

\# time:physicāl

\# as.factor (I Cat2) SAC:emotion

\# as.factor (I_Cat2) VAP:emotion

\# time:emotion

\# as.factor (I Cat2) SAC:self

\# as.factor (I_Cat2) VAP:self

\# time:self

\# as.factor (I Cat2) SAC: think

\# as.factor (I'Cat2) VAP: think

\# time:think

\# as.factor (I_Cat2) SAC:attitude

\# as.factor (I Cat2) VAP:attitude

\# time:attitūe

\# as.factor (I_Cat2) SAC: change

\# as.factor (I Cat2) VAP: change

\# time: change

\# as.factor (I_Cat2) SAC:time:live

\# as.factor (I Cat2) VAP:time:live

\# as.factor (I_Cat2) SAC:time:relation

\# as.factor (I Cat2) VAP:time:relation

\# as.factor (I Cat2) SAC:time:ete

\# as.factor (I Cat2) VAP:time: ete

\# as.factor (I Cat2) SAC:time:where

\# as.factor (I Cat2) VAP:time: where

\# as.factor (I Cat2) SAC:time:life

\# as.factor (I Cat2) VAP:time:life

\# as.factor (I_Cat2) SAC:time:drugs

\# as.factor ( I Cat2) VAP:time: drugs

\# as.factor(I_Cat2)SAC:time:physical

\# as.factor (I_Cat2) VAP:time:physical

\# as.factor (I Cat2) SAC:time: emotion

\# as.factor (I_Cat2) VAP:time: emotion

\# as.factor (I Cat2) SAC: time:self

\# as.factor (I Cat2) VAP:time:self

\# as.factor (I_Cat2) SAC:time: think

\# as.factor (I Cat2) VAP:time: think

\# as.factor ( I Cat2) SAC:time:attitude

\# as.factor (I-Cat2) VAP:time: attitude

\# as.factor (I Cat2) SAC:time: change

\# as.factor(I_Cat2) VAP:time: change

\# - - -

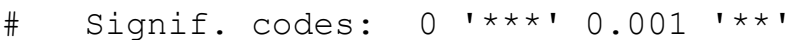

$-0.154801$

0.897084

$-1.226223$

0.030290

0.620042

3.031500

0.078644

0.380963

0.397904

0.058170

$-1.795189$

$-0.864645$

0.032043

0.044915

$-0.457527$

$-0.094117$

$-1.012361$

0.187958

$-0.159196$

$-0.253472$

2. 360754

0.065423

0.256100

0.384825

$-0.075963$

$-1.331381$

0.768872

$-0.061904$

0.048123

$-0.221061$

$-0.354845$

0.409128

0.260361

0.994206

$-0.283551$

0.445649

$-0.114294$

$-0.597604$

$-0.007908$

$-0.240944$

0.134442

0.006648

0.314734

0.455581

0.356921

0.011821

$-0.232814$

$-0.751560$

0.126537

$-0.518966$

0.167923

$-0.306902$
$-0.350280$

$-0.563835$

$-3.083528$

$-0.126869$

$-1.803513$

0.436828

$-0.257402$

$-1.101983$

$-1.539619$

$-0.128232$

$-3.841906$

$-3.450338$

$-0.215322$

$-1.556007$

$-2.159855$

$-0.335147$

$-3.082461$

$-2.047094$

$-0.418112$

$-2.462002$

$-0.711508$

$-0.211664$

$-1.893079$

$-2.933205$

$-0.376581$

$-4.311756$

$-1.766975$

$-0.339219$

$-0.299609$

$-0.781783$

$-0.793085$

$-0.222372$

$-0.076479$

0.409849

$-0.564248$

0.010154

$-0.600907$

$-1.196980$

$-0.358715$

$-0.640630$

$-0.319428$

$-0.700826$

$-0.025479$

$-0.017742$

$-0.081653$

$-0.494148$

$-0.749425$

$-1.455873$

$-0.371688$

$-1.395638$

$-0.378097$

$-0.811149$
0.036448

2.340939

0.683675

0.192574

2. 927807

5. 904368

0.427629

1. 852875

2.111969

0.265204

0.215249

1.666862

0.300231

1.693744

1.204326

0.128959

1.149123

2. 612903

0.101368

1. 974100

5.592396

0.327670

2. 417996

3. 469433

0.229499

1. 749860

3.111716

0.216316

0.404217

0.292283

0.062665

1. 120088

0.613711

1. 611091

0.021767

0.930464

0.382499

$-0.016437$

0.300204

0.165758

0.588156

0.761486

0.650132

0.978476

0.845260

0.496503

0.267215

$-0.122303$

0.635780

0.233665

0.764096

0.170330
$5038 \quad 0.1099$

48580.2160

$\begin{array}{lll}5314 & 0.1938\end{array}$

53140.7136

50570.6123

38280.0154 *

47360.6541

$4895 \quad 0.6105$

$\begin{array}{lll}5314 & 0.6752\end{array}$

53140.5736

50850.0813 .

$\begin{array}{lll}4968 & 0.5070\end{array}$

$\begin{array}{lll}5314 & 0.7986\end{array}$

$\begin{array}{lll}5314 & 0.9590\end{array}$

43090.6112

$\begin{array}{lll}5314 & 0.4324\end{array}$

$\begin{array}{lll}4808 & 0.3624\end{array}$

$4430 \quad 0.8728$

$\begin{array}{lll}5314 & 0.2209\end{array}$

48390.8479

49320.1393

$\begin{array}{lll}5314 & 0.6255\end{array}$

53140.8190

$5314 \quad 0.8344$

$5314 \quad 0.6116$

43440.3820

$\begin{array}{lll}5274 & 0.5167\end{array}$

$4524 \quad 0.6522$

$\begin{array}{lll}4948 & 0.7881\end{array}$

$\begin{array}{lll}5314 & 0.4230\end{array}$

45170.0997 .

$\begin{array}{lll}5314 & 0.2247\end{array}$

$\begin{array}{lll}5314 & 0.1385\end{array}$

$3148<2 e-04 * * *$

53140.0531 .

41350.0489 *

43940.6571

37870.0373 *

$\begin{array}{lll}5051 & 0.9710\end{array}$

$\begin{array}{lll}5078 & 0.2386\end{array}$

$4816 \quad 0.5706$

53140.9947

51050.0640 .

45000.0636 .

$\begin{array}{lll}5031 & 0.1234\end{array}$

$\begin{array}{lll}4948 & 0.9552\end{array}$

53140.3764

47770.0199 *

50550.6172

$4222 \quad 0.2047$

49010.5600

53140.2115

0.01 '*' 0.05 '.' 0.1 ' ' 1 
Trace Plots and Posterior Density Plots

Fixed Effects

Trace of (Intercept)

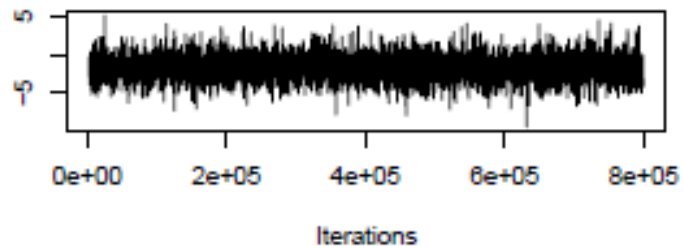

Trace of as.factor(I_Cat2)SAC

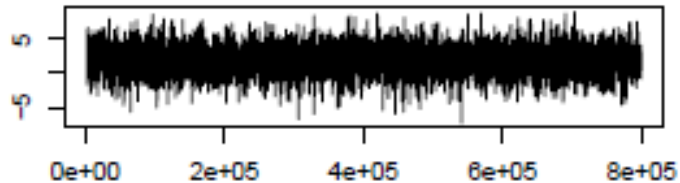

Iterations

Trace of as.factor(I_Cat2)VAP

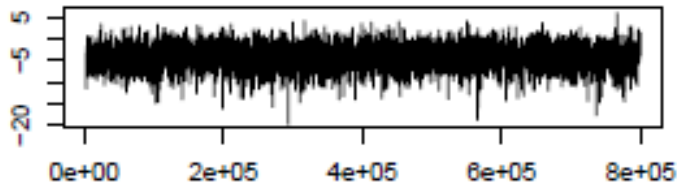

Iterations

Trace of time

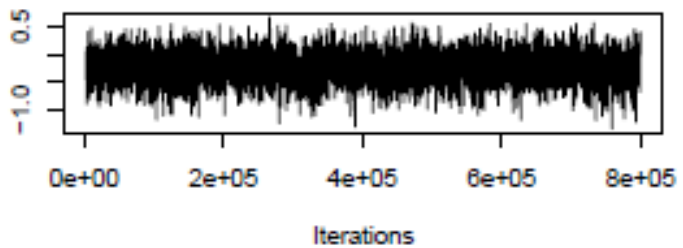

Trace of live

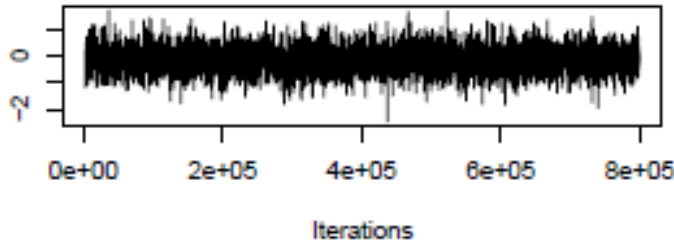

Trace of relation

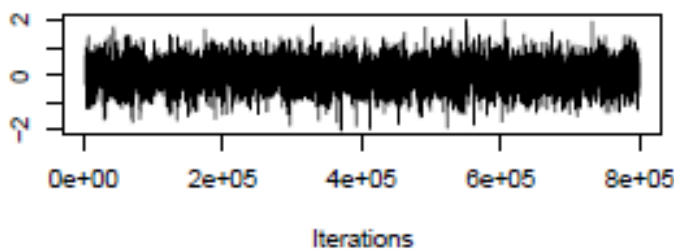

Density of (Intercept)

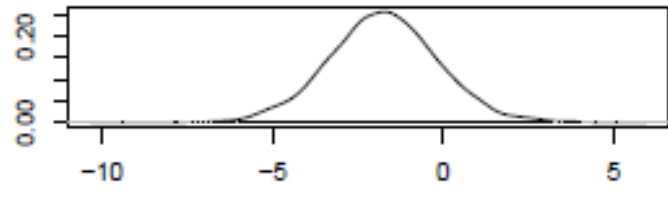

$\mathrm{N}=5314$ Bandwidth $=0.2957$

Density of as.factor(I_Cat2)SAC

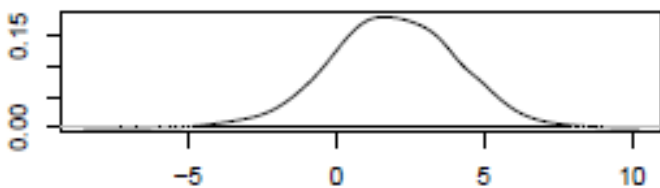

$\mathrm{N}=5314$ Bandwidth $=0.4066$

Density of as.factor(I_Cat2)VAP

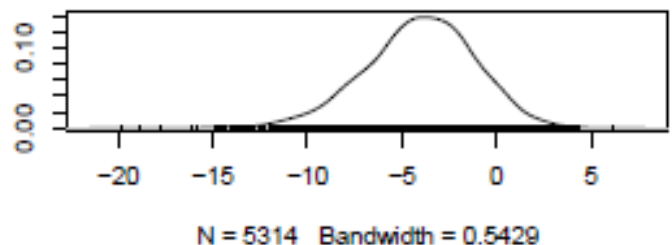

Density of time

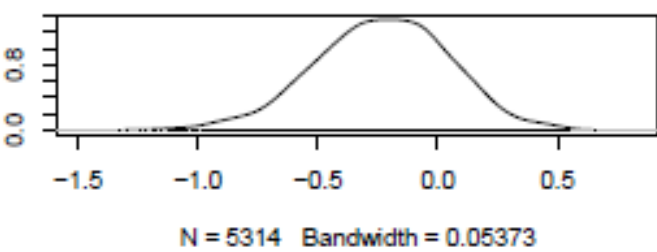

Density of live

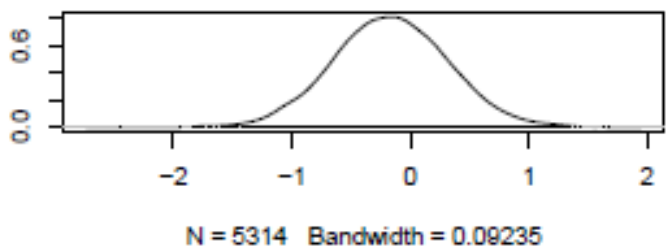

Density of relation

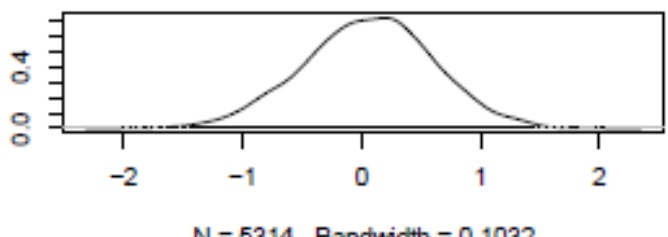

$N=5314$ Bandwidth $=0.1032$ 
Trace of ete

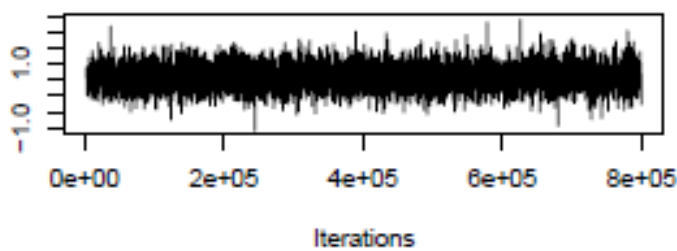

Trace of where

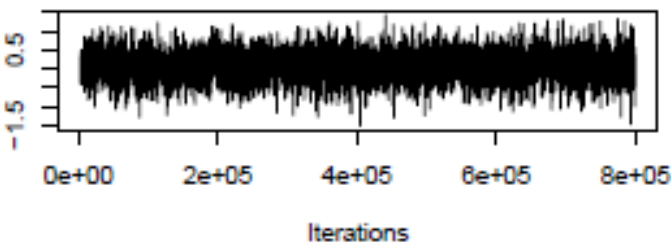

Trace of life

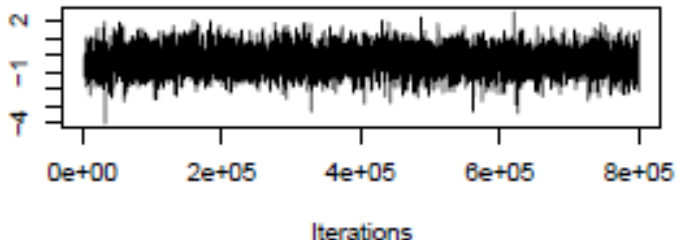

Trace of drugs

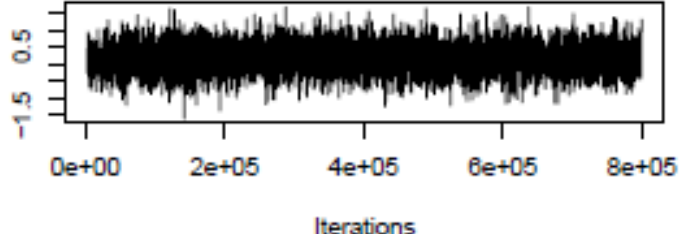

Trace of physical

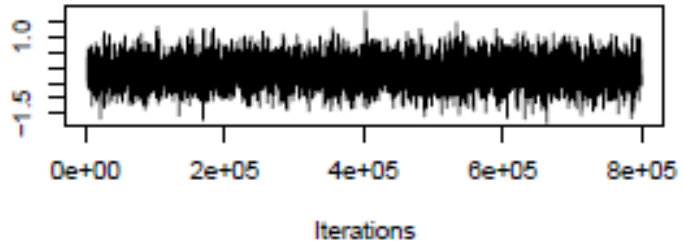

Trace of emotion

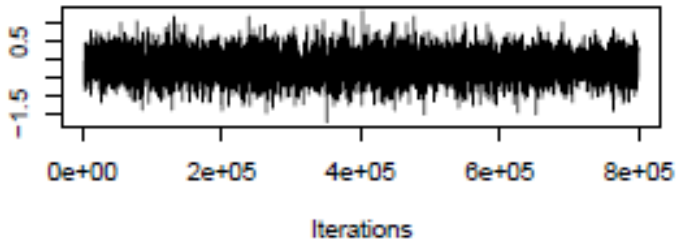

Density of ete

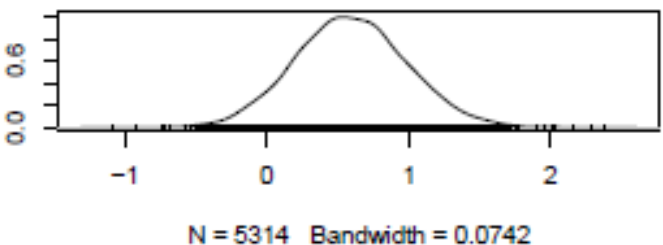

Density of where

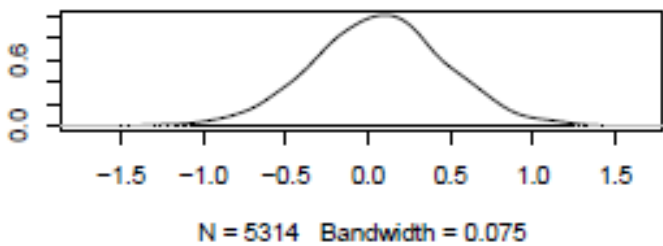

Density of life

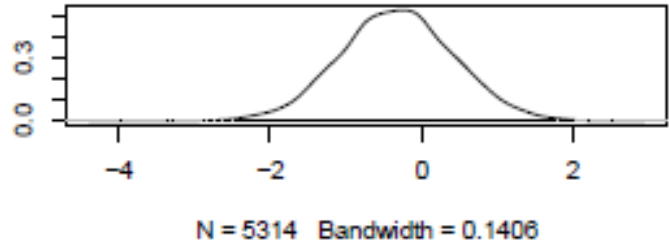

Density of drugs

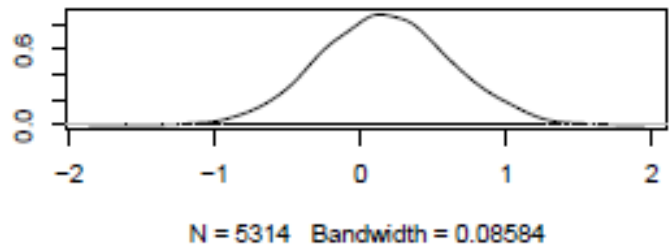

Density of physical

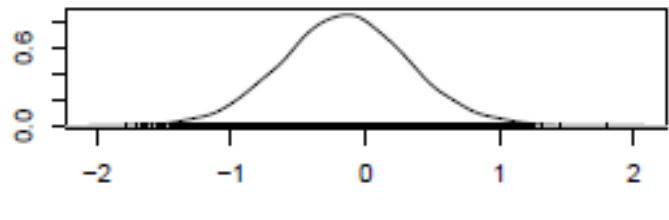

$\mathrm{N}=5314$ Bandwidth $=0.08866$

Density of emotion

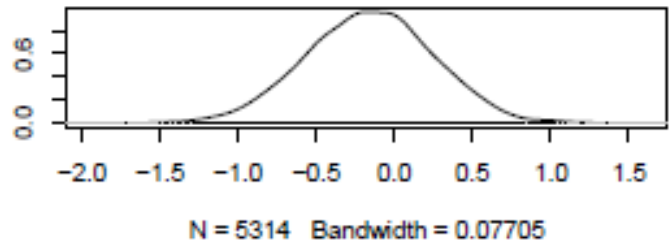


Trace of self

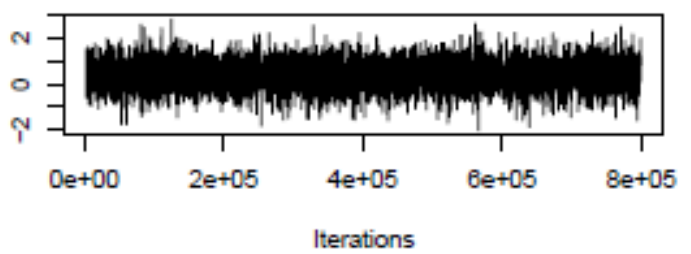

Trace of think

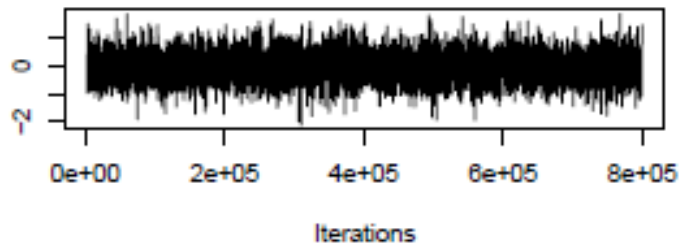

Trace of attitude

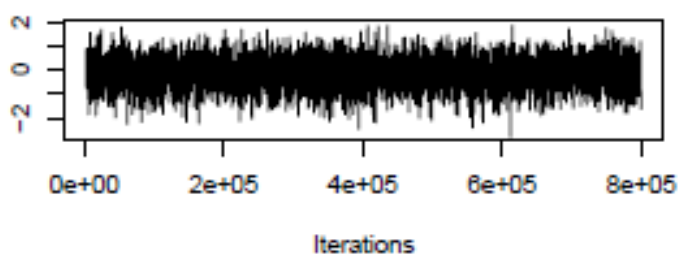

Trace of change

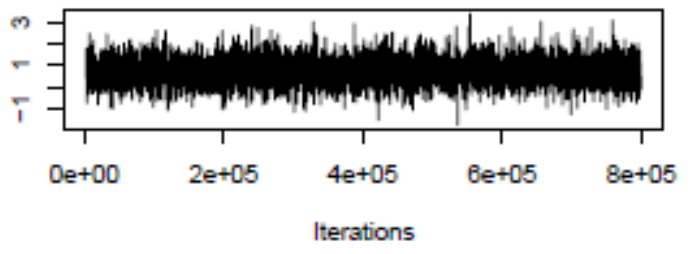

Trace of as.factor(I_Cat2)SAC:time

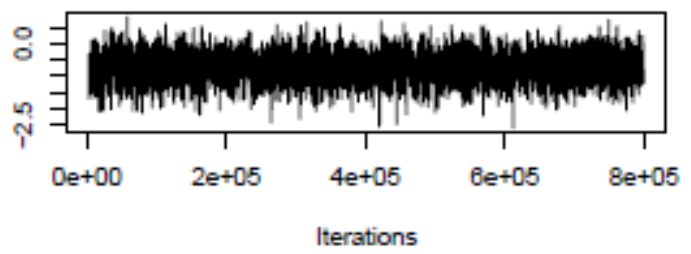

Trace of as.factor(I_Cat2)VAP:time

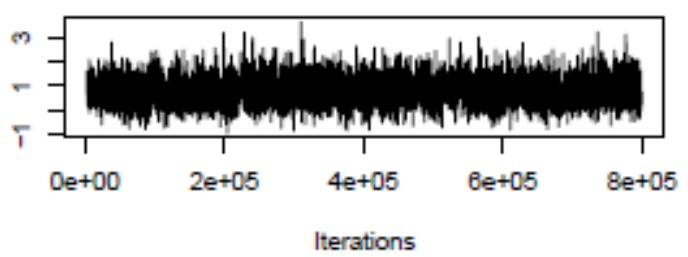

Density of self

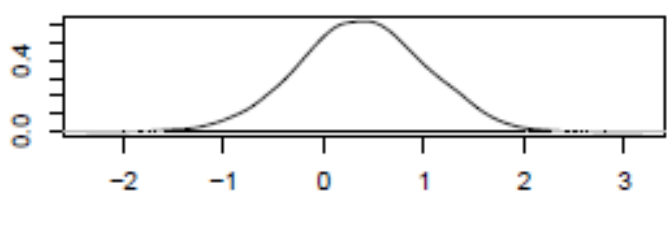

$N=5314$ Bandwidth $=0.1213$

Density of think

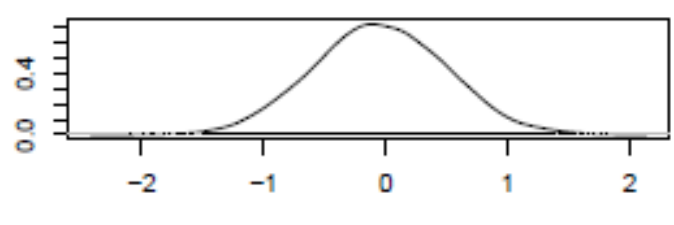

$N=5314$ Bandwidth $=0.1045$

Density of attitude

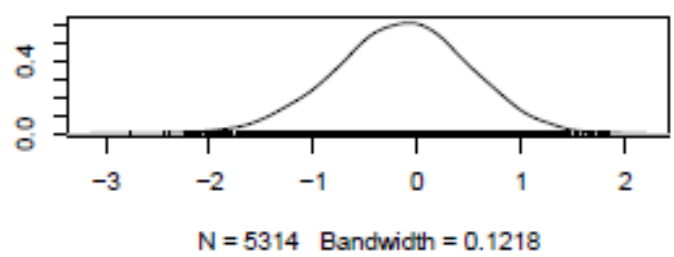

Density of change

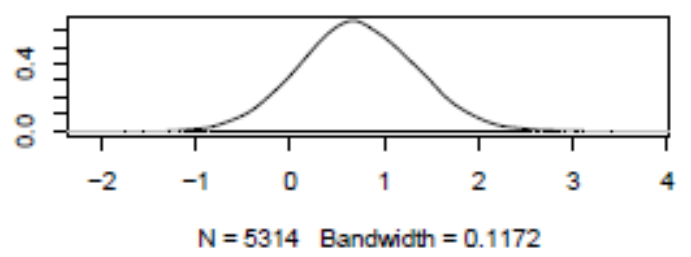

Density of as.factor(I_Cat2)SAC:time

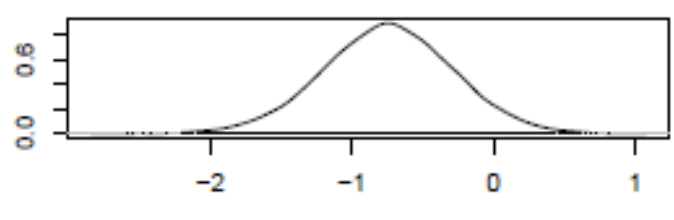

$\mathrm{N}=5314$ Bandwidth $=0.08604$

Density of as.factor(I_Cat2)VAP:time

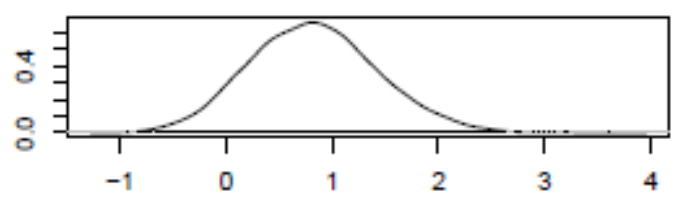

$N=5314$ Bandwidth $=0.1146$ 
Trace of as.factor(I_Cat2)SAC:Iive

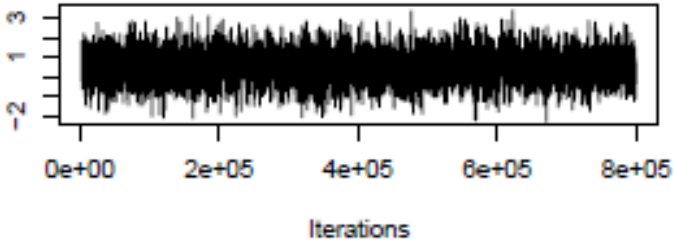

Trace of as.factor(I_Cat2)VAP:live

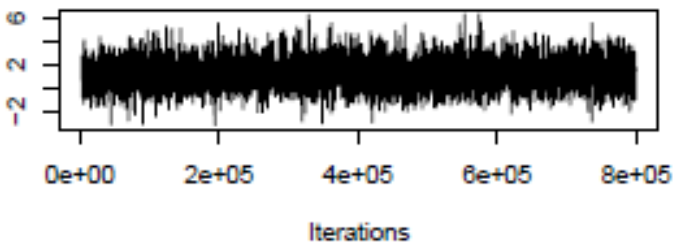

Trace of time:live

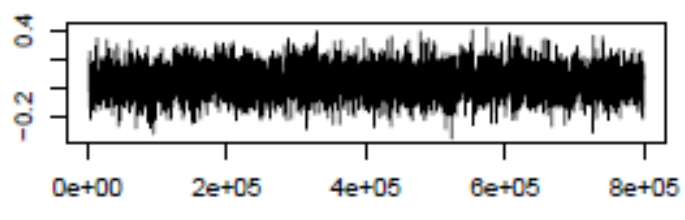
Iterations

Trace of as.factor(I_Cat2)SAC:relation

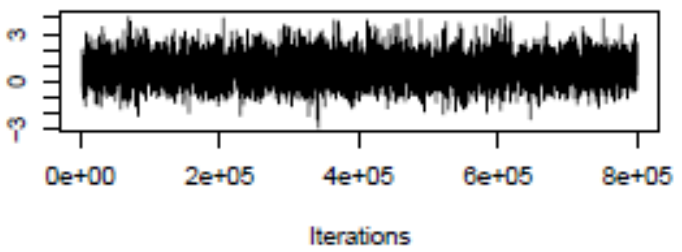

Trace of as.factor(I_Cat2)VAP:relation

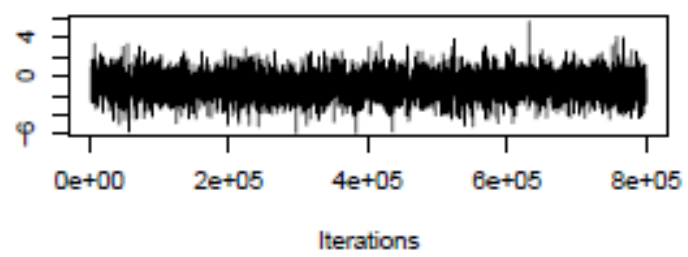

Trace of time:relation

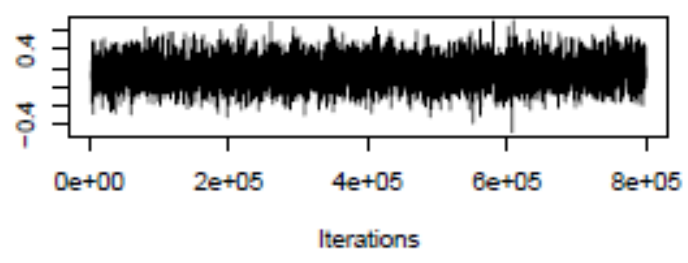

Density of as.factor(I_Cat2)SAC:live

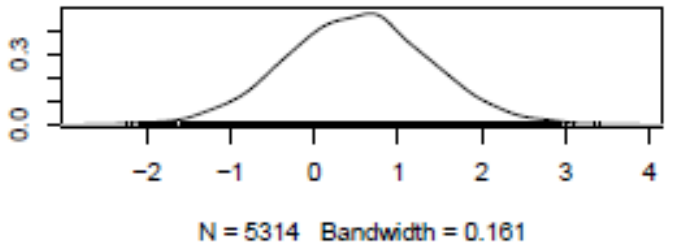

Density of as.factor(I_Cat2)VAP:Iive

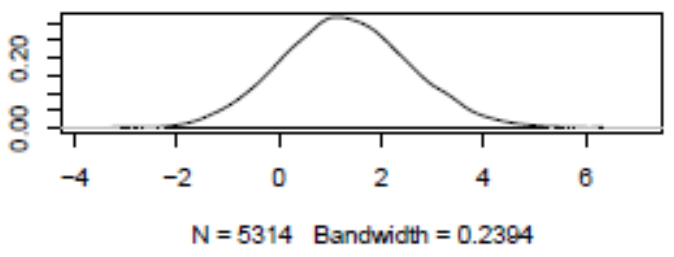

Density of time:live

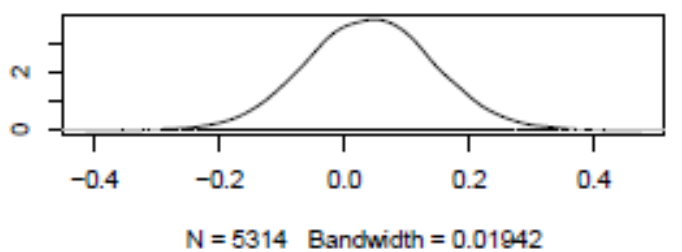

Density of as.factor(1_Cat2)SAC:relation

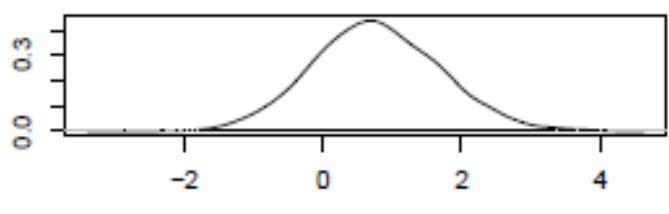

$\mathrm{N}=5314$ Bandwidth $=0.1774$

Density of as.factor(I_Cat2)VAP:relation

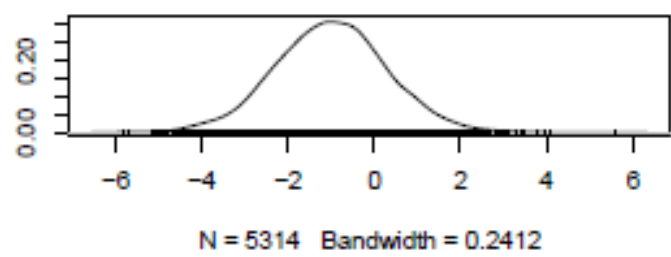

Density of time:relation

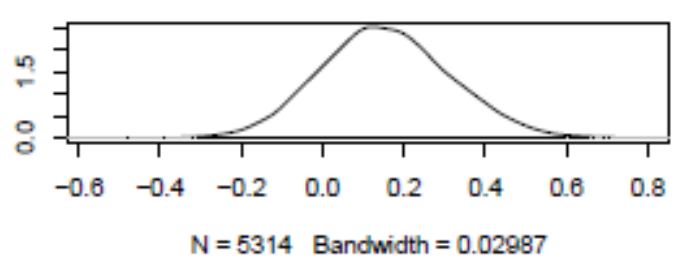


Trace of as.factor(I_Cat2)SAC:ete

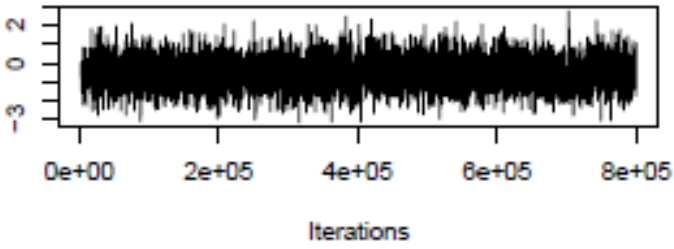

Trace of as.factor(1_Cat2)VAP:ete

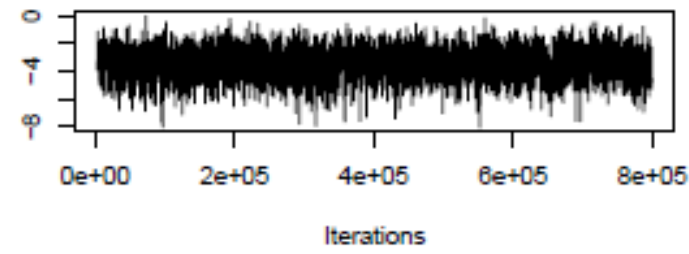

Trace of time:ete

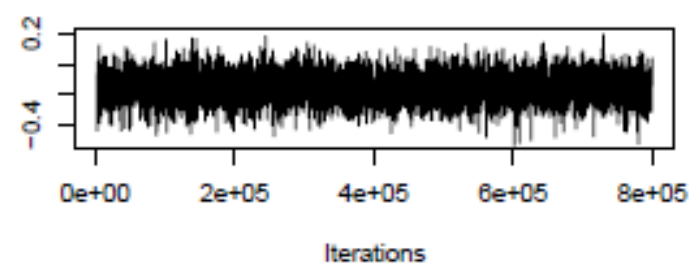

Trace of as.factor(I_Cat2)SAC:where

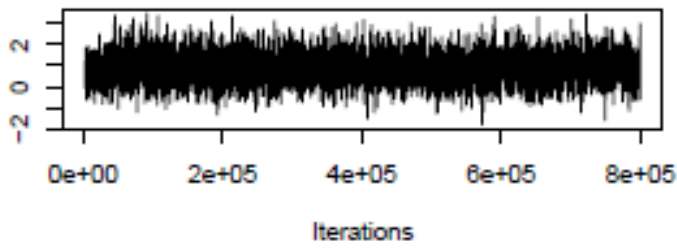

Trace of as.factor(I_Cat2)VAP:where

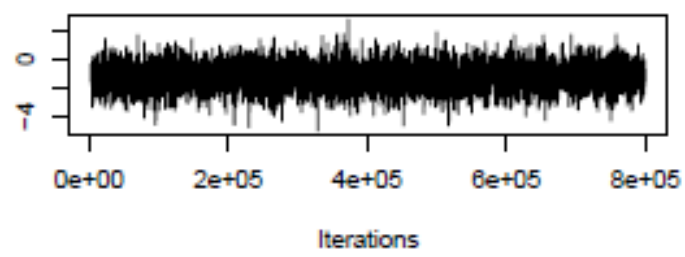

Trace of time:where

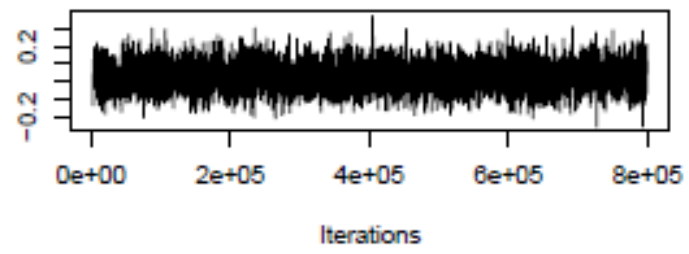

Density of as.factor(I_Cat2)SAC:ete

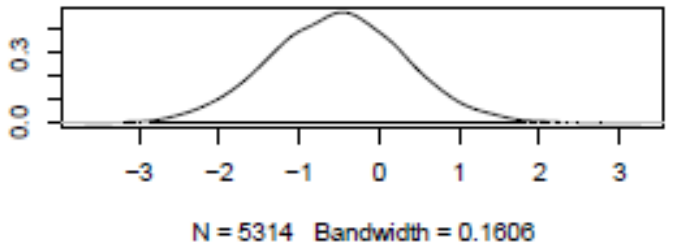

Density of as.factor(I_Cat2)VAP:ete

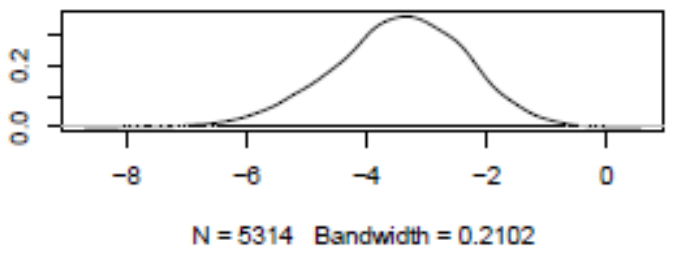

Density of time:ete

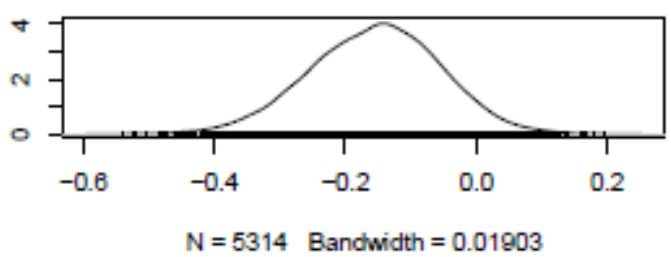

Density of as.factor(I_Cat2)SAC:where

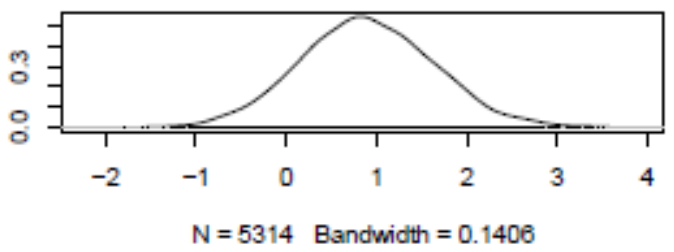

Density of as.factor(I_Cat2)VAP:where

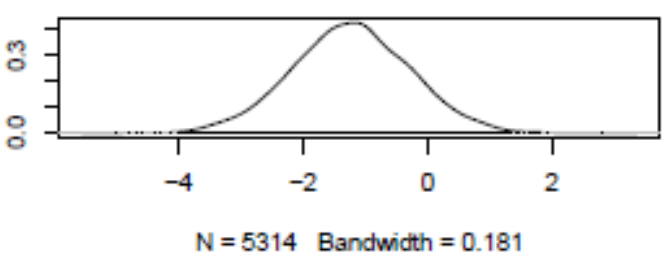

Density of time:where

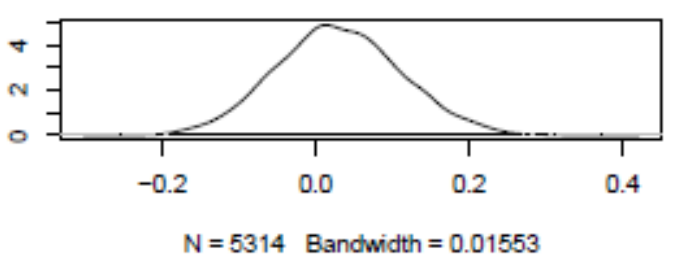


Trace of as.factor(I_Cat2)SAC:life

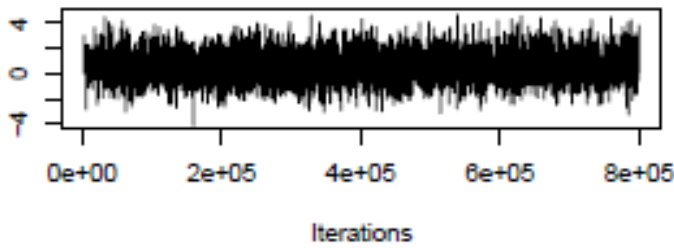

Trace of as.factor(1_Cat2)VAP:life

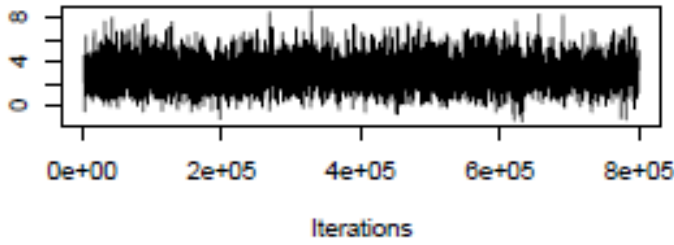

Trace of time:life

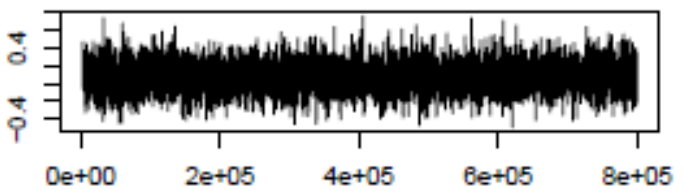

Iterations

Trace of as.factor(I_Cat2)SAC:drugs

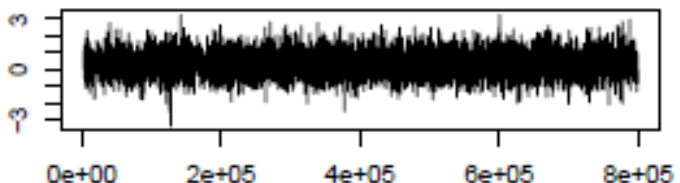

Iterations

Trace of as.factor(1_Cat2)VAP:drugs

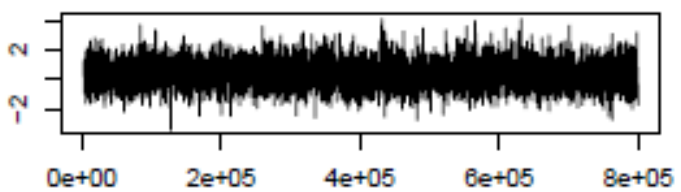

Iterations

Trace of time:drugs

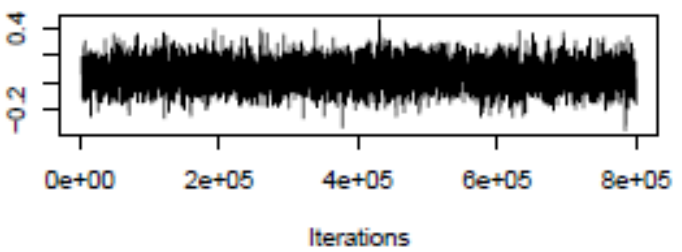

Density of as.factor(1_Cat2)SAC:life

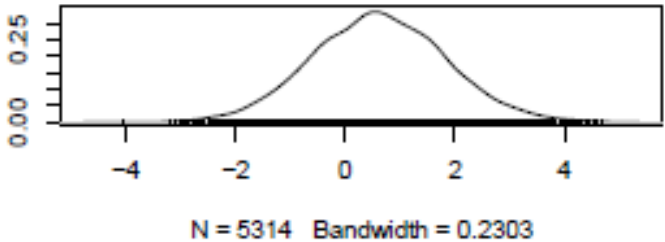

Density of as.factor(I_Cat2)VAP:life

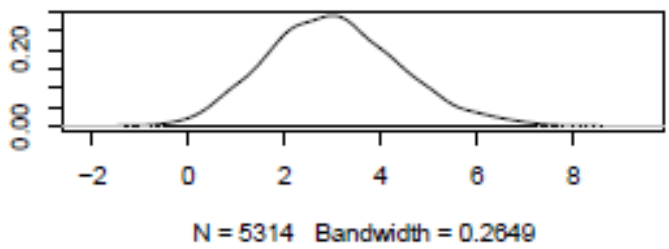

Density of time:life

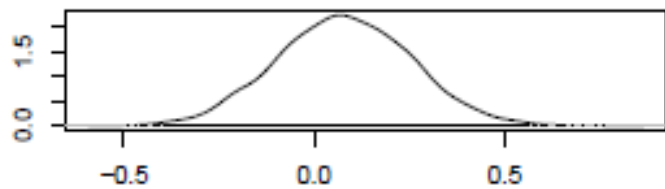

$\mathrm{N}=5314$ Bandwidth $=0.03367$

Density of as.factor(I_Cat2)SAC:drugs

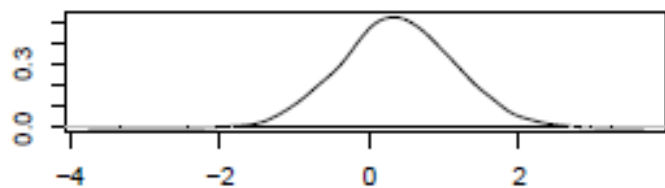

$\mathrm{N}=5314$ Bandwidth $=0.1424$

Density of as.factor(I_Cat2)VAP:drugs

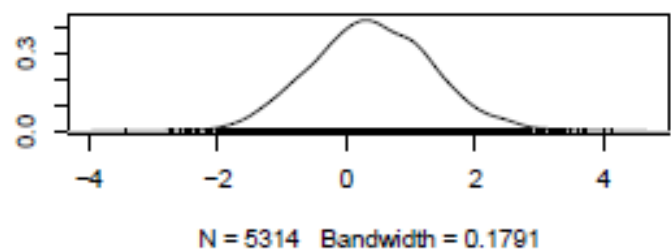

Density of time:drugs

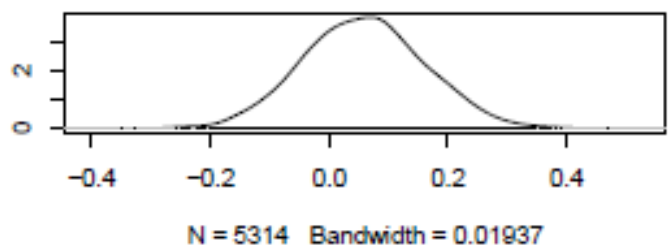


Trace of as.factor(I_Cat2)SAC:physical

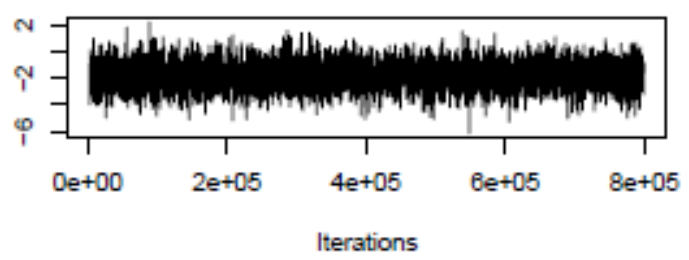

Trace of as.factor(I_Cat2)VAP:physical

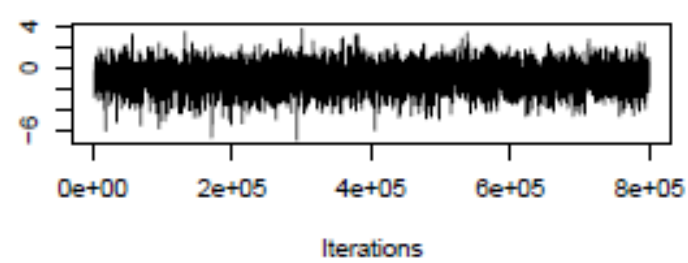

Trace of time:physical

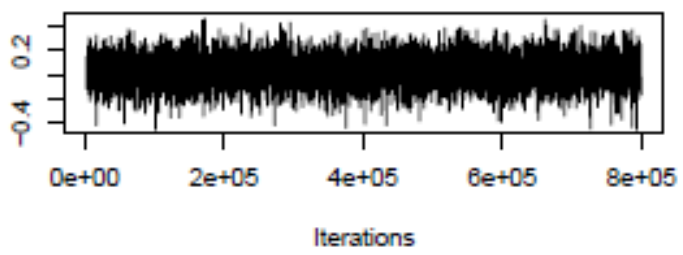

Trace of as.factor(I_Cat2)SAC:emotion

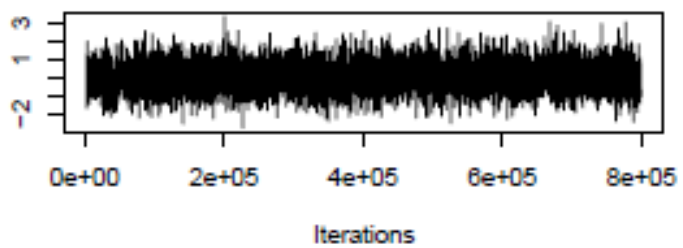

Trace of as.factor(I_Cat2)VAP:emotion

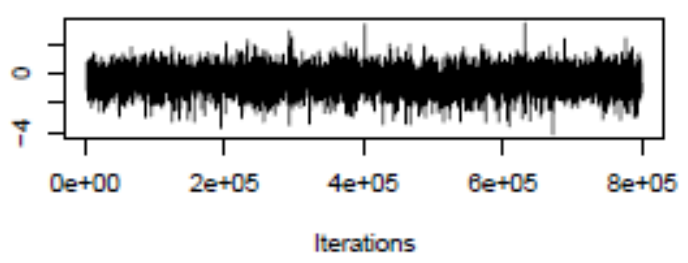

Trace of time:emotion

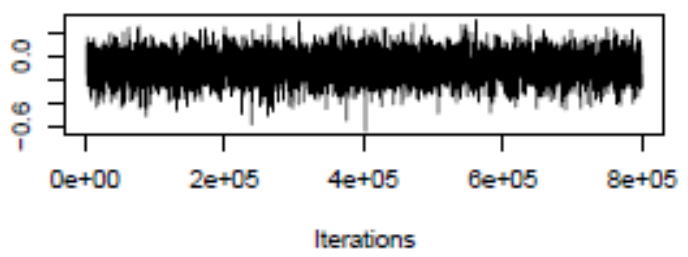

Density of as.factor(I_Cat2)SAC:physical

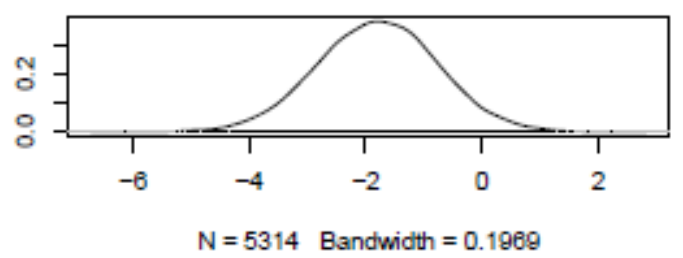

Density of as.factor(I_Cat2)VAP:physical

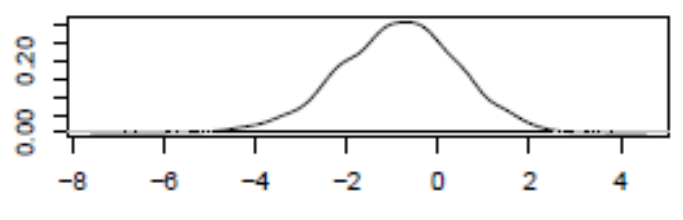

$\mathrm{N}=5314$ Bandwidth $=0.2457$

Density of time:physical

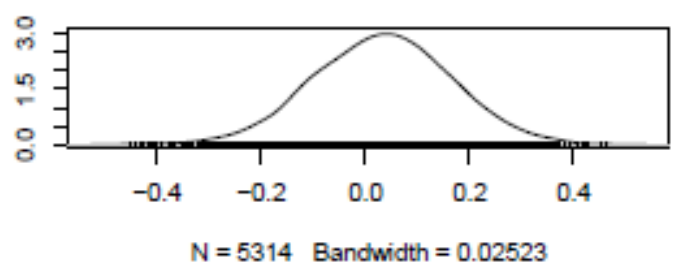

Density of as.factor(I_Cat2)SAC:emotion

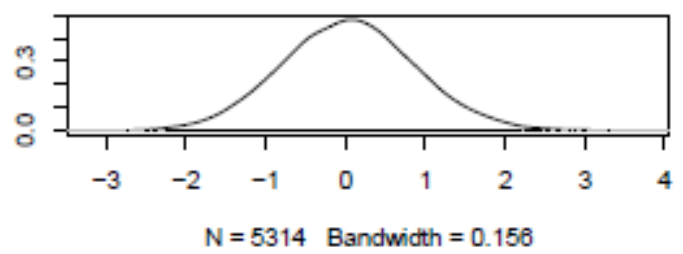

Density of as.factor(I_Cat2)VAP:emotion

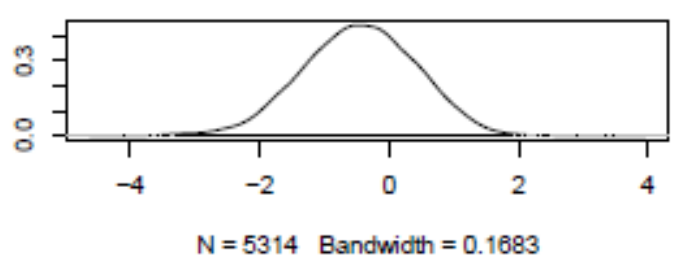

Density of time:emotion

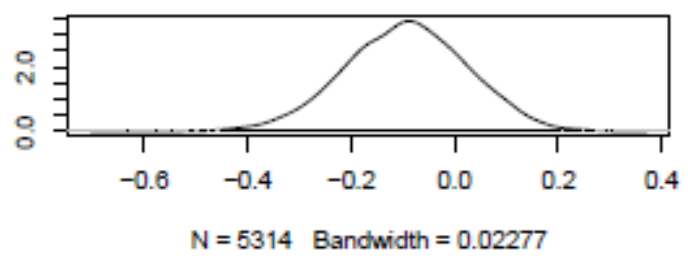


Trace of as.factor(I_Cat2)SAC:self

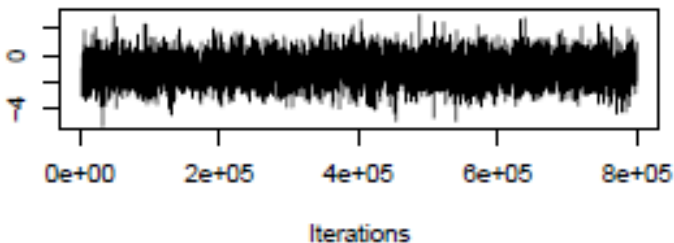

Trace of as.factor(I_Cat2)VAP:self

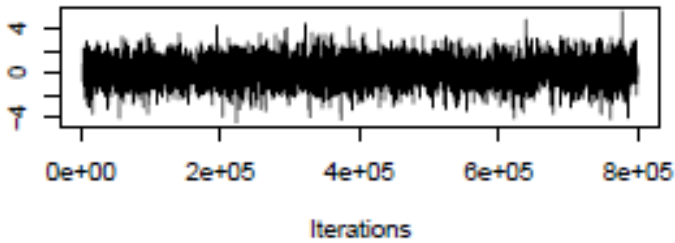

Trace of time:self

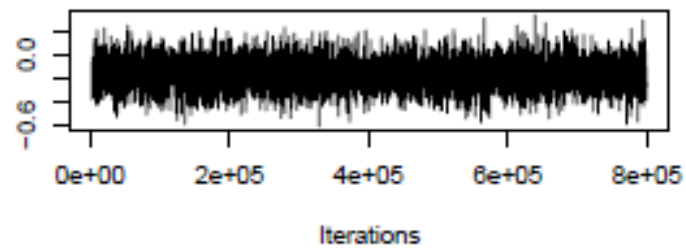

Trace of as.factor(I_Cat2)SAC:think

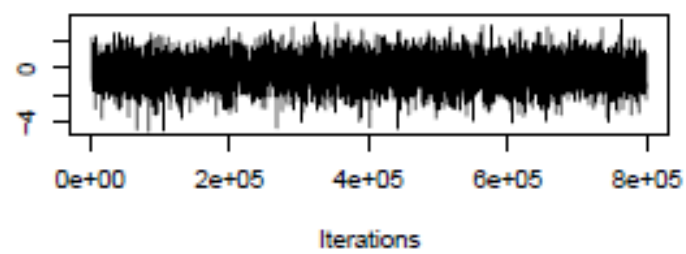

Trace of as.factor(I_Cat2)VAP:think

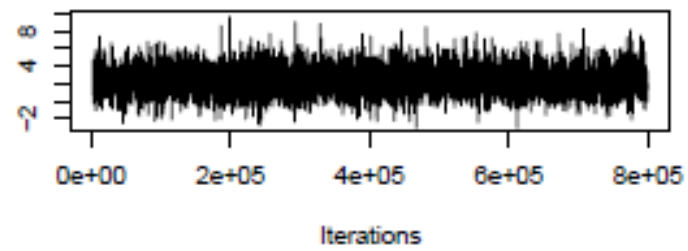

Trace of time:think

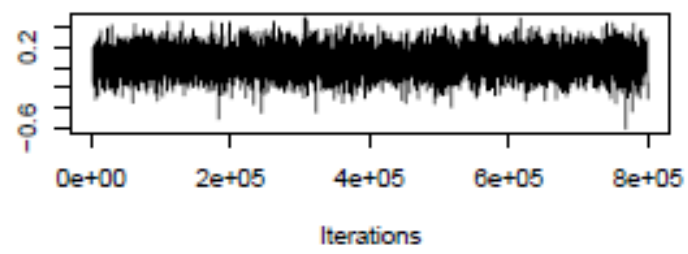

Density of as.factor(I_Cat2)SAC:self

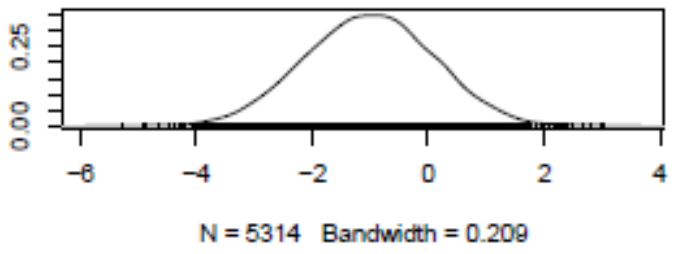

Density of as.factor(I_Cat2)VAP:self

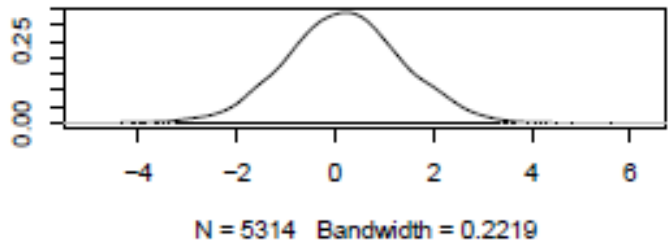

Density of time:self

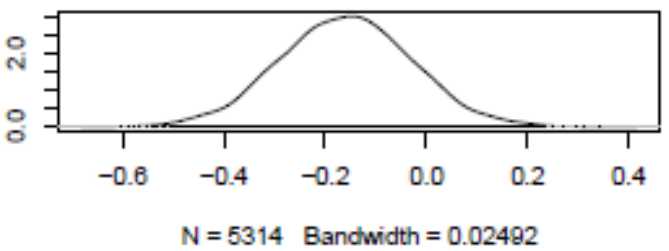

Density of as.factor(I_Cat2)SAC:think

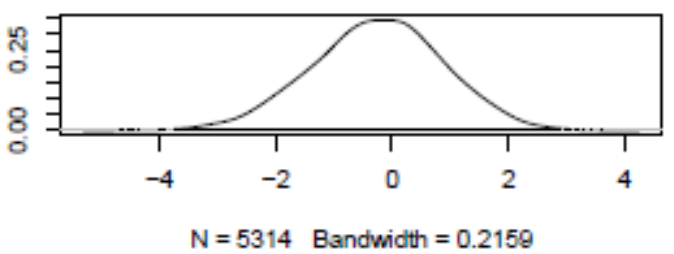

Density of as.factor(I_Cat2)VAP:think

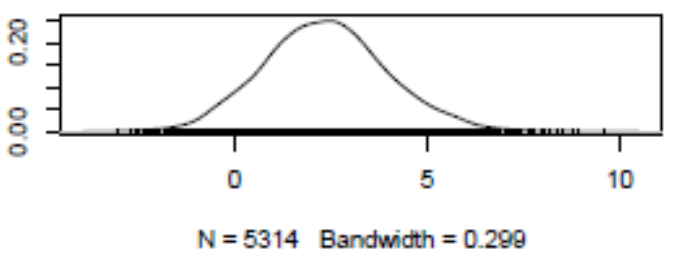

Density of time:think

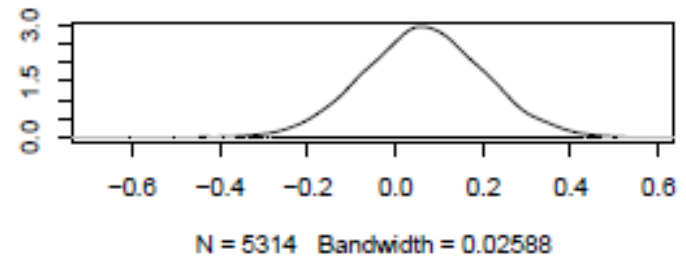


Trace of as.factor(I_Cat2)SAC:attitude

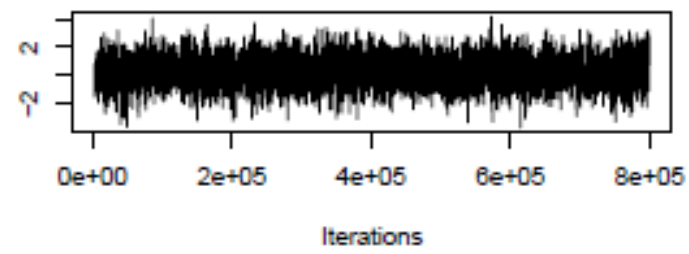

Trace of as.factor(I_Cat2)VAP:attitude

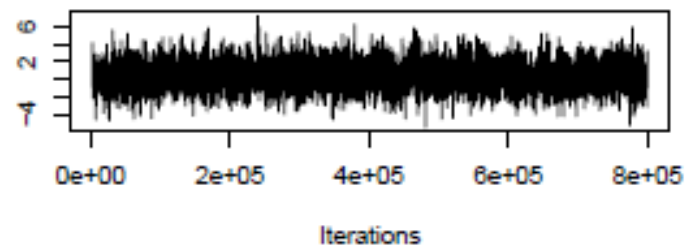

Trace of time:attitude

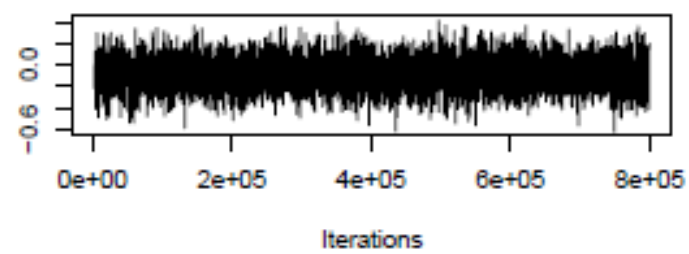

Trace of as.factor(I_Cat2)SAC:change

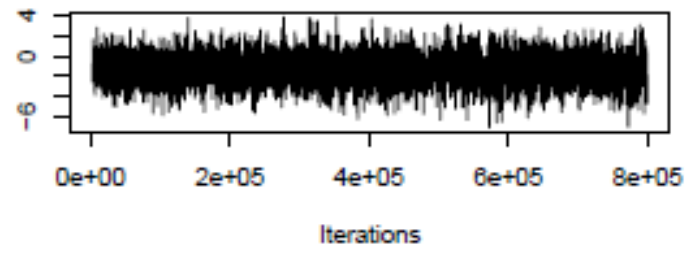

Trace of as.factor(I_Cat2)VAP:change

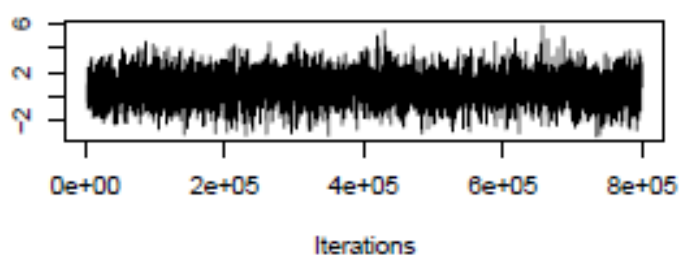

Trace of time:change

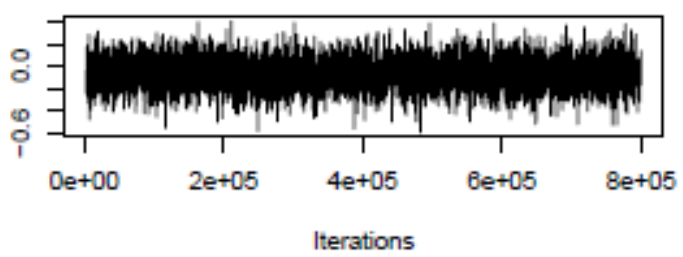

Density of as.factor(I_Cat2)SAC:attitude

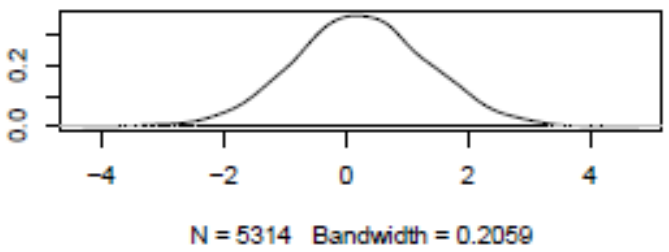

Density of as.factor(I_Cat2)VAP:attitude

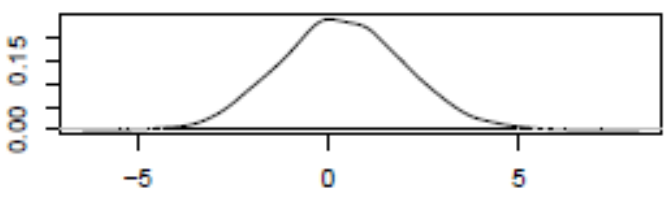

$\mathrm{N}=5314$ Bandwidth $=0.315$

Density of time:attitude

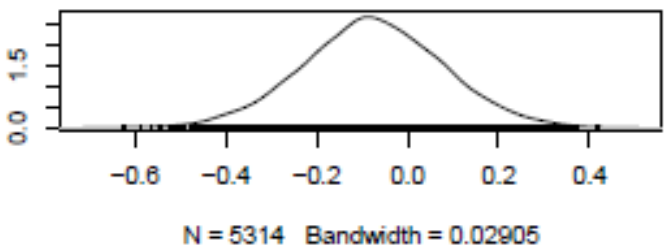

Density of as.factor(I_Cat2)SAC:change

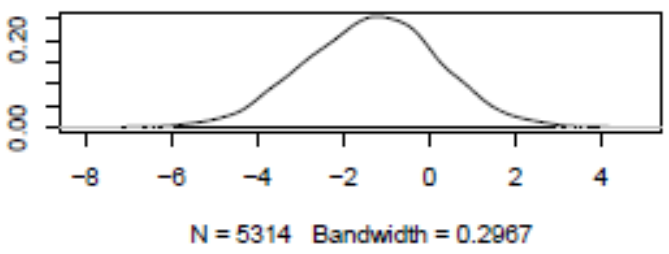

Density of as.factor(I_Cat2)VAP:change

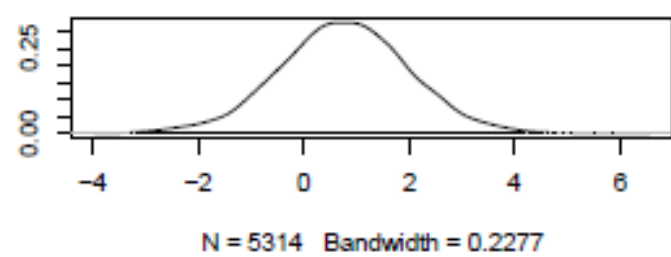

Density of time:change

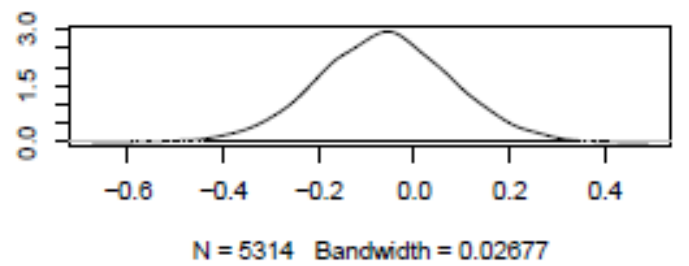


Trace of as.factor(I_Cat2)SAC:time:live

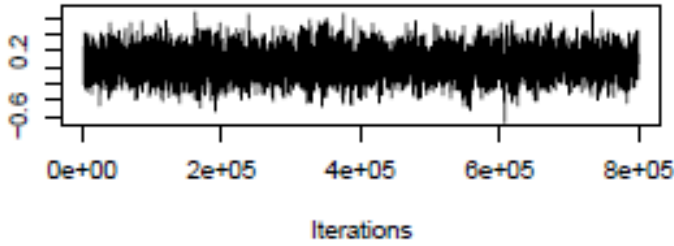

Trace of as.factor(I_Cat2)VAP:time:live

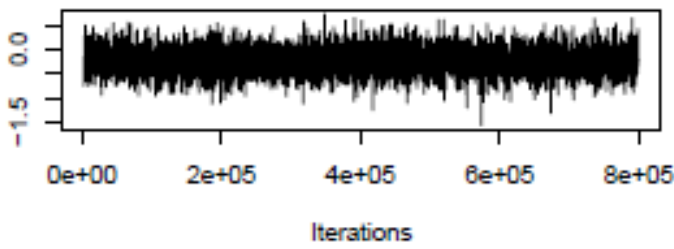

Trace of as.factor(I_Cat2)SAC:time:relation

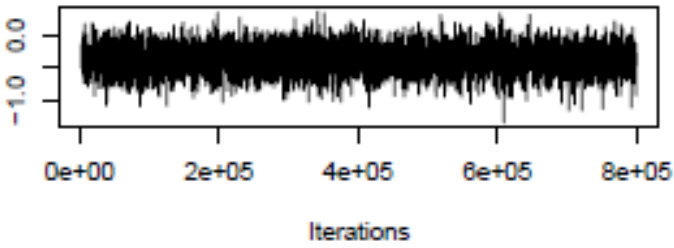

Trace of as.factor(I_Cat2)VAP:time:relation

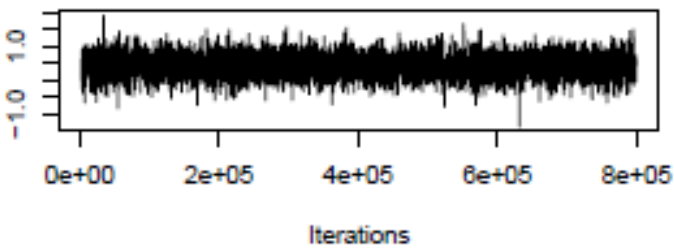

Trace of as.factor(I_Cat2)SAC:time:ete

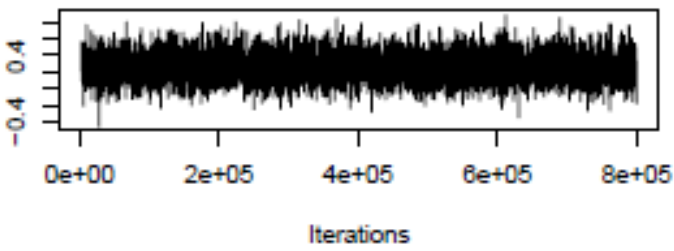

Trace of as.factor(I_Cat2)VAP:time:ete

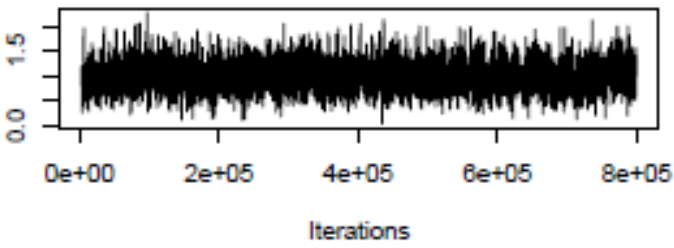

Density of as.factor(I_Cat2)SAC:time:live

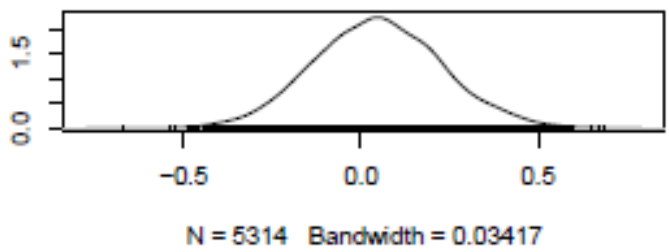

Density of as.factor(I_Cat2)VAP:time:live

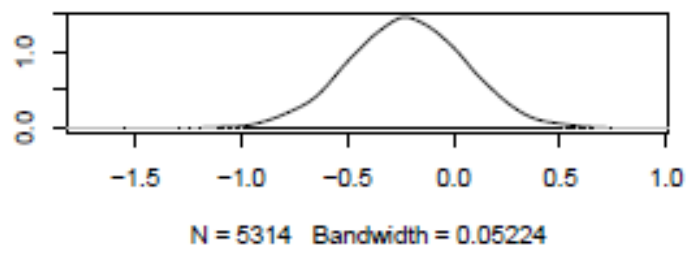

Density of as.factor(I_Cat2)SAC:time:relation

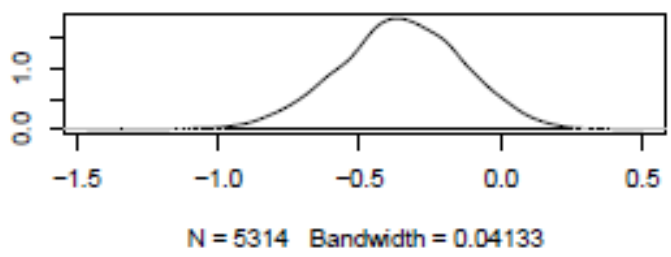

Density of as.factor(I_Cat2)VAP:time:relation

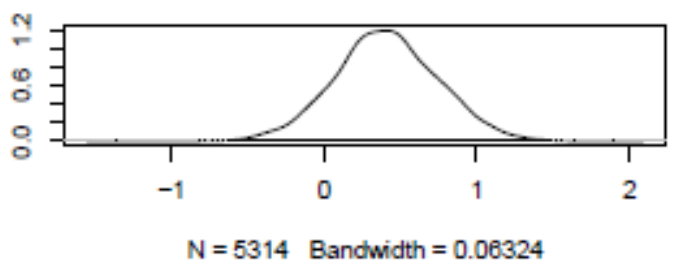

Density of as.factor(I_Cat2)SAC:time:ete

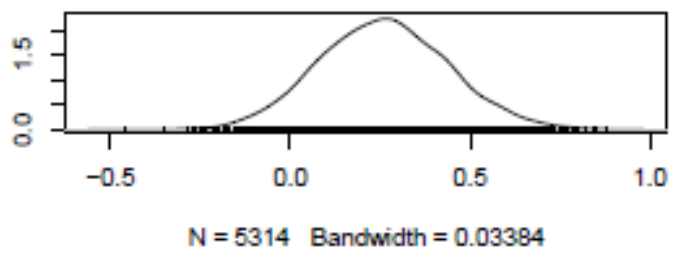

Density of as.factor(I_Cat2)VAP:time:ete

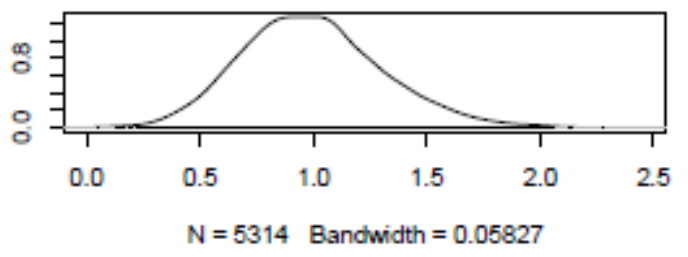


Trace of as.factor(I_Cat2)SAC:time:where

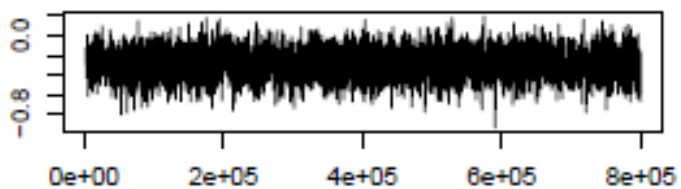

Iterations

Trace of as.factor(1_Cat2)VAP:time:where

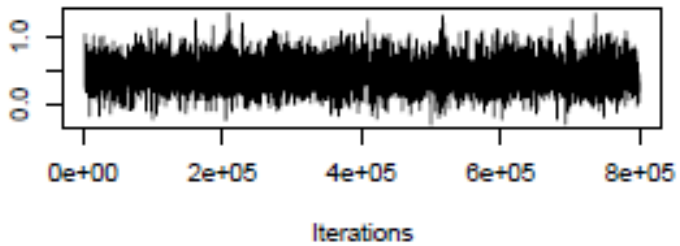

Trace of as.factor(I_Cat2)SAC:time:life

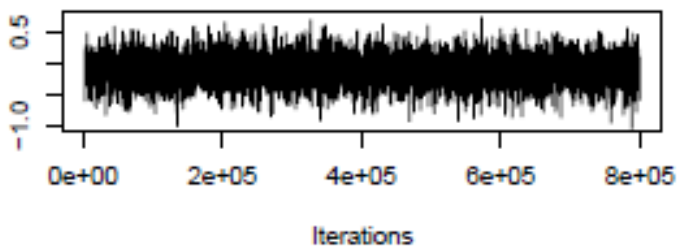

Trace of as.factor(I_Cat2)VAP:time:life

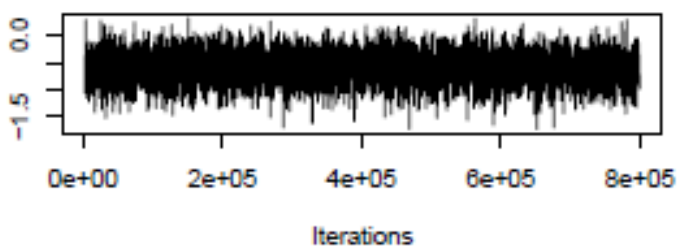

Trace of as.factor(I_Cat2)SAC:time:drugs

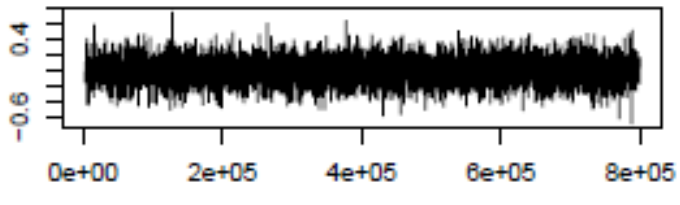

Iterations

Trace of as.factor(I_Cat2)VAP:time:drugs

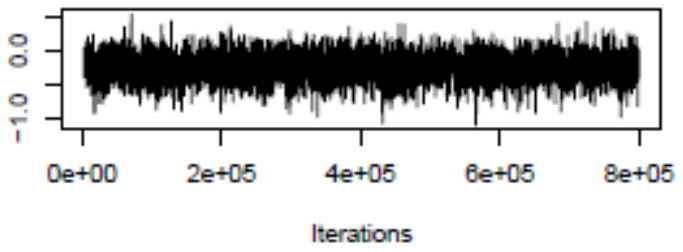

Density of as.factor(I_Cat2)SAC:time:where

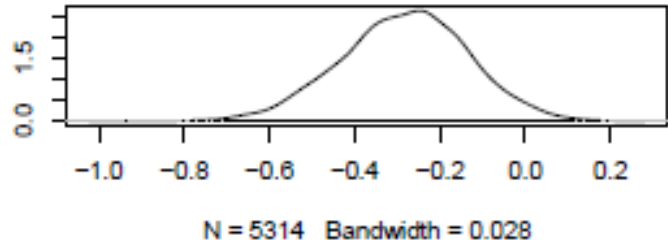

Density of as.factor(I_Cat2)VAP:time:where

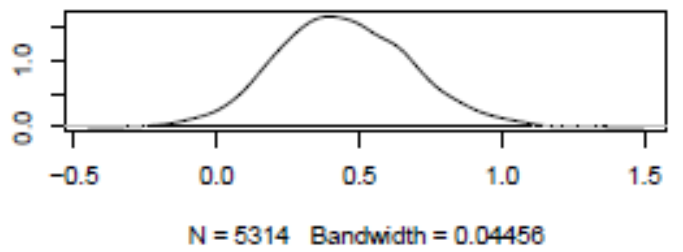

Density of as.factor(I_Cat2)SAC:time:life

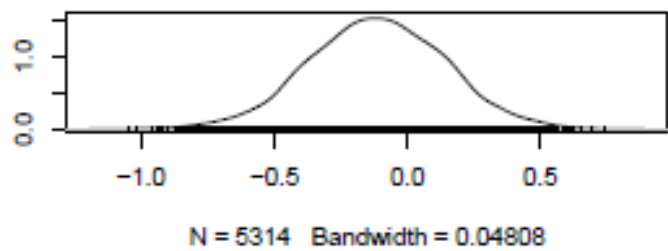

Density of as.factor(I_Cat2)VAP:time:life

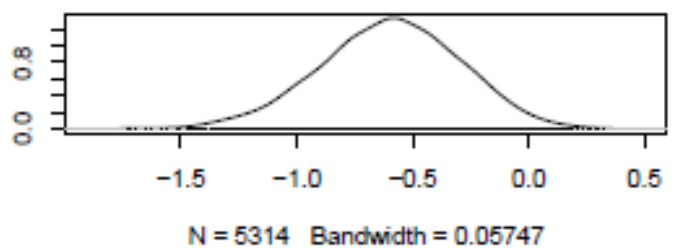

Density of as.factor(I_Cat2)SAC:time:drugs

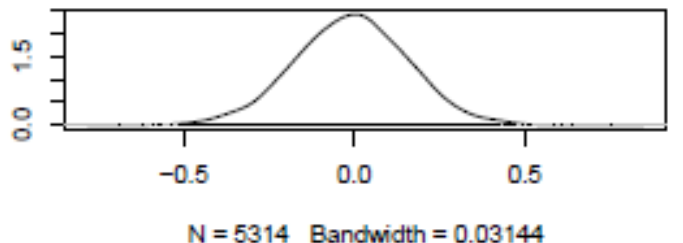

Density of as.factor(I_Cat2)VAP:time:drugs

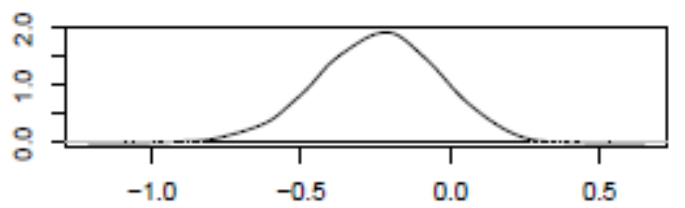

$\mathrm{N}=5314$ Bandwidth $=0.03932$ 
Trace of as.factor(1_Cat2)SAC:time:physical

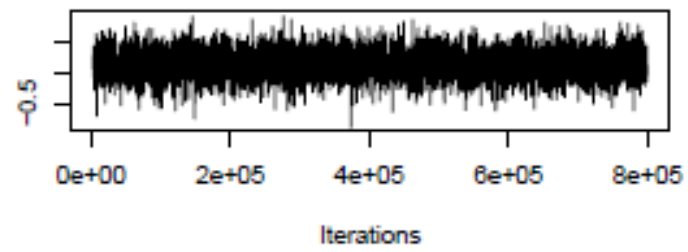

Trace of as.factor(I_Cat2)VAP:time:physical

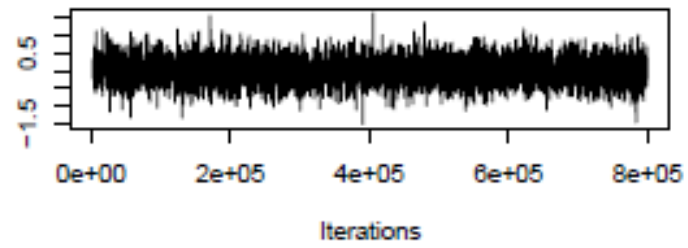

Trace of as.factor(I_Cat2)SAC:time:emotion

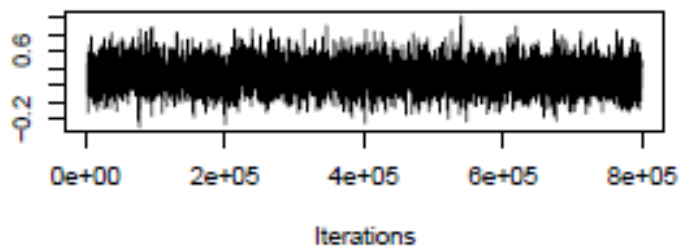

Trace of as.factor(I_Cat2)VAP:time:emotion

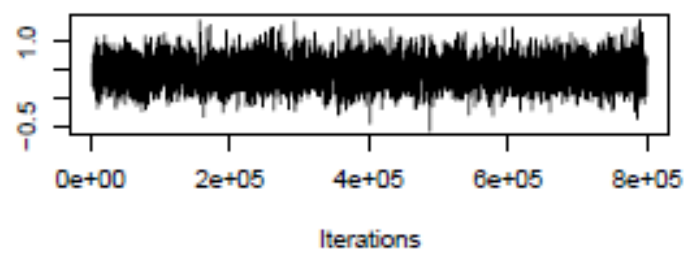

Trace of as.factor(I_Cat2)SAC:time:self

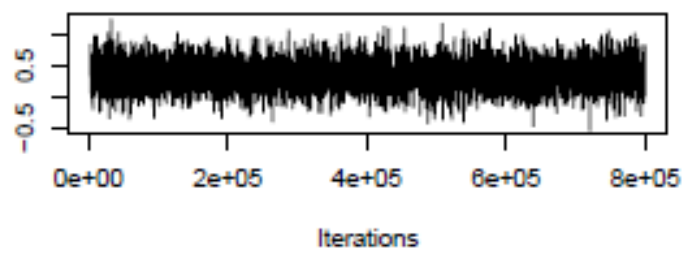

Trace of as.factor(I_Cat2)VAP:time:self

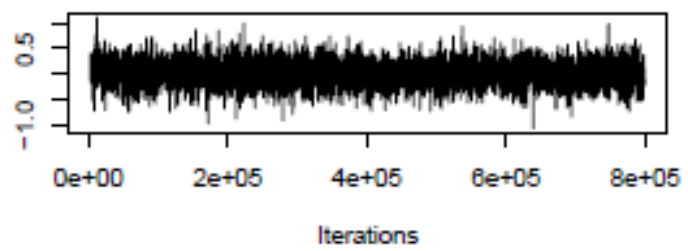

Density of as.factor(I_Cat2)SAC:time:physical

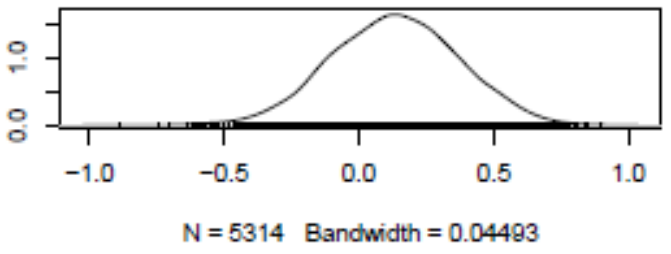

Density of as.factor(I_Cat2)VAP:time:physical

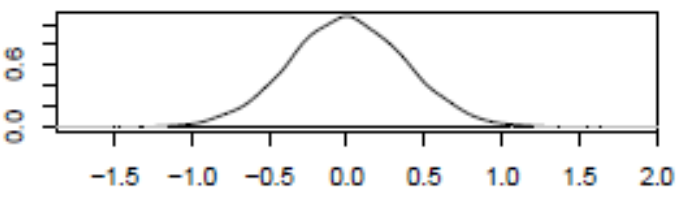

$\mathrm{N}=5314$ Bandwidth $=0.07111$

Density of as.factor(I_Cat2)SAC:time:emotion

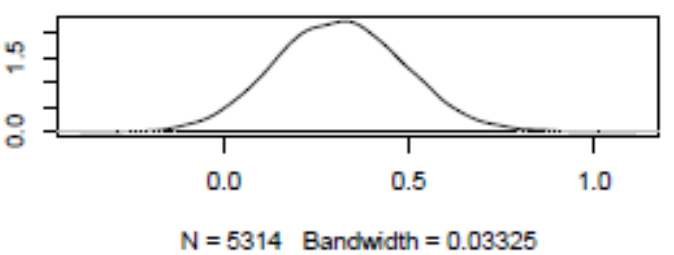

Density of as.factor(I_Cat2)VAP:time:emotion

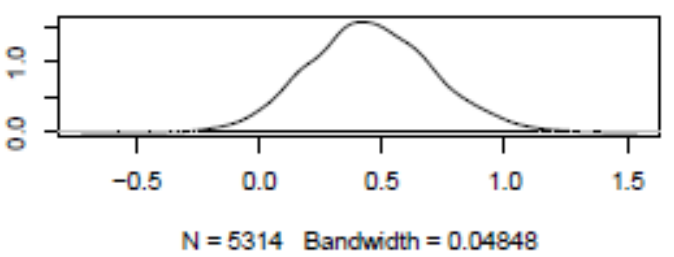

Density of as.factor(I_Cat2)SAC:time:self

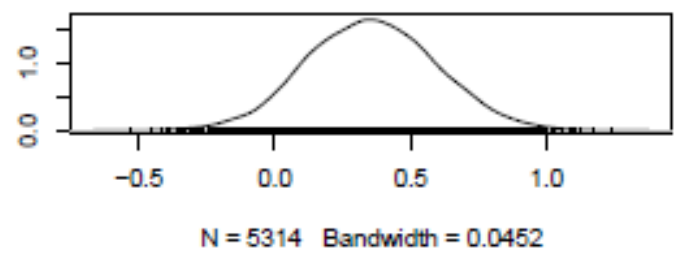

Density of as.factor(I_Cat2)VAP:time:self

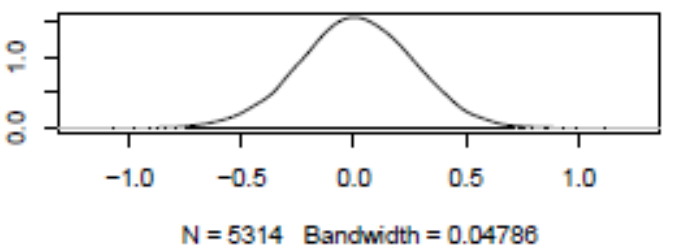


Trace of as.factor(I_Cat2)SAC:time:think

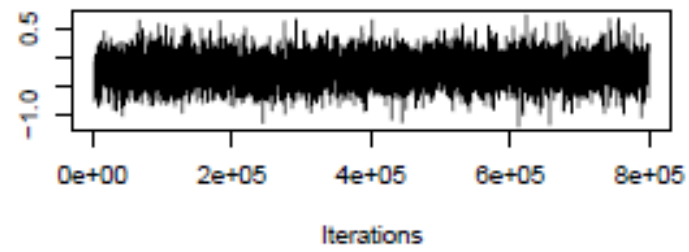

Trace of as.factor(I_Cat2)VAP:time:think

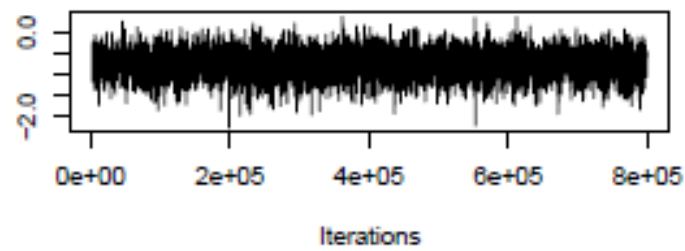

Trace of as.factor(1_Cat2)SAC:time:attitude

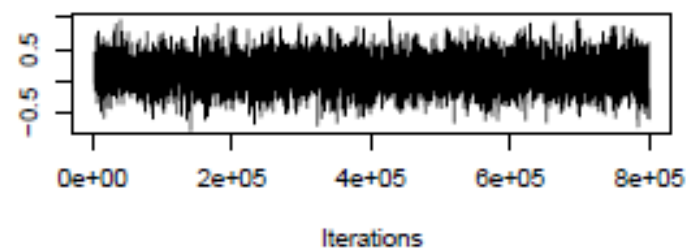

Trace of as.factor(I_Cat2)VAP:time:attitude

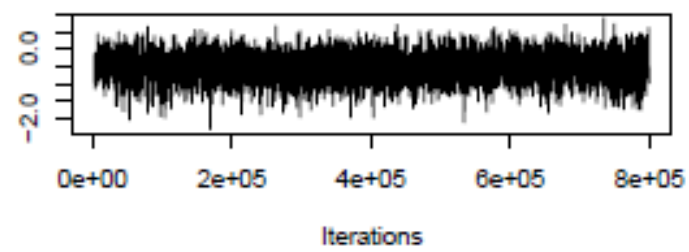

Trace of as.factor(I_Cat2)SAC:time:change

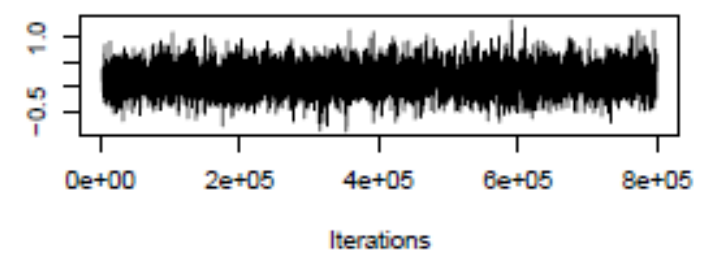

Trace of as.factor(I_Cat2)VAP:time:change

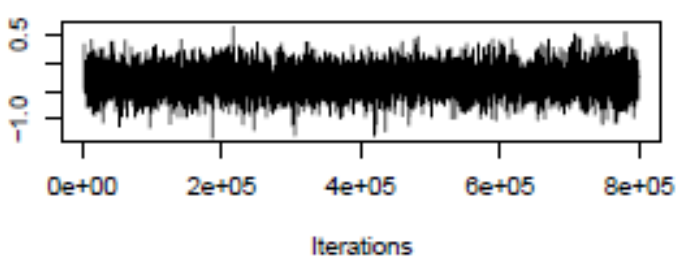

Density of as.factor(1_Cat2)SAC:time:think

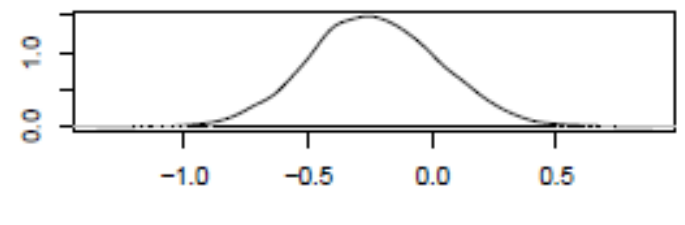

$\mathrm{N}=5314$ Bandwidth $=0.05015$

Density of as.factor(I_Cat2)VAP:time:think

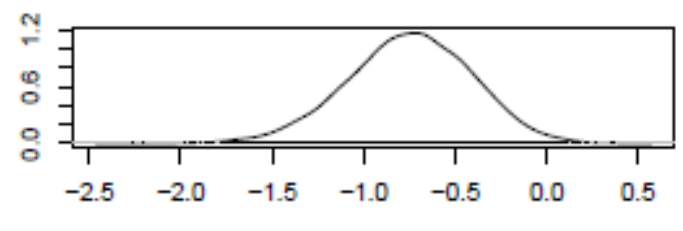

$\mathrm{N}=5314$ Bandwidth $=0.08522$

Density of as.factor(I_Cat2)SAC:time:attitude

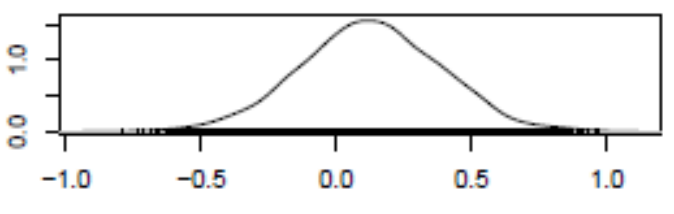

$\mathrm{N}=5314$ Bandwidth $=0.04908$

Density of as.factor(I_Cat2)VAP:time:attitude

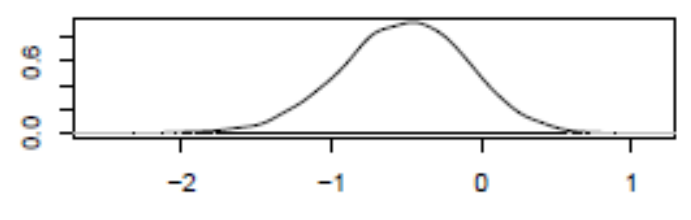

$\mathrm{N}=5314$ Bandwidth $=0.07973$

Density of as.factor(I_Cat2)SAC:time:change

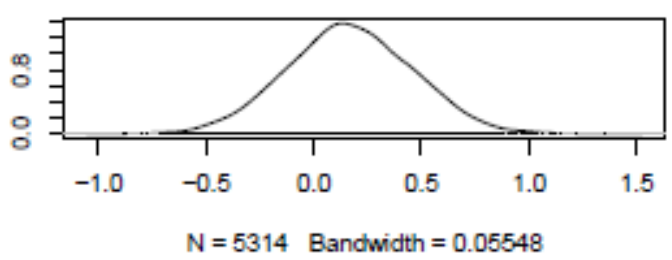

Density of as.factor(I_Cat2)VAP:time:change

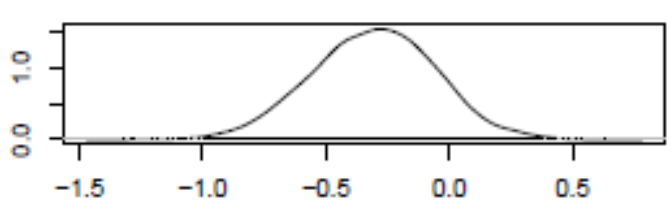

$\mathrm{N}=5314$ Bandwidth $=0.04816$ 
$\underline{\text { Random Effects }}$

Trace of time

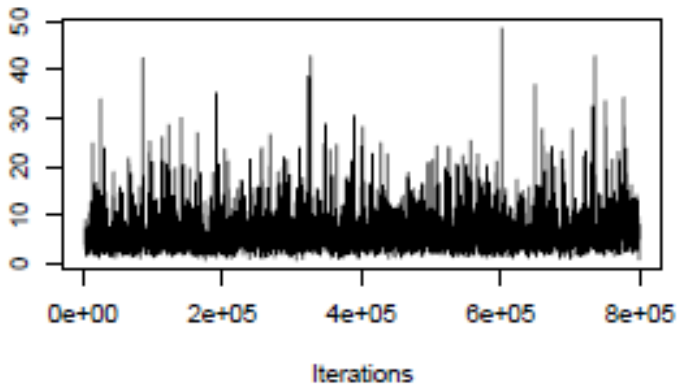

Trace of Research.ID

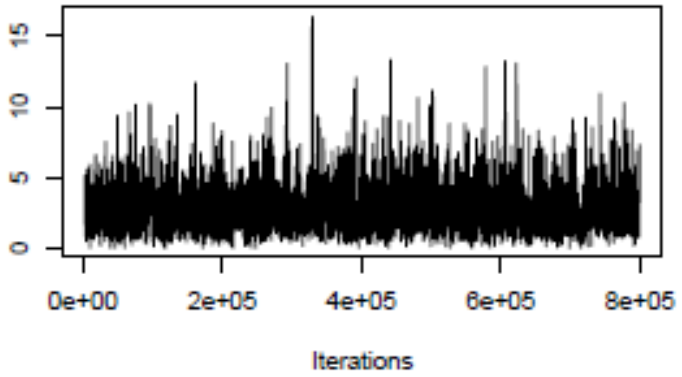

Density of time

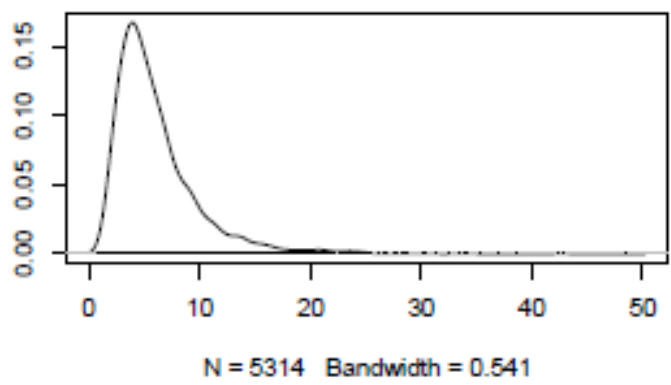

Density of Research.ID

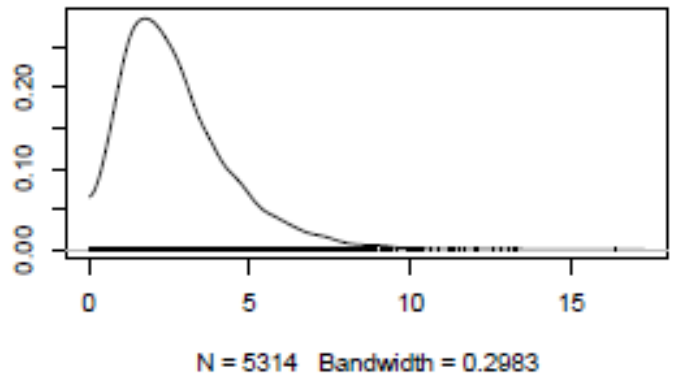


Dynamic Model involving YJB Gravity Score (Table 6.19)

Bayesian Model (BDm3_o2a)

\section{\#\# Define the model}

BDm3_o2a <- MCMCglmm(FO.bin I Seriousness2*time*live + I_Seriousness $2 *$ time*relation + $\bar{I}$ Seriousness $2 *$ time*ete + I Seriousness2*time*where + I Seriousness2*time*life + I-Seriousness2*time*drugs + I Seriousness2*time*physical + I_Seriousness2*time*emotion + I_Seriousness $2 *$ time*self + I_Seriousness2*time*think + I_seriousness $2 *$ time*attitude + I Seriousness $2 *$ time* change, rāndom= time+Research.ID, data=data3, family="ordinal", prior=priorD, slice=TRUE, nitt $=300000$, thin=50, burnin=3000)

\section{\#\# Checks for suitable convergence}

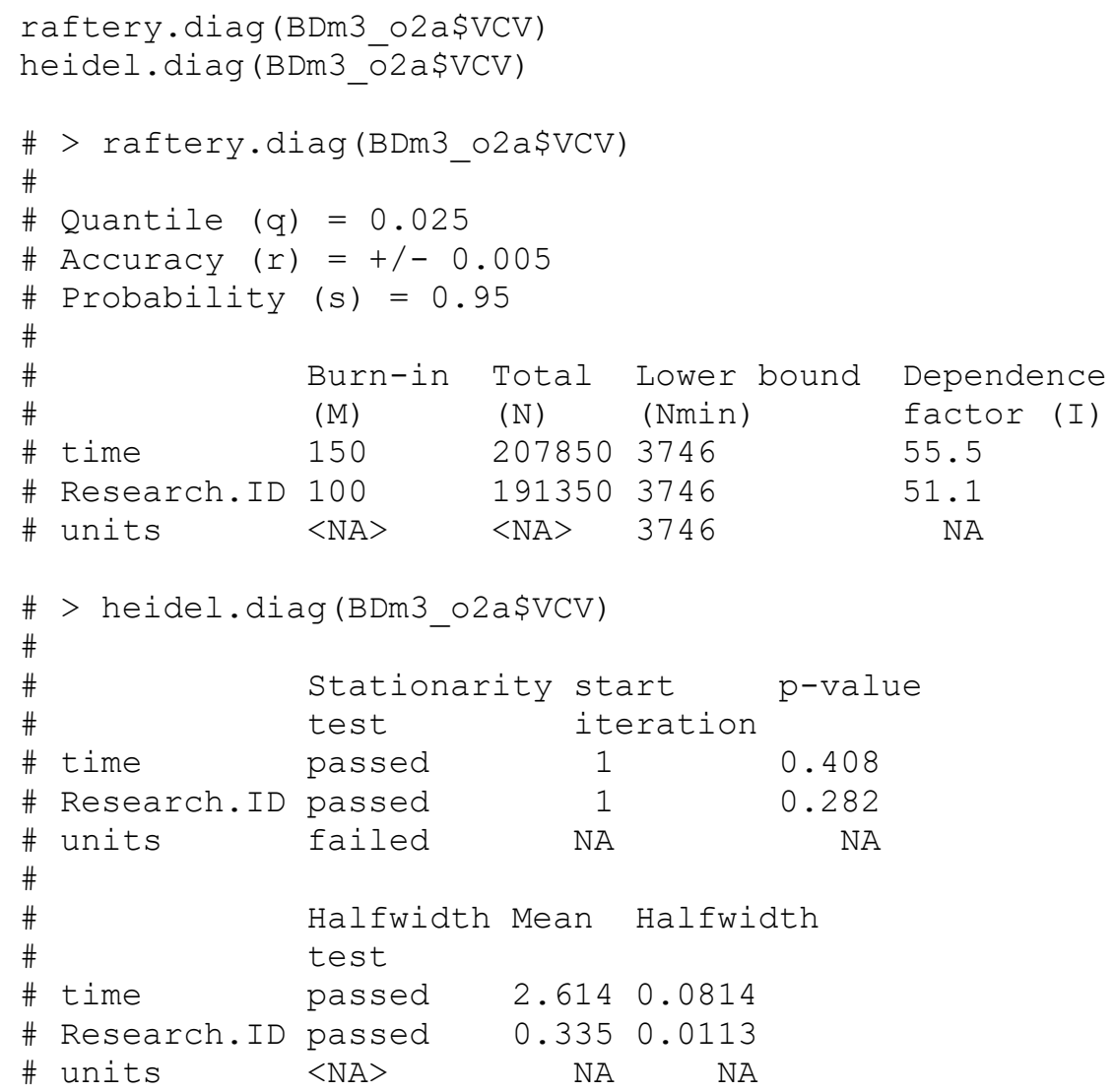




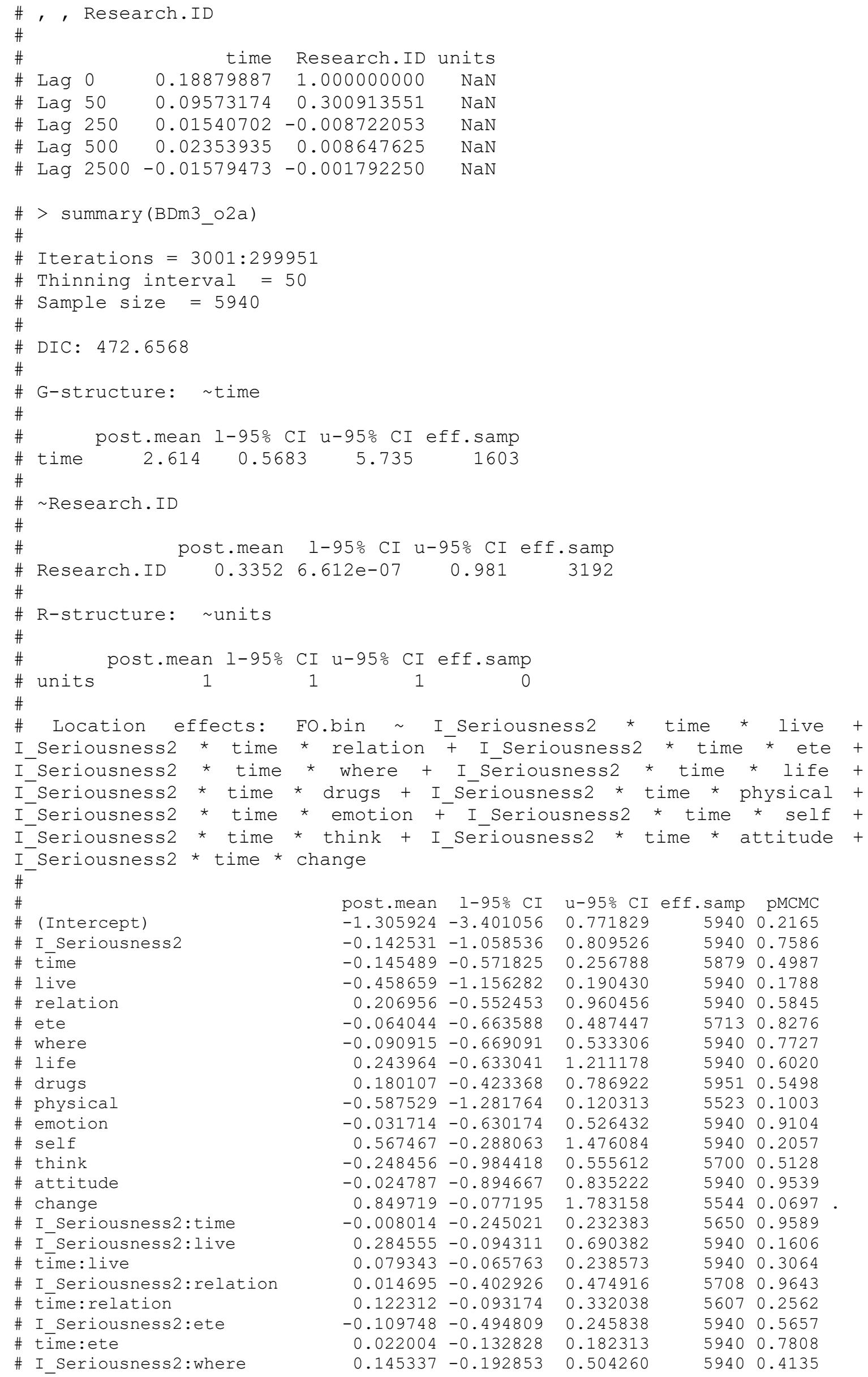


\# time:where

\# I Seriousness2:life

\# tīme:life

\# I Seriousness2:drugs

\# time:drugs

\# I Seriousness2:physical

\# time:physical

\# I Seriousness2:emotion

\# time:emotion

\# I_Seriousness2:self

\# time:self

\# I Seriousness2:think

\# time:think

\# I Seriousness2:attitude

\# time:attitude

\# I_Seriousness2: change

\# tīme:change

\# I_Seriousness2:time:live

\# I Seriousness2:time:relation

\# I-Seriousness2: time: ete

\# I_Seriousness2:time: where

\# I Seriousness2:time: life

\# I_-Seriousness2:time:drugs

\# I Seriousness2:time:physical

\# I_Seriousness2:time:emotion

\# I Seriousness2:time:self

\# I_Seriousness2:time:think

\# I-Seriousness2:time: attitude

\# I_Seriousness2: time: change

\# ---

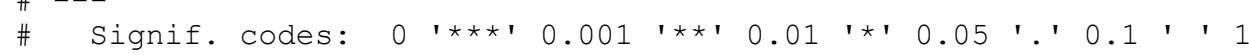

$5644 \quad 0.1360$

59400.6185

$5940 \quad 0.4003$

$\begin{array}{lll}5585 & 0.7259\end{array}$

56330.7185

$5940 \quad 0.7842$

$5940 \quad 0.0498$

$\begin{array}{lll}5940 & 0.7098\end{array}$

57220.1771

$\begin{array}{lll}5940 & 0.5347\end{array}$

$\begin{array}{lll}5940 & 0.0677\end{array}$

$5320 \quad 0.6993$

60520.1108

$5940 \quad 0.9515$

$\begin{array}{lll}5690 & 0.7818\end{array}$

52030.3229

54440.0542 .

$5717 \quad 0.7596$

$5258 \quad 0.1013$

$5940 \quad 0.2455$

54210.0333 *

$5940 \quad 0.6788$

$6019 \quad 0.8397$

$\begin{array}{lll}5655 & 0.3101\end{array}$

55450.0064 **

$5940 \quad 0.2768$

59400.0673 .

$\begin{array}{lll}5684 & 0.6933\end{array}$

$\begin{array}{lll}5112 & 0.1781\end{array}$ 
Trace of (Intercept)

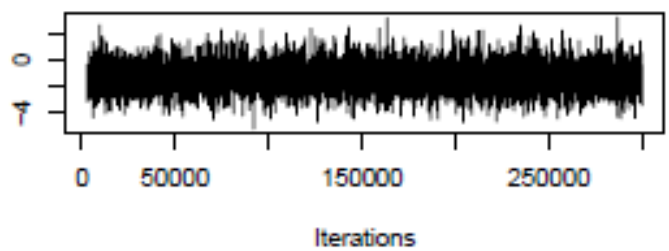

Trace of I_Seriousness2

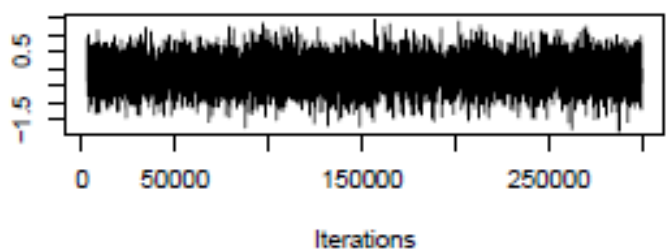

Trace of time

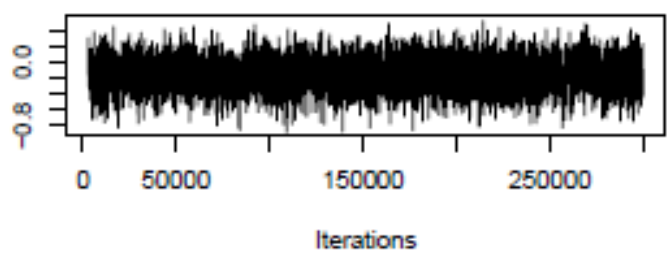

Trace of live

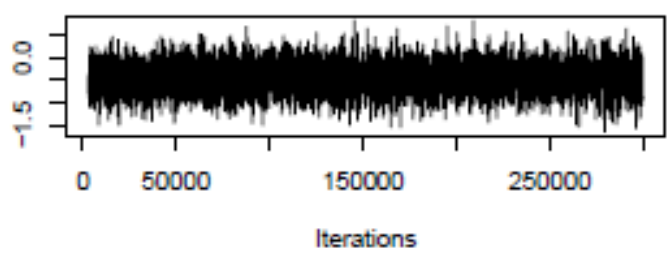

Trace of relation

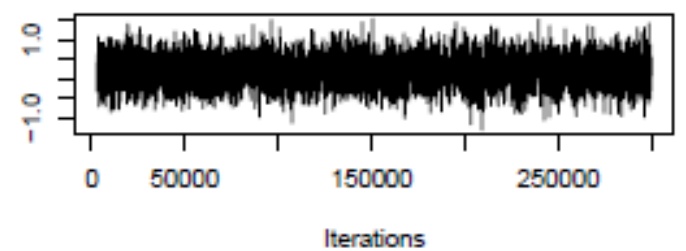

Trace of ete

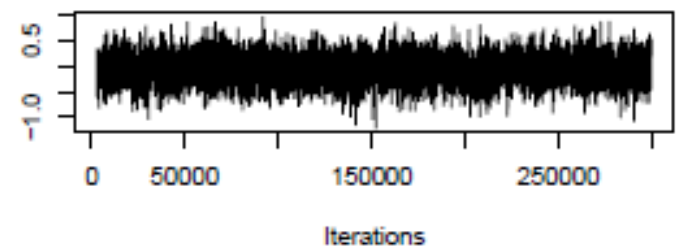

Density of (Intercept)

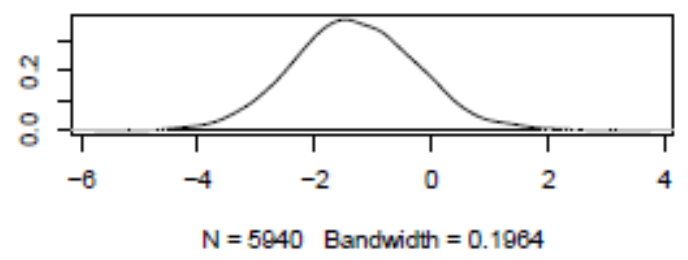

Density of I_Seriousness2

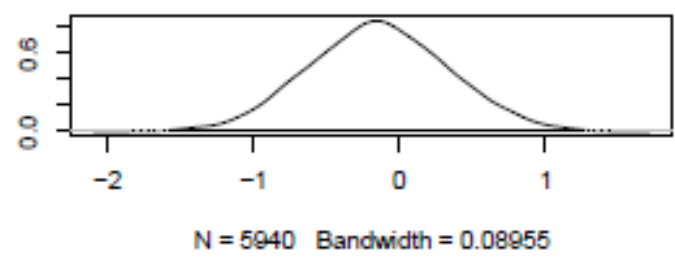

Density of time

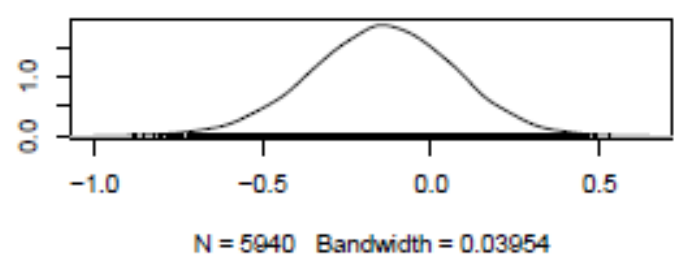

Density of live

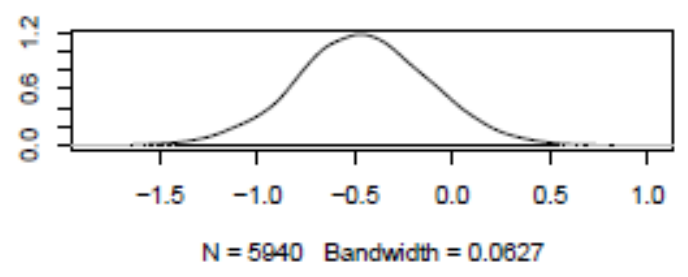

Density of relation

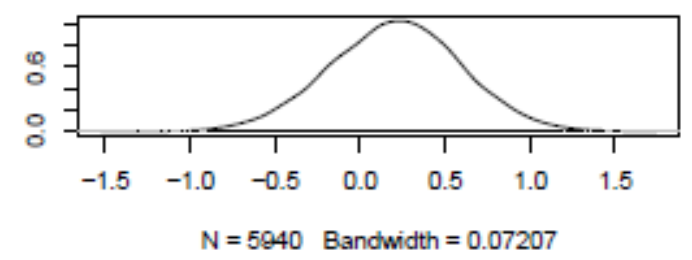

Density of ete

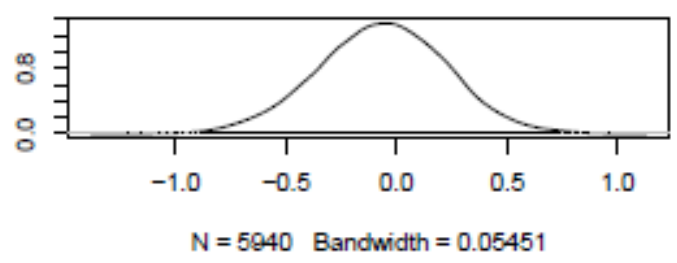


Trace of where

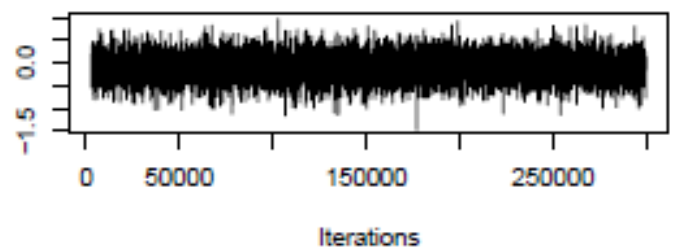

Trace of life

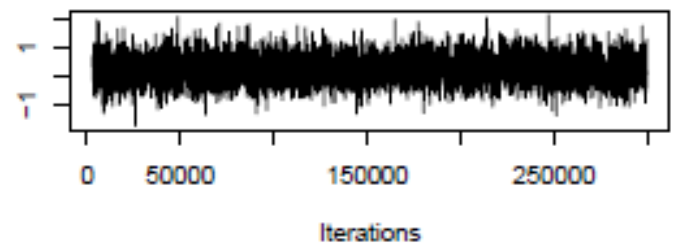

Trace of drugs

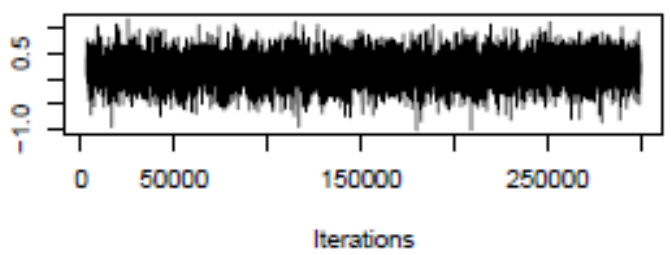

Trace of physical

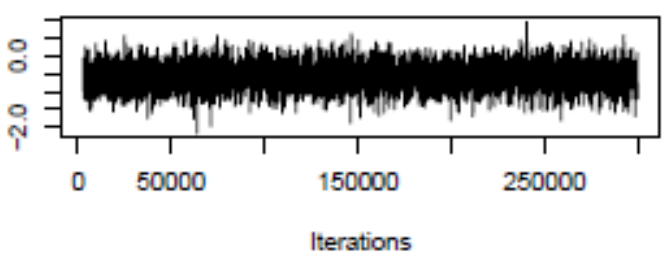

Trace of emotion

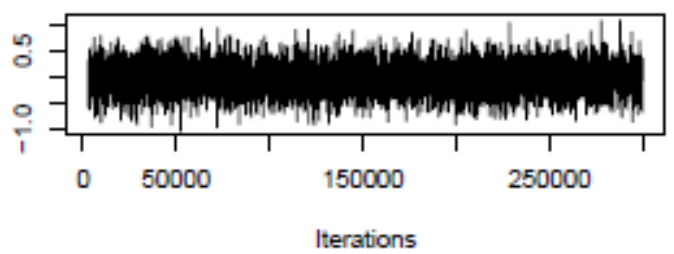

Trace of self

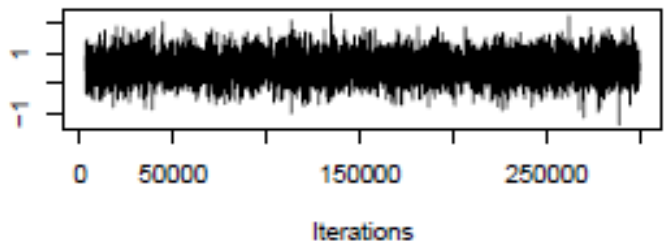

Density of where

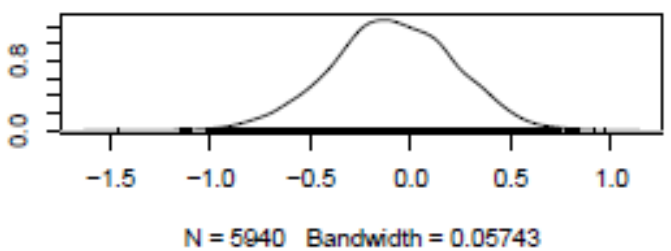

Density of life

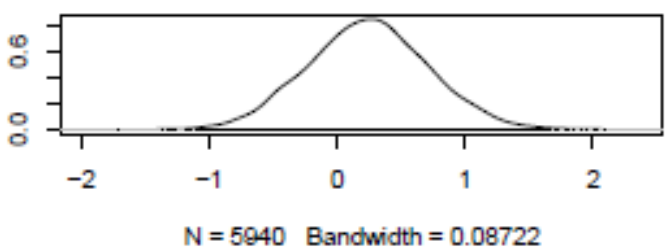

Density of drugs

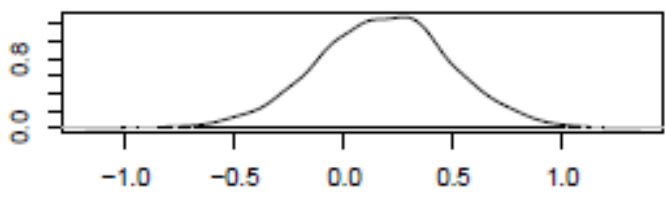

$\mathrm{N}=5940$ Bandwidth $=0.05688$

Density of physical

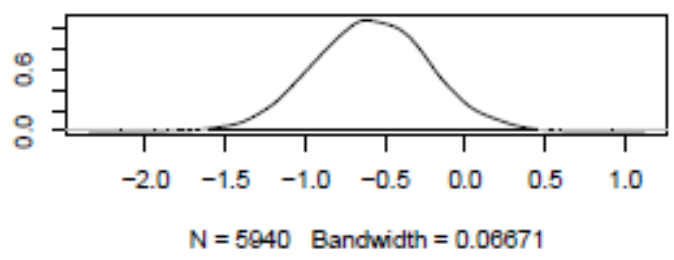

Density of emotion

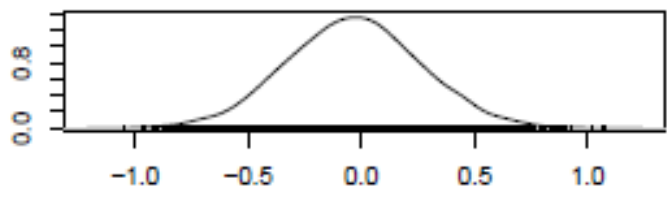

$\mathrm{N}=5940$ Bandwidth $=0.05496$

Density of self

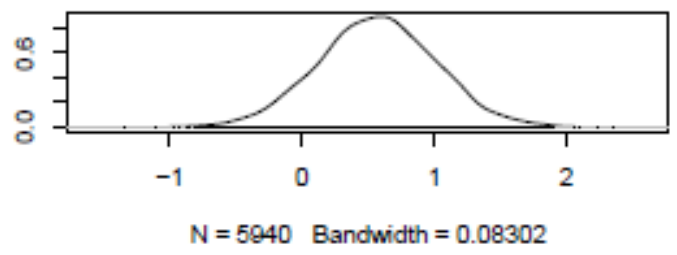


Trace of think

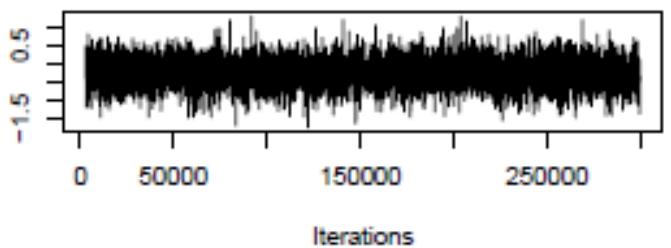

Trace of attitude

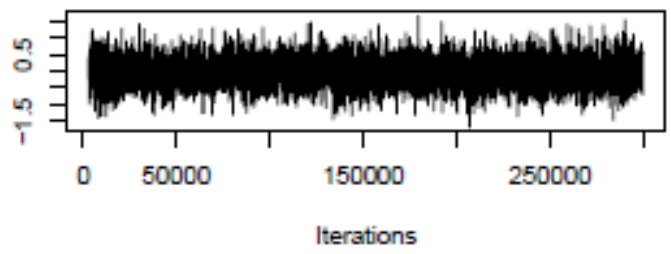

Trace of change

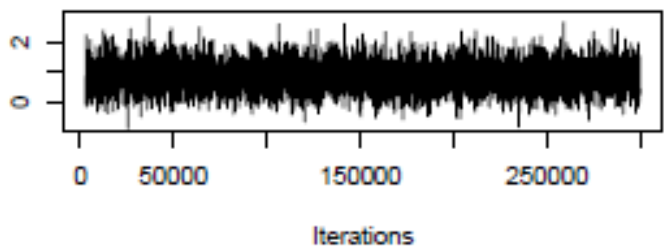

Trace of I_Seriousness2:time

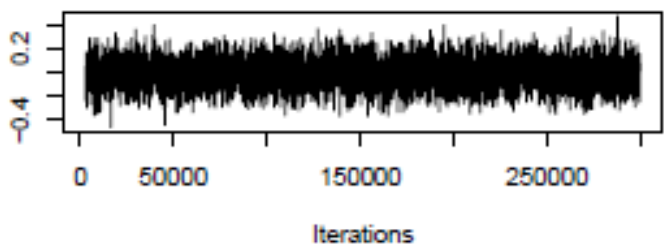

Trace of I_Seriousness2:live

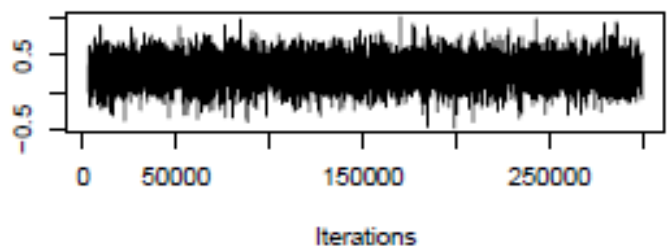

Trace of time:live

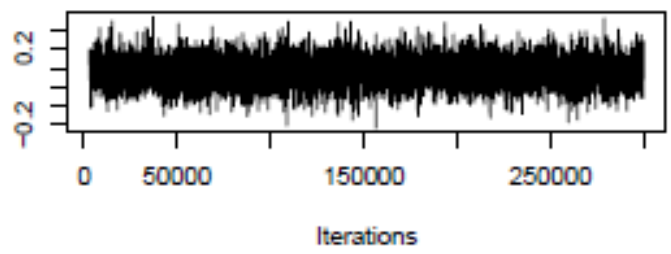

Density of think

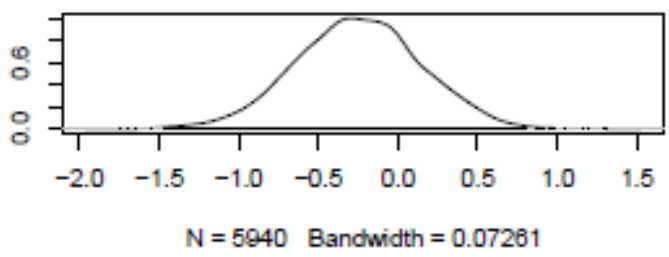

Density of attitude

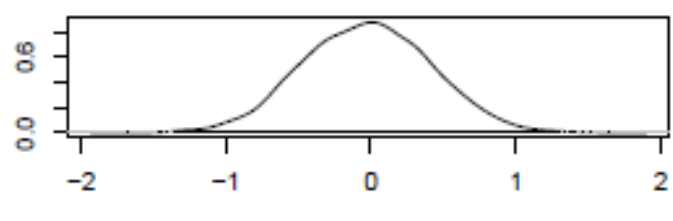

$\mathrm{N}=5940$ Bandwidth $=0.08296$

Density of change

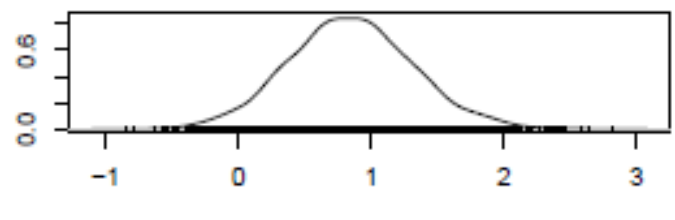

$\mathrm{N}=5940$ Bandwidth $=0.08559$

Density of I_Seriousness2:time

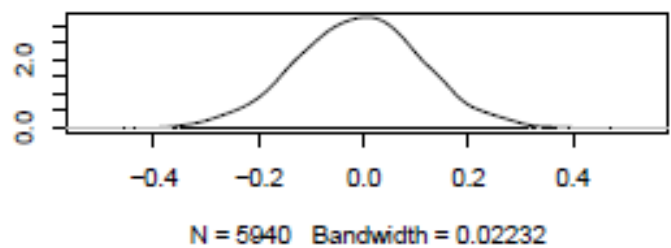

Density of I_Seriousness2:live

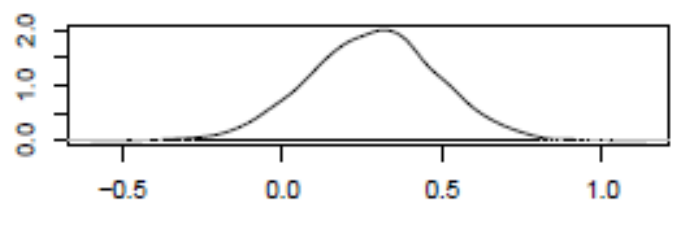

$\mathrm{N}=5940$ Bandwidth $=0.03685$

Density of time:live

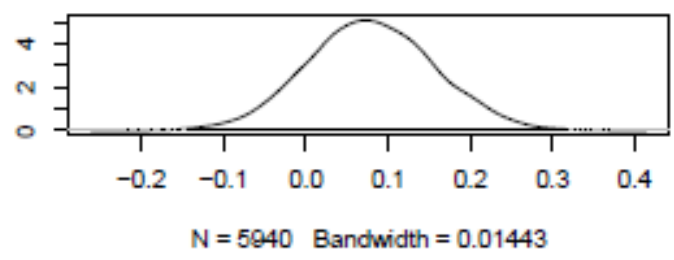


Trace of I_Seriousness2:relation

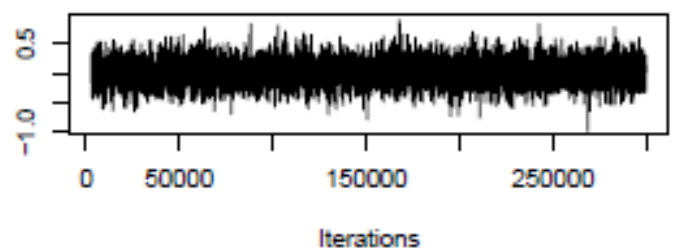

Trace of time:relation

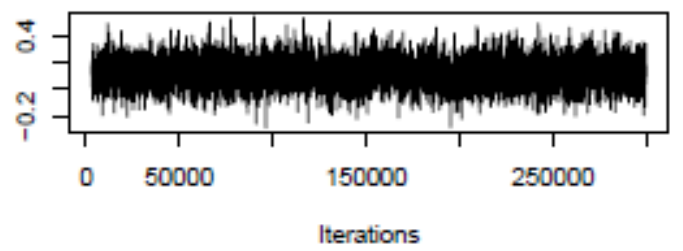

Trace of I_Seriousness2:ete

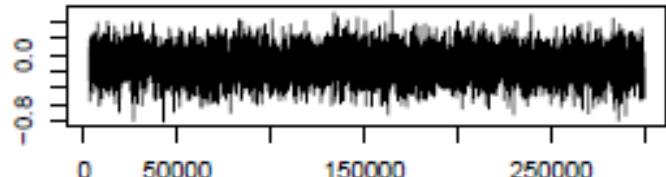

Iterations

Trace of time:ete

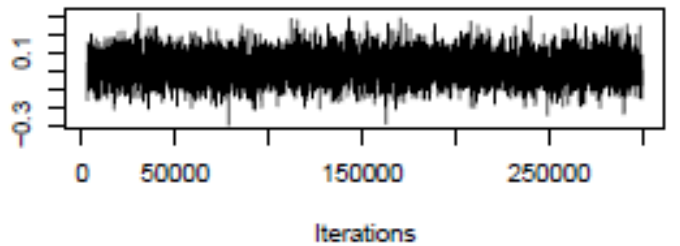

Trace of I_Seriousness2:where

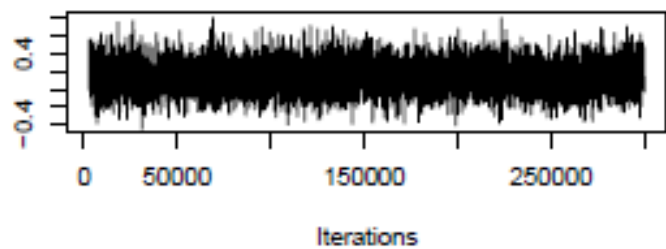

Trace of time:where

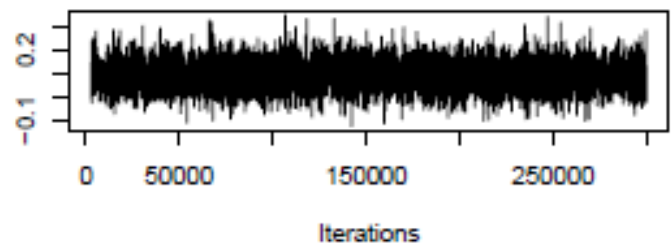

Density of I_Seriousness2:relation

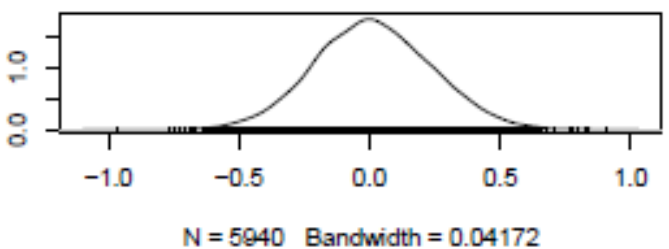

Density of time:relation

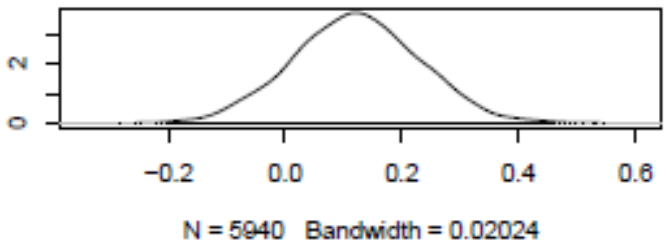

Density of I_Seriousness2:ete

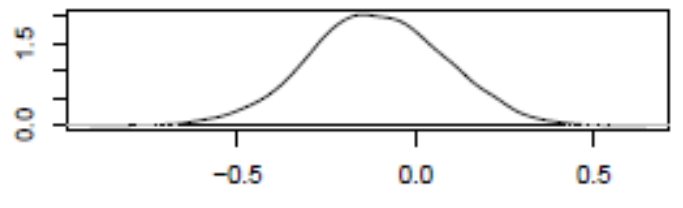

$\mathrm{N}=5940$ Bandwidth $=0.03549$

Density of time:ete

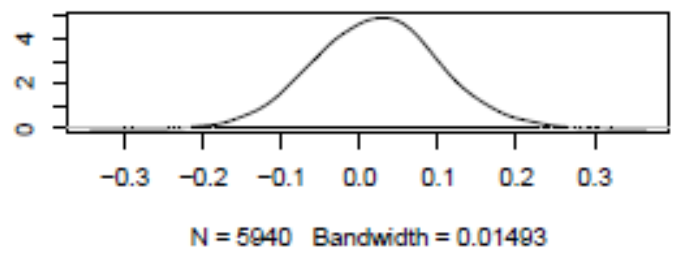

Density of I_Seriousness2:where

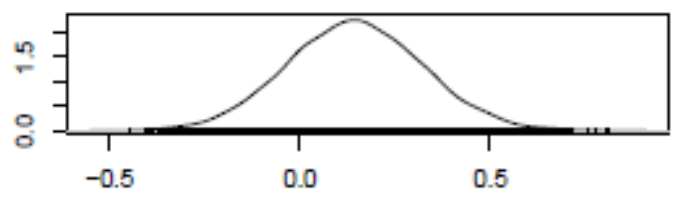

$\mathrm{N}=5940$ Bandwidth $=0.03324$

Density of time:where

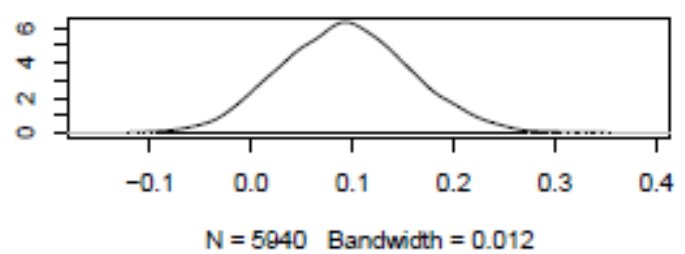


Trace of I_Seriousness2:life

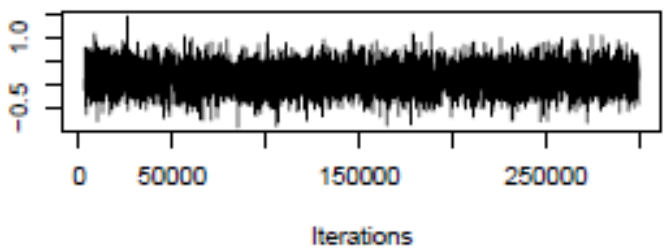

Trace of time:life

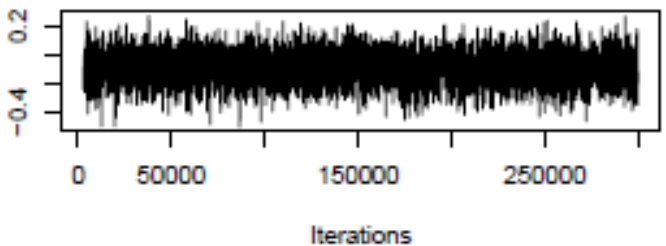

Trace of I_Seriousness2:drugs

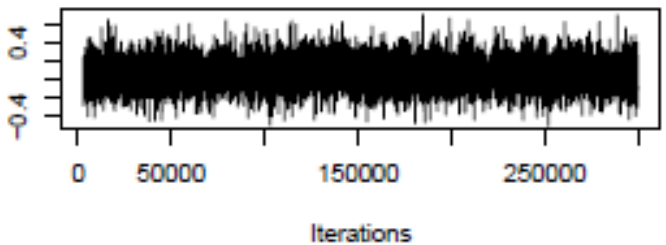

Trace of time:drugs

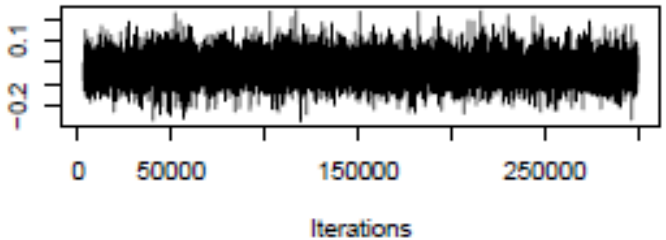

Trace of I_Seriousness2:physical

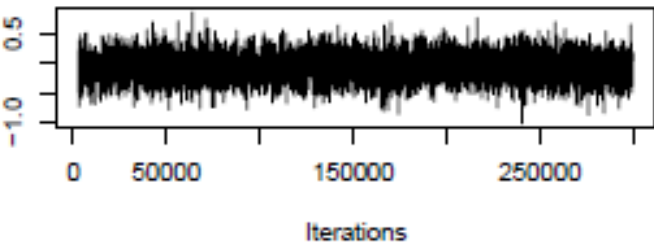

Trace of time:physical

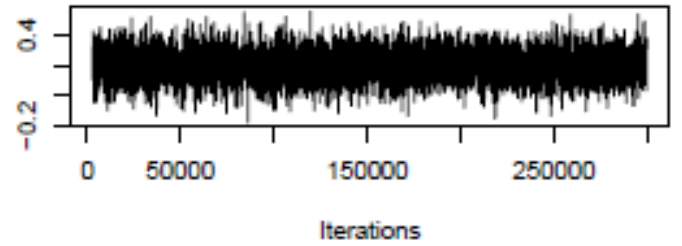

Density of I_Seriousness2:life

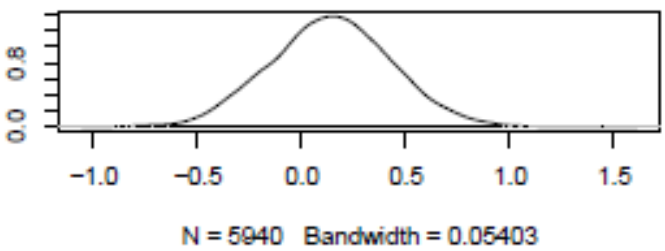

Density of time:life

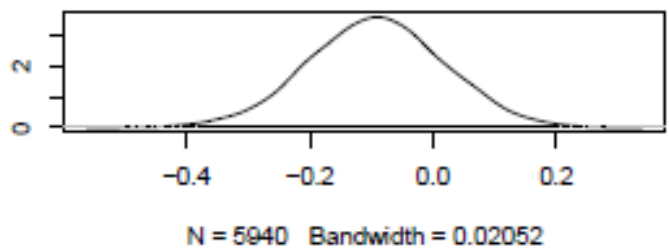

Density of I_Seriousness2:drugs

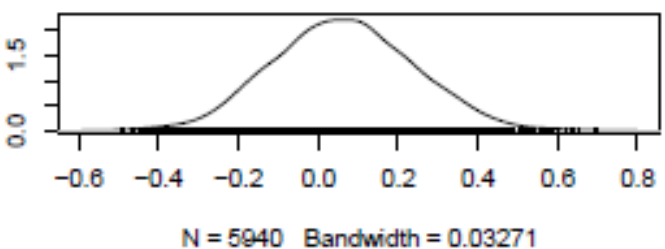

Density of time:drugs

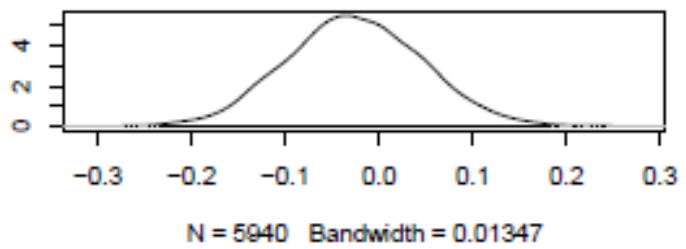

Density of I_Seriousness2:physical

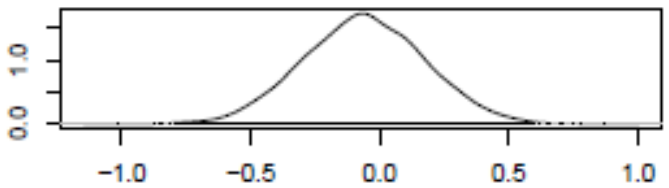

$\mathrm{N}=5940$ Bandwidth $=0.04387$

Density of time:physical

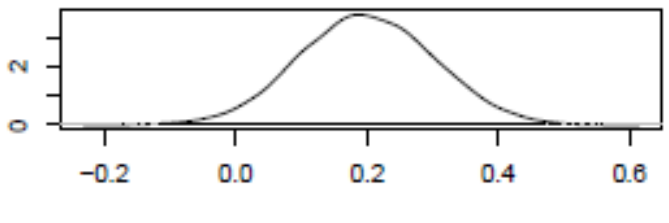

$\mathrm{N}=5940$ Bandwidth $=0.01917$ 
Trace of I_Seriousness2:emotion

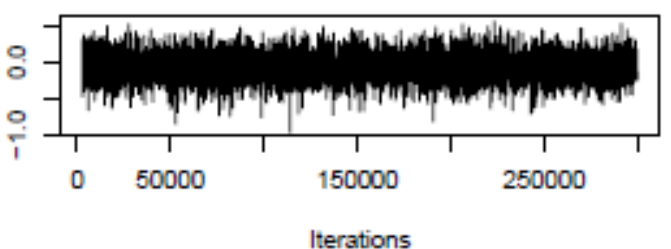

Trace of time:emotion

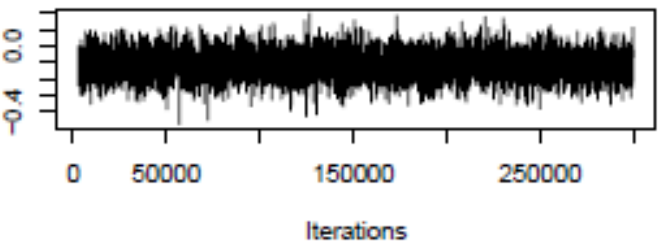

Trace of I_Seriousness2:self

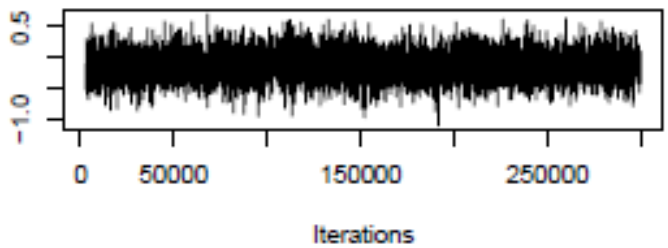

Trace of time:self

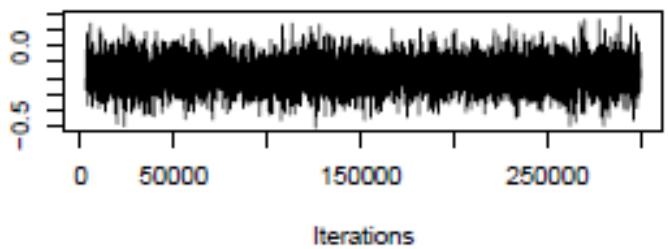

Trace of I_Seriousness2:think

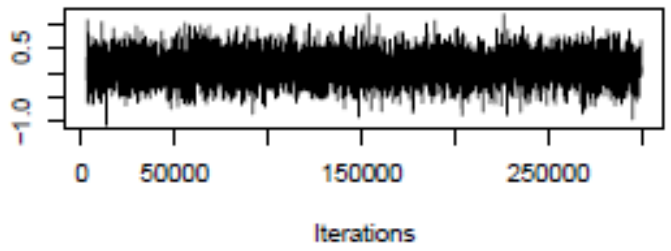

Trace of time:think

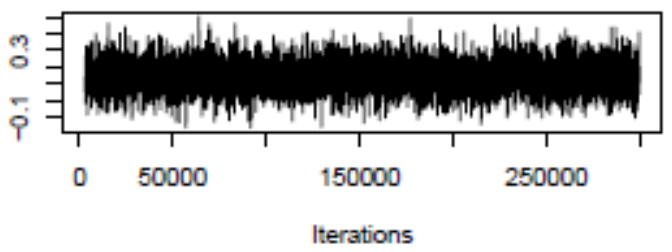

Density of I_Seriousness2:emotion

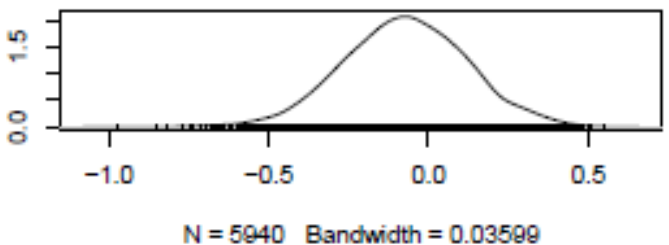

Density of time:emotion

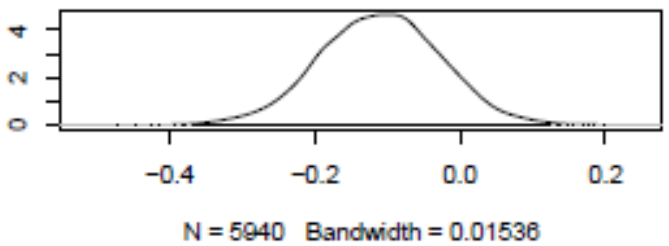

Density of I_Seriousness2:self

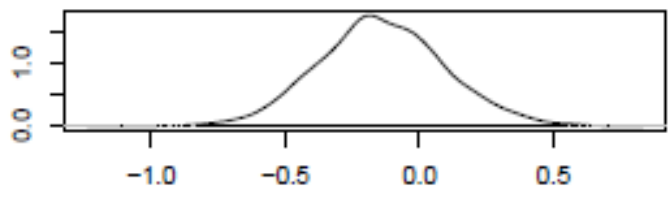

$\mathrm{N}=5940$ Bandwidth $=0.04311$

Density of time:self

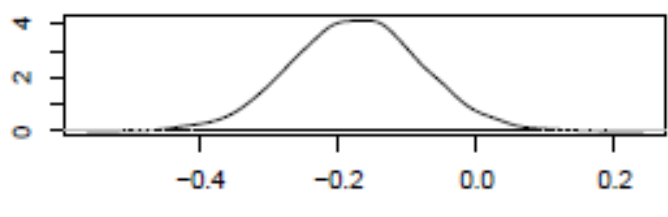

$\mathrm{N}=5940$ Bandwidth $=0.0175$

Density of I_Seriousness2:think

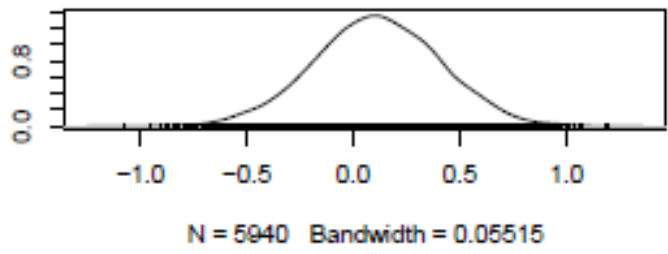

Density of time:think

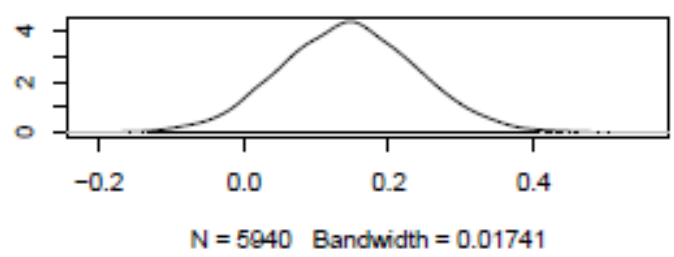


Trace of I_Seriousness2:attitude

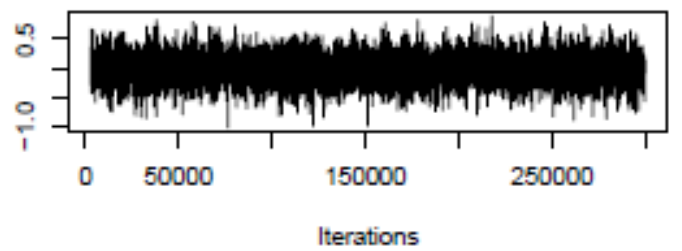

Trace of time:attitude

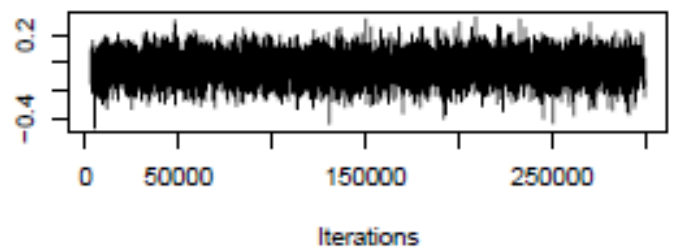

Trace of I_Seriousness2:change

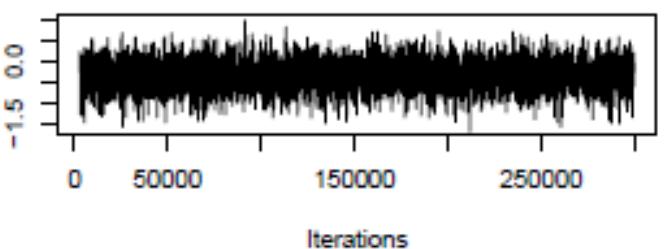

Trace of time:change

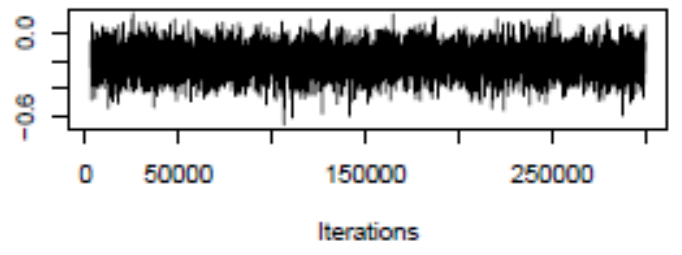

Trace of I_Seriousness2:time:live

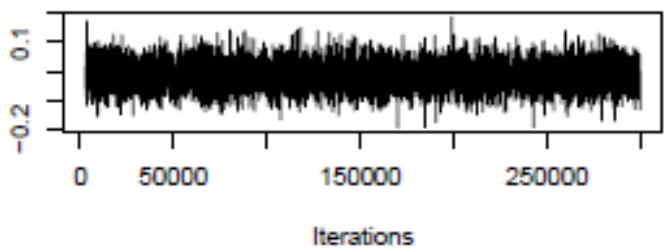

Trace of I_Seriousness2:time:relation

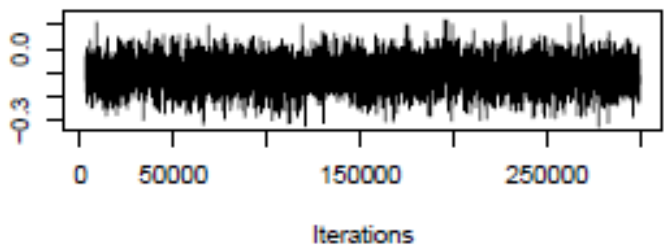

Density of I_Seriousness2:attitude

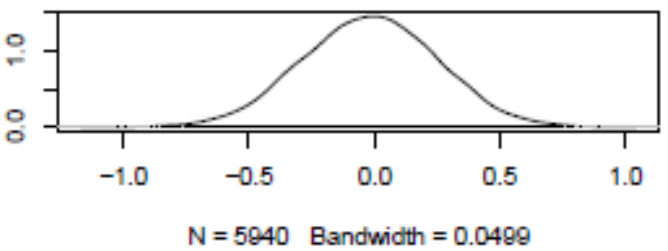

Density of time:attitude

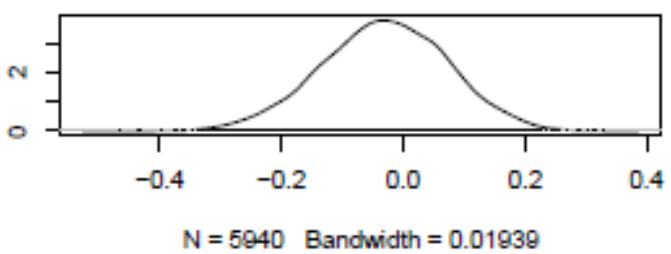

Density of I_Seriousness2:change

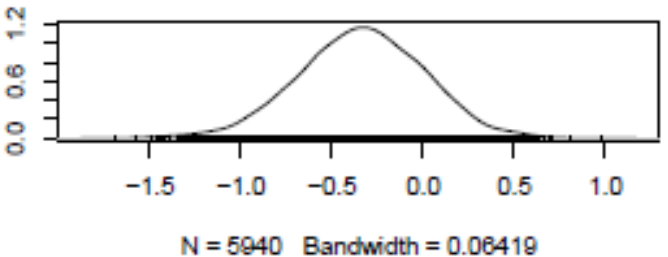

Density of time:change

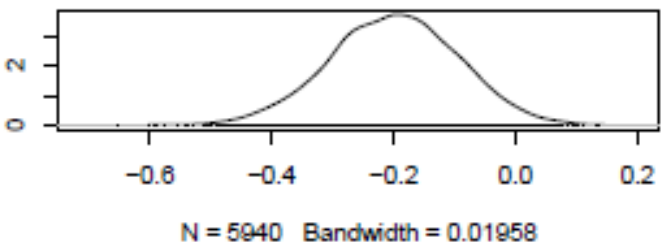

Density of I_Seriousness2:time:live

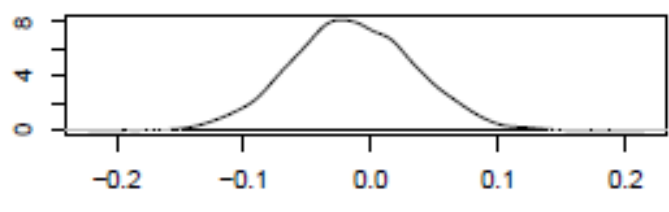

$\mathrm{N}=5940$ Bandwidth $=0.008976$

Density of I_Seriousness2:time:relation

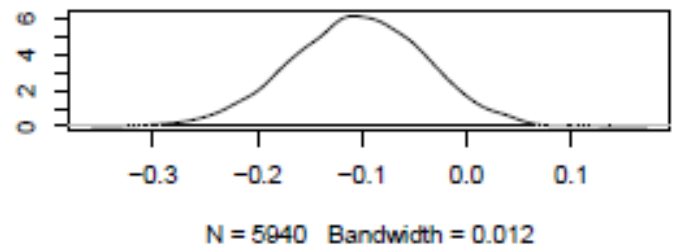


Trace of I_Seriousness2:time:ete

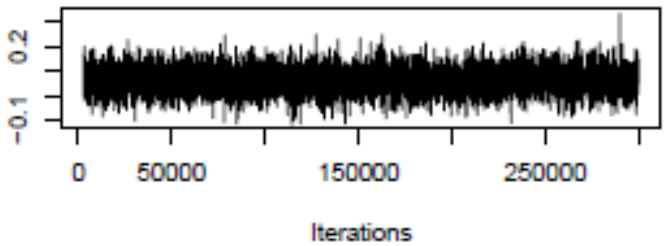

Trace of I_Seriousness2:time:where

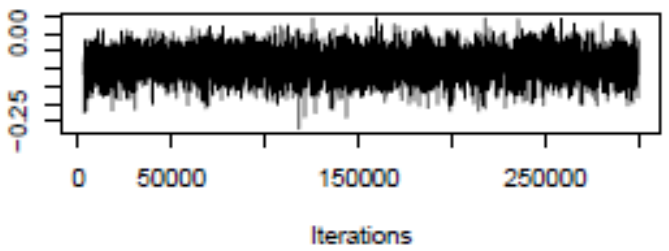

Trace of I_Seriousness2:time:life

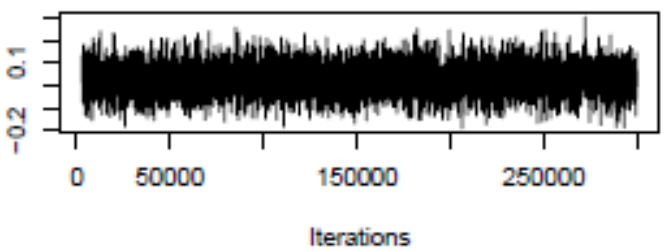

Trace of I_Seriousness2:time:drugs

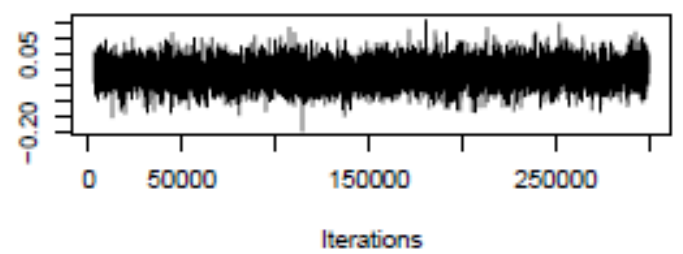

Trace of I_Seriousness2:time:physical

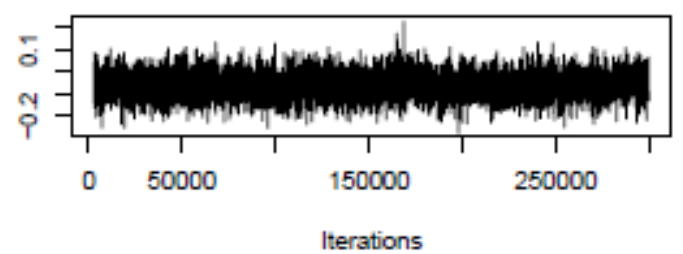

Trace of I_Seriousness2:time:emotion

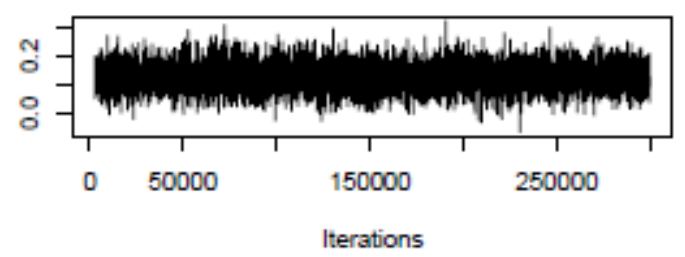

Density of I_Seriousness2:time:ete

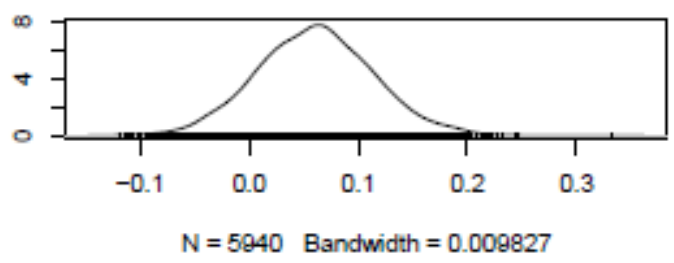

Density of I_Seriousness2:time:where

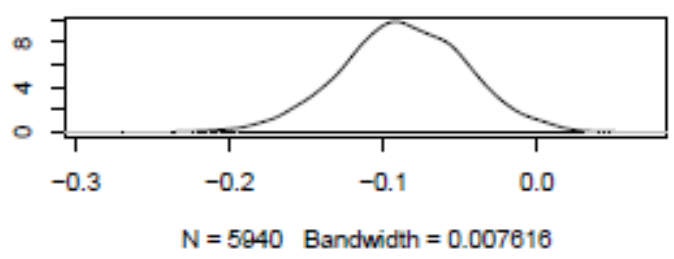

Density of I_Seriousness2:time:life

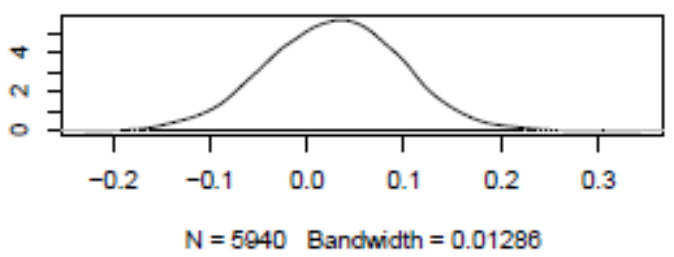

Density of I_Seriousness2:time:drugs

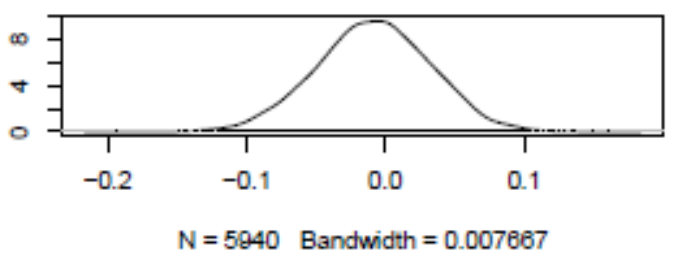

Density of I_Seriousness2:time:physical

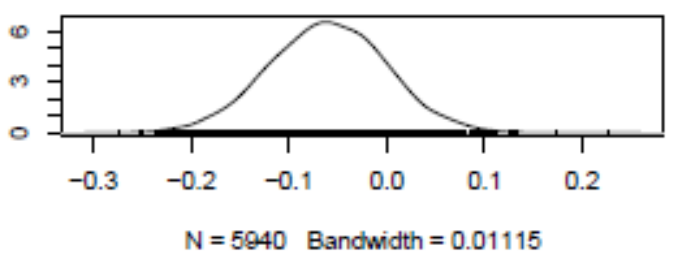

Density of I_Seriousness2:time:emotion

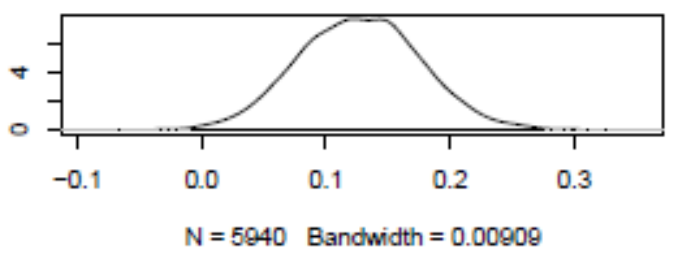


Trace of I_Seriousness2:time:self

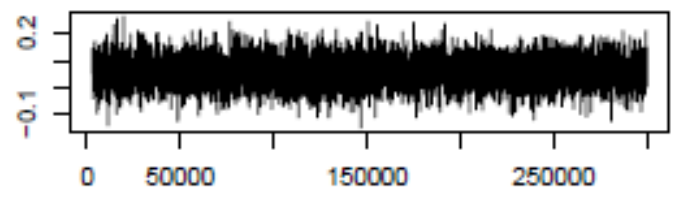

Iterations

Trace of I_Seriousness2:time:think

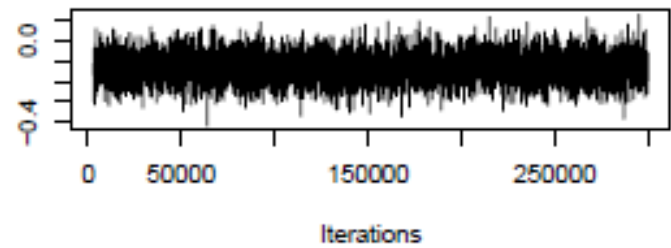

Trace of I_Seriousness2:time:attitude

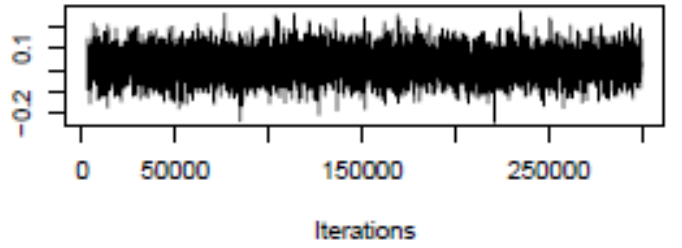

Trace of I_Seriousness2:time:change

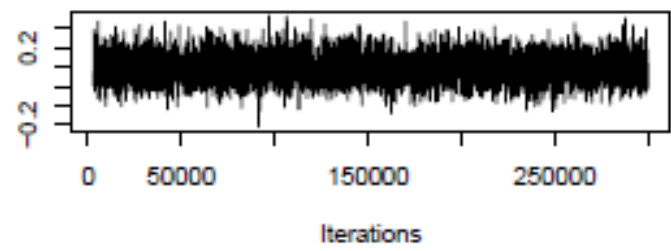

$\underline{\text { Random Effects }}$

Trace of time

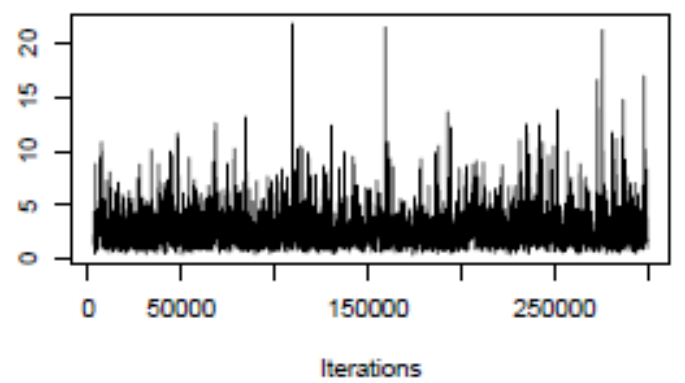

Density of I_Seriousness2:time:self

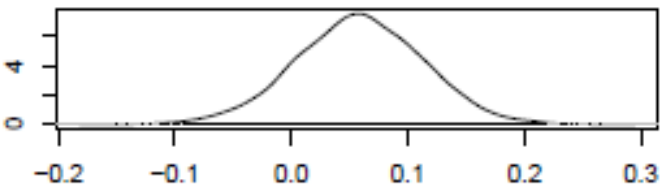

$\mathrm{N}=5940$ Bandwidth $=0.01015$

Density of I_Seriousness2:time:think

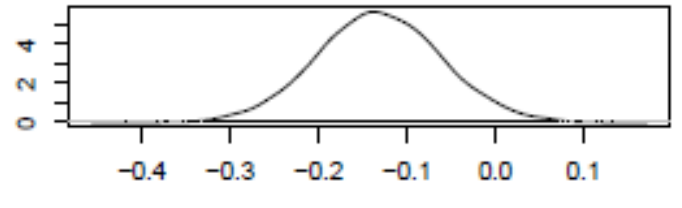

$\mathrm{N}=5940$ Bandwidth $=0.01313$

Density of I_Seriousness2:time:attitude

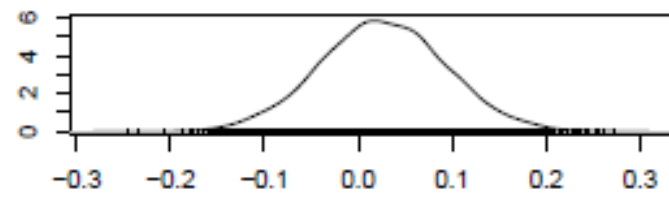

$\mathrm{N}=5940$ Bandwidth $=0.01245$

Density of I_Seriousness2:time:change

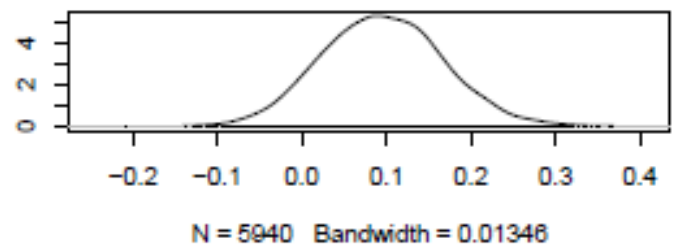

Density of time

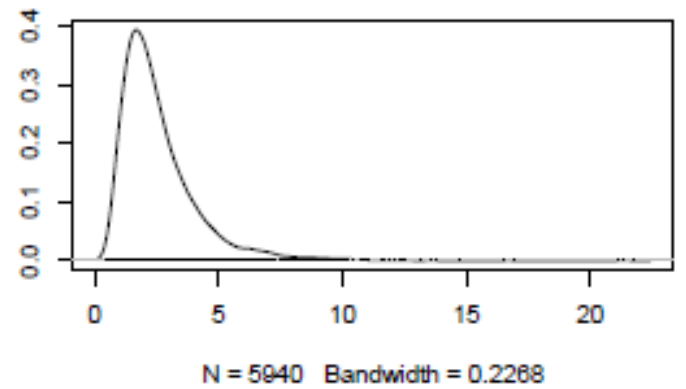


Trace of Research.ID

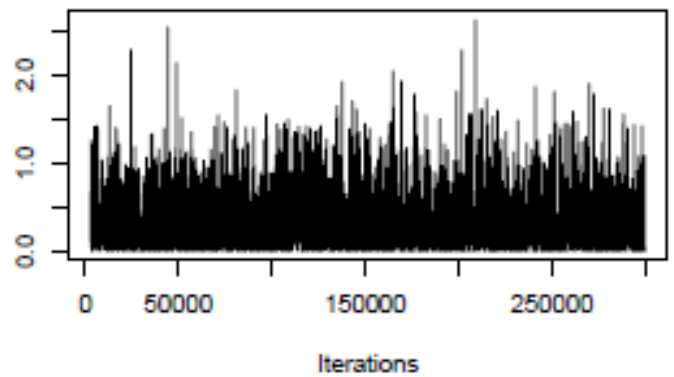

Density of Research.ID

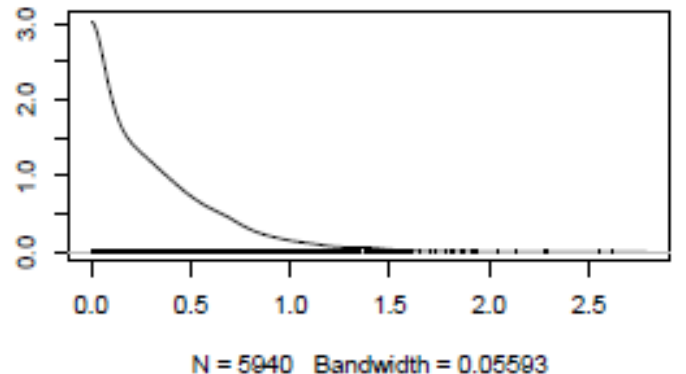


The Combined Model involving Offending History: Version 1

Bayesian Model (BDm3G_cc1202a)

\section{\#\# Define the Model}

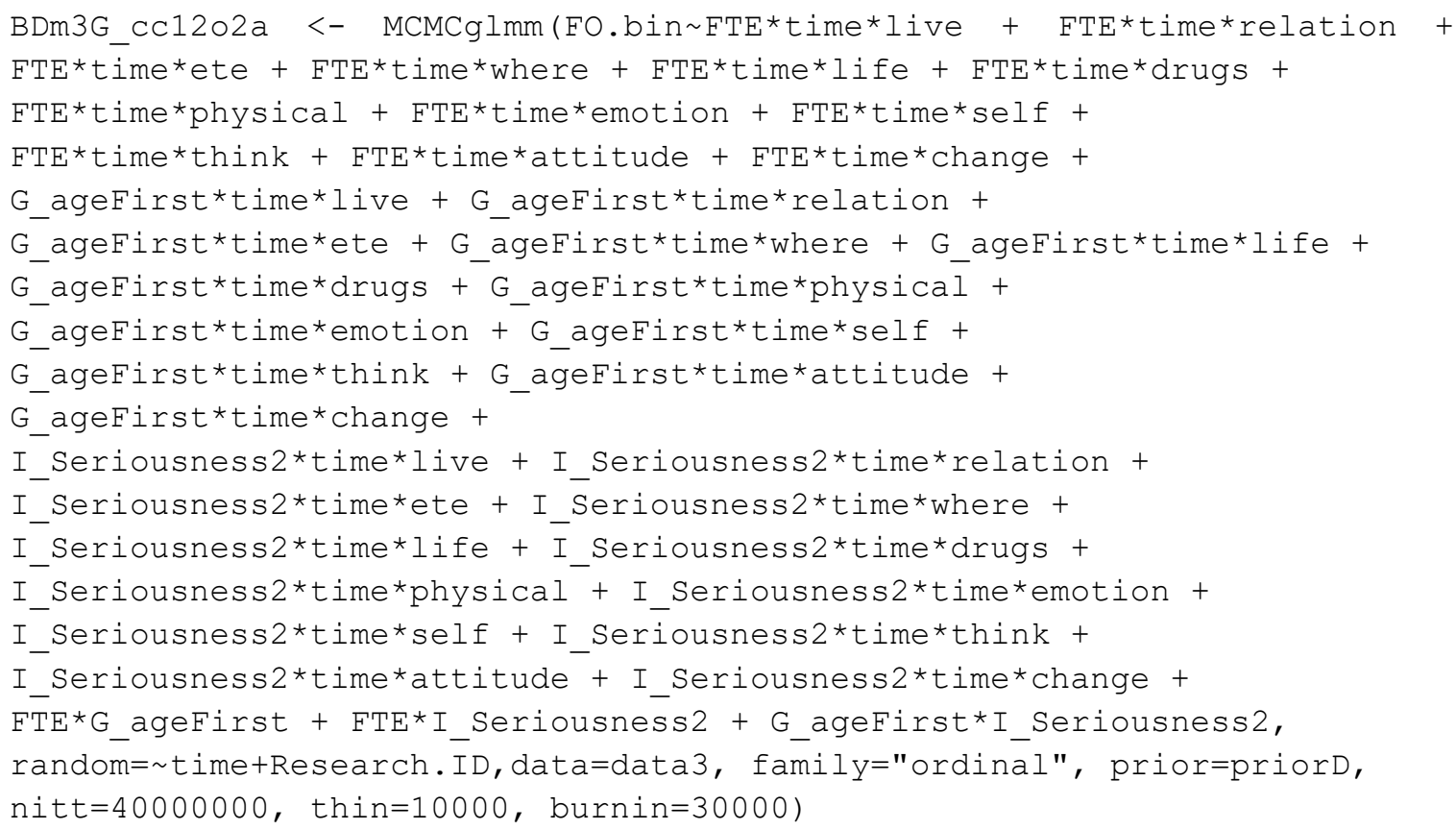

\section{\# Checks for suitable convergence}

raftery.diag (BDm3G_cC1202a\$VCV)

heidel.diag (BDm3G_CCC1202a\$VCV)

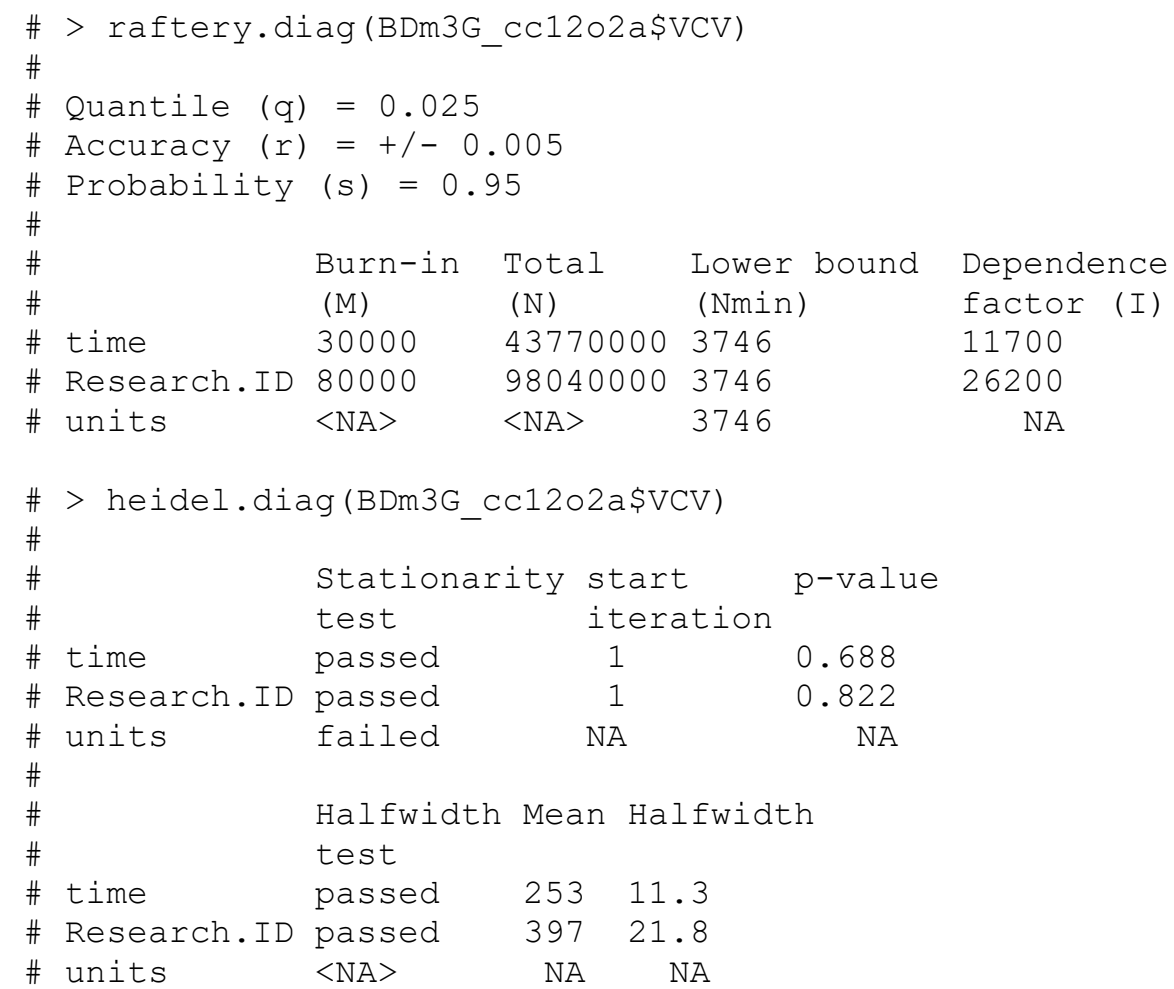




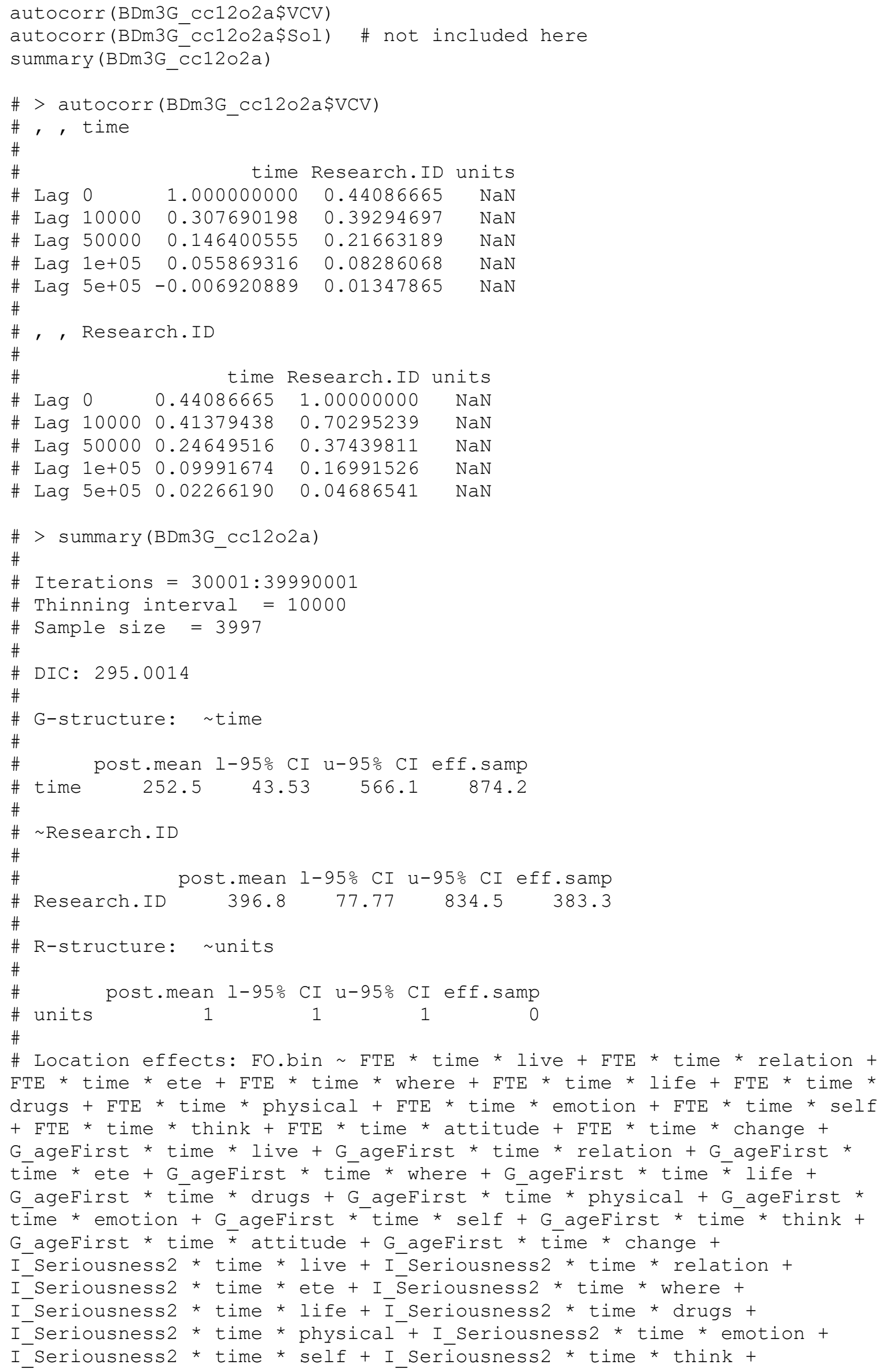


I_Seriousness2 * time * attitude + I_Seriousness 2 * time * change + FTE *G_ageFirst + FTE * I_Seriousness2 + G_ageFirst * I_Seriousness 2 $\#$

$\#$

\# (Intercept)

\# FTE

\# time

\# live

\# relation

\# ete

\# where

\# life

\# drugs

\# physical

\# emotion

\# self

\# think

\# attitude

\# change

\# G ageFirst13 to 17 years

\# I Seriousness2

\# FTE:time

\# FTE:live

\# time:live

\# FTE:relation

\# time:relation

\# FTE:ete

\# time:ete

\# FTE: where

\# time:where

\# FTE:life

\# time:life

\# FTE:drugs

\# time:drugs

\# FTE:physical

\# time:physical

\# FTE:emotion

\# time:emotion

\# FTE:self

\# time:self

\# FTE: think

\# time:think

\# FTE:attitude

\# time:attitude

\# FTE: change

\# time:change

\# time:G ageFirst13 to 17 years

\# live:G ageFirst13 to 17 years

\# relation:G_ageFirst13 to 17 years

\# ete:G_ageFirst13 to 17 years

\# where:G ageFirst13 to 17 years

\# life:G àgeFirst13 to 17 years

\# drugs: $\bar{G}$ ageFirst13 to 17 years

\# physical:G ageFirst13 to 17 years

\# emotion:G àgeFirst13 to 17 years

\# self:G agēFirst13 to 17 years

\# think: $\overline{\mathrm{G}}$ ageFirst13 to 17 years

\# attitude $: \mathrm{G}$ ageFirst13 to 17 years

\# change:G_ageFirst13 to 17 years

\# time: I sēeriousness2

\# live:I- Seriousness2

\# relation:I Seriousness2

\# ete:I_Seriousness2

\# where: I_Seriousness2

\# life:I Seriousness2

\# drugs: $\bar{I}$ Seriousness2

\# physical:I_Seriousness2

\# emotion:I Seriousness2

\# self:I Seriousness2

\# think: I Seriousness 2

\# attitude:I_Seriousness2

\# change:I Seriousness2

\# FTE:G_agēFirst13 to 17 years

\# FTE:I-Seriousness2

\# G age $\bar{F}$ irst13 to 17 years:I Seriousness2

\# FT̄E:time:live

\# FTE:time:relation

\# FTE:time:ete

\# FTE:time:where

\# FTE:time:life

\# FTE:time:drugs

\begin{tabular}{|c|c|c|c|c|c|}
\hline post.mean & $1-95 \% \mathrm{CI}$ & $u-95 \% \mathrm{CI}$ & eff.samp & $\mathrm{pMCMC}$ & \\
\hline-8.44750 & -30.73758 & 13.02716 & 3997.0 & 0.43132 & \\
\hline 16.17202 & -25.81242 & 61.55305 & 1247.0 & 0.48186 & \\
\hline 1.30756 & -1.33139 & 4.07595 & 3678.9 & 0.32324 & \\
\hline 0.50805 & -5.02432 & 5.52199 & 2979.1 & 0.85814 & \\
\hline 10.33569 & 4.10967 & 16.95180 & 2947.1 & 0.00100 & $\star \star$ \\
\hline-5.40679 & -9.29989 & -1.79054 & 3085.3 & 0.00450 & ** \\
\hline 6.73509 & 2.64381 & 10.48875 & 2892.3 & $<3 e-04$ & $\star \star \star$ \\
\hline-6.99846 & -15.23854 & 0.85251 & 1508.1 & 0.07055 & . \\
\hline-9.19871 & -14.32258 & -3.59913 & 1156.3 & $<3 e-04$ & $\star \star \star *$ \\
\hline 2.19807 & -2.59098 & 7.18762 & 2113.7 & 0.37428 & \\
\hline 1.54600 & -2.45487 & 5.47318 & 3599.5 & 0.44333 & \\
\hline-27.90316 & -39.21135 & -16.39462 & 709.9 & $<3 e-04$ & $\star \star \star \star$ \\
\hline 10.40967 & 4.12983 & 16.99035 & 1513.0 & $<3 e-04$ & $\star \star \star$ \\
\hline 7.46415 & -1.84720 & 16.94411 & 3997.0 & 0.11559 & \\
\hline 17.31457 & 6.84207 & 27.38253 & 1303.0 & $<3 e-04$ & $\star \star \star *$ \\
\hline 14.17181 & -12.13032 & 38.43889 & 3145.3 & 0.24869 & \\
\hline-31.54723 & -50.26978 & -13.83019 & 541.3 & $<3 e-04$ & $\star \star \star$ \\
\hline-11.25257 & -19.20095 & -4.64871 & 536.6 & $<3 e-04$ & $\star \star \star$ \\
\hline-6.17638 & -16.42788 & 4.14934 & 2163.7 & 0.23017 & \\
\hline-0.87303 & -1.94967 & 0.10321 & 1845.5 & 0.07656 & . \\
\hline-12.47750 & -27.35222 & -0.25586 & 619.8 & 0.03703 & * \\
\hline-2.73476 & -4.48587 & -1.13791 & 1479.6 & $<3 e-04$ & $\star \star \star \star$ \\
\hline-1.19977 & -10.03975 & 7.49297 & 3771.4 & 0.77758 & \\
\hline 0.99208 & -0.01728 & 2.05502 & 3192.6 & 0.05054 & . \\
\hline-24.25489 & -38.27035 & -10.52519 & 527.7 & $<3 e-04$ & $* \star \star$ \\
\hline-1.17454 & -1.93655 & -0.38984 & 2249.4 & 0.00050 & $\star \star \star *$ \\
\hline 22.28468 & 8.04872 & 35.72338 & 843.7 & $<3 e-04$ & $\star \star \star \star$ \\
\hline 0.87700 & -0.64423 & 2.46893 & 2284.6 & 0.24869 & \\
\hline-3.07929 & -11.98473 & 6.46785 & 3593.8 & 0.50238 & \\
\hline 2.16706 & 0.98851 & 3.32302 & 1019.8 & $<3 e-04$ & 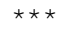 \\
\hline 4.75004 & -7.03414 & 16.32528 & 1743.2 & 0.41981 & \\
\hline-0.24577 & -1.19010 & 0.67903 & 3403.4 & 0.62497 & \\
\hline-5.54420 & -14.07013 & 2.41049 & 1383.3 & 0.16612 & \\
\hline-1.58783 & -2.91597 & -0.31037 & 714.4 & 0.00550 & $\star \star$ \\
\hline 0.42014 & -8.75807 & 10.31026 & 2849.7 & 0.91268 & \\
\hline 6.45773 & 3.99228 & 9.08888 & 531.3 & $<3 e-04$ & 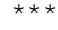 \\
\hline-5.94536 & -14.98144 & 3.01889 & 3335.1 & 0.19965 & \\
\hline-0.50773 & -1.55967 & 0.55633 & 4404.7 & 0.35427 & \\
\hline-8.42247 & -17.17194 & -0.31079 & 3061.7 & 0.03953 & * \\
\hline-2.04614 & -3.62134 & -0.49384 & 1836.5 & 0.00550 & ** \\
\hline 13.01145 & 1.16935 & 24.50985 & 3373.7 & 0.02152 & $\star$ \\
\hline-2.57358 & -4.38725 & -0.89461 & 1775.2 & 0.00050 & $\star \star \star$ \\
\hline-3.44781 & -6.71331 & -0 & 2878.9 & 0.04203 & * \\
\hline 10.97390 & 2.26640 & 20.20982 & 887.0 & 0.00450 & ** \\
\hline-18.28276 & -28.01374 & -8.57907 & 1642.7 & 0.00050 & $\star \star \star *$ \\
\hline 2.56777 & -4.43618 & 8.72230 & 3680.0 & 0.42732 & \\
\hline 0.04583 & -7.65008 & 7.06614 & 3367.4 & 0.99074 & \\
\hline 4.49353 & -6.29788 & 14.79465 & 2718.7 & 0.41831 & \\
\hline-7.55413 & -15.79449 & 0.58356 & 810.7 & 0.04253 & * \\
\hline-10.57770 & -20.71514 & -1.72729 & 1038.1 & 0.01001 & * \\
\hline-2.56672 & -8.14127 & 3.03106 & 3997.0 & 0.36227 & \\
\hline 32.39376 & 18.72654 & 45.74511 & 840.7 & $<3 e-04$ & $\star \star \star$ \\
\hline-0.67342 & -8.31531 & 7.56652 & 3498.5 & 0.84213 & \\
\hline-2.23790 & -13.87357 & 8.24089 & 3997.0 & 0.68752 & \\
\hline-14.42706 & -25.66251 & -2.82619 & 3165.4 & 0.01451 & * \\
\hline 3.98261 & 1.92625 & 6.15862 & 629.1 & $<3 e-04$ & 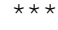 \\
\hline-2.99281 & -6.48954 & 0.17488 & 733.0 & 0.04754 & * \\
\hline 9.94485 & 3.83403 & 17.21775 & 419.6 & $<3 e-04$ & $\star \star \star \star$ \\
\hline 0.69141 & -1.86077 & 3.52406 & 1619.0 & 0.58794 & \\
\hline 1.30345 & -1.14932 & 3.90015 & 1570.2 & 0.30923 & \\
\hline-0.37537 & -5.32372 & 4.06987 & 1898.0 & 0.90668 & \\
\hline 10.81189 & 5.71745 & 16.60012 & 475.0 & $<3 e-04$ & $\star \star \star *$ \\
\hline-2.65002 & -6.95829 & 0.78216 & 2454.7 & 0.16562 & \\
\hline 0.04520 & -2.10683 & 2.10756 & 3997.0 & 0.97823 & \\
\hline 5.84416 & 1.52108 & 10.02360 & 931.7 & 0.00200 & $\star \star$ \\
\hline-2.82096 & -6.49552 & 0.60500 & 2574.1 & 0.11008 & \\
\hline-0.85787 & -4.30518 & 2.81751 & 3997.0 & 0.61046 & \\
\hline-7.30257 & -12.58698 & -2.67145 & 1312.6 & 0.00050 & $\star \star \star$ \\
\hline 14.18845 & -24.19119 & 53.26583 & 3488.9 & 0.44633 & \\
\hline 14.27908 & 3.76326 & 26.13142 & 686.2 & 0.00100 & $\star \star$ \\
\hline-2.40635 & $-13 \cdot 34167$ & 6.97468 & 4065.9 & 0.63147 & \\
\hline-1.59230 & -3.86018 & 0.65088 & 1681.5 & 0.1496 & \\
\hline 2.34144 & -0.31363 & 5.02442 & 739.4 & 0.06705 & . \\
\hline 3.49084 & 0.82962 & 6.33672 & 1115.8 & 0.00300 & $\star \star$ \\
\hline 2.81407 & 0.84091 & 4.73530 & 898.4 & $<3 e-04$ & $\star \star \star *$ \\
\hline-3.47073 & -6.42030 & -0.59878 & 1131.9 & 0.01051 & * \\
\hline 2.04837 & 0.05852 & 4.05554 & 1548.8 & 0.03403 & * \\
\hline
\end{tabular}




\begin{tabular}{|c|c|c|c|c|}
\hline & FTE:time:physical & -2.95480 & -5.83783 & -0.12907 \\
\hline \# & FTE:time:emotion & 1.34895 & -0.55633 & 3.54408 \\
\hline \# & FTE:time:self & -2.35135 & -4.49134 & -0.46138 \\
\hline \# & FTE:time:think & 3.46294 & 1.39021 & 5.74296 \\
\hline \# & FTE:time:attitude & 3.25390 & 0.84810 & 5.63711 \\
\hline \# & FTE:time: change & -3.97620 & -6.72157 & -1.5965 \\
\hline \# & time:live:G ageFirst13 to 17 years & 0.34392 & -1.19373 & 1.911 \\
\hline \# & time:relation: G ageFirst13 to 17 years & 4.47329 & 2.22524 & 000 \\
\hline \# & time:ete: $\mathrm{G}$ ageFirst13 to 17 years & -1.60184 & -3.06051 & -0.1571 \\
\hline \# & time: where:G_ageFirst13 to 17 years & 1.21788 & -0.05278 & 201010 \\
\hline \# & time:life:G ageFirst13 to 17 years & -1.22858 & -3.56421 & 1.2 \\
\hline \# & time:drugs: $\overline{\mathrm{G}}$ ageFirst 13 to 17 years & 0.23279 & -1.20777 & 1.63 \\
\hline \# & time:physical:G ageFirst 13 to 17 years & 2.08098 & 0.12354 & 18 \\
\hline \# & time:emotion:G_āgeFirst13 to 17 years & 0.22451 & -1.46971 & 1 \\
\hline \# & time:self:G agēFirst13 to 17 years & -6.06324 & -8.64022 & -3 \\
\hline \# & time:think: $\overline{\mathrm{G}}$ ageFirst 13 to 17 years & -2.47983 & -4.77106 & -0 \\
\hline \# & time:attitude: $\mathrm{G}$ ageFirst13 to 17 years & 0.13003 & -2.06392 & 2 \\
\hline \# & time:change:G aḡeFirst13 to 17 years & 3.545 & 1.29420 & 5 \\
\hline \# & time:live:I seriousness 2 & 0.66666 & -0.07011 & 1 \\
\hline \# & time:relation:I_Seriousness 2 & -1.46424 & -2.53489 & -0.5 \\
\hline \# & time:ete: I Seriousness2 & -0.25 & -0.81699 & 0 \\
\hline \# & time: where: I seriousness2 & -0.76809 & -1.43169 & -0.08 \\
\hline \# & time:life: I Seriousness 2 & -0.11589 & -1.00059 & 0 \\
\hline \# & time:drugs: $\bar{I}$ Seriousness 2 & -1.72829 & -2.70404 & -0.842 \\
\hline \# & time:physical': I Seriousness2 & 1.69601 & 0.54017 & 2.956 \\
\hline \# & time:emotion:I Seriousness2 & 1.14757 & 0.45166 & 1.82 \\
\hline \# & time:self:I Seriousness2 & -1.50970 & -2.62397 & -0.55 \\
\hline \# & time:think: $\overline{\mathrm{I}}$ Seriousness 2 & 0.349 & -0.26626 & \\
\hline \# & time:attitude: I Seriousness2 & -0.338 & -1.224 & 0.5 \\
\hline \# & time: change: I serriousness 2 & 1.42789 & 0.48066 & 2.30 \\
\hline \# & --- & & & \\
\hline & 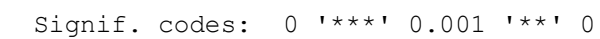 & $1 \star r^{\prime}$ & '. ' & \\
\hline
\end{tabular}

1084.50 .02402 *

$1146.6 \quad 0.17263$

2054.30 .01351 *

1477.10 .00200 **

$1506.00 .00300 *$

1525.80 .00050 ***

$3706.7 \quad 0.64548$

$1084.0<3 e-04 * * *$

1600.60 .01951 *

2555.60 .04704 *

$1548.4 \quad 0.31273$

$\begin{array}{lll}1521.6 & 0.77758\end{array}$

1670.90 .03152 *

3731.60 .78459

$787.3<3 e-04 * * *$

$739.10 .00650 \star \star$

$3997.0 \quad 0.90368$

$1062.3<3 e-04 * \star *$

834.80 .05954

$512.5<3 e-04$ ***

$3997.0 \quad 0.34926$

894.80 .00751 **

1999.20 .77208

$457.1<3 e-04 * * *$

$732.2<3 e-04 * \star *$

$777.6<3 e-04 * * *$

$730.3<3 e-04 * \star *$

$\begin{array}{lll}2485.3 & 0.26220\end{array}$

2179.50 .46385

2275.00 .00100 ** 
Trace Plots and Posterior Density Plots

Fixed Effects
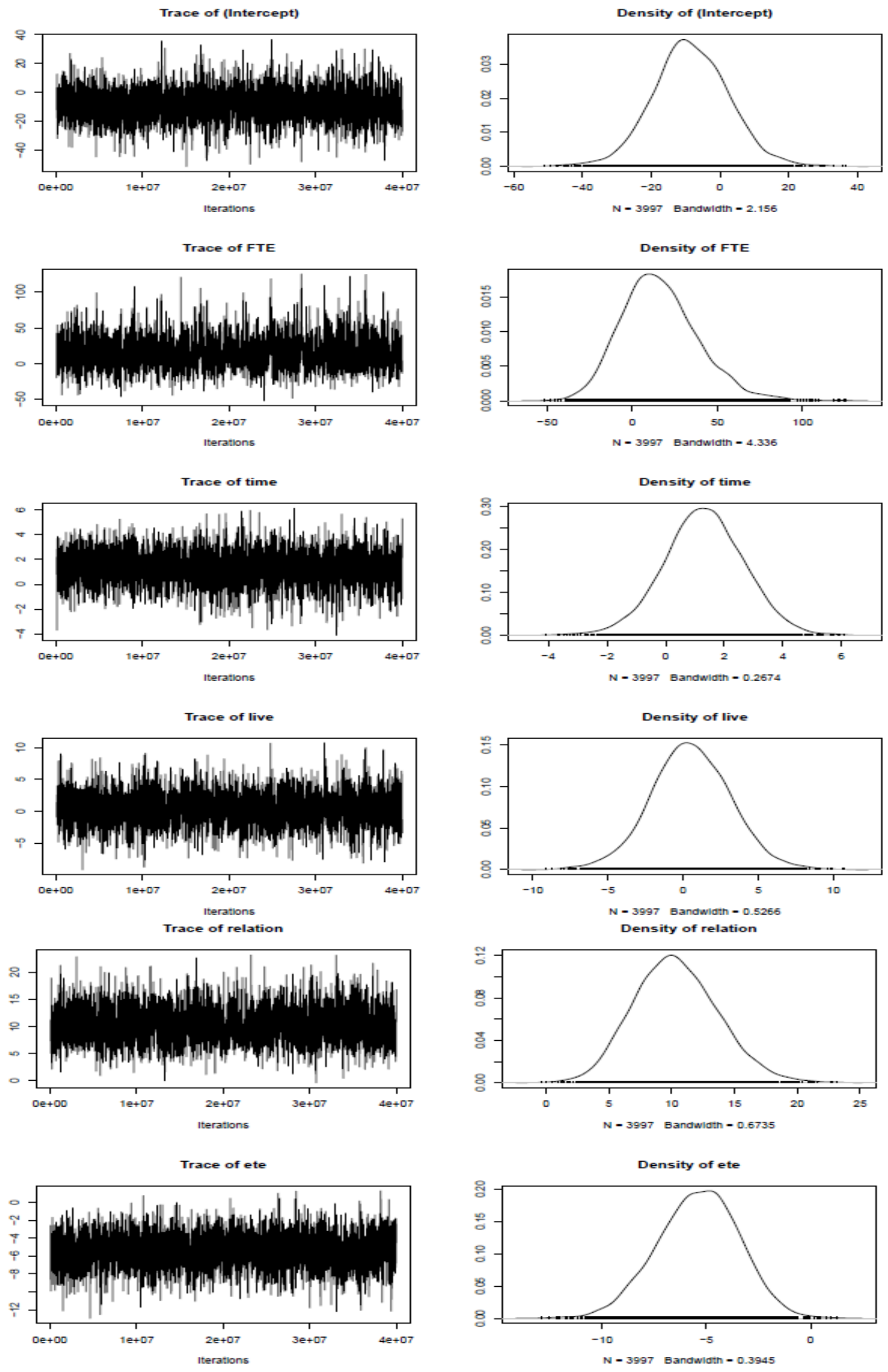

Page | 232 
Trace of where

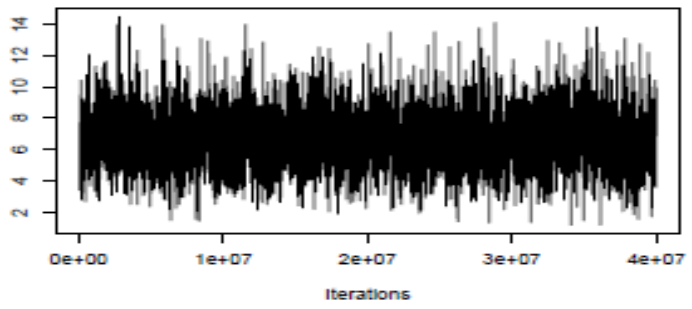

race of life
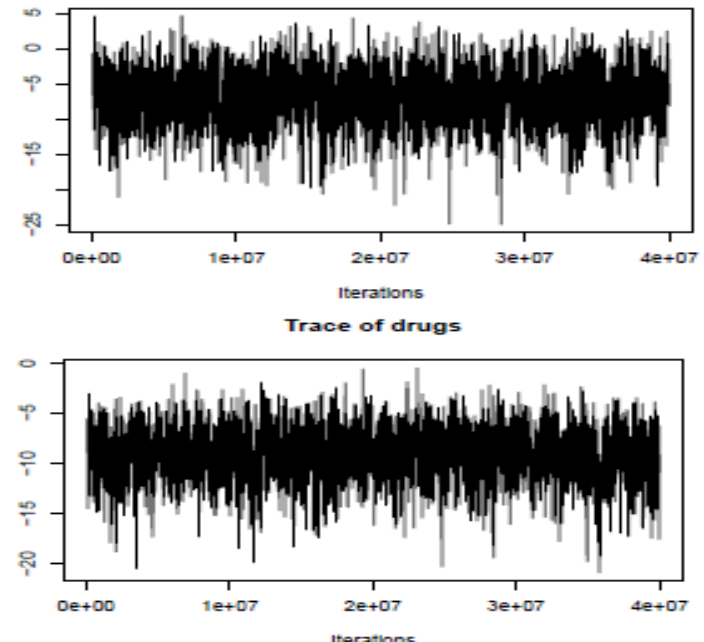

Trace of physical

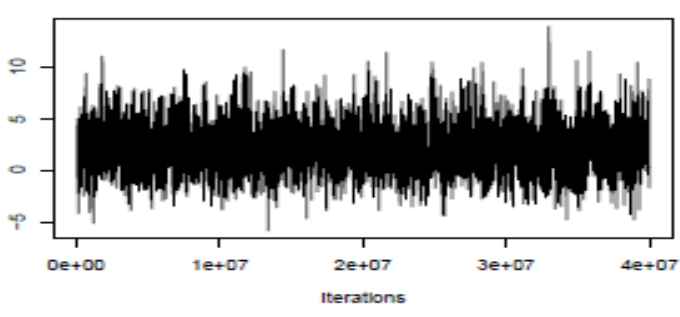

Trace of emotion

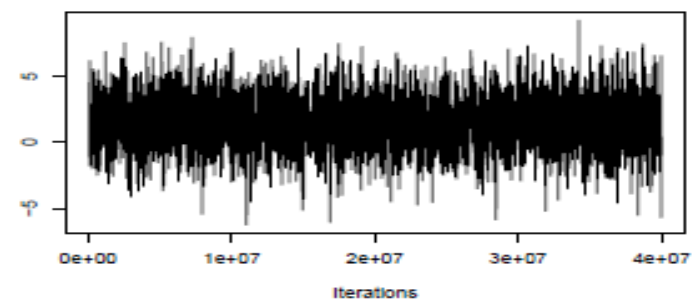

Trace of self

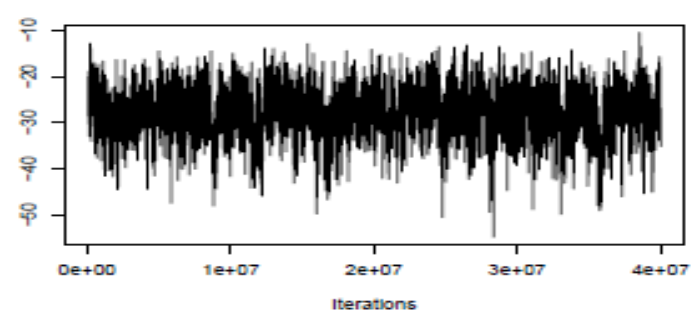

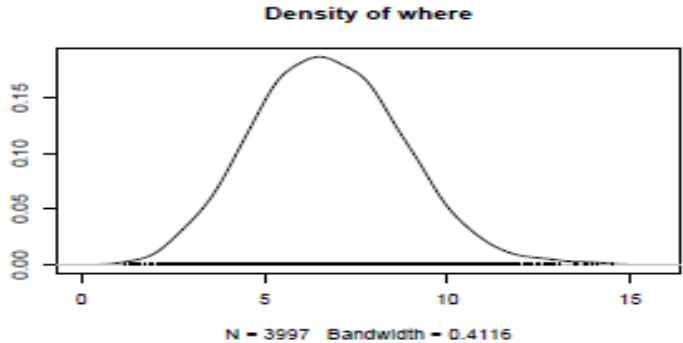

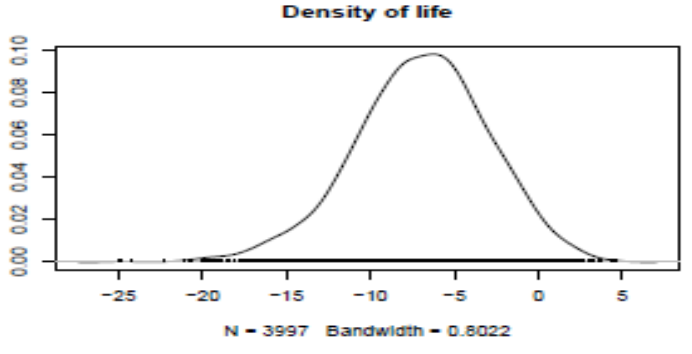

Density of drugs
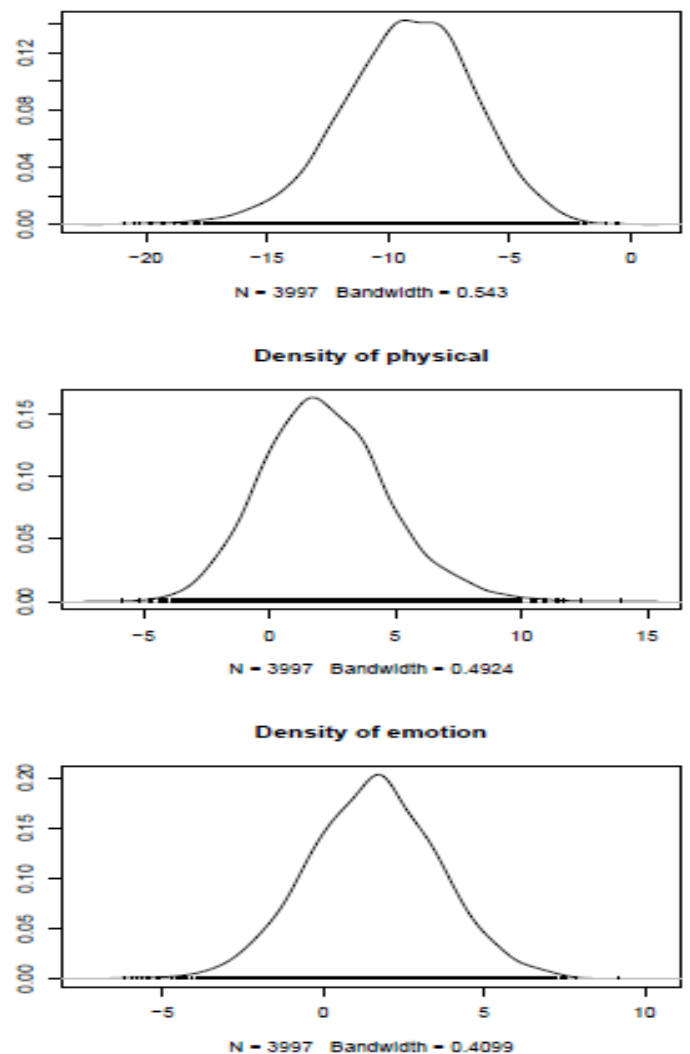

Density of self

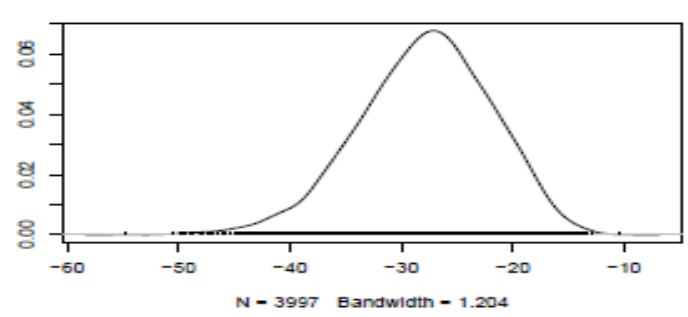



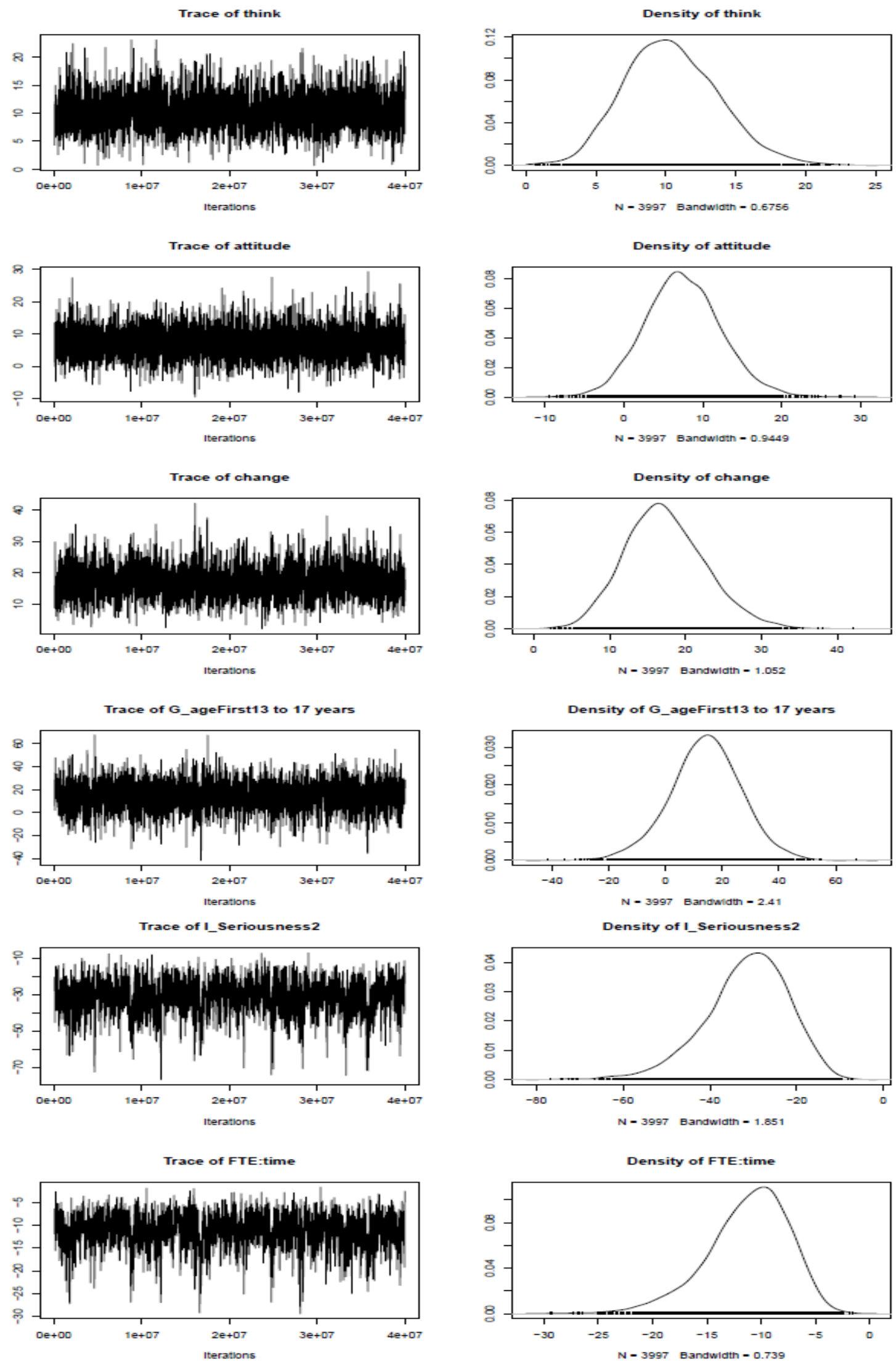
Trace of FTE:live

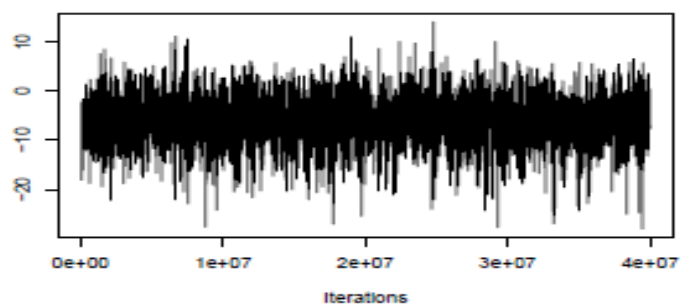

Trace of time:live

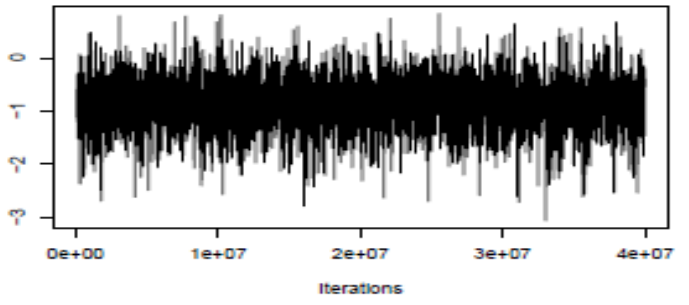

Trace of FTE:relation

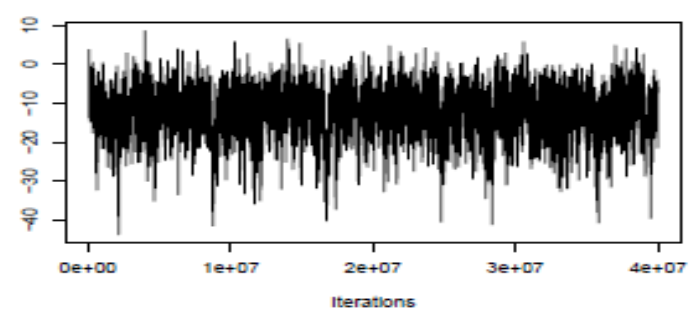

Trace of time:relation

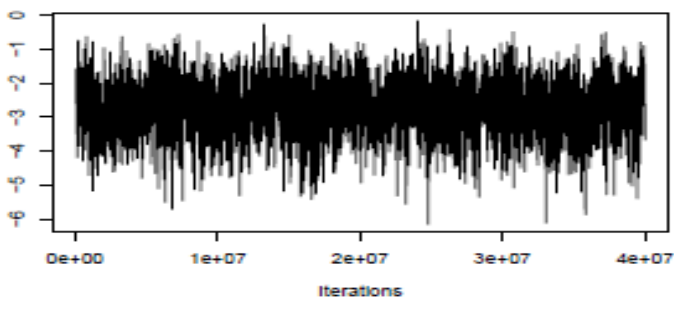

ace of FTE:ete

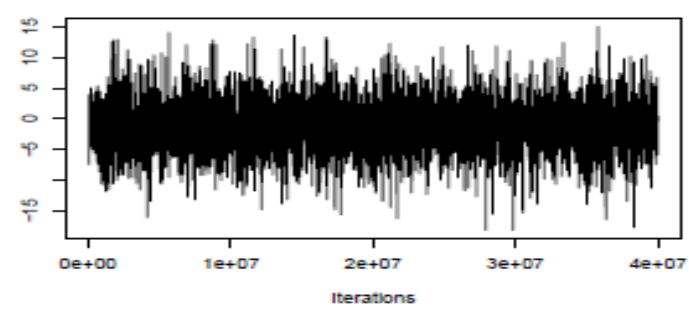

Trace of time:ete

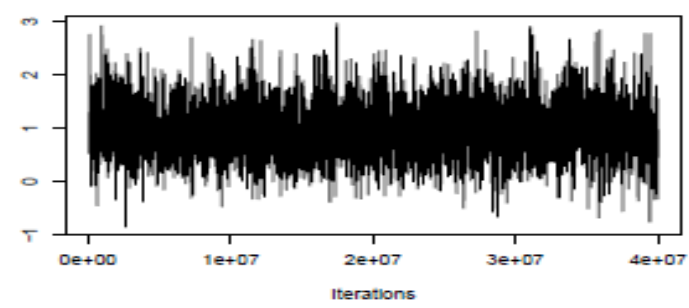

Density of FTE:live

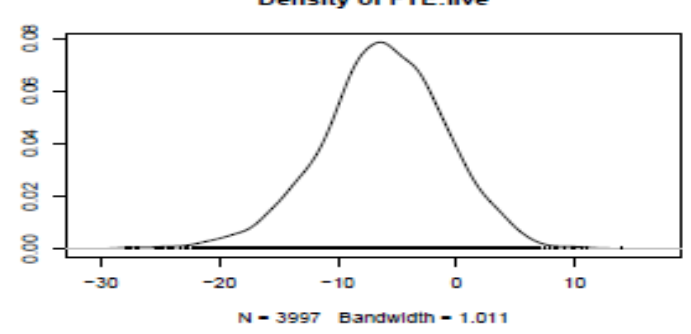

Density of time:live

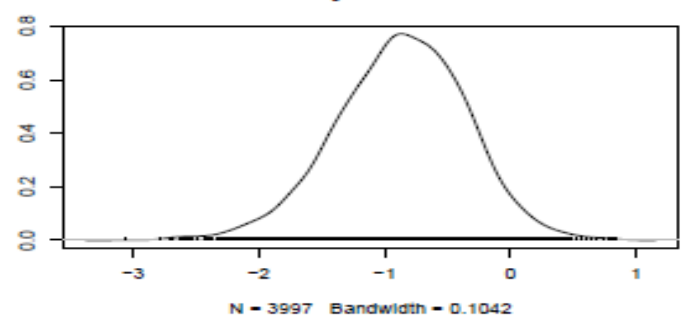

Density of FTE:relation

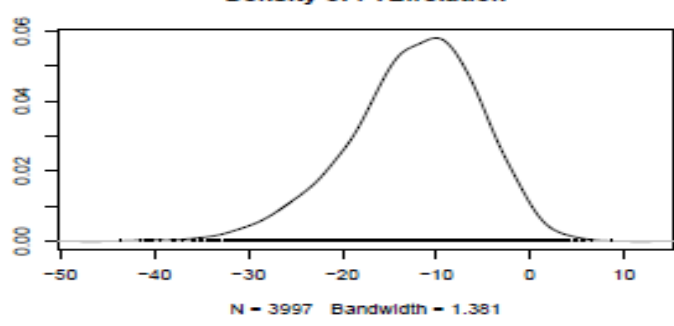

Density of time:relation
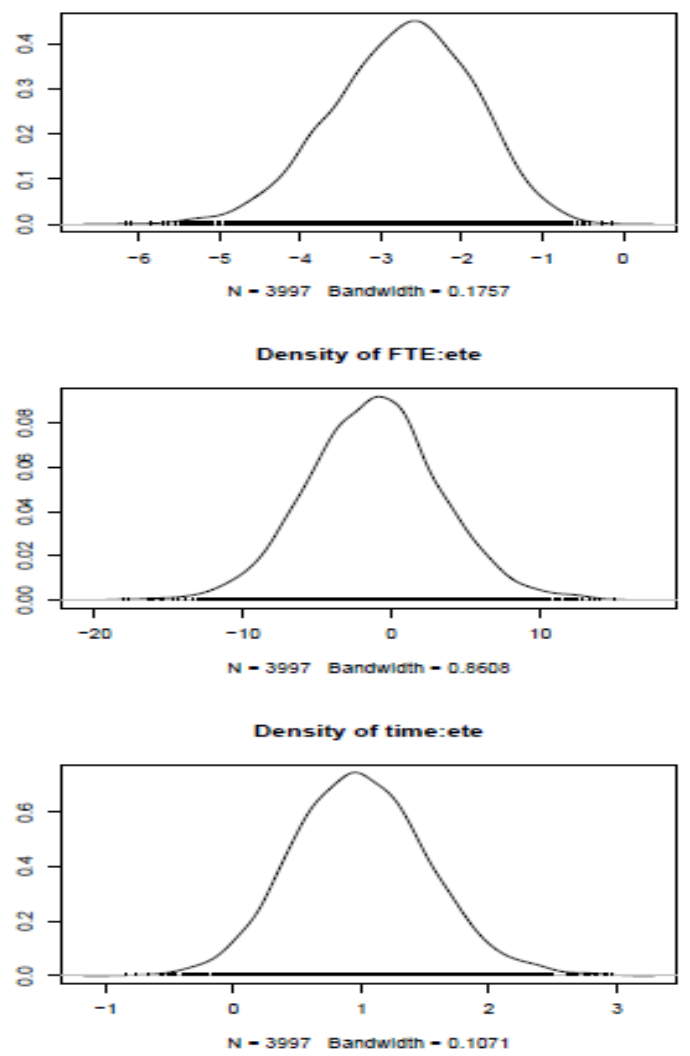

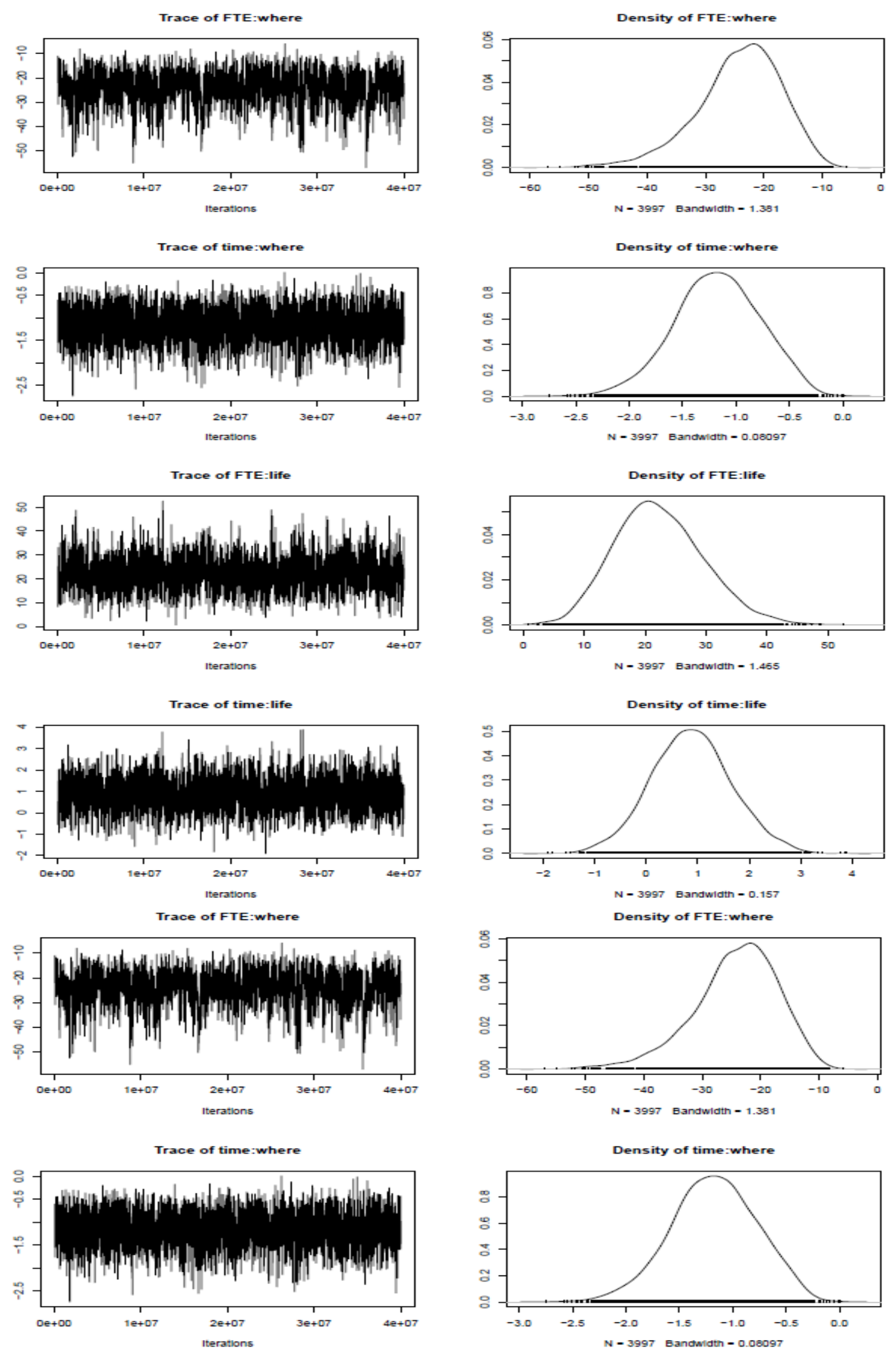

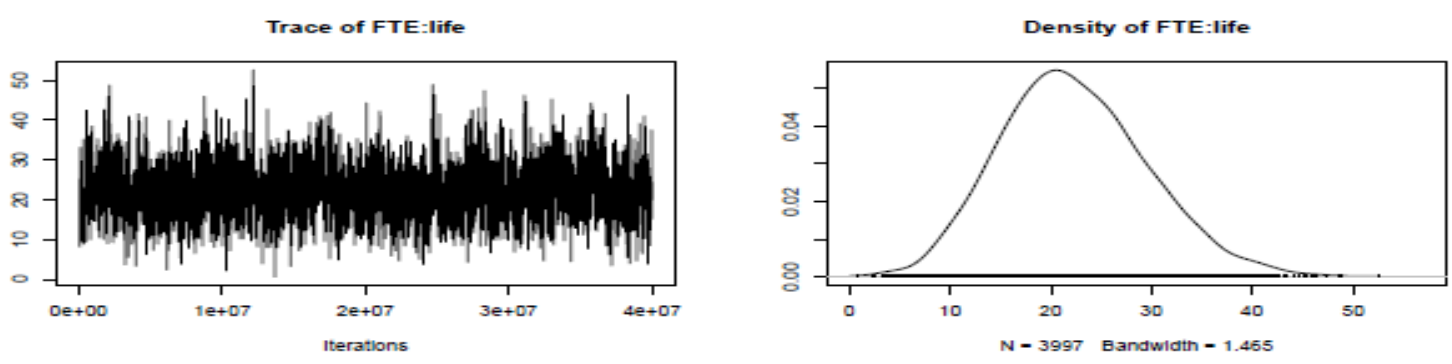

Trace of time:life
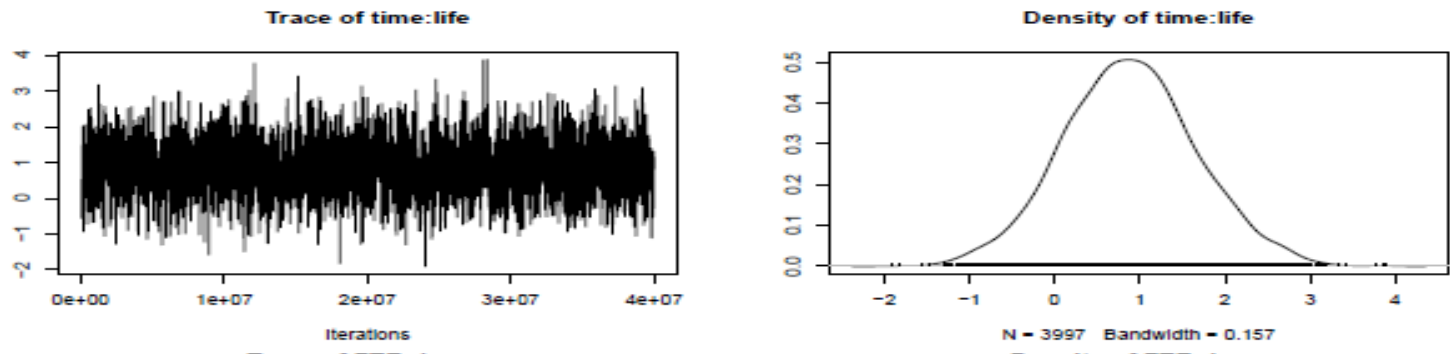

Trace of FTE-drugs
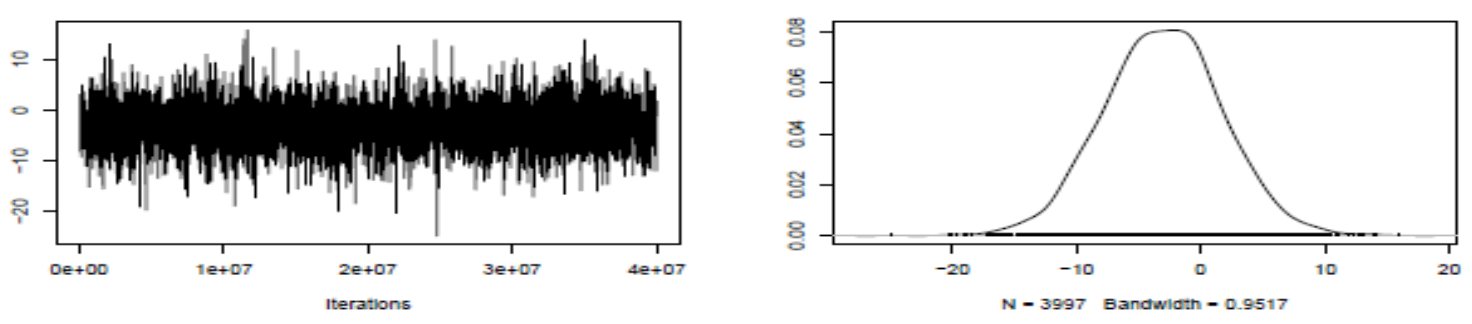

Trace of time:drugs
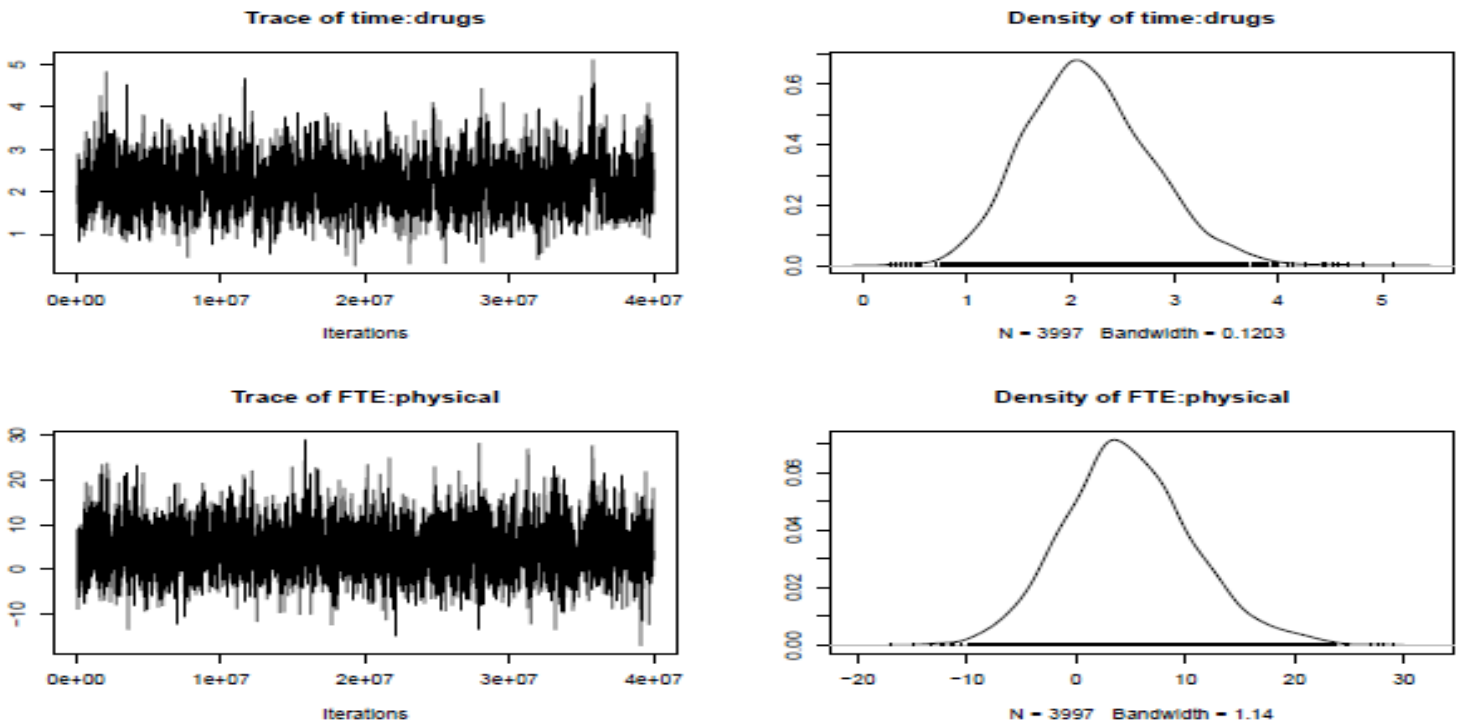

Trace of time:physical
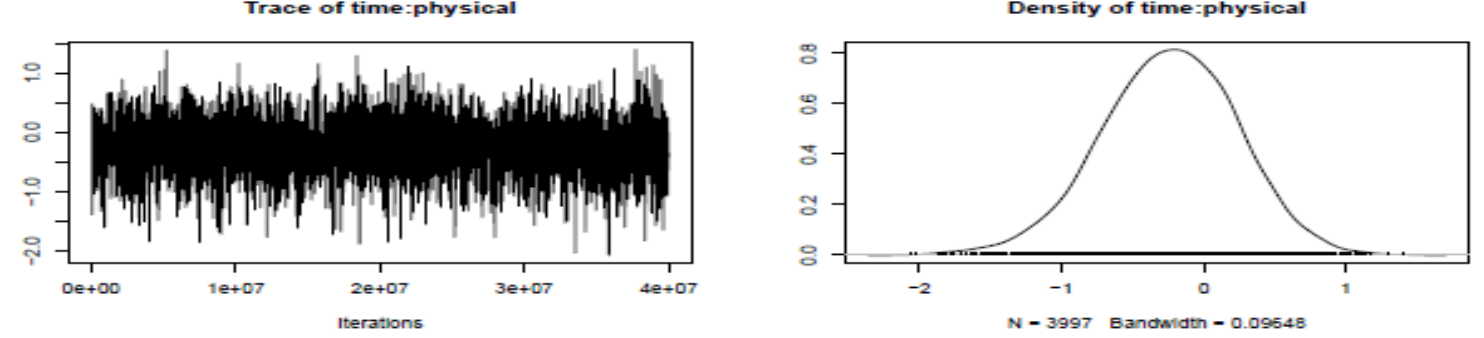

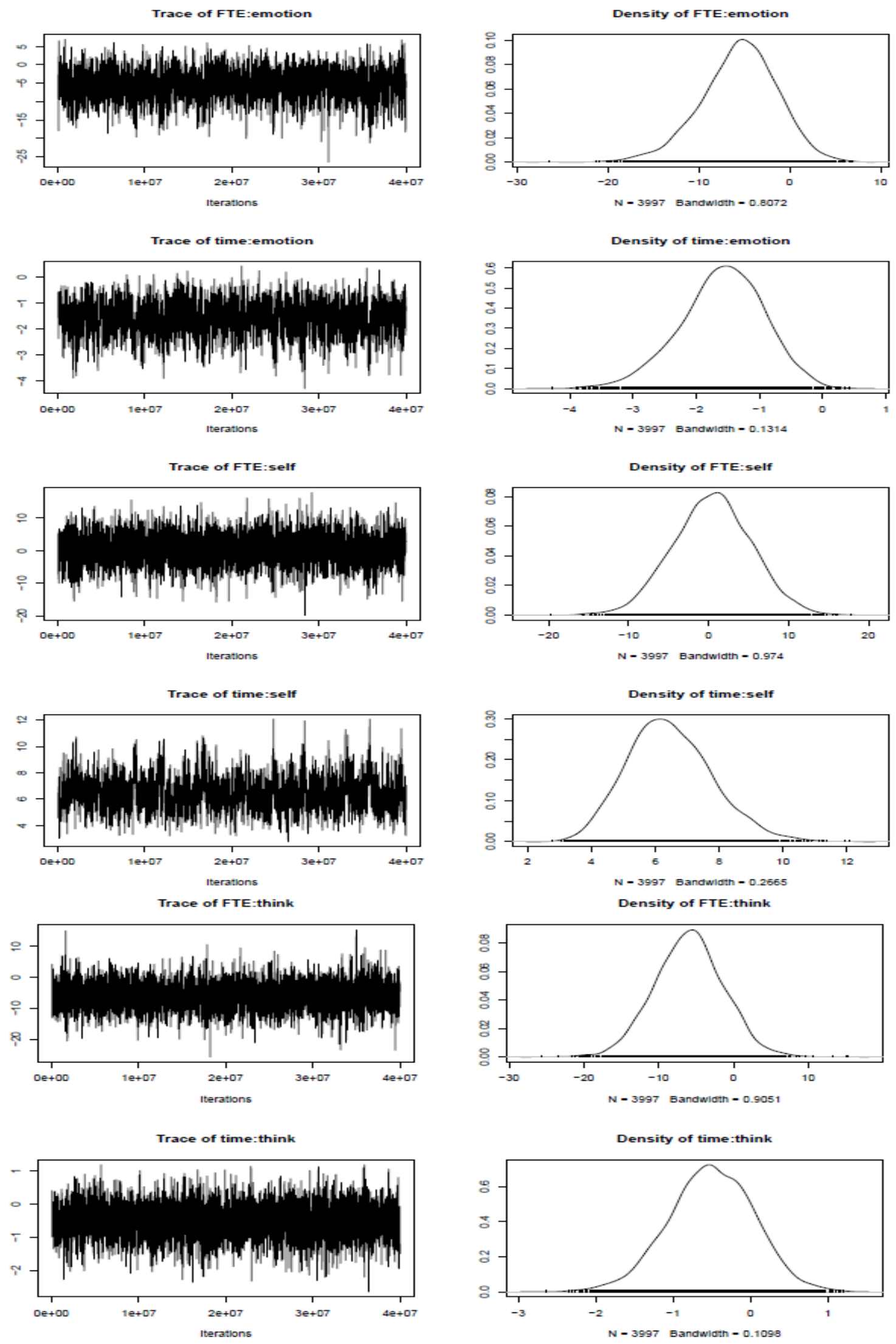

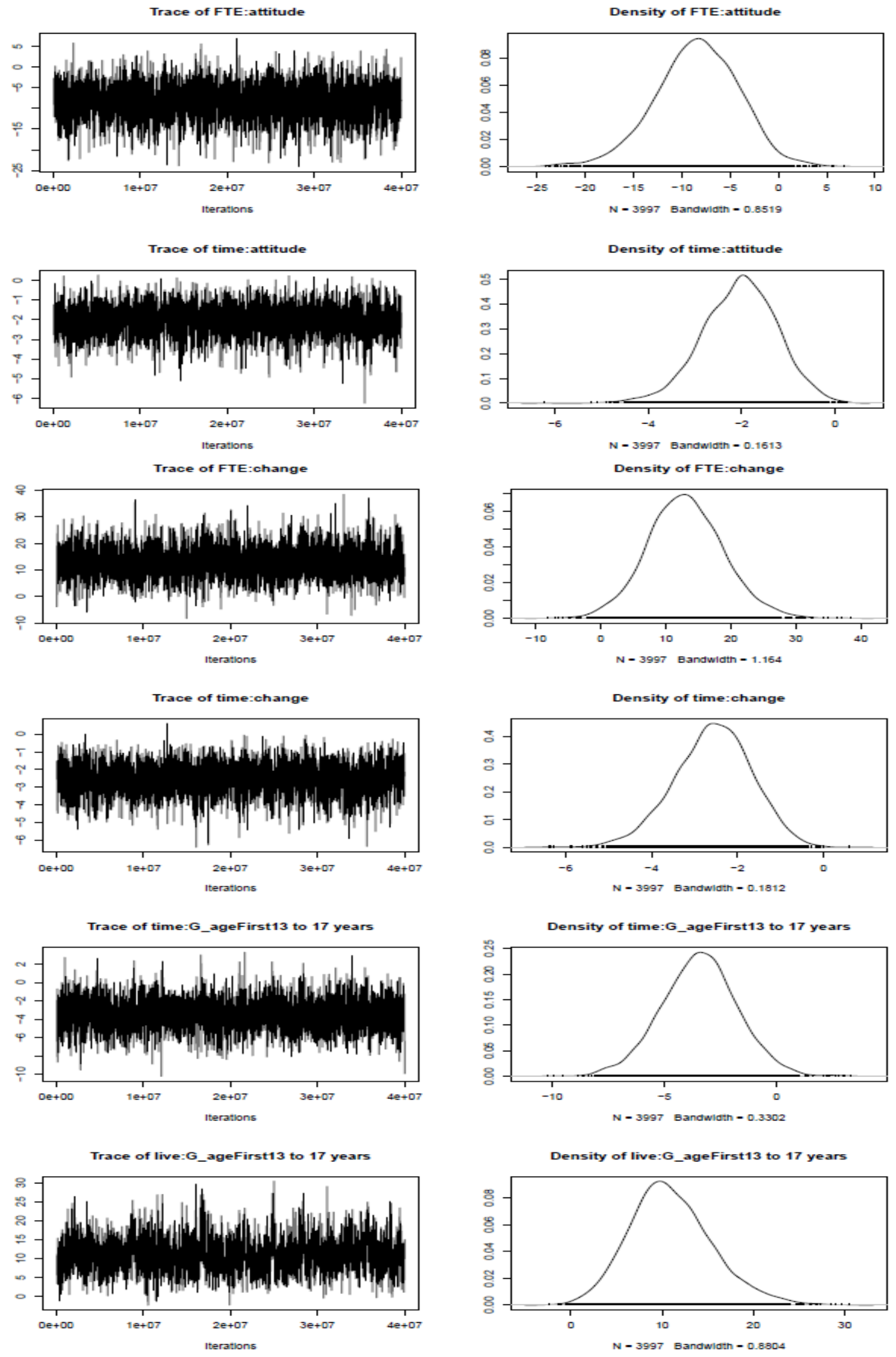

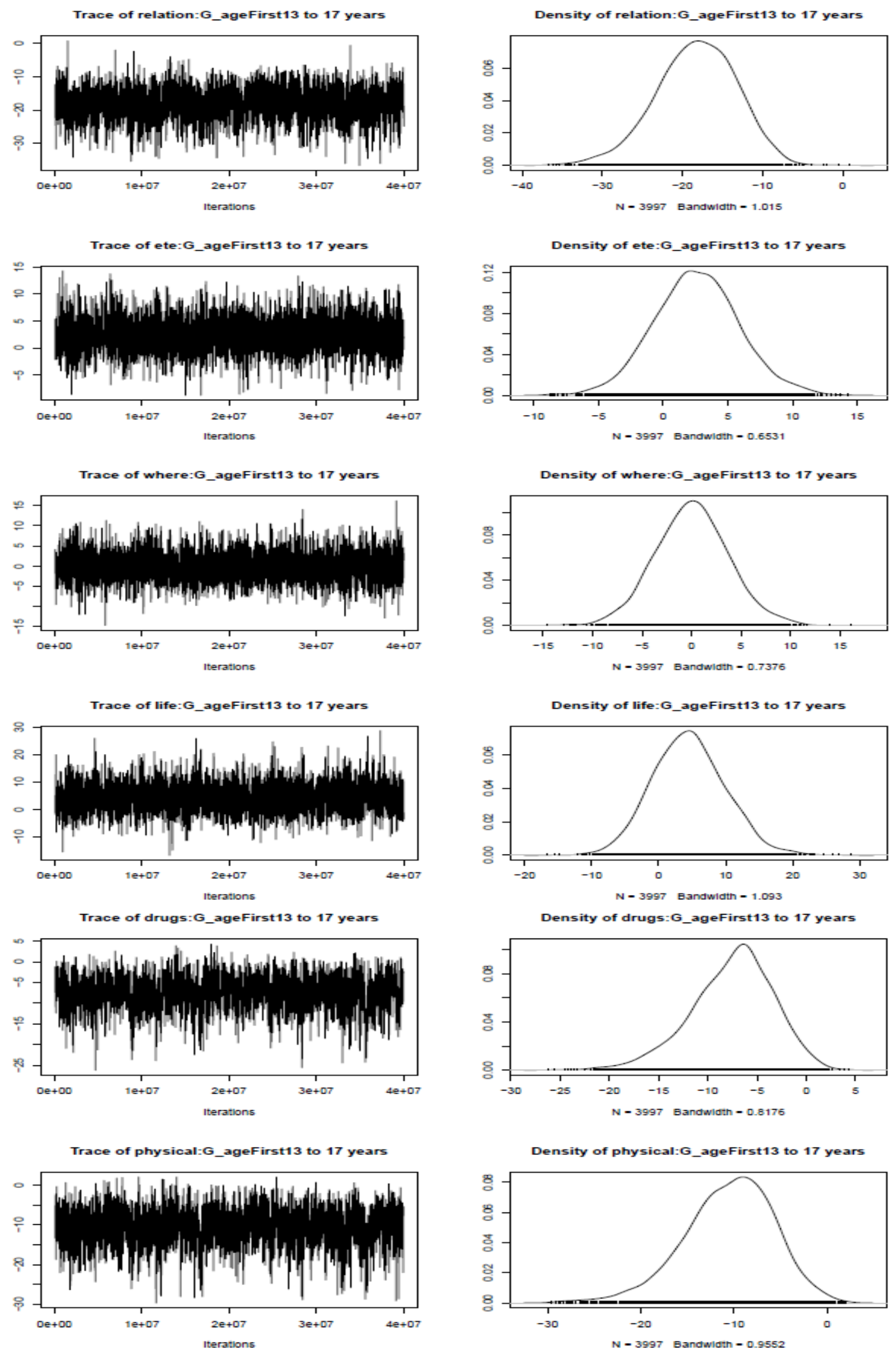

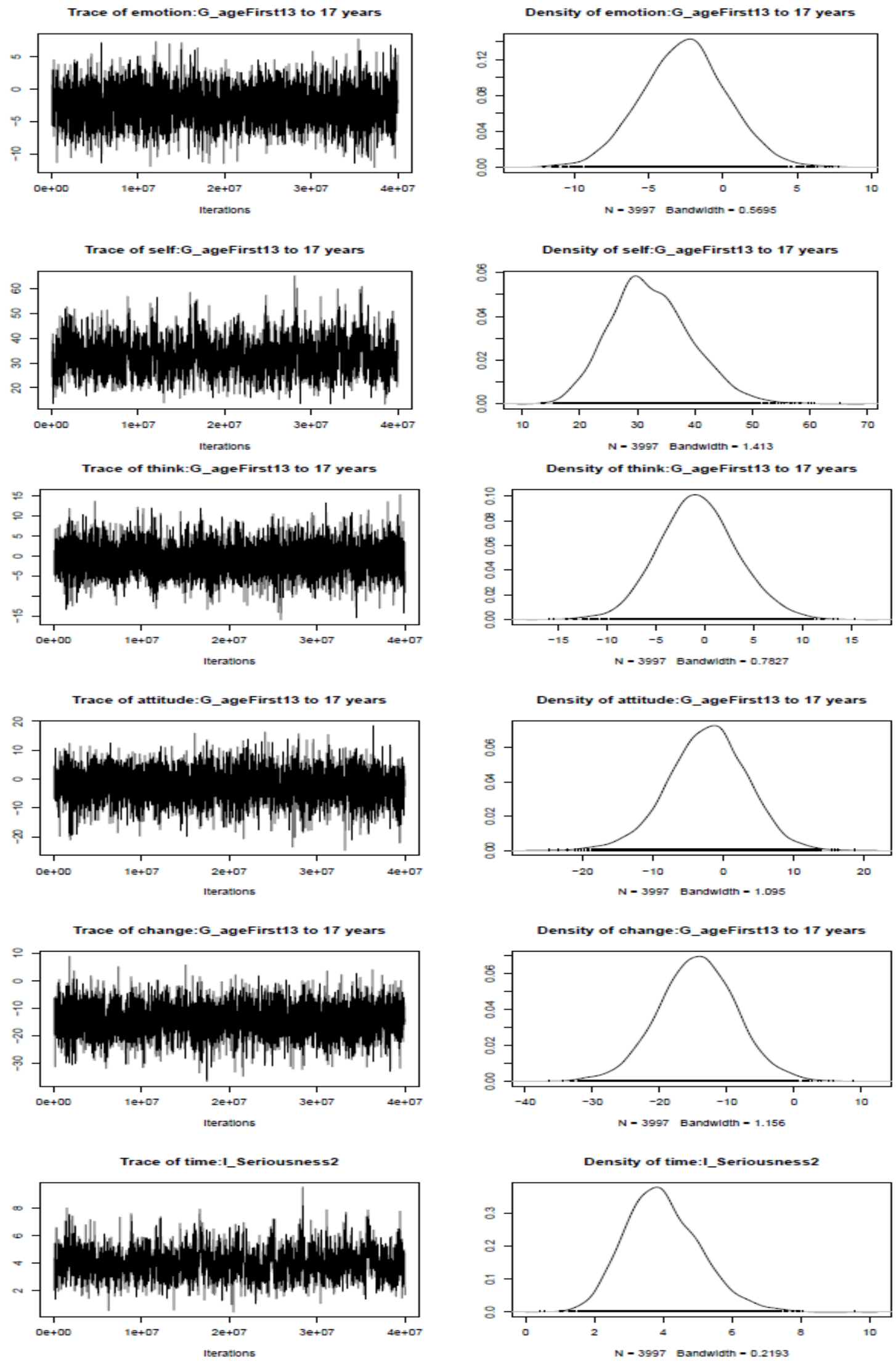
Trace of live:1_Seriousness2

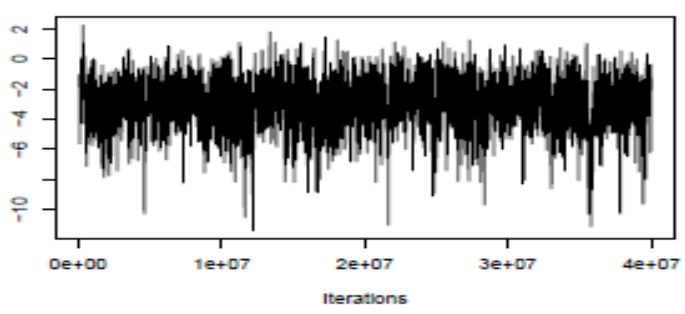

Trace of relation:I_Seriousness2

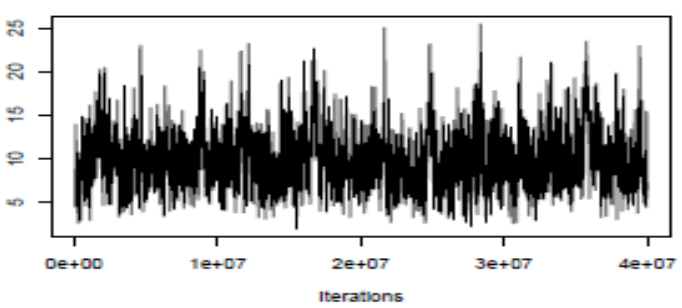

Trace of ete:1_Seriousness2

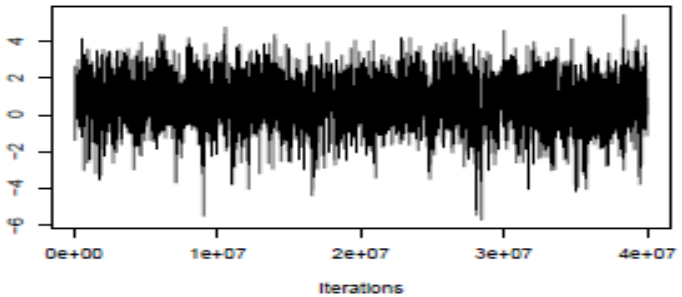

Trace of where:I_Seriousness2

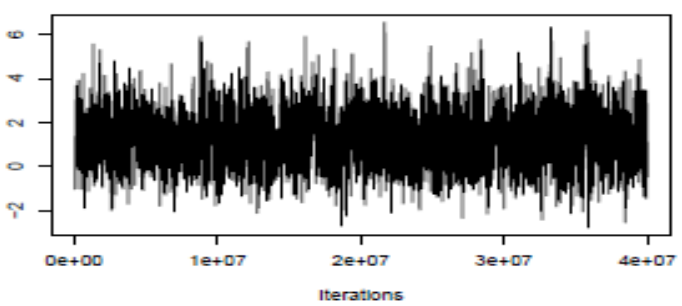

Trace of life:I_Seriousness2

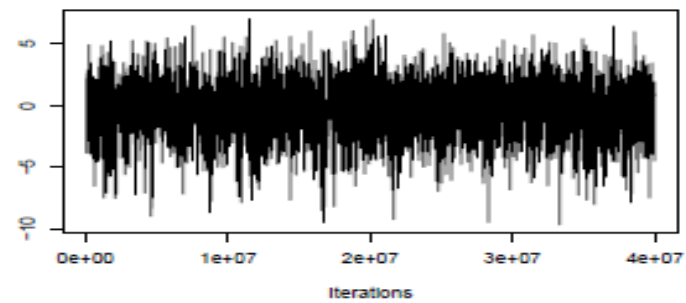

Trace of drugs:1_Seriousness2

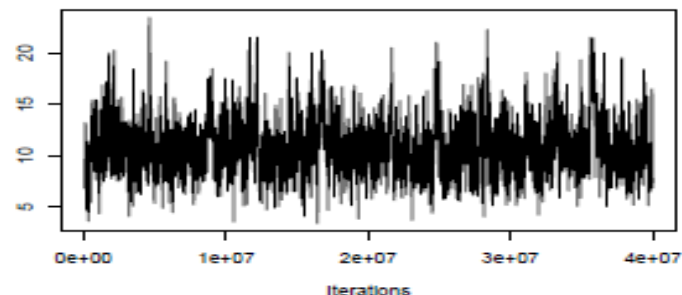

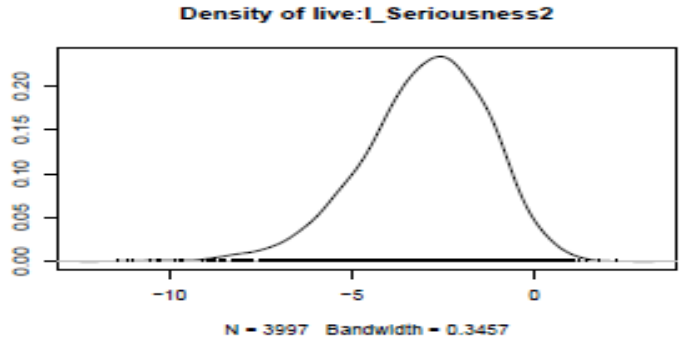
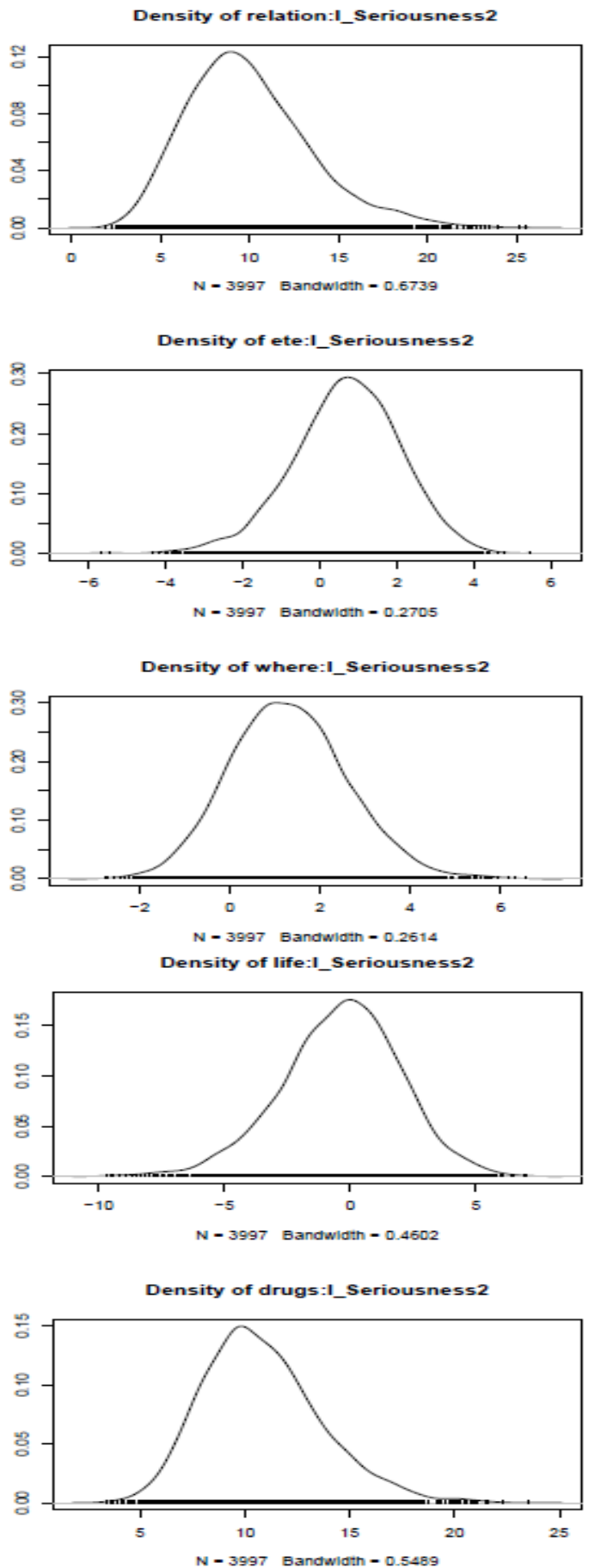

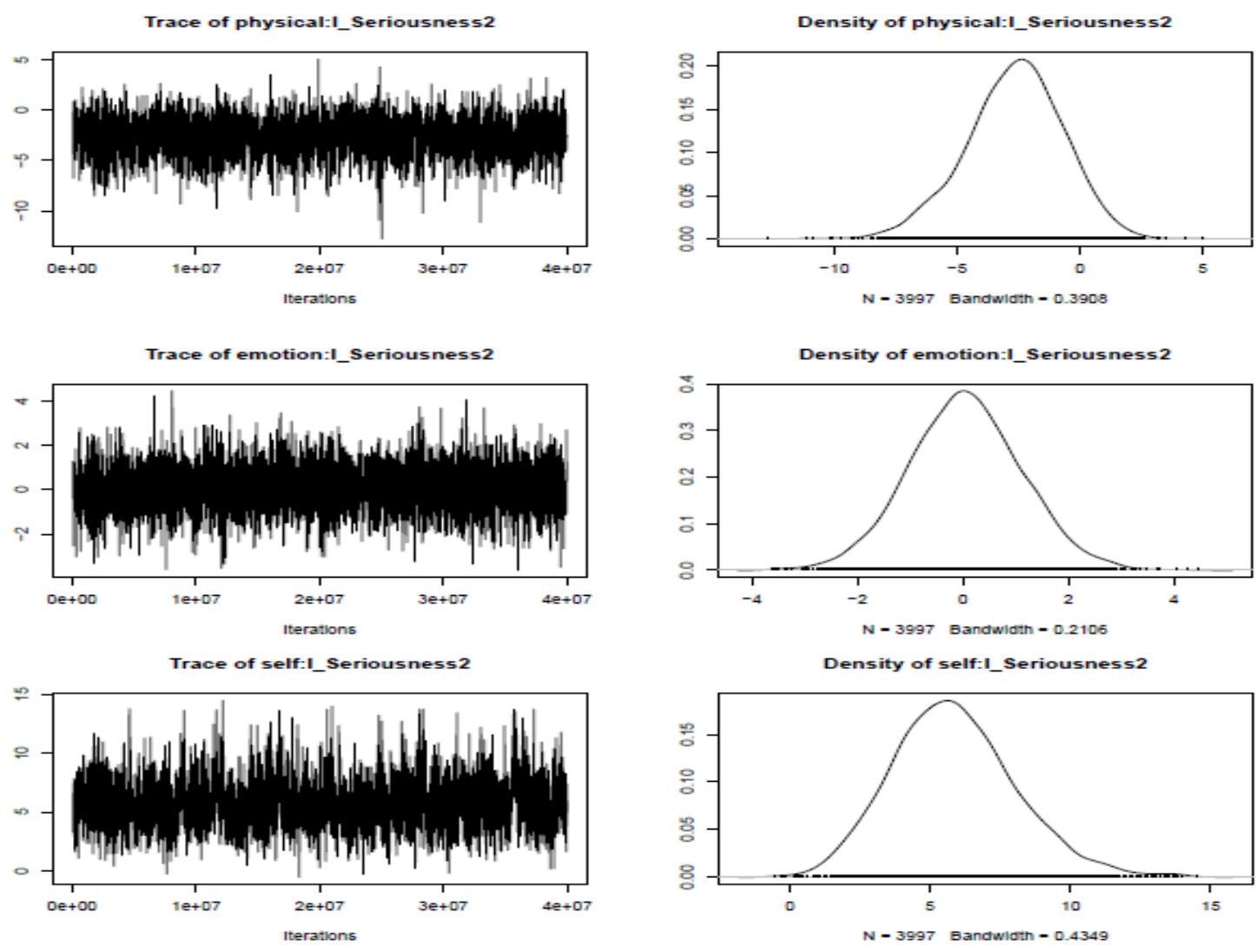

Trace of think:1_Seriousness2
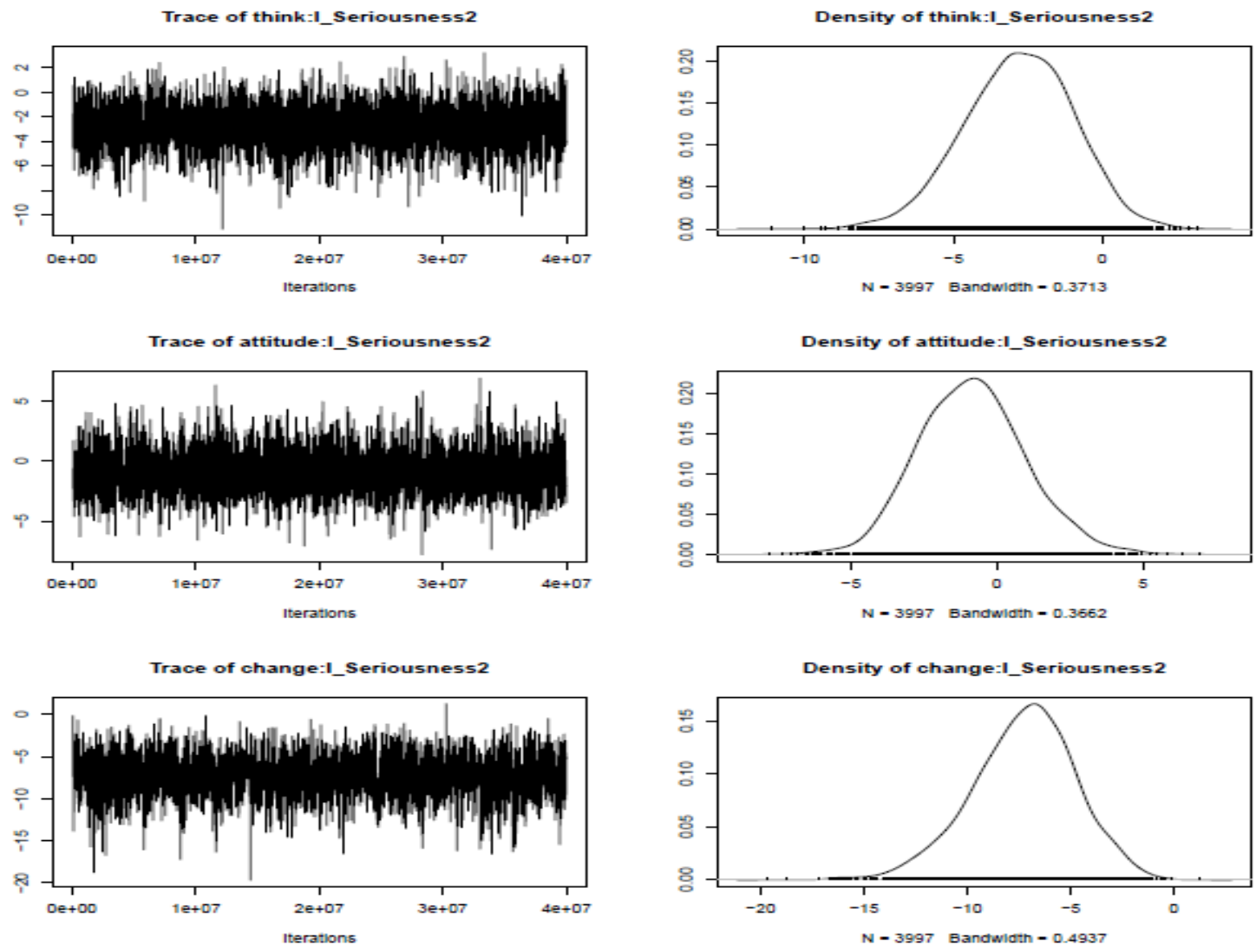

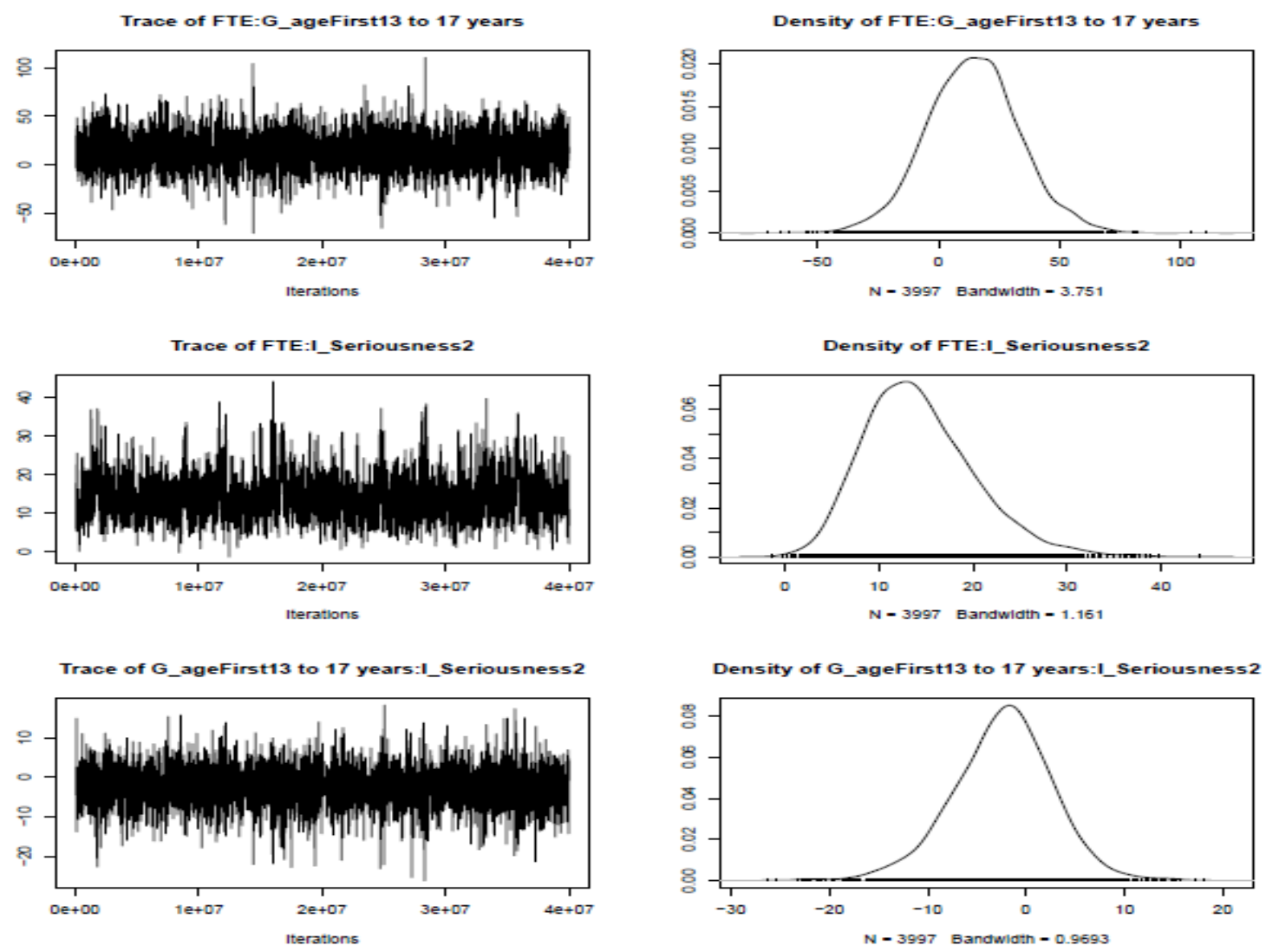

Trace of FTE:time:live
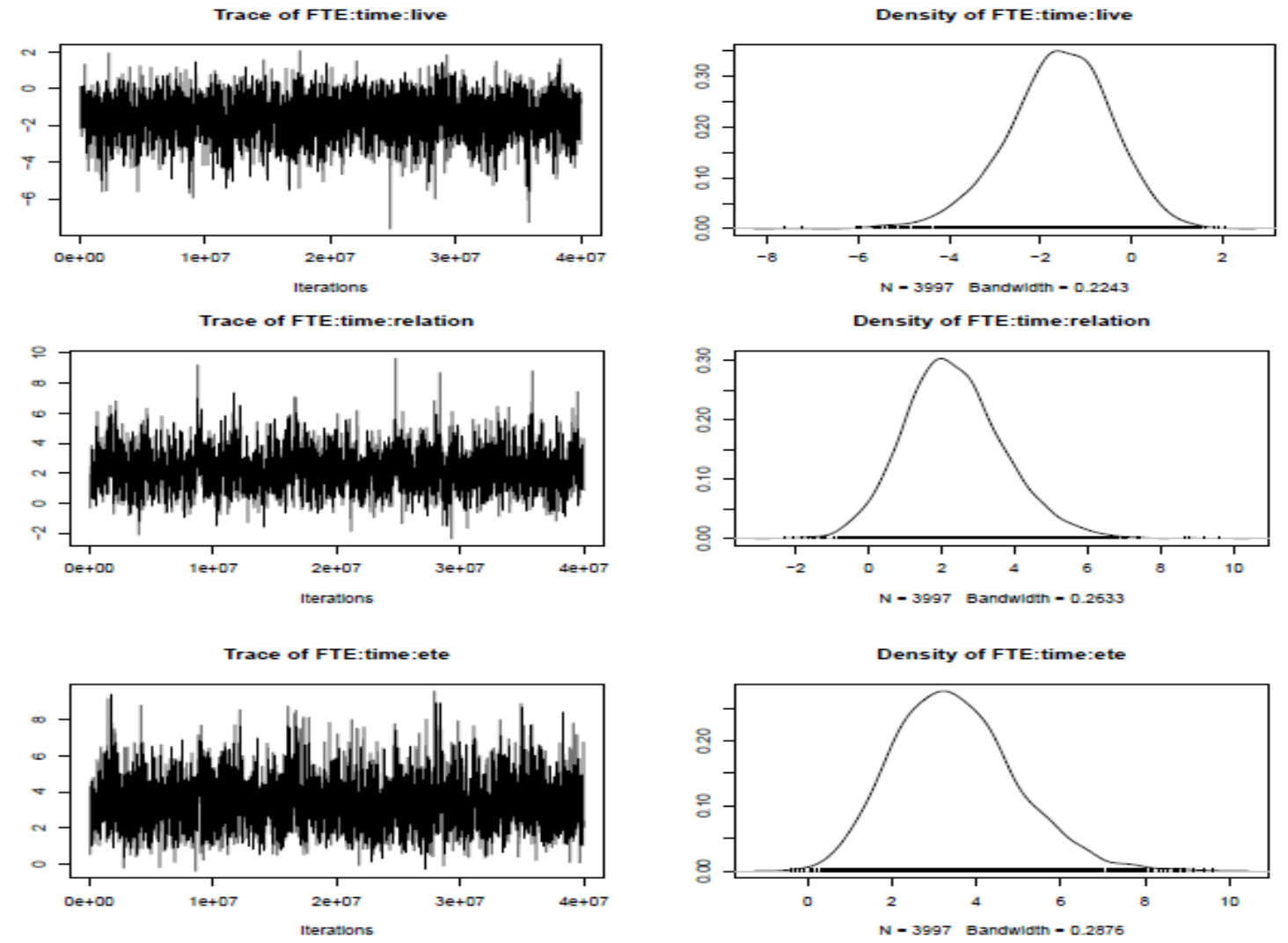
Trace of FTE:time:where

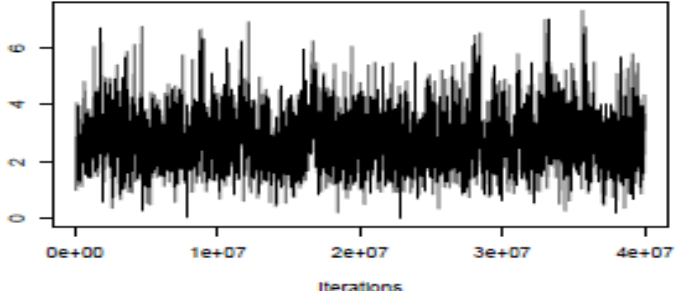

Trace of FTE:time:life

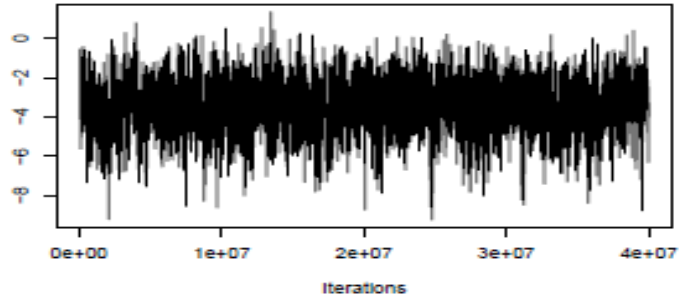

Trace of FTE:time:drugs

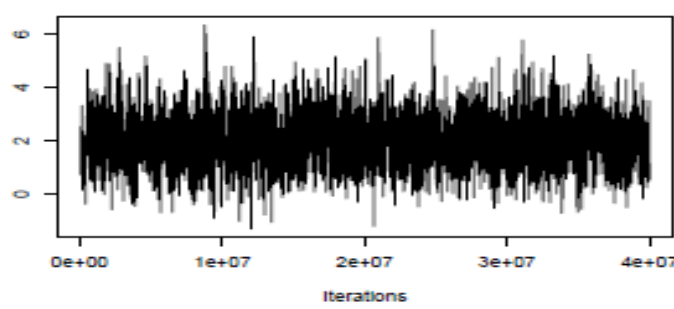

Trace of FTE:time:physical

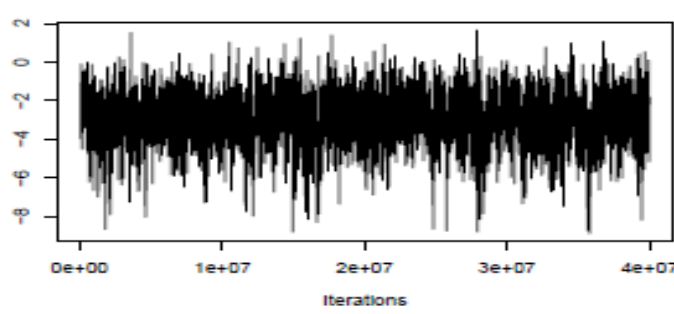

Trace of FTE:time:emotion

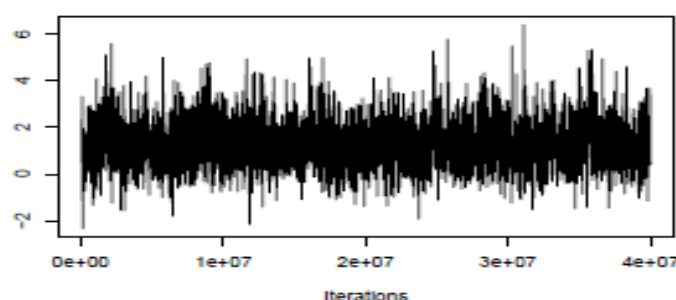

Trace of FTE:time:self

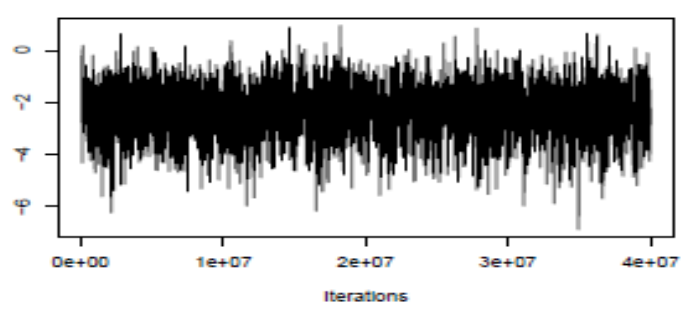

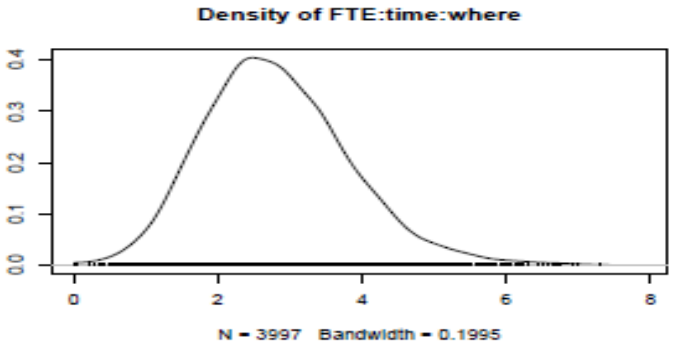

Density of FTE:time:life
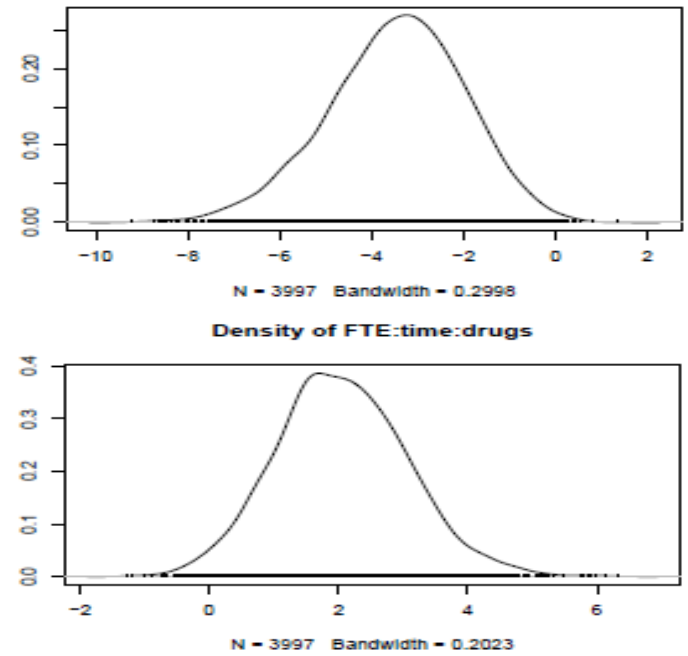

Density of FTE:time:physical
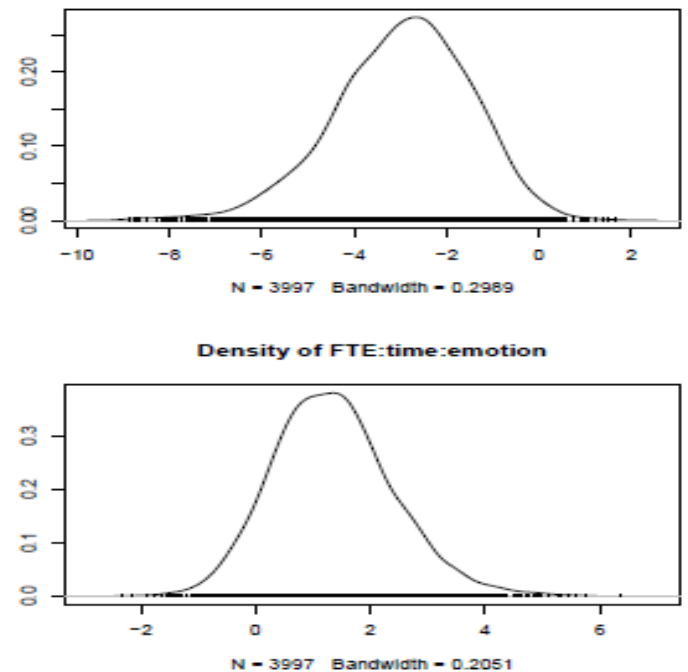

Density of FTE:time:self

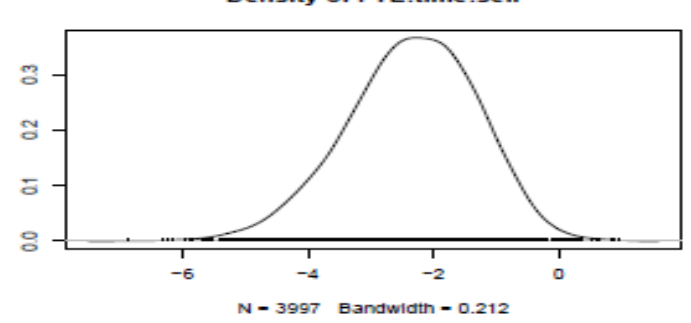


Trace of FTE:time:think

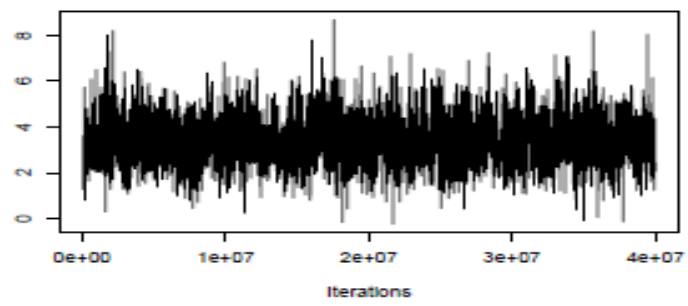

Trace of FTE:time:attitude

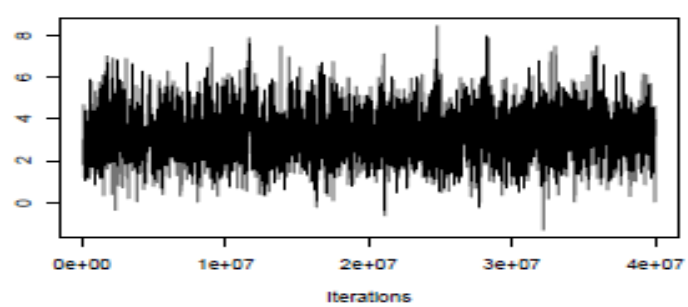

Trace of FTE:time:change

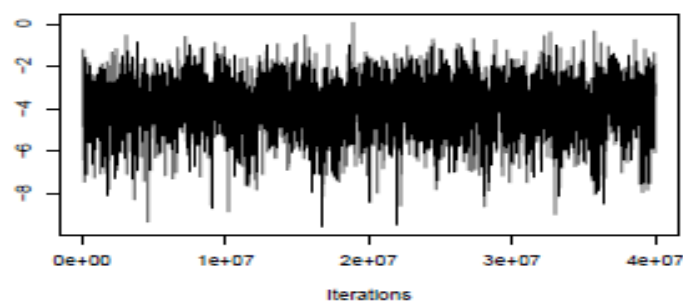

Trace of time:live:G_ageFirst13 to 17 years

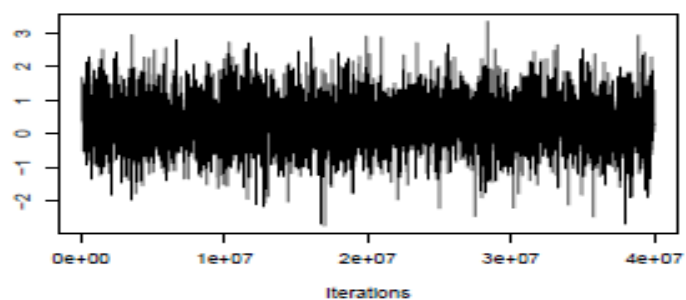

Iterations
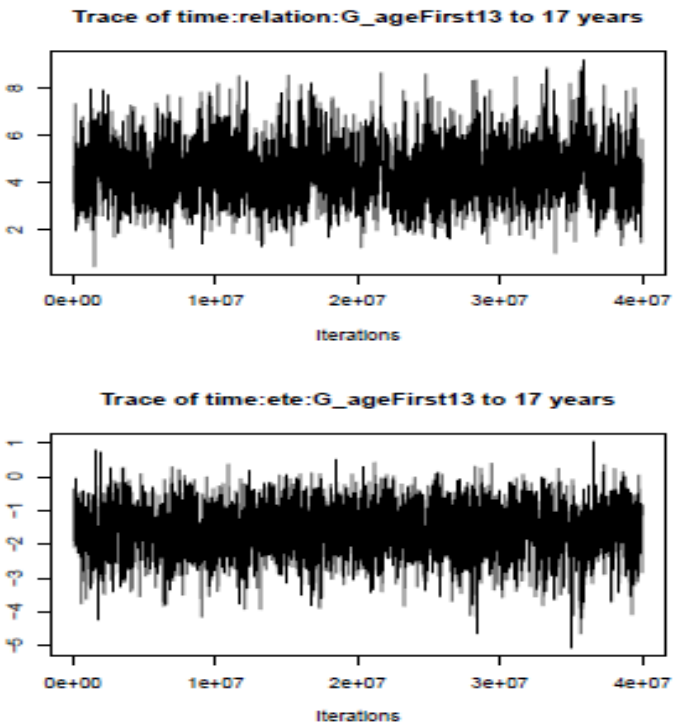
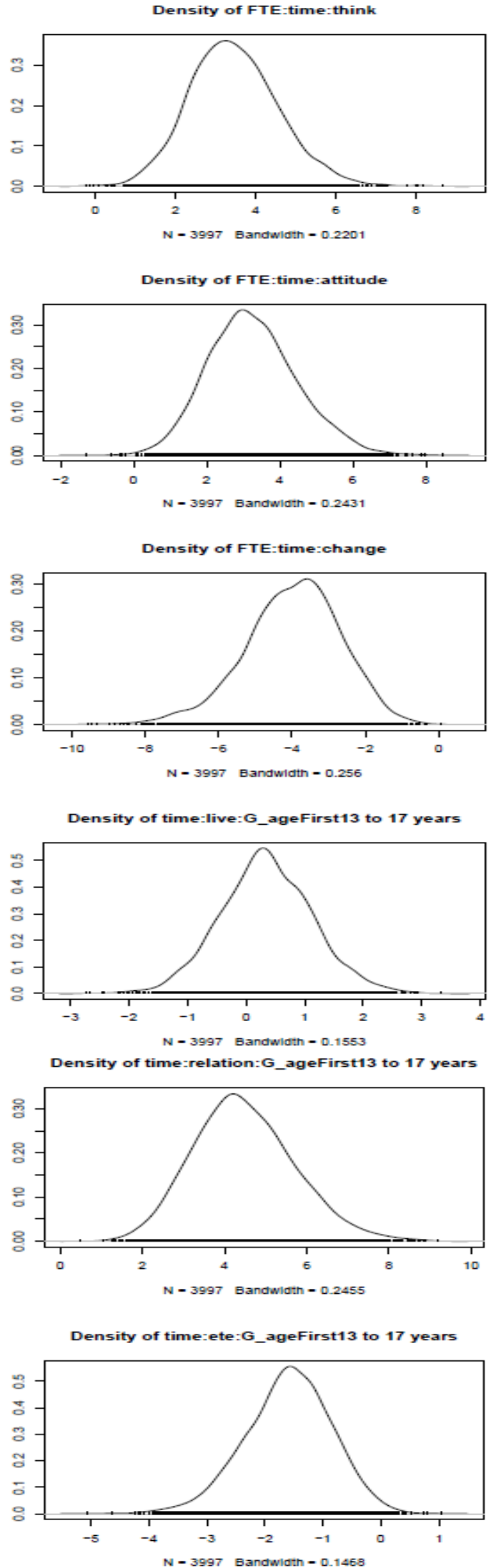

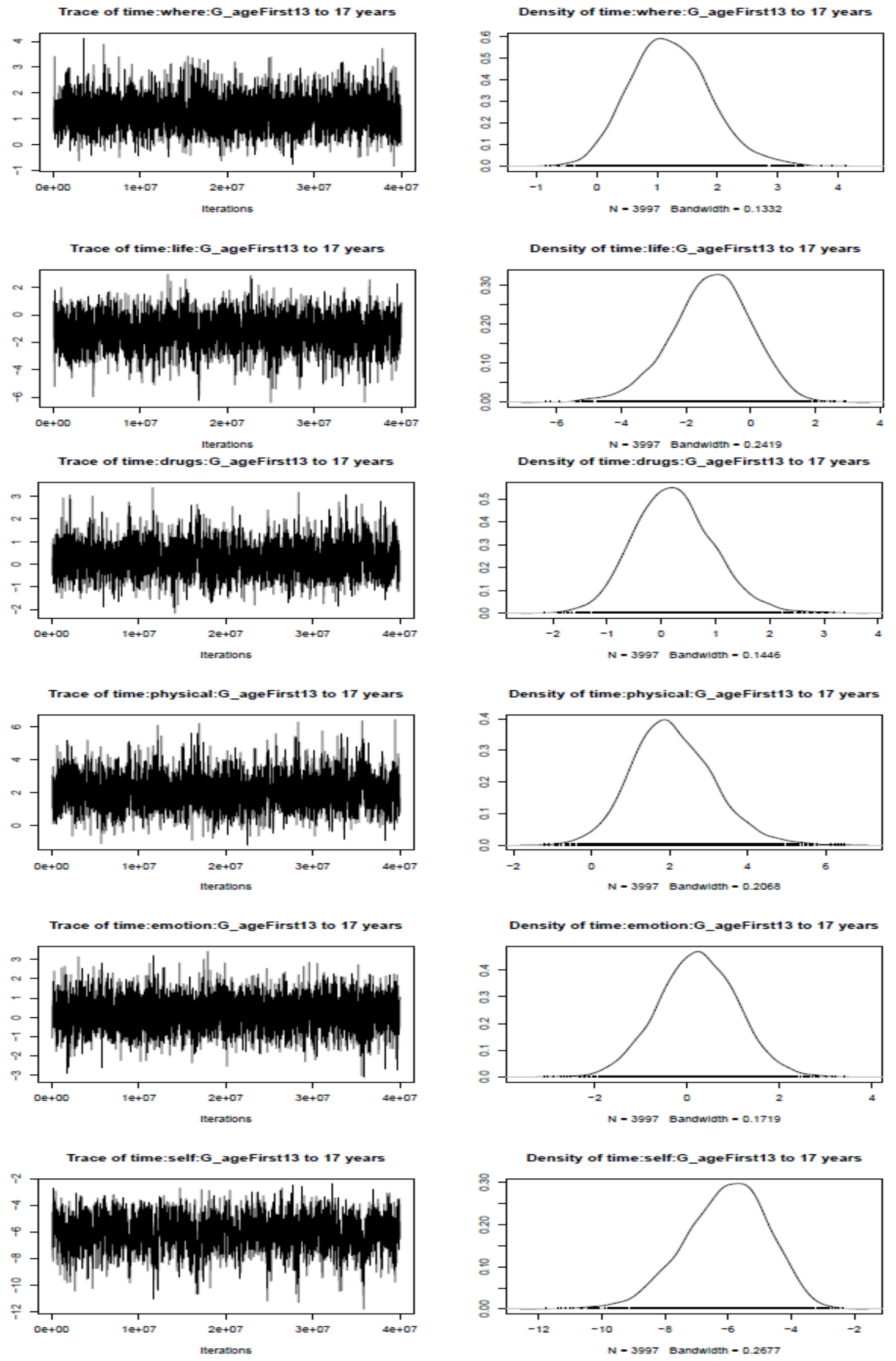

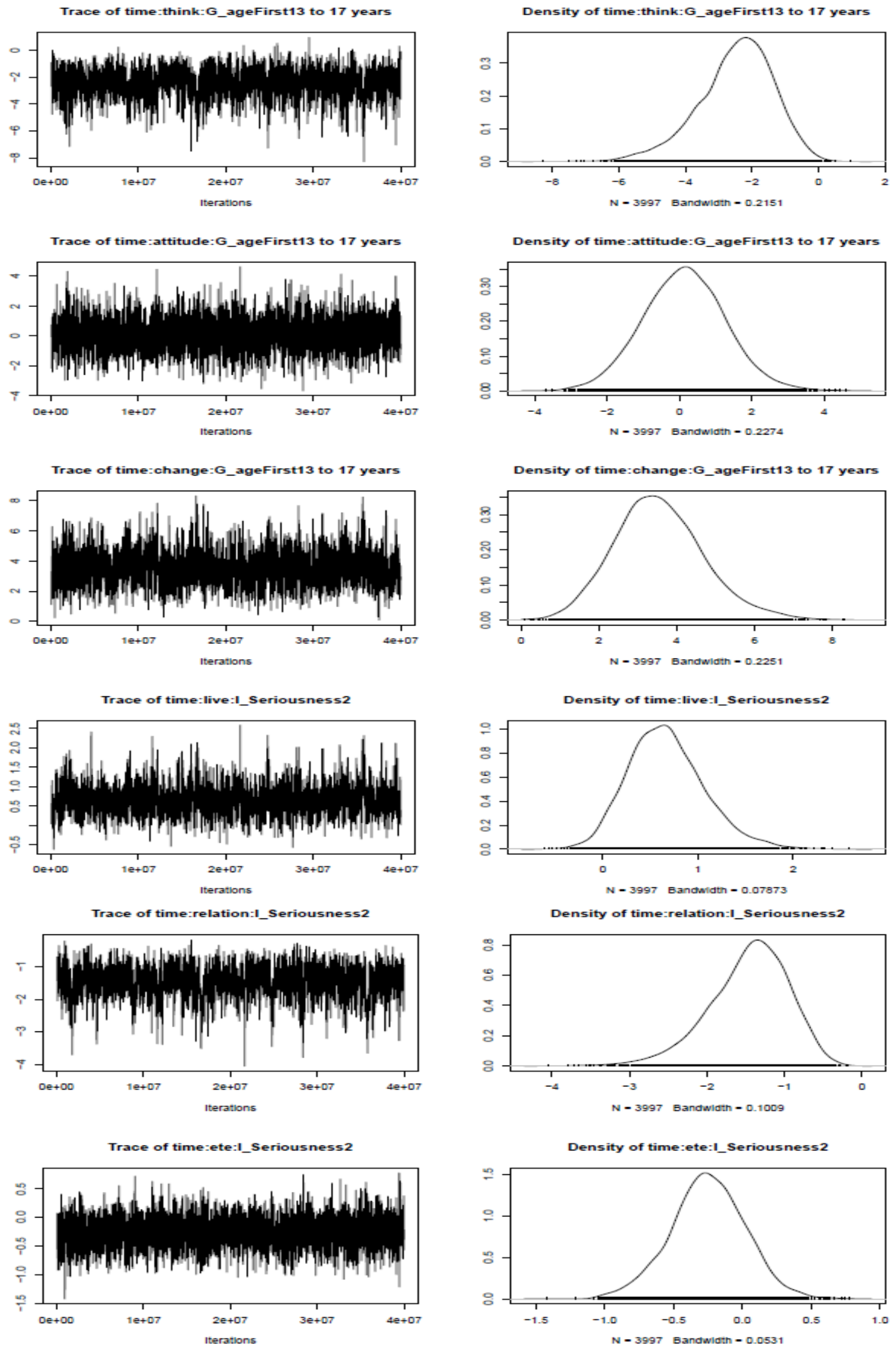

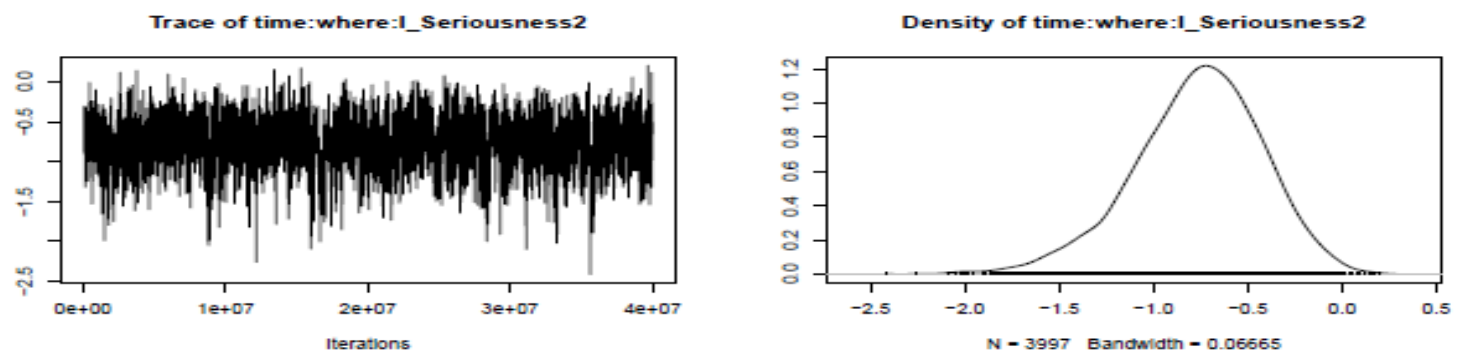

Trace of time:life:1_Seriousness2

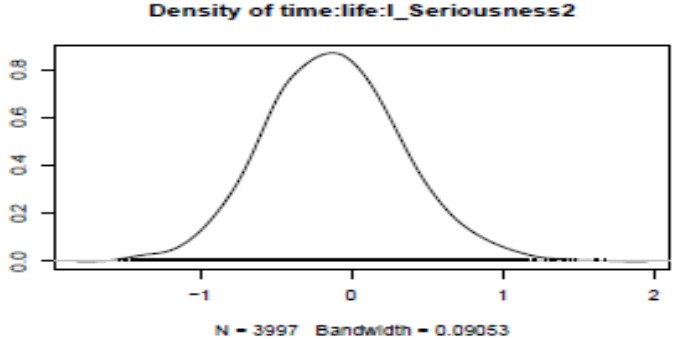

drugs:1_Seriousness2

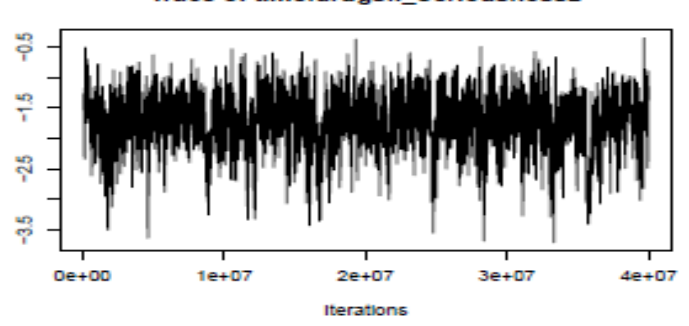

Density of time:drugs:I_Seriousness?

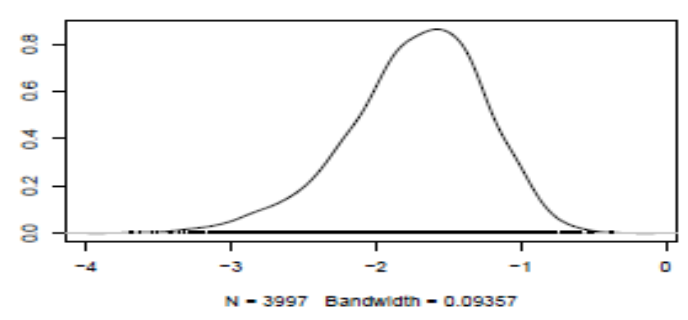

Trace of time:physical:I_Seriousness2
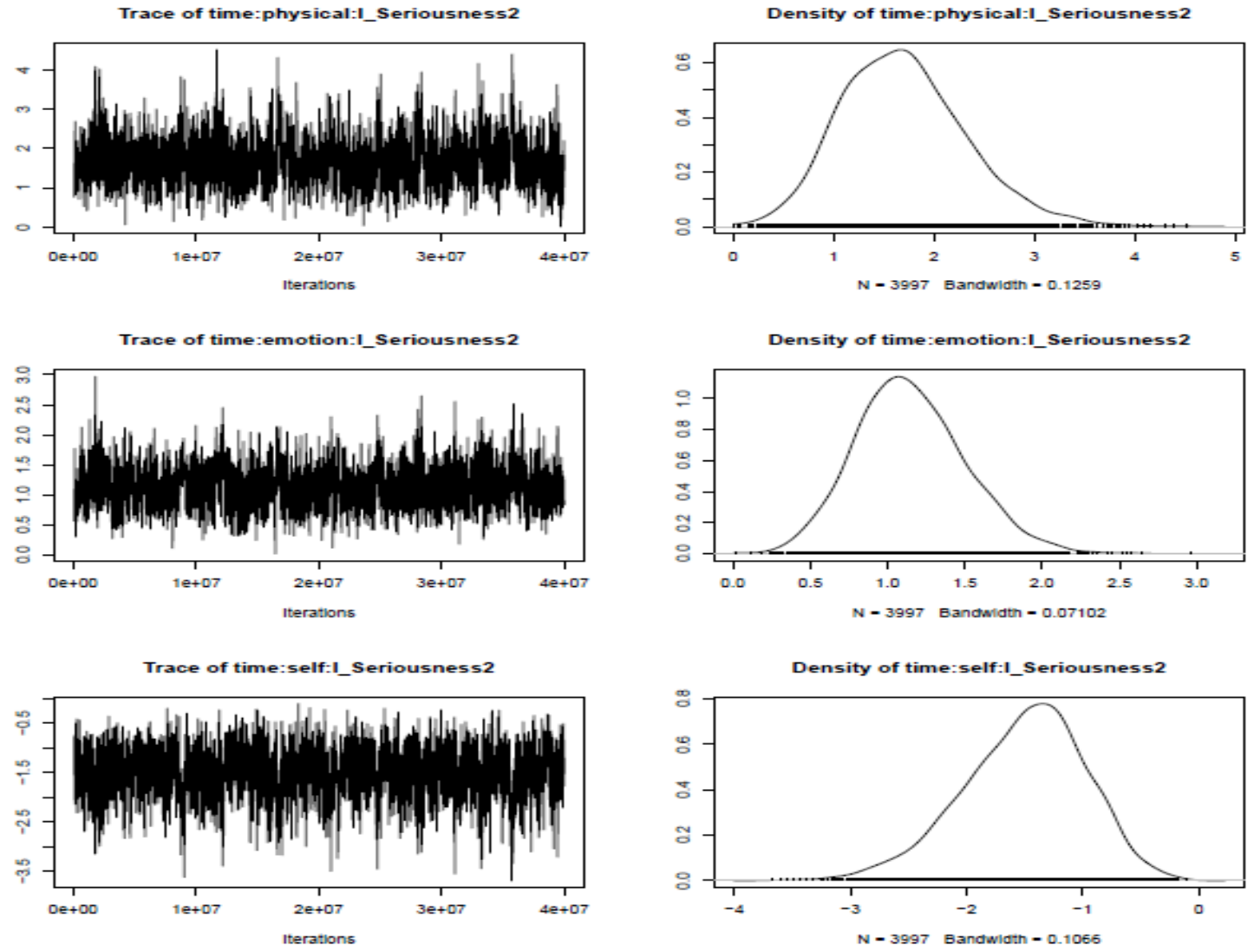

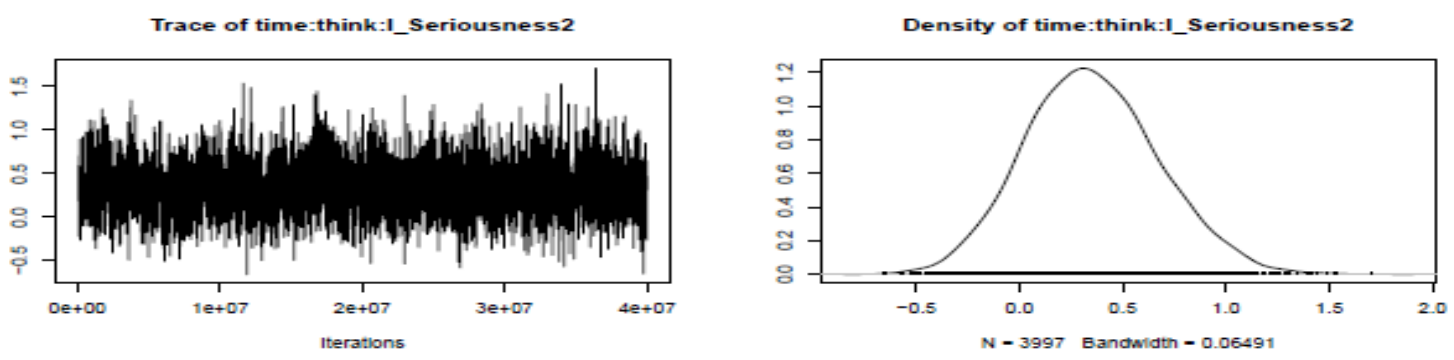

Trace of time:attitude:I_Seriousness2
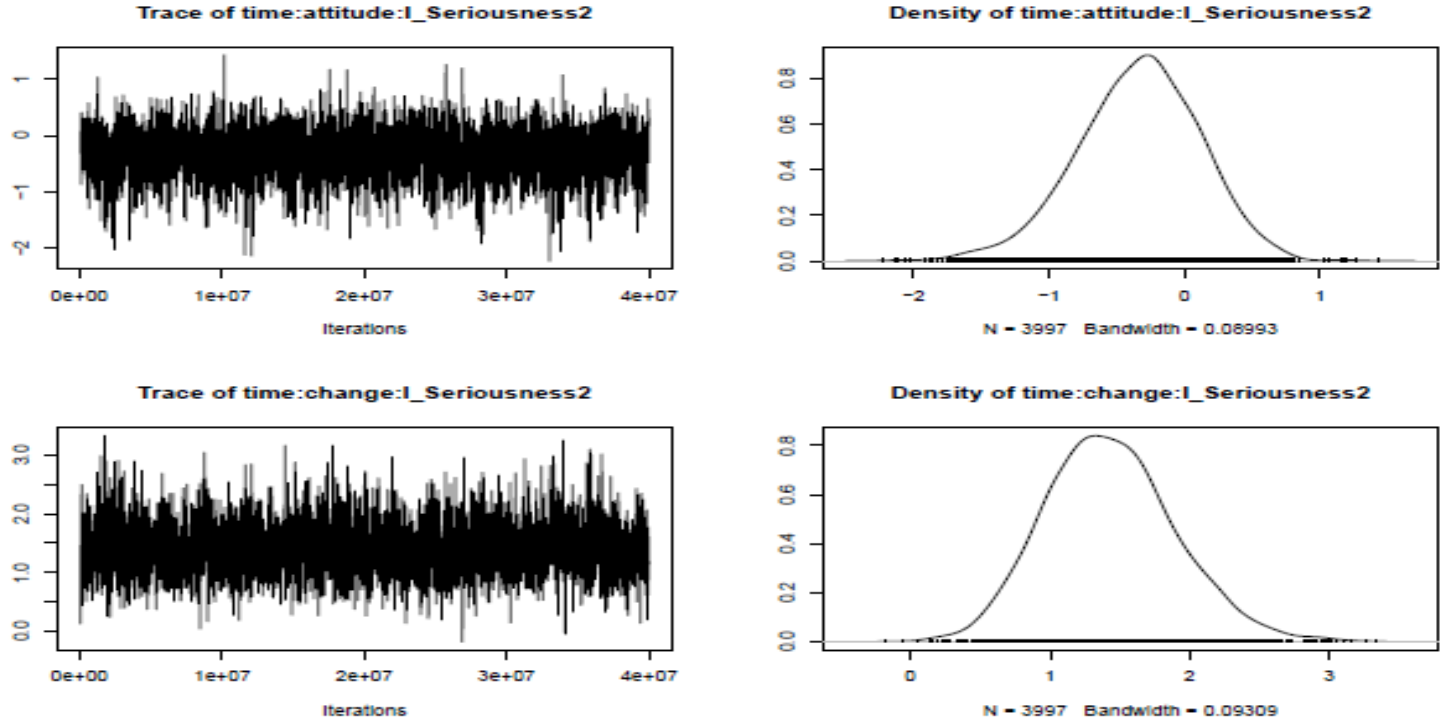

$\underline{\text { Random Effects }}$
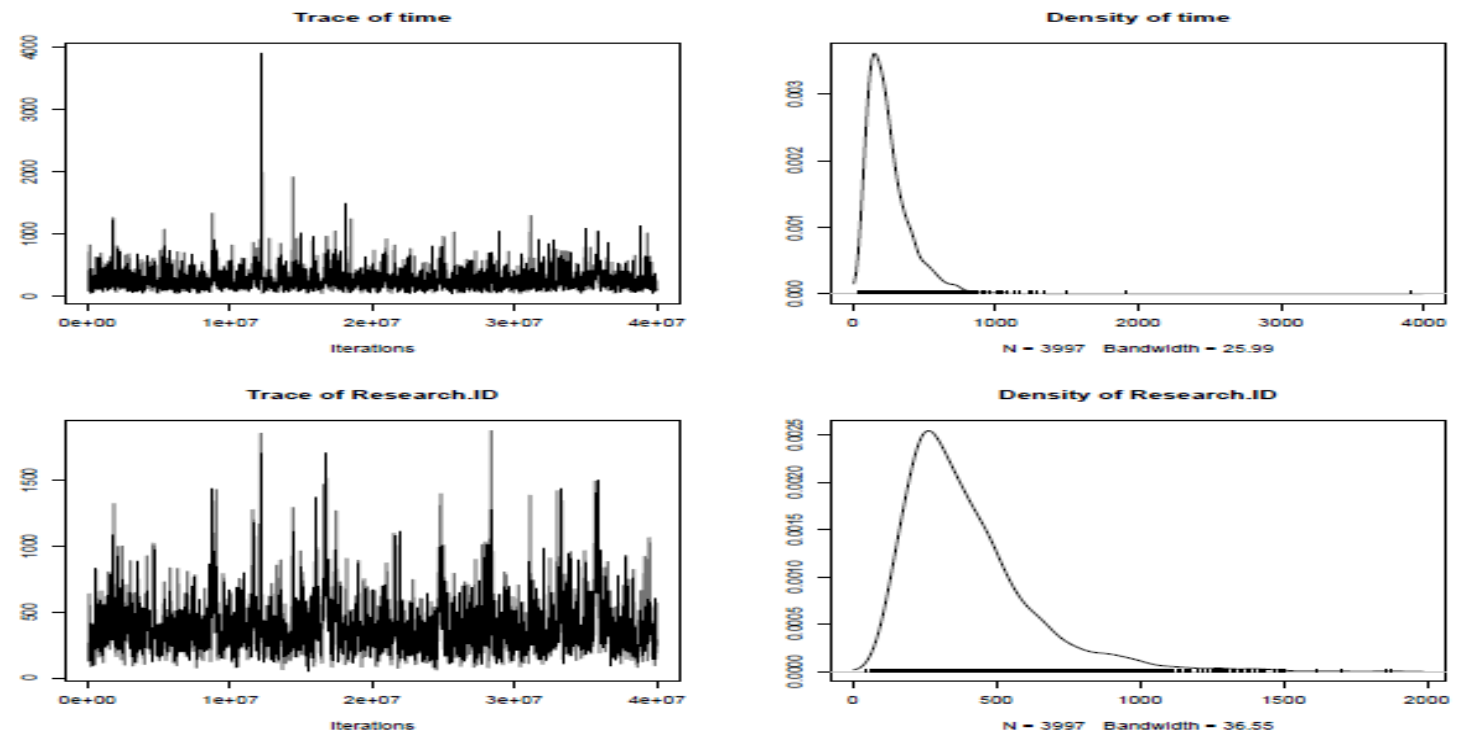
The Combined Model involving Offending History: Version 2a

Bayesian Model (BDm3G_cc12)

\section{\#\# Define the Model}

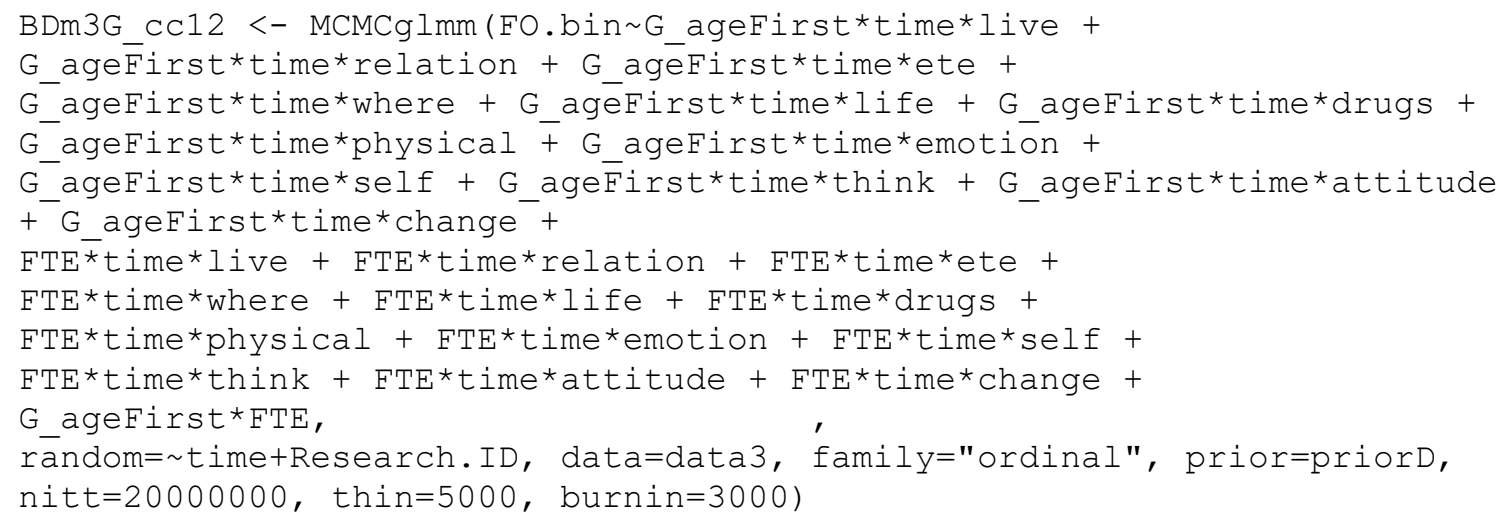

\section{\#\# Checks for suitable convergence}

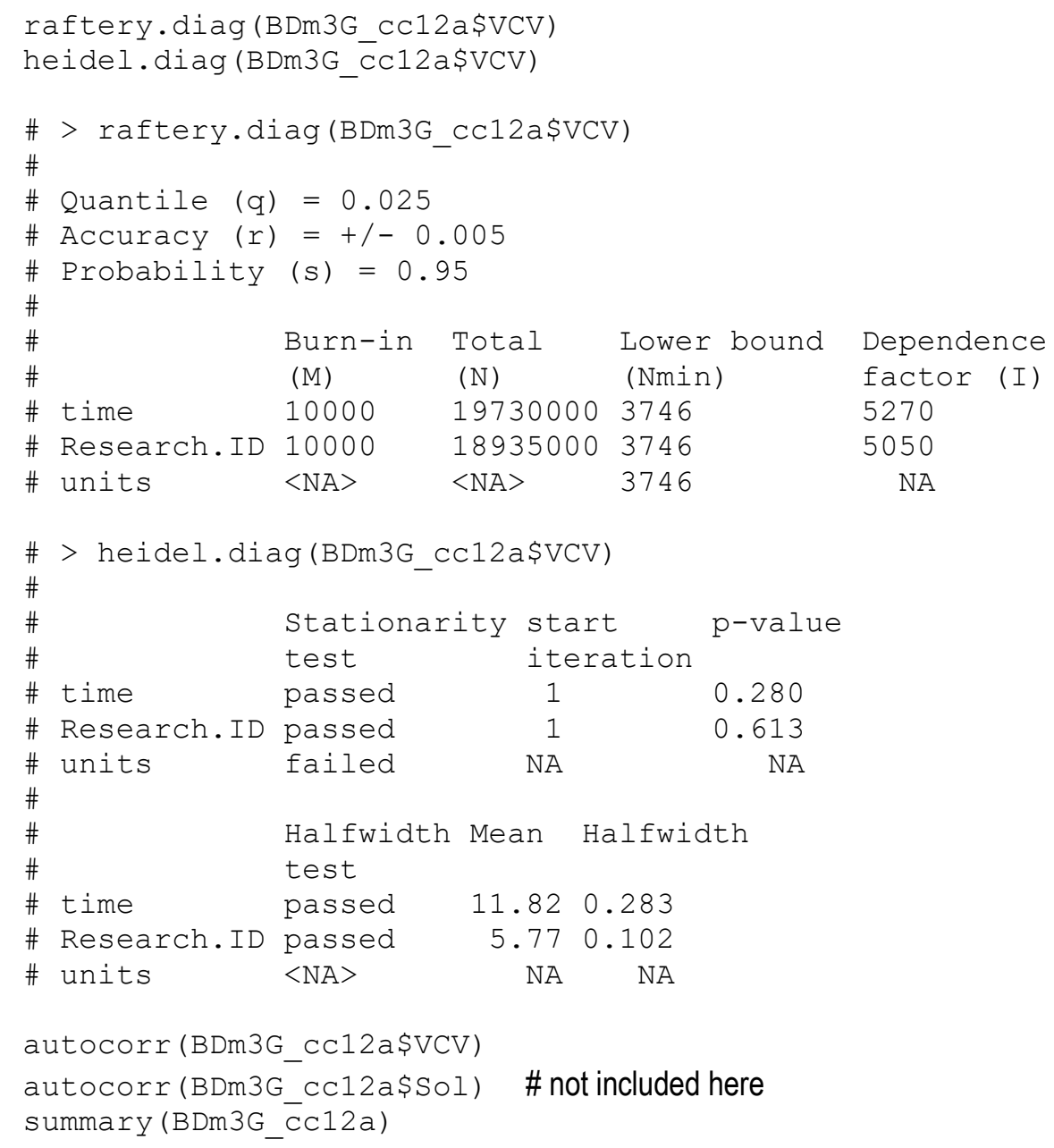




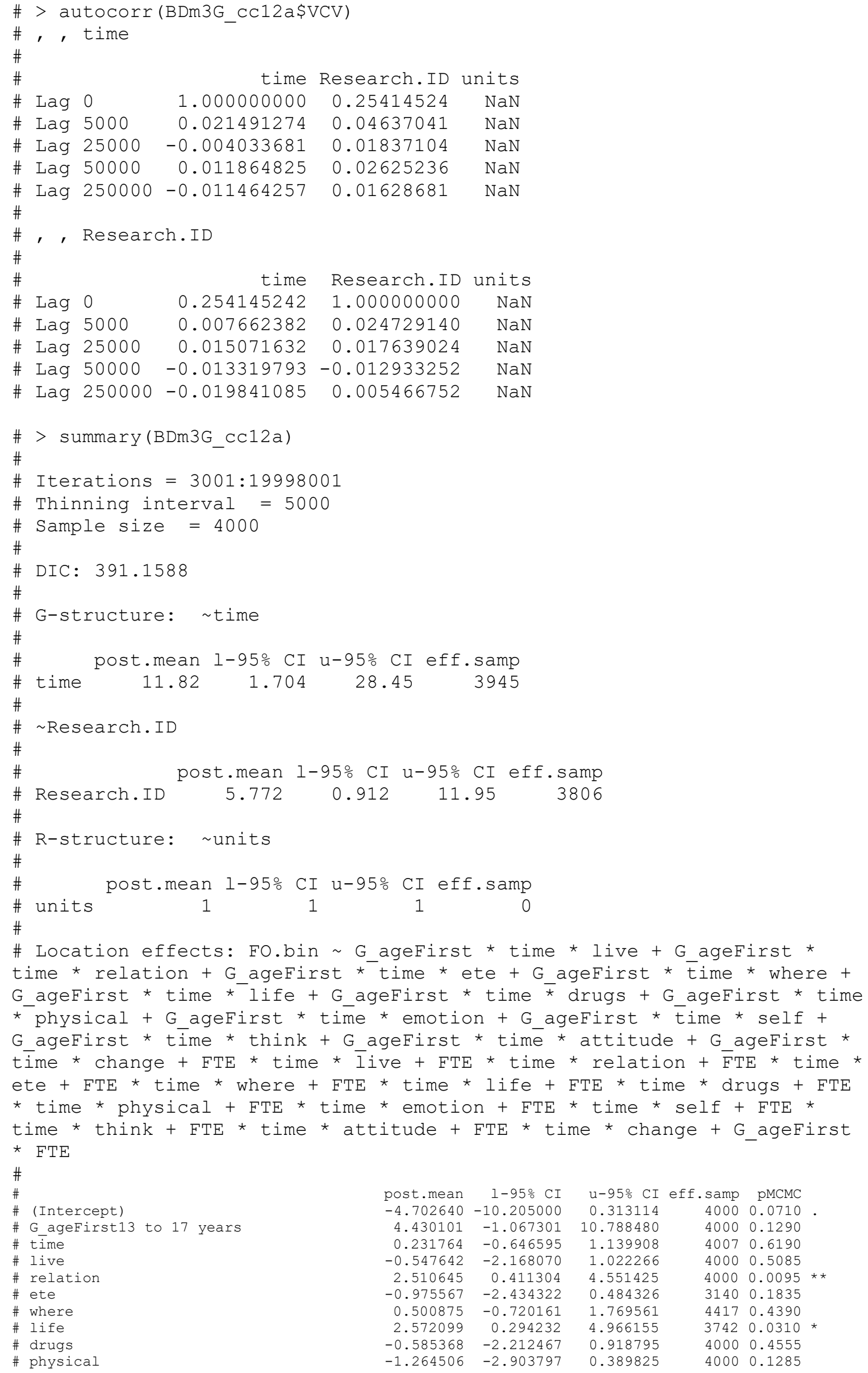




\begin{tabular}{|c|c|c|c|c|c|}
\hline \# & emotion & & -0.484623 & -1.829558 & 1.012913 \\
\hline \# & self & & -6.460357 & -9.678682 & -3.215738 \\
\hline \# & think & & 1.669118 & -0.704790 & 4.095740 \\
\hline \# & attitude & & 2.817919 & -0.291587 & 5.805046 \\
\hline \# & change & & 0.819301 & -1.721556 & 3.439919 \\
\hline$\#$ & FTE & & -4.847546 & -13.561338 & 4.455934 \\
\hline \# & G_ageFirst 13 to 17 & 17 years:time & -0.641117 & -1.928313 & 0.690669 \\
\hline \# & G_ageFirst 13 to 17 & 17 years:live & 1.115740 & -1.127051 & 3.368384 \\
\hline \# & time:live & & -0.155139 & -0.537235 & 0.203420 \\
\hline \# & G_ageFirst 13 to 17 & 17 years:relation & -3.813922 & -6.698231 & -1.011309 \\
\hline & time:relation & & -0.509886 & -1.007874 & -0.058906 \\
\hline \# & G_ageFirst 13 to 17 & 17 years:ete & 0.389873 & -1.441920 & 2.215190 \\
\hline \# & tīme:ete & & 0.190172 & -0.106355 & 0.518690 \\
\hline \# & G_ageFirst 13 to 17 & 17 years: where & 1.213332 & -0.736476 & 3.18305 \\
\hline \# & time:where & & -0.197742 & -0.480357 & 0.06053 \\
\hline \# & G_ageFirst 13 to 17 & 17 years:life & -2.511732 & -5.852417 & 0.716128 \\
\hline \# & time:life & & -0.521940 & -1.037114 & 0.056250 \\
\hline \# & G_ageFirst 13 to 17 & 17 years:drugs & 0.445641 & -1.513650 & 2.61 \\
\hline \# & time: drugs & & 0.344492 & 0.025170 & 0.67 \\
\hline \# & G_ageFirst 13 to 17 & 17 years:physical & -0.844165 & -3.195825 & 1.62 \\
\hline & tīme:physical & & 0.366109 & -0.04 & 0.76 \\
\hline \# & G_ageFirst 13 to 17 & 17 years:emotion & 0.053954 & -2.038371 & 1.919305 \\
\hline \# & tīme:emotion & & 0.360413 & 0.062408 & 0.67521 \\
\hline \# & G_ageFirst 13 to 17 & 17 years:self & 7.612935 & 3.84831 & 11.42241 \\
\hline \# & tīme:self & & 1.420245 & 0.78923 & 2.065964 \\
\hline \# & G_ageFirst 13 to 17 & 17 years:think & -0.458755 & -3.267825 & 2.433660 \\
\hline \# & time: think & & -0.349783 & -0.776270 & 0.105242 \\
\hline & G_ageFirst 13 to 17 & 17 years:attitude & -0.366802 & -4.182706 & 3.0 \\
\hline & tīme:attitude & & -0.825983 & -1.392150 & -0.2 \\
\hline \# & G_ageFirst 13 to 17 & 17 years: change & -2.899528 & -6.1 & 0.3 \\
\hline & time: change & 1 the & 0.122425 & -0.37812 & 0.613728 \\
\hline \# & time:FTE & & -0.892885 & -2.355309 & 0.574030 \\
\hline \# & live:FTE & & 1.071579 & -1.487255 & 4.14455 \\
\hline \# & relation:FTE & & -0.089557 & -2.834016 & 2.612893 \\
\hline \# & ete:FTE & & 1.041621 & -1.109479 & 3.288448 \\
\hline \# & where: FTE & & -4.046171 & -6.64 & -1.544 \\
\hline \# & life:FTE & & 2.175587 & -0 . & 5.6 \\
\hline & drugs: FTE & & -0.780049 & -3.3 & 1.7 \\
\hline \# & physical:FTE & & 1.036036 & -1.51 & 3.82 \\
\hline$\#$ & emotion:FTE & & -0.453750 & -2.45 & 1.701031 \\
\hline \# & self:FTE & & 2.172313 & -0.528967 & 4.841978 \\
\hline \# & think: FTE & & -2.247786 & -4.958328 & 0.372087 \\
\hline \# & attitude:FTE & & -3.612875 & -6.506613 & -1.077648 \\
\hline \# & change:FTE & & 3.066947 & 0.281370 & 6.043277 \\
\hline \# & G_ageFirst 13 to 17 & 17 years:FTE & 4.855018 & -3.669 & 13.759253 \\
\hline \# & G_ageFirst 13 to 17 & 17 years:time:live & 0.308343 & -0.289208 & 0.865401 \\
\hline \# & G_ageFirst 13 to 17 & 17 years:time:relation & 1.030753 & 0 & 1.6 \\
\hline \# & G_ageFirst 13 to 17 & 17 years:time:ete & -0.283843 & -0.7724 & 0.195 \\
\hline \# & G_ageFirst13 to 17 & 17 years:time: where & 0.032733 & -0.36 & 0.451480 \\
\hline \# & G_ageFirst 13 to 17 & 17 years:time:life & 0.367604 & -0.411213 & 1.212240 \\
\hline \# & G_ageFirst13 to 17 & 17 years:time:drugs & -0.622412 & -1.118620 & -0.106981 \\
\hline \# & G_ageFirst13 to 17 & 17 years:time:physical & 0.451169 & -0.294234 & 1.210187 \\
\hline \# & G_ageFirst13 to 17 & 17 years:time:emotion & -0.552947 & -1.172558 & 0.010831 \\
\hline \# & G_ageFirst13 to 17 & 17 years:time:self & -1.495552 & -2.288632 & -0.728005 \\
\hline \# & G_ageFirst13 to 17 & 17 years:time:think & -0.105656 & -0.76188 & 0.540139 \\
\hline \# & G_ageFirst 13 to 17 & 17 years:time:attitude & 0.071999 & -0.744118 & 0.831351 \\
\hline \# & G_ageFirst13 to 17 & 17 years:time:change & 0.598472 & 117981 & 1.367059 \\
\hline \# & tīme:live:FTE & & -0.548858 & -1.29 & 0.119 \\
\hline \# & time:relation:FTE & & -0.095832 & -0.806058 & 0.570128 \\
\hline \# & time:ete:FTE & & 0.422807 & -0.184195 & 1.054467 \\
\hline \# & time: where:FTE & & 0.571166 & 0.102918 & 1.033199 \\
\hline \# & time:life:FTE & & -0.497196 & -1.356102 & 0.283092 \\
\hline \# & time:drugs:FTE & & 0.655910 & 0.097017 & 1.267469 \\
\hline \# & time:physical:FTE & & -0.856352 & -1.701723 & -0.001359 \\
\hline \# & time:emotion:FTE & & 0.452900 & -0.160053 & 1.079197 \\
\hline$\#$ & time:self:FTE & & -0.771919 & -1.398545 & -0.135649 \\
\hline \# & time:think:FTE & & 0.753242 & 0.106954 & 1.468762 \\
\hline \# & time:attitude:FTE & & 1.065784 & 0.339676 & 1.868700 \\
\hline \# & time: change: FTE & & -0.949386 & -1.738856 & -0.210314 \\
\hline & ( & & & & \\
\hline & Signif. co & 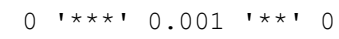 & & & 1 \\
\hline
\end{tabular}

$4000 \quad 0.5115$

$4000<3 e-04$ **

$4000 \quad 0.1660$

$4000 \quad 0.0670$.

$4000 \quad 0.5390$

$4000 \quad 0.2825$

$4000 \quad 0.3330$

$4000 \quad 0.3365$

$4000 \quad 0.4020$

40000.0050 **

40000.0260 *

$4000 \quad 0.6745$

$4000 \quad 0.2355$

$4000 \quad 0.2150$

44690.1390

$4000 \quad 0.1330$

40000.0600 .

$4000 \quad 0.6515$

38210.0340 *

$4000 \quad 0.4850$

$4000 \quad 0.0760$.

$4000 \quad 0.9370$

40000.0160 *

$4000<3 e-04 * * *$

$4000<3 e-04 * * *$

33330.7490

$4000 \quad 0.1160$

$4000 \quad 0.8350$

40000.0025 **

40000.0715 .

$4000 \quad 0.6315$

$4000 \quad 0.2150$

$4417 \quad 0.4535$

$4000 \quad 0.9580$

$4180 \quad 0.3315$

$4000<3 e-04 * * *$

$4000 \quad 0.1745$

$4000 \quad 0.5345$

$4000 \quad 0.4405$

44750.6665

43680.0970 .

$4000 \quad 0.0880$.

$40000.0055 * *$

42510.0370 *

$4216 \quad 0.2680$

$4543 \quad 0.2910$

40000.0015 **

$4000 \quad 0.2495$

$4413 \quad 0.8830$

$4000 \quad 0.3705$

39090.0130 *

$4000 \quad 0.2265$

40000.0610 .

$4000<3 e-04 * \star *$

36660.7600

$4000 \quad 0.8540$

$4000 \quad 0.1025$

$4234 \quad 0.1070$

$4000 \quad 0.7755$

40410.1710

37520.0090 **

$4000 \quad 0.2230$

$4000 \quad 0.0215$ *

40000.0380 *

$4000 \quad 0.1425$

40000.0085 **

40000.0250 *

$4000 \quad 0.0050$ **

41860.0135 * 
Trace of (Intercept)

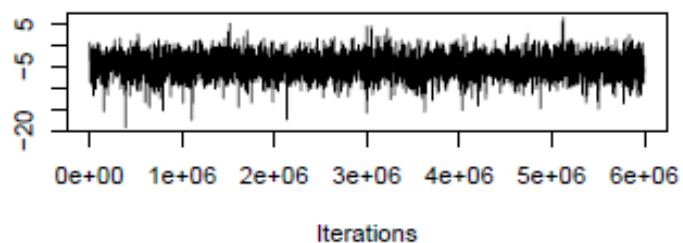

Trace of G_ageFirst13 to 17 years

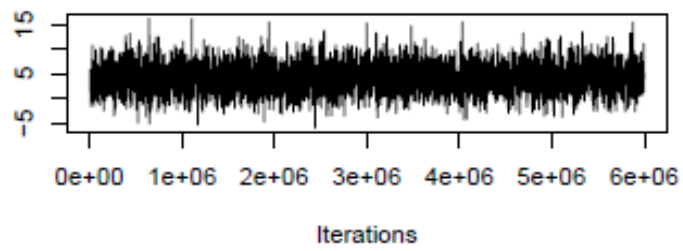

Trace of FTE

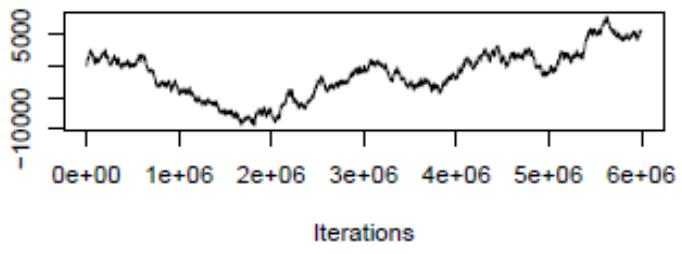

Trace of time

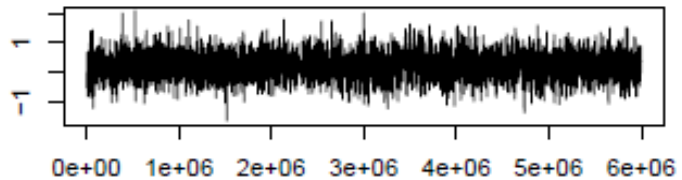

Iterations

Trace of live

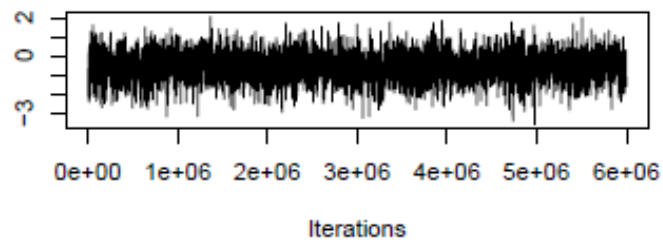

Trace of relation

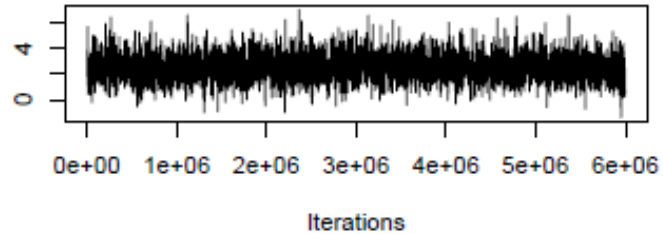

Density of (Intercept)

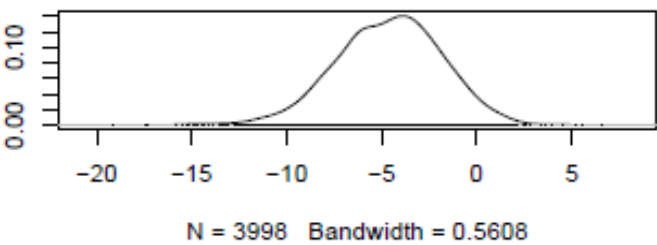

Density of G_ageFirst 13 to 17 years
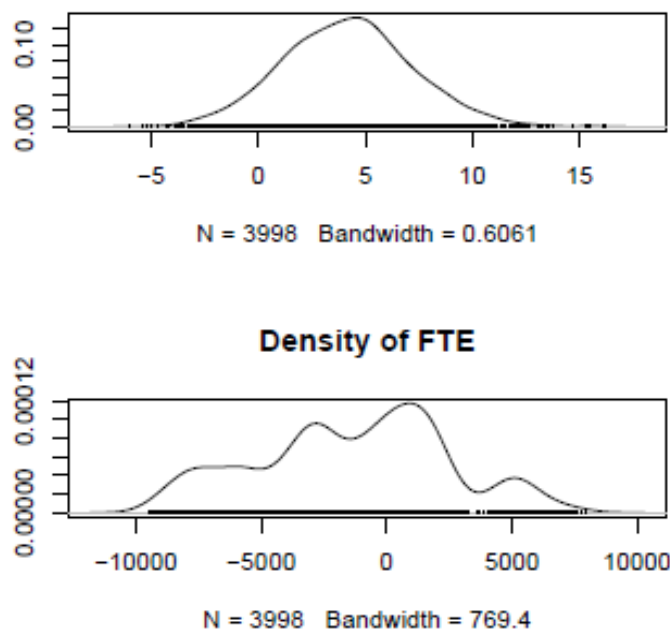

Density of time

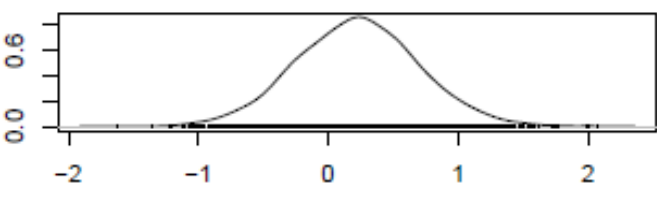

$\mathrm{N}=3998$ Bandwidth $=0.09461$

Density of live

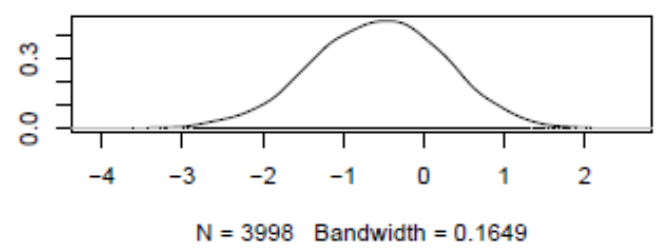

Density of relation

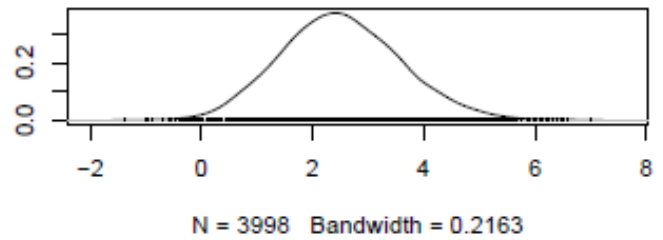


Trace of ete

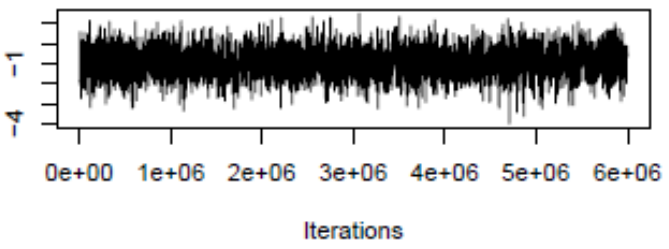

Trace of where

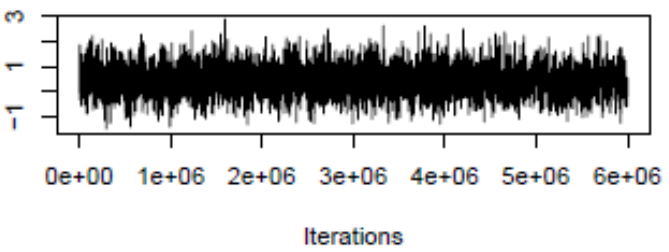

Trace of life

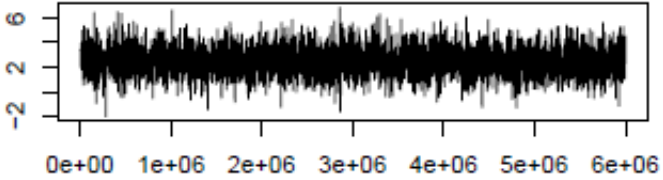

Iterations

\section{Trace of drugs}

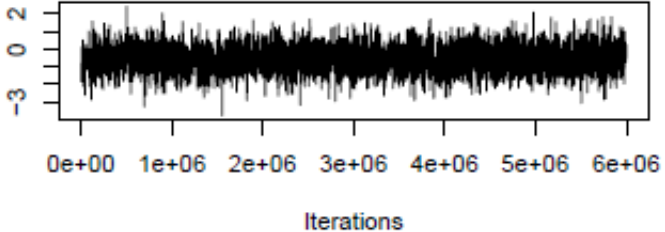

Trace of physical

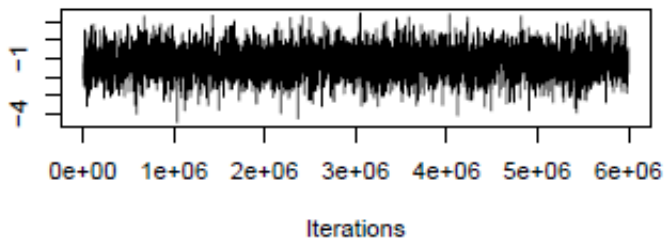

Trace of emotion

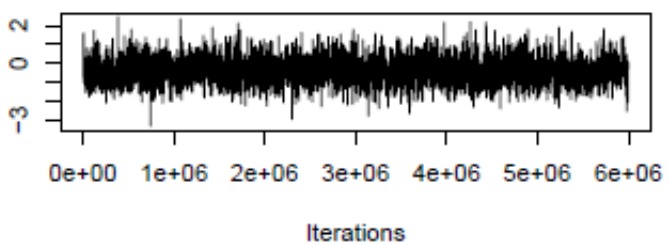

Density of ete

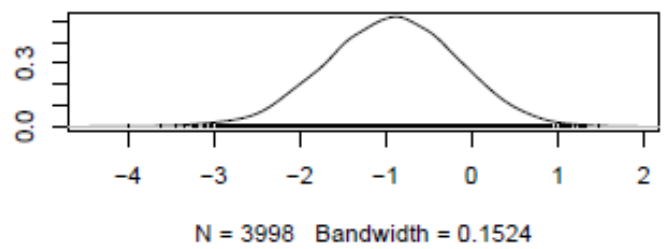

Density of where

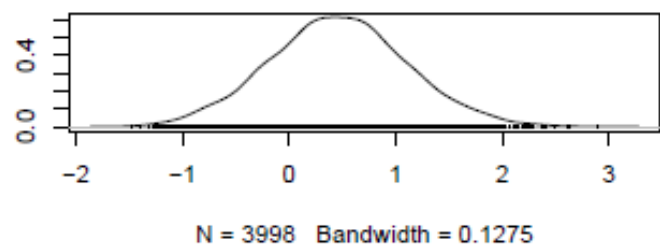

Density of life

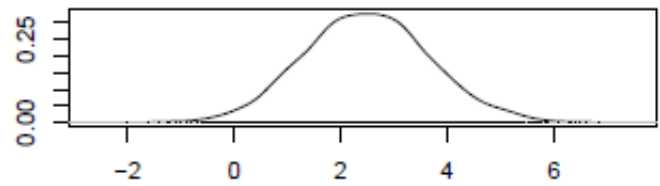

$\mathrm{N}=3998$ Bandwidth $=0.2345$

\section{Density of drugs}

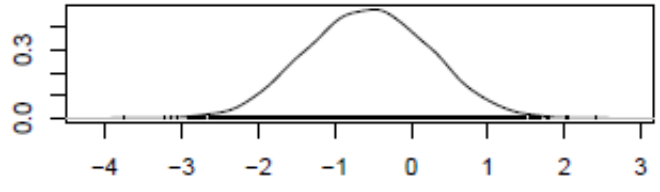

$\mathrm{N}=3998$ Bandwidth $=0.1616$

Density of physical

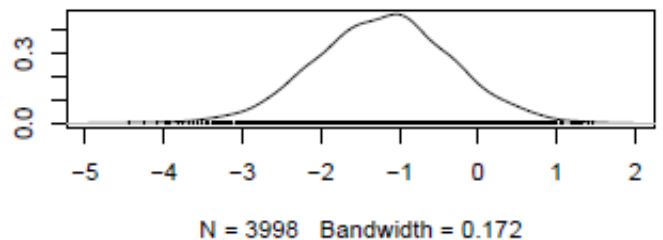

Density of emotion

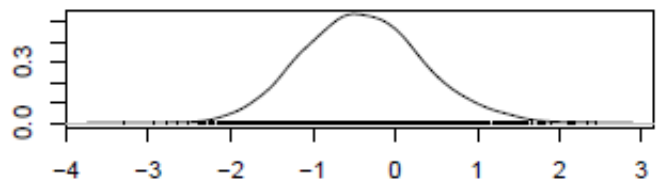

$\mathrm{N}=3998$ Bandwidth $=0.1465$ 
Trace of self

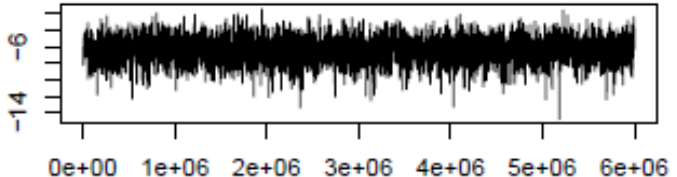

Iterations

\section{Trace of think}

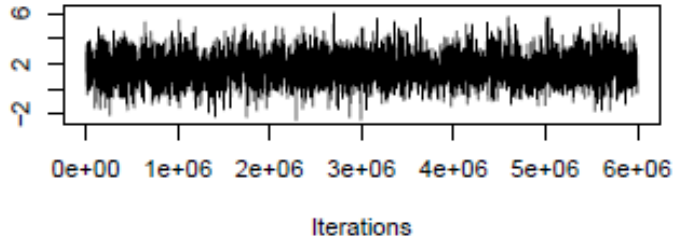

Trace of attitude

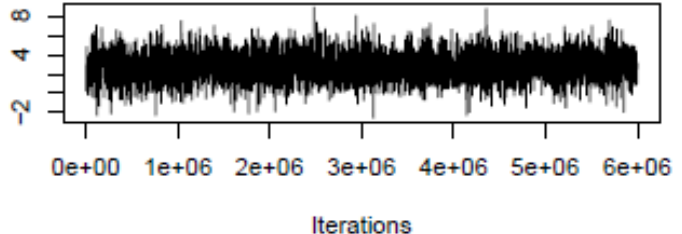

Trace of change

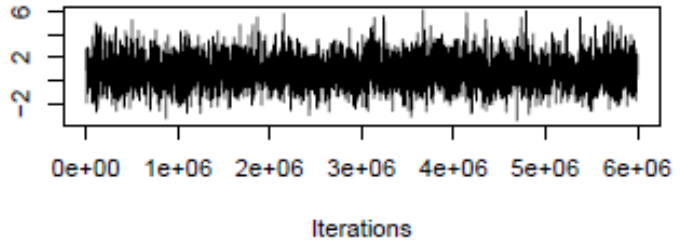

Trace of G_ageFirst13 to 17 years:FTE

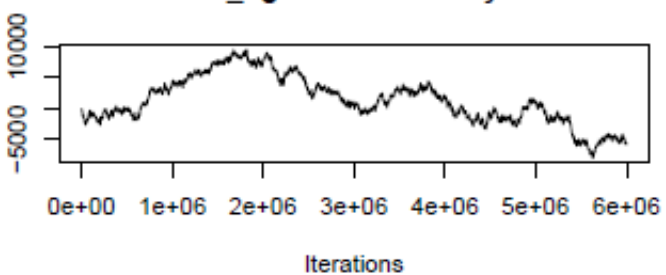

Trace of G_ageFirst13 to 17 years:time

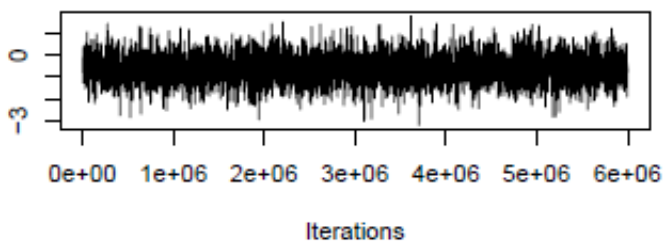

Density of self

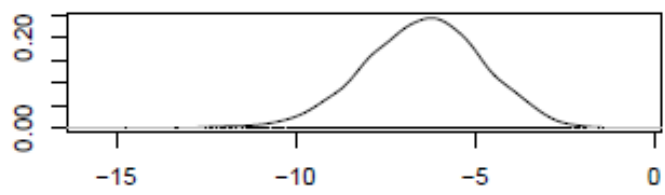

$\mathrm{N}=3998$ Bandwidth $=0.3331$

Density of think

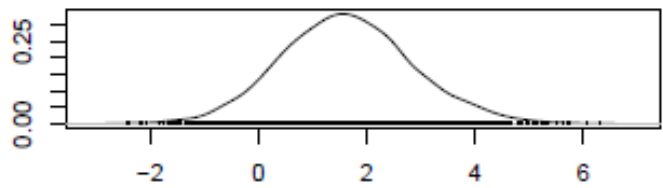

$\mathrm{N}=3998$ Bandwidth $=0.2412$

\section{Density of attitude}

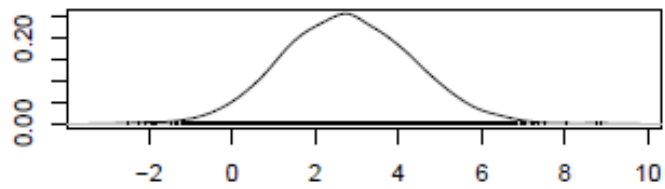

$\mathrm{N}=3998$ Bandwidth $=0.3106$

\section{Density of change}

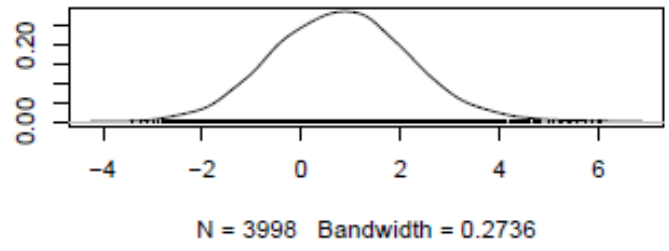

Density of G_ageFirst13 to 17 years:FTE

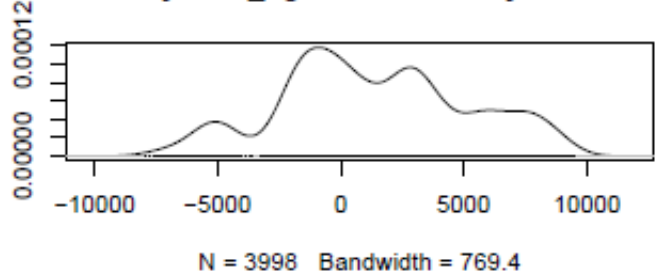

Density of G_ageFirst13 to 17 years:time

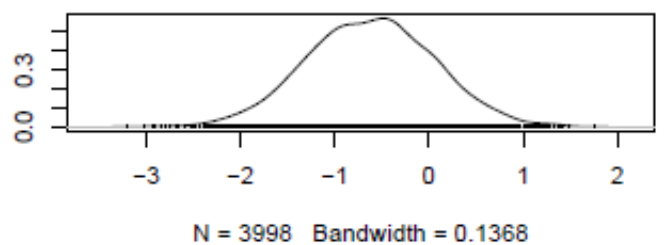


Trace of FTE:time

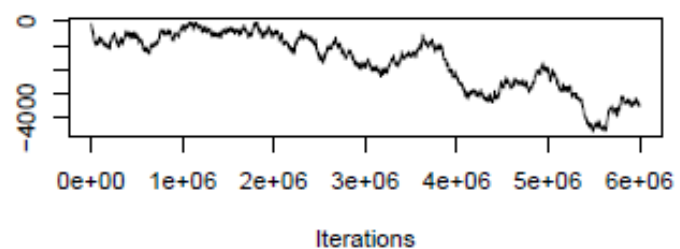

Trace of G ageFirst13 to 17 years:live

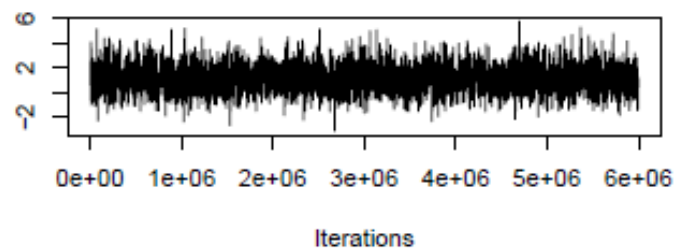

Trace of FTE:live

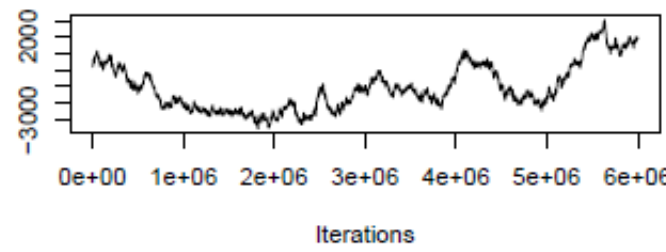

Trace of time:live

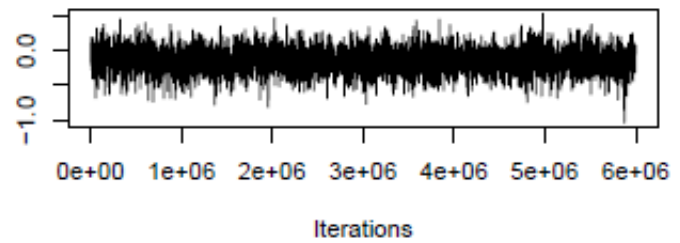

Trace of G_ageFirst13 to 17 years:relation

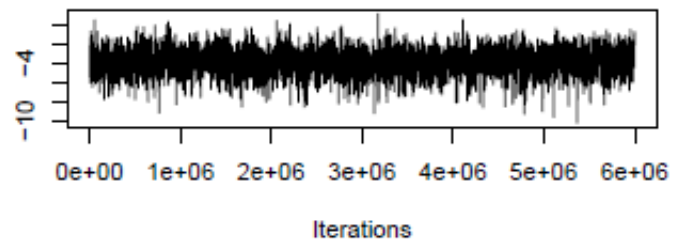

Trace of FTE:relation

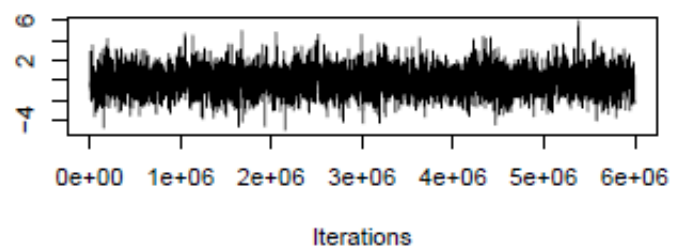

Density of FTE:time

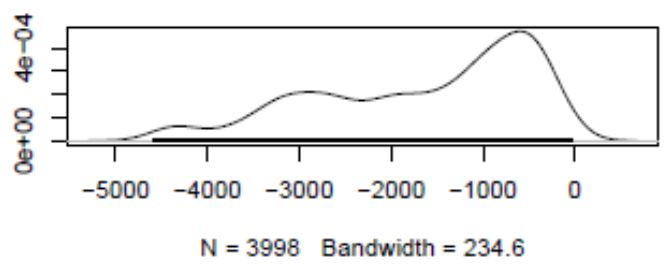

Density of G_ageFirst13 to 17 years:live

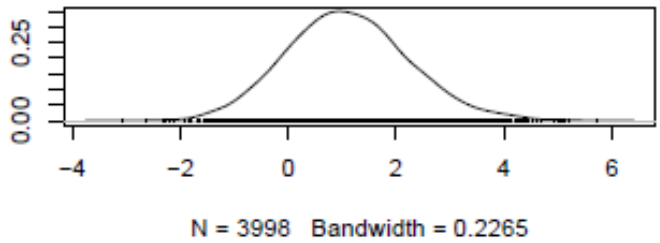

Density of FTE:live

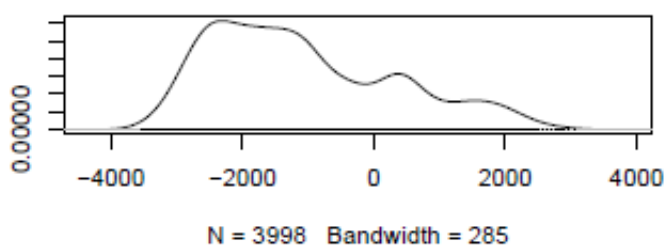

Density of time:live

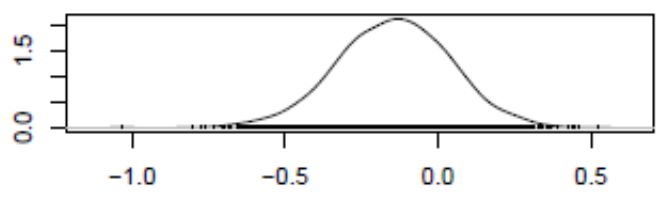

$\mathrm{N}=3998$ Bandwidth $=0.03749$

Density of G_ageFirst13 to 17 years:relation

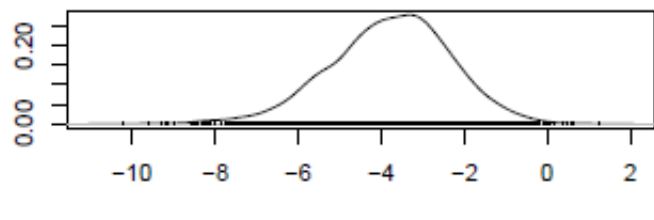

$\mathrm{N}=3998$ Bandwidth $=0.2833$

Density of FTE:relation

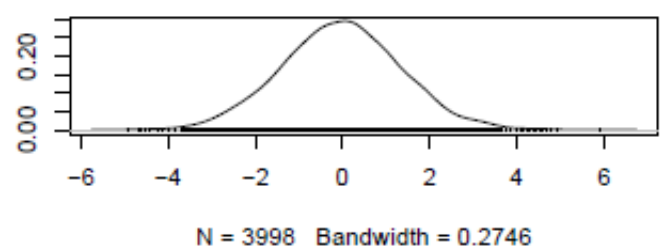


Trace of time:relation

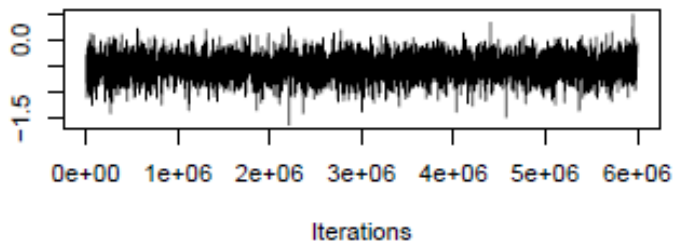

Trace of G_ageFirst13 to 17 years:ete

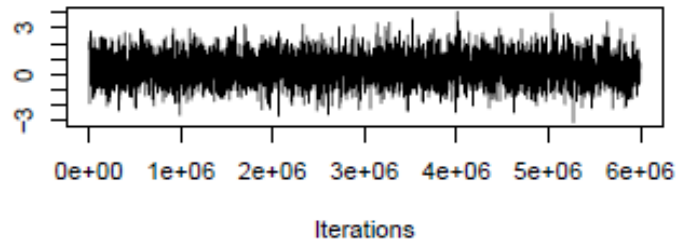

Trace of FTE:ete

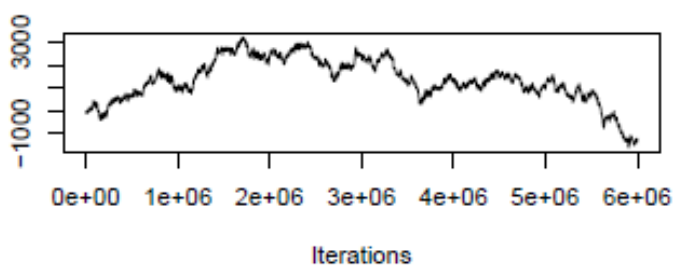

Trace of time:ete

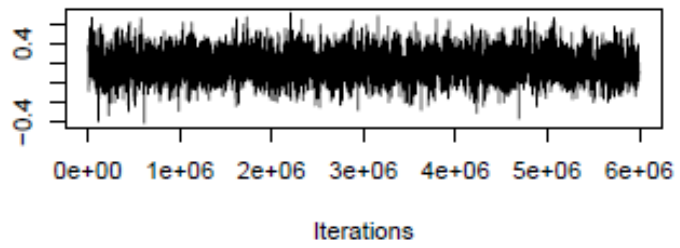

Trace of G_ageFirst13 to 17 years:where

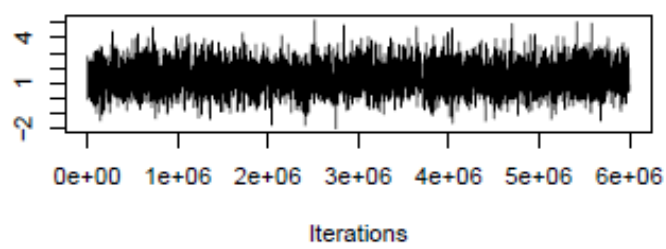

Trace of FTE:where

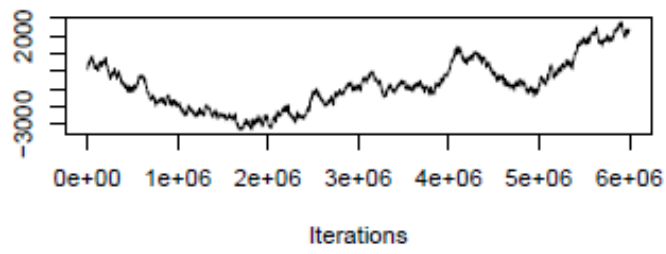

Density of time:relation

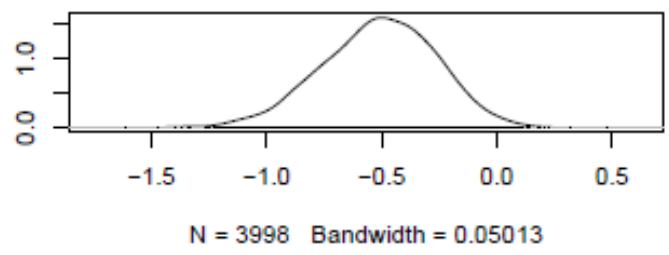

Density of G_ageFirst13 to 17 years:ete

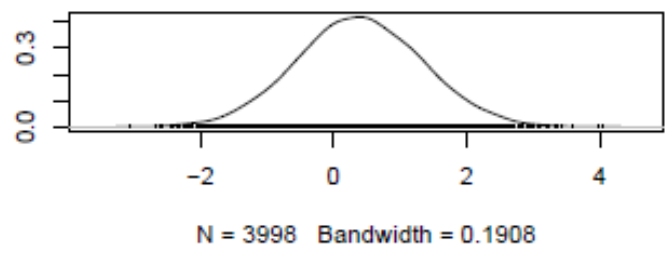

Density of FTE:ete

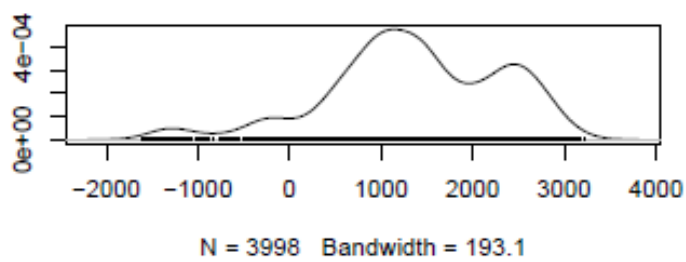

Density of time:ete

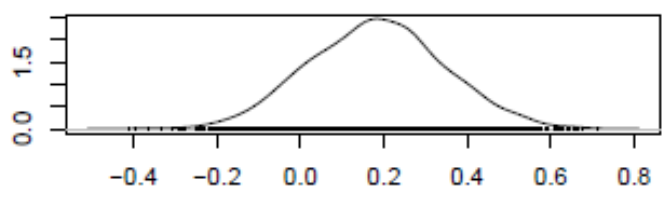

$\mathrm{N}=3998$ Bandwidth $=0.03256$

Density of G_ageFirst13 to 17 years:where

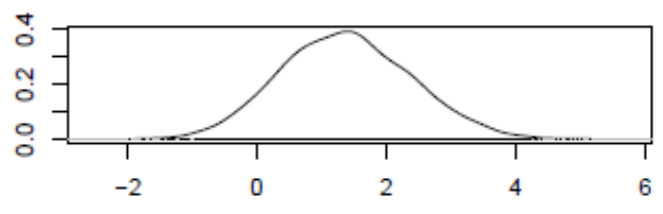

$\mathrm{N}=3998$ Bandwidth $=0.2039$

Density of FTE:where

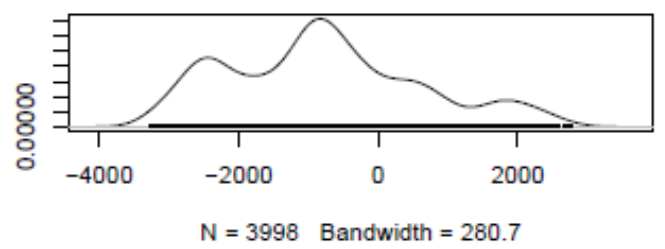


Trace of time:where

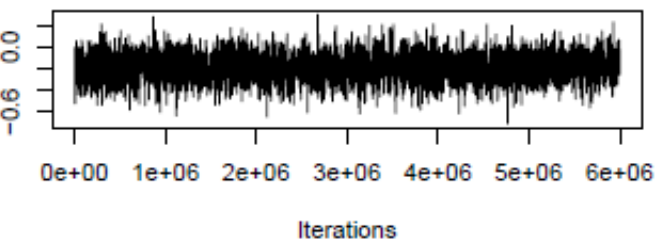

Trace of G_ageFirst13 to 17 years:life

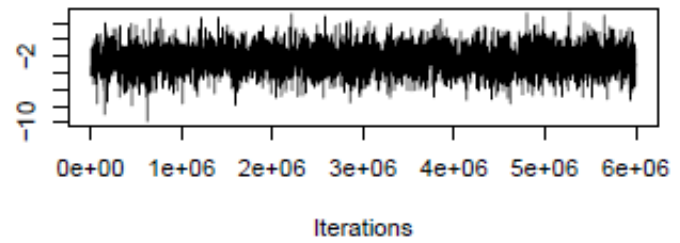

Trace of FTE:life

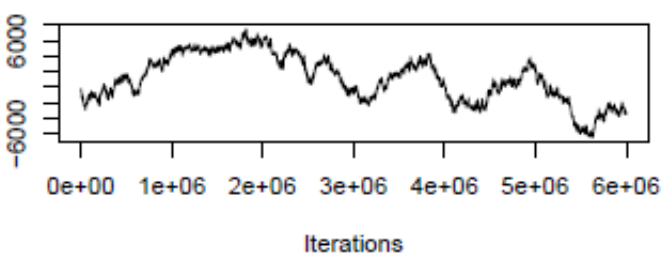

Trace of time:life

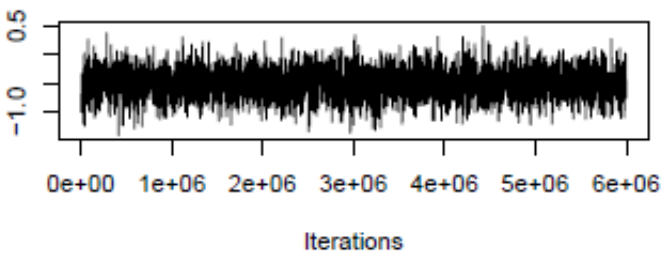

Trace of G_ageFirst13 to 17 years:drugs

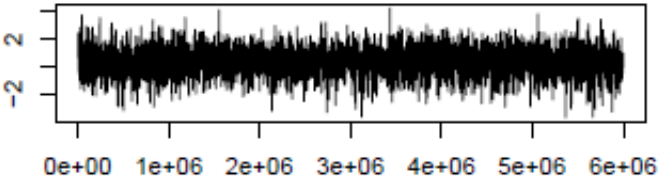

Iterations

\section{Trace of FTE:drugs}

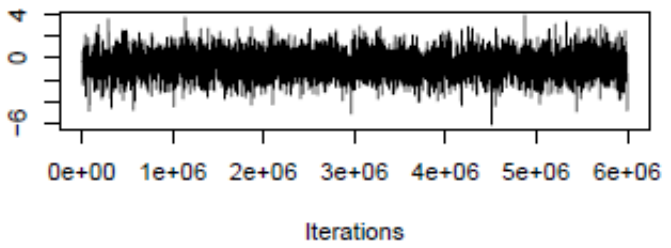

Density of time:where

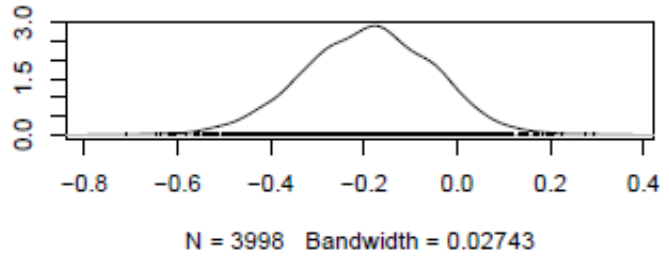

Density of G_ageFirst13 to 17 years:life

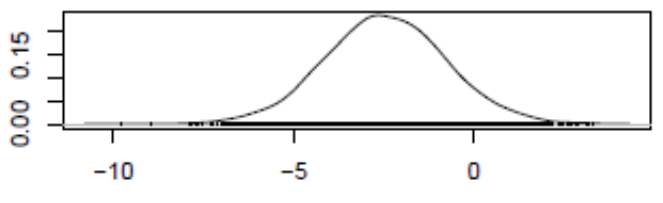

$\mathrm{N}=3998$ Bandwidth $=0.3336$

Density of FTE:life

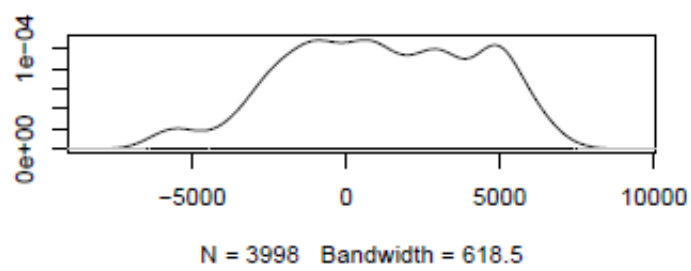

Density of time:life

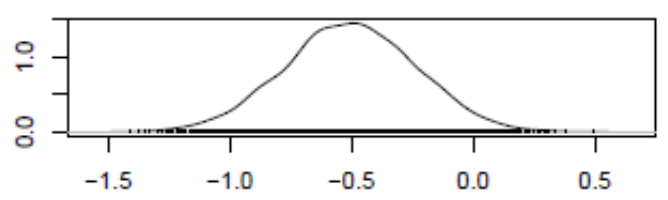

$\mathrm{N}=3998$ Bandwidth $=0.05433$

Density of G_ageFirst13 to 17 years:drugs

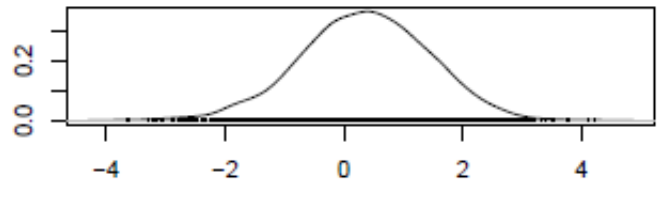

$\mathrm{N}=3998$ Bandwidth $=0.2146$

\section{Density of FTE:drugs}

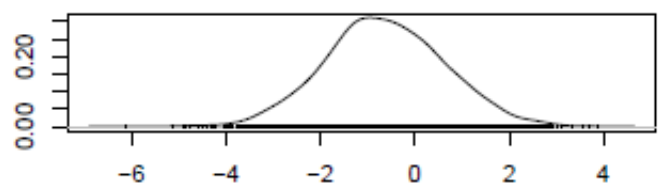

$\mathrm{N}=3998 \quad$ Bandwidth $=0.2531$ 
Trace of time:drugs

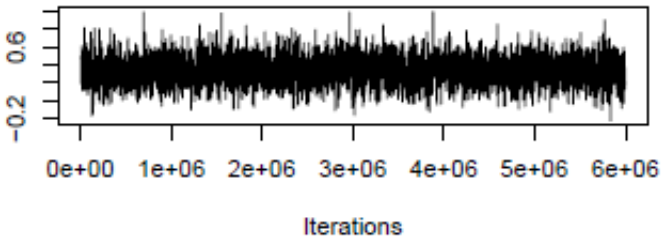

Trace of G_ageFirst13 to 17 years:physical

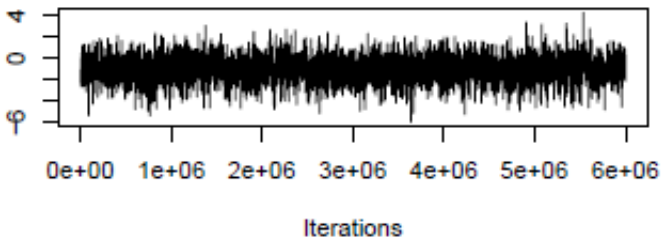

Trace of FTE:physical

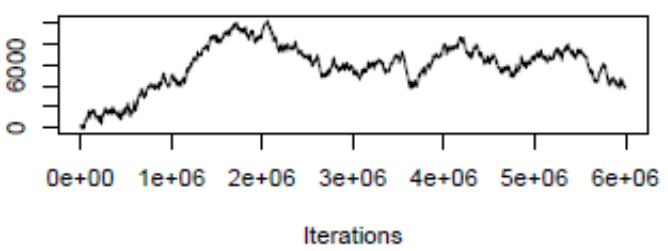

Trace of time:physical

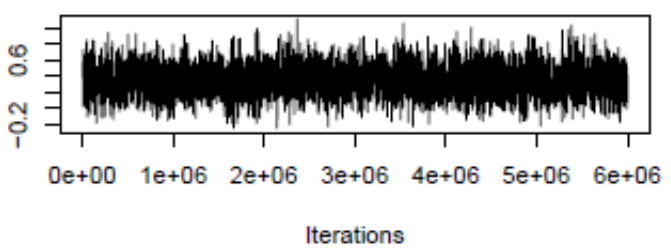

Trace of G_ageFirst 13 to 17 years:emotion

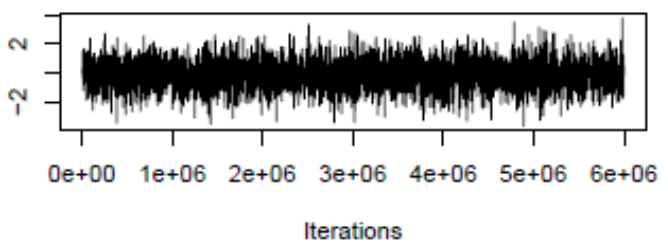

Trace of FTE:emotion

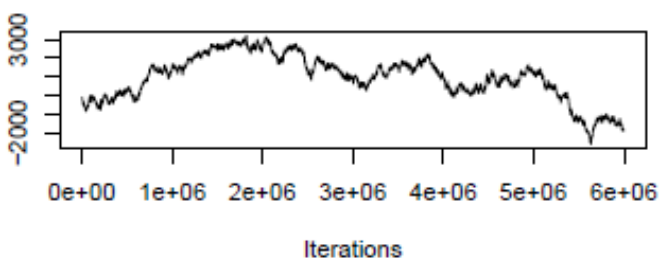

Density of time:drugs

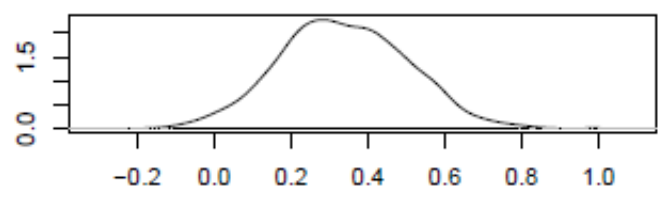

$\mathrm{N}=3998$ Bandwidth $=0.03355$

Density of G_ageFirst13 to 17 years:physical

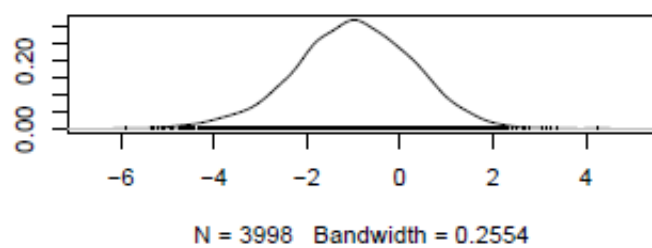

Density of FTE:physical

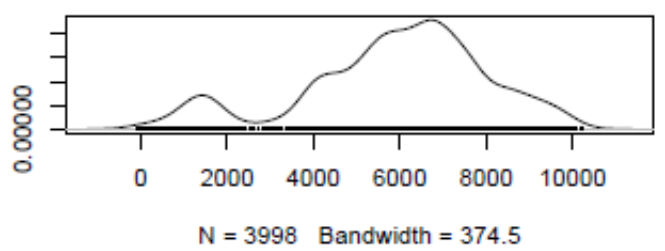

Density of time:physical

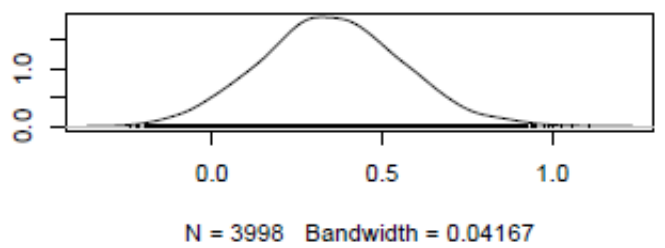

Density of G_ageFirst13 to 17 years:emotion

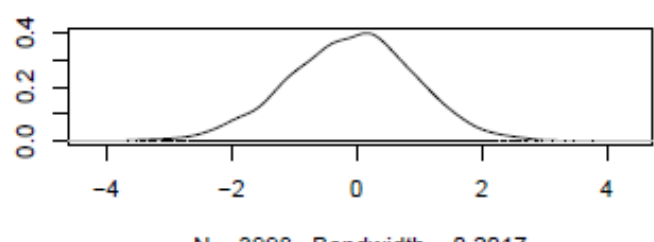

$\mathrm{N}=3998$ Bandwidth $=0.2017$

Density of FTE:emotion

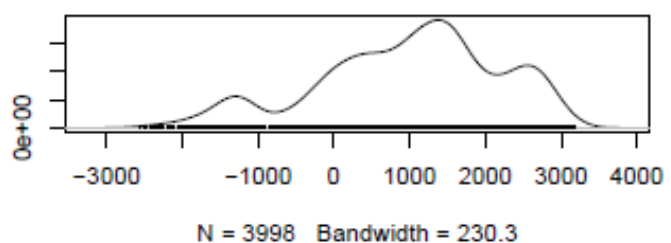


Trace of time:emotion

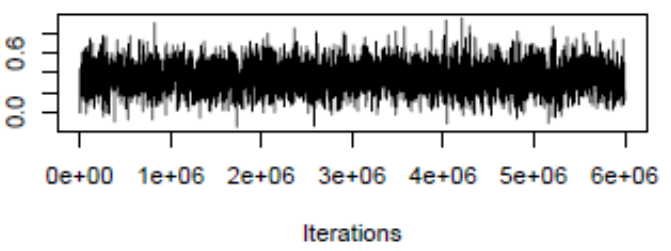

Trace of G_ageFirst13 to 17 years:self

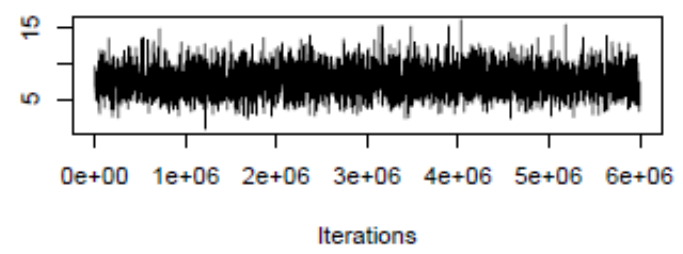

Trace of FTE:self

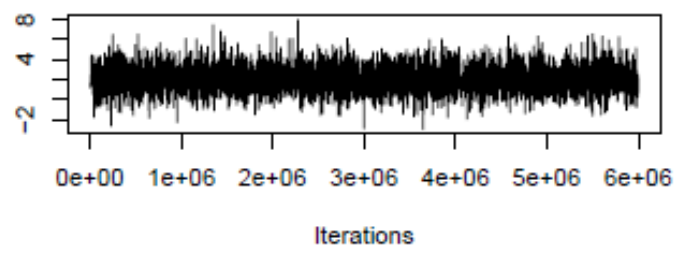

Trace of time:self

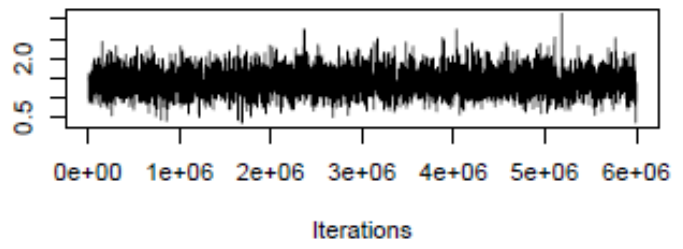

Trace of G_ageFirst13 to 17 years:think

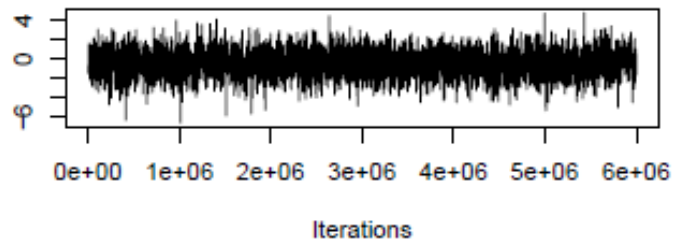

Trace of FTE:think

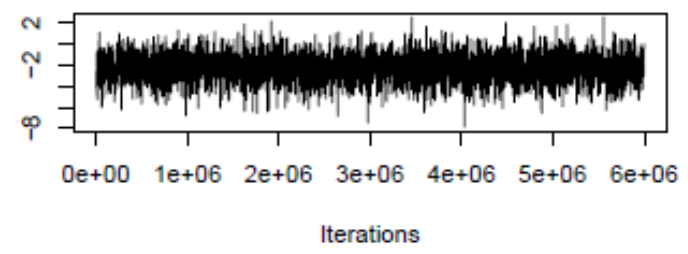

Density of time:emotion

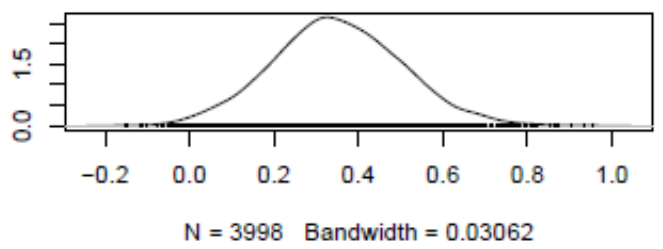

Density of G_ageFirst13 to 17 years:self

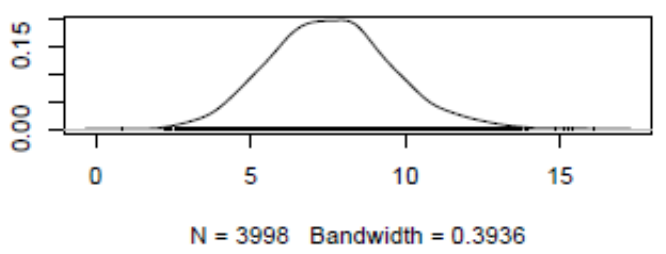

Density of FTE:self

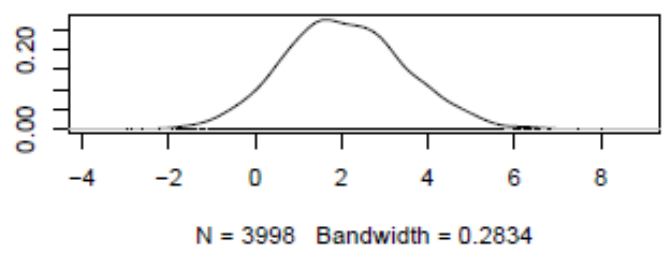

Density of time:self

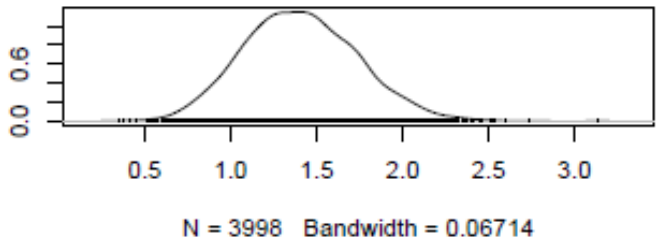

Density of G_ageFirst13 to 17 years:think

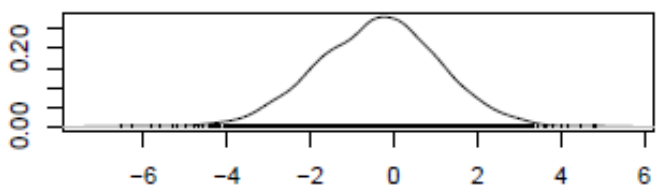

$\mathrm{N}=3998$ Bandwidth $=0.2913$

\section{Density of FTE:think}

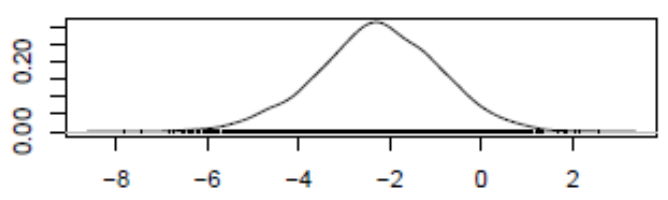

$\mathrm{N}=3998$ Bandwidth $=0.2653$ 
Trace of time:think

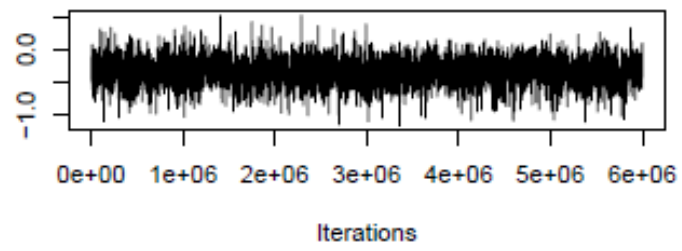

Trace of G_ageFirst 13 to 17 years:attitude

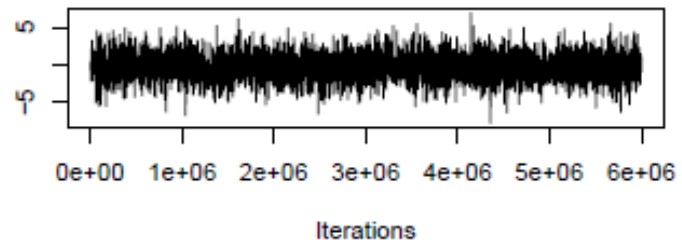

Trace of FTE:attitude

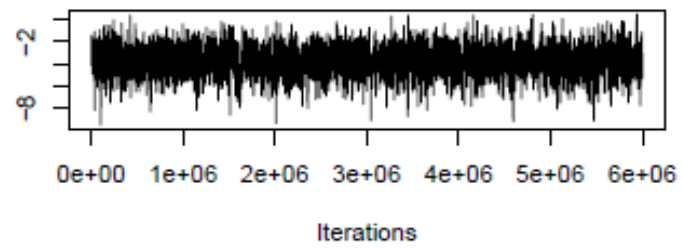

Trace of time:attitude

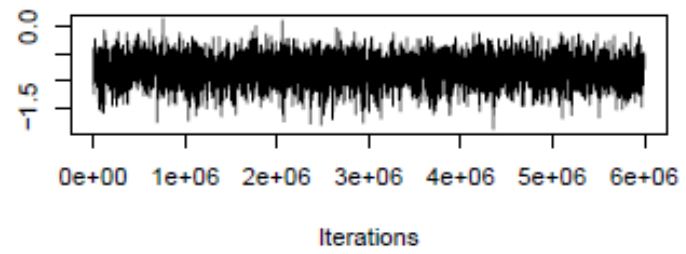

Trace of G_ageFirst13 to 17 years:change

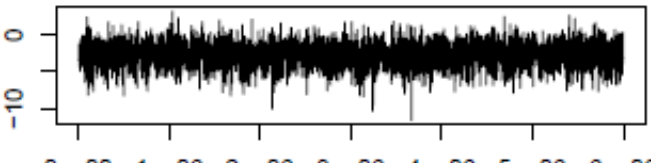

Iterations

\section{Trace of FTE:change}

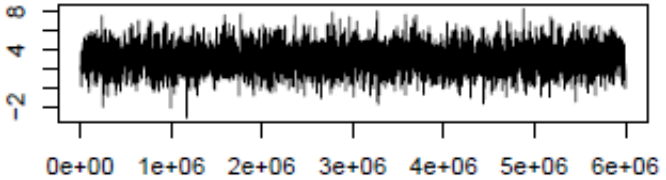

Iterations
Density of time:think

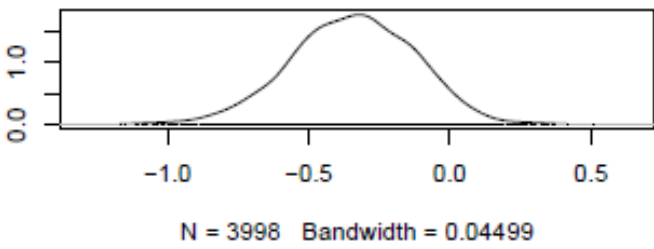

Density of G_ageFirst13 to 17 years:attitude

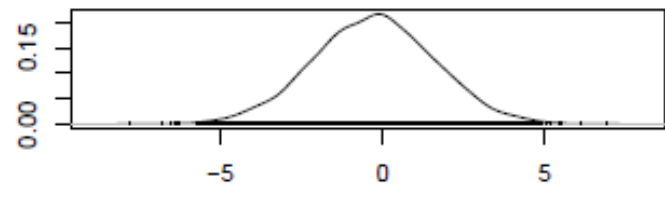

$\mathrm{N}=3998$ Bandwidth $=0.3708$

Density of FTE:attitude

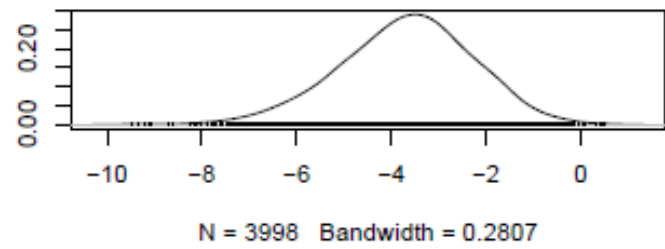

Density of time:attitude

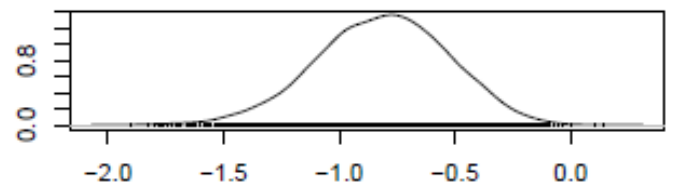

$\mathrm{N}=3998$ Bandwidth $=0.05711$

Density of G_ageFirst13 to 17 years:change

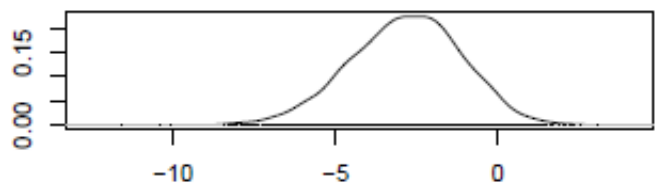

$\mathrm{N}=3998$ Bandwidth $=0.3466$

Density of FTE:change

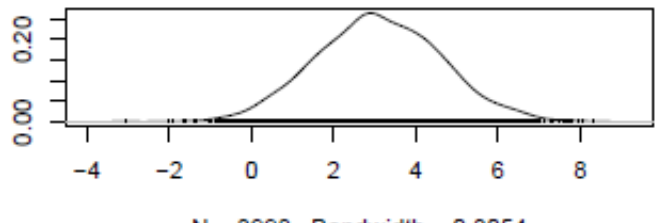

$\mathrm{N}=3998$ Bandwidth $=0.3054$ 
Trace of time:change

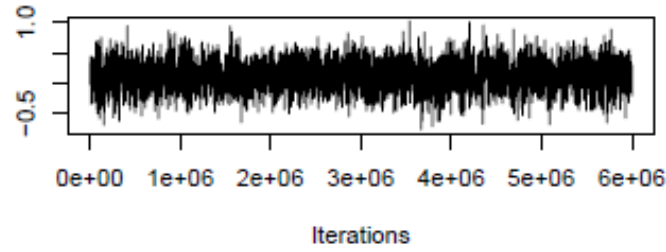

Trace of G_ageFirst13 to 17 years:FTE:time

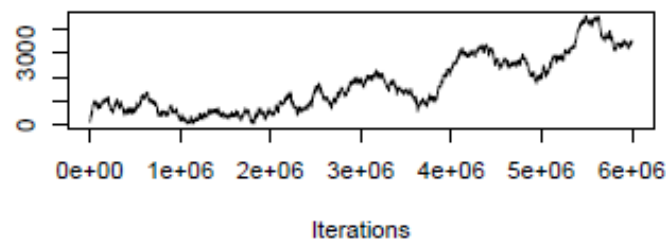

Trace of G_ageFirst 13 to 17 years:FTE:live

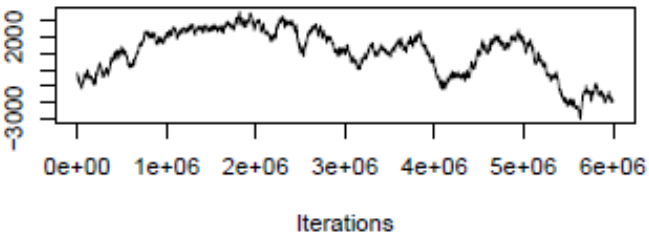

Trace of G_ageFirst13 to 17 years:time:live

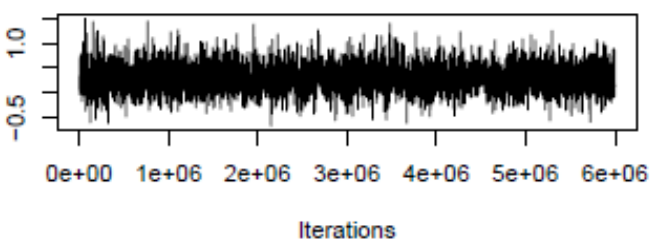

\section{Trace of FTE:time:live}

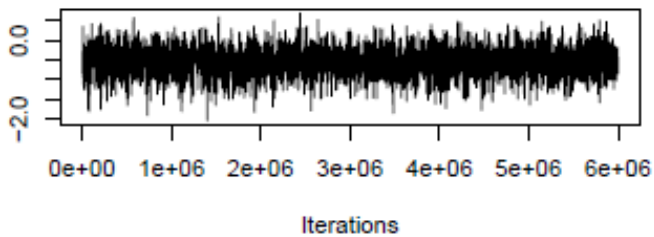

Trace of G_ageFirst13 to 17 years:time:relation

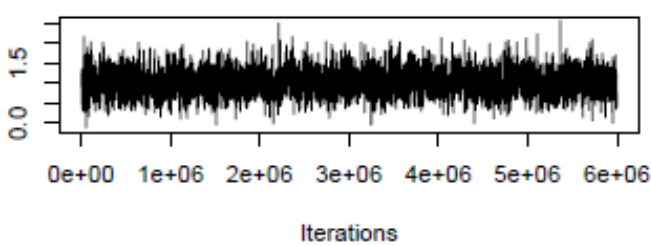

Density of time:change

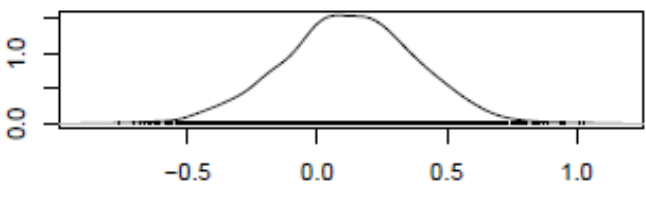

$\mathrm{N}=3998$ Bandwidth $=0.05003$

Density of G_ageFirst13 to 17 years:FTE:time

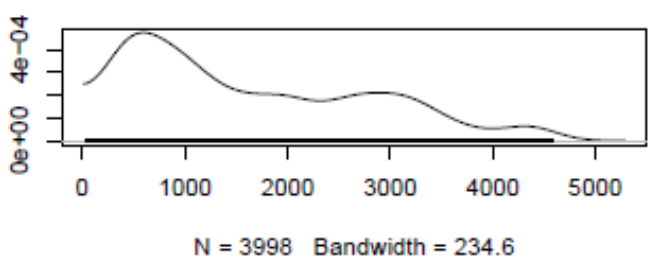

Density of G_ageFirst13 to 17 years:FTE:live

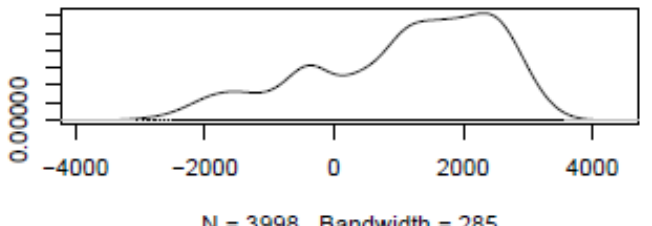

Density of G_ageFirst13 to 17 years:time:live

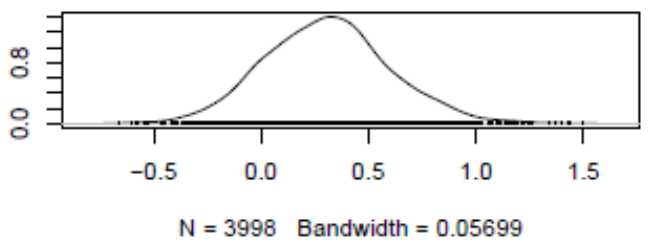

Density of FTE:time:live

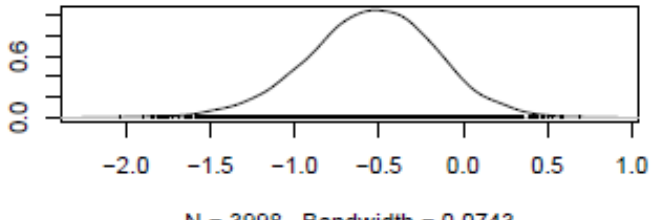

$\mathrm{N}=3998$ Bandwidth $=0.0743$

Density of G_ageFirst13 to 17 years:time:relation

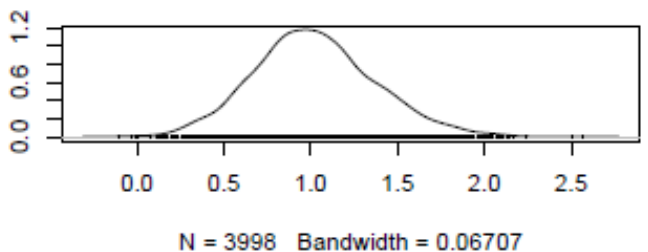


Trace of FTE:time:relation

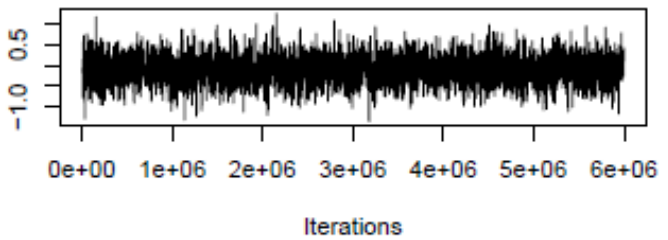

Trace of G_ageFirst13 to 17 years:FTE:ete

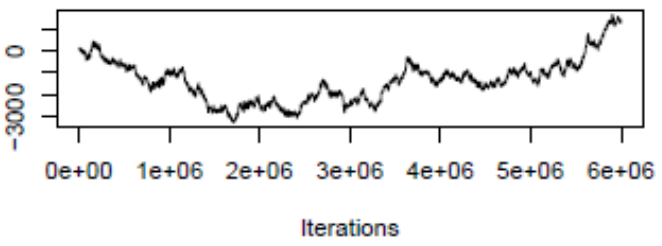

Trace of G_ageFirst13 to 17 years:time:ete

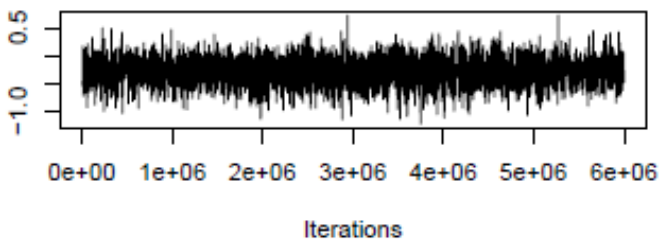

Trace of FTE:time:ete

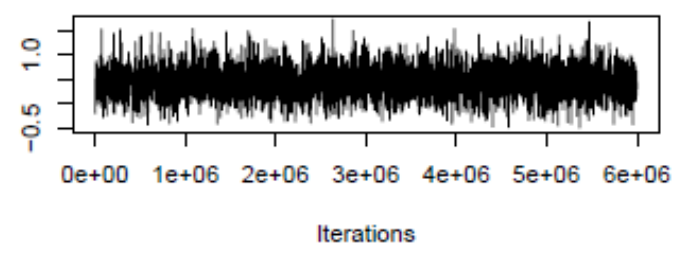

Trace of G_ageFirst13 to 17 years:FTE:where

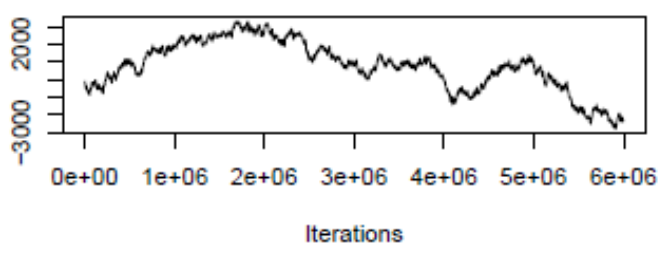

Trace of G_ageFirst13 to 17 years:time:where

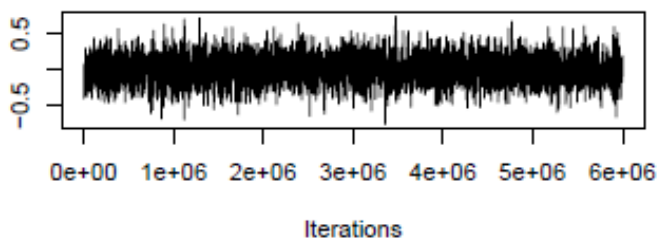

Density of FTE:time:relation

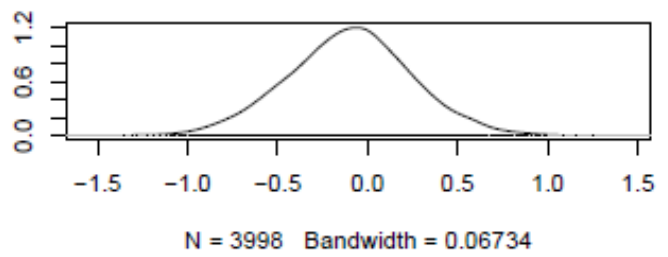

Density of G_ageFirst13 to 17 years:FTE:ete

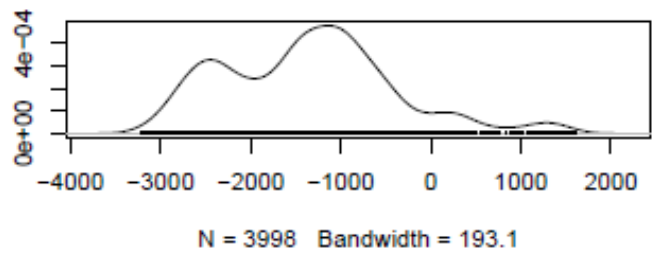

Density of G_ageFirst 13 to 17 years:time:ete

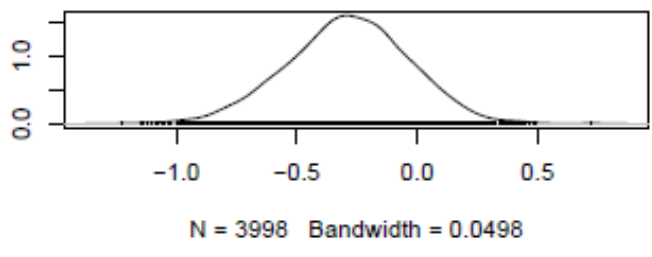

Density of FTE:time:ete

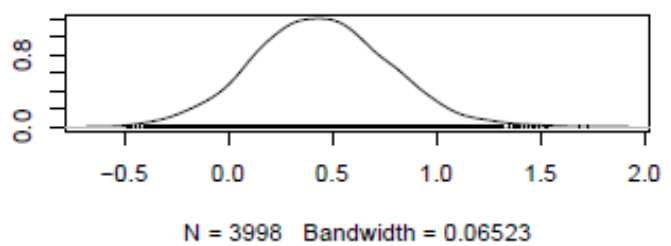

Density of G_ageFirst13 to 17 years:FTE:where

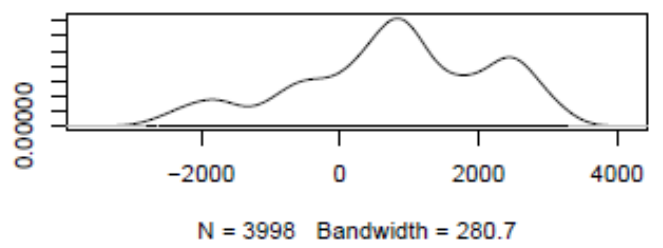

Density of G_ageFirst13 to 17 years:time:where

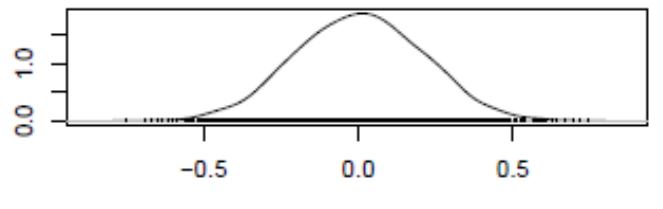

$\mathrm{N}=3998$ Bandwidth $=0.04175$ 
Trace of FTE:time:where

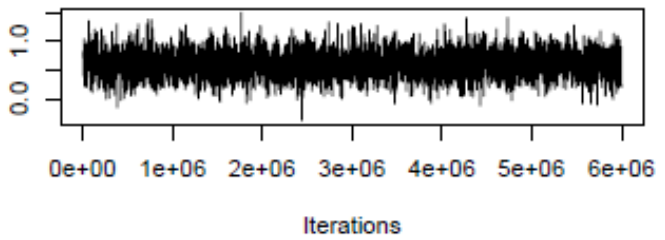

Trace of G_ageFirst13 to 17 years:FTE:life

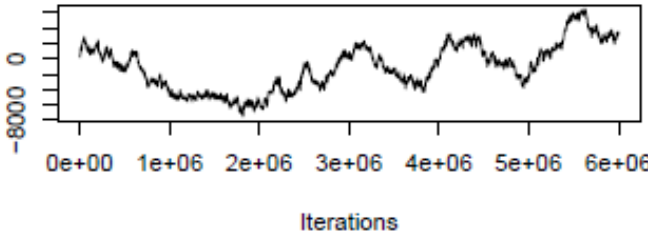

Trace of G_ageFirst13 to 17 years:time:life

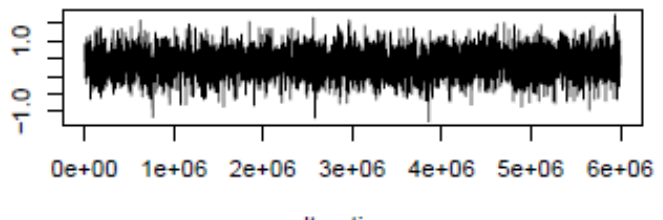

Iterations

Trace of FTE:time:life

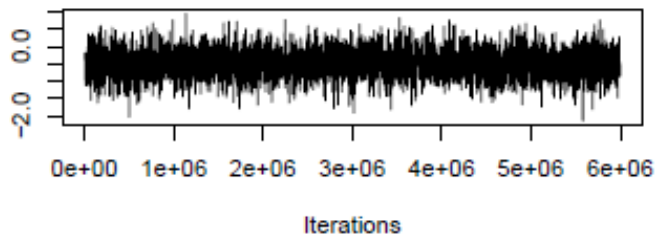

Trace of G_ageFirst13 to 17 years:time:drugs

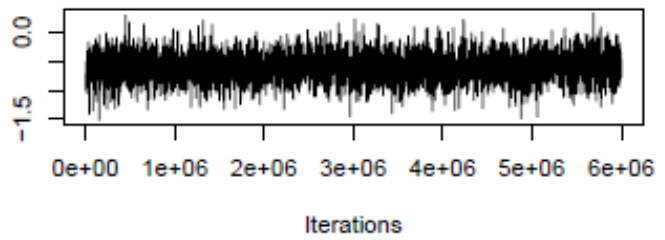

Trace of FTE:time:drugs

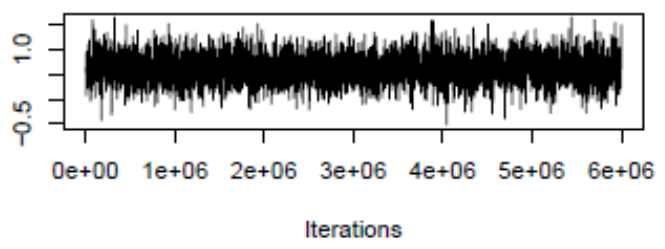

Density of FTE:time:where

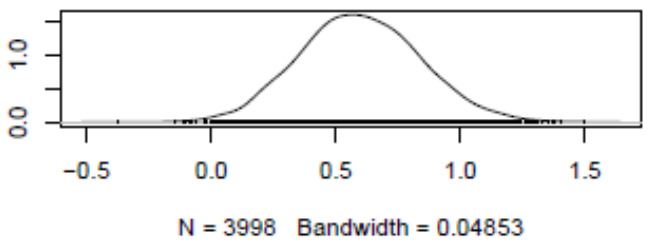

Density of G_ageFirst13 to 17 years:FTE:life

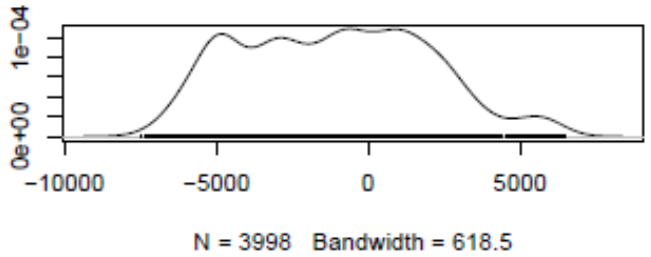

Density of G_ageFirst13 to 17 years:time:life

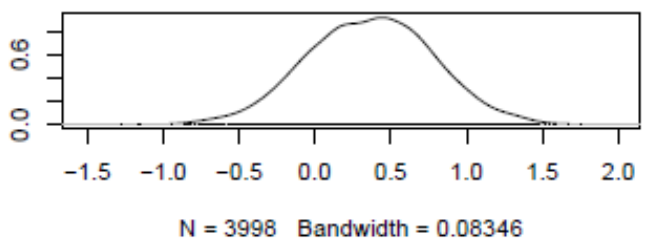

Density of FTE:time:life

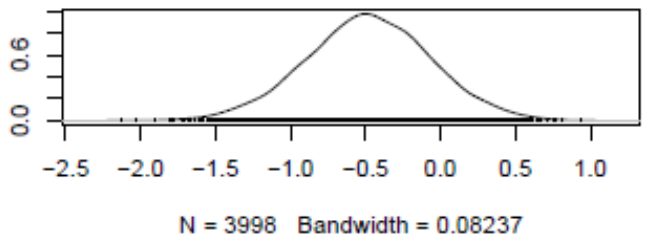

Density of G_ageFirst13 to 17 years:time:drugs

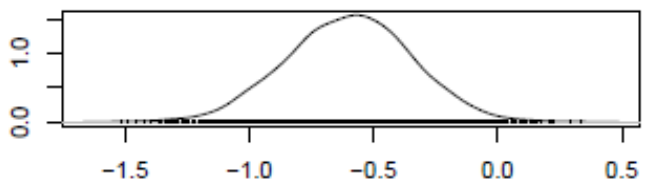

$\mathrm{N}=3998$ Bandwidth $=0.05085$

Density of FTE:time:drugs

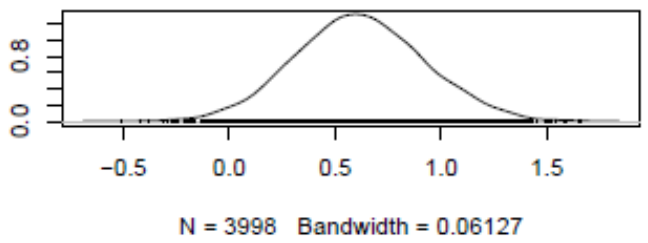


Trace of G_ageFirst13 to 17 years:FTE:physical

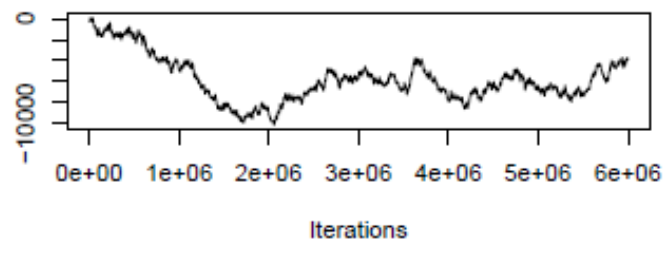

Trace of G_ageFirst13 to 17 years:time:physical

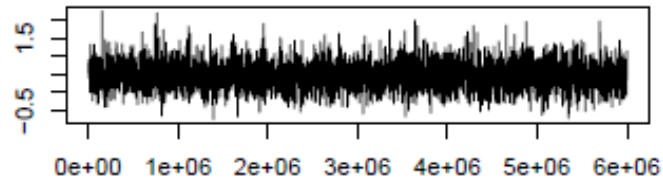

Iterations

Trace of FTE:time:physical

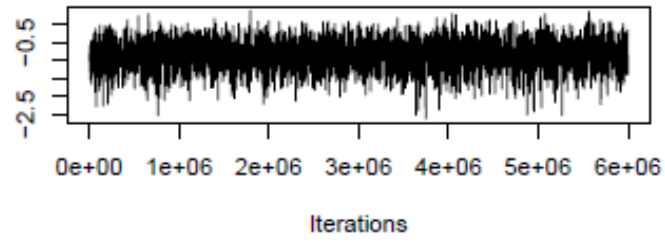

Trace of G_ageFirst13 to 17 years:FTE:emotion

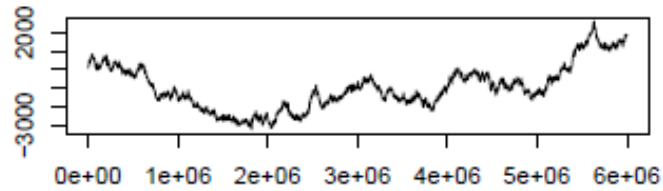

Iterations

Trace of G_ageFirst13 to 17 years:time:emotion

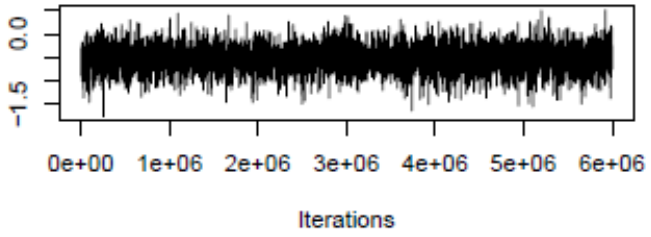

Trace of FTE:time:emotion

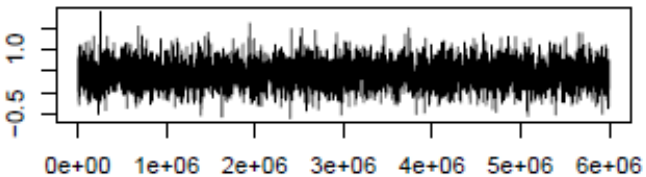

Iterations
Density of G_ageFirst13 to 17 years:FTE:physic:

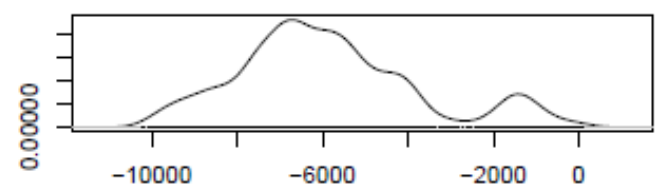

$\mathrm{N}=3998$ Bandwidth $=374.3$

Density of G_ageFirst13 to 17 years:time:physic:

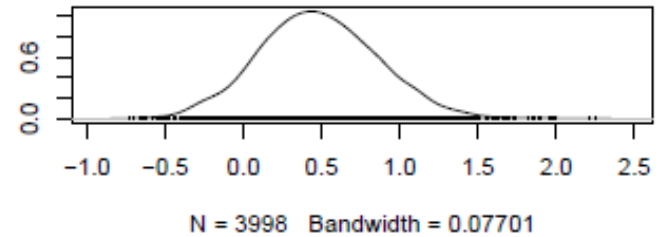

Density of FTE:time:physical

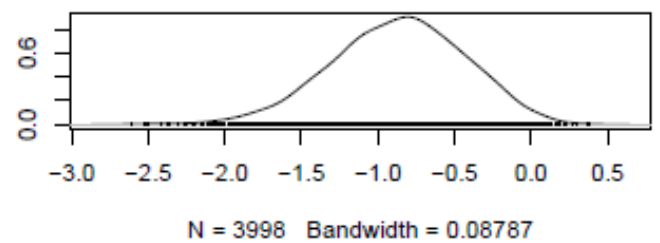

Density of G_ageFirst13 to 17 years:FTE:emotio

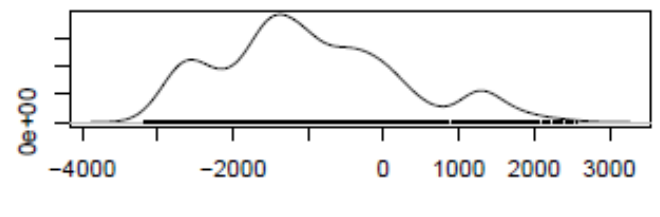

$\mathrm{N}=3998$ Bandwidth $=230.3$

Density of G_ageFirst13 to 17 years:time:emotior

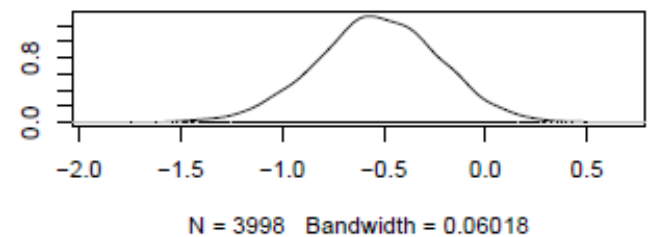

Density of FTE:time:emotion

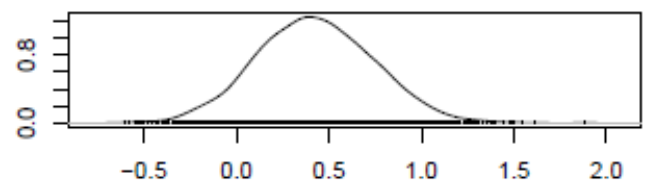

$\mathrm{N}=3998$ Bandwidth $=0.06392$ 
Trace of G_ageFirst13 to 17 years:time:self

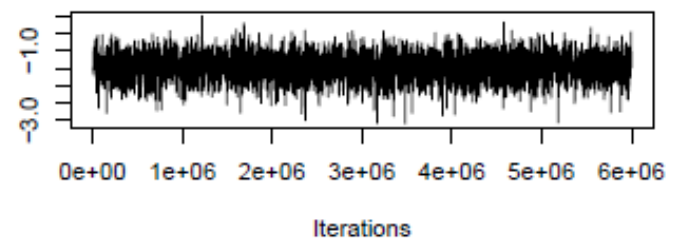

Trace of FTE:time:self

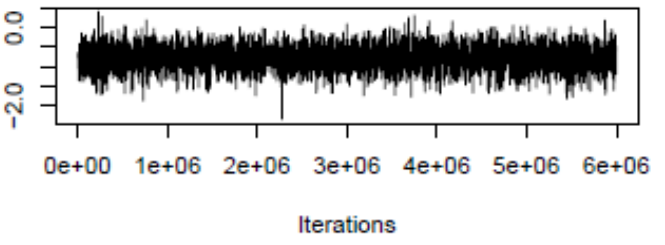

Trace of G_ageFirst13 to 17 years:time:think

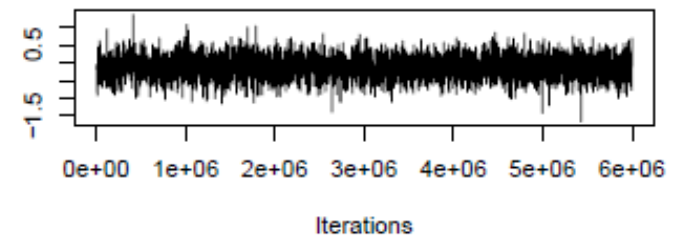

Trace of FTE:time:think

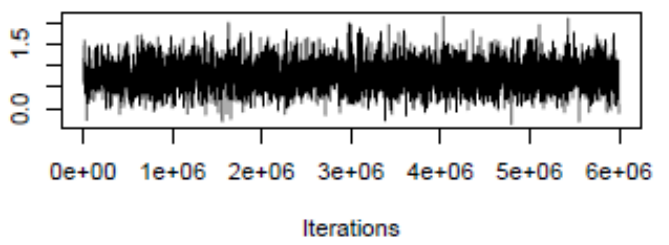

Trace of G_ageFirst13 to 17 years:time:attitude

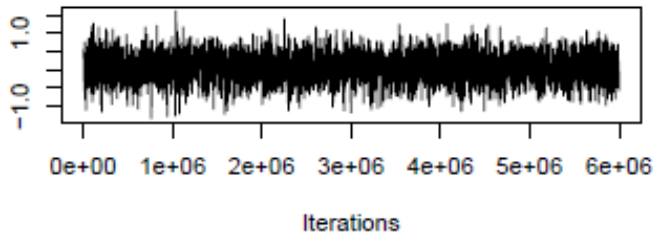

Trace of FTE:time:attitude

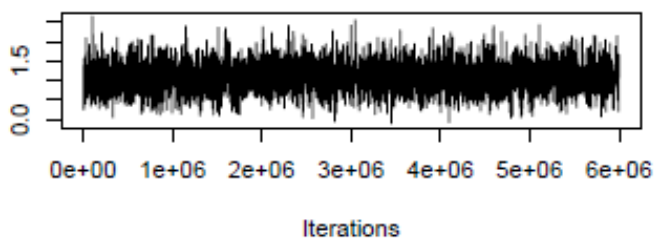

Density of G_ageFirst13 to 17 years:time:self

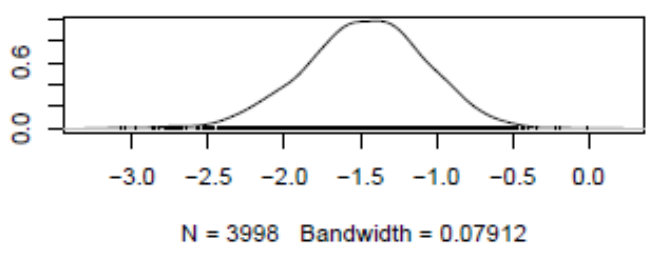

Density of FTE:time:self

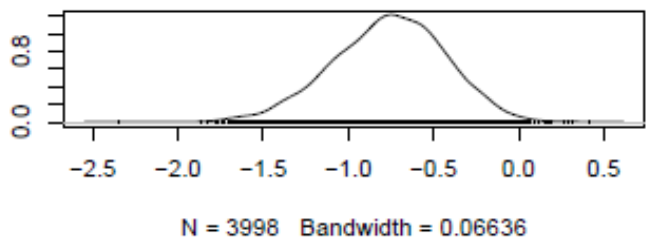

Density of G_ageFirst13 to 17 years:time:think

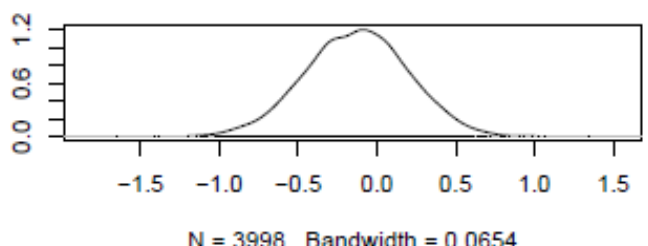

Density of FTE:time:think

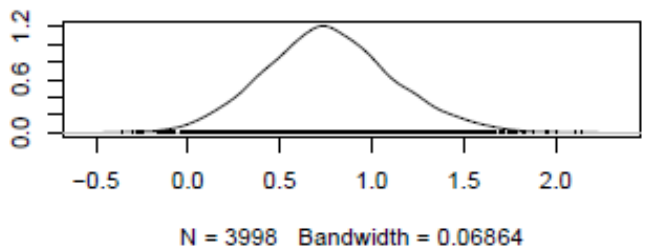

Density of G_ageFirst13 to 17 years:time:attitude

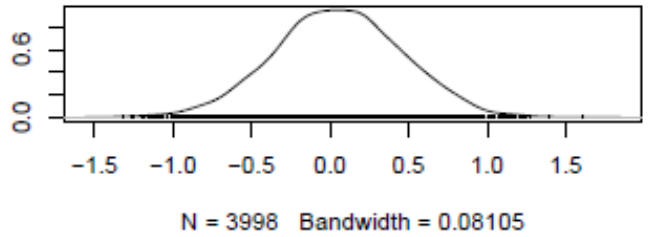

Density of FTE:time:attitude

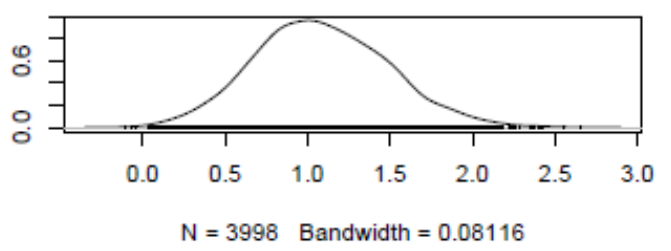


Trace of G_ageFirst13 to 17 years:time:change

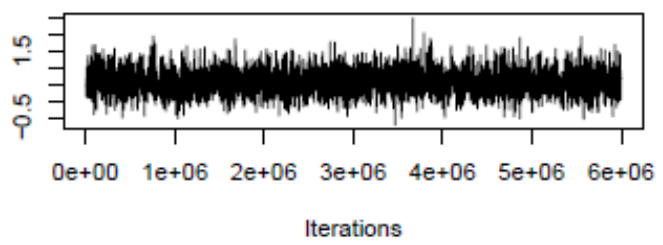

Trace of FTE:time:change

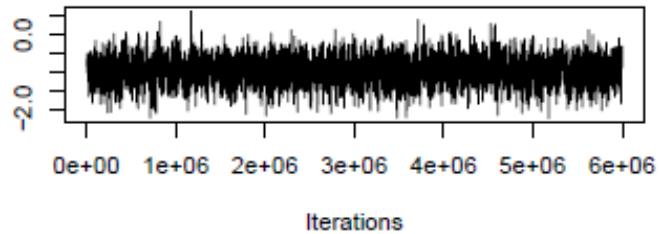

$\underline{\text { Random Effects }}$

Trace of time

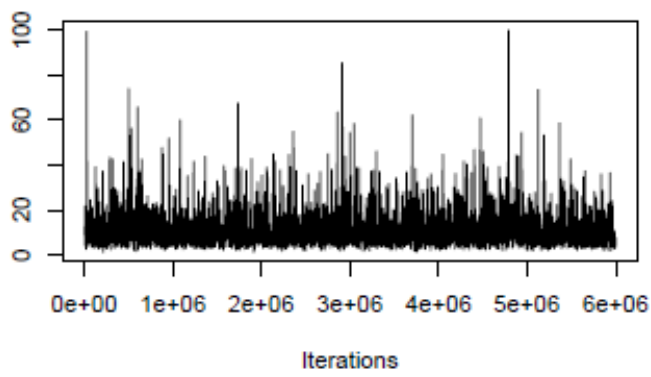

Trace of Research.ID

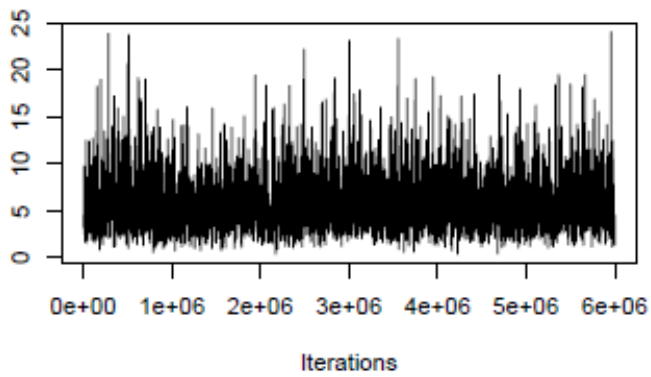

Density of G_ageFirst13 to 17 years:time:change

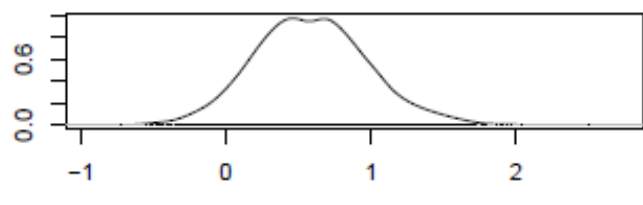

$\mathrm{N}=3998$ Bandwidth $=0.07836$

Density of FTE:time:change

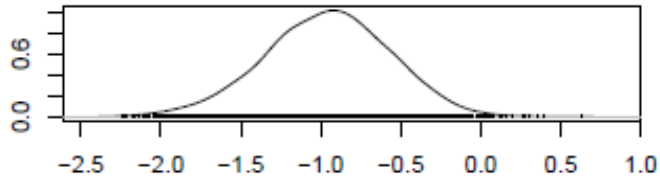

$\mathrm{N}=3998$ Bandwidth $=0.07897$

Density of time

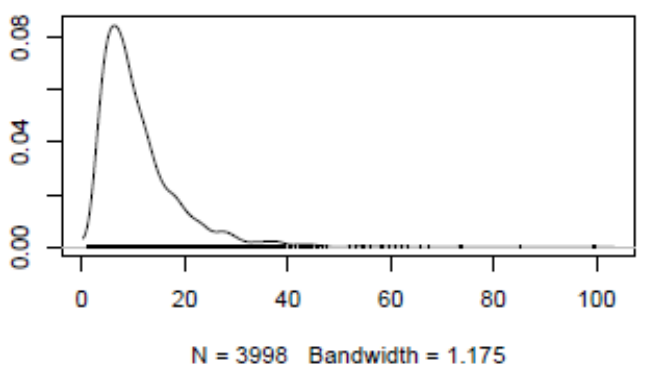

Density of Research.ID

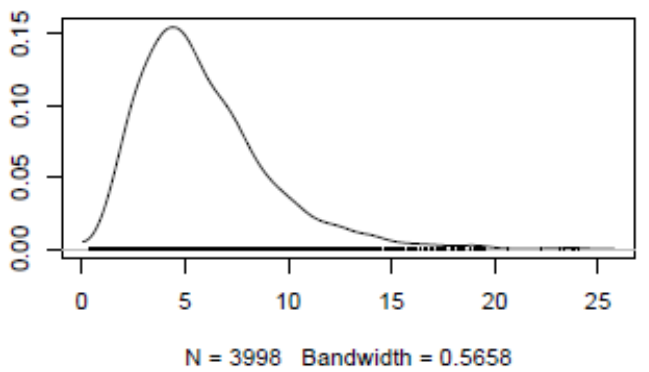


The Combined Model involving Offending History: Version 2b

Bayesian Model (BDm3G_cc102a)

\section{\#\# Define the Model}

BDm3 cclo2a <- MCMCglmm(FO.bin FTE*time*live + FTE*time*relation + FTE*time*ete + FTE*time*where + FTE*time*life + FTE*time*drugs + FTE*time*physical + FTE*time*emotion + FTE*time*self + FTE*time*think + FTE*time*attitude + FTE*time*change + I_Seriousness $2 *$ time*live + I_Seriousness $2 *$ time*relation + I_Seriousness2*time*ete + I_Seriousness2*time*where + I_Seriousness $2 *$ time*life $+\bar{I}$ _Seriousness2*time*drugs + $I^{-}$Seriousness $2 *$ time*physical ${ }^{-}$I Seriousness $2 *$ time*emotion + I_Seriousness2*time*self + I_Seriousness2*time*think + I_Seriousness2*time*attitude + I_Seriousness $2 *$ time*change + FTE*I_Seriousness2, random= time-tResearch.ID, data=data3, famil $\bar{y}=$ "ordinal", prior=priord, nitt $=4500000$, thin=1000, burnin=3000)

\section{\#\# Checks for suitable convergence}

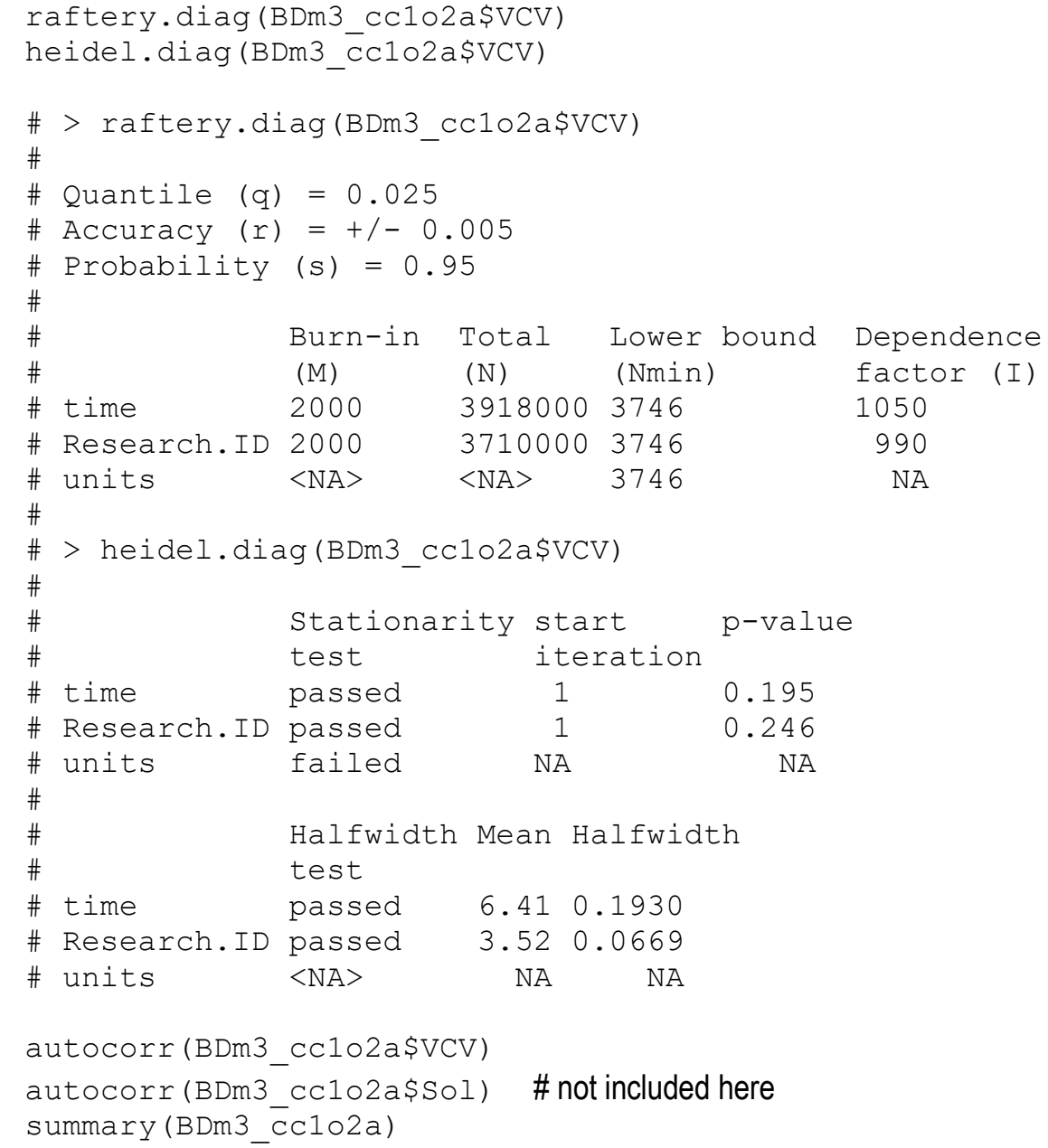




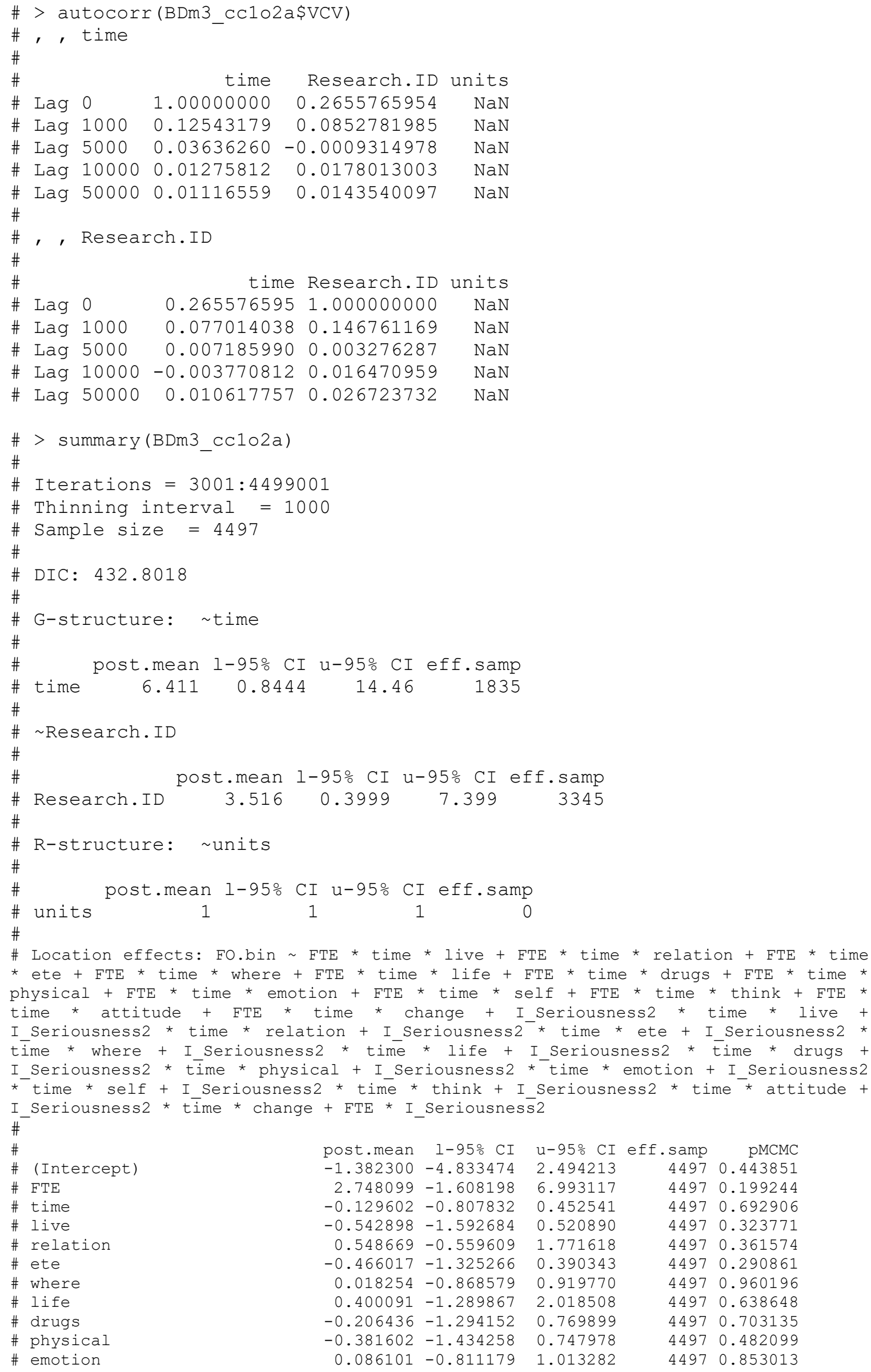

$\begin{array}{rrrrr}\text { post.mean } & 1-95 \% \text { CI } & \text { u-95\% CI } & \text { eff.samp } & \text { pMCMC } \\ -1.382300 & -4.833474 & 2.494213 & 4497 & 0.443851 \\ 2.748099 & -1.608198 & 6.993117 & 4497 & 0.199244 \\ -0.129602 & -0.807832 & 0.452541 & 4497 & 0.692906 \\ -0.542898 & -1.592684 & 0.520890 & 4497 & 0.323771 \\ 0.548669 & -0.559609 & 1.771618 & 4497 & 0.361574 \\ -0.466017 & -1.325266 & 0.390343 & 4497 & 0.290861 \\ 0.018254 & -0.868579 & 0.919770 & 4497 & 0.960196 \\ 0.400091 & -1.289867 & 2.018508 & 4497 & 0.638648 \\ -0.206436 & -1.294152 & 0.769899 & 4497 & 0.703135 \\ -0.381602 & -1.434258 & 0.747978 & 4497 & 0.482099 \\ 0.086101 & -0.811179 & 1.013282 & 4497 & 0.853013\end{array}$


\# self

\# think

\# attitude

\# change

\# I Seriousness 2

\# FTE:time

\# FTE:live

\# time:live

\# FTE:relation

\# time:relation

\# FTE : ete

\# time:ete

\# FTE:where

\# time:where

\# FTE:life

\# time:life

\# FTE:drugs

\# time:drugs

\# FTE:physical

\# time:physical

\# FTE:emotion

\# time:emotion

\# FTE:self

\# time:self

\# FTE:think

\# time:think

\# FTE:attitude

\# time:attitude

\# FTE : change

\# time:change

\# time:I Seriousness2

\# live:I_Seriousness 2

\# relation:I Seriousness2

\# ete:I_Seriousness 2

\# where: I Seriousness2

\# life:I Seriousness2

\# drugs: $\bar{I}$ Seriousness2

\# physical:I Seriousness2

\# emotion:I Seriousness2

\# self:I Seriousness2

\# think: $\bar{I}$ Seriousness2

\# attitude: I Seriousness2

\# change: I sēeriousness 2

\# FTE:I Seriousness2

\# FTE:time:live

\# FTE:time:relation

\# FTE:time:ete

\# FTE:time:where

\# FTE:time:life

\# FTE:time:drugs

\# FTE:time:physical

\# FTE:time:emotion

\# FTE:time:self

\# FTE:time:think

\# FTE:time:attitude

\# FTE:time:change

\# time:live:I Seriousness2

\# time:relation: I_Seriousness 2

\# time:ete:I Seriousness2

\# time:where:I Seriousness 2

\# time:life:I Seriousness2

\# time:drugs: $\bar{I}$ Seriousness 2

\# time:physical $:$ I Seriousness2

\# time:emotion:I Seriousness 2

\# time:self:I Seriousness2

\# time:think: $\bar{I}$ Seriousness 2

\# time:attitude: I Seriousness

\# time:change:I_seriousness2

\# ---

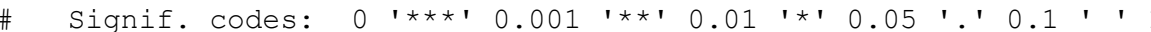

$\begin{array}{llll}-0.342930 & -1.925782 & 1.066532\end{array}$

$0.216209-1.005404 \quad 1.498252$

$0.238029-1.342149 \quad 1.561904$

$1.223136-0.242999 \quad 2.738791$

$-2.253587-4.370716-0.048567$

$-1.660161-2.842567-0.585947$

$1.135370-0.9750413 .189806$

$0.104465-0.131132 \quad 0.321750$

$-2.358624-4.491019-0.394325$

$0.089489-0.228527 \quad 0.401815$

$0.247655-1.489445 \quad 2.016588$

$0.025860-0.1825620 .245143$

$-3.055754-5.234707-1.165322$

$0.126564-0.046067 \quad 0.314463$

$1.907104-0.732942 \quad 4.403817$

$\begin{array}{llll}-0.016182 & -0.354350 & 0.348589\end{array}$

$0.015186-0.2303140 .252018$

$-0.707541-2.961240 \quad 1.375536$

$\begin{array}{llll}0.068348 & -0.255334 & 0.357237\end{array}$

$\begin{array}{llll}-1.537741 & -3.216871 & 0.010309\end{array}$

$\begin{array}{llll}-0.253510 & -0.517977 & 0.001654\end{array}$

$\begin{array}{lll}3.475428 & 1.239732 & 5.661227\end{array}$

$0.075353-0.2175690 .356569$

$\begin{array}{lll}-0.634879 & -2.831293 & 1.265667\end{array}$

$0.025417-0.260386 \quad 0.308615$

$-1.436785-3.241776 \quad 0.749092$

$-0.079201-0.383948$

$1.643750-0.807792$

$-0.277032-0.599596$

$0.257480-0.152208$

$0.162427-0.437151$

$0.542614-0.137583$

$0.038391-0.511244$

$0.340200-0.181482$

$0.056096-0.844746$

$0.462739-0.198586$

$-0.061651-0.875632$

$0.282737-0.306169$

$-0.120144-0.829189$

$-0.047151-0.928193$

$0.253458-0.539994$

$-1.090915-2.070497$

$1.210039-0.034627$

$-0.263340-0.751909$

$0.584765 \quad 0.123945$

$0.419638-0.126208$

$0.436195 \quad 0.024574$

$-0.663469-1.266730$

$0.375388-0.071661$

$-0.013302-0.560279$

$0.331637-0.044422$

$-0.937199-1.491810$

$0.305740-0.134129$

$0.372506-0.153163$

$-0.362468-0.918849$

$0.004346-0.133557$

$-0.231991-0.414573$

$0.030451-0.116756$

$-0.162123-0.302361$

$0.005614-0.184254$

$-0.073027-0.211623$

$0.055819-0.159365$

$0.019499-0.155689$

$-0.052948-0.229350$

$-0.065910-0.261099$

$0.251913 \quad 0.062418$

0.749092
0.247747

3.701927

0.023732

0.646677

0.820362

1. 338979

0.540541

0.930463

0.965582

1. 102590

0.688354

0.868801

0.640971

0.736806

1.003869

$-0.104056$

2.557973

0.230959

1. 094246

0.928547

$-0.054756$

0.827867

0.540833

0.740836

$-0.440383$

0.898032

0.190889

0.153397

$-0.041742$

0.164473

0.024723

0.206578

0.056374

0.274706

0.178772

0.131690

0.136127

0.454085 $\begin{array}{lll}-0.965043 & -2.807518 & 0.961111\end{array}$

0.1530520 .006180

0.806203

0.763320

0.293244
$4497 \quad 0.646209$

42350.736936

$4497 \quad 0.733378$

44970.096064 .

40490.027129 *

$40970.001334 * *$

$4497 \quad 0.267289$

$4497 \quad 0.357572$

41180.021792 *

44970.585724

41690.788970

44970.814765

$40250.000445 * * *$

$4497 \quad 0.169002$

$4276 \quad 0.131199$

$\begin{array}{lll}4741 & 0.913943\end{array}$

$4497 \quad 0.301090$

$\begin{array}{lll}4310 & 0.925951\end{array}$

$4497 \quad 0.511007$

49930.640872

38040.048922 *

44970.048477 *

$44970.000889 * \star *$

44970.619969

$\begin{array}{lll}4072 & 0.545697\end{array}$

$4240 \quad 0.841450$

$4746 \quad 0.149433$

$4497 \quad 0.620414$

$4497 \quad 0.145875$

44970.079609 .

$4497 \quad 0.194352$

$4497 \quad 0.604848$

$\begin{array}{lll}4178 & 0.137870\end{array}$

$4368 \quad 0.879253$

$4497 \quad 0.226373$

$4497 \quad 0.902824$

40850.146320

44970.869913

$\begin{array}{lll}4771 & 0.354459\end{array}$

$4497 \quad 0.720036$

$4257 \quad 0.917056$

$\begin{array}{lll}4801 & 0.512342\end{array}$

40850.023571 *

42510.051145 .

41270.293529

40790.016900 *

$4230 \quad 0.112519$

44980.018679 *

40490.026684 *

35370.097398 .

48550.994441

44970.087169 .

$4267<2 e-04 * \star *$

42930.171670

$4497 \quad 0.167223$

49650.192128

$4497 \quad 0.964643$

41760.005337 **

$4497 \quad 0.676451$

44970.014232 *

44970.956638

40860.283745

$4240 \quad 0.616411$

44970.032911 *

44970.809873

42370.557260

44970.521681

44970.008450 ** 
Trace Plots and Posterior Density Plots

Fixed Effects
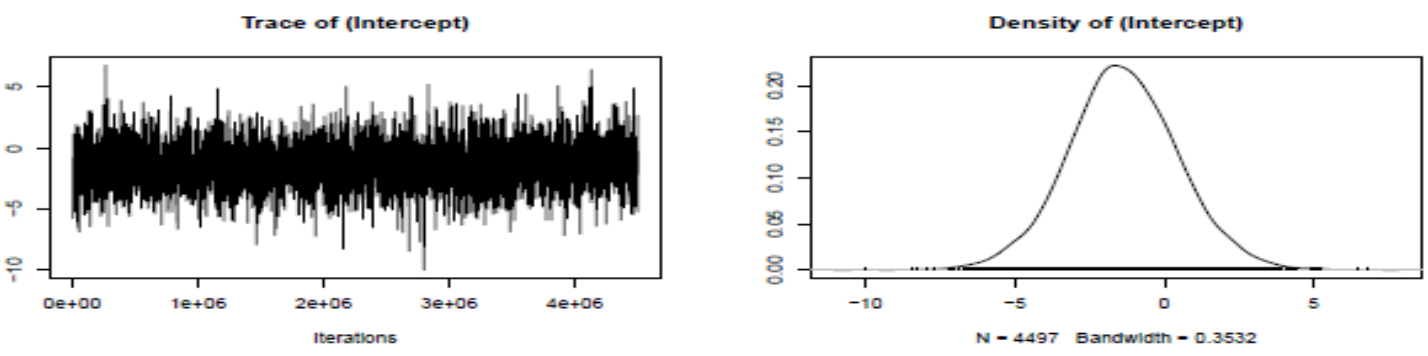

Trace of FTE
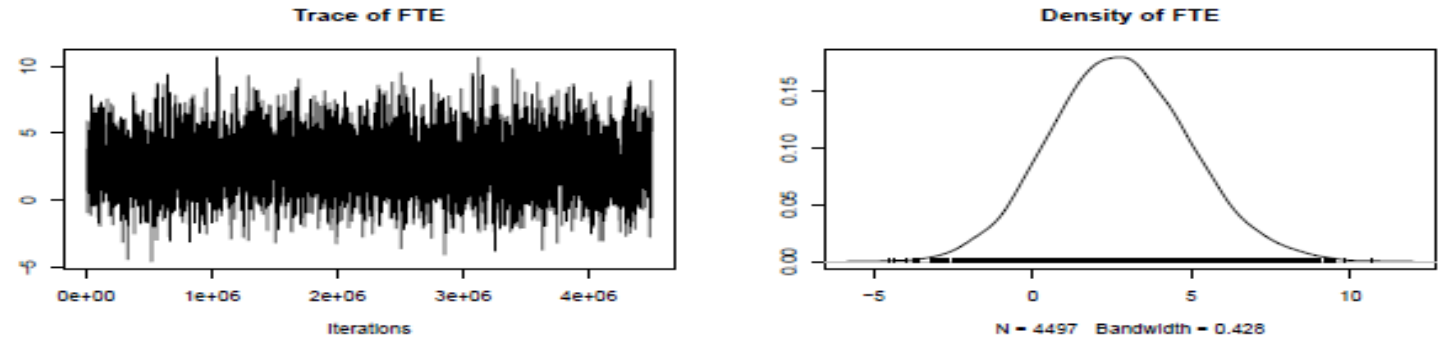

Trace of time
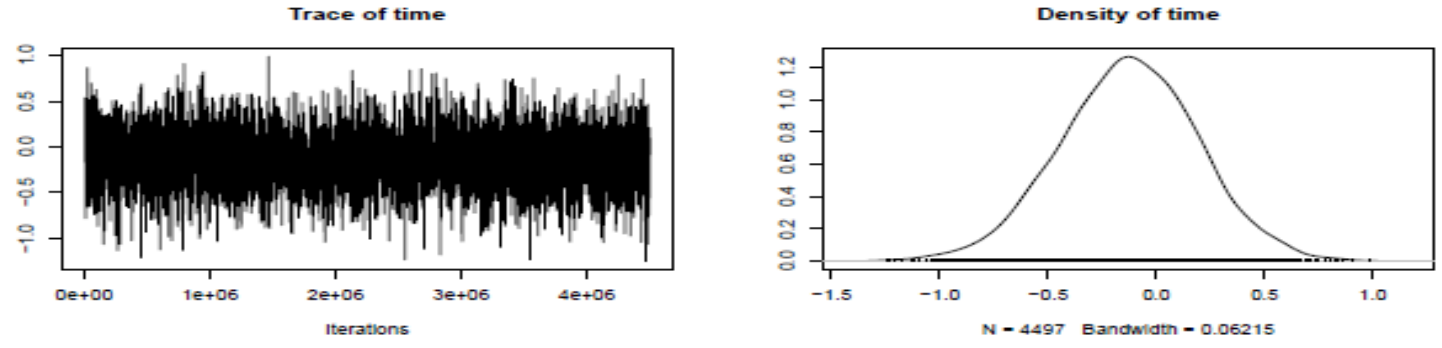

Trace of live
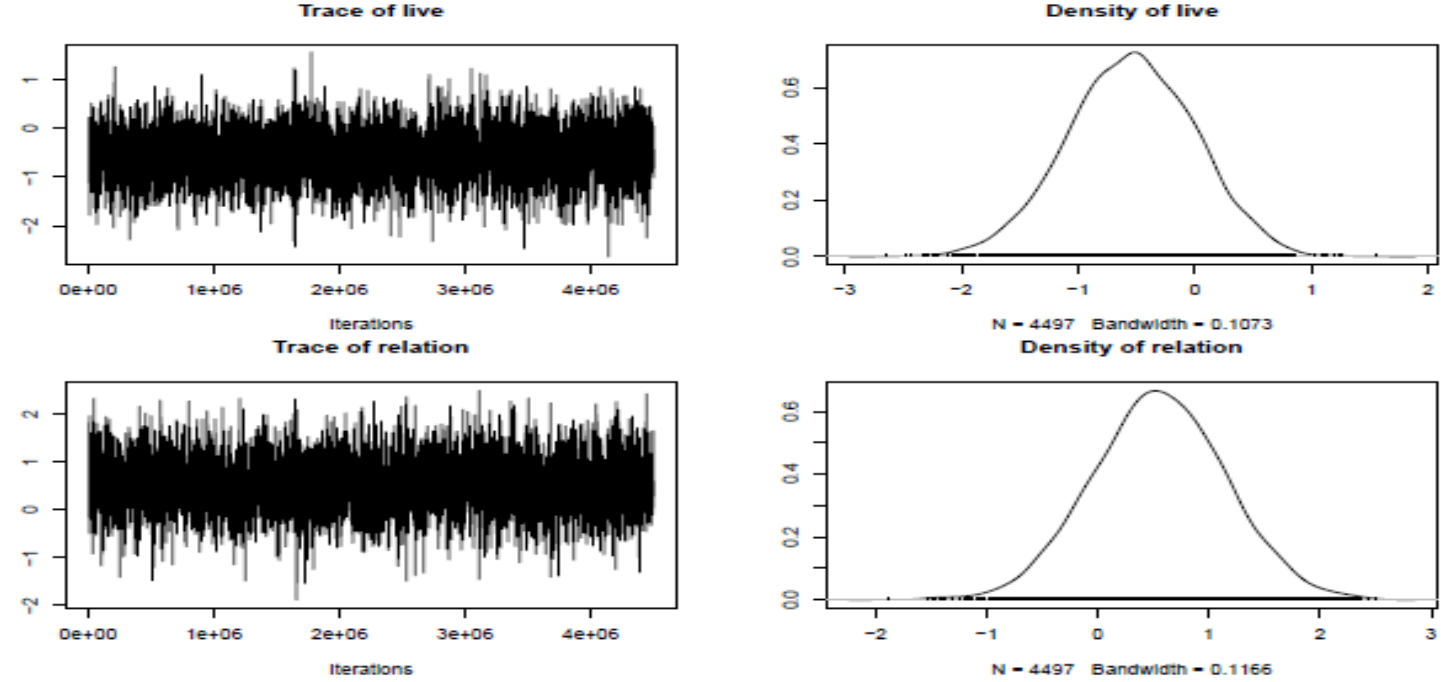

Trace of ete
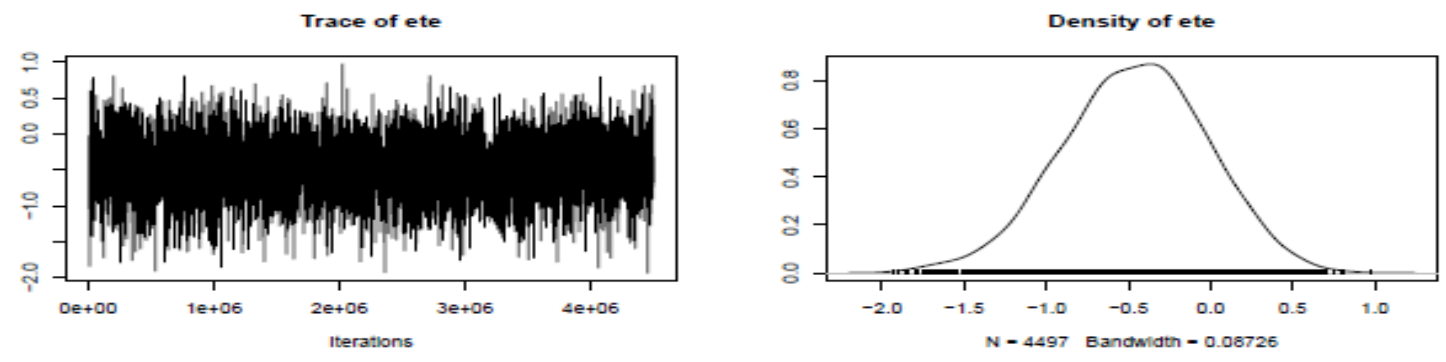
Trace of where

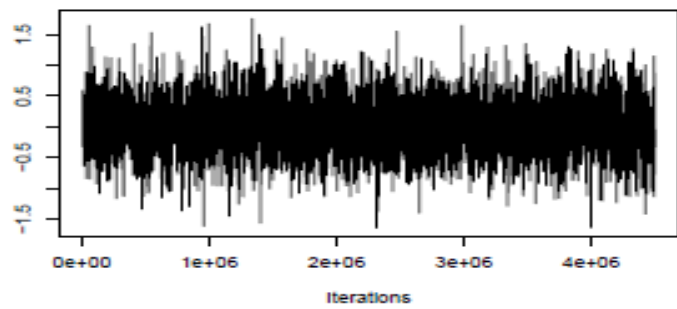

Trace of life

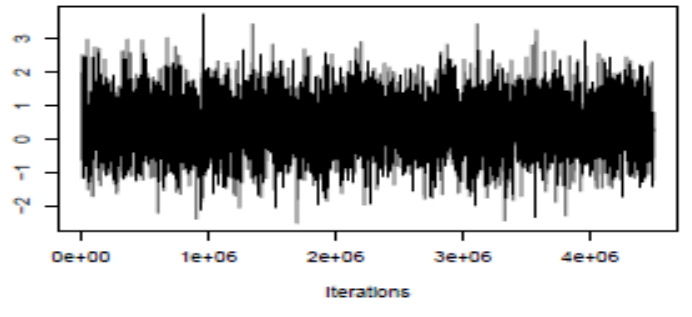

Trace of drugs

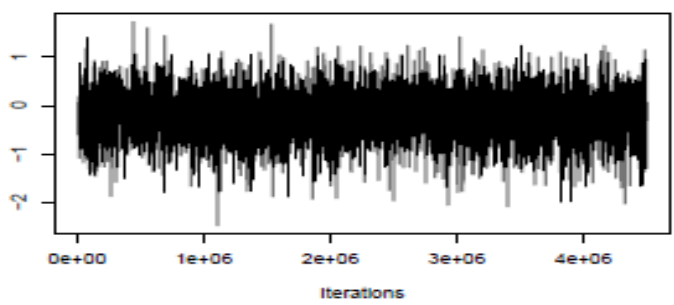

Trace of physical

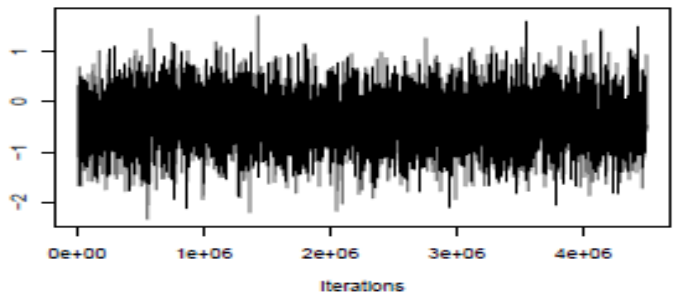

Trace of emotion

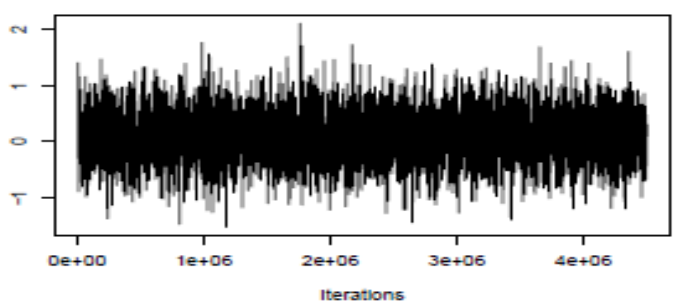

Trace of self

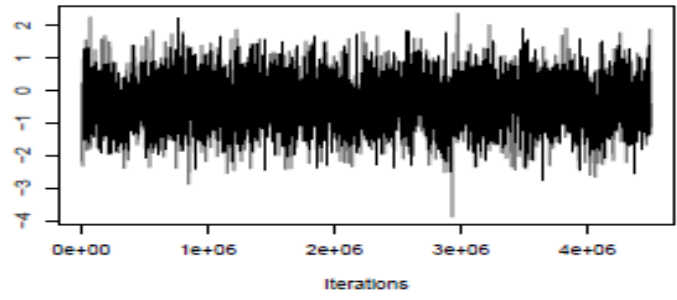

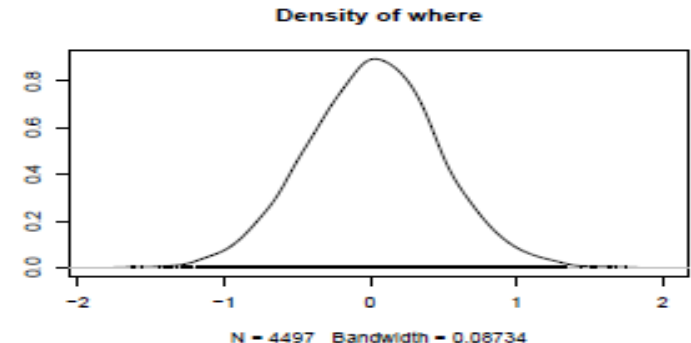

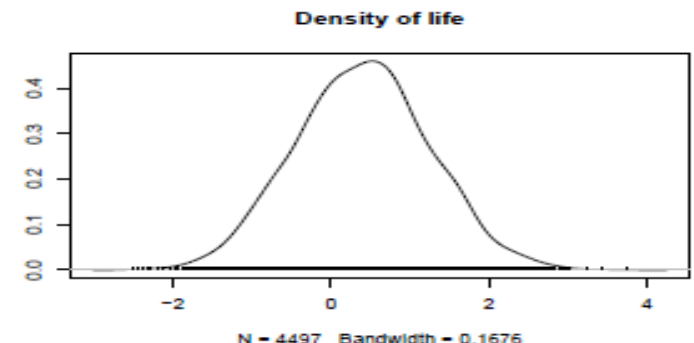

Density of drugs

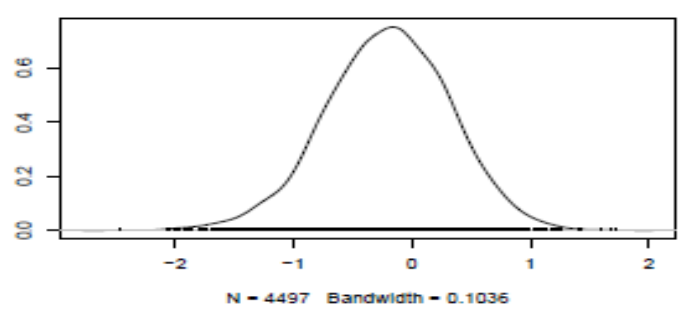

Density of physical
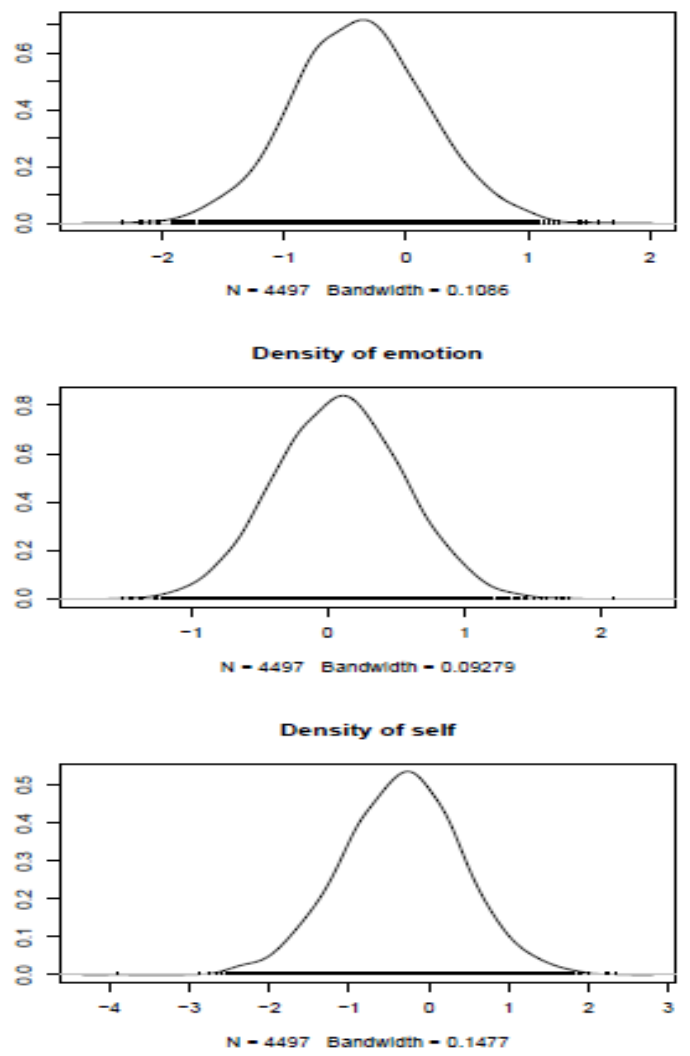

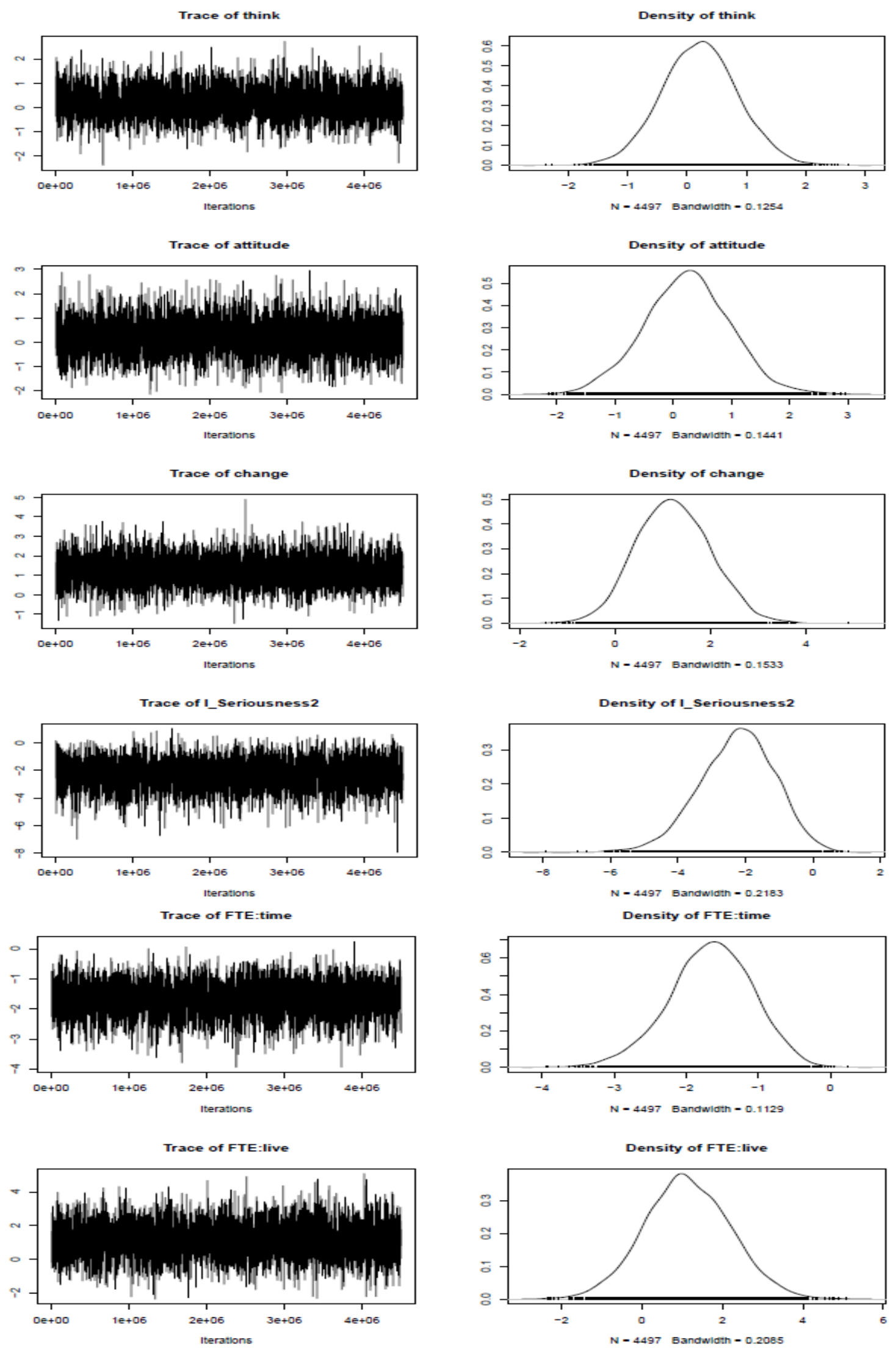

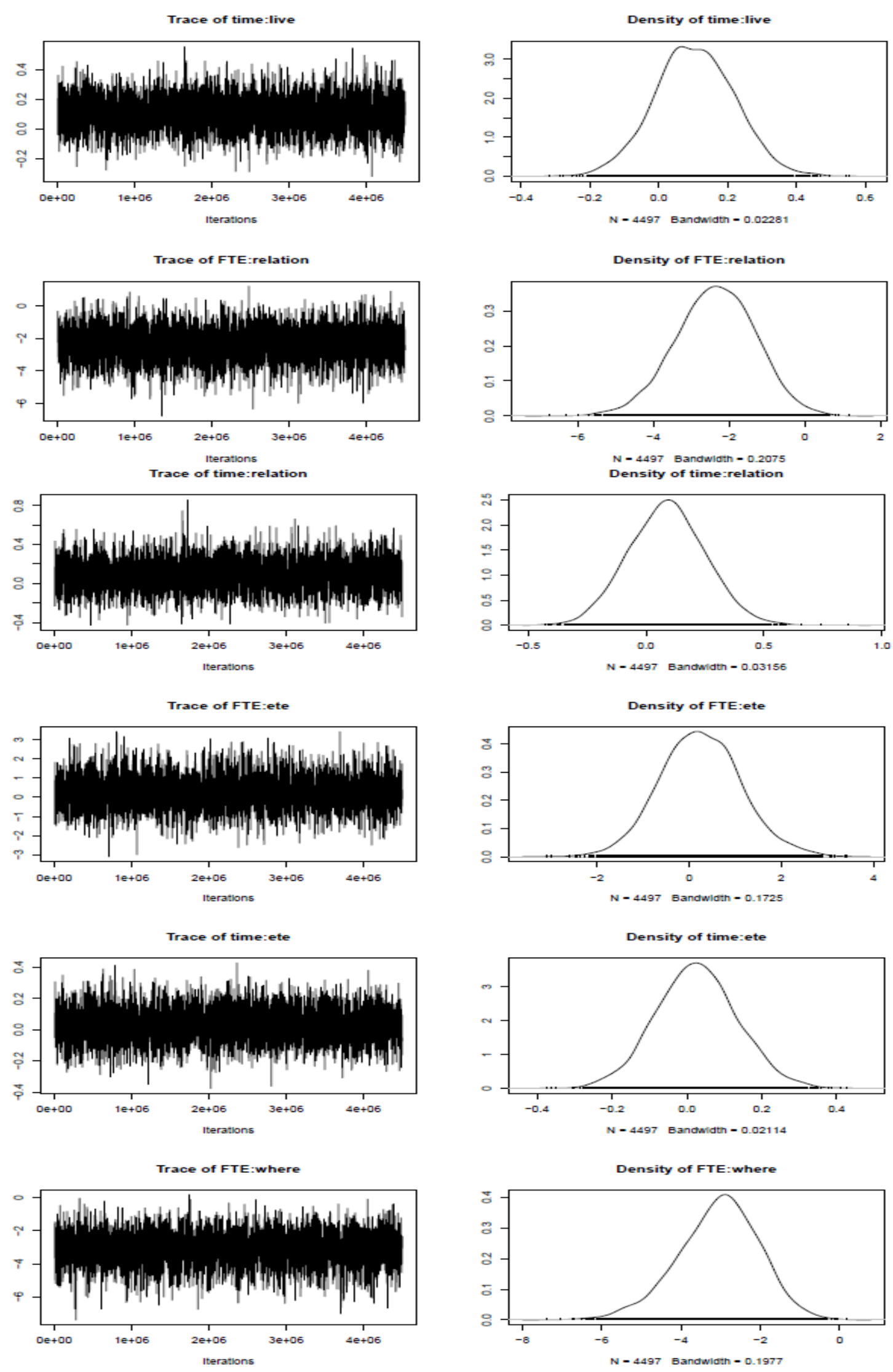
Trace of time:where

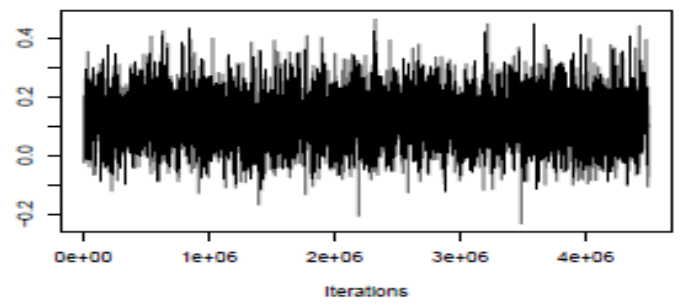

Trace of FTE:life

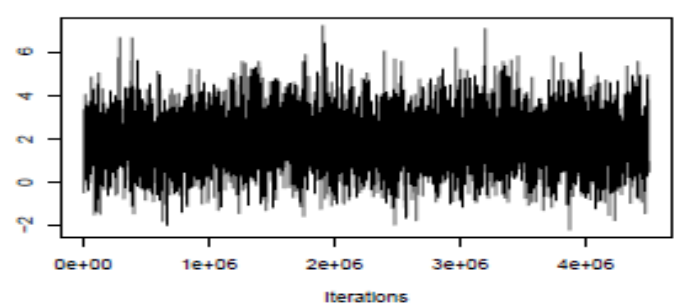

Trace of time:life

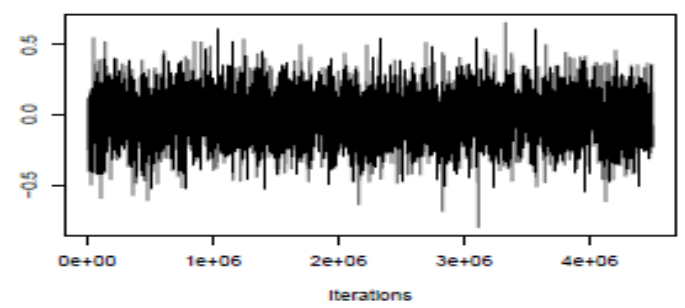

Trace of FTE:drugs
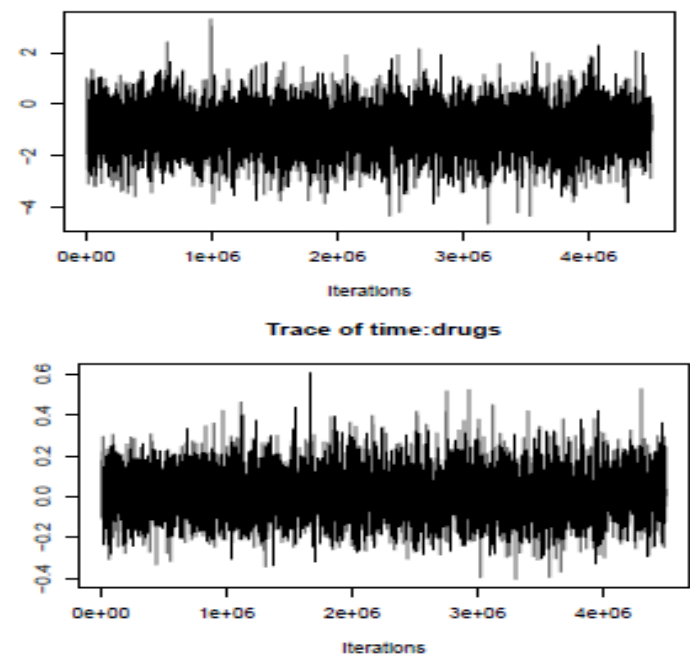

Trace of FTE:physical

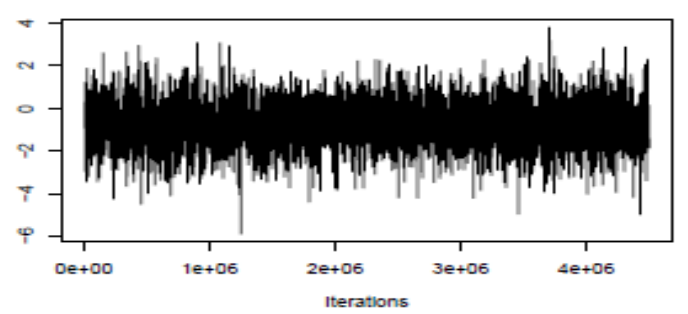

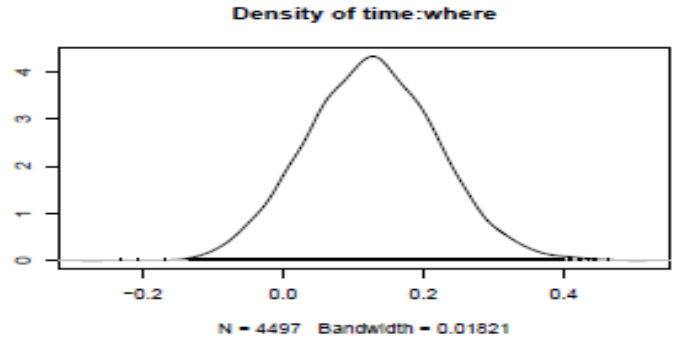
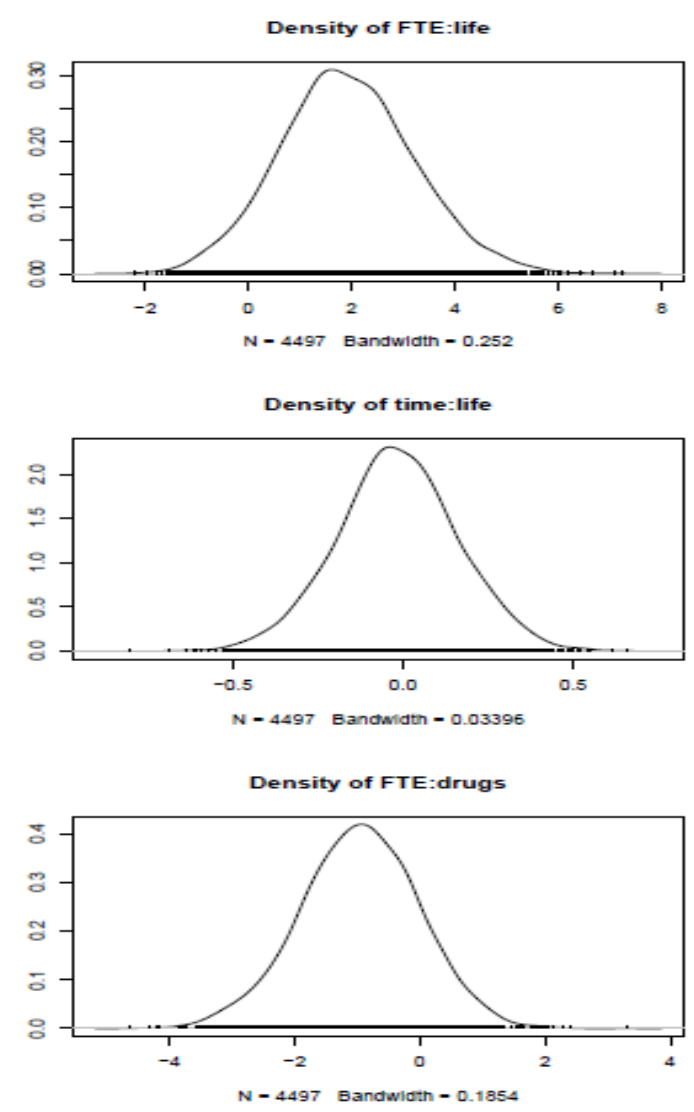

Density of time:drugs
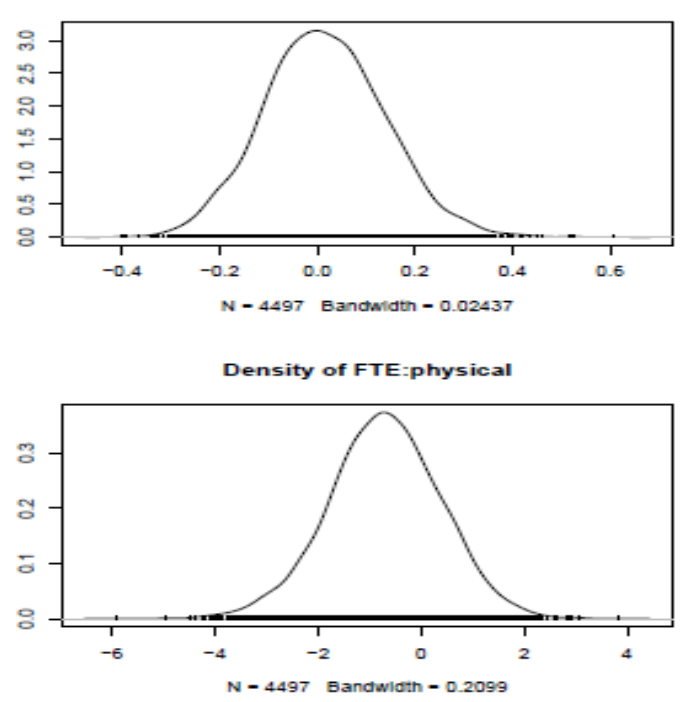

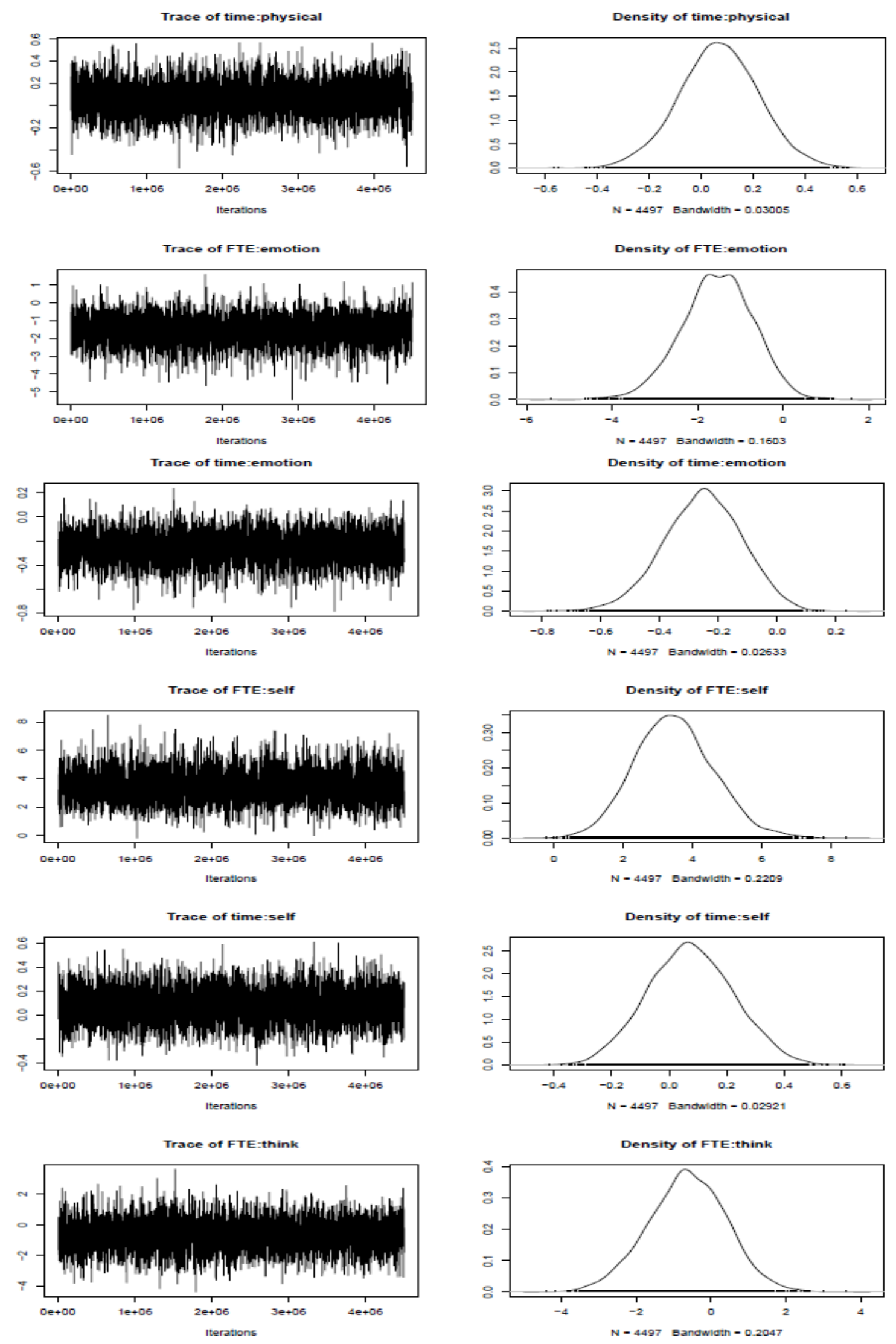
Trace of time:think

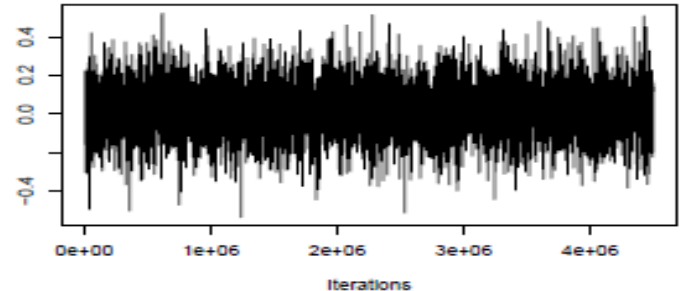

Trace of FTE:attitude

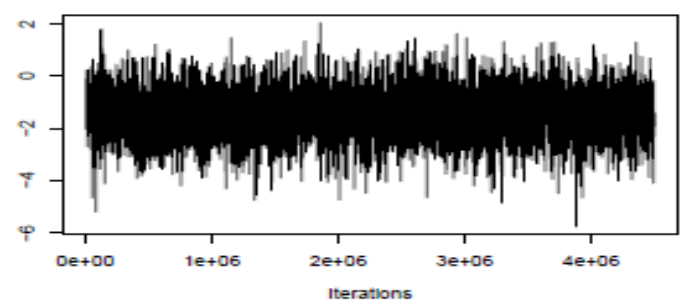

Trace of time:attitude

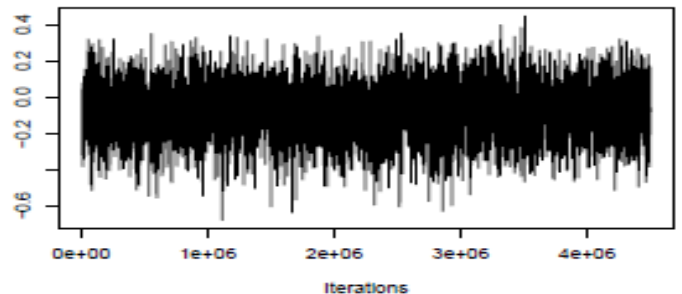

Trace of FTE:change

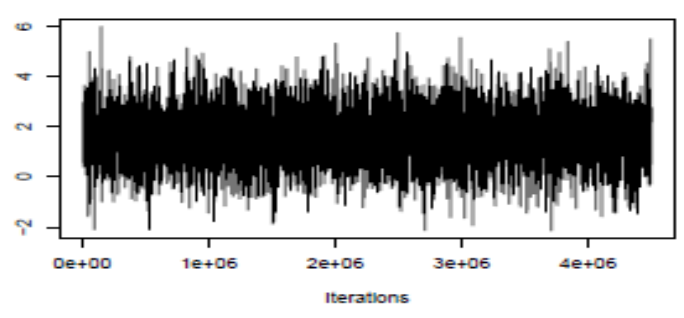

Trace of time:change
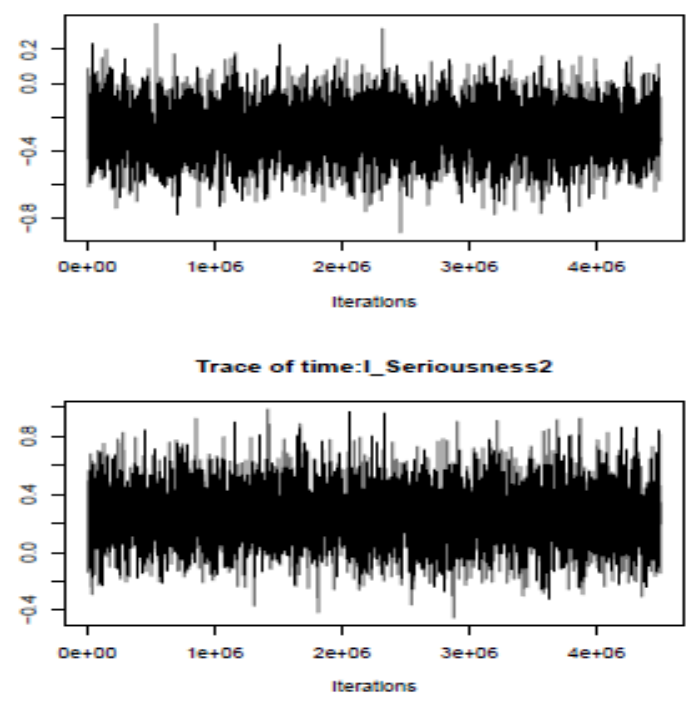

Density of time:think

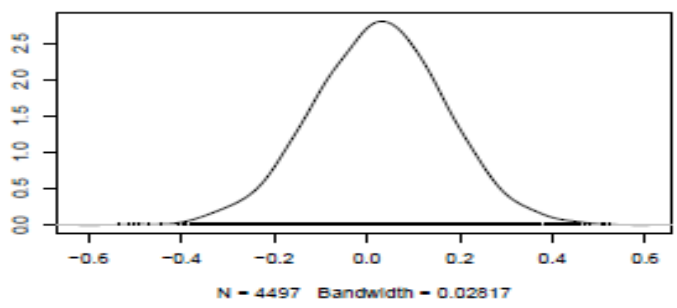

Density of FTE:attitude

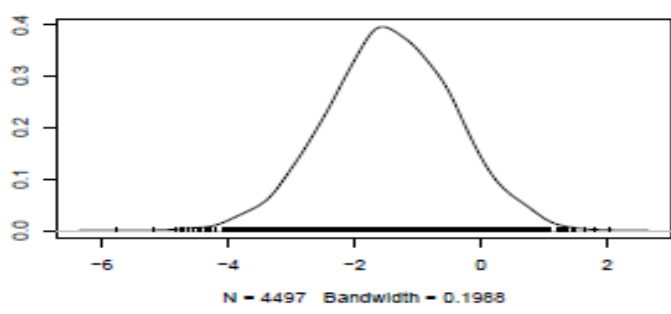

Density of time:attitude

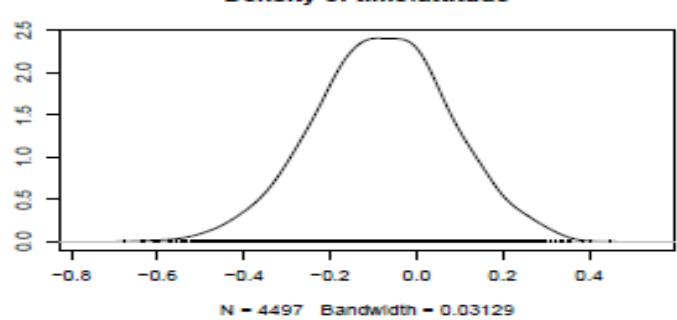

Density of FTE:change

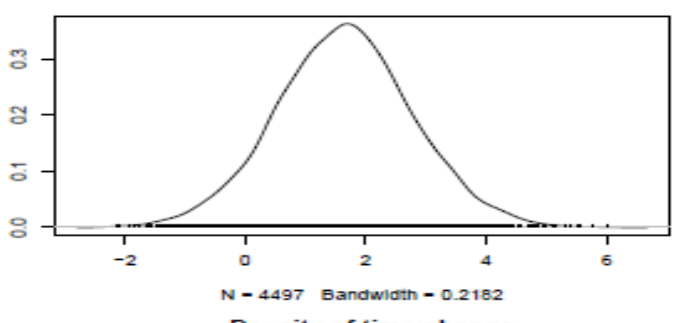

Density of time:change

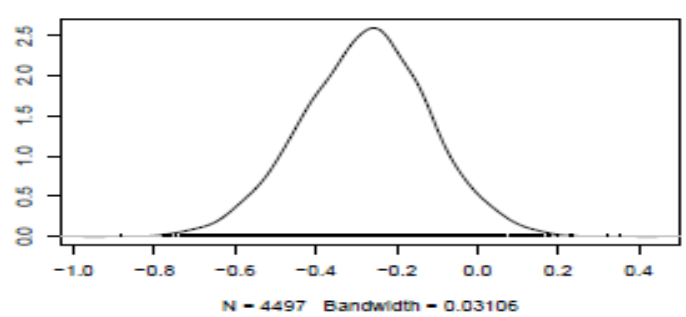

Density of time:1_Seriousness2

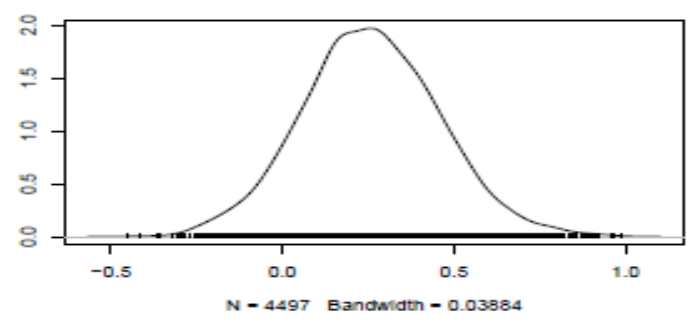


Trace of live:1_Seriousness2

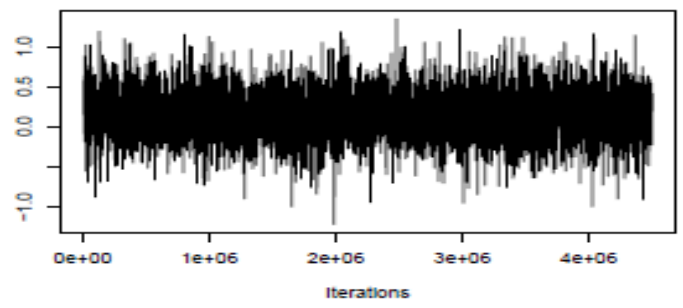

Trace of relation:1_Seriousness2

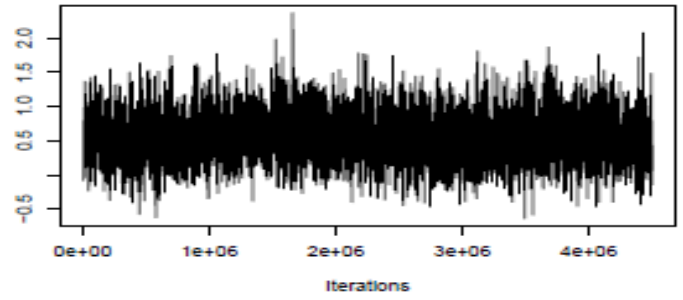

Trace of ete:I_Seriousness2

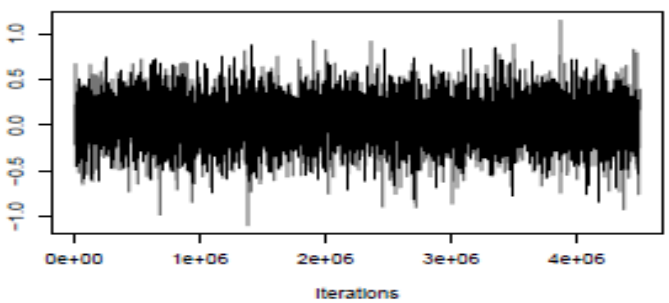

Trace of where:1_Seriousness2

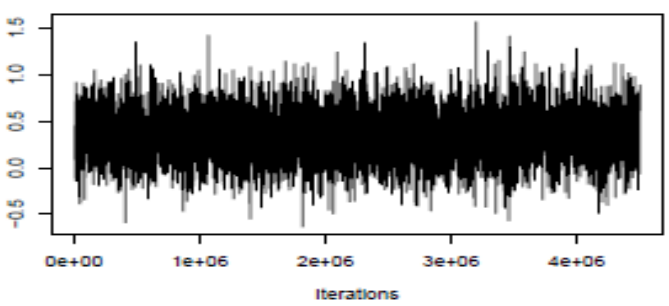

Trace of life:I_Seriousness2

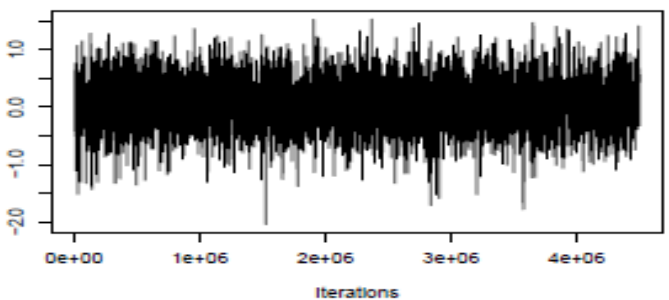

Trace of drugs:1_Seriousness2

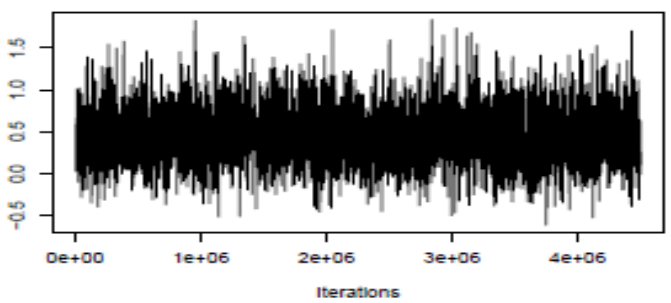

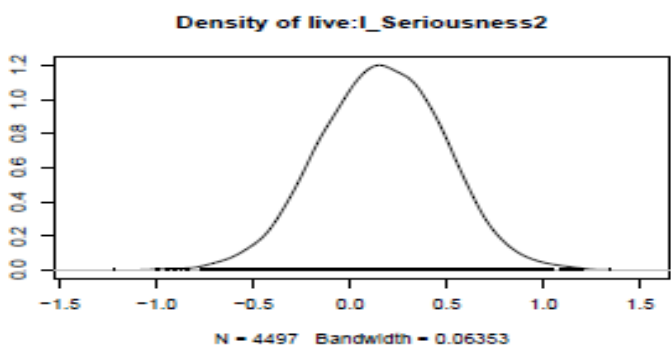
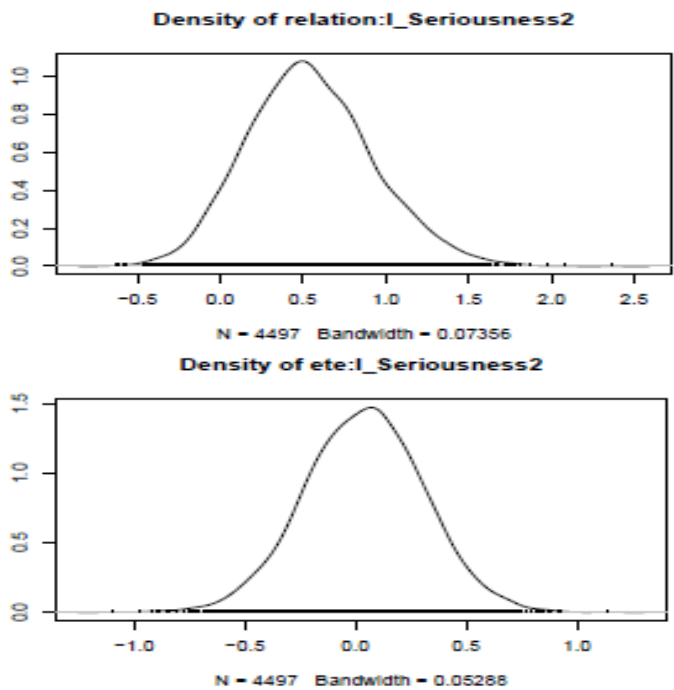

$N-4497$ Bandwidth -0.05288
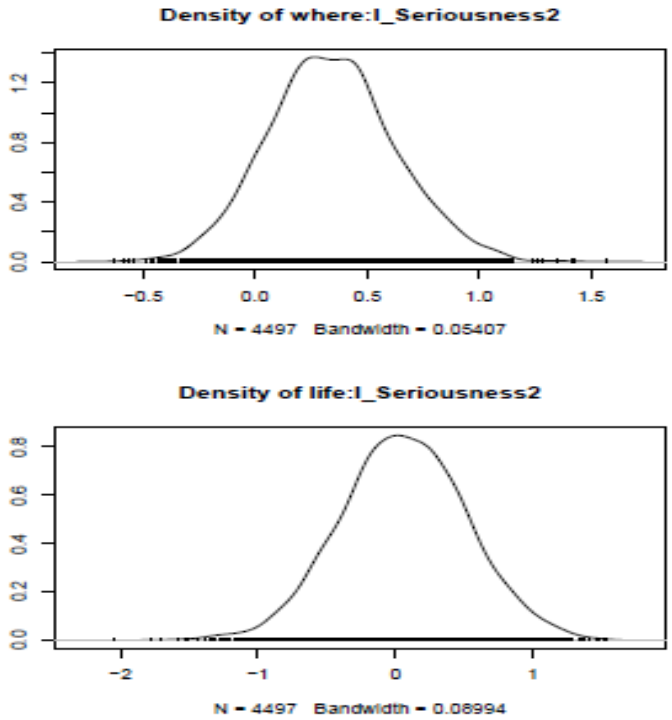

Density of drugs:1_Seriousness?

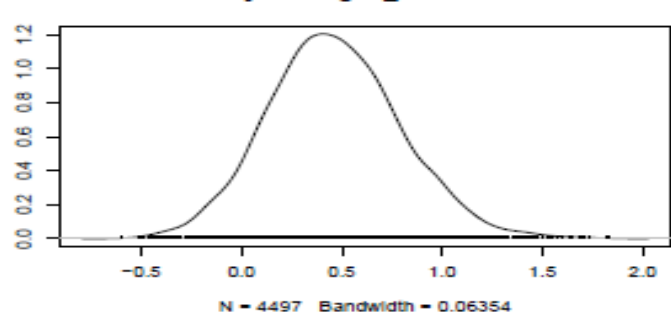



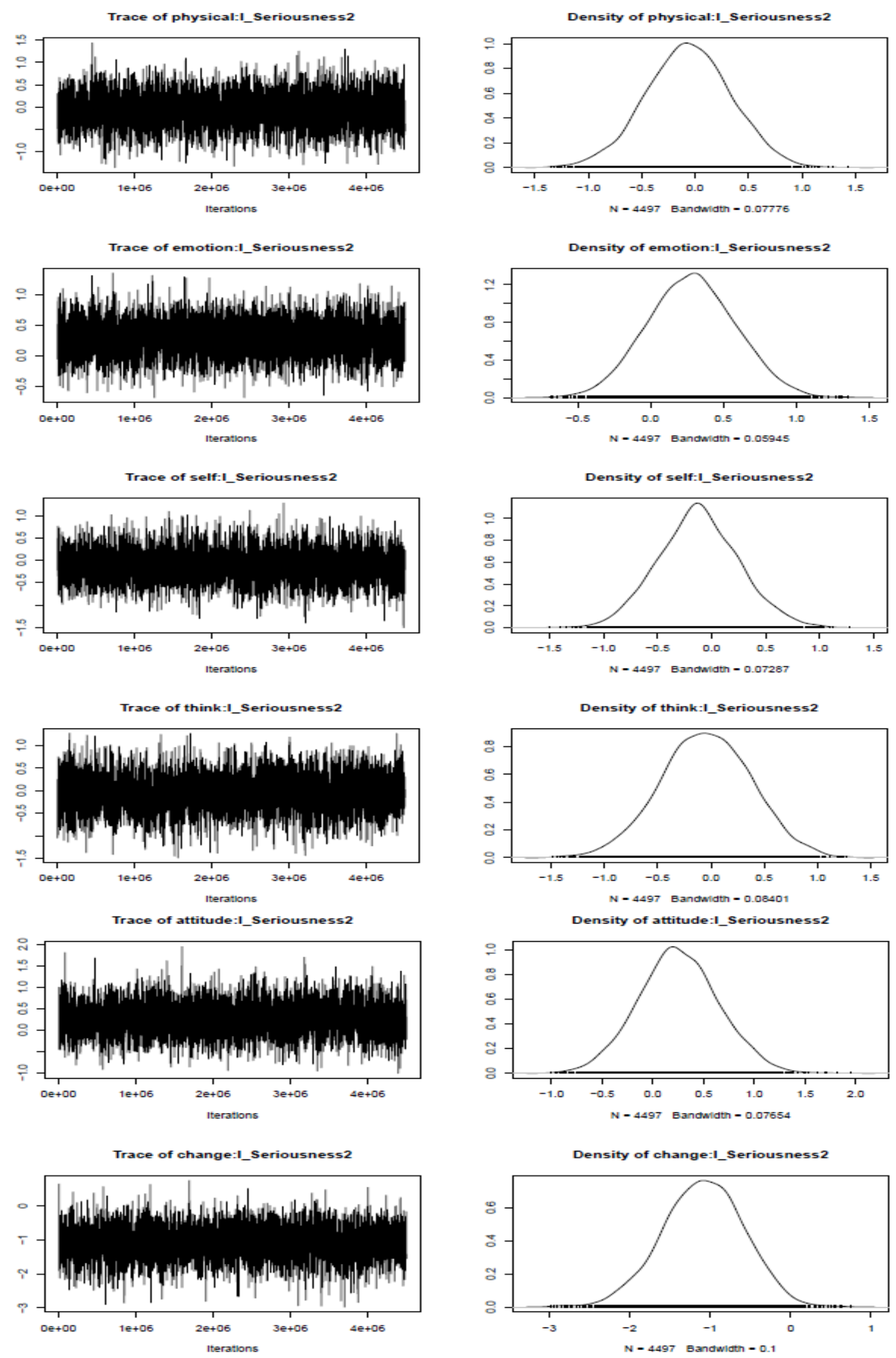

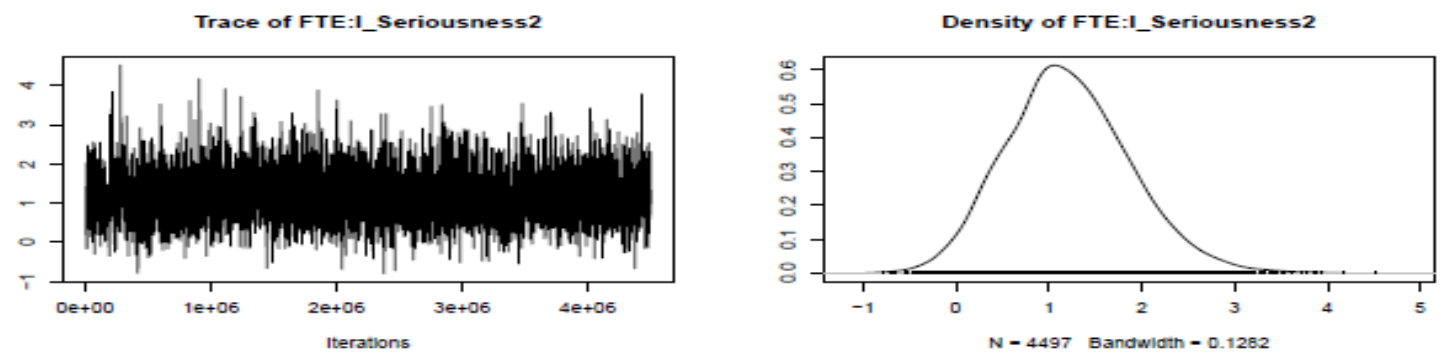

Trace of FTE:time:live
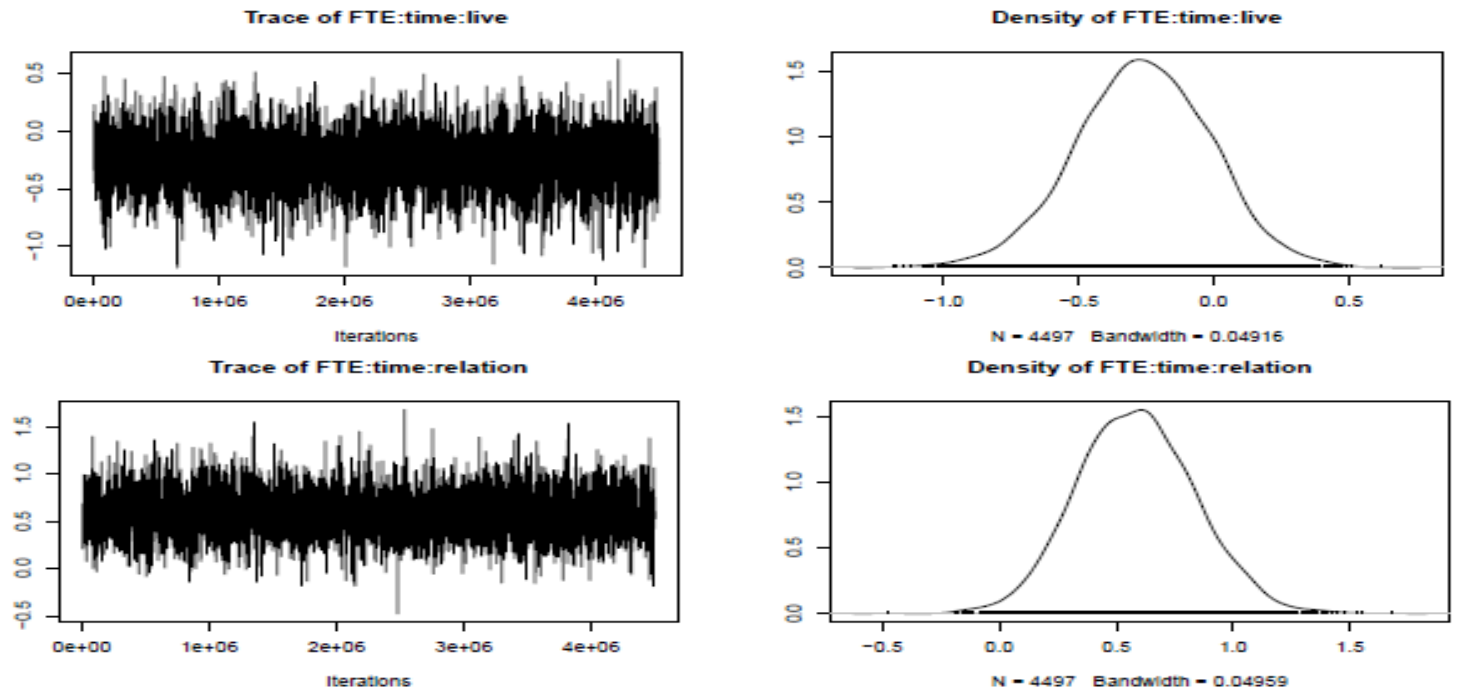

Trace of FTE:time:ete

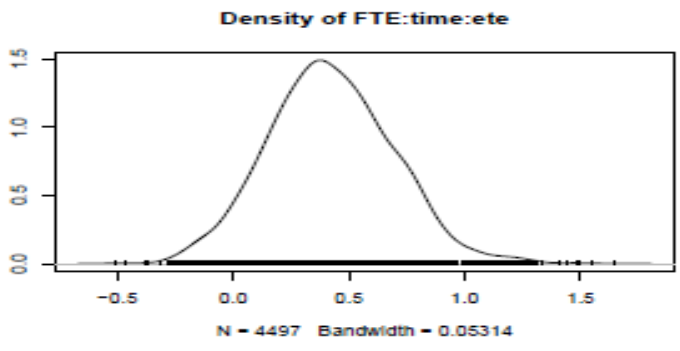

Trace of FTE:time:where

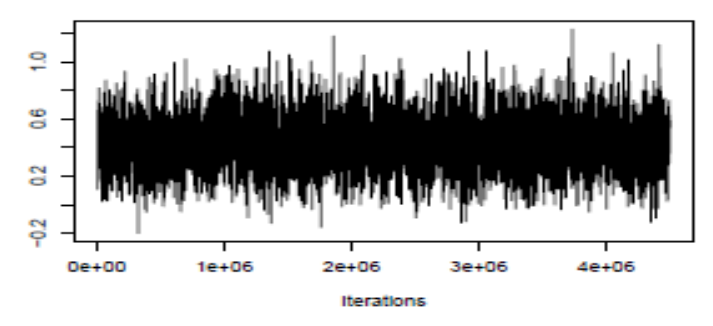

Density of FTE:time:where

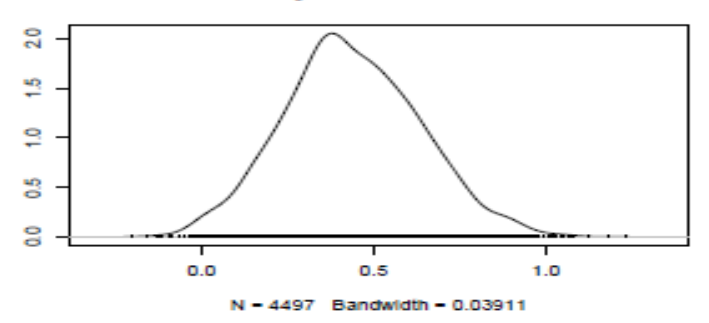

Trace of FTE:time:life
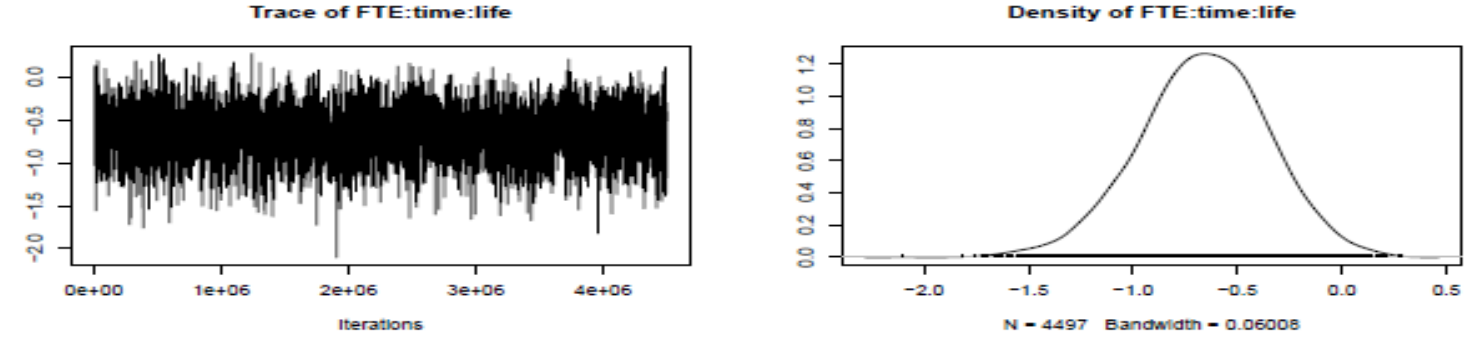

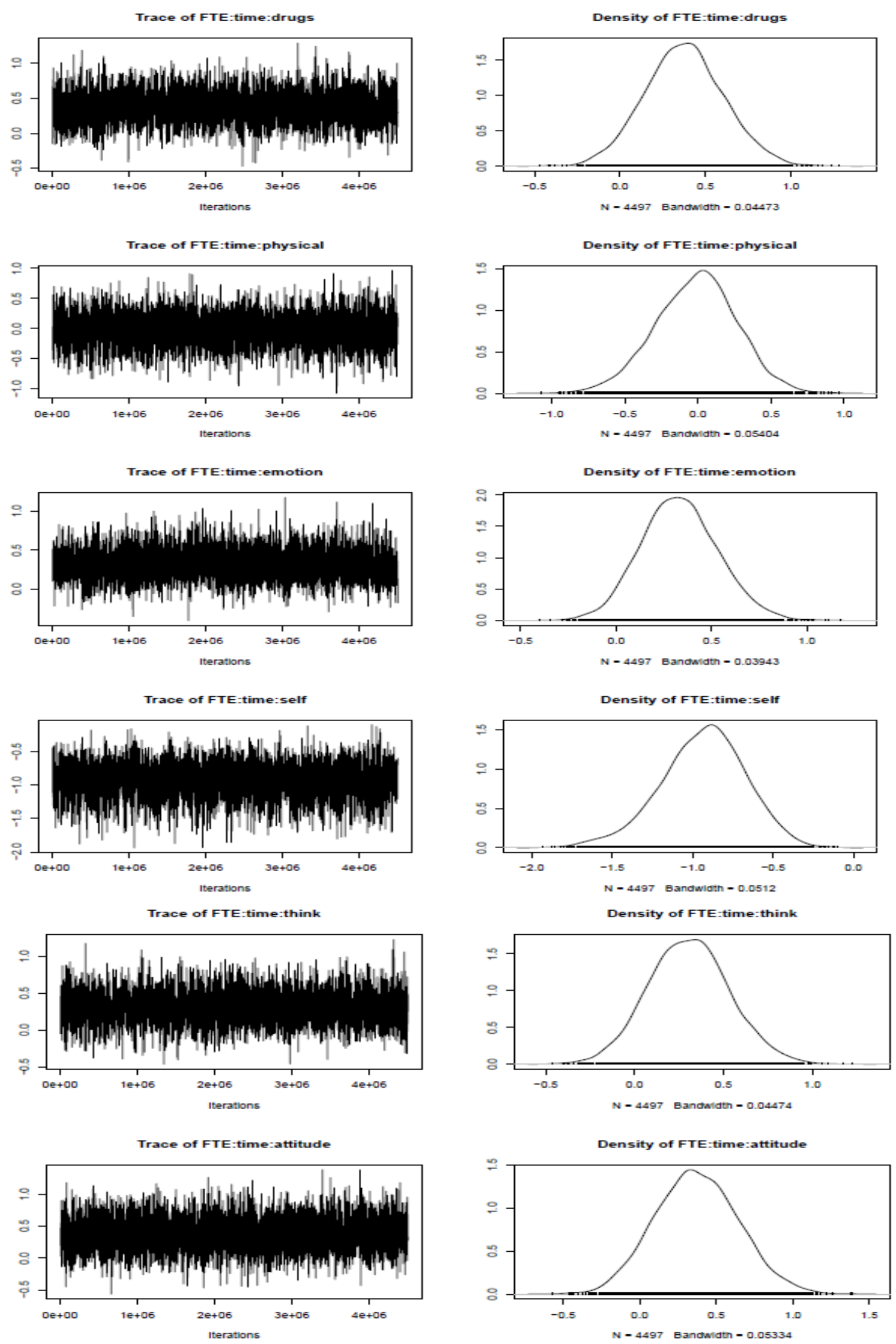

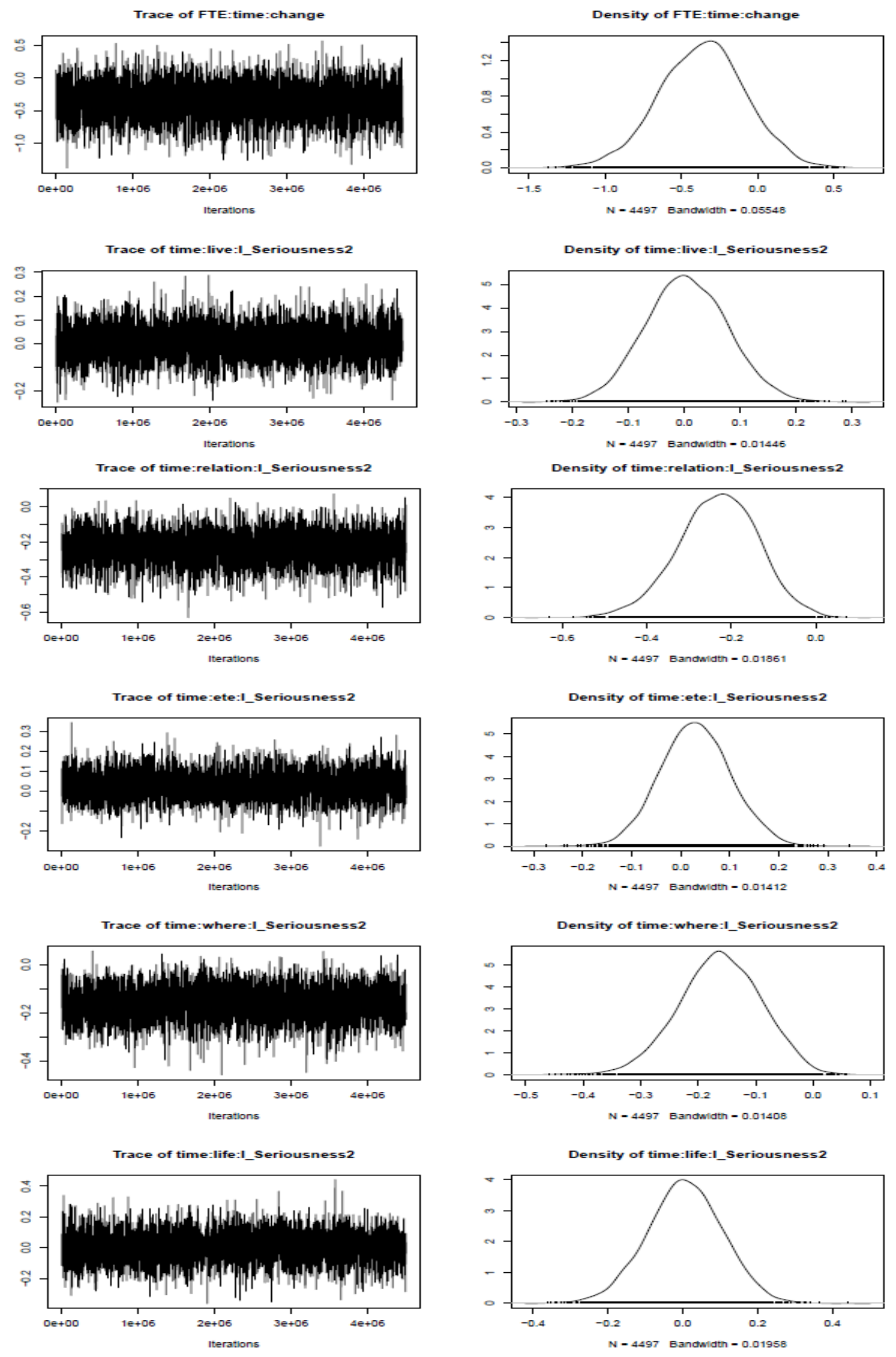

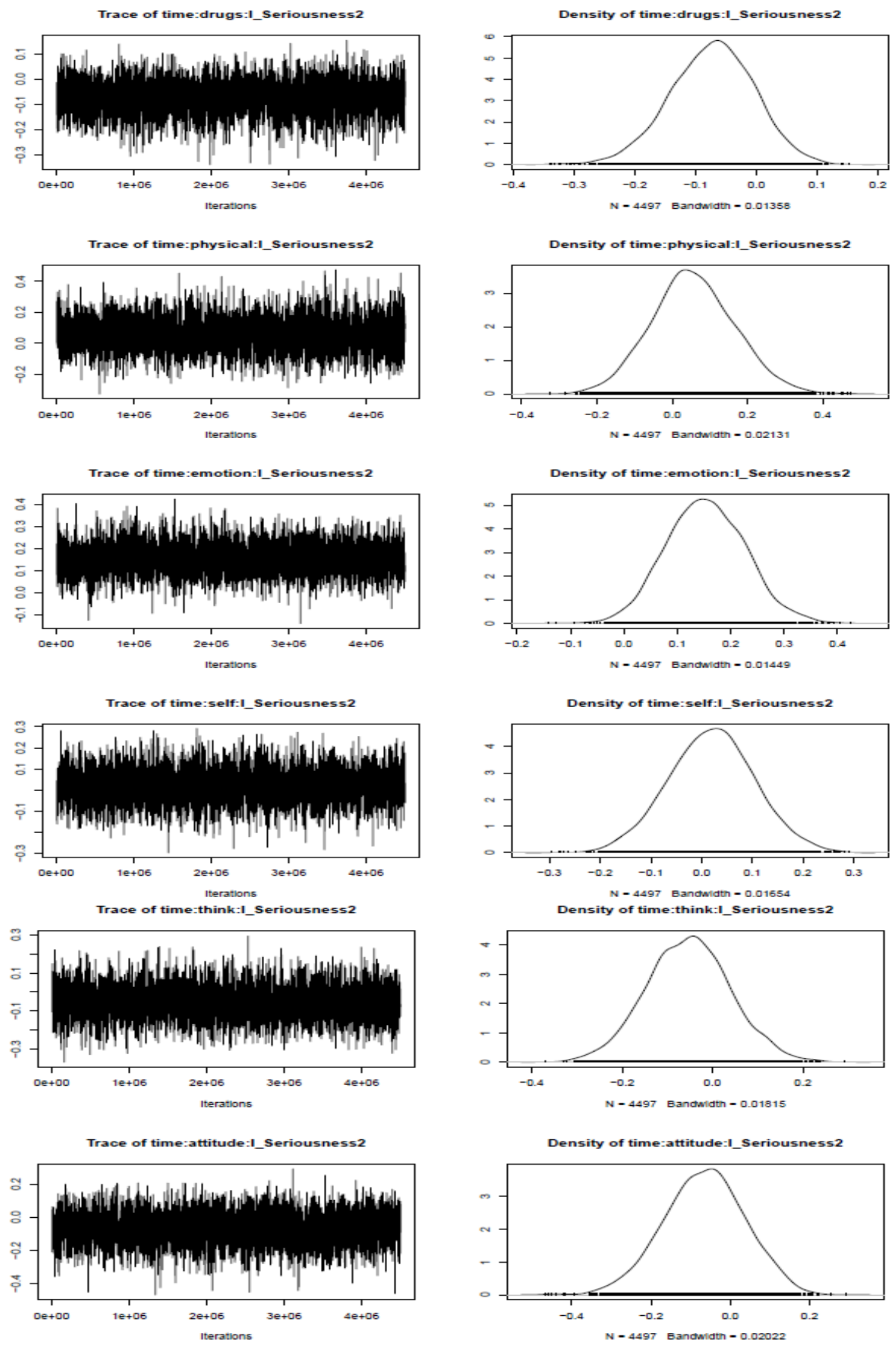

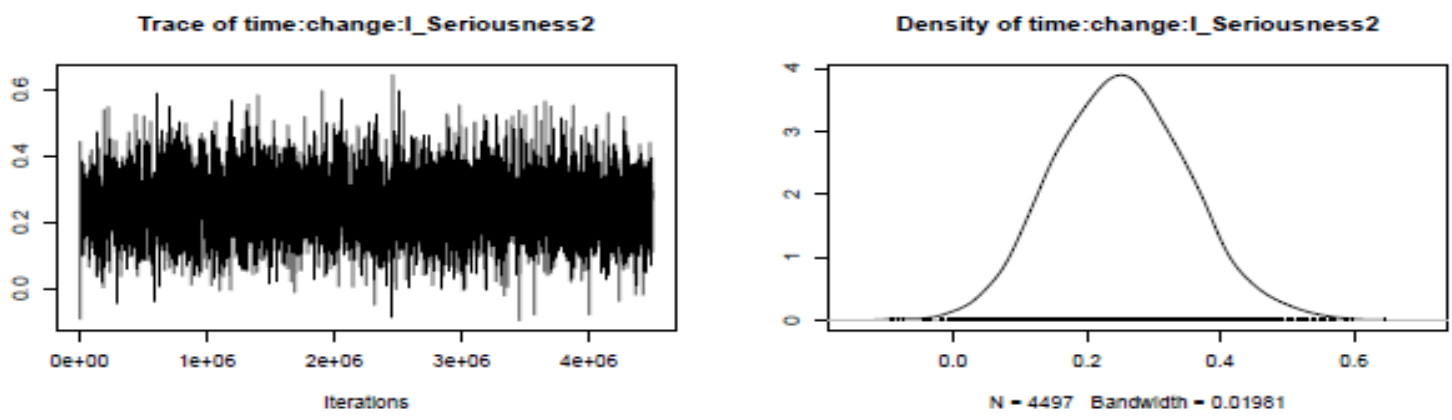

$\underline{\text { Random Effects }}$
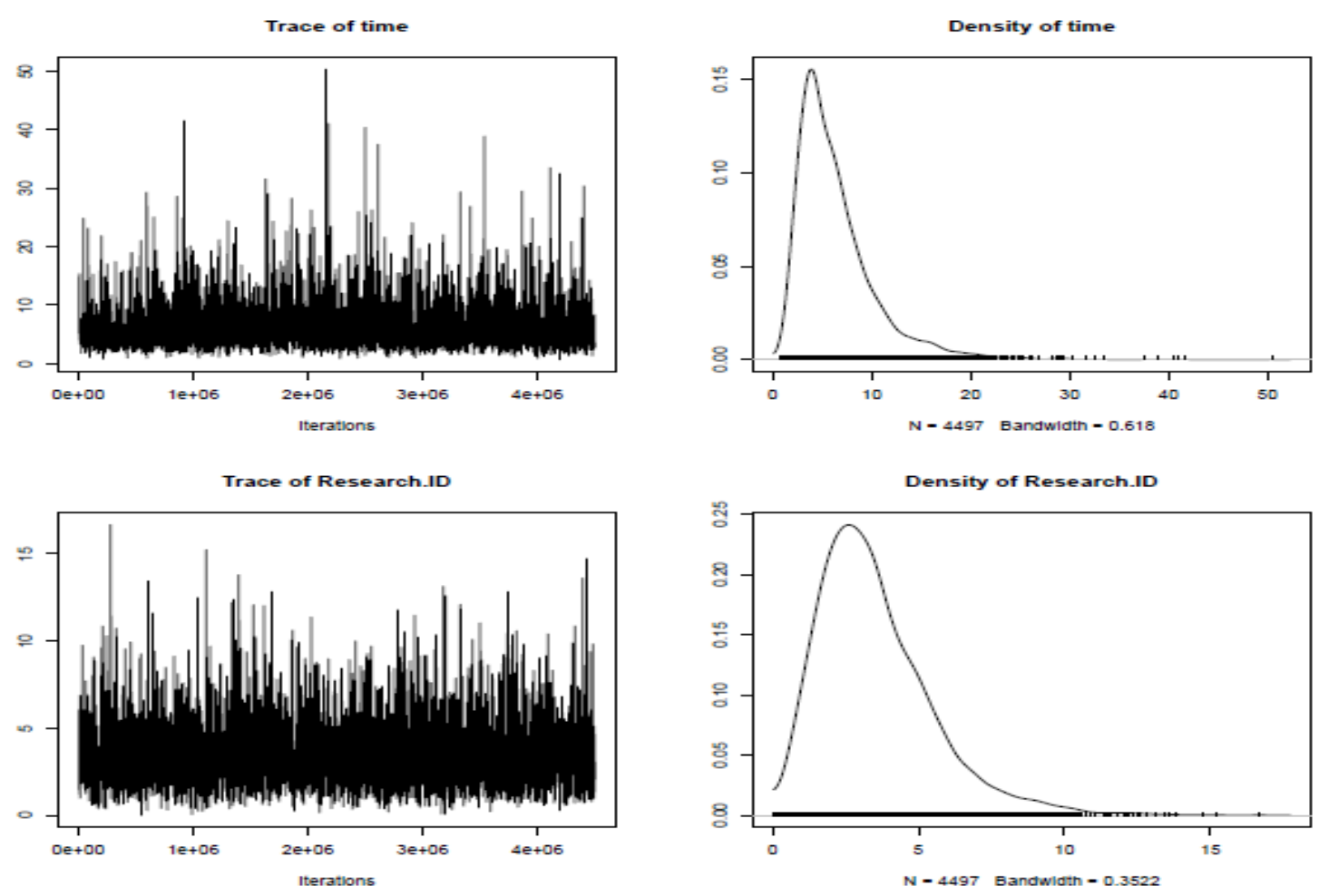
The Combined Model involving Offending History: Version 2c

Bayesian Model (BDm3G_cc2o2a)

\section{\# Define the Model}

BDm3G cC202a <- MCMCglmm(FO.bin G ageFirst*time*live + G_age First*time*relation + G_ageFirst*time*ete + G_ageFirst*time*where + G_ageFirst*time*life + G_ageFirst*time*drugs + G_ageFirst*time*physical

+ G agefirst*time*emotion + G ageFirst*time*self +

G ageFirst*time*think + G ageFirst*time*attitude +

G_ageFirst*time* change +

I Seriousness2*time*live + I Seriousness2*time*relation +

I Seriousness2*time*ete + I Seriousness $2 *$ time*where +

I Seriousness2*time*life + I Seriousness $2 *$ time*drugs +

I_Seriousness2*time*physical + I_Seriousness2*time*emotion +

I Seriousness2*time*self + I Seriousness2*time*think +

I Seriousness2*time*attitude ${ }^{-}+$I Seriousness $2 *$ time*change +

G_ageFirst*I Seriousness2,

rāndom= time+Research. ID, data=data3, family="ordinal", prior=priord, nitt $=4500000$, thin=1000, burnin=3000)

\section{\# Checks for suitable convergence}

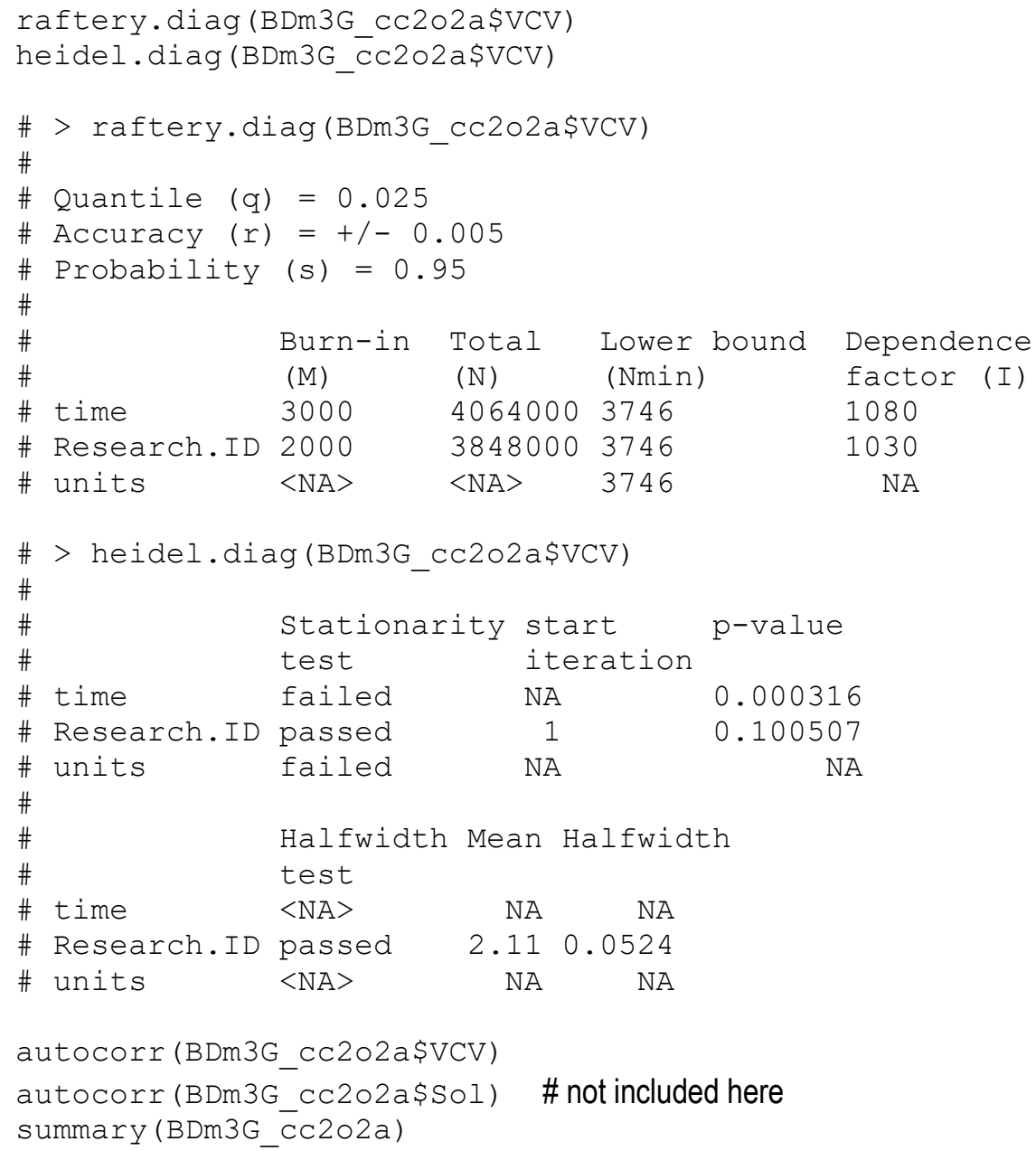




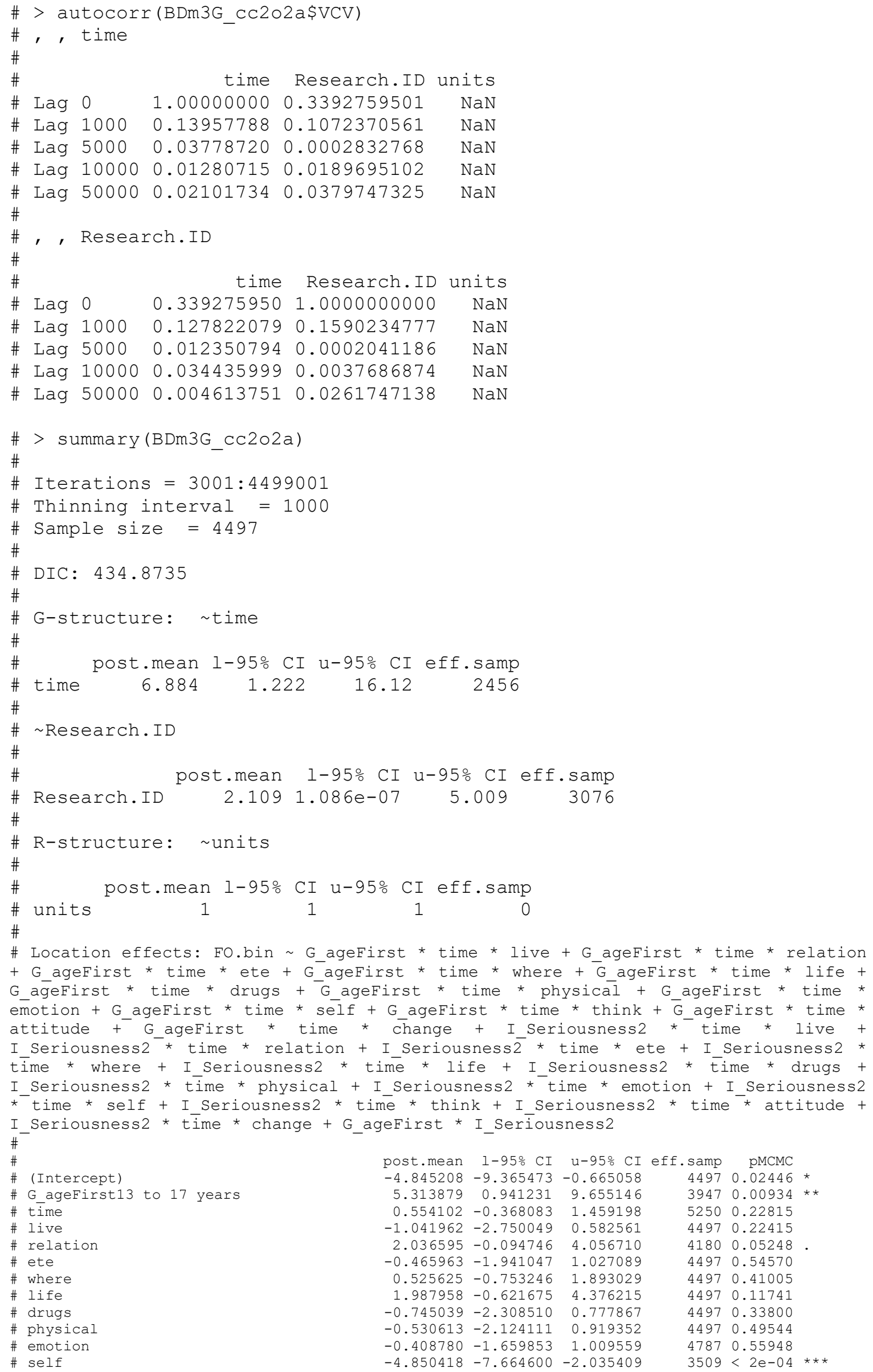


\# think

\# attitude

\# change

\# I Seriousness2

\# G_ageFirst13 to 17 years:time

\# G_ageFirst13 to 17 years:live

\# time:live

\# G ageFirst13 to 17 years:relation

\# time:relation

\# GageFirst13 to 17 years:ete

\# time:ete

\# G_ageFirst13 to 17 years:where

\# time:where

\# G ageFirst13 to 17 years:life

\# time:life

\# G_ageFirst13 to 17 years:drugs

\# time:drugs

\# G ageFirst13 to 17 years:physical

\# time:physical

\# G_ageFirst13 to 17 years:emotion

\# time:emotion

\# G ageFirst13 to 17 years:self

\# time:self

\# G ageFirst13 to 17 years:think

\# time:think

\# G_ageFirst13 to 17 years:attitude

\# time:attitude

\# G ageFirst13 to 17 years:change

\# time: change

\# time:I_Seriousness2

\# live:I- Seriousness2

\# relation:I Seriousness2

\# ete: I Seriousness2

\# where: I Seriousness2

\# life:I Seriousness2

\# drugs: $\bar{I}$ Seriousness2

\# physical:I_seriousness2

\# emotion:I Seriousness2

\# self:I Seriousness2

\# think: $\bar{I}$ Seriousness 2

\# attitude: I Seriousness2

\# change:I Seriousness2

\# G ageFirst13 to 17 years:I Seriousness2

\# G_ageFirst13 to 17 years:time:live

\# G-ageFirst13 to 17 years:time:relation

\# G ageFirst13 to 17 years:time:ete

\# G_ageFirst13 to 17 years:time:where

\# G ageFirst13 to 17 years:time:life

\# G ageFirst13 to 17 years:time:drugs

\# G_ageFirst13 to 17 years:time:physical

\# G ageFirst13 to 17 years:time:emotion

\# G ageFirst13 to 17 years:time:self

\# G ageFirst13 to 17 years:time:think

\# G_ageFirst13 to 17 years:time:attitude

\# G-ageFirst13 to 17 years:time:change

\# time:live:I Seriousness2

\# time:relatiōn:I Seriousness2

\# time:ete: I Seriousness2

\# time:where: I Seriousness2

\# time:life:I Seriousness2

\# time:drugs:I Seriousness 2

\# time:physical:I Seriousness2

\# time:emotion:I Seriousness 2

\# time:self:I Seriousness2

\# time:think:I Seriousness 2

\# time:attitude: I Seriousness2

\# time:change:I_Seriousness2

\# ---

$\#$ \#

Signif. codes:
$1.531073-0.837077 \quad 3.798752$

$\begin{array}{lll}1.648651 & -0.857748 & 4.245089\end{array}$

$1.561624-0.834507 \quad 3.837416$

$-0.529703-2.293308 \quad 1.160746$

$-1.019994-2.033596-0.158709$

$0.274137-1.550578 \quad 2.034567$

$\begin{array}{lll}-0.095794 & -0.503263 & 0.276487\end{array}$

$-2.523551-4.862332-0.345153$

$\begin{array}{lll}-0.293833 & -0.895886 & 0.227693\end{array}$

$0.035873-1.571953 \quad 1.591780$

$\begin{array}{llll}0.031108 & -0.352049 & 0.414621\end{array}$

$\begin{array}{lll}-0.751577 & -2.340717 & 0.746703\end{array}$

$\begin{array}{lll}-0.173479 & -0.453938 & 0.105196\end{array}$

$\begin{array}{llll}-2.222948 & -4.733746 & 0.293226\end{array}$

$\begin{array}{lll}-0.439546 & -1.037985 & 0.179820\end{array}$

$\begin{array}{llll}0.943723 & -0.691234 & 2.423550\end{array}$

$\begin{array}{lll}0.436618 & 0.062899 & 0.824812\end{array}$

$\begin{array}{lll}-0.432504 & -2.345595 & 1.461063\end{array}$

$0.127947-0.314793 \quad 0.576081$

$\begin{array}{lll}-0.424059 & -1.862574 & 1.091487\end{array}$

$\begin{array}{llll}0.103187 & -0.296401 & 0.481579\end{array}$

$\begin{array}{lll}7.274862 & 4.174170 & 10.584677\end{array}$

$\begin{array}{lll}1.149899 & 0.529328 & 1.819848\end{array}$

$\begin{array}{lll}-0.818664 & -3.148625 & 1.532716\end{array}$

$\begin{array}{lll}-0.157131 & -0.588816 & 0.316864\end{array}$

$\begin{array}{llll}-1.373094 & -4.366109 & 1.290830\end{array}$

$-0.690845-1.205314-0.192087$

$\begin{array}{lll}-1.271744 & -3.840756 & 1.180617\end{array}$

$\begin{array}{lll}-0.126111 & -0.634886 & 0.379490\end{array}$

$0.068583-0.267104 \quad 0.411008$

$\begin{array}{lll}0.729438 & 0.056152 & 1.375594\end{array}$

$\begin{array}{lll}0.105349 & -0.539488 & 0.764591\end{array}$

$\begin{array}{lll}0.030777 & -0.486260 & 0.617603\end{array}$

$\begin{array}{lll}0.152915 & -0.343264 & 0.696599\end{array}$

$\begin{array}{llll}0.579917 & -0.310903 & 1.419792\end{array}$

$\begin{array}{llll}0.198894 & -0.324971 & 0.814341\end{array}$

$\begin{array}{lll}-0.016765 & -0.754021 & 0.707431\end{array}$

$\begin{array}{lll}-0.115152 & -0.739543 & 0.445747\end{array}$

$\begin{array}{lll}-0.057963 & -0.820255 & 0.627269\end{array}$

$\begin{array}{lll}-0.584045 & -1.555725 & 0.337977\end{array}$

$\begin{array}{llll}-0.117701 & -0.959506 & 0.681988\end{array}$

$\begin{array}{lll}-0.516985 & -1.567937 & 0.491443\end{array}$

$\begin{array}{llll}-0.333040 & -1.280756 & 0.664171\end{array}$

$0.307286-0.107757 \quad 0.712230$

$0.502973-0.061281 \quad 1.019482$

$\begin{array}{lll}0.021505 & -0.321508 & 0.394904\end{array}$

$\begin{array}{lll}0.273128 & -0.038278 & 0.599362\end{array}$

$0.447158-0.111649 \quad 1.028163$

$-0.469318-0.835467-0.095394$

$\begin{array}{llll}0.115366 & -0.355544 & 0.651551\end{array}$

$\begin{array}{lll}-0.056832 & -0.439570 & 0.337727\end{array}$

$-1.624014-2.342584-0.973356$

$\begin{array}{llll}0.251063 & -0.192669 & 0.737697\end{array}$

$0.401481-0.1856851 .042453$

$\begin{array}{llll}0.047039 & -0.483663 & 0.562252\end{array}$

$\begin{array}{lll}-0.100628 & -0.258226 & 0.058768\end{array}$

$\begin{array}{lll}-0.124871 & -0.309567 & 0.060818\end{array}$

$\begin{array}{lll}0.080462 & -0.083779 & 0.240542\end{array}$

$\begin{array}{lll}-0.061447 & -0.185911 & 0.060174\end{array}$

$\begin{array}{lll}-0.112723 & -0.321087 & 0.104006\end{array}$

$\begin{array}{lll}-0.043295 & -0.177969 & 0.096658\end{array}$

$\begin{array}{lll}-0.034524 & -0.217524 & 0.153743\end{array}$

$\begin{array}{lll}0.166970 & 0.008951 & 0.329542\end{array}$

$\begin{array}{lll}-0.003704 & -0.193680 & 0.162753\end{array}$

$\begin{array}{llll}-0.051042 & -0.249519 & 0.155564\end{array}$

$\begin{array}{lll}0.125413 & -0.088931 & 0.356560\end{array}$

$\begin{array}{llll}0.127453 & -0.096407 & 0.357011\end{array}$
$4086 \quad 0.19924$

$4497 \quad 0.19524$

44970.18813

$4497 \quad 0.55281$

42320.03336

44970.77919

$4497 \quad 0.63509$

$39940.02135 *$

44970.30909

$4497 \quad 0.99755$

$4497 \quad 0.87169$

44970.32155

$4497 \quad 0.21570$

44970.08761 .

43340.14499

$4497 \quad 0.23927$

43090.01868 *

44970.66222

44970.60040

48230.57950

51330.59017

$3539<2 e-04 * \star *$

$3762<2 e-04 \star \star \star *$

40710.51101

42610.51768

44970.33533

42090.00400 **

$4497 \quad 0.31710$

$4497 \quad 0.63598$

44970.70758

42780.02757 *

$4497 \quad 0.77074$

$4497 \quad 0.90994$

$4497 \quad 0.55771$

$4497 \quad 0.17790$

$\begin{array}{lll}3510 & 0.52791\end{array}$

$4497 \quad 0.94908$

$4497 \quad 0.72359$

$4499 \quad 0.85479$

$4497 \quad 0.19924$

$4497 \quad 0.76051$

$\begin{array}{lll}4497 & 0.33178\end{array}$

44970.48299

$4497 \quad 0.14454$

$4497 \quad 0.06404$.

49960.89304

44970.08584 .

44970.12586

44970.01423

44970.66489

52660.76851

$3830<2 e-04 * \star \star$

$4497 \quad 0.27485$

$4497 \quad 0.19391$

$4497 \quad 0.86769$

37130.20102

36460.18145

$4497 \quad 0.34334$

$4497 \quad 0.32733$

44970.30064

$4250 \quad 0.54792$

$4497 \quad 0.70669$

42260.02846 *

44970.98866

$4497 \quad 0.61552$

$4497 \quad 0.26507$

$4497 \quad 0.27574$ 
Trace Plots and Posterior Density Plots

Fixed Effects

Trace of (Intercept)

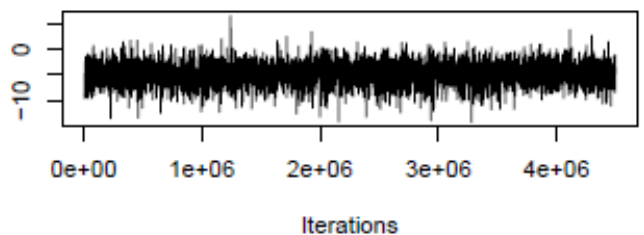

Trace of G_ageFirst13 to 17 years

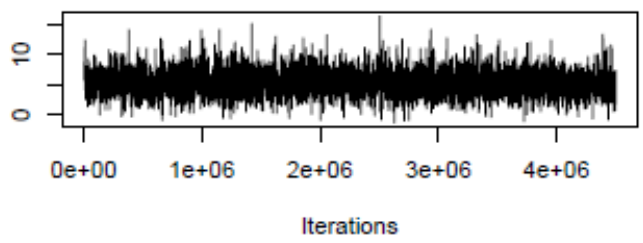

Trace of time

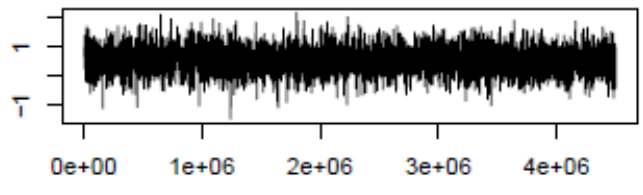

Iterations

Trace of live

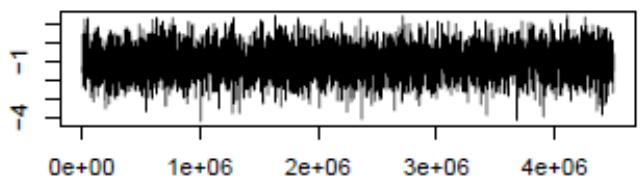

Iterations

Trace of relation

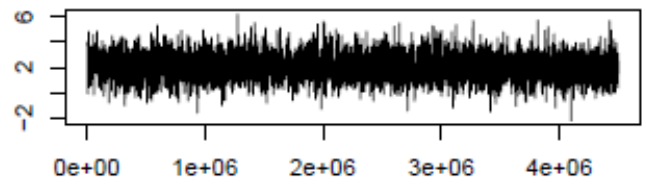

Iterations

Trace of ete

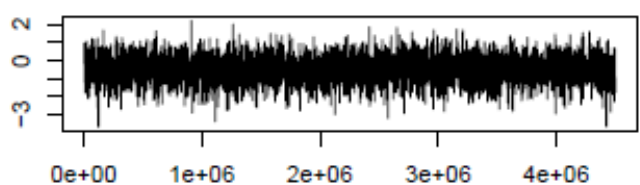

Iterations
Density of (Intercept)

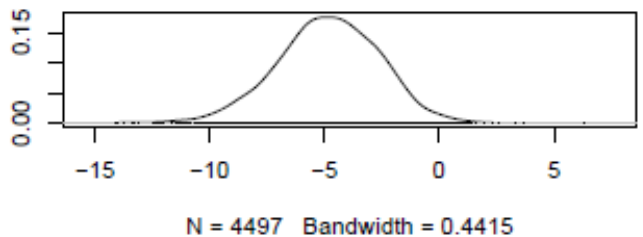

Density of G_ageFirst13 to 17 years

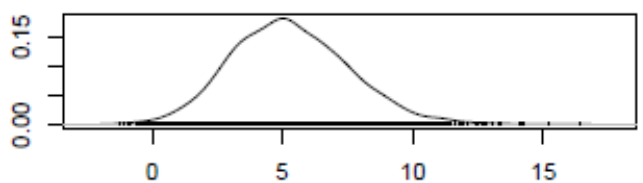

$\mathrm{N}=4497$ Bandwidth $=0.4458$

Density of time

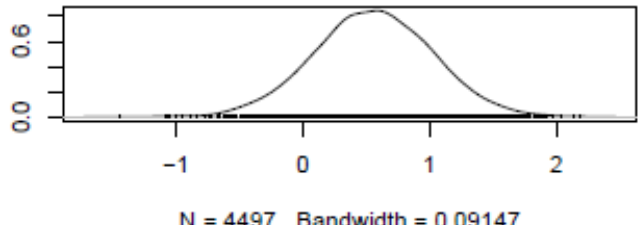

Density of live

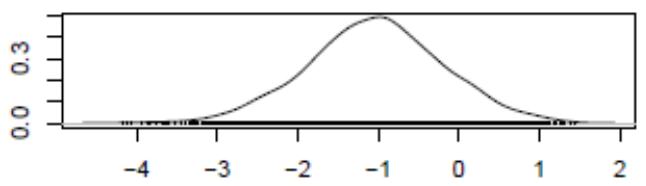

$\mathrm{N}=4497$ Bandwidth $=0.162$

Density of relation

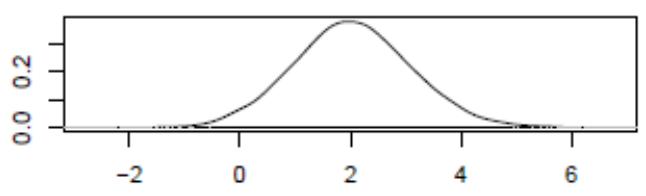

$\mathrm{N}=4497$ Bandwidth $=0.2077$

Density of ete

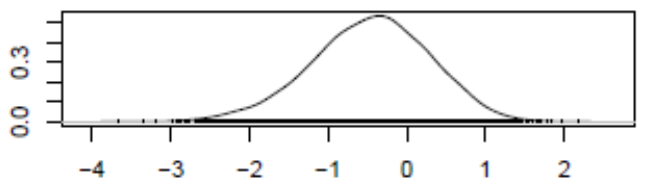

$\mathrm{N}=4497$ Bandwidth $=0.1486$ 
Trace of where

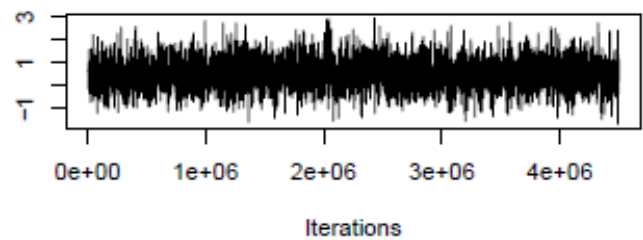

Trace of life

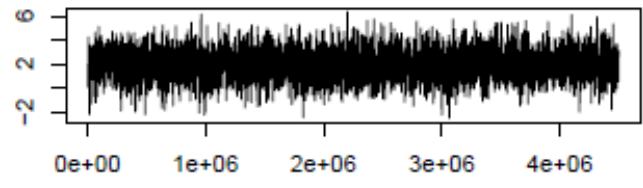

Iterations

Trace of drugs

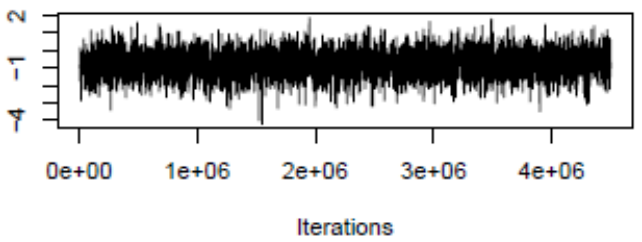

Trace of physical

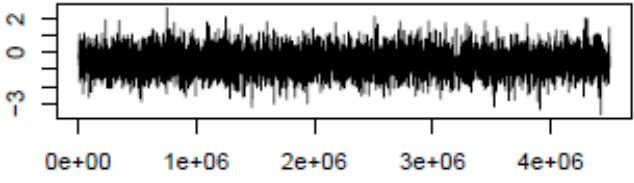

Iterations

Trace of emotion

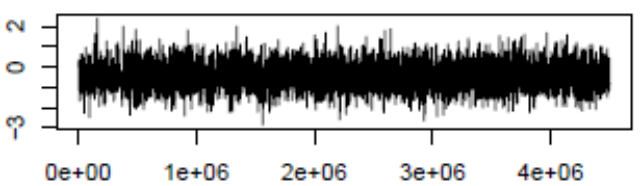

Iterations

Trace of self

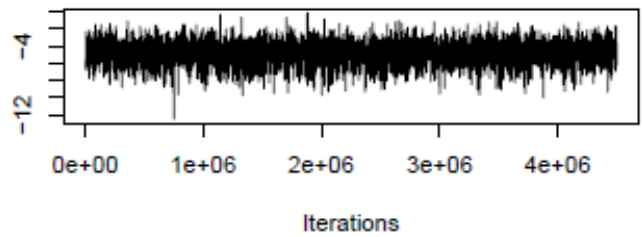

Density of where

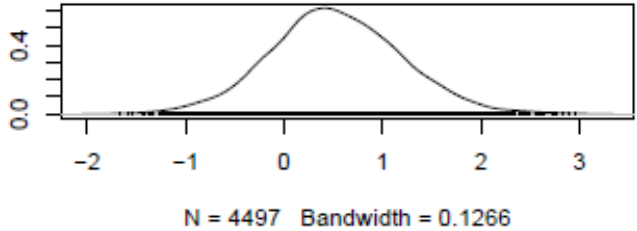

Density of life

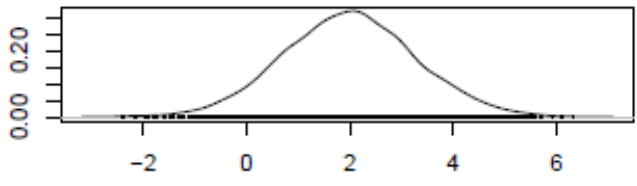

$\mathrm{N}=4497$ Bandwidth $=0.2506$

Density of drugs

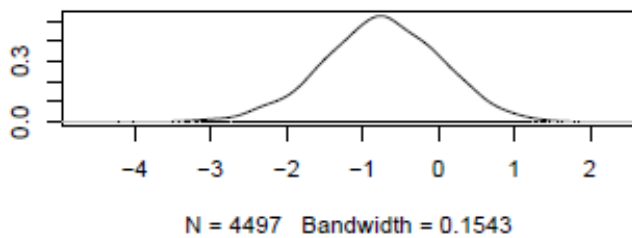

Density of physical

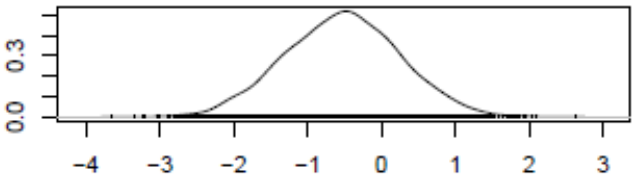

$\mathrm{N}=4497$ Bandwidth $=0.1546$

Density of emotion

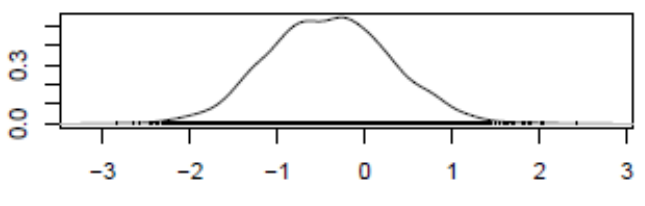

$\mathrm{N}=4497$ Bandwidth $=0.137$

\section{Density of self}

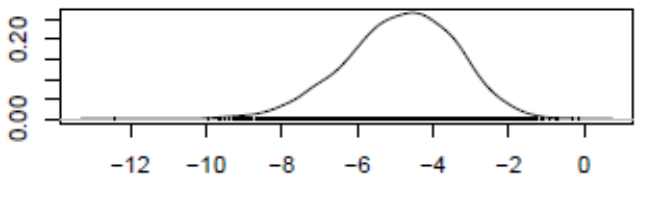

$\mathrm{N}=4497$ Bandwidth $=0.2862$ 
Trace of think

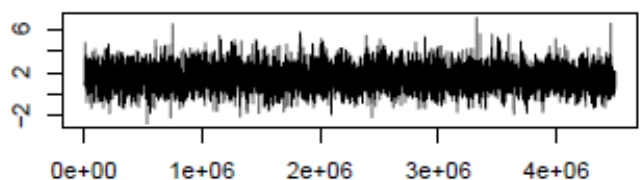

Iterations

Trace of attitude

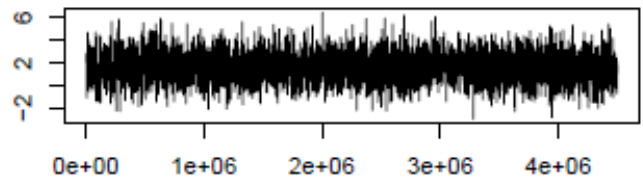

Iterations

Trace of change

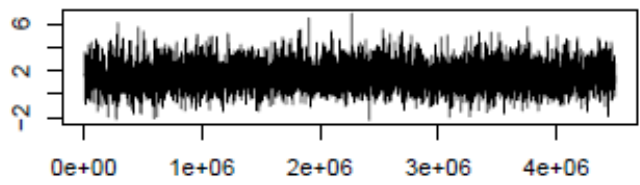

Iterations

Trace of I_Seriousness2

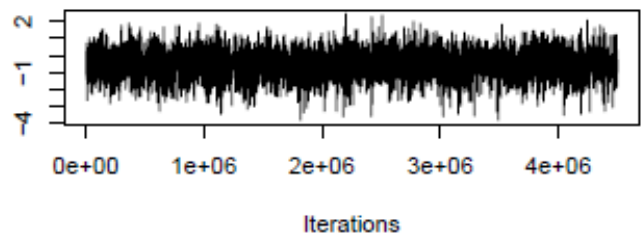

Trace of G_ageFirst13 to 17 years:time

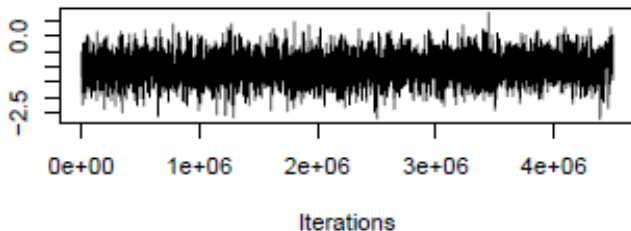

Trace of G_ageFirst13 to 17 years:live

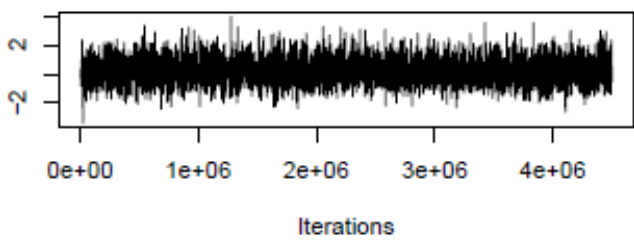

Density of think

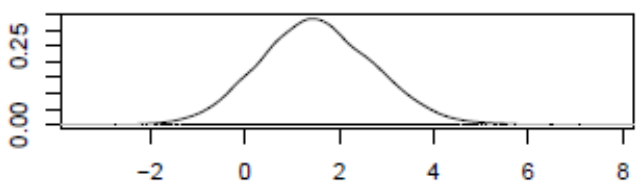

$\mathrm{N}=4497$ Bandwidth $=0.2365$

Density of attitude

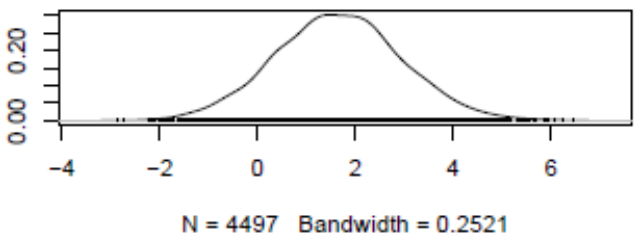

Density of change

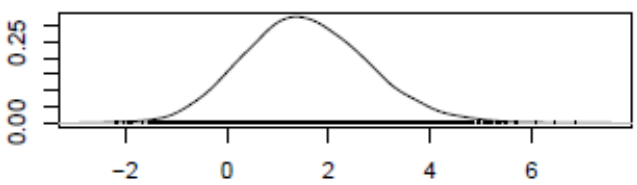

$\mathrm{N}=4497$ Bandwidth $=0.2372$

Density of I_Seriousness2

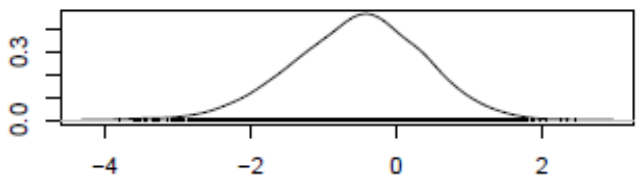

$\mathrm{N}=4497$ Bandwidth $=0.1747$

Density of G_ageFirst13 to 17 years:time

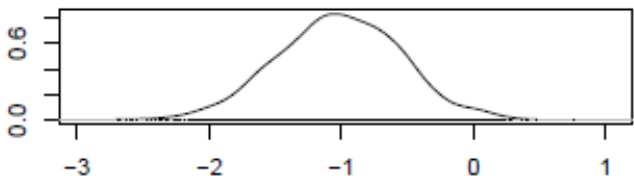

$\mathrm{N}=4497$ Bandwidth $=0.09291$

Density of G_ageFirst13 to 17 years:live

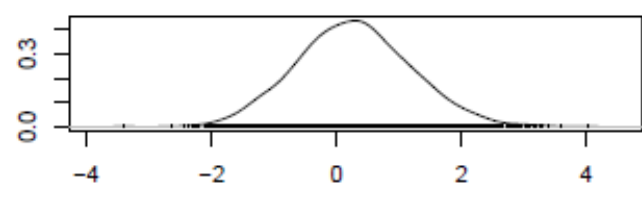

$\mathrm{N}=4497$ Bandwidth $=0.1794$ 
Trace of time:live

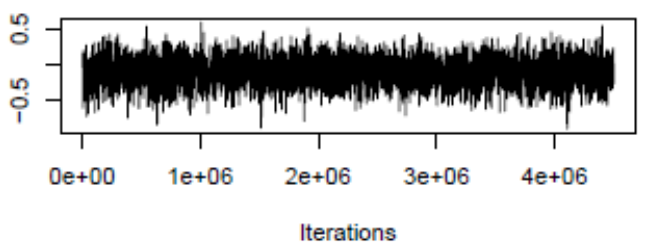

Trace of G_ageFirst 13 to 17 years:relation

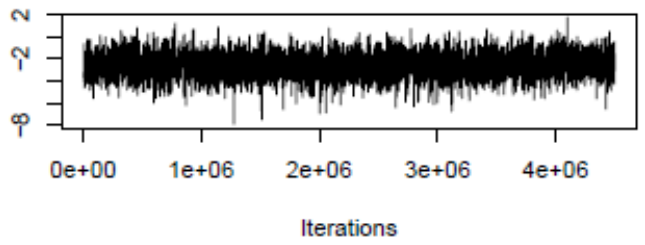

Trace of time:relation

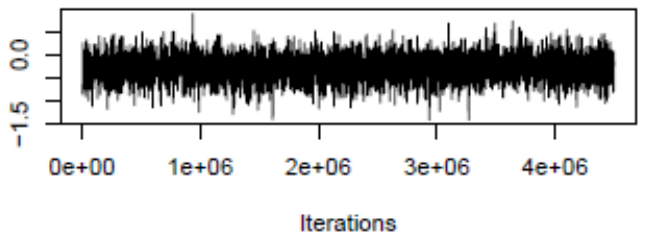

Trace of G_ageFirst13 to 17 years:ete

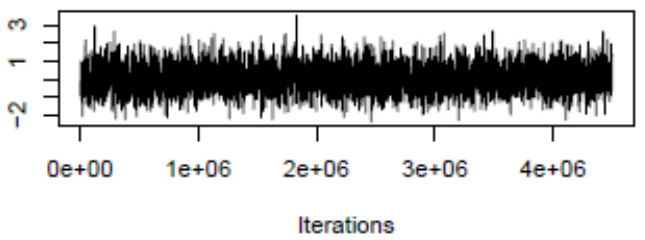

Trace of time:ete

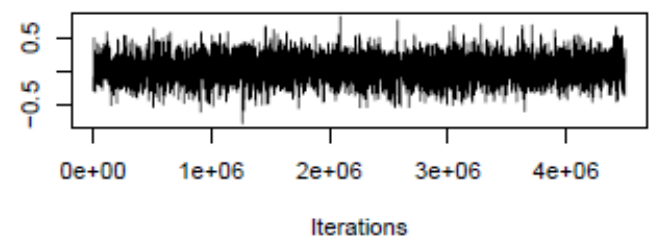

Trace of G_ageFirst13 to 17 years:where

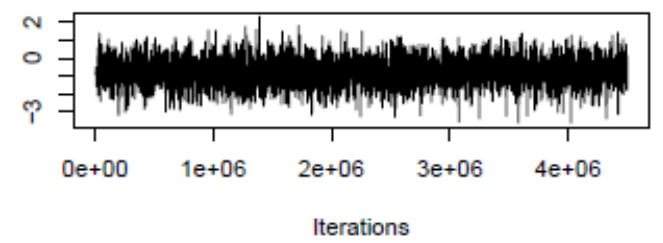

Density of time:live

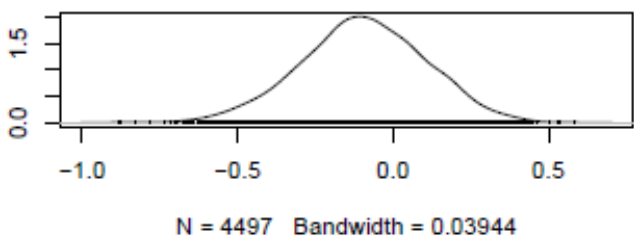

Density of G_ageFirst13 to 17 years:relation

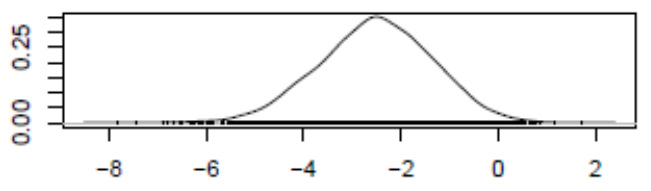

$\mathrm{N}=4497$ Bandwidth $=0.2278$

Density of time:relation

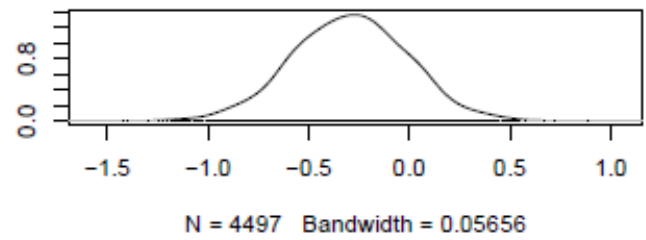

Density of G_ageFirst13 to 17 years:ete

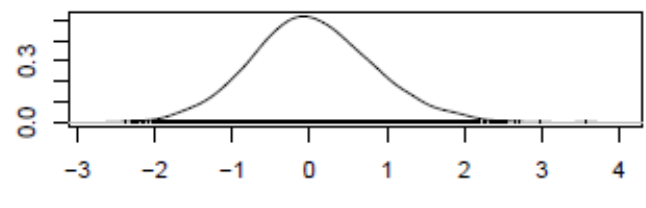

$\mathrm{N}=4497$ Bandwidth $=0.1527$

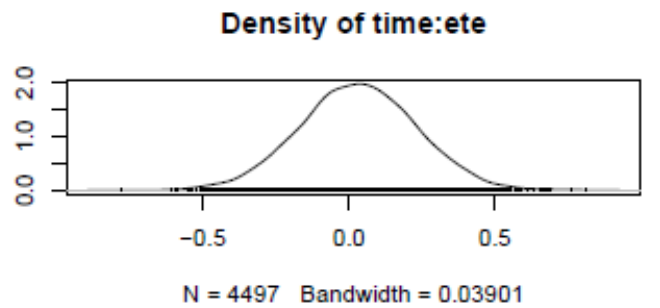

Density of G_ageFirst13 to 17 years:where

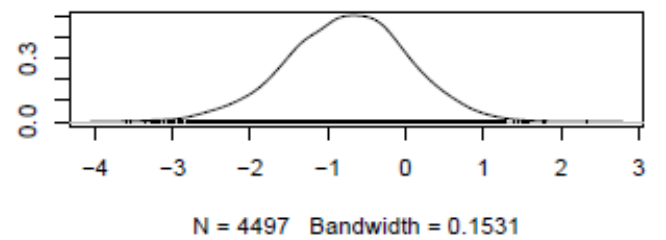


Trace of time:where

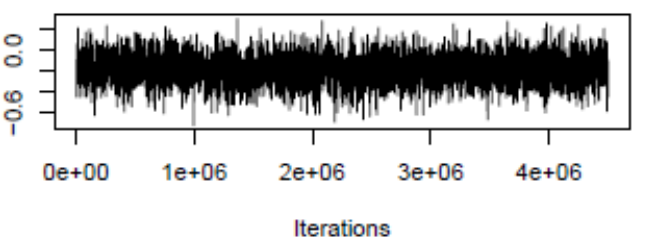

Trace of G_ageFirst13 to 17 years:life

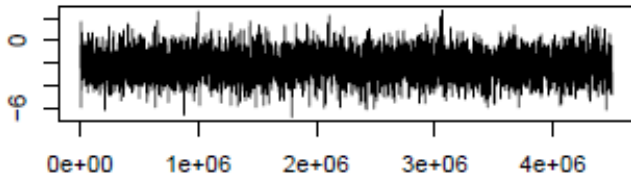

Iterations

Trace of time:life

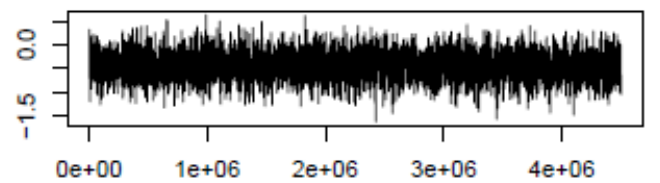

Iterations

Trace of G_ageFirst13 to 17 years:drugs

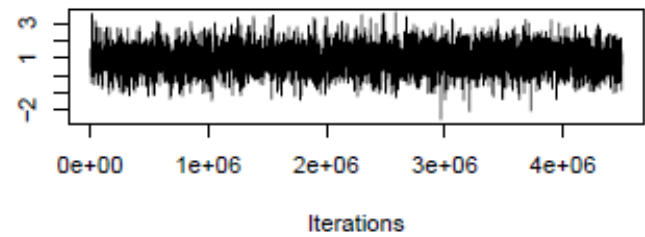

Trace of time:drugs

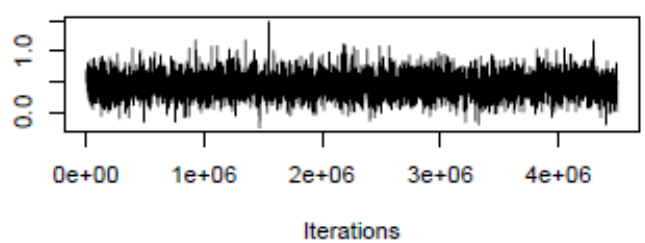

Trace of G_ageFirst13 to 17 years:physical

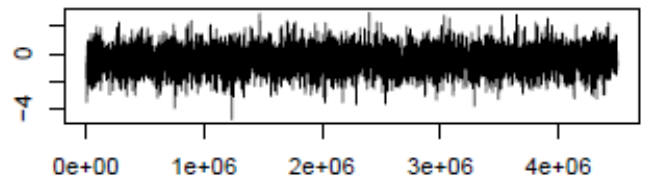

Iterations
Density of time:where

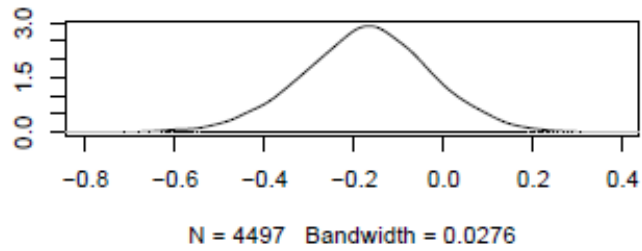

Density of G_ageFirst13 to 17 years:life

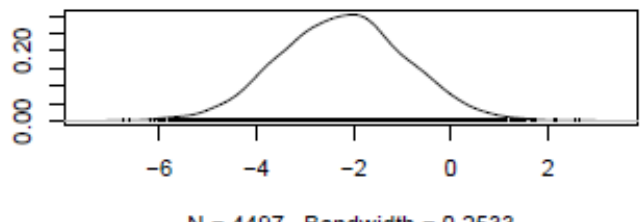

Density of time:life

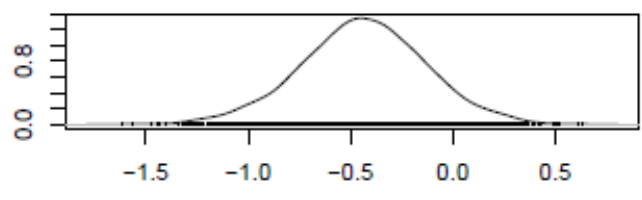

$\mathrm{N}=4497$ Bandwidth $=0.0586$

Density of G_ageFirst13 to 17 years:drugs

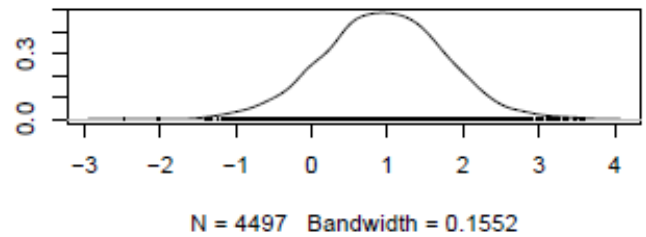

Density of time:drugs

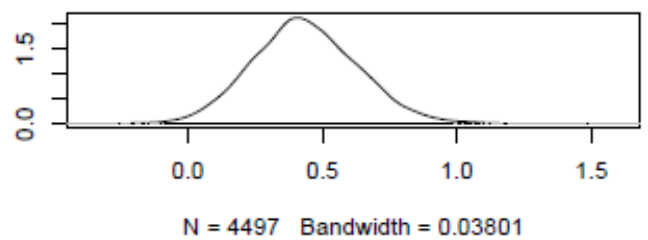

Density of G_ageFirst13 to 17 years:physical

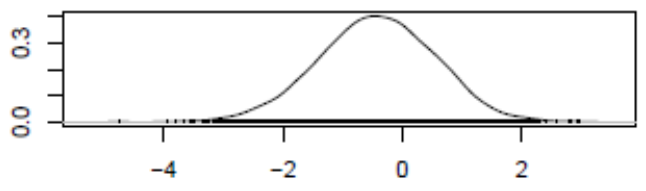

$\mathrm{N}=4497$ Bandwidth $=0.1924$ 
Trace of time:physical

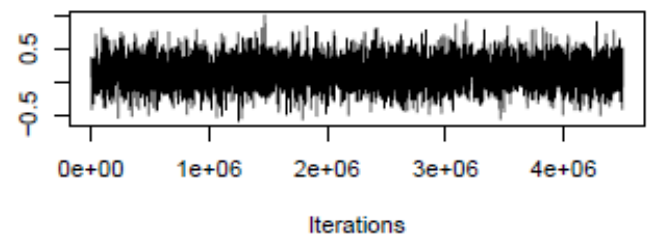

Trace of G_ageFirst 13 to 17 years:emotion

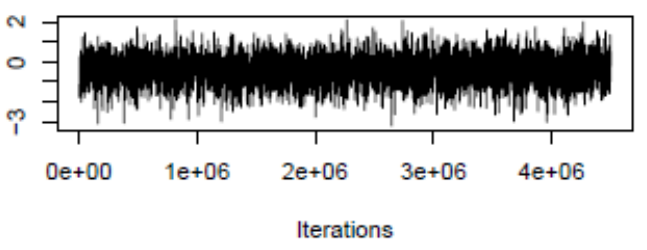

Trace of time:emotion

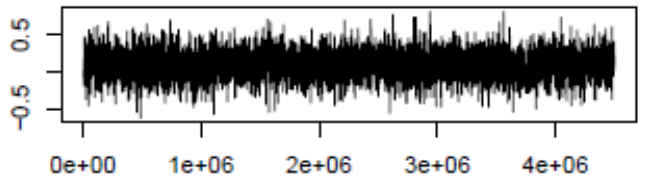

Iterations

Trace of G_ageFirst13 to 17 years:self

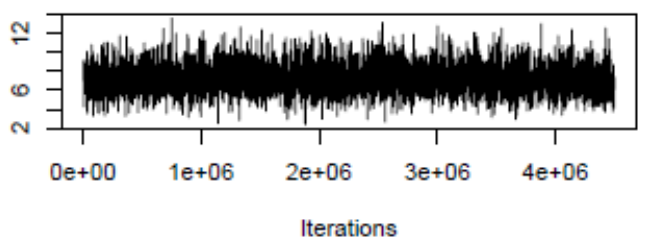

\section{Trace of time:self}

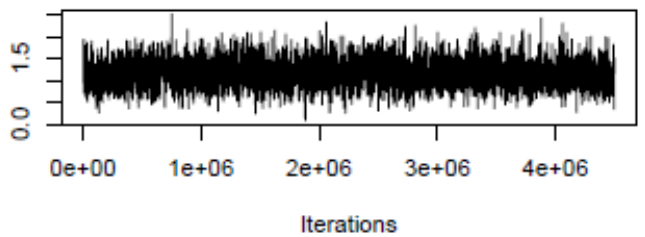

Trace of G_ageFirst13 to 17 years:think

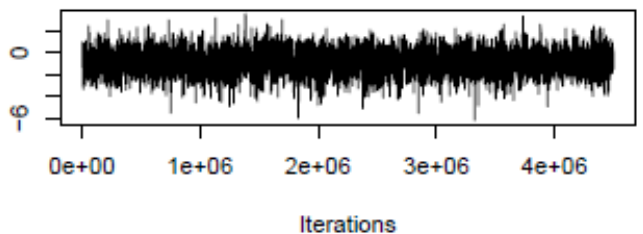

Density of time:physical

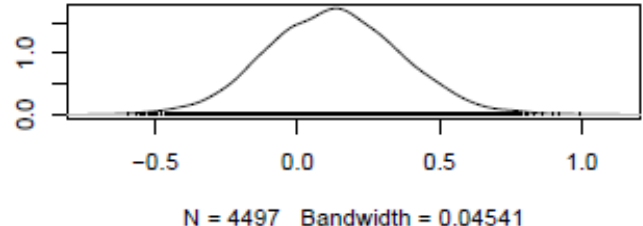

Density of G_ageFirst13 to 17 years:emotion

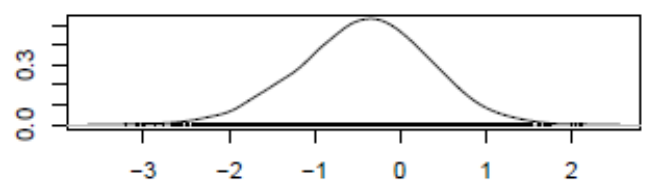

$\mathrm{N}=4497$ Bandwidth $=0.1477$

Density of time:emotion

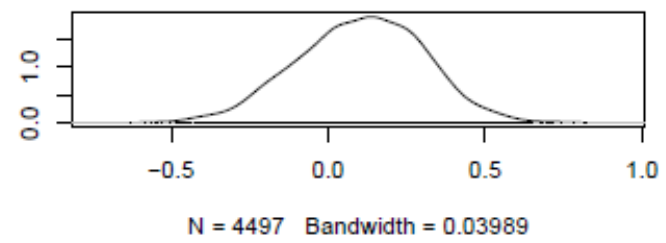

Density of G_ageFirst 13 to 17 years:self

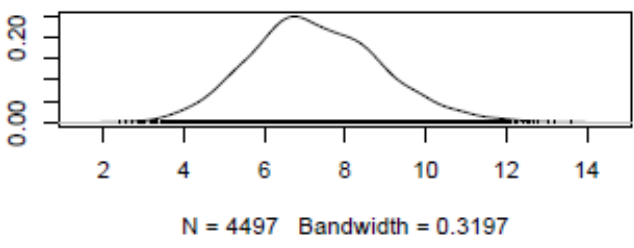

Density of time:self

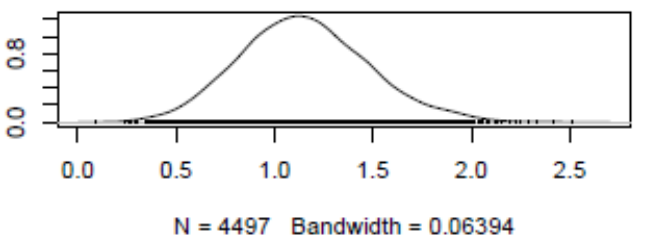

Density of G_ageFirst13 to 17 years:think

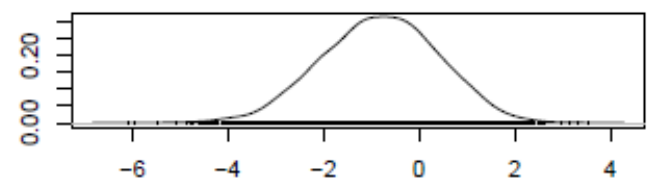

$\mathrm{N}=4497$ Bandwidth $=0.2426$ 
Trace of time:think

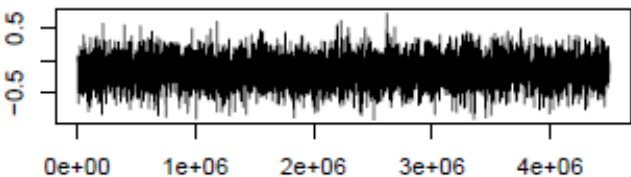

Iterations

Trace of G_ageFirst13 to 17 years:attitude

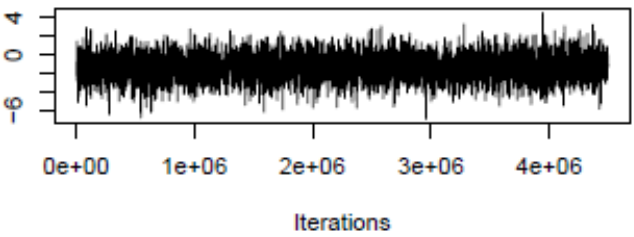

Trace of time:attitude

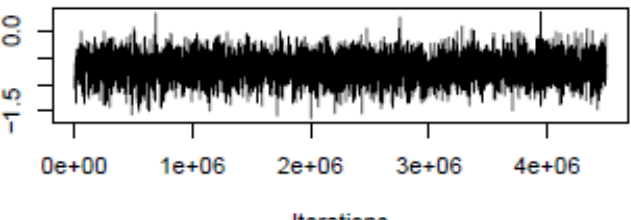

Trace of G_ageFirst13 to 17 years:change

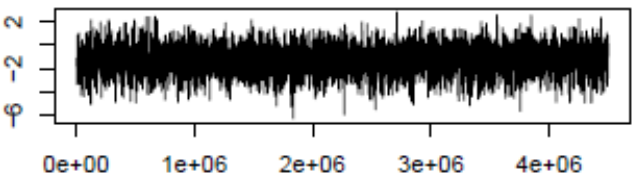

Iterations

Trace of time:change

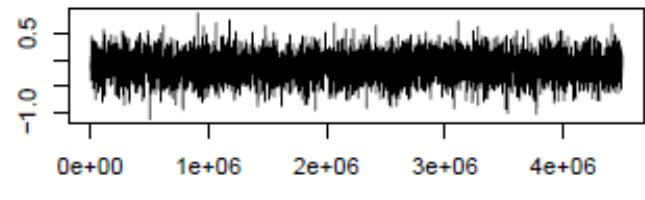

Iterations

Trace of time:I_Seriousness2

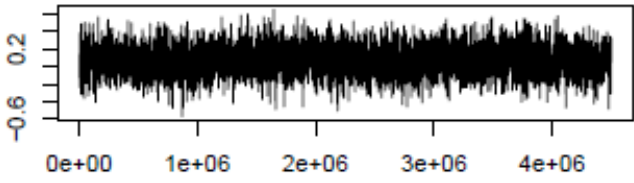

Iterations
Density of time:think

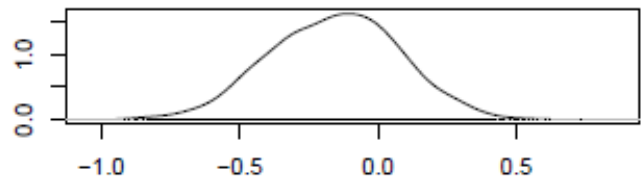

$\mathrm{N}=4497$ Bandwidth $=0.04643$

Density of G_ageFirst13 to 17 years:attitude

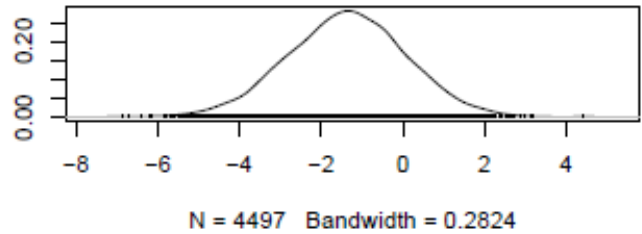

Density of time:attitude

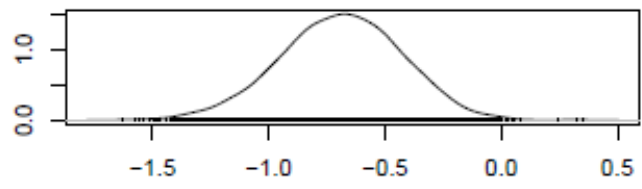

$\mathrm{N}=4497 \quad$ Bandwidth $=0.05151$

Density of G_ageFirst13 to 17 years:change

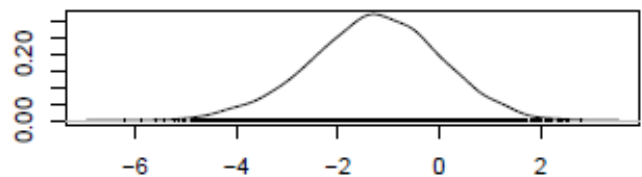

$\mathrm{N}=4497$ Bandwidth $=0.2471$

Density of time:change

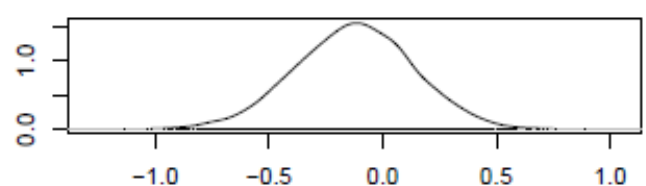

$\mathrm{N}=4497$ Bandwidth $=0.05128$

Density of time:I_Seriousness2

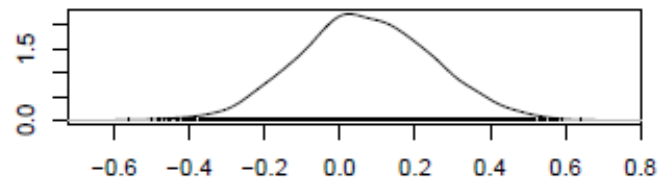

$\mathrm{N}=4497$ Bandwidth $=0.03466$ 
Trace of live:I Seriousness2

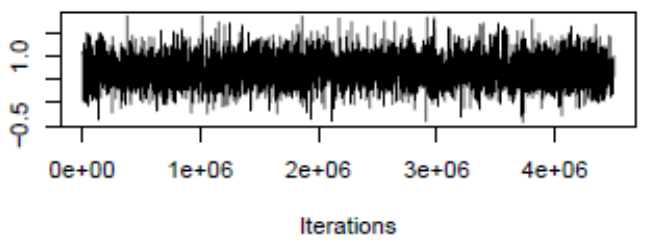

Trace of relation:I_Seriousness2

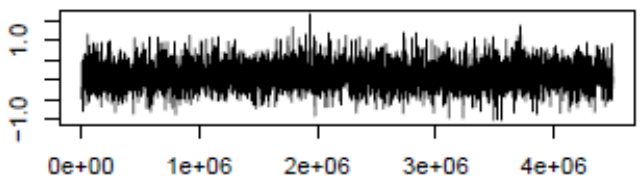

Iterations

Trace of ete:I_Seriousness2

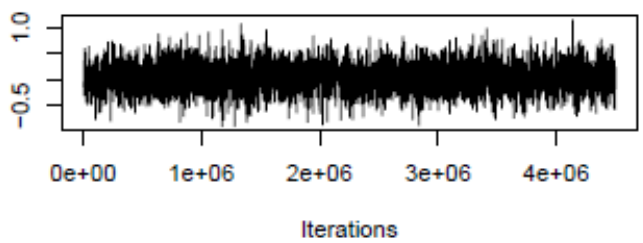

Trace of where:I_Seriousness 2

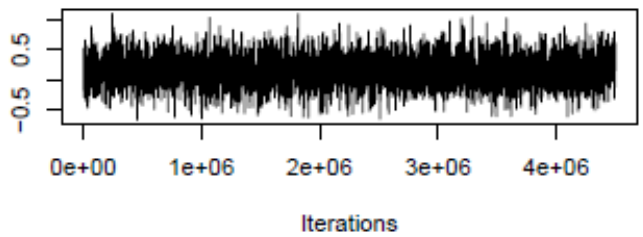

Trace of life:I_Seriousness2

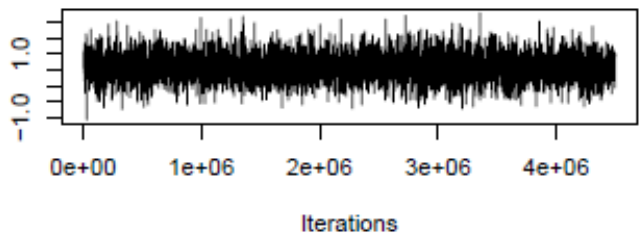

Trace of drugs:I_Seriousness2

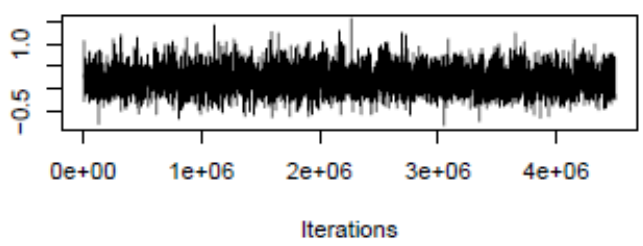

Density of live:I Seriousness2

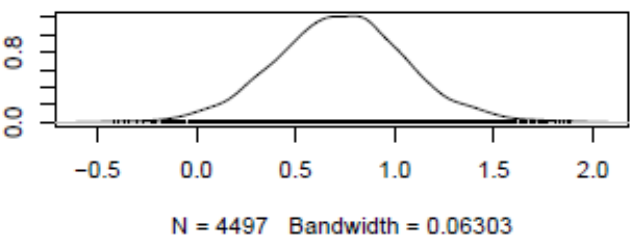

Density of relation:I_Seriousness2

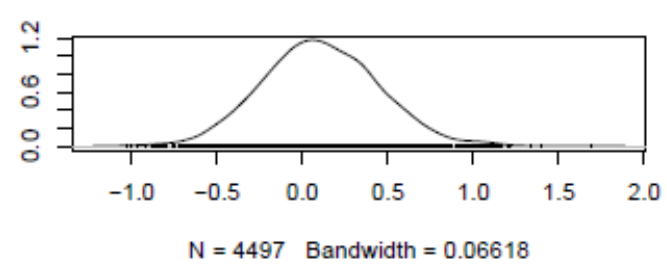

Density of ete:I_Seriousness2

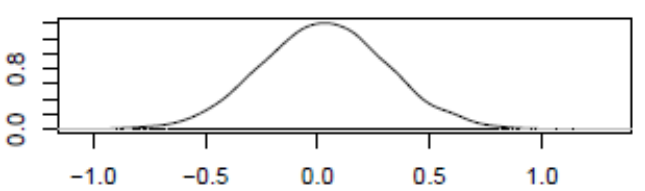

$\mathrm{N}=4497$ Bandwidth $=0.05518$

Density of where:I_Seriousness2

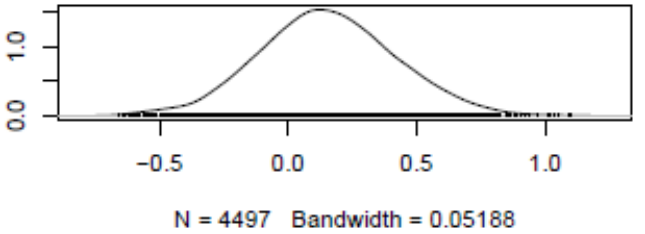

Density of life:I_Seriousness2

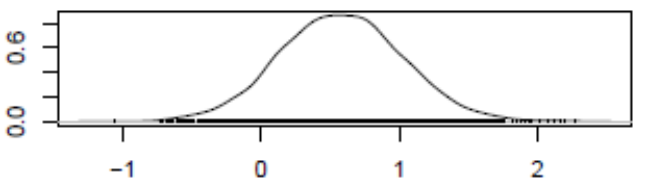

$\mathrm{N}=4497$ Bandwidth $=0.08709$

Density of drugs:I_Seriousness2

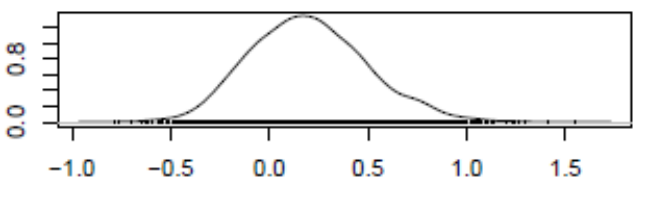

$\mathrm{N}=4497$ Bandwidth $=0.0585$ 
Trace of physical:I_Seriousness2

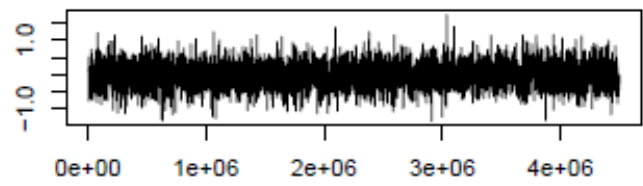

Iterations

Trace of emotion:I_Seriousness2

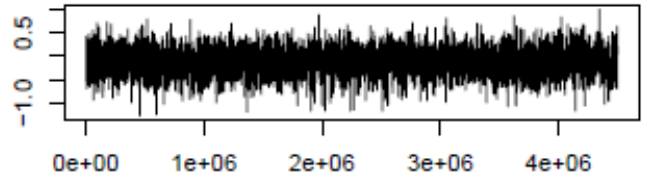

Iterations

Trace of self:I_Seriousness2

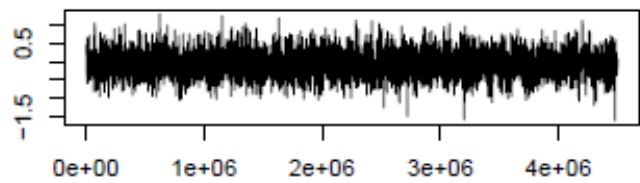

Iterations

Trace of think:I_Seriousness2

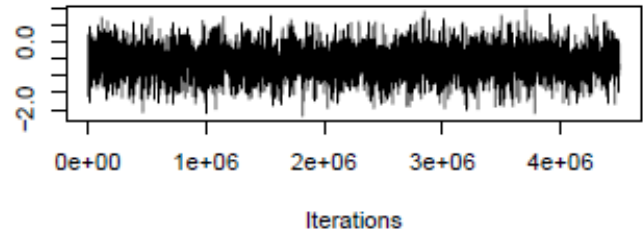

Trace of attitude:I_Seriousness2

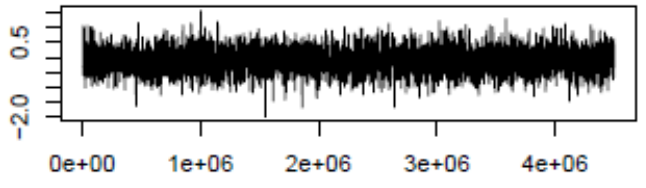

Iterations

Trace of change:I_Seriousness2

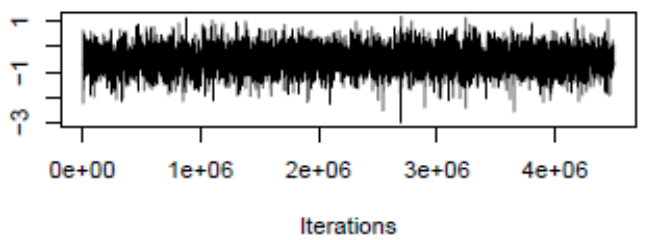

Density of physical:I_Seriousness2

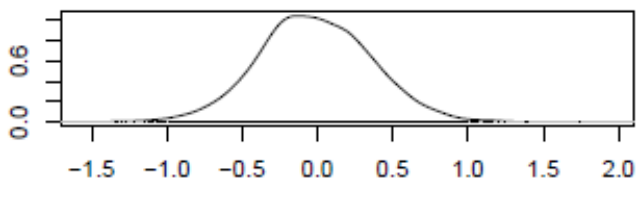

$\mathrm{N}=4497$ Bandwidth $=0.07288$

Density of emotion:I_Seriousness2

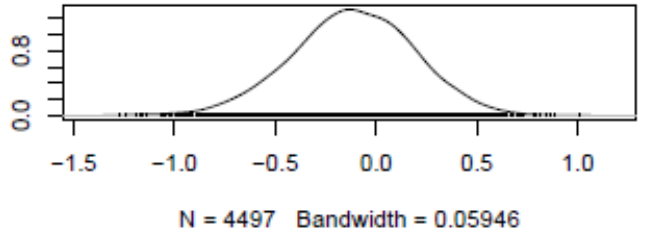

Density of self:I_Seriousness2

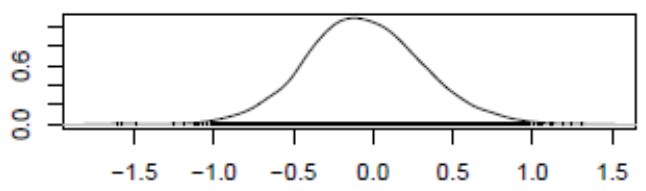

$\mathrm{N}=4497$ Bandwidth $=0.07103$

Density of think:I_Seriousness2

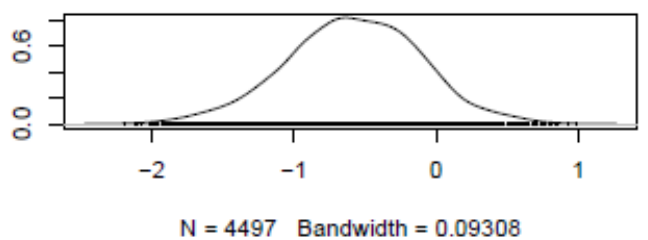

Density of attitude:I_Seriousness2

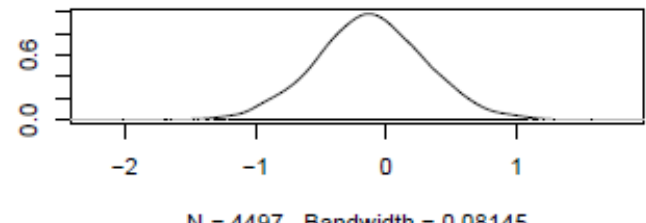

Density of change:I_Seriousness2

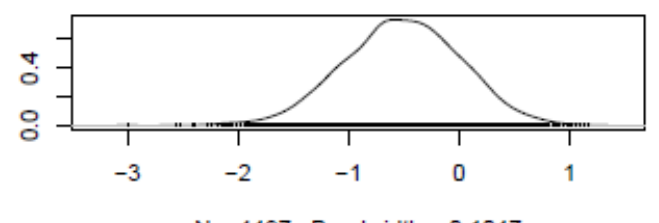

$\mathrm{N}=4497$ Bandwidth $=0.1047$ 
Trace of G_ageFirst13 to 17 years:I_Seriousness2

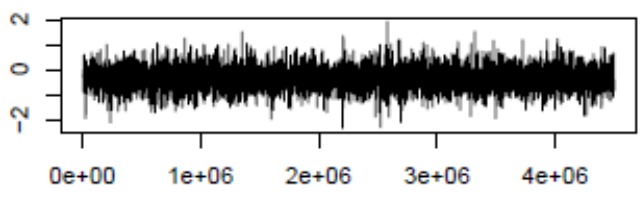

Iterations

Trace of G_ageFirst13 to 17 years:time:live

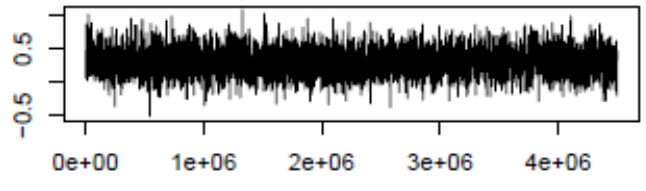

Iterations

Trace of G_ageFirst13 to 17 years:time:relation

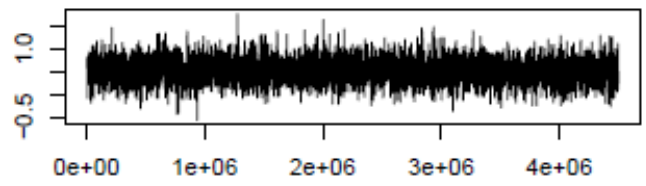

Iterations

Trace of G_ageFirst13 to 17 years:time:ete

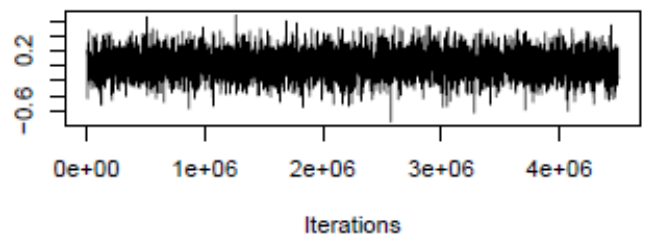

Trace of G_ageFirst13 to 17 years:time:where

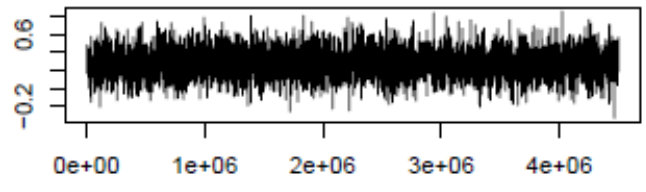

Iterations

Trace of G_ageFirst13 to 17 years:time:life

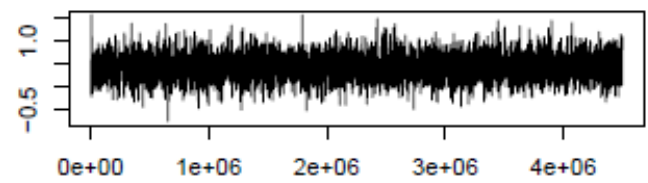

Iterations
Density of G_ageFirst13 to 17 years:I_Seriousness

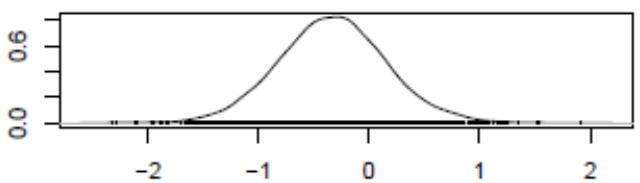

$\mathrm{N}=4497$ Bandwidth $=0.0939$

Density of G_ageFirst13 to 17 years:time:live

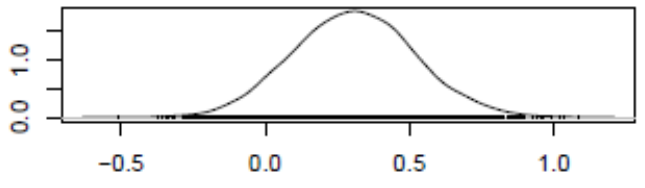

$\mathrm{N}=4497$ Bandwidth $=0.04141$

Density of G_ageFirst13 to 17 years:time:relatior

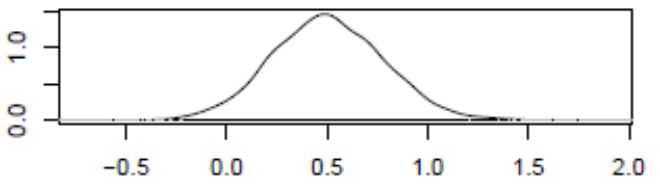

$\mathrm{N}=4497$ Bandwidth $=0.05445$

Density of G_ageFirst13 to 17 years:time:ete

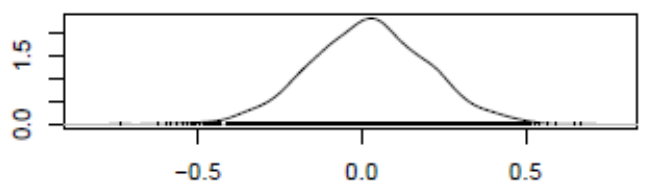

$\mathrm{N}=4497$ Bandwidth $=0.03523$

Density of G_ageFirst 13 to 17 years:time:where

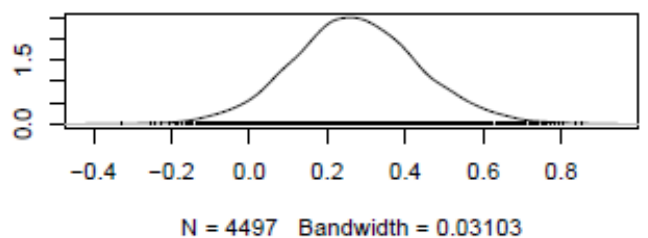

Density of G_ageFirst13 to 17 years:time:life

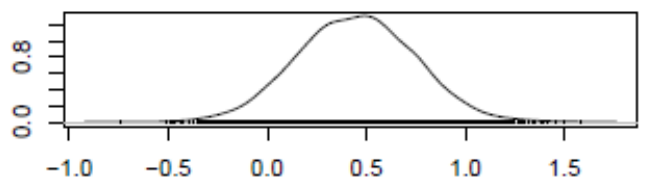

$\mathrm{N}=4497$ Bandwidth $=0.0583$ 
Trace of G_ageFirst13 to 17 years:time:drugs

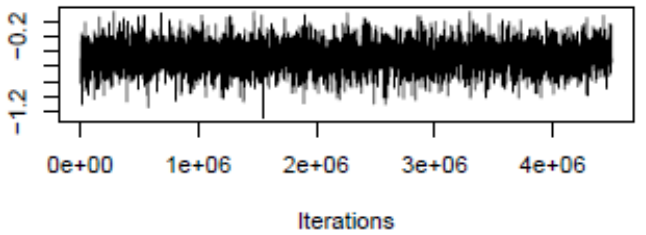

Trace of G_ageFirst13 to 17 years:time:physical

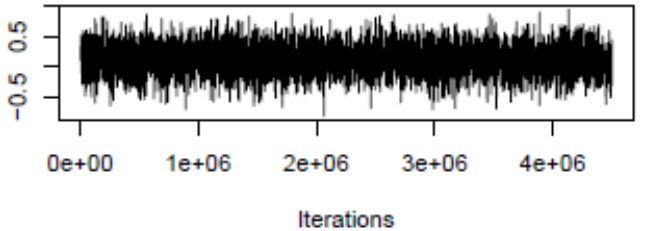

Trace of G_ageFirst13 to 17 years:time:emotion

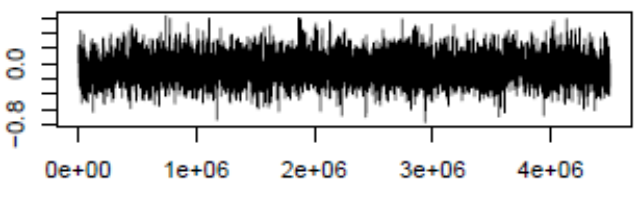

Iterations

Trace of G_ageFirst13 to 17 years:time:self

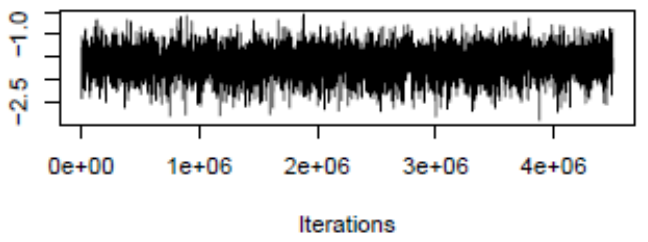

Trace of G_ageFirst 13 to 17 years:time:think

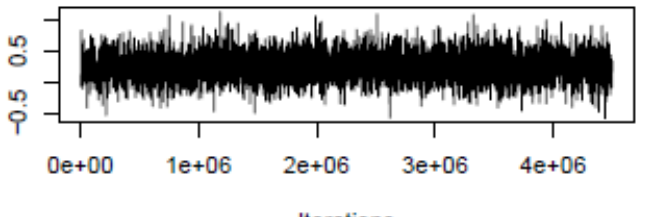

Trace of G_ageFirst13 to 17 years:time:attitude

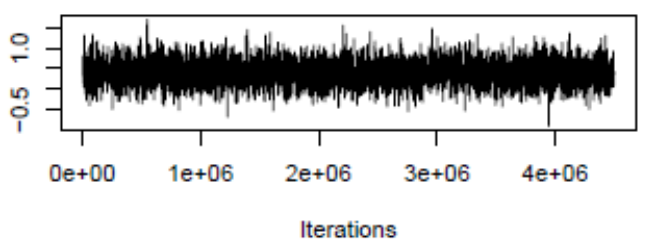

Density of G_ageFirst13 to 17 years:time:drugs

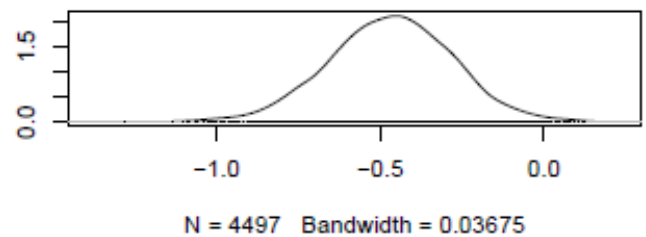

Density of G_ageFirst13 to 17 years:time:physica

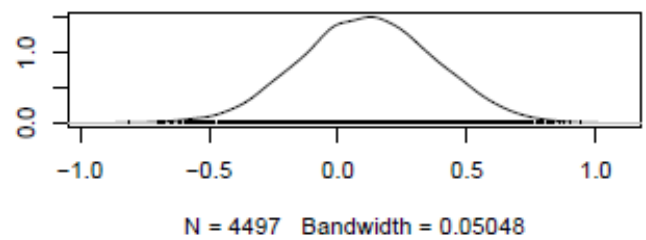

Density of G_ageFirst13 to 17 years:time:emotior

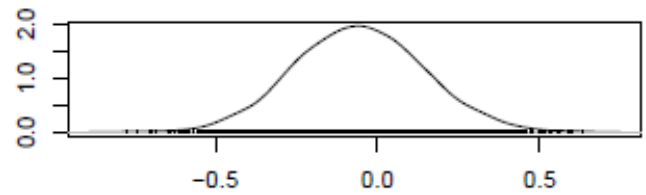

$\mathrm{N}=4497$ Bandwidth $=0.03914$

Density of G_ageFirst13 to 17 years:time:self

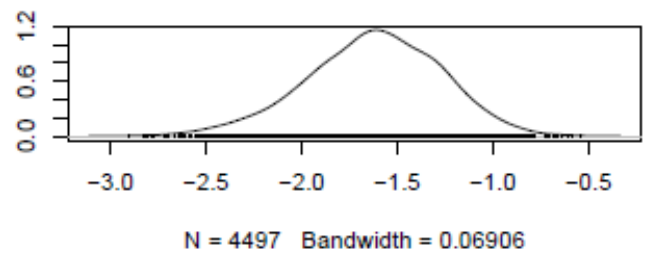

Density of G_ageFirst13 to 17 years:time:think

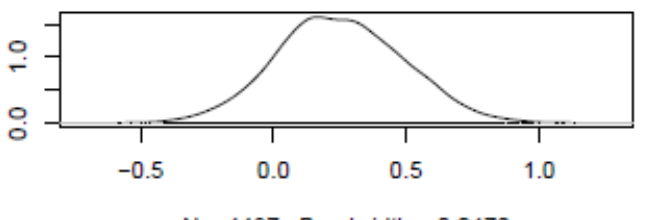

Density of G_ageFirst13 to 17 years:time:attitud $\epsilon$

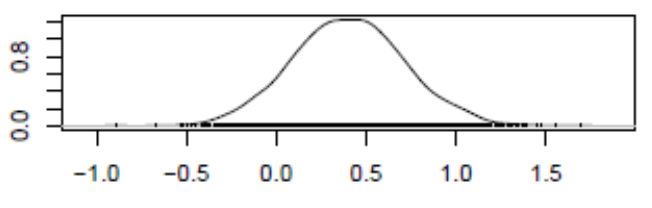

$\mathrm{N}=4497 \quad$ Bandwidth $=0.06154$ 
Trace of G_ageFirst13 to 17 years:time:change

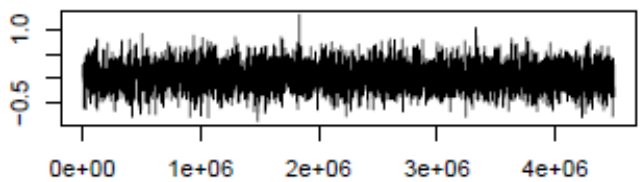

Iterations

Trace of time:live:I_Seriousness2

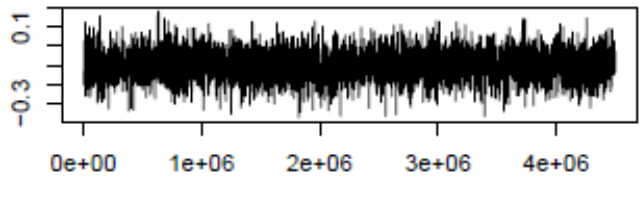

Iterations

Trace of time:relation:I_Seriousness2

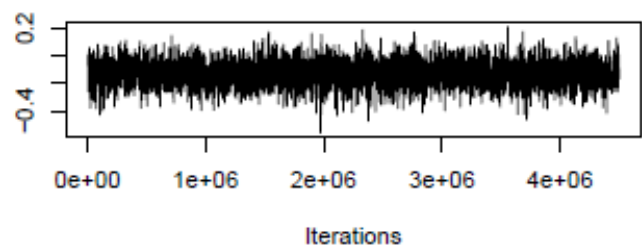

Trace of time:ete:I_Seriousness2

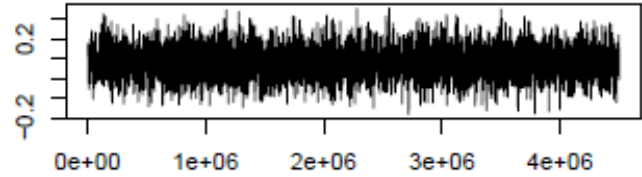

Iterations

Trace of time:where:I_Seriousness2

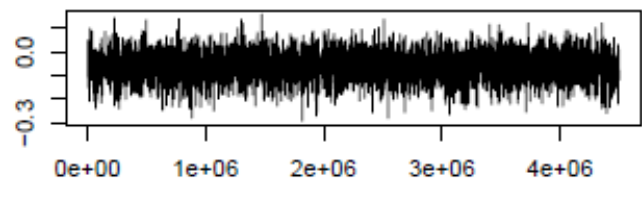

Iterations

Trace of time:life:I_Seriousness2

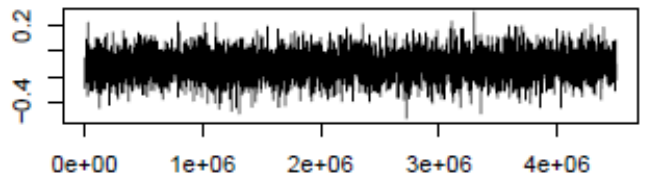

Iterations
Density of G_ageFirst13 to 17 years:time:change

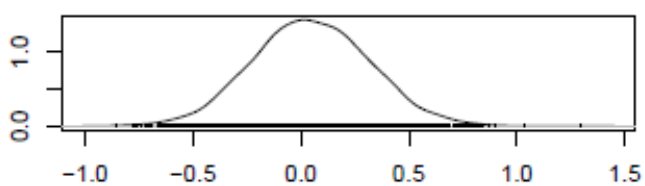

$\mathrm{N}=4497$ Bandwidth $=0.05277$

Density of time:live:I_Seriousness2

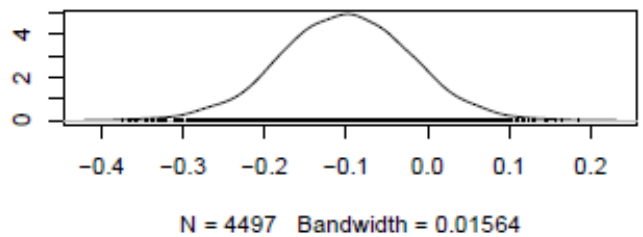

Density of time:relation:I_Seriousness2

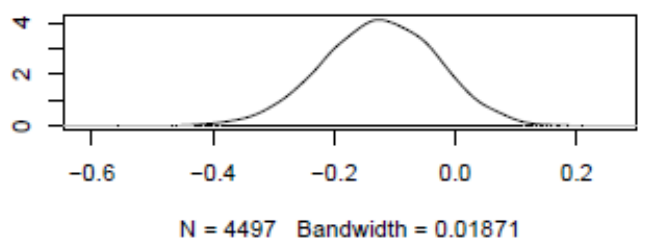

Density of time:ete:I_Seriousness2

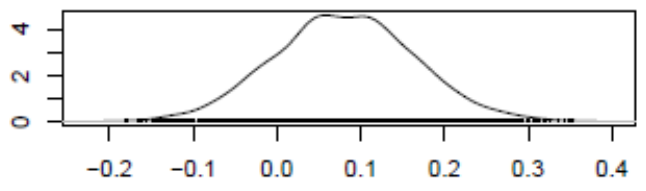

$\mathrm{N}=4497$ Bandwidth $=0.01622$

Density of time:where:I_Seriousness2

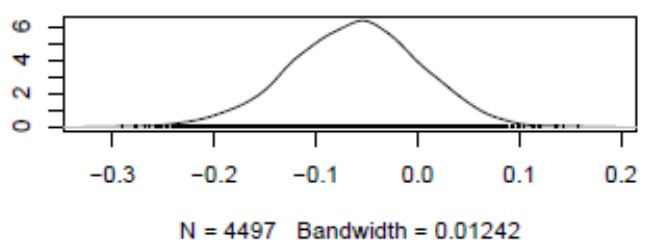

Density of time:life:I_Seriousness2

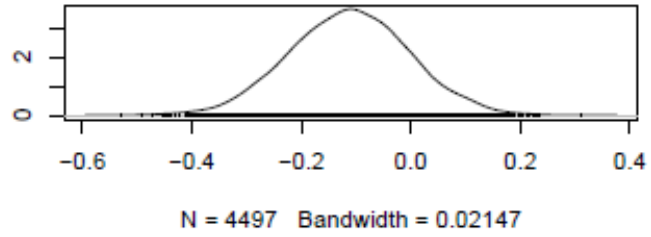


Trace of time:drugs:I_Seriousness2

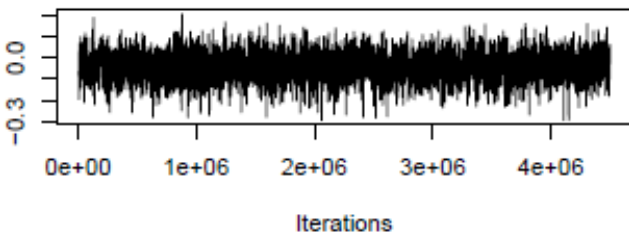

Trace of time:physical:I_Seriousness2

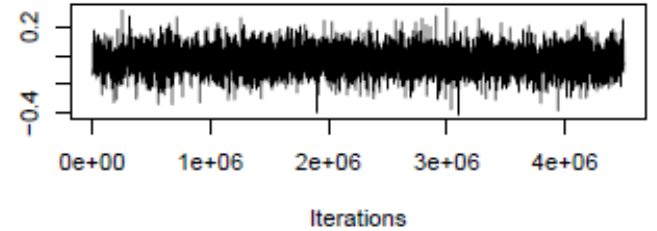

Trace of time:emotion:I_Seriousness2

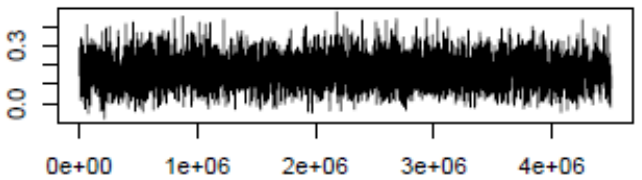

Iterations

Trace of time:self:I_Seriousness2

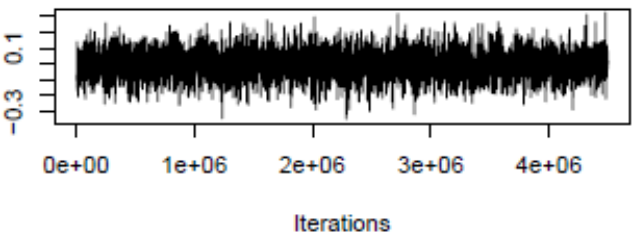

Trace of time:think:I_Seriousness2

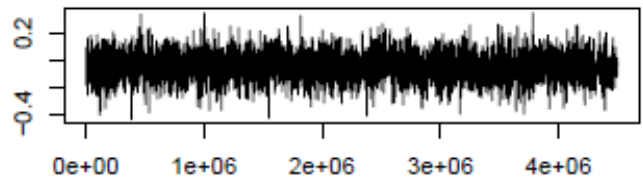

Trace of time:attitude:I_Seriousness2

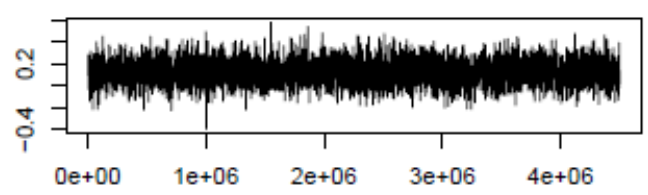

Iterations
Density of time:drugs:I_Seriousness2

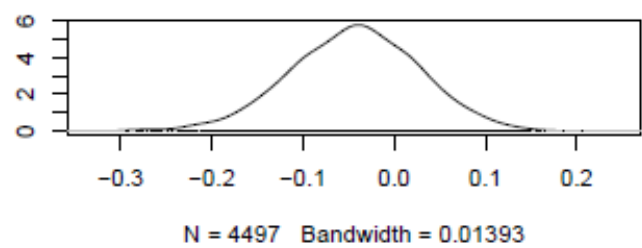

Density of time:physical:I_Seriousness2

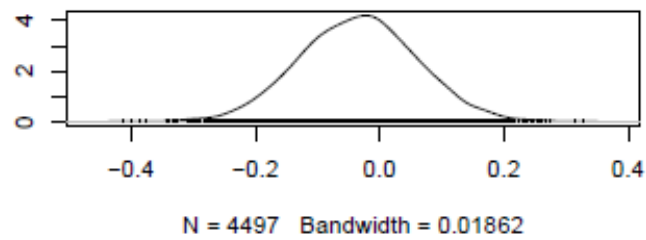

Density of time:emotion:I_Seriousness2

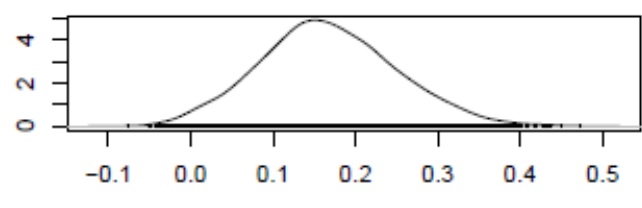

$\mathrm{N}=4497$ Bandwidth $=0.016$

Density of time:self:I_Seriousness2

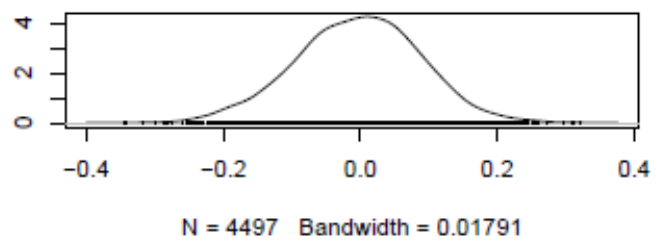

Density of time:think:I_Seriousness2

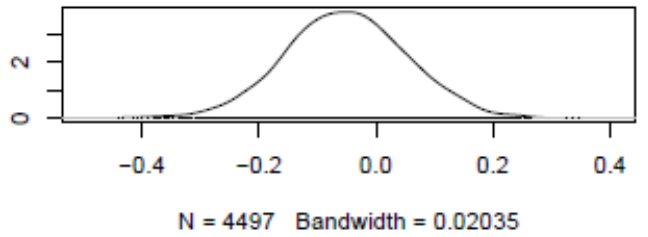

Density of time:attitude:I_Seriousness2

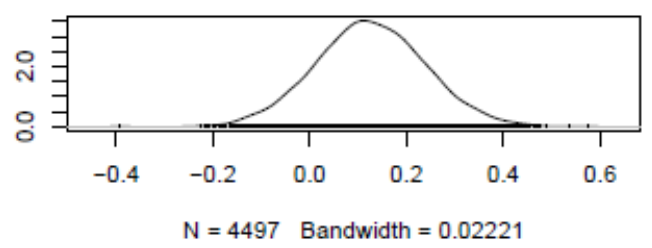


Trace of time:change:I_Seriousness2

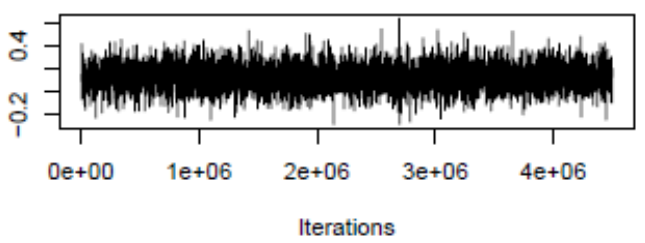

$\underline{\text { Random Effects }}$

Trace of time

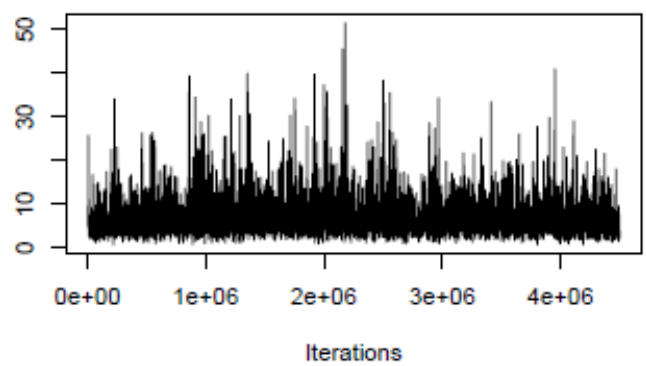

Trace of Research.ID

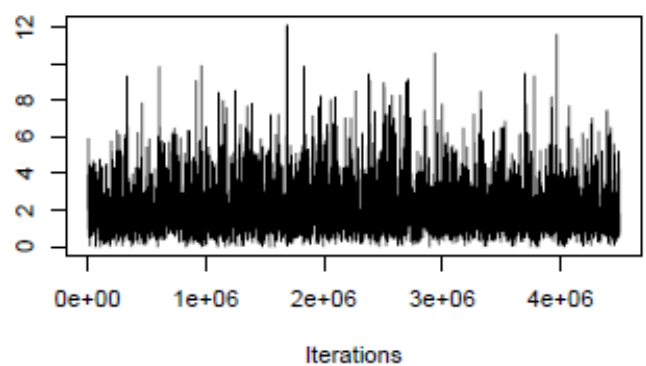

Density of time:change:I_Seriousness2

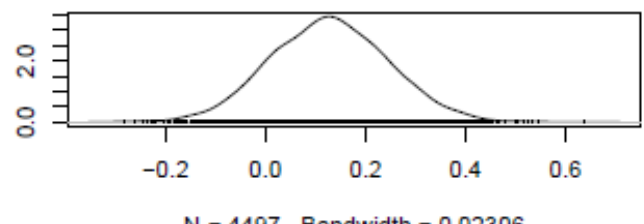

$\mathrm{N}=4497$ Bandwidth $=0.02306$

Density of time

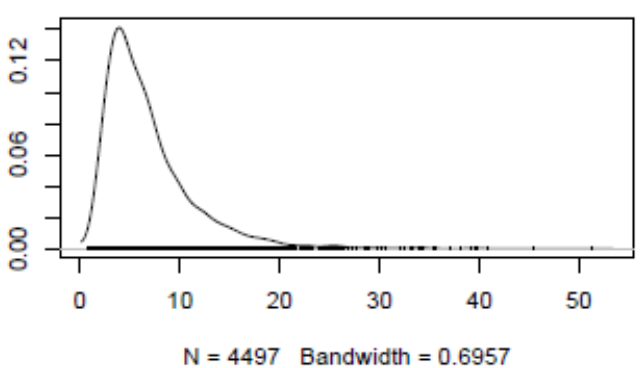

Density of Research.ID

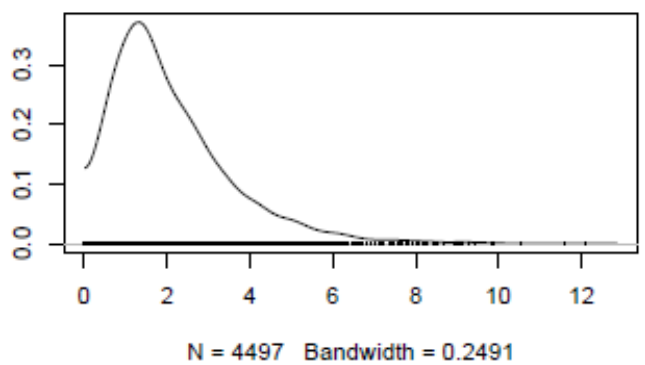


Dynamic Model 3 (Table 6.20)

Bayesian Model (BDm3G_cc12ao2a)

\section{\#\# Define the model}

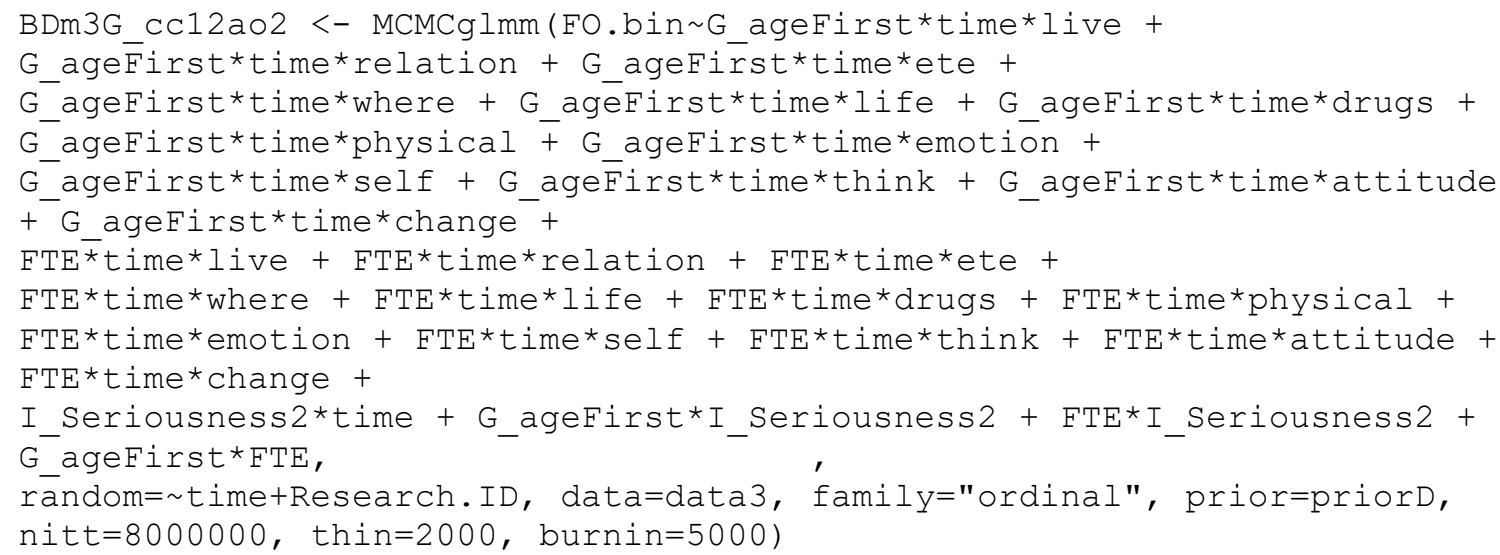

\section{\#\# Checks for suitable convergence}

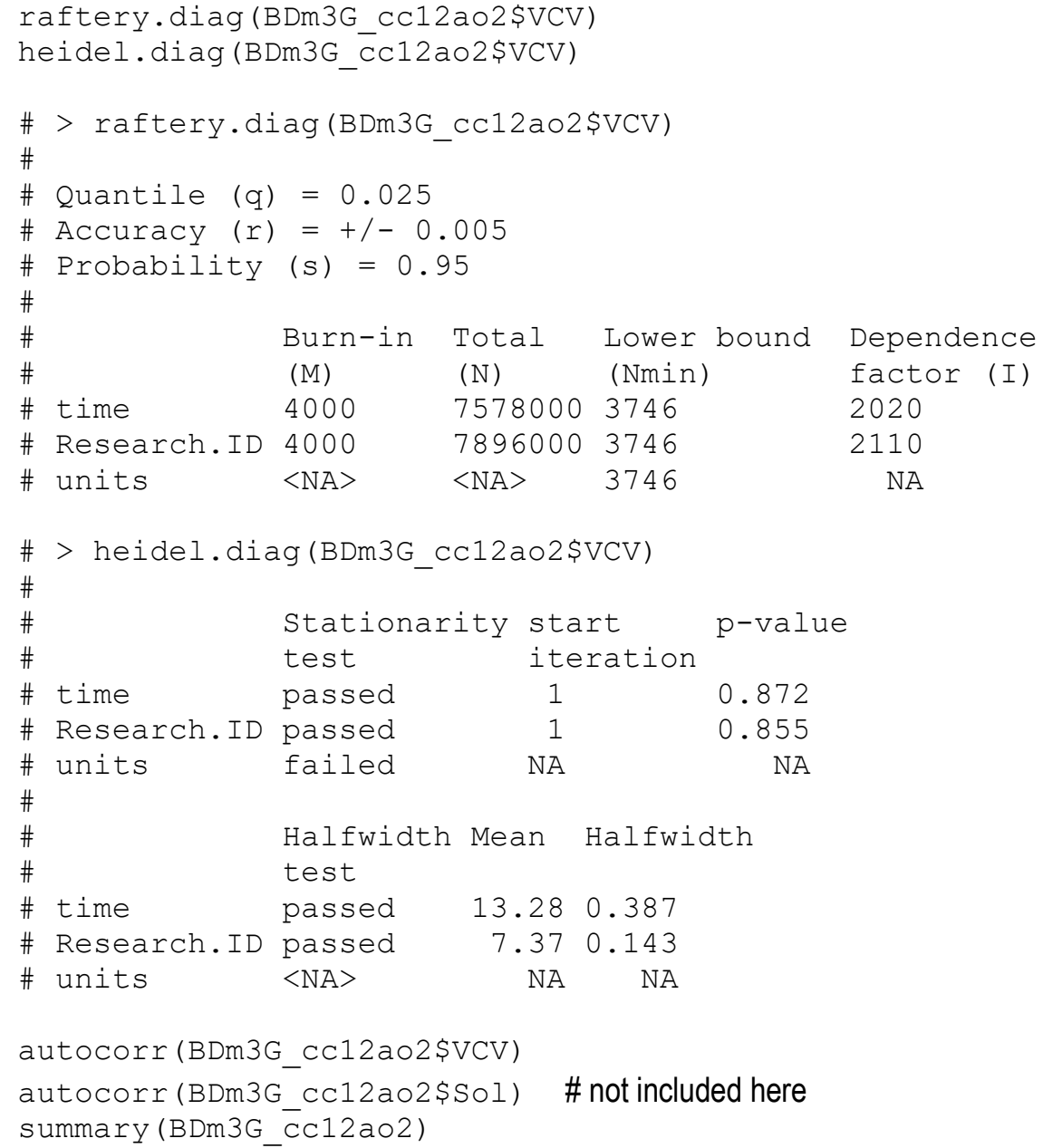




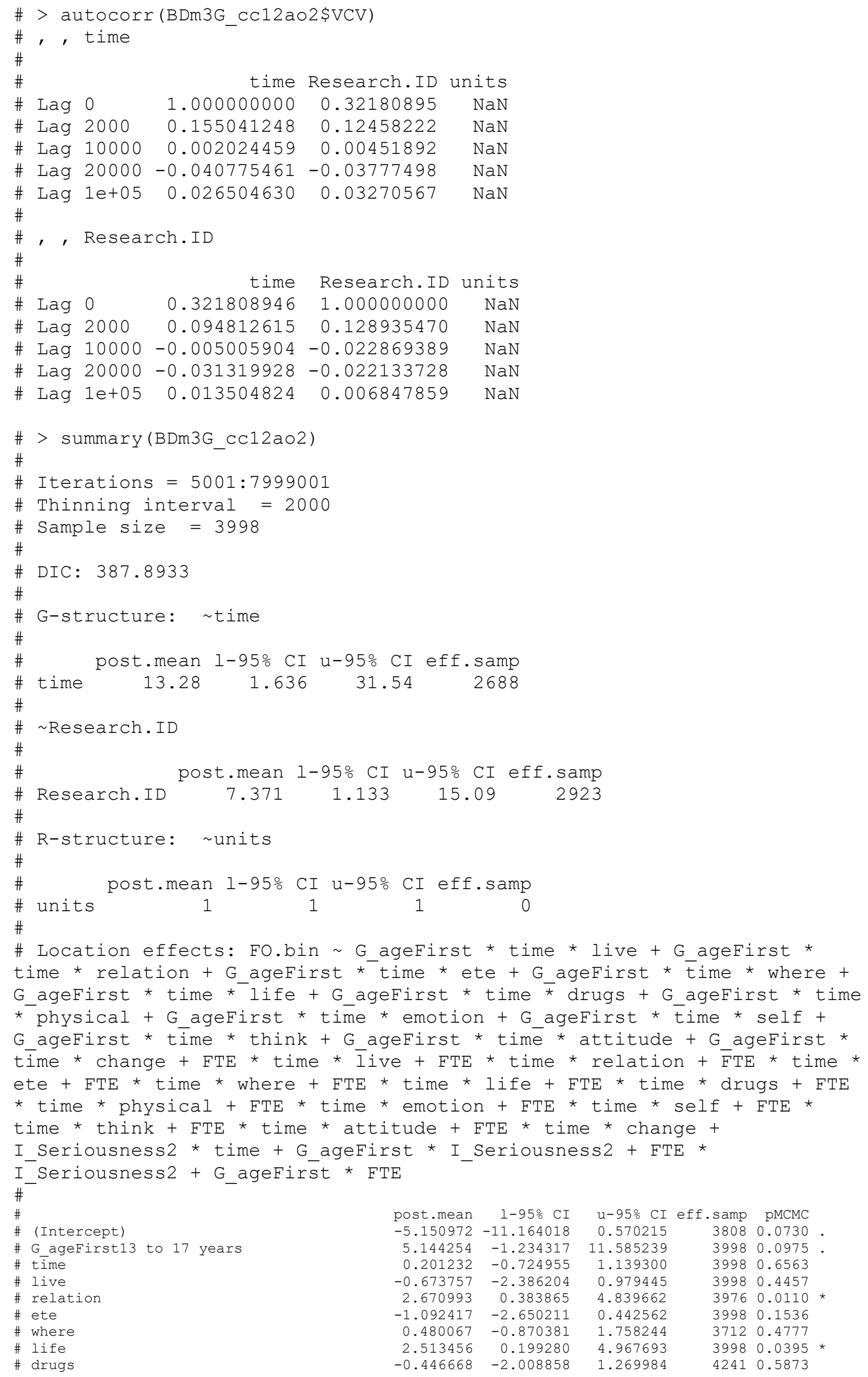


\# emotion

\# self

\# think

\# attitude

\# change

\# FTE

\# I Seriousness2

\# G-ageFirst13 to 17 years:time

\# G ageFirst13 to 17 years:live

\# time:live

\# G_ageFirst13 to 17 years:relation

\# time:relation

\# G ageFirst13 to 17 years:ete

\# time:ete

\# G_ageFirst13 to 17 years:where

\# time:where

\# G ageFirst13 to 17 years:life

\# time:life

\# G_ageFirst13 to 17 years:drugs

\# time:drugs

\# G_ageFirst13 to 17 years:physical

\# time:physical

\# G ageFirst13 to 17 years:emotion

\# time:emotion

\# G_ageFirst13 to 17 years:self

\# time:self

\# G ageFirst13 to 17 years:think

\# time:think

\# G_ageFirst13 to 17 years:attitude

\# time:attitude

\# G ageFirst13 to 17 years:change

\# time:change

\# time:FTE

\# live:FTE

\# relation:FTE

\# ete:FTE

\# where: FTE

\# life:FTE

\# drugs:FTE

\# physical:FTE

\# emotion:FTE

\# self:FTE

\# think:FTE

\# attitude:FTE

\# change:FTE

\# time:I_Seriousness 2

\# G_ageFirst13 to 17 years:I_Seriousness2

\# FTE:I Seriousness2

\# G age $\bar{F}$ irst13 to 17 years:FTE

\# G-ageFirst13 to 17 years:time:live

\# G ageFirst13 to 17 years:time:relation

\# G_ageFirst13 to 17 years:time:ete

\# G_ageFirst13 to 17 years:time:where

\# G ageFirst13 to 17 years:time:life

\# G ageFirst13 to 17 years:time:drugs

\# G_ageFirst13 to 17 years:time:physical

\# G-ageFirst13 to 17 years:time:emotion

\# G ageFirst13 to 17 years:time:self

\# G_ageFirst13 to 17 years:time:think

\# G_ageFirst13 to 17 years:time:attitude

\# G ${ }^{-}$ageFirst13 to 17 years:time:change

\# time:live:FTE

\# time:relation:FTE

\# time:ete:FTE

\# time:where:FTE

\# time:life:FTE

\# time:drugs:FTE

\# time:physical:FTE

\# time:emotion:FTE

\# time:self:FTE

\# time:think:FTE

\# time:attitude:FTE

\# time: change:FTE

\# ---

Signif. codes: $\begin{array}{lll}-1.159918 & -2.987216\end{array}$

$-0.471944-1.888879$

$-6.735600-10.170844$

$1.839929-0.618378$

$2.818820-0.321225$

$1.065015-1.535402$

$-4.720378-14.577722$

$0.084943-1.265054$

$-0.587150-1.873360$

$1.273958-1.151754$

$-0.142900-0.516844$

$-4.089522-7.297931$

$-0.518666$

0.332241

0.207592

1.477620

$-0.193267$

$-2.415914$

$-0.527517$

0.357525

0.339848

$-1.259509$

0.326911

0.170452

0.372673

8.071152

1. 471754

$-0.318265$

$-0.366022$

$-0.362018$

$-0.859489$

$-3.296657$

0.099450

$-1.046247$

1. 079842

$-0.024719$

1. 228447

$-4.412089$

2.333735

$-0.963615$

1. 310114

$-0.686621$

2.151453

$-2.604403$

$-3.706076$

3.256467

0.041573

$-0.672519$

0.509346

4. 642491

0.325173

1.049072

$-0.308003$

$-0.003797$

0.357633

$-0.622083$

0.554315

$-0.592757$

$-1.561500$

$-0.162584$

0.106286

0.653849

$-0.593345$

$-0.083004$

0.434190

0.606103

$-0.525872$

0.695160

$-0.920443$

0.498393

$-0.807315$

0.853626

1.087005

$-0.987037$

$-1.013080$

$-1.547266$

$-0.113639$

$-0.590788$

$-0.461103$

$-5.929684$

$-1.055041$

$-1.859566$

0.020407

$-3.939164$

$-0.118426$

$-1.771077$

0.075528

4.000871

0.820258

$-3.185917$

$-0.842472$

$-4.096083$

$-1.421434$

$-6.840479$

$-0.413371$

$-2.577325$

$-1.841439$

$-2.810608$

$-1.146733$

$-7.223455$

$-1.050389$

$-3.504742$

$-1.555944$

$-2.926955$

$-0.631508$

$-5.332442$

$-6.714992$

0.291315

$-0.080143$

$-2.240818$

$-0.708681$

$-4.262667$

$-0.282989$

0.391227

$-0.806831$

$-0.432438$

$-0.464132$

$-1.203937$

$-0.232537$

$-1.182620$

$-2.373767$

$-0.820154$

$-0.694704$

$-0.128593$

$-1.353827$

$-0.805392$

$-0.226843$

0.102222

$-1.400477$

0.065938

$-1.851233$

$-0.121621$

$-1.496655$

0.141123

0.331429

$-1.798955$
.610702

1.008432

$-3.456450$

4. 405593

6.040191

3. 995168

4.547239

.478074

0.828290

3.454466

0.257516

$-1.259992$

$-0.012912$

2. 373488

0.528399

3.578961

0.084899

.776649

0.038999

2.530974

0.700737

1.430843

0.756309

2.322582

.675698

12.024510

2.191141

.640217

0.068409

3. 346858

$-0.282483$

0.087031

0.604584

0.473854

4.082608

2.747417

3. 681512

$-1.938864$

5.869065

1.834314

4. 123877

.532065

4.936126

0.131680

$-1.076466$

6.520936

0.161113

0.841369

.935971

13.741457

0.940236

1.773237

0.198059

0.432732

1.197844

$-0.150852$

1. 365315

$-0.023141$

$-0.739141$

0.494323

0.907729

1. 402211

0.138757

0.602084

1.115738

1.064807

0.338078

1.285142

$-0.108412$

1.134819

$-0.160331$

1.529417

1. 921780

$-0.236410$

$3808 \quad 0.1956$

36690.5103

$3485<3 e-04 * \star *$ 
Trace Plots and Posterior Density Plots

Fixed Effects
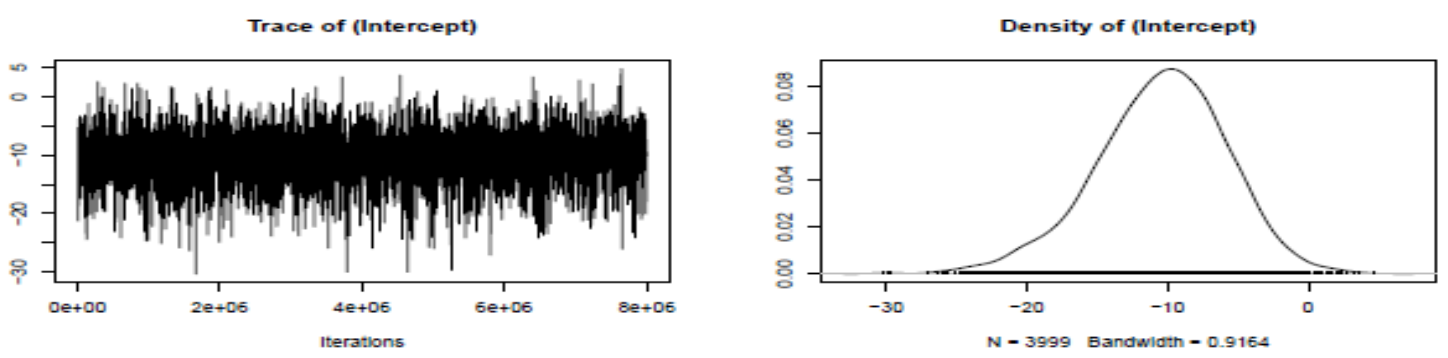

Trace of FTE

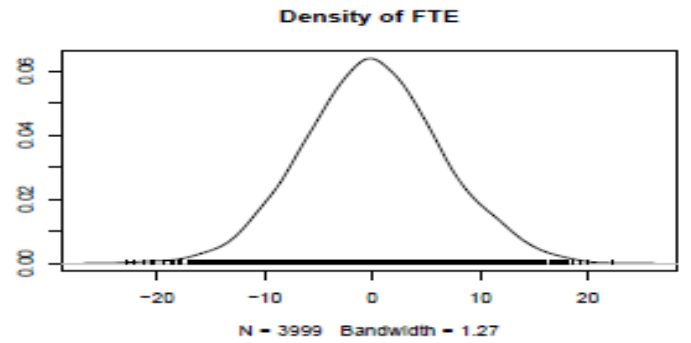

Trace of time
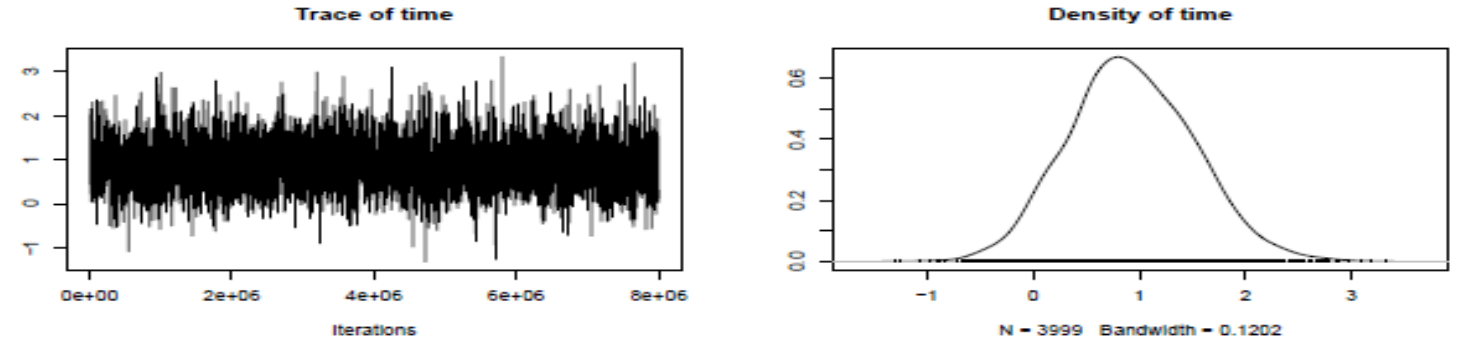

Trace of live
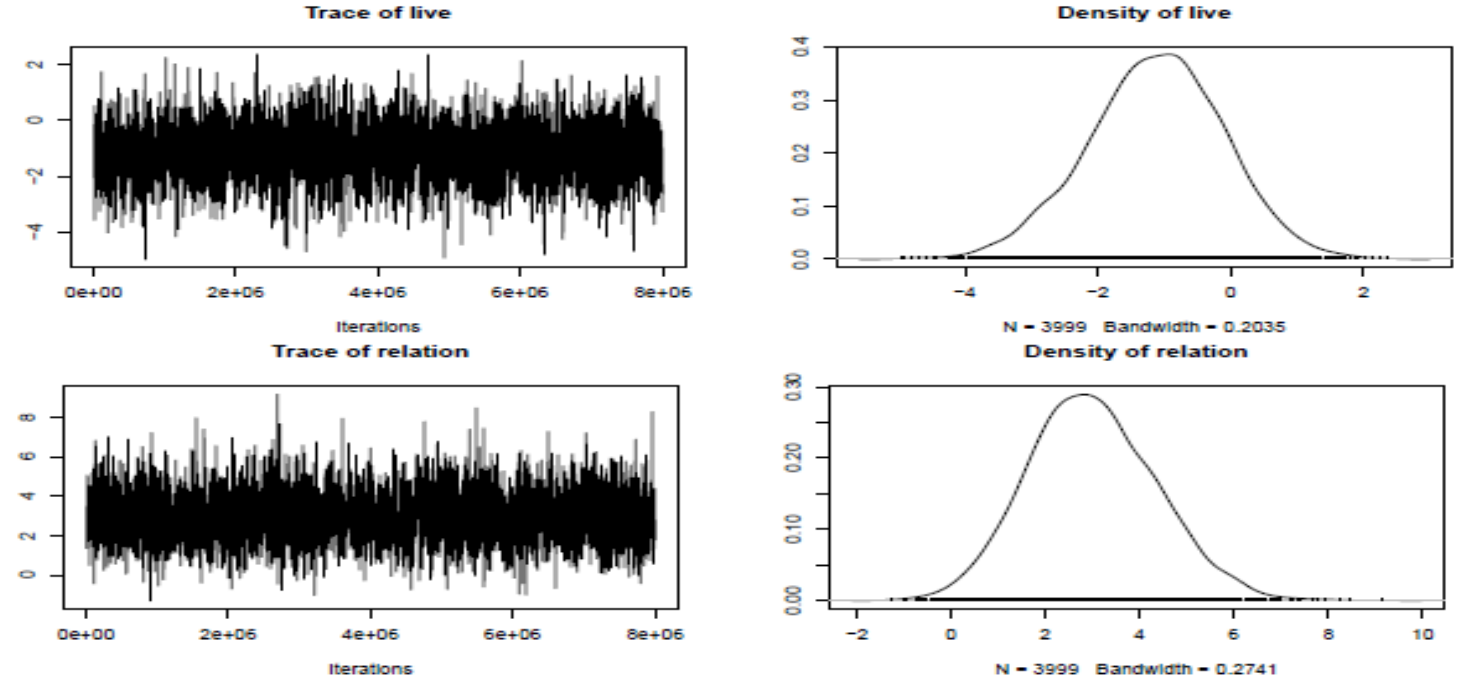

Trace of ete
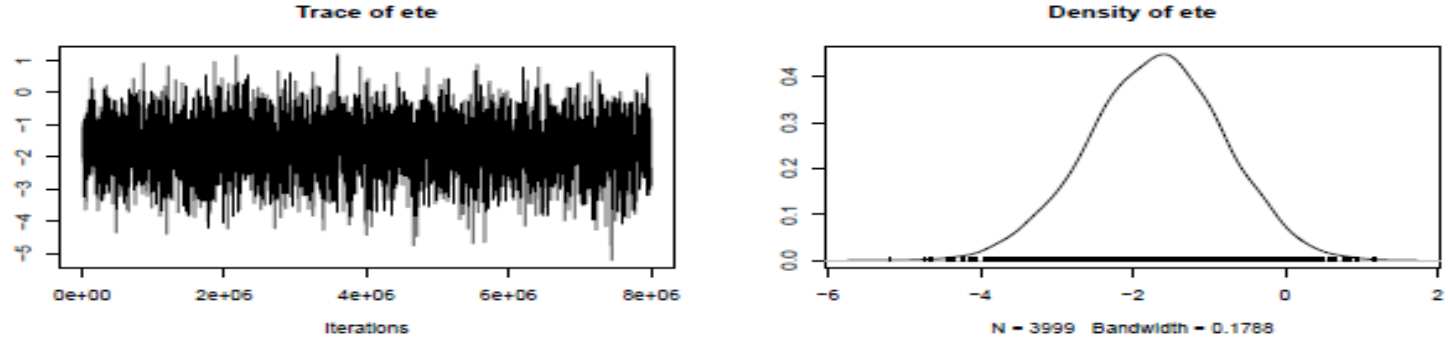
Trace of where

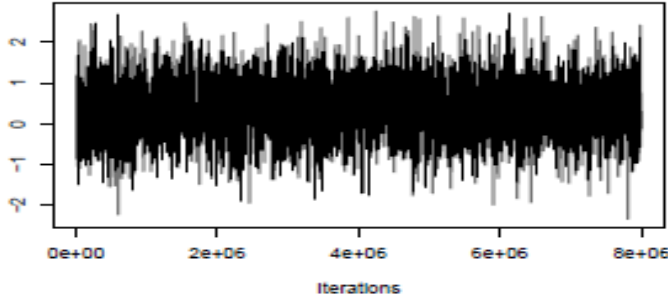

Trace of life

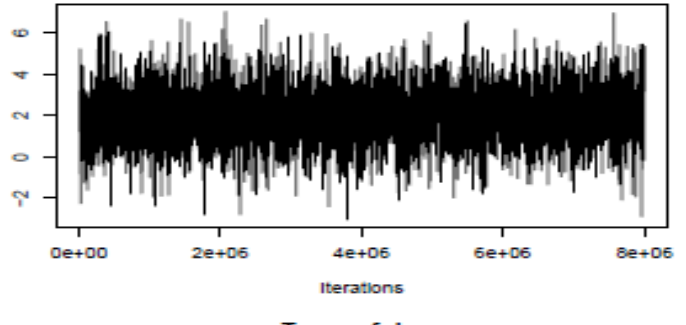

Trace of drugs

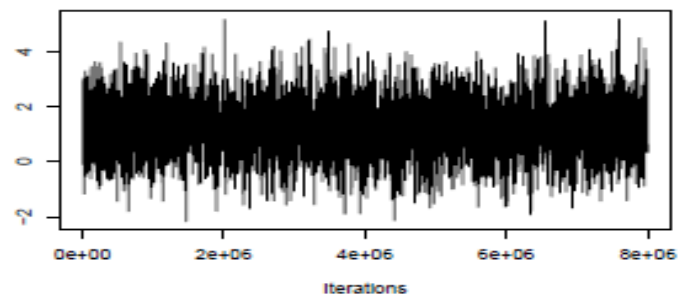

Trace of physical

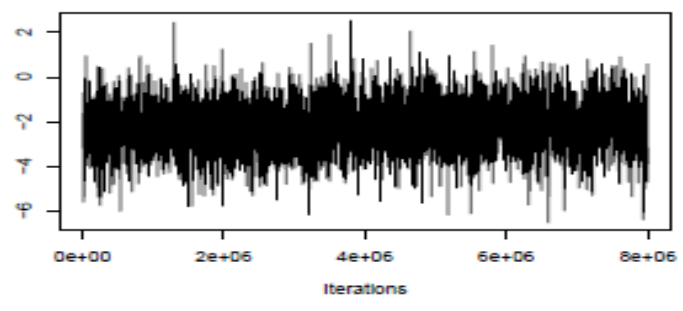

Trace of emotion

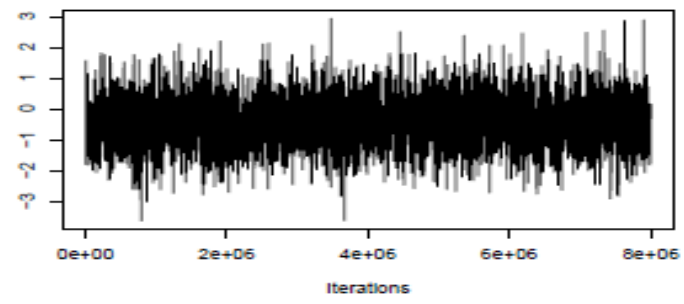

Trace of self

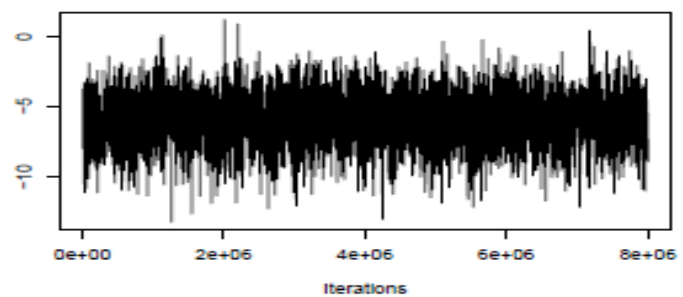

Density of where

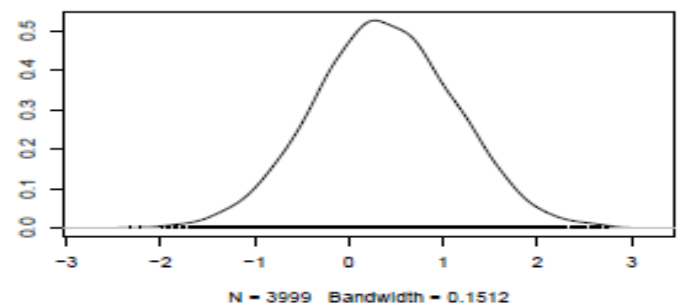

Density of life

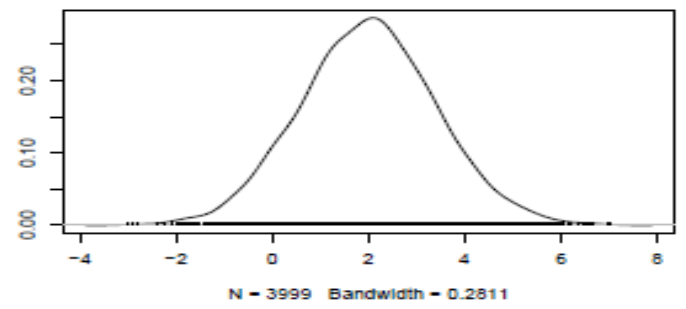

Density of drugs

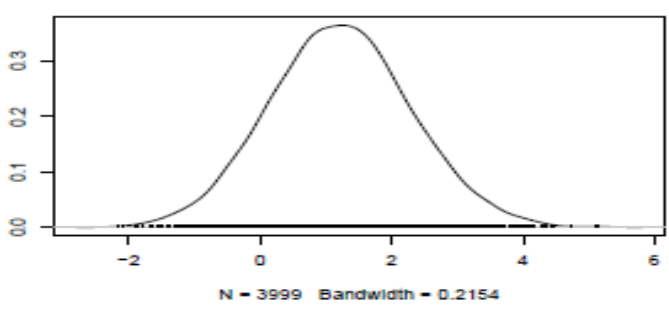

Density of physical

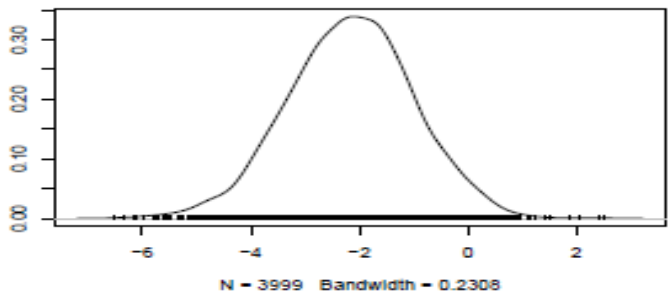

Density of emotion

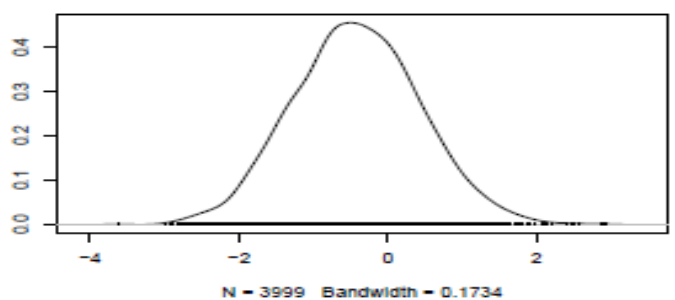

Density of self

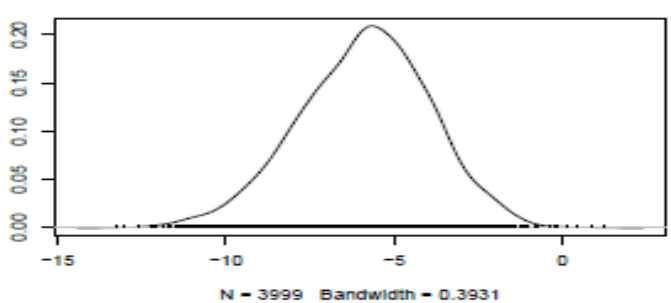


Trace of think

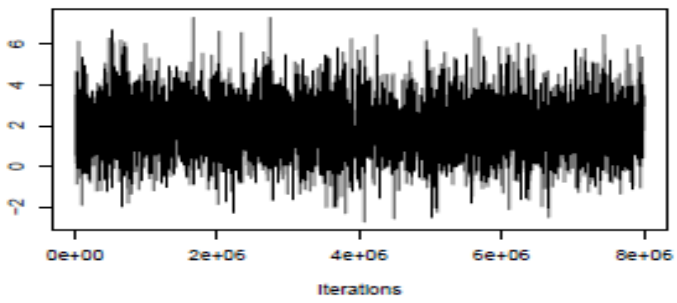

Trace of attitude

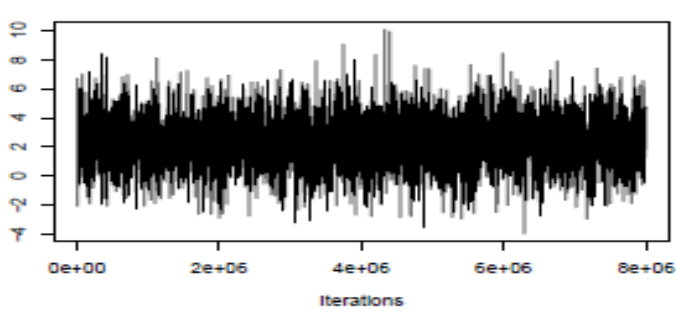

Trace of change
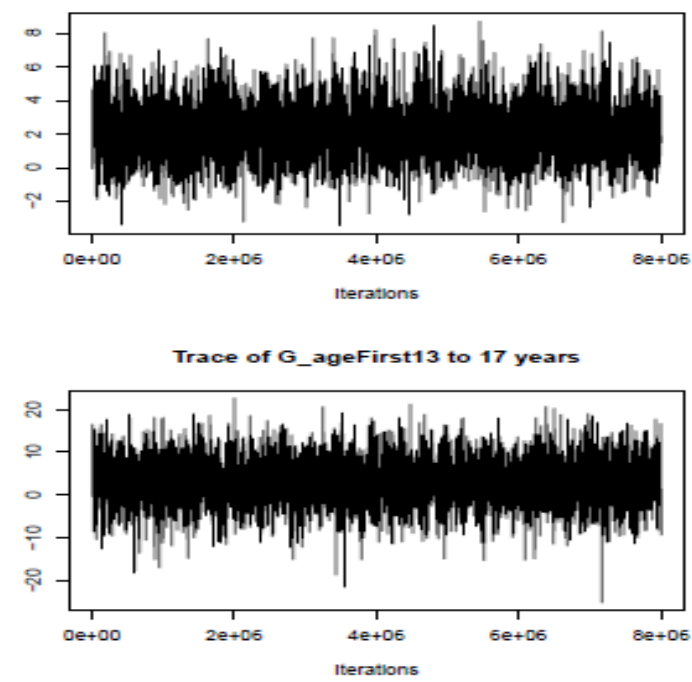

Trace of G_ageCon14 to 17 years

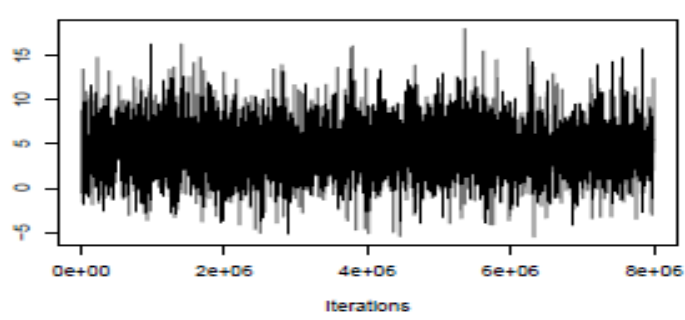

Trace of I_Seriousness 2

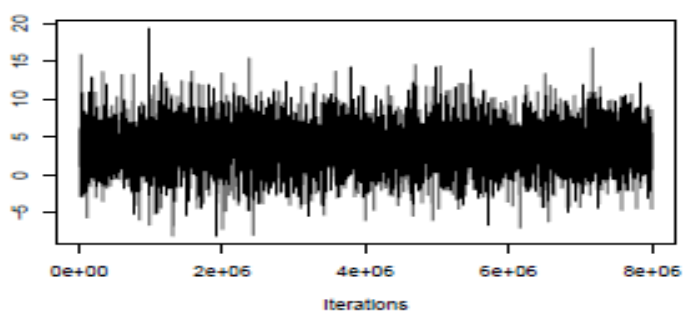

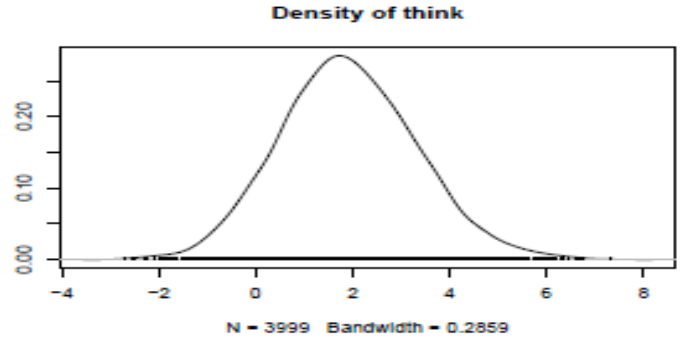
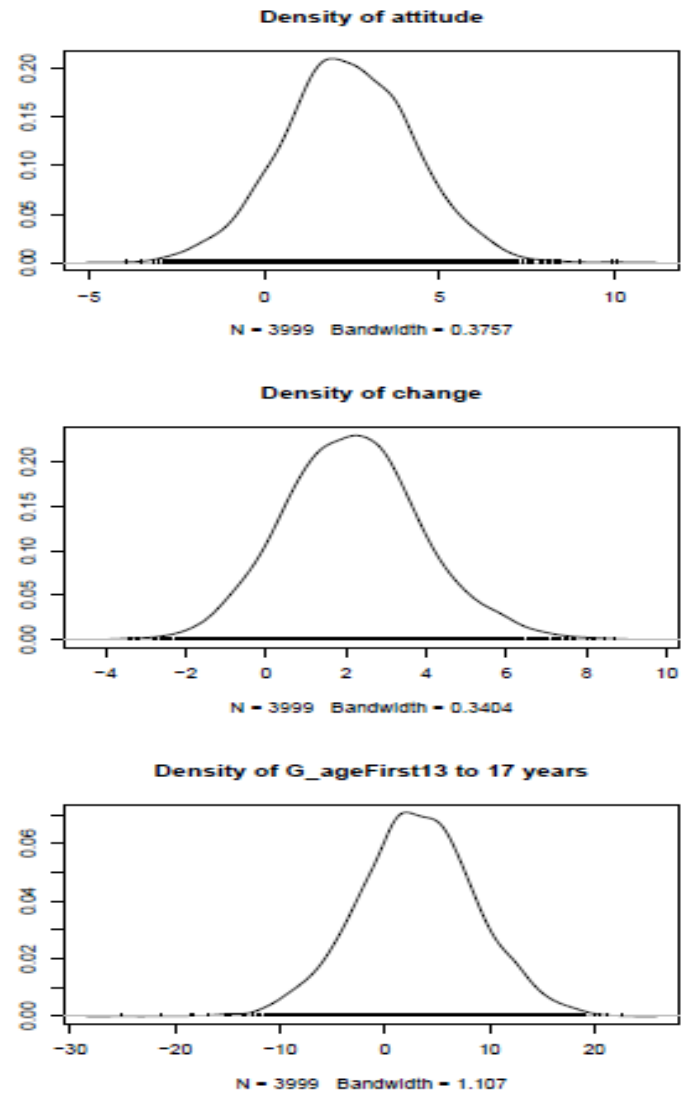

Density of G_ageCon14 to 17 years
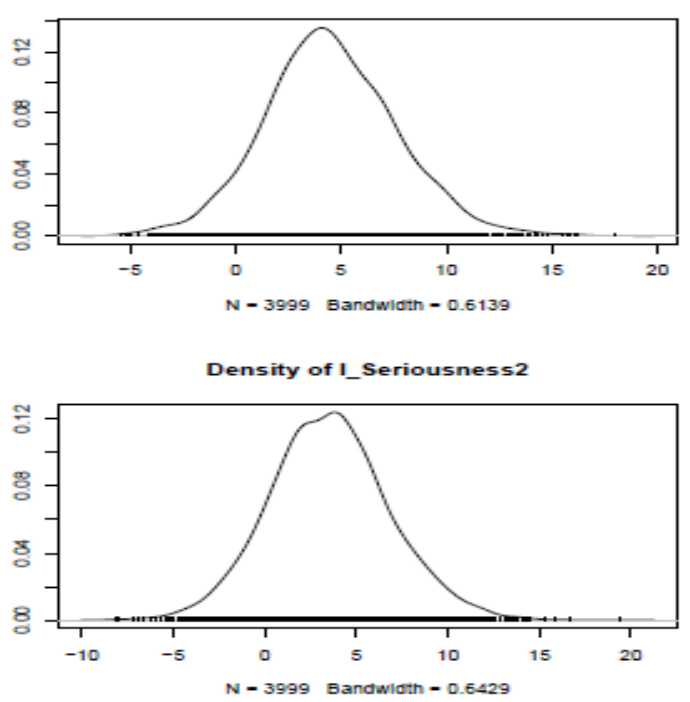
Trace of FTE:time

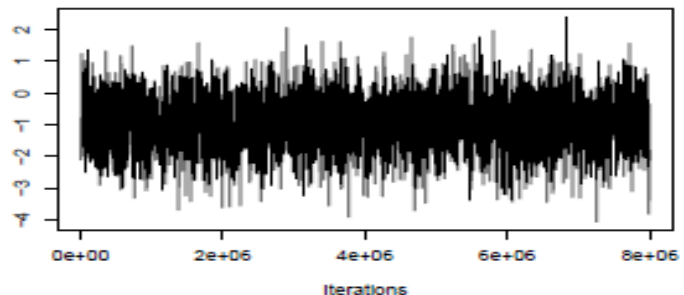

Trace of FTE:live

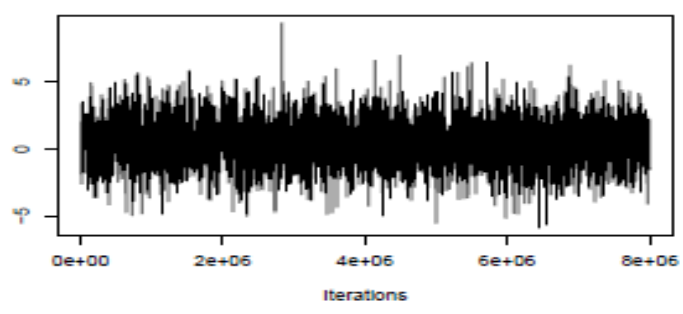

Trace of time-live

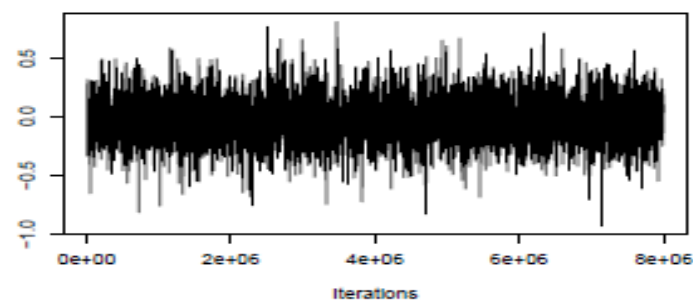

Trace of FTE:relation

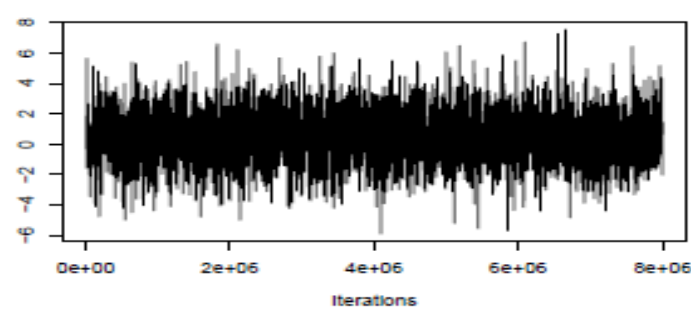

Trace of time: relation

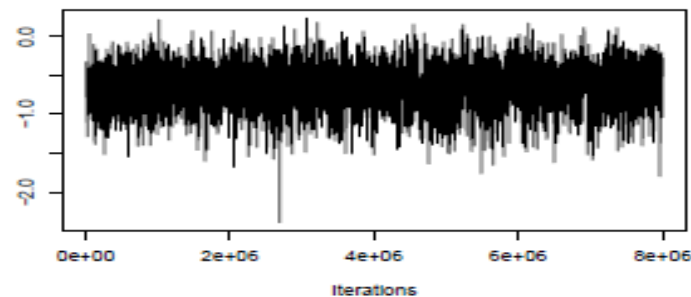

Trace of FTE:ete

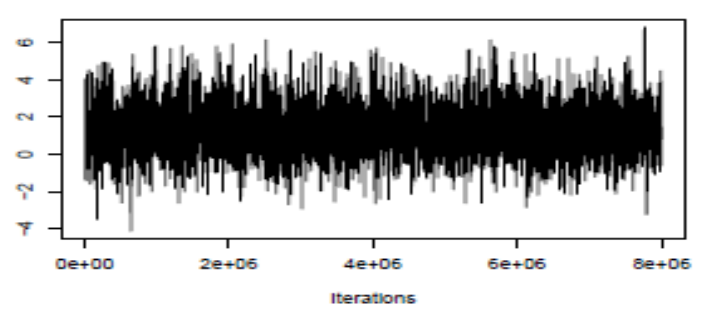

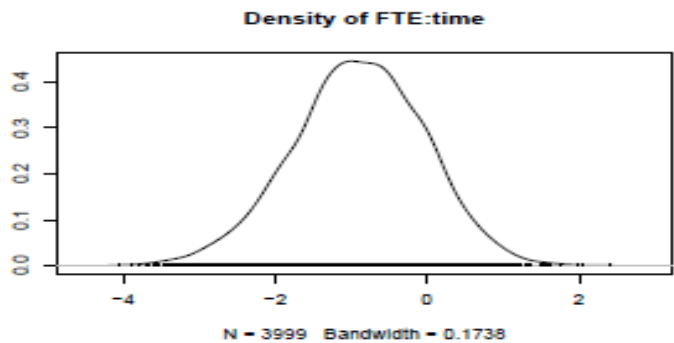
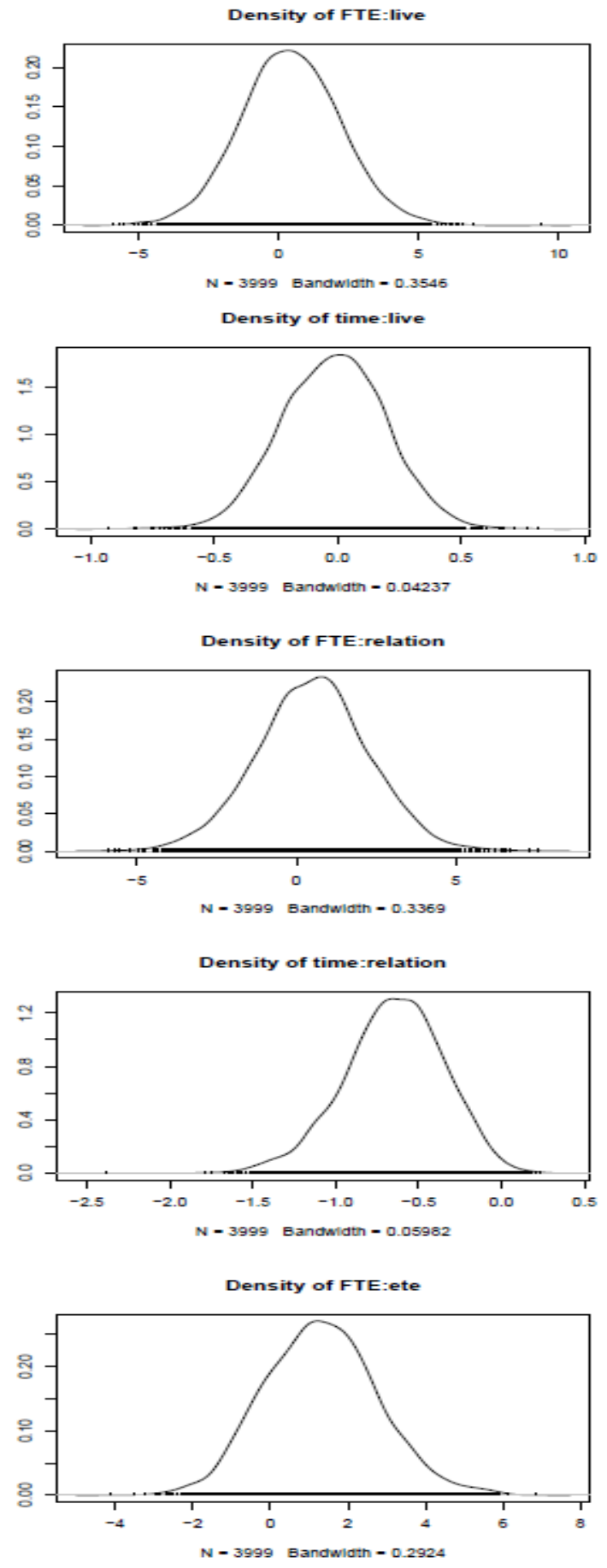
Trace of time:ete

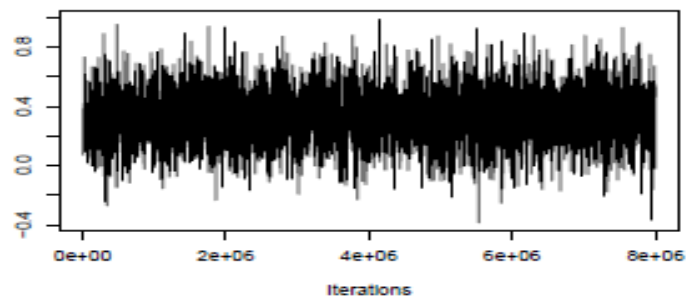

Trace of FTE:wher

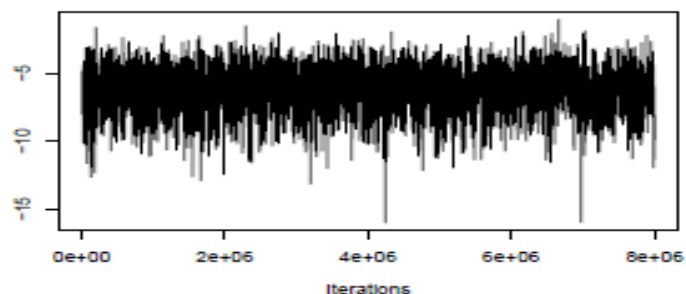

Trace of time:where

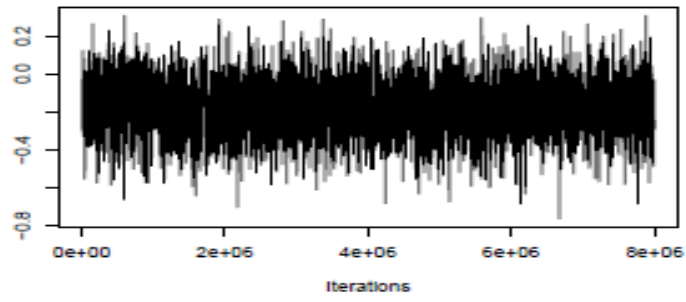

Trace of FTE-life
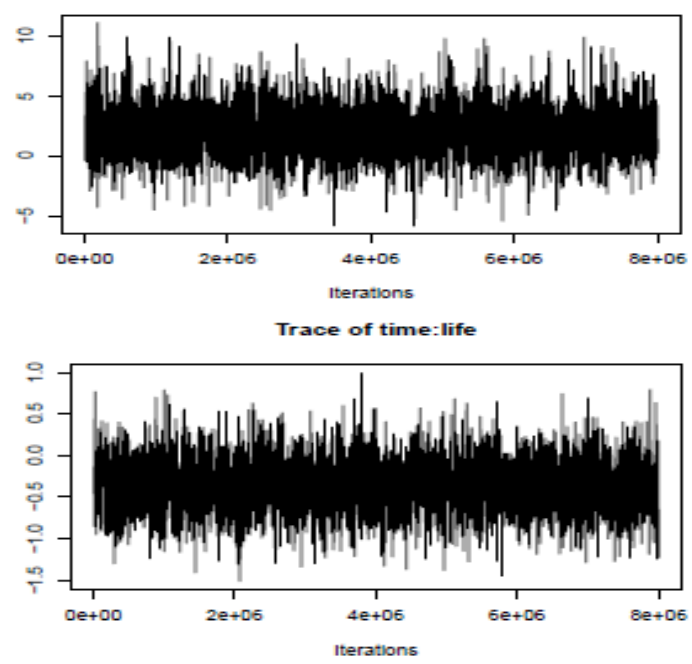

Trace of FTE:drug

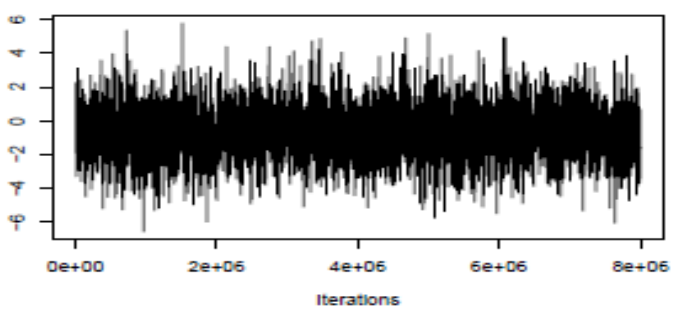

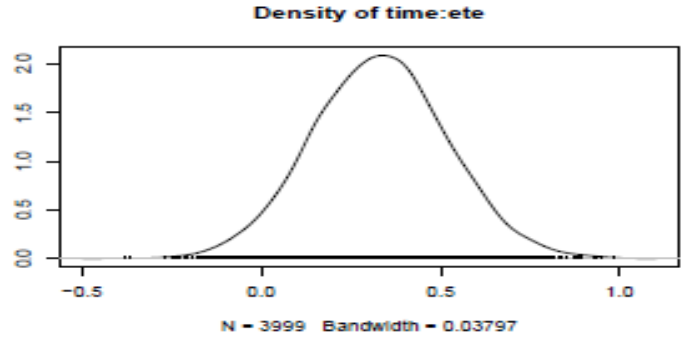
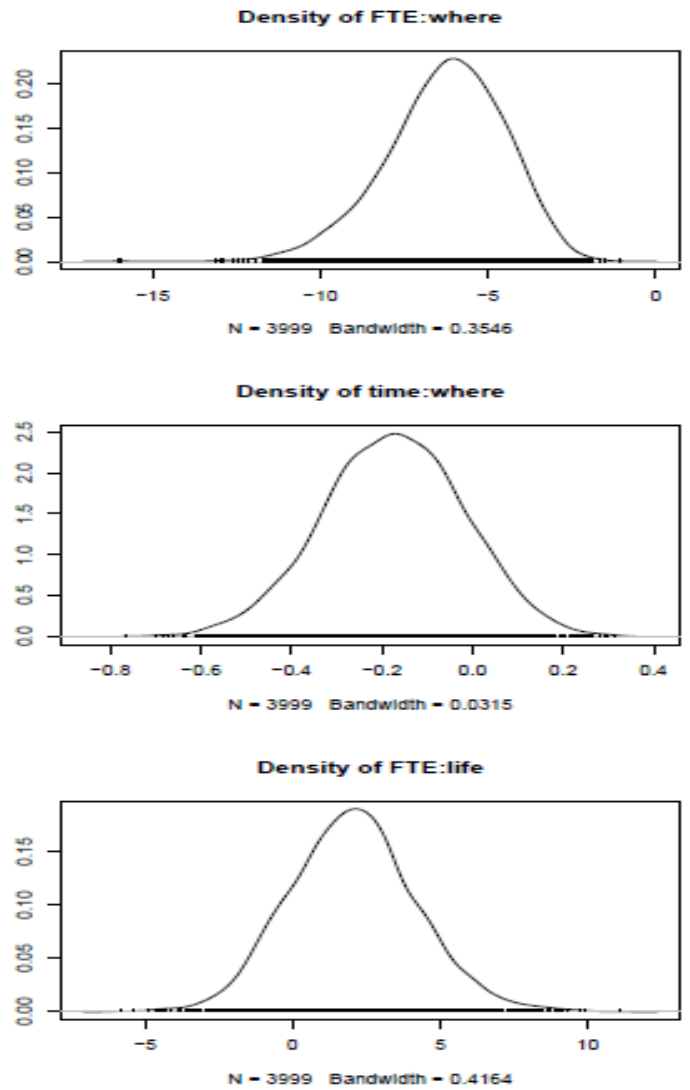

Density of time:life
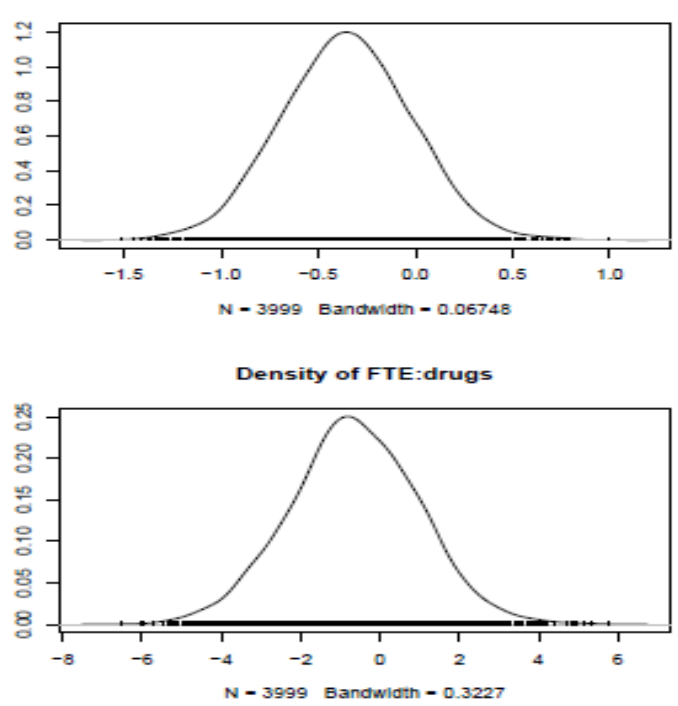

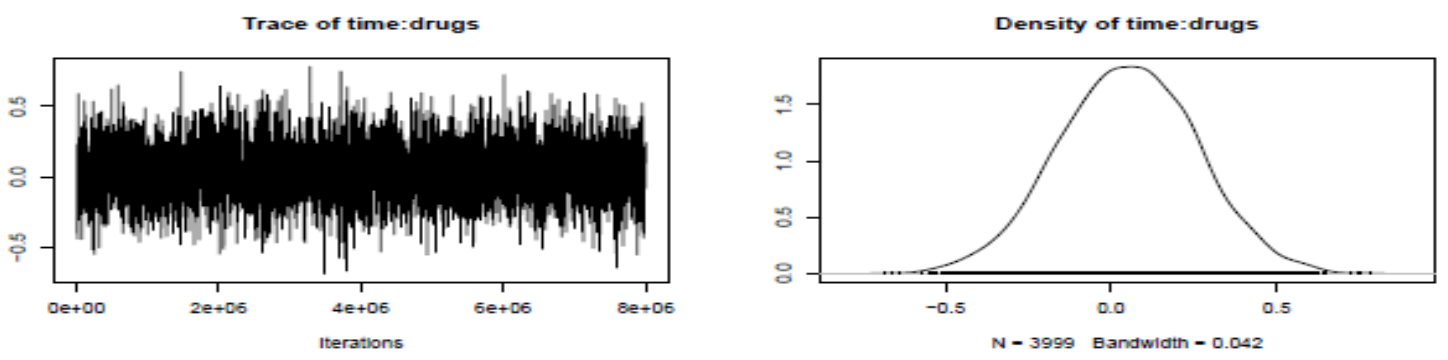

Trace of FTE:physical
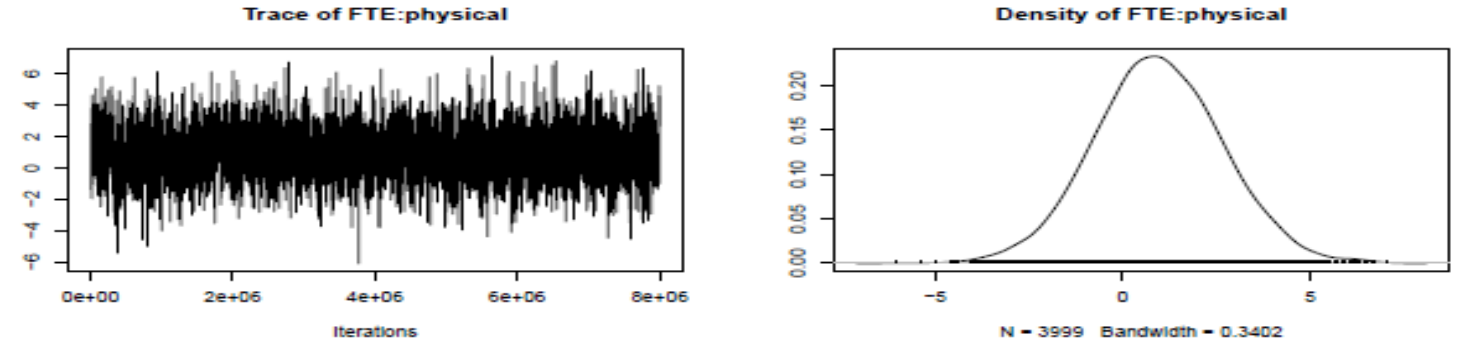

Trace of time:physical
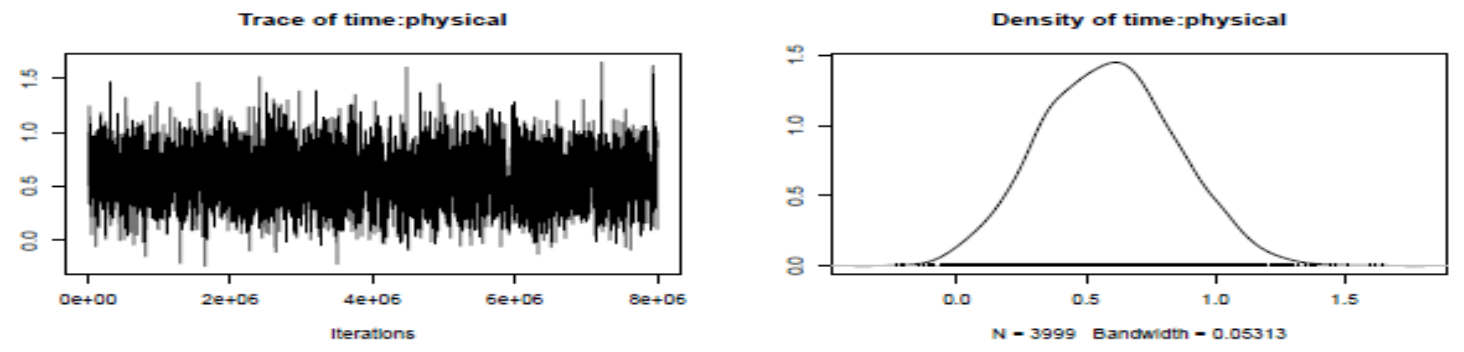

Trace of FTE:emotion
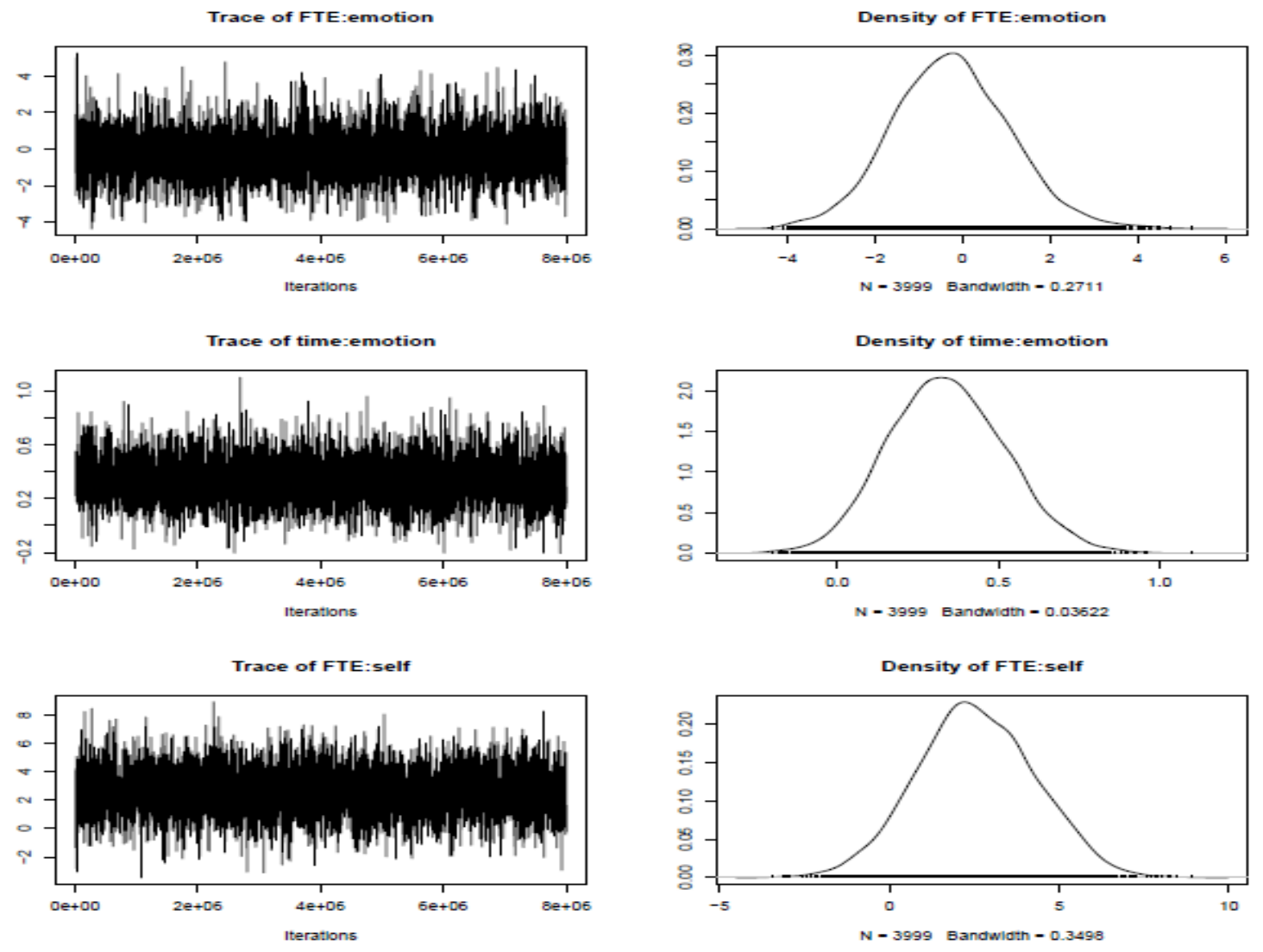

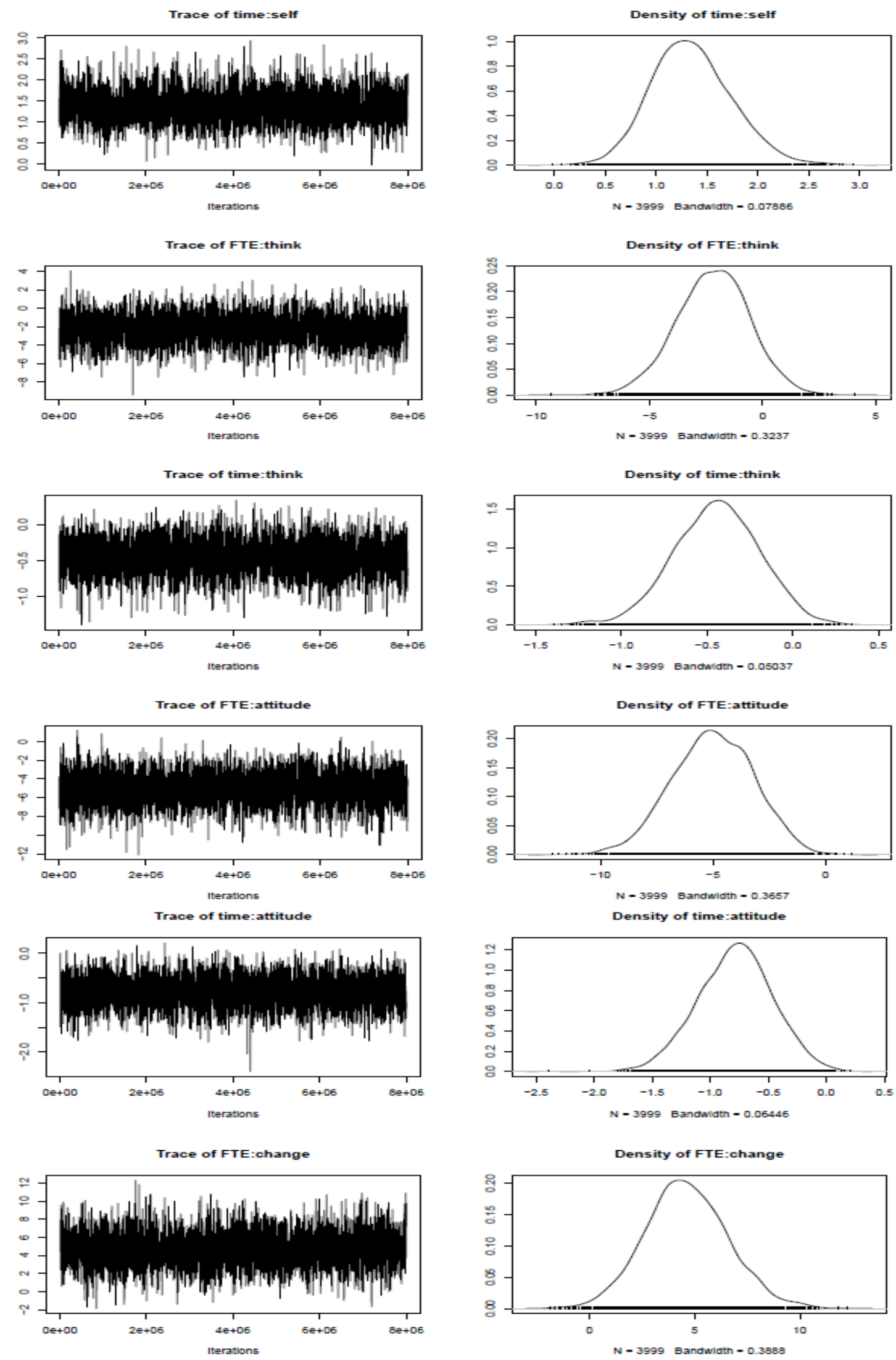

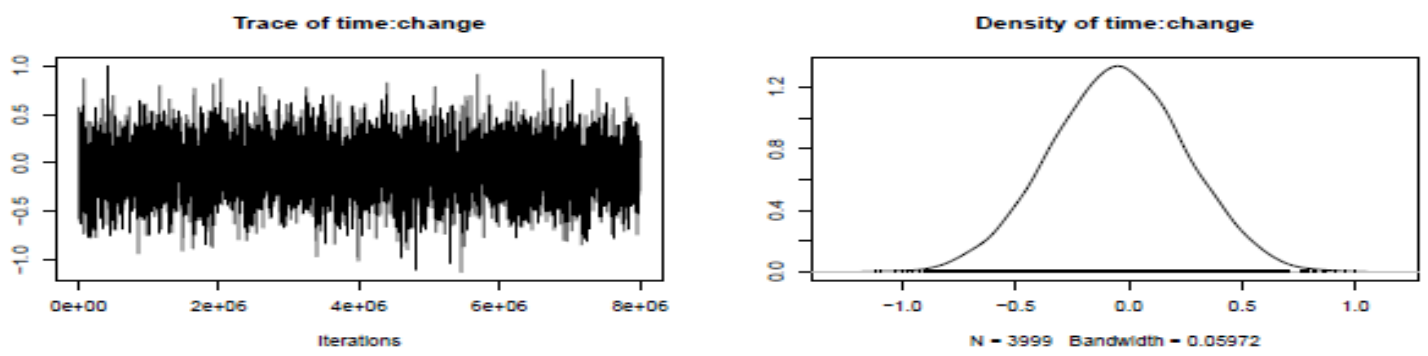

Trace of time:G_ageFirst 13 to 17 years
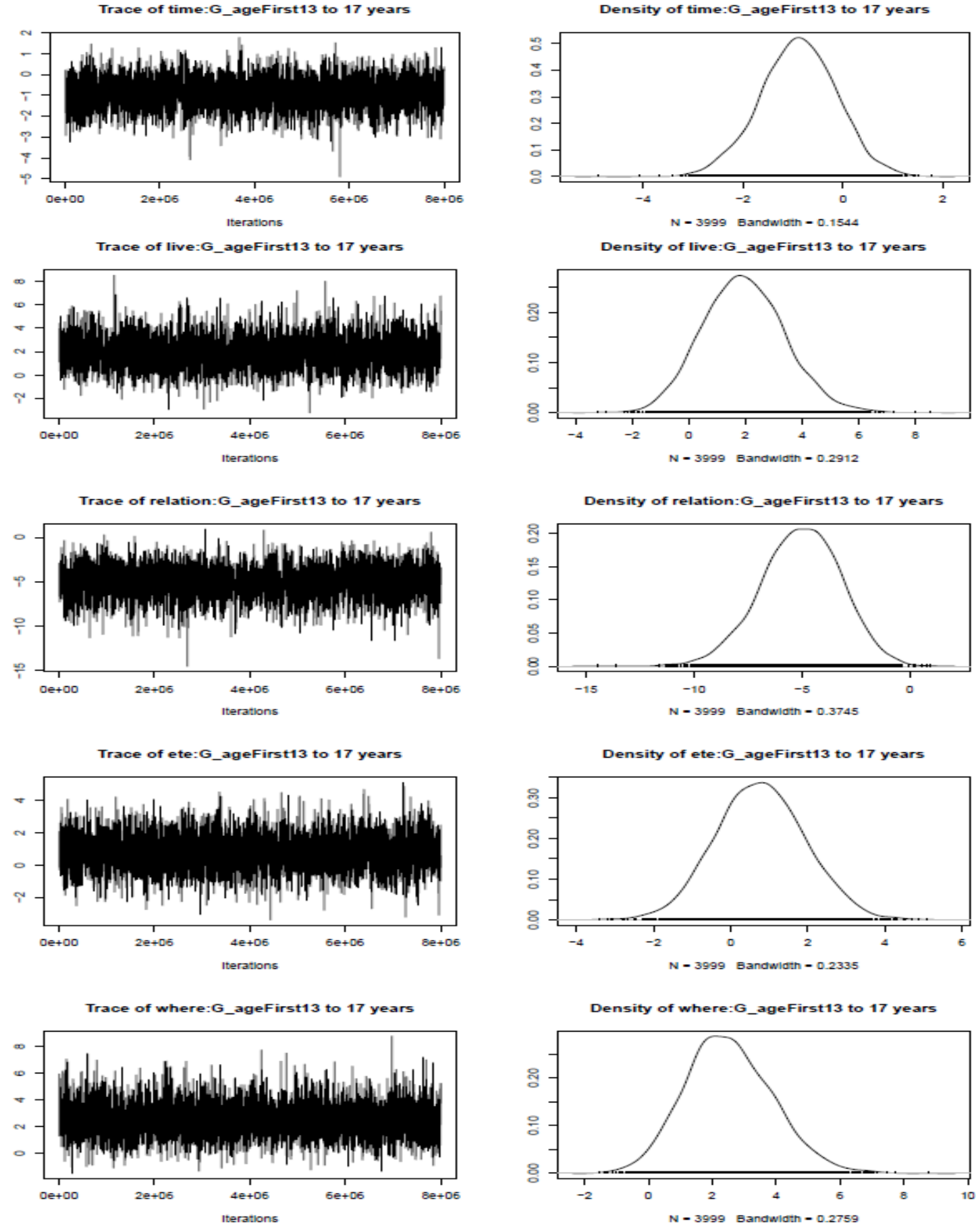

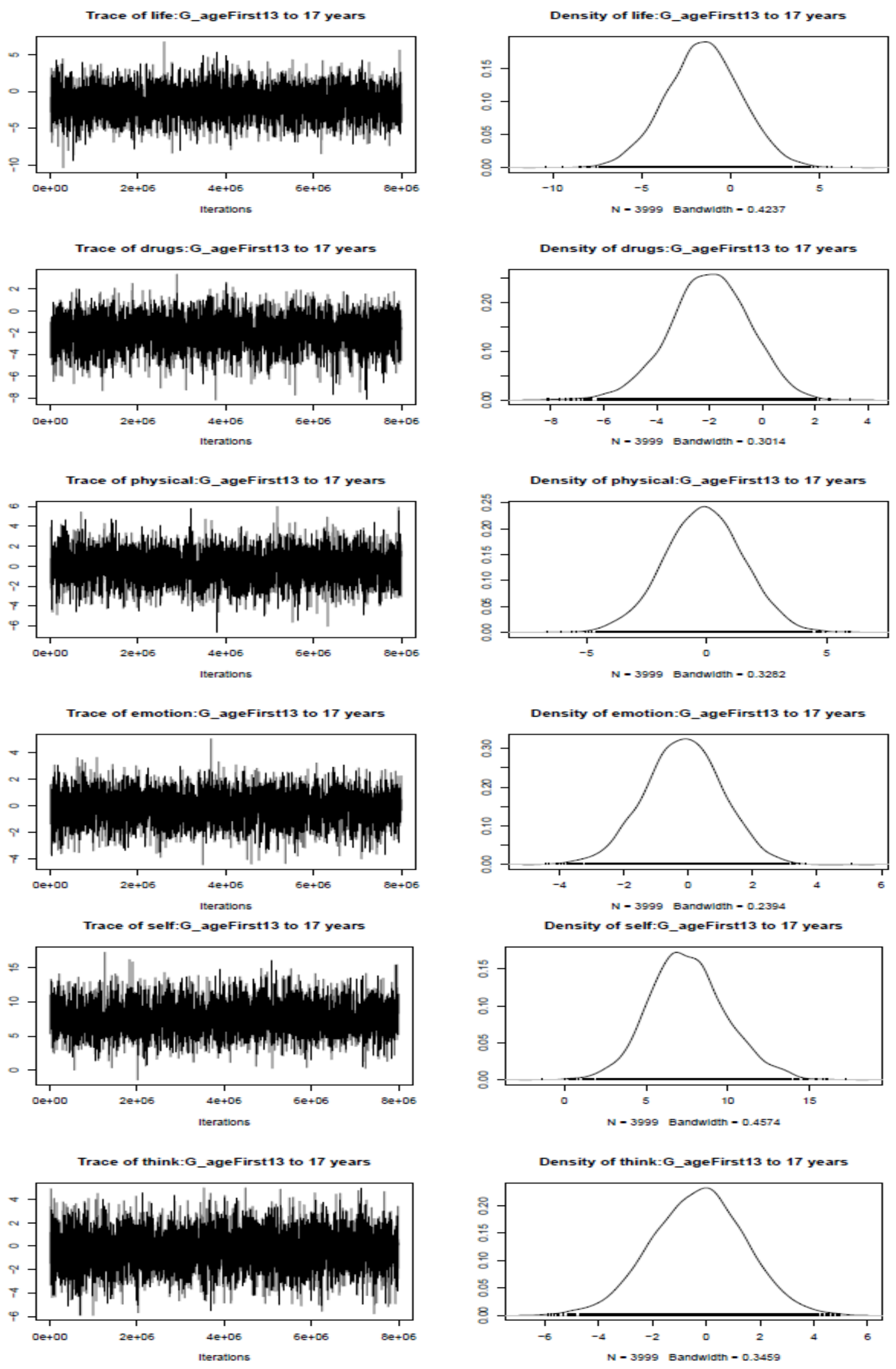

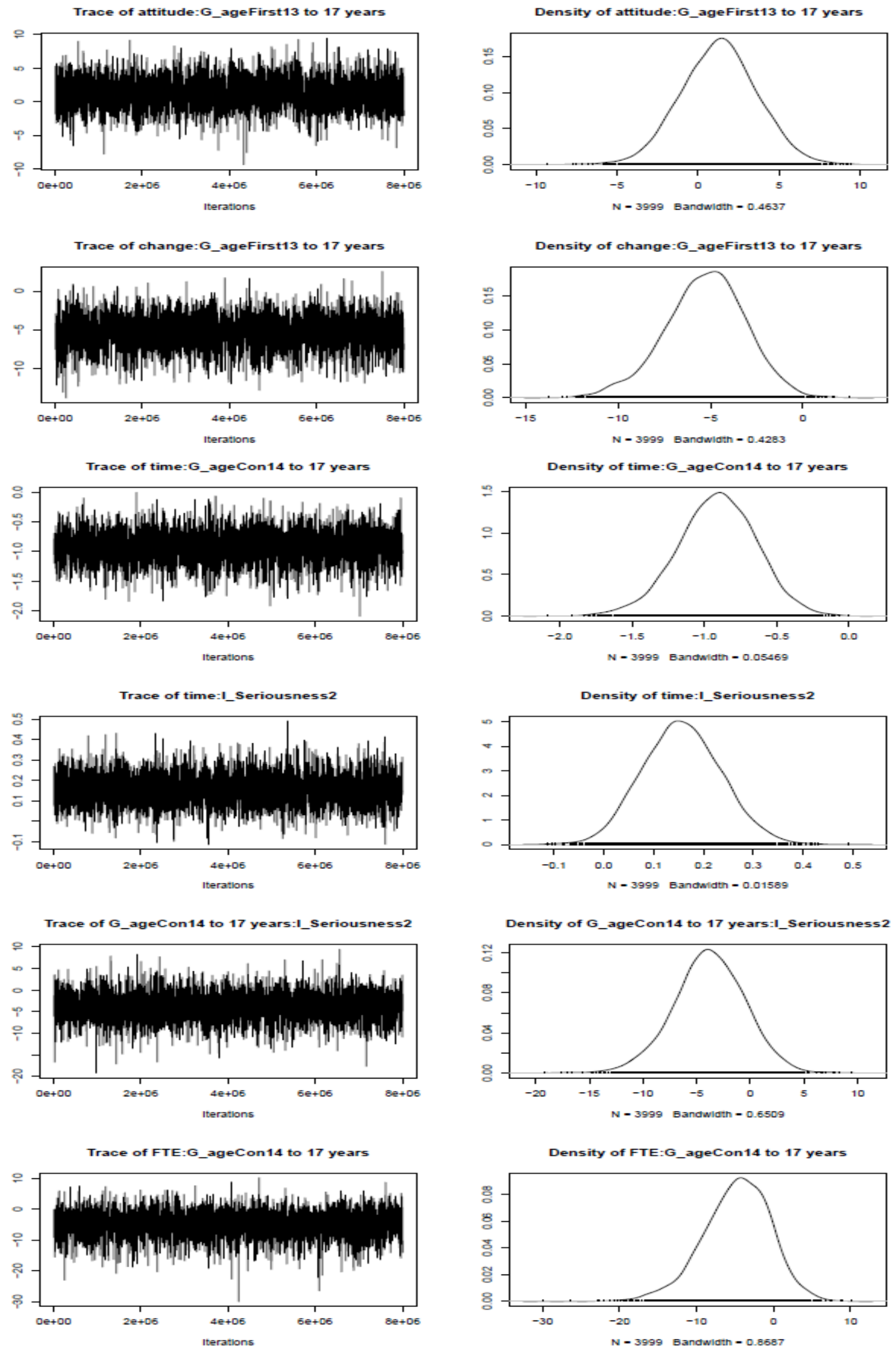


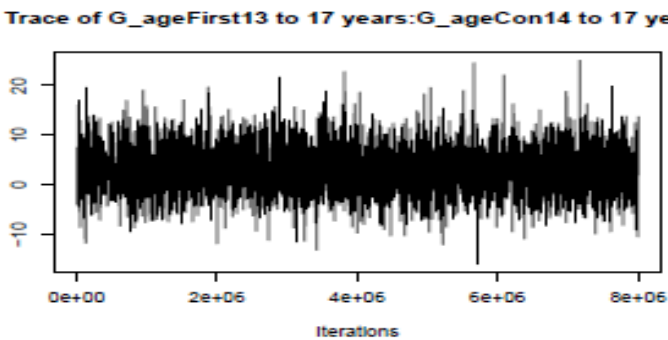

terations

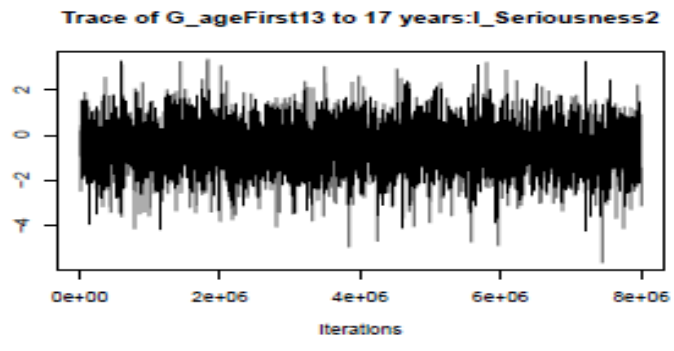

Trace of FTE:G_ageFirst13 to 17 years

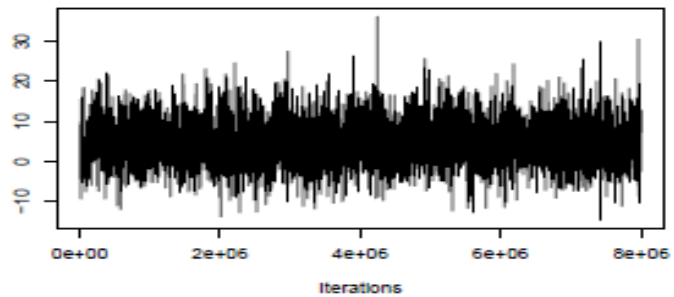

Trace of FTE:1_Seriousness2

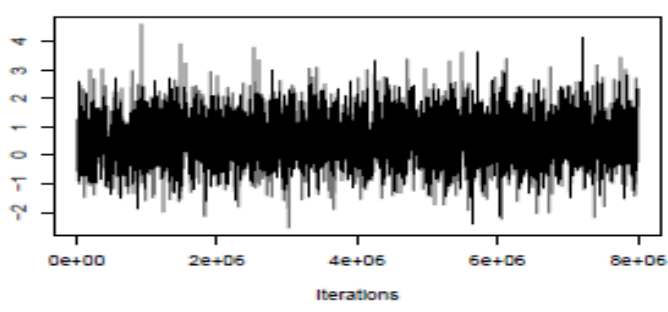

Trace of FTE:time:live

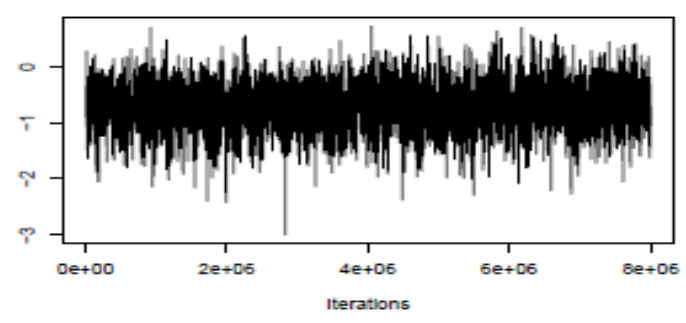

Trace of FTE:time:relation

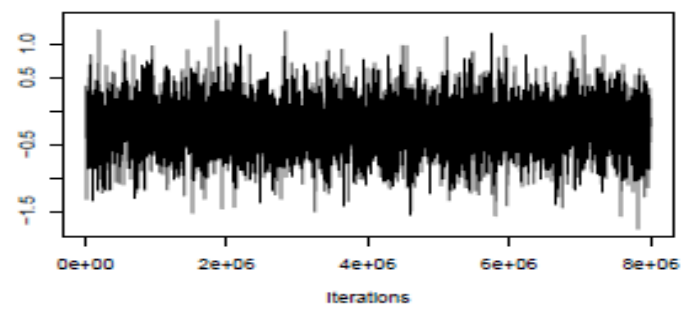

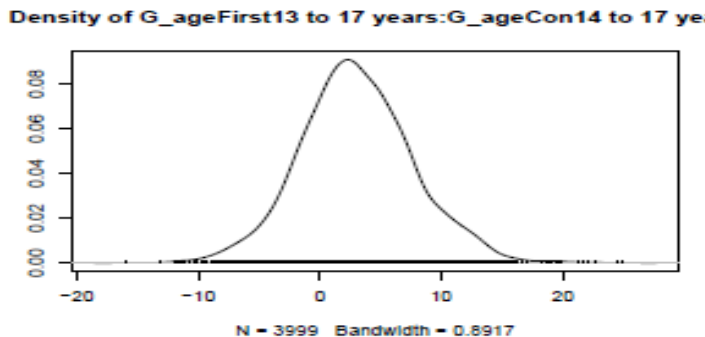

Density of G_ageFirst13 to 17 years:1_Seriousness2

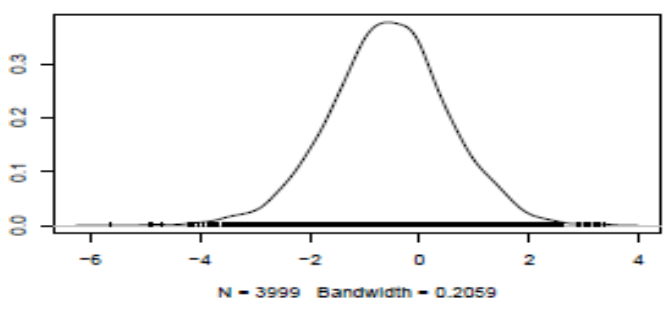

Density of FTE:G_ageFirst13 to 17 years

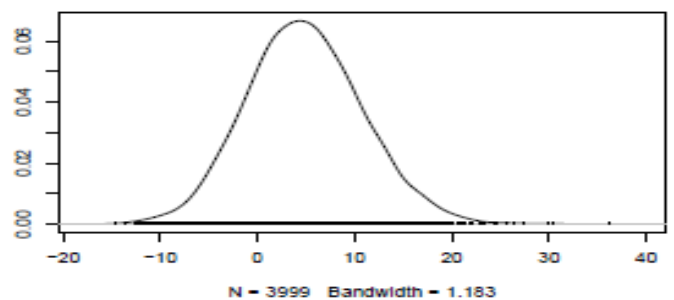

Density of FTE:1_Seriousness2
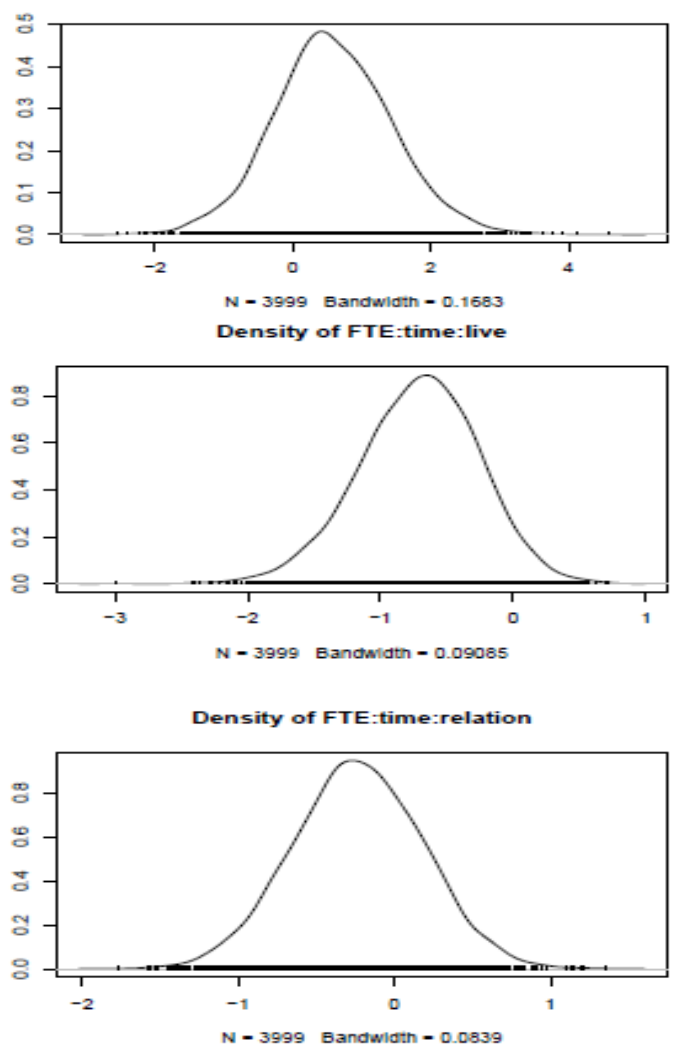
Trace of FTE:time:ete

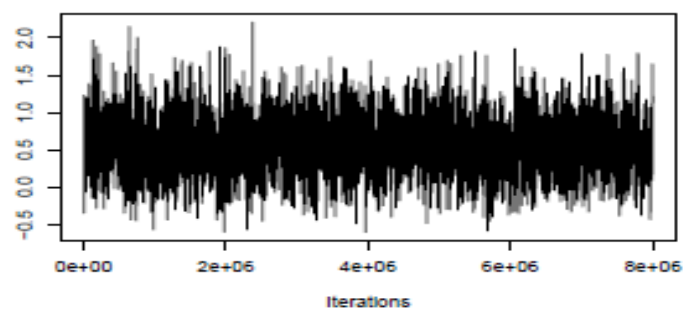

Trace of FTE:time:where

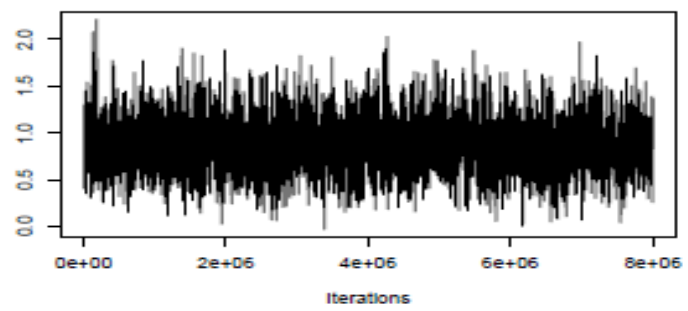

Trace of FTE:time:life

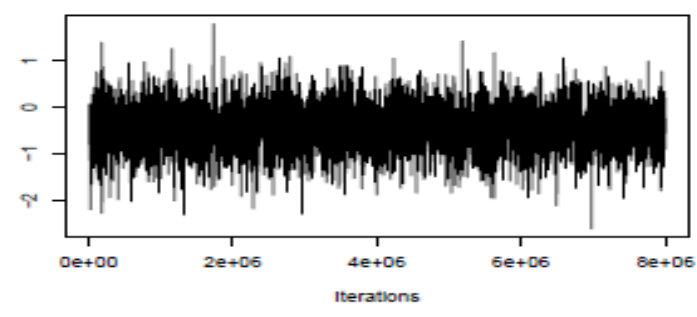

Trace of FTE:time:drugs

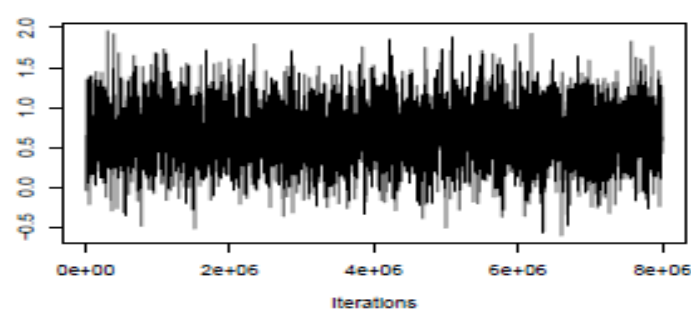

Trace of FTE:time:physical

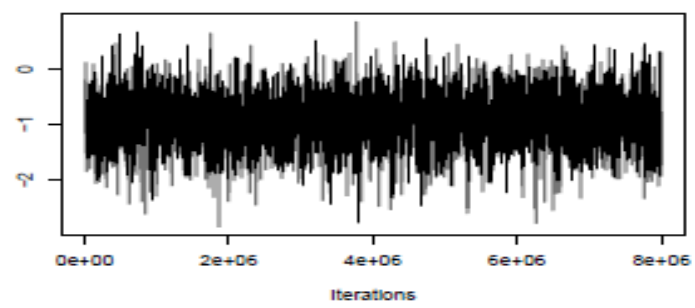

Trace of FTE:time:emotion

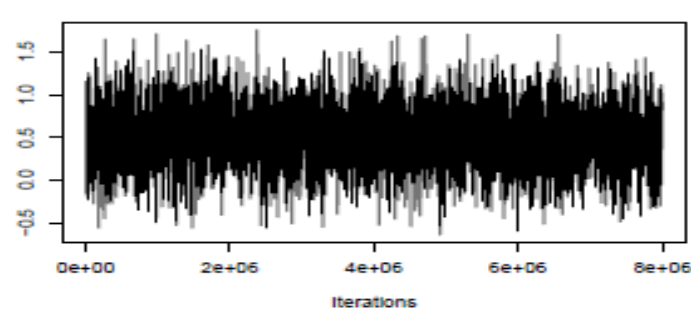

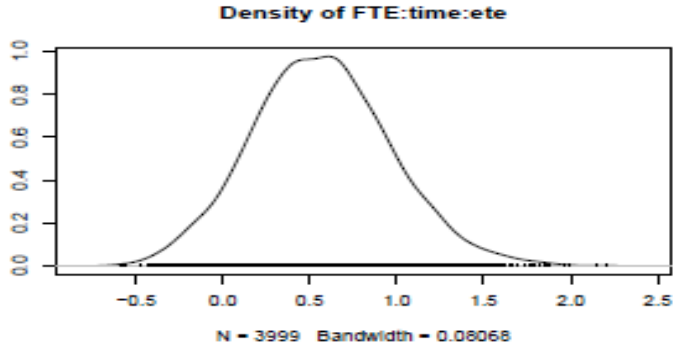

Density of FTE:time:where

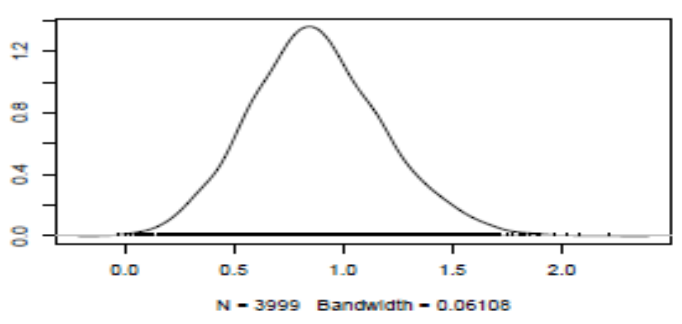

Density

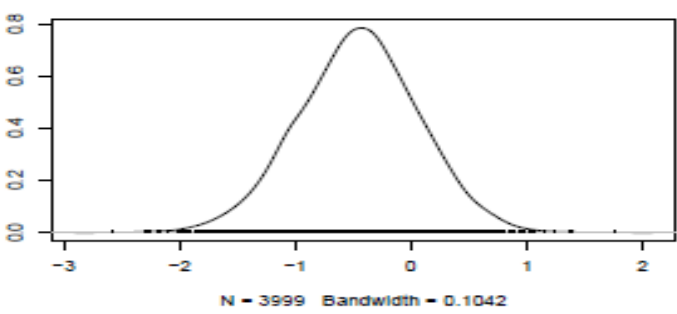

Density of FTE:time:drugs

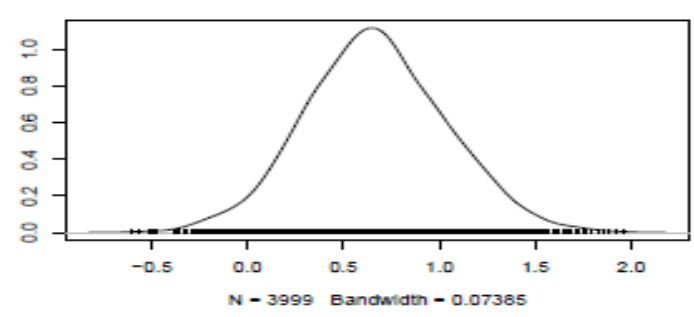

Density of FTE:time:physical

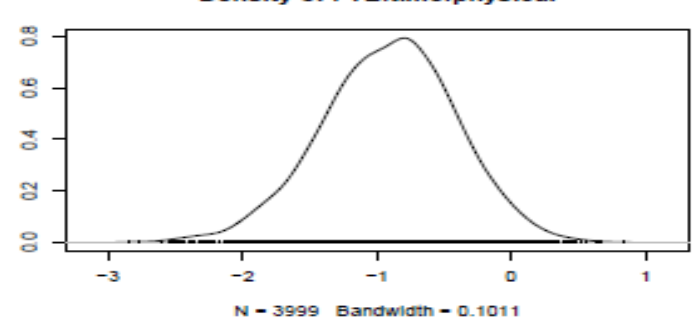

Density of FTE:time:emotion

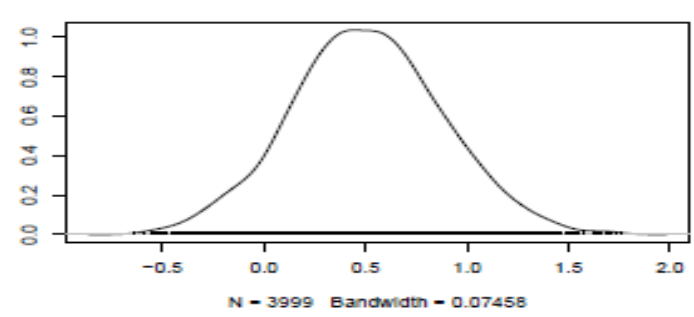



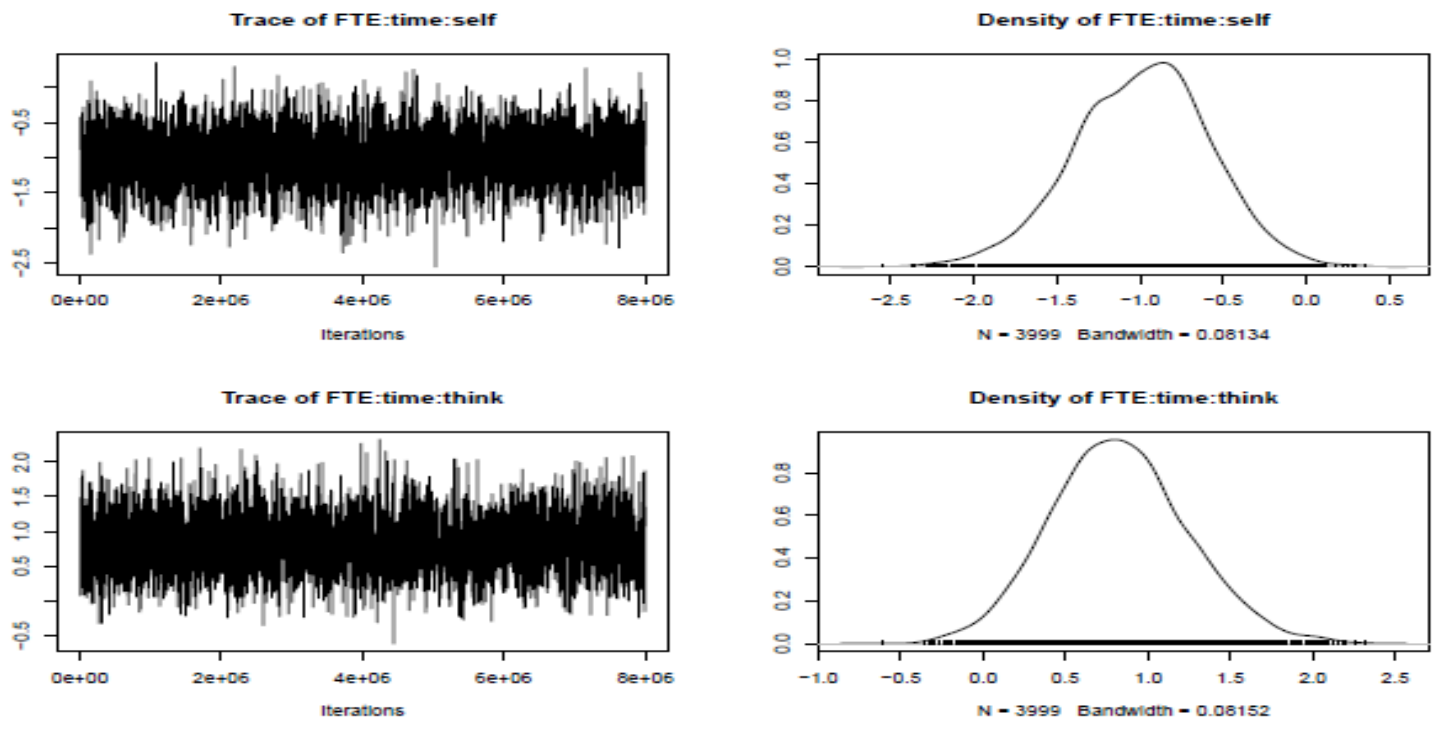

Trace of FTE:time:attitude
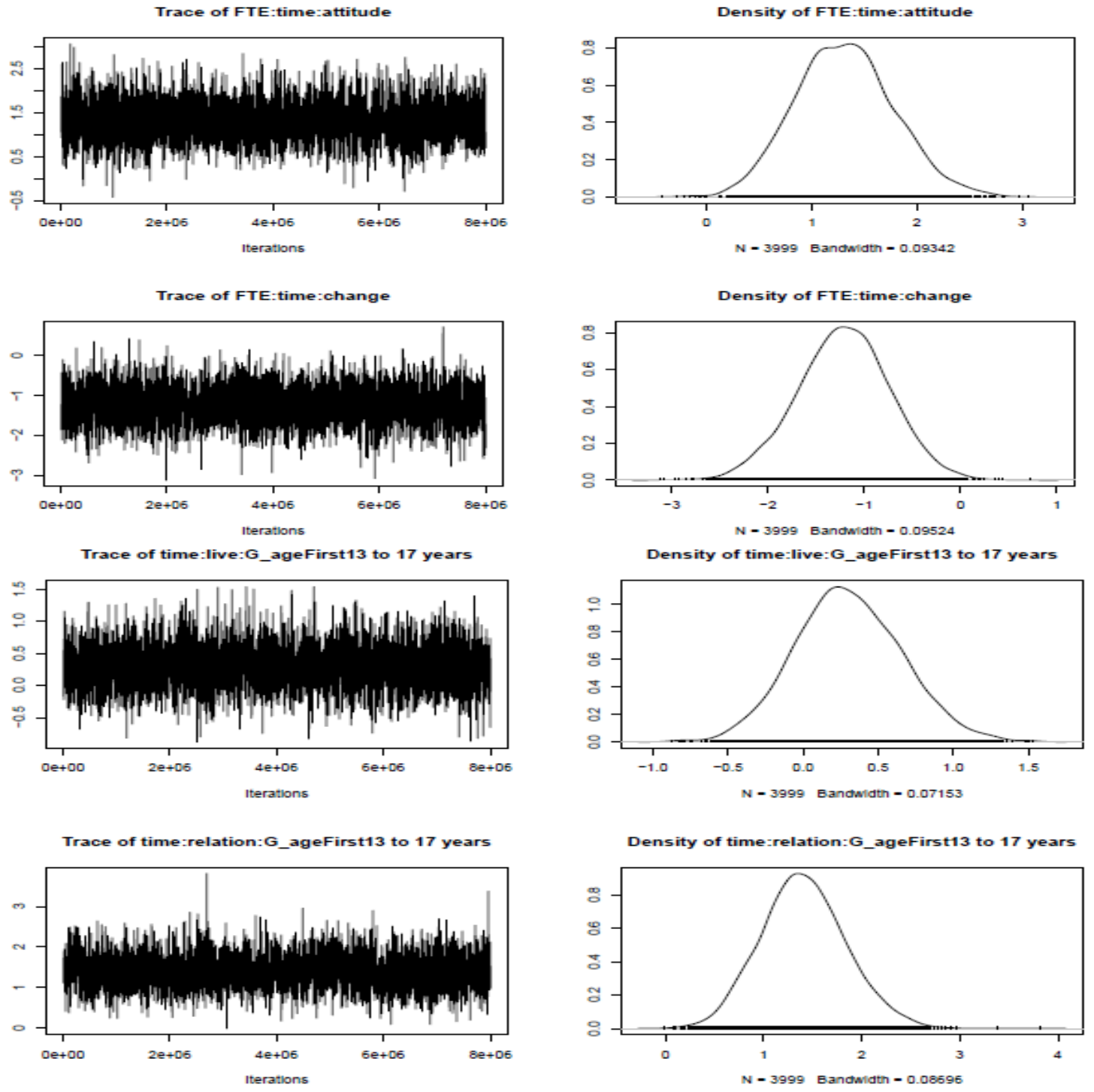

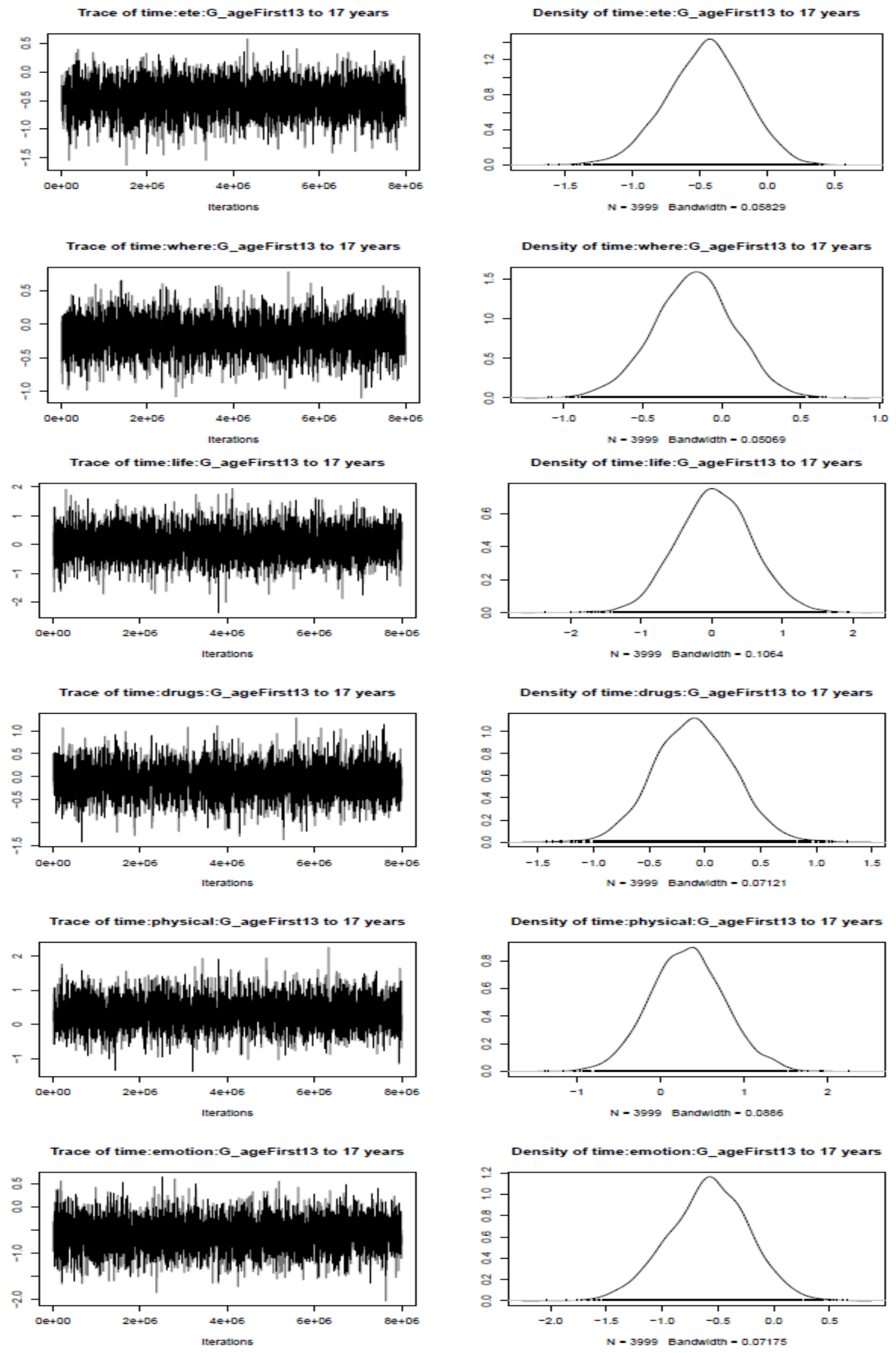

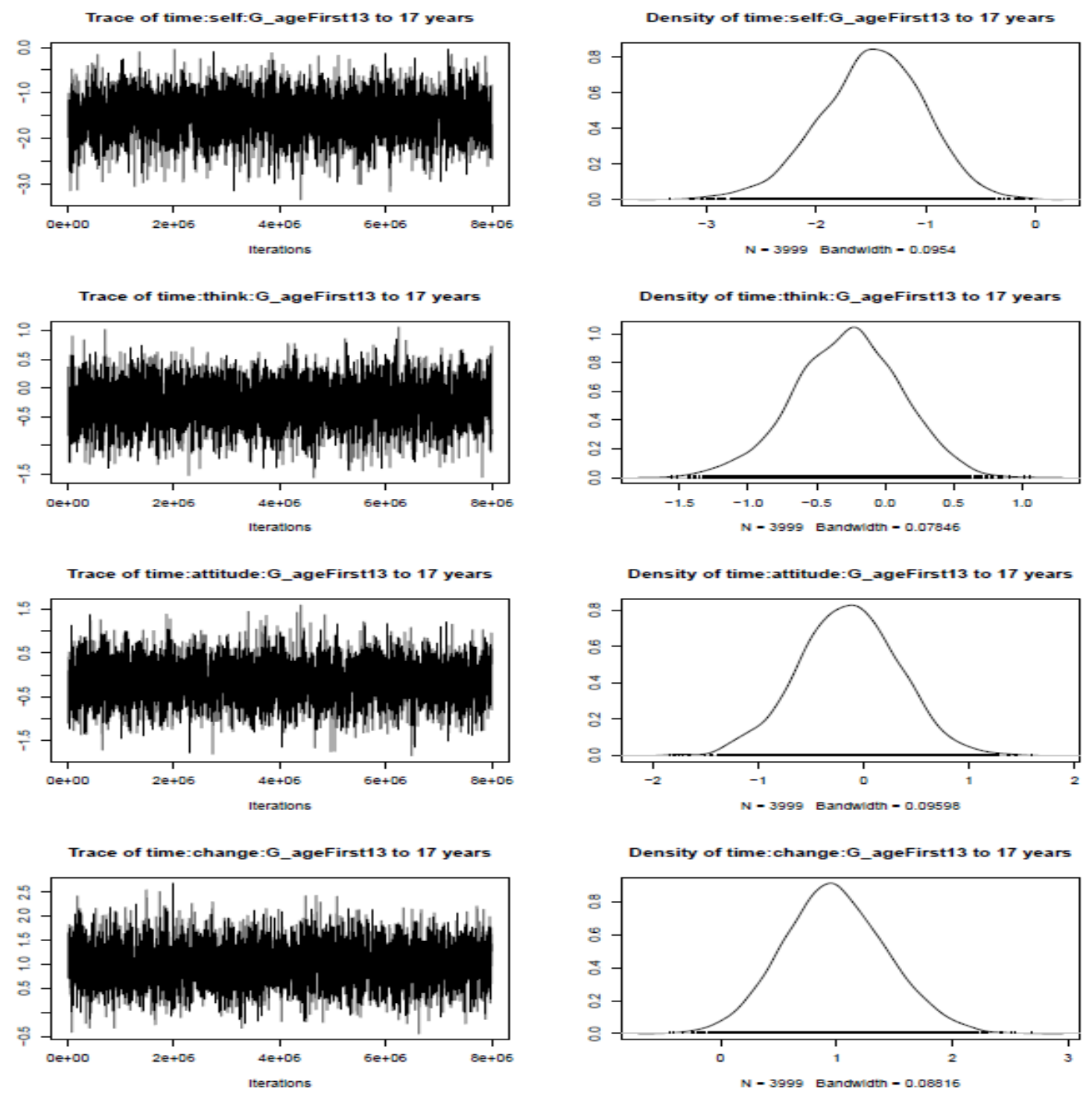


\section{$\underline{\text { Random Effects }}$}

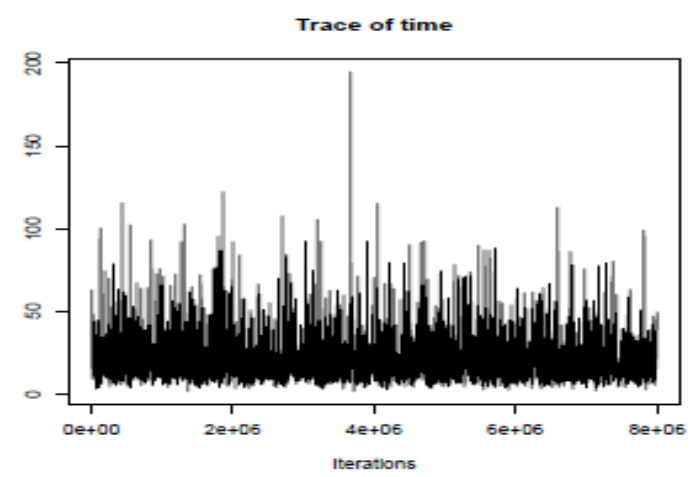

Trace of Research.ID

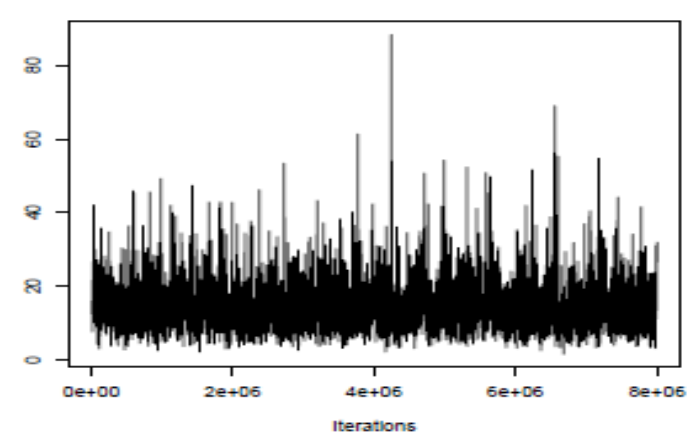

Density of time

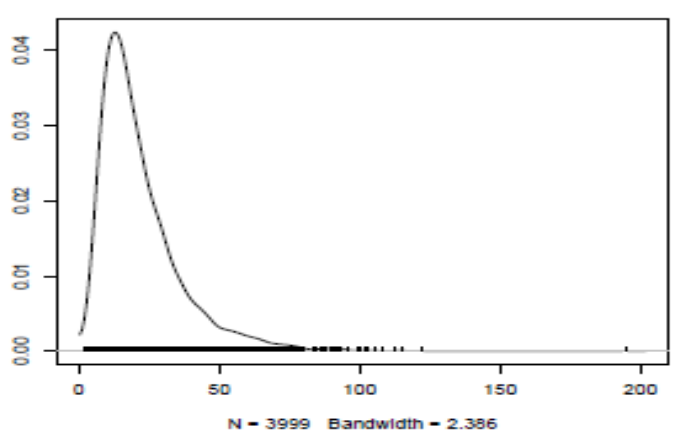

Density of Research.ID

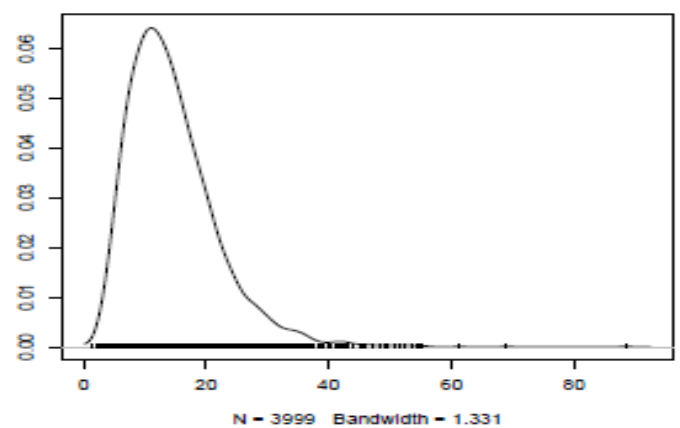


Dynamic Model involving Age at First Offence (Table 6.21)

Bayesian Model (BDm3_cc2)

\section{\#\# Define the model}

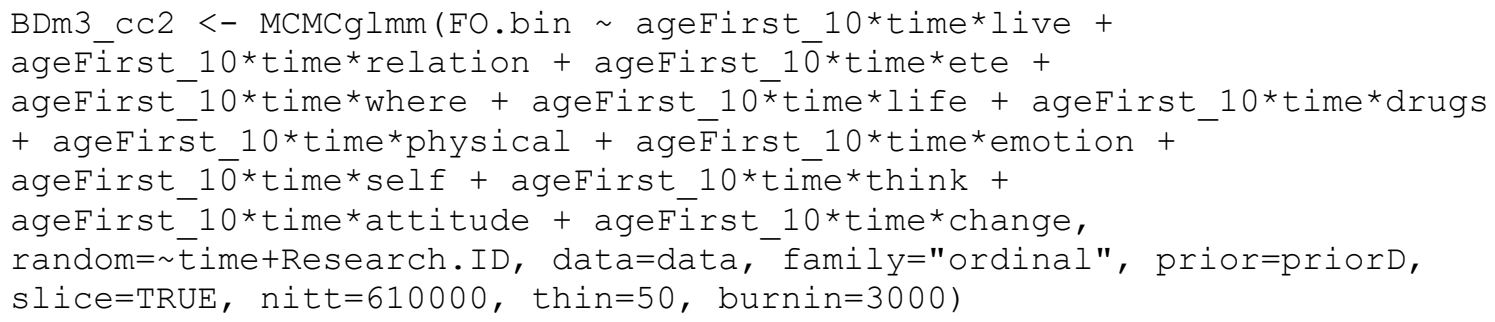

\section{\#\# Checks for suitable convergence}

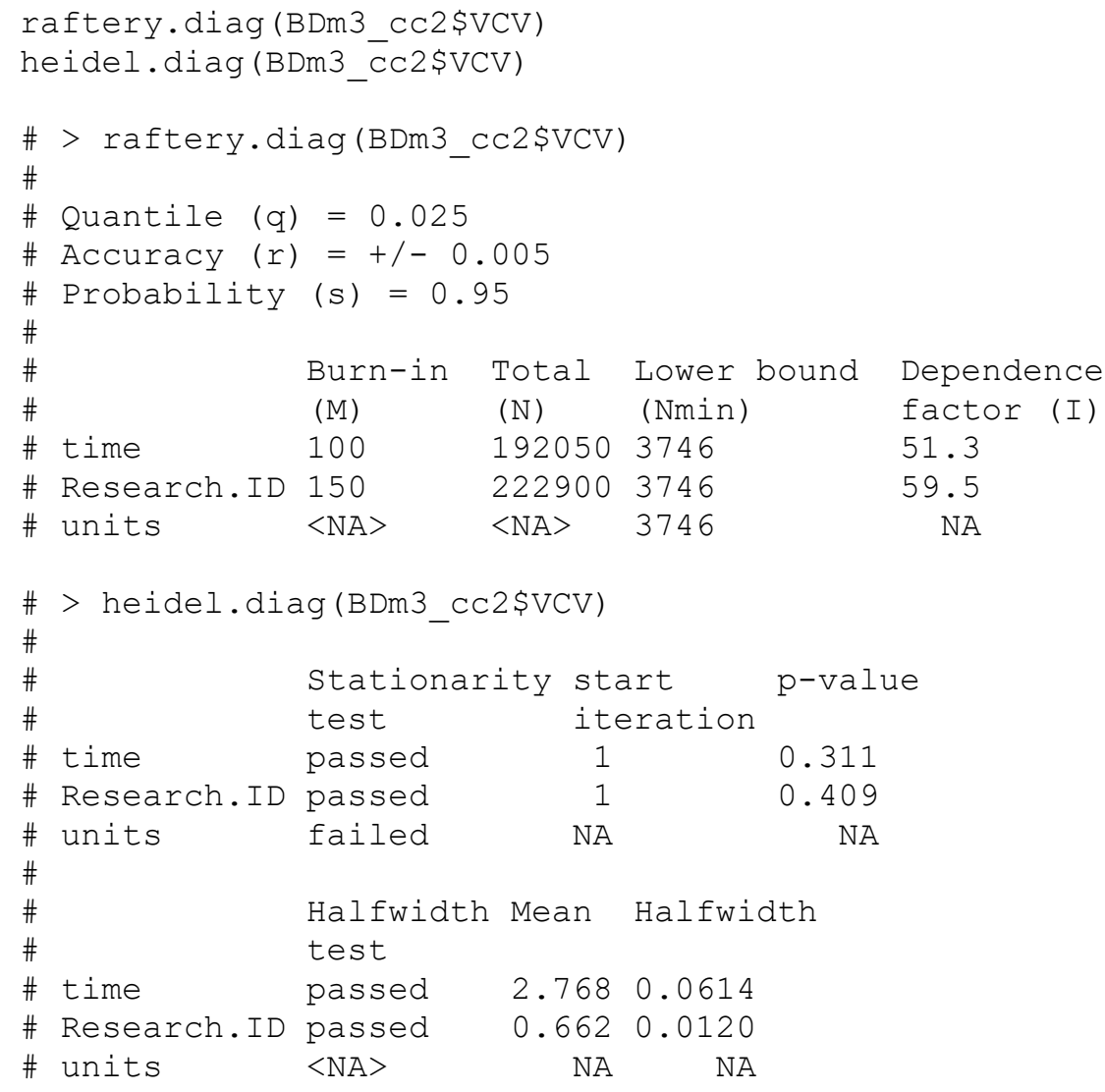




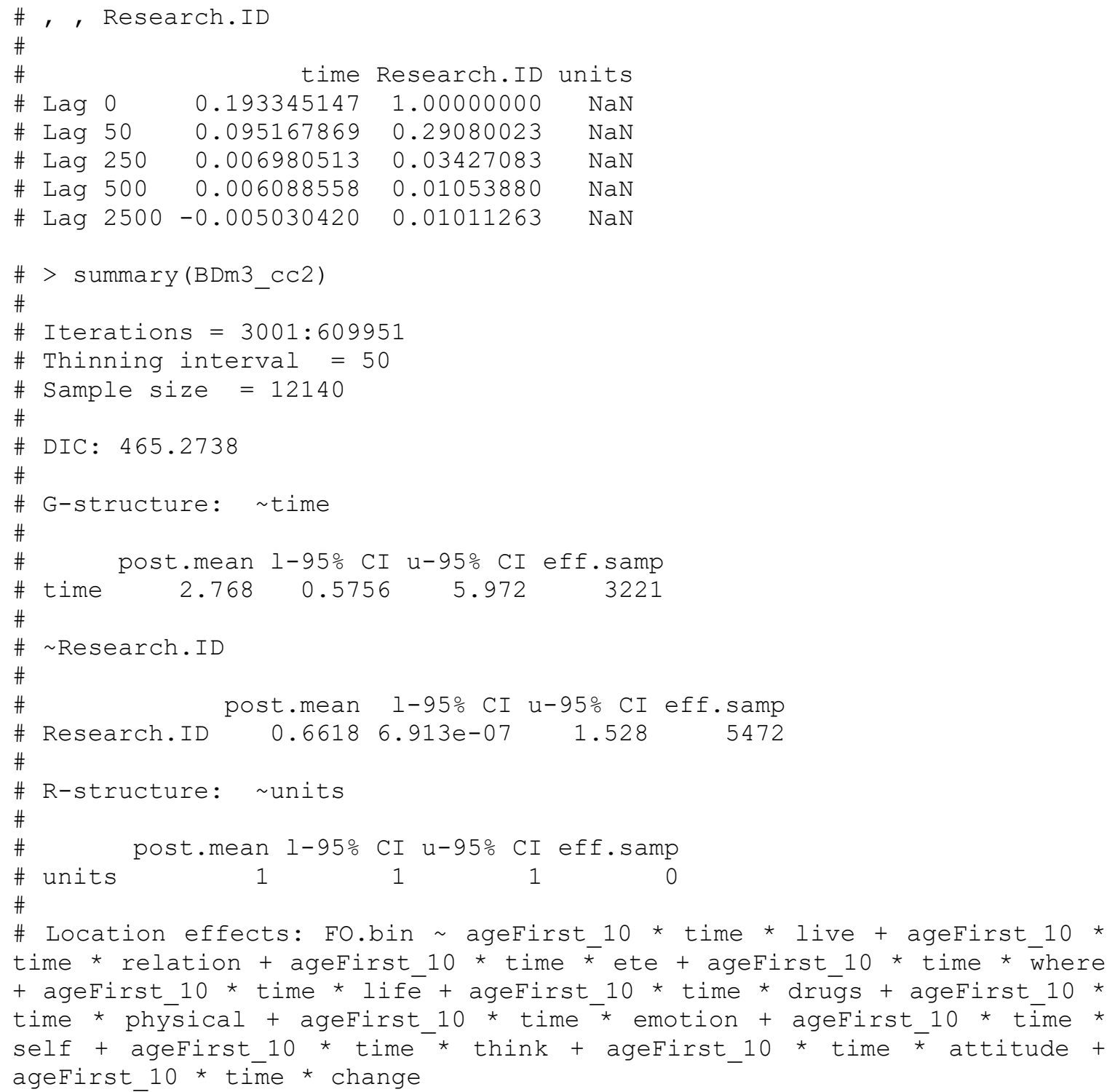

$\begin{array}{rrrrrr}\text { post.mean } & 1-95 \% \text { CI } & \text { u-95\% CI } & \text { eff.samp } & \text { pMCMC } \\ -2.530000 & -5.687846 & 0.408050 & 10640 & 0.100824 \\ 0.297198 & -0.368490 & 0.938716 & 10718 & 0.377595 \\ 0.220691 & -0.396946 & 0.807179 & 10728 & 0.470675 \\ -0.109482 & -1.405698 & 1.076946 & 12140 & 0.866886 \\ 1.606528 & 0.206527 & 3.021811 & 11669 & 0.025865 \\ -0.409371 & -1.571403 & 0.718282 & 11590 & 0.475453 \\ 0.251670 & -0.766253 & 1.219090 & 11364 & 0.621252 \\ 0.536263 & -1.115136 & 2.141893 & 12017 & 0.520593 \\ 0.606402 & -0.578611 & 1.692365 & 11630 & 0.287809 \\ -0.473916 & -1.602006 & 0.702729 & 11677 & 0.423394 \\ -0.360726 & -1.404536 & 0.672859 & 11098 & 0.494399 \\ -2.082001 & -3.783864 & -0.323285 & 11180 & 0.013839 \\ 0.351036 & -1.241713 & 1.997439 & 11535 & 0.679242 \\ 0.445100 & -1.324734 & 2.280710 & & 12140 & 0.629489 \\ 0.169732 & -1.430469 & 1.821562 & & 12140 & 0.837891 \\ -0.140295 & -0.312066 & 0.025096 & 11216 & 0.097529 \\ 0.026597 & -0.289357 & 0.365958 & 12140 & 0.875288 \\ -0.075220 & -0.350869 & 0.196760 & 12140 & 0.586820 \\ -0.364494 & -0.717212 & -0.012033 & 11574 & 0.042010 \\ -0.361232 & -0.703048 & -0.020496 & 11136 & 0.034102 \\ 0.020860 & -0.262475 & 0.315496 & 12140 & 0.899012 \\ 0.008325 & -0.253992 & 0.269855 & 11752 & 0.957002 \\ -0.045942 & -0.319690 & 0.207531 & 11453 & 0.732455\end{array}$ *




\begin{tabular}{|c|c|c|c|c|c|c|c|}
\hline \# & time: where & -0.042589 & -0.245877 & 0.167784 & 11620 & 0.680066 & \\
\hline \# & ageFirst_10:life & -0.052279 & -0.458204 & 0.369826 & 11623 & 0.807249 & \\
\hline$\#$ & time: life & 0.014039 & -0.353171 & 0.401648 & 10456 & 0.954201 & \\
\hline \# & ageFirst_10:drugs & -0.085978 & -0.375990 & 0.195714 & 11802 & 0.554201 & \\
\hline \# & time: drugs & -0.114764 & -0.380707 & 0.117230 & 11412 & 0.366886 & \\
\hline \# & ageFirst_10:physical & -0.047447 & -0.363590 & 0.258750 & 11793 & 0.768369 & \\
\hline \# & time:physical & 0.232502 & -0.063816 & 0.517579 & 11473 & 0.114992 & \\
\hline$\#$ & ageFirst_10:emotion & 0.005864 & -0.265216 & 0.291699 & 11329 & 0.967710 & \\
\hline$\#$ & time:emotion & 0.114801 & -0.125499 & 0.361792 & 11152 & 0.350906 & \\
\hline \# & ageFirst_10:self & 0.666313 & 0.237757 & 1.116004 & 10864 & 0.001318 & $\star \star$ \\
\hline$\#$ & time:sele & 0.514624 & 0.153033 & 0.902988 & 10932 & 0.004778 & $\star \star$ \\
\hline \# & ageFirst_10:think & -0.122879 & -0.543053 & 0.267296 & 12140 & 0.547776 & \\
\hline \# & time:think & -0.156506 & -0.482053 & 0.169959 & 11690 & 0.336079 & \\
\hline \# & ageFirst_10:attitude & -0.019507 & -0.470902 & 0.421149 & 11273 & 0.9428 & \\
\hline$\#$ & time:attitude & -0.096444 & -0.451299 & 0.251643 & 12140 & 0.601647 & \\
\hline \# & ageFirst_10:change & 0.013744 & -0.448135 & 0.451297 & 12140 & 0.948270 & \\
\hline$\#$ & time: change & 0.070945 & -0.277724 & 0.424002 & 12999 & 0.683361 & \\
\hline \# & ageFirst_10:time:live & 0.030960 & -0.048252 & 0.104150 & 11702 & 0.427018 & \\
\hline \# & ageFirst_10:time:relation & 0.093347 & -0.002207 & 0.181162 & 10891 & 0.04 & * \\
\hline \# & ageFirst_10:time:ete & 0.032027 & -0.044399 & 0.113027 & 11189 & 0.427018 & \\
\hline \# & ageFirst_10:time: where & 0.018543 & -0.038420 & 0.076280 & 11455 & 0.532 & \\
\hline \# & ageFirst_10:time:life & -0.023736 & -0.129972 & 0.085828 & 11090 & 0.67 & \\
\hline \# & ageFirst_10:time:drugs & 0.030245 & -0.044580 & 0.104906 & 11593 & 0.428830 & \\
\hline$\#$ & ageFirst_10:time:physical & -0.018817 & -0.101539 & 0.064733 & 11448 & 0.658320 & \\
\hline \# & ageFirst_10:time:emotion & 0.001546 & -0.072904 & 0.076484 & 11101 & 0.970675 & \\
\hline$\#$ & ageFirst_10:time:self & -0.182721 & -0.293139 & -0.083412 & 10297 & 0.000494 & $\star \star \star$ \\
\hline$\#$ & ageFirst_10:time:think & 0.040274 & -0.045860 & 0.127252 & 11716 & 0.353542 & \\
\hline \# & ageFirst_10:time:attitude & -0.008130 & -0.109456 & 0.091971 & 12140 & 0.872817 & \\
\hline \# & ageFirst_10:time: change & -0.015731 & -0.116063 & 0.089730 & 12140 & 0.754036 & \\
\hline
\end{tabular}




\section{Fixed Effects}

Trace of (Intercept)

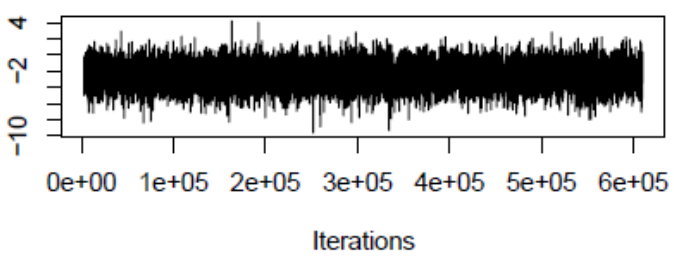

Trace of ageFirst_10

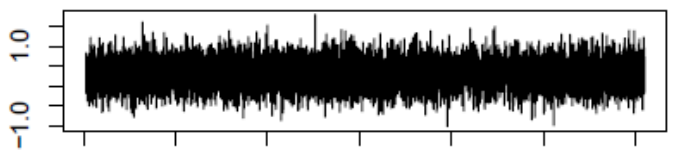

$\begin{array}{lllllll}0 e+00 & 1 e+05 & 2 e+05 & 3 e+05 & 4 e+05 & 5 e+05 & 6 e+05\end{array}$

Iterations

\section{Trace of time}

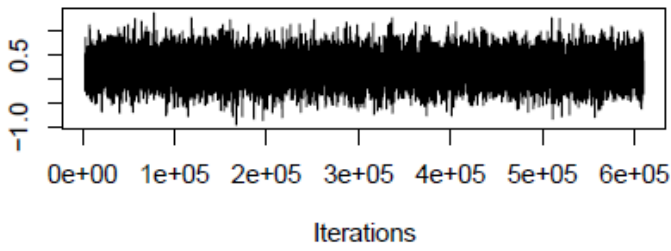

Trace of live

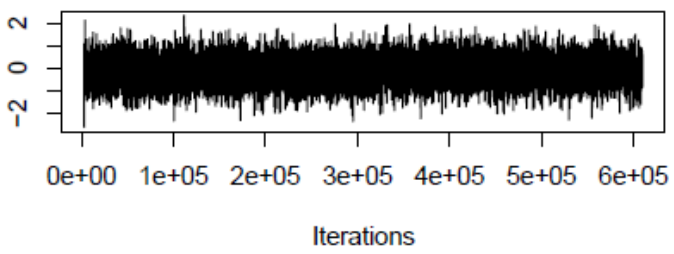

Trace of relation

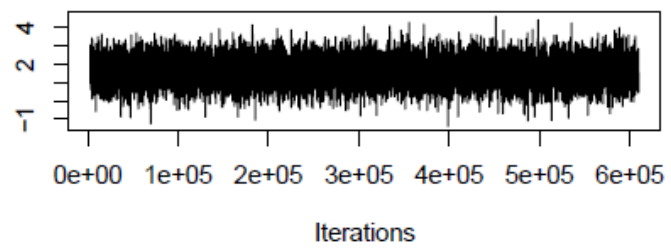

Trace of ete

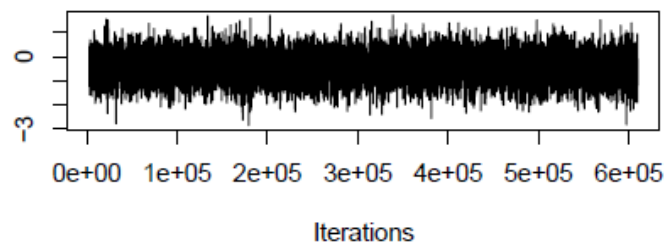

Density of (Intercept)

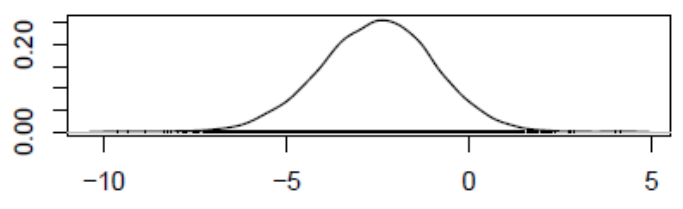

$\mathrm{N}=12140$ Bandwidth $=0.2501$

Density of ageFirst_10

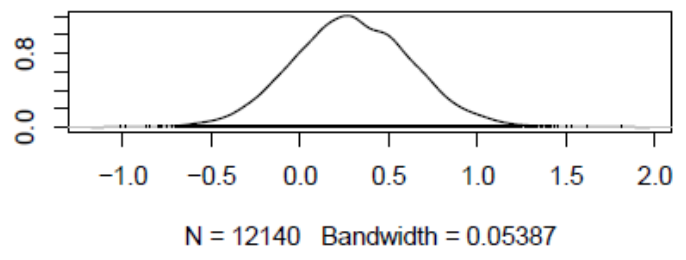

Density of time

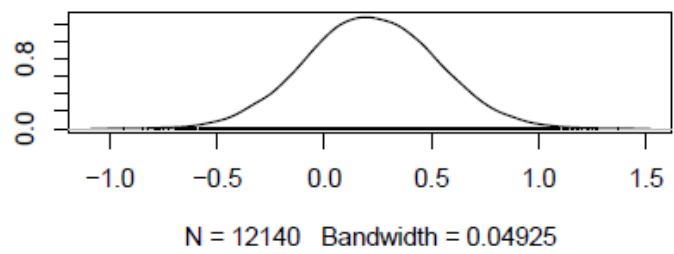

Density of live

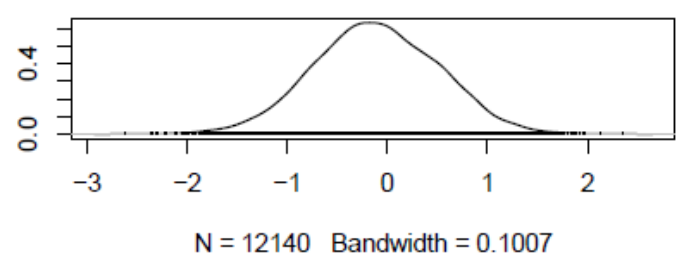

Density of relation

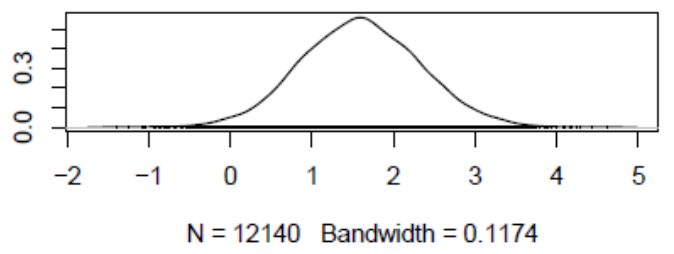

Density of ete

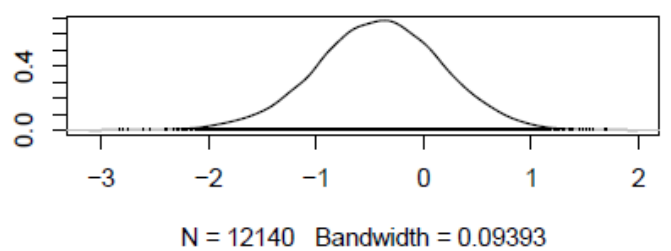

Page | 325 
Trace of where

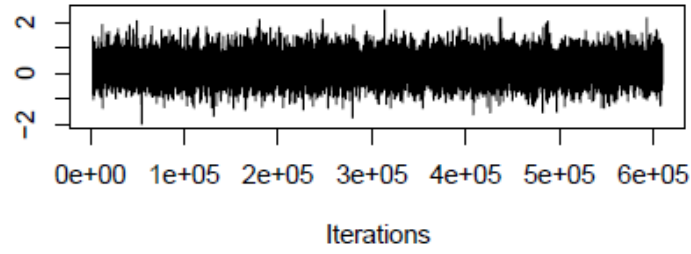

Trace of life

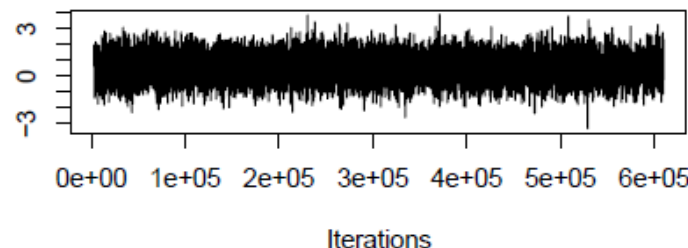

Trace of drugs

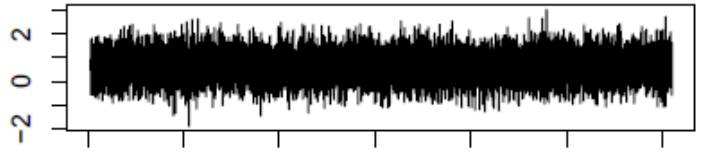

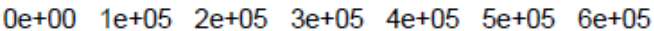

Iterations

Trace of physical

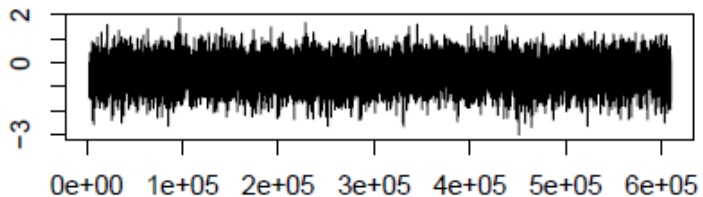

Iterations

Trace of emotion

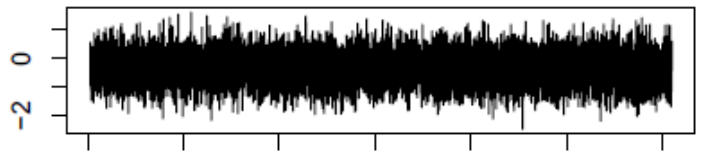

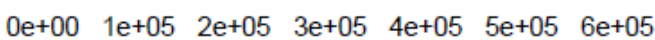

Iterations

Trace of self

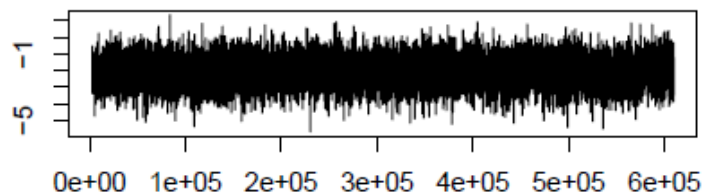

Iterations
Density of where

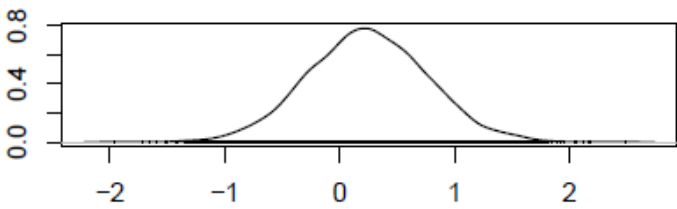

$\mathrm{N}=12140$ Bandwidth $=0.08388$

Density of life

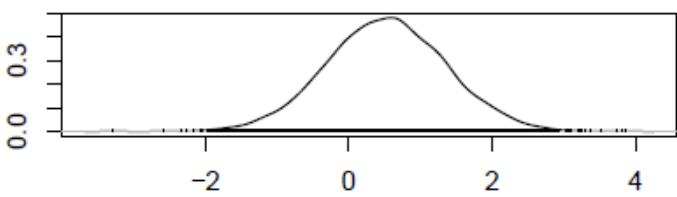

$\mathrm{N}=12140$ Bandwidth $=0.1335$

Density of drugs

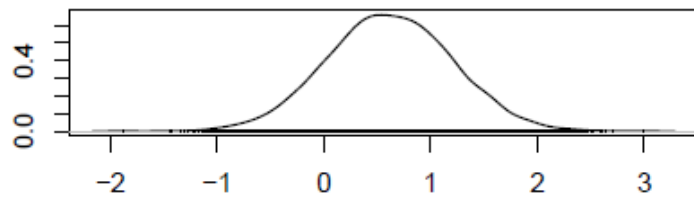

$\mathrm{N}=12140 \quad$ Bandwidth $=0.09467$

Density of physical

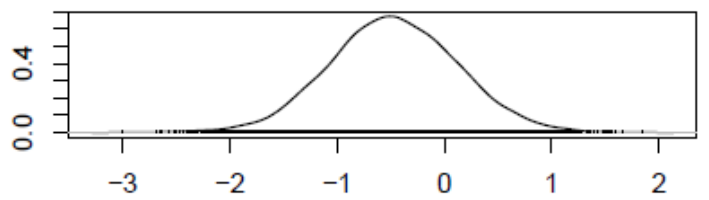

$\mathrm{N}=12140$ Bandwidth $=0.09545$

Density of emotion

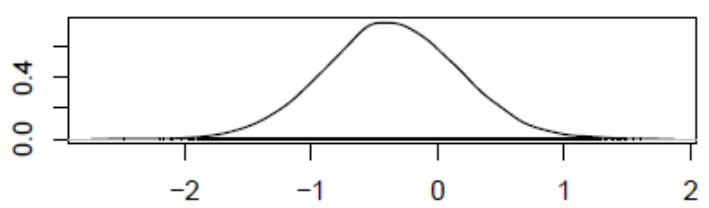

$\mathrm{N}=12140$ Bandwidth $=0.08488$

Density of self

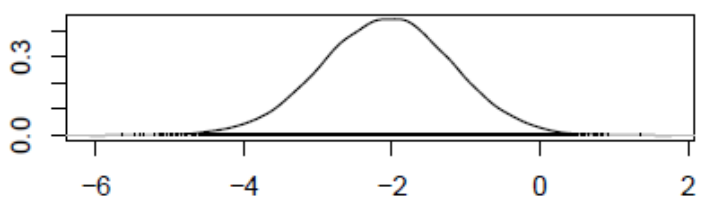

$N=12140$ Bandwidth $=0.1422$ 
Trace of think

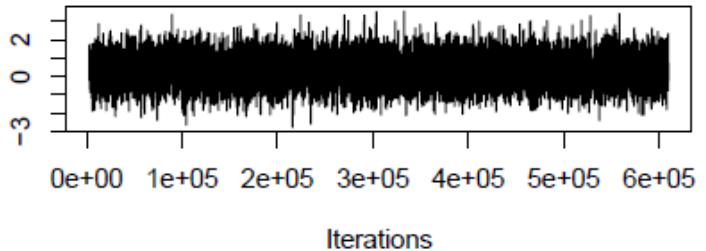

Trace of attitude

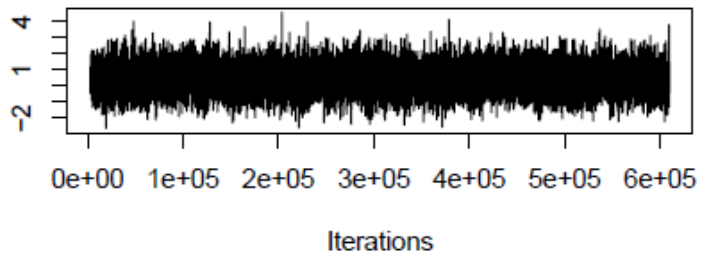

Trace of change

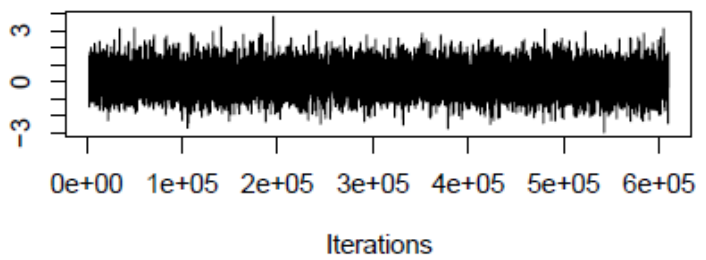

Trace of ageFirst_10:time

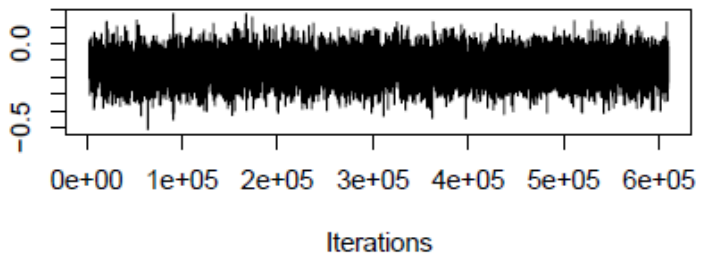

Trace of ageFirst_10:Iive

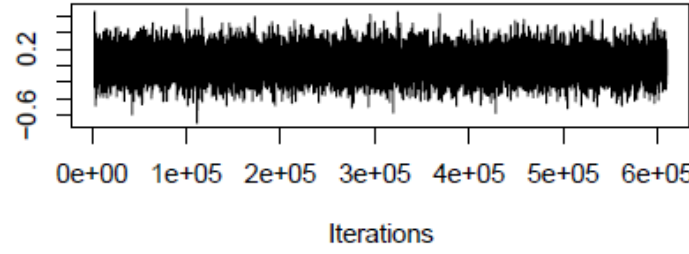

Trace of time:live

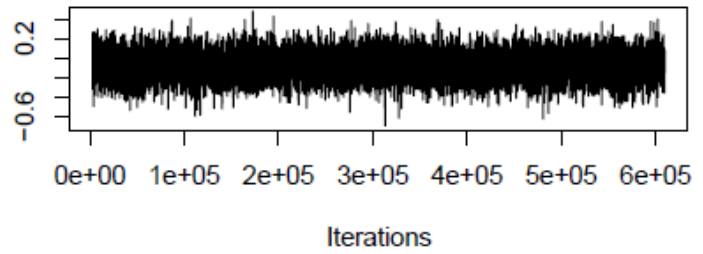

Density of think

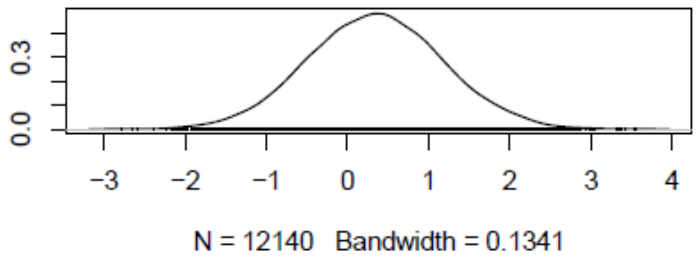

Density of attitude

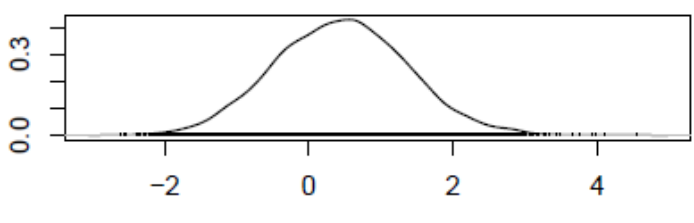

$N=12140$ Bandwidth $=0.1473$

Density of change

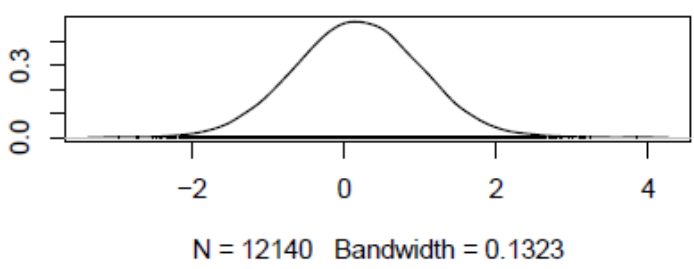

Density of ageFirst_10:time

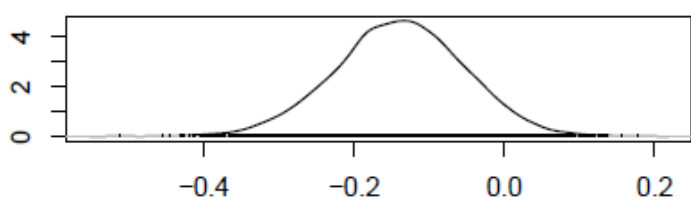

$\mathrm{N}=12140$ Bandwidth $=0.01362$

Density of ageFirst_10:live

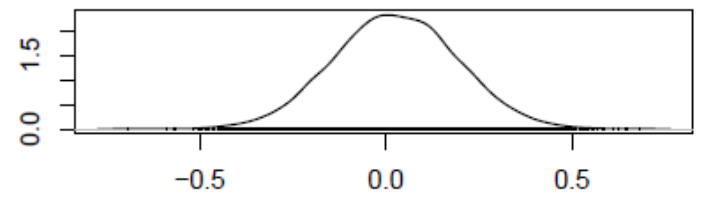

$\mathrm{N}=12140$ Bandwidth $=0.02692$

Density of time:live

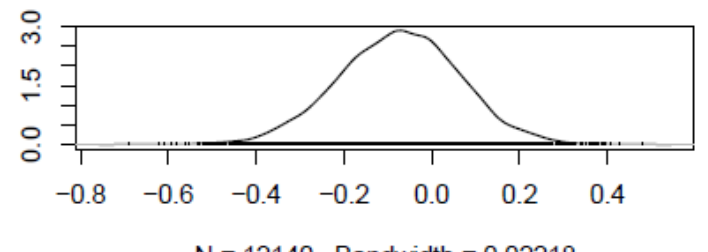

$\mathrm{N}=12140$ Bandwidth $=0.02218$ 
Trace of ageFirst_10:relation

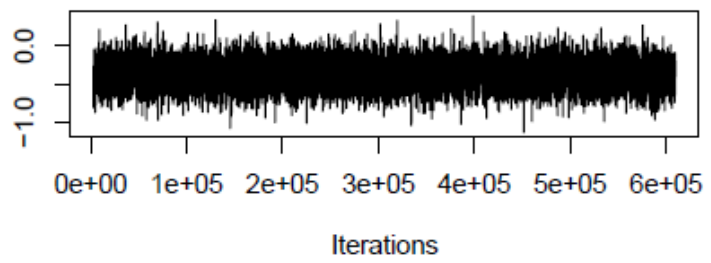

Trace of time:relation

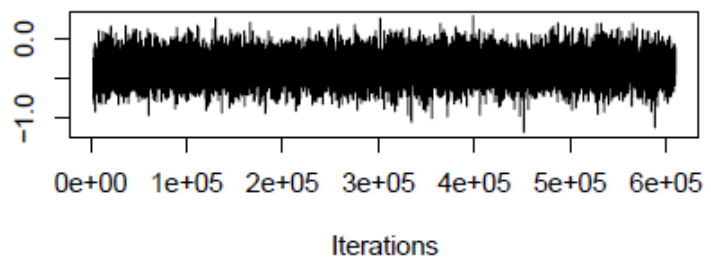

Trace of ageFirst_10:ete

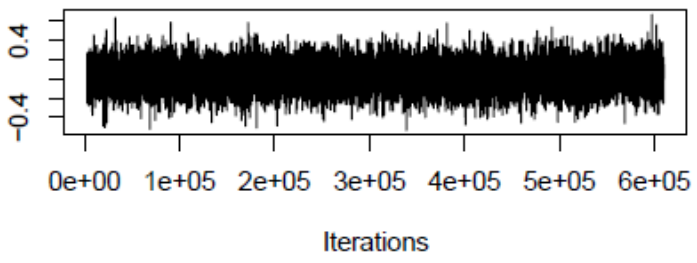

Trace of time:ete

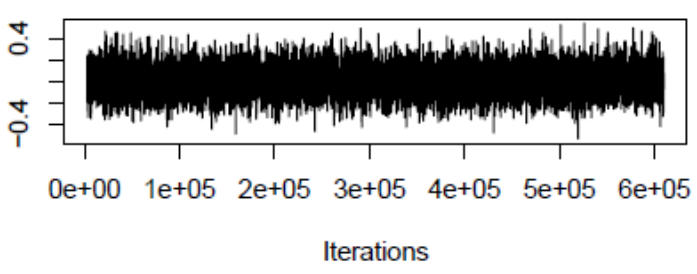

Trace of ageFirst_10:where

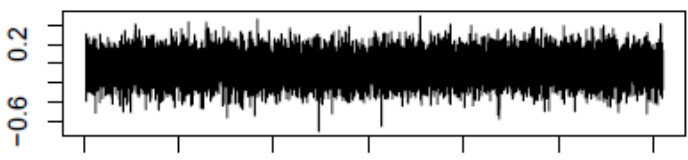

$0 \mathrm{e}+00 \quad 1 \mathrm{e}+05 \quad 2 \mathrm{e}+05 \quad 3 \mathrm{e}+05 \quad 4 \mathrm{e}+05 \quad 5 \mathrm{e}+05 \quad 6 \mathrm{e}+05$ Iterations

\section{Trace of time:where}

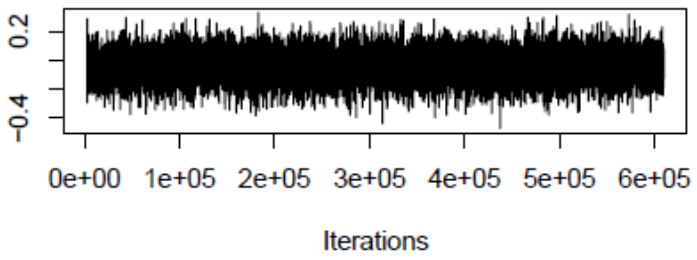

Density of ageFirst_10:relation

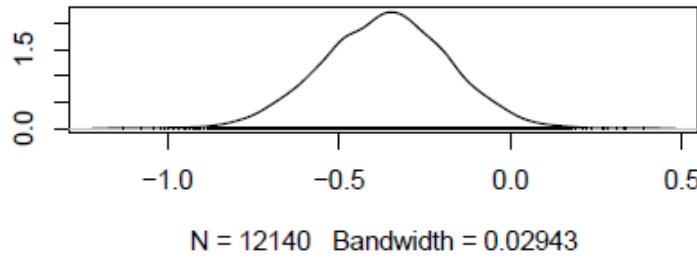

Density of time:relation

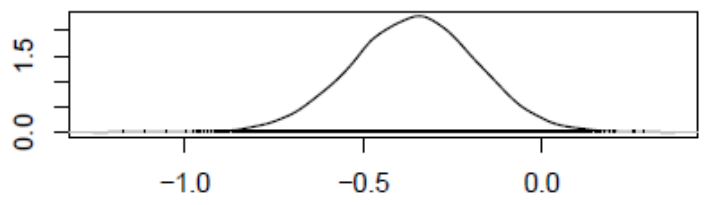

$\mathrm{N}=12140$ Bandwidth $=0.02822$

Density of ageFirst_10:ete

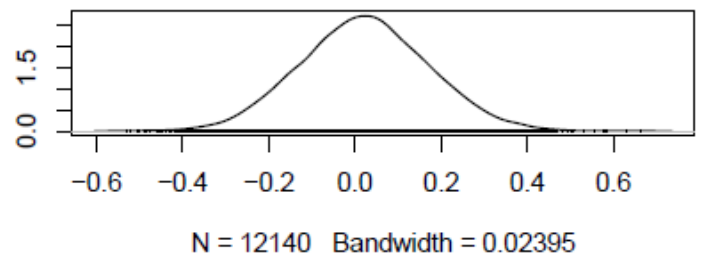

Density of time:ete

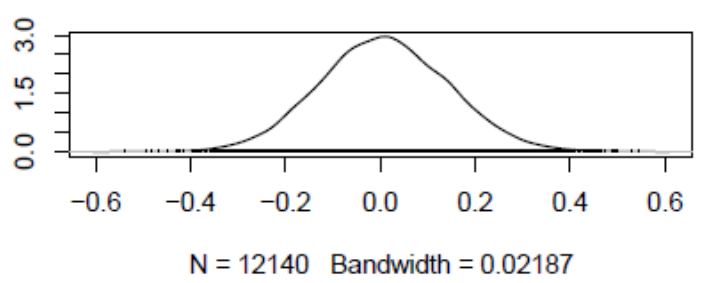

Density of ageFirst_10:where

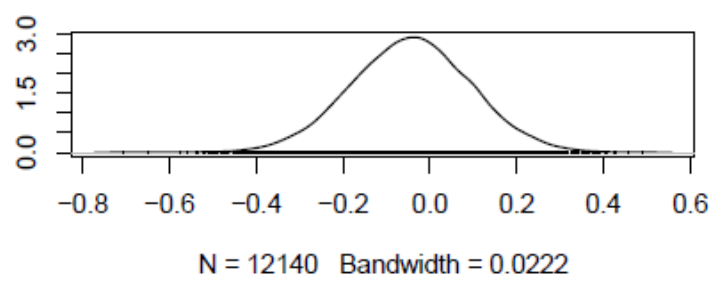

Density of time:where

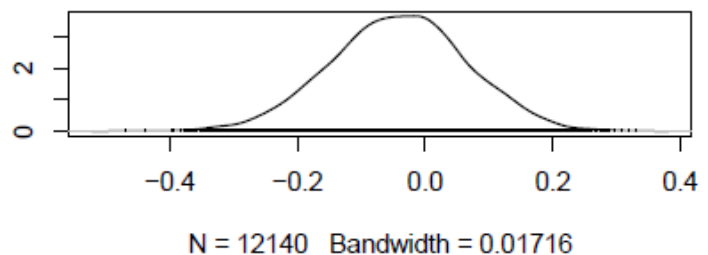

$\mathrm{N}=12140$ Bandwidth $=0.01716$ 
Trace of ageFirst_10:life

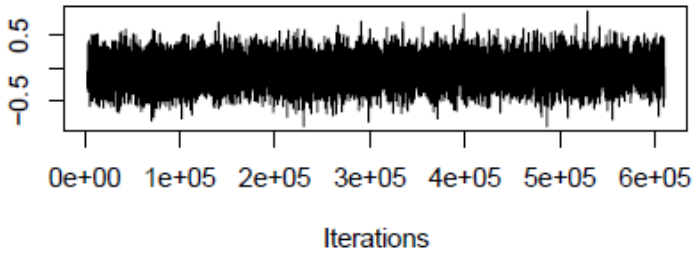

Trace of time:life

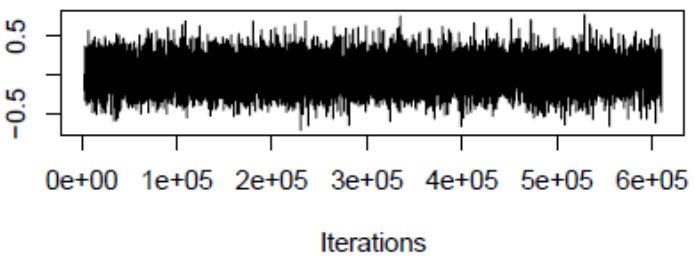

Trace of ageFirst_10:drugs

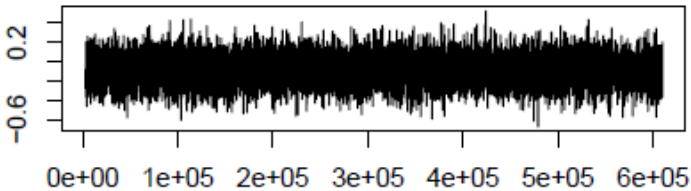

Iterations

\section{Trace of time:drugs}

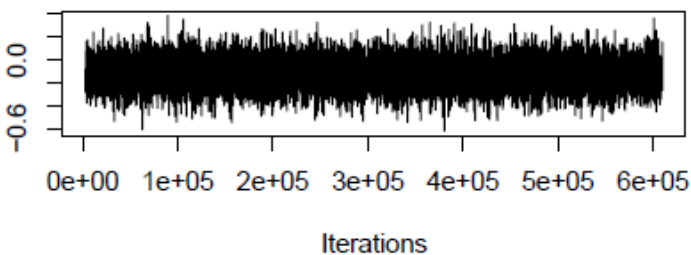

Trace of ageFirst_10:physical

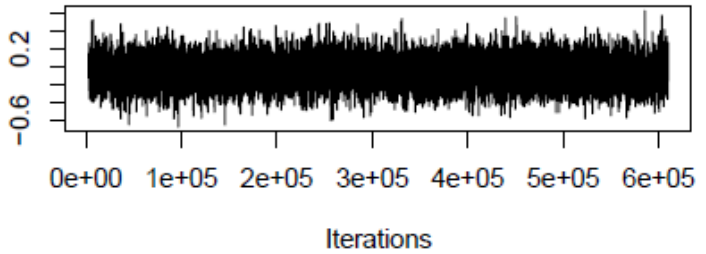

Trace of time:physical

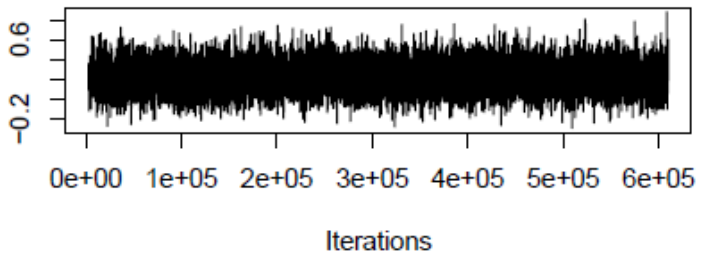

Density of ageFirst_10:life

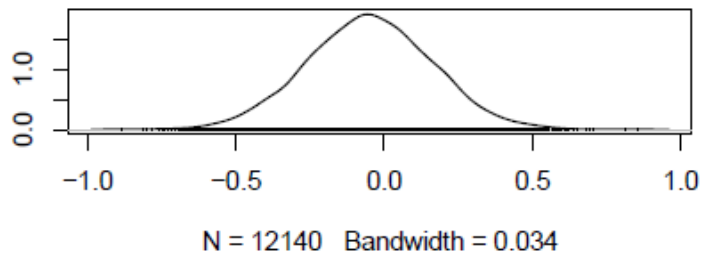

Density of time:life

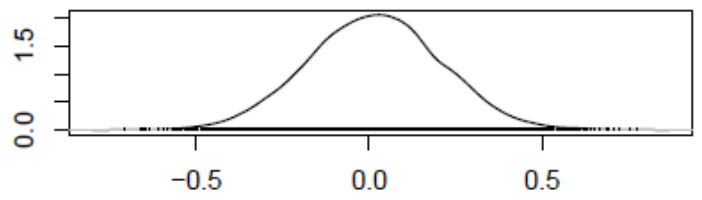

$\mathrm{N}=12140$ Bandwidth $=0.03106$

Density of ageFirst_10:drugs

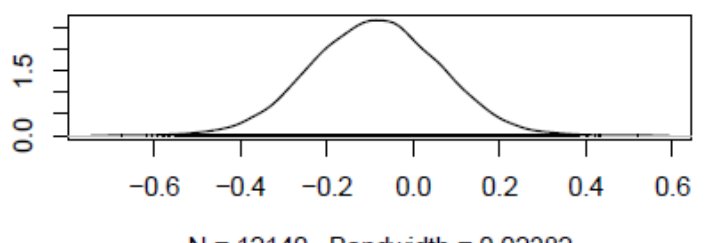

Density of time:drugs

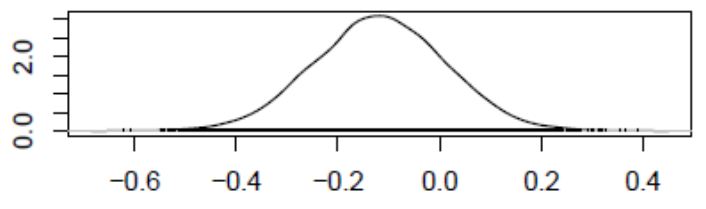

$\mathrm{N}=12140$ Bandwidth $=0.02076$

Density of ageFirst_10:physical

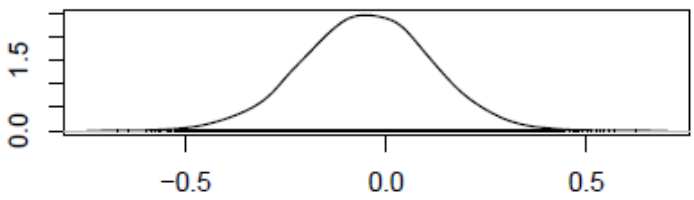

$\mathrm{N}=12140$ Bandwidth $=0.02574$

Density of time:physical

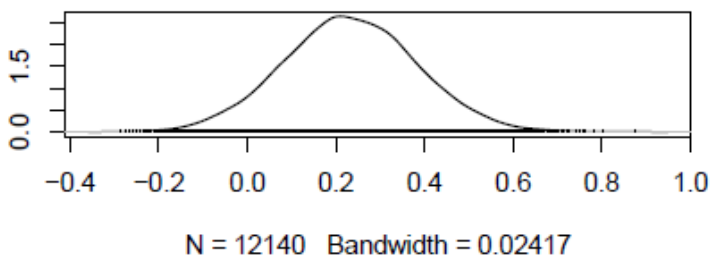


Trace of ageFirst_10:emotion

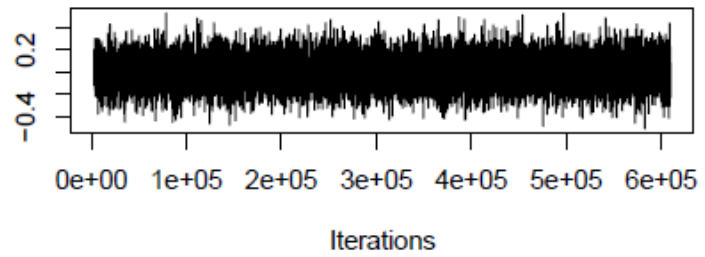

Trace of time:emotion

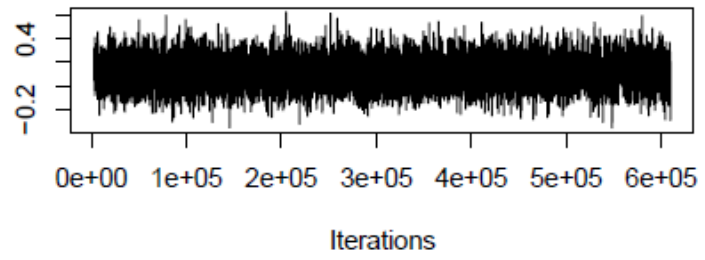

Trace of ageFirst_10:self

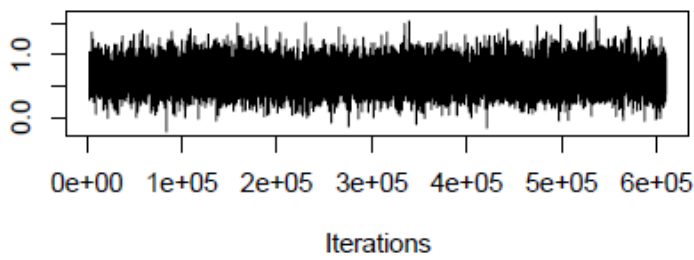

Trace of time:self

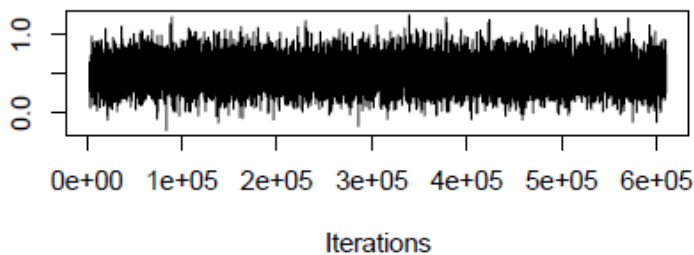

Trace of ageFirst_10:think

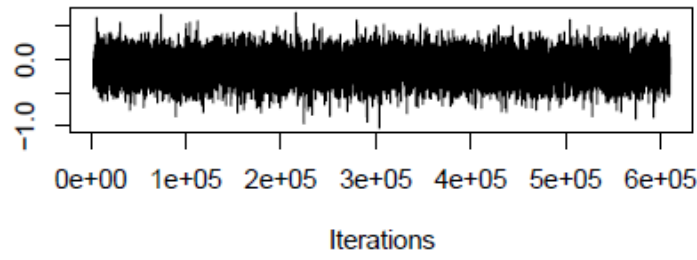

Trace of time:think

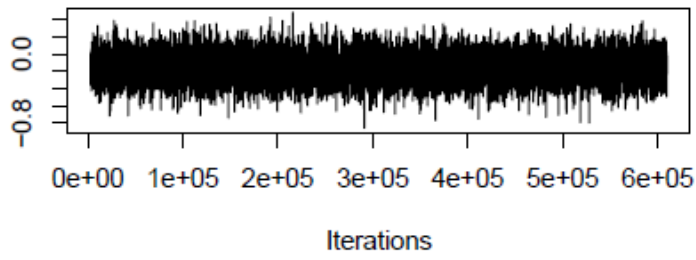

Density of ageFirst_10:emotion

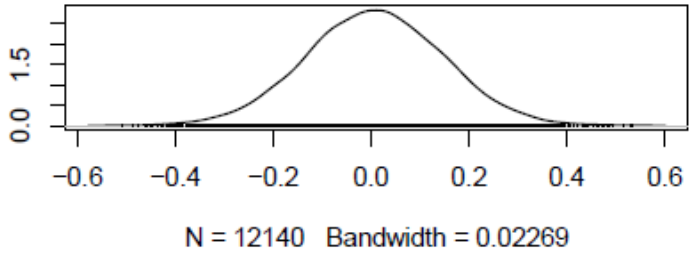

Density of time:emotion

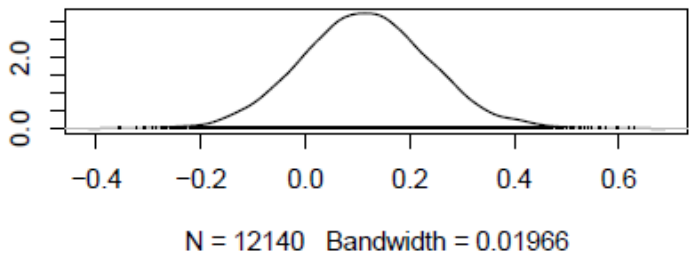

Density of ageFirst_10:self

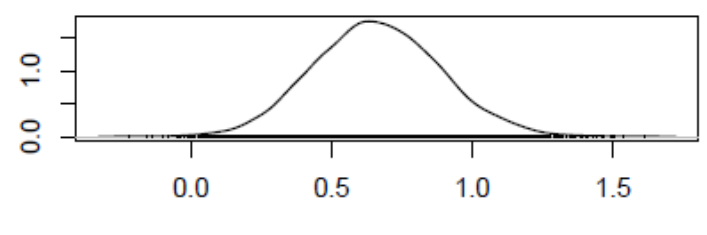

$\mathrm{N}=12140$ Bandwidth $=0.03637$

Density of time:self

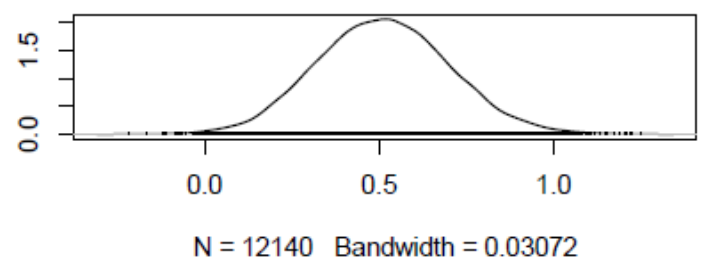

Density of ageFirst_10:think

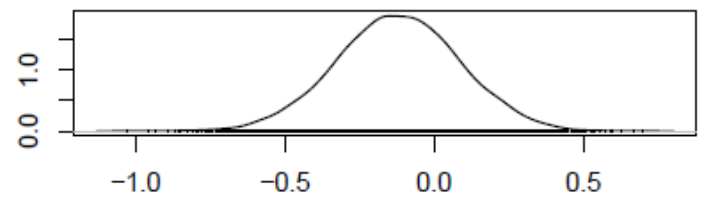

$\mathrm{N}=12140$ Bandwidth $=0.03349$

Density of time:think

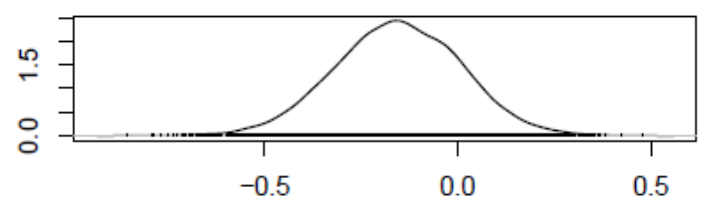

$\mathrm{N}=12140$ Bandwidth $=0.02646$ 
Trace of ageFirst_10:attitude

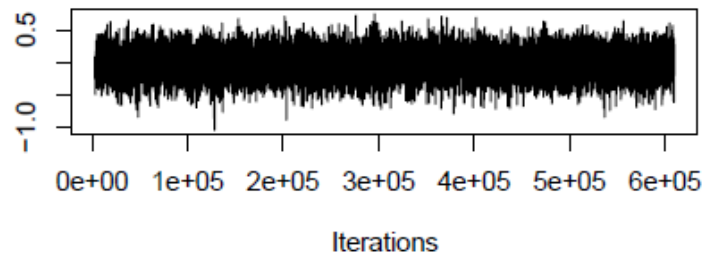

Trace of time:attitude

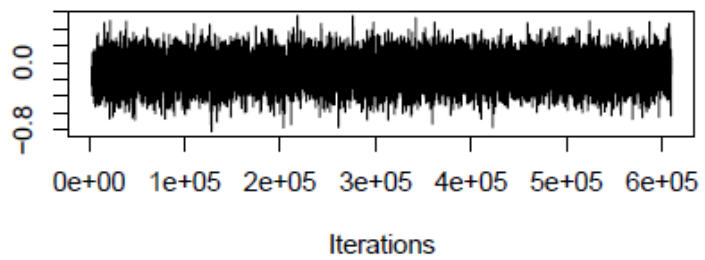

Trace of ageFirst_10:change

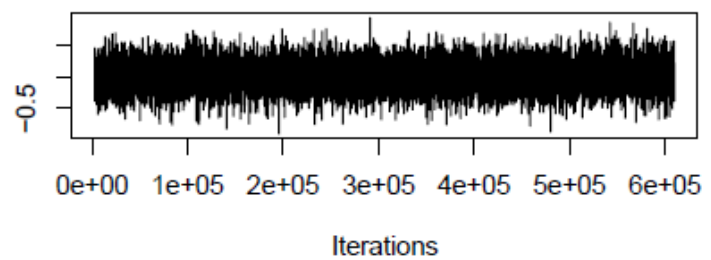

Trace of time:change

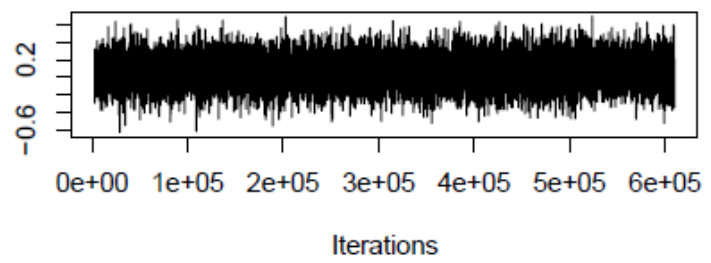

Trace of ageFirst_10:time:live

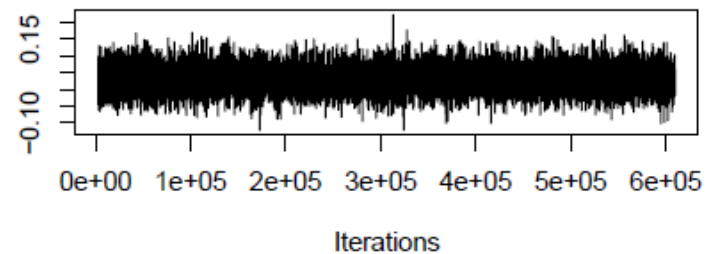

Trace of ageFirst_10:time:relation

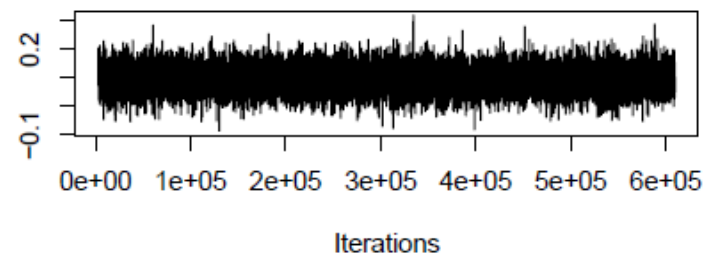

Density of ageFirst_10:attitude

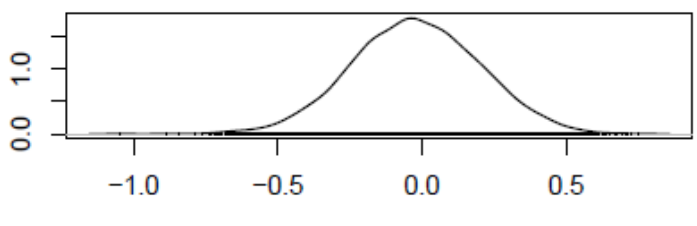

$\mathrm{N}=12140$ Bandwidth $=0.03585$

Density of time:attitude

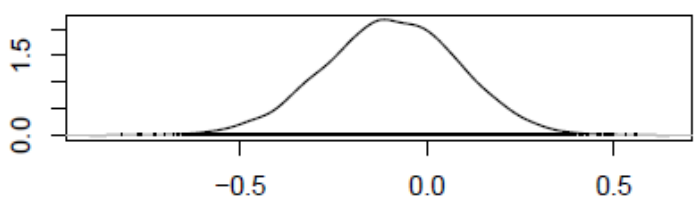

$\mathrm{N}=12140$ Bandwidth $=0.02912$

Density of ageFirst_10:change

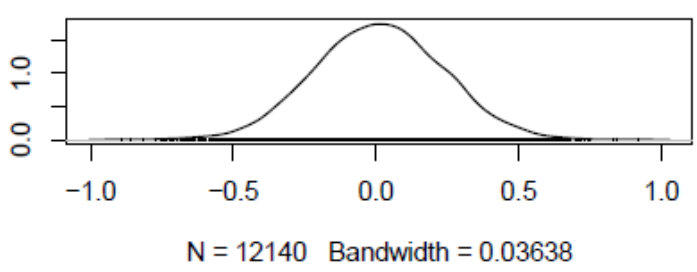

Density of time:change

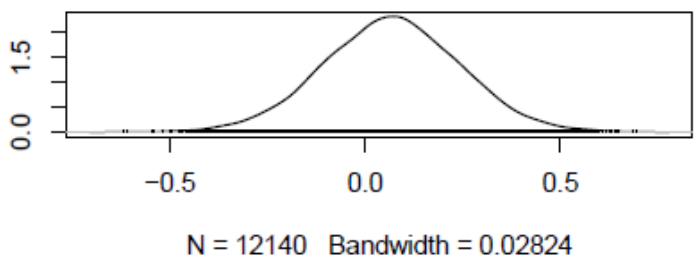

Density of ageFirst_10:time:live

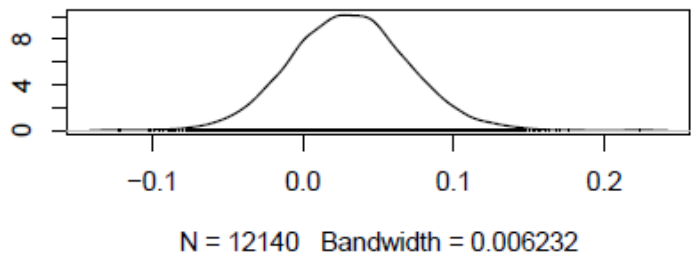

Density of ageFirst_10:time:relation

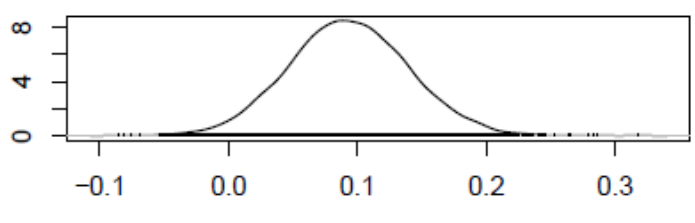

$\mathrm{N}=12140$ Bandwidth $=0.007474$ 
Trace of ageFirst_10:time:ete

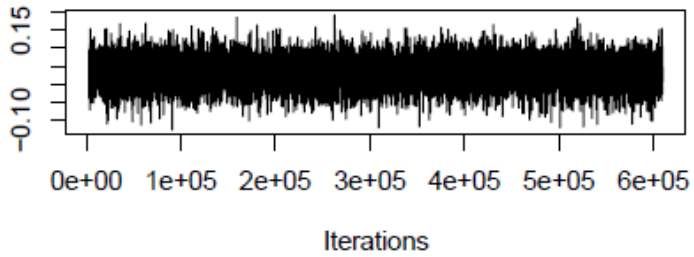

Trace of ageFirst_10:time:where

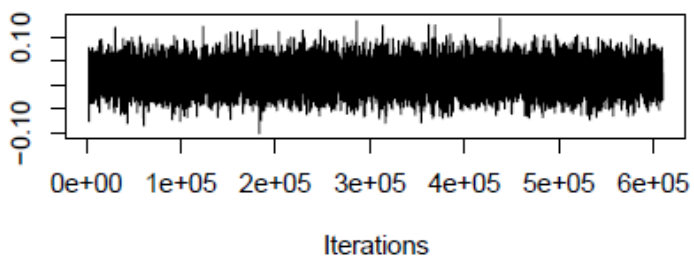

Trace of ageFirst_10:time:life

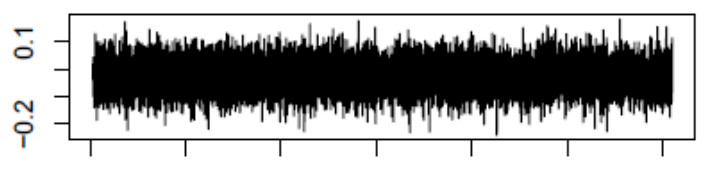

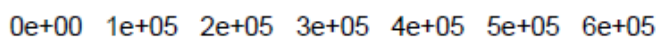
Iterations

Trace of ageFirst_10:time:drugs

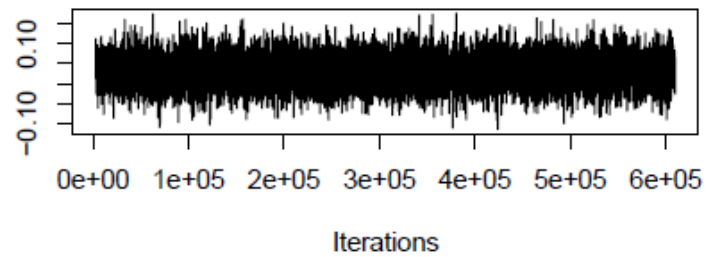

Trace of ageFirst_10:time:physical

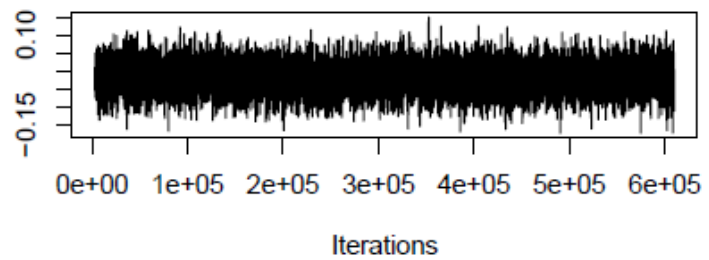

Trace of ageFirst_10:time:emotion

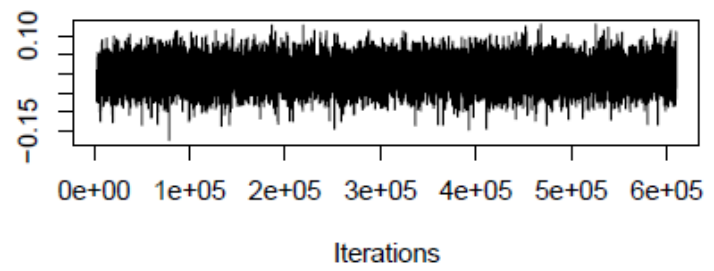

Density of ageFirst_10:time:ete

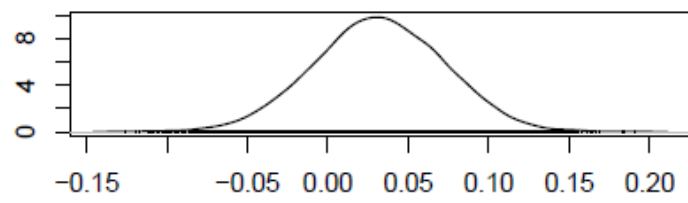

$\mathrm{N}=12140$ Bandwidth $=0.006566$

Density of ageFirst_10:time:where

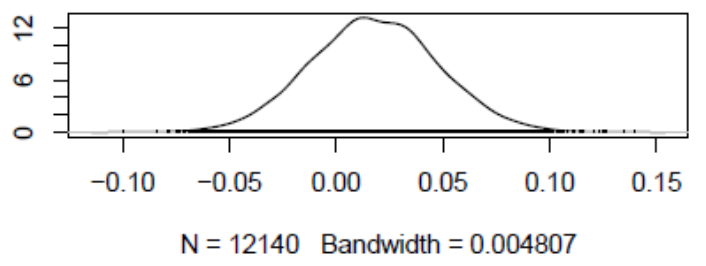

Density of ageFirst_10:time:life

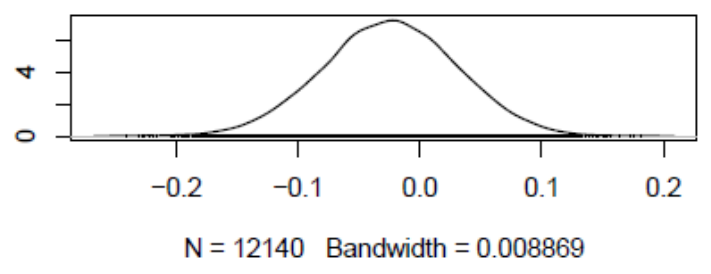

Density of ageFirst_10:time:drugs

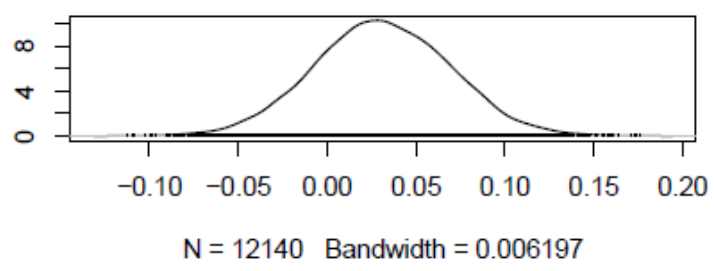

Density of ageFirst_10:time:physical

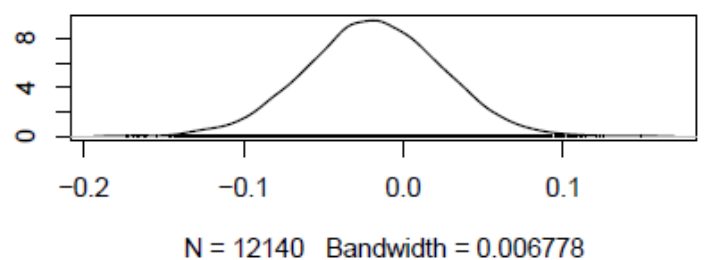

Density of ageFirst_10:time:emotion

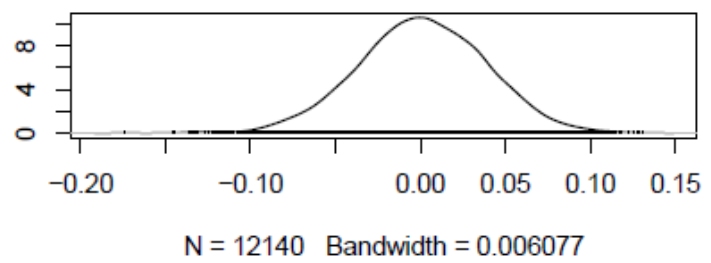


Trace of ageFirst_10:time:self

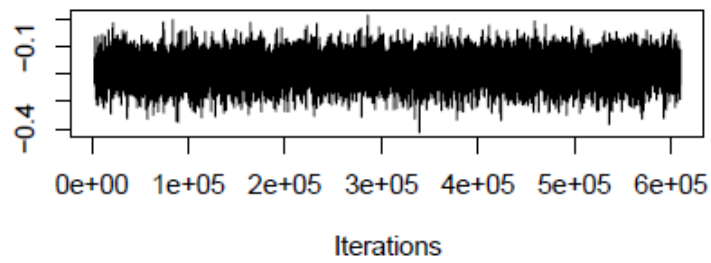

Trace of ageFirst_10:time:think

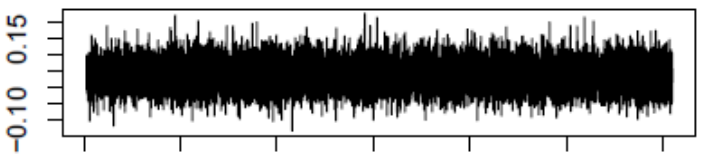

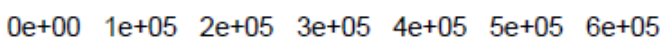
Iterations

Trace of ageFirst_10:time:attitude

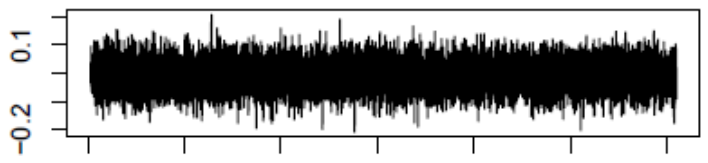

$\begin{array}{lllllll}0 e+00 & 1 e+05 & 2 e+05 & 3 e+05 & 4 e+05 & 5 e+05 & 6 e+05\end{array}$ Iterations

Trace of ageFirst_10:time:change

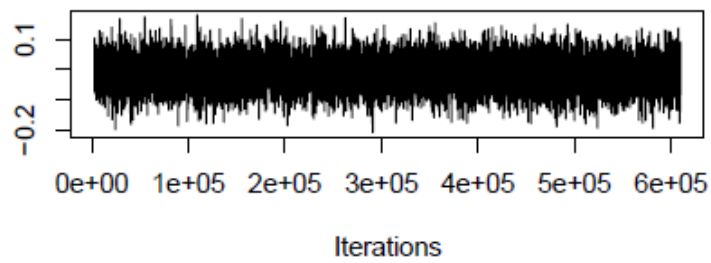

Density of ageFirst_10:time:self

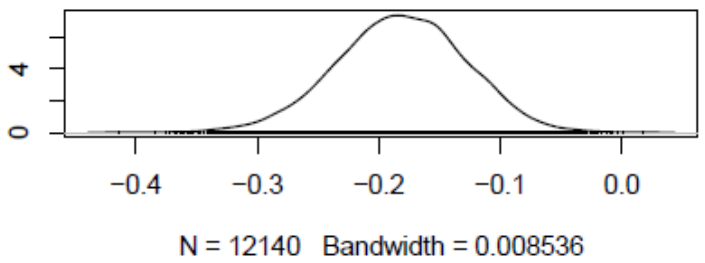

Density of ageFirst_10:time:think

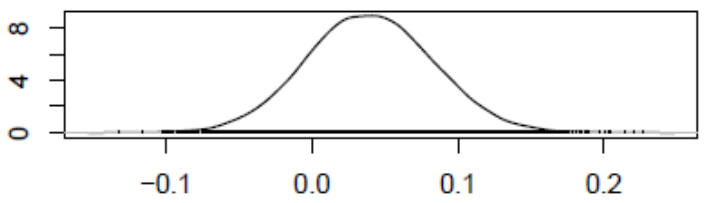

$\mathrm{N}=12140$ Bandwidth $=0.007037$

Density of ageFirst_10:time:attitude

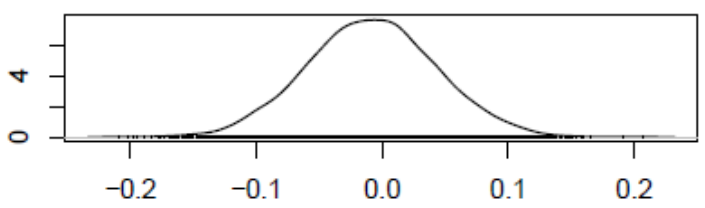

$\mathrm{N}=12140$ Bandwidth $=0.008276$

Density of ageFirst_10:time:change

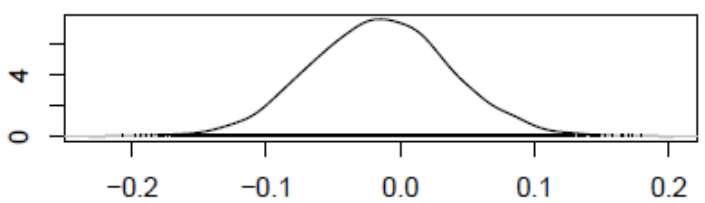

$\mathrm{N}=12140$ Bandwidth $=0.008324$ 
Trace of time

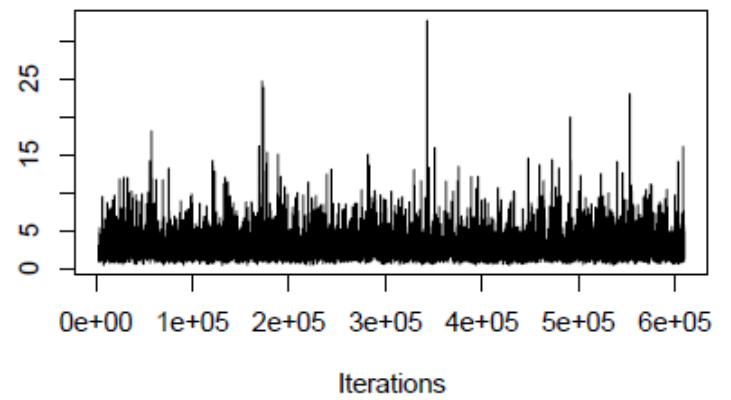

Trace of Research.ID

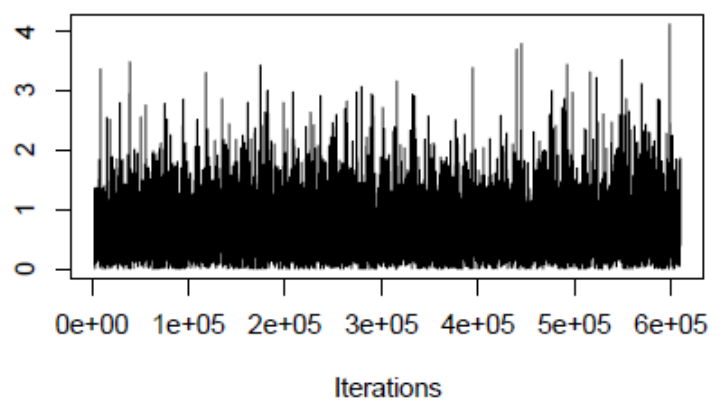

Density of time

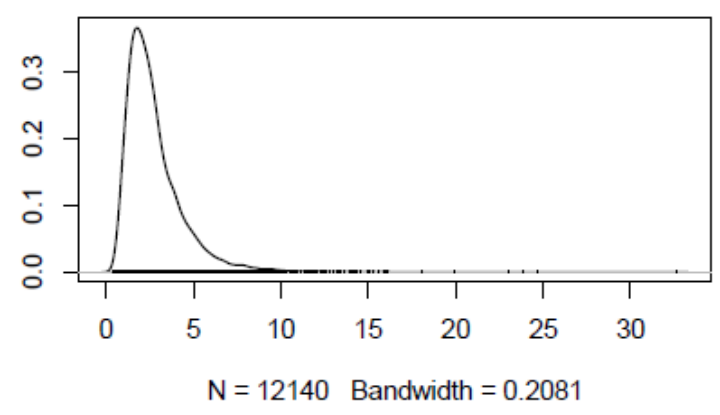

Density of Research.ID

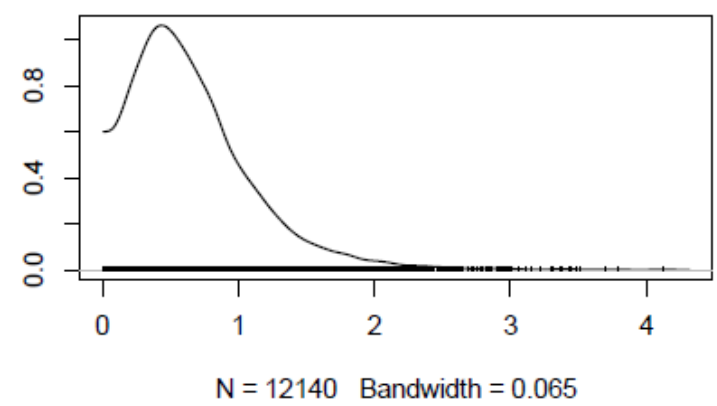


Model 1.11 - Basic Model + Grouped YJB Offence Category and YJB Gravity Score (Table 6.23)

Bayesian Model (Bm1G_o12a)

\section{\#\# Define the model}

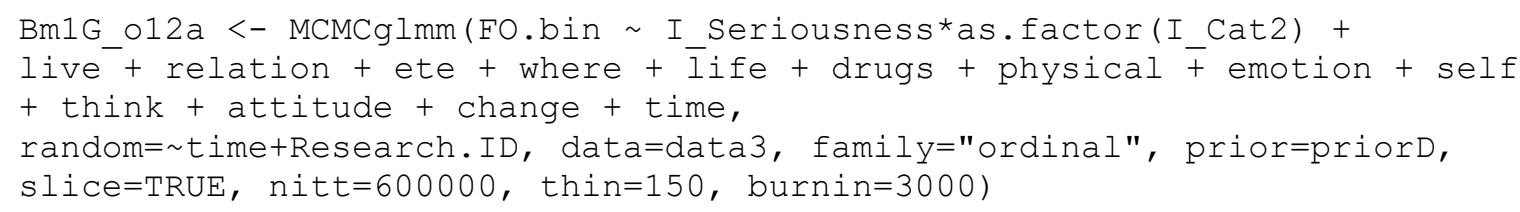

\section{\#\# Checks for suitable convergence}

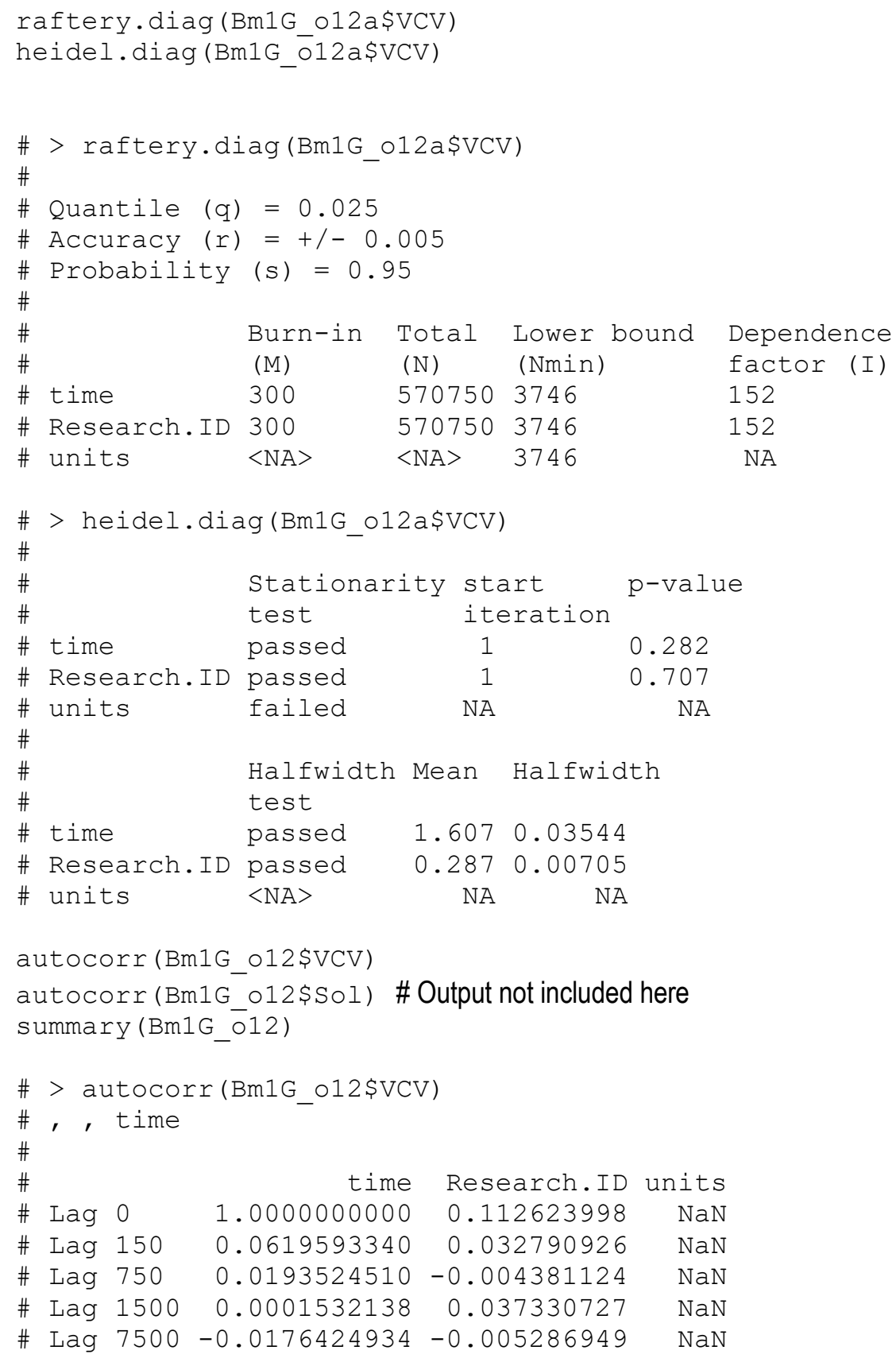




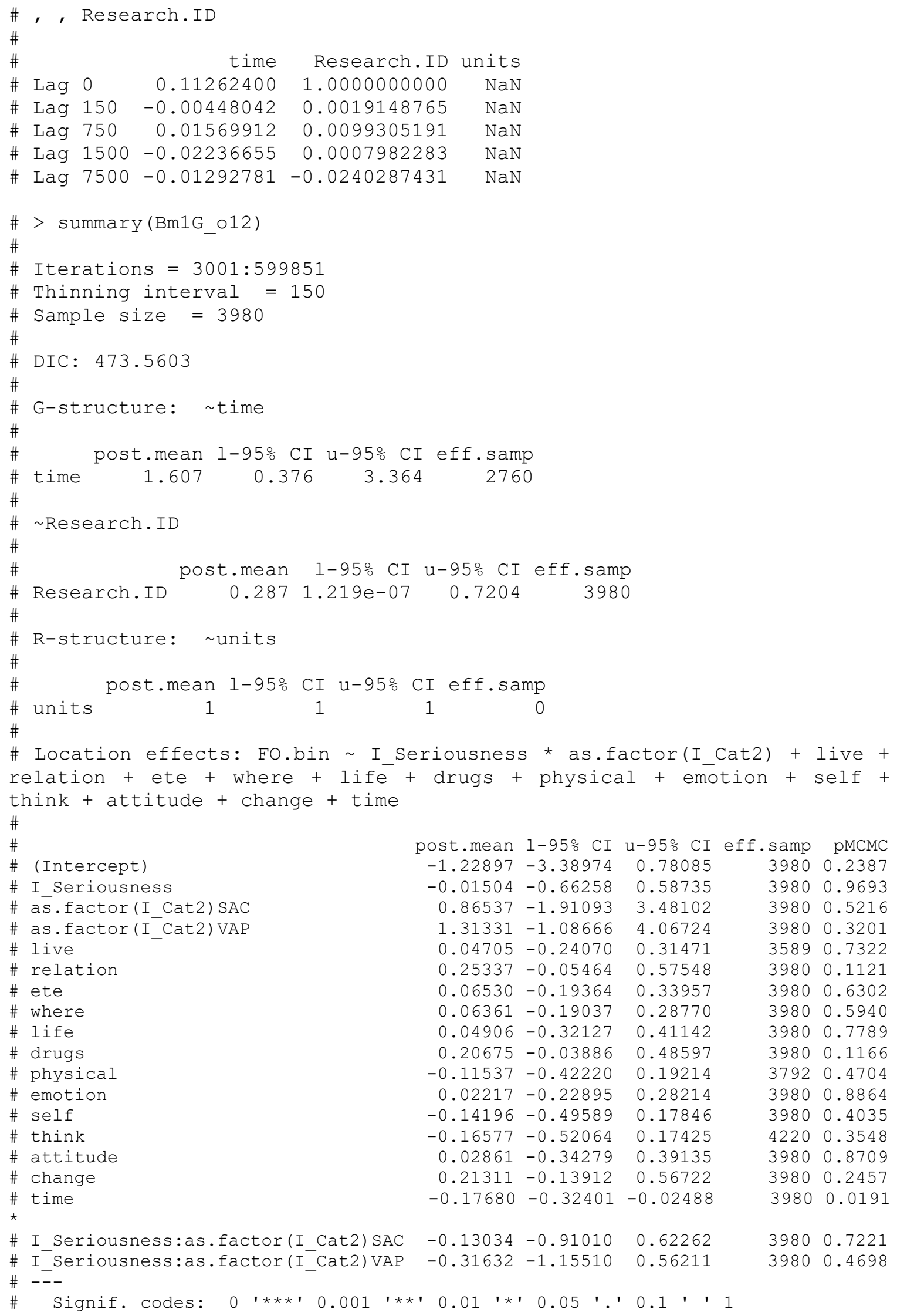

\begin{tabular}{|c|c|c|c|c|}
\hline nean & $1-95 \%$ & CI & $m p$ & \\
\hline-1.22897 & -3.38974 & 0.78085 & 3980 & 0.238 \\
\hline-0.01504 & -0.66258 & 0.58735 & 3980 & 0.969 \\
\hline 0.86537 & -1.91093 & 3.48102 & 3980 & 0.521 \\
\hline 1.31331 & -1.08666 & 4.06724 & 3980 & 0.320 \\
\hline 0.04705 & -0.24070 & 0.31471 & 3589 & 0.732 \\
\hline 0.25337 & -0.05464 & 0.57548 & 3980 & 0.112 \\
\hline 0.06530 & -0.19364 & 0.33957 & 3980 & 0.6302 \\
\hline 0.06361 & -0.19037 & 0.28770 & 3980 & 0.5940 \\
\hline 0.04906 & -0.32127 & 0.41142 & 3980 & 0.7789 \\
\hline 0.20675 & -0.03886 & 0.48597 & 3980 & 0.1166 \\
\hline-0.11537 & -0.42220 & 0.19214 & 3792 & 0.4704 \\
\hline 0.02217 & -0.22895 & 0.28214 & 3980 & 0.8864 \\
\hline-0.14196 & -0.49589 & 0.17846 & 3980 & 0.4035 \\
\hline-0.16577 & -0.52064 & 0.17425 & 4220 & $0.354 \varepsilon$ \\
\hline 0.02861 & -0.34279 & 0.39135 & 3980 & $0.870 s$ \\
\hline 0.21311 & -0.13912 & 0.56722 & 3980 & 0.2457 \\
\hline-0.17680 & -0.32401 & -0.02488 & 3980 & 0.019 \\
\hline-0.13034 & -0.91010 & 0.62262 & 3980 & 0.7 \\
\hline & -1.15510 & 0.56211 & 3980 & 0.469 \\
\hline
\end{tabular}


Frequentist Model (m1G_o12)

M1G_o12 <- glmer(FO.bin as.factor(offence 2$){ }^{*}$ serious + live + relātion + ete + where + life + drugs + physical + emotion + self + think + attitude + change + time + (timelIndividual), data=data3, family=binomial)

summary (m1G o12)

vcomps.icc (m1G_o12)

\section{Warning message:}

In checkConv(attr (opt, "derivs"), opt\$par, ctrl = control\$checkConv, : Model failed to converge with max $\mid$ gradl $=0.0838951$ (tol $=0.001$, comp onent 1)

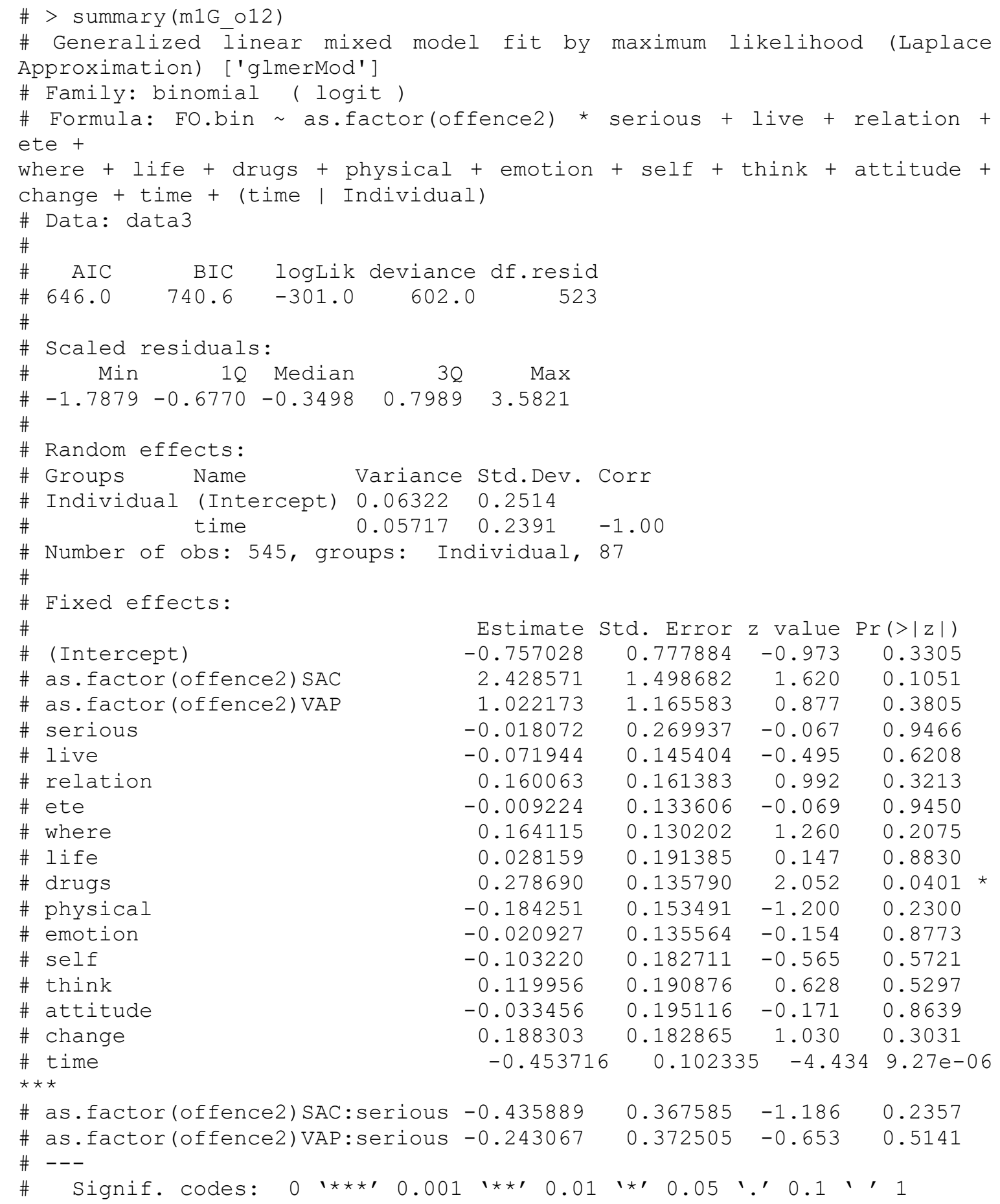

\begin{tabular}{|c|c|c|c|}
\hline Estimate & Std. Error & value & $\ln (>|z|)$ \\
\hline-0.757028 & 0.777884 & -0.973 & 0.3305 \\
\hline 2.428571 & 1.498682 & 1.620 & 0.1051 \\
\hline 1.022173 & 1.165583 & 0.877 & 0.3805 \\
\hline-0.018072 & 0.269937 & -0.067 & 0.9466 \\
\hline-0.071944 & 0.145404 & -0.495 & 0.6208 \\
\hline 0.160063 & 0.161383 & 0.992 & 0.3213 \\
\hline-0.009224 & 0.133606 & -0.069 & 0.9450 \\
\hline 0.164115 & 0.130202 & 1.260 & 0.2075 \\
\hline 0.028159 & 0.191385 & 0.147 & 0.8830 \\
\hline 0.278690 & 0.135790 & 2.052 & 0.0401 * \\
\hline-0.184251 & 0.153491 & -1.200 & 0.2300 \\
\hline-0.020927 & 0.135564 & -0.154 & 0.8773 \\
\hline-0.103220 & 0.182711 & -0.565 & 0.5721 \\
\hline 0.119956 & 0.190876 & 0.628 & 0.5297 \\
\hline-0.033456 & 0.195116 & -0.171 & 0.8639 \\
\hline $\begin{array}{l}0.188303 \\
-0.453716\end{array}$ & 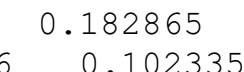 & $\begin{array}{l}1.030 \\
5 \quad-4.434\end{array}$ & $\begin{array}{l}0.3031 \\
9.27 e-06\end{array}$ \\
\hline-0.435889 & 0.367585 & -1.186 & 0.2357 \\
\hline-0.243067 & 0.372505 & -0.653 & 0.5141 \\
\hline
\end{tabular}




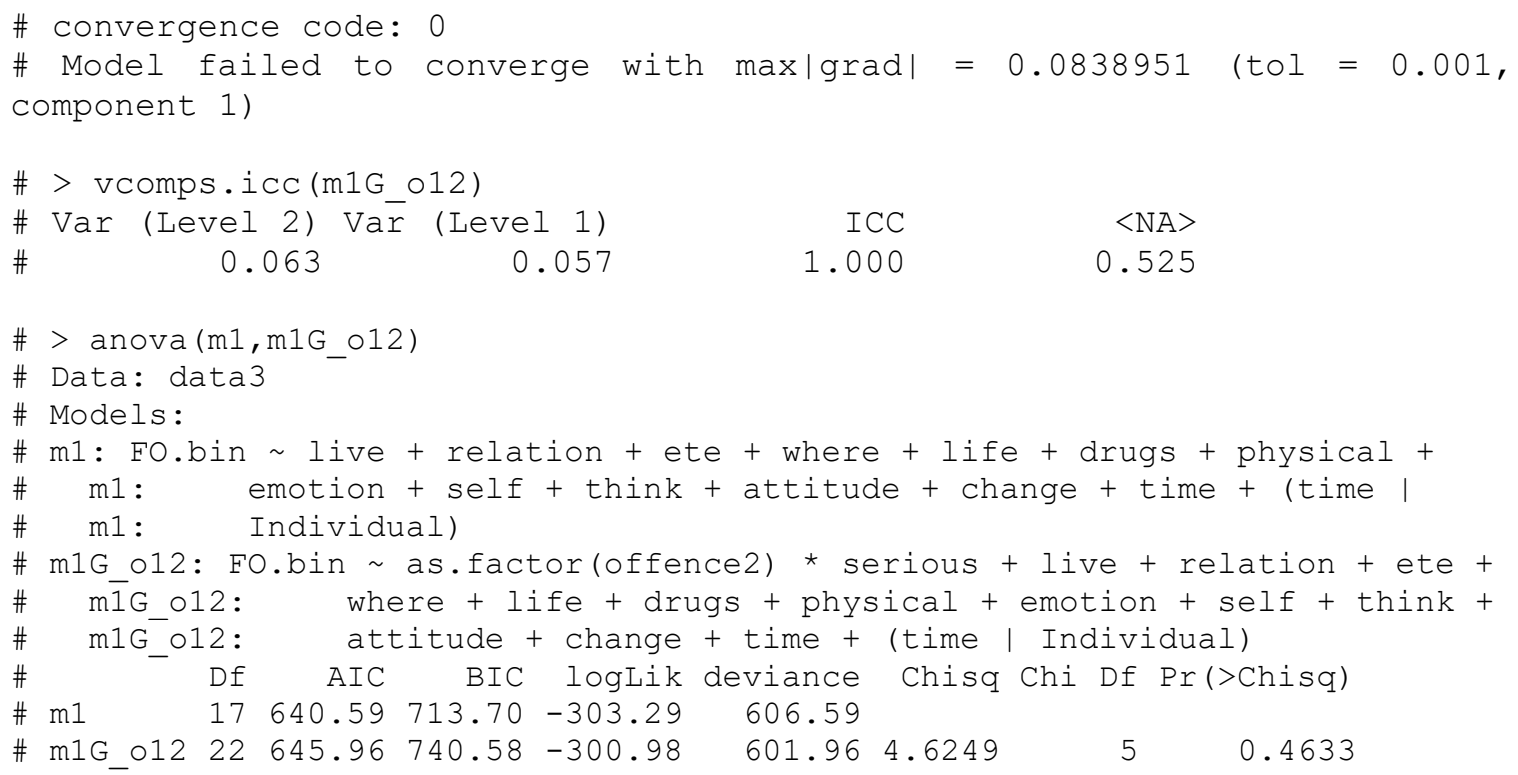




\section{Chapter Seven - System Contact}

Dynamic Model 4 (Table 7.1)

Bayesian Model (BDm4G_cc2_ch)

\section{\#\# Define the model}

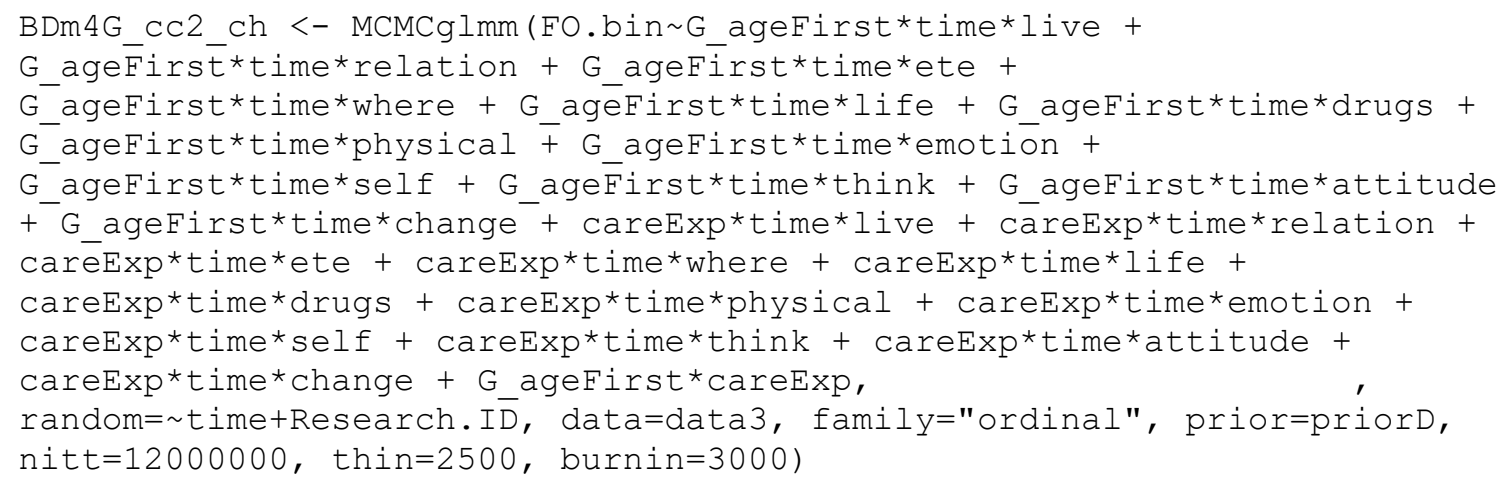

\section{\#\# Checks for suitable convergence}

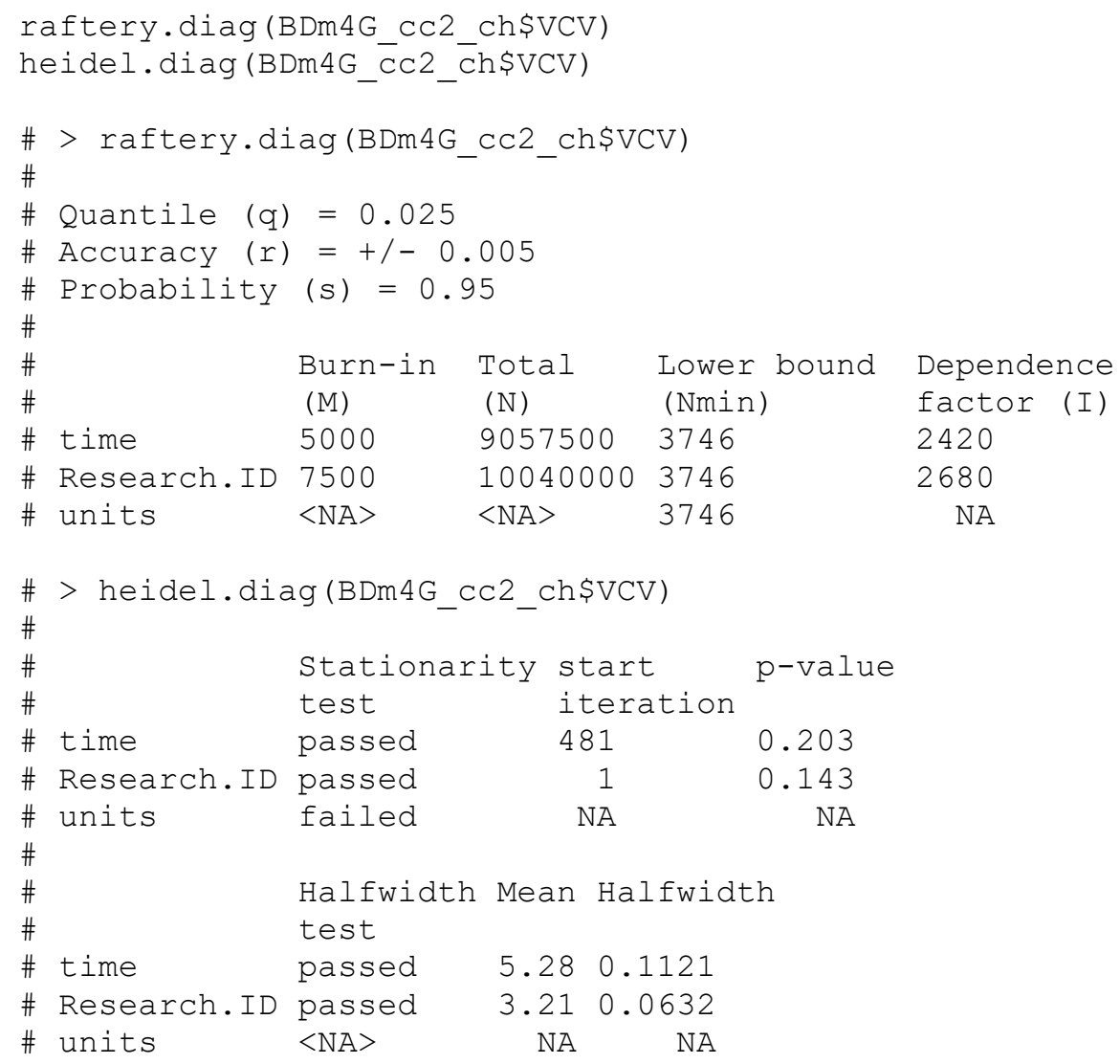




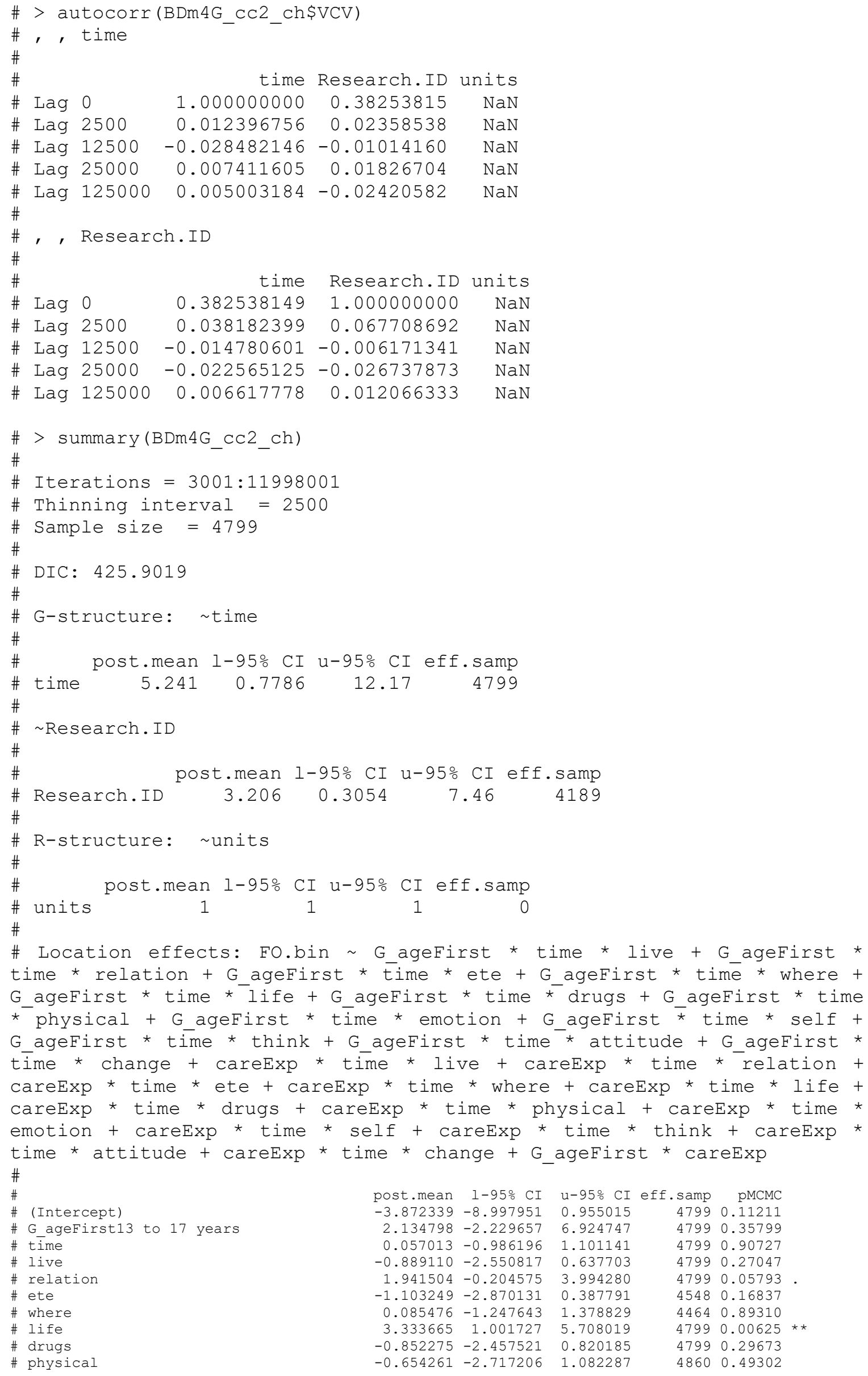

$\begin{array}{rrrrr}\text { post.mean } & 1-95 \% \text { CI } & \text { u-95\% CI eff.samp } & \text { pMCMC } \\ -3.872339 & -8.997951 & 0.955015 & 4799 & 0.11211 \\ 2.134798 & -2.229657 & 6.924747 & 4799 & 0.35799 \\ 0.057013 & -0.986196 & 1.101141 & 4799 & 0.90727 \\ -0.889110 & -2.550817 & 0.637703 & 4799 & 0.27047 \\ 1.941504 & -0.204575 & 3.994280 & 4799 & 0.05793 \\ -1.103249 & -2.870131 & 0.387791 & 4548 & 0.16837 \\ 0.085476 & -1.247643 & 1.378829 & 4464 & 0.89310 \\ 3.333665 & 1.001727 & 5.708019 & 4799 & 0.00625\end{array}$ **


\# emotion

\# self

\# think

\# attitude

\# change

\# careExp

\# G ageFirst13 to 17 years:time

\# G ageFirst13 to 17 years:live

\# time:live

\# G ageFirst13 to 17 years:relation

\# time:relation

\# G_ageFirst13 to 17 years:ete

\# time:ete

\# G ageFirst13 to 17 years:where

\# time:where

\# G_ageFirst13 to 17 years:life

\# time:life

\# G ageFirst13 to 17 years:drugs

\# tìme:drugs

\# G_ageFirst13 to 17 years:physical

\# time:physical

\# G ageFirst13 to 17 years:emotion

\# time:emotion

\# G ageFirst13 to 17 years:self

\# time:self

\# G_ageFirst13 to 17 years:think

\# time:think

\# G ageFirst13 to 17 years:attitude

\# time:attitude

\# G_ageFirst13 to 17 years:change

\# time: change

\# time:careExp

\# live:careExp

\# relation:careExp

\# ete:careExp

\# where:careExp

\# life:careExp

\# drugs:careExp

\# physical:careExp

\# emotion:careExp

\# self:careExp

\# think:careExp

\# attitude:careExp

\# change: careExp

\# G ageFirst13 to 17 years:careExp

\# G-ageFirst13 to 17 years:time:live

\# G_ageFirst13 to 17 years:time:relation

\# G ageFirst13 to 17 years:time:ete

\# G ageFirst13 to 17 years:time:where

\# G_ageFirst13 to 17 years:time:life

\# G_ageFirst13 to 17 years:time:drugs

\# G ageFirst13 to 17 years:time:physical

\# G ageFirst13 to 17 years:time:emotion

\# G_ageFirst13 to 17 years:time:self

\# G ageFirst13 to 17 years:time:think

\# G-ageFirst13 to 17 years:time:attitude

\# G_ageFirst13 to 17 years:time:change

\# time:live:careExp

\# time:relation:careExp

\# time:ete:careExp

\# time: where:careExp

* time:life:careExp

\# time:drugs:careExp

\# time:physical:careExp

\# time:emotion:careExp

\# time:self:careExp

\# time:think:careExp

\# time:attitude: careExp

time: change: careExp

\# ---

\# $\begin{array}{lll}-1.227014 & -2.673822 & 0.252411\end{array}$

$\begin{array}{llll}-5.759757 & -8.832532 & -2.634739\end{array}$

$\begin{array}{lll}1.797935 & -0.471976 & 4.183234\end{array}$

$\begin{array}{lll}3.033604 & 0.211281 & 5.908489\end{array}$

$\begin{array}{llll}0.467332 & -1.929188 & 2.911647\end{array}$

$-0.228025-5.271780 \quad 5.155326$

$\begin{array}{lll}-0.263512 & -1.194770 & 0.725694\end{array}$

$\begin{array}{llll}0.631514 & -1.052351 & 2.535050\end{array}$

$\begin{array}{lll}-0.239882 & -0.624241 & 0.127367\end{array}$

$\begin{array}{llll}-1.886166 & -4.096080 & 0.329907\end{array}$

$\begin{array}{llll}-0.411429 & -0.862590 & 0.057267\end{array}$

$0.947751-0.688462 \quad 2.781769$

$\begin{array}{lll}0.185148 & -0.148967 & 0.511745\end{array}$

$\begin{array}{lll}-0.084367 & -1.548711 & 1.334312\end{array}$

$\begin{array}{llll}-0.230428 & -0.504518 & 0.078273\end{array}$

$\begin{array}{llll}-2.566938 & -5.159708 & 0.005613\end{array}$

$-0.621558-1.171776-0.100854$

$\begin{array}{lll}1.200262 & -0.500819 & 2.914296\end{array}$

$\begin{array}{lll}0.479659 & 0.117203 & 0.837792\end{array}$

$\begin{array}{lll}0.654892 & -1.418091 & 2.663817\end{array}$

$0.314691-0.228012 \quad 0.821034$

$\begin{array}{lll}-0.204765 & -1.844180 & 1.463089\end{array}$

$\begin{array}{lll}0.396577 & 0.070341 & 0.720360\end{array}$

$\begin{array}{llll}6.720708 & 3.484180 & 10.099387\end{array}$

$\begin{array}{lll}1.497034 & 0.916763 & 2.226787\end{array}$

$\begin{array}{lll}-1.033804 & -3.532620 & 1.418097\end{array}$

$\begin{array}{lll}-0.437461 & -0.925407 & 0.012309\end{array}$

$\begin{array}{llll}-2.351456 & -5.354870 & 0.683452\end{array}$

$-0.952729-1.518737-0.418043$

$\begin{array}{lll}-1.599914 & -4.253427 & 1.068217\end{array}$

$\begin{array}{llll}0.288514 & -0.280780 & 0.807395\end{array}$

$\begin{array}{lll}-0.074282 & -1.013078 & 0.758561\end{array}$

$\begin{array}{lll}1.024483 & -0.689049 & 2.680836\end{array}$

$\begin{array}{lll}-0.405719 & -2.258436 & 1.661162\end{array}$

$\begin{array}{lll}0.053004 & -1.458192 & 1.546695\end{array}$

$\begin{array}{lll}0.434813-0.985248 & 1.842742\end{array}$

$\begin{array}{lll}-1.018860 & -3.178350 & 1.297538\end{array}$

$\begin{array}{lll}0.140209 & -1.247198 & 1.583807\end{array}$

$\begin{array}{lll}-1.778954 & -3.693988 & 0.284462\end{array}$

$\begin{array}{llll}1.582138 & -0.022036 & 3.174495\end{array}$

$\begin{array}{lll}1.492924 & -0.856122 & 3.921866\end{array}$

$\begin{array}{lll}-1.937133 & -4.050339 & 0.118227\end{array}$

$\begin{array}{lll}-0.277645 & -2.230124 & 1.610904\end{array}$

$\begin{array}{lll}0.460460 & -1.800147 & 2.657094\end{array}$

$\begin{array}{llll}2.550140 & -0.513954 & 5.636276\end{array}$

$\begin{array}{llll}0.324585 & -0.114959 & 0.783763\end{array}$

$\begin{array}{lll}0.374828 & -0.139287 & 0.892689\end{array}$

$\begin{array}{lll}-0.217400 & -0.611978 & 0.161938\end{array}$

$\begin{array}{lll}0.136712 & -0.163324 & 0.441435\end{array}$

$\begin{array}{llll}0.490657 & -0.090900 & 1.056123\end{array}$

$-0.390639-0.772823-0.024585$

$\begin{array}{lll}-0.435456 & -0.979248 & 0.039765\end{array}$

$\begin{array}{llll}-0.159673 & -0.558997 & 0.217374\end{array}$

$-1.643284-2.358392-0.984504$

$\begin{array}{llll}0.326859 & -0.181477 & 0.829892\end{array}$

$0.570583-0.067273-1.216288$

$\begin{array}{llll}0.164517 & -0.407990 & 0.739970\end{array}$

$\begin{array}{llll}0.019327 & -0.373352 & 0.442100\end{array}$

$0.009034-0.450543-0.424516$

$\begin{array}{llll}0.163132 & -0.143513 & 0.498032\end{array}$

$\begin{array}{llll}0.198704 & -0.110234 & 0.468676\end{array}$

$\begin{array}{llll}0.021968 & -0.430367 & 0.434656\end{array}$

$\begin{array}{llll}-0.169615 & -0.533720 & 0.156080\end{array}$

$\begin{array}{llll}0.165546 & -0.301081 & 0.617280\end{array}$

$\begin{array}{lll}-0.154553 & -0.525639 & 0.192321\end{array}$

$-0.546273-1.018585-0.069081$

$\begin{array}{lll}0.451540 & 0.002091 & 0.899078\end{array}$

$\begin{array}{lll}0.293294 & -0.196383 & 0.801338\end{array}$

$\begin{array}{lll}-0.389711 & -0.861326 & 0.090797\end{array}$
47990.09794

$4799<2 e-04 * * *$

47990.13086

47990.03251 *

47990.70890

$4917 \quad 0.93478$

47990.58721

$4799 \quad 0.48385$

47990.21088

47990.08418 .

47990.07001

$\begin{array}{lll}4799 & 0.27047\end{array}$

52210.26714

47990.91186

47990.12003

47990.04543 *

43640.02626 *

47990.16295

$47990.00750 * \star$

$4878 \quad 0.51386$

$4799 \quad 0.21880$

$4577 \quad 0.81517$

47990.01167 *

$4799<2 e-04 * \star *$

$4799<2 e-04 * \star *$

47990.42009

47990.06210 .

47990.12961

$4799<2 e-04 * \star *$

47990.23463

47990.30090

$4799 \quad 0.87101$

$4799 \quad 0.22796$

47990.69973

$4799 \quad 0.95020$

$4799 \quad 0.55470$

47990.36383

$4799 \quad 0.84684$

47990.06543 .

47990.04668 *

47990.21338

47990.06543 .

$4799 \quad 0.77433$

$4799 \quad 0.67097$

$4799 \quad 0.08377$.

44620.14295

47990.14128

$\begin{array}{lll}5218 & 0.26297\end{array}$

$4799 \quad 0.37675$

$4799 \quad 0.10294$

47990.03542 *

47990.08043 .

47990.42259

$4799<2 e-04$ ***

47990.20129

47990.07418 .

$4504 \quad 0.58846$

47990.93811

47990.97020

$4799 \quad 0.31840$

$4799 \quad 0.17337$

47990.91269

$4799 \quad 0.32632$

47990.48718

47990.39800

47990.01750 *

47990.04668 *

47990.24088

47990.10210 
Trace Plots and Posterior Density Plots

Fixed Effects
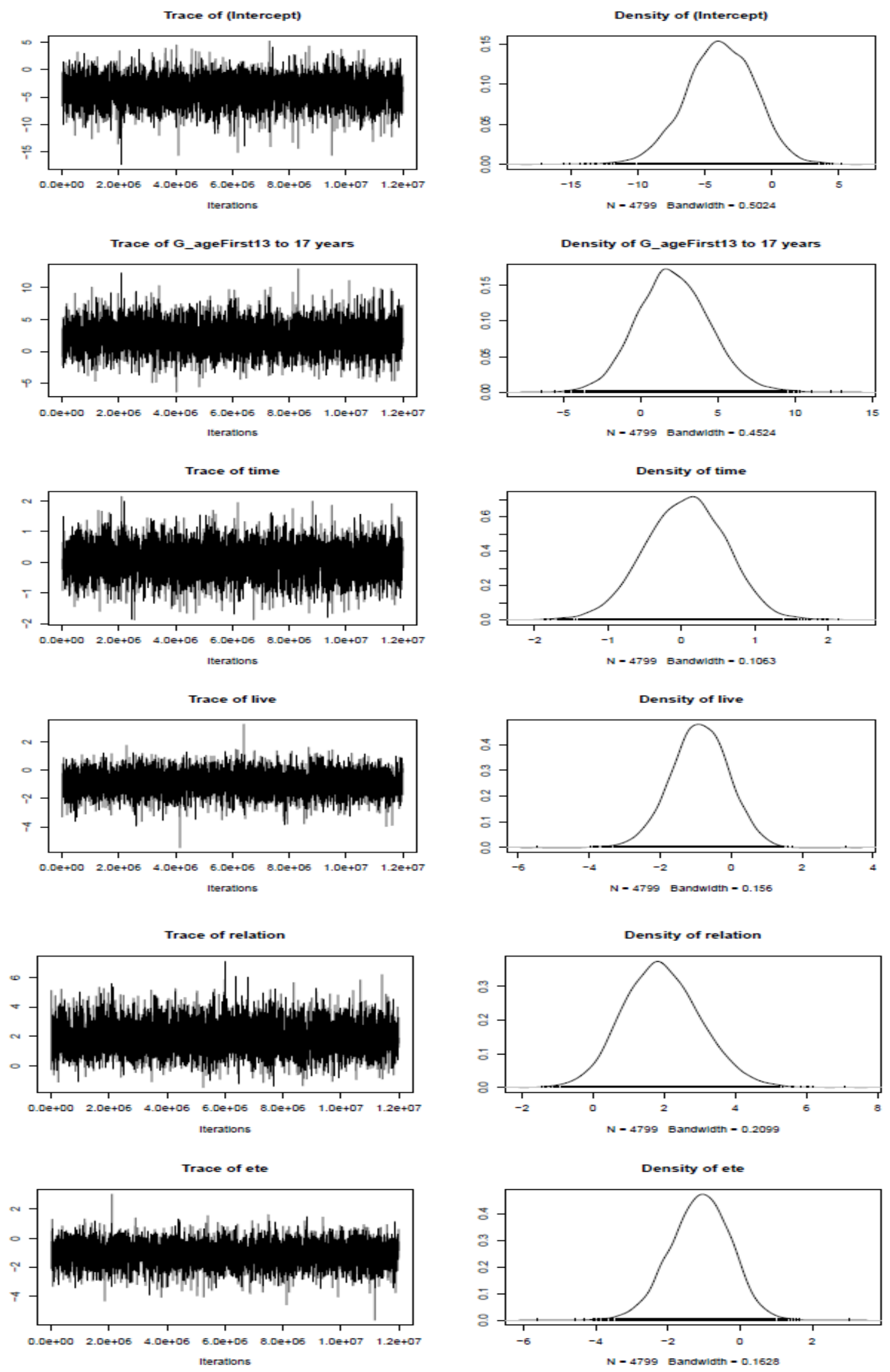
Trace of where

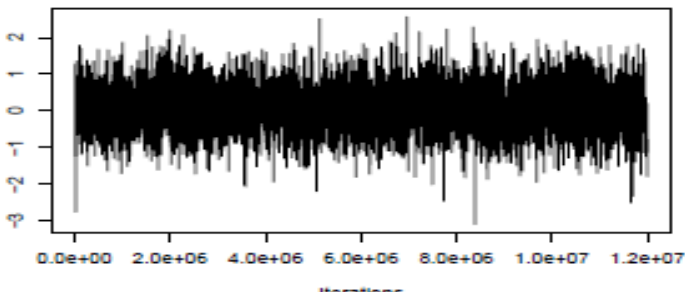

Iterations
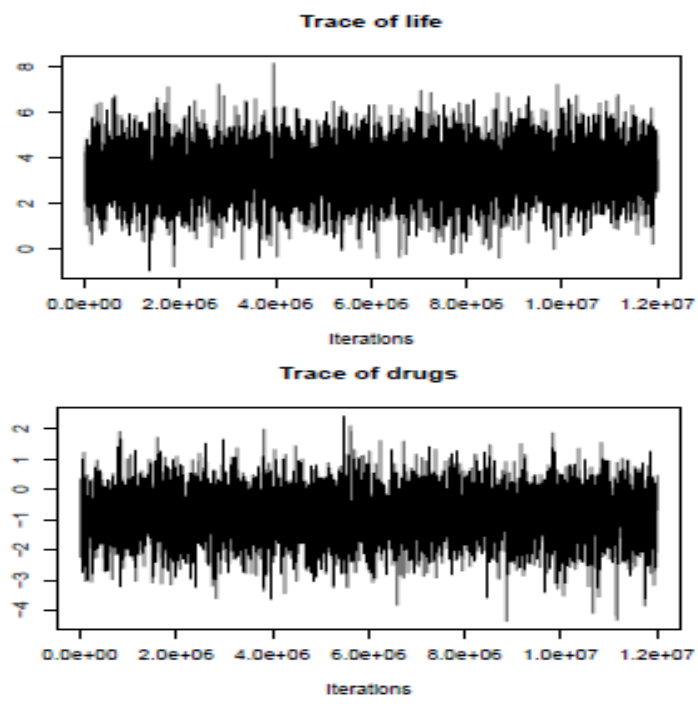

Trace of physical

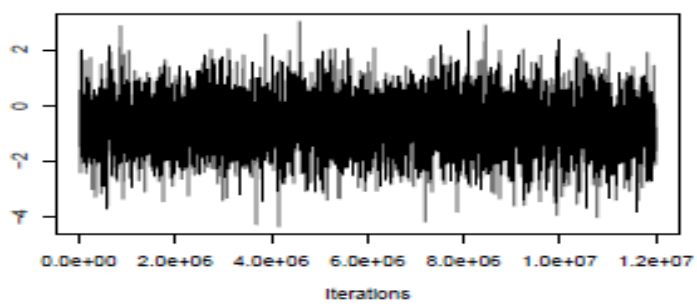

ace of emotion

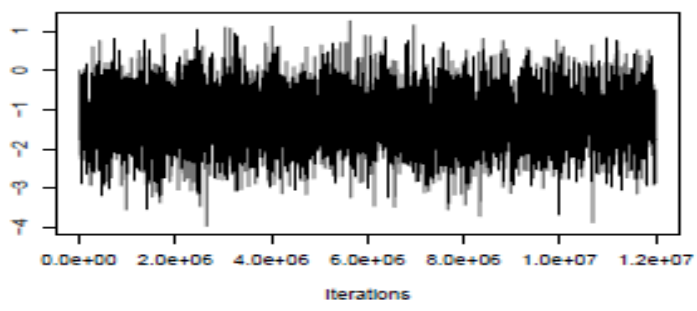

Trace of self

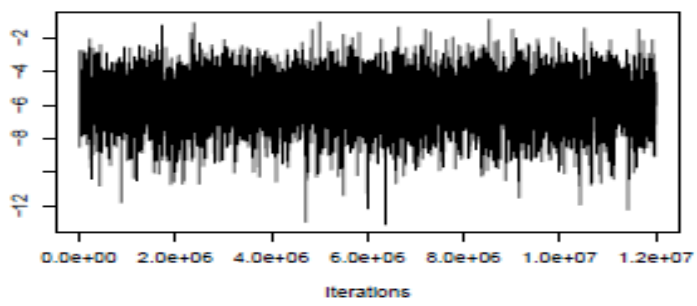

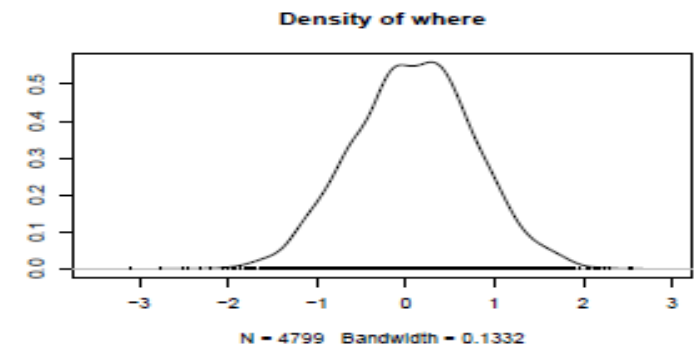
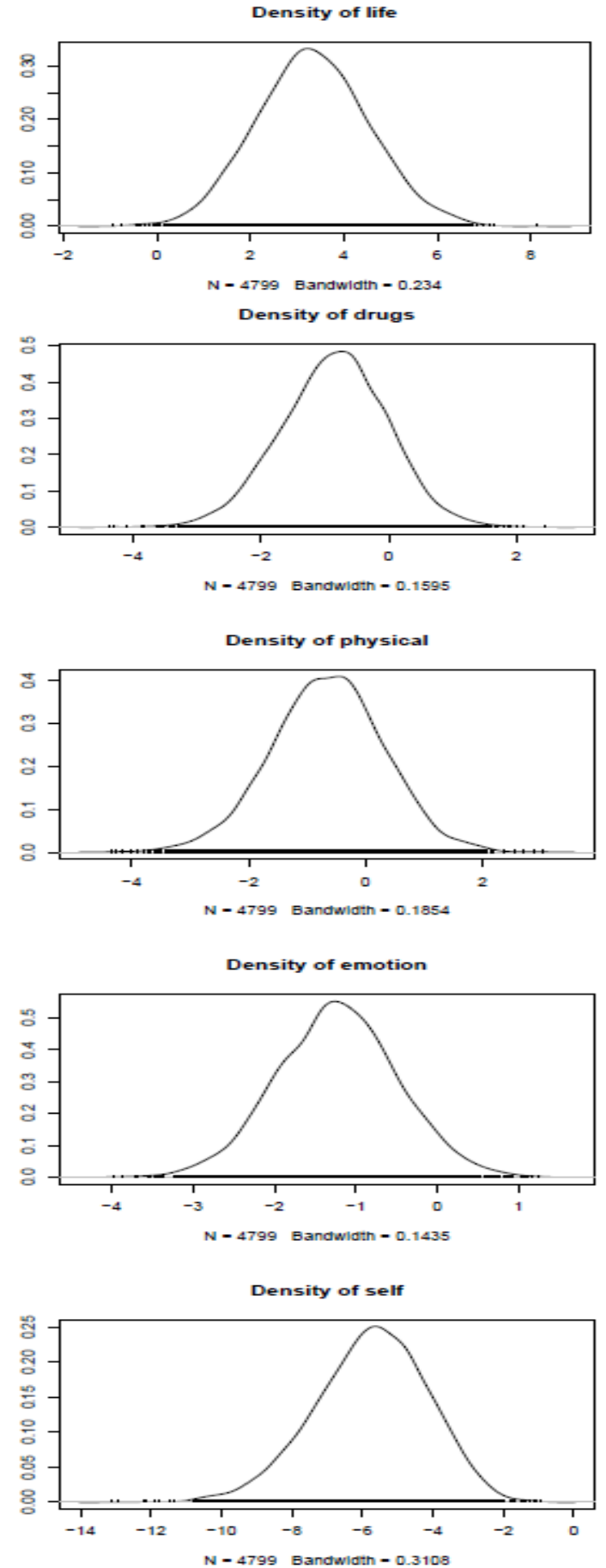

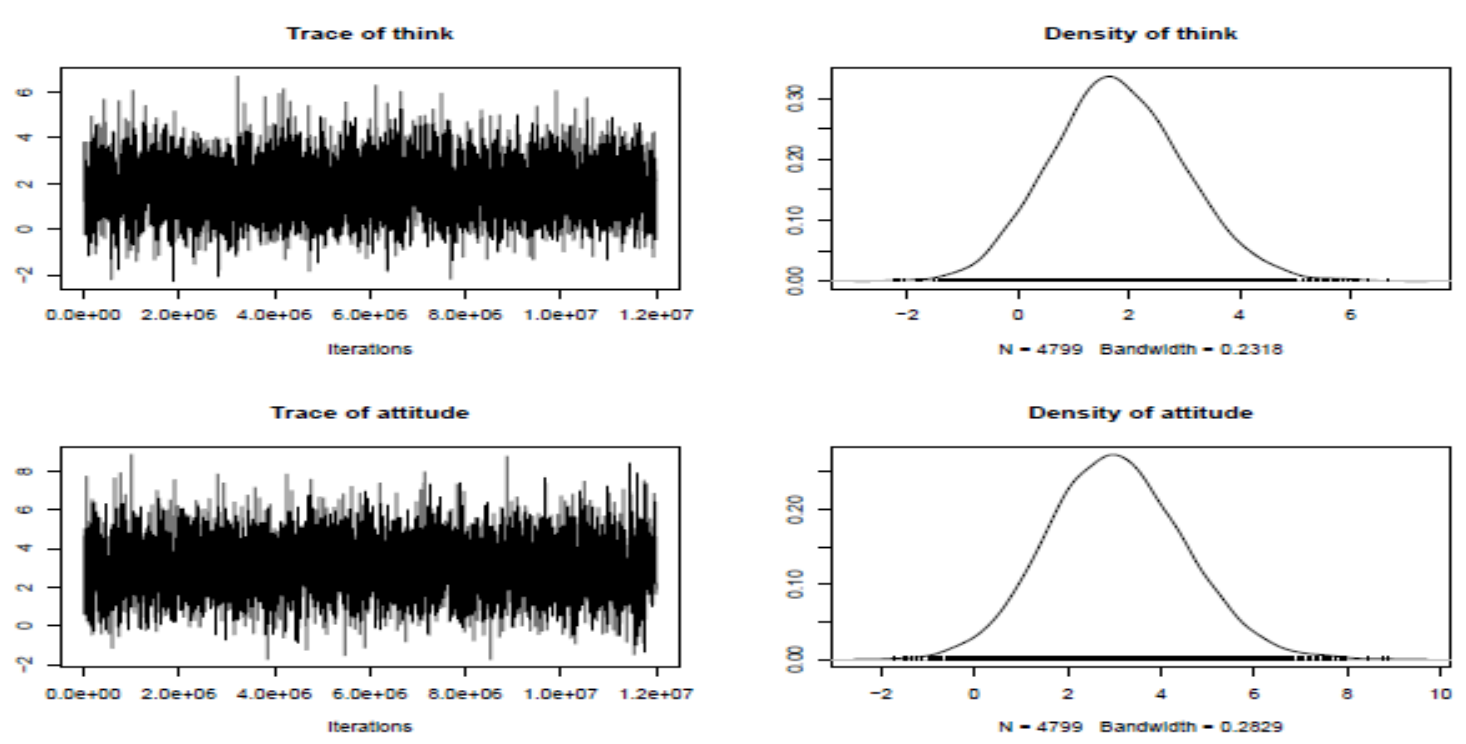

Trace of change
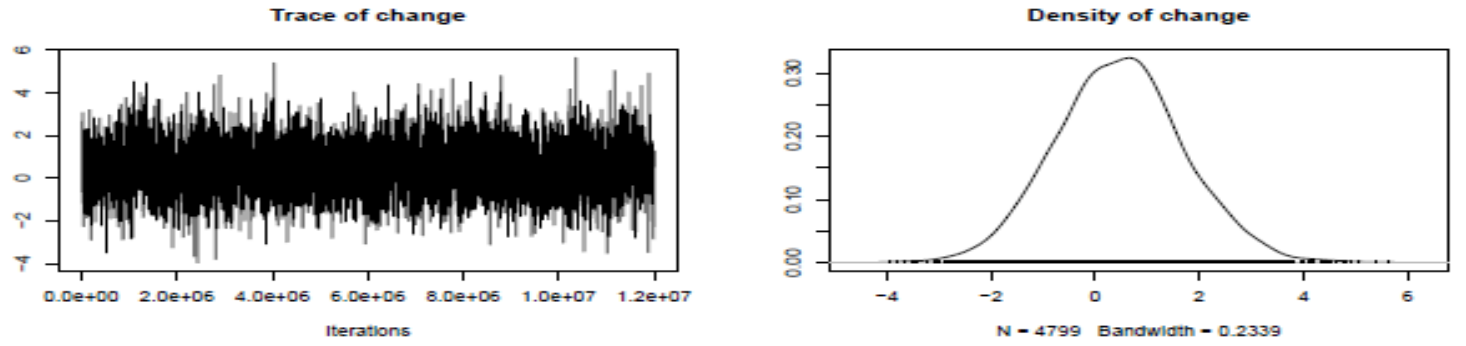

Trace of careExp
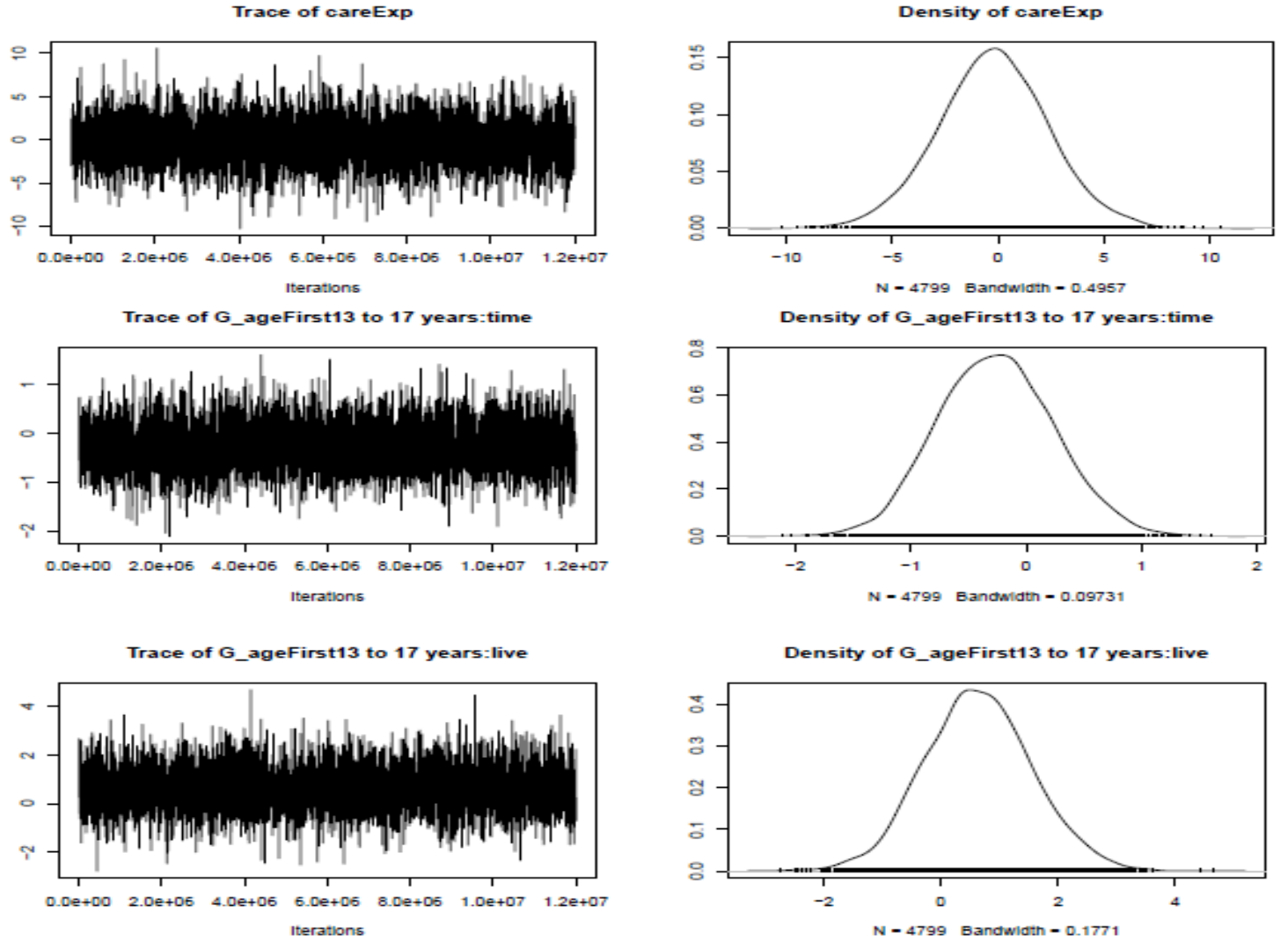
Trace of time:live

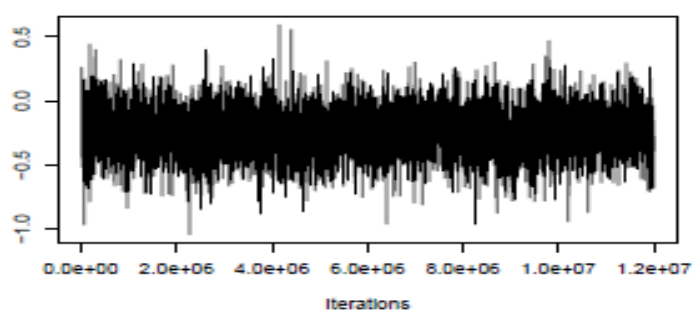

Trace of G_ageFirst13 to 17 years:relation

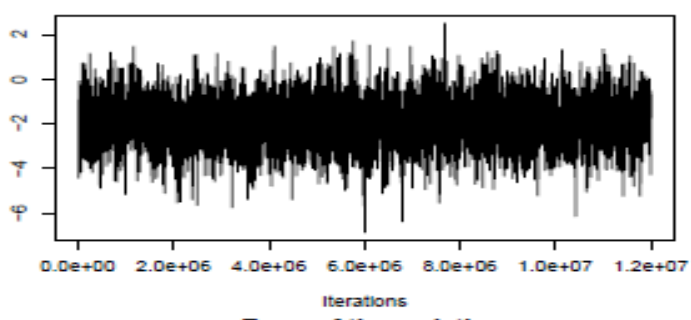

time:relation

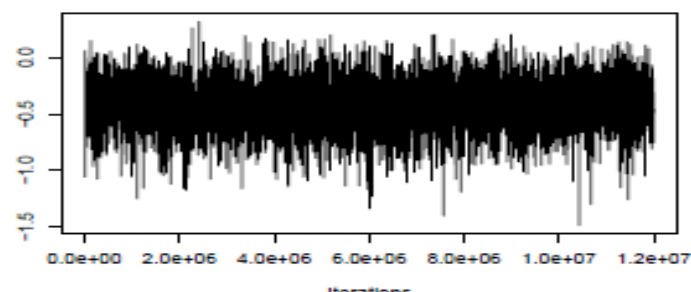

iterations

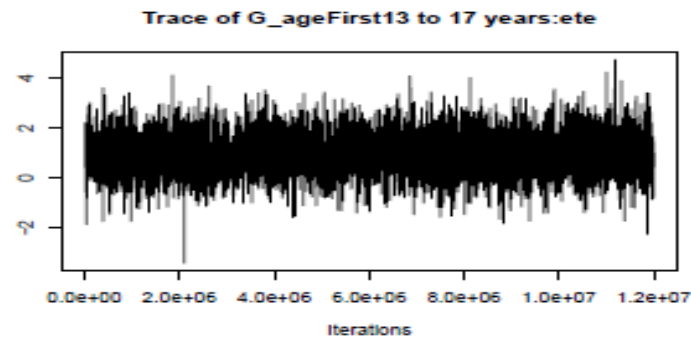

Trace of time:ete

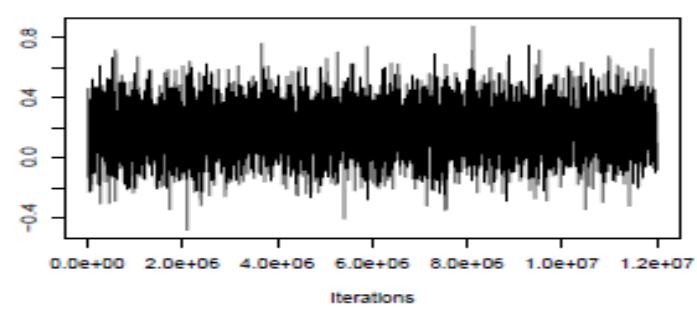

Trace of G_ageFirst13 to 17 years: where

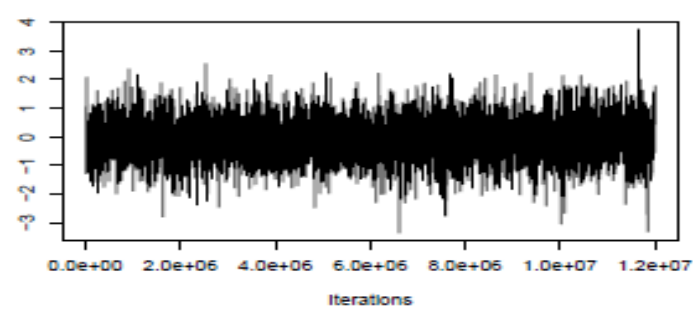

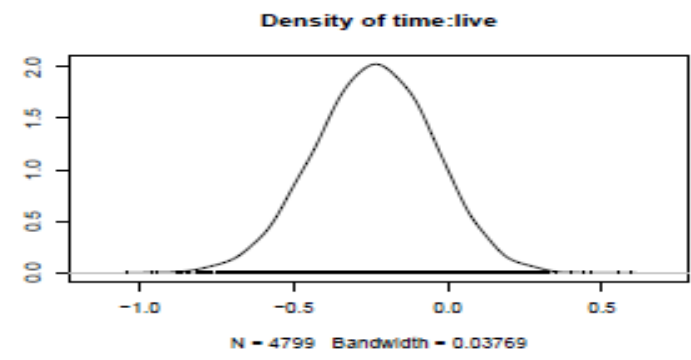
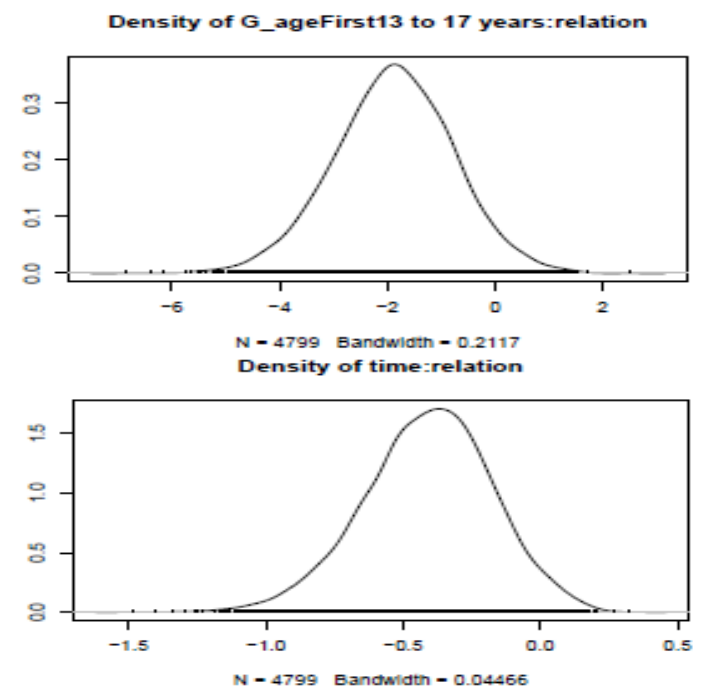

Density of G_ageFirst 13 to 17 years:ete

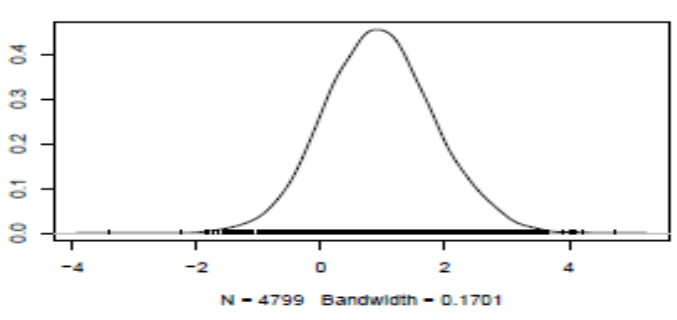

Density of time:ete

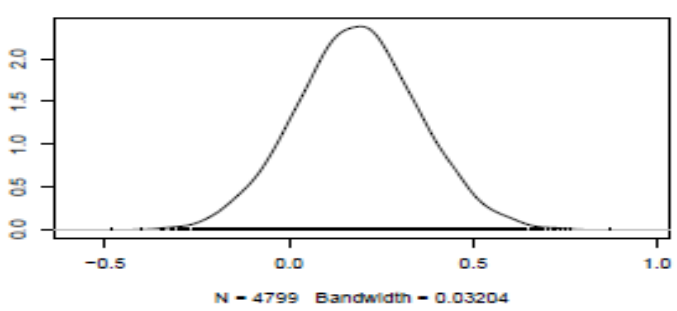

Density of G_ageFirst13 to 17 years:where

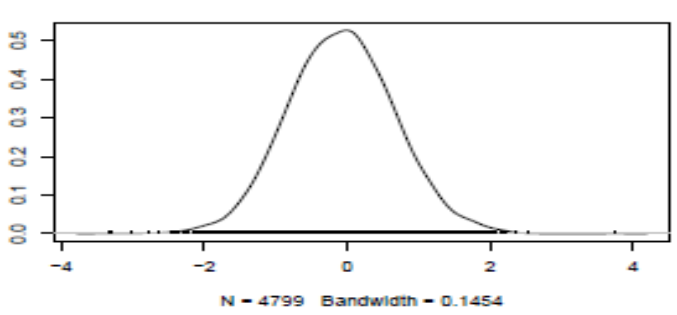


Trace of time:where

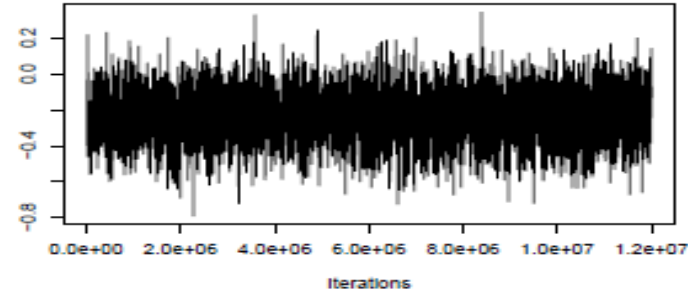

Trace of G_ageFirst13 to 17 years:life

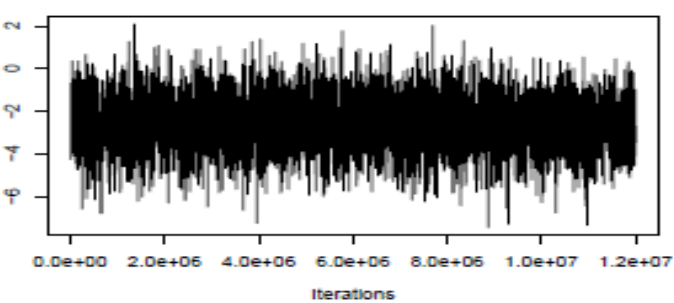

Trace of time:life

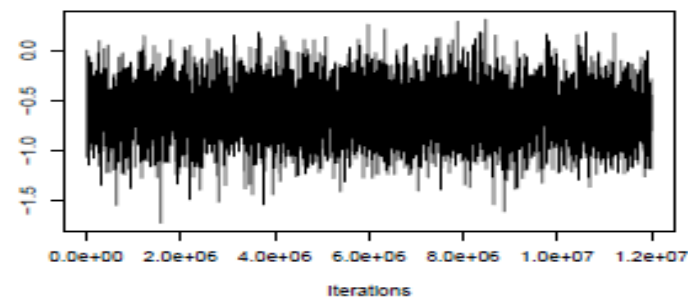

Iterations

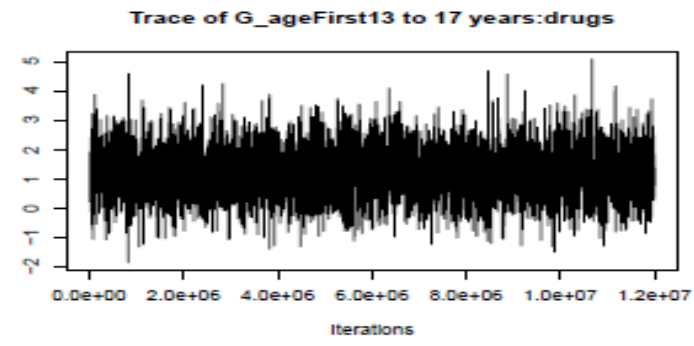

Trace of time:drugs

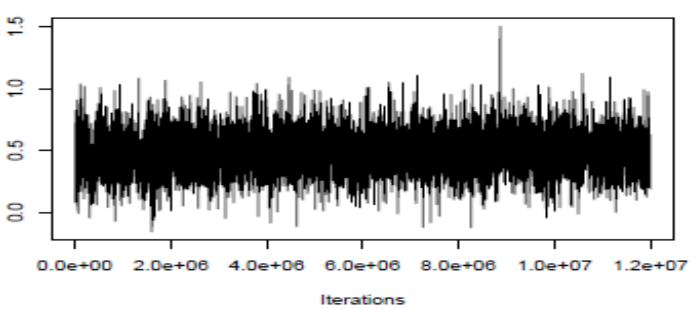

Trace of G_ageFirst13 to 17 years:physica

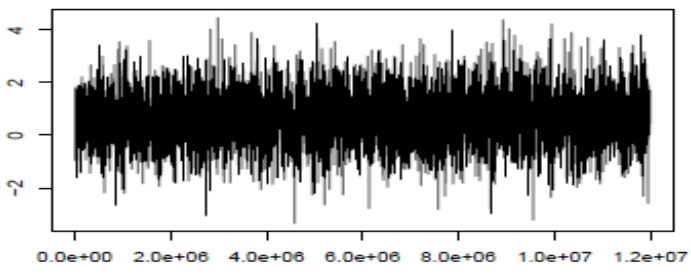

Iterations.
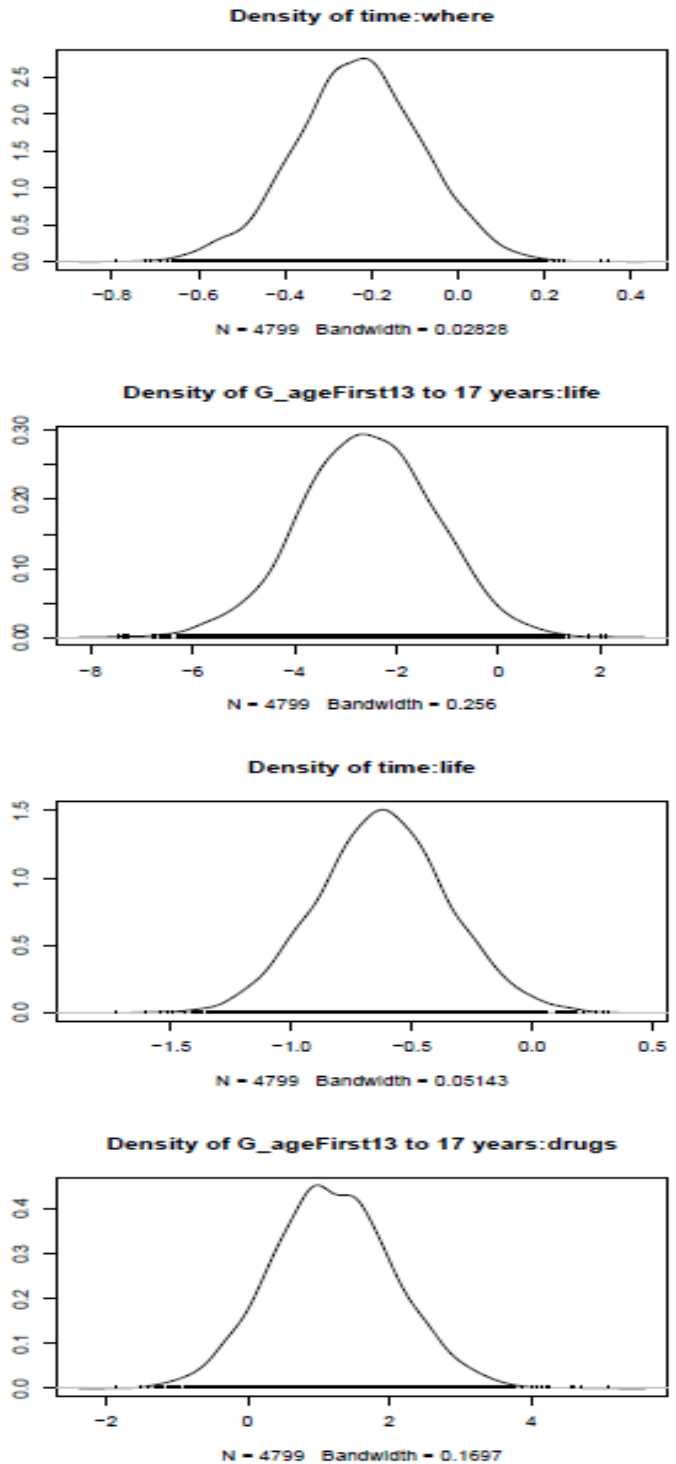

Density of time:drugs
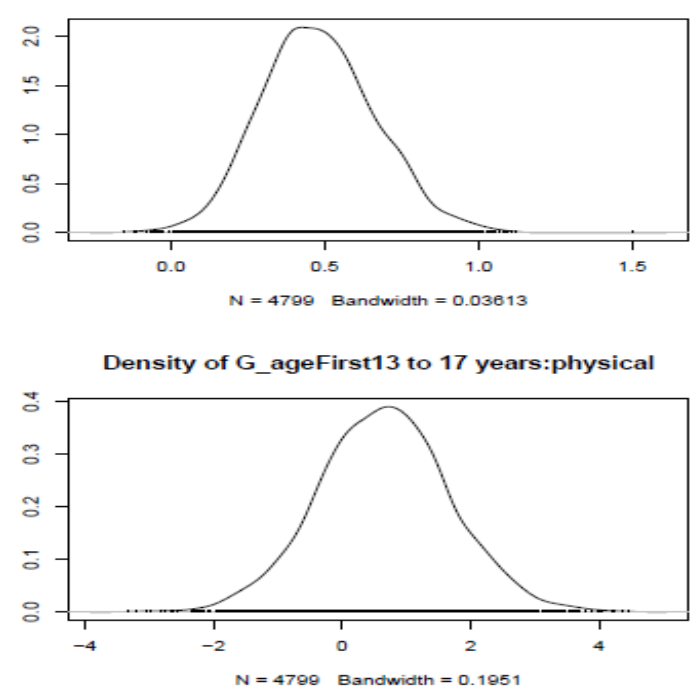

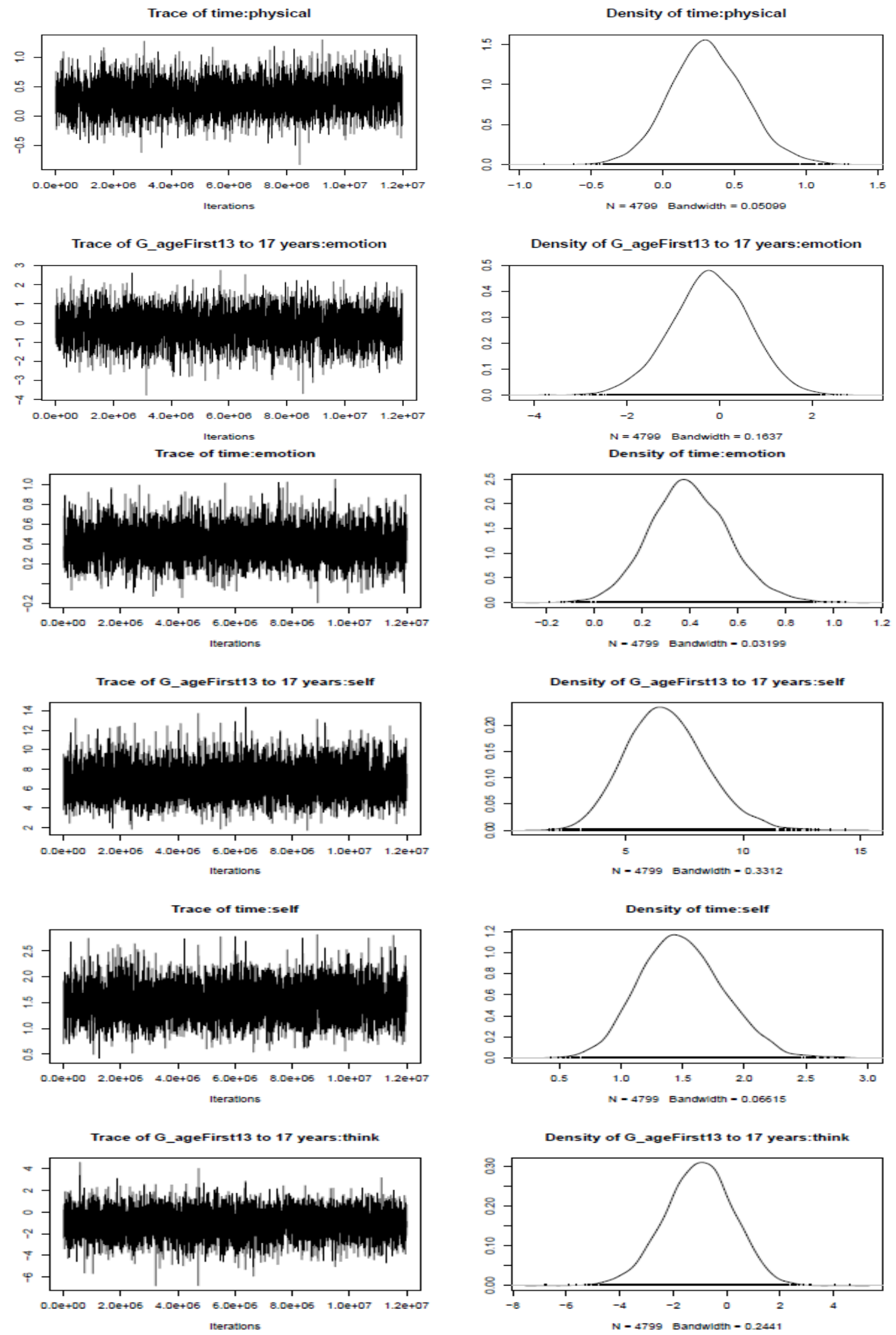
Trace of time:think

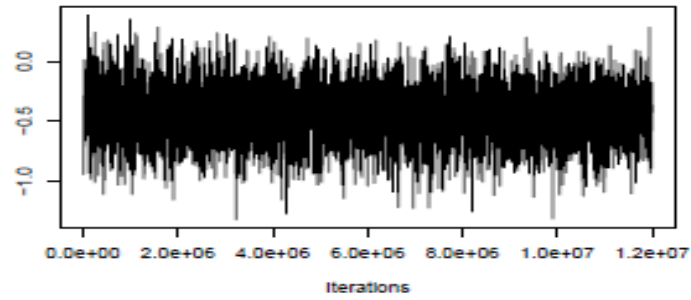

Iterations

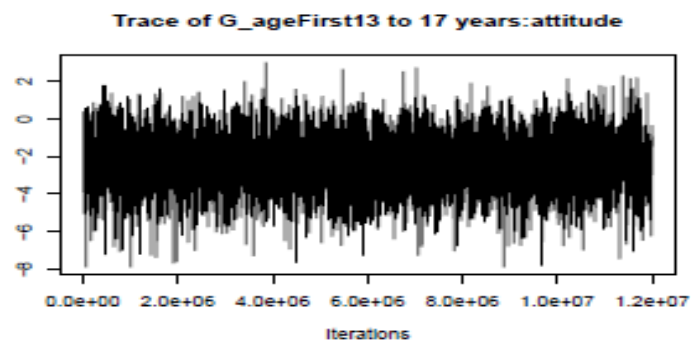

Trace of time:attitude

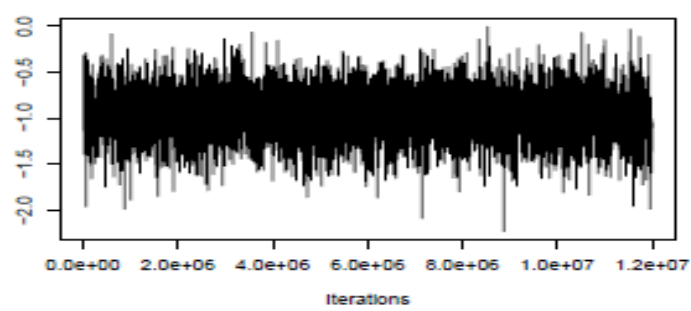

Trace of G_ageFirst13 to 17 years:change

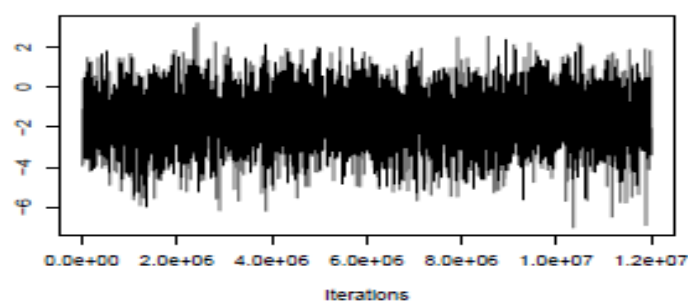

Trace of time:chang

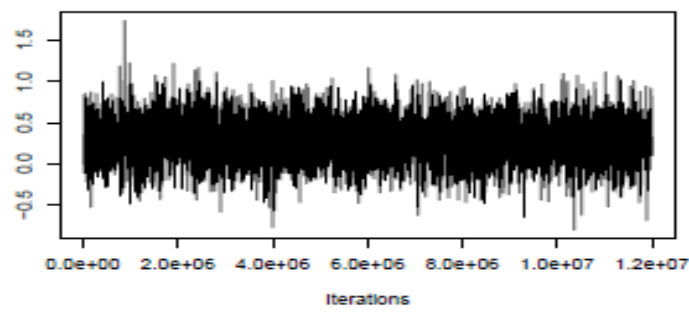

iterations

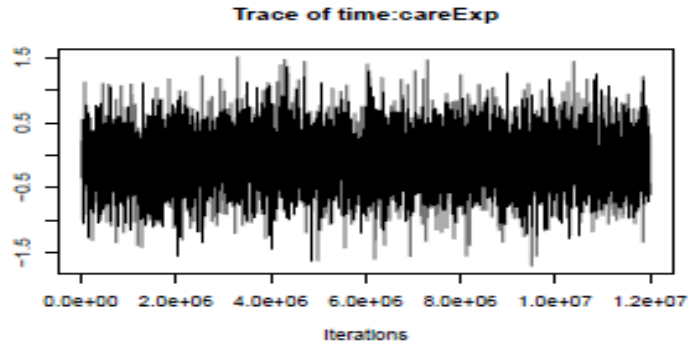

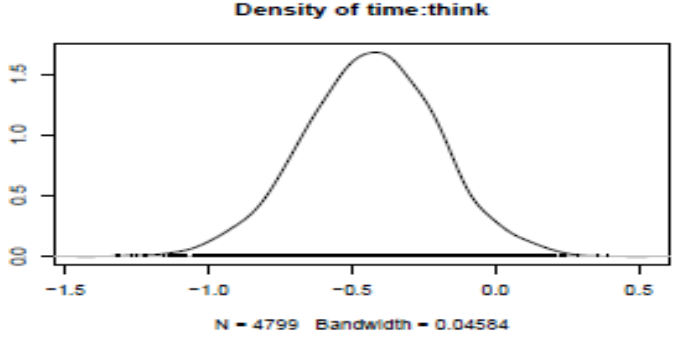
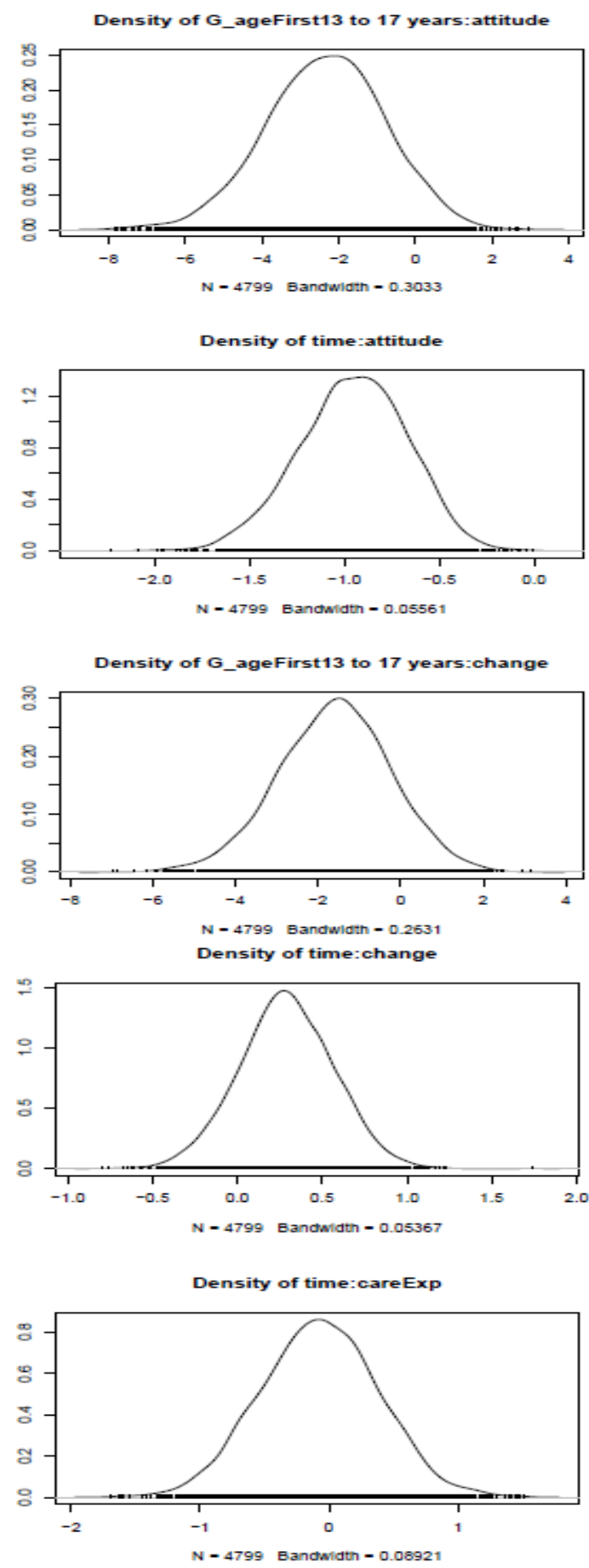
Trace of live:careExp

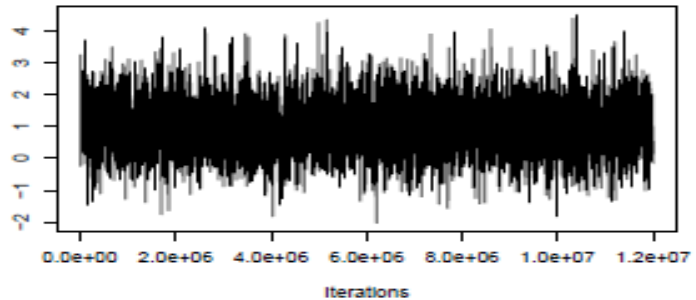

Iterations

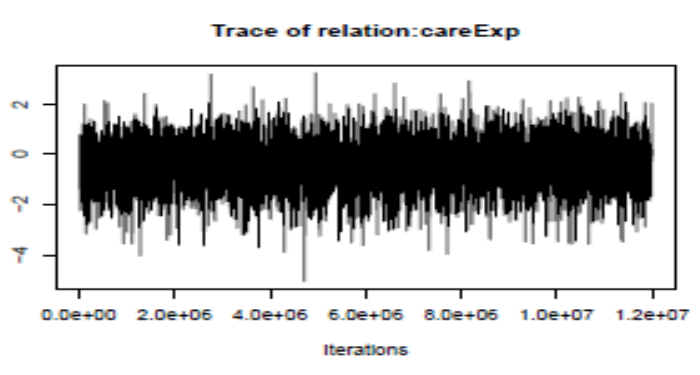

Trace of ete:careExp

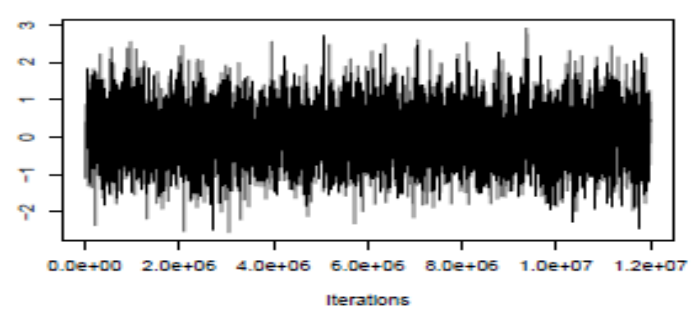

Trace of where:careExp

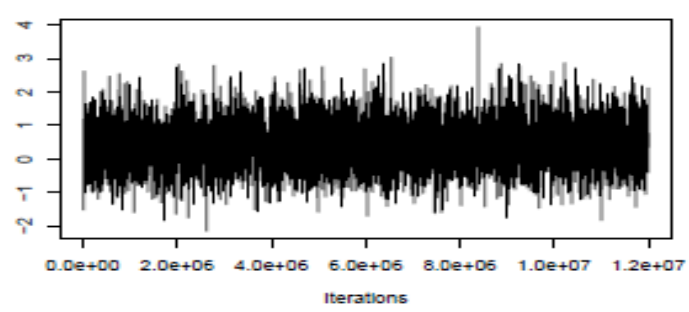
Iterations

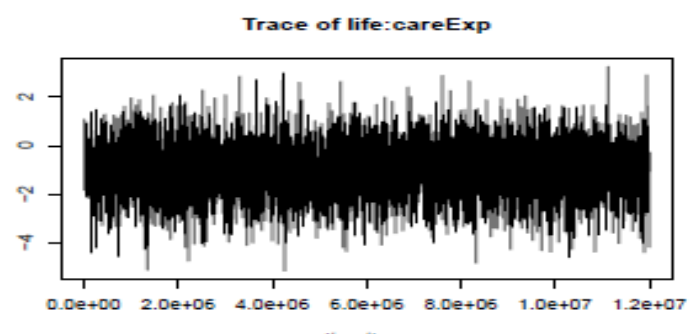

iterations

Trace of drugs:careExp

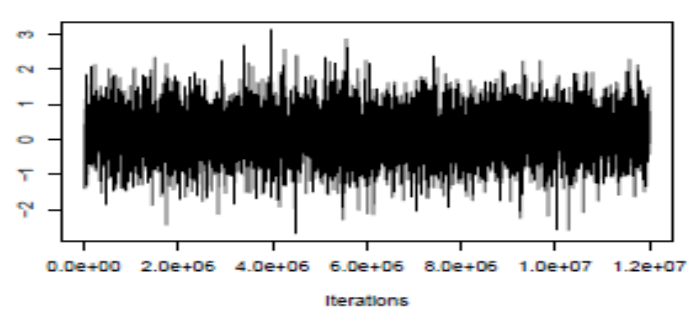

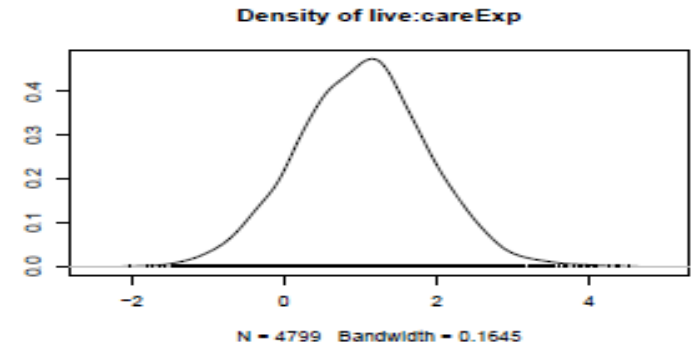
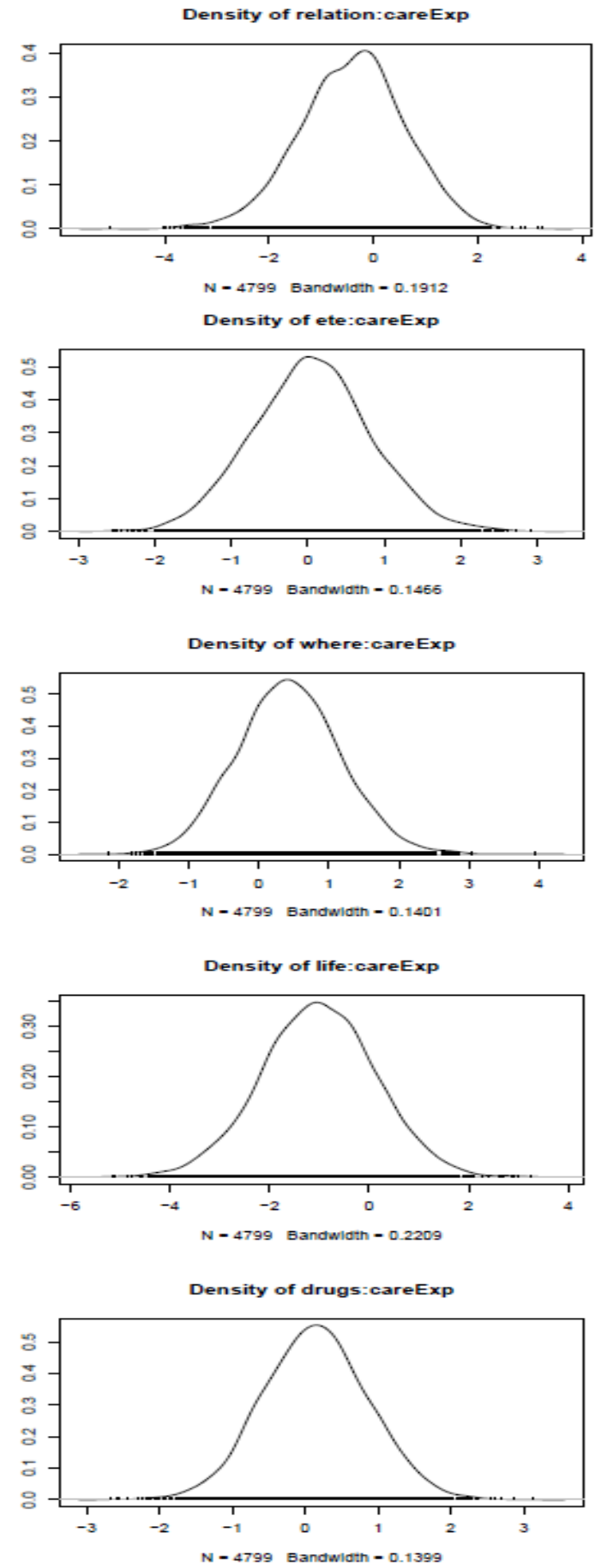

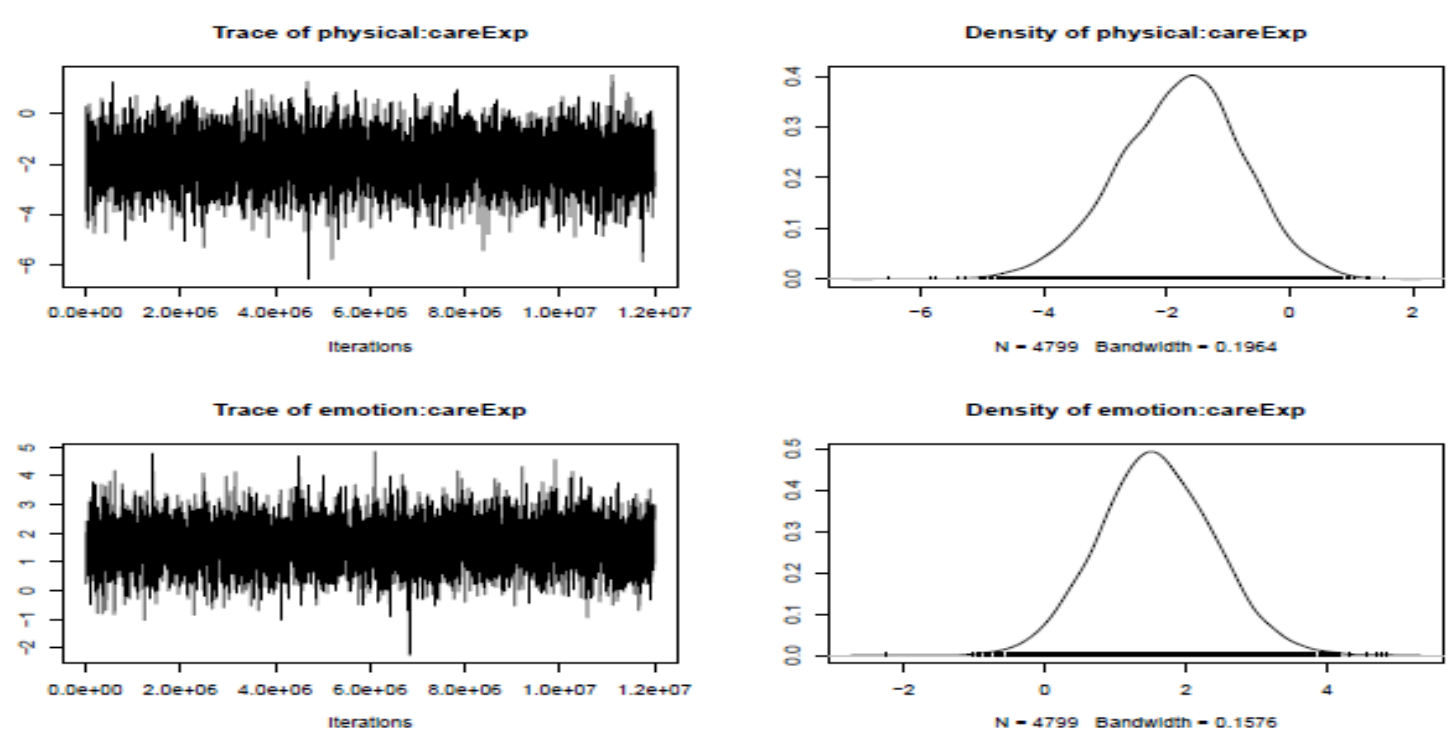

Trace of self:careExp
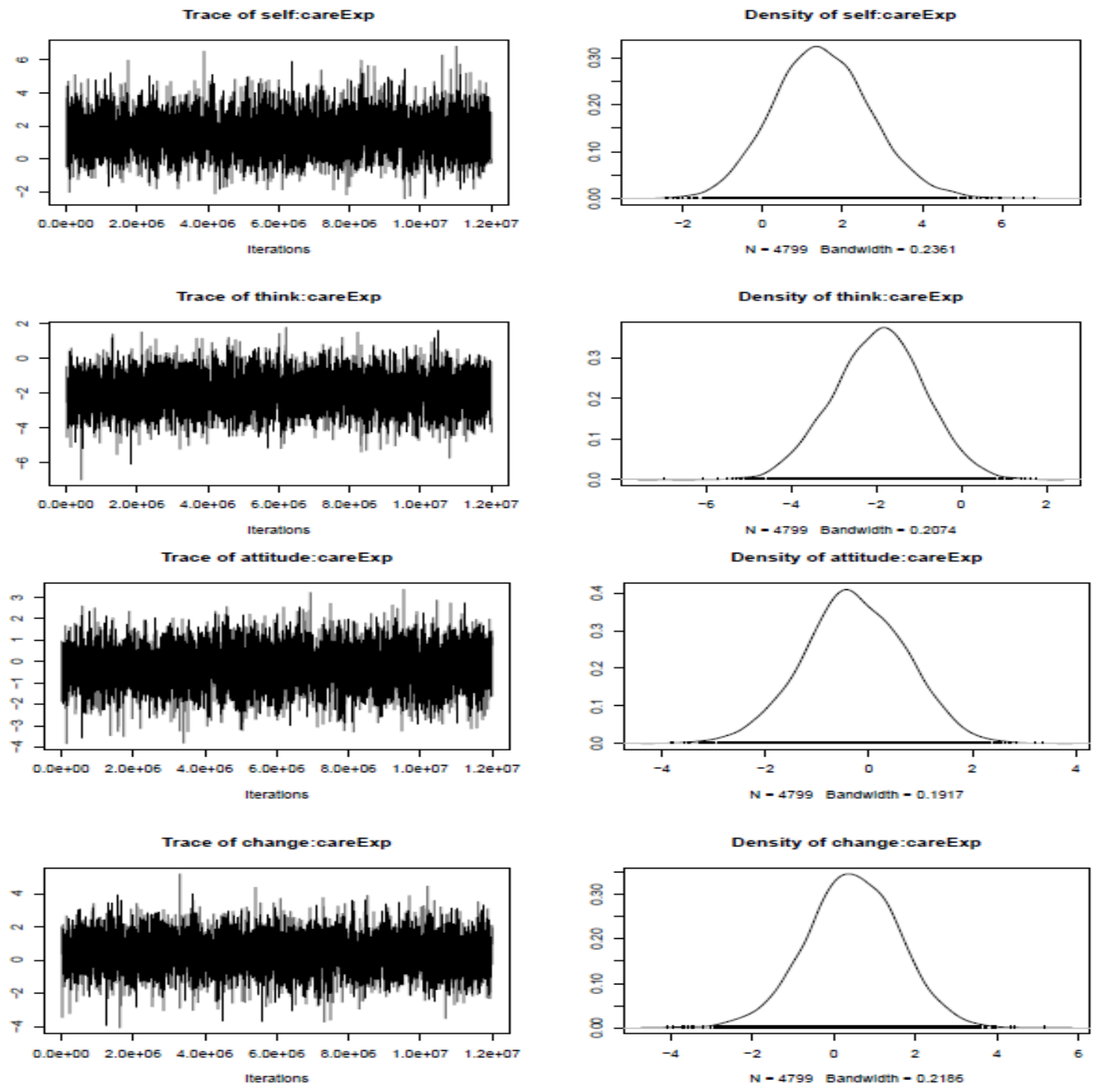

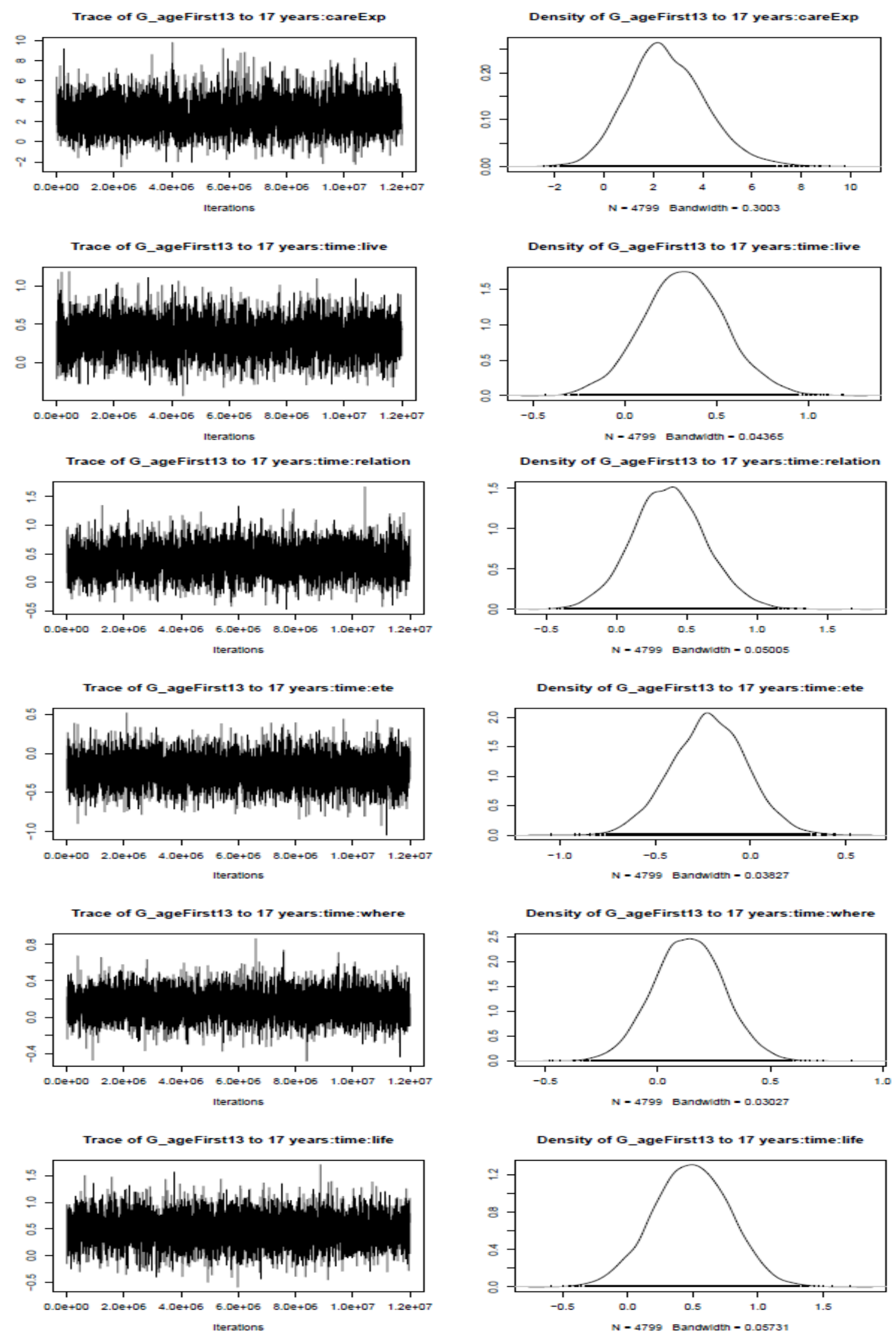

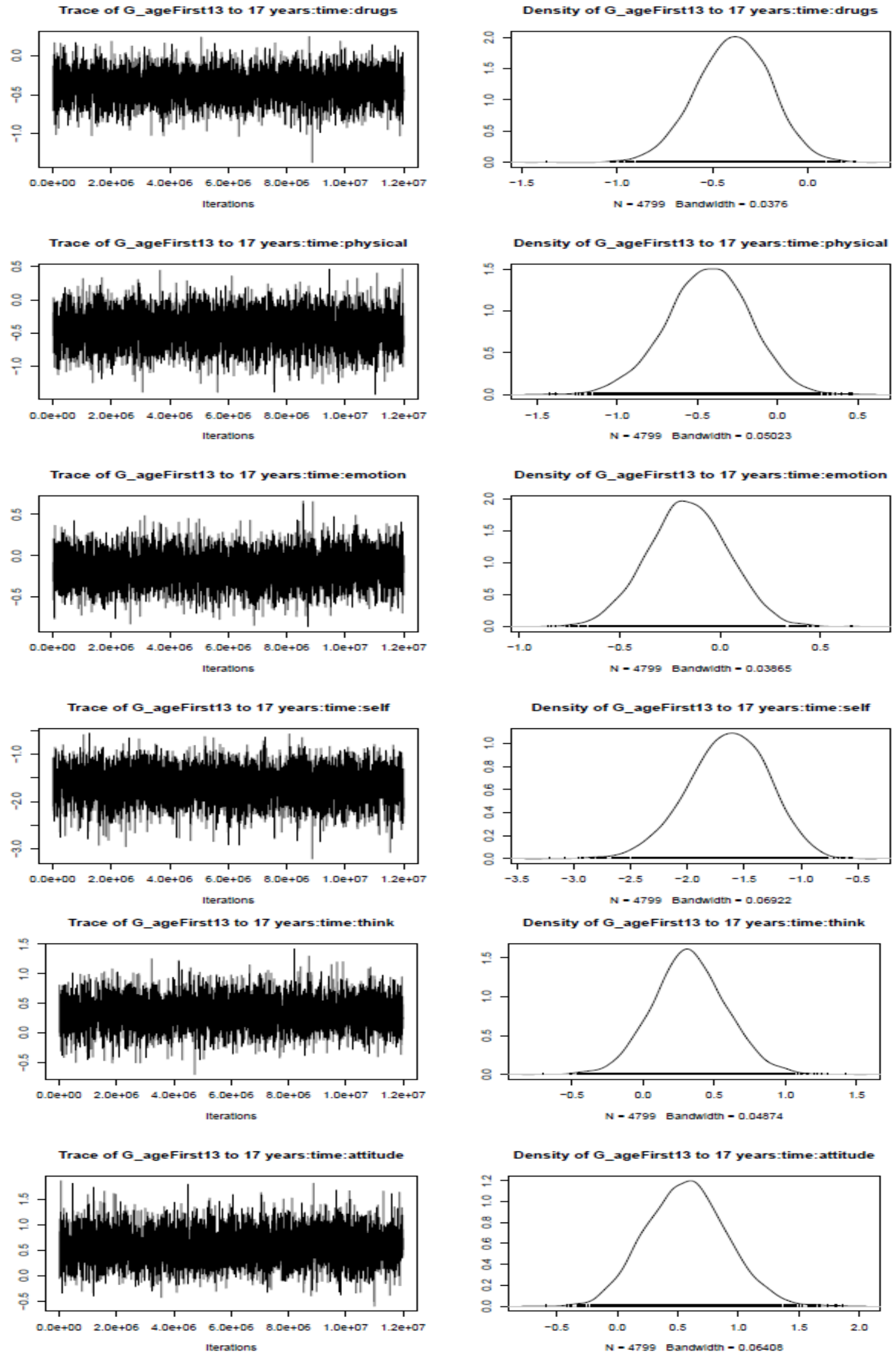

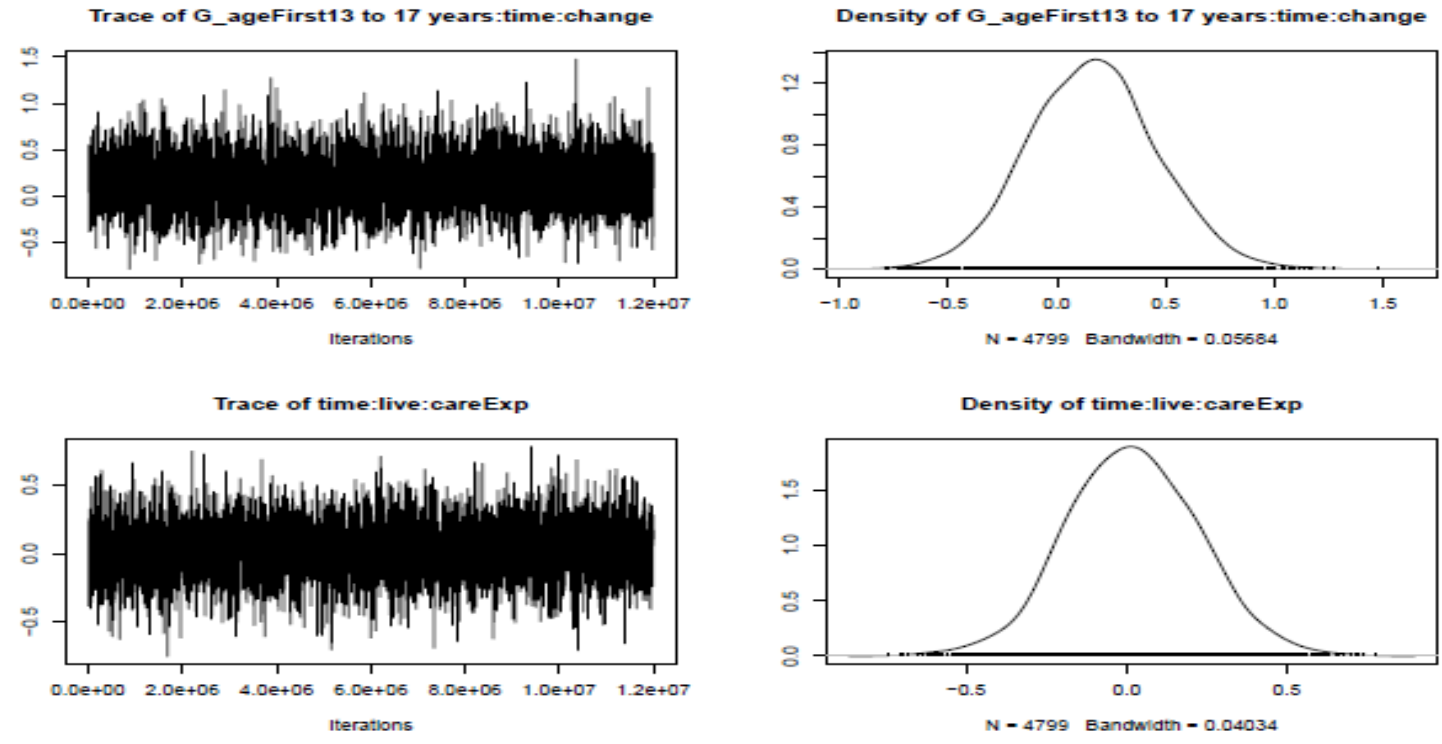

Trace of time:relation:careExp

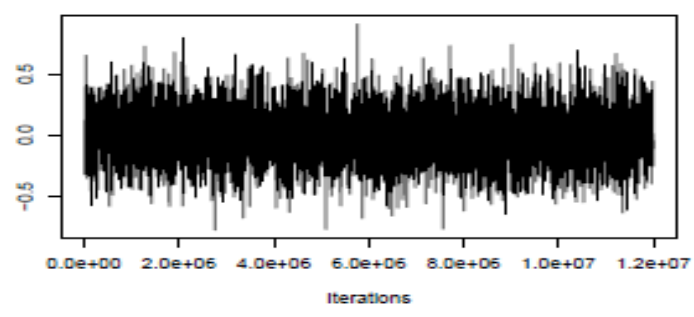

Density of time:relation:careExp

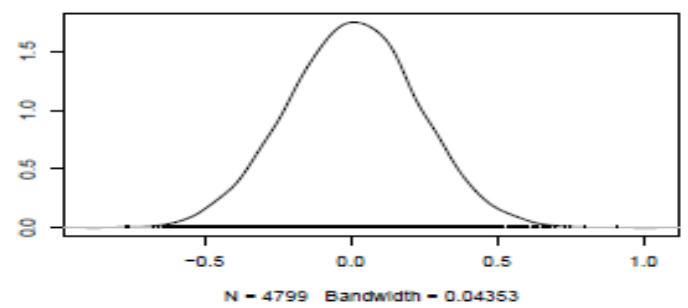

Trace of time:ete:careExp

Density of time:ete:careExp
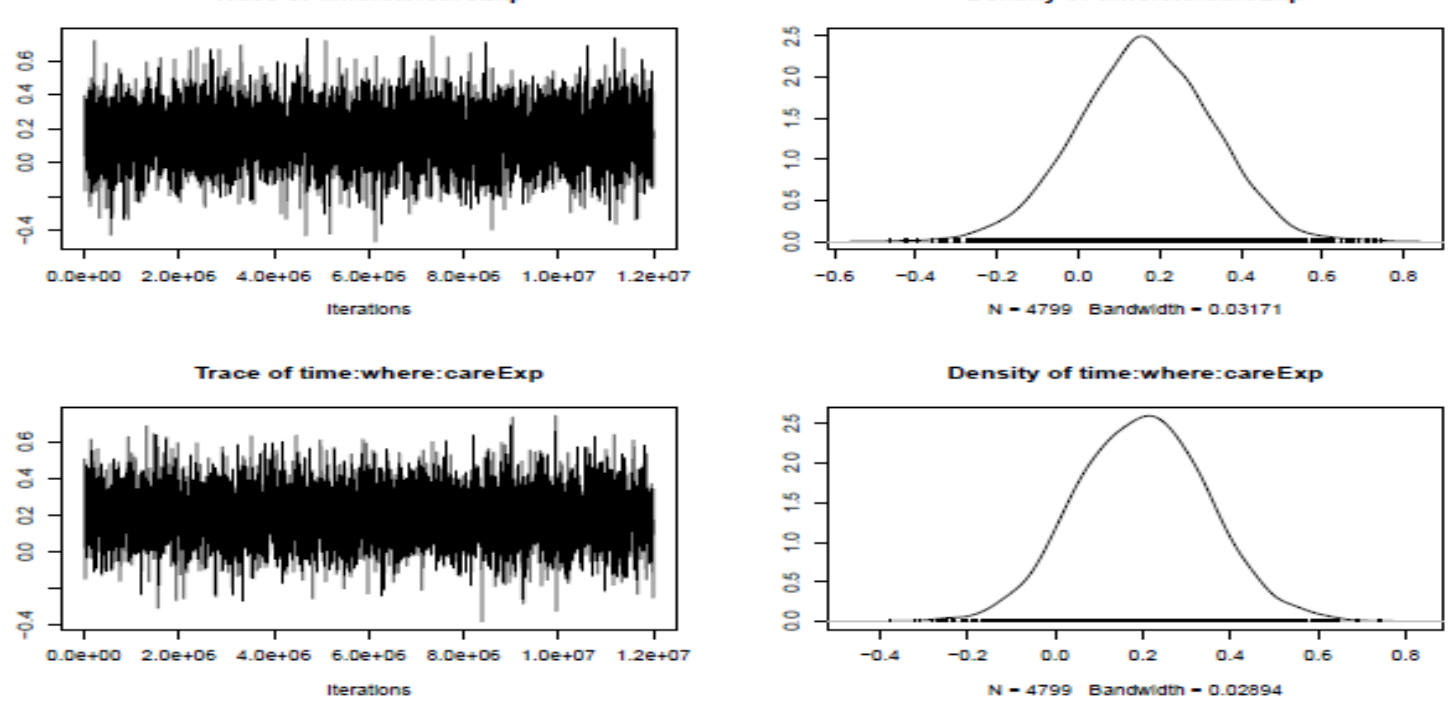

Trace of time:life:careExp
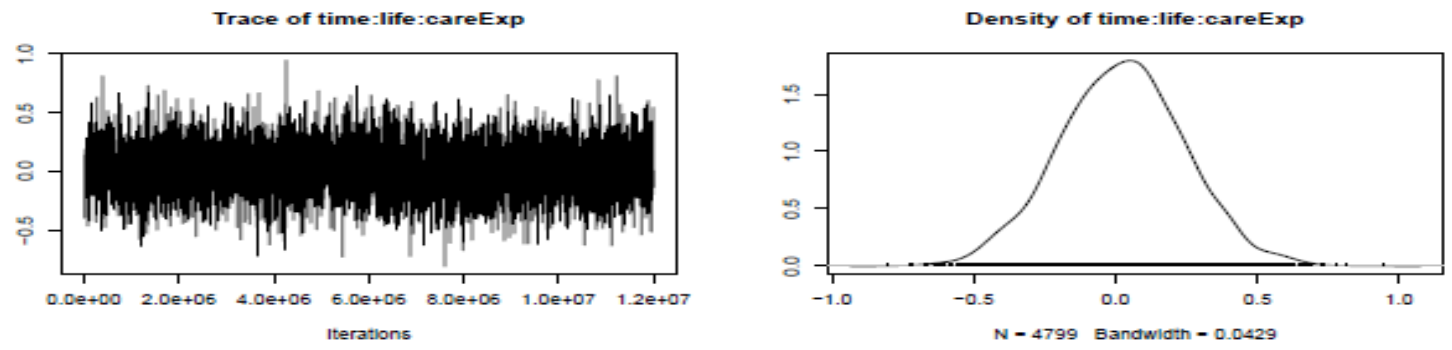

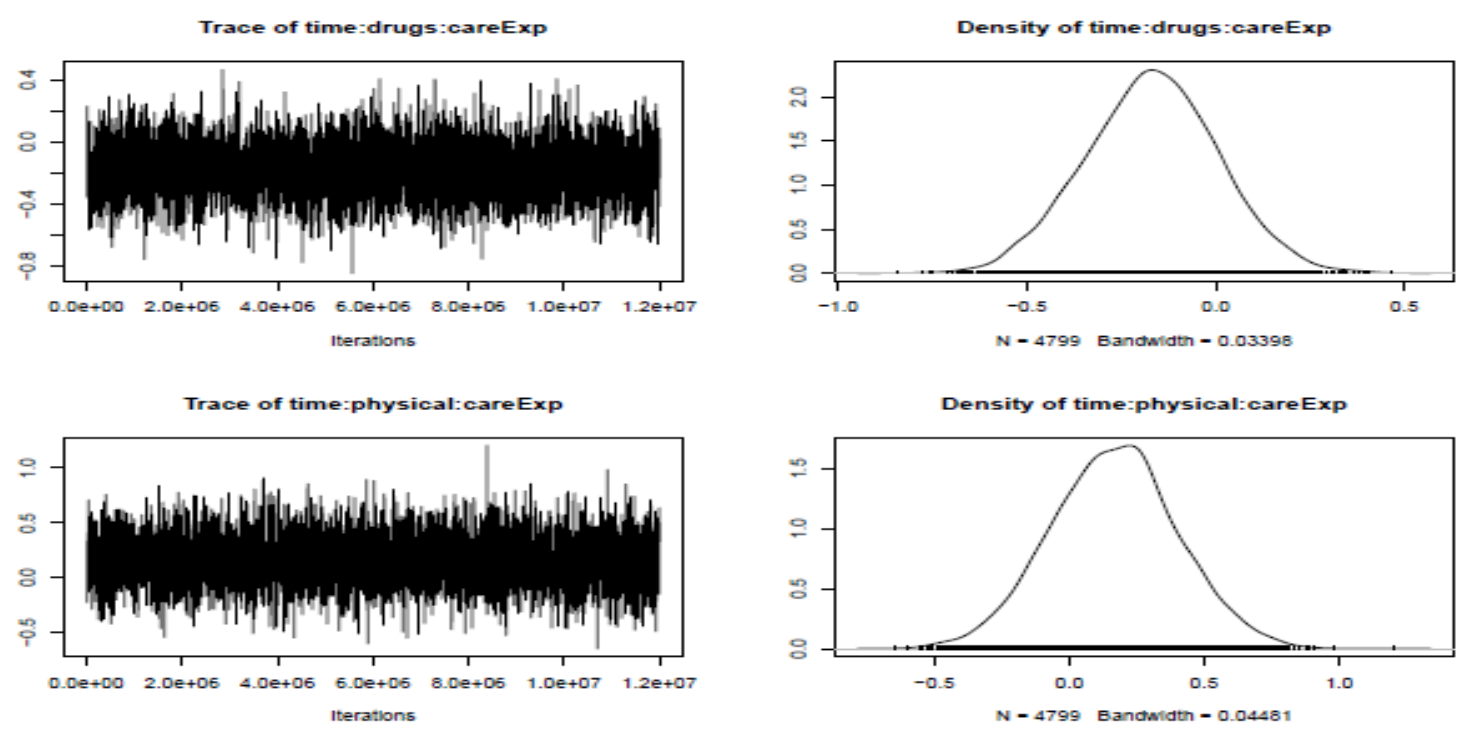

Trace of time:emotion:careExp

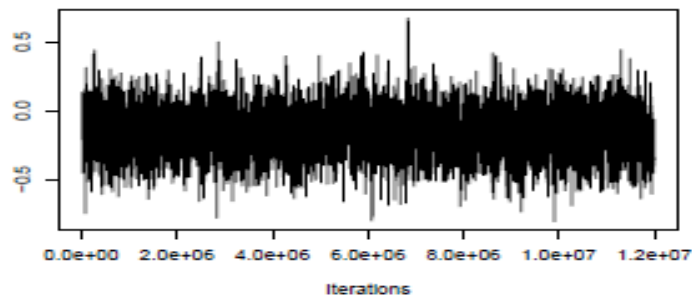

Density of time:emotion:careExp

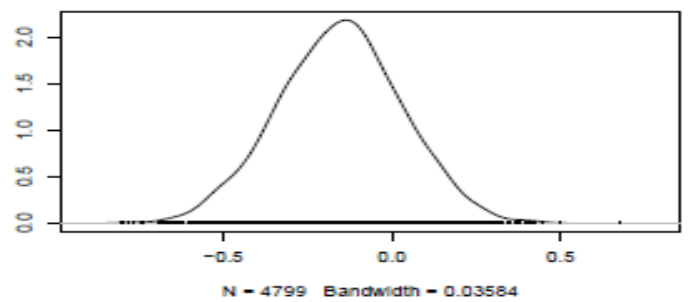

Trace of time:self:careExp

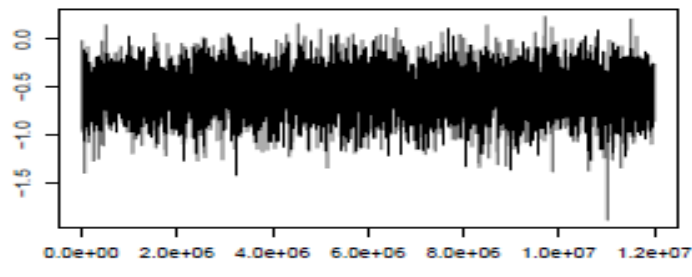

Density of time:self:careExp

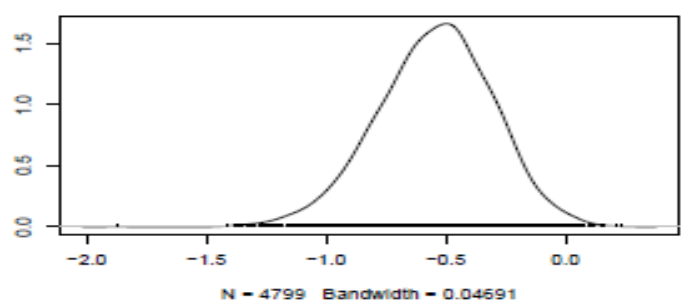

Iterations

Trace of time:think:careExp

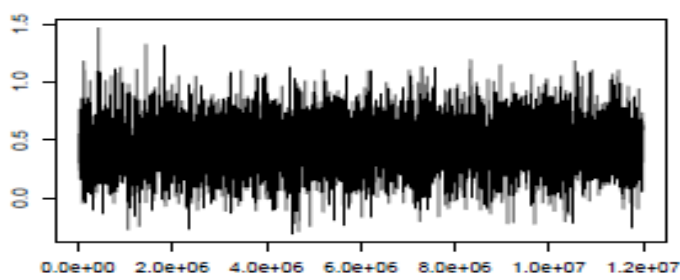

Density of time:think:careExp

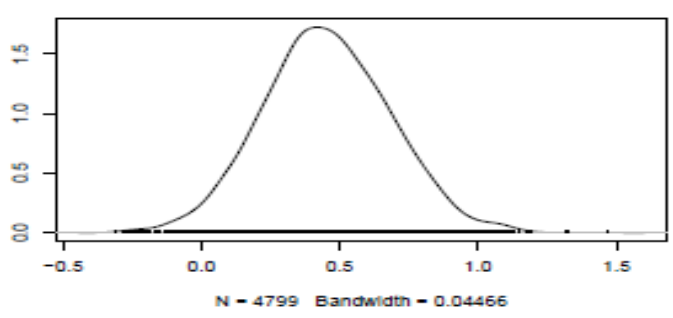

Trace of time:attitude:careExp
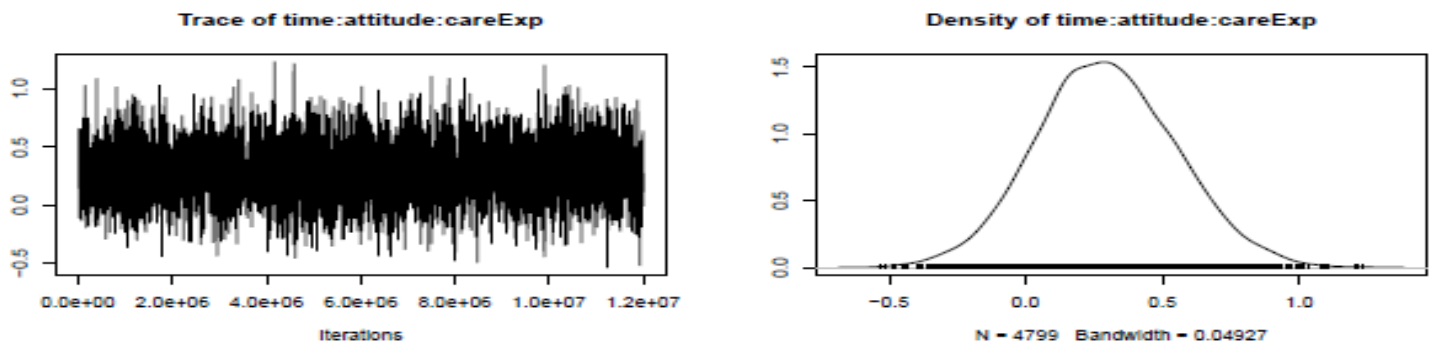

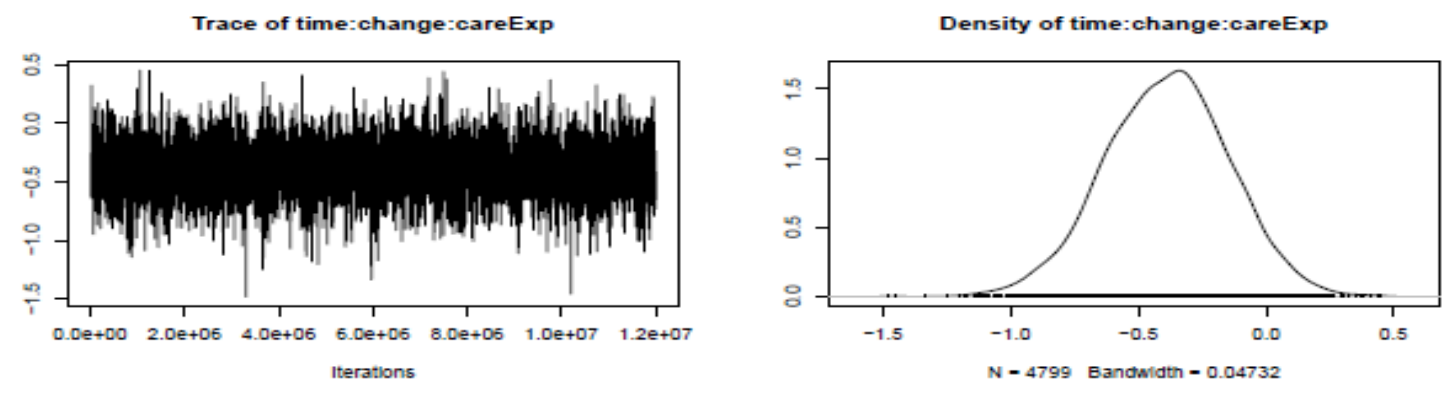

\section{$\underline{\text { Random Effects }}$}
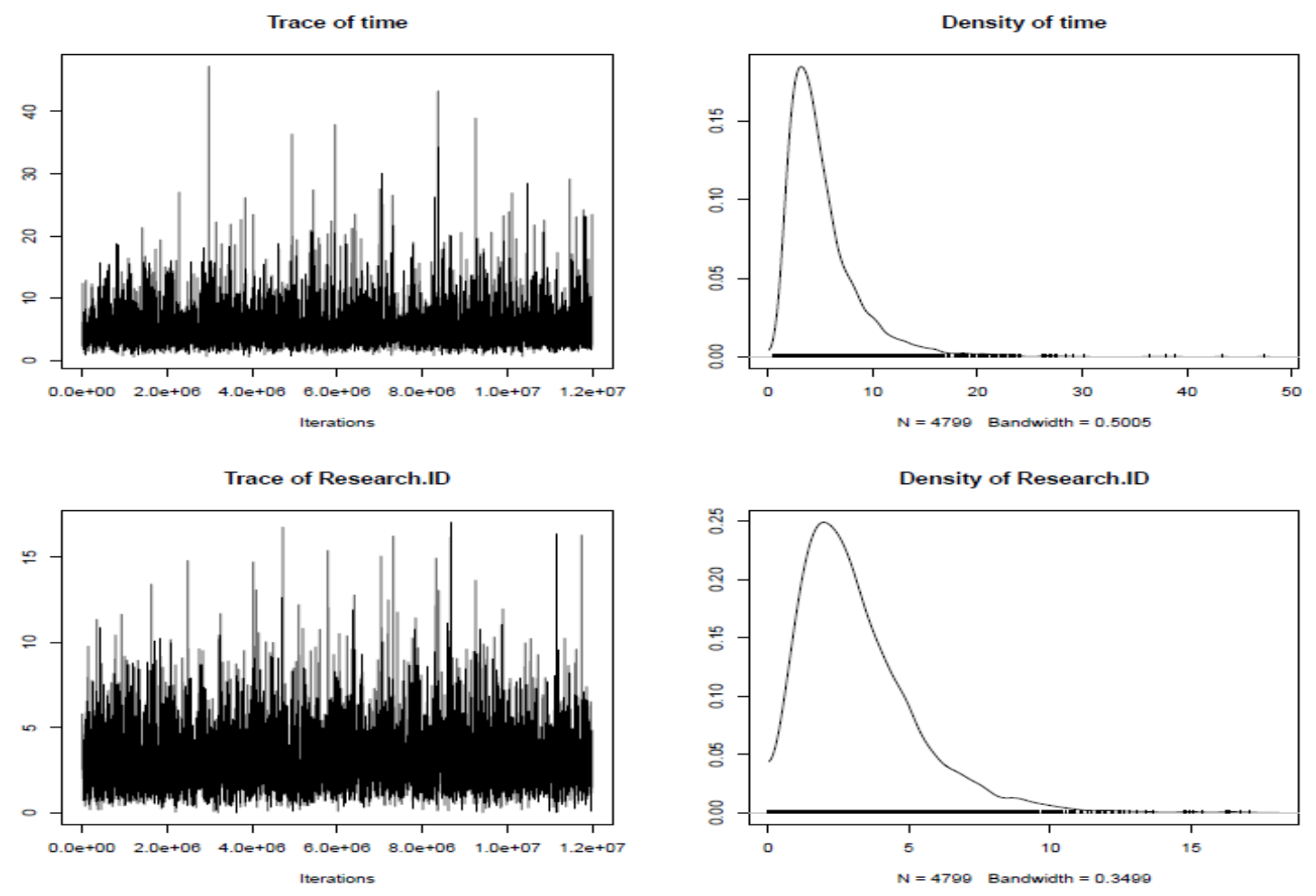
Model 1.12 - Basic Model + Breach (Table 7.4)

Bayesian Model (Bm1_cj1)

\section{\# Define the model}

Bm1_cj1 <- MCMCglmm(FO.bin breach + live + relation + ete + where + life

+ drugs + physical + emotion + self + think + attitude + change + time, random= time+Research.ID, data=data, family="ordinal", prior=prior2, nitt $=250000$, thin=10, burnin=3000)

\section{\# Checks for suitable convergence}

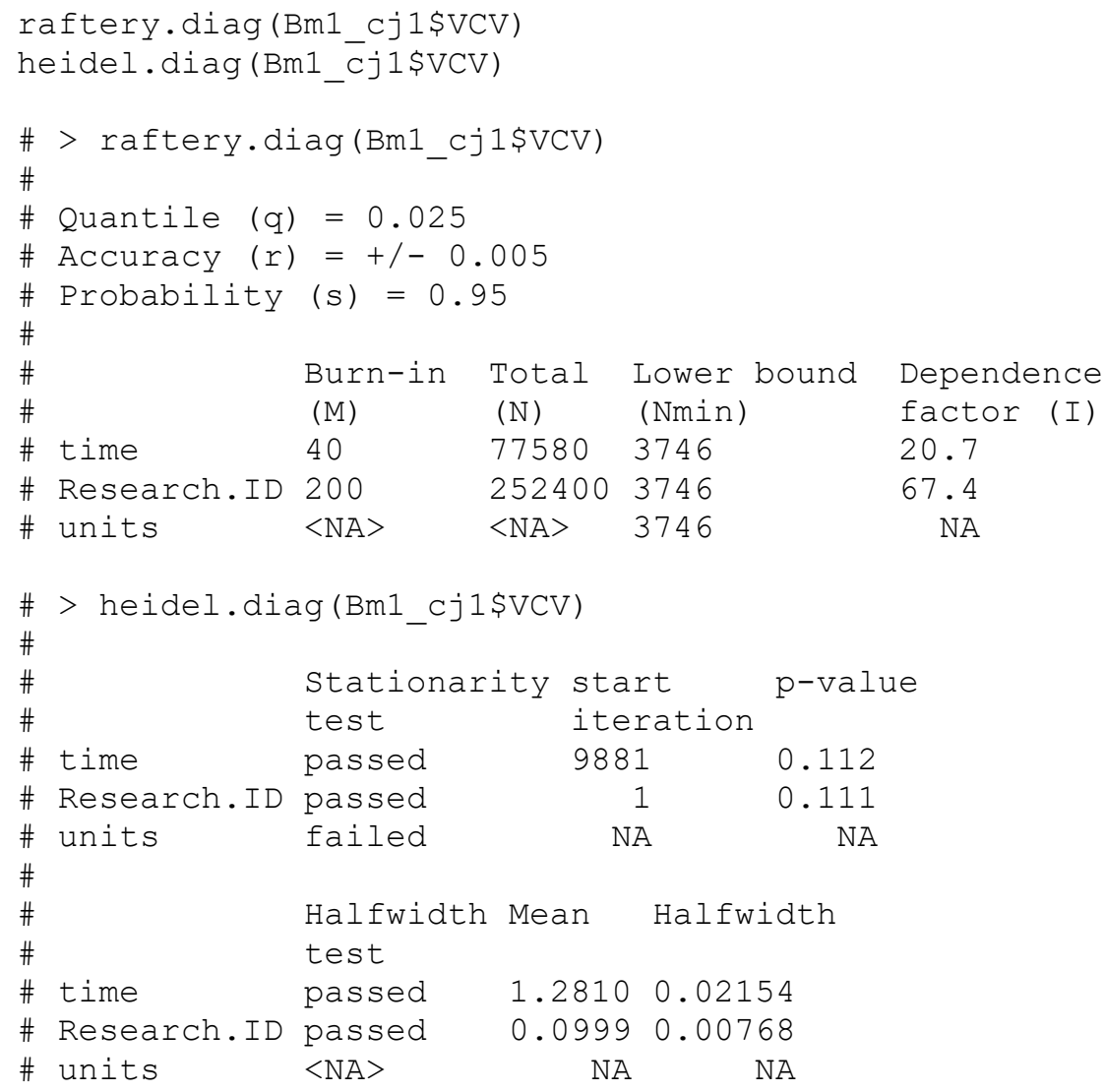




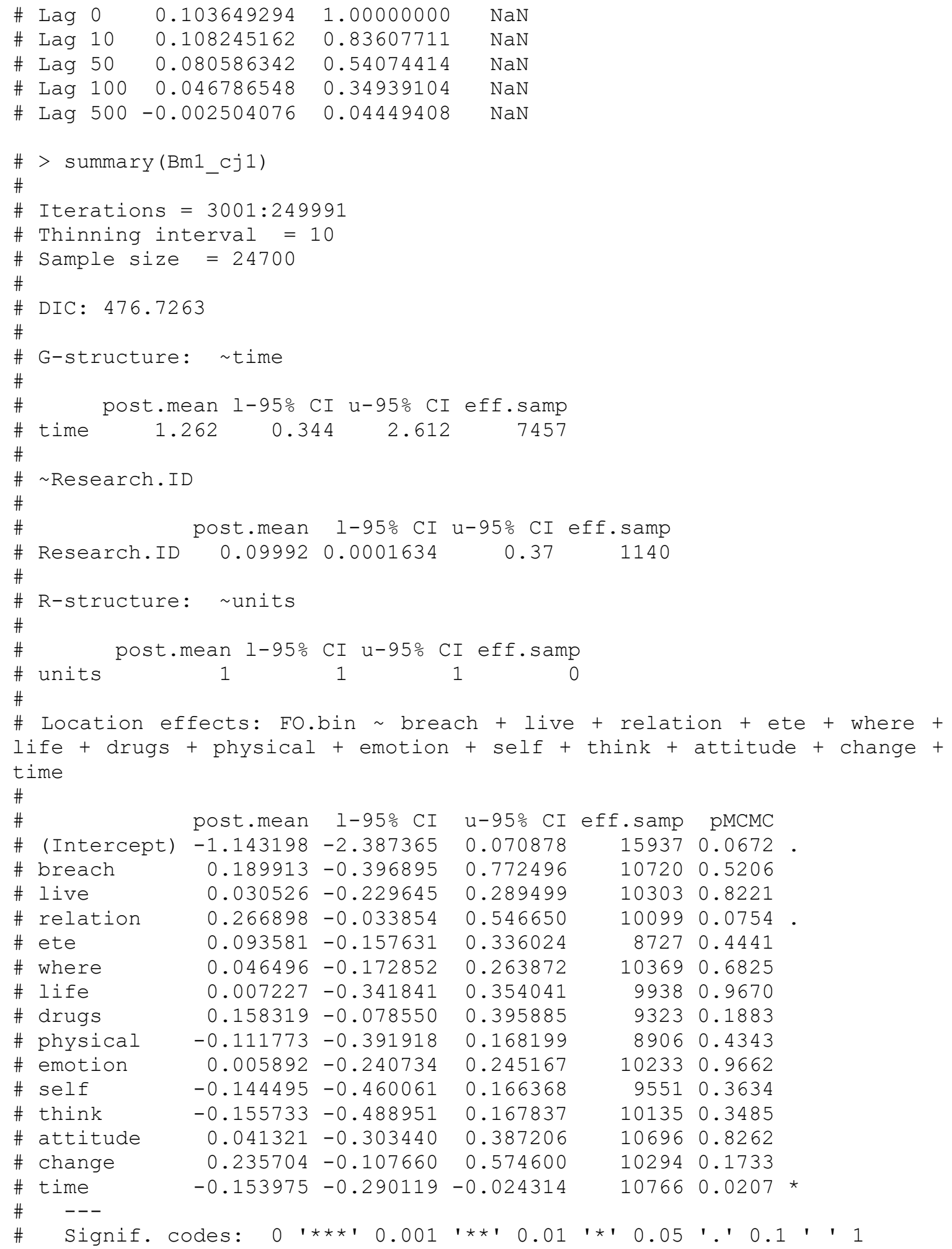




\section{Frequentist Model (m1_cj1)}

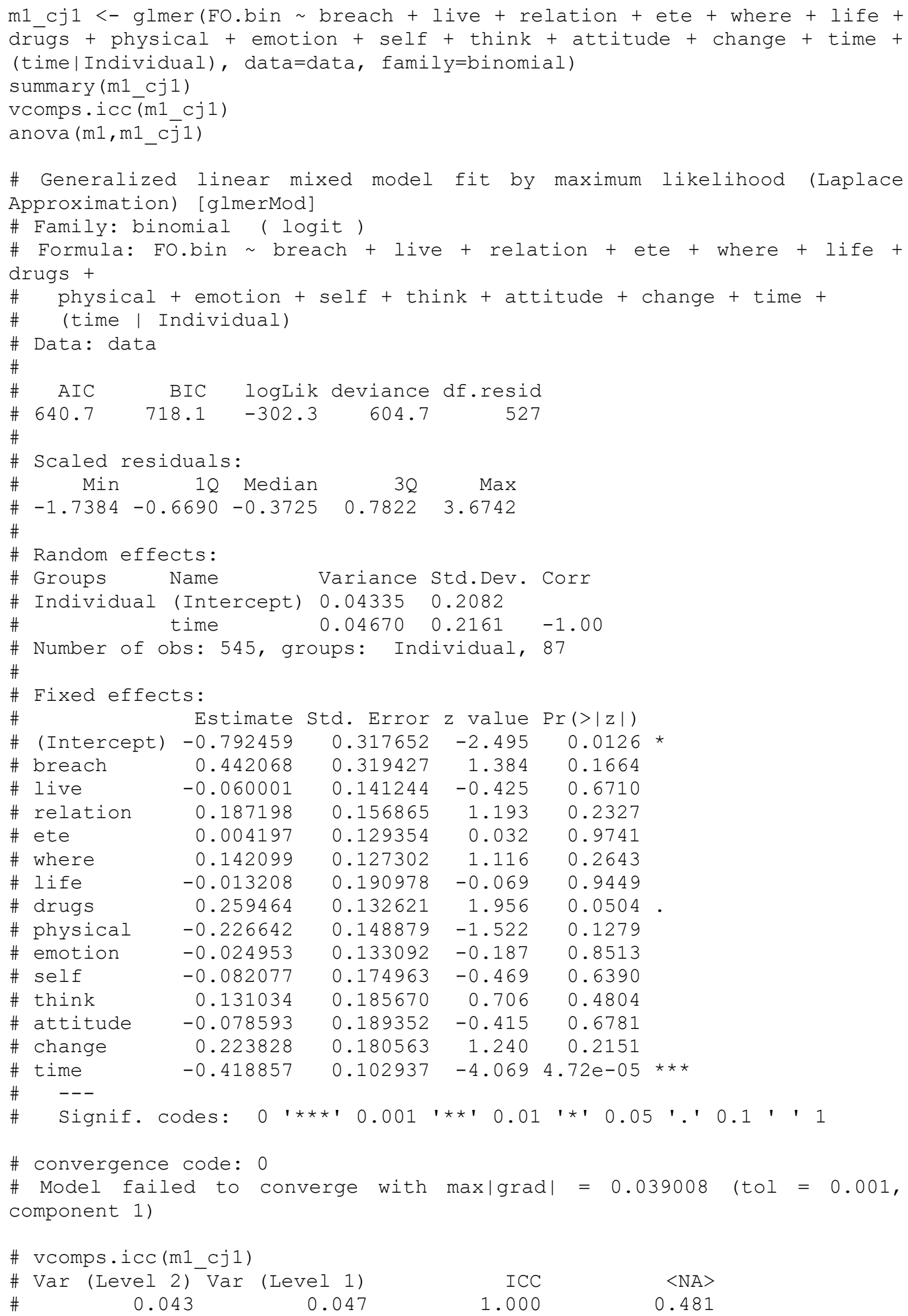




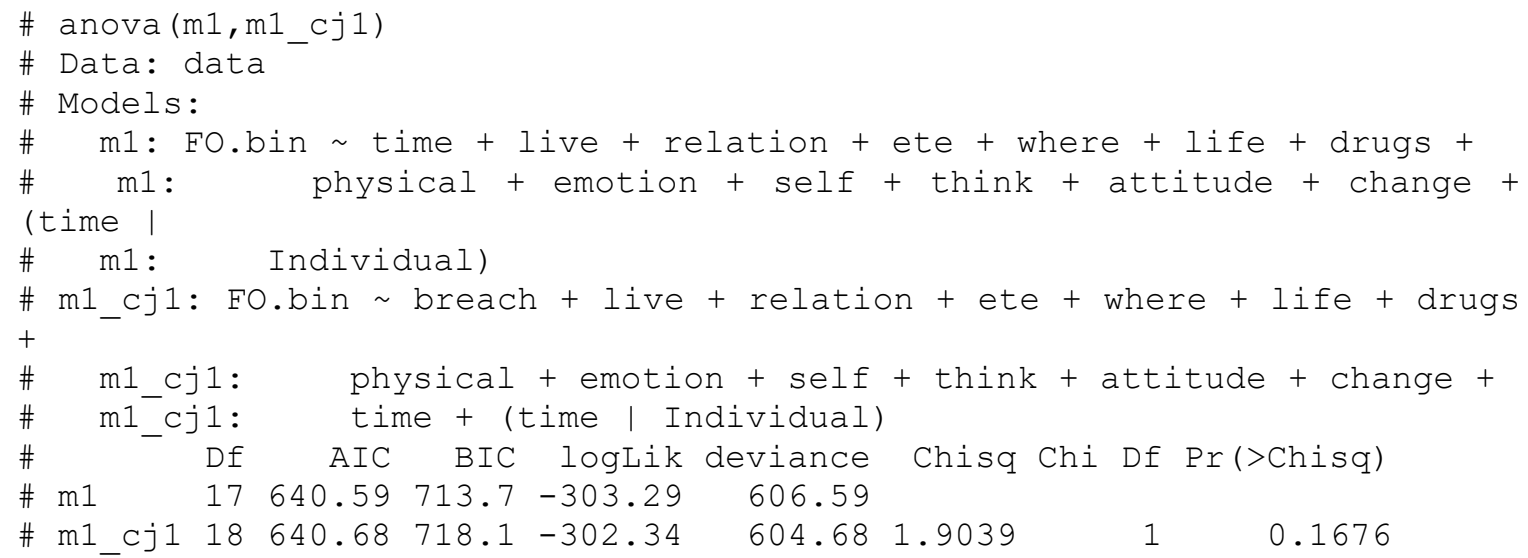


Model 1.13 - Basic Model + Court Appearance (Table 7.4)

Bayesian Model (Bm1_cj2)

\section{\# Define the model}

Bm1 cj2 <- MCMCglmm(FO.bin appear + live + relation + ete + where + life + drugs + physical + emotion + self + think + attitude + change + time, random= time+Research.ID, data=data, family="ordinal", prior=prior2, nitt $=250000$, thin=10, burnin=3000)

\section{\# Checks for suitable convergence}

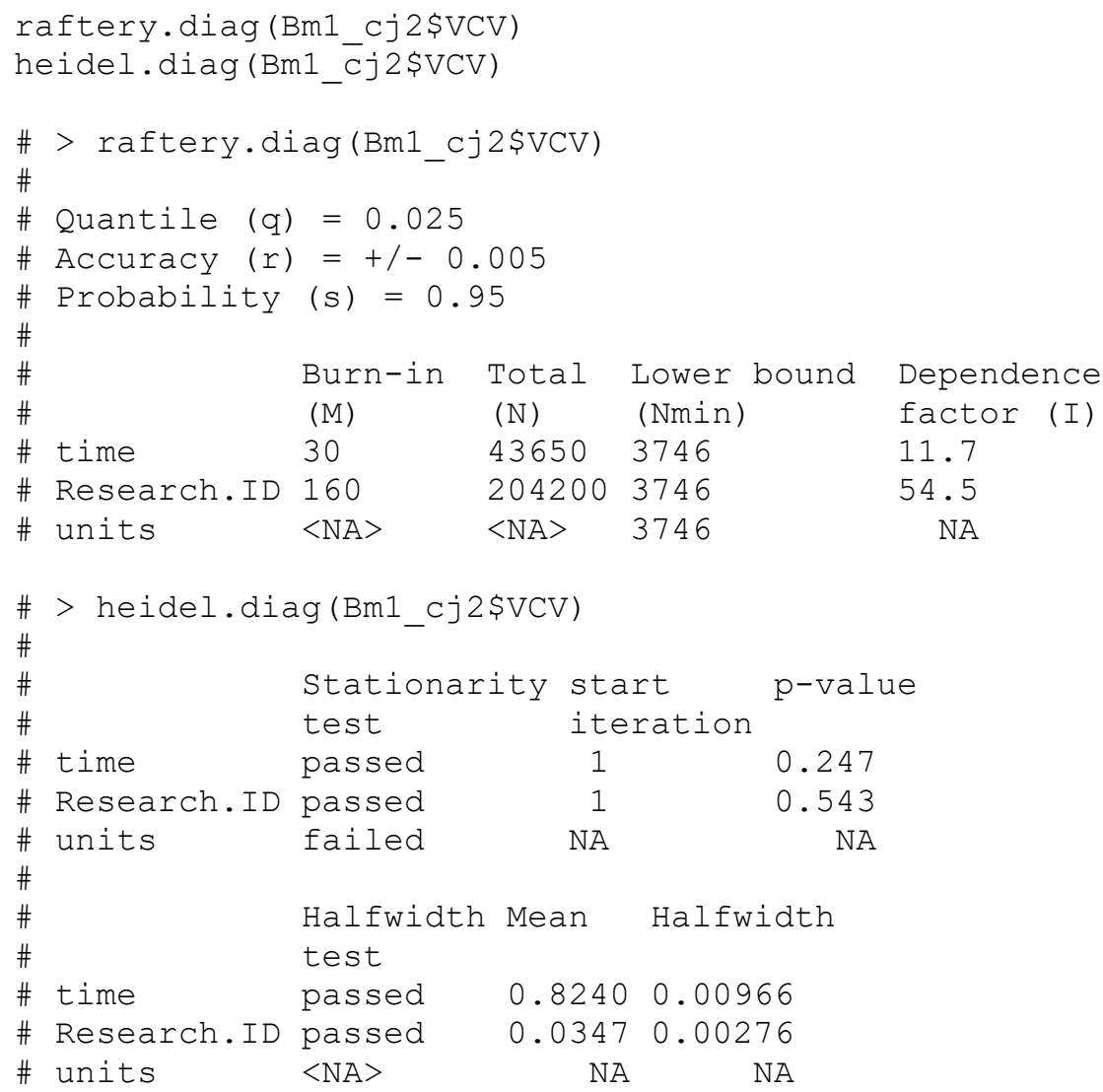




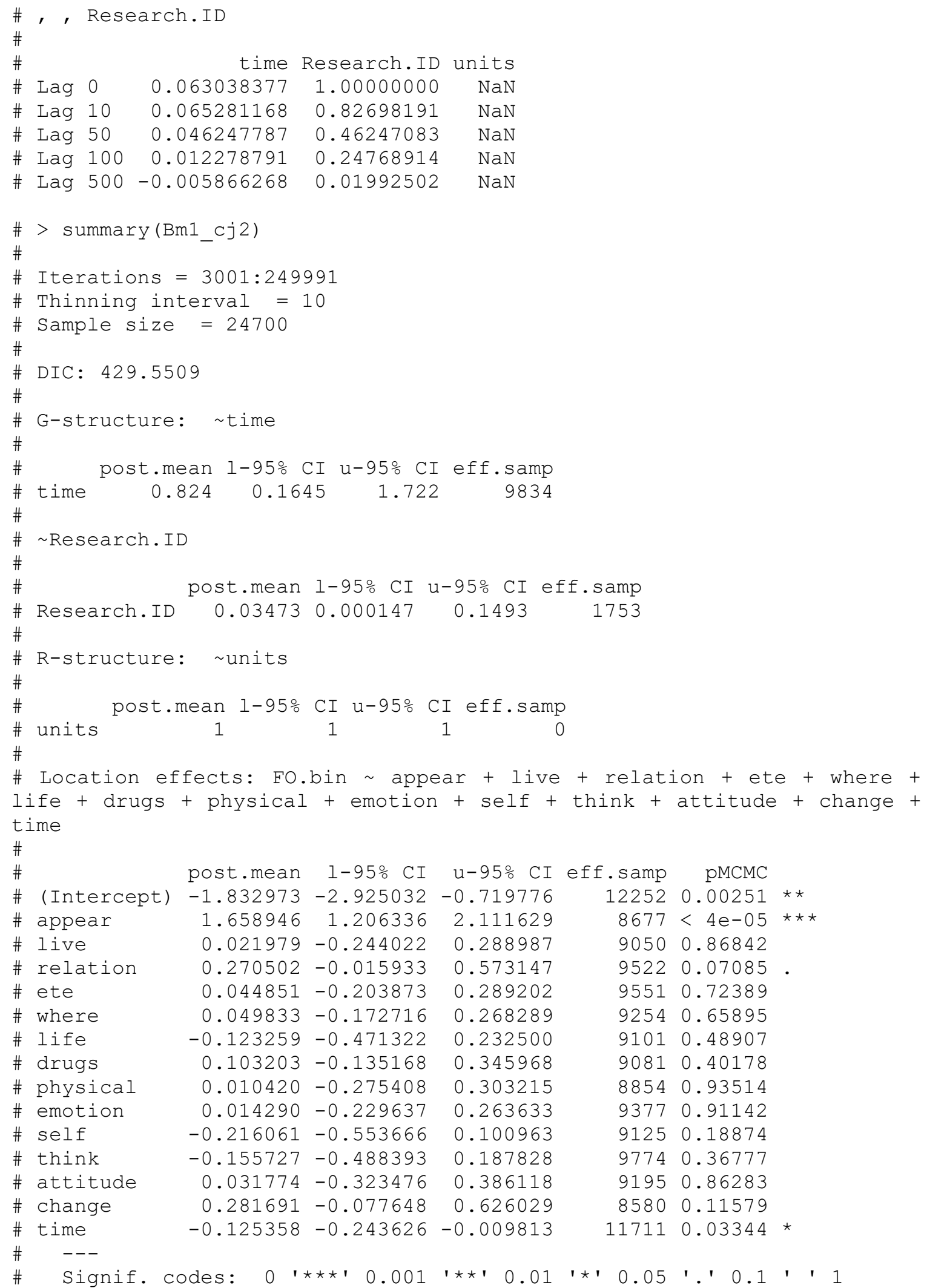




\section{Frequentist Model (m1_cj2)}

m1_cj2 <- glmer(FO.bin appear + live + relation + ete + where + life + drugs + physical + emotion + self + think + attitude + change + time + (timelIndividual), data=data, family=binomial)

summary (m1_cj2)

vcomps.icc (m1_cj2)

anova (m1, m1_cje)

\# Generalized linear mixed model fit by maximum likelihood (Laplace Approximation) [glmerMod]

\# Family: binomial ( logit)

\# Formula: FO.bin appear + live + relation + ete + where + life + drugs +

\# physical + emotion + self + think + attitude + change + time +

\# (time | Individual)

\# Data: data

\#

\# AIC BIC logLik deviance df.resid

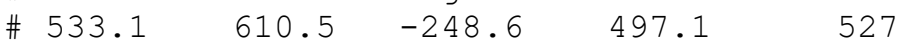

\#

\# Scaled residuals:

\# Min 12 Median 30 Max

$\begin{array}{llllll}\# & -1.7861 & -0.4465 & -0.2578 & 0.6134 & 9.4408\end{array}$

$\#$

\# Random effects:

\# Groups Name Variance Std.Dev. Corr

\# Individual (Intercept) 0.0382200 .19550

\# time $0.0062820 .07926-1.00$

\# Number of obs: 545, groups: Individual, 87

$\#$

\# Fixed effects:

\# $\quad$ Estimate Std. Error z value $\operatorname{Pr}(>|z|)$

\# (Intercept) $-2.034945 \quad 0.369552-5.507 \quad 3.66 \mathrm{e}-08$

\# live $\quad 0.004493 \quad 0.1510390 .030 \quad 0.976$

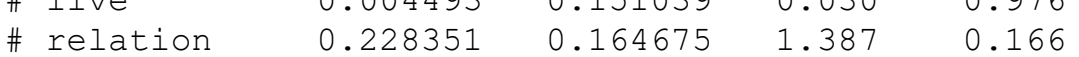

$\begin{array}{lllll}\# \text { ete } & -0.006867 & 0.141854 & -0.048 & 0.961\end{array}$

$\begin{array}{lllll}\text { \# where } & 0.036791 & 0.127525 & 0.288 & 0.773\end{array}$

$\begin{array}{lllll}\# \text { life } & -0.137637 & 0.201059 & -0.685 & 0.494\end{array}$

$\begin{array}{lllll}\text { \# drugs } & 0.077222 & 0.138560 & 0.557 & 0.577\end{array}$

$\begin{array}{lllll}\text { \# physical } & 0.031244 & 0.164426 & 0.190 & 0.849\end{array}$

$\begin{array}{lllll}\text { \# emotion } & -0.012031 & 0.139123 & -0.086 & 0.931\end{array}$

$\begin{array}{lllll}\# \text { self } & -0.178852 & 0.183423 & -0.975 & 0.330\end{array}$

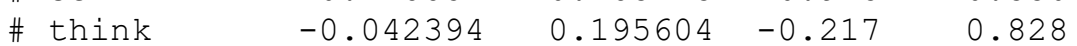

$\begin{array}{lllll}\text { \# attitude } & -0.067963 & 0.204140 & -0.333 & 0.739\end{array}$

$\begin{array}{lllll}\text { \# change } & 0.315790 & 0.196219 & 1.609 & 0.108\end{array}$

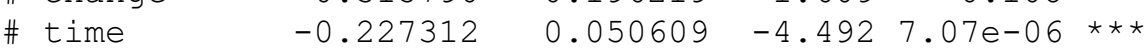

\# $\quad---$

\# Signif. codes: $0{ }^{\prime * \star * \prime} 0.001$ '**' 0.01 '*' 0.05 '.' 0.1 ' ' 1

\# convergence code: 0

\# Model failed to converge with max|grad $=0.0132078$ (tol = 0.001, component 1)
\# vcomps.icc (m1_cj2)
\# Var (Level 2) Var (Level 1) ICC <NA>
$\begin{array}{rrrrr}\# & 0.038 & 0.006 & 1.000 & 0.859\end{array}$ 


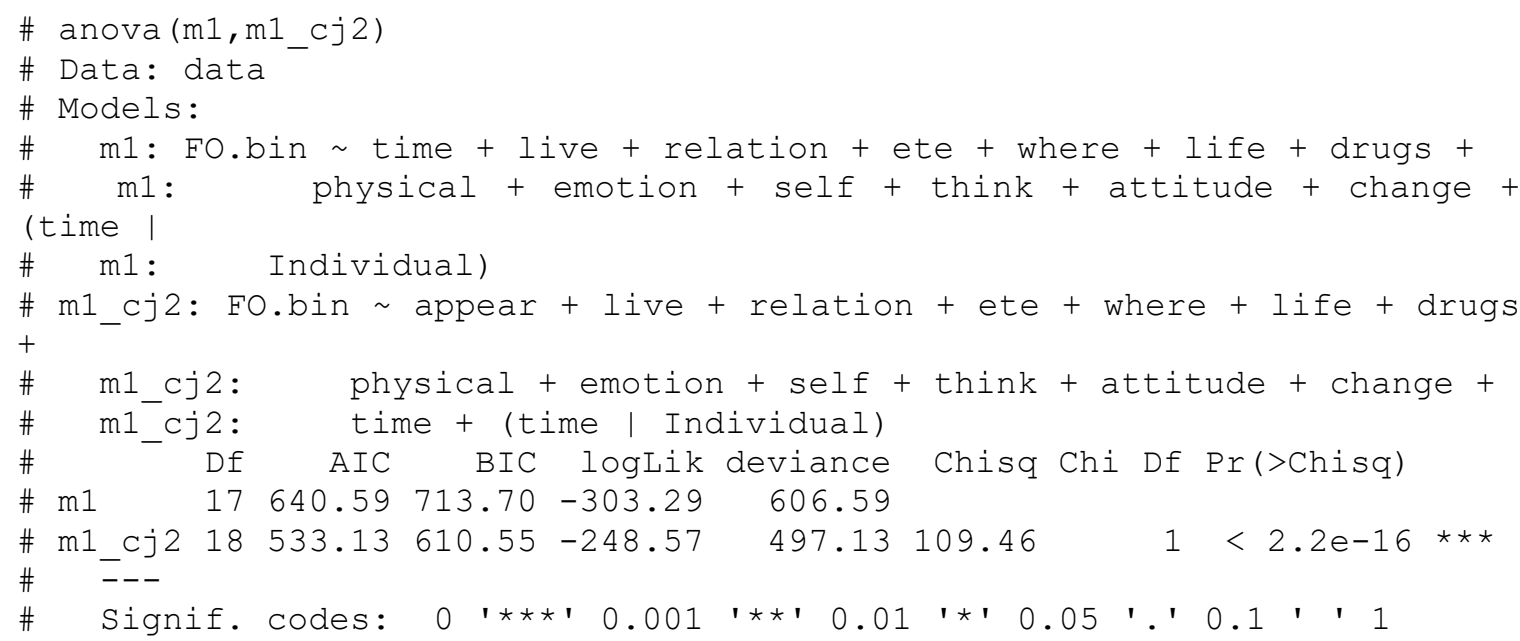


Model 1.14 - Basic Model + Custody (Table 7.4)

Bayesian Model (Bm1_cj3)

\section{\#\# Define the model}

Bm1_cj3 <- MCMCglmm(FO.bin custody + live + relation + ete + where + life + drugs + physical + emotion + self + think + attitude + change + time,

random= time+Research.ID, data=data, family="ordinal", prior=prior2, nitt $=210000$, thin=10, burnin=3000)

\section{\# Checks for suitable convergence}

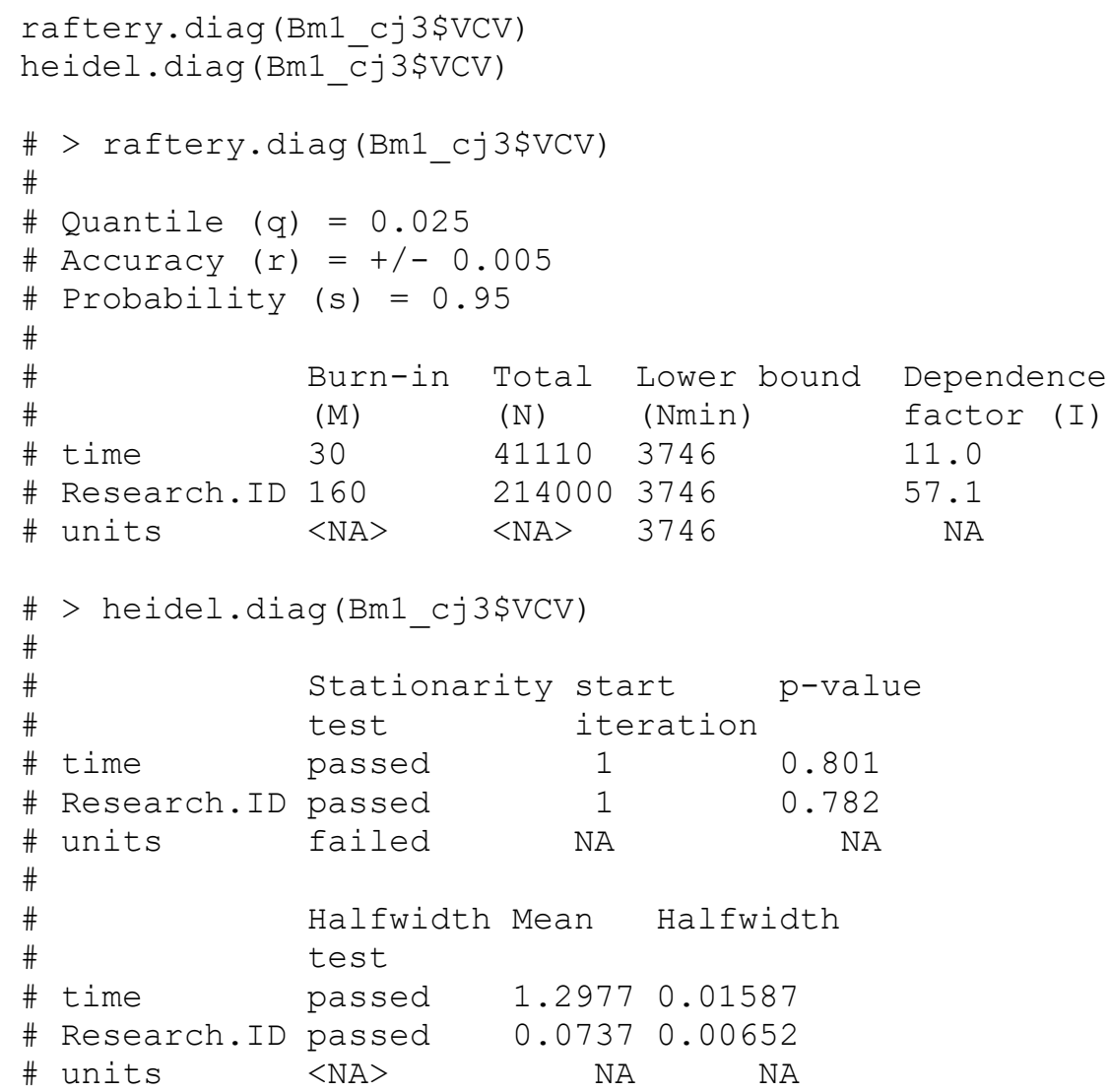




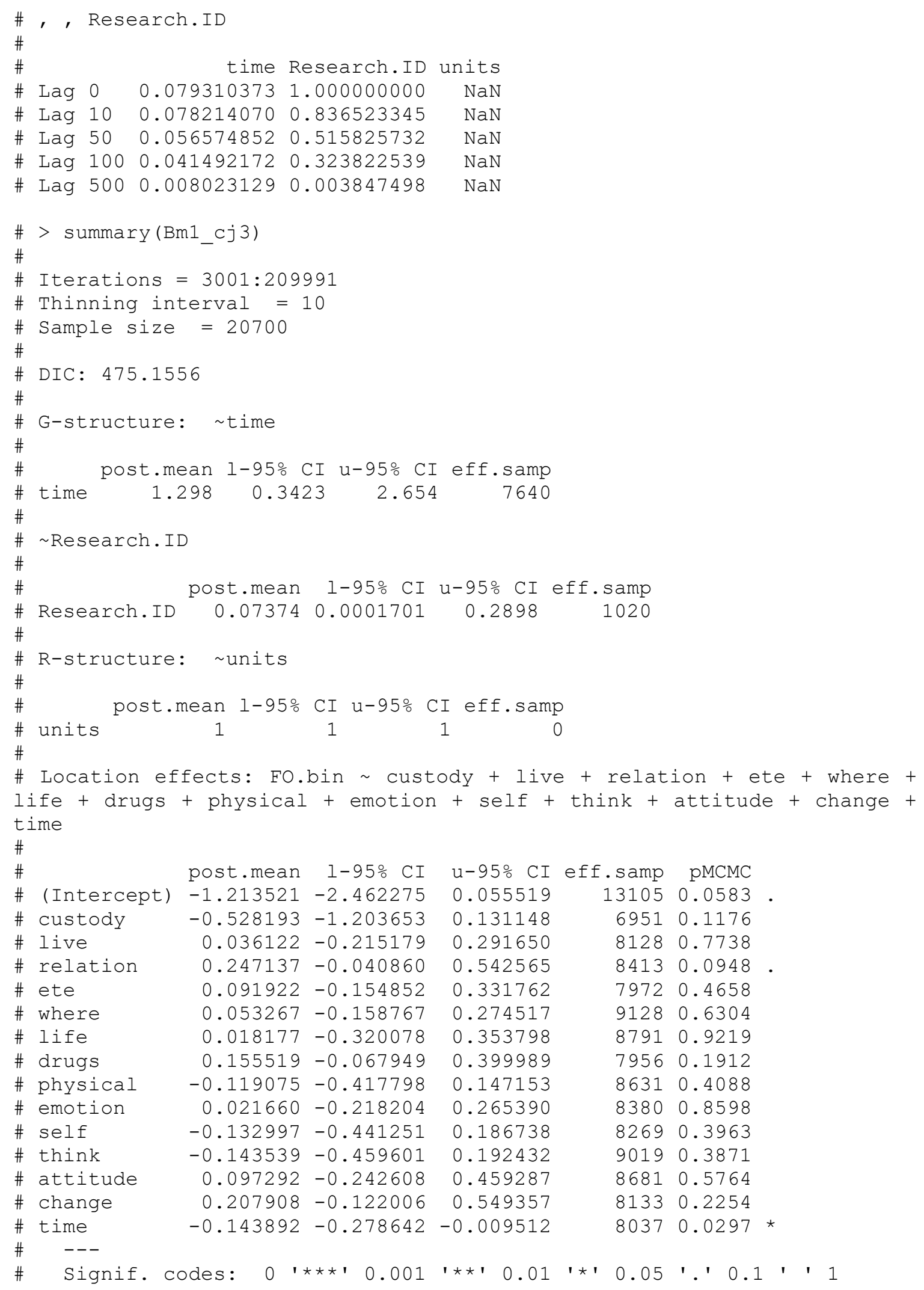




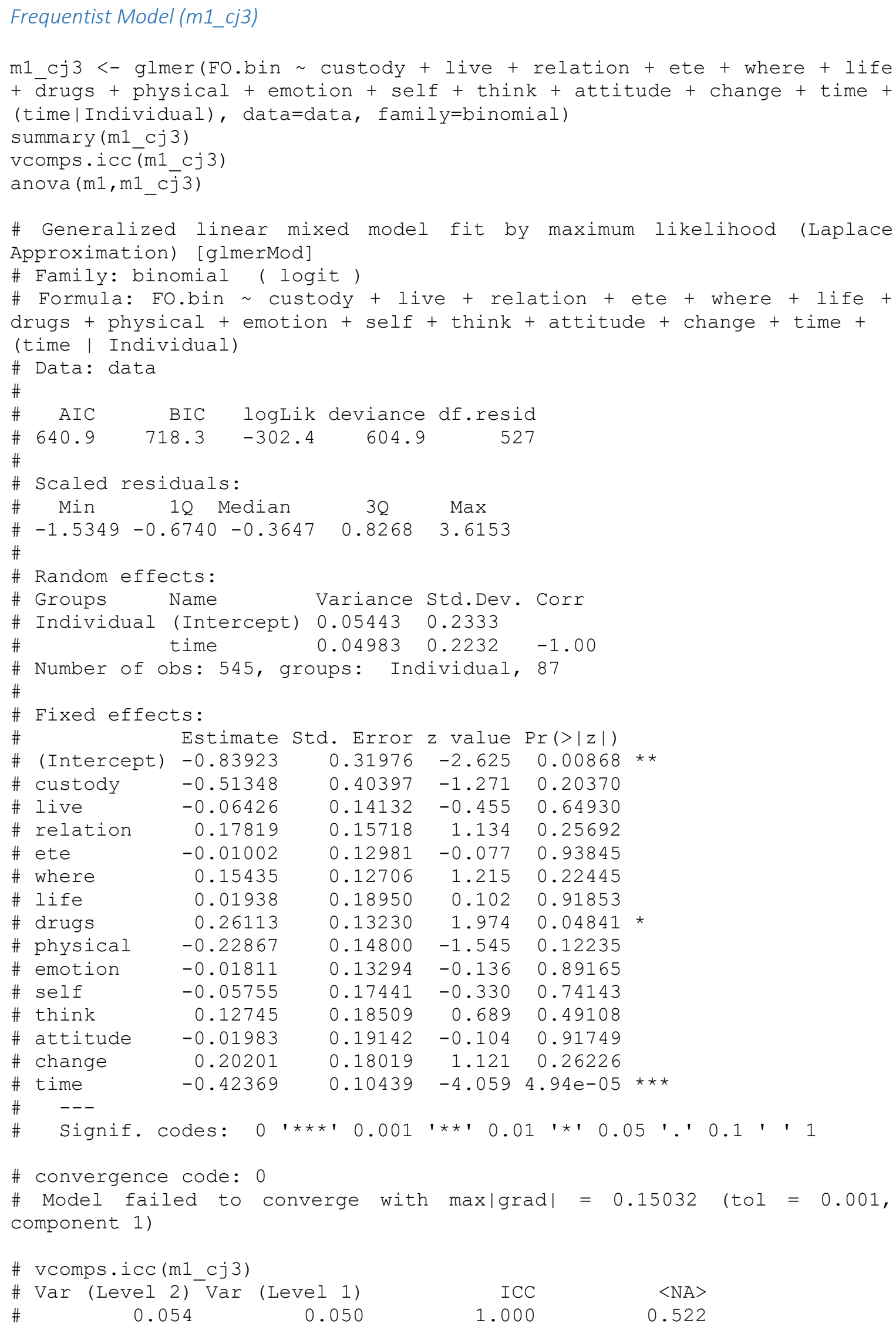




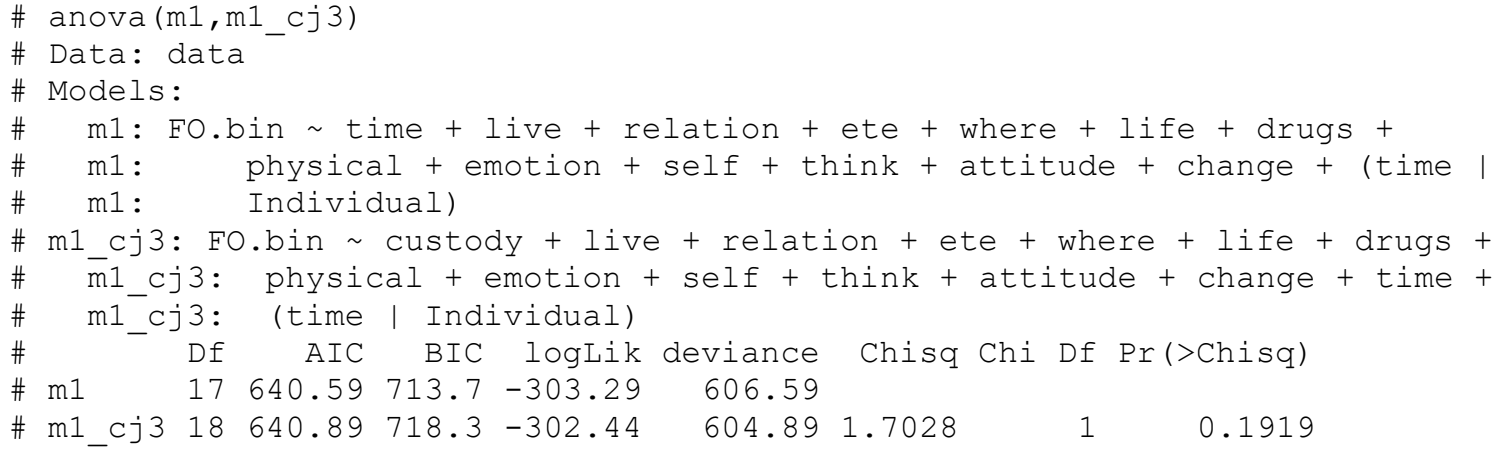


Dynamic Model 5: Breaches (Table 7.5)

Bayesian Model (BDm5 B)

\section{\# Define the model}

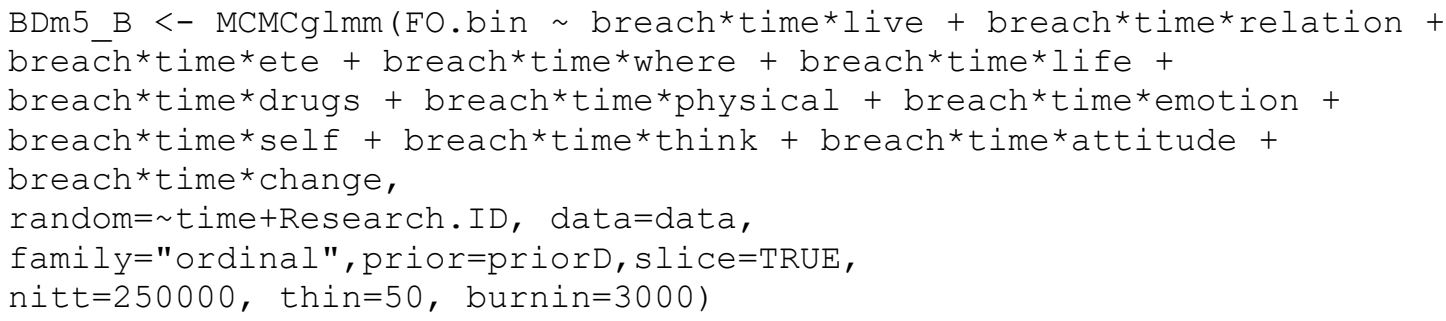

\section{\#\# Checks for suitable convergence}

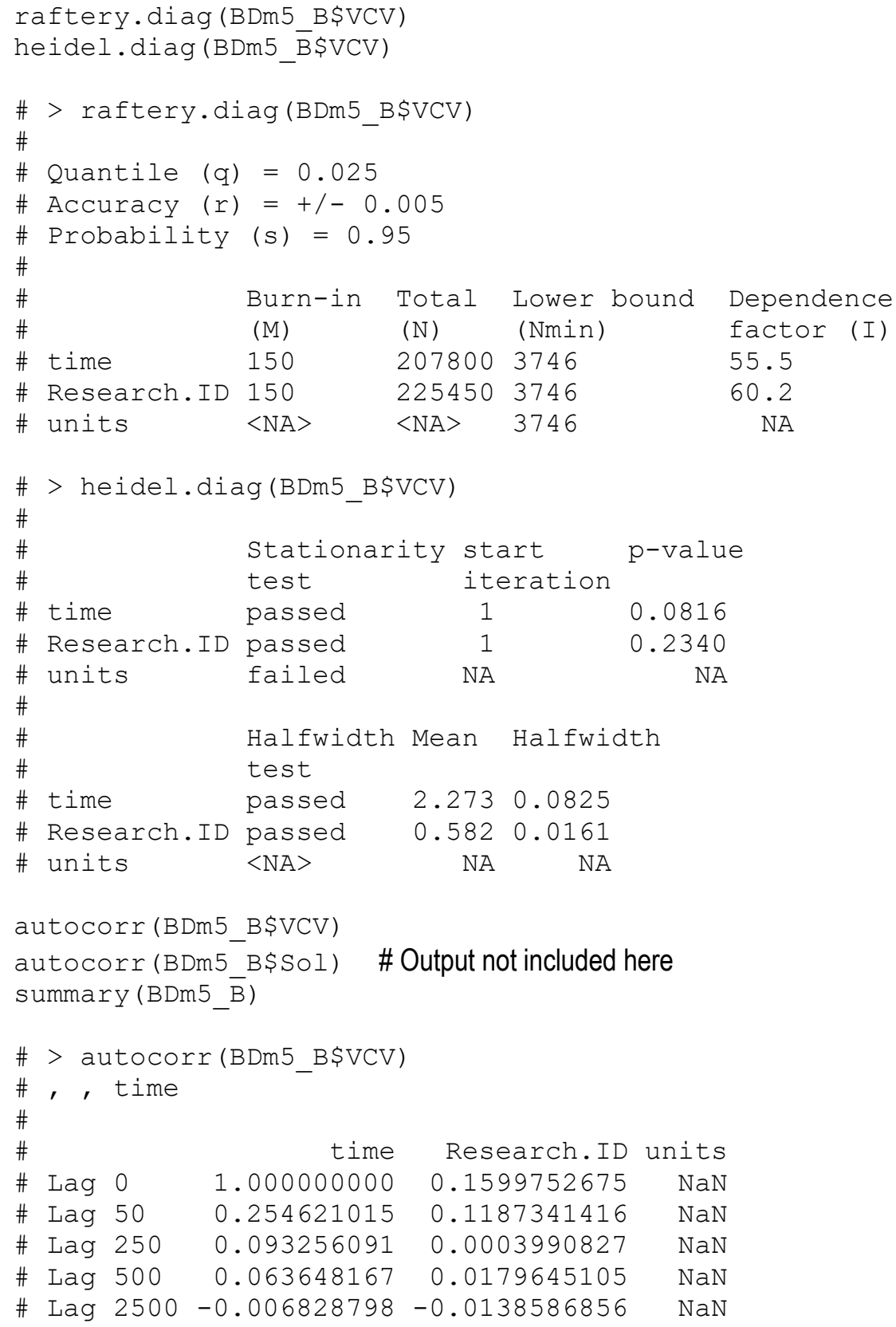




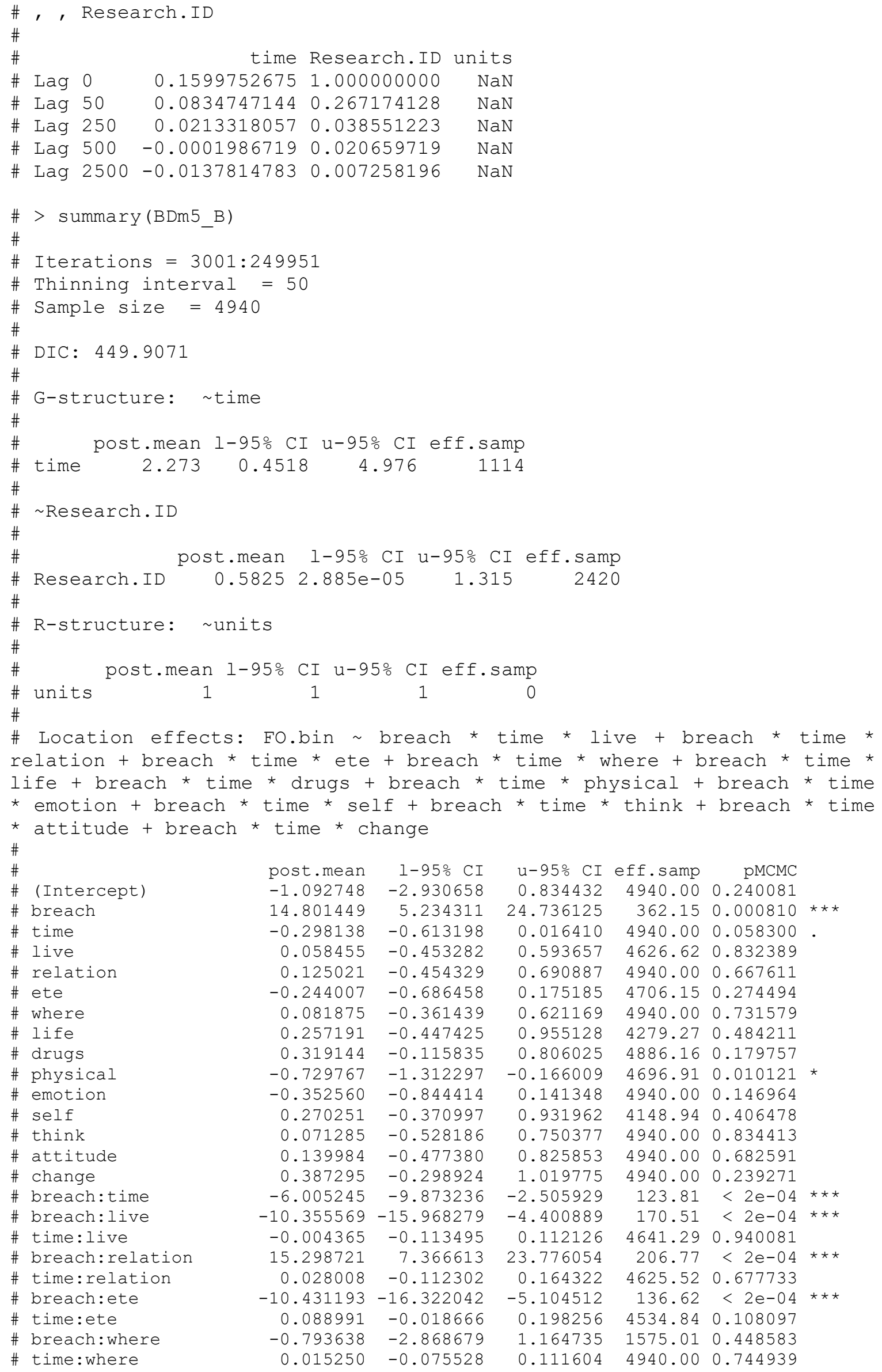




\begin{tabular}{|c|c|c|c|c|c|c|c|}
\hline$\#$ & breach:life & 4.784246 & -0.907616 & 10.398429 & 529.71 & 0.087854 & . \\
\hline \# & time:life & -0.056880 & -0.204665 & 0.093713 & 4421.70 & 0.455870 & \\
\hline \# & breach:drugs & -3.050524 & -6.599359 & 0.048901 & 966.40 & 0.059919 & . \\
\hline \# & time:drugs & -0.020533 & -0.120113 & 0.087057 & 4940.00 & 0.689474 & \\
\hline$\#$ & breach:physical & 4.932068 & 1.218155 & 9.212560 & 540.35 & 0.002834 & $\star \star$ \\
\hline \# & time:physical & 0.151894 & 0.019748 & 0.291163 & 4940.00 & 0.034818 & $\star$ \\
\hline \# & breach:emotion & 7.798455 & 3.609135 & 12.234344 & 171.73 & $<2 e-04$ & $\star \star \star$ \\
\hline \# & time: emotion & 0.118724 & 0.002507 & 0.233172 & 4644.83 & 0.037247 & $\star$ \\
\hline \# & breach:self & 6.533446 & 1.588917 & 11.396637 & 429.82 & 0.004453 & $\star \star$ \\
\hline \# & time:self & -0.096029 & -0.229016 & 0.037262 & 4940.00 & 0.1639 & \\
\hline \# & breach:think & $-19 \cdot 325248$ & -29.823146 & -7.733745 & 173.07 & $<2 e-04$ & $\star \star \star$ \\
\hline \# & time:think & -0.046171 & -0.190511 & 0.090131 & 4940.00 & 0.533603 & \\
\hline \# & breach:attitude & -4.25 & -9.508121 & 0.888145 & 1153.11 & 0.1 & \\
\hline \# & time:attitude & -0.05 & -0.212348 & 0.10 & 4940.00 & 0.5 & \\
\hline \# & breach: change & 10.73 & 4.120 & 17.668559 & 262.35 & 0.0 & $\star \star \star$ \\
\hline \# & time:change & -0.034837 & -0.192263 & 0.104689 & 4940.00 & 0.642 & \\
\hline \# & breach:time:live & 1.10 & 0.09 & 2.15 & 0.08 & 0.02 & * \\
\hline \# & breach:time:relation & -0.70 & -2.248 & 0.77 & 9.33 & 0.3623 & \\
\hline \# & breach:time:ete & 2.29 & 0.89 & 3.973414 & 2.44 & $<2 e-$ & $\star \star \star$ \\
\hline \# & breach:time: where & -1.07 & -1.857900 & -0.24 & 57.08 & 0.006478 & $\star \star$ \\
\hline \# & breach:time:life & 1.490473 & -0.087001 & 3.07 & 268.38 & 0.052632 & . \\
\hline \# & breach:time:drugs & 1.377608 & 0.64177 & 2.217526 & 312.88 & $<2 e-04$ & $\star \star \star$ \\
\hline \# & breach:time:physical & -1.865093 & -3.357332 & -0.635078 & 165.28 & 0.0012 & $\star \star$ \\
\hline \# & breach:time:emotion & -2.80 & -4.44 & -1.45 & 4.71 & $<$ & $\star \star \star$ \\
\hline \# & breach:time:self & -1.364682 & -2.244127 & -0.504141 & 271.59 & 0.000405 & $\star \star \star$ \\
\hline$\#$ & breach:time:think & 2.389414 & 0.680819 & 4.157169 & 251.71 & 0.002024 & $\star \star$ \\
\hline \# & breach:time:attitude & 1.432577 & 0.346706 & 2.469320 & 227.02 & 0.003 & $\star \star$ \\
\hline \# & breach:time: change & -3.625822 & -5.677445 & -1.557652 & 102.97 & $<2 e-04$ & $\star \star \star$ \\
\hline & Signif. codes: 0 & & & $005 \quad$ ' & $0.1 \quad$ & 1 & \\
\hline
\end{tabular}




\section{Fixed Effects}

Trace of (Intercept)

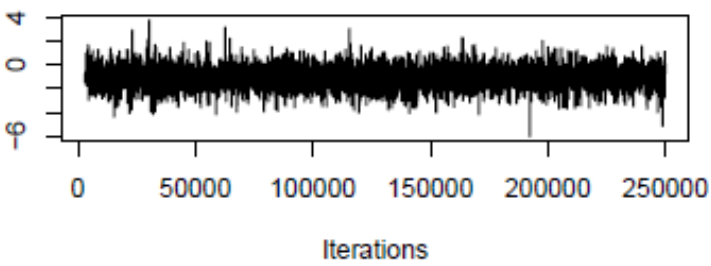

Trace of breach

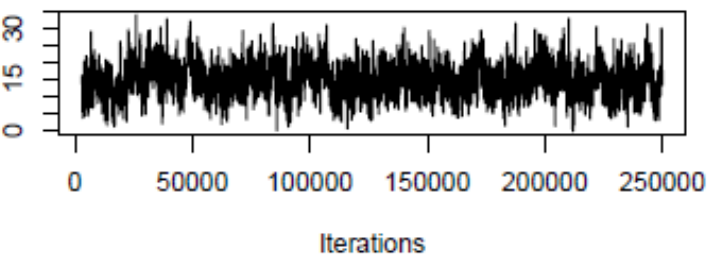

\section{Trace of time}

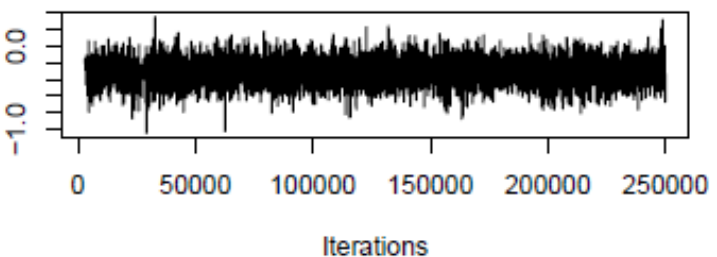

Trace of live

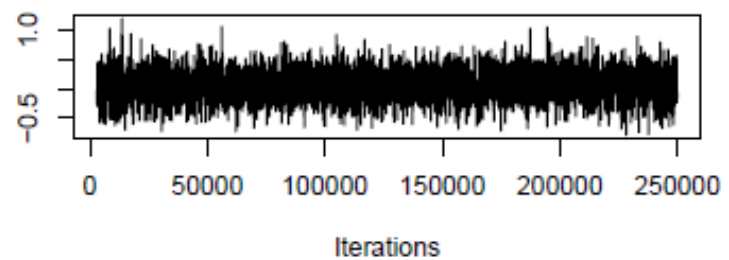

Trace of relation

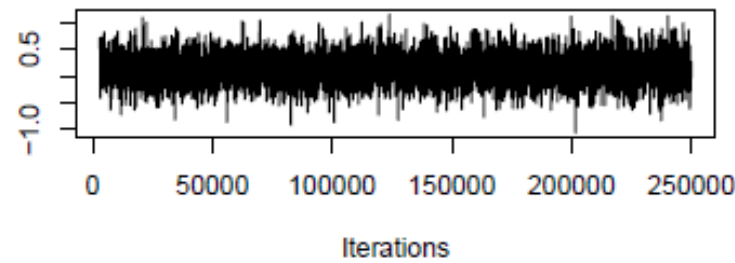

Density of (Intercept)

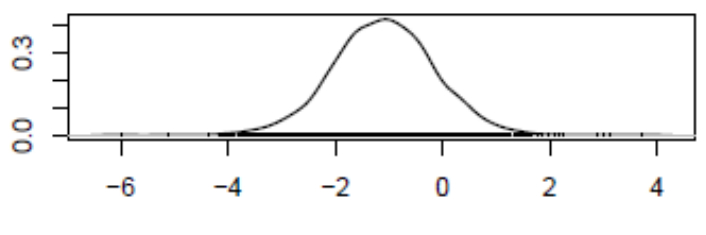

$\mathrm{N}=4940$ Bandwidth $=0.1797$

Density of breach

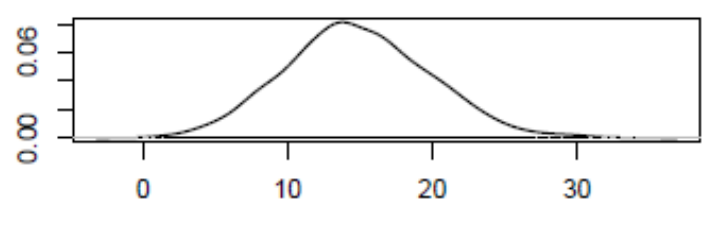

$\mathrm{N}=4940$ Bandwidth $=0.9651$

\section{Density of time}

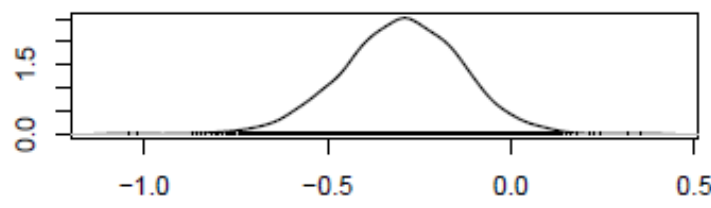

$\mathrm{N}=4940$ Bandwidth $=0.03113$

Density of live

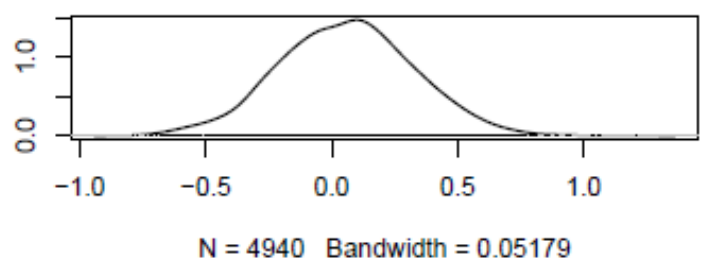

Density of relation

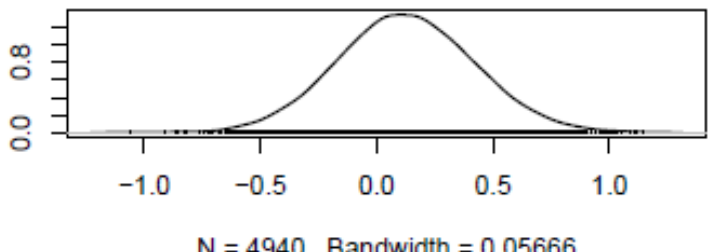

$\mathrm{N}=4940$ Bandwidth $=0.05666$ 
Trace of ete

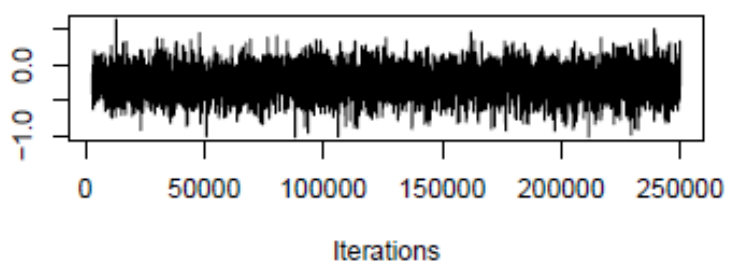

Trace of where

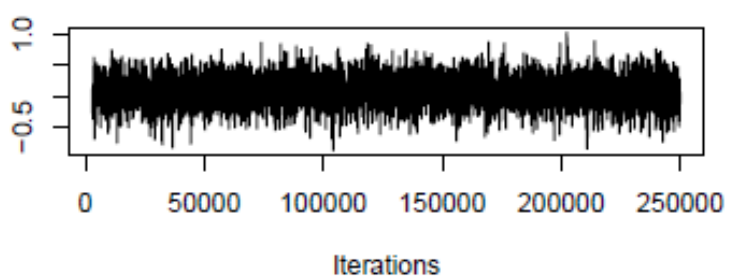

Trace of life

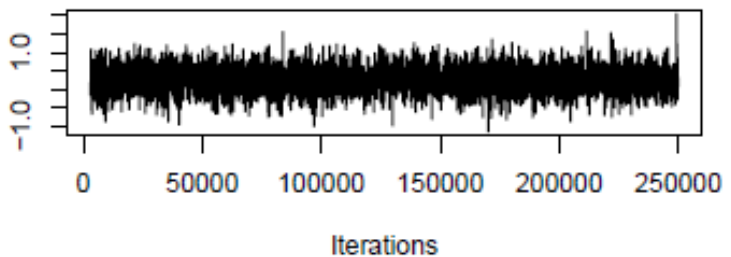

Trace of drugs

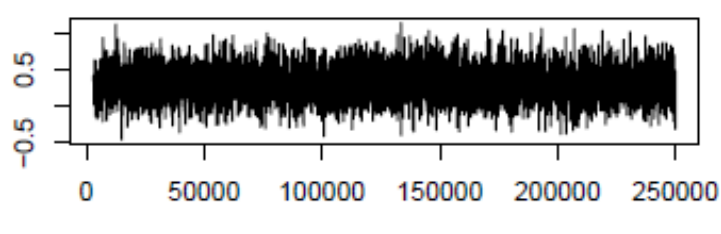

Iterations

Trace of physical

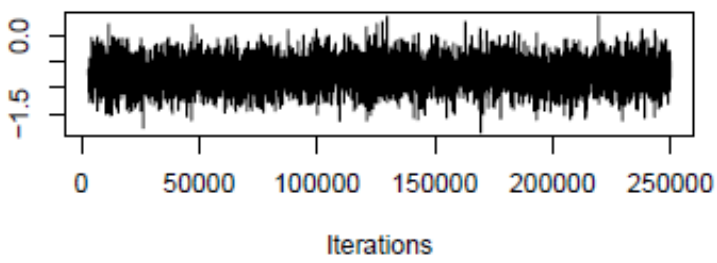

Trace of emotion

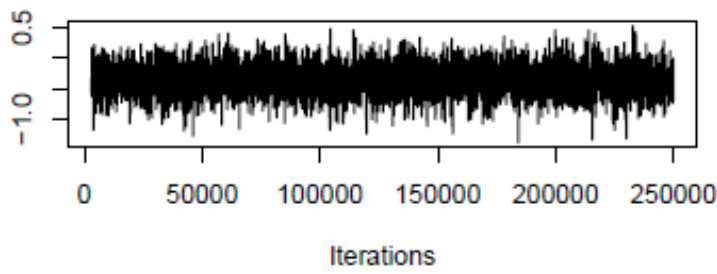

Density of ete

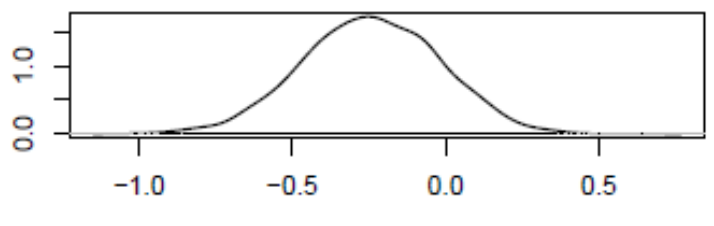

$\mathrm{N}=4940$ Bandwidth $=0.04343$

Density of where

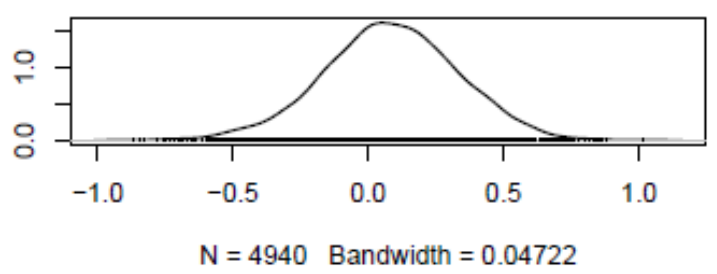

Density of life

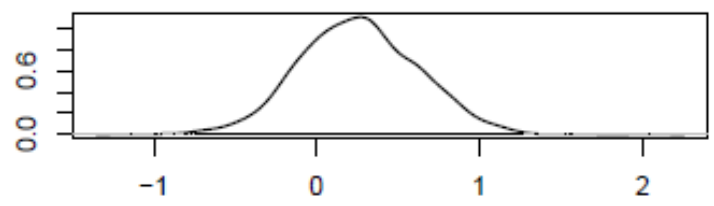

$\mathrm{N}=4940$ Bandwidth $=0.0699$

Density of drugs

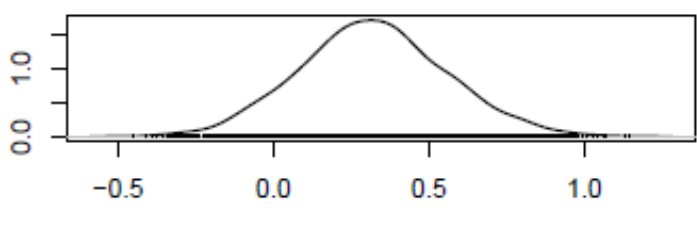

$\mathrm{N}=4940$ Bandwidth $=0.0449$

Density of physical

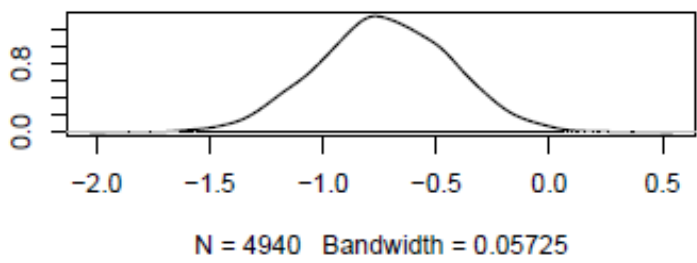

Density of emotion

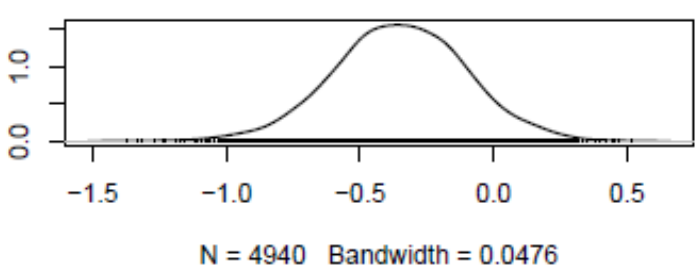


Trace of self

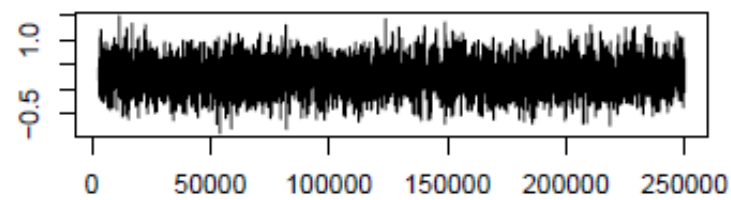

Iterations

Trace of think

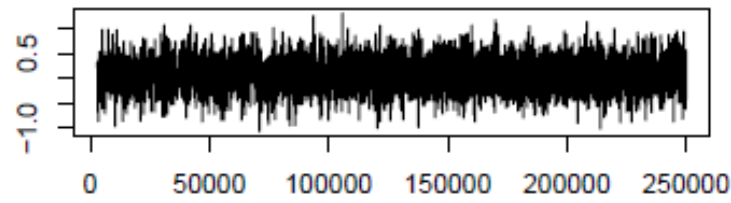

Iterations

Trace of attitude

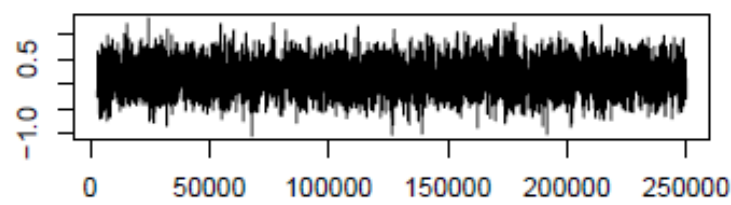

Iterations

Trace of change

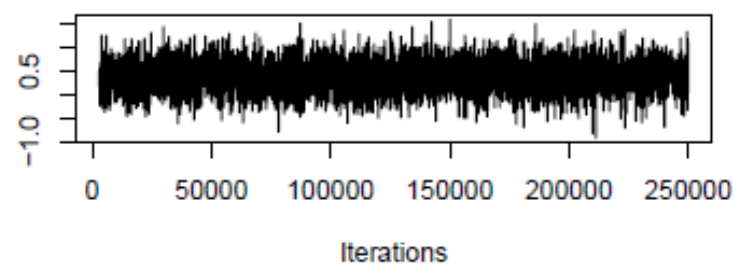

Trace of breach:time

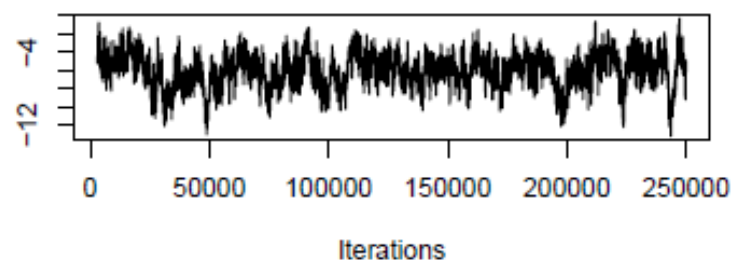

Trace of breach:live

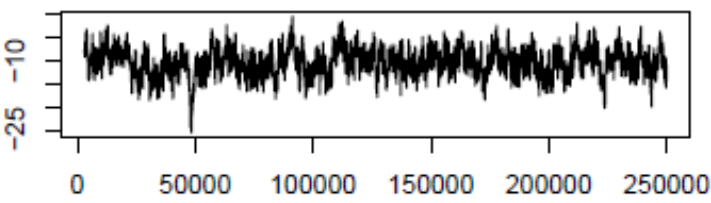

Iterations
Density of self

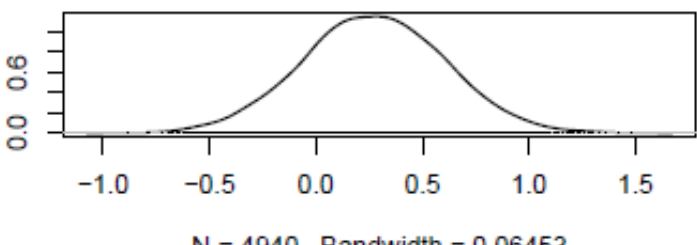

Density of think

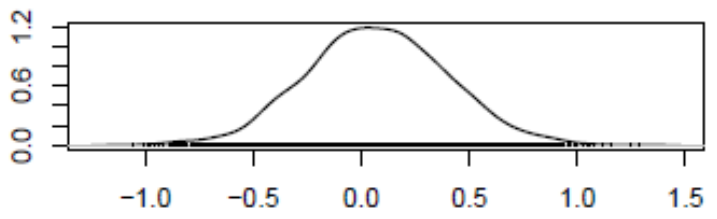

$\mathrm{N}=4940$ Bandwidth $=0.06313$

Density of attitude

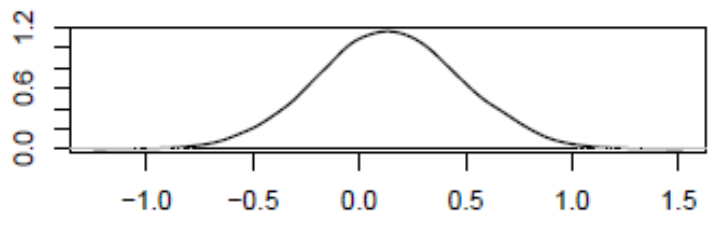

$\mathrm{N}=4940$ Bandwidth $=0.06468$

Density of change

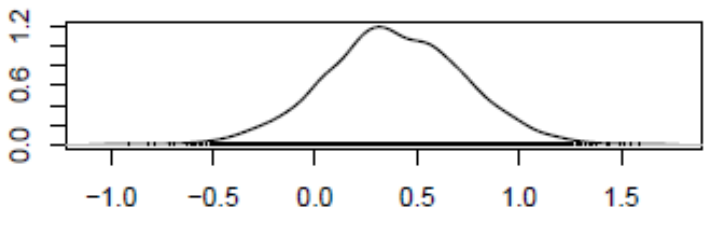

$\mathrm{N}=4940$ Bandwidth $=0.06434$

Density of breach:time

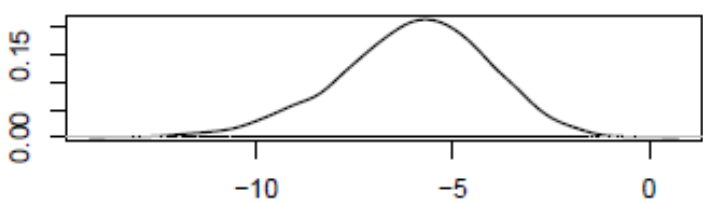

$\mathrm{N}=4940$ Bandwidth $=0.3626$

Density of breach:live

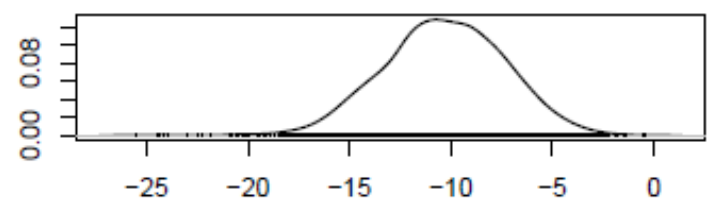

$\mathrm{N}=4940$ Bandwidth $=0.5839$ 
Trace of time:live

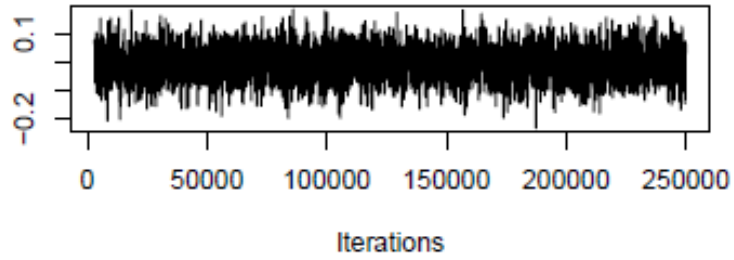

Trace of breach:relation

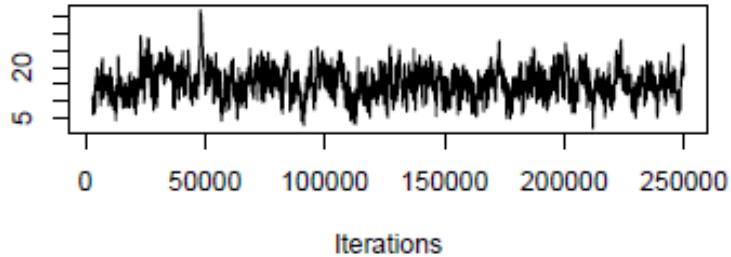

Trace of time:relation

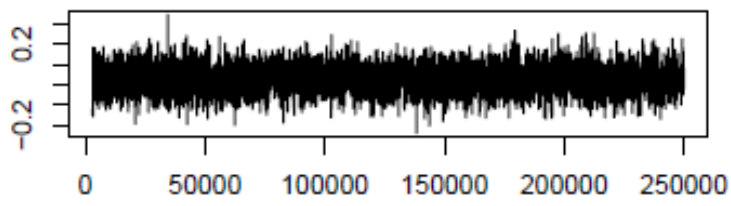

Iterations

\section{Trace of breach:ete}

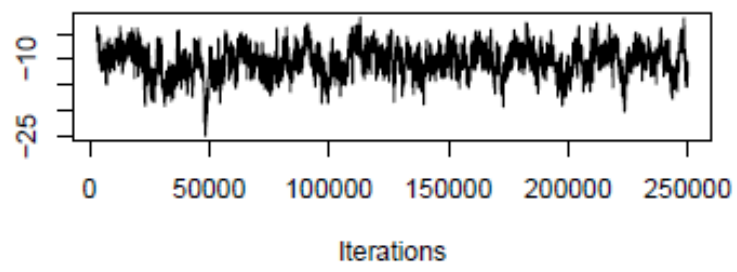

Trace of time:ete

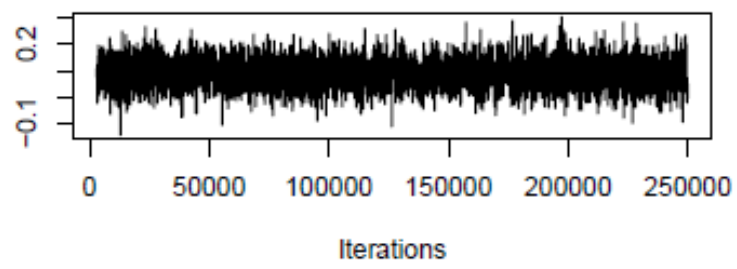

Trace of breach:where

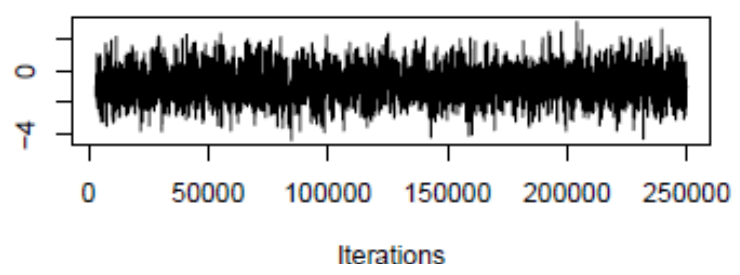

Density of time:live

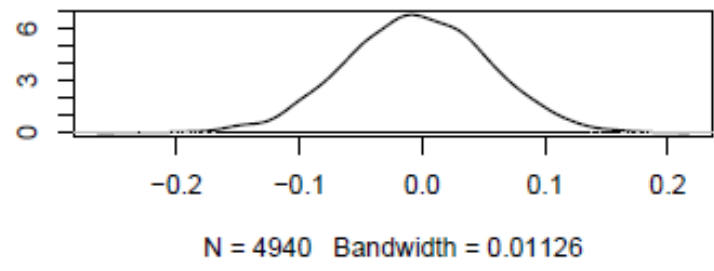

Density of breach:relation

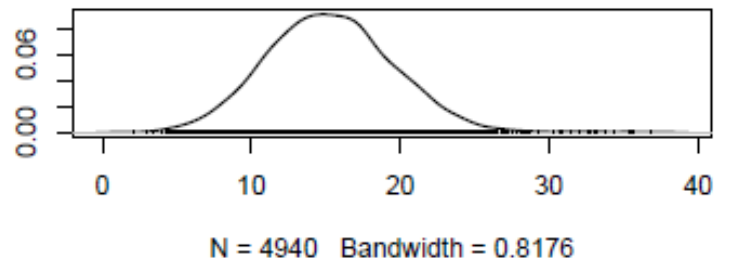

Density of time:relation

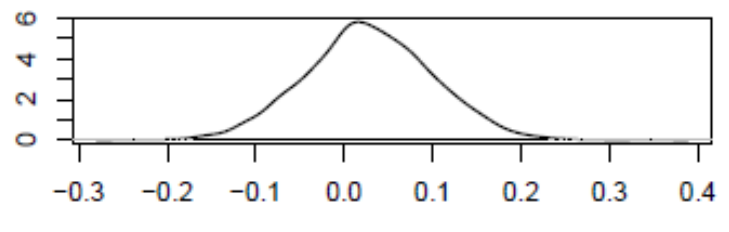

$\mathrm{N}=4940$ Bandwidth $=0.01362$

Density of breach:ete

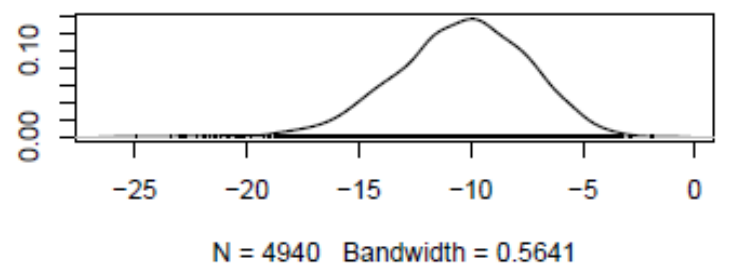

Density of time:ete

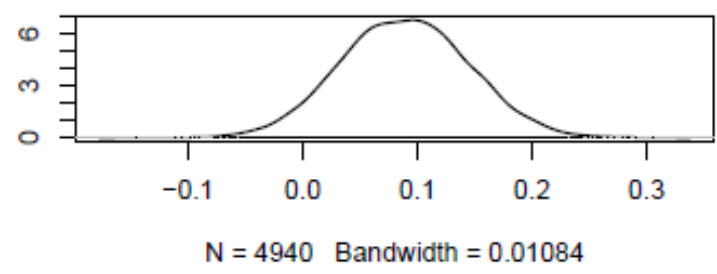

Density of breach:where

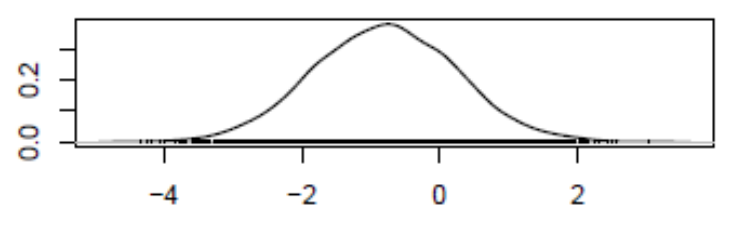

$\mathrm{N}=4940$ Bandwidth $=0.2001$ 
Trace of time:where

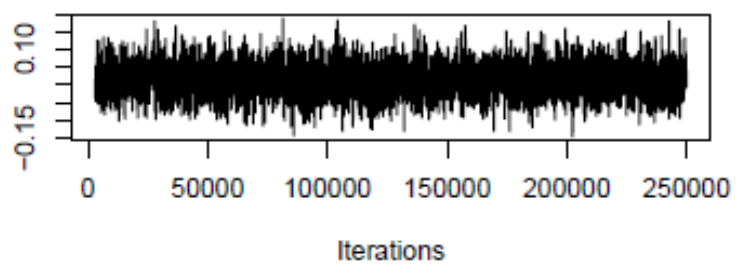

Trace of breach:life

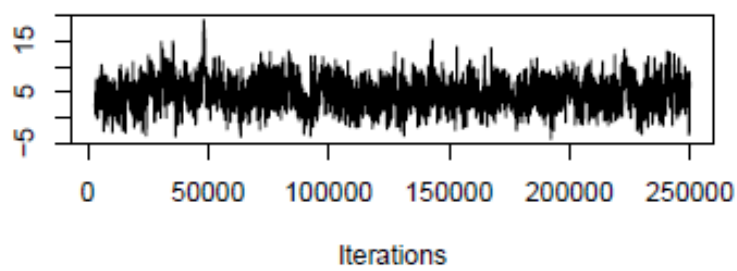

Trace of time:life

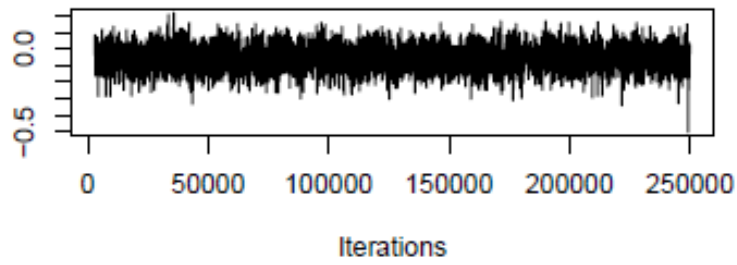

Trace of breach:drugs

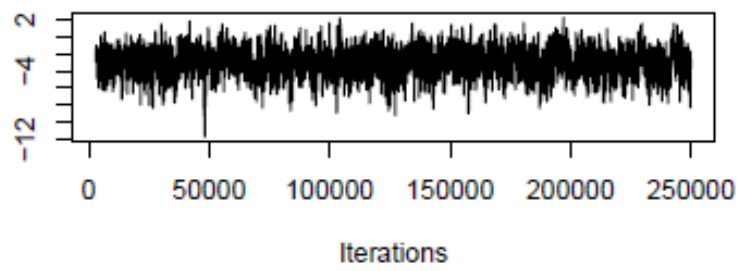

Trace of time:drugs

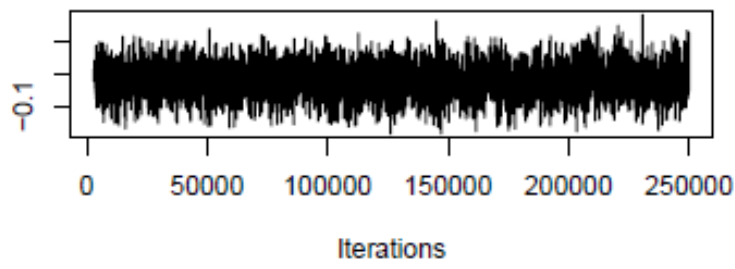

Trace of breach:physical

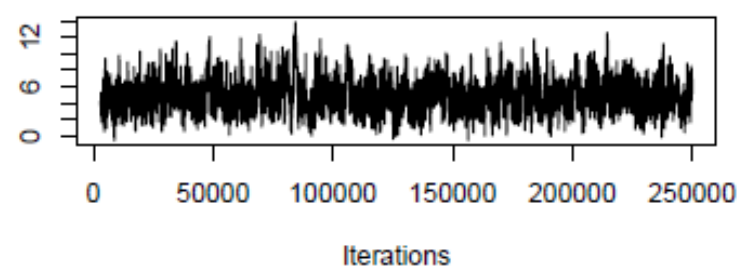

Density of time:where

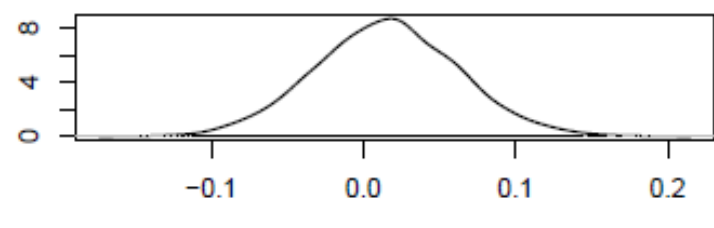

$\mathrm{N}=4940$ Bandwidth $=0.009111$

Density of breach:life

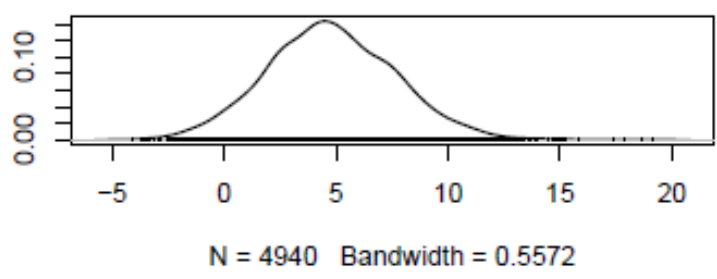

Density of time:life

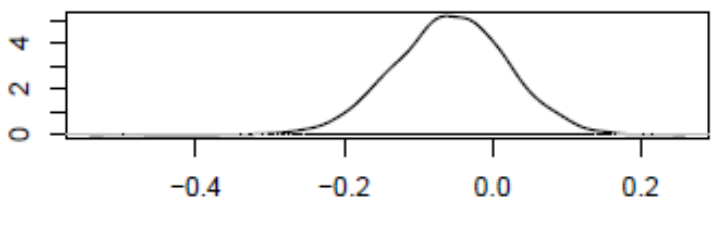

$\mathrm{N}=4940$ Bandwidth $=0.01477$

Density of breach:drugs

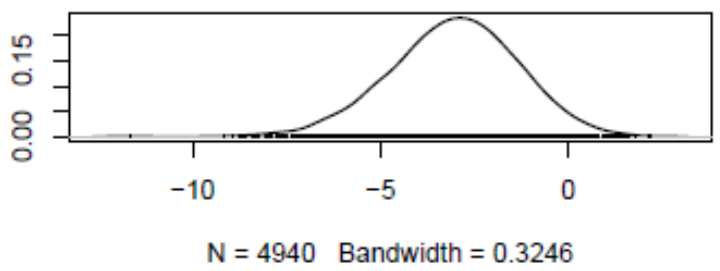

Density of time:drugs

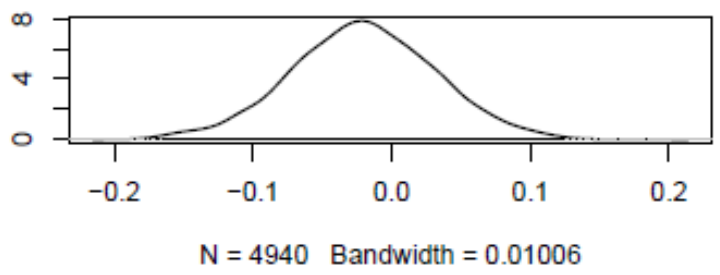

Density of breach:physical

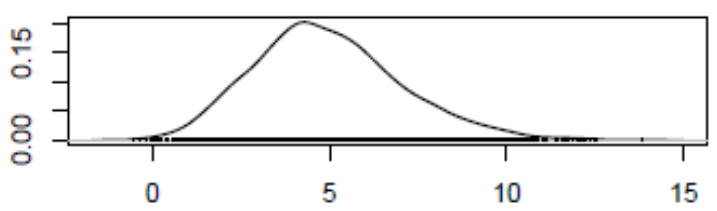

$\mathrm{N}=4940$ Bandwidth $=0.389$ 
Trace of time:physical

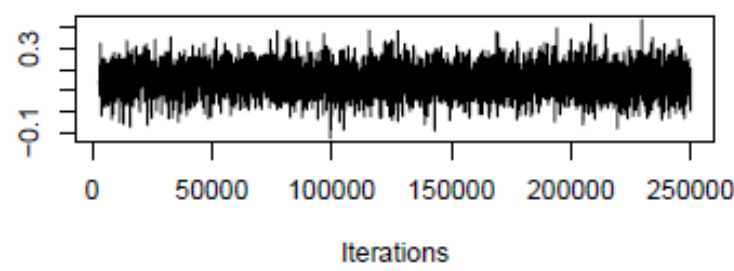

Trace of breach:emotion

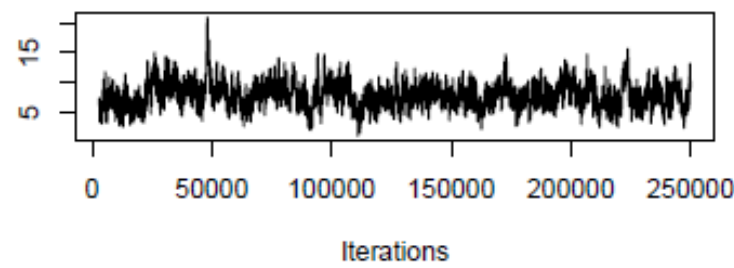

Trace of time:emotion

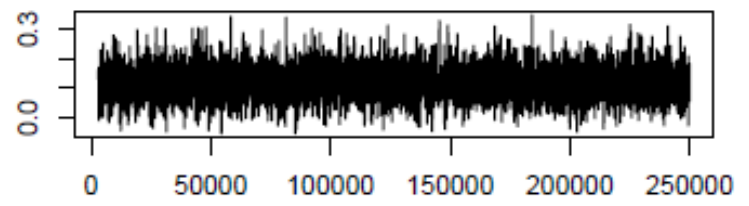

Trace of breach:self

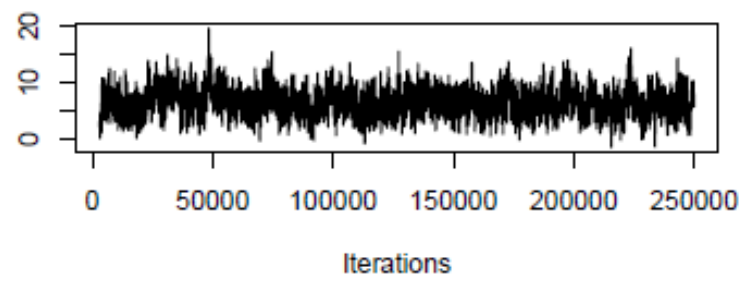

Trace of time:self

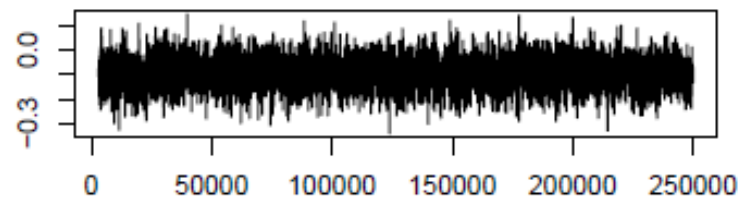

Iterations

Trace of breach:think

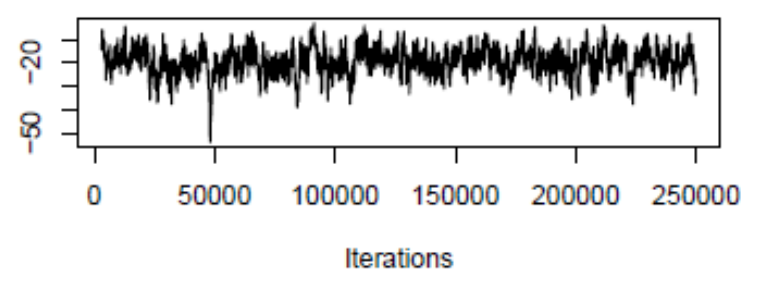

Density of time:physical

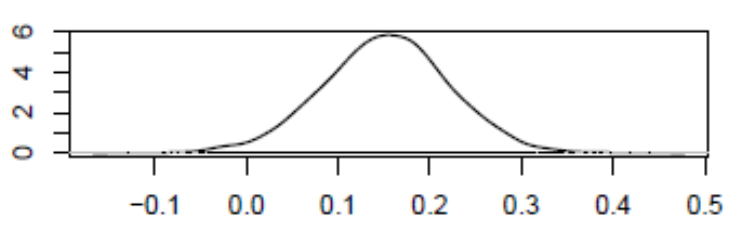

$\mathrm{N}=4940$ Bandwidth $=0.01294$

Density of breach:emotion

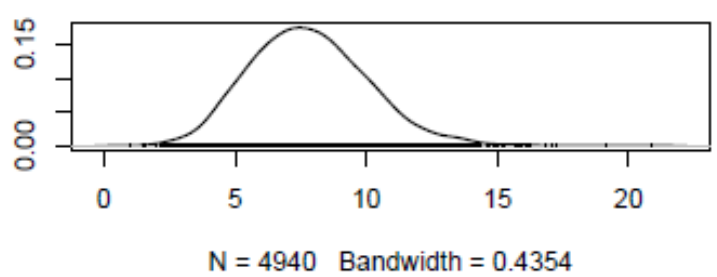

Density of time:emotion
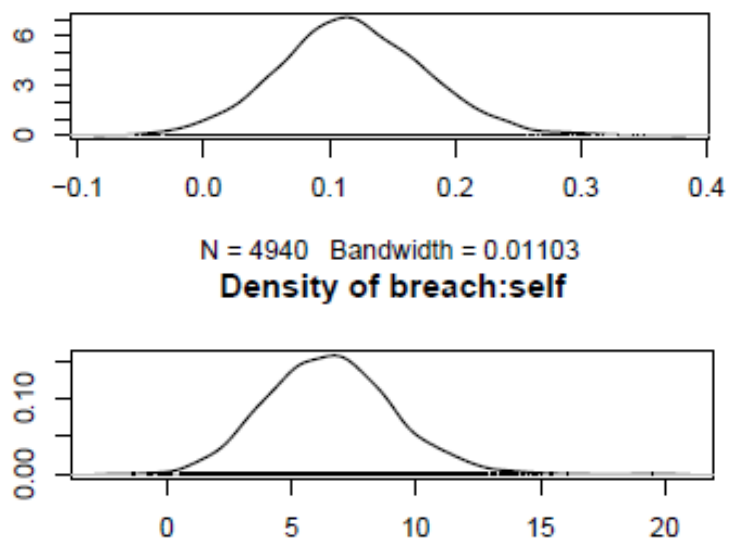

$\mathrm{N}=4940 \quad$ Bandwidth $=0.4831$

Density of time:self

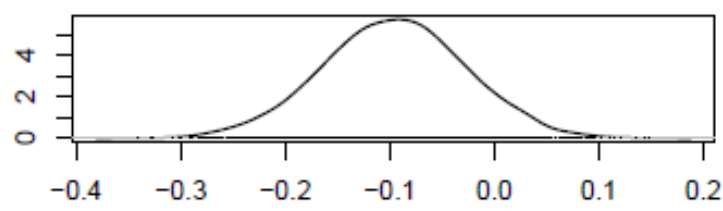

$\mathrm{N}=4940$ Bandwidth $=0.01321$

Density of breach:think

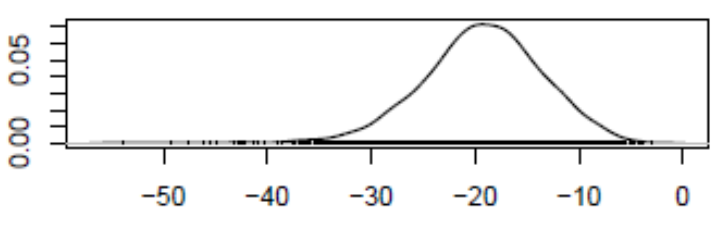

$\mathrm{N}=4940$ Bandwidth $=1.06$ 
Trace of time:think

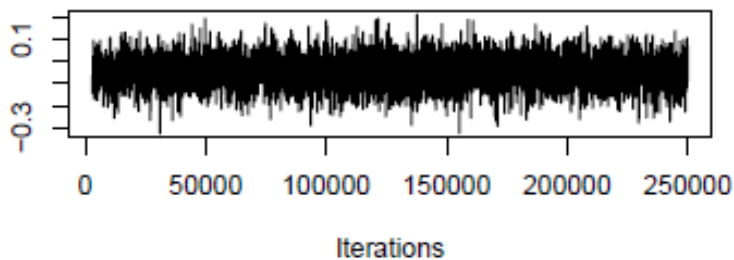

Trace of breach:attitude

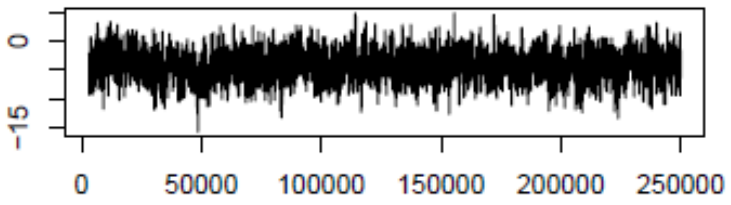

Iterations

Trace of time:attitude

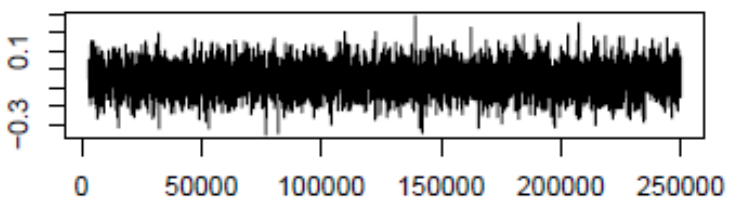

Iterations

Trace of breach:change

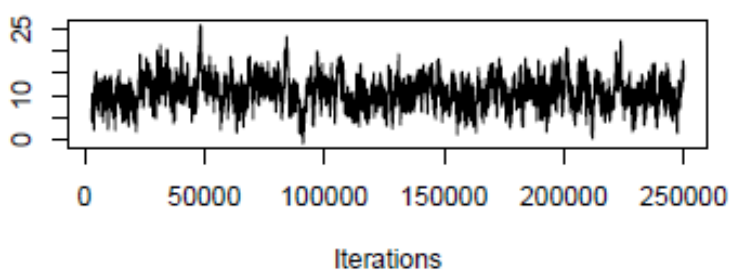

Trace of time:change

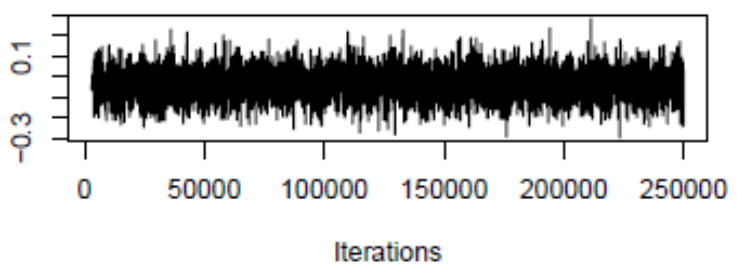

Trace of breach:time:live

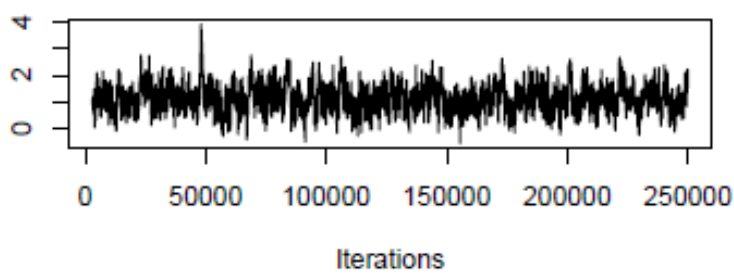

Density of time:think

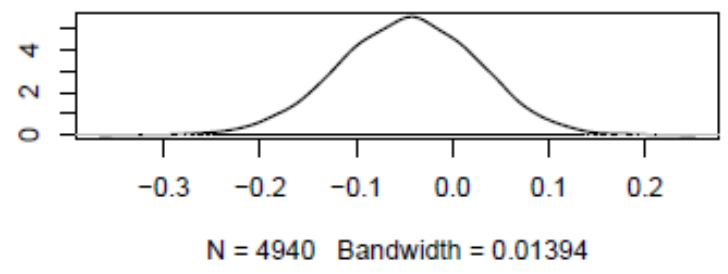

Density of breach:attitude

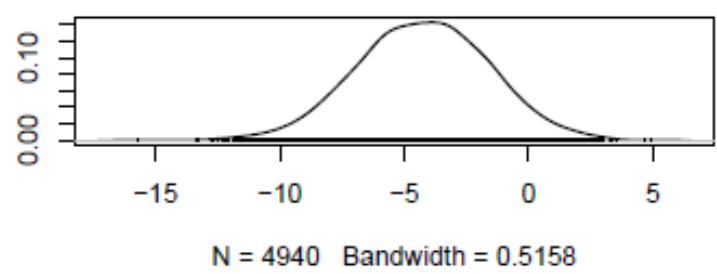

Density of time:attitude

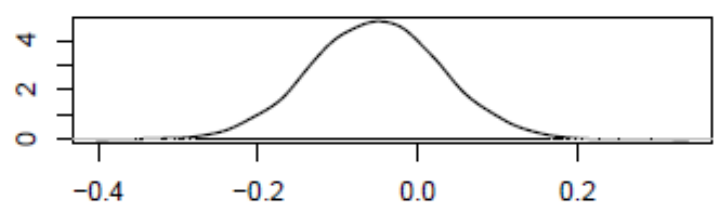

$\mathrm{N}=4940$ Bandwidth $=0.01588$

Density of breach:change

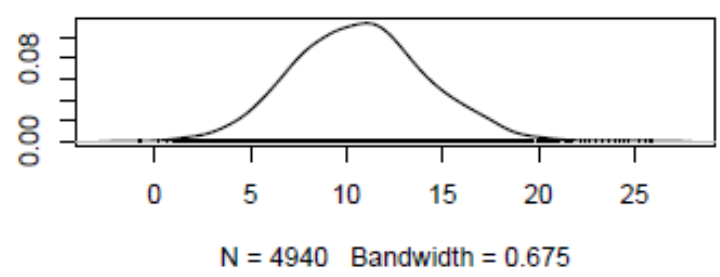

Density of time:change

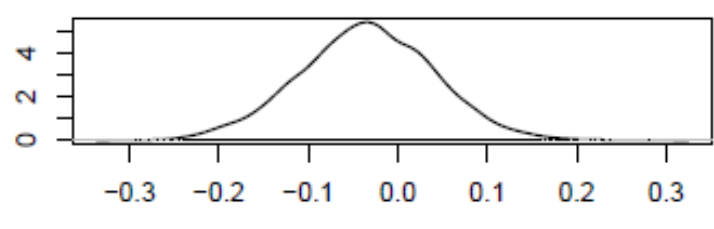

$\mathrm{N}=4940$ Bandwidth $=0.0144$

Density of breach:time:live

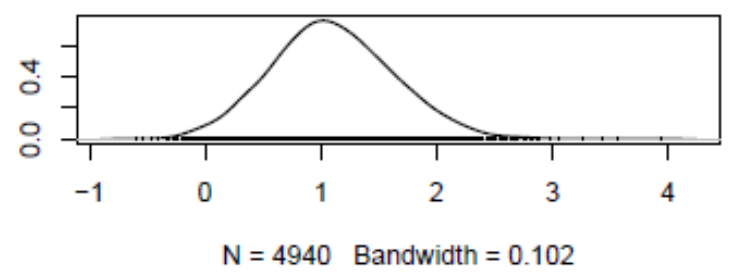

Page | 377 
Trace of breach:time:relation

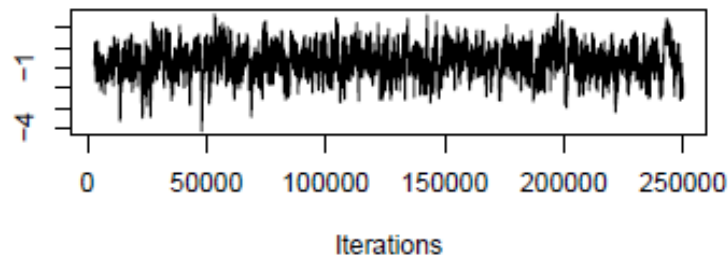

Trace of breach:time:ete

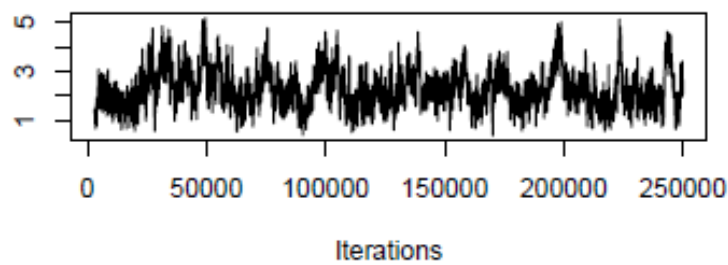

Trace of breach:time:where

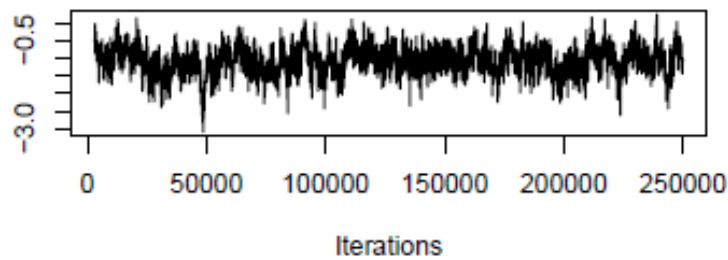

Trace of breach:time:life

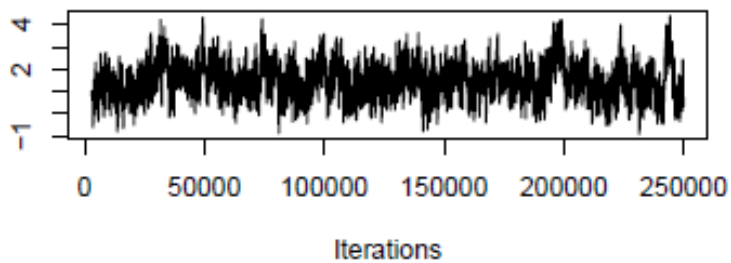

Trace of breach:time:drugs

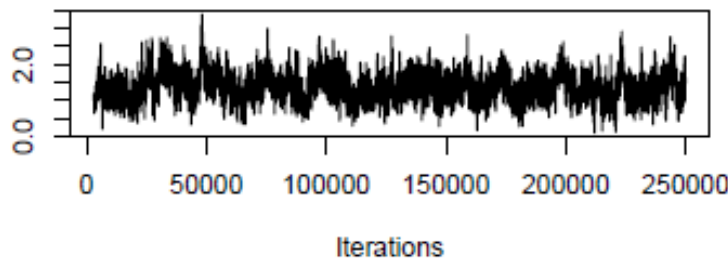

Trace of breach:time:physical

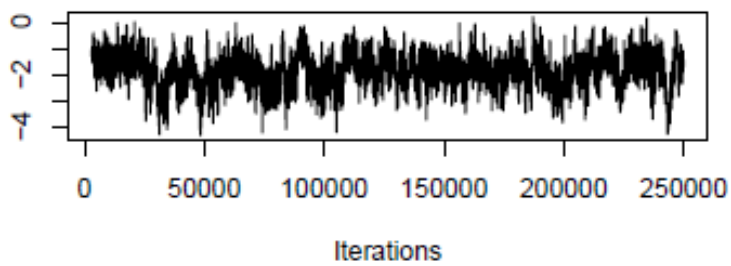

Density of breach:time:relation

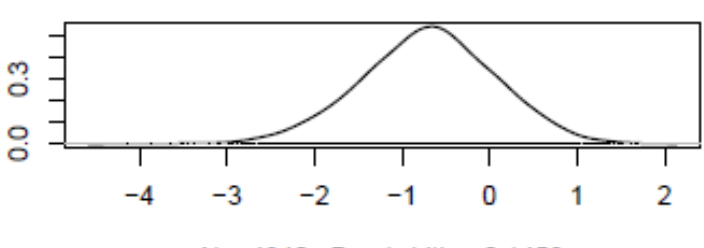

$\mathrm{N}=4940$ Bandwidth $=0.1458$

Density of breach:time:ete

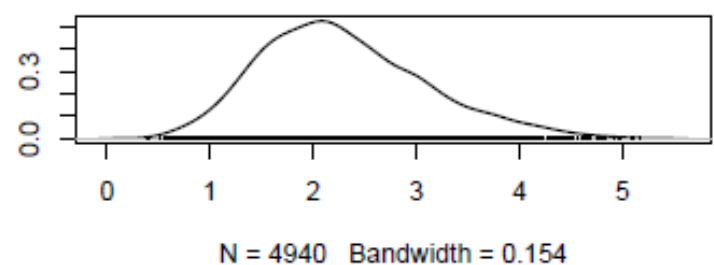

Density of breach:time:where

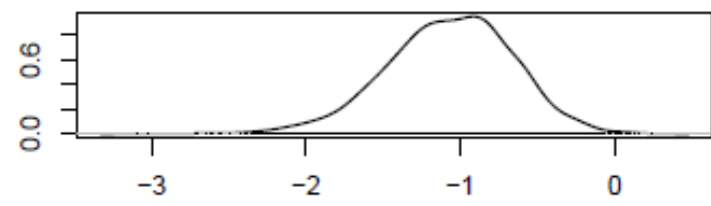

$\mathrm{N}=4940$ Bandwidth $=0.07909$

Density of breach:time:life

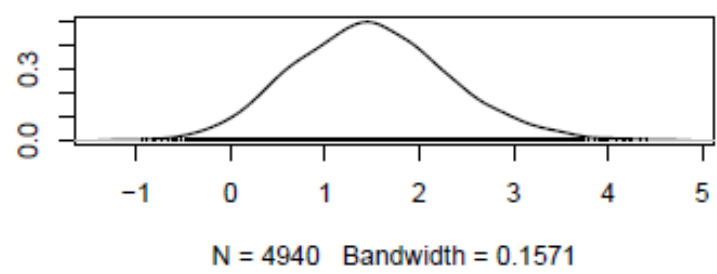

Density of breach:time:drugs

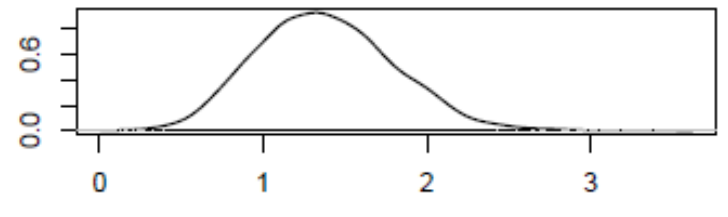

$\mathrm{N}=4940$ Bandwidth $=0.08096$

Density of breach:time:physical

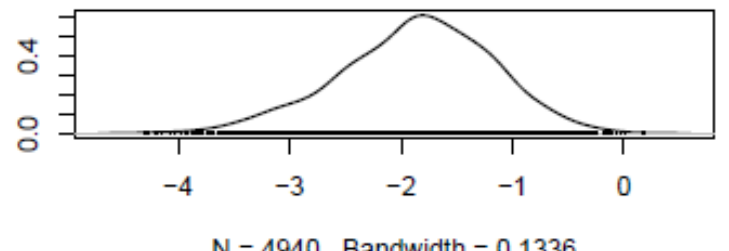

$\mathrm{N}=4940$ Bandwidth $=0.1336$ 


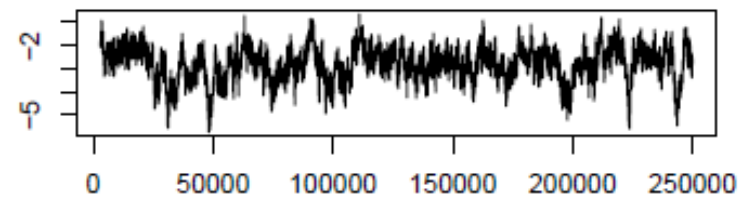

Iterations

Trace of breach:time:self

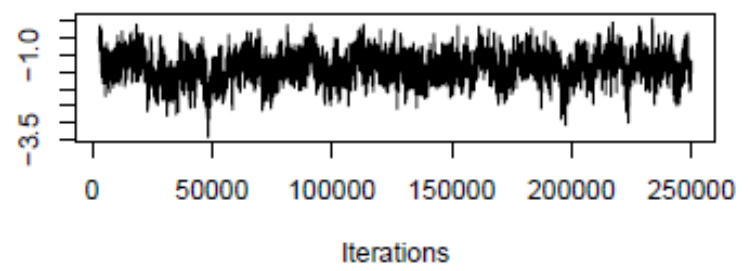

Trace of breach:time:think

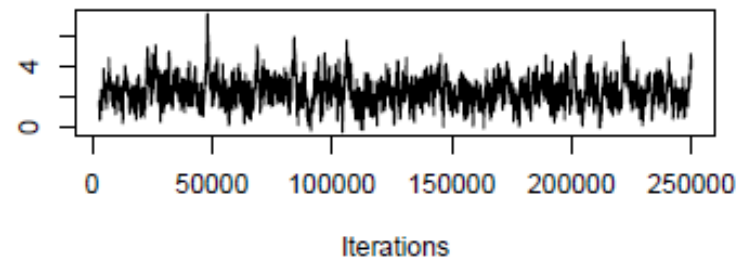

Trace of breach:time:attitude

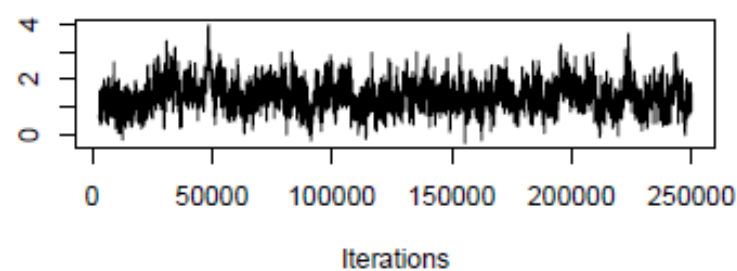

Trace of breach:time:change

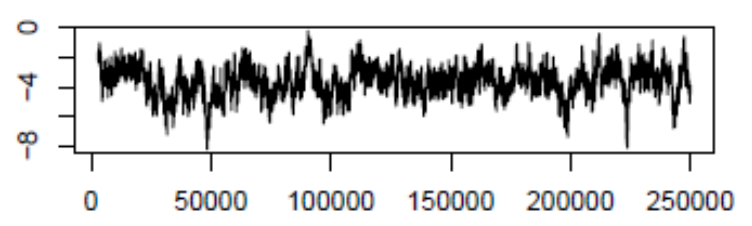

Iterations

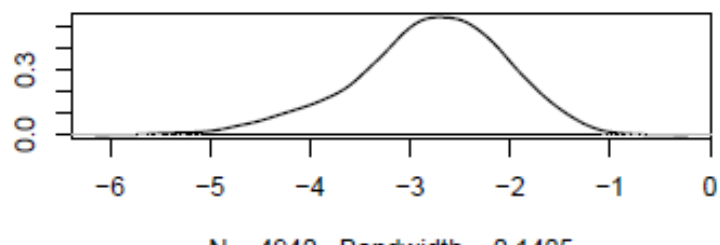

$\mathrm{N}=4940$ Bandwidth $=0.1405$

Density of breach:time:self

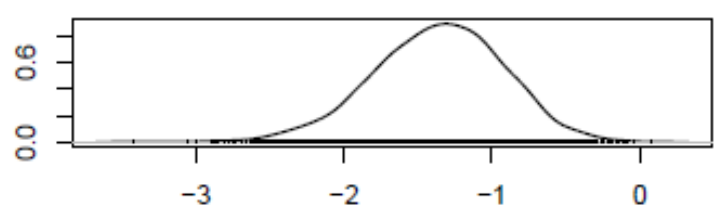

$\mathrm{N}=4940$ Bandwidth $=0.08541$

Density of breach:time:think

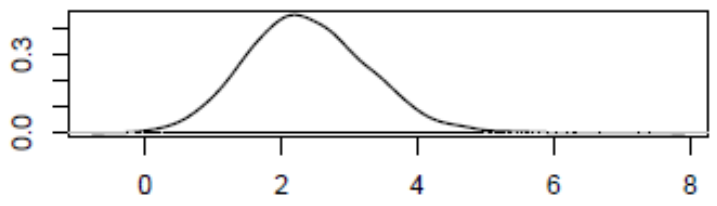

$\mathrm{N}=4940 \quad$ Bandwidth $=0.1737$

Density of breach:time:attitude

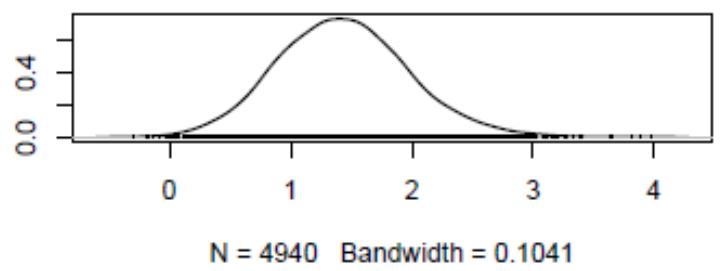

Density of breach:time:change

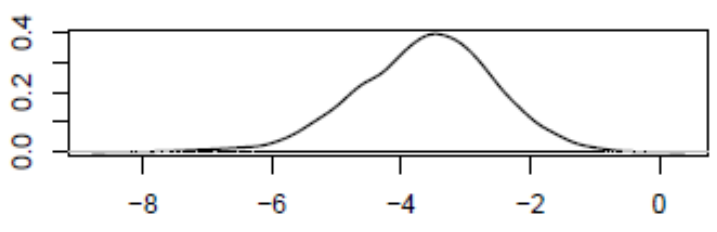

$\mathrm{N}=4940$ Bandwidth $=0.1999$ 
$\underline{\text { Random Effects }}$

Trace of time

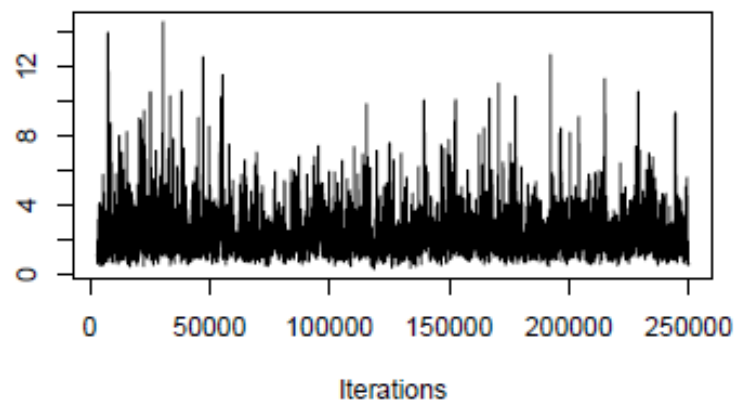

Trace of Research.ID

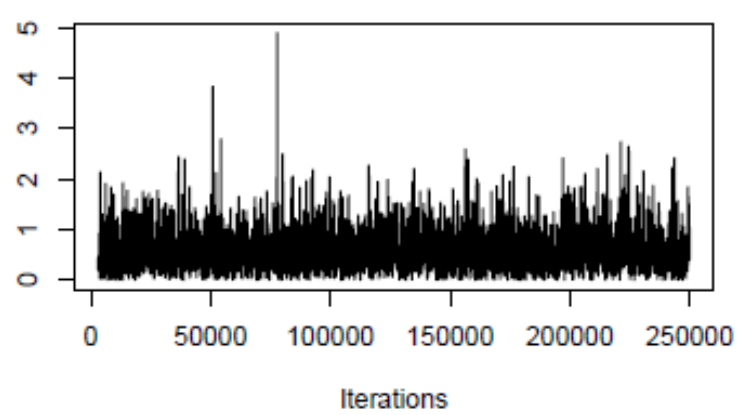

Density of time

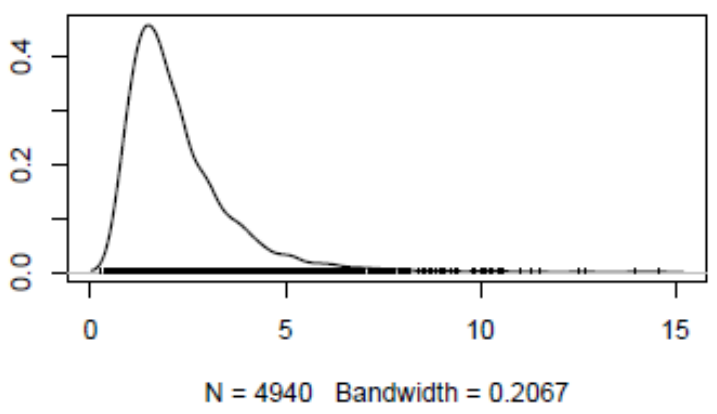

Density of Research.ID

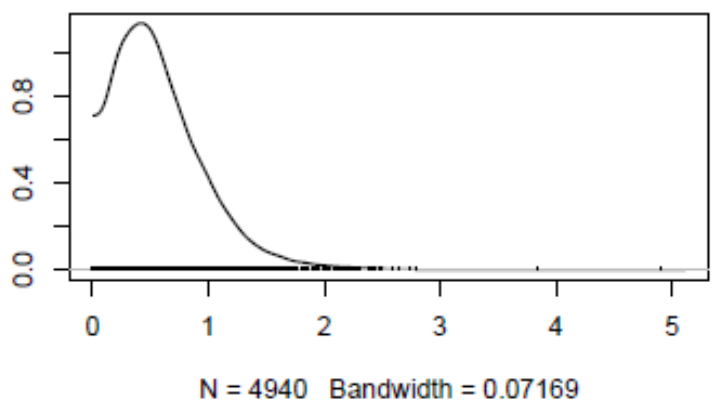


Dynamic Model 5: Court Appearances (Table 7.7)

Bayesian Model (BDm5_A)

\section{\# Define the model}

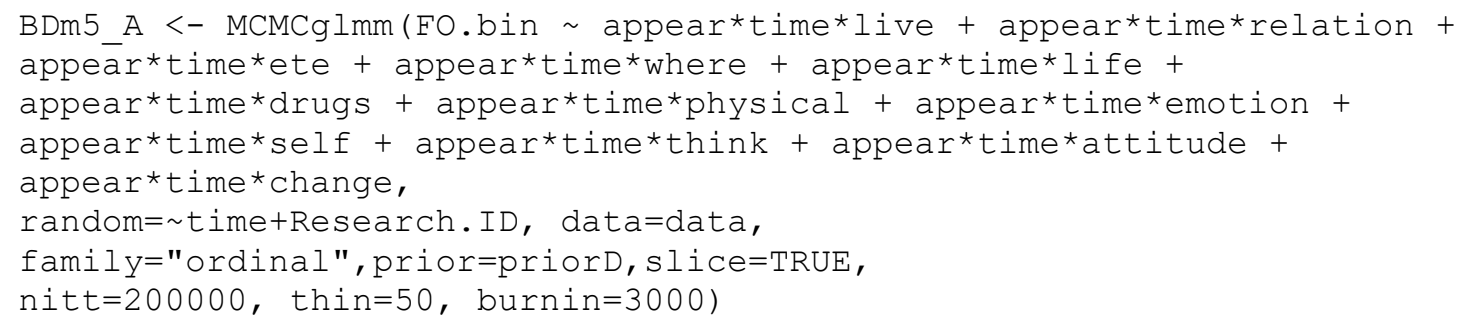

\section{\#\# Checks for suitable convergence}

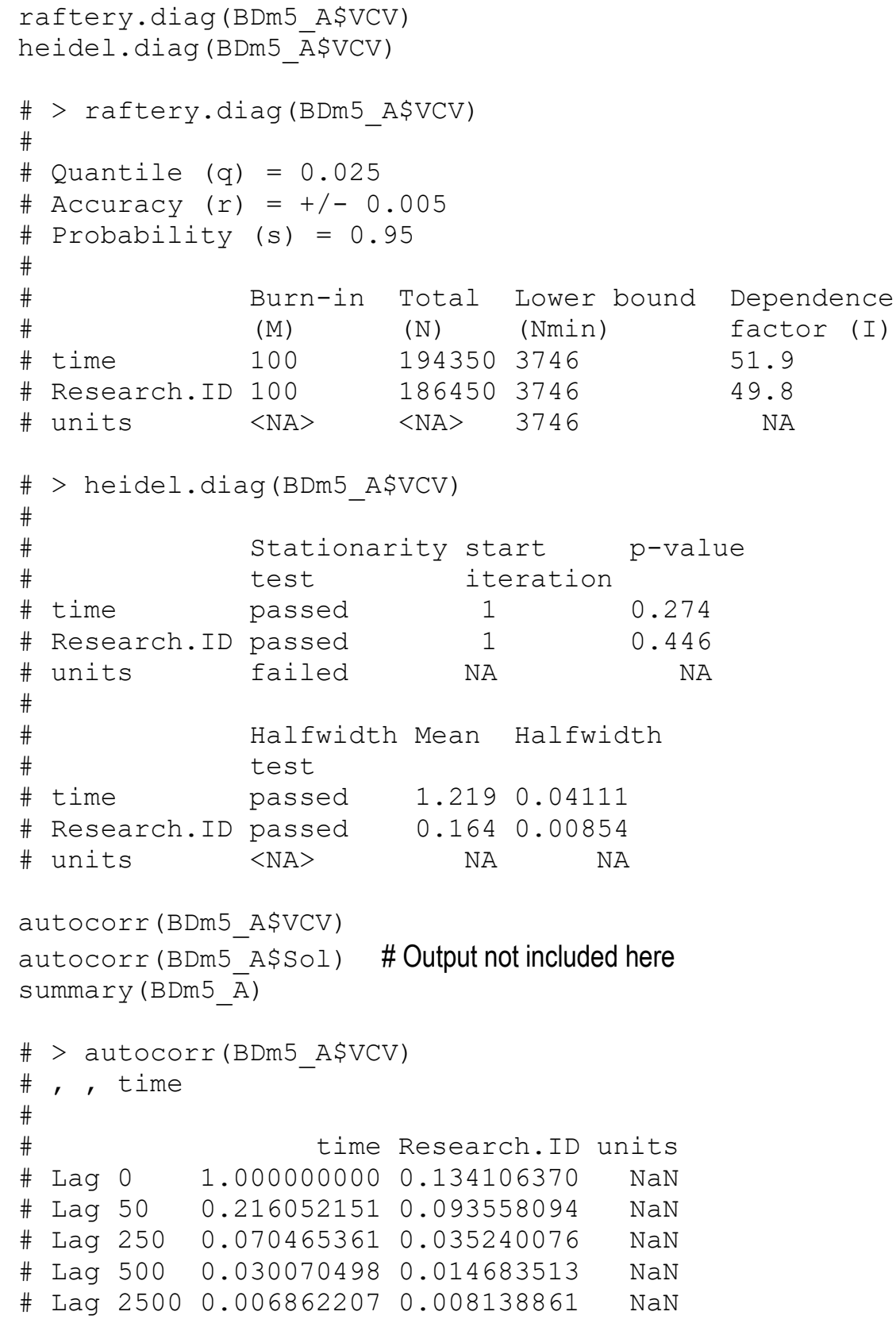




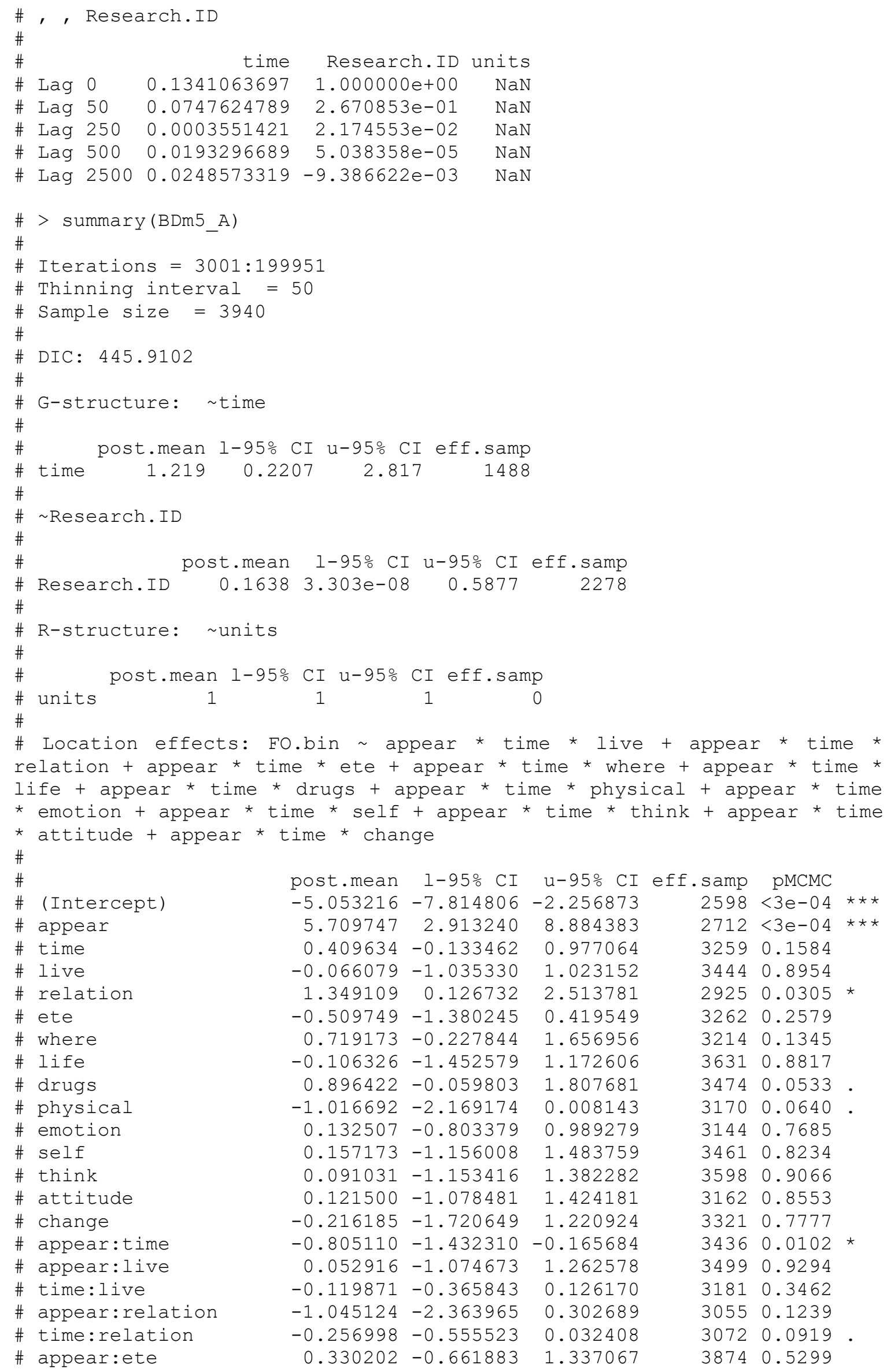




\begin{tabular}{|c|c|c|c|c|c|c|}
\hline \# & time:ete & 0.098178 & -0.114627 & 0.303793 & 3587 & 0.3477 \\
\hline \# & appear: where & -0.794658 & -1.853522 & 0.226605 & 3163 & 0.1254 \\
\hline \# & time: where & -0.013168 & -0.181236 & 0.169508 & 3390 & 0.8599 \\
\hline \# & appear:life & 0.107449 & -1.362381 & 1.656022 & 3067 & 0.9015 \\
\hline \# & time:life & 0.028882 & -0.244569 & 0.348722 & 321 & 0.8594 \\
\hline \# & appear:drugs & -0.789434 & -1.796985 & 0.247185 & 638 & 0.1447 \\
\hline \# & time:drugs & -0.115975 & -0.302226 & 0.079546 & 424 & 0.24 \\
\hline \# & appear:physical & 0.801711 & -0.438474 & 2.019193 & & 0.1 \\
\hline \# & time:physical & 0.196196 & -0.066303 & 0.447562 & & 0.1 \\
\hline \# & appear:emotion & -0.185342 & -1.265025 & 0.800439 & 08 & 0.73 \\
\hline \# & time:emotion & 0.052920 & -0.171298 & 0.27331 & & 0.6 \\
\hline \# & appear:self & -0.244089 & -1.696440 & 1.2536 & & 0.7 \\
\hline \# & time:self & -0.057703 & -0.318252 & 0.21602 & & 0.6 \\
\hline \# & appear: think & -0.378711 & -1.787579 & 1.0445 & & 0.6 \\
\hline \# & time:think & 0.012472 & -0.283208 & 0.30 & & 0.9 \\
\hline \# & appear:attitude & -0.126793 & -1.495381 & 1.35234 & & 0.8 \\
\hline \# & time:attitude & -0.110925 & -0.439534 & 0.21183 & & 0.5 \\
\hline \# & appear: change & 0.666199 & -1.005825 & 2.15 & & 0.4 \\
\hline \# & time: change & 0.070520 & -0.243854 & 0.378299 & & 0.667 \\
\hline \# & appear:time:live & 0.188389 & -0.077190 & 0.46 & & 0.1 \\
\hline \# & appear:time:relation & 0.226057 & -0.083488 & 0.56 & & 0.1 \\
\hline \# & appear:time:ete & -0.042766 & -0.274674 & 0.201501 & 98 & 0.72 \\
\hline \# & appear:time: where & 0.003518 & -0.200224 & 0.1867 & & 0.9 \\
\hline \# & appear:time:life & -0.046350 & -0.379604 & 0.286693 & & 0.7 \\
\hline \# & appear:time:drugs & 0.107363 & -0.092609 & 0.34017 & 9 & 0.32 \\
\hline \# & appear:time:physical & -0.094817 & -0.388504 & 0.1976 & & 0.53 \\
\hline \# & appear:time:emotion & -0.035068 & -0.279783 & 0.211832 & & 0.78 \\
\hline \# & appear:time:self & 0.010078 & -0.278015 & 0.31394 & & 0.953 \\
\hline \# & appear:time:think & -0.007101 & -0.336114 & 0.3286 & & 0.95 \\
\hline \# & appear:time:attitude & 0.143184 & -0.203426 & 0.5054 & & 0.43 \\
\hline \# & appear:time:change & -0.094426 & -0.421831 & 0.256820 & 867 & 0.5888 \\
\hline \# & --- & & & & & \\
\hline & Signif. codes: 0 & & & & & \\
\hline
\end{tabular}


Fixed Effects

Trace of (Intercept)

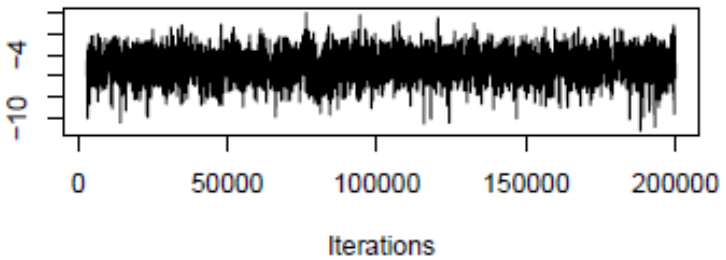

Trace of appear

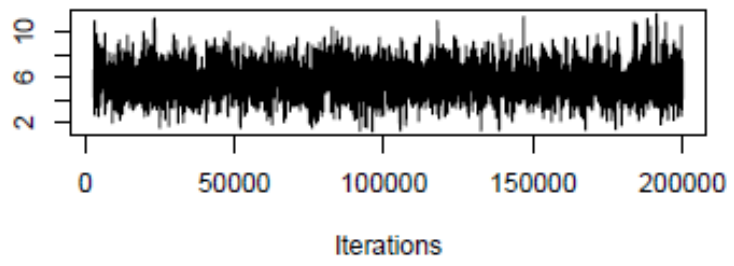

Trace of time

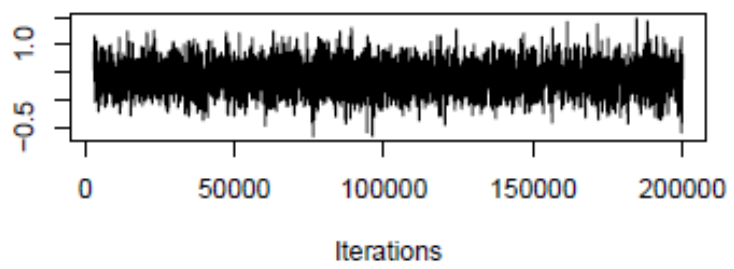

Trace of live

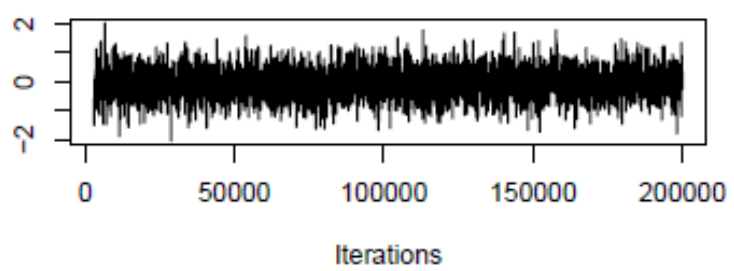

Trace of relation

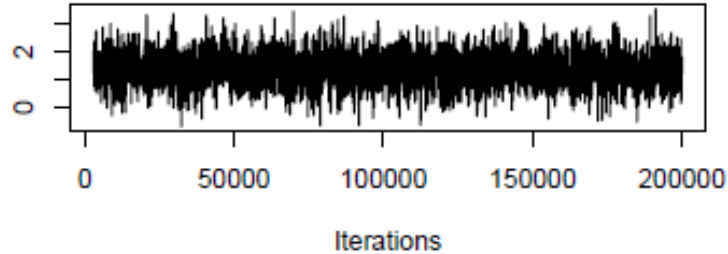

Density of (Intercept)

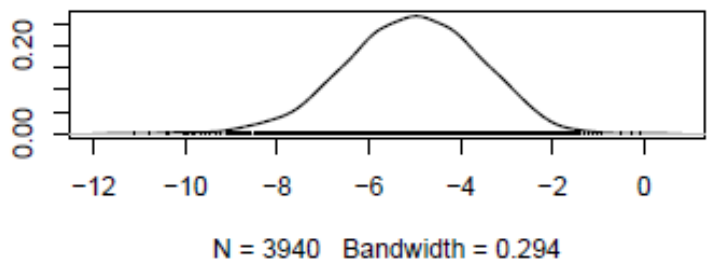

Density of appear

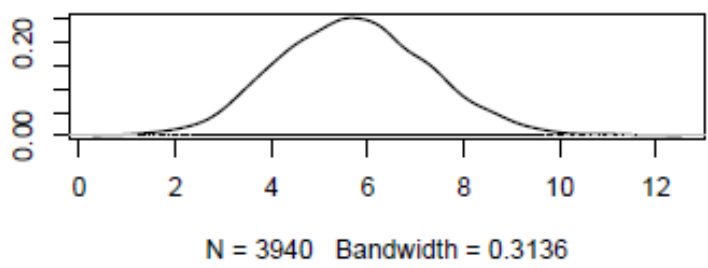

Density of time

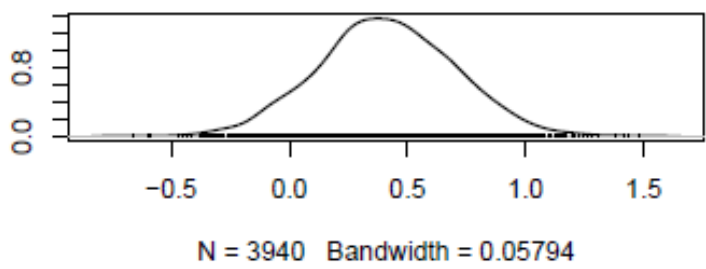

Density of live

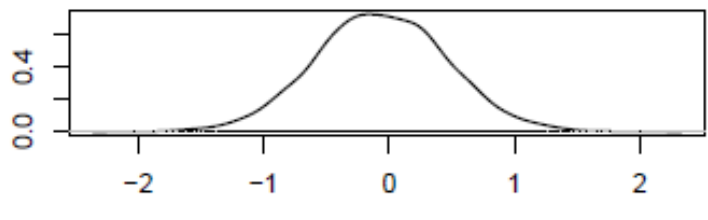

$\mathrm{N}=3940$ Bandwidth $=0.1065$

Density of relation

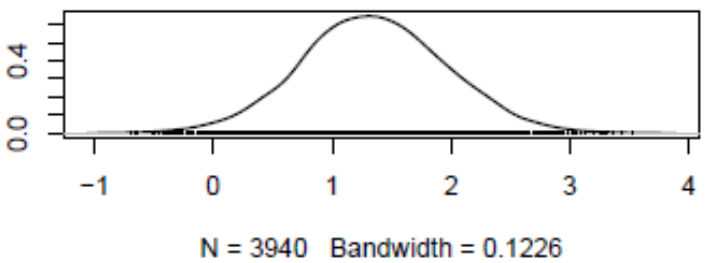


Trace of ete

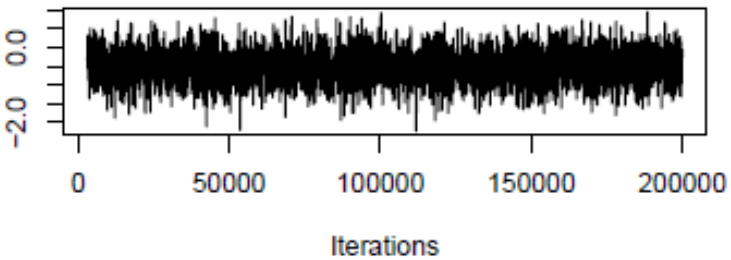

Trace of where

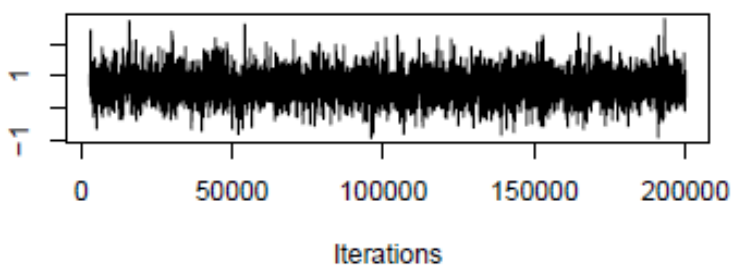

Trace of life

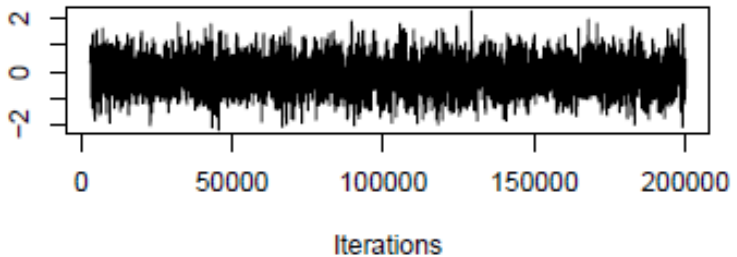

Trace of drugs

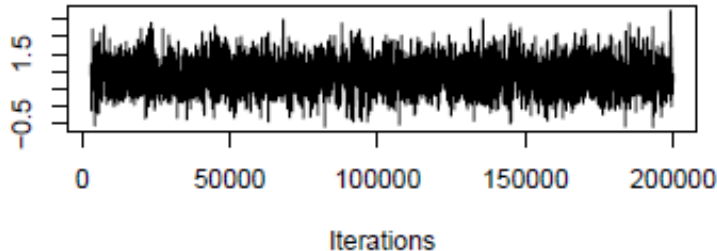

Trace of physical

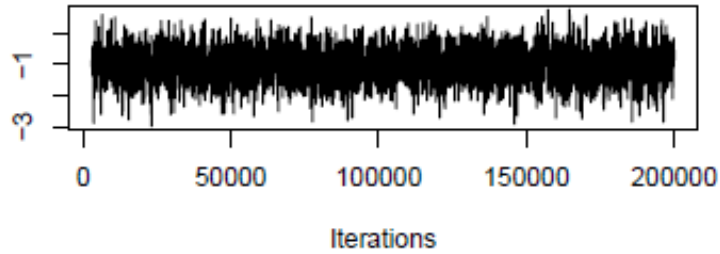

Trace of emotion

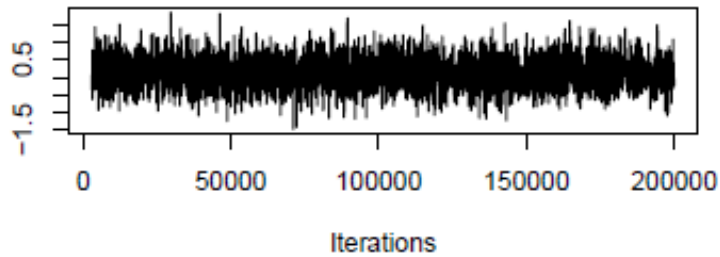

\section{Density of ete}

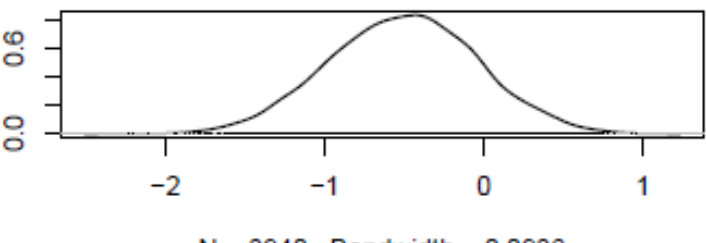

$\mathrm{N}=3940$ Bandwidth $=0.0936$

Density of where

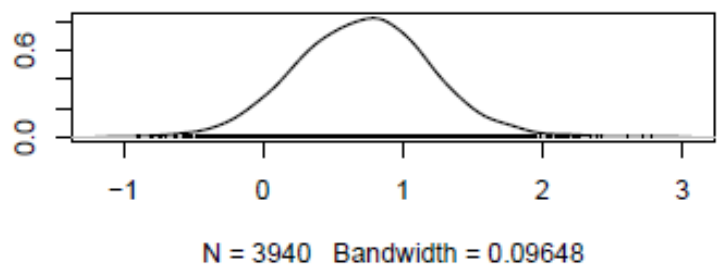

Density of life

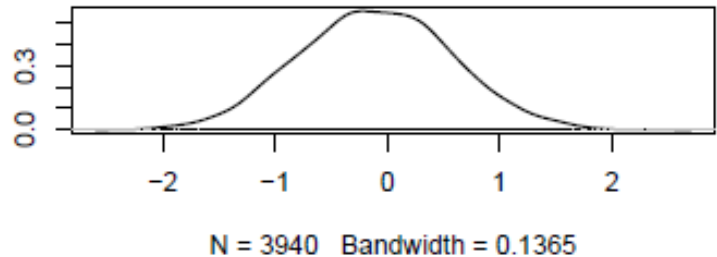

Density of drugs

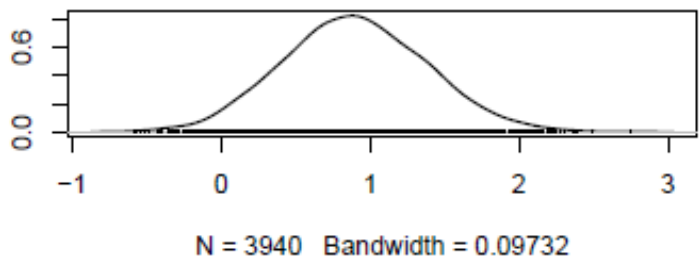

Density of physical

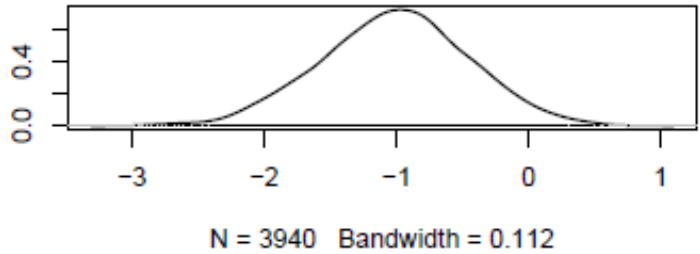

Density of emotion

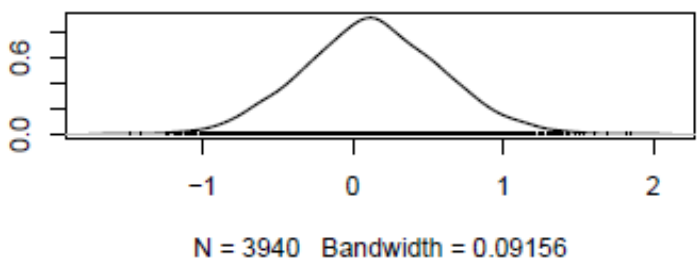

Page | 385 
Trace of self

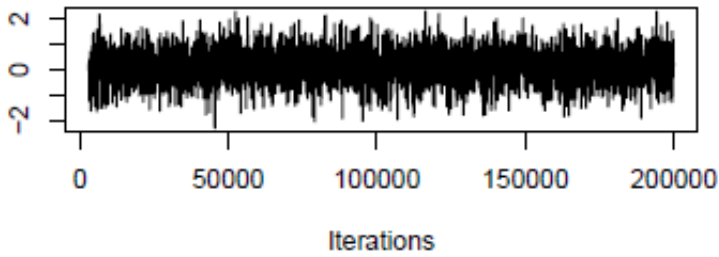

Trace of think

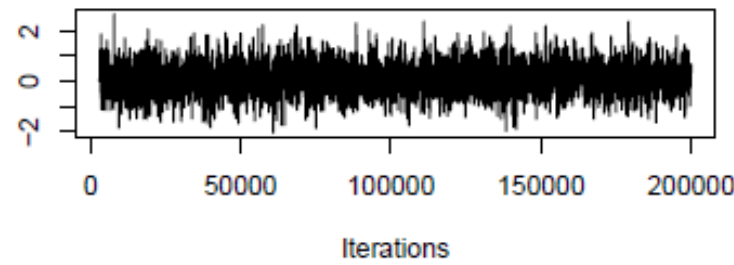

Trace of attitude

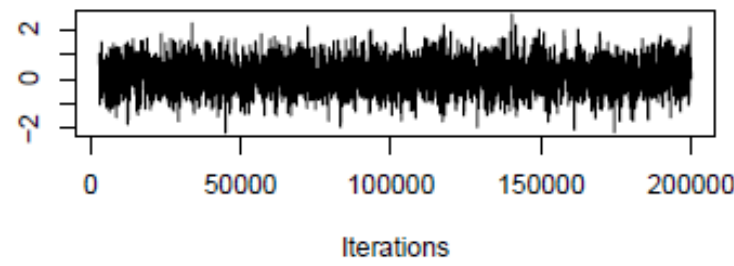

Trace of change

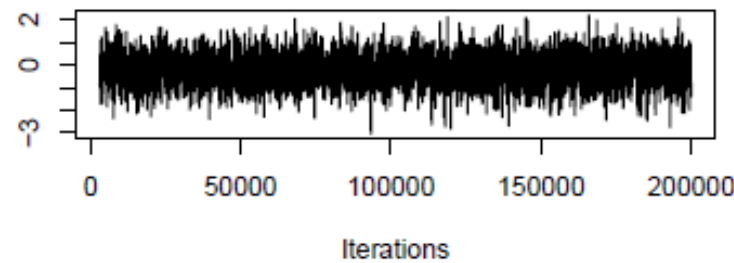

Trace of appear:time

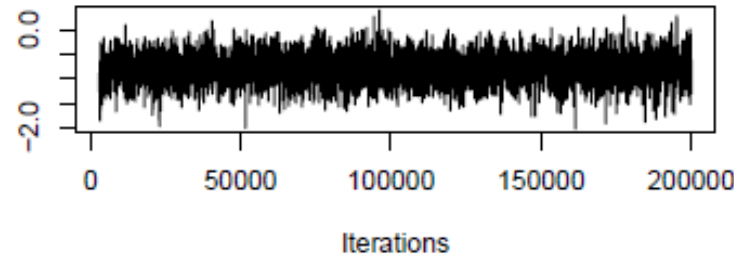

Trace of appear:live

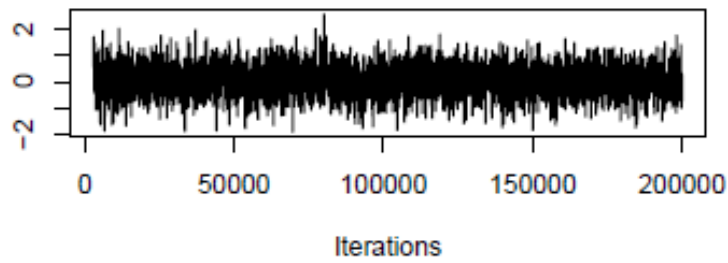

Density of self

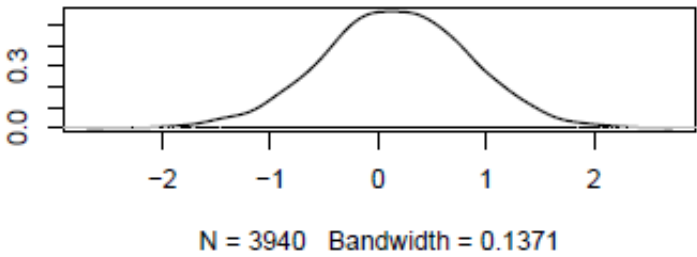

Density of think

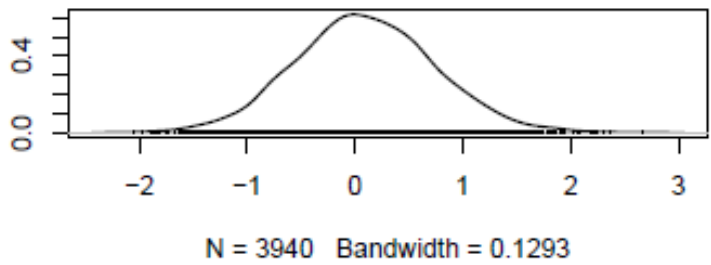

Density of attitude

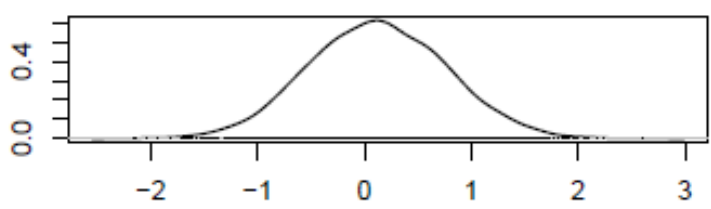

$\mathrm{N}=3940$ Bandwidth $=0.1299$

Density of change

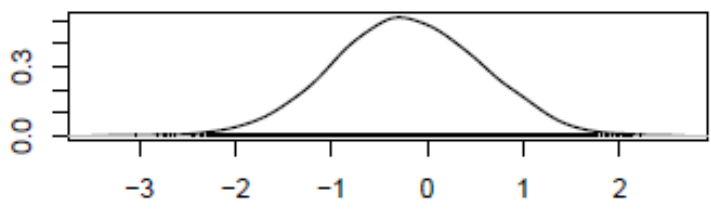

$\mathrm{N}=3940$ Bandwidth $=0.1542$

Density of appear:time

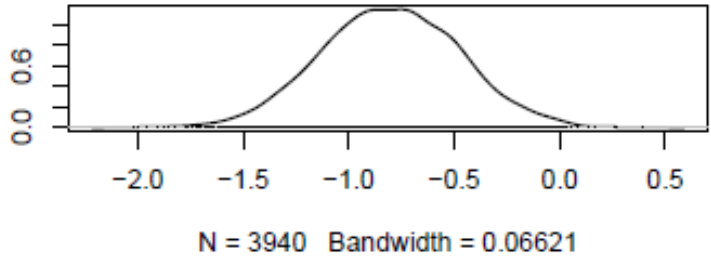

Density of appear:live

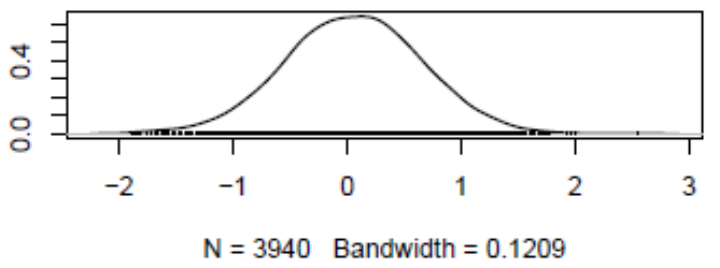


Trace of time:live

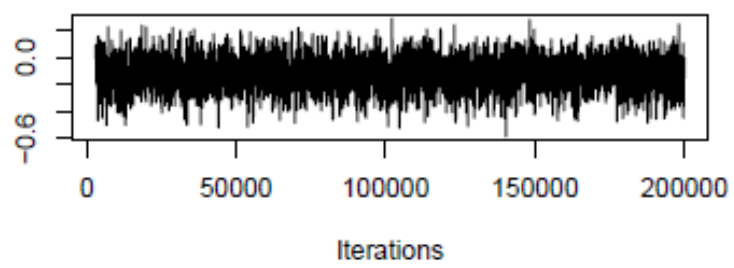

Trace of appear:relation

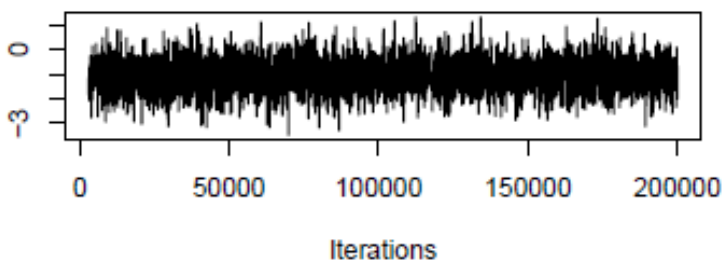

Trace of time:relation

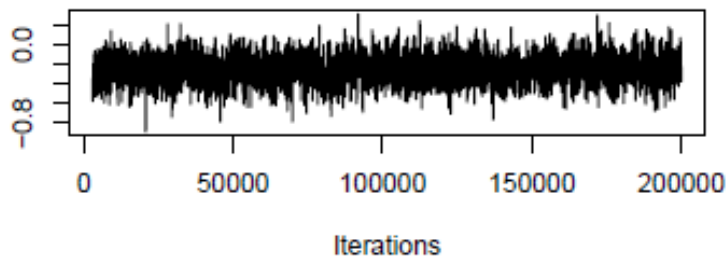

Trace of appear:ete

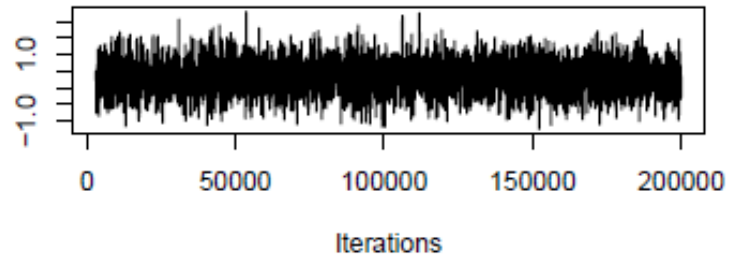

Trace of time:ete

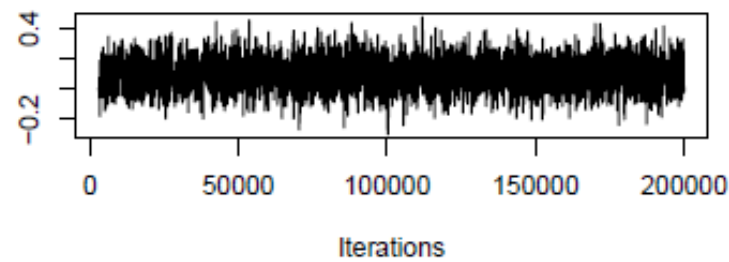

Trace of appear:where

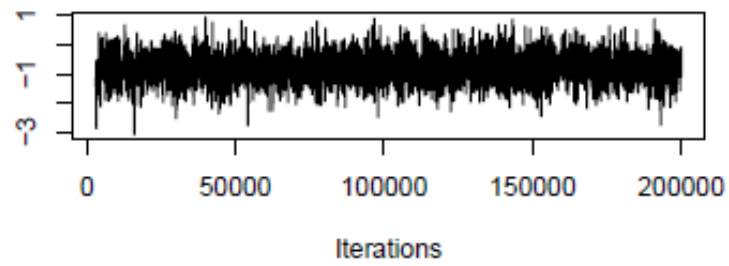

Density of time:live

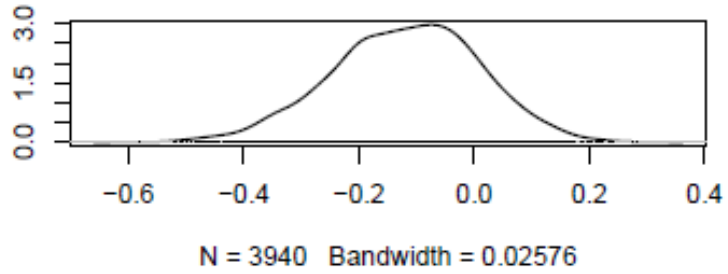

Density of appear:relation

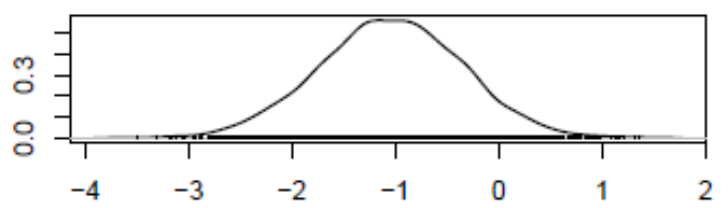

$\mathrm{N}=3940$ Bandwidth $=0.1394$

Density of time:relation

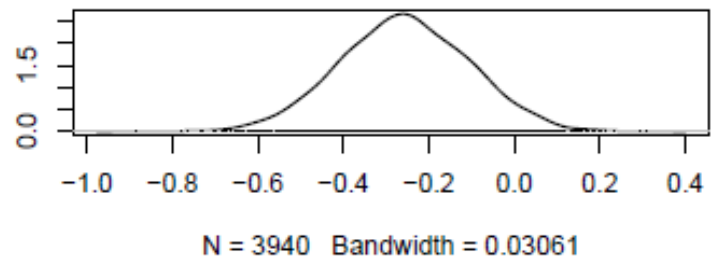

Density of appear:ete

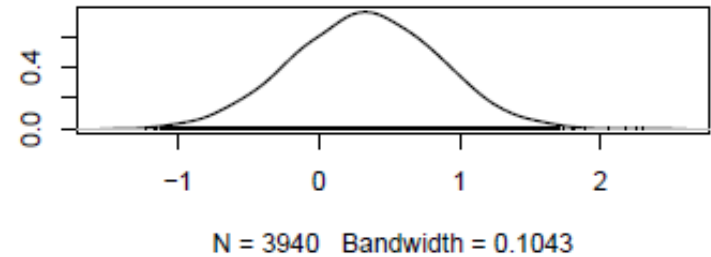

Density of time:ete

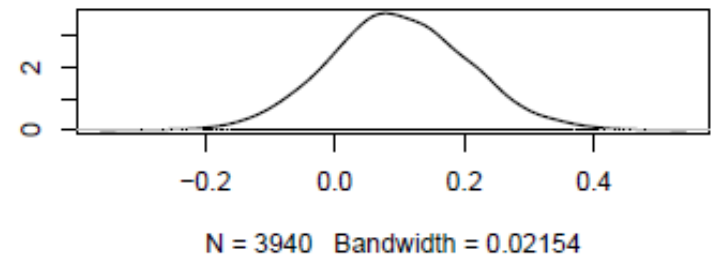

Density of appear:where

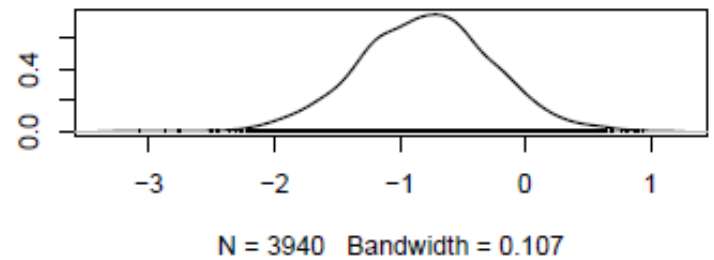


Trace of time:where

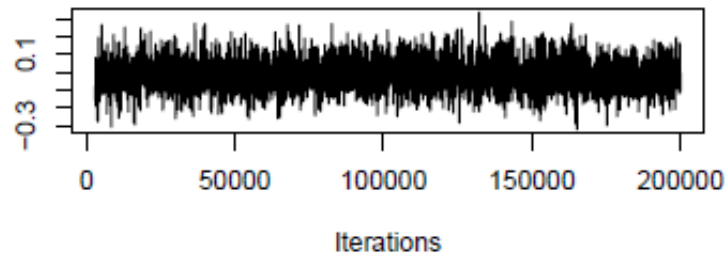

Trace of appear:life

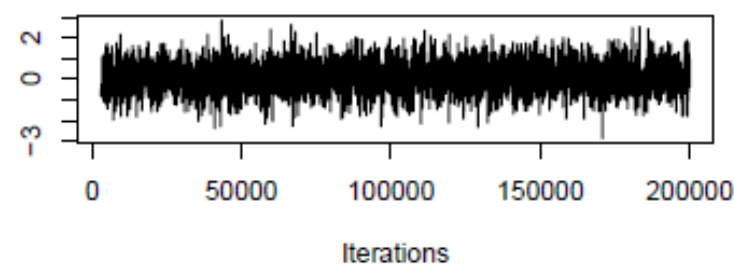

Trace of time:life

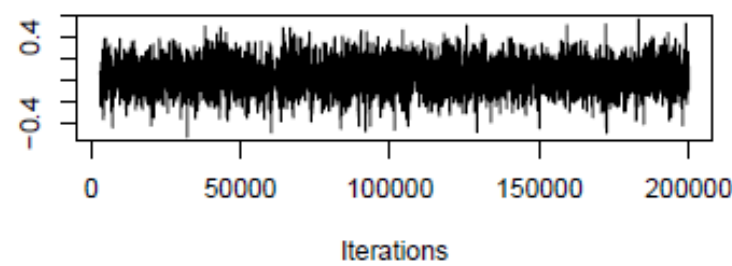

Trace of appear:drugs

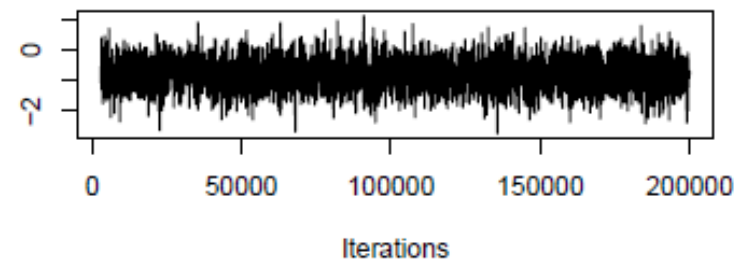

Trace of time:drugs

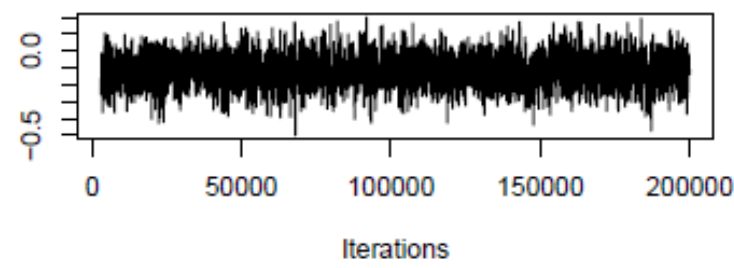

Trace of appear:physical

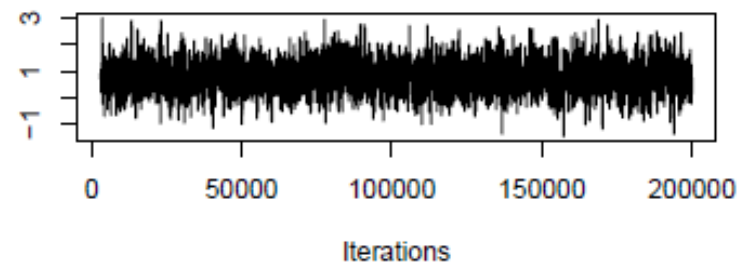

Density of time:where

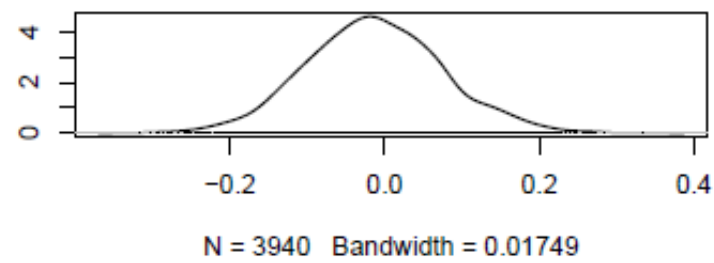

Density of appear:life

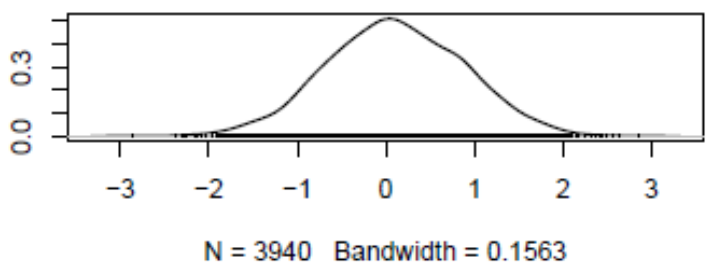

Density of time:life

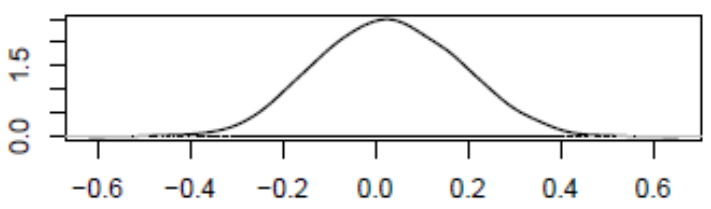

$\mathrm{N}=3940$ Bandwidth $=0.03133$

Density of appear:drugs

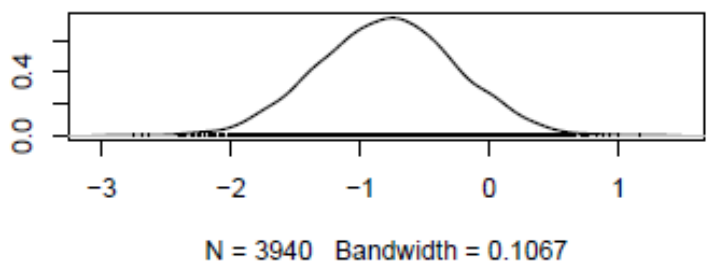

Density of time:drugs

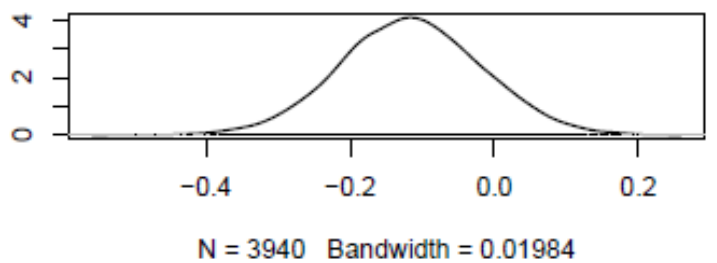

Density of appear:physical

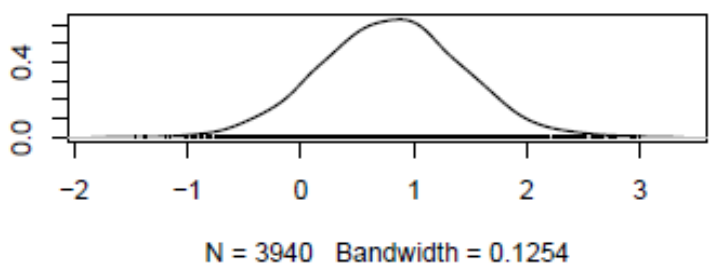


Trace of time:physical

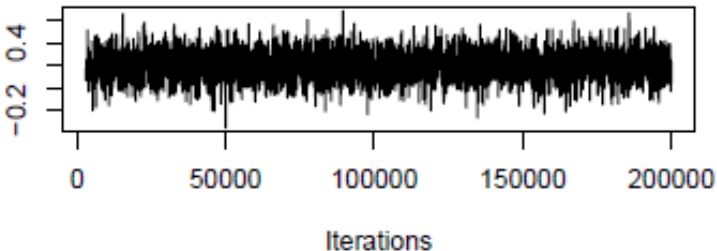

Trace of appear:emotion

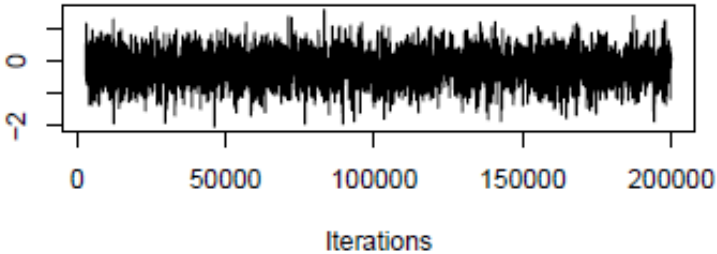

Trace of time:emotion

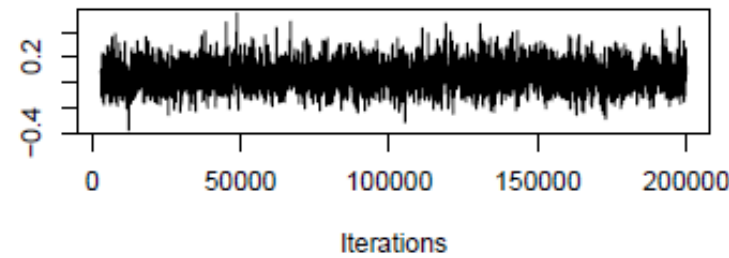

Trace of appear:self

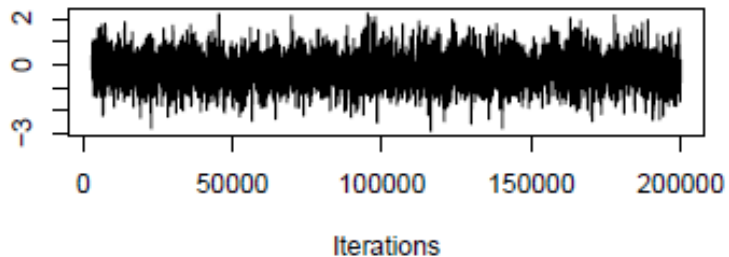

Trace of time:self

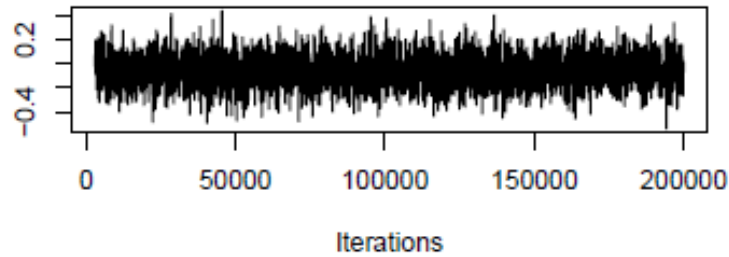

Trace of appear:think

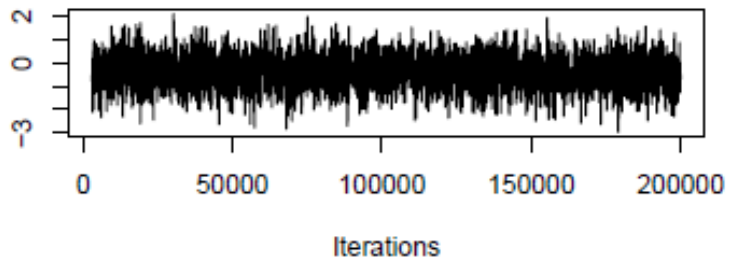

Density of time:physical

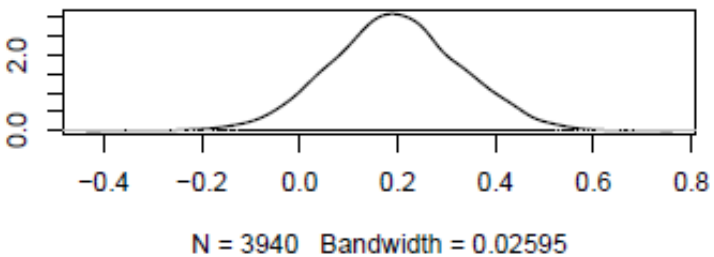

Density of appear:emotion

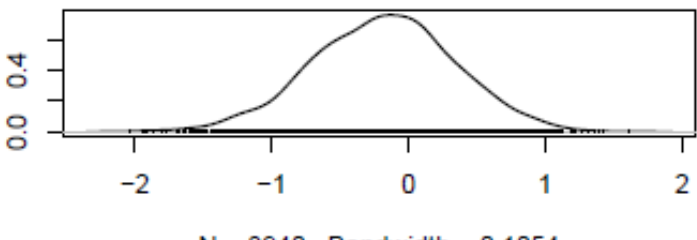

Density of time:emotion

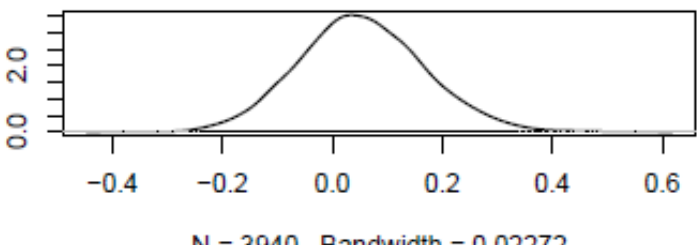

Density of appear:self

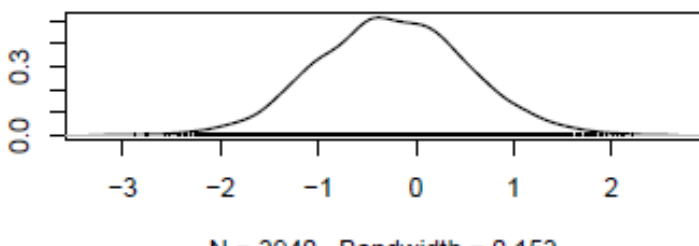

Density of time:self

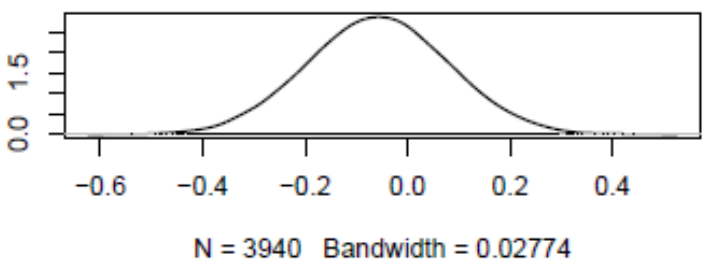

Density of appear:think

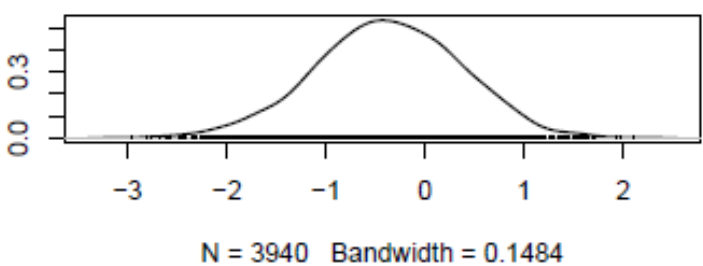


Trace of time:think

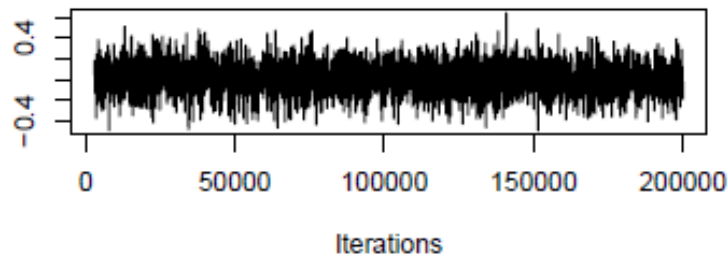

Trace of appear:attitude

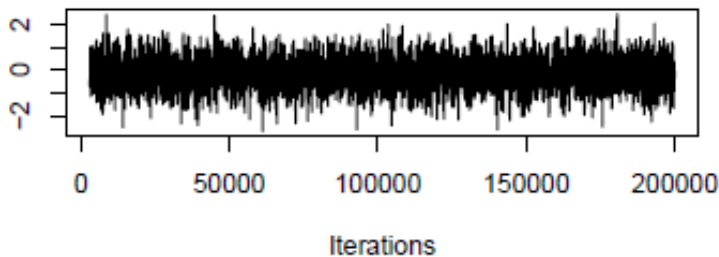

Trace of time:attitude

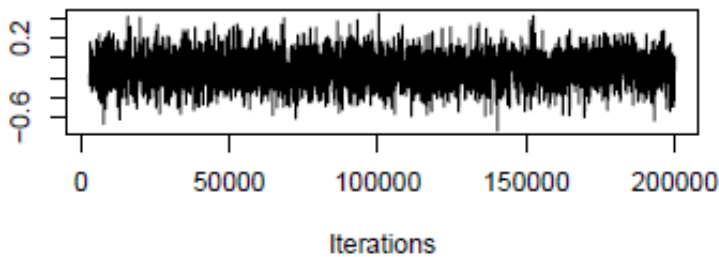

Trace of appear:change

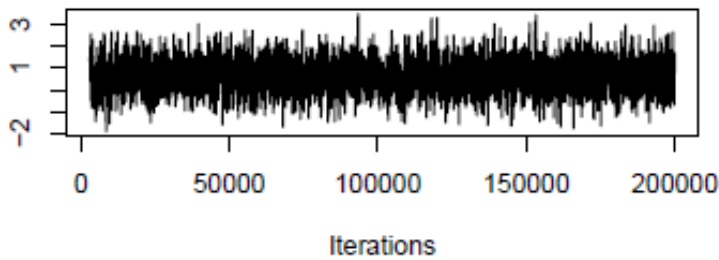

Trace of time:change

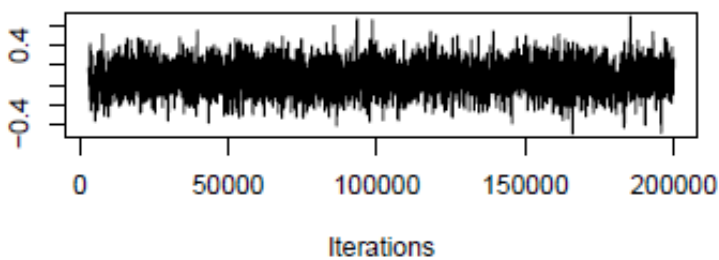

Trace of appear:time:live

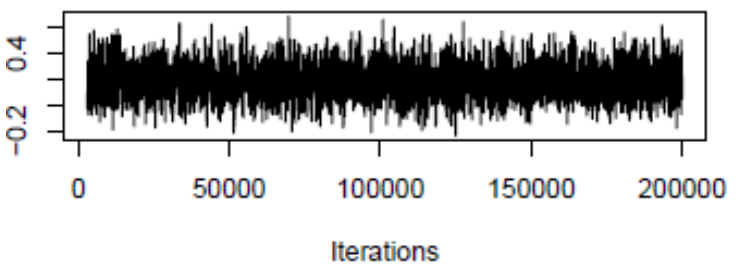

Density of time:think

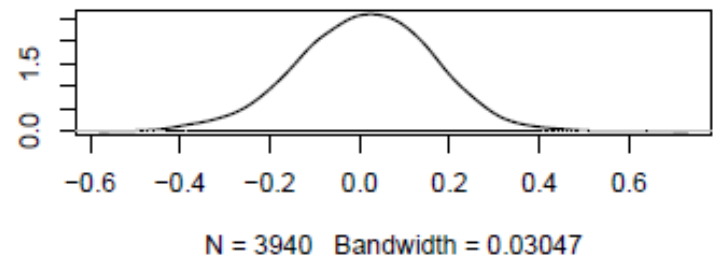

Density of appear:attitude

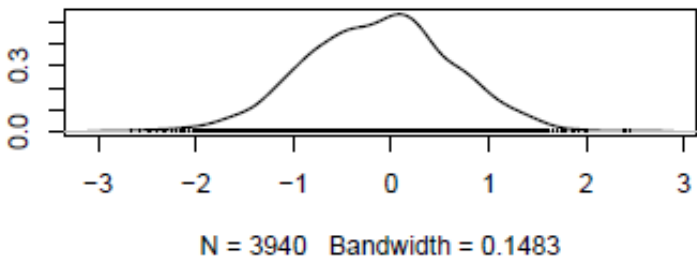

Density of time:attitude

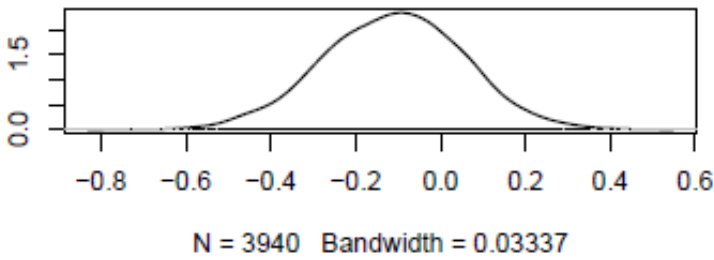

Density of appear:change

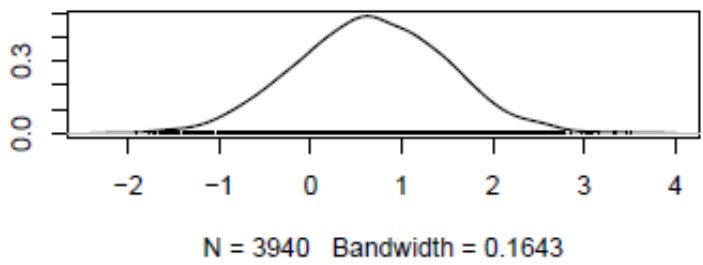

Density of time:change

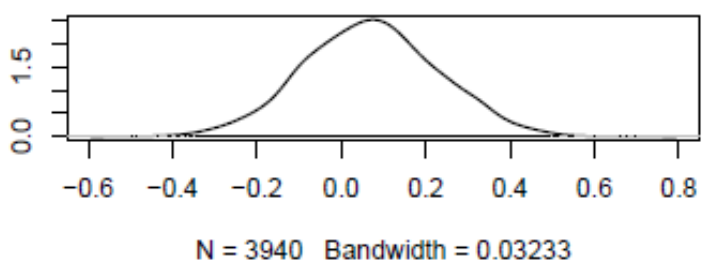

Density of appear:time:live

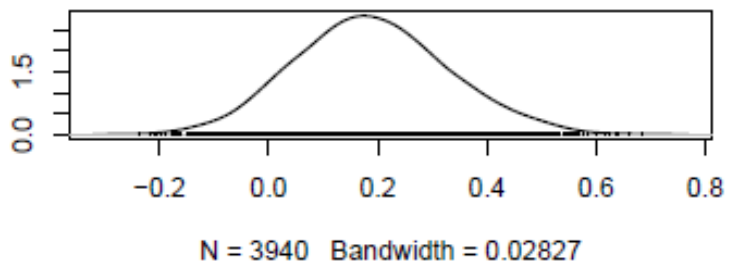


Trace of appear:time:relation

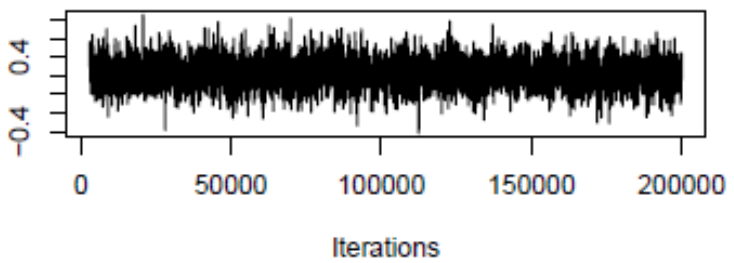

Trace of appear:time:ete

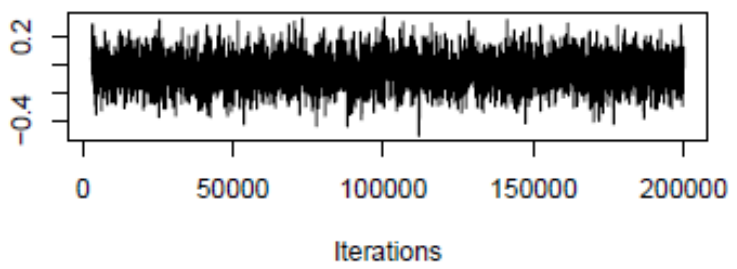

Trace of appear:time:where

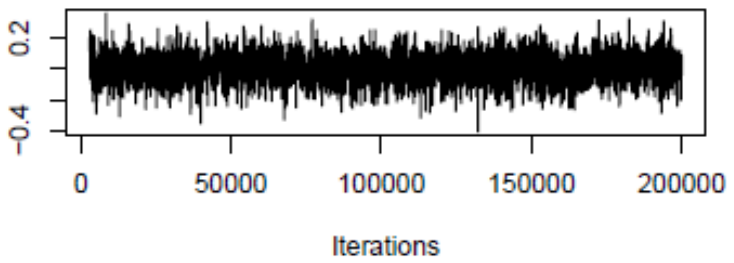

Trace of appear:time:life

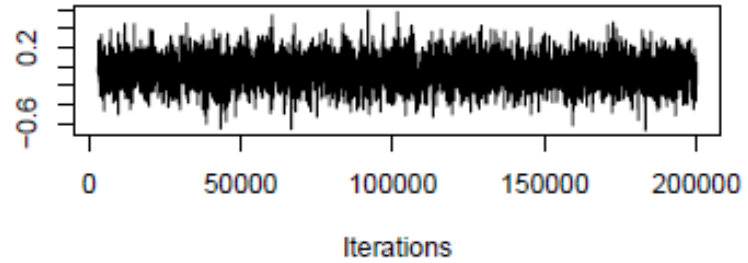

Trace of appear:time:drugs

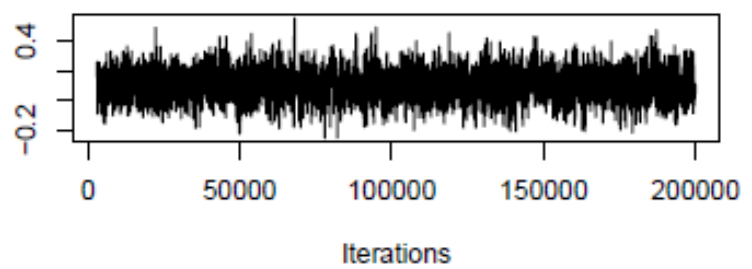

Trace of appear:time:physical

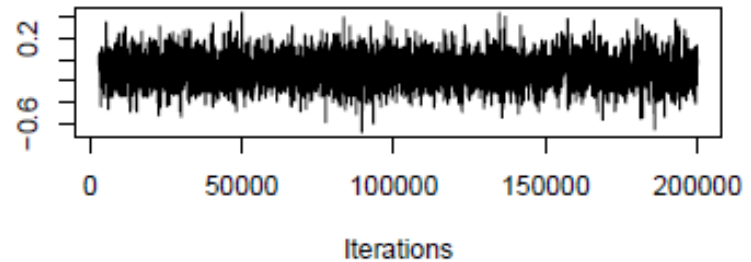

Density of appear:time:relation

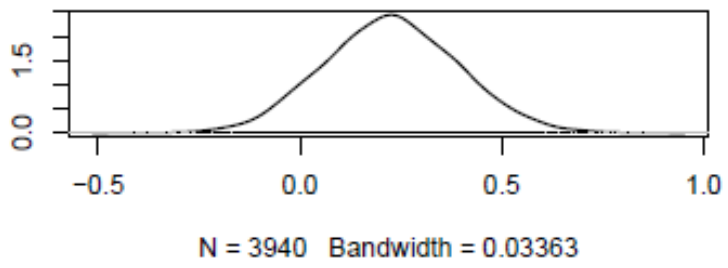

Density of appear:time:ete

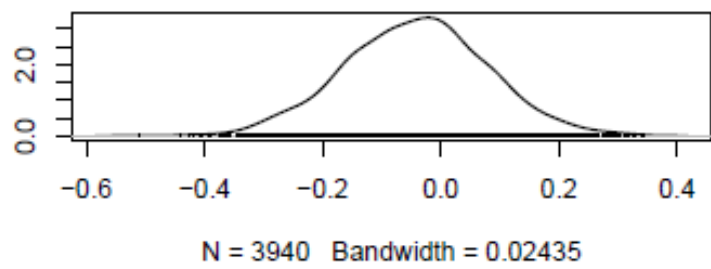

Density of appear:time:where

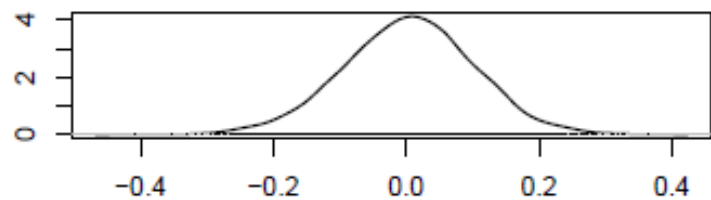

$\mathrm{N}=3940$ Bandwidth $=0.01941$

Density of appear:time:life

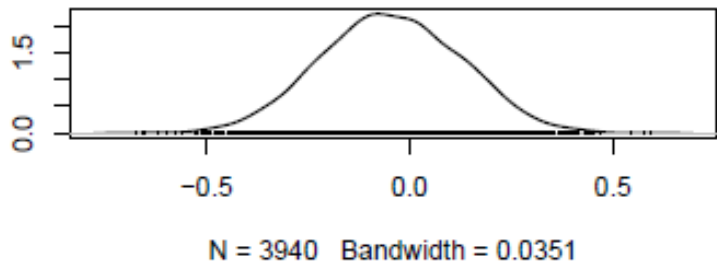

Density of appear:time:drugs

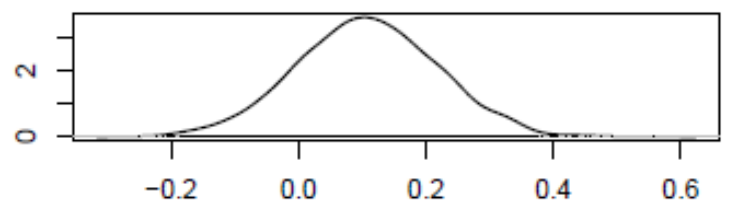

$\mathrm{N}=3940$ Bandwidth $=0.02216$

Density of appear:time:physical

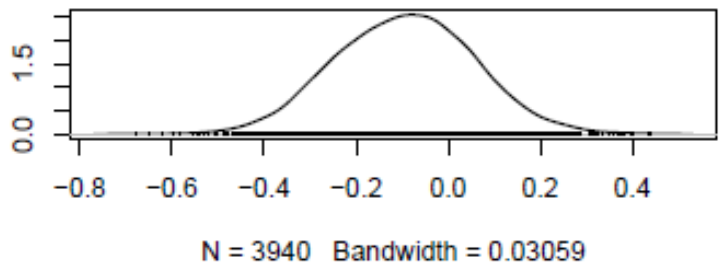

Page | 391 
Trace of appear:time:emotion

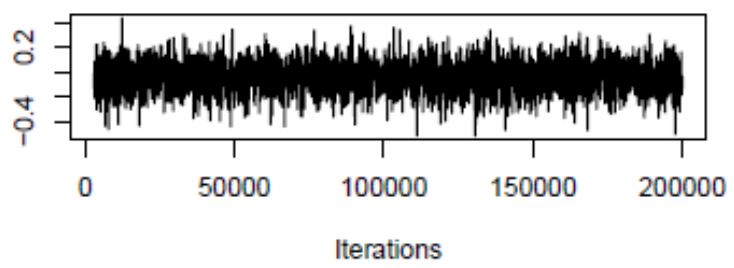

Trace of appear:time:self

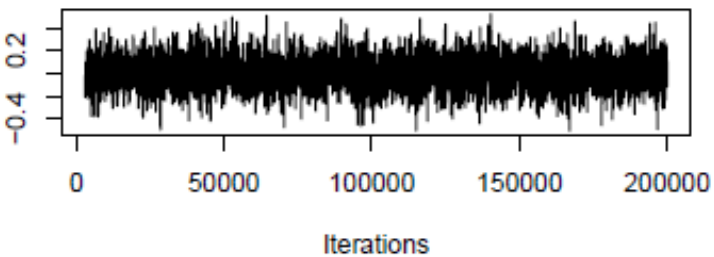

Trace of appear:time:think

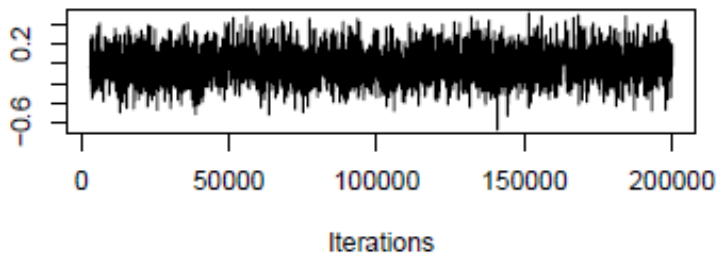

Trace of appear:time:attitude

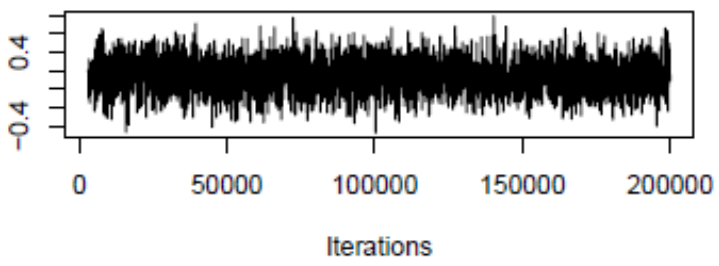

Trace of appear:time:change

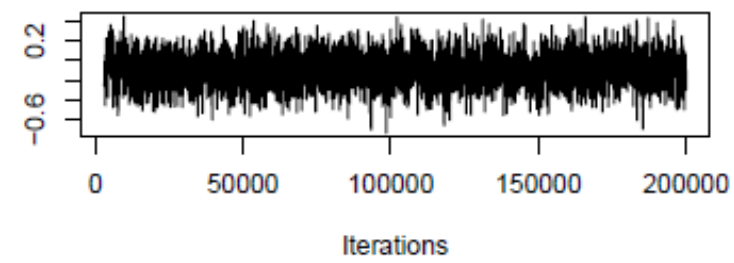

Density of appear:time:emotion

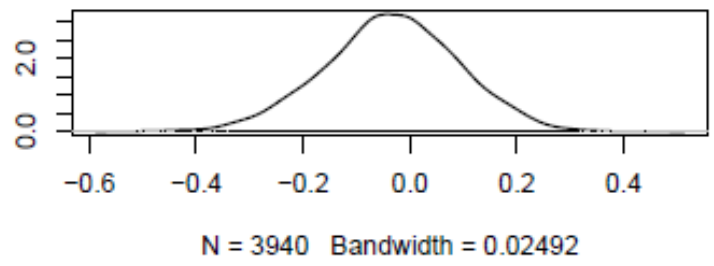

Density of appear:time:self

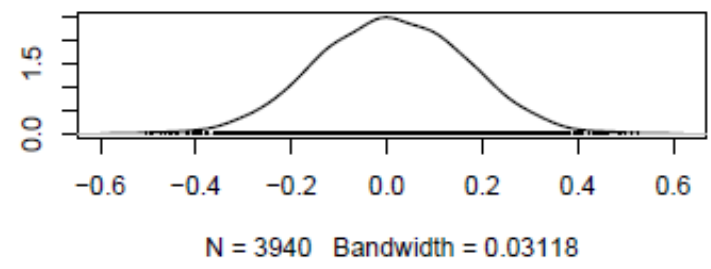

Density of appear:time:think

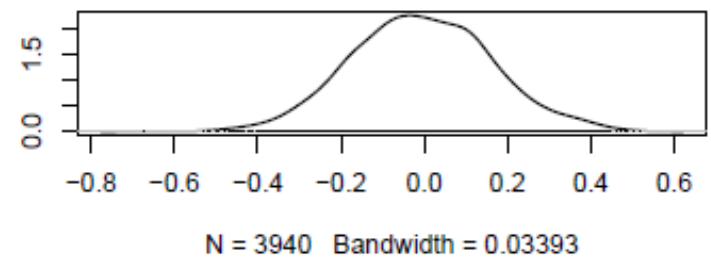

Density of appear:time:attitude

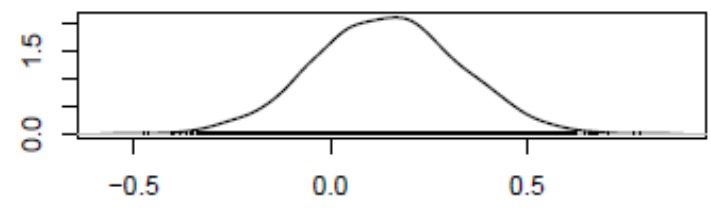

$\mathrm{N}=3940$ Bandwidth $=0.03685$

Density of appear:time:change

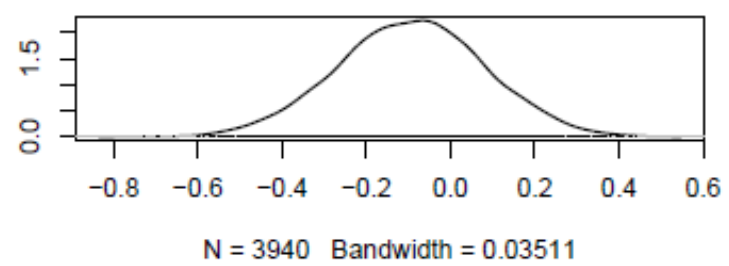


$\underline{\text { Random Effects }}$

Trace of time

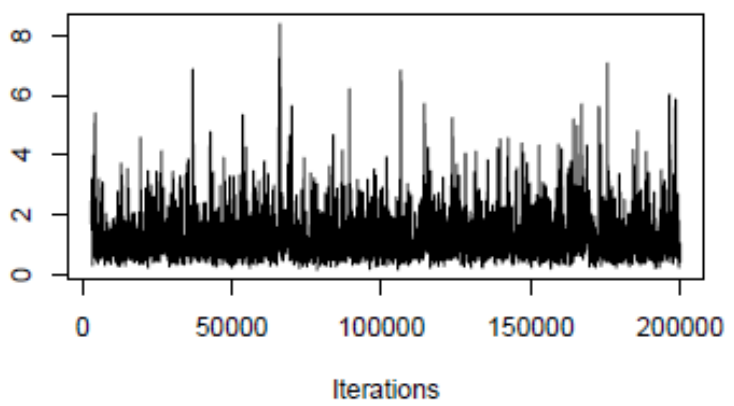

Trace of Research.ID

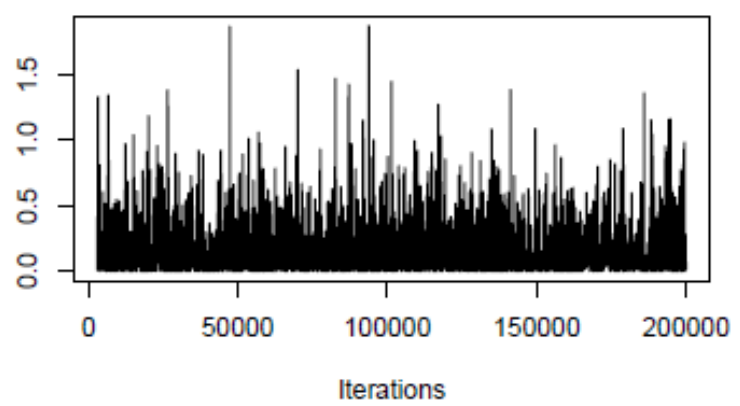

Density of time

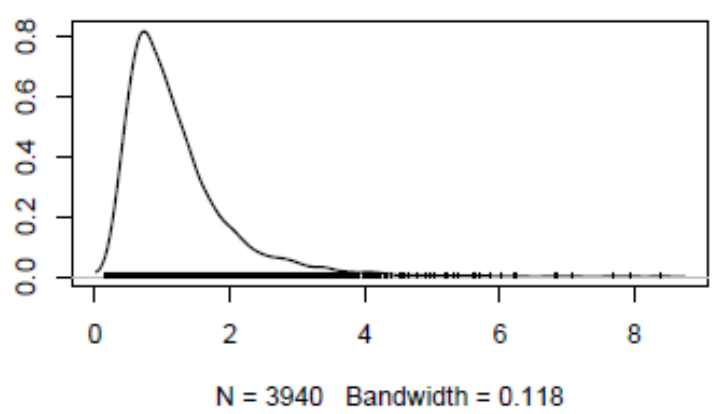

Density of Research.ID

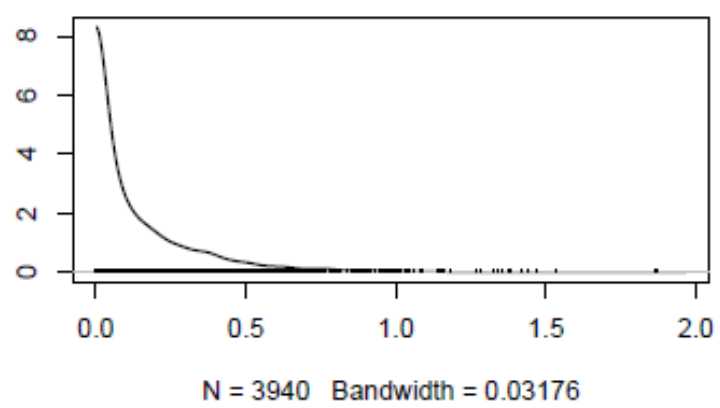


Dynamic Model 5: Custody (Table 7.9)

Bayesian Model (BDm5_C)

\section{\# Define the model}

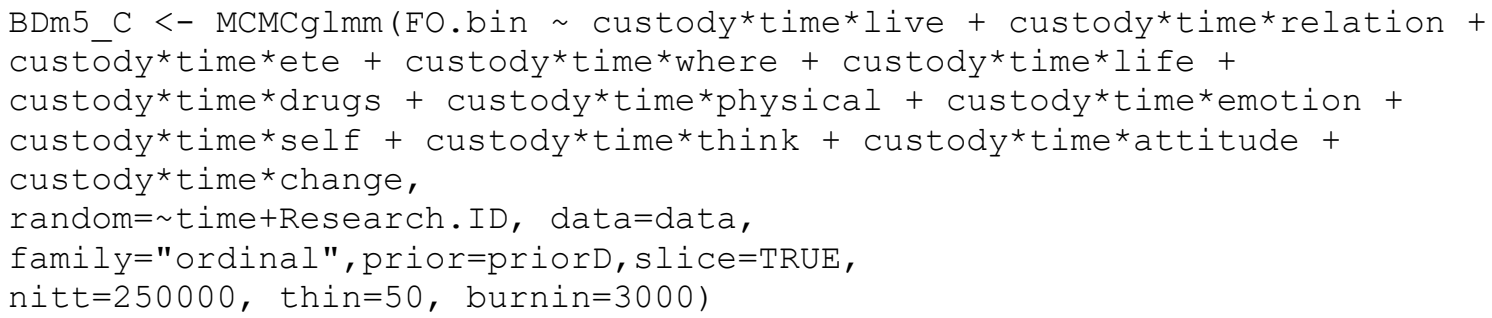

\section{\#\# Checks for suitable convergence}

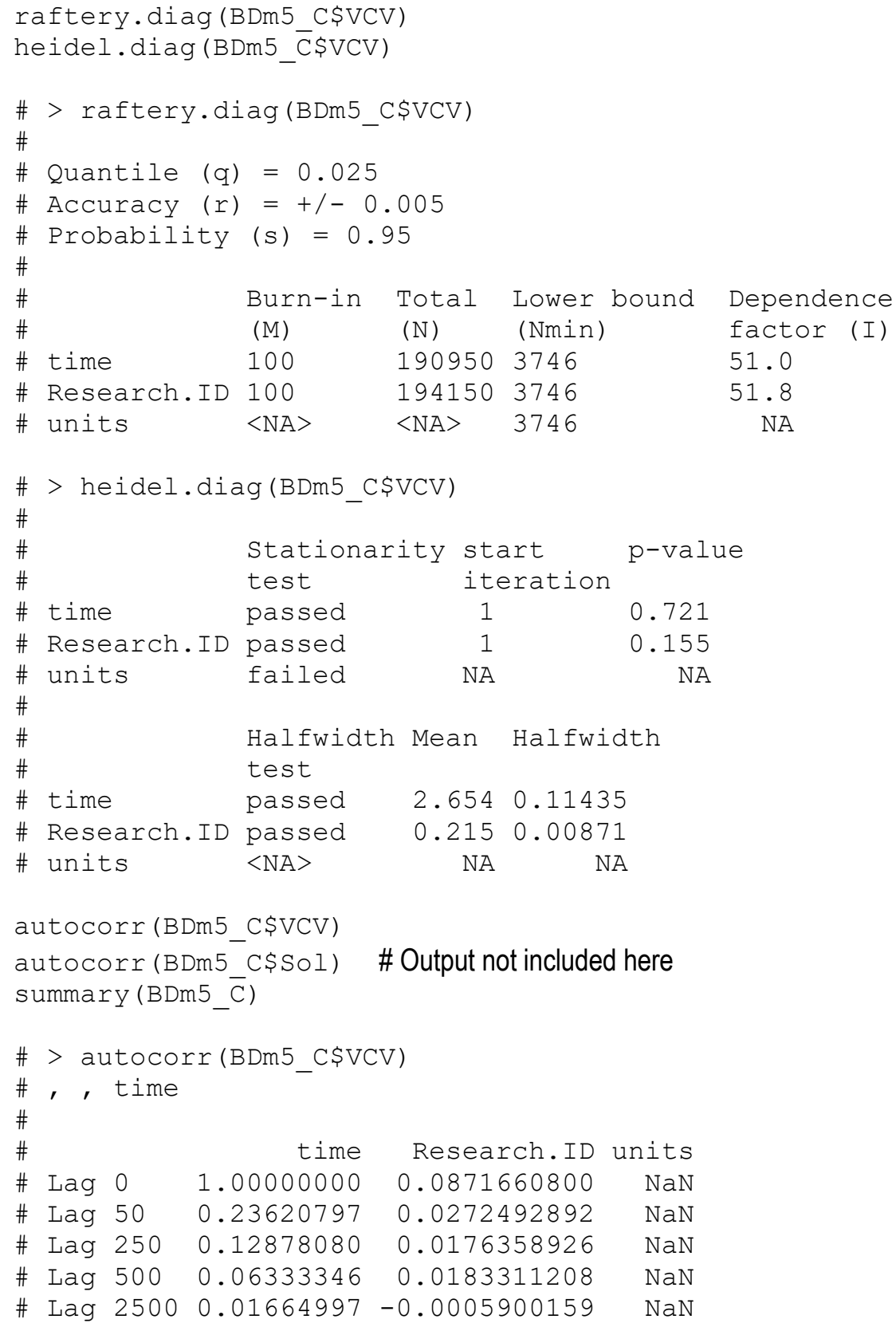




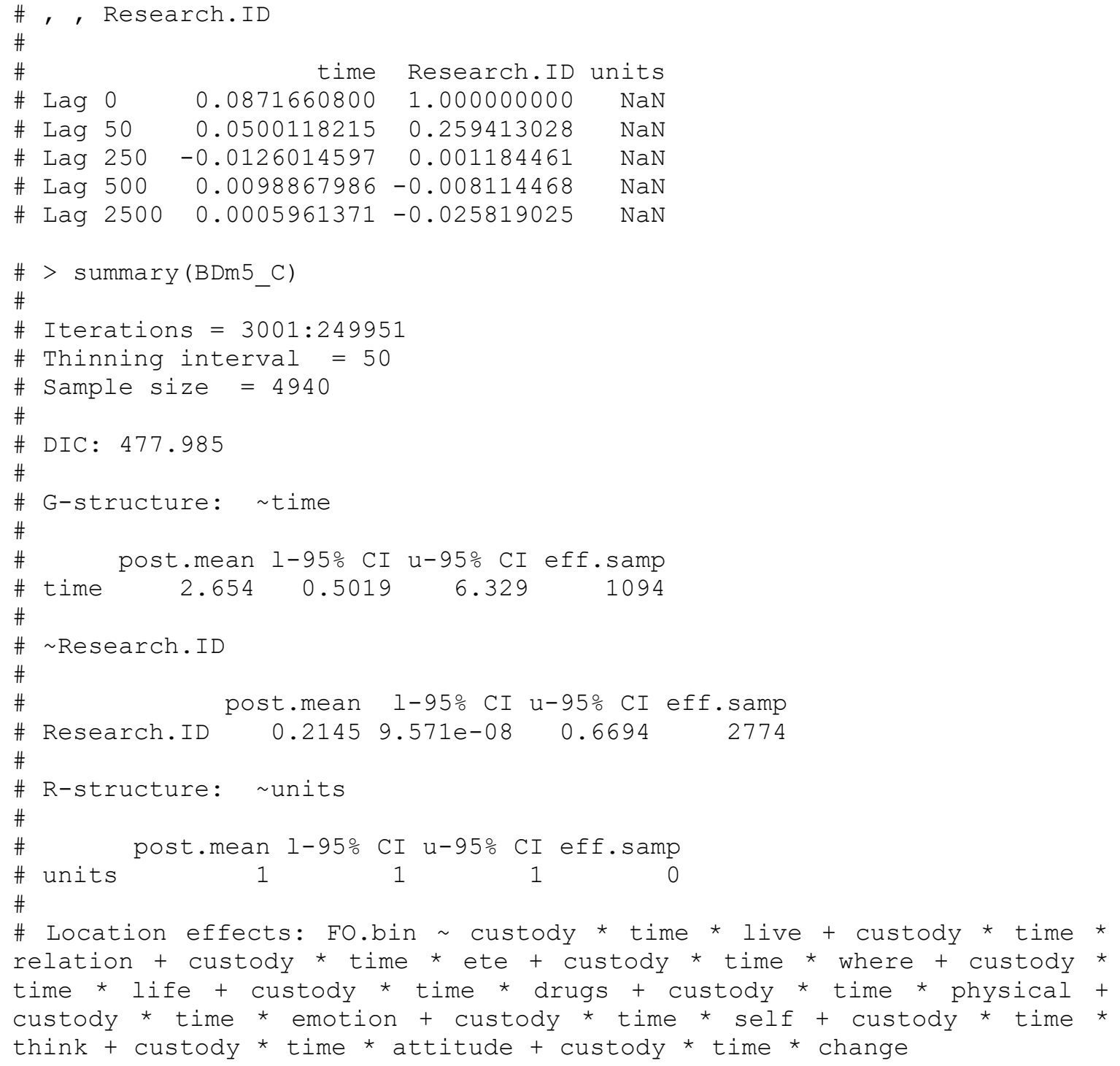

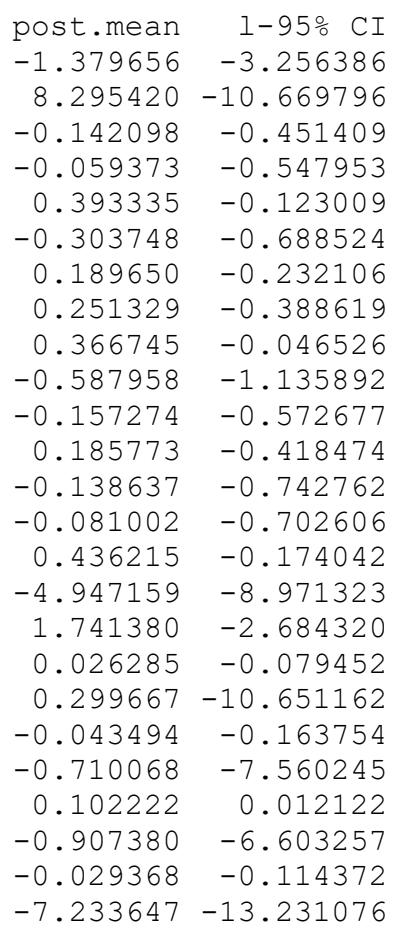

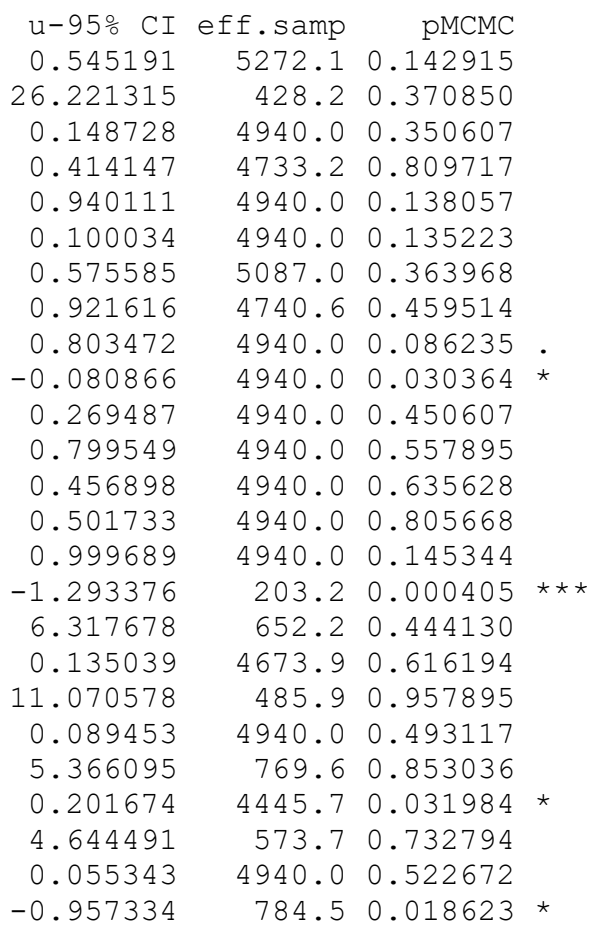




\begin{tabular}{|c|c|c|c|c|c|c|}
\hline \# & time:life & -0.031307 & -0.173771 & 0.104467 & 4723.0 & 0.675304 \\
\hline \# & custody: drugs & -1.733850 & -7.218663 & 3.397332 & 624.3 & 0.519433 \\
\hline \# & time:drugs & -0.054709 & -0.146487 & 0.035063 & 4940.0 & 0.250607 \\
\hline$\#$ & custody:physical & -1.560205 & -6.103669 & 2.726212 & 937.4 & 0.488259 \\
\hline \# & time:physical & 0.123609 & -0.014393 & 0.257073 & 4940.0 & 0.072065 \\
\hline \# & custody: emotion & 2.791973 & -8.356890 & 14.398819 & 545.2 & 0.658704 \\
\hline \# & time:emotion & 0.056767 & -0.038385 & 0.148771 & 4940.0 & 0.225101 \\
\hline \# & custody:self & 0.659167 & -8.023239 & 9.339297 & 280.2 & 0.866802 \\
\hline \# & time:self & -0.082914 & -0.206727 & 0.039614 & 4730.4 & 0.189474 \\
\hline \# & custody: think & 1.833132 & -9.165312 & 13.882505 & 374.5 & 0.780162 \\
\hline \# & time:think & 0.001207 & -0.139149 & 0.130715 & 4705.9 & 91498 \\
\hline \# & custody:attitude & 7.785652 & -4.112593 & 20.235339 & 681.3 & 00405 \\
\hline \# & time:attitude & 0.018583 & -0.112816 & 0.152234 & 4940.0 & 77328 \\
\hline \# & custody: change & -8.794357 & -24.027253 & 6.464864 & 439.0 & 3968 \\
\hline \# & time: change & -0.052021 & $-0.1 \mathrm{~s}-\mathrm{s}-\mathrm{s}$ & 176872 & 4940.0 & \\
\hline \# & custody:time:live & -0.781755 & $-1 \cdot 7$ & 0.132218 & 248.9 & 5020 \\
\hline \# & custody:time:relation & -0.359860 & $-1 \cdot 71$ & 0900 & 590.7 & 9271 \\
\hline \# & custody:time:ete & 0.940456 & -0.578471 & 2.380562 & 622.8 & 6356 \\
\hline \# & custody: time: where & 0.902648 & 0.05 & 1.709943 & 317.5 & 8219 \\
\hline \# & custody:time:life & 0.907317 & -0.440528 & 2.229964 & 466.6 & 0.176923 \\
\hline \# & custody:time:drugs & 0.988711 & -0.022727 & 1.912290 & 526.5 & 0.028340 \\
\hline \# & custody:time:physical & 0.812469 & -0.051338 & 1.712480 & 444.2 & 0.050607 \\
\hline \# & custody:time:emotion & -0.285715 & -1.736301 & 1.166276 & 628.1 & 0.714980 \\
\hline \# & custody:time:self & 0.050467 & -1.714904 & 1.804351 & 250.3 & 0.973684 \\
\hline \# & custody: time:think & -0.331905 & -2.214339 & 1.370768 & 366.4 & 0.742510 \\
\hline \# & custody:time:attitude & -1.338827 & -3.096636 & 0.214132 & 997.8 & 0.090688 \\
\hline \# & custody:time: change & 0.868017 & -1.457904 & 3.101199 & 390.9 & 0.461134 \\
\hline & & & & & & \\
\hline & cones: & 0.001 & 0.01 & 0.03 & & \\
\hline
\end{tabular}


Fixed Effects

Trace of (Intercept)

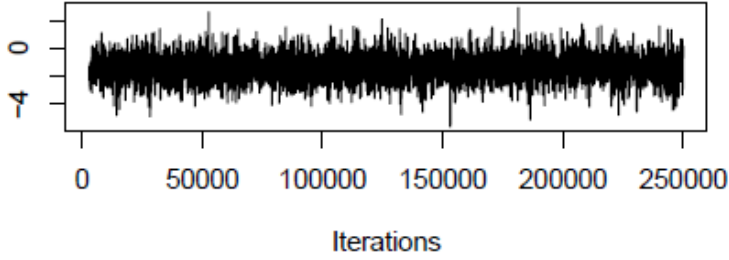

Trace of custody

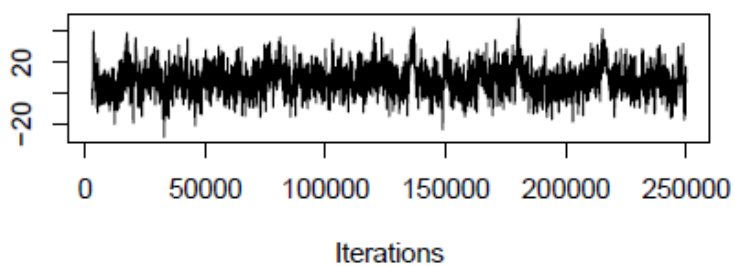

Trace of time

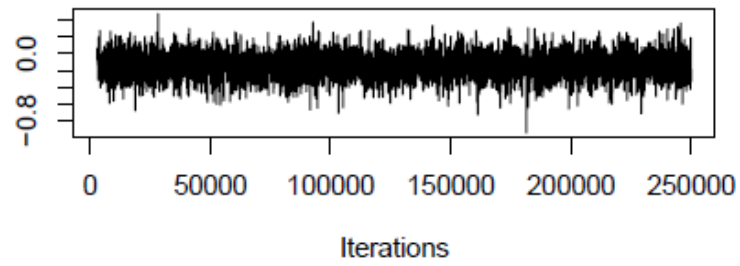

Trace of live

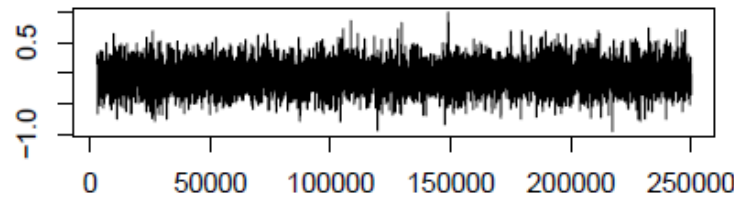

Iterations

Trace of relation

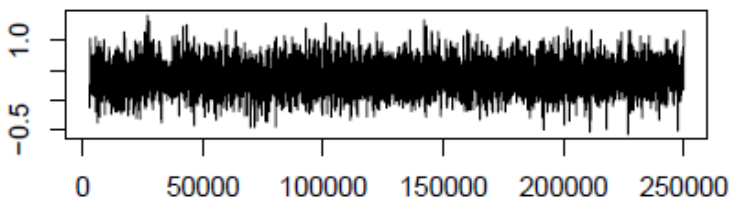

Iterations
Density of (Intercept)

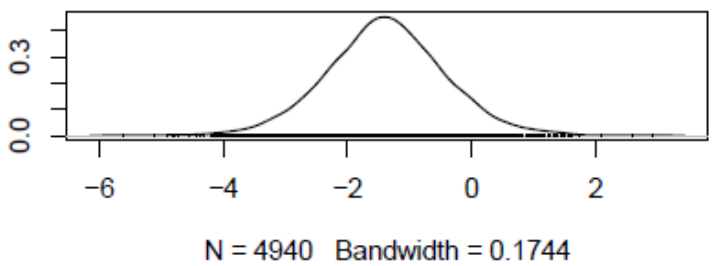

Density of custody

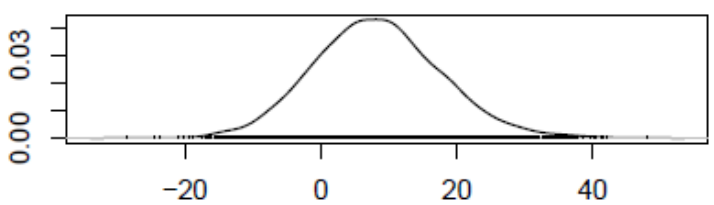

$\mathrm{N}=4940 \quad$ Bandwidth $=1.789$

Density of time

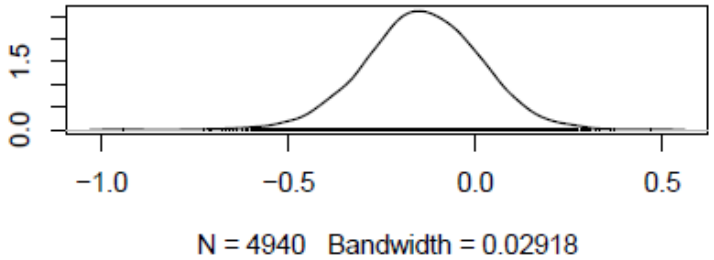

Density of live

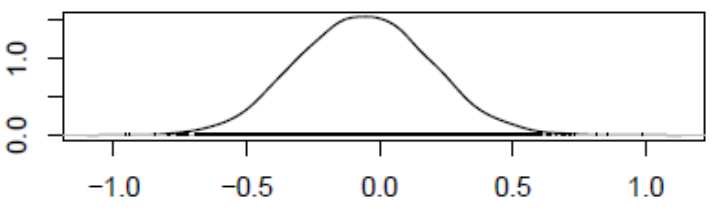

$\mathrm{N}=4940$ Bandwidth $=0.04793$

Density of relation

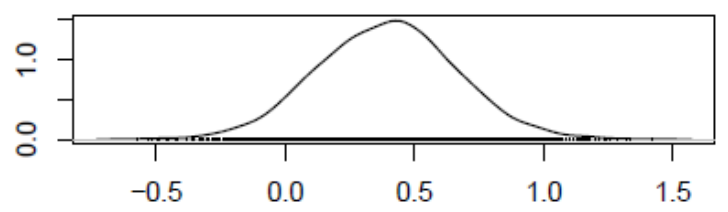

$\mathrm{N}=4940$ Bandwidth $=0.05146$ 
Trace of ete

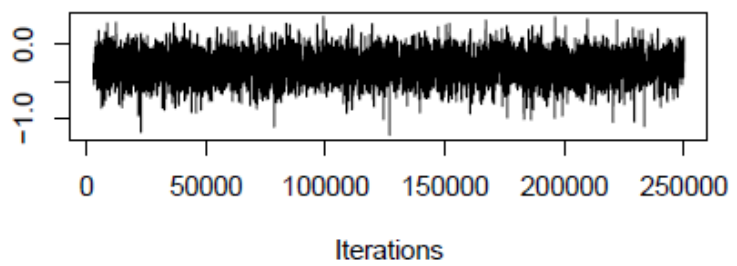

Trace of where

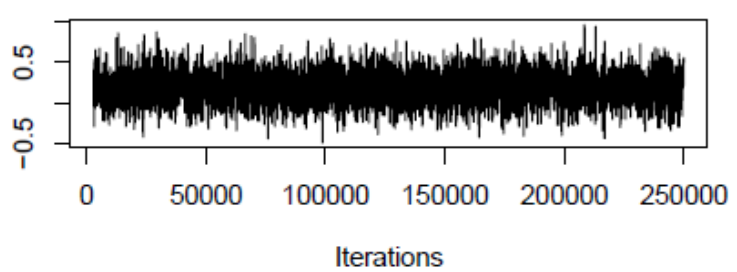

Trace of life

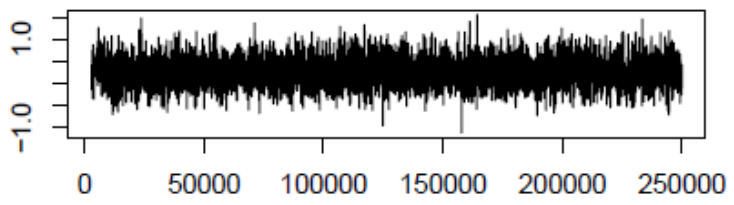

Iterations

Trace of drugs

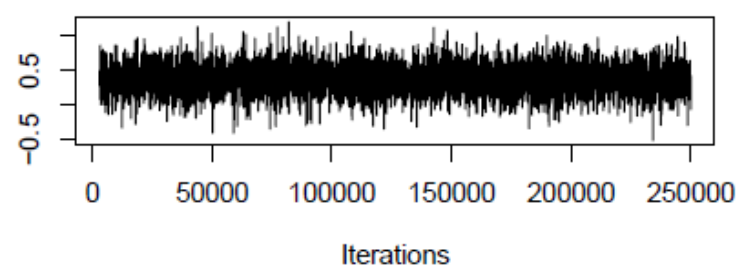

Trace of physical

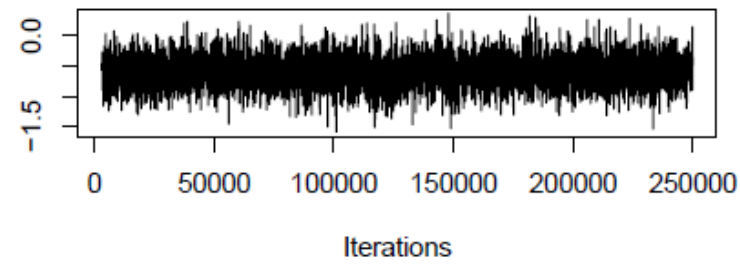

Trace of emotion

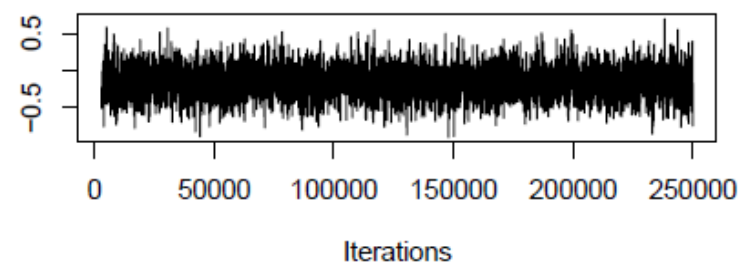

Density of ete

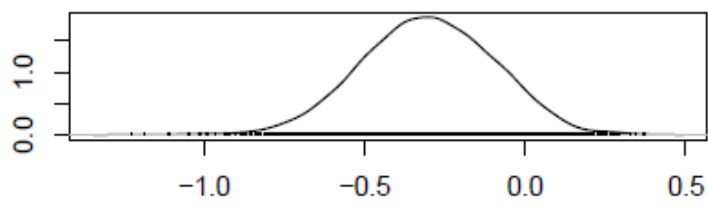

$\mathrm{N}=4940$ Bandwidth $=0.03997$

Density of where

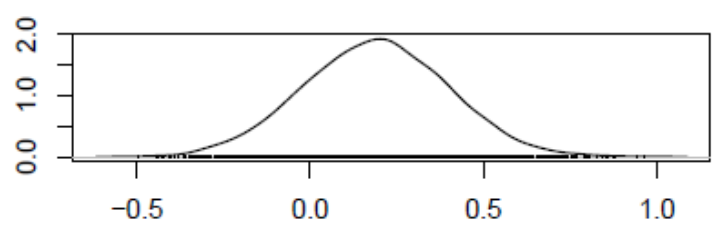

$\mathrm{N}=4940 \quad$ Bandwidth $=0.04007$

Density of life

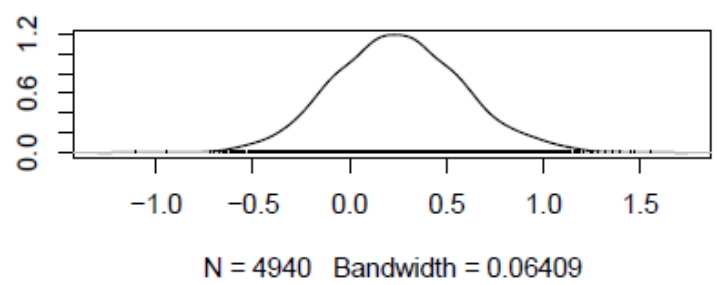

Density of drugs

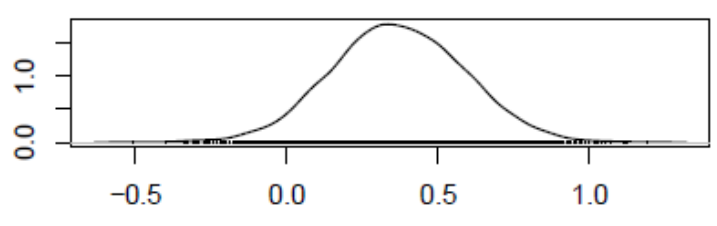

$\mathrm{N}=4940$ Bandwidth $=0.04225$

Density of physical

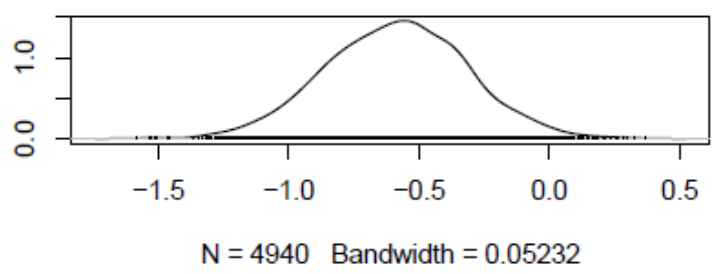

\section{Density of emotion}

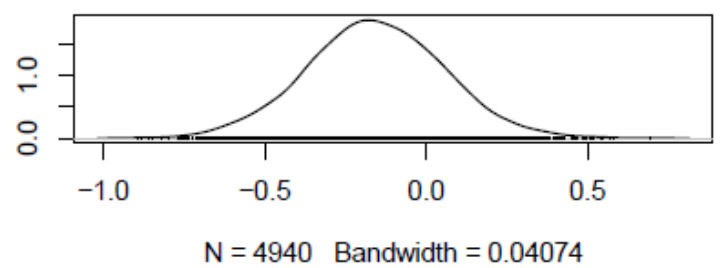


Trace of self

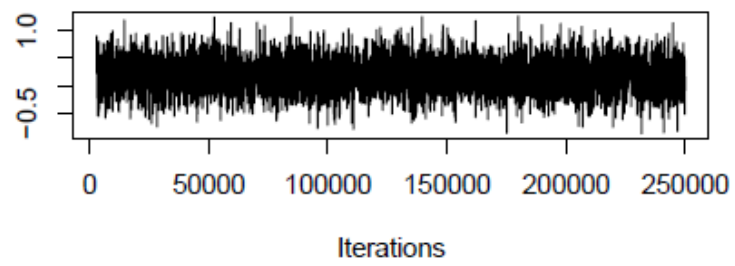

Trace of think

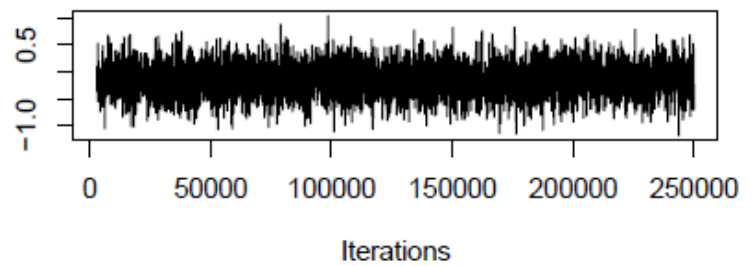

Trace of attitude

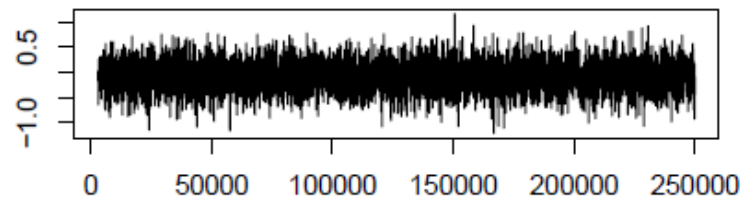

Iterations

Trace of change

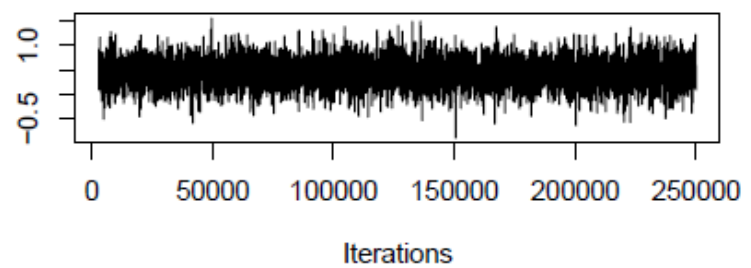

Trace of custody:time

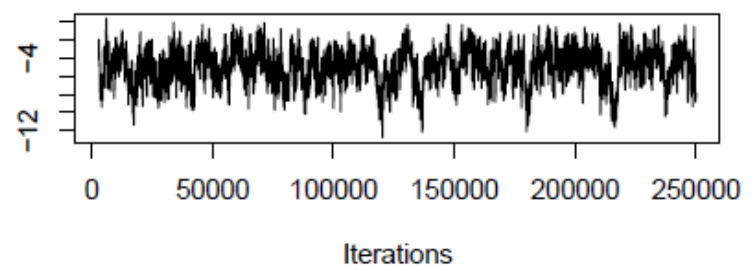

Trace of custody:live

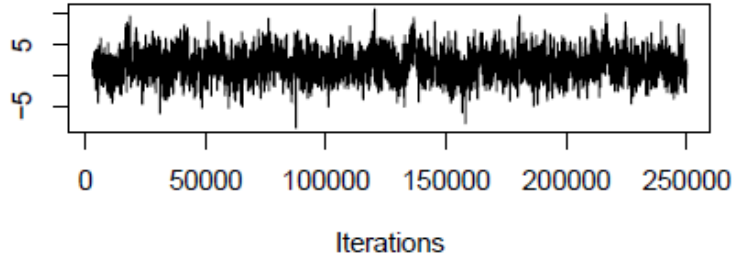

Density of self

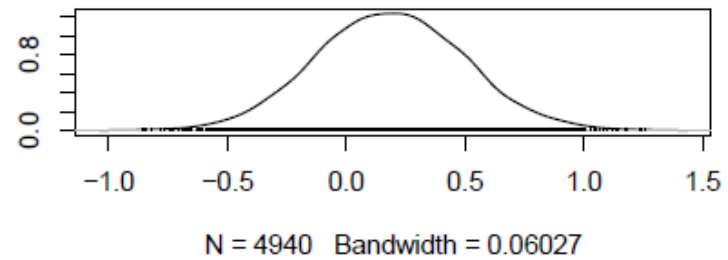

Density of think

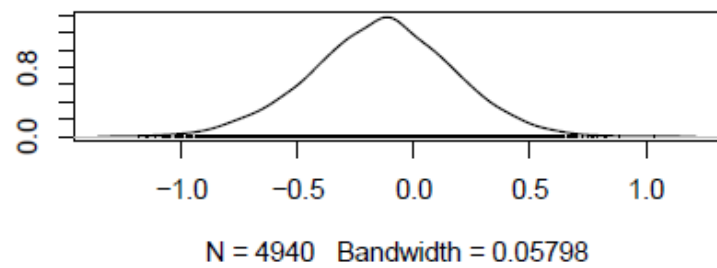

Density of attitude

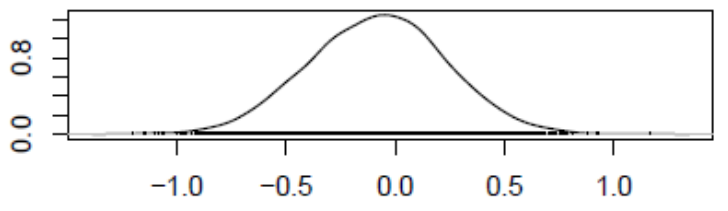

$N=4940$ Bandwidth $=0.06027$

Density of change

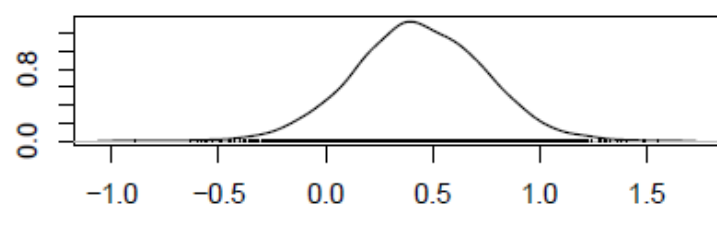

$\mathrm{N}=4940$ Bandwidth $=0.05828$

Density of custody:time

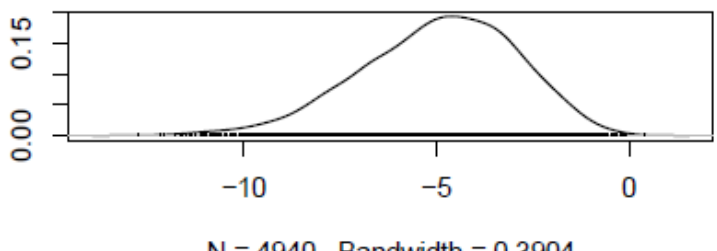

Density of custody:live

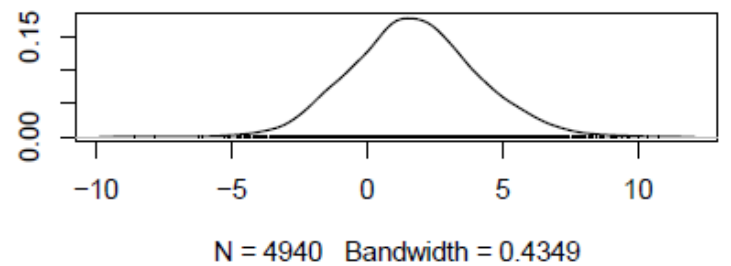


Trace of time:live

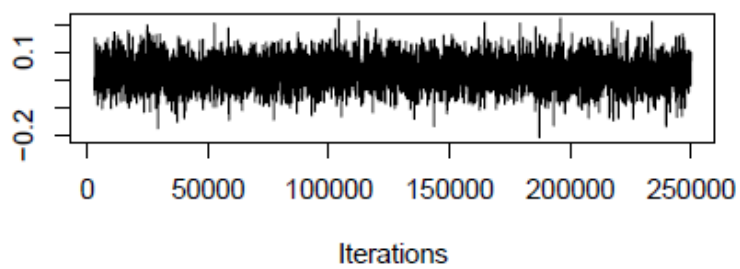

Trace of custody:relation

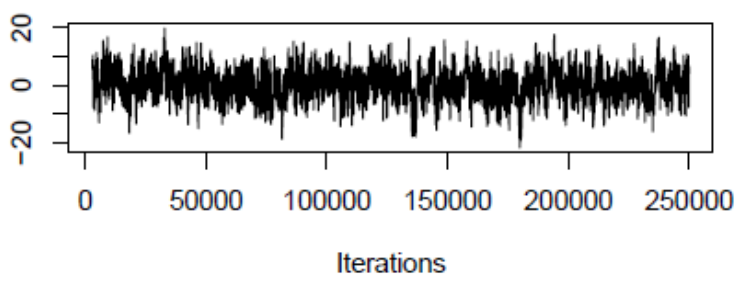

Trace of time:relation

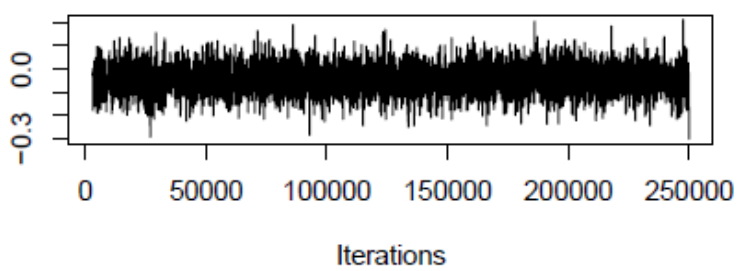

Trace of custody:ete

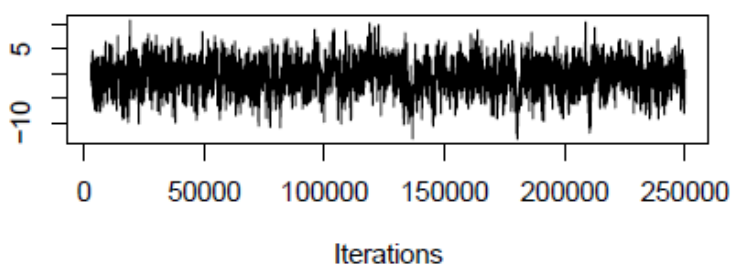

Trace of time:ete

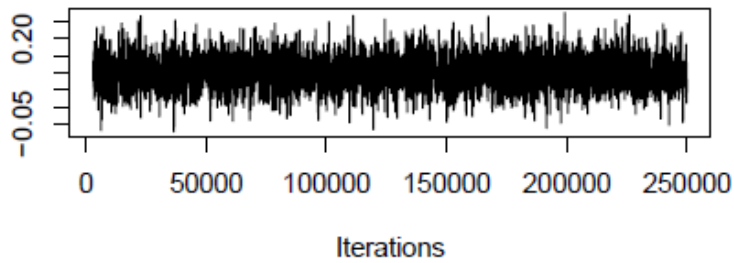

Trace of custody:where

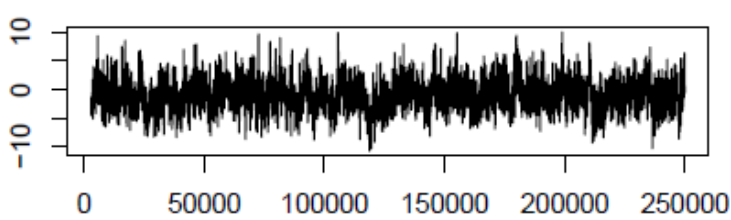

Iterations
Density of time:live

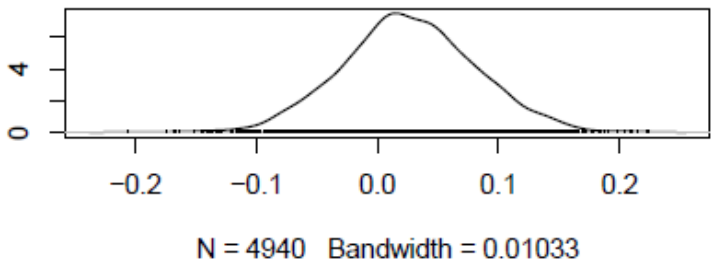

Density of custody:relation

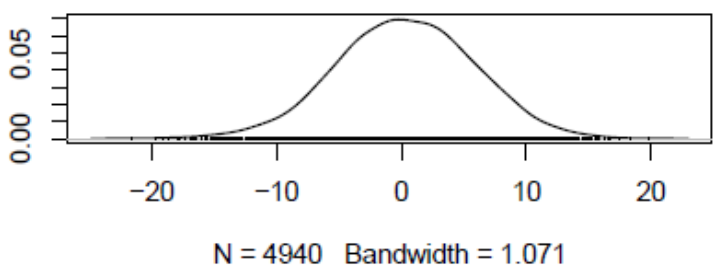

Density of time:relation

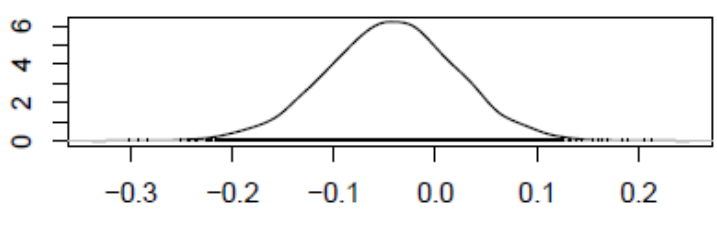

$\mathrm{N}=4940$ Bandwidth $=0.01226$

Density of custody:ete

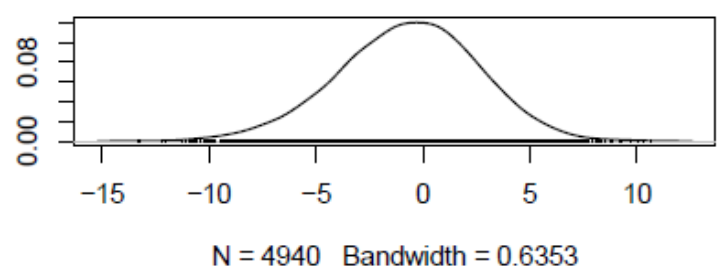

Density of time:ete

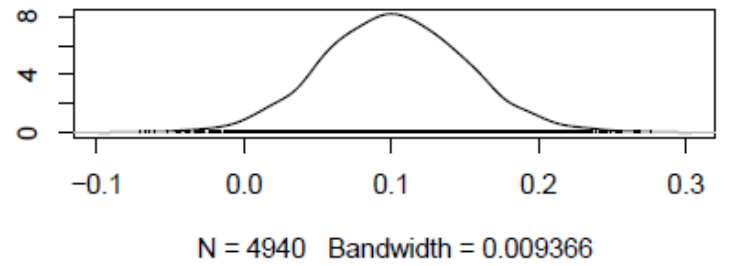

Density of custody:where

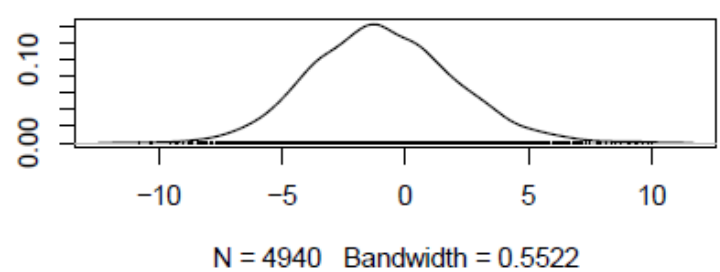


Trace of time:where

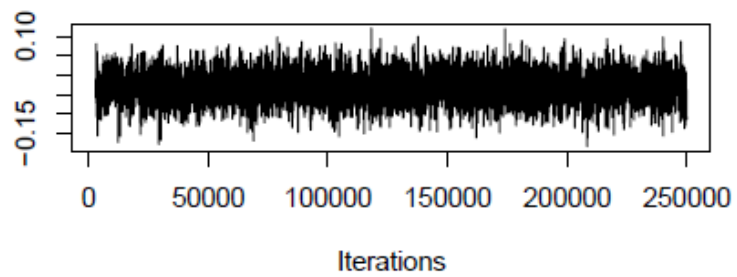

Trace of custody:life

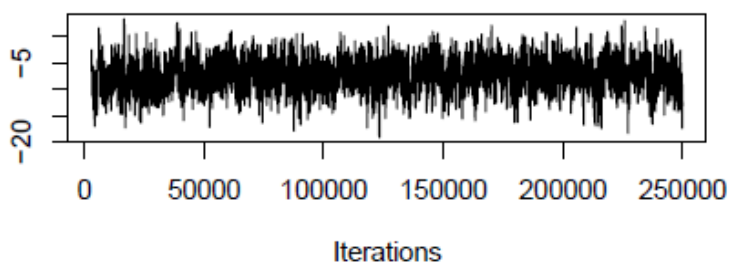

Trace of time:life

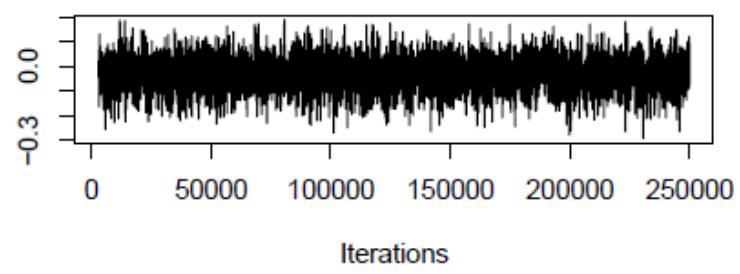

Trace of custody:drugs

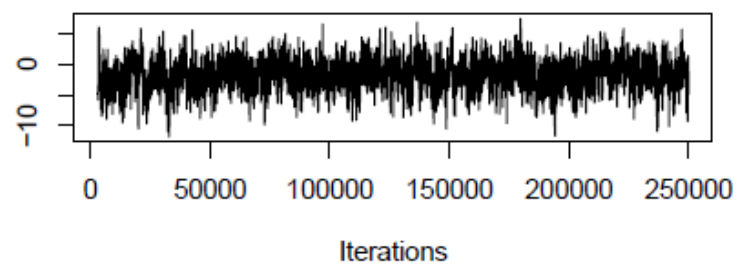

Trace of time:drugs

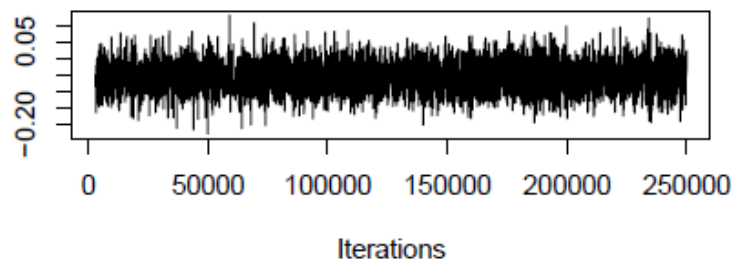

Trace of custody:physical

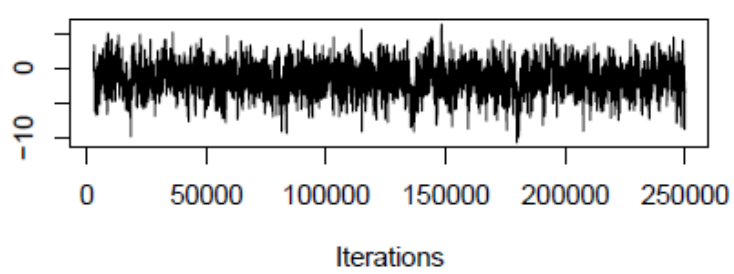

Density of time:where

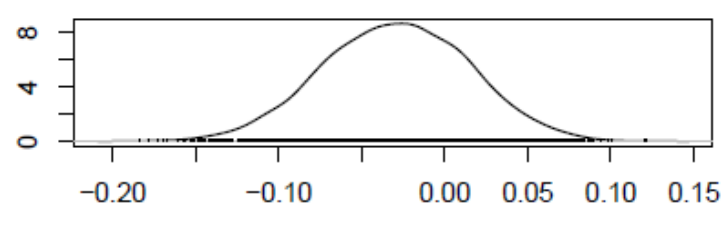

$\mathrm{N}=4940$ Bandwidth $=0.008463$

Density of custody:life

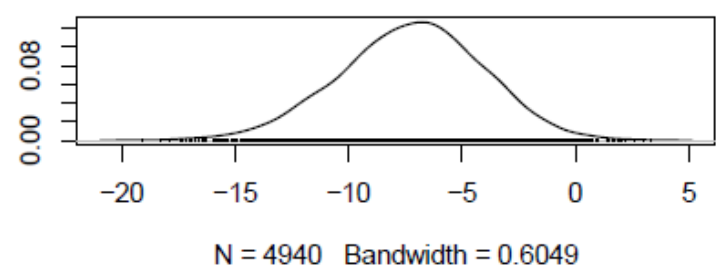

Density of time:life

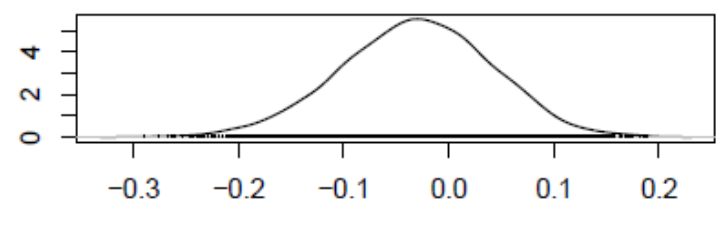

$\mathrm{N}=4940$ Bandwidth $=0.01387$

Density of custody:drugs

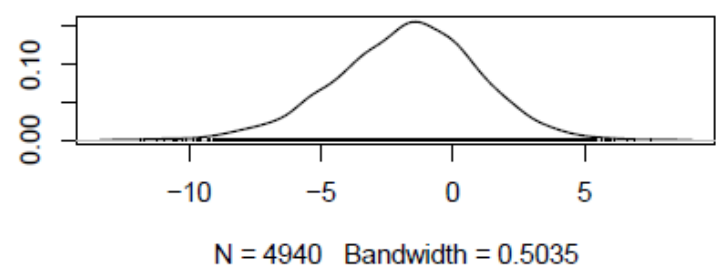

Density of time:drugs

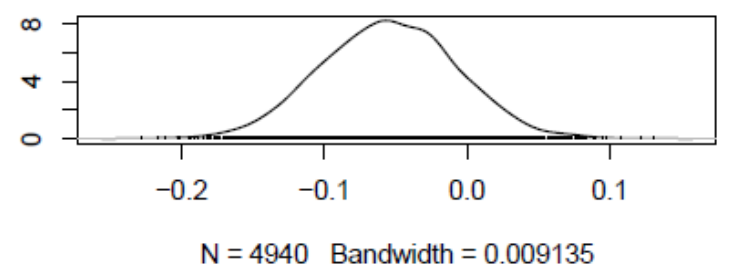

Density of custody:physical

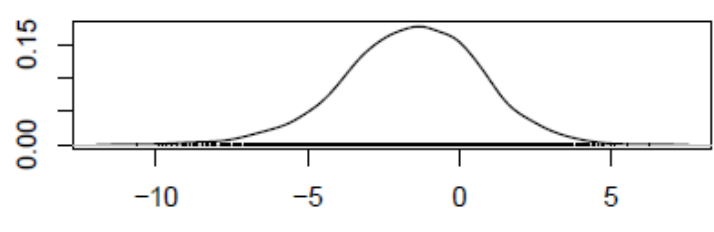

$\mathrm{N}=4940$ Bandwidth $=0.4289$ 
Trace of time:physical

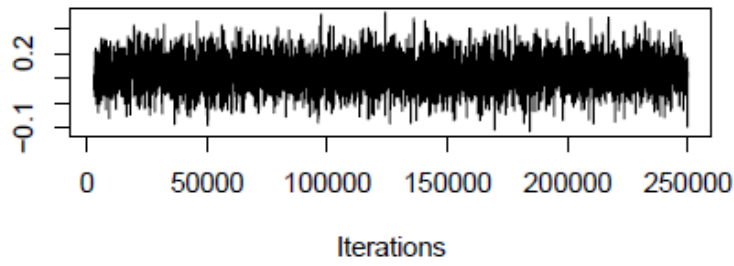

Trace of custody:emotion

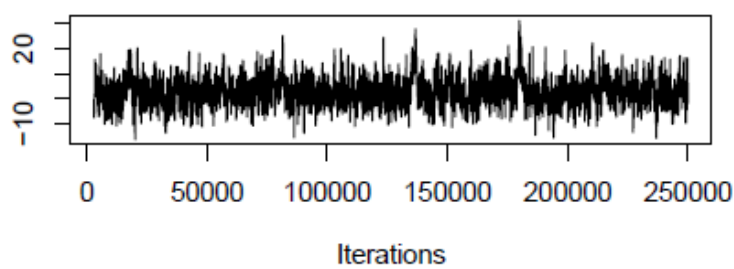

Trace of time:emotion

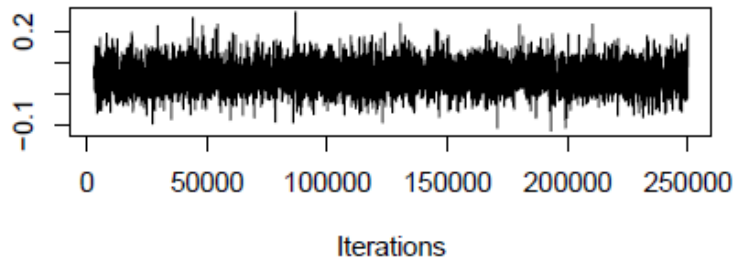

Trace of custody:self

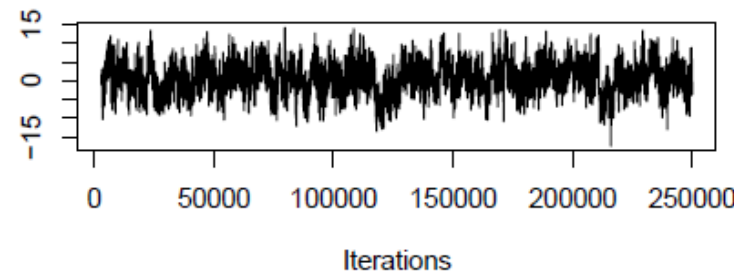

Trace of time:self

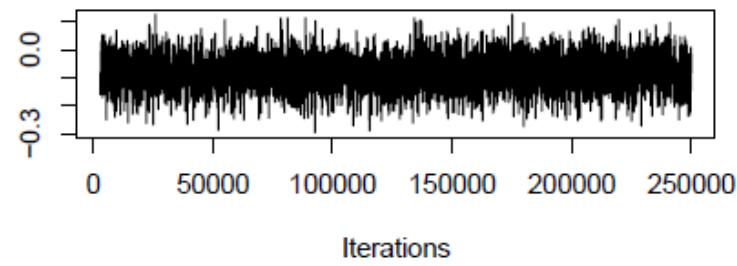

Trace of custody:think

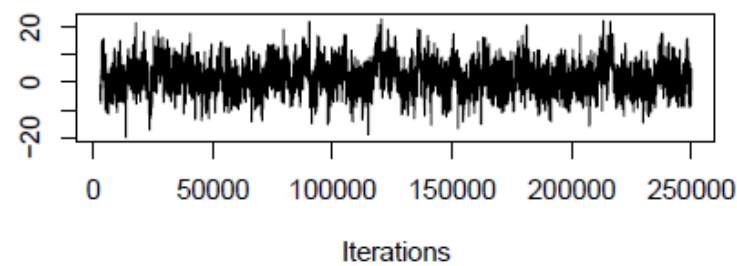

Density of time:physical

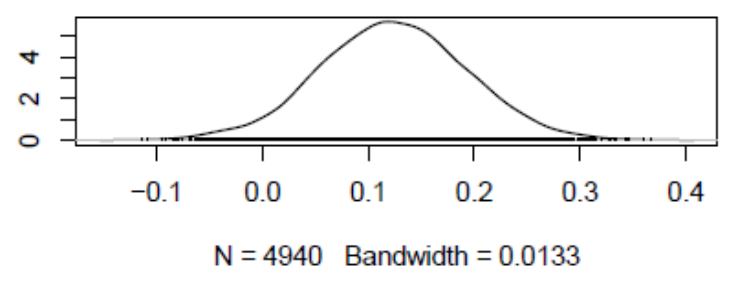

Density of custody:emotion

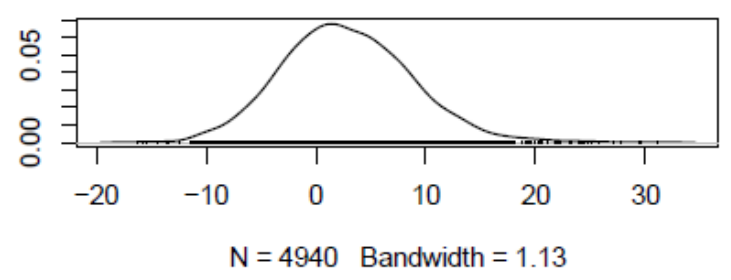

Density of time:emotion

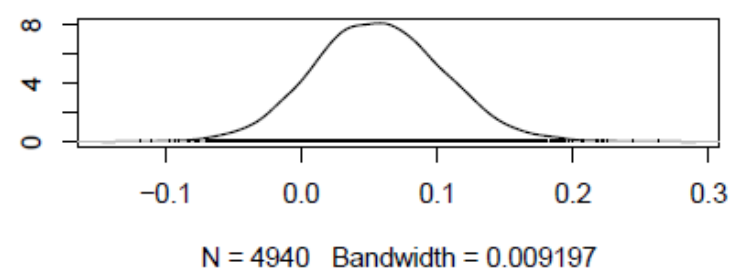

Density of custody:self

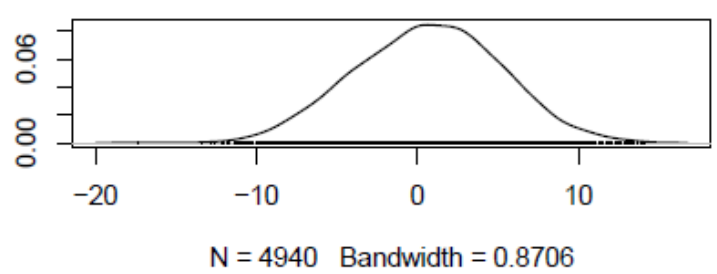

Density of time:self

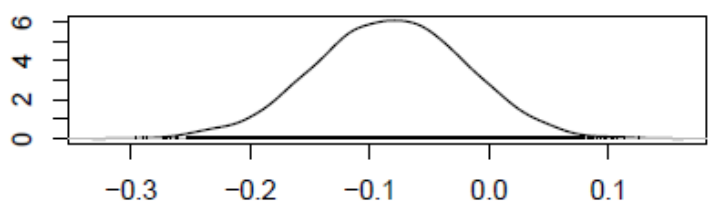

$\mathrm{N}=4940$ Bandwidth $=0.01218$

Density of custody:think

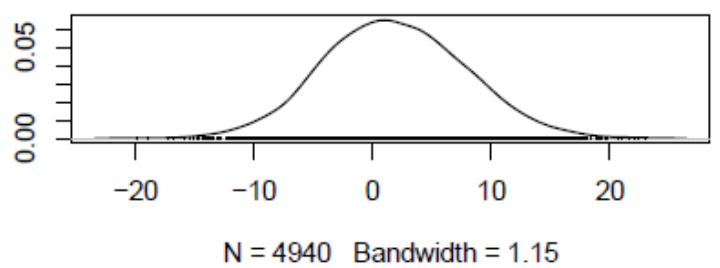


Trace of time:think

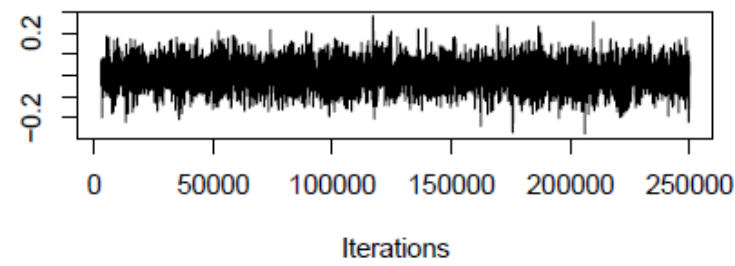

Trace of custody:attitude

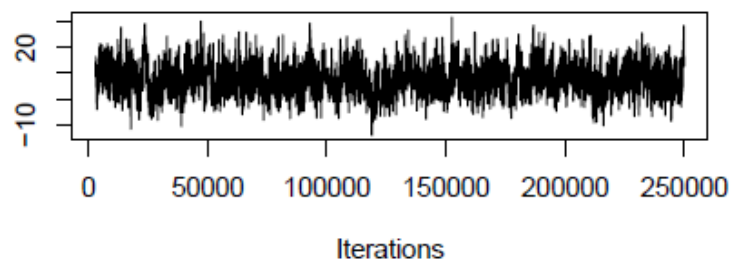

Trace of time:attitude

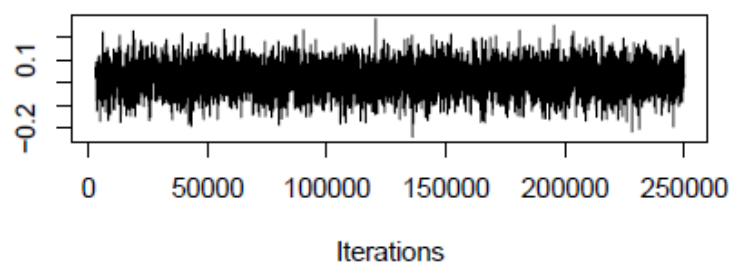

Trace of custody:change

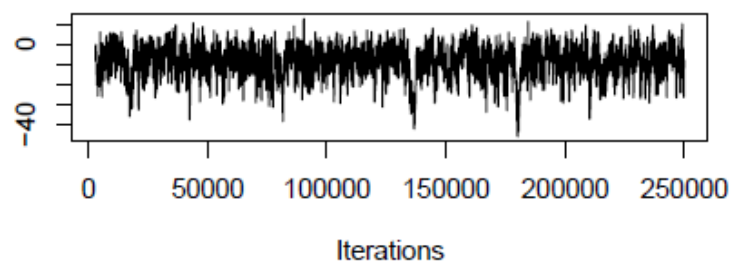

Trace of time:change

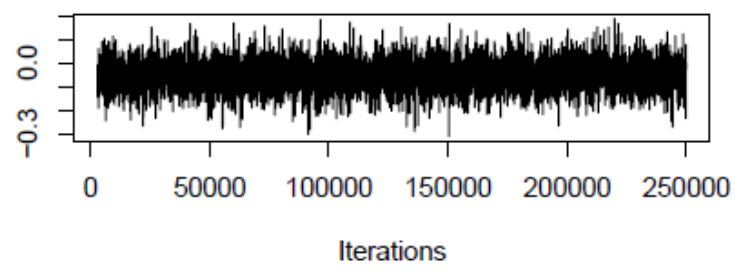

Trace of custody:time:live

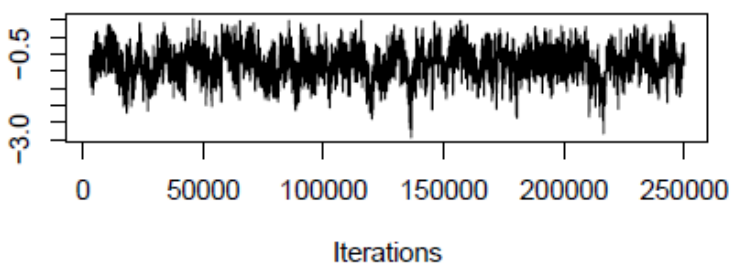

Density of time:think

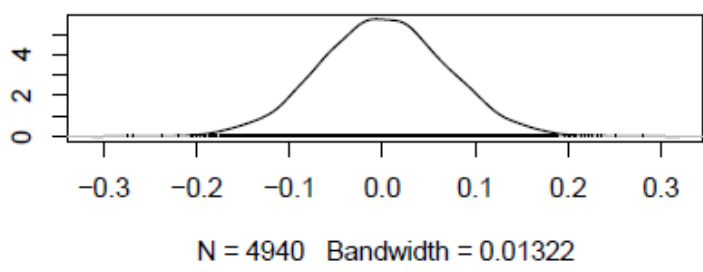

Density of custody:attitude

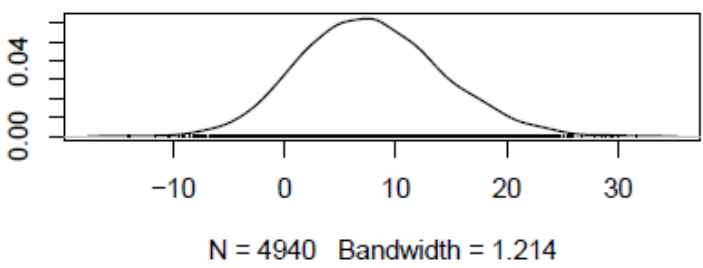

Density of time:attitude

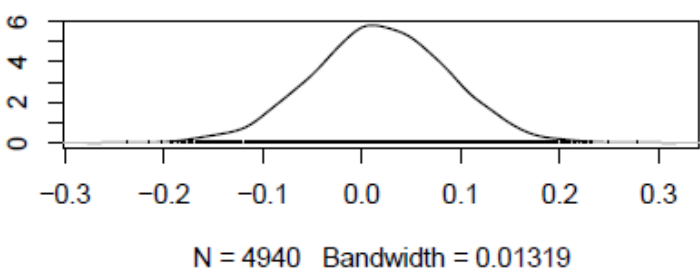

Density of custody:change

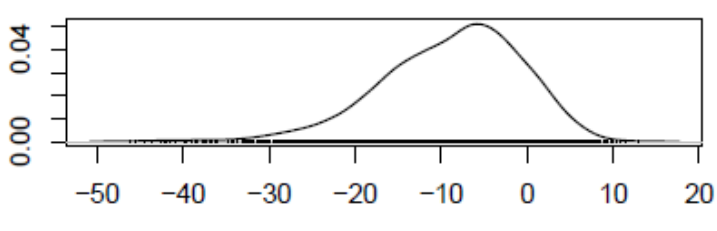

$\mathrm{N}=4940$ Bandwidth $=1.57$

Density of time:change

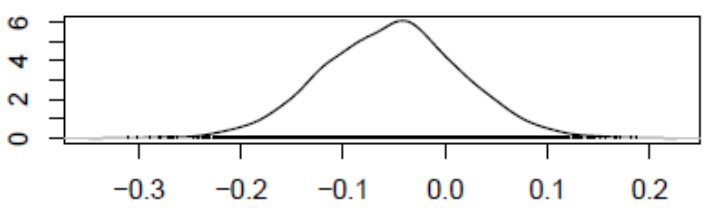

$\mathrm{N}=4940$ Bandwidth $=0.01301$

Density of custody:time:live

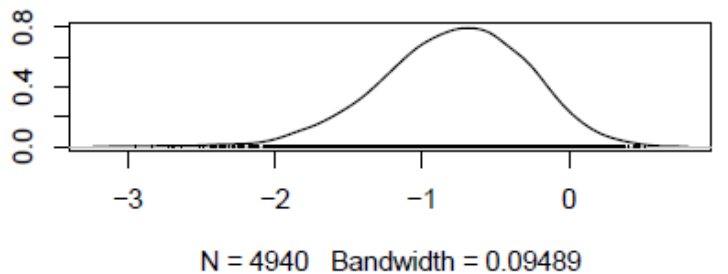


Trace of custody:time:relation

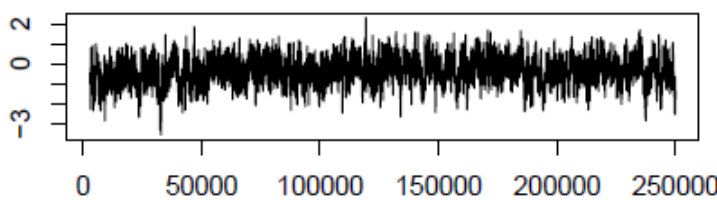

Iterations

Trace of custody:time:ete

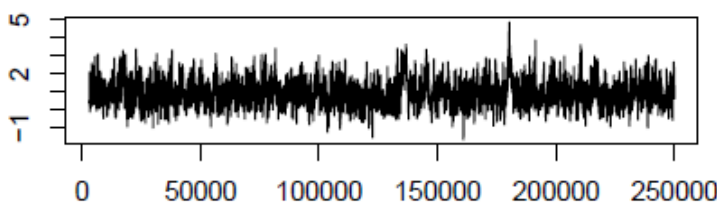

Iterations

Trace of custody:time:where

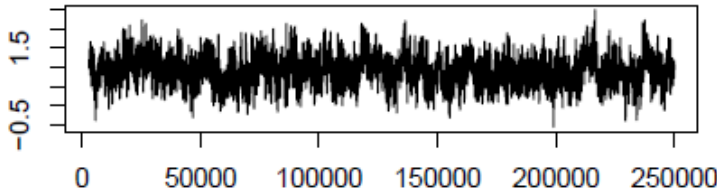

Iterations

Trace of custody:time:life

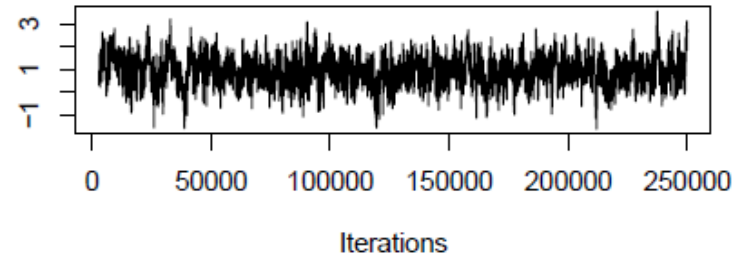

Trace of custody:time:drugs

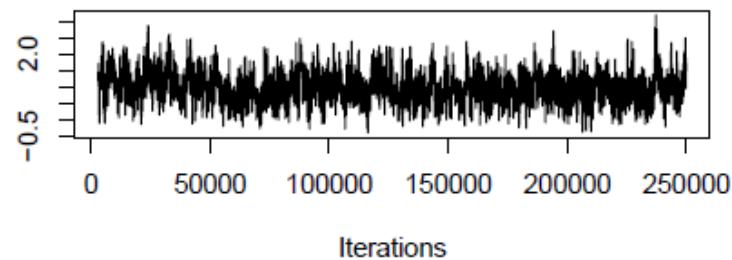

Trace of custody:time:physical

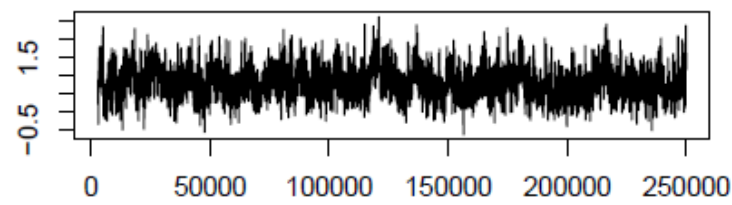

Iterations
Density of custody:time:relation

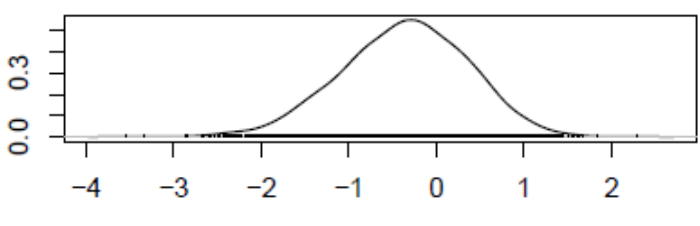

$\mathrm{N}=4940$ Bandwidth $=0.1402$

Density of custody:time:ete

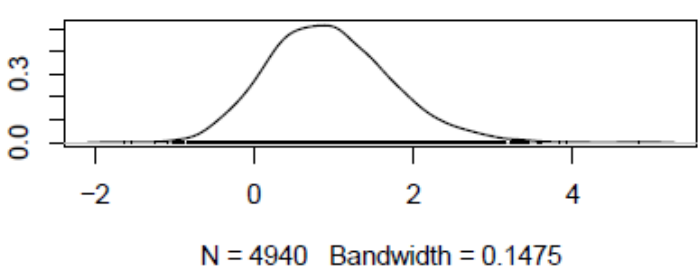

Density of custody:time:where

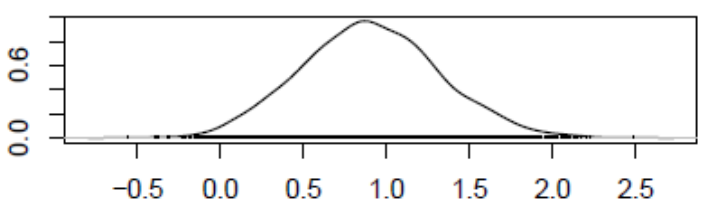

$\mathrm{N}=4940$ Bandwidth $=0.08006$

Density of custody:time:life

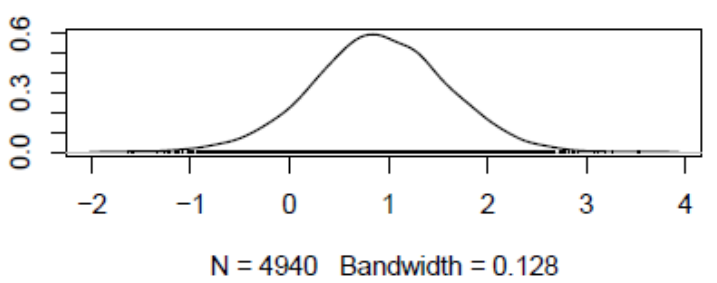

Density of custody:time:drugs

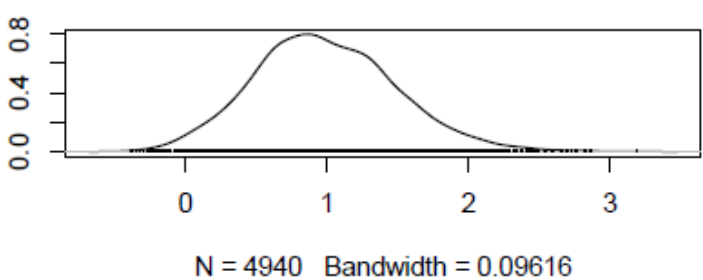

Density of custody:time:physical

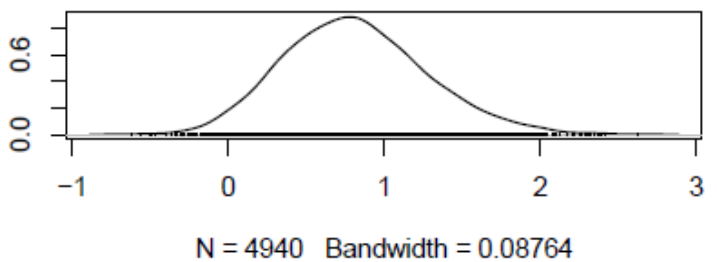


Trace of custody:time:emotion

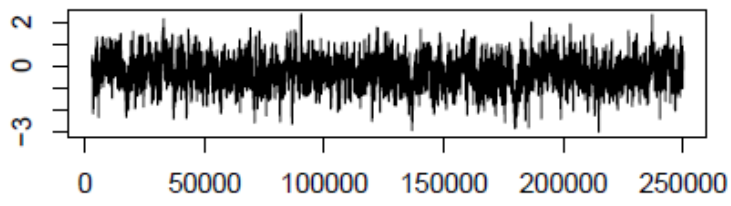

Iterations

Trace of custody:time:self

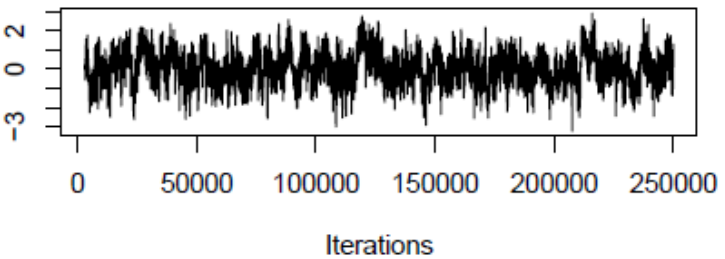

Trace of custody:time:think

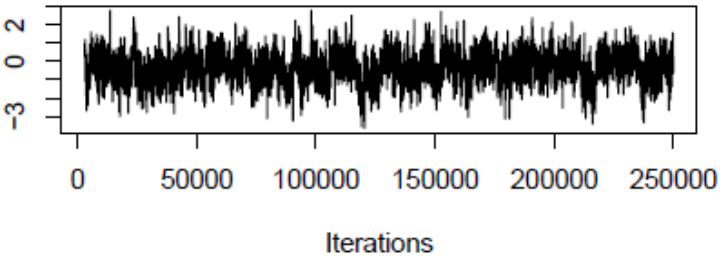

Trace of custody:time:attitude

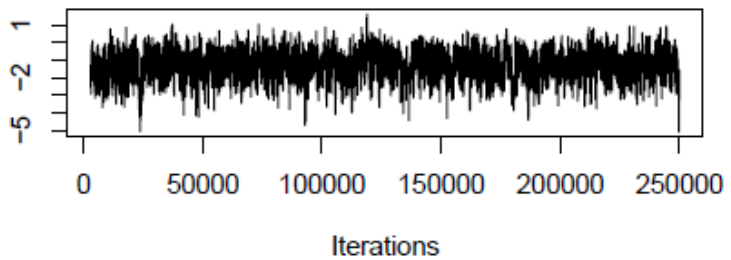

Trace of custody:time:change

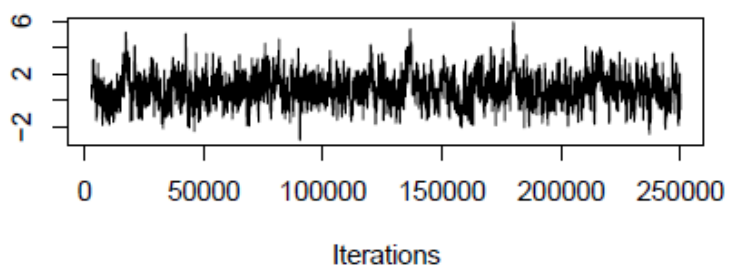

Density of custody:time:emotion

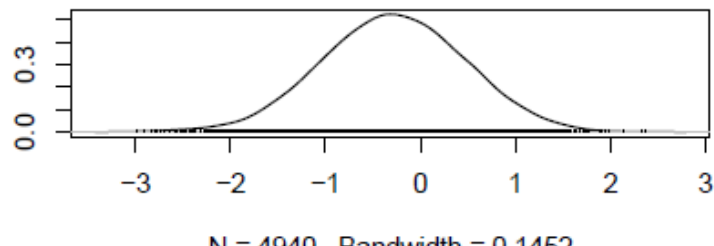

Density of custody:time:self

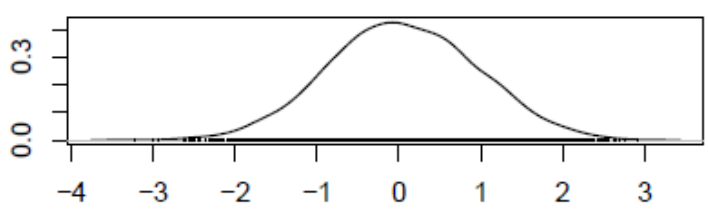

$\mathrm{N}=4940$ Bandwidth $=0.1747$

Density of custody:time:think

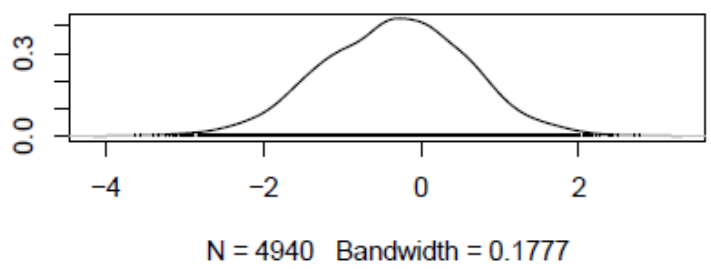

Density of custody:time:attitude

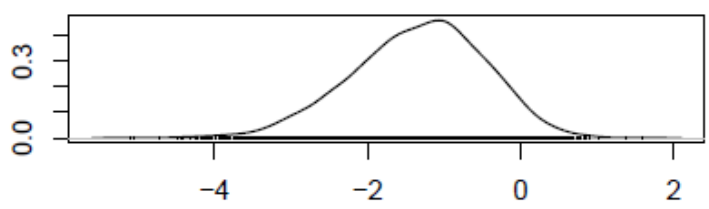

$\mathrm{N}=4940$ Bandwidth $=0.1675$

Density of custody:time:change

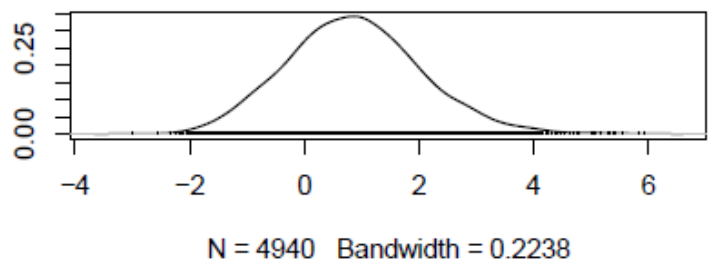


Trace of time

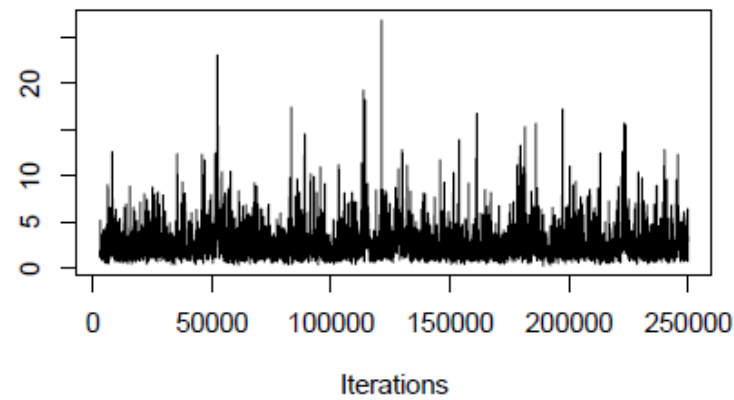

Trace of Research.ID

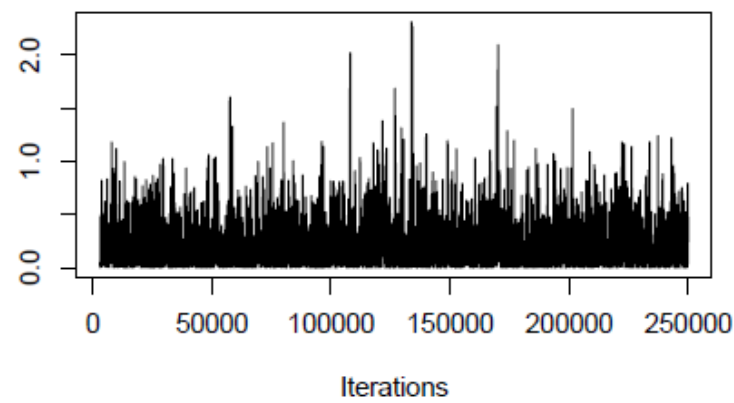

Density of time

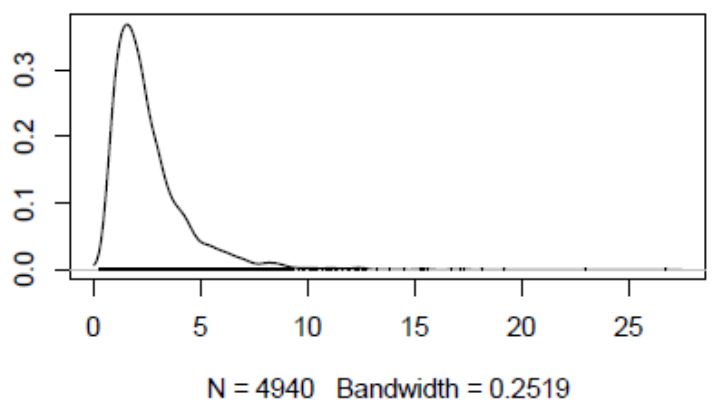

Density of Research.ID

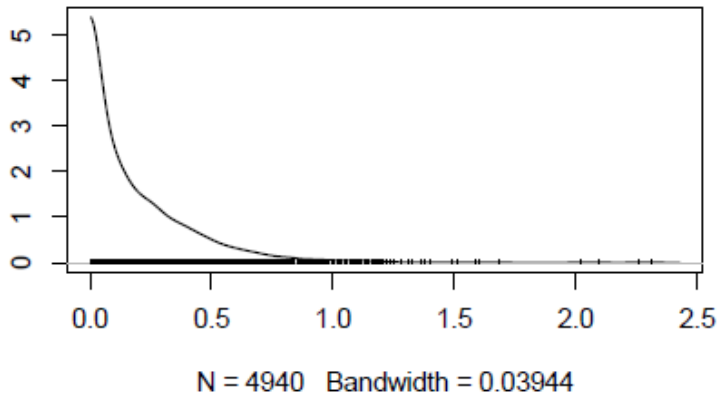


Dynamic Model 6 (Table 7.11)

Bayesian Model (BDm6)

\section{\#\# Define the Model}

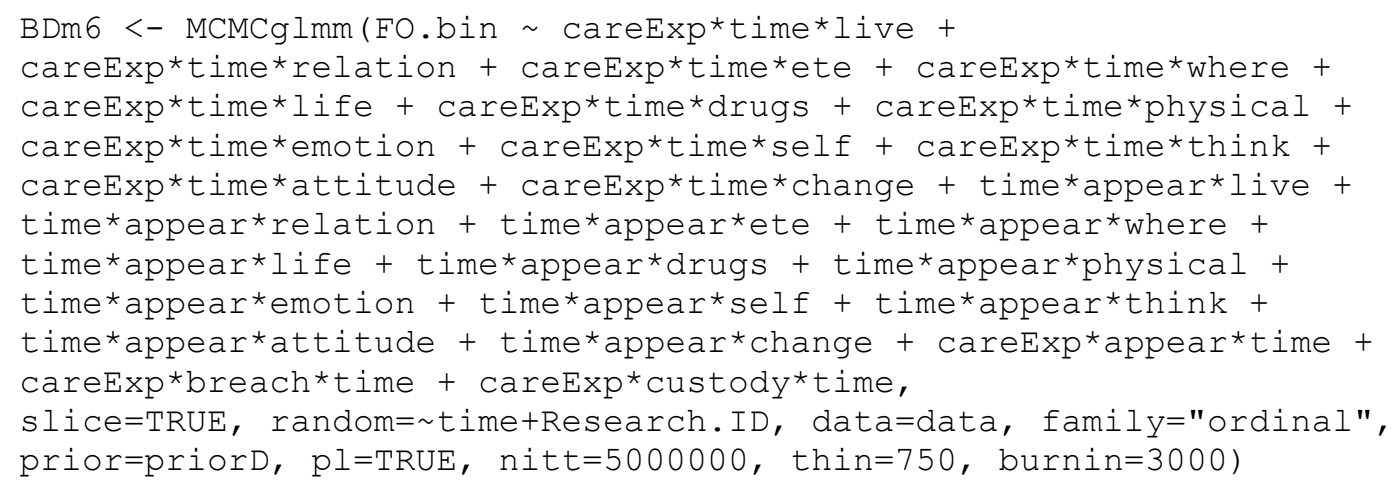

\section{\#\# Checks for suitable convergence}

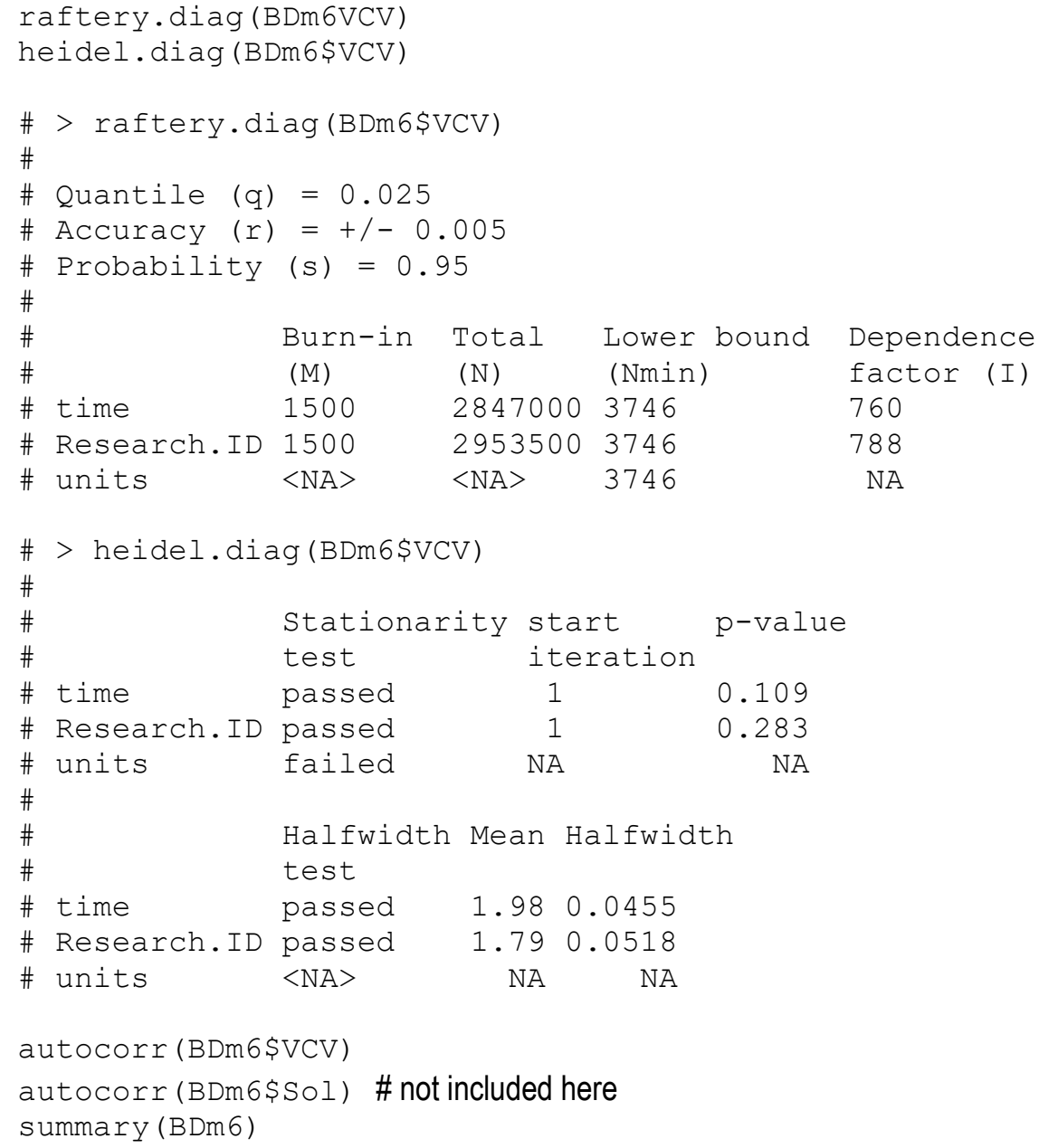




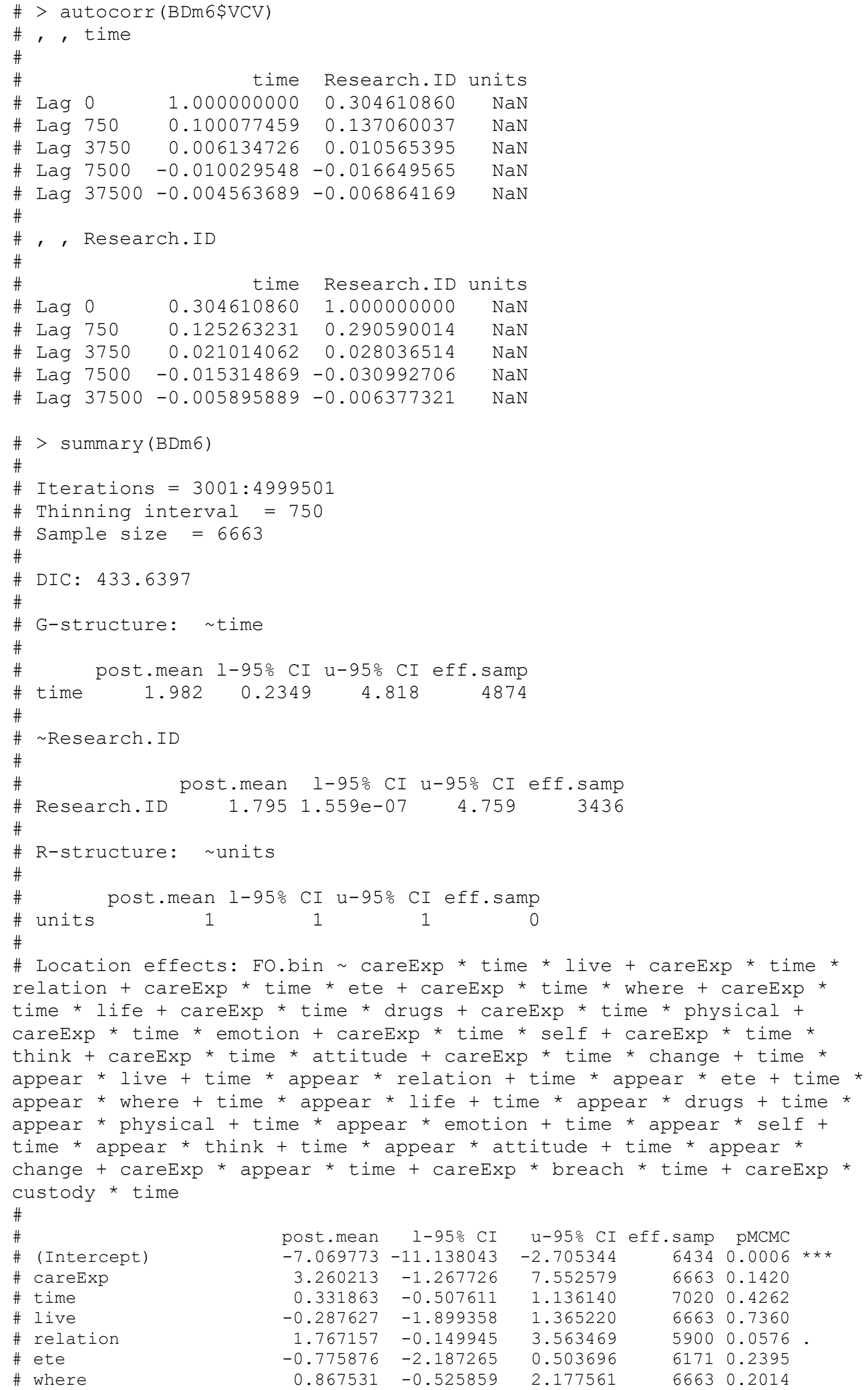

$\begin{array}{rrrrr}\text { post.mean } & 1-95 \% \text { CI } & \text { u-95\% CI eff.samp } & \text { pMCMC } \\ -7.069773 & -11.138043 & -2.705344 & 6434 & 0.0006 \\ 3.260213 & -1.267726 & 7.552579 & 6663 & 0.1420 \\ 0.331863 & -0.507611 & 1.136140 & 7020 & 0.4262 \\ -0.287627 & -1.899358 & 1.365220 & 6663 & 0.7360 \\ 1.767157 & -0.149945 & 3.563469 & 5900 & 0.0576 \\ -0.775876 & -2.187265 & 0.503696 & 6171 & 0.2395 \\ 0.867531 & -0.525859 & 2.177561 & 6663 & 0.2014\end{array}$




\begin{tabular}{|c|c|}
\hline & life \\
\hline \# & drugs \\
\hline & physical \\
\hline & emotion \\
\hline & self \\
\hline & think \\
\hline & attitude \\
\hline & change \\
\hline & appear \\
\hline & breach \\
\hline & custody \\
\hline & careExp:time \\
\hline & careExp:live \\
\hline & time:live \\
\hline \# & careExp:relation \\
\hline 开 & time:relation \\
\hline & careExp:ete \\
\hline & time:ete \\
\hline \# & careExp: where \\
\hline \# & time: where \\
\hline \# & careExp:life \\
\hline \# & time:life \\
\hline \# & careExp:drugs \\
\hline \# & time:drugs \\
\hline \# & careExp:physical \\
\hline \# & time:physical \\
\hline$\#$ & careExp:emotion \\
\hline \# & time:emotion \\
\hline \# & careExp:self \\
\hline \# & time:self \\
\hline$\#$ & careExp:think \\
\hline \# & time:think \\
\hline \# & careExp:attitude \\
\hline \# & time:attitude \\
\hline \# & careExp: change \\
\hline \# & time:change \\
\hline \# & time:appear \\
\hline \# & live:appear \\
\hline$\#$ & relation:appear \\
\hline \# & ete:appear \\
\hline \# & where: appear \\
\hline \# & life:appear \\
\hline \# & drugs:appear \\
\hline \# & physical :appear \\
\hline \# & emotion:appear \\
\hline \# & self:appear \\
\hline$\#$ & think:appear \\
\hline \# & attitude:appear \\
\hline \# & change:appear \\
\hline \# & careExp:appear \\
\hline \# & careExp:breach \\
\hline \# & time:breach \\
\hline \# & careExp:custody \\
\hline \# & time:custody \\
\hline \# & careExp:time:live \\
\hline \# & careExp:time:relation \\
\hline \# & careExp:time:ete \\
\hline \# & careExp:time: where \\
\hline \# & careExp:time:life \\
\hline \# & careExp:time:drugs \\
\hline & careExp:time:physical \\
\hline \# & careExp:time:emotion \\
\hline & careExp:time:self \\
\hline & careExp:time:think \\
\hline & careExp:time:attitude \\
\hline & careExp:time:change \\
\hline & time:live:appear \\
\hline & time:relation:appear \\
\hline
\end{tabular}

\begin{tabular}{|c|c|c|}
\hline $860 \Rightarrow$ & 1585451 & 356092 \\
\hline & -0.291584 & 625191 \\
\hline to & -2.529983 & 0.889509 \\
\hline 22118 & -1.716649 & 989895 \\
\hline 1894 & -1.884595 & 1.903803 \\
\hline 0.122643 & -1.597343 & 1.875663 \\
\hline 1.127322 & -0.758501 & \\
\hline-1.05961 & -3 & \\
\hline 7.794157 & & \\
\hline-0.8 & -2 . & \\
\hline-2 . & -5 . & \\
\hline-0 . & -1 & \\
\hline 0 . & -0 . & \\
\hline-0 . & -0 . & \\
\hline-0 . & -1 & \\
\hline-0 . & -0 . & \\
\hline 0 . & -1 & \\
\hline & -0 . & \\
\hline-0.2 & -1 & \\
\hline & -0 . & \\
\hline-0 . & -2 . & \\
\hline-0 . & -0 . & \\
\hline-0 . & -1 & \\
\hline-0 . & -0 . & \\
\hline-1.2 & -3 . & \\
\hline-0 & -0 . & \\
\hline 0.9 & -0 . & \\
\hline 0.1 & -0 . & \\
\hline 1.1 & -0 & \\
\hline 0.1 & -0 & \\
\hline-1.3 & -3 & \\
\hline 0.0 & -0 & \\
\hline-0 . & -2 & \\
\hline-0 & -1.0 & -0 . \\
\hline 1.1 & $-0 . \varepsilon$ & \\
\hline & -0 . & \\
\hline-1.2 & -2 . & -0 . \\
\hline 0.1 & -1. & \\
\hline-1.5 & -3. & \\
\hline 0.3 & $-1 \cdot 1$ & \\
\hline-0.9 & -2. & \\
\hline 0.2 & -1.7 & \\
\hline-1.0 & -2.5 & \\
\hline 1 & -0 . & \\
\hline-0.2 & -1.581978 & 1.2 \\
\hline-0.5 & -2.67 & \\
\hline & -1.8 & \\
\hline-1.14 & -3 & \\
\hline 1. & -0 . & \\
\hline-0.7 & -3.41 & \\
\hline 2. & -0 . & \\
\hline 0. & -0 . & \\
\hline 1.1 & -2 . & \\
\hline-0 . & -0 . & \\
\hline 0. & -0 . & \\
\hline-0 . & -0 . & 0.25 \\
\hline & -0 & \\
\hline 0.2 & -0.0 & 0. \\
\hline-0. & -0.458574 & 0.35129 \\
\hline$-0.1 \mathrm{C}-2$ & -0.402127 & 0.19720 \\
\hline & -0.004666 & 0.8255 \\
\hline-0.116 & -0.459221 & 0.19977 \\
\hline-0.630780 & -1.068681 & -0.19613 \\
\hline & & \\
\hline 0.379641 & -0.064960 & 0.817040 \\
\hline-0.409267 & -0.841456 & 0.01710 \\
\hline 0.272084 & -0.112738 & 0.65373 \\
\hline 0.32402 & -0.104088 & 0.76484 \\
\hline
\end{tabular}

$\begin{array}{ll}6663 & 0.6931\end{array}$

61920.0946.

$\begin{array}{ll}6418 & 0.3080\end{array}$

$\begin{array}{ll}6663 & 0.6472\end{array}$

$\begin{array}{ll}6252 & 0.9770\end{array}$

$\begin{array}{lll}6663 & 0.8945\end{array}$

62350.2440

$\begin{array}{lll}6663 & 0.3482\end{array}$

$5892<2 e-04 * \star \star$

$\begin{array}{ll}6663 & 0.3071\end{array}$

$\begin{array}{lll}6315 & 0.2245\end{array}$

$7040 \quad 0.5529$

$\begin{array}{lll}6308 & 0.4154\end{array}$

$\begin{array}{lll}6663 & 0.1447\end{array}$

60550.8450

63640.2176

$\begin{array}{lll}5939 & 0.6892\end{array}$

$\begin{array}{ll}6365 & 0.3857\end{array}$

$\begin{array}{lll}6663 & 0.5307\end{array}$

$\begin{array}{lll}6384 & 0.8954\end{array}$

$\begin{array}{lll}6663 & 0.3983\end{array}$

$\begin{array}{ll}6387 & 0.8351\end{array}$

$\begin{array}{ll}6663 & 0.9092\end{array}$

$\begin{array}{lll}6663 & 0.9248\end{array}$

52260.1645

$\begin{array}{ll}5851 & 0.9869\end{array}$

$\begin{array}{lll}6663 & 0.1831\end{array}$

$\begin{array}{lll}6293 & 0.3629\end{array}$

63120.2164

$\begin{array}{ll}6197 & 0.4959\end{array}$

66630.1906

$\begin{array}{ll}6382 & 0.9944\end{array}$

$\begin{array}{ll}6432 & 0.3410\end{array}$

56750.0402 *

$\begin{array}{ll}6663 & 0.2644\end{array}$

59030.1153

61020.0036 **

$\begin{array}{lll}6663 & 0.8618\end{array}$

$\begin{array}{lll}6304 & 0.1057\end{array}$

66630.6210

$\begin{array}{ll}6303 & 0.1624\end{array}$

$\begin{array}{ll}6663 & 0.8378\end{array}$

$\begin{array}{lll}6133 & 0.1573\end{array}$

$\begin{array}{lll}6378 & 0.1846\end{array}$

$\begin{array}{lll}6394 & 0.7591\end{array}$

$\begin{array}{lll}5654 & 0.6048\end{array}$

$\begin{array}{ll}6663 & 0.8975\end{array}$

$\begin{array}{lll}6275 & 0.2488\end{array}$

$\begin{array}{lll}6320 & 0.2137\end{array}$

$\begin{array}{lll}5718 & 0.5862\end{array}$

$\begin{array}{lll}6663 & 0.0897 .\end{array}$

$\begin{array}{lll}6663 & 0.4785\end{array}$

$\begin{array}{ll}6377 & 0.5694\end{array}$

$\begin{array}{lll}6663 & 0.9656\end{array}$

$\begin{array}{ll}6663 & 0.6069\end{array}$

$\begin{array}{ll}6663 & 0.5034\end{array}$

$\begin{array}{ll}7082 & 0.9272\end{array}$

66630.0825 .

$\begin{array}{ll}6663 & 0.8411\end{array}$

$6663 \quad 0.4824$

61000.0507.

$\begin{array}{ll}6663 & 0.4848\end{array}$

55480.0012 **

59910.0348 *

66630.0903.

66630.0543.

$\begin{array}{ll}6663 & 0.1474\end{array}$

$\begin{array}{lll}6663 & 0.1432\end{array}$ 


\begin{tabular}{|c|c|c|c|c|c|c|}
\hline \# & time:ete:appear & -0.122960 & -0.455313 & 0.216137 & 6663 & 0.4770 \\
\hline \# & time: where:appear & -0.115755 & -0.399078 & 0.172582 & 6357 & 0.4436 \\
\hline$\#$ & time:life:appear & 0.066307 & -0.405193 & 0.554893 & 6663 & 0.7792 \\
\hline$\#$ & time:drugs:appear & 0.108021 & -0.191315 & 0.420726 & 6648 & 0.4941 \\
\hline$\#$ & time:physical:appear & -0.176126 & -0.615083 & 0.251586 & 5611 & 0.4286 \\
\hline$\#$ & time:emotion:appear & -0.048471 & -0.410514 & 0.315329 & 6268 & 0.7975 \\
\hline \# & time:self:appear & 0.091462 & -0.353513 & 0.496388 & 5866 & 0.6910 \\
\hline \# & time:think:appear & -0.173315 & -0.607257 & 0.275383 & 6385 & 0.4460 \\
\hline \# & time:attitude:appear & 0.424585 & -0.067991 & 0.908028 & 925 & 0.0783 \\
\hline$\#$ & time: change:appear & -0.229760 & -0.742272 & 0.239993 & 6308 & 0.3683 \\
\hline$\#$ & careExp:time:appear & 0.435027 & -0.172613 & 1.074508 & 5786 & 0.1693 \\
\hline \# & careExp:time:breach & -0.668934 & -1.225926 & -0.055823 & 6663 & 0.0177 \\
\hline \# & careExp:time:custody & 0.093250 & -0.555543 & 0.700844 & 6663 & 0.7588 \\
\hline \# & & & & & & \\
\hline & Signif. codes: & & $\star 1$ & 0.05 & & \\
\hline
\end{tabular}


Trace Plots and Posterior Density Plots

Fixed Effects
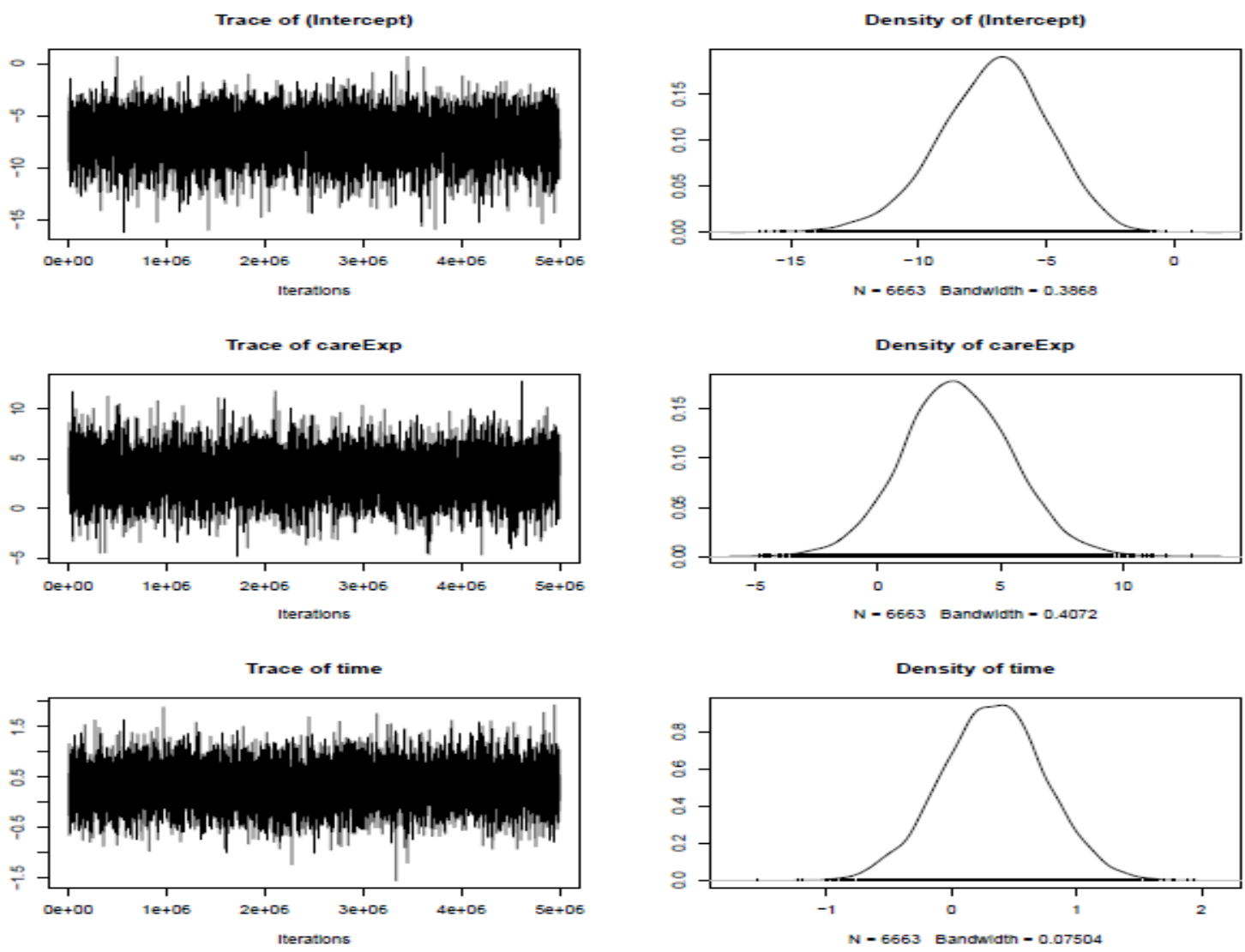

Trace of live
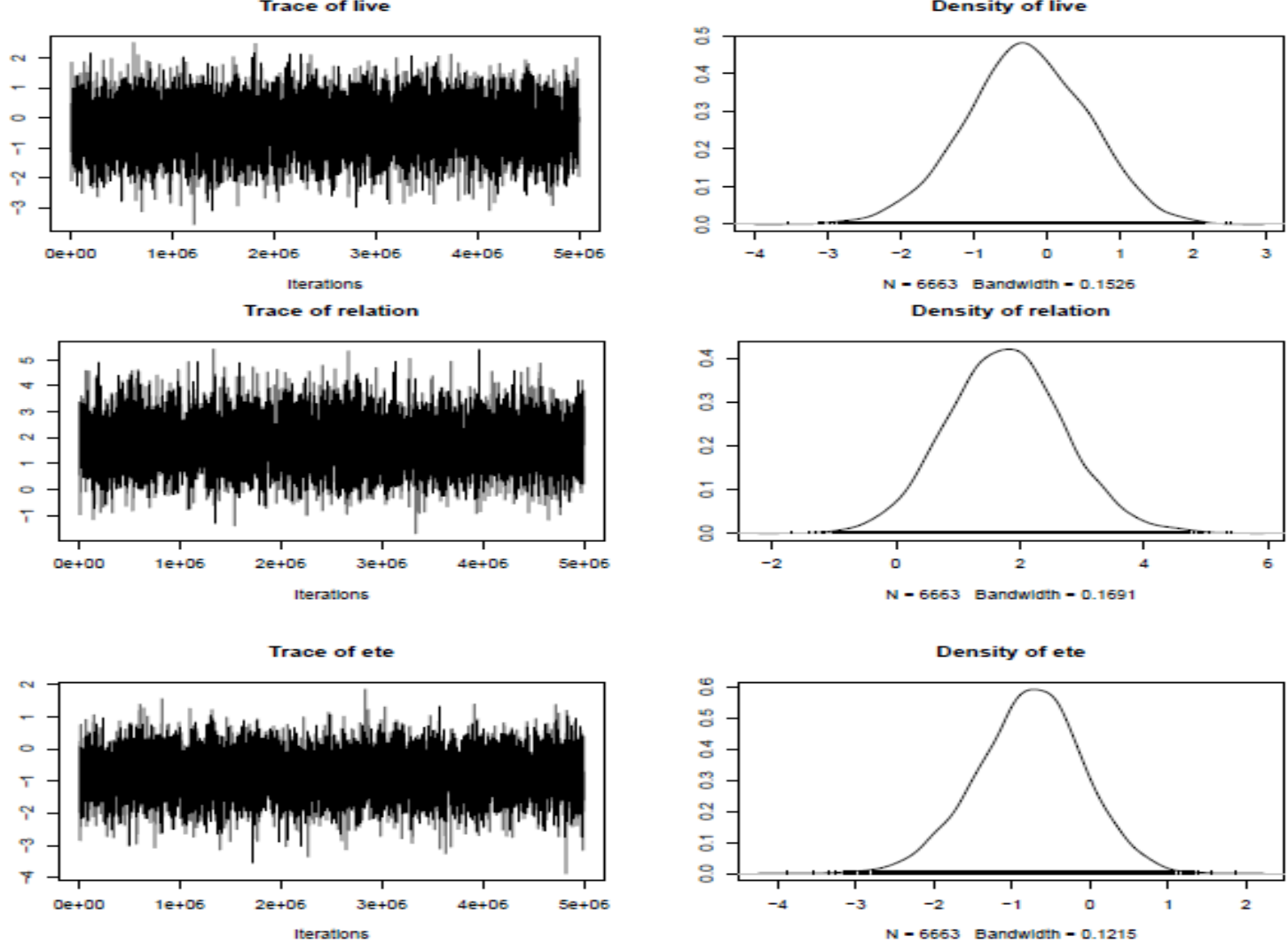

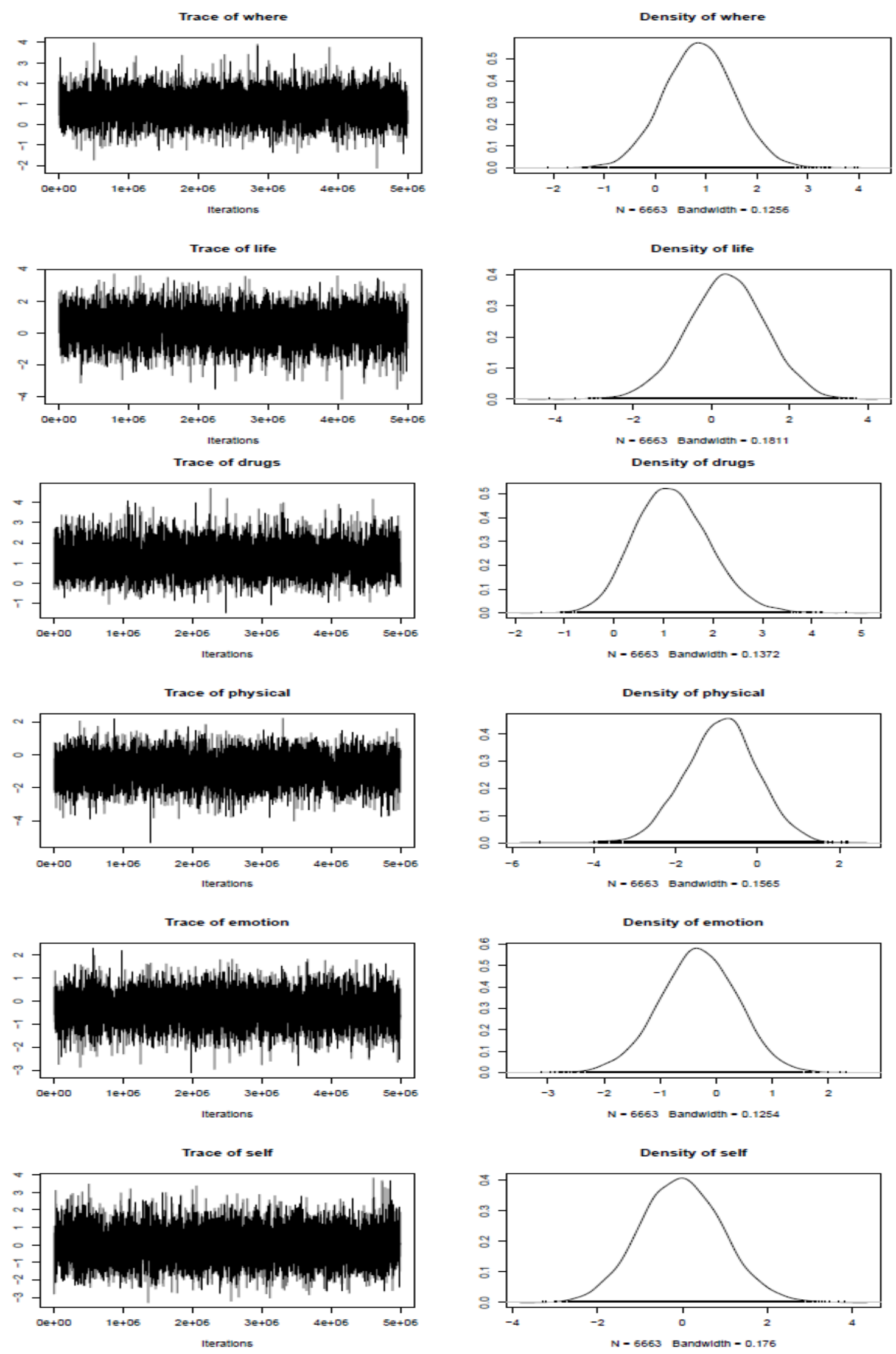
Trace of think

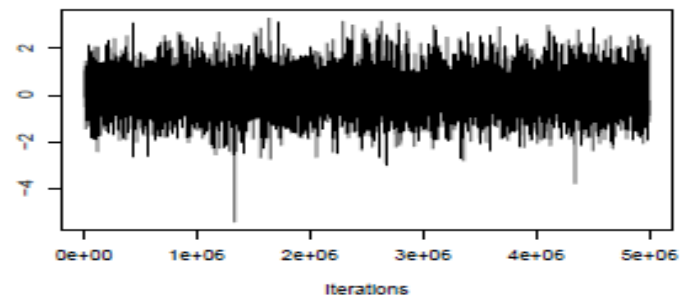

Trace of attitude

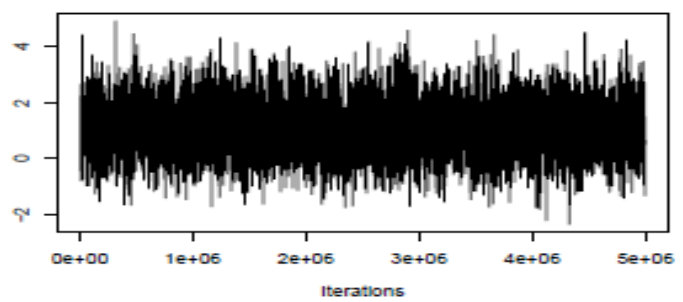

Trace of change

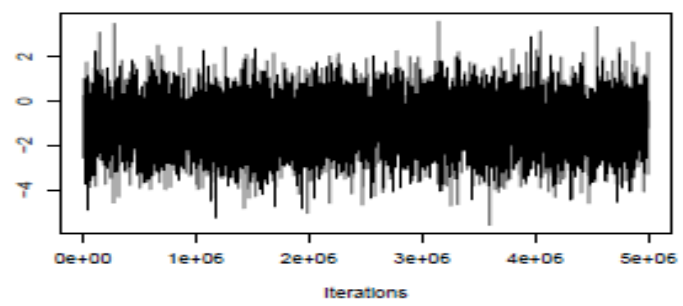

Trace of appear

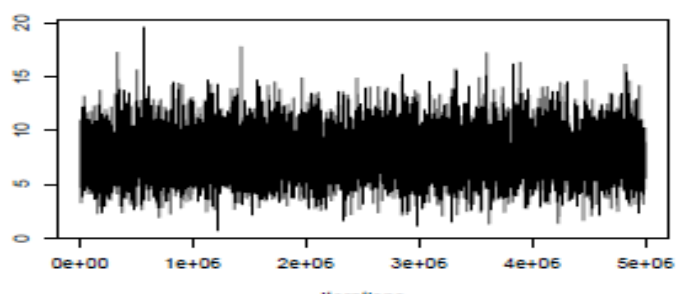
Iterations

Trace of breach

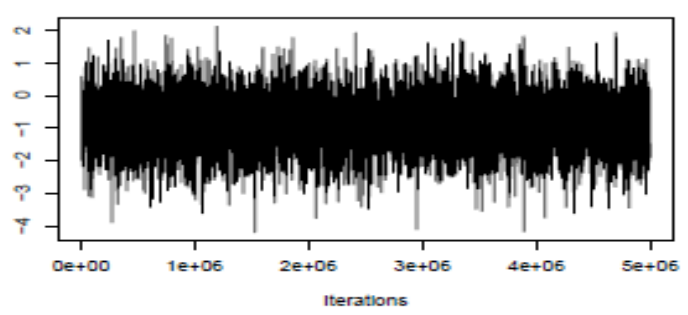

Trace of custody

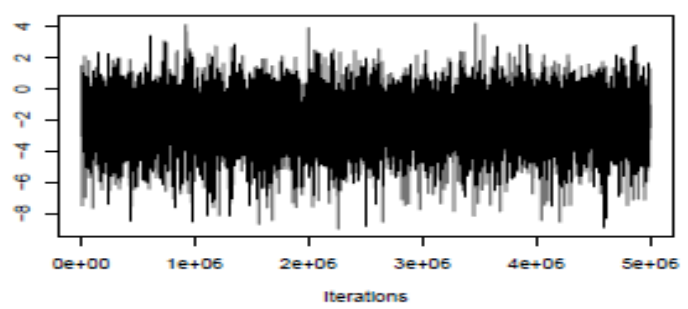

Density of think
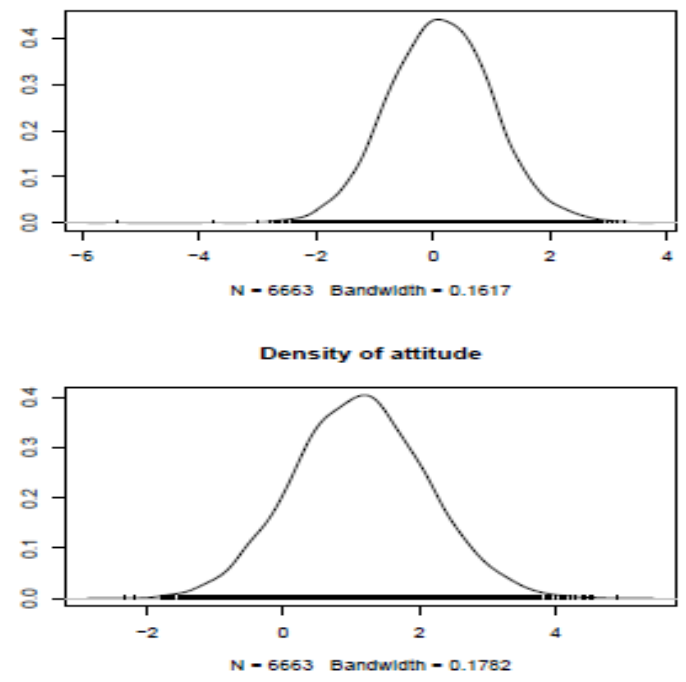

Density of change
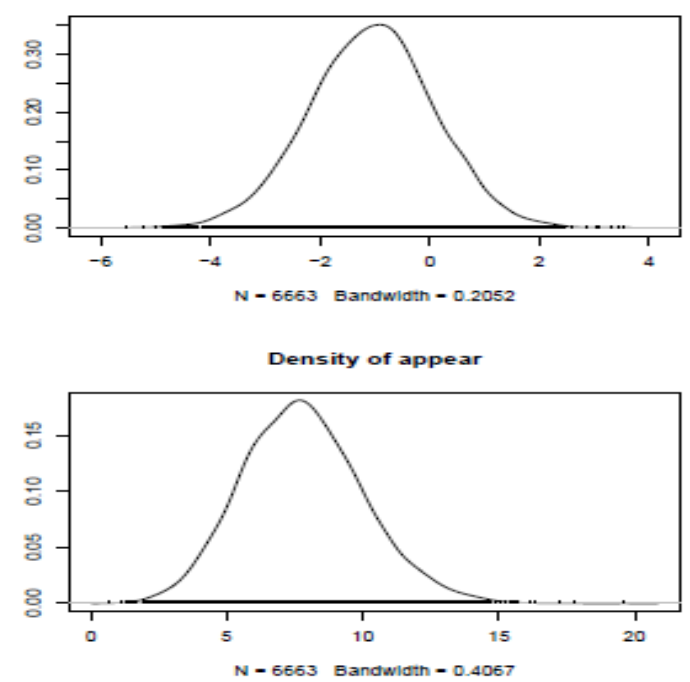

Density of breach
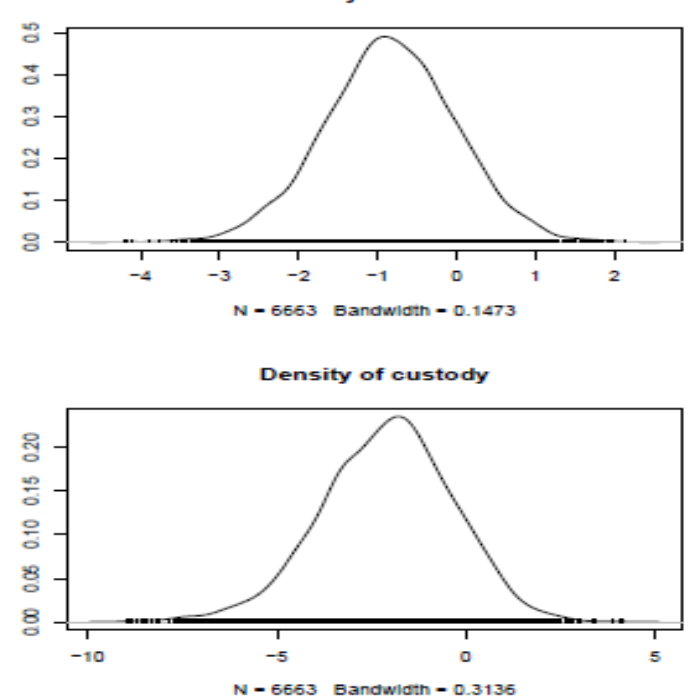


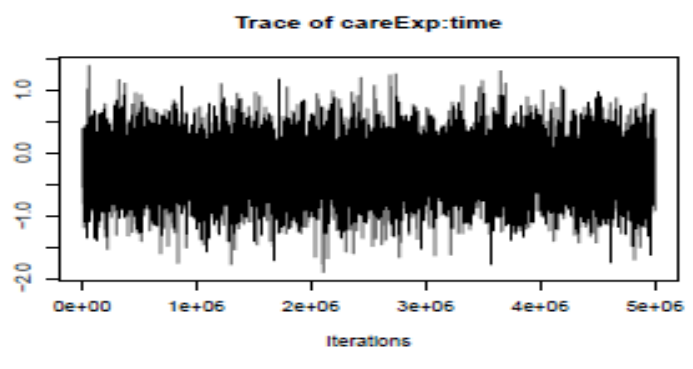

Trace of careExp:live

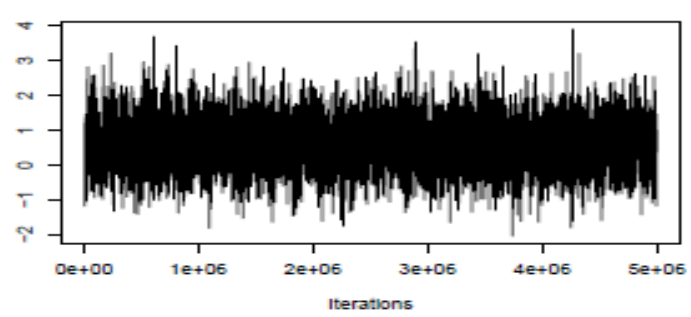

Trace of time:live

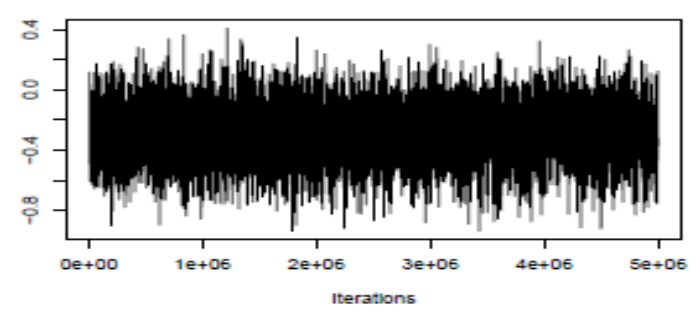

Trace of careExp:relation

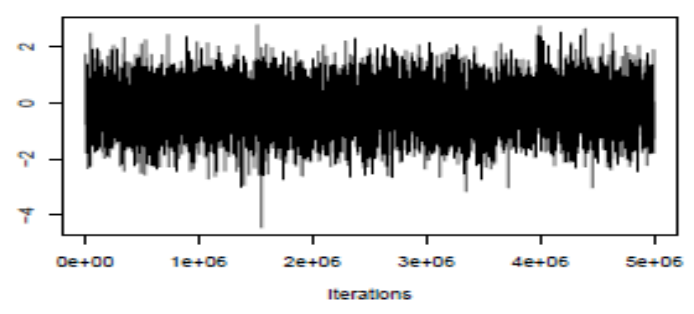

Trace of time:relation
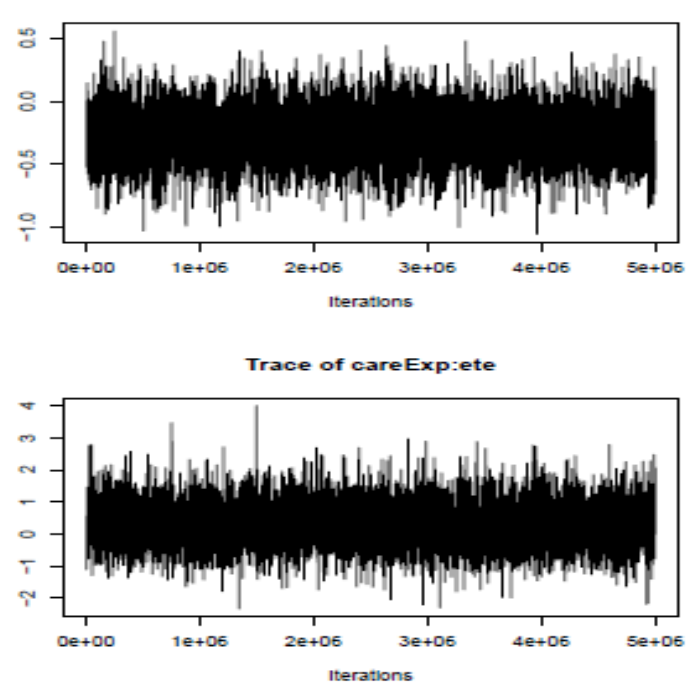
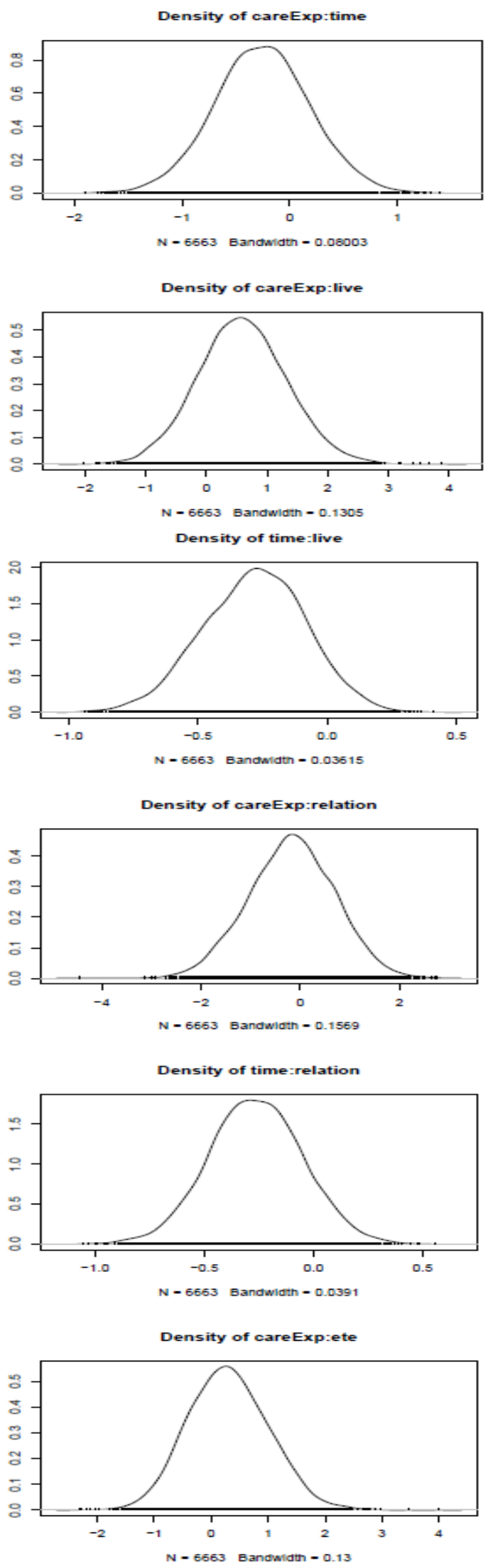
Trace of time:ete

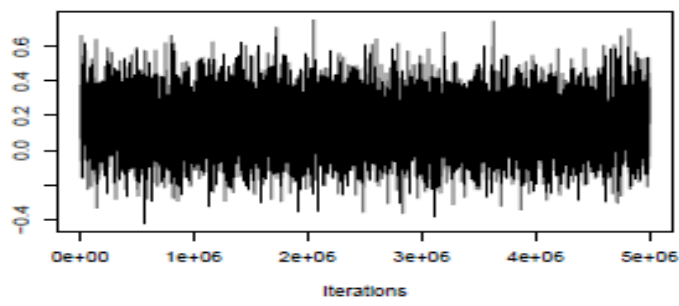

Trace of careExp:where

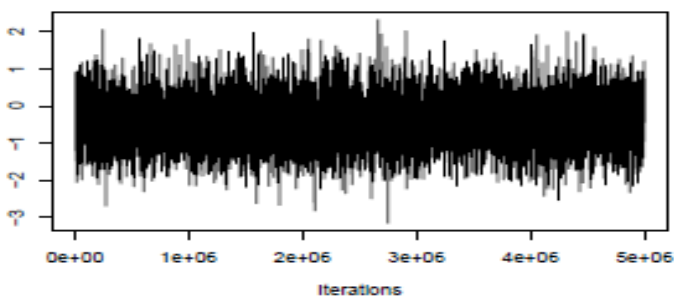

Trace of time:where
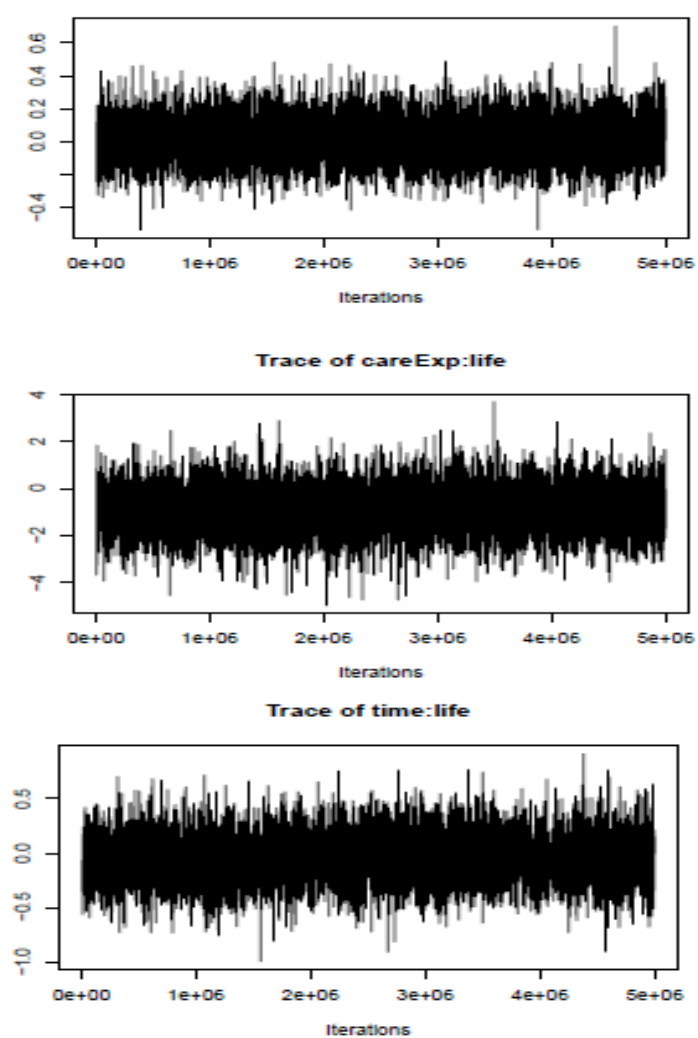

Trace of careExp:drugs

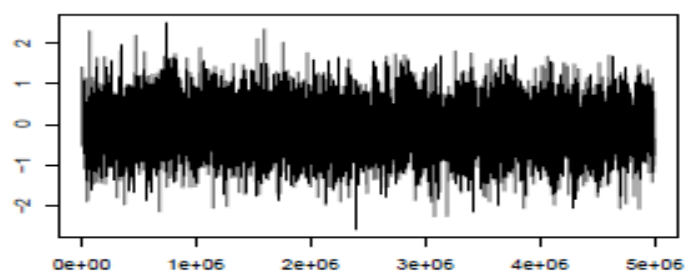

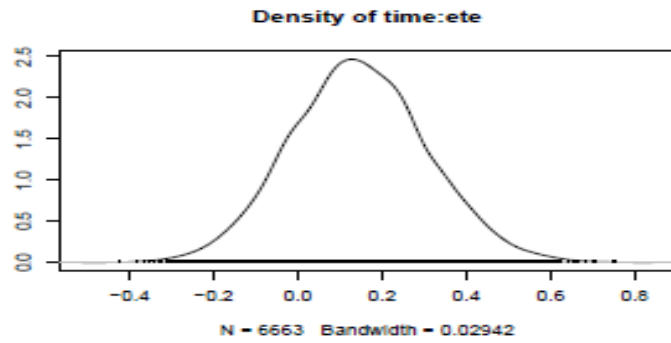

Density of careExp:where

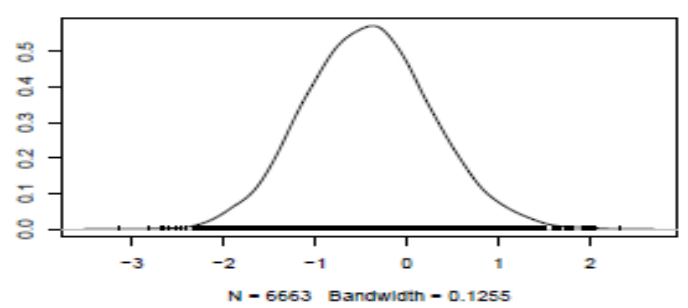

Density of time:where
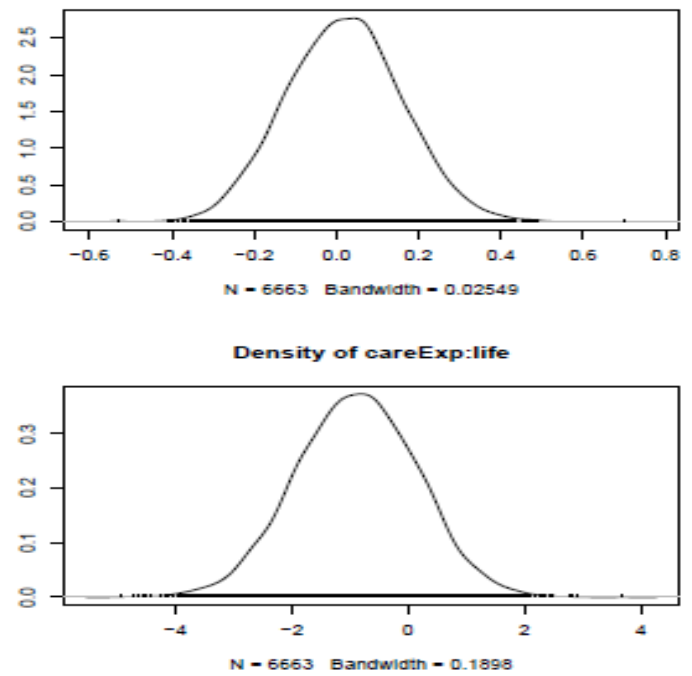

Density of time:life

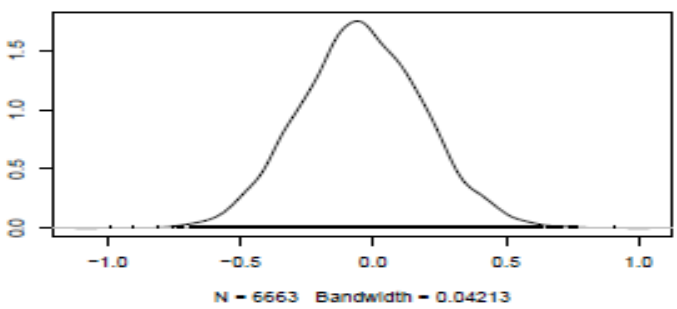

Density of careExp:drugs

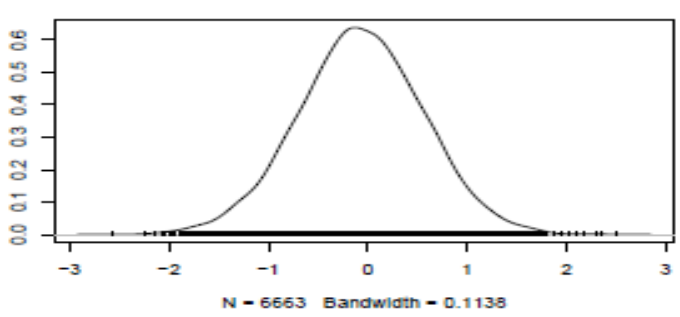



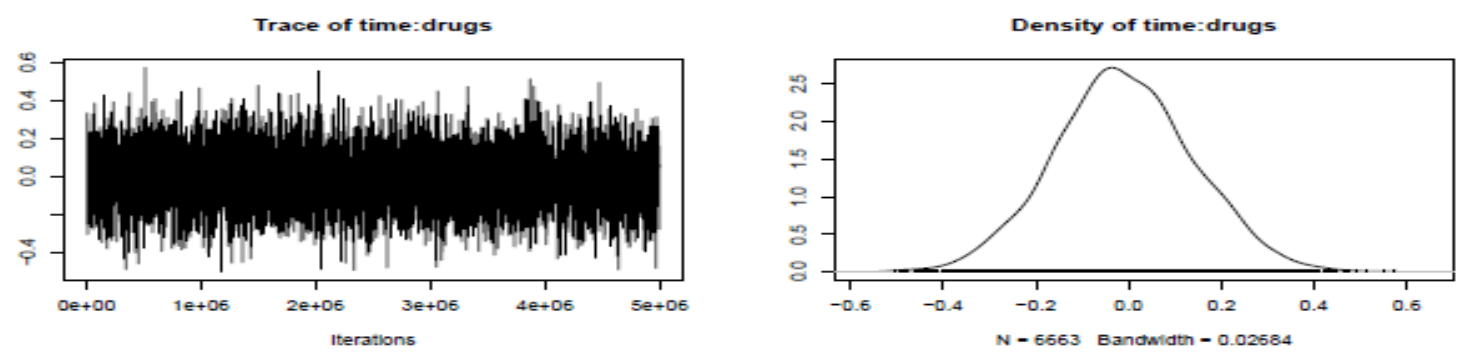

Trace of careExp:physical
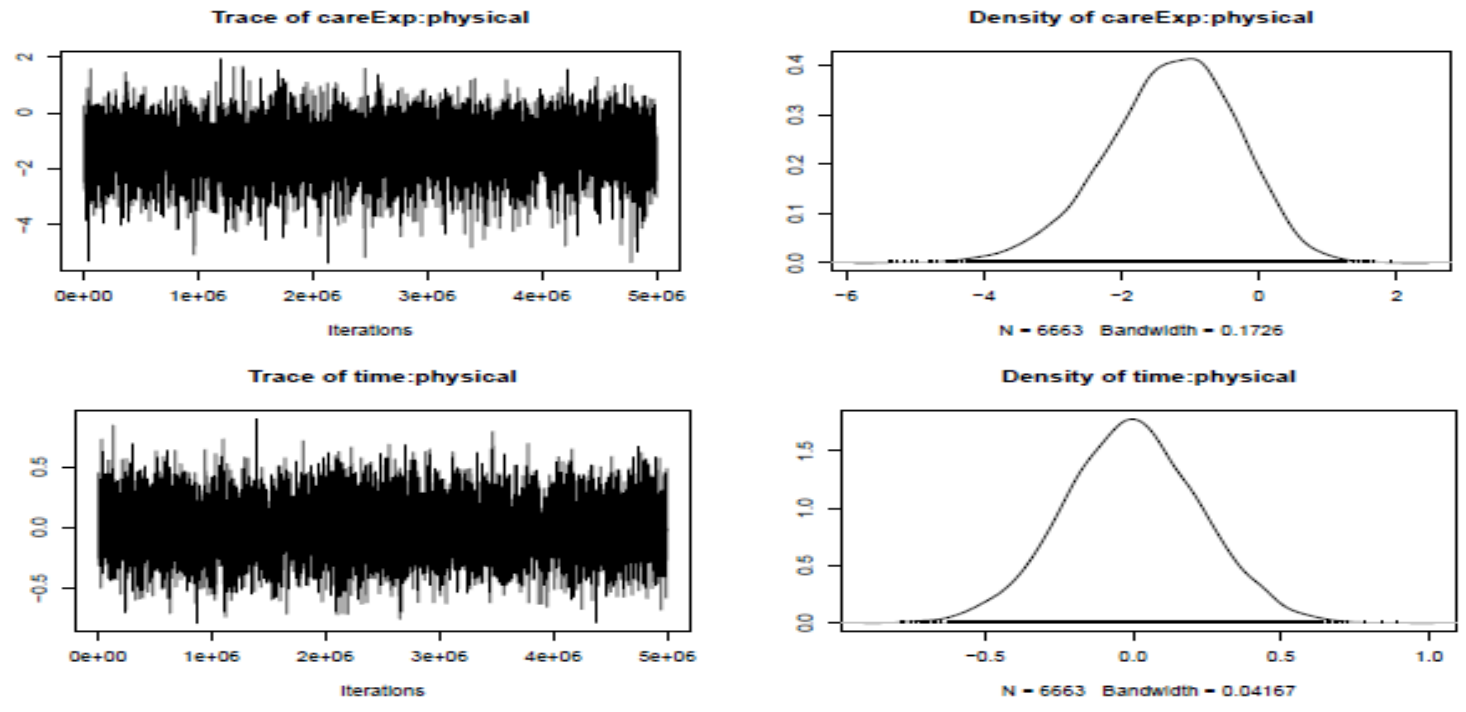

Trace of care Exp:emotion
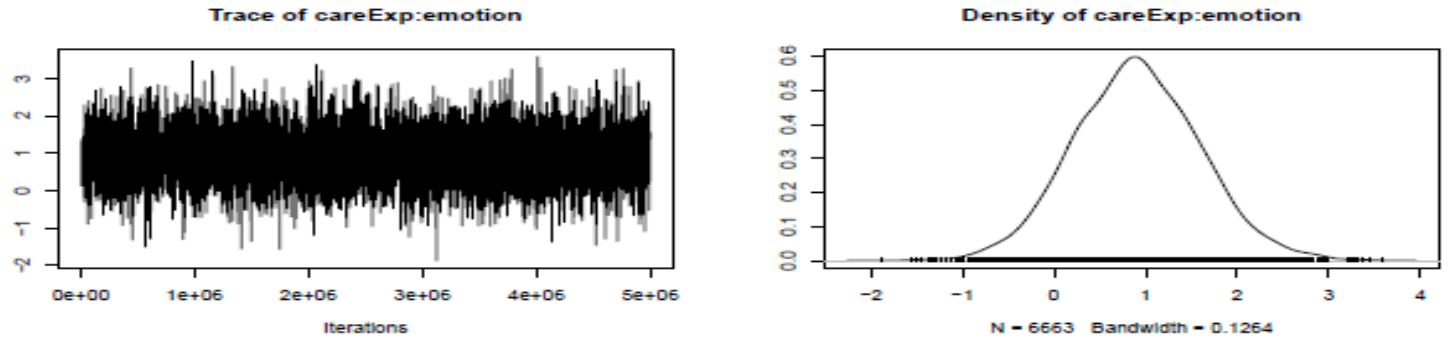

Trace of time:emotion
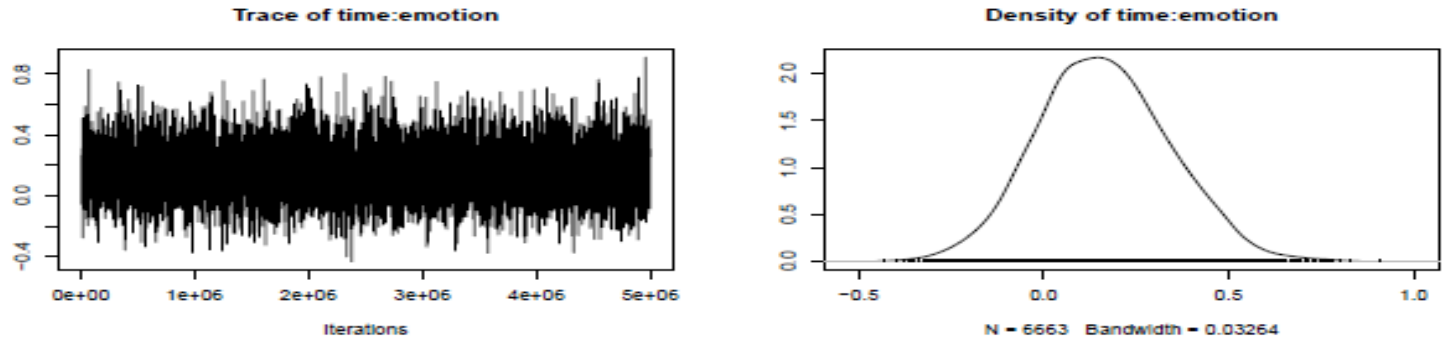

Trace of careExp:self
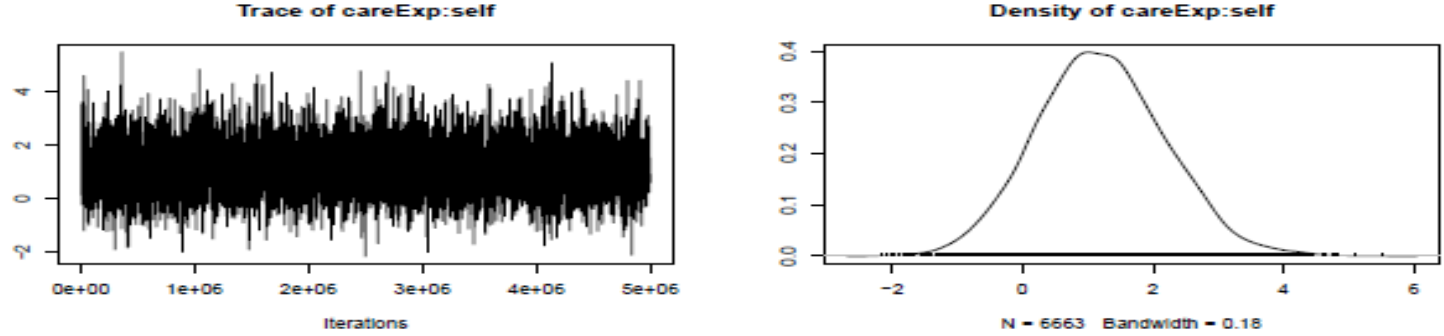

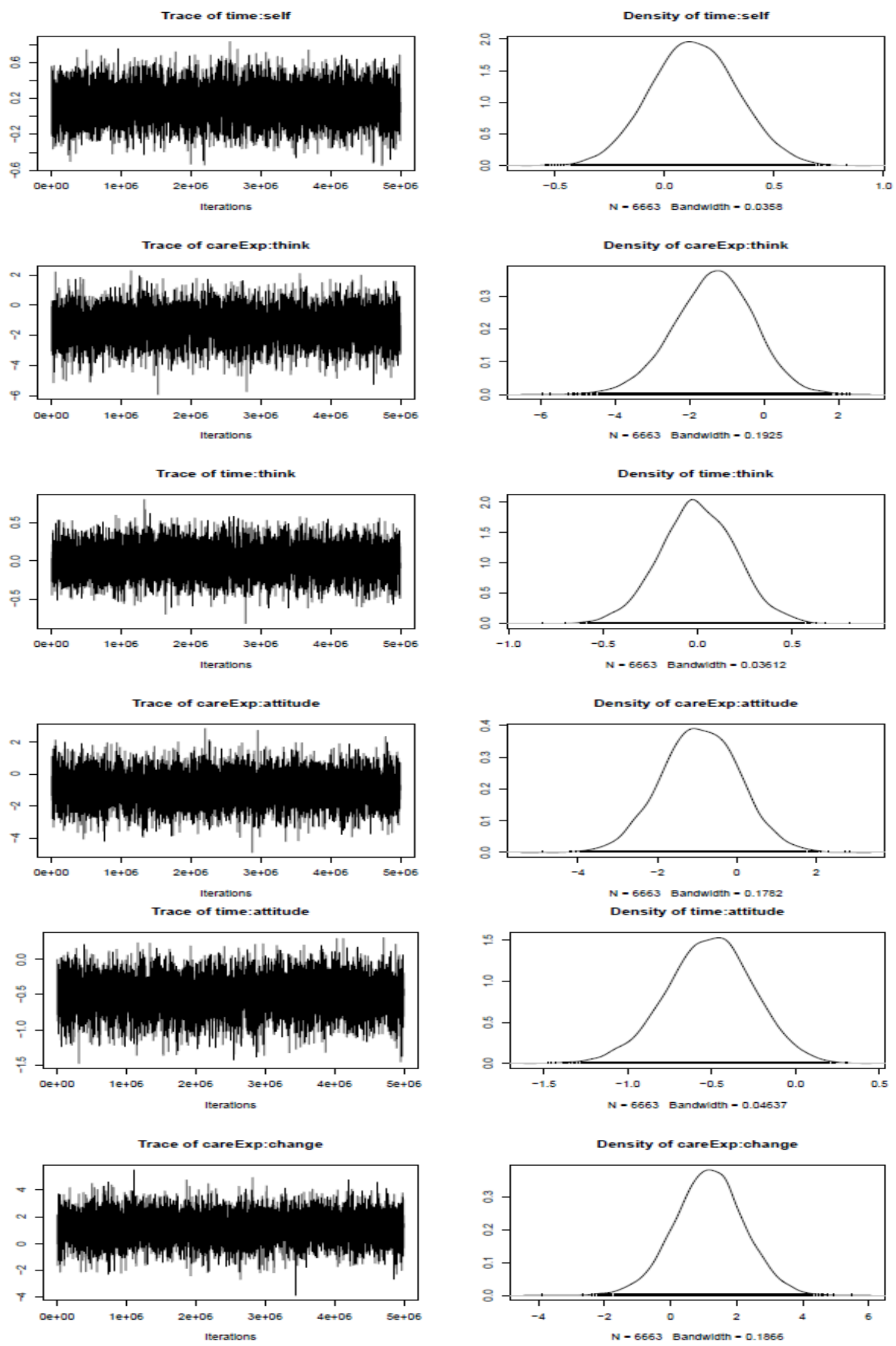
Trace of time:change

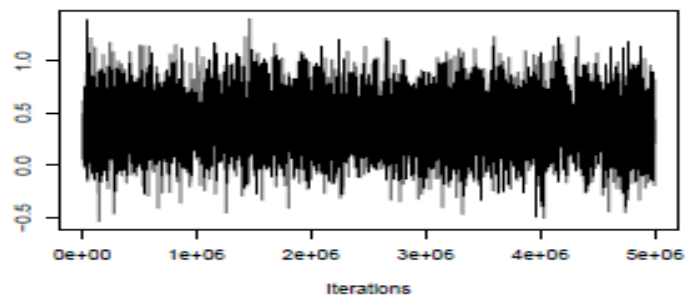

Trace of time:appear

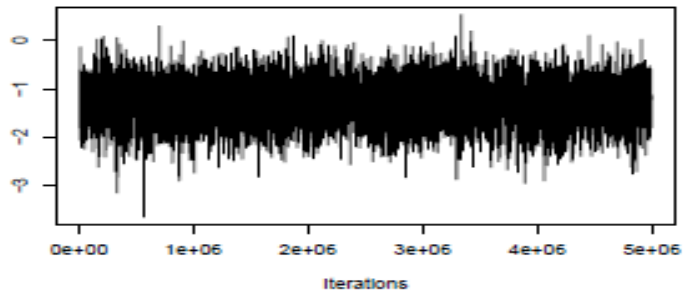

Trace of live:appear

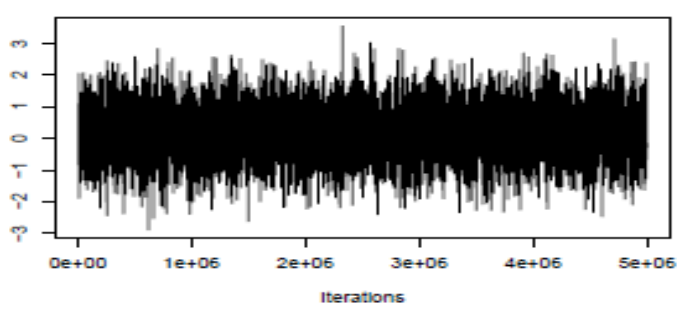

Trace of relation:appear

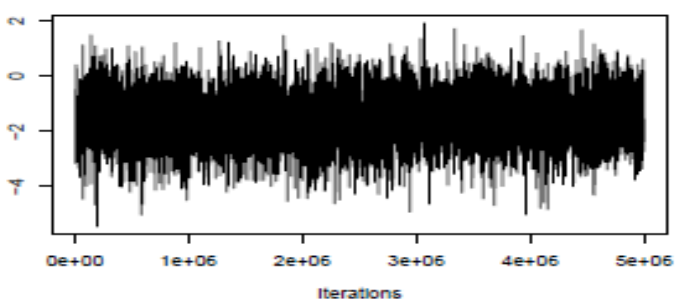

Trace of ete-appear

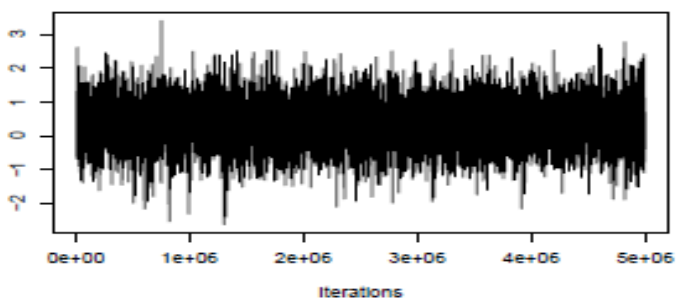

Iterations

Trace of where:appear

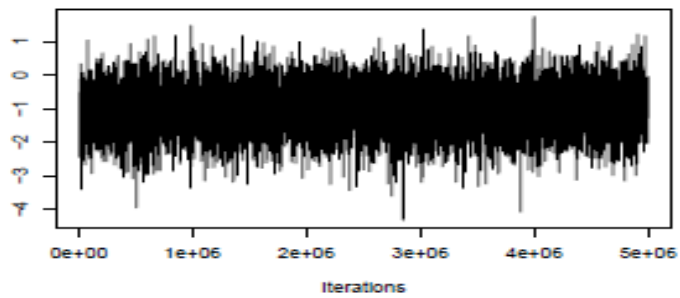

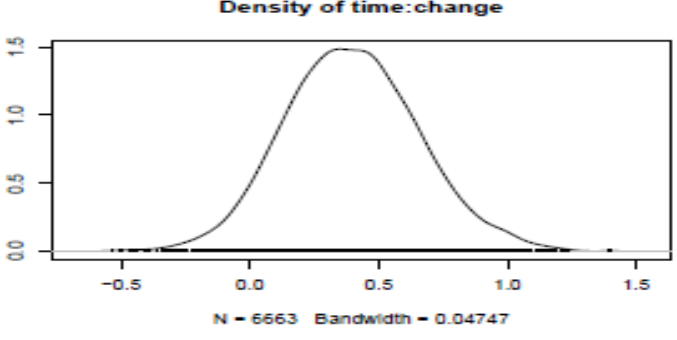

Density of time:appear

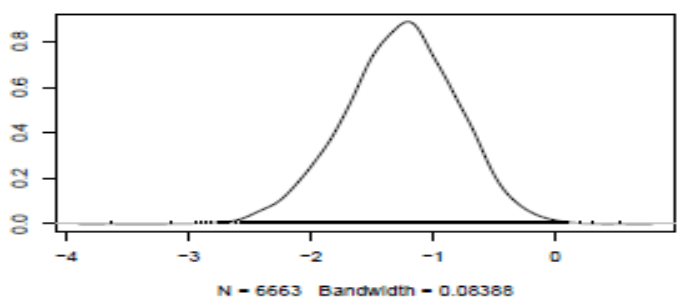

Density of live:appear

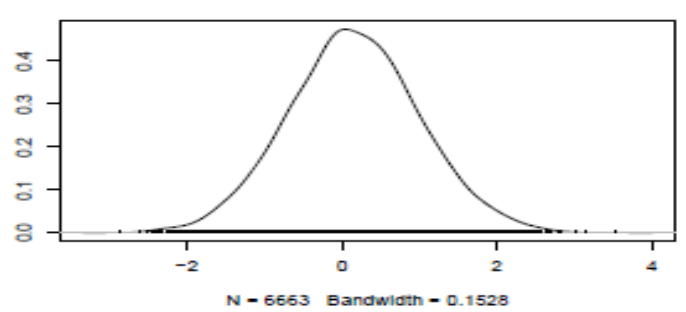

Density of relation:appear
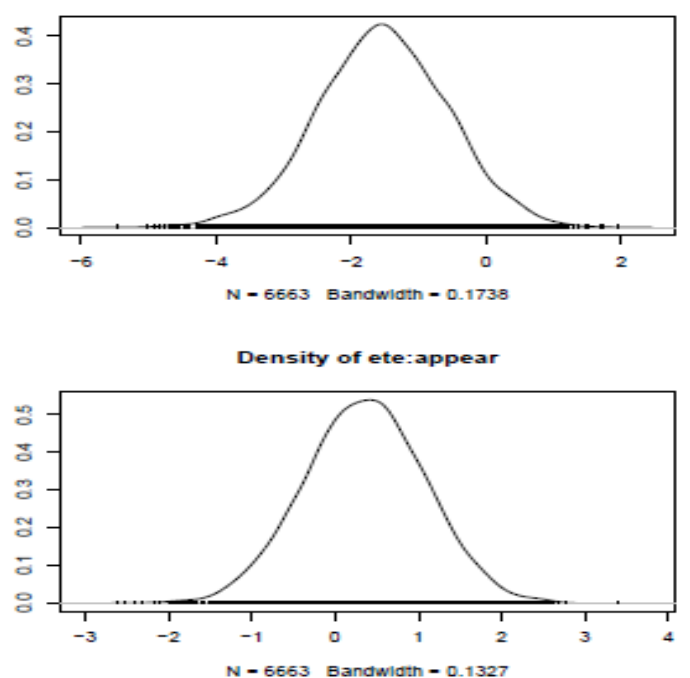

Density of where:appear

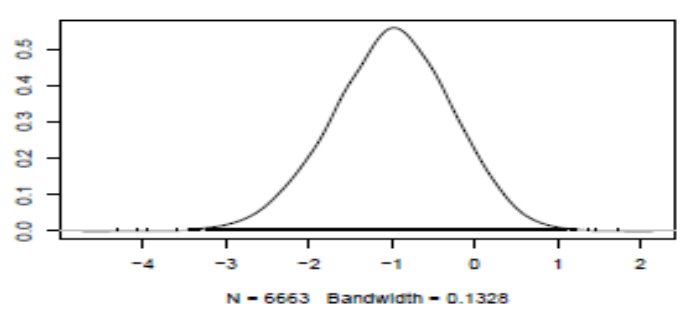


Trace of self:appear

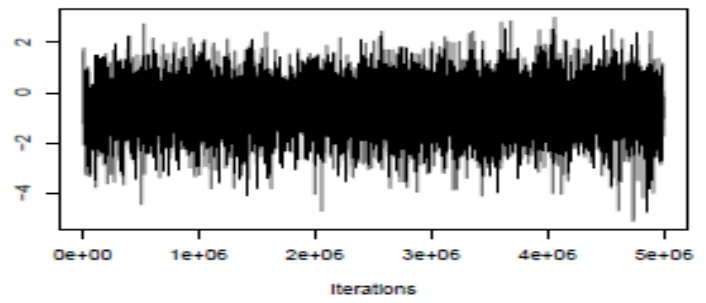

Trace of think:appear

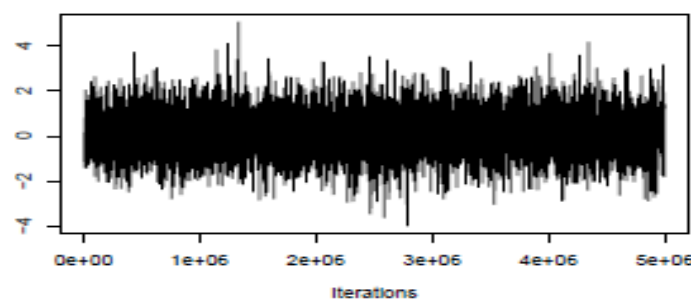

Trace of attitude:appear

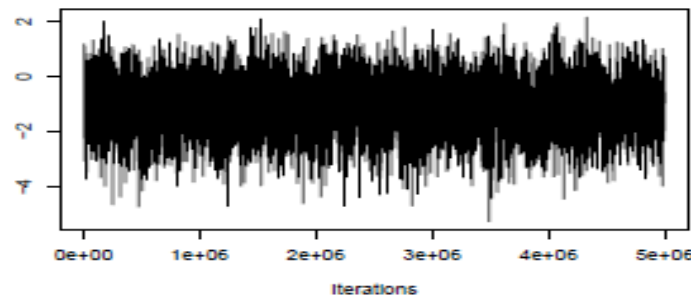

Trace of change:appear

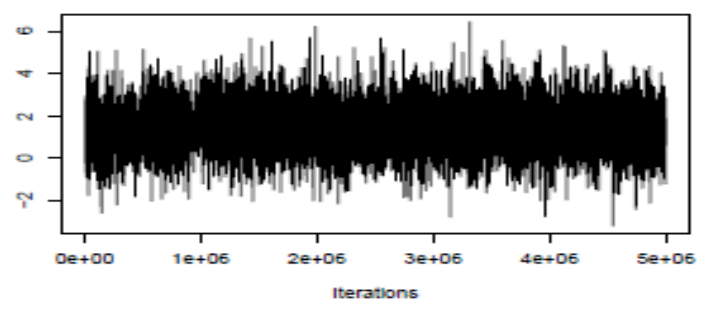

Trace of careExp:appear

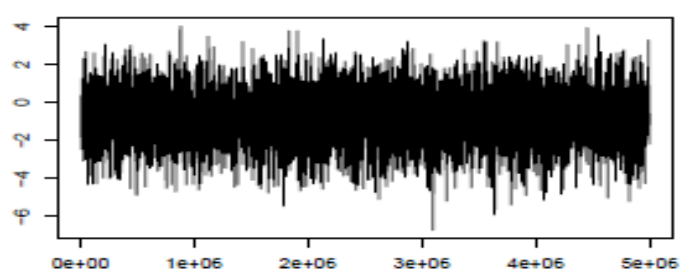$$
\text { Iterations }
$$

Trace of careExp:breach

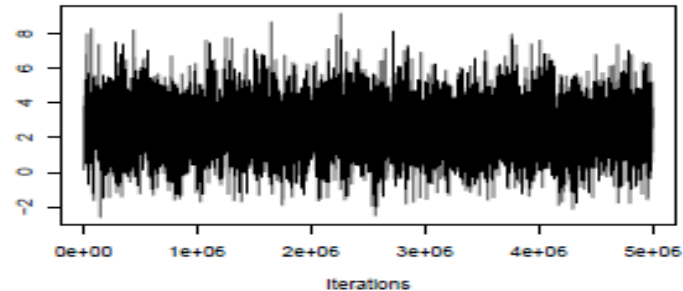

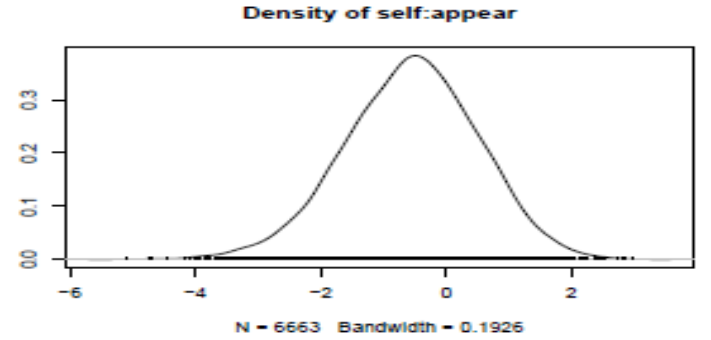
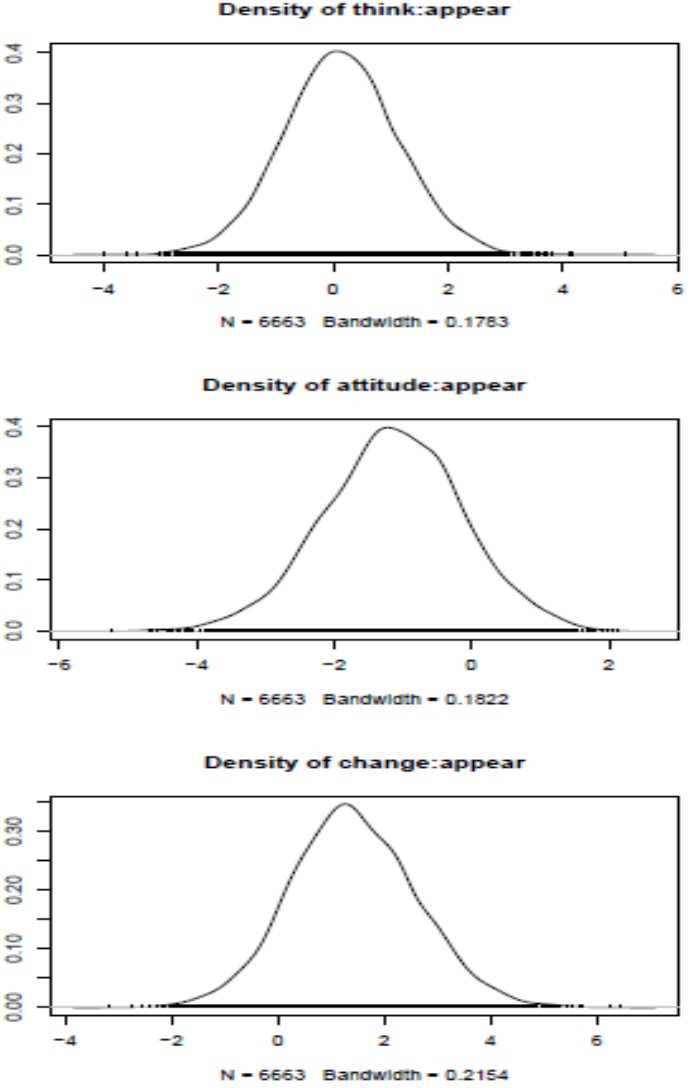

Density of careExp:appear

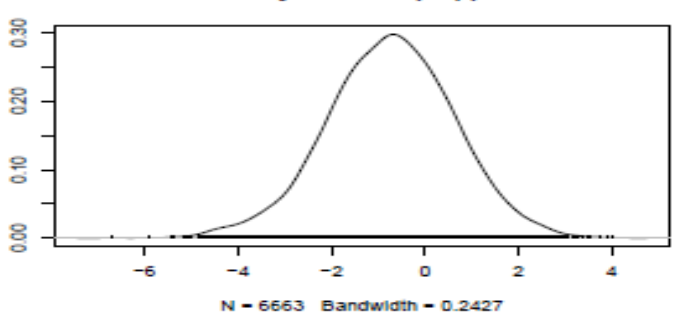

Density of careExp:breach

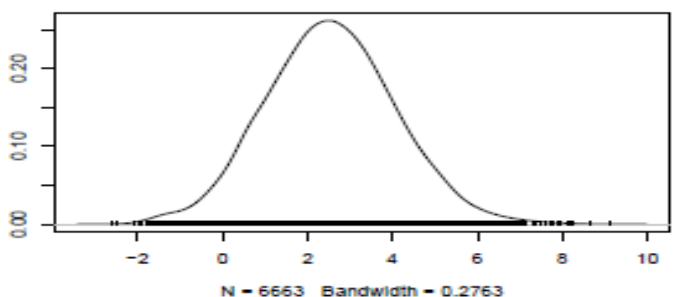



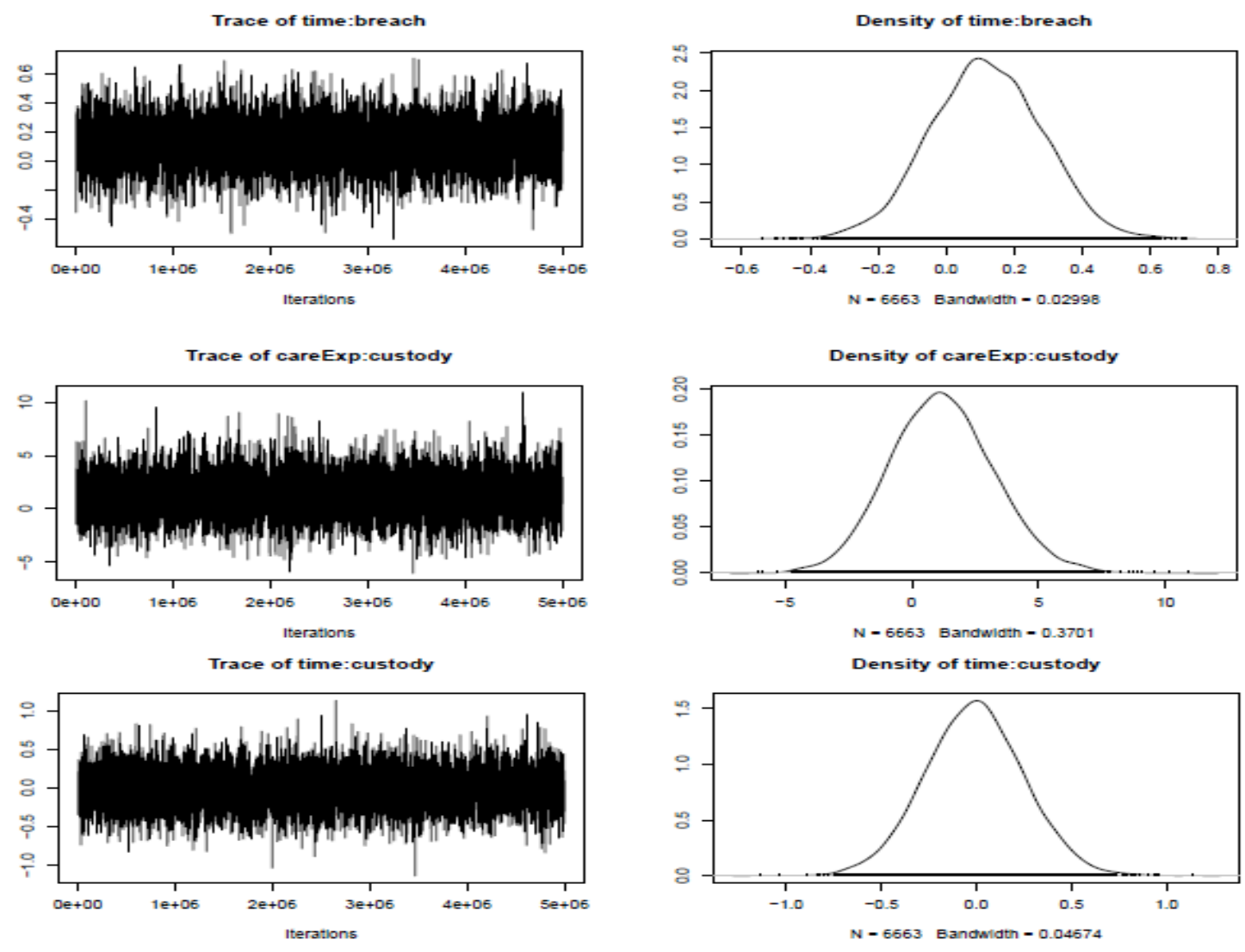

Trace of careExp:time:live
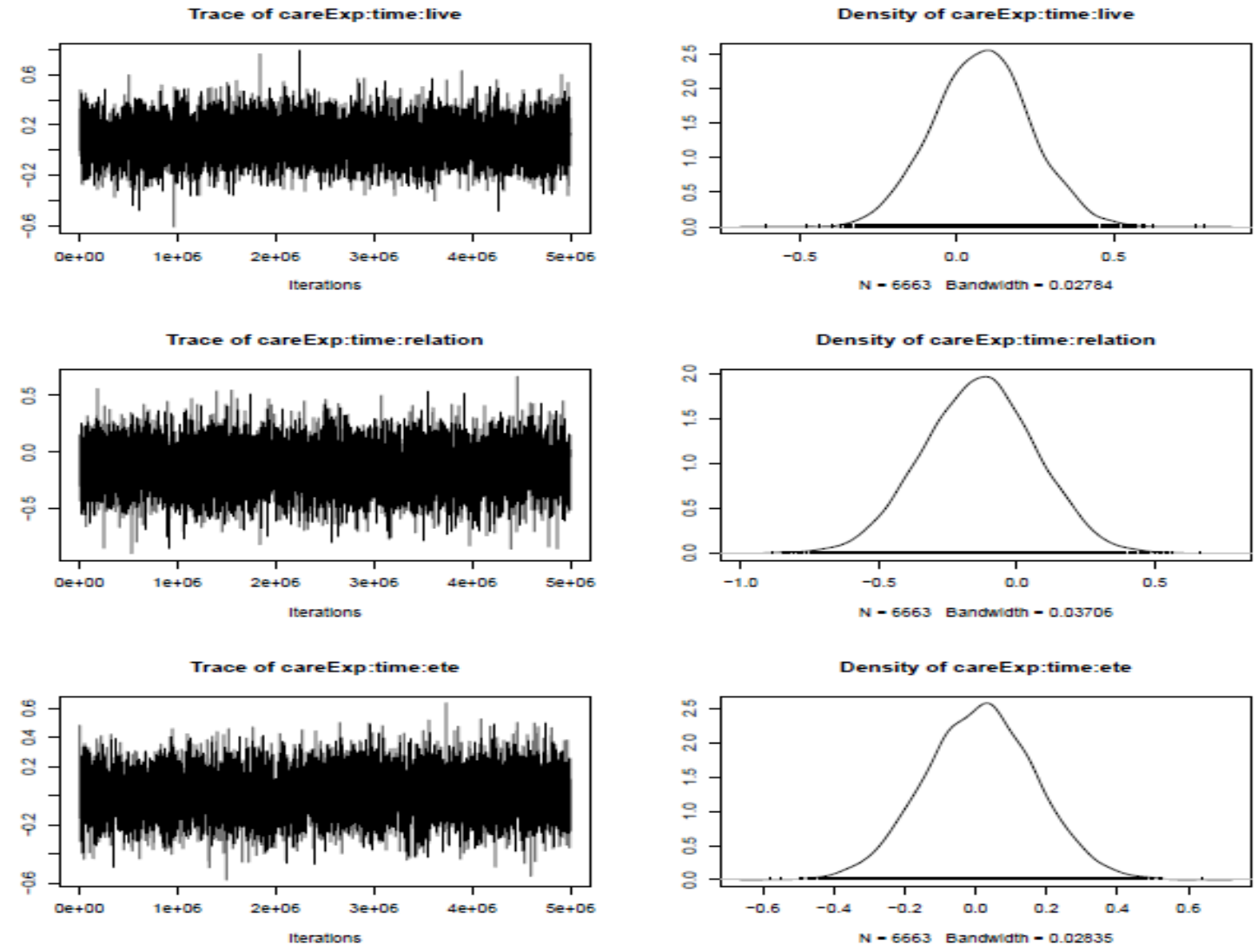
Trace of careExp:time:where

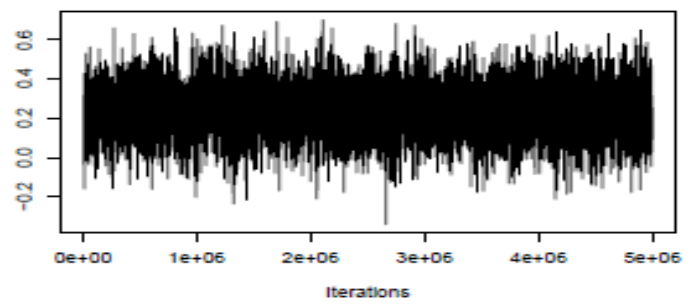

Trace of care Exp:time:life

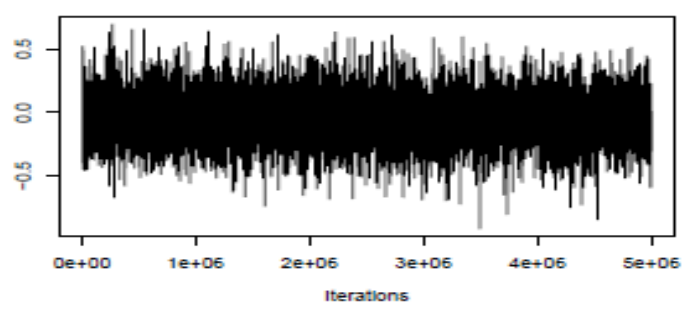

Trace of careExp:time:drugs

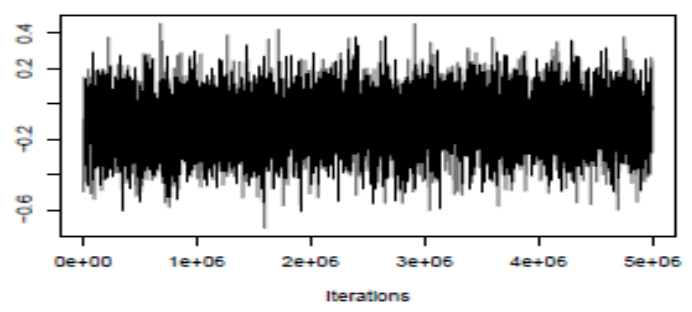

Trace of careExp:time:physical

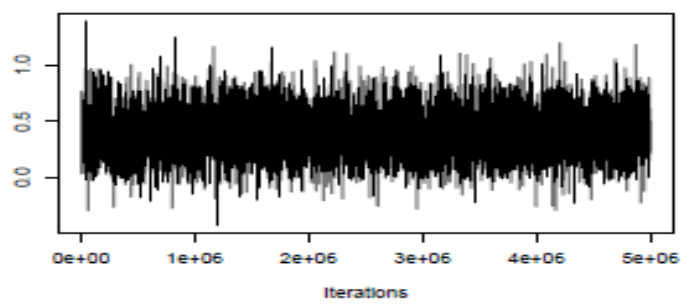

Trace of careExp:time:emotion

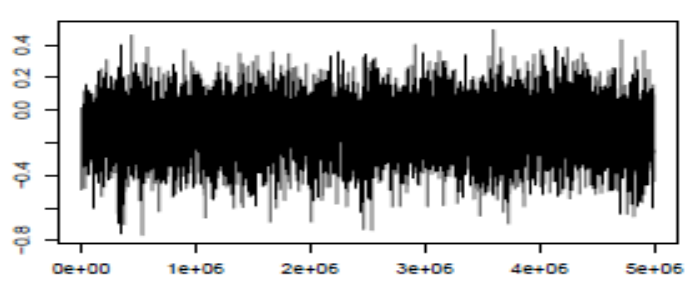
Iterations

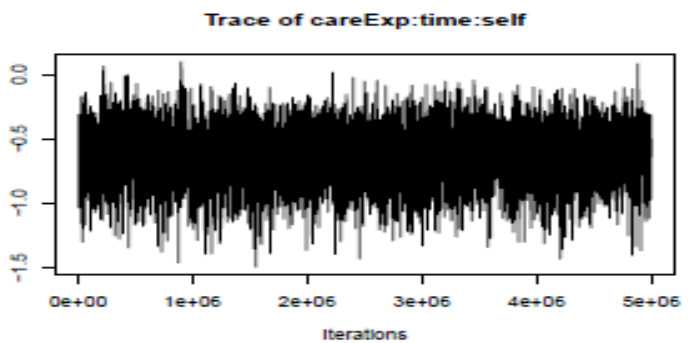

Density of careExp:time:where
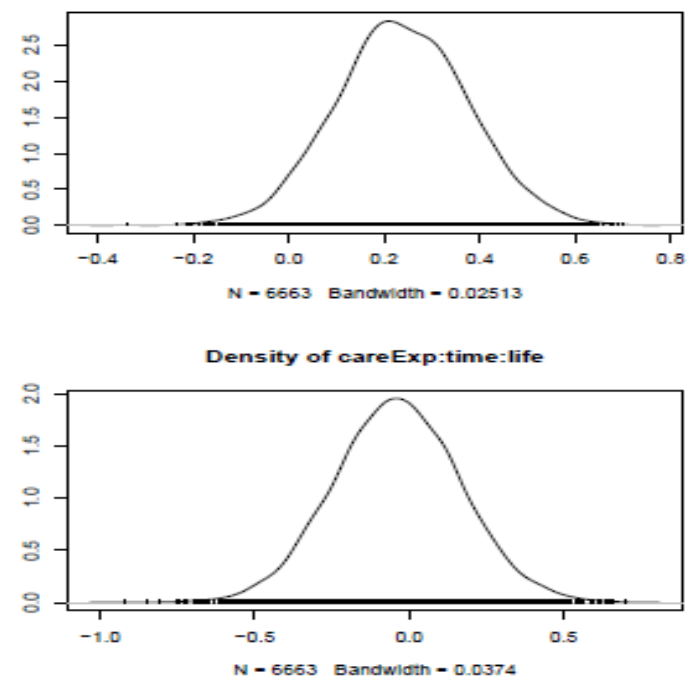

Density of careExp:time:drugs

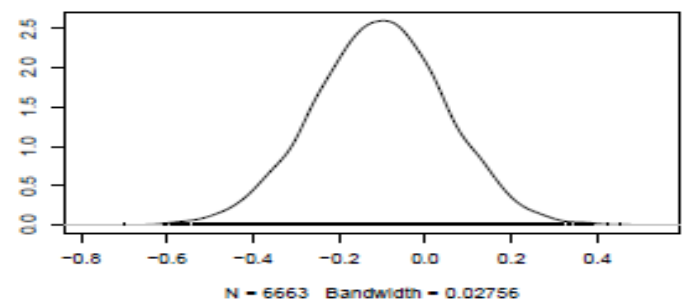

Density of careExp:time:physical

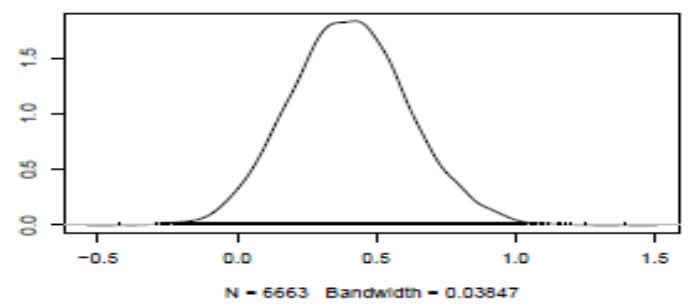

Density of careExp:time:emotion
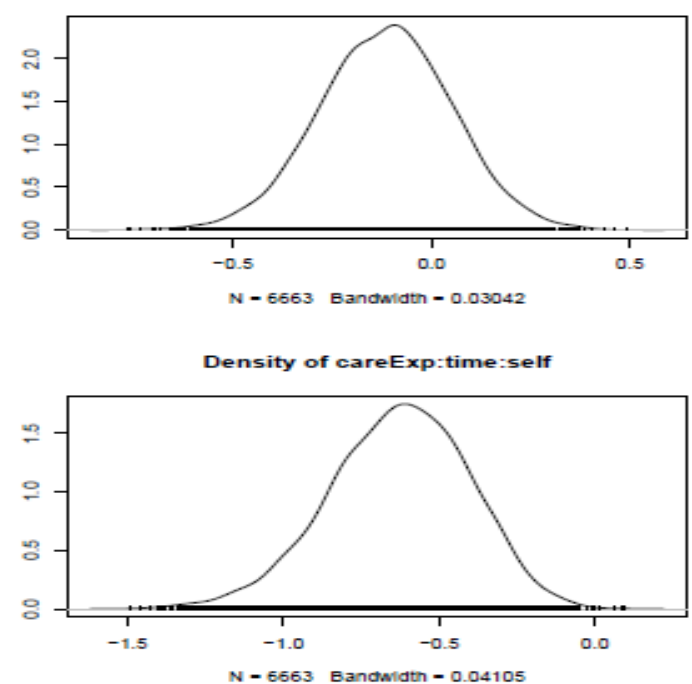
Trace of careExp-time:think

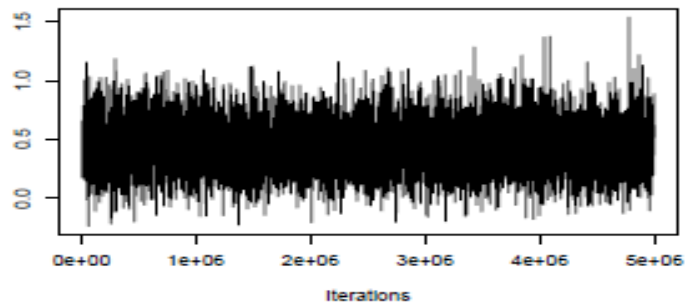

Trace of care Exp:time:attitude

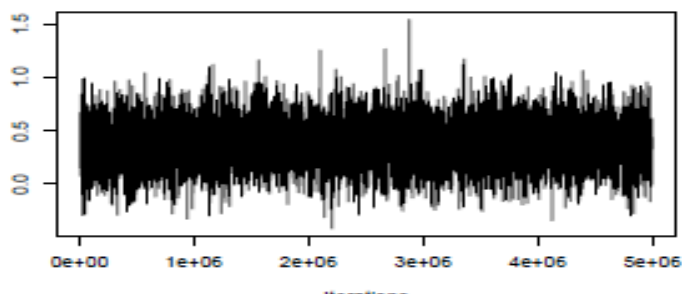
Iterations

Trace of careExp:time:change

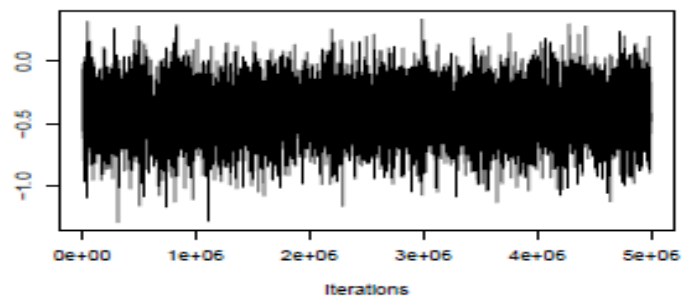

Trace of time:live:appear

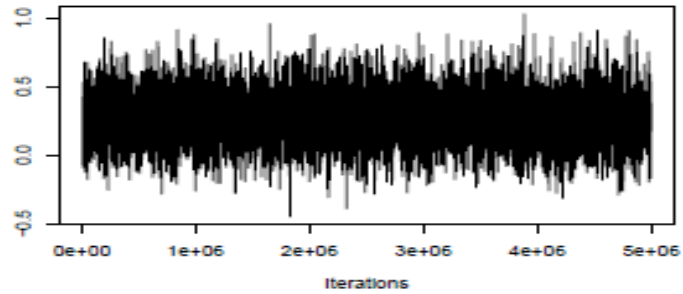

Trace of time:relation:appear

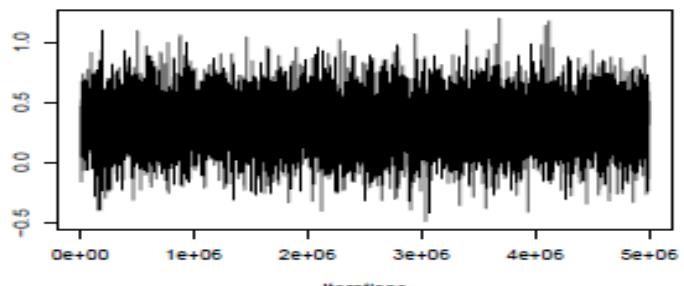
Iterations

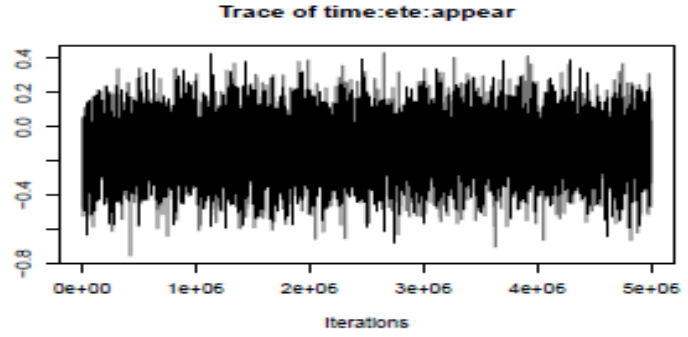

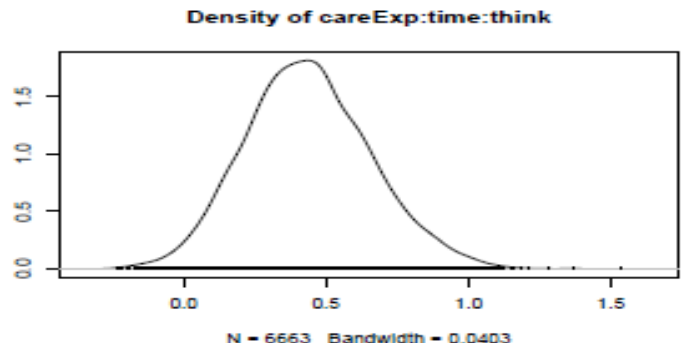

Density of careExp:time:attitude
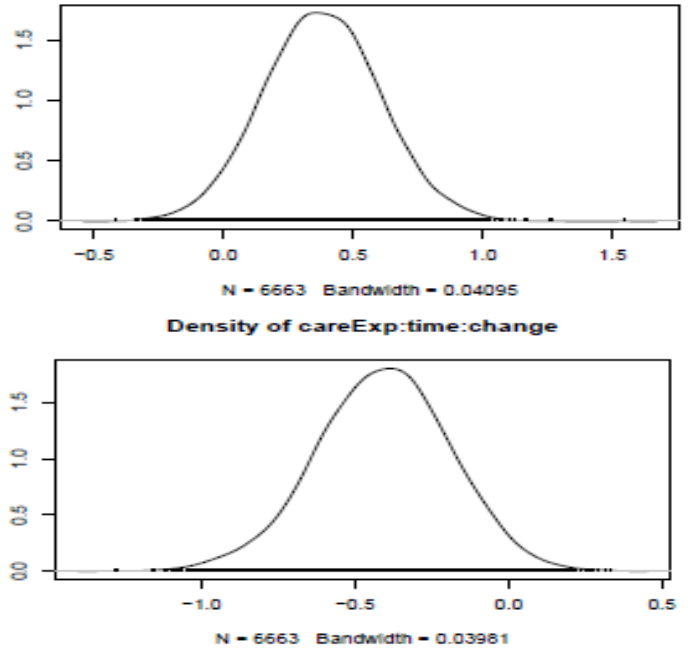

Density of time:live:appear
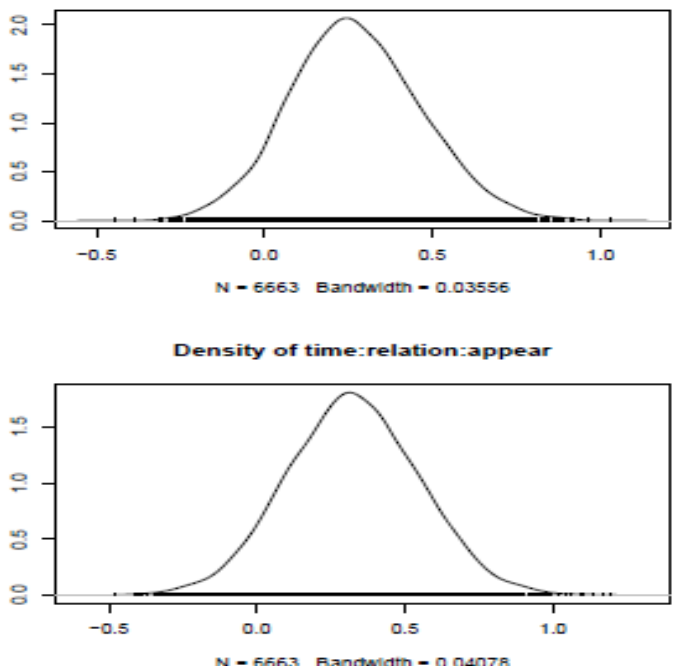

Density of time:ete:appear

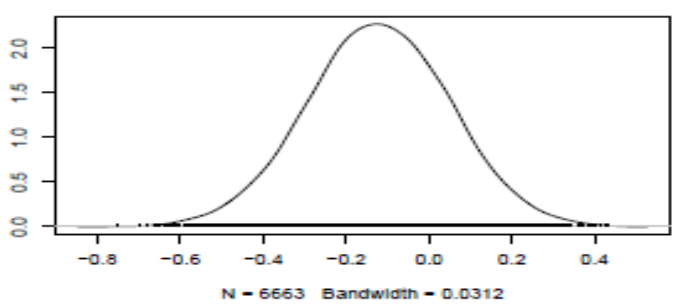


Trace of time:where:appear

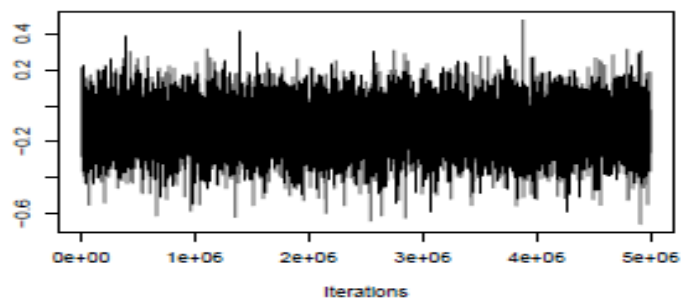

Trace of time:life:appear

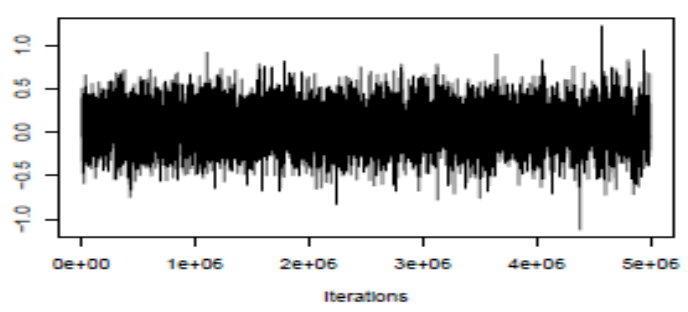

Trace of time:drugs:appear

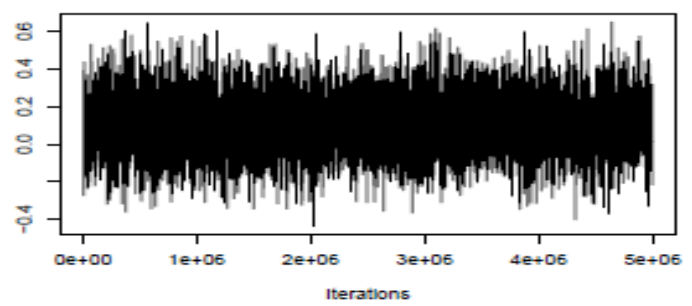

Trace of time:physical:appear

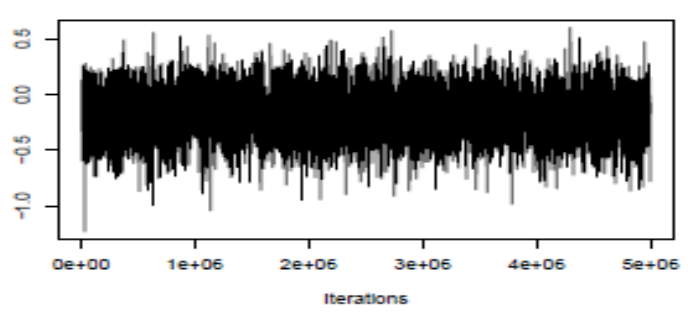

Trace of time:emotion:appear

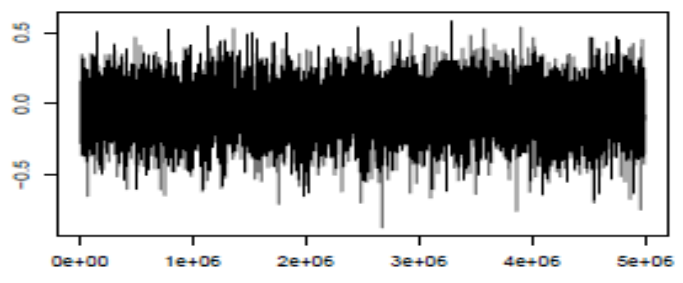
Iterations

Trace of time:self:appear

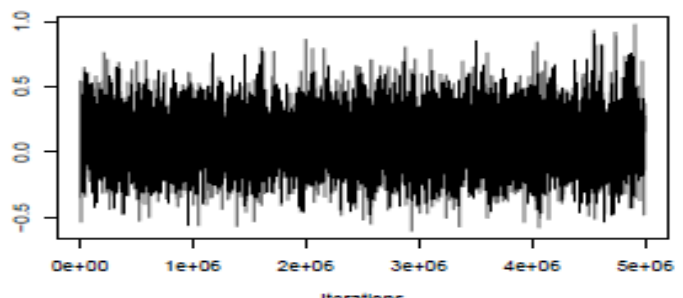

Density of time-where:appear

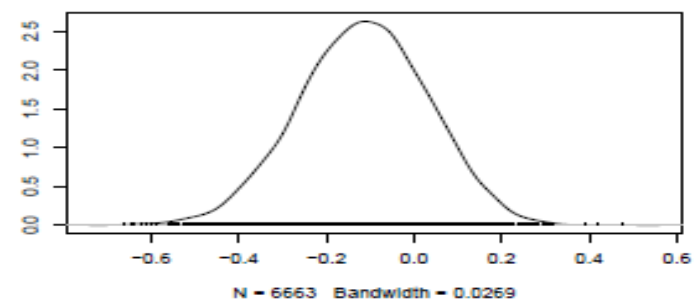

Density of time:life:appear

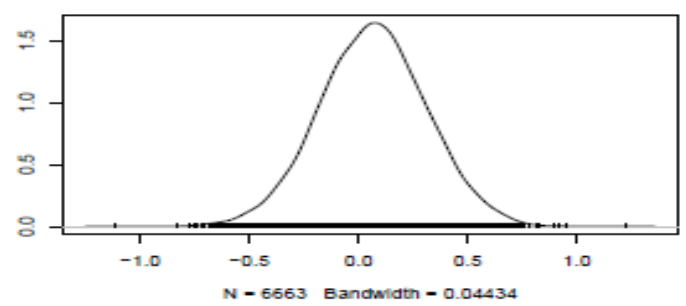

Density of time:drugs:appear

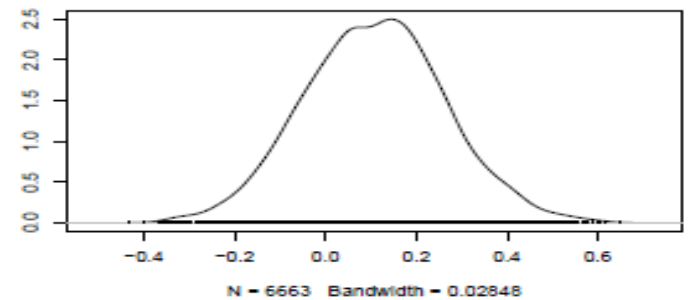

Density of time:physical:appear

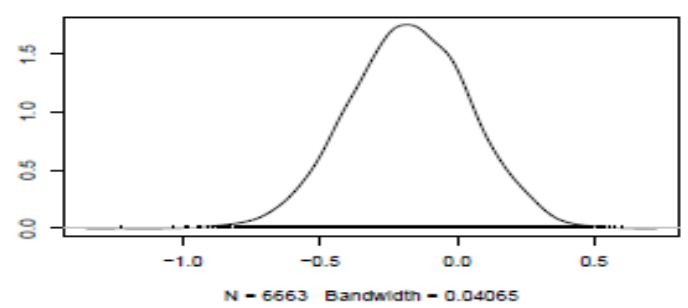

Density of time:emotion:appear

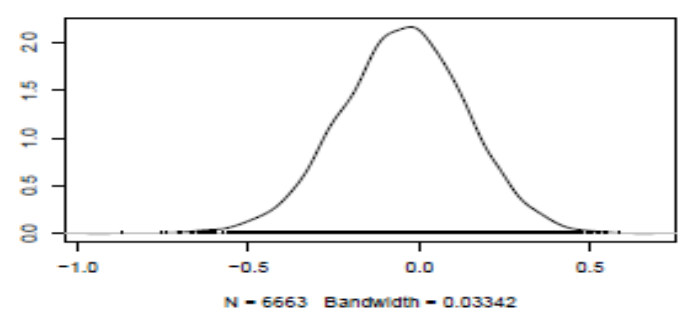

Density of time:self:appear

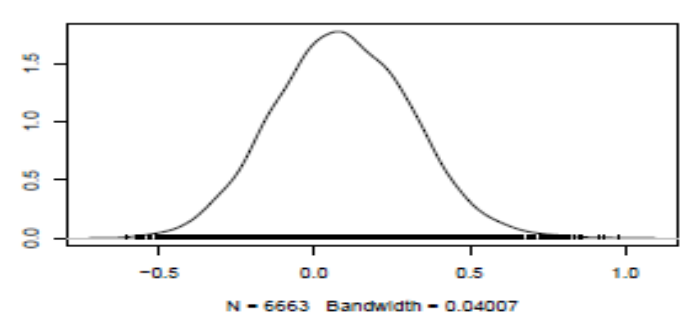


Trace of time:think:appear

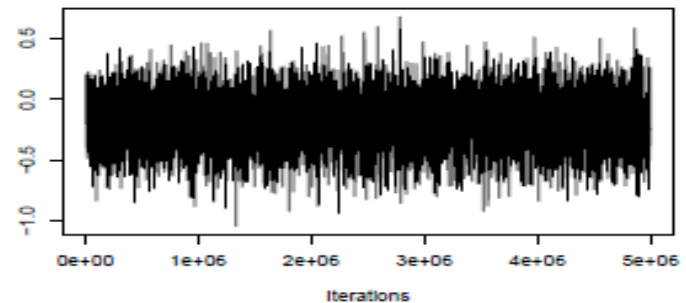

Trace of time:attitude:appear

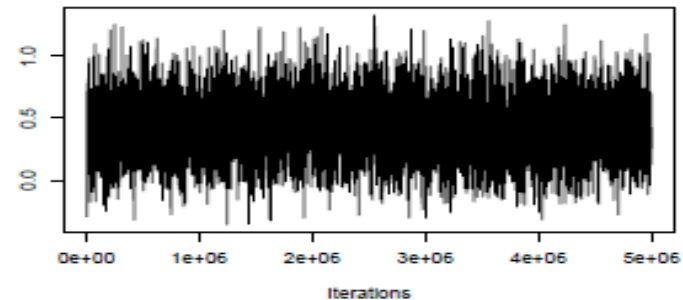

Trace of time:change:appear

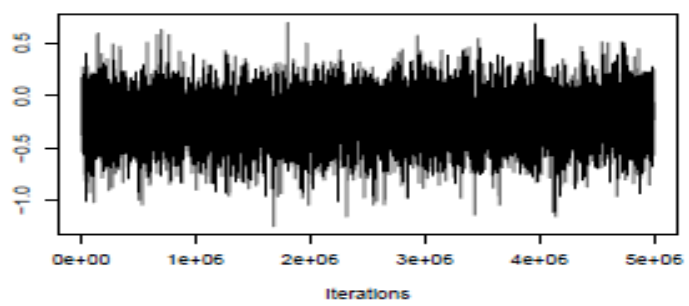

Trace of careExp:time:appear

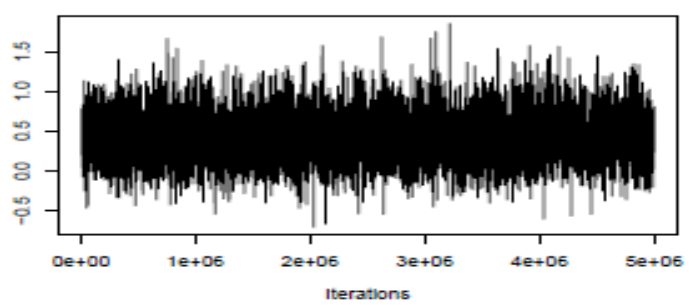

Trace of careExp:time:breach

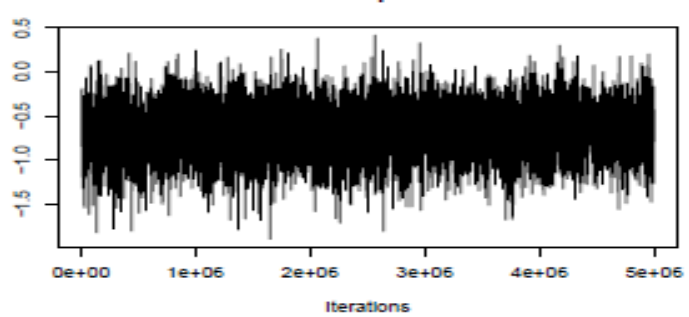

Trace of careExp:time:custody

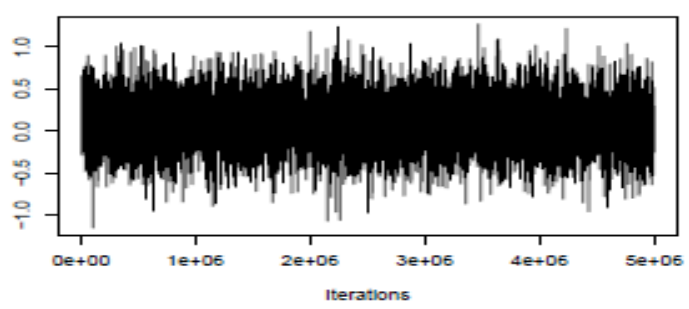

Density of time:think:appear

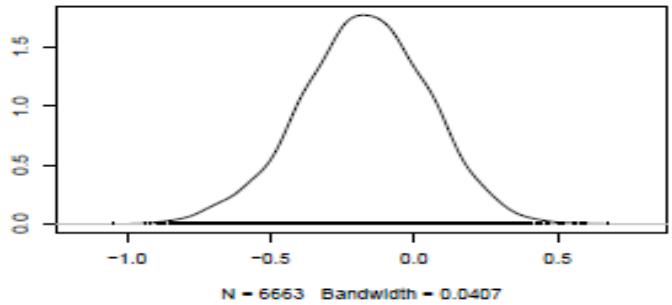

Density of time:attitude:appear
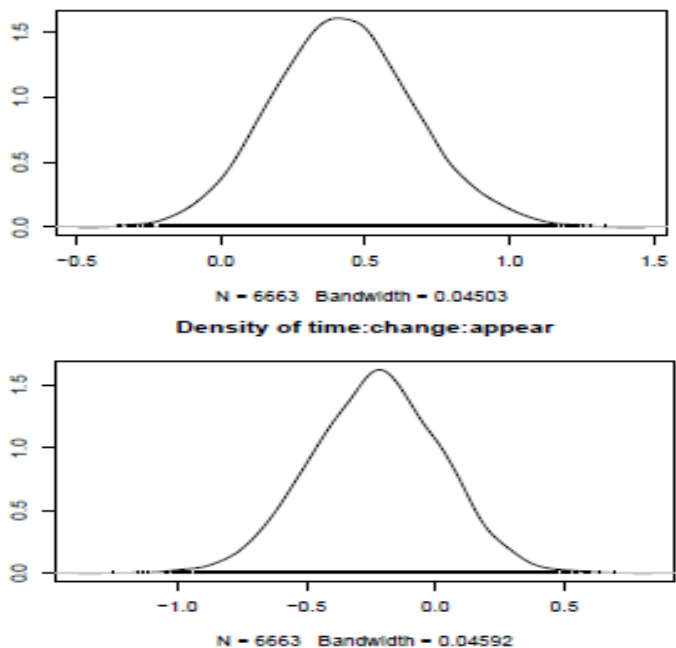

Eanonith - 0.04592

Density of careExp:time:appear

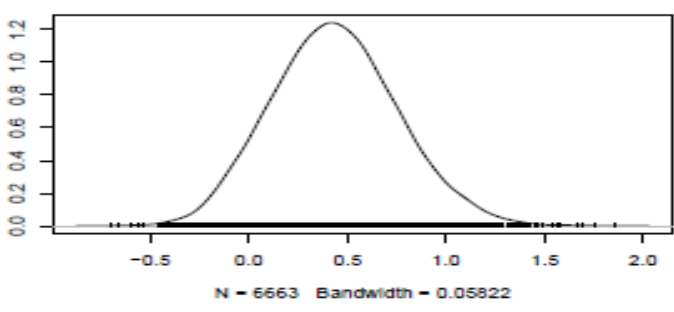

Density of careExp:time:breach

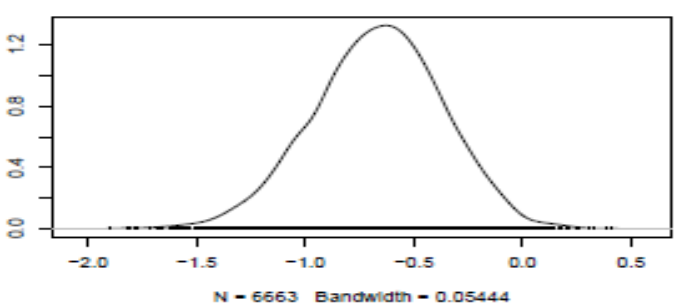

Density of careExp:time:custody

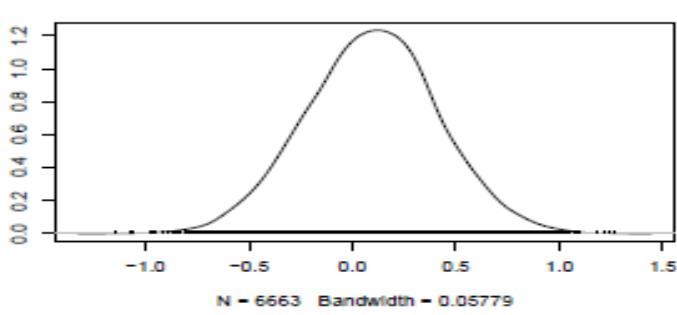


$\underline{\text { Random Effects }}$
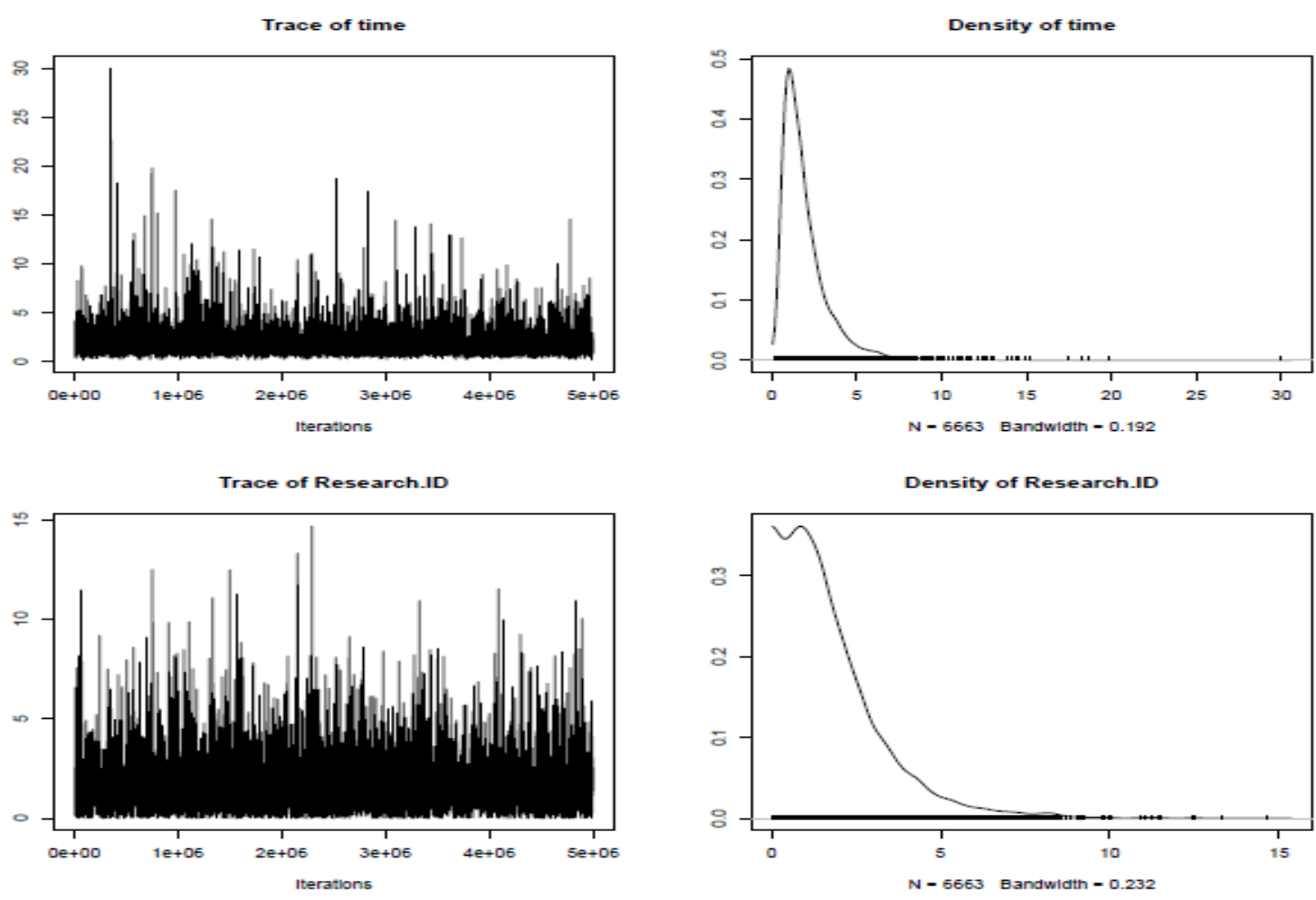


\section{References}

Finch WH, Bolin JE and Kelley K. (2014) Multilevel Modeling Using R, Boca Raton, FL: CRC Press.

Youth Justice Board. (2008a) Asset Core Profile - Guidance. Available at:

https://www.gov.uk/government/publications/asset-documents. (Accessed 31/1/18).

Youth Justice Board. (2008b) Asset Core Profile - Introduction. Available at:

https://www.gov.uk/government/publications/asset-documents. (Accessed 31/1/18). 